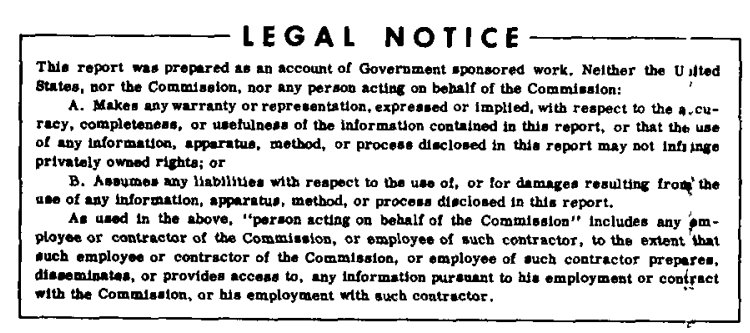

ORNT- 4464

Neutron Physics Division

NEUTRON AND SECONDARY GAMMA-RAY TRANSPORT

IN INFINTTE HOMOGENEOUS AIR

E. A. Straker and M. L. Gritzner*

* Computing Technology Center, Union Carbide Corporation, Oak Ridge, Tenn.

NOTE :

This Work Funded by

DEFENSE ATOMIC SUPPORT AGENCY

Under Subtask No. PB050

\title{
DECEMBER 1969
}

OAK RIDGE NATIONAL LABORATORY

Oak Ridge, Tennessee

operated by

UNION CARBIDE CORPORATION

for the

U. S. ATOMIC ENERGY COMMISSION 


\section{DISCLAIMER}

This report was prepared as an account of work sponsored by an agency of the United States Government. Neither the United States Government nor any agency Thereof, nor any of their employees, makes any warranty, express or implied, or assumes any legal liability or responsibility for the accuracy, completeness, or usefulness of any information, apparatus, product, or process disclosed, or represents that its use would not infringe privately owned rights. Reference herein to any specific commercial product, process, or service by trade name, trademark, manufacturer, or otherwise does not necessarily constitute or imply its endorsement, recommendation, or favoring by the United States Government or any agency thereof. The views and opinions of authors expressed herein do not necessarily state or reflect those of the United States Government or any agency thereof. 


\section{DISCLAIMER}

Portions of this document may be illegible in electronic image products. Images are produced from the best available original document. 


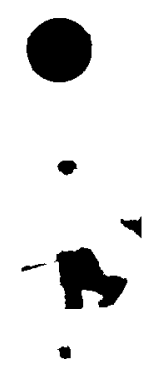

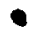

$-$

$s$

7

$\checkmark$ $\bullet$ 
NEUTRON AND SECONDARY GAMMA-RAY TRANSPORT

IN INFINITE HOMOGENEOUS AIR

E. A. Straker and M. L. Gritzner

\section{Abstract}

The detailed energy, angular, and spatial distribution of neutrons and their secondary gamma rays is given for nine source energy groups, a fission spectrum, and a typical thermonuclear spectrum. Results for ranges to 2000 meters for the eleven sources are supplemented with results to 5000 meters for a fission and 12.2- to 15-MeV source. In addition to the detailed description of the radiation field, the angular distributions of Henderson dose, Snyder-Neufeld dose, tissue kerma, mid-phantom dose, concrete kerma, air kerma, ionizing silicon kerma, and non-ionizing silicon kerma are given for neutrons, and the Henderson dose, concrete kerma, air kerma, and silicon kerma are given for secondary gamma rays. Some comparisons with air-over-ground results are made. 
The detailed radiation field from weapons detonated in the atmosphere is required as the source term for many shielding calculations. For low altitude detonations, less than approximately $20,000 \mathrm{ft}$, and for source and detector altitudes greater than approximately 2,000 ft, the radiation field may be determined from results of transport in an infinite homogeneous medium. In addition, infinite air results provide the upper limit for the effect of the ground on the radiation field. In order to provide infinite air data for comparison with those previously published ${ }^{1}$ for a source height of $50 \mathrm{ft}$ above an airground interface, tabular results are presented of discrete ordinates calculations of the energy, angular and spatial distribution of neutrons and their secondary gamma rays due to point isotropic sources in an infinite medium of air. The same cross sections, air density and method of calculation were used as in reference 1 .

The neutron sources for this problem were isotropic and uniformly distributed over each energy group; the groups considered are shown in Table 1. In addition to these band spectra, results are also given for a typical thermonuclear source (see Fig. 1 and Table 2 ) and for a fission source. The air composition was assumed to be $21 \%$ oxygen and $79 \%$ nitrogen at a density of $1.11 \mathrm{mg} / \mathrm{cc}$. The discrete ordinates code $\mathrm{ANISN}^{2}$ was used to determine the neutron and gamma-ray distribution in a 2,000-meter sphere; in two cases results for a 5,000-meter sphere are presented. The neutron cross sections were taken from $\mathrm{ENDF} / \mathrm{B},{ }^{3}$ and the secondary gamma-ray production cross sections were from various sources. 4 Twenty-two neutron groups and 18 gamma-ray groups were used to represent the cross sections in the $\mathrm{S}_{16}{ }^{\mathrm{P}} 5$ calculations. 
TABLE 1. SOURCE ENERGY BANDS

\begin{tabular}{c}
$\begin{array}{c}\text { Energy Range } \\
(\mathrm{MeV})\end{array}$ \\
\hline $12.2-15.0$ \\
$10.0-12.2$ \\
$8.18-10.0$ \\
$6.36-8.18$ \\
$4.06-6.36$ \\
$2.35-4.06$ \\
$1.108-2.35$ \\
$0.111-1.108$ \\
$1 / \mathrm{E}, 0.0033-0.111$ \\
\hline \hline
\end{tabular}




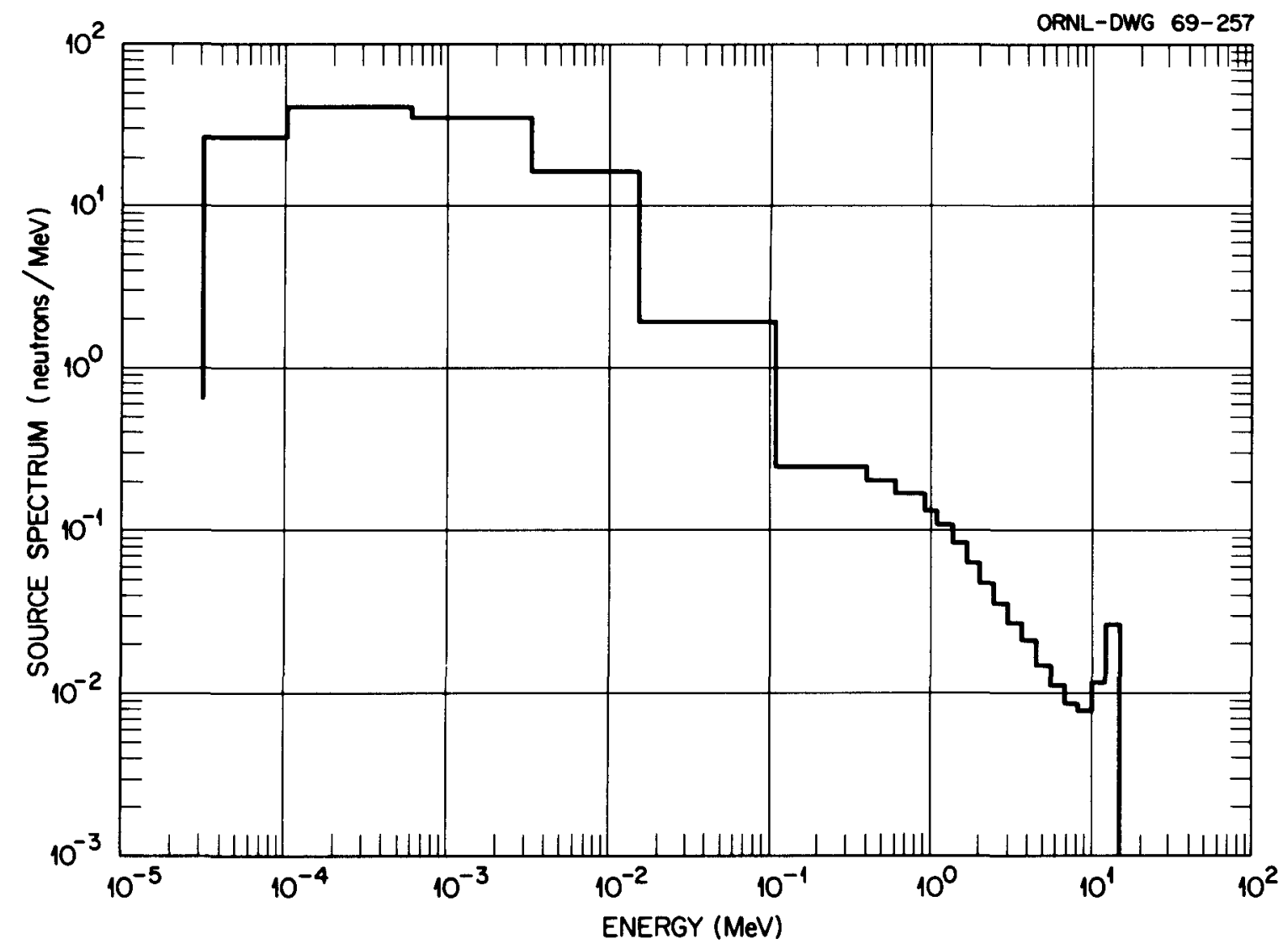

Fig. 1. Source Energy Spectrum for a Typical Thermonuclear Weapon. 
TABLE 2. ENERGY DISTRIBUTIONS FOR FISSION AND THERMONUCLEAR SOURCES

\begin{tabular}{ccc}
\hline $\begin{array}{c}\text { Energy } \\
(\mathrm{MeV})\end{array}$ & \multicolumn{2}{c}{ Fraction in Group } \\
$12.2-15.0$ & $1.568(-4)$ & $7.06(-2)$ \\
$10.0-12.2$ & $8.932(-4)$ & $2.56(-2)$ \\
$8.19-10.0$ & $3.480(-3)$ & $1.41(-2)$ \\
$6.36-8.19$ & $1.392(-2)$ & $1.47(-2)$ \\
$4.97-6.36$ & $3.457(-2)$ & $1.80(-2)$ \\
$4.07-4.97$ & $3.507(-2)$ & $1.70(-2)$ \\
$3.01-4.07$ & $1.072(-1)$ & $2.60(-2)$ \\
$2.46-3.01$ & $8.898(-2)$ & $1.90(-2)$ \\
$2.35-2.46$ & $2.323(-2)$ & $5.00(-3)$ \\
$1.83-2.35$ & $1.203(-1)$ & $2.80(-2)$ \\
$1.11-1.83$ & $2.181(-1)$ & $6.20(-2)$ \\
$0.55-1.11$ & $1.983(-1)$ & $8.50(-2)$ \\
$0.11-0.55$ & $1.403(-1)$ & $1.02(-1)$ \\
$0.0335-0.110$ & $1.550(-2)$ & $3.65(-1)$ \\
$5.83(-4)-3.35(-2)$ & 0 & $1.22(-1)$ \\
$1.01(-4)-5.83(-4)$ & 0 & $2.40(-2)$ \\
$2.90(-5)-1.01(-4)$ & .0 & $2.00(-3)$ \\
$<2.90(-5)$ & 0 & 0 \\
\hline \hline
\end{tabular}

$a_{\text {Read as } 1.568 \times 10^{-4}}$. 
Results for the energy-angular distribution of both neutrons and their secondary gamma rays from inelastic neutron scattering, fast neutron capture, and thermal neutron capture are given for the ranges listed in Table 3 . In addition, the angular fluence is integrated to give the scalar fluence at each range.

Several neutron and gamma-ray responses have been used to weight the energy fluence to provide angular-dependent response information. Specifically, the neutron responses were the Henderson dose, 5 the SnyderNeufeld dose, ${ }^{6}$ tissue kerma, ${ }^{7}$ the mid-phantom dose, ${ }^{8}$ concrete kerma, ${ }^{7}$ air kerma, ${ }^{7}$ and both ionizing and non-ionizing silicon kerma. ${ }^{9}$ The specific response functions are given in Table 4. The gamma-ray responses were Henderson dose, ${ }^{5}$ concrete kerma, ${ }^{10}$ air kerma, ${ }^{10}$ and silicon kerma ${ }^{10}$ (see Table 5). To illustrate the variation of the various responses with range, Fig. 2 shows the results for a 12.2- to 15-MeV source. The type of response has little effect on the shape of the curve for ranges greater than w 800 meters.

When the angular fluence varies with angle by a factor of approximately 500 or more, the angular fluence may be negative in the backward angles due to the truncation of the Legendre expansion of the scattering cross section at $\mathrm{P}_{5}$. These negative fluences are small and do not significantly affect the scalar fluence; however, if a negative value occurs for a direction which is important to the solution of a particular problem, then these results are probably inadequate.

An index to the tabulated results is given in Table 6. To illustrate some of the results, Fig. 3 shows the spatial distribution of $4 \pi R^{2}$ neutron and gamma-ray doses due to both a 12.2- to 15-MeV source and a fission source. Note that for ranges greater than about 2,000 meters the secondary gamma-ray dose dominates. 
TABLE 3. RANGE IN METERS FOR WHICH RESULTS ARE TABULATED

\begin{tabular}{lll}
\hline & & $\begin{array}{c}\text { Fission and 12.2- } \\
\text { to 15-MeV Sources }\end{array}$ \\
75 & 600 & 2400 \\
150 & 900 & 3600 \\
200 & 1200 & \\
300 & & 4800 \\
400 & 1500 & \\
500 & 1800 & \\
\hline
\end{tabular}


TABLE 4. NEUTRON RESPONSE FUNCTIONS

\begin{tabular}{|c|c|c|c|c|c|c|c|c|c|}
\hline Group & $\begin{array}{l}\text { Upper } \\
\text { Energy } \\
(\mathrm{eV})\end{array}$ & $\begin{array}{c}\text { Henderson } \\
\text { Tissue } \\
\text { Dosea }\end{array}$ & $\begin{array}{l}\text { Snyder- } \\
\text { Neufeld } \\
\text { Doseb }\end{array}$ & $\begin{array}{l}\text { Tissue } \\
\text { Kermac }\end{array}$ & $\begin{array}{c}\text { Mid- } \\
\text { Phantom } \\
\text { Dosed * }\end{array}$ & $\begin{array}{l}\text { Concrete } \\
\text { Kermac }\end{array}$ & $\underset{\text { Kermac }}{\text { Air }}$ & $\begin{array}{c}\text { Non- } \\
\text { Ionizing } \\
\text { Silicon } \\
\text { Kermae }\end{array}$ & $\begin{array}{l}\text { Ionizing } \\
\text { Silicon } \\
\text { Kermae }+\end{array}$ \\
\hline $\begin{array}{l}1 \\
2 \\
3 \\
4 \\
5\end{array}$ & $\begin{array}{r}15.0(+6) \\
12.2(+6) \\
10.0(+6) \\
8.18(+6) \\
6.36(+6)\end{array}$ & $\begin{array}{l}5.46(-9) \\
5.13(-9) \\
4.84(-9) \\
4.61(-9) \\
4.44(-9)\end{array}$ & $\begin{array}{l}7.0(-9) \\
7.0(-9) \\
7.08(-9) \\
6.72(-9) \\
6.03(-9)\end{array}$ & $\begin{array}{l}6.36(-7) \\
5.74(-7) \\
5.17(-7) \\
4.87(-7) \\
4.5(-7)\end{array}$ & $\begin{array}{ll}4.9 & (-9) \\
4.5 & (-9) \\
4.2 & (-9) \\
3.5 & (-9) \\
2.8 & (-9)\end{array}$ & $\begin{array}{l}1.58(-7) \\
1.17(-7) \\
8.2(-8) \\
7.05(-8) \\
5.75(-8)\end{array}$ & $\begin{array}{l}2.66(-7) \\
1.93(-7) \\
1.41(-7) \\
1.11(-7) \\
1.05(-7)\end{array}$ & $\begin{array}{ll}7.5 & (-9) \\
6.6 & (-9) \\
6.4 & (-9) \\
6.6 & (-9) \\
5.5 & (-9)\end{array}$ & $\begin{array}{ll}8.6 & (-8) \\
9.0 & (-8) \\
8.1 & (-8) \\
5.0 & (-8) \\
1.6 & (-8)\end{array}$ \\
\hline $\begin{array}{r}6 \\
7 \\
8 \\
9 \\
10\end{array}$ & $\begin{array}{l}4.96(+6) \\
4.06(+6) \\
3.01(+6) \\
2.46(+6) \\
2.35(+6)\end{array}$ & $\begin{array}{l}4.13(-9) \\
4.01(-9) \\
3.39(-9) \\
3.15(-9) \\
3.09(-9)\end{array}$ & $\begin{array}{l}5.43(-9) \\
4.83(-9) \\
4.48(-9) \\
4.33(-9) \\
4.23(-9)\end{array}$ & $\begin{array}{l}4.21(-7) \\
3.98(-7) \\
3.43(-7) \\
3.15(-7) \\
3.05(-7)\end{array}$ & $\begin{array}{l}2.3(-9) \\
1.75(-9) \\
1.25(-9) \\
1.15(-9) \\
7.00(-10)\end{array}$ & $\begin{array}{ll}5.4 & (-8) \\
5.8 & (-8) \\
4.1 & (-8) \\
3.2 & (-8) \\
3.5 & (-8)\end{array}$ & $\begin{array}{l}1.20(-7) \\
1.06(-7) \\
5.38(-8) \\
3.09(-8) \\
3.37(-8)\end{array}$ & $\begin{array}{l}5.1(-9) \\
4.8(-9) \\
4.8(-9) \\
4.7(-9) \\
4.2(-9)\end{array}$ & $\begin{array}{ll}9.0 & (-9) \\
5.4 & (-9) \\
3.6 & (-9) \\
3.0 & (-9) \\
2.7 & (-9)\end{array}$ \\
\hline $\begin{array}{l}11 \\
12 \\
13 \\
14 \\
15\end{array}$ & $\begin{array}{l}1.83(+6) \\
1.11(+6) \\
5.50(+5) \\
1.11(+5) \\
3.35(+3)\end{array}$ & $\begin{array}{l}2.64(-9) \\
1.97(-9) \\
1.12(-9) \\
2.29(-10) \\
0\end{array}$ & $\begin{array}{l}3.96(-9) \\
3.30(-9) \\
1.73(-9) \\
7.0(-10) \\
6.07(-10)\end{array}$ & $\begin{array}{l}2.63(-7) \\
2.05(-7) \\
1.27(-7) \\
4.0(-8) \\
1.96(-9)\end{array}$ & $\begin{array}{l}5.30(-10) \\
2.8(-10) \\
2.0(-10) \\
1.2(-10) \\
1.05(-10)\end{array}$ & $\begin{array}{l}3.12(-8) \\
2.61(-8) \\
1.48(-8) \\
3.55(-9) \\
1.58(-10)\end{array}$ & $\begin{array}{l}2.95(-8) \\
1.61(-8) \\
9.84(-9) \\
2.67(-9) \\
3.61(-10)\end{array}$ & $\begin{array}{ll}3.4 & (-9) \\
3.1 & (-9) \\
2.0 & (-9) \\
0 & \\
0 & \end{array}$ & $\begin{array}{ll}2.1 & (-9) \\
1.7 & (-9) \\
1.4 & (-9) \\
0 & \\
0 & \end{array}$ \\
\hline $\begin{array}{l}16 \\
17 \\
18 \\
19 \\
20\end{array}$ & $\begin{array}{l}5.83(+2) \\
1.01(+2) \\
2.90(+1) \\
1.07(+1) \\
3.06(+0)\end{array}$ & $\begin{array}{l}0 \\
0 \\
0 \\
0 \\
0\end{array}$ & $\begin{array}{l}6.72(-10) \\
5.35(-10) \\
3.88(-10) \\
3.42(-10) \\
3.27(-10)\end{array}$ & $\begin{array}{l}3.67(-10) \\
1.17(-10) \\
1.11(-10) \\
1.62(-10) \\
2.65(-10)\end{array}$ & $\begin{array}{l}1.10(-10) \\
1.15(-10) \\
1.10(-10) \\
1.0(-10) \\
8.5(-11)\end{array}$ & $\begin{array}{l}2.85(-11) \\
7.1(-12) \\
5.0(-12) \\
6.35(-12) \\
1.02(-11)\end{array}$ & $\begin{array}{l}5.62(-10) \\
1.28(-9) \\
2.26(-9) \\
3.70(-9) \\
6.71(-9)\end{array}$ & $\begin{array}{l}0 \\
0 \\
0 \\
0 \\
0\end{array}$ & $\begin{array}{l}0 \\
0 \\
0 \\
0 \\
0\end{array}$ \\
\hline $\begin{array}{l}21 \\
22\end{array}$ & $\begin{array}{l}1.12(+0) \\
0.414(+0)\end{array}$ & $\begin{array}{l}0 \\
0\end{array}$ & $\begin{array}{l}3.22(-10) \\
3.2(-10)\end{array}$ & $\begin{array}{l}4.26(-10) \\
9.36(-10)\end{array}$ & $\begin{array}{ll}7.9 & (-11) \\
5.5 & (-11)\end{array}$ & $\begin{array}{l}1.63(-11) \\
3.62(-11)\end{array}$ & $\begin{array}{l}1.13(-8) \\
2.43(-8)\end{array}$ & $\begin{array}{l}0 \\
0\end{array}$ & $\begin{array}{l}0 \\
0\end{array}$ \\
\hline
\end{tabular}

$a_{\text {From }}$ reference 5 .

brom reference 6 .

From reference 7 .

* Units of $\mathrm{rad} /\left(\mathrm{n} / \mathrm{cm}^{2}\right)$

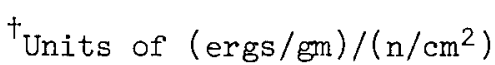

$\mathrm{d}_{\text {From reference } 8 \text {. }}$

Erom reference 9 . 
TABLE 5. GAMMA-RAY RESPONSE FUNCTIONS

\begin{tabular}{cccccc}
\hline \hline Group & $\begin{array}{c}\text { Upper } \\
\text { Energy } \\
(\text { MeV })\end{array}$ & $\begin{array}{c}\text { Henderson } \\
\text { Tissue } \\
\text { Dosea* }\end{array}$ & $\begin{array}{c}\text { Concrete } \\
\text { Kermabt }\end{array}$ & $\begin{array}{c}\text { Air } \\
\text { Kermabt }\end{array}$ & $\begin{array}{c}\text { Silicon } \\
\text { Kermabt }\end{array}$ \\
\hline 1 & 10.0 & $2.42(-9)$ & $2.65(-7)$ & $2.24(-7)$ & $2.80(-7)$ \\
2 & 8.0 & $2.07(-9)$ & $2.18(-7)$ & $1.90(-7)$ & $2.28(-7)$ \\
3 & 6.5 & $1.76(-9)$ & $1.80(-7)$ & $1.60(-7)$ & $1.83(-7)$ \\
4 & 5.0 & $1.59(-9)$ & $1.46(-7)$ & $1.34(-7)$ & $1.48(-7)$ \\
5 & 4.0 & $1.27(-9)$ & $1.18(-7)$ & $1.12(-7)$ & $1.20(-7)$ \\
6 & 3.0 & $1.08(-9)$ & $9.80(-8)$ & $9.52(-8)$ & $9.85(-8)$ \\
7 & 2.5 & $8.75(-10)$ & $8.40(-8)$ & $8.30(-8)$ & $8.40(-8)$ \\
8 & 2.0 & $7.35(-10)$ & $7.15(-8)$ & $2.13(-8)$ & $7.12(-8)$ \\
9 & 1.66 & $6.44(-10)$ & $6.15(-8)$ & $6.15(-8)$ & $6.10(-8)$ \\
10 & 1.33 & $5.30(-10)$ & $5.05(-8)$ & $5.05(-8)$ & $5.05(-8)$ \\
11 & 1.0 & $4.45(-10)$ & $4.10(-8)$ & $4.10(-8)$ & $4.10(-8)$ \\
12 & 0.8 & $3.50(-10)$ & $3.30(-8)$ & $3.26(-8)$ & $2.70(-8)$ \\
13 & 0.6 & $2.56(-10)$ & $2.42(-8)$ & $2.38(-8)$ & $2.37(-8)$ \\
14 & 0.4 & $1.77(-10)$ & $1.68(-8)$ & $1.63(-8)$ & $1.65(-8)$ \\
15 & 0.3 & $1.22(-10)$ & $1.20(-8)$ & $1.11(-8)$ & $1.17(-8)$ \\
16 & 0.2 & $6.60(-11)$ & $8.00(-9)$ & $5.92(-9)$ & $7.25(-9)$ \\
17 & 0.1 & $3.90(-11)$ & $1.20(-8)$ & $2.72(-9)$ & $9.75(-9)$ \\
18 & 0.05 & $8.37(-11)$ & $5.90(-8)$ & $4.40(-9)$ & $4.13(-8)$ \\
\hline \hline
\end{tabular}

$a_{\text {From reference }} 5$.

$\mathrm{b}_{\text {From reference }} 10$.

*Units of $\operatorname{Rad} /\left(\gamma / \mathrm{cm}^{2}\right)$

tUnits of $(\mathrm{ergs} / \mathrm{gm}) /\left(\gamma / \mathrm{cm}^{2}\right)$ 
$x i i$

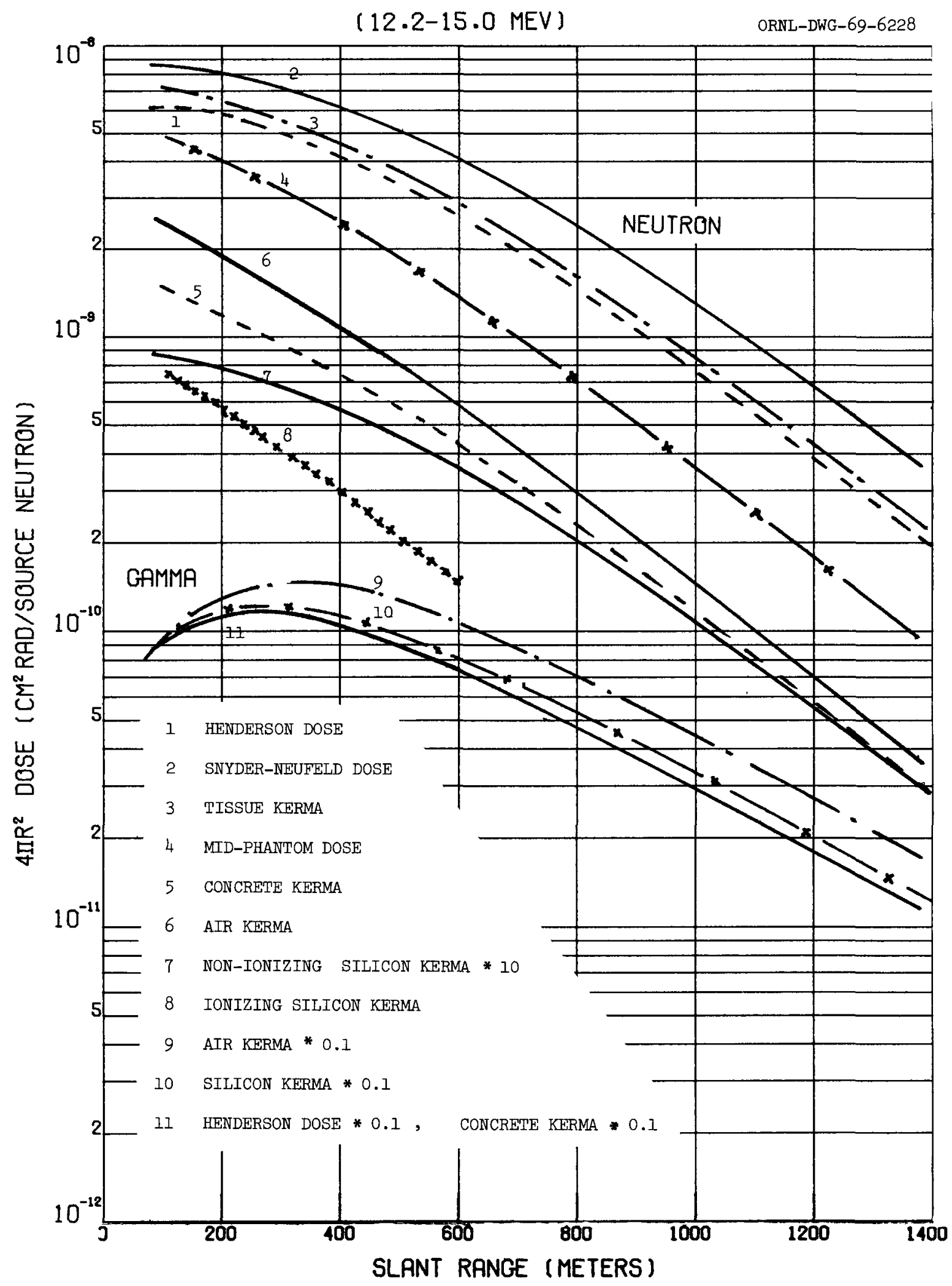

Fig. 2. $4 \pi R^{2}$ Dose Versus Range Due to a 12.2- to 15-MeV Source. 


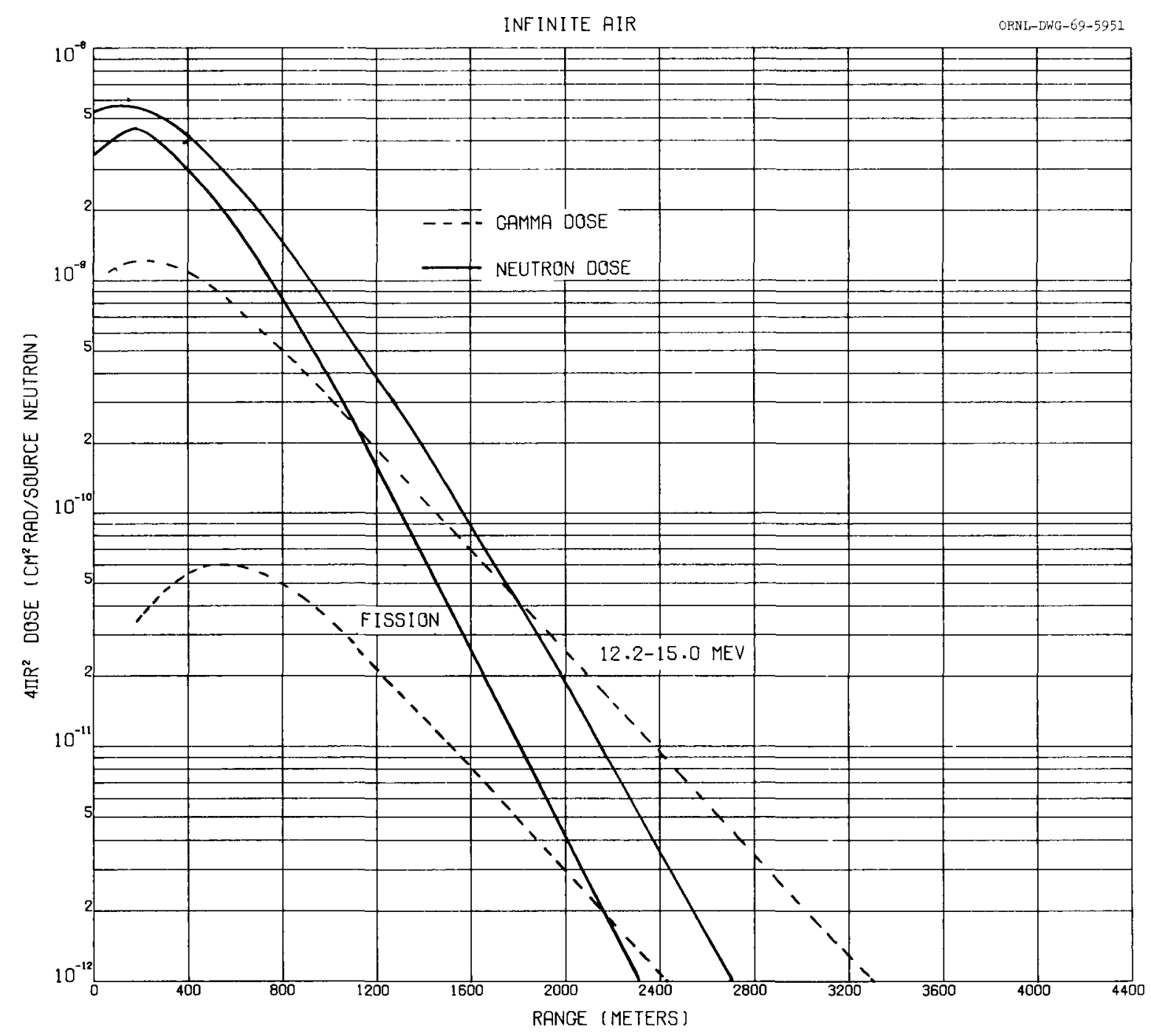

Fig. 3. $4 \pi R^{2}$ Dose Versus Range for both 12.2- to 15-MeV and Fission Sources. 


$$
x i v
$$

To compare these infinite air results with the results for a source height of $50 \mathrm{ft}$, a rotation of the coordinates of the air-over-ground results must be made. Figs. 4 and 5 show the comparison of infinite air results at 900 meters with those for source heights of 50 and 1125 ft and detector height of $3 \mathrm{ft}$. The angular variable is measured from the source-detector axis. At this range, the effect of the ground is to make the dose entering the ground slightly more peaked for both neutrons and secondary gamma rays.

These tabulated results provide the detailed energy angular distribution of both neutrons and secondary gamma rays in infinite air. The validity of the calculational technique and the input cross sections for the air-over-ground results has been established previously ${ }^{4}$ by comparing calculations with experimental results from $\mathrm{BREN}^{11}$ and HENRE. ${ }^{12}$ These calculations for infinite air utilized the same cross sections and the accuracy of the results should be essentially the same as for the air-over-ground results. 


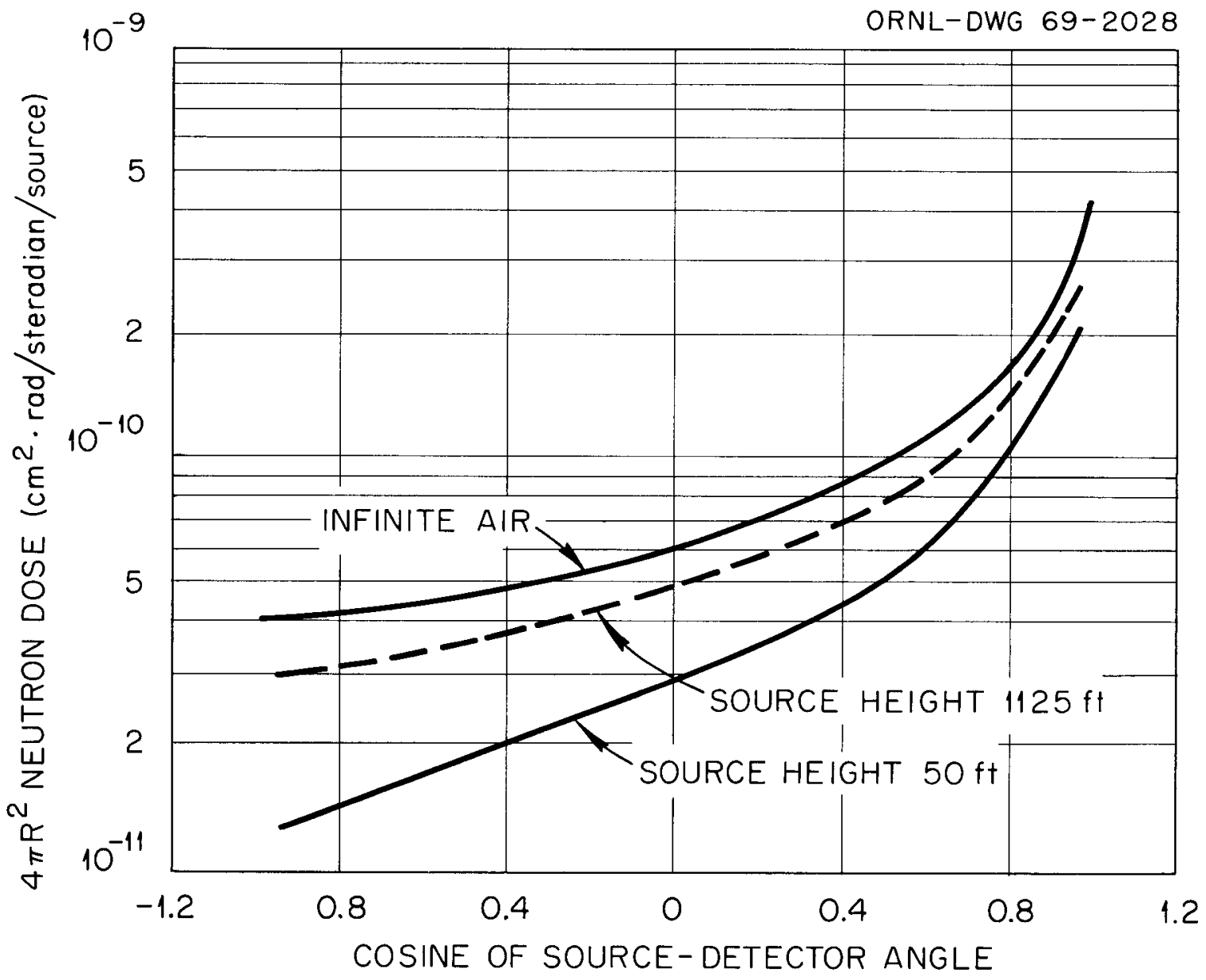

Fig. 4. Angular Distribution of Neutron Dose at a Range of 900 Meters Due to a 12.2- to 15-MeV Source. For the air-over-ground case, the detector is $3 \mathrm{ft}$ above the ground. 


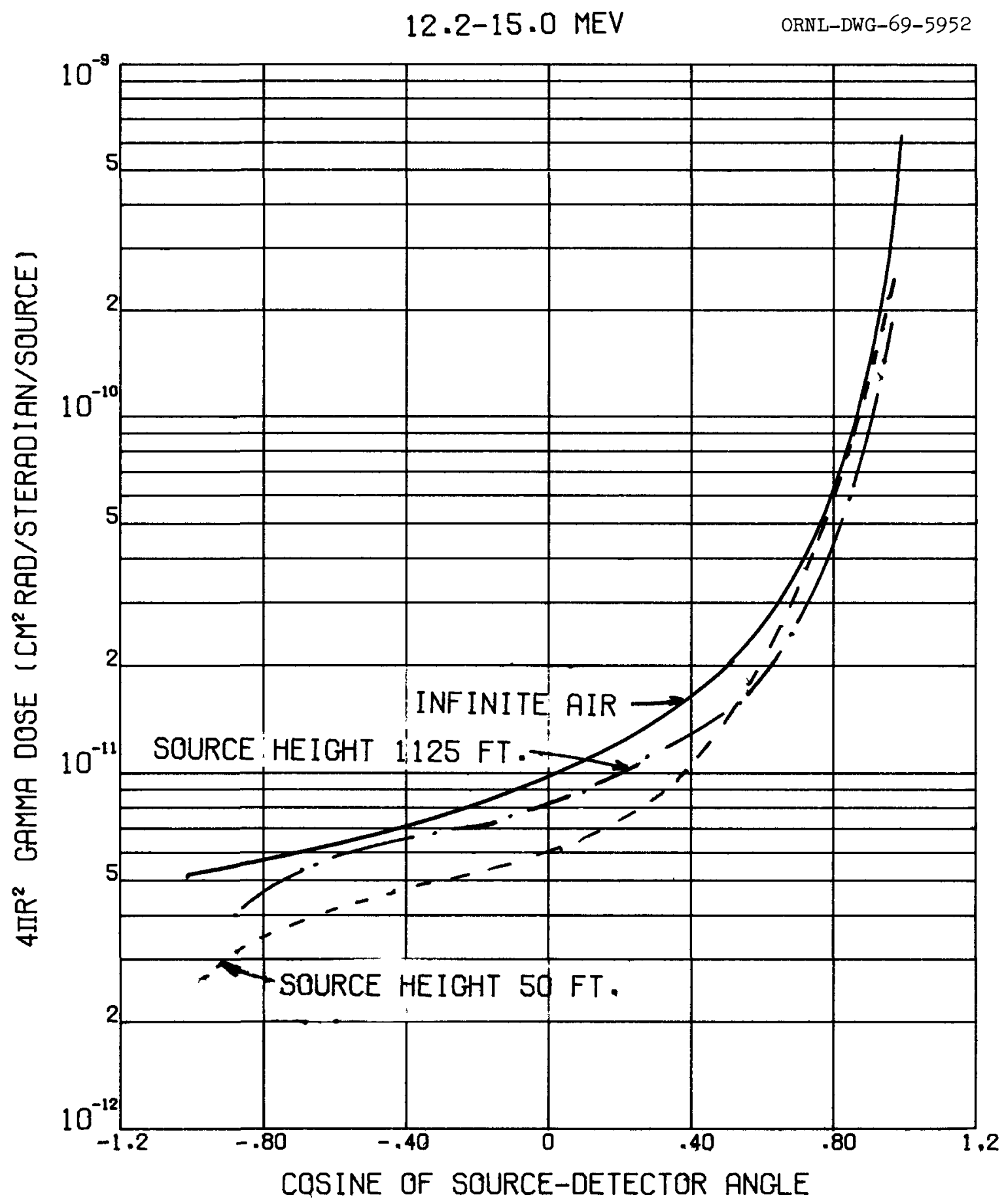

Fig. 5. Angular Distribution of Gamma-Ray Dose at a Range of 900 Meters Due to 12.2- to 15-MeV Source. For the air-over-ground case, the detector is $3 \mathrm{ft}$ above the ground. 
xvi i

REFERENCES

1. E. A. Straker, Time-Dependent Neutron and Secondary GammaRay Transport in an Air-Over-Ground Geometry, Volume II. Tabulated Data, ORNL-4289 (1968).

2. W. W. Engle, Jr., A User's Manual for ANISN - A One-Dimensional Discrete Ordinates Transport Code with Anisotropic Scattering, K-1693, Union Carbide Corporation (1967).

3. Evaluated Nuclear Data File of the National Neutron Cross Section Center.

4. E. A. Straker, Time-Dependent Neutron and Secondary Gamma-Ray Transport in an Air-Over-Ground Geometry, Vol. I, Calculational Techniques and Discussion of Results, ORNL-4289, Vol. I (to be published).

5. B. J. Henderson, Conversion of Neutron and Gamma-Ray Flux to Absorbed Dose Rate, XDC 59-8-179 (1959).

6. W. S. Snyder and C. Neufeld, Radiation Research, 6(1), 67 (1957).

7. E. Solomito, J. J. Ritts, and H. C. Claiborne, AVKER: A Fortran Program for Calculating Dose or Heat Generation Rates in Materials, ORNL-TM-2558 (Apri1 15, 1969).

8. J. A. Auxier, W. S. Snyder, and T. D. Jones, "Neutron Interaction and Penetration in Tissue," Radiation Dosimetry, Academic Press (1968).

9. Allan R. Sattler and Frederich L. Vook, "Partition of the Average Energy Deposited in Silicon as a Function of Incident Neutron Energy," Phys. Rev. 155, 211 (1967). 
10. D. K. Trubey, PRINT-SIG, An Ogre Routine to Print Gamma-Ray Total

Cross Sections and Energy Deposition Coefficients, ORNL-CF-68-10-19 (1968).

11. E. A. Straker and R. J. Rodgers, "Neutron Transport in the Atmosphere," ANS Trans. 10, 730 (1967).

12. J. H. Thorngate, et al., Energy and Angular Distribution of Neutrons and Gamma Rays - Operation HENRE, CEX 65.12, USAEC (to be published). 
O xix

TABULATED DATA 
TABLE 6. PAGE INDEX TO TABULATED DATA

\begin{tabular}{|c|c|c|c|c|c|c|c|c|c|c|c|c|c|}
\hline $\begin{array}{l}\text { Type of } \\
\text { Radiation }\end{array}$ & $\begin{array}{l}\text { Data } \\
\text { Tabulated }\end{array}$ & $\begin{array}{l}\text { Range } \\
\text { (Meters) }\end{array}$ & $\begin{array}{c}12.2-15 \\
\mathrm{MeV}\end{array}$ & $\begin{array}{c}10.0-12.2 \\
\mathrm{MeV}\end{array}$ & $\begin{array}{c}8.18-10.0 \\
\mathrm{MeV}\end{array}$ & $\begin{array}{l}6.36-8.18 \\
\mathrm{MeV}\end{array}$ & $\begin{array}{c}4.06-6.36 \\
\mathrm{MeV}\end{array}$ & $\begin{array}{c}2.35-4.06 \\
\mathrm{MeV}\end{array}$ & $\begin{array}{l}1.108-2.35 \\
\mathrm{MeV}\end{array}$ & $.111-1.108$ & $\begin{array}{c}.0033-.111 \\
\mathrm{MeV}\end{array}$ & Fission & $\begin{array}{l}\text { Thermo- } \\
\text { nuclear }\end{array}$ \\
\hline \multirow[t]{11}{*}{ Neutrons } & \multirow{11}{*}{$\begin{array}{l}\text { Energy-angular } \\
\text { distributions }\end{array}$} & 75 & 1 & 35 & 69 & 103 & 137 & 171 & 205 & 239 & 273 & 307 & 341 \\
\hline & & 150 & 2 & 36 & 70 & 104 & 138 & 172 & 206 & 240 & 274 & 308 & 342 \\
\hline & & 200 & 3 & 37 & 71 & 105 & 139 & 173 & 207 & 241 & 275 & 309 & 343 \\
\hline & & 300 & 4 & 38 & 72 & 106 & 140 & 174 & 208 & 242 & 276 & 310 & 344 \\
\hline & & 400 & 5 & 39 & 73 & 107 & 141 & 175 & 209 & 243 & 277 & 311 & 345 \\
\hline & & 500 & 6 & 40 & 74 & 108 & 142 & 176 & 210 & 244 & 278 & 312 & 346 \\
\hline & & 600 & 7 & 41 & 75 & 109 & 143 & 177 & 211 & 245 & 279 & 313 & 347 \\
\hline & & 900 & 8 & 42 & 76 & 110 & 144 & 178 & 212 & 246 & 280 & 314 & 348 \\
\hline & & 1200 & 9 & 43 & 77 & 111 & 145 & 179 & 213 & 247 & 281 & 315 & 349 \\
\hline & & 1500 & 10 & 44 & 78 & 112 & 146 & 180 & 214 & 248 & 282 & 316 & 350 \\
\hline & & 1800 & 11 & 45 & 79 & 113 & 147 & 181 & 215 & 249 & 283 & 317 & 351 \\
\hline \multirow[t]{11}{*}{ Gammas } & \multirow{11}{*}{$\begin{array}{l}\text { Energy-angular } \\
\text { distributions }\end{array}$} & 75 & 12 & 46 & 80 & 114 & 148 & 182 & 216 & 250 & 284 & 318 & 352 \\
\hline & & 150 & 13 & 47 & 81 & 215 & 149 & 183 & 217 & 251 & 285 & 319 & 353 \\
\hline & & 200 & 14 & 48 & 82 & 116 & 150 & 184 & 218 & 252 & 286 & 320 & 354 \\
\hline & & 300 & 15 & 49 & 83 & 117 & 151 & 185 & 219 & 253 & 287 & 321 & 355 \\
\hline & & 400 & 16 & 50 & 84 & 118 & 152 & 186 & 220 & 254 & 288 & 322 & 356 \\
\hline & & 500 & 17 & 51 & 85 & 119 & 153 & 187 & 221 & 255 & 289 & 323 & 357 \\
\hline & & 600 & 18 & 52 & 86 & 120 & 154 & 188 & 222 & 256 & 290 & 324 & 358 \\
\hline & & 900 & 19 & 53 & 87 & 121 & 155 & 189 & 223 & 257 & 291 & 325 & 359 \\
\hline & & 1200 & 20 & 54 & 88 & 122 & 156 & 190 & $22 L_{4}$ & 258 & 292 & 326 & 360 \\
\hline & & 1500 & 21 & 55 & 89 & 123 & 157 & 191 & 225 & 259 & 293 & 327 & 361 \\
\hline & & 1800 & 22 & 56 & 90 & 124 & 158 & 192 & 226 & 260 & 294 & 328 & 362 \\
\hline \multirow[t]{9}{*}{ Neutrons } & $\begin{array}{l}\text { Henderson } \\
\text { dose }\end{array}$ & $\begin{array}{c}75-1800 \\
1800-4800\end{array}$ & $\begin{array}{r}23 \\
381\end{array}$ & 57 & 91 & 125 & 159 & 193 & 227 & 261 & 295 & $\begin{array}{l}329 \\
399\end{array}$ & 363 \\
\hline & \multirow{2}{*}{\multicolumn{2}{|c|}{$\begin{array}{ll}\text { Snyder-Neufeld } & 75-1800 \\
\text { dose } & 1800-4800\end{array}$}} & $\begin{array}{r}24 \\
382\end{array}$ & \multirow[t]{2}{*}{58} & \multirow[t]{2}{*}{92} & \multirow[t]{2}{*}{126} & \multirow[t]{2}{*}{160} & \multirow[t]{2}{*}{194} & \multirow[t]{2}{*}{228} & \multirow[t]{2}{*}{262} & \multirow[t]{2}{*}{296} & $\begin{array}{l}330 \\
400\end{array}$ & \multirow[t]{2}{*}{364} \\
\hline & & & & & & & & & & & & & \\
\hline & $\begin{array}{l}\text { Tissue } \\
\text { kerma }\end{array}$ & $\begin{array}{c}75-1800 \\
1800-4800\end{array}$ & $\begin{array}{r}25 \\
383\end{array}$ & 59 & 93 & 127 & 161 & 195 & 229 & 263 & 297 & $\begin{array}{l}331 \\
401\end{array}$ & 365 \\
\hline & $\begin{array}{l}\text { Mid-phantom } \\
\text { dose }\end{array}$ & $\begin{array}{c}75-1800 \\
1800-4800\end{array}$ & $\begin{array}{r}26 \\
384\end{array}$ & 60 & 94 & 128 & 162 & 196 & 230 & 264 & 298 & $\begin{array}{l}332 \\
402\end{array}$ & 366 \\
\hline & $\begin{array}{l}\text { Concrete } \\
\text { kerma }\end{array}$ & $\begin{array}{c}75-1800 \\
1800-4800\end{array}$ & $\begin{array}{r}27 \\
385\end{array}$ & 61 & 95 & 129 & 163 & 197 & 231 & 265 & 299 & $\begin{array}{l}333 \\
403\end{array}$ & 367 \\
\hline & $\begin{array}{l}\text { Air } \\
\text { kerma }\end{array}$ & $\begin{array}{c}75-1800 \\
1800-4800\end{array}$ & $\begin{array}{r}28 \\
386\end{array}$ & 62 & 96 & 130 & 164 & 198 & 232 & 266 & 300 & $\begin{array}{l}334 \\
404\end{array}$ & 368 \\
\hline & $\begin{array}{l}\text { Ionizing } \\
\text { silicon kerma }\end{array}$ & $\begin{array}{c}75-1800 \\
1800-4800\end{array}$ & $\begin{array}{r}29 \\
387\end{array}$ & 63 & 97 & 131 & 165 & 199 & 233 & 267 & 301 & $\begin{array}{l}335 \\
405\end{array}$ & 369 \\
\hline & $\begin{array}{l}\text { Non-ionizing } \\
\text { silicon kerma }\end{array}$ & $\begin{array}{c}75-1800 \\
1800-4800\end{array}$ & $\begin{array}{r}30 \\
388\end{array}$ & 64 & 98 & 132 & 166 & 200 & 234 & 268 & 302 & $\begin{array}{l}336 \\
406\end{array}$ & 370 \\
\hline
\end{tabular}


TABLE 6. (CONT.)

\begin{tabular}{|c|c|c|c|c|c|c|c|c|c|c|c|c|c|}
\hline \multirow[t]{4}{*}{ Gammas } & $\begin{array}{l}\text { Henderson } \\
\text { dose }\end{array}$ & $\begin{array}{c}75-1800 \\
1800-4800\end{array}$ & $\begin{array}{r}31 \\
389\end{array}$ & 65 & 99 & 133 & 167 & 201 & 235 & 269 & 303 & $\begin{array}{l}337 \\
407\end{array}$ & 371 \\
\hline & $\begin{array}{l}\text { Concrete } \\
\text { kerma }\end{array}$ & $\begin{array}{c}75-1800 \\
1800-4800\end{array}$ & $\begin{array}{r}32 \\
390\end{array}$ & 66 & 100 & 134 & 168 & 202 & 236 & 270 & 304 & $\begin{array}{l}338 \\
408\end{array}$ & 372 \\
\hline & $\begin{array}{l}\text { Air } \\
\text { kerma }\end{array}$ & $\begin{array}{c}75-1800 \\
1800-4800\end{array}$ & $\begin{array}{r}33 \\
391\end{array}$ & 67 & 101 & 135 & 169 & 203 & 237 & 271 & 305 & $\begin{array}{l}339 \\
409\end{array}$ & 373 \\
\hline & $\begin{array}{l}\text { Silicon } \\
\text { kerma }\end{array}$ & $\begin{array}{c}75-1800 \\
1800-4800\end{array}$ & $\begin{array}{r}34 \\
392\end{array}$ & 68 & 102 & 136 & 170 & 204 & 238 & 272 & 306 & $\begin{array}{l}340 \\
410\end{array}$ & 374 \\
\hline Neutrons & $\begin{array}{l}\text { Energy-angular } \\
\text { distributions }\end{array}$ & $\begin{array}{l}2400 \\
3600 \\
4800\end{array}$ & $\begin{array}{l}375 \\
376 \\
377\end{array}$ & & & & & & & & & $\begin{array}{l}393 \\
394 \\
395\end{array}$ & \\
\hline Gammas & $\begin{array}{l}\text { Energy-angular } \\
\text { distributions }\end{array}$ & $\begin{array}{l}2400 \\
3600 \\
4800\end{array}$ & $\begin{array}{l}378 \\
379 \\
380\end{array}$ & & & & & & & & & $\begin{array}{l}396 \\
397 \\
398\end{array}$ & \\
\hline
\end{tabular}


(NEUTRONS/MEV/STERADIAN/SOURCE NEUTRON)

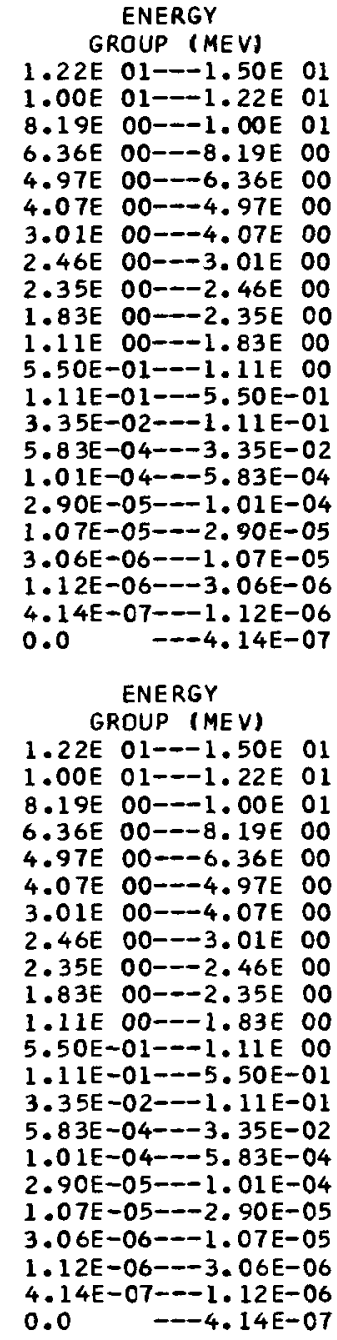
ANGLE 1 ANGLE 2 $-6.573 E-04 \quad-5.322 E-04$ $2.253 \mathrm{E}-03$ $9.432 \mathrm{E}-04$ $9.432 E-04$
$7.545 E-04$ $7.545 E-04$
$1.106 E-03$ $1.631 \mathrm{E}-03$ $1.911 E-03$ $3.081 E-03$ $3.734 \mathrm{E}-03$ $3.026 \mathrm{E}-03$ $3.291 E-03$ $4.510 E-03$ $5.660 E-03$ $2.751 E-02$ $2.162 \mathrm{E}-02$ $1.219 E 00$ $5.070 \mathrm{E}$ OO $1.442 E$ O1 $3.757 \mathrm{E} 01$ 9.868E 01 $\begin{array}{ll}2.123 E & 02 \\ 3.031 E & 02\end{array}$ ANGLE 10 $M U=0.0950$
$1.332 E-03$ $1.332 E-03$
$4.322 E-03$ $4.322 \mathrm{E}-03$
$1.718 \mathrm{E}-03$ $1.718 \mathrm{E}-03$
$1.320 \mathrm{E}-03$ $1.320 E-03$ $1.962 E-03$ $4.193 E-03$ $4.482 \mathrm{E}-03$ $4.482 E-03$ $4.700 E-03$ 4. $5.862 E-03$ $6.759 E-03$ $2.963 \mathrm{E}-02$ $2.260 \mathrm{E}-02$ $1.260 E$ OO $5.213 \mathrm{E} \mathrm{OO}$ $\begin{array}{lll}1.213 E & 00 \\ 1.478 E & 01\end{array}$ $3.843 E$ O 1 $1.007 \mathrm{E} 02$ $2.164 \mathrm{E} 02$ 3.084 E 02 $9.366 E-04$ $7.567 E-04$ $1.110 \mathrm{E}-03$ $1.637 E-03$ . $916 \mathrm{E}-03$ . . . $3.300 E-03$ ..519E-03 $5.668 E-03$ $2.752 E-02$ $2.163 \mathrm{E}-02$ $1.219 E 00$ $5.072 E$ OO $1.442 E$ O 1 $3.758 \mathrm{E} 01$
$9.870 \mathrm{E}$ O1 $2.124 \mathrm{E} 02$
$3.032 \mathrm{E} 02$

ANGLE 11

$M U=0.2816$ $1.490 E-03$ . $1.463 \mathrm{E}-03$ $2.490 \mathrm{E}-03$ 2.986E-03 2. $4.767 E-03$ 4.76 $6.030 E-03$ $5.514 E-03$ $6.362 E-03$ $7.086 \mathrm{E}-03$ $3.011 \mathrm{E}-02$ $2.280 \mathrm{E}-02$ $1.268 E$ OO $5.239 E 00$ $1.485 \mathrm{E} 01$ $3.858 \mathrm{E} 01$ $1.011 \mathrm{E} 02$ $2.172 \mathrm{E} \quad 02$ $\begin{array}{ll} & \\ & \end{array}$ $2.205 E-03 \quad 2.037 \mathrm{E}-03 \quad 1.796 \mathrm{E}-03$
ANGLE 5
$U=-0.7550$ $1.440 \mathrm{E}-04$
$1.607 \mathrm{E}-03$ $8.507 E-03$ $8.178 \mathrm{E}-04$ 8.1 $1.211 \mathrm{E}-0.03$ $1.787 \mathrm{E}-03$ $2.043 E-03$ $3.207 E-03$ $3.878 E-03$ $3.213 E-03$ $3.504 \mathrm{E}-03$ $4.726 E-03$ $5.854 \mathrm{E}-03$ $2.791 \mathrm{E}-02$ $2.182 \mathrm{E}-02$ $1.228 E 00$ $5.101 E$ OO $1.450 E$ Ol $3.776 E$ O1 $9.913 E$ O1 $2.132 E 02$ 3.045E 02 ANGLE 14 $\begin{aligned} & M U=0.7550 \\ & 1.160 \mathrm{E}-02\end{aligned}$ $1.160 \mathrm{E}-02$
$5.085 \mathrm{E}-03$ $3.084 \mathrm{E}-03$ $3.663 \mathrm{E}-03$ $4.210 \mathrm{E}-03$ $5.915 \mathrm{E}-03$ 6. $260 \mathrm{E}-03$ 8. $908 \mathrm{E}-03$ $1.035 \mathrm{E}-02$ $8.928 \mathrm{E}-03$ $8.285 E-03$ $9.158 \mathrm{E}-03$ $8.612 \mathrm{E}-03$ $3.172 \mathrm{E}-02$ $2.336 \mathrm{E}-02$ $1.288 \mathrm{E} 00$ 5.307E 00 1.502E 01 3.897E 01 $1.020 E$ O2 $3.117 E 02$
ANGLE 6 $M U=-0.6179$
$1.833 \mathrm{E}-04$ $1.833 \mathrm{E}-04$ $8.430 \mathrm{E}-04$ $8.430 E-04$ $.674 E-04$ $1.890 \mathrm{E}-03$ $.136 E-03$ $3.305 E-03$ $3.998 \mathrm{E}-03$ $3.348 E-03$ $3.648 E-03$ $4.870 E-03$ $5.976 E-03$ 2.816E-02 $2.194 \mathrm{E}-02$ $1.233 E 00$ $5.118 \mathrm{E} 00$ $1.454 \mathrm{E} \mathrm{OI}$ $3.786 \mathrm{E}$ Ol $9.939 E$ O1 $2.137 E$ O2 $3.051 \mathrm{E} 02$ ANGLE 15 $M U=0.8656$
$2.004 E-02$ $.164 E-03$ $3.836 \mathrm{E}-03$ . . . . . 1. 1.147E-02 $1.147 \mathrm{E}-02$ $9.721 \mathrm{E}-03$ 3.237E-02 . $351 \mathrm{E}-02$ $1.293 \mathrm{E} 00$ $5.323 E 00$ $1.506 E 01$ $3.906 E$ O1 $1.023 E 02$ 2.195E 02 $3.122 \mathrm{E} 02$
ANGLE 7 MU $=-0.2816$ $.126 E-05 \quad 7.541 E-05$ $.683 E-03 \quad 1.949 E-03$ $.665 \mathrm{E}-04 \quad 9.270 \mathrm{E}-04$ $.393 E-04 \quad 1.039 E-03$ $1.402 \mathrm{E}-03 \quad 1.552 \mathrm{E}-03$ $2.265 E-03 \quad 2.4$ $3.448 \mathrm{E}-03 \quad 3.655 \mathrm{E}-03$ $4.180 \mathrm{E}-03 \quad 4.443 \mathrm{E}-03$ $3.537 E-03 \quad 3.793 E-03$ $3.840 E-03 \quad 4.091 E-03$ $5.060 \mathrm{E}-03 \quad 5.306 \mathrm{E}-03$ $6.131 \mathrm{E}-03 \quad 6.322 \mathrm{E}-03$ $2.846 \mathrm{E}-02$ 2.881E-02

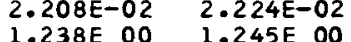
$5.139 \mathrm{E} 00$ $1.459 E$ O1 $3.799 E$
$9.969 E$ $2.143 E 02$ $3.059 E 02$

ANGLE 16 $M U=0.9446$ $.611 \mathrm{E}-02$
$1.464 \mathrm{E}-02$ $6.010 E-03$ $0.019 E-03$ 1.183E-02 (.) $1.424 E-02$ $1.718 E-02$ $1.615 \mathrm{E}-02$ 1.615E-02 $1.429 E-02$ $1.057 \mathrm{E}-02$ $3.287 \mathrm{E}-02$ $2.363 \mathrm{E}-02$ $5.334 \mathrm{E} 00$ $1.509 \mathrm{E} 01$ $3.912 \mathrm{E} \mathrm{O1}$ $1.024 \mathrm{E} 02$ $2.198 \mathrm{E} 02$ $3.125 E 02$
ANGLE 9 $A U=-0.0950$ $2.937 \mathrm{E}-04$ $2.293 \mathrm{E}-03$ 1.027E-03 $1.751 E-03$ $2.448 E-03$ $2.678 E-03$ $3.948 E-03$ $4,817 E-03$ $4.135 \mathrm{E}-03$ $4.417 E-03$ $5.620 E-03$ $6.553 E-03$ $2.920 \mathrm{E}-02$ $2.242 \mathrm{E}-02$
$1.252 \mathrm{E} 00$ $5.187 E 00$ $1.472 E$ OI $1.004 \mathrm{E} 02$ $2.157 \mathrm{E} 02$ SCALAR FLUX 2.814E-01 $5.077 E-02$ $2.285 E-02$ $2.628 E-02$ 5. $046 \mathrm{E}-02$ $5.375 E-02$ $7.347 \mathrm{E}-02$ $8.867 E-02$ $7.367 E-02$ $7.349 E-02$ $8.349 E-02$ $8.943 E-02$ $3.730 \mathrm{E}-01$ $2.835 \mathrm{E}-01$
$1.580 \mathrm{E} 01$ 6.537 E OI $1.854 \mathrm{E} \mathrm{O2}$ $4.820 E 02$ $1.264 \mathrm{E} 03$ $1.264 \mathrm{E} \mathrm{O3}$
$2.716 \mathrm{E} \mathrm{O3}$ $3.871 E$ O3 
(NEUTRONS/MEV/STERADIAN/SOURCE NEUTRON)

ENERGY GROUP (MEV) $1.22 \mathrm{E} 01--1.50 \mathrm{E} 01$ $1.00 E$ O1-D- $1.22 E$ O1 $8.19 \mathrm{E} 00--1.00 \mathrm{E} 01$ $6.36 \mathrm{E}$ 00--8.19E 00 4.97E $00--6.36 \mathrm{E} 00$ 4.07E 00---4.97E 00 $3.01 E$ 00--4.07E 00 $2.46 \mathrm{E} 00--3.01 \mathrm{E} 00$ $2.35 \mathrm{E} 00--2.46 \mathrm{E} 00$ $1.83 \mathrm{E} 00--2.35 \mathrm{E} 00$ $1.11 E$ 00-- $1.83 E$ OO 5.50E-01-- -1.11E 00 $1.11 E-01--5.50 E-01$ $3.35 \mathrm{E}-02--1.11 \mathrm{E}-01$ $5.83 \mathrm{E}-04-\cdots 3.35 \mathrm{E}-02$ $1.01 \mathrm{E}-04--5.83 \mathrm{E}-04$ $2.90 E-05--1.01 E-04$ $1.07 \mathrm{E}-05--2.90 \mathrm{E}-05$ $3.06 \mathrm{E}-06--1.07 \mathrm{E}-05$ $1.12 \mathrm{E}-06--3.06 \mathrm{E}-06$ $4.14 E-07--1.12 E-06$
$0.0 \quad--4.14 E-07$ ENERGY GROUP (MEV) $1.22 \mathrm{E}$ O1---1.50E 01 $1.00 E$ O1---1.22E 01
$8.19 E$ OO--1.00E 01 $8.19 E$ OO--1.00E 01
$6.36 E$ O0--8.19E 00

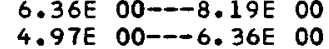

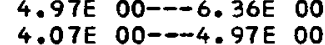
$3.01 E$ OO--4. $07 E$ OO 3.46E $00--3.01 E$ OO $2.36 \mathrm{E} 00--3.01 \mathrm{E}$ OO $1.83 \mathrm{E} 00-2.35 \mathrm{E}$ CO $1.11 E$ OO--1.83E 00 $5.50 E-01-1.11 E 00$ $1.11 \mathrm{E}-01=-5.50 \mathrm{E}-01$ 3.35E-02---1.11E-01 $5.83 \mathrm{E}-04---3.35 \mathrm{E}-02$ $1.01 \mathrm{E}-04--5.83 \mathrm{E}-04$ $2.90 E-05--1.01 E-04$ $1.07 E-05--2.90 E-05$ 3.06E-06---1.07E-05 $1.12 \mathrm{E}-06--3.06 \mathrm{E}-06$ $4.14 \mathrm{E}-07--1.12 \mathrm{E}-06$ $0.0 \quad--4.14 \mathrm{E}-07$
ANGLE 1 $M U=-1.0000$ $-6.143 E-04$ .1139E-03 .072E-03 .671E-04 $2.390 \mathrm{E}-03$ $2.888 E-03$ $4.966 \mathrm{E}-03$ $6.235 \mathrm{E}-03$ $5.352 \mathrm{E}-03$ $6.408 E-03$ $1.012 \mathrm{E}-02$ $1.521 E-02$ $8.730 \mathrm{E}-02$ $7.253 \mathrm{E}-02$ $4.222 \mathrm{E} 00$ $1.786 \mathrm{E} 01$ $5.129 \mathrm{E} 01$ $1.350 E$ O2 3.567E 02 $\begin{array}{ll}7.714 E & 02 \\ 1.107 E & 03\end{array}$ ANGLE 10 $M U=0.0950$ $6.479 E-04$ $3.229 \mathrm{E}-03$ $1.118 \mathrm{E}-03$ $2.095 \mathrm{E}-03$ $1.894 E-03$ $3.578 E-03$ $3.578 E-03$ $9.057 E-C 3$ 1.107E-02 $1.019 E-02$ $1.094 \mathrm{E}-\mathrm{C2}$ $1.727 E-02$ $9.585 \mathrm{E}-02$ 7.756E-02 $4.463 E 00$ $1.875 E$ O 1 $5.366 \mathrm{E}$ OI $5.366 E$
$1.407 E$ $3.707 E$ O2 $3.707 E$
$8.000 E$ 02 $8.000 E$
$1.144 E$ 03
ANGLE 3 ANGLE 4 $A U=-0.9894 \quad M U=-0.9446$ $-4.951 E-04 \quad-1.698 E-04$ $2.091 \mathrm{E}-03 \quad 1.922 \mathrm{E}-0$ $1.064 E-03 \quad 1.039 E-03$ $9.694 \mathrm{E}-04 \quad 9.795 \mathrm{E}-0$ $1.502 E-03$ 1.522E-O3 $2.894 \mathrm{E}-03$ $4.972 \mathrm{E}-03$ $6.242 E-03$ $5.364 E-03$ $6.425 E-03$ $1.014 \mathrm{E}-02$ $1.523 \mathrm{E}-02$ $8.738 \mathrm{E}-02$ $7.258 E-02$ $4.225 \mathrm{E} 00$ $1.787 \mathrm{E} 01$ $5.131 \mathrm{E}$ O1 $1.350 E$ O2 $3.568 \mathrm{E} 02$ $7.717 E \quad 02$
$1.108 E \quad 03$ ANGLE 11 $M U=0.2816$ $1.997 \mathrm{E}-03$
$3.970 \mathrm{E}-03$ $3.970 \mathrm{E}-03$ $2.158 \mathrm{E}-03$ $2.755 E-03$ $4.291 E-03$ 4.900E-03 S.241E-03 T. 7. O.574E-OS 1.689E-02 1.039E-02 . $839 E-02$ $9.839 E-02$
$7.857 E-02$ $4.509 E$ OO $1.892 \mathrm{E}$ OI $5.410 E$ OI $1.417 \mathrm{E} 02$ $3.733 E 02$ $8.051 \mathrm{E} 02$ $1.151 E 03$ $1.427 \mathrm{E} \mathrm{O2}$ $8.101 E$ O2 $M U=-0.8656$ $4.872 \mathrm{E}-05$ $1.685 \mathrm{E}-03$ $1.004 \mathrm{E}-03$ $1.003 E-03$ $1.566 \mathrm{E}-03$ $2.517 \mathrm{E}-03$ $2.976 \mathrm{E}-03$ $5.046 \mathrm{E}-03$ $6.348 \mathrm{E}-03$ $5.524 E-03$ $6.631 \mathrm{E}-03$ $1.040 \mathrm{E}-02$ 1. $552 \mathrm{E}-02$ $8.823 E-02$ $7.309 \mathrm{E}-02$ $4.250 \mathrm{E} 00$ $1.796 \mathrm{E} 01$ $5.156 \mathrm{E} \mathrm{O1}$ $1.356 \mathrm{E} 02$ $3.583 \mathrm{E} 02$ $7.748 \mathrm{E} \mathrm{O2}$ $1.112 \mathrm{E} \quad 03$ ANGLE 13 $M U=0.6179$ $3.988 \mathrm{E}-03$ $5.139 \mathrm{E}-03$ 3.186E-O3 $4.113 E-03$ 5.876E-03 $6.301 E-03$ $0.301 \mathrm{E}-03$ $9.442 E-03$ $1.479 E-02$ 1.110E-02 1.323E-02 $2.170 \mathrm{E}-02$ $1.022 \mathrm{E}-01$ $8.054 \mathrm{E}-02$ $4.597 E$ OO $1.923 \mathrm{E} \mathrm{O1}$ $5.492 \mathrm{E} 01$ $1.437 \mathrm{E} 02$ 3.780 E O2 $8.147 E$ O2 $1.163 \mathrm{E} \mathrm{O3}$
ANGLE 5 $1.854 \mathrm{E}-04$ $.514 E-03$ $.796 E-04$ $1.642 \mathrm{E}-03$ $2.631 E-03$ $3.062 \mathrm{E}-03$ $5.134 E-03$ .696E-03 . $1.066 \mathrm{E}-02$ $8.903 E-02$ $7.355 \mathrm{E}-02$ $4.272 E$ OO $1.804 E$ OI $5.179 E$ OI $1.362 \mathrm{E} \mathrm{O2}$ 3.597E 02 $7.775 \mathrm{E} \mathrm{O2}$ $1.116 \mathrm{E} 03$ ANGLE 14 $A N=0.7550$
$5.854 E-03$ $5.706 E-03$ $3.353 \mathrm{E}-03$ $4.131 E-03$ $6.683 E-03$ 1.067E-02 $1.110 E-02$ 1.661E-02 1.976E-02 1.671E-02 $1.671 \mathrm{E}-02$ $2.339 \mathrm{E}-02$ $1.043 \mathrm{E}-01$ $8.143 \mathrm{E}-02$ $4.636 \mathrm{E} \mathrm{OO}$ $5.527 E$ OI $1.445 \mathrm{E} \mathrm{O2}$ $3.800 E$ E2 $8.187 \mathrm{E} \quad 2$ $1.168 \mathrm{E} \quad 03$
ANGLE $\quad 6$
$M U=-0.6179$ $2.665 E-04$ $1.497 E-03$ $9.857 \mathrm{E}-04$ $1.112 \mathrm{E}-03$ $1.760 \mathrm{E}-03$ $2.786 \mathrm{E}-03$ $3.189 \mathrm{E}-03$ $5.275 \mathrm{E}-03$ $6.696 \mathrm{E}-03$ $5.950 \mathrm{E}-03$ $7.129 \mathrm{E}-03$ $1.101 \mathrm{E}-02$ $1.616 \mathrm{E}-02$ $9.006 \mathrm{E}-02$ $7.415 E-02$ 4.301E OO $1.815 \mathrm{E} 01$ $5.208 E$ OI $1.369 \mathrm{E} 02$ $3.614 E \quad 02$ $7.810 E$ O2 1.121E 03

ANGLE 15 1.641 E-02 $9.919 \mathrm{E}-03$ $6.105 \mathrm{E}-03$ $7.560 \mathrm{E}-03$ $1.026 \mathrm{E}-02$ $1.243 \mathrm{E}-02$ $1.260 E-02$ . $1.904 \mathrm{E}-02$ $.902 \mathrm{E}-02$ $2.310 \mathrm{E}-02$ 1.059E-0 $8.219 E-02$ $4.668 \mathrm{E} 00$ $1.948 \mathrm{E}$ O $5.556 \mathrm{E} 01$ $1.451 \mathrm{E} \mathrm{O2}$ $3.816 \mathrm{E} \mathrm{O2}$

1.172E 03

ANGLE 7 ANGLE 8 ANGLE 9 $M U=-0.4580 \quad M U=-0.2816 \quad M U=-0.0950$ $4.341 \mathrm{E}-05 \quad 9.858 \mathrm{E}-05 \quad 3.859 \mathrm{E}-04$ $\begin{array}{ll}1.981 E-03 & 2.372 E-03\end{array}$ $1.214 \mathrm{E}-03 \quad 1.359 \mathrm{E}-03 \quad 1.557 \mathrm{E}-03$ $1.928 \mathrm{E}-03 \quad 2.154 \mathrm{E}-03 \quad 2.447 \mathrm{E}-03$ $2.990 E-03 \quad 3.258 E-03 \quad 3.613 E-03$ $3.367 E-03 \quad 3.613 E-03 \quad 3.953 E-03$ $5.493 E-03 \quad 5.820 E-03 \quad 6.299 E-03$ $7.029 E-03 \quad 7.519 E-03 \quad 8.220 E-03$ $6.306 \mathrm{E}-03 \quad 6.791 \mathrm{E}-03 \quad 7.440 \mathrm{E}-03$ $8.017 \mathrm{E}-03$ 
(NEUTRONS/MEV/STERADIAN/SOURCE NEUTRON)

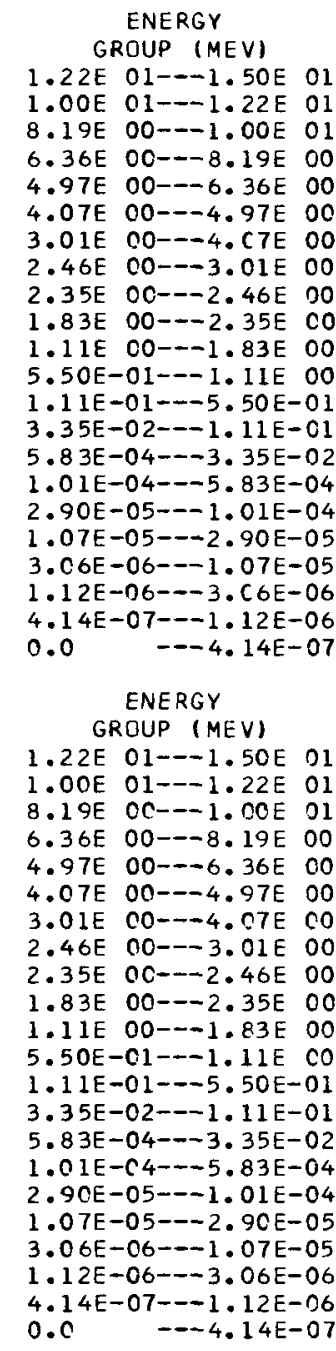
ANGLE 1 ANGLE 2 $M U=-1.0000$ $1.876 \mathrm{E}-\mathrm{C} 3$ $1.020 \mathrm{E}-\mathrm{C3}$ $9.825 E-04$ $1.572 \mathrm{E}-\mathrm{C} 3$ $2.597 \mathrm{E}-03$ $3.168 \mathrm{E}-03$ $5.531 E-C 3$ $7.041 \mathrm{E}-03$ $6.271 E-C 3$ $7.856 \mathrm{E}-\mathrm{C} 3$ $1.319 \mathrm{E}-\mathrm{C} 2$ $2.142 \mathrm{E}-02$ $1.319 \mathrm{E}-01$ $1.123 \mathrm{E}-\mathrm{C}$ $6.649 \mathrm{E} \mathrm{OO}$ $2.840 \mathrm{E} \mathrm{Cl}$ $8.211 E$ O 1 $2.175 E 02$ $5.777 \mathrm{E} \mathrm{C}$ $1.808 \mathrm{E} 03$

ANGLE 10 $M U=0.6950$ $1.153 \mathrm{E}-03$ $2.309 \mathrm{E}-03$ $1.736 E-C$ $1.757 \mathrm{E}-\mathrm{C3}$ $3.546 E-C 3$ $4.921 E-03$ $5.312 E-03$ $6.749 E-03$ $9.725 E-03$ $8.855 \mathrm{E}-\mathrm{C} 3$ $1.092 \mathrm{E}-\mathrm{C} 2$ $1.979 E-C 2$ $2.729 \mathrm{E}-02$ $1.472 \mathrm{E}-\mathrm{O} 1$ $1.213 \mathrm{E}-\mathrm{C} 1$ $7.104 E$ OO $3.014 \mathrm{E} 01$ $8.683 E$ C1 2.291E O2 6.065E 02 $1.884 \mathrm{E} \mathrm{O3}$ $4.136 E-04$ $1.835 E-03$ $1.014 E-03$ $1.576 \mathrm{E}-03$ $2.608 \mathrm{E}-03$ $3.175 \mathrm{E}-03$ $5.537 \mathrm{E}-03$ $7.050 \mathrm{E}-03$ $6.286 E-03$ $7.877 \mathrm{E}-03$ $1.322 \mathrm{E}-02$ $2.146 \mathrm{E}-02$ $1.320 \mathrm{E}-01$ $1.124 \mathrm{E}-01$ $6.653 \mathrm{E} 00$ 2.841E 01 $8.215 E \quad 01$ $2.176 \mathrm{E} \mathrm{O2}$ $5.779 \mathrm{E} 02$ $1.255 E 03$ $1.809 \mathrm{E} 03$ ANGLE 11 $M U=0.2816$ $1.517 \mathrm{E}-\mathrm{C} 3$ $3.135 \mathrm{E}-03$ $1.653 \mathrm{E}-03$ $2.413 E-03$ $3.173 \mathrm{E}-03$ $4.850 E-03$ $5.189 \mathrm{E}-03$ $9.982 E-03$ $1.326 \mathrm{E}-0$ $1.217 \mathrm{E}-02$ $1.369 \mathrm{E}-02$ $1.844 E-02$ $2.713 E-02$ $1.500 \mathrm{E}-0$ $1.231 \mathrm{E}-01$ $7.192 E$ OO $3.047 E$ OI $8.772 E$ O1 $2.313 E 02$ $1.324 E 03$ $1.898 \mathrm{E} 03$ $\begin{array}{ccc}\text { ANGLE } & 3 & \text { ANGLE } \quad 4 \\ M U=-0.9446 & M U=-0.8656\end{array}$

$-1.291 E-04$ $1.689 E-03$ 9. $910 E-04$ $9.944 \mathrm{E}-04$ 2. $252 E-03$ $3.202 E-03$ $5.562 \mathrm{E}-03$ $7.091 E-03$ $6.351 \mathrm{E}-03$ $7.965 \mathrm{E}-03$ $1.334 E-02$ 2. $160 E-02$ 1. $325 \mathrm{E}-0$ $1.127 E-01$ $6.669 E 00$ $2.848 E 0$ $8.233 E 0$ $2.181 E 0$ $5.790 E$ O2 $1.257 E 0$ $1.812 \mathrm{E} 03$ ANGLE 12 $M U=0.4580$ $2.465 \mathrm{E}-03$ $3.225 \mathrm{E}-03$
$2.414 \mathrm{E}-03$ $2.734 \mathrm{E}-03$ $4.813 E-03$ $6.624 \mathrm{E}-03$ $7.006 \mathrm{E}-03$ $9.831 \mathrm{E}-0$ $1.398 E-02$ $1.233 \mathrm{E}-02$ $1.411 \mathrm{E}-02$ $2.286 \mathrm{E}-02$ 2. $536 \mathrm{E}-01$ $1.536 \mathrm{E}-01$ $1.249 E-C 1$ $7.279 E$ OO $3.079 \mathrm{E}$ Ol $8.859 E 01$ 2.334E O2 $1.335 \mathrm{E} 03$ $1.912 \mathrm{E} 03$ $M U=-0.8656$ $3.126 \mathrm{E}-05$ $1.487 E-03$ $9.604 E-04$ $1.018 \mathrm{E}-03$ $1.643 \mathrm{E}-03$ $2.738 \mathrm{E}-03$ $5.617 E-03$ $7.182 \mathrm{E}-03$ $6.484 \mathrm{E}-03$ $8.139 E-03$ $1.358 \mathrm{E}-02$ $2.189 \mathrm{E}-02$ $1.334 \mathrm{E}-01$ $1.133 \mathrm{E}-01$ 列 $8.265 \mathrm{E} 01$ $5.810 E$ O2 $1.818 \mathrm{E} 03$ ANGLE 13 $M U=0.617$ $3.260 \mathrm{E}-03$ $4.291 \mathrm{E}-03$ $2.763 \mathrm{E}-03$ $3.750 \mathrm{E}-03$ $5.309 \mathrm{E}-03$ $7.612 \mathrm{E}-03$ $7.977 \mathrm{E}-03$ $1.387 \mathrm{E}-02$ $1.879 E-02$ $1.632 \mathrm{E}-\mathrm{C2} 2$ $1.734 E-02$ $2.316 \mathrm{E}-02$ $1.567 \mathrm{E}-01$ $1.567 E-01$ $1.267 \mathrm{E}-01$ $7.362 E$ OO $3.110 E$ OI $8.940 E$ OI $2.353 E$ E2 $1.345 \mathrm{E} O 3$ $1.925 \mathrm{E} \mathrm{O3}$

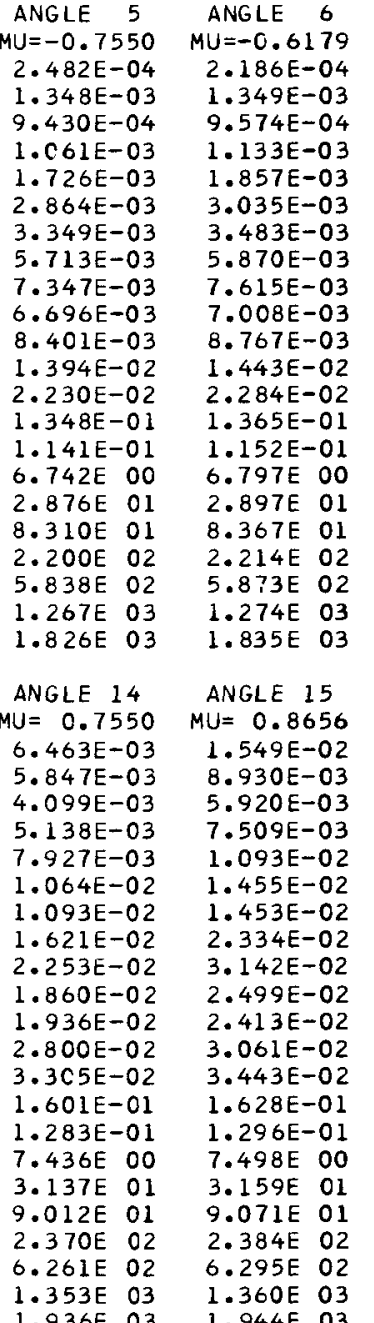

ANGLE 7 ANGLE 8 MU $=-0.0950$ $5.572 E-05 \quad 1.222 E-04 \quad 4.026 E-04$ $1.517 \mathrm{E}-03 \quad 1.819 \mathrm{E}-03 \quad 2.185 \mathrm{E}-03$ $1.018 \mathrm{E}-03 \quad 1.133 \mathrm{E}-03 \quad 1.303 \mathrm{E}-03$ $1.244 \mathrm{E}-03 \quad 1.402 \mathrm{E}-03 \quad 1.617 \mathrm{E}-03$ $2.045 \mathrm{E}-03 \quad 2.296 \mathrm{E}-03 \quad 2.622 \mathrm{E}-03$ $3.261 \mathrm{E}-03 \quad 3.559 \mathrm{E}-03 \quad 3.955 \mathrm{E}-03$ $3.674 E-03 \quad 3.942 E-03 \quad 4.318 E-03$ $6.117 \mathrm{E}-03 \quad 6.494 \mathrm{E}-03 \quad 7.050 \mathrm{E}-03$ $8.027 E-03 \quad 8.632 E-03 \quad 9.495 E-03$ $\begin{array}{lll}7.446 E-03 & 8.042 E-03 & 8.835 E-03\end{array}$ $9.254 E-03 \quad 9.885 E-03 \quad 1.069 E-02$ $1.507 E-02 \quad 1.589 E-02 \quad 1.692 E-02$ $2.353 E-02 \quad 2.436 E-02 \quad 2.534 E-02$ $1.386 \mathrm{E}-01 \quad 1.411 \mathrm{E}-01 \quad 1.439 \mathrm{E}-01$ $1.165 E-01$ 1.179E-01 1.196E-01 $6.862 E$ OO 6.936E 00 T.018E OO 2.922E 01 2.950E O1 2.981E OL $8.435 E$ OI $8.512 E$ OI $8.595 E$ OL 2.231E 02 2.249E 02 $2.270 E$ O2 5.915E O2 $5.962 E$ O2 $6.012 E$ O2

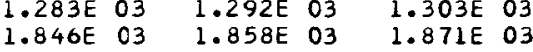
ANGLE 16 $M U=0.9446 \quad M U=0.9894$ $4.819 E-02 \quad 6.280 E-01$ $1.770 \mathrm{E}-02 \quad 4.899 \mathrm{E}-02$ $1.054 \mathrm{E}-02 \quad 2.465 \mathrm{E}-02$ $1.341 \mathrm{E}-02 \quad 3.190 \mathrm{E}-02$ $1.887 E-02 \quad 4.179 E-02$ $2.340 E-02 \quad 4.887 E-02$ $2.230 E-02 \quad 4.297 E-02$ $3.420 E-02 \quad 6.824 E-02$ $4.565 E-02$ $8.780 E-02$ $3.386 E-02 \quad 6.008 E-02$ $3.024 E-02$ $4.557 E-02$ $3.716 \mathrm{E}-02 \quad 3.994 \mathrm{E}-02$ $1.716 E-02$ $3.994 E-02$ $1.652 E-01$ 1.667E-01 $1.306 E-01$ 1.313E-OI 7.544E OO 7.57OE OD 3.176E O1 $3.185 E$ OI 2.114E 01 $9.139 E$ O1 2.395E O2 $2.401 E$ O2 6.320 E 02 $6.335 E$ O2 $\begin{array}{lll}1.365 \mathrm{E} \mathrm{O3} & 1.368 \mathrm{E} 03 \\ 1.949 \mathrm{E} 03 & 1.952 \mathrm{E} 03\end{array}$
SCALAR FLUX
1. 5 C1E-0

1.501E-01
4. $949 E-02$ $3.091 E-02$ $3.841 \mathrm{E}-02$ $5.812 \mathrm{E}-02$ $8.061 E-02$ $8.373 \mathrm{E}-02$ $1.335 E-01$ $1.795 \mathrm{E}-01$ $1.529 E-01$ $1.672 E-01$ . $389 E-01$ $1.842 E 00$ 1.519E OO 3.773E 02 $1.087 E_{03} 03$ $2.869 E$ E $7.596 E 03$ $\begin{array}{ll}1.645 E & 04 \\ 2.362 E & 04\end{array}$ 
(NEUTRONS/MEV/STERADIAN/SCURCE NEUTRON)

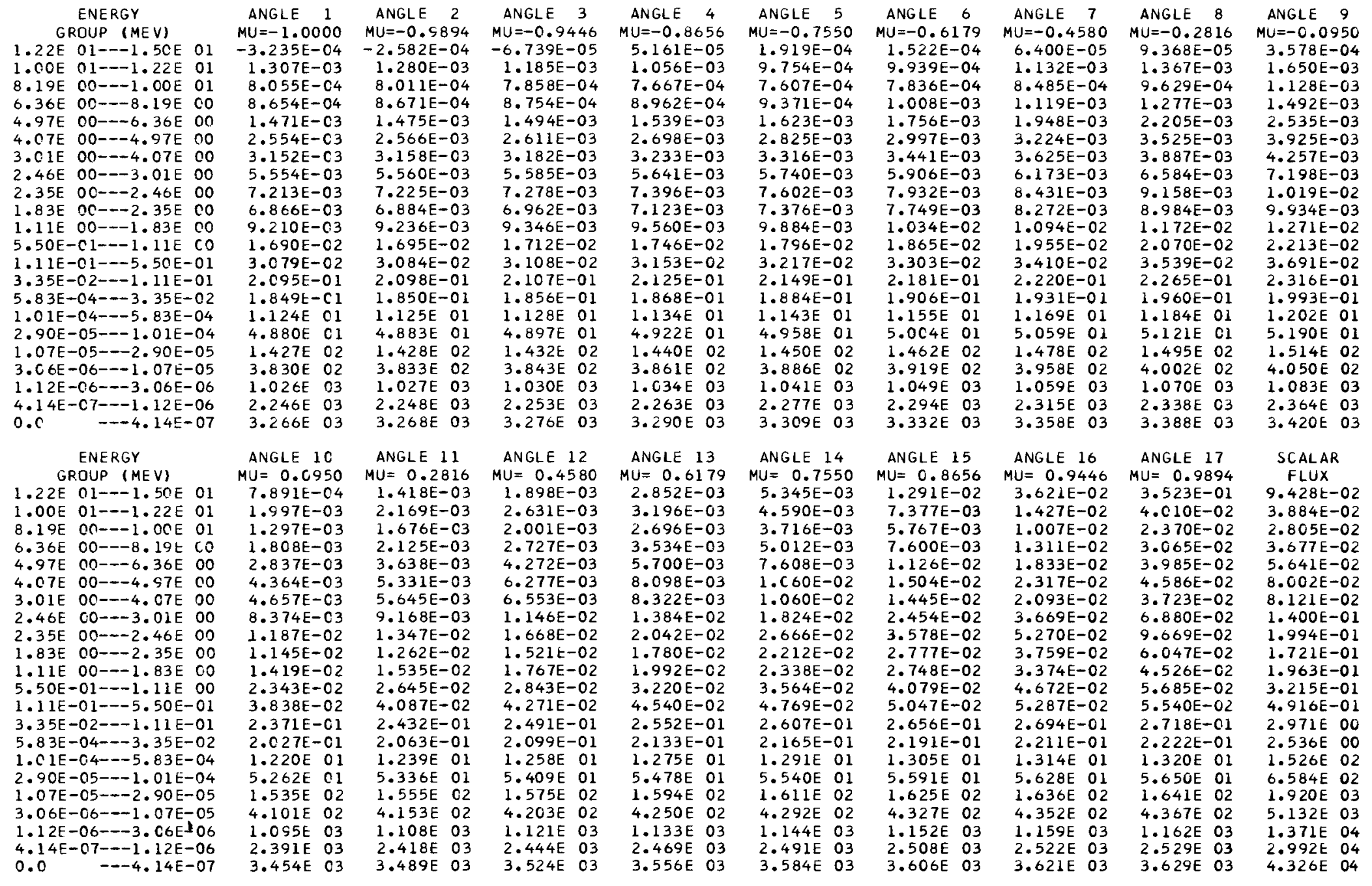


(NEUTRONS/MEV/STERADIAN/SOURCE NEUTRON)

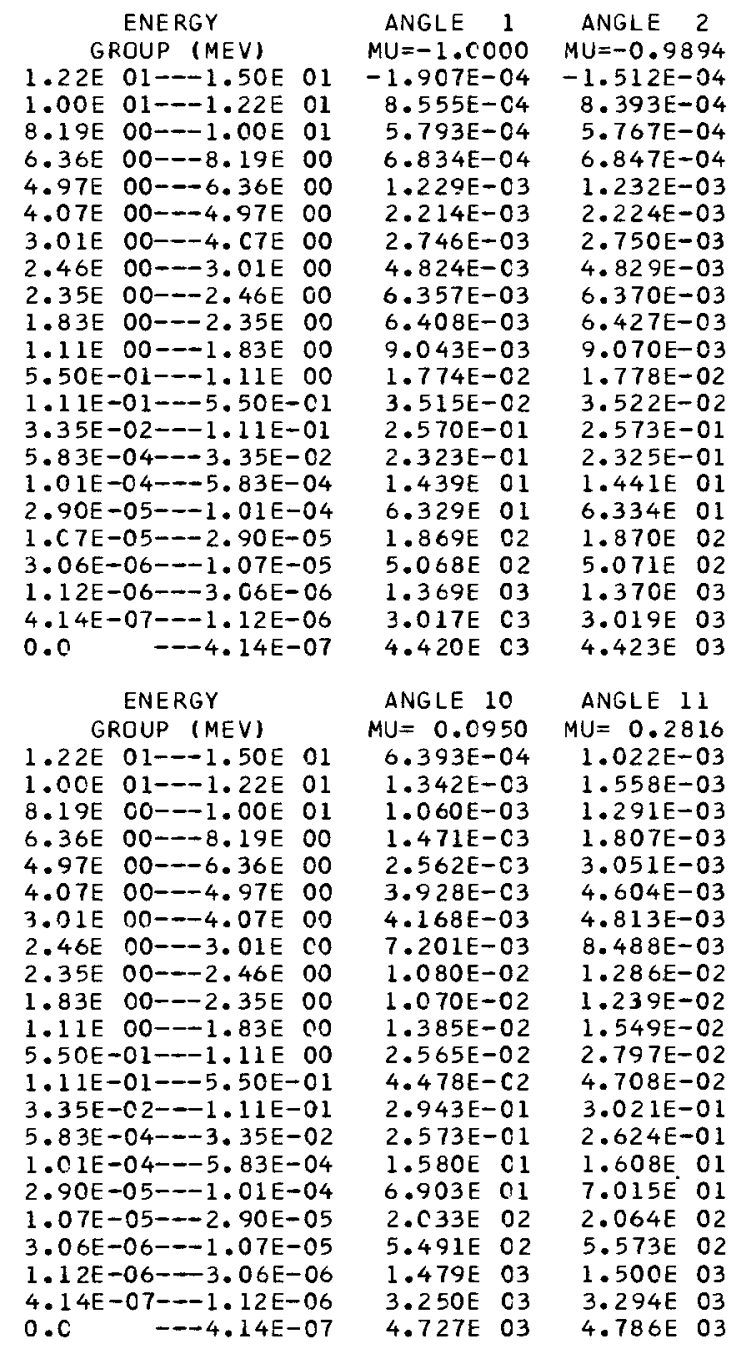

- ANGLE 3 ANGLE 4 ANGLE 5 $M U=-0.9446$ $-3.277 E-05$ $7.822 \mathrm{E}-04$ $6.013 E-04$ 6. $913 \mathrm{E}-04$ $1.248 \mathrm{E}-03$ $2.264 \mathrm{E}-03$
$2.770 \mathrm{E}-03$ $4.852 \mathrm{E}-03$ $6.429 \mathrm{E}-03$ $6.506 \mathrm{E}-03$ $9.506 \mathrm{E}-03$ $9.182 \mathrm{E}-03$
$1.798 \mathrm{E}-02$ $3.551 \mathrm{E}-02$ $2.586 \mathrm{E}-01$ $2.334 \mathrm{E}-01$ $1.446 \mathrm{E} 01$ $6.355 E$ ol $1.876 \mathrm{E} 02$ 5.087E 02 $1.374 E 03$ $3.028 \mathrm{E} 03$
$4.436 \mathrm{E}$

ANGLE 7

ANGLE 12 $M U=0.4580$ $1.432 \mathrm{E}-03$ $1.817 \mathrm{E}-03$ $1.635 \mathrm{E}-03$ $2.284 E-03$ $3.807 \mathrm{E}-03$ $5.634 \mathrm{E}-03$ $5.801 E-C 3$ $1.024 \mathrm{E}-02$ $1.570 E-02$ $1.453 \mathrm{E}-02$ $1.744 \mathrm{E}-02$ $3.097 \mathrm{E}-02$ 4.977E-02 3. $102 \mathrm{E}-01$ $2.674 \mathrm{E}-01$ $1.635 \mathrm{E} 01$ $7.125 E$ OI $2.096 \mathrm{E} \mathrm{O2}$ $5.653 E 02$ $1.520 E 03$ $4.843 \mathrm{E} 03$ $M U=-0.8656$ $M U=-0.7550$ 0.6179 . $5.576 \mathrm{E}-04$ 7.085E-04 $1.306 \mathrm{E}-04$ $5.582 \mathrm{E}-0$ $7.435 \mathrm{E}-04$ $288 \mathrm{E}-03$ $7.435 \mathrm{E}-04$ $2.341 E-03$ 4.904E-03 $6.555 \mathrm{E}-0$ $6.667 \mathrm{E}-03$ $9.401 \mathrm{E}-0$ $1.836 \mathrm{E}-02$ $3.606 \mathrm{E}-02$ $2.6009 \mathrm{E}-01$ 2.350 E-OI $1.455 \mathrm{E} \mathrm{OI}$ $6.393 E$ OI $1.887 \mathrm{E} \mathrm{O2}$ $5.115 \mathrm{E} \mathrm{O2}$ $1.381 \mathrm{E} 03$ $3.044 \mathrm{E} 03$
$4.458 \mathrm{E} 03$ $2.878 \mathrm{E}-03$ $4.996 \mathrm{E}-03$ $6.770 \mathrm{E}-03$ $6.921 \mathrm{E}-0$ $9.731 \mathrm{E}-03$ $1.892 \mathrm{E}-02$ $3.684 \mathrm{E}-0$ $2.642 \mathrm{E}-0$ $2.373 \mathrm{E}-01$ $\begin{array}{ll}1.468 \mathrm{E} & 01 \\ 6.446 \mathrm{E} & 01\end{array}$ $1.902 \mathrm{E} 02$ $5.155 \mathrm{E} 02$ $1.392 E 03$ $3.066 \mathrm{E} 03$
$4.488 \mathrm{E} 03$ $.803 E-05$ $5.815 E-04$ . $1.483 E-03$ $1.481 E-03$ $2.904 E-03$ $5.151 \mathrm{E}-03$ $7.108 \mathrm{E}-03$ $7.108 \mathrm{E}-03$ $7.294 E-03$ $1.019 E-02$ $3.788 \mathrm{E}-02$ $2.685 \mathrm{E}-01$ $2.403 \mathrm{E}-01$ $1.485 \mathrm{E} \mathrm{OI}$ $6.515 \mathrm{E} \mathrm{Ol}$ $1.922 E 02$ $5.2 C 6 E 02$ $1.405 \mathrm{E} 03$ $3.094 \mathrm{E} 03$
$4.526 \mathrm{E} 03$ $M U=-0.4580$ $7.832 \mathrm{E}-04$ $6.383 \mathrm{E}-04$ $9.025 \mathrm{E}-04$ $1.653 E-03$ $3.139 E-03$ $5.401 E-0$ $7.613 \mathrm{E}-03$ $7.815 \mathrm{E}-03$ $1.080 \mathrm{E}-02$ $2.071 E-02$ $3.918 \mathrm{E}-02$ $2.738 E-0$ $2.438 \mathrm{E}-0$ $1.505 \mathrm{E} \mathrm{OI}$ $1.945 \mathrm{E} \mathrm{O2}$ $5.267 \mathrm{E} \mathrm{O2}^{2}$ $1.420 \mathrm{E} 03$ $3.127 \mathrm{E} 03$

ANGLE 13

ANGLE 14 UU $0.6179 \quad M U=0.7550$ $.311 \mathrm{E}-03$ $2.165 \mathrm{E}-0$ $3.051 \mathrm{E}-03$ 4.937E-0 $7.154 \mathrm{E}-03$ $7.195 \mathrm{E}-03$ $1.290 \mathrm{E}-02$ $1.985 \mathrm{E}-02$ $1.746 \mathrm{E}-02$ $1.995 \mathrm{E}-02$ 3. $441 \mathrm{E}-02$ $5.253 \mathrm{E}-02$ $3.182 \mathrm{E}-01$ $2.722 \mathrm{E}-01$ $1.661 \mathrm{E} 01$ 7.230 E 01 $2.125 \mathrm{E} 02$ $5.729 \mathrm{E}$ O2 $1.540 \mathrm{E} \mathrm{O3}$ $4.897 \mathrm{E} 03$ 3. $319 E-03$ 3.109E-O $4.349 E-03$ 6.804E-O $9.547 E-03$ $9.279 E-03$ $2.590 \mathrm{E}-02$ $2.137 \mathrm{E}-02$ 2. $302 \mathrm{E}-02$ $3.860 \mathrm{E}-02$ $5.547 \mathrm{E}-02$ $3.256 \mathrm{E}-01$ $2.766 E-01$ $1.685 \mathrm{E}$
$7.324 \mathrm{E}$ $7.324 \mathrm{E} 01$ $2.152 \mathrm{E} 02$ $5.796 \mathrm{E} 02$ $1.557 \mathrm{E} 03$ $4.944 \mathrm{E} \mathrm{O3}$
ANGLE 15 $9.605 \mathrm{E}-03$ $5.509 \mathrm{E}-03$ $6.712 \mathrm{E}-0$ $6.712 \mathrm{E}-03$ 1. $1.239 \mathrm{E}-02$ $.294 \mathrm{E}-02$ 3. $532 \mathrm{E}-02$ $2.693 \mathrm{E}-02$ $2.695 \mathrm{E}-02$ $4.344 \mathrm{E}-02$ $5.828 \mathrm{E}-02$ 3. $319 \mathrm{E}-01$ $2.803 \mathrm{E}-01$ $1.705 \mathrm{E} 01$ $7.402 E$ OI 2.174E O2 $5.852 \mathrm{E}$ O2 1.571E O3 $4.981 \mathrm{E} 03$
ANGLE 16 $A N=0.9446$
$2.582 E-02$ $1.068 \mathrm{E}-0$ $8.616 \mathrm{E}-03$ $1.153 \mathrm{E}-02$ $1.610 \mathrm{E}-02$ $2.038 \mathrm{E}-02$ $1.750 \mathrm{E}-02$ $3.373 E-02$ $5.172 \mathrm{E}-02$ 3.549E-C2 $3.206 E-02$ $4.923 E-02$ $6.087 \mathrm{E}-02$ $3.368 \mathrm{E}-01$ $2.830 E-01$ $1.719 E$ OI

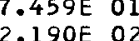
$2.190 \mathrm{E} 02$ $5.893 \mathrm{E} 02$ $1.581 \mathrm{E} 03$ $5.007 E 03$ $M U=-0.2816$ $9.698 E-05$ $7.351 \mathrm{E}-04$ $1.042 \mathrm{E}-03$ ( $882 \mathrm{E}-03$ 3.070E-O $3.365 \mathrm{E}-03$ $5.789 E-03$ $8.342 \mathrm{E}-03$ $8.524 \mathrm{E}-03$ $1.160 \mathrm{E}-02$ $2.199 \mathrm{E}-02$ $4.075 \mathrm{E}-02$ $2.799 \mathrm{E}-01$ $.479 E-C$ $1.528 \mathrm{E} 01$ $1.972 \mathrm{E} 02$ $5.336 \mathrm{E} \mathrm{O2}$ $1.438 \mathrm{E} \mathrm{O3}$ $3.165 E O 3$
$4.618 E \quad 03$

ANGLE 9 0.0950 $2.901 E-04$
$1.146 E-03$ $8.737 E-04$ $1.229 E-03$ 2.174E-O3 $3.427 \mathrm{E}-03$ $3.689 E-03$ $6.372 E-03$ $9.372 \mathrm{E}-03$ $9.471 E-03$ $1.261 \mathrm{E}-02$ $2.360 E-02$ $4.260 \mathrm{E}-02$ $2.867 \mathrm{E}-0$ .525E-0 $6.793 E$ O $2.001 E 02$ $5.411 \mathrm{E} 02$ $1.458 \mathrm{E} 03$ $3.206 E$ E
$4.671 E 03$ ANGLE 17 $M U=0.9894$
$1.973 E-01$ $2.935 \mathrm{E}-02$ $1.992 \mathrm{E}-02$ $2.600 \mathrm{E}-02$ . $336 \mathrm{E}-02$ .797E-02 ..872E-02 6.072E-02 5. . $4.015 E-02$ .685E-02 6. $3.398 \mathrm{E}-01$ $1.728 \mathrm{E} O 1$ $7.492 E 01$ 2.199E 02 5.916 E 02 $1.587 E 03$ $5.022 E \quad 03$ 
(NEUTRONS/MEV/STERADIAN/SOURCE NEUTRONI GROUP (MEV) $1.22 E$ O1---1.50E 01 $1.00 E$ O1- $-1.22 E$ OI $6.36 \mathrm{E} 00---8.19 \mathrm{E} 00$ $4.97 \mathrm{E} 00---6.36 \mathrm{E} \mathrm{CO}$ $4.07 \mathrm{E} 00---4.97 \mathrm{E} 00$ $3.01 \mathrm{E} 00---4.07 \mathrm{E} 00$ $2.46 \mathrm{E} 00--3.01 \mathrm{E}$ OO $2.35 \mathrm{E} 00---2.46 \mathrm{E} 00$ $1.83 \mathrm{E} \quad 00--2.35 \mathrm{E}$ CO $1.11 \mathrm{E} 00--1.83 \mathrm{E} 00$ $5.50 \mathrm{E}-01--1.11 \mathrm{E}$ OO $1.11 \mathrm{E}-01---5.50 \mathrm{E}-01$ $3.35 \mathrm{E}-02---1.11 \mathrm{E}-0$ $5.83 \mathrm{E}-04---3.35 \mathrm{E}-02$ $1.01 \mathrm{E}-04---5.83 \mathrm{E}-04$ $2.90 \mathrm{E}-05--1.01 \mathrm{E}-04$ $1.07 E-05---2.90 E-05$ $3.06 E-06--1.07 E-05$ $1.12 \mathrm{E}-06--3$. . C6E-06 $4.14 E-07--1.12 E-06$ $0.0 \quad--4.14 E-07$ ENERGY GROUP (MEV) $1.22 \mathrm{E}$ 01---1.50E 01 $1.00 \mathrm{E} \quad 01--1.22 \mathrm{E} \quad 01$ 8.19E 00---1.00E O $6.36 \mathrm{E}$ OO4.97E DO- 6.36 E 00 4.07E OO-4.97E 3.01 E $00--4.07 E$ OO 2.46E OO- 3 .01E $2.35 \mathrm{E} 00-2.46 \mathrm{E}$ $1.83 E$ OO- $2.35 E$ $5.50 E-01--1.11 E$ OO 1.11E-01-- 5 . $0 \mathrm{E}-0$ 1.35E-02$5.33 E-04--3.35 E-02$ 1.01E-04- $5.35 E-04$ $1.01 E-04-2.03 E-04$ 1.07E-05- $2.00 E-05$ $1.06 \mathrm{E}-\mathrm{C6}-\mathrm{C}-1.07 \mathrm{E}-05$ $1.12 \mathrm{E}-\mathrm{C6}-\mathrm{C}^{3} \mathrm{C6E}-06$ 4.14E-07-- $1.12 \mathrm{E}-06$ $4.14 E-07=-1.12 E-06$ $M=-1.0000$ $1 . \mathrm{C} 92 \mathrm{E}-04$
$5.416 \mathrm{E}-04$ $3.416 \mathrm{E}-04$ $5.085 E-04$ .644E-04 $2.222 E-03$ $3.872 E-03$ $5.164 E-03$ $8.037 \mathrm{E}-03$ $3.520 E-02$ $2.719 E-O$ $2.502 \mathrm{E}-\mathrm{C}$ $1.573 \mathrm{E} O 1$ $6.984 E$ CI 2.077E C2 $1.545 \mathrm{E} \mathrm{C}$ $3.425 \mathrm{E}$ $5.051 \mathrm{E} 03$

ANGLE 10 $M U=0.0950$ $4.487 E-04$ $8.988 E-04$ $7.760 E-04$ $1.148 \mathrm{E}-03$ $2.050 E-03$ 35 . 9.210E-03 - $210 E-O$ $1.246 \mathrm{E}-0$ $2.435 \mathrm{E}-02$ $4.525 \mathrm{E}-\mathrm{C} 2$

$3.139 \mathrm{E}-\mathrm{O}$ $2.793 \mathrm{E}-01$ $1.741 \mathrm{E} O 1$ $2.279 \mathrm{E}$ CD $6.211 \mathrm{E} 02$ $1.683 E \mathrm{O}$ $3.723 \mathrm{E}$ $3.723 E \mathrm{E}_{3}$
ANGLE 2 ANGLE 3 $-8.588 E-05 \quad-1.506 E-05$ $5.323 E-04$ $3.948 \mathrm{E}-04$ $5.094 \mathrm{E}-04$ $9.670 E-04$ $1.796 E-03$ $3.877 \mathrm{E}-0$ 5.177E-03 5.490E-03 $8.061 E-03$ $1.666 \mathrm{E}-02$ $3.528 \mathrm{E}-0$ $2.722 E-O$ $2.504 E-O 1$ $1.575 E$ O 6.990E OI $2.079 E \quad 02$ $1.546 E \quad 03$ $3.428 \mathrm{E} 03$ $\begin{array}{ll}3.428 E & 03 \\ 5.054 E & 03\end{array}$

ANGLE 11 $M U=0.2816$ $7.118 \mathrm{E}-04$ $1.04 \mathrm{CE}-03$ $9.601 \mathrm{E}-04$ 1.416E 03 $2.471 E-C 3$ 3. . 7.037E-03 $1.104 E-02$ $1.085 \mathrm{E}-02$ 1. $4.775 \mathrm{E}-02$ 3.775E-02 $2.851 \mathrm{E}-01$ $1.774 \mathrm{E} \mathrm{OI}$ $7.816 \mathrm{E} \mathrm{Ol}$ $2.318 \mathrm{E} 02$ $6.314 \mathrm{E} \mathrm{O2}$ $1.710 E 03$ $3.780 E \quad 03$ $5.524 \mathrm{E} \mathrm{O3}$
$-1.506 \mathrm{E}-05$
$4.996 \mathrm{E}-04$ $4.996 \mathrm{E}-04$
$3.901 \mathrm{E}-04$ $3.901 E-04$
$5.145 \mathrm{E}-04$
$0.799 E-04$ $9.799 E-04$ $1.829 E-03$ 2. $240 \mathrm{E}-03$ $3.897 \mathrm{E}-03$ $5.234 \mathrm{E}-03$
$5.563 \mathrm{E}-03$ $8.164 E-03$ $1.686 \mathrm{E}-02$ $3.558 \mathrm{E}-02$ $2.737 \mathrm{E}-01$ 2.515E-01 $1.581 \mathrm{E} 01$ $7.015 E 01$ $2.086 \mathrm{E} \mathrm{O2}$ $5.705 \mathrm{E} 02$ $1.551 \mathrm{E} 03$ $3.439 \mathrm{E} 03$ $M U=0.4580$ $1.229 \mathrm{E}-03$ $1.222 E-03$ $1.810 \mathrm{E}-03$ $3.086 E-03$ $4.689 E-03$ $4.68 \mathrm{E}-03$ $8.606 E-03$ $1.365 \mathrm{E}-02$ $1.569 \mathrm{E}-02$ $2.959 \mathrm{E}-02$ $5.048 E-02$ $2.910 E-01$ 2.910E-01 $7.951 \mathrm{E} \mathrm{O1}$ $2.357 E 02$ $6.415 \mathrm{E} 02$ $1.737 \mathrm{E} 03$ $3.836 \mathrm{E} 03$ $5.599 \mathrm{E} 03$
ANGLE 4 $=-0.8656$ $.458 E-05$ . $857 \mathrm{E}-04$ $5.280 E-04$ $1.012 \mathrm{E}-03$ $1.892 E-03$ $2.270 \mathrm{E}-03$ $3.942 \mathrm{E}-03$ $5.354 E-03$ 5.710E-03 $8.364 \mathrm{E}-03$ $1.723 \mathrm{E}-02$ $3.615 \mathrm{E}-02$ $2.763 \mathrm{E}-01$ $2.534 \varepsilon-01$ $1.592 \mathrm{E} 01$ $7.061 E 01$ $2.100 \mathrm{E} \mathrm{O2}$ $5.741 \mathrm{E} 02$
$1.560 \mathrm{E} 03$ $1.560 \mathrm{E} \mathrm{O3}$ $3.458 \mathrm{E} 03$
$5 . C 99 \mathrm{E} 03$ ANGLE 13 $M U=0.6179$ $1.495 \mathrm{E}-03$ $1.578 \mathrm{E}-03$ $1.648 \mathrm{E}-03$ .. $045 \mathrm{E}-03$ $4.048 E-03$ $5.922 E-03$ $1.031 E-03$ $1.090 E-02$ $1.742 E-02$ $1.540 \mathrm{E}-02$ 1.790E-02 $5.338 \mathrm{E}-02$ $3.409 E-01$ $3.409 E-01$ 2.966E-OI $1.838 E$ OI
$8.080 E$ Ol $2.394 E 02$ $6.511 \mathrm{E} 02$ $1.762 \mathrm{E} 03$ 3.889 E 03 $5.670 E 03$
ANGLE 5
$M U=-0.7550$ $8.377 \mathrm{E}-05$ 4. $345 E-04$ $5.561 E-04$ $1.073 E-03$ $1.983 E-03$ $2.322 E-03$ 4. $C 21 E-03$ $5.555 E-03$ $5.939 E-03$ $8.665 E-03$ $1.778 \mathrm{E}-02$ $3.657 \mathrm{E}-02$ $2.800 \mathrm{E}-01$ 2.560E-01 $1.607 \mathrm{E} 01$ $7.125 E 01$ $2.118 \mathrm{E} 02$ 5.790 E 02 $1.573 \mathrm{E} 03$ $3.486 \mathrm{E} 03$ $5.137 E 03$ ANGLE 14 $M U=0.7550$
$2.874 \mathrm{E}-03$ $2.317 \mathrm{E}-03$ $2.408 \mathrm{E}-03$ $3.540 E-03$ 5. 6 C E -03 $7.921 E-03$ $7.479 E-03$ $1.431 E-02$ $2.301 E-02$ 2.061E-02 $3.693 \mathrm{E}-02$ $3.492 \mathrm{E}-01$ $3.017 E-01$ $1.866 \mathrm{E} 01$ $2.427 \mathrm{E} 02$ $2.427 E ~ O 2$
$6.597 \mathrm{E} 02$ $1.784 \mathrm{E} 03$ $3.936 \mathrm{E} \mathrm{O3}$ $5.731 \mathrm{E} 03$

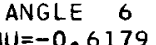
$6.150 E-05$ $4.518 E-04$ $.064 \mathrm{E}-0$ $1.172 \mathrm{E}-03$ $.106 \mathrm{E}-03$ $2.404 \mathrm{E}-03$ $4.156 \mathrm{E}-03$ .867E-03 $6.274 E-03$ $9.086 E-03$ $1.855 \mathrm{E}-02$ $3.806 \mathrm{E}-02$ $2.849 E-01$ $2.595 \mathrm{E}-01$ $1.627 E 01$ $7.208 E \quad 01$ $2.142 \mathrm{E} 02$

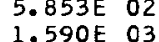
$3.522 \mathrm{E} 03$ $5.186 \mathrm{E} 03$ 
(NEUTRONS/MEV/STERADIAN/SOURCE NEUTRONI

\begin{abstract}
ENERGY
GROUP (MEV)

$1.22 E$ O1---1.5OE 01 $1.00 E$ O1-- $1.22 E 01$
$8.19 E$ OD---1.COE 01 $8.19 E$ OO-- 1. COE OI
$6.36 \mathrm{E} 00--8.19 \mathrm{E}$ CO 4.97E $00--6.36 \mathrm{E} 00$ 4.07E $00--4.97 E 00$ $3.01 \mathrm{E} 00--4.07 \mathrm{E} 00$ $2.46 \mathrm{E} \mathrm{O0--3.01E} 00$ $2.35 \mathrm{E} 00--2.46 \mathrm{E} 00$ $1.83 \mathrm{E} \mathrm{OO--2.35 \textrm {E } 0 0}$ $1.11 \mathrm{E} \mathrm{O0--1.83E} 00$ 5.50E-01--1.11E 00 $1.11 \mathrm{E}-01--5.50 \mathrm{E}-01$ 3.3 $5 \mathrm{E}-02--1.11 \mathrm{E}-01$ $5.83 \mathrm{E}-04---3.35 \mathrm{E}-02$ $1.01 \mathrm{E}-04--5.83 \mathrm{E}-04$ $2.90 E-05--1.01 E-04$ $1.07 E-05--2.90 \mathrm{E}-05$ 3.06E-06-- $1.07 E-05$ $1.12 E-08-1.07 E-05$ $4.14 \mathrm{E}-\mathrm{C7}---1.12 \mathrm{E}-06$
0.0
\end{abstract}

ENERGY

GROUP (MEV)

$1.22 \mathrm{E} 01--1.50 E$ O1 $1.00 \mathrm{E} 01--1.22 \mathrm{E} 01$ $8.19 E$ OO---1.00E OI $6.36 \mathrm{E} \mathrm{OO---8.19E} 00$ $4.97 \mathrm{E} \mathrm{OO---6.36E} \mathrm{OO}$ 4.07E 00-- 4.97E OO $3.01 \mathrm{E}$ OO- $-4.07 \mathrm{E}$ OO $2.46 \mathrm{E}$ CO-- 3.01E $\mathrm{CO}$ $2.35 E$ OO-- $2.46 E$ OO $1.83 \mathrm{E}$ 00---2.35E 00 $1.11 \mathrm{E} \mathrm{OO---1.83E}$ OO $5.50 \mathrm{E}-01--1.11 \mathrm{E} 00$
$1.11 \mathrm{E}-01--5.50 \mathrm{E}-01$

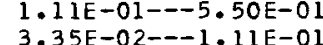
$3.35 \mathrm{E}-02---1.11 \mathrm{E}-01$
$5.83 \mathrm{E}-04--3.35 \mathrm{E}-02$ $5.83 E-04---3.35 E-02$ $1.01 E-04--5.83 E-04$ $2.90 E-05---1.01 E-04$
$1.07 E-05---2.90 E-05$ $1.07 E-05--2.90 E-05$ $3.06 \mathrm{E}-\mathrm{C6}---1.07 \mathrm{E}-05$
$1.12 \mathrm{E}-06---3.06 \mathrm{E}-06$ $1.12 E-06---3.06 E-06$
$4.14 \mathrm{E}-07---1.12 \mathrm{E}-06$ $4.14 \mathrm{E}-07---1.12 \mathrm{E}-06$
0.0 $M U=-1.0000$ $-6.143 E-C 5$
$3.359 E-04$ 2.626E-04 3.644E-04 $7.274 \mathrm{E}-04$ (1.381E-03 $1.716 \mathrm{E}-03$ $2.959 \mathrm{E}-\mathrm{C3}$ $3.987 \mathrm{E}-03$ $4.417 \mathrm{E}-\mathrm{C} 3$ $6.697 \mathrm{E}-\mathrm{C}$ $.446 \mathrm{E}-02$ $3.235 \mathrm{E}-02$ $2.611 \mathrm{E}-01$ $2.436 \mathrm{E}-\mathrm{C}$ $6.931 E$ OI $2.074 \mathrm{E} \mathrm{C}$ $5.711 E 02$ $1.561 \mathrm{E} \mathrm{O3}$ $3.478 \mathrm{E} \mathrm{O3}$

ANGLE $1 \mathrm{C}$ $M U=0.0950$ $3.025 \mathrm{E}-\mathrm{C} 4$ $5.824 \mathrm{E}-04$ $5.481 E-04$ 8.583E-04 $.577 \mathrm{E}-03$ $2.481 E-03$ $2.581 \mathrm{E}-\mathrm{C}$ $4.667 E-03$ $7.410 E-03$ $7.696 \mathrm{E}-03$ 1. $C 46 \mathrm{E}-02$ $2.147 \mathrm{E}-02$ $4.194 \mathrm{E}-\mathrm{C} 2$ $3.035 \mathrm{E}-01$ $2.735 \mathrm{E}-\mathrm{Cl}$ $1.725 \mathrm{E} O$ $7.670 \mathrm{E}$ Ol $2.290 \mathrm{E} O 2$ $6.286 E$ O2 $1.713 E 03$

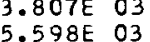
$-4.796 E-05$ $.307 \mathrm{E}-04$ $.620 E-04$ 7.65 E- 4 .294E-04 $1.387 E-03$ 1.719E-03

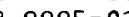
$4.431 E-03$ $4.718 E-03$ 1.450E-0 $3.242 E-02$ $2.615 \mathrm{E}-01$ $2.439 \mathrm{E}-0$ $1.551 \mathrm{E}$ OI $6.938 E$ OI $2.075 E$ O2 $5.716 \mathrm{E} 02$ $1.562 \mathrm{E} 03$ $3.481 \mathrm{E} O 3$ $3.481 E$
$5.161 E$ 03

ANGLE 11 $M U=0.2816$
$4.729 E-04$ $6.779 \mathrm{E}-04$ $6.832 E-04$ $1.065 E-03$ $1.908 \mathrm{E}-03$ $2.944 E-03$ $3.006 \mathrm{E}-03$ $5.555 \mathrm{E}-03$ $8.957 \mathrm{E}-03$ $8.949 \mathrm{E}-0$ $1.168 \mathrm{E}-02$ $2.362 \mathrm{E}-02$ $4.432 \mathrm{E}-02$ $3.125 \mathrm{E}-01$ $2.796 \mathrm{E}-0$ $1.760 E$ OI $7.815 \mathrm{E}$ OI $2.332 \mathrm{E} 02$ $6.398 E$ O2 $1.742 \mathrm{E} 03$ $5.684 \mathrm{E} O 3$ $.454 E-06$ J.126E-04 . 7.39 E 04 . 1. $729 \mathrm{E}-03$ $2.980 E-O B$ $6.805 \mathrm{E}-03$ $3.271 \mathrm{E}-02$ $2.629 \mathrm{E}-0$ $2.450 \mathrm{E}-0$ $1.557 \mathrm{E} 01$ $6.964 E 01$ 6.083E O2 $5.737 \mathrm{E} 02$ $3.493 \mathrm{E} \mathrm{O}$ $5.179 E \quad 03$

ANGLE 12 $M U=0.4580$ 作 $\mathrm{E}-04$
$\mathrm{E}-04$ . $373 E-03$ $2.397 E-03$ $3.629 E-03$ $3.619 E-03$ $6.844 \mathrm{E}-03$ $1.117 \mathrm{E}-02$ $1.060 \mathrm{E}-02$ $1.320 \mathrm{E}-02$ 2. $620 \mathrm{E}-02$ $4.691 \mathrm{E}-02$ $3.217 E-01$ $2.856 \mathrm{E}-01$ $1.795 \mathrm{E} 01$ $7.960 E$ O1 $2.374 \mathrm{E} 02$ $6.509 \mathrm{E} O 2$ $1.772 \mathrm{E} O 3$ $5.769 \mathrm{E} \mathrm{O3}$ $2.381 E-05$ 2.893 -04 $2.585 E-04$ . ..644E-04 $1.751 \mathrm{E}-03$ . 4.017E-03 4.618E-03 . $1.502 \mathrm{E}-02$ $3.326 \mathrm{E}-02$ $3.326 \mathrm{E}-02$ $2.469 \mathrm{E}-01$ $1.569 \mathrm{E} 01$ $7.013 E$ O1 $2.098 \mathrm{E} \mathrm{O2}$ $5.775 \mathrm{E} 02$ $1.578 \mathrm{E} 03$ $3.515 \mathrm{E} \quad 03$
$5.210 \mathrm{E} \quad 03$

$M U=-0.7550$
$5.206 E-05$
$5.206 E-05$ 2.780E- 04 .. 4.0C $3.082 \mathrm{E}-0$ 4. $7.232 \mathrm{E}-03$ $1.552 \mathrm{E}-0$ . $404 \mathrm{E}-02$ $2.693 \mathrm{E}-01$ $2.496 \mathrm{E}-01$ $1.585 \mathrm{E} 01$ $.081 E 01$ $.118 E 02$ ..828E 02 .592E 03 $3.546 \mathrm{E} \quad 03$

ANGLE 13 MU $=0.6179$ $1.052 \mathrm{E}-0$ $1.200 \mathrm{E}-0$ $1.878 \mathrm{E}-03$ $3.165 \mathrm{E}-03$ $4.664 \mathrm{E}-0$ $4.495 \mathrm{E}-03$
$8.729 \mathrm{E}-03$ $1.440 \mathrm{E}-02$ $1.278 \mathrm{E}-0$ $1.505 \mathrm{E}-02$ $2.924 \mathrm{E}-02$ $4.964 E-02$ 3.307E-01 $2.914 \mathrm{E}-01$ $1.827 \mathrm{E} \mathrm{OL}$ $8.096 E 01$ $2.414 E 02$ $6.614 \mathrm{E} 02$ 1.799 E 03 $5.848 \mathrm{E} 03$

ANGLE 14 $M U=0.7550$ 1. $572 \mathrm{E}-03$ . $755 \mathrm{E}-03$ $4.415 E-03$ $.257 E-03$ $1.153 \mathrm{E}-02$ . $153 \mathrm{E}-02$ $.567 \mathrm{E}-02$ $1.727 \mathrm{E}-02$ $3.273 \mathrm{E}-02$ $5.239 E-02$ $3.390 \mathrm{E}-01$ 2.966E-01 $1.857 E 01$ $8.219 E$ O1 $2.449 E \quad 2$ -.707E O2 $1.824 E \quad 03$ $5.918 E \quad 03$
ANGLE 6 $3.818 E-05$ 2.9OTE-O4 . 8.901E-04 $3.508 \mathrm{E}-0$ $2.742 \mathrm{E}-0$ $2.532 E-O$ $1.606 \mathrm{E} \mathrm{O}$ $7.169 E$ OI $2.143 \mathrm{E} 02$ 1.610 E 03 $3.585 \mathrm{E} \mathrm{O3}$ 5.306 E 03 


\begin{abstract}
ENERGY GROUP (MEV) $1.22 \mathrm{E} 01--1.50 \mathrm{E} 01$ 1.00 C C16. 36 E $00--8.19 E$ OO $4.97 E$ OO- $6.36 E 00$ $4.07 E$ OC- $4.367 \mathrm{E} 00$ $3.01 \mathrm{E}$ CO- 4 . C 7 E 00 2.46E $00--3.01 E$ CO $2.35 \mathrm{E}$ OO- $-2.46 \mathrm{E} 00$ $1.83 \mathrm{E} 00--2.35 \mathrm{E} 00$ $1.11 \mathrm{E} 00--1.83 \mathrm{E} 00$ $5.50 E-01-\cdots 1.11 E 00$ . $11 \mathrm{E}-01---5.50 \mathrm{E}-\mathrm{C}$ $3.35 \mathrm{E}-02-\cdots 1.11 \mathrm{E}-01$ $5.83 E-04--3.35 E-02$ $1.01 \mathrm{E}-04---5.83 \mathrm{~F}-04$ $2.90 E-05--1.01 E-04$ $1.07 \mathrm{E}-05--2.90 \mathrm{E}-05$ $3.06 E-06--1 . C 7 E-05$ $1.12 \mathrm{E}-06--3.06 \mathrm{E}-06$ $4.14 E-07--1.12 E-06$ 0.0
\end{abstract}

ENERGY

GROUP (MEV)

$1.22 E$ 01--1.50E 01 $1.00 E$ O1- $-1.22 \mathrm{E} \quad 01$ $8.19 E$ ON-- $1.00 E$ O1 $6.36 \mathrm{E}$ OO-- $8.19 \mathrm{E}$ OO 4.97E OC--6.36E OO 4.07E CO-- $4.97 E$ CO 3.C1E OC--4. 4.07E CO $2.46 \mathrm{E}$ 00-- $3.01 \mathrm{E}$ CO $2.35 \mathrm{E} \mathrm{OC}---2.46 \mathrm{E} C O$ 1.83 E $00--2.35$ E CO $1.11 \mathrm{E} \mathrm{O0--1.83E} \mathrm{CO}$ $5.50 E-01--1.11 E$ OO $1.11 \mathrm{E}-01---5.50 \mathrm{E}-0$ $3.35 \mathrm{E}-02--1.11 \mathrm{E}-0$ $5.83 \mathrm{E}-04---3.35 \mathrm{E}-02$ $1.01 E-04--5.83 E-04$ 2.9CE-05---1.01E-04 $1.07 E-05-2.90 E-05$ $3.06 \mathrm{E}-06-1.07 \mathrm{E}-05$ $1.12 \mathrm{E}-06-3.06 \mathrm{E}-06$ $4.14 \mathrm{E}-07---1.12 \mathrm{E}-06$
$0.0 \quad--4.14 \mathrm{E}-07$
ANGLE $11 \quad$ ANGLE 2
$M U=-1.0000 \quad M U=-0.9894$ $1.041 \mathrm{E}-\mathrm{C5}$ $6.868 \mathrm{E}-\mathrm{O}$ 1.182E-C $1.782 \mathrm{E}-\mathrm{C}$ $5.438 \mathrm{E}-0$. $6.718 \mathrm{E}-04$ 1.119E-OB $1.545 \mathrm{E}-\mathrm{C} 3$ $1.901 \mathrm{E}-\mathrm{C}$ $1.901 \mathrm{E}-\mathrm{C} 3$
$3 . \mathrm{C} 86 \mathrm{E}-03$ $7.331 \mathrm{E}-03$ $1.844 \mathrm{E}-\mathrm{C} 2$ $1.632 \mathrm{E}-01$ $1.566 \mathrm{E}-01$ $1.019 E$ OI $4.630 \mathrm{E}$ O1 $3.915 E \quad 02$ $1.082 \mathrm{E} \mathrm{O3}$ $2.435 \mathrm{E} \mathrm{C3}$ $3.651 \mathrm{E} \mathrm{O3}$

ANGLE 10 $M_{1} U=0.0950$ $7.858 \mathrm{E}-\mathrm{C5}$ $.684 \mathrm{E}-04$ $3.1 \mathrm{C} 8 \mathrm{E}-04$ $6.158 \mathrm{E}-04$ $9.911 \mathrm{E}-\mathrm{C} 4$ $1.002 E-03$ $1.880 \mathrm{E}-\mathrm{C}$ $3.165 E-03$ $3.451 \mathrm{E}-03$ $4.897 \mathrm{E}-\mathrm{C}$ $1.118 E-02$ $2.432 \mathrm{E}-\mathrm{C} 2$ $1.923 \mathrm{E}-01$ 1.779E-01 $1.148 \mathrm{E} C$ $5.185 \mathrm{E} O$ $1.567 \mathrm{E} \mathrm{O2}$ $4.365 \mathrm{E} O 2$ $1.203 E$
$2.701 E$
03 4.014E 03 $-7.922 E-06$ 7 $74 E-05$ $184 \mathrm{E}-0$ $2.729 E-0$ $.462 E-04$ $6.725 \mathrm{E}-04$ $1.120 \mathrm{E}-03$ $.551 \mathrm{E}-03$ $1.908 \mathrm{E}-03$ $3.096 \mathrm{E}-03$
$7.354 \mathrm{E}-03$ $1.848 \mathrm{E}-02$ $1.848 \mathrm{E}-02$ $1.635 \mathrm{E}-01$ $1.021 \mathrm{E} \mathrm{Ol}$ $4.635 E$ O1 $1.403 E$ C2 $3.919 \mathrm{E} 02$ $1.083 \mathrm{E} \mathrm{O3}$ $2.438 \mathrm{E} 03$
$3.655 \mathrm{E} 03$

ANGLE 11 $.199 E-04$ $2.143 E-04$ $3.920 E-04$ $7.515 E-04$ $1.186 E-03$ $1.168 \mathrm{E}-0$ $2.276 E=03$ $3.898 \mathrm{E}-\mathrm{O}$ $4.044 \mathrm{E}-03$ $5.482 E-03$ $1.236 \mathrm{E}-02$ $2.578 \mathrm{E}-02$ $1.985 \mathrm{E}-01$ $1.822 \mathrm{E}-01$ $1.174 \mathrm{E} 01$ $5.295 \mathrm{E} 01$ $1.600 \mathrm{E} \mathrm{O2}$ 4.454E 02
(NEUTRONS/MEV/STERADIAN/SOURCE NEUTRON) $M U=0.2816$ $1.227 \mathrm{E} 03$
$2.753 \mathrm{E} 03$ $4.085 \mathrm{E} 03$
ANGLE 3 $-6.444 E-08$ MU $=-0.8656$ ANGLE 5 $3.220 \mathrm{E}-03$ $7.635 \mathrm{E}-03$ $1.899 \mathrm{E}-02$ $3.669 \mathrm{E} \mathrm{O3}$ $M U=0.4580$ $708 E-04$
2 $2.824 \mathrm{E}-04$ $5.172 \mathrm{E}-04$ $9.568 \mathrm{E}-04$ $1.476 E-03$ $1.405 \mathrm{E}-03$ $2.853 \mathrm{E}-03$ $4.965 E-03$ $4.822 \mathrm{E}-03$ $6.197 E-03$ $1.376 \mathrm{E}-02$ $2.737 \mathrm{E}-02$ $2.048 \mathrm{E}-01$ $1.865 \mathrm{E}-01$ $1.200 \mathrm{E} 01$ $5.404 \mathrm{E}$ OI $1.632 \mathrm{E} 02$ $4.541 \mathrm{E} 02$ $1.250 E 03$ $2.805 \mathrm{E}$
$4.155 \mathrm{E}$
$\mathrm{CB}$ $6.199 \mathrm{E}-06$ $6.988 E-05$ . $240 E-04$ $2.871 \mathrm{E}-0$ $5.758 \mathrm{E}-04$ $6.829 E-04$ $1.146 \mathrm{E}-03$ $1.632 \mathrm{E}-03$ $1.998 \mathrm{E}-03$ $1.663 \mathrm{E}-01$ $1.663 \mathrm{E}-0$ $1.034 \mathrm{E} 01$ $4.691 \mathrm{E} \mathrm{O1}$ $1.420 \mathrm{E} \mathrm{O2}$ $3.965 \mathrm{E} 02$ $1.095 \mathrm{E} 03$ 2.465E O3 $3.694 \mathrm{E} 03$ $1.140 \mathrm{E}-05$
$6.658 \mathrm{E}-05$ $7.220 \mathrm{E}-05$ $1.325 \mathrm{E}-04$ 1. $066 \mathrm{E}-0$ $3.066 E-04$ $6.962 E-04$ $1.175 \mathrm{E}-03$ $1.718 \mathrm{E}-03$ $2.090 \mathrm{E}-03$ 3. $344 \mathrm{E}-03$ $7.909 \mathrm{E}-03$ $1.947 \mathrm{E}-02$ $1.689 \mathrm{E}-01$ $1.689 \mathrm{E}-01$ $1.045 \mathrm{E} \mathrm{OI}$ $4.742 \mathrm{E}$ OI $1.435 \mathrm{E} \mathrm{O2}$ 4.006 E 02 $1.106 E 03$ $2.489 \mathrm{E} 03$ $3.729 \mathrm{E} \quad 03$

ANGLE 6 $8.872 \mathrm{E}-06$ $7.777 \mathrm{E}-0 \mathrm{~S}$ 1. $479 \mathrm{E}-04$ $3.385 E-04$ $6.423 \mathrm{E}-04$ $6.423 \mathrm{E}-04$
$7.186 \mathrm{E}-04$ 1. $225 \mathrm{E}-03$ $1.247 \mathrm{E}-03$ $2.224 \mathrm{E}-03$ $2.224 \mathrm{E}-03$
$3.517 \mathrm{E}-03$ 3.517E-03 $8.286 E-03$ $1.722 \mathrm{E}-01$ $1.634 \mathrm{E}-01$ $1.061 E 0$ $4.808 E$ OI . $1.121 E$ O3 $2.521 \mathrm{E}$
$3.772 \mathrm{E}$ ANGLE 13

ANGLE 7 ANGLE 780 8.660 E-06 $8.884 E-05$ $1.722 E-04$ $3.840 E-C 4$ $6.936 E-04$ $7.538 \mathrm{E}-04$ $1.304 E-03$ $2.031 E-03$ $2.410 E-0$ $3.747 E-03$ $8.780 E-03$ $2.091 E-0$ $1.763 \mathrm{E}-01$ $1.079 E 01$ $4.887 E$ O1 $1.478 \mathrm{E} 02$ 4.124E 02 $2.559 \mathrm{E} \mathrm{C3}$ 3.823E 03

ANGLE 14 .
$.710 E-04$ $2.840 E-04$ $4.009 \mathrm{E}-04$ $7.299 E-04$ $1.285 \mathrm{E}-03$ $1.915 \mathrm{E}-03$ $1.737 \mathrm{E}-03$ $3.701 \mathrm{E}-03$ $6.552 E-03$ $5.842 \mathrm{E}-03$ $7.055 \mathrm{E}-03$ $1.541 E-02$ $2.902 \mathrm{E}-02$ $2.109 \mathrm{E}-01$ $1.906 \mathrm{E}-01$ $1.224 \mathrm{E} 01$ $5.508 \mathrm{E} 01$ $1.663 \mathrm{E} \mathrm{O2}$ $4.625 \mathrm{E} \mathrm{O2}$ $1.272 \mathrm{E} 03$ $4.222 \mathrm{E} 03$

ANGLE 8 $J=-0.2816$ $1.817 E-0$ $1.072 \mathrm{E}-04$ $1.072 E-04$ $4.435 E-04$ $7.626 \mathrm{E}-04$ $8.072 E-04$ $1.426 \mathrm{E}-03$ $2.290 \mathrm{E}-03$ $2.663 \mathrm{E}-03$ $4.045 \mathrm{E}-0$ $9.412 \mathrm{E}-0$ 
(NEUTRONS/MEV/STERADIAN/SOURCE NEUTRON)

\begin{tabular}{|c|c|c|c|}
\hline \multirow{2}{*}{\multicolumn{2}{|c|}{ ENERGY }} & & \\
\hline & & $\begin{array}{c}\text { ANGLE } 1 \\
M U=-1.0000\end{array}$ & $\begin{array}{c}\text { ANGLE } 2 \\
M U=-0.9894\end{array}$ \\
\hline & $01--1.50 \mathrm{E}$ & $-1.702 E-06$ & $-1.256 E-06$ \\
\hline & $01--1.22 \mathrm{E}$ & $1.556 \mathrm{E}-05$ & $6 E-$ \\
\hline $19 \mathrm{E}$ & $00--1.00 \mathrm{E} \quad 01$ & $1.642 E-05$ & $50 E-$ \\
\hline $36 E$ & $O O---8.19 E$ CO & $3.462 E-05$ & $3.473 E-$ \\
\hline $97 E$ & $00---6.36 E \quad 00$ & & \\
\hline $07 F$ & $0 C---4.97 E \quad 00$ & $1.877 E-04$ & \\
\hline - CIE & $00---4.07 \mathrm{E} \quad 00$ & $2.296 \mathrm{E}-04$ & $8 E-0$ \\
\hline $.46 \mathrm{E}$ & $C 0---3.01 E 00$ & 3. & \\
\hline $.35 \mathrm{E}$ & CO- - 2. 46E 00 & $5.215 E-04$ & $O E-$ \\
\hline $.83 \mathrm{E}$ & $C 0--2.35 \mathrm{E} \quad 00$ & $6.922 E-C 4$ & \\
\hline & $00---1$ & 1.1 & \\
\hline .50 & $-01--1.11 E 00$ & $1 E-03$ & $1 \mathrm{E}-$ \\
\hline & $-01---5.5$ & & \\
\hline & & & \\
\hline & $-c 4---3$ & 7. & \\
\hline & -04 & & \\
\hline & & 2 . & \\
\hline & -05 & & $\mathrm{BE}$ \\
\hline & -06 & & \\
\hline & $-06---3$ & $\begin{array}{ll}7 E & 02\end{array}$ & $2 E$ \\
\hline & $-07--1$ & $\begin{array}{lll}6 E & 03\end{array}$ & 1. \\
\hline & ---4 & LE 03 & \\
\hline & ENERGY & & \\
\hline & ROUP $\quad(M$ & .0950 & .2816 \\
\hline $22 \mathrm{E}$ & $01---1$ & $1.807 E-05$ & $9 E-05$ \\
\hline & $01--1.22 \mathrm{E}$ & 3. & \\
\hline & $00---1.00 E$ & 4. & \\
\hline & CO- $-8.19 E$ & 1. & \\
\hline & $00---6.36 E$ & & \\
\hline $7 E$ & $00--4.97 E$ & L- 04 & \\
\hline & $00--4.07 E \quad 00$ & & \\
\hline & $00---3.01 \mathrm{E} 00$ & $E-04$ & \\
\hline & $C O---2.46 E$ & & \\
\hline & $00---2 \cdot 3$ & $E-O$ & E- \\
\hline $1.11 E$ & $00---1.83 \mathrm{E}$ & 1. & \\
\hline & & & \\
\hline & & $E-O$ & $1 E-0$ \\
\hline & $-02---1$ & $E-C$ & \\
\hline & & & \\
\hline & $-04-$ & 5. & \\
\hline & & E 0 & \\
\hline & & E $\quad 0$ & $4 \mathrm{E}$ \\
\hline & -06 & $1 \mathrm{E} 02$ & $7 E \quad 02$ \\
\hline & & $5 \mathrm{E} \mathrm{C}$ & $1 \mathrm{E} 02$ \\
\hline & 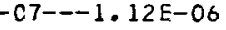 & $8 \mathrm{E} C 3$ & \\
\hline & & $2.0 .16 \mathrm{E} \quad 03$ & $2.054 E$ \\
\hline
\end{tabular}

ANGLE 3 $M U=-0.9446$ 3. $231 \mathrm{E}-08$ $1.512 E-05$ $1.678 \mathrm{E}-05$ $3.526 \mathrm{E}-05$ $9.260 \mathrm{E}-05$ $1.921 \mathrm{E}-04$ 2.307E-04 3. $745 \mathrm{E}-04$ $5.349 E-04$ $7.069 E-04$ $1.195 E-03$ $3.022 E-03$ $8.165 \mathrm{E}-03$ $7.621 E-02$ $7.423 E-02$ $4.89 E$ O $6.837 E$ OI 1. $926 \mathrm{E} \mathrm{O2}$ $5.355 \mathrm{E} 02$ $1.213 E \quad 03$ $1.830 \mathrm{E} \mathrm{O3}$

ANGLE 12 $M U=0.4580$ $3.939 \mathrm{E}-05$ $5.046 E-05$ $8.108 E-05$
$1.726 E-04$ $3.339 \mathrm{E}-04$ $1.339 E-04$
$5.198 E-04$ $5.198 \mathrm{E}-04$
$4.754 \mathrm{E}-04$ $4.754 E-04$
$1.016 E-03$
$1.845 E-03$ $1.016 \mathrm{E}-03$ $1.821 \mathrm{E}-03$ $2.383 E-03$ $5.703 \mathrm{E}-03$ $1.214 \mathrm{E}-02$ 9. $584 \mathrm{E}-02$ 8.857E-02 $5.778 E 00$ $2.627 \mathrm{E} \mathrm{O1}$ $7.992 \mathrm{E} \mathrm{O1}$ 2. $243 E \quad 02$ $6.216 \mathrm{E} 02$ $2.092 \mathrm{E} \quad 03$

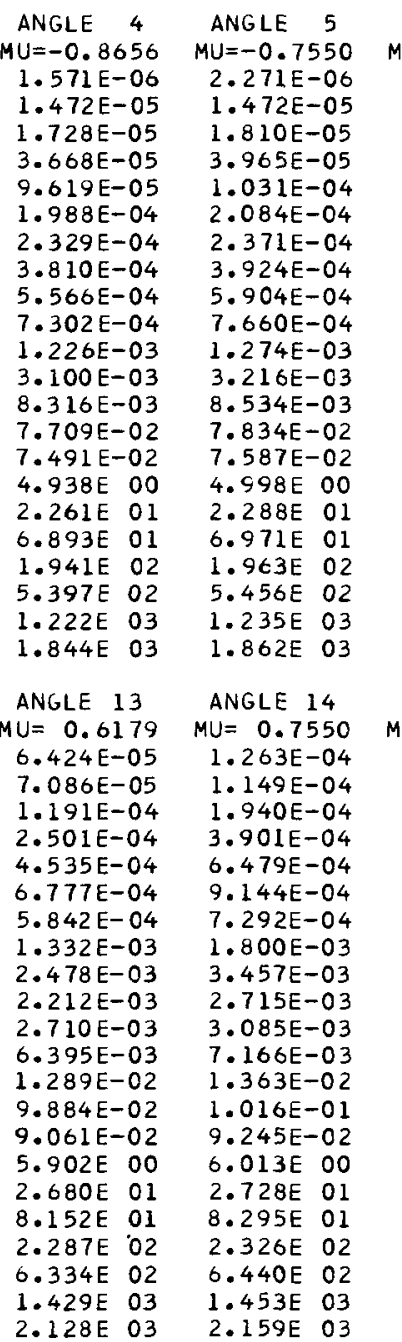

ANGLE 6 $M U=-0.6179$ $.564 \mathrm{E}-05$ 1. $974 \mathrm{E}-05$ $4.494 \mathrm{E}-05$ $1.144 \mathrm{E}-04$ $2.218 \mathrm{E}-04$ $2.443 \mathrm{E}-04$ $4.110 E-04$ $6.403 E-04$ $8.179 E-04$ $1.342 \mathrm{E}-03$ $3.376 \mathrm{E}-03$ $8.825 \mathrm{E}-03$ $7.998 \mathrm{E}-02$ . 2.075E 2.321E OI 1. 1.532 02 $1.532 E$ E $1.886 \mathrm{E} 03$ ANGLE 15 $2.765 E-04$ $2.080 E-04$ $3.435 \mathrm{E}-04$ $6.433 \mathrm{E}-04$ $9.433 \mathrm{E}-04$ $1.265 \mathrm{E}-03$ $1.265 \mathrm{E}-03$ $2.500 \mathrm{E}-03$ $5.500 E-03$ $3.011 E-03$ $3.493 E-03$ $7.976 \mathrm{E}-03$ $1.430 \mathrm{E}-02$ $1.039 \mathrm{E}-01$ $9.400 E-02$ $6.106 \mathrm{E} 00$ $2.768 E \quad 01$ $8.415 \mathrm{E} \mathrm{OI}$ $2.359 \mathrm{E} \mathrm{O2}$ $6.528 \mathrm{E} \mathrm{O2}$ $\begin{array}{ll}1.472 \mathrm{E} & 03 \\ 2.185 \mathrm{E} & 03\end{array}$
ANGLE 7 $M U=-0.4580$ $2.445 E-06$ $2.798 E-05$ $5.322 \mathrm{E}-05$ 1. $304 \mathrm{E}-04$ $2.397 E-04$ 2.560 E- 04 $4.404 E-04$ $7.109 E-04$ $8.896 \mathrm{E}-04$ $1.432 E-03$ . . $7.1960-02$ $5.167 E 00$ 2.362E 01 $7.194 \mathrm{E} 01$ $2.024 \mathrm{E} \mathrm{O2}$ $5.623 E \mathrm{E} 02$
$1.272 \mathrm{E} 03$ $1.272 E 03$

ANGLE 16 $M U=0.9446$ $6.294 \mathrm{E}-04$ $6.434 E-04$ $1.103 E-03$ $1.475 \mathrm{E}-03$ $1.475 E-03$ $1.775 E-03$
$1.140 E-03$ $1.140 \mathrm{E}-03$ $7.575 E-03$ $4.137 E-03$ $3.893 E-03$ $8.738 \mathrm{E}-03$
$1.484 \mathrm{E}-02$ $1.057 E-01$ $9.514 \mathrm{E}-02$ $6.175 \mathrm{E} \mathrm{CO}$ $2.798 \mathrm{E} 01$ $8.503 E \quad 01$ $2.383 E 02$ $6.592 E 02$ $\begin{array}{ll}1.486 \mathrm{E} & 03 \\ 2.204 \mathrm{E} & 03\end{array}$
ANGLE 8 $4.630 \mathrm{E}-06 \quad 1.015 \mathrm{E}-05$ $2.191 \mathrm{E}-05 \quad 2.712 \mathrm{E}-05$ $2.827 E-05 \quad 3.607 E-05$ $6.489 E-05 \quad 8.022 E-05$ $1.512 E-04 \quad 1.773 E-04$ $2.639 \mathrm{E}-04 \quad 2.974 \mathrm{E}-04$ $2.740 E-04 \quad 3.008 E-04$ $4.857 E-04 \quad 5.537 E-04$ 8.100E-04 $9.485 E-04$ $1.872 E-04 \quad 1.118 E-03$ $1.548 E-03 \quad 1.696 E-03$ 1. $190 \mathrm{E}-03$ 9.632E-03 $1.015 E-02$ $0.033 E-02 \quad 0.690 E-02$ $5.274 E$ OO $5.324 E-02$ 2.408E O1 $2.360 E$ OI $7.335 \mathrm{E} \mathrm{OI} 7.489 \mathrm{E} O 1$ $2.063 \mathrm{E} 02 \quad 2.105 \mathrm{E} 02$ $5.727 E$ O2 2.842 O2 $1.295 \mathrm{E} \mathrm{O3} 1.321 \mathrm{E} 03$ $1.944 \mathrm{E} \mathrm{O3} \quad 1.979 \mathrm{E} \mathrm{O3}$

ANGLE 17 1.979E

SCALAR $M U=0.9894$ 1.951E-03 $8.811 E-04$
$1.330 E-03$ $2.046 \mathrm{E}-03$ $2.395 E-03$
$2.534 E-03$ $2.534 \mathrm{E}-03$
$1.389 \mathrm{E}-03$ $1.389 \mathrm{E}-03$
$5.342 \mathrm{E}-03$ $1.231 \mathrm{E}-02$ $4.208 \mathrm{E}-03$ $9.302 \mathrm{E}-03$ $1.518 \mathrm{E}-02$ $1.067 \mathrm{E}-01$
$9.581 \mathrm{E}-02$ $6.214 E 00$ $2.815 \mathrm{E} \mathrm{O1}$ $8.554 E$ OI $6.630 \mathrm{E} 02$ $1.495 E \quad 03$ $2.214 \mathrm{E} \quad 03$

\section{$1.022 E-03$} $8.475 \mathrm{E}-04$ $1.310 E-03$ $2.497 \mathrm{E}-03$
$4.220 \mathrm{E}-03$ $6.184 E-03$ $5.343 \mathrm{E}-03$ $1.206 \mathrm{E}-02$ $2.257 \mathrm{E}-02$ $1.952 E-02$ $2.546 E-C 2$ $6.125 E-02$ .359E-C1 $1.122 \mathrm{E} 00$ $6.891 E$ Ol $3.141 E 02$ $9.560 \mathrm{E} 02$ $2.686 E 03$ $7.451 \mathrm{E} 03$ $2.520 \mathrm{E} 04$ 
(NEUTRONS/MEV/STERADIAN/SOURCE NEUTRON)

ENERGY GROUP (MEV) $1.22 E$ O1--- $1.50 E$ O1 . COE $01--1.22 E$ OI $8.19 \mathrm{E} \mathrm{OO---1.COE} 01$ $4.97 E$ O0- - $6.36 \mathrm{E} 00$ 4.07E $00---4.97 E$ DO $3.01 E$ 00---4.C7E 00 $2.46 E$ O0-- $3.01 E$ OO $2.35 \mathrm{E} 00---2.46 \mathrm{E}$ OO $1.83 E$ O0--2.35E 00 $1.11 \mathrm{E} \mathrm{O0--1.83E} 00$ .5CE-01---1.11E OO $11 \mathrm{E}-01---5.50 \mathrm{E}-0$ $3.35 \mathrm{E}-02--1.11 \mathrm{E}-01$ $5.83 \mathrm{E}-04---3.35 \mathrm{E}-0$ $1.01 E-04--5.83 E-04$ $2.90 E-05--1.01 E-04$ $1.07 E-05--2.90 \mathrm{E}-0$ $3.06 \mathrm{E}-06--1.07 \mathrm{E}-05$ $1.12 E-06--3.06 E-06$ $4.14 E-07--1.12 E-06$
$0.0 \quad---4.14 E-07$ ENERGY

GROUP (MEV)

$1.22 \mathrm{E} \mathrm{01--1.50E} 01$ 1.00 E $01--1.22 \mathrm{E} 01$ 8.19E AC---1. COE 01 $4.97 \mathrm{E} 00--6.36 \mathrm{E} 00$ 4.97 E CO--4.97.E OO $3.01 \mathrm{E}$ 00---4.07E 00 2.46E OO---3.01E ON $2.35 \mathrm{E} 00--2.46 \mathrm{E} \mathrm{OO}$ $1.83 \mathrm{E} 00--2.35 \mathrm{E} 00$ $1.11 \mathrm{E} \mathrm{O0--1.83E} \mathrm{CO}$ $5.50 \mathrm{O}-\mathrm{Cl}-\mathrm{C}-1.11 \mathrm{E}$ OO $1.11 \mathrm{E}-01--5$. $50 \mathrm{E}-0$ $3.35 \mathrm{E}-02--1.11 \mathrm{E}-01$ $5.83 \mathrm{E}-04--3.35 \mathrm{E}-02$ .01E $04-5$ - $03 E-04$ . 1.07E- C5 $2.90 E-05$ $1.06 \mathrm{E}-06-1.07 \mathrm{E}-05$ $4.14 \mathrm{E}-\mathrm{C7}-\mathrm{C}-1.12 \mathrm{E}-06$ $0.0 \quad--4.14 E-07$
ANGLE 1 ANGLE 2 $M U=-1.0000 \quad M U=-0.9894$ ..734E-07 -1.943E-07 .130E-06 .637E-C6 $2.856 \mathrm{E}-\mathrm{C} 5$ $.024 E-05$ $.148 \mathrm{E}-04$ $1.638 \mathrm{E}-04$ $2.298 \mathrm{E}-\mathrm{C}^{4}$ $4.020 \mathrm{E}-04$ $1.063 E-03$ $3.038 \mathrm{E}-\mathrm{C}$ $2.961 E-02$ 2.920E-02 $1.943 E$ OO $8.958 E 00$ $2.743 E$ CI 7.769E OI 4.170E 02 $4.934 \mathrm{E} \mathrm{OL}$

ANGLE 10 $M U=0.0950$ $3.812 \mathrm{E}-\mathrm{C} 6$ $7.279 E-06$ $1.2 \mathrm{C} 8 \mathrm{E}-05$ $3.041 E-05$ $6.767 E-05$ $1.114 \mathrm{E}-04$ $1.073 \mathrm{E}-04$ $2.101 E-04$ .778E-04 . $375 E-04$ 1.471E-04 $1.664 E-03$ ..077E-03 $3.532 E-02$ . 2.2 $12 E$ E 1. $8.760 E$ OI .536E 02 $8.312 \mathrm{E} \mathrm{C} 2$
ANGLE 3

$3 \quad$ ANGLE 4 $3.094 E-06$ $3.888 \mathrm{E}-06$ $2.911 E-05$ $7.321 E-05$ $1.161 E-04$ $1.686 \mathrm{E}-04$ 2. $349 \mathrm{E}-04$ 政 $3.077 \mathrm{E}-03$ $2.985 \mathrm{E}-02$ $2.939 \mathrm{E}-02$ $1.955 \mathrm{E} 00$ $9.010 E$ OO $2.759 \mathrm{E} 01$ $7.813 \mathrm{E} 01$ $2.182 E \quad 02$ 7.519E 02 ANGLE 12 $M U=0.4580$ 8.517E-06 $1.162 E-05$ $2.198 \mathrm{E}-05$ $5.402 E-05$ $1.082 E-04$ $1.690 \mathrm{E}-04$ $1.495 E-04$ $3.318 E-04$ $6.215 E-04$ $6.206 E-04$ $2.069 E-03$ $2.069 E-03$ $4.614 E-03$ 3.778E-02 . 2. $061 \mathrm{E} O$ $1.061 E$ OI 9.252E 01 $2.547 E 02$ $8.640 E$ O2 $3.351 E-07$ $3.066 \mathrm{E}-06$ .07 $3.029 E-05$ $6.378 \mathrm{E}-0$ $7.385 E-0$ $1.184 \mathrm{E}-04$ $1.762 \mathrm{E}-04$ $2.431 E-04$ $4.200 E-04$ $1.110 \mathrm{E}-03$ 3.136E-0 $3.021 E-02$ $2.966 \mathrm{E}-02$ $1.973 E 00$ $9.087 E 00$ $2.782 E$ O1 7.878 E 01 2.199E 02 $7.576 \mathrm{E} O 2$ ANGLE 13 $M U=0.6179$ $1.422 \mathrm{E}-05$ $1.685 \mathrm{E}-05$ $3.330 E-05$ $8.003 E-05$ $1.481 \mathrm{E}-04$ $2.208 \mathrm{E}-04$ $1.827 E-04$ $8.460 \mathrm{E}-04$ $8.460 E-04$ $9.325 E-04$

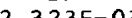
2.323E-03 4. 3.89 E- 02 2.371E 00 I. $083 \mathrm{E}$ 1.083E OI $9.337 \mathrm{E}$ OI . $5.655 \mathrm{E} 02 \quad 5.773 \mathrm{E} 02 \quad 5.885 \mathrm{E} O 2$ $5.655 \mathrm{E} O 2 \quad 5.773 \mathrm{E} 02 \quad 5.885 \mathrm{E} 02$ $8.794 \mathrm{E} \mathrm{O2}$ $4=-0.7550$
$4.316 E-07$ $3.103 E-06$ $1.128 \mathrm{E}-05$ $3.257 E-05$ $6.689 E-05$ $7.511 \mathrm{E}-05$ $1.223 \mathrm{E}-04$ $1.880 \mathrm{E}-04$ $2.556 E-04$ 4. $367 E-04$ $1.153 E-03$ 3.220E-03 $3.071 E-02$ $1.997 \mathrm{E} 00$ $9.196 \mathrm{E} \mathrm{OO}$ $2.815 \mathrm{E} 01$ $7.969 E 01$ $2.224 \mathrm{E} 02$ $5.056 \mathrm{E} \mathrm{O2}$ $7.655 \mathrm{E} 02$ ANGLE 14 $M U=0.7550$ $2.792 E-05$ $2.8 \mathrm{C} 9 \mathrm{E}-05$ $5.618 \mathrm{E}-05$ $1.274 \mathrm{E}-04$ $2.127 E-04$ $2.976 E-04$ $2.259 E-04$ I.954E-04 $1.199 E-03$ $1.059 \mathrm{E}-03$ $1.059 E-03$ $5.188 \mathrm{E}-03$ $4.0 C 9 E-02$ $4.0 C 9 E-O 2$ $2.417 E 00$ $2.417 E$ OO $3.370 E$ OI $.503 E 0$ $5.942 E$ O2 $8.929 E 02$ $M=-0.6179$ $4.464 E-07$ $3.311 E-06$ $4.759 \mathrm{E}-06$ $3.625 E-05$ $7.118 \mathrm{E}-05$ $7.732 E-05$ $1.286 E-04$ $2.052 E-04$ $4.736 E-04$ 1. $212 \mathrm{E}-04$ 1.212E-03 $3.333 \mathrm{E}-03$ $3.056 \mathrm{E}-02$ $2.029 E 00$ $9.336 E 00$ $2.857 E$ O1 8.087 E 01 $2.257 E$ O2 $7.755 \mathrm{E} 02$

ANGLE 15

$M U=0.8656$
$5.984 E-05$ $5.984 E-05$
$5.158 E-05$ $1.023 \mathrm{E}-04$ $2.128 \mathrm{E}-04$ $3.159 \mathrm{E}-04$ 4.087E-04 $2.794 E-04$ $8.284 E-04$ $1.766 \mathrm{E}-03$ $1.138 E-03$ $2.894 E-03$ $5.446 E-03$ $4.104 \mathrm{E}-02$ . 2.455E 00 $3.420 \mathrm{E} O 1$ $9.641 \mathrm{E} \mathrm{OI}$ $2.679 E \quad 02$ $9.040 E \quad 02$

ANGLE 7 ANGLE 8 $5=-0.4580 \quad M U=09.2816$ $3.813 E-06 \quad 4.675 E-06$ $5.605 E-06 \quad 7.061 E-06$ $1.558 E-05 \quad 1.926 E-05$ $4.143 E-C 5 \quad 4.814 E-05$ . $8.480 E-05$ $1.385 E-04 \quad 1.537 E-04$ $2.294 E-04 \quad 2.632 E-04$ $2.984 E-04 \quad 3.322 E-04$ $1.289 E-03 \quad 1.388 E-03$ $3.473 E-03 \quad 3.645 \mathrm{E}-0$ $3.217 E-02$ 3.311E-02 $3.117 E-02$ 3.188E-02 $2.067 E$ OO $2.111 E$ OO 9.504 O 1.658 O 0 
(NEUTRONS/MEV/STERADIAN/SOURCE NEUTRON)

ENERGY GROUP (MEV) $1.22 \mathrm{E} \mathrm{O1---1.50E} 01$ 8.19E $00-1.22 \mathrm{E} 01$ 6. 4.97E $00--6.36 E$ OD $4.07 \mathrm{E} 00--4.36 \mathrm{E}$ OO $3.01 E$ OO- $-4.07 E 00$ $2.46 E$ OO- $-3.01 E$ OO $2.35 \mathrm{E} 00---2.46 \mathrm{E}$ OO 1.83E $00-0-2.35 E$ 00 $1.11 E$ OC-D- 1.83E 00 $5.50 \mathrm{E}-01--1.11 \mathrm{E}$ OO $1.11 \mathrm{E}-01-\cdots 5.50 E-01$ $3.35 E-02-\cdots 1.11 E-01$ $5.83 E-04--3.35 E-02$ $1.01 E-04--5.83 E-04$ $2.9 \mathrm{CE}-05---1.01 \mathrm{E}-04$ 1.C7E-05--2. 90E-05 $3.06 \mathrm{E}-06---1.07 \mathrm{E}-05$ $1.12 E-06--3.06 E-06$ $4.14 \mathrm{E}-07--1.12 \mathrm{E}-06$
0.0

ENERGY

GROUP (MEV)

$1.22 E \mathrm{Cl}--1.50 \mathrm{E} O 1$ $1.00 E$ O1-- $1.22 \mathrm{E} O 1$ $8.19 \mathrm{E} \mathrm{O0--1.00E} 01$ 6.36 E $00--8.19$ E 00 4.97E $00--6.36 \mathrm{E} \quad 00$ 4.07E 00---4.97E CO $3.01 E$ OO-- 4 . C7E NO $2.46 E$ OO- $-3.01 \mathrm{E} \mathrm{OO}$ 2.35 E $00---2.46 E$ OO $1.83 \mathrm{E} 00--2.35 \mathrm{E} \mathrm{CO}$ .11E 00-- $1.83 E$ OO .50E-01- - $1.11 \mathrm{E} 00$ $1.11 E-01--5.50 E-0$ $3.35 \mathrm{E}-02--1.11 \mathrm{E}-01$ $5.83 \mathrm{E}-04--3.35 \mathrm{E}-02$ $1.01 \mathrm{E}-04--5.83 \mathrm{E}-04$ $2.9 \mathrm{CE}-05--1.01 \mathrm{E}-04$ $1.07 E-05--2.90 E-05$ $3.06 E-06--1.07 E-05$ $1.12 E-06--3.06 E-06$ $4.14 \mathrm{E}-07--1.12 \mathrm{E}-06$
0.0
$---4.14 \mathrm{E}-07$
ANGLE 1 $M U=-1.0000$ $-4.340 \mathrm{E}-08$ .1 $40 \mathrm{E}-07$ $8.223 \mathrm{E}-07$ $2.604 \mathrm{E}-06$ $8.628 E-06$ $1.847 E-05$ $2.211 \mathrm{E}-05$ $3.418 \mathrm{E}-05$ $4.930 \mathrm{E}-05$ $1.287 \mathrm{E}-\mathrm{C} 4$ $3.511 \mathrm{E}-\mathrm{C} 4$ $3.511 E-04$
$1.042 \mathrm{E}-03$ 1.C43E-02 $1.036 \mathrm{E}-\mathrm{C2} 2$ $6.935 \mathrm{E}-01$ 3.209 E CO $9.856 \mathrm{E} \mathrm{OO}$ $2.802 \mathrm{E} 01$ $7.847 \mathrm{E}$ OI $2.720 \mathrm{E} 02$

ANGLE 10 $7.789 E-07$ $1.556 \mathrm{E}-\mathrm{C6}$ $3.050 \mathrm{E}-06$ $8.899 \mathrm{E}-06$ $2.072 \mathrm{E}-05$ $3.427 \mathrm{E}-05$ $3.244 E-05$ $6.430 \mathrm{E}-05$ $1.178 \mathrm{E}-04$ $1.392 E-04$ 2. $080 \mathrm{E}-04$ $5.539 \mathrm{E}-04$ $1.404 \mathrm{E}-03$ $1.248 \mathrm{E}-02$ $1.191 \mathrm{E}-02$ $7.915 \mathrm{E}-\mathrm{C} 1$ $3.642 \mathrm{E} \mathrm{OO}$ $1.117 E \quad 01$ $3.168 E$ OI $8.850 E$ OL $\begin{array}{ll}2 . C 13 \mathrm{E} & 02 \\ 3.031 \mathrm{E} & 02\end{array}$
ANGLE 2 $M U=-0.9894$ $-2.947 \mathrm{E}-0$ . $2.616 \mathrm{E}-06$ $8.656 \mathrm{E}-06$ $1.855 \mathrm{E}-05$ $2.213 \mathrm{E}-05$ $3.426 E-05$ $4.960 \mathrm{E}-05$ $7.230 \mathrm{E}-05$ $1.292 \mathrm{E}-04$ $3.523 \mathrm{E}-04$ $1.044 \mathrm{E}-03$ $1.044 \mathrm{E}-02$ $1.037 \mathrm{E}-02$ $6.943 E-01$ $3.213 \mathrm{E} 00$ $9.867 \mathrm{E} \mathrm{OO}$ $2.805 \mathrm{E} \mathrm{O1}$ $7.855 \mathrm{E}$ OI $1.791 \mathrm{E} 02$ $2.723 \mathrm{E} \mathrm{O2}$ ANGLE 11 $M U=0.2816$ $1.177 \mathrm{E}-06$
$1.962 \mathrm{E}-06$ .092E-O .092E-06 $2.568 \mathrm{E}-05$ . $3.147 E-05$ $7.985 \mathrm{E}-05$ $1.493 \mathrm{E}-04$ $1.648 \mathrm{E}-04$ $2.333 E-04$ $6.163 \mathrm{E}-04$ $1.494 E-03$ 1. $291 \mathrm{E}-02$ $1.223 \mathrm{E}-02$ $8.111 \mathrm{E}-01$ $3.728 E 00$ $1.143 E$ OI $3.241 \mathrm{E} 01$ $9.048 E$ OI $2.058 \mathrm{E} O 2$
$3.092 \mathrm{E} O 2$
ANGLE 3

3 ANGLE 4
$446 \quad M U=-0.8656$ . 229E-08 $8.737 E-07$ $2.675 E-06$ $8.675 \mathrm{E}-06$ $8.803 E-06$
$1.890 E-05$ $2.220 \mathrm{E}-05$ $3.461 \mathrm{E}-05$ $5.088 \mathrm{E}-05$ $5.088 \mathrm{E}-05$ 1. $310 E-04$ $3.574 E-04$ $1.055 E-03$ $1.051 E-02$ $1.043 E-02$ $6.978 \mathrm{E}-01$ $3.228 \mathrm{E} 00$ $9.914 \mathrm{E} 00$ $2.818 E \quad 01$ $7.892 \mathrm{E} O 1$ $1.799 E \quad 02$ $2.735 \mathrm{E} \quad 02$

ANGLE 12 $M U=0.4580$ $2.598 \mathrm{E}-06$ $5.763 E-06$ $1.627 \mathrm{E}-05$ $3.343 \mathrm{E}-0$ $5.223 E-05$ $4.498 \mathrm{E}-05$ $1.027 E-0$ $1.968 \mathrm{E}-04$ $1.982 \mathrm{E}-04$ $2.638 \mathrm{E}-04$ $6.902 \mathrm{E}-04$ $1.591 \mathrm{E}-0$ $1.336 \mathrm{E}-02$ $1.255 \mathrm{E}-02$ $8.307 E-01$ $3.814 \mathrm{E}$ OO $1.169 \mathrm{E} \mathrm{O} 1$ $3.313 \mathrm{E} \mathrm{O}$ $9.245 E$ OI 2.101E 02 $6.565 \mathrm{E}-08$ $6.204 \mathrm{E}-07$ $2.311 E-07$ $9.174 \mathrm{E}-06$ $1.954 \mathrm{E}-05$ $2.238 E-05$ $3.534 E-05$ $5.334 E-05$ $7.633 \mathrm{E}-05$ $7.633 E-05$
$1.346 E-04$ $3.670 \mathrm{E}-04$ $1.075 \mathrm{E}-03$ $1.064 \mathrm{E}-02$ $1.053 \mathrm{E}-02$ $7.041 E-01$ $3.256 \mathrm{E} 00$ $1.000 \mathrm{E} \mathrm{OI}$ $2.842 \mathrm{E} O$ $7.957 \mathrm{E} 0$ $1.814 \mathrm{E} \quad 02$ $2.756 \mathrm{E} 02$ ANGLE 13 $M U=0.02 \mathrm{E}-06$ $3.873 \mathrm{E}-06$ $8.984 \mathrm{E}-06$ $2.455 \mathrm{E}-05$ 4.600E-05 $6.830 \mathrm{E}-05$ $5.471 \mathrm{E}-\mathrm{C5}$ $1.363 \mathrm{E}-04$ $2.708 \mathrm{E}-04$ $2.413 E-04$ $2.995 \mathrm{E}-04$ $7.754 E-04$ $1.692 \mathrm{E}-03$ $1.379 E-02$ $1.285 E-02$ $8.44 E-01$ 1.19LE OI $9.432 \mathrm{E} \mathrm{O1}$ $2.143 \mathrm{E} \mathrm{O}$ $3.211 \mathrm{E} \mathrm{O2}$
ANGLE $8.488 \mathrm{E}-08$ $6.340 \mathrm{E}-07$ $9.992 \mathrm{E}-07$ $3.115 E-06$ $9.884 E-06$ $2.050 E-05$ $2.275 E-05$ $3.657 \mathrm{E}-05$ $5.712 \mathrm{E}-05$ $8.039 \mathrm{E}-05$ $1.400 E-04$ $3.814 \mathrm{E}-04$ 1. $105 \mathrm{E}-0$ . $82 E-02$ $.067 \mathrm{E}-02$ $3.296 \mathrm{E} 00$ $1.012 \mathrm{E} 0$ $.050 E$ OL $2.786 \mathrm{E} 02$ ANGLE 14 $U=0.7550$ $5.883 E-06$ $6.005 \mathrm{E}-06$ $3.970 \mathrm{E}-05$ $6.628 E-0$ $85 E-05$ $6.714 \mathrm{E}-05$ $1.857 \mathrm{E}-0$ 3.8 $85 E-04$ $2.956 E-04$ $3.396 \mathrm{E}-04$ $8.689 E-04$

ANGLE 6 $9.295 \mathrm{E}-08$ 6.790E-07 1. $112 \mathrm{E}-06$ 3.627E-06 $1.102 \mathrm{E}-05$ $2.181 \mathrm{E}-05$ $2.341 E-05$ $3.858 E-05$ $8.623 E-05$ $1.475 E-04$ $4.013 \mathrm{E}-0$ $1.144 \mathrm{E}-03$ $1.106 \mathrm{E}-02$ $1.085 \mathrm{E}-02$ $7.247 \mathrm{E}-01$ $3.348 \mathrm{E} 00$ $1.028 \mathrm{E} \mathrm{OI}$ $2.919 E$ OI $8.169 \mathrm{E} 01$ $1.861 \mathrm{E} \mathrm{O}$ 2.823E 02 
(GAMMAS/MEV/STERADIAN/SOURCE NEUTRON)

ENERGY GROUP (MEV) 8. 00 E $00--1.00 E$ OI 6.5CE $00--8$. COE 00 $5.00 E$ OO--6.50E 00 4. 3 . OOE OO- - 5. COE OO 3. OCE OO---4. OCE OO $2.5 C E$ OO---3.00E 00 $2.00 \mathrm{E} O 0---2.50 E$ OO
$1.66 \mathrm{E} 00--2.00 \mathrm{E} 00$ $1.66 \mathrm{E}$ OO---2.00E 00
$1.33 \mathrm{E} 00--1.66 \mathrm{E} 00$ $1.33 \mathrm{E} O 0--1.66 \mathrm{E} \quad 00$ $1.0 C E$ OC---1.33E CO
$8.00 E-01--1.00 E$ CO
$6.00 E-01--2.00 E-O 1$ $8.00 E-01---1.00 E$ CO
$6.00 E-01---8.00 E-01$ $6.00 E-01--8.00 E-01$
$4.00 E-01--6.00 E-01$ $4.00 E-01---6.00 E-01$
$3.00 \mathrm{~F}-01---4.00 \mathrm{E}-01$ $3.00 \mathrm{E}-01---4.00 \mathrm{E}-01$
$2.00 \mathrm{E}-01---3.00 \mathrm{E}-01$ $2.00 \mathrm{E}-01---3.00 \mathrm{E}-01$
$1.00 \mathrm{E}-01 \mathrm{1}---2.00 \mathrm{E}-01$ $1.00 \mathrm{E}-01---2.00 \mathrm{E}-01$
$5.00 \mathrm{E}-02+--1.00 \mathrm{E}-01$ $2.00 \mathrm{E}-02--5.00 \mathrm{E}-02$

\section{ENERGY}

GROUP (MEV)
8.0OE OO---1.00E 8.00E $60-50$ OO- $0.00 E$. CO 5. COE OO---6.5CE CO $4.00 E$ OO- $-5.00 E$ OO $3.00 E$ OC-C.4. 2.5OE $00-3.00 E$ CO 2.0OE $00-2.50$ EO 00 $1.66 E$ DO- $2.00 E$ OO $1.00 E$ OO- $-1.33 E$ OO 1. 6.0OE $01-12-0.00 E-O 1$ $4.00 E-01-2-6.00 E-O$ 3.00E $01-2.00 E-01$ $2.00 E-01--3.00 E-0$ $1.00 \mathrm{E}-01--2.00 \mathrm{E}-01$ $5.00 \mathrm{E}-02-1.00 \mathrm{E}-01$ 2. OCE-02---5.00E-02 $M U=-1 . \cos 0$ $1.768 \mathrm{E}-04$ $8.648 \mathrm{E}-\mathrm{C} 4$ $1.821 \mathrm{E}-\mathrm{C}$ $1.406 \mathrm{E}-\mathrm{C3}$ $9.118 \mathrm{E}-04$ $3.206 \mathrm{E}-03$ $1.565 \mathrm{E}-03$ $3.042 \mathrm{E}-03$ $4.256 \mathrm{E}-\mathrm{C} 3$ $2.830 \mathrm{E}-\mathrm{C} 3$ $1.374 \mathrm{E}-03$ $4.686 \mathrm{E}-03$ $7.188 \mathrm{E}-03$ $9.275 E-03$ $3.422 E-02$ $7.084 \mathrm{E}-\mathrm{C2} 2$ $1.349 \mathrm{E}-01$
$3.678 \mathrm{E}-02$

ANGLE 10 $M U=0.0950$ $3.466 \mathrm{E}-04$ $.706 E-03$ . . $1.96 \mathrm{E}-03$ 6. $4.380 E-03$ $7.653 E-03$ 5. 5.161E-O $1.074 \mathrm{E}-02$ $1.546 \mathrm{E}-02$ $2.289 \mathrm{E}-02$ $4.393 E-C 2$ $8.414 E-02$ $1.518 \mathrm{E}-0$ $3.895 \mathrm{E}-02$
ANGLE 2 $M U=-0.9894$ $1.778 E-04$ $8.706 \mathrm{E}-04$ . $835 E-03$ $1.423 E-03$ .249E-04 . $230 E-03$ $1.577 E-03$ . . $268 E-03$ . $822 E-03$ $1.362 E-03$ $4.755 E-03$ . $231 E-03$ . . $1.350 \mathrm{E}-01$ $3.680 \mathrm{E}-02$

ANGLE 11 $M U=0.2816$ $6.251 E-04$ .074E-C3 $6.375 E-03$ . 1. 3.447E-03 $3.447 E-03$ $1.014 E-02$ . $6.865 E-03$ $1.63 \mathrm{E}-03$ $1.461 \mathrm{E}-02$ $1.573 E-02$ $4.643 E-02$ $8.825 \mathrm{E}-02$ 1. $558 \mathrm{E}-0$ 3. $942 E-C 2$ $M U=-0.9446$ $1.820 \mathrm{E}-04$ $8.930 \mathrm{E}-04$ $1.888 \mathrm{E}-03$ $1.482 \mathrm{E}-03$ $9.720 E-C 4$ $3.324 \mathrm{E}-\mathrm{C} 3$ $1.621 \mathrm{E}-0$ $3.095 \mathrm{E}-03$ 4. $324 \mathrm{E}-03$ 2.806E-03 $1.327 \mathrm{E}-03$ $5.008 \mathrm{E}-03$ $7.393 E-03$ $9.392 \mathrm{E}-03$ $3.495 \mathrm{E}-02$ $7.119 \mathrm{E}-02$ 3.687E-02 ANGLE 12 $M U=0.4580$ $6.001 E-C 4$ 2.948E-0 $4.891 E-03$ $4.026 E-03$ $3.063 E-03$ $1.066 \mathrm{E}-02$ 7. $774 \mathrm{E}-03$ ..774E-03 $1.084 E-02$ 5.464E-03 1. $793 \mathrm{E}-\mathrm{OL}$ $1.79 \mathrm{E}-02$ 2. 31 E 02 5.030E-02 $9.313 E-02$ 1. $01 E-01$ $3.991 E-02$
ANGLE $U=-0.8656$ $1.900 E-04$
$9.348 E-04$ $9.348 \mathrm{E}-04$ $1.985 \mathrm{E}-03$ $1.584 \mathrm{E}-03$ $1.050 \mathrm{E}-03$ $3.492 \mathrm{E}-03$ $1.701 \mathrm{E}-03$ $3.187 \mathrm{E}-03$ $4.446 \mathrm{E}-03$ $2.816 \mathrm{E}-03$ 1. $304 \mathrm{E}-03$ $5.431 \mathrm{E}-03$ $7.694 \mathrm{E}-03$ $9.781 \mathrm{E}-03$ $3.594 \mathrm{E}-02$ $7.178 \mathrm{E}-02$ $3.700 \mathrm{E}-0$

ANGLE 13 $U=0.6179$
$7.514 E-04$ $7.514 E-04$ $6.156 \mathrm{E}-03$ . $156 E-03$ $5.082 E-03$ 1.5 1.328E-02 . $322 E-03$ 1. $1.382 \mathrm{E}-02$ 1.032E-02 7.156E 03 $2.774 \mathrm{E}-02$ 3. $301 \mathrm{E}-02$ $5.475 \mathrm{E}-02$ $9.475 \mathrm{E}-02$ $1.645 \mathrm{E}-01$ $4.038 \mathrm{E}-02$

ANGLE 5
MU=-0.7550
$2.016 \mathrm{E}-04$
$9.937 \mathrm{E}-04$
$2.115 \mathrm{E}-03$
$1.7 C 9 \mathrm{E}-03$
$1.142 \mathrm{E}-03$
$3.718 \mathrm{E}-03$
$1.806 \mathrm{E}-03$
$3.341 \mathrm{E}-03$
$4.656 \mathrm{E}-03$
$2.905 \mathrm{E}-03$
$1.345 \mathrm{E}-03$
$5.933 \mathrm{E}-03$
$8.129 \mathrm{E}-03$
$1.068 \mathrm{E}-02$
$3.710 \mathrm{E}-02$
$7.269 \mathrm{E}-02$
$1.379 \mathrm{E}-01$
$3.719 \mathrm{E}-02$
$\mathrm{ANGLE} 14$
$\mathrm{MU}=0.7550$
$8.348 \mathrm{E}-04$
$4.090 \mathrm{E}-03$
$1.082 \mathrm{E}-02$
$9.031 \mathrm{E}-03$
$5.946 \mathrm{E}-03$
$1.476 \mathrm{E}-02$
$8.079 \mathrm{E}-03$
$1.743 \mathrm{E}-02$
$2.453 \mathrm{E}-02$
$1.729 \mathrm{E}-02$
$1.003 \mathrm{E}-02$
$3.380 \mathrm{E}-02$
$3.348 \mathrm{E}-02$
$3.746 \mathrm{E}-02$
$6.072 \mathrm{E}-02$
$1.047 \mathrm{E}-01$
$1.688 \mathrm{E}-01$
$4.083 \mathrm{E}-02$

$M U=$ $\begin{array}{ll}A N G L E & 6 \\ M U=-0.6179\end{array}$ $2.172 E-04$ $2.279 \mathrm{E}-03$ $1.853 \mathrm{E}-03$ $1.242 \mathrm{E}-0$ $4.003 E-03$ $1.938 \mathrm{E}-03$ 3. $575 \mathrm{E}-03$ $4.982 \mathrm{E}-03$ $3.111 \mathrm{E}-0$ $1.476 \mathrm{E}-03$ $6.478 \mathrm{E}-03$ $8.749 \mathrm{E}-0$ $1.224 \mathrm{E}-02$ 3. $830 \mathrm{E}-02$ $7.398 \mathrm{E}-02$ $1.398 \mathrm{E}-01$ $3.744 \mathrm{E}-0$

ANGLE 15 $M U=0.8656$ $1.585 E-03$ $1.578 \mathrm{E}-02$ $1.327 E-02$ 8.721E-03 1.165E-02 $1.165 \mathrm{E} 02$ 3. $598 \mathrm{E}-02$ . 2.536E-02 1.397E-02 $4.704 E-02$ $4.339 E-02$ $6.915 \mathrm{E}-02$ $1.115 \mathrm{E}-01$ $1.729 \mathrm{E}-\mathrm{O}$ $4.122 \mathrm{E}-02$

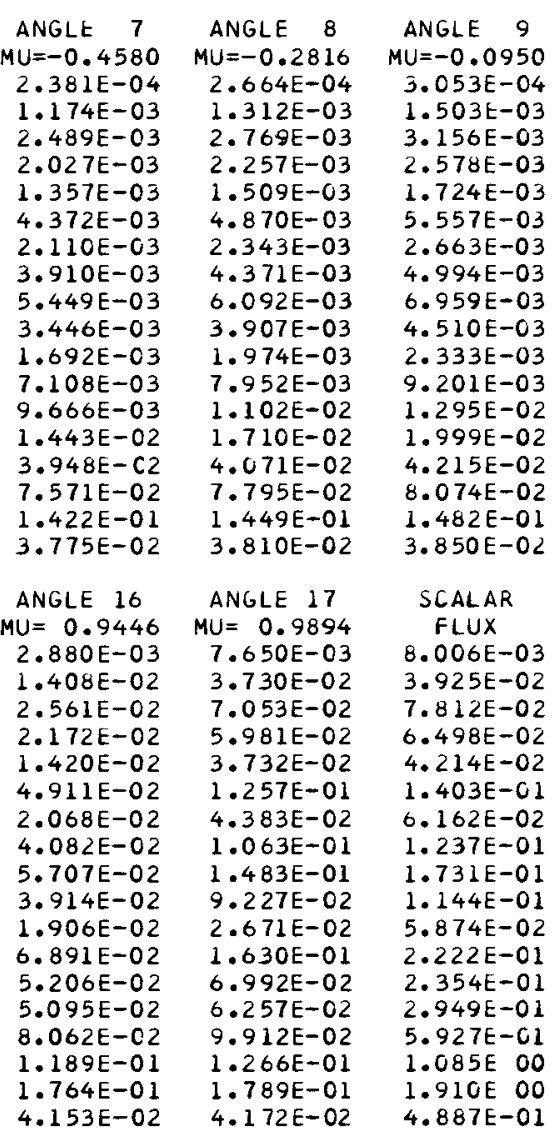


(GAMMAS/MEV/STERADIAN/SOURCE NEUTRON) 5. OCE-C2--1. OOE-O $2.00 E-02--5.00 E-02$

ENERGY GROUP (MEV) 8.0CE OO---1.00E O 6.50E OO---8. COE OO $5.00 \mathrm{E} \mathrm{CO---6.50E} \mathrm{OO}$ $4.00 E$ OO-- 5. OCE 00 $3.00 \mathrm{E}$ OS- $-4.00 \mathrm{E}$ $2.5 C E$ CO---3.00E OO $2.00 E$ OO-D-2.50E CO $1.66 \mathrm{E} \mathrm{OO---2.00E} 00$ $1.33 \mathrm{E} 00--1.66 \mathrm{E} 00$ $1.00 E$ O0-- $1.33 E$ OO $8.00 E-01--1$. COE OO 6.00E-CI---8.00E-01 $4.00 E-C 1--6.00 E-01$ $3.00 E-01--4.00 E-O 1$ $2.00 \mathrm{E}-\mathrm{C} 1--3.00 \mathrm{C}-\mathrm{OI}$ 1.00 E $01-D 2.00 E-01$ $2.00 \mathrm{E}-02--5.00 \mathrm{E}-02$
ANGLE 1 $M U=-1.0000$ $1.873 \mathrm{E}-04$ $2.045 \mathrm{E}-03$ $1.456 \mathrm{E}-03$ $1.456 \mathrm{E}-03$
$9.722 \mathrm{E}-04$
$3.461 E-03$ $9.722 \mathrm{E}-\mathrm{C4}$
$3.461 \mathrm{E}-03$ $3.461 E-03$
$2.020 E-03$ $2.020 \mathrm{E}-03$
$3.566 \mathrm{E}-0$ $4.979 \mathrm{E}-\mathrm{C}$ 3. $528 \mathrm{E}-03$ $2.072 \mathrm{E}-\mathrm{C3}$ $4.998 \mathrm{E}-03$ $1.179 \mathrm{E}-0$ $1.593 \mathrm{E}-\mathrm{C} 2$ $6.8 \in 1 \mathrm{E}-02$ $1.722 \mathrm{E}-0$ $3.905 \mathrm{E}-\mathrm{O}$ $1.125 \mathrm{E}-01$

ANGLE 2 ANGLE IC $M U=0 . C 950$

ANGLE 3 $5.114 \mathrm{E}-04$ $2.514 \mathrm{E}-03$ $5.528 E-C 3$ $4.482 E-C 3$ $3.151 \mathrm{E}-\mathrm{O}$ $9.416 E-C_{3}$ $3.450 E-C 3$ $8.674 E-C 3$ $1.194 \mathrm{E}-\mathrm{C2}$ $8.303 E-03$ $4.665 \mathrm{E}-03$ $1.795 E-02$ $2.941 \mathrm{E}-\mathrm{C2}$
$4.865 \mathrm{E}-02$ $8.818 \mathrm{E}-02$ $2.156 \mathrm{E}-0$

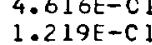
$M U=-0.9894$ $0.178 \mathrm{E}-0$ $2.071 E-03$ $2.071 E-03$ $1.002 \mathrm{E}-03$ $3.504 \mathrm{E}-03$ $3.504 E-03$ $2.042 E-03$ $4.979 E-03$ $3.492 E-03$ $5.134 E-03$ . $597 \mathrm{E}-02$ $6.896 \mathrm{E}-02$ $1.724 \mathrm{E}-0$ $3.910 \mathrm{E}-0$
$1.126 \mathrm{E}-0$ ANGLE 11 4. 212816 $4.214 E-04$
$2.070 E-03$ $4.68 \mathrm{CE}-\mathrm{C}$ $3.776 \mathrm{E}-\mathrm{C}$ $3.375 \mathrm{E}-0$ $7.797 \mathrm{E}-03$ $5.597 \mathrm{E}-03$ $7.295 \mathrm{E}-03$ $9.971 E-03$ $9.211 \mathrm{E}-0$ $7.45 \mathrm{CE}-03$ $2.092 \mathrm{E}-02$ $3.917 \mathrm{E}-02$ $5.683 \mathrm{E}-02$ $9.656 \mathrm{E}-02$ $2.291 E-01$ $1.240 E-01$ $5.194 \mathrm{E}-01$
$1.286 \mathrm{E}-01$ $M U=-0.9446$ 1. $949 E-04$ $2.165 E-03$ $1.611 \mathrm{E}-03$ $1.611 \mathrm{E}-03$
$1.107 \mathrm{E}-03$ $3.661 \mathrm{E}-03$ $2.124 E-03$ $3.603 \mathrm{E}-03$ $4.994 E-03$ 3. $383 \mathrm{E}-03$ $1.967 E-03$ $5.627 \mathrm{E}-03$ $1.220 \mathrm{E}-02$ $1.626 \mathrm{E}-02$ $7.028 \mathrm{E}-02$ $1.734 \mathrm{E}-01$ $3.931 E-01$
$1.129 E-01$ ANGLE 12 $M=-0.8656$ $2.060 E-04$ $1.013 \mathrm{E}-03$ $.324 E-03$ $1.267 E-03$ $3.922 \mathrm{E}-03$ $2.261 \mathrm{E}-03$ $1.733 E-02$ $7.246 \mathrm{E}-02$ 1. $754 \mathrm{E}-0$ $3.971 \mathrm{E}-01$
$1.134 \mathrm{E}-01$ $U=-0.7550$ $2.209 \mathrm{E}-04$ 2.516E-03 2. CO2E-03 $1.428 E-03$ $4.233 E-C 3$ $2.425 \mathrm{E}-03$ $3.855 E-03$ $5.279 E-03$ $3.336 \mathrm{E}-03$ $2.010 E-03$ $7.202 E-03$ $1.362 \mathrm{E}-02$
$1.974 \mathrm{E}-02$ $7.494 \mathrm{E}-02$ $1.785 \mathrm{E}-01$ $4.029 E-01$
$1.142 \mathrm{E}-01$ $M U=C .4580$ 7.506E-04 $7.865 \mathrm{E}-03$ $6.415 \mathrm{E}-0$ $3.160 \mathrm{E}-03$ 1. $358 \mathrm{E}-02$ $7.444 E-03$ $1.244 E-02$ $1.721 \mathrm{E}-02$ $9.374 \mathrm{E}-03$ $1.027 E-02$ $2.143 \mathrm{E}-02$ $4.678 E-02$ $6.291 \mathrm{E}-02$ $1.032 E-01$ $2.444 E-01$ 4.986E-01 ANGLE 13
$M U=0.6179$ $7.783 E-04$ $3.800 E-03$ $8.754 E-03$ $7.142 \mathrm{E}-03$ $6.764 \mathrm{E}-03$ $1.389 \mathrm{E}-02$ $7.715 \mathrm{E}-03$ $1.424 E-02$ $1.978 \mathrm{E}-02$ $2.051 E-02$ $1.571 \mathrm{E}-02$ 4. $337 \mathrm{E}-02$ $5.804 \mathrm{E}-02$ $7.216 \mathrm{E}-02$ $1.143 \mathrm{E}-01$ $2.630 \varepsilon-01$

\section{$M U=0.7550$}

$1.452 E-03$

$1.386 \mathrm{E}-0$

$1.386 \mathrm{E}-02$

$6.742 \mathrm{E}-03$

$2.551 E-02$

$1.452 \mathrm{E}-02$

2. $277 E-02$ $3.154 \mathrm{E}-\mathrm{C2}$ $2.343 E-02$ $4.337 \mathrm{E}-02$ $7.155 \mathrm{E}-02$ $8.353 \mathrm{E}-02$ $1.291 \mathrm{E}-0$ $2.837 \mathrm{E}-0$ $5.404 E-01$
$1.307 E-01$
ANGLE 6 $2.399 E-04$ $1.186 \mathrm{E}-03$ $2.732 \mathrm{E}-03$ $2.192 E-03$ $1.568 \mathrm{E}-03$ $4.584 \mathrm{E}-03$ $2.618 E-03$ $4.144 E-03$ $5.671 E-03$ $3.624 E-03$ $2.296 E-03$ . $941 E-03$ $2.383 E-02$ $7.740 E-02$ $1.828 \mathrm{E}-0$ $1.153 E-01$ ANGLE 15

ANGLE 7 $A U=-0.4580$
$2.648 E-04$ $1.307 E-03$ $2.992 E-03$ $2.393 \mathrm{E}-03$ $5.016 E-03$ $2.867 E-03$ $4.584 E-03$ $6.282 E-03$ 列 $2.742 E-03$ $8.720 E-03$ $2.944 \mathrm{E}-02$ $7.977 E-02$ $1.884 E-01$ $1.166 \mathrm{E}-01$ ANGLE 16 $2.050 \mathrm{E}-03$ $9.967 \mathrm{E}-03$

$2.186 \mathrm{E}-02$

$1.832 \mathrm{E}-0$

$1.336 \mathrm{E}-02$

$3.545 E-02$ 3.644E-02 $5.033 E-02$ $4.190 \mathrm{E}-02$ $3.318 \mathrm{E}-02$ $7.312 \mathrm{E}-02$ $8.808 \mathrm{E}-02$ $9.753 \mathrm{E}-0$ $1.465 E-0$ $3.070 E-O$ 1.327E-0

ANGLE 8 $U=-0.2816$ $2.987 \mathrm{E}-04$ $1.471 E-03$
$3.343 \mathrm{E}-03$ $3.343 \mathrm{E}-03$ $1.894 \mathrm{E}-03$ $5.611 \mathrm{E}-03$ $3.217 E-03$ $7.130 \mathrm{E}-03$ $4.846 \mathrm{E}-03$ $3.305 \mathrm{E}-03$ $9.855 E-03$ GOIE-02 $8.228 \mathrm{E}-0$ $1.956 \mathrm{E}-0 \mathrm{I}$ $4.320 \mathrm{E}-\mathrm{O}$
$1.181 \mathrm{E}-0$ ANGLE 17 
(GAMMAS/MEV/STERADIAN/SOURCE NEUTRON)

ENERGY

GROUP (MEV)

8.0OE OO---1.00E O

6.5OE DO---8. OCE OO

5.0CE $00---6.50 E$ CO

4.COE $00--5.0 C E$ OO

3.0OE OO-C- 4.OCE OD

$2.50 E$ OO- $-3.00 E$ OO

2.0CE OO- - 2.5OE OO

$1.66 \mathrm{E}$ ON-W-2.00E 00

$1.33 \mathrm{E}$ OO- $-1.66 \mathrm{E}$ กO

$1.00 E$ ON-D-1.33E OO

Q.OOE

6.00E-01- $-8.00 E-01$

4.0OE-C1---6.00E-01

3.OCE $01-2.00 E-01$

1.0OE-01-- $3.00 E-01$

$5.00 E-02-1.00 E-01$

$2.00 E-02---5.00 E-02$

$$
\begin{aligned}
& \text { ENERGY } \\
& \text { GROUP (MEV) }
\end{aligned}
$$

8.00E 00---1.00E 01

$6.5 \mathrm{CE}$ OO---8. COE OO

$5.0 O E$ OO- $-6.50 E$ CO

$4.00 E$ OO- $-5.00 E$

$3.00 \mathrm{E}$ 0O--4. $00 \mathrm{E}$ OO

$2.50 E$ OO-- 3. $00 E$ OO

$2.00 E$ OO-D. $2.50 E$ OO

$1.66 \mathrm{E} \mathrm{OO-D2.} \mathrm{COE} \mathrm{CO}$

$1.33 \mathrm{E} \mathrm{O0--1.66 \textrm {E } C O}$

1. . CCE DO--1.33E OO

8.00E-01---1. $00 E$ OO

6. OCE-01- - 8. OOE- 01

4. COE-01-- -6.0OE-O

$3.0 C E-01--4.00 E-01$

2.00E-01--3.00E-O

1 . กCE-ก1---2.00E-OI

5.CCE $-02---1.0 C E-01$
$2.00 E-02---5.00 E-02$ $M U=-1.0000$ $1.730 \mathrm{E}-04$ $8.332 E-04$ $1.944 E-03$ $1.305 \mathrm{E}-03$ 8. $801 E-04$ $3.226 \mathrm{E}-03$ $2.038 \mathrm{E}-03$ $3.504 \mathrm{E}-03$ ..910E-03 ..584E-C3 $4.569 \mathrm{E}-03$ $1.318 \mathrm{E}-\mathrm{C} 2$ $.819 \mathrm{E}-\mathrm{C} 2$ $8.436 E-02$ $5.524 \mathrm{E}-01$

. $624 E-01$

ANGLE 10 $M U=0.0950$ $3.545 \mathrm{E}-04$ $1.739 \mathrm{E}-\mathrm{C} 3$ $3.260 \mathrm{E}-03$ $2.437 \mathrm{E}-\mathrm{C} 3$ $6.681 E-03$ $6.681 E-03$
$5.185 E-C 3$ $6.361 E-03$ $8.6 C 3 E-0$ $6.383 \mathrm{E}-03$ $6.267 \mathrm{E}-03$ $1.441 E-02$ $3.833 \mathrm{E}-02$ $6.290 E-02$ $1.111 \mathrm{E}-0$ $2.889 E-01$ $6.664 E-01$ $1.780 \mathrm{E}-01$
ANGLE 2 $U=-0.9894$
$1.748 E-04$ $8.440 E-04$ $1.976 \mathrm{E}-03$ $1.349 \mathrm{E}-\mathrm{C} 3$ $9.202 E-04$ $3.277 E-03$ $2.065 \mathrm{E}-03$ $3.504 E-03$ $3.527 \mathrm{E}-03$ 3.527E-O3 $4.741 E-{ }^{2}$ 4.741E-03 $1.328 \mathrm{E}-02$ $8.480 E-02$ $2.401 E-01$ 5.532E-01 $1.630 E-01$

ANGLE 11 $M U=0.2816$ $5.48 \mathrm{CE}-0$ $2.689 E-03$
$S .130 E-03$ $4.949 E-03$ 3.623E-03 $1.015 \mathrm{E}-02$ 5. $324 \mathrm{E}-03$ $9.545 \mathrm{E}-03$ $1.306 \mathrm{E}-02$ $9.874 \mathrm{E}-03$ $8.352 \mathrm{E}-03$ $2.341 E-02$ $4.689 \mathrm{E}-\mathrm{C2}$ $7.007 E-02$ $1.172 \mathrm{E}-01$ 3.079E- 0 $1.815 \mathrm{E}-01$ $M U=-0.9446 \quad M U N G L E 4$ $8.842 E-04$ $2.092 \mathrm{E}-03$ $1.505 \mathrm{E}-03$ $1.059 \mathrm{E}-03$ $3.464 E-03$ $2.163 \mathrm{E}-03$ $4.864 E-03$ $4.864 E-03$ $2.056 E-03$ $5.056 E-03$ $1.367 E-02$ $1.367 E-02$ $8.647 \mathrm{E}-02$ $2.647 \mathrm{E}-02$ 5.56E 01

\section{ANGLE 12} $U=0.4580$ $6.151 \mathrm{E}-04$ $6.867 \mathrm{E}-03$ $6.867 \mathrm{E}-03$
$5.533 \mathrm{E}-03$ $4.089 \mathrm{E}-03$ $1.123 \mathrm{E}-\mathrm{O}$ $1.123 \mathrm{E}-02$ $1.083 \mathrm{E}-02$ $1.488 \mathrm{E}-02$ $1.237 \mathrm{E}-02$ $1.328 \mathrm{E}-02$ $2.935 \mathrm{E}-02$ $5.944 \mathrm{E}-02$ $7.972 \mathrm{E}-02$ $1.289 \mathrm{E}-0$ $3.306 E-01$ $7.272 \mathrm{E}-01$
$1.852 \mathrm{E}-01$ $1.266 \mathrm{E}-03$ $3.763 \mathrm{E}-03$ $2.321 \mathrm{E}-03$ . $572 E-03$ $4.880 E-03$ $3.166 \mathrm{E}-03$ $6.300 \mathrm{E}-03$ ..300E-03 $1.436 E-02$ 8. 2.306E-01 5.620E-01 $1.644 \mathrm{E}-01$

ANGLE 13 $9.352 E-04$ $4.560 \mathrm{E}-03$ $8.175 \mathrm{E}-03$ $8.175 \mathrm{E}-\mathrm{C}$
$5.987 \mathrm{E}-03$ $5.987 \mathrm{E}-03$
$1.674 \mathrm{E}-02$ $1.674 \mathrm{E}-02$
$9.685 \mathrm{E}-03$ $9.685 \mathrm{E}-03$
$1.647 \mathrm{E}-02$ $1.647 \mathrm{E}-02$
$2.284 \mathrm{E}-02$ $1.979 \mathrm{E}-02$ $2.001 \mathrm{E}-02$ $4.274 E-02$ $7.172 \mathrm{E}-02$ $9.002 E-02$ $1.414 \mathrm{E}-0$ $3.567 \mathrm{E}-0$ $7.611 \mathrm{E}-0$
ANGLE 4 ANGLE 5 $M U=-0.8656$ $1.937 \mathrm{E}-04$
$9.520 \mathrm{E}-04$ $2.281 E-03$ $1.742 \mathrm{E}-03$ $M U=0.6179$ $1.889 E-01$
$M U=-0.7550$ $2.092 \mathrm{E}-04$
$1.034 \mathrm{E}-03$ $2.493 E-03$ $1.974 \mathrm{E}-03$ $1.457 \mathrm{E}-03$ 4. $093 \mathrm{E}-03$ 2.501E-03 $3.723 E-03$ $3.183 E-03$ $7.212 \mathrm{E}-03$ 1. $536 \mathrm{E}-02$ $2.347 E-02$ $2.351 E-01$ $5.721 \mathrm{E}-0$ 1.657E-01

ANGLE 14 $M U=0.7550$ $6.331 \mathrm{E}-03$ 1. $375 \mathrm{E}-02$ $1.125 E-02$ $8.356 \mathrm{E}-03$ $2.305 \mathrm{E}-02$ $1.513 \mathrm{E}-02$ $2.335 \mathrm{E}-02$
$3.231 \mathrm{E}-02$ 2.946E-02 $3.041 E-02$ $5.625 E-02$ $8.847 E-02$ . $865 E-01$ $1.924 E-C 1$
ANGLE 6 $\mathrm{U}=-\mathrm{C} .6179$
$\angle .280 \mathrm{E}-04$ $1.128 \mathrm{E}-03$ $2.714 \mathrm{E}-03$ $2.166 \mathrm{E}-03$ $1.601 E-03$ $4.439 E 03$ $4.013 \mathrm{E}-03$ 5.013E 03 $3.506 \mathrm{E}-03$ $2.405 \mathrm{E}-03$ $7.970 \mathrm{E}-03$ $1.691 \mathrm{E}-02$ E-02 $9.514 E-02$ $2.413 E-01$ $1.673 \mathrm{E}-01$

ANGLE 15 $M U=0.8656$ $2.275 \mathrm{E}-03$ $1.102 \mathrm{E}-02$ $2.324 \mathrm{E}-02$ $1.945 \mathrm{E}-02$ $1.442 \mathrm{E}-02$ $3.943 E-02$
$2.398 E-02$ $3.944 E-02$ 3. $944 E-02$ $4.748 \mathrm{E}-02$ $4.368 \mathrm{E}-02$ (2E-02 $.092 E-01$ $1.956 \mathrm{E}-01$

ANGLE 7 ANGLE 8 ANGLE $M U=-0.4580 \quad M U=-0.2816 \quad M U=-0.095 \mathrm{C}$ $2.522 \mathrm{E}-04 \quad 2.855 \mathrm{E}-04 \quad 3.333 \mathrm{E}-04$ $1.404 E=03$ $1.637 E-03$ 2. $318 \mathrm{E}-03 \quad 3.836 \mathrm{E}-03$ 1.726E 03 2.604E-03 $3.020 E-03$ 4.85OE-03 $5.432 \mathrm{EE}-03$ $2.216 \mathrm{E}-03$ . $5.432 E-03$ O.JOOE-03 $4.476 E-03 \quad 5.128 E-03 \quad 5.995 E-03$ 5. $4.110 E-03 \quad 4.92 E-03$ $8.161 E-03$ 3.036E-03 $3.699 E-03$ $4.565 E-03$ B.725E-03 $1.945 E-02 \quad 2.353 E-02 \quad 2.954 E-02$ $3.659 E-02 \quad 4.524 E-02 \quad 5.402 E-02$ $9.791 \mathrm{E}-02 \quad 1.009 \mathrm{E}-0121.048 \mathrm{E}-01$ $2.495 E-012.599 E-01 \quad 2.729 E-G 1$ $5.998 E-01 \quad 6.186 E-01 \quad 6.408 E-01$ $1.694 \mathrm{E}-01 \quad 1.719 \mathrm{E}-01 \quad 1.748 \mathrm{E}-01$

ANGLE 16 ANGLE 17 SCALAR $M U=0.9446 \quad M U=0.9894 \quad$ FLUX $\begin{array}{lll}4.557 E-03 & 1.455 E-02 & 1.082 E-02\end{array}$ $2.201 E-02 \quad 6.966 E-02 \quad 5.249 E-02$ $4.454 \mathrm{E}-02 \quad 1.333 \mathrm{E}-01 \quad 1.121 \mathrm{E}-01$ $3.802 \mathrm{E}-02 \quad 1.124 \mathrm{E}-01 \quad 9.213 \mathrm{E}-02$ 
(GAMMAS/MEV/STERADIAN/SOURCE NEUTRON)

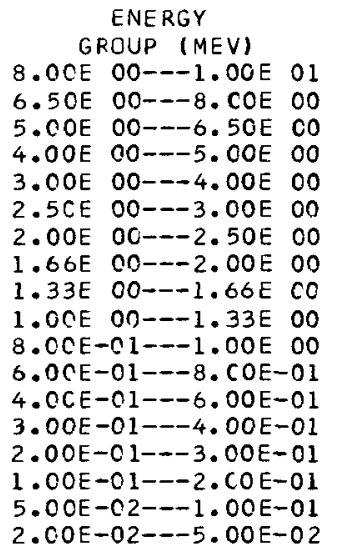

ANGLE 1
$M U=-1.0000$
$1.297 \mathrm{E}-04$
$6.115 \mathrm{E}-04$
$1.528 \mathrm{E}-03$
$8.819 \mathrm{E}-04$
$5.990 \mathrm{E}-04$
$2.447 \mathrm{E}-03$
$1.767 \mathrm{E}-03$
$2.980 \mathrm{E}-03$
$4.231 \mathrm{E}-03$
$3.228 \mathrm{E}-03$
$1.949 \mathrm{E}-03$
$3.236 \mathrm{E}-03$
$1.346 \mathrm{E}-02$
$1.900 \mathrm{E}-02$
$9.997 \mathrm{E}-02$
$2.859 \mathrm{E}-01$
$7.748 \mathrm{E}-01$
$2.355 \mathrm{E}-\mathrm{C1}$

ANGLE 2

$U=-0.9894$

ANGLE 3

$6.243 E-04$

$6.243 E-04$

$0.513 \mathrm{E}-04$

$6.541 \mathrm{E}-04$

$2.508 \mathrm{E}-03$

$1.797 \mathrm{E}-03$

$2.967 \mathrm{E}-03$

$3.138 \mathrm{E}-03$

$3.138 \mathrm{E}-03$
$1.916 \mathrm{E}-03$

$3.454 \mathrm{E}-03$

$3.454 \mathrm{E}-03$
$1.357 \mathrm{E}-\mathrm{C2}$

$1.357 \mathrm{E}-\mathrm{C} 2$
$1.908 \mathrm{E}-02$

$1.005 \mathrm{E}-01$

$2.865 \mathrm{E}-0$

$2.865 E-01$
$7.761 E-01$

$2.357 \mathrm{E}-\mathrm{O}$

ENERGY

GROUP (MEV)

8. CCE OO---1. COE OI

6.50E CC---8. COE CO

5.00 E CO-- 6.50 E OO

4.0OE OC-- -5. OOE OO

3. COE CO- - 4.0OE OO

. .5OE CO--3. COE CO

$2.00 E$ OO- -2.50 E 00

$1.66 E$ OO- $-2.00 E$ E

$1.33 \mathrm{E} 00--1.66 \mathrm{E} \mathrm{OO}$

$1.00 \mathrm{E}$ OC---1.33E OO

8.OCE-01-- 1. OOE OO

$6.00 E-01--8.00 E-01$

4.0OE-01---6. 0 . E E-O

$3.00 E-01--4.00 E-01$

2. OCE-C1---3. 00 E-O

$1.00 \mathrm{E}-\mathrm{C1}---2.00 \mathrm{E}-\mathrm{C1}$

$2.00 \mathrm{E}-02--5.00 \mathrm{E}-02$

$A N G L E 11$

ANGLE 10

$M U=0.0950$

$M U=-0.9446 \quad M U=-0.8656$

$1.393 \mathrm{E}-04$

$M U=-0.8656$

ANGLE 5

1. $711 \mathrm{E}-03$ - $330 \mathrm{E}-03 \quad 2.216 \mathrm{E}-04$

1.9

$1.110 \mathrm{E}-03$

1.326E-03

2. $725 \mathrm{E}-03$

$2.072 \mathrm{E}-03$

$4.031 \mathrm{E}-03 \quad 2.919 \mathrm{E}-03$ $3.011 \mathrm{E}-03$

$4.054 E-03$

$3.931 E-03$

$3.958 E-03$

$2.533 \mathrm{E}-03$

$3.958 E-03$

1.801E-03

$4.227 E-03$

$1.801 \mathrm{E}-03$
$5.340 \mathrm{E}-03$

$1.988 E-03$

$1.971 E-02 \quad 2.185 E-02$

$584 \mathrm{E}-02$

$1.056 \mathrm{E}-01$

$1.552 E-02$
$1.089 E-01$

$2.887 \mathrm{E}-01$

$7.814 \mathrm{E}-01 \quad 7.916 \mathrm{E}-01$

$2.994 E-01$

$8.063 \mathrm{E}-01$

ANGLE 12

ANGLE 13

ANGLE 14

$5.723 \mathrm{E}-04 \quad 7.902 \mathrm{E}-04$

$230 E-03$

$5.926 \mathrm{E}-03$

$4.149 E-04$
$2.027 \mathrm{E}-03$

$2.787 \mathrm{E}-03$

$8.968 \mathrm{E}-03$

$1.347 \mathrm{E}-02$

3. $962 \mathrm{E}-03$

$5.329 \mathrm{E}-03$

$7.125 \mathrm{E}-03$

$1.089 E-02$

$1.056 \mathrm{E}-02$

$5.547 \mathrm{E}-03$

$2.190 \mathrm{E}-02$

$7.843 E-03$

$7.136 \mathrm{E}-03$

$1.028 \mathrm{E}-02$

$1.589 \mathrm{E}-02$

$4.277 \mathrm{E}-0$

$5.616 \mathrm{E}-03$

$1.054 \mathrm{E}-02$
$1.451 \mathrm{E}-02$

2.397E-02

$3.317 \mathrm{E}-02$

$8.60 \mathrm{CE}-03$

$1.327 \mathrm{E}-02 \quad 2.085 \mathrm{E}-02$

$2.561 E-02$
$4.683 E-02$

$6.397 \mathrm{E}-\mathrm{C}$

$2.315 \mathrm{E}-02$

$3.413 E-02$

$8.613 E-02$

$1.037 \mathrm{E}-01$

$9.433 \mathrm{E}-0$

$1.074 \mathrm{E}-01$

$1.244 \mathrm{E}-0$

$7.515 \mathrm{E}-02$

$8.450 E-02$

1. $508 \mathrm{E}-01$

$1.673 E-01$

$1.386 \mathrm{E}-\mathrm{C1}$ 1.385E-01

$3.753 E-01$ 4.021E-01

$1.059 \mathrm{E} 00$

$4.699 \mathrm{E}-01$

$1.007 \mathrm{E} \mathrm{OD}$

$2.715 \mathrm{E}-01$

$1.116 \mathrm{E} 00$
$2.775 \mathrm{E}-01$

$1.872 \mathrm{E}-0$

5.1 $1.173 E-00$

2.832E-01

ANGLE 6

ANGLE 7 $2.010 \mathrm{E}-04$
$9.884 \mathrm{E}-04$
$2.543 \mathrm{E}-03$ $9.884 E-04$
$2.543 E-03$

ANGLE ANGLE 9

$\begin{array}{lll}1.942 \mathrm{E}-03 & 1.694 \mathrm{E}-03 & 1.352 \mathrm{E}-03\end{array}$

(1.964E-03

$1.445 \mathrm{E}-03$

$1.515 \mathrm{E}-03$

2.422E-03

$3.952 \mathrm{E}-03$

$4.35 E-03$

$2.658 \mathrm{E}-03$

$5.024 E-03$

$2.848 \mathrm{E}-03$ 3. $528 \mathrm{EE}-03$

$3.528 \mathrm{E}-03$

$\begin{array}{ll}6.299 \mathrm{E}-03 & 6.946 \mathrm{E}-03\end{array}$

$3.002 E-03$
$7.521 E-03$

$1.769 \mathrm{E}-02$

$3.412 E-02$

$3.083 \mathrm{E}-0$

$8.259 \mathrm{E}-01$

$2.093 \mathrm{E}-02$

$4.409 E-02$

$3.199 \mathrm{E}-01$

$8.508 \mathrm{E}-01$

$2.460 E-01$

ANGLE 16

ANGLE 15

ANGLE 16

$M U=0.7550 \quad M U=0.8656$

$2.137 E-03$

$1.027 \mathrm{E}-02$

$4.682 E-03$

$2.240 E-C 2$

$4.683 \mathrm{E}-02$

..528E-02

$3.178 \mathrm{E}-0$

$7.615 E-02$

-1
-10

$5.181 \mathrm{E}-02$
$7.576 \mathrm{E}-02$

$4.039 \mathrm{E}-02$

$.452 E-02$

$9.869 \mathrm{E}-02$

$5.379 \mathrm{E}-02$

$8.816 \mathrm{E}-0$

$5.623 \mathrm{E}-02$

$7.829 E-02$

$1.271 \mathrm{E}-01$

$1.205 \mathrm{E}-01$

$1.608 \mathrm{E}-01$

$1.462 \mathrm{E}-01 \quad 1.725 \mathrm{E}-0$

$2.431 \mathrm{E}-01$

$2.122 \mathrm{E}-0$

$5.542 \mathrm{E}-01$

$5.986 \mathrm{E}-01$

$2.883 \mathrm{E}-01$

$2.922 \mathrm{E}-01$

1.094E-04

.833E-03 3.305E-03

1.648E-03

$4.428 E-03 \quad 5.203 E-03$

3.027E-03

$4.346 E-03$ 5.157E-03

$5.896 E-03 \quad 6.968 E-03$

$4.354 E-03 \quad 5.253 E-03$

$3.693 \mathrm{E}-03 \quad 4.651 \mathrm{E}-03$

$2.628 \mathrm{E}-02 \quad 3.125 \mathrm{E}-02$

$5.511 E-02 \quad 6.574 E-02$

$1.179 \mathrm{E}-01 \quad 1.223 \mathrm{E}-01$

$3.346 E-01 \quad 3.529 E-01$

$8.812 \mathrm{E}-01 \quad 9.173 \mathrm{E}-01$

ANGLE 17 SCALAR

$M U=0.9894 \quad F L U X$

$\begin{array}{ll}1.645 E-02 & 1.038 E-02\end{array}$

$1.488 \mathrm{E}-01 \quad 1.098 \mathrm{E}-01$

$1.252 \mathrm{E}-01 \quad 8.984 \mathrm{E}-02$

..750E-02 6.870E-02

$2.222 \mathrm{E}-01 \quad 1.734 \mathrm{E}-01$

$1.176 \mathrm{E}-01 \quad 1.149 \mathrm{E}-01$

$2.332 \mathrm{E}-01 \quad 2.264 \mathrm{E}-01$

$1.708 \mathrm{E}-01 \quad 1.966 \mathrm{E}-01$

$1.098 \mathrm{E}-01 \quad 1.923 \mathrm{E}-01$

$2.065 E-C 1 \quad 3.584 E-01$

$2.136 E-01 \quad 6.697 \mathrm{E}-01$

$2.048 \mathrm{E}-01 \quad 9.401 \mathrm{E}-01$

$\begin{array}{ll}2.82 \mathrm{E}-01 & 1.760 \mathrm{E} 00 \\ 6.376 \mathrm{E}-01 & 4.890 \mathrm{E} 00\end{array}$

$1.310 \mathrm{E} 00 \quad 1.217 \mathrm{E} \mathrm{OI}$

$3.264 \mathrm{E} 00$ 
(GAMMAS/MEV/STERADIAN/SOURCE NEUTRON)

ENERGY GROUP (MEV) 8.00E OO--1. 1. OOE O 6.50 E $00---8.00 E$ CO 5.00 E $00---6.50 \mathrm{E}$ OO $4.0 \mathrm{CE}$ OS- $-5.00 \mathrm{E}$ OO 3.00E OO---4. 00 E CO $2.5 \mathrm{CE} 00--3$. . COE OO 1.66 E OC---2. COE OO $1.33 \mathrm{E} \mathrm{OO--1.66E} \mathrm{OO}$ 1.0 COE CO-D-1.33E CO 8.0OE-O1---1. ONE OD $6.00 E-01--8.00 E-0$ $4.00 E-01--6.00 E-01$ $3.00 E-O 1--4.00 E-O$ $2.00 E-01--3 \cdot 3 \cdot C O E-01$ $1.00 E-01-2.00 E-31$ $5.00 E-02--1.00 E-01$ 2.00E-02 --5.00E-02 ENERGY

GROUP (MEV)

8.00E OC-D-1.00E OI $6.50 E$ OO---8. COE CO $5.00 E$ OO-- -6.5OE OO 4. CCE OO---5.0CE OO 3.0OE OO- $4.00 E$ OO 2.50 E CO--3.00E OD $2.00 \mathrm{E}$ OO--2.50E CO 1.66 E OO--2. 2 . OOE OO $1.33 \mathrm{E}$ CO--1.66E 00 1 . OOE OO--1. 33 E CO 6. OCE $01-1.00$ COE 6.CCE-CI--8. OOE-O $3.00 E-01-4.00 E-O 1$ $3.00 E-01-4.00 E-O 1$ 2.0NE $01-3.00 E-01$ $5.00 E-02-1.00 E-01$ $5.00 E-02--1.00 E-01$
$2.00 E-02---5.00 \mathrm{E}-02$ $M U=-1.0000$ $8.853 \mathrm{E}-05$ $4.041 E-04$ $1.086 \mathrm{E}-\mathrm{C} 3$ $4.934 \mathrm{E}-04$ $3.289 E-C 4$ $1.680 \mathrm{E}-\mathrm{C} 3$ $1.377 \mathrm{E}-\mathrm{C}$ $2.347 E-03$ $3.392 E-C 3$ $2.673 \mathrm{E}-03$ $1.500 \mathrm{E}-\mathrm{C}$ $1.944 \mathrm{E}-03$ $1.209 E-02$
$1.717 E-02$ $1.006 \mathrm{E}-0$ $2.947 E-0$ $8.507 \mathrm{E}-\mathrm{O}$ $2.631 \mathrm{E}-\mathrm{Cl}$ ANGLE 10 $M U=0.0950$ $2.480 \mathrm{E}-04$ $1.203 E-03$ $3.2 \mathrm{C} 7 \mathrm{E}-03$ $2.443 \mathrm{E}-\mathrm{C} 3$ $2.001 E-03$ $4.859 E-03$ 3.65 E-C3 $4.860 E-03$ $5.476 E-03$ $5.898 \mathrm{E}-03$ $1.455 E-C 2$ $7.584 \mathrm{E}-02$ $7.584 \mathrm{E}-02$ 1.2 EE . $21 E-01$ $2.917 \mathrm{E}-01$
ANGLE 2 $A=-0.9894$ $4.174 E-04$ $1.130 \mathrm{E}-\mathrm{C} 3$ 5.6 C9E-04 $3.926 \mathrm{E}-04$ $1.742 \mathrm{E}-03$ $1.406 \mathrm{E}-03$
$2.322 \mathrm{E}-03$ $25 \mathrm{E}-\mathrm{O}$ $2.564 \mathrm{E}-03$ $1.475 \mathrm{E}-03$ $2.180 \mathrm{E}-0$ $1.218 \mathrm{E}-02$ $1.728 \mathrm{E}-02$ $1.011 \mathrm{E}-01$ $2.953 \mathrm{E}-0$ $8.522 \mathrm{E}-\mathrm{O}$ $2.633 E-01$ ANGLE 11 $M U=0.2816$ $3.245 \mathrm{E}-04$
$1.577 \mathrm{E}-03$ $4.133 E-03$ 3.233E-03 $2.642 \mathrm{E}-03$ $6.239 E-03$ $4.622 \mathrm{E}-03$ $6.173 \mathrm{E}-03$ $8.318 \mathrm{E}-03$ $7.443 E-03$ $9.691 E-03$ $2.261 \mathrm{E}-02$ $5.777 E-02$ $0.401 E-02$ 1.361E$1.125 \mathrm{E} 00$ $2.983 \mathrm{E}-01$
ANGLE 3 $U=-0.9446$ $9.817 E-05$ $4.648 E-04$ $1.283 E-03$ $6.081 E-04$ $1.961 E-03$ $1.507 \mathrm{E}-03$ $2.244 \mathrm{E}-03$ $3.109 \mathrm{E}-03$ 2. 212E-03 $1.415 E-03$ ..008E-03 $1.255 E-02$ $1.030 \mathrm{E}-01$ 2. $978 E-01$ (

ANGLE 12 $M U=0.4580$ 4. $401 \mathrm{E}-04$ . . $431 E-03$ $3.447 E-03$ 8. $182 \mathrm{E}-03$ (.) $1.175 E-02$ 1.175E-02 $1.653 \mathrm{E}-02$ $3.334 \mathrm{E}-02$ . $337 \mathrm{E}-02$ $9.337 E-02$ 4.546E-O 1.546E-01 $3.053 E-01$

ANGLE 4
$M U=-0.8656$
$1.099 E-04$
$5.356 E-04$
$1.506 E-03$
$1.104 E-03$
$8.970 E-04$
$2.272 E-03$
$1.653 E-03$
$2.169 E-03$
$2.874 E-03$
$1.822 E-03$
$1.428 E-03$
$4.148 E-03$
$1.320 E-02$
$2.055 E-02$
$1.060 E-01$
$3.026 E-01$
$8.703 E-01$
$2.658 E-01$
ANGLE 13
$M U=0.6179$
$6.377 E-04$
$3.059 E-03$
$7.533 E-03$
$5.871 E-03$
$4.790 E-03$
$1.156 E-02$
$9.080 E-03$
$1.303 E-02$
$1.835 E-02$
$2.027 E-02$
$2.706 E-02$
$4.673 E-02$
$8.581 E-02$
$1.058 E-01$
$1.643 E-01$
$4.930 E-01$
$1.254 E 00$
$3.123 E-01$

ANGLE 5 $M U=-0.7550$ $1.221 E-04$ 6.034E-04 $1.706 E-03$ $1.341 \mathrm{E}-03$ $2.538 \mathrm{E}-03$ $1.791 E-03$ $2.201 E-03$ $2.857 \mathrm{E}-03$ $1.745 E-03$ $1.641 E-03$ $5.026 E-03$ $1.424 E-02$ $2.583 E-02$ $1.089 E-01$ $3.096 \mathrm{E}-01$ $8.876 \mathrm{E}-01$ $2.682 E-01$ ANGLE 14 $M U=0.7550$ $1.015 E-03$
$4.842 E-03$ $4.842 E-03$ $1.148 E-02$ $9.174 \mathrm{E}-03$ $7.708 \mathrm{E}-03$ $1.826 \mathrm{E}-02$ $1.499 E-02$ $2.165 \mathrm{E}-02$ $3.010 E-02$ $3.359 E-02$ $4.143 E-02$ $6.259 E-02$ $1.022 E-01$ $1.225 E-01$ $1.832 \mathrm{E}-01$ $1.34 \mathrm{E} 0 \mathrm{O}$ 3.189E-01
$A N G L E \quad 6$
$M U=-0.0179$ $1.338 \mathrm{E}-04$ $6.600 E-04$ $1.853 E-03$ $1.449 E-03$ $1.184 \mathrm{E}-03$ $2.732 E-03$ $1.926 \mathrm{E}-03$ $2.410 \mathrm{E}-03$ $3.171 E-03$ $2.100 E-03$ $2.049 E-03$ $5.484 \mathrm{E}-03$ $1.614 E-02$ $3.421 E-02$ $1.115 \mathrm{E}-01$ $3.193 \mathrm{E}-01$ $9.108 \mathrm{E}-01$ $2.714 E-01$ ANGLE 15 $M U=0.8656$ $1.864 \mathrm{E}-03$
$8.878 \mathrm{E}-03$ $2.023 \mathrm{E}-02$ $1.704 E-02$ $1.467 \mathrm{E}-02$ $3.293 \mathrm{E}-02$ $2.713 E-02$ $3.809 \mathrm{E}-02$ $5.095 E-02$ $5.383 E-02$ $5.928 \mathrm{E}-02$ $8.224 \mathrm{E}-02$ $1.231 \mathrm{E}-01$ $1.430 \mathrm{E}-01$ $5.050 \mathrm{E}-01$ . 1.387E 00
ANGLE 7 ANGLE 8 ANGLE 9 $1.471 \mathrm{E}-04 \quad 1.067 \mathrm{E}-04 \quad 1.986 \mathrm{E}-04$ $\begin{array}{lll}7.179 E-04 & 8.066 \mathrm{E}-04 & 9.599 \mathrm{E}-04\end{array}$ $\begin{array}{lll}1.985 E-03 & 2.195 E-03 & 2.583 E-03\end{array}$

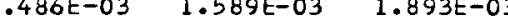

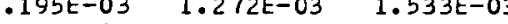
. $3.276 E-03 \quad 3.904 E-03$ $2.932 \mathrm{E}-03$ $3.360 \mathrm{E}-03$ 4.563E-03 $5.423 E-03$ . $3.513 E-03 \quad 4.236 E-03$ $3.146 E-03 \quad 4.037 E-03$

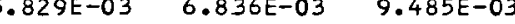
$1.962 E-02 \quad 2.545 E-02 \quad 3.400 E-02$ $\begin{array}{lll}1.632 E-02 & 6.683 E-02\end{array}$ $\begin{array}{lll}1.163 \mathrm{E}-01 & 1.203 \mathrm{E}-01 \\ 320 \mathrm{E}-01 & 3.480 \mathrm{E}-01 & 3.679 \mathrm{E}-01\end{array}$ $.401 \mathrm{E}-01 \quad 9.760 \mathrm{E}-01 \quad 1.019 \mathrm{E} 00$ $2.753 E-01 \quad 2.801 E-01 \quad 2.855 E-01$

ANGLE 16 ANGLE 17 SCALAR $M U=0.9446 \quad M U=0.9894 \quad$ FLUX $4.311 E-03$ $1.650 E-02 \quad 9.069 E-03$ $4.043 E-02 \quad 7.665 E-02 \quad 4.304 E-02$ $4.385 E-02$ 1.473E-01 $9.716 E-02$ $3.826 E-02$ 1.236E-01 7.921E-02 $6.921 E-02$ 2.021E-02 $6.350 E=02$ 6.921E-02 $2.049 E-01 \quad 1.470 E-01$ 7.289E-02 1.196E-C1 $1.074 \mathrm{E}-01$ 7.179E-02 1.705E-01 1.492E-01 $9.117 E-02$ 2.053E-01 1.961E-O $1.54 E-02$ 1.558E-01 1.830E-01 $1.100 E-01$ 1.TOOE-O1 $1.529 E-01$ 1. $1.664 \mathrm{E}-01 \quad 1.921 \mathrm{E}-01 \quad 9.312 \mathrm{E}-01$ 2.319E-01 2.615E-01 $1.730 E$ OO $1.442 \mathrm{E} 00$ 1.479E 00 1.357E 01

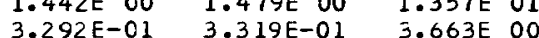


(GAMMAS/MEV/STERADIAN/SOURCE NEUTRON)

ENERGY

GROUP (MEV)

8.0OE OO---1.00E OI $6.50 E$ OO---8. COE CO
5. COE OO---6.5OE 00 $5.0 \cap E$
$4.00--6.50 E$ 4.OCE $00--5.00 E$ OO
$3.00 E$ OO---4.0OE CO
$2.50 E$ OO--3.0OE OD

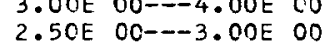
$2.50 E$ OO- $-3.00 E$ OD
$2.00 E$ OO- $-2.50 E$ OD 1.66 E $00--2.00 E$ EO $1.33 \mathrm{E} 00--1.66 \mathrm{E} 00$ $1.33 E$ OO---1.66E 00
$1.00 E 00--1.33$ E 00 $8.00 \mathrm{E}-01--1.00 \mathrm{E}$ OO $6.00 \mathrm{E}-01--8.00 \mathrm{E}-01$ 4.0OE-O1-C-6.00E-O 3.00E-01-- 4. $00 \mathrm{E}-\mathrm{O}$ 2.0OE-OI- $3.00 E-O$ $1.00 \mathrm{E}-1-2.00 \mathrm{E}-01$ $5.00 E-02--1.00 E-01$
$2.00 E-02---5.00 E-02$ ENERGY GROUP (MEV)

8. OOE DO---1. OCE OI 8.0OE DO---1. OCE O1
$6.50 E$ OO---8.0OE 00 5.0OE OO---6.50E OO $4.00 E$ OO-- $5.00 E$ OO 3.0OE OO---4. COE OO 2.5 CE $00--3.00 E$ OO 2.00 E OO-- $2.50 E$ OO $1.66 \mathrm{E} O 0---2.00 E$ DO $1.33 \mathrm{E} 00--1.66 \mathrm{E}$ OD $1.00 E$ OO-- - $1.33 E 00$ 8.00E-01-- $1.00 \mathrm{E}$ OO 6.00E-01---8.00E-01 $3.00 E-01--4.00 E-0$ $2.00 \mathrm{E}-01-\cdots 3.00 \mathrm{E}-0$ $1.00 \mathrm{E}-01--2.00 \mathrm{E}-01$ $5.00 \mathrm{E}-02--1.00 \mathrm{E}-0$ $2.00 E-02--5.00 E-02$ $M U=-1.0000 \quad M U=-0.9894$ $5.690 \mathrm{E}-05$ $2.468 \mathrm{E}-04$ $7.232 \mathrm{E}-04$ $2.053 E-04$ $1.229 \mathrm{E}-04$ $1.078 \mathrm{E}-03$ $1.018 E-03$ $1.790 \mathrm{E}-03$ $2.640 E-03$ . $130 \mathrm{E}-03$ 1.C53E-03 $9.535 E-04$ .021E-02 $1.442 E-02$ $2.728 \mathrm{E}-01$ $8.199 \mathrm{E}-0$ $2.561 E-O 1$ ANGLE 10 $1.777 \mathrm{E}-04$ $2.437 E-03$ $2.437 E-03$ $1.814 E-C 3$ $3.550 \mathrm{E}-\mathrm{C}$ $2.823 E-03$ $3.610 \mathrm{E}-\mathrm{C} 3$ $4.765 \mathrm{E}-03$ $3.997 E-03$ $5.011 E-03$ $1.269 \mathrm{E}-\mathrm{C} 2$ $4.177 \mathrm{~F}-02$ $6.936 \mathrm{E}-02$ $1.138 \mathrm{E}-\mathrm{Cl}$ $3.654 E-C 1$ $1.038 \mathrm{E} \mathrm{O0}$ $2.845 \mathrm{E}-\mathrm{Ol}$ ANGLE 3 $5.892 \mathrm{E}-05$ $2.599 E-04$ $7.666 \mathrm{E}-04$ $2.774 \mathrm{E}-04$ $1.916 \mathrm{E}-04$ $1.137 E-03$ $1.042 \mathrm{E}-03$ $1.757 \mathrm{E}-03$ $2.558 \mathrm{E}-03$ $2.016 E-03$ $1.038 \mathrm{E}-03$ . $189 \mathrm{E}-03$ $1.028 E-02$ $1.455 E-02$ ..734E-01 $8.214 \mathrm{E}-\mathrm{O}$ $2.563 \mathrm{E}-01$

$A J=-0.9446$ $6.611 \mathrm{E}-05$ $3.056 \mathrm{E}-04$ $9.178 E-04$ $5.102 E-04$ $4.127 E-04$ . $343 \mathrm{E}-03$ $1.126 \mathrm{E}-03$ $1.648 \mathrm{E}-03$ 2.290E-03 $1.641 E-03$ $1.013 E-03$ $2.008 \mathrm{E}-03$ 1. 54 E 02 $1.540 E-02$ $9.758 E-01$ $2.758 E-01$ $2.572 E-01$ $\triangle A N G L E \quad 4$ $M U=-0.8656$ $7.675 \mathrm{E}-05$ 3.711E-0 $1.130 \mathrm{E}-03$ $8.112 \mathrm{E}-04$ $0.942 E-04$ $1.622 \mathrm{E}-03$ $1.243 E-03$ $1.527 E-03$ $1.985 E-03$ $1.226 \mathrm{E}-03$ $1.065 \mathrm{E}-03$ $3.088 E-03$ 1.107E-02 $1.799 E-02$ $9.749 E-02$ $2.804 \mathrm{E}-01$ $2.588 E-01$

ANGLE 11 ANGLE 12 ANGLE 13 $M U=0.2816$ 2.360E-04 $1.138 \mathrm{E}-03$ $3.184 \mathrm{E}-03$
$2.459 \mathrm{E}-03$ $2.099 \mathrm{E}-03$ $4.596 \mathrm{E}-03$ $3.617 E-03$ $4.586 \mathrm{E}-03$ $6.161 \mathrm{E}-03$ $5.942 \mathrm{E}-0$ $8.828 \mathrm{E}-03$ $2.047 \mathrm{E}-02$ $5.357 E-02$ $7.605 \mathrm{E}-02$ $1.222 \mathrm{E}-01$ $3.925 \mathrm{E}-01$ $1.094 \mathrm{E} 00$ 2.911E-01 $M U=0.4580$ 3. $261 \mathrm{E}-04$ 1.563E-03 $3.253 E-03$ $2.744 E-03$ $6.097 E-03$ $4.914 E-03$ $6.552 E-03$ $9.188 E-03$ 1. C31E-02 1. $565 \mathrm{E}-02$ $3.074 \mathrm{E}-02$ $6.569 \mathrm{E}-02$ $8.385 E-02$ $1.332 \mathrm{E}-01$ 4.239E-01 $1.157 E 00$ $2.980 \mathrm{E}-01$
$M U=0.6179$ $4.815 \mathrm{E}-04$ $5.912 E-03$ $4.502 E-03$ $3.838 E-03$ $8.784 E-03$ $7.517 \mathrm{E}-03$ $1.062 \mathrm{E}-02$ $1.521 \mathrm{E}-02$ $1.837 E-02$ $2.603 E-02$ $4.298 \mathrm{E}-02$ $7.794 \mathrm{E}-02$ $9.468 \mathrm{E}-02$ $1.468 \mathrm{E}-01$ 4. 594E-01 $1.223 \mathrm{E} 00$
$3.048 \mathrm{E}-01$
ANGLE 5

ANGLE $5 \quad$ ANGLE 6
$M U=-0.7550 \quad M U=-0.6179$ $8.669 \mathrm{E}-05$ $4.271 \mathrm{E}-0$ $1.304 E-03$ $1.023 E-03$ . . $35=03$ 1. 5 E -03 . . $136 E-03$ . $279 E-03$ 6E- 03 $107 E-02$ 330E-02 . 8.568E-0 $2.612 E-01$

ANGLE 14
$M U=0.7550$ $7.943 \mathrm{E}-04$ $.745 E-03$ $9.253 E-03$ $6.479 E-03$ $1.452 \mathrm{E}-02$ 1. $310 \mathrm{E}-02$ $1.863 \mathrm{E}-02$ $2.611 E-02$ $3.114 \mathrm{E}-02$ $3.980 \mathrm{E}-02$ $5.691 \mathrm{E}-02$ $9.143 \mathrm{E}-02$ 1. C93E-01 $1.628 \mathrm{E}-01$ $4.980 E-01$ 1.290E 00
$3.113 \mathrm{E}-01$ $9.474 \mathrm{E}-05$ $4.646 \mathrm{E}-04$ $1.405 \mathrm{E}-03$ $1.091 \mathrm{E}-03$ 5. $294 \mathrm{E}-04$ $1.954 \mathrm{E}-03$ $1.436 \mathrm{E}-03$ $1.680 \mathrm{E}-03$ $2.183 \mathrm{E}-03$ $1.470 \mathrm{E}-03$ 1.628E-03 $4.111 E-03$ 1.965E-01 8.960 01 $2.644 E-01$

ANGLE 15 $M U=0.8656$ $1.534 \mathrm{E}-03$ $7.235 \mathrm{E}-03$ $1.444 E-02$ $1.319 E-02$ $2.756 \mathrm{E}-02$ $2.489 E-02$ $3.388 \mathrm{E}-02$ $4.497 \mathrm{E}-02$ $4.978 \mathrm{E}-02$ $5.627 E-02$ $7.307 \mathrm{E}-02$ $1.086 \mathrm{E}-01$ $1.268 E-01$ $1.811 \mathrm{E}-01$ $5.378 \mathrm{E}-01$ $3.170 \mathrm{E}-01$

ANGLE 7 $M U=-0.4580$ $1.029 E-04$ 4.964E-04 (2.05 E 04 . . $.031 E-01$ $.086 E-01$ $2.683 E-01$ ANGLE 16 $M U=0.9446$ $1.767 \mathrm{E}-02$ 
(GAMMAS/MEV/STERADIAN/SOURCE NEUTRON)

ENERGY GROUP (MEV) 8.00E CO-D-1.00E 01 5. COE DO--6.00E CO $4.00 E$ OO- - 5. OOE OO $3.00 E$ OO-- - 4. OOE OO 2.50 E ON-- 3. 00 E 00 2.00 E OO---2.50E OO $1.66 \mathrm{E} \mathrm{OC-D-2.00E} \mathrm{CO}$ $1.33 \mathrm{E} 00--1.66 \mathrm{E} \mathrm{OO}$ 1. COE OO---1.33E CO $8.00 E-01-1.00 E 00$ 6.00E-01---8. OCE-01 $4.00 \mathrm{E}-01--6.00 \mathrm{E}-01$ 3. OCE-D1---4.00E-01 2. CCE-01-- -3.00E-01 1. OCE $-01---2.00 E-01$
$5.00 E-C 2=-1.00 E-01$ $5.00 E-C 2--1.00 E-01$
$2.00 E-02---5.00 E-02$

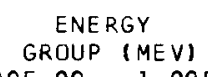

ANGLE 1 $M U=-1.0000$ $3.468 \mathrm{E}-05$ 1.384E-C4 $6.517 E-C 4$ $7.3 \mathrm{C} 9 \mathrm{E}-\mathrm{C4}$ $1.349 E-03$ 2. $C 30 E-03$ $1.666 \mathrm{E}-\mathrm{C} 3$ $6.846 \mathrm{E}-\mathrm{C4}$ $2.780 E-04$ $8.336 \mathrm{E}-\mathrm{C}$ $1.163 \mathrm{E}-\mathrm{C2}$ $8.140 E-02$ 2.370E-C1 $7.309 E-O$ $2.255 \mathrm{E}-01$ ANGLE 10 $M U=0.0950$ $1.235 \mathrm{E}-\mathrm{C} 4$ $5.861 E-04$ $1.30 \mathrm{E}-03$ $1.309 E-03$ 2.511E-03 2.511E-03 2.1C1E-O $2.573 E-C 3$ 2.908E-03 2.90E 03 1.07E-0 1.073E-02 . . $3.184 \mathrm{E}-01$ $9.288 \mathrm{E}-\mathrm{Cl}$ $2.551 \mathrm{E}-\mathrm{Cl}$
ANGLE 2 $M U=-0.9894$
$3.657 E-05$ . $508 \mathrm{E}-04$ . $9.286 \mathrm{E}-0 \mathrm{~S}$ $5.837 \mathrm{E}-05$ $7.053 \mathrm{E}-04$ $7.499 \mathrm{E}-04$ $1.311 \mathrm{E}-03$ $1.943 E-03$ $1.554 \mathrm{E}-03$ $6.802 \mathrm{E}-04$ $5.089 \mathrm{E}-04$ $8.382 \mathrm{E}-03$ $1.176 \mathrm{E}-02$ $8.178 \mathrm{E}-02$ $2.376 \mathrm{E}-01$ $.323 \mathrm{E}-01$ ANGLE 11 $M U=0.2816$ $1.671 \mathrm{E}-04$ .986E-04 $2.38 \mathrm{E}-03$ $1.821 \mathrm{E}-03$ $1.618 E-03$ $3.287 E-03$ $2.699 E-03$ $4.395 E-03$ $4.592 E-03$ 7. . 78 EE-O3 $4.700 E-02$ $6.504 E-02$ $1.038 \mathrm{E}-01$ $3.418 \mathrm{E}-01$ $9.798 \mathrm{E}-01$ 2. $610 E-01$
ANGLE 3 $4.321 \mathrm{E}-0$ $1.934 E-04$ $3.049 E-04$ $2.620 E-04$ $8.896 \mathrm{E}-04$ $8.153 E-04$ $1.184 E-03$ $1.652 \mathrm{E}-0$ $1.187 E-03$ . $865 E-04$ $1.275 E-03$ 1. $261 \mathrm{E}-02$ $1.261 E-02$ $8.315 \mathrm{E}-\mathrm{C2}$ 2. $397 \mathrm{E}-01$ .380E-O $M U=0.4580$ $2.338 \mathrm{E}-04$ $1.109 E-03$ ..187E-03 . 4. 370E-O 4. $379 E-03$ 3.72SE-03 . $8.600 \mathrm{E}-03$ 1. 1.407E-02 $5.710 E-02$ . 1.117E-O2 $3.688 \mathrm{E}-01$ $1.036 \mathrm{E} 00$ $2.672 E-01$
$A N G L E \quad 4$
$M U=-0.8656$ $5.264 \mathrm{E}-05$ ..318E-04 $5.819 \mathrm{E}-04$ $5.235 \mathrm{E}-0$ $1.131 \mathrm{E}-03$ $9.010 E-04$ $1.036 \mathrm{E}-03$ $1.316 \mathrm{E}-03$ $7.829 \mathrm{E}-04$ $7.679 E-04$ $2.245 \mathrm{E}-03$ $8.937 \mathrm{E}-03$ $1.511 \mathrm{E}-02$ $8.510 \mathrm{E}-02$ $2.439 E-01$ $.488 \mathrm{E}-01$ $2.320 \mathrm{E}-01$ ANGLE 13 $M U=0.6179$ $3.510 E-04$ $1.641 E-03$ $4.475 E-03$ $3.314 E-03$ $2.944 E-03$ $6.444 E-03$ . $8.406 E-03$ $1.230 \mathrm{E}-02$ $1.603 E-02$ $3.768 \mathrm{E}-02$ $6.768 E-02$ . $1.24 \mathrm{E}-0$ $3.991 E-01$ $1.095 \mathrm{E} 00$ $2.733 E-01$
ANGLE 5

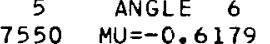
$6.058 E-05$ 2. $969 E-04$ $9.764 E-04$ $7.789 E-04$ $6.996 E-04$ $1.295 E-03$ . $680 E-04$ $9.956 \mathrm{E}-04$ $1.215 E-03$ 6. $930 E-04$ $9.693 E-04$ . $867 E-03$ ..688E-03 (.010E-02 $8.677 E-C 2$ $2.499 \mathrm{E}-0$ 2.341E-01 作 ANGLE 14 $M U=0.7550$ $5.984 E-04$ $2.783 E-03$ 7.169E-03 5.563E-03 . $223 \mathrm{E}-03$ $1.118 E-02$ $1.09 E-02$ . $198 \mathrm{E}-02$ 2.198E-02 $7.734 \mathrm{E}-02$ $9.207 E-O 2$ . $367 E-01$ $4.315 \mathrm{E}-01$ $1.155 E$ OO
$2.790 E-01$ $6.578 \mathrm{E}-05$
$3.198 \mathrm{E}-04$ $3.198 E-04$ .086E-0 $7.147 E-04$ $1.358 E-03$ $1.028 \mathrm{E}-03$ $1.127 E-03$ $1.448 \mathrm{E}-03$ $9.974 \mathrm{E}-04$ $1.247 \mathrm{E}-03$ $2.998 \mathrm{E}-03$ $1.130 E-02$ $2.770 \mathrm{E}-02$ $8.789 \mathrm{E}-02$ $2.582 \mathrm{E}-01$ $7.856 \mathrm{E}-01$ $2.370 \mathrm{E}-0$

ANGLE 15 $M U=0.8656$ $1.218 \mathrm{E}-03$ $5.683 \mathrm{E}-03$ $1.382 \mathrm{E}-02$ $1.180 E-02$ $1.140 E-02$ $2.239 E-02$ 2.179E-02 $3.835 E-02$ $3.835 E-02$ $4.380 E-02$ 5.005E-02 $6.184 E-02$ $9.061 \mathrm{E}-01$ $1.061 \mathrm{E}-01$ $4.644 E-01$

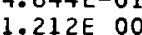
$2.840 \mathrm{E}-01$

ANGLE 7 ANGLE 8 ANGLE 9 $M U=-0.4580 \quad M U=-C .2816 \quad M U=-0.0950$ $\begin{array}{lll}7.019 E-05 & 7.028 E-05 & 9.491 E-05\end{array}$ $\begin{array}{lll}3.327 E-C 4 & 3.643 E-04 & 4.433 E-04\end{array}$ $7.295 \mathrm{E}-04 \quad 7.209 \mathrm{E}-04 \quad 9.133 \mathrm{E}-04$ $6.299 E-04 \quad 6.259 E-04 \quad 8.143 E-04$ $\begin{array}{lll}1.398 E-03 & 1.552 E-03 & 1.921 E-03\end{array}$ $1.129 \mathrm{E}-03 \quad 1.331 \mathrm{E}-03 \quad 1.658 \mathrm{E}-03$ $1.419 \mathrm{E}-03 \quad 1.792 \mathrm{E}-03 \quad 2.167 \mathrm{E}-03$ $1.933 \mathrm{E}-03 \quad 2.463 \mathrm{E}-03 \quad 2.892 \mathrm{E}-03$ $1.531 \mathrm{E}-03 \quad 2.013 \mathrm{E}-03 \quad 2.348 \mathrm{E}-03$ $1.521 \mathrm{E}-03 \quad 1.817 \mathrm{E}-03 \quad 2.446 \mathrm{E}-03$ $2.982 \mathrm{E}-03 \quad 3.673 \mathrm{E}-03 \quad 6.034 \mathrm{E}-03$ $1.446 \mathrm{E}-02 \quad 1.977 \mathrm{E}-02 \quad 2.737 \mathrm{E}-02$ $3.695 \mathrm{E}-02 \quad 4.622 \mathrm{E}-02 \quad 5.402 \mathrm{E}-02$ $8.863 \mathrm{E}-02 \quad 8.978 \mathrm{E}-02 \quad 9.228 \mathrm{E}-02$ $2.688 \mathrm{E}-01 \quad 2.821 \mathrm{E}-01 \quad 2.986 \mathrm{E}-01$ $8.122 E-01 \quad 8.449 E-01 \quad 8.837 E-01$ $2.405 E-01 \quad 2.448 E-01 \quad 2.497 E-01$ 
(GAMMAS/MEV/STERADIAN/SOURCE NEUTRON)

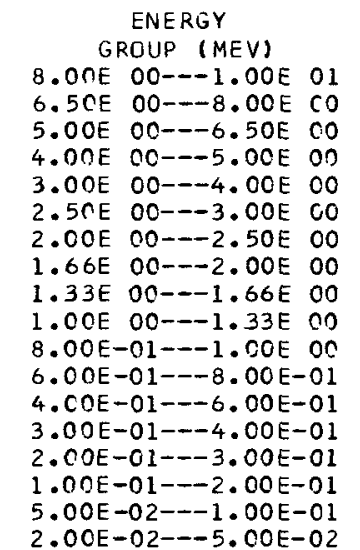

ANGLE 1 $A N G L E \quad 3$
$M U=-0.9446 \quad M U E$ $4.503 \mathrm{E}-\mathrm{C6}$ $-4.278 E-C 8$ $6.078 \mathrm{E}-\mathrm{C5}$ 9.108E-05 $7.836 \mathrm{E}-\mathrm{C} 5$ $2.576 E-04$ $04 \quad 5.504 E-04$ $9.237 E-04$ $7.632 E-C 4$ $8.651 \mathrm{E}-\mathrm{C5}$ $4.408 E-04$ $4.158 \mathrm{E}-03$ $4.022 E-03$ $1.287 \mathrm{E}-\mathrm{C}$ $4.089 \mathrm{E}-0$ $1.286 \mathrm{E}-0$ ANGLE 10 ENERG GROUP (MEV)
$.00 E$ OO-

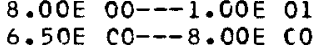
$6.50 E$ CO---8.00E CO
$5.00 E$ OD- $-6.50 E$ OO 4.0OE OO- - - . COE CO 3.00 E $00--4.00 E$ C.O 2.5CE OC---3.0OE OO 2.00E CO-- 2.5CE OO $1.66 E$ OO--2.00E CO $1.33 \mathrm{E} \mathrm{OC-D-1.66E} \mathrm{OO}$ $1.00 \mathrm{E}$ OO--1.33E CO 8.0CE-01--1.00E CO $6.00 E-01--8$. COE-0 4.DOE-O1-- 6 . COE-OI $3.00 E-01-2.00 E-01$ $2.00 E-01--3.00 E-01$ $1.00 E-01-2.00 E-01$ $5.00 \mathrm{E}-02-\cdots-1.00 \mathrm{E}-\mathrm{CI}$ $U=0.1950$ 3.879E-05 $1.756 E-04$ $6.747 \mathrm{E}-04$ $4.645 \mathrm{E}-04$ 4. $8 \mathrm{C} 8 \mathrm{E}-\mathrm{C}$ $8.247 E-0$ $7.578 \mathrm{E}-\mathrm{C}_{4}$ $8.044 \mathrm{E}-04$ $1.010 \mathrm{E}-03$ $9.5 \mathrm{C} 6 \mathrm{E}-04$ $2.010 E-03$ $5.972 \mathrm{E}-03$ $2.123 \mathrm{E}-02$ $3.251 \mathrm{E}-02$ $5.072 \mathrm{E}-02$ $5.187 E-01$ $1.187 \mathrm{E}-01$
$1.426 \mathrm{E}-\mathrm{C} 1$
ANGLE 2 $1.058 \mathrm{E}-05$ $1.8306-05$ $2.321 \mathrm{E}-05$ $3.412 \mathrm{E}-05$ $2.265 \mathrm{E}-04$ $2.832 \mathrm{E}-04$ 4. $240 \mathrm{E}-04$ $5.931 E-04$ $3.808 \mathrm{E}-04$ 1. $580 \mathrm{E}-04$ $1.995 E-04$ 4. $165 \mathrm{E}-03$ $6.195 E-03$ $4.732 E-02$ 1. $302 \mathrm{E}-01$ 4. $129 \mathrm{E}-01$ $1.292 E-01$ $1.287 \mathrm{E}-01$ ANGLE 11 $M U=0.2816$ $2.573 \mathrm{E}-04$ $9.435 \mathrm{E}-04$ $7.122 \mathrm{E}-04$ $6.956 \mathrm{E}-04$ $1.097 E-C 3$ $9.515 \mathrm{E}-04$ $9.987 E-04$ $1.360 \mathrm{E}-03$ $1.946 \mathrm{E}-03$ $4.454 \mathrm{E}-03$ 1.052E 02 $2.664 \mathrm{E}-02$ $3.424 E-02$ . $5.463 E-01$ $1.458 \mathrm{E}-01$ ANGLE 12

\section{ANGLE 13} $7.897 \mathrm{E}-05$ $3.568 \mathrm{E}-04$ 1. $243 \mathrm{E}-03$ $8.808 \mathrm{E}-04$ $8.335 \mathrm{E}-04$ $1.433 E-03$ 1. $377 \mathrm{E}-03$ $1.786 \mathrm{E}-03$ $2.818 \mathrm{E}-03$ $4.578 \mathrm{E}-03$ $8.644 \mathrm{E}-03$ $1.590 \mathrm{E}-02$ $3.139 \mathrm{E}-02$ $3.673 \mathrm{E}-02$ $5.825 \mathrm{E}-02$ $5.764 \mathrm{E}-01$ 1. $490 \mathrm{E}-01$ $M U=0.6179$
$1.203 \mathrm{E}-04$ $5.273 E-04$ $1.709 \mathrm{E}-03$ 1. $115 \mathrm{E}-03$ $1.119 \mathrm{E}-03$ $2.255 \mathrm{E}-03$ $2.587 \mathrm{E}-03$ $3.874 E-03$ $6.127 E-03$ $9.401 \mathrm{E}-03$ $1.453 \mathrm{E}-02$ $2.145 \mathrm{E}-02$ $4.0415-02$ $4.088 \mathrm{E}-02$ $6.313 E-02$ $2.125 E-01$ $1.522 \mathrm{E}-01$ ANGLE 4 $1.652 \mathrm{E}-05$ $3.155 \mathrm{E}-04$ $2.368 \mathrm{E}-04$ $2.372 E-04$ $1.216 E-04$ $2.677 E-04$ $8.747 E-04$ $4.255 E-03$
$7.951 E-03$ $4.804 \mathrm{E}-02$ $1.325 \mathrm{E}-01$
ANGLE 5 2.C53E-05 $1.005 E-04$ 3. $925 E-04$ 201E-0 $4.289 E-04$ $3.126 \mathrm{E}-04$ $2.089 E-04$ $2.051 E-04$ $1.231 \mathrm{E}-04$ $4.039 E-04$ $1.137 E-03$ $4.630 \mathrm{E}-03$ $1.125 E-02$ $4.841 E-02$ $1.359 \mathrm{E}-01$ $4.278 \mathrm{E}-01$
$1.312 \mathrm{E}-01$ ANGLE 14 $M U=0.7550$
$2.242 E-04$ 2.242E-04 $9.836 \mathrm{E}-04$ $2.910 \mathrm{E}-03$
$2.118 \mathrm{E}-03$ $2.375 \mathrm{E}-03$ $4.679 E-03$ $5.681 \mathrm{E}-03$ $8.256 E-03$ $1.199 \mathrm{E}-02$ $1.642 \mathrm{E}-02$ $2.149 E-02$
$2.674 E-02$ $2.674 E-02$ $3.943 E-02$ $4.656 \mathrm{E}-02$ $6.845 \mathrm{E}-02$ $2.278 \mathrm{E}-01$ $1.552 \mathrm{E}-01$
ANGLE 6
$M U=-0.6179$ $2.147 \mathrm{E}-05$ $1.004 \mathrm{E}-04$ $3.989 \mathrm{E}-04$ $2.892 E-04$ $2.831 \mathrm{E}-04$ $3.236 \mathrm{E}-04$ $2.851 E-04$ 3.855E-04 $2.853 E-04$ $5.105 E-04$ $1.051 \mathrm{E}-03$ $5.641 E-03$ $1.601 E-02$ 4.832E-02 $1.404 \mathrm{E}-01$ $4.396 \mathrm{E}-01$
$1.328 \mathrm{E}-01$ ANGLE 15 $M U=0.8656$ $5.392 E-04$
$2.426 E-03$ $6.503 \mathrm{E}-03$ $5.740 E-03$ $6.447 \mathrm{E}-03$ $1.106 \mathrm{E}-02$ 1. $260 \mathrm{E}-02$ $1.640 \mathrm{E}-02$ 2.119E-02 $2.521 \mathrm{E}-02$ 2.853 E-02 $3.180 \mathrm{E}-02$ $4.466 E-02$ $5.259 \mathrm{E}-02$ $7.414 \mathrm{E}-02$ $2.428 \mathrm{E}-01$ $1.578 \mathrm{E}-01$
ANGLE 7 $M=-0.4580$ $2.057 \mathrm{E}-05$
$8.767 \mathrm{E}-05$ $3.652 E-C 4$ $1.966 E-04$ $1.888 \mathrm{E}-04$ $3.806 E-04$ $3.650 E-04$ $4.652 E-04$ $6.483 E-04$ $5.806 \mathrm{E}-04$ $5.467 E-C 4$ $8.697 E-04$ $7.706 \mathrm{E}-03$ $2.147 \mathrm{E}-02$ $4.796 \mathrm{E}-02$ $1.461 \mathrm{E}-01$ $4.544 \mathrm{E}-01$
$1.347 \mathrm{E}-01$ ANGLE 16 $M U=0.9446$ $7.684 \mathrm{E}-03$ $1.813 \mathrm{E}-02$ $1.707 E-02$ $1.736 \mathrm{E}-02$ $2.688 E-02$ $2.689 \mathrm{E}-02$ $3.100 \mathrm{E}-02$ $3.541 \mathrm{E}-02$ 3.560E-02 $3.484 \mathrm{E}-02$ $5.186 \mathrm{E}-02$ $5.747 E-02$ $7.98 \mathrm{E}-02$ . $556 E-01$ $1.597 \mathrm{E}-01$

ANGLE 8 ANGLE 9 $M U=-0.2816 \quad M U=-0.0950$ $2.132 E-05 \quad 2.703 E-05$ $8.737 E-05 \quad 1.157 E-04$ $3.662 \mathrm{E}-\mathrm{C} 4 \quad 4.668 \mathrm{E}-04$ $1.487 \mathrm{E}-04 \quad 2.386 \mathrm{E}-04$ $1.541 E-04 \quad 2.603 E-04$ $4.142 E-04 \quad 5.727 E-04$ $6.501 \mathrm{E}-04 \quad 7.566 \mathrm{E}-04$ $9.027 E-04 \quad 1.023 E-03$ $7.784 \mathrm{E}-04 \quad 7.866 \mathrm{E}-04$ $5.881 E-04 \quad 9.122 E-04$ $2.830 E-03$ $1.111 E-02 \quad 1.579 E-02$ $2.651 E-02 \quad 3.021 E-02$ $4.788 E-02 \quad 4.867 E-02$ $1.532 E-01 \quad 1.619 E-01$ $\begin{array}{ll}4.726 E-01 & 4.940 E-01 \\ 1.370 E-01 & 1.397 E-01\end{array}$ ANGLE 17 SCALAR $\begin{array}{ll}A=0.9894 & \text { FLUX } \\ 9.187 \mathrm{E}-03 & 3.170 \mathrm{E}-03\end{array}$ $3.950 E-02 \quad 1.391 E-02$ $7.487 E-02 \quad 3.317 E-02$ $6.225 \mathrm{E}-02 \quad 2.706 \mathrm{E}-02$ 
(GAMMAS/MEV/STERADIAN/SOURCE NEUTRON)

ENERGY

GROUP (MEV)

8. OOE OO--1. $1.00 E \quad 01$

6.5CE OC- - 8. COE 00

4.0OE OO---5.00E OD $3.0 \mathrm{CE}$ DO-- -4.0OE DO 2.5 CE $00--3.00$ E CO 2. COE OO- - - 2.50E 00 $1.66 \mathrm{E} 00---2.00 \mathrm{E} C 0$ $1.33 \mathrm{E} 00-\rightarrow-1.66 \mathrm{E} 00$

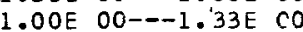
$8.0 \mathrm{EE}-01--1.00 \mathrm{E} \mathrm{NO}$ 6.0OE-C1---8.00E-01 $4.00 E-01--6.00 E-01$ $3.00 E-01--4.00 \mathrm{E}-01$ $2.00 E-01--3.00 E-01$ $1.00 E-01--2.00 E-01$ $5.00 \mathrm{E}-02---1.00 \mathrm{E}-\mathrm{C}$ $2.00 E-02--5.00 E-02$

ENERGY

GROUP (MEV)

8. COE OO---1. COE OI $6.50 E$ OO-- $8.00 E$ OO 5. CCE OO---6.50E OO 4.OCE OO- $-5.00 E O O$ 3. तOE OO--4. OOE OO 2.5กE OO---3.00E OO 2.DOE OO-- 2.50E OO 1.66 E CO---2.00E DO $1.33 \mathrm{E} 00---1.66 \mathrm{E} 00$ 1.00 E $00-\cdots 1.33 E$ OO $8.00 E-01--1.00 E \mathrm{CO}$ 6. COE-01--8. $00 E-01$ $4.00 E-01--6.00 E-0$ 3. COE-O1---4. COE-O $2.00 \mathrm{E}-01--3.00 \mathrm{E}-01$ $1.00 E-01--2.00 E-01$ $5.00 \mathrm{E}-02-1.00 \mathrm{E}-01$ $2.00 \mathrm{E}-\mathrm{C} 2---5.00 \mathrm{E}-02$
ANGLE 1 ANGLE 2 $M U=-1.0000 \quad M U=-0.9894$ $-2.074 E-05-1.420 E-05$ $-3.150 E-05-1.019 E-05$ $-1.446 E-04-1.105 E-04$ $-1.270 E-C 4 \quad-9.431 E-05$ $-3.257 E-C 5$ - $1.117 E-05$ $9.649 E-05 \quad 9.662 E-05$ $2.743 E-04 \quad 2.478 E-04$ $4.352 E-04 \quad 3.861 E-04$ $.461 E-04 \quad 3.001 E-04$ $-6.314 E-05 \quad-4.621 E-05$ 3.975E-04 -2.98CE-04 $1.990 \mathrm{E}-03 \quad 1.977 \mathrm{E}-03$ $2.466 \mathrm{E}-03$ $2.377 \mathrm{E}-\mathrm{C} 2 \quad 2.383 \mathrm{E}-0$ $6.218 \mathrm{E}-0$ $\begin{array}{ll}1.973 \mathrm{E}-01 & 1.977 \mathrm{E}-01 \\ 6.167 \mathrm{E}-02 & 6.172 \mathrm{E}-02\end{array}$ ANGLE 10 $M U=0.0950$ $1.219 E-05$ $5.311 \mathrm{E}-\mathrm{O}$ $2.477 E-04$ $1.796 \mathrm{E}-04$ $2.016 E-04$ $2.704 E-04$ $2.472 E-04$ $2.061 E-04$ $2.143 E-C 4$ . $9.590 E-04$ $3.151 \mathrm{E}-\mathrm{C}$ $1 . \mathrm{C} 88 \mathrm{E}-02$ $1.571 \mathrm{E}-\mathrm{C} 2$ $2.400 \mathrm{E}-\mathrm{C2}$ $8.223 \mathrm{E}-02$ $2.480 E-01$ $6.805 \mathrm{E}-\mathrm{C} 2$ ANGLE 11 $M U=0.2816$
ANGLE 3 $M U=-0.9446$ $1.695 \mathrm{E}-06$ $2.198 \mathrm{E}-06$ $-5.293 \mathrm{E}-06$ $2.437 \mathrm{E}-06$ $4.332 \mathrm{E}-0$ $9.587 \mathrm{E}-0$ $1.504 \mathrm{E}-04$ $1.877 \mathrm{E}-04$ $1.229 E-04$ $9.454 \mathrm{E}-06$ $9.350 E-06$ $1.936 E-03$ $2.878 \mathrm{E}-03$ $2.405 E-02$ $6.280 E-02$ $1.992 \mathrm{E}-01$ $6.193 E-02$ ANGLE 12 $M U=0.458$ $2.576 \mathrm{E}-05$ $1.087 \mathrm{E}-04$ $4.563 E-04$ $3.048 E-04$ $2.979 E-0$ $4.177 \mathrm{E}-04$ 4. $412 \mathrm{E}-04$ $6.178 E-04$ $1.146 \mathrm{E}-0$ $2.337 \mathrm{E}-03$ 4. 693E-0 $8.283 \mathrm{E}-03$ 1. $530 \mathrm{E}-02$ $1.707 \mathrm{E}-02$ $2.715 \mathrm{E}-02$ $9.343 E-02$ $2.738 \mathrm{E}-0$ 7.090 E- 02
ANGLE 4 $M U=-0.8656$ $5.663 E-06$ $2.609 \mathrm{E}-05$ $1.218 \mathrm{E}-04$ $8.835 \mathrm{E}-0$ $8.835 \mathrm{E}-05$ $9.133 \mathrm{E}-05$ 3. $766 \mathrm{E}-05$ $2.613 E-05$ $9.883 E-05$ $3.097 \mathrm{E}-04$ $3.908 \mathrm{E}-03$ $2.424 \mathrm{E}-0$ $6.392 \mathrm{E}-02$ $2.021 \mathrm{E}-0$ 6.231 E-02 $M U=0.6179$ $1.452 \mathrm{E}-04$ $5.790 \mathrm{E}-04$ $2.978 \mathrm{E}-04$ $3.467 \mathrm{E}-04$ $7.197 \mathrm{E}-04$ $1.054 \mathrm{E}-03$ $1.768 \mathrm{E}-03$ $3.008 \mathrm{E}-0$ $5.031 \mathrm{E}-03$ $7.755 \mathrm{E}-03$ $1.077 \mathrm{E}-0$ $1.671 \mathrm{E}-0$ $1.886 \mathrm{E}-0$ $2.908 \mathrm{E}-02$ $9.973 \mathrm{E}-02$ $2.876 \mathrm{E}-01$ $7.230 E-02$
ANGLE 5 $7.498 E-06 \quad 7.066 E-06$ $3.437 \mathrm{E}-05$ $1.569 \mathrm{E}-04$ $1.472 E-04$ $1.389 E-04$ $1.412 E-04$ $8.465 E-05$ $-1.072 E-05$ $-1.505 E-05$ $1.700 \mathrm{E}-04$ 4.716E-04 (1) $2.418 \mathrm{E}-02$ $6.553 E-02$ $2.062 E-01$ $6.286 E-02$ ANGLE 14 $M U=0.7550$ $7.459 E-05$
$2.970 E-04$ $1.044 E-03$ $1.044 \mathrm{E}-03$
$6.889 \mathrm{E}-04$ $9.690 \mathrm{E}-04$ $1.906 \mathrm{E}-03$ $2.752 E-03$ $4.179 E-03$ $6.172 E-03$
$8.688 E-03$ $1.107 \mathrm{E}-02$ $1.283 E-02$ $1.805 \mathrm{E}-02$ $2.130 \mathrm{E}-02$ $3.111 \mathrm{E}-02$ $1.061 E-0$ $7.359 E-02$

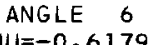
$7.066 E-06$ $3.137 E-05$
$1.462 E-04$ $1.092 \mathrm{E}-04$ $1.064 \mathrm{E}-04$ $1.194 \mathrm{E}-04$ $8.598 \mathrm{E}-05$ $5.626 \mathrm{E}-05$ $7.161 \mathrm{E}-05$ $7.643 \mathrm{E}-05$ $2.060 \mathrm{E}-04$ $3.272 E-04$ $6.359 E-02$

ANGLE 15 $M U=0.8656$ $2.178 E-04$
$9.397 E-04$ $2.790 \mathrm{E}-03$ $2.597 E-03$ $3.324 \mathrm{E}-03$ $6.577 \mathrm{E}-03$ $8.487 \mathrm{E}-03$ $1.074 \mathrm{E}-02$ $1.276 \mathrm{E}-02$ $1.399 \mathrm{E}-02$ $1.457 \mathrm{E}-02$ $1.997 E-02$ $2.365 \mathrm{E}-02$ $3.323 E-02$ $1.122 \mathrm{E}-01$ $3.131 \mathrm{E}-0$ $7.470 E-02$
$A N G L E \quad 7$
$M U=-0.4580$ $U=-0.4580$
$5.412 E-06$
$1.658 E-C 5$ $1.105 E-04$ $1.360 E-05$ $1.360 E-05$ $2.560 E-05$
$8.471 E-05$ $8.471 E-05$ $1.558 \mathrm{E}-04$ $2.328 E-04$ $7.035 E-02$ $2.186 \mathrm{E}-0 \mathrm{I}$ $6.448 E-02$

ANGLE 16 $M U=0.9446$ $3.667 \mathrm{E}-03$ $9.048 E-03$ $8.983 \mathrm{E}-03$
$9.795 \mathrm{E}-03$ $1.423 \mathrm{E}-02$ $1.567 \mathrm{E}-02$ $1.603 \mathrm{E}-02$ $1.621 \mathrm{E}-02$ $2.272 \mathrm{E}-02$ $2.517 \mathrm{E}-02$ $3.530 \mathrm{E}-02$ $1.172 \mathrm{E}-01$ $7.553 \mathrm{E}-02$

ANGLE 8 ANGLE 9 $M U=-0.2816 \quad M U=-0.0950$ $4.739 E-06 \quad 6.934 E-06$ $1.517 \mathrm{E}-05 \quad 2.684 \mathrm{E}-05$ $9.721 \mathrm{E}-05 \quad 1.438 \mathrm{E}-04$ $6.079 E-06 \quad 5.757 E-05$ $2.657 E-05 \quad 7.838 E-05$ $9.167 \mathrm{E}-05 \quad 1.645 \mathrm{E}-04$ $1.542 E-04 \quad 2.084 E-04$ $3.534 \mathrm{E}-04 \quad 3.147 \mathrm{E}-04$ 2.873E-04 $2.271 \mathrm{E}-04$ $1.362 E-04 \quad 3.083 E-04$ $3.872 E-04 \quad 1.372 E-03$ $5.710 E-03 \quad 8.174 E-03$ $1.347 E-02 \quad 1.500 E-02$ $2.302 E-02 \quad 2.319 E-02$ 7.364E-02 7.759E-02 
(GAMMAS/MEV/STERADIAN/SOURCE NEUTRON)

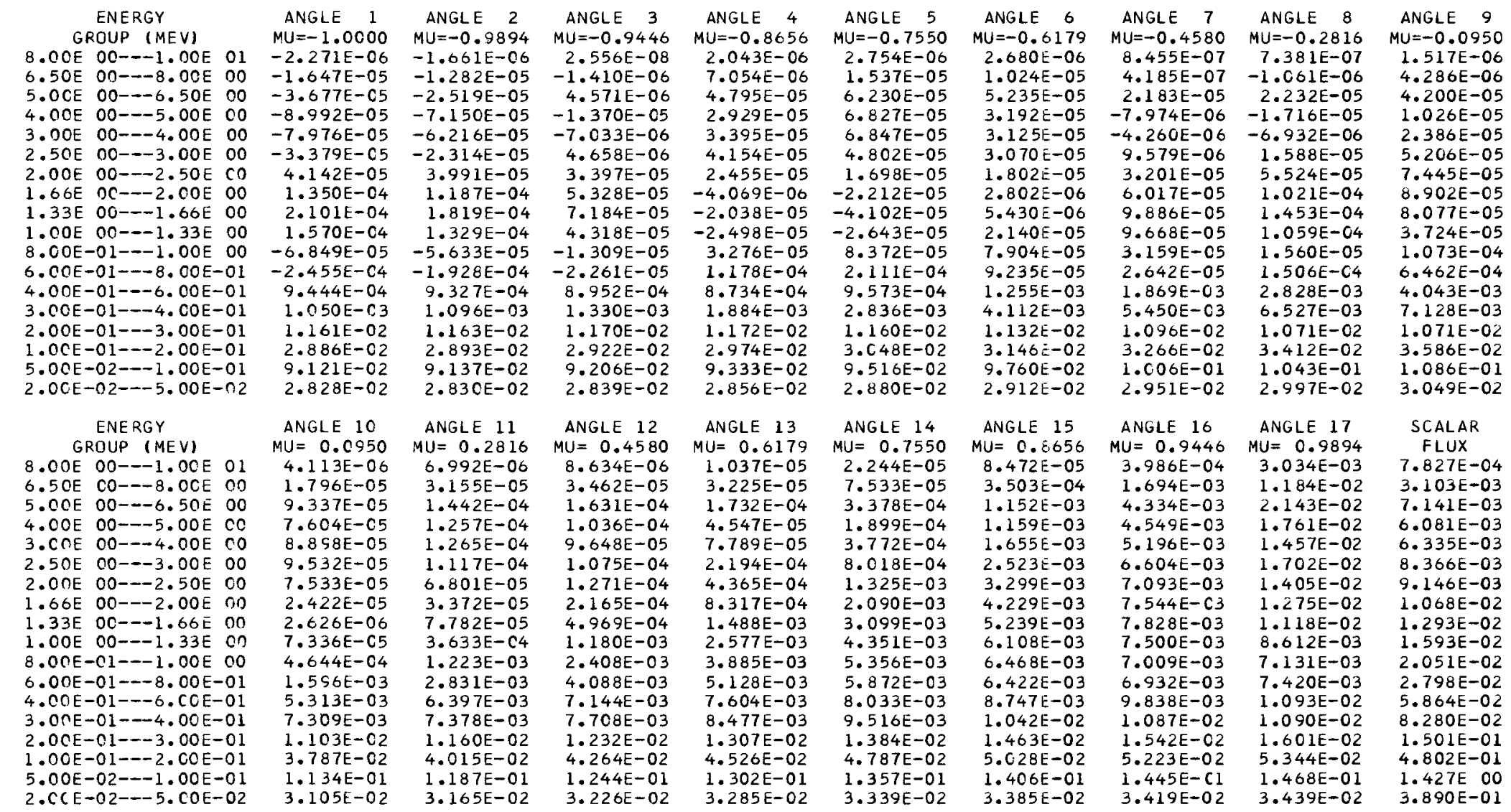


(GAMMAS/MEV/STERADI AN/SOURCE NEUTRON)

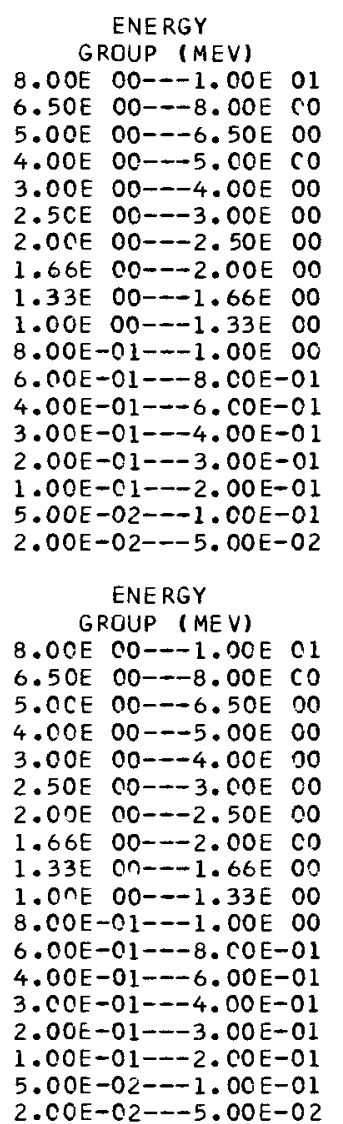

ANGLE 1 $-1.575 E-C 6$ $-1.002 E-C 5$ -4.956E-C5 $6.767 E-05$ $1.022 \mathrm{E}-04$ $7.121 \mathrm{E}-05$ 1. $324 E-04$ $4.484 E-C 4$ $4.667 \mathrm{E}-\mathrm{C4}$ $5.568 E-03$ $1.333 E-02$ $4.179 E-02$ $1.286 \mathrm{E}-\mathrm{C} 2$ ANGLE 10 $M U=0.0950$ $1.6 \mathrm{C} 8 \mathrm{E}-\mathrm{C6}$ $6.250 \mathrm{E}-\mathrm{C6}$ $3.658 \mathrm{E}-\mathrm{C}$ $2.941 \mathrm{E}-\mathrm{C5}$ $4.040 E-05$ $3.539 E-05$ 2.C52E-05 $-1.355 E-C 5$ $1.647 \mathrm{E}-\mathrm{C5}$ $2.287 E-04$ $343 E-03$ 2.543E-03 (2.0 $1.733 \mathrm{E}-\mathrm{C}$ $5.149 \mathrm{E}-02$

$1.407 E-02$ $-1.237 \mathrm{E}-06 \quad-1.726 \mathrm{E}-07$ $-8.061 E-06 \quad-1.875 E-06$ $-1.917 \mathrm{E}-05 \quad-8.155 \mathrm{E}-10$ $-0.155 E-10$
$-9.848 E-06$ $-3.511 E-05 \quad-6.632 E-06$ $-1.525 E-05$ $1.850 E-05$ $5.843 E-05$ $8.704 E-05$ . $3.894 \mathrm{E}-05$
$1.058 \mathrm{E}-04$ $1.058 \mathrm{E}-04$
$4.408 \mathrm{E}-04$ $4.920 E-C 4$ $5.575 E-03$ $1.336 \mathrm{E}-0$ $4.286 E-02$ $1.287 \mathrm{E}-\mathrm{C2}$ ANGLE 11 $M U=0.2816$ $2.882 E-06$ $1.357 \mathrm{E}-05$ $5.853 E-C 5$ $5.536 E-05$ $5.454 E-05$ $3.540 E-05$ $7.739 E-06$ $-9.470 E-06$ $1.285 E-05$ (175E-04 $1.396 E-03$ $3.009 E-C 3$ $3.351 E-03$ $5.287 E-03$ $1.831 \mathrm{E}-02$ $5.376 \mathrm{E}-02$ $1.432 \mathrm{E}-02$
ANGLE 3 $A N G L E \quad 4$
$M U=-0.8656$ $6.326 \mathrm{E}-07$ $2.801 \mathrm{E}-06$ $1.489 \mathrm{E}-05$
$1.314 \mathrm{E}-05$ $1.314 \mathrm{E}-05$
$1.496 \mathrm{E}-05$ $1.496 \mathrm{E}-05$
$1.316 \mathrm{E}-05$ $1.316 E-05$
$4.512 E-06$ $1.302 E-05$ $2.241 E-05-9.688 E-06$ $2.948 E-05-1.944 E-05$ $\begin{array}{rr}1.582 \mathrm{E}-05 & -1.734 \mathrm{E}-05 \\ -1.278 \mathrm{E}-05 & 1.406 \mathrm{E}-05\end{array}$ $-1.958 E-05 \quad 5.161 E-05$ 4.159E-04 3.987E-04 $6.177 \mathrm{E}-04 \quad 9.039 \mathrm{E}-04$ $5.592 E-03 \quad 5.577 E-03$ $1.349 E-02 \quad 1.373 E-02$ 4. $217 E-02 \quad 4.273 E-02$ $1.292 \mathrm{E}-02 \quad 1.299 \mathrm{E}-02$ ANGLE 12 $M U=0.458 \mathrm{C}$ $3.018 \mathrm{E}-06$ $1.162 \mathrm{E}-05$ 5.703E-05 2.780E-05 $2.086 \mathrm{E}-05$ $2.052 E-05$ $3.175 E-05$ $8.212 E-05$ $2.29 \mathrm{E}-04$ . $1.19 E-03$ $3.294 E-03$ $3.29 \mathrm{E}-03$ . $1.937 \mathrm{E}-02$ $1.458 \mathrm{E}-02$

ANGLE 13

$M U=0.6179$ $2.364 E-06$ 4. $138 E-05$ 1. $512 \mathrm{E}=05$ $6.468 E-05$ 1. $892 E-04$ $4.029 E-04$ $1.88 \mathrm{E}-03$ $3.437 \mathrm{E}-03$ . . $2.047 E-02$ $5.857 E-02$ $1.483 \mathrm{E}-02$
ANGLE 5 $M U=-0.7550$ $1.393 E-06$ $3.230 \mathrm{E}-05$ $3.149 \mathrm{E}-05$ $1.822 \mathrm{E}-05$ $5.416 \mathrm{E}-07$ $-1.951 E-05$ $-2.990 E-05$ $-1.723 E-05$ $9.266 \mathrm{E}-05$ $4.390 E-04$ $1.379 E-03$ $5.482 E-03$ $1.407 E-02$ $4.354 \mathrm{E}-02$ $1.310 \mathrm{E}-02$ ANGLE 14 $M U=0.7550$ 5. 7 C6E-06 $1.329 E-05$ $9.402 \mathrm{E}-05$ $4.840 E-05$
$1.456 E-04$ $1.456 \mathrm{E}-04$ $3.536 \mathrm{E}-04$ $6.429 E-04$ $1.038 \mathrm{E}-03$ 1. $2.119 E-03$ 2.650E-03 $3.571 E-03$ 4. $251 E-03$ $6.171 \mathrm{E}-03$ $2.154 \mathrm{E}-02$ $6.086 \mathrm{E}-02$ $1.505 \mathrm{E}-02$ $\begin{array}{lll}A N G L E & 6 & \text { ANGLE } \quad 7 \\ U U=-0.6179 & M U=-0.4580\end{array}$ $1.844 E-05$ $.141 E-05$ $1.050 E-05$ $2.283 E-06$ $-1.725 \mathrm{E}-06$ $3.163 E-05$ 2.756E-05 $5.916 E-04$ $1.998 E-03$ $1.451 E-02$ $4.462 \mathrm{E}-02$ $4.462 \mathrm{E}-02$
$1.323 \mathrm{E}-02$ ANGLE 15 $M U=0.8656$ $3.255 \mathrm{E}-05$
$1.292 \mathrm{E}-04$ $1.292 \mathrm{E}-04$ $4.695 \mathrm{E}-04$ $5.222 E-04$ $8.126 E-04$ l.626E-03 $2.066 \mathrm{E}-03$ $2.853 \mathrm{E}-03$ 2.9 2.815E-03 $4.849 E-03$ $6.482 \mathrm{E}-03$ $6.482 E-03$ $2.25 \mathrm{E}-02$ $1.525 \mathrm{E}-02$ ANGLE 16
ANGLE 7
$M U=-0.4580$$\quad$ ANGLE 8 A $\quad$ ANGLE 9 $1.749 E-08 \quad-2.022 E-07 \quad 3.010 E-07$ $-1.352 E-06 \quad-2.767 E-06 \quad 1.339 E-06$ $1.313 E-06 \quad 3.019 E-08 \quad 1.089 E-05$ $-1.034 E-05-1.524 E-05 \quad 6.503 E-06$ $-8.625 E-06 \quad-9.941 E-06 \quad 6.612 E-06$ - $.139 E-06 \quad 1.348 E-06 \quad 1.655 E-05$ $9.932 \mathrm{E}-06 \quad 2.181 \mathrm{E}-05 \quad 2.778 \mathrm{E}-05$ $3.588 E-05 \quad 6.391 E-05 \quad 1.649 E-05$ $4.164 E-05 \quad 3.906 E-05 \quad-9.555 E-07$ 9.198E-07 - $1.031 E-05 \quad 3.877 E-05$ $-7.972 E-06 \quad 5.752 E-05 \quad 3.170 E-04$ $9.031 E-04 \quad 1.378 E-03 \quad 1.960 E-03$ $2.026 E-C 3 \quad 3.105 E-03 \quad 3.337 E-03$ $5.100 E-03 \quad 4.947 E-03 \quad 4.922 E-03$ $1.505 E-02 \quad 1.570 E-02 \quad 1.646 E-02$ $1.340 E-02 \quad 1.350 E-02 \quad 4.940 E-02$ $M U=0.9446 \quad M U=0.9894$ $1.871 \mathrm{E}-04 \quad 1.648 \mathrm{E}-03$ $2.041 E-03 \quad 1.076 E-02$ $2.263 \mathrm{E}-03 \quad 8.791 \mathrm{E}-03$ $2.661 E-03 \quad 7.312 E-03$ $3.203 E-03 \quad 7.751 E-03$ $3.433 E-03 \quad 6.468 E-03$ $3.549 E-03 \quad 5.596 \mathrm{E}-03$ 3. 3. 2.0 $4.300 E-03 \quad 4.742 E-03$ $4.734 E-03 \quad 4.688 E=03$ $6.794 E-03 \quad 7.029 E-03$ $2.331 E-02 \quad 2.378 E-02$ $6.444 E-02 \quad 6.536 E-02$ $1.539 \mathrm{E}-02 \quad 1.547 \mathrm{E}-02$ 
4 PI R* 2 HENDERSON DOSE (NEUTRONS) ( CM* *2 RAD/STERADIAN/SOURCE NEUTRON)
12.200 TO 15.090 MEV NEUTRON SOURCE

RANGE (METERS)

$\begin{array}{lcccc}150.0 & 200.0 & 250.0 & 300.0 & 400.0 \\ 1.151 \mathrm{E}-10 & 1.285 \mathrm{E}-10 & 1.345 \mathrm{E}-10 & 1.35 \mathrm{CE}-10 & 1.252 \mathrm{E}-10 \\ 1.165 \mathrm{E}-10 & 1.298 \mathrm{E}-10 & 1.356 \mathrm{E}-10 & 1.36 \mathrm{CE}-10 & 1.258 \mathrm{E}-10 \\ 1.202 \mathrm{E}-10 & 1.333 \mathrm{E}-1 \mathrm{C} & 1.388 \mathrm{E}-10 & 1.389 \mathrm{E}-10 & 1.281 \mathrm{E}-10 \\ 1.224 \mathrm{E}-10 & 1.354 \mathrm{E}-1 \mathrm{~V} & 1.413 \mathrm{E}-10 & 1.415 \mathrm{E}-10 & 1.307 \mathrm{E}-10 \\ 1.253 \mathrm{E}-10 & 1.404 \mathrm{E}-1 . & 1.464 \mathrm{E}-10 & 1.465 \mathrm{E}-10 & 1.353 \mathrm{E}-10 \\ 1.307 \mathrm{E}-10 & 1.451 \mathrm{E}-1 . & 1.515 \mathrm{E}-10 & 1.521 \mathrm{E}-10 & 1.409 \mathrm{E}-10 \\ 1.356 \mathrm{E}-10 & 1.521 \mathrm{E}-10 & 1.600 \mathrm{E}-10 & 1.608 \mathrm{E}-10 & 1.495 \mathrm{E}-10 \\ 1.491 \mathrm{E}-10 & 1.671 \mathrm{E}-10 & 1.748 \mathrm{E}-10 & 1.757 \mathrm{E}-10 & 1.633 \mathrm{E}-10 \\ 1.700 \mathrm{E}-10 & 1.897 \mathrm{E}-10 & 1.981 \mathrm{E}-10 & 1.986 \mathrm{E}-10 & 1.836 \mathrm{E}-10 \\ 1.930 \mathrm{E}-10 & 2.253 \mathrm{E}-10 & 2.344 \mathrm{E}-10 & 2.288 \mathrm{E}-10 & 2.110 \mathrm{E}-10 \\ 2.596 \mathrm{E}-10 & 2.582 \mathrm{E}-10 & 2.668 \mathrm{E}-10 & 2.698 \mathrm{E}-10 & 2.452 \mathrm{E}-10 \\ 2.617 \mathrm{E}-10 & 3.148 \mathrm{E}-10 & 3.254 \mathrm{E}-10 & 3.187 \mathrm{E}-10 & 2.963 \mathrm{E}-10 \\ 3.652 \mathrm{E}-10 & 3.839 \mathrm{E}-10 & 3.957 \mathrm{E}-10 & 3.955 \mathrm{E}-10 & 3.572 \mathrm{E}-10 \\ 4.812 \mathrm{E}-10 & 5.337 \mathrm{E}-10 & 5.432 \mathrm{E}-10 & 5.304 \mathrm{E}-10 & 4.743 \mathrm{E}-10 \\ 7.917 \mathrm{E}-10 & 8.301 \mathrm{E}-10 & 8.365 \mathrm{E}-10 & 8.116 \mathrm{E}-10 & 7.043 \mathrm{E}-10 \\ 1.747 \mathrm{E}-09 & 1.694 \mathrm{E}-09 & 1.608 \mathrm{E}-09 & 1.495 \mathrm{E}-09 & 1.227 \mathrm{E}-09 \\ 1.471 \mathrm{E}-08 & 1.168 \mathrm{E}-08 & 9.232 \mathrm{E}-09 & 7.318 \mathrm{E}-09 & 4.618 \mathrm{E}-09 \\ 6.070 \mathrm{E}-09 & 5.810 \mathrm{E}-09 & 5.458 \mathrm{E}-09 & 5.055 \mathrm{E}-09 & 4.190 \mathrm{E}-09\end{array}$

\section{RANGE (METERS)}

1200.0

1500.0

1800.0
-1. CCONOE OO $-9.894 \cap 1 E-01$ $-9.44575 \mathrm{E}-01$ $-8.65631 \mathrm{E}-01$ $-7.55044 \mathrm{E}-01$ $-6.17876 E-01$ $-4.58017 E-01$ $-2.81605 \mathrm{E}-01$ $-9.50125 E-02$ $2.81605 \mathrm{E}-01$ $2.81605 \mathrm{E}-01$ $4.58017 E-01$ $7.55044 \mathrm{E}-\mathrm{Ol}$ $7.55044 E-01$ $8.65631 \mathrm{E}-01$ $9.89401 \mathrm{E}-01$

TOTAL
1.C $78 \mathrm{E}-10$ $1.083 \mathrm{E}-10$ $1.100 \mathrm{E}-10$ $1.124 \mathrm{E}-10$ $1.164 \mathrm{E}-10$ $1.214 \mathrm{E}-1 \mathrm{C}$ $1.291 \mathrm{E}-10$ $1.408 E-10$ $1.577 E-10$ $1.799 E-10$ $2.083 E-10$ $2.457 \mathrm{E}-1 \mathrm{C}$ $3.013 E-1 C$ $3.957 E-10$ S.7 $38 \mathrm{E}-1 \mathrm{C}$ $9.553 \mathrm{E}-10$

$3.346 \mathrm{E}-09$

$8.836 E-11$ $8.872 E-11$ $9.004 E-11$ $9.206 E-11$ $9.529 \mathrm{E}-11$ $9.958 \mathrm{E}-11$ $1.060 \mathrm{E}-10$
$1.155 \mathrm{E}-10$ $1.155 \mathrm{E}-10$ $1.284 \mathrm{E}-10$ $1.464 E-10$ $1.688 \mathrm{E}-10$ $2.426 E-10$ . $155 E-10$ . $155 E-10$ $4.483 E-10$ 1. $189 \mathrm{E}-10$

$2.598 \mathrm{E}-09$

\section{4. $043 \mathrm{E}-11$} 4.057E-1 4. $111 E-11$ 4. $208 \mathrm{E}-1$ 4. $357 \mathrm{E}-1$ 4. $566 \mathrm{E}-11$ 4. $865 \mathrm{E}-11$ $5.284 \mathrm{E}-11$ $6.849 E-11$ $6.583 E-11$ 7.53 1. $062 \mathrm{E}-11$ $1.064 E-10$ $1.352 E-10$ $2.708 \mathrm{E}-10$ $2.708 E-10$

$1.076 \mathrm{E}-09$

\section{$1.562 \mathrm{E}-11$} $1.567 \mathrm{E}-11$ $1.587 E-11$ $1.684 E-11$ . $766 \mathrm{E}-11$ $1.882 \mathrm{E}-11$ .039E- 11 .247E-11 $2.516 E-11$ 2.863E-11 3. $330 \mathrm{E}-11$ $4.990 E-11$ $6.564 \mathrm{E}-11$ $9.178 \mathrm{E}-11$ $1.487 \mathrm{E}-10$

\section{$5.451 \mathrm{E}-12$} $5.468 \mathrm{E}-12$ $5.538 E-12$ $5.674 \mathrm{E}-12$ $5.877 E-12$ $6.169 E-12$ $6.569 \mathrm{E}-12$ $7.107 E-12$ ..80 E-12 $8.714 \mathrm{E}-12$ $.886 \mathrm{E}-12$ $1.365 \mathrm{E}-11$ $1.365 E-11$ 1.685 E 11 $2.924 \mathrm{E}-11$ $4.287 \mathrm{E}-11$

$3.938 E-10$
$1.777 \mathrm{E}-12$

$1.782 \mathrm{E}-12$

$1.806 \mathrm{E}-12$

1. $850 \mathrm{E}-12$

$1.917 \mathrm{E}-12$

$2.012 \mathrm{E}-12$

(1)

.

.

. $200 \mathrm{E}-12$

.696E-12

4. $383 E-12$

. $364 \mathrm{E}-12$

$6.803 E-12$

$8.937 E-12$

4.241E-11 
COSINE

75.0

$1.111 E-10$ $1.129 \mathrm{E}-10$

$1.17 \mathrm{CE}-10$

$1.199 E-1 C$

. $208 \mathrm{E}-10$

.243E-10

$1.286 \mathrm{E}-1 \mathrm{C}$

$1.411 \mathrm{E}-10$

$1.611 \mathrm{E}-1 \mathrm{C}$

$2.448 \mathrm{E}-1 \mathrm{C}$

$2.334 \mathrm{E}-1 \mathrm{C}$

$2.952 \mathrm{E}-10$

$3.498 \mathrm{E}-10$

$5.833 \mathrm{E}-10$

$9.256 E-1 C$

$2.298 \mathrm{E}-09$

$8.614 E-C 9$

TOTAL

COSINE

-1. OCOCOE 00

$9.89401 E-0$

$-9.44575 E-0$

$-7.55044 \mathrm{E}-0$

-7.55044E-OL

- 17876 E-OI

$-2.81605 E-01$

$-2.81605 E-01$

$-9.5 C 125 E-02$

.

. $81605 E-01$

6.58176E-O1

$7.55044 \mathrm{E}-01$

$8.65631 \mathrm{E}-0$

$9.44575 \mathrm{E}-0$

. $89401 \mathrm{E}-01$

TOTAL

500.0

$1.83 C E-10$

$1.837 \mathrm{E}-10$

$1.862 \mathrm{E}-1 \mathrm{C}$

$1.89 \mathrm{E}-1 \mathrm{C}$

$1.957 E-10$
100.0

150.0

200.0
200 )

250.0

300.0

400.0

\section{$.361 E-1 C$}

$1.406 \mathrm{E}-10$

$1.438 \mathrm{E}-10$

$1.453 \mathrm{E}-10$

$1.497 \mathrm{E}-10$

1. $555 \mathrm{E}-10$

$1.703 \mathrm{E}-10$

1. $940 \mathrm{E}-10$

$2.174 \mathrm{E}-10$

$2.881 \mathrm{E}-10$

$3.072 \mathrm{E}-1$

4.C71E-10

.

(2.

$2.326 \mathrm{E}-\mathrm{CO}$

$8.376 \mathrm{E}-\mathrm{C} 9$

$1.706 \mathrm{E}-10$
$1.724 \mathrm{E}-10$

$1.945 \mathrm{E}-10$

$2.079 E-10$

$2.13 C E-10$

$2.052 E-10$

$\begin{array}{ll}.799 \mathrm{E}-10 & 2.034 \mathrm{E}-10 \\ .838 \mathrm{E}-10 & 2.103 \mathrm{E}-10\end{array}$

$1.912 E-10 \quad 2.171 \mathrm{E}-10$

$1.986 \mathrm{E}-10 \quad 2.273 \mathrm{E}-10$

$2.172 \mathrm{E}-10 \quad 2.483 \mathrm{E}-10$

$2.460 \mathrm{E}-10 \quad 2.796 \mathrm{E}-10$

$2.774 \mathrm{E}-10 \quad 3.282 \mathrm{E}-10$

$3.688 E-10 \quad 3.734 E-10$

$3.694 \mathrm{E}-10$ 4. $408 \mathrm{E}-10$

$5.129 E-10 \quad 5.452 E-10$

$6.664 \mathrm{E}-10$ $7.476 \mathrm{E}-10$

$1.082 \mathrm{E}-09$ 1.143E-0

$2.337 E-09$ 2.286E-09

1.906 E-0

$2.286 \mathrm{E}-09$

$2.093 \mathrm{E}-10$
$2.135 \mathrm{E}-10$

$2.169 \mathrm{E}-10$

$2.240 \mathrm{E}-10$

$2.439 E-10$

$2.648 E-10$

$2.973 \mathrm{E}-10$

$3.472 \mathrm{E}-10$

$3.921 \mathrm{E}-10$

$4.725 E-10$

7.687E-10

$1.161 E-09$

2.183E-09

$8.245 \mathrm{E}-\mathrm{C} 9$

$8.027 E-09$

$7.667 E-09$

$142 E-10$

.218E-10 $2.131 \mathrm{E}-10$

$2.289 \mathrm{E}-10 \quad 2.198 \mathrm{E}-10$

$2.282 E-10$

2. $212 \mathrm{E}-10 \quad 2.609 \mathrm{E}-10$

$3.033 \mathrm{E}-10 \quad 2.898 \mathrm{E}-10$

$3.452 \mathrm{E}-10 \quad 3.283 \mathrm{E}-10$

$4.020 E-10$ 3.762E-1O

$4.697 E-10$ 4.392E-10

$5.752 E-10 \quad 5.318 E-10$

$1.584 E-10 \quad 6.918 E-10$

$1.136 E-09 \quad 1.002 E-09$

$\begin{array}{ll}2.044 E-09 & 1.099 E-09 \\ 9.605 E-09 & 6.124 E-09\end{array}$

$7.218 E-09 \quad 6.171 E-09$

RANGE (METERS)

600.0

900.0

1200.0

1500.0

1800.0

$1.549 E-10$

7. $649 \mathrm{E}-11$

$3.119 E-11$

$1.131 \mathrm{E}-11$

3. $790 \mathrm{E}-12$

1. $574 \mathrm{E}-10$

$1.134 E-11$

$3.800 \mathrm{E}-12$

$3.841 \mathrm{E}-12$

$3.918 \mathrm{E}-12$

$4.034 E-12$

$4.414 \mathrm{E}-12$

$8.155 E-11$

$1.204 \mathrm{E}-1$

$1.253 \mathrm{E}-1$

$3.459 \mathrm{E}-1$

$1.319 \mathrm{E}-11$

$4.414 \mathrm{E}-12$

$1.960 \mathrm{E}-10$

$3.645 E-11$

$1.406 \mathrm{E}-11$

$1.518 \mathrm{E}-11$

$5.059 E-12$

$1.046 \mathrm{E}-10$

$4.217 E-11$

$1.838 \mathrm{E}-11$

$5.514 E-12$

. $408 \mathrm{E}-10$

$5.152 \mathrm{E}-1$

$1.838 \mathrm{E}-11$

$6.092 \mathrm{E}-12$

$1.295 \mathrm{E}-10$

(542E-11

$2.073 \mathrm{E}-11$

3. $153 \mathrm{E}-10$

$1.743 \mathrm{E}-10 \mathrm{O} 2.802 \mathrm{E}-11$

$2.394 E-11$

$7.857 E-12$

$4.777 E-10$

$2.147 \mathrm{E}-10$ $8.228 \mathrm{E}-11$

$1.042 \mathrm{E}-10$

$1.402 E-10$

$3.537 \mathrm{E}-11$

$0.276 E-12$

8.298E-1C $0.586 E-10$

l. $023 \mathrm{E}-09$

$2.565 \mathrm{E}-\mathrm{C} 9$

$\begin{array}{lll}4.004 E-10 & 1.402 E-10 & 4.587 E-11 \\ 7.352 E-10 & 2.175 E-10 & 6.459 E-11\end{array}$

列

$1.431 E-11$

$7.878 E-11$

$6.879 E-10$

$2.406 E-10$ 
4 PI R\#\#2 TISSUE KERMA (NEUTRCNS) (CM**2 ERGS/GRAM/STERADIAN/SOURCE NEUTRON)
12.2CO TO 15.000 MEV NEUTRON SOURCE
COSINE

$-1.00000 E 00$ $-9.89401 E-01$ $-9.44575 E-01$ $-8.65631 \mathrm{E}-01$ $-6.5787 \mathrm{E}-01$ $-4.58017 E-01$ $-2.81605 \mathrm{E}-01$ $-9.50125 \mathrm{E}-02$ $9.50125 E-02$ $2.81605 \mathrm{E}-01$ 4.58017E-01 $6.17876 \mathrm{E}-01$ $7.55044 E-01$ $8.65631 \mathrm{E}-\mathrm{Cl}$ $9.44575 \mathrm{E}-\mathrm{C}$ $9.89401 E-0$

TOTAL

COSINE

$-1.0 C \operatorname{COOE} 00$ $-9.89401 \mathrm{E}-01$ $-8.65631 \mathrm{E}-01$ $-8.65631 E-01$ -6.17876 E-01 $-4.58017 E-01$ -4.58017E-O1 - $50125 E-02$ $-90125 E-02$ $9.50125 E-02$ $4.58017 \mathrm{E}-01$ $4.57876 \mathrm{E}-01$ $7.55044 E-01$ $8.65631 \mathrm{E}-01$ $8.44575 E-O 1$ $9.89401 E-01$

TOTAL

$\begin{array}{lc}75.0 & 100.0 \\ 8.080 E-09 & 9.654 E-C 9 \\ 8.250 E-09 & 9.829 E-09 \\ 8.636 E-09 & 1.025 E-08 \\ 8.914 E-09 & 1.055 E-08 \\ 8.978 E-C 9 & 1.066 E-C 8 \\ 9.247 \mathrm{E}-09 & 1.099 \mathrm{E}-08 \\ 9.525 \mathrm{E}-09 & 1.138 \mathrm{E}-08 \\ 1.050 \mathrm{E}-08 & 1.251 \mathrm{E}-08 \\ 1.209 \mathrm{E}-08 & 1.439 \mathrm{E}-08 \\ 1.894 \mathrm{E}-08 & 1.645 \mathrm{E}-\mathrm{C} 8 \\ 1.794 \mathrm{E}-08 & 2.192 \mathrm{E}-\mathrm{C} 8 \\ 2.283 \mathrm{E}-08 & 2.337 \mathrm{E}-08 \\ 2.743 \mathrm{E}-08 & 3.142 \mathrm{E}-08 \\ 4.740 \mathrm{E}-08 & 4.576 \mathrm{E}-08 \\ 7.609 \mathrm{E}-08 & 8.135 \mathrm{E}-08 \\ 1.970 \mathrm{E}-07 & 1.960 \mathrm{E}-07 \\ 2.456 \mathrm{E}-06 & 2.152 \mathrm{E}-06 \\ 7.342 \mathrm{E}-07 & 7.016 \mathrm{E}-07\end{array}$

150.0
$1.203 E-08$
$1.220 \mathrm{E}-08$
$1.263 \mathrm{E}-08$
$1.288 \mathrm{E}-08$
$1.318 \mathrm{E}-\mathrm{C} 8$
$1.375 \mathrm{E}-08$
$1.423 \mathrm{E}-08$
$1.565 \mathrm{E}-08$
$1.790 \mathrm{E}-08$
$2.038 \mathrm{E}-08$
$2.765 \mathrm{E}-08$
$2.759 \mathrm{E}-08$
$3.903 \mathrm{E}-08$
$5.123 \mathrm{E}-08$
$8.59 \mathrm{E}-08$
$1.930 \mathrm{E}-07$
$1.691 \mathrm{E}-06$
$6.699 \mathrm{E}-07$

RANGE (METERS)

250.0

$1.348 \mathrm{E}-08$

$1.363 E-08$
$1.404 E-08$

$1.426 E-08$

$1.481 \mathrm{E}-08$

$1.529 E-08$

$1.599 \mathrm{E}-08$

$1.757 \mathrm{E}-08$

$1.999 \mathrm{E}-08$
$2.383 \mathrm{E}-08$

$2.383 \mathrm{E}-08$

$2.734 \mathrm{E}-08$
$3.337 \mathrm{E}-08$

$4.072 \mathrm{E}-08$

$5.693 \mathrm{E}-08$

$8.955 E-08$

$1.861 \mathrm{E}-07$

$1.337 \mathrm{E}-06$

$6.357 \mathrm{E}-07$

RANGE (METERS)

500.0

600.0

$1.157 \mathrm{E}-08$

1. $162 \mathrm{E}-08$

$1.181 \mathrm{E}-08$
$1.207 \mathrm{E}-08$

$1.207 E-08$

$1.301 \mathrm{E}-08$

$1.381 \mathrm{E}-08$

$1.381 \mathrm{E}-08$
$1.505 \mathrm{E}-08$

$1.683 \mathrm{E}-08$

$1.683 E-08$
$1.919 E-08$

$2.218 \mathrm{E}-08$

$2.611 E-08$

3.195E-C8

4. 2 COE-O 8

$0.127 \mathrm{E}-08$

$6.127 \mathrm{E}-08$
$1.031 \mathrm{E}-07$

$3.281 E-07$

$9.550 E-09$

$9.589 \mathrm{E}-\mathrm{C} 9$
$9.734 \mathrm{E}-09$

$9.950 E-C 9$

$1.029 E-C 8$

1.074E-C8

$1.141 E-C 8$

1. $382 \mathrm{E}-08$

$1.568 \mathrm{E}-08$

$1.803 \mathrm{E}-\mathrm{C} 8$

$2.115 E-C 8$

$2.577 E-08$
$3.351 E-08$

4. $350 E-C 8$

$4.780 E-C 8$
$7.734 E-08$

$2.092 E-07$

$2.792 \mathrm{E}-\mathrm{C} 7$

$$
\begin{aligned}
& \text { RANGE } \\
& 900.0
\end{aligned}
$$

1200.0

$4.447 \mathrm{E}-09$

$4.462 \mathrm{E}-09$
$4.521 \mathrm{E}-09$

$4.625 \mathrm{E}-09$

$4.784 \mathrm{E}-09$

$5.004 E-C 9$

$5.763 E-09$

$6.359 \mathrm{E}-09$

$7.132 E-09$

$8.125 \mathrm{E}-09$

$9.138 \mathrm{E}-08$

$1.441 \mathrm{E}-08$

$1.954 \mathrm{E}-08$

$2.896 \mathrm{E}-08$

$5.665 \mathrm{E}-08$

$1.740 E-09$

$1.746 \mathrm{E}-09$

$1.768 E-09$

$1.809 \mathrm{E}-09$

$1.871 E-09$
$1.959 E-09$

$2.082 \mathrm{E}-09$

$2.082 \mathrm{E}-09$

$2.468 E-09$

$2.752 \mathrm{E}-09$

$3.116 \mathrm{E}-09$

$3.603 E-09$

$4.294 \mathrm{E}-09$
$5.339 \mathrm{E}-09$

7.000E-09
$9.786 E-09$

$7.000 E-09$
$9.786 E-09$

$1.599 \mathrm{E}-08$

$1.161 E-07$

$4.275 E-08$
$1.416 \mathrm{E}-08$

$1.429 E-08 \quad 1.438 E-08 \quad 1.341 E-08$

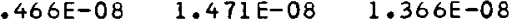

$\begin{array}{lll}1.492 \mathrm{E}-08 & 1.499 \mathrm{E}-08 & 1.395 \mathrm{E}-08\end{array}$

$\begin{array}{lll}1.547 \mathrm{E}-08 & 1.553 \mathrm{E}-08 & 1.444 \mathrm{E}-08 \\ 1.601 \mathrm{E}-08 & 1.610 \mathrm{E}-08 & 1.500 \mathrm{E}-08\end{array}$

$1.686 \mathrm{E}-08 \quad 1.7 \mathrm{COE}-08 \quad 1.590 \mathrm{E}-08$

$1.841 \mathrm{E}-08 \quad 1.856 \mathrm{E}-08 \quad 1.735 \mathrm{E}-08$
0.08

$2.091 \mathrm{E}-08 \quad 2.100 \mathrm{E}-08 \quad 1.951 E-08$

$2.481 E-08 \quad 2.422 E-08 \quad 2.241 E-08$

$2.825 \mathrm{E}-08 \quad 2.860 \mathrm{E}-08 \quad 2.6 \mathrm{C} 3 \mathrm{E}-08$

$3.448 \mathrm{E}-08 \quad 3.375 \mathrm{E}-08 \quad 3.077 \mathrm{E}-08$

4.191E-08 4.188E-08 3.783E-08

$5.038 \mathrm{E}-08 \quad 5.036 \mathrm{E}-08$

$\begin{array}{lll}9.000 E-08 & 8.713 E-08 & 7.536 E-08\end{array}$

$1.052 E-06$
$1.304 E-07$

5.935E-07 5.474E-07 4.513E-07

1500.0

1800.0 
COSINE

-1. COONOE OO $-9.89401 \mathrm{E}-01$ $-9.44575 \mathrm{E}-0$ $-8.65631 E-01$ $-7.55044 \mathrm{E}-\mathrm{O}$ $-6.17876 E-0$ $-4.58017 \mathrm{E}-01$ $-2.81605 E-O$ $-9.50125 \mathrm{E}-02$ $9.50125 \mathrm{E}-0$ $4.58017 \mathrm{E}-\mathrm{O}$ $6.17876 \mathrm{E}-\mathrm{Cl}$ $7.55044 \mathrm{E}-01$ $8.65631 E-n$ $9.84575 \mathrm{E}-01$

TOTAL

COSINE

$-1.00000 \mathrm{E} 00$ $-9.89401 \mathrm{E}-0$ $-9.44575 E-01$ $-8.65631 \mathrm{E}-0$ $-7.55044 \mathrm{E}-0$ $-6.17876 \mathrm{E}-0$ $-4.58017 \mathrm{E}-0$ $-2.81605 \mathrm{E}-0$ - $9.50125 E-02$ $9.50125 \mathrm{E}-02$ 2.81605E-01 4.58017E-O1 $6.17876 \mathrm{E}-\mathrm{C}$ $7.55044 \mathrm{E}-0$ $8.65631 \mathrm{E}-0$ $9.445401 \mathrm{E}-01$

TOTAL
75.0 $4.351 \mathrm{E}-11$ $4.752 \mathrm{E}-1$ $4.924 \mathrm{E}-11$ $5.037 \mathrm{E}-11$ $5.144 \mathrm{E}-11$ $5.752 \mathrm{E}-11$ $6.787 \mathrm{E}-11$ $1.159 \mathrm{E}-1 \mathrm{C}$ $1.077 E-10$ $1.375 \mathrm{E}-10$ $1.698 \mathrm{E}-10$ $3.142 \mathrm{E}-10$ $5.14 \mathrm{CE}-10$ $1.419 \mathrm{E}-09$ $1.871 \mathrm{E}-08$

$5.211 \mathrm{E}-09$

500.0

$4.257 \mathrm{E}-1$ $4.287 \mathrm{E}-11$ $4.383 \mathrm{E}-11$ $4.481 \mathrm{E}-1$ $4.652 \mathrm{E}-1$ $4.840 E-11$ . $170 \mathrm{E}-1$ $5.747 \mathrm{E}-1$ $0.634 \mathrm{E}-1$ $7.828 \mathrm{E}-11$ $.323 \mathrm{E}-11$ $1.130 E-10$ $1.442 \mathrm{E}-10$ $2 . C 32 E-10$ $3.263 E-10$ $6.111 \mathrm{E}-10$

$1.769 \mathrm{E}-09$
100.0 $4.946 \mathrm{E}-11$ $5.552 \mathrm{E}-11$ 5. $766 \mathrm{E}-11$ $5.863 E-11$ $6.557 E-11$ $7.759 \mathrm{E}-11$ $9.045 \mathrm{E}-1$ $1.291 E-10$ $1.916 \mathrm{E}-10$ $2.768 E-10$ $5.448 E-10$ $.376 \mathrm{E}-\mathrm{C} 9$
$.633 \mathrm{E}-08$

$4.831 E-09$

600.0

$3.404 E-11$ $3.424 E-11$ 3. $491 E-11$ $3.570 \mathrm{E}-1$ 3. $703 \mathrm{E}-1$ $3.862 \mathrm{E}-11$ 4. $131 E-11$ 4.577E-1 $5.243 E-11$ $6.131 E-11$ $7.255 \mathrm{E}-11$ $8.765 \varepsilon-11$ $1.116 E-10$ $1.559 \varepsilon-10$ $2.455 \mathrm{E}-10$ $4.437 E-10$

$1.302 \mathrm{E}-09$

\begin{tabular}{|c|c|c|c|c|}
\hline 150.0 & $\begin{array}{c}\text { IGE IMETERS } \\
200.0\end{array}$ & 250.0 & 300.0 & 400.0 \\
\hline & $6.024 E-11$ & $6.029 E-11$ & $5.838 E-11$ & $5.127 \mathrm{E}-11$ \\
\hline $825 E-11$ & $6.127 \mathrm{E}-11$ & $6.114 \mathrm{E}-11$ & $5.907 \mathrm{E}-11$ & $5.172 \mathrm{E}-11$ \\
\hline & $6.396 \mathrm{E}-11$ & $6.348 \mathrm{E}-11$ & $6.106 \mathrm{E}-11$ & $5.312 \mathrm{E}-11$ \\
\hline $239 \mathrm{E}-11$ & $6.480 \mathrm{E}-11$ & $6.452 \mathrm{E}-11$ & $6.220 \mathrm{E}-11$ & $5.424 E-11$ \\
\hline $366 \mathrm{E}-11$ & $6.770 E-11$ & $6.730 \mathrm{E}-11$ & $6.479 \mathrm{E}-11$ & $5.639 \mathrm{E}-11$ \\
\hline & $6.957 E-11$ & $6.933 \mathrm{E}-11$ & $6.691 \mathrm{E}-11$ & $5.848 \mathrm{E}-11$ \\
\hline $824 E-11$ & $7.253 E-11$ & $7.312 \mathrm{E}-11$ & $7.085 \mathrm{E}-11$ & $6.228 E-11$ \\
\hline & $8.150 E-11$ & $8.139 E-11$ & $7.895 \mathrm{E}-11$ & $6.938 E-11$ \\
\hline$E-11$ & $9.588 E-11$ & $9.582 \mathrm{E}-11$ & $9.262 \mathrm{E}-11$ & $8.076 E-11$ \\
\hline-10 & $1.196 E-10$ & $1.193 \mathrm{E}-1 \mathrm{C}$ & $1.108 \mathrm{E}-10$ & $9.633 \mathrm{E}-11$ \\
\hline $2 E-10$ & $1.396 \mathrm{E}-10$ & $1.379 \mathrm{E}-10$ & $1.355 \mathrm{E}-10$ & $1.154 \mathrm{E}-10$ \\
\hline $29 E-10$ & $1.760 \mathrm{E}-10$ & $1.747 \mathrm{E}-10$ & $1.638 E-10$ & $1.403 E-10$ \\
\hline $84 E-10$ & $2.190 E-10$ & $2.170 E-10$ & $2.106 E-10$ & $1.793 E-10$ \\
\hline $935 E-10$ & $3.243 E-10$ & $3.186 \mathrm{E}-10$ & $3.007 \mathrm{E}-10$ & $2.549 E-10$ \\
\hline$F-1$ & $5.456 \mathrm{E}-10$ & $5.346 \mathrm{E}-10$ & $5.056 \mathrm{E}-10$ & $4.180 \mathrm{E}-10$ \\
\hline & $1.235 E-09$ & $1.141 \mathrm{E}-09$ & $1.037 \mathrm{E}-09$ & $8.154 \mathrm{E}-10$ \\
\hline & $9.954 E-09$ & $7.751 \mathrm{E}-09$ & $6.050 E-09$ & $3.693 E-09$ \\
\hline $4.362 \mathrm{E}-09$ & $3.934 E-09$ & $3.505 \mathrm{E}-09$ & $3 .(96 \mathrm{E}-09$ & $2.366 E-09$ \\
\hline
\end{tabular}

RANGE (METERS)

$900.0 \quad 1200.0$

1500.0

1800.0

$\begin{array}{llll}1.507 \mathrm{E}-11 & 5.793 \mathrm{E}-12 & 2.031 \mathrm{E}-12 & 6.667 \mathrm{E}-13 \\ 1.513 \mathrm{E}-11 & 5.811 \mathrm{E}-12 & 2.037 \mathrm{E}-12 & 6.686 \mathrm{E}-13 \\ 1.535 \mathrm{E}-11 & 5.885 \mathrm{E}-12 & 2.061 \mathrm{E}-12 & 6.763 \mathrm{E}-13 \\ 1.570 \mathrm{E}-11 & 6.021 \mathrm{E}-12 & 2.107 \mathrm{E}-12 & 6.910 \mathrm{E}-13 \\ 1.625 \mathrm{E}-11 & 6.22 \mathrm{CE}-12 & 2.175 \mathrm{E}-12 & 7.131 \mathrm{E}-13 \\ 1.700 \mathrm{E}-11 & 6.509 \mathrm{E}-12 & 2.275 \mathrm{E}-12 & 7.454 \mathrm{E}-13 \\ 1.817 \mathrm{E}-11 & 6.939 \mathrm{E}-12 & 2.418 \mathrm{E}-12 & 7.905 \mathrm{E}-13 \\ 1.993 \mathrm{E}-11 & 7.549 \mathrm{E}-12 & 2.617 \mathrm{E}-12 & 8.523 \mathrm{E}-13 \\ 2.241 \mathrm{E}-11 & 8.387 \mathrm{E}-12 & 2.884 \mathrm{E}-12 & 9.340 \mathrm{E}-13 \\ 2.568 \mathrm{E}-11 & 9.484 \mathrm{E}-12 & 3.232 \mathrm{E}-12 & 1.041 \mathrm{E}-12 \\ 2.992 \mathrm{E}-11 & 1.092 \mathrm{E}-11 & 3.694 \mathrm{E}-12 & 1.182 \mathrm{E}-12 \\ 3.580 \mathrm{E}-11 & 1.295 \mathrm{E}-11 & 4.346 \mathrm{E}-12 & 1.383 \mathrm{E}-12 \\ 4.508 \mathrm{E}-11 & 1.611 \mathrm{E}-11 & 5.348 \mathrm{E}-12 & 1.687 \mathrm{E}-12 \\ 6.138 \mathrm{E}-11 & 2.144 \mathrm{E}-11 & 6.987 \mathrm{E}-12 & 2.172 \mathrm{E}-12 \\ 9.182 \mathrm{E}-11 & 3.084 \mathrm{E}-11 & 9.754 \mathrm{E}-12 & 2.965 \mathrm{E}-12 \\ 1.524 \mathrm{E}-10 & 4.807 \mathrm{E}-11 & 1.452 \mathrm{E}-11 & 4.264 \mathrm{E}-12 \\ 3.479 \mathrm{E}-10 & 9.032 \mathrm{E}-11 & 2.414 \mathrm{E}-11 & 6.550 \mathrm{E}-12 \\ 4.828 \mathrm{E}-10 & 1.661 \mathrm{E}-10 & 5.409 \mathrm{E}-11 & 1.689 \mathrm{E}-11\end{array}$


4 PI R**2 CONCRETE KERMA (NEUTRCNS) (CM**2 ERGS/GRAM/STERADIAN/SOURCE NEUTRON )
12. 200 TO 15.COO MEV NEUTRON SOURCE

\section{COSINE}

$-1.00000 \mathrm{E} 00$ $-9.89401 \mathrm{E}-01$ $-8.65631 \mathrm{E}-01$ $-7.55044 E-01$ $-6.17876 \mathrm{E}-01$ $-4.58017 \mathrm{E}-01$ $-9.8605 \mathrm{E}-01$ $9.50125 \mathrm{E}-02$ $2.81605 E-01$ $4.58017 E-01$ $6.17876 E-01$ $7.55044 \mathrm{E}-01$ $8.65631 \mathrm{E}-01$ $9.44575 \mathrm{E}-01$

$$
\text { TOTAL }
$$

COSINE

$-1 . C C O C O E 0 O$ $-9.89401 E-01$ $-9.44575 \varepsilon-01$ $-8.65631 \mathrm{E}-01$ $-7.55044 E-01$ $-6.17876 E-01$ $-4.58017 E-01$ $-2.81605 E-01$ $9.50125 E-02$ $9.50125 \varepsilon-02$ 2.81605E-01 $6.17876 E-01$ $6.17876 E-01$ $8.65631 E-01$ $9.44575 \mathrm{E}-01$ $9.89401 \mathrm{E}-01$

TOTAL
75.0

$1.136 \mathrm{E}-\mathrm{CQ}$ $1.180 \mathrm{E}-09$ $1.281 \mathrm{E}-09$ $1.354 \mathrm{E}-09$ $1.361 \mathrm{E}-09$ $1.400 E-09$ $1.416 \mathrm{E}-09$ $1.579 E-C 9$ $1.867 \mathrm{E}-09$ $3.195 \mathrm{E}-09$ $2.993 E-09$ $3.847 \mathrm{E}-09$ 4.789E-09 $9.211 \mathrm{E}-\mathrm{C} 9$ $1.516 \mathrm{E}-08$ $4.346 \mathrm{E}-08$ $6.0 C 3 E-C 7$

1.610 E-07

500.0

$1.453 E-09$ $1.464 \mathrm{E}-09$ $1.50 \mathrm{CE}-09$ $1.539 \mathrm{E}-09$ $1.600 \mathrm{E}-09$ $1.662 \mathrm{E}-09$ $1.765 \mathrm{E}-09$ $1.941 \mathrm{E}-09$ $2.214 E-09$ $2.581 \mathrm{E}-\mathrm{Cg}$ $3.037 \mathrm{E}-\mathrm{C}$ $3.624 E-C 9$ $4.528 \mathrm{E}-\mathrm{C} 9$ $9.730 E-09$ $9.730 E-09$ $1.792 \mathrm{E}-08$ 6.856E-08

$5.537 E-08$
160.0

$1.334 E-09$ $1.379 \mathrm{E}-\mathrm{C} 9$ $1.565 \mathrm{E}-09$ $1.579 \mathrm{E}-09$ $1.624 E-09$ $1.653 \mathrm{E}-09$ $1.840 \mathrm{E}-\mathrm{C} 9$ $2.177 E-09$ $2.641 \mathrm{E}-09$ 3. 595E-09 $3.725 \mathrm{E}-09$ $5.334 \mathrm{E}-09$ $7.851 \mathrm{E}-09$ $1.600 E-C 8$ 4. $158 E-0$ $5.219 E-07$

$1.482 \mathrm{E}-07$

600.0

$1.185 \mathrm{E}-\mathrm{C9}$ $1.193 \mathrm{E}-\mathrm{CI}$ $1.218 \mathrm{E}-09$ $1.249 E-09$
$1.297 E-09$ $1.297 \mathrm{E}-09$
$1.351 \mathrm{E}-09$ $1.351 \mathrm{E}-09$ $1.437 \mathrm{E}-09$ $1.578 \mathrm{E}-09$ $1.787 \mathrm{E}-09$ $2.065 \mathrm{E}-09$ $2.414 E-C 9$ 2.869E-O 3.568E-09 $7.375 \mathrm{E}-09$ 1. $301 \mathrm{E}-0$ 1.301E-08

$4.125 E-08$

\begin{tabular}{ccccc}
\multicolumn{5}{c}{ RANGE (METERS) } \\
150.0 & 200.0 & 250.0 & 300.0 & 400.0 \\
$1.626 \mathrm{E}-09$ & $1.793 \mathrm{E}-09$ & $1.860 \mathrm{E}-09$ & $1.853 \mathrm{E}-09$ & $1.700 \mathrm{E}-09$ \\
$1.669 \mathrm{E}-09$ & $1.830 \mathrm{E}-09$ & $1.890 \mathrm{E}-09$ & $1.878 \mathrm{E}-09$ & $1.716 \mathrm{E}-09$ \\
$1.778 \mathrm{E}-09$ & $1.929 \mathrm{E}-09$ & $1.976 \mathrm{E}-09$ & $1.952 \mathrm{E}-09$ & $1.768 \mathrm{E}-09$ \\
$1.833 \mathrm{E}-09$ & $1.972 \mathrm{E}-09$ & $2.024 \mathrm{E}-09$ & $2.000 \mathrm{E}-09$ & $1.813 \mathrm{E}-09$ \\
$1.885 \mathrm{E}-09$ & $2.075 \mathrm{E}-09$ & $2.122 \mathrm{E}-09$ & $2.091 \mathrm{E}-09$ & $1.889 \mathrm{E}-09$ \\
$1.974 \mathrm{E}-09$ & $2.131 \mathrm{E}-09$ & $2.183 \mathrm{E}-09$ & $2.157 \mathrm{E}-09$ & $1.956 \mathrm{E}-09$ \\
$2.004 \mathrm{E}-09$ & $2.203 \mathrm{E}-09$ & $2.285 \mathrm{E}-09$ & $2.267 \mathrm{E}-09$ & $2.069 \mathrm{E}-09$ \\
$2.233 \mathrm{E}-09$ & $2.453 \mathrm{E}-09$ & $2.517 \mathrm{E}-09$ & $2.499 \mathrm{E}-09$ & $2.280 \mathrm{E}-09$ \\
$2.6277 \mathrm{E}-09$ & $2.865 \mathrm{E}-09$ & $2.935 \mathrm{E}-09$ & $2.900 \mathrm{E}-09$ & $2.621 \mathrm{E}-09$ \\
$3.067 \mathrm{E}-\mathrm{C} 9$ & $3.552 \mathrm{E}-09$ & $3.620 \mathrm{E}-09$ & $3.442 \mathrm{E}-09$ & $3.092 \mathrm{E}-09$ \\
$4.429 \mathrm{E}-09$ & $4.147 \mathrm{E}-09$ & $4.180 \mathrm{E}-09$ & $4.170 \mathrm{E}-09$ & $3.664 \mathrm{E}-09$ \\
$4.244 \mathrm{E}-09$ & $5.173 \mathrm{E}-09$ & $5.222 \mathrm{E}-09$ & $4.988 \mathrm{E}-09$ & $4.395 \mathrm{E}-09$ \\
$6.466 \mathrm{E}-09$ & $6.397 \mathrm{E}-09$ & $6.434 \mathrm{E}-09$ & $6.319 \mathrm{E}-09$ & $5.513 \mathrm{E}-09$ \\
$8.496 \mathrm{E}-09$ & $9.386 \mathrm{E}-09$ & $9.321 \mathrm{E}-09$ & $8.893 \mathrm{E}-09$ & $7.672 \mathrm{E}-09$ \\
$1.576 \mathrm{E}-08$ & $1.59 \mathrm{E}-08$ & $1.563 \mathrm{E}-08$ & $1.483 \mathrm{E}-08$ & $1.237 \mathrm{E}-08$ \\
$3.902 \mathrm{E}-08$ & $3.644 \mathrm{E}-08$ & $3.358 \mathrm{E}-08$ & $3.047 \mathrm{E}-08$ & $2.393 \mathrm{E}-08$ \\
$4.035 \mathrm{E}-07$ & $3.134 \mathrm{E}-07$ & $2.422 \mathrm{E}-07$ & $1.877 \mathrm{E}-07$ & $1.130 \mathrm{E}-07$ \\
$1.327 \mathrm{E}-07$ & $1.195 \mathrm{E}-07$ & $1.066 \mathrm{E}-07$ & $9.457 \mathrm{E}-08$ & $7.310 \mathrm{E}-08$
\end{tabular}

RANGE (METERS)

$5.382 \mathrm{E}-10$ $5.492 \mathrm{E}-10$ $5.628 \mathrm{E}-10$ $5.833 E-10$ $6.104 \mathrm{E}-10$ $6.507 \mathrm{E}-10$ $7.099 E-10$ $7.924 E-10$ $9.002 E-10$ $1.038 \mathrm{E}-09$ $1.222 \mathrm{E}-09$ $1.498 \mathrm{E}-09$ 2.814E-0 $4.485 \mathrm{E}-09$ $4.485 E-C 9$ 1. $579 \mathrm{E}-08$

\section{$2.074 \mathrm{E}-10$} $2.110 \mathrm{E}-10$ $2.163 \mathrm{E}-10$ $2.240 \mathrm{E}-10$ $2.348 \mathrm{E}-10$ $2.503 \mathrm{E}-10$ $2.718 \mathrm{E}-10$ $3.009 E-10$ $3.386 E-10$ $3.870 E-10$ $4.523 E-10$ $5.482 E-10$ . ..601E-10 $2.578 \mathrm{E}-09$

5. $542 E-09$
1500.0

$7.230 \mathrm{E}-11$ $7.254 \mathrm{E}-11$ $7.351 \mathrm{E}-11$ $7.534 \mathrm{E}-11$ $7.801 E-11$ 8.183E-11 $.713 E-11$ $.438 E-11$ 1.039E- 10 作 . $4.327 E-10$ $6.848 E-10$ 1.827 E-09
1800.0

2. $357 \mathrm{E}-11$ $2.364 \mathrm{E}-11$
$2.396 \mathrm{E}-11$ $2.454 \mathrm{E}-11$ $2.542 E-11$ $2.838 \mathrm{E}-11$ $3.068 \mathrm{E}-11$ $3.368 \mathrm{E}-11$ $4.254 \mathrm{E}-11$ $4.923 E-11$ $5.869 \mathrm{E}-11$ 9.429E-11 $1.280 \mathrm{E}-10$ $858 \mathrm{E}-10$

$5.746 \mathrm{E}-10$ 
4 PI R*\#2 AIR KERMA (NEUTRONS)

(CM* 2 ERGS/GRAM/STERADIAN/SOURCE NEUTRON)
12.200 TO 15.0CO MEV NEUTRON SOURCE

\section{COSINE \\ $-1.000 C O E 0 O$ $-9.89401 \mathrm{E}-0$ $-8.65631 \mathrm{E}-0$ $-8.65631 \mathrm{E}-01$ $-6.17876 \mathrm{E}-01$ $-4.58017 \mathrm{E}-0$ $-4.58017 \mathrm{E}-01$ $-9.50125 \mathrm{E}-02$ $-9.50125 \mathrm{E}-02$
$9.50125 \mathrm{E}-02$ $9.50125 \mathrm{E}-02$
$2.81605 \mathrm{E}-0$ $2.81605 \mathrm{E}-01$ $6.17876 \mathrm{E}-01$ $7.55044 \mathrm{E}-01$ $8.65631 E-01$ $9.44575 \mathrm{E}-01$}

TOTAL

COSINE

$-1.000 C D O E O O$ $-9.89401 E-01$ $-9.44575 \mathrm{E}-01$ $-8.65631 \mathrm{E}-01$ $-6.17876 \mathrm{E}-0$ $-4.58017 \mathrm{E}-01$ $-2.81605 E-0$ $-9.50125 \mathrm{E}-02$ $9.50125 \mathrm{E}-0$ $2.81605 \mathrm{E}-0$ 4.58017E-0 $6.17876 \mathrm{E}-0$ $7.55044 \mathrm{E}-0$ $8.65631 \mathrm{E}-0$ $9.44575 \mathrm{E}-0$ $9.89401 \mathrm{E}-01$

TOTAL
75.0 $1.720 \mathrm{E}-09$ $1.794 \mathrm{E}-09$
$1.963 \mathrm{E}-09$ $1.963 \mathrm{E}-09$ $2.084 E-09$ . 2.174E-09 $2.437 \mathrm{E}-09$ $2.437 E-09$ $5.146 E-09$ $4.751 \mathrm{E}-09$ $4.751 E-09$ $6.131 \mathrm{E}-09$
$7.751 \mathrm{E}-09$ $1.505 \mathrm{E}-08$ $2.492 \mathrm{E}-08$ $7.225 \mathrm{E}-08$ $7.225 \mathrm{E}-08$ $2.661 \mathrm{E}-07$

500.0

$1.830 E-09$ $1.847 \mathrm{E}-09$ $1.901 \mathrm{E}-09$ $1.952 \mathrm{E}-09$ $2.034 \mathrm{E}-\mathrm{CS}$ $2.111 \mathrm{E}-0$ $2.246 \mathrm{E}-\mathrm{C} 9$ $2.494 \mathrm{E}-0$ $2.891 \mathrm{E}-09$ $3.432 E-=9$ $4.107 E-C 9$ $4.983 \mathrm{E}-\mathrm{C}$ $6.372 \mathrm{E}-09$ $9.049 \mathrm{E}-\mathrm{C} 9$ $1.477 E-0$ $2.828 \mathrm{E}-0 \mathrm{~B}$ $1.127 \mathrm{E}-07$

$8.079 E-08$
100.0 .C53E-09 $2.235 E-09$ . $381 \mathrm{E}-\mathrm{C} 9$ $2.449 \mathrm{E}-09$ $2.488 \mathrm{E}-0$ $2.488 E-C 9$
$2.786 \mathrm{E}-\mathrm{C} 9$ $3.333 \mathrm{E}-\mathrm{C}$ $4.062 \mathrm{E}-09$ $5.665 \mathrm{E}-\mathrm{C}$ $5.665 \mathrm{E}-\mathrm{C} 9$
$5.845 \mathrm{E}-09$ $8.501 \mathrm{E}-09$ $1.259 \mathrm{E}-\mathrm{C}$ $2.628 \mathrm{E}-\mathrm{C} 8$ $6.878 \mathrm{E}-0$ $8.878 E-08$ 2.434E-07

600.0

$1.469 \mathrm{E}-\mathrm{Cg}$ $1.480 \mathrm{E}-\mathrm{C} 9$ $1.556 \mathrm{E}-\mathrm{C}$ $1.618 \mathrm{E}-\mathrm{C} 9$ $1.683 E-C$ $1.795 \mathrm{E}-09$ $1.987 \mathrm{E}-0$ $2.284 \mathrm{E}-0$ $2.684 E-0$ $3.188 \mathrm{E}-0$ $3.856 \mathrm{E}-\mathrm{C}$ $4.914 \mathrm{E}-\mathrm{C}$ $6.905 \mathrm{E}-0$ $1.101 \mathrm{E}-\mathrm{C}$ $2.029 \mathrm{E}-0$ $6.849 E-C 8$ $5.871 E-08$

\begin{tabular}{ccccc}
\multicolumn{5}{c}{ RANGE (METERS) } \\
150.0 & 200.0 & 250.0 & 300.0 & 400.0 \\
$2.326 \mathrm{E}-09$ & $2.493 \mathrm{E}-09$ & $2.524 \mathrm{E}-09$ & $2.464 \mathrm{E}-09$ & $2.189 \mathrm{E}-09$ \\
$2.397 \mathrm{E}-09$ & $2.554 \mathrm{E}-09$ & $2.574 \mathrm{E}-09$ & $2.505 \mathrm{E}-09$ & $2.216 \mathrm{E}-09$ \\
$2.578 \mathrm{E}-09$ & $2.716 \mathrm{E}-09$ & $2.713 \mathrm{E}-09$ & $2.623 \mathrm{E}-09$ & $2.296 \mathrm{E}-09$ \\
$2.666 \mathrm{E}-09$ & $2.781 \mathrm{E}-09$ & $2.784 \mathrm{E}-09$ & $2.693 \mathrm{E}-09$ & $2.358 \mathrm{E}-09$ \\
$2.745 \mathrm{E}-09$ & $2.942 \mathrm{E}-09$ & $2.933 \mathrm{E}-09$ & $2.828 \mathrm{E}-09$ & $2.465 \mathrm{E}-09$ \\
$2.882 \mathrm{E}-09$ & $3.018 \mathrm{E}-09$ & $3.014 \mathrm{E}-09$ & $2.912 \mathrm{E}-09$ & $2.550 \mathrm{E}-09$ \\
$2.916 \mathrm{E}-09$ & $3.115 \mathrm{E}-09$ & $3.155 \mathrm{E}-09$ & $3.064 \mathrm{E}-09$ & $2.701 \mathrm{E}-09$ \\
$3.275 \mathrm{E}-09$ & $3.502 \mathrm{E}-09$ & $3.504 \mathrm{E}-09$ & $3.408 \mathrm{E}-09$ & $3.005 \mathrm{E}-09$ \\
$3.905 \mathrm{E}-09$ & $4.151 \mathrm{E}-09$ & $4.156 \mathrm{E}-09$ & $4.024 \mathrm{E}-09$ & $3.516 \mathrm{E}-09$ \\
$4.539 \mathrm{E}-09$ & $5.285 \mathrm{E}-09$ & $5.277 \mathrm{E}-09$ & $4.856 \mathrm{E}-09$ & $4.229 \mathrm{E}-09$ \\
$6.867 \mathrm{E}-09$ & $6.156 \mathrm{E}-09$ & $6.074 \mathrm{E}-09$ & $6.005 \mathrm{E}-09$ & $5.094 \mathrm{E}-09$ \\
$6.471 \mathrm{E}-09$ & $7.857 \mathrm{E}-09$ & $7.788 \mathrm{E}-09$ & $7.262 \mathrm{E}-09$ & $6.209 \mathrm{E}-09$ \\
$1.013 \mathrm{E}-08$ & $9.767 \mathrm{E}-09$ & $9.659 \mathrm{E}-09$ & $9.382 \mathrm{E}-09$ & $7.951 \mathrm{E}-09$ \\
$1.343 \mathrm{E}-08$ & $1.470 \mathrm{E}-08$ & $1.440 \mathrm{E}-08$ & $1.354 \mathrm{E}-08$ & $1.141 \mathrm{E}-08$ \\
$2.551 \mathrm{E}-08$ & $2.543 \mathrm{E}-08$ & $2.476 \mathrm{E}-08$ & $2.331 \mathrm{E}-08$ & $1.909 \mathrm{E}-08$ \\
$6.417 \mathrm{E}-08$ & $5.960 \mathrm{E}-08$ & $5.461 \mathrm{E}-08$ & $4.924 \mathrm{E}-08$ & $3.822 \mathrm{E}-08$ \\
$6.735 \mathrm{E}-07$ & $5.226 \mathrm{E}-07$ & $4.033 \mathrm{E}-07$ & $3.119 \mathrm{E}-07$ & $1.869 \mathrm{E}-07$ \\
$2.148 \mathrm{E}-07$ & $1.906 \mathrm{E}-07$ & $1.675 \mathrm{E}-07$ & $1.462 \mathrm{E}-07$ & $1.097 \mathrm{E}-07$
\end{tabular}

RANGE (METERS)

$900.0 \quad 1200.0$

1500.0

1800.0

$\begin{array}{llll}6.522 \mathrm{E}-10 & 2.504 \mathrm{E}-10 & 8.758 \mathrm{E}-11 & 2.870 \mathrm{E}-11\end{array}$ $6.663 \mathrm{E}-10 \quad 2.547 \mathrm{E}-10 \quad 8.894 \mathrm{E}-11 \quad 2.812 \mathrm{E}-11$ $6.826 \mathrm{E}-10 \quad 2.608 \mathrm{E}-10 \quad 9.097 \mathrm{E}-11 \quad 2.976 \mathrm{E}-11$ $7.072 \mathrm{E}-10 \quad 2.695 \mathrm{E}-10 \quad 9.392 \mathrm{E}-11 \quad 3.072 \mathrm{E}-11$ $7.388 \mathrm{E}-10 \quad 2.818 \mathrm{E}-10 \quad 9.822 \mathrm{E}-11 \quad 3.21 \mathrm{CE}-11$ $7.883 \mathrm{E}-10 \quad 3.002 \mathrm{E}-10 \quad 1.043 \mathrm{E}-1 \mathrm{C} \quad 3.403 \mathrm{E}-11$ $8.643 \mathrm{E}-10 \quad 3.264 \mathrm{E}-10 \quad 1.129 \mathrm{E}-10 \quad 3.669 \mathrm{E}-11$ $9.735 \mathrm{E}-10 \quad 3.630 \mathrm{E}-10 \quad 1.245 \mathrm{E}-10 \quad 4.022 \mathrm{E}-11$ $1.118 \mathrm{E}-09 \quad 4.112 \mathrm{E}-10 \quad 1.397 \mathrm{E}-10 \quad 4.486 \mathrm{E}-11$ $1.306 \mathrm{E}-09 \quad 4.742 \mathrm{E}-10 \quad 1.598 \mathrm{E}-10 \quad 5.102 \mathrm{E}-11$ $1.563 E-09 \quad 5.624 \mathrm{E}-10 \quad 1.879 \mathrm{E}-10 \quad 5.962 \mathrm{E}-11$ $1.967 \mathrm{E}-09 \quad 6.982 \mathrm{E}-10 \quad 2.306 \mathrm{E}-10 \quad 7.246 \mathrm{E}-11$ $2.680 \mathrm{E}-09 \quad 9.264 \mathrm{E}-10 \quad 2.995 \mathrm{E}-10 \quad 9.260 \mathrm{E}-11$ $4.027 \mathrm{E}-09 \quad 1.329 \mathrm{E}-09 \quad 4.151 \mathrm{E}-10 \quad 1.250 \mathrm{E}-10$ $6.743 \mathrm{E}-09 \quad 2.072 \mathrm{E}-09 \quad 6.134 \mathrm{E}-10 \quad 1.776 \mathrm{E}-10$ $1.603 \mathrm{E}-08 \quad 3.969 \mathrm{E}-09 \quad 1.023 \mathrm{E}-09 \quad 2.706 \mathrm{E}-10$ $2.124 \mathrm{E}-08 \quad 7.195 \mathrm{E}-09 \quad 2.322 \mathrm{E}-09 \quad 7.208 \mathrm{E}-10$ 

4 PI R*\#2 ICNIZING SILICON KERMA (NEUTRONS)
(CM\#\#2 ERGS/GRAM/STERADIAN/SOURCE NEUTRON)
COSINE

$-1.00000 \mathrm{E} O \mathrm{C}$ $-9.89401 E-01$ $-9.44575 E-01$ $-8.65631 \mathrm{E}-01$ $-7.55044 \mathrm{E}-\mathrm{Cl}$ $-4.58017 E-01$ $-2.81605 \mathrm{E}-01$ $-9.5 \mathrm{C} 125 \mathrm{E}-02$ $9.50125 \mathrm{E}-02$ $2.81605 E-01$ $6.17876 \mathrm{E}-01$ $6.17876 \mathrm{E}-1$ $8.65631 \mathrm{E}-01$ $0.44575 \mathrm{E}-01$ $98901 E-01$

TOTAL

CCSINE

$-1.00000 \mathrm{E} 00$ $-9.89401 \mathrm{E}-01$ $-9.44575 E-01$ $-7.55044 E-01$ $-6.17876 \mathrm{E}-01$ $-4.58017 \mathrm{E}-01$ $-2.81605 \mathrm{E}-01$ $-9.50125 E-02$ 9.50125E-02 $2.81605 \mathrm{E}-01$ 4. $58017 \mathrm{E}-\mathrm{Ol}$ $6.17876 \mathrm{E}-01$ $7.55044 \mathrm{E}-01$ $8.65631 \mathrm{E}-01$ $9.44575 \mathrm{E}-01$ $9.89401 E-01$

TOTAL
75.0

$5.683 E-10$ $5.883 \mathrm{E}-10$ $6.294 \mathrm{E}-10$ $6.474 \mathrm{E}-10$ $6.311 \mathrm{E}-10$ $6.384 E-10$ $6.418 \mathrm{E}-10$ $7.306 E-10$ $8.882 \mathrm{E}-10$ $1.683 E-C 9$ $1.519 \mathrm{E}-09$ $2.387 E-09$ $2.387 E-09$ $8.032 \mathrm{E}-09$ $2.340 E-C 8$ $3.253 \mathrm{E}-07$

$8.567 \mathrm{E}-08$

500.0

$3.0 C 9 \mathrm{E}-1 \mathrm{C}$ 3. $C 48 \mathrm{E}-1 \mathrm{C}$ $3.165 \mathrm{E}-1 \mathrm{C}$ $3.234 \mathrm{E}-1 \mathrm{C}$ $3.383 \mathrm{E}-10$ $3.504 \mathrm{E}-1 \mathrm{C}$ $3.802 \mathrm{E}-10$ $4.443 \mathrm{E}-10$ $5.5 \mathrm{C} 5 \mathrm{E}-10$ $6.933 \mathrm{E}-10$ $1.083 E-C 9$ $1.449 \mathrm{E}-09$ $2.223 \mathrm{E}-09$ $3.990 \mathrm{E}-09$ $8.350 E-09$ $3.610 E-08$

$2.041 E-08$
100.0

$6.097 E-10$ $6.300 E-10$ $6.740 E-10$ $6.922 \mathrm{E}-10$ $6.774 E-10$ $6.858 \mathrm{E}-10$ $6.346 E-10$ 9.745E-10 $9.745 E-10$ $1.176 \mathrm{E}-09$ $1.650 E-09$ $2.726 E-09$ $8.360 \mathrm{E}-09$ $2.226 \mathrm{E}-0 \mathrm{~B}$ 2. $827 \mathrm{E}-07$

7.747E-08

600.0

2. $179 \mathrm{E}-10$ 2. $279 E-10$ 2. $336 \mathrm{E}-10$ $2.444 E-10$ $2.548 \mathrm{E}-10$ $2.785 \mathrm{E}-10$ $3.255 E-10$ $4.010 \mathrm{E}-10$ $5.015 E-10$ $6.231 \mathrm{E}-10$ $7.830 \mathrm{E}-10$ $1.054 \mathrm{E}-09$ 1. $619 \mathrm{E}-09$ $2.873 \mathrm{E}-09$
$5.854 \mathrm{E}-09$ $5.854 \mathrm{E}-0$ 2.17.5E-08 $1.396 \mathrm{E}-08$

$\begin{array}{ccc} & \text { RANGE (METERS) } \\ 150.0 & 200.0 & 250.0 \\ 6.385 E-10 & 6.208 E-10 & 5.768 E-10 \\ 6.571 E-10 & 6.365 E-10 & 5.896 E-10 \\ 7.005 E-10 & 6.7511 E-10 & 6.223 E-10 \\ 7.059 E-10 & 6.745 E-10 & 6.256 E-10 \\ 7.095 E-10 & 7.055 E-10 & 6.544 E-10 \\ 7.390 E-10 & 7.146 E-10 & 6.651 E-10 \\ 7.440 E-10 & 7.378 E-10 & 7.003 E-10 \\ 8.599 E-10 & 8.584 E-10 & 8.048 E-10 \\ 1.063 E-09 & 1.061 E-09 & 1.003 E-09 \\ 1.314 E-09 & 1.380 E-09 & 1.309 E-09 \\ 2.069 E-09 & 1.685 E-09 & 1.585 E-09 \\ 1.676 E-09 & 2.14 C E-09 & 2.034 E-09 \\ 3.126 E-09 & 2.732 E-09 & 2.599 E-09 \\ 3.832 E-09 & 4.252 E-09 & 4.032 E-09 \\ 8.039 E-09 & 7.676 E-09 & 7.344 E-09 \\ 2.044 E-08 & 1.885 E-08 & 1.705 E-08 \\ 2.185 E-07 & 1.694 E-07 & 1.307 E-07 \\ 6.683 E-08 & 5.765 E-08 & 4.921 E-08\end{array}$

300.0

5. $216 E-10$ $5.319 E-10$ $5.59 \mathrm{CE}-10$ $5.647 \mathrm{E}-10$ $5.906 \mathrm{E}-10$ $6.025 E-10$ $6.393 E-10$ $7.390 E-10$ $9.218 \mathrm{E}-10$ $1.170 E-O 9$ $1.481 E-09$ $2.426 \mathrm{E}-09$ $2.426 \mathrm{E}-09$ $6.776 E-09$ $1.521 \mathrm{E}-0$
$1.009 \mathrm{E}-07$

$4.173 E-08$ 400.0

$4.047 E-10$ $4.111 E-10$ $4.291 \mathrm{E}-10$ $4.364 E-10$ $4.564 \mathrm{E}-10$ $5.044 E-10$ $5.044 E-10$ 7. $13 E-10$ $1.159 E-09$ $1.449 \mathrm{E}-09$ $1.925 \mathrm{E}-09$ $5.346 \mathrm{E}-0$ $1.154 \mathrm{E}-08$

$2.945 E-08$
RANGE (METERS)

7.581E-11 $7.641 \mathrm{E}-11$ $8.086 \mathrm{E}-11$ $8.472 \mathrm{E}-\mathrm{I}$ 8. $957 E-11$ 9. $886 E-11$ $1.149 E-10$ $1.390 \mathrm{E}-10$ $1.707 \mathrm{E}-10$ $2.104 E-10$ 2. $660 \mathrm{E}-10$ $3.619 E-10$ $5.531 \mathrm{E}-10$ $9.512 E-10$ 1. $813 \mathrm{E}-0$ 4. $905 \mathrm{E}-0$

4. $260 E-09$
1200.0

$2.454 \mathrm{E}-11$ $2.524 \mathrm{E}-11$ $2.614 E-11$ $2.736 \mathrm{E}-11$ $2.915 \mathrm{E}-11$ $3.227 \mathrm{E}-11$ $3.715 \mathrm{E}-11$ $4.429 \mathrm{E}-1$ $5.364 E-11$ $6.564 \mathrm{E}-1$ $8.318 \mathrm{E}-1$ $1.134 \mathrm{E}-10$

$1.716 \mathrm{E}-10$

$2.868 \mathrm{E}-10$ $1.154 \mathrm{E}-0$

$1.248 \mathrm{E}-09$
1500.0

$7.614 \mathrm{E}-12$ $7.656 \mathrm{E}-12$ $7.813 \mathrm{E}-12$
$8.095 \mathrm{E}-12$ $8.479 E-12$ $9.076 \mathrm{E}-12$ $1.002 \mathrm{E}-11$ $1.146 \mathrm{E}-11$ $1.349 \mathrm{E}-11$ $1.616 \mathrm{E}-1$ $1.969 \mathrm{E}-11$ $2.493 E-11$ $3.393 \mathrm{E}-1$

$5.076 \mathrm{E}-11$

$8.275 E-11$ $1.436 \mathrm{E}-10$
$2.812 \mathrm{E}-10$

$3.578 \mathrm{E}-10$
1800.0

$2.292 E-12$ $2.304 \mathrm{E}-12$ $2.349 \mathrm{E}-12$ $2.432 \mathrm{E}-12$ $2.734 \mathrm{E}-12$ $3.012 E-12$ $3.426 \mathrm{E}-12$ $3.996 \mathrm{E}-1$ $5.759 E-12$ .871E- 12

$1.460 E-11$

$2.332 E-1$

$7.026 \mathrm{E}-11$

$1.012 \mathrm{E}-10$ 
4 PI $R *$ *2 NON IONIZING SILICON KERMA (NEUTRONS) (CM* 2 ERGS/GRAM/STERADIAN/SOURCE NEUTRON)
12.200 TO 15.000 MEV NEUTRON SOURCE
COSINE

$-1 . \operatorname{crccos}$ DO $-9.89401 \mathrm{E}-01$ $-9.44575 \mathrm{E}-01$ $-7.55044 \mathrm{E}-01$ $-6.17876 E-01$ $-4.58017 \mathrm{E}-01$ $-2.81605 E-01$ $-9.50125 E-02$ $9.5 C 125 E-02$ $2.81605 \mathrm{E}-01$ 4.58017E-01 $6.17876 \mathrm{E}-01$ .5 $9.44575 \mathrm{E}-01$ $9.894 \mathrm{C} 1 \mathrm{E}-01$

TOTAL

COSINE

-1.rCOOOE 00 $-9.89401 \mathrm{E}-01$ $-9.44575 \mathrm{E}-01$ $-8.65631 E-01$ $-7.55044 E-01$ $-4.58 C 17 E-01$ $-2.81605 \mathrm{E}-01$ $-9.50125 \mathrm{E}-02$ $9.50125 \mathrm{E}-02$ $.81605 \mathrm{E}-01$ 4.58C1 7E-0 1 $6.17876 \mathrm{E}-01$ $7.55044 \mathrm{E}-01$ $8.65631 \mathrm{E}-01$ $9.44575 E-01$

TOTAL

75.0
$.021 \mathrm{E}-1 \mathrm{C}$
$.042 \mathrm{E}-10$
$.089 \mathrm{E}-10$
$.123 \mathrm{E}-10$
$.134 \mathrm{E}-10$
$.169 \mathrm{E}-10$
$.206 \mathrm{E}-10$
$.326 \mathrm{E}-10$
$.521 \mathrm{E}-10$
$.338 \mathrm{E}-10$
$.235 \mathrm{E}-10$
$.846 \mathrm{E}-10$
$.396 \mathrm{E}-10$
$.807 \mathrm{E}-10$
$.268 \mathrm{E}-10$
$.364 \mathrm{E}-09$
$.965 \mathrm{E}-08$
$.833 \mathrm{E}-09$

100.0

$1.227 \mathrm{E}-10$ $1.299 \mathrm{E}-10$ $1.338 \mathrm{E}-10$ $1.354 \mathrm{E}-10$ $1.397 E-10$ $1.448 \mathrm{E}-10$ $1.589 \mathrm{E}-10$ $1.820 \mathrm{E}-10$ $2.072 \mathrm{E}-10$ $2.741 \mathrm{E}-10$ $2.939 E-10$ $3.914 E-10$ $5.687 \mathrm{E}-10$ $9.940 \mathrm{E}-10$ $2.367 \mathrm{E}-0$ $2.549 E-08$ 8. $458 E-09$

500.0

600.0

$1.475 E-10$ $1.481 \mathrm{E}-10$ $1.506 \mathrm{E}-10$ $1.540 \mathrm{E}-10$ $1.594 \mathrm{E}-10$ $1.663 \mathrm{E}-10$ $1.768 E-10$ $1.929 E-10$ $2.158 E-10$ $2.461 E-10$ $3.353 \mathrm{E}-10$ $4.101 E-10$ $5.369 \mathrm{E}-10$ $7.762 E-10$ 7. $3.987 \mathrm{E}-09$

4.554E-09

$1.211 E-10$
$1.216 E-10$

$1.234 \mathrm{E}-10$

. $308 \mathrm{E}-10$

$1.455 \mathrm{E}-10$

$008 \mathrm{E}-10$

$2.717 \mathrm{E}-10$

. 293E-10
RANGE (METERS)

$$
200.0 \quad 250.0
$$

$1.540 E-10$ $1.560 \mathrm{E}-10$
$1.613 \mathrm{E}-10$ $1.613 E-10$ $1.685 \mathrm{E}-10$ $1.758 \mathrm{E}-10$ $1.822 \mathrm{E}-10$ $2.000 E-10$ $2.279 E-10$ $2.592 \mathrm{E}-10$ $3.501 E-10$ $4.894 E-10$ $6.423 \mathrm{E}-10$ $1.061 \mathrm{E}-0$ $2.349 E-09$ $2.008 \mathrm{E}-08$

8.202E-09

$1.730 \mathrm{E}-10$

$30 C .0$ 400.0 $1.748 \mathrm{E}-10$ $1.798 \mathrm{E}-10$ $1.897 E-10$ $1.961 \mathrm{E}-10$ $2.053 E-10$ $2.253 E-10$ $2.556 \mathrm{E}-10$ $3.030 \mathrm{E}-10$ $3.477 \mathrm{E}-10$ $4.229 \mathrm{E}-10$ $5.155 \mathrm{E}-10$ $7.153 \mathrm{E}-10$ $1.113 E-09$
$2.278 E-09$ $2.278 E-09$
$1.592 E-08$ $7.850 E-09$ $1.835 \mathrm{E}-10$ $1.880 \mathrm{E}-10$ $1.915 \mathrm{E}-10$ $2.057 \mathrm{E}-1 \mathrm{C}$ $2.168 \mathrm{E}-10$ $2.366 \mathrm{E}-10$ $2.68 \mathrm{CE}-10$ $3.165 \mathrm{E}-10$ $3.606 \mathrm{E}-10$ $4.386 \mathrm{E}-10$ $5.329 \mathrm{E}-10$ $7.299 \mathrm{E}-1 \mathrm{C}$ $1.124 \mathrm{E}-09$ $2.162 \mathrm{E}-09$ $1.257 E-08$

$7.380 E-09$

$\begin{array}{ll}1.833 \mathrm{E}-10 & 1.707 \mathrm{E}-10 \\ 1.846 \mathrm{E}-10 & 1.717 \mathrm{E}-10 \\ 1.887 \mathrm{E}-10 & 1.748 \mathrm{E}-10 \\ 1.924 \mathrm{E}-10 & 1.786 \mathrm{E}-1 \mathrm{C} \\ 1.994 \mathrm{E}-1 \mathrm{C} & 1.849 \mathrm{E}-10 \\ 2.069 \mathrm{E}-10 & 1.925 \mathrm{E}-1 \mathrm{C} \\ 2.186 \mathrm{E}-10 & 2.042 \mathrm{E}-10 \\ 2.386 \mathrm{E}-10 & 2.229 \mathrm{E}-10 \\ 2.695 \mathrm{E}-10 & 2.504 \mathrm{E}-10 \\ 3.102 \mathrm{E}-10 & 2.875 \mathrm{E}-10 \\ 3.655 \mathrm{E}-10 & 3.337 \mathrm{E}-10 \\ 4.312 \mathrm{E}-10 & 3.945 \mathrm{E}-10 \\ 5.338 \mathrm{E}-10 & 4.843 \mathrm{E}-10 \\ 7.144 \mathrm{E}-10 & 6.414 \mathrm{E}-10 \\ 1.092 \mathrm{E}-09 & 9.502 \mathrm{E}-10 \\ 2.012 \mathrm{E}-09 & 1.653 \mathrm{E}-09 \\ 9.951 \mathrm{E}-09 & 6.268 \mathrm{E}-09 \\ & \\ 6.843 \mathrm{E}-09 & 5.687 \mathrm{E}-09\end{array}$

\section{RANGE (METERS)}

$$
900.0
$$$$
1200.0
$$

1500.0

1800.0

5. $546 E-11$

$2.140 E-11$

$7.451 \mathrm{E}-12$

$2.424 \mathrm{E}-1$

5.565E-11

$2.146 E-11$

$2.175 \mathrm{E}-11$

$7.475 \mathrm{E}-12$

$7.575 \mathrm{E}-12$

$5.778 \mathrm{E}-11$

$5.987 E-11$

$8.054 \mathrm{E}-12$

$8.465 E-12$

.

(1)

$2.589 \mathrm{E}-11$

$9.028 \mathrm{E}-12$

$7.276 \mathrm{E}-11$

$9.782 \mathrm{E}-12$

.069 E- 11

$3.471 E-11$

$1.076 \mathrm{E}-1$

$1.037 \mathrm{E}-10$

952E-11

$1.203 E-11$

$1.582 \mathrm{E}-11$

$1.461 \mathrm{E}-10$

$4.594 \mathrm{E}-11$

$1.582 \mathrm{E}-1$

$1.883 \mathrm{E}-11$

$8.856 \mathrm{E}-11$

$2.321 E-11$

$2.502 \mathrm{E}-10$

1.25 E 11

2.979E-11

$9.718 \mathrm{E}-10 \quad 3.679 \mathrm{E}-10 \quad 1.251 \mathrm{E}-10 \quad 4.000 \mathrm{E}-11$

$5.411 E-10$

$1.829 E-10$

$2.527 \mathrm{E}-12$
$2.622 \mathrm{E}-12$
$2.757 \mathrm{E}-12$

(2.02

$3.183 \mathrm{E}-12$

$3.497 E-12$

(1)

$5.109 E-12$

$6.055 \mathrm{E}-12$

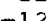

$358 \mathrm{E}-12$

$1.225 \mathrm{E}-11$

$1.475 E-09$

$5.836 E-11$ 
4 PI R**2 HENDERSON DOSE (GAMMAS) (CM* 2 RAD/STERADIAN/SOURCE NEUTRCN)
75.

$1.841 \mathrm{E}-11$ $1.855 \mathrm{E}-11$

$.905 \mathrm{E}-11$

$1.997 \mathrm{E}-11$

$2.122 \mathrm{E}-11$

$2.283 E-11$

$2.490 \mathrm{E}-11$

$2.769 \mathrm{E}-11$

$3.151 \mathrm{E}-11$

$3.628 \mathrm{E}-11$

$5.923 E-11$

$5.344 E-11$

$6.627 E-11$

$9.8 \mathrm{C} \mathrm{E}-11$

$1.523 E-10$

$6.584 \mathrm{E}-10$

$7.657 E-10$

500.0

$1.193 \mathrm{E}-11$
$1.232 \mathrm{E}-11$

$1.366 \mathrm{E}-11$

$1.55 \mathrm{EE}-11$

$1.725 \mathrm{E}-11$

$.852 E-11$

$2.184 E-11$

$2.562 \mathrm{E}-1$

$3.164 \mathrm{E}-11$

$4.024 E-11$

$5.261 \mathrm{E}-11$

$7.275 \mathrm{E}-11$

$1.110 \mathrm{E}-10$

$1.929 \mathrm{E}-1 \mathrm{C}$

$3.984 \mathrm{E}-1 \mathrm{C}$

$1.217 \mathrm{E}-09$

$8.980 E-10$
100.0

$.039 E-11$ $2.056 \mathrm{E}-11$

$2.121 \mathrm{E}-11$

$2.385 \mathrm{E}-11$

2. $572 \mathrm{E}-11$

2.809E-11

. $129 \mathrm{E}-11$

. $572 E-11$

.618E-11

(

.

116E- 10

. 11 E 10

.

$8.166 \mathrm{E}-10$

$9.077 \mathrm{E}-10$

600.0

$8.799 \mathrm{E}-12$

$9.187 \mathrm{E}-12$

$1.040 \mathrm{E}-1$

$1.352 \mathrm{E}-1$

. $441 \mathrm{E}-11$

$1.514 \mathrm{E}-11$

$1.663 \mathrm{E}-1$

$1.965 \mathrm{E}-1$

$2.455 \mathrm{E}-11$

$3.147 \mathrm{E}-1$

$4.128 \mathrm{E}-11$

$5.746 \mathrm{E}-11$

$8.935 \mathrm{E}-1$

$.602 \mathrm{E}-10$

$3.415 \mathrm{E}-10$

$9.44575 \mathrm{E}-0$

TOTAL

$7.4 C 8 E-10$
12.200 TO 15.000 MEV NEUTRON SOURCE

\begin{tabular}{|c|c|c|c|c|}
\hline 150.0 & $\begin{array}{c}\text { NGE (METERS) } \\
200.0\end{array}$ & 250.0 & $30 c .0$ & 400.0 \\
\hline $230 E-11$ & $2.232 E-11$ & $2.123 \mathrm{E}-11$ & $1.958 E-11$ & $1.567 E-11$ \\
\hline $254 \mathrm{E}-11$ & $2.262 \mathrm{E}-11$ & $2.157 \mathrm{E}-11$ & $1.994 \mathrm{E}-11$ & $1.606 \mathrm{E}-11$ \\
\hline $2.343 \mathrm{E}-11$ & $2.370 \mathrm{E}-11$ & $2.279 \mathrm{E}-11$ & $2.125 \mathrm{E}-11$ & $1.744 E-11$ \\
\hline $2.495 \mathrm{E}-11$ & $2.549 \mathrm{E}-11$ & $2.475 \mathrm{E}-11$ & $2.330 \mathrm{E}-11$ & $1.949 \mathrm{E}-11$ \\
\hline $2.682 \mathrm{E}-11$ & $2.758 \mathrm{E}-11$ & $2.692 \mathrm{E}-11$ & $2.546 \mathrm{E}-11$ & $2.146 \mathrm{E}-11$ \\
\hline $2.901 \mathrm{E}-11$ & $2.987 \mathrm{E}-11$ & $2.916 \mathrm{E}-11$ & $2.756 \mathrm{E}-11$ & $2.315 \mathrm{E}-11$ \\
\hline $73 E-11$ & $3.264 \mathrm{E}-11$ & $3.180 \mathrm{E}-11$ & $2.996 \mathrm{E}-11$ & $2.496 \mathrm{E}-11$ \\
\hline $3.541 \mathrm{E}-11$ & $3.646 \mathrm{E}-11$ & $3.550 \mathrm{E}-11$ & $3.342 \mathrm{E}-11$ & $2.774 \mathrm{E}-11$ \\
\hline $4.065 \mathrm{E}-11$ & $4.202 \mathrm{E}-11$ & $4.107 E-11$ & $3.879 \mathrm{E}-11$ & $3.238 \mathrm{E}-11$ \\
\hline $5.609 \mathrm{E}-11$ & $4.603 \mathrm{E}-11$ & $4.500 \mathrm{E}-11$ & $4.766 \mathrm{E}-11$ & $3.948 \mathrm{E}-11$ \\
\hline $206 E-11$ & $6.520 \mathrm{E}-11$ & $6.484 E-11$ & $5.757 \mathrm{E}-11$ & $4.985 E-11$ \\
\hline $854 F-11$ & $7.476 \mathrm{E}-11$ & $7.453 E-11$ & $7.574 \mathrm{E}-11$ & $6.467 \mathrm{E}-11$ \\
\hline $335 E-11$ & $1.070 \mathrm{E}-10$ & $1.078 \mathrm{E}-10$ & $1.010 \mathrm{E}-10$ & $8.871 \mathrm{E}-11$ \\
\hline $1.429 \mathrm{E}-10$ & $1.462 \mathrm{E}-10$ & $1.489 \mathrm{E}-10$ & $1.49 \mathrm{CE}-10$ & $1.325 E-10$ \\
\hline & $2.398 E-10$ & $2.462 \mathrm{E}-10$ & $2.425 \mathrm{E}-10$ & $2.233 E-10$ \\
\hline $06 E-10$ & $4.437 E-10$ & $4.669 \mathrm{E}-10$ & $4.727 \mathrm{E}-1 \mathrm{C}$ & $4.472 \mathrm{E}-10$ \\
\hline & $1.231 \mathrm{E}-09$ & $1.322 E-09$ & $1.359 \mathrm{E}-09$ & $1.328 E-09$ \\
\hline $1.075 \mathrm{E}-09$ & $1.156 \mathrm{E}-09$ & $1.180 \mathrm{E}-09$ & $1.165 \mathrm{E}-09$ & $1.052 E-09$ \\
\hline
\end{tabular}

RANGE (METERS)

$900.0 \quad 1200.0$

1500.0

1800.0

$3.186 \mathrm{E}-12 \quad 1.060 \mathrm{E}-12 \quad 3.344 \mathrm{E}-13 \quad 1.004 \mathrm{E}-13$

$\begin{array}{llll} & 1.232 \mathrm{E}-12 & 4.261 \mathrm{E}-13 & 1.473 \mathrm{E}-13\end{array}$

$2.232 \mathrm{E}-12 \quad 9.505 \mathrm{E}-13 \quad 4.049 \mathrm{E}-13$

$6.024 \mathrm{E}-12 \quad 2.610 \mathrm{E}-12 \quad 1.15 \mathrm{LE}-12 \quad 5.212 \mathrm{E}-13$

$6.227 \mathrm{E}-12 \quad 2.595 \mathrm{E}-12 \quad 1.060 \mathrm{E}-12 \quad 40495 \mathrm{E}-13$

$6.246 \mathrm{E}-12 \quad 2.406 \mathrm{E}-12 \quad 9.336 \mathrm{E}-13 \quad 3.730 \mathrm{E}-13$

$6.711 \mathrm{E}-12 \quad 2.616 \mathrm{E}-12 \quad \mathrm{l} 2.039 \mathrm{E}-12 \quad 4.164 \mathrm{E}-13$

$8.123 \mathrm{E}-12 \quad 3.264 \mathrm{E}-12 \quad 1.327 \mathrm{E}-12 \quad 5.676 \mathrm{E}-13$

$1.056 \mathrm{E}-11$ 4.430E-12 $1.889 \mathrm{E}-12 \quad 8.187 \mathrm{E}-13$

$1.380 \mathrm{E}-11 \quad 5.860 \mathrm{E}-12 \quad 2.522 \mathrm{E}-12 \quad 12107 \mathrm{E}-12$

$1.802 \mathrm{E}-11 \quad 7.469 \mathrm{E}-12 \quad 3.095 \mathrm{E}-12 \quad 120284 \mathrm{E}-12$

$2.516 \mathrm{E}-11 \quad 1.024 \mathrm{E}-11 \quad 4.097 \mathrm{E}-12 \quad 12047 \mathrm{E}-12$

$4.111 \mathrm{E}-11 \quad 1.745 \mathrm{E}-11 \quad 7.252 \mathrm{E}-12 \quad 3.044 \mathrm{E}-12$

$8.079 \mathrm{E}-11 \quad 3.746 \mathrm{E}-11 \quad 1.695 \mathrm{E}-11 \quad 7.656 \mathrm{E}-12$

$\begin{array}{llll}1.892 \mathrm{E}-10 & 9.540 \mathrm{E}-11 & 4.629 \mathrm{E}-11 & 2.211 \mathrm{E}-11 \\ 6.410 \mathrm{E}-10 & 3.465 \mathrm{E}-10 & 1.782 \mathrm{E}-10 & 8.911 \mathrm{E}-11\end{array}$

$3.751 E-10 \quad 1.782 E-10 \quad 8.317 E-11 \quad 3.873 E-11$ 
COSINE

-1.0 COOOE 00 $-9.89401 \mathrm{E}-01$ $-9.44575 E-01$

$-8.65631 \mathrm{E}-0$

$-7.55044 E-01$

$-6.17876 E-0$

$-4.58017 \mathrm{E}-0$

$-2.81605 \mathrm{E}-01$

$0.50125 E-02$

.

$2.81605 \mathrm{E}-\mathrm{C}$

$4.58017 E-01$

$7.55044 E-01$

$7.55044 E-01$

$9.44575 \mathrm{E}-01$

$9.89401 \mathrm{E}-01$

TOTAL

CCSINE

\section{-1. CAOCOE OO} $-9.89401 \mathrm{E}-0$ $75 \mathrm{E}-0$ $-8.65631 \mathrm{E}-0$ $.17876 \mathrm{E}-\mathrm{O}$ $-4.58017 \mathrm{E}-01$ $-2.81605 \mathrm{E}-01$ $-9.50125 \mathrm{E}-02$ $9.50125 \mathrm{E}-02$ $2.81605 \mathrm{E}-01$ $4.58017 \mathrm{E}-01$ $6.17876 \mathrm{E}-01$ $7.55044 \mathrm{E}-01$ $8.65631 \mathrm{E}-01$ $9.89401 \mathrm{E}-\mathrm{O}$

TOTAL

\begin{abstract}
75.0
\end{abstract}
100.0

$1.883 \mathrm{E}-0$ $1.896 \mathrm{E}-09$

$1.946 \mathrm{E}-09$

$2.037 \mathrm{E}-09$

$2.162 \mathrm{E}-09$

$2.321 \mathrm{E}-09$

$2.528 \mathrm{E}-09$

$2.804 \mathrm{E}-09$

$3.184 \mathrm{E}-09$

$3.655 E-C 9$

$5.944 E-09$

$5.361 E-C 9$

$0.635 \mathrm{E}-09$

$9.759 \mathrm{E}-09$

$1.516 E-08$

$6.542 \mathrm{E}-\mathrm{C} 8$

$7.668 \mathrm{E}-08$

500.0

$527 \mathrm{E}-\mathrm{C}$

$1.527 \mathrm{E}-\mathrm{C}$

$1.700 \mathrm{E}-09$

$1.892 \mathrm{E}-09$

$2.065 \mathrm{E}-09$

$2.201 E-09$

$2.335 \mathrm{E}-09$

2.555E-C9

$2.944 E-C 9$

$3.556 \mathrm{E}-09$

$4.424 \mathrm{E}-09$

$5.665 \mathrm{E}-09$

$7.671 \mathrm{E}-\mathrm{C} 9$

$1.146 \mathrm{E}-\mathrm{C} 8$

$1.955 \mathrm{E}-\mathrm{C} .8$

$3.992 \mathrm{E}-08$

$1.217 \mathrm{E}-07$

$9.425 \mathrm{E}-08$

600.0

$1.217 \mathrm{E}-09$

$1.51 \mathrm{CE}-\mathrm{CO}$

$1.657 \mathrm{E}-09$

$1.837 \mathrm{E}-\mathrm{C9}$

2. $31 \mathrm{CE}-09$

$2.809 \mathrm{E}-09$

$4.495 \mathrm{E}-\mathrm{C} 9$

$6.106 \mathrm{E}-09$

$1.072 \mathrm{E}-07$
RANGE (METERS)
200.0

250.0

$30 \mathrm{c} .0$

400.0

$2.114 \mathrm{E}-\mathrm{C} 9$

$2.131 \mathrm{E}-09$

$2.195 \mathrm{E}-09$

. $308 \mathrm{E}-\mathrm{C} 9$

$2.458 \mathrm{E}-\mathrm{C} 9$

$2.643 \mathrm{E}-\mathrm{C} .9$
$2.88 \mathrm{CE}-09$

$3.198 \mathrm{E}-09$

$3.639 E-C 9$

$5.671 E-09$

$4.946 \mathrm{E}-09$

$6.930 E-09$

1.117E-08

1.711E-08

1.

$8.120 E-C B$

150.0

$2.447 E-09$

. $393 \mathrm{E}-09$

$\begin{array}{ll}2.267 E-09 & 1.911 E-09 \\ 2.304 E-09 & 1.950 E-09\end{array}$

$2.435 \mathrm{E}-09 \quad 2.088 \mathrm{E}-09$

$2.641 \mathrm{E}-09 \quad 2.295 \mathrm{E}-09$

$2.860 \mathrm{E}-09 \quad 2.496 \mathrm{E}-09$

$3.075 E-09 \quad 2.672 E-09$

$3.323 E-09 \quad 2.863 E-09$

$3.677 \mathrm{E}-09 \quad 3.152 \mathrm{E}-09$

$4.221 \mathrm{E}-09 \quad 3.626 \mathrm{E}-09$

$5.112 \mathrm{E}-09 \quad 4.345 \mathrm{E}-09$

$6.107 \mathrm{E}-09 \quad 5.389 \mathrm{E}-09$

$7.920 E-09 \quad 6.873 E-09$

$1.043 \mathrm{E}-08 \quad 9.265 \mathrm{E}-09$ $1.519 E-08$ 1.361E-08 $2.444 E-08$
$4.727 E-08$

$1.356 \mathrm{E}-07 \quad 1.328 \mathrm{E}-07$

$9.123 \mathrm{E}-08$

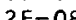

3.891E-08

$1.071 \mathrm{E}-07$

1.478E-08

$4.427 \mathrm{E}-08$

1. $227 E-07$

..328E-07

$1.179 E-07$

$1.212 E-07$

$1.203 \mathrm{E}-07$

1.097E-07

\section{RANGE (METERS)}

1200.0

1500.0

1800.0

$1.882 E-10$

$7.163 E-11$

$2.761 \mathrm{E}-11$

$5.154 E-10$

$2.053 \mathrm{E}-10$

$8.074 \mathrm{E}-11$
$1.064 \mathrm{E}-10$

$3.226 \mathrm{E}-11$

$7.022 \mathrm{E}-10$

$3.057 \mathrm{E}-10$

$1.064 \mathrm{E}-10$

$5.805 \mathrm{E}-11$

$7.748 \mathrm{E}-10$

$3.446 E-10 \quad 1.537 E-10$

$1.459 \mathrm{E}-10$

$1.345 \mathrm{E}-10$

$3.296 \mathrm{E}-10$

$6.987 E-11$

$6.328 \mathrm{E}-11$

$5.618 \mathrm{E}-11$

$6 . \mathrm{C} 96 \mathrm{E}-11$

$8.605 \mathrm{E}-10$

$3.532 E-10$
$4.204 E-10$
5.36

$2.329 E-10 \quad 7.647 \mathrm{E}-11$

$1.007 E-09$

$5.389 E-10$

$2.329 E-10$

$1.018 \mathrm{E}-10$

$1.585 \mathrm{E}-09$

$6.837 \mathrm{E}-10$

$2.968 E-10$
$3.545 E-10$

$1.488 \mathrm{E}-10$

$2.009 \mathrm{E}-09 \quad 8.456 \mathrm{E}-10$

$1.120 E-09$

$1.841 \mathrm{E}-10$

$4.290 \mathrm{E}-09 \quad 1.826 \mathrm{E}-09$

$1.826 E-09$
$3.79 C E-09$

$7.603 \mathrm{E}-10$

$1.841 \mathrm{E}-10$
$3.194 \mathrm{E}-10$

$3.423 \mathrm{E}-08 \quad 8.197 \mathrm{E}-09$

$1.709 E-09$
0

$3.194 \mathrm{E}-10$
$7.695 \mathrm{E}-10$

$\begin{array}{llll}1.893 \mathrm{E}-08 & 9.525 \mathrm{E}-09 & 4.611 \mathrm{E}-09 & 2.199 \mathrm{E}-09 \\ 6.429 \mathrm{E}-08 & 3.480 \mathrm{E}-08 & 1.792 \mathrm{E}-08 & 8.975 \mathrm{E}-09\end{array}$

$8.975 E-09$

$8.811 E-09$

4.C.97E-09

$1.890 E-08$ 
4 PI $R * 2$ AIR KERMA (GAMMAS)

(CM*2 ERGS/GRAM/STERADIAN/SOURCE NEUTRON)
12.200 TO 15.000 MEV NEUTRON SOURCE

RANGE (METERS)

$\begin{array}{lccc}900.0 & 1200.0 & 1500.0 & 1800.0 \\ 1.601 \mathrm{E}-09 & 7.284 \mathrm{E}-10 & 3.234 \mathrm{E}-10 & 1.439 \mathrm{E}-10 \\ 1.628 \mathrm{E}-09 & 7.451 \mathrm{E}-10 & 3.322 \mathrm{E}-10 & 1.483 \mathrm{E}-10 \\ 1.706 \mathrm{E}-09 & 7.909 \mathrm{E}-10 & 3.576 \mathrm{E}-10 & 1.623 \mathrm{E}-10 \\ 1.826 \mathrm{E}-09 & 8.494 \mathrm{E}-10 & 3.858 \mathrm{E}-10 & 1.744 \mathrm{E}-10 \\ 1.919 \mathrm{E}-09 & 8.976 \mathrm{E}-10 & 4.102 \mathrm{E}-10 & 1.877 \mathrm{E}-10 \\ 1.977 \mathrm{E}-09 & 9.153 \mathrm{E}-10 & 4.107 \mathrm{E}-10 & 1.852 \mathrm{E}-10 \\ 2.031 \mathrm{E}-09 & 9.225 \mathrm{E}-10 & 4.101 \mathrm{E}-10 & 1.831 \mathrm{E}-10 \\ 2.136 \mathrm{E}-09 & 9.707 \mathrm{E}-10 & 4.326 \mathrm{E}-10 & 1.927 \mathrm{E}-10 \\ 2.339 \mathrm{E}-09 & 1.064 \mathrm{E}-09 & 4.735 \mathrm{E}-10 & 2.128 \mathrm{E}-10 \\ 2.649 \mathrm{E}-09 & 1.209 \mathrm{E}-09 & 5.415 \mathrm{E}-10 & 2.429 \mathrm{E}-10 \\ 3.045 \mathrm{E}-09 & 1.384 \mathrm{E}-09 & 6.186 \mathrm{E}-10 & 2.776 \mathrm{E}-10 \\ 3.546 \mathrm{E}-09 & 1.583 \mathrm{E}-09 & 6.932 \mathrm{E}-10 & 3.034 \mathrm{E}-10 \\ 4.326 \mathrm{E}-09 & 1.893 \mathrm{E}-09 & 8.094 \mathrm{E}-10 & 3.473 \mathrm{E}-10 \\ 5.916 \mathrm{E}-09 & 2.609 \mathrm{E}-09 & 1.122 \mathrm{E}-09 & 4.853 \mathrm{E}-10 \\ 9.660 \mathrm{E}-09 & 4.485 \mathrm{E}-09 & 2.026 \mathrm{E}-09 & 9.137 \mathrm{E}-10 \\ 1.963 \mathrm{E}-08 & 9.796 \mathrm{E}-09 & 4.708 \mathrm{E}-09 & 2.232 \mathrm{E}-09 \\ 6.082 \mathrm{E}-08 & 3.267 \mathrm{E}-08 & 1.672 \mathrm{E}-08 & 8.332 \mathrm{E}-09 \\ & & & \\ 5.574 \mathrm{E}-08 & 2.649 \mathrm{E}-08 & 1.227 \mathrm{E}-08 & 5.661 \mathrm{E}-09\end{array}$

$\begin{array}{ccccc}150.0 & \text { RANGE (METERS) } & & \\ & 200.0 & 250.0 & 300.0 & 400.0 \\ 3.539 E-09 & 4.073 E-09 & 4.373 E-09 & 4.484 E-09 & 4.293 E-09 \\ 3.562 E-09 & 4.102 E-09 & 4.407 \mathrm{E}-09 & 4.521 \mathrm{E}-09 & 4.333 \mathrm{E}-09 \\ 3.651 \mathrm{E}-09 & 4.213 \mathrm{E}-09 & 4.534 \mathrm{E}-09 & 4.658 \mathrm{E}-09 & 4.479 \mathrm{E}-09 \\ 3.805 \mathrm{E}-09 & 4.399 \mathrm{E}-09 & 4.742 \mathrm{E}-09 & 4.880 \mathrm{E}-09 & 4.705 \mathrm{E}-09 \\ 4.000 \mathrm{E}-09 & 4.625 \mathrm{E}-09 & 4.985 \mathrm{E}-09 & 5.129 \mathrm{E}-09 & 4.944 \mathrm{E}-09 \\ 4.232 \mathrm{E}-09 & 4.884 \mathrm{E}-09 & 5.253 \mathrm{E}-09 & 5.395 \mathrm{E}-09 & 5.181 \mathrm{E}-09 \\ 4.524 \mathrm{E}-09 & 5.201 \mathrm{E}-09 & 5.577 \mathrm{E}-09 & 5.710 \mathrm{E}-09 & 5.454 \mathrm{E}-09 \\ 4.915 \mathrm{E}-09 & 5.631 \mathrm{E}-09 & 6.019 \mathrm{E}-09 & 6.146 \mathrm{E}-09 & 5.843 \mathrm{E}-09 \\ 5.460 \mathrm{E}-09 & 6.239 \mathrm{E}-09 & 6.655 \mathrm{E}-09 & 6.783 \mathrm{E}-09 & 6.430 \mathrm{E}-09 \\ 6.948 \mathrm{E}-09 & 6.726 \mathrm{E}-09 & 7.167 \mathrm{E}-09 & 7.768 \mathrm{E}-09 & 7.273 \mathrm{E}-09 \\ 6.679 \mathrm{E}-09 & 8.615 \mathrm{E}-09 & 9.156 \mathrm{E}-09 & 8.881 \mathrm{E}-09 & 8.450 \mathrm{E}-09 \\ 9.209 \mathrm{E}-09 & 9.657 \mathrm{E}-09 & 1.025 \mathrm{E}-08 & 1.078 \mathrm{E}-08 & 1.007 \mathrm{E}-08 \\ 1.069 \mathrm{E}-08 & 1.279 \mathrm{E}-08 & 1.353 \mathrm{E}-08 & 1.336 \mathrm{E}-08 & 1.257 \mathrm{E}-08 \\ 1.536 \mathrm{E}-08 & 1.659 \mathrm{E}-08 & 1.755 \mathrm{E}-08 & 1.805 \mathrm{E}-08 & 1.690 \mathrm{E}-08 \\ 2.232 \mathrm{E}-08 & 2.536 \mathrm{E}-08 & 2.672 \mathrm{E}-08 & 2.690 \mathrm{E}-08 & 2.552 \mathrm{E}-08 \\ 3.830 \mathrm{E}-08 & 4.421 \mathrm{E}-08 & 4.714 \mathrm{E}-08 & 4.822 \mathrm{E}-08 & 4.626 \mathrm{E}-08 \\ 1.01 \mathrm{CE}-07 & 1.163 \mathrm{E}-07 & 1.253 \mathrm{E}-07 & 1.293 \mathrm{E}-07 & 1.268 \mathrm{E}-07 \\ 1.217 \mathrm{E}-07 & 1.381 \mathrm{E}-07 & 1.472 \mathrm{E}-07 & 1.504 \mathrm{E}-07 & 1.433 \mathrm{E}-07\end{array}$

$2.649 E-08$

$1.227 E-08$

$5.661 E-09$

\section{$-1 . C C O O O E O O$ $-9.89401 E-C 1$
$-9.44575 E-01$ $-8.65631 \mathrm{E}-01$ $-7.55044 E-01$ $-4.58017 \mathrm{E}-01$ $-2.81605 E-01$ $9.50125 \mathrm{E}-02$ $2.81605 E-01$ $4.58017 \mathrm{E}-01$ $6.17876 E-01$ $8.65631 \mathrm{E}-01$ $8.65631 E-01$ $9.89401 E-01$}

TOTAL

$75 . \mathrm{C}$
$2.259 \mathrm{E}-09$
$2.272 \mathrm{E}-09$
$2.320 \mathrm{E}-09$
$2.408 \mathrm{E}-09$
$2.529 \mathrm{E}-05$
$2.685 \mathrm{E}-09$
$2.887 \mathrm{E}-09$
$3.157 \mathrm{E}-09$
$3.526 \mathrm{E}-09$
$3.986 \mathrm{E}-09$
$6.1 \mathrm{C} 8 \mathrm{E}-09$
$5.611 \mathrm{E}-09$
$6.819 \mathrm{E}-09$
$9.762 \mathrm{E}-09$
$1.477 \mathrm{E}-08$
$2.400 \mathrm{E}-08$
$6.106 \mathrm{E}-08$
$7.853 \mathrm{E}-08$

100.0

$2.748 \mathrm{E}-09$ $2.764 \mathrm{E}-09$
$2.827 \mathrm{E}-09$ $2.938 \mathrm{E}-09$ $3.087 \mathrm{E}-09$ . $274 \mathrm{E}-09$ $3.513 \mathrm{E}-09$
$3.832 \mathrm{E}-09$ $4.271 E=0$ $5.568 \mathrm{E}-09$ $7.433 \mathrm{E}-\mathrm{C}$ $8.981 E-09$ $1.706 E-08$ . $9.638 \mathrm{E}-08$

500.0

$3.791 \mathrm{E}-09$ 3.831E-09 $3.973 E-C 9$ $4.184 E-09$ $4.396 \mathrm{E}-09$ $4.593 E-09$ $4.811 \mathrm{E}-09$ 5.132E$6.372 \mathrm{E}-09$ $7.376 E-09$ $8.760 \mathrm{E}-09$ 1.089E-0 $1.469 \mathrm{E}-08$ $2.247 \mathrm{E}-\mathrm{CB}$ $4.149 \mathrm{E}-08$ $4.149 \mathrm{E}-08$
$1.162 \mathrm{E}-07$

1.268E-07
600.0

3.181E-09 $3.220 E-09$ 3.536E-09 3.719E-09 $4.873 E-09$ $4.033 E-09$ 4.700E-O $5.312 E-09$ $6.137 \mathrm{E}-09$ $7.255 \mathrm{E}-09$ $8.983 \mathrm{E}-\mathrm{C} 9$ $1.216 \mathrm{E}-08$ $1.889 \mathrm{E}-08$ $3.564 E-O B$ 1. $022 \mathrm{E}-0$

1.C7OE-07 
CCSINE

$-1.00000 E 00$ $-9.89401 \mathrm{E}-\mathrm{Cl}$ $-9.44575 \mathrm{E}-01$ $-8.65631 \mathrm{E}-0 \mathrm{l}$ $-7.55044 \mathrm{E}-01$ $-6.17876 \mathrm{E}-01$ $-4.58017 \mathrm{E}-01$ $-2.81605 E-01$ $0.50125 E-02$ $9.50125 E-02$ $2.81605 \mathrm{E}-01$ $4.57876 \mathrm{E}-01$ $7.55044 \mathrm{E}-01$ $8.65631 \mathrm{E}-01$ $8.44575 \mathrm{E}-01$ $9.89401 E-01$

TOTAL

COSINE

$-1 .(\operatorname{CONOE} 00$ $-9.894 \mathrm{CE}-\mathrm{Cl}$ $-9.44575 E-01$ $-8.65631 E-01$
$-7.55044 E-01$ $-6.17876 \mathrm{E}-01$ $-6.17876 E-01$ $-2.881605 \mathrm{E}-01$ $-9.50125 \mathrm{E}-02$ $0.50125 E-02$ . $81605 \mathrm{E}-01$ $4.81615 \mathrm{E}-01$ $6.17876 \mathrm{E}-01$ $7.55044 \mathrm{E}-01$ $8.65631 \mathrm{E}-01$ $9.44575 \mathrm{E}-01$ $9.89401 E-01$

TOTAL
75.0

100.0

$1.940 \mathrm{E}-09$ $1.953 \mathrm{E}-09$ $2.004 \mathrm{E}-09$ $2.098 \mathrm{E}-09$ $2.225 \mathrm{E}-09$ $2.388 \mathrm{E}-09$ $2.599 \mathrm{E}-09$ $2.881 \mathrm{E}-09$ $3.269 \mathrm{E}-09$ $3.749 \mathrm{E}-09$ $6.097 \mathrm{E}-09$ $5.496 \mathrm{E}-\mathrm{C}$ $6.799 E-09$ $9.981 \mathrm{E}-\mathrm{C}$ $1.551 \mathrm{E}-08$ $2.575 E-08$

$7.861 E-08$

500.0 $1.702 \mathrm{E}-0.9$ $1.880 \mathrm{E}-09$ $2.076 \mathrm{E}-09$ $2.253 \mathrm{E}-0$ $.39 \mathrm{CE}-\mathrm{C}$ $2.525 \mathrm{E}-09$ 3.141E-OS $3.761 E-C$ $4.641 E-09$ $5.899 \mathrm{E}-0$ $7.934 \mathrm{E}-0$ $1.179 \mathrm{E}-0$ $2.005 \mathrm{E}-08$ $4.090 E-08$ 4.090E-08

$9.792 \mathrm{E}-08$
RANGE (METERS) 200.0

250.0

300.0

400.0

\subsection{E-C9} $2.204 \mathrm{E}-\mathrm{C} 9$
$2.269 \mathrm{E}-\mathrm{C} 9$ $2.369 \mathrm{E}-\mathrm{C} 9$
$2.385 \mathrm{E}-09$ $2.538 \mathrm{E}-\mathrm{CO}$ $2.727 \mathrm{E}-09$ $2.969 \mathrm{E}-09$ $3.293 \mathrm{E}-09$ $3.743 \mathrm{E}-0$ $5.824 \mathrm{E}-09$ $5.075 \mathrm{E}-09$ 7.110E-O9 $8.721 E-C 9$ $1.145 \mathrm{E}-08$ $1.751 E-O B$ $3.279 \mathrm{E}-08$ $9.360 \mathrm{E}-08$

\section{$2.482 E-09$} $2.581 E-09$ $2.506 \mathrm{E}-09 \quad 2.611 \mathrm{E}-09$ $2.596 \mathrm{E}-09$ $2.751 E-09$ 3.166E- 99 $3.444 E-09$ $3.820 E-09$

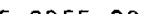
5.925E-0 ( (2) 3. $1.097 \mathrm{E}-07$ $2.722 \mathrm{E}-09$ $2.905 \mathrm{E}-09$ $3.119 \mathrm{E}-09$ $3.355 \mathrm{E}-09$ $3.639 E-09$ $4.030 E-09$ (.) $5.006 E-09$ (7.95E 1. (.) ..533E 08 $4.533 E-08$
$1.258 E-07$ $2.551 \mathrm{E}-09$ D 2.710E-09 $2.911 E-09$ $3.366 \mathrm{E}-09$ $3.639 \mathrm{E}-09$ $4.022 E-09$ $4.592 \mathrm{E}-09$ $4.994 E-09$ $7.014 \mathrm{E}-09$ $7.992 \mathrm{E}-09$ $1.136 \mathrm{E}-08$ $1.551 E-08$ $2.536 E-08$ 1. $352 \mathrm{E}-07$

$1.119 E-07$

$1.214 E-07$

$1.250 \mathrm{E}-07$

RANGE (METERS)

600.0

900.0

1200.0

1500.0

1800.0

$1.333 \mathrm{E}-09$ $1.497 \mathrm{E}-09$ $1.672 \mathrm{E}-09$ $1.823 \mathrm{E}-0$ $2.003 \mathrm{E}-\mathrm{CO}$ $2.164 \mathrm{E}-09$ $2.480 \mathrm{E}-09$ 2. $986 \mathrm{E}-09$ $3.695 \mathrm{E}-09$ $4.693 \mathrm{E}-\mathrm{CO}$ $6.325 \mathrm{E}-09$ $9.532 \mathrm{E}-0$ $1.667 \mathrm{E}-08$ $3.567 \mathrm{E}-\mathrm{O}$

$1.102 \mathrm{E}-07$

$5.991 E-10$

$2.265 \mathrm{E}-10$ $6.75 E-10 \quad 2.907 E-10 \quad 10245 E-10$ $8.648 \mathrm{E}-10 \quad 3.869 \mathrm{E}-10 \quad 1.729 \mathrm{E}-10$ $8.896 \mathrm{E}-10 \quad 3.874 \mathrm{E}-10 \quad 1.648 \mathrm{E}-10$ $8.964 \mathrm{E}-10 \quad 3.704 \mathrm{E}-10 \quad 1.526 \mathrm{E}-10$ $1.641 \mathrm{E}-10$ $1.348 \mathrm{E}-09 \quad 5.815 \mathrm{E}-10 \quad 2.510 \mathrm{E}-10$ $1.681 \mathrm{E}-09 \quad 7.277 \mathrm{E}-10$ 3.164E-10 $2.109 \mathrm{E}-09 \quad 8.899 \mathrm{E}-10 \quad 3.738 \mathrm{E}-10$ $2.824 \mathrm{E}-09 \quad 1.165 \mathrm{E}-09 \quad 4.715 \mathrm{E}-10$ $4.418 E-09 \quad 1.879 E-09 \quad 7.817 E-10$ $8.401 \mathrm{E}-\mathrm{Cg} \quad 3.881 \mathrm{E}-09 \quad 1.748 \mathrm{E}-09$ $1.940 \mathrm{E}-08 \quad 9.758 \mathrm{E}-09 \quad 4.723 \mathrm{E}-09$ $6.611 \mathrm{~F}-08 \quad 3.582 \mathrm{E}-08 \quad 1.846 \mathrm{E}-08$ $8.128 E-08$

$1.971 E-08$

$9.186 \mathrm{E}-09$ 
(NEUTRJNS/MEV/STERADIAN/SOURCE NEUTRON)

ENERGY

GROUP (MEV)

$1.22 E$ O1--1.50E 01 $1.00 \mathrm{E}$ 01- - $1.22 \mathrm{E}$ 01 $8.19 \mathrm{E} \quad 00--1.00 \mathrm{E}$ 01 $6.36 \mathrm{E}$ 00-- $-8.19 \mathrm{E} 00$ $4.97 \mathrm{E}$ 00-- $-6.36 \mathrm{E} 00$ $4.07 \mathrm{E}$ 00- $-4.97 \mathrm{E} 00$ $3.01 \mathrm{E} 00--4.07 \mathrm{E}$ OO $2.46 \mathrm{E} 00--3.01 \mathrm{E} 00$ $2.35 \mathrm{E} 00--2.46 \mathrm{E} 00$ $1.83 \mathrm{E} \quad 00--2.35 \mathrm{E} \quad 00$ $1.11 \mathrm{E} 00--1.83 \mathrm{E} 00$ $5.50 \mathrm{E}-01--1.11 \mathrm{E} 00$ $1.11 \mathrm{E}-01--5.50 \mathrm{E}-01$ $3.35 \mathrm{E}-02--1.11 \mathrm{E}-01$
$5.83 \mathrm{E}-04---3.35 \mathrm{E}-02$ $1.01 \mathrm{E}-04--5.83 \mathrm{E}-04$ $2.90 \mathrm{E}-05--1.01 \mathrm{E}-04$ $1.07 E-05--2.90 E-05$ $3.06 E-06--1.07 E-05$
$1.12 E-06---3.06 E-06$ $1,12 E-06---3.06 E-06$ $4.14 \mathrm{E}-07---1.12 \mathrm{E}-06$
$0.0 \quad--4.14 \mathrm{E}-07$ ENFRGY

GROUP (MEV)

$1.22 \mathrm{E} 01--1.50 \mathrm{E}$ O1 $1.00 E$ O1-- $1.22 \mathrm{E}$ OI O.1 6.36E $00--8.19 E$ OO $4.07 E$ OO- -4.36 E 4.07E $00-24.97 E$ OD $3.01 F$ OD- $-4.07 E$ OO 2.46E $00-3.31 \mathrm{E}$ OO 2.35E $00-2.46 E$ OO 1.1IE $00-2.35 E$ OO 5.5OE-01- $1.11 \mathrm{E} 00$ 1. $3.35 E-02--1.11 E-01$ . 1.01E-04- $5.33 E-04$ . 1.07E-05- $1.01 E-04$ 3.07E-06- $1.90 E-05$ $1.12 \mathrm{E}-06-2.06 \mathrm{E}-06$ $4.14 E-07--1.12 E-06$ $4.14 \mathrm{E}-07--1.12 \mathrm{E}-06$
0.0
ANGLE 1 ANGLE 2 $M U=-1$.

$-7.568 \mathrm{E}-04$
0.0 $1.896 \mathrm{E}-03$ $2.475 \mathrm{E}-03$ $1.060 \mathrm{E}-03$ $1.381 \mathrm{E}-03$ $1.740 \mathrm{E}-03$ $2.870 \mathrm{E}-03$ $3.735 \mathrm{E}-03$ $3.051 \mathrm{E}-03$ 3.297E-03 4.610E-03 $5.927 E-03$ $2.998 \mathrm{E}-02$ $2.368 \mathrm{E}-02$ $1.338 \mathrm{E}$ OO $5.573 \mathrm{E} O 0$ $1.586 \mathrm{E}$ O1 $4.136 \mathrm{E}$ O1 $1.087 \mathrm{E}$ O2 $2.339 E$ O2

ANGLE 10 MU $=0.0950$ 0.0 $7.459 E-04$ $5.869 E-03$ $2.138 \mathrm{E}-03$ $2.674 E-03$ $3.451 E-03$ $4.226 E-03$ $5.942 E-03$ . $4.798 E-03$ $6.93 E-03$ 7.245E-03 (.152E 03 $2.471 \mathrm{E}-02$ $2.471 \mathrm{E}-02$ $5.726 \mathrm{E} O 0$ $1.725 \mathrm{E} O 1$ $1.625 E$ OI $1.109 E$ O2 $2.384 \mathrm{E} O 2$ $3.398 \mathrm{E} 02$

$-6.179 E-04$

$1.857 \mathrm{E}-03$

$2.449 \mathrm{E}-03$

$1.063 \mathrm{E}-03$

$1.386 \mathrm{E}-03$ $1.744 \mathrm{E}-03$ $2.876 \mathrm{E}-03$ $3.741 E-03$ $3.059 E-03$ $3.306 \mathrm{E}-03$ $4.619 E-03$ $5.935 \mathrm{E}-03$ $3.000 E-02$ $2.369 \mathrm{E}-02$ $1.338 \mathrm{E} \quad 00$ $5.574 E \quad 00$ $1.586 E$ OI 4.137E OI $1.087 E$ O2 $2.339 E$ OD

ANGLE 11 0.0 $8.817 \mathrm{E}-04$ ..647E-03 $2.202 E-03$ $2.183 E-03$ 3.360E-O3 $4.973 E-03$ 6. $531 E-03$ 5.654E-0 $5.731 \mathrm{E}-03$ $6.918 E-03$ $7.558 \mathrm{E}-03$ 3.393E-02 $2.491 \mathrm{E}-02$ $1.390 \mathrm{E} 00$ $5.754 \mathrm{E} 00$ $4.245 E$ OI $4.245 E$
$1.113 \mathrm{E} 02$ $2.392 \mathrm{E} 02$
ANGLE 3 UU $=-0.9446 \quad M U$

$-2.740 E-04$
$1.727 E-03$
0.0 $1.727 E-03$ $1.075 \mathrm{E}-03$ $1.405 \mathrm{E}-03$ $1.764 \mathrm{E}-03$ $2.901 E-03$ $3.765 \mathrm{E}-03$ $3.090 E-03$ $3.341 \mathrm{E}-03$ $4.656 \mathrm{E}-03$ $5.968 \mathrm{E}-03$ 3.007E-02 $2.373 E-02$ $1.340 \mathrm{E} \mathrm{OO}$ $5.580 E$ OO $1.588 \mathrm{E} \quad 1$ $4.14 J E$ OI $1.088 E$ O2 $2.341 \mathrm{E} \mathrm{O2}$
$3.343 \mathrm{E} 02$

ANGLE 12 0.0 $1.683 E-03$ $6.107 E-03$ 4. $51 \mathrm{E}-03$ 2.51JE-03 . . $7.632 \mathrm{E}-03$ . $6.234 \mathrm{E}-03$ $7.244 E-03$ $7.865 \mathrm{E}-03$ $3.408 \mathrm{E}-02$ 2.510E-02 $2.510 \mathrm{E}-02$ $5.780 \mathrm{E} O 0$ $1.637 \mathrm{E} \mathrm{O1}$ 4.630E OI $1.1170^{2}$ $2.397 \mathrm{E} \mathrm{O2}$ $3.417 \mathrm{E} 02$ $A N G L E$
$U=-0.8656$ $3.698 E-05$ $1.565 E-03$ $2.200 E-03$ $1.101 \mathrm{E}-03$ $1.445 \mathrm{E}-03$ $1.804 \mathrm{E}-03$ $2.950 \mathrm{E}-03$ $3.816 \mathrm{E}-03$ $3.154 \mathrm{E}-03$ 4.727E-03 $6.031 \mathrm{E}-03$ $3.021 E-02$ $2.379 E-02$ $1.343 E 00$ $5.591 \mathrm{E} 00$ 2.5 E 01 4.147E 01 $1.089 E$ $3.348 \mathrm{E} \mathrm{O2}$ 0.0 $1.176 \mathrm{E}-04$ $1.499 E-03$ $2.037 E-03$ $1.145 \mathrm{E}-03$ $1.504 E-03$ $1.866 \mathrm{E}-03$ $3.028 \mathrm{E}-03$ $3.899 E-03$ $3.254 \mathrm{E}-03$ 3.515E-03 4. $835 \mathrm{E}-03$ $6.124 E-03$ 3.04OE-02 $2.389 E-02$ $1.347 E$ OO 5.606E 00 1.594E $4.156 E$ OI 1.091E O2 $3.354 \mathrm{E} 02$

ANGLE 6 $M U=-0$ 0.0

1.74 . E-04 $1.630 E-03$ $1.906 \mathrm{E}-03$ $1.211 \mathrm{E}-03$ $1.587 \mathrm{E}-03$ $1.955 \mathrm{E}-03$ 3.140E-03 $4.028 \mathrm{E}-03$ $3.396 \mathrm{E}-03$ 3.661E-03 $4.984 E-03$ $6.248 \mathrm{E}-03$ $3.366 \mathrm{E}-02$ $2.401 E-02$ $1.352 E 00$ . 1.S9E 01 1.157E 01 $1.094 \mathrm{E} 02$ $3.362 \mathrm{E} 02$ ANGLE 14 0.6179 0.0 $6.798 \mathrm{E}-03$ $6.851 E-03$ $2.866 E-03$ $3.435 \mathrm{E}-03$ 3.435E-03 $4.162 E-03$ $6.135 E-03$ $6.673 E-03$ $6.673 \mathrm{E}-03$ $6.633 E-03$ 7.813E-0 $0.133 E-03$ 3.183 $1.528 E-02$ $5.804 E 00$ $1.645 \mathrm{E}$ O1 $1.645 E$
$4.274 E$ O1 $1.120 \mathrm{E} 02$ $2.406 \mathrm{E} \mathrm{O2}$ $3.426 \mathrm{E} 02$

ANGLE 7 ANGLE 8 ANGLE 9
$94=-0.0950$ 0.0

$1.187 E-05$ $1.999 E-03$ $1.837 E-03$ $1.303 E-03$ $1.696 \mathrm{E}-03$ $2.076 E-03$ $3.297 \mathrm{E}-03$ $4.215 \mathrm{E}-03$ 
ENERGY GROUP (MEV) $1.22 E$ O1---1.5OE 01 $1.00 E$ O1- $-1.22 E$ O 6.1 SE OON-1.OOE OI $4.37000-6.36 E 0$ $4.07 E$ 00-- $4.97 E$ $3.07 E$ OO- $4.97 E$ OO 2.46E $00--3.01 E$ EO $2.36 \mathrm{E}$ OO- $2.46 \mathrm{E}$ OO 1. $33 \mathrm{E}$ 00--2. $35 \mathrm{E}$ OO 1.11E $00--1.83 \mathrm{E} O$ $5.50 E-01--1.11 \mathrm{E} O 0$ $1.11 E-01--5.50 E-01$ $3.35 \mathrm{E}-02-\cdots 1.11 \mathrm{E}-01$ $5.83 E-04--3.35 E-02$ $1.01 E-04--5.83 E-04$ $2.90 E-05--1.01 E-04$ $1.07 E-05--2.90 E-05$ $3.06 E-06-\cdots-1.07 E-05$ $1.12 \mathrm{E}-06--3.06 \mathrm{E}-06$ $4.14 \mathrm{E}-07--1.12 \mathrm{E}-06$

\section{ENERGY}

GROUP (MEV)

$1.22 E 01--1.50 E$ O1 $1.00 E$ 01---1.22E O1 8.1 9E 00---1.00E 01 $6.36 E$ 0O---8.19E OD $4.97 \mathrm{E}$ 00-- $-6.36 \mathrm{E}$ OO 4.07E $00--4.97 E$ EO $3.01 \mathrm{~F} 00---4.07 \mathrm{E} 00$ $2.46 \mathrm{E}$ 0O-- $3.01 \mathrm{E} \mathrm{OO}$ $2.35 E$ OO---2.46E 00 $1.83 \mathrm{E} 00--2.35 \mathrm{E} 00$ $1.11 \mathrm{E} 00--1.83 \mathrm{E} 00$ $5.50 E-01 \cdots-1.11 E$ OO 1.11E-01-- $5.50 E-01$ 3. $35 \mathrm{E}-02--1.11 \mathrm{E}-01$ $5.83 E-04--3.35 E-02$ $1.01 \mathrm{E}-04--5.83 \mathrm{E}-04$ $2.90 \mathrm{E}-05--1.01 \mathrm{E}-04$ $1.07 \mathrm{E}-05--2.90 \mathrm{E}-05$ $3.06 E-06--1.07 E-05$ $1.12 \mathrm{E}-06--0.06 \mathrm{E}-\mathrm{C} 6$ $4.14 \mathrm{E}-07---1.12 \mathrm{E}-06$
0.0
ANGLE 1 $M U=-1.0000$ $-8.683 E-04$ (.) $1.613 \mathrm{E}-03$ $1.613 E-03$ $2.680 E-03$ $4.733 E-03$ $6.431 E-03$ $5.446 \mathrm{E}-03$ $6.433 \mathrm{E}-03$ $1.044 \mathrm{E}-02$ $1.608 \mathrm{E}-02$ $9.596 \mathrm{E}-02$ $7.991 E-02$ $4.652 \mathrm{E}$ OO $1.968 \mathrm{E}$ O1 $5.652 \mathrm{E} 01$ $1.488 \mathrm{E} \quad 02$ $3.932 \mathrm{E} \mathrm{O2}$ $8.505 \mathrm{E} \mathrm{O2}$ $1.221 \mathrm{E} 03$

ANGLE 10 0.0 $8.934 E-04$ $3.420 E-03$ $3.778 E-03$ $2.367 \mathrm{E}-0$ $2.967 E-03$ $3.359 \mathrm{E}-03$ $5.855 E-03$ $1.248 E-02$ $1.122 E-02$ $1.198 \mathrm{E}-02$ $1.609 E-02$ $2.062 E-02$ $1.105 \mathrm{E}-01$ $8.533 \mathrm{E}-02$ $4.912 \mathrm{E} 00$ $2.064 \mathrm{E}$ O1 $5.910 \mathrm{E} 01$ $1.550 \mathrm{E} \quad 22$ $4.086 \mathrm{E}$ 02 $8.817 E \quad 02$
$1.261 \mathrm{E} 03$
ANGLE 2 0.0 $-7.084 \mathrm{E}-04$ $1.890 \mathrm{E}-03$ $2.871 \mathrm{E}-03$ $3.616 \mathrm{E}-03$ $2.166 \mathrm{E}-03$ $2.686 E-03$ $4.741 E-03$ $6.439 E-03$ $5.460 \mathrm{E}-03$ $6.450 \mathrm{E}-03$ $1.046 \mathrm{E}-02$ $1.046 E-02$
$1.611 E-02$ $9.604 E-02$ 7.995E-02 $4.654 \mathrm{E} \mathrm{CO}$ $1.969 \mathrm{E} 01$ $5.655 \mathrm{E} 01$ $1.488 \mathrm{E} 02$ $3.934 E 02$ $8,507 E$ C2 $1.221 \mathrm{E} 03$ ANGLE 11 0.0 $1.154 E-03$ $6.329 E-03$ $2.311 \mathrm{E}-0$ $3.770 E-0$ 4.732E-03 $5.512 \mathrm{E}-03$ $8.810 \mathrm{E}-03$ $8.939 E-03$ $8.0605-03$ $9.293 \mathrm{E}-03$ $1.438 \mathrm{E}-02$ $2.007 \mathrm{E}-02$ $1.071 \mathrm{E}-01$ $8.639 E-02$ $4.962 E 00$ 2.082E 01 $5.958 \mathrm{E} \quad 01$ $1.561 \mathrm{E} 02$ $4.113 E 02$ $8.873 \mathrm{E} O 2$
$1.268 \mathrm{E} 03$
(NEUTRJNS/MEV/STERADIAN/SOURCE NEUTRON)

ANGLE 3 ANGLE 4 ANGLE 5 ANGLE 6 $M U=-0.9446 \quad M U=-0.8656$ $0.0 \quad 0.0$ $2.753 \mathrm{E}-04 \quad 1.004 \mathrm{E}-04$
$1.724 \mathrm{E}-03$ $1.724 \mathrm{E}-03$
$2.751 \mathrm{E}-03$ $1.632 \mathrm{E}-03$ 2.198E-03 $2.711 E-03$ $4.774 \mathrm{E}-03$ $4.774 E-03$
$6.472 E-03$ $5.517 E-03$ $6.522 E-03$ $1.055 \mathrm{E}-02$ $1.055 \mathrm{E}-02$
$1.621 \mathrm{E}-02$ $9.636 E-02$ $9.636 \mathrm{E}-02$
$8.014 \mathrm{E}-02$ $8.014 E-02$
$4.663 E ~ 00$ $1.972 \mathrm{E} 01$ $5.664 \mathrm{E} 01$ $1.490 E 02$ $3.939 \mathrm{E} 02$ $8.519 E$ O2 $1.223 E \quad 03$

ANGLE 12

$1.253 E-03$ $8.280 \mathrm{E}-03$ 4.777E-03 94E-0 $5.020 E-03$ $8.515 E-03$ $1.30 \mathrm{BE}-0$ $1.145 \mathrm{E}-02$ $1.220 E-02$ $1.710 \mathrm{E}-02$ $2.169 \mathrm{E}-02$ $1.372 E-01$
$8.749 E-02$ $5.010 \mathrm{E} 00$ $2.100 E$ O1 $6.004 \mathrm{E} \mathrm{OL}$ $1.572 \mathrm{E} 02$ $4.140 E 02$

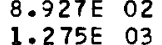

ANGLE 5 0.0

$2.083 E-04$ $2.563 E-03$ $1.671 \mathrm{E}-03$ $2.262 \mathrm{E}-03$ $4.843 \mathrm{E}-03$ $6.843 E-03$ $5.632 \mathrm{E}-03$ $6.664 \mathrm{E}-03$ $6.664 E-03$ $1.073 \mathrm{E}-02$ $9.697 E-02$ 8.051E-02 $4.681 \mathrm{E} \mathrm{OO}$ $1.979 E$ OI $5.682 E$ OI $1.495 \mathrm{E} \mathrm{O2}$ $3.950 E 02$ $8.541 \mathrm{E} 02$ $1.226 E 03$ ANGLE 13 $U=0.617$ $2.647 E-03$
$8.281 E-03$ 4.262E-0 $5.333 \mathrm{E}-0$ $6.599 E-03$ $7.704 \mathrm{E}-03$ $1.205 \mathrm{E}-02$ $1.626 \mathrm{E}-02$ $1.401 \mathrm{E}-02$ $1.445 \mathrm{E}-02$
$1.930 \mathrm{E}-02$ 2.307E-02 $1.154 \mathrm{E}-01$
$8.852 \mathrm{E}-02$ $5.057 \mathrm{E} 00$ 2.117E O1 $6.047 \mathrm{E}$ O1 1. $582 \mathrm{E} 02$ $4.165 E$ O2 1.282E 03
$1.449 \mathrm{E}-03$

$1.740 \mathrm{E}-03$

$2.357 E-03$

$2.844 \mathrm{E}-03$

$4.954 \mathrm{E}-03$
$6.678 \mathrm{E}-03$

$6.678 \mathrm{E}-03$
$5.816 \mathrm{E}-03$ $6.878 E-03$ $6.878 E-03$ $1.100 E-02$
$1.670 E-02$ $9.784 \mathrm{E}-02$ $8.101 E-02$ $4.706 E 00$ $1.988 \mathrm{E} 01$ $5.707 E$ O1
$1.501 \mathrm{E} 02$ 3.965E 02 $8.572 \mathrm{E} \quad 02$ 1. $231 \mathrm{E} 03$ ANGLE 14 $M U=0.7550$

$6.155 \mathrm{E}-03$ $1.427 E-02$ $6.536 E-03$ $6.141 \mathrm{E}-03$ $1.386 \mathrm{E}-02$ 1. $828 \mathrm{E}-02$ $1.526 E-02$ $1.544 \mathrm{E}-02$
$2.062 \mathrm{E}-02$ $2.393 \mathrm{E}-02$ $1.112 \mathrm{E}-01$ $5.097 \mathrm{E}$ OO $2.131 \mathrm{E}$ ol $6.085 \mathrm{E} 01$ $1.591 \mathrm{E} 02$ $4.187 \mathrm{E} \quad 02$ $1.287 E 03$

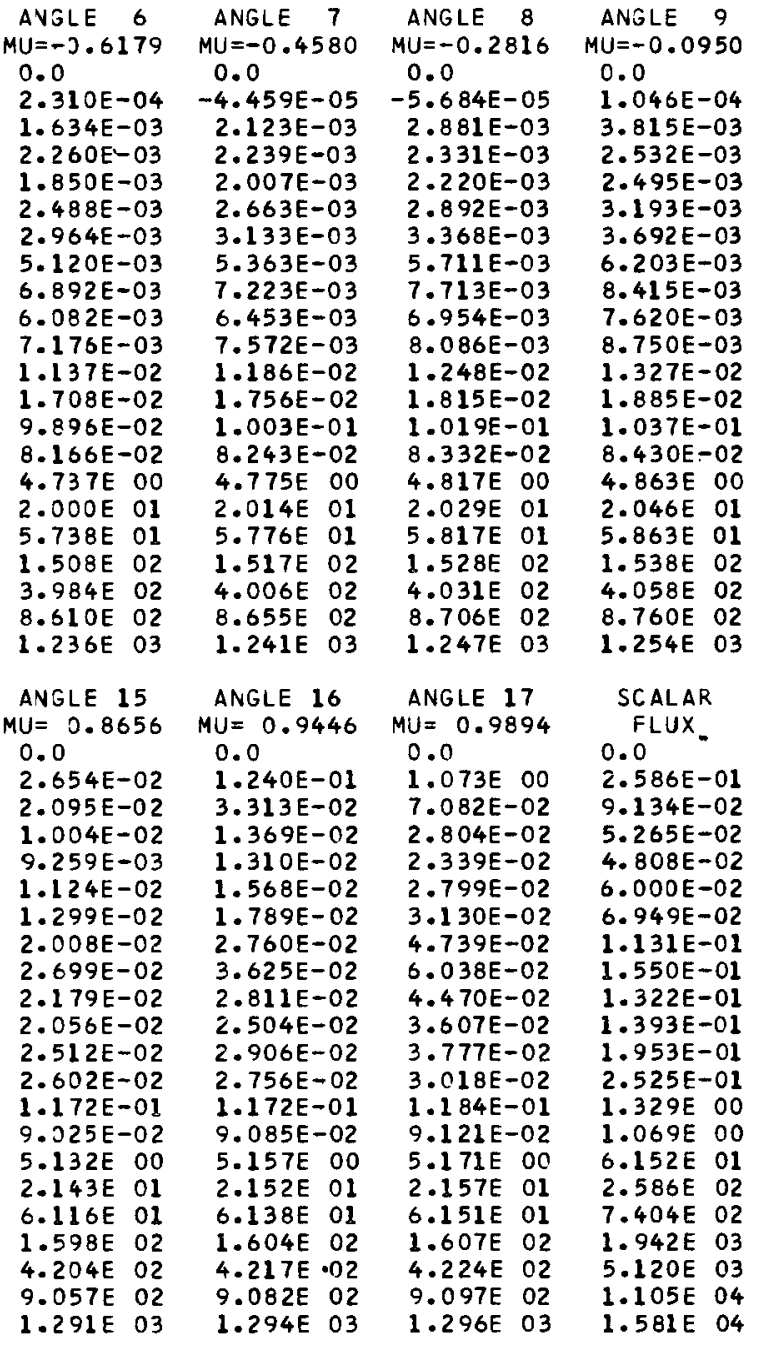


(NEUTRJNS/MEV/STERADIAN/SOURCE NEUTRON)

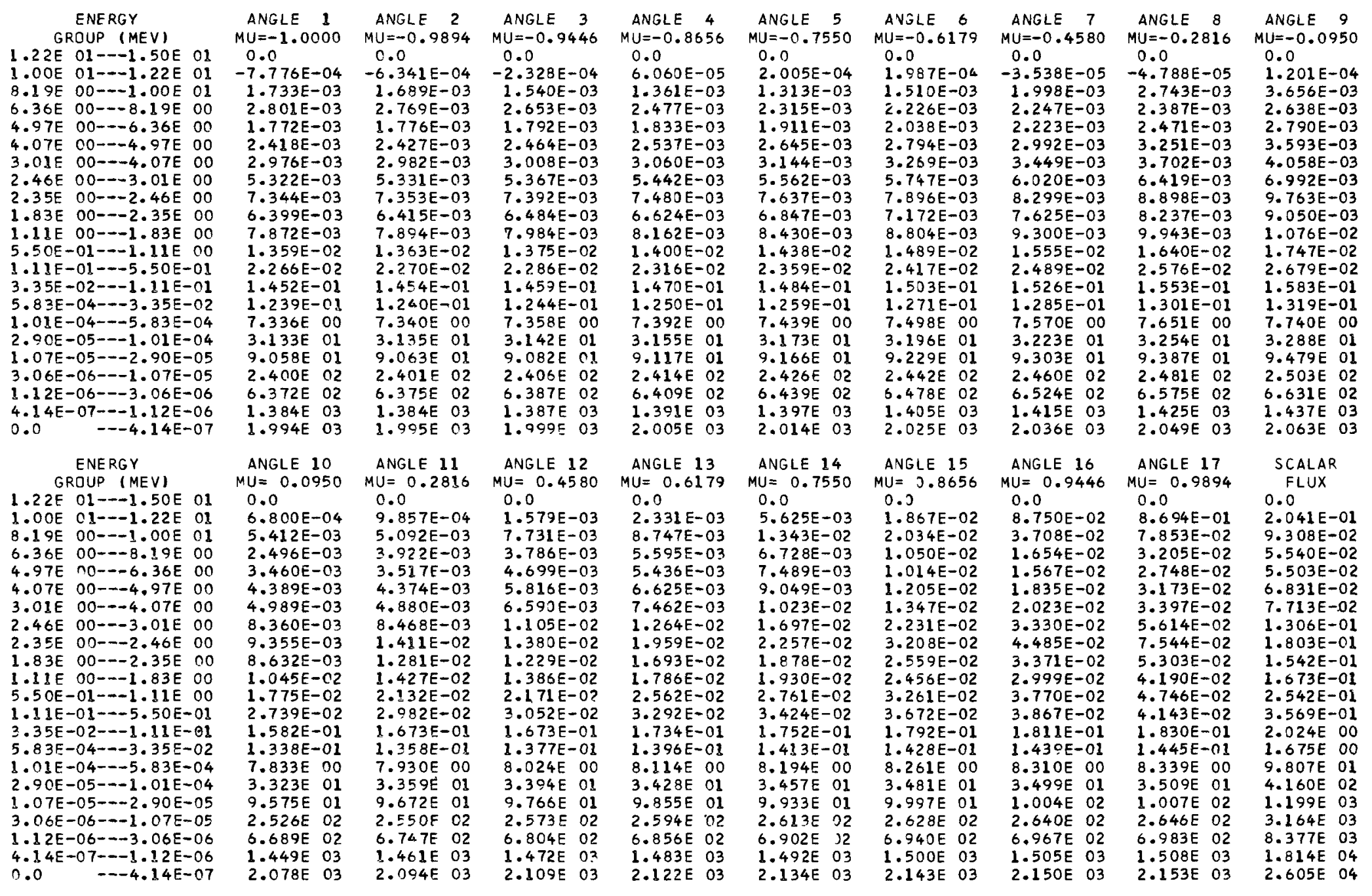


ENERGY $1.22 \mathrm{E} 01--1.50 \mathrm{E}$ O1 $1.00 E$ O1---1.22E O1 $8.19 \mathrm{~F}$ OO- $-1.00 \mathrm{E}$ OI $6.36 \mathrm{E} \quad 00---8.19 \mathrm{E}$ OO $4.97 \mathrm{E} \quad 00---6.36 \mathrm{E}$ OO 3.01E $00--4.07 E$ OO .46E $00--3.01 \mathrm{E} 00$ $2.35 E$ EO--2.46E 00 $1.83 E$ OO- $-2.35 E$ E $1.11 \mathrm{E} 00-\cdots-1.83 \mathrm{E} 00$ $5.50 E-01--1.11 E 00$ $1.11 \mathrm{E}-01--5.50 \mathrm{E}-01$ $3.35 E-02--1.11 E-01$ $5.83 E-04---3.35 E-02$ $1.01 E-04--5.83 E-04$ $2.90 E-05--1.01 E-04$ $1.07 E-05--2.90 E-05$ $3.06 E-06--1.07 E-05$ $1.12 \mathrm{E}-06=--3.06 \mathrm{E}-06$ $4.14 \mathrm{E}-07--1.12 \mathrm{E}-06$

ENE RGY

GROUP (MEV)

$1.22 E$ O1--1.50E OI $1.00 E$ O1---1.22E OI 8.1 EE OO---1.00E OI 6.36 E OO- $-8.19 E$ OO $4.97 E$ OO- $-6.36 \mathrm{E} \mathrm{OO}$ 4.07E 00---4.97E 00 $3.01 \mathrm{E} 00--4.07 E$ OO $2.46 E$ OO-D-3.01E OO $2.35 \mathrm{E} 00--2.46 \mathrm{E} 00$ $1.83 E$ EO--2.35E 00 $1.11 \mathrm{E}$ OO-C-1.83E OO $5.50 E-01-1.11 \mathrm{E}$ OO $1.11 E-01--5.50 E-01$ . 5.83E-04-C- $3.35 E-02$ $1.01 E-04--5.83 E-C 4$ $2.90 E-05--1.01 E-04$ $1.07 E-05=-2.90 E-05$ .06E-06- $1.07 E-05$ $4.14 E-07-0-1.12 E-06$ $\begin{aligned} & 4.14 \mathrm{E}-07--1.12 \mathrm{E}-06 \\ & 0.0\end{aligned}--4.14 \mathrm{E}-07$ $\begin{array}{ll}A N G L E & 1\end{array} \quad$ ANGLE 2 0.0 $.344 E-04$ $1.240 \mathrm{E}-03$ $1.772 \mathrm{E}-0$ $2.508 \mathrm{E}-03$ $3.043 \mathrm{E}-03$ $5.451 \mathrm{E}-03$ $7.667 \mathrm{E}-03$ $7.054 \mathrm{E}-03$ $9.211 \mathrm{E}-03$ $1.739 \mathrm{E}-02$ $3.253 \mathrm{E}-02$ 2.311E-01 $2.044 \mathrm{E}-01$ $1.242 E$ OI 5.390E 01 $1.576 \mathrm{E} \mathrm{O2}$ 4.229E 02 $1.133 \mathrm{E} \quad 03$ $3.605 \mathrm{E} \mathrm{O3}$ ANGLE 10 0.0

4. $853 \mathrm{E}-04$ $3.557 E-03$ $2.964 \mathrm{E}-03$ $3.255 \mathrm{E}-03$ $4.147 E-03$ $4.471 E-03$ $8.018 \mathrm{E}-0$ $1.245 \mathrm{E}-02$ $1.187 \mathrm{E}-02$ $1.442 \mathrm{E}-02$ $2.497 \mathrm{E}-02$ 4.111E-02 $2.634 \mathrm{~F}-01$ $2.241 E-0$ $1.348 E$ Ol 5.812E O1 $1.695 \mathrm{E} 02$ $4.528 E$ E2 $1.209 E$ O $3.812 \mathrm{E} 03$
$A N G L E ?$
$M U=-0.9894$ 0.0 4.351E-04 $1.211 \mathrm{E}-03$ $2.256 \mathrm{E}-0$ $1.775 \mathrm{E}-03$ $2.518 \mathrm{E}-03$ $5.459 \mathrm{E}-03$ $7.679 E-03$ $7.073 E-03$ $9.238 \mathrm{E}-\mathrm{O} 3$ $1.743 \mathrm{E}-02$ . $314 \mathrm{E}-0 \mathrm{I}$ $.243 E$ OI $.232 E$ O2 $1.134 E 03$ $3.607 \mathrm{E} 03$ ANGLE 11 0.0

$9.658 \mathrm{E}-04$ $4.787 \mathrm{E}-0$ $3.251 \mathrm{E}-03$ $3.958 \mathrm{E}-03$ 4. $970 \mathrm{E}-03$ $5.402 E-03$ $9.560 \mathrm{E}-03$ $1.380 \mathrm{E}-02$ $1.284 \mathrm{E}-02$ $1.531 \mathrm{E}-02$ $2.670 E-02$ 4. $284 \mathrm{E}-02$ $2.671 \mathrm{E}-01$ $2.280 E-01$ $1.369 E$ Ol 1.89E OI $1.717 E$ O2 4.584E 02 $2.669 \mathrm{E} 03$ $3.851 \mathrm{E} \mathrm{O3}$
(NEUTRONS/MEV/STERADIAN/SOURCE NEUTRON)

ANGLE 3 ANGLE 4 ANGLE 5 ANGLE

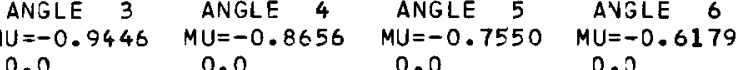
$1063 E-04$

$-1.463 E-04$
$1.113 E-03$ $2.166 \mathrm{E}-03$ $1.790 \mathrm{E}-03$ $1.790 \mathrm{E}-03$
$2.558 \mathrm{E}-03$ $3.070 \mathrm{E}-03$ $5.496 \mathrm{E}-03$ $7.727 \mathrm{E}-03$ $7.155 E-03$ $9.349 \mathrm{E}-03$ $1.761 E-02$ $2.324 E-O 1$ (2) 政

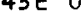
$1.137 E$ O3

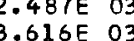
ANGLE 12 $M=0.4580$

0.0
$1.278 E-03$ $5.645 \mathrm{E}-03$ $4.161 \mathrm{E}-03$ $4.679 \mathrm{E}-03$ $5.781 E-03$ $6.257 \mathrm{E}-03$ $1.121 \mathrm{E}-02$ $1.731 \mathrm{E}-02$ $1.564 \mathrm{E}-02$ $1.789 \mathrm{E}-02$ $2.989 \mathrm{E}-02$ $4.545 \mathrm{E}-02$ $2.755 \mathrm{E}-01$ 2.320 E-OL $1.389 \mathrm{E}$ Ol 1.739E 02 .739E 02 238E 03 $.698 \mathrm{E} 03$ $3.889 \mathrm{E} \mathrm{C3}$
$2.841 E-05 \quad 0.0$ $2.035 E-03$ $1.831 \mathrm{E}-03$ 2.636E-0 $3.116 E-03$ $7.837 E-O 3$ $7.321 \mathrm{E}-03$ $9.567 \mathrm{E}-03$ $1.796 \mathrm{E}-02$ $3.332 \mathrm{E}-02$ $2.343 \mathrm{E}-01$ $2.065 \mathrm{E}-0$ $1.254 \mathrm{E} 01$ $5.437 E$ O1 1.590 E O2 $4.263 \mathrm{E} O 2$ $2.498 E$ O3 $3.631 \mathrm{E} 03$ ANGLE 13 0.

$2.029 E-03$ $7.697 \mathrm{E}-03$ $5.121 \mathrm{E}-03$ $5.981 \mathrm{E}-0$ 7.280 E-03 $7.902 \mathrm{E}-03$ $1.409 \mathrm{E}-02$ 2.091E-02 $1.812 \mathrm{E}-0$ $3.287 \mathrm{E}-02$ $4.776 \mathrm{E}-02$ $2.807 \mathrm{E}-01$ .358E-01 $1.409 \mathrm{E}$ OI $6.050 \mathrm{E} \mathrm{O1}$ $1.760 E$ O2 $4.692 \mathrm{E} O 2$ $2.725 \mathrm{E} O 3$ $3.925 \mathrm{E} 03$ $1.931 E-03$ $1.914 E-03$
$2.751 E-03$ $2.751 E-03$ $5.696 \mathrm{E}-03$ $8.030 \mathrm{E}-03$ $7.585 \mathrm{E}-03$ $9.896 \mathrm{E}-03$ $1.849 E-02$ $2.371 E-01$ $2.083 \mathrm{E}-01$ $1.263 \mathrm{E} 01$ $1.601 \mathrm{E} \mathrm{O2}$ 4.291E 02 $\begin{array}{lll}1.149 \mathrm{E} & 03 \\ 2.513 \mathrm{E} & 03\end{array}$ $3.652 \mathrm{E} 03$ $U=0.7550$ 0.0

4. $781 \mathrm{E}-03$ $1.083 \mathrm{E}-02$ $7.802 \mathrm{E}-03$ $9.296 \mathrm{E}-03$ $1.000 \mathrm{E}-02$ $1.797 \varepsilon-02$ 2.739E-02 $2.262 \mathrm{E}-02$ $3.721 \mathrm{E}-02$ $5.060 \mathrm{E}-02$ $2.879 E-01$ $2.392 E-01$ $1.426 \mathrm{E} 01$ $6.117 E$ O1 1.779E O2 $4.738 \mathrm{E} 02$ 1.742 O $3.956 \mathrm{E} \mathrm{O3}$
2.429E-04
0.0
$1.110 E-04$

ANGLE 7

AN $=-0.4580$ 0.0 $9.967 \mathrm{E}-04 \quad 1.177 \mathrm{E}-03$ 1. $901 \mathrm{E}-03$ $2.053 \mathrm{E}-03$
$2.908 \mathrm{E}-03$ $2.908 E-03$
$3.310 E-03$ $5.890 E-03$ $8.345 E-03$ $7.970 \mathrm{E}-03$ $1.035 E-02$ $1.920 \mathrm{E}-02$ $2.406 \mathrm{E}-01$ $2.107 \mathrm{E}-01$ 1.276E OL $5.528 \mathrm{E} 01$ $1.615 E 02$ 4.327E 02 $\begin{array}{lll}1.158 E & 03 \\ 2.533 E & 03\end{array}$ $3.677 \mathrm{E} 03$ ANGLE 15

0.0

$1.429 \mathrm{E}-02$ $1.779 \mathrm{E}-02$ $1.035 \mathrm{E}-02$
$1.098 \mathrm{E}-02$ $1.098 \mathrm{E}-02$ 
(NEUTRJNS/MEV/STERADIAN/SOURCE NEUTRON)

\begin{abstract}
ENERGY
GROUP (MEV)

$1.22 E$ O1---1.5OE 01

$1.00 \mathrm{E} 01---1.22 \mathrm{E}$ O1

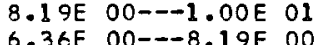
$6.36 \mathrm{E}$
$4.97 \mathrm{E}$ O0- $00-6.36 \mathrm{E}$ OO $4.97 E$ OO- $00.36 \mathrm{E} 00$
$4.07 \mathrm{E} 00---4.97 \mathrm{E}$ OO $3.01 \mathrm{E} \mathrm{OO--4.07E} 00$ $2.46 \mathrm{E}$ DO---3.01E 00 $2.35 \mathrm{E} 00--2.46 \mathrm{E}$ OO $1.83 \mathrm{E} 00--2.35 \mathrm{E} 00$ $1.11 \mathrm{E} 00--1.83 \mathrm{E} 00$ 1.11E $00--1.83 E$ OO . $11 \mathrm{E}-01-5.11 \mathrm{E}$ OO $3.35 \mathrm{E}-02--1.11 \mathrm{E}-01$ $3.35 \mathrm{E}-02-1.11 \mathrm{E}-01$ $5.83 \mathrm{E}-04--3.35 \mathrm{E}-02$ $1.03 E-04-25.33 E-04$ $2.90 E-05-1.01 E-04$ 3.06E-06---1.07E-05 $1.12 \mathrm{E}-06-03.06 \mathrm{E}-06$ $4.14 E-07--1.12 E-06$ $0.14 \mathrm{E}-07--1.12 \mathrm{E}-06$ ENERGY

GROUP (MEY)
\end{abstract}

$1.22 \mathrm{E} 01--1.50 \mathrm{E}$ OI $1.00 F$ 01---1.22E O1 $8.19 E$ OO- - - 1.00E 01

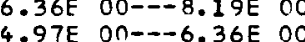
$4.07 \mathrm{E} 00--4.97 \mathrm{E}$ OO $3.01 E$ OO- $-4.07 E$ OO $2.46 \mathrm{E} 00--3.01 \mathrm{E} 00$ $2.35 \mathrm{E} 00---2.46 \mathrm{E} 00$ $1.83 \mathrm{E} 00--2.35 \mathrm{E} 00$ $1.11 \mathrm{E} 00--1.83 \mathrm{E}$ OO 5.50E-01---1.11E OO $1.11 E-01--5.50 E-01$ $3.35 \mathrm{E}-02---1.11 \mathrm{E}-01$ $5.83 \mathrm{E}-04---3.35 \mathrm{E}-02$ $1.01 E-04--5.83 E-04$ $2.90 E-05--1.01 E-04$ $1.07 E-05--2.90 E-05$ $3.06 \mathrm{E}-06--1.07 \mathrm{E}-05$ $1.12 E-06--3.06 E-06$ $4.14 E-07--1.12 E-06$ $0.0 \quad--4.14 \mathrm{E}-\mathrm{C}$ ?
ANGLE 1 ANGLE 2 $M U=-1.0000$ $3.390 E-04$ . $308 \mathrm{E}-04$ $1.703 \mathrm{E}-03$ $1.550 E-03$ $2.271 E-03$ 2.724E-03 $4.834 E-03$ $6.886 \mathrm{E}-03$ $0.642 E-03$ $1.819 E-02$ 1.704E-O2 $3.704 \mathrm{E}-0$ $2.831 E-01$ $2.566 \mathrm{E}-01$ $6.989 E$ OI $2.063 \mathrm{E} 02$ $5.595 \mathrm{E} O 2$ $5.595 E$
$1.511 E$ 1.511E 03 $3.331 \mathrm{E}$
$4.8393 \mathrm{E}$

ANGLE 10 $M U=$

0.0
$4.090 E-04$ $2.753 E-03$ $2.378 \mathrm{E}-\mathrm{C}$ $3.002 \mathrm{E}-03$ $3.871 E-03$ 4.091E-03 7.411E-03 $1.133 \mathrm{E}-0$ $1.102 \mathrm{E}-02$ $1.388 \mathrm{E}-02$ $2.630 \mathrm{E}-02$ $4.713 E-02$ $3.238 \mathrm{E}-01$ $2.844 \mathrm{E}-01$ $1.745 \mathrm{E} \mathrm{O}$ $7.624 \mathrm{E}$ OI $2.245 E$ O2 $6.063 E \quad 02$ $1.632 \mathrm{E} \mathrm{O}$ $3.588 \mathrm{E} 03$ $5.218 E \quad 03$
ANGLE 2 ANGLE 3 $M U=-0$ - $8.671 E-05$ 1. $365 \mathrm{E}-03$ . (.728E-03 6. $6.661 E-03$ 6.061E-03 $1.824 E-0$ $3.711 \mathrm{E}-02$ $.834 E-0$ $2.568 \mathrm{E}-01$ $1.591 \mathrm{E} 01$
$6.995 \mathrm{E}$ 01 $2.065 \mathrm{E} 02$ $5.509 E \quad 2$ 1.512E O3 $3.333 \mathrm{E} \mathrm{O3}$ $4.882 \mathrm{E} \mathrm{O3}$

ANGLE 11

0.0 $.243 E-04$ $2.854 E-03$ $3.536 \mathrm{E}-03$ . . $277 \mathrm{E}-02$ . $888 E-02$ 4. $973 E-02$ 3. $333 \mathrm{E}-01$ .9COE-OI $1.776 E$ OI $7.748 E$ OI $2.280 E$ O $6.154 E$ O2 1.656E 03 $3.637 E$
$5.283 E 03$

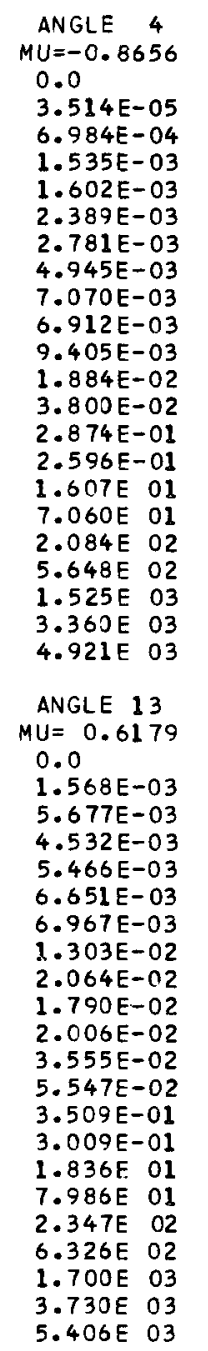

ANGLE 5
$M U=-0.7550$ 0.0 $1.695 \mathrm{E}-04$ $7.120 \mathrm{E}-04$ $1.476 E-03$ $1.680 E-03$ $2.495 E-03$ $2.844 E-03$ $7.061 E-03$ $7.275 E-03$ . 73 EE 03 $1.942 E-O 2$ 1. $882 \mathrm{E}-02$ 2. $11 E-01$ $2.911 E-01$ $2.622 \mathrm{E}-01$ $7.119 E$ OI $2.101 E$ O2 $5.692 E$ O2 $5.692 \mathrm{E} O 2$
$1.536 \mathrm{E} \mathrm{O3}$ $1.536 E$ E3 $4.954 \mathrm{E} \quad 03$ ANGLE 14 ANGLE 14 0.0 3.752E-03 $8.388 E-03$ $6.256 E-03$
$7.273 E-03$

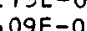
$8.914 \mathrm{E}-03$ $1.691 \mathrm{E}-0$ ? $2.679 E-02$ $2.183 \mathrm{E}-02$ $2.315 E-02$ $3.983 \mathrm{E}-02$ $5.850 E-02$ 3.588E-01 $3.058 \mathrm{E}-01$ $1.862 \mathrm{E}$ OL $8.090 \mathrm{E} \mathrm{OL}$ $2.377 \mathrm{E} \mathrm{O2}$ $6.400 E \quad 02$ $1.719 \mathrm{E} \quad 03$ $3.770 E$ O3 $5.458 \mathrm{E} \quad 03$ $U=-0.6179$ 60 $6.662 E-05$ $8.537 E-04$ $1.484 E-03$ $1.811 E-03$ $2.640 E-03$ $2.943 E-03$ $7.601 E-03$ $7.601 E-03$ 1.020E-02 . $020 \mathrm{E}$ . . 2.655E-01 $2.655 E-01$ $7.195 \mathrm{E}$ $2.122 E 02$ $5.748 E$ O2 1. $551 \mathrm{E} \mathrm{O} 03$ 3.415 E 03 $4.996 \mathrm{E} \mathrm{O3}$

AVGLE 15

0.0 $1.394 E-02$ $9.352 \mathrm{E}-03$ 1. $C 20 \mathrm{E}-02$ $1.166 \mathrm{E}-02$ $1.180 E-02$ $2.293 E-02$ $3.634 \mathrm{E}-02$ $2.746 E-02$ $2.713 \mathrm{E}-02$ $3.098 \mathrm{E}-01$ $1.883 E$ ol $8.176 E$ OI $2.401 \mathrm{E} 02$ $6.462 \mathrm{E} 02$ $1.734 \mathrm{E} 03$ $3.802 \mathrm{E} O$ $5.499 E_{03}$
ANGLE 7 ANGLE 8 ANGLE $M U=-0.4580 \quad M U=-0.2816 \quad M U=-0.0950$ 1.152E 03 . .093 8 8.096E-0 1.082E-0 $2.127 E-02$ $4.130 E-0$ $3.017 \mathrm{E}-01$ $2.694 \mathrm{E}-01$ $1.662 \mathrm{E}$ OI $7.286 \mathrm{E}$ O1 $5.815 E$ E 02 $5.815 \mathrm{E}$ O2
$1.568 \mathrm{E} 03$ $\begin{array}{ll}1.568 \mathrm{E} & 03 \\ 3.452 \mathrm{E} & 03\end{array}$ $5.043 \mathrm{E} \mathrm{O3}$

ANGLE 16 $3.535 \mathrm{E}-02$ $2.694 \mathrm{E}-02$ $1.546 \mathrm{E}-02$ $1.692 \mathrm{E}-02$ $1.652 \mathrm{E}-02$ $3.346 E-02$ $5.274 E-02$ $3.605 E-02$ $3.226 \mathrm{E}-02$
$5.087 \mathrm{E}-02$ $6.421 \mathrm{E}-02$ 3. $713 \mathrm{E}-01$ $3.129 E-01$ $1.899 \mathrm{E}$ OI
$8.240 \mathrm{E}$ OI $2.419 E$ O2 $6.507 \mathrm{E} 02$ $1.746 \mathrm{E} 03$ $\begin{array}{lll}3.827 E & 03 \\ 5.528 E & 03\end{array}$

0 $1.595 \mathrm{E}-03$ $1.040 E-04$ $2.585 E-03$ $3.415 E-03$ $3.627 E-03$ . $8.823 E-03$ 1. $264 E-02$ $1.264 E-02$ $2.428 E-02$ 4. $392 \mathrm{E}-02$ $3.160 E-01$ 
ENERGY

(MEV) $1.22 E$ O1---1.5OE 01 .OCE $01--1.22 E$ OI 6.36 OO--8.1 01 $4.07 E$ O0-- $6.36 \mathrm{E}$ OD $4.07500-04.07 E 00$ $3.01 \mathrm{E} 00--4.07 E \mathrm{E} 00$ $46 E$ 00- $3.01 E$ EO $2.35 E$ EO-- $2.46 E$ EO $1.83 E 00--2.35 E$ OO $1.11 \mathrm{E} 00-1.83 \mathrm{E} 00$ $5.50 \mathrm{E}-01--1.11 \mathrm{E} 00$ $1.11 \mathrm{E}-01--5.50 \mathrm{E}-01$ $3.35 \mathrm{E}-02--1.11 \mathrm{E}-01$ $5.83 E-04-\cdots 3.35 E-02$ $.01 E-04---5.83 E-04$ $3.90 E-05--1.01 E-04$ $1.07 E-05---2.90 E-05$ $3.06 \mathrm{E}-06--1.07 \mathrm{E}-05$ $1.12 E-06--3.06 E-06$ 4.1 $4 E-07--1.12 E-06$

ENERGY

GROUP (MEV)

$1.22 \mathrm{E} \mathrm{O1---1.50E} 01$ $1.00 E$ 01---1.22E 01 8.1 OE $00--1.00 E$ OI $6.36 E$ 0O- $-8.19 E$ OO 4.97E OO--6.36E 00 4.07E DO--4.97E 00 3.01E $00--4.07 E$ OO $2.46 \mathrm{E} \quad 00--3.01 \mathrm{E} 00$ $2.35 \mathrm{E}$ 00-- $-2.46 \mathrm{E}$ OO $1.83 E$ E $00--2.35 \mathrm{E} 00$ $1.11 \mathrm{E}$ 00-- $1.83 \mathrm{E}$ OO $5.50 E-01--1.11 E$ OO $1.11 \mathrm{E}-0 .--5.50 \mathrm{E}-0$ $3.35 E-02-1.11 E-01$ $5.83 E-04--3.35 E-02$ $1.01 F-04--5.83 E-04$ $2.90 E-05-D-1.01 E-04$ $1.07 E-05-2.0105$ $3.06 E-C 6-1.07 E-05$ $1.12 \mathrm{E}-06---3.06 E-06$ $4.14 E-07---1.12 E-06$
$0.0 \quad---4.14 E-n 7$
ANGLE 3 ANGLE $M U=-1.0000$ 0.0 $-2.077 E-04$ $5.384 E-04$ $1.214 \mathrm{E}-03$ $1.260 \mathrm{E}-03$ $1.903 E-03$ 2.262E-03 $3.965 E-03$
$5.701 E-03$ $5.732 \mathrm{E}-03$ $8.051 E-03$ $1.701 E-02$ $3.698 \mathrm{E}-02$ $2.989 \mathrm{E}-01$ $2.759 E-01$ $1.735 \mathrm{E} 01$ $7.702 E$ OI $2.291 E 02$ $6.266 E$ O2 $1.704 \mathrm{E} 03$ $3.777 \mathrm{E} O 3$

ANGLE 10 0.0

$3.001 E-04$ $1.907 \mathrm{E}-03$ $1.874 E-03$ $2.497 \mathrm{E}-03$ $3.257 \mathrm{E}-03$ $3.370 \mathrm{E}-03$ $6.211 E-03$ $9.820 \mathrm{E}-03$ $9.734 \mathrm{E}-03$ $1.250 E-02$ 2.501 E-02 $4.758 \mathrm{E}-02$ $3.454 \mathrm{E}-\mathrm{Cl}$ $3.021 \mathrm{E}-01$ $1.921 \mathrm{E} 01$ $8.473 E$ OI $2.514 \mathrm{E} O 2$ $6.853 E$ O2 4.107E 03 6.009503 0.0 0.0
(NEUTRJNS/MEV/STERADIAN/SOURCE NEUTRON) 2 ANGLE 3 0.0 865 $1.687 \mathrm{E}-04$ $5.291 E-04$ $1.202 \mathrm{E}-03$ $1.261 \mathrm{E}-03$ $1.910 E-03$ $2.265 \mathrm{E}-03$ $3.972 \mathrm{E}-03$ $5.714 E-03$ $5.749 E-03$ $8.076 \mathrm{E}-03$ $1.706 \mathrm{E}-02$ $3.706 \mathrm{E}-02$ $2.992 \mathrm{E}-01$ $2.762 \mathrm{E}-01$ $1.737 \mathrm{E}$ OI $.709 E$ OL $2.293 E$ O2 $6.271 \mathrm{E} 02$ ( $5.574 \mathrm{E} \mathrm{O3}$

$4.710 E-04$

$4.710 E-04$
$1.106 E-03$

$1.106 \mathrm{E}-03$

$2.003 E-03$

$2.305 E-03$ $4.061 \mathrm{E}-03$ $5.882 \mathrm{E}-03$ $5.978 \mathrm{E}-03$ $8.381 \mathrm{E}-03$ $1.765 \mathrm{E}-02$ $3.798 \mathrm{E}-02$ $3.037 \mathrm{E}-01$ $2.794 E-01$ $1.756 \mathrm{E}$ OI $7.788 E$ OI $2.316 \mathrm{E}$ O2 $6.332 \mathrm{E} \mathrm{O2}$ $1.721 \mathrm{E} 03$ $3.814 \mathrm{E} \mathrm{O3}$ $5.624 \mathrm{E} 03$ ( $5.592 \mathrm{E} \quad 03$

$5.228 \mathrm{E}-04$ $2.382 \mathrm{E}-03$ $2.249 E-03$ $2.965 \mathrm{E}-03$ $3.789 \mathrm{E}-03$ $3.909 E-03$ $7.312 \mathrm{E}-03$ $1.173 \mathrm{E}-02$ $1.124 \mathrm{E}-02$ $1.395 \mathrm{E}-02$ $2.747 E-02$ $5.021 \mathrm{E}-02$ 3.551E-01 $3.146 \mathrm{E}-01$ $1.957 E$ OI $8.625 \mathrm{~F}$ O1 $2.558 E$ O2 $6.967 E$ O2 $4.170 E$ O3 $6.093 \mathrm{E} 03$ ANGLE 13

ANGLE 12

0.0

$7.175 E-04$ $M U=0.6179$ 0.0 $2.792 E-03$ $3.633 E-03$ $4.549 E-03$ $4.674 E-03$ $8.894 \mathrm{E}-03$ $1.445 \mathrm{E}-02$ $1.323 E-02$ $1.576 \mathrm{E}-02$ $3.044 E-02$ . $3.653 \mathrm{E}-\mathrm{O}$ $3.211 E-01$ . $2.601 \mathrm{E} O 2$ T.079E O2 $1.916 \mathrm{E} 03$ $6.177 \mathrm{E} \quad 03$
ANGLE 5 $1.104 \mathrm{E}-04$ 4.903E-04 $1.370 E-03$ $2.093 \mathrm{E}-03$ $2.354 \mathrm{E}-03$ $4.161 E-03$ $4.161 E-03$
$6.076 E-03$ $6.217 E-03$ $8.685 E-03$ $1.822 \mathrm{E}-02$ $3.885 E-02$ 3.079E-01 2.824E-01 $1.773 E$ OI $7.859 E$ OI $6.386 \mathrm{E} \mathrm{O2}$ $1.735 \mathrm{E} \mathrm{O3}$ $3.845 \mathrm{E} \mathrm{O3}$ $5.666 \mathrm{E} \mathrm{O3}$

3.893E-05 $5.927 \mathrm{E}-04$ $1.101 \mathrm{E}-03$
$1.484 \mathrm{E}-03$ $2.216 \mathrm{E}-03$ $4.318 E-03$ $6.381 E-03$ $6.565 E-03$ $9.110 E-03$ $1.901 E-02$ DOE-O2 $3.133 \mathrm{E}-01$ $.862 \mathrm{E}-01$ $.795 E$ OI 作 $1.754 E \quad 03$ $3.885 \mathrm{E} \quad 03$

ANGLE 7 $M U=-0.4580$ 0.0 $2.411 E-05$ $7.996 \mathrm{E}-04$ $1.194 \mathrm{E}-03$
$1.650 \mathrm{E}-03$ $2.379 E-03$ $2.554 E-03$ 4. $559 E-03$ $6.839 E-03$ $7.049 \mathrm{E}-03$ $9.674 E-03$ $2.003 E-02$ $4.144 E-02$ $2.907 E-01$ $1.821 E$ OI $8.061 E$ o1 $2.395 \mathrm{E} 02$ $6.540 \mathrm{E} 02$ $1.776 \mathrm{E} \quad 3$ $3.932 \mathrm{E} 03$
$5.781 \mathrm{E} 03$ ANGLE 14 0.0
$2.772 E-03$ $6.127 E-0$ $5.155 E-03$ $6.221 E-03$ $7.328 \mathrm{E}-03$ 7. $330 \mathrm{E}-03$ $1.458 \mathrm{E}-02$ $2.408 E-02$ $1.937 E-02$ $2.071 E-02$ 3. $806 \mathrm{E}-02$ $5.926 E-02$ 3. $843 \mathrm{E}-01$ 3. $330 \mathrm{E}-01$ $2.060 E$ OI $9.044 E$ OI $2.678 \mathrm{E} \mathrm{O2}$ $7.279 \mathrm{E} \mathrm{O2}$ $1.968 \mathrm{E} 03$ $\begin{array}{ll}4.343 E & 03 \\ 6.323 E & 03\end{array}$ $M U=-0.2816 \quad M U=-0.0950$ 0.0 $4.847 E-06$ $1.106 E-03$ $1.359 \mathrm{E}-03$ $1.870 E-03$
$2.592 E-03$ $2.734 \mathrm{E}-03$ $4.918 E-03$ $7.508 E-03$ $7.706 E-03$ $1.041 \mathrm{E}-02$ $2.135 \mathrm{E}-02$ $3.274 E-01$ 


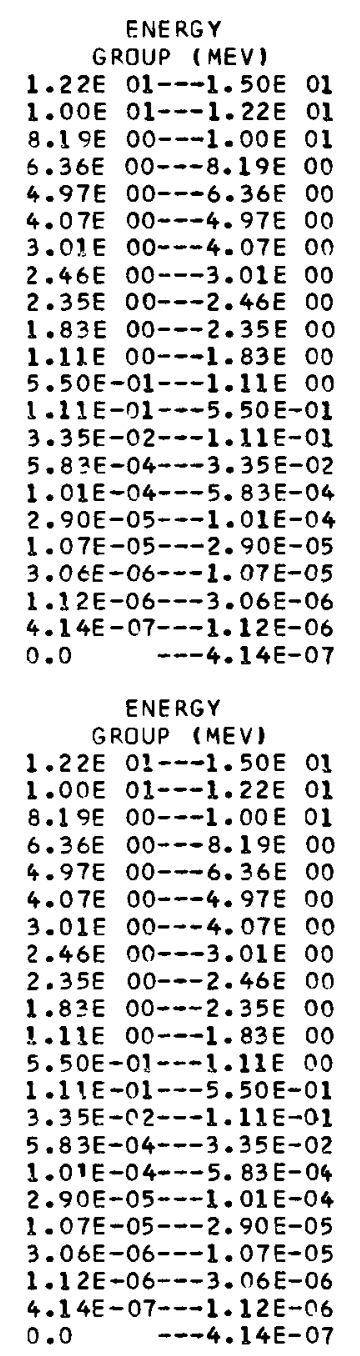

AU
0
-1

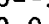

$-1.250 E-04$ $3.420 \mathrm{E}-04$ $8.416 \mathrm{E}-04$ $9.767 \mathrm{E}-04$ $1.516 \mathrm{E}-03$ $1.790 \mathrm{E}-03$ $3.097 \mathrm{E}-03$ $4.488 E-03$ $4.677 E-03$ $6.731 E-03$ $1.479 \mathrm{E}-02$ $3.389 E-02$ $2.864 E-01$ $2.681 E-01$ $1.706 \mathrm{E}$ OI 7.031E 01 1.283E 02 1.719E O3

$5.680 \mathrm{E} 03$

ANGLE 10 $M U=0.0550$

$2.114 \mathrm{E}-04$ $1.290 \mathrm{E}-03$ $1.979 \mathrm{E}-03$ $2.611 \mathrm{E}-03$ $2.659 E-03$ $4.958 \mathrm{E}-03$ $8.027 \mathrm{E}-03$ $8.069 E-03$ $1.052 E-02$ $2.202 E-02$ 4.396E-02 3. $331 \mathrm{E}-01$ $3.012 \mathrm{E}-01$ $1.900 E 01$ $8.449 E$ O1 $2.522 \mathrm{E} 02$ $6.925 \mathrm{E} 02$ $1.887 E \quad 03$ 4.104E 03
ANGLE $M U=-0.9894$ 0.0 $-1.013 E-04$ $3.374 E-04$
$8.339 E-04$ $8.339 E-04$ $9.780 E-04$ $1.523 \mathrm{E}-03$ $1.792 \mathrm{E}-03$ $3.102 \mathrm{E}-03$ $4.49 \mathrm{E}-03$ $4.693 E-03$ $6.752 E-03$ $1.484 E-02$ $3.397 E-C 2$ $2.867 E-01$ $2.684 E-O 1$ $1.708 E$ OI 7.639 OL $6.205 E$ O $3.721 E \quad 03$ $5.085 E$

ANGLE 11 $M U=0.2816$ 0.0

$3.603 E-04$
$1.617 E-03$ $1.617 E-03$
$1.700 E-03$ $2.358 \mathrm{E}-03$ $3.044 E-03$ $3.085 E-03$ $5.873 \mathrm{E}-03$ $9.665 \mathrm{E}-03$ $9.349 E-03$ $1.175 \mathrm{E}-02$ $2.424 \mathrm{~F}-02$ $4.647 \mathrm{E}-\mathrm{C2}$ $3.430 E-01$ $3.079 E-01$ $1.939 E$ O1 $8.610 \mathrm{OE}$ O1 $2.569 E \quad 2$ $7.049 E \quad 2$ $1.920 \mathrm{E} \mathrm{O3}^{2}$ $4.265 E \quad 03$
$6.263 E$
(NEUTRJNS/MEV/STERADIAN/SOURCE NEUTRON)

ANGLE 0.0

$2.880 E-05$ $3.225 \mathrm{E}-04$ $8.072 \mathrm{E}-\mathrm{C4}$ $9.864 \mathrm{E}-04$ $1.548 E-03$ $1.801 \mathrm{E}-03$ $3.126 \mathrm{E}-03$ $4.548 \mathrm{E}-03$ $4.758 E-03$ $6.841 \mathrm{E}-03$ $1.502 \mathrm{E}-02$ $3.427 E-02$ $2.883 E-01$ $2.696 \mathrm{E}-01$ $1.715 E$ E 7.668 E OI $6.318 E$ O2 .. $1.727 E$ O3
$2.847 E 03$ $5.704 E 03$ $M U=-0.8656$ 0.0 $2.190 \mathrm{E}-05$ $3.111 E-04$ 7.743E-04 $1.012 \mathrm{E}-03$ 1.597E-03 $1.821 \mathrm{E}-03$ . 4.651 E 03 $7.012 \mathrm{E}-03$ 1.537E-02 $2.484 E-02$ $2.913 E-01$ $2.717 E-01$ $7.727 \mathrm{E}$ OI $2.310 E$ 2.310E 1.738E 03 $1.738 E$ O3
$3.872 \mathrm{E} 03$ 5.739 E

ANGLE 12

ANGLE 13 0.0

ANGLE 13 0.0

$4.975 \mathrm{E}-04 \quad 8.193 \mathrm{E}-04$ $2.060 E-03$ $2.906 \mathrm{E}-03$ $3.966 \mathrm{E}-03$
3.6663 $3.666 E-03$
$3.691 E-03$ $3.691 \mathrm{E}-03$
$7.191 \mathrm{E}-03$ $1.200 \mathrm{E}-02$ $1.103 E-02$ 1.327E-02 $2.690 \mathrm{E}-\mathrm{n2}$ 4.919E-02 3. $532 \mathrm{E}-01$ $3.146 \mathrm{E}-01$ $1.977 E 0$ $8.770 \mathrm{E} \mathrm{OI}$ 2.616E 02 $7.172 \mathrm{E} 02$ $1.952 \mathrm{E} 03$ $6.357 E \quad 03$

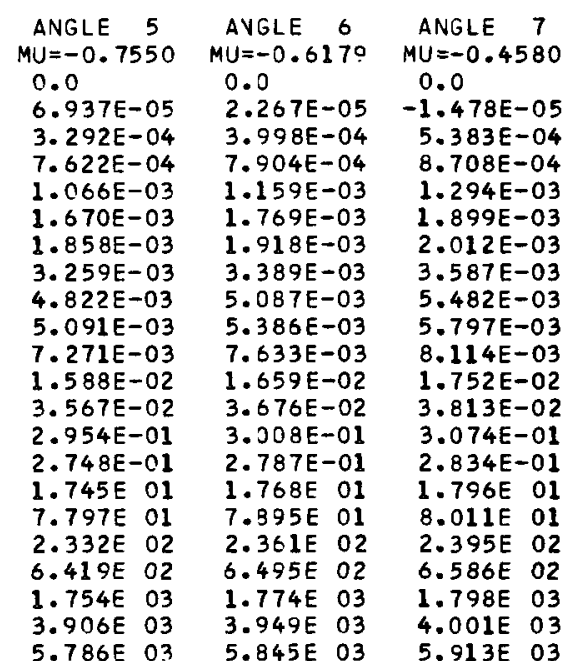

ANGLE

ANGLE $8 \quad$ ANGLE 9
$M=-0.2816 \quad M U=-0.0950$ 0.0

1. $926 E-06$ $7.440 \mathrm{E}-04$ $1.004 \mathrm{E}-03$ $1.473 E-03$
$2.072 E-03$ $2.072 E-03$
$2.153 E-03$ $2.153 E-03$
$3.885 E-03$ $6.054 E-0$ $1.871 E-02$ $3.978 E-02$ 3.1 5OE-OI 2.888E-01 $1.827 E$ OI $8.145 E$ OI 2.434E . 690 E $1.825 E 03$ $5.091 \mathrm{E} 03$

AVGLE 15 0.0 O. $\begin{array}{ll}0.0 & 0.0 \\ 1.970 E-03 & 5.526 E-03\end{array}$ $\begin{array}{ll}1.970 E-03 & 5.526 E-03 \\ 4.347 E-03 & 7.561 E-03 \\ 4.045 E-03 & 6.216 E-03\end{array}$ $5.047 E-03 \quad 7.138 E-03$ $5.924 \mathrm{E}-03 \quad 7.958 \mathrm{E}-03$ $5.750 \mathrm{E}-03 \quad 7.451 \mathrm{E}-03$ $1.192 \mathrm{E}-02 \quad 1.621 \mathrm{E}-02$ $2.036 \mathrm{E}-02 \quad 2.800 \mathrm{E}-02$ $1.617 \mathrm{E}-02 \quad 2.012 \mathrm{E}-02$ $1.737 E-02 \quad 2.002 E-02$ $3.368 E-02 \quad 3.773 E-02$ $5.496 \mathrm{E}-02 \quad 5.769 \mathrm{E}-02$ $3.722 \mathrm{E}-01 \quad 3.801 \mathrm{E}-01$ $3.267 E-01 \quad 3.316 E-01$ $2.046 E$ o1 $2.074 E$ OI $9.057 \mathrm{E}$ O1 $9.173 E$ OI $2.699 \mathrm{E} 02 \quad 2.732 \mathrm{E} 02$ $7.392 E \quad 02 \quad 7.478 E$ O2 $2.010 \mathrm{E} \mathrm{O3}$ 2.032E O3 $4.458 E$ O3 $4.506 E$ O3 $6.522 E \quad 03 \quad 6.583 E 03$
ANGLE 16 $M U=0.9446$

$1.616 \mathrm{E}-02$ $1.494 \mathrm{E}-02$
$1.035 \mathrm{E}-02$ $1.035 E-02$
$1.068 E-02$ $1.068 \mathrm{E}-02$
$1.118 \mathrm{E}-02$ $9.934 \mathrm{E}-03$ $2.330 E-02$ 4.073E-02 $2.571 \mathrm{E}-02$ $2.310 E-02$ $4.205 E-02$ $5.998 \mathrm{E}-02$ 3.860E-OL 3.352E-01 $2.094 E 01$ 253E 01 $2.756 E$ E $7.541 E$ O2 (.049E $6.627 E \quad 03$
ANGLE 17 0.9894 0.0 $1.073 \mathrm{E}-01$ $3.707 E-02$
$2.021 E-02$ $2.021 \mathrm{E}-02$
$1.793 \mathrm{E}-02$ $1.721 \mathrm{E}-02$ $1.410 \mathrm{E}-02$ $3.778 E-02$ $6.694 E-02$ $3.484 E-02$ $2.665 \mathrm{E}-02$ $4.632 E-02$ $6.151 E-02$ $3.895 \mathrm{E}-01$ $3.373 E-01$ $2.106 \mathrm{E} \mathrm{Ol}$ $9.301 E$ O $2.770 E$ OL $7.578 E$ O ..058E 03 $6.652 \mathrm{E} 03$ $6.513 E-05$ $1.001 \mathrm{E}-03$ $1.182 \mathrm{E}-03$ $1.696 \mathrm{E}-03$ $2.300 \mathrm{E}-03$ $2.360 \mathrm{E}-03$ $4.323 E-03$ $6.871 E-03$ 0.520E-03 . 4.173E-02 $3.237 \mathrm{E}-01$ 2.948E-OI $1.862 \mathrm{E}$ OI $8.292 E$ OI $8.292 E$ OI $1.855 E 03$ $4.125 \mathrm{E} 03$ $6.077 E 03$

SCALAR 0.0 $0.015 \mathrm{E}-02$ 
(NEUTRJNS/MEV/STERADIAN/SOURCE NEUTRONI

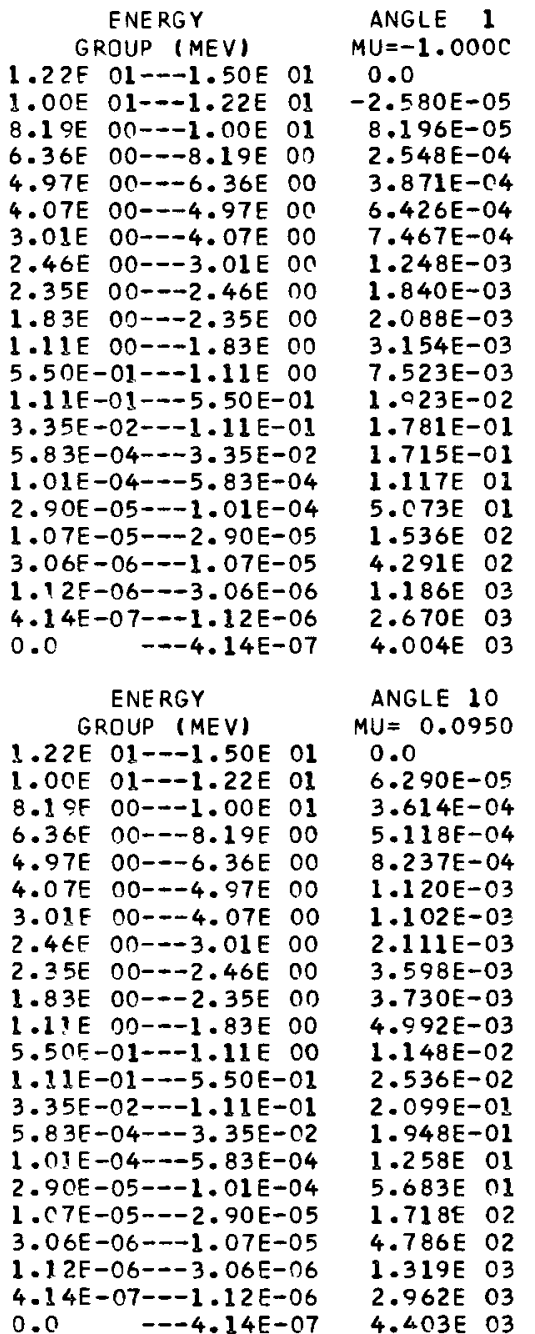

ENERGY $1.22 E 01--1.50 E$ 5.83E-04- $1.11 \mathrm{E}-01$ $-05--2.90 \mathrm{E}-0 \mathrm{~S}$ .12E-06--3.06E-06 $0.0 \quad---4.14 E-07$ ENERGY

$1.22 \mathrm{E} 01-21.50 \mathrm{E}$ O1 $1.00 E$ OL---1.22E OI 8.1 OF $00--1.00 E$ OI $4.97 \mathrm{E} \quad 00---6.36 \mathrm{E}$ 0.0 (1.12E-06 $\triangle A N G L E ?$ 0.0

$-2.084 E-05$ $8.174 E-05$ $2.530 \mathrm{E}-04$ $3.877 \mathrm{E}-04$ $6.452 \mathrm{E}-04$ $7.473 \mathrm{E}-04$ $1.251 \mathrm{E}-03$ 1.847E-03 $2.096 \mathrm{E}-03$ $3.164 \mathrm{E}-03$ $1.028 \mathrm{E}-02$ $1.784 \mathrm{E}-01$ $1.717 \mathrm{E}-01$ $1.118 \mathrm{E} 01$ $5.078 \mathrm{E} 01$ $1.537 \mathrm{E} \mathrm{O2}$ $4.255 E 02$ $1.187 \mathrm{E} 03$ $2.673 E \quad 03$ $4.008 \mathrm{E} \quad 0$ ANGLE 11 $M U=0.2816$ 0.0

$1.023 E-04$ $4.605 E-04$ 6.290E-04 $9.909 E-04$ $1.313 E-03$ $1.281 E-03$ 2.537E-03 $4.408 E-03$ $4.347 E-C 3$ $5.583 E-03$ . $2.687 E-02$ $1.905 \mathrm{E}-01$ 1.28EE O1 $5.804 E$ OI 1.754E O2 4.883E 02 1.345E 03 3.020803 $4.481 \mathrm{E}^{03}$ 0.0 $5.267 E-06$ $8.137 E-05$ $2.469 E-04$
$3.913 E-04$ $6.564 E-04$ $7.503 E-04$ $1.262 E-03$ $1.874 \mathrm{E}-03$ $2.129 E-03$ $7.646 E-03$ $7.646 \mathrm{E}-03$ $1.946 \mathrm{E}-02$
$1.795 \mathrm{E}-01$ $1.725 \mathrm{E}-01$ $1.123 \mathrm{E} 01$ $5.100 E 01$ $1.544 \mathrm{E} \mathrm{O2}$ $4.313 \mathrm{E} 02$ $1.192 \mathrm{E} 03$ $2.683 \mathrm{E} \mathrm{O3}$ 4.023E 03 ANGLE 12 $M U=$
0.0

1.450 E- 04 $6.013 E-04$ $8.073 E-04$ $1.238 E-03$ (.5) (.) 5.154E-03 政 $2.852 E-02$ 2.235E-01 $2.042 \mathrm{E}-01$ $1.315 \mathrm{E} 01$ $5.924 E 01$ $4.985 \mathrm{E} 02$ 1.371E 03 $3.076 \mathrm{E} \mathrm{O3}$ $4.558 \mathrm{E} 03$
ANGLE $\quad 4$
$M U=-0.8656$ 0.0 $6.409 E-06$ $8.295 E-05$ $2.410 E-04$ $4.025 \mathrm{E}-04$ $6.776 E-04$ $7.572 E-04$ $1.285 E-03$ $1.929 \mathrm{E}-03$ $2.193 E-03$ $3.291 E-03$ $7.836 E-03$ $1.981 \mathrm{E}-02$ . $814 \mathrm{E}-01$ $1.740 E-01$ $1.132 E$ OI $1.556 \mathrm{E} \mathrm{O} 02$ $4.346 \mathrm{E} 02$ $1.201 \mathrm{E} 03$ $2.702 E 03$ 4.051E 03 ANGLE 13 $M U=0.6179$ 0.0 $2.535 E-04$ $8.507 E-04$ 1.111E-03 $1.622 E-03$ $1.998 E-03$ $1.876 E-03$ 4. त45E-03 . T.168E-03 $1.583 \mathrm{E}-02$ 3. $024 \mathrm{E}-02$ 2.303E- 1 $1.341 \mathrm{E} 01$ $1.341 E$ O $1.033 \mathrm{E} 02$ $1.823 E 02$ $1.395 \mathrm{E} 03$ $3.130 \mathrm{E} 03$ $3.130 \mathrm{E} 03$
$4.631 \mathrm{E} 03$
$A N G L E \quad 5$
$M U=-0.7550$ 0.0 1. $556 \mathrm{E}-05$ $9.084 \mathrm{E}-05$ $2.438 \mathrm{E}-04$ $4.266 E-04$ 7.087E-04 $7.707 E-04$ $1.323 E-03$ $2.018 \mathrm{E}-03$ $2.292 E-03$ $3.416 E-03$ $8.118 \mathrm{E}-03$ $2.031 E-02$ $1.843 E-01$ $1.762 E-01$ $1.145 E$ Ol $5.196 \mathrm{E} 01$ $1.573 \mathrm{E} \mathrm{O2}$ $4.392 E 02$ $1.213 \mathrm{E} \quad 03$ $2.730 E^{03}$ $4.089 E_{03}$ ANGLE 14 $M U=0.7550$ $6.112 E-04$ $1.403 \mathrm{E}-03$ $1.653 \mathrm{E}-03$ $2.226 \mathrm{E}-03$ 2.5 2.341E-03 . $7.54 E-03$ $7.564 E-03$ $8.175 E-03$ $1.774 E-02$ $3.195 E-02$ $2.128 \mathrm{E}-01$ 1. $366 \mathrm{E} 01$ $6.140 E$ OI $1.854 \mathrm{E}$ O2 $5.153 E_{02}$ $1.417 \mathrm{E} \quad 03$ $3.178 E$
$4.696 \mathrm{E}$
ANGLE 6 0.3

4.678E-06 $1.106 \mathrm{E}-04$ $2.614 \mathrm{E}-04$ $4.678 \mathrm{E}-04$ $7.941 E-04$ $1.383 E-03$ 2.153E-03 $2.435 E-03$ $3.592 E-03$ $8.507 E-03$ $2.097 \mathrm{E}-02$ 1. $879 \mathrm{E}-01$ $1.789 \mathrm{E}-01$ 1. $162 \mathrm{E} 01$ $5.268 \mathrm{E} 01$ $4.450 E$ O2 $1.229 \mathrm{E} 03$ $2.765 \mathrm{E} 03$ $4.137 E 03$ ANGLE 15 $M U=0.8656$ 0.3
$1.642 E-03$ $2.565 \mathrm{E}-03$ $2.628 \mathrm{E}-03$ $3.176 \mathrm{E}-03$ $3.443 \mathrm{E}-03$ 2.958E-03 $7.301 E-03$ $1.386 \mathrm{E}-02$ $9.322 E-03$ $9.302 E-03$ $3.353 \mathrm{E}-02$ $3.353 \mathrm{E}-02$ $2.417 \mathrm{E}-01$ $1.386 \mathrm{E}$ OI 1.326E 01 $1.226 E$ OI 5.879 O2 $1.435 \mathrm{E} 03$ $1.435 E$
$3.218 E$ 03 $4.748 \mathrm{E} \mathrm{OS}$

ANGLE $\quad{ }^{7}$
$M U=-0.4580$ 0.0 $-2.314 E-06$ $1.476 E-04$ $.977 E-04$
$.274 E-04$ $8.080 E-04$ $8.321 E-04$ $1.475 E-03$ $2.349 \mathrm{E}-03$ $2.633 E-03$ . $826 E-03$ $9.015 \mathrm{E}-03$ $.180 \mathrm{E}-02$ $1.924 E-01$ $1.182 \mathrm{E}$ O1 $5.355 E$ O1 $1.620 \mathrm{E} \mathrm{O2}$ $4.521 E 02$ $1.248 E 03$ 4.193E 03 ANGLE 16 $M U=0.9446$ $4.450 E-03$ $5.165 \mathrm{E}-03$ 
(NEUTRONS/MEV/STERADIAN/SOURCE NEUTRON)

ENERGY GROUP (MEV) $1.22 E 01--1.50 E$ OI $1.00 E$ O1---1.22E OI 8.1 GE $00--1.00 E$ O1 $6.36 \mathrm{E}$ 00---8.19E 00 $4.97 \mathrm{E} 00--6.36 \mathrm{E}$ 00 4.07E $00--4.97 E$ O $2.46 \mathrm{E} \quad 00--3.01 \mathrm{E} O 0$ $2.35 \mathrm{E} \quad 00---2.46 \mathrm{E}$ OO $1.83 E \quad 00-2.35 E \quad 00$ $1.11 \mathrm{E} 00--1.83 \mathrm{E} 00$ $5.50 E-01--1.11 E$ OO $1.11 E-01---5.50 E-01$ $3.35 \mathrm{E}-02--1.11 \mathrm{E}-01$ $5.83 \mathrm{E}-04---3.35 \mathrm{E}-02$ $1.01 E-04--5.83 E-04$ .90E-05--1.01 E-04 1.07E-05-- 2. 90E-O5 $3.06 \mathrm{E}-06--1.07 \mathrm{E}-0$ 1.12E-06-- -3.06E-06 $4.14 E-07--1.12 E-06$ $0.0 \quad--4.14 \mathrm{E}-07$

\section{ENERGY}

GROUP (MEV) $1.22 \mathrm{E}$ 01---1.50E OI $1.0 \mathrm{OE}$ C1---1.22E O $8.19 \mathrm{~g}$ 00--1.00E 01 $6.36 \mathrm{E} 00--8.19 \mathrm{E} 00$ 4.97 E $00--6.36$ E OD 4.07E $00--4.97 E$ DO 3.03 E $00--4.07 E$ OO $2.46 \mathrm{E}$ 00-- $-3.01 \mathrm{E}$ OO $2.35 \mathrm{E} 00--2.46 \mathrm{E} 00$ $1.83 E$ EO- $2.35 \mathrm{E} 00$ $1.11 \mathrm{E} 00--1.83 \mathrm{E}$ OO 5.50E-01-- $1.11 E$ OO $1.11 E-01-2-5.50 E-01$ $3.35 E-02--1.11 E-01$ . $8 E-04-3.35 E-02$ 1. 2.90E-05- $-1.01 E-04$ $1.07 E-05--2.90 E-C 5$ $1.06 \mathrm{E}-06-2.07 E \mathrm{E}$ 1.12E-06- $3.06 E-06$ $4.14 E-07--1.12 E-06$
$0.0 \quad---4.14 E-07$
ANGLE 1 0.0 $5.141 \mathrm{E}-06$ $7.151 E-05$ $1.341 \mathrm{E}-04$ $2.339 E-04$ $2.688 \mathrm{E}-04$ 4.380E-O $6.543 E-04$ $7.888 \mathrm{E}-04$ $1.227 E-03$ $3.080 \mathrm{E}-03$ $8.426 E-03$ $8.247 E-0$ $8.070 \mathrm{E}-02$
$5.324 \mathrm{E} 00$ $2.440 E$ OI $7.439 E$ OI $2.096 \mathrm{E} 02$ $5.829 E$ O2 $1.320 \mathrm{E} O 3$ $1.993 \mathrm{E} 03$ ANGLE 10 $M U=0.0950$ 0.0

$1.614 \mathrm{E}-05$ $9.388 \mathrm{E}-05$ $1.648 \mathrm{E}-04$ $2.953 \mathrm{E}-04$ $4.108 \mathrm{E}-04$ $3.955 \mathrm{E}-04$ $7.718 \mathrm{E}-04$ $1.361 \mathrm{E}-03$ $1.442 \mathrm{E}-03$ $1.956 \mathrm{E}-03$ $4.769 E-03$ $1.121 \mathrm{E}-02$ $9.786 \mathrm{E}-02$ $9.221 \mathrm{E}-02$ $6.036 E$ DO $2.751 \mathrm{E}$ OL $8.374 E$ OI 2.35E O2 ..527E 02 $1.475 \mathrm{E} \quad 03$
$2.206 \mathrm{E} 03$
ANGLE 2
$M U=-0.9894$ 0.0 $-4.138 \mathrm{E}-06$ $1.868 E-05$ 7.112E-05 $1.343 \mathrm{E}-04$ $2.349 E-04$ $2.690 \mathrm{E}-04$ $4.390 E-04$ $6.570 E-04$ $7.919 E-04$ $1.231 \mathrm{E}-0$ $3.090 \mathrm{E}-0$ $8.446 \mathrm{E}-0$ $8.259 E-02$ $8.07 \% \mathrm{E}-02$ $5.33 \mathrm{CE} \mathrm{OO}$ $2.443 E$ OI $7.447 \mathrm{E}$ OI $2.098 \mathrm{E} \mathrm{O2}$ $5.835 \mathrm{E} \mathrm{C2}$ $1.322 \mathrm{E} 03$ $1.905 E$ O3

ANGLE 11 $U=0.2816$ $2.577 E-05$ $1.218 \mathrm{E}-04$ $2.056 E-04$ $3.579 E-04$ 4.837E-O4 4.5 .6E-0 $9.372 E-04$ $1.688 E-03$ $1.687 E-03$ $2.188 E-03$ $5.227 E-03$ $1.11 \mathrm{E}-01$ $1.011 E-01$ ..454E-C2 O.178E 2.813E OI . 55 E $2.404 E$ OL $6.665 E$ C2 $2.248 E \quad 3$
$\begin{array}{ll}A N G L E & 3 \\ M U=-0.9446\end{array}$

.451E-07 $1.933 E-05$ $6.992 \mathrm{E}-05$ . $357 E-04$ $2.390 E-04$ 2.6934E-04 $4.434 E-04$ $8.686 E-04$ 1.052E-04 $3.133 \mathrm{E}-03$ $8.531 E-03$ $8.312 E-02$ $8.120 \mathrm{E}-02$ $5.356 \mathrm{E} 00$ $2.454 \mathrm{E}$ OI $7.480 \mathrm{E}$ OI $2.107 E \quad 02$ $5.860 E \quad 02$ $1.327 E \quad 03$ $2.003 E$ O3 ANGLE 12 $U U=0.4580$ 0.0

3.785E-05 $1.633 \mathrm{E}-04$ $2.699 \mathrm{E}-04$ 4. $520 \mathrm{E}-04$ $5.890 E-04$ 1.4 $176 \mathrm{E}=03$ 2.007E-0 $2.470 \mathrm{E}-03$ $5.903 E-03$ $1.265 E-02$ $1.045 E-01$ $6.381 \mathrm{E} 00$ 6.321E OO $8.744 \mathrm{E}$ O1 $8.744 E$ OI $6.802 E$ E $1.802 E$ O2 $2.289 \mathrm{E} \mathrm{O3}$
$\begin{array}{ll}A N G L E & 4 \\ M U=-0.8656\end{array}$ 0.0

$1.557 E-06$ 2.062E-05 $6.93 \mathrm{E}-05$ ( $2.468 \mathrm{E}-04$ $4.523 \mathrm{E}-04$ $6.917 E-04$ $8.310 \mathrm{E}-04$ $1.281 E-03$ $3.213 E-03$ $8.687 E-03$ .407E-02 $8.194 E-02$ $474 \mathrm{E}$ Ol $7.541 \mathrm{E} \mathrm{Ol}$ $2.124 \mathrm{E} \quad 02$ $5.906 \mathrm{E} \mathrm{O2}$ $1.337 \mathrm{E} 03$ $2.018 E \quad 03$ ANGLE 13 MU $=0$ $6.936 E-05$ $2.437 \mathrm{E}-0$ $3.826 E-04$ $5.998 \mathrm{E}-0$ $6.687 \mathrm{E}-04$ $1.524 \mathrm{E}-03$ $2.882 \mathrm{E}-03$ $2.419 E-03$ $2.803 E-03$ $6.616 E-03$ $1.377 \mathrm{E}-02$ 1.077 E-01 $9.910 \mathrm{E}-02$ (. 2.932E O1 8 . 2.502E . $931 \mathrm{E}$ 2.329 E 3
$A N G$
$M U=-0$ 0.0 $3.281 E-06$ 2. $307 \mathrm{E}-05$ $7.160 E-05$ $2.582 E-04$ $2.766 E-04$ $7.282 E-04$ 706E-04 1. $331 E-03$ . $34 E-03$ . $544 E-02$ $8.299 E-02$ $8.299 E-02$ 5.467E OO $2.503 \mathrm{E} 01$
$7.628 \mathrm{E} 01$ $2.148 \mathrm{E} 02$ $5.970 \mathrm{E} \mathrm{O2}$ $1.352 \mathrm{E} 03$
$2.038 \mathrm{E} 03$ ANGLE 14 $M U=0.7550$ $1.665 E-04$ $4.166 E-04$ $5.874 \mathrm{E}-04$ $8.325 E-04$ $9.598 \mathrm{E}-04$ 2.030E-03 . $3.967 E=03$ 2. $44 E-03$ $7.431 E-03$ 1.419E-O $1.107 E-0$ $6.577 E 00$ 2. 085 E 2.985E 2.545E 02 $2.545 E$ O2 1.5 TOE 03 $2.363 \mathrm{E} 03$

AVGLE $\quad 6$
$M U=-0.6179$ 0.0 1. 040 E-06 2.802E-05 7. $370 \mathrm{E}-05$ $1.644 E-04$ $2.739 E-04$ 4. $903 \mathrm{E}-04$ $4.329 E-04$
$9.278 E-04$ (1) $1.401 \mathrm{E}-03$
$3.499 \mathrm{E}-03$ $9.217 E-0$ $8.722 E-0$ $8.435 E-02$ $5.551 E 00$ 
(NEUTRJNS/MEV/STERADIAN/SOURCE NEUTRON)

ENERGY GROUP (MEV) $1.22 \mathrm{E}$ O1-- -1.50E 01 $1.00 E$ O1- $-1.22 \mathrm{E} \mathrm{O1}$ 6.36 EO--1.00E O $4.97 E$ OO- $-6.36 \mathrm{E}$ OD $4.07 E$ OO- $-4.07 E$ OO $3.01 \mathrm{E} 00--4.07 \mathrm{E}$ OO $2.46 E$ OO- $-3.01 \mathrm{E} 00$ $2.35 \mathrm{E} 00-0-2.46 \mathrm{E}$ OO $1.83 \mathrm{E} 00-\cdots 2.35 \mathrm{E} 00$ $1.11 \mathrm{E} 00---1.83 \mathrm{E} 00$ $5.50 E-01--1.11 \mathrm{E}$ OO $1.11 \mathrm{E}-01---5.50 \mathrm{E}-01$ $3.35 \mathrm{E}-02---1.11 \mathrm{E}-0$ $5.83 \mathrm{E}-04--3.35 \mathrm{E}-02$ $1.01 E-04---5.83 E-04$ $2.90 E-05--1.01 E-04$ 1.07E-05---2.90E-05 3.06E-06---1.07E-0 $1.12 E-06---3.06 E-06$ $4.14 E-07---1.12 E-06$
0.0 ENERGY GROUP (MEV) $1.22 \mathrm{E} 01---1.50 \mathrm{E}$ $1.00 E$ O1- -1.22E O $8.19 \mathrm{E} \mathrm{DO--1.00E} \mathrm{OI}$ 6.36 E $00---8.19 E$ OO $4.97 \mathrm{E}$ OO- $-6.36 \mathrm{E}$ OO 4.07E $00---4.97 E$ OD $3.01 \mathrm{E} \mathrm{OO-C-4.07E} 00$ $2.46 \mathrm{E} 00--3.01 \mathrm{E} \mathrm{OO}$ $2.35 \mathrm{E} 00=-2.46 \mathrm{E} \mathrm{OO}$ $1.83 \mathrm{E}$ 00-- $-2.35 \mathrm{E}$ OO $1.11 E$ DO-- $1.83 E$ OD ..50E-01---1.11E 00 $1.11 E-01-2.50 E-01$ $3.35 E-02-1.11 E-01$ $5.83 E-04--3.35 E-02$ $1.01 E-04--5.83 E-04$ $2.90 \mathrm{E}-05-1.01 E-04$ $1.07 E-05--2.90 E-05$ 1.06E $06-1.07 E-05$ $4.14 E-07--1.12 E-06$ $4.14 \mathrm{E}-07--1.12 \mathrm{E}-06$
$0.0 \quad---4.14 E-07$

ANGLE 1 $M=-1.000$ $1.010 E-06$ $4.036 \mathrm{E}-06$ $1.929 E-05$ $4.319 E-05$ $8.909 E-05$ $1.426 \mathrm{E}-04$ $2.151 E-04$ $2.713 \mathrm{E}-04$ 1.118E-0 3.199E-03 $3.207 E-02$ $2.134 E \quad O 0$ $9.834 \mathrm{E}$ OD $3.011 E$ OI $8.526 E$ OI $2.381 E 02$ $5.413 E \quad 02$ $8.204 \mathrm{E} O$ ANGLE 10 $U=0.095$

0.0
$3.856 \mathrm{E}-06$ $2.348 \mathrm{E}-05$ $4.981 \mathrm{E}-05$ $9.755 \mathrm{E}-05$ $1.381 \mathrm{E}-04$ $1.308 \mathrm{E}-04$ $2.591 E-04$ $4.681 E-04$ $5.046 \mathrm{E}-\mathrm{n} 4$ $6.896 \mathrm{E}-04$ $1.748 \mathrm{E}-03$ $3.867 E-02$ $3.678 \mathrm{E}-02$ $8 \mathrm{E} 00$ $1.113 \mathrm{E} 01$ $3.402 E$ OI $9.610 \mathrm{E}$ O1 $6.072 \mathrm{E} \mathrm{O2}$ $9.115 \mathrm{E} 02$
ANGLE 2 ANGLE 3 0.0 $-8.10 G E-07$ 4.121E-06 $1.922 E-05$
$4.327 E-05$ $4.327 \mathrm{E}-05$
$7.851 \mathrm{E}-05$ $8.014 E-C 5$ $1.429 \mathrm{E}-04$ $2.161 E-04$ $2.724 \mathrm{E}-04$ $4.322 E-04$ $1.122 \mathrm{E}-03$
$3.207 \mathrm{E}-03$ $3.250 E-02$ $3.211 \mathrm{E}-02$ $2.126 \mathrm{E}$ तO $9.845 \mathrm{E} \mathrm{OO}$ $3.014 E$ EI $8.525 E$ OI 2.383E 22 $5.419 E$ O2
$8.213 \mathrm{E} 02$ ANGLE 11 $M U=0.2816$ 0.0 $6.123 E-06$ $3.104 E-05$ $6.304 \mathrm{E}-05$ $1.189 \mathrm{E}-04$ $1.630 \mathrm{E}-04$ $1.520 \mathrm{E}-04$ $3.170 E-04$ 5.85E 04 7. $92 \mathrm{BE}-04$ 1. . 4.000E-02 $3.773 E-C 2$ 1.138E OI $1.138 \mathrm{E} C 1$
$3.479 \mathrm{E} O 1$ $3.479 \mathrm{E}$ OI $9.824 E$ O1 $6.202 E 02$ $6.202 E$
$9.295 E$
$M U=-0.0$

$-1.679 E-07$ $4.434 \mathrm{E}-06$ $1.903 \mathrm{E}-05$ $4.375 E-05$ $7.989 E-05$
$8.940 E-05$ $8.940 E-05$
$1.445 E-04$ $2.204 E-04$ $2.772 E-04$ $4.383 E-04$ $1.138 \mathrm{E}-03$ $3.240 E-03$ 3.2 71 E- 02 $3.228 \mathrm{E}-02$ $2.147 E 00$ $9.891 \mathrm{E} \mathrm{OO}$ $3.028 \mathrm{E} 01$ $8.574 \mathrm{E} 01$ $2.394 \mathrm{E} 02$ $5.443 \mathrm{E} O 2$
$8.248 \mathrm{E} 02$

ANGLE 12 $M U=0.4580$ 0.0

$9.305 \mathrm{E}-06$ $4.273 \mathrm{E}-05$ $8.445 E-05$ $1.514 \mathrm{E}-04$ $1.990 E-04$ $1.811 \mathrm{E}-04$ $4.008 \mathrm{E}-04$ $7.602 E-04$ $7.054 \mathrm{E}-04$ 4. $4.838 E-03$ $4.135 E-02$ $3.868 E-02$ (164E 01 $3.557 \mathrm{E} 01$ $1.004 \mathrm{E} \mathrm{O2}$ 2.793 E 02 $6.331 \mathrm{E}$
0.42
$\begin{array}{ll}A N G L E & 4 \\ M U=-0.8656\end{array}$ 0.0 $3.516 E-07$ 4.920E-06 $1.911 \mathrm{E}-05$ $4.522 \mathrm{E}-05$ $8.251 E-05$ $9.007 E-05$ $1.476 E-04$ $2.288 \mathrm{E}-04$ $2.865 E-04$ 4. $500 \mathrm{E}-04$ $1.168 \mathrm{E}-03$ $3.301 E-03$ $3.313 \mathrm{E}-02$ $3.258 E-02$ $2.166 \mathrm{E} 00$ $9.975 \mathrm{E} 00$ $3.053 \mathrm{E} \mathrm{O1}$ $8.645 \mathrm{E}$ O1 $2.413 \mathrm{E} 02$ $8.311 E 02$ ANGLE 13 $M U=0.6179$ 0.0 $1.764 E-05$ $6.636 E-05$ $1.229 \mathrm{E}-04$ $2.029 E-04$ $2.509 E-04$ $2.159 \mathrm{E}-04$ $5.227 E-04$ $1.024 E-03$ $8.508 E-04$ $9.868 E-04$ $2.433 E-03$ $5.138 E-03$ $4.266 E-02$ $3.959 \mathrm{E}-02$ $2.601 \mathrm{E} 00$ $3.630 \mathrm{E} 01$ $1.024 \mathrm{E} \mathrm{O2}$ $2.848 \mathrm{E} 02$ $6.453 \mathrm{E} 02$ $9.642 \mathrm{E} 02$
ANGLE $5 \quad$ ANGLE 6 0.0 . $0764 E-07 \quad 2.890 E-07$ $5.589 E-06 \quad 6.759 E-06$ $2.010 E-05-2.252 E-05$ $4.832 E-05 \quad 5.354 E-05$ $8.634 \mathrm{E}-05 \quad 9.164 \mathrm{E}-05$ $9.149 E-05 \quad 9.412 E-05$ $1.527 E-04 \quad 1.609 E-04$ 2.420E-04 2.617E-04 $3.212 \mathrm{E}-04$ 2.2 $275 E-03$ $3.506 E-03$ $3.437 \mathrm{E}-02$ $3.301 \mathrm{E}-02 \quad 3.356 \mathrm{E}-02$ $2.193 E 00$ $1.009 E$ O1 $8.745 E$ ol $2.441 E 02$ $8.398 E 02$ ANGLE 14. $M U=0.7550$ 0.0 4. $202 E-05$ $1.180 E-04$ $1.935 \mathrm{E}-04$ $2.838 \mathrm{E}-04$ $3.243 E-04$ $2.697 E-04$ $6.996 \mathrm{E}-\mathrm{C} 4$ $1.429 \mathrm{E}-03$ $1.034 \mathrm{E}-03$ $1.117 \mathrm{E}-03$ $2.725 E-03$ $5.433 E-03$ $4.387 E-02$ $4.041 E-02$ $2.652 \mathrm{E} 00$ $1.210 E$ OI $3.696 \mathrm{E}$ O1 $2.897 \mathrm{E} 02$ $6.561 \mathrm{E} \quad 02$
$9.790 \mathrm{E} 02$

ANGLE 7 $M U=-0$ 0.0 1.582E-08 $8.913 \mathrm{E}-06$ $2.671 E-05$
$6.097 E-05$ $9.864 E-05$ $9.851 \mathrm{E}-05$ $1.733 E-04$ 2.899E-04 $3.493 E-04$ $5.256 E-04$ $1.356 \mathrm{E}-03$ $3.653 \mathrm{E}-03$ $3.524 E-02$ $3.423 \mathrm{E}-02$ $2.269 E 00$ $1.043 E$ O1 $3.191 E$ O1 $9.028 E$ OI 
(NEUTRJNS/MEV/STERAOIAN/SOURCE NEUTRON)

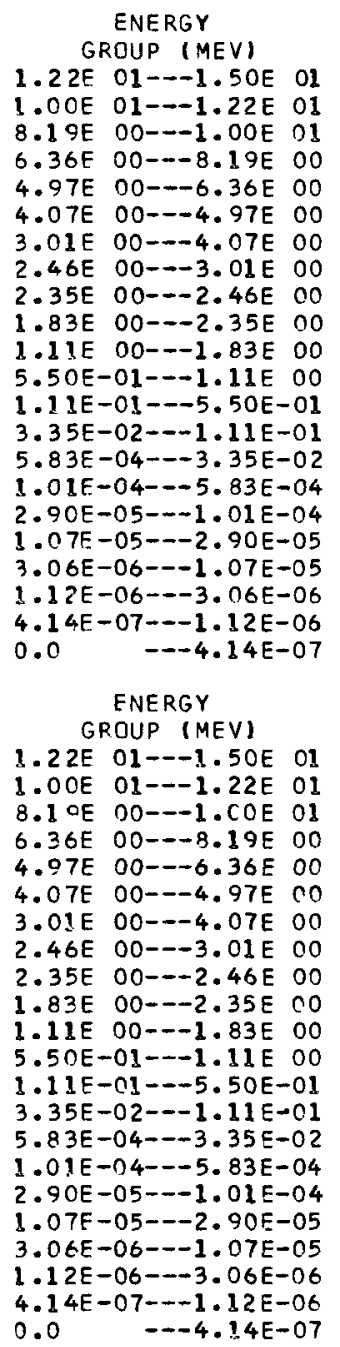

\begin{tabular}{|c|c|c|c|}
\hline $\begin{array}{l}\text { ANGLE } 1 \\
A U=-1.0000\end{array}$ & $\begin{array}{l}\text { ANGLE }{ }^{2} \\
M U=-0.9894\end{array}$ & $\begin{array}{l}\text { ANGLE } 3 \\
M U=-0.9446\end{array}$ & $\begin{array}{l}\text { ANGLE }{ }^{4} \\
M U=-0.8656\end{array}$ \\
\hline $0.0^{\circ}$ & 0.0 & 0.0 & 0.0 \\
\hline $0 E-07$ & $-1.574 \mathrm{E}-07$ & $-2.965 E-08$ & $7.635 E-08$ \\
\hline $8.566 E-07$ & $8.860 E-07$ & $9.925 E-07$ & $1.143 E-06$ \\
\hline $5.081 E-06$ & $5.07 C F-06$ & $5.053 E-C$ & $5.136 E-06$ \\
\hline $1.328 \mathrm{E}-05$ & $1.330 E-05$ & $1.346 E-05$ & $1.394 E-05$ \\
\hline $2.474 E-05$ & $2.484 E-05$ & $2.528 E-05$ & $2.611 E-05$ \\
\hline $2.800 E-05$ & $2.801 \mathrm{E}-0$ & $2.808 E-05$ & \\
\hline $4.424 E-05$ & $4.436 E-05$ & $4.486 E-05$ & $4.588 E-05$ \\
\hline $6.728 E-05$ & $6.762 E-05$ & $6.908 \mathrm{E}-\mathrm{C}$ & $7.192 E-05$ \\
\hline $8.781 E-05$ & $8.818 E-05$ & $8.980 E-05$ & $9.292 \mathrm{E}-05$ \\
\hline $1.417 E-04$ & $1.422 E-04$ & $1.442 E-04$ & $1.480 E-04$ \\
\hline $3.766 E-04$ & $3.779 E-04$ & $3.833 E-C$ & $36 E-04$ \\
\hline $1.110 E-03$ & $1.113 E-03$ & $1.125 E-03$ & $1.146 E-03$ \\
\hline 1.15 & 1.1 & $3 E-02$ & $1.177 \mathrm{E}-02$ \\
\hline $1.148 \mathrm{E}-0$ & $1.14 \circ \mathrm{E}-02$ & 1.1 & $57 E-02$ \\
\hline $7.680 E-01$ & $7.689 E-01$ & $7.728 E-01$ & $7.797 E-01$ \\
\hline $2=00$ & & & \\
\hline $1.090 E \quad 0 !$ & $1.052 E 01$ & $1.097 \mathrm{E}$ & $1.106 \mathrm{E} \quad 01$ \\
\hline $3.099 E \quad 01$ & $3.102 E 01$ & $3.117 \mathrm{E} \quad 01$ & $3.143 E \quad 01$ \\
\hline $8.675 E$ ol & $8.685 E$ ol & $8.725 E$ ol & 8.796501 \\
\hline $1.977 \mathrm{E} \quad 02$ & $1.979 E \quad 02$ & $1.988 \mathrm{E} \quad 02$ & $2.004 \mathrm{E} \quad 02$ \\
\hline $3.005 \mathrm{E} 02$ & $3.008 E \quad 02$ & $3.022 \mathrm{E} \quad 02$ & $3.045 \mathrm{E} \quad 02$ \\
\hline ANGLE 10 & ANGLE & ANGLE 12 & ANGLE \\
\hline & $M U=0$ & $M U=0$ & $M U=0$ \\
\hline 0.0 & 0.0 & 0.0 & 0.0 \\
\hline $8.833 \mathrm{E}$ & $1.404 \mathrm{E}-0 \mathrm{C}$ & 2.19 & 4.2 \\
\hline $5.747 E-06$ & $7.747 E-06$ & $1.097 \mathrm{E}-\mathrm{C}$ & $1.763 \mathrm{E}-\mathrm{C}$ \\
\hline $1.448 E-05$ & $1.858 \mathrm{E}-05$ & $2.538 E-05$ & $3.777 E-05$ \\
\hline $3.059 E-05$ & $3.746 E-05$ & $4.802 E-05$ & $6.479 E-05$ \\
\hline $4.385 E-05$ & $5.188 E-C 5$ & $6.346 \mathrm{E}-\mathrm{C}$ & $8.006 \mathrm{E}-\mathrm{C}$ \\
\hline $4.107 E-05$ & $4.771 \varepsilon-05$ & $5.677 E-05$ & $6.872 \varepsilon-05$ \\
\hline $8.233 E-05$ & $1.013 E-04$ & 1.2 & \\
\hline $1.514 E-04$ & $1.909 E-04$ & & $8 E-04$ \\
\hline $1.656 \mathrm{E}-04$ & $1.946 E-04$ & $2.323 E-04$ & $3 E-04$ \\
\hline $2.274 \mathrm{E}-$ & & & \\
\hline $5.924 \mathrm{E}-$ & $6.584 E-$ & & \\
\hline 1.4 & $1.586 \mathrm{E}-$ & $37 E-C$ & $2 E-03$ \\
\hline $1.379 \mathrm{E}$ & & & \\
\hline $1.319 E-02$ & $1.354 E-02$ & $9 E-02$ & $22 E-02$ \\
\hline $8.757 E-01$ & $8.973 \mathrm{E}-0$ & $8 E-01$ & $2 E-01$ \\
\hline TPर & $4.122 \mathrm{E} \quad 0$ & $4.216 E \quad 00$ & $4.306 E \quad 00$ \\
\hline $1.235 E$ & $1.264 E \quad 01$ & $1.292 E 01$ & $1.319 E$ O1 \\
\hline $3.501 E$ & $3.581 \mathrm{E}$ Ol & $3.660 E \quad 01$ & $3.735 \mathrm{E} \quad 01$ \\
\hline $9.776 \mathrm{E}$ ol & $9.994 \mathrm{E} \quad 01$ & $1.021 E 02$ & $1.041 E \quad 02$ \\
\hline $2.223 \mathrm{EO}$ & $2.272 \mathrm{E} \quad 02$ & $2.323 E \quad 02$ & $2.366 \mathrm{E} 02$ \\
\hline & & $3.481 \mathrm{E} \quad 02$ & $3.544 E \quad 02$ \\
\hline
\end{tabular}

ANGLE 5 $A N=-0.7550$
0.0
$1.379 E-07$ $1.379 \mathrm{E}-07$
$1.314 \mathrm{E}-06$ $1.314 \mathrm{E}-06$
$5.485 \mathrm{E}-06$ $1.494 E-05$ $2.733 E-05$ $2.872 E-05$ 4.757E-05 7. $633 \mathrm{E}-05$ $9.769 \mathrm{E}-05$ $1.539 E-04$ $4.090 \mathrm{E}-04$ $1.177 E-03$ $1.197 E-02$ $1.182 \mathrm{E}-02$ $7.896-01$ $3.648 \mathrm{E} 00$ $3.180 E$ OI $8.898 \mathrm{E}$ OI $2.027 E 02$ 3.077E 02

ANGLE 14 $M U=0.7550$ 0.0 1.008E-05 $1.008 E-05$
$3.241 E-05$ $3.241 E-05$
$6.069 E-05$ $6.069 E-05$
$0.114 E-05$ $9.114 E-05$
$1.033 E-04$ $8.380 E-05$ $2.266 E-04$ $2.266 E-04$
$4.799 E-04$ $3.401 E-04$ $3.673 E-04$ $9.244 E-04$ $1.895 \mathrm{E}-03$ $1.566 \mathrm{E}-02$ $1.451 E-02$ $9.576 \mathrm{E}-01$ $4.386 \mathrm{E} 00$ 1.344E 01 $3.802 E$ O1 $1.060 \mathrm{E} 02$

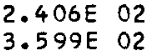
$M U=-0.6179$ 0.0 $6.217 \mathrm{E}-08$ $1.582 \mathrm{E}-06$ $6.245 \mathrm{E}-06$ $1.661 \mathrm{E}-05$ 2. $901 E-05$ $2.953 E-05$ 5.023E-05 $8.286 E-05$ $1.045 \mathrm{E}-04$ $1.622 \mathrm{E}-04$ $4.302 E-04$ $1.219 E-03$ $1.223 E-02$ 1.202E-02 $8.023 E-01$ 3.704E 00 $3.228 \mathrm{E} 01$ $9.029 E$ OI $2.057 E 02$ $3.118 E$ O2

ANOLE 15 0.0

0.0 $2.470 E-05$
$6.423 E-05$ $6.423 E-05$
$1.020 E-04$ $1.020 E-04$
$1.313 E-04$ 1. $345 E-04$ $1.345 E-04$
$1.017 E-04$ $1.017 E-04$
$3.098 E-04$ $7.017 \mathrm{E}-04$ $4.123 E-04$ $4.113 E-04$ $1.025 E-03$ $1.988 E-03$ $1.503 E-02$ $1.476 E-02$ $9.730 E-02$ $4.453 E 00$ $1.364 \mathrm{~F} \mathrm{OI}$ $3.858 \mathrm{E} 01$ $1.075 \mathrm{E} \mathrm{O2}$ $3.645 \mathrm{E} 02$ $U=-0.4580$ 0.0
$2.372 E-08$ $2.082 E-06$ $7.514 E-06$ $1.897 \mathrm{E}-05$ $3.123 E-05$ $3.091 E-05$ $5.428 \mathrm{E}-05$ $9.221 E-05$ $1.139 E-04$ 1.731E 04 $1.270 E-03$ $1.255 E-02$ $1.226 \mathrm{E}-02$ $8.176 E-01$ $1.157 \mathrm{E} 01$ $3.285 \mathrm{E} 01$ 2.186 01 $3.166 \mathrm{E} \quad 02$

ANGLE 16 0.0

$5.886 E-05$ $1.307 E-04$
$1.739 E-04$ $1.739 \mathrm{E}-04$ $1.893 E-04$ $1.732 E-04$
$1.209 E-04$ $1.209 E-04$
$4.268 E-04$
$1.056 E-03$ $1.056 E-03$ $4.923 E-04$ $4.517 E-04$
$1.116 E-03$ $2.062 \mathrm{E}-03$ $1.631 \mathrm{E}-02$ $1.495 E-02$ $9.843 E-01$ $4.502 \mathrm{E} 00$ $1.379 E$ O1 $3.899 E$ OI $1.086 \mathrm{E} \mathrm{O2}$ $3.677 \mathrm{E} 02$
ANGLE 8 ANGLE 9

$1.179 \mathrm{E}-07$ $2.942 E-06$ $9.299 E-06$ $3.420 E-C 5$ $3.308 \mathrm{E}-05$ $6.035 E-05$ $1.055 \mathrm{E}-04$ $1.265 E-04$ $1.873 E-04$ $1.333 E-03$ $1.292 E-02$ $1.255 \mathrm{E}-02$ $8.352 \mathrm{E}-01$ $1.181 E$ O1 $3.351 \mathrm{E}$ O1 (2) $2.132 E 02$

ANGLE 17 $=0.9894$ 0.0

$1.677 \mathrm{E}-04$ $2.830 \mathrm{E}-04$
$2.972 \mathrm{E}-04$ $2.972 \mathrm{E}-04$
$2.669 \mathrm{E}-04$ $2.669 \mathrm{E}-04$
$2.142 \mathrm{E}-04$ $1.372 E-04$ $5.841 E-04$ $1.631 E-03$
$5.681 E-04$ $5.681 E-04$
$4.801 E-04$ $1.179 E-03$ $2.107 E-03$ $1.647 \mathrm{E}-02$ $9.909 E-01$ $4.530 E$ OO $1.387 E$ OI

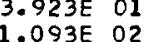
$2.479 E \quad 02$ $3.696 \mathrm{E} 02$
$M U=-0.0950$ 0.0 $4.276 E-07$ $4.181 E-06$ 1.158E-05 $2.575 E-05$ 3. $823 \mathrm{E}-05$ $3.635 \mathrm{E}-05$ $6.929 E-05$ $1.244 E-04$ $1.433 E-04$ $2.052 E-04$
$5.378 E-04$ . $1.333 E-02$ $1.286 E-02$ $8.547 E-01$ $3.936 E$ OO $3.423 \mathrm{E} 01$ 9.564 E $3.281 E$ O2

SCALAR FL 0

8. $415 E-05$ $2.215 E-04$
$3.768 E-04$ $3.768 E-04$
$5.800 E-04$ $7.163 E-04$
6.162 $7.163 E-04$
$6.227 E-04$ $1.485 \mathrm{E}-03$ $3.057 E-03$
$2.450 E-03$ $3.051 E-03$ $7.861 \mathrm{E}-03$ $1.885 \mathrm{E}-02$ $1.647 E-01$ $1.093 \mathrm{E} \mathrm{O1}$

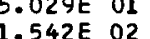
S42E O2 $1.221 E \quad 3$ $4.182 E 03$ 
(GAMYAS/MEV/STERADIAN/SOURCE NEUTRON)

\begin{tabular}{|c|c|c|c|c|c|c|c|c|c|c|}
\hline 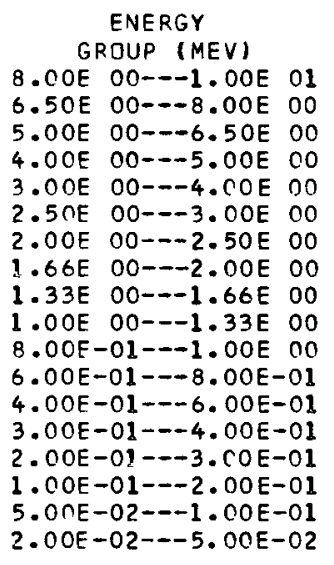 & $\begin{array}{l}\text { ANGLE } 1 \\
M U=-1.0000 \\
1.143 \mathrm{E}-04 \\
5.968 \mathrm{E}-04 \\
2.056 \mathrm{E}-03 \\
1.370 \mathrm{E}-03 \\
1.026 \mathrm{E}-03 \\
3.081 \mathrm{E}-03 \\
3.351 \mathrm{E}-03 \\
3.209 \mathrm{E}-03 \\
4.140 \mathrm{E}-03 \\
1.924 \mathrm{E}-03 \\
1.311 \mathrm{E}-03 \\
3.120 \mathrm{E}-03 \\
6.493 \mathrm{E}-03 \\
8.867 \mathrm{E}-03 \\
3.033 \mathrm{E}-02 \\
5.954 \mathrm{E}-02 \\
1.163 \mathrm{E}-01 \\
3.165 \mathrm{E}-02\end{array}$ & $\begin{array}{l}A N G L E \quad 2 \\
M U=-0.9894 \\
1.149 E-04 \\
6.002 E-04 \\
2.068 E-03 \\
1.382 E-03 \\
1.036 E-03 \\
3.101 E-03 \\
3.372 E-03 \\
3.226 E-03 \\
4.154 E-03 \\
1.917 E-03 \\
1.307 E-03 \\
3.164 E-03 \\
6.532 E-03 \\
8.898 E-03 \\
3.042 E-02 \\
5.961 E-02 \\
1.164 E-01 \\
3.166 E-02\end{array}$ & $\begin{array}{l}\text { ANGLE } 3 \\
M U=-0.9446 \\
1.174 E-04 \\
6.137 E-04 \\
2.115 E-03 \\
1.424 E-03 \\
1.071 E-03 \\
3.177 E-03 \\
3.455 E-03 \\
3.292 E-03 \\
4.215 E-03 \\
1.898 E-03 \\
1.301 E-03 \\
3.326 E-03 \\
6.683 E-03 \\
9.057 E-03 \\
3.077 E-02 \\
5.989 E-02 \\
1.168 E-01 \\
3.172 E-02\end{array}$ & $\begin{array}{l}\text { ANGLE } 4 \\
M U=-0.8656 \\
1.222 E-04 \\
6.397 E-04 \\
2.205 E-03 \\
1.502 E-03 \\
1.133 E-03 \\
3.319 E-03 \\
3.609 E-03 \\
3.420 E-03 \\
4.345 E-03 \\
1.895 E-03 \\
1.314 E-03 \\
3.603 E-03 \\
6.968 E-03 \\
9.476 E-03 \\
3.135 E-02 \\
6.044 E-02 \\
1.176 E-01 \\
3.183 E-02\end{array}$ & $\begin{array}{l}\text { ANGLE } 5 \\
M U=-0.7550 \\
1.294 E-04 \\
6.778 E-04 \\
2.334 E-03 \\
1.605 E-03 \\
1.214 E-03 \\
3.519 E-03 \\
3.826 E-03 \\
3.611 E-03 \\
4.559 E-03 \\
1.947 E-03 \\
1.377 E-03 \\
3.945 E-03 \\
7.383 E-03 \\
1.028 E-02 \\
3.205 E-02 \\
6.127 E-02 \\
1.187 E-01 \\
3.198 E-02\end{array}$ & $\begin{array}{l}\text { ANGLE } 6 \\
M U=-0.6179 \\
1.393 E-04 \\
7.297 E-04 \\
2.507 E-03 \\
1.733 E-03 \\
1.311 E-03 \\
3.784 E-03 \\
4.110 E-03 \\
3.876 E-03 \\
4.881 E-03 \\
2.087 E-03 \\
1.511 E-03 \\
4.325 E-03 \\
7.966 E-03 \\
1.155 E-02 \\
3.279 E-02 \\
6.239 E-02 \\
1.202 E-01 \\
3.217 E-02\end{array}$ & $\begin{array}{l}\text { ANGLE } 7 \\
M U=-0.4580 \\
1.526 E-04 \\
7.991 E-04 \\
2.735 E-03 \\
1.896 E-03 \\
1.433 E-03 \\
4.132 E-03 \\
4.486 E-03 \\
4.236 E-03 \\
5.337 E-03 \\
2.322 E-03 \\
1.718 E-03 \\
4.801 E-03 \\
8.789 E-03 \\
1.324 E-02 \\
3.354 E-02 \\
6.385 E-02 \\
1.221 E-01 \\
3.241 E-02\end{array}$ & $\begin{array}{r}\text { ANGLE } \\
M U=-0.2 \\
1.706 \mathrm{E} \\
8.924 \mathrm{E} \\
3.040 \mathrm{E} \\
2.111 \mathrm{E} \\
1.594 \mathrm{E} \\
4.600 \mathrm{E} \\
4.990 \mathrm{E} \\
4.725 \mathrm{E} \\
5.960 \mathrm{E} \\
2.652 \mathrm{E} \\
1.997 \mathrm{E} \\
5.424 \mathrm{E} \\
9.945 \mathrm{E} \\
1.524 \mathrm{E} \\
3.439 \mathrm{E} \\
6.567 \mathrm{E} \\
1.243 \mathrm{E}\end{array}$ & $\begin{array}{c}8 \\
2816 \\
E-04 \\
E-04 \\
E-03 \\
E-03 \\
E-03 \\
E-03 \\
E-03\end{array}$ & $\begin{array}{l}\text { ANGLE } 9 \\
\text { MU=-0.0950 } \\
1.950 \mathrm{E}-04 \\
1.019 \mathrm{E}-03 \\
3.451 \mathrm{E}-03 \\
2.404 \mathrm{E}-03 \\
1.813 \mathrm{E}-03 \\
5.235 \mathrm{E}-03 \\
5.676 \mathrm{E}-03 \\
5.389 \mathrm{E}-03 \\
6.800 \mathrm{E}-03 \\
3.088 \mathrm{E}-03 \\
2.368 \mathrm{E}-03 \\
6.317 \mathrm{E}-03 \\
1.151 \mathrm{E}-02 \\
1.738 \mathrm{E}-02 \\
3.543 \mathrm{E}-02 \\
6.788 \mathrm{E}-02 \\
1.268 \mathrm{E}-01\end{array}$ \\
\hline 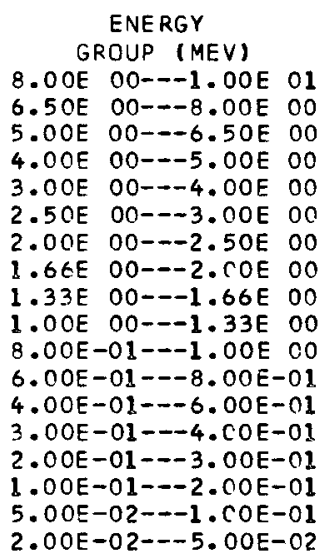 & $\begin{array}{l}3.209 \mathrm{E}-04 \\
1.232 \mathrm{E}-03 \\
4.134 \mathrm{E}-03 \\
2.896 \mathrm{E}-03 \\
2.027 \mathrm{E}-03 \\
6.300 \mathrm{E}-03 \\
6.822 \mathrm{E}-03 \\
6.490 \mathrm{E}-03 \\
8.185 \mathrm{E}-03 \\
5.628 \mathrm{E}-03 \\
2.921 \mathrm{E}-03 \\
1.171 \mathrm{E}-02 \\
1.359 \mathrm{E}-02 \\
1.958 \mathrm{E}-02 \\
3.692 \mathrm{E}-02 \\
7.051 \mathrm{E}-02 \\
1.296 \mathrm{E}-01 \\
3.334 \mathrm{E}-02\end{array}$ & $\begin{array}{l}2.504 E-04 \\
1.372 E-03 \\
5.572 E-03 \\
3.933 E-03 \\
3.814 E-03 \\
1.146 E-02 \\
9.256 E-03 \\
8.801 E-03 \\
1.110 E-02 \\
4.734 E-03 \\
3.798 E-03 \\
9.882 E-03 \\
1.622 E-02 \\
2.189 E-02 \\
3.910 E-02 \\
7.362 E-02 \\
1.326 E-01 \\
3.370 E-02\end{array}$ & $\begin{array}{l}5.104 \mathrm{E}-04 \\
2.669 \mathrm{E}-03 \\
6.685 \mathrm{E}-03 \\
4.731 \mathrm{E}-03 \\
3.043 \mathrm{E}-03 \\
9.181 \mathrm{E}-03 \\
1.112 \mathrm{E}-02 \\
1.060 \mathrm{E}-02 \\
1.338 \mathrm{E}-02 \\
5.848 \mathrm{E}-03 \\
4.779 \mathrm{E}-03 \\
1.212 \mathrm{E}-02 \\
1.872 \mathrm{E}-02 \\
2.391 \mathrm{E}-02 \\
4.122 \mathrm{E}-02 \\
7.708 \mathrm{E}-02 \\
1.358 \mathrm{E}-01 \\
3.406 \mathrm{E}-02\end{array}$ & $\begin{array}{l}3.993 \mathrm{E}-04 \\
2.098 \mathrm{E}-03 \\
6.945 \mathrm{E}-03 \\
4.912 \mathrm{E}-03 \\
3.592 \mathrm{E}-03 \\
1.022 \mathrm{E}-02 \\
1.152 \mathrm{E}-02 \\
1.111 \mathrm{E}-02 \\
1.407 \mathrm{E}-02 \\
7.104 \mathrm{E}-03 \\
6.010 \mathrm{E}-03 \\
1.408 \mathrm{E}-02 \\
2.130 \mathrm{E}-02 \\
2.600 \mathrm{E}-02 \\
4.344 \mathrm{E}-02 \\
8.078 \mathrm{E}-02 \\
1.389 \mathrm{E}-01 \\
3.441 \mathrm{E}-02\end{array}$ & $\begin{array}{l}5.345 \mathrm{E}-04 \\
2.776 \mathrm{E}-03 \\
9.125 \mathrm{E}-03 \\
6.491 \mathrm{E}-03 \\
4.897 \mathrm{E}-03 \\
1.395 \mathrm{E}-02 \\
1.522 \mathrm{E}-02 \\
1.477 \mathrm{E}-02 \\
1.871 \mathrm{E}-02 \\
1.009 \mathrm{E}-02 \\
8.613 \mathrm{E}-03 \\
1.860 \mathrm{E}-02 \\
2.628 \mathrm{E}-02 \\
3.005 \mathrm{E}-02 \\
4.862 \mathrm{E}-02 \\
8.499 \mathrm{E}-02 \\
1.420 \mathrm{E}-01 \\
3.473 \mathrm{E}-02\end{array}$ & $\begin{array}{l}8.987 \mathrm{E}-04 \\
4.544 \mathrm{E}-03 \\
1.534 \mathrm{E}-02 \\
1.102 \mathrm{E}-02 \\
8.123 \mathrm{E}-03 \\
2.397 \mathrm{E}-02 \\
2.572 \mathrm{E}-02 \\
2.480 \mathrm{E}-02 \\
3.121 \mathrm{E}-02 \\
1.550 \mathrm{E}-02 \\
1.186 \mathrm{E}-02 \\
2.707 \mathrm{E}-02 \\
3.223 \mathrm{E}-02 \\
3.476 \mathrm{E}-02 \\
5.559 \mathrm{E}-02 \\
8.963 \mathrm{E}-02 \\
1.450 \mathrm{E}-01 \\
3.502 \mathrm{E}-02\end{array}$ & $\begin{array}{l}1.528 \mathrm{E}-03 \\
7.869 \mathrm{E}-03 \\
2.468 \mathrm{E}-02 \\
1.784 \mathrm{E}-02 \\
1.290 \mathrm{E}-02 \\
3.892 \mathrm{E}-02 \\
4.138 \mathrm{E}-02 \\
3.955 \mathrm{E}-02 \\
4.939 \mathrm{E}-02 \\
2.226 \mathrm{E}-02 \\
1.323 \mathrm{E}-02 \\
3.785 \mathrm{E}-02 \\
3.431 \mathrm{E}-02 \\
3.622 \mathrm{E}-02 \\
5.866 \mathrm{E}-02 \\
9.359 \mathrm{E}-02 \\
1.472 \mathrm{E}-01 \\
3.522 \mathrm{E}-02\end{array}$ & & & $\begin{array}{l}3.938 \mathrm{E}-02 \\
1.165 \mathrm{E}-01 \\
1.237 \mathrm{E}-01 \\
1.181 \mathrm{E}-01 \\
1.487 \mathrm{E}-01 \\
7.090 \mathrm{E}-02 \\
5.127 \mathrm{E}-02 \\
1.357 \mathrm{E}-01 \\
1.905 \mathrm{E}-01 \\
2.456 \mathrm{E}-01 \\
4.880 \mathrm{E}-01 \\
8.997 \mathrm{E}-01 \\
1.527 \mathrm{E} 00 \\
4.182 \mathrm{E}-01\end{array}$ \\
\hline
\end{tabular}


(GAMMAS/MEV/STERAOIAN/SOURCE NEUTRON)

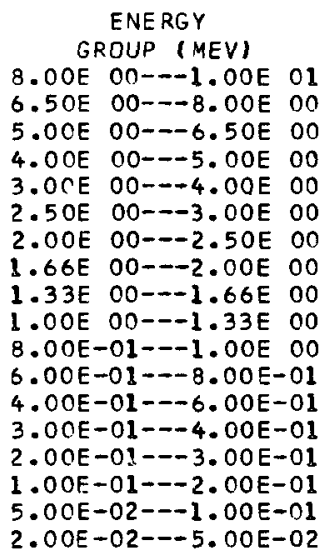

ENERGY

GROUP (MEV)

8.0OE OO---1.0CE 01

$6.50 E$ OO- $-8.00 E$ OO

$5.00 \mathrm{~F}$ OO---6.50E 00

4.0CE $00--5$. OOE OO

3.00 OO---3.0OE 00

$2.00 E$ OO- $-2.50 E$ OO

$1.66 E$ EO---2.00E 00

$1.32 \mathrm{E} 00---1.66 \mathrm{E}$ OO

$1.00 E$ OO- $-1.33 \mathrm{E} 00$

$8.00 E-01--1.00 E 00$

$6.00 E-01--8.00 E-01$

$4.00 E-01--6.00 E-01$

$3.00 \mathrm{E}-01--4.00 \mathrm{E}-0$

2.00E-01-- 3. 00E-O

$1.00 E-01---2.00 E-01$

$5.0 \mathrm{CE}-02-1.00 \mathrm{E}-01$

2.ONE-02-- 5. 00 E-02
ANGLE 1 ANGLE ? $U=-1.0000$ $1.199 \mathrm{E}-04$ 2.264E -04 $1.448 \mathrm{E}-03$ $1.448 E-03$ 1.141E 03 $3.724 E-03$ 3.724E-03 $3.640 \mathrm{E}-03$ $4.819 \mathrm{E}-03$ $2.662 \mathrm{E}-03$ $2.040 \mathrm{E}-03$ $3.433 \mathrm{E}-03$ $1.085 \mathrm{E}-02$
$1.545 \mathrm{E}-02$ $1.545 \mathrm{E}-02$
$6.393 \mathrm{E}-02$ $6.393 \mathrm{E}-02$ $1.516 \mathrm{E}-01$
$3.455 \mathrm{E}-01$ $9.901 \mathrm{E}-02$

ANGLE 10 $M U=0.0950$ $1.881 \mathrm{E}-04$ $1.097 \mathrm{E}-03$ 2.811E-03 $3.362 \mathrm{E}-03$ $5.832 \mathrm{E}-03$ $6.530 \mathrm{E}-03$ $6.253 E-03$ $7.874 E-03$ $4.068 \mathrm{E}-03$ $5.799 \mathrm{E}-03$ $9.125 \mathrm{~F}-03$ 2. $908 \mathrm{E}-02$ $4.549 \mathrm{E}-02$ $8.086 E-02$ $1.895 \mathrm{E}-01$ $4.050 \mathrm{E}-0$ $1.068 \mathrm{E}-01$ $1.208 E-04$ $6.317 E-04$ $2.380 \mathrm{E}-03$ $1.473 \mathrm{E}-03$ $1.164 \mathrm{E}-03$ $3.761 E-03$ $3.662 \mathrm{E}-03$ $4.825 \mathrm{E}-03$ $2.622 \mathrm{E}-03$ $2.020 E-03$ $3.538 \mathrm{E}-03$ $1.093 \mathrm{E}-02$ $1.093 \mathrm{E}-02$ $6.419 \mathrm{E}-02$ 1. $518 \mathrm{E}-01$ $3.459 \mathrm{E}-01$
$9.907 \mathrm{E}-02$ ANGLE 11 $M U=0.2816$ $3.447 E-04$ $6.803 E-03$ $4.727 E-03$ $2.935 \mathrm{E}-03$ $7.826 \mathrm{E}-03$ $1.101 \mathrm{E}-02$ $1.056 \mathrm{E}-02$ $1.332 \mathrm{E}-02$ $7.874 \mathrm{E}-03$ $6.961 E-03$ $1.768 \mathrm{E}-02$ $3.406 E-C 2$ $4.966 \mathrm{E}-02$ $8.364 \mathrm{E}-02$ $2.002 E-01$ $4.194 E-01$ $1.085 \mathrm{E}-01$
ANGLE 3 $1.243 \mathrm{E}-04$ . . . (1) . $3.750 E-03$ $4.859 \mathrm{E}-03$ $2.498 E-03$ $1.967 \mathrm{E}-03$ $3.915 \mathrm{E}-03$ $1.125 \mathrm{E}-02$ $1.587 E-02$ $6.515 \mathrm{E}-02$ $1.528 \mathrm{E}-01$ $3.477 \mathrm{E}-01$ $9.932 \mathrm{E}-02$ ANGLE 12 $M U=0.4580$ $4.702 \mathrm{E}-04$ $7.048 \mathrm{E}-03$ $4.862 \mathrm{E}-03$ $4.787 \mathrm{E}-03$ $1.441 E-02$ $1.101 \mathrm{E}-02$ 1.376E-02 $1.359 E-02$ $8.582 E-03$ $1.024 E-02$ $1.904 \mathrm{E}-02$ 5.556E-02 $9.035 \mathrm{E}-02$ $2.129 E-01$ $4.350 E-O 1$ $1.103 \mathrm{E}-01$
ANGLE 4 $1.308 \mathrm{E}-04$ $6.887 \mathrm{E}-04$ $2.605 \mathrm{E}-03$ $1.715 \mathrm{E}-03$ $3.701 \mathrm{E}-03$ $4.137 E-03$ $4.137 E-03$
$3.916 E-03$ . $562 E-03$ 2.366E-03 $1.946 E-03$ $4.513 E-03$ $4.513 E-03$ $1.183 E-02$
$1.696 E-02$ $6.672 \mathrm{E}-02$ $1.547 \mathrm{E}-01$ 3.511E-01 $9.979 E-02$ ANGLE 13 $M U=0.6179$ $5.069 E-04$ $1.254 E-02$ $8.820 E-03$ $5.412 E-03$ $1.321 E-02$ 2.053E-02 $1.999 \mathrm{E}-02$ $2.545 \mathrm{E}-02$ $1.456 \mathrm{E}-02$ 1.550E-02 $2.980 E-02$ $5.118 \mathrm{E}-02$ $6.317 E-02$ $1.005 \mathrm{E}-01$
$2.277 \mathrm{E}-01$ $4.516 \mathrm{E}-01$ $1.122 E-01$
ANGLE 5 ANGLE 6 $\begin{array}{rr}1.399 E-04 & 1.520 E-04\end{array}$ $7.386 E-04 \quad 8.024 E-04$ $3.025 E-03$
$-1.060 E-03$ $1.884 E-03 \quad 2.060 E-03$ $3.978 \mathrm{E}-03$ $4.435 E-03$ $4.153 E-03$ $5.186 \mathrm{E}-03$ $2.367 \mathrm{E}-03$ $2.367 \mathrm{E}-03$ $5.146 \mathrm{E}-03$ $1.267 E-02$ $1.923 E-02$ $6.848 \mathrm{E}-02$ $6.848 E-02$
$1.574 E-01$ $3.560 \mathrm{E}-01$ $1.005 \mathrm{E}-01$ ANGLE 14 $M U=0.7550$ $8.832 E-04$ $1.213 \mathrm{E}-02$ $8.524 E-03$ $8.812 \mathrm{E}-03$ $2.210 E-02$ $1.978 \mathrm{E}-02$ $1.999 \mathrm{E}-02$ $2.561 \mathrm{E}-02$ $1.994 \mathrm{E}-02$ $2.097 \mathrm{E}-02$ $3.591 \mathrm{E}-02$ $5.926 \mathrm{E}-02$ $7.043 E-02$ $1.090 \mathrm{E}-01$ $2.436 \mathrm{E}-01$ $4.679 E-01$ $1.139 E-01$
ANGLE 7 $M U=-0.2816 \quad M U=-0.0950$ 3.719E-03 $1.159 E-03$ $2.258 \mathrm{E}-032.524 \mathrm{E}-03 \mathrm{Z} 2.912 \mathrm{E}-03$ $1.818 E-03$ 2.020E-03 $2.33 \mathrm{E}-03$ $4.728 E-03 \quad 5.302 E-03 \quad 6.114 E-03$ $5.254 E-03$ (5.889E-03 $4.939 E-03 \quad 5.578 E-03 \quad 6.466 E-03$ $6.182 \mathrm{E}-03 \quad 7.020 \mathrm{E}-03 \quad 8.14 \mathrm{BE}-03$ $3.005 E-03 \quad 3.584 E-03 \quad 4.303 E-03$ $2.755 E-03 \quad 3.319 E-03 \quad 4.077 E-03$ $6.400 \mathrm{E}-03 \quad 7.358 \mathrm{E}-03 \quad 8.067 \mathrm{E}-03$ $1.577 \mathrm{E}-02 \quad 1.863 \mathrm{E}-02 \quad 2.967 \mathrm{E}-03$ $\begin{array}{lll}1.5770-02 & 1.363 E-02 & 2.269 E-02 \\ 2.780 E-02 & 3.343 E-02 & 3.917 E-02\end{array}$ $2.183 \mathrm{E}-02 \quad 7.362 \mathrm{E}-02 \quad 7.597 \mathrm{E}-02$
$1.662 \mathrm{E}-01$ $1.662 \mathrm{E}-01 \quad 1.724 \mathrm{E}-01 \quad 1.800 \mathrm{E}-01$ $\begin{array}{lll}1.662 E-01 & 1.724 E-01 & 1.800 E-01 \\ 3.707 E-01 & 3.804 E-01 & 3.919 E-01\end{array}$ $1.024 E-01$ 1.037E-01 1.052E-01 ANGLE 16 $M U=0.9446$ $2.328 E-03$ $1.208 E-02$ $3.986 \mathrm{E}-02$ $2.215 E-02$ $5.833 E-02$ $6.493 E-02$ $6.393 E-02$ $7.904 E-02$ 4. $716 \mathrm{E}-02$ $3.937 E-02$ $6.844 E-02$ $8.928 \mathrm{E}-02$ $9.527 E-02$ $1.418 \mathrm{E}-01$
ANGLE 17 SCALAR

$5.523 E-03 \quad 6.084 E-03$
$2.808 E-02 \quad 3.168 E-02$ $8.694 \mathrm{E}-02 \quad 1.078 \mathrm{E}-01$ $6.307 E-02 \quad 7.580 E-02$ $4.652 \mathrm{E}-02 \quad 6.018 \mathrm{E}-02$ $1.294 \mathrm{E}-01 \quad 1.573 \mathrm{E}-01$ $1.362 \mathrm{E}-01 \quad 1.732 \mathrm{E}-01$ $1.295 E-01 \quad 1.681 E-01$ $1.562 E-01 \quad 2.106 E-01$ $5.074 \mathrm{E}-02 \quad 1.165 \mathrm{E}-01$ $1.083 E-01 \quad 2.237 E-01$ $1.116 E-01 \quad 4.121 \mathrm{E}-01$ $1.105 E-01 \quad 5.605 E-01$ $2.937 E-01$ 1.074E 00

$\begin{array}{lllll}2.614 E-01 & 2.789 E-01 & 2.937 E-01 & 2.443 E & 00 \\ 4.836 E-01 & 4.968 E-01 & 5.055 E-01 & 5.108 E & 00 \\ 1.154 E-01 & 1.166 E-01 & 1.173 E-01 & 1.341 E & 00\end{array}$

$\begin{array}{lllll}2.614 E-01 & 2.789 E-01 & 2.937 E-01 & 2.443 E & 00 \\ 4.836 E-01 & 4.968 E-01 & 5.055 E-01 & 5.108 E & 00 \\ 1.154 E-01 & 1.166 E-01 & 1.173 E-01 & 1.341 E & 00\end{array}$

$\begin{array}{lllll}2.614 E-01 & 2.789 E-01 & 2.937 E-01 & 2.443 E & 00 \\ 4.836 E-01 & 4.968 E-01 & 5.055 E-01 & 5.108 E & 00 \\ 1.154 E-01 & 1.166 E-01 & 1.173 E-01 & 1.341 E & 00\end{array}$

$\begin{array}{lllll}2.614 E-01 & 2.789 E-01 & 2.937 E-01 & 2.443 E & 00 \\ 4.836 E-01 & 4.968 E-01 & 5.055 E-01 & 5.108 E & 00 \\ 1.154 E-01 & 1.166 E-01 & 1.173 E-01 & 1.341 E & 00\end{array}$

$\begin{array}{lllll}2.614 E-01 & 2.789 E-01 & 2.937 E-01 & 2.443 E & 00 \\ 4.836 E-01 & 4.968 E-01 & 5.055 E-01 & 5.108 E & 00 \\ 1.154 E-01 & 1.166 E-01 & 1.173 E-01 & 1.341 E & 00\end{array}$ 
(GAMMAS/MEV/STERADIAN/SOURCE NEUTRON)

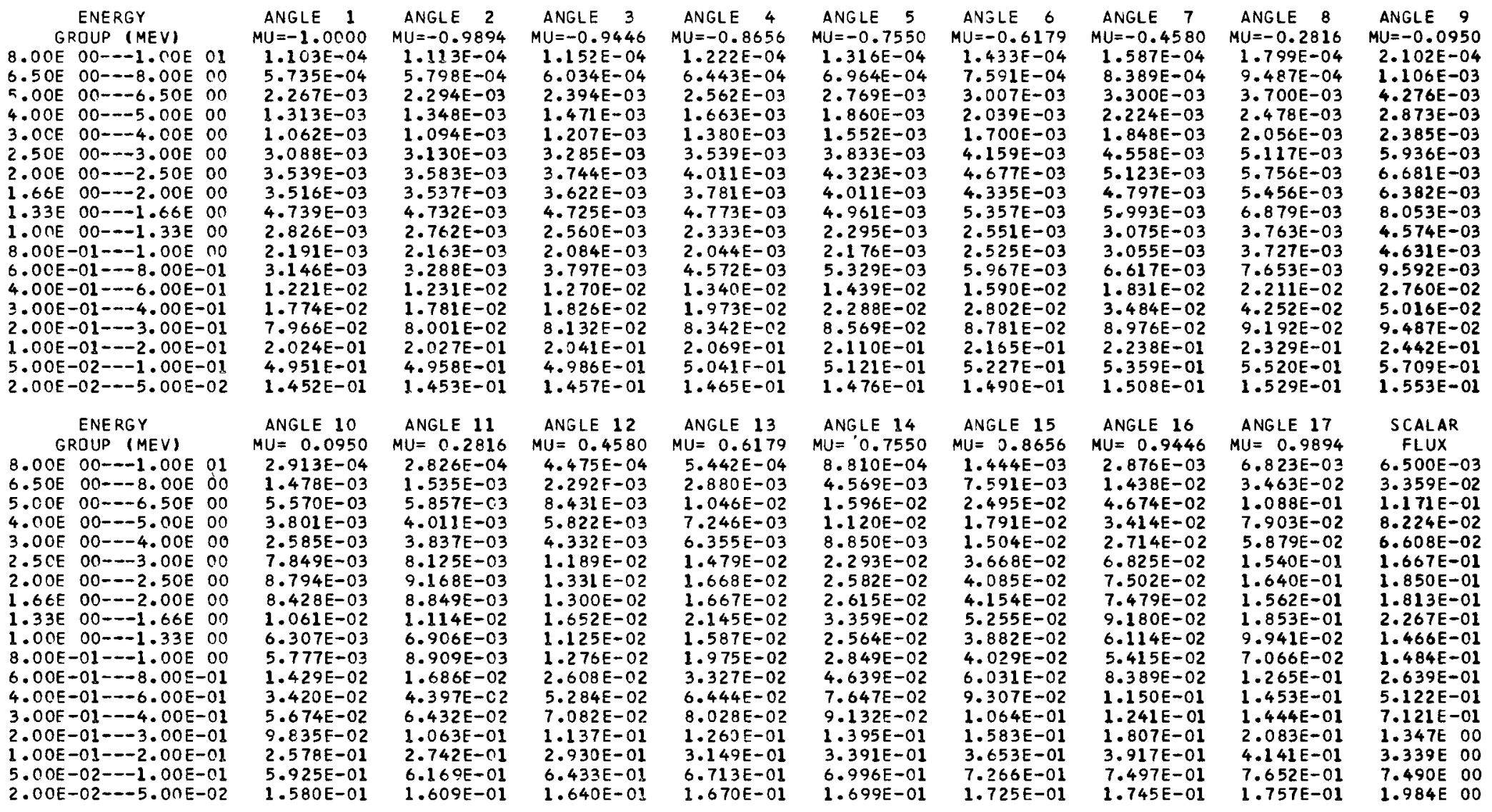


IGAMMAS/MEV/STERADIAN/SOURCE NEUTRONI

ENERGY GROUP (MEV) 8.0OE OO--1. $5.00 E$ OO- $0.6 .50 E$ EO 4.0OE O0---5. OOE 00 $3.0 C E$ OD- $-4.00 E$ OO 2.5 CE $00--3.00 E$ DO 2. .OOE OD--2.5OE OO 1.66 E $00-2.00 E 00$ $1.33 \mathrm{E} \quad 00--1.66 \mathrm{E} \quad 00$ $1.00 E$ OO- $-1.33 E 00$ $8.00 E-02--1.00 E 00$ $6.00 E-01--8.00 E-01$ $4.00 E-01--6.00 E-01$ $3.00 \mathrm{E}-01--4.00 \mathrm{E}-01$ 2.0OE-01--3. COE-O1 $1.00 E-01--2.00 E-C 1$ $5.00 E-02--1.00 E-01$
$2.00 E-02=--5.00 E-02$

\section{ENERGY}

GROUP (MEV)

8. OCE OO---1.0OE 01 $6.50 E$ OD-- $8.00 E$ OO 5.OOE OO- $5.50 E$ OO 4.OOE OO$2.50 E$ OO- $-3.00 E$ OO 2.0OE OO$1.66 \mathrm{E}$ OOD- $2.00 \mathrm{E}$ OO 1.33 E $00-1.66 E^{2} 00$ $1.00 E$ OO-- 1. 33E OO $8.00 E-01--1.00 E$ OO $6.00 E-01--8.00 E-01$ $4.0 C E-01--6$. COE-O1 3.00E-01-- $4.00 \mathrm{E}-01$ 2. OOE-01---3. OOE-O1 $1.00 \mathrm{E}-01--2.00 \mathrm{E}-01$ $5.00 E-02--1.00 E-01$ 2. OCE-02---5.00E-02
ANGLE 1 $M U=-1.0000$ $8.341 E-05$ 1.262E-04 $9.267 E-04$ 7. $2.364 E-03$ $2.843 \mathrm{E}-03$ $2.933 E-03$ 4. $C 96 E-03$ $2.725 \mathrm{E}-03$ 2.725E-03 $2.049 E-03$ $2.225 E-03$ $1.259 \mathrm{E}-02$ $1.879 E-02$ 政 7.080 $2.143 E-01$

ANGLE 10 $M U=0.0950$ $2.010 E-04$ $4.424 E-03$ $2.924 E-03$ $2.709 \mathrm{E}-03$ $5.846 E-03$ $6.730 \mathrm{E}-03$ $6.470 E-03$ $8.121 \mathrm{E}-03$ $5.281 E-C 3$ $6.621 E-03$ $1.333 \mathrm{E}-02$ $4.222 E-02$ $7.050 \mathrm{E}-02$ $1.192 \mathrm{E}-01$ $3.435 \mathrm{E}-01$ $8.716 E-01$ 2.357E-01
ANGLE 2 $M U=-0.9894$ $8.458 E-05$ $4.337 E-04$ $9.875 E-04$ . $758 E-04$ $2.416 E-03$ $2.492 \mathrm{E}-03$ $2.947 E-03$ $4.063 E-03$ $2.625 \mathrm{E}-03$ $2.012 E-03$ 2. $417 \mathrm{E}-03$ $1.271 E-02$ $1.887 E-02$ $9.643 E-02$ $2.633 E-01$ $2.144 E-01$

ANGLE 11 $M U=0.2816$ $2.724 \mathrm{E}-04$ 5.748E-03 S.748E-03 $3.304 E-03$ $3.304 E-03$ $8.62 \mathrm{E}-03$ $8.526 \mathrm{E}-03$ $1.074 \mathrm{E}-02$ $7.610 E-03$ $9.865 \mathrm{E}-03$ $2.073 \mathrm{E}-02$ $5.319 \mathrm{E}-02$ 7.831 E-02 1. $268 \mathrm{E}-01$ $3.670 E-01$ $9.129 E-01$ 2.406 F-01 ${ }^{3}$ $8.892 \mathrm{E}-05$ $4.612 E-04$ $1.908 \mathrm{E}-03$ $1.147 E-03$ $9.948 \mathrm{E}-04$ $2.603 \mathrm{E}-03$ 3.070E-03 $3.004 E-03$ $3.966 \mathrm{E}-03$ $2.303 E-03$ $1.908 \varepsilon-03$ $3.100 E-03$ $1.315 E-02$ $1.948 E-02$ . $812 \mathrm{E}-02$ $2.654 E-01$ 2.151E-01 ANGLE 12 $M U=0.4580$ . $480 E-04$ 1. $826 E-03$ $4.952 E-03$ $4.401 E-03$ $0.402 E-03$ $1.115 \mathrm{E}-02$ $1.104 E-02$ $1.417 E-02$ $1.140 E-02$ $1.595 E-02$ 2. $939 E-02$ $6.571 E-02$ $8.691 E-02$ $1.381 E-01$ $3.946 E-01$ $9.583 E-01$ $2.457 E-01$
ANGLE 4 $M U=-0.8656$ .616E-05 . . 1.39E-03 $2.887 E-03$ $3.344 E=03$ $3.115 E-03$ $3.890 E-03$ $1.934 E-03$ $1.858 E-03$ 4. $083 \mathrm{E}-03$ $1.392 \mathrm{E}=02$ $2.153 E-02$ $1.007 E-01$ $2.694 E-01$ $2.164 \mathrm{E}=01$ ANGLE 13 $M U=0.6179$ $2.621 \mathrm{E}-03$ $1.010 E-02$ $6.897 E-03$ $5.911 E-03$ 1. $581 \mathrm{E}-02$ $1.623 \mathrm{E}-02$ $2.121 E-02$ $1.884 \mathrm{E}-02$ $2.503 \mathrm{E}-02$ $2.503 E-02$ $1.853 \mathrm{E}-02$ $9.755 \mathrm{E}-02$ $1.517 \mathrm{E}-01$ $4.260 E-01$ $1.007 E$ OO 2.509E-01
ANGLE 5 $M U=-0.7550$ $5.549 E-04$ $2.404 E-03$ $1.616 \mathrm{E}-03$ $1.425 \mathrm{E}-03$ $3.172 \mathrm{E}-03$ 3.630E-03 $3.289 E-03$ $3.984 E-03$ $1.840 \mathrm{E}-03$ $2.027 E-03$ $4.930 E-03$ $1.503 E-02$ $2.596 \mathrm{E}-02$ $1.034 \mathrm{E}-01$ 2.754E-01 $2.182 \mathrm{E}-01$ ANGLE 14 $M U=0.7550$ 7.691E-04 1.976E-03 $1.030 \mathrm{E}-02$ 1.030E-02 . $049 E-02$ $2.049 E-02$ $2.513 \mathrm{E}-02$ $3.291 E-02$ $3.029 \mathrm{E}-02$ $3.766 \mathrm{E}-02$ $5.476 \mathrm{E}-02$ $9.338 \mathrm{E}-02$ $1.121 E-01$ $1.692 E-01$ $4.608 E-01$ $1.056 \mathrm{E} 00$ $2.558 E-01$ $M U=-0.6179$

ANGLE 7 ANGLE 8 $M U=-0.4580 \quad M U=-0.2816$

ANGLE 9 $1.767 E-03$ $1.554 E-03$ $3.438 E-03$ $3.562 E-03$ 4. $343 \mathrm{E}-03$ $2.151 E-03$ $2.449 E-03$ $5.501 \mathrm{E}-03$ $1.682 \mathrm{E}-02$ $3.314 \mathrm{E}-02$ $1.057 E-01$ $2.834 E-01$ 2.205E-01

ANGLE 15 $M U=0.8656$ $7.052 \mathrm{E}-03$ $.495 \mathrm{E}-02$ $.801 \mathrm{E}-02$ . $573 \mathrm{E}-02$ 4. $4.304 E-02$ $4.478 \mathrm{E}-02$ 4.806E-02 $5.323 \mathrm{E}-02$ $5.323 \mathrm{E}-02$ $1.119 \mathrm{E}-01$ $1.301 \mathrm{E}-01$ $1.899 \mathrm{E}-01$ 4.977E-01 2.601E-01 (2.441E-04 $1.699 \mathrm{E}-04$

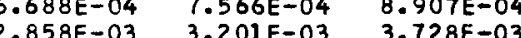
$1.889 E-03 \quad 3.201 E-03 \quad 3.728 E-03$ $1.653 \mathrm{E}-03 \quad 1.815 \mathrm{E}-03 \quad 2.132 \mathrm{E}-03$ $3.742 E-03 \quad 4.199 E-03 \quad 4.925 E-03$ $4.281 E-03 \quad 4.827 E-03 \quad 5.673 E-03$ $3.981 E-03 \quad 4.594 E-03 \quad 5.453 E-03$ $4.976 E-03 \quad 5.840 E-03 \quad 6.917 E-03$ $2.788 E-03 \quad 3.554 E-03 \quad 4.355 E-03$ $3.049 E-03 \quad 3.768 E-03 \quad 4.758 E-03$ $6.012 E-03 \quad 7.044 E-03 \quad 9.371 E-03$ $1.991 E-02 \quad 2.495 E-02 \quad 3.236 E-02$ $4.247 E-02 \quad 5.265 E-02 \quad 6.230 E-02$ $1.077 E-01$ $1.100 E-01$ 1.135E-01 2.939E-C1 3.072E-01 $3.236 E-01$ $\begin{array}{lll}7.235 E-01 & 2.270 E-01 & 2.311 E-01\end{array}$

ANGLE 16 $A N G L E 17$

SCALAR $M U=0.9446 \quad M U=0.9894$ 
(GAMMAS/MEV/STERADIAN/SOURCE NEUTRON)

\begin{abstract}
ENERGY
GROUP (MEV)

8.00E $00--1.00 E 01$ $6.50 E$ OO- - 8.00 E 00 5.COE OO-- -6.50E OO 4. OOE OO- -5. OOE OO $3.00 E$ OO- $-4.00 E$ OO $2.50 E$ OO- - - 3. DOE OO $2.00 E$ OO---2.5OE OO $1.66 \mathrm{E} 00---2.00 E 00$ $1.33 \mathrm{E} 00--1.66 \mathrm{E} \quad 00$ $1.00 \mathrm{E}$ 00---1.33E 00 $8.00 \mathrm{E}-01--1.00 \mathrm{E}$ OO 6. 0 CEE $-01--8.00 E-01$ $3.00 E-01---4.00 E-01$ $2.00 \mathrm{E}-01 \mathrm{1}-\mathrm{-3} .00 \mathrm{E}-01$ 1. $00 E-01--2.00 E-01$ 5. $00 E-02---1.00 E-01$
\end{abstract}

ENERGY

GROUP (MEV)

8.0OE $00--1.00 E$ OI $6.50 E$ OO- $-8.00 E$ DO $5.00 E$ OO- $-6.50 E$ OO $4.00 E$ OO- $-5.00 E$ OO $3.00 E$ OO- $-4.00 E$ OO $2.50 E$ OO- $-3.00 E$ OO $2.00 \mathrm{E}$ 00---2.50E OO 1.66 E $00--2.00 E$ OO $1.23 \mathrm{~F}$ 00--1.66E 00 $1.00 E$ O0- $-1.33 \mathrm{E}$ OO 8. ONE-OL- - - . OOE OO 6.00E-01---8.00E-O1 4.0CE-01---6.00E-O $3.00 E-01--4.00 E-01$ 2. तOE-01--3.00E-01 1. .0 E $-01-C-2.00 E-01$ $5.00 E-02---1.00 E-01$
$2.00 E-02---5.00 E-02$
ANGLE 1 ANGLE 2 M.854E-05 MU $5.974 E-05$ ( $2.899 \mathrm{E}-04$
$1.368 \mathrm{~F}-03$ $5.647 E-04$ $5.050 \mathrm{E}-04$ $1.656 \mathrm{E}-03$ $2.116 \mathrm{E}-03$ $2.288 \mathrm{E}-03$ $3.305 \mathrm{E}-03$ $2.370 \mathrm{E}-03$ $1.655 \mathrm{E}-03$ $1.322 \mathrm{E}-03$ $1.142 \mathrm{E}-02$ $1.722 \mathrm{E}-02$ $9.764 \mathrm{E}-02$ $2.760 E-01$ $7.914 E-01$ $2.435 \mathrm{E}-01$

ANGLE 10 $M U=0.0950$ $8.247 \mathrm{E}-04$ . $2.395 \mathrm{E}-03$ .195E-03 $4.671 E-03$ $5.469 E-03$ $5.265 E-03$ $6.605 E-03$ $4.623 E-03$ $6.029 E-03$ $1.307 \mathrm{E}-02$ 4.295E-02 $7.230 \mathrm{E}-02$ $1.191 \mathrm{E}-01$ $3.655 E-01$ $9.878 E-01$ $2.692 E-01$

ANGLE 11 $047 E-04$ $1.067 \mathrm{E}-03$ $4.636 E-03$ $3.120 E-03$ $2.860 E-03$ $5.927 E-03$ $6.907 E-03$ $6.682 E-03$ $8.412 \mathrm{E}-03$ $6.595 \mathrm{E}-03$ $2.042 E-02$ $5.490 E-02$ $7.949 E-02$ $1.274 \mathrm{E}-01$ $3.915 \mathrm{E}-01$ $1.038 E$
$2.751 E-01$
ANGLE 3 ANGLE 4 ANGLE 5 $M U=-0.9446$
$6.412 E-05$
$3.264 E-04$ $\begin{array}{lll}3.264 \mathrm{E}-04 & 3.597 \mathrm{E}-04 & 4.128 \mathrm{E}-04 \\ 1.537 \mathrm{E}-03 & 1.735 \mathrm{E}-03 & 1.927 \mathrm{E}-03\end{array}$ $1.537 \mathrm{E}-03$
$8.181 \mathrm{E}-04$ $7.485 E-04$ $7.485 E-04$
$1.906 E-03$ 2.32BE-03 $2.308 \mathrm{E}-03$ $3.080 \mathrm{E}-03$ $1.870 E-07$ $1.526 \mathrm{E}-03$ $2.287 E-03$ $1.192 \mathrm{E}-02$ $1.805 \mathrm{E}-02$ $9.982 \mathrm{E}-02$ $2.789 E-01$ $7.984 E-01$ 2. $445 E-01$ ANGLE 12 $8.093 E-01$ $M U=0.4580$ 2.781E-04 $.099 E-03$ $4.111 E-03$ $3.724 E-03$ $7.816 E-03$ $9.148 E-03$ $9.159 \mathrm{E}-0$ $1.190 \mathrm{E}-02$ $1.090 \mathrm{E}-02$ $1.645 \mathrm{E}-02$ $3.045 E-02$ $6.732 E-02$ $8.742 \mathrm{E}-02$ $1.384 E-01$ 4.217E-OI $1.094 \mathrm{E}-00$
$2.812 \mathrm{E}-01$

ANGLE 13 $M U=0.7550$ 列 . $431 E-03$ $8.431 E-03$ $5.165 \mathrm{E}-0$ $1.099 \mathrm{E}-0$ $1.303 \mathrm{E}-02$ $1.374 \mathrm{E}-02$ $1.839 \mathrm{E}-02$ $1.875 \mathrm{E}-02$ $2.673 \mathrm{E}-02$ 4. $252 \mathrm{E}-02$ $8.003 E-0$ $9.813 \mathrm{E}-02$ $1.523 \mathrm{E}-01$ $4.560 \mathrm{E}-0$ $1.152 \mathrm{E}-00$
$2.874 \mathrm{E}-01$ $273 \mathrm{E}-03$ $1.280 E-02$ $8.826 \mathrm{E}-03$ $8.158 E-03$ $2.730 E-02$ $2.263 E-02$ $3.018 E-0$ $4.050 E-02$ $9.385 E-02$ $1.125 \mathrm{E}-01$ $4.934 E-01$ $1.212 \mathrm{E}$ OO
ANấle 6 $M U=-0.6179$ $4.513 E-04$ $4.513 E-04$
$2.092 E-03$ 2.092E-0 $1.411 E-03$ $2.620 E-03$ 3.011E-03 $2.676 E-03$ $3.225 E-03$ $1.650 \varepsilon-03$ $2.099 E-03$ 4. $555 \mathrm{E}-03$ $1.552 \mathrm{E}-02$ $3.345 \mathrm{E}-02$ $1.069 \mathrm{E}-01$ 2. $990 E-01$ $8.459 E-01$ $2.510 E-01$ 2. $481 E-01$

ANज́LE 15 $M U=0.8656$
$1.177 \mathrm{E}-03$ $3.134 \mathrm{E}-02$ $5.643 E-02$ $1.688 \mathrm{E}-01$
ANGLE 7 $M U=-0.4580$ $4.928 E-04$ $4.928 E-04$ $1.462 \mathrm{E}-03$ $1.462 \mathrm{E}-03$ $1.334 E-03$
$2.819 E-03$ $3.275 E-03$ $3.033 \mathrm{E}-03$ $3.814 E-03$ $2.303 E-03$ $2.650 E-03$ $4.878 \mathrm{E}-03$ $1.885 \mathrm{E}-02$ 4. 364E-02 $1.085 \mathrm{E}-01$ $3.107 E-01$ $8.724 E-01$ 2.545E-01 ANGLE 16 $M U=0.9446$ . $384 \mathrm{E}-02$ 4.711E-O2 3.550E-02 $6.419 E-02$ $7.326 \mathrm{E}-02$ $7.630 \mathrm{E}-02$ $9.146 \mathrm{E}-02$ $7.743 \mathrm{E}-02$ $7.634 \mathrm{E}-02$ $9.238 \mathrm{E}-02$ $1.353 \mathrm{E}-01$ $1.500 E-01$ $2.105 E-01$ $5.696 \mathrm{E}-01$ $1.316 \mathrm{E} 00$
$3.023 \mathrm{E}-01$
ANGLE 8 $M U=-0.2816$ $5.552 \mathrm{E}-04$ $2.527 E-03$ $1.577 E-03$ 1. $441 E-03$ $3.157 \mathrm{E}-03$ 3. $710 E-03$ $3.560 \mathrm{E}-03$ $4.580 E-03$ $3.022 \mathrm{E}-03$ $3.280 E-03$ $5.813 \mathrm{E}-03$ $2.440 E-02$ $5.442 \mathrm{E}-02$ $1.104 E-01$ $3.254 \mathrm{E}-01$ $2.588 E-01$ ANGLE 17 $8.945 \mathrm{E}-03$ $4.395 \mathrm{E}-02$ $1.367 \mathrm{E}-0$ $9.977 \mathrm{E}-02$ $7.883 E-02$ $1.686 \mathrm{E}-01$ $1.779 E-01$ $1.693 \mathrm{E}-0$ $1.885 \mathrm{E}-\mathrm{O}$ $1.236 \mathrm{E}-01$ $9.877 \mathrm{E}-02$ $1.252 \mathrm{E}-01$ $1.685-01$ $1.699 \mathrm{E}-01$ $2.342 \mathrm{E}-01$ $1.988 E-01$ $1.347 \mathrm{E}$ OO
$3.046 \mathrm{E}-01$

ANGLE 9 $=-0.0950$ $1.271 E-04$
$6.590 E-04$ 2. $965 \mathrm{E}-03$ 1.871E-03 $1.871 E-03$
$1.719 E-03$ $1.719 E-03$
$3.750 E-03$ $3.750 E-03$ $4.279 E-03$ 5. $460 E-03$ $5.460 E=03$ 4. $200 E-03$ 
(GAMYAS/MEV/STERADIAN/SOURCE NEUTRON)

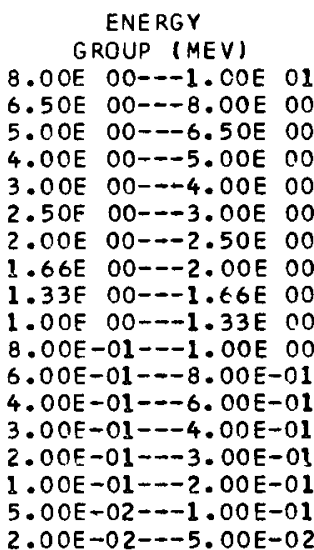

ENERGY

GROUP (MEV)

8. OOE OC-D-1.0OE O1

6.50 E $00---8.00 E$ OO

$5.00 E$ OD- $-6.50 F$ OO

$4.00 E$ DO- -5. . COE OO

$3.00 E$ OO- - - 4. OOE OO

2.5 CE OO-- $3.00 E$ OO

$2.00 E$ OO---2.50E OO

$1.66 \mathrm{E}$ DO---2.00E 00

$1.33 \mathrm{E} 00--1.66 \mathrm{~F} 00$

$1.00 E$ OO- $-1.33 E$ OO

$8.00 E-01--1.00 E \quad 00$

6.00E-01---8.00E-O

$4.00 E-01--6.00 E-01$

$3.00 F-01=-4.00 E-0$

$2.00 E-01--3.00 E-01$

$1.00 \mathrm{E}-01--2.00 \mathrm{E}-01$

$2.00 E-02---5.00 E-02$
ANGLE 1 $M U=-1,0000$
$3.932 \mathrm{E}-05$ $1.856 \mathrm{E}-04$ $9.643 \mathrm{E}-04$ $9.643 \mathrm{E}-04$ $2.887 E-04$ $2.786 \mathrm{E}-04$
$1.096 \mathrm{E}-03$ $1.514 \mathrm{E}-03$ $1.514 \mathrm{E}-03$ $2.587 \mathrm{E}-03$ $1.961 \mathrm{E}-03$ $1.561 E-03$ $6.228 \mathrm{E}-03$ $9.721 E-03$ $1.467 \mathrm{E}-02$ $9.087 \mathrm{E}-02$ $2.593 \mathrm{E}-01$ $7.736 E-01$ $2.405 E-01$

ANGLE 10 $M U=0.0950$ $5.929 \mathrm{E}-04$ $2.808 \mathrm{E}-03$ $1.812 \mathrm{E}-03$ $3.455 \mathrm{E}-03$ $4.107 E-03$ $3.945 \mathrm{E}-03$ $4.925 E-03$ $3.639 \mathrm{E}-03$ $5.182 \mathrm{E}-03$ $1.170 \mathrm{E}-02$ $4.023 E-02$ $6.698 \mathrm{E}-02$ $1.086 \mathrm{E}-0$ $3.458 E-01$ $9.739 E-01$ $2.665 \mathrm{E}-01$
ANGLE 2 $M U=-0.9894$ $1.934 E-04$ $1.934 E-04$ $1.001 \mathrm{E}-03$
$3.492 \mathrm{E}-04$ $3.369 E-04$ $3.369 E-04$ $1.554 E-03$ $1.554 E-03$
$1.728 E-03$ 2.520E-03 $2.520 \mathrm{E}-03$
$1.840 \mathrm{E}-03$ $1.202 \mathrm{E}-03$ $8.381 \mathrm{E}-04$ $9.804 \mathrm{E}-03$ 1. $478 E-02$ $9.129 E-O 2$ $2.599 E-O 1$ $7.750 \mathrm{E}-01$ 2.407E-01

ANGLE 11 $M U=0.2816$ $1.522 E-04$ $3.624 \mathrm{E}-\mathrm{C} 3$ $2.425 \mathrm{E}-03$ $2.304 \mathrm{E}-03$ $4.458 \mathrm{E}-03$ $5.253 \mathrm{E}-03$ $5.064 \mathrm{E}-03$ $6.376 \mathrm{E}-03$ $5.464 E-03$ $8.919 E-03$ $.908 E-02$ $5.144 E-02$ $7.293 \mathrm{E}-02$ $1.161 E-01$ 3.707E-01 $1.025 \mathrm{E} \mathrm{OO}$ $2.725 \mathrm{E}-01$
ANGLE 3 $\begin{array}{lll}3 & \text { ANGLE } \quad 4 \\ 4 & \end{array}$ $4.471 \mathrm{E}-05 \quad 5.103 \mathrm{E}-05$ 2.212E-04 $1.131 E-03$ $5.525 \mathrm{E}-04$ $5.342 E-04$ $1.335 \mathrm{E}-03$ $1.694 E-03$
$1.707 E-03$ $1.707 E-03$
$2.300 E-03$ $1.300 E-03$
$1.443 E-03$ $1.443 E-03$ $1.592 \mathrm{E}-03$ $1.013 E-02$ . $283 E-02$ $2.622 E-01$ $7.808 \mathrm{E}-01$ $2.415 E-01$

ANGLE 12 $U=0.4580$ $2.087 E-04$ $1.07 J E-03$ 3. $200 \mathrm{E}-03$ $3.312 E-03$ $5.905 E-03$ $7.021 \mathrm{E}-03$ $7.110 \mathrm{E}-0$ $8.392 E-03$ $9.643 \mathrm{E}-0$ $1.562 \mathrm{E}-02$ $2.879 \mathrm{E}-02$ $6.273 E-02$ $7.974 \mathrm{E}-02$ $1.260 \mathrm{E}-01$ 3.994E-01 $1.082 \mathrm{E} \quad 0$ $2.787 \varepsilon-01$ 1.319E-03 $8.222 E-04$ $7.942 \mathrm{E}-04$ $1.591 \mathrm{E}-03$ $1.890 E-03$ $2.056 \mathrm{E}-03$ $9.969 E-04$ $1.133 \mathrm{E}-03$ $2.590 E-03$ $1.070 \mathrm{~F}-02$ $1.800 E-02$ $9.508 \mathrm{E}-02$ $2.666 \mathrm{E}-01$ 2.430 E-O1

ANGLE 13 $M U=0.6179$ $3.062 E-04$
$1.558 E-03$ $6.708 \mathrm{E}-0$ 4. $430 E-03$ 4. $202 \mathrm{E}-03$ $6.473 E-03$ $1.030 E-02$ $1.119 \mathrm{E}-02$ $1.535 \mathrm{~F}-02$ $1.741 \mathrm{E}-02$ $2.582 \mathrm{E}-02$ 4. $024 E-02$
$7.378 E-02$ $7.378 E-02$
$8.924 E-02$ $8.924 \mathrm{E}-02$
$1.383 \mathrm{E}-01$ 1.383E-01 $1.141 \mathrm{E} 00$ 2. $849 \mathrm{E}-01$

$\begin{array}{cc}\text { ANGLE } 5 & \text { ANGLE } \\ \text { MU=-0.7E50 } & \text { MU=-0.6179 } \\ 5.712 E-05 & 6.235 E-05 \\ 2.974 E-04 & 3.237 E-04 \\ 1.484 E-03 & 1.604 E-03 \\ 1.013 E-03 & 1.079 E-03 \\ 9.720 E-04 & 1.026 E-03 \\ 1.791 E-03 & 1.912 E-03 \\ 2.061 E-03 & 2.203 E-03 \\ 1.750 E-03 & 1.912 E-03 \\ 2.016 E-03 & 2.281 E-03 \\ 8.809 E-04 & 1.203 E-03 \\ 1.323 E-03 & 1.690 E-03 \\ 3.290 E-03 & 3.562 E-03 \\ 1.163 E-02 & 1.337 E-02 \\ 2.309 E-02 & 3.104 E-02 \\ 9.710 E-02 & 9.856 E-02 \\ 2.730 E-01 & 2.818 E-01 \\ 8.077 E-01 & 8.291 E-01 \\ 2.452 E-01 & 2.481 E-01 \\ & \\ \text { ANGLE 14 } & \text { ANGLE 15 } \\ M U=0.7550 & M U=0.8656 \\ 5.019 E-04 & 9.658 E-04 \\ 2.542 E-03 & 4.888 E-03 \\ 1.040 E-02 & 1.874 E-02 \\ 7.107 E-03 & 1.378 E-02 \\ 6.909 E-03 & 1.349 E-02 \\ 1.385 E-02 & 2.594 E-02 \\ 1.707 E-02 & 3.151 E-02 \\ 1.932 E-02 & 3.527 E-02 \\ 2.622 E-02 & 4.529 E-02 \\ 2.970 E-02 & 4.732 E-02 \\ 3.923 E-02 & 5.494 E-02 \\ 5.285 E-02 & 6.657 E-02 \\ 8.551 E-02 & 1.001 E-01 \\ 1.021 E-01 & 1.176 E-01 \\ 1.526 E-01 & 1.688 E-01 \\ 4.665 E-01 & 5.021 E-01 \\ 1.202 E 00 & 1.258 E 00 \\ 2.907 E-01 & 2.958 E-01\end{array}$

ANGLE $M U=-0.4580$ $6.794 \mathrm{E}-05$ $3.482 E-04$ $1.715 \mathrm{E}-03$ $1.076 E-03$ $1.017 E-03$
$2.025 E-03$ $2.025 E-03$
$2.388 E-03$ $2.210 E-03$ $2.808 E-03$ 2.137E-03 $3.717 \mathrm{E}-03$ $1.664 E-02$ $4.094 E-02$ $2.931 E-01$ $8.561 E-0$ $2.517 E-01$

ANGLE 16 $A=0.9446$
$2.385 E-03$ $2.385 E-03$ $4.164 E-02$ $3.198 E-02$ 3.198E-02 $5.561 E-02$ $6.430 \mathrm{E}-02$ $6.793 E-02$ $8.047 E-02$ $7.228 \mathrm{E}-02$ $7.215 \mathrm{E}-02$ $8.261 \mathrm{E}-02$ $1.201 \mathrm{E}-01$ $1.338 \mathrm{E}-01$ 1.867E-01 $5.352 \mathrm{E}-01$ $1.306 \mathrm{E} 00$ $2.997 E-01$
ANGLE 8 $U=-0.2816$ $7.661 \mathrm{E}-05$ $1.900 E-03$ $1.131 E-03$ $1.076 \mathrm{E}-03$ $2.260 \mathrm{E}-03$ $2.722 \mathrm{E}-03$ $2.646 \mathrm{E}-03$ $3.453 E-03$ $2.427 E-03$ $2.629 \mathrm{E}-03$ $4.527 E-03$ $2.214 E-02$ $5.112 \mathrm{E}-02$ 1.010E-0 $8.892 \mathrm{E}-0$ 2.560E-01

ANGLE 17 $A N=0.9894$
$8.649 E-03$ $8.649 E-03$
$4.193 E-02$ 1.193E-02 $1.299 E-01$ $7.666 \mathrm{E}-02$ $1.504 \mathrm{E}-0$ $1.578 \mathrm{E}-0$ $1.496 \mathrm{E}-0$ $1.619 \mathrm{E}-0$ $1.121 \mathrm{E}-01$ $1.064 \mathrm{E}-01$ $1.466 \mathrm{E}-01$ $1.486 \mathrm{E}-01$ $2.041 \mathrm{E}-01$ $5.598 \mathrm{E}-01$ $1.336 \mathrm{E}$ ก $3.020 E-01$
ANGLE 9 $U=-0.0950$ $9.158 \mathrm{E}-05$ $4.656 \mathrm{E}-04$ $2.246 E-03$ $1.365 E-03$
$1.311 E-03$ 1. $311 E-03$ $3.284 E-03$ $3.284 E-03$ $3.210 E-03$
$4.117 E-03$ $2.923 E-03$ $3.419 E-03$ $6.939 E-03$ $3.014 E-02$ $1.037 \mathrm{E}-01$ $3.247 E-01$ $9.284 E-01$

SCALAR .629E-0 $2.313 E-02$ $8.744 E-02$ $6.168 E-02$ $5.642 \mathrm{E}-02$ $1.101 \mathrm{E}-01$ $1.313 E-01$ $1.624 \mathrm{E}-01$ $1.477 \mathrm{E}-01$ $1.780 \mathrm{E}-01$ $8.002 \mathrm{E}-01$ $1.477 \mathrm{E} 00$ $4.473 E 00$

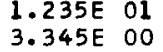


(GAMYAS/MEV/STERADIAN/SOURCE NEUTRON)

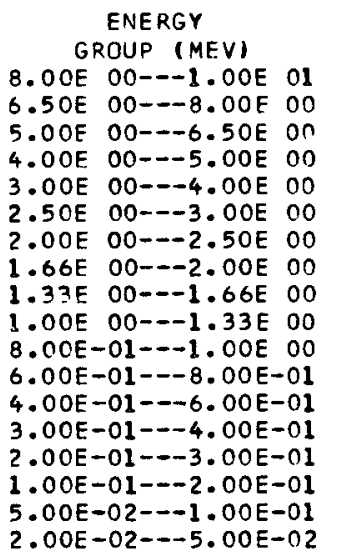

ENERGY

GROUP (MEV)

8.0OE OO---1.0OE 01

6.5OF OO---8.00E OC

$5.00 E$ OO- $-6.50 E$ OO

4.0OE OO- - -5.0OE OO

$3.00 E$ OO- - 4.00E 00

$2.00 E$ OO- $-2.50 E$ OO

$1.66 \mathrm{E} 00--2.00 \mathrm{E}$ OO

$1.33 \mathrm{E} \quad 00--1.66 \mathrm{E}$ OO

$1.00 F$ DO-- $1.33 E$ OO

$8.00 \mathrm{E}-01--1.00 \mathrm{E}$

$6.00 E-01--8.00 E-01$

4.00E-01---6.00E-OI

$3.00 E-01--4.00 E-01$

$1.00 E-01=-2.00 E-01$

$5.00 \mathrm{E}-02---1.00 \mathrm{E}-01$

2. $00 \mathrm{E}-02---5.00 \mathrm{E}-02$
ANGLE 1 ANGLE ? $M U=-1.0000 \quad M U=-0.9894$ $2.554 E-05 \quad 2.665 E-05$ 6.5 $0.533 E-04$ 1.157E-04 $6.92 \mathrm{E}-04$ 1.059E$1.303 E-03$ $1.303 \mathrm{E}-03$ $1.577 \mathrm{E}-03$ $1.577 E-03$
$8.571 E-04$ $1.348 \mathrm{E}-04$ $1.348 \mathrm{E}-04$
$7.987 \mathrm{E}-03$ $7.987 E-03$ $8.011 E-02$ $8.011 E-02$
$2.281 E-01$ $2.281 E-01$ $2.182 \mathrm{E}-01$

ANGLE 10 $M U=0.0950$ $8.289 E-05$ $4.164 E-04$ $2.103 E-03$ $1.335 \mathrm{E}-0$ $1.338 \mathrm{E}-0$ $2.496 E-03$ $3.002 E-03$ $2.868 E-0$ $3.554 \mathrm{E}-0$ $2.746 E-O 3$ $4.263 E-03$ $1.013 E-02$ $3.567 E-02$ $5.845 \mathrm{E}-02$ $9.356 \mathrm{E}=02$ $3.051 E-01$ $8.826 \mathrm{E}-01$
$2.421 \mathrm{E}-01$
ANGLE 3 $M U=-0.944 t$ 3.057E-05 $1.456 E-04$ $1.456 E-04$ $8.103 E-04$
$3.568 E-04$ $3.670 E-04$ . $1.203 \mathrm{E}-03$ $1.237 \mathrm{E}-03$ $1.237 \mathrm{E}-03$ $1.081 \mathrm{E}-03$ $1.081 E-03$ $8.023 \mathrm{E}-04$
$1.057 \mathrm{E}-03$ $1.057 \mathrm{E}-03$
$8.281 \mathrm{E}-03$ $8.281 \mathrm{E}-03$
$1.286 \mathrm{E}-02$ $1.286 \mathrm{E}-02$
$8.175 \mathrm{E}-02$ $8.175 E-02$
$2.307 E-01$ $2.307 E-01$
$7.047 E-01$ $2.192 \mathrm{E}-01$

ANGLE 12 $M U=0.4580$ 1. $531 \mathrm{E}-04$ 7. $726 \mathrm{E}-04$ $3.664 \mathrm{E}-03$ $2.417 \mathrm{E}-03$ $2.347 E-03$ 4. $328 E-03$ $5.216 E-03$ $5.347 E-03$ $7.209 E-03$ $1.406 \mathrm{E}-02$ $2.584 E-02$ 5.509E-02 $6.856 E-02$ $1.083 E-01$ $3.521 E-01$ $9.815 \mathrm{E}-01$ 2.532E-O1
ANGLE 4 $U=-0.8656$ $3.621 E-05$
$1.819 E-04$ $9.809 E-04$ 作 1.1 $33 E-03$ $1.353 \mathrm{E}-03$ $1.185 \mathrm{E}-03$ $1.185 \mathrm{E}-03$
$1.405 \mathrm{E}-03$ $6.578 E-04$ $8.311 E-04$ $8.311 E-04$
$1.960 E-03$ $1.960 \mathrm{E}-03$ 1.522E-02 $8.357 E-02$ $2.347 E-01$ $7.149 \mathrm{E}-01$ 2.206E-01 ANGLE 13 $M U=0.6179$ $2.273 E-04$ $1.137 \mathrm{E}-03$ $5.151 \mathrm{E}-03$ $3.333 E-03$ $3.282 E-03$ $7.888 \mathrm{E}-03$ $8.860 E-03$ $1.248 E-02$ $1.544 E-02$ $2.348 \mathrm{E}-02$ $3.595 E-02$ $6.410 \mathrm{~F}-02$ $7.651 E-02$ $1.184 \mathrm{E}-01$ 3. 800 E-01 $1.036 \mathrm{E} 00$ 2. $588 \mathrm{E}-01$ 4.114E-05 4.467E-05 .110E-04 $1.118 E-03$ $7.734 \mathrm{E}-04$ $7.653 \mathrm{E}-04$ $1.290 E-03$ $1.472 E-03$ $1.197 E-03$ $1.335 \varepsilon-03$ $5.525 E-04$ $1.011 E-03$ $2.552 \mathrm{E}-03$ $2.001 E-02$ $8.508 E-02$ $2.405 E-01$ $7.296 E-01$
$2.226 E-01$ ANGLE 14 $M U=0.7550$ 3.824E-04 1. $908 \mathrm{E}-03$ $1.079 \mathrm{E}-02$ $1.367 E-02$ $1.603 E-02$ $2.211 \mathrm{E}-02$ $2.668 \mathrm{E}-02$ $3.559 \mathrm{E}-02$ $4.668 \mathrm{E}-02$ 7.340E-02 8. $735 \mathrm{E}-02$ $1.299 \mathrm{E}-01$ 4.099E-01 $1.091 E 00$
$2.641 \mathrm{E}-01$ $2.277 E-04$ 1.200-0 $8.119 E-04$ 7. $915 E-04$ $1.359 E-03$
$1.564 E-03$ $1.564 \mathrm{E}-03$ $1.321 E-03$
$1.562 E-03$ $1.562 E-03$ $8.514 E-04$
$1.312 E-03$ $2.312 E-03$ $2.696 E-03$ $2.736 E-02$ $8.601 E-02$ $2.483 \mathrm{E}-01$ $7.493 E-01$ 2. $252 \mathrm{E}-01$ ANGLE 15 $M U=0.8656$ $3.849 E-03$ $1.525 \mathrm{E}-02$
$1.135 \mathrm{E}-02$ $1.166 E-02$ $2.118 \mathrm{E}-02$ $2.625 E-02$ $3.009 E-02$ 3. $869 \mathrm{E}-02$ $4.233 E-02$ 4.930E-02 $5.783 E-02$ $8.493 E-02$ $9.998 \mathrm{E}-02$ $1.428 \mathrm{E}-01$ $4.399 \mathrm{E}-01$ $1.142 E 00$
$2.687 E-01$
ANGLE 7 $M U=-0.4580$ 4.795E-05 $397 E-04$ $1.261 E-03$ $7.664 E-04$ $7.486 E-04$ $1.410 E-03$ $1.571 \mathrm{E}-03$ 1.571E-03 $1.023 E-03$ $1.644 \mathrm{E}-03$ $1.644 E-03$
$2.718 E-03$ $1.718 \mathrm{E}-03$
$1.411 \mathrm{E}-02$ $3.633 \mathrm{E}-02$ $8.653 \mathrm{E}-02$ $2.584 \mathrm{E}-01$ $7.742 E-01$ 2.285E-01 ANGLE 16 $M U=0.9446$ $2.009 E-03$ $3.545 \mathrm{E}-02$ $2.778 E-02$ $2.687 E-02$ $5.453 \mathrm{E}-02$ $5.819 E-02$ $6.810 E-02$ $6.352 E-02$ $6.357 E-02$ 7.005E-02 $1.006 \mathrm{E}-01$ $1.124 E-01$ $1.564 E-01$ 4.671E-01 $1.184 \mathrm{E} 00$ $2.721 E-01$

ANGLE 8 AU $=-0.2816$ MUELE 9 $5.362 E-05$ G.454E $2.643 E-04 \quad 3.196 E-04$ $1.385 \mathrm{E}-03 \quad 1.651 \mathrm{E}-03$ $7.739 E-04 \quad 1.651 E-03$ $7.700 E-04 \quad 9.651 E-04$ $1.925 E-03$ $1.942 E-032.376 E-03$ $1.923 E-02 \quad 2.346 E-03$ $2.548 \mathrm{E}-03 \quad 3.018 \mathrm{E}-03$ $1.883 \mathrm{E}-03 \quad 2.218 \mathrm{E}-03$ $1.999 \mathrm{E}-03 \quad 2.650 \mathrm{E}-03$ $3.402 E-03 \quad 5.647 E-03$ $1.922 E-02$ 2.656E-02 $4.532 E-02 \quad 5.284 E-02$ $8.738 E-02 \quad 8.948 E-02$ $2.710 \mathrm{E}-01 \quad 2.865 \mathrm{E}-01$ $8.047 \mathrm{E}-01$
$2.325 \mathrm{E}-01$ ANGLE 17 SCALAR $M J=0.9894 \quad$ FLUX 7.967E-03 3.808E-03 $1.174 \mathrm{E}-01 \quad 7.172 \mathrm{E}-02$ $8.624 \mathrm{E}-02 \quad 5.082 \mathrm{E}=02$ $7.066 \mathrm{E}-02 \quad 4.789 \mathrm{E}-02$ $1.282 \mathrm{E}-01 \quad 8.756 \mathrm{E}-02$ $1.336 \mathrm{E}-01 \quad 1.024 \mathrm{E}-01$ $1.260 \mathrm{E}-01$ $1.327 E-01$ $9.550 E-02$ $1.568 E-01$ $8.639 \mathrm{E}-02 \quad 2.258 \mathrm{E}-01$ $1.205 E-01$ $1.226 E-01 \quad 6.909 E-01$ $1.687 E-01$ 1.271E 00 $4.865 E-01 \quad 3.937 \mathrm{E}$ OO

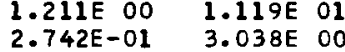


(GAMYAS/MEV/STERADIAN/SOURCE NEUTRON)

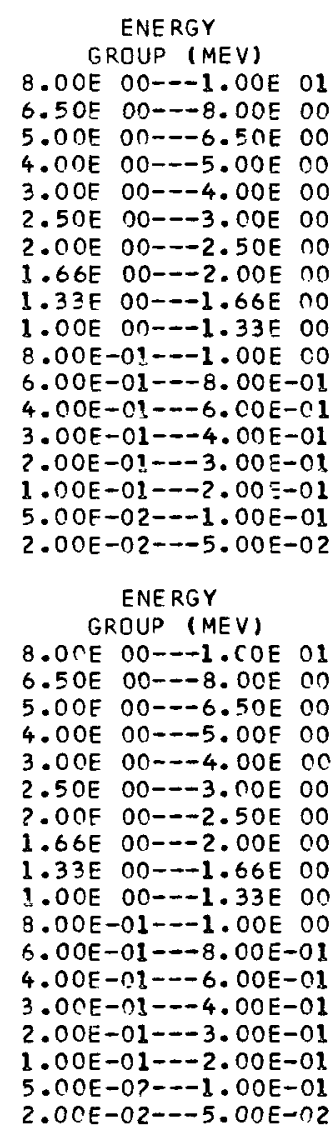

ANGLE 1

2.197E-05

$1.587 \mathrm{E}-04$

$-1.177 E-04$

$-8.878 E-05$

$1.153 \mathrm{E}-04$

$3.453 E-04$

$5.577 E-04$

$9.082 E-04$

$7.554 E-04$

$1.983 E-04$
$-3.851 E-04$

$-3.851 E-04$

$5.708 E-03$

$4.617 E-02$

$1.268 E-01$

$4.006 E-01$

$1.257 E-01$

ANGLE 10

$M U=0.0950$

$U=0.0950$

$2.914 E-05$
$1.348 E-04$

$8.145 E-04$

$8.145 E-04$

$5.629 E-04$

$8.757 \mathrm{E}-04$

$8.757 \mathrm{E}-04$
$1.062 \mathrm{E}-03$

$9.062 \mathrm{E}-03$

$1.148 \mathrm{E}-03$

$1.000 E-03$

$2.125 E-03$

$5.864 E-03$

2.080E-02

$3.225 E-02$

5.019E-02

$1.692 \mathrm{E}-01$

5. C67E-01

1. $392 E-01$
ANGLE 3

$6.263 E-06$

$1.768 \mathrm{E}-05$

$1.834 E-C 4$

$-6.518 \mathrm{E}-05$

.

. $476 \mathrm{E}=04$

.

(6.

. $028 \mathrm{E}-04$

D5 $5 E-04$

(

. $313-C 3$

$4.634 E-02$

.271E-01

.

$1.258 E-01$

ANGLE 11 $M U=0.2816$ $4.070 E-05$

$1.930 E-04$
$1.110 E-03$

$1.110 E-03$
$7.510 E-04$

$7.941 \mathrm{E}-04$

$1.162 \mathrm{E}-03$

$1.359 \mathrm{E}-03$

$.238 \mathrm{E}-03$

$1.576 E-03$

$1.973 E-03$

4. $505 E-03$

$1.028 \varepsilon-02$

$2.615 \mathrm{E}-02$

$3.397 E-02$

$5.331 E-02$

1. $808 \mathrm{E}-01$

$5.333 E-01$

$1.422 E-01$ $.053 E-06$ 3.595E-05 $2.584 E-04$ $5.519 E-05$ $8.283 E-05$ 2.55 E-04 $4.003 E-04$ $4.500 E-04$ ..250E-C4 $3.767 E-04$ $2.281 E-04$ $2.05 E-04$ 6. $424 E-03$ $4.693 E-02$ $4.045 \mathrm{E}-01$ 1. $262 \mathrm{E}-01$

ANGLE 12 $5.678 \mathrm{E}-05$ $2.665 E-04$ $1.476 E-03$ $9.423 E-04$ $9.782 \mathrm{E}-04$ $1.525 \mathrm{E}-03$ 1. $898 \mathrm{~F}-03$ . $045 E-03$ .535E-03 .632E-03 $.558 E-0$ $.085 E-02$

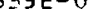
$5.736 \mathrm{E}-02$ $1.939 \mathrm{E}-0$ $1.453 \mathrm{E}-01$ $M U=-0.8656$ $1.271 E-0$ $5.906 E-05$ 3. $783 \mathrm{E}-04$ $2.474 E-04$ . . .5 . $725 E-04$ . $018 E-04$ . $341 \mathrm{E}-04$

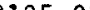
. 4.765E-02 $1.270 \mathrm{E}-01$ ANGLE 5 $M U=-0.7550$ $1.521 E-05$
$7.419 E-05$ $4.490 E-04$ $3.360 \mathrm{E}-04$ $3.520 E-04$ $4.536 E-04$ $4.810 E-04$ $3.155 E-04$ $2.915 E-04$ $1.165 E-04$ $4.244 E-04$ . 0 EE-O3 $4.612 E-03$ $4.805 \mathrm{E}-02$ $1.338 \mathrm{E}-01$ 1. 1. $282 \mathrm{~F}-01$ ANGLE 13

ANGLE 14 $A=0.6179$ $3.922 \mathrm{E}-04$ . $067 \mathrm{E}-03$

$1.220 \mathrm{E}-03$

$1.334 \mathrm{E}-03$

$2.355 \mathrm{E}-03$

3. $225 \mathrm{E}-03$

4.081E-03 $6.252 \mathrm{E}-03$ $9.259 E-03$ $3.449 \mathrm{E}-02$ $2.110 E-02$ $3.477 \mathrm{E}-02$ $4.024 \mathrm{E}-02$ 6.204E-02 2.081E-01 $\begin{array}{ll}5.922 \mathrm{E}-01 & 6.219 \mathrm{E}-01 \\ 1.484 \mathrm{E}-01 & 1.513 \mathrm{E}-01\end{array}$ $1.527 E-04$ 3.451E-03 $2.233 \mathrm{E}-03$ $2.632 E-03$ $4.685 \mathrm{E}-0$ $6.487 E-03$ $8.405 \mathrm{E}-03$ $1.206 \mathrm{E}-02$ $1.620 \mathrm{E}-02$ $2.148 E-02$ $2.634 E-02$ $3.857 E-02$ $4.558 \mathrm{E}-02$ $6.712 \mathrm{E}-02$ $2.228 \mathrm{E}-01$

AVILE 6 $\begin{aligned} & M U=-0.6179 \\ & 1.599 \mathrm{E}-05\end{aligned}$ $1.599 E-05$
$7.655 E-05$ $7.655 E-05$ 4. $643 E-04$ $3.126 E-04$ 3.269E-04 $4.474 E-04$ 4. $997 \mathrm{E}-04$ 3.79JE-04 4. $418 E-04$ .520E-04 . $1.382 E-01$ $1.297 E-01$

AVGLE 15 $M U=0.8656$ $3.510 E-C 4$ $1.682 E-03$
$7.278 E-03$ $5.671 E-03$ $6.598 E-03$ $6.598 E-03$
$1.057 E-02$ $1.383 \mathrm{E}-02$ $1.569 \mathrm{E}-02$ . $2.495 E-02$ $2.854 E-02$ 3.118E-02 4. $339 E-02$ $5.126 E-02$ $7.251 E-02$ $2.370 E-01$ $6.491 \mathrm{E}-01$
$1.537 \mathrm{E}-01$
ANGLE 7 $M U=-0.4580$ $1.592 \mathrm{E}-05$
$7.124 \mathrm{E}-05$ $4.533 E-04$ $2.298 E-04$ $2.493 E-04$ $4.205 E-04$ $5.422 E-04$ 5.287E-04 $7.314 E-04$ 6.357E-04 $1598 \mathrm{E}-03$ 1. $438 \mathrm{E}-02$ $1.316 E-01$

ANGLE 16 $M U=0.9446$ $1.081 \mathrm{E}-03$ $1.932 \mathrm{E}-02$

$1.932 \mathrm{E}-02$
$1.616 \mathrm{E}-02$

$1.701 \mathrm{E}-02$ $2.529 \mathrm{E}-02$ $2.972 \mathrm{E}-02$ $3.209 \mathrm{E}-02$ $3.612 \mathrm{E}-02$ $3.523 E-02$ $3.473 \mathrm{E}-02$ $3.577 \mathrm{E}-02$ 4. $995 E-02$ $5.584 \mathrm{E}-02$ $7.781 \mathrm{E}-02$ $2.492 \mathrm{E}-01$ $6.709 \mathrm{E}-01$
$1.556 \mathrm{E}-01$

ANGLE 8 ANGLE 9 $M U=-0.2816 \quad M U=-0.0950$ 1.248E-05 $9.274 E-05$ $5.946 E-04$ . $2.279 E-04 \quad 3.396 E-04$ $4.606 E-04 \quad 6.208 E-04$ 7.053E-04 8.291E-04 1. $082 E-03$ 7. 7. $1.059 E-03$ $2.833 E-03$ $1.546 E-02$ $2.993 E-02$ $1.508 \mathrm{E}-01$ $4.530 E-01$ $1.338 \mathrm{E}-01 \quad 1.364 \mathrm{E}-01$

ANGLE 17 $M U=0.9894 \quad F L U X$ $5.345 \mathrm{E}-03 \quad 1.987 \mathrm{E}-03$ $2.442 E-02 \quad 9.242 E-03$ $7.385 \mathrm{E}-02 \quad 3.586 \mathrm{E}-02$ $5.470 E-02 \quad 2.593 E-02$ 4.625E-02 2.613E-02 
IGAMMAS/MEV/STERADIAN/SOURCE NEUTRONI

ENERGY GROUP (MEV) 8. 0 CE $00--1.00 E$ OI 6.50E $00---8.00 E$ OO OOE OO-- -6.50E OO 4.0OE $00---5.00 E$ OO $3.50 E$ OO- $3.00 E$ OO $2.00 E$ OO- $-2.50 E$ EO 1.66E $00--2.00 E 00$ $1.33 \mathrm{E} 00--1.66 \mathrm{E} 00$ $1.00 \mathrm{E} 00---1.33 \mathrm{E} 00$ $8.00 \mathrm{E}-01-\cdots 1.00 \mathrm{E} 00$ $6.00 E-01-\cdots-8.00 E-01$ $4.00 E-01--6.00 E-01$ $3.00 E-01--4.00 E-01$ 2. $00 \mathrm{~F}-01---3.00 \mathrm{E}-01$ $1.00 \mathrm{E}-01-2.00 \mathrm{E}-01$ $5.00 E-02---1.00 E-01$ $2.00 E-02---5.00 E-02$

$$
\text { ENERGY }
$$$$
\text { GROUP (MEV) }
$$

8.00E 00--1. 1.00E 01 6.50E 00---8.00E 00 5.0CE 00---6.50E OO 4.0OE $00---5.00 E$ OO $3.00 E$ OO- $-4.00 E$ OO 2.5 CE 00-- 3. OOE OO $2.00 E$ OO- $-2.50 E$ EO $1.66 \mathrm{~F} \mathrm{00---2.00E} 00$ $1.33 \mathrm{E}$ 00---1.66E 00 $1.00 \mathrm{E}$ 00---1.33E 00 B. ONE-01--1. OOE DO $6.00 E-01--8.00 E-01$ $4.00 E-01-2.00 E-O 1$ $3.00 E-01--4.00 E-01$ 2.00 E $01--3.00 E-O 1$ $1.00 E-01--2.00 E-01$ $2.00 E-02---5.00 E-02$ $U=-1.0000$ $-8.509 \mathrm{E}-06$ $1.127 E-05$ $-1.172 \mathrm{E}-04$ $-1.601 \mathrm{E}-05$ $1.154 \mathrm{E}-04$ $2.543 E-04$ $2.543 E-04$ $3.449 \mathrm{E}-04$ $-9.528 E-06$ .157E-02 6.100E-02 ANGLE 10 $M U=0.0950$ $1.018 \mathrm{E}-05$ $4.317 \mathrm{E}-05$ $2.992 \mathrm{E}-04$ $2.028 \mathrm{E}-04$ $2.332 E-04$ 2. $982 E-04$ $3.472 \mathrm{E}-\mathrm{C} 4$ $2.750 \mathrm{E}-04$ $2.973 E-04$ $3.147 \mathrm{E}-04$ $1.008 \mathrm{E}-\mathrm{n}$ $3.096 E-03$ $1.064 \mathrm{E}-02$ $1.565 E-02$ $2.392 \mathrm{E}-02$ $8.156 \mathrm{E}-02$ $2.454 \mathrm{E}-01$
$6.730 \mathrm{E}-02$ $\triangle A N G L E \quad 2$ $6.235 E-07$ $.435 E-06$ $2.710 E-05$ $-8.639 E-05$ $3.893 E-06$ $3.893 E-06$ $1.186 E-04$ $2.341 E-04$
$3.770 E-04$ $3.004 E-04$ 1.823E-06 列 $6.171 E-02$ 6.105E-02 ANGLE 11 $M U=0.2816$ $1.498 E-05$ $6.672 E-05$ $3.055 E-04$ $3.346 E-04$ 3. $934 E-04$ $4.242 E-04$ $3.461 E-04$ $4.608 E-04$ $8.576 E-04$ 2.377E-03 $5.486 E-03$ $1.313 E-02$ $1.615 \mathrm{E}-\mathrm{C2}$ $2.528 \mathrm{E}-02$ . $2.576 \mathrm{E}-01$
$6.86 \mathrm{gE}-02$
ANGLE 3 $M U=-0.9446$ $2.308 E-06$ $5.408 \mathrm{E}-06$ $7.738 \mathrm{E}-05$ $8.925 \mathrm{E}-07$ $7.118 \mathrm{E}-06$ $5.998 E-05$ $1.288 \mathrm{E}-04$ $1.644 E-04$
$2.152 E-04$ $2.152 E-04$ $4.017 E-05$ $7.957 \mathrm{E}-05$ $1.877 \mathrm{E}-03$ $2.976 E-03$ $2.379 E-02$ 6.232E-02 $1.973 \mathrm{E}-01$
$6.126 \mathrm{E}-02$ ANGLE 12 $M U=0.4580$ $2.042 E-05$ $8.785 E-05$ $3.442 E-04$ $3.642 E-04$ 4.839E-04 $6.209 E-04$ $7.315 \mathrm{E}-04$ $1.241 \mathrm{E}-03$ 2.330E-03 $4.651 E-03$ $8.152 \mathrm{E}-03$ $1.510 \mathrm{E}-02$ $1.706 E-02$ $2.700 \mathrm{E}-02$ $9.263 \mathrm{E}-02$ $2.707 E-01$
$7.010 E-02$ $M U=-0.8656$ ANGLE 5 $M U=-0.7550$ 2.730E-05 $2.730 E-05$ $1.748 E-04$ $1.325 E-04$
$1.494 E-04$ $1.494 E-04$
$1.583 E-04$ $1.583 E-04$ $1.398 E-04$
$5.392 E-05$ $2.891 E-05$ $-6.720 E-06$ $1.796 E-04$ $4.421 E-04$ $2.093 E-03$ $5.716 E-03$ $2.398 \mathrm{E}-02$ $6.502 E-02$ $2.041 E-01$ $6.218 \mathrm{E}-02$ $2.001 \mathrm{E}-01$ $6.164 E-02$ ANGLE 14 $.954 E-05$
$.201 E-04$ . ..404E-04 $4.532 \mathrm{E}-04$ $8.024 \mathrm{E}-04$ $1.258 \mathrm{E}-03$ $1.839 \mathrm{E}-03$ $3.041 E-03$ $4.959 \mathrm{E}-03$ 7.698 E-03 $1.068 \mathrm{E}-02$ $1.655 E-02$ $1.879 \mathrm{E}-02$ 2.891 E-02 $9.884 \mathrm{E}-02$ $2.842 \mathrm{E}-01$
$7.148 \mathrm{E}-02$ $M U=0.7550$
$5.596 \mathrm{E}-05$ ( $2.343 E-04$
$1.302 E-03$ $7.861 E-04$ $1.100 E-03$ $1.957 E-03$ $2.970 E-03$ $4.190 E-03$ $6.149 E-03$ $8.578 \mathrm{E}-03$ $1.105 E-02$ $1.281 \mathrm{E}-02$ $2.114 E-02$ $3.091 \mathrm{E}-02$ $1.051 E-01$ $2.974 E-01$ $7.275 E+02$
ANजLE 6 $M U=-0.6179$ $518 \mathrm{E}-05$ $2.518 E-05$ $1.703 E-04$ $1.256 E-04$
$1.224 E-04$ $1.224 E-04$ $1.349 E-04$
$1.421 E-04$ $9.376 E-05$ 1. $057 \mathrm{E}-04$ $8.344 E-05$ $2.181 \mathrm{E}-04$ $3.772 E-04$ $2.644 E-03$ $8.186 \mathrm{E}-03$ $2.369 \mathrm{E}-02$ $6.714 E-02$ $6.290 E-02$ AVGLE 15
ANGLE 7 $M U=-0.4580$ $1.841 E-05$ $1.841 \mathrm{E}-05$
$1.455 \mathrm{E}-04$ $3.747 E-05$ $3.747 E-05$
$4.755 E-05$ $1.078 E-04$ $1.610 E-04$ $1.610 E-04$
$1.727 E-04$ 2.662E-04 2.412 E-04 2.115E-04 $2.487 E-04$ $3.765 \mathrm{E}-03$ $1.091 E-02$ $2.325 E-02$ $6.979 E-02$ $2.164 E-01$ $6.378 E-02$ ANGLE 16 $M U=0.9446$
$5.386 \mathrm{E}-04$ $2.493 E-03$ $9.568 \mathrm{E}-03$ $8.570 E-03$ $9.549 E-03$ $1.281 \mathrm{E}-02$ $1.488 E-02$ $1.597 E-02$ $1.728 \mathrm{E}-02$ $1.682 E-02$ $1.617 E-02$ $1.612 \mathrm{E}-02$ $2.234 E-02$ $2.492 E-02$ $3.499 E-02$ $1.160 \mathrm{E}-01$ $3.185 \mathrm{E}-01$ 7.464E-02

ANGLE 8 $M U=-0.2816$ $1.548 \mathrm{E}-05$ $1.430 \mathrm{E}-04$ $2.702 E-05$ $5.434 E-05$ $1.167 \mathrm{E}-04$ $1.167 E-04$
$2.086 E-04$ $2.726 E-04$ $3.784 \mathrm{E}-04$ 3.068E-04 $2.077 E-04$ $5.574 E-03$ $1.329 E-02$ 
(GAMMAS/MEV/STERADIAN/SOURCE NEUTRON)

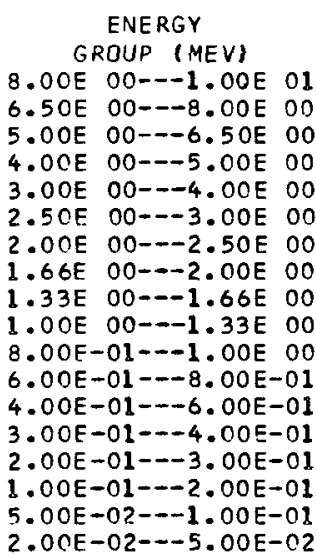

ENERGY

GROUP (MEV)

8.0OE O0---1.00E OI

$6.50 E$ OO- $-8.00 E$ OO

$5.00 E$ OO- $-6.50 E$ OO

$4.00 E$ OO-- $-5.00 E$ OO

3.0CE OO-- 4.00E OO

2.5OE $00--3.00 E$. 00

2.00E $00---2.50 E$ EO

$1.66 E$ E0--2.00E 00

1.33 E $00--1.66 E$ OO

8.0OF $01--1.00 \mathrm{E}$ O

$6.00 E-01--8.00 E-01$

4. $00 E-01--6.00 E-01$

$3.00 E-01--4.00 E-01$

$2.00 \mathrm{E}-01--3.00 \mathrm{E}-01$

$1.00 E-01---2.00 E-01$

$2.00 E-02--5.00 E-02$
ANGLE 1 $M U=-1.0000$ $-8.785 E-06$ $1.785-06$ $-7.604 E-05$ $-6.824 E-05$ $1.225 \mathrm{E}-04$ $1.089 \mathrm{E}-04$ $1.553 \mathrm{E}-04$ $1.553 E-04$
$-4.396 E-05$ $-4.396 \mathrm{E}-05$ $8.862 \mathrm{E}-04$ $1.089 \mathrm{E}-03$ $1.136 \mathrm{E}-02$ $2.851 E-02$ $9.012 \mathrm{E}-02$ $2.796 E-02$

ANGLE 10 $M U=0.0950$

$3.578 \mathrm{E}-06$

$.505 E-05$

1.087E-04

$8.128 \mathrm{E}-0$

$9.781 \mathrm{E}-0$

$1.064 \mathrm{E}-04$

$1.078 E-04$

$6.488 E-05$

$4.982 \mathrm{E}-05$

$8.773 E-05$

$4.780 E-04$

$1.562 E-03$

$5.153 \mathrm{E}-03$

$7.235 \mathrm{E}-03$

$1.094 \mathrm{E}-02$

$1.122 \mathrm{E}-01$

$3.072 \mathrm{E}-02$ $\triangle N G L E 2$ $5.548 \mathrm{E}-07$ $6.503 E-06$ $6.02 E-05$ -5.021E-05 1.748E-05 $4.297 E-05$ $1.738 \mathrm{E}-04$ $1.323 \mathrm{E}-04$ $-3.473 E-05$ $-3.473 E-05$
$-1.663 E-04$ $-1.663 E-04$
$8.779 E-04$ $8.779 E-04$
$1.132 E-03$ $1.139 \mathrm{E}-02$ $2.858 \mathrm{E}-02$ $9.028 \mathrm{E}-02$ 2. $798 \mathrm{E}-02$

ANGLE 11 $=0.2816$ $5.673 E-06$ $1.619 E-04$ $1.311 E-04$ $1.397 \mathrm{E}-0$ $1.311 E-C 4$ $1.171 \mathrm{E}-0$ $6.947 E-05$ $1.143 E-04$ $3.797 \mathrm{E}-04$ $1.204 E-03$ $2.757 E-03$ $6.241 E-03$ $7.344 \mathrm{E}-03$ $1.151 \mathrm{E}-02$ 3.072E-02 $1.175 \mathrm{E}-01$
$3.131 \mathrm{E}-02$
ANGLE 3 $3.623 E-07$ $1.346 \mathrm{E}-07$ $1.632 E-05$ $4.362 E-07$ $9.821 E-06$ 4.236E-0 $4.236 \mathrm{E}-05$ $7.575 \mathrm{E}-05$ $4.623 \mathrm{E}-05$ $-1.093 \mathrm{E}-06$ $-1.077 E-05$ $8.524 \mathrm{E}-04$ $1.350 \mathrm{E}-03$ $1.146 E-02$ $2.886 \mathrm{E}-02$ $9.096 \mathrm{E}-02$ $U=-0.8656$ $6.973 E-06$ . $973 E-06$ S.297E-0 3.166E-05 $4.656 E-05$ $3.961 E-05$ 8.957E-06 $-6.059 E-06$ $-1.938 \mathrm{E}-05$ $3.695 \mathrm{E}-05$ $3.695 \mathrm{E}-05$
$1.192 \mathrm{E}-04$ $1.192 \mathrm{E}-04$
$8.449 \mathrm{E}-04$ $1.873 \mathrm{E}-03$ $1.150 \mathrm{E}-02$ $2.938 \mathrm{E}-02$ $9.222 \mathrm{E}-0$ $2.824 \mathrm{E}-02$

ANGLE 12 $M J=0.4580$ $7.315 \mathrm{E}-06$ $2.918 E-05$ $1.964 E-04$ $1.197 E-04$ $1.234 \mathrm{E}-04$ $1.394 E-04$ $2.619 E-04$ $5.279 E-04$ $1.166 \mathrm{E}-03$ 2.357E-O3 4.J01E-03 $7.015 \mathrm{E}-03$ $7.686 \mathrm{E}-0$ $1.222 \mathrm{E}-02$ 4. $220 \mathrm{E}-0$ $1.231 \mathrm{E}-01$ $3.191 E-02$

ANGLE 13 $U=0.6179$ $3.219 E-05$ $2.401 E-04$ $8.273 E-05$ $1.251 \mathrm{E}-04$ $2.598 E-0$ $4.973 E-04$ $1.479 E-03$ $2.517 E-03$ $3.819 \mathrm{E}-03$ $5.067 \mathrm{E}-03$ $7.510 \mathrm{E}-03$ $8.435 \mathrm{E}-03$ $1.297 \mathrm{E}-0$ $4.481 \mathrm{E}-02$ $1.288 \mathrm{E}-01$ 3. 250 E-02
ANGLE $5 \quad$ ANGLE 6 $2.287 E-06$ $1.018 E-05$ $6.716 E-05$ $7.082 E$ $5.445 E-05$ . $5.531 E-06$ $-5.531 E-06$
$-2.392 E-05$ $-2.392 \mathrm{E}-05$ $-116 E-05$
$8.199 E-05$ 2. $083 \mathrm{E}-04$ $2.083 E-04$
$9.344 E-04$ $2.780 E-03$ $1.140 E-0$ 3.011E-02 $9.405 E-02$ $2.848 \mathrm{E}-02$ $2.042 E-06$ $9.479 E-O$ $6.017 E-0$ 3. 03 E 05 $4.037 E-05$ 3. $583 \mathrm{E}-05$ $3.572 \mathrm{E}-05$ $1.52 E-O 5$ 2.545E-05 $8.486 E-05$ $8.486 E-05$ $1.213 \mathrm{E}-04$
$1.222 \mathrm{E}-03$ 4.008E-0 $1.116 E-02$ $3.107 \mathrm{E}-02$ $9.647 \mathrm{E}-02$ ANGLE 14 ANJLE 15 $M U=0.7550$ $1.916 E-05$ $0.858 E-05$ ( $497 \varepsilon-04$ $2.431 E-04$ $4.329 E-04$ $1.369 E-03$ $2.058 \mathrm{E}-03$ $3.043 \mathrm{E}-03$ 4. $264 E-03$ $5.307 E-03$ $5.867 E-03$ $7.960 \mathrm{E}-03$ $9.442 E-03$ $1.374 \mathrm{E}-02$ 4. $740 \mathrm{E}-02$ $1.343 E-01$ $3.304 E-02$ $6.106 \mathrm{E}-05$ $2.624 E-04$ $1.306 E-03$ $1.177 \mathrm{E}-03$ $1.667 E-03$ $3.322 E-03$ $4.179 E-03$ 5.191E-03 $6.049 E-03$ $6.472 E-03$ $6.459 \mathrm{E}-03$ $8.665 \mathrm{E}-03$ $1.033 \mathrm{E} \rightarrow 02$ $1.454 \mathrm{E}-02$ 4. $980 E-02$ $1.392 \mathrm{E}-01$ $3.349 E-02$ $\triangle N G L E ?$ AU $=-0.4580 \quad$ ANGLE 8 $1.351 E-06 \quad 1.017 E-06$ $4.167 E-05$ $-3.435 E-07$ $2.278 E-05$ 6.495E-05 $1.052 E-04$ $1.048 E-04 \quad 1.513 E-04$ $1.006 \mathrm{E}-04 \quad 1.157 \mathrm{E}-04$ $5.984 E-05 \quad 4.188 E-05$ $5.417 \mathrm{E}-05 \quad 1.686 \mathrm{E}-04$ $1.808 E-03 \quad 2.728 E-03$ $5.311 E-03 \quad 6.382 E-03$ $1.084 E-02 \quad 1.062 E-02$ $3.227 E-02 \quad 3.372 E-02$ $9.949 \mathrm{E}-02$ $2.918 E-02$ $1.031 E-01$

ANGLE 16 $U=0.9446$ $2.599 E-04$ $4.528 \mathrm{E}-03$ 4. $352 \mathrm{E}-03$ $5.034 E-03$ $6.300 E-03$ $7.170 \mathrm{E}-03$ $7.575 \mathrm{E}-03$ $7.894 E-03$ $7.540 E-03$ $7.083 \mathrm{E}-03$ $6.962 E-03$ $9.713 \mathrm{E}-03$ $1.080 \mathrm{E}-02$ 
IGAMMAS/MEV/STERADIAN/SOURCE NEUTRONI

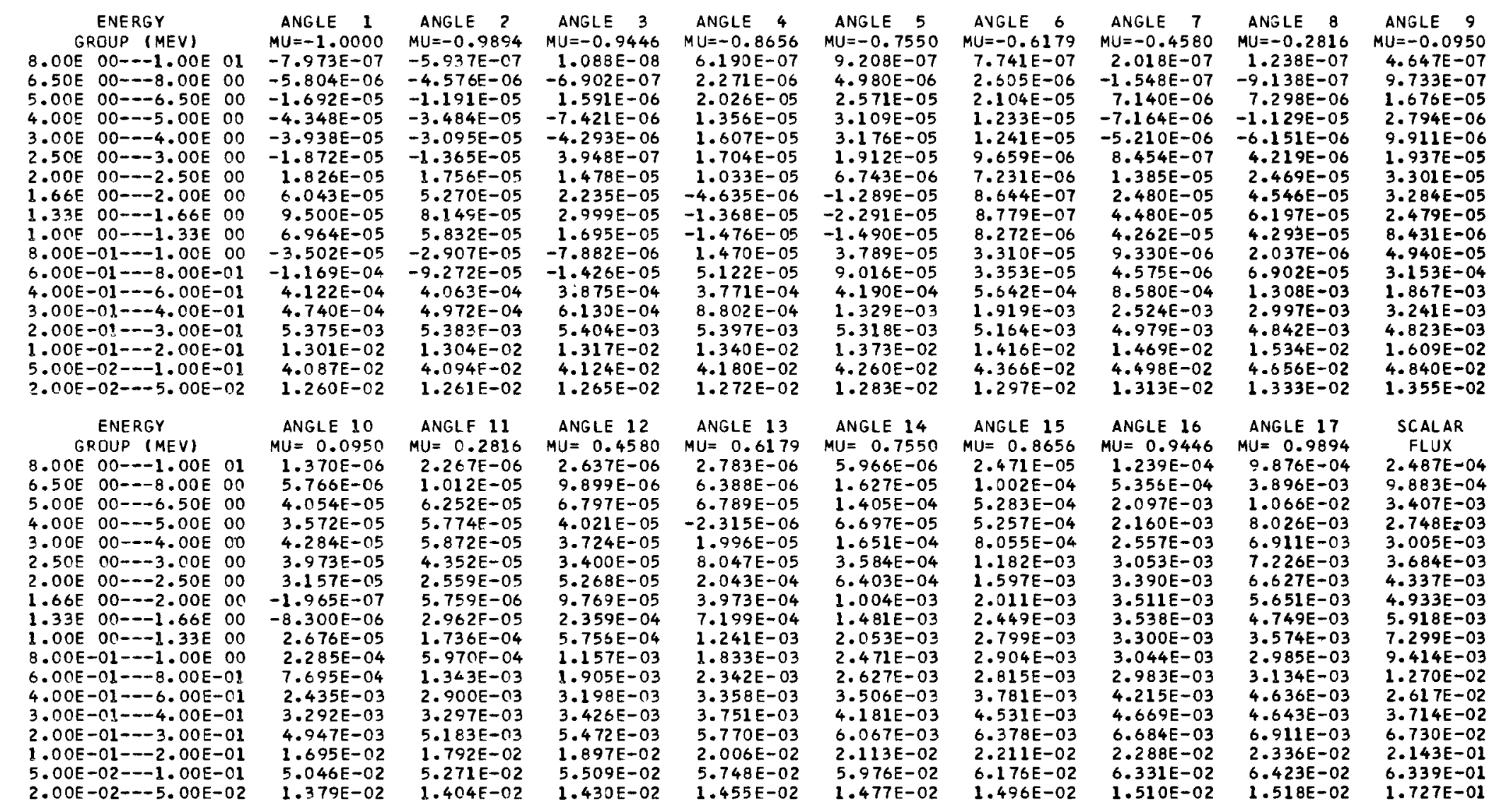



4 PI R**2 HENDER SON DOSE (NEUTRONS)
10.00C TO $12.20 C$ MEV NEUTRON SOUFCE

$\begin{array}{ll}75.0 & 100.0 \\ 7.437 E-11 & 9 .(61 E-11 \\ 7.549 E-11 & 9.185 \mathrm{~F}-11 \\ 7.789 E-11 & 9.471 E-11 \\ 7.962 E-11 & 9.684 E-11 \\ 8.033 E-11 & 9.764 E-11 \\ 8.280 E-11 & 1 . C 11 E-10 \\ 8.643 E-11 & 1 . C 56 E-1 C \\ 9.536 E-11 & 1.167 E-10 \\ 1.086 E-11 & 1.335 E-1 C \\ 1.663 E-1 C & 1.589 E-1 C \\ 1.770 E-10 & 1.834 E-1 C \\ 1.979 E-1 C & 2.278 E-1 C \\ 2.590 E-10 & 3.286 E-10 \\ 5.336 E-10 & 5.186 E-1 C \\ 1.513 E-C 9 & 1 . C 14 E-C 9 \\ 2.795 E-C 9 & 2.668 E-C 9 \\ 1.570 E-C 8 & 1.526 E-C 8 \\ 6.51 C E-C 9 & 6.296 E-C 9\end{array}$

500.0

$1.132 \mathrm{E}-10$

$1.137 \mathrm{E}-10$

$1.155 \mathrm{E}-10$

$1.178 \mathrm{E}-10$

$1.219 \mathrm{E}-10$

$1.270 E-10$

$1.354 E-10$

$1.484 \mathrm{E}-10$

$1.660 \mathrm{E}-10$

$1.893 E-10$

$2.183 E-10$

2.562E-1C

$3.124 E-10$

$4 . C 92 E-10$

$5.958 E-10$

$1 . C 15 E-C 9$
$3.386 E-C 9$

$3.555 \mathrm{E}-\mathrm{C} 9$

600.0

$758 \mathrm{E}-1 \mathrm{C}$

$2.229 E-C 9$

\begin{tabular}{|c|c|c|c|c|}
\hline 150.0 & $\begin{array}{l}\text { NGE (METERS) } \\
200.0\end{array}$ & $25 c . c$ & $3 c 0 . c$ & 400.0 \\
\hline & $1.290 E-10$ & $1.365 E-10$ & $1.382 \mathrm{E}-10$ & $1.300 E-10$ \\
\hline & $1.302 E-10$ & $1.375 \mathrm{E}-10$ & $1.391 \mathrm{E}-1 \mathrm{O}$ & $1.306 \mathrm{E}-10$ \\
\hline-10 & $1.334 \mathrm{E}-10$ & $1.405 E-10$ & $1.419 \mathrm{E}-10$ & $1.329 E-10$ \\
\hline-10 & $1.356 \mathrm{E}-10$ & $1.423 \mathrm{E}-10$ & $1.440 \mathrm{E}-1 \mathrm{C}$ & $1.353 \mathrm{E}-10$ \\
\hline & $1.384 E-10$ & $1.473 \mathrm{E}-10$ & $1.491 \mathrm{E}-1 \mathrm{C}$ & $1.400 \mathrm{E}-10$ \\
\hline-10 & $1.438 E-10$ & $1.519 E-10$ & $1.541 E-10$ & $1.454 \mathrm{E}-10$ \\
\hline-10 & $1.517 \mathrm{E}-10$ & $1.611 \mathrm{E}-10$ & $1.638 \mathrm{E}-10$ & $1.549 \mathrm{E}-10$ \\
\hline & $1.674 \mathrm{E}-10$ & $1.776 E-10$ & $1.803 \mathrm{E}-10$ & $1.701 \mathrm{E}-10$ \\
\hline 10 & $1.9 C 1 \mathrm{E}-10$ & $2.01 C E-10$ & $2.035 \mathrm{E}-10$ & $1.910 \mathrm{E}-10$ \\
\hline & $2.234 \mathrm{E}-10$ & $2.352 \mathrm{E}-10$ & $2.343 E-1 C$ & $89 E-10$ \\
\hline & $2.571 E-10$ & $2.698 \mathrm{E}-1 \mathrm{C}$ & $2.737 \mathrm{E}-10$ & $2.535 \mathrm{E}-10$ \\
\hline & $3.095 \mathrm{E}-10$ & $3.237 \mathrm{E}-1 \mathrm{C}$ & $3.222 \mathrm{E}-1 \mathrm{C}$ & $2.982 \mathrm{E}-1 \mathrm{C}$ \\
\hline $13 E-10$ & $3.790 \mathrm{E}-10$ & $3.944 \mathrm{E}-10$ & $3.964 \mathrm{E}-1 \mathrm{C}$ & $3.648 \mathrm{E}-10$ \\
\hline & $5.180 E-10$ & $5.347 \mathrm{E}-10$ & $5.294 \mathrm{E}-1 \mathrm{C}$ & $4.823 E-10$ \\
\hline & $8.398 \mathrm{E}-10$ & $8.329 E-10$ & $8.111 \mathrm{E}-10$ & $7.178 \mathrm{E}-10$ \\
\hline & $1.955 E-09$ & $1.747 \mathrm{E}-09$ & $1.584 \mathrm{E}-09$ & $1.288 \mathrm{E}-\mathrm{C} 9$ \\
\hline $05 t-08$ & $1.157 E-08$ & $9.554 \mathrm{E}-09$ & $7.810 \mathrm{E}-09$ & $5.150 E-C 9$ \\
\hline $33 E-0$ & $5.872 E-09$ & $5.57 \mathrm{CE}-09$ & $5.210 E-C 9$ & $4.389 \mathrm{E}-\mathrm{Cg}$ \\
\hline
\end{tabular}

RANGE (METERS )

900.0

$1200 . \mathrm{C}$

$150 \mathrm{C} .0$

1800.0

$4.376 \mathrm{E}-11$

$4.391 \mathrm{E}-11$

$1.719 \mathrm{E}-11$

$1.725 \mathrm{E}-11$

$1.747 \mathrm{E}-1$

$1.788 \mathrm{E}-11$

$1.853 \mathrm{E}-11$

$1.944 E-11$
$2.075 E-11$

$2.075 E-11$

$2.253 E-11$

$2.488 \mathrm{E}-11$

$2.788 \mathrm{E}-11$

$3.174 E-11$

$3.689 \mathrm{E}-11$

$4.431 E-11$

$5.576 E-11$

$1.071 \mathrm{E}-10$

$1.071 \mathrm{E}-10$
$1.885 \mathrm{E}-10$

$6.10 \mathrm{CE}-12$

$6.119 E-12$

$6.198 \mathrm{E}-1$

$6.346 \mathrm{E}-12$

$6.577 \mathrm{t}-12$

$6.907 \mathrm{E}-12$

$7.366 \mathrm{E}-12$

$7.986 \mathrm{E}-12$

$8.794 \mathrm{E}-12$

. $116 E-11$

$1.116 E-11$

1.294E-11

1. $928 \mathrm{E}-11$

.

3. $504 \mathrm{E}-11$

$5.514 E-11$

$6.445 \mathrm{E}-10$

$4.435 E-10$

$1.525 \mathrm{E}-10$
2. $023 E-12$

$2.030 \mathrm{E}-12$

$2.056 \mathrm{E}-12$

2.105E-12

$2.292 \mathrm{E}-12$

$2.444 E-12$

$2.647 \mathrm{E}-12$

.

.

$3.678 E-12$

$4.258 E-12$

6.275E-12

.

$1.095 \mathrm{E}-11$

1. 6 C 1E-11

$4.952 E-11$ 
COSINE

-1. COOOOE 00 $-9.89401 E-01$ $-9.44575 E-01$ $-8.65631 \mathrm{E}-01$ -7.55044E-DI $-6.17876 E-01$ - $.58017 E-01$ $-2.81605 E-01$ 9.50125E-C2 $9.50125 \mathrm{E}-\mathrm{C2}$ $4.58017 \mathrm{E}-01$ $4.17876 \mathrm{E}-01$ $7.55 \mathrm{C} 44 \mathrm{E}-01$ $8.65631 \mathrm{E}=01$ $8.65631 \mathrm{E}-01$ $9.89401 E-01$

TOTAL

COSINE

-1.00C00E GS $-9.39421 \mathrm{E}-31$ $-9.44575 \mathrm{E}-01$
$-8.65631 \mathrm{E}-01$ $-8.65631 E-01$ $-6.17876 \mathrm{E}-01$ $-4.58017 E-01$ $-2.81605 E-01$ $-9.50125 E-52$ $9.5 \mathrm{C} 125 \mathrm{E}-02$
$2.81605 \mathrm{E}-01$ 2.81605E-01 $4.58017 E-01$ $6.17876 \mathrm{E}-01$ $7.55044 \mathrm{E}-01$ $8.65631 E-01$ $9.44575 E-01$ $9.89401 E-J 1$

TOTAL

75.0
$1.087 \mathrm{E}-10$
$1.102 \mathrm{E}-10$
$1.133 \mathrm{E}-10$
$1.154 \mathrm{E}-10$
$1.158 \mathrm{E}-10$
$1.196 \mathrm{E}-10$
$1.249 \mathrm{E}-10$
$1.376 \mathrm{E}-1 \mathrm{C}$
$1.564 \mathrm{E}-10$
$2.362 \mathrm{E}-10$
$2.538 \mathrm{E}-10$
$2.822 \mathrm{E}-10$
$3.652 \mathrm{E}-1 \mathrm{C}$
$7.419 \mathrm{E}-10$
$2.084 \mathrm{E}-\mathrm{C} 9$
$3.843 \mathrm{E}-\mathrm{C} 9$
$2.146 \mathrm{E}-\mathrm{C} 8$
$9.018 \mathrm{E}-\mathrm{C} 9$

100.0

$1.337 \mathrm{E}-1 \mathrm{C}$ $1.354 \mathrm{E}-1 \mathrm{C}$
$1.391 \mathrm{E}-1 \mathrm{C}$ $1.391 \mathrm{E}-1 \mathrm{C}$ $1.417 \mathrm{E}-1 \mathrm{C}$ $1.427 \mathrm{E}-10$ $1.475 E-10$ $1.541 E-10$ $1.037 E-1 C$ 2. $2.629 E-10$ $3.277 \mathrm{E}-1 \mathrm{C}$ $4.668 \mathrm{E}-10$ $7.280 \mathrm{E}-10$ $1.408 \mathrm{E}-\mathrm{C} 9$ $3.678 \mathrm{E}-\mathrm{CO}$ 2. $C 87 E-C 8$

$8.777 E-C 9$

502.0

$1.939 \mathrm{E}-1 \mathrm{C}$ $1.946 \mathrm{E}-10$ 1. $972 \mathrm{E}-1 \mathrm{O}$ $2.007 E-10$ $2.147 \mathrm{E}-10$ $2.275 \mathrm{E}-10$ $2.468 \mathrm{E}-10$ $2.727 E-1 C$ $3.065 \mathrm{E}-1 \mathrm{C}$ $3.484 E-10$ $4 . C 27 E-10$ $4.826 \mathrm{E}-10$ $6.190 E-10$ $8.802 \mathrm{E}-10$ $1.464 \mathrm{E}-\mathrm{C} 9$ $4.731 E-C 9$

$5.481 E-C 9$

$1.652 \mathrm{E}-1 \mathrm{C}$ $1.658 \mathrm{E}-10$ $1.678 \mathrm{E}-10$ $1.762 \mathrm{E}-1 \mathrm{C}$ $1.832 \mathrm{E}-1 \mathrm{r}$ $1.940 E-10$ 2. C98E-1C $2.307 E-1 C$ $2.580 E-1 C$ $2.919 E-1 C$ $3.360 E-1 C$ $4.006 E-1 C$ $5.084 E-1 C$ $1.130 E-C 9$
$3.137 E-C .9$

$4.402 E-C 9$
150.0 RANGE (METERS)
200.0

$1.720 \mathrm{E}-10$ $1.779 E-10$ $1.810 \mathrm{E}-10$ $1.831 \mathrm{E}-10$ $1.896 \mathrm{E}-10$ $1.985 \mathrm{E}-10$ $2.186 \mathrm{E}-10$ $2.480 E-10$ $3.337 E-10$ $4.0805=10$ $4.903 E-1 C$ $6.782 E-10$ $1.247 E-09$ $3.086 E-C 9$ $1.872 \mathrm{E}-\mathrm{C} B$

$8.587 E-09$ $1.998 \mathrm{E}-10$ $2.04 C E-10$ 2.060 10 $2.108 E-10$ 2.302E -10 $2.302 E-10$ 2.525E-10 $3.314 E-10$ $3.314 \mathrm{E}-10$ 3. $830 E-10$ $5.525 \mathrm{E}-10$ $7.455 \mathrm{E}-10$ $1.194 E-09$ $1.194 E-69$
$2.732 E-C 9$ $1.590 \mathrm{E}-08$

$8.393 E-C 9$

RANGE (METERS)

$900 . C$
$8.280 E-11$
$8.304 E-11$
$8.397 E-11$
$8.562 E-11$
$8.823 E-11$
$9.186 E-11$
$9.710 E-11$
$1.043 E-10$
$1.138 E-10$
$1.258 E-10$
$1.410 E-10$
$1.609 E-10$
$1.895 E-10$
$2.347 E-10$
$3.120 E-10$
$4.575 E-10$
$9.290 E-10$

$120 \mathrm{C} .0$ $9.290 E-10$

\section{$3.416 \mathrm{E}-11$} 3. $425 \mathrm{E}-11$ $3.462 \mathrm{E}-11$ 3. $531 E-11$ $3.638 E-11$ $3.998 E-11$ $4.279 E-11$ $4.643 E-11$ $5.103 E-11$ $5.684 E-11$ $6.447 E-11$ $7.527 E-11$ $9.168 \mathrm{E}-11$ $1.182 \mathrm{E}-10$ $1.642 \mathrm{E}-10$ $2.782 E-10$

$7.708 E-10$
$250 . C$ $2.138 \mathrm{E}-10$ $2.152 \mathrm{E}-10$ 2.19E 10 $2.218 E-10$ $2.288 E-10$ 2. $2.491 \mathrm{E}-10$ 2.73CE-10 $3.54 C E-10$ $4.05 \mathrm{E}-10$ $4.797 E-10$ 5.797E-10 $7.759 \mathrm{E}-1 \mathrm{C}$ $1.192 \mathrm{E}-09$ $457 E-09$ $1.315 \mathrm{E}-08$

$8.066 E-09$

$150 C .0$

1. $254 \mathrm{E}-11$ $1.257 \mathrm{E}-11$ $1.271 E-11$
$1.296 E-11$ $1.335 \mathrm{E}-11$ 1. $39 C E-11$ $1.465 E-11$ $1.565 E-11$ $1.692 \mathrm{E}-11$ $1.852 \mathrm{E}-11$ $2.055 E-11$ $2.322 \mathrm{E}-11$ $2.693 \mathrm{E}-11$ $3.242 E-11$ $4.093 E-11$ $5.475 E-11$ $8.304 \mathrm{E}-11$

$2.731 E-1 C$
300.0

400.0 $2.208 E-1 C \quad 2.154 E-10$ $2.220 \mathrm{E}-1 \mathrm{C} \quad 2.163 \mathrm{E}-10$ $2.258 E-1 C$ 2.194E- 10 $2.288 E-10$ 2.230E-10 $2.361 E-10$ 2.3OOE-10 $2.437 E-10$ 2.382E-10 $2.570 E-10$ 2. $3.154 E-10 \quad 2.747 E-10$ $3.599 \mathrm{E}-10 \quad 3.453 \mathrm{E}-10$ $4.153 E-1 C$ C $3.950 E-10$ $4.845 E-10 \quad 4.587 E-10$ $5.887 E-10 \quad 5.530 E-10$ $7.755 E-10 \quad 7.181 E-10$ $1.168 \mathrm{E}-\mathrm{C} 9$ 1. $\mathrm{C} 47 \mathrm{E}-\mathrm{CO}$ $2.239 E-C Q \quad 1.040 E-09$ $1.078 \mathrm{E}-\mathrm{C} 8 \mathrm{~T} \quad \mathrm{7} .150 \mathrm{E}-09$

$7.644 E-C 9 \quad 6.606 E-09$

$4.263 \mathrm{E}-12$

4.273E-12

$4.319 \mathrm{E}-12$

$4.406 E-12$

$4.537 \mathrm{E}-12$

. $723 E-12$

$4.973 E-12$

$.303 \mathrm{E}-12$

$5.721 \mathrm{E}-12$

$.248 E-12$

$.914 E-12$

.787E-12

$.073 \mathrm{E}-11$

$.334 \mathrm{E}-11$

$1.737 \mathrm{E}-11$

$2.453 E-11$

9. C68E-11 
4 PI R* 2 TISSUE KERMA (NEUTRONS) (CM* 2 ERGS/GRAM/STERADIAN/SOURCE NEUTRON I
IC.000 TO 12.200 MEV NEUTRON SOURCE
COSINE

$-1.00000 E$ OO $-9.89401 E-01$ $-9.44575 \mathrm{E}-01$ $-8.65631 E-01$ $-7.55044 E-01$ $-6.17876 \mathrm{E}-01$ $-4.58017 \mathrm{E}-01$ $-2.81605 E-01$ $-9.50125 \mathrm{E}-02$ $9.50125 E-102$ 2.81605E-01 4.58017E-O1 $6.17876 \mathrm{E}-01$ $7.55044 E-01$ $8.65631 E-01$ $9.44575 E-01$
$9.89401 E-01$

TOTAL

COSINE

-1. CCCOOE 00 $-9.89401 E-01$ $-8.64531 E-01$ $-7.55044 \mathrm{E}-01$ $-6.17876 E-01$ $-4.58017 E-01$ 4. $-9.50125 E-02$ 9.50125E-D2 $2.81605 E-01$ $4.58017 \mathrm{E}-01$ $6.17876 \mathrm{E}-01$ 7.55044E-OI $8.65631 E-01$ $8.44575 E-J 1$ $9.89401 E-01$

TOTAL

$\begin{array}{lc}75.0 & 1 C C . n \\ .679 E-C 9 & 9.369 E-09 \\ .806 E-C 9 & 9.511 E-C 9 \\ 8.081 E-C 9 & 9.837 E-C 9 \\ 8.279 E-C 9 & 1 . C 08 E-08 \\ .322 E-09 & 1.016 E-08 \\ 8.610 E-C 9 & 1.053 E-C 8 \\ .973 E-C 9 & 1.097 E-C 8 \\ .9 C 6 E-C 9 & 1.213 E-C 8 \\ .130 E-C 8 & 1.389 E-08 \\ 1.734 E-C 8 & 1.659 E-C 8 \\ 1.853 E-08 & 1.915 E-C 8 \\ 2.073 E-C 8 & 2.384 E-08 \\ 2.752 E-08 & 3.458 E-C 8 \\ 5.784 E-C 8 & 5.552 E-08 \\ .665 E-C 7 & 1.103 E-07 \\ 3.085 E-C 7 & 2.931 E-C 7 \\ .750 E-C 6 & 1.698 E-C 6 \\ 7.123 E-C 7 & 6.849 E-07\end{array}$

$500 . n$

600.0

$1.213 \mathrm{E}-08$ $1.219 E-C 8$

$1.263 \mathrm{E}-08$ $1.307 \mathrm{E}-08$

$1.359 E-C 8$

$1.583 E-C B$

$1.767 E-C 8$

2 $10 E-C B$

$2.313 E-C 8$

$2.7 C 6 E-C B$

$3.290 E-C 8$

$4.307 E-08$

$6.288 \mathrm{E}-\mathrm{C} 8$

$1.079 E-C 7$

$3.690 E-C 7$

$3.787 E-C 7$

$4.999 E-C 8$

$8.238 E-r 8$

$2.418 \mathrm{E}-07$

$2.978 E-C 7$
150.0 RANGE (METERS)
200.0

250.0

300.0

$40 C \cdot C$

$1.186 E-C 8$

$1.201 E-08$

$1.237 \mathrm{E}-08$

$1.265 \mathrm{E}-08$

$1.282 \mathrm{E}-08$

$1.329 \mathrm{E}-\mathrm{C} 8$

$1.389 \mathrm{E}-08$

$1.535 \mathrm{E}-\mathrm{C} 8$

$1.752 \mathrm{E}-08$

$2.065 E-08$

$2.4 C 4 E-C 8$

$2.931 E-08$

$3.564 E-08$

$4.987 E-08$

$9.438 E-C 8$

1. $516 \mathrm{E}-06$

$1.516 E-06$

$1.347 E-08$
$1.36 C E-C 8$

$1.431 E-08$

$1.443 E-08$

$\begin{array}{ll}1.396 E-08 & 1.477 E-08 \\ .420 E-08 & 1.497 E-08\end{array}$

$1.420 E-08$

$1.45 C E-08$

1. $5 \mathrm{C} 6 \mathrm{E}-08$

$1.586 \mathrm{E}-08$

$1.749 E-08$

$1.987 \mathrm{E}-08$

2.34CE-08

$2.688 E-08$

$3.239 E-08$

$3.963 E-08$

$5.435 E-08$

$8.894 E-08$

$2.111 E-07$

$1.55 C E-08$

$1.597 E-08$

$1.690 \mathrm{E}-08$

$1.862 E-08$

$2.108 \mathrm{E}-08$

$2.468 \mathrm{E}-08$

2. $826 E-08$ $3.391 E-08$ $4.126 E-08$ $5.610 E-08$ $8.8 C 2 E-08$ $1.055 E-06$

$6.559 E-07$

6.297E-07

$5.953 E-07$

$1.456 E-C 8$ $.467 \mathrm{E}-\mathrm{CB}$

$.497 \mathrm{E}-\mathrm{CB}$

$.521 \mathrm{E}-\mathrm{C} 8$

$.575 E-C 8$

$1.626 \mathrm{E}-08$

$1.725 \mathrm{E}-\mathrm{C} 8$

$1.897 \mathrm{E}-\mathrm{C} 8$

$2.140 E-C 8$

2.463E

.

政

$8.565 E-C 8$

$1.694 \mathrm{E}-\mathrm{C} 7$

$8.598 E-C 7$

$5.556 \mathrm{E}-\mathrm{C} 7$

$1.382 \mathrm{E}-\mathrm{C} 8$

$1.389 \mathrm{E}-\mathrm{C} 8$

$1.440 E-C 8$

$1.490 E-08$

$1.545 \mathrm{E}-08$

$1.643 \mathrm{E}-08$

$1.802 \mathrm{E}-\mathrm{C} 8$

$2.021 E-C 8$

2.313E-08

.

3.139E-08

5. $C 67 \mathrm{E}-08$

$7.574 \mathrm{E}-\mathrm{C} 8$

5.640E-07

RANGE (METERS)

900.0

$1200 . \mathrm{C}$

1500.0

1800.0

$4.805 E-09$

$1.910 E-09$

$6.835 \mathrm{E}-10$

$4.674 E-C 7$

$2.281 \mathrm{E}-10$

$2.316 \mathrm{E}-10$

$\begin{array}{llll}4.884 E-09 & 1.941 E-C 9 & 6.941 E-10 & 2.316 E-10 \\ 4.993 E-09 & 1.985 \mathrm{E}-\mathrm{Cg} & 7.101 \mathrm{E}-10 & 2.370 \mathrm{E}-10 \\ 5.165 \mathrm{E}-09 & 2.054 \mathrm{E}-09 & 7.349 \mathrm{E}-10 & 2.453 \mathrm{E}-10\end{array}$

$\begin{array}{llll}4.884 E-09 & 1.941 E-C 9 & 6.941 E-10 & 2.316 E-10 \\ 4.993 E-09 & 1.985 E-C 9 & 7.101 E-10 & 2.370 E-10 \\ 5.165 E-09 & 2.054 E-09 & 7.349 E-10 & 2.453 E-10\end{array}$

$\begin{array}{lllll}1 . C 88 E-C 8 & 5.165 E-C 9 & 2.054 E-09 & 7.349 E-10 & 2.453 E-10 \\ 1.134 E-C 8 & 5.403 E-09 & 2.151 \mathrm{E}-09 & 7.701 \mathrm{E}-1 \mathrm{C} & 2.571 \mathrm{E}-10 \\ 1.208 \mathrm{E}-\mathrm{C} 8 & 5.755 \mathrm{E}-\mathrm{Cg} & 2.289 \mathrm{E}-09 & 8.189 \mathrm{E}-10 & 2.732 \mathrm{E}-10\end{array}$

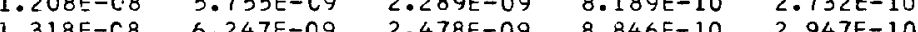

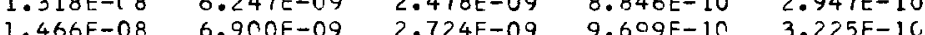

$1.66 C \mathrm{E}-\mathrm{C} 8 \mathrm{7} \quad 7.740 \mathrm{E}-09 \quad 3.03 \mathrm{EE}-\mathrm{CO} \quad 1.078 \mathrm{E}-09 \quad 3.578 \mathrm{E}-10$

$1.603 \mathrm{E}-\mathrm{C} 8 \mathrm{8} \quad 8.804 \mathrm{E}-09 \quad 3.442 \mathrm{E}-\mathrm{Cg} \quad 1.218 \mathrm{E}-09 \quad 4.59 \mathrm{E}-10$

$\begin{array}{lllll}2.221 \mathrm{E}-\mathrm{C} 8 & 1 . \mathrm{C} 22 \mathrm{E}-08 & 3.978 \mathrm{E}-09 & 1.2103 \mathrm{E}-09 & 4.036 \mathrm{E}-10\end{array}$

$2.691 \mathrm{E}-\mathrm{C} 8 \mathrm{1} \quad 1.227 \mathrm{E}-\mathrm{C} 8 \quad 4.749 \mathrm{E}-09 \quad 1.667 \mathrm{E}-09 \quad 5.484 \mathrm{E}-10$

$\begin{array}{lllll}2.691 \mathrm{E}-\mathrm{C} 8 & 1.227 \mathrm{E}-\mathrm{C} 8 & 4.749 \mathrm{E}-09 & 1.667 \mathrm{E}-09 & 5.484 \mathrm{E}-10 \\ 3.491 \mathrm{E}-\mathrm{C} 8 & 1.559 \mathrm{E}-08 & 5.942 \mathrm{E}-09 & 2.063 \mathrm{E}-09 & 6.733 \mathrm{E}-10\end{array}$

$2.136 \mathrm{E}-18$

$3.240 \mathrm{E}-\mathrm{CB} \quad 1.906 \mathrm{E}-09$

$6.923 E-08 \quad 2.013 E-08$

$2.063 E-09$

$3.717 E-09$

$5.866 E-09$

$6.733 E-10$
$8.639 E-10$

$8.639 E-10$
$1.163 E-09$

1. 7 C IE-C 9

1.275E-07

$4.794 E-C 8$

$1.657 E-08$ 
COSINE

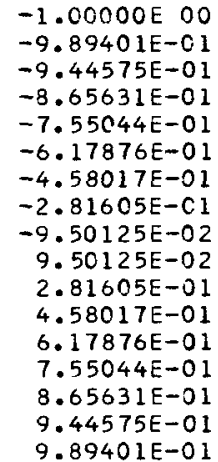

$9.89401 E-01$

TOTAL

COSINE

\section{$-1.00000 E$ OC} $-9.89401 E-01$ $-9.44575 E-01$ $-8.65631 \mathrm{E}-01$ $-7.55044 E-01$ $-6.17876 E-01$ $-4.58017 E-01$ $-2.81605 E-01$ $-9.50125 E-02$ 9.50125E-02 $2.81605 E-01$ 4.58017E-O1 $6.17876 \mathrm{E}-\mathrm{O}$ $065631 E-C 1$ $8.65631 E-C 1$ $9.89401 E-01$

TOTAL

$\begin{array}{lc}75.0 & 10 C .0 \\ 3.994 E-11 & 4.647 E-11 \\ 4.090 E-11 & 4.752 E-11 \\ 4.289 E-11 & 4.986 E-11 \\ 4.412 E-11 & 5.133 E-11 \\ 4.396 E-11 & 5.131 E-11 \\ 4.553 E-11 & 5.32 C E-11 \\ 4.743 E-11 & 5.542 E-11 \\ 5.346 E-11 & 6.272 E-11 \\ 6.266 E-11 & 7.412 E-11 \\ 1.022 E-10 & 8.936 E-11 \\ 1.129 E-10 & 1.081 E-1 C \\ 1.251 E-10 & 1.415 E-1 C \\ 1.767 E-10 & 2.105 E-10 \\ 4.059 E-10 & 3.682 E-1 C \\ 1.239 E-09 & 7.842 E-1 C \\ 2.329 E-C 9 & 2.177 E-C 9 \\ 1.360 E-C 8 & 1.314 E-C 8 \\ 5.154 E-09 & 4.797 E-C 9\end{array}$

150.0
$5.453 E-11$
$5.559 E-11$
$5.813 E-11$
$5.970 E-11$
$6.001 E-11$
$6.228 E-11$
$6.510 E-11$
$7.397 E-11$
$8.745 E-11$
$1.025 E-10$
$1.297 E-10$
$1.625 E-10$
$1.948 E-10$
$2.989 E-10$
$6.135 E-10$
$1.739 E-09$
$1.163 E-08$
$4.324 E-C 9$

RANGE (ME TERS) $2 \mathrm{CO} . \mathrm{C}$

$25 \mathrm{C} .0$

300.0

$400 . c$

$\begin{array}{ll}5.857 E-11 & 5.96 C E-11 \\ 5.952 E-11 & 6.042 E-11 \\ 6.195 E-11 & 6.26 C E-11 \\ 6.305 E-11 & 6.324 E-11 \\ 6.416 E-11 & 6.6 C 2 E-11 \\ 6.671 E-11 & 6.756 E-11 \\ 7.054 E-11 & 7.2 C 7 E-11 \\ 8.008 E-11 & 8.174 E-11 \\ 9.441 E-11 & 9.601 E-11 \\ 1.191 E-10 & 1.2 C 8 E-10 \\ 1.337 E-10 & 1.347 E-10 \\ 1.71 C E-10 & 1.722 E-10 \\ 2.104 E-10 & 2.107 E-10 \\ 3.092 E-10 & 3.089 E-10 \\ 5.487 E-10 & 5.254 E-10 \\ 1.459 E-09 & 1.256 E-09 \\ 9.748 E-C 9 & 7.949 E-09 \\ 3.743 E-09 & 3.556 E-09\end{array}$

5. $860 E-11$ $5.928 E-11$ $6.118 \mathrm{E}-11$ $6.466 \mathrm{E}-11$ $6.641 E-11$ $7.106 \mathrm{E}-11$ $8.045 E-11$ $9.407 \mathrm{E}-11$ $1.116 \mathrm{E}-10$ $1.362 \mathrm{E}-1 \mathrm{C}$ $1.625 \mathrm{E}-10$ 2.080E-10 $2.951 E-10$ $4.998 E-10$ $1.105 E-C 9$ $6.417 E-C 9$

3. $178 E-C 9$ $5.284 E-11$ $5.331 \mathrm{E}-11$ $5.565 E-11$ 5.793E-11 $5.986 E-11$ $6.429 E-11$ $7.246 E-11$ 8.401E-11 9.979E-11 $1.187 E-10$ $1.430 \mathrm{E}-10$ $1.812 \mathrm{E}-10$
$2.567 \mathrm{E}-10$ $4.232 \mathrm{E}-10$ $8.599 \mathrm{E}-10$ 4.120 E-09

$2.488 E-09$

1800.0

EOC. 0

RANGE
9OO.

$12 \mathrm{CC}$.

$150 \mathrm{c} .0$

$4.482 E-11$

$3.646 E-11$
$3.667 E-11$

$1.670 \mathrm{E}-11$

$1.676 \mathrm{E}-11$

6.552E-12

2. $333 \mathrm{E}-12$ 2. $34 C E-12$ $2.369 E-12$ $2.421 \mathrm{E}-12$ $2.5 \mathrm{C} 3 \mathrm{E}-12$ $2.622 \mathrm{E}-12$ $2.795 \mathrm{E}-12$ $3.038 \mathrm{E}-12$ $3.362 \mathrm{E}-12$ 3. $779 E-12$ 5. $095 \mathrm{E}-12$ $5.095 \mathrm{E}-12$ . $354 E-12$ 1.196E-11 $1.849 \mathrm{E}-11$

$\begin{array}{llll}1.778 E-10 & 5.898 \mathrm{E}-11 & 1.849 \mathrm{E}-11 & 5.586 \mathrm{E}-12 \\ 4.492 \mathrm{E}-10 & 1.221 \mathrm{E}-10 & 3.357 \mathrm{E}-11 & 9.272 \mathrm{E}-12\end{array}$

$7.775 E-13$

$7.796 \mathrm{E}-13$

$7.889 \mathrm{E}-13$

$8.060 \mathrm{E}-13$

$8.328 \mathrm{E}-13$

$.719 \mathrm{E}-13$

$3.277 \mathrm{E}-13$

$1.004 \mathrm{E}-12$

$1.106 \mathrm{E}-12$

$1.236 E-12$

1.654E-12

$1.654 E-12$

$2.034 E-12$

$1.177 E-10$

$3.664 \mathrm{E}-12$

$6.829 \mathrm{E}-11$

$5.586 \mathrm{E}-12$

$6.543 \mathrm{E}-1 \mathrm{O}$

1.22IE- 10

9. $272 \mathrm{E}-12$

1. $900 E-C 9$

$1.425 E-C 9$

$5.521 E-10$

1.956E-10

$0.511 E-11$

$2.069 E-11$ 
COS INE

$$
\begin{aligned}
& -1.00000 E \quad O O \\
& -9.89401 \mathrm{E}-01 \\
& -9.44575 \mathrm{E}-01 \\
& -8.65631 \mathrm{E}-01 \\
& -7.55044 \mathrm{E}-01 \\
& -6.17876 \mathrm{E}-01 \\
& -4.58 \mathrm{C} 17 \mathrm{E}-01 \\
& -2.81605 \mathrm{E}-01 \\
& -9.50125 \mathrm{E}-02 \\
& 9.50125 \mathrm{E}-02 \\
& 2.81605 \mathrm{E}-\mathrm{C} 1 \\
& 4.58017 \mathrm{E}-01 \\
& 6.17876 \mathrm{E}-01 \\
& 7.55 \mathrm{C} 44 \mathrm{E}-01 \\
& 8.55631 \mathrm{E}-01 \\
& 9.44575 \mathrm{E}-01
\end{aligned}
$$
$9.89401 E-01$

TOTAL

COSINE

$-1 \cdot \operatorname{COOOOE~OO~}$ $-9.89401 E-01$ $-9.44575 \mathrm{E}-01$ $-8.65631 \mathrm{E}-01$ $-7.55044 E-J 1$ $-6.17876 \mathrm{E}-01$ $-4.58017 E-01$ $-2.81605 E-01$ $-9.50125 \mathrm{E}-02$ $9.50125 E-02$ $2.81605 E-01$ $4.58017 \mathrm{E}-01$ $7.55044 E-01$ $7.55044 \mathrm{E}-01$ $8.65631 \mathrm{E}-01$ $9.44575 E-01$

TOTAL
75.0

$9.925 E-1 C$ 1. $C 2 O E-C 9$ $1 . C 83 \mathrm{E}-\mathrm{C} 9$ $1.131 \mathrm{E}-09$ 1. $14 C E-C 9$ 1.184E-09 $1.224 \mathrm{~F}-\mathrm{Cg}$ $1.361 \mathrm{E}-\mathrm{CO}$ $1.572 \mathrm{E}-09$ $2.492 E-C 9$ $2.702 \mathrm{E}-09$ 3. $C 43 E-09$ $4.406 E-C 9$ $1.028 \mathrm{E}-08$ $3.165 E-C 8$ $5.953 \mathrm{E}-\mathrm{CB}$ $3.521 E-07$

$1.318 E-C 7$

$500 . \mathrm{C}$

$1.488 \mathrm{E}-\mathrm{C9}$ $1.498 E-C 9$ $1.532 \mathrm{E}-\mathrm{Cg}$ $1.568 \mathrm{E}-09$ $1.630 E-C 9$ $1.691 \mathrm{E}-\mathrm{C} 9$ $1.801 \mathrm{E}-\mathrm{C} 9$ $1.984 E-C 9$ $2.238 \mathrm{E}-\mathrm{C} 9$ 2.581E-C9 3.rग2E-C9 $3.542 E-C 9$ $5.367 E-C 9$ 5.895E-C9 $1.674 \mathrm{E}-\mathrm{C}$ $1.674 E-C 8$

$5.383 E-C 8$
600.0

\section{0} $1.231 \mathrm{E}-09$ $3.3 C 5 \mathrm{E}-09$ $1.362 \mathrm{E}-\mathrm{C} 9$ $.377 \mathrm{E}-00$ $1.430 \mathrm{E}-\mathrm{C} 9$ $1.478 \mathrm{E}-\mathrm{C} 9$ $1.911 \mathrm{E}-\mathrm{C} 9$ $2.329 \mathrm{E}-\mathrm{C} 9$ $2.732 \mathrm{E}-\mathrm{C} 9$ $5.223 E-C 9$ $9.246 E-C 9$ $1.991 E-C 8$ $3.394 E-C 7$

$1.229 E-C 7$

$1.232 E-C 9$ $1.239 E-C 9$ $1.263 E-C 9$

$1.293 \mathrm{E}-\mathrm{CO}$

$1.343 \mathrm{E}-\mathrm{C} 9$

$1.397 \mathrm{E}-\mathrm{C} 9$

$1.491 \mathrm{E}-\mathrm{C} 9$

$1.638 \mathrm{E}-09$

$1.838 \mathrm{E}-09$ $2.107 E-C 9$ . 2.871E-C $3.528 \mathrm{E}-09$ $4.715 \mathrm{E}-09$ $1.253 E-C 8$ $1.253 E-C 8$

$4.125 E-C 8$
150.0 RANGE (METERS)
2 CO.

$25 C .0$

$300 . C$

400.0

\section{$\begin{array}{lll}.502 \mathrm{E}-\mathrm{C} 9 & 1.694 \mathrm{E}-09 & 1.791 \mathrm{E}-09\end{array}$} .534E-C9 $1.723 \mathrm{E}-09$ $1.615 \mathrm{E}-\mathrm{C} 9$ 1.8C1E-09

$1.7 C 4 \mathrm{E}-09$

$1.768 \mathrm{E}-09$

$1.924 E-09$

$1.972 \mathrm{E}-09 \quad 2.063 \mathrm{E}-09$

$2.066 \mathrm{E}-09$

2.296E-CQ $2.417 E-09$

$1.833 \mathrm{E}-\mathrm{Cg}$

$2.362 \mathrm{E}-09$

$3.2 \mathrm{C} 6 \mathrm{E}-09$

$2.473 \mathrm{E}-09$

3.343E-09

3. $809 E-09$

$3.376 \mathrm{E}-09$

$4.521 E-C 9$
$5.559 E-09$

. C 76E-09

$\begin{array}{lll}.445 E-C 9 & 7.922 E-09 & 8.067 E-09\end{array}$

$\begin{array}{lll}1.534 \mathrm{E}-08 & 1.385 \mathrm{E}-\mathrm{C} 8 & 1.342 \mathrm{E}-08\end{array}$

$4.404 E-C 8 \quad 3.668 E-08 \quad 3.155 E-38$

2.500E-07 2. $532 \mathrm{E}-07$

$1.03 C E-07$

$9.412 E-08$

$1.814 \mathrm{E}-\mathrm{C} 9$

$1.835 \mathrm{E}-00$

$1.938 \mathrm{E}-\mathrm{C} 9$

$2.024 \mathrm{E}-\mathrm{C} 9$

$2.080 E-09$

$2.202 E-C 9$

$.441 \mathrm{E}-09$

$2.788 \mathrm{E}-09$

$3.869 E-09$

$4.571 E-C$

$.7 C 6 \mathrm{E}-\mathrm{C} 9$

$.876 E-C 9$

$.635 \mathrm{E}-\mathrm{C} 7$

$8.539 E-C 8$

.707E-09

$1.722 \mathrm{E}-09$
$1.768 \mathrm{E}-09$

$.808 \mathrm{E}-0.9$

$1.883 \mathrm{E}-09$

$1.946 \mathrm{E}-09$

$.946 E-09$
$2.068 E-69$

$2.285 \mathrm{E}-\mathrm{C} 9$

. $592 \mathrm{E}-09$

$3.012 \mathrm{E}-09$

.524E-09

$.172 E-C 9$
$.161 E-09$

7. $C 48 \mathrm{E}-\mathrm{C} 9$

$1.114 \mathrm{E}-08$

$1.182 E-C 8$
$1.044 E-07$

$6.871 E-C 8$

\section{RANGE (METERS}

$900.0 \quad 1200.0$

$150 C .0$

$1800 . \mathrm{C}$

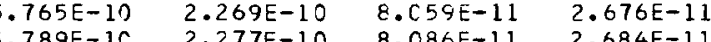

$\begin{array}{llll}2.269 E-10 & 8.059 E-11 & 2.676 E-11\end{array}$

$5.879 E-10 \quad 2.309 E-10 \quad 8.195 E-11 \quad 2.720 E-11$

$6.021 E-10$ 2.365E-10 $8.393 E-1122.785 E-11$

$6.243 E-10 \quad 2.451 E-10 \quad 8.697 \mathrm{E}-11 \quad 2.886 \mathrm{E}-11$

$6.53 C E-10 \quad 2.569 E-10 \quad 9.125 E-11 \quad 3.029 E-11$

7.607E-10 $2.741 E-10$ $9.728 E-11 \quad 3.228 E-11$

7.607E-10 $2.980 E-10$ 1.055E-10 $3.495 E-11$

3.298E-10 1.163E-10 3.844E-11

4.7C7E-10 1. $3 C 2 E-10$ 4.289E-11

1.20 $1.228 E-10$ 1.48CE-10 $4.863 E-11$

$5.927 E-10$ 1.

2.043E-09 $7.634 E-10 \quad 2.613 E-10 \quad 6.748 E-11$

2. $1.053 E-C 2 \quad 3.512 E-10 \quad 1.113 E-10$

$4.698 \mathrm{E}-\mathrm{C} 9 \mathrm{1} .58 \mathrm{EE}-09 \quad 5.066 \mathrm{E}-1 \mathrm{C} \quad 1.113 \mathrm{E}-10$

$1.129 E-08 \quad 3.589 E-09 \quad 5.066 E-1 C$ 1.552E-10

$.401 \mathrm{E}-1 \mathrm{C}$

$1.677 \mathrm{E}-08$

$6.116 E-C 9$

$2.074 E-09$

$6.676 \mathrm{E}-1 \mathrm{C}$ 
COSINE

$-1.0 C O O C E$ OO $-9.89401 E-01$ $-9.44575 E-01$ $-8.65631 \mathrm{E}-01$ $-7.55044 E-01$ $-6.17876 \mathrm{E}-\mathrm{Cl}$ $-4.58017 E-01$ $-2.81605 E-01$ $-9.50125 E-02$ $9.50125 \mathrm{E}-\mathrm{C2}$ $2.81605 E-01$ $4.58017 E-01$ $6.17876 \mathrm{E}-01$ $8.65631 E-01$

$9.894 C 1 E-01$

TOTAL

COSINE

-1.00000 E OC $-9.89401 E-01$ $-9.44575 E-C 1$ $-8.65631 \mathrm{E}-01$ $-7.55044 \mathrm{E}-01$ $-6.17876 \mathrm{E}-01$ $-4.58017 E-01$ $-2.81605 E-01$ $-9.50125 E-C 2$ $2.81605 E-01$ 4.58017E-O1 $6.17876 \mathrm{E}-01$ 7. $55044 \mathrm{E}-01$ 8.65631E-01 $9.44575 \mathrm{E}-01$
$9.89401 \mathrm{E}-01$

TOTAL
75.0

$1.469 \mathrm{E}-\mathrm{C} 9$

$1.515 E-09$

$1.617 \mathrm{E}-\mathrm{C} 9$

$1.694 \mathrm{E}-\mathrm{Cg}$

$1.706 E-C 9$
$1.777 E-C 9$

$1.842 \mathrm{E}-\mathrm{C} 9$

$2.065 \mathrm{E}-\mathrm{Cg}$

$2.410 \mathrm{E}-\mathrm{C} 9$

$3.975 \mathrm{E}-\mathrm{C} 9$

$4.271 E-C 9$

. $044 \mathrm{E}-09$

$7.044 E-O 9$

$1.672 \mathrm{E}-\mathrm{CB}$

$5.195 \mathrm{E}-\mathrm{C} 8$

$5.806 \mathrm{E}-\mathrm{CP}$

$2.15 C E-C 7$

500.0

$1.873 \mathrm{E}-\mathrm{C} 9$

$1.888 \mathrm{E}-09$
$1.938 \mathrm{E}-\mathrm{Cg}$

$1.984 \mathrm{E}-\mathrm{C} 9$

$2.068 \mathrm{E}-\mathrm{Cg}$

$2.143 \mathrm{E}-09$

$2.291 \mathrm{E}-09$

$2.549 \mathrm{E}-\mathrm{C} 9$

$2.912 \mathrm{E}-\mathrm{C} 9$

$4.022 E-09$

$4.814 \mathrm{E}-09$

$6.061 E-C 9$

$8.456 E-C 9$

$1.358 E-C B$

$2.611 \mathrm{E}-\mathrm{C}$

$1.081 \mathrm{E}-\mathrm{C} 7$

$7.761 \mathrm{E}-\mathrm{C} 8$
100.0

$1.738 E-C 9$

$1.788 \mathrm{E}-\mathrm{Cg}$

$1.908 \mathrm{E}-\mathrm{C} 9$

2. OCCE-09

$2.020 \mathrm{E}-\mathrm{C} 9$

$2.178 \mathrm{E}-\mathrm{C} 9$

$2.448 E-09$

$2.877 E-09$

$3.465 E-09$

列

.

$487 E-08$

$3.244 E-C 8$

$5.595 E-C 7$

$1.989 E-07$

600.0

$1.536 \mathrm{E}-\mathrm{C} 9$

$1.546 E-C 9$
$1.580 E-C 9$

$1.618 E-C 9$

$1.683 \mathrm{E}-\mathrm{Cg}$

$1.750 \mathrm{E}-09$

$1.872 \mathrm{E}-\mathrm{C} 9$

2.C75E-C9

$2.356 \mathrm{E}-\mathrm{Cg}$

$3.211 \mathrm{E}-\mathrm{C} 9$

$3.833 \mathrm{E}-\mathrm{C} 9$

$4.815 E-C 9$

6.66CE-C 9

$1.933 E-08$

$6.863 E-C 8$

$5.828 \mathrm{E}-\mathrm{C} 8$

$\begin{array}{ccccc}150.0 & \text { RANGE (METERS) } & & \\ 200.0 & 250.0 & 300 . C & 40 C .0 \\ 2.098 E-09 & 2.304 E-09 & 2.385 E-09 & 2.376 E-C 9 & 2.183 E-C 9 \\ 2.150 E-09 & 2.351 E-C 9 & 2.425 E-09 & 2.410 E-C 9 & 2.205 E-09 \\ 2.281 E-C 9 & 2.475 E-09 & 2.536 E-09 & 2.506 E-C 9 & 2.275 E-09 \\ 2.378 E-C 9 & 2.551 E-C 9 & 2.588 E-09 & 2.562 E-09 & 2.329 E-09 \\ 2.414 E-09 & 2.617 E-09 & 2.723 E-09 & 2.688 E-C 9 & 2.434 E-09 \\ 2.512 E-09 & 2.725 E-09 & 2.785 E-09 & 2.758 E-09 & 2.511 E-C 9 \\ 2.607 E-09 & 2.861 E-09 & 2.951 E-09 & 2.930 E-C 9 & 2.679 E-09 \\ 2.936 E-C 9 & 3.217 E-09 & 3.315 E-09 & 3.287 E-09 & 2.994 E-09 \\ 3.446 E-09 & 3.763 E-09 & 3.862 E-09 & 3.813 E-09 & 3.445 E-09 \\ 4 . C 23 E-09 & 4.739 E-09 & 4.849 E-09 & 4.499 E-09 & 4.073 E-09 \\ 5.160 E-C 9 & 5.290 E-09 & 5.373 E-09 & 5.483 E-C 9 & 4.827 E-09 \\ 6.239 E-09 & 6.773 E-09 & 6.874 E-09 & 6.516 E-C 9 & 5.800 E-09 \\ 7.759 E-09 & 8.292 E-09 & 8.361 E-09 & 8.314 E-09 & 7.312 E-09 \\ 1.166 E-08 & 1.221 E-08 & 1.226 E-08 & 1.175 E-C 8 & 1.03 C E-08 \\ 2.459 E-C 8 & 2.183 E-08 & 2.091 E-08 & 1.993 E-C 8 & 1.692 E-08 \\ 7.191 E-C 8 & 5.949 E-C 8 & 5.079 E-08 & 4.445 E-08 & 3.442 E-08 \\ 4.929 E-07 & 4.114 E-07 & 3.339 E-07 & 2.683 E-C 7 & 1.706 E-07 \\ 1.779 E-C 7 & 1.615 E-C 7 & 1.453 E-07 & 1.297 E-07 & 1.015 E-07\end{array}$

RANGE (METERS)

$12 \mathrm{CO} . \mathrm{C}$

1500.0

1800.0

\begin{tabular}{|c|c|c|c|}
\hline $\begin{array}{l}7.114 \mathrm{E}-10 \\
7.145 \mathrm{E}-10 \\
7.261 \mathrm{E}-10 \\
7.431 \mathrm{E}-10 \\
7.701 \mathrm{E}-10 \\
8.044 \mathrm{E}-10 \\
8.599 \mathrm{E}-10 \\
9.426 \mathrm{E}-10 \\
1.057 \mathrm{E}-\mathrm{C} \\
1.207 \mathrm{E}-\mathrm{C} 9 \\
1.398 \mathrm{E}-\mathrm{C} \\
1.658 \mathrm{E}-\mathrm{C} \\
2.066 \mathrm{E}-\mathrm{C} \\
2.791 \mathrm{E}-\mathrm{C} \\
4.177 \mathrm{E}-09 \\
7.046 \mathrm{E}-09 \\
1.788 \mathrm{E}-\mathrm{C} 8\end{array}$ & $\begin{array}{l}2.8 \mathrm{C} 3 \mathrm{E}-10 \\
2.812 \mathrm{E}-10 \\
2.851 \mathrm{E}-10 \\
2.916 \mathrm{E}-10 \\
3.017 \mathrm{E}-10 \\
3.156 \mathrm{E}-10 \\
3.365 \mathrm{E}-10 \\
3.665 \mathrm{E}-10 \\
4.071 \mathrm{E}-10 \\
4.600 \mathrm{E}-10 \\
5.285 \mathrm{E}-10 \\
6.233 \mathrm{E}-10 \\
7.702 \mathrm{E}-10 \\
1.02 \mathrm{CE}-\mathrm{C} \\
1.471 \mathrm{E}-09 \\
2.330 \mathrm{E}-09 \\
4.783 \mathrm{E}-09\end{array}$ & $\begin{array}{l}9.996 \mathrm{E}-11 \\
1.003 \mathrm{E}-10 \\
1.015 \mathrm{E}-10 \\
1.038 \mathrm{E}-10 \\
1.073 \mathrm{E}-10 \\
1.122 \mathrm{E}-10 \\
1.194 \mathrm{E}-10 \\
1.295 \mathrm{E}-10 \\
1.429 \mathrm{E}-10 \\
1.604 \mathrm{E}-10 \\
1.832 \mathrm{E}-10 \\
2.151 \mathrm{E}-10 \\
2.638 \mathrm{E}-10 \\
3.442 \mathrm{E}-10 \\
4.824 \mathrm{E}-10 \\
7.289 \mathrm{E}-10 \\
1.301 \mathrm{E}-09\end{array}$ & $\begin{array}{l}3.332 \mathrm{E}-11 \\
3.342 \mathrm{E}-11 \\
3.382 \mathrm{E}-11 \\
3.455 \mathrm{E}-11 \\
3.569 \mathrm{E}-11 \\
3.733 \mathrm{E}-11 \\
3.966 \mathrm{E}-11 \\
4.286 \mathrm{E}-11 \\
4.710 \mathrm{E}-11 \\
5.260 \mathrm{E}-11 \\
5.985 \mathrm{E}-11 \\
7.000 \mathrm{E}-11 \\
8.535 \mathrm{E}-11 \\
1.100 \mathrm{E}-10 \\
1.509 \mathrm{E}-10 \\
2.200 \mathrm{E}-10 \\
3.571 \mathrm{E}-10\end{array}$ \\
\hline $70 F-0$ & $.076 \mathrm{E}-\mathrm{Cg}$ & $.6997 E-C 9$ & $8.593 \mathrm{E}-10$ \\
\hline
\end{tabular}


COSINE

$-1.00000 E 00$ $-9.894 C 1 E-01$ $-9.44575 \mathrm{E}-01$ $-8.65631 E-01$ $-7.55044 E-01$ $-6.17876 \mathrm{E}-01$ $-4.58017 \mathrm{E}-01$ $-2.81605 E-01$ $-9.50125 \mathrm{E}-\mathrm{C} 2$ 9.50125E-02 $2.81605 E-01$ $4.58017 \mathrm{E}-01$ $6.17876 \mathrm{E}-01$ $7.55044 E-01$ $8.65631 \mathrm{E}-01$ $9.44575 E-01$
$9.89401 E-C 1$

TOTAL

COSINE

$-1.000 C O E$ OC $-9.89401 \mathrm{E}-01$ $-8.65631 E-01$ $-7.55044 E-01$ $-6.17876 \mathrm{E}-01$ $-4.58017 E-01$ $-2.816 C 5 E-01$ $90125 E-02$ 2.0125 $4.58017 E-01$ $6.17876 \mathrm{E}-01$ 7. $55044 \mathrm{E}-01$ $8.65631 E-01$ $9.44575 \mathrm{E}-01$ $9.89401 \mathrm{E}-01$

TOTAL

$\begin{array}{cc}75.0 & 10 C .0 \\ 4.236 E-10 & 4.652 E-1 C \\ 4.431 E-10 & 4.868 E-10 \\ 4.843 E-10 & 5.347 E-1 C \\ 5.095 E-10 & 5.642 E-1 C \\ 5.034 E-1 C & 5.591 E-10 \\ 5.254 E-10 & 5.842 E-1 C \\ 5.457 E-10 & 6.05 C E-10 \\ 6.385 E-10 & 7.146 E-10 \\ 7.834 E-1 C & 8.917 E-1 C \\ 1.354 E-C 9 & 1.133 E-C 9 \\ 1.627 E-C 9 & 1.397 E-09 \\ 1.747 E-09 & 1.972 E-09 \\ 2.772 E-C 9 & 3 . C 41 E-09 \\ 7.156 E-C 9 & 6 . C 47 E-C 9 \\ 2.334 E-08 & 1.404 E-C 8 \\ 4.446 E-08 & 4.082 E-C 8 \\ 2.692 E-C 7 & 2.586 E-07 \\ 9.452 E-C 8 & 8.526 E-08\end{array}$

150.0
$4.955 E-10$
$.172 \mathrm{E}-10$
$.688 \mathrm{E}-10$
$.986 \mathrm{E}-10$
$.969 \mathrm{E}-10$
$6.234 \mathrm{E}-10$
$.471 \mathrm{E}-10$
$.754 \mathrm{E}-10$
$.773 \mathrm{E}-10$
$.197 \mathrm{E}-09$
$.593 \mathrm{E}-\mathrm{C}$
$.128 \mathrm{E}-09$
$.448 \mathrm{E}-09$
$.268 \mathrm{E}-\mathrm{C}$
$9.767 \mathrm{E}-\mathrm{C} 9$
$3.137 \mathrm{E}-08$
$2.266 \mathrm{E}-\mathrm{C}$
$.238 \mathrm{E}-08$

RANGE (METERS)
200.0

250.0

$300 . n$

$40 \mathrm{c} .0$

4. $931 E-10$

$5.124 E-10$

$4.698 E-1 C$

$4.861 \mathrm{E}-1 \mathrm{C}$

$5.286 \mathrm{E}-10$

$5.795 \mathrm{E}-10 \quad 5.362 \mathrm{E}-10$

$5.901 \mathrm{E}-10$

$6.175 \mathrm{E}-10$

$6.545 E-10$

$7.872 \mathrm{E}-10$

$9.947 \mathrm{E}-10$

$1.379 E-09$

$1.542 \mathrm{E}-09$

2.086E-09

2.6CC.E-09

$4.141 E-09$

8.220E-09

$2.512 E-08$

OE-07

$6.260 E-C 8$

$5.777 \mathrm{E}-10$
$5.814 \mathrm{E}-1 \mathrm{C}$

$6.277 E-10$

$7.577 E-10$

$9.576 \mathrm{E}-1 \mathrm{C}$

$1.475 E-09$

$2.0 C S E-09$

$2.489 \mathrm{E}-\mathrm{C} 9$

$7.586 E-09$

2.083E-08

1. $516 \mathrm{E}-07$

5. $371 E-08$

RANGE (METERS)

$500 . c$

600.0

900.0

1200.0

$150 C .0$

E

$4.489 E-10$

$4.850 \mathrm{E}-10$
$4.947 \mathrm{E}-10$

5. 320E-1C

$5.377 E-10$

$5.846 E-10$

7. $C 70 E-10$

$8.918 E-10$

$1.466 E-09$

$1.782 E-C 9$

$2.387 E-C 9$

7.024E 09

$1.778 E-08$

1.210E-C7

4. $580 E-C 8$

$3.626 \mathrm{E}-10$

$3.874 E-10$
$3.981 E-10$

$4.265 \mathrm{E}-10$

$4.353 E-10$

$4.786 E-10$

$5.788 \mathrm{E}-10$

$7.263 E-10$

$1.165 E-09$

$1.929 E-09$

$3.009 \mathrm{E}-09$

$5.663 E-09$

$7.578 \mathrm{E}-08$

$3.287 E-08$

1800.0

$2.742 \mathrm{E}-1 \mathrm{O}$

$7.808 \mathrm{E}-11 \quad 2.681 \mathrm{E}-11$

$8.677 E-12$ 2.200E-10 $8.513 E-1122.797 E-1129.320 E-12$ 2.428E-1C $9.022 \mathrm{E}-1123.063 \mathrm{E}-11$ $9.3239 \mathrm{E}-12 \quad 3.042 \mathrm{E}-12$ $3.365 \mathrm{E}-1 \mathrm{O} 2.524 \mathrm{E}-10 \quad 9.538 \mathrm{E}-113.26 \mathrm{EE}-1101.055 \mathrm{E}-1123.266 \mathrm{E}-12$ $3.365 E-10$ 2.524E-10 $3.407 E-10 \quad 3.372 E-10 \quad 1.259 E-10$ $4.260 E-11 \quad 1.358 E-11-4.165 E-12$ 4.494E-10 $3.372 E-10$ 1.25EE-10 4.26CE-11 $1.358 E-11$ 4.165E-12 $\begin{array}{llllll}7.128 \mathrm{E}-10 & 5.277 \mathrm{E}-10 & 1.906 \mathrm{E}-10 & 6.259 \mathrm{E}-11 & 1.951 \mathrm{E}-11 & 5.883 \mathrm{E}-12\end{array}$ $\begin{array}{llllll}8.897 \mathrm{E}-10 & 6.561 \mathrm{E}-10 & 2.349 \mathrm{E}-10 & 7.672 \mathrm{E}-11 & 2.383 \mathrm{E}-11 & 7.164 \mathrm{E}-12\end{array}$ $1.109 \mathrm{E}-\mathrm{C9} \quad 8.2 \mathrm{C} 3 \mathrm{E}-1 \mathrm{C} \quad 2.958 \mathrm{E}-10 \quad 9.708 \mathrm{E}-11 \quad 3.025 \mathrm{E}-11 \quad 9.108 \mathrm{E}-12$ $\begin{array}{llllll}1.487 \mathrm{E}-C 9 & 1.107 \mathrm{E}-09 & 4 . C 47 \mathrm{E}-10 & 1.338 \mathrm{E}-10 & 4.180 \mathrm{E}-11 & 1.259 \mathrm{E}-11\end{array}$ $4.339 \mathrm{E}-\mathrm{Cg} \quad 3.209 \mathrm{E}-09 \quad 1.143 \mathrm{E}-\mathrm{C} 9 \quad 3.663 \mathrm{E}-10 \quad 1.11 \mathrm{CE}-10 \quad 3.248 \mathrm{E}-11$ $\begin{array}{llllll}4.332 \mathrm{E}-\mathrm{C} 9 & 6.93 \mathrm{CE}-\mathrm{C} 9 & 2.309 \mathrm{E}-09 & 7.038 \mathrm{E}-10 & 2.04 \mathrm{E}-10 & 5.248 \mathrm{E}-11\end{array}$ $\begin{array}{llllll}4.721 \mathrm{E}-\mathrm{C} 8 & 2.941 \mathrm{E}-\mathrm{CB} & 7.183 \mathrm{E}-\mathrm{CO} & 1.789 \mathrm{E}-09 & 4.5428 \mathrm{E}-1 \mathrm{C} & 1.160 \mathrm{E}-1 \mathrm{C}\end{array}$

$2.320 E-C 8 \quad 1.617 E-C 8 \quad 5.198 E-C 9 \quad 1.591 E-09 \quad 4.719 E-10 \quad 1.369 E-10$ 
COSINE $-9.89401 \mathrm{E}-01$ $-8.65631 \mathrm{E}-01$ $-7.55044 \mathrm{E}-01$ $-6.17876 E-01$ $-4.58017 E-01$ -2.81605 E-01 $-9.5 C 125 E-02$ $9.50125 \mathrm{E}-02$ $2.81605 E-01$ 4. $58017 E-01$ $6.17876 \mathrm{E}-01$ $7.55044 \mathrm{E}-01$ $8.65631 E-01$ $9.44575 \mathrm{E}-J 1$
$9.89401 \mathrm{E}-01$

TOTAL

COSINE

-1.00000 E 00 $-9.89401 \mathrm{E}-01$ $-9.44575 E-01$ $-8.65631 E-01$ $-7.55044 \mathrm{E}-01$ $-6.17876 E-01$ $-4.58017 \mathrm{E}-0$ $-2.81605 E-01$ -9.50125E-02 $9.50125 \mathrm{E}-02$ $2.81605 E-01$ 4.58017E-O I $6.17876 E-01$ $7.55044 \mathrm{E}-01$ $8.65631 \mathrm{E}-01$ $9.88401 \mathrm{E}-01$

TOTAL

\begin{tabular}{|c|c|c|c|c|c|c|}
\hline 75.0 & $1 C C . C$ & 150.0 & $\begin{array}{c}\text { NGE }(M E T E R S) \\
2 C C \cdot C\end{array}$ & 250.0 & 300.0 & 400.6 \\
\hline $016 \mathrm{E}-10$ & $1.240 E-10$ & $1.565 E-10$ & $1.770 \mathrm{E}-10$ & $1.873 \mathrm{E}-10$ & $1.897 E-10$ & $1.785 E-10$ \\
\hline & $1.255 E-10$ & $1.581 \mathrm{E}-10$ & $1.785 \mathrm{E}-10$ & $1.886 \mathrm{E}-1 \mathrm{C}$ & $1.909 \mathrm{E}-1 \mathrm{C}$ & $1.793 \mathrm{E}-10$ \\
\hline $.060 \mathrm{E}-10$ & $1.29 \mathrm{CE}-1 \mathrm{C}$ & $1.621 \mathrm{E}-10$ & $1.825 \mathrm{E}-1 \mathrm{C}$ & $1.924 \mathrm{E}-10$ & $1.944 \mathrm{E}-10$ & $1.822 \mathrm{E}-10$ \\
\hline $.080 \mathrm{E}-1 \mathrm{C}$ & $1.315 \mathrm{E}-1 \mathrm{C}$ & $1.651 \mathrm{E}-10$ & $1.851 E-10$ & $1.947 \mathrm{E}-10$ & $1.972 \mathrm{E}-10$ & $1.854 \mathrm{E}-10$ \\
\hline & $1.324 \mathrm{E}-1 \mathrm{C}$ & $1.670 E-10$ & $1.887 \mathrm{E}-10$ & $2.012 E-10$ & $2.038 \mathrm{E}-10$ & $1.917 \mathrm{E}-10$ \\
\hline-10 & $1.368 \mathrm{E}-1 \mathrm{C}$ & $1.729 E-10$ & $1.958 \mathrm{E}-10$ & $2.074 \mathrm{E}-10$ & $2.107 \mathrm{E}-10$ & $1.991 \mathrm{E}-10$ \\
\hline$E-10$ & $1.428 E-10$ & $1.810 E-10$ & $2.065 \mathrm{E}-10$ & $2.198 \mathrm{E}-1 \mathrm{C}$ & $2.238 E-10$ & $2.121 \mathrm{E}-10$ \\
\hline & $1.575 \mathrm{E}-1 \mathrm{C}$ & $1.997 \mathrm{E}-10$ & $2.275 \mathrm{E}-10$ & $2.419 E-10$ & $2.461 \mathrm{E}-$ & $2.328 \mathrm{E}-1 \mathrm{C}$ \\
\hline $.459 \mathrm{E}-10$ & $1.796 \mathrm{E}-1 \mathrm{C}$ & $2.272 \mathrm{E}-10$ & $2.579 E-10$ & $2.734 E-10$ & $2.773 \mathrm{E}-1 \mathrm{C}$ & $2.611 E-10$ \\
\hline $.207 \mathrm{E}-1 \mathrm{C}$ & $2.145 \mathrm{E}-1 \mathrm{C}$ & $2.688 \mathrm{E}-10$ & $3.0 \mathrm{CgE}-10$ & $3.175 \mathrm{E}-10$ & $3.190 E-10$ & $2.986 \mathrm{E}-10$ \\
\hline & $2.446 \mathrm{E}-10$ & $3.074 \mathrm{E}-10$ & $3.482 E-10$ & $3.665 E-10$ & $3.712 \mathrm{E}-10$ & $3.454 E-10$ \\
\hline .648 & $3.053 \mathrm{E}-1 \mathrm{C}$ & $3.777 \mathrm{E}-10$ & $4.159 E-10$ & $4.362 E-10$ & $4.366 \mathrm{E}-10$ & $4.055 \mathrm{E}-10$ \\
\hline-10 & $4.370 \mathrm{E}-1 \mathrm{C}$ & $4.561 \mathrm{E}-10$ & $5.1 C 2 \mathrm{E}-10$ & $5.325 \mathrm{E}-10$ & $5.353 \mathrm{E}-10$ & $4.947 \mathrm{E}-10$ \\
\hline & $6.829 \mathrm{E}-1 \mathrm{C}$ & $6.322 E-10$ & $6.921 \mathrm{E}-10$ & $7.164 \mathrm{E}-10$ & $7.120 \mathrm{E}-1 \mathrm{C}$ & $6.510 E-10$ \\
\hline $.963 \mathrm{E}-\mathrm{Cg}$ & $1.324 \mathrm{E}-\mathrm{CO}$ & $1.169 \mathrm{E}-09$ & $1.115 \mathrm{E}-09$ & $1.110 E-09$ & $1.082 \mathrm{E}-09$ & $9.619 \mathrm{E}-10$ \\
\hline & $3.463 E-09$ & $2.9 C 2 E-C 9$ & $2.564 E-09$ & $2.301 \mathrm{E}-09$ & $2.092 \mathrm{E}-09$ & $1.710 \mathrm{E}-09$ \\
\hline & $1.967 \mathrm{E}-\mathrm{C} 8$ & $1.764 \mathrm{E}-08$ & $1.497 E-C 8$ & $1.238 \mathrm{E}-08$ & $1.014 \mathrm{E}-\mathrm{C} 8$ & $6.714 \mathrm{E}-09$ \\
\hline $1 \bar{E}$ & $8.238 \mathrm{E}-09$ & $8.015 E-C 9$ & $7.777 E-C 9$ & $7.412 E-09$ & $6.960 E-09$ & $5.900 \mathrm{E}-\mathrm{Cg}$ \\
\hline
\end{tabular}

\section{RANGE (METERS)}

500.0

600.0

$1.554 \mathrm{E}-1 \mathrm{C}$

$1.561 \mathrm{E}-1 \mathrm{C}$

$1.584 E-10$

$2.615 E-10$

1.670 E-1C

$1.741 \mathrm{E}-1 \mathrm{C}$

$1.857 \mathrm{E}-10$

$2.034 E-10$

$2.273 E-1 C$

$2.588 E-10$

2.981E-1C

$3.493 E-1 C$

$4.249 \mathrm{E}-1 \mathrm{C}$

$5.541 E-10$

$8 . C 11 E-10$

4. $431 E-C 9$

4.799E-C 9
$1.285 \mathrm{E}-10$

$1.29 \mathrm{CE}-1 \mathrm{~S}$

$1.308 E-10$

$1.335 E-10$

$1.382 \mathrm{E}-1 \mathrm{C}$

$1.443 \mathrm{E}-10$

$1.541 \mathrm{E}-1 \mathrm{C}$

$1.686 \mathrm{E}-1 \mathrm{C}$

$1.878 \mathrm{E}-10$

$2.131 E-10$

$2.448 E-1 C$

$2.863 E-1 C$

$3.473 E-1 C$

$4.494 \mathrm{E}-1 \mathrm{C}$

$6.383 \mathrm{E}-1 \mathrm{C}$

$1.032 E-C 9$

$3.782 E-C O$
900.0

1200.0

1500.0

$5.984 E-11$
$6.003 E-11$

6. $(83 \mathrm{E}-11$

$6.224 E-11$

$6.448 E-11$

$6.763 E-11$

$7.227 E-11$

$7.873 \mathrm{E}-11$

$8.730 E-11$

$9.832 \mathrm{E}-11$

$1.309 E-10$

$1.578 \mathrm{E}-10$

2. CC5E-10

$2.738 \mathrm{E}-10$

$8.564 E-10$

$2.344 E-11$

$2.351 \mathrm{E}-11$

$2.382 \mathrm{E}-11$

$2.439 E-11$

$2.529 \mathrm{E}-11$

$2.657 \mathrm{E}-11$

$2.84 C E-11$

$3.087 \mathrm{E}-11$

$3.412 \mathrm{E}-11$

$3.827 \mathrm{E}-11$

$4.358 E-11$

$6.077 E-11$

$7.627 E-11$

$1.014 \mathrm{E}-10$

$2.528 \mathrm{E}-10$

$1.617 \mathrm{E}-\mathrm{C} 9$

$6.057 E-10$
$8.293 E-12$

$8.319 \mathrm{E}-12$

$8.429 E-12$

.637E-12

. $960 \mathrm{E}-12$

$9.422 E-12$

$1.006 E-11$

$1.093 \mathrm{E}-11$

$1.205 E-11$

1. $533 \mathrm{E}-11$

. $778 \mathrm{E}-11$

$2.125 E-11$

$2.642 E-11$

$3.449 E-11$

$7.442 \mathrm{E}-11$

$2.085 E-1 C$
1800.0

$2.745 \mathrm{E}-12$

$2.753 E-12$

$2.790 E-12$

$2.860 \mathrm{E}-12$

.968E-12

.

.

$3.617 \mathrm{E}-12$

3. $983 E-12$

4. $450 E-12$

.

.

.

$10 \mathrm{E}-11$

$1.493 E-11$

$2.172 \mathrm{E}-11$

$6.775 E-11$ 
4 PI R**2 HENDERSCN DOSE (GAMMAS)
(CM**2 RAD/STERADIAN/SOURCE NEUTRON)

COSINE

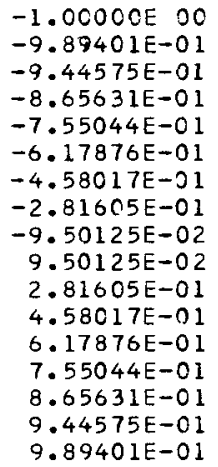

TOTAL

COSINE

$-1.00000 E$ OO $-9.89401 \mathrm{E}-01$

$-9.44575 \mathrm{E}-01$

$-8.65631 \mathrm{E}-01$

$-7.55044 \mathrm{E}-01$

$-6.17876 E-01$

$-4.581605 \mathrm{E}-01$

$-2.81605 E-01$

$-9.50125 \mathrm{E}-02$

$2.81605 \mathrm{E}-01$

$4.58017 E-01$

$4.58017 E-01$

7.55044E-0

$8.65631 \mathrm{E}-\mathrm{C}$

$44575 \mathrm{E}-\mathrm{C}$

$9.89401 \mathrm{E}-01$

TOTAL

\section{0}

$1.823 \mathrm{E}-1$ $1.834 \mathrm{E}-11$

$1.876 \mathrm{E}-11$

.069E- 11

$2.069 \mathrm{E}-11$
$2.221 \mathrm{~F}-11$

$2.422 \mathrm{E}-11$

$2.422 \mathrm{E}-1$

$3.053 \mathrm{E}-11$

$3.732 \mathrm{E}-11$

$5.01 \mathrm{CE}-11$

$5.972 \mathrm{E}-11$

6.C77E- 11

8.C34E-11

1. $327 \mathrm{E}-10$

$2.114 \mathrm{E}-10$

$3.170 \mathrm{E}-10$

$6.537 E-10$

500.0

. 252E-11

$1.285 \mathrm{E}-11$

$1.402 \mathrm{E}-11$

$1.572 \mathrm{E}-1$

$1.729 E-11$

$1.856 \mathrm{E}-1$

$1.992 \mathrm{E}-1$

$2.211 \mathrm{E}-1$

$2.589 \mathrm{E}-1$

$3.177 \mathrm{E}-11$

4.C18E-1

$5.235 \mathrm{E}-1$

$7.214 E-11$

1. C91E-10

$1.870 E-1 C$

$3.791 \mathrm{E}-10$

$1.032 E-C 9$

$8.543 E-10$
100.0

$2.107 E-11$

$2.206 \mathrm{E}-11$

$2.345 E-11$

$2.526 E-11$

$3.084 \mathrm{E}-11$

$3.523 E-11$

5.C6OE-11

5.1C3E-11

$5.418 E-11$

$9.268 \mathrm{E}-11$

$1.454 \mathrm{E}-1 \mathrm{C}$

$2.745 E-1 C$

$4.768 \mathrm{E}-1 \mathrm{C}$

$7.996 \mathrm{E}-1 \mathrm{C}$

600.0

$9.449 E-12$

$9.767 E-12$

$1.085 \mathrm{E}-11$
$1.236 \mathrm{E}-11$

. $367 \mathrm{E}-11$

$1.461 \mathrm{E}-11$

$1.551 E-11$

$1.713 \mathrm{E}-11$

$2.016 \mathrm{E}-11$

$2.499 \mathrm{E}-11$

$3.184 E-11$

$4.163 \mathrm{E}-11$

$5.769 E-11$

$8.869 \mathrm{E}-11$

$1.559 \mathrm{E}-1 \mathrm{C}$

$3.249 E-1 C$

$9.196 \mathrm{E}-1 \mathrm{C}$
2.C $39 E-11$

$1.108 E-10$

$150 . \mathrm{C}$

RANGE (METERS)
$200 . \mathrm{C}$

$25 C .0$

$30 \mathrm{C.C}$

$40 C \cdot C$

$2.214 \mathrm{E}-11$

$2.220 \mathrm{E}-11$

$2.336 E-11$

$2.820 E-11$

C

3. $455 \mathrm{E}-11$

. $065 \mathrm{E}-11$

4. (39E-11

$6.019 \mathrm{~F}-11$

$6.918 \mathrm{E}-1$

. $065 \mathrm{E}-10$

1.207E-10

$2.203 E-10$

7.45 SE-10

$7.306 E-13$

$2.490 \mathrm{E}-1$

$2.9 \mathrm{C} 1 \mathrm{E}-11$

$3.176 \mathrm{E}-11$

$3.555 E-11$
$4.097 E-11$

$4.097 E-11$
$5.196 E-11$

$5.196 \mathrm{E}-11$

$5.674 \mathrm{E}-11$
$7.865 \mathrm{E}-11$

$9.907 \mathrm{E}-11$

$1.475 E-10$

$2.291 E-10$

$4.117 E-10$

$9.041 E-10$

$9.709 E-10 \quad 1.071 E-09$

$2.122 \mathrm{E}-11$

$2.254 E-11$

$2.425 E-11$

$2.622 E-11$

$3.103 E-11$

$3.472 E-11$

$4.015 E-11$

$5.145 E-11$

$7.858 E-11$

$9.95 \mathrm{CE}-11$

$1.492 \mathrm{E}-10$

$2.356 \mathrm{E}-1 \mathrm{C}$

$4.41 C E-10$

$1.019 E-09$

$1.102 \mathrm{E}-09$

RANGE (METERS)

900.0

1200.0

1500.0

$3.662 E-12$

$3.902 E-12$

$5.554 \mathrm{E}-12$

$6.212 \mathrm{E}-12$

$6.472 \mathrm{E}-12$

$6.620 E-12$

$.194 \mathrm{E}-12$

$8.644 E-12$

$1.1 \mathrm{C8E-11}$

$1.437 \mathrm{E}-11$

$1.877 E-11$

$2.617 \mathrm{E}-11$

4.203E-11

$7.986 E-11$

5.581E-10

$1.299 \mathrm{E}-12$

$1.442 \mathrm{E}-12$

$1.832 \mathrm{E}-12$

$2.36 \mathrm{EE}-12$

$2.718 \mathrm{E}-12$

$2.599 \mathrm{E}-12$

$2.848 \mathrm{E}-12$

$3.515 \mathrm{E}-12$

$4.678 E-12$

$0.128 \mathrm{E}-12$

$7.893 \mathrm{E}-12$

$1.091 \mathrm{E}-11$

$1.822 \mathrm{E}-11$

$3.741 \mathrm{E}-11$

$3.020 \mathrm{E}-10$

$4.396 E-13$

$5.186 E-13$

$7.393 E-13$

$.783 \mathrm{E}-13$
$.157 \mathrm{E}-12$

$1.098 \mathrm{E}-12$

$1.025 \mathrm{E}-12$

$1.114 E-12$

$1.415 E-12$

$1.964 \mathrm{E}-12$

$2.595 \mathrm{E}-12$

$3.252 \mathrm{E}-12$

7. $402 E-12$

$7.641 \mathrm{E}-12$

$1.694 E-11$

$4.386 E-11$

$1.547 \mathrm{E}-10$

$1.721 E-10$

7. $952 E-11$

$1.972 \mathrm{E}-11$

$.002 E-11$

$2.115 \mathrm{E}-11$

$2.294 \mathrm{E}-11$

$2.492 \mathrm{E}-11$

$.939 E-11$

3. $285 \mathrm{E}-11$

3. $808 \mathrm{E}-11$

$4.513 \mathrm{E}-11$

5. $749 E-11$

$7.260 \mathrm{E}-11$

$9.938 \mathrm{E}-11$

$1.440 \mathrm{E}-10$

$.353 E-10$

$1.084 E-09$

$1.088 \mathrm{E}-\mathrm{C} 9$

$1.608 \mathrm{E}-1$

$1.762 \mathrm{E}-11$

$1.943 \mathrm{E}-11$

$2.125 \mathrm{E}-11$

$2.292 E-11$
$2.481 E-11$

$2.481 E-11$

$2.764 E-11$
$3.221 E-11$

$3.919 \mathrm{E}-11$

$4.905 E-11$

$8.695 \mathrm{E}-11$

$1.293 \mathrm{E}-10$

$2.159 \mathrm{E}-10$

$4.254 E-10$

1800.0

$.0 C 9 E-13$

$4.186 \mathrm{E}-13$

5.025E-1

$4.539 \mathrm{E}-13$

. $996 \mathrm{E}-13$

4. $467 \mathrm{E}-13$

$5.787 \mathrm{E}-13$

8. $398 \mathrm{E}-13$

1.115E-12

$1.343 \mathrm{E}-12$

$1.753 E-12$

$3.183 \mathrm{E}-12$

. S97E-12

$7.688 \mathrm{E}-11$

$3.648 E-11$ 
COS I NE

$-1.00000 E 00$ $-9.89401 E-01$ $-9.44575 E-01$ $-8.65631 \mathrm{E}-01$ $-7.55044 E-01$ $-6.17876 E-01$ $-4.58017 E-01$ $-2.81605 \mathrm{E}-01$ $-9.50125 \mathrm{E}-02$ $9.50125 E-02$ $2.816 \mathrm{C} 5 \mathrm{E}-01$ 4.58017E-01 $7.17876 E-01$ $7.55044 E-C 1$ $8.65631 E-01$ $9.89401 E-01$

TOTAL

COSINE

$-1.00000 E$ OC $-9.894 \mathrm{ClE}-01$ $-9.44575 E-01$ $-7.55044 E-01$ $-6.17876 \mathrm{E}-01$ $-4.58017 E-01$ $-2.81605 E-01$ $-9.50125 \mathrm{E}-02$ $9.50125 \mathrm{E}-02$ $2.81605 E-01$ 4. $58017 E-01$ $6.17876 E-01$ 7.55044E-01 $8.65631 \mathrm{E}-01$ $9.44575 E-01$ $9.89401 E-C 1$

TOTAL
75.0

100.0

$150 . \mathrm{C}$

RANGE (METERS)
200.0

$250 . \mathrm{C}$

300.0

$400 . \mathrm{C}$

1. $849 E-09$

$1.859 \mathrm{E}-09$

$1.901 E-C 9$

$1.979 \mathrm{E}-\mathrm{C} 9$

2.C92E-09

$2.242 \mathrm{E}-\mathrm{C} 9$
$2.441 \mathrm{E}-\mathrm{C} 9$

$2.706 \mathrm{E}-09$

3.064E-09

$3.737 E-C 9$

4.967E-C9

$5.971 E-09$

$6.048 E-C 9$

$7.974 \mathrm{E}-\mathrm{C} 9$

$1.314 E-C 8$

$3.134 E-C 8$

$2.093 E-C 9$
$2.107 E-09$

$2.160 E-09$

$2.258 \mathrm{E}-\mathrm{C} 9$

$2.395 \mathrm{E}-\mathrm{C} 9$

$2.575 E-09$

$2.810 E-C 9$

$3.128 E-09$

$3.562 E-09$

.

.

$4.716 E-08$

$4.715 E-C B$

$6.514 E-C 8$

$7.996 E-C 8$

$500 . \mathrm{C}$

600.0

$1.563 E-C 9$

$1.884 \mathrm{E}-\mathrm{C} 9$

$2.045 \mathrm{E}-\mathrm{C} g$

$2.179 E-C 9$

$2.324 \mathrm{E}-\mathrm{r} 9$

$2.552 \mathrm{E}-\mathrm{C} 9$

$2.939 E-C 9$

$3.534 E-09$

$4.378 \mathrm{E}-\mathrm{C} 9$

$5.594 E-09$

$7.556 \mathrm{E}-\mathrm{C} 9$

$1.120 E-C 8$

$1.887 \mathrm{E}-\mathrm{CB}$

$3.783 E-C 8$

$1.027 E-07$

$8.926 E-08$
$2.333 E-09$

$2.353 E-09$

$2.426 \mathrm{E}-09$

$2.556 \mathrm{E}-09$

$2.726 \mathrm{E}-09$

$2.936 \mathrm{E}-09$

$3.204 E-09$

4. $C 74 E=09$

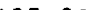

6.

1. $207 \mathrm{E}-0 \mathrm{~B}$

$2.191 \mathrm{E}-08$

(.429E-08

$2.403 E-09$
$2.427 E-09$

$2.427 \mathrm{E}-09$

$2.518 E-09$
$2.672 E-C 9$

$2.672 \mathrm{E}-\mathrm{C} 9$

$2.861 \mathrm{E}-09$

$3.083 \mathrm{E}-09$

$3.359 \mathrm{E}-\mathrm{C9}$

$3.738 E-C 9$

$4.279 \mathrm{E}-09$

$5.373 E=09$

$5.843 E-C 9$

(2)

$1.003 E-C 8$

$2.481 \mathrm{E}-08$

$2.285 E-C 8$

$4.058 E-08$

$9.784 E-C 8$

$1.087 E-07$

RANGE (METERS)

900.0

1200.0

1500.0

$.358 E-09$
$.387 E-09$

$2.49 C \mathrm{E}-09$

$2.660 E-09$

$2.858 \mathrm{E}-09$

$3.078 \mathrm{E}-09$

.

.717E-09

4.261E-0

4.

(

$.014 \mathrm{E}-08$

1.506E-08

.

1.01CE-07

$1.125 E-07$

$2.247 E-C 9$

$1.923 E-09$

$2.076 \mathrm{E}-09$

$2.570 \mathrm{E}-\mathrm{C} 9 \quad 2.258 \mathrm{E}-09$

$2.769 \mathrm{E}-\mathrm{C9} \quad 2.443 \mathrm{E}-09$

$2.977 E-C 9 \quad 2.617 E-09$

$3.226 \mathrm{E}-\mathrm{Cg} \quad 2.813 \mathrm{E}-09$

$3.577 E-C 9 \quad 3.105 E-09$

$4.104 E-C 9 \quad 3.570 E-09$

6.8CGE-CQ $4.272 E-C 9$

7.541E-CQ $6.715 E-09$

$1.019 E-C B$.

$1.459 E-C B \quad 1.320 E-0 B$

$1.320 E-08$

$4.453 E-C 8 \quad 4.241 E-C B$

$1.076 \mathrm{E}-\mathrm{C} 7 \mathrm{1} \quad \mathrm{.096 \textrm {E } - \mathrm { C } 7}$

$1.118 E-C 7 \quad 1.030 E-07$

1800.0
$1.228 \mathrm{E}-\mathrm{C} 9$

$8.176 E-11$

$3.166 \mathrm{E}-11$

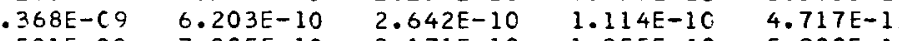

$1.521 E-C 9$

$1.756 E-C 9$

$1.855 E-C 9$

2.C26E-Cg

$2.337 \mathrm{E}-09$

$2.827 E-09$

$3.517 \mathrm{E}-\mathrm{C} 9$

$4.495 \mathrm{E}-09$

$6.089 E-09$

$9.144 E-C 9$

$1.576 \mathrm{E}-08$

3. $244 E-08$

$7.205 \mathrm{E}-10$

$3.171 \mathrm{E}-10$

$1.114 \mathrm{E}-1 \mathrm{C}$

5.900E-11

$.397 E-10 \quad 3.476 E-10 \quad 1.431 E-10$

$9.030 E-10 \quad 3.750 E-10$

$1.53 \mathrm{E}-09 \quad 4.439 \mathrm{E}-10 \quad 1.841 \mathrm{E}-1 \mathrm{C} \quad 7.715 \mathrm{E}-11$

$\begin{array}{ll}1.301 \mathrm{E}-\mathrm{C} 9 & 5.618 \mathrm{E}-10 \quad 2.395 \mathrm{E}-10 \\ 1.633 \mathrm{O}-09 & 1.034 \mathrm{E}-10\end{array}$

$1.633 \mathrm{E}-09 \quad 7.081 \mathrm{~F}-10 \quad 3.031 \mathrm{E}-1 \mathrm{C} \quad 1.311 \mathrm{E}-10$

$\begin{array}{llll}1.074 \mathrm{E}-09 & 8.852 \mathrm{E}-10 & 3.692 \mathrm{E}-10 & 1.541 \mathrm{E}-10\end{array}$

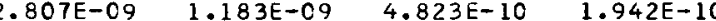

$\begin{array}{llll}4.365 E-09 & 1.899 E-09 & 7.979 E-10 & 3.329 E-10\end{array}$

$\begin{array}{ll}4.078 \mathrm{E}-09 \quad 3.776 \mathrm{E}-09 & 1.7 \mathrm{C} 6 \mathrm{E}-\mathrm{C} 9\end{array}$

$\begin{array}{llll}1.797 E-08 & 9.036 E-09 & 4.358 E-09 & 2.062 E-C 9 \\ 5.567 E-08 & 3.016 E-08 & 1.547 E-08 & 7.696 E-09\end{array}$

$3.838 \mathrm{E}-\mathrm{C} 8$

$1.823 E-08$

$8.414 E-09$

$3.855 \mathrm{E}-\mathrm{C} 9$ 
4 PI R**2 AIR KERMA (GAMMAS)
$($ CM**2 ERGS/GRAM/STERADIAN/SOURCE NEUTRON)

\section{COSINE

$-1.00000 E 00$
$-9.89401 \mathrm{E}-01$
$-9.44575 \mathrm{E}-01$
$-8.65631 \mathrm{E}-01$
$-7.55044 \mathrm{E}-01$
$-6.17876 \mathrm{E}-01$
$-4.58017 \mathrm{E}-01$
$-2.81605 \mathrm{E}-01$
$-9.50125 \mathrm{E}-02$
$9.50125 \mathrm{E}-02$
$2.81605 \mathrm{E}-01$
$4.58017 \mathrm{E}-01$
$6.17876 \mathrm{E}-01$
$7.55044 \mathrm{E}-01$
$8.65631 \mathrm{E}-01$
$9.44575 \mathrm{E}-01$
$9.894 \mathrm{C} 1 \mathrm{E}-01$

TOTAL

COS INE

-1.0 COCOE 50 $-9.89401 E-01$ $-9.44575 \mathrm{E}-01$ $-8.65631 E-01$ $-7.55044 E-C 1$ $-6.17876 E-01$ $-4.58017 \mathrm{E}-01$ $-2.81605 E-01$ $-9.50125 E-02$ $9.50125 \mathrm{E}-\mathrm{C2} 2$ 2. $81605 \mathrm{E}-01$ $4.58017 \mathrm{E}-01$ 6.17876E-01 $7.55044 E-01$ $8.65631 \mathrm{E}-\mathrm{Cl}$ $9.44575 \mathrm{E}-01$
$9.89401 \mathrm{E}-01$

TOTAL

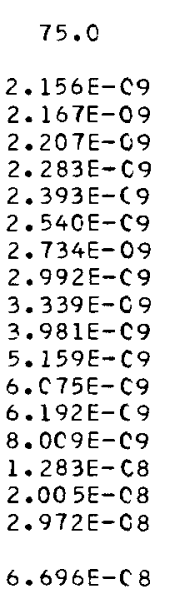

100.0

$2.627 E-C 9$
$2.641 E-09$
.64 $2.790 \mathrm{E}-\mathrm{C} 9$ $2.927 \mathrm{E}-\mathrm{CO}$ 3.1C7E-09 $3.343 E-09$ $3.659 \mathrm{E}-\mathrm{C} 9$ $4.088 E-C$ $5.522 \mathrm{E}-\mathrm{C} 9$ $5.607 E-C 9$ $5.922 \mathrm{E}-\mathrm{C} 9$ $9.507 \mathrm{E}-\mathrm{C}$ $1.121 \mathrm{E}-\mathrm{C}$ $1.443 \mathrm{E}-\mathrm{C} 8$ $2.628 \mathrm{E}-\mathrm{C} 8$ $4.481 E-08$ $8.457 E-C 8$

$500 . \mathrm{C}$

600.0

$3.7 \mathrm{ClE}-\mathrm{Cg}$ $3.735 \mathrm{E}-\mathrm{C} 9$ $3.861 \mathrm{E}-\mathrm{C} 9$ 4. C52E-C $4.249 E-C 9$ $4.441 \mathrm{E}-\mathrm{C} 9$ $4.663 \mathrm{E}-\mathrm{C} 9$ $4.983 E-09$ $5.475 E-C 9$ $6.184 \mathrm{E}-09$ $7.153 \mathrm{E}-09$ $8.498 \mathrm{E}-\mathrm{C} 9$ $1.057 E-C 8$ $1.422 \mathrm{E}-\mathrm{C} 8$ $2.161 \mathrm{E}-\mathrm{CB}$ $3.938 E-C 8$
$9.905 E-08$

\section{$3.141 \mathrm{E}-\mathrm{C} 9$} $3.174 E-09$ $3.289 \mathrm{E}-\mathrm{C} 9$ $3.460 \mathrm{E}-00$ $3.629 E-C 9$ $3.783 E-C 9$ $3.954 \mathrm{E}-\mathrm{C} 9$ $4.210 E-C 9$ $4.618 E-C 9$ $5.213 E-C 9$ 6.C $18 \mathrm{E}-\mathrm{C}$ $7.118 E-C 9$ $8.816 \mathrm{E}-\mathrm{C} 9$ $1.189 E-C 8$ $1.827 E-C 8$ $3.389 \mathrm{E}-\mathrm{C} 8$ $8.821 \mathrm{E}-\mathrm{C} 8$

$1.202 E-C 7 \quad$ I.C $23 E-C 7$
10.000 TO 12.200 MEV NEUTRON SOURCE

$\begin{array}{ccccc} & \text { RANGE (METERS) } & & & \\ 150.0 & 20 C . C & 250.0 & 300.0 & 400 . C \\ 3.348 E-C 9 & 3.85 C E-09 & 4.146 E-09 & 4.272 E-09 & 4.140 E-09 \\ 3.367 E-09 & 3.875 E-09 & 4.176 E-09 & 4.304 E-C 9 & 4.174 E-09 \\ 3.442 E-09 & 3.969 E-09 & 4.284 E-09 & 4.423 E-C 9 & 4.302 E-C 9 \\ 3.575 E-09 & 4.131 E-09 & 4.467 E-09 & 4.619 E-C 9 & 4.504 E-09 \\ 3.752 E-09 & 4.336 E-09 & 4.688 E-09 & 4.847 E-C 9 & 4.725 E-09 \\ 3.973 E-09 & 4.581 E-C 9 & 4.943 E-09 & 5.100 E-C 9 & 4.954 E-09 \\ 4.257 E-09 & 4.89 C E-09 & 5.258 E-09 & 5.408 E-C 9 & 5.225 E-09 \\ 4.638 E-C 9 & 5.307 E-09 & 5.687 E-C 9 & 5.832 E-C 9 & 5.607 E-09 \\ 5.161 E-09 & 5.888 E-09 & 6.294 E-09 & 6.440 E-09 & 0.173 E-09 \\ 5.292 E-C 9 & 6.997 E-09 & 7.461 E-09 & 7.239 E-C 9 & 6.987 E-09 \\ 7.191 E-09 & 7.550 E-09 & 8.044 E-C 9 & 8.551 E-C 9 & 8.094 E-09 \\ 8.085 E-C 9 & 9.695 E-09 & 1.028 E-08 & 1.014 E-08 & 9.667 E-09 \\ 1.162 E-09 & 1.172 E-08 & 1.239 E-08 & 1.283 E-C 8 & 1.206 E-08 \\ 1.3 C 1 E-08 & 1.632 E-08 & 1.716 E-C 8 & 1.716 E-C 8 & 1.623 E-08 \\ 2.225 E-08 & 2.395 E-08 & 2.528 E-C 8 & 2.578 E-08 & 2.442 E-08 \\ 3.384 E-08 & 4.082 E-08 & 4.427 E-08 & 4.540 E-C 8 & 4.382 E-08 \\ 6.908 E-C 8 & 8.593 E-C 8 & 9.719 E-08 & 1.037 E-C 7 & 1.058 E-07 \\ 1.093 E-07 & 1.267 E-07 & 1.361 E-C 7 & 1.395 E-C 7 & 1.345 E-07\end{array}$

\section{RANGE (METERS)}

SCO.O

$1200 . \mathrm{C}$

$150 \mathrm{C} .0$

$18 \times C . C$

$1.621 \mathrm{E}-09$
$1.645 \mathrm{E}-09$

$1.645 \mathrm{E}-09$
$1.714 \mathrm{E}-09$

$1.823 \mathrm{E}-\mathrm{C} 9$

$1.911 \mathrm{E}-\mathrm{C} 9$

$1.974 \mathrm{E}-\mathrm{C} 9$

2.C38E-09

$2.152 \mathrm{E}-09$

$2.356 E-C 9$

$2.663 \mathrm{E}-09$

$3.060 E-C 9$

$3.573 E-09$

$5.342 \mathrm{E}-09$

$5.947 E-09$

$9.517 \mathrm{E}-\mathrm{C} 9$
$1.875 \mathrm{E}-\mathrm{C} 8$
$5.320 \mathrm{E}-\mathrm{C}$

$1.875 E-C 8$
$5.320 t-C 8$

$7.445 \mathrm{E}-10$

$7.586 E-10$

$7.985 \mathrm{E}-10$

$8.554 \mathrm{E}-10$

$8.961 \mathrm{E}-10$

$9.205 \mathrm{E}-10$

$9.852 \mathrm{E}-1 \mathrm{C}$

$1.079 \mathrm{E}-09$

$1.224 E-59$

$1.401 \mathrm{E}-09$

$1.613 E-09$

$1.944 E-09$

$2.668 E-09$

$4.468 E-C 9$

$2.859 \mathrm{E}-08$

$3.294 \mathrm{E}-1 \mathrm{C}$
$3.37 \mathrm{E}-1 \mathrm{C}$

$3.37 C E-10$
$3.59 C E-10$

$3.848 \mathrm{E}-10$

$4.072 E-10$

$4.105 \mathrm{E}-10$

$4.146 \mathrm{E}-10$

$4.355 E-10$

$4.777 E-10$

6.

7.

.

..

2. $21 \mathrm{E}-09$

$2.021 E-09$

$1.456 E-C 8$

$5.418 \mathrm{E}-\mathrm{CB}$

$2.583 E-08$

$1.188 \mathrm{E}-08$

$1.447 E-10$

$1.486 E-10$

$1.600 \mathrm{E}-1 \mathrm{C}$

$1.725 \mathrm{E}-10$

1.829E-1C

$1.822 \mathrm{E}-10$

$1.921 \mathrm{E}-1 \mathrm{C}$

$2.1 \mathrm{C} 4 \mathrm{E}-1 \mathrm{C}$
$2.414 \mathrm{E}-1 \mathrm{C}$

$2.414 E-1 C$

$2.050 E-10$

3. $531 \mathrm{E}-1 \mathrm{C}$

9.041E-10

$9.041 E-10$

$2.108 \mathrm{E}-\mathrm{C}$

$5.41 C E-C 9$ 
COSINE

$-1.00000 E$ C.C. $-9.89401 \mathrm{E}-01$ $-9.44575 E-01$
$-8.65631 E-C 1$ $-7.55044 E-01$ $-7.55044 E-01$
$-6.17876 E-01$ $-4.58017 E-01$ $-2.81605 E-01$ $-9.50125 E-C 2$ 9. $50125 \mathrm{E}-02$ $2.816 \mathrm{C} 5 \mathrm{E}-01$ 4.58017E-C1 . $17876 \mathrm{E}-01$ $8.65631 \mathrm{E}-01$ $9.44575 E-01$

.894C1E-

TOTAL

COSINE

$-1.0000 C E$ CO $-9.89401 \mathrm{E}-01$
$-9.44575 \mathrm{E}-01$ $-9.44575 E-01$ $-8.65631 \mathrm{E}-01$ $-7.55044 \mathrm{E}-01$ $-6.17876 E-01$ $-4.58017 \mathrm{E}-01$ $-2.81605 E-C 1$ $-9.50125 E-92$ $9.50125 \mathrm{E}-02$ $2.816 C 5 E-01$ 4. $58017 \mathrm{E}-01$ $6.17876 \mathrm{E}-01$ $7.55044 \mathrm{E}-01$ 8. $65631 \mathrm{E}-\mathrm{Cl}$ $9.44575 \mathrm{E}-01$ $9.89401 \mathrm{E}-0$

TOTAL
75.0

$1.899 E-09$ $1.910 E-C 9$ $1.910 E-C$ $1.952 \mathrm{E}-\mathrm{C} 9$
$2.032 \mathrm{E}-\mathrm{C} 9$ $2.032 E-C 9$ $2.147 E-C$ $2.503 \mathrm{E}-\mathrm{C} 9$ 2. $773 \mathrm{E}-\mathrm{C}$ $3.138 \mathrm{E}-\mathrm{C}$ $3.825 \mathrm{E}-09$ $5.074 E-C 9$ $6.113 \mathrm{E}-0$ $6.181 \mathrm{E}-\mathrm{C} 9$ $8.146 \mathrm{E}-\mathrm{C} 9$ $1.342 \mathrm{E}-\mathrm{C}$ $2.136 E-08$ $3.203 E-08$

$6.663 E-08$

$100 \cdot 0$

$2.158 \mathrm{E}-\mathrm{C} 9$ $2.326 \mathrm{E}-\mathrm{C} .9$ $2.466 \mathrm{E}-0 \mathrm{O}$ $2.649 \mathrm{E}-\mathrm{C}$

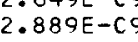
$3.212 \mathrm{E}-\mathrm{C}$ $3.654 \mathrm{E}-\mathrm{C} 9$ $5.193 \mathrm{E}-\mathrm{C} 9$ $5.268 \mathrm{E}-\mathrm{C}$ $5.553 \mathrm{E}-\mathrm{C}$ $0.443 E-C$ $1.125 \mathrm{E}-\mathrm{C} 8$ $1.473 \mathrm{E}-\mathrm{C} 8$ $2.774 E-C 8$ $4.819 E-C 8$

$8.186 E-C 8$

$50 \mathrm{C} .0$

600.0

$1.729 E-C 9$ $1.763 E-C 9$ $1.882 \mathrm{E}-\mathrm{C}$ $.057 \mathrm{E}-\mathrm{C}$ $2.220 E-C 9$ $2.356 \mathrm{E}-\mathrm{C}$ . $502 E-C$ $2.732 E-C$ $3.124 \mathrm{E}-\mathrm{C} 9$ 3. 72 6E-C $4.581 \mathrm{E}-09$ $5.812 E-C 9$ $7.800 E-C$ $1.151 \mathrm{E}-\mathrm{C}$ $1.931 E-C$ $3.869 \mathrm{E}-\mathrm{C}$ $1.051 \mathrm{E}-07$

$9.256 E-C 8$

$1.376 E-C 9$

1. $468 E-C 9$

$1.675 E-C$

$1.812 E-C 9$

$2.013 \mathrm{E}-\mathrm{C} Q$

$2.013 \mathrm{E}-\mathrm{C} 9$
$2.184 \mathrm{E}-\mathrm{Cg}$ $2.499 \mathrm{E}-\mathrm{C}$ $2.995 \mathrm{E}-\mathrm{C}$ $3.693 \mathrm{E}-\mathrm{C}$ $4.682 \mathrm{E}-\mathrm{C}$ $6.295 \mathrm{E}-\mathrm{C}$ $9.396 \mathrm{E}-\mathrm{C}$ $1.613 E-C 8$ $3.318 \mathrm{E}-\mathrm{O}$ $9.382 E-C 8$

$7.736 E-08$
150.0 RANGE (METERS)
200.0

$2.427 E-C 9$
$2.447 E-C 9$ $2.447 E-C 9$ $2.521 \mathrm{E}-\mathrm{C} 9$
$2.654 \mathrm{E}-09$ $2.827 E-09$ 3.C4OE-C. $3.313 E-C 9$ $3.683 E-C 9$ $4.199 E-09$ $4.264 E-C 9$ $6.282 \mathrm{E}-09$ $7.153 E-09$ .234E-08 $2.239 E-C 8$ $3.503 E-08$

$2.523 E-09$
$2.548 E-09$ $2.540 E-09$ $2.797 \mathrm{E}-09$ $2.990 \mathrm{E}-09$ $3.215 \mathrm{E}-09$ $3.496 \mathrm{E}-09$ $3.881 \mathrm{E}-09$ $4.431 E-09$ $5.545 \mathrm{E}-09$ $6.019 \mathrm{E}-09$ $1.028 \mathrm{E}-08$ $1.515 \mathrm{E}-08$ $2.335 \mathrm{E}-08$ $4.177 E-08$ $9.158 E-08$

$1.117 \mathrm{E}-07$

R.ANGE (METERS)

Q. $0.0 \quad 12 C 0.0$

$2.498 \mathrm{E}-1$

$0.133 E-10$

-10
$E-10$ 3.042 $3.584 \mathrm{E}-10$ 7. $C 47 \mathrm{E}-10$ $8.0 .71 E-1 C$ 作 $9.070 E-10$ 9.267 E-10 $9.897 E-10$ $1.392 \mathrm{E}-09$ $1.727 E-C 9$ $2.171 E-C 9$ $2.911 \mathrm{E}-09$ $4.490 E-09$ $8.269 E-C 9$ $1.838 \mathrm{E}-08$ $5.71 \mathrm{CE}-08$ $3.980 E-10$ $3.884 E-10$ $4.155 E-1 C$ $4.849 E-10$ 6. $642 E-10$ $7.519 E-10$ $9.295 \mathrm{E}-10$ $1.229 E-09$ $1.953 \mathrm{E}-09$ $3.864 \mathrm{E}-09$ $9.243 \mathrm{E}-09$ $3.096 E-08$
250.0 $2.501 E-09$ $2.635 E-09$ 2.809E-09 $3.011 \mathrm{E}-09$ 3. $233 E-09$ 3. $505 \mathrm{E}-09$ 3. $882 E-09$ $4.434 E-09$ $5.582 \mathrm{E}-09$ $6.051 E-09$ $8.318 E-09$ $1.041 \mathrm{E}-08$ $1.541 E-08$ $2.409 \mathrm{E}-08$ $4.481 E-08$ 1. $C 33 E-07$

$1.158 \mathrm{E}-07$

1500.0

9. $895 \mathrm{E}-11$ $.0294 \mathrm{E}-10$ $.541 \mathrm{E}-10$ $1.727 E-10$ $1.678 \mathrm{E}-10$ $1.613 E-10$ $1.710 \mathrm{E}-10$ $2.024 E-10$ $2.584 \mathrm{E}-1 \mathrm{C}$ $3.226 \mathrm{E}-1 \mathrm{C}$ $3.885 \mathrm{E}-1 \mathrm{C}$ $5.014 E-10$ $8.202 E-10$ $1.744 \mathrm{E}-\mathrm{C} 9$ $4.458 E-09$ $1.589 E-\cap 8$

$8.765 E-09$
300.0

400.0

$2.406 E-C 9 \quad 2.095 E-09$ $2.437 E-C 9 \quad 2.129 E-C 9$ $2.552 \mathrm{E}-\mathrm{C} 9 \quad 2.251 \mathrm{E}-\mathrm{C} 9$ $2.938 \mathrm{E}-09 \quad 2.625 \mathrm{E}-0$. $3.149 E-C 9 \quad 2.801 E-09$ $3.401 \mathrm{E}-\mathrm{Cg} \quad 3.000 \mathrm{E}-\mathrm{Cg}$ $3.756 \mathrm{E}-\mathrm{C} 9 \quad 3.295 \mathrm{E}-09$ $4.291 \mathrm{E}-09 \quad 3.765 \mathrm{E}-09$ $5.0 C 7 E-C 9 \quad 4.477 E-09$ $6.258 E-C 9 \quad 5.478 E-C 9$ $7.780 \mathrm{E}-\mathrm{C} 9 \quad 6.953 \mathrm{E}-09$ $1.047 \mathrm{E}-\mathrm{C} 8 \quad 9.294 \mathrm{E}-09$ $1.495 \mathrm{E}-\mathrm{C} 8 \quad 1.355 \mathrm{E}-08$ $2.413 \mathrm{E}-\mathrm{C} 8 \quad 2.223 \mathrm{E}-08$ $\begin{array}{ll}4.551 E-C 8 & 4.336 E-08 \\ 1.101 E-C 7 & 1.122 E-07\end{array}$

$1.153 E-C 7$

$1.066 E-07$

1800.0

3.921E-11 . $328 E-11$ .515E-11 $6.638 \mathrm{E}-11$ .149E-11

$.118 \mathrm{E}-1 \mathrm{C}$

$1.397 \mathrm{E}-1 \mathrm{C}$

$1.624 E-10$

$2.021 \mathrm{E}-10$

$3.419 E-10$

$7.794 \mathrm{E}-10$

$2.109 E-C 9$

$.914 \mathrm{E}-\mathrm{C} 9$

4.C $15 E-C 9$ 
(NEUTRONS/MEV/STERADIAN/SOURCE NEUTRON)

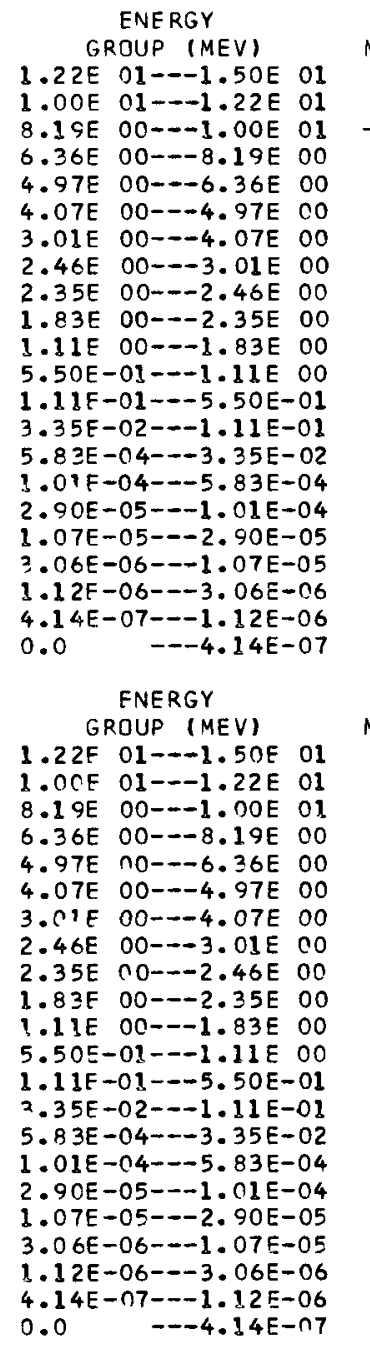

ANGLE 1 ANGLE 2 $=-1.0000$ 0.0

$-6.622 \mathrm{E}-04$ $2.006 \mathrm{E}-03$ $1.45 \mathrm{E}-03$ $1.392 \mathrm{E}-03$
$2.198 \mathrm{E}-03$ $2.198 \mathrm{E}-03$
$2.846 \mathrm{E}-03$ $2.846 E-03$ $2.808 \mathrm{E}-03$ $4.168 \mathrm{E}-03$ $4.168 \mathrm{E}-03$ $2.609 \mathrm{E}-02$ $2.609-02$ $1.181 \mathrm{E} \mathrm{CO}$ $4.937 E 00$ $1.408 \mathrm{E}$ OI $3.683 \mathrm{E}$ OI $9.693 \mathrm{E}$ OI $2.089 E 02$ 2.987E 02 ANGLE 10 0.0 0.0 1. $010 \mathrm{E}-03$ $5.866 \mathrm{E}-03$ $2.779 \mathrm{E}-03$ 2.244E-03 2. $977 E-03$ $4.269 \mathrm{E}-03$ $6.530 E-03$ $4.882 \mathrm{E}-03$ $5.529 \mathrm{E}-03$ $5.801 \mathrm{E}-03$ $2.796 \mathrm{E}-02$ $2.159 \mathrm{E}-02$ $1.217 E 00$ $5.064 \mathrm{E} 00$ $1.441 \mathrm{E}$ 01 $\begin{array}{ll}3.761 \mathrm{E} & 01 \\ 9.883 \mathrm{E} & 01\end{array}$ $9.883 \mathrm{E}$ OI $3.037 \mathrm{E}$ O2

0.0

.

$1.463 E-03$

$1.305-03$

2.

$2.817 \mathrm{E}-03$

$2.817 E-03$

$4.176 \mathrm{E}-03$

$5.111 E-03$

$5.111 \varepsilon-03$

$2.610 E-02$

2. $178 \mathrm{E}-02$

4.939800

$1.409 E 01$

$3.683 \mathrm{E}$ तl

$9.604 E 01$

$2.090 E$ O2

$2.988 \mathrm{~F} 22$

ANGLE 11

$M U=0$
0.0
0.0

$1.150 E-03$

$7.792 E-03$

$3.275 E-03$

$2.631 E-03$

$2.446 E-03$

$3.819 \mathrm{E}-03$

$5.033 E-C 3$

$5.363 E-C 3$

$6.057 \mathrm{E}-03$

$6.057 E-03$
$5.716 E-03$

$2.893 E-02$

$2.175 \mathrm{E}-02$

$1.223 E 00$

$5.087 \mathrm{E}$ CO

$1.447 \mathrm{E}$ OI

$3.774 \mathrm{E} 01$

$9.916 \mathrm{E} \mathrm{Cl}$

$2.134 \mathrm{E} 02$

$3.046 E 02$
$M U=-0.08 \times 4 \quad M U=-0.9446$

$-5.373 E-04 \quad-2.348 E-04$

3.077E-03
0.0
0.0

$1.975 \mathrm{E}-03$

$3.408 E-03$

$2.223 E-03$

$2.873 E-03$

$3.246 \mathrm{E}-03$

$4.206 E-03$

$4.206 E-03$

2. $615 E-02$

$2.08 J E-02$

$4.943 E$ OO

$1.410 E$ O1

$3.686 \mathrm{E} 01$

$9.702 \mathrm{E}$ O1

$2.091 \mathrm{E} 02$

ANGLE 4 0.

$3.367 \mathrm{E}-05$ $2.839 \mathrm{E}-03$ $1.947 E-03$ (1) (. (2) (1) $3.315 \mathrm{E}-03$ $4.267 E-03$ $5.186 E-03$ $2.627 E-02$ $2.627 \mathrm{E}-02$
$2.086 \mathrm{E}-02$ $2.086 \mathrm{E}-02$
$1.185 \mathrm{E} 00$ $4.952 \mathrm{E} 00$ $1.412 \mathrm{E} 01$ $3.692 \mathrm{E}$ 01 $0.715 \mathrm{E}$ 01 $2.094 E \quad 02$

ANGLE 12 0.0

$2.161 E-03$ $8.977 E-03$ $3.590 \mathrm{E}-03$ $2.891 E-03$ $2.766 E-03$ $4.318 E-03$ $5.563 E-03$ $6.102 E-03$ $6.169 F-03$ $6.470 E-03$ 2.308 $2.912 E-02$
$2.190 E-02$ $1.230 E 00$ $5.109 \mathrm{E}$ OO $1.453 \mathrm{E} 01$ $3.788 \mathrm{E} 01$ $9.948 \mathrm{E} 01$ $2.141 E$ O2 $3.054 \mathrm{E} 02$
$M U=0.6179$ 0.0 8.560E-03 $8.710 E-03$ $3.857 \mathrm{E}-03$ $3.090 \mathrm{E}-03$ 2.977E-03 $4.673 E-03$ $5.987 E-03$ $6.574 E-03$ $6.578 E-03$ $6.955 E-03$ $7.471 \mathrm{E}-03$ $2.785 \mathrm{E}-02$ $2.204 \mathrm{E}-02$ $1.235 \mathrm{E} 00$ $5.128 E 00$ $1.458 \mathrm{E}$ ol $3.799 \mathrm{E}$ Ol $9.977 \mathrm{E} 01$ $2.146 \mathrm{E} 02$ $3.061 E 02$
ANGLE 5 0.7550 $\quad M U=-0.6179$ $1.004 E-04$ $2.725 \mathrm{E}-03$ .940E-O3 .5 . $324 \mathrm{E}-03$

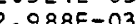
. $3.022 E-03$
$3.420 E-03$ $4.359 E-03$ $4.359 E-03$
$5.258 E-03$ $2.642 \mathrm{E}-02$ $2.094 E-02$
$1.189 E 00$ $4.965 E 00$ $1.416 E$ Ol $3.700 E$ O1 $9.734 E \quad 01$ $3.000 E \quad 02$

ANGLE 14 0.0 3. $316 E-02$ $1.084 E-02$ 4. $833 E-03$ $3.799 E-03$ $3.747 \varepsilon-03$ $5.899 \mathrm{E}-03$ $7.445 E-03$ $8.307 E-03$ $7.897 \varepsilon-03$ $7.746 \mathrm{E}-03$ $7.814 \varepsilon-03$ 2. $840 E-02$ $1.240 E 00$ $5.146 E$ OO $1.462 \mathrm{E} 01$ $3.810 \mathrm{E}$ O1 $1.000 E$ O2 $2.151 E \quad 02$ $3.068 E 02$
ANGLE 6 0.0

$1.513 \mathrm{E}-04$ $2.860 E-03$ $1.974 E-03$ $1.531 \mathrm{E}-03$ . $3.094 \mathrm{E}-03$ . $3.565 \mathrm{E}-03$ $4.485 E-03$ $4.485 E-03$
$5.355 E-03$ $2.662 E-02$ $2.104 \mathrm{E}-02$ $1.193 E 00$ $4.980 E$ OO $1.420 E$ OI $3.709 \mathrm{E}$ OI $9.758 \mathrm{E}$ Ol $2.102 E 02$ $3.006 \mathrm{E} 02$

ANGLE 15 0.

$1.256 \mathrm{E}-01$ $1.799 E-02$ $6.811 E-03$ $5.314 E-03$ $5.449 E-03$ $1.574 \mathrm{E}-03$ $1.054 \mathrm{E}-02$ $1.218 E-02$ $0.05 E-02$ $7.121 E-03$ $3.109 E-02$ $2.230 E-02$ $1.245 \mathrm{E} 00$ $5.160 E 00$ $1.466 E$ OI $3.818 \mathrm{E} 01$ $1.002 \mathrm{E} 02$ $2.155 \mathrm{E} 02$ $3.073 E \quad 02$

ANGLE $?$ $3.289 \mathrm{E}-03$ .063E-03 $1.705 E-03$ $1.611 \mathrm{E}-03$ $534 \mathrm{E}-03$ $.245 E-03$ $3.757 \mathrm{E}-03$ $4.650 E-03$ $5.475 E-03$ $2.686 E-02$ $2.115 E-02$ $1.198 \mathrm{E} 00$ $4.999 E 00$ $4.999 E$
$1.424 E$ $3.721 \mathrm{E}$ O1 $9.785 \mathrm{E} 01$ $3.013 E 02$

ANGLE 16 0.0

2.510E-01 $2.845 E-02$ $7.315 \mathrm{E}-03$ $7.728 E-03$ 
ENERGY GROUP (MEV) $1.22 E$ O1-D-1.50E 01 $1.00 E$ O1---1.22E 01 $6.36 E$ O0- $-8.19 E$ OO $4.97 E$ 00-- $6.36 \mathrm{E}$ OO 4.07E $00--4.97 E$ OO $3.01 E$ OD- $-4.07 E$. 00 $2.46 \mathrm{E} \quad 00--3.01 \mathrm{E} 00$ $2.35 \mathrm{E} \quad 00--2.46 \mathrm{E} 00$ $1.83 \mathrm{E} \quad 00--2.35 \mathrm{E} 00$ $1.11 E$ E0-- $1.83 E 00$ $5.50 E-01--1.11 E 00$ $1.11 E-01--5.50 E-01$ $3.35 \mathrm{E}-02---1.11 \mathrm{E}-01$ $5.83 \mathrm{E}-04--3.35 \mathrm{E}-02$ 1.01E-04---5.83E-04 $2.90 E-05--1.01 E-04$ $1.07 E-05--2.90 E-05$ $3.06 E-06--1.07 E-05$ $1.12 E-06---3.06 E-06$ $4.14 \mathrm{E}-07---1.12 \mathrm{E}-06$
0.0 ENERGY GROUP (MEV) $1.22 E$ O1---1.5OE Ol $1.00 E$ 01---1.22E OI

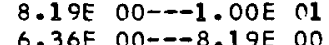

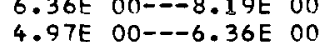
$4.97 E$
$4.00-07 E$ OO-- $0.36 E$

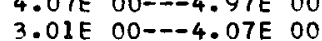

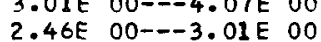

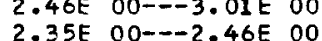
$1.83 \mathrm{E} 00--2.35 \mathrm{E}$ OO $1.11 \mathrm{E}$ 00---1.83E 00 $5.50 E-01-1.11 \mathrm{E} 00$ $1.11 \mathrm{E}-01=-5$. $50 \mathrm{E}-01$ $3.35 \mathrm{E}-02-\cdots-1.11 \mathrm{E}-01$ $5.83 E-04 \cdots-3.35 E-02$ 1. $01 \mathrm{E}-04--5.83 \mathrm{E}-04$ 2.90E-05--1.01E-04 $1.07 E-05--2.90 \mathrm{E}-05$ 3.06E-06--1.07E-05 $1.12 \mathrm{E}-06--3.06 \mathrm{E}-06$ $4.14 \mathrm{E}-07=-1.12 \mathrm{E}-06$ $0.0 \quad--4.14 E-07$
ANGLE 1 0.0 0.0
$-8.042 E-04$ $3.042 \mathrm{E}-04$
$3.636 \mathrm{E}-03$ $3.034 \mathrm{E}-03$ $2.646 \mathrm{E}-03$ $2.469 \mathrm{E}-03$ $3.857 \mathrm{E}-03$ $5.190 \mathrm{E}-03$ $4.836 E-03$ $6.100 E-03$ $9.611 E-03$ $1.431 \mathrm{E}-02$ $8.514 \mathrm{~F}-02$ $7.101 E-02$ 4.144E 00 $1.756 \mathrm{E} 01$ $5.052 \mathrm{E} \mathrm{OI}$ $1.332 \mathrm{E} \mathrm{O2}$ $3.524 \mathrm{E} \mathrm{O2}$ $7.630 \mathrm{E} \mathrm{O2}$ $1.096 \mathrm{E} 03$ ANGLE 10 $M U=0.0950$ 0.0
0.0 0.0
$5.601 E-04$ $5.582 E-03$ $6.116 E-03$ $5.257 E-03$ $3.009 E-03$ 4.817E-03 $6.585 \mathrm{E}-03$ $6.585 E-03$ $1.157 \mathrm{E}-02$ $1.423 \mathrm{E}-02$ $1.362 E-02$ $9.607 E-02$ 7.5 $4.363 E$ O $5.271 \mathrm{E}$ O1 $1.385 \mathrm{E} 02$ $3.656 \mathrm{E} 02$ 1.131E 03
(NEUTRONS/MEV/STERADIAN/SOURCE NEUTRON)

$\begin{array}{ll}A N G L E & 2 \\ M U=-0.9894 & \end{array}$ 0.0 $\begin{array}{ll}A N G L E & 4 \\ M U=-0.8656 & \end{array}$ 0.0 0.0
$9.521 E-05$ 2. $983 \mathrm{E}-03$ $2.967 E-03$ $2.736 \mathrm{E}-03$ $2.525 \mathrm{E}-03$ $3.953 \mathrm{E}-03$ $5.304 E-03$ $5.018 \mathrm{E}-03$ $6.321 E-03$ $9.874 \mathrm{E}-03$ $1.459 \mathrm{E}-02$ $8.598 \mathrm{E}-02$ 7.151 E-02 $4.169 \mathrm{E} 00$ $1.766 \mathrm{E} 01$ $5.078 E$ O1 $1.338 \mathrm{E}$ O2 $3.540 E$ O2 $7.662 \mathrm{E} \mathrm{O2}$ $1.101 E 03$ ANGLE 13 $M U=0.6179$ 0.0 $3.669 \mathrm{E}-03$ $1.280 \mathrm{E}-02$ $7.853 \mathrm{E}-03$ $6.544 \mathrm{E}-03$ $5.979 E-03$ $9.544 E-03$ $1.288 \mathrm{E}-02$ $1.337 \mathrm{E}-02$ $1.395 \mathrm{E}-02$ $1.729 \mathrm{E}-02$ $1.895 \mathrm{E}-02$ $7.80 \mathrm{E}$ $4.483 E 00$ $4.483 E$ O $1.882 E$ OI $1.412 E \quad 02$ $3.723 \mathrm{E} 02$ $3.723 \mathrm{E} 02$
$8.036 \mathrm{E} 02$ $1.149 E \quad 3$
ANGLE 5
$M U=-0.7550 \quad M U=-0.6179$ 0.0 $1.888 \mathrm{E}-04$ $2.847 \mathrm{E}-03$ $2.991 E-03$ $2.824 E-03$ $2.582 \mathrm{E}-03$ $4.048 \mathrm{E}-03$ $5.426 E-03$ $5.195 \mathrm{E}-0$ $6.526 \mathrm{E}-03$ $1.011 \mathrm{E}-02$ $1.483 \mathrm{E}-02$ $8.671 E-02$ $7.193 \mathrm{E}-02$ $4.190 E \quad 00$ $1.773 \mathrm{E}$ O1 $5.099 E$ OI $1.343 E$ O2 $3.552 \mathrm{E} 02$ $7.688 E$ O2 $1.105 E \quad 03$

ANGLE 14 MU $=$ 0.0 $8.249 E-03$ $1.801 E-02$ $8.763 \mathrm{E}-03$ $7.165 E-03$ $6.623 E-03$ $1.082 E-02$ $1.433 E-02$ $1.480 \mathrm{E}-02$ $1.494 \mathrm{E}-02$ 1.860E-02 2.213E-02 7.81 E 02 . 4.516E OO $5.418 \mathrm{E}$ OI $5.418 E$
$1.420 E$ O2 $3.742 \mathrm{E} 02$ $3.742 E$
$8.073 E \quad 02$ $1.153 \mathrm{E} 03$
0.0

$2.113 E-04$

$3.062 E-03$

.1 $00 E-03$

$2.667 \mathrm{E}-03$

$4.188 \mathrm{E}-03$

$5.615 \mathrm{E}-03$

$5.450 E-03$

$6.812 E-03$

$1.044 \mathrm{E}-02$

$8.764 \mathrm{E}-02$

$7.247 E-02$

$4.217 E$ O

$1.783 \mathrm{E}$ O1
$5.125 \mathrm{E}$ ol

1.350 O2

$3.569 \mathrm{E} \quad 02$

$7.721 \mathrm{E} 02$

2.109E 03

ANGLE 15 $M U=0.8656$ 0.0

$3.404 \mathrm{E}-02$

$2.305 \mathrm{E}-02$

$1.303 \mathrm{E}-02$

$1.031 E-02$

. $555 \mathrm{E}-02$

$2.094 \mathrm{E}-02$

2.125E-O2

2.011E-02

$2.216 E-02$

1.021 E-01

$.952 \mathrm{E}-02$

$4.544 E$ O

$.444 E$ OI

$1.426 E$ O2

8.103E 02

1.157E 03
NGLE 7 0.0

$1.342 E-05$ $3.680 \mathrm{E}-03$ 3. $314 \mathrm{E}-03$ $3.117 \mathrm{E}-03$ $2.790 E-03$ $4.389 E-03$ $5.895 \mathrm{E}-03$ 5. $801 E-03$ $7.193 \mathrm{E}-03$ $1.088 \mathrm{E}-02$ . $556 \mathrm{E}-02$ $8.876 \mathrm{E}-02$ $7.312 \mathrm{E}-02$ $4.248 \mathrm{E}$ OI
$5.157 \mathrm{E}$ OI 5.157E OI $1.357 \mathrm{E}$ O2 $3.588 E$ O2 7.760 E 02 $1.114 E 03$

ANGLE 16 MU $=$
0.0 0.0

$1.591 E-01$ $4.059 \mathrm{E}-02$ $1.736 \mathrm{E}-02$ 1. 1. . . $2.473 \mathrm{E}-02$ $2.539 \mathrm{E}-02$ .565E OO .911E OI $1.430 \mathrm{E}$ O2 3.767E 02 $8.125 \mathrm{E} 02$ 1.159E 03

$\begin{array}{ll}\text { ANGLE } & 8 \\ M U=-0.2816 & \text { ANGLE } 9 \\ \text { MU }=-0.0950\end{array}$ 0.0 $5.335 E-05$ $4.618 E-03$ $3.633 \mathrm{E}-03$ $3.341 \mathrm{E}-03$ .963E-03 $6.295 E-03$ $.688 E-03$ $1.143 \mathrm{E}-02$ $007 \mathrm{E}-02$ $9.007 \mathrm{E}-02$
$7.386 \mathrm{E}-02$ $4.284 E O O$ $1.808 E$ O1 $5.193 E$ O1 $1.366 E$ O2 $3.609 E$ O2 $7.803 \mathrm{E} \mathrm{O2}$ 

ENERGY
GRDUP (MEV) $1.22 \mathrm{E} 01--1.50 \mathrm{E}$ $1.00 \mathrm{E}$ 01-- $1.22 \mathrm{E}$ 01 $8.19 E$ OO--1. COE OI $4.36 E$ OO- $-1.19 E$ OO 4.07E $00-4.97 \mathrm{E} 00$ $3.01 E$ OO-- $4.07 E 00$ $2.46 E$ OO- $-3.01 E$ DO $2.35 E$ OO-D-2.46E 00 $1.83 \mathrm{E} 00---2.35 \mathrm{E} 00$ $1.11 \mathrm{E} \quad 00--1.83 \mathrm{E} \quad 00$ $5.50 E-01--1.11 \mathrm{E}$ OO $1.11 \mathrm{E}-01--5 \cdot 50 \mathrm{E}-01$ $3.35 E-02--1.11 E-01$ $5.83 E-04---3.35 E-0$ ? $2.90 E-05--1.0$ $1.07 E-05--2.90 E-05$ $3.06 \mathrm{E}-06--1.07 \mathrm{E}-0 \mathrm{~F}$ $1.12 \mathrm{E}-06---3.06 \mathrm{E}-06$ $4.14 E-07--1.12 E-06$
$0.0 \quad--0.14 E-07$ ENERGY GROUP (MEV)

$1.22 \mathrm{E}$ O1---1.50E 01 $1.00 E$ O1- - 1.22E OI $8.19 E$ OO---1.00E 01 6.36E $00--8.19 E \quad 00$ $4.97 E$
$4.00--07 E$ OD-36E OO 4.07E $00--4.97 E$ DO 3.01E $00---4.07 E 00$ $2.46 \mathrm{E} \quad 00--3.01 \mathrm{E} \quad 00$

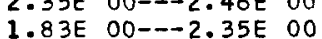
$\begin{array}{lll}1.83 \mathrm{E} & 00---2.35 \mathrm{E} & 00 \\ 1.13 \mathrm{E} & 00--1.83 \mathrm{E} & 00\end{array}$ 5.50E-01---1.11E 00 $1.11 E-01--5.50 E-01$ $3.35 \mathrm{E}-02-1.11 \varepsilon-01$ $5.83 E-04--3.35 E-n 2$ $5.83 E-04---3.35 E-12$
$1.01 E-04--5.83 E-04$ $2.90 \mathrm{E}-05-\cdots 1.01 \mathrm{E}-04$ $1.07 \mathrm{E}-05--2.90 \mathrm{E}-05$ $3.06 E-06--1.07 E-05$ $1.12 \mathrm{E}-06--3.06 \mathrm{E}-06$ $4.14 E-07--1.12 E-06$ $4.14 E-07---1.12 E-06$
$0.0 \quad---4.14 E-07$
ANGLE 1
MU $=-1.0000$ 0.0 0.0 $3.399 \mathrm{E}-03$ $3.269 E-03$ $3.109 \mathrm{E}-03$ $2.916 \mathrm{E}-03$ $4.517 \mathrm{E}-03$ $6.159 E-03$ $5.702 E-03$ $7.421 \mathrm{E}-03$ $1.258 \mathrm{E}-02$ $2.043 \mathrm{E}-02$ $1.301 \mathrm{E}-01$ $1.110 \mathrm{E}-01$ $6.579 E 00$ $2.812 \mathrm{E} 01$ $8.137 \mathrm{E} 01$ $2.158 E$ O2 $5.735 \mathrm{E} 02$ $\begin{array}{ll}1.246 E & 03 \\ 1.797 E & 03\end{array}$ ANGLE 10 $M U=0.0950$ 0.0
0.0

$1.034 E-03$ $7.492 \mathrm{E}-03$ $4.359 E-03$ $4.108 E-03$ $4.424 E-03$ $7.013 E-03$ $7.958 \mathrm{E}-03$ $9.803 E-03$ 1.655E-02 $1.655 E-02$ $2.728 E-02$ $1.420 E-01$ $3.194 \mathrm{E} 01$ T. $2.975 E$ OI $2.267 \mathrm{O}^{2}$ $2.267 E 02$ $1.302 E 03$ $\begin{array}{ll}1.302 E & 03 \\ 1.870 E & 03\end{array}$
[NEUTRONS/MEV/STERADIAN/SOURCE NEUTRON]

ANGLE 2 0.0 $0.0 \quad 0.0$ $-6.037 E-04$ $3.258 E-03$ $3.117 \mathrm{E}-03$ $2.920 \mathrm{E}-03$ $4.525 \mathrm{E}-03$ $6.168 E-03$ $5.718 \mathrm{E}-03$ $7.442 \mathrm{E}-03$ $1.261 E-02$ $2.046 \mathrm{E}-02$ $1.302 E-01$ $1.111 E-01$ $6.582 \mathrm{E} \mathrm{OO}$ $2.814 \mathrm{E} \mathrm{Ol}$ $8.141 \mathrm{E}$ O1 $2.159 E \quad 02$ $5.737 E$ O2

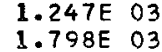
ANGLE 11 ANGLE 11 ANGLE 12
$M U=0.2816 \quad M U=0.4580$ MUU $=0.4580$ 0.0

$\begin{array}{ll}0.0 & 0.0 \\ 1.383 F-03 & 2.239 E-03\end{array}$ $7.414 \mathrm{E}-03$ $6.694 E-03$ $5.985 E-03$ $4.421 \varepsilon-03$ $7.137 E-03$ $1.166 E-02$ $9.953 E-03$ $1.353 \mathrm{E}-02$ $1.933 E-02$ 2.443E-02 $1.483 F-01$ $1.211 E-01$ $3.005 \mathrm{E} O 1$ $8.063 \mathrm{~F} 01$ $2.287 F \quad 02$ $6.058 E 02$ $1.312 E_{03}$ $1.312 E$ O

$$
0.0
$$

0.0
$-2.139 E-04$ $3.080 E-03$ $3.227 \mathrm{E}-03$ $3.150 E-03$ $2.937 E-03$ 4. $559 \mathrm{E}-03$ $6.209 E-03$ $5.784 E-03$ $7.527 E-03$ $1.272 E-02$ $2.060 \mathrm{E}-02$ 1.307E-01 $1.114 \mathrm{E}-01$
$6.598 \mathrm{E} 00$ $2.820 E \quad 01$ $8.158 \mathrm{E}$ ol $2.163 \mathrm{E} 02$ $5.747 \mathrm{E} 02$ $.249 E 03$ $1.801 \mathrm{E} 03$ 0.0
0.0 $1.013 E-02$
$6.422 E-03$ $5.752 \mathrm{E}-03$ $5.643 E-03$ $9.122 \mathrm{E}-03$ $1.143 E-02$ $1.275 E-02$ 1.302E-02 2. $002 E-02$ $2.886 E-02$ $1.491 E-01$ $1.227 E-01$ 3. $35 \mathrm{E}$ O $8.743 \mathrm{E} O 1$ 2.743E O1 2.306E 02 $6.106 E$ O2 $1.896 E_{03}$
ANGLE $\quad 4$
$M U=-0.8656$ 0.0 $6.063 E-05$ $2.779 E-03$ $3.206 E \sim 03$ $3.219 E-03$ $2.973 \mathrm{E}-03$ 4.627E-03 $6.298 E-03$ $5.919 E-03$ $7.696 \mathrm{E}-03$ $1.295 E-02$ $2.086 E-02$ $1.316 \mathrm{E}-01$ $1.120 \mathrm{E}-01$ $6.626 E 00$ $2.831 \mathrm{E}$ Ol $8.188 \mathrm{E}$ O1 $2.170 E \quad 02$ $5.766 \mathrm{E} 02$ $1.253 E$ O3 $1.807 E 03$ ANGLE 13 $M U=0.6179$ 0.0

$3.281 E-03$ $1.160 \mathrm{E}-02$ $9.188 \mathrm{E}-03$ $7.916 E-03$ $6.323 E-03$ $1.035 \mathrm{E}-02$ 1. $588 E-02$ $1.430 E-02$ 1. $2.318 E-02$ 2.769E-02 $1.536 E-08$ $7.243 E-01$ 3.23 01 $8.819 \mathrm{E}$ OI . $2.325 E$ O2 1.332E 03 $1.332 \mathrm{E} \mathrm{O3}$
$M U=-0.7550$ 0.0 $0.0000-04$ $2.671 \mathrm{E}=03$ $3.249 E-03$ 3. $324 \mathrm{E}-03$ 3.034E-03 $4.736 \mathrm{E}-03$ $6.448 \mathrm{E}-03$ $6.129 E-03$ $7.950 E-03$ 1. $329 E-02$ $2.124 E-02$ $1.329 E-01$ $1.127 E-01$ $6.667 E 00$ $2.846 E$ OI $8.230 E$ O1 $2.181 \mathrm{E} 02$ $5.793 \mathrm{E} 02$ $1.258 E \quad 03$ $1.814 \mathrm{E} 03$ ANGLE 14 $M U=0.7550$ 0.0 $7.928 E-03$ $1.708 E-02$ $1.058 E-02$
$8.881 E-03$ $8.881 E-03$
$8.253 E-03$ 1.362E-02 $1.812 \mathrm{E}-02$ $1.860 \mathrm{E}-02$ $1.843 E-02$ $2.514 E-02$ 1.554E-O1 $1.554 E-01$ $1.257 E-01$ $3.088 E$ O1 $8.088 \mathrm{E}$ O1 . $341 E$ OD $2.341 E$ O2 $1.340 E \quad 03$ $1.918 E 03$

ANज́LE 6 $M U=-0.6179$ 0.0 $2.814 E-04$ $2.917 E-03$ $3.398 E-03$ $3.476 E-03$ $3.127 \mathrm{E}-03$ $4.899 E-03$ $6.682 E-03$ $6.434 E-03$ $8.304 E-03$ $1.375 E-02$ $2.174 E-02$ $1.345 \mathrm{E}-01$ 1.137E-01 $2.866 E 01$ $8.284 E$ O1 $2.194 \mathrm{E} 02$ $5.326 \mathrm{E} \mathrm{O2}$ $1.265 E \quad 03$ $1.823 E$ O?

ANGLE 15 
(NEUTRJNS/MEV/STERADIAN/SOURCE NEUTRON)

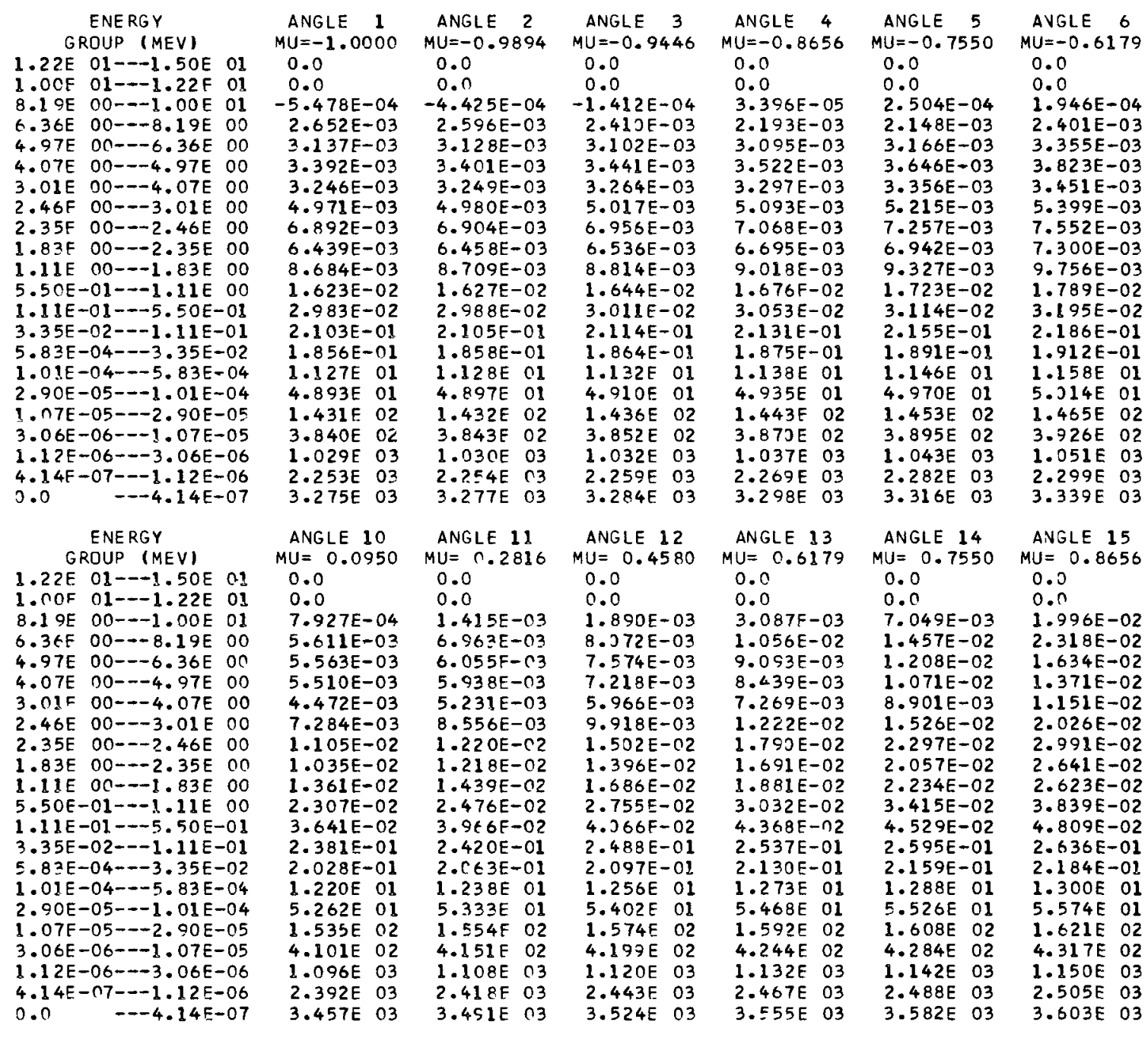

ANGLE 7

$M U=-0.4580$ 0.0

$0.007 E-05$

$\triangle N$ ANGE 8 $-1.004 E-05$ 0.0

$0.076-0$ 4.062E-03 $4.125 E-03$ $3.597 E-03$ $5.669 \mathrm{E}-03$ $7.991 \mathrm{E}-03$ $7.991 E-03$ $7.795 \mathrm{E}-03$ $1.033 E-02$ $1.874 E-02$ $3.295 \mathrm{E}-02$
$2.224 \mathrm{E}-01$ $2.224 \mathrm{E}-01$ $1.936 \mathrm{E}-01$
$1.171 \mathrm{E} 01$ $5.067 E$ OI $1.480 E 02$ $3.964 \mathrm{E} 02$ $1.061 \mathrm{E} 03$ 2.319E 03

4. $6.058 \mathrm{E}-03$ 8.623E-03 $8.463 E-03$ $1.107 \mathrm{E}-02$ $1.107 \mathrm{E}-0$ $3.417 E-02$ $3.417 E-02$ $2.268 E-01$ $1.964 \mathrm{E}-01$
$1.186 \mathrm{E}$ ol $5.127 E$ OI $1.497 \mathrm{E} 02$ $4.006 \mathrm{E} 02$ $1.072 E 03$ $2.342 E \quad 03$ $3.393 \mathrm{E} 03$ $=-0.0950$ 0.0 $3.099 \mathrm{E}-04$ 4. $789 \mathrm{E}-03$ $4.681 E-03$
$4.793 E-03$ $4.121 E-03$ $4.121 E-03$
$6.609 E-03$ $9.512 \mathrm{E}-03$ $9.347 \mathrm{E}-03$ 1.201E-02 $1.201 E-02$ $2.119 E-02$
$3.559 E-02$ $2.317 E-01$ $2.317 \mathrm{E}-01$
$1.995 \mathrm{E}-01$ $1.995 \mathrm{E}-01$
$1.202 \mathrm{E} 01$ $5.193 E$ O1 $1.515 E 02$ $4.053 \mathrm{E} 02$ $1.083 E \quad 03$ $2.366 \mathrm{E} \mathrm{O3}$

ANGLE 16 ANGLE 17 SCALAR $0.9446 \quad M U=0.9894$ 0.0 7.202E-02 $4.186 \mathrm{E}-02$ $2.411 E-02$ $1.893 \mathrm{E}-0$ $1.558 \mathrm{E}-0$ $2.888 E-02$ $4.262 E-02$ $3.605 E-02$ $3.249 E-02$ 4.397E-O2 4. $988 \mathrm{E}-02$ $2.675 \mathrm{E}-01$ $2.202 E-01$ $1.310 E$ O1 $5.610 \mathrm{E} 01$ 1.631E 02 $4.341 \mathrm{E}$ O2 2.517E 03 3.617E 03 
ENERGY GROUP IMEVI $1.22 \mathrm{E} \quad 01-\cdots 1.50 \mathrm{E}$ O1 $1.00 \mathrm{E}$ O1- - $1.22 \mathrm{E}$ OI $6.36 \mathrm{~F} 00--1.00 \mathrm{E} O 1$ $4.97 E$ OO- $6.36 E$ OO 4. $07 E$ OO- $-4.97 F$ OO $3.01 \mathrm{E} 00-\cdots 4.07 E \mathrm{O}$ $2.46 E$ O0- - 3.01E 00 $2.35 \mathrm{E} 00-\ldots 2.46 \mathrm{E} 00$ $1.83 \mathrm{E} 00-2-35 \mathrm{E} 00$ $1.11 \mathrm{E}$ ON-- $1.83 \mathrm{E} 00$ $5.50 E-01 \cdots-1.11 E$ OO $1.11 \mathrm{E}-01--5.50 \mathrm{E}-01$ $3.35 \mathrm{E}-02--1.11 \mathrm{E}-01$ $5.83 E-04--3.35 E-02$ $1.01 \mathrm{E}-04-\cdots-5.83 \mathrm{E}-04$ $2.90 E-05--1.01 F-04$ $1.07 E-05-2.90 E-05$ $3.06 E-06-1.07 E-05$ $1.12 E-06-3.06 E-06$ $4.14 E-07--1.12 E-06$
0.0 ENERGY GROUP (MEV) $1.22 \mathrm{E}$ O1- - 1.50E 01

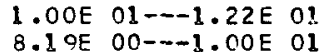
8.1 OE OD- - 1. OOE OI
6.36 OO

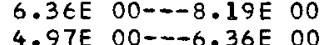
$4.97 E$
$4.00--6.36 E$ 00

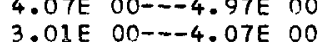

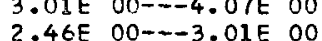
$\begin{array}{lll}2.46 E & 00--3.01 E & 00 \\ 2.35 E & 00---2.46 E & 00\end{array}$

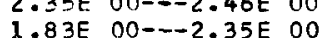
$1.83 E$ OO- -2.35E 00 $1.11 \mathrm{E}$
$5.50--1.83 \mathrm{E}-01-2.11 \mathrm{E} 00$ $5.50 E-01-2.11 E$
$1.11 E-01-5.50 E-01$ $1.11 E-01--5.50 E-01$ $5.83 E-04--3.35 E-02$ $5.83 E-04--3.35 E-02$
$1.01 \mathrm{E}-04---5.83 \mathrm{E}-04$ $2.00 \mathrm{~F}-05--1.01 \mathrm{E}-04$ $1.07 \mathrm{E}-05-2.90 \mathrm{E}-05$ $3.06 \mathrm{E}-06--1.07 E-05$ $1.12 E-06-\cdots 3.06 \mathrm{E}-06$ $4.14 \mathrm{E}-07--1.12 \mathrm{E}-06$ $4.14 \mathrm{E}-07--1.12 \mathrm{E}-06$
$0.0 \quad-0.14 \mathrm{E}-07$
ANGLE 1 $M U=-1.0000$ 0.0 0.0
$-3.704 E-04$ $-3.704 E-04$
$1.930 E-03$ $2.652 E-03$ $2.652 E-03$ $3.074 E-03$ $4.681 \mathrm{E}-03$ $6.564 \mathrm{E}-03$ $6.254 E-03$ $8.605 \mathrm{E}-03$ $1.715 \mathrm{E}-02$ $2.608 \mathrm{E}-01$ $2.359 E-01$ $1.460 E \quad 01$ $6.415 F \quad 01$ $1.893 \mathrm{E} \mathrm{O2}$ 5.132E O2 $1.386 \mathrm{E} \mathrm{O3}$ $3.054 \mathrm{E} \mathrm{O3}$ $4.472 E \quad 03$ ANGLE 10 $M U=0.0950$ 0.0
0.0 0.0
$6.936 E-04$ $4.495 E-03$ $4.749 \mathrm{E}-03$ $5.028 \mathrm{E}-03$ 4. $321 \mathrm{E}-03$ $7.112 \mathrm{E}-03$ $1.062 \mathrm{E}-02$ $1.037 \mathrm{E}-02$ $1.312 \mathrm{E}-02$ $2.461 \mathrm{E}-02$ $4.373 \mathrm{E}-02$ $2.606 \mathrm{E}-01$ $2.606 \mathrm{E}-01$ 1.598 OI $2.054 E$ O2 2.054E O2 I.5U8E 02 $3.493 E$ O $\begin{array}{ll}3.282 E & 3\end{array}$
(NEUTRONS/MEV/STERADIAN/SOURCE NEUTRON)

ANGLE 2 0.0 $\begin{array}{ll}0.0 & 0.0 \\ 0.0 & 0.0\end{array}$ $-2.985 E-04 \quad-8.746 E-05$

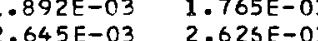
$2.645 \mathrm{E}-03$
$3.153 \mathrm{E}-03$
3.03 $3.076 \mathrm{E}-03 \quad 3.088 \mathrm{E}-03$ $4.689 E-03$ $6.577 E-03$ $6.273 \mathrm{E}-03$ $8.631 E-03$ $1.719 \mathrm{E}-02$ $3.447 \mathrm{E}-02$ $2.611 \mathrm{~F}-01$ $2.361 \mathrm{E}-01$ $1.461 \mathrm{~F} 01$ $6.420 E 01$ $1.805 \mathrm{E} 02$ $5.135 \mathrm{E} \quad 02$ $1.387 E \quad 03$ $3.056 \mathrm{E} 03$ $4.475 \mathrm{E} 03$ ANGLE 11 $=0.2816 \quad$ ANGLE 12 0.0 $1.130 E-03$ $5.252 E-03$ $5.587 E-03$ $5.788 \mathrm{E}-03$ $4.897 \mathrm{E}-03$ $8.1 \circ 8 \mathrm{E}-03$ $1.248 \mathrm{E}-02$ $1.187 \mathrm{E}-\mathrm{C} 2$ $1.471 \mathrm{E}-02$ $2.606 E-02$ $4.572 E-C 2$ $3.055 \mathrm{E}-01$ $2.656 E-01$ $1.626 E$ E $2.085 E$ E 2.085E 02 $3.628 E$ O2 1.514E .
ANGLE $\quad 4$
$M U=-0.8655$ 0.0 4.350E-05 $1.625 E-03$ $2.631 E-03$
$3.273 E-03$ $3.115 \mathrm{E}-03$ $4.799 E-03$ $6.755 E-03$ $6.514 E-03$ 8.946E-03 1.774E-02 $3.527 E-02$ 2.647E-01 2.3BSE-01 $1.475 \mathrm{E}$ ol $6.478 \mathrm{E}$ OI $1.911 E$ O2 5.179E 02 $1.398 \mathrm{E} 03$ $3.080 E \quad 03$ $4.510 E 03$ ANGLE 13 $M U=0.6179$ 0.0 2.536E-03 $8.289 E-03$ $8.436 E-03$ $8.244 E-03$ $6.880 \mathrm{E}-03$
$1.193 \mathrm{E}-02$ $1.193 \mathrm{E}-02$
$1.848 \mathrm{E}-02$ $1.848 \mathrm{E}-02$ $1.657 \mathrm{E}-02$ $1.892 \mathrm{E}-02$ $3.304=-02$ $5.088 \mathrm{E}-02$ $3.211 \mathrm{E}-01$ 2.752E$1.678 \mathrm{E}$ OI $7.299 \mathrm{~F}$ O1 $5.345 E$. $1.553 \mathrm{E} \mathrm{O3}$ $3.553 E$ O3 $4.940 \mathrm{E} \mathrm{O3}$
$M U=-0.7550$ 0.0 $1.899 E-04$ $1.620 E-03$ $2.710 E-03$
$3.395 E-03$ $3.166 \mathrm{E}-03$ 4. $919 \mathrm{E}-03$ $6.958 \mathrm{E}-03$ $6.763 \mathrm{E}-03$ $9.259 E-03$ $1.828 E-02$ $3.602 \mathrm{E}-02$ $2.680 E-01$ $2.409 \mathrm{E}-01$ $1.488 \mathrm{E} 01$ $6.531 \mathrm{E} 01$ $1.926 \mathrm{E} 02$ $5.218 \mathrm{E} 02$ $1.408 E \quad 03$ $3.101 \mathrm{E} 03$ $4.539 E^{03}$

ANGLE 14 $M U=0.7550$ 0.0
0.0 $5.862 E-03$ $.188 \mathrm{E}-02$ $1.110 \mathrm{E}-02$ $1.031 \mathrm{E}-02$ $8.498 \mathrm{E}-03$ $1.510 \mathrm{E}-02$ $2.346 \mathrm{E}-02$ $2.024 \mathrm{E}-02$ $2.182 \mathrm{E}-02$ $3.693 \mathrm{E}-02$ . $368 E-02$ $3.281 E-01$ $2.795 E-01$ 7.701E O1 . 371 E 02 $5.845 E$ O2 $1.570 \mathrm{E} 03$ 1.570E 03 4.985 E 03
ANGLE 6 $J=-C .6179$ 0.0 $1.323 E-04$ . $840 E-03$ $2.900 E-03$ $3.567 E-03$ $3.252 E-03$ $5.101 \mathrm{E}-03$ $7.271 E-03$ $7.125 E-03$ $9.695 E-03$ $1.902 E-02$ $2.723 E-01$ $.438 E-01$
$.535 E ~ 01$ $6.598 \mathrm{E}$ O1 $1.946 \mathrm{E} 02$ $5.258 \mathrm{E} 02$ $3.421 E 03$ $\begin{array}{lll}3.129 \mathrm{E} & 03 \\ 4.576 \mathrm{E} & 33\end{array}$ ANGLE 15 $U=0.8656$ 0.0 $1.615 \mathrm{E}-02$ 1. $900 \mathrm{E}-02$ $1.542 \mathrm{E}-02$ 1. $339 \mathrm{E}-02$ $1.078 \mathrm{E}-02$ $1.987 E-02$ 3.1 $00 \mathrm{E}-02$ 2.54 $2.562 \mathrm{~F}-02$ $4.152 \mathrm{E}-02$ $5.622 E-02$ $3.344 E-01$ $2.831 E-01$ 1.720E 01 .466E 01 $2.192 E$ O2 $1.594 \mathrm{E} 03$ $1.584 \mathrm{E} O 3$ 5. O21E 03

ANGLE 7 $M U=-0.4580$ 0.0 0.0
$-0.320 E-06$ $2.309 E-03$ $3.210 \mathrm{E}-03$ $3.799 \mathrm{E}-03$ $3.388 \mathrm{E}-03$ $5.370 \mathrm{E}-03$ $7.625 \mathrm{E}-03$ $1.027 \mathrm{E}-02$ $1.999 E-02$ $3.826 E-02$ $2.774 E-01$ $2.473 E-01$
$1.525 E$ OI 
(NEUTRONS/MEV/STERADIAN/SOURCE NEUTRON)

ENERGY GROUP (MEVI $1.22 \mathrm{E} 01--1.50 E 01$ $1.00 \mathrm{E}$ O1---1.22E OI $8.19 E$ OO-- 1. $00 \mathrm{E}$ O1 $4.97 \mathrm{E} 00--2.36 \mathrm{E}$ OO $4.07 E$ OD- $-4.07 E$ OO $3.01 \mathrm{E} 00--4.07 E$ O 00 $2.46 \mathrm{E} 00--3.01 \mathrm{E} 00$ $2.35 \mathrm{E} 00--2.46 \mathrm{E}$ OO $1.83 E$ OD- $-2.35 E$ OO $1.11 \mathrm{E} 00--1.83 \mathrm{E}$ OC $5.50 \mathrm{E}-01--1.11 \mathrm{E} 00$ $1.11 E-01--5.50 E-01$ $3.35 E-02--1.11 E-01$
$5.83 E-04---3.35 E-02$ $1.01 E-04---5.83 E-04$ $2.90 E-05--1.01 E-C 4$ $1.07 E-05--2.90 E-05$ $3.06 E-06--1.07 E-05$ $1.12 \mathrm{E}-06---3.06 \mathrm{E}-06$ $4.14 E-07---1.12 E-06$
$0.0 \quad---4.14 E-07$ ENERGY

$$
\text { GROUP (MEV) }
$$

$1.22 \mathrm{E}$ O1-- 1.50E oI $1.00 E$ O1---1.22E 01 $8.19 \mathrm{E} \quad 00--1.00 \mathrm{E}$ OI $6.36 \mathrm{E}$ 00---8.19E 00 4.97 E $00--6.36$ E 00 4.07E $00-2.97 E$ OO 3.01E 0O--4. OTE OO 2.46E $00--3.01 \mathrm{E} 00$ $2.35 \mathrm{E} 00--2.46 \mathrm{E}$ DO 1.83 E $00-2.35 E$ OO 1.12 E $0-1.83 E$ OO 1. $11 \mathrm{E}-01-1.11 \mathrm{E}$ $1.11 E-01-15.50 E-01$ 3.30 $1.01 E-04-5.35 E-04$ 2.0OF-05-- $1.01 \mathrm{E}-04$ 2.9CF-05--1.01E-04 3.06E-06--1.07E-05 $1.12 \mathrm{E}-06--3.06 \mathrm{E}-06$ $4.14 \mathrm{E}-07--1.12 \mathrm{E}-06$ $0.0 \quad--4.14 \mathrm{E}-07$
ANGLE 1 ANGLE 2

$-2.415 E-04$ $2.415 E-04$
$1.356 E-03$ $2.095 \mathrm{E}-03$ $2.095 E-03$ $2.658 \mathrm{E}-03$ $5.711 \mathrm{E}-03$ ..768E-03 $1.621 \mathrm{E}-02$ $2.784 \mathrm{E}-01$ $2.564 E-01$ 1.611 El $7.143 E$ O1 $2.124 \mathrm{E} O 2$ $5.805 \mathrm{E} 02$ $1.577 E$ O3 $\begin{array}{lll}3.496 \mathrm{~F} & 03 \\ 5.153 \mathrm{E} & 03\end{array}$ ANGLE 10 $M U=0.0950$ 0.0 0.0 $5.379 E-04$ $3.309 E-n 3$ $3.917 E-03$ $4.345 E-C_{3}$ (.607E-02 $1.196 \mathrm{E}-02$ $4.443 E-02$ $2.856 \mathrm{E}-01$ 1.778E OI $7.838 E$ OI $2.325 \mathrm{E} 02$ $6.332 \mathrm{E} 02$ $1.716 \mathrm{E} \mathrm{O3}$ $3.793 E \quad 03$
0.0

0.0
$-1.942 \mathrm{E}-04$

1. $33 \mathrm{LE}-03$ $2.090 \mathrm{E}-03$ $2.676 \mathrm{E}-03$ $2.660 E-03$ $4.044 E-C 3$ $5.585 E-03$ $7.792 \mathrm{E}-03$ $1.626 \mathrm{E}-02$ $3.482 \mathrm{E}-02$ $2.788 \mathrm{E}-01$ $2.567 \mathrm{E}-01$ $1.612 \mathrm{E} 01$ $7.149 \mathrm{E}$ Ol $2.125 \mathrm{E} O 2$ 5.8C9E 02 $1.579 \mathrm{E} \mathrm{O3}$ $3.499 \mathrm{E} C 3$
$5.157 \mathrm{E} 03$ ANGLE 11 $M U=0.2816$ 0.0
0.0 $8.673 E-04$ $3.929 E-03$ $4.598 \mathrm{E}-03$ 4. $99 \mathrm{EE}-03$ 4.260E-03 $7.272 E-C 3$ $1.133 \mathrm{E}-02$ $1.074 \mathrm{E}-02$ $1.331 E-02$ 2.5 3. $04 \mathrm{E}-01$ 2. $914 \mathrm{E}-01$ $1.811 \mathrm{E}$ OI $7.974 E ~ C 1$
$2.364 E ~ C 2$ $6.435 \mathrm{E} \mathrm{O2}$ $6.435 \mathrm{E}$ O2
$1.742 \mathrm{E} O 3$ $1.742 \mathrm{E} \mathrm{O3}$
$3.849 \mathrm{E} \mathrm{O3}$ $5.624 \mathrm{E} 03$
ANGLE 3 0.0 0.0 1.248E-03 $2.079 E-03$
$2.711 E-03$ $2.668 \mathrm{E}-03$ 4.076E-03 $5.780 \mathrm{E}-03$ $5.659 E-03$ $7.890 \mathrm{E}-03$ $1.645 \mathrm{E}-02$ $3.511 E-02$ $2.802 E-01$ $2.577 E-01$ $1.618 \mathrm{E}$ O1 $7.175 \mathrm{E}$ O1 $2.133 \mathrm{E}$ O2 5.829E O2 $\begin{array}{lll}1.584 E & 03 \\ 3.510 E & 03\end{array}$ 5.173E 03 ANGLE 12 $M U=0.4580$ 0.0 $1.204 E-03$ 4.786E-03 $5.578 E-03$ 5. $911 E-03$ $4.992 \mathrm{E}-03$ $8.702 E-03$ $1.375 E-02$ $1.257 E-02$ 2.864E-02 $2.864 E-02$ $4.945 E-02$ $2.973 \mathrm{E}-01$ $1.844 \mathrm{E}$ O1 $2.402 \mathrm{E} 02$ $6.535 \mathrm{~F} \mathrm{O2}$ $1.768 \mathrm{E} 03$ $1.768 \mathrm{E} 03$
$3.905 \mathrm{E} 03$ $5.698 \mathrm{E} 03$
ANGLE 4 0.0 0.0 $1.164 \mathrm{E}-03$ $2.089 \mathrm{E}-03$ $2.782 \mathrm{E}-03$ $2.690 \mathrm{E}-03$ $4.142 \mathrm{E}-03$ $5.898 \mathrm{E}-03$ $5.808 E-03$ $8.082 E-03$ $1.681 \mathrm{~F}-02$ $3.566 \mathrm{E}-02$ $2.829 E-01$ 2.596E-01 $1.629 \mathrm{E} \mathrm{Ol}$ $7.221 \mathrm{E}$ ol $2.146 \mathrm{E} \mathrm{O2}$ $5.864 E \quad 02$ $1.593 \mathrm{E} 03$ $3.530 \mathrm{E} 03$ $5.201 \mathrm{E} 03$ ANGLE 13 $M U=0.6179$ 0.0
0.0

$1.985 E-03$ $6.286 E-03$ 7.C79E-03 $7.214 E-03$ 1.007E-03 $1.072 E-02$ $1.711 E-02$ $1.498 E-02$ $1.708 E-02$ 3.187E-02 $3.273 E-01$ $3.028 \mathrm{E}-01$ $1.875 \mathrm{E} 01$ $8.235 \mathrm{E}$ OI $2.439 E$ O2 $6.630 \mathrm{E} \mathrm{O2}$ $1.793 E$ O3 $3.959 E$ O3 $5.768 E \quad 3$
$M U=-0.7550$ 0.0

..341E-04 $1.341 \mathrm{E}-04$
$1.177 \mathrm{E}-03$ $1.177 E-03$
$2.165 E-03$ $2.165 E-03$ $2.731 \mathrm{E}-03$ $4.250 E-03$ $6.094 \mathrm{E}-03$ $6.039 E-03$ 3.3 $70 E-03$ $1.734 \mathrm{E}-02$ $3.646 E-02$ $2.866 E-01$ $2.623 E-01$ $1.644 \mathrm{E} 01$ $7.285 E$ OI $2.165 \mathrm{E} 02$ $5.913 E 02$ $1.606 E$ O3 $5.240 E \quad 33$ ANGLE 14 $M U=0.7550$ 0.0 4. $594 E-03$ $9.163 E-03$ $9.442 E-03$ $9.089 E-03$ $7.392 \mathrm{E}-03$ $1.362 E-02$ $2.193 E-02$ 1.820E-02 $3.562 \mathrm{E}-02$ 5.500E-02 . . $1.903 \mathrm{E} 01$ $8.349 E$ O1 $2.471 E$ O2 $6.714 E 02$ $1.815 \mathrm{E} \quad 03$ $4.004 \mathrm{E} 03$ $5.829 E \quad 03$

ANGLE 6 0.0 0.0 $8.634 E-05$
$1.352 E-03$ $2.333 \mathrm{E}-03$ $2.333 E-03$ $2.803 E-03$ $4.415 E-03$ $6.394 E-03$ $6.372 E-03$ $8.772 \mathrm{E}-03$ $1.807 \mathrm{E}-02$ 3.752E-02 2.715E-01 $2.657 E-01$ $7.664 \mathrm{E}$ OI .368E O1 $5.976 \mathrm{E} 02$ $1.623 \mathrm{E} 03$ $5.288 \mathrm{E} 03$ ANGLE 15 $U=0.8656$ 


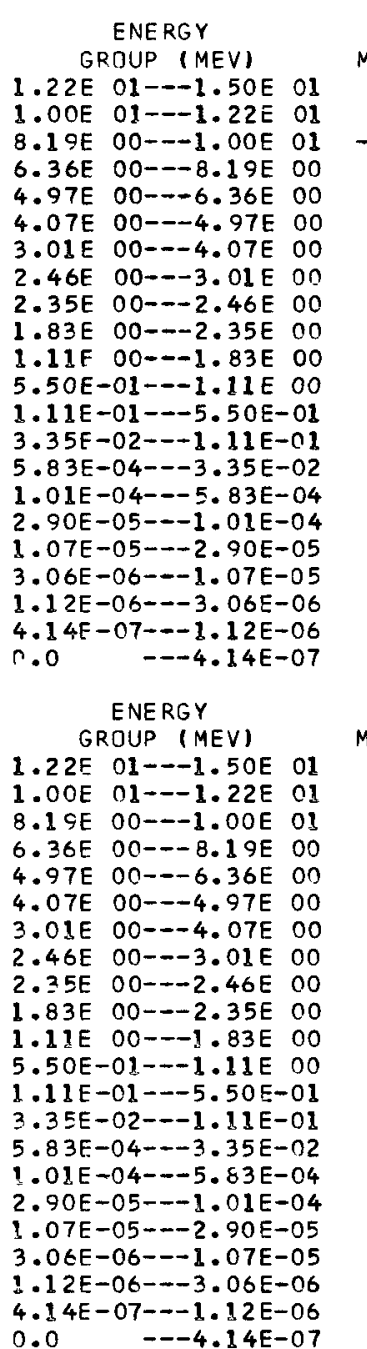

ANGLE 1

0.0

$-1.545 \mathrm{E}-04$ $9.322 E-04$ $1.588 \mathrm{E}-03$ $2.140 \mathrm{E}-03$ $2.168 \mathrm{E}-03$ $3.288 E-03$ $4.688 \mathrm{E}-03$ $4.679 E-03$ $6.603 E-03$ $1.428 \mathrm{E}-02$ $3.221 E-02$ $2.697 \mathrm{E}-0$ $2.519 \mathrm{E}-\mathrm{O}$ $1.600 E 01$ $7.150 E$ O $2.138 \mathrm{E}$ O2 $5.884 E \quad 02$ $1.607 E 03$ $3.580 E$ O3 $5.304 E \quad 03$ ANGLE 10 $M U=0.0950$ 0.0 0.0 $4.007 E-04$
$2.373 E-03$ $2.373 E-03$
$3.061 E-03$ $3.529 E-03$ $3.529 E-03$ $3.053 E-03$ $5.195 \mathrm{E}-03$ $8.146 E-03$ 1.021E-02 $1.021 E-02$ ..1 $4.35 \mathrm{E}-02$ . $125 \mathrm{E} 01$ $2.821 \mathrm{E}-01$ $7.895 E$ OI $2.356 \mathrm{E}$ O2 $6.462 \mathrm{~F}$ O2 $1.462 E$ OR $3.711 \mathrm{E} 03$ $5.748 E \quad 03$
ANGLE 2
$M U=-0.9894$ $0 . n$

$-1.239 E-04$ $9.164 E-04$ 1. $585 \mathrm{E}-03$ $2 \cdot 147 \mathrm{E}-03$ $2.169 \mathrm{E}-03$ $3.294 \mathrm{E}-03$ $4.700 E-03$ $4.694 E-03$ $6.623 E-03$ $1.432 E-C 2$ $2.700 \mathrm{E}-01$ $2.521 E-01$ $1.601 E$ O1 $7.156 E$ OI $2.14 C E$ O2 $5.889 \mathrm{E} \quad 02$ $1.609 \mathrm{E} 03$ $3.583 \mathrm{E} 03$ $5.309 \mathrm{E} 03$ ANGLE 18 $M U=0.2816$ 0.0

$0.070 \mathrm{E}-04$ $2.827 E-03$ $3.610 \mathrm{E}-03$ $4.074 \mathrm{E}-03$ $3.492 \mathrm{E}-03$ $6.077 E-03$ $9.082 E-03$ $1.137 \mathrm{E}-02$ 1.13 E-02 $2.312 E-C 2$ $4.383 E-02$ . $2.882 \mathrm{E}-01$
$1.812 \mathrm{E} 01$ $8.041 \mathrm{E} \mathrm{Cl}$ $2.398 \mathrm{E}$ O2 $6.575 E$ O2 $1.790 E^{03}$ $3.075 E$ N 3 $5.834 \mathrm{E} \quad \mathrm{O} 3$
(NEUTRONS/MEV/STERADIAN/SOURCE NEUTRON)

ANGLE $3 \quad$ ANGLE 4
$M U=-0.9446 \quad M U=-0.8656$

5
$0=-0.7550 \quad$ ANGLE 0.0 0.3 $5.553 E-05$ $9.669 \mathrm{E}-04$ $1.795 E-03$ $2.451 \mathrm{E}-03$ $2.282 \mathrm{E}-03$ $3.610 E-03$ $5.301 E-03$ $5.377 \mathrm{E}-03$ $7.469 \mathrm{E}-03$ $1.597 \mathrm{E}-02$ $3.487 \mathrm{E}-02$ $2.329 \mathrm{E}-01$ $2.615 E-01$ $1.657 \mathrm{E} 01$ $7.390 \mathrm{E} 01$ $2.208 \mathrm{E} 02$ $6.071 E 02$ $1.657 \mathrm{E} \quad 03$ $\begin{array}{ll}3.688 \mathrm{E} & 03 \\ 5.455 \mathrm{E} & 03\end{array}$

AVGLE 15

ANGLE 12 $U=0.4580 \quad M U=0.6179$ 0.0

8.0 SE- 04 $3.474 E-03$ $4.407 E-03$ $4.844 E-03$ $4.102 E-03$ .319E-03 $1.183 E-02$ $1.072 \mathrm{E}-02$ $1.280 \mathrm{E}-02$ $2.560 E-02$ $4.633 \mathrm{E}-02$ 2. $1.847 E$ OI $8.186 \mathrm{E}$ O1 $2.440 E 02$ $1.819 E$ O3 $4.037 \mathrm{E} 03$ 5. 519 E 03 0.0

$1.494 \mathrm{E}-03$ $4.626 \mathrm{E}-03$ $5.643 E-03$ $5.944 E-03$ $4.938 E-03$ $9.073 E-03$ $1.486 E-02$ $1.454 E-02$ $1.454 E-02$ $2.850 E-02$ $4.896 E-02$ 3. $1.001 E$ OI 1.830 OL . $324 E$ OL $2.480 \mathrm{E}$ O2 $1.792 E$ O2 $4.097 E 03$ 5.990 E 03
ANGLE 14
$M U=0.7550$ 0.0

$3.463 E-03$ $6.858 \mathrm{E}-03$ $7.600 E-03$ $7.522 E-03$ $6.068 E-03$ $1.157 E-02$ 1.920E-02 1. 663502 $1.663 E-02$ $3.185 E-02$ 3. $3.481 \mathrm{E}-01$ 1. $910 \mathrm{E} 01$ $8.447 E$ OI $2.516 E 02$
$6.885 \mathrm{E} 02$ 1.871 E 03 $4.149 E 03$ $6.068 \mathrm{E} 03$
$9.260 E-03$ $1.129 \mathrm{E}-02$ $1.073 E-02$ $7.794 \mathrm{E}-03$ 1. $524 \mathrm{E}-02$ $2.562 \mathrm{E}-02$ $1.911 \mathrm{E}-02$ $1.911 \mathrm{E}-02$ $3.55 \mathrm{E}-02$ $3.407 E-02$ $3.097 \mathrm{E}-01$ $1.935 \mathrm{E} 01$ $8.549 E$ O1 $2.546 E$ O2 1.95 02 $4.193 E 03$ $6.124 E \quad 03$ 0.00

ANGLE 7 8.31OE-07 $3.684 E-05$ $1.222 E-03 \quad 1.571 E-03$ $2.013 E-03 \quad 2.299 E-03$ $2.619 E-03 \quad 2.841 E-03$ $5.693 \mathrm{E}-03 \quad 6.255 \mathrm{E}-03$ $5.775 E-03 \quad 6.310 E-03$ $7.928 E-03 \quad 8.521 E-03$ $1.685 E-02 \quad 1.796 E-02$ $3.614 \mathrm{E}-02 \quad 3.768 \mathrm{E}-02$ $2.890 \mathrm{E}-01 \quad 2.960 \mathrm{E}-01$ $1.682 \mathrm{E}$ O1 $1.711 \mathrm{E}$ O1 $7.496 \mathrm{E}$ OI $2.239 E 02$ $6.154 \mathrm{E} \quad 02$ $1.679 \mathrm{E} 03$ $3.735 \mathrm{E} \quad 03$ $5.517 \mathrm{E} \quad 03$ $2.275 \mathrm{E} 02$ $6.249 \mathrm{E} \quad 02$ $1.704 E 03$ $\begin{array}{ll}3.789 E & 03 \\ 5.588 E & 03\end{array}$

ANGLE 16

ANGLE $16 \quad$ ANGLE 17
$M U=0.9446 \quad M U=0.9894$ 0.0 1. (. . . 3 $5.614 \mathrm{E}-02$ 3.606E 01 $1.953 E 01$ $8.624 \mathrm{E}$ 01 $2.567 E$ O2 $1.907 \mathrm{E} 03$ $4.225 \mathrm{E} \quad 03$ $6.164 E_{03}$ 0.0

$1.882 \mathrm{E}-01$ $4.751 E-02$
$2.558 E-02$ $1.846 \mathrm{E}-02$ $1.271 E-02$ . $215 E-02$ 3.340E-02 $3.340 E-02$ $4.330 E-02$ $5.750 E-02$ $3.638 \mathrm{E}-01$ $3.149 E-01$ $1.964 \mathrm{E} \mathrm{O1}$ $8.668 \mathrm{E} 01$ $2.580 E$ O2 $1.915 E 03$ $4.243 E 03$ $6.186 \mathrm{E} \mathrm{O3}$

ANGLE 9 MU $=-0.09$ 0.0 1. $739 E-04$ $1.965 \mathrm{E}-\mathrm{C}$ $2.644 E-03$ $3.134 E-03$ $2.741 E-03$ $4.568 \mathrm{E}-03$ 
ENERGY $1.22 E$ O1- - 1 1.50 EI $1.00 \mathrm{E}$ 01---1.22E 01 $8.19 \mathrm{E} \quad 00--1.00 \mathrm{E} \quad 01$ $4.97 E$ O0-- $0.19 \mathrm{E}$ OO $4.97 E$ OO-- 6.36E OO $3.01 \mathrm{E} 00--4.07 \mathrm{E} 00$ $2.46 E$ OO- $-3.01 \mathrm{E} 00$ $2.35 \mathrm{E} 00--2.46 \mathrm{E} 00$ $1.83 \mathrm{E} 00---2.35 \mathrm{E} 00$ $1.11 \mathrm{E} 00--1.83 \mathrm{E} 00$ $5.50 E-01--1.11 \mathrm{E} 00$ $1.11 \mathrm{E}-01---5.50 \mathrm{E}-01$ $3.35 \mathrm{E}-02--1.11 \mathrm{E}-01$ $5.83 \mathrm{E}-04--3.35 \mathrm{E}-02$ $1.01 E-04--5.83 E-04$ $2.90 E-05--1.01 E-04$ 1.07E-05--2.90E-05 $3.06 E-06--1.07 E-05$ $1.12 \mathrm{E}-06---3.06 \mathrm{E}-06$ $4.14 \mathrm{E}-07--1.12 \mathrm{E}-06$
$0.0 \quad--4.14 \mathrm{E}-07$ ENERGY GROUP (MEV) $1.2 \angle E$ O1---1.50E 01 $1.00 E$
$8.19 E$
$00--1.22 E$ $8.19 \mathrm{E}$ OO- -1. COE OI $6.36 \mathrm{E}$
$4.90---8.19 E$ 4.97E $00--6.36 E$ OO 3.01E OD---4. $07 E$ OO 2.46E $00--3.01 \mathrm{E}$ OO $2.46 \mathrm{E} \quad 00--3.01 \mathrm{E}$ O0 $1.83 E$ EO---2.35E 00 $1.83 \mathrm{E} 00--2.35 \mathrm{E}$ 00 5.50E-01--1.11E 00 $1.11 \mathrm{E}-01--5.50 \mathrm{E}-01$ $3.35 \mathrm{E}-02--1.11 \mathrm{E}-01$ 1. $01 E-04-3$. $35 E-04$ $1.01 E-04-2.83 E-04$ 2. $07 E-05-1.01 E-04$ $1.07 E-C 5-2.90 E-05$ 1.12E-06--3.06E-06 4.14E-07- $1.12 E-06$ 0.0 -

ANGLE 1 $M U=-1.0000$ 0 0.0 $-3.817 E-05$ $2.820 E-04$
$6.014 E-04$ $9.139 E-04$ $9.580 E-04$ $1.453 \mathrm{E}-03$ $2.110 E-03$ $2.251 \mathrm{E}-03$ $3.266 \mathrm{E}-03$ $7.583 \mathrm{E}-03$ $1.896 \mathrm{E}-02$ $1.734 \mathrm{E}-01$ $1.664 \mathrm{E}-01$ 1.081E 01 $4.903 E$ OI $\begin{array}{lll}1.483 \mathrm{E} & 02 \\ 4.139 \mathrm{E} & 02\end{array}$ 4.139E 02 $1.143 E \quad 03$ $\begin{array}{ll}2.571 E & 03 \\ 3.851 E & 03\end{array}$ ANGLE 10 $M U=0.0950$ 0.0

0.0
$1.410 E-04$ $7.915 E-04$ $1.237 \mathrm{E}-03$ I. 2.360E-03 $2.413 E-03$ $3.971 E-03$ $3.935 \mathrm{E}-03$ S.100E-03 $1.144 \mathrm{E}-02$ $2.483 E-02$ 1.884E-OI $1.214 \mathrm{E}$ 01 $5.477 \mathrm{E}$ OI $1.654 \mathrm{E} 02$ $4.603 \mathrm{E} 02$ $1.268 \mathrm{E} \quad 03$ $4.225 \mathrm{E} 0.3$
(NEUTRJNS/MEV/STERADIAN/SOURCE NEUTRON)

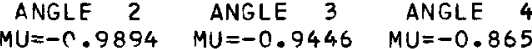
0.0 0.0 - $.042 E-05-6.336 E-06$ $2.783 E-04$ $6.007 E-04$ $9.172 E-04$ $1.456 \mathrm{E}-03$ $2.117 E-03$ $2.259 E-03$ $3.276 \mathrm{E}-03$ $7.606 \mathrm{E}-03$ $1.901 \mathrm{E}-02$ $1.736 E-01$ $1.666 \mathrm{E}-01$ $1.082 E \quad 1$ $4.908 E \quad 01$ $1.484 \mathrm{E} \quad 02$ 4.143E C2 $1.144 E$ O3 $\begin{array}{ll}2.573 \mathrm{E} & 03 \\ 3.855 \mathrm{E} & 03\end{array}$ ANGLE 11 $M U=0.2816$ 0.0

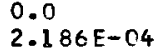
$9.569 E-04$ $1.474 \mathrm{E}-03$ $1.800 \mathrm{E}-03$ $1.564 \mathrm{E}-03$ $2.865 E-03$ $4.8 C 7 E-03$ $4.554 E-03$ $5.681 \mathrm{~F}-03$ $1.261 E-02$ $2.627 E-02$ $1.928 E-01$ $1.241 \mathrm{E} 01$ $5.591 \mathrm{E} 01$ $\begin{array}{ll}1.688 E & 02 \\ 4.695 E & 02\end{array}$ $1.292 E$ O3 1.292E O3 $\begin{array}{ll}2.899 E & 03 \\ 4.298 E & 03\end{array}$ 0.0
0.0
$-6.336 E$

0.0

0

$1.124 \mathrm{E}-05$ $2.589 E-04$
$6.099 E-04$ $9.583 \mathrm{E}-04$ $9.668 \mathrm{E}-04$ $1.497 E-03$ $2.206 \mathrm{E}-03$ $2.361 E-03$ $3.405 \mathrm{E}-03$ $7.892 \mathrm{E}-03$ $1.952 \mathrm{E}-02$ $1.765 E-01$ $1.688 \mathrm{E}-01$ $1.095 \mathrm{E} \mathrm{O1}$ 4.966E 01 $1.502 \mathrm{E} \mathrm{O2}$ 4.190E O2 $1.157 E \quad 03$ 2.601 E 03 $3.896 \mathrm{E} \mathrm{C3}$ $2.583 \mathrm{E} \quad 03$ $3.870 E$ O3

ANGLE 12 0.0

$3.145 E-04$ $1.208 \mathrm{E}-03$ $1.828 E-03$ $2.163 \mathrm{E}-03$ $2.843 E-03$ $3.506 E-03$ 5. $5.354 E-03$ $1.400 \mathrm{E}-02$ $2.783 \mathrm{E}-02$ 1.163E-01 $1.973 \mathrm{E}-01$ $5.704 E$ OI $1.721 E$ O2 $1.721 E$
$4.785 E$ 1.316E 03

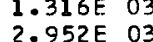
$4.371 \mathrm{E} \mathrm{O3}$ ANGLE 13 0.0 $5.539 E-04$ $1.673 \mathrm{E}-03$ $2.389 E-03$ $2.684 E-03$ $2.225 E-03$ $4.413 E-03$ $6.63 E-03$ $6.383 E-03$ $7.220 E-03$ 1.561E-O2 $2.946 \mathrm{E}-02$ 2.015E-OI 2.015E-OI $5.811 \mathrm{E} 01$ $1.753 \mathrm{E} \mathrm{O2}$ $4.871 E$ O2 1.339E 03 $1.339 \mathrm{E} 03$
ANGLE
$M U=-0.7550$ 0.0 $0.072 E-05$ $2.728 E-04$ $2.728 E-04$
$6.417 E-04$ $9.988 E-04$ $9.802 E-04$ $1.542 E-03$ $2.302 E-03$ $2.464 E-03$ 3. $532 \mathrm{E}-03$ $8.168 \mathrm{E}-03$ $2.000 E-02$ 1.792E-01 $1.708 \mathrm{E}-01$ $1.108 E$ OI $5.019 \mathrm{E} 01$ $1.518 E$ O2 4. $233 \mathrm{E} \mathrm{O2}$ $1.168 \mathrm{E} \mathrm{O3}$ .627E 03 $3.932 \mathrm{E} 03$ $M U=0.7550$ $4.439 E^{03}$
ANGLE 6 $M U=-0.6179$ 0.0 0.0
$1.457 E-05$ $3.204 \mathrm{E}-04$ $3.204 \mathrm{E}-04$
$7.036 \mathrm{E}-04$ $1.055 \mathrm{E}-03$ $1.005 E-03$ $1.612 E-03$ $2.447 E-03$ $3.709 E-03$ $8.550 \mathrm{E}-03$ .064E-02 $1.827 \mathrm{E}-01$ $1.734 E-01$ $1.124 E$ OI $5.088 \mathrm{E}$ 01 $1.538 \mathrm{E} \mathrm{O2}$ $4.289 \mathrm{E} 02$ $1.183 E$ O 3 $3.976 \mathrm{E} 03$ ANGLE 15 $M U=0.8656$ 0.0 $3.310 E-03$ $4.366 E-03$ $4.699 E-03$ 4. $466 \mathrm{E}-03$ $3.354 E-03$ $7.539 E-03$ $1.381 \mathrm{E}-02$ $9.371 E-03$ $9.262 E-03$ $1.935 E-02$ $3.255 \mathrm{E}-02$ $2.334 E-01$ 1. $334 \mathrm{E} O 1$ 5.334 E $1.986 \mathrm{E} \mathrm{OL}$ 1.811E 02 $\begin{array}{ll}\text { 5.011E } 02 \\ 1.377 \mathrm{E} & 03\end{array}$ 3. $3.083 \mathrm{E} 03$ 4.547E 03

ANGLE 7 0.0

$5.285 E-06$ 4.057E-04 7.967E-04 $1.131 E-03$ $1.048 \mathrm{E}-03$ $1.716 \mathrm{E}-03$ $2.657 \mathrm{E}-03$ $2.818 E-03$
$3.943 E-03$ $9.047 E-03$ $2.143 E-02$ $1.869 \mathrm{E}-01$ $1.765 E-01$ $1.142 \mathrm{E} \mathrm{Ol}$ $5.169 E$ OI $1.562 \mathrm{E} \mathrm{O2}$ 
ENERGY GROUP (MEV) $1.22 E$ O1- $1.50 E$ OL S.1 0 OO--1.00E 01 6.36E $00--8.19 E$ OO $4.97 E$ OO- $-6.36 E$ OO 4.07E $00--4.97 E$ OO $3.01 \mathrm{E} 00--4.07 \mathrm{E} 00$ $2.46 \mathrm{E} \quad 00---3.01 \mathrm{E}$ OO $2.35 \mathrm{E} 00--2.46 \mathrm{E} O 0$ $1.11 \mathrm{E} 00--1.33 \mathrm{E} O 0$ $5.50 E-01--1.11 \mathrm{E}$ OO $1.11 E-C 1--5.50 E-01$ $3.35 \mathrm{E}-02--1.11 \mathrm{E}-01$ $5.83 \mathrm{E}-04--3.35 \mathrm{E}-02$ $1.01 \mathrm{E}-04---5.83 \mathrm{E}-04$ $2.90 E-05---1.01 \mathrm{E}-04$ $1.07 E-05--2.90 E-05$ $3.06 \mathrm{E}-06--1.07 \mathrm{E}-05$ $1.12 \mathrm{E}-06-3.06 \mathrm{E}-06$ $4.14 \mathrm{E}-07---1.12 \mathrm{E}-06$ $0.0 \quad--4.14 E-07$ ENERGY GROUP (MEV) $1.22 E$ O1---1.50E OI $1.00 E$ 01- - $1.22 \mathrm{E}$ 8.1 9E $00--1.00 E$ E $6.36 \mathrm{E} \quad 00--78.19 \mathrm{E}$ OO 4.97E 00---6.36E 4.07E $00---4.97 E$ OO $3.01 E$ OO-- $4.07 E$ OO $2.46 \mathrm{E}$ ON---3.01E 0 $2.35 \mathrm{E} 00--2.46 \mathrm{E} 00$ $1.83 \mathrm{E} 00--2.35 \mathrm{E} 00$ 1.1 IE $00--1.83 E 00$ $5.50 \mathrm{E}-01--1.11 \mathrm{E}$ OO $1.11 \mathrm{E}-01--5.50 \mathrm{E}-01$ $3.35 \mathrm{E}-02--1.11 \mathrm{E}=0$ $5.83 \mathrm{E}-04--3.35 \mathrm{E}-02$ $1.01 E-04=-5.83 E-04$ $2.9 C E-05--1.01 E-04$ $1.07 E-05--2.90 E-05$ $3.06 E-06-2.07 E-05$ $1.12 E-06--3.06 E-06$ $4.14 E-07--1.12 E-06$
$0.0 \quad---4.14 E-07$

ANGLE 1 $U=-1.0000$ 0.0

$-9.083 E-06$ $-9.083 E-06$
$8.039 E-05$ 2.032E-04 $3.334 E-04$ $3.564 E-04$ $3.564 E-04$
$5.413 E-04$ $5.413 \mathrm{E}-04$ $7.975 E-04$
$9.004 F-04$ $9.004 \mathrm{E}-04$
$1.338 \mathrm{E}-03$ $1.338 \mathrm{E}-03$
$3.250 \mathrm{E}-\mathrm{OC}$ $8.648 \mathrm{E}-03$ $8.327 E-02$ $8.113 E-02$ $5.338 \mathrm{E} 00$ $2.442 \mathrm{E} O 1$ $2.091 \mathrm{E} \mathrm{O2}$ 5.809 E 02 $1.314 E$ O 3 ANGLE 10 $M U=0.0950$ 0.0

4. $354 \mathrm{E}-05$ $2.439 E-04$ $4.360 E-04$ $5.097 \mathrm{E}-04$ $9.343 \mathrm{E}-04$ $1.554 \mathrm{E}-03$ $1.610 \mathrm{E}-03$ $2.105 \mathrm{E}-03$ $4.972 \mathrm{E}-03$ $1.142 \mathrm{E}-02$ $9.837 E-02$ $9.238 \mathrm{E}-0$ ? $6.031 \mathrm{E} 00$ $2.744 \mathrm{E}$ OI $8.342 E$ OL 2.341E O2 $1.464 \mathrm{E} \quad 03$ $2.187 E \quad 03$
(NEUTRONS/MFV/STERADIAN/SOURCE NEUTRON)

$\triangle N G L E \quad 2$
$M U=-0.9894$ 0.0

$-7.189 E-06$ $7.963 \mathrm{E}-05$ $2.031 E-04$ $3.346 E-04$ $3.566 \mathrm{E}-04$ $5.426 E-04$ $8.005 E-04$
$9.039 E-04$ $1.343 E-03$ $1.343 E-03$
$3.260 E-03$ $8.260 E-03$ $8.668 E-03$
$8.339 E-02$ $8.123 \mathrm{E}-\mathrm{C} 2$ $5.343 E 00$ $2.444 \mathrm{E}$ OI $7.441 E$ C 1 $2.093 \mathrm{E} \mathrm{C2}$ $5.814 \mathrm{E} \quad 02$ $1.316 \mathrm{E} \mathrm{O3}$ $1.983 E$
1.93

ANGLE 34 0.0

$-0.0$ $7.744 E-05$ $2.036 E-04$ $3.403 E-04$
$3.573 E-04$ $3.573 E-04$
$5.481 E-04$ $8.136 E-04$ $9.136 \mathrm{E}-04$
$9.186 \mathrm{E}-04$ $1.361 \mathrm{E}-03$ $1.361 E-03$
$3.305 E-03$ $3.305 E-03$
$8.753 E-03$ $8.391 \mathrm{E}-02$ $8.163 E-02$ $5.368 \mathrm{E} 00$ $2.455 \mathrm{E} 01$ $7.474 \mathrm{E} O 1$ $2.102 \mathrm{E} 02$ 5.839 E O2 $1.321 \mathrm{E} 03$ $1.991 \mathrm{E} 03$ ANGLE 4 0.0 3. $377 \mathrm{E}-06$ 7.679E-05 $2.081 \mathrm{E}-04$
$3.503 \mathrm{E}-04$ $3.503 E-04$
$3.595 E-04$ $5.591 \mathrm{E}-04$ $8.397 E-04$ $9.471 E-04$ $1.396 \mathrm{E}-03$ $3.388 \mathrm{E}-03$ $8.910 E-03$ $8.485 \mathrm{E}-02$ $8.235 E-02$ $5.413 E 00$ $2.475 E$ Ol $7.533 \mathrm{E} \mathrm{O1}$ $2.118 \mathrm{E} 02$
$5.883 \mathrm{~F} 02$ $1.331 \mathrm{E} 03$ $2.006 \mathrm{E} 03$

$\triangle N G L E$ 0.0 $6.783 E-06$ $8.242 E-05$ $2.205 E-04$ $3.656 \mathrm{E}-04$ $3.644 E-04$ $5.773 E-04$ $8.811 E-04$
$9.908 E-04$ $9.908 E-04$
$1.449 E-03$ $3.511 \mathrm{E}-03$ $9.137 E-03$ $8.619 \varepsilon-02$ $8.338 \mathrm{E}-02$ $5.477 E 00$ $2.503 E 01$ $2.142 \mathrm{E} \quad 02$ 5. $046 E 02$ $1.345 \mathrm{E} 03$ $2.025 \mathrm{E} 03$

AVGLE 6 0.3 0.0 $3.963 E-06$ $9.764 E-05$ $2.764 E-05$ $2.434 E-04$
$3.869 E-04$ $3.738 \mathrm{E}-04$ $3.738 E-04$
$6.056 E-04$ $6.056 E-04$
$9.431 E-04$ $9.431 E-04$
$1.054 E-03$ $1.054 E-03$
$1.524 E-03$ $3.682 \mathrm{~F}-03$ $9.439 \mathrm{E}-03$ $8.794 E-02$ $8.471 E-02$ $5.559 E$ OO $2.539 \mathrm{E}$ ol $7.725 \mathrm{E}$ 01 $2.171 \mathrm{E} 02$
$6.027 \mathrm{E} 02$ $1.363 \mathrm{E} 03$ $2.050 E 03$ ANGLE 14 $A N G$ LE 11
$U=0.2816$ 0.0
0.0 $6.6 \subseteq 4 E-05$ $3.000 E-04$ $5.230 E-04$ $6.709 E-04$ $5.881 E-04$ $1.122 \mathrm{E}-03$ $1.955 \mathrm{E}-03$ $1.870 \mathrm{E}-\mathrm{C} 3$ $2.346 E-03$ $5.403 \mathrm{E}-03$ $1.210 E-02$ $1.016 \mathrm{E}-\mathrm{Cl}$ $9.465 \mathrm{E}-02$ $6.17 C E$ OO $2.804 E$ O1 $8.522 E$ OI $2.390 \mathrm{E} 02$ $6.619 E$ D2 $1.494 \mathrm{E}$
$2.228 \mathrm{E} \quad 03$

ANGLE 13 $J=0.4580$ 0.0 $9.942 E-05$ $3.891 \mathrm{E}-04$ $6.578 E-04$ $8.117 E-04$ $6.954 \mathrm{E}-04$ $1.389 \mathrm{E}-03$ $2.475 E-03$ $2.205 \mathrm{E}-03$ $2.635 E-03$ $6.108 E-03$ $1.284 E-02$ $1.048 \mathrm{E}-01$ $9.692 E-02$ $6.308 E$ OD 8.701 E 01 2.439 E 02 S.751E 02 $2.268 \mathrm{E} \quad 03$
0.0

0.

$1.817 E-04$ $5.571 E-04$ $8.716 \mathrm{E}-04$ $8.382 \mathrm{E}-04$ $1.769 E-03$ $3.234 E-03$ $2.634 \mathrm{E}-03$ $2.973 E-03$ $6.816 E-03$ $1.360 \mathrm{E}-02$ $1.080 \mathrm{E}-01$ $9.909 E-02$ $6.439 E \quad 00$ 2.920E 01 政 ( $.550 \mathrm{E} 03$ $2.305 \mathrm{E} 03$
$M U=0.7550 \quad M U=0.8656$

0.0

4. $198 \mathrm{E}-04$

8. $853 E-04$

1. $212 E-03$

$1.021 \mathrm{E}-03$

$2.308 \mathrm{E}-03$

4. 352E-03

$3.173 E-03$

$7.599 \mathrm{E}-03$

$1.435 \mathrm{E}-02$ $1.109 \mathrm{E}-01$ $6.556 \mathrm{E}$ OO $2.970 \mathrm{E} \mathrm{O1}$ $9.021 E$ O1 2.526 E 02 $6.987 E$ O2 2. $339 \mathrm{E} 03$

ANGLE 7 $M=-0.4580$ $2.512 \mathrm{E}-06$ $1.236 \mathrm{E}-04$ $4.152 E-04$ 
(NEUTRONS/MEV/STERADIAN/SOURCE NEUTRON)

ENERGY $1.22 E$ O1---1.50E O1 $1.00 E$ OL-1.22E 01 $6.36 \mathrm{E} 00-2-8.19 \mathrm{E}$ OO $4.97 E$ OO- $-6.36 E$ OO $4.07 \mathrm{E} \quad 00---4.97 \mathrm{E} \quad 00$ $3.01 \mathrm{~F} 00---4.07 \mathrm{E} 00$ $2.46 \mathrm{E} \quad 00--3.01 \mathrm{E} 00$ $2.35 \mathrm{E} \quad 00--2.46 \mathrm{E} \quad 00$ $1.83 \mathrm{E} 00--2.35 \mathrm{E} 00$ $1.11 \mathrm{~F} 00--1.83 \mathrm{E} 00$ $5.50 \mathrm{E}-01--1.11 \mathrm{E}$ OO $1.11 \mathrm{E}-0 \mathrm{l}--5.50 \mathrm{E}-01$ $3.35 E-02--1.11 E-01$ $5.83 \mathrm{E}-04---3.35 \mathrm{E}-0$ $1.01 E-04---5.83 E-04$ $2.90 \mathrm{E}-05--1.01 \mathrm{E}-04$ 1.07E-05--2. 90E-0 $3.06 \mathrm{E}-06--1.07 \mathrm{E}-0$ $1.12 \mathrm{E}-06--3.06 \mathrm{E}-06$ $4.14 E-07--1.12 E-06$ $0.0 \quad--4.14 \mathrm{E}-07$ ENERGY GROUP (MEV) $1.22 \mathrm{E}$ 01---1.50E OI $1.00 E$ OI---1.22E O $8.19 E$ OO--1.COE OI 6.36E $00-2.19 E$ CO 4.97E $00--6.36 E$ OO 4.07E $00-24$. $97 E$ OO . $.01 E$ OO- 0 . OTE OD $2.46 \mathrm{E}$ OO- $3.01 \mathrm{E}$ OO 2.35E $00-2.46 E$ OO $1.83 E$ OO- $2.35 E$ OO $1.11 \mathrm{E} 00-1.83 \mathrm{E}$ $1.5 \mathrm{E}-01--5.50 \mathrm{E}-01$ $3.35 E-02--1.11 E-01$ $5.83 E-04--3.35 E-02$ 1. $01 \mathrm{E}-04--5.83 \mathrm{E}-04$ $1.01 E-05-\cdots 1.01 E-04$ 1.07E-05--2. $90 \mathrm{E}-05$ $3.06 E-06--1.07 E-05$ $1.12 \mathrm{E}-06--3.06 \mathrm{E}-06$ $4.14 \mathrm{E}-07---1.12 \mathrm{E}-06$ $0.0 \quad-04.14 E-07$
ANGLE 1 ANG

0.0

$2.127 E-06$ $2.216 \mathrm{E}-0$ $6.447 \mathrm{E}-05$ $1.116 \mathrm{E}-04$ $1.207 E-04$ $1.835 \mathrm{E}-04$ $2.734 E-04$ $3.237 E-04$ $4.918 \mathrm{~F}-04$ $1.233 E-03$ $3.421 E-03$ $3.406 \mathrm{E}-\mathrm{O}$ $3.350 \mathrm{E}-02$ $2.222 \mathrm{E} 00$ $1.022 \mathrm{E} 01$ $3.123 \mathrm{E}$ OI $8.827 \mathrm{E}$ OI $2.461 \mathrm{E} \mathrm{O2}$ $5.589 E$ O2 $8.458 \mathrm{E} 02$ ANGLE 10 $M U=0.0950$ 0.0

$1.256 \mathrm{E}-05$ $7.200 \mathrm{E}-05$ $1.427 E-04$ $1.938 \mathrm{E}-04$ $1.736 E-04$ $5.724 E-04$ $5.724 E-04$ $7.778 \mathrm{E}-\mathrm{C} 4$ 1.905E-03 $4.543 E-03$ $4.040 E-02$ 3. $28 E-02$ $2.519 E$ OO $1.152 E$ OI $3.517 E$ OI $9.917 E$ OI $2.758 \mathrm{E} 02$ $6.250 E 02$ $9.371 \mathrm{E} 02$
ANGLE 2 0.0

$-1.671 E-06$ 2.2C $2 E-05$ $6.447 E-05$ . $120 \mathrm{E}-04$ 207E-04 $2.746 \mathrm{E}-04$ $3.250 E-04$ $4.934 E-04$ $1.237 E-03$ $3.429 E-03$ $3.411 \mathrm{E}-02$ $3.355 E-02$ $2.224 E 00$ $1.023 \mathrm{E}$ O1 $3.126 \mathrm{E}$ OI $8.836 \mathrm{E} \mathrm{Ol}$ $2.464 \mathrm{E} \mathrm{O2}$ $5.504 E$ C2

ANGLE 11 $M=0.2816$ 0.0 $1.931 \mathrm{E}-05$ S. $015 \mathrm{E}-05$ $1.727 E-04$ $2.279 E-04$ $2.006 E-04$ 3.956E-04 $6.870 \mathrm{E}-04$ $8.673 E-04$ 2.673E- 04 $4.818 E-03$ .175E-02 $3.924 \mathrm{E}-02$ $1.178 E$ OI $3.595 \mathrm{E}$ OI $1.013 E 02$ $2.817 E 02$ $6.380 \mathrm{E} 02$ $9.550 E 02$
ANGLE 3 0.0

$-2.197 E-07$ $2.166 \mathrm{E}-05$ $6.483 E-05$ $1.139 \mathrm{E}-04$ $1.210 E-04$ $1.859 \mathrm{E}-04$ $2.796 E-04$ 3.305E-04 5. $003 \mathrm{E}-0$ $1.255 E-03$ $3.464 E-03$ $3.433 \mathrm{E}-02$ $3.372 E-02$ $2.235 \mathrm{E} 00$ $1.027 E$ OI $3.140 E$ OI $8.875 E$ OI $2.475 \mathrm{~F}$ O2 $5.618 \mathrm{E} \mathrm{O2}$ $8.502 E$ O2 ANGLE 12 $M U=$
0.0 0.0 $2.957 \mathrm{E}-05$ $1.200 \mathrm{E}-04$ $2.188 E-04$ $2.374 \mathrm{E}-04$ $2.374 E-04$ $4.943 E-04$ $9.095 \mathrm{E}-04$ 9.125F-04 . $347 E-03$ $5.115 E-03$ $4.311 E-02$ $4.020 \mathrm{E}-02$ $2.637 \mathrm{E}$ OO $1.204 \mathrm{E} 01$ $3.672 \mathrm{E}$ OI $\begin{array}{ll}3.672 \mathrm{E} & 01 \\ 1.035 \mathrm{E} & 02\end{array}$ $2.875 \mathrm{E} 02$ $6.509 \mathrm{E} 02$ $9.729 \mathrm{E} 02$

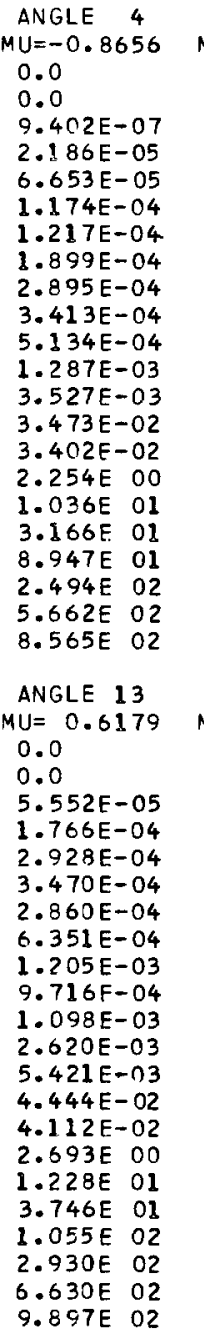

$A N G$ 0.0 0.0

1. $742 \mathrm{E}-06$ $2.380 E-05$ 7. $087 \mathrm{E}-05$ $1.226 E-04$
$1.233 E-04$ $1.964 E-04$ $3.052 E-04$ $3.577 \mathrm{E}-04$ $5.332 E-04$ $1.335 E-03$ $3.529 E-02$ $3.529 E-02$
$3.446 E-02$ $2.281 \mathrm{E} \mathrm{OO}$ $1.048 \mathrm{E} 01$ $3.202 E \quad 01$ $9.048 \mathrm{E} \mathrm{Ol}$ $2.522 E \quad 02$ $\begin{array}{ll}5.723 E & 02 \\ 8.652 E & 02\end{array}$ ANGLE 14 $M U=0.7550$ 0.0

0.0
$1.274 E-04$ $2.867 E-04$ 4.1 04E-04 $4.452 E-04$ $3.473 E-04$ $8.349 E-04$ $1.64 B E-03$ $1.269-03$ $2.920 \mathrm{E}-03$ $5.720 \mathrm{E}-03$ 4.566E-02 $2.744 \mathrm{E} 0 \mathrm{O}$ $1.250 E$ OI $3.812 \mathrm{E}$ 01 $1.073 E$ O2 $2.979 E \quad 02$ $1.739 E$ O2
$1.004 E$ O3
ANGLE 6
$M U=-3.6179$ $M U=-3$
0.0
0.0

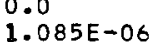
$2.837 E-05$ $7.860 E-05$ $1.299 E-04$ $1.266 E-04$
$2.067 E-04$ $3.284 E-04$ $3.812 E-04$ $5.609 E-04$ $1.402 E-03$
$3.741 E-03$ $3.741 E-03$ $3.502 E-02$ $2.317 E$ OO $1.063 E 01$ 3.249E 01 $9.177 \mathrm{E} 01$ $2.557 \mathrm{E} \mathrm{O2}$ $5.802 E$
$8.761 E$ ANGLE 15 $M U=0.8656$ 0.0

3.095E-04 4. $962 \mathrm{E}-04$ 5. $923 E-04$ $4.206 E-04$ $1.115 E-03$ $2.316 E-03$ $1.406 E-03$ $1.381 \mathrm{E}-03$ $3.227 E-03$ $5.991 E-03$ $4.264 E-02$ $2.786 \mathrm{E} 0 \mathrm{O}$ $1.268 \mathrm{E} 01$ $3.866 \mathrm{E}$ OI $1.088 E$ O2 3.020 E 02 $6.829 E \quad 02$ $1.016 E 03$

ANGLE 7 $U=-0.4580$ 0.0 $9.514 E-07$ $3.594 E-05$ $8.980 E-05$ $1.396 E-04$ $2.221 E-04$ $3.619 E-04$ $4.134 E-04$ $5.975 E-04$ $1.488 E-03$ $3.894 E-03$ $3.691 \mathrm{E}-02$ $2.359 \mathrm{E} 0 \mathrm{O}$ $3.082 E$ OI $9.332 \mathrm{E} 01$ $2.599 E$ O2 $5.896 E \quad 02$ 
(NEUTRONS/MEV/STERADIAN/SOURCE NEUTRONI

ENERGY GROUP (MEV) $1.22 E 01--1.50 E$ $1.00 E$ O1--1.22E 01 $8.19 E$ OO- - $1.00 E$ OI $4.97 E$ O0- $-6.36 E$ OO 4.07E $00--4.97 E$ OD $3.01 \mathrm{E} 00---4.07 \mathrm{E}$ 00 $2.46 \mathrm{E} \quad 00--3.01 \mathrm{E} 00$ 2.3 EE $00--2.46 E$ OO $1.83 E$ OO- $-2.35 E$ EO $1.11 \mathrm{E} \quad 00--1.83 \mathrm{E} 00$ $5.50 E-01--1.11 E$ OO $1.11 \mathrm{E}-01--5.50 \mathrm{E}-01$ $3.35 \mathrm{E}-02---1.11 \mathrm{E}-01$ $5.83 E-04---3.35 E-02$ $1.01 E-04---5.83 E-04$ $2.90 E-05---1.01 E-04$ 1. $07 \mathrm{E}-05--2.90 \mathrm{E}-05$ $3.06 \mathrm{E}-06---1.07 \mathrm{E}-05$ $1.12 \mathrm{E}-06--3.06 \mathrm{E}-06$ $4.14 \mathrm{E}-07--1.12 \mathrm{E}-06$
$0.0 \quad--4.14 \mathrm{E}-07$ ENERGY GROUP (MEV) $1.22 \mathrm{E} 01--1.50 \mathrm{E} 01$ $1.00 E$ O1-- $1.22 E$ OI

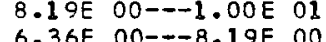
$6.36 \mathrm{E}$
$4.90--8.19 E$ $4.97 E$
$4.00---6.36 E$ 00

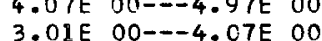

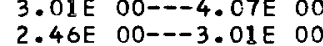

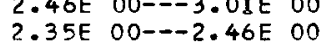

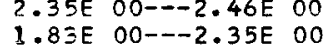

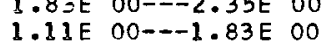
5.5OF-01--1.11E OO 1. $11 \mathrm{E}-01--5.50 \mathrm{E}-01$ 3. $35 \mathrm{E}-02--1.11 \mathrm{E}-01$ $5.83 \mathrm{E}-04---3.35 \mathrm{E}-02$ $1.01 \mathrm{E}-04--5.83 \mathrm{E}-04$ $1.907 E-05-1.01 E-04$ $1.07 E-05-2.90 E-05$ $1.12 \mathrm{E}-06--3.06 \mathrm{E}-06$ $1.32 \mathrm{E}-06 \mathrm{~T}-3.06 \mathrm{E}-06$ $\begin{array}{ll}4.14 E-07---1.12 E-06 \\ 0.0 & --4.14 E-07\end{array}$
ANGLE 1 ANGLE 2 $-4.939 E-07$ $5.974 \mathrm{E}-06$ $1.964 \mathrm{E}-05$ $3.536 \mathrm{E}-05$ $3.849 E-05$ $5.858 \mathrm{E}-05$ $8.811 \mathrm{E}-05$ $1.085 E-04$ $1.681 \mathrm{E}-04$ 4. $321 E-04$ $1.235 \mathrm{E}-03$ $1.259 E-02$ $1.246 \mathrm{E}-02$ $8.308 E-01$ $3.834 \mathrm{E} 00$ $1.175 E$ O1 $3.332 \mathrm{E} 01$ $9.315 \mathrm{E} 01$ $2.120 E$ O2
$3.217 E$ O2 ANGLE 10 $M U=0.0950$ 0.0 0.0
$3.479 E-06$ $3.479 E-06$
$2.068 E-05$ $4.452 E-05$
$6.188 \mathrm{E}-05$ $6.188 \mathrm{E}-05$ $5.562 \mathrm{E}-05$ $1.069 \mathrm{E}-04$ $1.914 E-04$ $2.006 \mathrm{E}-04$ $2.671 E-04$ $6.723 E-04$ $1.647 \mathrm{E}-03$ $1.498 E-02$ $1.427 E-02$ $9.441 \mathrm{E}-01$ $4.333 E$ OO $1.326 \mathrm{E}$ O1 1.753E OI $1.046 E$ OL $3.572 \mathrm{E} \quad 02$ $\triangle N$ ANGLE $2 \quad 3$ 0.0 $-3.853 E-07-3.629 E-08$ $5.952 \mathrm{E}-06$ . .850E-05

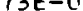
.853E-05 $4.335 E-04$ $1.238 \mathrm{E}-03$ $1.248 \mathrm{E}-02$ $.318 E-01$ $3.838 \mathrm{E}$ OO $1.176 \mathrm{E}$ OI $3.336 \mathrm{E} \mathrm{OI}$ $9.325 E$ Ol $2.122 \mathrm{E} \quad 02$ $3.220 E$ O2 ANGLE 11 $M U=0.2816$ 0.0 $5.372 E-0 B$ $2.635 \mathrm{E}-05$ $5.419 E-05$ $7.298 E-C 5$ $6.436 E-05$ $1.303 E-04$ $2.393 E-04$ 2.344E- 04 $2.980 E-04$ $7.448 E-04$ $1.748 E-03$ $1.548 E-02$ $1.463 E-02$ $9.667 E-C 1$ $4.432 E$ EO 1.35E OI 1.836 E $1.069 E$ O2 $3.642 \mathrm{E} O 2$
0.0

$-3.629 E-08$ $1.981 E-05$ $3.609 E-05$ $3.858 E-05$ $5.939 E-05$ $9.029 E-05$ $1.109 E-04$ $1.710 E-04$ $4.397 \mathrm{E}-04$ $1.251 E-03$ $1.269 \mathrm{E}-02$ $1.254 \mathrm{E}-02$ $8.359 E-01$ $3.856 \mathrm{E} \mathrm{OO}$ $1.182 \mathrm{E} 01$ $3.351 \mathrm{E} \mathrm{O1}$ $9.366 \mathrm{E}$ OI $2.132 \mathrm{E}$ O2 $3.234 \mathrm{E} 02$ ANGLE 12 $M U=0.4580$ 0.0
0.0

. $446 E-06$ 3.588E-05 $6.918 E-05$ $8.893 E-05$ $7.620 E-05$ $1.640 E-04$ $3.097 \mathrm{~F}-04$ 2.779E-04 $3.348 \mathrm{E}-04$ $8.300 \mathrm{E}-04$ $1.856 E-03$ $1.599-02$ 1.500 $9.892 E-01$ 4.530E OO 1.38 1.910 . $1.002 \mathrm{E}$ OL $3.711 \mathrm{E} 02$
ANGLE $\quad 4$
$M U=-0.8656$ 0.0 0.0
$2.516 E-07$ $0.516 E-07$
$6.055 E-06$ $2.040 \mathrm{E}-05$ $3.722 E-05$ 3. $881 \mathrm{E}-05$ $6.073 E-05$ $9.375 E-05$ $1.146 \mathrm{E}-04$ $1.756 \mathrm{E}-04$ $4.513 E-04$ $1.274 E-03$ $1.284 \mathrm{E}-02$ $1.266 E-02$ $8.432 E-01$ $3.888 \mathrm{E} 00$ $1.192 \mathrm{E} 01$ $3.378 E$ ol $9.442 E$ OI $2.148 \mathrm{E} \quad 02$ $3.259 \mathrm{E} 02$ ANGLE 13 $M U=0.6179$ 0.0

1.617E-05 $5.406 E-05$ $9.324 \mathrm{E}-05$ $1.117 \mathrm{E}-04$ $9.171 \mathrm{E}-05$ $2.123 E-04$ $4.154 E-04$ $3.329 \mathrm{E}-04$ $3.773 E-04$ $9.271 E-04$ 1. $.535 \mathrm{E}-02$ $1.011 \mathrm{~F} 00$ $4.414 \mathrm{E}$ $1.414 E$ OI 1.13E 02 1.113E 02 $3.777 \mathrm{E} 02$
ANGLE 5 ANGLE 6 0.0 $4.415 \mathrm{E}-07 \quad 2.966 \mathrm{E}-07$ $6.667 E-06$ .182E-05 . $890 E-05$ 6. $35 E-05$ $9.916 E-05$ $1.203 E-04$ $1.824 \mathrm{E}-04$ . $398 E-03$ $1.305 E-02$
$1.283 E-02$ $8.536 E-01$ $3.935 E 00$ $1.206 \mathrm{E} 01$ $\begin{array}{ll}3.417 E & 01 \\ 9.548 E & 01\end{array}$ $9.548 \mathrm{E} \mathrm{Ol}$ $2.172 E \quad 02$
$3.292 E \quad 02$ ANGLE 14 $M U=0.7550$ 0.0

3.679E-05 $8.921 \mathrm{E}-05$ $1.313 \mathrm{E}-04$ $1.433 \mathrm{E}-04$ $1.111 \mathrm{E}-04$ $2.807 E-04$ $5.756 E-04$ (.) $1.033 E-03$ 1.077E-03 $1.695 \mathrm{E}-02$
$1.566 \mathrm{E}-02$ $1.566 E-02$ $1.030 E$ OO 1.7OTE OO 4.066E O1 $1.132 \mathrm{E} \mathrm{O2}$ 2.566502 $3.834 \mathrm{E}$ O2

$A N G$
$U=-0$
0.0
0.0
2.9
7.968 $2.966 \mathrm{E}-07$
$7.988 \mathrm{E}-06$ $2.427 \mathrm{E}-05$ $4.124 E-05$ 4. $039 \mathrm{E}-05$ $6.636 \mathrm{E}-05$ 1. $072 \mathrm{E}-04$ $1.285 E-04$ $1.920 E-04$ . $353 E-03$ $1.333 \mathrm{E}-02$ $8.670 \mathrm{E}-01$ $3.094 \mathrm{E} \mathrm{OO}$ $1.224 E$ OI $3.467 \mathrm{E} 01$ $9.685 \mathrm{E} \mathrm{OL}$ $2.203 E$ O2 $3.335 \mathrm{E} \mathrm{O2}$

ANGLE 15 
(GAMMAS/MEV/STERADIAN/SOURCE NEUTRON)

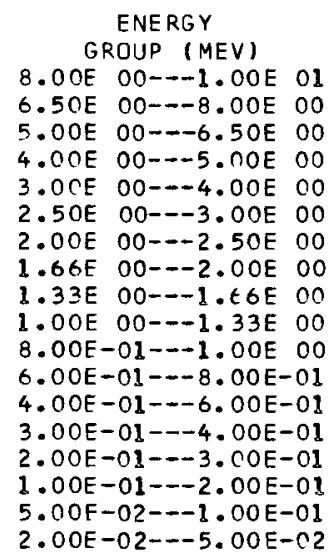

ENERGY

GROUP (MEV

8. OOE OO---1.00E O1 $6.50 \mathrm{E} \quad 00---8.00 \mathrm{E}$ OO $5.00 E$ OO- -6.50 E 00 $4.00 E$ OO- -5. . COE 00 $3.00 E$ OO $-4.00 E$ OO 2.50 E $00--3.00 \mathrm{E}$ OO $2.00 E$ OO--2.50E OO $1.66 \mathrm{E} 00--2.00 \mathrm{E} 00$ $1.32 \mathrm{E}$ OO- $-1.66 \mathrm{E}$ OO $1.00 E \quad 00--1.33 E \quad 00$ $8.00 E-01--1.00 E$ OO 6.00E-01--8.00E-01 4.00E-01--6.00E-01 $3.00 E-01--4.00 E-01$
$2.00 E-01--3.00 E-01$ $2.00 E-01--3.00 E-01$ 1. 0 CEE-01--2. 00 EO 01 $2.00 E-02--5.00 E-02$
ANGLE 1 ANGLE 2 $2.483 E-05$ MUN 2.096804 $1.483 E-05$ $1.9158 E-03$ $1.758 E-03$ $8.364 E-04$ $8.364 E-04$ $1.996 \mathrm{E}-03$ $1.820 \mathrm{E}-03$ $2.271 E-03$ $2.271 E-03$ $7.033 E-04$
$7.505 E-04$ $8.233 E-04$ $3.988 \mathrm{E}-03$ $5.221 E-03$ $1.708 \mathrm{E}-02$ $3.293 \mathrm{E}-02$ $6.581 \mathrm{E}-02$ 1.790E-02

ANGLE 10 $M U=0.0950$ $5.063 \mathrm{E}-05$ $1.773 \mathrm{E}-03$ $1.601 E-03$ $3.199 \mathrm{E}-03$ $3.679 E-03$ $3.604 E-02$ $4.401 E-03$ $1.891 E-03$ $1.676 \mathrm{E}-03$ $2.523 \mathrm{E}-03$ $7.931 E-03$ $1.093 \mathrm{E}-02$ $1.987 \mathrm{E}-02$ $3.882 \mathrm{E}-\mathrm{O} 2$ $7.279 E-02$ $1.878 \mathrm{E}-02$
ANGLE $.496 E-05$ $1.922 E-04$ $8.488 E-04$ $8.434 E-04$ $8.434 E-04$
$1.592 E-03$ 源 $1.828 \mathrm{E}-03$ $2.277 \mathrm{E}-03$ $6.964 E-04$ $7.504 \mathrm{E}-04$ $8.444 E-04$ $4.01 \mathrm{CE}-03$ $5.239 E-03$ $1.712 \mathrm{E}-02$ $3.297 E-02$ $6.586 \mathrm{E}-02$ 1.791E-02

ANGLE 11 $M U=0.2816$ $.636 E-05$
$4.361 E-04$ $4.777 E-03$ $2.384 E-03$ $2.866 E-03$ $4.319 E-03$ $6.955 E-03$ $4.842 \mathrm{E}-03$ $5.908 \mathrm{E}-03$ $1.471 \mathrm{E}-03$ $2.158 E-03$ $2.972 E-03$ . $333 \mathrm{E}-03$ $1.205 E-02$ 2. $092 \mathrm{E}-02$ $4.040 \mathrm{E}-02$ $7.436 \mathrm{E}-\mathrm{O2}$ $1.897 E-02$
ANGLE 4 $.548 \mathrm{E}-05$ $.964 E-04$ $8.768 E-04$ $8.768 \mathrm{E}-04$ $1.631 \mathrm{E}-\mathrm{C3}$ $2.052 \mathrm{E}-03$ $1.861 \mathrm{E}-03$ $2.306 \mathrm{E}-03$ $6.757 E-04$ $7.537 \mathrm{~F}-04$ $9.206 E-04$ $4.093 \mathrm{E}-03$ $5.332 \mathrm{~F}-03$ $1.727 \mathrm{E}-02$ $3.314 \mathrm{E}-02$ $6.608 E-0$ ? $1.794 \mathrm{E}-02$ ANGLE 12 $M U=0.4580$ $.480 E-04$ $5.738 \mathrm{E}-03$ .859E-03 . $353 E-03$ .1 $80 E-03$ $5.485 \mathrm{E}-03$ $5.818 E-03$ $7.110 \mathrm{E}-03$ $1.884 E-03$ $2.706 E-03$ $3.780 \mathrm{E}-03$ 1. $065 \mathrm{E}-02$ $1.303 E-02$ $2.199 \mathrm{E}-02$ $4.216 \mathrm{E}-02$ $7.599 \mathrm{E}-02$ $1.916 E-02$ $2.651 E-05$ $M U=-0.7550$ 2.803E-05 $2.166 E-04$ 1. $990 \mathrm{E}-03$ $9.918 \mathrm{E}-04$ $1.881 E-03$ 9.266E-04 $1.703 \mathrm{E}-03$ $2.136 \mathrm{E}-03$ $1.928 \mathrm{E}-03$ $2.369 \mathrm{E}-03$ $6.549 E-04$ $7.711 E-04$ $1.046 \mathrm{E}-03$ $4.252 E-03$ $5.575 E-03$ 1. $753 \mathrm{E}-02$ $3.346 E-02$ $6.650 E-02$ $1.799 \mathrm{E}-02$

ANGLE 13 $M U=0.6179$ $6.651 E-04$ $5.930 E-03$ $2.983 E-03$ $2.775 E-03$ $5.372 E-03$ $6.431 E-03$ $6.137 \mathrm{E}-03$ 7.53 E-03 . . $5.332 \mathrm{E}-03$ $1.195 \mathrm{E}-02$ $1.403 E-02$ $2.310 E-02$ 4.403E-02 $7.758 \mathrm{E}-02$ $1.933 \mathrm{E}-02$ $9.765 E-04$ $2.256 E-03$ $2.029 \mathrm{E}-03$ $2.479 E-03$ $6.589 E-04$ $6.589 E-04$ $1.189 E-03$ $4.484 E-03$ $6.034 E-03$ $1.782 \mathrm{E}-02$ $3.392 E-02$ $6.711 E-02$ 1. $807 \mathrm{E}-02$

ANGLE 14 $M U=0.7550$ $135 E-04$
$.777 E-04$ $7.787 \mathrm{E}-03$ $3.924 \mathrm{E}-03$ $3.730 E-03$ ..063E-03 .128E-03 $4.979 E-03$ 4.062E-03 4.716E-03 6.566E-03 $1.437 \mathrm{E}-02$ $1.596 E-02$ $2.551 E-02$ $4.608 \mathrm{E}-02$ $7.913 E-0$ $1.950 E-02$
AYGLE 6 $M U=-0.6179$ $3.014 \mathrm{E}-05$ $2.330 E-04$ $1.071 \mathrm{E}-03$ 1.071E-O $1.818 \mathrm{E}-02$ 865 $1.899 \mathrm{E}-04$ $1.469 \mathrm{E}-03$ $1.319 E-02$ $1.188 E-0$ $1.346 \mathrm{E}-02$ S.169E-O3 6.322E-03 1.727E-02 1.828E-02 $2.881 \mathrm{E}-02$ $4.829 E-02$ $1.064 E-02$ $M U=-0.4580$ $3.297 \mathrm{E}-05$ . $1.170 E-03$ $1.145 \mathrm{E}-03$ $1.145 \mathrm{E}-03$ $2.632 \mathrm{E}-03$ $2.372 \mathrm{E}-03$ $2.892 \mathrm{E}-03$ $7.929 \mathrm{E}-04$ $7.929 E-04$
$1.010 E-03$ $1.495 E-03$ $5.278 \mathrm{E}-03$ $7.675 \mathrm{E}-03$ $1.839 \mathrm{E}-02$ 3. $533 \mathrm{E}-02$ $6.888 \mathrm{E}-02$ 1. $830 \mathrm{E}-02$

ANGLE 8 $M U=-0.2816$ $2.845 E-04$ $2.590 E-03$ $2.349 E-03$ $2.919 E-03$ $3.225 \mathrm{E}-03$ 9.1 $91 \mathrm{E}-04$ $1.164 \mathrm{E}-03$ $1.705 E-03$ $5.928 \mathrm{E}-03$ $8.740 E-03$ $1.873 E-02$ $3.630 \mathrm{E}-02$ $7.003 \mathrm{E}-02$
$1.845 \mathrm{E}-02$

ANGLE 16

ANGLE 9 $U=-0.0950$ $4.194 \mathrm{E}-05$ $2.941 E-03$ $1.477 \mathrm{E}-03$ $1.435 \mathrm{E}-03$ $2.668 \mathrm{E}-03$ $3.308 \mathrm{E}-03$ 
(GAMMAS/MEV/STERADIAN/SOURCE NEUTRON)

\begin{abstract}
ENERGY
GROUP (MEV)

8.0OE OO- - 1.00E Ol E.5OE $00---8.00 E$ OO 5.0OE OO--6.5OE OO 4.00 EO3.00 E $00--4.00 E$ OO . 00 OO $00-3.50 E$ OD 1.6EE DO- 2.5OE OD $1.33 E$ OO- $2.00 E$ OO $1.00 E$ OO- $1.33 E$ EO $1.00 E$ OO-D. $33 E$ OO . OOE OI. 3.0CE- $01--6.00 E-01$ $2.00 E-01--3.00 E-01$ $1.00 E-01--2.00 E-01$ $5.00 E-02--1.00 E-01$ 2.0OE-02-- $5.005-02$
\end{abstract}

ENERGY

GROUP (MEV)

8.00E 00--1.00E OI 6.5OE OO---8.0OE OO $5.00 E$ OO---6.50E OO 4.0OE 00-- -5. OOE 00 3.0CE DO---4. DOE OO 2.5OF OO- $-3.00 E$ OO $2.0 \mathrm{CE} 00---2.50 E 00$ $1.66 \mathrm{E} 00--2.00 E 00$ $1.33 E$ OD-D-1.66E OO $1.00 E$ OO---1.33E 00 8.00E-01-- -1.00E OO $6.0 C E-01--8.00 E-01$ 4.00E-01---6.00E-0 3.00E-01---4. $00 E-01$ $2.00 \mathrm{E}-01--3.00 \mathrm{E}-01$ $1.00 E-01--2.00 E-01$ $5.00 E-02-1.00 E-01$ $2.00 E-02---5.00 E-02$
ANGLE 1 $M U=-1.0000$ $2.752 \mathrm{E}-05$ $2.062 \mathrm{E}-04$ 2.024E-03 $1.020 E-03$ 1.816E-03 2.417E-O3 2.209E-03 (2.8 1.210E-03 $1.21 . E-03$ 1.131E-03 $6.999 \mathrm{E}-03$ $6.999 \mathrm{E}-03$ 3.786E-02 $8.698 E-02$ $1.999 \mathrm{E}-01$ $5.705 E-02$

ANGLE 10 $M U=0.0950$ .702E-05 $.565 \mathrm{E}-04$
$.485 \mathrm{E}-03$ $1.837 E-03$ $2.757 \mathrm{E}-03$ $3.213 E-03$ $6.037 \mathrm{E}-03$ $3.725 E-03$ 2.CO7E-C3 $3.486 \mathrm{E}-03$ $5.813 F-03$ $1.754 \mathrm{E}-02$ $2.635 \mathrm{E}-02$ 4.549E-02 $1.079 \mathrm{E}-01$ $2.322 \mathrm{E}-01$ $6.125 \mathrm{E}-02$ $\triangle N G L E \quad 2$ $U=-0.9894$ $2.772 E-05$ $2.078 E-04$ $2.041 E-03$ 9.500E-C4 $1.037 \mathrm{E}-03$ $2.436 E-C 3$ $2.436 \mathrm{E}-\mathrm{C3}$ $2.212 \mathrm{E}-0$ $1.192 \mathrm{E}-03$ $1.192 E-03$ $1.1 \mathrm{CE}-0$ $7.045 \mathrm{E}-03$ $9.656 \mathrm{E}-03$ $9.656 \mathrm{E}-03$ $8.712 \mathrm{E}-02$ 2. $022 E-01$ $2.702 E-01$
$5.708 E-02$

ANELE 11 $M U=0.2816$ $6.154 \mathrm{E}-04$ $5.769 E-03$ $2.990 \mathrm{E}-03$ $2.476 \mathrm{E}-03$ $5.297 \mathrm{E}-0$ 5.2 C5E-0 $6.106 \mathrm{E}-03$ 6.486E-C3 $3.114 E-03$ $4.239 E-03$ ..117E-O3 $2.036 \mathrm{E}-\mathrm{C} 2$ $2.840 E-02$ 4.701E-02 $1.136 E-01$ $6.217 E-02$
ANGLE 3 $M U=-0.9446$ $2.848 E-05$ $2.141 \mathrm{E}-04$ $2.105 E-03$ $1.014 E-03$ $1.05 \mathrm{E}-03$ $2.509 E-03$ $2.257 E-03$ . $1.8 \mathrm{E}-03$ $1.108 E-03$ . $404 \mathrm{E}-03$ $1.404 \mathrm{E}-03$
$7.224 \mathrm{E}-03$ $9.899 \mathrm{E}-03$ $9.899 E-03$ $8.768 \mathrm{E}-02$ $2.011 E-01$ 5.722E-02 $U=-0.8656$ $2.257 E-04$ $2.221 \mathrm{E}-03$ $1.118 E-03$ $1.197 E-03$ 2.640E-O3 2.874E-03 $1.010 \mathrm{E}-03$ 1.225E-03 $1.733 E-03$ $7.556 \mathrm{E}-03$ $1.059 E-02$ $3.916 \mathrm{E}-02$ 8.877F-02 $2.030 \mathrm{E}-01$
$5.747 \mathrm{E}-02$ ANGLE 12 ANGLE 13 $M U=0.4580$ $.202 E-04$
$.452 E-04$ $5.925 E-03$ $3.154 \mathrm{E}-03$ $3.911 E-03$ $5.391 \mathrm{E}-03$ 8.507E-O $6.348 E-03$ $7.838 E-0$ $4.846 E-03$ $6.10 J F-03$ $1.043 \mathrm{E}-02$ $2.447 E-02$ $3.130 \mathrm{E}-02$ $5.045 E-02$ $1.203 \mathrm{E}-0$ $2.481 \mathrm{E}-01$ 6.313E-02 $M U=0.7550$ $.075 \mathrm{E}-04$ $1.093 \mathrm{E}-03$ $1.071 \mathrm{E}-02$ 5.474E-03 4.409E-03 .757E-03 1. $38 E-02$ $5.861 E-03$ ..008E-03 $1.152 E-02$ $2.931 E-02$ $5.566 \mathrm{E}-02$ $1.281 \mathrm{E}-01$ . $568 \mathrm{E}-01$ $6.409 E-02$ $6.001 \mathrm{E}-02$ $1.363 \mathrm{E}-01$ $2.653 E-01$ $6.498 \mathrm{E}-02$ ANGLE $3.189 \mathrm{E}-05$ $2.374 E-03$ 2. $374 E-03$ $1.232 E-03$ 1. $177 \mathrm{E}-0 \mathrm{O}$ $2.812 \mathrm{E}-03$ $2.462 E-03$ $2.462 E-03$ $9.865 E-04$ $1.311 E-03$ $2.062 E-03$ $8.048 \mathrm{E}-03$ $1.196 \mathrm{E}-02$ $3.993 E-02$ $9.037 E-02$ 2.057E-01 $5.784 E-02$

ANGLE 6 $3.452 \mathrm{E}-05$ E-04

ANGLE 7 $M U=-0.4580$ $3.801 E-05$ $2.817 E-03$ $2.817 E-03$ 1. $553 \mathrm{E}-03$ $2.580 \mathrm{E}-03$ 3.316E-O C.921 E-0 $3.921 E-0$ $1.291 \mathrm{E}-03$ $1.291 \mathrm{E}-03$
$1.730 \mathrm{E}-0$ $2.627 E-03$ $9.916 \mathrm{E}-03$ $1.691 \mathrm{E}-02$ $4.127 E-02$ $9.527 \mathrm{E}-02$ $2.137 \mathrm{E}-01$ 5. $890 E-02$ ANGLE 14

ANGLE 8 $M U=-0.2816$ $.249 E-04$ $.154 E-03$ $.638 \mathrm{E}-03$ $.727 E-03$ $.887 \mathrm{E}-03$ $.709 E-03$ $3.299 E-03$ 5E-03 $1.573 E-03$ $2.056 \mathrm{E}-0$ $3.052 \mathrm{E}-03$ $1.161 \mathrm{E}-02$ $2.003 E-02$
$4.201 E-02$ $4.201 \mathrm{E}-02$
$9.868 \mathrm{E}-02$ $2.190 \mathrm{E}-01$ $5.959 \mathrm{E}-02$ ANGLE 16 $M U=0.9446$ 4. $971 E-04$
$3.822 E-03$ 3.397E-02 ANGLE 17 $M U=0.9894$ $8.891 E-03$ $7.488 \mathrm{E}-02$ $3.776 E-02$ $3.362 \mathrm{E}-02$ $6.429 \mathrm{E}-02$ $7.572 \mathrm{E}-02$ $7.028 \mathrm{E}-02$ $8.199 E-02$ $2.783 \mathrm{E}-02$ $2.645 \mathrm{E}-02$ $2.909 \mathrm{E}-02$ $5.948 \mathrm{E}-02$ $5.885 E-02$ $8.687 E-02$ $1.611 E-01$ $2.845 \mathrm{E}-0$

ANGLE 9 $U=-0.0950$ 4. $941 E-05$ $3.625 E-03$ . 1. $979 \mathrm{E}-03$ 
(GAMMAS/MEV/STERADIAN/SOURCE NEUTRON)

\begin{abstract}
ENERGY
GROUP (MEV)
8.0OE OO---1.00E 01

$6.50 E$ OO- $-8.00 E$ OO

4.0OE $00--5.00 E$ OO

3.OOE $00--4.00 E$ OO

. 50 E $00--3.00 E^{\circ}$ OO

2.00E $00--2.50 E$ OO

$3.66 E$ OO-D-2.00E 00

$1.33 \mathrm{E} 00-0-1.66 \mathrm{E}$ OO

$1.00 E$ 00- $-1.33 \mathrm{E}$ O0 $8.00 E-01-\cdots 1.00 E$ OO $6.00 E-01---8.00 E-01$ $4.00 E-01--6.00 E-01$ .00E-01---4.00E-01 $2.00 E-01--3$. . COE-OI $1.00 E-01---2.00 E-01$ $5.00 E-02--1.00 E-01$ $2.00 E-02---5.00 E-02$
\end{abstract}

$$
\text { ENERGY }
$$$$
\text { GROUP (MEV) }
$$

8.00E 00---1.00E OI $6.50 E$ OO- - 8.00E OO $5.00 E$ OO-- $6.50 \mathrm{E}$ OC $4.00 E$ OO- - -5. OOE OO 3. OOE OO---4. COE 00 $2.50 E$ OO- 3 . OOE OO $2.00 E$ OO-D-2.5OE OO 1.66 E $00--2.00 E$ OO 1.33E OO- $1.66 E$ EO $1.00 E$ OO- $-1.33 E$ OO C.OE OL 1 $6.00 E-O 1=-8.00 E-O 1$ 3. OOE $01-4.00 E-01$ . OOE $01-1-3.00 E-01$ . OOE OL $5.00 E-02--1.00 E-01$ $2.00 E-02---5.00 E-02$
ANGLE 1 $M U=-1.0000$ $2.648 E-05$ $1.033 \mathrm{~F}-04$ $8.964 E-04$ $9.977 \mathrm{E}-04$ $1.751 \mathrm{E}-03$ $1.751 E-03$ $2.403 E-03$ $2.883 E-03$ $1.406 \mathrm{E}-03$ $1.339 \mathrm{E}-03$ $1.122 \mathrm{E}-03$ $8.069 \mathrm{E}-03$ $1.134 \mathrm{E}-02$ $4.834 \mathrm{E}-\mathrm{C2}$ $1.185 \mathrm{E}-0$ $8.475 \mathrm{E}-02$ ANGLE 10 $M U=0.0950$ $6.419 E-05$ 4.834E-04 $4.726 \mathrm{E}-03$ $2.498 E-03$ $2.267 E-03$ $4.361 E-03$ $4.642 \mathrm{E}-03$ $5.071 E-03$ $6.212 E-03$ $2.976 \mathrm{E}-03$ $5.883 \mathrm{E}-03$ $5.883 E-03$ $2.125 E-02$ $5.699 \mathrm{E}-02$ $1.497 \mathrm{E}-01$ $3.444 E-01$ $9.179 \mathrm{E}-02$
ANGLE 2 $M U=-0.9894$ $2.671 \mathrm{E}-05$ $1.984 \mathrm{E}-03$ $8.943 E-04$ $1.021 \mathrm{E}-03$ $1.775 \mathrm{E}-03$ $2.426 \mathrm{E}-03$ $2.426 E-03$ $2.873 \mathrm{E}-03$ $1.365 \mathrm{E}-03$ $1.331 \mathrm{E}-03$ $1.207 E-03$ $8.126 \mathrm{E}-03$ $1.140 \mathrm{E}-02$ 4. $852 E-02$ $1.187 \mathrm{E}-01$ $2.910 \mathrm{E}-01$ $8.481 F-02$

\section{ANGLE 11} $M U=0.281$ $6.682 \mathrm{E}-05$ $5.026 E-04$ 4. $951 \mathrm{E}-03$ 2. $659 \mathrm{E}-03$ $3.239 \mathrm{E}-03$ $4.576 \mathrm{E}-03$ $6.795 E-03$ . 36 E-O $6.600 E-03$ $3.564 E-03$ $0.302 E-03$ - $302 E-03$ $2.668=-02$ $3.748 E-02$ 6.112E-C2 $1.576 \mathrm{E}$ $9.328 \mathrm{E}-\mathrm{C2}$
ANGLE 3 $M U=-0.9446$ $2.758 \mathrm{E}-05$ 2.060E-03 9. $18 \mathrm{E}-0$ $1.105 \mathrm{E}-03$ $1.863 \mathrm{E}-03$ $2.512 \mathrm{E}-03$ $2.254 E-03$ $2.851 \mathrm{E}-03$ $1.233 \mathrm{E}-03$ $1.316 \mathrm{E}-03$ $1.510 E-03$ $8.346 \mathrm{E}-03$ $1.172 \mathrm{E}-02$ $4.916 E-02$ $1.195 E-01$
$2.925 E-01$ $8.503 E-02$ ANGLE 12 $M U=0.4580$ $9.859 \mathrm{E}-05$ $7.434 E-04$
$7.129 \mathrm{E}-03$ $3.793 \mathrm{E}-03$ $3.680 \mathrm{E}-03$ $6.554 \mathrm{E}-03$ $7.618 \mathrm{E}-03$ $7.765 E-03$ $9.630 \mathrm{E}-03$ $5.616 E-03$ $7.830 \mathrm{~F}-03$ $1.219 E-02$ $4.076 \mathrm{E}-02$ 4.076E-02 $1.688 \mathrm{E}-01$ 3.719E-01 $9.501 E-02$
ANGLE 4 $M U=-0.8656$ $154 E-O$ $2.192 E-03$ $1.117 \mathrm{E}-03$ $1.117 \mathrm{E}-03$
$1.235 \mathrm{E}-03$ $2.008 \mathrm{E}-03$ $2.659 \mathrm{E}-03$ $2.326 E-03$ $2.855 E-03$ $1.080 E-03$ $1.337 \mathrm{E}-03$ 1. $962 \mathrm{E}-03$ $8.751 \mathrm{E}-03$ $1.268 \mathrm{E}-02$ $5.016 \mathrm{E}-02$ $1.212 \mathrm{E}-01$ $8.545 \mathrm{E}-02$

ANGLE 5 $M U=-0.7550$

ANGLE 6 $M U=-0.6179$ 3.387E-05 $.319 \mathrm{E}-04 \quad 2.522 \mathrm{E}-04$ 1.25E $2.557 \mathrm{E}-03$ $1.369 E-03 \quad 1.372 E-03$ $2.174 \mathrm{E}-03 \quad 2.357 \mathrm{E}-03$ $2.843 \mathrm{E}-03 \quad 3.066 \mathrm{E}-03$ $2.448 E-03 \quad 2.041 E-03$ $2.951 E-03 \quad 3.182 E-03$ $1.037 E-03 \quad 1.167 E-03$ $1.448 \mathrm{E}-03 \quad 1.668 \mathrm{E}-03$ $2.383 \mathrm{E}-03 \quad 2.706 \mathrm{E}-03$ $9.352 \mathrm{E}-03 \quad 1.029 \mathrm{E}-02$ $1.463 E-02 \quad 1.770 E-02$ $5.118 \mathrm{E}-02 \quad 5.206 \mathrm{E}-02$ $1.267 E-01$ $8.606 \mathrm{E}-02 \quad 8.686 \mathrm{E}-02$ ANGLE 14

ANGLE 7 3. $733 \mathrm{E}-05$ $2.782 \mathrm{E}-04$ $2.806 \mathrm{E}-03$ $1.491 \mathrm{E}-03$ $1.622 \mathrm{E}-03$
$2.579 \mathrm{E}-03$ $3.359 \mathrm{E}-03$ $2.928 E-03$ $3.528 E-03$ $3.564 E-03$ 1. $978 \mathrm{E}-03$ $3.008 \mathrm{E}-03$ $1.178 \mathrm{E}-02$ $2.167 \mathrm{E}-02$ $5.283 \mathrm{E}-02$ $1.308 \mathrm{E}-01$ $3.134 \mathrm{E}-\mathrm{O}$ $8.783 E-02$ ANGLE 16 $M U=0.6179$ $M U=0.7550$ ANGLE 15 $1.228 E-04$ M. $027 E-04 \quad 3.129 E-04$ ..302E-04 $1.464 E-03 \quad 2.418 E-03$ $8.834 \mathrm{E}-03$ $4.696 \mathrm{E}-03$ 4.256E-03 1.162E-03 $1.115 E-02$ 12525 1.

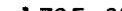
$1.705 \mathrm{E}-02$ . $4.565 \mathrm{E}-02$ $7.158 \mathrm{E}-02$ $3.869 \mathrm{E}-01$

$3.869 \mathrm{E}-01$
$9.665 \mathrm{~F}-02$

$1.348 \mathrm{E}-0$ $7.152 \mathrm{E}-03$ 7. $203 \mathrm{E}-03$

$1.248 \mathrm{E}-02$

$1.531 \mathrm{E}-02$

$1.541 \mathrm{E}-02$ $1.930 E-02$ $1.299 E-02$ $1.660 \mathrm{E}-02$ $4.375 \mathrm{E}-02$ $4.375 E-02$ 5. $146 \mathrm{~F}-02$ $1.932 \mathrm{E}-01$ $4.019 E-01$

$9.820 \mathrm{E}-02$

ANGLE 8 ANGLE 9 AU $=-0.2816 \quad M U=-0.0950$ $3.207 E-05 \quad 4.877 E-05$ $3.146 E-03 \quad 3.631 E-03$ $1.654 E-03 \quad 1.910 E-03$ $1.801 E-03 \quad 2.077 E-03$ $2.890 E-03 \quad 3.343 E-03$ $3.768 \mathrm{E}-03 \quad 4.351 \mathrm{E}-03$ $3.333 E-03 \quad 3.887 E-03$ $4.091 \mathrm{E}-03 \quad 4.775 \mathrm{E}-03$ $1.813 \mathrm{E}-03 \quad 2.228 \mathrm{E}-03$ $2.375 E-03 \quad 2.930 E-03$ $3.515 E-03 \quad 4.521 E-03$ $1.409 \mathrm{E}-02 \quad 1.736 \mathrm{E}-02$ $2.602 \mathrm{E}-02 \quad 3.019 \mathrm{E}-02$ $5.515 E-02$ $1.360 \mathrm{E}-01$ $\begin{array}{ll}3.222 \mathrm{E}-01 & 3.326 \mathrm{E}-01 \\ 8.900 \mathrm{E}-02 & 9.032 \mathrm{E}-02\end{array}$

\section{ANGLE 17 SCALAR} GU $=0.9446 \quad M U=0.9894 \quad$ FLUX $6.083 \mathrm{E}-04 \quad 1.450 \mathrm{E}-03 \quad 1.432 \mathrm{E}-03$ $\begin{array}{lll}4.557 \mathrm{E}-03 & 1.096 \mathrm{E}-02 & 1.081 \mathrm{E}-02 \\ 3.960 \mathrm{E}-02 & 9.325 \mathrm{E}-02 & 9.935 \mathrm{E}-02\end{array}$ $\begin{array}{lll}3.960 E-02 & 9.325 \mathrm{E}-02 & 9.935 \mathrm{E}-02 \\ 2.119 \mathrm{E}-02 & 4.789 \mathrm{E}-02 & 5.251 \mathrm{E}-02\end{array}$ $\begin{array}{lll}2.119 \mathrm{E}-02 & 4.789 \mathrm{E}-02 & 5.251 \mathrm{E}-02 \\ 2.065 \mathrm{E}-02 & 4.301 \mathrm{E}-02 & 5.348 \mathrm{E}-02\end{array}$ $\begin{array}{lll}2.065 E-02 & 4.301 \mathrm{E}-02 & 5.348 \mathrm{E}-02 \\ 3.582 \mathrm{E}-02 & 7.872 \mathrm{E}-02 & 9.018 \mathrm{E}-02\end{array}$ $3.582 \mathrm{E}-02 \quad 7.872 \mathrm{E}-02 \quad 9.018 \mathrm{E}-02$ $4.362 \mathrm{E}-02 \quad 9.152 \mathrm{E}-02 \quad 1.128 \mathrm{E}-01$ $4.232 \mathrm{E}-02 \quad 8.585 \mathrm{E}-02 \quad 1.061 \mathrm{E}-01$ $5.052 \mathrm{E}-02 \quad 9.868 \mathrm{E}-02 \quad 1.292 \mathrm{E}-01$ $2.823 E-02$ 3. $32 E-02 \quad 7.045 E-02$ 2.971E-02 $3.738 \mathrm{E}-02$ $8.731 \mathrm{E}-02$ $3.313 E-02$ 4.042E-02 1.186E-01 6. $318 E-02$ $7.852 E-02$ $3.045 E-01$ $6.836 E-02$ $7.833 E-02$ 4.162E-O1 
(GAMMAS/MEV/STERADIAN/SOURCE NEUTRONI

ENERGY GROUP (MEV) 8. OOE OO-D-1.00E 01 6.5OE $00---8.00 E$ ON $4.00 E$ OO$3.00 E$ OO$3.50 E$ OO- 3 . OOE OO 2.00E $00---2.50 \mathrm{E}$ OO $1.66 \mathrm{E}$ OO- $-2.00 \mathrm{E}$ OO $1.33 \mathrm{E} 00--1.66 \mathrm{E}$ OO $1.00 \mathrm{E} 00--1.33 \mathrm{E} 00$ $8.00 E-01-1.00 E$ O $6.00 E-01--8.00 E-01$ $4.00 E-01---6.00 E-01$ $3.00 E-01--4.00 E-01$ $2.00 \mathrm{E}-01--3.00 \mathrm{E}-01$ $1.00 \mathrm{E}-01--2.00 \mathrm{E}-01$ $5.00 E-02--1.00 E-01$ $2.00 E-02---5.00 E-02$

ENERGY

GROUP (MEV)

8.00E 00---1. DOE OI 6.50E $00--8.00 F$ FO $5.00 E$ OO- $-6.50 E$ OO 4.0OE OO- - 5.00E NO $3.00 E$ OO- 4 . OOE 00 2.5OE OO---3.0OE DO $2.00 E$ OO---2.50E 00 1.66 E $00--2.00 E 00$ 1.33 E $00--1.66 E$ OO $1.00 E$ 00- $-1.33 \mathrm{E} 00$ $8.00 E-01--1.00 E$ OO $6.00 E-01--8.00 E-01$ $4.00 E-01--6.00 E-01$ $3.00 E-01--4.00 E-01$ $2.00 E-01--3.00 E-01$ $1.00 \mathrm{E}-01--2.00 \mathrm{E}-01$ $5.00 E-02--1.00 E-01$ 2. $00 E-02---5.00 E-02$
ANGLE 1 $2.239 \mathrm{E}-05$ $1.525 \mathrm{E}-04$ $1.637 \mathrm{E}-03$ (2.145E-04 $1.432 \mathrm{E}-03$ $1.432 E-03$ $2.087 \mathrm{E}-03$ $2.648 \mathrm{E}-03$ $.648 \mathrm{E}-03$
$1.509 \mathrm{E}-03$ $1.509 E-03$
$1.306 \mathrm{E}-03$ $8.783 E-04$ $8.658 \mathrm{E}-03$ $1.257 \mathrm{E}-02$ $6.055 \mathrm{E}-02$ $1.595 \mathrm{E}-01$ 4.279E-01 1.284E-01

ANGLE 10 $M U=0.0950$ $5.069 E-05$
$3.634 E-04$
$3.782 E-03$ $.782 \mathrm{E}-03$ $2.053 \mathrm{E}-03$ .41 $7 \mathrm{E}-03$ $3.513 E-03$ $4.876 \mathrm{E}-03$ $4.160 E-03$ .108E-03 $2.978 E-03$ $4.385 E-03$ $7.738 \mathrm{E}-03$ $2.694 \mathrm{E}-02$ $4.343 \mathrm{E}-02$ $7.184 \mathrm{E}-02$ $2.063 \mathrm{E}-01$ $5.206 \mathrm{E}-01$ $1.405 \mathrm{E}-0$ ?
ANGLE 3

ANGLE 2 $M U=-C .9894$ $1.548 \mathrm{E}-04$ $1.548 E-04$ $6.866 \mathrm{E}-04$ $8.488 \mathrm{E}-04$ $1.463 \mathrm{E}-03$ $2.113 \mathrm{E}-03$ $1.972 \mathrm{E}-\mathrm{C}$ $2.621 \mathrm{E}-03$ 2.621E-03 $1.445 E-03$ $1.001 \mathrm{E}-03$ $8.723 \mathrm{E}-03$ $1.264 \mathrm{E}-02$ $6.079 E-02$ $1.598 \mathrm{E}-\mathrm{Cl}$ $4.286 E-01$ $1.285 E-C 1$ $2.365 \mathrm{E}-05$ . $632 \mathrm{E}-04$ $1.754 E-03$ $9.682 \mathrm{E}-04$ $1.575 E-03$ $2.208 E-03$ $1.984 \mathrm{E}-03$ $2.539 \mathrm{E}-03$ $1.240 \mathrm{E}-03$ $1.275 \mathrm{E}-03$ $1.431 \mathrm{E}-03$ $8.975 \mathrm{E}-03$ $1.311 \mathrm{E}-02$ $6.165 \mathrm{E}-02$ $1.610 \mathrm{E}-01$ $4.313 \mathrm{E}-01$ 1. 289 E-O1

ANGLE $M U=-0.8656$ $2.532 E-05$
$1.771 E-04$ $1.902 \mathrm{E}-03$ $9.886 \mathrm{E}-04$ $1.142 \mathrm{E}-03$ $1.747 \mathrm{E}-03$ $2.361 \mathrm{E}-03$ $2.023 \mathrm{E}-03$ $2.465 \mathrm{E}-03$ $1.004 E-03$ $1.302 \mathrm{E}-03$ $2.044 \mathrm{E}-03$ $9.435 \mathrm{E}-03$ $1.449 \mathrm{E}-02$ $6.296 \mathrm{E}-02$ $1.635 E-01$ 4. $365 \mathrm{E}-01$ $1.296 \mathrm{E}-01$

ANGLE . $1.931 E-04$ 2.069E-03 $1.145 \mathrm{E}-03$ $1.298 E-03$ $1.018 E-03$ $2.536 \mathrm{E}-03$ $2.116 E-03$ $2.510 \mathrm{E}-03$ $9.384 E-04$ $.446 E-03$ . $554 \mathrm{E}-03$ $.014 E-02$ $1.732 E-02$ $6.421 \mathrm{E}-02$ 1.670E-01 4. $440 E-01$
$1.306 E-01$

ANGLE 11 $M U=0.2816$ $6.598 E-05$ ANGLE 12 ANC $\leq E 13$ ANGLE 14 (1) $.387 \mathrm{E}-05$
$.111 \mathrm{E}-04$ $M U=.6179$ DL 14 1. $760 \mathrm{E}-04$ $1.300 \mathrm{E}-03$ . $248 E-02$ $6.847 E-03$ $1.188 \mathrm{E}-02$ $1.567 E-02$ $1.562 \mathrm{E}-02$ $2.000 E-02$ $1.716 \mathrm{E}-02$ $2.275 E-02$ $2.939 E-02$ $5.519 \mathrm{E}-02$ $6.555 E-02$ $9.929 E-02$ $2.716 E-01$ $6.227 E-01$ 1. $517 \mathrm{E}-01$

AVGLE 6 ANGLE 7 $2.973 E-05$ $2.247 E-03$ $2.247 E-03$
$1.251 E-03$ $1.411 \mathrm{~F}-03$ $2.077 \mathrm{E}-03$ $2.735 \mathrm{E}-03$ $2.292 \mathrm{E}-03$ $2.736 \mathrm{E}-03$ $1.121 \mathrm{E}-03$ $1.717 \mathrm{E}-03$ $2.871 E-03$ $1.131 \mathrm{E}-02$ $2.175 \mathrm{E}-02$ $6.520 E-02$ $1.718 \mathrm{E}-01$ $4.541 \mathrm{E}-0$ 1.320E-01

ANGLE 7 $3.270 E-05$ $2.316 \mathrm{E}-04$ $2.458 \mathrm{E}-03$ $1.510 \mathrm{E}-03$ $2.259 E-03$ $2.997 \mathrm{E}-03$ $2.575 \mathrm{E}-03$ $3.145 \mathrm{E}-03$ $1.499 \mathrm{E}-03$ $2.080 \mathrm{E}-03$ $3.137 \mathrm{E}-03$ $1.331 \mathrm{E}-02$ $2.736 \mathrm{E}-02$ $6.600 E-02$ $1.779 E-01$ 4.666E-0I 1.337E-01

ANGLE 15 $3=0.8856$ $2.273 \mathrm{E}-03$ ANGLE 16 $M U=0.9446$ $6.442 E-04$ 4.200E-02 $2.372 E-02$ $2.011 E-02$ $3.874 E-02$ 4. $804 \mathrm{E}-02$ 4.724E-O2 $5.588 \mathrm{E}-0$ 3.892E-0 4.083E-02 $4.444 E-02$ $7.840 E-02$ $8.661 \mathrm{E}-02$ $3.337 \mathrm{E}-02$ $4.734 \mathrm{~F}-02$ $2.197 \mathrm{E}-01$ $5.438 \mathrm{E}-0$ $1.433 \mathrm{~F}-01$ $5.186 E-02$ $8.236 \mathrm{E}-02$ 2.35LE-01 $1.461 \mathrm{E}-01$ 1.490 E-O1 $7.533 \mathrm{E}-02$ $1.103 \mathrm{E}-01$ $2.915 E-01$ $6.481 \mathrm{E}-0$ $1.235 \mathrm{E}-01$ $3.109 E-01$ $6.696 \mathrm{E}-0$ $1.559 E-01$

ANGLE 8 $M U=-0.2816$ $2.613 E-04$ $2.755 \mathrm{E}-03$ $1.457 E-03$ $1.663 E-03$ . $531 \mathrm{E}-03$ $3.31 \mathrm{E}-03$ $2.980 E-03$ $3.694 \mathrm{E}-03$ $1.946 \mathrm{E}-03$ . $525 \mathrm{E}-\mathrm{C}$ $3.718 \mathrm{E}-03$ $1.650 \mathrm{E}-02$ $3.331 \mathrm{E}-02$ $4.819 E-01$
$1.357 \mathrm{E}-01$ ANGLE 17 
(GAMYAS/MEV/STERADIAN/SOURCE NEUTRON)

\begin{abstract}
ENERGY
GROUP (MEV)

8.00E 00---1. COE CI

6.50E $00--8.00 \mathrm{E}$ OO

$4.0 O E$ OO- $\rightarrow-5.00 E$ OO

4.0OE OO-C.

$5.50 E$ OO- $3.00 E$ EO 2.0OE $00---2.50 E$ OO .66E 00$1.33 E \quad 00--1.66 E \quad 00$ $1.00 E$ OO-D-1.33E 00 $8.00 E-01--1.00 E$ OO $6.0 \mathrm{CE}-01---8.00 \mathrm{E}-01$ $4.00 E-01--6.00 E-01$ $3.00 E-01--4.00 E-01$ $2.00 \mathrm{E}-01--3.00 \mathrm{E}-01$ $1.00 E-01--2.00 E-01$ $5.00 E-02--1.00 E-01$

$2.00 \mathrm{E}-02--5.00 \mathrm{E}-02$
\end{abstract}

ENERGY

GROUP (MEV)

8.0OE O0--1.00E OI

6.50E 00-- - 8.00E 00

5.0OE O0--.5.00E OD

3.00 E $00-\rightarrow-4.00 E$ OO

$2.50 E$ OO- - 3. 00 EO

$2.00 E$ OO-- 2.5OE OO

.66E $00---2.00 E$ OO

$1.33 E$ OO-D-1.66E 00 1.COE OC-D-1.33E OO 8.00 E-C1-D-1.00E OO $6.00 E-01-8.00 E-0$ 4.00E-01---6.00E-01 $3.00 E-01=-4.00 E-01$ $2.00 E-01--3.00 E-01$ $1.00 \mathrm{E}-\mathrm{O}-\mathrm{C}-2.00 \mathrm{E}-\mathrm{O}$ $2.00 \mathrm{E}-02--5.00 \mathrm{E}-02$ $M U=-1.0000$ $1.119 E-04$ $1.119 E-04$ $4.281 E-04$ $5.894 \mathrm{E}-04$ $5.894 \mathrm{E}-04$
$1.068 \mathrm{E}-03$
$1.661 \mathrm{E}-03$ $1.661 \mathrm{E}-03$ $2.244 E-03$ $1.408 \mathrm{E}-03$ $1.097 \mathrm{E}-03$ $5.536 E-O 4$ $8.098 \mathrm{E}-03$ $1.196 \mathrm{E}-02$ $6.352 \mathrm{E}-02$ $1.727 E-01$ $4.906 \mathrm{E}-01$ $1.497 E-01$

ANGLE 10 $4=0.0950$ $2.943 E-04$ $3.171 E-03$ $1.737 E-03$ $2.026 E-03$ $2.958 \mathrm{E}-03$ $3.950 E-03$ $3.550 E-03$ $4.362 E-03$ $2.804 E-03$ $4.199 E-03$ $7.892 \mathrm{E}-03$ $2.817 \mathrm{E}-02$ $4.565 \mathrm{E}-02$ $7.433 \mathrm{E}-02$ $2.264 E-01$ $6.059 \mathrm{E}-01$ $1.647 E-01$
ANGLE 2 $1.828 \mathrm{E}-05$ $1.144 \mathrm{E}-04$ $1.287 E-03$ $6.296 \mathrm{E}-04$ $6.296 E-04$ $1.685 \mathrm{E}-03$ $1.685 \mathrm{E}-03$
$1.618 \mathrm{E}-03$ $2.204 E-03$ $1.334 \mathrm{E}-03$ $1.088 \mathrm{E}-03$ $6.938 \mathrm{E}-04$ $8.157 \mathrm{E}-03$ $1.205 E-02$ $6.377 \mathrm{E}-02$ $1.731 \mathrm{E}-01$ 1.498E-01

ANGLE 11 $A N=0.2816$
$5.469 \mathrm{E}-05$
$3.756 \mathrm{E}-04$ $3.756 \mathrm{E}-04$ $3.996 \mathrm{E}-0$ $2.246 \mathrm{E}-03$ $2.595 E-O 3$ $3.730 \mathrm{E}-03$ $4.964 E-03$ $4.472 E-O 3$ $5.531 \mathrm{E}-03$ 4.058E-0 $6.670 E-03$ $1.243 \mathrm{E}-02$ $3.533 \mathrm{E}-02$ 4. $956 \mathrm{E}-02$ $7.911 \mathrm{E}-02$ $2.416 E-01$ $1.681 \mathrm{E}-01$ ANGLE 4 ANGLE 5 ANGLE 6 ANGLE 7 7
ANGLE 8 3.050E-05 $1.232 \mathrm{E}-04$ $1.383 E-03$ $6.115 \mathrm{E}-04$
$7.686 \mathrm{E}-04$ $1.225 \mathrm{E}-03$ $1.775 \mathrm{E}-03$ $1.775 \mathrm{E}-03$
$1.604 \mathrm{E}-03$ $1.604 E-03$ $1.091 E-03$ $1.074 E-03$ $1.185 \mathrm{E}-03$ $8.391 \mathrm{E}-03$ $1.261 \mathrm{E}-02$ $6.469 E-02$ $1.746 \mathrm{E}-01$ $4.948 E-01$ 1. $503 E-01$ $2.095 E-05$ $1.368 \mathrm{E}-04$ $1.531 \mathrm{E}-03$ $8.091 \mathrm{E}-04$ $9.625 \mathrm{E}-04$ $1.400 \mathrm{E}-03$
$1.912 \mathrm{E}-03$ $1.912 \mathrm{E}-03$
$1.605 \mathrm{E}-03$ $1.605 \mathrm{E}-03$ $1.936 \mathrm{E}-03$
$8.150 \mathrm{E}-04$ $8.150 \mathrm{E}-04$
$1.113 \mathrm{E}-03$ $1.857 \mathrm{E}-03$ $8.825 \mathrm{E}-03$ $1.425 \mathrm{E}-02$ $6.602 \mathrm{E}-02$ $1.773 \mathrm{E}-0$ $5.012 E-01$ $1.512 E-01$ $2.280 E-05$

$\begin{array}{ll}1.509 E-04 & 1.644 E-04 \\ 1.682 E-03 & 1.324 E-03\end{array}$ $9.641 E-04$ $1.117 \mathrm{E}-03$ $1.556 \mathrm{E}-03$ $2.058 \mathrm{E}-03$ $1.663 \mathrm{E}-03$
$1.937 \mathrm{E}-03$ $1.937 \mathrm{E}-03$
$7.428 \mathrm{E}-04$ $1.428 E-04$
$1.271 E-03$ $2.365 E-03$ $1.814 \mathrm{E}-01$ $1.105 E-01$
$1.525 E-01$ ANGLE 12 ANGLE 13 ANGLE 14 $M U=0.7550$ 7.204E-05 $M U=0.6179$ $5.009 \mathrm{E}-04$ $5.232 \mathrm{E}-03$ $2.927 \mathrm{E}-03$ $3.345 E-03$ $1.004 E-04$ $-004 E-04$
$.060 E-04$ $3.974 \mathrm{E}-03$ $4.568 E-03$ $1.537 \mathrm{E}-04$ 1. $097 \mathrm{E}-03$ $1.084 E-02$ $6.989 \mathrm{E}-03$ $1.058 \mathrm{E}-02$ $70 \mathrm{E}-03$ $6.097 E-03$ $7.782 \mathrm{E}-03$ $6.735 \mathrm{E}-03$ $1.088 \mathrm{E}-02$ $1.827 E-02$ $4.255 \mathrm{E}-02$ $5.392 E-02$ $8.546 \mathrm{E}-02$ $2.590 \mathrm{E}-01$ $6.667 \mathrm{E}-01$ $1.717 \mathrm{~F}-01$ $1.413 E-02$ $.123 E-03$ $9.075 E-03$ $1.190 \mathrm{E}-02$ $1.151 \mathrm{E}-02$ $1.713 \mathrm{E}-02$ $2.505 \mathrm{E}-02$ 4. $967 \mathrm{E}-02$ $5.993 \mathrm{E}-02$ $9.335 \mathrm{E}-02$ $2.786 \mathrm{E}-01$ 7.C02E-01 $1.469 \mathrm{E}-02$
$1.916 \mathrm{E}-02$ 1. $889 \mathrm{E}-02$ 2. $522 \mathrm{E}-02$ $3.228 \mathrm{E}-02$ $5.720 \mathrm{E}-02$ $6.808 E-02$ $1.026 \mathrm{E}-01$ $2.998 \mathrm{E}-0$ 1. $785 E-01$ $1.042 \mathrm{E}-03$ $1.04203 E-03$
$1.676 E-03$ $2.216 \mathrm{E}-03$ $1.812 \mathrm{E}-03$ $2.147 E-03$ $9.520 \mathrm{E}-04$ 1.545E-03 $2.618 \mathrm{E}-03$ $2.260 E-02$ $6.807 E-02$ .869E-01 $1.229 E-01$
$1.541 E-01$ AVGLE 15 $M U=0.8656$ $2.712 E-04$ $1.958 E-03$ $1.863 \mathrm{E}-02$ $1.233 E-02$ $1.855 \mathrm{E}-02$ $2.420 \mathrm{E}-02$ $2.523 E-02$ $3.158 \mathrm{E}-02$ 2. $914 \mathrm{E}-02$ $3.459 E-02$ $3.978 \mathrm{E}-02$ $6.652 E-02$ $7.803 \mathrm{E}-02$ $1.133 \mathrm{E}-0$ $3.216 \mathrm{E}-0$ $7.656 \mathrm{E}-01$ 1. $814 \mathrm{E}-01$ $2.713 E-05$ $1.983 \mathrm{E}-03$ $1.078 \mathrm{E}-03$ $1.259 \mathrm{E}-03$
$1.803 \mathrm{E}-03$ $2.427 \mathrm{E}-03$ $2.427 \mathrm{E}-03$ $2.547 \mathrm{E}-03$ $1.362 E-03$ 1.805E-03 DOE-02 $2.889 E-02$ $1.939 E-01$ $5.385 \mathrm{E}-01$ ANGLE 16 $M U=0.9446$ $4.439 \mathrm{E}-03$ $3.931 \mathrm{E}-02$ $2.332 E-02$ $2.489 \mathrm{E}-02$ $4.713 E-02$ $4.613 E-02$ $5.429 \mathrm{E}-02$ $4.289 \mathrm{E}-02$ $4.468 \mathrm{E}-02$ 4. $770 \mathrm{E}-02$ 
(GAMMAS/MEV/STERADI AN/SOURCE NEUTRON)

\begin{abstract}
ENERGY
GROUP (MEV)

8. OOE OO---1.0OE O1 $6.50 E$ OO---8. OOE CO
$5.00 E$ OO---6.50E OO $5.00 E$ OO- $-6.50 E$ OO 4.0OE $00---5$. COE OO $3.00 E$ OO- -4.
$2.00 E$ OO $2.50 E$ OO---3.00E ON

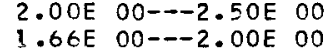
$1.66 \mathrm{E}$
$1.30---2.00 \mathrm{E}$ 00

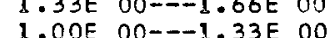
$1.00 E$ OO- $-1.33 E$ OO
$8.00 E-01--1.00 E O O$ $8.00 E-01--1.00 E$ OO
$6.00 \mathrm{E}-01---8.00 \mathrm{E}-01$ 6. $00 E-01---8.00 E-01$
$4.00 E-01--6.00 E-01$ $4.00 E-01---6.00 E-01$
$3.00 E-01---4.00 E-01$ $2.00 \mathrm{E}-01---3.00 \mathrm{E}-\mathrm{CI}$ $2.00 E-01---3.00 E-01$
$1.00 E-01--2.00 E-01$ $1.00 E-01--2.00 E-01$
$5.00 E-02---1.00 E-01$ $2.00 \mathrm{E}-02---5.00 \mathrm{E}-02$

\section{ENERGY \\ GROUP (MEV)}

8. OOE OO---1. OOE 01 $6.50 E$ OO- - - 8.00E OO 5.ONE NO---6.5OE OO 4. 00 OO- $00-5.00 E$ OO $3.00 E$ OO- -4 . COE OO $2.5 \mathrm{OE}$ OO---3.0OE OO 2. $.00 E$ OO---2.5OE 00 1.66 E $00---2.00 E$ OO $1.33 \mathrm{E} 00--1.66 \mathrm{E}$ CO $1.00 E$ 00--1.33E 00 8. COE-01---1.0OE OO $6.00 E-01--8.00 E-01$ 4. OOE-01---6.00E-0? 3. $00 \mathrm{E}-01--4.00 \mathrm{E}-0 \mathrm{I}$ 2. OOE-01-- - 3. COE-01 1. $00 \mathrm{E}-01---2.00 \mathrm{E}-01$ $5.00 E-02--1.00 E-01$ $M U=-1.0000$ $1.413 \mathrm{E}-05$ $7.896 E-05$ $9.282 \mathrm{E}-04$ $2.478 \mathrm{E}-04$ $3.903 E-04$ $7.512 E-04$ $1.261 \mathrm{E}-03$ $1.288 \mathrm{E}-03$ $1.828 \mathrm{E}-03$ $1.227 \mathrm{E}-03$ $8.479 E-04$ $2.627 E-04$ $7.065 E-03$ $1.052 \mathrm{E}-02$ ..065E-02 $1.669 E-01$ $1.516 \mathrm{E}-01$

ANGLE 2 $M U=-0.9894$ $.136 \mathrm{E}-05$ $9.544 \mathrm{E}-04$ $2.910 E-04$ $4.325 \mathrm{E}-04$ 7. $861 E-04$ $1.282 E-03$ $1.277 \mathrm{E}-03$ $1.770 \mathrm{E}-03$ $1.150 \mathrm{E}-03$ $8.422 \mathrm{E}-04$ 4.त $78 E-04$ 7.113E-03 $1.062 E-02$ $6.089 E-C 2$ $1.673 E-01$ $1.517 \mathrm{E}-01$

ANGLE 3 $A N=-0.9446$
$1.541 E-05$ $8.991 E-05$ $1.048 \mathrm{E}-0$ $4.384 E-04$ $5.776 \mathrm{E}-04$ $9.073 E-04$ $1.359 E-03$ $1.241 E-03$ $1.619 \mathrm{E}-03$ $8.961 E-04$ $8.390 E-C 4$ $9.096 E-04$ $7.305 \varepsilon-03$ $1.123 E-02$ $1.687 E-01$ $1.687 \mathrm{E}-01$ $4.962 \mathrm{E}-01$ ANGLE 4 $M U=-0.8656$
$1.696 E-05$ $1.026 \mathrm{E}-04$ $1.185 \mathrm{E}-03$ $6.361 E-04$ 7.731E-04 $1.074 E-03$ $1.472 E-03$ $1.210 \mathrm{~F}-03$ $1.441 \mathrm{E}-03$ $6.129 \mathrm{E}-04$ $8.916 \mathrm{E}-04$ $1.571 \mathrm{E}-03$ $7.674 E-03$ $1.295 \mathrm{E}-02$ . $293 E-02$ $1.715 E-01$ 1.532E-01 ANGLE 5

ANGLE 10 $M U=0.0950$ $.535 E-05$ ANGLE 11

\section{ANGLE 12} $U=0.2816$

ANGLE 13

ANGLE 14 $1.643 E-03$ 2.307E-03 $3.124 E-03$ $2.786 \mathrm{E}-03$ $3.417 E-O$ $2.374 \mathrm{E}-03$ $3.755 \mathrm{E}-03$ $7.542 E-03$ $2.697 \mathrm{E}-02$ $4.341 \mathrm{E}-02$ $6.986 \mathrm{E}-02$ $2.202 \mathrm{E}-01$ $6.127 E-01$ $1.672 \mathrm{E}-01$ $4.493 E-05$

(72E-03 $1.813 \mathrm{E}-03$ $2.134 \mathrm{E}-\mathrm{C} 3$ $2.954 E-C 3$ $3.938 \mathrm{E}-03$ $3.547 E-03$ $4.402 E-C 3$ $3.593 \mathrm{E}-03$ $6.278 E-03$ $1.219 E-02$ $3.386 \mathrm{~F}-02$ $4.677 \mathrm{E}-02$ 7.43 OE- 02 $2.352 E-01$ $6.432 \mathrm{E}-01$ $1.708 \mathrm{E}-01$
8.312E-05
$4.177 \mathrm{E}-03 \quad 5.804 \mathrm{E}-0$

$3.222 \mathrm{E}-03$

$3.860 \mathrm{E}-03 \quad 5.803 \mathrm{E}-03$

$5.208 \mathrm{E}-03 \quad 7.521 \mathrm{E}-03$

$4.953 \mathrm{E}-03 \quad 7.710 \mathrm{E}-03$

$6.432 \mathrm{E}-03 \quad 1.035 E-02$

$6.310 \mathrm{E}-03 \quad 1.122 \mathrm{E}-02$

$1.062 \mathrm{E}-02 \quad 1.699 \mathrm{E}-02$

$1.812 \mathrm{E}-02 \quad 2.481 \mathrm{E}-02$

4.062E-02 $4.701 \mathrm{E}-02$

5. $365 \mathrm{E}-02 \quad 5.618 \mathrm{E}-02$

$8.022 \mathrm{E}-02 \quad 8.739 \mathrm{E}-02$

$2.523 E-01 \quad 2.713 E-01$

$6.765 \mathrm{E}-01$

$2.713 E-01$
$7.2155-01$

1. $781 \mathrm{E}-01$ $.858 \mathrm{E}-05$ $1.146 \mathrm{E}-04$ $1.315 \mathrm{E}-03$ $7.768 \mathrm{E}-04$ $9.142 \mathrm{E}-04$ $1.206 \mathrm{E}-03$ 1.5 84E-03 $1.237 E-03$ $1.412 \mathrm{E}-03$ $5.442 E-04$ $1.048 \mathrm{E}-03$ $2.031 E-03$ $8.319 E-03$ $1.634 E-02$ $6.356 \mathrm{O}$ $1.756 \mathrm{E}-01$ $1.127 \mathrm{E}-01$

ANILE 6 $\mathrm{U}=-0.6179$
$2.015 \mathrm{E}-05$

1. $244 \mathrm{E}-04$

1. $421 E-03$

$8.260 E-04$

$9.704 E-04$

$1.288 E-03$

$1.701 \mathrm{E}-03$

1. $359 \mathrm{E}-03$

$1.601 \mathrm{E}-03$

7.579E-04

$1.295 E-03$

$2.207 E-03$

$9.544 E-03$

$2.147 \mathrm{E}-02$

$5.257 \mathrm{E}-01$

$1.552 \mathrm{E}-01$

AVGLE 15

$2.323 \mathrm{E}-04$

$1.533 \mathrm{E}-03$

$1.575 E-02$

5.048E-0

$6.018 E-03$

$8.81 \mathrm{E}-03$

$1.211 \mathrm{E}-0$ ?

$1.301=-02$

$1.727 E-02$

$1.871 E-02$

$2.507 E-02$

$3.175 E-02$

$5.360 E-02$

$6.374 \mathrm{E}-02$

$9.565 E-02$

$2.917 E-01$

$7.466 E-01$
$1.815 E-01$
ANGLE 7 $M=-0.4580$ $1.346 \mathrm{E}-04$ $1.531 \mathrm{E}-03$ $8.249 \mathrm{E}-04$ $9.874 E-04$ $2.367 E-03$ $1.864 \mathrm{E}-03$ $1.587 \mathrm{E}-03$ 1. $973 \mathrm{E}-03$ $1.155 \mathrm{E}-03$ $1.582 \mathrm{E}-03$ 2. $313 \mathrm{E}-03$ $1.178 E-02$ $2.772 E-02$ $5.420 E-01$ $1.584 \mathrm{E}-01$

\section{ANGLE 16} $M U=0.9446$ $3.863 E-03$ $3.461 \mathrm{E}-02$ $2.148 \mathrm{E}-02$ $2.355 E-02$ 3.354E-02 $4.168 \mathrm{E}-02$ $4.246 \mathrm{E}-02$ 4. $927 \mathrm{E}-02$ 4. $204 \mathrm{E}-02$ 4.344E-0 4.569E-02 7.274E-02 $8.165 \mathrm{E}-02$ $1.149 E-01$ 3. $310 E-01$ $8.063 E-01$ 1. $867 \mathrm{E}-01$

ANGLE $8 \quad$ ANGLE 9 $.402816 \quad M U=-0.0950$ $2.884 E-05$
$1.785 E-04$ .705E-03 2.006E-03 $8.676 \mathrm{E}-04 \quad 1.041 \mathrm{E}-03$ $1.061 \mathrm{E}-03 \quad 1.274 \mathrm{E}-03$ $1.525 E-03 \quad 1.832 E-03$ $2.129 E-03 \quad 2.540 E-03$ $1.904 \mathrm{E}-03 \quad 2.292 \mathrm{E}-03$ $2.420 \mathrm{E}-03 \quad 2.871 \mathrm{E}-03$ 
(GAMMAS/MEV/STERADIAN/SOURCE NEUTRON)

ENERGY GROUP (MEV) 8. OOE OO--1. 6.50 E $00--8.00 \mathrm{E}$ OO 5.OOE OO- -6.50E DO 4.00 E OO- $-5.00 E$ E 00 3.00 E $00--4.00 E 00$ $2.50 E \quad 00--3.00 E^{2} 00$ $2.00 E$ OO- $-2.50 E$ OO 1.6 EE $00---2.00 E$ OO $1.33 \mathrm{E} 00--1.66 \mathrm{E} 00$ $1.00 \mathrm{E} \quad 00--1.33 \mathrm{E} 00$ 8.00E-01---1.00E 00 $6.00 \mathrm{E}-01--8.00 \mathrm{E}-01$ 4.00E-01--6.00E-01 3. 0 CE-01-- 4. 00E-01 $2.00 E-01---3.00 E-01$ $1.00 E-01--2.00 E-01$
$5.00 E-02--1.00 E-01$ $2.00 \mathrm{E}-02--5.00 \mathrm{E}-02$ ENERGY GROUP (MEV) 8. OOE OO-D-1.00E 01 $6.50 \mathrm{E} 00---8.00 \mathrm{E} 00$ 5.00 OO-W. 0 . SOE OO $4.00 E$ OO- $-5.00 E$ OO $3.00 \mathrm{E}$ OO-- $-4.00 E$ OO 2. 50 E OO$2.00 E$ OO-D-2.50E NO $1.66 \mathrm{E}$ OO-C-2.00E 00 $1.33 \mathrm{~F}$ 00- $1.06 \mathrm{E}$ OO $1.00 E$ OD- 1.33 E 00 $8.00 E-01-2.00 E$ OO 6. OOE $01-1.00 E-O 1$ $4.00 E-O 1--6.00 E-01$ 2. OOE $-01--3.00 E-01$ 2.0OE-01-C-3.00E-O1 $5.00 \mathrm{E}-02-1.00 \mathrm{E}-01$ $5.00 E-02--1.00 E-01$
$2.00 E-02---5.00 E-02$
ANGLE 1 $M U=-1.0000$ $1.091 \mathrm{E}-05$ $6.632 E-04$ $1.168 \mathrm{E}-04$ $2.353 E-04$ $5.048 E-04$ $9.295 \mathrm{E}-04$ $1.002 \mathrm{E}-03$ $1.456 \mathrm{E}-03$ $1.027 \mathrm{E}-03$ $6.173 E-04$ $3.955 \mathrm{E}-05$ $5.918 \mathrm{E}-03$ $8.827 E-03$ $5.466 \mathrm{E}-02$ $1.506 \mathrm{E}-01$ $4.546 E-C 1$ 1.409E-01 ANGLE 10 $M U=0.0950$ $2.840 E-05$ $1.660 \mathrm{E}-04$ $1.895 E-03$ $1.048 \mathrm{E}-03$ $1.287 E-03$ $1.750 E-03$ $2.380 \mathrm{E}-03$ 2.115E-C3 2.582E-0 3 $6.834 E-03$ $6.834 E-03$ $2.437 E-02$ G.8 $1.01 E-02$ $1.93 E-01$ $5.689 \mathrm{E}-01$
$1.556 \mathrm{E}-01$
ANGLE 2 $.117 E-05$ $.378 E-04$ $9.844 E-04$ . $403 E-03$ $9 . \leq 26 E-04$ $6.157 \mathrm{E}-04$ $1.856 E-04$ $5.954 \mathrm{E}-03$ $8.932 E-03$ $5.486 E-02$ $1.509 \mathrm{E}-01$ $4.554 E-02$ 1.410E-01 ANGLE 11 $M U=0.2816$ $3.633 \mathrm{E}-05$ $2.180 E-04$ $2.448 E-03$ $1.421 \mathrm{E}-03$ $1.698 E-03$ $2.262 E-C 3$ $3.015 \mathrm{E}-03$ $3.365 \mathrm{E}-03$ $3.35 \mathrm{E}-03$ $5.620 E-03$ $5.620 E-03$ $1.129 E-C 2$ $4.150 E-C 2$ $6.568 \mathrm{E}-02$ $6.56 \mathrm{E}-02$ $5.077 E-01$ $5.977 E-01$
$1.500 E-01$ $M U=-0.9446 \quad M U=-0.8656$ $1.212 \mathrm{E}-05 \quad 1.354 \mathrm{E}-05$ $.433 E-05 \quad 7.575 E-05$
$.740 E-04$ $7.740 E-04$ 3.028E-04 .355E-04 $1.230 E-03$ $7.073 E-04$ $6.248 E-04$ $6.624 E-04$ $6.098 E-03$ 9.535 $5.559 \mathrm{E}-02$ $1.523 \mathrm{E}-01$ 4.587E-01

ANGLE 12 $M U=0.4580$ $4.811 E-05$ $3.241 E-03$ $1.851 \mathrm{E}-03$ $2.198 \mathrm{E}-03$ $2.984 E-03$ $3.035 \mathrm{E}-03$ $3.885 E-03$ $5.146 E-03$ $5.617 E-03$ $1.685 \mathrm{E}-02$ $1.685 E-02$ $3.646 \mathrm{E}-02$ $4.474 E-02$ 7.08 6.290E1. $624 \mathrm{E}-01$ $6.038 E-04$ . $024 E-04$ $1.097 \mathrm{E}-03$ .817E-04 $1.033 E-03$ $4.372 E-04$ $6.873 \mathrm{E}-04$ $1.272 \mathrm{E}-03$ $6.392 \mathrm{E}-03$ $1.122 \mathrm{E}-02$ $5.658 \mathrm{E}-02$ $1.548 \mathrm{E}-01$ $4.651 E-01$ $1.424 E-0$ ? ANGLE 13 $M U=0.6179$ $6.758 E-05$
$4.231 E-04$ $4.231 E-04$ $4.530 E-03$ 2.511E-03 $3.034 E-03$ 4. $312 \mathrm{E}-03$ (2) $6.332 E-03$ I. $715 E-03$ 1.580E-02 $1.580 \mathrm{E}-02$ 2.296E-02 $4.952 \mathrm{E}-02$ $7.690 \mathrm{E}-02$ . $2.452 E-01$ $6.619 E-01$
$1.658 E-01$ $\begin{array}{lll}\text { ANGLE } & 5 & \text { ANGLE } \\ M U & 6\end{array}$ $M U$
$1.492 \mathrm{E}-05$ $8.560 E-05$ 1.004E-03 $6.095 E-04$
$7.259 \mathrm{E}-04$ $9.101 E-04$ $1.179 \mathrm{E}-03$ $8.862 E-04$ $9.874 \mathrm{E}-04$ $3.765 E-04$ . $336 E-04$ . $672 E-03$ $6.954 \mathrm{E}-03$ $1.446 \mathrm{E}-02$ $5.734 E-02$ $1.586 \mathrm{E}-01$ $4.743 E-01$ $1.436 \mathrm{E}-01$ ANGLE 14 $M U=0.7550$ $1.055 \mathrm{E}-04$ $6.841 \mathrm{E}-04$ $.054 \mathrm{E}-03$ 4.037E-03 4. $964 \mathrm{E}-03$ $7.230 E-03$ $1.002 E-02$ $1.502 \mathrm{E}-02$ 1.502E-02 1.738E-02 . $4.723 E-02$ 5.606E-02 . $0.37 \mathrm{E}$ $6.946 \mathrm{E}-01$ $6.946 \mathrm{E}-01$
$1.690 \mathrm{E}-0$. $1.614 \mathrm{E}-05$
$9.245 \mathrm{E}-05$ $1.080 \mathrm{E}-03$ $6.371 \mathrm{E}-04$ $7.568 E-04$ .262 E-0 .155E-03 5.801E-04 $1.040 E-03$ $1.775 \mathrm{E}-03$ ..088E-03 $1.930 E-02$ $5.770 \mathrm{E}-02$ $1.636 E-01$ $4.866 E-01$ .

\section{ANGLE 15} $M U=0.8656$ . 1.316E $.047 E-03$ $9.699 E-03$ $1.384 E-02$ 1.839E-O2 $2.017 E-02$ $2.541 E-02$ $2.674 E-02$ 3.139E3.5 . 6. $2.810 \mathrm{E}-01$ $7.249 E-O 1$ $1.247 \mathrm{E}-01$

ANGLE 7 ANGLE 8 ANGLE 9 $M U=-0.4580$ $1.747 \mathrm{E}-05$ $1.151 \mathrm{E}-03$ $6.106 \mathrm{E}-04$ . $1.182 E-03$ .408E-04 $1.021 E-02$ 5.5005 S.780E-02 $1.700 E-01$ 
(GAMMAS/MEV/STERADIAN/SOURCE NEUTRON)

ENERGY 8. OOE OO- (MEV) 6.5 OE DO---8.0OE OO 5.00 E $00---6.50 E$ OO $4.00 E \quad 00---5.00 E \quad 00$ $3.00 E \quad 00---4.00 E \quad 00$ $2.50 E \quad 00---3.00 E \quad 00$ $2.00 E$ OO- - - 2.5OE 00 $1.66 \mathrm{E} 00---2.00 \mathrm{E} 00$ $1.33 E \quad 00--1.66 E 00$ $1.00 \mathrm{E} 00--1.33 \mathrm{E} 00$ $8.00 E-01--1.00 E$ OO $6.00 E-01--8.00 E-01$ $4.00 E-01---6.00 E-C 1$ $3.00 E-01--4.00 E-01$ $2.0 C E-01---3.00 E-01$ $1.00 E-01--2.00 E-01$ $5.00 E-02---1.00 E-01$ 2. OCE-02---5. $00 E-02$ ENERGY GROUP (MEV) 8. $.00 E$ OO---1.00E O1 $6.50 E$ OO- - 8. . COE 00 $5.00 E$ OO- $-6.50 E$ OO

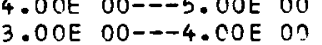

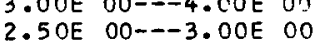
$2.00 E$ OO- - 2.5OE OC $1.66 \mathrm{E}$ OO---2.00E $\mathrm{OO}$ $1.33 \mathrm{E}$ O0- -1.66E 00 1. OOE OD--1.33E OD $8.00 E-01--1.00 E$ OD $6.00 E-01=-8.00 E-01$ 4. . OOE-01---6.00E-01 3.00E-01--4.00E-01 $2.00 E-01--3.00 E-0$ $1.00 E-01-\cdots 2.00 E-01$ $5.00 E-02--1.00 E-01$ $2.00 E-02--5.00 E-02$
ANGLE 1 $U=-1.0000$ $4.564 E-06$ $2.095 \mathrm{E}-04$ - $.491 E-05$ 1.348E-06 $1.133 \mathrm{E}-04$ $3.401 E-04$ $4.527 E-04$ $5.321 E-04$ $3.070 \mathrm{E}-03$ $4.486 \mathrm{E}-03$ $3.308 \mathrm{E}-02$ $8.891 \mathrm{E}-0$ $2.774 \mathrm{E}-0$ $8.641 \mathrm{E}-02$ ANGLE 10 $M U=0.0950$ $1.378 E-05$ $6.442 E-05$ $7.739 E-04$ $4.352 E-04$ $5.611 E-04$ $6.939 E-04$ (O) O. $9.55 E-04$ 1.717E 03 $1.77 E-03$ $4.278 E-03$ . $3.532 \mathrm{E}-02$ 1.177E- 01 $3.479 E-01$ $9.536 \mathrm{E}-02$
ANGLE 2 $4.758 \mathrm{E}-06$ $1.618 \mathrm{E}-05$ $2.262 E-04$ $1.684 E-05$ $3.277 E-05$ $1.361 E-04$ $4.340 E=04$ $6.504 E-04$ 4.795E-04 $1.805 E-04$ 1.368E-04 $4.572 E-03$ 3. $319 \mathrm{GE}-0$ $8.011 \mathrm{E}-02$ $2.779 E-01$ $8.648 \mathrm{E}-02$ ANGLF 11 $M U=0.2816$
$1.807 E-05$ $1.807 E-05$
$8.788 E-05$ $8.788 E-05$ $1.033 E-03$ $6.292 E-04$ $7.692 E-04$ $. .140 \varepsilon-04$ $1.030 E-03$ $1.030 E-03$ $1.303 E-03$ $3.424 E-03$ $7.424 E-03$ ?.311E-03 $2.378 \mathrm{E}-02$ $2.378 \mathrm{E}-02$ $1.254 \mathrm{E}-01$ $3.654 E-01$ $9.735 \mathrm{E}-02$ $\begin{array}{lc}\text { ANGLE } & 3 \\ M U=-0.9446\end{array}$ ANGLE $\quad 4$
$M U=-0.8656$ $.441 E-06$ $2.843 E-04$ $7.109 \mathrm{E}-05$ $1.348 E-04$ $2.135 E-04$ $3.733 E-04$ $3.707 E-04$ 4. $972 E-04$ $3.004 \mathrm{E}-04$ $2.120 E-04$ $1.821 E-04$ $3.114 \mathrm{E}-03$ 5. $344 E-03$ $3.354 E-02$ $8.996 \mathrm{E}-\mathrm{C2}$ 2.80JE-01 8.677E-02 $\mathrm{U}=-0.8656$ $.395 E-06$
$2.917 E-05$ $3.613 E-04$ $2.104 E-04$ $2.611 E-04$ $3.077 E-04$ $4.057 E-04$ $3.235 \mathrm{E}-04$ 1.140E-04 $2.803 E-04$ $6.188 E-04$ $3.228 E-03$ 6. 5 हE 03 $3.396 E-02$ 9.153E-02 $8.731 \mathrm{E}-02$ ANGLE 12 $M U=0.4580$
$2.415 E-05$ $2.415 E-05$
$1.193 E-04$ $1.374 \mathrm{E}-03$ $7.924 E-04$ $9.651 \mathrm{E}-04$ $1.197 E-03$ . $6.285 E-03$ $1.087 E-02$ $2.154 E-02$ 政-02 4.004E-02 3. $842 E-01$ $9.938 E-02$ ANGLE 13 $M U=0.6179$ $3.411 E-05$ $1.720 E-04$ $1.928 \mathrm{E}-03$ $1.026 \mathrm{E}-03$ . $1.024 E-02$ $1.448 \mathrm{E}-02$ $2.408 \mathrm{E}-02$ 2.787 E-02 $4.308 E-02$ $4.037 E-01$ $1.014 \mathrm{E}-01$ ANGLE 5
$M U=-0.7550 \quad M U=-0.6179$ $7.175 \mathrm{E}-06$ $3.424 E-05$ $3.334 E-04$ $3.581 E-04$ $4.303 E-04$ $9.208 E-05$ $3.764 \mathrm{E}-04$ $8.051 E-04$ $3.544 E-03$ 8. $488 E-03$ $9.378 \mathrm{E}-02$ $2.897 \mathrm{E}-01$ $8.808 E-02$ ANGLE 14

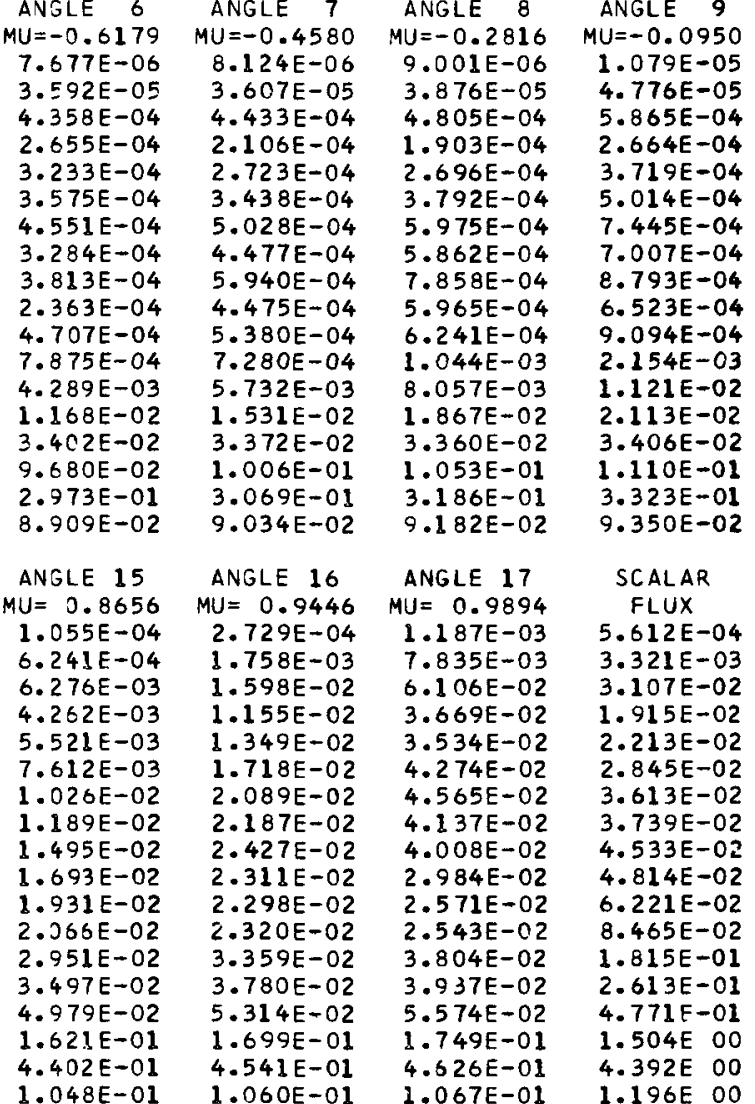


(GAMMAS/MEV/STERADIAN/SOURCE NFUTRON)

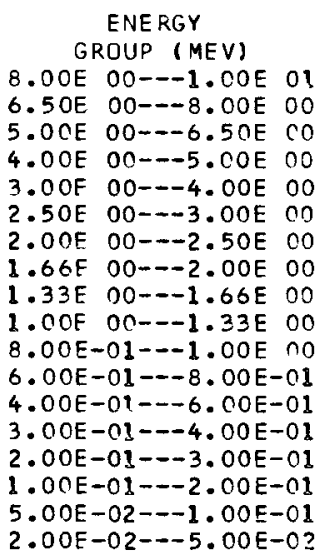

ENERGY

GROUP (MEV)

8.00E OO- -1. 1.00E OI

$6.50 \mathrm{E} \quad 00---8.00 \mathrm{E}$ ON

$5.00 \mathrm{E} \quad 00---6.50 \mathrm{E} \quad 00$

4. OOE OO- - -5. OOE OO

$3.00 E$ OO- $-4.00 E$ CO

$2.50 E$ OO- $-3.00 E$ OO

$2.00 \mathrm{E}$ OO---2.50E OO

1.66E $00--2.00 E$ OO

.33E $00---1.66 E$ EO

$1.00 \mathrm{E} \mathrm{OO-D-1.33E} \mathrm{OO}$

$8.00 E-01--1.00 E$ ON

$6.00 E-01---8.00 \mathrm{E}-01$

$4.00 E-01--6.00 E-01$

$3.00 E-01--4.00 E-0$

$2.00 \mathrm{E}-01--3.00 \mathrm{E}-01$

$1.00 E-01--2.00 E-01$

$5.00 E-02--1.00 E-01$
$2.00 E-02---5.00 E-02$
ANGLE 1

$M=-1.0000$ $1.607 \mathrm{E}-06$

$2.120 \mathrm{E}-06$
$5.010 \mathrm{E}-05$

$-7.057 \mathrm{E}-05$

$-4.8925-05$

$2.341 \mathrm{E}-06$

$1.183 \mathrm{E}-04$

$2.049 E-04$

$2.049 E-04$
$3.246 E-04$

$2.528 \mathrm{E}-04$

1.520E-05

$-2.117 E-04$

1. $4 \in 2 E-03$

$2.048 \mathrm{E}-03$

$1.734 E-02$

4.497E-02

$1.414 \mathrm{E}-0$

4.392E-02

ANGLE 10

$U=0.0950$

$6.071 \mathrm{E}-06$

$2.400 E-05$

$2.951 E-04$

$2.306 \mathrm{E}-04$

$2.306 E-04$
$2.597 E-04$

$3.308 \mathrm{E}-04$

$2.644 E-04$

$2.945 \mathrm{E}-04$

$3.001 E-04$

$8.401 E-04$

$2.336 F-03$

$7.802 \mathrm{E}-03$

$1.142 \mathrm{E}-02$

$1.754 \mathrm{E}-02$

$5.923 \mathrm{E}-02$

$1.765 \mathrm{E}-\mathrm{O}$

$4.833 E-02$ $\triangle N G L E 2$ $U=-0.9894$ $3.165 \mathrm{E}-06$ $6.001 \mathrm{~F}-05$ $4.831 E-05$ $4.651 \mathrm{E}-0$ $-2.651 E-05$
$1.653 E-05$ $1.653 E-05$ $1.206 E-04$ $1.004 \mathrm{E}-04$
$2.942 \mathrm{E}-04$ $2.92 E-04$ $2.353 E-05$ $-1.481 \mathrm{E}-04$ $1.458 E-03$ 1. $738 \mathrm{E}-02$ $4.507 \mathrm{E}-02$ $1.416 \mathrm{E}-01$
$4.396 \mathrm{E}-02$ ANGLE 11 $M U=0.2816$ $8.213 E-06$ $4.068 \mathrm{E}-04$ $2.698 \mathrm{E}-04$ $3.242 \mathrm{E}-0$ $3.403 E-04$ $4.099 \mathrm{~F}-\mathrm{C}$ 3.32 EE-O4 $4.305 \mathrm{E}-0$ $7.186 \mathrm{E}-04$ $2.843 E-03$ $4.035 \mathrm{E}-03$ $9.555 \mathrm{E}-0$ $1.179 \mathrm{E}-02$ $1.849 \mathrm{E}-02$ $6.293 \mathrm{~F}-02$ $4.930 E-02$
ANGLE 3 $2.165 E-06$ $6.608 \mathrm{E}-06$ $6.726 \mathrm{E}-06$ $2.917 E-05$ $2.917 E-05$ $1.280 \mathrm{E}-04$ $1.280 E-04$
$1.411 E-04$ $1.411 \mathrm{E}-04$
$1.901 \mathrm{E}-04$ $1.032 \mathrm{E}-04$ $1.032 \mathrm{E}-04$
$5.477 \mathrm{E}-05$ $2.891 \mathrm{E}-\mathrm{C5}$ $1.453 E-03$ $2.399 \mathrm{E}-03$ $1.753 E-02$ 4.551E-02 $1.427 \mathrm{E}-01$ 4. $410 E-02$ ANGLE 12 $M U=0.4580$ $1.110 E-05$
$4.574 E-05$ 5. $351 E-04$ $3.069 E-04$ $3.763 E-04$ 4. 269 E-04 $5.906 E-04$ $6.477 E-04$ $1.036 \mathrm{E}-03$ $1.794 \mathrm{E}-0$ $3.4 E 9 E-03$ $5.910 \mathrm{E}-03$ $1.094 E-02$ $1.969 \mathrm{E}-02$ $1.941 \mathrm{E}-01$ 5.028E-02
ANGLE 4 ANGLE 5 7550 $M U=-0.6179$ $1.342 E-05 \quad 1.345 E-05$ $1.367 E-04 \quad 1.617 E-04 \quad 1.634 E-04$ $8.937 E-05$ 1.114E-04 $1.356 \mathrm{E}-04$ $8.290 \mathrm{E}-05$ $8.290 E-05$ $6.382 E-05$
$1.538 E-05$ $1.538 E-05$ $2.735 \mathrm{E}-04$ $1.483 E-03$ $3.119 \mathrm{~F}-03$ $1.765 \mathrm{E}-02$ $4.631 \mathrm{E}-02$ $1.447 \mathrm{E}-01$ ANGLE 13 $M U=0.6179$ $1.582 E-05$
$6.429 E-05$ $7.393 \mathrm{E}-04$ $3.561 E-04$ $4.889 E-04$ $6.873 E-04$ $1.107 E-03$ $1.488 E-03$ $2.361 E-03$ $3.670 \mathrm{E}-03$ $5.613 E-03$ $7.658 \mathrm{E}-03$ $1.195 \mathrm{E}-02$ $1.364 \mathrm{E}-02$ $2.101 \mathrm{E}-02$ $7.133 \mathrm{E}-02$ $2.034 E-01$ $5.123 \mathrm{~F}-02$
$1.184 E-04$ $1.419 E-04$ $1.340 E-04$ $1.395 \mathrm{E}-04$ $6.191 E-05$ $5.718 \mathrm{E}-05$ $1.514 \mathrm{E}-05$ $1.608 E-04$ $3.534 E-04$ $4.746 E-02$ $1.476 \mathrm{E}-01$
$4.475 \mathrm{E}-02$ ANGLE 14 $\begin{aligned} & M U=0.7550 \\ & 2.614 E-05\end{aligned}$ $1.142 \mathrm{E}-04$ $1.254 E-03$ $6.903 E-04$ $1.034 E-03$ $2.407 \mathrm{E}-03$ $3.211 E-03$ $4.593 E-03$ $6.207 E-03$ $7.931 E-03$ $9.104 \mathrm{E}-03$ 1. $288 \mathrm{E}-02$ $1.526 \mathrm{E}-02$ $2.239 \mathrm{E}-02$ $7.569 \mathrm{E}-02$
$2.124 \mathrm{E}-01$ $2.124 \mathrm{E}-01$ 5. $211 \mathrm{E}-02$ $\triangle N$ NGLE 7 $M U=-0.4580$
$3.363 \mathrm{E}-06$ $3.363 E-06$ $1.213 E-05$
$1.546 E-04$ $4.722 E-05$ $8.339 E-05$ $8.339 E-05$ $1.654 \mathrm{E}-04$ $1.654 \mathrm{E}-04$
$1.634 \mathrm{E}-04$ $1.634 \mathrm{E}-04$
$2.304 \mathrm{E}-04$ $2.304 E-04$ $1.956 \mathrm{E}-04$
$1.954 \mathrm{E}-04$ $1.954 E-04$ $2.889 E-03$ $8.071 E-03$ $1.708 \mathrm{E}-02$ $5.087 E-02$ $1.562 \mathrm{E}-01$ 4.587E-02 ANGLE 16 $M U=0.9446$ $8.783 E-04$ $7.905 E-03$ $6.294 E-03$ $7.532 E-03$ $9.161 E-03$ $1.083 E-02$ $1.135 \mathrm{E}-02$ $1.217 E-02$ $1.167 \mathrm{E}-02$ $1.129 \mathrm{E}-02$ $1.121 \mathrm{E}-02$ $1.586 \mathrm{E}-02$ $1.780 E-02$ $2.517 E-02$ $8.309 \mathrm{E}-02$ $2.268 \mathrm{E}-01$ $5.341 E-02$
ANGLE 8 $M U=-0.2816$
$3.678 E-06$ $1.225 \mathrm{E}-05$ $1.620 \mathrm{E}-04$ 4. $457 \mathrm{E}-05$ $7.300 E-05$ $1.109 E-04$ $2.071 E-04$
$2.291 E-04$ $3.174 E-04$ 2. $502 E-04$ $2.115 E-04$ 4.264E-04 4.1 $97 E-03$ $9.746 E-03$ $1.687 E-02$ $5.321 E-02$ $1.620 E-01$ 4.660E-02

ANGLE 17 $A U=0.9894$
$7.280 E-04$ $7.280 E-04$ $.357 \mathrm{E}-02$ $2.099 E-02$ $2.005 E-02$ $.208 \mathrm{E}-02$ .250E-02 $1.999 \mathrm{E}-02$ . $836 \mathrm{E}-02$ $1.406 E-02$ $1.197 \mathrm{E}-02$ $1.761 \mathrm{E}-02$ $1.817 \mathrm{E}-02$ $2.617 E-02$ $8.519 E-02$ $2.307 E-01$ 5.374E-0?

ANGLE 9 0.0950 .542E-06 $2.080 E-04$ 
(GAMMAS/MEV/STERADIAN/SOURCE NEUTRONI

\begin{abstract}
ENERGY
GROUP (MEV) $8.00 E$ OO---1.00E 01 $6.50 E$ OO- - $8.00 E$ OO
$5.00 E$ OO- - 6.50E 4.0 OE OD--5.00E 00 $3.00 E$ OO- $004.00 E$ OO $? .50$ E $00--3.00 E$ E 00 $2.00 E$ OO- - 2.5OE 00 $1.66 \mathrm{E} 00-2.00 \mathrm{E} 00$ $1.33 \mathrm{E} 00--1.66 \mathrm{E}$ 00 $1.00 \mathrm{E} 00--1.33 \mathrm{E} 00$ 8.00E-01---1. $.00 E$ OO $6.00 \mathrm{E}-01-2-8.00 \mathrm{E}-01$ $4.00 E-01--6.00 E-01$
$3.00 E-01--4.00 E-01$ $2.00 \mathrm{E}-01--3.00 \mathrm{E}-\mathrm{C1}$ 1.00E-01--2.00E-01 $5.00 \mathrm{E}-02--1.00 \mathrm{E}-01$
\end{abstract}

\section{ENERGY}

ENERGY
GRDUP (MEVI

8.0OE 00---1.00E $6.50 E$ OO-- $-8.00 E$ OO $5.00 E$ OO- -6.50 E 00 $4.00 E$ OO- $-5.00 E$ OO $3.0 O E$ OO- $-4.00 E$ OO 2.50 E OC- -3.00 E OO $2.00 E$ 0O---2.50E OO $1.66 E$ OO-D-2.00E OO $1.33 \mathrm{E} 00--1.66 \mathrm{E} 00$ 1.00 E $00--1.33$ E $\mathrm{NO}$ $8.00 E-01--1.00 E$ OO $6.00 E-01=-8.00 E-01$ $4.00 F-01--6.00 E-01$ $3.00 E-01--4.00 E-01$ $2.00 E-01---3.00 E-01$
$1.005-01---2.00 E-01$ $1.00 E-01---2.00 E-01$
$5.00 E-02=-1.00 E-01$ $2.00 E-02-\cdots-5.00 E-02$ $M U=-1.0000$ $4.315 \mathrm{E}-07$ $-8.921 E-07$ $-4.991 E-05$ $-4.169 E-05$ $-1.590 E-05$ $4.180 \mathrm{E}-05$ $9.498 E-05$ $1.514 E-04$ $1.158 \mathrm{E}-04$ $-2.089 \mathrm{E}-05$ $-1.377 E-04$ $6.736 E-04$ $8.962 E-04$ $8.509 E-03$ $2.131 \mathrm{E}-0$ $6.697 E-02$ $2.072 E-02$

ANGLE 10 $M U=0.0950$ $2.467 \mathrm{E}-06$ $8.852 E-06$ 1.087E-04 $.242 E-05$ $9.464 E-05$ 7.414E-0 $7.127 E-05$ $9.941 \mathrm{E}-05$ $3.983 E-04$ $1.195 \mathrm{E}-03$ $3.828 \mathrm{E}-03$ $5.404 E-03$ $8.216 E-03$ 2.7 IE-O $8.311 E-02$
ANGLE 2 $M U=-0.9804$ $5.065 E-07$
$-2.017 E-07$ $9.600 E-06$ 列 $-2.954 E-05$ $-7.876 E-06$ $4.212 \mathrm{E}-05$ $8.576 \mathrm{E}-05$ $1.337 E-04$ 9.962E-0 $-1.438 E-05$
$-1.040 E-04$ $0.687 \mathrm{~F}-04$ $9.287 E-04$ $8.525 \mathrm{E}-03$ $2.136 \mathrm{E}-02$ 6.7C9E-0 $2.073 E-02$

ANGLE 11 $M U=0.2816$ 4E $1.323 E-05$ .130E-04 $1.333 \mathrm{E}-04$ $1.220 E-04$ $.284 \mathrm{E}-04$ $8.040 \mathrm{E}-05$ $1.326 \mathrm{E}-04$ $3.249 \mathrm{E}-04$ $9.355 \mathrm{E}-04$ $2.058 \mathrm{E}-03$ $4.623 E-03$ $5.501 \mathrm{E}-03$ $8.626 E-03$ $2.957 E-02$ $8.655 \mathrm{E}-02$ $M U=-0.9446$ $7.644 E-07$
$1.553 E-06$ $1.553 E-06$ 2.786E-05 $-1.292 E-05$ 1.323E-0 $4.31 E-05$ $5.284 E-05$ $5.430 E-05$ $3.880 \mathrm{E}-05$ $6.773 E-06$ $2.068 E-06$ $2.068 E-06$ $0.548 E-04$ $8.575 \mathrm{E}-03$ $2.157 \mathrm{E}-02$ $6.759 E-02$ $2.080 E-02$

ANGLE 4 1.088E-06 $.170 E-06$ $870 E-05$ .658E-05 $4.167 \mathrm{E}-05$ $4.243 E-05$ $.407 E-05$ $7.180 \mathrm{E}-06$ $4.176 \mathrm{E}-0 \mathrm{5}$ $1.051 E-04$ $6.576 \mathrm{E}-04$ $6.576 \mathrm{E}-04$ $8.599 E-03$ $2.195 E-02$ $6.852 \mathrm{E}-02$ $2.093 \mathrm{E}-02$

ANGLE 5 $1.282 E-06$ $5.272 \mathrm{E}-06$ $6.173 E-05$ . $486 \mathrm{E}-05$ 5.820E-05 $4.861 E-05$ 6.467E-06 $6.467 E-06$ $-7.448 E-06$ $6.742 E-05$ $1.552 \mathrm{E}-04$ $7.330 E-04$ $2.142 E-02$ $8.528 \mathrm{E}-03$ 2. $249 \mathrm{E}-02$ $6.986 E-02$ $2.110 E-02$

ANGLE 12 ANGLE 13 ANGLE 14 $=0.4580$
$4.587 E-06$ . $587 E-06$ $.695 \mathrm{E}-05$
$1.073 \mathrm{E}-04$ $.140 E-04$ $1.346 \mathrm{~F}-04$ $1.391 \mathrm{E}-04$ $1.976 E-04$ $2.453 \mathrm{E}-04$ $4.493 E-04$
$9.058 E-04$ $9.058 \mathrm{E}-04$ $1.777 E-03$ $2.962 \mathrm{E}-03$ $5.195 E-03$ $5.758 E-03$ $9.139 \mathrm{E}-03$ $3.138 E-02$ $9.101 E-02$ $M U=0.6179 \quad M U=0.7550$ . $.116 E-06 \quad 1.166 E-05$ $2.233 E-05 \quad 4.173 E-05$ .613E-04 1.015E-04 $.566 E-04$ $2.426 \mathrm{E}-04$ $4.470 E-04$ $6.889 E-04$ 1.155E-03 $1.89 J E-03$ 2. $835 \mathrm{E}-03$ $3.737 E-03$ $5.566 \varepsilon-03$ $6.298 \mathrm{E}-03$ . $3.327 E-02$ (.514E-02
ANGLE 6

$A N G L E \quad 6 \quad$ ANGLE 7
$M U=-0.6179$
MU $=-0.4580$ $1.305 \mathrm{E}-06$ . 3.786E-05 $2.300 E-05$ 2.760E-05 . $707 \mathrm{E}-05$ $7.644 \mathrm{E}-05$ $1.093 \mathrm{E}-04$ $9.529 E-04$ $3.033 E-03$ $8.353 E-03$ $2.321 E-02$ $7.163 \mathrm{E}-02$ $2.133 E-02$

ANGLE 15 $1.253 \mathrm{E}-06$ $3.699 \mathrm{E}-06$ $4.912 E-05$ . 1.358E-05 . 5.970E-05 . 8.303E-05 $6.337 E-05$ $8.005 \mathrm{E}-05$ $1.388 \mathrm{E}-03$ $3.976 E-03$ $8.131 E-03$ $2.409 \mathrm{E}-02$ $7.384 E-02$ $2.384 E-02$ ANGLE 16 $M U=0.9446$ $7.888 \mathrm{E}-05$ $4.238 E-04$
$3.729 E-03$ $3.729 \mathrm{E}-03$ $3.246 E-03$ $3.939 \mathrm{E}-03$ 4.641E-03 $5.536 \mathrm{E}-03$ $5.742 \mathrm{E}-03$ $5.443 E-03$ $5.149 E-03$ $5.073 E-03$ $7.136 E-03$ $7.984 \mathrm{E}-03$ $1.137 \mathrm{E}-02$ $3.827 E-02$ $2.498 \mathrm{E}-02$

ANGLE 8 ANGLE 9 $1.334 E-06 \quad M U=-0.0950$ 3.330E-06 $5.070 E=06$ ..3 2.568E-06 $2.310 E-05$ $4.226 E-05$ $2.773 E-05 \quad 5.641 E-05$ 5.641E-05 $9.089 E-05$ Q. $0505 E-05$ $1.273 E-04 \quad 1.080 E-04$ $9.840 \mathrm{E}-05 \quad 7.596 \mathrm{E}-05$ $5.965 E-05 \quad 1.373 E-04$ $1.602 E-04 \quad 5.378 E-04$ $2.062 E-03 \quad 2.920 E-03$ $4.756 E-03 \quad 5.222 E-03$ $7.974 E-03 \quad 7.988 E-03$ $2.517 \mathrm{E}-02 \quad 2.644 \mathrm{E}-02$ $7.651 \mathrm{E}-02 \quad 7.961 \mathrm{E}-02$
$2.195 \mathrm{E}-02$ ANGLE 17 SCALAR $M U=0.9894 \quad$ FLUX $2.445 \mathrm{E}-03 \quad 7.755 \mathrm{E}-04$ 
(GAMMAS/MEV/STERADIAN/SOURCE NEUTRON)

ENERGY GROUP (MEV) 8.00E $00--1.00 E 01$ 6.50E $00---8.00 \mathrm{E}$ OO $5.00 E$ OO- -6.50E DO 促 2.5OE OO- - 3 . 00 COE $2.00 \mathrm{E}$ ON---2.50E 00 $1.66 \mathrm{E} \quad 00--2.00 \mathrm{E} 00$ $1.33 \mathrm{E} 00--1.66 \mathrm{E} 00$ 1.0OE $00--1.33 E$ OO 8. OCE $-01--1.00 E$ DO $6.00 E-01--8.00 E-01$ $4.00 E-01--6.00 E-01$ $3.00 E-01---4.00 E-01$ $2.00 E-01--3.00 E-01$ $1.00 \mathrm{E}-01---2.00 \mathrm{E}-01$ $5.00 E-02--1.00 E-0$ 2.00E-02---5.00E-02

$$
\text { ENERGY }
$$

GROUP (MEV) 8.0OE OO---1.00E OI 6.5 NE $00---8.00 E$ OO 5.00 E DO--6.50E 00 4.00 F $00--5.00 E$ CO . CNE OO-Z4. OOE 00 $2.50 E$ OO- -3.00 E $0 O$ $2.00 E$ OO- $2.50 E$ OO $1.66 E$ CO-C2.00E 00 $1.32 E$ OO-D-1.66E OO 1. OOE OO- $1.33 E$ OO C.ONE OI . OOE-OI- $-8.00 E-O 1$ $3.00 E-01--4.00 E-01$ . OOE $-01--3.00 E-01$ $1.00 E-01--2.00 E-01$ $5.00 E-02-1.00 \mathrm{E}-01$ $2.00 E-02--5.00 \mathrm{~F}-02$
ANGLE 1 ANGLE 2 $M U=-1.0000 \quad M U=-0.58 \subseteq 4$ $4.466 \mathrm{E}-08$ $-1.143 E-06$ $-7.695 \mathrm{E}-07$ -1
-1 $-1.279 \mathrm{E}-05$ $1.573 \mathrm{E}-05$ $4.499 E-05$ $7.093 \mathrm{E}-05$ $5.230 \mathrm{E}-05$ $-2.023 E-05$ $3.062 \mathrm{E}-04$ $3.865 E-04$ $4.039 E-03$ $9.809 E-03$ $9.468 \mathrm{E}-\mathrm{C}$ ANGLE 10 $M U=0.0950$ $9.490 E-07$ $3.325 E-06$ $3.956 \mathrm{E}-05$ $3.077 E-05$ $3.947 E-05$ $3.630 E-05$ $3.488 \mathrm{E}-05$ $1.526 E-05$ $5.524 E-06$ $2.857 E-05$ $1.866 E-04$ . . $3.747 \mathrm{E}-03$ . 1.277E-02 $1.036 \mathrm{E}-02$ $U U=0.2816$
$1.383 \mathrm{E}-06$ $5.206 E-06$ $5.829 E-05$ $4.873 E-05$ $5.439 E-C 5$ $4.289 F-C 5$ $3.557 E-05$ $1.609 E-05$ 1.83 2 E-O5 $1.472 E-04$ 1.007E-0 $1.007 E-03$ 2.162E-03 2.49 1.350E1. $1.055 \mathrm{E}-02$
ANGLE 3 ANGLE 3 $2.355 \mathrm{E}-07$ $2.138 \mathrm{E}-07$ $6.508 \mathrm{E}-06$ $-3.376 \mathrm{E}-06$ $-1.751 E-07$ $1.857 \mathrm{E}-06$ $1.446 \mathrm{E}-05$ $1.876 \mathrm{E}-05$ $2.501 \mathrm{E}-05$ 1. $447 \mathrm{E}-05$ $-1.765 E-06$ $-5.102 E-06$ $2.915 E-04$ 4. $902 \mathrm{E}-04$ 4.063E-03 $9.929 \mathrm{E}-03$ $3.102 \mathrm{E}-02$ $9.505 E-03$ ANGLE 4 $M U=-0.8656$ $4.132 E-07$ $1.623 E-06$ $1.183 \mathrm{E}-05$ $1.515 \mathrm{E}-05$ $1.568 \mathrm{E}-05$ 1. $249 \mathrm{E}-0.5$ $3.030 \mathrm{E}-07$ $-5.655 E-06$ . $186 \mathrm{E}-06$ $4.165 E-05$ $2.880 E-04$ $6.872 E-O 4$ $4.055 E-03$ $1.011 E-02$ $3.144 \mathrm{E}-02$

ANGLE 12 $U=0.4580$ $1.846 E-06$ $6.136 E-06$ . $4.412 E-05$ $4.054 E-05$ . 4. $443 E-04$ $8.443 E-04$ $2.391 \mathrm{E}-03$ $M U=0.6179$ $2.608 \mathrm{E}-06$ $7.027 E-06$ $8.460 \mathrm{E}-05$ $1.840 E-05$ $3.992 \mathrm{E}-05$ $8.101 F-05$ $1.817 E-04$ $3.197 E-04$ $5.579 E-04$ $9.342 E-04$ $1.371 E-03$ $2.520 \mathrm{E}-03$ 2.5 $4.360 E-03$ . $1.429 \mathrm{E}-02$ $1.510 \mathrm{E}-02$ $4.314 \mathrm{E}-0$ $4.136 \mathrm{E}-02$
$1.074 \mathrm{E}-02$ $A N G L E \quad 5$
$M U=-0.7550$ 5.099E-07 $2.086 \mathrm{E}-06$ $2.315 \mathrm{E}-05$ $2.459 E-05$ $2.709 \mathrm{E}-0$ . $-5.160 \mathrm{E}-06$ $-1.186 E-05$ $3.076 E-05$ $3.042 E-05$ $1.016 \mathrm{E}-03$ $1.035 \mathrm{E}-02$ $3.204 \mathrm{E}-0$ $9.639 E-03$

\section{ANGLE 14}

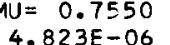
$4.823 E-06$
$1.412 E-05$ $1.412 \mathrm{E}-05$
$1.610 \mathrm{E}-04$ $7.229 E-05$ $1.599 \mathrm{E}-04$ $2.946 \mathrm{E}-04$ $5.136 \mathrm{E}-04$ . $718 \mathrm{E}-04$ $1.121 \mathrm{E}-03$ $1.530 E-O 3$ $1.843 E-03$ 2.637E-O 2.637E$4.15 \mathrm{E}-03$ 4.584E-03 $4.483 \mathrm{E}-02$ $4.483 E-02$
$1.109 E-02$
ANGLE 6 $M=-0.6179$ $4.703 \mathrm{E}-07$ 1. $759 \mathrm{E}-06$ $1.137 \mathrm{E}-05$ $1.314 E-05$ $1.027 \mathrm{E}-05$ $1.101 E-05$ 3.606E-06 5. $346 E-06$ $9.145 E-06$ $2.895 \mathrm{E}-05$ $3.749 E-05$ $4.332 E-04$ $1.448 \mathrm{E}-03$ $3.891 \mathrm{E}-03$ $1.068 \mathrm{E}-02$ $3.283 \mathrm{E}-02$ $9.741 E-03$ ANGLE 15 $M U=0.8656$
$1.221 \mathrm{E}-05$ $1.221 E-05$
$4.847 E-05$ 4. $847 E-05$
$4.806 E-04$ 4. $259 E-04$ $6.529 E-04$ $9.114 E-04$ $1.230 \mathrm{E}-03$ $1.518 E-03$ $1.835 E-03$ $2.165 \mathrm{E}-03$ $2.113 \mathrm{E}-0$ $2.113 E-03$ 2.840E-O $4.815 \mathrm{E}-03$ $4.815 E-03$ $4.6631 E-02$ $1.123 \mathrm{E}-02$

ANGLE 7 ANGLE 8 $7.583 E-07$ $2.323 E-05$ $6.509 \mathrm{E}-04$ $1.892 \mathrm{E}-03$ $3.761 E-03$ $1.108 \mathrm{E}-0$ $3.382 \mathrm{E}-02$ $9.866 E-03$

\section{ANGLE 16} $M U=0.9446$ $2.011 E-04$ $1.713 \mathrm{E}-0$ $1.620 \mathrm{E}-03$ $1.982 \mathrm{E}-03$ $2.281 \mathrm{E}-03$ $2.546 \mathrm{E}-03$ $2.609 \mathrm{E}-03$ $2.626 \mathrm{E}-03$ $2.445 E-03$ $2.273 E-03$ $2.235 E-03$ $3.1515 \mathrm{E}-03$ $5.040 E-03$ $1.719 \mathrm{E}-02$ $4.745 \mathrm{E}-02$ $1.133 \mathrm{E}-02$ 
4 PI R**2 HENDERSON DOSE (NEUTRCNS) (CM* 2 RAD/STERACIAN/SOURCE NEUTRON)
8.187 TO 10.000 MEV NEUTRON SOURCE

RANGE (METERS)

$200.0 \quad 250.0$

300.0

400.0

\section{$1.102 \mathrm{E}-10$}

$1.128 E-10$ 1.271E-10

$1.141 E-10$

$1.29 C E-10$

$1.302 \mathrm{E}-10$
$1.330 \mathrm{E}-10$

$1.330 \mathrm{E}-10$

$1.401 \mathrm{E}-10$

$1.493 \mathrm{E}-10$

$1.662 \mathrm{E}-10$
$1.889 \mathrm{E}-10$

$1.889 \mathrm{E}-10$

$2.521 \mathrm{E}-10$

$2.521 E-10$

$2.954 \mathrm{E}-10$
$3.617 \mathrm{E}-10$

$3.617 \mathrm{E}-10$
$4.895 \mathrm{E}-10$

$4.895 \mathrm{E}-10$

$1.854 \mathrm{E}-09$

$1.194 E-08$

$1.419 \mathrm{E}-09$

$.654 \mathrm{E}-09$
$1.528 \mathrm{E}-08$

$8.65631 E-01$

$9.89401 \mathrm{E}-\mathrm{Cl}$

TOTAL

$6.164 E-09$

$1.505 E-08$

$6.058 \mathrm{E}-09$

1. $378 \mathrm{E}-08$

$5.916 E-09$

$5.781 \mathrm{E}-09$

$1.351 \mathrm{E}-10$ $1.358 \mathrm{E}-10$ 1.388 E- 10 $1.434 \mathrm{E}-10$ $1.500 E-10$ $1.500 \mathrm{E}-10$ $1.780 \mathrm{E}-10$ $2.017 \mathrm{E}-10$ $2.313 \mathrm{E}-10$ $2.313 \mathrm{E}-10$ $2.673 E-10$ $3.813 \mathrm{E}-10$ $5.110 E-10$ $7.942 \mathrm{E}-10$ $1.675 \mathrm{E}-09$ $1.005 \mathrm{E}-08$

$5.545 \mathrm{E}-09$

$1.382 \mathrm{E}-10$ $1.342 \mathrm{E}-10$ $1.469 E-10 \quad 1.408 E-10$ $1.538 \mathrm{E}-10-1.476 \mathrm{E}-10$ $1.645 \mathrm{E}-10 \quad 1.580 \mathrm{E}-10$ $1.822 \mathrm{E}-10 \quad 10743 \mathrm{E}-1 \mathrm{C}$ $2.058 \mathrm{E}-10 \quad 1.958 \mathrm{E}-10$ $2.356 E-10 \quad 2.228 E-10$ $2.710 \mathrm{E}-10 \quad 2.556 \mathrm{E}-10$ $3.166 \mathrm{E}-10 \quad 2.980 \mathrm{E}-10$ $3.858 \mathrm{E}-10 \quad 3.625 \mathrm{E}-10$ $5.134 \mathrm{E}-1 \mathrm{C} \quad 4.778 \mathrm{E}-10$ $7.822 \mathrm{E}-10 \quad 7.093 \mathrm{E}-10$ $1.536 \mathrm{E}-09 \quad 1.278 \mathrm{E}-09$ $8.366 \mathrm{E}-09 \quad 5.700 \mathrm{E}-09$

$5.240 E-09 \quad 4.493 E-09$

$\begin{array}{lr}\text { RANGE } & \text { (METERS } \\ 900.0 & 1200\end{array}$

$500.0 \quad 600.0$

$120 \mathrm{C} .0$

1500.0

1800.0

$-1.0000 \mathrm{CE}$ DO $-9.44575 E-01$ $-8.65631 E-0$ $-7.55044 E-01$ $-6.17876 E-01$ $-4.58017 E-0$ $-2.81605 E-01$ $-9.50125 E-02$ $9.50125 \mathrm{E}-02$ $2.81605 \mathrm{E}-01$ $4.58 \cap 17 \mathrm{E}-01$ $6.17876 \mathrm{E}-01$ $7.55044 \mathrm{E}-01$ $8.65631 \mathrm{E}-\mathrm{O} 1$ $9.44575 E-01$ $9.89401 \mathrm{E}-01$

$1.1+6 E-10$ $4.726 \mathrm{E}-11$ $1.920 E-11$ $7.033 \mathrm{E}-12$ $2.403 \mathrm{E}-12$ $9.799 E-11$ $4.739 \mathrm{E}-11$ $1.925 \mathrm{E}-11$ $7.053 \mathrm{E}-12$ $2.410 \mathrm{E}-12$ $1.010 E-10$ $1.246 E-10$ 1.949E-11 $7.140 E-12$ $4.898 \mathrm{E}-11$ 1.993E-11 $7.306 \mathrm{E}-12 \quad 2.4912$ $5.074 \mathrm{E}-11 \quad 2.065 \mathrm{E}-11 \quad 7.572 \mathrm{E}-12 \quad 2.590 \mathrm{E}-12$ $\begin{array}{lllll}1.097 \mathrm{E}-10 & 5.329 \mathrm{E}-11 & 2.170 \mathrm{E}-11 & 7.957 \mathrm{E}-12 & 2.722 \mathrm{E}-12 \\ 1.175 \mathrm{E}-10 & 5.703 \mathrm{E}-11 & 2.319 \mathrm{E}-11 & 8.497 \mathrm{E}-12 & 2.904 \mathrm{E}-12\end{array}$ $\begin{array}{llllll}1.400 \mathrm{E}-10 & 1.175 \mathrm{E}-10 & 5.703 \mathrm{E}-11 & 2.319 \mathrm{E}-11 & 8.497 \mathrm{E}-12 & 2.904 \mathrm{E}-12 \\ 1.539 \mathrm{E}-10 & 1.288 \mathrm{E}-10 & 6.218 \mathrm{E}-11 & 2.521 \mathrm{E}-11 & 9.220 \mathrm{E}-12 & 3.147 \mathrm{E}-12\end{array}$ $\begin{array}{llllll}1.722 \mathrm{E}-10 & 1.437 \mathrm{E}-10 & 6.892 \mathrm{E}-11 & 2.783 \mathrm{E}-11 & 1.015 \mathrm{E}-11 & 3.460 \mathrm{E}-12 \\ 1.952 \mathrm{E}-10 & 1.624 \mathrm{E}-10 & 7.740 \mathrm{E}-11 & 3.115 \mathrm{E}-11 & 1.134 \mathrm{E}-11 & 3.859 \mathrm{E}-12\end{array}$ $\begin{array}{llllll}1.952 \mathrm{E}-10 & 1.624 \mathrm{E}-10 & 7.740 \mathrm{E}-11 & 3.115 \mathrm{E}-11 & 1.134 \mathrm{E}-11 & 3.859 \mathrm{E}-12 \\ 2.233 \mathrm{E}-10 & 1.855 \mathrm{E}-10 & 8.81 \mathrm{CE}-11 & 3.539 \mathrm{E}-11 & 1.287 \mathrm{E}-11 & 4.374 \mathrm{E}-12\end{array}$ $\begin{array}{llllll}2.233 \mathrm{E}-10 & 1.855 \mathrm{E}-10 & 8.81 \mathrm{CE}-11 & 3.539 \mathrm{E}-11 & 1.287 \mathrm{E}-11 & 4.374 \mathrm{E}-12 \\ 2.602 \mathrm{E}-10 & 2.159 \mathrm{E}-10 & 1.024 \mathrm{E}-10 & 4.110 \mathrm{E}-11 & 1.492 \mathrm{E}-11 & 5.066 \mathrm{E}-12\end{array}$ $4.132 \mathrm{E}-1 \mathrm{C}$ $6.018 \mathrm{E}-10$ $1.031 \mathrm{E}-\mathrm{C}$ $2.618 E-10$ $3.402 \mathrm{E}-10$ $4.877 E-10$ $8.051 \mathrm{E}-10$ $1.237 \mathrm{E}-10$ $1.582 \mathrm{E}-10$ $4.943 E-11$ $6.249 \mathrm{E}-11$ $1.788 \mathrm{E}-11$ $2.240 E-11$ $5.066 E-12$ $6.053 \mathrm{E}-12$ $7.524 E-12$ 1.582E-10 $6.249 E-11$ 2.240E-11 $7.524 E-12$ $3.351 E-10 \quad 1.227 E-10 \quad 4.161 E-11 \quad 1.340 E-11$

TOTAL

$3.695 E-09$

$2.942 \mathrm{E}-09$

$1.298 E-09$

$5.015 E-10$

$1779 E-10$

.072E-11

$5.947 E-11$ 
COSINE

$-1.0000 \mathrm{CE} 00$ $-9.89401 \mathrm{E}-01$ $-9.44575 E-01$ $-8.65631 \mathrm{E}-01$ $-7.55044 \mathrm{E}-01$ $-6.17876 \mathrm{E}-01$ $-4.58017 \mathrm{E}-01$ $-2.81605 E-01$ $-9.50125 E-02$ $2.81605 E-01$ 4.58017E-01 $6.17876 \mathrm{E}-01$ 7.55044E-O $8.65631 E-01$ $9.44575 \mathrm{E}-01$ $9.89401 E-01$

TOTAL

COSINE

-1.0 COODE CO $-9.44575 \mathrm{E}-01$ $-8.65631 \mathrm{E}-\mathrm{O}$ $-7.55044 E-0$ $-6.17876 \mathrm{E}-01$ $-4.58017 \mathrm{E}-\mathrm{O}$ $-2.81605 \mathrm{E}-01$ $-9.50125 \mathrm{E}-\mathrm{C} 2$ $9.50125 E-02$ $2.81605 \mathrm{E}-0$ $4.58017 \mathrm{E}-01$ $6.17876 \mathrm{E}-0$ $7.55044 \mathrm{E}-\mathrm{O}$ $8.65631 E-01$ $9.44575 \mathrm{E}-01$

TOTAL

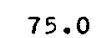
$9.978 \mathrm{E}-11$ $1.026 \mathrm{E}-10$ . $1.046 \mathrm{E}-10$ $.091 \mathrm{E}-10$ $1.168 \mathrm{E}-10$ $1.297 \mathrm{E}-10$ $1.484 E-10$ $1.937 \mathrm{E}-10$ $2.216 E-10$ $2.605 E-10$ $3.483 E-1 C$ $7.186 \mathrm{E}-10$ $2.063 E-09$ $3.863 \mathrm{E}-09$ $2.232 \mathrm{E}-08$

$8.958 \mathrm{E}-09$

500.0

$1.949 E-10$ $1.955 \mathrm{E}-10$ $1.977 \mathrm{E}-10$ $2.008 \mathrm{E}-10$ $2.072 \mathrm{E}-10$ $2.164 \mathrm{E}-10$ $2.301 \mathrm{E}-1 \mathrm{C}$ $2.505 \mathrm{E}-10$ $2.773 E-10$ $3.107 \mathrm{E}-10$ $3.513 \mathrm{E}-10$ $4.040 \mathrm{E}-10$ $4.832 E-10$ $6.217 E-10$ 1. $507 \mathrm{E}-0 \mathrm{O}$ $1.507 E-09$

$5.681 \mathrm{E}-09$
100.0

1. $246 \mathrm{E}-10$ . $257 \mathrm{E}-10$ . $279 \mathrm{E}-10$ . $254 \mathrm{E}-10$ 1. $365 \mathrm{E}-10$ 1. $455 \mathrm{E}-10$ $1.626 \mathrm{E}-10$ $1.860 \mathrm{E}-10$ $2.527 \mathrm{E}-10$ 2.514E- 10 $3.109 \mathrm{E}-10$ 4. $079 E-10$ $6.992 \mathrm{E}-10$ $1.375 \mathrm{E}-09$ $3.678 \mathrm{E}-\mathrm{C} 9$ 2.197E-08

8.802 E-09

600.0

$1.678 \mathrm{E}-10$ $1.683 \mathrm{E}-10$ $1.702 \mathrm{E}-10$ $1.786 \mathrm{E}-10$

$1.864 \mathrm{E}-10$

$1.981 \mathrm{E}-10$

$2.149 \mathrm{E}-10$

$2.368 \mathrm{E}-10$

$2.641 \mathrm{E}-10$

$2.975 E-10$

$3.413 E-10$

$4.067 E-10$

$5.183 \mathrm{E}-10$

$1.184 \mathrm{E}-0$ 1.784E-C

$4.604 E-09$
150.0 RANGE (METERS)
200.0

250.0

30.0 .0

400.0

$1.634 E-10$ $1.645 \mathrm{E}-10$

$1.672 \mathrm{E}-10$

$1.715 \mathrm{E}-10$

$1.792 \mathrm{E}-10$

$1.911 \mathrm{E}-10$

$2.127 \mathrm{E}-10$

$2.420 E-10$

$2.791 \mathrm{E}-10$

3. $279 \mathrm{E}-10$

$3.661 \mathrm{E}-10$

$4.885 \mathrm{E}-10$

$6.398 \mathrm{E}-10$

$1.148 \mathrm{E}-09$

3. $088 \mathrm{E}-09$

$2.010 E-08$

$1.908 \mathrm{E}-10$

$2.079 E-10$

$2.089 \mathrm{E}-10$

$2.135 \mathrm{E}-10$

$2.202 \mathrm{E}-10$

$2.298 \mathrm{E}-10$

$2.447 \mathrm{E}-10$

$2.701 E-10$
$3.041 E-10$

$3.468 \mathrm{E}-10$

$3.977 \mathrm{E}-10$

$4.621 \mathrm{E}-10$ 5.595E-10 $7.443 \mathrm{E}-10$ $1.150 E-09$ $2.424 E-09$ $1.464 \mathrm{E}-08$

$2.165 \mathrm{E}-10$ $2.174 \mathrm{E}-10$ $2.225 \mathrm{E}-10$ 2. $2.396 \mathrm{E}-10$ $2.551 \mathrm{E}-10$ $2.807 E-10$ $3.147 \mathrm{E}-10$ $3.575 \mathrm{E}-10$ $4.081 E-10$ 列 $5.709 \mathrm{E}-10$ $7.525 E-10$ $1.137 \mathrm{E}-09$ $1.224 \mathrm{E}-09$
$1.218 \mathrm{E}-08$

7.779E-09

$2.140 E-10$ $2.148 E-10$ $2.203 \mathrm{E}-10$ $2.273 E-10$ $2.373 \mathrm{E}-10$ $2.526 \mathrm{E}-10$ $2.763 \mathrm{E}-10$ $3.076 \mathrm{E}-10$ $3.466 \mathrm{E}-10$ $3.937 \mathrm{E}-10$ $4.540 E-10$ $5.456 E-10$ $.095 E-10$ $1.041 E-09$ $1.859 E-09$ $8.613 E-09$

8. $459 E-09$

8.169E-09

1800.0

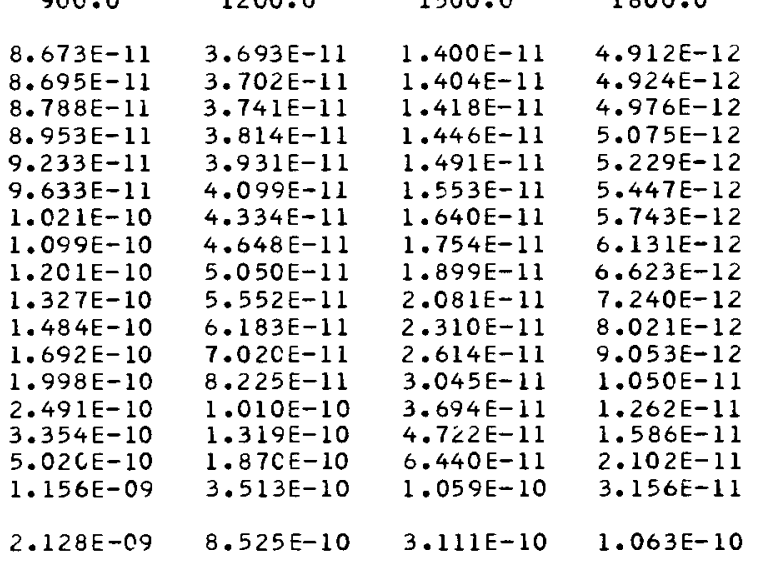




\begin{tabular}{|c|c|c|c|c|c|c|}
\hline COSINE & 75.0 & \multicolumn{5}{|c|}{$\begin{array}{l}\text { RANGE (METERS) } \\
200.0\end{array}$} \\
\hline $\begin{array}{l}-1 . C O C O O E O O \\
-9.89401 E-01 \\
-9.44575 E-01 \\
-8.65631 E-01 \\
-7.55044 E-01 \\
-6.17876 E-01 \\
-4.58017 E-01 \\
-2.81605 E-01 \\
-9.50125 E-02 \\
9.50125 E-02 \\
2.81605 E-01 \\
4.58017 E-01 \\
6.17876 E-01 \\
7.55044 E-01 \\
8.65631 E-01 \\
9.44575 E-01 \\
9.89401 E-01\end{array}$ & $\begin{array}{l}7.142 \mathrm{E}-09 \\
7.213 \mathrm{E}-09 \\
7.349 \mathrm{E}-09 \\
7.436 \mathrm{E}-09 \\
7.492 \mathrm{E}-09 \\
7.822 \mathrm{E}-09 \\
8.384 \mathrm{E}-09 \\
9.317 \mathrm{E}-09 \\
1.068 \mathrm{E}-08 \\
1.404 \mathrm{E}-08 \\
1.602 \mathrm{E}-08 \\
1.886 \mathrm{E}-08 \\
2.528 \mathrm{E}-08 \\
5.234 \mathrm{E}-08 \\
1.505 \mathrm{E}-07 \\
2.820 \mathrm{E}-07 \\
1.630 \mathrm{E}-06\end{array}$ & $\begin{array}{l}8.850 \mathrm{E}-C 9 \\
8.930 \mathrm{E}-09 \\
9.094 \mathrm{E}-09 \\
9.205 \mathrm{E}-09 \\
9.297 \mathrm{E}-09 \\
9.716 \mathrm{E}-09 \\
1.037 \mathrm{E}-08 \\
1.162 \mathrm{E}-08 \\
1.332 \mathrm{E}-08 \\
1.814 \mathrm{E}-08 \\
1.810 \mathrm{E}-08 \\
2.241 \mathrm{E}-\mathrm{C} 8 \\
2.954 \mathrm{E}-\mathrm{C} .8 \\
5 . \mathrm{C} 8 \mathrm{E}-08 \\
1.001 \mathrm{E}-\mathrm{C} \\
2.684 \mathrm{E}-\mathrm{C7} \\
1.6 \mathrm{C} 4 \mathrm{E}-06\end{array}$ & $\begin{array}{l}1.143 \mathrm{E}-08 \\
1.151 \mathrm{E}-08 \\
1.171 \mathrm{E}-08 \\
1.185 \mathrm{E}-08 \\
1.202 \mathrm{E}-08 \\
1.257 \mathrm{E}-08 \\
1.343 \mathrm{E}-08 \\
1.5 C 0 \mathrm{E}-08 \\
1.712 \mathrm{E}-08 \\
1.977 \mathrm{E}-08 \\
2.341 \mathrm{E}-08 \\
2.615 \mathrm{E}-08 \\
3.512 \mathrm{E}-08 \\
4.615 \mathrm{E}-08 \\
8.334 \mathrm{E}-08 \\
2.251 \mathrm{E}-07 \\
1.467 \mathrm{E}-06\end{array}$ & $\begin{array}{l}1.316 \mathrm{E}-08 \\
1.324 \mathrm{E}-08 \\
1.344 \mathrm{E}-08 \\
1.357 \mathrm{E}-08 \\
1.386 \mathrm{E}-08 \\
1.459 \mathrm{E}-08 \\
1.554 \mathrm{E}-08 \\
1.729 \mathrm{E}-08 \\
1.966 \mathrm{E}-08 \\
2.267 \mathrm{E}-08 \\
2.617 \mathrm{E}-08 \\
3.070 \mathrm{E}-08 \\
3.752 \mathrm{E}-08 \\
5.087 \mathrm{E}-08 \\
8.262 \mathrm{E}-08 \\
1.951 \mathrm{E}-07 \\
1.270 \mathrm{E}-06\end{array}$ & $\begin{array}{l}1.413 \mathrm{E}-08 \\
1.420 \mathrm{E}-08 \\
1.440 \mathrm{E}-08 \\
1.452 \mathrm{E}-08 \\
1.500 \mathrm{E}-08 \\
1.569 \mathrm{E}-08 \\
1.675 \mathrm{E}-08 \\
1.858 \mathrm{E}-08 \\
2.104 \mathrm{E}-08 \\
2.416 \mathrm{E}-08 \\
2.780 \mathrm{E}-08 \\
3.252 \mathrm{E}-08 \\
3.959 \mathrm{E}-08 \\
5.314 \mathrm{E}-08 \\
8.278 \mathrm{E}-08 \\
1.759 \mathrm{E}-07 \\
1.068 \mathrm{E}-06\end{array}$ & $\begin{array}{l}1.451 \mathrm{E}-08 \\
1.457 \mathrm{E}-08 \\
1.477 \mathrm{E}-08 \\
1.493 \mathrm{E}-08 \\
1.543 \mathrm{E}-08 \\
1.614 \mathrm{E}-08 \\
1.724 \mathrm{E}-08 \\
1.908 \mathrm{E}-08 \\
2.153 \mathrm{E}-08 \\
2.461 \mathrm{E}-08 \\
2.829 \mathrm{E}-08 \\
3.297 \mathrm{E}-08 \\
4.014 \mathrm{E}-08 \\
5.340 \mathrm{E}-08 \\
8.154 \mathrm{E}-08 \\
1.610 \mathrm{E}-07 \\
8.885 \mathrm{E}-07\end{array}$ \\
\hline TOTAL & $6.524 E-07$ & $6.395 E-07$ & $6.223 E-07$ & $6.071 E-07$ & $5.820 E-07$ & $5.499 E-07$ \\
\hline COSINE & 500.0 & $6 \mathrm{C} 0.0$ & $\begin{array}{l}\text { RANGE } \\
900.0\end{array}$ & $\begin{array}{l}\text { TERS) } \\
1200.0\end{array}$ & 1500.0 & 1800.0 \\
\hline $\begin{array}{l}-1.00000 \mathrm{E} 00 \\
-9.89401 \mathrm{E}-01 \\
-9.44575 \mathrm{E}-01 \\
-8.65631 \mathrm{E}-01 \\
-7.55044 \mathrm{E}-01 \\
-6.17876 \mathrm{E}-01 \\
-4.58017 \mathrm{E}-01 \\
-2.81605 \mathrm{E}-01 \\
-9.50125 \mathrm{E}-02 \\
9.56125 \mathrm{E}-02 \\
2.81605 \mathrm{E}-01 \\
4.58017 \mathrm{E}-01 \\
6.17876 \mathrm{E}-01 \\
7.55044 \mathrm{E}-01 \\
8.65631 \mathrm{E}-01 \\
9.44575 \mathrm{E}-01 \\
9.89401 \mathrm{E}-01\end{array}$ & $\begin{array}{l}1.242 \mathrm{E}-\mathrm{C} 8 \\
1.247 \mathrm{E}-\mathrm{C} 8 \\
1.262 \mathrm{E}-\mathrm{C} 8 \\
1.283 \mathrm{E}-08 \\
1.327 \mathrm{E}-08 \\
1.390 \mathrm{E}-08 \\
1.486 \mathrm{E}-08 \\
1.631 \mathrm{E}-08 \\
1.821 \mathrm{E}-08 \\
2.060 \mathrm{E}-08 \\
2.350 \mathrm{E}-08 \\
2.730 \mathrm{E}-08 \\
3.306 \mathrm{E}-\mathrm{C} 8 \\
4.315 \mathrm{E}-08 \\
6.285 \mathrm{E}-08 \\
1.079 \mathrm{E}-07 \\
4.077 \mathrm{E}-07\end{array}$ & $\begin{array}{l}1.048 E-08 \\
1.051 E-08 \\
1.063 E-C 8 \\
1.083 E-08 \\
1.120 E-08 \\
1.174 E-08 \\
1.255 E-08 \\
1.373 E-08 \\
1.528 E-08 \\
1.721 E-08 \\
1.960 E-08 \\
2.274 E-08 \\
2.748 E-08 \\
3.561 E-08 \\
5.099 E-08 \\
8.431 E-08 \\
2.743 E-07\end{array}$ & $\begin{array}{l}5.147 \mathrm{E}-09 \\
5.162 \mathrm{E}-09 \\
5.222 \mathrm{E}-09 \\
5.330 \mathrm{E}-09 \\
5.516 \mathrm{E}-09 \\
5.785 \mathrm{E}-09 \\
6.176 \mathrm{E}-09 \\
6.715 \mathrm{E}-09 \\
7.418 \mathrm{E}-09 \\
8.300 \mathrm{E}-09 \\
9.408 \mathrm{E}-09 \\
1.089 \mathrm{E}-08 \\
1.308 \mathrm{E}-08 \\
1.666 \mathrm{E}-08 \\
2.296 \mathrm{E}-08 \\
3.515 \mathrm{E}-08 \\
8.293 \mathrm{E}-08\end{array}$ & $\begin{array}{l}2.115 \mathrm{E}-09 \\
2.121 \mathrm{E}-09 \\
2.146 \mathrm{E}-09 \\
2.192 \mathrm{E}-09 \\
2.269 \mathrm{E}-09 \\
2.380 \mathrm{E}-09 \\
2.537 \mathrm{E}-09 \\
2.749 \mathrm{E}-09 \\
3.024 \mathrm{E}-09 \\
3.370 \mathrm{E}-09 \\
3.810 \mathrm{E}-09 \\
4.400 \mathrm{E}-09 \\
5.261 \mathrm{E}-09 \\
6.614 \mathrm{E}-09 \\
8.866 \mathrm{E}-09 \\
1.29 \mathrm{E}-08 \\
2.491 \mathrm{E}-08\end{array}$ & $\begin{array}{l}7.812 \mathrm{E}-10 \\
7.834 \mathrm{E}-10 \\
7.927 \mathrm{E}-10 \\
8.105 \mathrm{E}-10 \\
8.390 \mathrm{E}-10 \\
8.798 \mathrm{E}-10 \\
9.369 \mathrm{E}-10 \\
1.013 \mathrm{E}-09 \\
1.111 \mathrm{E}-09 \\
1.235 \mathrm{E}-09 \\
1.394 \mathrm{E}-09 \\
1.607 \mathrm{E}-09 \\
1.913 \mathrm{E}-09 \\
2.381 \mathrm{E}-09 \\
3.127 \mathrm{E}-09 \\
4.382 \mathrm{E}-09 \\
7.415 \mathrm{E}-09\end{array}$ & $\begin{array}{l}2.687 \mathrm{E}-10 \\
2.695 \mathrm{E}-10 \\
2.727 \mathrm{E}-10 \\
2.790 \mathrm{E}-10 \\
2.888 \mathrm{E}-10 \\
3.028 \mathrm{E}-10 \\
3.221 \mathrm{E}-10 \\
3.478 \mathrm{E}-10 \\
3.808 \mathrm{E}-10 \\
4.225 \mathrm{E}-10 \\
4.761 \mathrm{E}-10 \\
5.480 \mathrm{E}-10 \\
6.502 \mathrm{E}-10 \\
8.024 \mathrm{E}-10 \\
1.038 \mathrm{E}-09 \\
1.414 \mathrm{E}-09 \\
2.184 \mathrm{E}-09\end{array}$ \\
\hline TOTAL & $3.891 E-07$ & $3.106 E-07$ & $1.382 \mathrm{E}-07$ & $5.375 E-08$ & $1.918 E-08$ & $6.442 E-09$ \\
\hline
\end{tabular}

400.0

$1.397 \mathrm{E}-08$ $1.402 E-08$ $1.420 E-08$ $1.440 \mathrm{E}-08$ $1.489 \mathrm{E}-08$ $1.560 \mathrm{E}-08$ $1.667 \mathrm{E}-08$ $1.836 E-08$ $2.06 \mathrm{CE}-08$ $2.340 E-08$ 2.679E-C8 $3.782 E-08$ $4.979 \mathrm{E}-08$ $7.399 \mathrm{E}-08$ $1.339 \mathrm{E}-07$ $6.044 E-07$ $4.721 E-07$ 
COSINE

$$
\begin{aligned}
& -1.0000 C \mathrm{E} 00 \\
& -9.894 C 1 \mathrm{E}-01 \\
& -9.44575 \mathrm{E}-01 \\
& -8.65631 \mathrm{E}-01 \\
& -7.55044 \mathrm{E}-01 \\
& -6.17876 \mathrm{E}-01 \\
& -4.58017 \mathrm{E}-01 \\
& -2.81605 \mathrm{E}-01 \\
& -9.50125 \mathrm{E}-02 \\
& 9.50125 \mathrm{E}-02 \\
& 2.81605 \mathrm{E}-01 \\
& 4.58017 \mathrm{E}-01 \\
& 6.17876 \mathrm{E}-01 \\
& 7.55044 \mathrm{E}-01 \\
& 8.65631 \mathrm{E}-01 \\
& 9.44575 \mathrm{E}-01 \\
& 9.89401 \mathrm{E}-01
\end{aligned}
$$

TOTAL

COSINE

$-1 . \operatorname{COOCOE} 00$ $-9.89401 \mathrm{E}-01$ $-9.44575 \mathrm{E}-01$ $-8.65631 \mathrm{E}-0 \mathrm{I}$ $-7.55044 \mathrm{E}-01$ $-6.17876 E-01$ $-4.58017 \mathrm{E}-01$ $-2.81605 E-01$ $-9.50125 E-n 2$ $9.50125 \mathrm{E}-02$ $2.81605 E-01$ $4.58017 \mathrm{E}-01$ $7.55044 E-01$ $7.55044 E-01$ $8.65631 E-01$ $9.89401 \mathrm{E}-01$

TOTAL
75.0

100.0

$3.564 \mathrm{E}-11$ $3.623 \mathrm{E}-11$ $3.799 \mathrm{E}-1$ 3.79

$3.992 \mathrm{E}-1$

$4.312 \mathrm{E}-1$

$4.877 \mathrm{E}-11$

$5.736 \mathrm{E}-11$

$7.675 \mathrm{E}-1$

$9.130 \mathrm{E}-11$

$1.100 \mathrm{E}-1 \mathrm{C}$

$1.596 \mathrm{E}-10$

$3.695 \mathrm{E}-10$

$1.145 \mathrm{E}-09$

$2.182 \mathrm{E}-09$

$1.310 \mathrm{E}-08$

$4.805 E-09$

$500 . \mathrm{C}$

600.0

$4.243 E-11$ $4.443 \mathrm{E}-11$

$4.519 \mathrm{E}-11$

$4.540 \mathrm{E}-11$

5. $120 \mathrm{E}-11$

$5.874 \mathrm{E}-11$

$6.927 \mathrm{E}-11$

1.01CE-10

$9.904 \mathrm{E}-11$

$1.275 \mathrm{E}-10$

$1.728 \mathrm{E}-10$

$3.348 \mathrm{E}-10$

$2.194 \mathrm{E}-10$

1. $282 \mathrm{E}-\mathrm{CB}$

$4.544 E-09$

900.0

150.0

RANGE (METERS)
200.0

250.0

300.0

400.0

\section{$5.143 \mathrm{E}-11 \quad 5.653 \mathrm{E}-11 \quad 5.855 \mathrm{E}-11$}

. $211 E-11$

$.715 \mathrm{E}-11$

$5.910 \mathrm{E}-1$

$445 \mathrm{E}-1$

$5.920 \mathrm{E}-11$

$6.078 \mathrm{E}-11$

$5.498 \mathrm{E}-11$

$6.228 \mathrm{E}-1$

$7.142 \mathrm{E}-1$

$8.417 \mathrm{E}-11$

$1.232 E-10$

1.370E- 10

$1.912 \mathrm{E}-10$

$2.679 E-10$

$5.343 E-10$

$161 \mathrm{E}-0$

$6.038 \mathrm{E}-1$

$6.428 \mathrm{E}-11$

$7.881 \mathrm{E}-1$

$9.256 \mathrm{E}-11$

$1.116 \mathrm{E}-10$

$1.290 \mathrm{E}-10$

$1.567 \mathrm{E}-10$

$1.946 \mathrm{E}-10$

$2.818 \mathrm{E}-10$

$5.021 E-10$

$1.355 E-09$

$9.954 \mathrm{E}-09$

$6.330 \mathrm{E}-11$

$6.646 \mathrm{E}-11$

$8.167 \mathrm{E}-11$

$9.558 \mathrm{E}-11$

$1.148 \mathrm{E}-10$

$1.322 E-10$

$1.603 E-10$

$1.987 E-10$

$2.856 \mathrm{E}-10$

$1.178 E-09$

3.50GE-09

$3.829 E-09$

1500.0

$4.648 \mathrm{E}-11$

$4.672 E-1$

$3.834 E-11$

$3.852 \mathrm{E}-11$

$1.815 \mathrm{E}-1$

1200.0

$2.681 \mathrm{E}-12$

$2.689 \mathrm{E}-12$
$2.721 \mathrm{E}-12$

$2.779 \mathrm{E}-12$

$2.877 E-12$

$1.845 E-11$ 7.438E-12

$1.882 E-11$

$7.595 \mathrm{E}-1$

(2)

$4.136 E-11$

$4.353 E-11$

4.703E-11

$5.259 E-11$

$6.005 E-11$

$6.937 E-11$

8.

$.186 \mathrm{E}-1 \mathrm{C}$

.492E-10

3.

$3.378 \mathrm{E}-10$

6.551E-10

$1.204 \mathrm{E}-10$

$1.679 \mathrm{E}-10$

$4.990 E-10$

$4.990-10$

$2.055 \mathrm{E}-11$

$2.213 \mathrm{E}-11$

$2.444 \mathrm{E}-11$

$2.752 E-11$

$3.622 E-11$

4. $293 \mathrm{E}-1$

$5.384 \mathrm{E}-11$

$7.387 \mathrm{E}-11$

$1.055 \mathrm{E}-10$

$5.577 \mathrm{E}-10$

$8.272 \mathrm{E}-12$

$8.877 \mathrm{E}-12$

$9.728 \mathrm{E}-12$

$1.085 \mathrm{E}-11$

$1.227 \mathrm{E}-11$

$1.666 \mathrm{E}-11$

$2.080 \mathrm{E}-11$

$2.812 \mathrm{E}-11$

$4.169 E-11$

6.83 E-11

$1.513 \mathrm{E}-09$

$6.127 E-10$

$2.247 E-10$

.021E-12

$3.231 E-12$

3.522E-12

$3.903 \mathrm{E}-12$

$4.387 E-12$

5.020

$9.816 E-12$

$1.420 \mathrm{E}-11$

$4.427 \mathrm{E}-1 \mathrm{l}$

$7.703 \mathrm{E}-11$

$5.888 \mathrm{E}-11$

$6.063 \mathrm{E}-1$

$6.314 \mathrm{E}-1$

$6.634 E-11$

$7.154 \mathrm{E}-11$

$9.488 \mathrm{E}-11$

$1.116 \mathrm{E}-10$

1. $320 \mathrm{E}-10$

$1.563 \mathrm{E}-10$

$1.970 \mathrm{E}-10$

$2.789 E-10$

$4.693 \mathrm{E}-10$

(

$3.172 E-09$

1800.0

. $202 E-13$

.

.509E-13

$9.838 \mathrm{E}-13$
$1.032 \mathrm{E}-12$

$1.101 \mathrm{E}-12$

$1.195 E-12$

$1.475 \mathrm{E}-12$

$1.683 \mathrm{E}-12$

$1.980 \mathrm{E}-12$

2.448E-12

. $231 E-12$

$6.949 E-12$

$2.514 E-11$ 


CCSINE
$-1.0 C 0 C O E 00$
$-9.89401 E-01$
$-9.44575 E-01$
$-8.65631 E-01$
$-7.55044 E-01$
$-6.17876 E-01$
$-4.58017 E-01$
$-2.81605 E-01$
$-9.50125 E-02$
$9.50125 E-02$
$2.81605 E-01$
$4.58 C 17 E-01$
$6.17876 E-01$
$7.55044 E-01$
$8.65631 E-01$
$9.44575 E-01$
$9.89401 E-01$

TOTAL

COSINE

$-1.00 C C J E 00$ $-9.89401 E-01$ $-9.44575 E-01$

$-8.65631 \mathrm{E}-01$

$-7.55044 E-01$

$-4.58017 \mathrm{E}-01$

$-2.81605 E-01$

$-9.50125 E-02$

$9.50125 \mathrm{E}-02$

$2.816 C 5 E-0)$

$4.58017 E-01$

$6.17876 \mathrm{E}-01$

$7.55044 E-0$

$8.65631 \mathrm{E}-01$

$9.44575 E-01$

TOTAL

$\begin{array}{lc}75.0 & 1 C 0.0 \\ 9.346 \mathrm{E}-10 & 1.149 \mathrm{E}-\mathrm{C} 9 \\ 9.465 \mathrm{E}-1 \mathrm{C} & 1.162 \mathrm{E}-09 \\ 9.659 \mathrm{E}-10 & 1.190 \mathrm{E}-09 \\ 9.851 \mathrm{E}-10 & 1.209 \mathrm{E}-09 \\ 9.921 \mathrm{E}-10 & 1.221 \mathrm{E}-09 \\ 1.038 \mathrm{E}-09 & 1.278 \mathrm{E}-09 \\ 1.114 \mathrm{E}-09 & 1.365 \mathrm{E}-09 \\ 1.243 \mathrm{E}-09 & 1.537 \mathrm{E}-09 \\ 1.435 \mathrm{E}-09 & 1.775 \mathrm{E}-\mathrm{C} 9 \\ 1.904 \mathrm{E}-09 & 2.457 \mathrm{E}-09 \\ 2.189 \mathrm{E}-09 & 2.455 \mathrm{E}-09 \\ 2.600 \mathrm{E}-09 & 3.063 \mathrm{E}-09 \\ 3.607 \mathrm{E}-09 & 4.097 \mathrm{E}-09 \\ 7.832 \mathrm{E}-09 & 7.378 \mathrm{E}-09 \\ 2.328 \mathrm{E}-08 & 1.510 \mathrm{E}-\mathrm{C} 8 \\ 4.397 \mathrm{E}-08 & 4.145 \mathrm{E}-08 \\ 2.59 \mathrm{E}-07 & 2.541 \mathrm{E}-07 \\ 9.956 \mathrm{E}-08 & 9.610 \mathrm{E}-08\end{array}$

500.0

$1.532 \mathrm{E}-09$ $1.538 \mathrm{E}-09$ $1.560 E-09$ $1.587 \mathrm{E}-\mathrm{C} 9$
$1.645 \mathrm{E}-09$ $1.724 \mathrm{E}-09$ $1.847 \mathrm{E}-09$ $2.037 \mathrm{E}-09$ $2.290 E-09$ 2.608 E-09 $2.993 E-09$ $3.495 \mathrm{E}-09$ $4.269 E-09$ $5.674 E-09$ $8.505 \mathrm{E}-\mathrm{C9}$ $1.518 \mathrm{E}-08$ $6.198 E-D 8$

$5.206 E-08$

$\begin{array}{lcc} & \text { RANGE (METERSI } \\ 150.0 & 200.0 & 250.0 \\ .466 E-09 & 1.673 E-09 & 1.784 \mathrm{E}-09 \\ .480 \mathrm{E}-09 & 1.686 \mathrm{E}-09 & 1.796 \mathrm{E}-09 \\ .513 \mathrm{E}-09 & 1.719 \mathrm{E}-09 & 1.828 \mathrm{E}-09 \\ .535 \mathrm{E}-09 & 1.739 \mathrm{E}-09 & 1.844 \mathrm{E}-09 \\ .557 \mathrm{E}-09 & 1.777 \mathrm{E}-09 & 1.911 \mathrm{E}-09 \\ .630 \mathrm{E}-09 & 1.876 \mathrm{E}-09 & 1.999 \mathrm{E}-09 \\ .744 \mathrm{E}-09 & 1.997 \mathrm{E}-09 & 2.136 \mathrm{E}-09 \\ .958 \mathrm{E}-09 & 2.236 \mathrm{E}-09 & 2.383 \mathrm{E}-09 \\ .253 \mathrm{E}-09 & 2.562 \mathrm{E}-09 & 2.720 \mathrm{E}-09 \\ .591 \mathrm{E}-09 & 2.997 \mathrm{E}-09 & 3.167 \mathrm{E}-09 \\ .149 \mathrm{E}-09 & 3.446 \mathrm{E}-09 & 3.629 \mathrm{E}-09 \\ .497 \mathrm{E}-09 & 4.092 \mathrm{E}-09 & 4.299 \mathrm{E}-09 \\ .754 \mathrm{E}-09 & 5.01 \mathrm{CE}-09 & 5.244 \mathrm{E}-09 \\ .379 \mathrm{E}-09 & 6.939 \mathrm{E}-09 & 7.188 \mathrm{E}-09 \\ .197 \mathrm{E}-08 & 1.162 \mathrm{E}-08 & 1.152 \mathrm{E}-08 \\ 3.410 \mathrm{E}-08 & 2.896 \mathrm{E}-08 & 2.571 \mathrm{E}-08 \\ .313 \mathrm{E}-07 & 1.993 \mathrm{E}-07 & 1.667 \mathrm{E}-07 \\ 113 \mathrm{E}-08 & 8.717 \mathrm{E}-08 & 8.218 \mathrm{E}-08\end{array}$
$1.832 \mathrm{E}-09$ $1.883 E-09$ $1.88 \mathrm{E}-09$ $1.952 E-09$ $2.185 \mathrm{E}-09$ $2.431 E-09$ $2.764 \mathrm{E}-09$ 2.764E 09 $3.686 \mathrm{E}-09$ $4.309 E-09$ $5.294 \mathrm{E}-0 \mathrm{~S}$ $7.162 \mathrm{E}-09$ $7.162 E-09$ $2.326 \mathrm{E}-08$ $2.326 E-08$

$7.656 \mathrm{E}-08$

400.0

$1.737 \mathrm{E}-09$ $1.745 \mathrm{E}-09$ $1.797 \mathrm{E}-09$ $1.863 \mathrm{E}-09$ $1.863 E-09$ 1.952E-09 . $2.314 E-09$ $2.992 \mathrm{E}-09$ $3.445 E-09$ $4.028 \mathrm{E}-09$ $4.028 E-09$
$4.929 E-09$ $6.606 \mathrm{E}-09$ $6.606 \mathrm{E}-09$
$1.011 \mathrm{E}-08$ $1.011 \mathrm{E}-08$
$1.904 \mathrm{E}-08$ $9.283 \mathrm{E}-08$

$6.428 E-08$ $\begin{array}{rrr}600.0 & \text { RANGE } & \text { (METERS) } \\ 600.0 & 1200\end{array}$

$1.283 E-09$ $1.288 E-09$ $1.305 \mathrm{E}-09$ $1.330 \mathrm{E}-09$ $1.378 \mathrm{E}-09$ $1.446 \mathrm{E}-0$ $1.549 \mathrm{E}-09$ $1.702 E-09$ $1.906 \mathrm{E}-09$ $2.162 \mathrm{E}-\mathrm{C} 9$ $2.475 \mathrm{E}-0$ $2.888 \mathrm{E}-09$ $3.522 E-09$ $4.647 E-09$ $6.845 E-09$ $1.175 \mathrm{E}-08$ $4.126 E-08$ 4. C99E-08
$6.211 \mathrm{E}-10$ $6.309 \mathrm{E}-10$ $6.444 \mathrm{E}-10$ $6.677 E-10$ $7.011 \mathrm{E}-10$ $7.503 \mathrm{E}-10$ $8.192 \mathrm{E}-10$ $9.10 C E-10$ $1.024 E-09$ $1.167 \mathrm{E}-09$ $1.359 E-09$ $1.649 E-09$ $3.024 \mathrm{E}-09$ $3.024 \mathrm{E}-09$ 4.
$1.212 \mathrm{E}-08$ $1.772 \mathrm{E}-08$
1200.0

1500.0

$.526 \mathrm{E}-10$
$2.534 \mathrm{E}-10$ $2.565 \mathrm{E}-10$ $2.623 E-10$ $2.718 E-10$ $2.854 \mathrm{E}-10$ $3.050 \mathrm{E}-10$ $3.318 E-10$ $3.668 E-10$ $4.109 E-10$ $4.670 \mathrm{E}-10$ $5.429 E-10$ $6.558 \mathrm{E}-10$ $8.383 E-10$ $1.152 \mathrm{E}-09$ $1.730 \mathrm{E}-0$ 3.547 E-09 $6.768 E-09$

1800.0 
$4 P I R * 2$ AIR KERMA (NEUTRONS)

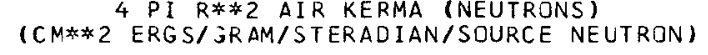

COSINE
$-1.00000 E$ NO
$-9.89401 E-01$
$-9.44575 E-01$
$-8.65631 E-C 1$
$-7.55044 E-01$
$-6.17876 E-01$
$-4.58017 E-C 1$
$-2.81605 E-01$
$-9.50125 E-02$
$9.50125 E-02$
$2.81605 E-01$
$4.58017 E-01$
$6.17876 E-01$
$7.55044 E-01$
$8.65631 E-01$
$9.44575 E-01$
$9.89401 E-01$

TOTAL

CCSINE

$-1.00000 \mathrm{E} 00$ $-9.894 \mathrm{C} 1 \mathrm{E}-\mathrm{O} 1$ $-9.44575 E-01$ $-7.55044 \mathrm{E}-01$ $-6.17876 \mathrm{E}-01$ $-4.58017 \mathrm{E}-01$ $-2.81605 \mathrm{E}-01$ $-9.50125 \mathrm{E}-02$ $9.50125 \mathrm{E}-02$ $2.81605 \mathrm{E}-01$ $4.58017 E-01$ $6.17876 \mathrm{E}-01$ $7.55044 \mathrm{E}-01$ $8.65631 \mathrm{E}-01$ $9.44575 E-01$

TOTAL

$\begin{array}{cc}75.0 & 100.0 \\ 1.377 \mathrm{E}-09 & 1.671 \mathrm{E}-09 \\ 1.358 \mathrm{E}-09 & 1.694 \mathrm{E}-09 \\ 1.440 \mathrm{E}-09 & 1.744 \mathrm{E}-09 \\ 1.467 \mathrm{E}-09 & 1.777 \mathrm{E}-09 \\ 1.476 \mathrm{E}-09 & 1.792 \mathrm{E}-\mathrm{C} 9 \\ 1.545 \mathrm{E}-\mathrm{C} 9 & 1.878 \mathrm{E}-09 \\ 1.660 \mathrm{E}-09 & 2.006 \mathrm{E}-09 \\ 1.858 \mathrm{E}-09 & 2.272 \mathrm{E}-09 \\ 2.159 \mathrm{E}-09 & 2.644 \mathrm{E}-09 \\ 2.889 \mathrm{E}-09 & 3.746 \mathrm{E}-09 \\ 3.351 \mathrm{E}-09 & 3.701 \mathrm{E}-\mathrm{C} 9 \\ 4.007 \mathrm{E}-09 & 4.657 \mathrm{E}-\mathrm{C9} \\ 5.707 \mathrm{E}-09 & 6.372 \mathrm{E}-09 \\ 1.283 \mathrm{E}-08 & 1.184 \mathrm{E}-\mathrm{C} 8 \\ 3.899 \mathrm{E}-08 & 2.488 \mathrm{E}-08 \\ 7.40 \mathrm{CE}-08 & 6.937 \mathrm{E}-08 \\ 4.4 \mathrm{C} 6 \mathrm{E}-07 & 4.317 \mathrm{E}-07 \\ 1.651 \mathrm{E}-07 & 1.577 \mathrm{E}-07 \\ & \end{array}$

500.0

$2.001 E-09$ 2.010E-09 $2.041 E-09$ $2.075 E-09$ $2.260 \mathrm{E}-09$ $2.428 \mathrm{E}-09$ $2.428 \mathrm{E}-09$
$2.699 \mathrm{E}-09$ 3.065E-09 $3.528 \mathrm{E}-09$ $4.091 \mathrm{E}-\mathrm{CO}$ $4.834 \mathrm{E}-09$ $6.014 \mathrm{E}-\mathrm{CO}$ $8.230 \mathrm{E}-09$ $1.281 \mathrm{E}-08$ $2.381 \mathrm{E}-08$ $7.616 \mathrm{E}-08$
600.0

$1.658 \mathrm{E}-09$ $1.665 \mathrm{E}-09$
$1.688 \mathrm{E}-09$ $1.719 \mathrm{E}-09$ $1.783 \mathrm{E}-09$ $1.871 \mathrm{E}-09$ 1.871E-09 2.226E-09 $2.516 E-09$ 2. $2162 \mathrm{E}-09$ $3.333 \mathrm{E}-\mathrm{C} 9$ 3. $936 \mathrm{E}-09$ 4. $895 \mathrm{E}-09$ $6.660 E-09$ $1.021 \mathrm{E}-\mathrm{C} 8$ 1. $827 \mathrm{E}-08$ 6. $799 \mathrm{E}-08$

$5.891 E-08$
RANGE (METERS)
200.0
150.0

$.332 E-09$

2.083E-09

2.107E-09

$2.164 \mathrm{E}-09$
$2.201 \mathrm{E}-09$

$2.201 E-C 9$

$2.230 \mathrm{E}-09$
$2.339 \mathrm{E}-09$ 2.504E-09 $2.831 \mathrm{E}-09$ $3.288 \mathrm{E}-09$ $3.852 E-09$ $4.660 \mathrm{E}-09$ $5.232 E-09$ $7.209 E-09$ $9.822 \mathrm{E}-09$ 5.639E-08 $5.639 E-08$
$3.918 E-07$ $2.355 \mathrm{E}-09$ $2.411 \mathrm{E}-09$ $2.440 \mathrm{E}-09$ $2.494 E-09$ $2.641 \mathrm{E}-09$ $2.814 \mathrm{E}-09$ $3.175 \mathrm{E}-09$ $3.674 E-09$ $4.326 \mathrm{E}-09$ $5.057 \mathrm{E}-09$ $6.023 E-09$ $7.489 E-09$ $1.056 \mathrm{E}-08$ $4.728 E-08$ $4.728 E-08$
$3.366 E-07$

1.466E-07 $1.377 \mathrm{E}-07$ 250.0 300.0 400.0

\section{RANGE (METERS)}

7. $883 \mathrm{E}-10$ 1200.0 $2.448 E-09$ $2.468 \mathrm{E}-09$ 2.521E-09 $2.542 \mathrm{E}-09$ $2.641 E-09$ $2.766 \mathrm{E}-09$ $2.960 \mathrm{E}-09$ $3.331 E-09$ $3.841 \mathrm{E}-09$ . 5.24 $7.744 \mathrm{E}-09$ $1.703 E-08$ $1.083 \mathrm{E}-08$ $4.153 \mathrm{E}-08$ $2.808 \mathrm{E}-07$ $2.465 \mathrm{E}-09$ $2.483 \mathrm{E}-09$ $2.532 E-09$ $2.559 E-09$ $2.658 E-09$ $2.786 \mathrm{E}-09$ $2.986 \mathrm{E}-09$ $3.351 \mathrm{E}-09$ $3.851 \mathrm{E}-09$ $4.486 E-09$ $5.244 E-09$ $7.720 \mathrm{E}-09$ $1.070 \mathrm{E}-08$ $1.739 \mathrm{E}-08$ $3.729 \mathrm{E}-08$ $3.729 E-08$
$2.316 E-07$ $1.278 \mathrm{E}-07$

1.173E-07 2.302E-09 $2.315 \mathrm{E}-09$ $2.355 \mathrm{E}-09$ $2.388 E-09$ $2.480 \mathrm{E}-09$ $2.601 \mathrm{E}-09$ $2.793 \mathrm{E}-09$ 3.119E-09 $3.562 \mathrm{E}-09$ $4.123 E-09$ $4.797 E-09$ $7.055 \mathrm{E}-09$ $7.0713 \mathrm{E}-09$ $9.713 \mathrm{E}-09$ $3.016 E-08$ $3.016 E-08$

$9.603 E-08$

1800.0

$\begin{array}{llll}7.883 \mathrm{E}-10 & 3.182 \mathrm{E}-10 & 1.163 \mathrm{E}-10 & 3.974 \mathrm{E}-11 \\ 7.908 \mathrm{E}-10 & 3.191 \mathrm{E}-10 & 1.166 \mathrm{E}-10 & 3.985 \mathrm{E}-11 \\ 8.007 \mathrm{E}-10 & 3.229 \mathrm{E}-10 & 1.180 \mathrm{E}-10 & 4.030 \mathrm{E}-11 \\ 8.168 \mathrm{E}-10 & 3.296 \mathrm{E}-10 & 1.205 \mathrm{E}-10 & 4.115 \mathrm{E}-11 \\ 8.461 \mathrm{E}-10 & 3.411 \mathrm{E}-10 & 1.246 \mathrm{E}-10 & 4.253 \mathrm{E}-11 \\ 8.881 \mathrm{E}-10 & 3.578 \mathrm{E}-10 & 1.306 \mathrm{E}-10 & 4.455 \mathrm{E}-11 \\ 9.522 \mathrm{E}-10 & 3.826 \mathrm{E}-10 & 1.393 \mathrm{E}-10 & 4.743 \mathrm{E}-11 \\ 1.044 \mathrm{E}-09 & 4.172 \mathrm{E}-10 & 1.513 \mathrm{E}-10 & 5.135 \mathrm{E}-11 \\ 1.168 \mathrm{E}-09 & 4.630 \mathrm{E}-10 & 1.670 \mathrm{E}-10 & 5.648 \mathrm{E}-11 \\ 1.324 \mathrm{E}-09 & 5.214 \mathrm{E}-10 & 1.872 \mathrm{E}-10 & 6.309 \mathrm{E}-11 \\ 1.522 \mathrm{E}-09 & 5.969 \mathrm{E}-10 & 2.136 \mathrm{E}-10 & 7.181 \mathrm{E}-11 \\ 1.795 \mathrm{E}-09 & 7.024 \mathrm{E}-10 & 2.507 \mathrm{E}-10 & 8.409 \mathrm{E}-11 \\ 2.224 \mathrm{E}-09 & 8.664 \mathrm{E}-10 & 3.078 \mathrm{E}-10 & 1.028 \mathrm{E}-10 \\ 2.978 \mathrm{E}-09 & 1.144 \mathrm{E}-09 & 4.017 \mathrm{E}-10 & 1.327 \mathrm{E}-10 \\ 4.392 \mathrm{E}-09 & 1.639 \mathrm{E}-09 & 5.621 \mathrm{E}-10 & 1.823 \mathrm{E}-10 \\ 7.283 \mathrm{E}-09 & 2.575 \mathrm{E}-09 & 8.480 \mathrm{E}-10 & 2.664 \mathrm{E}-10 \\ 1.955 \mathrm{E}-08 & 5.599 \mathrm{E}-09 & 1.597 \mathrm{E}-09 & 4.528 \mathrm{E}-10 \\ & & & \\ 2.450 \mathrm{E}-08 & 9.122 \mathrm{E}-09 & 3.157 \mathrm{E}-09 & 1.037 \mathrm{E}-09\end{array}$




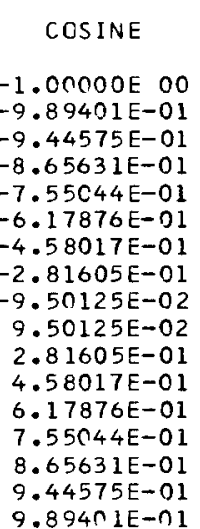

TOTAL

CCSINE

\section{-1.0 OCOOE 00} $-9.89401 E-01$ $-8.65631 \mathrm{E}-01$ $-7.55044 \mathrm{E}-01$ $-6.17876 \mathrm{E}-01$ $-4.58017 \mathrm{E}-01$ $-2.81605 E-01$ $-9.5 C 125 \mathrm{E}-02$ $9.5 \mathrm{C} 125 \mathrm{E}-\mathrm{C} 2$ $2.81605 \mathrm{E}-01$ $4.58017 \mathrm{E}-01$ $6.17876 \mathrm{E}-\mathrm{Cl}$ $7.55044 E-01$ $8.65631 \mathrm{E}-01$ $9.44575 \mathrm{E}-01$ 9.894ClE-01

TOTAL

$\begin{array}{cc}75.0 & 100.0 \\ 2.920 E-10 & 3.273 E-10 \\ 3.651 E-1 C & 3.420 E-10 \\ 3.325 E-10 & 3.744 E-10 \\ 3.503 E-10 & 3.953 E-10 \\ 3.506 E-1 C & 3.967 E-10 \\ 3.729 E-10 & 4.226 E-10 \\ 4.061 E-1 C & 4.533 E-10 \\ 4.721 E-1 C & 5.436 E-10 \\ 5.828 E-1 C & 6.763 E-10 \\ 8.177 E-1 C & 1.091 E-09 \\ 1.024 E-09 & 1.077 E-09 \\ 1.29 E E-C 9 & 1.44 E E-09 \\ 2.221 E-C 9 & 2.117 E-09 \\ 6.064 E-C 9 & 4.988 E-C 9 \\ 2.049 E-08 & 1.207 E-08 \\ 3.98 C E-08 & 3.628 E-C 8 \\ 2.494 E-C 7 & 2.426 E-07 \\ 8.342 E-C 8 & 7.583 E-08\end{array}$

150.0
$3.611 E-10$
$3.765 E-10$
$4.125 E-10$
$4.341 E-10$
$4.380 E-10$
$4.666 E-10$
$5.021 E-10$
$6.077 E-10$
$7.628 E-10$
$8.502 E-10$
$1.298 E-09$
$1.386 E-09$
$2.062 E-09$
$3.249 E-09$
$7.660 E-09$
$2.778 E-08$
$2.172 E-07$
$6.437 E-08$

RANGE (METERS)
200.0

250.0

300.0

400.0

$3.710 \mathrm{E}-10$ $3.851 \mathrm{E}-10$ $4.199 \mathrm{E}-1 \mathrm{C}$ $4.339 E-10$ $4.466 \mathrm{E}-10$ $4.898 E-10$ S.20CE-10 $6.305 E-10$ 1.930E-10 $1.07 C E-09$ $1.19 C E-09$ $1.574 \mathrm{E}-09$ $1.958-09$ . $210 \mathrm{E}-09$ $0.623 \mathrm{E}-09$ 2.169E-08 $3.638 \mathrm{E}-10$ $3.760 \mathrm{E}-10$ $4.076 \mathrm{E}-10$ $4.138 \mathrm{E}-10$ $4.488 \mathrm{E}-10$ $4.740 E-10$ 5.102 E-10 . $189 E-10$ 1.055E-09 $1.055 E-09$ $1.541 \mathrm{E}-09$ $1.54 \mathrm{E}-09$ $1.914 E-09$ $3.135 \mathrm{E}-09$ $1.797 \mathrm{E}-08$ $1.515 \mathrm{E}-07$

5.605E-08

$4.848 E-08$

$3.462 E-10$ $3.566 \varepsilon-10$ $3.842 \mathrm{E}-10$ $3.920 E-10$ $4.245 E-10$ $4.484 E-10$ $4.856 E-10$ 5. $885 E-10$ . $210 E-10$ ..210E-10 $1.165 E-09$ $1.869 E-09$ $1.869 E-09$ 2.73 E 09 $1.541 \mathrm{E}-08$ $1.232 \mathrm{E}-07$

$4.174 E-08$ $2.950 E-10$
$3.022 E-10$ $3.022 \mathrm{E}-10$ 3. $309 \mathrm{E}-10$ 3. $570 \mathrm{E}-10$ $3.778 \mathrm{E}-10$ $4.134 \mathrm{E}-10$ $4.992 E-10$ $7.863 \mathrm{E}-10$ $7.863 E-10$ $9.620 E-10$ $1.181 E-09$ $2.493 E-09$ $2.493 E-09$
$4.818 E-09$ $4.818 E-09$ $7.995 \mathrm{E}-08$ $3.058 E-08$

\section{RANGE (METERS)}

500.0

600.0

900.0

1200.0

1500.0

1800.0

$\begin{array}{llllll}2.383 \mathrm{E}-10 & 1.857 \mathrm{E}-10 & 7.701 \mathrm{E}-11 & 2.831 \mathrm{E}-11 & 9.672 \mathrm{E}-12 & 3.140 \mathrm{E}-12 \\ 2.431 \mathrm{E}-10 & 1.889 \mathrm{E}-10 & 7.791 \mathrm{E}-11 & 2.857 \mathrm{E}-11 & 9.744 \mathrm{E}-12 & 3.160 \mathrm{E}-12 \\ 2.570 \mathrm{E}-10 & 1.985 \mathrm{E}-10 & 8.088 \mathrm{E}-11 & 2.946 \mathrm{E}-11 & 1.001 \mathrm{E}-11 & 3.239 \mathrm{E}-12 \\ 2.651 \mathrm{E}-10 & 2.052 \mathrm{E}-10 & 8.387 \mathrm{E}-11 & 3.057 \mathrm{E}-11 & 1.039 \mathrm{E}-11 & 3.359 \mathrm{E}-12 \\ 2.850 \mathrm{E}-10 & 2.200 \mathrm{E}-10 & 8.938 \mathrm{E}-11 & 3.246 \mathrm{E}-11 & 1.100 \mathrm{E}-11 & 3.552 \mathrm{E}-12 \\ 3.023 \mathrm{E}-10 & 2.338 \mathrm{E}-10 & 9.552 \mathrm{E}-11 & 3.48 \mathrm{CE}-11 & 1.181 \mathrm{E}-11 & 3.817 \mathrm{E}-12 \\ 3.334 \mathrm{E}-10 & 2.593 \mathrm{E}-10 & 1.068 \mathrm{E}-10 & 3.887 \mathrm{E}-11 & 1.316 \mathrm{E}-11 & 4.243 \mathrm{E}-12 \\ 4.066 \mathrm{E}-1 \mathrm{C} & 3.099 \mathrm{E}-10 & 1.255 \mathrm{E}-10 & 4.528 \mathrm{E}-11 & 1.521 \mathrm{E}-11 & 4.871 \mathrm{E}-12 \\ 4.982 \mathrm{E}-10 & 3.821 \mathrm{E}-10 & 1.526 \mathrm{E}-10 & 5.427 \mathrm{E}-11 & 1.804 \mathrm{E}-11 & 5.729 \mathrm{E}-12 \\ 6.219 \mathrm{E}-10 & 4.751 \mathrm{E}-10 & 1.864 \mathrm{E}-10 & 6.552 \mathrm{E}-11 & 2.161 \mathrm{E}-11 & 6.825 \mathrm{E}-12 \\ 7.606 \mathrm{E}-10 & 5.796 \mathrm{E}-10 & 2.263 \mathrm{E}-10 & 7.942 \mathrm{E}-11 & 2.618 \mathrm{E}-11 & 8.263 \mathrm{E}-12 \\ 9.348 \mathrm{E}-10 & 7.148 \mathrm{E}-10 & 2.819 \mathrm{E}-10 & 9.972 \mathrm{E}-11 & 3.305 \mathrm{E}-11 & 1.047 \mathrm{E}-11 \\ 1.253 \mathrm{E}-09 & 9.643 \mathrm{E}-10 & 3.86 \mathrm{E}-10 & 1.376 \mathrm{E}-10 & 4.576 \mathrm{E}-11 & 1.451 \mathrm{E}-11 \\ 1.995 \mathrm{E}-\mathrm{Cg} & 1.538 \mathrm{E}-09 & 6.145 \mathrm{E}-10 & 2.175 \mathrm{E}-10 & 7.163 \mathrm{E}-11 & 2.248 \mathrm{E}-11 \\ 3.817 \mathrm{E}-09 & 2.913 \mathrm{E}-09 & 1.130 \mathrm{E}-09 & 3.887 \mathrm{E}-10 & 1.248 \mathrm{E}-10 & 3.832 \mathrm{E}-11 \\ 8.796 \mathrm{E}-09 & 6.487 \mathrm{E}-09 & 2.346 \mathrm{E}-\mathrm{C} 9 & 7.663 \mathrm{E}-10 & 2.358 \mathrm{E}-10 & 6.981 \mathrm{E}-11 \\ 5.143 \mathrm{E}-08 & 3.297 \mathrm{E}-\mathrm{C} 8 & 8.638 \mathrm{E}-09 & 2.261 \mathrm{E}-09 & 5.923 \mathrm{E}-10 & 1.554 \mathrm{E}-10 \\ 2.206 \mathrm{E}-08 & 1.572 \mathrm{E}-08 & 5.386 \mathrm{E}-09 & 1.743 \mathrm{E}-09 & 5.423 \mathrm{E}-10 & 1.638 \mathrm{E}-10\end{array}$




\begin{abstract}
COSINE
-1. CCOCOE OO $-9.89401 \mathrm{E}-\mathrm{Cl}$ $-9.44575 \mathrm{E}-0$. $-8.65631 E-01$ $-6.17876 \mathrm{E}-\mathrm{Ol}$ $-4.17876 E-n 1$ $-2.81605 E-01$ $-9.50125 E-02$ $9.5 \mathrm{C} 125 \mathrm{E}-02$ $2.81605 \mathrm{E}-\mathrm{OI}$ $4.58017 \mathrm{E}-01$ $6.17876 \mathrm{E}-01$ $6.17876 \mathrm{E}-01$ $8.65631 E-01$ $9.45675 \mathrm{E}-01$ $9.89401 \mathrm{E}-01$
\end{abstract}

TOTAL

COSINE

$-1.0 C O C O E$ OO $-9.89401 \mathrm{E}-01$ $-9.44575 \mathrm{E}-01$ $-8.65631 \mathrm{E}-01$ $-7.55044 \mathrm{E}-01$ $-6.17876 \mathrm{E}-\mathrm{O} 1$ $-4.58017 \mathrm{E}-\mathrm{n} 1$ $-2.816 C 5 E-C 1$ $-9.50125 \mathrm{E}-02$ $9.50125 t-02$ $2.81605 \mathrm{E}-01$ $4.58017 \mathrm{E}-01$ $6.17876 E-01$ $7.550 .44 E-01$ $8.65631 \mathrm{E}-01$ $9.44575 \mathrm{E}-01$
$9.89401 \mathrm{E}-01$

TOTAL

75.0
$9.447 \mathrm{E}-11$
$9.530 \mathrm{E}-11$
$9.682 \mathrm{E}-11$
$9.771 \mathrm{E}-11$
$9.836 \mathrm{E}-11$
$1.027 \mathrm{E}-10$
$1.103 \mathrm{E}-10$
$1.227 \mathrm{E}-10$
$1.407 \mathrm{E}-10$
$1.844 \mathrm{E}-10$
$2.107 \mathrm{E}-10$
$2.477 \mathrm{E}-10$
$3.275 \mathrm{E}-10$
$6.658 \mathrm{E}-10$
$1.890 \mathrm{E}-09$
$3.532 \mathrm{E}-09$
$2.024 \mathrm{E}-08$
$8.230 \mathrm{E}-09$

100.0

150.0

RA

(METERS)
$20 \mathrm{C} .0$

250.0

300.0

400.0

$1.168 \mathrm{E}-10$

$1.195 \mathrm{E}-10$

1. $207 \mathrm{E}-10$

1. $218 \mathrm{E}-10$

1. $273 \mathrm{E}-10$

1. $362 \mathrm{E}-10$

1. $527 \mathrm{E}-10$

1. $752 \mathrm{E}-10$

2. $379 \mathrm{E}-10$

2. $381 E-10$

$2.956 \mathrm{E}-10$

$3.842 \mathrm{E}-10$
$6.534 \mathrm{E}-10$

$1.534 E-10$
$1.270 E-09$

$3.375 E-09$

$1.994 \mathrm{E}-08$

$8.107 E-09$

$1.501 E-10$
$1.511 E-10$

$1.533 \mathrm{E}-10$

$1.548 \mathrm{E}-10$

$1.569 \mathrm{E}-10$

$1.642 \mathrm{E}-10$

$1.759 \mathrm{E}-10$

$1.960 E-10$
$2.246 E-10$

$2.246 E-10$
$2.574 E-10$

$2.574 E-10$
$3.08 C E-10$

$3.425 \mathrm{E}-10$

$4.594 \mathrm{E}-10$

$4.594 \mathrm{E}-10$
$6.032 \mathrm{E}-10$

$1.073 \mathrm{E}-09$

$2.850 \mathrm{E}-09$

$1.828 \mathrm{E}-08$

$1.720 \mathrm{E}-10$

$1.729 \mathrm{E}-10$

1. $84 \mathrm{CE}-10$

$1.848 \mathrm{E}-10$
$1.872 \mathrm{E}-10$

$1.767 \mathrm{E}-10 \quad 1.886 \mathrm{E}-10$

$1.804 \mathrm{E}-10 \quad 1.947 \mathrm{E}-10$

$2.901 \mathrm{E}-10 \quad 2.038 \mathrm{E}-10$

$2.261 \mathrm{E}-10 \quad 2.423 \mathrm{E}-10$

$2.572 E-10 \quad 2.747 E-10$

$2.978 E-10 \quad 3.16 \mathrm{EE}-10$

$3.416 \mathrm{E}-10 \quad 3.621 \mathrm{E}-10$

$4.025 \mathrm{E}-10 \quad 4.258 \mathrm{E}-10$

$4.900 \mathrm{E}-10 \quad 5.165 \mathrm{E}-10$

$6.640 E-10 \quad 6.931 E-10$

$1.070 \mathrm{E}-09 \quad 1.073 \mathrm{E}-09$

$2.487 E-09 \quad 2.253 E-0$

1.586 E-08 $1.336 \mathrm{E}-08$

$7.939 E-09$

$7.778 \mathrm{E}-09$

7.474 E- 09

$1.881 \mathrm{E}-10$

$10 \quad 1.805 E-10$

$1.826 \mathrm{E}-1 \mathrm{C}$

$1.996 E-10 \quad 1.852 E-10$

$2.091 E-10 \quad 2.009 E-10$

$2.239 E-10 \quad 2.153 E-10$

$2.482 \mathrm{E}-10 \quad 2.376 \mathrm{E}-10$

$2.804 \mathrm{E}-10 \quad 2.67 \mathrm{CE}-10$

$3.204 \mathrm{E}-10 \quad 3.038 \mathrm{E}-10$

$3.692 \mathrm{E}-10 \quad 3.482 E-10$

4.300E-10 4.055E-10

$5.240 \mathrm{E}-10 \quad 4.924 \mathrm{E}-10$

$6.954 \mathrm{E}-10 \quad 6.477 \mathrm{E}-10$

$1.057 \mathrm{E}-09 \quad 9.590 \mathrm{E}-10$

$\begin{array}{ll}2.067 \mathrm{E}-09 & 1.723 \mathrm{E}-09 \\ 1.114 \mathrm{E}-08 & 7.006 \mathrm{E}-09\end{array}$

$7.072 E-09 \quad 6.078 E-09$

\section{RANGE (METERS)}

$500.0 \quad 600.0$

900.0

1200.0

1530.0

1800.0

$1.593 \mathrm{E}-10$

1. $334 \mathrm{E}-10$

$6.428 E-11$

$2.608 \mathrm{E}-11$

$9.543 \mathrm{E}-12$

$3.258 \mathrm{E}-12$

. $350 E-10$

$6.525 \mathrm{E}-1$

$2.616 \mathrm{E}-11$

$9.572 \mathrm{E}-12$

$3.268 \mathrm{E}-12$

$1.375 E-10$
$1.424 E-10$

$2.71 \mathrm{CE}-11$

$2.810 \mathrm{E}-11 \quad 1.030 \mathrm{E}-12$

$3.310 E-12$

3.391E-12

3. $519 \mathrm{E}-12$

$3.704 E-12$

$\begin{array}{lllll}1.603 E-10 & 7.781 E-11 & 3.163 E-11 & 1.159 E-11 & 3.958 E-12 \\ 1.759 E-10 & 8.493 E-11 & 3.443 E-11 & 1.259 E-11 & 4.296 E-12\end{array}$

$\begin{array}{lllll}1.759 E-10 & 9.493 E-11 & 3.443 E-11 & 1.259 E-11 & 4.296 E-12\end{array}$

$2.217 \mathrm{E}-10 \quad 1.058 \mathrm{E}-10 \quad 4.262 \mathrm{E}-11 \quad 1.552 \mathrm{E}-11 \quad 5.284 \mathrm{E}-12$

$2.531 E-10 \quad 1.204 E-10 \quad 4.844 E-11 \quad 1.762 E-11 \quad 5.994 E-12$

$2.944 \mathrm{E}-10 \quad 1.399 \mathrm{E}-10 \quad 5.623 \mathrm{E}-11 \quad 2.044 \mathrm{E}-11 \quad 6.947 \mathrm{E}-12$

$\begin{array}{lllll}3.563 \mathrm{E}-10 & 1.687 \mathrm{E}-10 & 6.756 \mathrm{E}-11 & 2.448 \mathrm{E}-11 & 8.296 \mathrm{E}-12\end{array}$

$4.620 \mathrm{E}-10 \quad 2.154 \mathrm{E}-10 \quad 8.525 \mathrm{E}-11 \quad 3.061 \mathrm{E}-11 \quad 1.030 \mathrm{E}-11$

$6.604 \mathrm{E}-10 \quad 2.969 \mathrm{E}-10 \quad 1.145 \mathrm{E}-10 \quad 4.035 \mathrm{E}-11 \quad 1.338 \mathrm{E}-11$

$\begin{array}{lllll}1.087 \mathrm{E}-09 & 4.537 \mathrm{E}-10 & 1.666 \mathrm{E}-10 & 5.662 \mathrm{E}-11 & 1.827 \mathrm{E}-11 \\ 3.474 \mathrm{E}-09 & 1.058 \mathrm{E}-09 & 3.193 \mathrm{E}-10 & 9.540 \mathrm{E}-11 & 2.817 \mathrm{E}-11\end{array}$

$\begin{array}{ll}1.391 \mathrm{E}-09 \\ 5.149 \mathrm{E}-09 & 3.474 \mathrm{E}-09\end{array}$

$.058 E-09$

$6.837 E-10$

$2.429 E-10$

$8.125 E-11$ 
4 PI R**2 HENDERSCN DOSE (GAMMAS)

(CM* 2 RAD/STERADIAN/SOURCE NELTRON)
8.187 TO 10.000 MEV NEUTKON SOURCE

\section{RANGE (METERS)}

250.0

$30 \mathrm{C} .0$

400.0

$1.450 E-11$

$1.462 \mathrm{E}-11$

$1.485 \mathrm{E}-11 \quad 1.448 \mathrm{E}-11$

$1.501 E-11$

$1.467 \mathrm{E}-11$

$1.534 \mathrm{E}-11$

$1.645 \mathrm{E}-11$

$1.775 E-11$

$1.660 \mathrm{E}-11$

$1.919 E-11$

$1.702 \mathrm{E}-11$

$1.783 \mathrm{E}-11$

$1.927 E-11$

$2.095 \mathrm{E}-11$

$2.009 \mathrm{E}-11$

$2.34 \mathrm{CE}-11$

$1.757 \mathrm{E}-11$

$1.956 \mathrm{E}-11$

.242E-11

$2.696 \mathrm{E}-11$

$2.258 \mathrm{E}-11$

$3.359 \mathrm{E}-11$

$3.170 \mathrm{E}-11$

3.807E-11

$5.133 E-11$

(

$6.576 E-11$

$3.780 E-11$

$5.734 \mathrm{E}-11$

E 11

$9.652 \mathrm{E}-11$

$8.995 E-11$

8.13 SE-11

$1.288 \mathrm{E}-10$

$1.513 \mathrm{E}-10$

$2.785 \mathrm{E}-10$

$6.366 \mathrm{E}-10$

$7.151 E-10$

$6.864 E-10$

4.C29E-10

4. $581 E-10$

$6.151 E-10$
-1. กOCCOE OO

$-9.89401 \mathrm{E}-01$

$-9.44575 \mathrm{E}-01$

$-8.65631 \mathrm{E}-01$

$-7.55044 \mathrm{E}-01$

$-6.17876 E-01$

- $.58017 E-01$

$-2.81605 E-01$

$-9.50125 \mathrm{E}-02$

$9.50125 E-02$

$2.81605 \mathrm{E}-01$

4.58017E-O1

$6.17876 \mathrm{E}-01$

$7.55044 \mathrm{E}-01$

$8.65631 E-01$

$9.89401 \mathrm{E}-\mathrm{Cl}$

TOTAL
1500.0

$\begin{array}{cc}500.0 & 600.0 \\ 9.333 \mathrm{E}-12 & 7.279 \mathrm{E}-12 \\ 9.552 \mathrm{E}-12 & 7.487 \mathrm{E}-12 \\ 1.033 \mathrm{E}-11 & 8.213 \mathrm{E}-12 \\ 1.148 \mathrm{E}-11 & 9.248 \mathrm{E}-12 \\ 1.257 \mathrm{E}-11 & 1.016 \mathrm{E}-11 \\ 1.349 \mathrm{E}-11 & 1.086 \mathrm{E}-11 \\ 1.451 \mathrm{E}-11 & 1.159 \mathrm{E}-11 \\ 1.611 \mathrm{E}-11 & 1.283 \mathrm{E}-11 \\ 1.880 \mathrm{E}-11 & 1.505 \mathrm{E}-11 \\ 2.293 \mathrm{E}-11 & 1.852 \mathrm{E}-11 \\ 2.880 \mathrm{E}-11 & 2.342 \mathrm{E}-11 \\ 3.725 \mathrm{E}-11 & 3.040 \mathrm{E}-11 \\ 5.08 \mathrm{E}-11 & 4.170 \mathrm{E}-11 \\ 7.566 \mathrm{E}-11 & 6.258 \mathrm{E}-11 \\ 1.269 \mathrm{E}-10 & 1.080 \mathrm{E}-10 \\ 2.507 \mathrm{E}-10 & 2.182 \mathrm{E}-10 \\ 6.628 \mathrm{E}-10 & 5.958 \mathrm{E}-10 \\ 5.853 \mathrm{E}-10 & 4.944 \mathrm{E}-10\end{array}$

RANGE (METERS)

\section{3. $083 \mathrm{E}-12$}

$4.332 E-13$

$4.839 \mathrm{E}-13$

$6.288 \mathrm{E}-13$

8.037E-13

$9.189 \mathrm{E}-13$

$9.114 E-13$

$8.638 \mathrm{E}-13$

$9.563 E-13$

$1.199 \mathrm{E}-12$

$1.620 E-12$

$2.124 E-12$

2.703E-12

$3.711 \mathrm{E}-12$

$6.278 E-12$

$3.372 \mathrm{E}-11$

$1.030 \mathrm{E}-10$

$5.933 E-11$
1800.0

1.159E-11

$.391 \mathrm{E}-11 \quad 1.181 \mathrm{E}-11$

1. $585 E-111.260 E-11$

$1.505 E-11$

$1.853 \mathrm{E}-1 \mathrm{1} \quad 1.623 \mathrm{E}-11$

1.757E-1

1.956E-11

$\begin{array}{ll}.002 \mathrm{E}-11 & 2.272 \mathrm{E}-11 \\ 3.887 \mathrm{E}-11 & 2.747 \mathrm{E}-11\end{array}$

$3.871 E-11 \quad 3.420 E-11$

$4.403 \mathrm{E}-11$

$5.958 \mathrm{E}-11$

$8.744 E-11$

$1.434 E-10$

$\begin{array}{ll}2.856 E-10 & 2.767 E-10 \\ 6.815 E-10 & 7.012 E-10\end{array}$

$7.154 E-10 \quad 0.672 E-10$

$.537 E-13$

$1.801 \mathrm{E}-13$

$2.559 E-13$

$3.372 \mathrm{E}-13$

.

.

.

4.

6. $846 E-13$

$.022 \mathrm{E}-13$

$1.118 \mathrm{E}-12$

.

.

$943 \mathrm{E}-12$
$515 \mathrm{E}-11$

$5.101 \mathrm{E}-11$

2. $710 E-11$ 
COSINE

-1.00000 E 00 $-9.89401 \mathrm{E}-01$ $-9.44575 E-01$ $-8.65631 \mathrm{E}-01$ $-7.55044 E-01$ $-6.17876 \mathrm{E}-01$ . $-2.81605 \mathrm{E}-01$ $-9.50125 \mathrm{E}-02$ $9.5 C 125 \mathrm{E}-02$ 2.81605E-01 $4.58017 E-C 1$ $6.17876 \mathrm{E}-01$ $0.65631 \mathrm{E}-01$ $8.65631 E-C 1$ $9.89401 \mathrm{E}-\mathrm{O} 1$

TOTAL

\section{COSINE}

$-1.000 C \mathrm{CE} 00$ $-9.89401 \mathrm{E}-01$
$-9.44575 \mathrm{E}-01$ $-8.65631 \mathrm{E}-\mathrm{Cl}$ $-7.55044 \mathrm{E}-01$ $-6.17876 \mathrm{E}-01$ $-4.58017 \mathrm{E}-01$ $-2.816 C 5 E-01$ $-9.50125 \mathrm{E}-02$ $9.50125 E-02$ 2.81605E-01 4. $58017 \mathrm{E}-01$ $6.17876 \mathrm{E}-01$ $7.55044 \mathrm{E}-0 \mathrm{I}$ $8.65631 \mathrm{E}-01$ $9.44575 \mathrm{E}-01$
$9.894 \mathrm{Cl}-\mathrm{Cl}$

TOTAL

75.0
$1.15 S E-09$
$1.16 E \mathrm{E}-09$
$1.191 \mathrm{E}-C 9$
$1.24 C \mathrm{E}-09$
$1.3 C 9 \mathrm{E}-0.9$
$1.4 C 2 \mathrm{E}-C 9$
$1.524 \mathrm{E}-C 9$
$1.688 \mathrm{E}-09$
$1.967 \mathrm{E}-C 9$
$2.254 \mathrm{E}-C 9$
$3.110 \mathrm{E}-09$
$3.594 \mathrm{E}-09$
$3.752 \mathrm{E}-\mathrm{C} 9$
$4.917 \mathrm{E}-09$
$8.041 \mathrm{E}-09$
$1.272 \mathrm{E}-08$
$1.963 \mathrm{E}-08$
$4.005 \mathrm{E}-08$

$50 \mathrm{C} .0$

$1.128 \mathrm{E}-09$ $1.150 E-09$
$1.227 \mathrm{E}-09$ $1.342 \mathrm{E}-09$ $1.342 \mathrm{E}-09$ $1.54 \mathrm{SE}-\mathrm{CS}$ $1.655 \mathrm{E}-\mathrm{Cg}$ $1.820 \mathrm{E}-09$ $2.093 \mathrm{E}-09$ $2.507 \mathrm{E}-09$ $3.093 \mathrm{E}-\mathrm{C} 9$ $3.932 E-09$ $5.27 \mathrm{CE}-\mathrm{C} 9$ $7.712 \mathrm{E}-\mathrm{C} 9$ $1.274 \mathrm{E}-08$ $2.491 E-08$ $6.566 \mathrm{E}-08$

$6.067 E-08$

$1 C 0.0$
$1.329 \mathrm{E}-09$
$1.337 \mathrm{E}-09$
$1.371 \mathrm{E}-09$
$1.432 \mathrm{E}-09$
$1.518 \mathrm{E}-\mathrm{C} 9$
$1.630 \mathrm{E}-09$
$1.777 \mathrm{E}-09$
$1.974 \mathrm{E}-09$
$2.243 \mathrm{E}-09$
$3.262 \mathrm{E}-09$
$3.175 \mathrm{E}-09$
$3.306 \mathrm{E}-09$
$5.702 \mathrm{E}-09$
$6.855 \mathrm{E}-09$
$8.904 \mathrm{E}-\mathrm{C} 9$
$1.664 \mathrm{E}-08$
$2.872 \mathrm{E}-08$
$4.966 \mathrm{E}-08$

$600 . \mathrm{C}$

9. $100 E-10$ $9.305 \mathrm{E}-10$ $1.003 E-C 9$ $1.106 E-09$
$1.199 E-09$ 1. $273 E-09$ $1.351 \mathrm{E}-09$ $1.480 \mathrm{E}-\mathrm{C} 9$ $1.7 C 6 \mathrm{E}-09$ 2.C56E-09 $2.546 \mathrm{E}-09$ 3. $240 E-09$ $4.355 \mathrm{E}-09$ $6.444 \mathrm{E}-09$ $1.085 \mathrm{E}-08$ $2.168 E-08$ $5.904 E-C .8$

$5.151 E-08$
RANGE (METERS)
200.0

250.0

$30 \mathrm{C} .0$

400.0

\section{$1.513 \mathrm{E}-09 \quad 1.586 \mathrm{E}-09 \quad 1.583 \mathrm{E}-09$} $.525 \mathrm{E}-\mathrm{C9} \quad 1.602 \mathrm{E}-09$ $1.572 \mathrm{E}-09$ 1. $1.650 \mathrm{E}-09$ 1.7.8 $1.896 \mathrm{E}-09$ $2.067 \mathrm{E}-09$ $2.298 \mathrm{E}-09$ $2.618 \mathrm{E}-09$ $2.781 \mathrm{E}-09$ $4.402 \mathrm{E}-09$ $4.402 E-09$ $6.818 E-09$
$7.505 E-C 9$ 7.505E-C9 $1.360 E-08$ $4.130 \mathrm{E}-08$ $1.601 \mathrm{E}-09$ $1.668 \mathrm{E}-09$ $1.778 \mathrm{E}-09$ $1.908 \mathrm{E}-09$ $2.053 \mathrm{E}-09$ $2.229 E-09$ $2.474 E-09$ $2.830 E-09$ $3.490 E-09$ $3.928 \mathrm{E}-09$ $6.659 E-09$ $9.689 \mathrm{E}-09$ $9.689 E-09$ $2.760 \mathrm{E}-08$ $6.296 \mathrm{E}-08$

$7.262 E-08$

$1.532 \mathrm{E}-09$ $1.552 \mathrm{E}-09$ $1.625 \mathrm{E}-09$ $1.742 \mathrm{E}-09$ $1.874 \mathrm{E}-09$ $2.014 E-09$ $2.181 E-09$ $2.417 E-09$ $2.767 E-09$ 3.249E-09 $5.032 \mathrm{E}-09$ . . $560 \mathrm{E}-09$ $9.560 E-09$ $2.832 \mathrm{E}-0 \mathrm{~B}$ $6.744 E-08$ $5.548 \mathrm{E}-08$

$6.172 E-08$

$6.930 E-08$

$7.303 E-08$

$1.350 \mathrm{E}-09$ $1.372 \mathrm{E}-09$ $1.450 \mathrm{E}-09$ $1.571 E-09$ $1.696 \mathrm{E}-09$ $1.81 \mathrm{EE}-09$ 1. . 2. $951 \mathrm{E}-09$ $4.595 \mathrm{E}-09$ 6.129E-09 $1.436 \mathrm{E}-08$ $2.747 \mathrm{E}-08$ (943E-08

\section{RANGE (METERS)}

900.0

1200.0

1500.0

1800.0

$6.871 E-08$

$7.144 \mathrm{E}-11$

$2.838 \mathrm{E}-11$ $4.8785-10-1.774 E-10$ $4.827 \mathrm{E}-10 \quad 2.118 \mathrm{E}-10$ $5.492 \mathrm{E}-10 \quad 2.495 \mathrm{E}-10$ $5.984 E-10 \quad 2.722 E-10$ $6.254 \mathrm{E}-10 \quad 2.788 \mathrm{E}-10$ $6.476 E-10 \quad 2.804 E-10$ $3.017 \mathrm{E}-10 \quad 3.032 \mathrm{E}-10$ $8.174 \mathrm{E}-10 \quad 3.583 \mathrm{E}-10$ $1.003 \mathrm{E}-09 \quad 4.480 \mathrm{E}-10$ $1.254 \mathrm{E}-09 \quad 5.638 \mathrm{E}-10$ $1.592 \mathrm{E}-09 \quad 7.084 \mathrm{E}-10$ $2.148 \mathrm{E}-09$ $3.279 E-09$ $5.860 \mathrm{E}-09$ $3.020 \mathrm{E}-10 \quad 1.263 \mathrm{E}-10$ 4.012E-10 $1.648 \mathrm{E}-10$ $2.847 \mathrm{E}-09 \quad 1.318 \mathrm{E}-09 \quad 5.955 \mathrm{E}-10$ $\begin{array}{lll}6.425 \mathrm{E}-09 & 3.145 \mathrm{E}-09 & 1.497 \mathrm{E}-09 \\ 1.992 \mathrm{E}-08 & 1.022 \mathrm{E}-\mathrm{C8} & 5.064 \mathrm{E}-09\end{array}$ $3.649 \mathrm{E}-08 \quad 1.992 \mathrm{E}-08 \quad 1.022 \mathrm{E}-\mathrm{C} 8 \quad 5.064 \mathrm{E}-09$

$2.763 \mathrm{E}-08 \quad 1.343 \mathrm{E}-08 \quad 6.249 \mathrm{E}-09 \quad 2.853 \mathrm{E}-09$

$3.841 \mathrm{E}-11$

$1.081 \mathrm{E}-10 \quad 4.655 \mathrm{E}-11$

$1.200 \mathrm{E}-10 \quad 5.028 \mathrm{E}-11$

$1.164 \mathrm{E}-10 \quad 4.848 \mathrm{E}-11$

$1.264 \mathrm{E}-10 \quad 5.235 \mathrm{E}-11$

$1.512 \mathrm{E}-10 \quad 6.341 \mathrm{E}-11$

$1.935 \mathrm{E}-10 \quad 8.291 \mathrm{E}-11$ 


COSINE
-1. CONOOE 00
$-9.89401 \mathrm{E}-01$
$-9.44575 \mathrm{E}-01$
$-8.65631 \mathrm{E}-01$
$-7.55644 \mathrm{E}-01$
$-6.17876 \mathrm{E}-01$
$-4.58017 \mathrm{E}-01$
$-2.81605 \mathrm{E}-01$
$-9.5 \mathrm{C} 125 \mathrm{E}-02$
$9.50125 \mathrm{E}-02$
$2.81605 \mathrm{E}-01$
$4.58 \mathrm{C} 17 \mathrm{E}-01$
$6.17876 \mathrm{E}-01$
$7.55044 \mathrm{E}-01$
$8.65631 \mathrm{E}-01$
$9.44575 \mathrm{E}-01$
$9.89401 \mathrm{E}-01$

TOTAL

COSINE

-1 . COOCCE DO $-9.894 C 1 E-01$ $-9.44575 E-01$ $-8.65631 \mathrm{E}-01$ $-6.17876 E-01$ $-4.58017 E-01$ $-2.81605 E-01$ $-9.50125 E-02$ $9.5 \mathrm{C} 125 \mathrm{E}-02$ $2.81605 E-01$ $4.58017 \mathrm{E}-01$ $6.17876 \mathrm{E}-01$ $7.55044 E-01$ $8.65631 E-01$ $9.44575 \mathrm{E}-01$
$9.89401 \mathrm{E}-01$

TOTAL

$\begin{array}{ll}75.0 & 100.0 \\ 1.320 \mathrm{E}-09 & 1.619 \mathrm{E}-09 \\ 1.327 \mathrm{E}-09 & 1.627 \mathrm{E}-09 \\ 1.352 \mathrm{E}-09 & 1.660 \mathrm{E}-09 \\ 1.399 \mathrm{E}-09 & 1.720 \mathrm{E}-09 \\ 1.46 \mathrm{E}-09 & 1.805 \mathrm{E}-09 \\ 1.556 \mathrm{E}-\mathrm{CO} & 1.91 \mathrm{EE}-\mathrm{C} 9 \\ 1.675 \mathrm{E}-09 & 2.062 \mathrm{E}-09 \\ 1.832 \mathrm{E}-\mathrm{CS} & 2.257 \mathrm{E}-09 \\ 2.044 \mathrm{E}-09 & 2.520 \mathrm{E}-09 \\ 2.374 \mathrm{E}-09 & 3.483 \mathrm{E}-09 \\ 3.184 \mathrm{E}-09 & 3.415 \mathrm{E}-09 \\ 3.629 \mathrm{E}-09 & 3.569 \mathrm{E}-09 \\ 3.796 \mathrm{E}-09 & 5.80 \mathrm{E}-09 \\ 4.891 \mathrm{E}-\mathrm{C} 9 & 6.960 \mathrm{E}-09 \\ 7.797 \mathrm{E}-09 & 8.829 \mathrm{E}-\mathrm{C} 9 \\ 1.213 \mathrm{E}-08 & 1.6 \mathrm{C} 1 \mathrm{E}-08 \\ 1.797 \mathrm{E}-08 & 2.718 \mathrm{E}-08 \\ 4.072 \mathrm{E}-\mathrm{C} 8 & 5.184 \mathrm{E}-08\end{array}$

$\begin{array}{ccccc}150.0 & \text { RANGE (METERS) } & & & \\ & 200.0 & 250.0 & 300.0 & 400 . \mathrm{C} \\ 2.084 \mathrm{E}-09 & 2.422 \mathrm{E}-09 & 2.636 \mathrm{E}-\mathrm{C} 9 & 2.746 \mathrm{E}-09 & 2.721 \mathrm{E}-09 \\ 2.097 \mathrm{E}-09 & 2.438 \mathrm{E}-09 & 2.655 \mathrm{E}-09 & 2.766 \mathrm{E}-09 & 2.743 \mathrm{E}-09 \\ 2.144 \mathrm{E}-09 & 2.497 \mathrm{E}-09 & 2.724 \mathrm{E}-09 & 2.843 \mathrm{E}-09 & 2.827 \mathrm{E}-09 \\ 2.228 \mathrm{E}-09 & 2.601 \mathrm{E}-09 & 2.842 \mathrm{E}-09 & 2.970 \mathrm{E}-09 & 2.959 \mathrm{E}-09 \\ 2.339 \mathrm{E}-09 & 2.731 \mathrm{E}-09 & 2.984 \mathrm{E}-09 & 3.118 \mathrm{E}-09 & 3.105 \mathrm{E}-09 \\ 2.478 \mathrm{E}-09 & 2.887 \mathrm{E}-09 & 3.148 \mathrm{E}-09 & 3.283 \mathrm{E}-09 & 3.258 \mathrm{E}-09 \\ 2.656 \mathrm{E}-09 & 3.083 \mathrm{E}-09 & 3.350 \mathrm{E}-09 & 3.483 \mathrm{E}-09 & 3.440 \mathrm{E}-09 \\ 2.894 \mathrm{E}-09 & 3.347 \mathrm{E}-09 & 3.625 \mathrm{E}-09 & 3.759 \mathrm{E}-09 & 3.695 \mathrm{E}-09 \\ 3.220 \mathrm{E}-09 & 3.714 \mathrm{E}-09 & 4.013 \mathrm{E}-09 & 4.153 \mathrm{E}-09 & 4.070 \mathrm{E}-09 \\ 3.413 \mathrm{E}-09 & 4.352 \mathrm{E}-09 & 4.691 \mathrm{E}-09 & 4.683 \mathrm{E}-09 & 4.607 \mathrm{E}-09 \\ 4.440 \mathrm{E}-09 & 4.815 \mathrm{E}-09 & 5.187 \mathrm{E}-09 & 5.501 \mathrm{E}-09 & 5.341 \mathrm{E}-09 \\ 5.000 \mathrm{E}-09 & 6.057 \mathrm{E}-09 & 6.494 \mathrm{E}-09 & 6.546 \mathrm{E}-09 & 6.376 \mathrm{E}-09 \\ 7.279 \mathrm{E}-09 & 7.408 \mathrm{E}-09 & 7.924 \mathrm{E}-09 & 8.245 \mathrm{E}-09 & 7.948 \mathrm{E}-09 \\ 7.994 \mathrm{E}-09 & 1.019 \mathrm{E}-08 & 1.085 \mathrm{E}-08 & 1.102 \mathrm{E}-08 & 1.065 \mathrm{E}-08 \\ 1.369 \mathrm{E}-08 & 1.495 \mathrm{E}-08 & 1.597 \mathrm{E}-08 & 1.644 \mathrm{E}-08 & 1.592 \mathrm{5}-08 \\ 2.088 \mathrm{E}-08 & 2.529 \mathrm{E}-08 & 2.768 \mathrm{E}-08 & 2.868 \mathrm{E}-08 & 2.821 \mathrm{E}-08 \\ 4.224 \mathrm{E}-08 & 5.303 \mathrm{E}-08 & 6.038 \mathrm{E}-08 & 6.483 \mathrm{E}-08 & 6.694 \mathrm{E}-08 \\ 6.779 \mathrm{E}-08 & 7.930 \mathrm{E}-08 & 8.607 \mathrm{E}-08 & 8.917 \mathrm{E}-08 & 8.775 \mathrm{E}-08\end{array}$

\section{RANGE (METERS) \\ $900.0 \quad 1200.0$}

500.0

600.0

$2.487 \mathrm{E}-09$
$2.510 \mathrm{E}-09$

$2.592 \mathrm{E}-09$

$2.719 E-C 9$

$2.852 \mathrm{E}-09$

$2.983 E-09$

$3.136 \mathrm{E}-\mathrm{C} 9$

$3.356 \mathrm{E}-\mathrm{C} 9$

$3.690 \mathrm{E}-09$
$4.171 \mathrm{E}-\mathrm{Cg}$

$4.827 E-09$

$5.736 E-09$

$7.126 E-09$

$9.556 \mathrm{E}-09$

$1.439 \mathrm{E}-08$

$2.580 E-08$

6. $337 E-08$

$7.997 \mathrm{E}-08$
$1.177 \mathrm{E}-09$

$1.193 E-09$

1. $314 \mathrm{E}-09$

$1.377 E-09$

1.426 E-09

$1.477 \mathrm{E}-09$

$1.564 \mathrm{E}-09$

$1.715 \mathrm{E}-\mathrm{C} 9$

$1.939 \mathrm{E}-09$

$2.232 E-09$

$2.615 \mathrm{E}-09$

$3.209 E-09$

4. $045 E-09$

1. $300 \mathrm{E}-08$

3.507E-08

$3.851 E-08$
$5.032 E-10$
$5.724 E-10$ $5.987 E-10$ $6.395 E-10$ $6.092 \mathrm{E}-10$ $6.873 E-10$
$7.038 E-10$ $7.434 \mathrm{E}-10$ $8.162 \mathrm{E}-10$ $9.245 \mathrm{E}-10$ $1.061 E-09$

$1.061 E-09$
$1.493 E-09$

$1.493 E-09$

$3.336 E-09$

$6.678 \mathrm{E}-09$

$1.903 E-08$

$1.894 \mathrm{E}-08$
1500.0

$2.551 E-10$
$2.600 E-10$ $2.746 \mathrm{E}-10$ $2.935 E-10$ $3.085 \mathrm{E}-10$ $3.142 E-10$ $3.178 \mathrm{E}-10$ $3.357 E-10$ $3.686 E-10$ $4.192 \mathrm{E}-10$ $4.791 E-10$ $5.480 \mathrm{E}-10$ $0.576 E-10$ $9.093 E-10$ $3.252 \mathrm{E}-09$ $9.719 \mathrm{E}-09$

$8.842 E-09$
1800.0

$1.131 \mathrm{E}-10$ $1.232 \mathrm{E}-10$ $1.319 \mathrm{E}-10$ $1.393 \mathrm{E}-10$
$1.398 \mathrm{E}-10$ $1.413 \mathrm{E}-10$ $1.487 E-10$ $1.634 \mathrm{E}-10$
$1.864 \mathrm{E}-10$ $2.124 \mathrm{E}-10$ $2.392 E-10$ $2.828 \mathrm{E}-10$ $3.949 \mathrm{E}-10$ $7.003 E-10$ $1.540 \mathrm{E}-09$ $4.793 \mathrm{E}-09$

$4.036 E-09$ 
COSINE

$-1.0 \mathrm{COCOE} 00$ $-9.894 \cap 1 E-01$ $-9.44575 \mathrm{E}-01$ $-8.65631 \mathrm{E}-01$ $-4.58017 \mathrm{~F}-01$ $-2.81605 \mathrm{E}-01$ $-9.5 n 125 \mathrm{E}-02$ $9.50125 \mathrm{E}-02$ $2.81605 \mathrm{E}-01$ 4.58017E-01 $0.17876 \mathrm{E}-01$ $7.55044 \mathrm{E}-01$ $8.65631 \mathrm{E}-\mathrm{C} 1$ $9.44575 \mathrm{E}-01$ $9.89401 \mathrm{E}-\mathrm{n} 1$

TOTAL

COSINE

$-1.00000 \mathrm{E} 00$ $-9.89401 \mathrm{E}-\mathrm{C} 1$ $-8.65631 E-01$ $-7.55044 E-01$ $-6.17876 \mathrm{E}-01$ $-4.58017 E-01$ $-2.81605 E-01$ $-9.50125 \mathrm{E}-02$ . $50125 \mathrm{E} 02$ $2.81605 \mathrm{E}-01$ $4.58017 E-01$ $6.17876 \mathrm{E}-01$ . $8.65631 E-01$ $9.89401 \mathrm{E}-\mathrm{Cl}$

TOTAL
75.0 $1.196 \mathrm{E}-09$ $1.222 \mathrm{E}-09$ $1.343 \mathrm{E}-09$ $1.437 E-09$ $1.562 \mathrm{E}-09$ $1.728 \mathrm{E}-09$ $1.952 \mathrm{E}-09$ 2.306E-ก $3.176 \mathrm{E}-09$ $3.676 \mathrm{E}-\mathrm{C} 9$ $3.832 \mathrm{E}-09$ $5.020 \mathrm{E}-09$ $8.210 E-09$ $1.299 \mathrm{E}-08$ $1.943 \mathrm{E}-08$

4.095E-08

500.0

$1.234 \mathrm{E}-09$ $1.25 \mathrm{EE}-09$ $1.335 E-09$ $1.452 E-C 9$ $1.564 \mathrm{E}-09$ $1.662 \mathrm{E}-09$ $1.769 \mathrm{E}-09$ $1.935 E-09$ $2.211 \mathrm{E}-09$ $\angle .631 E-09$ $3.224 E-09$ $4.073 \mathrm{E}-\mathrm{C9}$ $5.428 E-09$ 7.9C7E-09 $1.302 \mathrm{E}-08$ $2.544 E-08$ $0.277 E-08$
100.0

$1.368 \mathrm{E}-\mathrm{C} 9$ $1.377 \mathrm{E}-09$ $1.410 \mathrm{E}-09$ $1.473 \mathrm{E}-\mathrm{C} 9$ $1.560 \mathrm{E}-09$ $1.674 \mathrm{E}-09$ $1.824 \mathrm{E}-09$ $2.025 E-09$ $2.299 E-09$ $3.338 \mathrm{E}-09$ $3.250 E-09$ 3. $380 \mathrm{E}-09$ $5.827 \mathrm{E}-09$ $7.001 \mathrm{E}-\mathrm{C} 9$ 9. C $90 E-C 9$ $1.699 \mathrm{E}-08$ $2.933 \mathrm{E}-08$

$5.080 E-08$

600.0

$1.006 \mathrm{E}-09$ 1.C27E-09 $1.101 \mathrm{E}-\mathrm{C} 9$ $1.207 \mathrm{E}-\mathrm{C}$ $1.302 \mathrm{E}-09$ $1.376 E-09$ $1.455 \mathrm{E}-09$ $1.584 \mathrm{E}-\mathrm{CS}$ $1.813 \mathrm{E}-09$ 2. $166 \mathrm{E}-0$ $2.662 \mathrm{E}-09$ 3. $364 \mathrm{E}-09$ $4.492 E-C 9$ $6.611 \mathrm{E}-\mathrm{C} 9$ $1.110 E-C 8$ $2.214 E-08$ $5.337 E-C 8$
RANGE (METERS)

150.0 200.0

250.0

300.0

400.0

$1.569 \mathrm{E}-09 \quad 1.659 \mathrm{E}-09$

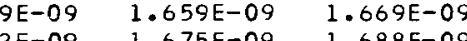
$1.734 E-09 \quad 1.756 E-09$ 1.713E-09 $1.835 \mathrm{E}-09 \quad 1.868 \mathrm{E}-09$ $1.823 \mathrm{E}-09 \quad 1.959 \mathrm{E}-09 \quad 2.000 \mathrm{E}-09$ $1.959 E-092.105 E-09 \quad 2.147 E-09$ $2.133 \mathrm{E}-092.287 \mathrm{E}-09 \quad 2.327 \mathrm{E}-0$ $2.368 \mathrm{E}-09 \quad 2.536 \mathrm{E}-09 \quad 2.575 \mathrm{E}-09$ $2.694 \mathrm{E}-09 \quad 2.889 \mathrm{E}-09 \quad 2.936 \mathrm{E}-09$ $2.855 \mathrm{E}-09 \quad 3.537 \mathrm{E}-09 \quad 3.608 \mathrm{E}-09$ $3.958 \mathrm{E}-09 \quad 3.964 \mathrm{E}-09 \quad 4.049 \mathrm{E}-09$ $4.5 n 7 E-09 \quad 5.258 E-09 \quad 5.388 E-09$ $6.973 \mathrm{E}-09 \quad 6.638 \mathrm{E}-09 \quad 6.825 \mathrm{E}-09$

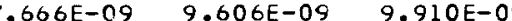
$1.389 \mathrm{E}-08 \quad 1.472 \mathrm{E}-08 \quad 1.539 \mathrm{E}-08$ $2.175 E-08 \quad 2.603 E-08 \quad 2.818 E-08$ $4.535 E-08 \quad 5.668 E-08 \quad 6.433 E-08$

$6.323 E-08$

$7.111 E-08$

$7.464 E-08$

$1.629 \mathrm{E}-09$ $1.724 E-09 \quad 1.559 E-09$ $1.843 \mathrm{E} \rightarrow 09 \quad 1.683 \mathrm{E}-09$ $1.977 \mathrm{E}-09 \quad 1.81 \mathrm{GE}-09$ $2.119 E-09 \quad 1.932 E-09$ $2.289 \mathrm{E}-09 \quad 2.071 \mathrm{E}-09$ $2.527 E-09 \quad 2.277 \mathrm{E}-09$ $2.883 E-09 \quad 2.599 E-09$ $3.371 E-09 \quad 3.081 E-09$ $4.163 \mathrm{E}-09 \quad 3.759 \mathrm{E}-09$ $5.181 \mathrm{E}-09 \quad 4.747 \mathrm{E}-09$ $6.901 \mathrm{E}-09 \quad 6.302 \mathrm{E}-09$ $9.783 E-09 \quad 9.083 E-09$ $1.557 \mathrm{E}-08 \quad 1.468 \mathrm{E}-08$ $\begin{array}{ll}2.892 \mathrm{E}-08 & 2.805 \mathrm{E}-08 \\ 6.891 \mathrm{E}-08 & 7.097 \mathrm{E}-08\end{array}$ 7.518E-08 7.093E-08

\section{RANGE (METERS)}

1500.0

1800.0

$2.056 E-10$

$4.948 E-10 \quad 2.15 C E-10$

. $412 E-10$ 2.40SE-10

$6.091 \mathrm{E}-10 \quad 2.794 \mathrm{E}-10$

$3.024 \mathrm{E}-10$

$6.862 E-10 \quad 3.090 E-10$

$7.081 E-10$ 3.101E-10

$7.621 E-10 \quad 3.328 E-10$

$8.789 E-10 \quad 3.884 E-10$

1.067E-09 4.790E-10

1.320E-09 $5.959 E-10$

$1.661 E-09$. $413 E-10$

$2.222 E-09 \quad 9.868 E-10$

2. $310 \mathrm{E}-09$

.

3. $734 \mathrm{E}-08$

2.910E-09

$6.562 E-09$

$8.443 \mathrm{E}-11$

$3.419 E-11$

$3.684 \mathrm{E}-1$

4.447E-11

$1.220 \mathrm{E}-10$ $5.281 \mathrm{E}-11$

$1.340 \mathrm{E}-10 \quad 5.889 \mathrm{E}-11$

1.340E-10 5.652E-11

1.300E-10 $5.457 E-11$

$1.650 \mathrm{E}-10 \quad 6.954 \mathrm{E}-1$

$2.078 \mathrm{E}-10 \quad 8.927 \mathrm{E}-11$

$2.587 \mathrm{E}-10 \quad 1.112 \mathrm{E}-10$

$3.167 E-10$ 1.328E-10

$4.162 \mathrm{E}-10$ 10 $1.711 \mathrm{E}-10$

$6.689 E-10 \quad 2.834 \mathrm{E}-10$

$1.346 E-09 \quad 6.082 E-10$

$3.212 E-09$ 1.529E-0

90E-09

$2.870 E-08$

$1.396 E-08$

$6.499 E-09$

$2.967 E-C 9$ 
(NEUTRJNS/MEV/STERADIAN/SOURCE NEUTRON)

ENERGY GROUP (MEV) $1.22 \mathrm{E}$ O1- $1.50 \mathrm{E}$ $1.00 E$ OI- $1.22 E$ 8.1 OE OO-A-1.00E O1 $6.36 E$ 00---8.1.9E 00 $4.97 \mathrm{E}$ OO--- $-3.36 \mathrm{E} 00$ 3.01E 00- $4.07 \mathrm{E} 00$ 2.46E OO---3.01E 00 $2.35 \mathrm{E} 00--2.46 \mathrm{E}$ O0 $1.83 \mathrm{E} 00--2.35 \mathrm{E} 00$ $1.11 \mathrm{E} 00--1.83 \mathrm{E}$ 00 $5.50 E-01--1.11 E$ OO 1.11E-01-- $-5.50 \mathrm{E}-01$ $3.35 \mathrm{E}-02--1.11 \mathrm{E}-01$ $5.83 \mathrm{E}-04--3.35 \mathrm{E}-02$ $2.90 \mathrm{E}-05--1.01 \mathrm{E}-04$ $1.07 \mathrm{E}-05--2.00 \mathrm{E}-05$ $3.06 \mathrm{E}-06--1.07 \varepsilon-05$ $1.12 E-06---3.06 E-06$ $4.14 E-07--1.12 E-06$
0.0 ENERGY GROUP (MEV) $1.22 E \quad 01-\cdots 1.50 E$ OI 1.0OE 01---1.22E OI $8.19 E$ OO-W-1.00E 03 $6.36 \mathrm{E} 00--8.19 \mathrm{E} 00$ 4.97E $00---6.36 E$ EO 4.07E $00--4.97 E$ OO 3.01 E $90--4.07 E O 0$ $2.46 \mathrm{E} 00---3.01 \mathrm{E} 00$ 2.35 E $00-2.46 E 00$ $\begin{array}{lll}1.83 \mathrm{E} & 00---2.35 \mathrm{E} \quad 00 \\ 1.11 \mathrm{E} & 00-\cdots 1.83 \mathrm{E} & 00\end{array}$ $1.11 E$ E0- - 1.83 E 00 $1.11 \mathrm{E}-01--5.50 \mathrm{E}-01$ $3.25 \mathrm{E}-02--1.11 \mathrm{E}-\mathrm{Cl}$ $5.83 E-04-\cdots 3.35 E-02$ $1.01 E-04--5.83 E-04$ $1.07 E-05--2.00 E-05$ $3.06 \mathrm{E}-06-\cdots 1.07 \mathrm{E}-05$ $3.06 E-06-1.06 E-05$ $4.14 E-07---1.12 E-06$ $4.14 E-07---1.12 E-06$
$0.0 \quad---4.14 E-07$ $\triangle A N G L 1$ 0.0 0.0 $-2.021 E-04$ $6.287 \mathrm{E}-03$
$4.287 \mathrm{E}-03$ $4.287 E-03$ $2.243 E-03$ $2.120 \mathrm{E}-03$ $1.757 \mathrm{E}-03$ $1.778 \mathrm{E}-03$ $2.778 \mathrm{E}-03$ $3.895 \mathrm{E}-03$ $1.998 \mathrm{E}-02$ $1.586 \mathrm{E}-02$ $9.006 \mathrm{E}-01$ $3.764 \mathrm{E} 00$ $1.074 E$ O1 $2.808 \mathrm{E}$ OI $7.394 E$ O1 $1.594 \mathrm{E} \quad 2$ ANGLE 10 $M U=0 . C 950$

0.0

0.0

$1.283 \mathrm{E}-03$ $1.033 \mathrm{E}-02$ $5.146 \mathrm{E}-03$ $2.851 E-03$ $2.902 E-03$ 3.537E-03 $2.477 E-03$ $2.575 \mathrm{E}-03$ $3.760 \mathrm{E}-03$ $4.720 \mathrm{E}-03$ 2.155E-02 $1.653 \mathrm{E}-02$ $9.287 E-01$ 1.862E OA 1.C99 01 $2.868 \mathrm{E}$ of $1.623 \mathrm{E} 02$ $\begin{array}{ll}1.623 E & 02 \\ 2.319 E & 02\end{array}$
ANGLE 2 0.0 0.0 $-1.507 E-04$ $6.203 E-03$ $4.269 \mathrm{E}-03$ $2.243 E-03$ $2.124 \mathrm{~F}-\mathrm{C3}$ $2.666 \mathrm{E}-03$ $1.783 \mathrm{E}-03$ $2.784 E-C 3$ $3.900 E-03$ 2. COOE-02 $1.587 E-02$ $0.009 E-01$ $3.765 \mathrm{E}$ OD $1.074 \mathrm{E}$ Ol $2.809 E$ OI $7.395 E \quad 01$ $\begin{array}{lll}1.595 \mathrm{E} & 02 \\ 2.281 \mathrm{E} & 02\end{array}$ ANGLE 11 $M U=0.2816$ 0.0
0.0
0.0 $1.651 \mathrm{E}-03$ $1.330 E-02$ $5.905 E-03$ $3.209 E-03$ $3.264 \mathrm{E}-03$ $3.961 \mathrm{E}-03$ $2.798 \mathrm{E}-03$ $2.930 E-03$ $4.174 E-03$ $5.004 E-03$ $2.240 E-02$ $1.666 E-02$ $9.339 E-01$ 1.80 E $1.204 E$ CI $7.563 E$ OI $7.563 \mathrm{E} \mathrm{Cl}$ $2.325 \mathrm{E} \mathrm{O2}$ $\begin{array}{ccc}\text { ANGLE } & 3 & \text { ANGLE } \quad 4 \\ M U=-0.94 & 46 & M U=-0.8656\end{array}$ 0.0

0.0

$-2.700 E-05$ $5.931 \mathrm{E}-03$ $2.246 \mathrm{E}-03$
$2.138 \mathrm{E}-03$ $2.138 E-03$
$2.679 E-03$ $1.775 \mathrm{E}-03$ $1.799 E-03$ $2.805 E-03$ $3.922 E-03$ 2.004E-02 1. $589 E-02$ $9.019 E-01$ $3.768 \mathrm{E} 00$ $1.075 E$ OI $2.811 \mathrm{E} 01$ $7.401 \mathrm{E}$ Ol $1.596 \mathrm{E}$
2.28 ANGLE 12 $M U=0.4580$
0.0 0.0 0.0 $4.502 E-03$ $1.505 \mathrm{E}-02$ $6.356 \mathrm{E}-03$ $3.474 \mathrm{E}-03$ 3.538E-03 $4.291 \mathrm{E}-03$ $3.024 \mathrm{E}-03$ 3.164E-03 $4.461 \mathrm{E}-03$ $5.211 E-03$ 1.254E-02 $1.673 E-02$ $3.390 E-01$ 1.897E 0 $3.108 E$ OS $2.888 \mathrm{E}$ OI $1.633 \mathrm{E} \mathrm{O2}$ $2.331 \mathrm{E} 02$
0.0

0.0

$3.299 E-05$ $5.603 \mathrm{E}-03$ 4.115E-03 2.255E-03 $2.167 \mathrm{E}-03$ 2.706 E-03 $1.803 \mathrm{E}-03$ $1.833 \mathrm{E}-03$ 2.848E-03 3. $963 \mathrm{E}-03$ 2. $013 \mathrm{E}-02$ $0.038 E-01$ $3.775 \mathrm{E} 00$ $1.077 E 01$ $2.815 \mathrm{E} 01$ $7.411 \mathrm{E}$
$1.598 \mathrm{E}$ 2.286E 02 ANGLE 13 $M U=0.6179$ 0.0 0.0 $1.094 \mathrm{E}-02$ $1.434 E-02$ $6.746 E-03$ 3. 722 E-03 3.783E-03 $4.593 \mathrm{E}-03$ $3.200 E-03$ 3. $328 \mathrm{E}-03$ $4.682 E-03$ $5.381 \mathrm{E}-03$ 2.142E-02 $1.690 \mathrm{E}-02$ $9.436 E-01$ 1.912E 00 $1.112 E$ O1 $7.509 E$ O1 T.509E O1 2.337E 02
ANGLE 5
$M U=-0.7550$ 0.0 0.0 1. $451 E-04$ $5.498 E-03$ $4.045 E-03$ 2. $273 E-03$ 2. $212 E-03$ 2.750E-03 $1.847 \mathrm{E}-03$ $1.883 E-03$ 4. $025 E-03$ $2.026 \mathrm{E}-02$ $1.600 \mathrm{E}-02$ $9.065 E-01$ $3.785 \mathrm{E} 00$ $1.079 E \quad 01$ $2.821 \mathrm{E} \quad 01$ $7.425 \mathrm{E} 01$ 2.291E 02 ANGLE 14 $M U=0.7550$ 0.0 0.0 3.681E-02 1. $778 \mathrm{E}-02$ $8.268 \mathrm{E}-03$ $4.416 \mathrm{E}-03$ $4.438 \mathrm{E}-03$ $5.366 E-03$ 3. $733 \mathrm{E}-03$ $3.396 \mathrm{E}-03$ 5. $342 \mathrm{E}-03$ $2.196 \mathrm{E}-02$ $1.702 E-02$
$9.477 E-01$ $9.477 E-01$ $1.925 \mathrm{E} 01$ $1.115 E$ O1 7.628 E $7.628 E 01$ $2.342 \mathrm{E} O 2$

ANGLE 6 $M U=-0.6179$ 0.0 2. $240 E-04$ 2.240E-04 $4.037 \mathrm{E}-03$ 2. $309 \mathrm{E}-03$ 2. $277 \mathrm{E}-03$ 2.817E-03 $1.909 E-03$ 1. $953 E-03$ 3.000E-03 4.107E-03 $2.042 E-02$ $1.608 \mathrm{E}-02$ 9.100 E-01 $3.797 E 00$ $1.082 \mathrm{E} 01$ $2.828 \mathrm{E} 01$ 7.443 E Ol $1.604 \mathrm{E} \mathrm{O2}$
$2.295 \mathrm{E} 02$ ANGLE 15 $M U=0.8656$ 0.0 
ENERGY GROUP (MEV)

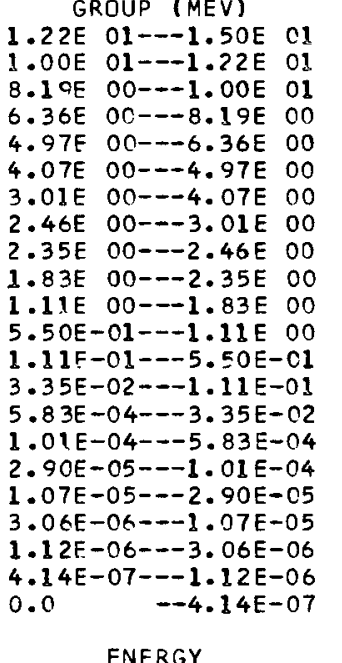
ENERGY GROUP (MEV)
$1.22 E$ O1--1.50E 0.1
1.00 O1---1.22E 01 $8.19 E$ OO--1. COE OI $6.36 \mathrm{E}$ 00- $-8.19 \mathrm{E}$ OO $4.97 E$ OO- - $6.36 E$ OO 4.07E $00--4.97 E$ OO 3.01E $00---4 . C 7 E$ OO $2.46 E$ OO- $-3.01 E$ OO $2.35 \mathrm{E} 00--2.46 \mathrm{E} 00$ $1.83 \mathrm{E} 00--2.35 \mathrm{E}$ OO $1.11 \mathrm{E} 00--1.83 \mathrm{E}$ OO $5.50 \mathrm{E}-01--1.11 \mathrm{E}$
$1.11 \mathrm{E}-\mathrm{O} 1---5.50 \mathrm{E}-01$ $3.35 \mathrm{E}-02--1.11 \mathrm{E}-01$ 5.3 $3 \mathrm{E}-04-2-3.35 \mathrm{E}-02$ $1.01 E-04--5.83 E-04$ $2.90 \mathrm{E}-05--1.01 \mathrm{E}-04$ $1.07 E-05 \cdots-2.90 E-05$ $3.06 \mathrm{E}-06--1.07 \mathrm{E}-05$ $1.12 \mathrm{E}-06---3.06 \mathrm{E}-06$ $4.14 \mathrm{E}-07--1.12 \mathrm{E}-06$ $4.14 \mathrm{E}-07---1.12 \mathrm{E}-06$
$0.0 \quad--04.14 \mathrm{E}-07$
ANGLE 1 $M U=-1.0000$ 0.0 0.0 $-2.342 E-04$ 7.064E-03 $6.442 \mathrm{E}-03$ $4.273 \mathrm{E}-03$ $4.273 E-03$
$4.433 E-03$ $5.763 \mathrm{E}-03$ $3.914 \mathrm{E}-03$ $3.925 \mathrm{E}-03$ $6.569 E-03$ $1.064 E-02$ $6.447 E-02$ $5.393 \mathrm{E}-02$ $3.153 \mathrm{E} 00$ $1.338 \mathrm{E} \mathrm{O1}$ $3.852 \mathrm{E}$ OI $1.016 \mathrm{E} 02$ $2.692 E 02$ $5.832 \mathrm{E} \mathrm{O2}$ $8.385 \mathrm{E} \mathrm{O2}$ ANGLE 10 $M U=0.0950$

0.0

0.0

2.288E-03 $9.996 \mathrm{E}-03$ $1.102 \mathrm{E}-02$ $6.273 E-03$ $6.985 \mathrm{E}-03$ $8.755 \mathrm{E}-03$ $6.402 E-03$ $6.627 E-03$ $9.955 \mathrm{E}-03$ $7.319 E-02$ $5.740 E-02$ $3.320 E 00$ .400 E 01 4.017E O1 1.0 $6.033 E$ E $6.033 E$ O2
$8.644 E$ O2 $\triangle N G L E \quad 2$ $M U=-0.9894$ 0.0 0.0 $0.070 E-04$ $6.947 \mathrm{E}-03$ $6.428 \mathrm{E}-\mathrm{C} 3$ $4.272 \mathrm{E}-03$ $4.440 E-03$ $5.769 \mathrm{E}-03$ $3.921 \mathrm{E}-0$ $3.935 \mathrm{E}-03$ $6.583 \mathrm{E}-03$ $1.066 \mathrm{E}-02$ $6.452 E-02$ $5.396 \mathrm{E}-\mathrm{O} 2$ $3.155 E$ EO $1.238 E$ O1 $3.853 \mathrm{E} \mathrm{O1}$ $1.017 E$ O2 $5.834 \mathrm{E} \quad 02$ $8.388 \mathrm{~F} 02$

ANGLE 11 0.0 0.0 0.0 2.073E-03 $1.528 \mathrm{E}-02$ $8.077 E-03$ $5.242 \mathrm{E}-03$ $6.139 \mathrm{E}-03$ $7.924 \mathrm{E}-03$ $5.528 \mathrm{E}-03$ $5.398 \mathrm{E}-03$ $8.772 \mathrm{E}-03$ $1.311 E-02$ $7.164 \mathrm{E}-02$ $5.808 \mathrm{E}-02$ $3.352 E$ O $1.411 E$ CI $4.048 \mathrm{E}$ O1 $1.064 \mathrm{E} 02$ 6.060 O 02 $8.690 E$ C2
(NEUTRONS/MEV/STERADIAN/SOURCE NEUTRON)

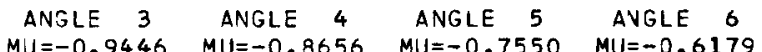
0.0 0.0 $-6.050 E-06$ $6.564 \mathrm{E}-03$ $6.382 \mathrm{E}-03$ $4.267 \mathrm{E}-03$ $4.468 \mathrm{E}-03$
$5.793 \mathrm{E}-03$ $5.793 E-03$
$3.954 E-03$ $3.973 \mathrm{E}-03$ $6.637 \mathrm{E}-03$ $1.072 E-02$ $6.473 \mathrm{E}-02$ $5.408 E-02$ $3.161 \mathrm{E} \quad 00$ $1.341 \mathrm{E} 01$ $3.859 \mathrm{E}$ O1 $2.696 \mathrm{E} \mathrm{O2}$ 5.84LE O2 $8.400 E$ O2

0.0 0.0 $8.652 E-05$ $8.652 E-05$ $6.115 E-03$
$6.336 \mathrm{E}-03$ $4.367 E-03$ $4.527 \mathrm{E}-03$ 4.527E-03 $4.020 \mathrm{E}-03$ $4.020 E-03$ $6.743 \mathrm{E}-03$ $1.085 E-02$ $6.511 E-02$ $5.431 E-02$ $3.172 E$ OO $1.345 \mathrm{E}$ 01 $3.871 \mathrm{E} 01$ $1.021 \mathrm{E} 02$ $1.703 \mathrm{E}$ O2
$5.855 \mathrm{E} 02$ $8.420 E$ O2 ANGLE 12 0.0 0.0 0.0 $3.868 \mathrm{E}-03$ $1.492 E-02$
$1.161 \mathrm{E}-02$ $1.161 \mathrm{E}-02$
$6.765 \mathrm{E}-03$ 7.707E-03 $9.832 \mathrm{E}-03$ $6.860 \mathrm{E}-03$ $6.775 \mathrm{E}-03$ $1.421 \mathrm{E}-02$ $7.194 \mathrm{E}-02$ $5.878 E-02$ $3.383 E$ OO $4.423 E$ OI
4.077 $1.071 E 02$ $2.825 E$ O2 $\begin{array}{ll}6.104 E & 02 \\ 8.735 E & 02\end{array}$ ANGLE 13 0.0 0.0 5.762E-03 $2.094 \mathrm{E}-0$ $1.381 \mathrm{E}-02$ 7. $964 \mathrm{E}-03$ $8.995 E-03$ $1.143 \mathrm{E}-02$ $7.809 E-03$ $1.169 \mathrm{E}-02$ $1.512 \mathrm{E}-02$ $7.652 E-02$ 3. 1.43 $4.105 E$ O1 1.077 O2 $2.841 \mathrm{E} 02$ $6.136 E$ O2
8.777 E

6
$M U=-0.7550$ 0.0 0.0 2. $306 E-04$ 6. $003 E-03$ $6.349 E-03$ $4.283 E-03$ $4.263 E-03$ 5. $941 E-03$ $4.123 \mathrm{E}-03$ $4.160 E-03$ 6. $901 E-03$ $1.103 E-02$ $6.567 E-02$ $5.463 E-02$ $3.188 \mathrm{E} \quad 00$ $1.351 \mathrm{E} O 1$ $1.025 E 02$ $2.713 \mathrm{E} 02$ $5.875 E$ E2

ANGLE 14 0.0 0.0 $3.035 E-04$ $6.465 E-03$ $6.473 E-03$ 4.334F-03 $4.755 \mathrm{E}-03$ $6.090 E-03$ $4.270 E-03$ 4.315E-03 $7.118 E-03$ $1.127 E-02$ $6.638 E-02$ $5.504 \mathrm{E}-02$ $3.208 \mathrm{E} 00$ $1.358 E$ O1 $3.907 \mathrm{E} 01$ $1.030 E_{02}$ $2.725 \mathrm{E} 02$ $5.899 \mathrm{E} \mathrm{O2}$ $8.480 E 02$

ANGLE 15 $M U=0.7550$ 0.0 1.233E-02 $.995 E-02$ . $531 E-02$ . $1.003 E-02$ $1.279 E-02$ 8.554E-03 1.266E-03 $1.239 E-02$ $7.453 E=02$ $6.004 E-02$ 3.430E 02 $1.442 E$ ol $4.129 E$ OI 2.855E 02 $6.164 \mathrm{E} 02$ $8.812 \mathrm{E} 02$ $M U=0.8656$ 0.0

ANGLE 7 $M=-0.4580$ 0.0 0.0 4. $038 \mathrm{E}-04$ 7. $514 \mathrm{E}-03$ $6.741 E-03$ 4. $436 E-03$ 4. $946 \mathrm{E}-03$ $6.317 E-03$ 4. $471 \mathrm{E}-03$ 7.400 E- 03 $1.158 \mathrm{E}-02$ $6.724 E-02$ 5. $554 \mathrm{E}-02$ $3.232 \mathrm{E} 00$ $1.367 E$ O1 $3.931 \mathrm{E} \mathrm{O1}$ 1.035 E 02 $2.739 E 02$ $5.929 E \quad 2$ 8.517 E 02 ANGLE 16 
ENERGY GROUP (MEV $1.22 E$ O1--1.5OE $1.00 E$ 01--1.22E $8.19 E$ O0-- $1.00 E$ OI $4.36 E$ OO- $4.8 .19 E$ O $4.97 E$
$4.007 E$
3 $3.01 \mathrm{E} 00--4.07 \mathrm{E} 00$ $2.46 \mathrm{E} 00--3.01 \mathrm{E} 00$ $2.35 \mathrm{E} 00--2.46 \mathrm{E} 00$ $1.83 \mathrm{E} 00--2.35 \mathrm{E} 00$ $1.11 \mathrm{E} 00--1.83 \mathrm{E} 00$ $5.50 E-01--1.11 E 00$ $1.11 E-01---5.50 E-01$ $3.35 \mathrm{E}-02---1.11 \mathrm{E}-01$ $5.82 E-04--3.35 E-02$ $1.01 E-04---5.83 E-04$ $2.90 E-05--1.01 E-04$ $1.07 E-05--2.90 E-05$ $3.06 E-06--1.07 E-05$ $1.12 \mathrm{E}-06---3.06 \mathrm{E}-06$ $4.14 \mathrm{E}-07---1.12 \mathrm{E}-06$
$0.0 \quad--4.14 \mathrm{E}-07$ ENERGY GROUP (MEV)

$1.22 \mathrm{E}$ 01-- $1.50 \mathrm{E}$ O1 $1.00 E$ O1-- $1.22 \mathrm{E}$ O1

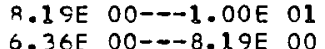

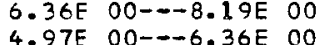
$\begin{array}{llll}4.97 E & 00--6.36 E & 00 \\ 4.07 E & 00--4.97 E & 00\end{array}$ $4.07 E$
$3.00--4.97 E$ 00

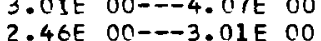
$2.46 E$ OO-- $3.01 E$ EO $2.35 \mathrm{E} 00---2.46 E$
$1.83 \mathrm{E} 00---2.35 \mathrm{E}$ OO $1.83 \mathrm{E}$ ON---1.83E 00 $5.50 E-01--1.11 \mathrm{E}$ OO $1.11 E-01--5.50 E-01$ $3.35 \mathrm{~F}-02--1.11 \mathrm{E}-01$ 5.8 $2 E-04--3.35 E-02$ $1.01 E-04--5.83 E-04$ 2.90E-05-C $1.07 E-05-2-2.00 E-05$ $1.12 E-06-03.06 E-06$ $\begin{array}{ll}4.14 \mathrm{E}-07---1.12 \mathrm{E}-06 \\ 0.0 & ---4.14 \mathrm{E}-07\end{array}$
ANGLE 1 0.0

0.0

0.0

$-2.051 E-04$ $6.768 E-03$ $6.908 \mathrm{E}-03$ $5.033 E-03$ $5.487 \mathrm{E}-03$ $7.242 \mathrm{E}-03$ $5.073 E-03$ $5.157 E-03$ $8.871 \mathrm{E}-03$ $1.521 E-02$ $9.846 E-02$ $8.430 E-02$ $5.009 \mathrm{E} 00$ $2.145 E$ E 01 $6.213 E$ O1 4.388E 02 $9.541 \mathrm{E} \mathrm{O2}$ $1.377 \mathrm{E} 03$ ANGLE 10 $M U=0.0950$ 0.0 0.0
0.0 0.0
$1.599 E-03$ $1.308 \mathrm{E}-02$ $8.311 \mathrm{E}-03$ $5.710 \mathrm{E}-03$ $7.129 \mathrm{E}-03$ $9.440 E-03$ $6.798 E-03$ $6.650 E-03$ $1.126 E-02$ $1.810 E-02$ $1.071 E-01$ $9.961 E-02$ S.327E 00 $2.266 E$ O1 1.731E 02 $1.731 E 02$ 4.591202 $1.431 E \quad 03$
(NEUTRONS/MEV/STERADIAN/SOURCE NEUTRON) ANGLE
$M U=-0.9894$$\quad$ ANGLE $3 \quad$ ANGLE 4 0.0 $-1.459 E-04$ $6.656 \mathrm{E}-03$ $6.900 \mathrm{E}-\mathrm{CB}$ $5.030 E-03$ $5.496 \mathrm{E}-03$ $7.249 E-03$ 5. $084 \mathrm{E}-03$ $5.170 \mathrm{E}-03$ $8.890 E-03$ $1.524 \mathrm{E}-02$ $9.855 \mathrm{E}-02$ $8.436 E-02$ $5.012 E 00$ $2.146 E$ O1 $6.216 E 01$ $1.650 \mathrm{OE} 02$ 4.390E 02 $\begin{array}{ll}9.545 \mathrm{E} & 02 \\ 1.378 \mathrm{E} & 03\end{array}$ ANGLE 11 $M U=0.2815$ 0.0 0.0 $2.864 E-03$ $1.269 E-02$ $1.207 F-02$ $7.437 \mathrm{E}-03$ $8.935 \mathrm{E}-03$ $1.164 E-02$ $8.462 E-03$ $8.480 E-03$ $1.344 E-02$ $1.975 E-02$ $3.123 E-01$ ?.186E-02 $5.389 E 00$ $6.606 E$ OI $6.606 E$ OS $1.746 E$ O2 $4.629 \mathrm{E} 02$ $1.441 \mathrm{E} \mathrm{O}^{3}$
0.0
0.0

$1.073 E-05$ $6.289 E-03$ $6.877 \mathrm{E}-03$ $5.022 \mathrm{E}-03$ $5.531 E-03$ $281 E-03$ $5.128 \mathrm{E}-03$ $5.221 \mathrm{E}-03$ 8.967F-03 $1.534 \mathrm{E}-02$ $9.893 \mathrm{E}-02$ $8.458 E-02$ $5.023 \mathrm{E} 00$ $2.153 E 01$ $6.228 E 01$ $1.653 E 02$ $4.397 E \quad 02$ $.561 \mathrm{E} 02$ $1.380=03$

ANGLE 12 $M U=0.4580$ 0.0
0.0 0.0 $3.475 \mathrm{E}-03$ $1.685 E-02$ $1.170 \mathrm{E}-02$ $7.561 E-03$ $9.309 E-03$ $1.232 \mathrm{E}-02$ $8.640 F-03$ $8.349 \mathrm{E}-03$ $1.355 E-02$ $2.010 F-02$ $1.126 E-01$ 5.449E 00 5.449E 0 2.312E O1 $6.667 \mathrm{E}$ O 1.761 E 02 $4.665 \mathrm{E} 02$ $\begin{array}{ll}1.011 E & 03 \\ 1.451 E & 03\end{array}$
0.0

0.0

1.080 E- 04 $5.875 \mathrm{E}-03$ $6.873 \mathrm{E}-03$ $5.015 \mathrm{E}-03$ $5.604 E-03$ $7.352 \mathrm{E}-03$ $5.219 E-03$ $5.323 E-03$ $9.118 \varepsilon-03$ 1. $553 \mathrm{E}-02$ $9.956 \mathrm{E}-02$ $8.500 \mathrm{~F}-02$ $5.045 \mathrm{E} 00$ $2.158 \mathrm{E}$ O1 $6.250 E$ OI $1.659 \mathrm{E} 02$ $4.4 I I E ~ 02$ $9.590 E 02$ $1.384 \mathrm{E} 03$

ANGLE 13 $M U=0.6179$ 0.0 0.0 $6.284 \mathrm{E}-03$ $1.936 \mathrm{E}-02$ $1.616 \mathrm{E}-02$ $9.705 \mathrm{E}-03$ $1.165 \mathrm{E}-02$ $1.524 \mathrm{E}-02$ $1.056 \mathrm{E}-02$ $1.025 E-02$ $1.587 \mathrm{E}-02$ $2.169 \mathrm{E}-02$ $1.163 E-03$ $5.506 \mathrm{E} 00$ $2.333 \mathrm{E}$ O1 $6.724 E$ OI 4.775 E 02 $4.699 \mathrm{E}$ O2 $\begin{array}{ll}1.018 E & 03 \\ 1.460 E & 03\end{array}$
$M U=-0.7550$ 0.0 0.0
$2.509 E-04$ $5.814 \mathrm{E}-03$ $6.944 E-03$ $5.030 E-03$ 5.72OE-03 $7.477 E-03$ $5.361 F-03$ $5.474 \mathrm{E}-03$ $9.342 E-03$ $1.580 E-02$ $.005 E-01$ $8.558 \mathrm{E}-02$ $5.075 E 00$ $2.170 E$ O1 $6.282 E$ OL $1.667 E 02$ $4.431 \mathrm{E} 02$ $.631 E 02$ $1.390 E \quad 03$ ANGLE 14 0.0 $.256 E-02$ $2.830 \mathrm{E}-02$ $.840 \mathrm{E}-02$ $1.101 \mathrm{E}-02$ $1.326 E-02$ 1.743E-02 $1.160 \mathrm{E}-02$ $1.09 \mathrm{E}-02$ . $2.249 \mathrm{E}-02$ 1.176E-01 5.558E 00 . $6.774 E$ OI $1.787 \mathrm{E} 02$ 1. $024 \mathrm{E} 03$ $1.024 \mathrm{E} \mathrm{O3}$

AVGLE 6 0.0 0 $3.210 E-04$ $6.333 E-03$ $7.138 \mathrm{E}-03$ $5.085 E-03$ $5.892 \mathrm{E}-03$ $7.680 E-03$ $5.564 E-03$ $5.682 E-03$ $.646 E-03$ 1. $017 \mathrm{E}-01$ $8.633 E-02$ $5.113 E 00$ $2.185 E$ O1 $6.322 \mathrm{E} 01$ 4.456E O2 $9.582 \mathrm{E} 02$ $1.397 E \quad 03$ AVGLE 15 0.3 
ENERGY GROUP IMEV $1.22 \mathrm{E} \quad 03--1.50 E$ O1 $1.00 E$ O1--1.22E 01 $6.36 \mathrm{~F}$ 00---8.19E 00 $4.97 E$ OC- $-6.36 E$ OD $4.07 E$ DO- $-4.97 E$ OO $3.01 \mathrm{E} 00---4.07 E 00$ $2.46 \mathrm{E}$ O0-- $-3.01 \mathrm{E}$ Oก $2.35 \mathrm{E} \quad 00--2.46 \mathrm{E} \quad 00$ $1.83 \mathrm{E}$ On-- $-2.35 \mathrm{E} 00$ $1.11 \mathrm{E} 00---1.83 \mathrm{E}$ 00 $5.50 E-01--1.11 \mathrm{E}$ 0O $1.11 \mathrm{E}-01--5.50 \mathrm{E}-01$ $3.35 E-02--1.11 E-01$ $5.83 E-04--3.35 E-02$ $1.01 E-04---5.83 E-04$ $2.90 F-05--1.01 E-04$ 1. $07 E-05---2.90 \mathrm{E}-05$ $3.06 \mathrm{E}-06--1.07 \mathrm{E}-05$ $1.12 E-06--3.06 E-06$ $4.14 E-07---1.12 E-06$ 0.0

ENERGY$$
\begin{aligned}
& \text { ENERGY } \\
& \text { GROUP (MEV) }
\end{aligned}
$$

$1.22 E 01--1.50 E$ OI $1.00 E$ Ol--1.22E OI $8.19 F$ ON---1.00E O1 6.36 E ON-- $8.19 E$ OO 4.97E 00--6.36E 00 $3.01 E$ OD- $4.07 E 0$ 3.02 E OD- $4.07 E$ OO 2.46E 00- $3.01 \mathrm{E}$ 00 2.35E OO- $2.46 E$ OO $1.11 \mathrm{E} 00-2.35 \mathrm{E} 00$ $5.50 E-01-1.11 E 00$ 5.50E-O1-D.11E 00 $3.35 \mathrm{E}-02-1.11 \mathrm{E}-01$ $5.35 E-02-1.11 E-01$ $1.01 E-04--5.83 E-04$ $2.90 E-05--1.01 E-04$ $1.07 E-05--2.00 E-05$ $3.06 \mathrm{E}-06--1.07 \mathrm{E}-05$ $1.12 \mathrm{E}-06---3.06 \mathrm{E}-06$ $4.14 \mathrm{E}-07-0-1.12 \mathrm{E}-06$ $0.0 \quad--4.14 \mathrm{E}-07$ $\triangle N G L E \quad 1$ 0.0

$-1.325 E-04$ $5.538 \mathrm{E}-03$ $6.594 \mathrm{E}-03$ $5.428 \mathrm{E}-03$ $6.381 E-03$ $8.609 E-03$ $6.423 E-03$ $6.789 \mathrm{E}-03$ $1.221 \mathrm{~F}-02$ $2.269 E-02$ $1.607 \mathrm{E}-01$ $1.421 \mathrm{E}-01$ $8.650 E$ OO $3.760 E$ O1 $1.101 \mathrm{E} 02$ $2.957 E$ O2 $7.932 E \quad 02$ $\begin{array}{ll}1.737 E & 03 \\ 2.527 E & 03\end{array}$ ANGLE 10 $M U=0.0950$ 0.0
0.0 0.0 $1.810 E-03$ $1.810 E-03$ $1.012 E-02$ $1.015 \mathrm{E}-02$ 1.020E-03 1.252E-03 9.695E-03 $9.65 \mathrm{E}-03$ $2.693 E-02$ $1.693 \mathrm{E}-02$ $1.811 \mathrm{E}-01$ $1.547 \mathrm{E}-01$ $9.333 E 00$ $4.032 \mathrm{E} 01$ $1.177 E_{02}$ $3.150 E$ O2 8.42 EE 0 ? $1.841 E \quad 03$ $2.662 E 03$ $\begin{array}{cc}\triangle N G L E & 2 \\ M U=-0.9894\end{array}$ 0.0

$-8.757 E-05$ $5.451 E-03$ $6.595 E-03$ $5.425 E-03$ $6.391 E-03$ $8.619 E-03$ $6.438 \mathrm{E}-03$ $6.807 E-03$ $1.224 E-02$ 2.273E-02 $1.609 \mathrm{E}-\mathrm{Cl}$ $1.422 \mathrm{E}-01$ $8.656 \mathrm{E} \mathrm{OO}$ $3.762 E$ OI $1.102 E 02$ $2.959 \mathrm{~F} \mathrm{O2}$ $7.936 \mathrm{E} \mathrm{O2}$ $1.738 \mathrm{~F} 03$ $2.529 \mathrm{EO}^{03}$ ANGLE 11 0.0 0.0 0.0 $2.394 E-03$ $1.206 \mathrm{E}-02$ $7.615 E-03$ $1.015 E-03$ $1.043 \mathrm{E}-02$ $1.413 E-02$ $1.050 E-02$ $1.794 \mathrm{E}-02$ $2.010 \mathrm{E}-02$ $1.838 \mathrm{E}-01$ $1.838 \mathrm{E}-01$ $9.46 E E$ OO $4.084 \mathrm{E} 01$ $1.192 E 02$ $3.187 E \quad 02$ $8.518 E 02$ $1.860 F 03$ $2.687 \mathrm{E} \quad 03$
(NEUTRJNS/MEV/STERADIAN/SOURCE NEUTRON)

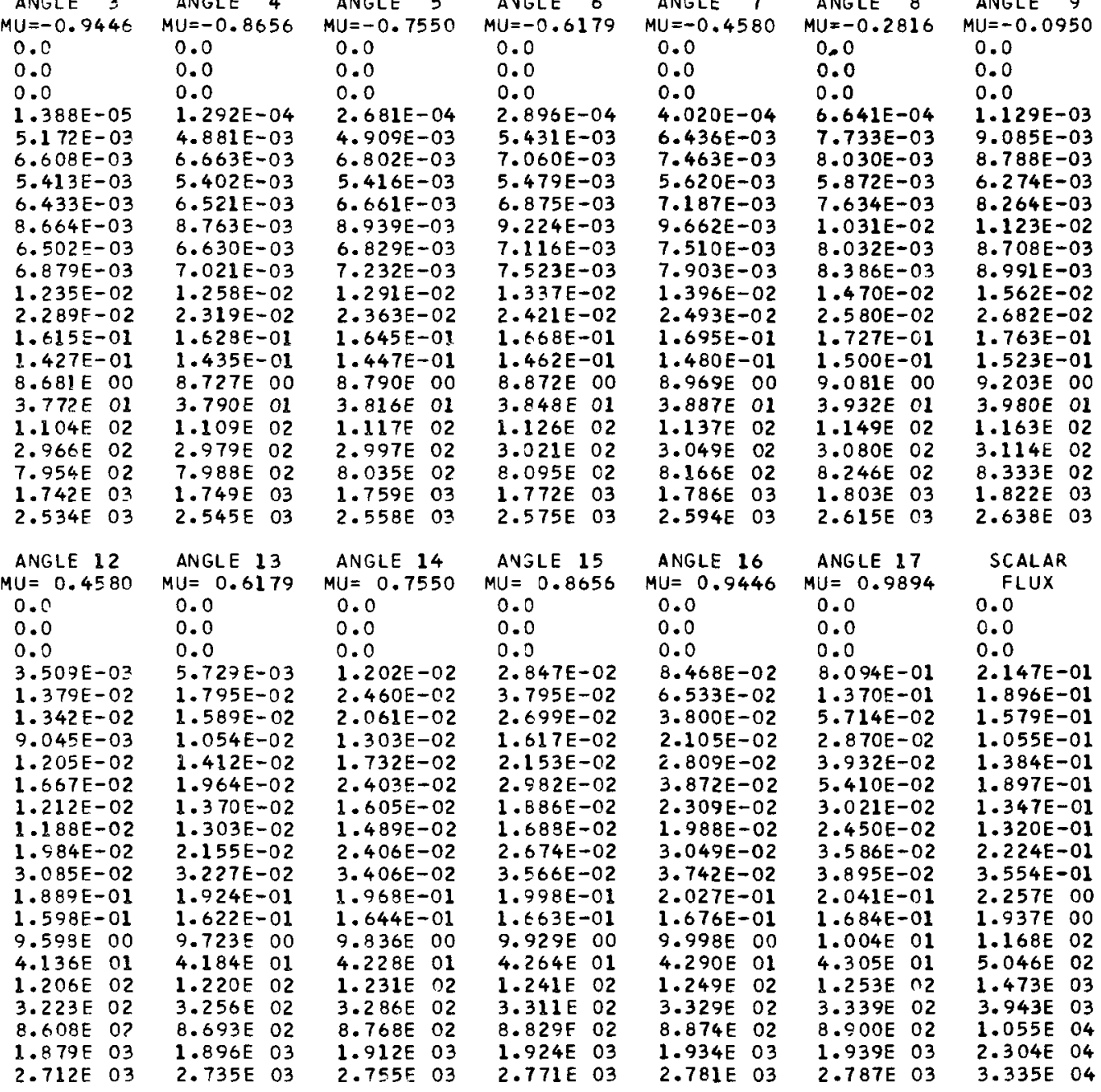


(NEUTRONS/MEV/STERADIAN/SOURCE NEUTRON)

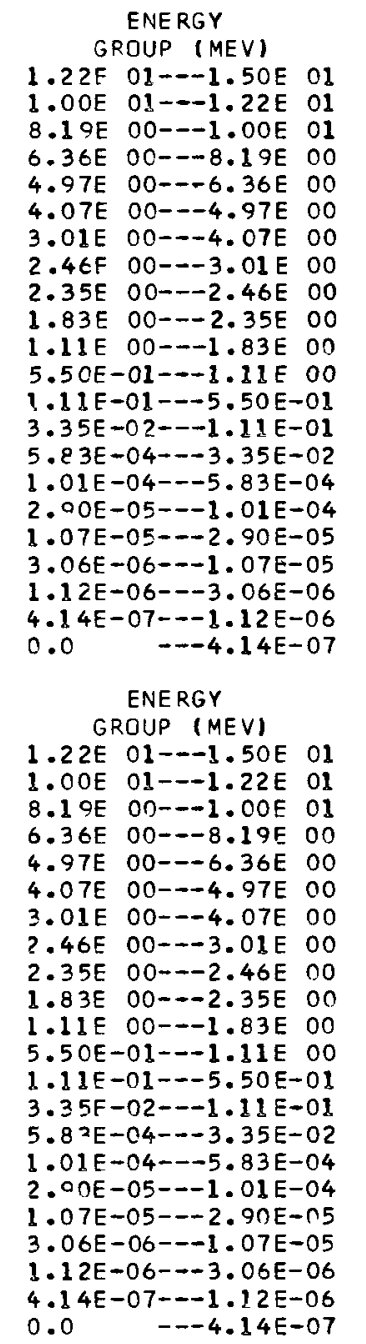

ANGLE 1

0.0

0.0

$-7.645 E-05$

$4.210 \mathrm{E}-03$

$5.557 E-03$

$6.128 \mathrm{E}-03$

$8.404 E-03$

$6.648 \mathrm{E}-03$

$7.326 \mathrm{E}-03$

$1.369 \mathrm{E}-02$

$2.700 E-02$

$2.033 \mathrm{E}-01$

$1.837 \mathrm{E}-01$

$5.000 \mathrm{~F}$ OI

$5.000 \mathrm{~F}$ OI

$4.003 E \quad 02$

$1.081 \mathrm{E} 03$

$2.384 E \quad O 3$

$3.492 \mathrm{E} \quad 03$

ANGLE 10

$M U=0.0950$

0.0

0.0
0.0

0.0
$1.452 E-03$

$1.452 \mathrm{E}-03$
$8.252 \mathrm{E}-03$

$8.252 \mathrm{E}-03$
$8.540 \mathrm{E}-03$

$6.349 E-03$

$9.009 E-03$

$1.281 \mathrm{E}-\mathrm{C2}$

$1.021 E-02$

$1.007 \mathrm{E}-02$

$1.907 E-02$

3.361E-02

$2.300 E-01$

$1.019 E-01$

$1.239 E$ O

1.505E

$4.311 E$ O2

4.31

1.161E

$2.553 \mathrm{E} 03$
$3.716 \mathrm{E} 03$
$A N G L E \quad 2$
$M U=-0.9894$

$M U=-0.9$
0.0
0.0 0.0

$-4.506 E-05$

4.15CE-C3

$5.562 E-03$

$4.939 \mathrm{E}-03$

$6.138 \mathrm{E}-03$

$8.415 \mathrm{E}-03$
$6.665 \mathrm{E}-03$

$7.346 \mathrm{E}-03$

$.372 \mathrm{E}-02$

$2.705 E-02$

$2.036 E-01$

$1.839 E-01$

$1.138 \mathrm{E} 01$
$5.003 \mathrm{E}$ O1

.477E 02

$4.006 E 02$

$1.082 \mathrm{E} 03$

$2.385 \mathrm{E} \quad 03$

$3.494 \mathrm{E} \mathrm{O3}$

ANGLE 11

MU $=0.2$
0.0
0.0

0.0

0.0
$2.049 E-03$

$2.049 \mathrm{E}-03$
$9.381 \mathrm{E}-03$

$9.381 \mathrm{E}-03$

$9.887 E-03$

$7.231 \mathrm{E}-03$

$1.033 \mathrm{E}-02$

$1.482 \mathrm{E}-02$

$1.148 \mathrm{E}-02$

$1.170 E-02$

$2.071 E-02$

2.360E-OI

..360E-01

$1.259 \mathrm{E} O \mathrm{C}$

1.259 OI

$618 E$

$4.370 E$ O2

$1.176 E$ O3

$2.585 \mathrm{E} \quad 03$

$3.758 E \quad 33$
$\begin{array}{lcl}A N G L E & 3 & \text { ANGLE } \\ M U=-0.9446 & M U=-0.8656\end{array}$

0.0

$1.376 \mathrm{E}-04$

$3.959 \mathrm{E}-03 \quad 3.777 \mathrm{E}-03$

$5.671 \mathrm{E}-03$

$4.918 \mathrm{E}-03$

$6.180 E-03$

$6.267 E-03$
$8.582 \mathrm{E}-0$

$6.881 \mathrm{E}-\mathrm{C}$

$7.589 \mathrm{E}-03$

$1.414 E-02$

$725 \mathrm{E}-02$

$2.764 \mathrm{E}-02$

$2.062 E-01$

$1.845 \mathrm{E}-01$

$1.142 \mathrm{E}$ OI

$5.018 \mathrm{E}$ O1

$1.149 \mathrm{E}$ OI

$5.046 \mathrm{E}$ OI

$1.490 \mathrm{E} 02$

$4.017 E$ O2 4.038E 02

$1.085 E$ O3

$2.392 \mathrm{E} C 3$

$1.090 \mathrm{E} 03$

$2.403 E \quad 03$

$3.520 \mathrm{E} 03$

$M U=-0.7550$

0.0 0.0

$2.044 E-04$

$3.848 \mathrm{E}-03$
$5.824 \mathrm{E}-03$

$5.824 E-03$

$6.408 E-03$

$8.782 E-03$

$7.105 E-03$
$7.827 E-03$

$.827 E-03$

$2.819 \mathrm{E}-02$

$2.086 \mathrm{E}-01$

$1.158 E$ E 01

$5.085 E$ OI

1.501E 02

$1.098 \mathrm{E} 03$

$2.419 E$ O2

$3.542 \mathrm{E} \quad 03$

ANGLE 12

$M U=0.4580 \quad M U=0.6179$

0.0

0.0

$2.918 E-03$

$1.121 \mathrm{E}-0$ ?

$1.169 \mathrm{E}-02$

$1.210 \mathrm{E}-02$

$1.751 \mathrm{E}-02$

$1.304 \mathrm{E}-02$

$2.257 \mathrm{E}-02$

$3.702 E-02$

$2.416 E-01$

$1.279 E$ OI

$5.576 E$ E 1

$4.428 \mathrm{E} 02$

$1.191 E 03$

$2.617 \mathrm{E} 03$

ANGLE 14
$M U=0.7550$

0.0

0.0

4. $941 \mathrm{E}-03$

$1.440 \mathrm{E}-02$

$1.448 E-02$

$1.458 \mathrm{E}-02$

$2.121 \mathrm{E}-02$

$1.504 \mathrm{E}-02$
$1.442 \mathrm{E}-02$

$2.481 \mathrm{E}-02$

3.89 E- 02

$2.474 E-01$

2.125E-01

$5.651 \mathrm{E} 01$

$4.483 E 02$

$1.205 \mathrm{E} 03$

$2.646 \mathrm{E} 03$

.0

$1.023 E-02$

2. $030 E-02$

$1.856 E-02$

$1.796 \mathrm{E}-02$

$2.621 \mathrm{E}-02$

$1.752 E-02$

$2.735 E-02$

$2.524 \mathrm{E}-01$

$2.524 E-01$

2.157E-

$5.718 E$ O1

$1.681 \mathrm{E} 02$

$1.218 E \quad 03$

$2.672 E 03$

$3.873 \mathrm{E} \mathrm{O3}$

ANGLE 6
$M U=-0.6179$

0.0

0.0

2. $494 E-04$

$4.298 E-03$

$6.080 E-03$
$4.994 E-03$

$6.625 \mathrm{E}-03$

$9.105 \mathrm{E}-03$

$7.429 E-03$

$8.156 \mathrm{E}-03$

$2.992 E-02$

$2.117 \mathrm{E}-01$

$1.895 \mathrm{E}-01$

$1.171 \mathrm{E} 01$

$5.135 \mathrm{E} 01$
$1.515 \mathrm{E} \mathrm{O2}$

4.104E 02

$1.108 E 03$

3.569E 03

ANGLE 15

$M U=0.8656$
0.3

0.3

0.0

$2.386 \mathrm{E}-02$

$3.114 \mathrm{E}-02$

$2.487 E-02$

作

$3.317 E-02$

2.072E-02

$3.033 \mathrm{E}-02$

$274 \mathrm{E}-02$

$2.570 \mathrm{E}-01$

$2.183 \mathrm{E}-01$

$5.774 \mathrm{E}$ OI

$1.597 \mathrm{E} 02$

$4.571 E \quad 02$

4.571E O2

$2.694 \mathrm{E} 03$

$3.899 \mathrm{E} 03$

ANGLE 7

0.0

0.0

$3.415 E-04$

$5.111 E-03$

$6.456 \mathrm{E}-03$

$5.132 E-03$

$6.947 E-03$

$9.600 E-03$
$7.873 E-03$

$8.588 \mathrm{E}-03$

$1.579 E-02$
$2.982 E-02$

$2.155 \mathrm{E}-01$

$1.921 \mathrm{E}-01$

$5.194 \mathrm{E} 01$

$\begin{array}{lll}5.194 E & 01 \\ 1.532 E & 02\end{array}$

$4.148 E$ O2

$1.119 E$ E 3

$3.601 \mathrm{E} 03$

ANGLE 8

0.0

0.0

$5.653 E-04$

$6.138 \mathrm{E}-03$

$6.971 E-03$

$5.379 E-03$
$7.415 E-03$

$1.033 \mathrm{E}-02$

$8.465 E-03$

$9.136 E-03$

$3.091 \mathrm{E}-02$

$2.199 \mathrm{E}-01$

$1.951 E-01$

$5.262 E$ OI

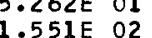

$4.198 \mathrm{E} 02$

$1.132 E 03$

$2.492 \mathrm{E} \quad 03$

$3.636 E \quad 03$

ANGLE $16 \quad$ ANGLE 17
$M U=0.9446 \quad M U=0.9894$

$M U=0.9446$
0.0

0.0

0.0

MU $=$

0.0

$6.407 E-02 \quad 5.176 E-01$

$5.356 \mathrm{E}-02 \quad 1.135 \mathrm{E}-01$

$5.256 \mathrm{E}-02$

$2.661 \mathrm{E}-02$

$4.122 \mathrm{E}-02$

(2.08

$3.376 E-02$

$3.156 \mathrm{E}-0$

$2.448 \mathrm{E}-02$
$3.813 \mathrm{E}-02$

$3.813 E-02$

$465-01$

$2.214 \mathrm{E}-01$

1.339E

. 
(NEUTRJNS/MEV/STERADIAN/SOURCE NEUTRON)

ENERGY GROUP (MEV) $1.00 E$ O1- $-1.22 \mathrm{E}$ OI 8.1 E $00--1.00 E$ OI 6.36 E $00--7.19 E \quad 00$ $4.97 E$ OO-- $6.36 E$ OO OO- $0-4.97 \mathrm{E}$ OO $3.01 \mathrm{E} 00 \rightarrow-\rightarrow 4.07 \mathrm{E} 00$ $.46 E$ OO-- 3.01E 00 $2.35 \mathrm{E} \quad 00---2.46 \mathrm{E} \quad 00$ $1.83 \mathrm{E} 00---2.35 \mathrm{E} 00$ $1.11 \mathrm{E} 00--1.83 \mathrm{E}$ OO $5.50 \mathrm{E}-01--1.11 \mathrm{E}$ OO $1.11 \mathrm{E}-01---5.50 E-01$ $3.35 \mathrm{E}-02--1.11 \mathrm{E}-0$ $5.83 \mathrm{E}-04---3.35 \mathrm{E}-0$ $1.01 E-04--5.83 E-04$ $2.90 E-05--1.01 E-04$ $1.07 E-05--2.90 E-05$ $3.06 \mathrm{E}-06-2-1.07 \mathrm{E}-05$ $1.12 \mathrm{E}-06-0-3.06 \mathrm{E}-06$ $4.1 \angle E-07-=-1.12 E=06$ $0.0 \quad-4.14 \mathrm{E}-07$

$$
\text { ENERGY }
$$$$
\text { GROUP (MEV }
$$

$1.22 \mathrm{E}$ O1-- $1.50 \mathrm{E} \quad$ $1.00 E$ O1---1.22E OI $8.19 \mathrm{E} 00--1.00 \mathrm{E}$ OI 6.36E $00---8.19 E$ OO $4.97 \mathrm{E} 00--6.36 \mathrm{E}$ OO 4.07E $00-4.97 E$ OD 3.01E 00---4.07E OO $2.46 \mathrm{E}$ 00-- $-3.01 \mathrm{E}$ OO $2.35 \mathrm{E} 00-2-2.46 \mathrm{E}$ OO $1.83 E$ OO- - 2.35E 00 $1.11 E$ OO-CD. $1.83 E$ OO .5OE-O1-- $1.11 E$ OO 1. 11 E $01--5$. . $35 E-02-1.11 E-01$ . $83 E-04--3.35 E-02$ $1.01 E-05-1.01 E-04$ $2.90 E-05-1.01 E-04$ $3.06 E-06=-1.07 E-05$ $1.12 \mathrm{~F}-06-0-3.06 \mathrm{E}-06$ $4.14 E-07-2-1.12 E-06$ $0.0 \quad--4.14 E-07$
ANGLE 1 $=-1.0000$ 0.0 0.0 $-4.065 E-05$ $3.080 E-03$ 4.381E-0 $4.112 \mathrm{E}-03$ $5.303 E-03$ $7.366 \mathrm{E}-03$ $6.145 E-03$ $7.046 \mathrm{E}-03$ $1.364 \mathrm{E}-02$ 2.823E-02 $2.227 E-01$ $1.281 \mathrm{E}$ OI $1.678 E$ O1 .687E 02 ..610E $1.253 E$
$2.776 E$ 4.089E 03 ANGLE 10
$M U=0.0950$ 0.0
0.0
0.0
1.14 0.0
$1.143 E-03$ $6.202 \mathrm{E}-03$ $6.942 \mathrm{E}-03$ $5.376 E-03$ $8.000 \mathrm{E}-03$ $1.175 E-02$ $9.697 \mathrm{E}-03$ $1.039 \mathrm{E}-02$ $1.932 \mathrm{~F}-02$ $3.549 \mathrm{E}-02$ $2.541 E-01$ $2.260 E-01$ $1.406 E$ OI $6.196 \mathrm{E}$ Cl
$1.837 \mathrm{E} 02$ $5.005 E$ E $1.356 \mathrm{E} \mathrm{O}$ $2.997 E$ O3 $4.385 E^{03}$ $\triangle N G L E \quad 2$
$M U=-0.9894$ 0.0 0.0 $-1.958 E-05$ $3.040 E-03$ $4.389 E-03$ 4.109E-03 $5.311 \mathrm{~F}-0$ $7.378 \mathrm{E}-0$ $6.162 \mathrm{E}-\mathrm{O}^{2}$ $7.066 \mathrm{E}-03$ $1.368 \mathrm{E}-02$ $2.829 \mathrm{E}-02$ $2.229 \mathrm{E}-0$ $2.045 \mathrm{E}-\mathrm{CI}$ $1.282 \mathrm{E} O$ $5.683 \mathrm{E}$ OI $1.689 \mathrm{E} \mathrm{O2}$ $4.614 \mathrm{E} \mathrm{O2}$ $1.253 \mathrm{E} 03$ $2.778 \mathrm{E} \quad \mathrm{B}$ $4.092 E \mathrm{CB}$

$\triangle N G L E 3$ $M U=-0.9446$
0.0 0.0 0.0 2.932E-05 $2.915 \mathrm{E}-03$ 4. $423 \mathrm{E}-03$ 4.099E-03 $5.349 \mathrm{E}-03$ $6.233 \mathrm{E}-0$ $7.149 \mathrm{E}-03$ $1.382 \mathrm{E}-02$ $2.851 \mathrm{E}-02$ $2.240 E-01$ $2.053 E-01$ $1.287 \mathrm{E} 01$ $5.702 E$ OI $4.628 \mathrm{E}$ O $1.257 E \quad 03$ $2.786 E$ O3 $4.104 \mathrm{E} \mathrm{O3}$

ANGLE 11 ANGLE 12 $M U=C .2816$ $M U=0$
0.0
0.0 $1.606 \mathrm{E}-03$ $7.117 E-03$ $8.020 \mathrm{E}-\mathrm{C}$ $6.131 \mathrm{E}-03$ $9.241 \mathrm{E}-03$ $1.374 \mathrm{E}-02$ $1.095-02$ $1.141 E-02$ $2.098 E-02$ $2.605 \mathrm{E}-01$ $2.303 \mathrm{E}-01$ 6.2397 OI $6.257 E$ OI $1.866 \mathrm{E}$ O2 $1.376 \mathrm{E} O 3$ $3.040 \mathrm{E} \mathrm{O3}$ $4.441 E$ O3
$M U=0.4$
0.0
0.0

0.0

$2.325 \mathrm{E}-03$

$8.544 \mathrm{E}-03$

$9.598 \mathrm{E}-0$

$7.199 \mathrm{E}-03$

1. ก97E-02

$1.647 \mathrm{E}-02$
$1.254 \mathrm{~F}-02$

$1.265 \mathrm{E}-02$

$2.294 \mathrm{E}-02$ $2.673 E-01$ $2.346 \mathrm{E}-01$ $1.455 \mathrm{E}$ Ol . $397 \mathrm{E}$ OL $1.89 E$ O2 $1.155 \mathrm{E} 02$ 3.081 E 03 $4.497 \mathrm{CB}^{2}$

\begin{tabular}{|c|c|c|}
\hline $\begin{array}{l}\text { ANGLE } 4 \\
M U=-0.8656\end{array}$ & $\begin{array}{l}\text { ANGLE } \\
M U=-0.7550\end{array}$ & $M U=-0.6179$ \\
\hline 0.0 & 0.0 & 0.0 \\
\hline 0.0 & 0.0 & 0.0 \\
\hline 0.0 & 0.0 & 0.0 \\
\hline $1.107 E-04$ & $1.585 E-04$ & $1.964 E-04$ \\
\hline $2.809 E-03$ & $2.892 E-03$ & $3.249 E-03$ \\
\hline $4.502 \mathrm{E}-\mathrm{C}$ & $4.643 E-03$ & $4.864 E-03$ \\
\hline $4.093 \mathrm{E}-03$ & $4.107 E-03$ & $4.162 \mathrm{E}-03$ \\
\hline $5.427 E-03$ & $5.555 E-03$ & $5.753 E-03$ \\
\hline $7.549 E-03$ & $7.751 \mathrm{E}-03$ & $8.072 E-03$ \\
\hline $6.376 E-03$ & $6.598 \mathrm{E}-03$ & $6.918 \mathrm{E}-03$ \\
\hline $7.309 E-03$ & $7.548 E-03$ & $7.877 E-03$ \\
\hline $1.411 \mathrm{E}-02$ & $1.453 E-02$ & $1.510 E-02$ \\
\hline $2.893 E-02$ & $2.953 E-02$ & $3.033 E-02$ \\
\hline $2.260 E-01$ & $2.288 E-01$ & $2.325 E-01$ \\
\hline $2.067 \mathrm{E}-01$ & $2.087 E-01$ & $2.113 E-01$ \\
\hline $1.295 \mathrm{E} 01$ & 1.307E 01 & $1.321 \mathrm{E} 01$ \\
\hline $5.736 E \quad 01$ & $5.784 E \quad 01$ & $5.846 E$ Ol \\
\hline $1.704 \mathrm{E} 02$ & $1.718 \mathrm{E} \quad 02$ & $1.736 \mathrm{E} \quad 02$ \\
\hline $4.655 \mathrm{E} \quad 02$ & $4.692 \mathrm{E} \quad 02$ & $4.739 E \quad 02$ \\
\hline $1.264 E \quad 03$ & $1.274 \mathrm{E} \quad 03$ & $1.286 \mathrm{E} 03$ \\
\hline $2.801 E 03$ & $2.822 \mathrm{E} \quad 03$ & $2.848 \mathrm{E} \quad 3$ \\
\hline $4.126 \mathrm{E} \quad 03$ & $4.154 E \quad 03$ & $4.190 E \quad 03$ \\
\hline ANGLE 13 & ANGLE 14 & ANGLE \\
\hline$M U=0.6179$ & $M U=0.7550$ & $M U=0.8656$ \\
\hline 0.0 & 0.0 & 0.0 \\
\hline 0.0 & 0.0 & 0.0 \\
\hline 0.0 & 0.0 & 0.0 \\
\hline $3.975 E-03$ & $8.215 E-$ & $1.887 \mathrm{E}-02$ \\
\hline $1.112 \mathrm{E}-02$ & $1.581 E-02$ & $2.435 E-02$ \\
\hline $1.194 \mathrm{E}-02$ & $1.546 E-02$ & $2.080 E-02$ \\
\hline $8.674 E-03$ & $1.069 E-02$ & $1.340 E-02$ \\
\hline $\begin{array}{l}1.336 E-02 \\
2.023 E-02\end{array}$ & $\begin{array}{l}1.667 E-02 \\
2.540 E-02\end{array}$ & $\begin{array}{l}2.131 E-02 \\
3.261 E-02\end{array}$ \\
\hline $1.453 \mathrm{E}-02$ & $3.703 E-02$ & $2.016 E-02$ \\
\hline $1.410 \mathrm{E}-02$ & $1.581 \mathrm{E}-02$ & $1.776 \mathrm{E}-02$ \\
\hline & & $3.064 E-02$ \\
\hline $4.115 E-02$ & $4.316 E-02$ & $4.507 E-02$ \\
\hline $2.737 E-01$ & $2.797 E-01$ & $2.849 \mathrm{E}-01$ \\
\hline $2.387 \mathrm{E}-01$ & $2.424 E-01$ & $2.455 E-01$ \\
\hline $1.478 \mathrm{E} 01$ & $1.498 \mathrm{E} \quad 01$ & $1.515 \mathrm{E} 01$ \\
\hline $6.491 \mathrm{E} 01$ & $6.575 \mathrm{E}$ & $6.645 \mathrm{E} 01$ \\
\hline $1.922 E 02$ & $1.946 \mathrm{E} 02$ & $1.966 \mathrm{E} 02$ \\
\hline $5.226 E \quad 02$ & $5.289 E \quad 02$ & $5.340 E \quad 02$ \\
\hline $1.413 E 03$ & $1.430 E 03$ & $1.443 \mathrm{E} \quad 03$ \\
\hline $3.120 \mathrm{E} \mathrm{O3}$ & $3.155 \mathrm{E} 03$ & $3.184 E \quad 03$ \\
\hline & $4.595 E_{03}$ & \\
\hline
\end{tabular}

ANGLE 7 $M U=-0.4580$ 0.0 0.0 2.741E- 04 3. $867 \mathrm{E}-03$ $5.180 E-03$ $4.285 E-03$ $6.050 E-03$ $8.562 E-03$ $7.358 \mathrm{E}-03$ $8.310 E-03$ 1. $584 \mathrm{E}-02$ $3.132 \mathrm{E}-02$ $2.369 E-01$ $2.143 E-01$ $1.339 \mathrm{E} \mathrm{O1}$ $5.920 E \quad 1$ $1.758 \mathrm{E} 02$ $4.795 \mathrm{E} \mathrm{O2}$ $1.301 E 03$ $2.880 E$ O3 $4.232 \mathrm{E} \mathrm{O3}$ ANGLE 16 $M U=0.9446$ 0.0
0.0 0.0
0.0 0.0
$4.811 E-02$ 4. $411 E-02$ $4.145 E-02$
$2.918 E-02$ $1.708 \mathrm{E}-02$ 2. $800 E-02$ $4.299 E-02$ $2.414 \mathrm{E}-02$ $1.998 E-02$ 3.373E-02 $4.671 E-02$ $2.888 \mathrm{E}-01$ $2.478 E-01$ $1.528 E$ O1 1.696E O1 $1.981 E$ O2 $1.379 \mathrm{E} 03$ $1.453 E$ O $4.656 \mathrm{E} 03$
ANGLE 8 $M U=-0$ 0.0 4. $526 E-04$ $4.635 E-03$ $5.607 E-03$ $4.503 E-03$ $6.486 \mathrm{E}-03$ $9.281 E-03$ $7.945 \mathrm{E}-03$ $8.861 E-03$ $1.677 \mathrm{E}-02$ $3.251 E-02$ $2.420 E-01$ $2.179 E-01$ $1.360 E$ Ol $6.005 E$ OL $1.782 \mathrm{E} 02$ $4.860 \mathrm{E} \mathrm{O2}$ $1.318 E \quad 03$

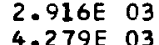
ANGLE 17 $M U=0.9894$ 0.0
0.0 0.0 $3.289 E-01$ $8.682 E-02$ $4.349 \mathrm{E}-02$ $2.226 \mathrm{E}-02$ $3.853 \mathrm{E}-02$ $5.959 E-02$ $2.970 \mathrm{E}-02$ $2.259 \mathrm{E}-02$ $3.709 \mathrm{E}-02$
$4.785 \mathrm{E}-02$ $4.785 E-02$ $2.911 \mathrm{E}-01$ $1.535 \mathrm{E} O 1$ $1.535 E$ OI . $726 E$ OI $5.401 E 02$ $1.459 E$ E 03 $3.217 E \quad 03$ 4.670 E O3

ANGLE 9 $M U=-0.0950$ 0.0 7. $529 E-04$ $5.425 E-03$ $6.173 E-03$ $4.852 E-03$ $7.112 E-03$ $1.031 E-02$ $8.712 E-03$ $9.548 E-03$ $1.792 E-02$ $3.390 \mathrm{E}-02$ 


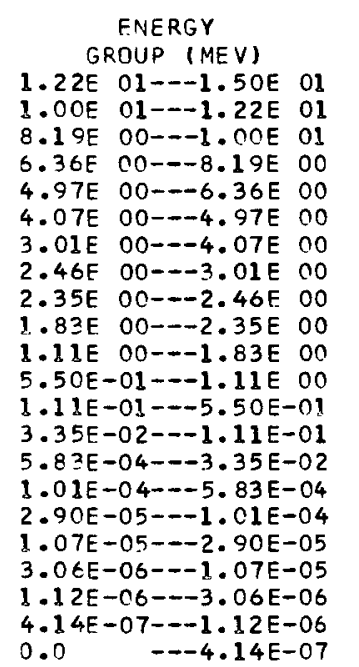

ENERGY

GROUP (MEV)

$1.22 \mathrm{E} 01---1.50 \mathrm{E} 01$

$1.00 E$ O1- $-1.22 E$ OI

$8.19 E$ OO---1.0OE 01

$6.36 E$ O0-- $8.19 E 00$

$4.97 E$ O0---6.36E OO

4.07E 00---4.97E 00

3.01E $00--4.07 E$ OO

$2.35 \mathrm{E} 00 \cdots-2.46 \mathrm{E} 00$

1.83E $00--2.35 E$ nO

1.11E 00--1.83E 00

$5.5 \mathrm{CF}-01=-1.11 \mathrm{E} 00$

1.11E-01-- $5.50 \mathrm{E}-01$

$3.35 \mathrm{E}-02--1.11 \mathrm{E}-01$
$5.83 \mathrm{E}-04--3.35 \mathrm{E}-02$

$5.83 \mathrm{E}-04---3.35 \mathrm{E}-02$
$1.01 \mathrm{E}-04--5.83 \mathrm{E}-04$

$2.90 E-05=-1.01 \mathrm{E}-04$

$1.07 E-05--2.90 E-05$

$3.06 \mathrm{E}-06---1.07 \mathrm{E}-05$
$1.12 \mathrm{E}-06--3.06 \mathrm{E}-06$

$1.12 E-06--3.06 E-06$
$4.14 E-07--1.12 E-06$

$4.14 \mathrm{E}-07--1.12 \mathrm{E}-06$
$0.0 \quad--4.14 \mathrm{E}-07$
ANGLE $1 \quad$ ANGLE 2
$M U=-1.0000 \quad M U=-0.5894$ $M U=-1.0000$ 0.0

0.0

$-1.968 \mathrm{E}-05$ $-1.968 \mathrm{E}-05$
$2.199 \mathrm{E}-03$ $3.315 \mathrm{E}-03$ 3.234E-03 $4.297 \mathrm{E}-03$ $6.034 \mathrm{E}-03$ $5.283 \mathrm{E}-03$ $6.283 \mathrm{E}-03$ $1.256 \mathrm{E}-02$ $2.710 E-02$ $2.710 \mathrm{E}-02$
$2.218 \mathrm{E}-01$ $2.059 \mathrm{E}-01$ $2.059 \mathrm{E}-01$ $1.304 \mathrm{E} O 1$
$5.818 \mathrm{E} O 1$ $1.737 \mathrm{E} 02$ $4.776 E$ O2 $1.303 \mathrm{E} 03$ $2.901 \mathrm{E}^{03}$

ANG LE 10 $M U=0.0950$

0.0

0.0

$8.610 E-04$

$4.538 \mathrm{E}-03$

. $.35 \therefore E-03$

6.631E-03

$6.631 \mathrm{E}-03$

$1.002 \mathrm{E}-02$
$8.524 \mathrm{E}-03$

$9.373 E-03$

$1.803 E-02$

$3.433 \mathrm{E}-02$

$2.545 \mathrm{E}-01$
$2.290 \mathrm{E}-01$

$1.439 \mathrm{E} 01$

$6.384 \mathrm{E}$ O1

$1.903 \mathrm{E} 02$ $5.215 \mathrm{E}$ O2 $\begin{array}{ll}1.420 F & 03 \\ 3.152 E & 03\end{array}$ $4.631 \mathrm{E} 03$ 0.0

0.0

$-6.074 E-06$

$6.074 E-06$

2.174E-

$3.232 E-03$

$4.304 E-03$

$6.046 E-03$

$5.298 \mathrm{E}-03$

$6.301 \mathrm{E}-03$

6. $301 E-03$

$2.715 \mathrm{E}-02$

$2.221 \mathrm{E}-\mathrm{C} 1$

$2.061 \mathrm{E}-01$

$1.305 E$ OI

$5.822 \mathrm{E}$ O1

$1.739 E 02$

$4.780 \mathrm{E} \mathrm{O2}$

$1.304 \mathrm{E} O 3$

$2.903 E O 3$
$4.297 E$

ANGLE 11

0.0

0.0

.0

$1.214 E-03$

$5.230 \mathrm{E}-03$

$6.213 \mathrm{E}-03$

4.907E-0

$7.725 \mathrm{E}-03$

$1.184 \mathrm{E}-02$

$9.678 \mathrm{E}-03$

$1.032 \mathrm{E}-02$

$1.963 \mathrm{E}-02$

$3.607 E-02$

$2.614 \mathrm{E}-01$

$2.336 \mathrm{E}-01$ .465E OL $6.494 \mathrm{E}$ 01 1.935 E? 1. (.442E O3 $4.696 \mathrm{E} \quad 03$
(NEUTRONS/MEV/STERADIAN/SOURCE NEUTRON)

\begin{tabular}{|c|c|c|c|c|c|c|}
\hline ANGLE 3 & ANGLE 4 & ANGLE 5 & ANGLE 6 & ANGLE 7 & ANGLE 8 & ANGLE $\quad 9$ \\
\hline$M U=-0.9446$ & $M U=-0.8656$ & $M U=-0.7550$ & $M U=-0.6179$ & $M U=-0.4580$ & $M U=-0.2816$ & $M U=-0.0950$ \\
\hline 0.0 & 0.0 & 0.0 & 0.0 & 0.0 & 0.0 & 0.0 \\
\hline 0.0 & 0.0 & 0.0 & 0.0 & 0.0 & 0.0 & 0.0 \\
\hline 0.0 & 0.0 & 0.0 & 0.0 & 0.0 & 0.0 & 0.0 \\
\hline $2.700 \mathrm{E}-05$ & $8.514 E-05$ & $1.182 E-04$ & $1.487 \mathrm{E}-04$ & $2.115 E-04$ & $3.480 E-04$ & $5.714 E-04$ \\
\hline $2.095 \mathrm{E}-03$ & $2.036 E-03$ & $2.114 \mathrm{E}-03$ & $2.384 E-03$ & $2.836 \mathrm{E}-03$ & $3.392 \mathrm{E}-03$ & $3.965 E-03$ \\
\hline 3.35 & $3.423 E-03$ & $3.540 E-03$ & $3.719 E-03$ & 3. $968 \mathrm{E}-03$ & $4.303 E-03$ & $4.747 \mathrm{E}-03$ \\
\hline $3.225 \mathrm{E}-03$ & $3.221 \mathrm{E}-03$ & $3.234 E-03$ & $3.281 \mathrm{E}-03$ & $3.383 E-03$ & $3.563 E-03$ & $3.851 E-03$ \\
\hline $4.336 E-03$ & $4.40 ? E-03$ & $4.510 E-03$ & $4.679 E-03$ & $4.934 E-03$ & $5.310 \mathrm{E}-0$ & $5.855 \mathrm{E}-03$ \\
\hline 6.09 & $6.207 \mathrm{E}-03$ & $6.393 E-03$ & $6.687 E-03$ & $7.131 \mathrm{E}-03$ & $7.781 E-0$ & $8.710 E-03$ \\
\hline 5.36 & $5.494 \mathrm{E}-03$ & $5.695 \mathrm{E}-03$ & $5.986 E-03$ & $6.386 E-03$ & $6.921 \mathrm{E}-0$ & $7.621 \mathrm{E}-03$ \\
\hline 6.37 & $6.524 E-03$ & $6.744 E-03$ & $7.048 \mathrm{E}-03$ & $7.447 E-03$ & $7.956 \mathrm{E}-03$ & $8.592 \mathrm{E}-03$ \\
\hline 1.2 & $1.301 E-02$ & $1.342 \mathrm{E}-02$ & $1.397 \mathrm{E}-02$ & $1.468 \mathrm{E}-02$ & $1.558 \mathrm{E}-\mathrm{C}$ & $1.669 \mathrm{E}-02$ \\
\hline 2.7 & $2.779 E-02$ & $2.839 E-02$ & $2.919 E-02$ & $3.017 E-02$ & $3.136 \mathrm{E}-0$ & $3.275 E-02$ \\
\hline-01 & $2.253 \mathrm{E}-01$ & $2.282 E-01$ & $2.320 E-01$ & $2.366 \mathrm{E}-01$ & $2.420 E-01$ & $2.480 E-01$ \\
\hline 2.070 & $2.085 E-01$ & $2.106 \mathrm{E}-01$ & $2.133 \mathrm{E}-01$ & $2.166 E-01$ & $2.204 E-01$ & $2.245 \mathrm{E}-01$ \\
\hline $1.310 \mathrm{E} \quad 01$ & $1.319 \mathrm{E} \mathrm{O1}$ & $1.331 \mathrm{E} \mathrm{O1}$ & $1.348 \mathrm{E} \quad 01$ & $1.367 \mathrm{E}$ & $1.389 \mathrm{E} \quad 01$ & $1.413 E$ O1 \\
\hline $5.843 E$ & $5.881 \mathrm{E}$ & $5.933 E$ ol & $6.001 E 01$ & $6.082 \mathrm{E} 01$ & $6.174 \mathrm{E}$ & $6.276 E$ o1 \\
\hline $1.745 \mathrm{E} \quad 02$ & $1.756 \mathrm{E} \quad 02$ & $1.771 \mathrm{E} \quad 02$ & $1.791 \mathrm{E} \quad 02$ & $1.815 E \quad 02$ & $1.842 \mathrm{E}$ & $1.871 \mathrm{E} 02$ \\
\hline $4.796 \mathrm{E} \quad 02$ & $4.825 \mathrm{E} 02$ & $4.866 \mathrm{E}$ & $4.919 E \quad 02$ & $4.981 \mathrm{E}$ & $5.053 \mathrm{E}$ & $5.132 \mathrm{E} \quad 02$ \\
\hline $1.309 \mathrm{E}$ & $1.316 E 03$ & $1.327 \mathrm{E}$ & $1.341 \mathrm{E} 03$ & $1.358 \mathrm{E} \quad 03$ & $1.377 \mathrm{E} 03$ & $1.398 \mathrm{E} 03$ \\
\hline $2.912 E 03$ & $2.929 \mathrm{E} \quad 03$ & $2.953 \mathrm{E} \quad 03$ & $2.983 E \quad 03$ & 3.0195 & $3.060 E \quad 03$ & $3.105 E \quad 03$ \\
\hline $4.310 E \quad 03$ & $4.335 \mathrm{~F} \quad 03$ & $4.367 E \quad 03$ & $4.408 \mathrm{E} \quad 03$ & $4.456 \mathrm{E} \quad 03$ & $4.509 E \quad 03$ & $4.568 E \quad 03$ \\
\hline ANGLE 12 & ANGLE 13 & ANGLE 14 & ANGLE 15 & ANGLE & ANGLE 17 & SCALAR \\
\hline$M U=0.4580$ & $M U=0.6170$ & $M U=0.7550$ & $M U=0.8656$ & $M U=0.9446$ & $M U=0.9894$ & FLUX \\
\hline 0.0 & 0.0 & 0.0 & 0.0 & 0.0 & 0.0 & 0.0 \\
\hline 0.0 & 0.0 & 0.0 & 0.0 & 0.0 & 0.0 & 0.0 \\
\hline $0 . n$ & 0.0 & 0.0 & 0.0 & 0.0 & 0.0 & 0.0 \\
\hline 1.780 & $3.075 E-03$ & $6.3365-03$ & $1.435 F-02$ & $3.536 E-02$ & $2.086 E-01$ & $7.176 E-02$ \\
\hline 6.32 & $8.315 E-03$ & $1.191 E-02$ & $1.838 E-02$ & $3.096 \mathrm{E}-02$ & $6.340 E-0$ & $8.712 E-02$ \\
\hline 7.475 & $9.360 \mathrm{E}-03$ & $1.218 \mathrm{E}-02$ & $1.643 E-02$ & $2.298 \mathrm{E}-$ & $3.378 \mathrm{E}-0$ & $8.936 E-02$ \\
\hline 5.78 & $7.003 E-03$ & $8.646 E-03$ & $1.083 \mathrm{E}-02$ & $1.370 E-C$ & $1.748 \mathrm{E}-0$ & $6.667 \mathrm{E}-02$ \\
\hline $9.255 E-03$ & $1.139 E-02$ & $1.437 E-02$ & $1.854 E-02$ & $2.448 \mathrm{E}-0$ & 3.34 & $1.062 E-01$ \\
\hline $1.436 \mathrm{E}-0$ & $1.787 \mathrm{E}-02$ & & $2.959 \mathrm{E}-02$ & $3.940 E-C$ & & $1.631 \mathrm{E}-01$ \\
\hline $1.114 \mathrm{E}-02$ & $1.298 \mathrm{E}-02$ & $E-02$ & $1.812 \mathrm{E}-02$ & $2.162 \mathrm{E}-0$ & 2.61 & $1.212 E-01$ \\
\hline $1.145 E-02$ & $1.278 \mathrm{E}-02$ & $1.431 E-02$ & $1.603 \mathrm{E}-02$ & $1.786 \mathrm{E}-0$ & 1.9 & $B E-01$ \\
\hline $2.150 E-02$ & $2.366 \mathrm{E}-02$ & 2.608 & $2.868 \mathrm{E}-02$ & $3.135 \mathrm{E}-\mathrm{C}$ & & $2.366 \mathrm{E}-01$ \\
\hline 3.796 & $3.992 \mathrm{E}-02$ & $4.187 \mathrm{E}-02$ & $4.368 \mathrm{E}-02$ & $4.519 E-0$ & 4.61 & $4.332 E-01$ \\
\hline 2.68 & $2.751 \mathrm{E}-01$ & $2.813 \mathrm{E}-0$ & $2.866 \mathrm{E}-01$ & $2.906 \mathrm{~F}-$ & & \\
\hline $2.381 E-01$ & $2.425 E-01$ & $2.464 E-0$ & $2.497 \mathrm{E}-01$ & & 2.53 & $2.862 E \quad 00$ \\
\hline $1.492 E$ ol & $1.516 \mathrm{E} 01$ & $1.539 \mathrm{E} \mathrm{O1}$ & $1.557 \mathrm{E} 01$ & $1.571 \mathrm{E} 0$ & $1.579 \mathrm{E}$ & $1.799 \mathrm{E} 02$ \\
\hline $6.604 \mathrm{E} \mathrm{O1}$ & $6.707 E$ O1 & $6.799 E \quad 01$ & $6.876 E$ Ol & $6.932 \mathrm{E} \mathrm{O1}$ & & $11 \mathrm{E} \quad 02$ \\
\hline $67 E \quad 02$ & $1.997 E \quad 02$ & $2.024 E \quad 02$ & $2.046 E \quad 02$ & $2.062 E \quad 02$ & E 02 & $9 E 03$ \\
\hline $5.385 \mathrm{E} 02$ & $5.464 \mathrm{E} \quad 02$ & 5.534 & $5.593 E \quad 02$ & $5.636 E \quad 02$ & 5.6 & $6.521 E 03$ \\
\hline $1.464 E \quad 03$ & $1.485 \mathrm{E} 03$ & $1.503 E$ & $1.519 \mathrm{E} 03$ & $1.530 \mathrm{E}$ & $1.536 \mathrm{E} 03$ & $1.775 \mathrm{E} \quad 04$ \\
\hline $3.248 \mathrm{E} \quad 03$ & $3.292 \mathrm{E}_{03}$ & $3.332 \mathrm{E}$ & $3.365 \mathrm{E} 03$ & $3.389 \mathrm{E}$ & $3.403 E \quad 03$ & $3.941 E \quad 04$ \\
\hline $4.760 E \quad 03$ & $4.820 E \quad 03$ & $4.873 E$ & $4.915 E \quad 03$ & $4.944 E$ & $4.961 E \quad 03$ & $5.795 E$ \\
\hline
\end{tabular}


(NEUTRONS/MEV/STERAOIAN/SOURCE NEUTRON)

ENERGY GROUP (MEV)

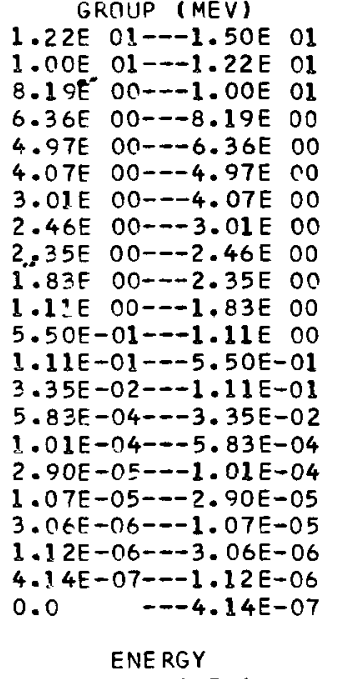

\section{ENERGY}

$1.22 E$ O1-- $1.50 E$ OI

$1.00 E$ 01- $-1.22 \mathrm{E}$ O

$8.19 E$ OD-C1. OOE OL $4.36 \mathrm{E}$ OO$4.97 E$ OO- $-3.36 E$ OO 4.07E $00-4.97 E$ OO 2.46E $00-2.3 .01 E$ OO 2. $35 \mathrm{E}$ OO- $3.01 \mathrm{E}$ OO 1. $33 \mathrm{E} 00--2.35 \mathrm{E} 00$ 1.11E $00-2.33 \mathrm{E} 00$ $5.50 \mathrm{E}-01--1.11 \mathrm{E}$ OO $1.1 \mathrm{E}-01-0-5.50 \mathrm{E}-01$ $3.35 \mathrm{E}-02-0-1.11 \mathrm{E}-01$ $5.83 E-04--3.35 E-02$ $1.01 E-04=-5.83 E-04$ $2.00 E-05--1.01 E-04$ $1.07 E-05--2.90 E-05$ $3.06 E-06--1.07 E-05$ $1.12 \mathrm{E}-06--3.06 \mathrm{E}-\mathrm{CG}$ $4.14 \mathrm{E}-07---1.12 \mathrm{E}-06$
ANGLE 1 $M=-1.0000$ 0.0 0.0 5.055E-07 $7.376 \mathrm{E}-04$ $1.247 \mathrm{E}-03$ $1.303 \mathrm{E}-03$ $1.829 \mathrm{E}-03$ $2.634 \mathrm{E}-03$ $2.601 E-03$ 3. $383 \mathrm{E}-03$ $7.348 \mathrm{E}-03$ $1.747 \mathrm{E}-02$ $1.550 \mathrm{E}-01$ $1.475 \mathrm{E}-01$ $9.530 E$ DO $4.308 E$ O1 $1.300 E$ O2 $3.617 \mathrm{E}$ O2 $9.965 \mathrm{E} \mathrm{O2}$ $2.238 \mathrm{E}$ O3 $3.344 E \quad 0$ ANGLE 10 $M=0.0950$ 0.0 0.0 $3.209 \mathrm{E}-04$ $1.602 E-03$ $1.775 E-03$ $2.991 E-03$ $4.831 E-03$ $4.417 E-03$ $5.180 \mathrm{E}-03$ $1.088 \mathrm{E}-02$ $2.255 \mathrm{E}-02$ $2.255 \mathrm{E}-02$
$1.802 \mathrm{E}-01$ $1.658 \mathrm{E}-01$ $1.058 \mathrm{E}-01$ $1.063 E$
$4.782 \mathrm{E}$ $1.441 \mathrm{E} O 2$ $3.998 E$ O2 $1.099 \mathrm{E} \mathrm{O3}$ $2.462 \mathrm{E} \quad 3$ 3.649 E 03
ANGLE 2 $U=-0.9894$ 0.0 4.013E-06 $7.314 E-04$ $1.251 \mathrm{E}-03$ $1.302 \mathrm{E}-03$ $1.833 \mathrm{E}-03$ $2.641 \mathrm{E}-\mathrm{C} 3$ $2.610 E-03$ $3.393 \mathrm{E}-03$ 7.370E-03 $1.751 \mathrm{E}-02$ $1.552 \mathrm{E}-01$ $1.477 \mathrm{E}-01$ $9.540 E$ OD $4.312 E \quad 01$ $1.301 \mathrm{E} 02$ $3.620 E$ O2 2. $3.347 \mathrm{E} 03$ ANGLE 11 $\mathrm{U}=0.2816$ 0.0 0.0 $4.550 E-04$ $1.873 E-C 3$ $2.448 \mathrm{E}-03$ $2.051 \mathrm{E}-03$ $3.553 E-03$ $5.074 E-03$ $5.733 \mathrm{E}-03$ $1.101 \mathrm{E}-\mathrm{C}$ $2.377 \mathrm{E}-02$ $1.377 \mathrm{E}-02$ $1.695 \mathrm{E}-01$ $1.085 \mathrm{E} 01$ $4.875 \mathrm{~F}$ OI $1.468 \mathrm{E} 02$ 4.073E 02 $1.119 \mathrm{E} 03$ $2.505 E \quad O$ $3.709 E_{03}$
ANGLE 3 ANGLE 4 ANGLE 5 AVOLE 6 0.0 0.0 $1.614 E-05 \quad 3.160 E-05$

$1.267 E-03$

$1.848 \mathrm{E}-03$

$1.848 E-03$
$2.673 E-03$

$2.647 E-03$

$3.437 \mathrm{E}-03$

$7.462 \mathrm{E}-03$

$1.766 \mathrm{E}-02$

$1.561 \mathrm{E}-01$

$1.483 E-01$

$4.330 E$ OI

$1.306 \mathrm{E} O$

$3.634 \mathrm{E} \mathrm{O2}$

$1.001 \mathrm{E} 03$

$3.359 \mathrm{E} \mathrm{O3}$

ANGLE $\quad 4$
$M=-0.8656$

4.394E-05

$7.078 E-04 \quad 7.469 E-04$

$1.879 E-03$

$2.719 E-03$

$3.521 E-03$

$1.637 \mathrm{E}-03$
$1.796 \mathrm{E}-02$

$1.577 \mathrm{E}-01$

$1.495 \mathrm{E}-01$
$9.652 \mathrm{E} 00$

$4.361 E 01$

$1.315 E$ E 2

3.660 E 02

1.008 E 03

$3.381 \mathrm{E} 03$

ANGLE 12

ANGLE 13

$J=0.4580$

0.0
0.0

$6.962 E-04$

$2.315 \mathrm{E}-03$

$2.978 \mathrm{E}-03$

$U=0.6179$

0.0

0.0

$1.231 E-03$

$3.106 \mathrm{E}-03$

$3.770 E-03$

4. $352 \mathrm{E}-03$

$2.967 E-03$
$5.483 E-03$

$9.348 \mathrm{E}-03$

$5.913 E-03$

.

$7.160 \mathrm{E}-03$

$1.313 \mathrm{E}-02$

$2.508 \mathrm{E}-0$ ?

$1.909 \mathrm{E}-01$

$1.451 \mathrm{E}-02$

$2.644 E-02$

1.766E-01

$1.127 \mathrm{E}$ Ol

$1.521 \mathrm{E} 02$

$1.107 E$ O1

$1.495 \mathrm{E} \mathrm{O2}$

$4.146 E$ O2

$1.133 \mathrm{E} \mathrm{O} 03$

$3.767 E \quad 03$

$3.822 \mathrm{E} 03$ U $=-0$

0.0

0.0

1. $351 \mathrm{E}-03$

$1.308 E-03$

$1.931 \mathrm{E}-03$

$2.844 E-03$
$2.831 E-03$

$3.647 E-03$

$3.647 E-03$
$7.895 E-03$

1. $838 \mathrm{E}-02$

$1.512 \mathrm{E}-01$

$9.754 E 00$

$4.405 E 01$

$3.695 \mathrm{E} 02$

$1.017 \mathrm{E} 03$

$2.284 E \quad 03$

ANGLE 14

$M U=-0.6179$ 0.0

0.0

$5.744 E-05$

$1.425 E-03$

$1.330 E-03$

3. $009 \mathrm{E}-03$

$2.992 E-03$

$3.823 E-03$

$8.249 E-03$

$1.893 \mathrm{E}-02$

$1.629 \mathrm{E}-01$

$9.885 \mathrm{E} 00$

$4.461 E$ O1

$1.345 \mathrm{E} 02$

1.030 E 03

$.310 E \quad 03$

$3.447 \mathrm{E} 03$

AVGLE 15

$U=0.7550$

0.0

(2)

$2.510 E-03$

$2.510 E-03$
$4.509 E-03$

$4.946 \mathrm{E}-03$

$3.667 E-03$

$7.081 \mathrm{E}-03$

1.230E-02

$8.285 E-03$

$1.602 \mathrm{E}-02$

$2.776 \mathrm{E}-02$

$2.008 E-01$
$1.797 E-01$

$1.146 \mathrm{E}$ O1

$1.544 \mathrm{E}$ O2

4.277E 02

$1.173 E \quad 03$

$3.624 E$ OS
$3.870 E$ O3
ANGLE 7 $U=-0.4580$ 0.0

$8.354 E-05$ $1.004 E-03$

$1.526 E-03$

$1.376 \mathrm{E}-03$

$2.138 E-03$

$3.253 \mathrm{E}-03$

$3.214 E-03$

$8.707 E-03$

$1.963 E-02$

$1.560 \mathrm{E}-01$

$1.004 E$ OI

$4.529 \mathrm{E} 01$

$3.795 \mathrm{E} 02$

$.044 E 03$

$2.342 E \quad 03$

OE 03 ANGLE
$M U=-0.2816 \quad$ ANGLE 9
$0.0=-0.0950$ 0.0

$2.155 E-04$

$1.193 E-03$

$1.660 E-03 \quad 1.840 E-03$

$1.457 E-03 \quad 1.584 E-03$

$2.324 E-03 \quad 2.597 E-03$

$3.608 E-03 \quad 4.114 E-03$

$3.513 E-03 \quad 3.906 E-03$

4. $723 E-03$

$1.001 E-02$

$2.046 E-02$ 2.144E-02

$1.705 E-01 \quad 1.752 E-01$

$1.590 E-01$ 1.623E-01

1.022E OI 1.042E OI

4.606E OL 4.591E OL 
(NEUTRONS/MEV/STERADIAN/SOURCE NEUTRON) $1.22 \mathrm{E} \quad 01--1.50 \mathrm{E}$ O $1.00 E$ 01-T-1.22E O1 $8.19 E$ O0--1.00E 01 $4.36 \mathrm{E} 00--8.19 \mathrm{E}$ CO 4.97 E $00---4.36$ E 00 4.07E $00--4.97 E 00$ $2.46 \mathrm{E} 00--3.01 \mathrm{E} 00$ $2.46 E$ OO- $-3.01 E 00$ $1.83 \mathrm{E} 00--2.35 \mathrm{E} 00$ $1.11 \mathrm{E} 00--1.83 \mathrm{E} 00$ $5.50 E-01--1.11 E 00$ $1.11 E-01--5.50 E-01$ $3.35 \mathrm{E}-02--1.11 \mathrm{E}-01$ $5.83 E-04--3.35 E-02$ $1.01 \mathrm{E}-04---5.83 \mathrm{E}-04$ $2.90 E-05--1.01 E-04$ 1.07E-05--2. $20 \mathrm{E}-05$ $3.06 E-06--1.07 E-05$ $1.12 \mathrm{E}-06---3.06 \mathrm{E}-06$ $4.14 E-07---1.12 E-06$
0.0 ENERGY GROUP IMEV

$1.22 \mathrm{E} 01--1.50 \mathrm{E}$ Ol $1.00 E$ 01- -1.22E OI

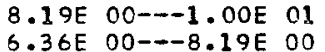
$5.36 \mathrm{E} \quad 00--8.19 \mathrm{E} \quad 00$ $4.97 E$ OO---6.36E 00 4.07E $00--4.97 \mathrm{E} 00$

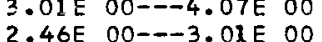
$2.46 \mathrm{E} 00--3.01 \mathrm{E} 00$

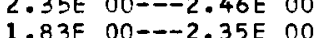
$1.83 E$ OO- - 2.35E 00 $1.11 \mathrm{E} 00--1.83 \mathrm{E} 00$
$5.50 \mathrm{E}-01-\cdots-1.11 \mathrm{E} 00$ $5.50 \mathrm{E}-01--1.11 \mathrm{E} 00$ $1.12 \mathrm{E}-01--5.50 \mathrm{E}-01$
$3.35 \mathrm{E}-02-0-1.11 \mathrm{E}-01$ $5.83 \mathrm{E}-04---3.35 \mathrm{E}-02$ $5.83 E-04---3.35 E-02$
$1.01 E-04---5.83 E-04$ $2.90 \mathrm{E}-05--1.01 \mathrm{E}-04$ $2.90 \mathrm{E}-05-1-1.01 \mathrm{E}-04$
$1.07 \mathrm{E}-05--2.90 \mathrm{E}-05$ $3.06 \mathrm{~F}-0 \mathrm{E}--1.07 \mathrm{E}-05$ $1.12 E-06--3.06 E-06$ $4.14 \mathrm{~F}-07-\cdots 1.12 \mathrm{E}-06$ $4.14 \mathrm{E}-07---1.12 \mathrm{E}-06$
$0.0 \quad--4.14 \mathrm{E}-07$
ANGLE 1 $M U=-1.0000$ 0.0 0.0 $0.0752 \mathrm{E}-06$ $2.304 E-04$ $2.304 E-04$ $4.175 E-04$ $6.503 E-04$ $9.541 E-04$ $1.033 E-03$ $3.326 \mathrm{E}-03$ $7.966 \mathrm{E}-02$ $7.704 E-02$ $5.041 E 00$ $2.298 \mathrm{E} 01$ $6.978 E$ OI $1.957 E$ O2 $\begin{array}{ll}5.424 E & 02 \\ 1.225 \mathrm{E} & 03\end{array}$ $1.842 \mathrm{E} \mathrm{O3}$ ANGLE 10 $M U=0.0950$ 0.0
0.0
0.0 $1.069 E-04$ $5.184 E-04$ $7.140 \mathrm{E}-04$ $6.251 E-04$ $1.114 \mathrm{E}-03$ $1.888 \mathrm{E}-03$ $1.819 E-03$ $2.236 \mathrm{E}-03$ 1.111E-02 . (5) $5.665 E$ OO $7.791 \mathrm{E}$ OI $2.179 \mathrm{E}$ O2 $1.025 \mathrm{E} 02$ $1.358 \mathrm{E} \mathrm{O3}$
$2.024 \mathrm{E} \mathrm{O}$ $\triangle N G L E \quad 2$
$M U=-0.9894$ 0.0 0.0 $2.723 E-06$
$2.290 E-04$ $2.290 E-04$ $4.501 E-04$ $6.516 E-04$ 9.574E-04 $1.037 E-03$ $1.440 \mathrm{E}-03$ $3.336 E-03$ $8.517 E-03$ 7.977E-02 $7.713 \mathrm{E}-02$ $5.046 E 00$ $2.300 E$ OI 6.985 E 01 1.959E 02 $5.429 E 02$ $1.226 E 03$ $1.843 E 03$

ANGLE 11 $M U=0.2816$ 0.0 0.0 $1.553 E-04$ $6.139 \mathrm{E}-04$ $8.415 \mathrm{E}-04$ $7.251 E-04$ $1.342 \mathrm{E}-03$ $2.323 F-03$ $2.107 E-C 3$ 2. $484 \mathrm{E}-03$ $5.534 \mathrm{E}-03$ $1.174 \mathrm{E}-02$ $9.636 E-02$ 8.927E-02 $5.789 E$ CO $2.622 E$ E $7.950 E$ OI $2.223 E$ O2 $1.383 E \quad 03$ $2.059 E \quad 03$
ANGLE 3

0.0

0

$6.141 E-06$ 2.256E-04 $4.249 E-04$ $4.496 \mathrm{E}-04$ $6.576 E-04$ $9.717 E-04$ 1. $053 \mathrm{E}-03$ $1.457 \mathrm{E}-03$ $3.380 \mathrm{E}-03$ $8.508 \mathrm{E}-03$ $8.025 \mathrm{E}-02$ $7.749 E-02$ 5.069 E DO 2.310E 01 7.015E O1 $5.451 \mathrm{E} 02$ $1.231 \mathrm{E} 03$ 1.851E 03 ANGLE 12 $M U=0.4580$ 0.0 0.0 $2.420 E-04$ $7.711 E-04$ $1.030 E-03$ $8.645 E-04$ $1.669 E-03$ $2.953 \mathrm{E}-03$ $2.475 \mathrm{E}-03$ $2.779 E-03$ $6.125 E-03$ 1.242E-02 $9.929 \mathrm{E}-02$ $9.130 \mathrm{E}-02$ $5.912 \mathrm{E} 00$ 2.675 E 01 $8.108 E$ O1 $2.266 E$ O $1.409 \mathrm{E} \quad 03$ $2.095 \mathrm{E} 03$
ANGLE 4
$M U=-0.8656$

0.0

$1.063 E-05$

$2.268 \mathrm{E}-04$

$4.368 E-04$ $4.368 E-04$
$4.500 E-04$ $6.698 \mathrm{E}-04$ $1.001 \mathrm{E}-03$ $1.085 E-03$ $1.496 \mathrm{E}-03$ $3.464 \mathrm{E}-03$ $8.747 \mathrm{E}-03$ 8.111E-02 $7.815 E-02$ $5.110 E$ OO $2.328 \mathrm{E} 01$ $7.068 \mathrm{E} 01$ $1.982 \mathrm{E} 02$ $5.491 \mathrm{E} 02$ $1.239 \mathrm{E} 03$ 1.863E 03 ANGLE 13 $M U=0.6179$ 0.0 0.0 4.341E-04 $1.049 E-03$ $1.311 \mathrm{E}-03$ $1.053 E-03$ 2.137E-03 $3.872 \mathrm{E}-03$ $2.941 E-03$ 3.121E-03 $6.797 E-03$ $1.312 E-02$ $1.021 \mathrm{E}-01$ . 324 E 02 $6.028 E$ OO $2.725 E$ OI $8.257 \mathrm{E} 01$ 2.307E 02 S.368E O2 $2.128 \mathrm{E} 03$
ANGLE 5
$M U=-0.7550$

0.0

0.0

0.0
$1.467 E-05$ $1.467 E-05$
$2.416 E-04$ $2.416 E-04$ $4.533 E-04$ $6.902 \mathrm{E}-04$ $1.047 \mathrm{E}-03$ $1.133 E-03$ $.552 \mathrm{E}-03$ $3.587 E-03$ $8.963 E-03$ $8.235 \mathrm{E}-02$ $.909 \mathrm{E}-02$ $5.167 E 00$ $2.353 \mathrm{E}$ 01 $7.144 \mathrm{E}$ O1
$2.002 \mathrm{E} 02$ $5.547 \mathrm{E} \quad 02$ $1.252 \mathrm{E} 03$ $1.881 \mathrm{E} 03$ ANGLE 14 $M U=0.7550$ 0.0
0.0 0.0 8. $741 E-04$ $1.530 E-03$ $1.722 \mathrm{E}-03$
$1.298 \mathrm{E}-03$ $2.804 E-03$ $5.226 \mathrm{E}-03$ 3.517E-03 3. $502 E-03$ $1.380 \mathrm{E}-02$ $1.047 \mathrm{E}-01$ $9.498 E-02$ $6.133 E 00$ 2. $391 \mathrm{E}$ OI $8.391 \mathrm{E} 01$ $2.343 E 02$ $6.466 E 02$ $2.157 E 03$

ANGLE 6 .6179 0.0 0.0 $1.968 E-05$ $2.744 E-04$ $2.744 E-04$
$4.812 E-04$ $4.812 E-04$
$4.620 E-04$ $4.620 E-04$
$7.225 \mathrm{E}-04$ $1.118 \mathrm{E}-03$ $1.202 E-03$ $1.630 \mathrm{E}-03$ $3.757 E-03$ $9.249 E-03$ $8.395 E-02$ $8.029 E-02$ $5.241 E 0 O$ $2.385 \mathrm{E} 01$ $7.240 E$ OI 
(NEUTRJNS/MEV/STERADIAN/SOURCE NEUTRON) ENERGY

$1.22 E$ 01---1.50E $1.00 \mathrm{E}$ 01---1.22E 01 $8.19 \mathrm{E}$ 00---1.0OE 01 $6.36 E$ O0- $-8.19 E$ E 00 4.97E $00--6.36 \mathrm{E} 00$ $4.07 E$ O0- $-4.97 E$ OO $2.46 E 00-0-3.01 E 00$ $2.35 \mathrm{E} 00--2.46 \mathrm{E} 00$ $1.83 \mathrm{E} 00--2.35 \mathrm{E} 00$ $1.11 \mathrm{~F} 00--1.83 \mathrm{E} 00$ $5.50 E-01--1.11 \mathrm{E} 00$ $1.11 \mathrm{E}-01--5.50 \mathrm{E}-01$ $3.35 \mathrm{E}-02--1.11 \mathrm{E}-01$ $5.83 E-04--3.35 E-02$ $1.01 \mathrm{E}-04--5.83 \mathrm{E}-04$ $2.90 E-05--1.01 E-04$ 1.07E-05--2. $90 \mathrm{E}-05$ $3.06 E-06--1.07 E-05$ $1.12 \mathrm{E}-06--3.06 \mathrm{E}-06$ $4.14 E-07--1.12 E-06$
$0.0 \quad-0-14 E-07$

ENERGY

GROUP (MEV)

$1.22 E$ O1--1.50E 01 $1.00 E$ O1---1.22E 01

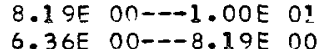
6.36E $00--8.19 E$ E 00

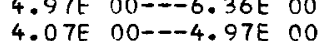
4.07E $00---4.97 E$ OO
$3.01 E$ OO-- $4.07 E 00$ 2.46E $00--3.01 \mathrm{E} 00$ $2.46 \mathrm{E} 00--3.01 E$ E

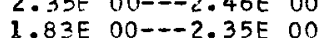
1.83 E $00--2.35 E$ OO $5.50 E-01--1.11 E 00$ $1.11 \mathrm{E}-01-\cdots 5.50 \mathrm{E}-01$ $3.35 \mathrm{E}-02--1.11 \mathrm{E}-01$ $5.83 \mathrm{E}-04-\cdots-3.35 \mathrm{E}-02$ $1.01 \mathrm{E}-04-\cdots 5.83 \mathrm{E}-04$ $2.90 E-05--1.01 E-04$ $1.075-05-2.90 E-05$ $3.06 E-06--1.07 E-05$ $1.12 \mathrm{~F}-06--3.06 \mathrm{E}-06$ $4.14 E-07--1.12 \mathrm{E}-06$ $0.0 \quad---4.14 E-07$
ANGLE 1 0.0 0.0
$8.686 \mathrm{E}-07$ $6.901 \mathrm{E}-05$ 1.901E-05 $1.434 E-04$ $2.105 E-04$ $3.133 E-04$ $3.647 \mathrm{E}-04$ $5.322 E-04$ $1.296 \mathrm{E}-03$ $3.499 \mathrm{E}-03$ $3.416 \mathrm{E}-02$ $3.342 \mathrm{E}-02$ $2.207 E 00$ $1.012 \mathrm{E} 01$ 3.087E 01 $8.704 \mathrm{E}$ 01 $2.422 \mathrm{E} \quad 02$ $5.491 E$
$8.293 E$ ANGLE 10 $M U=0.0950$ 0.0 0.0 3.352E-05 $1.594 E-04$ $2.269 E-04$ $2.017 E-04$ $3.740 E-04$ $6.573 E-04$ $6.597 E-04$ $8.389 E-04$ $1.991 \mathrm{E}-03$ $4.620 E-03$ $4.034 \mathrm{E}-02$ $3.803 E-02$ 2.4 $1.137 \mathrm{E}$ OI $9.464 \mathrm{E}$ Od $2.705 \mathrm{E} 02$ $6.119 E 02$ $9.158 \mathrm{E} 02$
ANGLE $2 \quad$ ANGLE 3

$1.132 E-06$ 6.874E-05 $1.313 E-04$ $1.434 E-04$ $2.109 E-04$ $3.146 E-04$ $3.661 E-04$ $5.340 E-04$ $1.301 E-C_{3}$ $3.507 E-03$ $3.346 \mathrm{E}-02$ $2.209 E$ CO $1.013 E$ OI $3.090 E^{01}$ $8.713 E$ O1 $2.425 \mathrm{E} \quad 02$ $8.302 \mathrm{E} \mathrm{O2}$ ANGLE 11 $M U=0.2816$ 0.0 0.0 $4.939 \mathrm{E}-\mathrm{n} 5$ $1.908 \mathrm{E}-04$ 2. $685 \mathrm{E}-04$ $2.344 \mathrm{E}-\mathrm{C} 4$ $4.555 E-04$ $8.150 E-04$ $7.684 E-04$ $9.344 E-04$ $2.198 \mathrm{E}-03$ $4.8 .2 E-03$ $4.164 E-02$ $3.896 E-02$ 1.54 $1.162 E$ O 9.538 O $2.761 E 0 ?$ $2.761 E ~$
$6.241 E$
9.32 $9.327 E \quad 2$
0.0 $2.074 \mathrm{E}-06$ $6.819 E-05$ $1.334 \mathrm{E}-04$ $1.433 E-04$
$2.130 E-04$ $3.201 E-04$ 3. $722 E-04$ $5.413 E-04$ $1.318 \mathrm{E}-03$ $3.542 E-03$ $3.443 \mathrm{E}-02$ $3.362 E-02$ 2.219E 00 $1.018 E$ O1 $3.104 E 01$ $8.751 \mathrm{E}$ 01 $2.435 \mathrm{E} \mathrm{O2}$ $8.336 \mathrm{E} \mathrm{O2}$ ANGLE 12 $M U=0.4580$ 0.0 0. 7.853E-05 $2.426 E-04$ $3.299 \mathrm{E}-04$ $2.797 E-04$ $5.730 E-04$ $1.056 E-03$ 1. $048 \mathrm{E}-03$ 1.04EE-03 $2.441 E-03$ $5.184 E-03$ $4.296 E-02$ 3. $2.606 E$ EO $1.186 \mathrm{E}$ OI $3 . E 12 E$ OL $2.815 \mathrm{E} \quad 02$ $5.363 \mathrm{E} 02$
ANGLE 4

0.0

0.0

$3.352 E-06$ $6.919 E-05$ $1.373 \mathrm{E}-04$ $1.435 \mathrm{E}-04$ $2.173 E-04$ $3.311 E-04$ $3.840 E-04$ $5.554 E-04$ $1.352 \mathrm{E}-03$ $3.606 \mathrm{E}-03$ $3.481 E-02$ 3.392E-02 $2.238 \mathrm{E} 00$ $1.026 E 01$ $3.128 \mathrm{E} 01$ $8.819 \mathrm{E} 01$ $2.454 E \quad 02$ $5.561 \mathrm{E} \mathrm{O2}$ 8.396 E 02 ANGLE 13 $U=0.6179$ 0.0 $1.419 E-04$ $3.327 \mathrm{E}-04$ 4. $206 E-04$ $3.404 E-04$ $7.423 \mathrm{E}-04$ $1.410 \mathrm{E}-03$ $1.085 E-03$ $1.179 \mathrm{E}-03$ $2.717 E-03$ $4.423 \mathrm{E}-02$ $4.423 E-02$ . 2.65 $3.581 \mathrm{~F} 01$ $1.034 \mathrm{E} 02$ $1.034 E$ O2 $6.477 \mathrm{E} 02$ $9.653 \mathrm{E} 02$
$M U=-0.7550$ 4.0 $4.616 E-06$ $7.416 \mathrm{E}-05$ $1.432 \mathrm{E}-04$ $1.448 \mathrm{E}-04$ $2.245 E-04$ $3.485 E-04$ $4.021 E-04$ $5.766 \mathrm{E}-04$ $1.402 \mathrm{E}-03$ .698E-03 $3.536 \mathrm{~F}-02$ $.434 E-02$ $.264 E$ OO
$1.037 E$ OI $3.163 \mathrm{E} 01$ $8.916 E$ OI 2.480 E 02 $5.619 \mathrm{E} \mathrm{O2}$ $8.478 E \quad 02$ ANGLE 14 $U=0.7550$ 0.0 . $826 E-04$ 4. $864 \mathrm{E}-04$ $5.519 E-04$ $4.182 E-04$ $9.848 \mathrm{E}-04$ $1.941 E-03$ $1.302 \mathrm{E}-03$ . $5.775 \mathrm{E}-03$ $4.540 \mathrm{E}-02$ $4.540 E-02$ 2.75E 02 $2.707 E$ O ..230E OI . 2.013E . $579 E$ O2 $9.791 \mathrm{E} \mathrm{O2}$

AVGL $M U=-0.6179$ 0.0 0.0 $6.298 E-06$ $8.425 \mathrm{E}-05$ $1.516 E-04$ $1.478 E-04$ $2.358 E-04$ $3.748 \mathrm{E}-04$ $4.281 E-04$ $6.064 E-04$ $3.820 E-03$ $3.608 E-02$ $.489 E-02$ $2.298 \mathrm{E} 00$
$1.352 \mathrm{E} 01$ $3.208 \mathrm{E} 01$ 
(NEUTRJNS/MEV/STERADIAN/SOURCE NEUTRONI

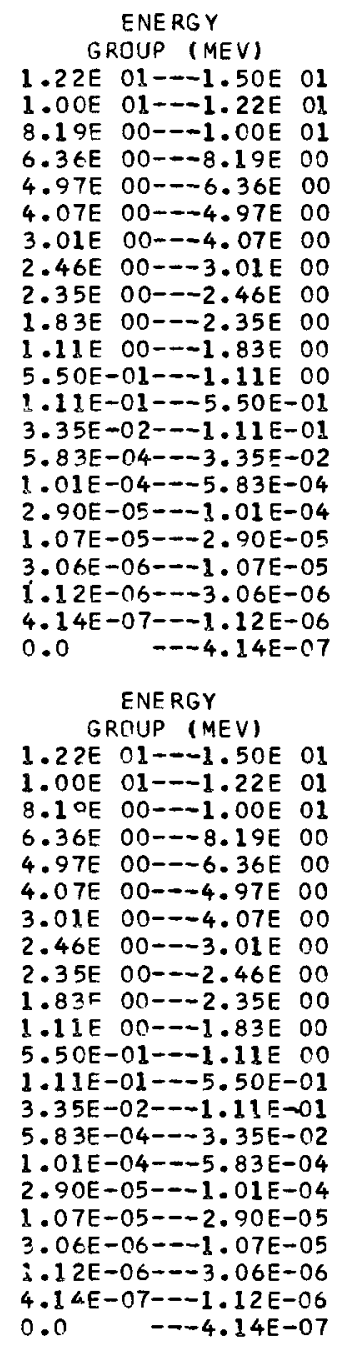

ANGLE 1
$M U=-1.0000$ 0.0

0.0

0.0

$3.277 E-07$

$2.009 E-05$
$3.933 E-05$

$3.933 \mathrm{E}-05$
$4.344 \mathrm{E}-05$

$4.344 E-05$
$6.437 \mathrm{E}-05$

$6.437 E-05$
$9.696 E-05$

$1.195 \mathrm{E}-04$

$1.811 \mathrm{E}-04$

$4.584 E-04$

$1.291 \mathrm{E}-03$

20

$1.282 E-02$

$8.523 E-01$

1.926E 00

$1.202 \mathrm{E} 01$

$3.402 E$
$9.497 E$

$2.159 \mathrm{E} 02$

$3.271 \mathrm{E} \quad 02$

ANGLE 10 $M U=0.0950$ 0.0 0.0

$1.010 \mathrm{E}-05$ $4.735 E-05$ $6.883 \mathrm{E}-05$ $6.167 E-05$ $1.179 \mathrm{E}-04$ $2.130 E-04$ $2.207 E-04$ $2.878 \mathrm{E}-04$ $7.120 \mathrm{E}-\mathrm{C} 4$ $1.542 \mathrm{E}-02$ $1.465 \mathrm{E}-02$ $9.664 \mathrm{E}-01$ $4.427 E$ OO $1.353 E 01$ $3.822 \mathrm{E}$ O1 $1.065 \mathrm{E} \mathrm{O2}$ $2.415 E$
$3.625 E$
ANGLE 2. ANGLE 3 $M U=-0.9894$
0.0 0.0 0.0 0.0
$3.984 E-07$ $2.004 \mathrm{E}-05$
$3.548 \mathrm{E}-05$ $3.548 \mathrm{E}-05$ $4.343 E-05$ $6.452 \mathrm{E}-05$ $9.741 E-05$ $1.200 \mathrm{E}-04$ .600E-04 ( . $1.284 E-02$ $8.533 \mathrm{E}-01$ $1.203 \mathrm{E} 01$ $1.203 E$
$3.4 C 5 E$ $9.507 E$ OI $2.161 E$ O2 $3.275 E$ E 02

ANGLE 11 0.0 0.0

$1.506 \mathrm{E}-05$ $5.719 \mathrm{E}-05$ $8.165 E-05$ $7.174 E-05$ $1.447 \mathrm{E}-04$ $2.678 E-04$ $2.582 \mathrm{E}-\mathrm{C} 4$ $3.211 \mathrm{E}-\mathrm{C} 4$ $7.881 E-04$ $1.820 E-03$ $1.593 E-02$ 1. $502 \mathrm{E}-02$ 9.891 E-01 $4.526 \mathrm{E} 00$ $1.383 \mathrm{E}$
3.1 $3.505 E$ O1 $1.087 \mathrm{E} \mathrm{O2}$ $2.465 \mathrm{E} \mathrm{O2}$
$3.694 \mathrm{E} 02$
0.0

0.0

0.0

$6.547 \mathrm{E}-07$

$1.999 \mathrm{E}-05$

$4.011 E-05$

$4.343 E-05$

$6.521 E-05$

$9.933 \mathrm{E}-05$

$1.221 E-04$

列

1.307E-0

$1.310 \mathrm{E}-02$

$1.290 E-O 2$

$3.948 E 00$

$1.208 E$ OI

$3.421 \mathrm{E}$ OI

$9.549 \mathrm{E} O 1$

$2.171 E$ OL

$3.288 \mathrm{E} 02$

ANGLE 12 $M U=$

0.0

0.0

$2.432 E-05$

7.337E-05

$1.005 \mathrm{E}-04$

$8.563 \mathrm{E}-05$

$1.836 E-04$

$3.494 E-04$

$3.065 E-04$

$3.608 \mathrm{E}-04$

$8.774 E-04$

$1.931 E-03$
$1.645 E-02$

$1.538 \mathrm{E}-02$

$1.012 E 00$

$4.625 E 00$

$1.413 \mathrm{E}$ ol

$3.987 \mathrm{E}$ O1

$1.109 \mathrm{E}$ O2

$2.514 E \quad 02$
$3.763 E$ O2

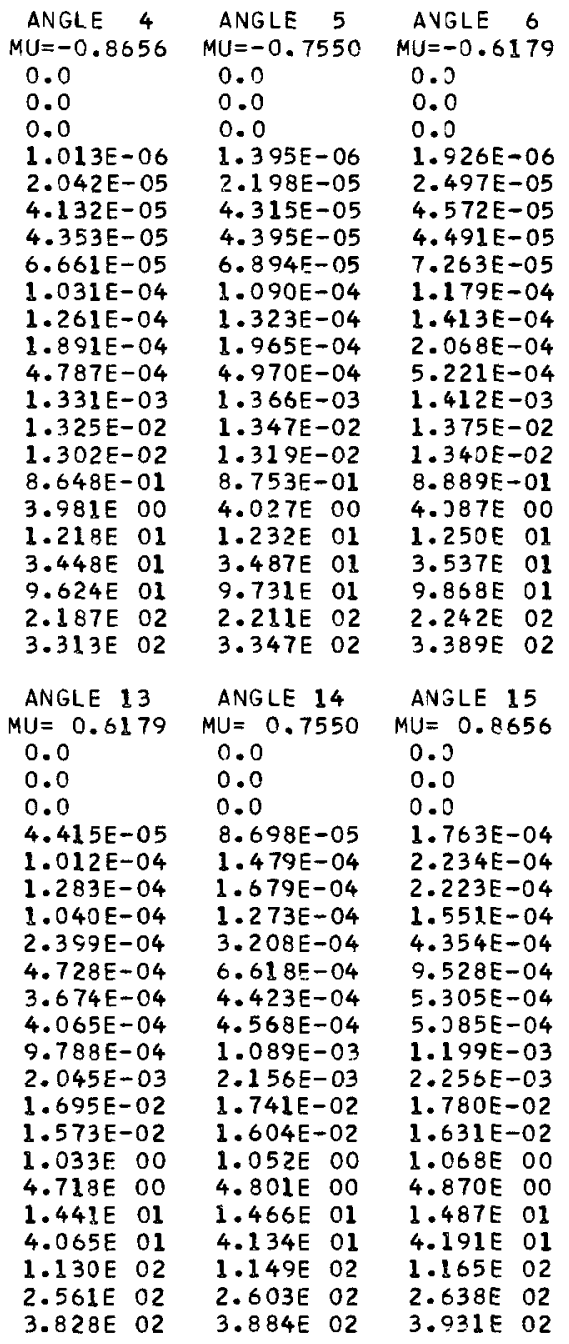

ANGLE 7 $M U=-0.4580$ 0.0 0.0 0.0 $2.849 E-06$ $2.929 E-05$ 4.915E-05 $4.674 E-05$ $7.830 E-05$ $1.307 E-04$ 1.535E-04 2. $204 E-04$ 1.470E-03 $1.409 \mathrm{E}-02$ $1.409 E-02$ $1.366 \mathrm{E} 02$ $4.051 E-01$ $4.272 \mathrm{E} 01$ $1.272 \mathrm{E}$ O1
$3.597 \mathrm{E}$ ol $3.597 \mathrm{E}$ O1 $2.278 E$ O2 $3.439 \mathrm{E} \mathrm{O2}$

ANGLE 16 $M U=0.9446$ 0.0 0.0

3.601E-04 $3.415 E-04$ $2.919 E-04$ 1. $847 E-04$ $5.912 \mathrm{E}-04$ $1.394 \mathrm{E}-03$ $6.256 E-04$ $5.550 E-04$ 1.297E-03 $2.334 E-03$ $1.809 \mathrm{E}-02$ $1.650 \mathrm{E}-02$ $1.080 E \quad 00$ $4.921 \mathrm{E} 00$ $1.502 \mathrm{E} 01$ $4.234 E$ OI $1.176 E$ O2 $2.663 E$ O2 $3.964 E \quad 02$
ANGLE 8 0.0 $4.435 E-06$ $3.453 E-05$ S.375E-05 $4.981 E-05$ $8.683 E-05$ $1.491 E-04$ $1.700 E-04$ $2.381 E-04$ 1.541E-0 $.449 \mathrm{E}-02$ $9.237 E-01$ $4.240 E$
$1.297 E$ 1.666 01 $1.606 \mathrm{E}$ O1 $2.320 E \quad 22$ $3.495 \mathrm{E} 02$

ANGLE 17 $M U=0.9894$ 0.0 $8.969 E-04$ 5.303E-04 $3.683 E-04$ $2.095 E-04$ $7.788 \mathrm{E}-04$ $2.008 \mathrm{E}-03$ $7.081 E-04$ $5.868 \mathrm{E}-04$ $1.362 E-03$ . $382 E-03$ $.826 \mathrm{E}-02$ $1.661 \mathrm{E}-02$ $1.087 E 00$ $4.950 \mathrm{E} 00$ $1.511 E$ O1 $4.258 E$ OI $1.183 E$ O2 $2.678 \mathrm{E}$ O2 $3.982 \mathrm{E} \mathrm{O2}$
$M U=-0.0950$ 0.0 $6.806 E-06$ $4.039 E-05$ $6.001 E-05$ $5.460 E-05$ $1.753 \mathrm{E}-04$ 1.919E-04 6.603E-04 6. $485 E-04$ $1.623 E-03$ $493 \mathrm{E}-02$ $420 \mathrm{E}-02$ . $443 E-01$ 4. $331 E O D$ $1.324 \mathrm{E} 01$ $3.742 \mathrm{E}$ OI $1.043 \mathrm{E} 02$ $2.366 \mathrm{E}$ O2 SCALAR FLUX 0.0 0.0 0.0
$5.841 E-04$ $9.515 \mathrm{E}-04$ $1.154 \mathrm{E}-03$ $9.437 E \div 04$ $2.102 E-03$ $4.184 E-03$ $3.226 \mathrm{E}-03$ $3.837 \mathrm{E}-03$ $.386 \mathrm{E}-03$ $2.165 E-02$ $1.926 \mathrm{E}-01$ . $1.207 E$ OL $5.528 \mathrm{E}$ O1 $1.690 \mathrm{E} 02$ $4.773 \mathrm{E} O 2$ $1.329 \mathrm{E} 03$

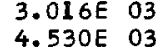


(GAMMAS/MEV/STERADIAN/S OURCE NEUTRON)

ENERGY GROUP (MEV) 8.0OE OO---1.0OE 01 6.5 OE $00---8.00 E$ OO $5.00 E \quad 00--6.50 E \quad 00$ 4. COE $00---5.00 E$ OO $3.00 E$ OO- - 4. OOE 00 2.50E OD---3.00E 00 $2.00 E$ OO- - 2.50E OO $1.66 \mathrm{E} \quad 00--2.00 E 00$ $1.33 \mathrm{E} 00--1.66 \mathrm{E}$ CO $1.00 E$ OO-D-1.33E 00 $8.00 E-01--1.00 E 00$ $6.00 E-01--8.00 \mathrm{E}-01$ $4.00 F-01--6.00 E-01$ $3.00 E-01--4.00 E-01$ $2.00 E-01--3.00 E-01$
$1.00 F-01--2.00 E-01$ $1.00 F-01--2.00 E-01$
$5.00 E-02--1.00 E-01$ $5.00 E-02--1.00 E-01$
$2.00 E-02--5.00 E-02$ ENERGY GROUP (MEV) 8.0OE OO---1.00E 01 $6.50 F$ OC---8. OOE 00 $5.00 E$ OO-C.6.50E OO 4.00E CO-C-5. OOE $D O$ 3.OOF OO-C. OOE OO 2.5CE OO-T-3.0OE OO 2.ONE OO-C. $1.66 \mathrm{E}$ OO- - $2.00 \mathrm{E}$ OO $1.33 E$ OOD- $1.66 E$ OO $1.00 E$ OD $-1.33 E$ OD $6.00 E-01--8.00 E-01$ $4.00 E-01--0.00 E-0 I$ 4. OOE $-01-2-6.00 E-O D$ $3.00 E-01-3.00 E-01$ 2.00E-OL--3.00E-01 5.OOE-O2- $1.00 E-01$ $2.00 E-02---5.00 E-02$
ANGLE 1 ANGLE 2 $M U=-1.0000 \quad M U=-0.9894$ $3.056 E-07$ 6.199E-04 $7.384 \mathrm{E}-04$ $8.958 \mathrm{E}-04$ $1.125 E-03$ $4.097 E-04$ $4.289 \mathrm{E}-04$ $2.177 \mathrm{E}-03$ $3.004 E-03$ $9.830 \mathrm{E}-03$ $1.874 \mathrm{E}-02$ $3.753 E-02$ $1.021 \mathrm{E}-02$ ANGLE 10 $M U=0.0950$ $3.444 \mathrm{E}-07$ $1.192 \mathrm{E}-05$ $1.657 E-03$ $9.897 \mathrm{E}-04$ $2.041 E-03$ $1.451 \mathrm{E}-03$ $1.665 \mathrm{E}-03$ $2.012 \mathrm{E}-03$ $2.012 E-03$ $7.460 E-04$ $9.700 E-04$ $4.380 \mathrm{E}-03$ $4.37 \mathrm{E}-03$ $1.134 \mathrm{E}-02$ $2.209 \mathrm{E}-02$ $2.209 \mathrm{E}-\mathrm{O} 2$
$4.149 \mathrm{E}-02$ $1.071 \mathrm{E}-02$
ANGLE 3 $6.577 \mathrm{E}-06$ .244E-04 $5.1 \mathrm{C} 2 \mathrm{~F}-04$ $9.818 \mathrm{~F}-04$ $7.444 \mathrm{E}-04$ $1.403 E-03$ . $996 \mathrm{E}-04$ $1.127 E-03$ $4.051 E-04$ $4.293 E-04$ $4.537 \mathrm{E}-04$ $2.189 E-03$ $3.014 \mathrm{E}-03$ $9.853 \mathrm{E}-03$ $1.877 E-02$ $3.756 E-C 2$ $1.021 E-02$ ANGLE 11 $M U=0.2816$
$2.522 \mathrm{E}-07$ $2.275 E-05$ $2.242 \mathrm{E}-03$ $1.887 E-03$ $2.780 E-03$ $2.630 \mathrm{~F}-03$ $4.816 E-03$ $3.027 E-03$ $3.654 E-03$ I. $529 E-04$ $1.248 \mathrm{E}-03$ 5. $0.163 \mathrm{E}-03$ $6.8 \mathrm{E}-\mathrm{O}$ $2.299 E-02$ $4.238 \mathrm{E}-02$ $1.081 \mathrm{E}-02$ $6.706 \mathrm{E}-06$ $.423 \mathrm{E}-04$ $5.256 \mathrm{E}-04$ ..009E-03 .67OE-O4 .1150E-04 1.135E-03 . 91 JE-O4 4. $330 E-04$ $5.005 E-04$ $2.235 E-03$ .066E-03 . $39 E-03$ 1.886E-02 ..769E-O2

ANGLE 12 $1.778 \mathrm{E}-05$ $2.687 \mathrm{E}-03$ $1.486 \mathrm{E}-03$ $3.335 E-03$ $2.121 \mathrm{E}-0$ $3.834 E-03$ $2.481 E-03$ 1.286E-03 1..56E-03 2. $7.433 \mathrm{E}-03$ 1.43 $2.399 \mathrm{E}-02$ $4.330 \mathrm{E}-02$ $4.330 \mathrm{E}-02$
$1.092 \mathrm{E}-02$ ANGLE 4 AN $=-0.9446 \quad M U=-0.8656$ $3.072 \mathrm{E}-07$ $M U=0.4580$ $3.597 \mathrm{E}-07$
ANGLE 5 $M U=-0.7550$ 7. $329 \mathrm{E}-06$ $9.270 \mathrm{E}-04$ 5.907E-04 $1.128 \mathrm{E}-03$ $8.606 \mathrm{E}-04$ $1.579 \mathrm{E}-03$ $9.948 \mathrm{E}-04$ $1.206 \mathrm{E}-03$ $3.757 E-04$ $4.739 E-04$ $6.615 E-04$ $2.451 E-03$ $3.469 \mathrm{E}-03$

$1.024 \mathrm{E}-02$ $1.931 \mathrm{E}-02$ $3.827 E-02$ $1.031 \mathrm{E}-02$ ANGLE 14 $M U=0.7550$
$3.729 \mathrm{E}-07$ 3. $729 \mathrm{E}-07$ $2.753 E-05$ $3.598 E-03$ $2.324 E-03$ $4.462 E-03$ $3.333 \mathrm{E}-03$ 5. $975 E-03$ 4. $011 E-03$ $4.872 E-03$ 2.183E$2.713 E-03$ $7.949 \mathrm{E}-03$ $9.109 \mathrm{E}-0$ $1.452 \mathrm{E}-02$ $2.621 \mathrm{E}-02$ $4.507 E-0$ 1.111E-02 ANGLE 6
$M U=-0.6179$ $3.179 \mathrm{E}-07$ $7.841 \mathrm{E}-06$ $6.373 E-04$ $1.216 \mathrm{E}-03$ $.268 E-04$ $1.692 \mathrm{E}-03$ $1.066 \mathrm{E}-03$ 1. $287 \mathrm{E}-03$ 4.006E-04 $5.211 \mathrm{E}-04$ $7.457 \mathrm{E}-04$ $2.635 \mathrm{E}-03$ $3.879 \mathrm{E}-03$ $1.039 \mathrm{E}-02$ $1.966 \mathrm{E}-02$ $3.872 E-02$ $1.037 \mathrm{E}-02$ ANGLE 15 $M U=0.8656$
$3.784 \mathrm{E}-07$ $3.784 E-07$ $4.634 E-05$ $6.076 E-03$ $3.887 E-03$ $7.594 E-03$ 5. $769 \mathrm{E}-03$ $9.769 E-03$ $6.461 E-03$ .762E-03 . $4.269 E-03$ $9.496 E-03$ $1.040 E-02$ $1.637 \mathrm{E}-02$ $2.745 \mathrm{E}-02$ $4.589 \mathrm{E}-02$
ANGLE 7 ANGLE 8 ANGLE 9 $M U=-0.4580 \quad M U=-0.2816 \quad M U=-0.0950$ $\begin{array}{lll}3.235 E-07 & 3.299 E-07 & 3.369 \mathrm{E}-07\end{array}$ $8.529 E-06 \quad 9.457 E-06 \quad 1.072 E-05$ $\begin{array}{lll}1.087 E-03 & 1.211 E-03 & 1.379 E-03\end{array}$ $1.330 E-03 \quad 1.483 E-03 \quad 1.692 E-03$ $1.123 E-03$ $1.843 \mathrm{E}-03$ $1.164 \mathrm{E}-03 \quad 1.300 \mathrm{E}-03 \quad 1.483 \mathrm{E}-03$ $1.407 \mathrm{E}-03 \quad 1.573 \mathrm{E}-03 \quad 1.794 \mathrm{E}-03$ $4.518 E-04 \quad 5.244 E-04 \quad 6.163 E-04$ $5.881 E-04 \quad 6.763 E-04 \quad 7.940 E-04$ $8.354 E-04 \quad 9.527 E-04 \quad 1.128 E-03$ $2.896 \mathrm{E}-03 \quad 3.264 \mathrm{E}-03 \quad 3.756 \mathrm{E}-03$ $4.416 E-03 \quad 5.028 E-03 \quad 5.655 E-03$ $1.05 \mathrm{C}-02$ 1.072E-02 $1.096 E-02$ 2.011E-02 $2.066 E-02$ 2.132E-02 $1.927 E-02 \quad 3.992 E-02 \quad 4.067 E-02$ $1.061 \mathrm{E}-02$

ANGLE 16 $M U=0.9446$ $7.499 \mathrm{E}-05$ $9.838 \mathrm{E}-03$ $6.246 E-03$ $1.236 \mathrm{E}-02$ $8.573 E-03$
$1.537 E-02$ $1.537 E-02$ . $972 \mathrm{E}-03$ 3 $3.930 E-03$ $1.003 \mathrm{E}-02$ $1.003 \mathrm{E}-02$ $1.728 \mathrm{E}-02$ $2.852 E-02$ $2.852 \mathrm{E}-02$
$4.650 \mathrm{E}-02$ $4.650 E-02$

\section{ANGLE 17 SCALAR} $M U=0.9894$ 1.143E-04 . $143 \mathrm{E}-04$ $1.496 E-02$ $1.880 \mathrm{E}-02$ $1.270 E-02$ $2.275 E-02$ $1.448 E-02$ $4.70 E-02$ 
(GAMMAS/MEV/STERADIAN/SOURCE NEUTRON)

ENERGY GROUP (MEV) 8.00E OO--1.00E O $5.00 \mathrm{~F} \mathrm{OO-2.0.50E} 00$ 4.0OE 00-- 5.00 E 00 3.OOE $00--4.00 E$ OO 50 . $00--3.00 E$ OO $.00 \mathrm{~F} 00---2.50 E 00$ $.66 \mathrm{E} 00--2.00 \mathrm{E} 00$ $1.33 \mathrm{E} 00--1.66 \mathrm{E} 00$ $1.00 \mathrm{E} 00--1.33 \mathrm{E}$ OO $8.00 E-01--1.00 E$ OO $6.00 \mathrm{E}-01--8.00 \mathrm{E}-0$ $4.00 \mathrm{E}-01--06.00 \mathrm{E}-01$ $3.00 E-01--4.00 E-01$ . $00 E-01--3.00 E-0$ . ONE-01---2.00E-O1 $5.00 E-02--1.00 E-01$
$2.00 E-02--5.00 E-02$

\section{ENERG Y}

\section{GROUP (MEV)} 8. OOE OO---1. COE O1
$6.50 E$ OO---8.0OE OO $5.00 E$ OO---6.5OE $C O$ $4.00 E$ OO-- $-5.00 E$ CO $3.00 E$ OO---4.00E $O 0$ $2.50 E$ OO---3.00E OD . $.00 E$ DO-- $2.50 E$ OO $3.66 E$ OO---2. OCE OO $1.33 \mathrm{E} \quad 00--1.66 \mathrm{E} 00$ $1.00 E$ O0- -1.33 E 00 $8.00 E-01--1.00 E$ OO $6.00 E-01--8.00 E-01$ 4. $00 \mathrm{E}-01--6.00 \mathrm{E}-01$ $3.00 E-01--4.00 E-01$ . OOE $-01--3.00 E-01$ $1.00 E-01--2.00 E-01$ $2.00 E-02-1.00 E-02$
ANGLE 1 ANGLE 2 UU $=-1.0000$ $8.712 \varepsilon-06$ $9.437 \mathrm{E}-04$ $5.596 \mathrm{E}-\mathrm{C}$ $1.048 E-03$ $1.048 E-03$ $8.757 E-04$ $1.689 E-03$ $1.481 \mathrm{E}-03$ $7.251 E-04$ $7.017 E-04$ $6.205 E-04$ $3.805 \mathrm{E}-03$ $5.492 E-03$ $2.187 E-02$ 4. $961 E-02$ $1.142 \mathrm{E}-01$ ANGLE 10 $M U=0.0950$

$1.998 \mathrm{E}-05$ $1.612 \mathrm{E}-0$ $1.576 \mathrm{E}-03$ $1.912 \mathrm{E}-0$ $2.359 E-03$ $4.200 E-03$ $2.781 E-03$ $3.367 \mathrm{E}-03$ $1.519 \mathrm{E}-0$ 2.020E-0 $3.056 \mathrm{E}-03$ $9.721 \mathrm{E} 03$ $1.512 E-02$ $2.598 \mathrm{E}-02$ $6.153 \mathrm{E}-02$ $1.325 \mathrm{E}-01$
$3.496 \mathrm{E}-02$
$1.044 E-06$

$9.511 \mathrm{E}-04$

$9.511 \mathrm{E}-04$

$5.686 \mathrm{E}-04$
$1.062 \mathrm{E}-0$

$1.062 \mathrm{E}-03$
$8.888 \mathrm{E}-04$

$1.703 E-03$

$1.140 \mathrm{E}-03$

$1.476 \mathrm{E}-03$

$7.079 E-04$ $6.002 E-04$ $5.517 \mathrm{E}-\mathrm{C}$ . 1 4E- 02 $1.143 E-01$
$3.259 E-02$ ANGLE 11 $M U=0.2816$ $1.361 E-06$ $1.695 \mathrm{E}-05$ $2.649 \mathrm{E}-03$ 1.33 OE-03 $3.207 E-03$ $2.045 \mathrm{E}-0$ $3.579 E-03$ $2.441 \mathrm{E}-03$ $2.964 E-03$ $1.728 E-03$ $2.468 \mathrm{E}-03$ $3.934 \mathrm{E}-03$ $1.136 \mathrm{E}-02$ $1.627 E-02$ $2.683 E-02$ $6.479 E-02$ $3.548 E-02$
ANGLE 3

$\begin{array}{lll}3 & \text { ANGLE } 4 \\ & 4\end{array}$ $1.052 E-06$ $8.964 \mathrm{E}-06$ $9.793 E-04$
$6.013 E-04$ $6.013 \mathrm{E}-04$
$1.114 \mathrm{E}-03$ $1.114 E-03$
$9.362 E-04$ $9.362 E-04$ $1.158 \mathrm{E}-03$ $1.464 E-03$ 列 $7.867 \mathrm{E}-04$ $3.926 \mathrm{E}-03$ $5.659 \mathrm{E}-03$ $2.220 E-02$ $5.001 E-02$ $1.149 \mathrm{E}-0$ 3.267E-02 ANGLE 12 $U=0.4580$ $1.422 E-06$
$2.730 E-05$ $2.707 \mathrm{E}-03$ $2.222 \mathrm{E}-03$ $3.455 E-03$ $3.337 \mathrm{E}-03$ $5.874 \mathrm{E}-03$ $4.000 E-03$ $4.881 \mathrm{E}-03$ $2.495 \mathrm{E}-03$ $5.399 \mathrm{E}-03$ $1.365 E-02$ $1.788 \mathrm{E}-02$ $2.875 \mathrm{E}-02$ $6.861 \mathrm{E}-02$ $3.603 \mathrm{E}-02$ $1.067 E-06$ $9.336 E-06$
$1.030 E-03$ $1.030 E-03$
$6.546 E-04$ $6.546 \mathrm{E}-04$
$1.201 \mathrm{E}-03$ $1.201 E-03$
$1.013 E-03$ $1.013 E-03$ $1.848 E-03$ $1.465 E-03$ $5.889 E-04$ $7.184 E-04$ $9.849 E-04$ $4.106 E-03$ $6.061 E-03$ 2.260E-02 $5.064 \mathrm{E}-0$ $1.159 E-01$
$3.282 E-02$ ANGLE 13 $M U=0.6179$ $1.481 \mathrm{E}-06$ $4.972 E-03$ $2.441 E-03$ $5.057 E-03$ $3.716 \mathrm{E}-03$ $6.489 E-03$ $4.620 E-03$ $5.703 E-03$ $3.883 E-0$ $5.238 \mathrm{E}-03$ $7.261 \mathrm{E}-03$ $1.630 \mathrm{E}-02$ $2.005 E-02$ $3.171 \mathrm{E}-02$ $7.300 \mathrm{E}-02$ $1.465 \mathrm{E}-01$
$3.657 \mathrm{E}-02$
ANGLE 5 $1.090 \mathrm{E}-06$ $9.858 \mathrm{E}-06$ $1.099 E-03$ $7.155 E-04$ $1.303 E-03$ $1.101 E-03$
$1.969 E-03$ $1.258 \mathrm{E}-03$ $1.508 E-03$ $7.730 E-04$ $1.180 E-03$ $4.376 E-03$ $6.861 \mathrm{E}-03$ $2.302 E-02$ $1.175 E-0$ 3. 302E-02 ANGLE 14 $M U=0.7550$ $4.870 E-05$ $4.668 E-03$ $4.037 E-03$ $7.725 \mathrm{E}-03$ $1.050 E-02$ $7.519 E-03$ $9.232 \mathrm{E}-03$ $6.960 E-03$ $8.956 E-03$ 1. $857 \mathrm{E}-02$ $2.216 \mathrm{E}-02$ $3.421 \mathrm{E}-02$ $7.768 \mathrm{E}-0$ $3.708 \mathrm{E}-02$
AVGLE 6 $1.119 E-06$ $1.054 \mathrm{E}-05$ $1.187 \mathrm{E}-03$ $7.792 \mathrm{E}-04$
$1.415 \mathrm{E}-03$ $1.415 E-03$ $2.121 \mathrm{E}-03$ $1.354 \mathrm{E}-03$ $1.617 \mathrm{E}-03$ $6.228 E-04$ 8. $721 E-04$ $1.341 E-0$ 4.787E-O $8.117 \mathrm{E}-03$ 2. $339 E-0$ $5.278 \mathrm{E}-0$ $1.195 \mathrm{E}-01$
$3.329 \mathrm{E}-02$

ANGLE 15 $M U=3.8656$ $1.582 E-06$
$7.184 E-05$ $9.965 E-03$ $6.052 E-03$ $1.059 E-02$ $8.936 \mathrm{E}-03$ $1.544 \mathrm{E}-02$ $1.116 \mathrm{E}-02$ $1.349 \mathrm{E}-02$ $9.626 \mathrm{E}-03$ $1.135 \mathrm{E}-02$ $2.246 \mathrm{E}-02$ $2.572 \mathrm{E}-02$ $3.879 E-02$ $8.277 \mathrm{E}-0$

3.752E-02

ANGLE 7 $M U=-0.4580$
$1.156 E-06$ $1.144 E-05$ $1.301 E-03$ $8.519 \mathrm{E}-04$ $1.548 \mathrm{E}-03$ $1.303 E-03$ $2.317 E-03$ $1.495 \mathrm{E}-03$ $1.798 E-03$ $7.441 E-04$ $1.013 E-03$ $1.499 E-03$ $5.429 E-03$
$9.743 E-03$ $2.372 \mathrm{E}-02$ $5.434 \mathrm{E}-0$ $1.220 \mathrm{E}-01$
$3.363 \mathrm{E}-02$

ANGLE 16 $M U=0.9446$ $1.617 E-06$ $1.571 E-02$ $1.017 \mathrm{E}-02$ $1.955 E-02$ $1.475 E-02$ $1.799 \mathrm{E}-02$ $2.114 E-02$ $1.131 \mathrm{E}-02$ $1.215 \mathrm{E}-02$
$1.355 \mathrm{E}-02$ $2.669 E-02$ $2.923 \mathrm{E}-02$ 
(GAMYAS/MEV/STERADIAN/SOURCE NEUTRON)

ENERGY GROUP (MEV) 8.00E $00--1.00 E \quad 01$ .5OE $00--8.00 \mathrm{E}$ CO 4.0OE OC- $-6.50 \mathrm{OE}$ OO 4.0OE $00-5.00 E$ OO 50 OO- $-3.00 E$ OO 2.00E $00-2-2.50 E$ EO 1.66E $00-0-2.00 E$ OO .33E $00--1.66 E^{2} 00$ . COF $00--1.33 E 00$ $8.00 E-01-\cdots-1.00 E$ OO $6.00 \mathrm{E}-01-\cdots-8.00 \mathrm{E}-01$ $4.00 E-01---6.00 E-01$ .00E-01-- -4.00E-01 $.00 E-01-\cdots 3.00 E-01$ . OCE-01-- $2.00 E-01$ $5.00 E-02-\cdots 1.00 E-01$ $2.00 E-02-25.00 E-02$

ENERGY GROUP (MEV) 8.00E OC- - 1. OOE 01 6.5 OE OO- - 8.00 E 00 $5.00 E$ OO- $-6.50 E$ OO 4. NOE OO- - 5. OOE OO 3. OCE OO- - 4.0OE 00 $2.50 E$ OO- - 3.00E DO $2.00 E$ OO-D-2.5OE OO $1.66 E$ OO- - 2. OOE OO $1.33 \mathrm{E} \quad 00-1.66 \mathrm{E} 00$ $1.00 E$ OD---1.33E 00 $8.00 E-01-1.00 E$ OD 6.0 0 E-01- - -8.00E-01 4.00E-01- $-6.00 E-01$ 3.00E-01-C-4.00E-01 2.OOE $01-C$. $1.00 E-01-2.00 E-O 1$ $5.00 E-02--1.00 E-01$
$2.00 E-02-\cdots-5.00 E-02$
ANGLE 1 ANGLE 2 $M U=-1.0000$ $1.641 \mathrm{E}-06$ $9.685 \mathrm{E}-06$ $9.678 \mathrm{E}-06$ $5.300 E-04$ $9.852 E-04$ $8.583 E-04$ $1.686 \mathrm{E}-03$ $1.165 \mathrm{E}-03$ $1.554 E-03$ $8.486 E-04$ $7.824 E-04$ $6.200 E-04$ 4.3 B6E-03 $6.505 E-03$ $2.796 \mathrm{E}-02$ $6.769 E-02$ $1.662 \mathrm{E}-01$
$4.844 \mathrm{E}-02$ ANGLE 10 $2.182 \mathrm{E}-06$ $1.672 \mathrm{E}-05$ $2.184 \mathrm{E}-03$ $.179 \mathrm{E}-03$ $2.587 \mathrm{E}-03$ $1.861 \mathrm{E}-03$ $3.233 \mathrm{E}-03$ $2.210 E-03$ $2.677 \mathrm{E}-03$ 1. $530 \mathrm{E}-03$ $2.155 \mathrm{E}-03$ $3.551 \mathrm{E}-03$ $1.189 \mathrm{E}-02$ $1.937 E-02$ 3.2 2 E -02 $8.549 E-02$ $1.968 \mathrm{E}-01$ $M U=-C .9894$
$1.645 \mathrm{~F}-06$ $9.748 \mathrm{E}-0 \mathrm{~S}$ $9.367 \mathrm{E}-04$ $5.423 E-04$ $5.423 E-04$ $1.004 E-03$
$8.761 E-04$ $1.703 \mathrm{E}-03$ $1.168 \mathrm{E}-03$ 1. $543 \mathrm{E}-03$ 8. $219 E-04$ $7.789 \mathrm{E}-04$ $6.723 E-04$ $4.4 ? 7 F-03$ $6.534 E-03$ $2.806 E-02$ $6.781 E-02$ $1.664 \mathrm{E}-01$ 4.847E-02

ANGLE 11 $M U=0.2816$ 2.312E-06 $2.348 E-05$ $1.762 \mathrm{E}-03$ $2.706 \mathrm{E}-03$ $2.712 \mathrm{E}-03$ $4.724 \mathrm{E}-03$ 4.
$3.236 \mathrm{E}-03$ $3.928 \mathrm{E}-03$ $2.230 E-03$ $3.226 \mathrm{E}-03$ $5.250 E-03$ $1.4 \div 2 \mathrm{E}-02$ $2.149 \mathrm{E}-02$ $3.492 E-02$ $9.057 E-02$ $2.043 E-01$
ANGLE 3 $M U=-0.9446$ $9.991 \mathrm{E}-06$ $9.701 \mathrm{~F}-04$ $5.860 \mathrm{E}-04$ $1.071 \mathrm{E}-03$ $9.394 \mathrm{E}-04$ $1.767 \mathrm{E}-03$ $1.184 \mathrm{E}-\mathrm{O}^{3}$ $1.512 \mathrm{E}-03$ $7.357 \mathrm{E}-04$ $7.741 \mathrm{E}-04$ $8.572 \mathrm{E}-04$ 4.537F-03 $6.716 E-03$ $2.843 \mathrm{E}-02$ $6.829 \mathrm{E}-0$ ? $1.673 E-01$ $4.860 \mathrm{E}-02$

ANGLE 12 $M U=0.4580$ .45 E- 06 2.573E-OS 1.957F-03 1.900 -03 . $5.254 \mathrm{E}-03$ $3.725 E-03$ $4.586 \mathrm{E}-03$ $.102 \mathrm{E}-03$ 4.5 $77 \mathrm{E}-03$ $7.176 \mathrm{E}-03$ $.768 \mathrm{E}-02$ $.368 \mathrm{E}-02$
.3302 $3.718 \mathrm{E}-02$ $9.636 E-02$ $2.124 E-01$
ANGLE 4 $M U=-0.8656$ $1.691 \mathrm{E}-06$ $1.028 \mathrm{E}-03$ $6.544 E-04$ $6.544 E-04$ $1.178 E-03$ $1.874 \mathrm{E}-03$ $1.220 E-03$ $1.489 E-03$ $6.341 E-04$ $7.914 \mathrm{E}-04$ $1.131 \mathrm{E}-03$ $6.922 \mathrm{E}-0$ $4.884 \mathrm{E}-02$ ANGLE 13 $M U=0.6179$ $2.590 E-06$ .650E- 05 $2.056 \mathrm{E}-03$ $4.814 \mathrm{E}-03$ 4. $415 E-03$ $7.658 E-03$ $5.574 E-03$ $6.949 \mathrm{E}-03$ $4.930 \mathrm{E}-03$ $6.876 E-03$ $9.737 \mathrm{E}-03$ $2.101 \mathrm{E}-02$ $2.602 \mathrm{E}-02$ $4.082 E-02$ $1.030 \mathrm{E}-01$ $2.209 E-01$
ANGLE 5 $M U=-0.7550$ $1.736 \mathrm{E}-06$ $1.103 \mathrm{E}-03$ $7.258 \mathrm{E}-04$ 7.258E-04 $1.141 \mathrm{E}-03$ $2.006 \mathrm{E}-03$ $1.282 \mathrm{E}-03$ 1. $521 E-03$ $6.009 \mathrm{E}-04$ 8.596E-04 . $382 \mathrm{E}-03$ $5.090 E-03$ $8.394 \mathrm{E}-03$ 2. $956 \mathrm{E}-02$ $7.058 E-02$ 1. $716 \mathrm{E}-01$
$4.918 \mathrm{E}-02$

ANGLE 14 $M U=0.7550$ 4. $833 E-05$ $4.833 E-05$ 3.909E-03 $7.433 E-03$ $6.071 E-03$ $1.042 \mathrm{E}-0$ 7. $906 E-O$ $9.854 \mathrm{E}-03$ $7.434 \mathrm{E}-03$ $9.647 \mathrm{E}-03$ 1. $239 \mathrm{E}-02$ 2.438E-O2 $2.928 \mathrm{E}-02$ $4.480 E-02$ $1.102 \mathrm{E}-0$ $2.294 \mathrm{E}-01$
$5.608 \mathrm{E}-02$
ANGLE 6 $M U=-0.6179$ $1.796 \mathrm{E}-06$ $1.193 \mathrm{E}-03$ $7.920 \mathrm{E}-04$ $1.407 \mathrm{E}-03$ $1.238 E-03$ 2.163E-03 $1.639 \mathrm{E}-03$ . $567 \mathrm{E}-03$ $.611 E=03$ $1.019 E-0$ $7.241 E-02$ I. $750 \mathrm{E}-01$ $4.954 E-02$

ANGLE 15 $M U=0.8656$ $7.957 \mathrm{E}-05$ $0.654 E-03$ $6.644 E-03$ $1.211 \mathrm{E}-02$ $1.015 \mathrm{E}-02$ $1.717 \mathrm{E}-02$ $1.309 E-02$ 1.590E-02 $1.118 \mathrm{E}-02$ 1.547E-O2 . $1.180 E-01$ $5.685 \mathrm{E}-02$

ANGLE 7 $M U=-0.4580$ $1.870 \mathrm{E}-06$ $1.308 E-03$ $8.618 \mathrm{E}-04$ $1.533 \mathrm{E}-03$ $1.345 \mathrm{E}-03$ $2.363 E-03$ $1.537 E-03$ $.849 E-03$ $6.458 \mathrm{E}-03$ $.250 \mathrm{E}-02$ $7.475 \mathrm{E}-02$ $1.791 \mathrm{E}-01$ 5.019E-02 ANGLE 16 $2.930 \mathrm{E}-06$ $1.436 \mathrm{E}-04$ $1.836 \mathrm{E}-02$ 1. $219 \mathrm{E}-02$ 
(GAMMAS/MEV/STERADIAN/SOURCE NEUTRON)

\begin{abstract}
ENERGY GROUP (MEV) 8.00E $00--1.00 E$ O1 6.50E 00---8.00E DO 5. OOE OOC-6.5OE OO $4.00 E$ OO$3.0 O E$ OO2.5OE OO- 3. COE OO 1. $1.33 \mathrm{~F} \mathrm{OO-D1.66E} 00$ $1.00 E$ OO- $1.33 E$ OD $1.0 O E-O 1-1.33 E$ OO $6.00 E-01--8.00 E-01$ 4. $.00 E-01=-0.00 E-01$ $3.00 \mathrm{E}-01---4.00 \mathrm{E}-01$ 2. $00 E-01--3.00 E-01$ 1. $00 \mathrm{E}-01--2.00 \mathrm{E}-01$ $5.00 E-02--1.00 E \sim 01$ $2.00 E-02---5.00 E-02$
\end{abstract}

ENERGY

GROUP (MEV)

8.00E OD-- -1.00E 01 6.5OE OO---8. OOE OO $5.00 E$ OO- $-6.50 E$ OO $4.00 E$ OO- $-5.00 E$ OO $3.00 F$ NO- $-4.00 E$ OO $2.50 E$ OO- $-3.00 E$ OO 2.0OE OO---2.5OE OO 1.66 E $00-O 2.00 E 00$ 1.33 E $00--1.66 E$ E 00 1.00 E $00--1.33 E 00$ $8.00 E-01-1.00 E$ OO $6.00 F-01--8.00 E-01$ $4.00 E-01--6.00 E-01$ 3. OOE - 01---4. OOE-OI $2.00 E-01--3.00 E-01$ 1.0OE-01---2.0OE-01 $2.00 \mathrm{E}-02---5.00 \mathrm{E}-02$
ANGLE 1 $M U=-1.0000$ $2.816 E-06$ 096E-05 .005E-04 $.161 \mathrm{E}-04$ $7.735 \mathrm{E}-04$ $7.162 \mathrm{E}-04$ $1.469 \mathrm{E}-03$ $1.067 \mathrm{E}-03$ $1.482 \mathrm{E}-03$ $9.271 \mathrm{E}-04$ $7.837 E-04$ $4.910 E-03$ 7.263E-03 $3.510 E-02$ $9.140 \mathrm{E}-02$ $2.453 E-01$ $7.355 \mathrm{E}-02$ ANGLE 10 $M U=0.0950$ $4.161 E-06$ $1.789 \mathrm{E}-03$ 1.236E-03 $2.038 \mathrm{E}-03$ $.988 E-03$ . $419 E-03$ 2.387E-03 $2.003 E-03$ $1.791 \mathrm{E}-03$ $2.571 E-03$ $4.458 E-03$ $1.513 \mathrm{E}-02$ $2.505 \mathrm{E}-02$ $4.124 \mathrm{E}-02$ $1.182 \mathrm{E}-01$ $2.981 E-01$ $8.046 \mathrm{E}-02$
ANGLE 2 $M U=-0.9894$ $2.825 \mathrm{E}-06$ $8.112 \mathrm{E}-04$ ..112E-04 $4.332 E-C 4$ . $405 \mathrm{E}-04$ .405E-04 1.486E-03 1. $461 \mathrm{E}-03$ $8.861 \mathrm{E}-04$ $7.775 \mathrm{E}-04$ $5.724 \mathrm{E}-04$ $4.747 \mathrm{E}-03$ $7.300 \mathrm{E}-03$ $3.524 E-02$ $9.157 \mathrm{E}-02$ $2.456 \mathrm{E}-01$ $7.360 \mathrm{E}-\mathrm{C} 2$ ANGLF 11 $M U=0.2816$ $2.426 \mathrm{~F}-05$ $2.299 \mathrm{E}-03$ $1.491 \mathrm{E}-03$ $2.660 \mathrm{E}-03$ $2.389 E-03$ $4.041 E-03$ 2.870E-03 $3.492 \mathrm{E}-03$ $2.443 F-03$ $3.8 \mathrm{COE}-03$ $6.691 E-03$ $1.882 \mathrm{E}-02$ $2.723 \mathrm{E}-02$ 4. $362 \mathrm{E}-02$ $1.258 \mathrm{E}-01$ $3.113 E-01$
$8.202 E-02$
ANGLE 3 ANGLE $M U=-0.8656$ $2.931 \mathrm{E}-06$ $1.190 E-05$ 5.813E-04 $8.875 E-04 \quad 1.019 E-03$ 9.255E-04 9.498E-04

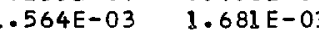
$1.076 \mathrm{E}-03 \quad 1.099 \mathrm{E}-03$ $1.395 E-03 \quad 1.327 E-03$ $7.526 E-04 \quad 5.974 E-04$ $7.657 E-04 \quad 7.826 E-04$ 8.335E-04 1.204E-03 $4.890 \mathrm{E}-03 \quad 5.148 \mathrm{E}-03$ $.549 \mathrm{E}-03 \quad 8.330 \mathrm{E}-03$ $3.574 \mathrm{E}-02 \quad 3.649 \mathrm{E}-02$ $2.472 \mathrm{E}-01 \quad 2.502 \mathrm{E}-01$ $7.382 \mathrm{E}-02 \quad 7.423 \mathrm{E}-02$ ANGLE 12 $M U=0.4580$ $3.034 E-05$ $2.886 E-03$ $1.976 \mathrm{E}-03$ $3.331 E-03$ $3.144 \mathrm{E}-03$ 5.326E-03 3. $910 \mathrm{E}-03$ 4.873E-03 $3.859 \mathrm{E}-03$ $5.971 \mathrm{E}-03$ .703E-03 $2.278 \mathrm{E}-02$ $2.972 \mathrm{E}-02$ 4.715E-02 $1.346 \mathrm{E}-01$ $8.365 \mathrm{~F}-02$ ANGLE 13 $M U=0.6179$ . $370 E-06$ .832E-05 . $2.609 E-03$ $4.182 \mathrm{E}-03$ $.096 \mathrm{E}-03$ $5.522 \mathrm{E}-03$ 7.057E-03 6.279E-03 $9.116 E-03$ $1.318 \mathrm{E}-02$ $2.666 \mathrm{E}-02$ $3.292 \mathrm{E}-02$ $5.140 \mathrm{E}-02$ . $445 \mathrm{E}-01$ $.409 E-01$ $8.528 \mathrm{E}-02$

\begin{tabular}{cc} 
ANGLE 5 & \multicolumn{1}{c}{ ANGLE 6} \\
MU $=-0.7550$ & $M U=-0.6179$ \\
$3.035 E-06$ & $3.175 E-06$ \\
$1.260 E-05$ & $1.344 E-05$ \\
$9.905 E-04$ & $1.073 E-03$ \\
$6.615 E-04$ & $7.202 E-04$ \\
$1.141 E-03$ & $1.238 E-03$ \\
$1.062 E-03$ & $1.148 E-03$ \\
$1.811 E-03$ & $1.951 E-03$ \\
$1.150 E-03$ & $1.247 E-03$ \\
$1.334 E-03$ & $1.459 E-03$ \\
$5.491 E-04$ & $6.578 E-04$ \\
$8.681 E-04$ & $1.027 E-03$ \\
$1.508 E-03$ & $1.591 E-03$ \\
$5.541 E-03$ & $6.197 E-03$ \\
$9.960 E-03$ & $1.253 E-02$ \\
$3.720 E-02$ & $3.773 E-02$ \\
$9.572 E-02$ & $9.846 E-02$ \\
$2.545 E-01$ & $2.602 E-01$ \\
$7.482 E-02$ & $7.560 E-02$
\end{tabular}

ANGLE 14 ANजLE 15 $M U=0.7550 \quad M U=0.8656$ $5.815 E-06 \quad 6.228 E-06$ 5.762E-03 $9.591 E-03$ $6.561 E-03$ $1.162 E-02$ $1.060 E-02$ $1.063 E-02 \quad 1.734 E-02$ $1.734 E-02$
$.063 E-02$ $1.107 E-02 \quad 1.776 E-02$ $1.007 E-02$ 1.531E-02 $1.329 E-02 \quad 1.816 \mathrm{E}-02$ $3.094 \mathrm{E}-02 \quad 3.618 \mathrm{E}-02$ $3.737 \mathrm{E}-02 \quad 4.288 \mathrm{E}-02$ $5.670 \mathrm{E}-02 \quad 6.293 \mathrm{E}-02$ $1.553 E-01 \quad 1.667 E-01$ $3.562 E-01 \quad 3.706 E-01$ $8.681 E-02 \quad 8.815 E-02$ MU $=-0.4580$ $3.354 \mathrm{E}-06$ $1.451 E-05$
$1.173 E-03$ $1.711 \mathrm{E}-04$ I.711E-04 $1.330 E-03$ $1.232 E-03$ $1.1203 \mathrm{E}-03$ $1.403 E-03$ $8.881 E-04$ $1.239 E-03$ $1.836 E-03$ $7.328 \mathrm{E}-03$ $1.581 \mathrm{E}-02$ $3.813 \mathrm{E}-02$ $3.813 \mathrm{E}-02$
$1.020 \mathrm{E}-01$ $2.674 E-01$ $2.674 E-01$
$7.655 \mathrm{E}-02$

ANGLE 8 ANGLE 9 3.576E-06 $M U=-0.0950$ $1.594 E-05 \quad 3.844 E-06$ $1.313 E-03 \quad 1.521 E-03$ 1.521E-03 $1.471 \mathrm{E}-03 \quad 1.712 \mathrm{E}-03$ $1.364 E-03 \quad 1.593 E-03$ $2.389 \mathrm{E}-03$ 2.779E-03 $2.779 E-03$ $1.009 E-03 \quad 2.374 E-03$ $1.157 \mathrm{E}-03 \quad 1.423 \mathrm{E}-03$ $1.496 \mathrm{E}-03 \quad 1.871 \mathrm{E}-03$ $2.163 \mathrm{E}-03 \quad 2.954 \mathrm{E}-03$ $9.151 \mathrm{E}-03 \quad 1.177 \mathrm{E}-02$ $1.927 \mathrm{E}-02 \quad 2.242 \mathrm{E}-02$ $3.862 E-02 \quad 3.954 E-02$ $1.064 \mathrm{E}-01 \quad 1.117 \mathrm{E}-01$ $2.761 E-01 \quad 2.863 \mathrm{E}-01$ ANGLE 16 ANGLE 17 SCALAR $M U=0.9446 \quad M U=0.9894$ $6.564 \mathrm{E}-06 \quad 6.769 \mathrm{E}-06$ $1.593 E-04 \quad 4.160 E-04$ $1.926 E-02 \quad 5.179 E-02$ $2.357 E-02 \quad 6.390 E-02$ $2.050 E-02$ $3.243 E-02 \quad 7.202 E-02$ $2.610 E-C 2 \quad 5.229 E-02$ $3.015 \mathrm{E}-02$ $5.659 \mathrm{E}-02$ $2.237 E-02 \quad 3.163 E-02$ $2.357 E-02 \quad 2.937 E-02$ $2.543 E-02 \quad 3.033 E-02$ $4.364 E-02 \quad 5.397 E-02$ $4.922 E-02 \quad 5.571 E-02$ 7.042E-02 7.871E-02 $1.777 \mathrm{E}-01 \quad 1.865 \mathrm{E}-01$ $3.828 E-01 \quad 3.909 E-01$ 8.982E-02
FLUX $4.365 E-04$ 4. $555 \mathrm{E}-02$ .049E-02 $5.333 E-02$ 4. $727 E-02$ $5.904 E-02$ 7.095E-02 $5.194 E-02$ $6.564 E-02$ $8.858 \mathrm{E}-02$ $2.093 E-01$ 3.016E-01 5.589E-01 . $1.010 \mathrm{E} 00$ 
(GAMMAS/MEV/STERADIAN/SOURCE NEUTRON) GROUP (MEV) 8.00E CO-D-1.0OE OI $6.50 E$ OO- - - 8.00E 00 $5.00 E$ 00---6.50E 00 $-5.00 \mathrm{E} 00$ .0OF $00--4.00 E \quad 00$ $2.50 E$ OO- - 3. COE 00 $2.50 \mathrm{E} 00$ $00-\cdots 2.00 E 00$ $1.33 \mathrm{E} \quad 00--1.66 \mathrm{E} \mathrm{CO}$ $1.00 E$ O0-- - 1. 33E 00 $8.00 E-01--1.00 E 00$ $6.00 E-01---8.00 E-01$ $4.00 \mathrm{E}-01--6.00 \mathrm{E}-0$ ? $3.00 E-01---4.00 E-n 1$ $2.00 E-01-\rightarrow-3.00 E-01$ $1.00 E-01---2.00 E-01$ $5.00 \mathrm{E}-02$ . NOE-02---5.00E-02

$$
\text { ENERGY }
$$

$$
\text { GROUP (MEV) }
$$

8.00E 00---1.00E 01 $6.50 \mathrm{E}$ OO-- -8.00E OO 5. . OE OO- $6.50 E$ OO 4.00E OO- - -5.00E 00 $3.00 E$ OO- $-4.00 E$ OO $2.50 E$ OO--3.00E 00 $2.00 E$ NO-- $2.50 E$ OO $1.66 \mathrm{~F}$ OO--2.00E 00 $1.33 \mathrm{E} 00--1.66 \mathrm{E} 00$ $1.00 \mathrm{E}$ 00- $-1.33 \mathrm{E}$ OO $8.00 E-01-2.1 .00 E$ OO 6.0OE-O1- $6.00 E-O 1$ 4.0OE $01-2.00 E-O 1$ 2.0OE-01- $3.00 E-01$ $1.00 E-01--2.00 E-01$ $1.00 \mathrm{E}-0 \mathrm{C}-2.0 \mathrm{E}-\mathrm{O}$ 2.0OE $-02-=5.00 \mathrm{E}-02$
ANGLE 1 ANGLE 2 $U=-1.0000$ $3.701 E-06$ $6.426 \mathrm{E}-04$ $2.950 \mathrm{E}-04$ $5.587 E-04$ $5.398 \mathrm{E}-04$ $1.168 \mathrm{E}-03$ $8.929 \mathrm{E}-04$ $1.284 E-03$ $8.756 E-04$ $6.793 E-04$ $3.307 E-04$ $4.411 E-03$ $6.960 E-03$
$3.687 \mathrm{E}-02$ $9.931 \mathrm{E}-02$ $2.819 E-01$ $8.596 E-02$ ANGLE 10 $M U=0.0950$ $5.958 \mathrm{E}-06$ $2.195 E-05$ $1.537 \mathrm{E}-03$ $1.002 \mathrm{~F}-03$ $1.706 \mathrm{E}-03$ $1.646 \mathrm{E}-03$ $2.782 \mathrm{E}-03$ $3.415 E-03$ $2.415 \mathrm{E}-03$ 1.655E-03 $2.481 E-03$ $4.621 E-03$ 2.641E-02 $2.641 \mathrm{E}-02$ $4.283 \mathrm{E}-02$ 1.3 $9.452 \mathrm{E}-0$ ?
$A N G L E \quad 3$
$M U=-0.9446$ $3.715 E-06$ $1.1 \in 1 E-05$ $6.538 \mathrm{E}-04$ $3.146 E-04$
$5.867 E-04$ $5.667 \mathrm{E}-04$ $1.188 \mathrm{E}-03$ $8.914 E-04$ $1.256 \mathrm{E}-03$ $8.277 E-04$ $6.726 E-04$ $4.145 E-04$ 4.447E-03 7. $005 \mathrm{E}-03$ $3.702 E-C 2$ $9.951 E-02$ $2.823 E-01$
$8.602 E-02$ ANGIE 1 ANGLE 11
$M U=C .2816$ $6.635 \mathrm{E}-06$ $2.603 \mathrm{E}-05$ $1.921 E-03$ 1.290E-03 $2.165 \mathrm{E}-03$ 2.095E-O3 2.578E-03 3. (.) 3. 7. $2.002 \mathrm{E}-02$ $2.859 E-C 2$ 1.55E-02 $3.644 E-01$ $9.644 E-01$ $3.771 E-06$ $1.197 E-05$ $6.947 E-04$
$3.825 E-04$ $6.842 E-04$ $6.602 E-04$ $1.262 \mathrm{E}-03$ $8.890 E-04$ $1.166 \mathrm{E}-03$ $6.707 E-04$ $6.597 E-04$ $7.084 E-04$ 4. $584 E-03$ $7.301 \mathrm{E}-03$ $3.756 E-02$ $1.004 E-01$ 2.843E-01 $8.629 E-02$ ANGLE 12 $M U=0.4580$
$7.428 E-06$ $7.428 \mathrm{E}-06$
$3.164 \mathrm{E}-05$ $2.495 E-03$ $1.668 \mathrm{E}-03$ $2.808 \mathrm{E}-03$ $2.699 E-03$ $4.486 \mathrm{E}-03$ $3.410 E-03$ $4.312 E-03$ $3.942 E-03$ $6.393 E-03$ 2. $413 \mathrm{E}-02$ 2.413E-0? . $4.997 E-01$ $3.485 \mathrm{E}-01$ $9.849 \mathrm{E}-0$ ? ANGLE 4 $M U=-0.8656$ $3.881 E-06$ $1.258 \mathrm{E}-05$ . $4.782 E-04$
$8.222 E-04$ 7. $907 E-04$ . $373 \mathrm{E}-03$ . $962 E-04$ $1.068 \mathrm{E}-03$ $4.913 E-04$ $6.781 E-04$ $1.111 E-03$ 4. $834 E-03$ $8.214 E-03$ $3.834 E-02$ $1.020 E-01$ $2.883 E-01$ $8.680 E-02$ ANGLE 13 $M U=0.6179$ $8.326 E-06$
$4.012 E-05$ $3.405 \mathrm{E}-03$ $2.264 E-03$ $3.825 \mathrm{E}-03$ $3.714 \mathrm{E}-03$ $6.226 \mathrm{E}-03$ $5.101 E-03$ $6.675 E-03$ $1.006 \mathrm{E}-02$ $1.006 \mathrm{E}-02$ $1.464 \mathrm{E}-02$ 2. $311 E-02$ $3.435 \mathrm{E}-02$ 1.355E$1.599 E-01$ $4.016 E-01$
$1.005 E-01$

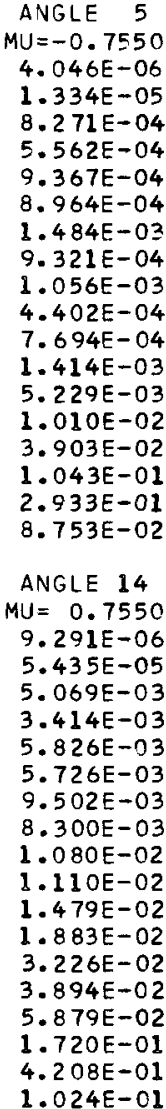
$M U=-0.7550$

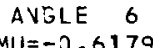
4. $270 \mathrm{E}-06$ $1.424 \mathrm{E}-05$ $8.950 \mathrm{E}-04$ $6.008 E-04$ $1.008 \mathrm{E}-03$ $9.609 E-04$ $1.595 E-03$ $1.015 \mathrm{E}-03$ $1.176 \mathrm{E}-03$ $5.670 E-04$ $9.324 E-04$ $1.562 \mathrm{E}-03$ 5. $931 E-03$ $1.304 \mathrm{E}-02$ 3.949E-02 1. $074 \mathrm{E}-01$ $3.004 E-01$ $8.849 E-02$ $\triangle N G L E 15$ $\begin{aligned} & M U=0.8656 \\ & 1.024 \mathrm{E}-05\end{aligned}$ $8.291 \mathrm{E}-05$ $8.592 E-03$ $6.022 \mathrm{E}-03$ $1.030 \mathrm{E}-02$ $1.010 E-02$ $1.614 \mathrm{E}-02$ $1.436 \mathrm{E}-02$ $1.780 \mathrm{E}-02$ $1.706 E-02$ $2.021 E-02$ $2.311 E-02$ $3.736 E-02$ $4.455 E-2$ $1.844 \mathrm{E}-01$ $4.388 E-01$ $1.040 E-01$
ANGLE 7 ANGLE 8 ANGLE 9 $M U=-0.4580 \quad M U=-0.2816 \quad M U=-0.0950$ $4.563 E-06 \quad 4.933 E-06 \quad 5.394 E-06$ $1.537 E-05 \quad 1.690 E-05 \quad 1.902 E-05$ $9.738 \mathrm{E}-04 \quad 1.088 \mathrm{E}-03 \quad 1.266 \mathrm{E}-03$ $6.291 \mathrm{E}-04 \quad 6.824 \mathrm{E}-04 \quad 8.012 \mathrm{E}-04$ $1.063 \mathrm{E}-03 \quad 1.164 \mathrm{E}-03 \quad 1.369 \mathrm{E}-03$ $1.735 E-03 \quad 1.955 E-03 \quad 2.296 E-03$ $1.159 E-03 \quad 1.366 E-03 \quad 1.637 E-03$ $1.414 E-03 \quad 1.713 E-03 \quad 2.033 E-03$ $\begin{array}{lll}8.196 E-04 & 1.091 E-03 \quad 1.332 E-03\end{array}$ $\begin{array}{lll}1.138 E-03 & 1.379 E-03 & 1.749 E-03\end{array}$ $1.664 E-03 \quad 1.995 E-03 \quad 2.893 E-03$ $\begin{array}{lll}7.188 E-03 & 9.247 E-03 & 1.220 E-02\end{array}$ $1.671 E-02 \quad 2.050 E-02 \quad 2.381 E-02$ $3.980 E-02 \quad 4.022 E-02 \quad 4.112 E-02$ $1.115 E-01$ 1.165E-01 1.227E-01 $3.093 E-01 \quad 3.202 E-01 \quad 3.330 E-01$ 8.968E-02 $9.109 E-02 \quad 9.272 E-02$ ANGLE 16 $M U=0.9446$
$1.106 E-05$ ANGLE 17 $M U=0.9894$
$1.159 E-05$ $4.419 E-04$ $5.299 \mathrm{E}-02$ $3.525 E-02$ $5.984 E-02$ 4. $779 \mathrm{E}-02$ $7.071 E-02$ $5.320 E-02$ $5.598 E-02$ $3.502 E-02$ $3.238 \mathrm{E}-02$ $3.238 E-02$ $5.416 E-02$ $5.622 \mathrm{E}-02$ . $050 \mathrm{E}-01$ $4.636 \mathrm{E}-01$ $4.636 E-01$

SCALAR

FLUX

7.868E-05 $4.530 E-04$

$4.118 \mathrm{E}-02$ $2.777 \mathrm{E}-02$ 4.722E-02 4. $369 \mathrm{E}-02$ $7.013 \mathrm{E}-02$ $5.558 \mathrm{E}-02$ $6.681 \mathrm{E}-02$ $5.511 E-02$ $7.009 E-02$ . . . 
(GAMMAS/MEV/STERADIAN/SOURCE NEUTRON)

ENERGY 8. OOE $00--1.00 E$ OI 6.50E 00---8.00E OO 作 $00=-6.50 E$ OO $3.0 O E$ OO-- $-4.00 E$. 00 $2.50 E$ OO- - 3. OOE OO $2.00 E$ OO---2.5OE 00 $1.66 \mathrm{E} 00-\cdots 2.00 \mathrm{E}$ OO $1.33 \mathrm{E} 00---1.66 \mathrm{E} 00$ $1.00 \mathrm{E} 00---1.33 \mathrm{E} O 0$ $8.00 E-01---1.00 E$ OO $6.00 E-01--8.00 E-01$ $4.00 E-01---6.00 E-01$ $3.00 E-01---4.00 E-01$ 2.00E-01-- $3.00 E-01$ $1.00 \mathrm{CE}-01--2.00 \mathrm{E}-01$ $5.00 E-02--1.00 E-01$ $2.00 E-02--5.005-02$

ENERGY$$
\text { GROUP (MEV) }
$$

8.0OE OD---1.00E O1 6.5OE 00- - 8. OOE OO 5.00 E $00--6.50 E$ OO $4.00 E$ OO- $-5.00 E$ EO 3. 00 OO $00--4$. $00 E$ OO 2.50E OO---3.00E DO $2.00 E$ OO- $-2.50 E$ OO $1.66 \mathrm{E} \mathrm{OO-D-2.00E} \mathrm{OO}$ $1.33 \mathrm{E} 00-1.66 \mathrm{E}$ OO $1.00 E$ 00---1.33E CO $8.00 \mathrm{~F}-01--1.00 \mathrm{E}$ OO $6.00 E-01--8.00 E-01$ $4.00 E-01--6.00 E-01$ 3.00E-01- $-4.00 \mathrm{E}-01$ $2.00 E-01--3.00 E-01$ $1.00 E-01--2.00 E-C 1$ $5.00 E-02--1.00 E-01$
ANGLE 1 ANGLE 2 $4.172 E-06 \quad 4.100 E-06$ $1.139 \mathrm{E}-05 \quad 4.190 \mathrm{E}-06$ $4.972 E-04 \quad 5.082 E-04$ $1.940 E-04 \quad 2.141 E-04$ $3.823 E-04 \quad 4.105 E-C 4$ 3.812E-04 $4.081 E-04$ $8.829 E-04 \quad 9.017 E-04$ $7.137 E-04 \quad 7.098 E-04$ $1.059 \mathrm{E}-03 \quad 1.028 \mathrm{E}-03$ $7.687 E-04 \quad 7.195 E-04$ $5.444 \mathrm{E}-04 \quad 5.385 \mathrm{E}-04$ $1.785 E-04 \quad 2.635 E-04$ $3.855 E-03 \quad 3.885 E-03$ $6.164 E-03 \quad 6.213 E-03$ $3.522 \mathrm{E}-02 \quad 3.536 \mathrm{E}-02$ $9.622 \mathrm{E}-02 \quad 9.642 \mathrm{E}-02$ $2.833 E-01 \quad 2.837 E-01$ $8.725 \mathrm{E}-02$ ANGLE 10 $M U=0.0950$ $7.212 E-06$ $2.224 \mathrm{E}-05$ $1.238 E-03$ .979E-04 $1.334 \mathrm{E}-03$ $1.323 \mathrm{E}-03$ $2.207 \mathrm{E}-03$ $1.602 \mathrm{E}-\mathrm{C} 3$ $1.942 \mathrm{E}-03$ $1.418 \mathrm{E}-03$ $2.227 \mathrm{E}-03$ $4.420 \mathrm{E}-03$ $1.528 E-02$ $2.517 E-02$ $4.037 E-02$ $1.268 E-01$ $3.525 E-01$ $8.732 E-02$ ANGLE 11 $M U=0.2816$
$8.203 E-06$ $2.640 \mathrm{E}-05$ $1.565 \mathrm{E}-03$ $1.043 E-03$ $1.722 E-03$ $1.701 \mathrm{E}-03$ $2.769 E-03$ $2.036 \mathrm{E}-03$ $2.484 \mathrm{E}-03$ $2.115 \mathrm{E}-03$ $3.693 \mathrm{E}-\mathrm{C3}$ $7.141 \mathrm{E}-03$ $1.926 \mathrm{E}-02$ $2.706 \mathrm{E}-02$ $4.287 \mathrm{E}-\mathrm{C2}$ 1. $354 E-01$ $3.700 E-01$
ANGLE $4=-0.9446$ $4.262 E-06$ $5.476 \mathrm{E}-04$ $2.836 \mathrm{E}-04$ $5.079 \mathrm{E}-04$ $5.007 E-04$ $9.683 \mathrm{E}-04$ $6.986 \mathrm{E}-04$ $9.260 E-04$ $5.576 \mathrm{E}-04$ $5.283 \mathrm{E}-04$ $5.596 \mathrm{E}-04$ $4.004 E-03$ $6.531 \mathrm{E}-03$ $3.587 E-02$ $9.727 \mathrm{E}-02$ $2.858 \mathrm{E}-01$

$8.760 \mathrm{E}-02$ ANGLE $\quad 4$
$M U=-0.8656$ $4.403 E-06$
$1.251 E-05$ $6.072 E-04$ $3.783 E-04$ $6.409 E-04$ $6.254 E-04$ $1.064 E-03$ $6.920 E-04$ $8.115 E-04$ $3.755 E-04$ $5.496 E-04$ $9.523 E-04$ $4.222 \mathrm{E}-03$ $7.484 E-03$ $3.658 \mathrm{E}-02$ $9.888 \mathrm{E}-02$ $8.813 \mathrm{E}-02$

ANGLE 5 $4.614 \mathrm{E}-06$ $4.614 E-06$
$1.329 E-05$ $6.660 E-04$ $4.487 E-04$ $7.409 E-04$ $7.170 \mathrm{E}-04$ $1.154 \mathrm{E}-03$ $7.132 \mathrm{E}-04$ $7.881 \mathrm{E}-04$ $3.281 E-04$ $6.386 \mathrm{E}-04$ $1.226 E-03$ $4.588 \mathrm{E}-03$ $9.418 \mathrm{E}-03$ $3.717 E-02$ $1.012 \mathrm{E}-01$ $2.952 \mathrm{E}-01$ $8.889 E-02$ $M U=-0.6179$ $4.904 \mathrm{E}-06$ $1.422 E-05$
$7.188 E-04$ $4.188 E-04$ $4.787 E-04$
$7.883 E-04$ $7.597 \mathrm{E}-04$ $1.235 \mathrm{E}-03$ \begin{tabular}{l}
$7.817 \mathrm{E}-04$ \\
\hline
\end{tabular} $8.988 \mathrm{E}-04$ $4.588 E-04$ $7.883 \mathrm{E}-04$ $1.331 E-03$ $5.274 E-03$ $1.239 \mathrm{E}-02$ $3.751 E-02$ $1.043 E-0.1$ $3.027 E-01$ $8.989 E-02$

ANGLE 7 $M U=-0.4580$ $5.287 \mathrm{E}-06$ $1.537 \mathrm{E}-05$ $7.772 \mathrm{E}-04$ $4.878 E-04$
$8.134 E-04$ $7.867 E-04$ $1.339 E-03$ $9.072 E-04$ $1.121 \mathrm{E}-03$ 7.055E-04 ( $1.391 E-03$ $0.538 E-03$ . $769 E-02$ $3.120 \mathrm{E}-01$
$9.114 \mathrm{E}-02$

ANGLE 8 $M U=-0.2816$ $5.783 E-06$ $.695 \mathrm{E}-05$ $8.658 \mathrm{E}-04$ $8.807 E-04$ $8.620 \mathrm{E}-04$ $1.514 \mathrm{E}-03$ $1.087 E-03$ $1.385 \mathrm{E}-03$ $9.510 E-04$ $1.170 E-03$ $1.703 E-03$ $8.621 E-03$ $1.973 E-02$ $3.798 \mathrm{E}-02$ $1.134 \mathrm{E}-01$ $3.235 \mathrm{E}-01$ ANGLE 12 ANGLE 13

ANGLE 15 $.413 E-06 \quad 1.085 E-05$ $2.040 \mathrm{E}-03$ $1.357 \mathrm{E}-03$ $2.234 E-03$ $2.208 \mathrm{E}-03$ $3.613 \mathrm{E}-03$ $2.827 E-03$ $3.633 \mathrm{E}-03$ $3.702 \mathrm{E}-03$ $6.237 \mathrm{E}-03$ $1.062 \mathrm{E}-02$ $2.313 \mathrm{E}-02$ $2.922 \mathrm{E}-02$ $4.623 \mathrm{E}-02$ $1.452 \mathrm{E}-01$ $1.003 \mathrm{E}-01$
$4.048 E-0$

$2.804 E-03$

$1.843 \mathrm{E}-03$

$3.057 E-03$

$3.071 E-03$

$5.105 E-03$

$4.393 \mathrm{E}-03$

5.8 $6.598 \mathrm{E}-03$

$1.954 E-02$ $1.454 E-02$ . (2) S.029E-OL $4.000 \mathrm{E}-01$ $1.024 \mathrm{E}-01$ $248 E-05$ $5.420 E-05$ $4.227 E-03$ $2.840 E-C 3$ 4.758E-03 $4.902 E-03$ . $7.460 E-03$ $1.102 E-02$ $1.474 \mathrm{E}-02$ . . . $658 E-02$ . $48 \mathrm{E}-02$ $4.290 \mathrm{E}-0$ $1.044 \mathrm{E}-01$
$M=0.8656$

$.419 E-05$ $8.102 E-05$ . $225 \mathrm{E}-03$ $8.749 E-03$ $9.038 \mathrm{E}-03$

$1.416 \mathrm{E}-02$

$1.329 \mathrm{E}-02$

$1.657 \mathrm{E}-02$ $1.697 \mathrm{E}-02$ $2.002 E-02$ $2.258 E-02$ $3.484 E-02$ $4.163 \mathrm{E}-02$ $6.023 \mathrm{E}-02$ $1.795 E-01$ 1.061E-01
ANGLE 16 
(GAMMAS/MEV/STERADIAN/SOURCE NEUTRON) ENERGY
GROUP (MEV) 8. OOE OO---1. COE O1 6.50E $00-.8 .00 E$ OO $5.00 E$ ON-- $6.50 E 00$ $4.00 E$ OO- $-5.00 E 00$ $3.00 \mathrm{E} 00--4.00 \mathrm{E} 00$ $2.50 E$ OO- -3.00 E 00 $2.00 E$ OO---2.5OE 00 $1.33 \mathrm{E} 00--1.66 \mathrm{E} 00$ $.00 E$ O0---1.33E 00 8.00E-01---1. COE CO $6.00 E-01---8.00 E-01$ $.00 E-01--4.00 E-01$ $2.00 E-01--3.00 E-01$ $1.00 E-01---2.00 E-01$ $2.00 \mathrm{E}-02---5.00 \mathrm{E}-02$

$$
\text { ENERGY }
$$

8.0OE 00---1. $1.00 E$ O $6.50 E$ OO- - 8. OOE CO 5.0OE OD---6.50E DO 4.0OE OO---5.0OE 00 $3.00 F$ OO- $-4.00 E$ OO 2.50E OO--3. 00 E 00 2.00 E $00---2.50 E 00$ $1.66 E$ OO-D-2.00E OO $1.33 E$ O0---1.66E 00 $1.0 \mathrm{OE} 00--1.33 \mathrm{E} 00$ 8.0OE-01--1. $6.00 \mathrm{E}-01--8.00 \mathrm{E}-01$ $4.00 E-01--6.00 E-01$ $3.00 E-01--4.00 E-01$ $2.00 \mathrm{E}-01--3.00 E-01$ $1.00 \mathrm{E}-01--2.00 \mathrm{E}-01$ $2.00 E-02--5.00 E-02$
ANGLE 1 $M U=-1.0000$ $4.251 E-06$
$1.070 E-05$ $3.761 \mathrm{E}-04$ $1.179 E-04$ $1.179 E-04$ $2.498 E-04$
$2.555 E-04$ $6.466 E-04$ $5.560 E-04$ $8.491 E-04$ $4.143 E-04$ $6.081 E-05$ $3.235 \mathrm{E}-03$ $5.207 E-03$ $3.173 E-02$ $8.699 E-02$ $2.623 E-01$ $8.127 E-02$ ANG LE 10 $M U=0.0950$ $7.804 E-06$ $2.151 E-05$ $9.765 E-04$ $6.175 E-04$ $1.022 \mathrm{E}-03$ $1.027 \mathrm{E}-03$ $1.688 \mathrm{E}-03$ $1.240 \mathrm{E}-03$ $1.495 \mathrm{E}-03$ $1.149 E-03$ . $383 \mathrm{E}-02$ 1.150E-O 1.250 01 $3.280 \mathrm{E}-01$ $\triangle N G L E ?$ $M U=-0.9894$ $4.271 E-06$
$1.080 E-05$ $1.080 \mathrm{E}-05$
$3.863 \mathrm{E}-04$ $3.863 \mathrm{E}-04$
$1.373 \mathrm{E}-04$ $1.373 E-04$
$2.764 E-04$ $2.764 E-04$
$2.806 E-04$ $6.628 E-04$ $5.504 E-04$ 8.174E-04 5.996E-04 $4.100 E-04$ $1.427 E-04$ $3.259 \mathrm{E}-03$ 5.258E-03 $3.185 \mathrm{E}-02$ $8.718 E-02$ $2.628 \mathrm{E}-01$
$8.133 \mathrm{E}-02$ ANGLE 11 $M U=0.2816$ $9.046 E-06$ $1.243 \mathrm{E}-03$ $8.217 E-04$ $1.336 \mathrm{E}-03$ $1.335 \mathrm{E}-03$ $2.130 \mathrm{E}-03$ $1.582 \mathrm{E}-03$ $1.921 \mathrm{E}-03$ $1.785 \mathrm{E}-03$ $3.303 E-03$ $6.604 E-03$ $1.743 E-02$ $2.407 E-02$ $3.800 E-C 2$ $3.445 \mathrm{E}-01$ $9.162 \mathrm{E}-02$
ANGLE 3 4.351 .9446 4. 351 E-06 $1.117 \mathrm{E}-05$
$4.226 \mathrm{E}-04$ $2.040 \mathrm{E}-04$ $3.681 \mathrm{E}-04$ $3.681 \mathrm{E}-04$
$3.667 \mathrm{E}-04$ $7.197 \mathrm{E}-04$ $5.326 E-04$ $7.127 \mathrm{E}-04$ $4.448 E-04$ $4.044 \mathrm{E}-04$ $4.220 E-04$ $3.354 E-03$ 5.574E-03 $3.230 E-02$ $8.797 E-02$ $2.647 E-01$ $8.160 E-02$

ANGLE 12 $M U=0.4580$ $1.062 E-05$ $3.144 \mathrm{E}-05$ $1.627 \mathrm{E}-03$ $1.369 \mathrm{E}-03$ $1.730 E-03$
$1.735 E-03$ $1.735 E-03$
$2.80 O E-02$ $2.800 E-0$ ? $2.250 E-03$ $2.944 \mathrm{E}-03$ $3.295 \mathrm{E}-03$ $5.733 E-03$ $9.869 E-03$ $2.083 E-02$ $2.589 E-02$ 1. $317 E-01$ 1.317E-01 $9.359 E-02$
ANGLE 4 4. $509 \mathrm{E}-06$ $1.181 \mathrm{E}-05$ 4. $759 E-04$ $2.925 E-04$ $4.896 E-04$ $4.792 E-04$ $7.990 \mathrm{E}-04$ $5.157 E-04$ $5.941 E-04$ $2.734 E-04$ 4. $284 E-04$ $7.809 E-04$ $3.534 E-03$ $6.499 E-03$ $3.290 E-02$ $8.945 \mathrm{E}-02$ $2.684 E-01$ $8.211 E-02$ ANGLE 13 $M U=0.6179$ $1.257 \mathrm{E}-05$ $3.968 E-05$ $2.244 E-03$ $2.368 E-03$ $2.440 E-03$ $4.038 \mathrm{E}-03$ $3.642 E-03$ $5.005 E-03$ $6.063 E-03$ $9.281 E-03$ $1.347 E-02$ $2.388 \mathrm{E}-02$ $2.858 E-02$ $4.438 E-02$ $1.414 E-01$ $9.5133 \mathrm{E}-02$
ANGLE 5
$M U=-0.7550$ 4. $745 \mathrm{E}-06$ $1.258 E-05$ $5.248 \mathrm{E}-04$
$3.530 \mathrm{E}-04$ $3.530 E-04$
$5.735 E-04$ 5. $547 \mathrm{E}-04$ $5.547 E-04$
$8.669 E-04$ $5.254 E-04$ $5.649 E-04$ $2.325 E-04$ $5.103 E-04$ $1.016 E-03$ $3.855 E-03$ $8.342 E-03$ $.335 E-02$ $9.159 \mathrm{E}-02$ $.282 E-02$ ANGLE 14 $M U=0.7550$ . $286 \mathrm{E}-05$ $.422 E-03$ $2.279 E-03$ $3.771 \mathrm{E}-03$ $6.594 E-03$ $6.434 \mathrm{E}-03$ $8.675 E-03$ $1.024 E-02$ $1.370 \mathrm{E}-02$ $1.709 \mathrm{E}-02$ $2.689 E-02$ $3.228 \mathrm{E}-02$ $4.830 E-02$ $1.517 E-01$ $4.001 E-01$
$9.735 E-02$
AVGLE $M U=-0.6179$ $5.073 E-06$ $1.348 E-05$
$5.644 E-04$ $3.708 E-04$ $3.708 E-04$ $5.795 E-04$ $9.238 E-04$ 5. $805 \mathrm{E}-04$ $6.636 E-04$ $3.573 E-04$ 6. $387 E-04$ $1.082 E-03$ 1.489E-03 .357E-02 $2.807 E-01$
$8.377 E-02$

ANGLE 15 $M U=0.8656$ $.748 E-05$
$7.785 E-05$ $6.071 E-03$ 4. $381 E-03$ $7.218 E-03$ $7.772 E-03$ 1.197E-02 $1.176 \mathrm{E}-02$ $1.471 \mathrm{E}-02$ $1.574 \mathrm{E}-02$ $1.846 \mathrm{E}-02$ $2.054 E-02$ $3.055 \mathrm{E}-02$ $3.653 E-02$ $5.259 E-02$ $1.619 \mathrm{E}-01$ $9.892 E-02$

ANGLE 7 $M U=-0.4580$ $5.511 E-06$ . 462E-05 $6.058 \mathrm{E}-04$ . $662 E-04$ $.050 E-04$
$5.871 E-04$ $9.985 \mathrm{E}-04$ $t .874 E-04$ $8.620 E-04$ $5.821 E-04$ $7.817 E-04$ .106E-03 . .362E-02 $2.896 E-01$
$8.495 E-02$ ANGLE 16 $M U=0.9446$
$1.997 E-05$ $1.415 E-04$ 
(GAMMAS/MEV/STERADIAN/SOURCE NEUTRON)

\begin{abstract}
ENERGY
GROUP (MEV)

8.0OE 00---1.0OE OI $6.50 \mathrm{E}$ 00-- $-8.00 \mathrm{E}$ OO $5.00 E$ OO- $-6.50 E$ OO 4.0OE OO--5. OOE OO . . . . . . 6.00E $-01-=-8.00 E-01$ $4.00 E-01--8.00 E-01$ . $00 E-01-2.00 E-01$ 2.00E-01- $3.00 E-01$ $1.0 \mathrm{EE}-01-2-2.00 \mathrm{E}-01$ $5.00 E-02--1.00 E-01$ 2.00E-02---5.00E-02
\end{abstract}

ENERGY

GROUP (MEV)

8.0OE $00--1.00 E$ OI $6.50 E$ OO- $-8.00 E$ OO 5.00 E $00--6.50$ E 00 $4.00 E$ OD- $-5.00 E$ OO 3.00 E $00--4.00 E$ OO 2.50E $00--3.00 E 00$ $2.00 E$ OO--2.50E 00 $1.66 \mathrm{E} 00--2.00 \mathrm{E}$ OO $1.33 \mathrm{E} \quad 00--1.66 \mathrm{E}$ กO $1.00 \mathrm{~F} \quad 00--1.33 \mathrm{E}$ OO $8.00 E-01--1.00 E$ OO $6.00 E-01-2.00 E-01$ $4.00 E-01--6.00 E-01$ $3.00 E-01--4.00 E-01$ $2.00 E-01--3.00 E-01$ $1.00 \mathrm{E}-01--2.00 \mathrm{E}-01$ $5.00 E-02--1.00 E-01$ 2. $00 \mathrm{E}-02--5.00 \mathrm{E}-02$ $M U=-1.0000$ (3.109E-06 $6.035 E-06$ $1.486 \mathrm{E}=04$ $4.491 E-06$ $5.329 E-05$ $2.275 \mathrm{E}-04$ $4.038 E-04$ $1.468 E-04$ $-9.136 \mathrm{E}-0 \mathrm{5}$ $1.1685 \mathrm{E}-03$
$1.6397 \mathrm{E}-03$ $1.685 E-03$
$2.697 E-03$ $2.697 E-03$
$1.911 E-02$ $1.911 \mathrm{E}-02$
$5.153 \mathrm{E}-02$ $1.606 \mathrm{E}-0$ $5.002 \mathrm{E}-02$

ANGLE 10 $M U=0.0950$ $6.549 \mathrm{E}-06$ $1.543 E-05$ $4.434 \mathrm{E}-04$ $2.651 \mathrm{E}-04$ $4.302 \mathrm{E}-04$ $4.306 E-04$ $6.719 E-04$ $5.010 \mathrm{E}-\mathrm{C} 4$ $5.856 E-04$ . $114 E-04$ $1.025 E-03$ $2.493 E-03$ $8.431 \mathrm{E}-0$ $1.318 \mathrm{E}-0$ $2.055 E-02$ $6.819 E-02$ $2.014 \mathrm{E}-01$
$5.520 \mathrm{E}-02$
ANGLE 2 $3.127 \mathrm{E}-06$ $7.015 E-06$ $1.554 E-04$ $6.5 C 4 E-05$ 6.5 6. . $3.788 E-04$ $3.035 \mathrm{E}-04$ $1.471 \mathrm{E}-04$ $1.471 \mathrm{E}-04$ $1.993 E-05$ $2.739 \mathrm{E}-03$ $2.739 E-03$
$1.917 \mathrm{~F}-02$ $5.165 E-02$ $1.609 \mathrm{E}-01$ 5.006E-02

ANGLE 11 $M U=0.2816$ $7.960 E-06$
$1.900 E-05$ $5.770 \mathrm{E}-04$ $3.713 E-04$ $5.815 E-04$ $5.734 E-04$ $8.545 E-04$ $6.406 F-04$ $7.851 \mathrm{E}-04$ $9.204 E-04$ $1.994 \mathrm{E}-03$ 4.242E-03 $1.053 \mathrm{E}-02$ $1.385 \mathrm{E}-02$ $2.172 \mathrm{E}-0$ $7.265 E-02$ $2.115 E-01$ $5.634 E-02$ $=-9.946$ 7.315E-OS $1.795 \mathrm{E}-04$ 1.656E-05 $1.276 E-04$ $2.650 \mathrm{E}-04$ $2.650 E-04$ $2.948 E-04$ $2.948 E-04$ $1.934 E-04$ $1.534 \mathrm{E}-04$
$1.388 \mathrm{E}-04$ $1.388 \mathrm{E}-04$
$1.728 \mathrm{E}-03$ $2.982 \mathrm{E}-03$ $2.982 E-03$
$1.940 E-02$ $5.214 E-02$ $1.621 \mathrm{E}-01$ $1.621 E-01$
$5.023 E-02$

ANGLE 12 $M U=0.4580$ $2.395 \mathrm{E}-05$ $7.583 \mathrm{E}-04$ $4.735 \mathrm{E}-04$ $7.358 \mathrm{E}-04$ $7.356 E-04$ $1.145 \mathrm{E}-03$ $9.947 E-04$ $1.393 E-03$ $1.964 \mathrm{E}-03$ $3.65 l E-03$ $6.328 E-03$ $1.236 \mathrm{E}-0$ $1.474 \mathrm{E}-02$ $2.325 \mathrm{E}-02$ $7.765 \mathrm{E}-0$ $2.224 \mathrm{E}-01$ $5.752 \mathrm{E}-02$ 3. $342 E-06$ $7.803 E-06$ $2.124 E-04$ . $250 E-04$ $2.050 E-04$ $1.943 E-04$ $3.018 \mathrm{E}-04$ $1.868 \mathrm{E}-0$ 2.001 E- 04 $8.377 E-05$ $3.858 \mathrm{E}-04$ $3.858 E-04$ $1.808 E-03$
$3.640 E-03$ $3.640 \mathrm{E}-03$
$1.967 \mathrm{E}-02$ $1.967 \mathrm{E}-02$
$5.304 \mathrm{E}-02$ $5.304 \mathrm{E}-0$
$1.644 \mathrm{E}-01$ $1.644 E-01$
$5.054 E-02$ ANGLE 13 $M U=0.6179$ $1.264 E-05$ $1.049 \mathrm{E}-03$ $6.222 \mathrm{E}-04$ $9.917 \mathrm{E}-04$ $1.064 \mathrm{E}-03$ $1.772 \mathrm{E}-03$ $1.84 J \mathrm{E}-03$ $2.718 E-03$ $3.860 \mathrm{E}-03$ $5.980 \mathrm{E}-03$ $8.478 E-03$ $1.384 \mathrm{E}-02$ $1.619 E-02$ $2.500 E-02$ $8.303 E-02$ $2.336 \mathrm{E}-\mathrm{O}$ 5.867E-02
ANGLE 5 3.550E-06 $8.372 E-06$ $2.378 \mathrm{E}-04$ $1.599 \mathrm{E}-04$ $2.482 E-04$ . $303 E-04$ $1.267 E-04$ 1. $729 \mathrm{E}-04$ $6.378 \mathrm{E}-05$ $2.321 \mathrm{E}-04$ $4.321 E-04$ $1.993 E-03$ $4.888 \mathrm{E}-03$ $1.982 \mathrm{E}-02$ $1.982 \mathrm{E}-02$
$5.434 \mathrm{E}-02$ $1.677 E-01$ $5.098 \mathrm{E}-02$

ANGLE 14 $M U=0.7550$ $1.634 \mathrm{E}-05$
$4.249 \mathrm{E}-05$ $1.643 E-03$ $1.040 \mathrm{E}-03$ $1.699 E-03$ $1.975 E-03$ $3.226 \mathrm{E}-03$ $3.609 E-0$ $5.061 E-03$ $6.603 E-03$ $8.712 \mathrm{E}-03$ 1. $\cap 47 E-02$ $1.522 \mathrm{E}-02$ $1.817 \mathrm{E}-02$ $2.689 \mathrm{E}-02$ $8.856 \mathrm{E}-02$ $2.447 \mathrm{E}-0$ $5.974 E-02$
ANGLE 6 3.841E-06 $9.025 \mathrm{E}-06$ $2.520 \mathrm{E}-04$ .. 2.468E-04 ..275E- 04 3.421E-04 . $1.502 E-04$ $2.502 E-04$ $4.968 \mathrm{E}-04$ $2.408 E-03$ $6.708 \mathrm{E}-03$ $1.978 \mathrm{E}-02$ $1.978 \mathrm{E}-02$
$5.509 \mathrm{E}-02$ $1.722 E-01$ 5.157F-02 ANGLE 15 $M=0.8656$ $2.113 \mathrm{E}-05$ . $3.102 \mathrm{E}-0$ 3.692E-03 $4.326 \mathrm{E}-03$ . $961 \mathrm{E}-03$ $1.139 E-02$ $1.693 \mathrm{E}-02$ $2.025 E-02$ 2.886E-02 $9.385 \mathrm{E}-02$ $2.547 \mathrm{E}-01$ $6.065 \mathrm{E}-02$

ANGLE 7 AN $=-0.4580 \quad$ ANGLE 8 $4.238 E-06$ . $863 E-06$ 2.114E-04 2.665E-04 ( $3.511 E-04$ 4. $721 E-04$ 
(GAMMAS/MEV/STERADIAN/SOURCE NEUTRON)

ENERGY GROUP (MEV) 8.00E OC- - -1.00E O1 6.50 E $00--8.00 E$ OO $4.00 E$ OO- $-6.50 E$ OO $3.00 E$ OO- 0 . 00 E 00 $2.50 E$ OO- $-3.00 E$ OO $2.00 \mathrm{~F} 00---2.50 \mathrm{E}$ OO $1.66 \mathrm{E} \quad 00---2.00 \mathrm{E}$ OO $1.33 \mathrm{E}$ OC- $-1.66 \mathrm{E}$ OO $1.00 \mathrm{E} \quad 00--1.33 \mathrm{E} \quad 00$ 8.00E-01---1.00E 00 $6.00 E-01---8.00 E-01$ $4.00 E-01---6.4$ $3.00 E-01--4.00 E-01$ $2.00 E-01--3.00 E-01$ 1.00E-01---2. $00 \mathrm{E}-01$ $5.00 E-02--1.00 E-01$
$2.00 E-02---5.00 E-02$ ENERGY GROUP (MEV) 8. DOE OO---1. OOE OI $6.50 E$ OO- - 8. OOE OO 5.0CE OO- - 6.50E OO $4.00 E$ OO-- . 3. COE OO- 4 . OOE OO 2.5OE OO- 3 . OOE DO 1.6OE OO- $2.00 E$ OO $1.33 E$ OO- $1.06 E$ OO $1.33 E$ OD -1.66 E 00 $1.00 E-01-1.33 E$ OD G. OOE $-01-1-2.00 E-O L$ $4.00 E-01-0-6.00 E-O 1$ $4.00 E-01-4.00 E-01$ 2. OOE $-01--3.00 E-O 1$ 1.0OE-01---2.0OE-O $5.00 \mathrm{E}-02--1.00 \mathrm{E}-01$ $2.00 E-02--5.00 E-02$
ANGLE 1 $M U=-1.0000$ $1.636 \mathrm{E}-06$ $3.445 \mathrm{E}-06$ $-1.949 E-05$ $-8.060 E-06$ $-3.029 E-06$ $7.339 E-05$ $1.071 E-04$ $1.834 \mathrm{E}-04$ $1.572 \mathrm{E}-04$ $3.697 \mathrm{E}-05$ $-9.399 E-05$ $7.997 E-04$ $1.246 \mathrm{E}-03$ $9.918 \mathrm{E}-03$ $2.599 \mathrm{E}-02$ $8.168 \mathrm{E}-02$ $2.539 E-02$ ANGLE 10 $M U=0.0950$ $3.818 \mathrm{E}-06$ $8.498 E-06$ 1. $095 E-04$ $1.090 E-04$ $1.734 E-04$ 1.681E-04 . $734 \mathrm{E}-04$ $1.776 E-04$ 1.976E-04 $4.906 \mathrm{E}-04$ $1.344 E-03$ $4.405 E-03$ $6.617 \mathrm{E}-03$ $1.019 \mathrm{E}-02$ $1.019 \mathrm{E}-02$
$3.425 \mathrm{E}-02$ $3.425 \mathrm{E}-02$
$1.021 \mathrm{E}-01$ $2.795 \mathrm{E}-02$ $M U=-0.9894 \quad M U=-0.9446 \quad M U=-0.8656$ $3.498 E-06 \quad 3.697 E-06 \quad 1.786 E-06$ $5.562 \mathrm{E}-05$ $-9.423 E-08$ $3.762 \mathrm{~F}-06$ $6.790 E-06$ $7.705 E-05$ $1.013 E-04$ $1.676 \mathrm{E}-04$ $1.392 \mathrm{E}-04$ $3.896 \mathrm{~F}-05$ $-5.985 \mathrm{E}-05$ $8.007 E-04$ $1.274 \mathrm{E}-03$ $9.946 E-03$ $2.605 \mathrm{E}-02$ $8.183 E-02$

ANGLE 11 $M U=0.2816$ $4.821 \mathrm{E}-\mathrm{C6}$ $1.080 E-05$ $2.483 E-04$ $1.596 E-04$ $2.407 E-04$ 2.251E-04 作 2.8 ..202E-04 1.2 $2 E-04$ $1.050 E-03$ . $434 \mathrm{E}-03$ $5.434 \mathrm{E}-03$ $6.858 \mathrm{E}-03$ $3.639 \mathrm{E}-02$ $3.639 \mathrm{E}-02$
$1.070 \mathrm{E}-01$ 2.851E-02
ANGLE 3 ANGLE 4

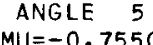
1. $911 \mathrm{E}-06$ 4.329E-06 9. $951 \mathrm{E}-05$ $6.845 E-05$ $1.541 \mathrm{E}-05 \quad 5.304 \mathrm{E}-05$ $8.936 E-05$ $8.158 \mathrm{E}-05$ 1.141E-04 $6.898 \mathrm{E}-05$ $4.864 E-05$ $3.646 \mathrm{E}-05$ $8.078 \mathrm{E}-04$ $1.425 \mathrm{E}-03$ $1.004 \mathrm{E}-02$ $2.630 \mathrm{E}-02$ $8.245 E-02$

ANGLE 12 $M U=0.4580$
$6.315 \mathrm{E}-06$ $.315 \mathrm{E}-06$
$1.412 \mathrm{E}-05$ $1.412 \mathrm{E}-05$
$3.261 \mathrm{E}-04$ $3.261 \mathrm{E}-04$ $1.930 E-04$ (2.092E-04 4 3 6.130E-04 (.). $1.034 \mathrm{E}-03$ $3.402 E-03$ $6.260 E-03$ $7.241 \mathrm{E}-03$ $142 \mathrm{E}-02$ $3.1476 \mathrm{E}-02$ $1.123 E-0$ ? $2.908 E-02$ ..036E-04 $5.110 E-05$ $1.678 E-05$ 6.945E-05 $1.703 E-04$ $8.371 E-04$ $1.810 E-03$ $1.014 E-02$ $2.675 \mathrm{E}-02$ $8.361 \mathrm{E}-02$ ANGLE 13 $M U=0.6179$
$8.597 \mathrm{E}-06$ $8.597 E-06$
$1.924 E-05$ $4.497 \mathrm{E}-04$ $2.365 \mathrm{E}-04$ .7 4.1 7.15IE- 04 $1.355 \mathrm{E}-03$ . $2.107 E-03$ $4.452 E-03$ $6.452 E-03$ $6.872 \mathrm{E}-03$ $1.219 \mathrm{E}-0$ 1.219E-02 $4.127 \mathrm{E}-02$
$1.177 \mathrm{E}-01$ 2. $963 \mathrm{E}-02$
$8.915 \mathrm{E}-05$

$1.109 E-04$ $5.061 \mathrm{E}-05$ $3.865 E-05$ $1.654 E-05$ $9.793 E-05$ $2.186 E-04$ 9. $314 \mathrm{E}-04$ 2. $509 \mathrm{E}-03$ 1. $014 \mathrm{E}-0$ ? 2.742E-02 $8.528 \mathrm{E}-02$ $2.588 \mathrm{E}-02$ ANGLE 14 $M U=0.7550$
$1.210 E-05$ $1.210 E-05$
$2.773 E-05$ $2.773 E-05$ $7.241 E-04$ $4.255 E-04$ 7.079E-04 1. $430 E-04$ $1.466 E-03$ $2.634 \mathrm{E}-03$ $3.586 \mathrm{E}-03$ $4.586 E-03$ 5. $346 E-03$ $7.421 \mathrm{E}-03$ $7.421 E-03$ $8.850 \mathrm{E}-03$ $1.299 \mathrm{E}-02$
$4.380 \mathrm{E}-02$ 1.? $29 E-01$ 3. $014 \mathrm{E}-02$
ANGLE 6 $2.081 \mathrm{E}-06$ 4.677E-06 $6.218 \mathrm{E}-05$ . $075 E-05$ $1.135 E-04$
$6.545 E-05$ $1.545 E-05$
$7.780 E-05$ $5.438 \mathrm{E}-05$ $1.233 E-04$ $2.025 \mathrm{E}-04$ $1.163 E-03$ $3.497 E-03$ $1.005 \mathrm{E}-02$ $2.830 \mathrm{E}-02$ $8.751 \mathrm{E}-02$
$2.617 \mathrm{E}-02$ ANGLE 15 $M U=0.8656$ $1.732 E-05$
$4.293 E-05$ $1.458 \mathrm{E}-03$ $.109 \mathrm{E}-0$ $1.767 E-03$ . $3.154 E-03$ $4.536 \mathrm{E}-03$ $5.261 \mathrm{E}-03$ 6. $6.060 \mathrm{E}-03$ $8.136 E-03$ $9.742 \mathrm{E}-03$ 1.382E-O2 4.616E-02 1.276E-01 $3.057 \mathrm{E}-02$

ANGLE 7 ANGLE 8 ANGLE 9 $M U=-0.4580$ $2.317 E-06$ $5.123 E-06$ $4.441 E-05$ $7.428 E-05$ $6.474 \mathrm{E}-05$ $1.212 \mathrm{E}-04$ $9.918 \mathrm{E}-05$ $1.367 \mathrm{E}-04$ $1.262 E-04$ $1.366 \mathrm{E}-04$ $1.744 \mathrm{E}-04$ $1.618 \mathrm{E}-03$ 4.600E-03 9. $890 \mathrm{E}-03$ $2.940 \mathrm{E}-02$ $9.030 E-02$ $2.653 \mathrm{E}-02$ ANGLE 16 $M U=0.9446$
$2.428 \mathrm{E}-05$ $2.428 \mathrm{E}-05$ $7.488 E-05$ $3.785 \mathrm{E}-03$ $3.213 E-03$ $4.69 \mathrm{E}-03$ 
IGAMMAS/MEV/STERADIAN/SOURCE NEUTRONI GROUP (MEV)

8.0OE OO---1.0OE OI 6.50E OO-- 8. 00 E 00 $5.00 E$ OO---6.50E 00 $3.00 E$ OO- $-4.00 E$ OO $2.50 E$ OO- $-3.00 E$ OO $2.00 E$ OO- - 2.50E 00 $1.66 \mathrm{E} 00--2.00 \mathrm{E} D 0$ $1.33 E 00--1.66 E 00$ $1.00 E$ O0--1.33E OO $8.00 E-01--1.00 E$ OO $6.00 E-01--8.00 E-01$ $4.00 E-01---6.00 E-01$ $3.00 E-01--4.00 E-01$ $2.00 E-01--3.00 E-01$ $1.00 E-01--2.00 E-01$ $5.00 E-02--1.00 E-01$
$2.00 F-02---5.00 E-02$

\section{ENERGY}

$$
\text { GROUP (MEV) }
$$

8.00E OO---1.00E 01 $6.50 E$ OO- -8.00 E 00 5.0 NE CO---6.5OE OO 4.0OF 00---5.0OE OO 3.00 E OO-- $4.00 E$ OO 2.50 E ON---3.0OE 00 2.00 E $00---2.50 E$ OO $1.66 E$ EO---2.00E OO 1.32 E $00--1.66 \mathrm{E}$ OO 1.00 E $00--1.33 E$ OO $8.00 E-01--1.00 E 00$ $6.00 E-01---8.00 E-01$ $4.00 E-01--6.00 E-01$ $3.00 E-01--4.00 E-01$ $2.00 E-01--3.00 E-01$ $1.00 E-01--2$. NOE-01 $5.00 E-02--1.00 E-01$
$2.00 E-02--5.00 E-02$
ANGLE 1
$M U=-1.0000$
ANGLE 2 7.060E-07 MU $=-0.9894$ $1.413 \mathrm{~F}-06 \quad 1.447 \mathrm{E}-06$ $1.502 \mathrm{E}-05 \quad 1.718 \mathrm{E}-05$ $-1.761 E-05 \quad-1.22 C E-05$ $-1.491 \mathrm{E}-05 \quad-8.371 \mathrm{E}-06$ $-1.080 E-05 \quad-5.521 E-06$ $2.299 E-05 \quad 2.437 E-C 5$ $4.740 E-05 \quad 4.362 E-05$ $7.362 E-05$ . $2.357 E-06$ $3.638 E-04$ 4.799E-03 $1.220 E-02$ $1.189 \mathrm{E}-02$ ANGLE 10 $M U=0.0950$ $1.801 \mathrm{E}-06$ .912E-06 $.379 E-05$ $4.460 E-05$ $6.905 E-05$ $6.380 E-05$ $8.357 \mathrm{E}-05$ $5.676 F-05$ $5.645 E-05$ $7.037 \mathrm{E}-05$ $2.339 E-04$ $6.735 \mathrm{E}-04$ $2.137 E-03$ $3.100 E-03$ $4.730 \varepsilon-03$ $1.601 \mathrm{E}-02$ 1.306E-02 2816 $2.348 E-06$ $5.123 \mathrm{E}-06$ $1.006 \mathrm{E}-04$ $6.727 E-05$ $9.671 \mathrm{E}-05$ $8.439 \mathrm{E}-05$ $1.027 \mathrm{E}-04$ $7.056 \mathrm{E}-05$ $9.318 \mathrm{E}-05$ $1.933 \mathrm{E}-04$ $5.280 \mathrm{E}-04$ $1.158 \mathrm{E}-03$ $2.602 E-03$ $3.175 E-03$ 1. $697 E-02$ $1.697 E-02$ $1.331 \mathrm{E}-02$
ANGLE 3 $\begin{array}{ccc}\text { ANGLE } & 3 & \text { ANGLE } \\ M U=-0.9446 & M U=-C .8656\end{array}$ $7.417 \mathrm{E}-07$ $1.569 E-06$ $2.465 \mathrm{E}-05$ $1.847 \mathrm{E}-06$ $8.756 E-06$ $8.500 E-06$ 2.890E-05 $3.063 \mathrm{E}-05$ $3.875 \mathrm{E}-05$ $2.622 E-05$ $1.240 \mathrm{E}-05$ $5.110 F-06$ $3.612 E-04$ $6.463 \mathrm{E}-04$ 4.844E-03 $1.235 \mathrm{E}-02$ $1.194 \mathrm{E}-02$ ANGLE 12
$M U=0.4580$

$3.211 \mathrm{E}-06$

$6.926 \mathrm{E}-06$

$7.450 \mathrm{E}-05$

1. $371 \mathrm{E}-04$

$9.675 E-05$

$1.450 \mathrm{E}-04$

$1.547 \mathrm{E}-04$

2.651 E- 04 $5.136 \mathrm{E}-04$ 1.001 E-03 $1.679 E-03$ $2.948 E-03$ $3.332 E-03$ 5 . $231 \mathrm{E}-02$ $5.231 \mathrm{E}-02$ 7.897E-07 $.744 E-06$ $3.393 E-05$ $2.197 E-05$ $3.307 E-05$ . $763 \mathrm{E}-05$ $3.359 E-05$ $1.478 E-05$ $9.463 E-06$ $-2.450 E-07$ $2.605 E-05$ $7.214 E-05$ $3.699 E-04$ $8.504 E-04$ $4.870 \mathrm{E}-03$ $1.257 \mathrm{E}-02$ $3.929 \mathrm{E}-02$ $1.201 \mathrm{E}-02$

\section{ANGLE 13} $M U=0.6179$ $9.895 E-06$ $1.795 \mathrm{E}-04$ $8.032 \mathrm{E}-05$ $1.306 \mathrm{E}-04$ $1.504 \mathrm{E}-04$ $2.812 \mathrm{E}-04$ $3.919 E-04$ $6.518 \mathrm{E}-04$ $1.066 E-03$ $1.611 \mathrm{E}-03$ $2.146 \mathrm{E}-03$ $3.182 E-03$ $3.639 E-03$ 1.5 $3.912 \mathrm{E}-02$ ..470F-C2
$A N G L E \quad 5$
$M=-0.7550$ $8.508 \mathrm{E}-07$ $1.906 \mathrm{E}-0 \mathrm{~S}$ $3.925 \mathrm{E}-05$ $2.826 \mathrm{E}-05$ $4.092 \mathrm{E}-05$ $3.281 E-05$ $3.509 E-05$ $1.012 \mathrm{E}-05$ $3.850 \mathrm{E}-06$ $-7.724 E-07$ .043E-05 . $167 E-05$ 1.209E-03 $1.209 E-03$ 4.846E-03 . $1.211 \mathrm{E}-02$ ANGLE 14 $M U=0.7550$ $7.122 \mathrm{E}-06$
$1.530 \mathrm{E}-05$ 1. $530 \mathrm{E}-05$ $2.996 E-04$ $1.615 \mathrm{E}-04$ 2. $826 \mathrm{E}-04$ 3.825E-04 $6.529 E-04$ 8. $907 E-04$ $1.297 \mathrm{E}-03$ $1.791 \mathrm{E}-03$ $2.246 E-03$ $2.511 E-03$ $3.387 E-03$ $4.045 E-03$ $5.909 E-03$ $2.021 E-02$ $5.699 \mathrm{E}-02$
ANGLE 6 $\begin{aligned} & M U=-0.6179 \\ & 9.291 \mathrm{E}-07\end{aligned}$ 2.050E-06 $3.959 E-05$ $2.332 E-05$ $3.460 E-05$ $2.701 E-05$ $3.492 E-05$ $1.892 E-05$ 2. $385 E-05$ $1.944 E-05$ 4.867E-05 $7.733 E-05$ $5.351 E-04$ $1.701 \mathrm{E}-03$ $4.766 E-03$ $1.329 E-02$ $4.109 E-02$ .225E-02

ANGLE 15

$M U=0.8656$ $2.568 \mathrm{E}-05$ $6.527 \mathrm{E}-04$ $5.134 \mathrm{E}-04$ $8.222 E-04$ $1.055 \mathrm{E}-03$ $1.493 \mathrm{E}-03$ $1.784 E-03$ 2.203E-03 $2.549 E-03$ $2.755 E-03$ 2.781E-03 $3.678 E-03$ $4.411 E-03$ $6.242 E-03$ $5.821 E-02$ $5.902 E-02$

ANGLE 7 $M U=-0.4580$ $1.037 \mathrm{E}-06$ $3.800 \mathrm{E}-05$ $9.098 E-06$ $2.138 \mathrm{E}-05$ $1.727 \mathrm{E}-05$ $3.764 \mathrm{E}-05$ $3.544 \mathrm{E}-05$ $5.489 E-05$ $5.324 E-05$ $4.822 \mathrm{E}-05$ $5.814 E-05$ $7.703 E-04$ $2.233 E-03$ $1.380 \mathrm{E}-02$ .241E-02 ANGLE 16 $M U=0.9446$ $4.734 E-05$ $4.734 E-05$ $1.637 \mathrm{E}-03$ . $2.623 \mathrm{E}-03$ $3.159 \mathrm{E}-03$ 
(GAMMAS/MEV/STERADIAN/SOURCE NEUTRON I

ENERGY GROUP (MEV) 8.0OE $00---1.00 F 01$ 6.5 5 EOE $00--8.00 E$ OO $5.00 E$
$4.00 E$ OO- $00--5.50 E$ OD $3.00 E$ OO- -4 . OOE 00 $2.50 E$ OO-- 3. 00 E 00 $2.00 E$ OD- - 2.5OE 00 1.66 E $00---2.00 E \quad 00$ $1.33 \mathrm{E} \quad 00---1.66 \mathrm{E} \quad 00$ $1.00 E$ OO- $-1.33 E 00$ $8.00 E-01--1.00 E$ OO $6.00 E-01--8.00 E-01$ $4.00 E-01---6.00 E-01$ $3.00 E-01-2-4 . C O E-01$ $? .00 \mathrm{E}-01--3.00 \mathrm{E}-01$ 1. OOE-01--2.00E-01 $2.00 \mathrm{E}-02---5.00 \mathrm{E}-02$

ENERGY GROUP (MEV)

8.0OE OO---1.00E 01 6.50E OO---8.00E 00 $5.00 E$ OO-- 6.5OE DO 4. $.00 E$ OO- - - 5. OOE OO 3.00 E OO- $-4.00 E$ OO 2.5OE OO---3.0OE OO $2.00 E$ OO- $2.50 E$ OO $1.66 \mathrm{E} \mathrm{OO---2.00E} \mathrm{OO}$ 1.33E DO- -1.66E 00 $1.00 E$ OO---1.33E OO $8.00 E-01--1.00 E$ OD $6.00 E-01-\cdots-8.00 E-01$ 4.00E-01 ---6.00E-01 $3.00 \mathrm{E}-01--4.00 \mathrm{E}-\mathrm{C}$ I.OOE OI- 3 . OOE-OI $5.00 E-02-1.00 E-1$ $5.00 E-C 2--1.00 E-01$
$2.00 E-02---5.00 E-02$
ANGLE 1 ANGLE 2 $2.635 E-07$ $2.678 E-07$ $4.904 E-07 \quad 5.678 E-07$ (2.989E-0 $-1.138 \mathrm{E}-05$ - $1.608 \mathrm{E}-06$ $7.361 \mathrm{E}-06 \quad 7.812 \mathrm{E}-06$ .150E-05 $3.256 E-05$ $3.075 \mathrm{E}-05 \quad 2.626 \mathrm{E}-05$ $-4.787 E-06 \quad-3.316 \mathrm{E}-06$ $-3.590 E-05 \quad-2.726 E-05$ $1.621 E-04 \quad 1.610 \mathrm{E}-04$ $2.334 E-04 \quad 2.41 \% E-04$ $2.238 E-03 \quad 2.242 \mathrm{E}-03$ 1.739E-02 $5.366 \mathrm{E}-03 \quad 5.370 \mathrm{E}-03$ ANGLE 10 $M U=0.0950$ ANGLE 11 $7.394 \mathrm{E}-07$ $1.594 \mathrm{E}-06$ $2.778 \mathrm{E}-05$ $1.827 \mathrm{E}-05$
$2.751 \mathrm{E}-05$ $2.751 \mathrm{E}-05$ $2.427 \mathrm{E}-05$ $2.765 \mathrm{E}-05$ 1. $553 \mathrm{E}-05$ $1.235 E-05$ $2.362 \mathrm{E}-05$ $1.076 E-04$ $3.233 E-04$ $9.967 E-04$ . $7.226 \mathrm{E}-03$ 2 $2.150 E-02$
$5.876 E-03$ $M U=0.2816$ $.147 E-08$ .8 .805E-C5 $3.826 \mathrm{E}-05$ $3.080 E-05$ $3.205 \mathrm{E}-05$ $1.885 \mathrm{E}-05$ $2.852 \mathrm{E}-05$ $8.618 E-05$ $2.541 E-04$ $5.557 E-04$ $1.199 E-03$ $1.423 \mathrm{E}-03$ $2.226 E-03$ $7.643 E-C 3$ 5. $985 E-03$
ANGLE 3 $M U=-0.9446$ $2.841 \mathrm{E}-07$ $5.849 E-07$ $7.951 E-06$
$1.387 E-07$ $1.387 E-07$
$1.114 E-06$ $1.357 E-06$ 1.35TE-06 $1.108 \mathrm{E}-05$ $1.453 \mathrm{E}-05$ $9.715 \mathrm{E}-06$ $1.685 E-06$ $7.682 \mathrm{E}-07$ $1.580 \mathrm{E}-04$ $2.856 \mathrm{E}-04$ $2.254 E-03$ $5.604 \mathrm{E}-03$ 1.755E-O2 $5.387 E-03$

ANGLE 12

ANGLE 4
$M U=-0.8656$ 3.090E-07 $6.827 \mathrm{E}-07$ $1.254 \mathrm{E}-05$ $1.254 E-05$ $1.310 \mathrm{E}-05$ $1.031 \mathrm{E}-05$ $1.043 E-05$ $2.34 \mathrm{JE}-06$ $-6.293 E-07$ $-2.090 \mathrm{E}-06$ 1. 001 E-05 $2.586 \mathrm{E}-05$ $1.598 \mathrm{E}-04$ $3.883 E-04$ $2.257 \mathrm{E}-03$ $5.703 \mathrm{E}-03$ $1.779 E-02$ $5.418 \mathrm{E}-03$ ANGLE 13 $M U=0.4580$ $1.406 \mathrm{E}-06$ .966E-O5 $2.728 \mathrm{E}-05$ $3.728 \mathrm{E}-05$ $.127 \mathrm{E}-05$ 4.764E-0 $5.938 \mathrm{E}-05$ $1.152 E-04$
$2.471 E-04$ $2.471 E-04$
$4.821 E-04$ $4.821 \varepsilon-04$ $7.920 E-04$ $1.339 \mathrm{E}-\mathrm{C}$ 1.485E-0 $2.350 E-03$ 2.340E-OP $6.094 \mathrm{E}-03$ $M U=0.6179$ $2.153 E-06$ $4.434 \mathrm{E}-06$ $6.651 E-05$ $2.311 \mathrm{E}-05$ 4. $060 \mathrm{E}-05$ $5.202 E-05$ $1.108 E-04$ 1. $780 \mathrm{E}-04$ $3.080 E-04$ $5.164 E-04$ $7.652 E-04$ $9.908 E-04$ $1.424 E-03$ $1.619 E-03$ $2.481 E-03$ 2. $451 E-02$ $6.200 \mathrm{E}-03$ $\triangle A N G L E \quad 5$ $3.357 \mathrm{E}-07$ $-3.657 E-06$ 7.567E-07 .486E-05 $1.486 E-05$ $1.610 E-05$ $1.211 \mathrm{E}-05$ $1.039 \mathrm{E}-05$ $3.211 \mathrm{E}-07$ $-3.084 \mathrm{E}-06$ .073E-05 $5.630 E-04$ $2.235 E-03$ 作 $1.813 E-02$
$5.464 \mathrm{E}-03$ ANGLE 14 $M=0.7550$ $3.599 E-06$ $7.418 \mathrm{E}-06$ $1.166 \mathrm{E}-04$ $5.680 E-05$ $1.095 \mathrm{E}-04$ $1.644 \mathrm{E}-04$ $2.907 \mathrm{E}-04$ $4.231 E-04$ $6.185 E-04$ $8.538 E-04$ $1.043 E-03$ $1.132 E-03$ $1.498 \mathrm{E}-03$ $1.794 E-03$ $2.612 E-03$ $2.54 B E-02$ $6.296 \mathrm{E}-03$
ANGLE 6 $3.655 \mathrm{E}-07$ $8.028 E-07$ $1.437 E-05$ 8. $322 \mathrm{E}-06$ 1.216E-05 $8.466 E-06$ $9.978 \mathrm{E}-06$ 4.341E-0 $5.043 E-06$ $6.706 \mathrm{E}-06$ $1.886 \mathrm{E}-05$ $2.653 \mathrm{E}-05$ $2.399 E-04$ $7.975 E-04$ $2.184 \mathrm{E}-0$ $6.027 E-03$ 1.859E-02 $5.522 \mathrm{E}-03$ ANŚLE 15

ANGLE 7 $4=-0.4580$ $8.537 \mathrm{E}-07$ $1.270 \mathrm{E}-0$ $7.756 E-07$ $8 \mathrm{E}-06$ 1.120E-05 E-OS ( .201E-05 $3.558 E-04$ $2.121 E-03$ $1.915 \mathrm{E}-0$ $5.594 E-03$ ANGLE 16 $M U=0.9446$ $1.378 \mathrm{E}-05$ $2.814 E-04$ $2.328 E-04$ $3.775 \mathrm{E}-04$ $4.997 \mathrm{E}-04$ $6.945 \mathrm{E}-04$ $8.466 \mathrm{E}-04$ 1.031E-03 1.244E 03 1.244E 03 ( (. 1.746E-O . $2.634 \mathrm{E}-02$ $6.377 \mathrm{E}-03$

ANGLE 8 ANGLE 9 $4.708 E-07 \quad 5.759 E-07$ $9.710 E-07 \quad 1.209 E-06$ $1.318 E-05 \quad 1.829 E-05$ 6.614E-07 5.813E-06 $4.360 \mathrm{E}-06 \quad 1.218 \mathrm{E}-05$ $4.389 E-06 \quad 1.227 E-05$ $1.560 E-05 \quad 2.211 E-05$ $2.087 E-05 \quad 2.095 E-05$ $3.047 E-052.365 E-05$ $2.521 E-05 \quad 1.787 E-05$ $3.627 E-05$ $4.520 E-05 \quad 1.463 E-04$ $5.344 E-04 \quad 7.603 E-04$ $1.362 E-03$ $2.073 E-03$ $6.853 E-03$ $\begin{array}{ll}1.983 E-02 & 2.062 E-02 \\ 5.678 E-03 & 5.773 E-03\end{array}$

ANGLE 17 
COSINE

75.0

100.0

150.0

RANGE (METERS)
$200 . \mathrm{C}$

$25 C .0$

300.0

400.0

-1. COCOOE CO $-9.89401 E-01$ $-8.44575 E-01$ $-7.55044 E-01$ $-6.17876 \mathrm{E}-01$ $-4.58017 E-01$ $-2.81605 \mathrm{E}-01$ $-2.81605 E-01$ $9.50125 \mathrm{E}-02$ $9.50125 \mathrm{E}-02$
$2.81605 \mathrm{E}-01$ $4.58017 \mathrm{E}-01$ $6.17876 \mathrm{E}-01$ $7.55044 E-C 1$ $8.65631 \mathrm{E}-01$ $9.44575 \mathrm{E}-01$ $9.894 \mathrm{ClE}-01$

TOTAL

$$
\begin{aligned}
& 7.898 E-11 \\
& 7.886 E-11 \\
& 7.812 E-11 \\
& 7.654 E-11 \\
& 7.703 E-11 \\
& 8.018 E-11 \\
& 8.679 E-11 \\
& 9.695 E-11 \\
& 1.101 E-10 \\
& 1.282 E-1 C \\
& 1.566 E-10 \\
& 1.961 E-10 \\
& 2.500 E-1 C \\
& 5.024 E-10 \\
& 1.194 E-C 9 \\
& 2.869 E-09 \\
& 1.459 E-08
\end{aligned}
$$
$1.214 \mathrm{E}-10$

$1.368 \mathrm{E}-10$ $1.444 E-10$ 1.212E-10 1.367E-10 $1.443 E-10$ $1.203 E-10 \quad 1.360 E-10 \quad 1.435 E-10$ $1.202 E-10$ $350 E-10$ $1.373 \mathrm{E}-10$ (2) $9.796 \mathrm{E}-11$ $1.061 E-1 C$ $1.186 \mathrm{E}-10$ $1.346 \mathrm{E}-10$ $1.875 E-10$ $1.713 E-10$ $1.713 E-1 C$
$2.310 E-1 C$ $2.718 \mathrm{E}-1 \mathrm{C}$ $4.486 \mathrm{E}-10$ $7.748 \mathrm{E}-10$ $2.668 \mathrm{E}-09$ $1.477 \mathrm{E}-08$

$6.054 E-09$

$5.982 E-C 9$

$1.256 E-10$

$1.359 E-10$

$1.514 E-10$

$1.712 \mathrm{E}-10$

2. $565 \mathrm{E}-10$

$2.148 \mathrm{E}-10$

$2.581 E-10$

$3.330 E-10$

$4.587 E-10$

$7.671 E-10$

$2.055 E-09$

$1.554 E-10$

$1.725 E-10$

$1.941 \mathrm{E}-10$

$2.127 \mathrm{E}-10$
$2.559 \mathrm{E}-10$

$2.872 \mathrm{E}-10$

$3.675 E-10$

$4.979 E-10$

$8.099 E-10$

$1.805 \mathrm{E}-09$

$1.180 E-08$

1.466E-10

$1.537 E-10$

$1.659 \mathrm{E}-1 \mathrm{C}$

$1.834 E-10$

$2.245 \mathrm{E}-10$

$2.703 \mathrm{E}-10$

$3.033 E-1 C$

$3.872 \mathrm{E}-10$

$5.197 \mathrm{E}-10$

$8.208 E-10$

$9.915 E=09$

$5.784 E-09$

$5.561 E-09$

$1.462 \mathrm{E}-10$

$1.379 E-10$

$-10 \quad 1.381 E-10$

$1.463 \mathrm{E}-10 \quad 1.394 \mathrm{E}-10$

$\begin{array}{ll}1.499 \mathrm{E}-10 & 1.430 \mathrm{E}-10 \\ 1.568 \mathrm{E}-10 & 1.500 \mathrm{E}-10\end{array}$

$1.692 \mathrm{E}-10 \quad 1.5014 \mathrm{E}-10$

$1.868 \mathrm{E}-10 \quad 1.773 \mathrm{E}-10$

$2.089 \mathrm{E}-10 \quad 1.974 \mathrm{E}-10$

$2.371 E-10 \quad 2.214 E-10$

$2.659 \mathrm{E}-10 \quad 2.521 \mathrm{E}-10$

$3.138 \mathrm{E}-10 \quad 2.945 \mathrm{E}-10$

$3.859 E-10 \quad 3.643 E-10$

$5.267 E-10 \quad 4.893 E-10$

$8.069 E-10 \quad 7.329 E-10$

$\begin{array}{ll}1.545 \mathrm{E}-\mathrm{C} 9 & 1.299 \mathrm{E}-09 \\ 8.257 \mathrm{E}-\mathrm{C} 9 & 5.640 \mathrm{E}-\mathrm{C} 9\end{array}$

$5.903 E-09$

$5.268 \mathrm{E}-\mathrm{Cg}$

$4.524 E-09$

$500 \cdot C$

600.0

RANGE
9ON.C

1200.0

1.210E-10 1. $211 E-10$ 1. $215 \mathrm{E}-10$ $1.231 E-1 C$ $1.265 \mathrm{E}-10$ $1.328 \mathrm{E}-10$ $1.426 \mathrm{E}-10$ $1.561 E-10$ $1.732 \mathrm{E}-10$ $1.942 \mathrm{E}-10$ $2.207 \mathrm{E}-10$ $2.583 \mathrm{E}-10$ $3.184 E-10$ $4.239 E-10$ $6.219 E-10$ 3. $820 \mathrm{E}-\mathrm{C}$

$3.727 \mathrm{E}-\mathrm{Cg}$

$1.011 E-10$
$1.012 E-1 C$ $1 .(18 E-10$ $1.033 \mathrm{E}-1 \mathrm{C}$ 1. $C 64 \mathrm{E}-1 \mathrm{C}$ $1.117 \mathrm{E}-10$ $1.197 E-10$ $1.308 \mathrm{E}-1 \mathrm{C}$ $1.447 \mathrm{E}-1 \mathrm{C}$ $1.62 \mathrm{CE}-10$ $1.842 \mathrm{E}-10$

$2.155 \mathrm{E}-10$

$2.650 \mathrm{E}-10$

$3.497 \mathrm{E}-1 \mathrm{C}$

$5.042 \mathrm{E}-10$ $8.230 E-10$ $2.577 \mathrm{E}-\mathrm{C}$

$2.976 E-O 9$

\section{$4.911 E-11$}

$4.962 E-11$
$15 C C \cdot C$

1800.0

$.014 E-11 \quad 7.4 C 3 E-12$

$2.034 E-11$

$2.077 E-11$

$7.485 E-12 \quad 2.555 E-12$

$7.651 \mathrm{E}-12-2.613 \mathrm{E}-12$

$2.148 E-11 \quad 7.919 E-12 \quad 2.706 E-12$

$5.483 E-11 \quad 2.256 E-11 \quad 8.317 E-12 \quad 2.842 E-12$

$6.370 E-11$ 2.61CE-11 $9.597 E-12 \quad 3.274 E-12$

$7.019 E-11$ 2.869E-11 $1.053 E-11 \quad 3.589 E-12$

$1.173 E-11-3.994 E-12$

$1.007 E-11$ 3.631E-11 1.33 CE-11 $4.523 E-12$

$1.041 E-104.233 E-11 \quad 1.546 E-11 \quad 5.246 E-12$

$1.269 E-10$ 5.123E-11 $1.86 C E-11 \quad 6.276 E-12$

$1.639 E-10$ 6.5C3E-11 2.331E-11 $7.788 E-12$

$2.267 E-10 \quad 8.729 E-11 \quad 3.060 E-11 \quad 1.006 E-11$

$\begin{array}{llll}3.430 E-10 & 1.255 E-10 & 4.24 C E-11 & 1.356 E-11 \\ 7.832 E-10 & 2.354 \mathrm{E}-10 & 6.993 \mathrm{E}-11 & 2.050 \mathrm{E}-11\end{array}$

$1.326 E-09 \quad 5.16 .7 E-10 \quad 1.84 C E-10 \quad 6.136 E-11$
TOTAL 


COSINE
$-1.00000 E O Q$
$-9.89401 E-01$
$-9.44575 E-01$
$-8.65631 E-C 1$
$-7.55044 E-01$
$-6.17876 E-01$
$-4.58017 E-01$
$-2.81605 E-01$
$-9.50125 E-02$
$9.50125 E-02$
$2.81605 E-01$
$4.58017 E-01$
$6.17876 E-01$
$7.55044 E-01$
$8.65631 E-01$
$9.44575 E-01$
$9.89401 E-01$

TOTAL

COSINE

$$
\begin{array}{r}
-1.000 C 0 E C C \\
-9.89401 \mathrm{E}-\mathrm{C} 1 \\
-9.44575 \mathrm{E}-01 \\
-8.65631 \mathrm{E}-01 \\
-7.55044 \mathrm{E}-01 \\
-6.17876 \mathrm{E}-01 \\
-4.58017 \mathrm{E}-01 \\
-2.81605 \mathrm{E}-01 \\
-9.50125 \mathrm{E}-02 \\
9.50125 \mathrm{E}-02 \\
2.81605 \mathrm{E}-01 \\
4.58017 \mathrm{E}-01 \\
6.17876 \mathrm{E}-01 \\
7.55044 \mathrm{E}-01 \\
8.05631 \mathrm{E}-01 \\
9.44575 \mathrm{E}-01 \\
9.89401 \mathrm{E}-01
\end{array}
$$

TOTAL

$\begin{array}{cc}75.0 & 1 C 0.0 \\ 1.083 E-10 & 1.328 E-1 C \\ 1.081 E-10 & 1.326 E-1 C \\ 1.073 E-10 & 1.316 E-1 C \\ 1.052 E-10 & 1.292 E-1 C \\ 1 . C 60 E-10 & 1.304 E-10 \\ 1.104 E-10 & 1.359 E-1 C \\ 1.195 E-10 & 1.471 E-1 C \\ 1.335 E-10 & 1.642 E-10 \\ 1.516 E-10 & 1.864 E-1 C \\ 1.764 E-10 & 2.592 E-10 \\ 2.153 E-1 C & 2.371 E-10 \\ 2.713 E-10 & 3.191 E-10 \\ 3.497 E-1 C & 3.771 E-10 \\ 7.140 E-10 & 6.289 E-10 \\ 1.712 E-C 9 & 1.099 E-C 9 \\ 4.139 E-C 9 & 3.835 E-09 \\ 2.121 E-C 8 & 2.144 E-08 \\ 8.665 E-C 9 & 8.529 E-C 9\end{array}$

\section{0}

$1.915 \mathrm{E}-1 \mathrm{C}$ $1.917 \mathrm{E}-10$ $1.924 \mathrm{E}-10$ $1.949 \mathrm{E}-10$ $2.001 E-1 C$ $2.233 E-10$ $2.425 \mathrm{E}-10$ $2.668 \mathrm{E}-10$ $2.964 t-10$ $3.336 \mathrm{E}-1 \mathrm{C}$ $3.858 \mathrm{E}-10$ $4.693 \mathrm{E}-10$ $6.160 E-10$ $8.931 E-10$ $1.500 \mathrm{E}-\mathrm{C} 9$ $5.491 E-C 9$

$5.537 E-C 9$

600.0
RANGE (MEIERS)
$2 C C .0$

$25 \mathrm{C.C}$

300.0

400.0

\section{$1.704 \mathrm{E}-10 \quad 1.955 \mathrm{E}-10$}

$\begin{array}{ll}1.703 E-10 & 1.954 E-10 \\ 1.693 E-10 & 1.947 E-10\end{array}$

$1.696 \mathrm{E}-10 \quad 1.936 \mathrm{E}-10$

$1.770 \mathrm{E}-10 \quad 2.060 \mathrm{E}-10$

$1.913 \mathrm{E}-10$

$2.126 E-10$

$2.757 \mathrm{E}-10$

$3.015 E-10$

$3.610 E-10$

$4.041 E-10$

$6.967 \mathrm{E}-10$

$1.135 \mathrm{E}-09$

$.560 E-09$

$1.978 \mathrm{E}-68 \quad 1.706 \mathrm{E}-08 \quad 1.432 \mathrm{E}-08$

$2.101 E-10$

$2.097 \mathrm{E}-10$

$2.095 E-10$

$2.14 C E-10$

$2.239 E-10$

$2.408 \mathrm{E}-10$

$2.652 E-10$

$2.96 \mathrm{CE}-10$

3.223E-10

$3.856 E-1 C$

4.310E-1C

$5.469 E-1 C$

$7.314 E-1 C$

$2.931 \mathrm{E}-09 \quad 2.560 \mathrm{E}$

$7.957 E-09$

$2.165 E-1 C$

$2.165 \mathrm{E}-10$

$2.163 \mathrm{E}-10$

$2.172 \mathrm{E}-10$

$2.225 \mathrm{E}-10$

$2.323 \mathrm{E}-10$

$2.496 E-10$

$2.741 E-10$

$3.04 \mathrm{~F}-10$

$3.442 E-10$

.

.

7. $455 E-10$

. $185 \mathrm{E}-\mathrm{C} 9$

1.19OE-C8

$8.397 E-09$

$8.242 E-09$

$7.582 \mathrm{E}-\mathrm{C} 9$

$2.113 \mathrm{E}-1 \mathrm{C}$

$2.115 \mathrm{E}-10$

$2.118 E-10$

$2.140 \mathrm{E}-10$

$2.193 \mathrm{E}-10$

$2.294 E-10$

$2.455 E-10$

$2.679 E-10$

$2.961 E-10$

$3.726 \mathrm{E}-10$

. $726 E-1 C$

$5.278 \mathrm{E}-10$

$7.016 \mathrm{E}-10$

$1.043 E-C 9$

$1.843 E-09$

$6.608 E-09$

RANGE (METERS)

900.0

1200.0

1500.0

1800.0
$8.648 E-11$
$8.664 E-11$

$8.887 \mathrm{E}-113.87 \mathrm{CE}-1$

$9.146 \mathrm{E}-11$

$9.546 \mathrm{E}-11$

1. $C 87 E-10$

$1.183 \mathrm{E}-10$

1. $302 \mathrm{E}-10$

$1.456 \mathrm{E}-10$

$1.670 E-10$

$1.992 \mathrm{E}-10$

$2.509 E-10$

3. $389 E-10$

5.C $27 E-10$

$1.134 \mathrm{E}-09$

$3.87 C E-11$

$3.985 E-11$

$4.390 \mathrm{CE}-11$

$4.699 E-11$

$5.090 \mathrm{E}-11$

$5.582 \mathrm{E}-11$

$6.217 E-11$

7.086E-11

$8.353 E-11$

$1.343 \mathrm{E}-10$

$1.881 \mathrm{E}-10$

$3.706 E-09$

$2.112 E-C 9$
$1.446 \mathrm{E}-11$

$1.45 C E-11$

$1.492 \mathrm{E}-11$

$1.536 \mathrm{E}-11$

$1.536 E-11$

$1.60 C E-11$

$1.8 \cap 2 \mathrm{E}-11$

$1.946 \mathrm{E}-11$

$2.128 \mathrm{E}-11$

$2.363 E-11$

$2.679 \mathrm{E}-11$

3.13CE-11

.799E-11

4.83 CE- 1

$1.042 \mathrm{E}-10$

$3.181 E-10$

$5.114 \mathrm{E}-12$

$5.126 \mathrm{E}-12$

$5.177 \mathrm{E}-12$
$5.278 \mathrm{E}-12$

$5.434 \mathrm{E}-12$

$5.434 \mathrm{E}-12$
$5.658 \mathrm{E}-12$

$5.959 \mathrm{E}-12$

$6.351 E-12$

$6.846 \mathrm{E}-12$

$7.470 \mathrm{E}-12$

$8.271 E-12$

$9.341 \mathrm{E}-12$

$.084 \mathrm{E}-11$

$1.300 \mathrm{E}-11$

$1.622 \mathrm{E}-1$

3.105E-11

$1.093 \mathrm{E}-10$ 
4 PI R**2 TISSUE KERMA INEUTRONSI
ICM**2 ERGS/GRAM/STERADIAN/SOURCE NEUTRONI

COS INE

$-1.00000 E$ OC
$-9.89401 \mathrm{E}-01$
$-9.44575 \mathrm{E}-01$
$-8.65631 \mathrm{E}-01$
$-7.55044 \mathrm{E}-01$
$-6.17876 \mathrm{E}-01$
$-4.58017 \mathrm{E}-01$
$-2.81605 \mathrm{E}-01$
$-9.50125 \mathrm{E}-02$
$9.50125 \mathrm{E}-02$
$2.81605 \mathrm{E}-01$
$4.58017 \mathrm{E}-01$
$6.17876 \mathrm{E}-01$
$7.55044 \mathrm{E}-01$
$8.65631 \mathrm{E}-01$
$9.44575 \mathrm{E}-01$
$9.89401 \mathrm{E}-01$

TOTAL

COSINE

$-1.00000 \mathrm{E} C \mathrm{CO}$ $-9.89401 \mathrm{E}-01$ $-9.44575 \mathrm{E}-01$

$-8.65631 E-01$ $-7.55044 E-01$ $-6.17876 E-01$ $-4.58017 \mathrm{E}-01$ $-9.8105 \mathrm{E}-01$ $-9.50125 \mathrm{E}-02$ $9.50125 E-02$ $2.81605 E-01$ $6.17876 \mathrm{E}-01$ $6.17876 \mathrm{E}-01$ $7.55044 E-91$ $8.65631 E-91$ $9.89401 \mathrm{E}-01$

TOTAL
75.0

100.0

$9.793 \mathrm{E}-09$ $9.778 E-C 9$ $9.693 \mathrm{E}-\mathrm{C} 9$ $9.512 \mathrm{E}-09$ $9.995 \mathrm{E}-\mathrm{CO}$ $1.083 E-08$ $1.210 \mathrm{E}-08$ $1.374 \mathrm{E}-08$ $1.915 \mathrm{E}-08$ $1.75 \mathrm{CE}-08$ $2.359 \mathrm{E}-\mathrm{C} 8$ $2.785 E-C 8$ $4.628 \mathrm{E}-08$ $8.051 \mathrm{E}-\mathrm{C} 8$ $2.795 E-07$
$1.556 \mathrm{E}-\mathrm{C} 6$ $6.231 E-C 7$

2.57

$5.228 E-08$

3. $12 \mathrm{E}-\mathrm{C} 7$

1. $539 E-06$

$6.325 \mathrm{E}-07$

500.0

$1.270 \mathrm{E}-\mathrm{C} 8$

$1.271 \mathrm{E}-\mathrm{C} 8$

$1.275 \mathrm{E}-\mathrm{C} 8$

$1.292 \mathrm{E}-08$

$1.328 E-C 8$

$1.393 \mathrm{E}-\mathrm{C} 8$

$1.493 E-C 8$

$1.632 \mathrm{E}-\mathrm{C8}$

$1.808 \mathrm{E}-\mathrm{C} 8$

$2.294 \mathrm{E}-\mathrm{CB}$

$2.294 \mathrm{E}-\mathrm{CB}$

3.

. $294 \mathrm{E}-08$

$4.378 E-C 8$

6. $423 E-C 8$

1. $3.995 \mathrm{E}-\mathrm{C})$

$$
600.0
$$

$1.069 E-08$

$1.07 \mathrm{CE}-\mathrm{C} 8$
$1.076 \mathrm{C}-\mathrm{CB}$

$1.0765-08$
$1.092 \mathrm{E}-C 8$

$1.092 E-C 8$

$1.124 E-08$

$1.179 E-08$

$1.262 E-08$

$1.375 E-08$

$1.519 E-C 8$

$1.923 E-08$

$2.244 E-C 8$

2.751E-C8

$3.621 \mathrm{E}-\mathrm{C} 8$
$5.216 \mathrm{E}-\mathrm{C} 8$

$5.216 E-C 8$

$8.521 E-C 8$
$2.693 E-C 7$

$3.877 \mathrm{E}-\mathrm{C} 7$

$3.105 E-C 7$

$\begin{array}{ccccc}150.0 & \text { RANGE (METERS) } & & & \\ 200.0 & 25 C .0 & 300.0 & 400.0 \\ 1.241 E-08 & 1.404 E-08 & 1.488 E-08 & 1.512 E-C 8 & 1.437 E-08 \\ 1.239 E-08 & 1.403 E-08 & 1.487 E-08 & 1.512 E-C 8 & 1.438 E-08 \\ 1.231 E-08 & 1.396 E-08 & 1.483 E-08 & 1.509 E-C 8 & 1.439 E-08 \\ 1.215 E-08 & 1.387 E-08 & 1.480 E-08 & 1.514 E-08 & 1.454 E-08 \\ 1.231 E-C 8 & 1.411 E-08 & 1.512 E-08 & 1.551 E-C 8 & 1.490 E-08 \\ 1.285 E-08 & 1.476 E-08 & 1.584 E-08 & 1.623 E-C 8 & 1.563 E-08 \\ 1.391 E-C 8 & 1.596 E-C 8 & 1.709 E-08 & 1.749 E-C 8 & 1.679 E-08 \\ 1.549 E-08 & 1.77 C E-08 & 1.888 E-08 & 1.928 E-08 & 1.842 E-08 \\ 1.752 E-08 & 1.992 E-08 & 2.115 E-08 & 2.155 E-08 & 2.048 E-08 \\ 2.114 E-08 & 2.182 E-08 & 2.308 E-08 & 2.444 E-C 8 & 2.294 E-08 \\ 2.197 E-08 & 2.623 E-08 & 2.778 E-08 & 2.738 E-08 & 2.608 E-08 \\ 2.642 E-08 & 2.943 E-08 & 3.114 E-08 & 3.228 E-C 8 & 3.042 E-C 8 \\ 3.408 E-08 & 3.767 E-08 & 3.975 E-08 & 3.967 E-08 & 3.756 E-08 \\ 4.7 C 5 E-C 8 & 5.110 E-08 & 5.339 E-08 & 5.416 E-08 & 5.042 E-C 8 \\ 7.915 E-08 & 8.341 E-08 & 8.454 E-08 & 8.313 E-08 & 7.559 E-08 \\ 2.143 E-07 & 1.875 E-07 & 1.723 E-07 & 1.599 E-C 7 & 1.344 E-07 \\ 1.437 E-06 & 1.240 E-C 6 & 1.041 E-06 & 8.664 E-C 7 & 5.907 E-07 \\ 6.129 E-07 & 5.996 E-07 & 5.761 E-07 & 5.459 E-C 7 & 4.694 E-07\end{array}$

RANGE (METERS)

$$
900.0 \quad 1200 . \mathrm{C}
$$

1500.0

$5.286 E-C 9$ $2.200 E-09$ $5.41 \mathrm{E}-\mathrm{CO}$ (5) (5. 6.880 6.807E-C9 $7.478 E-09$ 8. $9.422 \mathrm{E}-09$ $1.096 \mathrm{E}-08$ $1.331 E-08$ $2.71 C E-08$ $2.357 E-08$
$3.561 E-08$ $8.169 \mathrm{E}-08$ $2.222 \mathrm{E}-09$ $2.268 \mathrm{E}-09$ $2.343 \mathrm{E}-09$ $2.456 \mathrm{E}-09$ $2.615 \mathrm{E}-09$ $2.826 E-09$ $3.095 \mathrm{E}-09$ $3.436 E-09$ $3.883 E-09$ $5.416 E-09$ . $6.835 E-C 9$ $1.308 \mathrm{E}-08$ $1.308 E-08$
$2.456 E-08$

$5.499 E-08$
$8.164 \mathrm{E}-10$ $8.185 \mathrm{E}-10$ $8.273 E-1 C$ $8.450 E-10$ $8.735 E-10$ $9.156 \mathrm{E}-10$ $9.736 \mathrm{E}-10$ $1.050 E-09$ $1.148 E-09$ $1.272 \mathrm{E}-09$ $1.435 E-09$ $1.658 E-09$ $1.981 \mathrm{E}-09$ $2.465 E-09$ $4.436 \mathrm{E}-09$ $4.436 \mathrm{E}-09$

$1.974 E-08$ 
COSINE

$-1.00000 E 00$ $-9.89401 E-01$ $-9.44575 \mathrm{E}-01$ $-8.65631 \mathrm{E}-01$ $-7.55044 \mathrm{E}-01$ $-6.17876 E-01$ $-4.58017 E-01$ $-2.81605 \mathrm{E}-01$ $-9.50125 \mathrm{E}-02$ $9.50125 \mathrm{E}-02$ $2.816 \mathrm{C} 5 \mathrm{E}-01$ 4. $58017 \mathrm{E}-01$ $6.17876 \mathrm{E}-01$ $7.55044 \mathrm{E}-01$ $8.65631 \mathrm{E}-01$ $9.44575 \mathrm{E}-01$
$9.89401 \mathrm{E}-01$

TOTAL

COS INE

$-1.00000 E$ OO $-9.89401 E-01$ $-9.44575 E-01$ $-7.55044 E-01$ $-6.17876 \mathrm{E}-0$ $2.81605 \mathrm{E}-01$ $-9.50125 \mathrm{E}-02$ $9.50125 \mathrm{E}-0$ $2.81605 \mathrm{E}-01$ 4.58017E-01 $6.17876 \mathrm{E}-01$ $7.55044 E-01$ $8.65631 \mathrm{E}-01$ $9.65631 \mathrm{E}-01$ $9.89401 E-01$

TOTAL
75.0

$4.067 E-11$ 4.064E-11 $4.028 \mathrm{E}-11$ $3.928 \mathrm{E}-11$ 3.957E- 11 $4.143 E-11$ 4. $537 E-11$ 5.153E-11 $5.958 \mathrm{E}-11$ $7.034 E-11$ $8.722 E-11$

$1.142 \mathrm{E}-1 \mathrm{O}$

$1.543 \mathrm{E}-10$

$3.390 E-10$

$8.463 E-10$

$2.094 \mathrm{E}-\mathrm{Cg}$

1.C97E-C8

$4.206 \mathrm{E}-\mathrm{C} 9$

500.0

$4.825 E-11$ $4.828 \mathrm{E}-11$ $4.890 \mathrm{E}-11$ $5.032 \mathrm{E}-11$ $5.320 \mathrm{E}-11$ $5.320 E-11$ $6.754 E-11$ $7.295 \mathrm{E}-11$ $8.308 \mathrm{E}-11$ $9.585 \mathrm{E}-11$ $1.146 \mathrm{E}-10$ $1.470 E-10$ $2.089 \mathrm{E}-10$ $3.334 \mathrm{E}-10$ $6.207 \mathrm{E}-10$ $2.649 \mathrm{E}-09$

$1.895 E-09$
100.0

4.801E-11 4.796E-11 $4.750 E-11$ $4.633 E-11$ $4.673 E-11$ $4.898 \mathrm{E}-11$ $5.375 E-11$ $6.120 E-11$ $7.087 \mathrm{E}-11$ $1.022 \mathrm{E}-10$ $9.351 \mathrm{E}-11$ $1.305 \mathrm{E}-1 \mathrm{C}$ $1.551 \mathrm{E}-10$ $2.798 E-1 C$ $5.165 \mathrm{E}-1 \mathrm{C}$ $1.914 \mathrm{E}-\mathrm{C} 9$ $4.024 E-09$

\section{0} $3.937 E-11$ $3.94 C E-11$
$3.953 E-11$ . $4.132 \mathrm{E}-11$ $4.365 \mathrm{E}-11$ $4.738 \mathrm{E}-11$ . (5. $6.718 \mathrm{E}-11$ $7.749 \mathrm{E}-11$ $9.270 \mathrm{E}-11$ $1.188 \mathrm{E}-10$ $1.675 \mathrm{E}-1 \mathrm{C}$ $2.633 \mathrm{E}-10$ $4.740 E-10$ 1. $751 \mathrm{E}-09$

$1.452 \mathrm{E}-09$
150.0 RANGE 2 (METERS)
$2 \mathrm{CO} 0.0$

250.0

300.0

$400 . C$ 5.764E-11 $.711 E-11$ $6.242 \mathrm{E}-11$ $6.289 E-11$ $5.680 E-11$ $.600 E-11 \quad 6.121 E-1$ $5.676 \mathrm{E}-11 \quad 6.236 \mathrm{E}-11$ (2)E-11 $6.287 E-11$ $5.681 E-11$ $6.556 E-11 \quad 7.221 E-11$ $.457 \mathrm{E}-11 \quad 8.191 \mathrm{E}-11$ $1.036 \mathrm{E}-10$ $1.13 \mathrm{CE}-10$ $1.361 \mathrm{E}-10$ $1.807 E-10$ $2.641 E-10$ $4.731 E-10$ $1.413 \mathrm{E}-\mathrm{C}$ $1.013 E-C 8$

$.424 E-11$ 1. $663 \mathrm{E}-10$ $1.274 \mathrm{E}-10$ $1.475 \mathrm{E}-10$ $1.926 \mathrm{E}-10$ $2.770 E-10$ $4.825 E-10$
$1.193 E-09$ 1.16

$3.761 E-09$

$3.528 E-09$

$7.47 \mathrm{CE}-11$ $8.438 \mathrm{E}-11$

$9.666 \mathrm{E}-11$

$1.086 \mathrm{E}-10$

$1.305 \mathrm{E}-10$

$1.511 \mathrm{E}-10$

$1.973 \mathrm{E}-10$

$2.819 E-10$

$4.776 \mathrm{E}-10$

$1.067 \mathrm{E}-09$

$2245-09$

$3.261 E-09$

$5.669 E-11$ $6.255 E-11 \quad 5.718 E-11$ $5.871 E-11$ $6.768 \mathrm{E}-11 \quad 6.206 \mathrm{E}-11$ $7.593 \mathrm{E}-11$ $8.626 \mathrm{E}-11$ $1.102 E-10 \quad 9.847 E-11$ $1.138 \mathrm{E}-10$ $1.511 E-10 \quad 1.357 E-10$ $1.928 \mathrm{E}-10 \quad 1.742 \mathrm{E}-10$ $2.786 E-10-2.492 E-10$ $4.612 E-10-4.048 E-10$ $9.692 \mathrm{E}-10 \quad 7.883 \mathrm{E}-10$ $2.982 E-09 \quad 2.414 E-09$

RANGE (METERS) 900.0 1200.0

1500.0

$1800 . \mathrm{C}$

$2.732 E-12$ $9.373 \mathrm{E}-13$ $\begin{array}{llll}1.840 E-11 & 7.442 E-12 & 2.737 E-12 & 9.394 \mathrm{E}-13 \\ 1.853 \mathrm{E}-11 & 7.507 \mathrm{E}-12 & 2.763 \mathrm{E}-12 & 9.487 \mathrm{E}-13\end{array}$ $1.886 \mathrm{E}-11 \quad 7.650 \mathrm{E}-12 \quad 2.818 \mathrm{E}-12 \quad 9.678 \mathrm{E}-13$ $1.948 \mathrm{E}-11 \quad 7.905 \mathrm{E}-12 \quad 2.911 \mathrm{E}-12 \quad 9.693 \mathrm{E}-13$ 2.212E-11 $8.904 \mathrm{E}-12 \quad 3.260 \mathrm{E}-12 \quad 12114 \mathrm{E}-12$ $2.212 \mathrm{E}-11 \quad 9.705 \mathrm{E}-12 \quad 3.534 \mathrm{E}-12 \quad 1203 \mathrm{E}-12$ 2.704E-11 $1.073 E-11 \quad 3.887 E-12 \quad 1.318 E-12$ $3.051 E-11 \quad 1.2 C 4 E-11 \quad 4.342 E-12 \quad 1.467 E-12$ $3.511 \mathrm{E}-11 \quad 1.38 \mathrm{CE}-11 \quad 4.959 \mathrm{E}-12 \quad 12069 \mathrm{E}-12$ $4.194 \mathrm{E}-11 \quad 1.542 \mathrm{E}-11 \quad 5.867 \mathrm{E}-12 \quad 12065 \mathrm{E}-12$ $5.333 \mathrm{E}-11 \quad 2.067 \mathrm{E}-11 \quad 7.312 \mathrm{E}-12 \quad 2.427 \mathrm{E}-12$

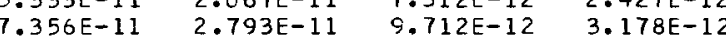

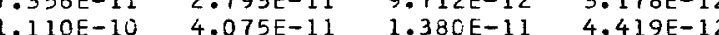
$\begin{array}{llll}1.856 E-10 & 6.463 E-11 & 2.099 E-11 & 6.507 E-12\end{array}$ $4.998 \mathrm{E}-10 \quad 1.415 \mathrm{E}-10 \quad 3.986 \mathrm{E}-11 \quad 1.116 \mathrm{E}-11$

$5.929 E-10 \quad 2.189 E-10 \quad 7.536 E-11 \quad 2.462 E-11$ 


\begin{tabular}{|c|c|c|c|c|c|c|}
\hline $\operatorname{COSINE}$ & 75.0 & \multicolumn{3}{|c|}{$\begin{array}{c}\text { RANGE (METERS) } \\
200.0\end{array}$} & 250.0 & 300.0 \\
\hline $\begin{array}{l}-1.00000 \mathrm{E} 00 \\
-9.89401 \mathrm{E}-01 \\
-9.44575 \mathrm{E}-01 \\
-8.65631 \mathrm{E}-01 \\
-7.55044 \mathrm{E}-01 \\
-6.17876 \mathrm{E}-01 \\
-4.58017 \mathrm{E}-01 \\
-2.81605 \mathrm{E}-01 \\
-9.50125 \mathrm{E}-02 \\
9.50125 \mathrm{E}-02 \\
2.81605 \mathrm{E}-01 \\
4.58017 \mathrm{E}-01 \\
6.17876 \mathrm{E}-01 \\
7.55044 \mathrm{E}-01 \\
8.65631 \mathrm{E}-01 \\
9.44575 \mathrm{E}-01 \\
9.89401 \mathrm{E}-01\end{array}$ & $\begin{array}{l}1.628 \mathrm{E}-09 \\
1.028 \mathrm{E}-09 \\
1.021 \mathrm{E}-09 \\
1.001 \mathrm{E}-09 \\
1.009 \mathrm{E}-\mathrm{C} 9 \\
1.052 \mathrm{E}-\mathrm{C} 9 \\
1.140 \mathrm{E}-09 \\
1.276 \mathrm{E}-09 \\
1.454 \mathrm{E}-09 \\
1.697 \mathrm{E}-09 \\
2.075 \mathrm{E}-09 \\
2.646 \mathrm{E}-09 \\
3.476 \mathrm{E}-09 \\
7.279 \mathrm{E}-\mathrm{C9} \\
1.768 \mathrm{E}-\mathrm{C} 8 \\
4.312 \mathrm{E}-\mathrm{C} 8 \\
2.231 \mathrm{E}-07\end{array}$ & $\begin{array}{l}1.252 E-C 9 \\
1.251 E-C 9 \\
1.242 E-C 9 \\
1.220 E-C 9 \\
1.232 E-09 \\
1.284 E-C 9 \\
1.392 E-C 9 \\
1.559 E-09 \\
1.775 E-09 \\
2.492 E-C 9 \\
2.274 E-C 9 \\
3 . C 81 E-C 9 \\
3.682 E-C 9 \\
6.266 E-C 9 \\
1.116 E-08 \\
3.975 E-08 \\
2.251 E-C 7\end{array}$ & $\begin{array}{l}1.580 \mathrm{E}-09 \\
1.579 \mathrm{E}-09 \\
1.571 \mathrm{E}-09 \\
1.551 \mathrm{E}-09 \\
1.573 \mathrm{E}-09 \\
1.644 \mathrm{E}-09 \\
1.780 \mathrm{E}-09 \\
1.987 \mathrm{E}-09 \\
2.253 \mathrm{E}-09 \\
2.737 \mathrm{E}-09 \\
2.839 \mathrm{E}-09 \\
3.439 \mathrm{E}-09 \\
4.453 \mathrm{E}-09 \\
6.222 \mathrm{E}-09 \\
1.671 \mathrm{E}-08 \\
3.001 \mathrm{E}-08 \\
2.071 \mathrm{E}-07\end{array}$ & $\begin{array}{l}1.781 \mathrm{E}-09 \\
1.780 \mathrm{E}-09 \\
1.774 \mathrm{E}-09 \\
1.762 \mathrm{E}-09 \\
1.795 \mathrm{E}-09 \\
1.879 \mathrm{E}-09 \\
2.032 \mathrm{E}-09 \\
2.26 \mathrm{E}-09 \\
2.550 \mathrm{E}-09 \\
2.799 \mathrm{E}-09 \\
3.386 \mathrm{E}-09 \\
3.805 \mathrm{E}-09 \\
4.9 C 9 \mathrm{E}-09 \\
6.731 \mathrm{E}-09 \\
1.118 \mathrm{E}-08 \\
2.592 \mathrm{E}-08 \\
1.782 \mathrm{E}-07\end{array}$ & $\begin{array}{l}1.879 \mathrm{E}-09 \\
1.879 \mathrm{E}-09 \\
1.875 \mathrm{E}-09 \\
1.872 \mathrm{E}-09 \\
1.914 \mathrm{E}-09 \\
2.006 \mathrm{E}-09 \\
2.166 \mathrm{E}-09 \\
2.398 \mathrm{E}-09 \\
2.695 \mathrm{E}-09 \\
2.947 \mathrm{E}-09 \\
3.57 \mathrm{E}-09 \\
4.009 \mathrm{E}-09 \\
5.158 \mathrm{E}-09 \\
7.006 \mathrm{E}-09 \\
1.129 \mathrm{E}-08 \\
2.362 \mathrm{E}-08 \\
1.491 \mathrm{E}-07\end{array}$ & $\begin{array}{l}1.902 \mathrm{E}-\mathrm{C} 9 \\
1.902 \mathrm{E}-\mathrm{C} 9 \\
1.9 C O \mathrm{E}-\mathrm{C} 9 \\
1.907 \mathrm{E}-\mathrm{C} 9 \\
1.955 \mathrm{E}-\mathrm{C} 9 \\
2.045 \mathrm{E}-\mathrm{C} 9 \\
2.207 \mathrm{E}-\mathrm{C} 9 \\
2.439 \mathrm{E}-09 \\
2.735 \mathrm{E}-09 \\
3.114 \mathrm{E}-\mathrm{C} 9 \\
3.499 \mathrm{E}-\mathrm{C} 9 \\
4.143 \mathrm{E}-09 \\
5.126 \mathrm{E}-\mathrm{C} 9 \\
7.084 \mathrm{E}-\mathrm{C} 9 \\
1.106 \mathrm{E}-\mathrm{C} 8 \\
2.178 \mathrm{E}-\mathrm{C} 8 \\
1.236 \mathrm{E}-\mathrm{C} 7\end{array}$ \\
\hline TOTAL & $8.903 E-C 8$ & $8.683 E-C 8$ & $8.415 E-C 8$ & $8.138 E-08$ & $7.744 E-08$ & $7.277 \mathrm{E}-\mathrm{C} 8$ \\
\hline COSINE & 500.0 & 600.0 & $\begin{array}{l}\text { RANGE I } \\
9 C 0.0\end{array}$ & $\begin{array}{l}\text { TERSI } \\
120 \mathrm{C} .0\end{array}$ & $150 \mathrm{C} .0$ & 1800.0 \\
\hline $\begin{array}{l}-1.00000 E ~ 00 \\
-9.89401 E-01 \\
-9.44575 E-01 \\
-8.65631 E-01 \\
-7.55044 E-01 \\
-6.17876 E-01 \\
-4.58017 E-01 \\
-2.81605 E-01 \\
-9.50125 E-02 \\
9.50125 E-02 \\
2.81605 E-01 \\
4.58017 E-01 \\
6.17876 E-01 \\
7.55044 E-01 \\
8.65631 E-01 \\
9.44575 E-01 \\
9.89401 E-01\end{array}$ & $\begin{array}{l}1.573 E-C 9 \\
1.575 E-C 9 \\
1.580 E-C 9 \\
1.602 E-C 9 \\
1.646 E-C 9 \\
1.728 E-09 \\
1.855 E-C 9 \\
2.033 E-09 \\
2.259 E-09 \\
2.537 E-09 \\
2.888 E-09 \\
3.388 E-C 9 \\
4.199 E-09 \\
5.652 E-C 9 \\
8.436 E-C 9 \\
1.461 E-C 8 \\
5.623 E-08\end{array}$ & $\begin{array}{l}1.315 E-09 \\
1.317 E-09 \\
1.324 E-09 \\
1.344 E-C 9 \\
1.384 E-C 9 \\
1.452 E-C 9 \\
1.557 E-C 9 \\
1.701 E-C 9 \\
1.885 E-09 \\
2.113 E-09 \\
2.406 E-C 9 \\
2.821 E-09 \\
3.486 E-09 \\
4.648 E-C 9 \\
6.812 E-09 \\
1.137 E-08 \\
3.765 E-08\end{array}$ & $\begin{array}{l}6.399 \mathrm{E}-10 \\
6.411 \mathrm{E}-10 \\
6.467 \mathrm{E}-10 \\
6.588 \mathrm{E}-10 \\
6.801 \mathrm{E}-10 \\
7.139 \mathrm{E}-10 \\
7.628 \mathrm{E}-10 \\
8.290 \mathrm{E}-10 \\
9.137 \mathrm{E}-10 \\
1.021 \mathrm{E}-\mathrm{C} 9 \\
1.160 \mathrm{E}-09 \\
1.358 \mathrm{E}-09 \\
1.662 \mathrm{E}-\mathrm{C} 9 \\
2.163 \mathrm{E}-09 \\
3.033 \mathrm{E}-\mathrm{C} 9 \\
4.68 \mathrm{CE}-09 \\
1.121 \mathrm{E}-08\end{array}$ & $\begin{array}{l}2.626 \mathrm{E}-10 \\
2.632 \mathrm{E}-10 \\
2.658 \mathrm{E}-10 \\
2.713 \mathrm{E}-10 \\
2.805 \mathrm{E}-10 \\
2.944 \mathrm{E}-10 \\
3.14 \mathrm{CE}-10 \\
3.402 \mathrm{E}-10 \\
3.738 \mathrm{E}-10 \\
4.166 \mathrm{E}-10 \\
4.728 \mathrm{E}-10 \\
5.514 \mathrm{E}-10 \\
6.692 \mathrm{E}-10 \\
8.550 \mathrm{E}-10 \\
1.161 \mathrm{E}-09 \\
1.697 \mathrm{E}-\mathrm{C} 9 \\
3.311 \mathrm{E}-09\end{array}$ & $\begin{array}{l}9.677 \mathrm{E}-11 \\
9.701 \mathrm{E}-11 \\
9.808 \mathrm{E}-11 \\
1.002 \mathrm{E}-10 \\
1.037 \mathrm{E}-10 \\
1.088 \mathrm{E}-10 \\
1.159 \mathrm{E}-10 \\
1.253 \mathrm{E}-10 \\
1.374 \mathrm{E}-10 \\
1.529 \mathrm{E}-10 \\
1.733 \mathrm{E}-10 \\
2.015 \mathrm{E}-10 \\
2.427 \mathrm{E}-10 \\
3.057 \mathrm{E}-10 \\
4.052 \mathrm{E}-10 \\
5.696 \mathrm{E}-10 \\
9.702 \mathrm{E}-10\end{array}$ & $\begin{array}{l}3.309 \mathrm{E}-11 \\
3.317 \mathrm{E}-11 \\
3.355 \mathrm{E}-11 \\
3.431 \mathrm{E}-11 \\
3.550 \mathrm{E}-11 \\
3.726 \mathrm{E}-11 \\
3.966 \mathrm{E}-11 \\
4.284 \mathrm{E}-11 \\
4.691 \mathrm{E}-11 \\
5.214 \mathrm{E}-11 \\
5.899 \mathrm{E}-11 \\
6.838 \mathrm{E}-11 \\
8.190 \mathrm{E}-11 \\
1.020 \mathrm{E}-10 \\
1.328 \mathrm{E}-10 \\
1.813 \mathrm{E}-10 \\
2.813 \mathrm{E}-1 \mathrm{C}\end{array}$ \\
\hline TOTAL & $5.041 E-C 8$ & $3.998 E-08$ & $1.761 E-C 8$ & $6.822 E-09$ & $2.422 E-09$ & $8.069 E-10$ \\
\hline
\end{tabular}


4 PI R**2 AIR KERMA INEUTRONS)
(CM**2 ERGS/GRAMISTERADIAN/SOURCE NEUTRI

COSINE

$-1.00000 E$ OC $-9.894 \mathrm{C} 1 \mathrm{E}-01$ $-9.44575 \mathrm{E}-01$ $-8.65631 \mathrm{E}-01$ $-7.55044 \mathrm{E}-01$ $-6.17876 \mathrm{E}-\mathrm{Cl}$ - $.58017 E-01$ $-2.81605 E-01$ $-9.50125 \mathrm{E}-02$
$9.50125 \mathrm{E}-02$ $9.50125 \mathrm{E}-02$ $2.81605 E-01$ $4.58017 E-01$ 6.17876E-C1 $7.55044 E-01$ $8.65631 E-01$ $9.44575 E-01$
$9.89401 E-01$

TOTAL

\section{COS INE}

$-1.00600 E 00$ $-9.89401 \mathrm{E}-01$ $-9.44575 \mathrm{E}-01$ $-7.55044 \mathrm{E}-01$ $-6.17876 \mathrm{E}-01$ $-4.58017 E-01$ $-2.81605 E-01$ $-9.50125 E-02$ $9.50125 \mathrm{E}-02$ $2.81605 E-01$ 4.58017E-01 $6.17876 E-01$ $7.55044 E-01$ $8.65631 \mathrm{E}-01$ $9.44575 E-C 1$
$9.89401 E-01$

TOTAL

$\begin{array}{cc}75.0 & 100.0 \\ 1.784 E-C 9 & 2.134 E-C 9 \\ 1.781 E-09 & 2.130 E-C 9 \\ 1.761 E-C 9 & 2.106 E-C 9 \\ 1.720 E-C 9 & 2 . C 57 E-09 \\ 1.726 E-C 9 & 2.068 E-09 \\ 1.795 E-C 9 & 2.154 E-C 9 \\ 1.945 E-C 9 & 2.339 E-09 \\ 2.180 E-09 & 2.625 E-C 9 \\ 2.486 E-09 & 2.996 E-C 9 \\ 2.905 E-C 9 & 4.226 E-09 \\ 3.567 E-C 9 & 3.845 E-C 9 \\ 4.501 E-09 & 5.248 E-C 9 \\ 5.793 E-09 & 6.193 E-09 \\ 1.184 E-C 8 & 1.041 E-C 8 \\ 2.843 E-C 8 & 1.822 E-C 8 \\ 6.864 E-C 8 & 6.365 E-C 8 \\ 3.507 E-C 7 & 3.547 E-C 7 \\ & \\ 1.433 E-07 & 1.404 E-C 7\end{array}$

$1.433 E-07$

500.0

2. $217 E-C 9$ 2. $218 \mathrm{E}-\mathrm{C} 9$ $2.220 \mathrm{E}-\mathrm{C} 9$ $2.302 \mathrm{E}-\mathrm{C} 9$ $2.422 \mathrm{E}-\mathrm{CO}$ $2.617 \mathrm{E}-09$ $2.889 \mathrm{E}-\mathrm{C} 9$ $3.235 E-09$ $3.658 \mathrm{E}-\mathrm{C} 9$ $4.200 \mathrm{E}-\mathrm{C} 9$ $4.988 \mathrm{E}-\mathrm{C} 9$ $6.299 E-C 9$ $8.681 E-C 9$ $1.327 \mathrm{E}-\mathrm{C} 8$ $2.341 \mathrm{E}-\mathrm{C} 8$
$8.976 \mathrm{E}-\mathrm{C} 8$

$7.621 E-08$

600.0

$1.804 E-09$

$1.805 \mathrm{E}-\mathrm{C} 9$

$1.810 \mathrm{E}-\mathrm{C}$

$.983 E-C 9$

$.626 E-09$
RANGE (METERS)
$20 \mathrm{C} .0$

150.0

$20 \mathrm{C} .0$
$2.848 E-09$

$2.6 C 4 E-C 9$

$2.599 E-09$

$2.843 \mathrm{E}-09$

$2.529 E-09$

$2.555 E-C 9$

$2.670 E-09$

$2.902 \mathrm{E}-\mathrm{C} 9$

$3.255 E-09$

$3.706 E-09$

$4.497 E-09$

$5.698 \mathrm{E}-\mathrm{C} 9$

$7.434 E-09$

$1.041 \mathrm{~F}-\mathrm{C} 8$

$1.773 E-08$

$4.860 E-08$

$3.274 E-07$

$2.823 \mathrm{E}-09$

$2.789 E-09$

$2.832 \mathrm{E}-09$

$2.968 E=09$

$3.226 E-09$

$3.6 C 9 E-C 9$

$4.095 E-09$

4.519E-09

$5.475 E-09$

$6.198 E-09$

$1.111 \mathrm{E}-08$

$1.849 \mathrm{E}-08$

$4.229 E-08$
$2.824 E-07$

$1.310 E-07$

1.361E-C 7

250.0

300.0

400.0

$2.925 \mathrm{E}-09$ $2.922 \mathrm{E}-09$
$2.905 \mathrm{E}-09$ 2. $905 \mathrm{E}-09$ $2.886 \mathrm{E}-09$ $2.943 E-09$ $3.09 \mathrm{CE}-09$ 3. $355 E-09$ 3.741E-09 4. $231 \mathrm{E}-09$ $4.652 E-09$ $5.657 E-09$ ..405E 09 $8.313 \mathrm{E}-09$ $1.142 E-08$ $3.861 \mathrm{E}-08$ 2. $369 \mathrm{E}-07$

2.890E-C9 $2.887 \mathrm{E}-\mathrm{C} \quad 2.614 \mathrm{E}-09$ $2.614 \mathrm{E}-09$ $2.608 \mathrm{E}-09$ $2.626 \mathrm{E}-09$ $3.030 \mathrm{E}-\mathrm{C} 2.69 \mathrm{E}-09$ 3.080E-C $2.829 E-09$ 3. $723 E-C 9$ (3.062E-09 $4.202 E-C O$. $3.315 E-09$ 4.813E-09 4.317E-09 $5.441 E-C O \quad 4.963 E=C 9$ $6.497 \mathrm{E}-\mathrm{C} 9 \quad 5.876 \mathrm{E}-09$ $8.135 \mathrm{E}-09 \quad 7.426 \mathrm{E}-09$ $1.140 E-08 \quad 1.029 E-08$ $1.800 E-C 8 \quad 1.598 E-08$ $3.555 E-C 8 \quad 2.941 E-C 8$ $1.968 \mathrm{E}-\mathrm{C} 7 \quad 1.336 \mathrm{E}-07$

$1.150 E-C 7 \quad 9.545 E-08$

RANGE (METERS)

$1.236 \mathrm{E}-07$

$150 \mathrm{C} .0$

1800.0

$8.316 \mathrm{E}-10$

$12 \mathrm{CO} 0$

1. $209 \mathrm{E}-10$

$4 \cdot 116 \mathrm{E}-11$

$3.327 E-10$

$1.212 \mathrm{E}-10$
$1.223 \mathrm{E}-10$

$1.246 \mathrm{E}-10$

$1.286 \mathrm{E}-10$

$3.525 E-10$

$1.348 \mathrm{E}-10$

$1.435 \mathrm{E}-10$

$9.232 \mathrm{E}-10$

$1.435 \mathrm{E}-10$
$1.553 \mathrm{E}-1 \mathrm{C}$

1.08E-CO $3.951 E-10$

$1.705 \mathrm{E}-10$

$4.730 E-10$

$1.9 C 2 E-10$

$5.295 E-1 C$

$2.168 \mathrm{E}-10$

$1.545 \mathrm{E}-09$

$6 . C 58 E-10$

$2.554 \mathrm{E}-10$

4.C47E-C9 $1.835 \mathrm{E}-09 \quad 7.168 \mathrm{E}-10$

$2.300 E-C 9 \quad 8.908 E-10$

$3.146 E-10$

$\begin{array}{ll}1.176 \mathrm{E}-09 & 4.09 \mathrm{CE}-10 \\ 1.657 \mathrm{E}-09 & 5.629 \mathrm{E}-10\end{array}$

$6.993 \mathrm{E}-\mathrm{C} 9 \quad 3.091 \mathrm{E}-09 \quad 1.176 \mathrm{E}-09$

$\begin{array}{lllll}1.801 E-C 8 & 7.163 E-09 & 2.517 E-09 & 8.225 E-10 & 2.562 E-10 \\ 6.000 E-08 & 1.762 E-08 & 5.100 E-09 & 1.461 E-09 & 4.145 E-10\end{array}$

$\begin{array}{lllll}1.801 E-C 8 & 7.163 E-09 & 2.517 E-09 & 8.225 E-10 & 2.562 E-10 \\ 6.000 E-08 & 1.762 E-08 & 5.100 E-09 & 1.461 E-09 & 4.145 E-10\end{array}$

$4.125 \mathrm{E}-11$

$4.165 \mathrm{E}-11$

$4.382 \mathrm{E}-11$

4. $587 \mathrm{E}-11$

$4.874 \mathrm{E}-1$

$5.257 E-11$

5.753E-11

$7.273 E-11$

$8.527 E-11$

$1.042 \mathrm{E}-10$

$1.338 \mathrm{E}-10$

$4.145 E-10$

$2.465 E-08$

$9.182 E-09$

$3.174 E-09$

$1.039 E-C 9$ 
COSINE

\section{-1. OOOCCE OO $-9.89401 \mathrm{E}-01$ \\ $-8.65631 \mathrm{E}-01$ \\ $-7.55044 E-01$ \\ $-6.17876 \mathrm{E}-01$ \\ $-4.58017 E-01$ \\ $-2.81605 E-01$ \\ $-9.50125 E-02$ \\ $9.50125 \mathrm{E}-02$ \\ $2.81605 E-01$ \\ 4. $58017 \mathrm{E}-01$ \\ $6.17876 \mathrm{E}-01$ \\ $7.55044 E-01$ \\ $8.65631 \mathrm{E}-01$ \\ $9.44575 \mathrm{E}-\mathrm{O}$ \\ $9.89401 \mathrm{E}-01$}

TOTAL

COS INE

$-1.00000 E 00$ $-9.89401 \mathrm{E}-01$

$-9.44575 E-01$

$-8.65631 \mathrm{E}-01$

$-7.55044 \mathrm{E}-01$

$-6.17876 E-01$

$-4.58017 E-01$

$-2.81605 E-01$

$-9.50125 E-02$

$9.50125 \mathrm{E}-02$

$2.81605 E-01$

$4.58 C 17 \mathrm{E}-01$

6. $17876 \mathrm{E}-01$

$7.55044 E-01$

$8.65631 E-01$

$9.89401 E-01$

TOTAL

\section{0}

$1.842 \mathrm{E}-1 \mathrm{O}$

$1.869 E-10$

$1.918 \mathrm{E}-1 \mathrm{C}$

1. $895 \mathrm{E}-10$

$1.973 \mathrm{E}-10$

$2.118 \mathrm{E}-10$

$2.388 \mathrm{E}-10$

$2.834 \mathrm{E}-10$

$3.459 E-10$

$4.258 \mathrm{E}-10$

5. $363 \mathrm{E}-10$

$8.428 \mathrm{E}-10$

$1.422 \mathrm{E}-09$

$3.886 \mathrm{E}-\mathrm{C} 9$

$1.062 \mathrm{E}-\mathrm{C} 8$

$2.775 E-08$
$1.538 E-07$

$5.140 E-C 8$

100.0

$2.123 \mathrm{E}-1 \mathrm{C}$

$2.153 \mathrm{E}-10$
$2.21 \mathrm{CE}-1 \mathrm{C}$

$2.185 \mathrm{E}-1 \mathrm{C}$

$2.277 E-10$

$2.442 \mathrm{E}-1 \mathrm{C}$

$2.755 E-10$

$3.281 \mathrm{E}-1 \mathrm{C}$

4. $024 E-10$

$6.417 E-10$

$6.417 E-10$
$8.843 E-10$
$.082 E-10$

$.682 E-10$
$.174 E-C 9$

$2.654 \mathrm{E}-\mathrm{C} 9$

$5.768 \mathrm{E}-09$

$2.458 \mathrm{E}-\mathrm{C} 8$

1. $536 \mathrm{E}-07$

$4.661 E-08$

500.0

600.0

$1.863 E-10$

$1.876 \mathrm{E}-10$

$1.901 \mathrm{E}-10$

$1.970 E-10$

2.061E-10

$2.222 E-10$

$2.496 \mathrm{E}-10$

2.921E-10

$3.494 E-10$

4. $187 E-10$

$5.036 \mathrm{E}-10$

6. $315 E-10$

..816E-10

$1.432 E-C 9$

$2.678 E-C 9$

$3.275 E-08$

$1.478 E-C 8$

$1.072 E-C 8$
150.0 RANGE
200.0

250.0

300.0

$400 . c$

$2.472 E-10$

$2.503 \mathrm{E}-10$
$2.568 \mathrm{E}-10$

$2.554 \mathrm{E}-10$

$2.671 E-10$

$2.625 E-10$

$2.654 E-10$
$2.717 E-10$

$2.717 E-10$
$2.720 E-10$

$2.720 E-10$
$2.855 E-10$

$2.855 E-10$
$3.068 E-10$

$3.068 \mathrm{E}-10$

$4.123 E-10$

$.852 \mathrm{E}-10$

$5.036 \mathrm{E}-10$

$5.958 \mathrm{E}-10$

$7.556 \mathrm{E}-10$

9. $333 E-10$

$8.756 \mathrm{E}-10$

$1.279 \mathrm{E}-09$

2.C $86 \mathrm{E}-09$

$4.485 \mathrm{E}-09 \quad 4.267 \mathrm{E}-09$

$1.316 \mathrm{E}-08$

1. 170 E-07

$2.637 \mathrm{E}-10$

$2.663 \mathrm{E}-10$
$2.717 \mathrm{E}-10$

$2.738 \mathrm{E}-10$

$2.889 E-10$

$3.113 \mathrm{E}-10$

. $506 E-10$

$.149 E-10$

5.04CE-10

$5.936 E-10$

$.564 E-10$
$.038 \mathrm{E}-10$

$1.282 \mathrm{E}-09$

$2.079 E-09$

$4.123 E-09$

$1.118 \mathrm{E}-08$
$9.595 \mathrm{E}-08$

$4.018 E-0.8$

$3.530 E-C 8$

$3.086 E-08$

$2.558 \mathrm{E}-10$

$2.581 \mathrm{E}-10$

$.618 \mathrm{E}-10$

$2.672 E-10$

$3.015 E-10$

$3.411 \mathrm{E}-10$

$4.037 E-10$

4. $897 \mathrm{E}-10$

$5.976 \mathrm{E}-10$

$7.099 \mathrm{E}-10$

8. $905 \mathrm{E}-10$

$1.226 \mathrm{E}-\mathrm{C} 9$

$2.020 E-09$

3. $912 \mathrm{E}-\mathrm{C}$

$9.812 E-09$
$7.796 E-08$

$2.690 E-08$

$.249 E-10$

$.266 E-10$

$2.375 E-10$

$2.483 E-10$

$2.673 \mathrm{E}-1.0$

3.009E-10

$3.541 \mathrm{E}-10$

.265E-10

$.132 E-10$

.182E-10

$.710 E-10$

$1.074 E-09$

..334E-C9

$7.650 E-0 S$

$5.071 \mathrm{E}-08$

$2.011 E-08$

1800.0

$\begin{array}{lllll}1.485 E-1 C & 6.500 E-11 & 2.485 E-11 & 8.717 E-12 & 2.875 E-12\end{array}$

$\begin{array}{lllll}1.494 E-1 C & 6.529 E-11 & 2.496 E-11 & 8.753 E-12 & 2.887 E-12\end{array}$

$\begin{array}{lllll}1.516 E-1 C & 6.647 E-11 & 2.540 E-11 & 8.907 E-12 & 2.937 E-12\end{array}$

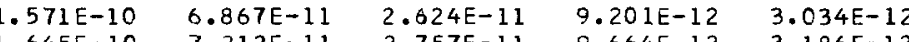

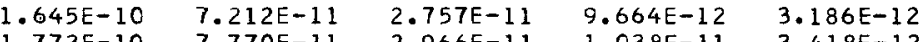

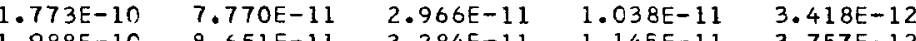

1.98BE-1C $8.651 E-11$ 3.284E-11 $1.145 E-11$ 3.757E-12

2.315E-1C $9.90 E-11$ 3.739E-11 $1.295 E-11$ 4.229E-12

2. $1.165 E-10$ 4.3

1.378E-10 5.105E-11 $1.749 E-11$ 5.669E-12

2.65e

$6.953 E-1 C \quad 2.932 E-10 \quad 1.086 E-10 \quad 3.694 E-11 \quad 1.6387 E-11$

2.651E-10 1.685E-10 $5.638 \mathrm{E}-11 \quad 1.1786 \mathrm{E}-11$

$2.067 \mathrm{E}-09 \quad 8.223 \mathrm{E}-10 \quad 2.880 \mathrm{E}-10 \quad 9.367 \mathrm{E}-11 \quad 2.901 \mathrm{E}-11$

$\begin{array}{lllll}4.365 E-C 9 & 1.615 E-09 & 5.361 E-10 & 1.671 E-10 & 4.999 E-11 \\ 2.108 E-08 & 5.6 C 1 E-09 & 1.486 E-09 & 3.948 E-10 & 1.049 E-10\end{array}$

$3.849 E-09$

$4.135 E-10$

$1.275 E-10$ 


COSINE
$-.0000 C E O 0$
$9.89401 E-01$
$9.44575 E-01$
$-8.65631 E-01$
$-7.55044 E-01$
$6.17876 E-01$
$4.58017 E-01$
$-2.81605 E-01$
$9.50125 E-02$
$9.50125 E-02$
$2.81605 E-01$
$4.58017 E-01$
$6.17876 E-01$
$7.55044 E-01$
$8.65631 E-01$
$9.44575 E-01$
$9.89401 E-01$

TOTAL

\section{COSINE}

$-1.00000 E$ OC $-9.89401 \mathrm{E}-01$ $-9.44575 E-01$ $-8.65631 E-01$ $-7.55044 E-01$ $-6.17876 \mathrm{E}-01$ $-2.81605 \mathrm{E}-01$ $-2.81605 E-01$ $9.50125 \mathrm{E}-02$ 2.81605E-C1 4.58017E-01 $6.17876 \mathrm{E}-01$ $7.55044 \mathrm{E}-01$ $8.65631 \mathrm{E}-\mathrm{C} 1$ $9.89401 \mathrm{E}-01$

TOTAL

$\begin{array}{lc}75.0 & 100.0 \\ 1.001 \mathrm{E}-10 & 1.225 \mathrm{E}-10 \\ 1.001 \mathrm{E}-10 & 1.224 \mathrm{E}-10 \\ 9.937 \mathrm{E}-11 & 1.216 \mathrm{E}-10 \\ 9.756 \mathrm{E}-11 & 1.195 \mathrm{E}-1 \mathrm{C} \\ 9.842 \mathrm{E}-11 & 1.207 \mathrm{E}-10 \\ 1.025 \mathrm{E}-10 & 1.259 \mathrm{E}-1 \mathrm{C} \\ 1.110 \mathrm{E}-10 & 1.363 \mathrm{E}-10 \\ 1.241 \mathrm{E}-10 & 1.523 \mathrm{E}-1 \mathrm{C} \\ 1.411 \mathrm{E}-10 & 1.732 \mathrm{E}-10 \\ 1.644 \mathrm{E}-10 & 2.419 \mathrm{E}-10 \\ 2.607 \mathrm{E}-10 & 2.209 \mathrm{E}-10 \\ 2.545 \mathrm{E}-10 & 2.979 \mathrm{E}-1 \mathrm{C} \\ 3.320 \mathrm{E}-10 & 3.551 \mathrm{E}-10 \\ 6.874 \mathrm{E}-10 & 5.978 \mathrm{E}-10 \\ 1.660 \mathrm{E}-09 & 1.056 \mathrm{E}-09 \\ 4.033 \mathrm{E}-\mathrm{CO} & 3.726 \mathrm{E}-09 \\ 2 . \mathrm{C} 9 \mathrm{E}-08 & 2.099 \mathrm{E}-08 \\ 8.379 \mathrm{E}-\mathrm{C} 9 & 8.209 \mathrm{E}-09\end{array}$

$8.379 E-C 9$

\section{0}

$1.610 \mathrm{E}-10$ $1.612 \mathrm{E}-10$ $1.619 \mathrm{E}-10$ $1.642 E-10$ $1.689 E-10$ $1.73 \mathrm{E}-10$ $1.903 E-10$ $2 . C 32 E-10$ 2. $509 E-10$ $2.539-10$ $3.441 E-10$ $5.646 E-10$ $8.311 \mathrm{E}-10$ $5.309 \mathrm{E}-\mathrm{C} 9$

$5.013 E-C 9$

600.0
150.0
RAN
GE (METERS)
200.6

$1.561 \mathrm{E}-10$

$1.56 C E-10$

$1.535 \mathrm{E}-10$

. $557 \mathrm{E}-10$

$1.627 \mathrm{E}-10$

$1.760 E-10$

$1.980 \mathrm{E}-10$

$2.217 E-10$

$2.690 \mathrm{E}-10$

$2.779 \mathrm{E}-10$

$3.361 \mathrm{E}-10$

$4.332 E-10$

6.CO6E-10

$1.024 \mathrm{E}-\mathrm{C} 9$

$2.829 E-09$

$1.934 E-C 8$

$8 . C 21 E-C 9$

$1.773 \mathrm{E}-10$

$1.772 E-10$

$1.767 \mathrm{E}-10$

$1.758 \mathrm{E}-10$

$1.874 \mathrm{E}-10$

$2 . C 25 \mathrm{E}-10$

$2.247 \mathrm{E}-10$

. $529 \mathrm{E}-10$

$2.768 \mathrm{E}-10$

$3.343 \mathrm{E}-10$

$3.744 E-10$

$4.809 \mathrm{E}-10$

$6.539 E-10$

$1.075 \mathrm{E}-09$

$.457 \mathrm{E}-0$

$1.665 \mathrm{E}-\mathrm{C} 8$

$7.815 E-09$

300.0

400.0

RANGE (METERS)

900.0

$1200 . \mathrm{C}$

$1.883 E-10$

$1.883 E-10$

$1.881 \mathrm{E}-10$
$1.88 \mathrm{CE}-10$

$1.923 E-10$

$2.015 E-10$

$2.401 \mathrm{E}-10$

$2.691 \mathrm{E}-10$

$2.935 \mathrm{E}-1 \mathrm{C}$

$3.548 \mathrm{E}-10$

$3.971 \mathrm{E}-10$

$5.084 E-10$

$6.843 \mathrm{E}-1 \mathrm{C}$

1.09 CE- 09

$2.249 E-09$

1. $395 \mathrm{E}-08$

7. $489 \mathrm{E}-09$

$1.917 \mathrm{E}-10$ $1.917 E-10$ $1.916 E-10$ $1.975 \mathrm{E}-1 \mathrm{C}$ $2.066 \mathrm{E}-10$ $2.227 \mathrm{E}-10$ $2.456 \mathrm{E}-10$

$2.747 \mathrm{E}-10$

.120E-10

$3.498 E-10$

. 13 CE-10

$5.080 E-10$

$1.073 E-C 9$

2.08

$7.083 E-09$

$824 E-10$
$825 E-10$

$.825 \mathrm{E}-10$
$1.829 \mathrm{E}-10$

$1.850 E-10$

1. $898 \mathrm{E}-10$

$1.991 \mathrm{E}-10$

$2.141 \mathrm{E}-10$

$2.350 \mathrm{E}-10$

$2.935 \mathrm{E}-10$

$3.342 \mathrm{E}-10$

$3.902 E-10$

$4.824 E-1 C$

$9.768 \mathrm{E}-10$

$6.078 \mathrm{E}-09$

1800.0

$1.354 \mathrm{E}-10 \quad 6.638 \mathrm{E}-11$ 2.72E-11 1.C01E-11 $3.413 \mathrm{E}-12$

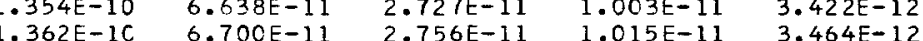
$1.385 \mathrm{E}-10$ 6.700E-11 2.756E-11 $1.015 \mathrm{E}-113.464 \mathrm{E}-12$ $\begin{array}{lllll}1.427 \mathrm{E}-10 & 7.061 \mathrm{E}-11 & 2.915 \mathrm{E}-11 & 1.076 \mathrm{E}-11 & 3.677 \mathrm{E}-12\end{array}$

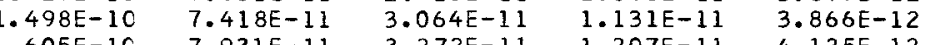
$\begin{array}{lllll}1.605 E-1 C & 7.931 E-11 & 3.272 E-11 & 1.207 E-11 & 4.125 E-12\end{array}$ $1.752 E-1 C \quad 8.620 E-11 \quad 3.549 E-11 \quad 1.368 E-11 \quad 4.467 E-12$

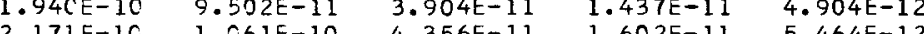
$2.171 \mathrm{E}-1 \mathrm{C} \quad 1.061 \mathrm{E}-1 \mathrm{O} \quad 4.356 \mathrm{E}-11 \quad 1.602 \mathrm{E}-11 \quad 5.464 \mathrm{E}-12$ 2.468E 10 1C $1.206 E-10$ 4.947E-11 $1.818 E-11 \quad 6.196 \mathrm{E}-12$ $3.885 \mathrm{E}-1 \mathrm{C} \quad 1.4 \mathrm{CE}-10 \mathrm{5}, 763 \mathrm{E}-11 \quad 2.114 \mathrm{E}-11 \quad 7.188 \mathrm{E}-12$ $4.544 E-1 C \quad 1.715 E-10$ 6.96EE-11 2.540E-11 $8.595 E-12$ $6.756 \mathrm{E}-10$ 2.21E-10 $3.831 \mathrm{E}-11$ 3.179E-11 $1.066 \mathrm{E}-11$ $\begin{array}{lllll}6.756 \mathrm{E}-10 & 3.059 \mathrm{E}-10 & 1.185 \mathrm{E}-10 & 4.171 \mathrm{E}-11 & 1.375 \mathrm{E}-11\end{array}$ $\begin{array}{lllll}1.109 E-C 9 & 4.641 E-10 & 1.7 C 5 E-10 & 5.782 E-11 & 1.855 E-11 \\ 3.57 C E-C 9 & 1.078 E-09 & 3.234 E-1 C & 9.606 E-11 & 2.818 E-11\end{array}$

4.COQE-C9 1.797E-C9 7.C31E-10 2.51CE-10 $8.388 E-11$ 
COS INE

-1. COOCOE OO
$-9.89401 E-C 1$
$-9.44575 E-01$
$-8.65631 E-01$
$-7.55044 E-01$
$-6.17876 E-01$
$-4.58017 E-01$
$-2.81605 E-01$
$-9.50125 E-C 2$
$9.50125 E-02$
$2.81665 E-01$
$4.58017 E-01$
$6.17876 E-01$
$7.55044 E-01$
$8.65631 E-01$
$9.44575 E-01$
$9.89401 E-01$

TOTAL

COSINE

$-1.0000 C E$ OC $-9.89401 E-C$ $-9.44575 E-0$ $-8.65631 \mathrm{E}-0$ $-7.55044 E-0$ $-6.17876 E-0$ $-4.58017 E-0$ $-2.81605 E-0$ $-9.50125 \mathrm{E}-0$ $9.50125 E-02$ 2. $81605 E-01$ 4. $58017 \mathrm{E}-\mathrm{C}$ $6.17876 \mathrm{E}-01$ $7.55044 E-01$ $8.65631 E-01$ $9.44575 E-01$ $9.89401 E-C 1$

TOTAL
75. C

100.0

$6.303 \mathrm{E}-12$ $6.340 \mathrm{E}-12$ $6.483 E-12$

$7.145 E-12$

$7.656 \mathrm{E}-12$

$8.356 \mathrm{E}-12$

$9.281 E-12$

$1.054 \mathrm{E}-11$

$1.228 \mathrm{E}-11$

$1.861 E-11$

$1.905 \mathrm{E}-11$

$2.062 E-11$

2. 7 C 9E-11

$4.448 \mathrm{E}-11$

$7.016 E-11$

$1.049 E-10$

$2.213 E-1 C$

500.0

$5.476 E-12$

$5.59 C E-12$

$5.998 \mathrm{E}-12$

$6.607 \mathrm{E}-12$

$7.193 \mathrm{E}-12$

$8.301 \mathrm{E}-12$

9. $214 \mathrm{E}-12$

1.C $72 \mathrm{E}-11$

. $300 \mathrm{E}-11$

$1.622 \mathrm{E}-1$

2.C83E-11

$2.817 E-11$

4. $145 E-11$

$6.846 E-11$

$3.466 E-10$

$3.195 \mathrm{E}-10$
$7.072 E-12$

7. $\mathrm{C} 72 \mathrm{E}-12$

$7.302 \mathrm{E}-12$

$7.638 \mathrm{E}-12$

$8.107 \mathrm{E}-12$

$9.519 \mathrm{E}-12$

$1 . C 6 C E-11$

1. $207 \mathrm{E}-1$

$1.822 \mathrm{E}-11$

$.682 \mathrm{E}-1$

2.158E-11

$2.951 E-11$

$3.772 \mathrm{E}-11$

$4.907 E-11$

$9.190 E-11$
$1.58 C E-10$

$2.733 \mathrm{E}-1 \mathrm{C}$

600.0

4. $368 E-12$

$4.474 E-12$
$4.85 C E-12$

$5.395 \mathrm{E}-12$

$5.889 E-12$

$6.291 \mathrm{E}-12$

$6.729 E-12$

$7.454 \mathrm{E}-12$

$8.711 \mathrm{E}-12$

1. $C 64 E-11$

$1.336 \mathrm{E}-11$

$1.721 \mathrm{E}-11$

$2.336 \mathrm{E}-11$

$3.476 E-11$

$5.841 \mathrm{E}-11$

$3.083 \mathrm{E}-10$

$2.706 E-10$
150.0 RANGE (MLTERS)
$2 C O . \mathrm{C}$

25 C. C

300.0

$400 . C$

$7.927 \mathrm{E}-12$

$7.994 \mathrm{E}-12$

$8.698 \mathrm{E}-12$

$0.286 \mathrm{E}-12$

CC1E- 11

$1 . C 94 E-11$

$1.219 E-11$

$1.394 E-1$

$1.613 \mathrm{E}-11$

$1.981 \mathrm{E}-11$

$3.435 \mathrm{E}-11$

$4.488 \mathrm{E}-11$

$7.241 E-11$

$1.173 E-10$

$2.433 E-10$

$8.19 \mathrm{CE}-12$

$8.261 \mathrm{E}-12$

$8.16 \mathrm{CE}-12$

$.591 E-12$

$9.130 \mathrm{E}-12$
$9.794 \mathrm{E}-12$

$1.057 \mathrm{E}-11$

$1.154 E-11$

$1.287 \mathrm{E}-11$

$1.476 \mathrm{E}-11$
$1.756 \mathrm{E}-11$

$2.114 \mathrm{E}-11$

$2.689 \mathrm{E}-11$

3. 53 CE-11

$5.028 \mathrm{E}-11$

$7.903 E-11$

$1.395 \mathrm{E}-10$

3.02 CE-10

$9.122 \mathrm{E}-12$

1. $C 6 \mathrm{CE}-11$

$1.156 \mathrm{E}-11$

$1.289 E-11$

$1.482 \mathrm{E}-11$

$1.772 E-11$

2. $74 \mathrm{CE}-11$

$3.616 E-11$

$5.172 \mathrm{E}-11$

8.18CE-11

$3.414 E-10$

$3.350 E-10$

$3.721 E-10$

$3.878 E-10$

$7.708 \mathrm{E}-12$

$8.844 \mathrm{E}-12 \quad 7.836 \mathrm{E}-12$

$8.554 \mathrm{E}-12 \quad 8.504 \mathrm{E}-12$

$1.031 \mathrm{E}-11 \quad 9.152 \mathrm{E}-12$

$1.121 \mathrm{E}-11 \quad 0.904 \mathrm{E}-12$

$1.249 \mathrm{E}-11 \quad 1.101 \mathrm{E}-11$

$1.440 \mathrm{E}-11 \quad 1.276 \mathrm{E}-11$

$2.115 \mathrm{E}-11 \quad 1.903 \mathrm{E}-11$

$2.686 \mathrm{E}-11 \quad 2.434 \mathrm{E}-11$

$3.587 \mathrm{E}-11 \quad 3.272 \mathrm{E}-11$

$5.143 \mathrm{E}-11 \quad 4.758 \mathrm{E}-11$

$8.209 E-11 \quad 7.721 E-11$

$\begin{array}{ll}1.534 \mathrm{E}-1 \mathrm{C} & 1.475 \mathrm{E}-10 \\ 3.639 \mathrm{E}-10 & 3.706 \mathrm{E}-10\end{array}$

$3.888 E-10 \quad 3.633 E-10$

RANGE (MFTERS)

$900.0 \quad 12 \mathrm{CO} . \mathrm{C}$

1500.0

1800.0

.C53E-12 $8.579 E-13 \quad 3.391 E-13 \quad 1.295 E-13$

$2.296 E-12 \quad 9.844 E-13 \quad 4 . C 25 E-13 \quad 1.636 E-13$

$2.626 \mathrm{E}-12 \quad 1.169 \mathrm{E}-12 \quad 4.993 \mathrm{E}-13 \quad 2.054 \mathrm{E}-13$

2.885E-12 $1.292 \mathrm{E}-12 \quad 5.494 \mathrm{E}-13 \quad 2.309 \mathrm{E}-13$

1.043E-12 $1.337 \mathrm{E}-12 \quad 5.605 \mathrm{E}-13 \quad 2.287 \mathrm{E}-13$

3.192E-12 $1.373 E-12 \quad 5.574 E-13 \quad 2.196 E-13$

1.51CE-12 $6.171 \mathrm{E}-13 \quad 2.470 \mathrm{E}-13$

4.176E-12 $1.827 \mathrm{E}-12 \quad 7.62 \mathrm{CE}-13 \quad 3.103 \mathrm{E}-13$

$5.221 E-12$ 2.34CE-12 1.0N1E-12 4.183E-13

$6.644 \mathrm{E}-12 \quad 3.011 \mathrm{E}-12 \quad 1.299 \mathrm{E}-12 \quad 5.456 \mathrm{E}-13$

$1.50 E-12$ 3.887E-12 $1.667 E-12 \quad 6.919 E-13$

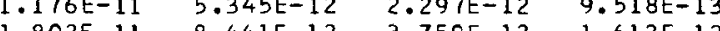

$1.802 E-11$ 8.441E-12 3.75CE-12 $1.612 \mathrm{E}-12$

$3.182 \mathrm{E} 11.565 \mathrm{E}-11 \quad 7.327 \mathrm{E}-12 \quad 3.333 \mathrm{E}-12$

$\begin{array}{llll}6.579 E-11 & 3.379 E-11 & 1.649 E-11 & 7.814 E-12 \\ 1.849 E-10 & 9.827 E-11 & 4.924 E-11 & 2.386 E-11\end{array}$

$1.448 E-10 \quad 7.021 E-11 \quad 3.246 E-11 \quad 1.463 E-11$ 
COSINE

$-1.000 C O E$ OC $-9.894 C 1 E-0$ $-9.44575 E-01$ $-8.65631 \mathrm{E}-01$ $-7.55044 \mathrm{E}-0$ $-6.17876 \mathrm{E}-01$ $-4.58017 E-01$ $-2.81605 \mathrm{E}-01$ $-9.50125 E-02$ $9.50125 \mathrm{E}-\mathrm{C} 2$ $2.81605 \mathrm{E}-01$ $4.58017 \mathrm{E}-01$ $6.17876 \mathrm{E}-01$ $7.55044 \mathrm{E}-\mathrm{Cl}$ $8.65631 \mathrm{E}-01$ $9.89401 E-01$

TOTAL

COSINE

$-1.00000 E C C$ $-9.89401 \mathrm{E}-01$ $-8.65631 \mathrm{E}-01$ $-8.65631 E-01$ $-6.17876 E-01$ $-4.58017 E-0$ 2. $50125 E-C 2$ $9.50125 \mathrm{E}-02$ $2.81605 E-01$ $4.58017 \mathrm{E}-01$ $6.17876 E-01$ $7.55044 \mathrm{E}-01$ $8.65631 \mathrm{E}-0$ $9.44575 E-C 1$ $9.89401 \mathrm{E}-\mathrm{Cl}$

TOTAL

\begin{abstract}
75.0
\end{abstract}
100.0

$6.297 E-10$ $6.333 E-10$ $6.473 E-10$ $6.737 E-10$ 7. $117 \mathrm{E}-10$ $7.624 E-1 C$ $8.297 E-10$ $9.198 \mathrm{E}-10$ $1.042 E-09$ $1.212 E-C 9$ $1.825 E-C 9$ $1.872 \mathrm{E}-\mathrm{C} 9$ $2.023 E-09$ $2.651 E-09$ $4.341 E-C 9$ $6.838 \mathrm{E}-09$

$2.173 E-C 8$

500.0

$6.573 E-10$ $6.685 \mathrm{E}-10$ $7.086 E-10$ $8.281 E-10$ 9.821E 10 $1.037 E-09$ 1. $189 E-09$ $1.416 E-C 9$ $1.735 \mathrm{E}-\mathrm{C} 9$ $2.190 \mathrm{E}-\mathrm{C} g$ $2.909 E-C 9$ $4.204 E-C 9$ $6.828 \mathrm{E}-\mathrm{C} 9$ 1. $310 \mathrm{E}-08$ $3.392 E-C 8$

$3.296 \mathrm{E}-08$

$60 c .0$
150.0 RANGE 2 METERS)
$2 \mathrm{CO} 0 . \mathrm{C}$

250.0

300.0

$400 . n$

$7.152 E-1 C$ $7.198 \mathrm{E}-1 \mathrm{C}$ $7.375 \mathrm{E}-10$ $7.703 E-1 C$ $8.16 C E-10$ $8.757 \mathrm{E}-1 \mathrm{C}$ $9.540 E-1 C$ $1 . C 59 E-C 9$ $1.203 E-c 9$ $1.800 E-09$ $1.666 E-C 9$ $2.115 E-C 9$ $2.908 \mathrm{E}-\mathrm{C} 9$ $3.699 E-C 9$ $4.799 \mathrm{E}-\mathrm{C}$ $8.963 E-C$

$2.693 \mathrm{E}-\mathrm{C} 8$

$8.220 E-10$ $9.788 \mathrm{E}-10$ $9.626 \mathrm{E}-10$ $9.549 \mathrm{E}-10 \quad 1.028 \mathrm{E}-\mathrm{C}$ $1.026 E-C .9$ 1.1C5E-09 $1.117 \mathrm{E}-\mathrm{C} 9$ 1.2C1E-C $1.240 E-C 9$ 1.332E-C $1.411 \mathrm{E}-09 \quad 1.518 \mathrm{E}-\mathrm{C} 9$ $1.623 \mathrm{E}-\mathrm{C} 9 \quad 1.794 \mathrm{E}-0$ $1.986 E-C 9 \quad 2.141 E-09$ $2.456 E-C 9$ 2.705E-C $3.408 E-09 \quad 3.522 E-09$ $4.4 C 7 E-09 \quad 4.981 E-09$ $7.111 E-C 9$ T.771E-O $1.146 E-C 8$ 1.366E-08

\section{$8.776 E-1 C$} $8.873 E-10$ $9.228 \mathrm{E}-1 \mathrm{C}$ $9.817 E-10$ $1.051 E-09$ $1.125 E-09$ $1.224 E-09$ $1.356 \mathrm{E}-09$ $1.547 E-09$ $1.834 E-09$ $2.196 E-09$ $2.782 \mathrm{E}-09$ S.15CE-O9 $1.472 E-0 B$ $3.334 \mathrm{E}-0 \mathrm{~B}$

3. $906 \mathrm{E}-0$

$3.723 E-C 8$

$8.582 E-10$ $7.829 E-10$
$670-10-8.241 E-10$ $9.699 E-10 \quad 8.880 \mathrm{E}-10$ $1.041 \mathrm{E}-\mathrm{C} 9 \quad 9.55 \mathrm{CE}-10$ $1.116 \mathrm{E}-\mathrm{C} 9 \quad 1.021 \mathrm{E}-09$ $1.207 \mathrm{E}-\mathrm{C} 9 \quad 1.098 \mathrm{E}-\mathrm{C} 9$ $1.335 \mathrm{E}-\mathrm{C} 9 \quad 1.211 \mathrm{E}-09$ $1.525 \mathrm{E}-\mathrm{rg} \quad 1.385 \mathrm{E}-09$ $1.798 \mathrm{E}-09 \quad 1.643 \mathrm{E}-09$ $2.190 \mathrm{E}-09 \quad 2.006 \mathrm{E}-09$ $2.749 \mathrm{E}-\mathrm{C} 9 \quad 2.529 \mathrm{E}-09$ $3.629 \mathrm{E}-\mathrm{CS} \quad 3.349 \mathrm{E}-\mathrm{C} 9$ $5.144 \mathrm{E}-\mathrm{Cg} \quad 4.796 \mathrm{E}-09$ 1.122E-O $7.675 E-09$ $1.505 E-C 8$ 1.450E-C8 $3.940 E-C 8 \quad 3.719 E-08$

RANGE (METERS ) OOC.O

$12 \mathrm{CO} . \mathrm{C}$

1500.0

1800.0
$2.633 \mathrm{E}-10$ $2.7 C 4 E-10$ $2.943 E-10$ $3.536 \mathrm{E}-10$ $3.736 E-10$ 3.710E-10 $4.226 E-10$ $4.899 \mathrm{E}-1 \mathrm{C}$ $5.949 \mathrm{E}-10$

7. $369 E-10$

$9.301 E-10$

$9.361 E-10$
$1.242 E-C 9$

$1.242 E-C 9$
$1.852 E-09$ $3.191 \mathrm{E}-\mathrm{n} 9$ $6.491 E-09$ $1.813 E-C 8$

\section{$1.152 \mathrm{E}-10$} $1.195 \mathrm{E}-10$

4. $765 \mathrm{E}-11$ $4.992 \mathrm{E}-11$ 5 C 2E-10 $\quad 6.572 \mathrm{E}-1$ . $628 E-19$ $1.731 \mathrm{E}-10$ $1.731 E-10$ $1.879 E-10$ $2.273 E-10$ $3.388 \mathrm{E}-10$ $4.260 E-10$ $5.695 \mathrm{E}-10$ $8.712 \mathrm{E}-10$ $1.571 \mathrm{E}-09$ $3.333 E-09$ $9.645 \mathrm{E}-\mathrm{C} 9$

$.092 \mathrm{E}-1$

$7.249 E-11$

$7.281 \mathrm{E}-11$

$7.928 \mathrm{E}-11$
$9.405 \mathrm{E}-11$

$1.180 \mathrm{E}-1 \mathrm{C}$

$1.478 \mathrm{E}-10$

$1.845 \mathrm{E}-10$

$2.465 \mathrm{E}-10$

$3.880 E-10$

$7.348 \mathrm{E}-10$

$1.625 \mathrm{E}-09$

$4.836 \mathrm{E}-09$

$1.913 E-11$ $2.026 \mathrm{E}-11$ $2.774 \mathrm{E}-11$ 3. $037 \mathrm{E}-1$ .038E-11 $2.977 E-11$ $3.271 \mathrm{E}-11$ $3.915 E-11$ $4.996 \mathrm{E}-11$ $6.269 E-11$ $7.732 \mathrm{E}-11$ $1.029 \mathrm{E}-10$ $1.672 \mathrm{E}-10$ $3.339 \mathrm{E}-10$ $7.694 \mathrm{E}-10$
$2.346 \mathrm{E}-\mathrm{C} 9$

$1.516 E-C 8 \quad 7.38 C E-09 \quad 3.415 E-09 \quad 1.539 E-09$ 
$\left(\begin{array}{l}4 \text { PI R**2 AIR KERMA (GAMMAS) } \\ \text { (CM**2 } \text { ERGS/GRAM/STERADIAN/SOURCE NEUTRON) }\end{array}\right.$

COSINE

$-1.00000 E 00$ $-9.89401 \mathrm{E}-01$ $-9.44575 E-01$ $-8.65631 E-01$ $-7.55044 E-01$ $-4.58017 \mathrm{E}-01$ $-2.81605 E-01$ $-9.50125 \mathrm{E}-02$ $9.50125 \mathrm{E}-02$ $2.81605 \mathrm{E}-01$ 4. $58017 \mathrm{E}-01$ $6.17876 \mathrm{E}-01$ 7. $55044 \mathrm{E}-01$ $8.65631 \mathrm{E}-01$ $9.44575 \mathrm{E}-01$ $9.894 C 1 E-01$

TOTAL

COSINE

$-1.00000 E \mathrm{CO}$ $-9.89401 \mathrm{E}-01$ $-9.44575 E-C 1$ $-8.65631 \mathrm{E}-01$ $-7.55044 E-01$ $-6.17876 E-01$ $-4.58017 \mathrm{E}-01$ $-2.81605 \mathrm{E}-01$ $-9.50125 \mathrm{E}-02$ $9.50125 \mathrm{E}-02$ $2.81605 \mathrm{E}-01$ $4.58017 E-01$ $6.17876 E-01$ 7.55044E-0 1 $8.05631 E-01$ $9.89401 \mathrm{E}-01$

TOTAL

$75 . \mathrm{C}$
$7.285 \mathrm{E}-10$
$7.320 \mathrm{E}-10$
$7.457 \mathrm{E}-10$
$7.716 \mathrm{E}-10$
$8.088 \mathrm{E}-10$
$8.586 \mathrm{E}-10$
$9.245 \mathrm{E}-10$
$1.012 \mathrm{E}-09$
$.131 \mathrm{E}-09$
$1.295 \mathrm{E}-\mathrm{C} 9$
$.878 \mathrm{E}-\mathrm{C} 9$
$1.922 \mathrm{E}-09$
$2.073 \mathrm{E}-\mathrm{C} 9$
$2.669 \mathrm{E}-\mathrm{C} 9$
$4.255 \mathrm{E}-09$
$6.592 \mathrm{E}-\mathrm{C} 9$
$9.743 \mathrm{E}-09$
$2.237 \mathrm{E}-\mathrm{C} 8$

$1 C C . \mathrm{C}$

150.0

RANGE (METERS)
2 CO.0

250.0

300.0

400.0

$8.885 \mathrm{E}-10$ $8.931 E-1 C$ $9.435 \mathrm{E}-10$ $9.894 \mathrm{E}-10$ 1.C $49 E-09$ $1.128 \mathrm{E}-\mathrm{C} 9$ 1. $233 \mathrm{E}-09$ $1.376 \mathrm{E}-\mathrm{C} 9$ $1.946 \mathrm{E}-09$ $1.827 E-C 9$ 2. $271 \mathrm{E}-09$ 3. $C 10 E-09$ $3.768 \mathrm{E}-09$ $4.815 E-09$ $8.717 E-09$ $1.472 \mathrm{E}-08$

$1.156 \mathrm{E}-09 \quad 1.356 \mathrm{E}-\mathrm{C9}$ $1.163 \mathrm{E}-09$ $1.188 \mathrm{E}-\mathrm{C} 9$ $1.294 \mathrm{E}-09$ $1.369 \mathrm{E}-09$ $1.465 E-09$ $1.594 \mathrm{E}-\mathrm{C} 9$ $1.771 \mathrm{E}-09$ $1.994 \mathrm{E}-\mathrm{C} 9$ $2.346 \mathrm{E}-09$ $2.821 \mathrm{E}-09$ $3.727 E-C 9$ $4.718 \mathrm{E}-09$ $7.259 E-C 9$ $1.136 \mathrm{E}-08$ $1.364 \mathrm{E}-09$ $1.396 \mathrm{E}-09$ $1.523 E-09$ $1.608 \mathrm{E}-09$ $1.715 E-09$ $1.858 E-09$ $2.057 E-09$ 2.342E-09 $2.7 \mathrm{C} 8 \mathrm{E}-\mathrm{C} 9$ $3.272 E-09$ 4. $187 E-09$ $5.50 C E-09$ $8.169 E-09$ $1.372 \mathrm{E}-08$
$2.849 \mathrm{E}-08$ 2. $849 E-C 8$

$3.713 E-C 8$

4. $339 E-08$

$1.487 \mathrm{E}-09$ $1.535 \mathrm{E}-09$ $1.598 \mathrm{E}-09$ $1.675 E-09$ $1.765 \mathrm{E}-09$ $1.876 \mathrm{E}-09$ 2.027E-09 .239E-09 $2.542 E-09$ $2.934 \mathrm{E}-09$ $3.530 E-09$ 4. 394E-09 $5.877 \mathrm{E}-\mathrm{C} 9$ $8.685 \mathrm{E}-09$ $3.236 \mathrm{E}-08$

4. $721 E-08$

1. $559 \mathrm{E}-\mathrm{C9}$ $1.612 \mathrm{E}-\mathrm{C} 9$ $1.680 \mathrm{E}-\mathrm{C} 9$ $1.761 \mathrm{E}-\mathrm{C} 9$ $1.852 \mathrm{E}-0$ $1.963 E-09$ $2.115 \mathrm{E}-09$ $2.331 \mathrm{E}-\mathrm{CO}$ $2.634 \mathrm{E}-\mathrm{C} 9$ $3.054 \mathrm{E}-09$ $3.642 \mathrm{E}-09$ $4.537 \mathrm{E}-09$ $6.036 \mathrm{E}-\mathrm{CS}$ $.908 \mathrm{E}-\mathrm{C} 9$ $3.461 E-08$

$4.909 E-08$

$1.562 \mathrm{E}-09$ $1.574 E-09$
$1.619 E-09$ $1.619 \mathrm{E}-09$
$1.690 \mathrm{E}-09$ $1.770 E-C 9$ $1.856 \mathrm{E}-09$ $1.958 \mathrm{E}-09$ $2.101 \mathrm{E}-09$ $2.310 E-09$ $2.605 \mathrm{E}-09$ $3.009 \mathrm{E}-09$ $3.572 \mathrm{E}-09$ $4.423 E-09$ $5.871 \mathrm{E}-09$ $8.663 \mathrm{E}-09$ 3.542E-08

\section{RANGE (METERS)}

500.0

$600 . \mathrm{C}$

900.0

1200.0

1500.0

1800.0 $1.454 E-09$ $1.497 \mathrm{E}-\mathrm{C}$ $1.565 \mathrm{E}-\mathrm{C} 9$ $1.638 \mathrm{E}-\mathrm{C} 9$ $1.713 E-C 9$ $1.801 \mathrm{E}-09$ $1.926 E-09$ $2.114 \mathrm{E}-09$ $2.381 \mathrm{E}-09$ $2.744 \mathrm{E}-09$ $3.244 E-09$ 4. $5.304 E-C 9$ 1.35 E 09 $1.380 E-08$ $1.271 \mathrm{E}-09$ $1.312 E-C 9$ $1.373 \mathrm{E}-\mathrm{C} 9$ $1.436 \mathrm{E}-\mathrm{C} 9$ $1.498 E-09$ $1.570 E-09$ $1.676 \mathrm{E}-\mathrm{C} 9$ $1.837 E-C 9$ $2.068 \mathrm{E}-\mathrm{C} 9$ $2.379 E-C 9$ $2.802 \mathrm{E}-\mathrm{Cg}$ $3.444 E-09$ $4.567 E-C 9$ $1.204 E-08$ $1.204 E-08$ $6.996 E-10 \quad 3.370 E-10$ $7.071 \mathrm{E}-10 \quad 3.415 \mathrm{E}-10$ $7.329 E-10 \quad 3.548 E-10$ $7.703 E-10 \quad 3.753 E-10$ $8.049 E-10 \quad 3.921 E-10$ $8.347 E-10 \quad 4 . C 40 E-10$ $8.679 E-10$ 4.171E-10 $9.213 E-10$ 4.415E-10 1. COSE-09 $1.137 E-09 \quad 5.466 E-10$ $1.3 C 4 E-09 \quad 6.259 E-10$ $1.524 E-09 \quad 7.263 E-10$ 1.8. $2.478 E-C 9 \quad 1.187 E-09$ $3.779 E-09 \quad 1.864 E-O 9$ $1.771 \mathrm{E}-\mathrm{CO} \quad 9.369 \mathrm{E}-09$ $1.523 \mathrm{E}-10$ 1. $546 \mathrm{E}-10$ $6.671 \mathrm{E}-11$ $6.785 \mathrm{E}-11$ $7.133 \mathrm{E}-11$ $7.588 \mathrm{E}-11$ $7.939 E-11$ $8.082 E-11$ . $205 E-11$ $8.695 \mathrm{E}-11$ $9.545 E-11$ $1.084 \mathrm{E}-10$ $1.235 \mathrm{E}-10$ $1.409 E-10$ $1.691 E-10$ $2.337 \mathrm{E}-10$ . $8.026 E-10$

4. $441 E-08$ $3.867 E-C 8$

2.16CE-08

$1.062 E-08$

2. $220 E-09$ 
COSINE

$-1.000 C 0 E 00$ $-9.89401 \mathrm{E}-01$ $-9.44575 \mathrm{E}-31$ $-8.65631 E-01$ $-7.55044 \mathrm{E}-J 1$ $-6.17876 E-01$ $-4.58017 \mathrm{E}-01$ $-2.81605 E-01$ $-9.50125 \mathrm{E}-02$ $9.50125 \mathrm{E}-02$ $2.81605 \mathrm{E}-\mathrm{GI}$ $4.58017 \mathrm{E}-\mathrm{Cl}$ $6.17876 \mathrm{E}-01$ $7.55044 \mathrm{E}-01$ $8.65631 \mathrm{E}-01$ $9.89401 E-01$

TOTAL

COSINE

$-1.00000 E 00$ $-9.89401 E-01$ $-9.44575 E-01$ $-7.55044 \mathrm{E}-01$ $-6.17876 \mathrm{E}-01$ $-4.58017 E-01$ $-2.816 C 5 \mathrm{E}-0$ $-9.50125 E-02$ $9.50125 \mathrm{E}-02$ 2.81605E-01 4. $58017 \mathrm{E}-01$ $6.17876 \mathrm{E}-01$ $7.55044 E-01$ $8.65631 \mathrm{E}-01$ $9.44575 E-01$ $9.894[1 E-01$

TOTAL

75.0
$6.447 E-10$
$6.484 E-10$
$6.626 E-1 C$
$6.895 E-10$
$7.281 E-10$
$7.795 E-1 C$
$8.480 E-10$
$9.396 E-1 C$
$1.064 E-09$
$1.237 E-C 9$
$1.859 E-C 9$
$1.908 E-C 9$
$2.061 E-C 9$
$2.699 E-C 9$
$4.419 E-C 9$
$6.963 E-C 9$
$1.04 C E-C 8$
$2.215 E-C 8$

100.0

$7.348 \mathrm{E}-1 \mathrm{C}$ .395E-1C $7.575 E-1 C$ $7.908 \mathrm{E}-1 \mathrm{C}$ . $373 E-1 C$ $8.979 E-10$ $9.774 E-1 C$ 1. C84E-CQ $1.230 \mathrm{E}-\mathrm{C} 9$ $1.837 \mathrm{E}-\mathrm{C} 9$ 1. $7 \mathrm{ClE}-09$ $2.154 E-C S$ $2.965 E-C 9$ $3.768 \mathrm{~F}-09$ $4.885 \mathrm{E}-09$ $1.568=C 8$ E-C 8 $2.747 E-C 8$

$500 . \mathrm{C}$

$60 \mathrm{c} .0$

$7.176 \mathrm{E}-1 \mathrm{C}$ $7.290 E-1 C$ 7.69E $8.914 E-1 C$ $8.914 E-1 C$ $1.007 \mathrm{E}-\mathrm{C} 9$ 1. $102 \mathrm{E}-\mathrm{CO}$ $1.484 E-C 9$ $1.487 \mathrm{E}-\mathrm{C}$ 2. $266 \mathrm{E}-\mathrm{C} 9$ $2.993 \mathrm{E}-\mathrm{C} 9$ 4. $303 \mathrm{E}-\mathrm{CO}$ $6.965 \mathrm{E}-\mathrm{C} \theta$ 1. $334 \mathrm{E}-\mathrm{C} 8$ $3.457 \mathrm{E}-\mathrm{C} 8$ $3.405 E-C 8$

$5.958 \mathrm{E}-1 \mathrm{C}$ $6 . C \in 3 E-1 C$ $6.992 \mathrm{E}-1 \mathrm{IC}$ $7.500 \mathrm{E}-1 \mathrm{C}$ 7.00 (.) . $150 \mathrm{E}-1 \mathrm{C}$ . $1.043 \mathrm{E}-\mathrm{C} 9$
$1.239 \mathrm{E}-\mathrm{CQ}$ $1.511 \mathrm{E}-\mathrm{CO}$ $1.895 \mathrm{~F}-\mathrm{CO}$ $2.505 \mathrm{E}-09$ $3.629 \mathrm{E}-09$ $5.958 E-C 9$ $1.158 \mathrm{E}-\mathrm{C} 8$ $3.078 \mathrm{E}-\mathrm{C} 8$

$2.905 E-08$
RANGE (METERS)
$2 O C . C$

$25 C \cdot C$

300.0

430.0

8.513E-10 9.r94E-10 $8.58 C E-10$ $9.179 E-10$ $9.249 E-1 C$ $9.347 \mathrm{E}-10$ $9.708 \mathrm{E}-10$ $.279 E-10 \quad 1 . C C 3 E-C 9$ l.C31E-C $9.864 E-12 \quad 1.069 E-C 9$ $1.059 E-09 \quad 1.147 E-C 9$ $\begin{array}{lll}1.151 E-C 9 & 1.245 E-C 9 & 1.276 E-09\end{array}$ $1.276 \mathrm{E}-09 \quad 1.377 \mathrm{E}-09 \quad 1.41 \mathrm{CE}-09$ $\begin{array}{lll}1.449 E-09 & 1.566 E-09 & 1.603 E-09\end{array}$ $\begin{array}{lll}1.662 \mathrm{E}-09 & 1.846 \mathrm{E}-09 & 1.894 \mathrm{E}-\mathrm{C} 9\end{array}$ $2.034 \mathrm{E}-\mathrm{C} \quad 2.196 \mathrm{E}-\mathrm{CO} \quad 2.259 \mathrm{E}-09$ $\begin{array}{lll}2.507 E-09 & 2.77 C E-09 & 2.854 E-09\end{array}$ $3.479 E-09$ 3.597E-CO $3.716 E-0$ $4.487 E+C 9 \quad 5 . C 79 E-09 \quad 5.256 E-09$ $7.242 E-09 \quad 7.913 E-09 \quad 8.221 E-09$

$1.167 E-C 8$ 1.30CE-O8 $1.499 E-C 8$

$3.400 \mathrm{E}-08$

$3.812 F-08$

4. CCTE-C 8

RANGE (METERS)
$12 \mathrm{CC} . \mathrm{C}$

$150 C \cdot C$

$2.968 \mathrm{E}-10 \quad 1.318 \mathrm{E}-1 \mathrm{n}$ $.64 \cap E-10 \quad 1.362 E-10$ 3.610E-10 $1.676 E-10$ $487 E-10 \quad 1.8 C 4 E-10$ 4.C62E-10 1.858E-10 $4.232 E-10 \quad 1.9 C 5 E-10$ $4.577 E-10 \quad 2.053 E-10$ 4.57E 10 2.053E 10 6.315E-10 $2.899 \mathrm{E}-10$ $7.747 \mathrm{E}-10 \quad 3.575 \mathrm{E}-10$ $1.603 \mathrm{E}-10 \quad 4.452 \mathrm{E}-10$ $1.283 E-C 9 \quad 5.896 E-10$ 1.9กCE-CQ $8.944 E-10$ $3.258 \mathrm{~F}-\mathrm{CO} \quad 1.6 \mathrm{C} 4 \mathrm{E}-0$ $1.850 \mathrm{E}-08 \quad 9.843 \mathrm{E}-\mathrm{C} 9$

$5.53 \mathrm{CE}-11$ $5.763 E-11$ $6.4 C 1 E-11$ $7.903 \mathrm{E}-11$ B. $565-11$ $8 . C 77 E-11$ $8.723 \mathrm{E}-11$

$1.021 \mathrm{E}-1 \mathrm{C}$

$1.263 \mathrm{E}-1 \mathrm{C}$

$563 \mathrm{E}-1 \mathrm{C}$

$1.932 \mathrm{E}-1 \mathrm{C}$

$2.555 \mathrm{E}-1 \mathrm{C}$

$3.984 \mathrm{E}-1 \mathrm{C}$

$7.503 \mathrm{E}-1 \mathrm{C}$

$1.65 \in E-09$

$4.938 \mathrm{E}-0.9$

$1.574 E-C 8 \quad 7.669 E-C 9$ $6.615 \mathrm{E}-\mathrm{C} 9 \quad 3.397 \mathrm{E}-\mathrm{CO}$
$1800 . \mathrm{C}$ $\begin{array}{ll}9.119 \mathrm{E}-10 & 8.321 \mathrm{E}-10 \\ 9.227 \mathrm{E}-10 & 8.437 \mathrm{E}-10\end{array}$ $9.620 \mathrm{E}-10 \quad 8.856 \mathrm{E}-10$ $1.026 E-C 9 \quad 9.506 E-10$ $1.097 \mathrm{E}-\mathrm{C} 9 \quad 1.019 \mathrm{E}-09$ $1.174 \mathrm{E}-\mathrm{C} 9 \quad 1 . \mathrm{C} 85 \mathrm{E}-0$ $1.266 \mathrm{E}-\mathrm{C} 9 \quad 1.163 \mathrm{E}-\mathrm{C} 9$ $1.395 \mathrm{E}-\mathrm{C} 9 \quad 1.277 \mathrm{E}-09$ $1.587 E-C 9 \quad 1.453 E-C 9$ $1.863 \mathrm{E}-\mathrm{C9} \quad 1.713 \mathrm{E}-09$ $2.260 E-09 \quad 2.080 E-09$ $2.825 \mathrm{E}-\mathrm{C9} \quad 2.609 \mathrm{E}-09$ $3.717 \mathrm{E}-\mathrm{C} 9 \quad 3.438 \mathrm{E}-09$ $5.252 \mathrm{E}-\mathrm{C} 9 \quad 4.904 \mathrm{E}-\mathrm{C} 9$ $1.277 E-0926 E-09$ $3.622 E-C 8 \quad 3.693 E-08$

4. $C 49 E-C 8 \quad 3.833 E-08$

2. $253 \mathrm{E}-11$ .368E-11 3. $132 \mathrm{E}-11$ $3.399 \mathrm{E}-1$ $3.396 \mathrm{E}-11$ $3.328 \mathrm{E}-11$ $3.621 \mathrm{E}-1$ $4.271 \mathrm{E}-11$ $5.363 \mathrm{E}-1$ $8.11 \cap E-11$ $1.068 \mathrm{E}-10$

$1.716 E-10$ $3.409 \mathrm{E}-10$ $7.845 \mathrm{E}-10$

$1.600 E-C 9$ 
(NEUTRONS/MEV/STERADIAN/SOURCE NEUTRON)

ENERGY

GROUP (MEV)

$1.22 E$ O1---1.5OE 01 $1.00 \mathrm{E} 01--1.22 \mathrm{E}$ OI $8.19 E$ OO- $-1.00 E$ OI $4.36 E$ DO-- $8.19 E$ OO $4.97 E$ EO--6.36E 00 4.07E $00-4.97 E$ OO 2.01E $00-3.07 E$ OO 2.46E $00-2-3.01 \mathrm{E} 00$ 1.33E $00-2.35 E$ OO 1.83 E $00-2.35 E$ OO 5.50E-01--1.11E 00 $1.50-01--1.11 E$ OO $3.35 \mathrm{E}-02--1.11 \mathrm{E}-01$ $5.3 \mathrm{E}-04--3.35 \mathrm{E}-02$ 1.01E-04-- $5.83 E-04$ $2.90 \mathrm{E}-05--1.01 \mathrm{E}-04$ $1.07 E-05---2.90 E-05$ $3.06 \mathrm{E}-06--1.07 \mathrm{E}-05$ $1.12 \mathrm{E}-06---3.06 \mathrm{E}-06$ $4.14 \mathrm{E}-07--1.12 \mathrm{E}-06$ $0.0 \quad--4.14 \mathrm{E}-07$

ENERGY

GROUP (MEV)

$1.22 E$ O1-- $1.50 E$ OI $1.00 E$ O1- $-1.22 E$ OI 8.1 GE $00--1.00 E$ OI 6.36 E $00--8.19 E \quad 00$ $4.97 E$ DO---6.36E OO $4.07 E$ DO- - 4.97E 00 $3.01 E$ OO- $-4.07 E 00$ 2.46E $00--3.01$ E OO $2.35 \mathrm{E}$ 00---2.46E 00 $1.83 \mathrm{E} 00---2.35 \mathrm{E} \mathrm{OO}$ $1.11 E$ OO-- $1.83 E$ OO .50E-01---1.11E OO $1.11 E-01--5.50 E-0$ 3.35E-02--1.11 E-01 $5.83 E-04--3.35 E-02$ $1.01 E-04--5.83 E-04$ $2.90 E-05--1.01 E-04$ 1.07E-05-O-2. $00 E-05$ $3.06 \mathrm{E}-06--1.07 \mathrm{E}-05$ $1.12 E-06-2.06 E-06$ $4.14 \mathrm{E}-07---1.12 \mathrm{E}-06$
$0.0 \quad--4.14 \mathrm{E}-07$ $M U=-1.0000$ 0.0

0.0

0.0

$3.548 E-06$

1.433E-03

$1.092 E-02$

6.536E-03

$3.815 \mathrm{E}-03$

$2.269 \mathrm{E}-03$

$2.603 E-03$

1. $324 E-02$

$1.496 E-02$

$8.705 E-01$

3.689E 00

$1.062 \mathrm{E} \mathrm{O1}$

$2.806 E 01$

$7.436 \mathrm{E}$ Ol

$1.612 \mathrm{E} \mathrm{O2}$

$2.318 \mathrm{E} \mathrm{O} 2$

ANGLE 10 $M U=0.0950$ 0.0
0.0
0.0 0.0 0.0 $1.415 \mathrm{E}-03$ $1.155 \mathrm{E}-02$ $1.097 \mathrm{E}-0$ $8.744 E-03$ $1.348 \mathrm{E}-02$ $4.725 \mathrm{E}-03$ $2.910 E-03$ $3.145 \mathrm{E}-03$ $3.619 \mathrm{E}-03$ $1.912 \mathrm{E}-02$ $1.538 \mathrm{E}-02$ $8.900 E-01$ $3.761 E 00$ $1.082 \mathrm{E} 01$ $2.853 \mathrm{E} \mathrm{O1}$ $7.553 \mathrm{E}$ OL $\begin{array}{ll}1.636 E & 02 \\ 2.350 E ~ & 02\end{array}$ $\triangle A N G L 2 \quad$ ANGLE 3 ANGLE 4 $\begin{array}{ll}U V=-0.9894 \quad M U= \\ C .0 & 0.0\end{array}$ 0

0.0

$1.160 \mathrm{E}-05$

. $498 E-03$

$1.088 E-02$

$6.542 E-03$

$3.818 \mathrm{~F}-03$

$2.273 \mathrm{E}-03$

$2.606 \mathrm{E}-03$

1.825E-02

$1.825 E-02$
$1.496 E-02$

$8.707 \mathrm{E}-01$

$3.689 E$ OO

$1.063 \mathrm{E} 01$

$2.806 \mathrm{E}$ Ol

$1.613 \mathrm{~F} 02$

2.318E 02

ANGLE 11 $M=0.2816$

0.0

0.0

0.0

$1.844 E-03$

$1.242 \mathrm{E}-02$

$1.137 \mathrm{E}-02$

$9.070 E-03$

$9.503 E-03$

$4.856 E-03$

$3.140 \mathrm{E}-03$

$3.326 E-03$

$3.6 C 4 \mathrm{E}-03$

$1.951 \mathrm{E}-02$

1. $546 \mathrm{E}-02$

8.936 E- 01

$3.774 \mathrm{E} \quad 00$

$1.085 \mathrm{E}$ O1

$2.861 \mathrm{E} 01$

..573E OI

2.641E 02
0.0

0.0
0.0
0.0

4.010E-05

$5.746 E-03$

$1.071 \mathrm{E}-02$

$6.567 E-03$

6.

$3.831 \mathrm{E}-03$

$2.620 E-03$

$3.318 \mathrm{E}-03$

$1.828 E-02$

$1.828 \mathrm{E}-02$
$1.497 \mathrm{E}-0$ ?

$8.714 \mathrm{E}-01$

$3.692 \mathrm{E} 00$

$1.063 \mathrm{E} \mathrm{O1}$

$2.808 \mathrm{E} 01$

$7.442 \mathrm{E}$ OI

$1.614 E 02$

$2.320 \mathrm{E} 02$

ANGLE 12

0.0

0.0

.0

$5.019 \mathrm{E}-03$

. $378 E-02$

$1.190 E-02$
$. .937 E-03$

$1.042 \mathrm{E}-02$

$5.602 \mathrm{E}-03$

$3.325 \mathrm{E}-03$

$3.474 E-03$

$3.822 E-03$

$1.961 \mathrm{E}-02$

$1.553 \mathrm{E}-02$

$8.969 \mathrm{E}-01$

$3.786 \mathrm{E} 00$

$1.088 \mathrm{E} 01$

$2.869 \mathrm{E}$ O1

$7.592 \mathrm{E}$ OI

$2.360 \mathrm{E} \mathrm{O} 2$
0.0

0.0

0.0

$.663 E-05$

$6.188 E-03$

. .

$8.095 E-03$

.862E-0

$2.319 \mathrm{E}-0$

$3.341 E-03$

1. $333 \mathrm{E}-02$

$1.500 \mathrm{E}-02$

$8.728 \mathrm{E}-01$

$3.697 \mathrm{E} 00$

$1.065 \mathrm{E}$ Ol
$2.811 \mathrm{E}$ Ol

7.450 E Ol

$1.615 E$ O2

$2.323 \mathrm{E} 02$

ANGLE 13 $M U=0.6179$ 0.0 0.0 0.0

$1.082 \mathrm{E}-02$

$1.845 \mathrm{E}-02$

$1.550 \mathrm{E}-02$

1.301E-O2

$6.914 E-03$

3.480E-03

$3.607 E-03$

$4.233 \mathrm{E}-03$

1.919E-02

$1.560 \mathrm{E}-02$

$9.000 E-01$

3.797 O

$1.091 \mathrm{E} 01$

7.610E OI

$1.648 \mathrm{E} 02$

$2.365 E 02$
ANGLE 5
$M U=-0.7550$ MU $=-0$ 0.0

0.0

$1.474 E-04$

$6.756 E-03$

$1.013 E-02$

$069 E-03$

$3.918 \mathrm{E}-03$

2. $688 \mathrm{E}-03$

$3.373 \mathrm{E}-03$

$1.841 \mathrm{E}-02$

$1.504 E-02$
$8.747 E-01$

$8.747 \mathrm{E}-01$

$3.704 \mathrm{E} 00$

$1.067 \mathrm{E} 01$
$2.816 \mathrm{E} 01$

$7.462 E$ ol

$1.618 \mathrm{E} 02$

$2.326 \mathrm{E} 02$

ANGLE 14

0.0

0.0

0.0

3.195E-02

$2.949 E-02$

$1.913 E-02$

. $577 E-02$

$7.600 E-03$

$3.793 E-03$

$3.867 E-03$

$4.359 E-03$

$1.941 E-02$

$1.567 E-02$

$9.027 E-01$

$3.806 E$ OO

$1.094 E$ OI

.

$.651 \mathrm{E} \mathrm{O2}$

$2.369 \mathrm{E} 02$

ANGLE 6 $M=-0.6$ 0.0

0.0
0.0

2.389E-04

$7.412 E-03$
$9.876 E-03$

$9.876 E-03$

$082 E-03$

.009E-03

$2.428 \mathrm{E}-03$

$2.743 E-03$
$3.415 E-03$

1. $851 \mathrm{E}-02$

. $510 \mathrm{E}-02$

.771E-01

$3.713 E 00$

$1.069 E 01$

$2.822 \mathrm{E} 01$

$7.476 \mathrm{E}$ 01

$1.521 E \quad 02$
$2.331 \mathrm{E} 02$

ANGLE 15

$J=0.8656$

0.0

0.0

$8.723 E-02$

$8.284 \mathrm{E}-02$

$3.135 E-02$

$1.747 E-02$ 
(NEUTRONS/MEV/STERADIAN/SOURCE NEUTRON)

\begin{tabular}{|c|c|}
\hline & $\begin{array}{l}\text { FNERGY } \\
\text { FNHE }\end{array}$ \\
\hline & $\begin{array}{l}\text { ROUP (MEV) } \\
\text { O1---1.50E O1 }\end{array}$ \\
\hline $.00 F$ & $01---1.22 E$ Ol \\
\hline $8.19 E$ & $00---1.00 E \quad 01$ \\
\hline $6.36 \mathrm{~F}$ & $00---8.1$ QE 00 \\
\hline $4.97 \mathrm{E}$ & $00---6.36 \mathrm{E} \quad 00$ \\
\hline $4.07 E$ & $00---4.97 \mathrm{E} 00$ \\
\hline $3.01 E$ & $0 n-\cdots 4.07 E \quad 00$ \\
\hline $2.46 E$ & $00--3.01 \mathrm{E} \quad 00$ \\
\hline $2.35 E$ & $00---2.46 \mathrm{E}$ on \\
\hline $1.83 \mathrm{E}$ & $00---2.35 \mathrm{E} \quad 00$ \\
\hline $1.11 E$ & $00--1.83 F \quad 00$ \\
\hline $5.50 E-$ & $-01-\cdots-1.11 \mathrm{E} \quad 00$ \\
\hline $1.11 \mathrm{~F}-$ & $-01---5.50 E-01$ \\
\hline $\begin{array}{l}3.35 \mathrm{E}- \\
5.83 \mathrm{E}-\end{array}$ & $\begin{array}{l}-02---1.11 \mathrm{E}-n 1 \\
04---3.35 \mathrm{E}-02\end{array}$ \\
\hline $1.01 \mathrm{~F}-\mathrm{C}$ & $-04--5.83 E-04$ \\
\hline & \\
\hline $1.07 E-C$ & $-05---2.90 E-05$ \\
\hline $3.06 E-C$ & $-06---1.07 E-05$ \\
\hline $1.12 \mathrm{E}-\mathrm{r}$ & $-n 6---3.06=-06$ \\
\hline $4.1<F-$ & $-07---1.12 \mathrm{E}-06$ \\
\hline 3.0 & $---4.14 E-07$ \\
\hline & ENERGY \\
\hline & ROUP It \\
\hline $1.22 \mathrm{EC}$ & $01---1.50 \mathrm{E}$ 01 \\
\hline $1.00 E ?$ & $n 1---1.22 E \quad 01$ \\
\hline $8.19 E$ & $00---3.00 E \quad 01$ \\
\hline $6.36 \mathrm{E}$ & $00---8.19 \mathrm{E} \quad 00$ \\
\hline $4.97 \mathrm{E}$ & $0 C---6.36 \mathrm{E}$ OO \\
\hline $4 . C 7 F$ & $00---4.97 \mathrm{E} \quad 00$ \\
\hline $3.01 E$ & $00---4.07 E \quad 00$ \\
\hline $2.46 E$ & $00---3.01 E 0 n$ \\
\hline $2.35 \mathrm{E}$ & $n 0---2.46 E 00$ \\
\hline & $00--2.25 \mathrm{E} \quad 00$ \\
\hline $1.11 \mathrm{E} \mathrm{C}$ & $00---1.83 \mathrm{E} \quad 00$ \\
\hline $5.50 E-C$ & $-01---1.11 \mathrm{E} \quad 00$ \\
\hline & $-01---5.50 E-01$ \\
\hline $3.35 E-C$ & $-1.11 \mathrm{E}-\mathrm{C}$ \\
\hline & \\
\hline & \\
\hline & $\begin{array}{l}-05--1.01 \mathrm{E}-04 \\
-05---2.90 \mathrm{E}-05\end{array}$ \\
\hline & $-0 K---1.07 F-05$ \\
\hline $\begin{array}{l}1.12 E-C \\
4.14 E-C\end{array}$ & $-06---3.06 \mathrm{E}-06$ \\
\hline 0.0 & $---4.14 E-07$ \\
\hline
\end{tabular}

ANGLE 1 $M U=-1$

0.0

0.0

0.0

$3.003 E-05$

$5.864 \mathrm{E}-\mathrm{C} 3$

$1.238 \mathrm{~F}-02$

$1.084 \mathrm{~F}-0$

$1.435 \mathrm{E}-02$

$7.978 \mathrm{E}-02$

$5.704 \mathrm{~F}-03$

$7.347 \mathrm{E}-03$

$1.042 \mathrm{E}-02$

$6.322 \mathrm{~F}+\mathrm{C}$ ?

$5.335 \mathrm{E}-\mathrm{C} 2$
$3.155 \mathrm{E}$

$\begin{array}{ll}3.155 \mathrm{E} & 00 \\ 1.348 \mathrm{E} & 01\end{array}$

$\begin{array}{ll}1.348 \mathrm{E} & 01 \\ 3.903 \mathrm{E} & 01\end{array}$

$1.036 \mathrm{E} \mathrm{O2}$

2.755E O2

$5.99 ? E$
$8.643 F$

ANGLE 10

$M U=0.0950$ 0.0

0.0

0.0

$2.446 E-03$ $9.904 E-03$ $9.904 \mathrm{E}-03$
$1.040 \mathrm{E}-02$ $1.252 \mathrm{E}-02$ $1.526 E-02$ $8.328 \mathrm{E}-03$ $8.150 \mathrm{E}-0^{2}$ $9.579 \mathrm{E}-03$ $1.071 \mathrm{E}-02$ $6.864 E-02$ $5.589 \mathrm{~F}-02$ $3.282 \mathrm{E} 00$ $1.397 \mathrm{E} 01$ $4.036 E$ OI $1.069 \mathrm{~F} 02$ $2.838 E \quad 02$ $6.163 \mathrm{E} 02$ $8.868 \mathrm{E} 02$ $M U=-0.9894$ 0.0 $4.066 \mathrm{E}-05$ 5. $C 41 E-03$ $1.233 E-0$ ? $1.085 E-02$ $3.433 E-02$ $7.986 \mathrm{E}-02$ $5.716 \mathrm{E}-03$ $7.360 E-03$ $1.043=-02$ $6.326 \mathrm{~F}-02$ $5.327 E-02$ $3.356 \mathrm{E} \mathrm{OO}$ 1.348 E 1 $3.904 \mathrm{~F}$ O1 $1.026 \mathrm{E} 02$ 2.756E C2 $8.645 E \quad 02$ $U=-0.9446$ 0.0 0.0 0.0 $7.795 \mathrm{E}-05$ $6.238 \mathrm{E}-03$ $1.213 E-C 2$ $1.788 \mathrm{E}-02$ $1.427 \mathrm{E}-\mathrm{n} 2$ $8.023 E-02$ $5.764 E-03$ $7.411 E-03$
$1.048 E-02$ $1.048 \varepsilon-02$ $6.341 \mathrm{E}-02$ $5.346 \mathrm{E}-02$ $3.160 E$ OO $\begin{array}{ll}1.350 F & 01 \\ 3.909 E & 01\end{array}$ $1.509 E$ O1
$1.037 E$ O2 $2.750 \mathrm{~F} 02$ $6.000 E 02$ $8.656 \mathrm{E}$ O2

ANGLE 11

ANGLE 12 ANGLE $1216 \quad M U=0.4580 \quad M U=0$ $0.0 \quad 0.0$ $2.245 \mathrm{E}-03$ $1.61 E E-02$ $1.422 \mathrm{E}-\mathrm{C} 2$ $1.020 \mathrm{E}-02$ $1.270 \mathrm{E}-02$ $7.934 \mathrm{E}-03$ $9 . E I 3 F-03$ 1. $206 \mathrm{~F}-02$ $6.835 \mathrm{E}-02$ $5.6375-02$ $3.306 E$ OD $1.406 \mathrm{E}$ 01 4. $060 \mathrm{E} 01$ $1.075 \mathrm{E} 02$ 2.853502 $8.907 \mathrm{~F} 02$ 
(NEUTRONS/MEV/STERADIAN/SOURCE NEUTRONI

ENERGY GROUP (MEV) $1.22 E$ O1---1.5OE 01 $\begin{array}{lll}1.22 E & 01--1.50 E & 01 \\ 1.00 E & 01--1.22 E & 01\end{array}$ 8.1 OE $00--1.00 E$ O1 6.36E OO---8.19E OO 4.97E $00---6.36$ E 00 $4.07 E$ DO-- $4.97 E$ DO $3.01 \mathrm{E} 00--4.07 E$ OO $2.46 E$ OO-- $3.01 \mathrm{E} 00$ $2.35 \mathrm{~F} 00--2.46 \mathrm{E}$ 0O 1. $83 \mathrm{E} \quad 00-\cdots 2.35 \mathrm{E} 00$ $1.11 \mathrm{E} 00--1.83 \mathrm{E}$ 00 $5.50 E-01--1.11 \mathrm{E}$ OO $1.11 E-01--5.50 E-01$ $3.35 \mathrm{E}-02---1.11 \mathrm{E}-01$ $5.83 \mathrm{E}-04---3.35 \mathrm{E}-02$ $1.01 E-04---5.83 \mathrm{E}-04$ $2.90 \mathrm{E}-05--1.01 \mathrm{E}-04$ $1.07 E-05--2.90 E-05$ $3.06 E-06=-1.07 E-05$ $1.12 \mathrm{E}-06---3.06 \mathrm{E}-06$ $4.14 E-07---1.12 E-06$
$0.0 \quad---4.14 E-07$ ENERGY GROUP (MEV)

$1.22 E$ O1---1.50E OI $1.00 \mathrm{E}$ O1--1.22E OI

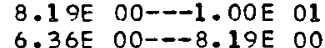
$\begin{array}{lll}6.36 E & 00---8.19 E & 00 \\ 4.97 E & 00---6.36 E & 00\end{array}$ 4.97E $00---6.36 E$
4.00 4.07E OO---4.97E OO
$3.01 E$ OO---4.07E 00 $3.01 \mathrm{E}$ 00---4.07E 00 $2.35 \mathrm{E} 00-\cdots 2.46 \mathrm{E} O 0$ $\begin{aligned} & 2.35 E \\ & 1.83 E\end{aligned} 00--2.35 E 00$ $1.11 E$ OO-- $1.83 E 00$ $5.50 \mathrm{E}-01--1.11 \mathrm{E}$ OO $1.13 E-01-2502$ 3. 5.83E-04- $-3.35 E-02$ $1.01 E-C 4-2.35 E-04$ $1.907-05-1.01 E-04$ $1.07 E-05-2.075$ $1.0 \mathrm{E}-06-2.07 \mathrm{E}-05$ $4.14 E-07=-3.06 E-06$ $4.14 E-07---1.12 E-06$
0.0
ANGLE 1 1.0000 0 0.0 $4.721 E-05$ $5.592 \mathrm{E}-03$ $1.184 \mathrm{E}-02$ $1.180 \mathrm{E}-02$ $1.594 \mathrm{E}-02$ $9.692 E-03$ $7.633 E-03$ $1.050 E-02$ $1.581 E-02$ $1.004 \mathrm{E}-01$ 8.601E-02 $5.135 E 00$ $2.207 E$ O1 $6.413 E$ o1 $1.709 \mathrm{E} 02$ $4.558 \mathrm{E} \mathrm{O2}$ $\begin{array}{ll}9.936 E & 02 \\ 1.437 E & 03\end{array}$ ANGLE 10 $M U=0.0950$ 0.0

0.0
0.0 0.0 $1.670 \mathrm{E}-03$ $1.303 E-02$ $1.342 E-02$ $1.665 E-02$ $2.090 E-02$ $1.526 \mathrm{E}-02$ $1.020 \mathrm{E}-02$ $1.331 E-02$ $1.924 \mathrm{E}-02$ $1.03 \mathrm{E}-02$ $5.103 E-02$ 2.308E OI $2.308 E$ O1 1.773 OI $1.735 E$ O2 4.735 O $1.485 \mathrm{E} \mathrm{O3}$
ANGLE 2 $2 \quad$ ANGLE 3 0.0 0.0 $5.771 E-05$ $5.666 \mathrm{E}-03$ $1.179 \mathrm{E}-02$ $1.181 \mathrm{E}-02$ $1.592 \mathrm{E}-02$ $9.704 \mathrm{E}-03$ $7.650 \mathrm{E}-03$ $1.052 \mathrm{E}-02$ $1.583 \mathrm{E}-02$ $1.005 E-01$ $8.606 \mathrm{E}-02$ $5.138 \mathrm{E} \quad 00$ $2.208 E \quad 01$ $6.416 E \quad 01$ $1.71 \mathrm{OE}$ O? 4.560 E 02 $9.94 \mathrm{CE} 02$ $1.438 \mathrm{E} \quad 03$ ANGLE 11 $M U=0.2816$ 0.0 0.0 $3.028 E-03$ . $268 \mathrm{E}-02$ $1.303 E-02$ $1.714 E-02$ $2.184 \mathrm{E}-02$ $1.417 E-02$ $1.159 \mathrm{E}-02$ $1.451 E-02$ 1.848E-02 $9.109 \mathrm{E}-02$ $5.444 E 00$ $2.444 E$ OO $6.745 \mathrm{E}$ Ol $1.792 \mathrm{E} O 2$ 4.767E 02 4.7ETE O2 $1.494 \mathrm{E} 03$ 0.0 0.0 0.0 $5.948 \mathrm{E}-03$ 1.161E-02 $1.184 \mathrm{E}-02$ $1.585 \mathrm{E}-02$ $9.757 \mathrm{E}-03$ $7.721 \mathrm{E}-03$ $1.060 \mathrm{E}-02$ 1.591E-02 $1.008 \mathrm{E}-01$ $8.624 E-02$ 2.147E 00 $6.426 \mathrm{E}$ OI $1.712 \mathrm{E} 02$ 4.566E 02 $9.954 \mathrm{E} 02$ ANGLE 12 0.0
0.0 0.0
0.0 0.0 3. $886 \mathrm{E}-03$ $1.822 E-02$ $1.780 \mathrm{E}-02$ $2.186 \mathrm{E}-02$ $2.760 \varepsilon-02$ $1.851 E-02$ $1.217 E-02$ $1.514 \mathrm{E}-02$ $116 \mathrm{E}-01$ $1.116 E-01$ $9.292 E-02$ 5.491E OO $6.794 \mathrm{E}$ OI $1.804 \mathrm{E} 02$ 1.804E O2 1.794E 03 $1.044 E$
$1.502 E$
ANGLE 4
$M U=-0.8656 \quad M U G L=-0.7550 \quad$ ANGLE 6

0.0
0.0 $9.491 E-05$ $M U=0.4580$
0.0

$1.530 E-04$

$6.44 \mathrm{EE}-03$

$1.131 \mathrm{E}-02$

$1.190 \mathrm{E}-02$

$9.878 \mathrm{E}-03$

$7.859 E-03$

$1.076 E-02$

1.607 E-02

$1.013 \mathrm{E}-01$

$.658 \mathrm{E}-02$
$5.165 \mathrm{E} 00$

$2.218 E \quad 01$

$6.446 E 01$

$1.717 \mathrm{E} \mathrm{O2}$

$4.579 E$ O2

$1.444 \mathrm{E} 03$

ANGLE 13 $U=0.6179 \quad$ ANGLE 14

$M U=0.61$
0.0
0.0
0.0

0.0

0.0

7.075E-03

2.255E-02

$2.106 E-02$

$2.537 E-02$

$3.182 \mathrm{E}-02$

$1.906 E-02$

$1.376 E-02$

$1.650 \mathrm{E}-02$

$.137 \mathrm{E}-01$

9. $381 \mathrm{E}-02$

.

$6.362 E$ OI

$6.8415 \mathrm{E} 02$

$4.827 E 02$

$1.049 \mathrm{E} 03$

1. 510 E 03
0.0

0.0

$2.254 \mathrm{E}-04$

$1.101 \mathrm{E}-02$

$1.202 \mathrm{E}-02$

. $569 \mathrm{E}-02$

E-02

.067E-03

$.630 \mathrm{E}-02$

$1.021 \mathrm{E}-01$

.706E-02

$5.190 E 00$

.4283 OI

$6.473 E$
$1.724 E$ 02

4.596E 02

$1.002 \mathrm{E} 03$

$U=0.7550$

0.0
0.0
0.0
0.0

0

$1.304 \mathrm{E}-02$ 3. $741 \mathrm{E}-02$ $3.009 E-02$ $3.342 E-02$ 4. 政 ( $1.149 E-01$ . $460 E-02$ .376E 01 $.881 \mathrm{E} 01$ $4.852 \mathrm{E} 02$ $1.055 \mathrm{E} 03$ 1.517E 03

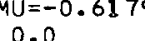
0.0 0 0.0 $3.376 E-04$ $7.755 \mathrm{E}-03$ $1.078 \mathrm{E}-02$ . $225 E-02$ 1. $576 \mathrm{E}-02$ 1. $044 \mathrm{E}-02$ $8.353 E-03$ $1.131 \mathrm{E}-02$ $1.660 E-02$ . 03 JE-01 $.766 \mathrm{E}-02$ $5.222 E \quad 00$ ..507E OI $1.733 E$ O2 4.618E 02 $1.306 \mathrm{E} \mathrm{O3}$ $1.455 \mathrm{E} 03$

ANGLE 15

MU $=$
0.0

0.0

0.0

2. $731 \mathrm{E}-02$ $6.371 \mathrm{E}-02$ $4.292 E-02$ 
(NEUTRJNS/MEV/STERAOIAN/SOURCE NEUTRON)

ENERGY

$1.22 E$ OL---1.5OE 01

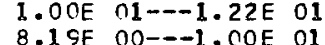
$6.36 \mathrm{E} \quad 00--8.19 \mathrm{E}$ OO $4.97 E$ EO-- $-6.36 E$ OO 4.07E $00---4.97 E$ OD $3.01 \mathrm{E}$ 00---4.07E OD $2.46 E$ OD- $-3.01 \mathrm{E} \mathrm{OO}$ $2.35 \mathrm{E} 00--2.46 \mathrm{~F} 00$ $1.82 E$ EO- $--2.35 E$ OO $1.11 \mathrm{~F} 00--1.23 E \mathrm{CO}$ $5.50 E-01--1.11 E$ OO $1.12 E-01--5.50 E-01$ $3.35 \mathrm{E}-02---1.11 \mathrm{E}-\mathrm{n} !$
$5.83 \mathrm{~F}-04--3.35 \mathrm{E}-02$ $1.01 E-04--5.83 E-04$ $2.00 E-05-2.01 E-04$ $1.07 E-05---2.90 E-05$ $3.06 E-06-1.07 E-0.05$ $1.12 E-06-0-3.06 E-06$ $\begin{array}{ll}4.14 \mathrm{E}-07---1.12 \mathrm{E}-06 \\ 0.0 & ---4.14 \mathrm{E}-07\end{array}$

$$
\text { ENERGY }
$$$$
\text { GROUP (MEV) }
$$

$1.22 E$ O1---1.50E 01 $1.00 E$ 01---1.22E 01 6.3ES $00---8.19 E$ OO $4.07 E$ DO- - 6.36E 00 4.07E $00---4.97 \mathrm{E}$ กO $3.01 \mathrm{E} 00--4.07 \mathrm{E}$ OO $2.46 \mathrm{E} 00---3.01 \mathrm{E}$ OO $2.35 \mathrm{~F}$ CO---2.46E OO $3.83 \mathrm{E} 00--2.35 \mathrm{E} \mathrm{CO}$ $1.11 \mathrm{~F} 00--1.83 \mathrm{E} 00$ $5.50 E-01--1.11 E$ OO $1.5 \mathrm{E}-01--5.50 \mathrm{E}-01$ $3.35 \mathrm{E}-02--1.11 \mathrm{E}-01$ $5.83 E-04--3.35 E-02$ $1.01 E-04-0-53 E-04$ $2.90 E-05--1.01 E-04$ $1.07 E-05--2.00 E-05$ 3.06E-06---1.07E-05 $1.12 \mathrm{E}-06-\cdots 3.06 \mathrm{E}-06$ $4.14 E-07---1.12 E-06$
$0.0 \quad--04.14 E-07$
ANGLE 1 $U=-1.0000$ 0.0 0.0 0.0 $6.340 E-05$ $4.532 \mathrm{E}-03$ $9.522 \mathrm{E}-\mathrm{O}$ $1.114 E-02$ $1.544 \mathrm{E}-02$ $1.082 \mathrm{E}-02$ $9.907 E-03$ $1.522 \mathrm{E}-02$ $2.541 E-02$ $1.741 \mathrm{E}-01$ $1.529 \mathrm{E}-01$ $9.283 \mathrm{E} 00$ $4.031 \mathrm{E} 01$ $1.180 \mathrm{E} 02$ $3.170 E$ N2 $8.505 E$ O $1.863 \mathrm{E} 03$
$2.711 \mathrm{E} 03$

ANGLE 10 $M U=0.0950$ 0.0

0.0

$1.834 \mathrm{E}-03$ $9.127 E-03$ $1.004 \mathrm{E}-02$ $1.513 \mathrm{~F}-02$ $1.513 \mathrm{~F}-02$ $2.076 \mathrm{E}-02$ $1.428 \mathrm{E}-02$ $1.428 \mathrm{E}-02$ $3.024 \mathrm{E}-02$ $1.924 E-01$ $1.924 E-01$
$1.644 E-01$ $9.909 E 00$ $4.281 \mathrm{E} 01$ $1.251 \mathrm{E} 02$ $3.350 \mathrm{E} 02$ $8.966 E 02$ $1.960 \mathrm{E} 03$ 2.838 E 03
ANGLE 2 0.0 0.0 0.0 0.0
$7.205 \mathrm{~F}-05$ $4.589 E-C 3$ $0.485 \mathrm{E}-0$ $1.114 \mathrm{E}-02$ $1.54 \mathrm{ZE}-02$ $1.084 E-02$ $9.932 \mathrm{E}-\mathrm{C} 3$ $1.525 \mathrm{E}-02$ $2.545 \mathrm{E}-02$ $1.742 \mathrm{E}-01$ $1.530 E-01$ $9.288 \mathrm{E} 00$ $4 . \Upsilon 33 E$ OI $1.181 E$ O2 $3.172 \mathrm{E} \mathrm{C2}$ $8.5 C 9 E$ OS $1.864 E \quad 03$
$2.712 E \quad D^{2}$

ANGLE 11 $M U=C .2816$ 0.0

0.0 0.0 $2.442 E-C 3$ l. $225 \mathrm{E}-02$ ].7C $3.775 \mathrm{E}-02$ $2.475 E-02$ i. $543 E-02$ $1.543 \mathrm{E}-02$ $3.191 E-02$ $1.955 \mathrm{E}-01$ $1.955 E-01$ $1.003 \mathrm{E} \mathrm{OI}$ 4.328E 01 $1.264 E 02$ $3.383 E$ O2 $9.051 \mathrm{~F} 02$ $1.078 \mathrm{~F} 03$ $\begin{array}{ll}\text { ANGLE } & 3 \\ 3 & \text { ANGLE } 4 \\ 0.0 .9446 & M U=-0.8656\end{array}$ MU $=-0.0$
0.0 0.0 0.0 0.0
0.0 $1.034 \mathrm{E}-04$ 4. $812 \mathrm{E}-03$ $9.345 \mathrm{E}-03$ $1.116 \mathrm{E}-02$ $1.529 \mathrm{E}-02$ $1.092 \mathrm{E}-02$ $1.004 E-02$ $1.539 E-02$ 2.561E-02 $1.749 \mathrm{E}-\mathrm{n} 1$ $1.534 \mathrm{E}-01$ $9.311 E 00$ $4.042 E$ O1 $1.183 \mathrm{E}$ O2 $3.178 \mathrm{E} 02$ $8.526 E$ O2 $\begin{array}{lll}1.868 \mathrm{E} & 03 \\ 2.717 \mathrm{E} & 03\end{array}$ 0.0 $1.535 \mathrm{E}-04$ $5.197 E-03$ $9.128 \mathrm{E}-0$ $1.122 \mathrm{E}-02$ $1.533 \mathrm{E}-02$ $1.109 \mathrm{E}-02$ $1.024 \mathrm{E}-02$ $1.566 \mathrm{E}-02$ $2.591 E-02$ $1.761 \varepsilon-01$ $1.542 \mathrm{E}-01$ $9.354 \mathrm{~F} 00$ 4.059E 01 $1.188 \mathrm{E} \quad 02$ $3.191 \mathrm{E} 02$ $8.558 \mathrm{E} 02$

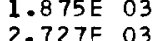
$\begin{aligned} M U & =-0.7 \\ 0.0 & \\ 0.0 & \end{aligned}$ 0.0 0.0 $2.189 E-04$ $5.681 E-03$ $8.915 E-03$ $1.134 E-02$ $1.536 \mathrm{E}-02$ $1.139 \mathrm{E}-02$ $1.054 \mathrm{E}-02$ $1.606 \mathrm{E}-02$ $2.635 \mathrm{E}-02$ $1.777 \mathrm{E}-01$ 1. $552 \mathrm{E}-01$ $9.413 E 00$ $4.083 \mathrm{E} 01$ $1.195 \mathrm{E} \quad 02$ $3.208 E 02$ $8.602 E$ O

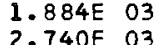

AVGLE 6 $M U=-3.6179$ 0.0 $3.222 E-04$ $6.229 E-03$ $8.770 E-03$ 1.157E-02 $1.555 \mathrm{E}-02$ $1.185 \mathrm{E}-02$ $1.096 \mathrm{E}-02$ $1.660 \mathrm{E}-02$ $2.593 E-02$ $1.798 \mathrm{E}-01$ $1.566 \mathrm{E}-01$ $9.489 \mathrm{E} 00$ $4.114 \mathrm{E}$ O1 $1.203 E$ O2 $3.230 \mathrm{E} 02$ $8.658 \mathrm{E} 02$ $1.896 E$
$2.756 E$

ANGLE 12 0.0

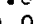
0.0

$3.779 E-03$ $1.396 \mathrm{E}-02$ $1.35 \mathrm{E}-02$ 2. $452 \mathrm{E}-0 \mathrm{O}$ $2.351 \mathrm{E}-\mathrm{OL}$ $2.094 \mathrm{E}-02$ $1.713 \mathrm{E}-02$ $2.364 E-02$ $3.282 \mathrm{E}-02$ $3.282 \mathrm{E}-02$
$1.905 \mathrm{E}-01$ $1.958 \mathrm{E}-01$ $1.014 \mathrm{E} \mathrm{O1}$ 4.374E 01 3.277E 02 $3.416 \mathrm{E} 02$ $9.133 E 02$ $1.905 \mathrm{E} 03$ 2.885E 03
ANGLE 13 0.0 0 0.0

$6.207 F-03$ $2.001 \mathrm{E}-02$ $1.927 \mathrm{E}-02$ $.693 \mathrm{E}-02$ $3.716 \mathrm{E}-0$ $2.496 \mathrm{E}-02$ $1.888 \mathrm{E}-02$ $2.547 \mathrm{E}-0$ $3.441 E-02$ $1.709 \mathrm{E}-01$ $1.025 \mathrm{E} \mathrm{Ol}$ 4.417E 01 $1.289 \mathrm{~F} 02$ $3.446 \mathrm{E} \mathrm{O2}$ $9.210 \mathrm{E} 02$ $2.011 E 03$ $2.906 E 03$
ANGLE 14 0.0 0.0

$1.194 \mathrm{E}-02$ 3. $057 \mathrm{E}-02$ $3.475 \mathrm{E}-02$ $4.749 \mathrm{E}-02$ $2.916 E-02$ $2.115 \mathrm{E}-02$ $2.764 \mathrm{E}-02$ 3. $547 \mathrm{E}-02$ $2.061 \mathrm{E}-0$ $2.061 \mathrm{E}-01$
$1.727 \mathrm{E}-01$ $1.035 \mathrm{E}$ OI $4.455 \mathrm{E}$ OI $1.299 \mathrm{E} 02$ 
(NEUTRONS/MEV/STERADIAN/SOURCE NEUTRON)

ENERGY GROUP (MEV)

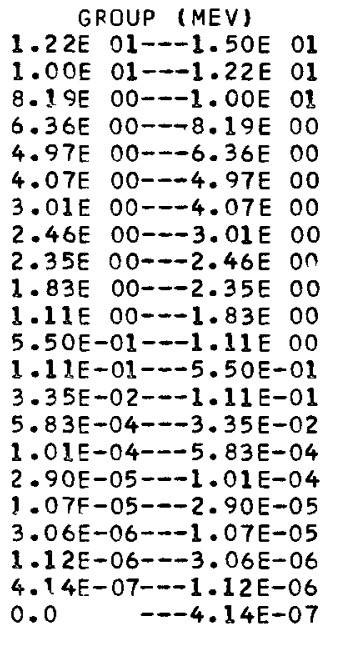

\begin{abstract}
ENERGY
\end{abstract}
GROUP (MEV)

$1.22 E$ OI-- $1.50 E$ OI
$1.00 E$ OI-- $1.22 E$ OI

$8.19 E$ OD- - 1 . OOE OI

$6.36 \mathrm{E} 00--8.19 \mathrm{E} \mathrm{OO}$

4.97E $00--6.36 E$ OO

4.07E $00--4.97 E$ OO

3.01 E $00--4.07 E$ OO

$2.46 E$ OO- $-3.01 E$ OO $2.35 \mathrm{E} 00--2.46 \mathrm{E} 00$ $1.83 \mathrm{E} 00--2.35 \mathrm{E}$ OO $1.11 \mathrm{~F} 00--1.83 \mathrm{E}$ 00 $5.50 \mathrm{E}-01--1.11 \mathrm{E}$ OO $1.11 \mathrm{E}-01-=-5.50 \mathrm{E}-01$ $3.35 \varepsilon-02--1.11 E \rightarrow 01$ 5.8 $E$ E $04--3.35 E-02$ $1.01 E-04--5.83 E-04$ $2.90 \mathrm{E}-05--1.01 E-\cap 4$ $1.07 E-05-2.90 E-05$ $1.12 \mathrm{E}-06-2=07 \mathrm{E}-05$ $1.12 \mathrm{E}-06-2.06 \mathrm{E}-06$ $4.14 E-07---1.12 E-06$
ANGLE 1 $U=-1.0000$ 0.0 0.0 0.0 0.0 $6.188 \mathrm{E}-05$ $3.391 \mathrm{E}-03$ $9.034 \mathrm{E}-0$ $9.034 \mathrm{E}-03$ $1.276 \mathrm{E}-02$ $1.002 \mathrm{E}-02$ $1.027 \mathrm{E}-02$ 1. $326 \mathrm{E}-02$ $2.293 \mathrm{E}-01$ $2.054 \mathrm{E}-01$ $1.265 \mathrm{E} 01$ $5.546 \mathrm{E}$ OI $1.635 \mathrm{E} \mathrm{O2}$ $4.425 E$ O2 $1.194 \mathrm{E} 03$ $2.629 E$ O3 ANGLE 10 $M U=0.0950$ 0.0 0.0 0.0 $1.408 \mathrm{E}-03$ $7.074 E-03$ $7.993 \mathrm{E}-03$ $1.291 E-02$ $1.879 \mathrm{E}-02$ $1.584 \mathrm{E}-02$ $1.512 \mathrm{E}-02$ $2.395 E-02$ $3.867 \mathrm{E}-02$ $2.567 E-01$ $2.237 E-01$ $1.368 \mathrm{E} 01$ $1.962 E$ OI $1.754 \mathrm{E} O 2$ $4.733 \mathrm{E}$ O2 $1.274 E$ OS $2.798 \mathrm{E} 03$ $\triangle N G L E \quad 2$
$M U=-0.9894$ $M U=-0$
0.0
0.0 0.0 0.0 $6.833 E-05$ $6.833 E-05$
$3.432 E-03$ $3.432 E-03$
$6.995 E-03$ $0.03 E-03$ $1.275 E-02$ $1.004 \mathrm{E}-02$ $3.029 \mathrm{E}-02$ $3.730 E-02$ $3.149 E-02$ $2.2 \subseteq 5 \mathrm{E}-01$ $2.055 E-01$ $1.266 E 01$ $5.549 \mathrm{E}$ OI $1.636 E$ O2 4.427E O2 $1.194 \mathrm{E} 03$ $2.630 E^{03}$ $3.848 \mathrm{E} 03$

ANGLE 3 0.0 0.0

0.0 $9.220 \mathrm{E}-05$ $3.593 E-03$ ..059E-03 $1.274 \mathrm{E}-02$ 1. $013 \mathrm{E}-02$ $1.013 \mathrm{E}-02$
$1.041 \mathrm{E}-02$ $1.041 \mathrm{E}-02$
$1.748 \mathrm{E}-02$ $3.171 \mathrm{E}-02$ 2. $305 E-01$ 2. $062 \mathrm{E}-01$ $1.270 E$ O1 $5.565 \mathrm{E} 01$ $1.640 E \quad 02$ 4.439E 02 $1.197 \mathrm{E} 03$ 2.637E O3 $3.857 \mathrm{E} 03$ ANGLE $\quad 4$
$M U=-0.8656$ 0.0 0.0 (1) 1. $314 E-04$ $3.872 \mathrm{E}-03$
$6.755 \mathrm{E}-03$ $9.711 \mathrm{E}-03$ $9.111 \mathrm{E}-03$ $1.032 \mathrm{E}-02$ $1.032 E-02$ $1.064 E-02$
$1.782 E-02$ $3.214 \mathrm{E}-02$ $2.323 E-01$ $2.074 \mathrm{E}-01$ $1.277 \mathrm{E} 01$ $5.593 E$ OI $1.648 \mathrm{E} \mathrm{O2}$ $.460 E 02$ $1.203 E 03$ $2.648 \mathrm{E} \mathrm{O3}$ $A N G L E \quad 5$
$M U=-0.7550$ 0.0 0.0 0.0 $1.845 E-04$ $4.220 \mathrm{E}-03$
$6.618 \mathrm{E}-03$ $9.6120 \mathrm{E}-03$ $1.286 \mathrm{E}-02$ $1.063 \mathrm{E}-02$ $1.063 \mathrm{E}-02$
$1.098 \mathrm{E}-02$ $1.098 \mathrm{E}-02$
$1.832 \mathrm{E}-02$ $3.275 \mathrm{E}-02$ $2.348 \mathrm{E}-01$ $2.091 \mathrm{E}-01$ $1.287 E 01$ $5.632 E$ OI $1.659 \mathrm{E} 02$ $4.489 E$ O2 $1.210 E 03$ $2.665 \mathrm{E} 03$ 3.897E 03

ANGLE 6
$M U=-0.6179$ $\begin{array}{rl}M U & =-0 \\ 0.0 & \\ 0.0 & 0 \\ 0.0\end{array}$ 0.0

2.693E-04 $4.618 \mathrm{E}-03$
$6.536 \mathrm{E}-03$ $9.426 \mathrm{E}-03$ $1.314 \mathrm{E}-02$ $1.112 E-02$ $1.112 \mathrm{E}-\mathrm{O} 2$ $1.145 \mathrm{E}-02$
$1.901 \mathrm{E}-02$ $3.355 \mathrm{E}-02$ $2.380 \mathrm{E}-01$ $2.113 \mathrm{E}-01$ $1.299 \mathrm{E} 01$ $5.583 E$ OI $1.674 \mathrm{E} \mathrm{O2}$ $4.526 E^{02}$ $1.220 \mathrm{E} 03$ $2.685 E$ O3 $3.924 \mathrm{E} 03$

ANGLE 7 $M U=-0.4580$ 0.0 0.0 0.0 $4.154 E-04$ $5.060 \mathrm{E}-03$ $6.559 \mathrm{E}-\mathrm{O}$ $9.792 \mathrm{E}-0$
$1.371 \mathrm{E}-02$ $1.371 \mathrm{E}-02$ $1.182 \mathrm{E}-02$
$1.208 \mathrm{E}-02$ $1.208 \mathrm{E}-02$ $1.989 E-02$
$3.454 E-02$ $2.419 \mathrm{E}-01$ $2.139 E-01$ $1.314 \mathrm{E}$ O1 $5.743 E$ OI $1.691 \mathrm{E} 02$ $4.571 \mathrm{E} 02$ $1.232 E \quad 03$ $2.710 E \quad 03$

ANGLE 8 $M U=-0.2816$ 0.0 0.0 0.0 $6.491 E-04$ $6.742 E-03$ $1.041 \mathrm{E}-02$ $1.472 \mathrm{E}-02$ $1.279 E-02$ $1.288 \mathrm{E}-02$ $2.099 \mathrm{E}-02$ $3.572 \mathrm{E}-02$ $2.464 \mathrm{E}-01$ $2.169 E-01$ $1.330 \mathrm{E} 01$ .810E OI $1.710 E$ O2 1.621E 02 $1.245 \mathrm{E} \mathrm{O}$ $2.737 \mathrm{E} \quad 03$
$3.991 \mathrm{E} 03$ ANGLE 16 $\mathrm{NU}=0.2816$ ANGLE 12
$U=0.4580$ ANGLE 13 0.0 0.0 0.0 0.0 $2.021 E-03$ $8.362 \mathrm{E}-03$ $9.295 \mathrm{E}-0$ $1.515 E-02$ $2.236 \mathrm{E}-02$ $1.801 \mathrm{E}-02$ $1.666 \mathrm{E}-02$ $2.588 \mathrm{E}-02$ $4.027 E-02$ $2.625 E-01$ $2.273 E-01$ $1.387 \mathrm{E} 01$ $1.0776 \mathrm{E} O 2$ $1.776 \mathrm{E} \mathrm{O2}$ $4.791 E$ O2 $3.830 E$ O3 $4.112 \mathrm{E} \mathrm{O} 3$
0.0

0.0

0.0

0.0

$3.026 \mathrm{E}-03$

$1.077 E-02$

$1.157 \mathrm{E}-02$

$1.857 \mathrm{E}-02$

$2.759 \mathrm{E}-02$

2.096E-02

$1.847 \mathrm{E}-02$

$2.808 \mathrm{E}-0$

4. $211 E-02$ $2.680 E-01$ 2.3OBE $1.406 E$ OL $6.119 E$ OI $1.798 E$ O2 1. $303 E$ E $1.361 \mathrm{E}$ $4.153 \mathrm{E} 03$ 0.0

0.0 0.0

$5.109 \mathrm{E}-03$

$1.513 \mathrm{E}-02$

1. $504 \mathrm{E}-02$ $2.360 \mathrm{E}-02$ $3.510 \mathrm{E}-02$ $2.469 \mathrm{E}-02$ $2.065 \mathrm{E}-02$ 3.060E-OL $4.391 E-02$ 2.735E-O1 10 
(NEUTRONS/MEV/STERADIAN/SOURCE NEUTRON)

\begin{tabular}{|c|c|}
\hline & $\begin{array}{l}\text { ENERGY } \\
\text { (MEV }\end{array}$ \\
\hline $1.22 \mathrm{GR}$ & $\begin{array}{l}\text { NOUP (MEV) } \\
0 \leq--1.50 \mathrm{E} \text { OI }\end{array}$ \\
\hline $1 . C O E$ & $01--1.22 E$ \\
\hline $8.19 \mathrm{E}$ & $O O--1.00 E$ OS \\
\hline $6.36 \mathrm{E}$ & $00---8.19 \mathrm{E}$ of \\
\hline $4.97 E$ & $00---6.36 \mathrm{E}$ OO \\
\hline $4.07 E$ & $00---4.97 E$ OO \\
\hline $3.01 \mathrm{E}$ & $00-\cdots-4.07 E 0 C$ \\
\hline $2.46 \mathrm{E}$ & $00---3.01 \mathrm{E}$ o \\
\hline $2.35 \mathrm{E}$ & $00--2.46 \mathrm{E}$ or \\
\hline 1.825 & $00---2.35 \mathrm{E}$ O \\
\hline $\begin{array}{l}1.1 ! \mathrm{E} \\
5.50 \mathrm{~F}-\end{array}$ & $\begin{array}{c}00---1.83 \mathrm{E} \text { oc } \\
-01--1.11 \mathrm{E} \text { of }\end{array}$ \\
\hline $1.11 \mathrm{~F}-$ & $\begin{array}{l}-01--5.50 E-0 \\
-02--1.11 E-0\end{array}$ \\
\hline $5.83 \mathrm{E}-$ & $n 4--3.35 E-0$ \\
\hline $1.01 \mathrm{E}-$ & $-04---5.83 E-0$ \\
\hline & $-05---1.01 E-0$ \\
\hline & $-05---2.90 \mathrm{E}-\mathrm{n}=$ \\
\hline $3.06 \mathrm{E}-$ & $-06---1.07 \mathrm{E}-0$ \\
\hline & $-06---3.0$ \\
\hline $\begin{array}{l}4.14 \mathrm{E}- \\
0.0\end{array}$ & $\begin{array}{r}-07--1.12 \mathrm{E}-0 \mathrm{O} \\
-=-4.14 \mathrm{E}-\mathrm{C}\end{array}$ \\
\hline & $E R G Y$ \\
\hline & ROUP (MEV) \\
\hline $1.22 \mathrm{~F}$ & $01--1.50 E$ \\
\hline $1.00 \mathrm{E}$ & $01--1.22 \mathrm{E} \quad 0$ \\
\hline $8.19 \mathrm{gE}$ & $00--1.00 E$ OI \\
\hline $6.36 \mathrm{E}$ & $00---8.19 \mathrm{E} \mathrm{OC}$ \\
\hline $4.97 \mathrm{E}$ & $00---6.36 \mathrm{E} \quad 00$ \\
\hline $4.07 E$ & $00---4.97 \varepsilon$ \\
\hline $3.01 \mathrm{E}$ & $00---4.07 E$ OC \\
\hline $2.46 \mathrm{E}$ & $00---3.01 \mathrm{E} 0$ \\
\hline $2.35 \mathrm{E}$ & $00---2.46 \mathrm{E}$ of \\
\hline $1.8^{2} \mathrm{E}$ & $00---2.35 \mathrm{E}$ \\
\hline $1.11 \mathrm{E}$ & $00---1.83 \mathrm{E}$ of \\
\hline $5.50 E-$ & $-01---1.11 \mathrm{E}$ \\
\hline $1.11 \mathrm{E}-$ & $-01---5.505$ \\
\hline & \\
\hline $5.83 \mathrm{E}-$ & \\
\hline $1.01 \mathrm{E}-$ & $-04---5.83 E-0$ \\
\hline & \\
\hline & \\
\hline & $-06---1.07$ \\
\hline & $-06---3$ \\
\hline $\begin{array}{l}.14 \mathrm{E}- \\
.0\end{array}$ & $--4.14 E-0$ \\
\hline
\end{tabular}
$M J=-1.0000$

0.0

0.0

0.0

$5.249 E-05$

$2.427 E-03$

$4.938 E-03$

$6.766 \mathrm{E}-03$

$5.693 E-03$

$8.386 \mathrm{~F}-03$

$9.389 \mathrm{E}-03$

$1.702 \mathrm{E}-02$

$3.341 E-0$ ?

$2.571 \mathrm{E}-01$

$2.341 \mathrm{E}-01$

$1.461 \mathrm{E}$ O1

6.454E OS

$5.216 \mathrm{E} 0$ ?

1.415E O3

$3.130 \mathrm{E} \mathrm{O3}$

$4.603 E$ O3

ANGLE 10

$M U=0.0950$ 0.0

0.0

0.0

$1.065 \mathrm{E}-03$

$5.016 \mathrm{E}-03$

$5.788 \mathrm{E}-03$

$9.998 \mathrm{E}-03$

$1.534 \mathrm{E}-02$

$1.370 E-02$

$1.415 \mathrm{E}-02$

$2.425 E-02$
$4.178 E-02$

$2.915 \mathrm{E}-01$

$2.576 E-01$

$1.595 \mathrm{E}$ OI

$7.007 E$ OL

$2.073 E \quad 02$

$5.634 \mathrm{E} \mathrm{O2}$

$1.524 \mathrm{E} 03$

$3.364 \mathrm{E} 03$

$4.915 E^{03}$
$M U=-0.9894$ 0.0

0

$5.707 E-05$

$2.455 \mathrm{E}-\mathrm{C}$

4.921E-03

$6.770 E-03$

$8.406 E-03$

$9.415 \mathrm{E}-03$

$1.7075-C 2$

3.347E-02

$2.344 \mathrm{E}-01$

$1.462 E$ OI

$6.459 \mathrm{E}$ O2

$5.220 \mathrm{E} 02$

$.415 \mathrm{E} \mathrm{C3}$

$3.132 E$ O3

ANGLE 11

$0.2816 \quad M U=0.4580$

(a)

0.0

0

$1.526 \mathrm{E}-03$
$6.019 \mathrm{E}-03$
$6.837 \mathrm{E}-03$

$6.837 \mathrm{E}-03$

$1.191 \mathrm{~F}-0$

$1.855 F-C 2$

$1.576 \mathrm{E}-02$

$1.565 \mathrm{E}-02$

$2.634 \mathrm{E}-\mathrm{C2}$

$4.376 \mathrm{E}-02$

$2.986 \mathrm{E}-01$

$2.622 \mathrm{E}-01$

$1.620 \mathrm{E} 01$

$7.113 E$ Ol

$2.104 \mathrm{E} \mathrm{C?}$

$5.714 \mathrm{E} \quad 2$
$1.545 \mathrm{E} 03$

$3.409 E \quad 03$

$4.974 \mathrm{E} \mathrm{O3}$
$U=-0.9446$

0.0

$7.434 E-05$

$2.567 \mathrm{~F}-03$

4. $859 E-03$

$6.789 \mathrm{~F}-03$

$8.492 E-03$

$9.527 \mathrm{E}-03$

$1.725 \mathrm{E}-02$

(1)

$.352 \mathrm{E}-01$

1. $467 E$ OI

$6.479 \mathrm{E}$ Ol

$1.921 \mathrm{E}$ O2

$.420 \mathrm{E} 03$

.14 03

ANGLE 12

$0.0 \quad 0$.

0.0
0.0

0.0

2.314E-03

$7.773 E-03$
$8.519 E-07$

$7.519 E-07$
$1.477 E-02$

$2.323 \mathrm{E}-02$

$1.845 \mathrm{E}-02$

$1.746 \mathrm{E}-02$

$2.878 \mathrm{E}-02$

$.587 E-02$
$.057 E-01$

$2.668 \mathrm{E}-01$

$1.646 \mathrm{E} \mathrm{Ol}$

$7.218 \mathrm{E}$ O1

2.134502

$5.792 \mathrm{E} 02$
$1.565 \mathrm{E} 03$

$3.452 E 03$

$5.032 \mathrm{E} 03$
$U=-0.8656$ 0

0.0

0.0

0.0

1.033E-04

$2.760 \mathrm{E}-03$

$4.766 E-03$
$6.834 E-03$

$6.834 \mathrm{E}-03$

$9.762 \mathrm{E}-03$

$8.671 \mathrm{E}-03$

$9.746 \mathrm{E}-03$

$1.762 \mathrm{E}-02$

$3.422 \mathrm{E}-02$

$2.608 \mathrm{~F}-01$

.

. $476 \mathrm{E}$ OI

(3) 02

$5.263 \mathrm{E} \mathrm{O}$

$1.427 E$ O3

4.04

ANGLE 13

0.6179

0.0

0.0

$3.915 \mathrm{E}-03$

$1.101 \mathrm{E}-02$

$1.115 \mathrm{E}-02$

$1.907 \mathrm{E}-02$

$3.009 \mathrm{E}-02$

$2.196 \mathrm{E}-02$

$3.156 \mathrm{E}-02$

$3.125 \mathrm{E}-01$

$2.711 \mathrm{E}-01$

$1.670 \mathrm{E}$ O1
$7.315 \mathrm{E}$ ol

$2.162 E$

$5.865 \mathrm{E} \mathrm{OZ}$

$1.584 \mathrm{E} 03$

$3.492 \mathrm{E} 03$

$5.086 \mathrm{E} 03$
ANGLE 5 ANGLE 6 0.0

0.0

$1.439 E-04$

$3.001 \mathrm{E}-03$

$4.682 \mathrm{E}-03$

$6.926 E-03$

.

$04 E-03$

1.

. $15 E-02$

$2.639 \mathrm{E}-01$

$2.389 \mathrm{E}-01$

. 588 E

DE

D3E 02

$4.673 E$ O3

ANGLE 14

0.7550

0.0

0.0

0.0

$7.376 E-03$

$1.711 \mathrm{E}-02$

$1.515 \mathrm{E}-02$

2.559E-02

$4.027 \mathrm{E}-02$

4.027E-02

.461E-02

作

. $749 E-01$

$1.691 \mathrm{E}$ 01

.402E O1

$1.601 E 03$

$3.528 \mathrm{~F} \quad 03$

$5.133 E \quad 03$
$M=-0.6179$

0.0

0.0

0.0

$2.090 E-04$

$3.278 E-03$

$4.639 \mathrm{E}-03$

$7.100 \mathrm{E}-03$

$1.021 \mathrm{E}-02$

$9.409 \mathrm{E}-03$

$1.054 E-02$

$1.888 \mathrm{E}-02$

.

.679E 01

$.534 \mathrm{E} 01$

$6.635 E$ OI

353E

. $51 \mathrm{E}$

$.207 \mathrm{E} 03$

$4.711 \mathrm{E} 03$

ANGLE 15

$U=0.8656$

0.0

0.0

0.3

$1.505 E-02$

$2.895 \mathrm{E}-02$

$2.895 \mathrm{E}-02$
$2.132 \mathrm{E}-02$

$2.132 \mathrm{E}-02$
$3.587 \mathrm{E}-02$

$5.590 \mathrm{E}-02$

$3.258 \mathrm{E}-02$

$2.492 E-02$

$3.781 E-02$

.209E-O2

$.240 E-01$

.781E-01

$1.709 \mathrm{E} 01$

$7.474 \mathrm{E}$ OI

$2.207 E$ O2

$5.983 E$ O2

$1.614 \mathrm{E} \quad 03$

5.170E 03

ANGLE 7 $U=-0.4580$

0.0

0.0

0.0

$3.194 E-04$

$3.588 \mathrm{E}-03$

$4.676 \mathrm{E}-03$

$7.406 \mathrm{E}-03$

$1.005 \mathrm{E}-02$

$1.115 \mathrm{E}-02$

$1.983 \mathrm{E}-02$

$3.701 \mathrm{E}-02$

.

.5

(1)

.714E O1

$413 E 02$

466E 03

4.75E

$3.241 E$ E

ANGLE 16 $U=0.9446$

0.0

0.0

0.0

$3.453 E-02$

$5.481 E-02$

$3.156 E-02$

$5.324 \mathrm{E}-02$

$8.155 \mathrm{E}-02$

$4.056 E-02$ 
ENERGY $1.22 \mathrm{E}$ 01---1.50E 01 $1.00 E$ 01--1.22E 01 $6.36 \mathrm{E} \quad 00-\cdots 8.19 \mathrm{E} 00$ $4.97 E 00--6.36 E 00$ 4.07E $00--4.97 E$ OO $3.01 \mathrm{E} 00--4.07 E$ OO $2.46 \mathrm{E} 00---3.01 \mathrm{E} 00$ $2.35 \mathrm{E} \quad 00-\cdots-2.46 \mathrm{E} \quad 00$ $1.83 \mathrm{E} \quad 00---2.35 \mathrm{E} \quad 00$ $1.11 \mathrm{E} 00--1.83 \mathrm{E} 00$ $5.5 \mathrm{CE}-01--1.11 \mathrm{E}$ OO $1.11 \varepsilon-01--5.50 E-01$ $3.35 \mathrm{E}-02---1.11 \mathrm{E}=01$ $5.83 E-04--3.35 E-02$ $1.01 E-04--5.83 E-04$ $2.90 \mathrm{E}-05---1.01 \mathrm{E}-04$ $1.07 E-05---2.90 E-05$ $3.06 \mathrm{E}-06--1.07 \mathrm{E}-0$ $1.12 \mathrm{E}-06---3.06 \mathrm{E}-06$ $4.14 E-07=-1.12 E-06$ $0.0 \quad---4.14 E-07$ ENERGY GROUP (MEV) $1.22 E$ 01--1.50E 01 $1.00 E$ O1---1.22E OI 8.19E 00---1.00E OI 6.36 E $00--8.1$ SE 00 4.97E $00--6.36 \mathrm{E}$ OO 4.07E $00--4.97 E$ OO $3.01 \mathrm{E}$ 00---4.07E 00 $2.46 E$ OO-- $3.01 E$ OO 2.35E $00--2.46 E$ OO $1.83 \mathrm{E} 00--2.35 \mathrm{E}$ OO $1.11 \mathrm{E} 00--1.83 E$ OO S. $0 E-01--3.11 E$ OO $1.11 E-01=-5.50 E-01$ $3.35-02--1.11 E-01$ . $83 E-04--3.35 E-02$ $1.01 E-04--5.83 E-04$ 1.907E-05-1.01E-04 1.07E-05 $3.06 E-06-1.07 E-05$ 3.12E-06- $-3.06 E-06$ $4.14 E-07=-1.12 E-06$ $\triangle A N G L E \quad 1$ 0.0 0.0 0.0 $(0.0$ 4.115E-05 $1.688 \mathrm{E}-03$ $3.371 \mathrm{E}-03$ $4.825 E-03$ $6.999 E-03$ $6.590 \mathrm{E}-03$ $7.929 E-03$ $1.533 E-02$ $3.210 \mathrm{E}-02$ $2.587 \mathrm{E}-01$ $2.391 \mathrm{E}-01$ $1.508 \mathrm{E} 01$ $6.708 E$ OI $1.999 \mathrm{E} \mathrm{O2}$ $5.484 \mathrm{E} 02$ $1.494 \mathrm{E} \quad 03$ $3.320 E \quad 03$ 4.906F 0 ANG LE 10
$M U=0.0950$ 0.0
0.0
0.0 0.0
0.0
0.0 0.0
$7.705 E-04$ $3.479 \mathrm{E}-03$ 4.065E-0 7.376E-03 $1.183 \mathrm{E}-02$ $1.108 E-02$ 1.2 $4.073 E=02$ $4.073 E=02$ 2.652 01 1.659E 01 1. $342 \mathrm{E} 01$ ग $2.18 \mathrm{E}$. .623E 03 $3.598 \mathrm{E} 03$ $5.278 E 03$ $\triangle N G L E \quad 2$
$M U=-0.9894$ 0.0 0.0 $4.431 E-05$ $1.707 E-03$ $3.361 E-03$ $4.82 \mathrm{SE}-03$ $7.004 \mathrm{E}-03$ $6.607 \mathrm{E}-03$ $7.952 \mathrm{E}-03$ $1.537 E-02$ $2.591 \mathrm{E}-01$ $2.393 \mathrm{E}-01$ $1.509 \mathrm{E} \mathrm{O1}$ $6.714 \mathrm{E} \mathrm{Ol}$ $2.001 E 02$ $5.488 \mathrm{E} \quad 2$ 1.495 O3 $\begin{array}{ll}3.323 E & 03 \\ 4.910 E \quad 03\end{array}$ ANGLE 11 $M U=0.2816$ 0.0

0.0 0.0

$1.110 E-03$ $4.195 \mathrm{E}-03$ $4.827 \mathrm{E}-03$ $8.883 E-03$ $1.448 \mathrm{E}-02$ $1.283 E-02$ $1.350 \mathrm{E}-02$ 4. $3.041 \mathrm{E}-01$ $2.704 \mathrm{E}-01$ $1.689 \mathrm{E}$ o1 $7.465 \mathrm{E}$ OI $2.220 \mathrm{~F}$ O2 $1.647 \mathrm{E} 03$ $5.349 \mathrm{E} 03$
(NEUTRINS/MEV/STERADIAN/SOURCE NEUTRON)

ANGLE $\quad 3$
$M U=-0.9446 \quad M U N=0$

$\begin{array}{ll}0.0 & 0.9446 \\ 0.0 & 0.0\end{array}$

$5.642 \mathrm{E}-05 \quad 7.715 \mathrm{E}-05$

$1.782 E-03 \quad 1.912 E-03$

$3.321 \mathrm{E}-03 \quad 3.263 \mathrm{E}-03$

$4.844 E-03$

$7.031 \mathrm{~F}-03$

$6.682 E-03$

$8.05 L E-03$

$3.244 \mathrm{E}-02$

$2.604 \mathrm{E}-01$

$2.402 \mathrm{E}-01$

$1.515 \mathrm{E}$ O1

$6.737 E 01$

5.506E 02

$5.506 E$
$1.500 E$ 03

$3.333 \mathrm{E} 03$

4.925E 03

ANGLE 12

$M U=0.4580$

$M U=0.4$
0.0
$C .0$
0.0

0.0

$1.697 E-03$

$5.452 \mathrm{E}-03$

$6.042 E-03$

1. $838 \mathrm{E}-02$

1. $512 \mathrm{E}-02$

$1.513 E-02$

$4.499 \mathrm{E}-02$

$3.119 \mathrm{E}-03$

$2.755 E-01$
$1.718 E ~ 01$

$7.585 \mathrm{E}$ O1

$2.25 E$ O2

$1.672 E$ E 03

$1.672 E 03$

5.419 E 03

$=-0.8656$
.0
.0
.0
.0
$.715 E-05$
$.912 E-03$
$.263 E-03$
$.881 E-03$
$.102 E-03$

$7.102 \mathrm{E}-03$

$6.837 E-03$

$1.500 \mathrm{E}-02$

$3.294 E-02$

$2.628 E-01$

$2.419 E-01$
$1.525 E 01$

$1.525 E$ OI

$2.020 E O 2$

$5.539 \mathrm{E} 02$

$1.509 E 03$

4.952E 03

ANGLE 13

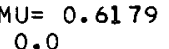

0.0
0.0

0.0

$2.880 E-03$

$7.748 \mathrm{E}-03$

$1.457 \mathrm{E}-02$

$2.416 E-02$

$1.812 E-02$

$1.707 E-02$

4. $725 \mathrm{E}-02$

$3.195 \mathrm{E}-01$

$2.803 \mathrm{E}-01$

$1.745 E$ O1 $2.287 E \quad 02$ $6.244 E 02$

3.751 E 03

5.485 E 03
$M U=-0.7550$ 0.0

$1.068 \mathrm{E}-04$ $3.213 \mathrm{E}-03$ $4.956 \mathrm{E}-03$ $7.255 E-03$ $7.087 \mathrm{E}-03$ $8.532 E-03$ $3.366 E-02$ $2.662 E-01$ $2.444 \mathrm{E}-01$ $1.539 \mathrm{E} \mathrm{O1}$ $6.839 \mathrm{E}$ OI $2.038 \mathrm{E} 02$ $5.585 \mathrm{E} 02$ $1.521 E \quad 03$ $3.378 \mathrm{E} 03$ $4.988 E \quad 03$

ANGLE 14 $M U=0.7550$ $M U=0.7$
0.0
0.0
0.0 0.0 $5.401 E-03$ 1. $200 \mathrm{E}-02$ $1.073 E-02$ $3.284 E-02$ $2.205 E-02$ 1. $931 \mathrm{E}-02$ $4.946 E-02$ $4.946 E-02$ . $1.770 E$ OI 7.800 E 01 2.317E 02 $6.321 E$ O2 $1.714 \mathrm{E} 03$ $5.541 E \quad 03$

ANILE 6 0 0.0 $1.547 E-04$ 2.264E-03 $3.192 E-03$ $8.939 E-03$ $3.461 \mathrm{E}-02$ $2.706 E-01$ $2.475 E-01$ $1.557 \mathrm{E} 01$ $6.915 E$ OI $2.060 E 02$ $5.644 E$ O2 $1.536 \mathrm{E} 03$ $3.411 E$ E
$5.034 E \quad 03$ ANGLE 15 $M U=0.8656$ MUNGLE
0.0
0.0
0.0
0.0 0.0

$1.089 E-02$ 2. $009 \mathrm{E}-02$ $1.499 E-02$ $2.801 E-02$ $4.633 E-02$ 2.719E-02 $3.563 E-02$ (.) 
ENERGY GROUP (MEV) $1.22 E$ O1--1.50E 01 8.1 OE OO6.36 E $00--8.1$ EE 00 $4.97 \mathrm{~F} 00---6.36 \mathrm{E} 00$ $4.07 E$ OO- $-4.97 E$ OO $3.01 \mathrm{E} 01--4.07 \mathrm{E}$ OO $2.4 E E$ OO---3.01E 00 $2.35 \mathrm{E} 00-2-26 \mathrm{E}$ OO $1.83 \mathrm{~F} \mathrm{O0-D2.35E} 00$ $1.11 \mathrm{E} \mathrm{OO--1.83 \textrm {E }} 00$ 5.50E-01---1.11E 00 $1.11 \mathrm{E}-01--5.50 \mathrm{E}-0$ $3.35 E-02--1.11 E-01$ $5.83 \mathrm{E}-04-\cdots 3.35 \mathrm{E}-0$ $1.01 E-04---5.83 E-04$ $2.90 E-05--1.01 E-04$ $1.07 E-05--2.90 E-05$ $3.06 \mathrm{E}-06--1.07 \mathrm{E}-05$ $1.12 E-06--3.06 E-06$ $4.14 \mathrm{~F}-07--1.12 \mathrm{E}-06$ ENERGY GROUP (MEV) $1.22 \mathrm{~F} 01---1.50 E$ OI $1.00 \mathrm{~F}$ 01---1.22E 01 $8.19 \mathrm{E}$ 00---1.00E O1 $6.36 \mathrm{E} 00--8.19 \mathrm{E}$ 00 $4.97 \mathrm{E}$ 00---6.36E 00 4.07E $00--4.97 E$ EO 3.03 E $00--4.07 E$ OO $2.46 E$ OO---3.01E OO $2.35 \mathrm{E} 00--2.46 \mathrm{E} 00$ 1.83 E $00--2.35 E$ OO $1.11 E$ CO- $-2.83 E$ OO $1.50 E-01--1.11 E$ OO $1.11 E-01--5.50 E-01$ $3.35-02--1.11 E-01$ $1.83 \mathrm{E}-04--3.35 \mathrm{E}-02$ $1.01 E-04-1$ OIE-O 1.07E-051.07E-051.06E $06-1.07 E-05$ 4.1 4E-07- $1.12 E-06$ $4.14 E-07--1.12 E-06$
0.0 $J=-1.0000$ 0.0 0.0 $1.553 E-05$ $5.130 E-04$ $9.676 \mathrm{E}-04$ $2.224 \mathrm{E}-03$ $2.572 \mathrm{E}-03$ $3.613 E-03$ $8.089 \mathrm{E}-03$ $1.970 E-02$ $1.769 E-01$ $1.688 \mathrm{E}-01$ $1.091 \mathrm{E} 01$ 4.936E O1 $1.489 E \quad 02$ $1.142 \mathrm{E} 03$ $2.564 \mathrm{E} 03$ $3.832 \mathrm{E} \mathrm{O3}$ ANGLE 10 $U=0.0950$ 0.0

0.0

0.0 $2.535 E-04$ $1.049 \mathrm{E}-03$ $1.237 \mathrm{E}-0$ $2.493 \mathrm{E}-03$ $4.427 E-03$ $4.620 E-03$ $5.733 E \rightarrow 03$ 1.230E-C2 $2.577 \mathrm{E}-\mathrm{O}$ ? $2.069 \mathrm{E}-01$ 1.205 01 $1.222 \mathrm{E} 01$ $5.495 \mathrm{E}$ OL $1.655 \mathrm{~F}$ O2 1. $1.262 E$ O2 $4.190 \mathrm{E}^{2}$
ANGLF 2 $0 . n$ 0.0 0.0 $1.647 E-05$ $5.182 \mathrm{E}-04$ $9.182 E-04$ $1.487 E-03$ $2.229 \mathrm{E}-\mathrm{C} 3$ $2.580 \mathrm{E}-03$ $3.624 \mathrm{E}-03$ $8.115 \mathrm{E}-03$ $1.975 E-02$ $1.771 \mathrm{E}-01$ 1.690E-01 $1.093 \mathrm{E}$ OI $4.941 E$ O1 $1.491 \mathrm{E} \mathrm{O2}$ $4.149 \mathrm{E} \quad 02$ $1.143 E 03$ $\begin{array}{lll}2.566 \mathrm{E} & 03 \\ 3.836 \mathrm{E} & 03\end{array}$

ANGLE 11 $U=0.2816$ 0.0

0.0 0.0 $3.710 \mathrm{E}-04$ 1. $279 \mathrm{E}-03$ $1.483 \mathrm{E}-03$ $3.084 \mathrm{E}-03$ $5.575 E-03$ $5.425 \mathrm{E}-\mathrm{C} 3$ $6.420 E-03$ $2.724 E-02$ $2.724 E-02$ $2.132 \mathrm{E}-01$ $1.248 E$ OI $5.605 E$ OL $1.688 \mathrm{E}$ C2 $4.681 E$ O2 2.878E 03 $2.878 E$ O3
(NEUTRONS/MEV/STERADIAN/SOURCE NEUTRON)

ANGLE 3$$
0.0
$$$$
\begin{aligned}
& 0.0 \\
& 0.0
\end{aligned}
$$

$2.022 E-05$ $5.393 E-04$
$9.557 E-04$ $1.494 \mathrm{E}-03$ $2.254 \mathrm{E}-03$ $2.617 \mathrm{E}-03$ $3.673 \mathrm{E}-03$ $8.222 E-03$ $1.993 E-02$ $1.782 F-01$ $1.698 E-01$
$1.097 E \quad 01$ 4.961E Ol $1.497 E 02$ $4.165 \mathrm{~F} 02$ $\begin{array}{lll}1.147 E & 03 \\ 2.576 E & 03\end{array}$ $3.85 \mathrm{OE} \quad 03$ ANGLE 4 0.0 0.0 $2.691 E-05$ $2.691 E-05$ $5.756 E-04$
$9.424 E-04$ $9.424 \mathrm{E}-04$
$1.511 \mathrm{E}-03$ $1.511 E-03$
$2.306 \mathrm{E}-03$ $2.691 \mathrm{E}-03$ $3.767 \mathrm{E}-03$ $8.426 \mathrm{E}-03$ $2.028 \mathrm{~F}-02$ $1.801 \mathrm{E}-01$ $1.712 \mathrm{E}-01$ $1.106 \mathrm{E} 01$ $4.098 \mathrm{E} 01$ $1.508 \mathrm{E} 02$ $4.195 E \quad 02$ $1.155 \mathrm{E} 03$ $\begin{array}{ll}2.593 E & 03 \\ 3.875 E & 03\end{array}$ ANGLE 5 ANOLE 6 ANGLE 7 $3.696 E-05$ $6.218 \mathrm{E}-04$ $9.324 E-04$ 1.542E-03 2. $807 E-03$ $3.909 E-03$ $8.727 E-03$ 2. $078 E-02$ $1.827 \mathrm{E}-01$ $1.732 E-01$ $1.118 \mathrm{E} 01$ $5.050 E$ O1 $1.523 \mathrm{E} 02$ $4.237 E \quad 02$ $1.167 E 03$ $2.618 \mathrm{E} \mathrm{O3}$
$3.910 \mathrm{E} \mathrm{O3}$ 0.0 0. 0.0 5. $316 E-05$ $6.761 E-04$ $6.761 E-04$
$9.322 E-04$ $1.599 E-03$ 2.547E-03 2.979E-03 4.111E-03 $9.143 E-03$ $2.144 E-02$ $1.862 \mathrm{E}-01$ $1.181 E 03$ $2.650 E ~ 03$
$3.953 E \quad 03$ ANGLE 14 ANGLE 15 $U=0.4580$ 0.0

0.0 0.0 0.0 $5.780 \mathrm{E}-04$ $1.679 \mathrm{E}-03$ $1.865 \mathrm{E}-03$ $7.301 \mathrm{E}-03$ $6.486 \mathrm{E}-03$ $7.254 E-03$ $1.507 \mathrm{E}-02$ $2.883 E-02$ $2.195 \mathrm{E}-01$ $1.792 E-01$ $1.273 E$ OI $5.713 E$ ol $4.767 E \quad 02$ 1.309E O3 $4.328 \mathrm{E}^{03}$
$U=0.6179$

0.0

0.0

0.0 0.0

$9.876 \mathrm{E}-04$ $2.388 \mathrm{E}-03$ $2.439 \mathrm{E}-03$ $5.351 E-03$ $9.937 \mathrm{E}-03$ . $8.248 E-03$ . $2.258 \mathrm{E}-01$ $2.033 E-01$ $1.298 \mathrm{E} 01$ $5.816 E$ OI

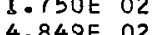
$4.849 \mathrm{E} 02$ $2.076 \mathrm{E} \mathrm{O3}$ 4.392E O3

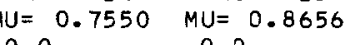

0.0

0.0

0.0

$1.828 E-03$

$3.650 \mathrm{E}-03$

3. $276 \mathrm{E}-03$

$7.467 E-03$

$1.404 \mathrm{E}-02$

$9.393 E-03$

1. $871 \mathrm{E}-02$

$3.207 E-02$ $2.314 \mathrm{E}-01$ $2.070 \mathrm{E}-01$ 1.319 E 1 1.977 E 02 $1.777 \mathrm{E} O 2$
$4.921 \mathrm{E} O 2$ 4. $921 E$ E $1.350 E$ E 3 $4.449 \mathrm{E} \quad 03$

0.0

0.0 
(NEUTRONS/MEV/STERADIAN/SOURCE NEUTRON)

ENERGY GROUP (MEV) $1.22 \mathrm{~F}$ O1---1.5OE OI $1.00 E$ O1---1.22E 01 8.1.E OO- -1. COE OI 6. $4.97 E$ O0- 6.36 E 00 4.07E $00-4.97 E$ O 2. 2.46E OO1.83F $00-2.35 \mathrm{E}$ DO $1.83 \mathrm{E} 00-2.35 \mathrm{E} 00$ $1.25 \mathrm{E}-1.00-1 .-1 \mathrm{E}$ O 1.5CE-01--1.11E $3.35 \mathrm{~F}-02-\cdots 1.11 \mathrm{E}-\mathrm{Cl}$ $5.83 E-04---3.35 E-02$ $3.01 \mathrm{E}-04--5.83 \mathrm{E}-04$ 2.90E-05---1.01E-04 $1.07 E-05--2.90 \mathrm{E}-05$ 3.06E-06-- - 1.07E-05 1.12E-06-- -3.06E-06 $4.14 E-07---1.12 E-06$ 0.0

ENERGY GROUP (MEV)

$1.22 \mathrm{E} \mathrm{O1---1.50E}$ 1.00 01---1.22E 01 8.1 OE OO---1. 00 OI 6.36 E $00--8.19 E \quad 00$ $4.97 \mathrm{E} \quad 00---6.36 \mathrm{E} \quad 00$ 4.07E $00--4.97 E$ EO 3. त1E DO---4.07E 00 $2.46 E$ O0-- $3.01 E 00$ $2.35 E \quad 00--2.46 E$ OO $1.83 \mathrm{E} \quad 00---2.35 \mathrm{E} \quad 00$ $1.11 E$ O0- $-1.83 E$ DO 5.5 CE $-01--1.11 \mathrm{E} O 0$ $1.11 \mathrm{E}-01-5.50 \mathrm{E}-01$ 3.35E-02---1.11E-.01 $1.01 \mathrm{E}-04---5.83 \mathrm{E}-04$ $2.90 E-05--1.01 E-04$ 1.07E-05-- $2.90 \mathrm{E}-0$ $3.06 \mathrm{E}-06---1.07 \mathrm{E}-\mathrm{C}$ $1.12 \mathrm{E}-06--3.06 \mathrm{E}-06$ $4.14 \mathrm{E}-07---1.12 \mathrm{E}-06$ $0.0 \quad--4.14 \mathrm{E}-07$ U. $=-1.0000$

0.0

$4.866 \mathrm{E}-06$ $1.425 E-04$ $2.548 E-04$ $4.021 E-04$ $8.379 E-04$ $8.379 E-04$ $3.330 E-03$ $8.750 E-03$ $8.474 \mathrm{E}-02$ $8.263 \mathrm{E}-02$ $5.434 E 00$ 2.485 E 01 2.485E O1 $2.126 E 02$ $5.904 \mathrm{E} 02$ $1.335 E \quad 03$ $2.012 \mathrm{E} \quad 03$ ANGLE 10 0.0 0.0 0.0 7.415E-05 $2.894 E-04$ $3.372 E-04$ $7.314 \mathrm{E}-04$ $1.398 \mathrm{E}-03$ $1.577 E-03$ $2.109 E-03$ $5.048 \mathrm{E}-03$ $1.166 \mathrm{E}-02$ $1.004 \mathrm{E}-01$ $9.427 \mathrm{E}-02$ $6.150 E$ OO $2.796 \mathrm{E} \mathrm{O1}$ $8.496 \mathrm{E}$ OI $2.382 E$ O2
$6.597 E$ O2 $6.597 \mathrm{E}$ O2 $\begin{array}{ll}1.488 E & 03 \\ 2.222 E & 03\end{array}$
$A N G L E \quad 2$
$M U=-0.9894$ 0.0 $0 . \mathrm{C}$ $0.025 E-06$ $5.125 \mathrm{E}-06$ $1.439 \mathrm{E}-04$ $2.542 E-C 4$ $4.027 E-04$ 6. $8.411 \mathrm{E}-04$ $3.241 E-03$ 3. $241 E-03$ $8.771 E-03$ $8.487 E-02$ $8.272 E-02$
$5.440 \mathrm{E} \mathrm{CO}$ $2.488 E 01$ $7.571 \mathrm{E}$ O1 2.120 O? 5.91 OE O2 1.337E 03 $2.014 E 03$ ANGLE 11 O. 0.0 0.0 1.099E-04 $3.548 E-04$ $4.057 E-04$ $9.236 E-04$ $1.796 E-03$ $1.870 E-03$ $2.372 E-03$ $5.607 E-03$ $1.237 E-02$ $1.038 \mathrm{E}-01$ $9.661 \mathrm{E}-02$ $6.292 \mathrm{E} 00$ $2.858 \mathrm{E} 01$ $8.681 \mathrm{E} \mathrm{O1}$ 2.433 E 02 $6.733 E 02$ $1.518 E \quad 03$ $2.263 \mathrm{E} 03$
ANGLE 3 $=-0.9446 \quad M U=-0.8656$ 0.0 0.0 0.0 .180E-06 $1.494 \mathrm{E}-04$ $4.054 E-04$ $4.05 E-04$ . $8.549 \mathrm{E}-04$
$1.325 \mathrm{E}-03$ $1.325 E-03$
$2.287 E-03$ $8.859 \mathrm{E}-03$ $8.540 \mathrm{E}-02$ $8.314 E-02$ $8.314 E-02$
$5.466 E 00$ $2.499 \mathrm{E}$ OI $7.605 \mathrm{E} \mathrm{O1}$ $2.138 E$ O2 $5.935 \mathrm{E} 02$ $1.342 E 03$ $2.022 E 03$

ANGLE 12 $M U=0.4580$

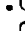
0.0 1. $734 E-04$ $4.667 E-04$ $5.100 E-04$ $1.217 \mathrm{E}-03$ $2.406 \mathrm{E}-03$ $2.256 \mathrm{E}-03$ $2.693 E-03$ $6.272 E-03$ $1.315 E-02$ 1.J71E-01 $9.896 \mathrm{E}-02$ $6.434 \mathrm{E} 00$ 2.919501 $8.863 \mathrm{E} \mathrm{Ol}$ $2.482 \mathrm{E} 02$ $6.867 \mathrm{E} \mathrm{O2}^{2}$ $1.548 E 03$ 2.304E 03 $M U=-0.8656$
0.0 0. 0.0 $.116 \mathrm{E}-06$ $1.589 \mathrm{E}-04$ $2.492 E-04$ $6.541 E-04$ $8.541 E-04$
$8.822 E-04$ $8.822 E-04$
$1.360 E-03$ 3. $373 E-03$ $9.023 E-03$ $8.638 \varepsilon-02$ $8.389 \mathrm{E}-02$ $8.389 \mathrm{E}-02$
$5.513 \mathrm{E} 00$ 2.519E 01 $\begin{array}{lll}2.519 E & 01 \\ 7.666 E & 01\end{array}$ 2.155 E 02 5.981 E त 1.352E 03 2.037E O3 ANGLE 13 0.0 0.0 0.0 2. $970 \mathrm{E}-04$ $6.607 E-04$ $6.634 \mathrm{E}-04$ $1.670 \mathrm{E}-03$ $3.363 \varepsilon-03$ $2.765 E-03$ $3.075 E-03$ $7.041 E-03$ $1.395 \mathrm{E}-02$ $1.104 E-01$ $1.012 E-01$ $6.569 \mathrm{E} \mathrm{OO}$ $2.977 \mathrm{E}$ OI $9.036 \mathrm{E} 01$
$2.529 \mathrm{E}$ $2.529 E$ O2 $\begin{array}{ll}6.994 \mathrm{E} & 02 \\ 1.576 \mathrm{E} & 03\end{array}$ 2.342E 03
ANGLE 5 0.0 0.0 $1.111 E-05$ 1. $710 E-04$ $4.420 E-04$ 4. $220 E-04$ 9. $9.247 E-04$ $3.502 \mathrm{E}-03$ $9.261 E-03$ $8.778 \mathrm{E}-02$ $8.495 \mathrm{E}-02$ $5.578 E$ OO $2.548 E$ OI 7.752E OI 2.178 O2 $6.045 E 02$ $1.367 \mathrm{E} 03$ 2.057 E 03

ANGLE 14 0.0

0.

0.0

0.0

5. $.34 E-04$ 9. $947 E-04$ $8.813 E-04$ $2.375 E-03$ $4.901 \mathrm{E}-03$ $3.427 E-03$ $3.512 E-03$ $7.992 E-03$ $1.474 \mathrm{E}-02$ $1.134 \mathrm{E}-01$ $6.690 E 00$ $3.028 E 01$ $9.191 E$ OL $2.572 E \quad 02$ $7.107 E \quad 02$ $1.601 E 03$ $2.376 E \quad 03$

AVGLE 6 ANGLE 7 0.0 $.500 E-05$ $1.856 \mathrm{E}-04$ $2.482 E-04$ $4.409 E-04$ $9.871 E-04$ $9.871 E-04$
$1.490 E-03$ 
ENERGY GROUP (MEV)

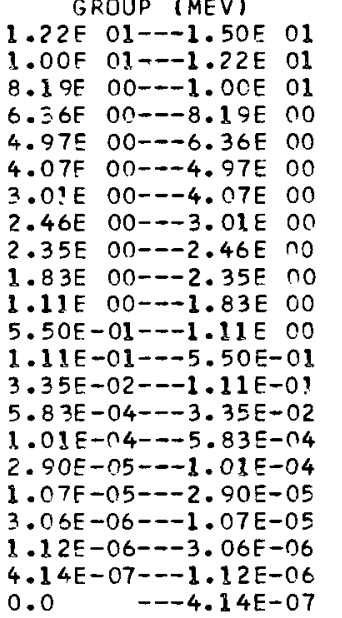

$$
\text { ENERGY }
$$
GROUP (MEV) $1.22 \mathrm{E} 01---1.50 E$ O1 1. COE $01--1.22 E$ OI $8.19 E$ ON $---1.00 E$ O? 6.36 E $00---8.19 E$ EO $4.97 E$ CO- $-6.36 E$ OO 4. . 7F $00--4$. $97 E$ OD 3.0 IE $00--4.07 E$ OO $2.46 \mathrm{E}$ OC-- $-3.01 \mathrm{E}$ OC $2.35 E$ EO- $02.46 \mathrm{E}$ OO $2.83 \mathrm{E}$ 00--2.35E 00 1.1 TE $00--1.83 E$ OC 5.5 1.11E 3.35E-C2- $1.11 E-01$ $5.83 E-04--3.35 E-02$ 2.90E-05-1.01E-04 2.90E-051.07E-05- $2.00 \mathrm{C}$ $1.12 \mathrm{E}-06-2-3.06 \mathrm{E}-06$ $4.14 F-07-0-1.12 E-06$ $4.14 \mathrm{~F}-07--1.12 \mathrm{E}-06$
0.0
ANGLE 1 $M=-1.0000$ 0.0 0.0 0.0 $1.386 \mathrm{E}-06$ $3.758 \mathrm{E}-05$ $6.403 E-05$ $1.021 E-04$ $1.610 E-04$ $2.474 E-04$ $4.134 E-04$ $1.104 \mathrm{E}-03$ $3.229 \mathrm{E}-03$ $3.304 \mathrm{E}-02$ $3.270 \mathrm{E}-02$ $2.177 E$ O $1.003 E$ O $3.072 \mathrm{E}$ O1 $8.699 E$ OI $2.429 E$ O $5.521 \mathrm{E} \mathrm{C}$ $8.367 \mathrm{E} \mathrm{O2}$ ANGLE $1 \mathrm{C}$ $M U=$
0.0

0.0
0.0
0.0

0.0
0.0
0.0

$2.028 E-05$ $7.576 \mathrm{E}-05$ $8.659 \mathrm{~F}-0$ $1.990 E-04$ 4.C 20E-04 $4.829 E-04$ $6.775 E-04$ $1.757 E-03$ 4.3 $3.757 \mathrm{E}-02$ 1.481E $1.137 E$ OI $9.415 E$ OI $9.813 E$ OI $6.198 \mathrm{E} 02$ $6.198 \mathrm{E} 02$
$9.302 \mathrm{E} 02$
(NEUTRONS/MEV/STERADIAN/SOURCE NEUTRON)

ANGLE
$M U=-0.98 \times 4$$\quad$ ANGLE $3 \quad$ ANGLE $4 \quad 0.9446 \quad M U=-0.8656 \quad M U=-0.7550$ 0.0 0.0
$1.454 E-06$ $3.702 E-05$ $6.3 \mathrm{COE}-05$ $1.023 E-C .4$ $1.619 \mathrm{~F}-04$ $2.484 F-04$ $4.14 P E-C 4$ $1.1 C 7 E-C 3$ $3.237 \mathrm{E}-03$ $3.3 \mathrm{C} 9 \mathrm{~F}-\mathrm{C} 2$ $3.274 E-02$ $2.179 E$ OO $1.004 \mathrm{E} 01$ $3.075 \mathrm{E}$ CI $8.708 \mathrm{E} 01$ $2.421 \mathrm{E} 02$ $5.527 E \quad 02$ $8.376 \mathrm{E} 02$

0.0

0.0

0.0

$1.735 E-06$ $3.930 E-05$ $6.342 E-05$ $1.032 \mathrm{E}-\mathrm{n} 4$ 1. $657 E-04$ $2.529 E-04$ 4.207E-04 $1.124 E-03$ $3.331 E-02$. $3.292 \mathrm{E}-02$ $2.190 \mathrm{E} 00$ $1.009 E 01$ $3.090 E$ O1 $8.749 \mathrm{E}$ 01 $2.442 \mathrm{E} \quad 02$ $8.413 \mathrm{E} 02$

0.0
0.0 $2.261 \mathrm{~F}-06$ . $731 E-04$ $2.518 E-04$ $4.320 E-04$ . $34 E-03$ $3.371 E-02$ $3.323 E-02$ $2.210 E$ OO $1.018 E$ OI $3.116 E$ E $8.821 \mathrm{E}$ O $2.462 E$ OL
$5.596 \mathrm{E} 02$ $8.477 \mathrm{E} \mathrm{O2}$ 0.0 $3.087 E-06$ 4.477E-05 $6.245 E-05$ $1.083 E-04$ $1.849 E-04$ $2.755 E-04$ 1. $200 \mathrm{E}-03$ $3.426 \mathrm{E}-03$ $3.428 \mathrm{E}-02$ $3.367 E-02$ $2.238 \mathrm{E} 00$ $1.030 E$ OI $3.153 \mathrm{E}$ OI $8.925 \mathrm{E} \quad 1$ $2.490 \mathrm{E} O 2$ 8.566502 $A V G L E$
$M U=-0.6179$ $M U=-0.6$
0.3
0.0 0.0 0.3 4. $403 E-06$ 4.847E-05 $6.285 \mathrm{E}-05$ $1.139 E-04$ 2. $027 E-04$ $2.954 \mathrm{E}-04$ 4.744E-04 $3.548 E-03$ $3.503 E-02$ $3.425 E-02$ ?.274E 00 $3.201 \mathrm{E}$ O1 $9.057 \mathrm{E} \mathrm{OI}$ $2.527 \mathrm{E} \mathrm{O2}$ $5.739 \mathrm{E} 02$ $8.677 \mathrm{E} 02$ ANGLE 11 ANGLE 12 $M U=0.2816$ 0.0 0.0 0.0 0.0
$3.026 E-05$ $3.026 E-05$
$9.308 E-05$ $9.308 E-05$
$1.042 E-04$ $1.042 E-04$
$2.553 E-04$ $5.244 E-04$ $5.761 \mathrm{E}-04$ $7.643 E-04$ 1.960E-O3 $4.636 E-03$ $4.087 E-02$ 3. $542 E-C 2$ $2.542 E$ CO $3.555 \mathrm{E}$ O $1.004 \mathrm{E} 02$ $1.004 \mathrm{E}$ O2 6.332502 $6.332 E \quad 02$
$9.486 E 02$
$M=0.4580 \quad M U=0.6179 \quad M U=0.7550$ 0.0

0.0

0.0

4. $833 E-05$

$1.223 E-04$

$1.307 \mathrm{E}-04$

$3.420 \mathrm{E}-04$
$7.152 \mathrm{E}-04$

$6.998 E-04$

$8.700 \mathrm{~F}-04$

$2.203 E-02$

$4.938 E-03$

$3.955 E-02$

$2.602 E$ OO

3.1935 o1

3.635 E

$2.853 E 02$

$6.464 E 02$

0.0 O.

0.0

$8.282 E-05$

$1.719 E-04$

$1.690 \mathrm{E}-04$

$4.705 E-04$

$8.624 \mathrm{E}-04$

$9.056 E-04$

$2.484 E-03$

.

4.049E-02

$2.660 \mathrm{E} 00$

$1.215 E 01$
$3.710 \mathrm{E} 01$

$3.710 E$ E

$2.909 \mathrm{E} 0$ ?

6.589 E 02

1. 500 E- 04
ANGLE 15

$9.841 \mathrm{E}$ O2
$? .551 \mathrm{E}-04$

2.22?E-04

$6.870 E-04$

1. $073 \mathrm{E}-03$

$1.139 E-03$

$2.795 \mathrm{E}-03$

$5.561 E-03$

$4.488 \mathrm{E}-02$

$4.134 E-02$

$2.712 E 00$

2.778 E 01

$1.065 \mathrm{E} \mathrm{O2}$

2.95E 02

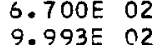

$M U=0.8656$

0.0

0.0

$2.809 E-04$ 3. $928 \mathrm{~F}-04$ $2.933 \mathrm{E}-04$ $1.022 \mathrm{E}-03$ 2. $393 E-03$ $1.339 E-03$ (1)

$M=-0.4580$

0.0

0.0

0.0

$6.458 \mathrm{E}-06$

$5.276 E-05$

$6.460 \mathrm{E}-05$

$1.233 E-04$

$2.286 E-04$

$3.232 E-04$

$1.347 E-03$

3. $700 E-03$

$3.593 E-02$

$3.494 \mathrm{E}-02$

$2.317 \mathrm{E} 00$

$1.065 \mathrm{E}$ O1
$3.258 \mathrm{E}$ 01

$9.216 E$ OI

$2.570 E$ O2

$8.808 \mathrm{E} \mathrm{O2}$

ANGLE 16 
(NEUTR JNS/MEVYSTERADIAN/SOURCE NEUTRON) GROUP (MEV)

$1.225 \quad 01--1.50 E$ OI $1.00 E$ O1+--1.22E O1 $8.19 E$ OO- $-1.00 \mathrm{E} 01$ $6.36 \mathrm{E} 00--8.19 \mathrm{E}$ 00 $4.97 E$ DO-- $6.36 E$ E 00 $4.07 E$ EO- $-4.97 \mathrm{E}$ DO $3.01 \mathrm{E} 00--4.07 \mathrm{E} 00$ $2.46 \mathrm{E} \quad 0 \mathrm{C}---3.01 \mathrm{E} 00$ $2.35 \mathrm{E} 00---2.46 E$ E 00 1.1.E $00-2.35 E$ EO $5.50 E-01--1.11 \mathrm{E}$ DO $1.11 E-01---5.50 E-01$ $3.35 \mathrm{E}-02--1.11 \mathrm{E}-\mathrm{Cl}$
$5.83 \mathrm{E}-04--3.35 \mathrm{E}-02$ $1.01 E-04--5.83 E-04$ $2.90 E-05--1.01 E-04$ $1.07 E-05--2.90 \mathrm{E}-05$ 3.06E-06-- $1.07 E-05$ $1.12 \mathrm{E}-06---3.06 \mathrm{E}-06$ $4.14 \mathrm{E}-07--1.12 \mathrm{E}-06$ $0.0 \quad-4.14 E-07$ ENERGY GROUP (MEV) $1.22 \mathrm{E} O 1--1.50 \mathrm{E} 01$ $1.00 \mathrm{~F}$ O1---1.22E Ol

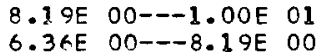
$\begin{array}{llll}6.36 E & 00--8.19 E & 00 \\ 4.97 E & 00---6.36 E & 00\end{array}$

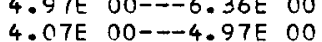
4.07E $00--4.97 E$
3.00 $3.01 \mathrm{E}$
$2.46 \mathrm{E} 00---3.07 \mathrm{E}$ OO $2.46 \mathrm{E} 00--3.01 \mathrm{E}$ OD $1.33 \mathrm{E} 00-\cdots 2.35 \mathrm{E} 00$

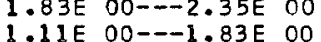
$1.11 \mathrm{E} 00---1.83 \mathrm{E}$ O0
$5.50 \mathrm{E}-01---1.11 \mathrm{E}$ OO $1.11 \mathrm{E}-01---5.50 \mathrm{E}-01$ $1.11 \mathrm{E}-01---5.50 \mathrm{E}-0 \mathrm{~L}$
$3.35 \mathrm{E}-02--1.11 \mathrm{E}-01$ $5.83 \mathrm{E}-04-\cdots-3.35 \mathrm{E}-0$ ? $5.83 \mathrm{E}-04---3.35 \mathrm{E}-02$
$1.01 \mathrm{IE}-04--5.83 \mathrm{E}-04$ $2.90 E-05-1.01 \mathrm{E}-04$ $1.07 E-05--2.90 \mathrm{E}-05$ $3.06 E-06--1.07 E-05$ 1.12E-06---3.06E-06 $4.14 E-07---1.12 E-06$ $4.14 \mathrm{E}-07---1.12 \mathrm{E}-06$
$0.0 \quad--4.14 \mathrm{E}-07$
ANGLE 1 $M U=-1.0000$ 0.0

0.0 0.0 3.725E-07 $9.570 \mathrm{E}-06$ $1.564 \mathrm{E}-05$ $2.503 E-0.5$ $4.032 E-05$ $6.863 E-05$ $1.211 E-04$ $3.415 \mathrm{E}-04$ $1.127 \mathrm{E}-02$ $1.1<8 \mathrm{E}-02$ $7.575 E-01$ $3.511 \mathrm{E} 00$ $1.080 \mathrm{E} \mathrm{OL}$ $3.073 E$ OL $8.615 E$ OI 1.966 E
2.92
O ANGLE 10 $M U=0.0950$ 0.0

0.0 0.0 5.311E-06 $1.916 E-05$ $2.144 E-05$ $5.168 E-05$ $1.091 \mathrm{E}-04$ $1.380 E-04$ $2.001 E-04$ $1.302 \mathrm{E}-02$ . $673 \mathrm{E}-01$ $3.097 E 00$ $1.227 E \quad 01$ 0.74 E 01 $9.741 E$ E $3.340 E 02$ $\begin{array}{ll}\text { ANGLE } & 2 \\ M U=-0.9894 & \text { ANGLE } \quad 3 \\ 0 U=-0.9446\end{array}$ 0.0

0.0

0.0

$3.898 E-07$ $9.653 E-06$ $1.561 E-05$ $2.5 \mathrm{C} 8 \mathrm{E}-05$ $4.059 E-05$ $6.895 E-05$ $1.215 \mathrm{E}-04$ $2.427 E-04$ $1.061 E-03$ $1.129 E-02$ $1.130 E-02$ $7.584 E-01$ $3.515 E$ OO $1.081 E 01$ $3.077 \mathrm{E} 01$ $8.625 \mathrm{E} \mathrm{O1}$ $1.968 E 02$ 2.995E 02 ANGLE 11 $M U=0.2816 \quad M J=0.458$ 0.0 0.0 0.0 $8.010 \mathrm{E}-\mathrm{C} 6$ $2.354 E-05$ $2.580 E-C 5$ $6.718 E-C 5$ $1.439 E-04$ $1.655 E-C 4$ $2.261 E-04$ 1. $164 E-04$ $1.405 E-02$ $1.338 \mathrm{E}-02$ $8.894 \mathrm{E}-01$ 4.094E CO $3.566 \mathrm{E}$ OI $0.964 E$ OI $9.964 E$ OI $3.409 E$ O2
0.0

0.0

4.622E-07 $9.990 E-06$ $1.550 E-05$ $2.533 E-05$ $4.175 E-05$ 7.032E-05 $1.233 \mathrm{E}-04$ $3.479 E-04$ $1.072 \mathrm{E}-03$ $1.137 E-02$ $1.136 \mathrm{E}-02$ $7.623 \mathrm{E}-01$ $3.533 E 00$ $1.086 \mathrm{E} \mathrm{O1}$ $3.091 \mathrm{E} 01$ 8.665 E OI

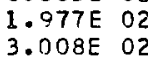
ANGLE 12
$M U=0.4580$ 0.0 0.0
0.0
0.0

$1.0325-05$ 1.283E-05 $3.086 \mathrm{E}-05$ $3.225 E-05$ . $112 E-05$ $1.992 E-04$ $2.020 E-04$ 2.5 $1.638 \mathrm{E}-03$ $1.454 \mathrm{E}-02$ $1.373 \mathrm{E}-02$ $9.114 \mathrm{E}-01$ 1.19OE OO $1.286 E$ OI $3.647 E$ OI $2.317 E$ O2 $3.478 E 02$
$A N G L E \quad 4$
$M U=-0.8656$ 0.0 0.0 0.0 5. $992 E-07$ 1.057E-05 $1.537 \mathrm{E}-05$ $2.584 E-05$ 4.399E-05 $7.296 \mathrm{E}-05$ $1.266 \mathrm{E}-04$ $3.577 E-04$ $1.094 \mathrm{E}-03$ $1.151 \mathrm{E}-02$ $1.147 E-02$ $7.694 \mathrm{E}-01$ $3.564 E$ OO $1.096 \mathrm{E} \mathrm{O1}$ $3.113 \mathrm{E}$ O1 $8.739 \mathrm{E}$ O1 $1.994 E \quad 02$ $3.032 \mathrm{E} \mathrm{O2}$ ANGLE 13 $M U=0.6179$ 0.0 0.0

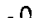
$2.198 \mathrm{E}-05$ 4.3 C6E-05 $4.145 E-05$ $1.284 E-04$ $2.900 E-04$ $2.499 E-04$ $2.954 E-04$ $7.858 E-04$ $1.745 E-03$ $1.503 E-02$ $1.407 E-02$ $9.324 E-01$ 4.282E 0 $1.314 \mathrm{E}$ O1 $3.040 \mathrm{E} 02$

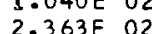
$3.543 E$ O2

ANGLE 5
MU=-0.7550
0.0
0.0
0.0
0.0
$8.163 E-07$
$1.133 E-05$
$1.531 E-05$
$2.676 E-05$
$4.745 E-05$
$7.703 E-05$
$1.318 E-04$
$3.723 E-04$
$1.124 E-03$
$1.171 E-02$
$1.163 E-02$
$7.795 E-01$
$3.609 E 00$
$1.109 E \quad 01$
$3.156 E \quad 01$
$8.843 E \quad 01$
$2.017 E \quad 02$
$3.065 E \quad 02$
ANGLE 14
$M U=0.7550$
0.0
0.0
0.0
0.0
$3.949 E-05$
$6.303 E-05$
$5.402 E-05$
$1.871 E-04$
$4.436 E-04$
$3.115 E-04$
$3.381 E-04$
$8.868 E-04$
$1.851 E-03$
$1.547 E-02$
$1.438 E-02$
$9.513 E-01$
$4.364 E 00$
$1.339 E 01$
$3.793 E 01$
$1.058 E 02$
$2.405 E 02$
$3.500 E 02$
0

3.500E O2
ANGLE 6
$M U=-0.6179$ 0.0 0.0 0.0 1.161 E-06 1. $225 \mathrm{E}-05$ $1.543 E-05$ 2.833 E-05 $5.259 \mathrm{E}-05$ $8.291 E-05$ 1. $392 E-04$ 3. $726 E-04$ $1.166 \mathrm{E}-03$ $1.198 \mathrm{E}-02$ $1.183 E-02$ 7.924E-01 $3.666 \mathrm{E} \mathrm{OO}$ $1.127 E$ OL $3.205 E$ O1 $8.976 E$ OI $2.047 E \quad 02$ $3.107 E 02$ ANGLE 15 $M U=0.8656$ 0.0
0.0 0.0 7.2 $79 E-05$ $9.507 E-05$ $7.037 E-05$ $2.805 E-04$ $7.118 E-04$ $3.888 E-04$ $3.836 E-04$ $1.946 \mathrm{E}-03$ $1.585 \mathrm{E}-02$ $1.464 E-02$ $9.670 E-01$ $4.433 E$ OD $3.350 \mathrm{E}$ OI $1.874 \mathrm{E} 02$ 1.074E O2 $\begin{array}{ll}2.44 J \mathrm{E} & 02 \\ 3.547 \mathrm{E} & 02\end{array}$

ANGLE $\quad 7$
$M U=-0.4580$ 0.0 0.0 0.0 0.0
$1.695 E-06$ i. $332 \mathrm{E}-05$ $1.590 E-05$ $3.092 E-05$ $5.999 E-05$ $9.107 E-05$ $1.492 E-04$ $4.193 E-04$ $1.217 E-03$ $1.230 E-02$ $1.208 \mathrm{E}-02$ $8.080 E-01$ 
(GAMMAS/MEV/STERADIAN/SOURCE NEUTRON) GROUP (MEV) 8. OCE $00--1.00 E$ O1 $5.00 E$ OOT- $6.50 E$ OO $4.00 E$ OO- $5.50 E$ OO $3.00 E$ OD- $4.00 E$ OO . 50 E $00--3.00 E$ OO $2.00 E \quad 00--2.50 E \quad 00$ $1.66 \mathrm{E} 00--2.00 \mathrm{E}$ OO $1.33 \mathrm{E} 00--1.66 \mathrm{E} \quad 00$ $1.00 E$ O0-- $-1.33 E$ OO $8.00 E-01---1.00 E$ OO $6.00 E-01--8.00 E-01$ $4.00 E-01---6.00 E-0$ ? $3.00 E-01--4.00 E-01$ .00E-01---3.00E-01 $1.00 E-01-\cdots-2.00 E-01$ E.00E $-02-\cdots 1.00 E-01$ $2.00 E-02---5.00 E-02$

$$
\text { ENERGY }
$$$$
\text { GROUP (ME }
$$

8.00 E OO-- $1.00 E$ OI $6.50 E$ OO- $-8.00 E$ OO 5.00 E $00---6.50 E$ OO $4.00 E$ OO- $-5.00 E$ OO 3.00 E $00---4.00 E$ OO $2.50 E$ OO-- $3.00 E$ OO $2.00 E$ OO-D-2.5OE OO 1.66 E $00--2.00 E$ EO $1.33 \mathrm{E}$ 00-D-1.66E OO $1.00 E$ EO- $00.33 E$ OO $8.00 \mathrm{E}-01--1.00 \mathrm{E}$ OO $6.00 E-01--8.00 E-01$ 4. COE-01- $-6.00 E-01$ $3.00 E-01--4.00 E-01$ $2.00 E-01-2$. COE-OI 5. $2.00 E-02---5.00 E-02$
ANGLE 1
$M U=-1.000 n \quad M U=-0.0894$ $6.651 E-07$ $8.439 \mathrm{E}-0$ $8.439 E-05$ $2.167 \mathrm{~F}-05$ $2.885 E-04$ $4.833 \mathrm{E}-04$ $2.488 \mathrm{E}-04$ $3.151 \mathrm{~F}-04$ $9.331 E-05$ $1.031 \mathrm{E}-04$ $9.842 E-05$ $3.473 E-04$ $5.984 E-04$ $2.030 E-03$ $3.770 E-03$ $7.505 \mathrm{E}-03$
$2.042 \mathrm{E}-03$ ANGLE 10 $M U=0.0950$

$3.600 \mathrm{E}-07$ $7.409 E-07$ $1.667 E-04$ $1.572 \mathrm{E}-04$ $7.199 \mathrm{E}-05$ $5.343 E-04$ $9.788 \mathrm{E}-04$ 4.866E-04 $5.871 \mathrm{E}-04$ $1.683 E-04$ $2.115 \mathrm{~F}-04$ $2.977 E-04$ $7.712 E-04$ $1.270 E-03$ $2.380 E-03$ $4.473 E-03$ $2.146 \mathrm{E}-03$ 11 $M U=0.2816$ $3.673 \mathrm{E}-07$
$7.557 \mathrm{E}-07$ $2.250 E-04$ $2.140 \mathrm{E}-04$ $5.831 \mathrm{E}-05$ $1.021 E-03$ $1.326 E-03$ $9.332 \mathrm{E}-04$ $1.125 \mathrm{E}-03$ $2.162 \mathrm{E}-04$ $2.728 \mathrm{E}-04$ $3.838 \mathrm{E}-04$ $9.228 \mathrm{E}-04$ $1.4 C 7 E-03$ $2.506 \mathrm{E}-03$ $4.664 E-03$ $8.5 C 9 E-03$
$2.167 E-03$
ANGLE 3 $M U=-0.9446$ $3.243 \mathrm{E}-07$ $6.683 \mathrm{E}-07$ $8.660 E-05$ $7.794 \mathrm{E}-0$ $2.965 E-04$ 4. $984 E-n 4$ $2.579 \mathrm{E}-04$ 3. $216 \mathrm{E}-04$ $8.846 E-05$ 1. $007 E-04$ $1.094 E-04$ 3.512E-04 6.047E-04 $2.057 E-03$ 3.794E-03 $7.537 E-03$ $2.046 E-03$ ANGLE 12 $7.701 \mathrm{E}-07$ $2.691 \mathrm{E}-04$ $2.568 E-04$ $6.902 E-05$ $8.018 \mathrm{E}-04$ $1.590 \mathrm{E}-03$ $7.386 E-04$ $8.918 \mathrm{E}-04$ $2.686 \mathrm{E}-04$ $3.432 \mathrm{E}-04$ $4.786 \mathrm{E}-04$ $1.068 \mathrm{E}-03$ $1.523 E-03$ $2.632 E-03$ $4.877 E-03$ 2.1 89E-03 ANGLE 4
$M U=-0.8656$
$3.266 E-07$
$6.731 \mathrm{E}-07$
$8.998 \mathrm{E}-05$
$8.180 \mathrm{E}-05$
$2.462 \mathrm{E}-05$
$3.086 \mathrm{E}-04$
$5.207 \mathrm{E}-04$
$2.709 \mathrm{E}-04$
$3.323 \mathrm{E}-04$
$8.428 \mathrm{E}-05$
$9.987 \mathrm{E}-05$
$1.238 \mathrm{E}-04$
$3.806 \mathrm{E}-04$
$6.254 \mathrm{E}-04$
$2.092 \mathrm{E}-03$
$3.831 \mathrm{E}-03$
$7.586 \mathrm{E}-03$
$2.053 \mathrm{E}-03$ ANGLE 13 $M U=0.6179$ $7.831 \mathrm{E}-07$ $2.755 E-04$
$2.633 E-04$ $2.633 \mathrm{E}-04$
$7.710 \mathrm{E}-05$ .359E-04 $1.634 \mathrm{E}-03$ $8.705 \mathrm{E}-04$ $1.057 \mathrm{E}-03$ $3.367 E-0$ 4. $326 \mathrm{E}-04$ $1.212 \mathrm{E}-03$ $1.642 \mathrm{E}-03$ 5. 2. $210 \mathrm{E}-03$ ANGLE 5 3. $301 \mathrm{E}-07$ $6.802 E-07$
$9.493 F-05$ $8.709 \mathrm{E}-05$ $8.709 E-05$ $3.260 E-04$ $5.518 E-04$ 2. $884 E-04$ $3.493 E-04$
$8.353 E-05$ $1.030 E-04$ $1.405 E-04$ $4.068 \mathrm{E}-04$ $6.728 E-04$ $2.132 E-03$ $3.887 \mathrm{E}-03$ $7.658 E-03$ $2.062 E-03$ ANGLE 14

ANGLE 6
$M U=-0.6179$ 3. $393 \mathrm{E}-07$ $1.017 E-04$ $9.387 \mathrm{E}-05$ $2.882 E-05$
$3.494 E-04$ $5.925 E-04$ $3.107 E-04$ $8.875 E-05$ $1.580 E-04$ $4.410 E-04$
$7.534 E-04$ $2.171 E-03$ $3.961 \mathrm{E}-03$ $7.751 E-03$
$2.074 E-03$ ANGLE 15

ANGLE 7 $M U=-0.4580$ $3.400 \mathrm{E}-07$ $7.003 E-07$ $1.026 \mathrm{E}-04$ 3.136E-05 $3.805 E-04$ $3.397 \mathrm{E}-04$ $1.003 \mathrm{E}-04$ $1.269 \mathrm{E}-04$
$1.774 \mathrm{E}-04$ $1.774 E-04$
$4.883 \mathrm{E}-04$ $8.649 E-04$ $2.208 E-03$ $4.055 \mathrm{E}-03$ $7.865 \mathrm{E}-0$ $2.089 E-03$

ANGLE 16 $M U=0.7550$ $7.948 \mathrm{E}-07$ $3.580 E-04$ 3. $442 E-04$ $1.049 \mathrm{E}-04$ 1. $242 \mathrm{E}-03$ $2.111 \mathrm{E}-03$
$1.177 \mathrm{E}-03$ $1.430 E-03$ $5.044 E-04$ $6.151 \mathrm{E}-04$ $1.458 E-03$ $1.877 \mathrm{E}-03$ $3.076 E-03$ ( ..074E-03 $2.230 E-03$
ANGLE 8 ANGLE 9 $M U=-0.2816 \quad M U=-0.0950$ $3.461 \mathrm{E}-07 \quad 3.528 \mathrm{E}-07$
$7.128 \mathrm{E}-07$ I. $228 E-04 \quad 1.393 E-04$ $1.144 E-04 \quad 3.304 E-04$ $3.468 \mathrm{E}-05 \quad 3.930 \mathrm{E}-05$ $4.222 E-04 \quad 4.787 E-04$ 7.176E-04 8.154E-04 $3.788 E-04 \quad 4.326 E-04$ $4.577 E-04 \quad 5.230 E-04$ $1.171 E-04 \quad 1.383 E-04$ $1.731 E-04$ $2.025 E-04$ $5.555 E-04$ $9.964 E-04 \quad 1.134 E-03$ $2.248 \mathrm{E}-03$ $4.171 E-03$ $\begin{array}{ll}7.999 E-03 & 8.153 E-03 \\ 2.106 E-03 & 2.125 E-03\end{array}$ ANGLE 17 SCALAR $\begin{array}{lll}M U=0.8656 & M U=0.9446 & M U=0.9894 \quad F L U X\end{array}$ $\begin{array}{llll}3.916 E-07 & 3.948 E-07 & 3.946 E-07 & 4.493 E-06\end{array}$ $\begin{array}{llll}6.063 E-04 & 9.851 E-04 & 1.502 E-03 & 2.982 E-03\end{array}$ $\begin{array}{llll}5.860 E-04 & 9.547 E-04 & 1.456 E-03 & 2.839 E-03\end{array}$ $\begin{array}{llll}1.681 E-04 & 2.586 E-04 & 3.753 E-04 & 8.416 E-04\end{array}$ $2.025 \mathrm{E}-03 \quad 3.193 \mathrm{E}-03 \quad 4.755 \mathrm{E}-03 \quad 1.017 \mathrm{E}-02$ $\begin{array}{llll}3.518 E-03 & 5.633 E-03 & 8.462 E-03 & 1.737 E-02\end{array}$ $1.940 E-03 \quad 3.068 E-03 \quad 4.549 E-03 \quad 9.444 E-03$ $2.338 \mathrm{E}-03 \quad 3.659 \mathrm{E}-03 \quad 5.374 \mathrm{E}-03 \quad 1.138 \mathrm{E}-02$ $\begin{array}{llll}7.451 E-04 & 8.905 E-04 & 1.132 E-03 & 3.087 E-03\end{array}$ $8.375 \mathrm{E}-04 \quad 9.262 \mathrm{E}-04 \quad 1.110 \mathrm{E}-03 \quad 3.682 \mathrm{E}-03$ $9.706 \mathrm{E}-04 \quad 1.025 \mathrm{E}-03 \quad 1.188 \mathrm{E}-03 \quad 4.740 \mathrm{E}-03$ $\begin{array}{llll}1.736 \mathrm{E}-03 & 1.827 \mathrm{E}-03 & 2.128 \mathrm{E}-03 & 1.060 \mathrm{E}-02\end{array}$ $2.150 \mathrm{E}-0 \mathrm{3} \quad 2.234 \mathrm{E}-03 \quad 2.506 \mathrm{E}-03 \quad 1.571 \mathrm{E}-02$ $3.497 E-03 \quad 3.689 E-03 \quad 4.071 E-03 \quad 3.152 E-02$ $5.622 E-03$ 5.855E-03 $5.976 E-03$ $5.693 E-02$ $\begin{array}{llll}2.247 E-03 & 2.259 E-03 & 2.264 E-03 & 2.691 E-02\end{array}$ 
(GAMMAS/MEV/STERADIAN/SOURCE NEUTRONI

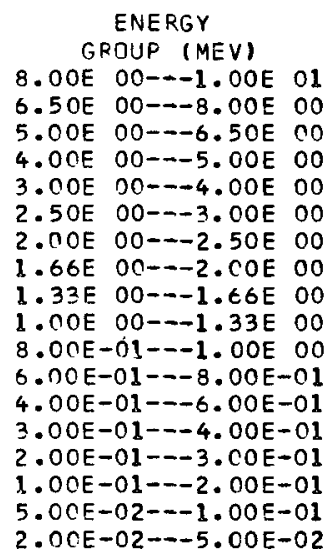

ENERGY

8.0OE OO--1.00E

$6.50 E$ OOM- 8 . COE OO $5.00 E$ OO-- $-6.50 E$ OO $4.00 E \quad 00--5.00 E \quad 00$ 3.00E $00---4.00 E$ OO $2.50 E$ OO- $-3.00 E$ EO 2.00E $00---2.50 E$ OO $1.66 E 00--2.00 E 00$ $1.33 E 00--1.66 E 00$ 1.00 E $00--1.33 \mathrm{E}$ OO $8.00 \mathrm{E}-01--1.00 \mathrm{E}$ OC $6.00 E-01--8.00 E-01$ $4.00 E-01--6.00 E-01$ $3.00 E-01--4.00 E-01$ $2.00 E-01--3.00 E-01$ $1.00 E-01--2.00 E-01$ $5.00 E-02--1.00 E-01$ 2. $00 E-02---5.00 E-.02$
ANGLE 1 $1.118 \mathrm{E}-06$ $2.306 E-06$ $085 E-04$ $8.327 \mathrm{E}-05$ $3.043 \mathrm{E}-05$ $3.352 \mathrm{E}-04$ $5.376 \mathrm{E}-04$ $2.782 \mathrm{E}-04$ $3.705 E-04$ $1.611 E-C 4$
$1.729 E-04$ $1.729 E-04$ $1.433 E-04$
$5.972 E-04$ $1.074 E-03$ $4.360 E-03$ $9.826 \mathrm{E}-03$ $2.262 \mathrm{E}-02$ $6.462 \mathrm{E}-03$

ANGLE 10 $M U=0.0950$ .375E-06 $.830 E-06$ $1.748 E-04$ $1.505 E-04$ $8.372 E-04$ $9.182 E-04$ $7.615 \mathrm{E}-04$ $9.205 E-04$ $3.324 E-04$ 4.259E-04 $6.268 \mathrm{E}-04$ $1.710 \mathrm{E}-03$ $3.010 E-03$ $5.289 E-03$ $1.226 E-02$ $2.635 E-02$ $6.950 E-03$ 0.9894 $1.120 \mathrm{E}-06$ . 1.430E-05 3. $2.379 E-04$ $5.429 E-04$ $2.818 E-04$ $2.818 E-04$
$3.717 E-04$ $3.717 E-04$
$1.571 E-04$ $1.571 E-04$
$1.703 E-04$ $1.703 E-04$ $6.040 E-04$ $1.074 E-03$ 4. $377 \mathrm{E}-03$ $9.842 E-03$ $2.264 E-02$ $6.466 E-03$

ANGLE 11 $M U=0.2816$ $.942 E-06$ . $770 E-04$ $2.499 E-04$ $9.863 E-05$ $6.972 E-C 4$ $1.527 \mathrm{E}-03$ $6.399 E-04$ $7.737 E-04$ $3.670 E-04$ $5.068 E-04$ $8.007 E-04$ $2.046 E-03$ 3.260E-03 $5.427 E-03$ $1.293 \mathrm{E}-02$ $2.724 E-02$ $7.057 \mathrm{E}-03$
ANGLE 3 $1.128 \mathrm{E}-06$ $2.326 \mathrm{E}-06$ $1.121 E-04$ $8.846 \mathrm{E}-05$ $3.395 \mathrm{E}-05$ $3.483 E-04$ $3.483 E-04$
$5.629 E-04$ $2.948 E-04$ $2.948 E-04$
$3.767 E-04$ $3.440 E-04$ $1.440 E-04$
$1.626 \mathrm{E}-04$ $1.717 E-04$ $6.289 E-04$ 1. $087 \mathrm{E}-03$ $4.440 E-03$ $9.906 \mathrm{E}-03$ $2.275 \mathrm{E}-02$ $6.481 E-03$

ANGLE 12 $M U=0.4580$ $.058 \mathrm{E}-06$ $2.794 E-04$ $.705 E-04$ $.458 E-05$ $.169 E-03$ $.625 E-03$ .. $097 \mathrm{E}-03$ $1.328 \mathrm{E}-03$ $5.335 E-04$ $7.412 \mathrm{E}-04$ $1.122 \mathrm{E}-03$ $2.507 E-03$ $3.601 E-03$ $5.838 E-03$ $1.373 \mathrm{E}-02$ $2.823 \mathrm{E}-02$
$769 \mathrm{E}-0.3$
ANGLE 4 $M U=-0.8656$
$1.143 E-06$ $1.143 \mathrm{E}-06$
$2.358 \mathrm{E}-06$ 1.1 $72 E-04$ $9.524 \mathrm{E}-05$ $3.815 E-05$ 3.666E-04 $3.163 E-04$ $3.163 E-04$
$3.879 E-04$ $3.879 E-04$
$1.283 E-04$ $1.253 E-04$ 2.065E-04 $6.711 E-04$ $1.142 \mathrm{E}-03$ $4.542 E-03$ 1. $003 E-02$ $2.297 E-02$ $6.511 E-03$ ANGLE 13 $M U=0.6179$ $3.170 E-06$ $4.960 E-04$ $3.934 \mathrm{E}-04$ $1.478 E-04$ $1.254 E-03$ $1.361 \mathrm{E}-03$ $1.490 \mathrm{E}-03$ $8.433 \mathrm{E}-04$ $1.121 \mathrm{E}-03$ 1. $548 \mathrm{E}-03$ 3. $021 E-C 3$ 4. $035 \mathrm{E}-03$ $6.465 \mathrm{E}-03$ $1.465 \mathrm{E}-02$ $2.923 E-02$
$7.282 E-03$ $M U=-0.7550$ $1.166 E-06$ $2.404 E-06$
$1.241 E-04$ $1.032 \mathrm{E}-04$ $4.238 E-05$ 3. $908 \mathrm{E}-04$ $6.398 \mathrm{E}-04$ $3.416 E-04$
$4.077 E-04$ $3.416 E-04$
$4.077 E-04$ $4.077 E-04$
$1.225 E-04$ 1. $595 E-04$ $2.430 E-04$ $7.252 E-04$ $1.280 E-03$ $4.648 E-03$ $1.021 \mathrm{E}-02$ 2. $328 \mathrm{E}-02$ $6.553 E-03$

ANGLE 14 $M U=0.7550$ $1.593 E-06$ $4.703 E-04$ $5.994 E-04$ $1.819 E-04$ $2.060 E-03$ $3.541 E-03$ $2.018 \mathrm{E}-03$ $2.481 E-03$ $1.196 \mathrm{E}-03$ $1.520 E-03$ $1.946 \mathrm{E}-03$ 4. $452 E-03$ $4.452 E-03$ $1.563 E-02$ $3.023 E-02$ $7.386 \mathrm{E}-03$ $M U=-0.6179$ $1.195 E-06$ 1. $329 \mathrm{E}-04$ 1.329E-04 $4.600 E-05$ $4.210 E-04$ $6.901 E-04$ 3. $700 E-04$ $4.389 E-04$ $4.389 E-04$
$1.335 E-04$ $1.335 E-04$
$1.785 E-04$ $2.758 \mathrm{E}-04$ $7.580 E-04$ $1.521 \mathrm{E}-03$ $4.742 E-03$ $2.369 E-02$ $6.608 \mathrm{E}-03$

ANGLE 15 $M U=0.8656$ $1.636 E-06$ $3.358 E-06$ $9.963 E-04$ . $223 E-04$ 3.13904 $3.011 E-03$ 3.763E -03 $3.697 \mathrm{E}-03$ $3.872 \mathrm{E}-03$ $2.175 E-03$ $2.531 E-03$ $4.171 E-03$ $5.181 \mathrm{E}-03$ T. $922 E-03$ $1.672 \mathrm{E}-02$ $3.118 E-02$ $7.480 \mathrm{E}-03$ $M U=-0.4580$ $1.232 \mathrm{E}-06$ $2.538 \mathrm{E}-06$ . $443 E-04$ 4. $945 E-05$ $4.598 \mathrm{E}-04$ $4.598 \mathrm{E}-04$
$7.529 \mathrm{E}-04$ $4.049 E-04$ $4.049 E-04$
$4.848 E-04$ $4.848 E-04$
$1.603 E-04$ $1.603 E-04$
$2.109 E-04$ $3.095 E-04$ $9.081 E-04$ $1.852 \mathrm{E}-03$ $4.822 \mathrm{E}-03$ $1.078 \mathrm{E}-02$ $2.420 E-02$ $6.676 \mathrm{E}-03$

ANGLE 16 $M U=0.9446$ $1.667 E-06$ $3.421 \varepsilon-06$ $1.565 E-03$ 1.524E-03 5. $4.980 E-03$ $8.655 E-03$
$5.075 E-03$ $6.016 \mathrm{E}-03$ $2.696 E-03$ $2.813 \mathrm{E}-03$ $3.059 \mathrm{E}-03$ $4.938 E-03$ 
(GAMMAS/MEV/STERADIAN/SOURCE NEUTRON)

ENERGY GROUP (MEV) 6.5OE OO-D- - . COE 00 $5.0 \mathrm{CF}$ 00---6.50E OO 4. COE $00--5.00 E$ OO $3.00 E$ OO- $-4.00 E$ OO 2.50 OE $00--3.00 E$ OO $2.00 E$ O0-- $2.50 E$ OO $1.66 \mathrm{E} \quad 00---2.00 \mathrm{E}$ $1.33 E$ E0---1. 66 E 00 $1.00 E$ 00- $-1.33 E 00$ 8.00E-01- -1.00E 00 $6.00 E-01--8.00 E-01$ $4.00 E-01---6.00 E-01$ $3.00 E-01--4.00 E-01$ $2.00 E-01---3.00 E-01$ $1.00 \mathrm{E}-01---2.00 \mathrm{E}-\mathrm{C}$ 5.0CE-02-- -1.00E-C $2.00 E-C 2---5.00 E-C 2$

$$
\text { ENERGY }
$$

GROUP (MEV)

8.00E OO---1.00E 01

$6.50 E$ OO- - 8.00E 00

$5.00 E$ OO-- 6.50 E 00

4.0NE OO--5. $3.00 E$ OO- $-4.00 E$ OO 2.50 E $00--3.00 F$ OO 2.OOE OO--2.5OE 00 $1.66 E$ OO- $2.00 E$ OO $1.33 E$ O0--1.66E OD 1.00E $01-1.00 E$ G.0OE $01-2.00 E$ S.0OE OI $-2.00 E-C 1$ $4.00 E-01-6.00 E-01$ 4.0OE $01-4.00 E-01$ $1.00 E-01--2.00 E-01$ $5.00 \mathrm{D}-02--1.00 \mathrm{E}-01$ 2. COE $-\mathrm{C} 2--5.00 \mathrm{E}-02$
ANGLE 1 ANGLE ? $M U=-1.0000 \quad M U=-0.0894$ $1.780 \mathrm{E}-08$ $3.674 \mathrm{E}-06$ $1.188 \mathrm{E}-04$ $3.485 \mathrm{E}-05$ $3.262 E-04$ $5.134 E-04$ $2.666 E-04$ $3.671 \mathrm{E}-04$ $1.968 \mathrm{E}-04$ $1.499 \mathrm{E}-04$ $6.900 E-C 4$ $1.269 E-03$ $5.479 \mathrm{E}-\mathrm{C}$ $1.330 \mathrm{E}-0$ $3.272 \mathrm{E}-0$ $9.558 \mathrm{E}-03$ ANGLE 10 $M U=0.0950$ $2.324 \mathrm{E}-06$ $4.781 \mathrm{E}-06$ $2.462 E-04$ $2.058 E-04$ ..192E-05 -. $66 E-04$ $5.592 \mathrm{E}-\mathrm{C}$ $5.592 \mathrm{E}-\mathrm{C}$ $6.755 E-04$ $3.231 E-04$ 4. 2.096 E-C3 .096 $6.85 E=03$ . 3.828E-02 $1.037 E-02$ $1.784 E-C 6$
$3.683 E-06$ $1.198 \mathrm{~F}-04$ 8.243E-05 $3.601 E-05$ $3.294 \mathrm{E}-\mathrm{C}$ $5.109 \mathrm{E}-0$ $2.710 F-04$ $3.679 \mathrm{E}-0$ $1.804 \mathrm{~F}-04$ 1. $928 \mathrm{~F}-04$ $1.584 \mathrm{E}-04$ $6.989 E-04$ $1.268 \mathrm{~F}-0$ $5.503 \mathrm{~F}-0$ $1.333 \mathrm{E}-\mathrm{C}$ $3.277 \mathrm{E}-0$ $9.564 \mathrm{E}-0$ ANGLE 11 $M U=0.2816$ $2.450 E-06$ $5.037 E-06$ $2.57 \mathrm{CE}-\mathrm{O}$ 2.157E-04 $.404 E-O E$ $9.132 \mathrm{E}-04$ $1.273 \mathrm{E}-\mathrm{C}$ 1.457E$4.0207 E-04$ $6.522 \mathrm{E}-04$ $6.522 E-04$ $1 . r 5 E-03$ $4.693 \mathrm{E}-\mathrm{CB}$ $4.273 E-03$ $1.792 \mathrm{E}-\mathrm{O}$ $4.041 \mathrm{E}-02$ $4.041 E-02$
$1.055 \mathrm{E}-02$
ANGLE 3 $\begin{array}{ccc}3 & \text { ANGLE } 4 \\ 4\end{array}$ $1.800 E-06$
$3.715 E-06$ 1. $232 E-04$ $8.774 \mathrm{~F}-05$ $4.001 E-05$ $3.417 \mathrm{E}-04$ $5.439 E-04$ $2.871 E-04$ 3. $717 E-04$ $1.608 E-04$ $1.807 E-04$ $1.889 E-04$ $7.316 E-04$ 1. $281 \mathrm{E}-03$ $5.593 E-03$ $1.342 E-02$ $3.295 \mathrm{E}-02$ $9.590 E-03$ ANGLE 12 $M U=0.4580$ $2.581 \mathrm{~F}-06$
$5.303 E-06$ $3.533 \mathrm{E}-04$ $3.081 \mathrm{E}-04$ $1.304 \mathrm{E}-04$ $1.002 \mathrm{E}-03$ $1.821 \mathrm{E}-\mathrm{O}^{2}$ $.481 \mathrm{E}-04$ 6. $6.411 E-04$ 1.263E-04 $3.255 E-03$ $4.645 E-03$ $7.428 \mathrm{E}-03$ $1.911 \mathrm{E}-02$ $4.206 \mathrm{E}-02$ $1.074 E-02$ $1.831 \mathrm{E}-06$ $3.779 E-06$ $1.293 E-04$ $.597 E-05$ $3.626 \mathrm{E}-04$ $5.831 \mathrm{E}-04$ $3.124 E-04$ $3.813 \mathrm{E}-04$ $1.272 \mathrm{E}-04$ $1.693 \mathrm{~F}-04$ $2.356 \mathrm{E}-04$ $7.850 \mathrm{E}-04$ $1.353 \mathrm{E}-03$ $5.733 \mathrm{E}-03$ $1.360 E-02$ $3.330 \mathrm{E}-02$ $9.638 \mathrm{E}-03$ ANGLE 13 $M U=0.6179$ $2.713 E-06$ $5.569 \mathrm{E}-06$ 4.287E-04 $3.788 E-04$ $3.913 E-03$ $8.181 E-03$ $2.048 E-02$ $4.381 E-02$ $1.093 \mathrm{E}-02$

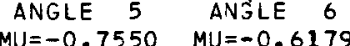
$1.877 \mathrm{E}-06$ . $873 E-06$ $1.370 E-04$ $5.151 E-05$ $6.285 E-04$ $3.398 E-04$ 4.001E-04 $1.284 \mathrm{E}-04$ $1.734 \mathrm{E}-04$ $2.814 \mathrm{E}-04$ $8.509 E-04$ $1.545 \mathrm{E}-03$ $5.877 \mathrm{E}-03$ $1.387 E-02$ $3.381 \mathrm{E}-02$ $9.708 \mathrm{E}-03$ ANGLE 14 $M U=0.7550$
$2.836 E-06$ $5.819 E-06$ $6.327 E-04$ $5.810 E-04$ $5.822 \mathrm{~F}-03$ $4.556 E-02$ 1.111E-02 $M U=-0.6179$ $3.997 \mathrm{E}-06$ $1.465 E-04$ $1.144 E-04$ $5.566 E-05$ $4.191 \mathrm{E}-04$ $6.781 E-04$ $3.679 E-04$ $4.320 E-04$ $1.435 E-04$ $1.986 \mathrm{E}-04$ $3.191 \mathrm{E}-04$ $9.400 E-04$ 3.883E-03 $5.999 \mathrm{E}-03$ $1.424 \mathrm{E}-02$ $3.448 E-02$
$9.799 E-03$ ANGLE 15 $M U=0.8656$ $2.943 E-06$ $6.034 E-06$ 9.750E-04 $3.600 E-04$ 政 $3.375 E-03$ $5.407 E-03$ $7 \mathrm{E}-02$ $2.361 \mathrm{E}-02$ $1.127 \mathrm{E}-02$

ANGLE 7 ANGLE 8 ANGLE 9 $M U=-0.4580 \quad M U=-0.2816 \quad M U=-0.0950$ $4.151 E-06 \quad 4.334 E-06 \quad 4.545 E-06$ $1.584 E-04 \quad 1.745 E-04 \quad 1.969 E-04$ $1.243 E-04 \quad 1.379 E-04 \quad 1.584 E-04$ $5.913 E-05 \quad 6.390 E-05 \quad 7.196 E-05$ $5.108 E-04 \quad 5.864 E-04$ $8.224 E-04 \quad 9.454 E-04$ $4.507 E-04 \quad 5.245 E-04$ $5.479 E-04 \quad 6.390 E-04$ $2.271 E-04 \quad 2.783 E-04$ $2.408 E-04 \quad 2.931 E-04 \quad 3.578 E-04$ $3.555 E-04 \quad 4.124 E-04 \quad 5.228 E-04$ $1.081 E-03 \quad 1.311 E-03 \quad 1.657 E-03$ $2.346 E-03$ 2.873E-03 $3.388 E-03$ $6.097 E-03$ 6.196E-03 $6.340 \mathrm{E}-03$ $1.471 E-02$ 1.530E-02 $1.602 E-02$ $3.011 E-02$ 3.633E-02 $3.752 E-02$ ANGLE 16 $M U=0.9446 \quad M U=0.9894$ 


\begin{abstract}
ENERGY
GROUP (MEV)

3.00E $00--1.00 E 01$ $6.50 E$ O0- $-8.00 E$ OO 5. OOE OO- - 6.50 E OO $4.00 E$ OOL 3. 2.00E $00-2.50 E$ OD 1.66E $00-2.00 E$ OO $1.33 E$ OO- 1 . 66 E 00 $1.00 E$ OO- $1.33 \mathrm{E} 00$ $8.00 \mathrm{E}-01--1.00 \mathrm{E}$ OO $6.00 \mathrm{E}-01---8.00 \mathrm{E}-01$ $4.00 E-01--2.00 E-01$ $3.00 E-01--4.00 E-01$ 2.00E-01-- 3. $00 \mathrm{E}-01$ $1.00 \mathrm{E}-01---2.00 \mathrm{E}-01$ 5.00E-02-- - $1.00 E-01$ $2.00 E-02---5.00 E-02$
\end{abstract}

ENERGY

GROUP (MEV)

8.00E 00---1.00E O1 $6.50 E \quad 00-7.00 E \quad 00$ 5.00E $00---6.50 E$ OO $4.00 E$ OO- $-5.00 E$ OO $3.00 E$ OO- - - 4. OOE OO $2.50 E$ OO- $-3.00 E$ OO 2.00 E $00--2.50 E 00$ $1.66 \mathrm{E} 00--2.00 \mathrm{E}$ OO $1.33 \mathrm{E} 00--1.66 \mathrm{E}$ OO $1.00 E$ O0- $-1.33 E 00$ $8.00 E-01--1.00500$ $6.00 \mathrm{E}-01--8.00 \mathrm{E}-01$ 4.0 ONE-01-- -6.00E-01 3.00E-01---4. COE-01 $2.00 E-01--3.00 E-01$ 1.00E-01--2.00E-01 $2.00 E-02--5.00 E-02$
ANGLE 1 ANGLE 2 $000 \quad M U=-0.9894$ $.119 \mathrm{E}-06$ $6.445 E-06$ . (.) $4.705 \mathrm{E}-04$ $148 \mathrm{E}-04$ 2.1 $85 E-04$ $3.194 \mathrm{E}-04$ $2.005 E-04$ $2.062 E-04$ $2.062 E-04$
$1.399 E-04$ $1.399 \mathrm{E}-04$
$7.635 \mathrm{E}-04$ $1.430 \mathrm{E}-03$ $6.710 \mathrm{E}-03$ $1.776 \mathrm{E}-02$ 4.783E-02 $1.438 \mathrm{E}-02$

ANGLE 10 $M U=0.0950$ $4.526 E-06$ $2.477 \mathrm{E}-04$ $1.738 \mathrm{E}-04$ $1.029 E-04$ $6.285 E-04$ $.422 E-04$ $5.817 \mathrm{E}-04$ $7.055 \mathrm{E}-04$ $3.748 E-04$ $5.136 E-04$ $8.598 \mathrm{E}-04$ $2.674 E-03$ $4.871 \mathrm{E}-03$ $8.102 E-03$ $2.303 E-02$ $1.575 E-02$ $1.439 \mathrm{E}-02$ ANGLE 11 $M U=0.2816$ $1.005 E-05$ $3.012 E-04$ $2.230 E-04$ $1.267 E-04$ $7.391 \mathrm{E}-\mathrm{C} 4$ . $219 \mathrm{E}-03$ $6.964 E-C 4$ $8.374 E-04$ $4.930 E-04$ $7.339 E-04$ $1.287 \mathrm{E}-03$ $3.408 \mathrm{E}-03$ $5.338 \mathrm{E}-03$ $8.550 \mathrm{E}-03$ $2.455 \mathrm{E}-02$ $1.606 \mathrm{E}-02$
(GAMMAS/MEV/STERADIAN/SOURCE NEUTRON)

ANGLE 4
$M U=-0.8656$
$3.242 E-06$
$6.696 E-06$
$1.458 E-04$
$9.037 E-05$
$5.901 E-05$
$3.109 E-04$
$4.914 E-04$
$2.697 E-04$
$3.249 E-04$
$1.307 E-04$
$1.669 E-04$
$2.516 E-04$
$8.796 E-04$
$1.545 E-03$
$7.049 E-03$
$1.820 E-02$
$4.880 E-02$
$1.451 E-02$

ANGLE 5

ANGLE 6 $1.443 \mathrm{E}-02$ $3.353 E-06$ $6.923 \mathrm{E}-06$ $1.548 \mathrm{E}-04$ $1.005 \mathrm{E}-04$ $6.640 \mathrm{E}-05$ $3.359 \mathrm{E}-04$ $5.343 E-04$ $5.393 \mathrm{E}-04$
$2.958-04$ $3.394 E-04$ $3.394 \mathrm{E}-04$
$1.194 \mathrm{E}-04$ 1. $125 \mathrm{E}-04$ $3.056 \mathrm{E}-04$ $9.564 E-04$ $1.815 \mathrm{E}-03$ $7.232 E-03$ $1.859 E-02$ 4. $964 E-02$ 1.463 E-02 $M U=-0.6179$ 3.502E-06 $7.227 \mathrm{E}-06$ $1.650 E-04$ $7.093 \mathrm{E}-05$ $3.626 E-04$ $5.753 \mathrm{E}-04$ $3.190 \mathrm{E}-04$ $3.685 E-04$ $1.411 E-04$ $1.411 E-04$
$2.056 E-04$ $3.444 \mathrm{E}-04$ $3.444 \mathrm{E}-04$
$1.064 \mathrm{E}-03$ $2.287 \mathrm{E}-03$ $7.379 \mathrm{E}-03$ $1.913 E-02$ $5.077 \mathrm{E}-02$ $1.478 \mathrm{E}-02$ ANGLE 12 ANGLE 13 ANGLE 14 $5.297 \mathrm{E}-06 \quad 5.720 \mathrm{E}-06$ $.087 \varepsilon-05$ $3.017 \mathrm{E}-04$ $2.765 E-04$ $1.503 E-C 4$ $9.733 E-04$ $1.508 \mathrm{E}-03$ $9.386 \mathrm{E}-04$ $1.151 \mathrm{E}-03$ $7.544 \mathrm{E}-04$ $1.161 E-03$ $1.906 \mathrm{E}-03$ 4.206E-03 $5.842 \mathrm{E}-03$ $9.240 \mathrm{E}-03$ $2.631 \mathrm{E}-02$ $1.639 \mathrm{E}-02$ $.748 E-04$ . $1.279 E-03$ $1.618 \mathrm{E}-03$ $1.250 E-03$ $1.823 E-03$ $2.668 E-03$ $4.988 E-03$ $6.458 E-03$ $1.008 E-02$ $6.831 \mathrm{E}-02$ $1.672 \mathrm{E}-02$ $M U=-0.4580$ $3.691 \mathrm{E}-08$ $1.773 \mathrm{E}-04$ $1.77 \mathrm{E}-04$ $7.399 \mathrm{E}-05$ $3.949 \mathrm{E}-04$ $6.229 E-04$ $3.462 E-04$ $4.153 \mathrm{E}-04$ $1.895 \mathrm{E}-04$ $2.895 \mathrm{E}-04$ $3.786 \mathrm{E}-04$ $1.245 \mathrm{E}-03$ $2.925 \mathrm{E}-03$ $7.490 \mathrm{E}-03$ $1.981 \mathrm{E}-02$ $5.219 E-02$ 1.497E-02

ANGLE 8 $M U=-0.2816$ $3.924 E-06$
$8.085 E-06$ 1. $941 E-04$ 281E-04 $4.412 E-04$ $6.933 E-04$ . $895 E-04$ $4.811 E-04$ $2.472 E-04$ $3.189 E-04$ $4.420 E-04$ $1.556 \mathrm{E}-03$ $3.632 \mathrm{E}-03$ $7.603 E-03$ $2.068 \mathrm{E}-02$ $5.392 \mathrm{E}-02$ $1.520 E-02$
ANGLE 15

ANGLE 16 $M U=0.9446$
$6.823 E-06$ $1.394 E-05$ $1.961 \mathrm{E}-03$ $1.872 \mathrm{E}-03$ $8.439 E-04$ $5.789 E-03$ $9.523 E-03$ $6.499 E-03$ $7.619 E-03$ $5.077 E-03$ $5.250 E-03$ $5.561 E-03$ $8.157 \mathrm{E}-03$ $9.664 E-03$ $1.402 \mathrm{E}-0$ $3.511 \mathrm{E}-02$ $7.540 E-02$
$1.751 E-02$

ANGLE 17 $M U=0.9894$ $.431 E-05$ $5.194 E-03$ $4.897 E-03$ $1.842 E-03$ $1.405 E-02$ $2.300 E-02$ $1.428 \mathrm{E}-02$ $1.569 E-02$ $7.623 E-03$ $6.760 E-03$ $6.688 E-03$ 1.002E-02 $1.104 E-02$ $1.579 E-02$ 3.696E-02 l.117E-O2 3. $051 E-02$ $1.703 E-02$

1.730 E-02

ANGLE 9 $M U=-0.0950$ $4.202 E-06$ $2.179 \mathrm{E}-04$ $1.474 E-04$ $8.874 E-05$ $5.106 E-04$ $8.038 \mathrm{E}-04$ $4.601 E-04$ $5.679 E-04$ $3.030 E-04$ $3.908 E-04$ $5.838 E-04$ $2.031 E-03$ $4.299 E-03$ $7.781 E-03$ $2.174 E-02$ $5.595 \mathrm{E}-02$ 
(GAMMAS/MEV/STERAOIAN/SOURCE NEUTRON)

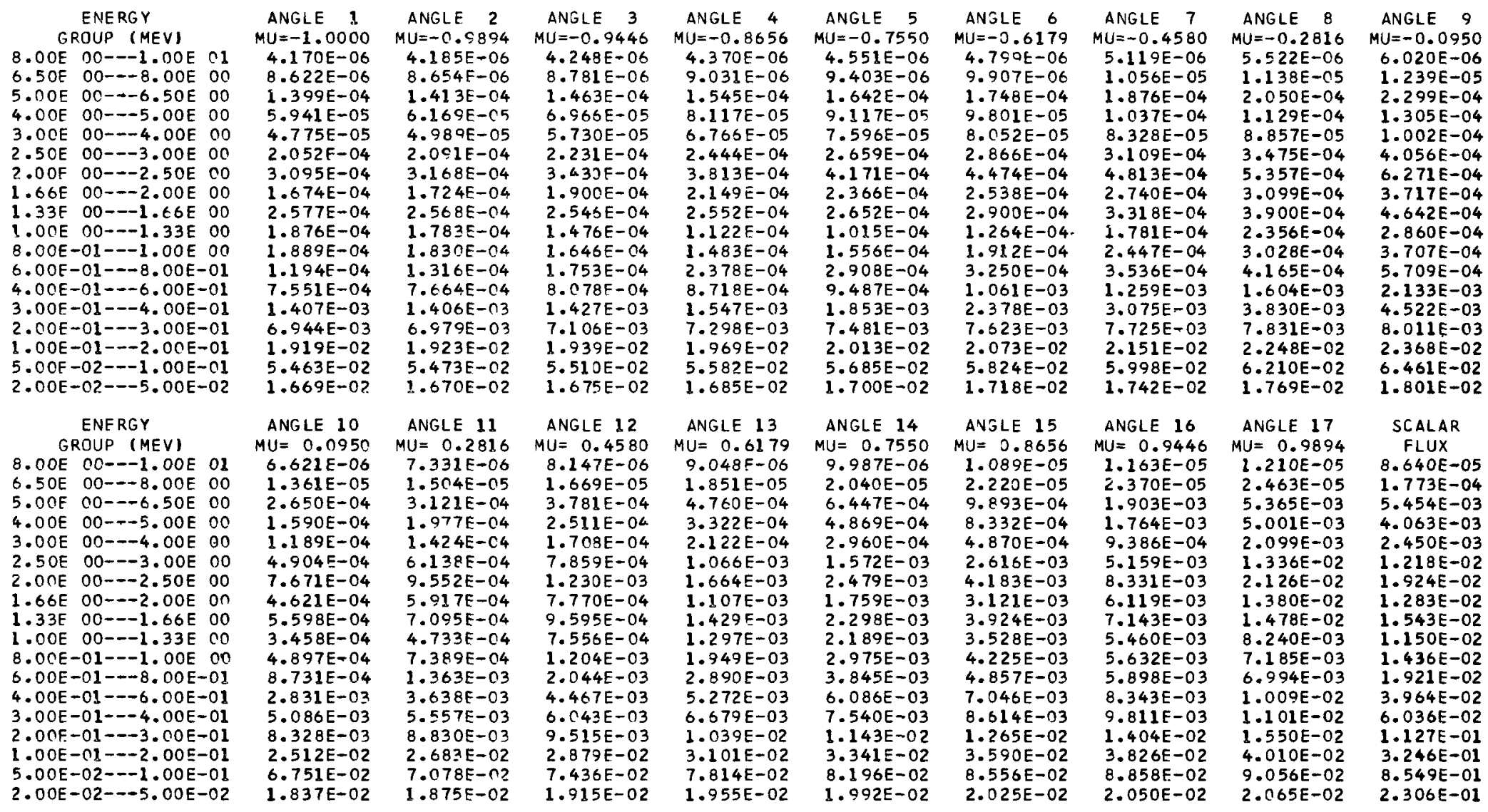


(GAMMAS/MEV/STERADIAN/SOURCE NEUTRON)

ENERGY

GROUP (MEV) 8. COE DO---1.0OE 01 6.50 E $00--8.00$ E OO $5.00 E$ OO- $-6.50 E$ EO 4.0OE OO- - 5. OOE OD $3.00 E$ OON- $4.00 E$ OO $2.50 E$ OO- $-3.00 E$ OO $2.00 E$ OO- $2.50 E$ OO $1.66 \mathrm{E} 00--2.00 \mathrm{E}$ OO $1.33 E$ OO- $1.66 E$ OO $1.00 E$ OO- $1.33 \mathrm{E}$ OO . 6.00E-01- $-8.00 E-01$ 4.00E-01-C. 3.00E-O1- $-4.00 E-O 1$ . $1.00 \mathrm{C}-01-2.00 \mathrm{E}-\mathrm{OI}$ $2.00 \mathrm{E}-02-02-0.00 \mathrm{E}-02$

$$
\text { ENERGY }
$$

GROUP (MEV)

8.00E $00--1.00 E$ OI 5.5OE OO--8.00E 00 $4.0 O E$ OO- $-5.00 E$ OO $3.00 E$ 0O- -4 COE 00 $2.50 E$ OO- $3.00 E$ OO $2.00 E$ OD- - 2.5OE 00 1.66 E $00--2.00 E 00$ $1.33 E$ O0-- 1. $66 E$ OO $1.00 \mathrm{E} 00-\cdots 1.33 \mathrm{E} 00$ $8.00 E-01--1.00 E 00$ $6.00 E-01---8.00 E-01$ $4.00 E-01--6.00 E-01$ $3.00 E-01--4.00 E-01$ $2.00 E-01--3.00 E-0$ $1.00 E-01--2$. COE-O $5.00 E-C 2---1.00 E-01$ $2.00 E-02---5.00 E-02$
ANGLE 1 $M U=-1.0000$ $4.754 E-06$ $9.835 \mathrm{E}-06$ $1.396 \mathrm{E}-04$ $4.931 E-C 5$ $4.987 E-05$ $1.484 \mathrm{E}-04$ $2.214 \mathrm{E}-04$ $1.246 \mathrm{E}-04$ $2.007 E-04$ $1.639 \mathrm{E}-04$ $1.617 E-04$ $9.785 E-05$ $7.073 \mathrm{E}-04$ $1.297 E-C 3$ $1.588 E-03$ $5.478 \mathrm{E}-02$ $1.688 \mathrm{E}-02$

ANGLE 10 $M U=0.0950$ $8.156 E-06$ $1.675 \mathrm{E}-05$ $2.686 E-04$
$1.395 E-04$ $1.395 E-04$
$1.266 E-04$
$3.782 E-04$ $3.782 \mathrm{E}-04$ $5.796 \mathrm{E}-04$ $3.661 E-04$ $4.423 \mathrm{E}-04$ $3.029 E-04$ $4.425 E-04$ $8.291 \mathrm{E}-04$ $2.749 E-03$ $4.827 \mathrm{E}-03$ $7.830 \mathrm{E}-03$ $2.442 E-02$ $6.817 E-02$ 1. $862 \mathrm{E}-02$
ANGLE 2 $M U=-0.9894$ $4.774 E-06$ $9.876 \mathrm{E}-06$ $1.410 \mathrm{E}-04$ $5.164 \mathrm{E}-05$ $5.217 \mathrm{E}-05$ $1.520 E-04$ $2.279 \mathrm{E}-04$ $1.289 \mathrm{E}-04$ $1.903 E-04$ $1.550 E-04$ $1.562 \mathrm{E}-04$ $1.097 E-04$ 7.177E-04 $1.298 E-03$ . $5.487 \mathrm{E}-02$ $1.487 \mathrm{E}-02$

\section{ANGLE 11} $M U=0.2816$ $9.234 \mathrm{E}-06$ $3.170 E-04$ $1.751 \mathrm{E}-\mathrm{C} 4$ $1.751 E-C 4$
$1.526 E-04$ $4.759 \mathrm{E}-04$ $7.317 \mathrm{E}-04$ $4.702 \mathrm{E}-04$ $5.613 \mathrm{E}-\mathrm{C4}$ 4.209E-04 $6.873 E-04$ $1.318 \mathrm{E}-03$ $3.523 \mathrm{E}-03$ $5.242 E-C$ $8.294 \mathrm{E}-03$ $2.608 \mathrm{E}-02$ $7.157 E-02$ $1.901 \mathrm{E}-02$ $M U=-0.9446$ $4.856 \mathrm{E}-06$ 1. $004 \mathrm{E}-05$ 1. $462 \mathrm{E}-04$ $5.977 \mathrm{E}-05$ $6.012 \mathrm{E}-05$ $1.649 \mathrm{E}-04$ $2.509 \mathrm{E}-04$ $1.439 \mathrm{E}-04$ $1.951 \mathrm{E}-04$ $1.253 \mathrm{E}-04$ $1.396 E-04$ $1.522 E-04$ 1. $1.325 E-03$ $6.738 E-03$ $5.527 \mathrm{E}-02$

ANGLE 12 $M U=0.4580$ $1.053 \mathrm{E}-05$ $3.819 \mathrm{E}-04$ $2.211 E-04$ 1.823E-04 $6.149 E-04$ $9.441 E-04$ $6.252 E-04$ $7.779 E-04$ $6.969 E-04$ $1.151 \mathrm{E}-03$ $1.991 \mathrm{E}-03$ $4.301 \mathrm{E}-03$ $5.679 \mathrm{E}-03$ $8.928 \mathrm{E}-03$ $2.799 E-02$ $7.528 \varepsilon-02$ $1.942 E-02$

\section{ANGLE 4
$M U=-0.8656$ $A N=-0.8656$
$5.015 E-06$ $5.015 \mathrm{E}-06$
$1.037 \mathrm{E}-05$ $1.548 \mathrm{E}-04$ $7.119 \mathrm{E}-05$ $7.109 \mathrm{E}-05$ $1.837 \mathrm{E}-04$ $2.833 \mathrm{E}-04$ $1.644 E-04$ $1.924 E-04$ $9.184 \mathrm{E}-05$ $1.258 E-04$ 2.117E-04 ..143E-04 $1.451 E-03$ $6.913 E-03$ $1.90 \mathrm{E}-02$ $5.601 E-02$}

ANGLE 13 $M U=0.6179$ $1.202 E-05$ $4.759 E-04$ $2.903 \mathrm{E}-04$ $2.246 E-04$ $8.397 \mathrm{E}-04$ $1.291 \mathrm{E}-03$ $9.069 \mathrm{E}-04$ $1.194 \mathrm{E}-03$ $1.230 E-03$ $1.887 E-03$ $2.812 \mathrm{E}-03$ $5.035 \mathrm{E}-03$ $6.262 E-03$ $9.714 \mathrm{E}-03$ $3.011 E-02$ $7.919 E-02$ $1.983 E-02$
ANGLE 5 U $=-0,7550$ $5.254 \mathrm{E}-06$
$1.086 \mathrm{E}-05$ $1.086 \mathrm{E}-05$ $1.647 \mathrm{E}-04$ $8.048 E-05$ $7.959 \mathrm{E}-05$ $2.013 E-04$ $3.113 E-04$ $1.811 \mathrm{E}-04$
$1.988 \mathrm{E}-04$ $1.988 \mathrm{E}-04$ $8.322 E-05$ $1.345 E-04$ $2.596 E-04$ $8.864 E-04$ $1.761 E-03$ $5.709 E-02$ $1.720 E-02$

ANGLE 14 $M U=0.7550$ $1.367 E-05$ $6.323 E-04$ $6.323 E-04$ $3.098 \mathrm{E}-04$ $1.263 E-03$ $1.960 E-03$ $1.960 E-03$ $1.983 \mathrm{E}-03$ $2.105 E-03$ $2.889 E-03$ 3. $722 E-03$ $5.760 E-03$ $7.051 \mathrm{E}-03$ $1.063 \mathrm{E}-02$ $3.239 \mathrm{E}-02$ $8.312 E-02$ $2.021 E-02$
AVGLE 6 $M U=-0.6179$ $5.582 E-06$
$1.153 E-05$ 1. $755 \mathrm{E}-04$ $8.597 \mathrm{E}-05$ $8.400 \mathrm{E}-05$ $2.163 E-04$ $3.325 \mathrm{E}-04$
$1.934 \mathrm{E}-04$ $1.934 E-04$ $2.194 \mathrm{E}-04$ $1.086 \mathrm{E}-04$ $1.688 E-04$ $2.879 E-04$ 7.107E-03 7. $5.852 \mathrm{E}-02$

$1.740 \mathrm{E}-02$

ANGLE 15 $M U=0.8656$ $1.532 E-05$ $3.115 \mathrm{E}-05$
$9.465 \mathrm{E}-04$ $9.465 E-04$ $7.344 E-04$
$5.070 E-04$ $2.157 \mathrm{E}-03$ $3.398 \mathrm{E}-03$ 2.718E-03 $3.450 E-03$ $3.400 \mathrm{E}-03$ $4.082 E-03$ $4.657 E-03$ .005E-0 3. $470 \mathrm{E}-02$ $8.680 E-02$ $2.054 \mathrm{E}-02$

ANGLE 7 $M U=-0.4580$ $1.241 E-05$ 1.886E-04 $8.993 E-05$ 8.666 E-05 $2.335 E-04$ $3.554 \mathrm{E}-04$ 2.084E-04 $2.555 E-04$ $1.581 \mathrm{E}-04$ $2.178 \mathrm{E}-04$ $3.110 \mathrm{E}-04$ 1.195E-03 
ENERGY GROUP (MEV) 8.0OE OO--1. DOE 01 5.5OE OO---8. OOE OO $4.00 E$ OO- $-5.00 E$ OO 3.00 E $00-2.00 E$ OO 2.50E $00-3.00 E$ OO $2.00 E$ OO- $2.50 E$ OO $1.66 E$ 00--2. $00 E$ OO $1.33 \mathrm{E} 00--1.66 \mathrm{E} 00$ $1.00 E$ OO- $-1.33 E$ OO $8.00 E-01--1.00 E$ OO $6.00 \mathrm{E}-01--8.00 \mathrm{E}-01$ $4.00 E-03--6.00 E-0$ $3.00 E-01--4.00 E-01$ $2.00 E-01--3.00 E-01$ $1.005-01--2.00 E-01$ $5.00 E-02--1$. COE -01
$2.00 E-02--5 . C O E-02$

$$
\text { ENERGY }
$$$$
\text { GROUP (MEVI }
$$

8.0OF OO- - 1.00E O 6.50 E OO---8. COE ON 5.00 E $00--6.50$ E 00 $4.00 E$ OO- $-5.00 E$ OO 3.00 E $00--4.00 E$ OO 2.50 E $00--3.00 E$ OO 2. COE OO---2.50E OO $1.66 \mathrm{E}$ OO- $-2.00 \mathrm{E}$ OO $1.32 E$ OO- - 1. $66 E$ OO 1.0 E $00-1.33 E$ OO $8.00 \mathrm{E}-01--1.00 \mathrm{E} O 0$ $6.00 \mathrm{E}-01--8.00 \mathrm{E}-01$ 4.00E-01- $-6.00 E-01$ $3.00 E-01--4$. OOE-0I $2.00 E-1--3.00 E-01$ $1.00 E-02-1.00 E-01$ $2.00 E-02--1.00 E-01$
ANGLE 1 ANGLE ?

$M U=-1.0000 \quad M U=-0.9894$ $4.869 E-06$ 1. CO7E-05 $1.320 \mathrm{E}-04$ $4.035 E-05$ $4.853 E-05$ $1.041 \mathrm{E}-04$ $1.545 E-04$ $9.165 E-05$ $1.374 \mathrm{E}-04$ $7.811 E-05$ $6.402 E-04$ $1.151 E-03$ $5.935 E-03$ $1.687 \mathrm{E}-02$ $5.083 \mathrm{E}-02$
$1.574 \mathrm{E}-02$

\section{ANGLE 10} $M U=0.0950$ 8.930 E-06 $1.833 \mathrm{E}-05$ $2.613 \mathrm{E}-04$ $1.212 \mathrm{E}-04$ $1.272 \mathrm{E}-04$ $2.843 E-04$ 4.293E-04 $2.834 E-04$ $3.410 \mathrm{E}-04$ $2.561 \mathrm{E}-04$ $3.862 \mathrm{E}-04$ $7.534 E-04$ 2.527E-03 $6.370 E-03$ $6.970 E-03$ $0.344 \mathrm{E}-02$ $1.344 \mathrm{E}-02$
$1.736 \mathrm{E}-02$ $1.012 \mathrm{E}-\mathrm{C}$ $3.334 E-04$ $4.259 E-05$ $5.081 E-05$ $1.073 E-04$ $1.599 E-04$ $9.505 E-C 5$ $1.518 \mathrm{E}-\mathrm{C} 4$ $1.294 E-04$ $1.281 \mathrm{E}-04$ $8.904 F-C 5$ $6.491 E-04$ $1.153 \mathrm{E}-03$ 5.963E-03 $1.691 \mathrm{E}-0$ $5.091 E-C 2$
$1.575 F-02$ ANGLE 11 $M U=0.281$ $1.032 \mathrm{~F}-\mathrm{C5}$ $2.114 \mathrm{E}-05$ $3.100 E-04$ $1.533 \mathrm{E}-04$ ?. 54EE-04 $3.612 \mathrm{E}-\mathrm{C} 4$ $5.463 \mathrm{E}-04$ $3.666 E-04$ $4.358 \mathrm{E}-04$ $3.625 \mathrm{E}-04$ $6.149 \mathrm{E}-04$ 1.2CSE-03 $3.221 \mathrm{E}-03$ $7.372 E-03$ 2. $6.36 \mathrm{EE}-02$ $6.662 E-02$
$1.773 E-02$
(GAMYAS/MEV/STERADIAN/SOURCE NEUTRON)

ANGLE 3 ANGLE 4 ANGLE 5 AVJLE 6 $4.983 E-06 \quad 5 U=-0.8656 \quad M U=-0.7550 \quad M U=-0.6179$ $1.031 E-05 \quad 1.069 E-05 \quad 1.124 E-05 \quad 1.201 E=05$ $1.386 E-04 \quad 1.470 E-04 \quad 1.566 E-04 \quad 1.673 E-04$ $5.337 \mathrm{E}-05$ 6.113E-05 $6.943 \mathrm{E}-05 \quad 7.376 \mathrm{E}-05$ $5.874 E-05 \quad 6.954 E-05 \quad 7.771 E-05 \quad 8.376 E-05$ $1.186 \mathrm{E}-04 \quad 1.346 \mathrm{E}-04 \quad 1.484 \mathrm{E}-04 \quad 1.52 \mathrm{E}-05$ $\begin{array}{llll}1.790 E-04 & 2.051 E-04 & 2.262 E-04 & 2.595-04\end{array}$ $1.069 E-04 \quad 1.227 E-04 \quad 1.350 E-04 \quad 1.438 E-04$ $1.463 \mathrm{~F}-04 \quad 1.416 \mathrm{E}-04 \quad 1.455 \mathrm{E}-04 \quad 1.626 \mathrm{E}-04$ $1.027 \mathrm{E}-04 \quad 7.325 \mathrm{E}-05 \quad 6.688 \mathrm{E}-05 \quad 9.097 \mathrm{E}-05$ $1.280 \mathrm{~F}-04$ $6.819 E-04$ $1.184 \mathrm{E}-03$ $6.064 E-03$ $1.705 \mathrm{E}-02$ $5.129 \mathrm{E}-02$
$1.580 \mathrm{E}-02$ ANGLE 12 $M U=0.4580$ $1.206 E-05$ $2.464 E-05$ $3.745 E-04$ $1.931 \mathrm{E}-04$ $1.854 \mathrm{E}-04$ $4.691 E-04$
$7.080 E-04$ $4.917 E-04$ $6.170 E-04$ $6.175 \mathrm{E}-04$ 1. $046 \mathrm{E}-03$ $1.826 \mathrm{E}-03$ $3.903 E-03$ $5.044 E-03$ $7.922 \mathrm{E}-\mathrm{C3}$ $2.538 E-02$ $7.009 E-02$
$1.811 E-02$ $1.773 E-02 \quad 1.827 E-02$ $1.225 E-03 \quad 2.289 E-03$ $1.040 E-04$ $1.816 \mathrm{E}-04$ $? .334 E-04$ $1.310 \mathrm{E}-03$ $6.212 \mathrm{E}-03$ $1.733 \mathrm{E}-02$ $5.199 \mathrm{E}-\mathrm{O}$ $1.590 E-02$ ANGLE 13

$1.134 \mathrm{E}-04 \quad 1.443 \mathrm{E}-04$ $2.231 E-04 \quad 2.459 E-04$ $7.989 E-04 \quad 9.030 E-04$ $1.603 E-03 \quad 2.082 E-03$ $6.346 E-03 \quad 6.441 E-03$ $1.300 \mathrm{E}-02$
$1.604 \mathrm{E}-02$
$1.622 \mathrm{E}-02$ ANGLE 15 $M U=0.6179$ $1.417 E-05$
$2.886 \mathrm{E}-05$ $4.654 \mathrm{E}-04$ $2.516 \mathrm{E}-04$ $2.282 \mathrm{E}-04$ $6.456 \mathrm{E}-04$ $9.761 \mathrm{~F}-04$ $7.269 E-04$ $9.7 \in 2 E-C$ $1.110 E-03$ 1.725E-03 2.567E-03 $4.530 E-03$ (2) 8. $7.373 \mathrm{E}-02$ $1.848 \mathrm{E}-02$
7550 MNGLE 15 $1.660 \mathrm{E}-05 \quad 1.918 \mathrm{E}-05$ $3.371 \mathrm{E}-05 \quad 3.885 \mathrm{E}-05$ $\begin{array}{ll}6.115 E-04 & 8.955 E-04 \\ 3.663 E-04 & 6.378 E-04\end{array}$ $3.133 E-04 \quad 5.089 E-04$ $9.880 E-04 \quad 1.730 E-03$ $1.511 \mathrm{E}-03 \quad 2.687 \mathrm{E}-03$ $1.662 \mathrm{E}-03 \quad 2.926 \mathrm{E}-03$ $1.910 E-03 \quad 3.072 E-03$ $2.631 E-03 \quad 3.685 E-03$ 5. 6. 2. $125 E-02$ 1.01 $7.737 \mathrm{E}-02 \quad 8.075 \mathrm{E}-02$ $1.884 \mathrm{E}-02 \quad 1.914 \mathrm{E}-02$ 6.
ANGLE 7 $M U=-0.4580$ $6.317 E-06$ $1.304 E-05$ $1.803 E-04$ $7.648 E-05$ $8.444 E-05$
$1.706 E-04$ $2.557 E-04$ $1.553 E-04$ $1.931 E-04$ $1.868 \mathrm{E}-04$ $2.648 \mathrm{E}-04$ $1.091 E-03$ $2.694 \mathrm{E}-03$ $1.897 \mathrm{E}-02$ $5.607 \mathrm{E}-02$ $1.644 \mathrm{E}-02$

ANGLE 8 $6.977 E-06$ 7.832E-OS $1.438 E-05 \quad 2.611 E-05$ $1.984 E-04 \quad 2.247 E-04$ $8.270 E-05 \quad 5.712 E-05$ $9.071 E-05 \quad 3.049 E-04$ $1.913 E-04 \quad 2.281 E-04$ $2.858 \mathrm{E}-04 \quad 3.424 \mathrm{E}-04$ $1.790 E-04 \quad 2.214 E-04$ $2.337 E-04 \quad 2.812 E-04$ $1.803 E-04 \quad 2.142 E-04$ 2.299E-04 2.822E-04 $3.203 E-04 \quad 4.665 E-04$ $1.416 E-03$ 1.903E-03 $3.334 E-03 \quad 3.892 E-03$ $6.571 E-03 \quad 6.710 E-03$ $1.985 E-02 \quad 2.091 E-02$ $\begin{array}{ll}5.815 \mathrm{E}-02 & 6.061 \mathrm{E}-02 \\ 1.671 \mathrm{E}-02 & 1.702 \mathrm{E}-02\end{array}$

ANGLE 16 $M U=0.9446$ 4.357E-05

$1.632 \mathrm{E}-03$ $1.387 E-03$ $9.658 \mathrm{E}-04$ $3.561 E-03$ $5.542 \mathrm{E}-03$ $4.582 \mathrm{E}-03$ S.296E-03 $4.677 E-03$ $4.787 E-03$ $6.736 E-03$ $6.736 E-03$ $1.105 E-02$ $8.352 \mathrm{E}-02$ $1.937 E-02$
ANGLE 17 SCALAR $M U=0.9894 \quad$ FLUX $2.319 E-05 \quad 1.251 E-04$ $\begin{array}{ll}4.680 E-05 & 2.558 E-04 \\ 4.720 E-03 & 5.092 E-03\end{array}$ $4.233 \mathrm{E}-03 \quad 3.174 \mathrm{E}-03$ $2.134 \mathrm{E}-03 \quad 2.562 E-03$ $9.756 \mathrm{E}-03 \quad 7.807 \mathrm{E}-03$ $1.470 \mathrm{E}-02 \quad 1.190 \mathrm{E}-02$ $1.052 E-02 \quad 1.064 E-02$ $6.892 E-03$ $9.607 E-03$ 5. P.84 $3.393 E-02$ $1.182 \mathrm{E}-02$ $3.427 E-02$ $2.356 E-02$ $.528 E-02 \quad 8.027 E-01$ $1.951 \mathrm{E}-02 \quad 2.178 \mathrm{E}-01$ $1.026 \mathrm{E}-02 \quad 8.841 \mathrm{E}-03$ $7.636 E-03 \quad 1.644 E-02$ 
(GAMMAS/MEV/STERADIAN/SOURCE NEUTRON)

ENERGY GROUP (MEV) 8.0OE $00---1.00 E$ O1 $6.50 E$ O0---8.00E 00

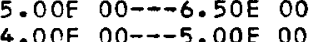
4.0OE OO-- -5. OOE OO $3.00 E$ OOA-
$2.50 E$ OO. OOE OO 2.50 E $00---2.50 E$ OO $1.66 \mathrm{E} 00--2.00 \mathrm{E} 00$ $1.66 E$ OO- $1.2 .00 E$ OO $1.00 \mathrm{E} 00--1.33 \mathrm{E}$ CO $8.00 E$ OD- $1.33 E$ CO

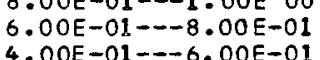
$3.00 E-01--4.00 E-01$ $2.00 E-01--3.00 E-01$ $1.00 E-01--2.00 E-01$ $5.00 \mathrm{E}-02--1.00 \mathrm{E}-01$ $2.00 E-02---5.00 E-02$

ENERGY GROUP (MEV)

8.00E 00---1.00E 01 6.50 OE $00---8.00 E$ OO 5.00 E $00---6.50 E \quad 00$ $4.00 E$ OO- -5.00 E 00 3.00 E $00--4.00 E 00$ $2.50 E$ OO---3.00E 00 $2.00 E$ OO---2.50E 00 1.66 E $00---2.00 E$ EO 1.33 E $00--1.66$ E 00 $1.00 E$ OO- 1.33 E 00 $8.00 E-01--1.00 E 00$ $6.00 E-01--8.00 E-01$ $4.00 E-01-\cdots-6.00 E-01$ $3.00 E-01--4.00 E-01$ 2.0OE-01---3.00E-01 $1.00 E-01-2.00 E-01$ $5.00 E-02--1.00 E-01$
$2.00 E-02---5.00 E-02$ $M U=-1.0000$ $3.487 \mathrm{E}-06$ (.205E-06 . $490 E-05$ .916E-05 4.186E-05 $4.858 E-05$ $6.991 E-05$ . $7.009 E-04$ (.689E-03 $1.037 \mathrm{E}-02$ $9.926 \mathrm{E}-03$ ANGLE 10 $M U=0.0950$ $7.479 E-06$ $1.533 \mathrm{E}-05$ $1.868 \mathrm{~F}-04$ $7.202 E-05$ $9.598 \mathrm{E}-05$ $1.145 \mathrm{E}-04$ $1.646 \mathrm{E}-04$ $1.251 \mathrm{E}-04$ $1.485 \mathrm{E}-04$ $1.413 \mathrm{E}-04$ $2.343 E-04$ $4.908 E-04$ $1.638 \mathrm{E}-03$ $2.603 E-03$ $4.153 \mathrm{E}-03$ $1.356 \mathrm{E}-02$ $3.988 \mathrm{E}-02$
$1.092 \mathrm{E}-02$
ANGLE 2 $3.5 \mathrm{C} 7 \mathrm{E}-0 \mathrm{~S}$ $8.249 E-06$ $8.594 E-05$ $3.330 \mathrm{E}-05$ $3.375 \mathrm{E}-05$ $5.113 E-05$ $3.739 E-05$ $6.392 \mathrm{E}-05$ $6.517 E-05$ $5.982 E-0.5$ $4.087 E-05$ $4.141 E-04$ $7.0 t 1 E-04$ $3.703 E-03$ $1.039 E-02$ $3.207 E-02$ $9.934 E-03$ ANGLE 11 $M U=0.2816$ $9.128 \mathrm{E}-\mathrm{C} 6$ $1.865 \mathrm{E}-05$
$2.285 \mathrm{E}-04$ $2.285 E-04$ $9.410 E-05$ $1.209 E-04$ $1.505 \mathrm{E}-04$ $2.150 E-04$ $1.656 \mathrm{E}-04$ $1.966 \mathrm{E}-04$ $2.137 \mathrm{E}-04$ $3.921 \mathrm{E}-04$ $7.865 E-04$ $2.041 E-03$ $2.777 E-03$ $4.375 E-03$ $1.442 E-02$ $4.182 \mathrm{E}-02$
$1.114 \mathrm{E}-02$
ANGLE 3 $M U=-0.9446$ $3.591 \mathrm{E}-06$
$7.429 \mathrm{E}-06$ $7.429 E-06$
$8.985 E-05$ $2.645 E-05$ $3.948 \mathrm{E}-05$ $4.033 \mathrm{E}-05$ $6.002 E-05$ $4.149 E-05$ $5.795 \mathrm{E}-05$ $4.955 E-05$ $5.470 E-05$ 6.597E-05 $4.322 \mathrm{E}-04$ $7.396 \mathrm{E}-04$ $3.752 \mathrm{~F}-03$ $1.048 \mathrm{E}-02$ $3.231 E-02$ $9.966 E-03$ ANGLE 12 $M U=0.4580$ $2.321 \mathrm{E}-05$ $2.848 E-04$ $1.201 \mathrm{E}-04$ $1.495 \mathrm{E}-04$ $1.992 \mathrm{E}-04$ $2.839 E-04$ $2.313 E-04$ $3.001 \mathrm{E}-04$ $3.852 E-04$ $6.746 \mathrm{E}-04$ $1.165 \mathrm{E}-03$ $2.415 \mathrm{E}-03$ 2.973E-03 $4.665 E-03$ $1.537 E-02$ $4.395 E-02$
$1.137 E-02$ ANGLE $\quad 4$
$M U=-0.8656$ 3.752E-06 $7.771 E-06$ $9.606 E-05$ $3.389 \mathrm{E}-05$ 4.751E-05 4.894E-05 $4.679 F-05$ $5.181 E-05$ $3.324 E-05$ $5.258 \mathrm{E}-05$ $9.896 E-05$ $4.628 E-04$ $8.389 E-04$ 3.820 E -03 $1.065 \mathrm{E}-02$ $3.275 E-02$ 1.003E-02 ANGLE 13 $M U=0.6179$ $1.455 E-05$
$2.945 E-05$ $3.642 E-04$ $1.571 E-04$ $1.903 E-04$ $2.822 \mathrm{E}-04$ 4.054E-04 3.638E 04 $5.119 E-04$ $7.028 \mathrm{E}-04$ $1.096 E-03$ 1.592E-03 2.7 $37 E-03$ $3.249 E-03$ $5.003 E-03$ $1.6406-02$ $4.606 E-02$
$1.159 E-02$
ANGLE 5
$M U=-0.7550$ 3. 991 E-06 8. $266 E-06$ $1.030 E-04$ 3. $897 E-05$ $5.317 E-05$ $5.502 \mathrm{E}-05$ 5. 090E-05 $5.216 \mathrm{E}-05$ $3.155 E-05$ 6.080E-05 1. $225 E-04$ $5.078 E-04$ $1.039 E-03$ 3.874E-03 $1.090 \mathrm{E}-02$ 3.339E-02 $1.011 E-02$ ANGLE 14 $M U=0.7550$ $1.874 E-05$
$3.773 E-05$ 4.861E-04 2. $306 \mathrm{E}-04$ $2.686 E-04$ $4.556 \mathrm{E}-04$ $6.660 \mathrm{E}-04$ $6.493 E-04$ $9.072 \mathrm{E}-04$ $1.191 \mathrm{E}-03$ $1.624 E-03$ $2.024 E-03$ $3.035 E-03$ $3.608 E-03$ $5.370 E-03$ $1.746 E-02$ $4.817 E-02$
$1.179 E-02$
ANGLE 6 4.326E-06 $8.950 E-06$ $1.108 \mathrm{E}-04$ $4.078 E-05$ $5.571 E-05$ 5. $809 E-05$ $5.461 E-05$ $6.176 E-05$ $4.764 E-05$ 7. $903 E-05$ $1.342 \mathrm{E}-04$ $5.844 E-04$ $1.343 E-03$ 3. $904 \mathrm{E}-03$ $1.124 \mathrm{E}-02$ $3.425 \mathrm{E}-02$ $1.022 \mathrm{E}-02$ ANGLE 15 $M U=0.8656$ 4.797E-05 7.000E-04 4. $058 E-04$ 4.376E-04 $8.468 \mathrm{E}-04$ $1.254 \mathrm{E}-03$ $1.246 \mathrm{E}-03$ $1.599 E-03$ $1.854 \mathrm{E}-03$ $2.191 \mathrm{E}-03$ $2.421 E-03$ 3. $370 \mathrm{E}-03$ 5.95E 03 1.75OE-03 $1.847 E-02$ $5.010 E-02$
$1.197 E-02$

ANGLE 7 $M U=-0.4580$ $4.787 E-06$ $9.884 E-06$ $4.136 \mathrm{E}-05$ $5.751 E-05$ $6.116 \mathrm{E}-05$ $8.790 E-05$ $6.094 E-05$ $7.938 \mathrm{E}-05$ $7.435 E-05$ $1.019 E-04$ $1.463 E-04$ $7.201 E-04$ $1.712 E-03$ $3.916 \mathrm{E}-03$ $1.166 \mathrm{E}-02$ $1.036 \mathrm{E}-02$ ANGLE 16 $\begin{aligned} & A U=0.9446 \\ & 2.958 E-05\end{aligned}$ $5.896 \mathrm{E}-05$ $1.188 \mathrm{E}-03$ B. $771 E-04$ 
(GAMMAS/MEV/STERADIAN/SOURCE NEUTRON)

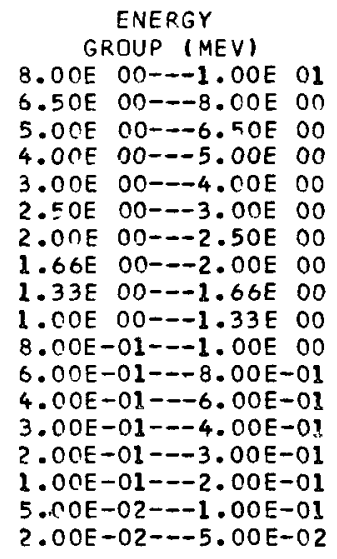

ENERGY

GROUP (MEV)

8. 00 OOD--1.00E 01 $6.50 E$ DO-- - 8. OOE 00 $5.00 E$ OO- - -6.50E 00 4.0OE OO- - 5. 00 CO $3.00 E$ OO- - 4.00E 00 $2.50 E$ O0--3.00E 00 $2.00 E^{00--2.50 E ~} \mathrm{CO}$ $1.66 \mathrm{E} 00--2.00 \mathrm{E} 00$ $1.33 E$
$1.00=-1.66 E$ 00 $1.00 E$ OO- -1.33 E 00
$8.00 E-01--1.00 E 00$ 8.00E-01--1.00E 00 $6.00 E-01--8.00 E-01$
$4.00 E-01---6.00 E-01$ $4.00 E-01--6.00 E-01$
$3.00 E-01--4.00 E-01$ $3.00 E-01--4.005-01$
$2.00 E-01---3.00 E-01$ $2.00 E-01--3.00 E-01$
$1.00 E-C 1--2.00 E-01$ $5.00 E-02--1.00 E-01$ 2. OOE-02---5.00E-02
ANGLE 1 ANGLE 2 $1.724 E-06$ .541E-06 6.569E-06 $1.386 \mathrm{E}-05$ $8.065 \mathrm{E}-06$ $8.065 \mathrm{E}-06$ $1.490 \mathrm{E}-05$ $2.783 E-05$ $3.123 E-05$ $2.325 \mathrm{E}-05$ $9.823 \mathrm{E}-06$ 2.211E-04 $3.679 E-04$ $2.025 \mathrm{E}-03$ $5.524 E-03$ $1.712 \mathrm{E}-0$ ? $5.288 E-03$

ANGLE 10 $M U=0.0950$ $4.154 E-06$ $8.506 \mathrm{E}-06$ $.826 E-05$ $3.585 E-05$ $5.226 E-05$ $4.450 E-05$ $6.078 \mathrm{~F}-05$ $5.196 \mathrm{E}-05$ $6.152 \mathrm{E}-05$ $7.107 \mathrm{E}-05$ $1.301 \mathrm{E}-04$ $2.826 \mathrm{E}-04$ $9.155 \mathrm{E}-04$ 1.3 $2.180 \mathrm{E}-03$ $7.183 \mathrm{E}-03$ $2.121 \mathrm{E}-02$
$5.799 \mathrm{E}-\mathrm{C3}$
ANGLE 3 1. $737 E-06$ $.572 \mathrm{E}-06$ .076E-05 $7.607 E-06$
$1.4 C 8 F-05$ $9.072 \mathrm{~F}-06$ $1.551 \mathrm{E}-05$ $1.501 \mathrm{E}-05$ $2.644 E-05$ $2.879 E-C 5$ .284E-05 $1.383 E-05$ $2.2 ? 1 F-04$ $3.730 E-04$ 2.031E-03 $5.536 \mathrm{E}-03$ $1.715 E-02$ $5.292 E-C 3$

ANGLE 11 $M U=0.2816$ $5.3 C 2 E-06$ .081E-05 $4.877 \mathrm{E}-05$ $0.831 E-05$ $6.056 E-05$ $8.205 E-05$ $7.161 \mathrm{E}-05$ $8.720 \mathrm{E}-05$ $1.174 E-04$ 2.263E-04 $4.480 E-04$ $1.118 \mathrm{E}-03$ $1.453 \mathrm{E}-03$ $2.288 \mathrm{E}-03$ $7.610 E-03$ $2.218 E-02$ ANGLE 12 8.770E-05 0.9446 $1.787 E-06$ . $311 E-05$ 4. $111 \mathrm{E}-05$
$1.18 \mathrm{O}-05$ $1.885 \mathrm{E}-05$ $1.253 \mathrm{E}-05$ $1.253 E-05$
$1.894 \mathrm{E}-05$ $1.543 E-05$ $2.181 F-05$ $2.077 \mathrm{E}-05$ $2.198 \mathrm{E}-05$ $2.797 E-05$ $2.316 E-04$ $3.997 E-04$ $2.050 E-03$ $5.585 \mathrm{E}-03$ 1.728E-02 5.309E-C3 ANGLE 4 $M U=-0.8656$ $3.912 \mathrm{E}-06$ $4.671 \mathrm{E}-05$ $1.566 E-05$ $2.375 E-05$ $1.678 E-05$ $2.315 E-05$ $1.605 \mathrm{E}-05$ $1.692 \mathrm{E}-05$ $1.275 E-05$ 2.301E-05 $4.580 \mathrm{E}-05$ $2.478 E-04$ $4.658 \mathrm{E}-04$ $2.073 E-03$ $5.677 E-03$ 5.340E-03 $M U=0.4580$ $7.035 E-06$
$1.424 E-05$ . . $315 \mathrm{E}-04$ .349E-05 1. $132 E-04$ $1.083 \mathrm{E}-04$ $1.472 \mathrm{E}-04$ $2.207 \mathrm{E}-04$ $3.864 E-04$ $6.463 E-04$ $1.296 \mathrm{E}-03$ $1.547 E-03$ $2.425 E-03$ $8.080 \mathrm{E}-03$ $2.323 E-02$ $6.022 \mathrm{E}-02$

ANGLE 13 $M U=0.6179$ $.704 E-06$ $2.173 \mathrm{E}-04$ 8. $648 E-05$ $1.179 E-04$ $.250 \mathrm{E}-04$ 1.730 E- 04 $1.848 \mathrm{E}-04$ $2.676 E-04$ $4.001 E-04$ 6.096 E-04 $8.548 \mathrm{E}-04$ $1.443 \mathrm{E}-03$ $1.682 \mathrm{E}-03$ 2.581E-03 $8.576 \mathrm{E}-03$ 2.429E-02 $6.131 \mathrm{E}-03$
ANGLE 5 ANGLE 6 $2.021 E-06 \quad 2.209 E-06$ $4.200 E-06$ $5.046 \mathrm{E}-05$ $1.834 E-05$
$2.684 E-05$ $2.684 E-05$
$1.926 E-05$ $1.926 \mathrm{E}-05$
$2.574 \mathrm{E}-05$ $2.574 E-05$
$1.700 E-05$ $1.700 E-05$
$1.642 E-05$ $1.642 E-05$
$1.255 E-05$ $2.812 \mathrm{E}-05$ $5.758 \mathrm{E}-05$ $.753 E-04$ $5.845 \mathrm{E}-04$ 2. $086 \mathrm{E}-03$ $5.810 E-03$ $1.785 E-02$ $5.384 E-03$ ANGLE 14 $M U=0.7550$ $1.380 \mathrm{E}-05$ $2.750 E-05$ $1.344 E-04$ $.790 E-04$ $2.167 E-04$ 3.039E-04 $3.421 E-04$ $4.788 \mathrm{E}-04$ $6.572 E-04$ $8.708 E-04$ $1.051 \mathrm{E}-03$ $1.575 \mathrm{E}-03$ $1.852 \mathrm{E}-03$ $2.744 E-03$ $9.073 \mathrm{E}-0$ $6.231 \mathrm{E}-03$
ANGLE 7 $M U=-0.4580$ $2.469 E-06$ $5.94 \mathrm{E}-05$ 5.944E- 05 $2.819 E-05$ $2.819 E-05$ $2.838 \mathrm{E}-05$ $2.286 \mathrm{E}-05$ $3.122 E-05$ $3.477 \mathrm{E}-05$ $4.708 \mathrm{E}-05$ $7.068 E-05$ $4.087 E-04$ $9.480 \mathrm{E}-04$ 2. $080 E-03$ $6.208 \mathrm{E}-03$ $1.886 \mathrm{E}-02$
$5.514 \mathrm{E}-03$

ANGLE 8 $M U=-0.2816$ $5.843 E-06$ $6.728 E-05$ $1.985 E-05$ $3.130 \mathrm{E}-05$ $2.348 E-05$ $3.362 \mathrm{E}-05$ $2.947 \mathrm{E}-05$ $4.138 E-05$ $4.636 E-05$ $5.952 E-05$ .856E-05 $.388 \mathrm{E}-04$ $2.082 E-03$ $6.480 \mathrm{E}-03$ $1.953 \varepsilon-02$ $5.599 E-03$ $5.443 \mathrm{E}-03$

ANGLE 16 $=0.8656$
$.976 E-05$ $3.907 \mathrm{E}-05$ $4.651 \mathrm{E}-04$ ..077E-04 5.901E-04 6.480E-04 . $193 E-04$ .77OE 04 1.127E 03 $1.218 E-03$ 1.724E 03 . . $2.623 \mathrm{E}-02$ $6.316 E-03$

$M J=0.9446$

ANGLE 17 $2.735 E-05$ $.363 \mathrm{E}-05$ .8 $312 E-04$ . $667 E-04$ $.914 \mathrm{E}-04$ . $218 E-03$ $.220 \mathrm{E}-03$ $1.349 \mathrm{E}-03$ $1.339 \mathrm{E}-03$ $1.335 \mathrm{E}-03$ $1.347 E-03$ $1.904 \mathrm{E}-03$ $2.150 E-03$ $3.057 E-03$ 9.911 E-03 $6.38 E-02$ 
(GAMMAS/MEV/STERADIAN/SOURCE NEUTRON)

\begin{abstract}
ENERGY
GROUP (MEV) 8. OOE OO---1. DOE OI $6.50 E$ OO- -8.00 E 00 $5.00 E$ OO- -6.50 E 00
4.00 OOD 4.0OE OO- - 5. DOE OO $3.00 E$ OO- - 4.0OE OO 2.5OE OO--3. 00 E . $1.66 E$ OO- $2.00 E$ OO 1. $33 E$ OO- $1.66 E$ OO 1. G.OOE $01-2.00 E-01$ . 3.00E-01-- $4.00 E-01$ 2.0OE-O1- $-3.00 E-01$ 1. OOE $-01---2.00 E-01$ $5.00 \mathrm{E}-02-\cdots 1.00 \mathrm{E}-01$ $2.00 \mathrm{E}-02---5.00 \mathrm{E}-02$
\end{abstract}

\section{ENE RGY}

. OOE 00-- - 1.00E $6.50 E$ OO- $-8.00 E$ OO $5.00 E$ OON-6.50E OO 4.00 E $00--5.00 E$ OO 3.00 E $00-\cdots 4$. COE OO .5OE OO-- 3 . NOE OO $2.00 \mathrm{E} \mathrm{OO--2.50E} \mathrm{OO}$ $1.66 \mathrm{E} \mathrm{OOL-2.00E} 00$ $1.33 \mathrm{E} 00---1.66 \mathrm{E} 00$ $1.00 F$ 00-- $1.33 E$ OO 8.00E-01---1.00E OO $6.00 E-01--8.00 E-01$ 4. OOE $-01-\cdots 6.00 E-01$ $3.00 E-01--4.00 E-01$ $2.00 E-01--3.00 E-01$ $1.00 \mathrm{E}-01--2.00 \mathrm{E}-0$ $5.00 \mathrm{E}-02--1.00 \mathrm{E}-01$ $2.00 E-02---5.00 E-02$ $M U=-1.0000$ $U=-1.0000$
$6.786 \mathrm{E}-07$ $.370 E-06$ $.524 \mathrm{E}-05$
$1.033 \mathrm{E}-06$ $179 \mathrm{E}-06$ .269E-06 $4.351 E-06$ $6.596 E-06$ $1.310 E-05$ $6.310 E-05$ 4.814E-08 I. $064 \mathrm{E}-04$ $1.734 \mathrm{E}-04$ $1.039 E-03$ $2.739 \mathrm{E}-03$ $8.499 \mathrm{E}-03$ $2.615 \mathrm{E}-03$

ANGLE 10 $M U=0.0950$ $1.811 \mathrm{E}-06$ $3,710 E-06$ $1.538 \mathrm{E}-05$ $2.312 \mathrm{E}-05$ $1.667 E-05$ $2.155 E-05$ $1.977 \mathrm{E}-0$ $2.357 E-05$ 3.253E-05 $6.681 E-05$ $1.504 E-04$ 4. $712 \mathrm{E}-04$ $6.804 \mathrm{E}-04$ $1.070 \mathrm{E}-03$ $3.550 \mathrm{E}-03$ $1.047 \mathrm{E}-02$ $2.860 \mathrm{E}-03$
ANGLE 2 $M U=-0.9894$ $6.861 \mathrm{~F}-07$ $1.301 E-06$ $1.560 E-05$ $1.682 E-06$ 4.847E-06 $1.796 E-06$ $4.680 E-06$ $6.379 E-06$ $1.188 E-05$ 1. $2.321 E-06$ $2.321 \mathrm{E}-06$ $1.771 \mathrm{E}-04$ $1.771 \mathrm{E}-04$
$1.041 \mathrm{E}-03$ $1.041 \mathrm{E}-03$
$2.745 \mathrm{E}-03$ $8.513 \mathrm{E}-03$ $2.617 \mathrm{E}-03$

ANGLE 11 $M U=0.2816$ $.401 E-06$ $5.473 E-05$ $2.174 \mathrm{E}-05$ $3.121 \mathrm{E}-05$ $2.369 E-05$ $3.018 \varepsilon-05$ $2.893 E-05$ $3.717 E-05$ $5.997 E-05$ $1.214 E-04$ $2.372 E-04$ $5.670 E-04$ $7.123 E-04$ $1.119 E-03$ $3.752 \mathrm{E}-03$ .094E-C $2.913 \mathrm{E}-03$

ANGLE $3 \quad$ ANGLE 4 $A U=-0.9446 \quad M U=-0.0656 \quad$ MUELE 5 $7.155 E-07 \quad 7.647 \mathrm{E}-07$
$1.470 E-06 \quad 1.593 E-06$ $1.470 E-06 \quad 1.593 E-06$ $1.689 E-05 \quad 1.875 E-05$ 7.806E-06 $6.359 E-06$ $7.131 E-06$ $9.886 E-06$ 3.562E-06 $5.606 E-06$ 5.800E-06 $4.105 E-06$ 8. $7.878 E-06$ $3.970-06$ $7.503 E-06$ (.978E-06 $9.839 E-06 \quad 1.895 E-05$ $1.106 \mathrm{E}-04 \quad 1.182 E-04$ $1.1048 E-04 \quad 2.382 E-04$ $1.047 \mathrm{E}-03 \quad 1.053 \mathrm{E}-03$ $2.047 E-03 \quad 1.053 E-03$ $1.771 E-03$
$8.575 E-03$ $\begin{array}{ll}2.575 E-03 & 8.690 E-03 \\ 2.625 E-03 & 2.640 E-03\end{array}$ ANG $=-0.7550$ ANGLE 6 $8.264 E-07$ $1.728 \mathrm{E}-06$ $2.044 E-05$ $7.715 E-06$ $1.136 \mathrm{E}-05$ $6.572 E-06$ $7.815 E-06$ $4.123 E-06$ $4.123 E-06$ 4.037E-06 $2.434 E-05$ 1.332E-04 $1.332 E-04$ $1.052 \mathrm{E}-03$ $2.883 \mathrm{E}-03$ $8.855 \mathrm{E}-03$ $2.662 \mathrm{E}-03$ $M U=-0.6179$ $9.051 \mathrm{E}-07$ $1.882 \mathrm{E}-06$ $2.196 \mathrm{E}-05$ 7.505E-06 $1.136 E-05$ $6.408 E-06$ $8.092 E-06$ $6.973 E-06$ $8.973 E-06$ $8.304 E-06$ 1.521E-05 2.621E-05 $3.893 E-04$ $1.044 E-03$ $2.970 \mathrm{E}-03$ $9.073 E-03$ $2.690 \mathrm{E}-0$

ANGLE 7

$\begin{array}{lccc}A N G L E & 7 & \text { ANGLE } 8 & \text { ANGLE } 9 \\ M U=-0.4580 & M U=-0.2816 & M U=-0.0950\end{array}$ $1.014 \mathrm{E}-06$ $2.089 E-06$ $2.386 E-05$ $6.681 E-06$ $1.102 E-05$ $8.123 E-06$ $8.767 E-06$ $8.294 E-06$ $1.189 E-05$ $1.448 E-05$ $1.019 E-05$ . $4.868 \mathrm{E}-04$ 1. $034 \mathrm{E}-03$ $1.034 E-03$
$3.079 E-03$ $3.079 E-03$
$9.345 E-03$ $2.725 \mathrm{E}-03$ ANGLE 12 ANGLE 13
$M U=0.6179$ ANGLE $14 \quad$ ANGLE 15
$M U=0.7550 \quad M U=0.8656$ $J=0.4580$ $3.354 \mathrm{E}-06$ 7.368E-05 $2.882 E-05$ 4. $109 \mathrm{E}-05$ $3.383 E-05$ $4.488 \mathrm{E}-0$ 4. $951 E-05$ $7.146 \mathrm{E}-0$ $1.185 E-04$ $2.065 E-04$ $3.349 E-04$ $6.468 \mathrm{E}-04$ $7.541 \mathrm{E}-04$ $1.182 \mathrm{E}-03$ 3. $973 \mathrm{E}-03$ $1.143 \mathrm{E}-02$
$2.965 \mathrm{E}-03$ 4.984E-06 $9.931 E-06$ 4. $067 \mathrm{E}-05$ $5.862 E-05$ $5.514 E-05$ $7.623 E-05$ $9.361 E-05$ $1.385 E-04$ $2.144 \mathrm{E}-04$ $3.178 \mathrm{E}-04$ 4. $303 E-04$ $7.096 E-04$ $8.168 \mathrm{E}-04$ $1.251 \mathrm{E}-03$ $4.202 \mathrm{E}-03$
$1.192 \mathrm{E}-02$ 7. $849 \mathrm{E}-06$ 1. $598 \mathrm{E}-04$ $6.949 E-05$ $9.840 E-05$ $1.055 \mathrm{E}-04$ $1.451 \mathrm{E}-04$ $1.793 \mathrm{E}-04$ $2.486 E-04$ $3.424 \mathrm{E}-04$ $4.390 \mathrm{E}-04$ $5.133 \mathrm{E}-04$ $7.657 \mathrm{E}-04$ $8.945 \mathrm{E}-04$ $1.322 \mathrm{E}-03$ $4.428 \mathrm{E}-03$ $1.239 \mathrm{E}-0$ $M U=0.8656$ $1.275 E-05$ $2.635 E-04$ $1.404 E-04$ 1. $852 E-04$ $2.168 E-04$ $2.876 E-04$ $.326 E-04$ .106E-04 $4.875 E-04$ . $8.303 E-04$ $9.679 \varepsilon-04$ 1. $393 \mathrm{E}-03$ $4.634 E-03$ $1.281 \mathrm{E}-0$ ANGLE 16 $M U=0.9446$ $2.012 E-05$
$3.875 E-05$ 4.713E-04 $3.093 E-04$ $3.544 \mathrm{E}-04$ $4.507 E-04$ $5.719 E-04$ $5.917 E-04$ $6.358 E-04$ $6.325 \mathrm{E}-04$ $6.227 E-04$ $6.274 \mathrm{E}-04$ $9.075 E-04$ $1.018 \mathrm{E}-03$ $1.455 E-03$ $4.798 \mathrm{E}-03$ $1.313 E-02$ $3.132 \mathrm{E}-03$ $\begin{array}{ll}1.178 \mathrm{E}-06 & 1.429 \mathrm{E}-06\end{array}$ $2.414 \mathrm{E}-06 \quad 2.927 \mathrm{E}-06$ $2.718 E-05 \quad 3.294 E-05$ 1.116E-06 1.008E-05 
(GAMMAS/MEV/STERADIAN/SOURCE NEUTRON)

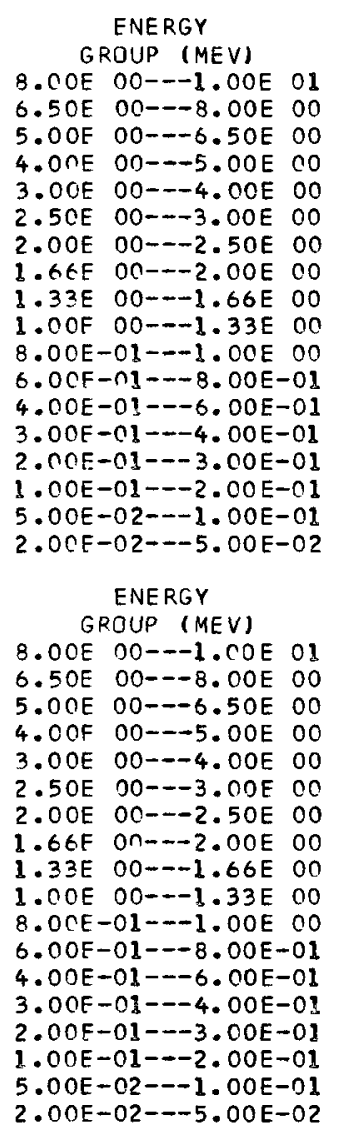

ANGLE 1 2
MU $2.249 E-07$ $4.338 E-07$ $4.824 E-06$ $6.094 \mathrm{E}-07$ $5.330 E-07$ $3.164 \mathrm{E}-06$ $5.545 \mathrm{E}-06$ $5.415 \mathrm{E}-06$ $1.286 \mathrm{E}-06$ $-2.268 \mathrm{E}-06$ $4.810 \mathrm{E}-05$ $7.608 E-05$ $5.106 \mathrm{~F}-04$ $1.300 E-03$ $4.037 \mathrm{E}-03$ 1.239E-03 ANGLE 10 $M U=0.0950$ $6.759 \mathrm{E}-07$ $1.390 E-06$ $1.549 E-05$ $6.009 E-06$ $9.022 E-06$ $6.055 E-06$ $7.228 E-06$ $6.699 E-06$ $8.056 E-06$ $3.362 E-05$ $7.527 E-05$ $2.305 E-04$ $3.230 E-04$ $5.032 \mathrm{E}-04$ $1.681 E-03$ $1.681 \mathrm{E}-03$
$4.956 \mathrm{E}-03$ $4.956 \mathrm{E}-03$
$1.352 \mathrm{E}-03$ $2.294 E-07$ $5.021 F-06$ $-1.018 \mathrm{E}-07$ $1.396 \mathrm{E}-08$ $1.488 E-06$ $2.924 E-06$ 4. $955 E-06$ $4.803 E-06$ 1. $453 E-06$ $-1.029 E-06$ $4.828 \mathrm{E}-05$ $7.830 \mathrm{E}-0 \mathrm{~S}$ $5.111 \mathrm{E}-04$ $1.303 E-03$ $4.043 E-03$ $1.24 \mathrm{CE}-\mathrm{C}$ ANGLE 1 ? $M U=0.2816$
$9.262 \mathrm{E}-07$ $9.262 E-07$
$1.803 E-06$ $2.084 \mathrm{E}-05$ $8.764 \mathrm{E}-06$ $1.245 E-05$ $8.699 E-06$ $1.045 E-05$ $1.066=-05$ $2.40 E-C 5$ $2.875 E-05$ 1.1 1.198E-C. $3.349 E-04$ $5.25 \mathrm{CE}-04$ $1.774 \mathrm{E}-03$ $1.774 \mathrm{E}-03$
$5.169 \mathrm{E}-03$ $1.169 \mathrm{E}-\mathrm{C}$
$1.376 \mathrm{E}-\mathrm{C}$
ANGLE 3 $4.993 E-07$ $5.720 E-C 6$ $9.288 E-07$ $2.218 E-06$
$8.553 E-07$ $2.100 E-06$ $2.796 E-06$ $2.650 E-06$ $2.125 \mathrm{E}-06$ $2.694 E-06$ 4. $932 E-05$ $8.870 \mathrm{r}-05$ $5.126 E-04$ 1. $316 E-03$ $4.073 E-03$
$1.243 E-03$ ANGLE 12 $M=0.4580$ $1.350 E-06$ $2.711 E-06$ $2.879 E-05$ $1.137 E-05$ $1.638 E-05$ $1.286 \mathrm{E}-05$ $1.727 E-05$ $3.36 \theta E-05$ ..36 1.02 1.665 E-04 $3.085 \mathrm{E}-\mathrm{C} 4$ $3.525 E-04$ $5.526 E-04$ $1.874 E-03$ $5.393 \mathrm{E}-03$ $1.400 \mathrm{E}-03$
ANGLE 4 ANGLE 5 $.494 E-06$
$3.471 E-06$ $1.188=-06$ $8.490 \mathrm{E}-07$ $3.262 E-06$ $7.551 \mathrm{E}-06$ 5.243E-05 $1.107 E-04$ $5.128 E-04$ 1. $338 E-03$ 1.250E-03 ANGLE 13 MU $=0.6179$ $2.149 E-06$ $4.239 E-06$ $1.643 F-05$ 1.643 (2. 6. $1.099 \mathrm{E}-04$ 1.591E-04 $2.088 E-04$ 3. $5.826 E-04$ $1.928 \mathrm{E}-03$ $5.517 \mathrm{E}-03$ $1.424 \mathrm{E}-03$ $6.281 E-07 \quad 6.744 E-07$ $2.190 \mathrm{E}-06$ $6.007 E-07$ $1.065 E-06$ $4.538 E-06$ 9.535E-06 5.991E-05 $1.457 E-04$ 5.091E-04 $1.369 E-03$ $4.204 E-03$

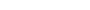
ANGLE 14 $M U=0.7550$ 3. $765 \mathrm{E}-06$ $7.343 \mathrm{E}-06$ $7.142 E-05$ $3.194 E-05$ $4.712 E-05$ 5.070E-05 9.105E-05 1.19E-05 $1.263 E-04$ 1.72 E-04 $2.138 \mathrm{E}-04$ $3.426 E-04$ $4.5757 E-04$ $4.157 E-04$
$6.133 E-04$ $6.133 E-04$ $2.079 E-03$ 1. $445 \mathrm{E}-03$ ANGLE 15 $M U=0.8656$ 7. $012 \mathrm{E}-06$ $1.352 \mathrm{E}-05$ $1.315 \varepsilon-04$ $1.680 \mathrm{E}-04$ 2. $025 E-04$ 2. $363 E-04$ 2.677E-04 $3.842 E-04$ $4.471 E-04$ 4.4 $634 E-04$ $6.434 E-04$
$2.170 E-03$ $2.170 E-03$
$6.014 E-03$ 1. $462 \mathrm{E}-03$
3.580E-07 4.174E-07 5.172E-07 $\begin{array}{llll}2.728 \mathrm{E}-06 & 2.016 \mathrm{E}-06 & 2.015 \mathrm{E}-06 & 3.432 \mathrm{E}-06\end{array}$ $1.947 \mathrm{E}-06 \quad 1.665 \mathrm{E}-06 \quad 2.029 \mathrm{E}-06 \quad 3.686 \mathrm{E}-06$ $2.267 E-06 \quad 2.606 E-06 \quad 3.532 E-06 \quad 5.100 E-06$ $2.990 E-06$
$4.369 E-06$ $1.975 E-06 \quad 4.500 E-06 \quad 6.315 E-06 \quad 6.786 E-06$ $2.895-06 \quad 5.597 E-06 \quad 7.407 E-06 \quad 8.706 E-06$

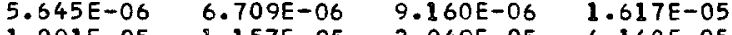
$1.001 E-05$ 1.157E-05 $2.049 E-05 \quad 4.148 E-05$

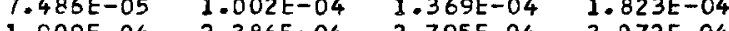
$1.909 E-04 \quad 2.386 E-04 \quad 2.795 E-04 \quad 3.072 E-04$ $\begin{array}{llll}1.016 E-04 & 4.928 E-04 & 4.875 E-04 & 4.903 E-04\end{array}$ $\begin{array}{llll}1.411 E-03 & 1.462 E-03 & 1.524 E-03 & 1.597 E+03\end{array}$ $\begin{array}{llll}4.306 E-03 & 4.433 E-03 & 4.585 E-03 & 4.760 E-03 \\ 1.274 E-03 & 1.290 E-03 & 1.308 E-03 & 1.329 E-03\end{array}$

ANGLE 16

ANGLE 17 $M U=0.9446 \quad M U=0.9894$ $1.281 E-05 \quad 2.024 E-05$ $2.422 E-05$ $2.580 \mathrm{E}-04$ $2.735 \mathrm{E}-04$ $2.846 E-04$ 2.972E-04 2.920E-04 $2.856 \mathrm{E}-04$ $4.176 E-04$ $4.176 E-04$ $4.665 E-04$ $6.703 E-04$ $2.242 E-C 3$
$6.157 E-03$ $1.475 \mathrm{E}-03$ 4.110E-06 3.571E-06 $3.951 \mathrm{E}-06 \quad 5.880 \mathrm{E}-06$ 
4 PI R*\#2 HENDERSON DUSE (NEUTRONS)
(CM**2 RAD/STERADI AN/SOURCE NEUTRON)
4.065 TO 6.360 MEV NEUTRON SOURCE
COS INE

$-1.0 C O O O E$ OC $-9.89401 E-01$ $-9.44575 \mathrm{E}-01$ $-8.65631 E-01$ $-7.55044 E-01$ $-6.17876 \mathrm{E}-\mathrm{O}$ $-4.58017 \mathrm{E}-01$ $-2.81605 \mathrm{E}-0$ $-9.50125 E-02$ $9.50125 E-02$ ..81605E-01 $4.58017 E-01$ $6.17876 \mathrm{E}-01$ 7.55044E-O $8.65631 E-01$ $9.44575 E-01$

TOTAL

COSINE

$-1 . \cap 0000 E$ OO $-9.89401 E-01$ $-9.44575 \mathrm{E}-01$ $-8.65631 \mathrm{E}-01$ $-7.55044 E-0$ $-6.17876 E-0$ 4.58017 E 01 $-2.81605 \mathrm{E}-01$ -9.50125E-02 . $4.58017 E-O$ 4.58017E-O1 $7.55044 \mathrm{E}-\mathrm{Cl}$ $8.65631 E-O 1$ $9.44575 \mathrm{E}-01$ $9.89401 E-01$

TOTAL

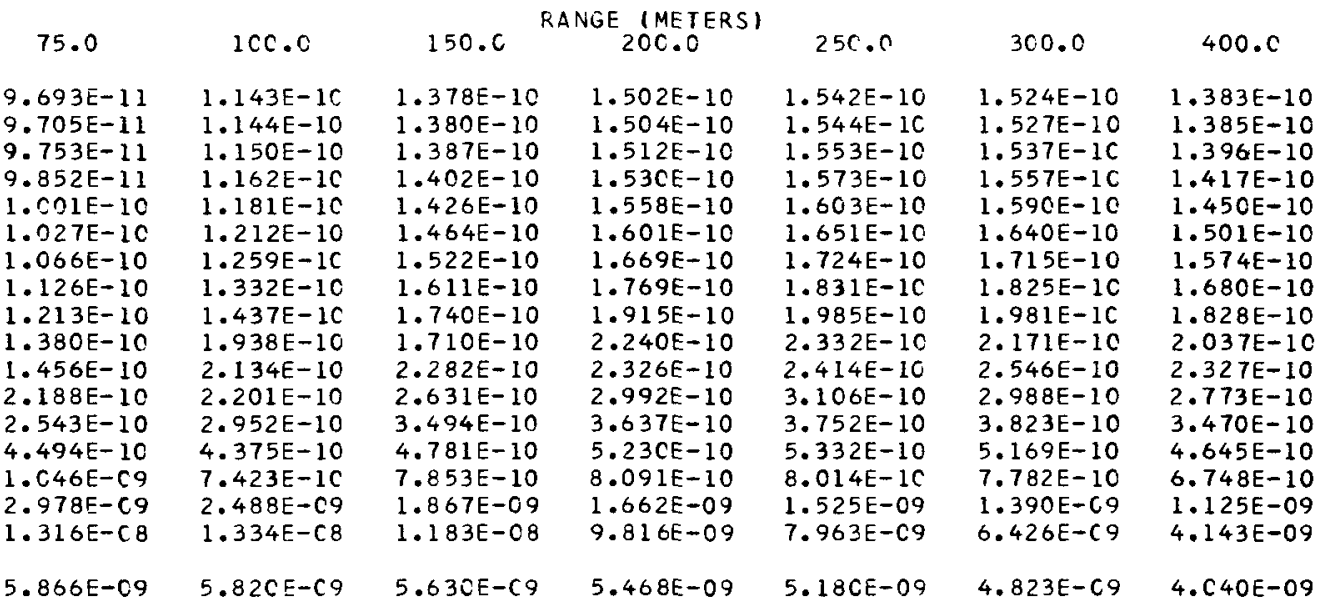

500.0

$60 \mathrm{C} .0$

RANGE (METERS)

900.0

$1200 . \mathrm{C}$

$1500 . \mathrm{C}$

1800.0

$1.174 \mathrm{E}-10$

$1.176 \mathrm{E}-10$

$1.186 \mathrm{E}-10$

$1.206 \mathrm{E}-10$

$1.237 \mathrm{E}-10$

$1.283 \mathrm{E}-1 \mathrm{C}$

1. $350 \mathrm{E}-10$

$1.443 E-10$

$1.573 \mathrm{E}-10$

$1.752 E-10$

$2.004 E-10$

$2.376 E-10$

$2.950 E-10$

$3.884 E-10$

$5.502 E-10$

. $740 E-10$

$3.251 E-09$

$523 E-11$

$.544 E-11$

$.632 E-11$

$9.807 \mathrm{E}-11$

$1.047 E-10$

$1.104 \mathrm{E}-10$

$1.182 \mathrm{E}-1 \mathrm{C}$

$1.289 \mathrm{E}-1 \mathrm{C}$

$1.436 \mathrm{E}-1 \mathrm{C}$

$1.641 E-10$

$1.938 E-10$

$2.387 E-10$

$3.099 E-1 C$

$4.295 \mathrm{E}-10$

$6.571 \mathrm{E}-1 \mathrm{C}$

2.536E-O

4. $251 \mathrm{E}-11$

$4.298 \mathrm{E}-11$

$4.387 \mathrm{E}-11$

4.524E-11

$4.720 E-11$

$5.360 \mathrm{E}-11$

$5.853 \mathrm{E}-11$

$6.513 \mathrm{E}-11$

$7.410 \mathrm{E}-11$

$8.660 E-11$

$1.046 E-10$

$1.315 \mathrm{E}-10$

$1.735 E-10$

$2.451 \mathrm{E}-10$
$4.703 \mathrm{E}-10$

$1.580 E-11$
$1.584 E-11$

$1.603 E-1$

$1.639 E-11$

$1.693 E-11$
$1.770 E-11$

$1.875 \mathrm{E}-11$

$2.015 E-11$

$2.200 E-11$

$2.444 E-11$

$2.769 \mathrm{E}-11$

$3.211 E-11$

$3.826 E-11$

$4.707 E-11$

$6.013 E-11$

8.07TE-11

$3.754 E-10$

$5.241 \mathrm{E}-12$

$5.257 E-12$

$5.321 \mathrm{E}-12$

$5.445 E-12$
$5.631 E-12$

$5.894 \mathrm{E}-12$

$6.248 \mathrm{E}-12$

$6.717 E-12$

$7.330 \mathrm{E}-12$

$8.129 E-12$

$9.18 C E-12$

$1.058 E-11$

. $513 \mathrm{E}-11$

$1.891 \mathrm{E}-11$

$2.454 E-11$

$1.213 E-10$

$1.603 E-12$

$1.607 \mathrm{E}-12$

$1.628 \mathrm{E}-12$

$1.666 \mathrm{E}-12$

$1.724 \mathrm{E}-12$

$1.806 \mathrm{E}-12$

$1.915 E-12$

$2.246 \mathrm{E}-12$

$2.487 \mathrm{E}-12$

$2.801 \mathrm{E}-12$

$3.214 \mathrm{E}-12$

3.75 E-12

$7.043 \mathrm{E}-12$
$9.562 \mathrm{E}-12$

$3.640 E-11$ 
COSINE

$-1.000 N O E$ OR $-9.89401 E-01$ $-9.44575 E-01$ $-8.65631 E-01$ $-7.55044 \mathrm{E}-\mathrm{C} 1$ $-6.17876 \mathrm{E}-01$ $-4.58017 E-01$
$-2.816 C 5 E-01$ $-2.81605 \mathrm{E}-01$ $-9.50125 \mathrm{E}-32$
$0.50125 \mathrm{E}-02$ $2.81605 \mathrm{E}-01$ 4. $58017 \mathrm{E}-01$ $6.17876 \mathrm{E}-01$ $7.55044 \mathrm{E}-01$ $8.65631 \mathrm{E} \rightarrow 01$ $9.44575 E-01$ $9.89401 E-01$

TOTAL

COS INE

- 1. OCCOCE CO $-9.89401 E-C 1$ $-9.44575 E-01$
$-8.65631 E-01$ $-7.55044 \mathrm{E}-01$ $-6.17876 E-01$ $-2.81605 E-01$ $-2.81605 E-01$ $9.50125 \mathrm{E}-02$ $9.50125 E-02$ $2.81605 E-01$ $6.17876 \mathrm{E}-\mathrm{Ol}$ $7.55044 \mathrm{E}-01$ 8.65 $8.65631 \mathrm{E}-01$ $9.89401 E-01$

TOTAL

75.0
$1.267 \mathrm{E}-10$
$1.268 \mathrm{E}-10$
$1.276 \mathrm{E}-10$
$1.290 \mathrm{E}-1 \mathrm{C}$
$1.314 \mathrm{E}-1 \mathrm{C}$
$1.349 \mathrm{E}-10$
$1.403 \mathrm{E}-10$
$1.483 \mathrm{E}-1 \mathrm{C}$
$1.599 \mathrm{E}-10$
$1.817 \mathrm{E}-10$
$1.918 \mathrm{E}-10$
$2.842 \mathrm{E}-10$
$3.358 \mathrm{E}-10$
$5.966 \mathrm{E}-1 \mathrm{C}$
$1.390 \mathrm{E}-\mathrm{C} 9$
$3.960 \mathrm{E}-\mathrm{C} 9$
$1.763 \mathrm{E}-08$
$7.797 \mathrm{E}-09$

100.0 $1.516 \mathrm{E}-10$
$1.518 \mathrm{E}-1 \mathrm{C}$ $1.527 \mathrm{E}-1 \mathrm{O}$ $1.545 \mathrm{E}-1 \mathrm{C}$ $1.572 \mathrm{E}-1 \mathrm{C}$ $1.615 \mathrm{E}-1 \mathrm{C}$ $1.68 \mathrm{CE}-1 \mathrm{C}$ $1.778 \mathrm{E}-1 \mathrm{C}$ $1.918 \mathrm{E}-1 \mathrm{C}$ $2.565 \mathrm{E}-10$ $2.813 E-1 C$ $2.925 \mathrm{E}-1 \mathrm{C}$ $3.888 \mathrm{E}-1 \mathrm{C}$ $5.784 \mathrm{E}-1 \mathrm{C}$ $9.840 \mathrm{E}-10$ $3.309 E-C$ $1.784 \mathrm{E}-\mathrm{C} 8$ $7.748 E-09$

500.0

$1.917 \mathrm{E}-1 \mathrm{C}$ $1.921 \mathrm{E}-1 \mathrm{C}$ $1.937 \mathrm{E}-10$ $1.968 \mathrm{E}-10$ $2.016 \mathrm{E}-1 \mathrm{C}$ $2.086 \mathrm{E}-10$ $2.185 E-10$ 2.322E-10 $2.762 \mathrm{E}-10$ $3.114 \mathrm{E}-10$ $3.624 \mathrm{E}-10$ 4.402E-10 $5.655 \mathrm{E}-10$ $7.811 \mathrm{E}-10$ $1.212 \mathrm{E}-09$ 3.611E-O9

$60 C .1$

$1.624 \mathrm{E}-1 \mathrm{C}$ $1.627 \mathrm{E}-1 \mathrm{C}$ $1.641 \mathrm{E}-1 \mathrm{C}$ $1.669 E-1 C$ $1.712 E-1 C$ $1.773 E-10$ $1.976 E-10$ $1.976 E-1 C$ $2.345 \mathrm{E}-1 \mathrm{C}$ $2.635 \mathrm{E}-1 \mathrm{C}$ $2.635 \mathrm{E}-1 \mathrm{C}$ $3.663 \mathrm{E}-10$ $4.626 E-10$ $0.229 E-1 C$ $0.229 E-1 C$ $2.351 \mathrm{E}-\mathrm{CO}$

$3.918 \mathrm{E}-\mathrm{Cg}$

$4.862 E-C 9$
150.0

200.0
20 (METES)

$.882 \mathrm{E}-10$
$.885 \mathrm{E}-10$

$.896 \mathrm{E}-10$

$1.918 \mathrm{E}-10$

$1.953 E-10$

2. CCSE-10

$2.208 \mathrm{E}-10$

$2.381 E-10$

3.C $90 \mathrm{E}-10$

3.564E-10

$4.670 E-10$

$6.380 \mathrm{E}-10$

$1 . C 4 \mathrm{CE}-\mathrm{C} 9$

$2.482 \mathrm{E}-\mathrm{CS}$

$1.581 E-n g$

$7.564 E-C 9$

$2.108 \mathrm{E}-10$

$2.111 \mathrm{E}-10$

$2.125 \mathrm{E}-10$

$2.151 \mathrm{E}-10$

$2.191 E-10$

$2.348 \mathrm{E}-10$

$2.487 \mathrm{E}-1 \mathrm{C}$

$2.684 \mathrm{E}-10$
$3.112 \mathrm{E}-10$

$3.235 E-10$

4.1 CBE-10

$4.962 \mathrm{~F}-10$

$7.049 \mathrm{E}-10$

$1.081 \mathrm{E}-09$

$2.213 E-09$

$1.312 \mathrm{E}-08$

$7.434 E-09$

$25 \mathrm{C} \cdot \mathrm{C}$

$400 \cdot C$

RANGE (METERS)

$900 . C$

$120 \mathrm{C} . \mathrm{C}$

$2.224 E-10$

2. $242 \mathrm{E}-1 \mathrm{C}$

$2.271 E-1 C$

$2.385 \mathrm{E}-10$

$2.488 \mathrm{E}-1 \mathrm{C}$

$2.849 E-1 C$

$3.3 C 6 E-1 C$

$3.434 \mathrm{E}-1 \mathrm{C}$

$4.34 \mathrm{CE}-1 \mathrm{C}$

$7.277 \mathrm{E}-1 \mathrm{C}$

$1.082 E-09$

$2.038 E-09$

$1.065 E-08$

$7.142 E-00$

2. 257E-10

$2.261 E-10 \quad 2.158 \mathrm{E}-10$

$2.277 E-1 C$ 2.175E-10

$2.3 C 8 E-1 C \quad 2.207 E-10$

$2.357 E-10 \quad 2.258 E-10$

$2.430 \mathrm{E}-10 \quad 2.333 \mathrm{E}-10$

$2.692 \mathrm{E}-10 \quad 2.592 \mathrm{E}-10$

$2.908 E-10 \quad 2.801 E-10$

$3.170 \mathrm{E}-1 \mathrm{C} \quad 3.092 \mathrm{E}-10$

$3.672 \mathrm{E}-10 \quad 3.490 \mathrm{E}-10$

$4.266 \mathrm{E}-1 \mathrm{C} \quad 4.094 \mathrm{E}-10$

5.372E-1C 5.C28E-10

$7.153 E-10 \quad 6.593 E-10$

$1.06 C E$ C. $982 E-10$

$8.605 E-C 9 \quad 1.535 E-09$

$6.753 E-C 9 \quad 5.845 E-09$

8. $C 54 \mathrm{E}-11$

$3.258 E-11$

$3.291 \mathrm{E}-11$

$3.353 E-11$

$3.447 E-11$

$3.578 E-11$

3.

$3.981 \mathrm{E}-11$
$4.276 \mathrm{E}-11$

$4.657 \mathrm{E}-11$

$5.152 \mathrm{E}-11$

$5.808 \mathrm{E}-11$

$6.699 \mathrm{E}-11$

$7.944 \mathrm{E}-11$

$9.754 \mathrm{E}-11$

$1.256 \mathrm{E}-10$

$6.659 \mathrm{E}-10 \quad 1.917 \mathrm{E}-10 \quad 5.465 \mathrm{E}-11 \quad 1.523 \mathrm{E}-11$

$150 C .0$

1800.6

$1.144 \mathrm{E}-11$

- 11

158E-11 $3.702 E-12$

$1.181 \mathrm{E}-11 \quad 3.773 \mathrm{E}-12$

$1.26 C E-11$ 4.026E-12

$1.321 E-11$ 4. $218 E-12$

1.40CE-11 $4.467 E-12$

1. 5 CCE-11 $4.781 E-12$

$1.629 E-11 \quad 5.177 E-12$

$1.793 E-11$ 5.679E-12

$2 . C C 6 E-11$ 6.320E-12

$2.288 \mathrm{E}-11 \quad 7.152 \mathrm{E}-12$

$2.669 E-11$ 8.248E-12

$3.201 E-11 \quad 9.730 E-12$

$3.975 \mathrm{E}-11$ 1. $179 \mathrm{E}-11$

1.769E-09

$6.772 E-10$

2. $3 C 6 E-10$

$7.206 E-11$ 
4 PI R**2 TISSUE KERMA INEUTRONS)
(CM*2 ERGS/GRAMISTERADIAN/SOURCE NEUTRON)

4.C65 TO 6.960 MEV NEUTRON SOURCE

COSINE
$-1.00 C 00 E 00$
$-9.89401 E-01$
$-9.44575 E-01$
$-8.65631 E-01$
$-7.55044 E-01$
$-6.17876 E-01$
$-4.58017 E-01$
$-2.81605 E-01$
$-9.50125 E-02$
$9.50125 E-02$
$2.81605 E-01$
$4.58017 E-01$
$6.17876 E-01$
$7.55044 E-01$
$8.65631 E-21$
$9.44575 E-01$
$9.89401 E-01$

TOTAL

COSINE

$-1.00000 E$ DO $-9.89401 \mathrm{E}-\mathrm{C} 1$ $-9.44575 E-01$ $-8.65631 E-01$ $-7.55044 E-01$ $-6.17876 E-01$ $-4.58017 E-01$ $-2.81605 E-01$ $-9.50125 \mathrm{E}-02$ $9.50125 \mathrm{E}-\mathrm{C} 2$ $2.81605 E-01$ 4.58017E-01 $6.17876 \mathrm{E}-01$ $7.55 \mathrm{C} 44 \mathrm{E}-01$ $8.65631 E-01$ $9.44575 E-01$
$9.89401 E-01$

TOTAL

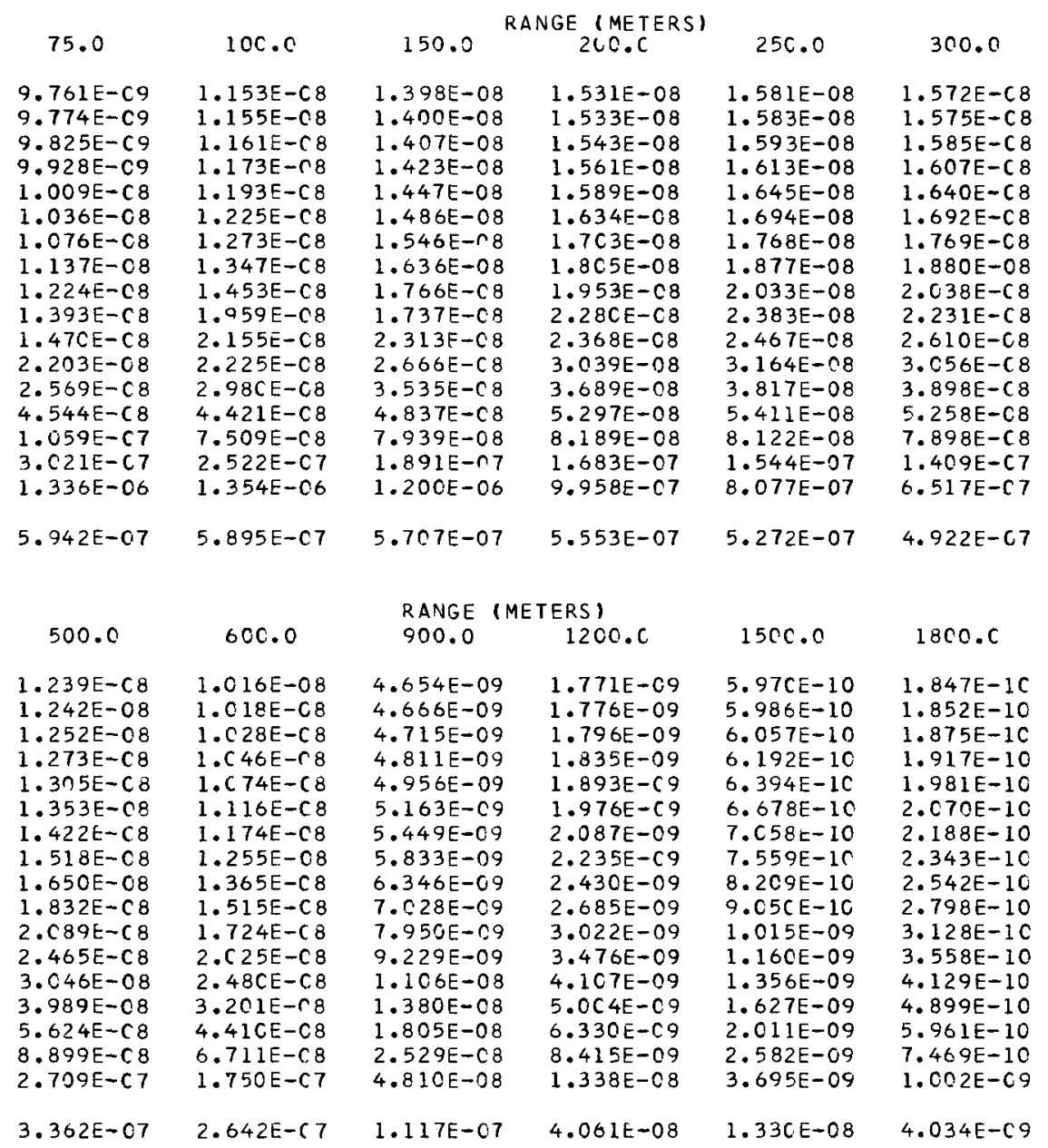




\begin{abstract}
COSINE

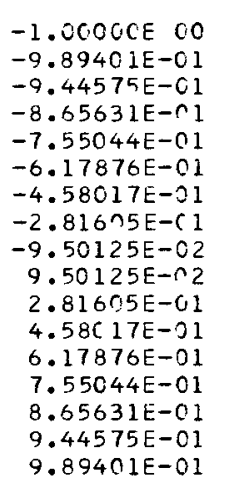

TOTAL

COSINE

-1. COCOOE CO $-9.894 C 1 E-01$ $-9.44575 E-01$ $-7.55044 E-01$ $-6.55044 E-C 1$ $-6.17876 \mathrm{E}-01$ $-2.81605 E-01$ - $9.50125 E-01$ $9.50125 \mathrm{E}-02$ $2.51605 E-01$ $4.58017 E-01$ $6.17876 \mathrm{E}-01$ $7.55644 \mathrm{E}-31$ $8.65631 \mathrm{E}-01$ $9.44575 \mathrm{E}-01$ $9.89401 \mathrm{E}-01$

TOTAL

$\begin{array}{ll}75 . C & 100.6 \\ 4.077 E-11 & 4.671 E-11 \\ 4 . C 85 E-11 & 4.681 E-11 \\ 4.119 E-11 & 4.721 E-11 \\ 4.184 E-11 & 4.795 \mathrm{E}-11 \\ 4.280 \mathrm{E}-11 & 4.905 \mathrm{E}-11 \\ 4.423 \mathrm{E}-11 & 5.068 \mathrm{E}-11 \\ 4.634 \mathrm{E}-11 & 5.313 \mathrm{E}-11 \\ 4.945 \mathrm{E}-11 & 5.678 \mathrm{E}-11 \\ 5.389 \mathrm{E}-11 & 6.2 \mathrm{C} 1 \mathrm{E}-11 \\ 6.199 \mathrm{E}-11 & 8.715 \mathrm{E}-11 \\ 6.61 \mathrm{CE}-11 & 9.679 \mathrm{E}-11 \\ 1.020 \mathrm{E}-10 & 9.937 \mathrm{E}-11 \\ 1.256 \mathrm{E}-10 & 1.388 \mathrm{E}-1 \mathrm{C} \\ 2.402 \mathrm{E}-10 & 2.186 \mathrm{E}-10 \\ 5.899 \mathrm{E}-10 & 3.922 \mathrm{E}-1 \mathrm{C} \\ 1.718 \mathrm{E}-\mathrm{C} 9 & 1.418 \mathrm{E}-\mathrm{C} 9 \\ 7.859 \mathrm{E}-\mathrm{C} 9 & 7.917 \mathrm{E}-\mathrm{C} 9 \\ 3.223 \mathrm{E}-\mathrm{C9} & 3.098 \mathrm{E}-\mathrm{C} 9\end{array}$

530.0

3. $918 E-11$ $3.927 E-11$ $3.962 E-11$ $4.135 E-11$ 4. $292 \mathrm{E}-11$ $4.292 E-11$ $4.851 E-11$ $5.318 \mathrm{E}-11$ $5.979 E-11$ $6.948 \mathrm{E}-11$ 8. $451 \mathrm{E}-11$ $1.093 \mathrm{E}-1 \mathrm{C}$ $1.525 \mathrm{E}-1 \mathrm{C}$ $2.320 \mathrm{E}-1 \mathrm{C}$ 4. CIIE-1C $1.446 E-C 9$

$1.291 \mathrm{E}-\mathrm{C} 9$

600.8

\begin{tabular}{|c|c|c|c|c|}
\hline 150.0 & $\begin{array}{l}\text { GF (METERS) } \\
200 . \mathrm{C}\end{array}$ & $25 C \cdot C$ & 300.0 & $400 . C$ \\
\hline $5.378 \mathrm{E}-11$ & $5.645 E-11$ & $5.617 \mathrm{E}-11$ & $5.412 E-11$ & 4.725E-11 \\
\hline $5.389 \mathrm{E}-1$ & $5.657 E-11$ & $5.629 E-11$ & $5.423 \mathrm{E}-11$ & $4.735 E-11$ \\
\hline $5.434 E-11$ & $5.7 C 4 E-11$ & $5.676 E-11$ & $5.469 E-11$ & $4.776 E-11$ \\
\hline $5.517 \mathrm{E}-11$ & $5.792 E-11$ & $5.765 E-11$ & $5.557 E-11$ & $4.856 E-11$ \\
\hline $5.641 E-11$ & $5.924 \mathrm{E}-11$ & $5.899 E-11$ & $5.689 \mathrm{E}-11$ & $4.978 \mathrm{E}-11$ \\
\hline 5.82 & $6.123 E-11$ & $6.101 \mathrm{E}-11$ & $5.889 \mathrm{E}-11$ & $5.160 \mathrm{E}-11$ \\
\hline $6.108 \mathrm{E}-11$ & $6.424 E-11$ & $6.4 C 8 E-11$ & $6.190 \mathrm{E}-11$ & $5.431 E-11$ \\
\hline$E-11$ & $6.874 \mathrm{E}-1 \mathrm{l}$ & $6.863 E-11$ & $6.635 E-11$ & $5.827 E-11$ \\
\hline$E-11$ & $7.525 E-11$ & $7.521 \mathrm{E}-11$ & $7.274 E-11$ & $6.391 E-11$ \\
\hline 7. $(87 E-1$ & $8.950 \mathrm{~F}-11$ & $9.001 E-11$ & $8.055 \mathrm{E}-11$ & $7.209 E-11$ \\
\hline $9.662 E-11$ & $9.397 E-11$ & $9.388 E-11$ & $9.646 E-11$ & $8.369 E-11$ \\
\hline & $1.241 \mathrm{~F}-10$ & $1.246 E-1 C$ & $1.160 \mathrm{E}-1 \mathrm{C}$ & $1.025 \mathrm{E}-1 \mathrm{C}$ \\
\hline 1.5 & $1.564 E-10$ & $1.561 E-10$ & $1.547 E-1 C$ & $1.337 \mathrm{E}-1 \mathrm{C}$ \\
\hline $2.229 \mathrm{E}-10$ & $2.361 \mathrm{E}-10$ & $2.339 \mathrm{E}-1 \mathrm{C}$ & & $1.896 \mathrm{E}-1 \mathrm{C}$ \\
\hline $2.900 E-10$ & $3.886 E-10$ & $3.749 \mathrm{E}-10$ & $3.554 \mathrm{E}-10$ & $2.954 \mathrm{E}-1 \mathrm{C}$ \\
\hline $1.017 \mathrm{E}-09$ & $8.697 E-10$ & $7.736 E-10$ & $6.886 \mathrm{E}-10$ & $5.351 \mathrm{E}-10$ \\
\hline & $5.729 E-09$ & $4.6 C C E-C 9$ & $3.669 E-C 9$ & $2.307 E-09$ \\
\hline $2.843 F-09$ & $2.635 E-C 9$ & $2.395 E-09$ & $2.148 \mathrm{E}-09$ & $1.688 E-09$ \\
\hline
\end{tabular}

RANGE (METERS)

I2CC.C

1500.0

$1800 . C$

$\begin{array}{lllll}3.139 \mathrm{E}-11 & 1.405 \mathrm{E}-11 & 5.405 \mathrm{E}-12 & 1.86 \mathrm{CE}-12 & 5.876 \mathrm{E}-13 \\ 3.146 \mathrm{E}-11 & 1.408 \mathrm{E}-11 & 5.418 \mathrm{E}-12 & 1.864 \mathrm{E}-12 & 5.890 \mathrm{E}-13 \\ 3.176 \mathrm{E}-11 & 1.422 \mathrm{E}-11 & 5.472 \mathrm{E}-12 & 1.883 \mathrm{E}-12 & 5.949 \mathrm{E}-13 \\ 3.232 \mathrm{~F}-11 & 1.449 \mathrm{E}-11 & 5.575 \mathrm{E}-12 & 1.918 \mathrm{E}-12 & 6.059 \mathrm{E}-13 \\ 3.319 \mathrm{E}-11 & 1.489 \mathrm{E}-11 & 5.730 \mathrm{E}-12 & 1.971 \mathrm{E}-12 & 6.225 \mathrm{E}-13 \\ 3.447 \mathrm{E}-11 & 1.547 \mathrm{E}-11 & 5.951 \mathrm{E}-12 & 2.045 \mathrm{E}-12 & 6.456 \mathrm{E}-13 \\ 3.631 \mathrm{~F}-11 & 1.629 \mathrm{E}-11 & 6.254 \mathrm{E}-12 & 2.146 \mathrm{E}-12 & 6.764 \mathrm{E}-13 \\ 3.895 \mathrm{E}-11 & 1.742 \mathrm{E}-11 & 6.665 \mathrm{E}-12 & 2.28 \mathrm{CE}-12 & 7.172 \mathrm{E}-13 \\ 4.265 \mathrm{E}-11 & 1.896 \mathrm{E}-11 & 7.216 \mathrm{E}-12 & 2.458 \mathrm{E}-12 & 7.705 \mathrm{E}-13 \\ 4.785 \mathrm{E}-11 & 2.110 \mathrm{E}-11 & 7.964 \mathrm{E}-12 & 2.695 \mathrm{E}-12 & 8.406 \mathrm{E}-13 \\ 5.541 \mathrm{E}-11 & 2.413 \mathrm{E}-11 & 9.000 \mathrm{E}-12 & 3.017 \mathrm{E}-12 & 9.346 \mathrm{E}-13 \\ 6.70 C \mathrm{E}-11 & 2.861 \mathrm{E}-11 & 1.049 \mathrm{E}-11 & 3.471 \mathrm{E}-12 & 1.064 \mathrm{E}-12 \\ 8.582 \mathrm{E}-11 & 3.557 \mathrm{E}-11 & 1.273 \mathrm{E}-11 & 4.132 \mathrm{E}-12 & 1.249 \mathrm{E}-12 \\ 1.178 \mathrm{E}-10 & 4.681 \mathrm{E}-11 & 1.62 \mathrm{CE}-11 & 5.126 \mathrm{E}-12 & 1.521 \mathrm{E}-12 \\ 1.753 \mathrm{E}-10 & 6.578 \mathrm{E}-11 & 2.178 \mathrm{E}-11 & 6.67 \mathrm{E}-12 & 1.931 \mathrm{E}-12 \\ 2.920 \mathrm{E}-1 \mathrm{C} & 1.6 \mathrm{C} \mathrm{E}-10 & 3.136 \mathrm{E}-11 & 9.181 \mathrm{E}-12 & 2.570 \mathrm{E}-12 \\ 9 . \mathrm{C} 60 \mathrm{E}-10 & 2.261 \mathrm{E}-10 & 5.757 \mathrm{E}-11 & 1.480 \mathrm{E}-11 & 3.802 \mathrm{E}-12 \\ 9.679 \mathrm{E}-10 & 3.733 \mathrm{E}-1 \mathrm{C} & 1.301 \mathrm{E}-10 & 4.185 \mathrm{E}-11 & 1.263 \mathrm{E}-11\end{array}$


4 PI R**2 CONCRETE KERMA (NEUTRONS)
(CM**2 ERGSIGRAM/STERADIAN/SOURCE NEUTRON

COS INE

$-1.0000 \mathrm{CE} 00$ $-9.89401 \mathrm{E}-01$ $-9.44575 E-01$ $-7.55044 E-0$ $-6.17876 \mathrm{E}-01$ $-4.58017 \mathrm{E}-0$ $-2.81605 \mathrm{E}-01$ $-9.50125 \mathrm{E}-02$ $9.50125 \mathrm{E}-02$ $2.81605 \mathrm{E}-0$ 4.58017E-O $6.17876 \mathrm{E}-0$ $7.55044 E-01$ $8.65631 \mathrm{E}-31$ $9.89401 \mathrm{E}-01$

TOTAL

$$
75.0
$$

$100 \cdot 0$

150.0

RA

$2(0.0)$

250.0

300.0

$400 . C$

$1.303 \mathrm{E}-09$ $1.310 \mathrm{E}-\mathrm{CQ}$ $1.321 \mathrm{E}-\mathrm{CO}$ $1.340 E-09$ $1.371 \mathrm{E}-\mathrm{C} 9$ $1.421 \mathrm{E}-09$ $1.498 \mathrm{E}-09$ $1.611 \mathrm{E}-\mathrm{C9}$ $1.829 E-09$ $1.934 \mathrm{E}-\mathrm{C}$ $2.951 \mathrm{E}-\mathrm{O} 9$ $3.363 \mathrm{E}-\mathrm{C} 9$ $5.922 \mathrm{E}-\mathrm{C}$ $1.379 \mathrm{E}-\mathrm{C}$ 3. $920 \mathrm{E}-08$ $1.724 \mathrm{E}-07$

7. $733 E-08$

$1.524 E-C 9$ . $526 \mathrm{E}-\mathrm{C} 9$ $1.532 \mathrm{E}-\mathrm{C} 9$ $1.568 \mathrm{E}-09$ $1.606 \mathrm{E}-\mathrm{C} 9$ $1.666 \mathrm{E}-\mathrm{C} 9$ $1.759 E-C 9$ $1.896 \mathrm{E}-\mathrm{C}$ $2.572 E-C$ $2.844 E-09$ $2.893 E-C 9$ $3.930 E-C 9$ $5.79 \mathrm{CE}-\mathrm{C}$ C. $785 E-C 9$ $3.277 \mathrm{E}-0$ $1.748 E-07$ $7.669 E-C 8$ $1.817 E-09$ $1.968 E-09 \quad 2.011 E-09$
$1.828 E-09$ $\begin{array}{lll}1.845 E-09 & 1.999 E-C 9 & 2.045 E-09\end{array}$ $\begin{array}{lll}1.873 E-09 & 2.032 E-C 9 & 2.082 E-09\end{array}$ $1.920 E-09 \quad 2.086 E-09 \quad 2.142 E-09$ $1.994 E-09 \quad 2.171 E-09 \quad 2.233 E-09$ $2.107 E-09 \quad 2.299 E-09 \quad 2.369 E-09$ $2.273 E-09 \quad 2.486 E-09 \quad 2.566 E-09$ $2.230 E-09 \quad 2.91 C E-09 \quad 3.016 E-09$ $2.991 E-09 \quad 3.017 E-09 \quad 3.116 E-09$ $3.422 E-09 \quad 3.892 E-C 9 \quad 4.022 E-09$ $4.616 \mathrm{E}-\mathrm{CO} \quad 4.740 \mathrm{E}-09 \quad 4.867 \mathrm{E}-09$ $6.270 \mathrm{E}-09 \quad 6.843 \mathrm{E}-09 \quad 6.945 \mathrm{E}-09$ $1.038 \mathrm{E}-08 \quad 1.063 \mathrm{E}-08 \quad 1.048 \mathrm{E}-08$ $2.457 E-08 \quad 2.186 E-08 \quad 2.000 E-08$ $1.551 E-r 7 \quad 1.287 E-07 \quad 1.044 E-07$ $7.389 E-08$

$7.150 E-08$

$6.749 E-08$

RANGE (METERS)

$$
500.0
$$

600.0

-1.00 OOOE 00 $-9.89401 E-01$ $-9.44575 E-01$ $-7.55044 E-01$ $-6.17876 \mathrm{E}-01$ $-4.58017 E-01$ $-2.91605 E-01$ $-9.50125 E-02$ $9.50125 E-02$ $4.58017 \mathrm{E}-01$ $6.17876 \mathrm{E}-0$ $7.55044 \mathrm{E}-0$ $8.65631 \mathrm{E}-0$ 9.44575E-O $9.89401 E-C 1$

TOTAL

\section{$1.518 \mathrm{E}-09$ $1.521 \mathrm{E}-\mathrm{C} 9$ $1.534 E-C 9$ $1.558 \mathrm{E}-\mathrm{C} 9$ $1.597 \mathrm{E}-\mathrm{C} 9$ $1.654 E-C 9$
$1.738 E-09$ $1.738 E-09$ $1.855 E-09$ $2.019 E-09$ $2.246 \mathrm{E}-\mathrm{C}$ 2.568E-C $3.784 \mathrm{E}-\mathrm{CO}$ . $7.091 E-09$ $1.131 E-C 8$ $3.475 E-08$} $1.233 E-09$ $1.235 E-C 9$ $1.246 E-C 9$ $1.268 E-09$ $1.302 E-C 9$ $1.351 E-c 9$ $1.422 E-c 9$ $1.521 \mathrm{E}-09$ $1.656 E-C 9$ $1.842 E-C 9$ $2.103 E-C 9$ 2.482E-CO 3.058 09 $3.974 E-C 9$
$5.518 E-09$ $8.518=09$ $2.235 E-C$ $3.263 E-C 8$

$\begin{array}{ll}1.979 E-C 9 & 1.789 E-09 \\ 1.982 E-C 9 & 1.792 E-09 \\ 1.994 E-C 9 & 1.805 E-09 \\ 2.019 E-C 9 & 1.831 E-09 \\ 2.059 E-09 & 1.872 E-09 \\ 2.121 E-09 & 1.935 E-09 \\ 2.215 E-C 9 & 2.027 E-09 \\ 2.354 E-C 9 & 2.160 E-09 \\ 2.553 E-C 9 & 2.348 E-09 \\ 2.794 E-09 & 2.615 E-09 \\ 3.280 E-C 9 & 2.986 E-09 \\ 3.853 E-C 9 & 3.561 E-09 \\ 4.943 E-C 9 & 4.463 E-09 \\ 6.705 E-C 9 & 5.992 E-09 \\ 1.014 E-C 8 & 8.735 E-09 \\ 1.817 E-C 8 & 1.463 E-08 \\ 8.415 E-C 8 & 5.413 E-08 \\ & \\ 6.263 E-C 8 & 5.221 E-08\end{array}$

18000

1800.

$2.121 E-11$
$2.127 E-11$ $2.153 \mathrm{E}-11$ $2.203 E-11$ $2.277 \mathrm{E}-11$ $2.382 \mathrm{E}-11$ 2.522E-11 $2.705 E-11$ 2. $943 E-11$ $3.250 \mathrm{E}-11$ $3.648 \mathrm{E}-11$
$4.170 \mathrm{E}-11$ $4.865 \mathrm{E}-11$ 4.865E-11 5. $104 \mathrm{E}-11$ $8.104 E-11$ 1.209E-10 4. $725 \mathrm{E}-10$ 
COS INE

$-1.00000 E 00$ $-9.89401 E-C 1$ $-9.44575 E-01$ $-8.65631 E-01$ $-7.55044 \mathrm{E}-01$ $-6.17876 \mathrm{E}-01$ $-4.58 \mathrm{C} 17 \mathrm{E}-01$ $-2.81605 \mathrm{E}-01$ $-9.50125 E-C 2$ $9.50125 \mathrm{E}-02$ $2.81605 \mathrm{E}-01$ 4.58017E-01 $6.17876 \mathrm{E}-01$ $7.55044 \mathrm{E}-01$

$8.65631 \mathrm{E}-01$ $9.44575 E-01$ $89401 E-0$

TOTAL

COSINE

$-1.00000 E$ OC $-9.89401 \mathrm{E}-0$ $-9.44575 E-01$ $-8.65631 E-C 1$ $-6.17876 \mathrm{E}-01$ $-4.58017 \mathrm{E}-0 \mathrm{I}$ $-2.81605 \mathrm{E}-01$ - $211005 \mathrm{E}$ 9.50125E-02 . 2.58017E-C $6.17876 \mathrm{E}-01$

$7.55044 \mathrm{E}-01$

$8.65631 \mathrm{E}-01$

$8.65631 \mathrm{E}-01$
$9.44575 \mathrm{E}-01$

$9.89401 \mathrm{E}-01$

TOTAL

$\begin{array}{cc}75.0 & 10 C .0 \\ 2.199 E-C 9 & 2.488 E-C 9 \\ 2.202 E-C 9 & 2.492 E-C Q \\ 2.216 E-C 9 & 2.508 E-C 9 \\ 2.242 E-C 9 & 2.538 E-C 9 \\ 2.283 E-C 9 & 2.585 E-09 \\ 2.347 E-C 9 & 2.659 E-C 9 \\ 2.444 E-C 9 & 2.771 E-C 9 \\ 2.588 E-C 9 & 2.94 C E-09 \\ 2.798 E-C 9 & 3.186 E-09 \\ 3.190 E-C 9 & 4.464 E-C 9 \\ 3.396 E-C 9 & 5.024 E-C 9 \\ 5.239 E-C 9 & 4.953 E-C 9 \\ 6.0 C 1 E-C 9 & 6.941 E-C 9 \\ 1.080 E-C 8 & 1.039 E-C 8 \\ 2.607 E-C 8 & 1.799 E-C 8 \\ 7.713 E-C 8 & 6.316 E-C 8 \\ 3.372 E-C 7 & 3.419 E-C 7 \\ 1.461 E-C 7 & 1.424 E-C 7\end{array}$

500.0

$1.880 \mathrm{E}-\mathrm{C} g$ $1.884 E-09$ $1.899 E-C 9$ 1. $777 \mathrm{E} C \mathrm{C}$ 2. $48 \mathrm{E}-\mathrm{CO}$ 2.048E-C9 $2.304 \mathrm{E}=0$ $2.521 \mathrm{E}-\mathrm{C} 9$ 2.521E-C9 $3.290 E-C 9$ 4. $004 E-09$ $5.178 E-C 9$ $7.191 E-C 9$ $1.085 \mathrm{E}-\mathrm{C} 8$ $1.085 E-C 8$ $6.301 \mathrm{E}-08$ $5.998 E-C 8$
RANGE (METERS)
$2 \mathrm{CO} 0.0$

$25 r .0$

300.0

400.0

$2.808 \mathrm{E}-09$ 2.9C1E-C9 $2.851 E-09$
$2.871 E-09$ $2.962 \mathrm{E}-09$ 3. C2CE-C9 2.969E-09 3.11CE-09 3.06CE-09 $3.248 \mathrm{E}-\mathrm{C} 9 \quad 3.2 \mathrm{CCE}-\mathrm{C}$ $3.455 \mathrm{E}-\mathrm{C} 9 \quad 3.41 \mathrm{CE}-0$ $3.758 \mathrm{E}-09 \quad 3.716 \mathrm{E}-09$ $4.474 E-09 \quad 4.46 C E-09$ $4.627 E-09 \quad 4.575 E-09$ $6.148 E-C 9 \quad 6.123 E-09$ $7.664 \mathrm{E}-\mathrm{C} 9 \quad 7.588 \mathrm{E}-\mathrm{O}$ $1.151 E-C B \quad 1.133 E-08$ $1.862 E-C 8 \quad 1.788 E-C 8$ $\begin{array}{ll}4 . C C 3 E-08 & 3.589 E-08 \\ 2.483 E-C 7 & 1.996 E-07\end{array}$

$1.121 E-07$

$2.709 \mathrm{E}-\mathrm{Cg}$ $2.733 \mathrm{E}-\mathrm{CS} \quad 2.335 \mathrm{E}-\mathrm{Cg}$ $2.771 E-C 9 \quad 2.37 C E-O$ 2.83 CE-09 $2.424 \mathrm{E}-09$ $2.921 E-C 9 \quad 2.507 E-09$ $3.058 \mathrm{E}-\mathrm{C} 9 \quad 2.631 \mathrm{E}-0$ $3.263 \mathrm{E}-09 \quad 2.813 \mathrm{E}-09$ 3.56OE-C $3.075 E-C 9$ $3.918 \mathrm{E}-\mathrm{C} 9 \quad 3.461 \mathrm{E}-0$ 4.699E-C9 4.011E-09 $5.628 E-C 9 \quad 4.913 E-09$ $7.496 \mathrm{E}-\mathrm{C} 9 \quad 6.396 \mathrm{E}-09$ $1.062 E-C 8 \quad 9.025 E-C 9$ $1.689 E-C 8 \quad 1.392 E-C 8$ $3.198 \mathrm{E}-\mathrm{C} 8 \mathrm{2} 2.478 \mathrm{E}-08$ $.595 E-C 7 \quad 1.005 E-07$ $1.231 E-C 7$

$1.006 \mathrm{E}-\mathrm{C} 7$

$7.885 E-08$

1800.0

$1.48 C E-C 9$
$1.483 E-C 9$
$1.496 E-09$
$1.521 E-09$
$1.560 E-C 9$
$1.619 E-09$
$1.703 E-C 9$
$1.824 E-09$
$1.994 E-09$
$2.238 E-C 9$
$2.594 E-C 9$
$3.142 E-09$
$4.025 E-09$
$5.51 C E-09$
$8.134 E-09$
$1.337 E-C 8$
$3.948 E-08$
$4.467 E-08$

$6.357 E-10$ $2.380 \mathrm{E}-10$ $6.371 E-10$ $6.432 E-10$ $6.731 E-10$ $6.731 E-10$ $7.363 E-10$ . ?.878 10 $8.585-10$ $1.098 E-C 9$ $1.307 E-09$ $1.629 \mathrm{E}-09$ $1.629 \mathrm{E}-09$
$2.142 \mathrm{E}-09$ $2.993 E-09$ $4.528 \mathrm{E}-09$ $9.805 E-09$

$1.688 E-08$
1500.0 $8.051 E-11$ $8.07 \mathrm{CE}-11$ $8.151 E-11$ . $8.54 C E-11$ $0.320 E-11$ $9320 \mathrm{E}-11$ $9.923 E-11$ $1.073 E-10$ $1.32 \mathrm{E}-10$ $1.328 \mathrm{E}-10$
$1.535 \mathrm{E}-10$ $1.834 \mathrm{E}-10$ $2.277 \mathrm{E}-10$ . $4.020 \mathrm{E}-1 \mathrm{C}$

$6.323 \mathrm{E}-1 \mathrm{C}$

$1.834 E-00$
$2.516 \mathrm{E}-11$ $2.522 \mathrm{E}-11$ $2.548 \mathrm{E}-11$ $2.596 \mathrm{E}-11$ $2.669 E-11$ $2.770 E-11$ 2.907E-11 $3.329 \mathrm{E}-11$ 3.646E-11 4. C73E-11 $4.661 E-11$ $5.491 \mathrm{E}-11$ $6.690 E-11$

$8.462 E-1$

$1.115 \mathrm{E}-10$

$1.614 E-10$

$5.481 E-I C$ 
COSINE

$-1.00000 \mathrm{E} 00$ $-9.89401 E-01$ $-9.44575 E-01$ $-8.65631 E-01$ $-7.55044 E-01$ $-6.17876 E-01$ $-4.58017 E-01$ $2.81605 E-01$ $9.50125 \mathrm{E}-02$ $.50125 E-02$ $2.81605 E-01$ $4.58017 \mathrm{E}-\mathrm{Cl}$ $6.17876 \mathrm{E}-\mathrm{OI}$ $.55044 E-01$ $8.65631 \mathrm{E}-01$ $9.89401 E-01$

TOTAL

$100 \cdot \mathrm{C}$ $1.556 \mathrm{E}-1 \mathrm{C}$ $1.576 \mathrm{E}-10$ $1.665 \mathrm{E}-10$ $1.739 \mathrm{E}-10$ $1.846 \mathrm{E}-10$ $2.004 \mathrm{E}-1 \mathrm{C}$ $2.227 E-10$ $3.226 \mathrm{E}-1 \mathrm{C}$ $3.565 E-1 C$ $3.773 E-10$ $5.467 E-10$ $9.361 E-10$ $1.779 E-09$ $6.816 E-C 9$ $3.964 E-C 8$

$1.505 E-C 8$

$1.41 C E-C 8$

COS INE

$-1.00000 E 00$ $-9.89401 E-01$ $-9.44575 E-01$ $-8.65631 \mathrm{E}-01$ $-7.55044 \mathrm{E}-01$ $-6.17876 \mathrm{E}-01$ -4.58017E-01 $-2.81605 E-01$ $-9.50125 E-02$ $9.50125 E-C 2$ 2.81605E-01 4.58017E-OI $6.17876 E-O 1$ $8.55631 E-01$ $8.65631 E-01$ $9.89401 \mathrm{E}-01$

TOTAL

$1.284 \mathrm{E}-10$

$1.288 \mathrm{E}-10$

1. $304 \mathrm{E}-10$

$1.334 \mathrm{E}-10$

$1.443 E-10$

$1.537 \mathrm{E}-10$

$1.671 E-10$

$2.117 E-10$

$2.493 E-10$

$3.080 \mathrm{E}-10$

$4.078 E-10$

$9.322 E-10$

$9.322 E-10$

$6.926 \mathrm{E}-09$

5.084E-09

75.0
$1.355 E-10$
$1.360 E-1 C$
$1.378 E-10$
$1.410 E-1 C$
$1.456 E-10$
$1.521 E-1 C$
$1.615 E-10$
$1.751 E-1 C$
$1.942 E-10$
$2.258 E-10$
$2.447 E-10$
$3.903 E-10$
$5.301 E-10$
$1.118 E-09$
$2.854 E-C 9$
$8.226 E-C 9$
$3.965 E-08$

\begin{tabular}{|c|c|c|c|c|}
\hline 150.0 & $\begin{array}{l}\text { IGE } \\
200.0 \\
200 \text { SE) }\end{array}$ & $25 c .0$ & $300 . C$ & 400.0 \\
\hline & $1.876 E-10$ & $1.869 E-10$ & $1.8 C O E-10$ & $1.565 E-10$ \\
\hline & $1.882 \mathrm{E}-10$ & $1.875 E-10$ & $1.806 E-10$ & $1.569 E-10$ \\
\hline $.814 E-10$ & $1.906 \mathrm{E}-10$ & $1.898 \mathrm{E}-10$ & $1.828 \mathrm{E}-10$ & $1.589 \mathrm{E}-10$ \\
\hline $.855 E-10$ & $1.949 \mathrm{E}-10$ & $1.941 \mathrm{E}-10$ & $1.869 \mathrm{E}-10$ & $1.625 \mathrm{E}-10$ \\
\hline & $2.010 E-10$ & $2.001 \mathrm{E}-10$ & $1.928 \mathrm{E}-10$ & $1.678 \mathrm{E}-10$ \\
\hline & $2.097 E-10$ & $2.089 E-10$ & $2.014 E-10$ & $1.754 \mathrm{E}-10$ \\
\hline & $2.227 E-10$ & $2.219 E-10$ & $2.141 \mathrm{E}-10$ & $1.867 \mathrm{E}-10$ \\
\hline & $2.417 E-10$ & $2.41 \mathrm{CE}-10$ & $2.326 \mathrm{E}-1 \mathrm{C}$ & $2.029 \mathrm{E}-10$ \\
\hline & $2.687 \mathrm{E}-10$ & $2.681 \mathrm{E}-1 \mathrm{C}$ & $2.587 \mathrm{E}-10$ & $2.257 \mathrm{E}-10$ \\
\hline & $3.207 E-10$ & $3.215 \mathrm{E}-1 \mathrm{C}$ & $2.912 E-10$ & $2.578 E-10$ \\
\hline-10 & $3.483 E-10$ & $3.471 \mathrm{E}-1 \mathrm{C}$ & $3.511 E-10$ & $3.031 \mathrm{E}-10$ \\
\hline & $4: 596 \mathrm{E}-10$ & $4.595 \mathrm{E}-10$ & $4.301 E-10$ & $3.766 E-10$ \\
\hline & $6.001 E-10$ & $5.965 \mathrm{E}-10$ & $5.850 \mathrm{E}-10$ & $5.025 E-10$ \\
\hline & $9.312 \mathrm{E}-10$ & $9.179 \mathrm{E}-10$ & $8.664 E-1 C$ & $7.367 E-10$ \\
\hline & $1.612 \mathrm{E}-09$ & $1.544 E-09$ & $1.453 E-C 9$ & $1.197 \mathrm{E}-09$ \\
\hline $.737 E-C$ & $3.913 E-09$ & $3.404 E-09$ & $2.992 E-09$ & $2.288 E-C 9$ \\
\hline & $2.844 E-08$ & $2.271 \mathrm{E}-08$ & $1.801 E-C 8$ & $1.119 \mathrm{E}-08$ \\
\hline $253 E-C$ & $1.132 \mathrm{E}-08$ & $1.009 E-08$ & $8.9 C 4 E-C 9$ & $6.807 E-09$ \\
\hline
\end{tabular}

\section{RANGE (METERS)}

SO0.0 $\quad 1200.0$

1500.0

1800.
4.26CE-11

\section{0}

.013E-10

$1.016 E-1 C$

$1.053 E-1 \mathrm{C}$

$1.089 E-10$

$1.142 \mathrm{E}-10$

$1.217 E-10$

$1.323 E-10$

$1.473 E-1 C$

$1.966 \mathrm{E}-1 \mathrm{C}$

$2.418 \mathrm{E}-1 \mathrm{O}$

$4.503 E-10$

6.982E-10

$1.223 \mathrm{E}-09$

$3.729 E-09$

$4.273 E-11$

$4.330 E-11$

$4.437 E-11$

$4.828 \mathrm{E}-11$

$5.151 \mathrm{E}-1$

$5.596 \mathrm{E}-11$

$6.202 E-11$

$8.203 E-1$

$9.956 E-11$

$1.274 E-10$

2.546E-10

$4.095 \mathrm{E}-10$

$1.027 \varepsilon-09$

$1.352 E-C 9$
$1.537 E-11$

$1.558 E-11$

$1.598 \mathrm{E}-11$
$1.657 \mathrm{E}-11$

$1.742 E-11$

$1.860 E-11$

$2.019 \mathrm{E}-11$

$2.232 E-11$

$2.521 E-11$

$2.922 E-11$

$5.820 E-11$

$8.179 \mathrm{E}-11$

$2.53 \mathrm{E}-10$

4. $448 E-10$

4. $965 E-12$

4. $981 E-12$

$5.05 \mathrm{CE}-12$

$5.182 E-12$

$5.658 \mathrm{E}-12$

$6.039 \mathrm{E}-12$

$6.55 \mathrm{CE}-12$

$8.139 E-12$

$9.384 \mathrm{E}-12$

$1.379 \mathrm{E}-11$

$1.785 E-11$

$2.431 E-11$

$6.168 E-11$

$1.361 E-10$

$493 E-12$

$1.498 \mathrm{E}-12$

$1.559 \mathrm{E}-12$

$1.619 \mathrm{E}-12$

$1.818 \mathrm{E}-12$

$1.971 E-12$

$2.440 E-12$

2. $801 \mathrm{E}-12$

3. $308 \mathrm{E}-12$

$5.045 E-12$

$6.848 \mathrm{E}-12$

$9.577 E-12$

3. $933 E-11$ 
COSINE

-1. COOOCE OO $-9.89401 \mathrm{E}-01$ $-9.44575 E-01$ $-8.65631 \mathrm{E}-01$ $-7.55044 \mathrm{E}-01$ $-6.17876 E-01$ $-4.58017 \mathrm{E}-01$ $-2.81605 E-01$ $-9.50125 E-02$ $9.50125 E-02$ $2.81605 E-01$ $4.58017 E-01$ $7.17870 \mathrm{E}-01$ 7.55044E-01 $8.65631 \mathrm{E}-01$ $9.89401 E-01$

TOTAL

COSINE

$-1.00000 E C C$ $-9.89401 E-01$ $-9.44575 \mathrm{E}-01$ $-8.65631 E-01$ $-6.17876 \mathrm{E}-01$ $-4.58017 \mathrm{E}-01$ $-2.81605 E-01$ $-9.50125 E-02$ $9.50125 E-02$ $2.81605 \mathrm{E}-01$ 4. $58 \mathrm{C} 17 \mathrm{E}-01$ $6.17876 \mathrm{E}-01$ $7.55044 E-01$ $8.65631 \mathrm{E}-\mathrm{Ol}$ $9.44575 E-01$ $9.89401 E-G 1$

TOTAL
75.0

$1.233 \mathrm{E}-10$ $1.235 \mathrm{E}-10$ $1.241 \mathrm{E}-1 \mathrm{C}$
$1.254 \mathrm{E}-1 \mathrm{C}$ $1.254 \mathrm{E}-1 \mathrm{C}$ $1.275 \mathrm{E}-10$ $1.308 \mathrm{E}-1 \mathrm{C}$ $1.359 \mathrm{E}-1 \mathrm{C}$ $1.435 \mathrm{E}-1 \mathrm{C}$ $1.545 \mathrm{E}-1 \mathrm{C}$ $1.758 E-10$ $1.850 E-10$ 3. $345 \mathrm{E}-10$ $3.212 E-10$ S. $335 \mathrm{E}-1 \mathrm{C}$ $3.688 E-C 9$ $1.688 \mathrm{E}-\mathrm{C} 9$

7.317E-09

100.5

150.0

RANGE (METERS)
2CC.C

$25 C .0$

$3 \mathrm{coc} .0$

$400 . c$

$1.468 \mathrm{E}-10$ $1.469 \mathrm{E}-1 \mathrm{C}$ $1.477 \mathrm{E}-1 \mathrm{C}$ $1.493 E-1 C$ $1.518 \mathrm{E}-10$ $1.558 \mathrm{E}-1 \mathrm{C}$ $1.619 E-1 C$ $1.712 \mathrm{E}-1 \mathrm{C}$ $1.846 E-10$ $2.470 \mathrm{E}-10$ 2.73E $2.821 \mathrm{E}-1 \mathrm{C}$ $5.732 E-1 C$  . $315 E-10$ $1.051 E-C B$

$7.294 E-r 9$

$500 . \mathrm{C}$

600.0 $1.7955-10$
$1.797 E-10$ $1.807 E-10$ $1.827 E-10$ $1.858 \mathrm{E}-10$ $1.908 E-10$ $1.984 E-10$ $2.099 E-10$ $2.265 E-10$ 2.226E-10 $2.950 E-10$ $4.461 E-10$ $6.112 E-10$ 6.112E-10 9. $10 E-10$ $1.466 \mathrm{E}-\mathrm{C} 8$

$7.128 E-C 9$

$1.977 E-10$ $1.979 \mathrm{E}-10$ $1.991 E-10$ $2.014 \mathrm{E}-10$ 2. $551 E-10$ $2.1 \mathrm{C} 9 \mathrm{E}-10$ . $2.33 C E-10$ $2.519 E-10$ 2.939E-10 . $4.715 E-10$ $4.715 E-10$ 03CE-O9 2.091E-CO 2.09E-C

$6.988 \mathrm{E}-09$

$2.046 \mathrm{E}-10$
$2.049 \mathrm{E}-10$ $2.049 \mathrm{E}-1 \mathrm{C}$ $2.062 \mathrm{E}-10$ $2.088 \mathrm{E}-10$ $2.13 \mathrm{CE}-1 \mathrm{C}$ $2.194 E-10$ $2.29 C E-10$ $2.432 \mathrm{E}-10$ $2.634 \mathrm{E}-10$ $3.083 \mathrm{E}-10$ . $4.082 E-10$ $4.904 E-10$ . $027 E-0$ $1.027 E-0$ . $905 \mathrm{E}-09$

$6.677 E-05$

RANGE (METERS)

$9 C 0.0 \quad 1200.0$

1500.0

1800.0
$1.597 \mathrm{E}-1 \mathrm{C}$ $1.600 \mathrm{E}-1 \mathrm{C}$ $1.642 \mathrm{E}-1 \mathrm{C}$ $1.685 E-10$ $1.749 \mathrm{E}-1 \mathrm{C}$ $1.840 E-1 C$ $1.967 \mathrm{E}-1 \mathrm{C}$ $2.142 E-10$ $2.383 E-1 \mathrm{C}$ $2.720 \mathrm{E}-1 \mathrm{C}$ $3.210 E-1 C$ $3.960 E-10$ $5.166 E-10$ $7.236 E-10$ $1.134 \mathrm{E}-\mathrm{C} 9$ $3.369 E-C 9$

$5.827 E-11$

$5.842 \mathrm{E}-11$

$5.908 E-11$
$6.0 .35 E-11$

$6.228 \mathrm{E}-11$

$6.506 \mathrm{E}-11$

$6.887 E-11$

$7.402 E-11$

8.C $88 E-11$

9. CCIE-11

$1.023 E-10$

$1.193 \mathrm{E}-10$

$1.435 E-10$

$1.792 \mathrm{E}-10$

$2.343 \mathrm{E}-1 \mathrm{C}$

$3.270 E-10$

$2.168 \mathrm{E}-11$

$2.174 E-11$

$2.252 \mathrm{E}-11$

$2.329 E-11$

$2.439 E-11$

$2.587 \mathrm{E}-11$

$2.785 E-11$

3.046E- 11

$3.387 \mathrm{E}-11$

$3.839 \mathrm{E}-11$

$4.447 \mathrm{E}-11$

$5.286 E-11$

$6.473 E-11$

$8.211 \mathrm{E}-11$

$5.695 \mathrm{E}-1 \mathrm{C}$

2.19 CE-C 9

$4.326 \mathrm{E}-\mathrm{C} 9$

$1.721 E-10$

$6.118 \mathrm{E}-10$

$5.158 E-10$
7. $165 \mathrm{E}-12$ $7.187 \mathrm{E}-12$

$.28 \mathrm{CE}-12$

$.456 \mathrm{E}-12$

$7.722 \mathrm{E}-12$

$8.601 E-12$

$9.268 \mathrm{E}-12$

C $14 E-11$

$1.126 \mathrm{E}-11$

$1.274 E-11$

$1.469 \mathrm{E}-11$

$1.731 \mathrm{E}-11$

2.092E-11

2. $6 C 1 E-11$

$2.037 \mathrm{E}-1 \mathrm{C}$ $2.041 \mathrm{E}-10$ $2.055 \mathrm{E}-1 \mathrm{C}$

.

. $127 E-10$

.

$2.441 \mathrm{E}-10$

$2.647 E-10$

O58E-10

$.028 \mathrm{E}-10$

.

$.769 \mathrm{E}-\mathrm{C}$

8. C12E-C9

$1.868 \mathrm{E}-1 \mathrm{C}$

$1.871 \mathrm{E}-10$

$1.886 \mathrm{E}-1 \mathrm{C}$

$1.915 E-10$

1.961E-10

.

作

.

.

. $131 E-10$

$3.714 \mathrm{E}-10$

$4.616 \mathrm{E}-10$

. $793 \mathrm{E}-10$

$.447 \mathrm{E}-09$

$196 \mathrm{E}-09$

$4.78 \mathrm{CE}-11$

$2.181 \mathrm{E}-12$

$2.188 \mathrm{E}-12$

. $217 \mathrm{E}-12$

$2.356 \mathrm{E}-12$

$2.356 \mathrm{E}-12$
$2.472 \mathrm{E}-12$

$2.628 \mathrm{E}-12$

$834 \mathrm{E}-12$

$3.099 \mathrm{E}-12$

$.442 E-12$

$.885 E-12$

.

$.262 \mathrm{E}-12$

$.677 \mathrm{E}-12$
$.676 \mathrm{E}-12$

$1.300 E-11$

$1.671 E-10$

$5.021 E-11$ 

4 PI R*\#2 HENDERSON DOSE (GAMMAS)
(CM\#\#2 RAD/STERADIAN/SOURCE NEUTRON)
4.065 TO 6.360 MEV NFUTRON SOURCE

150.0

RANGE (METERS)
2 CO.0

250.0

300.0

400.0

$\begin{array}{lllll}1.309 \mathrm{E}-12 & 1.405 \mathrm{E}-12 & 1.446 \mathrm{E}-12 & 1.451 \mathrm{E}-12 & 1.390 \mathrm{E}-12 \\ 1.318 \mathrm{E}-12 & 1.418 \mathrm{E}-12 & 1.460 \mathrm{E}-12 & 1.467 \mathrm{E}-12 & 1.406 \mathrm{E}-12 \\ 1.356 \mathrm{E}-12 & 1.464 \mathrm{E}-12 & 1.513 \mathrm{E}-12 & 1.523 \mathrm{E}-12 & 1.467 \mathrm{E}-12 \\ 1.422 \mathrm{E}-12 & 1.543 \mathrm{E}-12 & 1.6 \mathrm{C} \mathrm{E}-12 & 1.616 \mathrm{E}-12 & 1.563 \mathrm{E}-12 \\ 1.511 \mathrm{E}-12 & 1.643 \mathrm{E}-12 & 1.707 \mathrm{E}-12 & 1.726 \mathrm{E}-12 & 1.670 \mathrm{E}-12 \\ 1.622 \mathrm{E}-12 & 1.763 \mathrm{E}-12 & 1.830 \mathrm{E}-12 & 1.848 \mathrm{E}-12 & 1.785 \mathrm{E}-12 \\ 1.764 \mathrm{E}-12 & 1.915 \mathrm{E}-12 & 1.983 \mathrm{E}-12 & 1.998 \mathrm{E}-12 & 1.925 \mathrm{E}-12 \\ 1.958 \mathrm{E}-12 & 2.122 \mathrm{E}-12 & 2.195 \mathrm{E}-12 & 2.209 \mathrm{E}-12 & 2.124 \mathrm{E}-12 \\ 2.226 \mathrm{E}-12 & 2.414 \mathrm{E}-12 & 2.498 \mathrm{E}-12 & 2.514 \mathrm{E}-12 & 2.420 \mathrm{E}-12 \\ 2.508 \mathrm{E}-12 & 2.869 \mathrm{E}-12 & 2.972 \mathrm{E}-12 & 2.941 \mathrm{E}-12 & 2.843 \mathrm{E}-12 \\ 3.167 \mathrm{E}-12 & 3.363 \mathrm{E}-12 & 3.488 \mathrm{E}-12 & 3.566 \mathrm{E}-12 & 3.430 \mathrm{E}-12 \\ 3.843 \mathrm{E}-12 & 4.281 \mathrm{E}-12 & 4.44 \mathrm{CE}-12 & 4.425 \mathrm{E}-12 & 4.260 \mathrm{E}-12 \\ 5.267 \mathrm{E}-12 & 5.5 \mathrm{C} 5 \mathrm{E}-12 & 5.711 \mathrm{E}-12 & 5.786 \mathrm{E}-12 & 5.533 \mathrm{E}-12 \\ 7.084 \mathrm{E}-12 & 7.777 \mathrm{E}-12 & 8.054 \mathrm{E}-12 & 8.071 \mathrm{E}-12 & 7.712 \mathrm{E}-12 \\ 1.683 \mathrm{E}-11 & 1.201 \mathrm{E}-11 & 1.242 \mathrm{E}-11 & 1.250 \mathrm{E}-11 & 1.191 \mathrm{E}-11 \\ 1.771 \mathrm{E}-11 & 2.079 \mathrm{E}-11 & 2.226 \mathrm{E}-11 & 2.260 \mathrm{E}-11 & 2.159 \mathrm{E}-11 \\ 3.601 \mathrm{E}-11 & 4.410 \mathrm{E}-11 & 4.918 \mathrm{E}-11 & 5.177 \mathrm{E}-11 & 5.153 \mathrm{E}-11 \\ & & & & \\ 5.183 \mathrm{E}-11 & 5.795 \mathrm{E}-11 & 6.091 \mathrm{E}-11 & 6.175 \mathrm{E}-11 & 5.954 \mathrm{E}-11\end{array}$

$\begin{array}{rr}\text { RANGE } & \text { (METERS } \\ 9 \mathrm{C} 0.0 & 1200\end{array}$

$500.0 \quad 600.0$

1200.0

1500.0

1800.0

$-1.00000 E 00$ $-9.89401 E-01$ $-9.44575 E-01$ $-8.65631 \mathrm{E}-01$ $-7.55044 \mathrm{E}-01$ $-6.17876 E-01$ $-4.58017 \mathrm{E}-01$ $-2.81605 E-01$ $-9.50125 E-02$ $9.50125 \mathrm{E}-02$ $2.81605 \mathrm{E}-01$ $4.58017 E-01$ $6.17876 E-01$ $7.55044 \mathrm{E}-01$ $8.65631 \mathrm{E}-01$ $9.44575 E-01$
$9.89401 E-01$

$1.121 \mathrm{E}-12$ $6.478 E-13 \quad 3.068 E-13$ $1.136 E-12 \quad 6.578 E-13 \quad 3.126 E-13$ $1.267 E-13$ $1.299 E-13 \quad 4.966 E-14$ $6.943 E-13 \quad 3.335 E-13 \quad 1.411 E-13 \quad 5.532 E-14$ $\begin{array}{llll}7.492 E-13 & 3.637 E-13 & 1.566 E-13 & 6.334 E-14\end{array}$ $1.539 \mathrm{E}-12$ 1. $1.36 \mathrm{EE}-12$ $\begin{array}{llllll}1.643 \mathrm{E}-12 & 1.458 \mathrm{E}-12 & 8.602 \mathrm{E}-13 & 4.192 \mathrm{E}-13 & 1.8 \mathrm{C} 4 \mathrm{E}-13 & 7.229 \mathrm{E}-14\end{array}$ $\begin{array}{llllll}1.769 E-12 & 1.570 E-12 & 9.296 E-13 & 4.534 E-13 & 1.937 E-13 & 7.640 E-14\end{array}$ $\begin{array}{llllll}1.953 E-12 & 1.736 E-12 & 1 . C 38 E-12 & 5.107 E-13 & 2.192 E-13 & 8.634 E-14\end{array}$ $\begin{array}{llllll}2.230 \mathrm{E}-12 & 1.99 \mathrm{CE}-12 & 1.209 \mathrm{E}-12 & 6.058 \mathrm{E}-13 & 2.646 \mathrm{E}-13 & 1.062 \mathrm{E}-13\end{array}$ $2.627 E-12 \quad 2.354 E-12 \quad 1.459 E-12 \quad 7.476 E-13 \quad 3.34 C E-13 \quad 1.371 E-13$

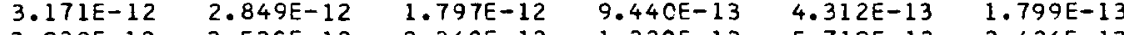

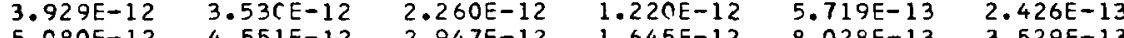

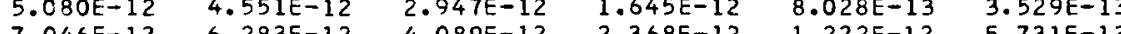
$7.046 E-12 \quad 6.283 E-12 \quad 4.089 E-12 \quad 2.368 E-12 \quad 1.222 E-12 \quad 5.731 E-13$ $1.083 \mathrm{E}-11$ 9.600E-12 $6.186 \mathrm{E}-12$ 3.675E-12 $2.008 \mathrm{E}-12$ 1.016E-12

$\begin{array}{llllll}1.951 \mathrm{E}-11 & 1.711 \mathrm{E}-11 & 1.064 \mathrm{E}-11 & 6.254 \mathrm{E}-12 & 3.510 \mathrm{E}-12 & 1.877 \mathrm{E}-12 \\ 4.731 \mathrm{E}-11 & 4.154 \mathrm{E}-11 & 2.481 \mathrm{E}-11 & 1.378 \mathrm{E}-11 & 7.445 \mathrm{E}-12 & 3.944 \mathrm{E}-12\end{array}$

TOTAL

$5.454 E-11$

$4.843 E-11$

$3.016 E-11 \quad 1.656 E-11$

$8.301 E-12$

3. $919 E-12$ 
COSINE

$-1.00000 E \cap C$ $-9.89401 E-01$ $-9.44575 \Xi-01$ $-8.65631 E-01$ $-7.55044 E-01$ $-6.17876 E-01$ $-4.58017 \mathrm{E}-01$ $-2.81605 \mathrm{E}-01$ $-9.50125 \mathrm{E}-02$ $9.50125 \mathrm{E}-02$ $2.81605 E-01$ $4.58017 \mathrm{E}-01$ $6.17876 \mathrm{E}-01$ $7.55044 \mathrm{E}-01$ $8.65631 E-01$ $9.894 \mathrm{ClE}-01$

TOTAL

COSINE

\section{$-1.00000 E$ OC} $-9.89401 \mathrm{E}-0$ $-8.65631 E-01$ $-8.65631 E-01$ $0.5784 \mathrm{E}-01$ $-4.58017 E-01$ $-2.81605 \mathrm{E}-\mathrm{C}$ - $50125 \mathrm{E}-02$ $-9.50125 \mathrm{E}-02$
$9.50125 \mathrm{E}-02$ . $4.58017 \mathrm{E}-0$ $6.17876 \mathrm{E}-01$ $7.55044 E-0$ $8.65631 t-0$

$9.44575 \mathrm{E}-\mathrm{Cl}$

9.894 C IE-O

TOTAL

\begin{abstract}
75.0
\end{abstract}
100.0

$9.952 \mathrm{E}-11$

1. $\mathrm{rONE}-10$

$1.022 \mathrm{E}-10$

1. $061 \mathrm{E}-10$

$1.119 \mathrm{E}-10$

$1.196 \mathrm{E}-10$

$1.298 \mathrm{E}-10$

$1.436 \mathrm{E}-1 \mathrm{C}$

$1.622 \mathrm{E}-10$

$1.919 E-10$

$2.778 \mathrm{E}-10$

$2.914 E-10$

$3.139 E-1 C$

$4.391 E-10$

$1.628 E-10$

$1.034 E-C 9$

$3.351 E-C 9$

$500 . \mathrm{C}$

$1.484 E-10$

$1.500 \mathrm{E}-10$

1.652E-10

1. $85 E-10$

$.993 \mathrm{E}-10$

$462 \mathrm{E}-10$

$2.462 E-10$

.

. $153 E-10$

$5.283 E-10$

$7.199 E-10$

$1.088 \mathrm{E}-\mathrm{C} 9$

$1.928 E-C 9$

$4.629 E-C 9$

$5.680 E-C 9$

$.337 E-1 C$

$.477 E-1 C$

.

$2.212 \mathrm{E}-10$

3.074E-10

$3.749 E-10$

$4.755 E-10$
RANGE (METERS)
$200 . \mathrm{C}$

$25 \mathrm{C} .0$

$300 . c$

400.0

$1.149 \mathrm{E}-10$

$1.156 \mathrm{E}-1 \mathrm{C}$

$1.182 E-10$

$1.232 \mathrm{E}-1 \mathrm{O}$

$1.301 \mathrm{E}-10$

$1.391 \mathrm{E}-10$

$1.511 \mathrm{E}-1 \mathrm{C}$

1.672E-1C

. 74 E 10

.

(6)

.

(

.

$.353 E-09$

.

4.156E-C9

$1.370 E-10$

$1.51 \mathrm{CE}-10$

$1.589 \mathrm{E}-10$

$1.603 \mathrm{E}-10$

$1.654 \mathrm{E}-1 \mathrm{C}$

$1.742 \mathrm{E}-10$

$1.848 \mathrm{E}-1 \mathrm{C}$

$1.97 \mathrm{CE}-1 \mathrm{C}$

$2.124 E-1 C$

$2.335 E-10$

$2.635 \mathrm{E}-10$

$3.106 \mathrm{E}-10$

$3.605 \mathrm{E}-1 \mathrm{C}$

$4.541 E-1 C$

$5.772 E-10$

$8.05 \mathrm{CE}-10$

$1.227 E-09$

$4.79 C \mathrm{C}-0 \mathrm{O}$

T.72CE-10

1.71E

1.726E-CO $2 . C 32 E-O Q$

$1.624 \mathrm{E}-1 \mathrm{C}$

$1.639 \mathrm{E}-1 \mathrm{C}$

$1.695 \mathrm{E}-10$

$1.788 \mathrm{E}-10$

$1.897 \mathrm{E}-10$

$2.021 E-10$

$2.173 E-1 C$

2.

.

$3.111 E-10$

$5.568=10$

1.113E-10

1.241E-C

2.218E-C

$6.176 E-09$

$6.3 C 8 E-C 9$

$1.597 E-10$

$1.673 \mathrm{E}-1 \mathrm{C}$

$1.770 \mathrm{E}-10$

$1.878 \mathrm{E}-10$

$2.64 \mathrm{CE}-10$

$3.063 \mathrm{E}-10$

$3.644 E-10$

$4.463 E-10$

$7.828 \mathrm{E}-10$

$1.19 \mathrm{CE}-09$

$5.034 E-09$

$6.153 E-C 9$

RANGE (METERS)

$900.0 \quad 1200.0$

1500.0

1800.0

\section{$1.322 \mathrm{E}-1 \mathrm{C}$}

$7.776 E-11$

$3.772 E-11$

$1.62 \mathrm{CE}-11$

$6.471 \mathrm{E}-12$

$3.829 \mathrm{E}-1$

$1.651 \mathrm{E}-11$

$7.213 E-12$

$4.038 E-11$

$1.918 \mathrm{E}-11$

(1)

..794E-11 4.342E-11 $1.918 E-11$

$2.17 C E-11$

8. $599 \mathrm{E}-12$

(5E-10

$4.632 E-11$

$\begin{array}{llll}9.949 E-11 & 4.923 E-11 & 2.17 C E-11 & 8.977 E-12 \\ 1 . C 68 E-10 & 5.285 E-11 & 2.314 E-11 & 9.439 E-12\end{array}$

$\begin{array}{llll}9.949 E-11 & 4.923 E-11 & 2.17 C E-11 & 8.977 E-12 \\ 1 . C 68 E-10 & 5.285 E-11 & 2.314 E-11 & 9.439 E-12\end{array}$

180E-10

$5.879 E-11$

$3.039 \mathrm{E}-1$

$1.048 E-11$

$1.355 \mathrm{E}-10$

$6.845 \mathrm{E}-11$

$3.039 \mathrm{E}=11$

$1.249 \mathrm{E}-11$

$1.945 E-10$

$1.024 \mathrm{E}-10$

$4.710 E-11$

$1.988 \mathrm{E}-11$

2.407E-10 $1.299 E-10$

$3 . C 87 E-10 \quad 1.721 E-10$

$8.40 C E-11 \quad 3.704 E-11$

$2.433 E-10 \quad 1.254 E-10 \quad 5.873 E-11$

6.252E-10 $3.712 E-10$

$1.254 E-10$

$5.873 \mathrm{E}-11$
$1.023 \mathrm{E}-1 \mathrm{C}$

$\begin{array}{llll}1 . C 58 E-C 9 & 6.229 E-10 & 3.496 E-10 & 1.023 E-1 C\end{array}$

4.C7OE-09 2.441E-09 1.359E-09

$3.496 \mathrm{E}-10$
$7.361 \mathrm{E}-10$

$3.906 \mathrm{E}-1 \mathrm{C}$

$8.72 C E-10$

4.117E-1C 
4 PI R*\#2 AIR KERMA (GAMMAS)
(CM**2 ERGS/GRAM/STERAOIAN/SOURCE NEUTRON)
4.065 TO 6.360 MEV NEUTRON SOURCE

RANGE (METERS)

$\begin{array}{ccccc}15 C .0 & 200.0 & 25 C .0 & 300.0 & 400.0 \\ 2.062 \mathrm{E}-10 & 2.488 \mathrm{E}-10 & 2.796 \mathrm{E}-10 & 2.996 \mathrm{E}-10 & 3.119 \mathrm{E}-1 \mathrm{C} \\ 2.072 \mathrm{E}-10 & 2.501 \mathrm{E}-10 & 2.812 \mathrm{E}-10 & 3.013 \mathrm{E}-1 \mathrm{C} & 3.137 \mathrm{E}-10 \\ 2.111 \mathrm{E}-10 & 2.551 \mathrm{E}-10 & 2.869 \mathrm{E}-10 & 3.076 \mathrm{E}-10 & 3.206 \mathrm{E}-10 \\ 2.182 \mathrm{E}-10 & 2.637 \mathrm{E}-10 & 2.968 \mathrm{E}-10 & 3.183 \mathrm{E}-10 & 3.319 \mathrm{E}-10 \\ 2.277 \mathrm{E}-10 & 2.750 \mathrm{E}-10 & 3.093 \mathrm{E}-1 \mathrm{C} & 3.315 \mathrm{E}-10 & 3.454 \mathrm{E}-10 \\ 2.398 \mathrm{E}-10 & 2.889 \mathrm{E}-10 & 3.242 \mathrm{E}-10 & 3.470 \mathrm{E}-10 & 3.610 \mathrm{E}-10 \\ 2.553 \mathrm{E}-10 & 3.065 \mathrm{E}-10 & 3.431 \mathrm{E}-10 & 3.665 \mathrm{E}-10 & 3.803 \mathrm{E}-10 \\ 2.76 \mathrm{E}-10 & 3.301 \mathrm{E}-10 & 3.684 \mathrm{E}-10 & 3.927 \mathrm{E}-1 \mathrm{C} & 4.066 \mathrm{E}-10 \\ 3.043 \mathrm{E}-10 & 3.624 \mathrm{E}-10 & 4.034 \mathrm{E}-10 & 4.292 \mathrm{E}-10 & 4.435 \mathrm{E}-10 \\ 3.346 \mathrm{E}-10 & 4.107 \mathrm{E}-10 & 4.554 \mathrm{E}-10 & 4.784 \mathrm{E}-10 & 4.939 \mathrm{E}-1 \mathrm{C} \\ 3.999 \mathrm{E}-10 & 4.637 \mathrm{E}-10 & 5.128 \mathrm{E}-10 & 5.474 \mathrm{E}-10 & 5.612 \mathrm{E}-10 \\ 4.679 \mathrm{E}-10 & 5.568 \mathrm{E}-10 & 6.116 \mathrm{E}-10 & 6.398 \mathrm{E}-10 & 6.529 \mathrm{E}-10 \\ 6.051 \mathrm{E}-10 & 6.793 \mathrm{E}-10 & 7.414 \mathrm{E}-10 & 7.800 \mathrm{E}-10 & 7.870 \mathrm{E}-10 \\ 7.800 \mathrm{E}-10 & 8.991 \mathrm{E}-10 & 9.709 \mathrm{E}-10 & 1.007 \mathrm{E}-\mathrm{C} 9 & 1.006 \mathrm{E}-09 \\ 1.131 \mathrm{E}-\mathrm{C} 9 & 1.300 \mathrm{E}-09 & 1.387 \mathrm{E}-09 & 1.431 \mathrm{E}-\mathrm{Cg} & 1.411 \mathrm{E}-09 \\ 1.772 \mathrm{E}-09 & 2.119 \mathrm{E}-\mathrm{C9} & 2.306 \mathrm{E}-09 & 2.375 \mathrm{E}-\mathrm{Cg} & 2.317 \mathrm{E}-09 \\ 3.460 \mathrm{E}-\mathrm{Cg} & 4.270 \mathrm{E}-09 & 4.79 \mathrm{CE}-09 & 5.066 \mathrm{E}-\mathrm{Cg} & 5.076 \mathrm{E}-09 \\ & & & & \\ 6.096 \mathrm{E}-09 & 7.213 \mathrm{E}-09 & 7.938 \mathrm{E}-09 & 8.347 \mathrm{E}-09 & 8.455 \mathrm{E}-09\end{array}$

RANGE (METERS)

$1200.0 \quad 1500.0 \quad 1800.0$
COSINE

$-1.00000 E D O$ $-9.89401 \mathrm{E}-01$ $-9.44575 \mathrm{E}-01$ $-8.65631 \mathrm{E}-01$ $-7.55044 E-01$ $-6.17876 E-01$ $-2.81605 \mathrm{E}-01$ $9.50125 E-02$ $2.81605 E-01$ 4.58017E-01 $6.17876 \mathrm{E}-01$ $7.55044 \mathrm{E}-01$ $8.65631 E-01$ $9.44575 E-01$
$9.89401 E-J 1$

TOTAL
$1.225 E-10$ $1.231 \mathrm{E}-10$ 1.292E- 10 $1.350 \mathrm{E}-10$ $1.532 \mathrm{E}-10$ . $671 \mathrm{E}-10$ $1.857 E-10$ $2.149 \mathrm{E}-10$ $2.988 \mathrm{E}-10$ $3.120 \mathrm{E}-10$ $4.277 E-10$ $6.709 \mathrm{E}-10$ $1.502 E-09$

$3.602 E-09$

600.0

$2.981 \mathrm{E}-10$ $2.999 E-10$ $3.066 \mathrm{E}-10$ $3.176 E-10$ 3. $304 \mathrm{E}-10$ $3.450 \mathrm{E}-10$ $3.631 E-10$ 3.881E- 10 $4.232 E-10$ 4.711E-10 $5.344 \mathrm{E}-10$ $6.191 \mathrm{E}-10$ $7.417 E-10$ $9.403 \mathrm{E}-10$ $1.307 E-09$ $2.119 \mathrm{E}-\mathrm{C} 9$ $4.678 \mathrm{E}-\mathrm{C}$

$2.693 E-10$ $2.709 E-10$ $2.772 E-10$ $2.989 \mathrm{E}-10$ $3.121 \mathrm{E}-10$ $3.284 E-10$ $3.510 E-10$ $3.832 E-10$ $4.271 E-10$ $4.846 \mathrm{E}-10$ $5.608 \mathrm{E}-1 \mathrm{C}$ $6.696 E-10$ $8.447 E-10$ $1.165 \mathrm{E}-\mathrm{C} 9$ $1.868 \mathrm{E}-09$ 4.113E-C 9

$7.952 E-C 9$

$7.142 E-09$
$1.628 \mathrm{E}-10$ $1.639 E-10$ $1.680 E-10$ $1.817 \mathrm{E}-10$ $1.897 E-10$ $1.997 \mathrm{E}-10$ $2.142 E-10$ $2.352 E-10$

$2.642 E-10$ $3.023 \mathrm{E}-10$ $3.526 \mathrm{E}-10$ $4.240 E-10$ $5.373 E-10$ $7.378 \mathrm{E}-10$ $1.152 \mathrm{E}-09$ OE-O9 $8.333 E-11$ $3.935 \mathrm{E}-11$ 4.057E-11 $4.235 E-1$ 4. $411 \mathrm{E}-1$ 4. 585E-11 4. $803 E-1$ $5.150 E-11$ $5.697 \mathrm{E}-11$ $6.482 \mathrm{E}-1$ $7.539 \mathrm{E}-11$ $9.011 \mathrm{E}-11$ $1.132 \mathrm{E}-1 \mathrm{C}$ $1.534 \mathrm{E}-10$ 2.267E-10 $3.647 \mathrm{E}-1$ 7. $23 \mathrm{CE}-10$

$1.740 \mathrm{E}-1$ $1.759 E-11$ $1.820 E-11$ $1.911 \mathrm{E}-11$ $2.055 \mathrm{E}-11$ $2.283 E-11$ $2.525 \mathrm{E}-11$ 2.877E-11 $3.346 \mathrm{E}-11$ 5. $103 \mathrm{E}-11$ 7.199E-11 $1.131 E-10$ $1.919 \mathrm{E}-10$ 3.797E-10 $5.624 E-10$ 
$\begin{array}{rl}4 & P I \\ \text { R**2 SILICON KERMA (GAMMAS) } \\ \text { (CM**2 ERGS/GRAM/STERADI AN/SOURCE NEUR }\end{array}$

COSINE

$-1.00000 E$ OO $-9.89401 \mathrm{E}-01$ $-9.44575 E-C 1$ $-8.65631 \mathrm{E}-01$ $-7.55044 \mathrm{E}-01$ $-6.17876 \mathrm{E}-01$ $-4.58017 \mathrm{E}-01$
$-2.81605 \mathrm{E}-01$ $-2.81605 E-01$ $-9.50125 \mathrm{E}-02$ $9.50125 \mathrm{E}-\mathrm{C} 2$ $2.81605 \mathrm{E}-01$ 4.58017E-O $7.55044 \mathrm{E}-0$ $7.55044 E-01$ $8.65631 E-01$ $9.89401 \mathrm{E}-01$

TOTAL

COS INE

$-1 . \operatorname{CCOOOE~CO}$ $-9.89401 E-C 1$ $-9.44575 \mathrm{E}-01$ $-8.65631 \mathrm{E}-01$ $-6.17876 \mathrm{E}-01$ $-4.58017 \mathrm{E}-01$ $-2.81605 \mathrm{E}-01$ $-9.53125 \mathrm{E}-02$ $9.50125 \mathrm{E}-02$ $2.81605 E-01$ 4.58017E-0 6.17876E-01 $7.55044 \mathrm{E}-01$ $8.65631 \mathrm{E}-0$ $9.89401 \mathrm{E}-01$

TOT AL

$\begin{array}{cc}75 . C & 10 C .0 \\ 1.016 E-10 & 1.178 E-1 C \\ 1.021 E-10 & 1.185 \mathrm{E}-1 C \\ 1.042 \mathrm{E}-10 & 1.212 \mathrm{E}-1 \mathrm{C} \\ 1.083 \mathrm{E}-10 & 1.262 \mathrm{E}-1 \mathrm{C} \\ 1.141 \mathrm{E}-10 & 1.331 \mathrm{E}-10 \\ 1.219 \mathrm{E}-10 & 1.423 \mathrm{E}-1 \mathrm{C} \\ 1.322 \mathrm{E}-10 & 1.544 \mathrm{E}-1 \mathrm{C} \\ 1.461 \mathrm{E}-10 & 1.706 \mathrm{E}-1 \mathrm{C} \\ 1.649 \mathrm{E}-10 & 1.927 \mathrm{E}-1 \mathrm{C} \\ 1.949 \mathrm{E}-10 & 2.789 \mathrm{E}-1 \mathrm{C} \\ 2.815 \mathrm{E}-10 & 2.654 \mathrm{E}-1 \mathrm{C} \\ 2.955 \mathrm{E}-10 & 3.492 \mathrm{E}-1 \mathrm{C} \\ 3.182 \mathrm{E}-1 \mathrm{C} & 4.397 \mathrm{E}-10 \\ 4.143 \mathrm{E}-10 & 5.761 \mathrm{E}-1 \mathrm{C} \\ 6.710 \mathrm{E}-10 & 7.445 \mathrm{E}-1 \mathrm{C} \\ 1.047 \mathrm{E}-\mathrm{C} 9 & 1.37 \mathrm{CE}-\mathrm{C} 9 \\ 1.554 \mathrm{E}-09 & 2.328 \mathrm{E}-\mathrm{C} 9\end{array}$

$1.554 \mathrm{E}-\mathrm{C} Q \quad 2.328 \mathrm{E}-\mathrm{CO}$

$3.399 E-C 9$

500.0

$1.607 E-10$

$1.623 \mathrm{E}-10$

$1.683 E-10$

$1.777 \mathrm{E}-10$

$1.880 E-10$

$1.989 E-10$

$2.122 \mathrm{E}-10$

2. $312 \mathrm{E}-10$

$2.595 \mathrm{E}-10$

$2.097 E-10$

$3.544 E-10$

4. $300 \mathrm{E}-10$

$5.438 \mathrm{E}-10$

$7.369 \mathrm{E}-1 \mathrm{C}$

$1.108 E-C 9$

$1.959 \mathrm{E}-09$

$4.700 E-C 9$

$5.873 E-C 9$

\section{0}

$1.437 E-1 C$

$1.452 E-1 C$

$1.508 E-1 C$

$1.595 \mathrm{E}-1 \mathrm{C}$

$1.785 \mathrm{E}-1 \mathrm{r}$

$1.904 \mathrm{E}-1 \mathrm{C}$

2.C77E-10

$2.338 \mathrm{E}-1 \mathrm{C}$

$2.708 \mathrm{E}-10$

$3.2 \mathrm{CBE}-10$

$3.889 E-10$

$.609 E-1 C$

$9.863 E-1 C$

$1.724 E-09$
$4.135 E-09$

$5.25 C E-C 9$
$150.0 \quad$ RANGE (METERS)
2 CC.0

256.0

300.6

$400 . \mathrm{C}$

$1.419 \mathrm{E}-10 \quad 1.580 \mathrm{E}-10 \quad 1.677 \mathrm{E}-10$

$1.429 \mathrm{E}-1) \quad 1.592 \mathrm{E}-10$

$1.466 \mathrm{E}-10 \quad 1.638 \mathrm{E}-10$

$1.532 \mathrm{E}-10$

$1.717 \mathrm{E}-10$

$1.816 \mathrm{E}-10$

$1.936 E-10$

$2.088 \mathrm{E}-10$

$2.294 E-10$

$2.584 \mathrm{E}-10$

3. $C 39 \mathrm{E}-10$

$3.514 E-10$

$4.425 \mathrm{E}-10$

$2.614 E-10$

$1.844 E-10$

1.198E-09

$4.346 E-09$

$1.691 E-10$

$1.743 E-10$

$1.832 \mathrm{E}-1 \mathrm{C}$

$1.939 E-10$

2.063E 10

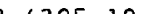

(..730E-10

(2.732E-10

. 7 C $7 E-10$

$4.653 \mathrm{E}-1 \mathrm{C}$

$5.891 E-10$

$8.191 E-10$

$2.2 C 9 E-0 O$

$2.209 E-09$

$6.325 E-09$

$1.727 E-10$

$1.742 \mathrm{E}-10$

$1.799 \mathrm{E}-10$

$1.893 E-10$

2.0C4E 10

2.128E-10

$2.494 \mathrm{E}-10$

$2.801 \mathrm{E}-10$

.

$4.693 E-10$

$6.031 \mathrm{E}-10$

.

$1.260 \mathrm{E}-\mathrm{C}$

$5.116 \mathrm{E}-\mathrm{CO}$

$5.950 E-09$

$6.477 E-C 9$

$1.717 \mathrm{E}-1 \mathrm{C}$

$1.734 \mathrm{E}-10$

$1.892 \mathrm{E}-10$

$2.002 \mathrm{E}-10$

$2.121 E-10$

$2.266 E-10$

$2.470 E-1 C$

$3.196 \mathrm{E}-10$

$3.782 \mathrm{E}-1 \mathrm{C}$

$4.606 E-10$

T.8OOE-10

T.997E-10

$1.211 E-09$

$5.106 \mathrm{E}-09$

$6.344 E-09$

RANGE (METERS)
$902.3 \quad 1200 . \mathrm{C} \quad 150 \mathrm{C} . \mathrm{C} \quad 1800 . ?$

$8.506 E-11$

$8.979 E-11$

$8.979 E-11$

$1.012 \mathrm{E}-10$

1. $C 71 E-10$

$1.145 E-10$

$1.259 \mathrm{E}-10$

$1.436 \mathrm{E}-10$

$1.690 \mathrm{E}-10$

$2.034 E-10$

$.562 E-10$

$4.324 \mathrm{E}-10$

$6.396 \mathrm{E}-10$

$1.079 E-C 9$
$2.485 E-C 9$

4.154E-11

$4.212 \mathrm{E}-11$

$4.424 \mathrm{E}-11$

$4.734 \mathrm{E}-11$

$5.323 \mathrm{E}-11$

$5.69 C E-11$

$6.291 \mathrm{E}-11$

$7.271 \mathrm{E}-11$

$8.719 E-11$

1.C. $72 \mathrm{E}-10$

$1.351 \mathrm{E}-10$

$1.779 \mathrm{E}-10$

$2.502 \mathrm{E}-10$

$3.802 \mathrm{E}-10$

$6.362 E-1$

$1.387 \mathrm{E}-09$

$3.291 E-C 9$

$1.8 C 8 E-C 9$

$1.802 E-11$

7. 3C $3 \mathrm{E}-12$

$7.486 E-12$

$8.062 E-12$
$8.882 E-12$

$9.473 \mathrm{E}-12$

$9.851 \mathrm{E}-12$

$1.031 E-11$

1. $136 E-11$

$1.340 \mathrm{E}-11$

$2.089 \mathrm{E}-11$

2.722E-11

$3.831 \mathrm{E}-11$

$1.048 \mathrm{E}-1 \mathrm{C}$

$1.912 E-1 C$

$3.995 E-1 C$

$4.279 E-1 C$ 
ENERGY GROUP (MEV) $1.22 E$ O1- $1.50 E$ OI $1.00 E$ 01-- $1.22 E$ OI $6.3 \in E$ OO$4.97 E$ O0-- $6.36 E$ OO $4.07 E$ O0- $-4.07 E$ E 00 $3.01 E$ OD- $-4.07 E$ OO $2.46 E$ E0- $3.01 E 00$ $2.35 E$ EO- $2.46 E 00$ $1.83 \mathrm{E} 00--2.35 \mathrm{E} 00$ $1.11 \mathrm{E} 00--1.83 \mathrm{E} 00$ $5.50 \mathrm{E}-01-21.11 \mathrm{E} 00$ $1.11 E-01--5.50 E-01$ $3.35 \mathrm{E}-02--1.11 \mathrm{E}-01$ $5.83 E-04--3.35 E-02$ $1.01 E-04---5.83 E-04$ $2.90 \mathrm{E}-05--1.01 \mathrm{E}-04$ $1.07 E-05--2.90 E-05$ $3.06 \mathrm{E}-06---1.07 \mathrm{E}-05$ $1.12 E-06---3.06 E-06$ $4.14 E-07--1.12 E-06$

\section{ENERGY}$$
\text { GROUP (ME }
$$

1.22E 01-D-1.50E O1 $1.00 E$ OL---1.22E OI 8.1 SE $00--1.00 E$ OI $6.36 \mathrm{E} \quad 00---8.19 \mathrm{E} \quad 00$ 4.97E $00--6.36 E$ OO 4.07E 00---4.97E 00 3.01 E $00--4.07 E$ OO $2.46 \mathrm{E} 00--3.01 \mathrm{E} 00$ $2.35 E \quad 00--2.46 E 00$ $1.83 \mathrm{E} \quad 00--2.35 \mathrm{E} 00$ $1.11 \mathrm{E}$ 00--1.83E 00 $5.50 \mathrm{E}-01--1.11 \mathrm{E} 00$ $1.11 E-01=-5.50 E-01$ $3.35-02=-1.11 E-01$ $5.83 E-04--3.35 E-02$ $1.01 E-04--5.83 E-04$ $2.90 E-05--1.01 E-04$ $1.07 E-05-2.90 E-05$ $3.06 E-06--1.07 E-05$ 1.12E-06-C $3.06 E-16$ $4.14 E-07--1.12 E-06$
ANGLE 1 $=-1.0000$ 0.0 0.0 0.0 0.0 0.0 $7.590 E-04$ $2.136 \mathrm{E}-02$ 3.232E-02 $2.066 \mathrm{E}-02$ $9.356 \mathrm{E}-03$ $8.144 E-03$ $8.774 E-03$ 3.784E-02 $2.184 E \quad O O$ $9.198 \mathrm{E} \quad 00$ $2.637 E$ OI $6.929 E$ OI $1.830 \mathrm{E} \mathrm{O2}$ $3.954 \mathrm{E} \mathrm{O}$ $5.665 \mathrm{E} 02$ ANGLE 10 0.0 0.0 0.0

$2.555 \mathrm{E}-02$ $1.253 \mathrm{E}-02$ $1.010 \mathrm{E}-02$ $9.957 E-03$ 4.896E-02 $3.899 \mathrm{E}-02$ $2.238 E$ OO $9.400 \mathrm{E}$ OO $2.691 \mathrm{E}$ OI 7.060 E OI $1.862 \mathrm{E} \mathrm{O2}$ $4.020 E$ O2
$5.753 E \quad 02$
(NEUTRONS/MEV/STERADIAN/SOURCE NEUTRON)

ANGLE
MU $=-0.9854 \quad M U=-0.9446$ 0.0

0.0

0.0

0.0

0.0

$7.886 E-04$

$2.131 \mathrm{E}-02$

$3.214 \mathrm{E}-02$

$2.062 \mathrm{E}-02$

$8.157 \mathrm{E}-\mathrm{C}$

$8.782 \mathrm{E}-03$

4.664E-02

$3.785 E-02$

$2.184 E$ EO

$9.200 E$ OO

OI

$1.830 \mathrm{E} \mathrm{C2}$

$5.666 \mathrm{E} 02$

0.0

0.0

0.0

0.0

0.0

$9.005 E-04$

$2.114 \mathrm{E}-02$

$3.147 \mathrm{E}-02$

$9.396 E-03$

$8.205 E-03$

$8.814 E-03$

$4.672 \mathrm{E}-02$

$2.186 \mathrm{E} \quad 00$

$9.208 \mathrm{E} 00$

$2.640 \mathrm{E} \mathrm{CI}$

$6.935 \mathrm{E} 01$

$\begin{array}{lll}1.831 E & 02 \\ 3.957 E & 02\end{array}$

$5.671 \mathrm{E} \mathrm{O2}$

ANGLE 4

ANGLE

ANBLE 6
MU $=-3.6179$

ANGLE 7 $0.0 \quad 0.0$

0.0

0.0

0.0

0.0

0.0
0.0
0.0

$1.095 \mathrm{E}-03$

$2.082 \mathrm{E}-02$

2.027E-02

$9.477 \mathrm{E}-03$

$8.874 E-03$

4.686E-02

$3.797 \mathrm{E}-02$

$9.222 \mathrm{E} \mathrm{OO}$

$2.644 \mathrm{E}$ Ol

$6.945 \mathrm{E}$ O1

1.833E 02

5.678 E 02

0.0

0.0

0.0
$1.339 E-03$

2. $040 \mathrm{E}-02$

2.896E-02

$2.019 E-02$

$8.633 E-03$

$8.962 E-03$

4.707E-02

$2.195 E 00$

$9.242 \mathrm{E} 00$

$2.649 \mathrm{E} 01$

$1.837 \mathrm{E} 02$

$3.968 \mathrm{E} 02$

$5.688 \mathrm{E} 02$

0.0

0.0

0.0

0.0

0.0

$1.616 E-03$

1. $998 \mathrm{E}-02$

$2.770 E-02$

$2.038 E-02$
$9.880 E-03$

$8.633 \mathrm{E}-03$

$9.076 \mathrm{E}-03$

$3.823 E-02$

$2.202 E 00$

$9.267 \mathrm{E} 00$

$2.656 E$ O1

$1.841 \mathrm{E} \mathrm{O2}$

$3.976 \mathrm{E} 02$

$5.699 E 02$

ANGLE 14 $M U=0.2816$

ANGLE 13

0.0

$0 . \mathrm{C}$

0.0

0.0

0.0

0.0

$5.191 \mathrm{E}-03$

2.782E-02

3.320E-02

$4.567 \mathrm{E}-02$
$1.430 \mathrm{GE}-02$

$1.43 \mathrm{E}-02$

$9.971 \mathrm{E}-03$

$4.996 E-02$
$3.921 E-02$

$3.921 E-02$

$2.248 E 00$

$9.435 E$ OO
$2.701 \mathrm{~F} 01$

$7.083 \mathrm{E} 01$

$3.868 \mathrm{E} \mathrm{O}$

$4.031 E$ O2
$5.768 \mathrm{E} 02$
0.0

0.0

0.0

0.0

0.0

0.0

0.0

$8.774 E-03$

3.003E-02

$3.947 \mathrm{E}-02$
$3.462 \mathrm{E}-02$

$1.538 \mathrm{E}-02$

$1.058 E-02$

$9.946 E-03$

$3.941 E-02$

$2.257 E 00$

$9.469 E$ OO

$2.710 E$ O1

$7.105 E$ OI

$1.873 E$ O2

$4.042 E$ O2
$5.782 \mathrm{E} 02$
0.0

0.0

0.0

$1.706 E-02$

$3.461 \mathrm{E}-02$

$5.812 \mathrm{E}-02$
$3.798 \mathrm{E}-02$

$1.476 \mathrm{E}-02$

$1.086 \mathrm{E}-02$

$1.062 \mathrm{E}-02$

$3.959 \mathrm{E}-02$

$2.265 E 00$

$9.500 E$ OO

$2.718 E$ O1

1.124E OI

$4.052 \mathrm{E} 02$

$5.795 \mathrm{E} \mathrm{O2}$

$\begin{array}{ll}0.0 & M U= \\ 0.0 & 0.0\end{array}$

0.0

0.0

0.0

0.0

4.267E-02

$5.766 E-02$

$9.043 \mathrm{E}-02$

$4.692 E-02$
$1.770 E-02$

$1.318 E-02$

$1.104 \mathrm{E}-02$

3.976E-02

$2.273 E 00$

$9.526 \mathrm{E} 00$

2.725E 01

1.882E 02

$4.061 \mathrm{E} 02$

$5.806 \mathrm{E} 02$

0.0

0.0
0.0

0.0

0.0

0.0

$1.320 \mathrm{E}-01$

$1.393 E-01$

$1.804 \mathrm{E}-01$
$7.108 \mathrm{E}-02$

$2.441 \mathrm{E}-02$

$1.339 \mathrm{E}-02$

$5.217 E-02$

$3.991 \mathrm{E}-02$

2.279E 00

9.548 O

$7.155 \mathrm{E}$ ol 

GROUP (MEV) $1.22 E 01--1.50 E$ O $3.00 \mathrm{E} 01--1.22 \mathrm{E} 01$ $8.19 F \quad 00--1.00 E$ OI $6.36 \mathrm{E} 00-0-8.19 \mathrm{E}$ 00 4.97E $00---6.36 \mathrm{E}$ OO 4. 07 F $00---4.97 E$ OO $3.03 \mathrm{E} 00--4.07 E$ OO $2.46 \mathrm{E} 00--3.01 \mathrm{E}$ CO $2.35 \mathrm{E} \quad 00--2.46 \mathrm{E} 00$ $1.83 E \quad 00-\cdots 2.35 E$ OO $1.11 \mathrm{E} 00--1.83 \mathrm{E}$ OO $5.50 E-01-\cdots-1.11 E$ OO $1.11 \mathrm{E}-01--5.50 \mathrm{E}-0$ $3.35 E-02--1.11 E-01$ $5.83 \mathrm{E}-04---3.35 \mathrm{E}-02$ $1.01 E-04---5.83 E-04$ $2.90 E-05--1.01 E-04$ $1.07 \mathrm{E}-05--2.90 \mathrm{E}-05$ $3.06 E-06-=-1.07 E-05$ $1.12 \mathrm{E}-06---3.06 \mathrm{E}-06$ $4.14 E=07-=-1.12 E-06$ $0.0 \quad--4.14 E-07$ ENERGY GROUP IMEVI $1.22 E$ O1- -1.50E OI $1.00 \mathrm{E}$ O1---1.22E OI 8.19E DO---1.00E O1 $6.36 E$ OO- $-8.19 E$ OO 4.97E $00-2.36$. 65 OD 4.07E $00--4.97 E$ OO 3.01E $00--4.07 E$ OO 2.46E $00--3.02 \mathrm{R}$ OO $2.35 \mathrm{E} 00--2.46 \mathrm{E}$ OO $1.83 \mathrm{E} 00-2.35 \mathrm{E}$ OO $1.11 \mathrm{E} 00-1.83 \mathrm{E}$ OO $5.5 \mathrm{E}-1 \mathrm{C}-11 \mathrm{E}$ 1 .11E-OL - 5 . $50 E-O$ 5. 1.01E-04- $5.33 E-04$ 2.01E $05-1.01 E-04$ 1.07E-05-D2.90E-0 3.06E-06- $1.07 E-05$ $1.12 E-06-0-3.06 E-06$ $4.14 E-07=-1.12 E-06$ $0.0 \quad--4.14 E-07$
ANGLE 1 ANG $M U=-1.0000$ 0.0 0.0 0.0 0.0 0.0
$7.703 E-04$ $1.995 \mathrm{E}-02$ $3.105 \mathrm{E}-02$ $2.499 E-02$ $1.785 \mathrm{E}-02$ $2.087 E-02$ $2.717 \mathrm{E}-02$ $1.595 \mathrm{E}-01$ $1.333 \mathrm{E}-01$ $7.817 E 00$ $3.322 \mathrm{E} O$ $9.579 \mathrm{E}$ OI $6.709 E$ O2 $1.455 \mathrm{E}$
2.093 ANGLE 10 $U=0.0950$ 0.0
0.0
0.0 0.0
0.0 0.0 0.0 $2.803 E-03$ $2.440 E-0$ $2.270 E-02$ 4.133E-OL 2.877E-02 3.534E-02 1. $347 \mathrm{E}-02$ $1.405 \mathrm{E}-01$ $.177 E$ OO $3.459 E$ OI $9.953 \mathrm{E} 01$ 2.623E O2 $1.502 \mathrm{E} O 3$ $1.502 E$
ANGLE 2 0.0 0.0 0.0 0.0 0.0 0.0 $8.014 E-C 4$ 1. $990 \mathrm{~F}-02$ 3. $089 \mathrm{E}-02$ 2.495E-02 $1.787 \mathrm{E}-02$ $2.001 \mathrm{E}-02$ $2.720 \mathrm{E}-02$ $1.596 \mathrm{E}-01$ $1.334 \mathrm{E}-\mathrm{Cl}$ $7.820 \mathrm{E}$ DO $3.323 \mathrm{E}$ Ol $9.582 \mathrm{E}$ OI $2.532 \mathrm{E} \mathrm{O2}$ $6.711 \mathrm{E} \mathrm{O2}$ $1.455 \mathrm{E} 03$ $2.093 \mathrm{E} 03$

ANGLE 11 $M U=0$
0.0
0.0 0.0
0.0 0.0 0.0 $5.659 E-03$ $3.182 E-02$ 4. $316 E-02$ $2.700 E-O 2$ 2.7 OE-02 2.842E-OR $3.006-C 2$ $1.741 E-01$ 1.41 E $3.484 \mathrm{E} O 1$ $1.402 E$ OL 1.63 6. $1.510 \mathrm{E} \mathrm{O3}$ 1. $1 \in 5 E$
(NEUTRJNS/MEV/STERADIAN/SOURCE NEUTRON)

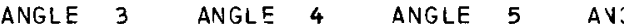

$M U=-0.9446 \quad M U=-0.8656$ 0.0

0.0

0.0

0.3

0.0

$9.200 E-04$

$1.971 E-02$

$3.025 \mathrm{E}-02$

$2.485 \mathrm{E}-02$

$1.799 \mathrm{E}-02$

$2.108 \mathrm{E}-02$

$1.336 \mathrm{E}-0$

$7.834 \mathrm{E} \quad 00$

$3.329 E$ OL

$9.596 \mathrm{E}$ OI

$2.535 \mathrm{E} O 2$
$6.720 \mathrm{E}$

$6.720 E$ O

2.096E 03

$M U=-0.0$
0.0

0.0

0.0

0.0

$1.122 E-03$

$1.934 \mathrm{E}-02$

$2.919 \mathrm{E}-02$

$1.825 \mathrm{E}-0$

$2.140 E-02$

2.762E-02

$1.609 E-01$

$1.341 \mathrm{E}-01$

$7.859 \mathrm{E} \mathrm{OO}$

$9.338 \mathrm{E}$ O1

$9.623 \mathrm{E}$ OL

$6.736 \mathrm{E} \mathrm{O2}$

$1.460 \mathrm{E} 03$

$A U=-0.7550$

0.0
0.0
0.0

0.0
0.0
0.0

0.0

0.0

1. $368 \mathrm{E}-03$

$1.887 \mathrm{E}-02$

2. $803 E-02$

$1.869 \mathrm{E}-02$

$2.189 E-02$

$2.802 \mathrm{E}-02$

$1.620 E-01$

$1.348 \mathrm{E}-01$

$\begin{array}{ll}7.893 \mathrm{E} & 00 \\ 3.352 \mathrm{E} & 01\end{array}$

$3.352 E$ OI
$9.659 E$ OI

O?

$6.759 \mathrm{E} \mathrm{O}$

$1.465 E \quad 03$

2.108E 03

ANGLE 12
$M U=0.4580$

$M U=0.4$
0.0
0.0
0.0

0.0
0.0
0.0
0.0

0.0

0.0

$6.413 E-03$

$2.386 \mathrm{E}-02$

$6.246 \mathrm{E}-02$

$5.356 \mathrm{E}-02$

3.JO7E-O2

3.028E-02

$3.600 \mathrm{E}-02$
$1.754 \mathrm{E}-01$

$1.754 E-01$
$1.432 E-01$

$.307 E$ OO

$508 E$ O

$1.008 \mathrm{E} 02$

$2.655 E$ O

ANGLE 13

ANGLE 14

0.0

0.0

0.0

0.0

0.0

0.0

0.0

0.0

0.0

0.0

0.0

4. $324 \mathrm{E}-02$

$5.892 \mathrm{E}-02$

2.253E-02

$7.621 \mathrm{E}-02$

$1.185 \mathrm{E}-01$

7. $978 E-02$

3.840E-02

$383 E-02$

$3.362 E-02$

$3.695 E-02$

1. $455 \mathrm{E}-01$

. $367 \mathrm{E} 00$

.

$419 E 00$

$3.550 \mathrm{E}$ O1

$1.020 E 02$

$2.683 E$
$7.087 E$ O2

1. $532 E \quad 03$

$1.519 \mathrm{E} \quad 03$

$2.194 \mathrm{E} \quad 03$ 0.0 0.5

0.0

0.0

0.0

0.0

0.0

0.0

0.0

AVSLE 6
$M U=-0.6179$ $M U=-0$
0.0
0.0

0.0
$1.644 E-03$

$1.838 \mathrm{E}-02$

$2.708 E-02$

$2.546 E-02$
$1.937 E-02$

$2.255 \mathrm{E}-02$

2. $855 \mathrm{E}-02$

$1.635 E-01$

$7.937 \mathrm{E} 00$

$3.368 \mathrm{E}$ OI

9 .705E O2

6.562E O2

1.787E 02

$2.115 \mathrm{E} 03$

ANOLE 15

$M=0.8656 \quad$ ANGLE 16

$4.680 E-02$

$1.034 \mathrm{E}-01$

$1.613 \mathrm{E}-01$

.

$4.313 E-02$

$3.724 E-02$

(3)

$1.465 E-01$

$3.566 E$ OI 
(NEUTRONS/MEV/STERADIAN/SOURCE NEUTRONI

ENERGY GROUP (MEV) $1.22 E \quad 01---1.50 E$ OI $1.00 E$ O1---1.22E OI $8.19 E$ OO-- -1. OOE OI 6.97E $00--8.19 \mathrm{E}$ OO $4.07 E$ 00- $-4.97 E$ OO $3.01 E 00--4.07 E$ OO $2.46 \mathrm{E} 00-0-3.01 \mathrm{E}$ OO $2.35 \mathrm{E} 00--2.46 \mathrm{E}$ OD $1.33 \mathrm{E} 00--2.35 \mathrm{E} 00$ $1.11 E$ OO- $-1.83 \mathrm{E} 00$ $5.50 E-01--1.11 \mathrm{E}$ OO $1.11 \mathrm{E}-01-2-5.50 \mathrm{E}-01$ $3.35 \mathrm{E}-02--1.11 \mathrm{E}-01$ $5.83 \mathrm{E}-04--3.35 \mathrm{E}-02$ $1.01 E-04--5.83 E-04$ $2.90 \mathrm{E}-05--1.01 \mathrm{E}-04$ $1.07 E-05-2.90 E-05$ $3.06 \mathrm{E}-06---1.07 \mathrm{E}-05$ $1.12 \mathrm{E}-06--3.06 \mathrm{E}-06$ $4.14 \mathrm{E}-07--1.12 \mathrm{E}-06$ ENERGY GRQUP (MEV) $1.22 E$ 01--1.50E 01 $1.00 E$ O1---1.22E $8.19 E$ OO- $-1.00 E$ OI 6.36E $00--8.19 E$ OO 4.97E $00--6.36 E$ OO 4.07E $00--4.97 E$. 00 $3.01 E$ OO- $4.07 E$ OO $2.46 \mathrm{E} 00--3.01 \mathrm{E} 00$ $2.35 \mathrm{~F} \mathrm{OO-D2.46E} 00$ $1.83 \mathrm{E} 00-2.35 \mathrm{E} 00$ 1.IIE 00-- $1.83 \mathrm{E}$ 00 $5.50 \mathrm{E}-01--1.11 \mathrm{E}$ on $1.11 E-01--5.50 E-0$. $3.35 E-02--1.11 E-.01$ $5.83 E-04--3.35 E-02$ $1.01 \varepsilon-04--5.83 \varepsilon-04$ 2.90E-05-- $1.01 E-04$ $1.07 E-05--2.90 E-05$ $3.06 E-06--1.07 E-05$ $1.12 F-06--3.06 \mathrm{E}-06$ $4.14 \mathrm{E}-0 \mathrm{C}---1.12 \mathrm{E}-06$
$0.0 \quad--4.14 \mathrm{E}-07$
ANGLE 1 0.0 0.0 0.0 0.0 0.0
$7.146 E-04$ $1.724 E-02$ $2.704 \mathrm{E}-02$ $2.453 \mathrm{E}-02$ $2.095 E-02$ $2.783 \mathrm{E}-02$ $4.005 \mathrm{E}-02$ $2.120 E-01$ $1.257 \mathrm{E}$ OI $5.376 \mathrm{E}$ OI $1.557 E \quad 02$ $4.132 \mathrm{E} \quad 02$ $1.099 \mathrm{E} \mathrm{O3}$ $2.389 E \quad 03$ ANGLE 10 $M U=0.0950$ 0.0 0.0 0.0 0.0 $3.708 E-03$ $1.655 \mathrm{E}-02$ 3.859E-02 $3.301 \mathrm{E}-02$ $2.883 \mathrm{E}-02$ $3.631 \mathrm{E}-02$ $4.579 E-02$ $2.709 E-01$ $2.262 \mathrm{E}-01$ $1.330 \mathrm{E}$ OI $5.659 \mathrm{E} \mathrm{OI}$ $1.635 E$ O $4.326 \mathrm{E} O 2$ $1.148 E$ O

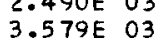

ANGLE

0.0

0.0

0.0

0.0

0.0

0.0
$7.417 E-C 4$

$1.719 \mathrm{E}-02$

$2.691 \mathrm{E} \rightarrow 02$

$2.451 \mathrm{~F}-02$
$2.098 \mathrm{E}-02$

$2.789 \mathrm{E}-02$

4. $011 \mathrm{E}-02$

$2.494 E-01$

$2.121 \mathrm{E}-01$

(.257E

$1.557 \mathrm{E} \mathrm{O2}$

$4.134 E \quad 02$

$1.099 \mathrm{E} 03$

$\begin{array}{lll}2.390 E & 03 \\ 3.449 E & 03\end{array}$

ANGLE 11
$M U=0.2816$

0.0

0.0

0.0

0.0

0.0

0.0

4.144F-0.3

$2.393 E-02$

3.533E-02

$4.516 \mathrm{E}-02$

$3.576 \mathrm{E}-02$

$4.171 \mathrm{E}-02$ $2.787 \mathrm{E}-01$ $2.289 E-01$ $1.344 E$ O1 $5.711 E$ OI $1.649 E$ O2 $4.361 \mathrm{E} \mathrm{C2}$ $1.150 \mathrm{E}$ O $3.603 \mathrm{E} \mathrm{O3}$

0.0

0.0

0.0

0.0

0.0

0.0

0.0

0.0

0.0

0.0

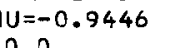

$8.457 E-04$

1. 702 E-02

$2.641 \mathrm{E}-02$

$2.447 E-02$

2.814E-02

$4.036 E-02$

2.126E-01

$1.260 E$ O1

$5.389 E \quad 01$

$4.141 E \quad 02$

$1.101 \mathrm{E} 03$

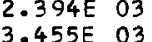

ANGLE 4

$M U=-0.8$
0.0
0.0

0.0

0.0

0.0

0.0

0.0
$1.022 E-03$

1. $670 \mathrm{E}-02$

$2.560 \mathrm{E}-02$

$2.451 E-02$

$2.150 \mathrm{E}-02$

$4.083 E-02$

$2.518 E-01$

$2.136 \mathrm{E}-01$

$1.265 \mathrm{E}$ O1

$1.566 \mathrm{E} 02$

$4.154 \mathrm{E} \quad 02$

$1.104 \mathrm{E} \quad 03$

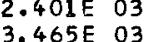

ANGLE 12

ANGLE 13

$7.182 E-03$

$2.773 E-02$

$5.787 E-02$

4.804E-02

$3.723 \mathrm{E}-02$
$4.306 \mathrm{E}-02$

5.040E-02

2.814E-01

$2.316 \mathrm{E}-01$

$1.357 \mathrm{E}$ OI

$5.762 \mathrm{E} 01$

$1.663 E$ O2

$4.395 \mathrm{E} 02$

$\begin{array}{lll}1.165 E & 03 \\ 2.525 E & 03\end{array}$

$3.626=03$

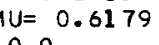

0.0

0.0

0.0

0.0

.0

$1.133 E-02$

4.160E-02

$6.962 \mathrm{E}-02$

$6.461 \mathrm{E}-02$

4. $887 \mathrm{E}-02$

$5.513 \mathrm{E}-02$

$2.874 \mathrm{E}-01$

$2.341 \mathrm{E}-01$

1.369E OI

$1.676 \mathrm{E} \mathrm{O2}$

4.427E 02

$1.173 E$ E3

$3.647 E \quad 03$

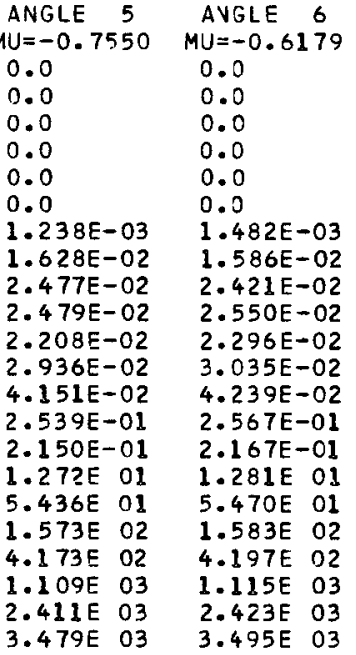

ANGLE 7 $M U=-0.4580$ ANGLE 8 ANGLE 9 $0.0 \quad 0.0$

$0.0 \quad 0.0$

0.0

0.0

0.0

2.107E-03

1.570E-02

$2.534 \mathrm{E}-02$

$2.892 \mathrm{E}-02$

$2.585 \mathrm{E}-02$

$3.325 \mathrm{E}-02$

$4.479 \mathrm{E}-02$

$2.210 E-01$

$1.303 E$ O1

$5.557 E$ OI

$1.607 E$ O2

$4.256 E 02$

$1.130 E \quad 03$

$2.454 \mathrm{E} 03$

$3.534 \mathrm{E} 03$

ANGLE 14 ANGLE 15

0.0

0.0

0.0

0.0

0.0

2.162E-02

$6.318 E-02$

$1.108 E-01$

7.820E-02

$5.202 E-02$

$2.906 \mathrm{E}-01$

2. $363 \mathrm{E}-01$

1.380 01

1.851E O1

$4.454 \mathrm{E} 02$

$1.180 \mathrm{E} 03$

$\begin{array}{ll}2.556 E & 03 \\ 3.665 E & 03\end{array}$

ANGLE 17

0.0

0.0

0.0

0.0

4.243E-02

$1.052 \mathrm{E}-01$

$1.682 \mathrm{E}-01$

$1.111 E-01$

$6.138 \mathrm{E}-02$

$5.867 \mathrm{E}-02$

$2.947 \mathrm{E}-01$

$2.382 \mathrm{E}-01$

$1.389 \mathrm{E} 01$

$5.884 E$ a

4.477E O2

$1.185 \mathrm{E} 03$

$2.568 E$ O3
$3.579 E$ O3

$J=0.9446$

0.0

0.0

$1.158 E-01$

2.271E-01

$3.422 E-01$ 


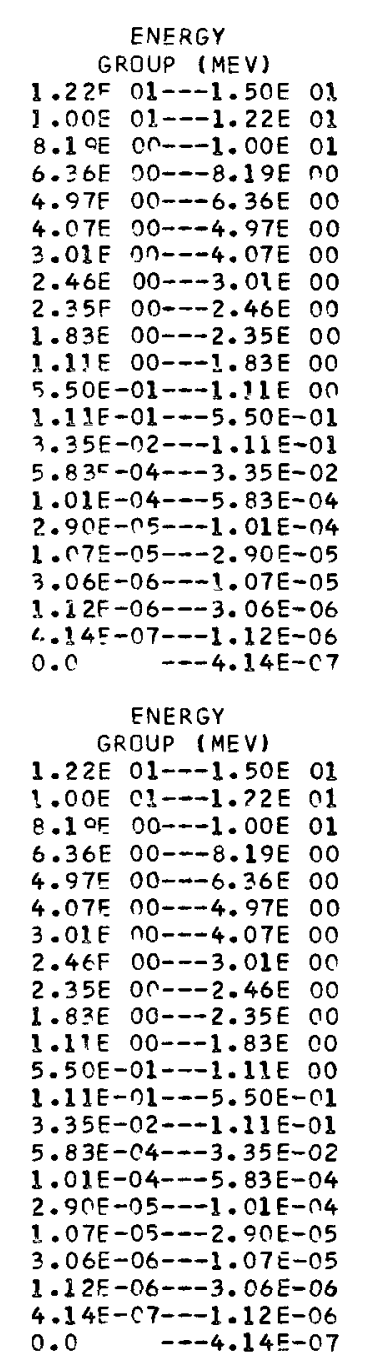
$J=-1.0000$ 0.0 0.0 0.0 0.0 $5.318 \mathrm{E}-04$ $1.152 \mathrm{E}-02$ $1.835 \mathrm{E}-02$ $2.043 E-02$ $2.225 \mathrm{E}-02$ $3.558 \mathrm{E}-02$ $6.012 F-C 2$ $4.132 \mathrm{E}-01$ $3.626 \mathrm{E}-02$ $2.195 E$ O1 $9.510 \mathrm{E}$ O1 $2.779 E \quad 02$ $7.448 E$ O2 $1.995 E$ O3 $4.364 E$ E $.340 \mathrm{E} \mathrm{O}$ ANGLE 10 $U=0.0950$ 0.0

0.0

0.0
0.0

0.0

0.0

$2.304 \mathrm{E}-03$

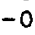
$2.535 \mathrm{E}-02$ $3.458 \mathrm{E}-02$ $5.088 \mathrm{E}-\mathrm{C2}$ $7.545 \mathrm{E}-02$ $4.643 E-01$ $3.946 \mathrm{E}-01$ $2.3 \in 7 E$ OI $1.019 E 02$ $2.971 E$ O2 $7.935 E 02$ 2.1198 O3 $4.624 \mathrm{E} 03$ $6.681 \mathrm{E} 03$
ANGLE? $M U=-C .5904$ C. 0 0.0 0.0 0.0 $5.407 E-04$ $1.140 \mathrm{~F}-0$ $1.828 \mathrm{E}-02$ $2.044 E-02$ $2.230 \mathrm{E}-02$ $3.567 F-02$ $6.023 \mathrm{~F}-02$ $4.12 E E-O$ $3.629 E-01$ $9.516 \mathrm{~F} \mathrm{Cl}$ $2.780 \mathrm{E} \mathrm{C2}$ $7.452 E$ N2 1. $6.366 E$ D

ANGLE 11 $\begin{array}{ll}A N G L F & 11 \\ M U=0.2816\end{array}$ $0 . n$ 0.0 0.0 0.0 0.0 $3.488 \mathrm{E}-03$ $1.632 E-02$ $3.792 \mathrm{E}-02$ $3.776 \mathrm{E}-02$ $3.776 E-02$
$5.472 E-C ?$ $7.757 E-02$ $4.731 E-\cap 1$ $4.000 \mathrm{E}-01$ $2.400 E$ CI $1.022 \mathrm{~F} 02$ $3.007 E$ C2 $8.026 \mathrm{E} \mathrm{O2}$ $2.142 F \quad n 3$ $4.672 \mathrm{E} \quad 03$ $6.744 \mathrm{E} 03$
(NEUTRJNS/MEV/STERADIAN/SOURCE NEUTRON)

$\begin{array}{cc}A N G L E & 3\end{array} \quad$ ANGLE 4
0.0 0.0 0.0 $6.192 \mathrm{E}-04$ $1.138 \mathrm{E}-02$ $1.804 \mathrm{E}-02$ $2.053 \mathrm{E}-02$ $2.251 \mathrm{E}-02$ $3.604 E-C 2$
$6.068 E-02$ $4.154 \mathrm{E}-\mathrm{O}$ $3.64 J F-0$ $2.203 \mathrm{E}$ o1 $9.542 E$ OI $2.788 \mathrm{E}$ O? $7.470 E$ O2 $2.000 E$ O3 $4.376 E$ E 0.0 0.0 0.0 0.0 0.0 $7.373 E-0.4$ $1.117 \mathrm{E}-02$ 1. $770 \mathrm{E}-02$ $2.059 \mathrm{E}-02$ $3.677 \mathrm{E}-02$ $3.677 E-02$ $4.186 \mathrm{E}-01$ $3.602 \mathrm{E}-01$ $2.214 \mathrm{E} 01$ 9.588 E 01 $2.801 \mathrm{E} 02$ $7.504 \mathrm{E} 02$ 2.009E 03 $4.394 \mathrm{E} 03$ $6.384 \mathrm{E}$ $A N G L E \quad 5$
$H=-0.7550$ 0.0 0.0 0.0 0.0 0.0 $8.827 E-04$ $1.091 \mathrm{E}-02$. 1. 744E-0? $2.112 E-02$ $3.785 \mathrm{E}-02$ $6.274 E-02$ $4.232 E-01$
$3.692 E-01$ $2.231 \mathrm{E} \mathrm{Ol}$ $9.653 \mathrm{E}$ 01 $2.819 E \quad 02$ 7.550 O2 2.021E 03 $4.419 E \quad 03$ ANGLE 13 $M U=0.4580$

ANGLE 14 $M U=0.6179 \quad M U=0.7550 \quad M U=0.8656$ 0.0 0.0 0.0 0.0 0.0 $5.163 \mathrm{E}-03$ 0.0 0.0 0.0 $8.949 E-03$ 3.2?3E-02 $6.543 \mathrm{E}-02$ $4.084 \mathrm{E}-02$ $6.663 \mathrm{E}-02$ $8.543 \mathrm{E}-02$ $4.937 E-01$ $4.129 \mathrm{~F}-01$ $2.463 \mathrm{E} \mathrm{O1}$ $1.057 \mathrm{E} \mathrm{O2}$ $3.075 \mathrm{E} \mathrm{O2}$ $8.197 E 02$ $2.185 E 03$ 4.763E 03 0.0 0.0 0.0 0.0 0.0 1.612E-02 5. $072 E-02$ $9.527 E-02$ $7.555 E-02$ 5.892E-02 $8.440 E-02$ $5.034 E-01$ $4.181 E-01$ $2.490 \mathrm{E} 01$ 1.067E 02 3.104E 02 $8.269 E \quad 02$ $4.801 E 03$ $6.913 E 03$ ANILE 6
$M U=-0.6179$ 0.0 0.0 0.0 0.0 0.0 1. $052 E-03$ 1.067E-02 $2.195 \mathrm{E}-02$ $2.474 \mathrm{E}-02$ $3.932 E-02$ $4.291 \mathrm{E}-01$ $3.730 E-01$ $2.251 E$ OI $2.842 E 02$ $2.842 E$
$7.609 E$
02 $2.036 E \quad 03$ $4.453 \mathrm{E} \mathrm{O3}$

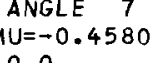

0.0 0.0
0.0
0.0 0.0 0.0 0.0 1.252E-03 $1.057 E-02$ $1.808 \mathrm{E}-02$ $2.622 E-02$ $4.125 E-02$ $6.634 E-02$ 4. $362 \mathrm{E}-01$ 3.776F-01 $2.276 E$ OI
$9.834 \mathrm{E} \mathrm{OI}$ $2.870 E$ O2 $7.679 E \quad 02$ $2.054 \mathrm{E} 03$ $\begin{array}{ll}4.488 \mathrm{E} & 03 \\ 6.508 \mathrm{E} & 03\end{array}$

ANGLE 16 0.0 0.0 0.0

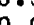
0.0 $3.139 E-02$ $0.139 \mathrm{E}-02$
$8.558 \mathrm{E}-02$ $1.543 \mathrm{E}-01$ 1. CI EE-01 $6.947 E-02$ $9.313 \mathrm{E}-\mathrm{C2}$ $5.108 \mathrm{E}-01$ 4.?24E-01 $2.512 \mathrm{E} 01$ $3.128 \mathrm{E} 02$ $8.329 E 02$ 2. $219 E 03$ $4.832 E 03$ $6.551 E 03$

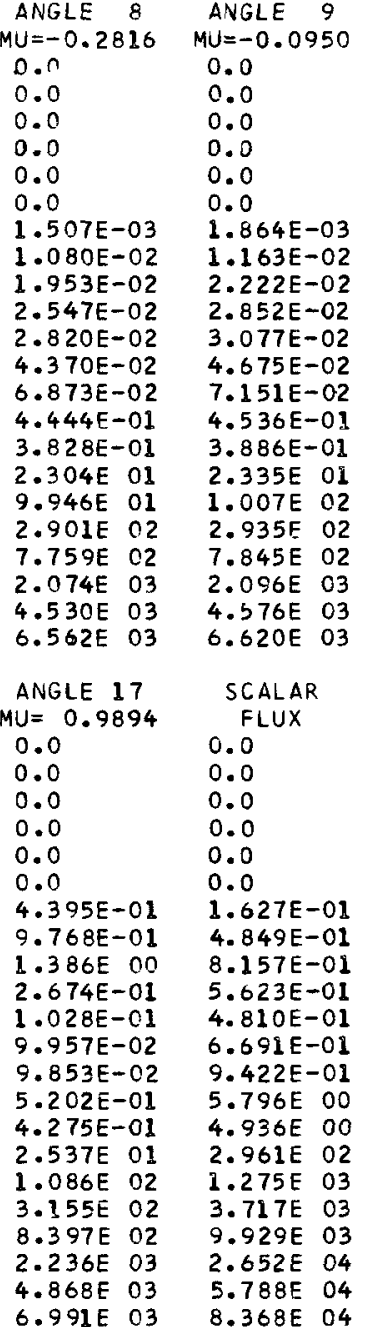


(NEUTRJNS/MEV/STERADIAN/SOURCE NEUTRON)

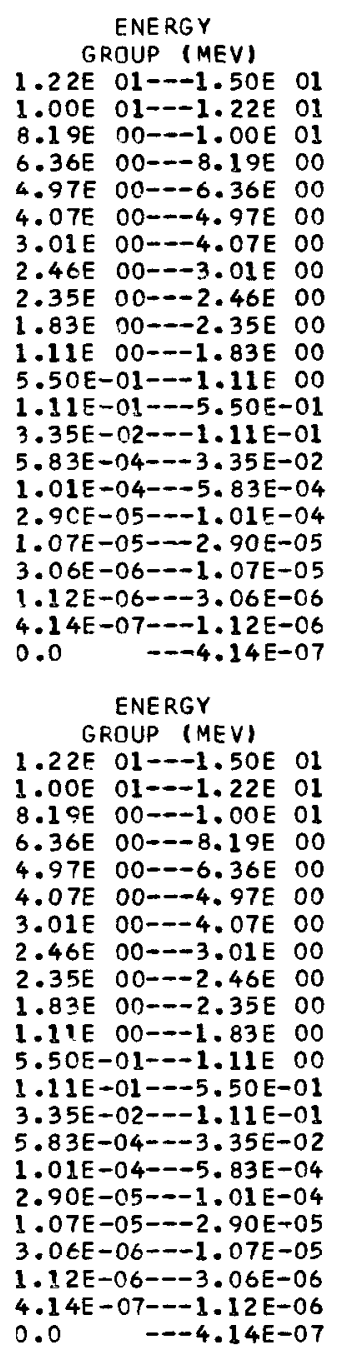

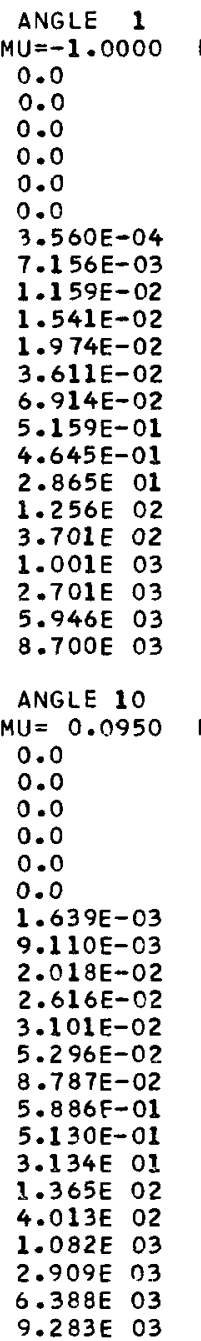

ANGLE
$M U=-0.9894$ 0.0 0.0 0.0 0.0 0.0 $3.669 E-04$ $3 . \in 69 E-04$ $1.140 \mathrm{E}-03$ $1.156 \mathrm{E}-02$ $1.543 E-02$ $3.975-02$ $3.621 E-02$ $5.165 E-C 1$ $4.649 \mathrm{E}-01$

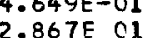
$2.867 E$
$1.257 E$ $3.703 E 02$ $1.002 E 03$ $1.002 \mathrm{E} 03$

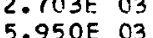
$8.706 E 03$

ANGLE 11 0.0 0.0
0.0
0.0 0.0
0.0
0.0 0.0 0.0 $2.592 \mathrm{E}-02$ $3.130 E-02$ $3.511 \mathrm{E}-02$ $5.828 E-02$ $9.270 E-02$ $6.042 \mathrm{E}-01$ $5.226 E-01$ $3.187 E$ O1 $1.387 \mathrm{E}$ ? $4.073 E \quad 02$ $1.097 \mathrm{E} 03$ $2.949 \mathrm{E} 03$ $6.471 E 03$ $9.393 F \quad 03$
ANGLE 3 $\begin{aligned} M U & =-0.9446 \\ 0.0 & \end{aligned}$ 0.0
0.0
0.0 0.0 0.0 $4.095 E-04$ $4.095 \mathrm{E}-04$ 1.075E-03 $1.149 E-02$ $1.553 \mathrm{E}-02$
$2.301 \mathrm{E}-02$ 2.663E-02 $3.063 \mathrm{E}-02$ 5. $4.666 \mathrm{E}-01$ 4.666E- 21 1.261E 02 $3.715 E \quad 02$ $1.005 \mathrm{E} 03$ $2.711 E 03$ 2.711 E 03
$5.967 E_{03}$ $8.730 E$ O3

ANGLE 12 0.0 0.0 0.0 0.0 0.0 0.0 $3.614 E-03$ $1.620 \mathrm{E}-02$ $3.559 \mathrm{E}-02$ 3.807E-02 4. $008 \mathrm{E}-02$ $6.453 E-02$ $9.752 \mathrm{E}-02$ $5.321 \mathrm{E}-01$ $3.238 E$ O1 $1.407 \mathrm{E} 02$ $4.131 \mathrm{E} 02$ $1.112 \mathrm{E} 03$ $2.987 E \quad 03$

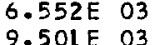
ANGLE 4
$M U=-0.8556$
0.0 0.0 0.0 0.0 4.821E- 04 $1.142 \mathrm{E}-02$ $1.576 \mathrm{E}-02$ $3.744 E-02$ $3.744 E-02$ $5.236 E-01$ $5.236 \mathrm{E}-01$ $4.698 E-01$ 2.895801
$1.268 E 02$ $3.736 E 02$ 1.011 E 03 2.725 E 03 $5.997 E 03$ 8.773 E 03

ANGLE 13 $A U=0.6179$ 0.0 0.0 0.0 0.0 0.0 6.10JE-03 $2.397 \mathrm{E}-02$ $5.085 E-02$ 4. $790 E-02$ 4. $557 \mathrm{E}-02$ $7.204 E-02$ 1. $029 \mathrm{E}-01$ $5.412 E-01$ $3.287 E$ OI $1.427 E 02$ $4.187 E 02$ $1.126 E 03$ $3.023 \mathrm{E} 03$ $6.628 \mathrm{E} 03$ $9.601 E$ O3

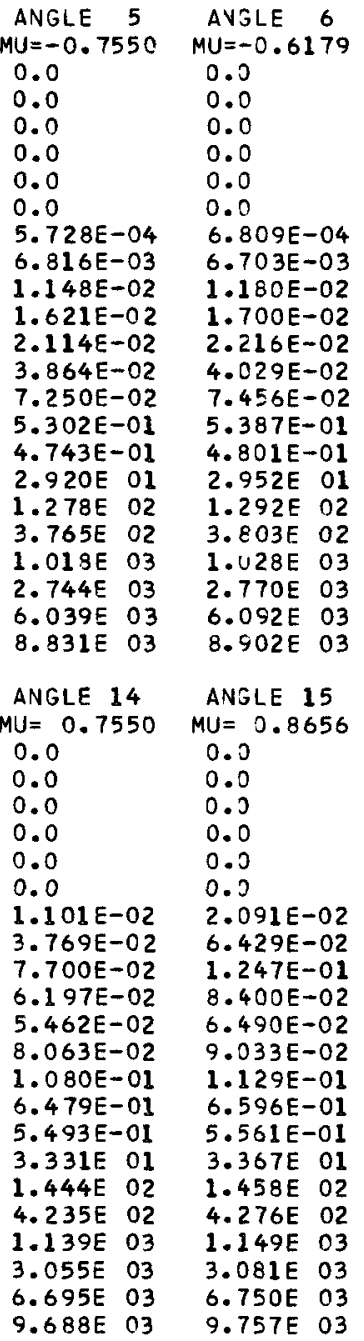
$M U=-0.4580$ 0.0 0.0
0.0
0.0
0.0 0.0 0.0 8.04 $6.713 E-03$ $6.713 E-03$ $1.256 E-02$ $1.823 E-02$
$2.359 E-02$ $2.35 \mathrm{E}-02$ 4. $714 \mathrm{E}-02$ $5.490 E-01$ 5.470E-01 $4.870 E-01$ $2.991 E$ OI $1.307 E 02$ $1.039 \mathrm{E} \quad 03$ 2.800 E 03 2.800E 03 $8.984 \mathrm{E} \mathrm{O3}$ ANGLE 16 $M U=0.9446$ 0.0 0.0 0.0 0.0 0.0 4. $448 E-02$ $1.280 E-01$ 2.337E-01 $1.231 \mathrm{E}-01$ $7.780 E-02$ $1.169 E-01$ $6.683 E-01$ $5.611 E-01$ $3.393 \mathrm{E} 01$ $1.469 \mathrm{E} 02$ 4.305E 02 $1.157 E 03$ $3.101 E 03$ $6.790 E \quad 03$ $9.804 E 03$

\begin{tabular}{|c|c|}
\hline $\begin{array}{l}\text { ANGLE } 8 \\
M U=-0.2816 \\
0.0 \\
0.0 \\
0.0 \\
0.0 \\
0.0 \\
0.0 \\
9.849 E-04 \\
6.984 E-03 \\
1.395 E-02 \\
2.005 E-02 \\
2.548 E-02 \\
4.524 E-02 \\
8.024 E-02 \\
5.610 E-01 \\
4.949 E-01 \\
3.035 E \quad 01 \\
1.325 E \quad 02 \\
3.899 E \quad 02 \\
1.053 E \quad 03 \\
2.834 E \quad 03 \\
6.228 E \quad 03 \\
9.076 E \quad 03\end{array}$ & $\begin{array}{l}\text { ANGLE } 9 \\
\text { MU=-0.0950 } \\
0.0 \\
0.0 \\
0.0 \\
0.0 \\
0.0 \\
0.0 \\
1.233 \mathrm{E}-03 \\
7.708 \mathrm{E}-03 \\
1.629 \mathrm{E}-02 \\
2.264 \mathrm{E}-02 \\
2.793 \mathrm{E}-02 \\
4.871 \mathrm{E}-02 \\
8.387 \mathrm{E}-02 \\
5.744 \mathrm{E}-01 \\
5.037 \mathrm{E}-01 \\
3.083 \mathrm{E} \quad 01 \\
1.345 \mathrm{E} \quad 02 \\
3.954 \mathrm{E} \quad 02 \\
1.067 \mathrm{E} \quad 03 \\
2.871 \mathrm{E} \quad 03 \\
6.306 \mathrm{E} \quad 03 \\
9.176 \mathrm{E} \quad 03\end{array}$ \\
\hline $\begin{array}{l}\text { ANGLE } 17 \\
M U=0.9894 \\
0.0 \\
0.0 \\
0.0 \\
0.0 \\
0.0 \\
0.0 \\
2.236 E-01 \\
6.247 \mathrm{E}-01 \\
9.855 \mathrm{E}-01 \\
2.137 \mathrm{E}-01 \\
9.396 \mathrm{E}-02 \\
1.098 \mathrm{E}-01 \\
1.197 \mathrm{E}-01 \\
6.735 \mathrm{E}-01 \\
5.640 \mathrm{E}-01 \\
3.409 \mathrm{E} \quad 01 \\
1.475 \mathrm{E} \quad 02 \\
4.322 \mathrm{E} \quad 02 \\
1.161 \mathrm{E} \quad 03 \\
3.112 \mathrm{E} \quad 03 \\
6.813 \mathrm{E} \quad 03 \\
9.831 \mathrm{E} \quad 03\end{array}$ & 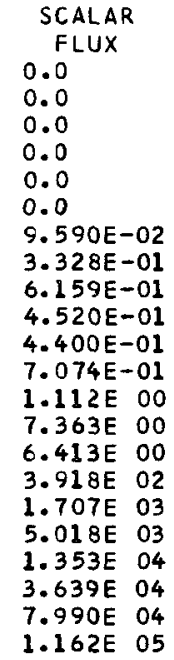 \\
\hline
\end{tabular}


(NEUTRJNS/MEV/STERADIAN/SOURCE NEUTRON)

ENERGY GROUP (MEV) 1.2 ?F $01 \rightarrow-1.50 E$ OI $1.00 E$ Ol---1.22E 0 $8.19 \mathrm{E}$ 00---1.00E 01 6.36 E $00---8.19 E$ EO $4.97 E$ OD- - -6.36E 00 4.07E $00 \cdots 4.97 E \quad 00$ $3.01 \mathrm{E} 00---4.07 \mathrm{E} 00$ $2.46 \mathrm{E} 00--3.01 \mathrm{E}$ CO $2.35 \mathrm{E} \quad 00---2.46 \mathrm{E} \mathrm{OO}$ $1.83 E \quad 00-\cdots 2.35 E$ OO $1.11 \mathrm{E} \quad 00--1.83 \mathrm{E} \quad 00$ 5.5OE-01-T-1.11E OO $1.11 E-01--5.50 E-01$ $3.35 E-02---1.11 E-01$ $5.83 E-04---3.35 E-02$ $1.01 \mathrm{E}-04--5.83 \mathrm{E}-04$ $2.90 \mathrm{E}-05--1.01 \mathrm{E}-04$ 1.07E-05--2. $90 \mathrm{E}-05$ $3.06 \mathrm{E}-06-1.07 \mathrm{E}-0$ $1.12 \mathrm{E}-05--3.06 \mathrm{E}-06$ $4.14 \mathrm{E}-07---1.12 \mathrm{E}-06$
$0.0 \quad---4.14 \mathrm{E}-07$

\section{ENERGY}

$$
\text { GROUP (MEV) }
$$

$1.22 E$ O1---1.50E 01 $1.00 E^{2}$ 01--1.22E 01 $8.19 E$ OO-C-1.00E O1 6.36E 0O--8.19E OO 4.97 E $00--6.36$ E 00 4.07E 00--4. $.97 E$ OO 3.01 E OO-O-4.07E 00 2.46E OO-C. 01 E 2.35E OD---2.46E DO $1.83 E$ O0---2.35E DO 1.21 E $00-1.83 E$ OO $1.11 E-01-0.50$. $1.11 \mathrm{E}-01 \mathrm{C}-5.5 \mathrm{E}-0$ $5.3 E-12-211$ E-OL $183 \mathrm{E}-04-3.35$ $2.90 \mathrm{E}-05--1.01 \mathrm{E}-04$ $1.07 E-05-2.2 .00 E-05$ $3.06 E-06-1.07 E-05$ $1.12 E-06=3.06 E-06$ $4.14 E-07--1.12 E-06$ $4.14 E-07--1.12 E-06$

ANGLE 1 $M U=-1.0000$ 0.0 0.0 0.0 0.0 $2.243 E-04$ $4.269 E-03$ $7.047 \mathrm{E}-03$ $1.101 \mathrm{E}-02$ $1.588 \mathrm{E}-02$ $3.224 \mathrm{E}-02$ $6.831 \mathrm{E}-02$ $5.459 \mathrm{E}-01$ $5.021 \mathrm{E}-01$ $3.146 \mathrm{E}$ O1 $1.394 \mathrm{E} \mathrm{O2}$ $4.139 E \quad 02$ $1.130 \mathrm{E} 03$ $3.067 E 03$ $6.794 \mathrm{E} 03$ $1.001 \mathrm{E} 04$ ANGLE 10 $M U=0.0050$ 0.0 0.0
0.0
0.0 0.0 0.0 $1.031 E-03$ $6.032 E-03$ $1.424 \mathrm{E}-02$ $1.968 \mathrm{E}-02$ 2.552E-02 $8.860 E-02$ $6.316 \mathrm{E}-01$ $5.608 \mathrm{E}-\mathrm{Cl}$ 1.482E 01 $1.532 E 02$ 1.53 $3.342 \mathrm{E} 03$

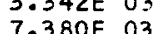
1.079E 04 $\triangle N G L E \stackrel{2}{M U=-0.9894}$
MU 0.0 0.0 0.0 0.0 0.0 0.0 $2.306 E-04$ 4. $261 E-03$ $7.046 E-C 3$ $1.103 F-02$ 1.593E-02 $3.234 E-02$ $6.846 E-02$ $5.466 \mathrm{E}-01$ $5.026 E-01$ $3.149 \mathrm{E}$ 01 $1.395 \mathrm{E} \quad 02$ 4.142 E ? $1.131 \mathrm{~F} 03$ 3.070E O3 1.001 E 04 ANGLE 13 $M U=0.2816$ 0.0
0.0
0.0
$n .0$ 0.0 n.c 0.0 0.0
$1.486 E-03$ $1.486 E-03$
$7.940 E-0^{2}$ 1. $875 E-02$ 2. $357 \mathrm{E}-02$ $2.890 \mathrm{E}-\mathrm{C2}$ $0.35 \mathrm{E}-02$ $6.406 \mathrm{E}-\mathrm{O}$ $6.43 \mathrm{E}-\mathrm{Cl}$ 3.726E 0 I . $4.617 E 02$ $4.617 E$ त2 $3.394 \mathrm{E} 03$ $3.492 \mathrm{E} O 3$ $1.093 E$ O4
$\begin{array}{lcc}A N G L E & 3 & \text { ANGLE } \quad 4 \\ M U=-0.9446 & M U=-0.8656\end{array}$ 0.0 0.0 0.0 0.0 0.0 $2.555 E-04$ 4. $226 E-03$ 7. $\cap 52 E-03$ $1.113 \mathrm{E}-02$ $1.611 \mathrm{E}-02$ $3.274 E-02$ 6.907E-02 $5.495 \mathrm{E}-01$ $5.047 E-01$ $3.161 \mathrm{E}$ ol $1.400 E 02$ $4.157 \mathrm{E}$ O2 $1.135 \mathrm{E} 03$ $3.080 E$ O3 $6.820 \mathrm{E} \mathrm{O3}^{0}$ $1.004 \mathrm{E} 04$

ANGLE 12 $M U=0.4580$ 0.0 0.0
0.0 0.0 0.0 0.0 $1.121 \mathrm{E}-02$ $2.594 \mathrm{E}-02$ $2.898 \mathrm{E}-02$ $3.321 \mathrm{E}-02$ $5.991 \mathrm{E}-02$ $9.903 E-02$ $6.679 E-01$ . . $4.693 \mathrm{E} 02$ 1. 275 E 03 $3.446 \mathrm{E} 03$ $3.602 \mathrm{E} 03$ $1.108 \mathrm{E} 04$
$M U=-0.0$
0.0
0.0

0.0

0.0

0.0

0.0

$2.984 \mathrm{E}-04$ 4.166E-03 $7.110 \mathrm{OE}-03$ 1.135 E- 02 $1.648 \mathrm{E}-02$ $3.351 \mathrm{E}-02$ $7.023 \mathrm{E}-02$ $5.549 E-01$ 5.085E-01 $3.184 \mathrm{E} \mathrm{O1}$ $1.409 \mathrm{E} \mathrm{O?}$ $4.183 E \quad 02$ $1.142 \mathrm{E} \quad 03$ $3.098 \mathrm{E} 03$ $6.860 E 03$ $1.010 E 04$

ANGLE 13 ANGLE $13 \quad$ ANGLE 14
$M U=0.4179 \quad M U=0.7550$ 0.0 0.0 0.0 0.0 0.0 $3.937 E-03$ $1.682 \mathrm{E}-02$ $3.774 E-02$ $3.657 \mathrm{E}-02$ $3.864 \mathrm{E}-02$ $1.047 \mathrm{E}-01$ $6.857 \mathrm{E}-01$ $3.675 \mathrm{E}$ O $3.671 \mathrm{E} 02$ $1.611 \mathrm{E}$ O I. $293 \mathrm{E} 03$ $3.494 \mathrm{E} 03$ $3.495 \mathrm{E}$ O3 1.7 . 04

0.0

0.0

0.0

0.0

$3.526 E-04$ 4.098E-03 $7.290 E-03$ $1.175 \mathrm{E}-02$ $1.708 \mathrm{E}-02$ $3.466 \mathrm{E}-02$ $7.189 \mathrm{E}-02$ $5.626 \mathrm{E}-01$ $5.139 \mathrm{E}-01$ $3.215 E$ OI 4.221E 02 $\begin{array}{ll}4.2151 \mathrm{E} & 02 \\ 1.151 & 03\end{array}$ $3.124 E \quad 03$ .915E 03 $1.018 E \quad 04$ $M U=$
0.0
0.0 0.0 0.0 0.0 2.681E-02 $4.546 E-02$ 1.580E-02 1.104E-OL 7.02OE-OI $6.054 E-01$ I.634E 02 $1.634 E$ OR 1. $310 \mathrm{E} \mathrm{O3}$ $3.537 E$ O3 $7.796 \mathrm{E} \mathrm{O3}$
$M U=-0.7550 \quad M U=-0.6179$ 0.0 $1.134 \mathrm{E} 04$ 0.0 0.0

0.0

0.0

0.0

4.186E-04 4.059E-03 $7.677 \mathrm{E}-03$ $1.239 E-02$ $1.795 E-02$ $3.625 \mathrm{E}-02$ $7.409 \mathrm{E}-02$ $5.726 \mathrm{E}-01$ $5.209 \mathrm{E}-01$ $3.255 \mathrm{E} \mathrm{O1}$ $1.439 \mathrm{E} \mathrm{O2}$ $4.259 \mathrm{E} \mathrm{O2}$ $1.164 \mathrm{E} 03$

$M U=-0.6179 \quad$ ANGLE ${ }^{7}$ 
(NEUTRJNS/MEV/STERADIAN/SOURCE NEUTRONI

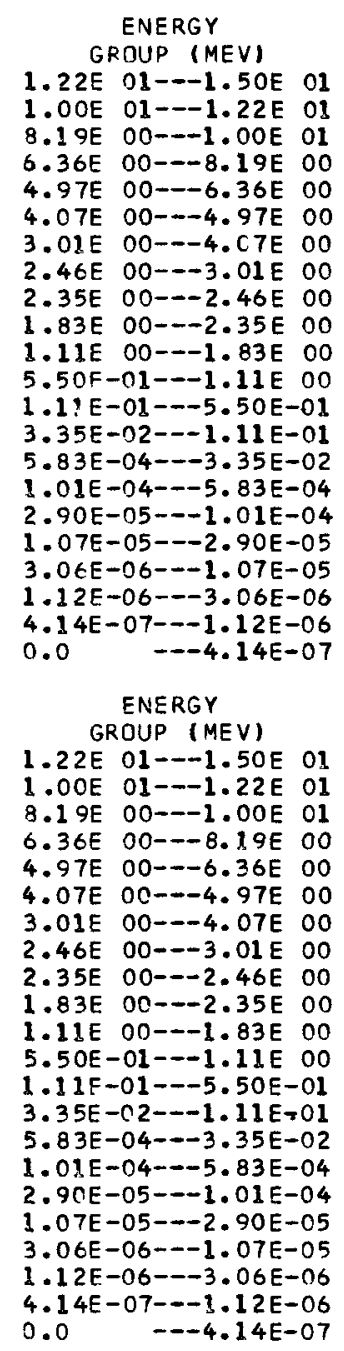

ANGLE 1 $M U=-1.0000$ 0.0 0.0 0.0 0.0 $1.358 E-04$ $1.358 E-04$
$2.481 E-03$ $2.481 E-03$
$4.189 E-03$ $7.594 \mathrm{E}-03$ $1.201 \mathrm{E}-02$ $2.653 E-02$ 6.113E-02 $5.177 \mathrm{E}-01$ 4.846E-01 3.079E OI $3.079 E$ OI 4.113E O2 I.132E O3 $3.091 \mathrm{E} 03$ $6.882 \mathrm{E} \quad 03$ $1.020 E 04$

ANGLE 10 $M U=0.0950$ 0.0

0.0

0.0

0.0

$6.298 \mathrm{E}-04$ $3.879 E-03$ $9.769 \mathrm{E}-03$ $1.412 \mathrm{E}-02$ $1.958 \mathrm{E}-02$ $4.079 E-02$ $8.041 E-02$ $6.053 \mathrm{E}-01$ $5.462 \mathrm{E}-01$ $3.438 \mathrm{E} 01$ $1.526 E 02$ $4.552 \mathrm{E} \mathrm{O2}$ $1.248 E 03$ $3.398 E 03$ $7.545 \mathrm{E} 03$
ANGLE 2 $M U=-0.9894 \quad M U=-0.9446$ 0.0 0.0 0.0 0.0

0.0 $1.394 \mathrm{E}-04$
$2.477 \mathrm{E}-03$ $4.157 \mathrm{E}-03$ $7.613 E-03$ $1.204 \mathrm{E}-02$ $2.661 E-02$ $.127 \mathrm{~F}-02$ $5.184 E-01$ $4.851 \mathrm{E}-\mathrm{Cl}$ .082E O1 $4.117 \mathrm{E} 02$ $1.133 E \quad 03$ $3.093 E$ OT $6.888 E \quad 03$ $1.021 \mathrm{E} 04$

ANGLE 11 0.0 0.0 0.0

0.0

0.0

$9.152 E-04$ $5.228 \mathrm{E}-03$

$1.7015-02$

$2.225 \mathrm{~F}-02$

4.531E-02 $8.522 \mathrm{E}-02$ $6.239 E-C 1$ $5.586 E-01$ $3.509 \mathrm{E} \mathrm{Cl}$ $1.556 \mathrm{E} \mathrm{O2}$ $4.638 \mathrm{E}$ O2 $1.271 \mathrm{E} 03$ $3.457 E$ O3 $7.672 E$ O3
0.0
0.0
0.0
0.0

0.0

0.0

$1.536 \mathrm{E}-04$ $2.461 \mathrm{E}-03$

$7.700 \mathrm{E}-03$

$1.70 \mathrm{E}-02$

$2.696 \mathrm{E}-02$

$6.185 \mathrm{E}-02$

$5.214 \mathrm{E}-01$

$3.095 \mathrm{E} 01$

$1.383 E$ O2

$4.133 E$ O2

$1.137 \mathrm{E} 03$

$3.105 E \quad 03$

6.91 ? E 03

$1.024 \mathrm{E} 04$

ANGLF 12 $J=0.4580$ 0.0

0.0

0.5

0.0

$1.441 E-03$

$.518 E-03$

$1.822 \mathrm{E}-02$

$2.103 E-02$

$2.563 \mathrm{E}-02$

$5.080 \mathrm{E}-02$

$9.045 \mathrm{E}-02$

$6.428 \mathrm{E}-01$

$5.709 \mathrm{E}-01$

$3.579 \mathrm{E} 01$

$1.585 \mathrm{E} 02$

$4.723 E$ O2

$1.293 E 03$

$3.516 E \quad 03$

$7.798 E$
$1.143 E$
ANGLE $\quad 4$
$M U=-0.8656$ $M U=-0.8$
0.0

0.0

0.0

0.0

0.0

$1.782 E-04$

$33 E-03$

.334E-03

$7.886 \mathrm{~F}-03$

$1.248 \mathrm{E}-02$

$2.763 E-02$

.269F-O1

4. $913 \mathrm{E}-01$

4.913E-01

$1.393 E \quad 02$

$4.162 \mathrm{E} 02$

$1.145 \mathrm{E} 03$

$3.125 \mathrm{E} 03$ $6.956 \mathrm{E} 03$

1.030 E 04

ANGLE 13 $M=0.6179$

0.0

0.0

.0

0.0

$2.430 \mathrm{E}-03$

$.145 \mathrm{E}-02$

$2.685 \mathrm{E}-02$

$2.665 \mathrm{E}-02$

$2.990 \mathrm{E}-02$

5.733 E-02

$9.595 \mathrm{E}-02$

$6.612 \mathrm{E}-01$

$3.646 \mathrm{E} 01$

$1.613 \mathrm{E} 02$

$4.803 \mathrm{E} 02$

$1.314 \mathrm{E} 03$

3.571E 03

1.158E 04
ANGLE 5 $=-0.7550 \quad$ MU $=-0.617$ 0.0 0.0 0.0

$.007 E-04$ $2.097 E-04$
$2.405 E-03$
$4.532 E-03$ . $201 \mathrm{E}-03$ (2) . $625 \mathrm{E}-02$ $6.451 \mathrm{E}-02$ . $347 \mathrm{E}-01$ 4. $970 \mathrm{E}-01$ $3.152 E$ OI $1.407 \mathrm{E} 02$ $4.203 E 02$ $1.156 \mathrm{E} 03$ $3.154 E 03$ $7.019 E$ O3 $1.039 E \quad 04$

ANGLE 14

.0

0.0

$4.302 E-03$ $1.843 \mathrm{E}-02$ $4.174 \mathrm{E}-02$ $3.471 \mathrm{E}-02$ $3.521 E-02$ $6.489 \mathrm{E}-02$ $1.015 \mathrm{E}-01$ $6.781 \mathrm{E}-01$ $5.933 \mathrm{E}-01$ $3.706 \mathrm{E} 01$ $1.638 \mathrm{E} 02$ 4.875E 02 $1.333 \mathrm{E} 03$ 3.620 E 03 $1.172 \mathrm{E} 04$
ANGLE 6
$M U=-0.6179$ 0.0 0.0 0.0 0.0

0.0 $2.489 E-04$ $2.402 E-03$
$4.882 E-03$ 4.8B2 03 1. $364 E-02$ $3.000 \mathrm{E}-02$ $6.660 \mathrm{E}-02$ $5.448 E-01$ $5.042 E-01$ $3.194 \mathrm{E}$ OI $1.425 \mathrm{E} \mathrm{O2}$ $4.255 \mathrm{E} 02$ $1.169 E 03$ $3.191 \mathrm{E} 03$ $7.098 E$ O3 1.050 E 04

ANGLE 15 0.0

0

0.0

0.0

7. $.29 E-03$ $3.170 \mathrm{E}-02$ $6.945 E-02$ $4.678 \mathrm{E}-02$ $4.171 E-02$ $7.330 \mathrm{E}-02$ $1.066 \mathrm{E}-01$ $6.926 \mathrm{E}-01$ 6.023E-01 $3.756 \mathrm{E} 01$ $1.658 \mathrm{E} 02$ 4. $934 E 02$ $1.348 \mathrm{E} 03$ $3.560 E 03$ $8.108 \mathrm{E} \mathrm{O3}$ $U=-0.4580$ 0.0 0.0
0.0 0.0 0.0

$.984 E-04$ $2.469 \mathrm{E}-03$ $9.441 \mathrm{E}-03$ $1.458 \mathrm{E}-02$ $3.182 \mathrm{E}-02$ $5.571 \mathrm{E}-01$ . $130 \mathrm{E}-01$ $3.245 \mathrm{E}$ 01 $1.446 \mathrm{E} \mathrm{O2}$ $1.446 \mathrm{E} \mathrm{O2}$
$4.318 \mathrm{E} 02$ $1.186 \mathrm{E} 03$ $3.234 E$ O3 $7.193 \mathrm{E} 03$ $1.062 \mathrm{E} 04$

ANGLE 16 0.0 0.0 0.0 0.0 
(NEUTRONS/MEV/STERADIAN/SOURCE NEUTRON)

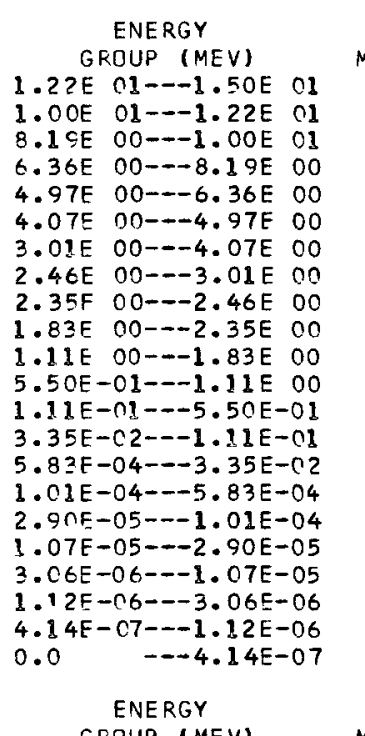

$1.22 E$ O1---1.50E 01

1.00 F $01--1.22 E$ OI

$8.19 \mathrm{E} \quad 00--1.00 \mathrm{E}$ 01

$4.97 \mathrm{~F}$ O0---6.36E 00

4. CTE $00--4.97 E 00$

$3.01 E$ E $00--4.07 E 00$

$? .46 \mathrm{E} \quad 00---3.01 \mathrm{E} \quad 00$

2.35 E $\cap 0---2.46 E 00$

$1.83 \mathrm{E} \quad 00--2.35 \mathrm{E} 00$

$1.11 \mathrm{E} \quad 00--1.83 \mathrm{E} 00$

$5.5 \mathrm{CE}-01---1.11 \mathrm{E}$ OO
$1.11 \mathrm{E}-01---5.50 \mathrm{E}-01$

$3.35 E-02---2.11 E-01$

$5.83 E-04---3.35 E-02$

$1.01 E-04---5.83 E-04$

2.90E $-05--1.01 \mathrm{E}-04$

$1.07 \mathrm{E}-05--2.90 \mathrm{E}-05$

$3.06 E-06--1.07 E-05$

1.12E-06---3.06E-06

$4.14 \mathrm{E}-07---1.12 \mathrm{E}-06$
$0.0 \quad---4.14 \mathrm{E}-07$
$A N G L E \quad 1$
$M U=-1.0000$

0.0

0.0

0.0

0.0

0.0

0.0

$2.612 \mathrm{E}-05$

$4.448 \mathrm{E}-04$

$8.207 E-04$
$2.205 E-03$

$4.180 \mathrm{E}-\mathrm{C} 3$

$1.108 E-02$

$3.072 E-02$

$2.961 E-01$
$2.879 E-01$

$1.885 \mathrm{E} \mathrm{Ol}$

$8.590 E$ OI

2.607E 02

$2.607 E$
$7.302 \mathrm{E} \mathrm{O2}$

$2.021 \mathrm{E} 03$

$6.549 \mathrm{O}^{2} 3$

ANGLE 10 $M U=0.0950$

0.0

0.0

0.0

0.0

$1.244 E-04$

$9.513 E-04$

$2.773 \mathrm{E}-03$

$4.473 E-03$

$7.010 \mathrm{E}-03$

$1.776 \mathrm{E}-02$
$4.156 \mathrm{E}-02$

$3.535 E-01$

$3.302 \mathrm{E}-01$

$2.142 \mathrm{E} 01$

$9.701 E$ OI

$2.938 E$ O2

$2.264 E \quad 03$

$5.091 \mathrm{E} 03$
$7.575 \mathrm{E}$
ANGLE ?
$M U=-C .9894$

0.0

0.0

0.0

0.0

0.0

$2.673 \mathrm{E}-05$

$4.447 E-C 4$

$8.2798-04$

$4.193 \mathrm{E}-03$

$1.112 \mathrm{E}-02$

$2.965 \mathrm{~F}-\mathrm{Cl}$

$2.882 E-01$

8.600 O 11

$8.600 E$ OI

$2.610 E$ C?

$2.024 E 03$

$2.024 E$ E
$4.563 E$

$6.856 \mathrm{E} \mathrm{O3}$

ANGLE 11.

0.0
0.0

0.0

0.0

0.0

0.5
$1.838 E-04$

$1.356 E-03$

$3.795 E-03$

$5.457 E-03$

$1.092 E-C 2$

4.4?2E-02

$3.659 \mathrm{E}-\mathrm{Cl}$

$3.388 \mathrm{E}-01$

$9.922=01$

$3.004 E$ O?

$8.381 \mathrm{E} 02$

$2.312 E \quad 03$

$\begin{array}{ll}5.196 E & 03 \\ 7.718 E & 03\end{array}$ $\begin{array}{ccc}\text { ANGLE } 3 & \text { ANGLE } 4 & \text { ANGLE } 5 \\ M U=-0.9446 & M U=-0.8656 & M U=-0.7550 \\ 0.0 & 0.0 & 0.0\end{array}$ 0.0

0.0

0.0

0.0

$.916 E-05$

$4.442 E-04$

$8.589 \mathrm{~F}-04$

$2.251 E-03$

$4.249 E-03$

3

$3.112 \mathrm{E}-02$

$2.897 \mathrm{E}-01$

$1.896 E$ E1

2.639 E 01

$2.621 E$ E 2

$3.35 \mathrm{E}$

$2.532 E$ O
$4.582 \mathrm{~F}$

4.582F O3

ANGLE 12 $\begin{array}{ll}0.0 & 0.0 \\ 0.0 & 0.0\end{array}$

$3.340 E-05$

$4.439 E-04$

$9.207 E-04$

$2.327 E-03$

$4.360 E-03$

$1.159 E-02$

$3.372 E-02$

$2.924 E-01$

$1.913 E$ O1

$8.712 \mathrm{E} 01$

$7.401 \mathrm{E} 02$

$2.048 E 03$

$6.934 E \quad 03$

ANGLE 13

0.0

0.0

0.0

0.0

0.0

$2.913 E-04$

$2.038 E-03$

GE-03

$2.255 \mathrm{E}-02$
$4.733 \mathrm{E}-02$

$3.785 E-01$

$3.475 E-01$

$2.245 \mathrm{E} 01$

$1.014 \mathrm{E}$ O?

$3.069 E \quad 02$

$\begin{array}{ll}8.558 E & 02 \\ 2.359 E & 03\end{array}$

$5.300 E$ O3

7.8 OE 03
0.

0.0

0.0

0.0

$4.876 \mathrm{E}-04$

$3.207 E-03$

$.328 E-03$

$8.715 \mathrm{E}-03$
$1.085 \mathrm{E}-02$

$2.570 E-02$

5.050E-02

$3.909 E-01$

$3.557 \mathrm{E}-01$

$1.035 E$ O2

$3.131 \mathrm{E} 02$

$8.726 \mathrm{E} 02$

$2.404 \mathrm{E} 03$

$5.398 \mathrm{E} 03$

$7.994 E \quad 03$
$7.004 \mathrm{E} 03$

ANGLE 14

$M U=-0.6179$
0.0

0.0

0.0
0.0

0.0

0.0

. $903 E-05$

4. 469E-04

$1.018 E-03$

$.535 E-03$

$1.204 \mathrm{E}-02$
$3.259 \mathrm{E}-02$

$.071 E-01$

$2.963 \mathrm{E}-01$

.814E O1

$.674 \mathrm{E} 02$

.

4.631E-05

$4.600 \mathrm{E}-04$

$1.164 \mathrm{E}-03$

$4.792 E-03$

1.268E-02

$3.376 E-02$

$3.137 E-01$

$1.757 \mathrm{E} \mathrm{OI}$

$8.945 \mathrm{E} \mathrm{O1}$

$2.713 E$ O2

2.059 E 03

$4.730 \mathrm{E}$ O3

$4.730 E 03$

ANGLE 15

0.0

0.0

0.0

0.0

0.0

8.460E-04

$5.281 E-03$

1. $345 \mathrm{E}-02$

$1.138 \mathrm{E}-02$

$1.278 \mathrm{E}-02$

$5.367 \mathrm{E}-\mathrm{n} 2$

$4.023 E-01$

$3.632 E-01$

$2.339 E$ Ol

$1.054 \mathrm{E}$ O2

$3.187 E 02$

$8.876 \mathrm{E} \mathrm{O2}$

$5.486 \mathrm{E} \mathrm{O3}$

$8.112 E 03$
$M U=0.8656$

0.0

0.5

0.0

0.0

1. $509 \mathrm{E}-03$

$9.181 \mathrm{E}-03$

2. $324 E-02$

$1.522 \mathrm{E}-02$

1. $506 \mathrm{E}-02$

5. $562 E-02$

$4.121 \mathrm{~F}-01$

$3.695 \mathrm{E}-01$

$2.376 \mathrm{E} 01$

$1.070 \mathrm{E} 02$

$3.234 \mathrm{E} \mathrm{O2}$

$9.002 F \quad 02$

2.478 E 03

8.207E 03 $M U=-0.4580$ 0.0

0.0

0.0

$5.590 E-05$

$4.952 E-04$

1.

$2.885 E-03$

$5.145 \mathrm{E}-03$

$1.353 \mathrm{E}-02$

17E-01

$.072 E-01$

$2.003 E$ OI

$2.103 E$ E

7.719E 02

$2.134 \mathrm{E} \quad 03$

.806 E 03

$.192 \mathrm{E} \quad 03$

ANGLE 16

0.0

0.0

0.0

0.0

$2.816 \mathrm{E}-03$

.759E-02

$4.477 E-02$

$.112 E-02$

.758E-02 
ENERGY GROUP (MEV) $1.22 E$ O1---1.50E 01 $1.00 \mathrm{E}$ O1---1.22E O1 6.36E $00--1.00$ E 01 $4.97 \mathrm{E} 00--0.36 \mathrm{E}$ OO $4.07 E$ OO- $-4.97 E$ CO $3.01 \mathrm{E} 00--4.07 \mathrm{E} 00$ $2.46 \mathrm{E} 00--3.01 \mathrm{E} 00$ $2.35 E$ E0- $-2.46 E$ OD $1.83 \mathrm{E} 00--2.35 \mathrm{E} 00$ $1.11 \mathrm{E} 00--1.83 \mathrm{E}$ O0 $5.50 \mathrm{E}-01--1.11 \mathrm{E}$ OO $1.11 E-01--5.50 E-01$ $3.35 E-02--1.11 E-01$ $5.83 E-04---3.35 E \rightarrow 02$ $1.01 \mathrm{E}-04--5.83 \mathrm{E}-04$ $2.90 \mathrm{E}-05--1.01 \mathrm{E}-04$ $1.07 E-05--2.90 E-05$ $3.06 E-06--1.07 E-05$ $1.12 \mathrm{E}-06---3.06 \mathrm{E}-06$ $4.14 \mathrm{E}-07---1.12 \mathrm{E}-06$ $0.0 \quad--4.14 E-07$

\section{ENERGY}

GROUP (MEV)

$1.22 \mathrm{E}$ 01---1.50E 01 1.00 E $01--1.22 E$ OI $8.19 E$ O0---1.00E O1 $6.36 \mathrm{E}$ 00-- $8.19 \mathrm{E}$ OO $4.97 \mathrm{E}$ OO- $-6.36 \mathrm{E}$ OO 4.07E DO-- 4.97E 00 3.01 E $00--4.07 E$ OO $2.46 \mathrm{E} 00--3.01 \mathrm{E}$ OO 2.35 E $00--2.46 E$ OO $1.83 \mathrm{E}$ 00--2.35E 00 $1.11 E$ OO--1.83E OO $5.50 E-01--1.11 E$ OO 1.11E-01-- 5 . 50E-O1 $3.35 E-02--1.11 E-01$ $5.83 E-04--3.35 E-02$ . $01 E-04--5.83 E-04$ . $90 \mathrm{E}-05--1.01 E-04$ 1.07E-05--2.90E-05 . .06E-06- $1.07 E-05$ $1.32 E-06-2.06 E-C 6$ $4.14 E-07--1.12 E-06$
$0.0 \quad--04.14 E-07$
ANGLE 1 (1) 0.0 0.0 0.0 0.0 0.0 $4.443 E-06$ $7.398 E-05$ $1.543 \mathrm{E}-04$ $5.775 \mathrm{~F}-04$ $1.220 E-03$ $3.635 E-03$ $1.138 \mathrm{E}-02$ $1.195 \mathrm{E}-01$ $1.190 \mathrm{E}-01$ $7.945 E 00$ $3.667 \mathrm{E} O$ $1.124 E 02$ $3.185 E$ O2 $8.896 \mathrm{E} 02$ $2.023 E \mathrm{C} 3$ $3.068 \mathrm{E} 03$ ANGLE 20
$M U=0.0950$ 0.0
0.0
0.0
0.0 0.0
0.0
0.0 0.0 0.0 $2.160 \mathrm{E}-05$ $2.189 \mathrm{E}-04$ 7.071E-04 $1.245 E-03$ $2.073 \mathrm{E}-03$ $5.972 \mathrm{E}-03$ $1.565 E-02$ $1.445 \mathrm{E}-01$ $1.379 E-01$ $9.122 E 00$ $4.184 E$ O $1.280 E$ O2 $3.616 E$ O2 $2.284 E \quad 03$ $3.428 \mathrm{E} 03$
(NEUTRINS/MEV/STERADIAN/SOURCE NEUTRON)

$3.336 E$ O
ANGLE 12 $M U=0.4580 \quad M U=0.6179$ 0.0

0.0

0.0

0.0

0.0

0.0

$5.040 \mathrm{E}-04$ $1.451 \mathrm{E}-03$ $2.753 E-03$ $1.795 E-02$ $1.554 E-01$ $1.457 \varepsilon-01$ $4.392 \mathrm{E} 01$ $1.342 \mathrm{E} 02$ $3.788 \mathrm{E} 02$ $1.054 \mathrm{E} \quad 03$ 3.570 E 03

0.0

0.0

0.0

0.0

0.0

$1.609 E-01$

政 02
$A N G L E \quad 5 \quad$ ANGLE 6
$M U=-0.7550 \quad M U=-0.6179$<smiles>[18OH]</smiles>

0.0

0.0

0.0

0.0

6. $518 E-06$

$7.806 E-05$

$2.193 \mathrm{E}-04$

$1.334 E-04$
$1.327 E-03$

$3.969 E-03$

$1.211 \mathrm{E}-02$

1.243E-01

$8.179 E$ OO

$3.771 \mathrm{E} 01$

$3.271 \mathrm{E} 02$

$9.133 E \quad 02$

$\begin{array}{ll}2.076 E & 03 \\ 3.144 \mathrm{E} & 03\end{array}$

$M U=-0.6$
0.0
0.0

0.0

0.0

0.0

0.0

$7.740 E-06$

$8.345 E-05$

$2.628 E-04$

$1.404 E-03$

$4.191 \mathrm{E}-03$

$1.257 \mathrm{E}-02$

$1.272 E-01$

$8.318 \mathrm{E} 00$

$3.832 \mathrm{E} \mathrm{O1}$

$1.173 \mathrm{E} 02$

(..32

2.107E 03

$\begin{array}{lll}2.107 E & 03 \\ 3.186 E & 03\end{array}$

ANGLE 14 ANGLE 15

$M U=0.7550$

0.0

0.0

0.0

0.0

0.0

1.456E-04

$1.356 \mathrm{E}-03$

$M U=0.8656$

0.0
0.0

0.0

0.0

0.0

0.0

$2.537 E-04$
$2.374 E-03$

$2.374 E-03$
$6.810 E-03$

$4.280 E-03$

$3.224 \mathrm{E}-03$

$3.790 E-03$

$4.444 E-03$

$1.149 E-02$

$1.659 E-01$

$1.557 \mathrm{E}-01$

$1.003 \mathrm{E} 01$

4.579E OI

$1.021 \mathrm{E} 01$

1.65 E 01

4.004E 02

政

1.096E 03

$1.076 E$ O3 $1.096 \mathrm{E} O 3$

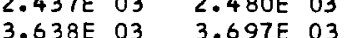

ANGLE 7 ANGLE 8 ANGLE

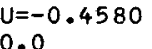

0.0

0.0

0.0

0.0

0.0

$9.389 E-06$

$9.480 E-05$

$3.234 E-04$

$7.836 E-04$

10E-03

$1.314 E-02$

$1.306 E-01$ 
INEUTRJNS/MEV/STERADIAN/SOURCE NEUTRONI

ENERGY GROUP (MEV) $1.22 E \quad 01---1.50 F$ OI $1.00 E$ O1---1.22E OI $8.19 \mathrm{E}$ OC- $-1.00 \mathrm{E}$ O1 $6.36 \mathrm{~F}$ 00-- $-8.19 \mathrm{E}$ OO 4.97E $00--6.36 \mathrm{E}$ OO 4.07E $00--4.97 E$ OO 3.01E $00--4.07 E$ EO $2.46 \mathrm{E}$ 00-- $-3.01 \mathrm{E}$ 0O $2.3 \mathrm{FE} 00--2.46 \mathrm{E}$ OO $1.83 \mathrm{E} 00--2.35 \mathrm{E}$ OO $1.11 \mathrm{E}$ O0- $-1.83 \mathrm{E}$ OO $5.50 E-01--1.11 \mathrm{E} 00$
$1.11 \mathrm{E}-01--5.50 \mathrm{E}-01$ $1.11 \mathrm{E}-01-2-5.50 \mathrm{E}-01$ $3.35 \mathrm{E}-02--1.11 \mathrm{E}-01$
$5.83 \mathrm{E}-04 \cdots-3.35 \mathrm{E}-02$ $1.01 E-04---5.83 E-04$ $2.90 \mathrm{E}-05--1.01 \mathrm{E}-04$ $1.07 E-05---2.90 \mathrm{E}-05$ $3.06 E-06--1.07 E-05$ $1.12 E-06--3.06 E-06$ $4.14 E-07---1.12 E-06$
$0.0 \quad--4.14 E-07$

\section{ENERGY}

GROUP (MEV)
1.22 (ME)

$1.00 E$ 11--1.22E OI

$8.19 \mathrm{E}$ O0---1.00E 01

6.36 $00--8.19 \mathrm{E}$ OO

4.075 O0- $00.367 \mathrm{E} 00$

4.07E $00-4.97 E$ OO

3.01E $00-4.07 E$ OO

$2.35 \mathrm{E} 00--2.46 \mathrm{E} 00$

$1.83 \mathrm{E} 00--2.35 \mathrm{E}$ OO

$1.11 \mathrm{E} 00-2-1.83 \mathrm{E} 00$

$5.50 \mathrm{E}-01-1.11 \mathrm{E}$ OO

$1.11 \mathrm{E}-01-0-5.50 \mathrm{E}-01$

$3.35 E-02--1.11 E-01$

$5.83 \mathrm{E}-04--3.35 \mathrm{E}-02$

$1.01 E-\cap 4---5.83 E-04$

$2.90 E-05--1.01 E-04$

$1.07 E-05--2.90 E-05$

$3.06 \mathrm{E}-06--1.07 \mathrm{E}-05$

$1.12 \mathrm{E}-06---3.06 \mathrm{E}-06$

$4.14 E-07---1.12 E-06$
$0.0 \quad--4.14 E-07$
ANGLE 1

$(0.0$

0.0

0.0

0.0
$7.046 E-07$

$1.189 \mathrm{E}-05$

$2.897 \mathrm{E}-05$

$1.429 \mathrm{E}-04$

$3.243 \mathrm{E}-04$
$1.046 \mathrm{E}-03$

$3.558 \mathrm{E}-03$

3.967E-02

$4.013 \mathrm{E}-02$

$2.713 E 00$

$1.263 \mathrm{E} 01$

$3.895 \mathrm{E}$ ol

$1.113 E$ O2

$3.127 E$ E2

$1.091 \mathrm{E} 03$

ANGLE 10

$M U=0 . C 950$

0.0

0.0

0.0

0.0

0.0
$3.481 E-06$

$4.898 \mathrm{E}-05$

$1.701 E-04$

$3.227 \mathrm{E}-04$

$5.548 \mathrm{E}-04$

$4.944 \mathrm{E}-03$

$4.831 \mathrm{E}-02$

$4.678 \mathrm{E}-02$

$3.135 \mathrm{E} 00$

$1.450 E 01$

$4.465 \mathrm{E} 01$

$3.563 \mathrm{~F} 02$

$8.128 \mathrm{E} 02$

$1.227 \mathrm{E} \mathrm{O} 3$
$M U=-0.9854$

0.0

0.0

0.0

0.0

0.0

$7.187 \mathrm{E}-\mathrm{CT}$

$1.194 \mathrm{~F}-05$

$2.974 E-05$

$1.437 E-C 4$

$3.253 \mathrm{E}-\mathrm{CS}$

$1.049 E-03$
$3.568 \mathrm{E}-03$

3.974E- 0

$4.018 \mathrm{E}-02$

$1.264 \mathrm{E} \cap$

$3.900 \mathrm{~F} O 1$

$1.114 \mathrm{E} 02$

$3.130 \mathrm{~F} \mathrm{C2}$

$1.092 \mathrm{E} 03$

ANGLE 11

0.0

0.0

0.0

0.0

$5.221 E-06$

7. $2121 E-06$
$2.506 E-05$

$2.404 E-04$

$3.980 E-04$

$6.358 E-04$

$6.358 E-04$
$1.975 E-03$

$5.301 E-03$

$5.01 \mathrm{SE}-0$ ?

4. $816 \mathrm{E}-\mathrm{C} 2$

$3.221 \mathrm{E} C O$

$4.580 \mathrm{E}$ OI

$1.304 \mathrm{E} \mathrm{O2}$

$3.650 \mathrm{~F} \mathrm{C2}$

$8.323 \mathrm{E} \mathrm{O2}$

$1.254 E \quad 3$
3
$U=-0.9446$

0.0

0.0

0.0

$7.767 E-07$

$1.215 \mathrm{E}-05$

$3.287 E-05$
$1.472 E-04$

$3.298 \mathrm{E}-04$

$4.003 \mathrm{E}-02$

$4.042 \mathrm{E}-0$ ?

$2.731 \mathrm{E} 00$

$3.920 \mathrm{E}$ OI

$1.125 \mathrm{E} \quad 22$

$3.146 \mathrm{E} \quad 02$

$1.098 \mathrm{E} 03$

4
$0-0.8656$

0.0

0.0

0.0

$8.795 E-07$

1.258E-05

$3.861 \mathrm{E}-05$

1. $540 E-04$

$3.388 \mathrm{E}-04$

$1.098 E-03$

$3.685 E-03$

$4.055 E-02$

4. $084 \mathrm{E}-02$

$2.7=8 F 00$

$3.957 \mathrm{E} 01$

$1.130 \mathrm{E}$ O2

$3.174 E$ E2

$1.1070^{03}$

$=-0.7550$

0.0

0.0

0.0

$1.022 E-06$

1. $339 \mathrm{E}-05$

$4.674 E-05$

$1.643 E-04$

$3.530 E-04$

$1.145 E-03$

$3.795 E-03$

$4.131 E-02$

$2.797 \mathrm{E} 00$

$1.300 E 01$

$4.009 E$ O1

$3.214 E 02$

$1.347 \mathrm{E} 02$

$1.119 E \quad 03$

ANGLE 13

0.0

0.0

0.0

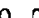

8.295E-06

$1.188 E-C 4$

5.040E-04

$7.375 E-04$

$2.256 \mathrm{E}-03$

$5.693 \mathrm{E}-03$

$5.212 E-02$

$3.307 E$ OO

$1.526 \mathrm{E}$ OI

$4.694 E 01$

$3.738 \mathrm{E}$ O2

$8.517 \mathrm{E} \quad 02$

ANGLE 14

$0.0 \quad 0.0$

0.0

0.0
0

$0.073 F-05$

$1.941 \mathrm{E}-04$

$5.799 \mathrm{E}-04$

$6.478 E-04$

$8.629 E-04$
$2.502 E-03$

$6.107 E-03$

$5.402 E-02$

$5.087 E-\cap 2$

$3.389 \mathrm{E} 00$

$1.562 F$ O1

$4.804 \mathrm{E}$ OI

3.820E 02

0.0

0.0

0.0

0.0

$2.324 E-05$

1.275E-04

$8.438 E-04$

$1.012 E-03$

$2.980 E-03$

6.521E-03

$5.577 \mathrm{E}-02$

5.207E-02

$3.463 \mathrm{E}$
$1.594 \mathrm{E}$

4. $902 \mathrm{E}$ O1

1.393E 02

$3.895 \mathrm{E} 02$

$8.702 \mathrm{~F} 02$

R. $868 E 02$
$1.329 E 03$

AVGLE 6
$M U=-0.6179$

0

0.0

0.0

1.215E-06

1. $498 \mathrm{E}-05$

$5.801 E-05$

3. $739 \mathrm{E}-04$

$1.211 E-03$

$3.942 \mathrm{E}-03$

4. $230 \mathrm{E}-02$

$4.222 E-02$

$1.322 \mathrm{E} 01$

$4.076 \mathrm{E}$ OI

$1.163 E 02$

$3.266 \mathrm{E} 02$

$1.463 \mathrm{~F}$
$1.136 \mathrm{~F}$

ANGLE $\quad 7$
IU $=-0.4580$

0.0

0.0

$1.479 E-06$

$1.805 E-05$

$7.347 \mathrm{E}-05$

$1.996 \mathrm{E}-04$

$4.027 E-04$

. $299 E-03$

$4.129 E-03$

$4.351 E-02$

4.316E-02

$2.906 \mathrm{E} 00$

4.157E 01

$1.186 \mathrm{E} 02$

$3.328 E$ E2

$1.155 \mathrm{E} 03$

ANGLE 8 $=-0.2816 \quad$ ANGLE 9

$0.0 \quad 0.0$

$0.0 \quad 0.0$

0.0 
(NEUTRONS/MEV/STERADIAN/SOURCE NEUTRON)

ENERGY C,ROUP (MEV) $1.22 \mathrm{E}$ 01-- $1.50 \mathrm{E}$ O1

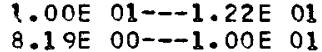

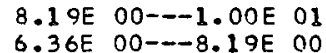

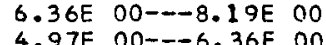
4.97E $00--6.36 E$ OO

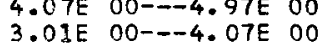
$2.46 E$ OO-- 3.01E 00 $\begin{array}{lll}2.46 E & 00--3.01 E & 00 \\ 2.35 E & 00---2.46 E & 00\end{array}$ $2.35 \mathrm{E}$
$1.83 \mathrm{E} 00---2.35 \mathrm{E}$ OO $1.83 \mathrm{E}$
$1.11 \mathrm{E} 00---2.35 \mathrm{E}$
5 $5.50 \mathrm{E}-01--1.11 \mathrm{E}$ OO $1.11 \mathrm{E}-01--5.50 \mathrm{E}-01$ 3.35E-02--1.11E-01 $5.83 \mathrm{E}-04--3.35 \mathrm{E}-02$ $1.01 \mathrm{~F}-04--5.83 \mathrm{E}-04$ $2.90 \mathrm{E}-05--1.01 \mathrm{E}-04$ $1.07 \mathrm{E}-05--2.90 \mathrm{E}-05$ 3.06E-06-- -1.07E-05 $1.12 \mathrm{E}-06--3.06 \mathrm{E}-06$ $4.14 \mathrm{E}-07---1.12 \mathrm{E}-06$ 0.0

ENERGY GROUP IMEV

$1.22 \mathrm{E}$ 01-- -1.50E 01 1. OOE $01--1.22 \mathrm{E}$ OI $8.19 \mathrm{E}$ OO- $-1.00 \mathrm{E}$ O1 $-6.36 \mathrm{E} O 0$ 4.07E $00---4.97 E$ OO $3.01 E$ EO--4.07E 00 $2.46 E$ O0-- $3.01 \mathrm{E} 00$ $2.35 \mathrm{E} \quad 00--2.46 \mathrm{E}$ 00 $1.83 \mathrm{E} 00--2.35 \mathrm{E}$ 00 $1.11 \mathrm{E} 00--1.83 \mathrm{E}$ 00 $5.50 \mathrm{E}-01--1.11 \mathrm{E} 00$ $1.11 \mathrm{E}-01--5.50 \mathrm{E}-01$ $3.35 \mathrm{E}-02--1.11 \mathrm{E}-01$
$5.83 \mathrm{E}-04-0-3.35 \mathrm{E}-02$ $5.83 E-04--3.35 E-02$ $1.01 E-04--5.83 E-04$ 2.90E-05--1.01E-04 1.07E-05--2.90E-05 $3.06 \mathrm{E}-06--1.07 \mathrm{E}-05$
$1.12 \mathrm{E}-06--3.06 \mathrm{E}-06$ $1.12 \mathrm{E}-06-0-3.06 \mathrm{E}-06$
$4.14 \mathrm{E}-07-0-1.12 \mathrm{E}-06$ $4.14 \mathrm{E}-07--1.12 \mathrm{E}-06$
$0.0 \quad--4.14 \mathrm{E}-07$
ANGLE $\quad$
$M U=-1.0000$ 0.0

0.0

0.0

0.0

0.0

0.0

$1.068 \mathrm{E}-07$

$1.893 E-06$

$5.532 F-06$

$3.412 F-05$

$8.154 E-05$

2.774E-04

$1.0164 E-02$

$1.190 \mathrm{E}-02$

$8.115 E-01$

$3.799 E$ OO

$1.177 \mathrm{E} \quad 01$

$3.380 E$ OI
$9.538 E$ OI

2.190E O2

3.356E O?

ANGLE 10 $M U=0.0950$ 0.0

0.0

0.0

0.0

0.0
$5.343 E-07$

$1.081 \mathrm{E}-05$

$3.948 \mathrm{E}-05$

$8.009 E-05$

$1.399 E-04$

$4.678 \mathrm{E}-04$

$1.400 E-03$

$1.424 \mathrm{E}-02$

$1.393 \mathrm{~F}-02$

$9.414 E-01$

$4.380 E 00$

$1.355 \mathrm{E} \mathrm{O1}$

$3.879 \mathrm{E}$ O1

$1.092 \mathrm{E} \mathrm{O2}$

$\begin{array}{ll}2.50 O E & 02 \\ 3.788 E & 02\end{array}$
ANGLE 2
$M U=-0.9894$

0.0

0.0

0.0

0.0

.

.

$434 E-05$

$80 E-05$

.

.

$1.166 E-02$

.19

.

$178 \mathrm{E} 01$

$3.384 E$ O1

9.549E 01

$2.192 E$ O?

$3.360 E 02$

ANGLE 11 $M U=0.2816$ 0.0

0.0

0.0

0.0

0.0

$8.043 E-07$

$1.693 E-05$

$5.638 \mathrm{E}-05$

$9.941 \mathrm{E}-\mathrm{C5}$

$.603 E-04$

$5.304 E-C 4$

$.503 E-03$

$1.481 E-02$

$1.435 E-02$

$9.679 E-01$

$4.497 E$ OO

$1.391 \mathrm{E} 01$

$3.980 \mathrm{E}$ ol

$1.119 \mathrm{~F} \mathrm{C2}$

$2.562 \mathrm{E} \mathrm{O2}$

$3.874 E$ O2
$M U=-0.9446 \quad M U=-0.8656$

0.0

0.0
0.0

0.0

0.0

$0.0745-07$

$1.174 E-07$

$1.968 E-06$

$6.544 E-06$

8.294E-05

.

.

1.

1.174E-02

$8.199 E-02$

$3.825 \mathrm{E} 00$

1.185E

$3.402 \mathrm{E}$ O1

. $599 \mathrm{E}$ OI

$2.204 E$ O2

$3.275 \mathrm{E} 02$

ANGLE 12

$M U=0.458 \mathrm{C}$

0.0

0.0

0.0

0.0

1.278E-06

2. $719 E-05$

8.590E-05

$1.263 E-04$

$1.859 E-04$

$6.071 \mathrm{E}-04$

$1.616 \mathrm{E}-03$

$1.539 \mathrm{E}-02$

$1.477 \mathrm{E}-0$ ?

$9.946 \mathrm{E}-01$

$4.615 E 00$

$1.427 E 01$

$4.081 \mathrm{E} 01$

$1.147 \mathrm{E} \mathrm{O2}$

$2.624 \mathrm{E} 02$

3.961E 02
0.0

0.0

0.0

0.0

$.326 E-07$

$2.092 \mathrm{E}-06$

$3.976 E-06$

$3.708 E-05$

$8.520 E-05$

$1.037 E-03$

$1.190 \mathrm{E}-02$

$.211 E-02$

8.254E-01

$1.196 \mathrm{E} \mathrm{O1}$

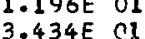

$0.434 E$ OI

$2.224 E$ E2

3.405 E 02

ANGLE 13

$U=0.6179$

0.0

0.0

0.0

$2.106 \mathrm{E}-06$ 4.485E-05 $1.416 \mathrm{E}-04$ $1.620 E-04$ $2.173 \mathrm{E}-04$ $6.988 \mathrm{E}-04$ $1.736 \mathrm{E}-03$ . $597 \mathrm{E}-02$ $1.517 \mathrm{E}-02$ $3.020 E 00$ $4.727 E$ OO $1.461 \mathrm{E} \mathrm{O1}$ $4.177 E^{\circ}$ OI $1.173 E 02$ $2.683 \mathrm{E} \mathrm{O2}$ $4.043 E 02$
ANG LE 5
$M U=-0.7550$ 0.0

0.0
0.0

0.0

0.0

0.0
$1.539 E-07$ 2.314E-06 $9.960 E-06$ $8.880 E-05$ $1.069 \mathrm{E}-03$ $1.065 E-03$ 1. $213 E-02$ $1.230 E-02$ $8.372 E-01$ 1. $3.480 E$ OI $9.814 \mathrm{E}$ ol $2.252 E 02$ $3.446 E$ O2

ANGLE 14 $U=0.7550$ 0.0
0.0 0.0 0.0 0.0 3.537E-06 $7.613 \mathrm{E}-05$ $2.516 E-04$ $2.105 E-04$ $2.543 \mathrm{E}-04$ $8.042 E-04$ $1.855 E-03$ $1.650 E-02$ $1.5043 \mathrm{E} 00$ 4.828E 00 $1.492 \mathrm{E} 01$ 4.263E 01 $1.197 E 02$ $2.736 \mathrm{E} 02$ 4.116E 02

ANGLE $\quad 6$
$U=-0.6179$ 0.3 0.0
0.0
0.0 0.0

$1.830 \mathrm{E}-07$ $2.728 \mathrm{E}-06$ $1.267 E-05$ $4.362 E-05$ $9.408 E-05$ I.224E-04 $1.111 E-03$ 1.243E-02 $1.253 E-02$ 8.524E-01 $1.233 \mathrm{E} 01$ 3.539E OI $9.976 \mathrm{E}$ O1 $2.289 E 02$ $3.497 E 02$

ANGLE 15 0.5
0.0
0.0 
(GAMMAS/MEV/STERADIAN/SOURCE NEUTRON)

FNERGY GROUP (MEV) 8.OCE $00--1.00 E$ OI 6.5 CE OO-D-8. COE OR 5.OSE OO---6.5OE OO 4. OOE 00---5. OOE OO $3.00 E$ ODT- $0.00 E$ OO $2.50 E$ OO-- -3. OOE OO 2. OCE OO-D-2.5OE OO $1.66 E$ OO-T-2.00E OO $1.33 E$ OO--1.66E 00 1.OCE OO---1.33E OO 3. OOE-01--1. 00 OO 00 $6.00 E-01--8.00 E-01$ $4.00 E-01---6.00 E-0$. 3.00E-01--4.00E-01 2. COE $-01--3.00 E-01$ $1.00 E-C)-C 2.00 E-01$

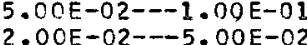

\section{ENERGY \\ GROUP (MEV)}

8.00E $00--1.00 E$ OI 5. S.OOE DO- - 6.5CE OO 4.0NF DO- 5. OOE OO . 2. DOE OO-TS.50E 1. 6 E $00--2$. COE 00 1.33E OO-O $1.00 E 00-1.33 E 00$ $8.00 E-01--1.00 E$ OO $6.00 E-01--8.00 E-01$ $4.005-01---6.005-01$ $3.00 E-01--4.00 E-01$ . $00 E-01--3.00 E-01$ $1.00 E-01--2.005-01$ $5.00 \mathrm{E}-02--1.00 \mathrm{E}-0$ $2.0 C E-02---5.00 E-02$
ANGLE 1 ANGLE 2 $M U=-1.000 \mathrm{O} \quad M U=-0.9894$ $7.563 E-07$ $1.559 E-06$ $1.733 E-05$ $4.976 E-06$ $1.004 \mathrm{E}-05$ $5.223 E-05$ $5.192 E-05$ $5.893 \mathrm{E}-\mathrm{C} 6$ $9.076 E-06$ $1.185 \mathrm{E}-05$ $1.343 E-05$ ]. $559 \mathrm{E}-05$ $5.511 \mathrm{E}-05$ $8 . ? 64 E-05$ $2.215 E-04$ $4.720 E-04$ $1.049 E-03$

ANGLE 10 $M U=0.0950$ $8.520 \mathrm{E}-07$ $1.756 \mathrm{E}-06$ $1.943 \mathrm{E}-0 \mathrm{E}$ $1.293 E-05$ $1.293 E-05$ $1.063 E-04$ $1.0304 E-05$ $1.361 E-05$ $1.715 \mathrm{E}-05$ $2.747 E-05$ $3.211 \mathrm{E}-05$ $8.765 \mathrm{E}-05$ $1.352 \mathrm{E}-04$ $2.459 F-04$ $5.281 E-04$ $1.117 \mathrm{E}-\mathrm{n3}$ $2.933 E-04$ $1.050 E-03$

ANGLE 11 $M=0.2816$
ANGLE 3 ANGLE 4 ANGLE 5 ANS̈LE

$\begin{array}{llll}1.742 E-05 & 1.755 E-05 & 1.775 E-05 & 1.800 E-05 \\ 5.305 E-06 & 5.049 E-06 & 5.112 E-06 & 5.195 E-06\end{array}$ $5.005 E-06 \quad 5.049 E-06$ $1.014 E-05 \quad 1.027 E-05$ $\begin{array}{ll}5.373 E-05 & 5.599 E-05 \\ 5.378 E-05 & 5.645 E-05\end{array}$ $6.303 \mathrm{E}-06 \quad 6.815 \mathrm{E}-06$ $8.823 \mathrm{E}-06 \quad 8.513 \mathrm{E}-06$ $1.133 \mathrm{E}-05 \quad 1.084 \mathrm{E}-05$ $1.340 \mathrm{E}-05 \quad 1.350 \mathrm{E}-05$ $1.663 E-05 \quad 1.796 E-05$ 5.612E-05 5.756E-05 $8.322 E-05 \quad 8.497 E-05$ $2.235 E-04 \quad 2.261 E-04$ $4.74 J E-04 \quad 4.771 E-04$ $1.052 E-03 \quad 1.056 E-03$ 1.047E-05 $5.926 \mathrm{E}-05$ $6.007 E-05$ $7.387 E-06$ $8.609 E-06$ 1. $066 \mathrm{E}-05$ 1.390E-05 $1.946 \mathrm{E}-05$ 5 (2) 4. $816 \mathrm{E}=04$ $1.062 E-03$ $1.062 E-03$

ANGLE I2 ANGLE 13 ANGLE 14 $M U=0.4580$ $8.896 \mathrm{E}-07$ $1.832 \mathrm{E}-06$ $M U=0.7550$ $9.216 \mathrm{E}-07$ $1.897 E-06$ $2.094 \mathrm{E}-05$ $6.162 \mathrm{E}-06$ 1.740E-05 $2.337 E-04$ $3.251 E-05$ $3.251 E-05$ $4.175 \mathrm{E}-05$ $5.221 E-05$ $6.720=-05$ $1.361 \mathrm{E}-04$ $1.361 E-04$
$1.797 E-04$ $1.797 E-04$
$2.965 F-04$ $2.965 \mathrm{E}-04$ $5.951 E-04$
$1.177 E-03$ 3.0 OOE-04

$5.195 \mathrm{E}-06$

$6.365 E-05$ $6.467 \mathrm{E}-05$ $7.967 \mathrm{E}-06$ $8.065 \mathrm{E}-06$ $1.103 E-05$ $1.469 E-05$ $2.094 E-05$ $6.221 E-05$ . 2.316E-04 $1.070 E-03$

ANGLE 15 $M U=0.8656$ $9.350 F-07$ $1.924 E-06$ $2.123 E-05$
$6.258 E-06$ $2.255 E-05$ $3.869 E-04$ $3.934 \mathrm{E}-04$ $4.777 E-05$ 5. $762 \mathrm{E}-05$ $5.762 E-05$
$6.733 E-05$ $8.122 \mathrm{E}-05$ $1.545 \mathrm{E}-04$ 2. $000 E-04$
$3.271 F-04$ $3.271 F-04$ 1.190E-03 $3.014 \mathrm{~F}-04$ ANGLE 8 8.001E-07 $\quad M U=-0.2816 \quad M U=-0.0950$ $\begin{array}{ll}.160 \mathrm{C}-07 & 8.335 \mathrm{E}-07 \\ & -1.718 \mathrm{E}-06\end{array}$ 5.294E-06 $5.408 E-06 \quad 5.532 E-06$ $1.107 E-05$ 1.149E-05 1.202E-05 $6.951 E-05 \quad 7.743 E-05 \quad 8.830 E-05$ $7.061 E-05$ 7.861E-05 8.968E-05 $8.620 E-06 \quad 9.492 E-06 \quad 1.075 E-05$ $9.719 E-06 \quad 1.082 E-05 \quad 1.223 E-05$ $1.195 E-05$ 1.327E-05 1.490E-05 $1.58 \mathrm{E}-05 \quad 1.740 \mathrm{E}-05 \quad 1.944 \mathrm{E}-05$ $2.250 E-05 \quad 2.450 E-05 \quad 2.748 E-05$ 2.0 $1.041 E-04 \quad 1.144 E-04 \quad 1.249 E+04$ L. $2.365 E-04 \quad 2.401 E-04$ 1.079E-03 $1.044 E-04$ $5.153 E-04$ $\begin{array}{lll}1.079 E-03 & 1.091 E-03 & 1.103 E-03 \\ 2.887 E-04 & 2.901 E-04 & 2.916 E-04\end{array}$ ANGLE 17

\section{SCALAR}

$1.063 \mathrm{E}-05$ $2.190 \mathrm{E}-05$ $2.425 E-04$ $1.771 E-04$ 1.771E-04 $1.929 E-03$ 1.929E$2.401 E-04$ $2.801 E-04$ $2.825 E-04$
$3.539 E-04$ 4. $730 E-04$ $4.730 E-04$ $1.671 E-03$ 3. $212 \mathrm{E}-03$ $6.212 E-03$ $6.683 E-03$
$1.402 E-02$ $1.402 E-02$
$3.681 E-03$ 
IGAMMAS/MEV/STERADIAN/SOURCE NEUTRONI

ENERGY GROUP (MEV 8.00E DO-- - 1. OOE OI 6.5OE $00--8.00 E$ OO $4.00 E$ OO- - - 5. 0 .0E 00 $3.00 E$ OO- $-4.00 E$ EO $2.50 E \quad 00--3.00 E$ OO . 00 E $00--2.50 E$ OO $1.66 \mathrm{E} 00--2.00 \mathrm{E} 00$ $1.33 \mathrm{E} 00--1.66 \mathrm{E} 00$ 1.00 E $00--1.33 E 00$ 8.00E-01---1.00E 00 $6.00 E-01---8.00 E-01$ $4.00 E-01--6.00 E-01$ .00E-01-- 4. 00E-01 $.00 E-01--3.00 E-01$ .00E-01---2.00E-01 $5.00 \mathrm{E}-02--1.00 \mathrm{E}-01$ 2.00E-02---5.00E-02

$$
\text { ENERGY }
$$$$
\text { GROUP (MEV) }
$$

$8.00 E$ OO- - - 1.00E 01 6.5OE OO---8.COE OO 5.0OE 0O- - 6.50E OO $4.00 E$ DO- $-5.00 E$ OO 3.0OE OO--4. 2.5OE $00--3.00 E \quad 00$ $2.00 E$ O0--2.50E 00 $1.66 E$ OO- $02.00 \mathrm{E}$ OO $1.33 \mathrm{E} 00--1.66 \mathrm{E} 00$ $1.00 E$ O0-D-1.33E OO $8.00 E-01--1.00 E$ DO . $4.00 E-01--6.00 E-01$ 3.00E-01-- $4.00 \varepsilon-01$ 2.00E-01--3.00E-01 $1.00 E-O 1-2.00 E-01$ $2.00 \mathrm{E}-02---5.00 \mathrm{E}-02$ $\triangle A N G L E \quad 1$ $M U=-1.0000$ $2.573 \mathrm{E}-06$ $5.314 \mathrm{E}-06$
$5.927 \mathrm{E}-05$ 1.927E-05 $3.083 E-05$ $5.755 E-05$ $5.673 \mathrm{E}-0.5$ $1.355 \mathrm{E}-05$ $2.124 E-05$ $2.883 E-05$ $3.379 \mathrm{E}-05$ 4. $047 E-05$ $1.555 \mathrm{E}-04$ $2.219 E-04$ $5.957 E-04$ $1.467 \mathrm{E}-03$ $3.567 \mathrm{E}-03$ $9.953 \mathrm{E}-04$ ANGLE 10 $M U=0.0950$ $3.232 E-06$ $6.658 \mathrm{E}-06$ . $363 \mathrm{E}-05$ $2.153 \mathrm{E}-05$ $4.397 E-05$ $9.548 \mathrm{E}-05$ $9.877 \mathrm{E}-05$ $3.628 \mathrm{E}-05$ $4.195 \mathrm{E}-05$ $4.365 E-05$ $5.833 E-05$ $8.534 \mathrm{E}-05$ 2.5 3.813E-04 $0.643 E-04$ $1.680 E-03$ $1.039 \mathrm{E}-03$ $\triangle N G L F ?$ $2.578 E-06$ $5.324 E-06$ $5.938 \mathrm{E}-05$ $1.687 E-05$ $3.074 \mathrm{E}-05$ $5.802 E-05$ $5.745 E-05$ $1.383 E-05$ $2.107 E-05$ 2. $843 E-05$ $3.369 E-05$ $4.111 \mathrm{E}-05$ $1.561 E-04$ $2.221 E-04$ $5.971 \mathrm{E}-04$ $1.469 E-03$ $3.570 E-03$ $9.956 \mathrm{E}-04$

\section{NGLE 11} $M U=0.2816$ $3.376 E-06$ $6.951 E-06$ $7.675 \mathrm{E}-05$ $2.256 \mathrm{E}-05$ $3.973 \mathrm{E}-05$ $1.593 E-04$ $1.640 E-04$ $2.670 E-05$ $3.005 E-05$ $4.759 E-05$ $6.631 E-05$ 2.007E-0 4 $2.804 E-04$ $4.009 E-04$ $6.767 E-04$ $3.981 E-03$ $1.048 \mathrm{E}-03$ ANGLE 3
MU $=-0.9446 \quad$ MNGLE $\quad 4$ $2.598 E-06$
$5.365 E-06$ $5.365 E-06$ $1.701 \mathrm{E}-05$ $3.102 E-05$ $5.981 E-05$ $6.008 E-05$ $1.481 \mathrm{E}-05$ $2.053 \mathrm{E}-05$ $2.714 \mathrm{E}-05$ $3.347 \mathrm{E}-05$ $4.344 E-05$ $1.585 E-04$ $2.234 E-04$ $6.021 \mathrm{E}-04$ $1.475 E-03$ $3.580 \mathrm{E}-03$ $9.971 \mathrm{E}-04$ $2.637 E-06$ $6.068 \mathrm{E}-05$ $1.729 E-05$ $3.157 E-05$ $6.303 E-05$ $6.444 E-05$ $1.631 E-05$ $1.997 E-05$ 2. $563 \mathrm{E}-05$ $3.355 E-05$ 4.712E-0 $1.625 E-04$ $2.286 \mathrm{E}-04$ $6.100 E-04$ $1.486 E-03$ $3.601 E-03$ $9.998 E-04$

ANGLE 5
$M U=-0.7550$ $2.694 E-06 \quad 2.769 E-06$ $1.770 \mathrm{E}-05 \quad 1.823 \mathrm{E}-05$ $3.235 E-05 \quad 3.335 E-05$ $6.717 E-05 \quad 7.235 E-05$ $7.504 E-05$ $1.912 E-05$ $2.002 \mathrm{E}-05 \quad 2.108 \mathrm{E}-05$ $2.518 E-05 \quad 2.645 E-05$ $3.673 E=05$ $5.097 \mathrm{E}-05 \quad 5.446 \mathrm{E}-05$ $1.679 E-04 \quad 1.751 E-04$ 2.611E-04 $0.252 E-04$ $3.630 E-03 \quad 3.668 E-03$ $1.004 \mathrm{E}-03 \quad 1.009 \mathrm{E}-03$ ANGLE 12 $U=0.4580$ $M U=0.617$ $3.525 \mathrm{E}-06$ $7.253 \mathrm{E}-06$ $7.995 \mathrm{E}-05$ $2.363 E-05$ $4.565 \mathrm{E}-05$ $1.695 \mathrm{E}-04$ $1.757 \mathrm{E}-04$ $4.939 E-05$ $5.632 E-05$ $6.170 \mathrm{E}-05$ $8.571 \mathrm{E}-05$ $1.268 E-04$ $3.166 E-04$ $4.277 E-04$ $7.089 E-04$ $4.063 E-03$ $4.063 \mathrm{E}-03$
$1.057 \mathrm{E}-03$ ANGLE 13 ANGLE 14

$M U=0.7550$

ANGLE 15

$7.549 E-06$

$8.309 \mathrm{E}-05$

$2.468 E-05$

$4.950 E-05$

$2.453 E-04$

2.519E-04

$4.988 E-05$ $5.854 \mathrm{~F}-05$ $8.458 E-05$ $1.139 E-04$ $1.585 E-04$ $3.533 E-04$ $4.596 E-04$ $7.539 E-04$
$1.871 E-03$ $1.871 E-03$ $1.066 \mathrm{E}-03$
$817 \mathrm{E}-06$

$8.593 \mathrm{E}-05$

2.563E-05

$5.122 \mathrm{E}-05$

$3.741 E-04$

7.

$7.966 E-05$

.

$1.103 E-04$ . $432 E-04$ . $841 \mathrm{E}-04$ 4.8 7. $08 \mathrm{E}-04$ 4. $229 \mathrm{E}-03$ $1.075 \mathrm{E}-03$
MU 0.8656

$8.049 \mathrm{E}-06$

$8.837 E-05$

$2.645 E-05$

$6.242 E-05$ $5.053 E-04$ 1. 1.184E-04 I. $504 \mathrm{E}-04$ 1.5 . . . . $666 \mathrm{E}-04$ . $4.305 \mathrm{E}-03$ $1.083 \mathrm{E}-03$

ANGLE 7 ANGLE 8 $2.862 E-06 \quad 2.971 E-06$ $5.904 \mathrm{E}-06$ $6.560 E-05$ $1.889 E-05$ $3.459 E-05$ $7.898 \mathrm{E}-05$ $8.162 E-05$ . $317 \mathrm{E}-05$ 2.926E-05 $3.993 E-05$ $5.794 E-05$ .309E-04 $1.554 \mathrm{E}-03$ $3.715 \mathrm{E}-03$
$1.015 \mathrm{E}-03$ ANGLE 16 $M U=0.9446$ 4.000E-06 $8.216 \mathrm{E}-06$

$2.704 \mathrm{E}-05$

7.361E-05 $9.327 \mathrm{E}-04$ $9.648 \mathrm{E}-04$ $1.964 \mathrm{E}-04$ $2.016 \mathrm{E}-04$ $2.115 \mathrm{E}-04$ $2.326 \mathrm{E}-04$ $2.671 E-04$ $4.861 \mathrm{E}-04$ 
(GAMMAS/MEV/STERADIAN/SOURCE NEUTRON)

ENERGY GROUP (MEV) 8.00E OO-D-1.00E C1 $6.50 \mathrm{E} 00---8.00 \mathrm{E} 00$ $5.00 E$ DO- $-6.50 E$ OO $4.00 E$ OO- $-5.00 E$ OO $3.00 \mathrm{E} 00--4.00 \mathrm{E} 00$ 2.5 OE OO---3.00E 00 2. OOE $00---2.50 E$ OO $1.66 E \quad 00---2.00 E \quad 00$ $1.23 \mathrm{E} \quad 00---1.60 \mathrm{E}$ OO $1.00 E \quad 00-\cdots-1.33 E D O$ $8.00 F-01--1$. . COE OO $6.00 E-01---8.00 E-01$ $4.00 E-01--6.00 E-01$ $3.00 E-01---4.00 E-01$ $2.00 E-01--3.00 E-01$ $1.00 E-01--2.00 E-01$ 5. DOE $-02--1.00 E-01$ 2.0OE-02---5.00E-02 ENERGY GROUP (MEV) 8.0OE OO---1.00E $O 1$ 6.505 O0-- $8.00 E$ OO $5.00 E$ OOW-6.50E OD $4.00 E$ DO---5.0OE OC 3.00 E $00--4.00 E$ OO $2.50 E$ EO--3.00E 00 $2.00 E$ OD---2.5OE OO $1.66 \mathrm{~F}$ DO- $-2.00 \mathrm{E}$ OO $1.33 \mathrm{E}$ 00-- $1.66 \mathrm{E}$ CO 1.00 E $00-1.33$ E DO 8.ONE O1-C1. DOE OD 6. COE-CL $-2.00 E-01$ $3.00 E-01-C-4.00 E-O 1$ $3.00 E-01-4.00 E-01$ 2.0OF $1-2.00 E-01$ 5. OOE $02-2.00 E-01$
ANGLE 1 ANGLE 2 $M U=-1.0000 \quad M U=-0.9894$ $4.041 E-06$ $.568 E-05$ $4.925 \varepsilon-C 5$ $5.906 E-05$ $2.353 E-04$ $3.308 \mathrm{E}-04$ . $698 \mathrm{E}-04$ $2.244 E-03$ $1.655 \mathrm{E}-03$ ( ANGLE 10 $M U=0.0950$ $5.418 \mathrm{E}-06$ $1.116 E-05$ $1.233 E-04$ $3.615 \mathrm{E}-05$ 6. $255 E-05$ 1. $305 E-04$ 1.357E-04 $3.717 \mathrm{E}-05$ $4.298 E-05$ $5.921 E-05$ 1.2065-04 $1.206 E-04$ . $9.582 E-04$ $3.58 E-04$ 2. 3. $255 \mathrm{E}-03$
ANGLE ? $A U=-0.9446$
$4.091 E-06$ $8.455 \mathrm{E}-06$ $9.443 E-05$ $2.669 E-05$ $4.787 E-05$ $5.929 E-05$ 5. $991 \mathrm{E}-05$ $2.078 E-05$ $2.836 \mathrm{E}-05$ $3.781 E-05$ 4. $766 E-05$ $6.346 E-05$ $2.397 E-04$ $3.329 E-04$ $8.792 E-04$ 2.257E-03 $5.679 \mathrm{E}-03$ $1.599 \mathrm{E}-03$

$2.247 \mathrm{E}-03$ $5.660 \mathrm{E}-03$ $1.596 E-03$ ANGLE 11 $M U=0.2816 \quad$ ANGLE 12 $5.745 \mathrm{E}-06$ $1.182 \mathrm{E}-05$ $1.304 E-04$ $3.849 \mathrm{E}-05$ 7. $051 E-05$ $1.338 E-04$ $1.390 E-04$ $5.166 E-05$ $7.122 E-05$ $7.1225-05$ $1.502 E-04$ $1.502 E-04$ 5. $233 E-04$ . $9.938 E-04$ $6.304 E-03$ $1.3942-03$

\section{$M=0.4580$} $6.088 \mathrm{E}-06$ $1.252 E-05$ $1.377 E-04$ 4. $096 \mathrm{E}-05$ $7.223 E-05$ $1.906 E-04$ $1.982 E-04$ 5. $515 E-05$ $6.381 E-05$ $8.713 E-05$ $1.232 E-04$ $4.740 E-04$ $4.740 E-04$ $1.240 E-04$ 1. $030 E-03$ $6.539 E-03$ 1. $706 \mathrm{E}-03$ $M U=-0.8656$ . $169 \mathrm{E}-06$ $8.614 E-06$ $2.725 E-05$ $6.515 \mathrm{E}-05$ $2.300 E-05$ $2.773 \mathrm{E}-05$ $3.580 \mathrm{E}-05$ $4.788 \mathrm{E}-05$ $6.887 E-05$ $2.463 E-04$ $3.406 E-04$ $8.908 \mathrm{E}-04$ $2.276 E-03$ $5.715 \mathrm{E}-03$ $1.604 \mathrm{E}-03$ ANGLE 13 $M U=0.6179$ $6.433 E-06$ $1.322 \mathrm{E}-05$ $1.451 E-04$ 4.345E-05 8.111E-05 $2.306 E-04$ 2.396E-04 $7.694 E-05$ $9.238 E-05$ $1.186 \mathrm{E}-04$ $1.631 \mathrm{E}-04$ $2.300 E-04$ $5.246 \mathrm{E}-04$ 6.661E-04 $1.087 E-03$ $6.87 E-03$ $6.690 \mathrm{E}-03$
$1.722 \mathrm{E}-03$
ANGLE $5 \quad$ ANGLE 6 $4.284 E-06$ $.849 E-06$ . 806E-05 $5.032 E-05$ $6.735 E-05$ $7.079 E-05$ .514E-05 . $806 \mathrm{E}-05$ .551E-05 $4.959 \mathrm{E}-05$ $7.448 \mathrm{E}-05$ $2.546 \mathrm{E}-04$ 3.587E-04 $9.027 E-04$ $2.303 E-03$ $5.767 E-03$ 1. $611 E-03$ ANGLE 14 $M U=0.7550$ $6.760 E-06$
$1.388 E-05$ $1.388 \mathrm{E}-05$ $4.581 E-05$ $8.512 \mathrm{E}-05$ $3.486 E-04$ $3.628 E-04$ $1.032 E-04$ $1.244 E-04$ $1.628 E-04$ $2.121 E-04$ $2.776 E-04$ 7.73 E-04 I.1 $50 \mathrm{E}-03$ 3. $6.838 \mathrm{E}-03$ $1.738 \mathrm{E}-03$ $4.437 E-06$
$4.165 E-06$ $9.16 J E-06$ $.020 E-04$ . $915 \mathrm{E}-05$ $5.220 E-05$ $7.261 E-05$ $7.638 \mathrm{E}-05$ .695E-05 $2.986 \mathrm{E}-05$ $5.314 \mathrm{E}-05$ $7.952 E-05$ $2.656 E-04$ $3.892 \mathrm{E}-04$ $9.128 E-04$ $2.339 E-03$ $5.834 \mathrm{E}-03$ $.619 E-03$

ANGLE 15 $M U=0.8656$ $1.445 \mathrm{E}-05$ $1.582 \mathrm{E}-04$ 4. $787 \mathrm{E}-05$ $9.697 E-05$ $5.546 E-04$ $5.806 E-04$ $1.684 E-04$ $1.919 E-04$ $2.269 E-04$ 2.731E-04 $3.312 E-04$ 7.327E-04 7.83 E-04 1.236E-03 $6.976 \mathrm{E}-03$ $1.751 \mathrm{E}-03$

ANGLE 7 ANGLE 8 ANGLE 9 $M U=-0.4580 \quad M U=-0.2816 \quad M U=-0.0950$ $4.627 E-06 \quad 4.855 E-06 \quad 5.120 E-06$ $9.548 \mathrm{E}-06 \quad 1.001 \mathrm{E}-05 \quad 1.055 \mathrm{E}-05$ $1.062 E-04 \quad 1.111 E-04 \quad 1.169 E-04$ $\begin{array}{lll}3.050 E-05 & 3.212 E-05 & 3.401 E-05 \\ 5.454 E-05 & 5.736 E-05 & 6.072 E-05\end{array}$

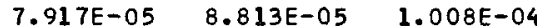
8.270E-05 9.148E-05 1.047E-04 $2.879 E-05 \quad 3.142 E-C 5 \quad 3.546 E-05$ $3.312 \mathrm{E}-05 \quad 3.738 \mathrm{E}-05 \quad 4.218 \mathrm{E}-05$ $4.242 E-05 \quad 4.804 E-05 \quad 5.379 E-05$ $5.817 E-05 \quad 6.419 E-05 \quad 7.144 E-05$ $8.453 E-05 \quad 9.143 E-05 \quad 1.030 E-04$ $2.815 E-04 \quad 3.048 E-04 \quad 3.374 E-04$ $4.300 E-04 \quad 4.756 E-04 \quad 5.198 E-04$ $9.210 E-04 \quad 9.294 E-04 \quad 9.417 E-04$ $2.385 E-03 \quad 2.441 E-03 \quad 2.508 E-03$ $.917 E-03 \quad 6.016 E-03 \quad 6.129 E-03$ 1.643E-03 $1.657 E-03$

ANGLE 16 $M U=0.9446 \quad$ ANGLE 17 7.379E-06 1.513E-05 $4.942 \mathrm{E}-05 \quad 5.029 \mathrm{E}-05$ $4.942 E-05$ $1.135 E-04$ $1.0058 E-03$ $2.797 E-04$ $2.882 \mathrm{E}-04$ $3.082 E-04$ 3. $7.080-04$ 
(GAMMAS/MEV/STERADIAN/SOURCE NEUTRON)

ENERGY GROUP IMEV I $8.00 E$ OO---1.00E 01 S. . 3.OOE OO. 50 OO $00-3.00 E$ OO 2.00E $00-2-2.50 E$ OO 1.66E $00--2.00 E$ OO $1.33 \mathrm{E} \quad 00--1.66 \mathrm{E} 00$ 1.00E $00--1.33 E$ E 00 $8.00 \mathrm{E}-01--1.00 \mathrm{E} 00$ $6.00 E-01--8.00 E-01$ $4.00 E-01--6.00 E-01$ 3.00E-01-- $-4.00 E-01$ $2.00 \mathrm{E}-01--3.00 \mathrm{E}-01$ $1.00 E-01--2.00 E-01$ $5.00 E-02--1.00 E-01$ $2.00 \mathrm{E}-02--5.00 \mathrm{E}-02$

ENERGY

GROUP (MEV)

8.0OE 00---1. OOE OI 6.5OE OO- - -8.00E OO 5.00 E $00--6.50$ E OD $4.00 E$ 00-- $5.00 E 00$ 3.0OE $00--4.00 E$ OO . .5OE OO- -3.00E OO . $.00 E$ OO- $-2.50 E$ OO 1.66 E $00--2.00 E$ OO $1.33 \mathrm{E}$ 00---1.66E 00 $1.00 E$ DO--1.33E CO $8.00 E-01-1.00 E$ OO 6.0NE-01-- - $.00 E-01$ $4.00 E-01--6.00 \mathrm{E}-01$ $3.00 E-01--4.00 E-01$ . $1.00 E-01--2.00 E-01$ 2.0OE-02---5. COE-0?
ANGLE 1 ANGLE 2 E-06 $.418 \mathrm{E}-05$ 1.436E-05 $7.885 \mathrm{E}-05$ $5.160 E-05$ $5.107 E-05$ $2.841 \mathrm{E}-\mathrm{C5}$ 4.8227E-O5 $5.872 \mathrm{E}-05$ $7.312 E-05$ $9.424 E-05$ $3.973 \mathrm{E}-04$ $5.535 \mathrm{E}-04$ $1.422 E-03$ $3.829 E-03$ $1.006 E-02$ $2.878 E-03$

ANGLE 10 $M U=0.0950$ $1.031 E-05$ $6.906 E-05$ $1.203 E-04$ $1.114 E-04$ $1.187 E-04$ $6.672 \mathrm{E}-05$ $7.882 E-05$ $1.002 \mathrm{E}-04$ $1.365 E-04$ $2.039 E-04$ $6.527 E-04$ $9.188 \mathrm{E}-04$ $1.572 E-03$ $4.450 \mathrm{E}-03$ $1.128 E-02$ $6.879 E-06$ $1.423 E-05$ $1.525 E-04$ $4.452 E-05$ $7.452 E-05$ $5.226 \mathrm{E}-05$ $5.222 \mathrm{E}-05$ $2.905 E-05$ $4.207 \mathrm{E}-05$ $5.802 E-05$ $7.298 E-05$ $9.569 E-05$ $3.991 E-04$ $5.542 E-04$ $1.425 E-03$ $3.833 E-03$ $1.007 \mathrm{E}-\mathrm{C2}$ $2.880 E-03$

ANGLE 11 $M U=0.2816$ .12 . $313 E-05$ $.580 \mathrm{E}-05$ $1.307 E-C 4$ $1.40 \% \mathrm{E}-04$ $1.504 \mathrm{E}-04$ $7.692 \mathrm{E}-05$ $8.907 E-05$ $1.161 \mathrm{E}-04$ $1.627 E-04$ $2.483 \mathrm{E}-04$ $7.286 \mathrm{E}-04$ $9.686 \mathrm{E}-04$ $1.617 \mathrm{E}-03$ $4.605 \mathrm{E}-03$ $1.156 \mathrm{E}-02$ $3.065 \mathrm{E}-03$ $M U=-0.9446$

.970E-06

$1.442 E-05$

$1.615 E-04$

$4.518 \mathrm{E}-05$

$8.027 E-05$

$5.47 L \mathrm{E}-05$

$5.631 \mathrm{E}-05$

$3.134 \mathrm{E}-05$

$4.154 \mathrm{E}-05$

$5.585 E-05$

7. $284 E-05$

.011E-04

.062E-04

$1.436 E-03$

3. $852 E-03$

$1.011 E-02$

$2.885 E-03$

$U=-0.8656$

$7.150 \mathrm{E}-06$

$.655 \mathrm{E}-0$

$4.647 \mathrm{E}-05$

$8.247 E-05$

$5.873 \mathrm{E}-05$

$6.250 \mathrm{E}-05$

$3.475 \mathrm{E}-05$

$4.139 \mathrm{E}-05$

$5.386 \mathrm{E}-05$

.3.4E-05

$1.096 \mathrm{E}-04$
$5.185 \mathrm{E}-04$

$5.740 E-04$

$1.453 E-03$

$3.888 \mathrm{E}-03$

$2.895 \mathrm{E}-03$

ANGLE 12

ANGLE 13

$M U=0.4580$

E- 05

$.334 E-05$

$1.441 E-04$

$1.714 E-04$

$1.821 E-04$

$9.338 \mathrm{E}-05$

$1.099 E-04$

$1.445 E-04$

2.047E-04

$3.064 E-04$

$8.067 \mathrm{E}-04$

$1.020 \mathrm{E}-03$

$1.680 E-03$

$4.777 E-03$

3.098E-03 .747E-05 .138E-05 $2.279 E-04$ .404E-04 .149E-04 1. $405 \mathrm{E}-04$ 1.900E-04 $2.639 E-04$ B. $133 E=04$ . 07 E- 04 $1.078 E-03$ 4. $963 E-03$ 4. $63 \mathrm{E}=03$ 3.1 $30 E-03$ ANGLE 5

ANGLE 6 $M U=-0.6179$ $418 E-06$ . $533 \mathrm{E}-05$ 1.714E-04 $4.837 E-05$ $6.330 E-05$ 6. $857 \mathrm{E}-05$ . $483 E-05$ $.778 \mathrm{E}-05$ $4.347 E-04$ 6.054E-04 $.939 E-03$ $1.029 E-02$ $.908 E-03$

ANGLE 14 $.970 E-05$ . $9.946 \mathrm{E}-05$ . I $62 E-04$ $3.350 E-04$ $1.587 \mathrm{E}-04$ $1.965 E-04$ $2.587 E-04$ $3.408 E-04$ $4.466 E-04$ $9.524 \mathrm{E}-04$ 1. $152 E-03$ $1.843 E-03$ $5.159 \varepsilon-03$ $1.244=-02$ $3.160 E-03$ $M U=0.8656$ $.551 E-05$ .175E-05 $.898 \mathrm{E}-04$ $5.073 \mathrm{E}-04$ .449E-04 $2.442 E-04$ . .5 . $288 E-04$ 1.031E-03 1.031E-03 $1.240 E-03$
ANGLE 7 $M U=-0.4580$
$8.239 E-06$ $1.701 E-05$ $1894 \mathrm{E}-04$ $5.420 \mathrm{E}-05$ . $530 \mathrm{E}-05$ $7.479 E-05$ $7.968 E-05$ $4.414 E-05$ $5.225 E-05$ $6.840 E-05$ $9.440 E-05$ $1.377 \mathrm{E}-04$ 4.856E-04 $7.204 \mathrm{E}-04$ $1.501 \mathrm{E}-03$ 4.090E-03 $1.060 E-02$ $2.948 E-03$

ANGLE 16

ANGLE 8 $M U=-0.2816$ $8.811 E-06$ 2.018E-04 $5.827 E-05$ 8. $348 \mathrm{E}-05$ $8.812 E-05$ $4.884 \mathrm{E}-05$ $5.971 E-05$ $7.844 E-05$ $1.056 E-04$ $1.510 \mathrm{E}-04$ $5.274 E-04$ $7.917 E-04$ $1.516 E-03$ $4.192 \mathrm{E}-03$ .080E-02 $2.973 E-03$ ANGLE 17 
(GAMMAS/MEV/STERADIAN/SOURCE NEUTRON) GROUP (MEV) 8.00E OO-D-1.00E 01 6.50 E $00---8.00 \mathrm{E}$ OD $5.00 E$ DO-D- $6.50 E$ OO $4.00 E$ OD- $-5.00 E$ OO $3.00 \mathrm{E} 00-2-4.00 \mathrm{E}$ CO $2.50 E$ OO- - 3.00E 00 $2.00 E$ OO- $-2.50 E$ OO $1.66 \mathrm{E} 00-2.00 \mathrm{E} 00$ $1.33 \mathrm{E} \quad 00--1.66 \mathrm{E} \quad 00$ $1.00 E$ OO-D-1.33E CO $8.00 E-01--1$. COE 00 $6.00 E-01--8.00 E-01$ $4.00 \mathrm{E}-01--6.00 \mathrm{E}-01$ $3.00 E-01--4.00 E-01$ $2.00 E-01--3.00 E-01$ 1. $C O E-01--2.00 E-01$ 5. OCE-O2- - - 1.0OE-OI 2. 0 OEE-02-D-5. OOE-02

ENERGY

GROUP (MEV)

8.00E OO--1.00E 01 $6.5 \mathrm{NE}$ 00-- $8.00 \mathrm{E} \mathrm{OO}$ $5.0 \mathrm{HE}$ DO-D-6.50E nO $4.00 E$ OO- $-5.00 E$ OO $3.0 \mathrm{CE}$ OO-- 4 . COE 00 2.5CE DO--3.00E 00 $2.00 E$ OO---2.5CE 00 $1.6 E E$ OC- 2 . NOE DO $3.33 E$ OO-D-1.66E 00 $1.00 E$ OD- $1.33 E$ EO . ODE-OL 4 OOE $O 1-C .005-01$ $3.00 E-C 1--4.00 E-01$ $3.00 E-C 1--4.00 E-O 1$ 1.0OE-01$5.0 O E-02-O-100 E-01$ $2.00 \mathrm{E}-\mathrm{C2}-\mathrm{2}-5.00 \mathrm{E}-02$
ANGLE 1
$M U=-1.0000$
$M U=-0.986204$ $.826 \mathrm{E}-05$ $.051 \mathrm{E}-04$ $5.670 \mathrm{~F}-05$ 4 $4.575 E-05$ $4.604 E-05$ $3.454 E-05$ $4.965 \mathrm{E}-05$ $.92 .3 E-05$ $.866 \mathrm{E}-05$ $5.294 \mathrm{E}-04$ $7.417 E-04$ $1.915 E-03$ $5.229 E-03$ $1.407 E-02$ $4.057 E-03$ ANGLE 10 $M U=0.0950$ $1.463 E-05$
$3.010 E-05$ $3.010 E-05$ $3.316 \mathrm{E}-04$ $9.833 \mathrm{E}-05$ $1.686 E-04$ $1.093 \mathrm{E}-04$ $1.189 E-04$ $1.7697 \mathrm{E}-04$ $1.047 \mathrm{E}-04$ $1.365 \mathrm{E}-04$ $1.877 E-C 4$ 2.816E-04 $1.338 \mathrm{E}-03$ $1.230 \mathrm{E}-0 \mathrm{~B}$ $2.116 E-03$ $1.592 \mathrm{E}-02$ $4.289 \mathrm{E}-03$
ANGLE 3 $8.855 E-C 6 \quad 8.957 E-06$ $1.833 E-05$ 2.C59E-04 $5.655 E-05$ $1.013 E-04$ $4.643 E-05$ $4.722 E-05$ $3.530 E-05$ $4.955 E-05$ $6.859 E-05$ $8.8 E 5 E-C 5$ $1.256 \mathrm{E}-04$ $5.321 E-04$ $7.437 E-C 4$ $1.018 \mathrm{E}-03$ $5.235 \mathrm{E}-03$ $1.408 \mathrm{E}-02$ $4.059 \varepsilon-03$ $\triangle N G L F 11$ $M U=0.2816$ $1.639 \mathrm{E}-05$
$3.366 \mathrm{E}-\mathrm{C5}$ $3.6 \subseteq 1 E-04$ $1.111 \mathrm{E}-04$ $1.892 E-04$ $1.336 E-04$ $1.459 E-04$ 2.C56E-04 1.239E-04 2. $265 E-04$ $3.454 E-04$ 1. $306 E-03$ $2.179 E-03$ $1.634 E-02$ $4.324 E-03$ 列 $5.799 E-C 5$ $1.0305-04$ 4.896E-05 $5.147 E-05$ $3.801 E-05$ $4.944 E-05$ $6.676 \mathrm{E}-05$ $8.911 E-05$ 1.274E-04 $5.429 E-04$ $7.537 \mathrm{E}-04$ $1.930 E-03$ $5.263 \mathrm{E}-03$ $.414 \mathrm{E}-02$ $4.067 E-03$ $\triangle N G L E 12$ $M U=0.4580$ $1.844 \mathrm{E}-05$
$3.781 \mathrm{E}-\mathrm{C5}$ $3.781 E-C 5$
$4.126 E-04$ $4.126 E-04$
$1.261 E-04$ $1.261 \mathrm{E}-04$ $2.131 E-04$ $3.673 E-04$ $1.813 E-04$ $1.271 E-04$ $1.510 E-04$ 2.006E-O4 2.820E-04 $1.114 E-03$ $1.114 E-03$ 1. C. 5 E $1.677 \mathrm{E}-02$ $4.385 E-03$ ANGLE $\quad 4$
$M U=-0.8656$ $0.278 E-06$ $1.920 \mathrm{~F}-05$ $2.153 E-04$ $6.002 E-05$ .064E-04 $5.306 \mathrm{E}-05$ $5.778 \mathrm{E}-05$ $4.213 E-05$ $5.013 \mathrm{E}-05$ $6.571 E-05$ $9.163 E-05$ 1.386E-04 $5.621 \mathrm{E}-04$ 7.785E-04 $1.950 \mathrm{E}-03$ . $316 E-13$ 作

ANGLE 13 $M U=0.6179$ $2.075 E-05$
$4.247 F-05$ $4.247 F-05$ $4.611 E-04$ $1.431 \mathrm{E}-04$ $2.403 E-04$ $2.155 E-04$ $2.313 E-04$ 1.578E-04 $1.936 E-04$ 3. $05 E-04$ $3.505 E-04$ 1. $211 \mathrm{E}-03$ 1.451E-03 1.451E-03 $6.031 \mathrm{E}-03$ $4.432 \mathrm{E}-03$
ANGLE 5
$M U=-0.7550$ 9.697E-06 $2.006 \mathrm{E}-05$ $2.245 E-04$ $6.302 \mathrm{E}-05$ $1.113 F-04$ $.381 \mathrm{E}-05$ 4.627E-05 $5.281 E-05$ $6.848 E-05$ $9.797 \mathrm{E}-05$ 1. $515 E-04$ $5.880 \mathrm{E}-04$ . 247E-04 $1.972 E-03$ $5.391 \mathrm{E}-03$ $.442 \mathrm{E}-02$ $4.103 E-03$

ANGLE 14

$U=0.7550$
$2.320 E-05$ 4. $741 E-05$ $5.122 E-04$ $1.612 E-04$ $2.699 E-04$ $2.964 E-04$ 3.2. $93 E-04$ ..1 02 E-04 2.613E-04 $4.464 E-04$ $4.560 E-04$ . $302 \mathrm{E}-03$ 1. $302 \mathrm{E}-03$ $2.451 E-03$ $7.451 E-03$ $1.763 E-02$ $4.475 E-03$
AVGLE 6 1. $027 \mathrm{E}-05$ $2.123 E-05$ $2.372 E-04$ $6.709 E-05$ $1.180 \mathrm{E}-04$ $6.278 \mathrm{E}-05$ $6.705 E-05$ 5.030E-05 $5.324 E-05$ $7.633 \mathrm{E}-05$ $.087 \mathrm{E}-04$ $1.655 \mathrm{E}-04$ $6.224 E-04$ . $932 E-04$ . $992 \mathrm{E}-03$ $5.493 E-03$ $1.463 \mathrm{E}-02$ 作

ANGLE 15 $M U=0.8656$
$2.558 \mathrm{E}-05$ $5.220 \mathrm{E}-05$ $5.615 \mathrm{E}-04$ $1.789 \mathrm{E}-04$ $3.013 E-04$ $4.574 \mathrm{E}-04$ 5.01 8E-04 $30 \mathrm{~F}-04$ $3.696 E-04$ 4. $585 \mathrm{E}-04$ $5.516 E-04$ $395-0$ $1.543 \mathrm{E}-\mathrm{n} 3$ $2.552 E-03$ 7. $201 \mathrm{E}-02$ 4. $512 \mathrm{E}-03$

ANGLE 7 $M U=-0.4580$ $1.122 E-05$
$2.276 E-05$ $2.536 E-04$ $7.241 \mathrm{E}-05$ $1.267 E-04$ $6.903 E-05$ $7.479 \mathrm{E}-05$ $5.515 \mathrm{E}-05$ $6.655 \mathrm{E}-\mathrm{CS}$ $8.854 E-05$ . $232 E-04$ $1.818 \mathrm{E}-04$ $6.687 \mathrm{E}-04$ $9.791 \varepsilon-04$ $2.012 E-03$ $5.614 \mathrm{E}-03$ $1.488 \mathrm{E}-02$ 4.162E-03 ANGLE 16 $M U=0.9446$
$2.759 E-05$ $2.759 \mathrm{E}-05$
$5.622 \mathrm{E}-05$ 
IGAMMAS/MEV/STERADIAN/SOURCE NEUTRONI

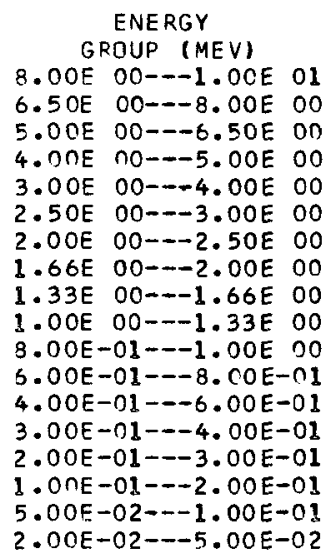

ENERGY$$
\text { GROUP (MEV) }
$$

8. OOE NO--1.

$6.50 E$ OO- - $8.00 E$ OO $5.00 E$ OO- $-6.50 E$ OO 4.0OE $00--5.00 E$ OO $3.00 E$ OO- $-4.00 E$ OO 2.50E OO- - $3.00 E$ OO $2.00 E$ EO- - 2.50E OO ?.66E $10--2.00 E$ OO $1.33 E$ COD- $1.66 E$ OO $1.00 \mathrm{~N}$ OO- - $1.33 \mathrm{E}$ O0 8.0DE-O1---1.00E OD $6.00 \mathrm{E}-01--8.00 \mathrm{E}-0$. 4. OOE $01---6.00 E-01$ $3.00 E-01-2.00 \mathrm{E}-01$ 1. $.00 E-01---2.00 E-01$ $1.00 E-01-2.00 E-01$ $2.00 \mathrm{E}-02--5.00 \mathrm{E}-02$ $M U=-1.0000$ $1.994 \mathrm{E}-05$ $2.294 \mathrm{E}-05$ $6.245 E-05$ l. $097 E-04$ $1.097 E-04$ $4.073 E-05$ $3.067 E-05$ $5.132 E-05$ $7.157 E-05$ $9.335 \mathrm{E}-05$ $1.287 E-04$ $6.087 E-04$ $8.638 \mathrm{E}-04$ $2.289 E-03$ $6.273 E-03$ $1.715 \mathrm{E}-02$ 4. $979 E-03$

ANGLE 10 $M U=0.0950$ $.736 E-05$
$3.569 E-05$ $3.925 E-04$ $1.171 E-04$ $1.996 \mathrm{E}-04$ $1.157 \mathrm{E}-04$ $1.242 E-04$ $1.639 E-04$ $2.280 E-04$ $3.443 E-04$ $1.104 \mathrm{E}-03$ $1.481 E-03$ $2.528 \mathrm{E}-03$ $7.427 \mathrm{E}-03$ $1.958 \mathrm{E}-02$ $5.280 E-03$
ANGLE 2 $9.673 E-06$ $2.003 E-05$ $2.254 \mathrm{E}-04$ $6.183 \mathrm{E}-05$ $1.103 E-04$ $1.103 E-04$
$4.032 E-05$ $4.183 \mathrm{E}-05$ $3.733 E-05$ $5.131 E-05$ $5.131 \mathrm{E}-05$
$7.108 \mathrm{E}-05$ $9.351 \mathrm{E}-05$ $9.351 E-05$ $6.122 E-04$ $8.674 \mathrm{E}-04$ $2.292 E-03$ $6.282 \mathrm{E}-03$ 1.717E-O2 4. $982 E-03$

ANGLE 11 $M U=0.2816$ 1.993E-05 $4.472 \mathrm{E}-04$ $1.360 E-04$ $2.294 E-04$ $1.302 E-04$ $1.447 E-04$ 1.271E-04 $1.507 E-04$ $1.986 E-C 4$ $2.703 \varepsilon-04$ 4. $217 E-04$ 1. $232 E-03$ $1.566 E-03$ $2.606 \mathrm{E}-03$ $7.699 \varepsilon-03$ $2.012 \mathrm{E}-02$ $5.342 E-03$
ANGLE 3 $M U=-0.9446$ $9.851 E-06$
$2.040 E-05$ $2.040 E-05$ $6.317 E-05$ . $125 \mathrm{E}-04$ $1.125 \mathrm{E}-04$
$4.276 \mathrm{E}-05$ 4.582E-05 4.011E-05 4.011E-05 $6.983 \mathrm{E}-05$ $.468 \mathrm{E}-05$ $9.468 \mathrm{E}-05$ $6.263 E-04$ $8.840 E-04$ $2.305 \mathrm{E}-03$ $6.318 \mathrm{E}-03$ $1.725 E-02$ 4. $992 E-03$

ANGLE 12 $M U=0.4580$ $.309 E-05$ . $135 \mathrm{E}-04$ .658E-04 . 812 E-04 $1.566 \mathrm{E}-04$ $1.875 E-04$ $2.497 E-04$ $3.505 E-04$ $5.138 E-04$ $.358 \mathrm{E}-03$ $1.652 \mathrm{E}-03$ $2.700 \mathrm{E}-03$ 7. $989 \mathrm{E}-03$ $2.067 E-02$ $5.403 E-03$
ANGLE 4 $M U=-0.8656$
$1.020 E-05$ 1.112E-05 $2.112 E-05$ $2.376 \mathrm{E}-05$ $1.167 \mathrm{E}-04$ $1.167 E-04$
$4.670 E-05$ $5.174 E-05$ $4.440 E-05$ $5.296 E-05$ $6.989 \mathrm{E}-05$ . $860 \mathrm{E}-05$ $9.860 \mathrm{E}-05$
$1.515 \mathrm{E}-04$ $6.520 E-04$ 9.206 E-04 $2.325 \mathrm{E}-03$ $6.386 \mathrm{E}-03$ 1.740E-02 5. $011 \mathrm{E}-03$ ANGLE 13 $M U=0.6179$ $2.684 E-05$
$5.481 \mathrm{E}-05$ $5.913 E-04$ $1.872 \mathrm{E}-04$ 3.090 E-04 $2.121 E-04$ $2.313 E-04$ $1.966 \mathrm{E}-04$ $2.411 E-04$ $3.235 \mathrm{E}-04$ $4.432 E-04$ $6.149 E-04$ $1.476 E-03$ $1.745 E-03$ $8.289 \mathrm{E}-03$ $2.123 E-02$ $5.463 E-03$
ANGLE 5 $1.073 \mathrm{E}-05$ $2.220 E-05$ $2.488 E-04$ $0.455 E-05$ 1. $229 E-04$ 5.119E-05 $5.745 \mathrm{E}-05$ $4.898 \mathrm{E}-05$ $5.662 \mathrm{E}-05$ $7.424 E-05$ $1.071 \mathrm{E}-04$ $1.675 E-04$ $6.876 E-04$ $9.815 \mathrm{E}-04$ $2.347 E-03$ $6.484 \mathrm{E}-03$ $1.761 E-02$ 5. $039 E-03$

ANGLE 14 $M U=0.7550$
$3.105 \mathrm{E}-05$ $6.327 E-05$ $6.779 E-04$ .187E-04 $3.577 \mathrm{E}-04$ $.132 \mathrm{E}-04$ .592E-04 .206E-04 4.227E-04 5. $27 E-04$ $7.170 \mathrm{E}-04$ $1.584 \mathrm{E}-03$ 1. $850 \mathrm{E}-03$ $8.586 \mathrm{E}-03$ $2.176 \mathrm{E}-02$ $5.517 E-03$
AVILE 6 1. $145 \mathrm{E}-05$
$2.369 \mathrm{E}-05$ $2.369 \mathrm{E}-05$ $2.649 \mathrm{E}-04$ $7.472 \mathrm{E}-05$ $7.472 E-05$
$1.313 E-04$ $1.313 \mathrm{E}-04$
$5.621 \mathrm{E}-05$ $5.621 E-05$ $6.263 E-05$
$5.388 E-05$ $6.340 E-05$ $6.340 E-05$
$8.419 \mathrm{E}-05$ $8.419 E-05$
$1.209 E-04$ $1.209 E-04$
$1.862 E-04$ $7.353 \mathrm{E}-04$ $1.067 \mathrm{E}-03$ $2.370 E-03$ $6.612 \mathrm{~F}-03$ $1.789 \mathrm{E}-02$ $5.074 E-03$

ANGLE 15 $M U=0.8656$ $3.539 \mathrm{E}-05$ $7.664 \mathrm{E}-04$ $2.512 \mathrm{E}-04$ $4.096 \mathrm{E}-04$ $4.262 \varepsilon-04$ .691E-04 $4.396 \mathrm{E}-04$ $6.682 \mathrm{E}-04$ $8.124 \mathrm{E}-04$ $1.690 E-03$ $1.959 E-03$ $3.034 E-03$ $8.864 E-03$ $.223 E-02$ $5.562 E-03$

$\triangle N G L E \quad 7$ $M U=-0.4580$ $1.242 E-05$ $2.566 E-05$ . $1.425 \mathrm{E}-04$
1.04 $1.425 E-04$
$6.254 E-05$ $6.866 E-05$ $6.021 \mathrm{E}-05$ $7.359 E-05$ $9.921 E-05$ $9.921 E-05$ $2.092 \mathrm{E}-04$ $7.986 \mathrm{E}-04$ $1.171 \mathrm{E}-03$ $2.395 E-03$ $6.771 E-03$ $1.823 E-02$ $5.117 E-03$

ANGLE 16 $M U=0.9446$ $3.926 \mathrm{E}-05$ $8.444 E-04$ $2.802 E-04$ $4.632 E-04$ $7.709 E-04$ $8.612 \mathrm{E}-04$ $5.737 E-04$ $6.211 E-04$ $6.923 E-04$ 7. $771 E-04$ $8.964 E-04$
$1.803 E-03$ $2.060 \mathrm{E}-03$ 
(GAMMAS/MEV/STERADIAN/SOURCE NEUTRON)

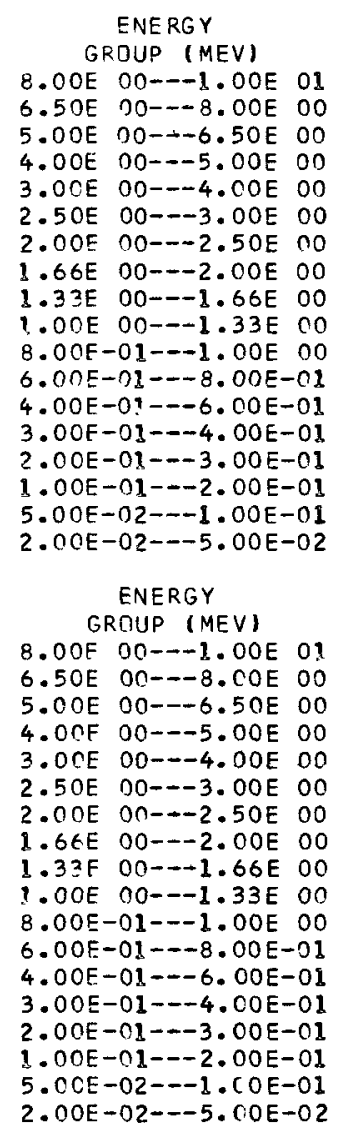

ANGLE 1 ANGLE 2

ANGLE 3

ANGLE 4 ANGLE 5 $A V G L E \quad 6$
$M U=-3.6179$ $9.409 E-06 \quad 9.450 E-06$ $M U=-0.9446$ $M U=-0.8656$ $M U=-0.7550$

ANGLE 7 $M U=-0.4580$ $1.948 \mathrm{E}-05 \quad 1.958 \mathrm{E}-05$ $2.197 E-04$ $5.964 \mathrm{E}-05$ $1.069 E-04$
$3.340 E-05$ $3.503 \mathrm{E}-05$ $3.503 E-05$ $3.517 \mathrm{E}-\mathrm{C5}$
$4.855 \mathrm{E}-05$ $6.754 \mathrm{E}-05$ $8.898 \mathrm{E}-05$ $1.253 E-04$ $6.318 \mathrm{E}-04$ $9.108 \mathrm{E}-04$ $2.515 \mathrm{E}-03$ $6.885 \mathrm{E}-03$ $1.908 \mathrm{E}-02$ 5. $573 E-03$ . $6.003 E-05$ $1.075 \mathrm{E}-04$ $3.400 E-05$ $3.509 \mathrm{E}-05$ $3.584 E-05$ $4.859 E-05$ $6.719 E-C 5$ $8.929 E-05$ 1.274E-04 $6.359 E-C 4$ 9.1 AIE-04 $2.518 E-03$ $6.895 \mathrm{E}-03$ $1.910 E-02$ ANGLE 10 $F-05$ $2.251 \mathrm{E}-04$ $6.155 E-05$
$1.100 E-04$ $1.100 E-04$
$3.624 E-05$ $3.624 E-05$
$3.949 E-05$ $3.838 E-05$ $3.838 E-05$
$4.902 E-05$ $4.902 E-05$ $9.105 E-05$ 1.356E-04 $6.523 E-04$ $9.393 E-04$ $2.530 E-03$ $6.938 \mathrm{E}-03$ $1.920 \mathrm{E}-02$
$5.588 \mathrm{E}-03$ $77 E-05$ 2. $336 E-04$ $6.447 \mathrm{E}-05$ $1.146 \mathrm{E}-04$ $3.987 E-05$ $4.471 E-05$ $4.240 E-05$
$5.072 \mathrm{E}-05$ $6.735 \mathrm{E}-05$ $9.597 E-05$ $1.496 E-04$ $6.828 E-04$ $9.873 E-04$ $2.548 \mathrm{E}-03$ 7.019E-03 $1.938 \mathrm{E}-02$ 5.611E-03 6. $2.03 \mathrm{E}$ $6.863 \mathrm{E}-05 \quad 2.637 \mathrm{E}-04$ 1.213E- 04 $1.213 E-04$
$4.405 E-05$ $4.405 \mathrm{E}-05$
$4.988 \mathrm{E}-05$ $4.696 E-05$ $4.696 E-05$
$5.484 E-05$ $7.265 E-05$ $1.057 \mathrm{E}-04$ $1.375 E-04$ $7.262 E-04$ $1.062 \mathrm{E}-03$ $2.568 \mathrm{E}-03$ $7.133 \mathrm{E}-03$ $1.963 E-02$
$5.643 E-03$

.426E-05 4. $885 \mathrm{E}-05$ $5.489 \mathrm{E}-05$ $5.229 \mathrm{E}-05$ $6.227 E-05$ $8.370 \mathrm{E}-05$ $1.213 E-04$ $1.896 \mathrm{E}-04$ $7.852 E-04$ $1.162 \mathrm{E}-03$ $2.591 \mathrm{E}-03$ $7.284 E-03$ $1.996 \mathrm{E}-02$
$5.685 \mathrm{E}-03$ ANGLE 11 ANGLE 12 ANGLE 13 1.821E-05 $4.743 E-05$ $1.234 \mathrm{E}-04$ $2.094 E-04$ $9.792 E-05$ $1.102 \mathrm{E}-04$ 1. $097 \mathrm{E}-04$ $1.333 E-04$ $1.784 \mathrm{E}-04$ $2.518 \mathrm{E}-04$ $3.844 E-04$ $1.223 E-03$ $1.628 E-03$ $2.772 E-03$ $8.238 E-03$ $2.1970-02$ $M U=0.2816$ $4.388 \mathrm{E}-05$ $4.782 \mathrm{E}-04$ $1.470 \mathrm{E}-04$ $2.463 E-04$ 1. $250 \mathrm{E}-04$ $1.409 E-04$ 1. $3 \subseteq 1 E-04$ $1.668 \mathrm{E}-04$ $2.225 E-04$ $3.150 E-04$ 4. $753 E-04$ $1.369 \mathrm{E}-03$ $1.727 E-03$ 2.861E-03 $8.553 E-03$ $2.260 E-02$ $2.549 E-05$ $5.211 \varepsilon-05$ $5.634 E-04$
$1.775 E-04$ 2. $934 E-04$ 1.612E-04 $1.803 E-04$ $1.765 \mathrm{E}-04$ $2.131 \mathrm{E}-04$ $2.852 E-04$ 3. $994 \mathrm{E}-04$ $5.806 \mathrm{E}-04$ $1.513 \mathrm{E}-03$ $1.826 E-03$ $2.967 E-03$ $8.886 \mathrm{E}-03$ $2.326 E-02$ ANGLE 14 ANGLE 15 0.6179 $M U=0.7550$ $3.061 \mathrm{~F}-05$ (2) $2.684 \mathrm{E}-04$ $3.518 E-04$ $2.111 E-04$ $2.333 E-04$ $2.267 E-04$ $2.779 \mathrm{E}-04$ $3.715 E-04$ $5.048 \mathrm{E}-04$ $6.930 E-04$ $1.647 \mathrm{E}-03$ $3.933 E-03$ (.085 $9.226 \mathrm{E}-03$ $6.142 E-03$ O. $\quad A U=0.8656$ $7.454 \mathrm{E}-05$ $7.917 \mathrm{E}-04$ $2.620 E-04$ 4. $210 E-04$ $2.851 E-04$ $3.147 E-04$ $3.000 E-04$ $3.685 \mathrm{E}-04$ $4.818 E-04$ $6.245 \mathrm{E}-04$ $8.034 \mathrm{E}-04$ $1.770 \mathrm{E}-03$ 2.048E-03 $3.206 \mathrm{E}-03$ $9.557 E-03$ $6.204 E-03$ $1.246 E-05$ 2.575E-05 $8.180 E-05$ $1.428 E-04$ $5.510 \mathrm{E}-05$ $6.110 \mathrm{E}-05$ $5.954 E-05$ $5.954 \mathrm{E}-05$ $1.002 \mathrm{E}-04$ $1.428 \mathrm{E}-04$ $2.181 E-04$ $8.629 E-04$ $1.280 \mathrm{E}-03$ $2.617 \mathrm{E}-03$ $7.471 \mathrm{E}-03$ $2.036 E-02$
$5.736 E-03$

ANGLE 16 $\begin{aligned} & M U=0.9446 \\ & 4.964 E-05\end{aligned}$ $1.003 \mathrm{E}-04$ $1.049 E-03$ $3.601 \mathrm{E}-04$ 5.740E-O $6.963 \mathrm{E}-04$ $7.858 \mathrm{E}-04$ $6.182 E-04$ $6.753 \mathrm{E}-04$ $7.580 E-04$ $8.535 E-04$ $9.85 I E-04$ $2.001 E-03$ $2.262 E-03$ $3.433 E-03$ $1.011 E-02$

$6.296 E-03$ $2.505 E-02$

ANGLE 8 $M U=-0.2816$ $1.388 \mathrm{E}-05$ .864E-05 $3.280 \mathrm{E}-04$ $1.592 E-04$ $6.431 \mathrm{E}-0 \mathrm{~S}$ $6.431 E-05$
$7.099 E-05$ $7.056 E-05$ $8.864 E-05$ $1.212 \mathrm{E}-04$ $2.573 E-04$ $9.627 E-04$ $2.652 E-03$ $7.693 \mathrm{E}-03$ $5.794 \mathrm{E}-03$ ANGLE 17 $5.403 E-05 \quad 2.637 E-04$ $1.090 \mathrm{E}-04 \quad 5.394 \mathrm{E}-04$ $3.929 \mathrm{E}-04 \quad 1.822 \mathrm{E}-03$ $6.611 E-04 \quad 3.036 E-03$ $1.761 E-03 \quad 2.021 E-03$ $1.821 E-03 \quad 2.233 E-03$ $9.800 E-04 \quad 1.964 E-03$ $9.384 E-04 \quad 2.314 E-C 3$ $9.163 E-04 \quad 2.949 E-03$ $9.396 \mathrm{E}-04 \quad 3.886 \mathrm{E}-03$ $1.049 E-03 \quad 5.376 E-03$ $2.111 \mathrm{E}-03 \quad 1.523 \mathrm{E}-02$ $2.333 E-03 \quad 1.967 E-02$ $3.515 E-03$ 3.551E-02 $1.027 E-02$ 1.036E-01

$6.319 \mathrm{E}-03 \quad 7.428 \mathrm{E}-02$ 
(GAMMAS/MEV/STERADIAN/SOURCE NEUTRON)

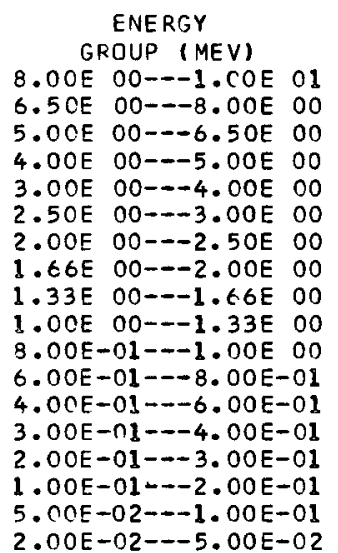

ENERGY

$$
\text { GROUP IMEVI }
$$

$8.00 E$ OO- - - 1.00E 01 $6.50 E$ OO---8.00E 00
$5.00 E$ OO- - 6.5OE NO $4.00 E$ OO- $-5.00 E$ OO $3.00 E$ OO- $-4.00 E$ OO $2.50 F \quad 00--3.00 E \quad 00$ 2.00 E $00---2.50 E \quad 00$ 1.66 E $00--2.00 E$ CO $1.33 \mathrm{E}$ 00---1.66E 00 $1.00 E$ OD---1.33E 00 $8.00 E-01--1.00 E 00$ $6.00 \mathrm{E}-01---8.00 \mathrm{E}-01$ $4.00 E-01--6.00 E-01$ $3.00 \mathrm{E}-01--4.00 \mathrm{E}-01$ $2.00 E-01--3.00 E-01$ $1.00 E-01--2.00 E-01$ $5.00 \mathrm{E}-02--1$. COE-OI $2.00 E-02---5.00 E-02$
ANGLE 1 $5.782 \mathrm{E}-06$ $1.194 \mathrm{E}-05$ $1.354 \mathrm{E}-04$ $0.475 E-05$ $1.615 \mathrm{E}-05$ $1.815 E-05$ $2.816 \mathrm{E}-05$ $2.216 \mathrm{E}-05$ $2.994 \mathrm{E}-05$ $4.029 E-05$
$5.186 E-05$ $5.186 \mathrm{E}-05$
$7.557 \mathrm{E}-05$ $7.557 E-05$
$4.809 E-04$ $4.809 \mathrm{E}-04$
$7.257 \mathrm{E}-04$ $2.387 E-03$ $2.387 E-03$
$6.437 E-03$ $6.437 E-03$
$1.840 E-02$ 1.849E-02 ANGLE 10 $M U=0.0950$ $1.320 E-05$
$2.707 E-05$ $2.707 E-05$ $9.061 \mathrm{E}-05$ $1.523 \mathrm{E}-04$ $6.543 \mathrm{E}-05$ $7.581 \mathrm{E}-05$ $8.694 \mathrm{E}-05$ $1.091 \mathrm{E}-04$ l. $540 E-04$ $2.310 E-04$ $3.718 \mathrm{E}-04$ $1.153 \mathrm{E}-03$ $1.538 \mathrm{E}-03$ $2.579 \mathrm{E}-03$ $7.929 \mathrm{E}-03$ $2.177 E-02$ $5.891 E-03$
ANGLE ? $5.820 E-06$ 1.202E-05 $1.363 E-04$ $3.566 E-05$ $1.656 E-05$ $1.854 E-05$ $2.237 \mathrm{E}-05$ $2.978 \mathrm{E}-05$ $4.978 \mathrm{E}-05$ $5.239 \mathrm{E}-05$ $7.747 \mathrm{E}-05$ $4.846 E-04$ $4.846 \mathrm{E}-04$
$7.341 \mathrm{E}-04$ $7.341 E-04$
$2.388 E-03$ $2.388 \mathrm{E}-03$ $6.449 E-03$
$1.851 E-02$ 5.491E-03 ANGLE 11 $M U=0.2816$ $1.646 \mathrm{E}-05$ $3.634 E-04$ 1.154E-04 $1.905 E-04$ 9.05 OE-05 $1.053 \mathrm{E}-04$ $1.207 E-04$ $1.507 E-04$ 2.116 F-04 3.123E-04 4. $809 E-04$ $1.310 \mathrm{E}-03$ $1.639 E-03$ $2.673 \mathrm{E}-03$ $8.277 \mathrm{E}-03$ $2.250 \mathrm{E}-02$ $5.973 \mathrm{E}-03$
ANGLE 3 $5.972 \mathrm{E}-06$ 5. $972 E-06$ $1.236 E-05$ $3.338 E-05$ $6.763 E-05$ $1.809 \mathrm{E}-05$ $2.063 \mathrm{E}-05$ $2.327 E-05$ $2.327 E-05$ $2.949 E-05$
$3.968 E-05$ $5.478 E-05$ $8.489 E-05$ 5.006E-04 $7.696 \mathrm{E}-04$ $7.696 \mathrm{E}-04$
$2.393 \mathrm{E}-03$ $6.496 \mathrm{E}-03$ $1.862 \mathrm{E}-02$ $5.505 E-03$

ANGLE 12 $M U=0.4580$ $4.306 E-05$ $4.588 \mathrm{E}-04$ $1.514 E-04$ $2.449 E-04$ 1.281 E- 04 $1.482 E-04$ $1.702 \mathrm{E}-04$ $2.124 E-04$ $2.935 \mathrm{E}-04$ $4.165 E-04$ $6.009 E-04$ $1.461 E-03$ $1.744 E-03$ $2.786 \mathrm{E}-03$ $8.644 E-03$ $2.324 \mathrm{E}-02$ $6.055 E-03$
ANGLE 4
ANGLE 5
$6=-0.8656$
$M U I=-0.7550$ $6.263 E-06$ ANGLE 5 ANGLE 6 7.290E-06 $1.464 E-04$ $7.021 E-05$ 2.040E-05 $2.325 \mathrm{E}-05$ $2.503 E-05$ $3.011 E-05$ $3.011 E-05$
$4.075 E-05$ $5.998 \mathrm{E}-05$ $9.740 E-05$ 5. $319 E-04$ $5.319 E-04$
$8.370 E-04$ $2.400 E-03$ $6.584 \mathrm{E}-03$ $1.882 \mathrm{E}-02$ 5. $531 E-03$ ANGLE 13 $M U=0.6179$ $5.653 \mathrm{E}-05$ $5.930 E-04$ 2.039E-04 $3.226 E-04$ $1.843 E-04$ $2.107 E-04$ $2.410 E-04$ $2.988 E-04$ 4. $008 \mathrm{E}-04$ $5.384 E-04$ $7.216 \mathrm{E}-04$ $1.602 \mathrm{E}-03$ $1.857 \mathrm{E}-03$ $2.909 \mathrm{E}-03$ $9.016 \mathrm{E}-03$ $2.398 \mathrm{E}-02$ $6.133 E-03$ $4.363 E-05$ $7.688 E-05$ $2.315 \mathrm{E}-05$ $2.618 \mathrm{E}-05$ $2.784 E-05$ 3.309E-05 $4.541 E-05$ $6.875 \mathrm{E}-05$ $1.135 E-04$
$5.801 E-04$ $9.801 E-04$ $2.408 E-03$ $6.710 E-03$ $1.911 E-02$ $5.568 E-03$ ANGLE 14 $M U=0.7550$ 7. $337 \mathrm{E}-05$ $7.774 \mathrm{E}-04$ $2.788 E-04$ $4.299 E-04$ $2.673 E-04$ $3.019 E-04$ $3.392 E-04$ $5.274 E-04$ $6.274 E-04$ $8.326 E-04$ $1.731 E-03$ $1.976 \mathrm{E}-03$ $3.034 E-03$ $9.371 E-03$ $2.466 E-02$ $6.203 E-03$ ANILE 15 $M U=0.8656$ 4. $999 E \rightarrow 05$ 1. $014 \mathrm{E}-03$ $3.774 E-04$ $5.669 E-04$ $3.881 E-04$ $4.357 E-04$ 4.71 $5 E-04$ $6.617 E-04$ $7.845 E-04$ $1.851 \mathrm{E}-03$ $1.851 E-03$ $3.152 E-03$ $9.686 E-03$ $2.525 \mathrm{E}-02$ $6.261 E-03$ $M U=-0.4580$ ANGLE 8 $1.511 E-05$ 1.673E-0 $2.913 E-0522.243 E-05$ 1.675E-05 $5.327 E-05$ 6.134E-05 $7.472 E-04$ 4. $375 E-05$ $0.305 E-05 \quad 1.063 E-04 \quad 1.254 E-04$ $2.326 E-05 \quad 3.059 E-05 \quad 3.7511 E-05 \quad 4.254 E-04$ $2.967 E-05 \quad 3.464 E-05 \quad 4.273 E-05 \quad 5.579 E-05$ $3.222 E-05 \quad 3.888 E-05 \quad 4.897 E-05$ T. $3.930 E-05 \quad 4.903 E-05 \quad 6.261 E-05 \quad 8.418 E-05$ $\begin{array}{llll}3.472 E-05 & 6.903 E-05 & 6.261 E-05 & 8.147 E-05 \\ & 6.891 E-05 & 8.833 E-05 & 1.151 E-04\end{array}$ $\begin{array}{llll}8.224 E-05 & 1.019 E-04 & 1.304 E-04 & 1.151 E-04\end{array}$ $\begin{array}{llll}1.352 E-04 & 1.666 E-04 & 2.337 E-04 & 1.718 E-04\end{array}$ $\begin{array}{llll}6.493 \mathrm{E}-04 & 7.425 \mathrm{E}-04 & 8.606 \mathrm{E}-04 & 1.000 \mathrm{E}-03\end{array}$ $\begin{array}{llll}1.051 \mathrm{E}-03 & 1.182 \mathrm{E}-03 & 1.312 \mathrm{E}-03 & 1.031 \mathrm{E}-03\end{array}$ $\begin{array}{llll}2.417 E-03 & 2.432 E-03 & 2.461 E-03 & 2.508 E-03\end{array}$ $6.876 \mathrm{E}-03 \quad 7.081 \mathrm{E}-03 \quad 7.326 \mathrm{E}-03 \quad 7.610 \mathrm{E}-03$ $1.948 \mathrm{E}-02 \quad 1.994 \mathrm{E}-02 \quad 2.048 \mathrm{E}-02 \quad 2.110 \mathrm{E}-02$ $5.615 E-03 \quad 5.672 \mathrm{E}-03 \quad 5.738 \mathrm{E}-03 \quad 5.812 \mathrm{E}-03$ ANGLE 16 $6.420 \mathrm{E}-05$ $1.277 E-04$ $1.277 E-03$ $4.872 E-04$ $5.801 E-04$ $6.488 \mathrm{E}-04$ $6.478 \mathrm{E}-04$ $7.080 \mathrm{E}-04$ $7.894 E-04$ $8.775 E-04$ $9.950 E-03$
$1.959 E-03$ $1.95 \mathrm{E}-03$ $3.249 \mathrm{E}-03$ $9.932 \mathrm{E}-03$ $2.570 E-02$ $6.304 E-03$ SCALAR

FLUX $2.321 E-04$ 4. $966 \mathrm{E}-03$ $1.663 E-03$ $2.655 \mathrm{E}-03$ $1.547 \mathrm{E}-03$ $1.740 \mathrm{E}-03$ $1.857 \mathrm{E}-03$ 2.207E-03 $2.831 E-03$ $3.736 E-03$ 
(GAMYAS/MEV/STERADIAN/SOURCE NEUTRON)

ENFRGY GROUP (MEV) 8.0OE OD---1.0OE 03 6.50E $00--8.00$ E 00 5.00 E ON-- 6.50 E 00 4.0OE O0---5.00E CD 3.00 E $00--4.00 E$ OO $2.50 \mathrm{E} 00---3.00 \mathrm{E}$ OO $2.00 E$ OO- $-2.50 E$ NO $1.66 \mathrm{E} 00---2.00 \mathrm{E} 00$ $1.33 \mathrm{E} 00--1.66 \mathrm{E} 00$ 1.00 E $00--1.33 E 00$ 8.0CE-01--1. 00 E 00 $6.00 E-01--8.00 E-01$ 4.00E-01-- - $6.00 \mathrm{E}-01$ $3.00 E-01--4.00 E-01$ 2. $00 \mathrm{E}-01--3.00 \mathrm{E}-01$ $3.00 E-01--2.00 E-01$
$5.00 E-02--1.00 E-01$ $5.00 \mathrm{E}-02--1.00 \mathrm{E}-01$
$2.00 \mathrm{E}-02---5.00 \mathrm{E}-02$

\section{ENERGY}

$$
\text { GRDUP (MEV) }
$$

$8.00 E$ OO--1. DOE 01 6.5OE DO---8.0OE OO $5.00 F$ DO---6.50E OO 4.0OE OO---5.0OE OO $3.00 E$ OO--C. $2.50 E$ DO-D-3.00E NO $2.00 E$ OD-C. $2.50 E$ CO $1.66 \mathrm{E}$ OO---2. OOE OO $1.00 F$ 00--1.33E 00 2.0OF $00-1.33 E$ OO $6.0 C E-02=-8.005-01$ $4.00 E-01-6.00 E-O 1$ $4.00 E-01-6.00 E-01$ 2.00E-01- $3.00 \mathrm{E}-01$ $2.00 E-01-2.00 E-01$ $5.00 \mathrm{E}-02-1.00 \mathrm{E}-02$ $2.00 E-02--5.00 E-02$
ANGLE 1 $2.430 \mathrm{~F}-06$ $7.933 E-06$ 1.114E-05 $1.507 E-05$ $1.832 \mathrm{E}-05$ $2.012 E-05$ $2.902 \mathrm{E}-05$ $2.637 E-04$ 4.096E-04 $1.661 \mathrm{~F}-03$ $4.372 \mathrm{E}-03$ $1.292 \mathrm{E}-02$ $3.884 \mathrm{E}-03$ ANGLE 10 $M U=0.0950$ $6.284 E-06$
$1.288 E-05$ $1.399 E-04$ $4.393 E-05$ 3.327E-05 $3.227 E-05$ $4.575 E-05$ $4.575 E-05$ ..955E-05 $1.486 \mathrm{E}-04$ $1.485 E-04$ 7. $1.056 \mathrm{E}-03$ $1.733 \mathrm{E}-03$ $5.495 E-03$ $1.547 E-02$ $4.197 E-03$
ANGLE 3 $2.452 F-06$ $.875 E-06$ $.043 F-06$ $1.095 \mathrm{E}-05$ $1.440 E-05$ $3.798 \mathrm{E}-05$ $2.063 E-05$ $3.055 E-05$ 2. $658 \mathrm{E}-04$ $4.172 F-04$ 1. $6 \in 1 E-03$ $4.380 \mathrm{E}-03$ $1.254 \mathrm{E}-02$ ANGLE 11 $M U=0.2816$
E.?31F-06 $1.680 \mathrm{~F}-05$ 1.797E-04 $5.020 \mathrm{E}-05$ $9.632 E-05$ $4.814 E-05$ 5.713E-05 . 97 E 05 I. $3885-04$ $2.388 E-04$ 2.1 $9 E-04$ 9. $228 E-04$ i. $122 \mathrm{E}-03$ 1. $801 \mathrm{E}-03$ $1.761 \mathrm{E}-03$
$1.504 \mathrm{E}-02$ 4.262E-03 ANGLE 5 2. $907 \mathrm{E}-06$ $6.062 \mathrm{E}-06$ $6.794 E-05$ $1.958 \mathrm{E}-05$ $3.389 \mathrm{E}-05$ $9.670 \mathrm{~F}-06$ $1.038 \mathrm{E}-05$ $1.101 E-05$ $1.310 E-05$ 1. $924 E-05$ $3.153 E-05$ $5.454 E-05$ 3. $298 \mathrm{E}-04$ $5.934 E-04$ 1.656E-03 4.576E-03 $1.340 E-02$
$3.945 E-03$

ANGLE 14 $M U=0,7550$ $2.462 \mathrm{E}-05$ $4.899 E-05$ 4.891E-04 $1.906 \mathrm{E}-04$ $2.856 E-04$ $1.973 E-04$ 2.289E-04 $2.703 E-04$ $3.294 E-04$ $4.195 E-04$ 6. $292 E-04$ 1. $201 \mathrm{E}-03$ $1.201 E-03$ $1.364 \mathrm{E}-03$ $6.602 \mathrm{E}-03$ $1.775 \mathrm{E}-02$ 4.441E-0?
ANGLE 6 $M U=-3.6179$ $3.189 \mathrm{E}-06$ $7.408 \mathrm{E}-05$ $2.122 \mathrm{E}-05$ $3.684 \mathrm{E}-05$ $1.092 \mathrm{E}-05$ $1.211 \mathrm{E}-05$ 1. $358 \mathrm{E}-05$ $1.688 \mathrm{E}-05$ $2.460 E-05$ $3.870 E-05$ $6.724 \mathrm{E}-05$ $3.829 \mathrm{E}-04$ $6.947 \mathrm{E}-04$ \$. $650 \mathrm{E}-03$ 4. $700 \mathrm{E}-03$ 1. $369 \mathrm{E}-02$ ANGLE 15 $M U=0.8656$
$3.770 E-05$ $.438 E-05$ $7.221 E-04$ $2.977 \mathrm{E}-04$ $4.273 E-04$ 3.130E-04 . $515 E-04$ 4.515E-04 . $6.954 E-04$ 1. $1.448 \mathrm{E}-03$ $1.448 \mathrm{E}-03$
$2.155 \mathrm{E}-\mathrm{n} 3$ $6.844 \mathrm{E}-03$ $6.844 \mathrm{E}-03$
$1.822 \mathrm{E}-02$ $4.487 \mathrm{E}-03$
ANGLE 7 $M U=-0.4580$ $3.581 \mathrm{E}-06$
$7.396 \mathrm{E}-06$ $8.254 E-05$ $2.327 E-05$ 4.084E-05 $.279 E-05$ $1.489 E-05$ 1.780E-05 $2.295 \mathrm{E}-05$ 3. $281 E-05$ $4.972 E-05$ 8.800E-05 $4.574 \mathrm{E}-04$ $8.008 E-04$ 1. $649 E-03$ $4.854 \mathrm{E}-03$ $1.404 \mathrm{E}-02$ 4.026E-03 ANGLE 16 $A N=0.9446$
$5.570 E-05$ $5.570 E-05$ $1.088 \mathrm{E}-04$ $1.031 E-03$ $4.385 E-04$ 4.725E-04 $4.725 E-04$ $5.143 E-04$ $5.389 E-04$ $6.261 E-04$ $6.749 E-04$ $7.433 E-04$ $1.333 E-04$ $1.367 \mathrm{E}-03$ $1.513 E-03$
$2.227 E-03$ $2.227 \mathrm{E}-03$
$7.033 \mathrm{E}-03$ $7.033 \mathrm{E}-03$
$1.857 \mathrm{E}-02$ $4.521 \mathrm{E}-03$
ANGLE 8 ANGLE 9 $M U=-0.2816 \quad M U=-0.0950$ $.154 \mathrm{E}-06$
$8.307 \mathrm{E}-06$ $9.488 E-05 \quad 1.131 E-04$ $3.362 E-05$ $4.731 E-05 \quad 5.765 E-05$ $2.239 E-05$ $2.368 E-05 \quad 3.208 E-05$ $3.081 E-05 \quad 4.162 E-05$ $9.934 E-05$ $1.240 E-04 \quad 1.805 E-04$ $5.532 E-04 \quad 6.654 E-04$ 9.852E-04 1.686E-03 5.039E-03 $5.253 E-C 3$ $1.4078 \mathrm{E}-03$
$4.135 \mathrm{E}-03$

ANGLE 17 $M U=0.9894$ $7.353 \mathrm{E}-05$
$1.424 \mathrm{E}-04$ $1.424 \mathrm{E}-04$ $1.326 \mathrm{E}-03$
$5.681 \mathrm{E}-04$
$7.7112 \mathrm{E}-04$ $5.681 \mathrm{E}-04$ $7.712 E-04$ 7. $7.714 E-04$ $1.423 E-03$ $1.550 \mathrm{E}-03$ $2.273 \mathrm{E}-03$ $2.273 E-03$
$7.146 E-03$ $7.146 \mathrm{E}-03$
$1.878 \mathrm{E}-02$ $4.541 E-03$

SCALAR FLUX $1.433 E-04$ $2.866 \mathrm{E}-04$ $2.913 \mathrm{E}-03$ 1.627E-03 $1.027 \mathrm{E}-\mathrm{C3}$ $1.163 \mathrm{E}-03$ $1.312 E-03$ 1.563E-03 3.639E-O 9. $437 \mathrm{E}-0$ $1.253 E-02$ 2. $259 \mathrm{E}-02$ 
[GAMMAS/MEV/STERADIAN/SOURCE NEUTRON)

ENERGY GROUP (MEV) 8.0OE OO- -1. COE 01 5.0 CE $00---6.50 E$ OO $4.00 E$ OO- $-5.00 E$ OO $3.00 E$ OO- $-4.00 E$ OO $? .50 E$ OO- - 3. COE OO $2.0 \mathrm{CE}$ OO---2.5OE OO $1.6 K E$ OO- - - 2. COE OO $1.33 \mathrm{E} \quad 00--1.66 \mathrm{E}$ OO $1.00 \mathrm{E} 00--1.33 \mathrm{E}$ OO 8.00E-01--1.00E 00 $6.00 E-01--8.00 E-01$ $3.00 E-01---4.00 E-01$ $2.00 E-01--3.00 E-01$ $1.00 \mathrm{E}-01-\rightarrow-2.00 \mathrm{E}-01$ $5.00 E-02--1.00 E-01$ 2.00E-02-- 5.00E-02

ENERGY

GROUP (MEV)

8.00E DO---1. OOE OI 6.5OE DO---8.0OE OO 5.0 EE OO- $-6.50 E$ OO $4.00 E$ OO- $-5.00 E$ OO 3.00 E $00-2.00 E$ OO 2.50 E $10-3.00 E$ OO $2.00 E$ OO--2.5OE OO 1.66 E $00-2.00 E$ OD $1.33 E$ ON--1.66E CO 1.0 E $00--1.33 E$ DO $8.00 E-01-2-1$. COE OO $6.00 E-01--8.00 E-01$ 4. COE-OL- - 6. OOE-OL $3.00 E-01--4.00 E-01$ 2.0CE OI 1.00E OI $2.00 E-02--5.00 E-02$
ANGLE 1 $U=-1.0000$ $8.023 E-07$ $1.574 \mathrm{E}-06$ $2.904 \mathrm{E}-06$ $7.556 \mathrm{E}-06$ $1.142 \mathrm{E}-06$ $3.167 E-06$ $5.584 E-06$ $7.638 \mathrm{E}-06$ $7.729 E-06$ $5.258 \mathrm{E}-06$ $6.644 E-06$ $1.235 E-04$ $1.926 \mathrm{E}-04$ $9.709 E-04$ $2.481 E-03$ $7.487 \mathrm{E}-03$ $2.267 \varepsilon-03$ ANGLE 10 $A U=0.0950$ $2.355 \mathrm{E}-06$ $4.836 E-06$ $5.221 E-05$ $1.705 \mathrm{E}-05$ $2.798 E-05$ $1.293 E-05$ $1.507 \mathrm{E}-05$ $1.846 \mathrm{E}-05$ $2.507 E-05$ $4.260 E-05$ $7.880 \mathrm{E}-05$ $1.502 E-04$ $4.489 E-04$ $6 . C 54 E-04$ $3.7156-04$ 9.046E-03 $2.459 E-03$
ANGLE 2 $8.156 \mathrm{E}-07$ $1.615 \mathrm{E}-06$ 1. $908 \mathrm{E}-05$ $3.327 E-06$ $7.960 E-06$ $1.367 E-06$ 3.138E-06 $5.245 \mathrm{E}-06$ $7.075 E-06$ $7.346 E-06$ $5.682 \mathrm{E}-06$ $7.829 E-C 6$ $1.243 E-04$ $1.978 E-04$ $9.707 E-04$ $2.487 \mathrm{E}-03$ $7.499 E-C$ $2.269 E-03$ ANGLE 11 $M U=0.2816$ $3.214 E-C 6$
$6.568 \mathrm{E}-\mathrm{C} 6$ $6.568 \mathrm{E}-\mathrm{C6}$ $6.948 \mathrm{E}-\mathrm{C}$ $2.407 \mathrm{E}-05$ $3.832 \mathrm{E}-05$ $2.039 \mathrm{E}-05$ $2.441 \mathrm{E}-05$ $3.112 E-C 5$ $4.376 E-05$ $1.375 \mathrm{E}-04$ $1.275 E-04$ $2.147 E-04$ $6.371 \mathrm{~F}-04$ 1.010E-0 $9.398 E-03$ $2.499 E-03$
ANGLE 3

$\begin{array}{lcc}\text { ANGLE } & 3 & \text { ANGLE } \quad 4 \\ M U=-0.9446 & M U=-0.8656\end{array}$ $8.652 E-07$ $1.763 E-06$
$2.030 E-05$ 4.737E-06 $9.362 E-06$ $2.118 \mathrm{E}-06$ $3.054 \mathrm{E}-08$ 4.129E-06 $5.222 \mathrm{E}-0 \mathrm{~S}$ $6.177 \mathrm{E}-\mathrm{nG}$ $7.277 \mathrm{E}-06$ $1.200 \mathrm{E}-05$ $1.284 E-04$ $2.201 E-04$ $9.694 \mathrm{E}-04$ $2.508 \mathrm{E}-03$ $7.549 E-03$ $2.275 E-03$

ANGLE 12 $M U=0.4580$ $4.664 E-06$
$0.401 E-06$ $9.401 E-06$
$9.727 E-05$ $3.496 \mathrm{E}-05$ 5.520 E-05 $3.451 E-05$ 4. $332 \mathrm{E}-05$ 5.716E-त 7.979E-O5 1. $1.912 \mathrm{E}-04$ 5. $6.743 E=04$ $1.058 E-03$ $1.058 E-03$ $9.763 \mathrm{E}-03$ $2.538 \mathrm{E}-\mathrm{n} 3$ $9.426 \mathrm{E}-07$
$1.974 \mathrm{E}-06$ $2.215 E-05$ $6.479 \mathrm{E}-06$ $1.116 E-05$ $3.020 E-06$ $3.026 \mathrm{E}-06$ $3.95 E-06$ $3.400 E-06$ $5.336 \mathrm{E}-06$
$9.671 \mathrm{E}-06$ 1.743E-05 $1.382 \mathrm{~F}-04$ $2.617 \mathrm{E}-04$ $9.653 E-04$ $2.548 \mathrm{E}-03$ $7.642 E-03$ $.287 E-03$

ANGLE 13 $M U=0.6179$ $7.327 \mathrm{E}-06$
$1.456 \mathrm{E}-05$ $1.461 \mathrm{E}-04$ $5.614 E-05$ $8.703 E-05$ $6.311 \mathrm{E}-05$ $7.947 \mathrm{E}-05$ $1.031 E-04$ $1.372 E-04$ $1.941 E-04$ $2.631 \mathrm{E}-04$ $6.342 E-04$ $6.342 E-04$ $7.216 \mathrm{E}-04$ 1.111E-03 $1.013 E-02$ $2.576 E-03$
ANGLE 5
$M U=-0.7550$ $1.028 \mathrm{E}-06 \quad 1.126 \mathrm{E}-06$ $2.171 \varepsilon-06$ . $1.243 E-05$ 3. $566 E-06$ $3.474 \mathrm{E}-06$ $6.212 E-06$ 1. $215 \mathrm{E}-05$ $2.203 E-05$ $1.574 E-04$ $3.197 \mathrm{E}-04$ $9.574 E-04$ $2.604 E-03$

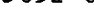

\section{ANGLE 14} $M U=0.7550$
$1.248 \mathrm{E}-05$ $2.454 \mathrm{E}-05$ $2.366 \mathrm{E}-04$ $1.003 \mathrm{E}-04$ $1.482 \mathrm{E}-04$ $1.175 E-04$ $1.415 \mathrm{E}-04$ ..141E-04 $6.820 E-04$ $7.750 E-04$ 1. $2.610 E-03$
$M U=-0.6179$ $2.353 \mathrm{E}-06$
$2.616 \mathrm{E}-05$ 7.780E-06 $1.316 \mathrm{E}-0$ 3.810E-O $3.989 \mathrm{E}-06$ $4.423 E-06$ $5.669 \mathrm{E}-06$ $1.489 \mathrm{E}-05$ $2.719 E-05$ $3.890 \mathrm{E}-04$ $9.469 E-0$ $2.679 \mathrm{E}-03$ 7.951E-03 327E-O3

ANGLE 15 $M U=0.8656$
$2.224 E-05$ $4.329 E-05$ 4.000E-O $1.838 \mathrm{E}-04$ $2.549 E-04$ 2.082E-04 $2.350 \mathrm{E}-04$ 2.660E-0 $3.026 E-04$ $3.483 E-04$ $3.882 \mathrm{E}-04$ $4.267 E-04$ 3. $76 E=03$ $2.639 E-03$

ANGLE 7 ANGLE 8 $1.259 \mathrm{E}-06 \quad 1.470 \mathrm{E}-06$ $2.588 E-06$ 2.895E-05 $7.866 E-06$ $1.408 E-05$ $4.253 E-06$ $5.312 E-06$ $9.108 E-06$ $1.920 E-05$ $3.731 E-05$ $2.374 E-04$
$4.595 \mathrm{E}-04$ $9.379 E-04$ $2.771 \mathrm{E}-03$ $8.167 E-03$ .354E-O3

ANGLE 16 $M U=0.9446$
$3.857 E-05$ $7.396 \mathrm{E}-05$ 
(GAMMAS/MEV/STERADIAN/SOURCE NEUTRON)

ENERGY GROUP (MEV) 8.0OE DO---1. OOE 03 6.50E $00--8.00 E$ DO $5.00 E 00--6.50 E \quad 00$ $4.00 E$ OO- $-5.00 E$ OO 3.00 OO $00--4.00 E$ EO $2.50 E$ OO- $-3.00 E$ OO $2.00 E$ OO- -2.50E 00 $1.6 \mathrm{EE} 00--2.00 E$ OO $1.33 \mathrm{E} \quad 00-1.66 \mathrm{E} 00$

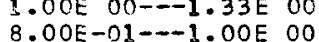
$4.00 E-01--6.00 E-01$ $3.00 F-01--4.00 E-01$ 2.00E-C1--3.00E-01 1.0OE-01--2.00E-01 $5.00 E-02--1.00 E-01$
$2.00 E-02--5.00 E-02$ ENERGY GROUP (MEV 8.0CE OO---1. DOE OI 6.5 OE $30---8.00$ E 00 5.0OE DO---6.50E 00 $4.00 E$ OO- $-5.00 F$ OO $3.00 E$ OO-C. $.00 E$ OO 2.5OE OD-D-3.0OE OO .OOE $00--2.50 E$ OO $1.66 \mathrm{E}$ OO-D-2.00E 00 $1.33 E$ DOT-1.66E 00 $1.00 E$ DO $-1.33 E$ OO $6.00 E-01-2.00 E-01$ $4.00 E-01--6.00 E-01$ 4. OOE-O1 $-6.00 E-01$ 3. OOE $O 1-4.00 E-O 1$ 2.0OE-O1-D-3.00E-O1 $5.00 E-02-1.00 E-O 1$ $2.00 \mathrm{E}-02--5.005-02$ ANGLE 1
$M U=-1.0000$$\quad$ ANGLE 2 $2.136 \mathrm{E}-07 \quad 2.220 \mathrm{E}-07$ $4.998 \mathrm{E}-06 \quad 5.2 \mathrm{C2E}-06$ $-3.122 E-07 \quad 4.301 E-08$ $1.225 \mathrm{E}-06 \quad 1.526 \mathrm{E}-06$ $-1.756 \mathrm{E}-07 \quad 2.337 \mathrm{E}-10$ $1.325 \mathrm{E}-06 \quad 1.265 \mathrm{E}-06$ $3.002 E-06 \quad 2.693 E-06$ $4.154 \mathrm{E}-06 \quad 3.676 \mathrm{~F}-06$ $3.411 \mathrm{E}-06 \quad 3.089 \mathrm{E}-06$ $3.182 E-07 \quad 6.476 E-07$ $6.476 E-07$
$0.668 E-07$ $8.256 \mathrm{E}-05 \quad 8.452 \mathrm{E}-05$ $5.132 \mathrm{E}-04 \quad 5.130 \mathrm{E}-04$ $1.272 \mathrm{E}-03 \quad 1.274 \mathrm{E}-03$ $3.885 \mathrm{E}-03$ $1.181 E-03$ ANGLE 10 $M U=0.0950$ $7.623 E-07$ $1.580 \mathrm{E}-06$ $1.688 E-15$ $6.018 \mathrm{E}-06$ $9.465 E-06$ $4.598 E-06$ $5.044 E-06$ $5.989 E-06$ 1.740E-05 $1.740 E-05$ $7.919 E-05$ 7.91 $4.32 E-04$ 3.128E-04 1.627E-03 $4.715 E-03$ 1. $283 \mathrm{E}-03$ $1.182 \mathrm{E}-03$ ANGLE 11 $1.080 E-06$ $2.227 E-06$ $2.336 \mathrm{~F}-05$ $8.706 \mathrm{E}-06$ $1.329 E-C 5$ $7.407 E-06$ 8.699E-06 $1.155 E-05$ $3.509 \mathrm{E}-05$ $3.5 C 9 E-05$ $1.170 \mathrm{E}-04$ $2.707 E-04$ $3.250 E-04$ $5.108 E-04$ $1.711 E-03$ $4.902 \mathrm{~F}-03$
ANGLE 3 $2.517 E-07 \quad 2.921 E-07$ 6.233E-07 $.924 E-06$ .015E-06 ..518E-06 $.845 E-06$ $2.051 E-06$ $1.763 \mathrm{E}-06$ $3.044 E-06$ $5.563 E-05$ $9.863 E-05$ $5.118 E-04$ $1.286 \mathrm{E}-03$ $3.918 E-03$ $1.185 \mathrm{E}-03$ $M U=0.2816 \quad M U=0.458$ 1.304E-03 $M U=0.458 n$ 1. $63\lfloor E-06$ 3.2 $75 E-06$ $3.337 E-05$ $1.243 E-05$ $1.946 E-05$ $1.340 E-05$ $1.795 E-05$ $2.572 E-05$ . $58 B \mathrm{E}-05$ $1.5053 \mathrm{E}-0 \mathrm{O}$ $1.053 E-04$ d. $563 E-04$ $3.417 E-04$ $5.35 \mathrm{JE}-04$ I.3 $5.097 \mathrm{E}-\mathrm{OB}$ 1. $325 \mathrm{E}-03$ $1.345 \mathrm{E}-03$ ANGLE 5
$M U=-0.7550$ $3.251 E-07$ $7.072 \mathrm{E}-07$ $7.616 \mathrm{E}-06$ $2.830 \mathrm{E}-06$ 4. $248 E-06$ $1.273 E-06$ $7.824 E-07$ $3.882 \mathrm{E}-07$ $1.506 \mathrm{E}-06$ $4.437 E-06$ 8.358E-06 $6.891 E-05$ 1.579E-04 5. $013 \mathrm{E}-04$ $1.337 E-03$ 4. $038 \mathrm{E}-03$ 1. 201E-0 ANGLE 14
ANGLE 6 $M U=-0.6179$ $3.498 \mathrm{E}-07$
$7.412 \mathrm{E}-07$ $8.110 E-06$ $2.540 E-06$ $4.171 E-06$ $1.167 \mathrm{E}-06$ $1.069 E-06$ $1.098 \mathrm{E}-06$ $1.454 \mathrm{E}-06$ $2.865 \mathrm{E}-06$ $5.140 \mathrm{E}-06$ $9.670 E-06$ $8.623 E-05$ $1.093 E-04$ 4. $919 \mathrm{E}-04$ $1.376 \mathrm{E}-03$ 1. $213 \mathrm{E}-03$ ANGLE 15 $M U=0.8656$
$1.125 E-05$ $1.125 E-05$
$2.163 E-05$ $2.163 E-05$ $1.893 E-04$ $9.757 E-05$ $1.324 \mathrm{E}-04$ $1.203 E-04$ $1.374 \mathrm{E}-04$ $1.566 E-04$ $1.772 E-04$ $2.004 E-04$ 2. $303 E-04$ $3.702 E-04$ $4.201 E-04$ $4.201 E-04$ $6.058 E-03$ $5.629 E-03$ $1.379 E-03$
ANGLE 7 ANGLE 8 ANGLE 9

$\begin{array}{rrr}M U=-0.4580 & M U=-0.2816 \quad M U=-0.0950 \\ 3.801 E-07 & 4.422 E-07 & 5.623 E-07\end{array}$ $3.801 E-07$ .703E-07 $.126 E-06$ $1.117 E-06$ . $372 \mathrm{E}-05$ $1.132 \mathrm{E}=04$ $2.402 \mathrm{~F}-04$ $4.828 E-04$ $M U=0.9446$ $4.376 E-05$ $3.644 \mathrm{E}-04$ $1.917 \mathrm{E}-04$ $2.395 \mathrm{E}-04$ $2.083 E-04$ $2.177 E-04$ $2.240 E-04$ . . . $5.748 E-03$ 1. $390 \mathrm{E}-03$ $8.798 \mathrm{E}-07 \quad 1.141 \mathrm{E}-06$ $1.004 E-05 \quad 1.266 E-05$ $4.618 \mathrm{E}-06 \quad 6.505 \mathrm{E}-06$ $1.579 \mathrm{E}-06 \quad 2.788 \mathrm{E}-06$ $2.547 \mathrm{E}-06 \quad 3.522 \mathrm{E}-06$ $3.717 E-06 \quad 4.423 E-06$ $4.893 E-06 \quad 5.783 E-06$ $9.453 E-06$ $1.935 E-05$ $4.672 E-05$ $1.910 E-04$ $2.980 E-04$ $4.778 E-04 \quad 4.803 E-04$ $1.550 E-03$ $1.244 E-03 \quad 1.263 E-03$

ANGLE 17 $M U=0.9894$ $4.053 E-05$ $7.434 \mathrm{E}-05$ $5.991 \mathrm{E}-04$ $3.038 \mathrm{E}-04$ $3.589 \mathrm{E}-04$ $3.121 E-04$ $3.028 \mathrm{E}-04$ (2.833E-04 $2.666 \mathrm{E}-04$ 2.472E-04 . 4 $4.146 E=04$ $5.818 \mathrm{E}-03$ $\begin{array}{ll}1.291 \mathrm{E}-03 & 5.472 \mathrm{E}-03 \\ 1.345 \mathrm{E}-03 & 1.364 \mathrm{E}-03\end{array}$
SCALAR
FLUX
$3.587 E-05$
$6.892 E-05$
$6.224 E-04$
$2.857 E-04$
$3.925 E-04$
$3.181 E-04$
$3.517 E-04$
$4.183 E-04$
$4.986 E-04$
$6.378 E-04$
$8.334 E-04$
$1.135 E-03$
$2.651 E-03$
$3.522 E-03$
$6.537 E-03$
$2.047 E-02$
$5.908 E-02$
$1.606 E-02$ 
COSINE

$-1.00000 E 00$
$-9.89401 E-01$
$-9.44575 E-01$
$-8.65631 \mathrm{E}-01$
$-7.55644 \mathrm{E}-01$
$-6.17876 \mathrm{E}-01$
$-4.58017 \mathrm{E}-01$
$-2.816 \mathrm{C} 5 \mathrm{E}-01$
$-9.50125 \mathrm{E}-02$
$9.50125 \mathrm{E}-02$
$2.816 \mathrm{C} 5 \mathrm{E}-01$
$4.58017 \mathrm{E}-01$
$6.17876 \mathrm{E}-01$
$7.55044 \mathrm{E}-01$
$8.65631 \mathrm{E}-01$
$9.44575 \mathrm{E}-01$
$9.89401 \mathrm{E}-01$

TOTAL

COSINE

$-1.00000 E$ OO $-9.89401 E-01$ $-9.44575 E-01$ $-8.65631 E=01$ $-7.55044 E-0$ -6.17876E-O $-4.58 C 17 \mathrm{E}-01$ $-2.81605 \mathrm{E}-01$ $-9.50125 E-02$ $9.50125 \mathrm{E}-02$ $2.81605 E-01$ 4. $58017 \mathrm{E}-01$ 6.17876E-01 $7.55044 E-01$ $8.65631 \mathrm{E}-01$ $9.44575 E-01$
$9.89401 E-01$

TOTAL
75.0

100.0 $1.193 E-10$ $1.191 \mathrm{E}-10$ $1.189 \mathrm{E}-10$ $1.191 \mathrm{E}-10$ $1.226 \mathrm{E}-10$ $1.275 E-10$ $1.360 \mathrm{E}-10$ $1.770 \mathrm{E}-10$ $2.043 E-10$ $2.093 E-10$ $2.644 E-10$ $4.501 E-10$ $1.065 \mathrm{E}-\mathrm{C} 9$ $2.578 \mathrm{E}-\mathrm{Cg}$ $1.089 E-08$

$5.554 \mathrm{E}-09$

500.0

$1.381 E-10$ $1.384 E-10$ $1.397 \mathrm{E}-10$
$1.424 \mathrm{E}-10$ $1.424 \mathrm{E}-10$ $1.466 E-10$ $1.526 \mathrm{E}-10$ $1.611 \mathrm{E}-10$ $1.726 \mathrm{E}-10$ $1.882 \mathrm{E}-10$ $2.090 \mathrm{E}-10$ $2.372 \mathrm{E}-10$ $2.761 E-10$ $3.312 E-10$ $4.131 E-10$ $5.438 \mathrm{E}-10$ $7.923 E-10$

$3.447 E-C 9$
$1.383 E-10$ $1.383 \mathrm{E}-10$ $1.382 \mathrm{E}-10$ $1.382 E-10$ $1.387 E-10$ $1.4 \mathrm{C} 4 \mathrm{E}-10$ $1.438 \mathrm{E}-10$ $1.501 \mathrm{E}-10$ $1.959 E-10$ $2 . C 88 \mathrm{E}-10$ $2.726 \mathrm{E}-1 \mathrm{C}$ $3.127 E-10$ $4.150 E-10$ $7.647 E-10$ $2.324 E-09$ 1. $.84 \mathrm{E}-\mathrm{C8}$

5.513E-C9

600.0

$1.1 \mathrm{C} 2 \mathrm{E}-10$ 1.1 $1.140 E-1 C$ $1.175 E-10$ $1.226 E-10$ $1.297 E-10$ $1.392 \mathrm{E}-1 \mathrm{C}$ $1.518 \mathrm{E}-1 \mathrm{C}$ $1.685 E-1 C$ $1.907 \mathrm{E}-10$ $2.209 E-10$ $2.628 E-10$ $3.233 E-10$ $4.169 \mathrm{E}-10$ $5.859 E-10$ $317 E-09$ $2.674 E-C 9$
150.0 RANGE (METERS)
$2 O C .0$

250.0

300.0

400.0

$1.645 E-10$ $1.786 \mathrm{E}-10 \quad 1.833 \mathrm{E}-10$ $1.648 E-10=1.793 E-10 \quad 1.844 E-10$ $\begin{array}{lll}.655 E-10 & 1.807 E-10 & 1.864 E-10\end{array}$ $\begin{array}{lll}1.833 E-10 & 1.898 E-10\end{array}$ $1.757 E-10 \quad 1.949 E-10 \quad 2.038 E-10$ $1.846 \mathrm{E}-10 \quad 2.060 \mathrm{E}-10 \quad 2.162 \mathrm{E}-10$ $1.985 \mathrm{E}-10 \quad 2.224 \mathrm{E}-10 \quad 2.342 \mathrm{E}-10$ $2.397 \mathrm{E}-10 \quad 2.352 \mathrm{E}-10 \quad 2.476 \mathrm{E}-10$ $2.550 E-10 \quad 2.913 E-10 \quad 3.07 C E-10$ $2.908 \mathrm{E}-10 \quad 3.275 \mathrm{E}-10 \quad 3.435 \mathrm{E}-10$ $3.790 E-10 \quad 4.258 E-10 \quad 4.426 E-10$ $5.386 E-10 \quad 5.592 E-10 \quad 5.685 E-10$ $7.808 \mathrm{E}-10 \quad 8.285 \mathrm{E}-10 \quad 8.249 \mathrm{E}-10$ $1.709 \mathrm{E}-09 \quad 1.539 \mathrm{E}-09 \quad 1.412 \mathrm{E}-09$ $9.530 \mathrm{E}-\mathrm{C} 9 \quad 7.758 \mathrm{E}-09 \quad 6.211 \mathrm{E}-09$

$5.543 E-09$

$5.458 E-C 9$

$5.269 E-09$

RANGE (METERS)

$900.0 \quad 120 C . C$

1500.0

1800.0 $1.523 E-11 \quad 4.502 E-12 \quad 1.224 E-12$ $\begin{array}{llll}4.598 E-11 & 1.543 E-11 & 4.562 E-12 & 1.2412 E-12 \\ 4.705 E-11 & 1.580 E-11 & 4.674 E-12 & 1.272 E-12\end{array}$ $4.867 E-11 \quad 1.636 \mathrm{E}-11 \quad 4.843 \mathrm{E}-12 \quad 1.318 \mathrm{E}-12$ $5.094 \mathrm{E}-11 \quad 1.715 \mathrm{E}-11 \quad 5.077 \mathrm{E}-12 \quad 1.382 \mathrm{E}-12$ $5.400 \mathrm{E}-11 \quad 1.819 \mathrm{E}-11 \quad 5.387 \mathrm{E}-12 \quad 1.466 \mathrm{E}-12$ $5.804 \mathrm{E}-11 \quad 1.955 \mathrm{E}-11 \quad 5.79 \mathrm{CE}-12 \quad 1.575 \mathrm{E}-12$ $6.329 \mathrm{E}-11 \quad 2.130 \mathrm{E}-11 \quad 6.3 \mathrm{C} 3 \mathrm{E}-12 \quad 1.714 \mathrm{E}-12$

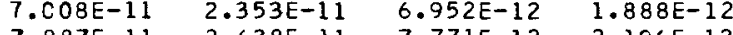

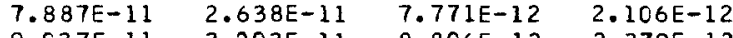
$\begin{array}{llll}9.037 \mathrm{E}-11 & 3.003 \mathrm{E}-11 & 8.806 \mathrm{E}-12 & 2.379 \mathrm{E}-12\end{array}$ $1 . C 57 E-10 \quad 3.475 E-11$ 1.012E-11 $2.723 E-12$ $1.266 \mathrm{E}-10$ 4.098E-11 $1.182 \mathrm{E}-11 \quad 3.158 \mathrm{E}-12$ $1.566 \mathrm{E}-10 \quad 4.953 \mathrm{E}-11 \quad 1.408 \mathrm{E}-11 \quad 3.725 \mathrm{E}-12$ $2.053 E-10$ 6.247E-11 $1.735 E-11 \quad 4.523 E-12$ 3.584E-10 9.570E-11 2.47CE-11 $6.161 \mathrm{E}-12$

$1.043 E-09$

$3.395 E-10$

$9.852 \mathrm{E}-11$

$2.646 \mathrm{E}-11$ 


COSINE
$-1.06000 E 00$
$-9.89401 E-01$
$-9.44575 E-01$
$-8.65631 E-01$
$-7.55044 E-01$
$-6.17876 E-01$
$-4.58017 E-01$
$-2.81605 E-C 1$
$-9.50125 E-02$
$9.50125 E-02$
$2.81605 E-01$
$4.58017 E-01$
$6.17876 E-01$
$7.55044 E-01$
$8.65631 E-01$
$9.44575 E-01$
$9.894 C 1 E-01$

TOTAL

COSINE

$-1.00 C O O E$ OC $-9.44575 \mathrm{E}-\mathrm{Cl}$ $-8.65631 E-C 1$ $-7.55044 E-01$ $-6.17876 E-01$ $-4.58017 E-01$ $-2.81605 E-01$ $-9.50125 E-02$ $9.50125 E-02$ 2.81605E-01 $4.58017 E-01$ $6.17876 \mathrm{E}-01$ $7.55044 \mathrm{E}-01$ $8.656315-0$ $9.44575 E-01$
$9.89401 E-01$

TOTAL

75.0
$1.797 \mathrm{E}-10$
$1.706 \mathrm{E}-10$
$1.703 \mathrm{E}-1 \mathrm{C}$
$1.700 \mathrm{E}-1 \mathrm{C}$
$1.702 \mathrm{E}-10$
$1.716 \mathrm{E}-10$
$1.751 \mathrm{E}-10$
$1.818 \mathrm{E}-1 \mathrm{C}$
$1.933 \mathrm{E}-10$
$2.474 \mathrm{E}-10$
$2.859 \mathrm{E}-10$
$2.901 \mathrm{E}-10$
$3.593 \mathrm{E}-10$
$5.953 \mathrm{E}-10$
$1.369 \mathrm{E}-\mathrm{C} 9$
$3.303 \mathrm{E}-\mathrm{C} 9$
$1.370 \mathrm{E}-\mathrm{C} 8$
$7.300 \mathrm{E}-\mathrm{C}$

500.0

$2.619 \mathrm{E}-10$ $2.624 \mathrm{E}-1 \mathrm{C}$ $2.647 \mathrm{E}-10$ $2.692 \mathrm{E}-1 \mathrm{C}$ $2.761 E-1 \mathrm{C}$ $2.861 E-10$ $2.997 \mathrm{E}-1 \mathrm{C}$ $3.181 \mathrm{E}-10$ $3.423 \mathrm{E}-10$ $3.743 E-10$ $4.167 E-1 C$ 4.740E-10 $5.534 E-10$ $8.484 E-10$ $1.182 E-09$ $2.808 E-C 9$

5.805E-C9
$10 C .0$ 2.C $22 E-10$ $2.021 \mathrm{E}-1 \mathrm{C}$ $2.02 C E-10$ $2.02 \mathrm{CE}-10$ $2.028 \mathrm{E}-1 \mathrm{C}$ 2.C51E-1C 2.10CE-10 2.18त E-10 $2.332 \mathrm{E}-10$ 2.82E2.9BOE-IC $4.388 \mathrm{E}-1 \mathrm{C}$ $5.68 C E-1 C$ $5.6 B C E-1 S$ $2.988 \mathrm{~F}-\mathrm{C} 9$ $2.988 \mathrm{~F}-\mathrm{C} 9$
$1.371 \mathrm{E}-\mathrm{CB}$

$7.392 E-C 9$

600.0

$2.19 C E-10$ $2.135 E-10$ $2.215 E-10$ 2. $255 \mathrm{E}-1 \mathrm{C}$ $2.315 E-10$ 2.401E-10 $2.517 E-1 C$ $2.671 \mathrm{E}-1 \mathrm{C}$ $2.872 \mathrm{E}-1 \mathrm{C}$
$3.134 \mathrm{E}-1 \mathrm{C}$ $3.475 \mathrm{E}-1 \mathrm{C}$ $3.927 E-1 C$ $4.541 \mathrm{E}-10$ $5.4 \mathrm{C} 6 \mathrm{E}-10$ $6.709 E-1$ $1.862 E-C 9$ $4.692 \mathrm{E}-\mathrm{CCO}^{\circ}$
150.0 RANGE (METERS)
$200 . \mathrm{C}$

$2.504 E-10$ $2.505 \mathrm{E}-10$ $2.509 E-10$ $2.520 E-10$ $2.544 E-10$ $2.590 E-10$ $2.799 E-10$ $2.799 E-10$ $2.99 \mathrm{E}-10$ . $759 E-10$ $4.271 \mathrm{E}-10$ $5.456 \mathrm{E}-10$ 7.54CE-10 $2.251 E-C O$ $1.215 \mathrm{E}-\mathrm{C} 8$

$2.823 E-10$ $2.825 \mathrm{E}-10$ $2.835 \mathrm{E}-10$ $2.857 \mathrm{E}-10$ $2.897 \mathrm{E}-10$ $2.965 E-10$ $3.072 E-10$ $3.234 E-10$ $3.471 E-10$ $3.646 E-10$ $4.449 E-10$ $6.281 E-10$ $8.042 E-10$ $0.042 E-10$ $2.075 E-09$

$9.976 \mathrm{E}-\mathrm{C} 9$

7. $842 E-C 9$

$7.706 \mathrm{E}-09$ $25 \mathrm{C} .0$

300.2 400.0

$3.052 E-10$ $3.006 \mathrm{E}-10$
$3.021 \mathrm{E}-1 \mathrm{C}$ $3.021 E-10$ $3.052 E-10$ 3.1C6E-1C $3.191 E-1 C$ $3.319 E-10$ 3. 5 C $4 E-10$ $3.768 E-10$ . $4.814 E-10$ 6. 8.363E-10 1.178 E-09 $1.941 E-09$ $8.06 \in E-09$

7. $809 E-09$

3. $071 \mathrm{E}-10$ $3.075 \mathrm{E}-1 \mathrm{C}$
$3 . \mathrm{C} 94 \mathrm{E}-10$ $3.133 E-10$ $3.196 \mathrm{E}-10$ $3.293 E-10$ $3.434 E-10$ 3. $12 \mathrm{E}-10$ $4.790 E-1 C$ $5.617 \mathrm{E}-1 \mathrm{C}$ $8.485 E-10$ 1. $146 E-C$ 1.808E-C 6. $514 \mathrm{E}-\mathrm{C}$

$7.628 \mathrm{E}-\mathrm{C} 9$ $2.952 \mathrm{E}-10$ $2.958 \mathrm{E}-10$ $2.981 \mathrm{E}-10$ $3.027 E-10$ . 3. 3.554E-10 3. $4.188 \mathrm{E}-10$ $4.695 \mathrm{E}-10$ $5.373 E-10$ $6.359 \mathrm{E}-10$ $7.822 \mathrm{E}-10$ $1.022 \mathrm{E}-09$ $1.497 \mathrm{E}-09$ $4.261 E-09$

$6.853 \mathrm{E}-\mathrm{Cg}$

RANGE (METERS)

$900.0 \quad 1200.0$

1500.0

1800.0

COSE-10 $3.630 E-11$

1.C $17 \mathrm{E}-10$ 3.676E-

$1.131 E-11$

$1.134 E-11$

$3.193 \mathrm{E}-12$
$3.201 \mathrm{E}-12$

3. $234 E-12$

$3.296 \mathrm{E}-12$ $. C 36 E-10$
.$=65 E-10$ $1.105 \mathrm{E}-10$ $3.852 E-11$ $3.998 E-11$ $4.188 \mathrm{E}-11$

$.168 E-11$

$1.245 \mathrm{E}-11$

$3.388 E-12$

$3.513 \mathrm{E}-12$

$.228 \mathrm{E}-10$

$1.316 \mathrm{E}-10$

$4.431 E-11$

$.304 \mathrm{E}-1$

$3.676 F-12$

$3.881 \mathrm{E}-12$

$4.136 E-12$

$1.584 \mathrm{E}-11 \quad 4.449 \mathrm{E}-12$

$5.593 E-11 \quad 1.725 E-11 \quad 4.830 E-12$

$6.185 \mathrm{E}-11 \quad 1.897 \mathrm{E}-11 \quad 5.294 \mathrm{E}-12$

$1.984 \mathrm{E}-10 \quad 6.930 \mathrm{E}-11 \quad 2.11 \mathrm{CE}-11 \quad 5.858 \mathrm{E}-12$

$7.881 \mathrm{E}-11$

$9.142 E-11 \quad 2.714 E-11 \quad 7.413 E-12$

$\begin{array}{llll}3.4 n 4 E-10 & 1.098 E-10 & 3.185 E-11 & 8.573 E-12 \\ 5.455 E-10 & 1.549 E-10 & 4.193 E-11 & 1.084 E-11\end{array}$

$2 \cdot C 18 E-C 9$

$7.042 E-1 C$

$2.147 E-10 \quad 5.975 E-11$ 
4 PI R**2 TISSUE KERMA INEUTRONSI
(CM**2 ERGS/GRAMISTERADIAN/SOURCE NEUTRON
2.350 TO 4.065 MEV NEUTRON SOURCE
CDSINE

$-1.00000 E 00$ $-9.894010$ $-9.44575 E-01$ $-8.65631 \mathrm{E}-01$ $-7.55044 E-01$ $-6.17876 E-01$ $-4.58017 E-01$ $-2.81605 E-01$ $-9.50125 \mathrm{E}-02$ $9.50125 \mathrm{E}-02$ $2.81605 \mathrm{E}-01$ $4.58017 \mathrm{E}-01$ $6.17876 \mathrm{E}-01$ $7.55044 \mathrm{E}-01$ $8.65631 \mathrm{E}-01$ $9.44575 E-01$ $9.89401 E-01$

TOTAL

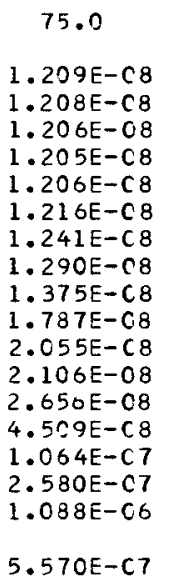

100.0

$1.408 \mathrm{E}-08$ $1.4 C 7 E-08$ $1.407 E-08$

$1.412 \mathrm{E}-\mathrm{C} 8$

$1.429 \mathrm{E}-\mathrm{C} 8$

$1.463 E-08$

$1.526 \mathrm{E}-08$

$1.630 \mathrm{E}-\mathrm{C} 8$
$1.983 \mathrm{E}-08$

$2.114 \mathrm{E}-08$

$2.754 E-C 8$

$3.153 E-08$

$4.171 E-C$

$7.663 \mathrm{E}-\mathrm{C}$

$2.324 E-C 7$

$1.083 E-C 6$

$5.543 E-07$

500.0

600.0

COS INE

$-1.00000 E$ OC $-9.89401 \mathrm{E}-01$ $-9.44575 E-01$ $-8.65631 E-01$ $-6.17876 \mathrm{E}-01$ $-6.17876 E-C 1$ $-2.81605 E-01$ $-9.50125 E-02$ . $50125 E-02$ $2.81605 \mathrm{E}-01$ $2.81605 E-01$ $4.57017 E-O 1$ 6. $55044 \mathrm{E}-01$ $8.65631 E-01$ $9.44575 \bar{E}-01$ $9.89401 \mathrm{E}-01$

TOTAL

$1.518 E-C 8$

$1.228 \mathrm{E}-\mathrm{C} 8$ $1.659 \mathrm{E}-\mathrm{C} 8 \mathrm{1} \quad 1.358 \mathrm{E}-08$ $1.758 E-08$ 1.433E-C8 1. $2.035 \mathrm{E}-\mathrm{C} 8$ $1.533 E-C 8$

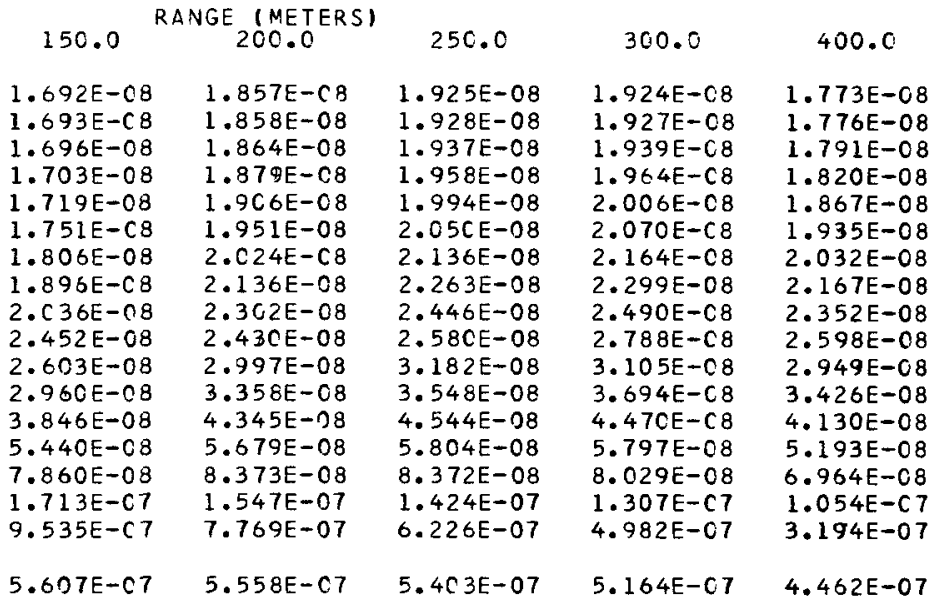

RANGE (METERS)

$$
900.0
$$

$120 \mathrm{C} .0$

1500.0

1800.0

5.196E-09 $1.778 \mathrm{E}-09$ $5.34 \mathrm{E}-10 \quad 1.469 \mathrm{E}-10$ $5.415 E-10 \quad 1.487 E-10$ $5.729 \mathrm{E}-10 \quad 1.574 \mathrm{E}-1 \mathrm{C}$ 5.99CE-10 1.646E-10 $6.332 \mathrm{E}-10 \quad 1.740 \mathrm{E}-10$ $6.774 E-10$ 1.86CE-10

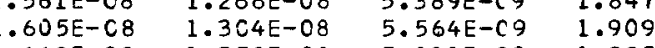
$5.811 \mathrm{E}-\mathrm{CQ} \quad 1.99$ $6.574 \mathrm{E}-09 \quad 2.257 \mathrm{E}-09$ $7.133 E-09 \quad 2.44$ $8.012 \mathrm{E}-10$ 8. $1212 \mathrm{E}-10 \quad 2.437 \mathrm{E}-10$

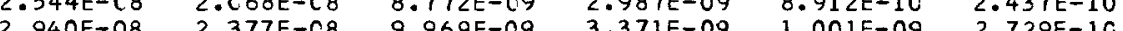
$3.500 \mathrm{E}-08 \quad 2.804 \mathrm{E}-\mathrm{CB} \quad 1.155 \mathrm{E}-08 \quad 3.853 \mathrm{E}-09 \quad 1.139 \mathrm{E}-09 \quad 3.092 \mathrm{E}-10$

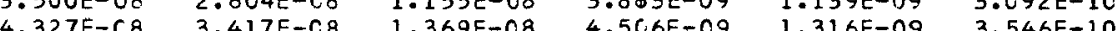
$5.642 \mathrm{E}-08$ 4.361E-CB $1.3674 \mathrm{E}-08 \quad 5.381 \mathrm{E}-09$ 1.3 $548 \mathrm{E}-09$ 3.546E-10 $\begin{array}{llllll}8.135 \mathrm{E}-\mathrm{C} 8 & 6.059 \mathrm{E}-\mathrm{C8} & 2.166 \mathrm{E}-\mathrm{C} 8 & 6.692 \mathrm{E}-09 & 1.548 \mathrm{EE}-09 & 4.945 \mathrm{E}-1 \mathrm{C}\end{array}$ $\begin{array}{llllll}8.1335-C 8 & 6 . C 59 E-C 8 & 2.166 E-C 8 & 6.692 E-09 & 1.88 C E-09 & 4.945 E-1 C \\ 2.063 E-C 7 & 1.339 E-07 & 3.704 E-08 & 1.004 E-08 & 2.621 E-09 & 6.598 E-10\end{array}$ $\begin{array}{llllll}3.655 E-C 7 & 2.867 E-07 & 1.149 E-07 & 3.814 E-08 & 1.122 E-08 & 3.043 E-09\end{array}$ 


\begin{abstract}
COSINE
$-1.00 C O O E$ CO $-9.89401 \mathrm{E}-01$ $-9.44575 E-01$ $-8.65631 \mathrm{E}-01$ $-7.55044 \mathrm{E}-01$ $-6.17876 \mathrm{E}-01$ $-4.58017 E-01$ $-2.81605 E-01$ $-9.50125 \mathrm{E}-\mathrm{G} 2$ $9.50125 E-02$ $2.81605 E-01$ $4.5817 E-01$ $6.17876 E-J 1$ $7.55044 E-01$ $8.65631 E-01$ $9.89401 E-01$
\end{abstract}

TOTAL

COSINE

-1. COOGGE OO $-9.89401 \mathrm{E}-01$ $-8.65631=-21$ $-7.55044 \mathrm{E}-\mathrm{O}$ $-6.17876 \mathrm{E}-01$ $-4.58017 \mathrm{E}-01$ $-2.81605 E-01$ $-9.50125 \mathrm{E}-02$

$9.50125 \mathrm{E}-02$ $2.81605 E-01$ $4.58017 \mathrm{E}-01$ $6.17876 E-01$ $7.55044 \mathrm{E}-01$ $8.65631 E-C 1$ $9.44575 \mathrm{E}-01$ $9.89401 E-01$

TOTAL

$\begin{array}{cc}75.0 & 1 C C . C \\ 3.639 E-11 & 4 . C 86 E-11 \\ 3.638 E-11 & 4.685 E-11 \\ 3.634 E-11 & 4 . C 83 E-11 \\ 3.629 E-11 & 4 . C 83 E-11 \\ 3.634 E-11 & 4 .(95 E-11 \\ 3.664 E-11 & 4.137 E-11 \\ 3.739 E-11 & 4.233 E-11 \\ 3.892 E-11 & 4.416 E-11 \\ 4.164 E-11 & 4.732 E-11 \\ 5.665 E-11 & 5.754 E-11 \\ 6.341 E-11 & 6.390 E-11 \\ 6.749 E-11 & 8.588 E-11 \\ 8.981 E-11 & 9.802 E-11 \\ 1.63 C E-10 & 1.385 E-1 C \\ 4.100 E-10 & 2.776 E-10 \\ 1 . C 08 E-C 9 & 8.98 C E-1 C \\ 4.422 E-C 9 & 4.355 E-C 9 \\ 2.057 E-C 9 & 1.964 E-C 9\end{array}$

500.0

600.0

$3.895 \mathrm{E}-11$ $3.902 \mathrm{E}-11$ $3.995 E-11$ 4. $4.231 E-11$ $4.430 \mathrm{E}-11$

$4.708 \mathrm{E}-11$ 5.C90E-11 $5.619 E-11$ $6.359 E-11$ $7.425 \mathrm{E}-11$ 9. $C 17 \mathrm{E}-11$ $1.152 \mathrm{E}-1 \mathrm{C}$ $1.576 \mathrm{E}-1 \mathrm{C}$ $2.434 \mathrm{E}-10$ $7.128 \mathrm{E}-10$

$9.841 E-10$ . $223 E-11$ $3.257 E-11$ $3.312 E-11$ $3.395 E-11$ $3.515 E-11$ $3.681 \mathrm{E}-11$ $3.9 C 8 E-11$ $4.215 \mathrm{E}-11$ $4.631 \mathrm{E}-11$ $5.202 \mathrm{E}-11$ $6.0 C 5 \mathrm{E}-11$ $7.177 E-11$ $8.968 \mathrm{E}-11$ $1.192 \mathrm{E}-10$ $1.763 E-1 C$ $4.474 \mathrm{E}-10$
150.0 RANGE (METERS)
$20 \mathrm{C} .0$

250.0

300.5

400.0

$4.650 E-11$ 4.650E-11 $4.655 E-11$ $4.670 E-11$ $4.705 \mathrm{E}-11$ $4.778 E-11$ $4.919 \mathrm{E}-11$ $5.163 E-11$ $5.563 E-11$ $6.641 E-11$ $7.508 E-11$ $8.415 E-11$ $1.733 E-10$ $1.733 E-10$ $2.601 E-10$ $6.283 E-10$ $1.856 \mathrm{E}-09$

$4.913 E-11$
$4.916 \mathrm{E}-11$ $4.929 E-11$ $4.959 E-11$ $5.016 \mathrm{E}-11$ $5.12 \mathrm{CE}-11$ $5.298 \mathrm{E}-11$ $5.587 \mathrm{E}-11$ $6.039 \mathrm{E}-11$ $6.484 E-11$ 8.01CE-11 $9.327 E-11$ $1.242 E-10$ $1.722 E-10$ $2.679 \mathrm{E}-10$ 5.393E-10 $4.974 E-11$ 4.978E-11 $4.998 \mathrm{E}-11$ $5.042 E-11$ $5.118 \mathrm{E}-11$ $5.245 \mathrm{E}-11$ $5.449 E-11$ $5.767 \mathrm{E}-11$ $6.245 E-11$ $6.691 \mathrm{E}-11$ $8.263 E-11$ $9.55 \mathrm{CE}-11$ $1.258 \mathrm{E}-1 \mathrm{C}$ $1.70 C E-10$ $4.786 E-10$ 4. $786 \mathrm{E}-10$

1.743E-09

$1.62 C E-09$

RANGE (METERS)

900.0 1200.0

1500.0

1800.0

$4.903 E-11$ 4.909E-11

4. $934 \mathrm{E}-11$

$4.988 \mathrm{E}-11$

S.077E-11

. $219 E-11$

$5.439 E-11$

$6.254 \mathrm{E}-11$

$7.960 E-11$

$.663 \mathrm{E}-1$

(.)

(1C 4. $260 \mathrm{E}-10$ 1. $86 \mathrm{IE}=\mathrm{C} 9$

$1.494 \mathrm{E}-\mathrm{C} 9$ $4.497 E-11$ $4.504 \mathrm{E}-11$ $4.536 E-11$ $4.598 \mathrm{E}-11$ $4.698 \mathrm{E}-11$ $4.849 E-11$ $5.071 E-11$ . . $838 E-11$ 7.396E 11 $.737 \mathrm{E}-11$ $1.082 E-10$ 1.008E-10 $.274 E-10$

$1.232 E-C 9$

$1.48 C E-11$

$5.43 C E-12$

$1.716 \mathrm{E}-12$ $5.442 E-12$ $5.593 \mathrm{E}-12$ $1.72 \mathrm{CE}-12$ $1.736 \mathrm{E}-12$ $1.812 \mathrm{E}-12$

$1.812 \mathrm{E}-12$
$1.874 \mathrm{E}-12$ $5.738 \mathrm{E}-12$ $6.261 \mathrm{E}-12$ $1.620 \mathrm{E}-11$ $1.694 E-11$ $1.792 \mathrm{E}-11$ $1.92 C E-11$ 2. $C 85 \mathrm{E}-11$ $2.303 E-11$ $2.594 \mathrm{E}-11$ $2.996 E-11$ $3.574 \mathrm{E}-11$ $6.025 E-11$ $1.149 \mathrm{E}-10$

641E-12 $6.972 \mathrm{E}-12$ $7.520 \mathrm{E}-12$ $8.219 \mathrm{E}-12$ $9.124 \mathrm{E}-12$ $1.033 E-11$ 1. $198 \mathrm{E}-11$ $1.441 E-11$ $1.343 E-11$ $3.002 E-11$

$2.058 \mathrm{E}-12$

$2.187 \mathrm{E}-12$

$348 \mathrm{E}-12$

$2.55 C E-12$

$.806 E-12$

3. $137 \mathrm{E}-12$

. $579 E-12$

$5.2 C \mathrm{CE}-12$
$7.726 \mathrm{E}-12$

4. $896 \mathrm{E}-13$ $4.954 E-13$ 5. $041 \mathrm{E}-13$ $5.169 \mathrm{E}-13$

$5.343 E-13$

$5.343 E-13$
$5.569 E-13$

$5.854 E-13$

$6.209 \mathrm{E}-13$

$6.647 E-13$

$7.189 \mathrm{E}-13$

$.865 E-13$

$9.846 \mathrm{E}-1$

$1.140 \mathrm{E}-12$

$1.380 E-12$
$1.938 E-12$

$3.112 E-10$

$1.07 \mathrm{CE}-10$

3. $257 E-11$

$9.084 E-12$ 
COSINE

$$
\begin{aligned}
& -1.00000 \mathrm{E} 00 \\
& -9.89401 \mathrm{E}-01 \\
& -9.44575 \mathrm{E}-01 \\
& -8.65631 \mathrm{E}-01 \\
& -7.55044 \mathrm{E}-01 \\
& -6.17876 \mathrm{E}-01 \\
& -4.58017 \mathrm{E}-01 \\
& -2.816 \mathrm{C}-01 \\
& -9.50125 \mathrm{E}-01 \\
& 9.50125 \mathrm{E}-02 \\
& 2.81605 \mathrm{E}-01 \\
& 4.55017 \mathrm{E}-01 \\
& 6.17876 \mathrm{E}-01 \\
& 7.55044 \mathrm{E}-01 \\
& 8.65631 \mathrm{E}-01 \\
& 9.44575 \mathrm{E}-01 \\
& 9.89401 \mathrm{E}-01
\end{aligned}
$$

TOTAL

COSINE

$-1.0000 C E$ CO $-9.89401 E-C 1$ $-9.44575 E-01$ $-8.65631 E-01$ $-7.55044 E-01$ $-6.17876 E-01$ $-4.58017 \mathrm{E}-01$ $-2.81605 E-01$ $-9.50125 E-02$ $9.50125 \mathrm{E}-02$ $2.81605 E-C 1$ 4.58017E-C I $6.17876 \mathrm{E}-01$ $7.55044 E-01$ $8.65631 E-01$ $9.89401 E-01$

TOTAL

$\begin{array}{lc}75.0 & 100.0 \\ .421 E-09 & 1.656 E-C 9 \\ .421 E-09 & 1.656 E-C 9 \\ .420 E-C 9 & 1.657 E-C 9 \\ .421 E-09 & 1.661 E-C 9 \\ .427 E-C 9 & 1.671 E-09 \\ .443 E-C 9 & 1.695 E-09 \\ .477 E-09 & 1.740 E-C 9 \\ .539 E-C 9 & 1.819 E-09 \\ .644 E-C 9 & 1.946 E-C 9 \\ .151 E-09 & 2.387 E-C 9 \\ .468 E-C 9 & 2.546 E-09 \\ .573 E-C 9 & 3.298 E-C 9 \\ .310 E-C 9 & 3.835 E-09 \\ .793 E-C 9 & 5.167 E-09 \\ .407 E-08 & 9.841 E-C 9 \\ .368 E-08 & 3.047 E-C 8 \\ .461 E-07 & 1.441 E-C 7 \\ .169 E-08 & 7.038 E-08\end{array}$

$50 C . C$

$1.763 E-09$ $1.767 \mathrm{E}-09$ $1.785 \mathrm{E}-\mathrm{C} 9$ $1.819 E-C 9$ $1.948 \mathrm{E}-\mathrm{C} 9$ $2.054 E-C 9$ $2.198 \mathrm{E}-\mathrm{C} 9$ $2.390 E-C 9$ $2.648 \mathrm{E}-09$ $2.595 E-C 9$ $3.474 E-C 9$ $4.152 E-09$ $5.160 E-C 9$ $6.773 E-C 9$ $9.845 \mathrm{E}-\mathrm{C} 9$ $2.541 \mathrm{E}-08$ $4.339 E-08$

$60 \mathrm{C} .0$
150.0 RANGE (METERS)
200.0

250.0

300.0

400.0

$1.993 \mathrm{E}-09$ $1.994 E-C 9$ 2.C11E-C9 2.011E-C $2.075 \mathrm{E}-09$ $2.145 \mathrm{E}-\mathrm{C} 9$ $2.256 E-C 9$ $2.426 E-C 9$ $2.923 \mathrm{E}-\mathrm{CO}$ $3.128 \mathrm{E}-\mathrm{Cg}$ $3.538 \mathrm{E}-\mathrm{C} 9$ $4.683 \mathrm{E}-09$ $6.637 E-09$ $9.771 \mathrm{E}-09$ $2.201 \mathrm{E}-08$ $1.255 \mathrm{E}-07$

$2.186 \mathrm{E}-09$ $2.188 \mathrm{E}-09$ $2.198 E-09$ $2.218 E-C 9$ $2.310 E-39$ . $2.537 \mathrm{E}-09$ $2.737 E-C 9$ $2.896 \mathrm{E}-\mathrm{C} 9$ $3.576 \mathrm{E}-09$ 4.027E-09 5.24CE-C9 $6.919 E-09$ $1.032 \mathrm{E}-08$ $1.950 E-08$ $1.012 \mathrm{E}-\mathrm{C} 7$

$6.991 E-08$

$6.845 E-08$

$2.265 \mathrm{E}-09$ $2.281 \mathrm{E}-09$ 2.281E-09 2.35 $2.423 \mathrm{E}-09$ $2.528 \mathrm{E}-09$ $2.682 E-09$ $2.902 \mathrm{E}-09$ 3. $068 \mathrm{E}-09$ $3.788 \mathrm{E}-09$ $4.243 E-09$ $4.243 \mathrm{E}-09$
$5.461 \mathrm{E}-09$ 5.461E-09 $1.026 \mathrm{E}-08$ $1.775 E-08$ $8.024 E-08$

$6.592 \mathrm{E}-08$

RANGE (METERS)

900.0

1200.0

$150 C .0$

1800.0 $\begin{array}{lllll}.423 E-C 9 & 5.959 E-10 & 2.02 C E-10 & 6.013 E-11 & 1.638 E-11\end{array}$ $1.438 E-09 \quad 6.030 E-10 \quad 2.045 E-10 \quad 6.09 C E-11 \quad 1.664 E-11$ $1.468 E-C 9 \quad 6.168 E-10 \quad 2.094 E-10 \quad 6.237 E-11 \quad 1.705 E-11$ $1.513 E-C 9 \quad 6.375 E-10 \quad 2.166 E-10 \quad 6.455 E-11 \quad 1.765 E-11$ $\begin{array}{lllll}1.578 \mathrm{E}-09 & 6.666 \mathrm{E}-10 & 2.267 \mathrm{E}-10 & 6.759 \mathrm{E}-11 & 1.848 \mathrm{E}-11\end{array}$ $1.666 E-09$ 7.055E-10 2.401E-10 7.159E-11 1.957E-11 $\begin{array}{lllll}1.785 E-09 & 7.567 \mathrm{E}-10 & 2.575 \mathrm{E}-10 & 7.676 \mathrm{E}-11 & 2.098 \mathrm{E}-11\end{array}$ $1.942 \mathrm{E}-09 \quad 8.228 \mathrm{E}-10 \quad 2.797 \mathrm{E}-10 \quad 8.332 \mathrm{E}-11 \quad 2.276 \mathrm{E}-11$ $2.149 \mathrm{E}-09 \quad 9 . \mathrm{C}^{2} 0 \mathrm{E}-10 \quad 3.080 \mathrm{E}-10 \quad 9.159 \mathrm{E}-11$ 1 $2.499 \mathrm{E}-11$ $2.425 \mathrm{E}-09 \quad 1.018 \mathrm{E}-09 \quad 3.438 \mathrm{E}-10 \quad 1.020 \mathrm{E}-10 \quad 2.776 \mathrm{E}-11$ $2.796 \mathrm{E}-\mathrm{C} 9 \quad 1.161 \mathrm{E}-\mathrm{C9} \quad 3.894 \mathrm{E}-10 \quad 1.15 \mathrm{CE}-10 \quad 3.121 \mathrm{E}-11$ $3.312 \mathrm{E}-\mathrm{C} 9 \quad 1.349 \mathrm{E}-\mathrm{C} 9 \quad 4.480 \mathrm{E}-10 \quad 1.314 \mathrm{E}-10 \quad 3.550 \mathrm{E}-11$ $4.055 \mathrm{E}-\mathrm{Cg} \quad 1.606 \mathrm{E}-09 \quad 5.244 \mathrm{E}-10 \quad 10 \mathrm{1}-522 \mathrm{E}-1 \mathrm{O} \quad 4.086 \mathrm{E}-1 \mathrm{I}$ $5.204 \mathrm{E}-\mathrm{C} 9 \quad 1.971 \mathrm{E}-09 \quad 6.282 \mathrm{E}-10 \quad 1.796 \mathrm{E}-1 \mathrm{C} \quad 4.774 \mathrm{E}-11$

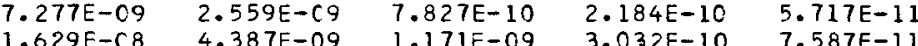
$1.629 \mathrm{E}-\mathrm{C} 8 \quad 4.387 \mathrm{E}-09 \quad 1.171 \mathrm{E}-09 \quad 3.032 \mathrm{E}-10 \quad 7.587 \mathrm{E}-11$ $3.382 E-C 8 \quad 1.337 E-08 \quad 4.304 E-09 \quad 1.284 E-09 \quad 3.466 E-10$ 


COSINE
-1. COOOEE Ci
$-9.89401 E-01$
$-9.44575 E-01$
$-8.65631 E-C 1$
$-7.55044 E-01$
$-6.17876 E-01$
$-4.58017 E-01$
$-2.81605 E-01$
$-9.50125 E-02$
$9.50125 E-02$
$2.81605 E-01$
$4.58017 E-01$
$6.17876 E-01$
$7.55044 E-C 1$
$8.65631 E-01$
$9.44575 E-01$
$9.89401 E-01$

TOTAL

$\operatorname{COSINE}$

-1. CCOOCE $0 O$ $-9.89401 E-01$ $-9.44575 E-01$ $-8.65631 E-01$ $-7.55044 E-01$ $-6.17876 E-01$ $-4.58017 E-01$ $-2.81605 E-01$ $-9.50125 E-02$ $9.50125 \mathrm{E}-02$ $2.81605 E-01$ $4.58017 \mathrm{E}-01$ 6.17876E-01 $7.55044 E-01$ $8.65631 \mathrm{E}-\mathrm{C} 1$ $9.44575 \mathrm{E}-01$ $9.89401 \mathrm{E}-\mathrm{Cl}$

TOTAL

$\begin{array}{cc}75 . C & 1 C C . C \\ 1.525 E-C 9 & 1.729 E-C 9 \\ 1.526 E-C 9 & 1.73 C E-09 \\ 1.530 E-C 9 & 1.735 E-C 9 \\ 1.538 E-C 9 & 1.746 E-C 9 \\ 1.552 E-C 9 & 1.765 E-C 9 \\ 1.577 E-C 9 & 1.796 E-C 9 \\ 1.622 E-C 9 & 1.851 E-09 \\ 1.701 E-C 9 & 1.943 E-C Q \\ 1.831 E-C 9 & 2.093 E-C 9 \\ 2.519 E-C 9 & 2.594 E-C 9 \\ 2.796 E-C 9 & 2.873 E-C 9 \\ 3.107 E-C 9 & 3.770 E-C 9 \\ 4.289 E-C 9 & 4.493 E-C 9 \\ 8.20 C E-C 9 & 6.553 E-C 9 \\ 2.152 E-C 8 & 1.395 E-C 8 \\ 5.135 E-C 8 & 4.630 E-C 8 \\ 2.354 E-C 7 & 2.285 E-C 7 \\ 1.634 E-C 7 & 9.696 E-C 8 \\ 1.69 & \end{array}$

500.0

$1.676 \mathrm{E}-\mathrm{C9}$ $1.679 E-C 9$ $1.695 E-09$ $1.724 \mathrm{E}-\mathrm{C} 9$ $1.770 \mathrm{E}-\mathrm{C} 9$ $1.836 \mathrm{E}-09$ $1.929 E-C 9$ $2.057 E-C 9$ $2.233 E-C 9$ $2.476 E-C 9$ $2.815 \mathrm{E}-\mathrm{C} 9$ 3. $305 \mathrm{E}-\mathrm{C} 9$ 4. C39E-C9 $5.195 \mathrm{E}-09$ $7.160 \mathrm{E}-\mathrm{C} 9$ $1.113 \mathrm{E}-08$ 3. $202 \mathrm{E}-\mathrm{C} 8$

$4.398 \mathrm{E}-\mathrm{C} 8$

$600 . \mathrm{C}$

\begin{tabular}{|c|c|c|c|c|}
\hline 150.0 & $\begin{array}{c}\text { E IMETER } \\
20 \mathrm{C} \cdot \mathrm{C}\end{array}$ & $25 r .0$ & $300 . \mathrm{C}$ & $40 \mathrm{C} \cdot \mathrm{C}$ \\
\hline $\begin{array}{l}1.997 \mathrm{E}-\mathrm{C9} \\
1.999 \mathrm{E}-09 \\
2.008 \mathrm{E}-09 \\
2.025 \mathrm{E}-09 \\
2.053 \mathrm{E}-\mathrm{C9} \\
2.099 \mathrm{E}-09 \\
2.173 \mathrm{E}-09 \\
2.293 \mathrm{E}-19 \\
2.480 \mathrm{E}-09 \\
2.968 \mathrm{E}-09 \\
3.394 \mathrm{E}-09 \\
3.743 \mathrm{E}-09 \\
5.333 \mathrm{E}-09 \\
7.966 \mathrm{E}-\mathrm{C9} \\
1.242 \mathrm{E}-\mathrm{C8} \\
3.152 \mathrm{E}-08 \\
1.947 \mathrm{E}-\mathrm{C} 9\end{array}$ & $\begin{array}{l}2.129 \mathrm{E}-09 \\
2.132 \mathrm{E}-09 \\
2.144 \mathrm{E}-09 \\
2.166 \mathrm{E}-09 \\
2.203 \mathrm{E}-09 \\
2.261 \mathrm{E}-09 \\
2.352 \mathrm{E}-09 \\
2.491 \mathrm{E}-09 \\
2.701 \mathrm{E}-09 \\
2.911 \mathrm{E}-09 \\
3.603 \mathrm{E}-09 \\
4.224 \mathrm{E}-09 \\
5.676 \mathrm{~F}-09 \\
7.987 \mathrm{E}-09 \\
1.265 \mathrm{E}-08 \\
2.629 \mathrm{E}-08 \\
1.535 \mathrm{E}-\mathrm{C}\end{array}$ & $\begin{array}{l}2.165 \mathrm{E}-09 \\
2.168 \mathrm{E}-09 \\
2.182 \mathrm{E}-09 \\
2.269 \mathrm{E}-09 \\
2.253 \mathrm{E}-09 \\
2.319 \mathrm{E}-09 \\
2.421 \mathrm{E}-09 \\
2.572 \mathrm{E}-09 \\
2.794 \mathrm{E}-09 \\
3.04 \mathrm{E}-09 \\
3.717 \mathrm{E}-09 \\
4.323 \mathrm{E}-09 \\
5.736 \mathrm{E}-09 \\
7.857 \mathrm{E}-09 \\
1.216 \mathrm{E}-08 \\
2.294 \mathrm{E}-08 \\
1.186 \mathrm{E}-07\end{array}$ & $\begin{array}{l}2.136 \mathrm{E}-\mathrm{C} 9 \\
2.139 \mathrm{E}-\mathrm{C} 9 \\
2.155 \mathrm{E}-\mathrm{C} 9 \\
2.185 \mathrm{E}-\mathrm{C} 9 \\
2.233 \mathrm{E}-\mathrm{C} 9 \\
2.305 \mathrm{E}-\mathrm{C} 9 \\
2.411 \mathrm{E}-\mathrm{C} 9 \\
2.567 \mathrm{E}-\mathrm{C} 9 \\
2.792 \mathrm{E}-\mathrm{C} 9 \\
3.148 \mathrm{E}-\mathrm{C} 9 \\
3.577 \mathrm{E}-\mathrm{C} 9 \\
4.362 \mathrm{E}-\mathrm{C} 9 \\
5.516 \mathrm{E}-\mathrm{C} 9 \\
7.569 \mathrm{E}-\mathrm{C} 9 \\
1.129 \mathrm{E}-\mathrm{C} 8 \\
2.018 \mathrm{E}-\mathrm{C} 8 \\
9.150 \mathrm{E}-\mathrm{C} 8\end{array}$ & $\begin{array}{l}1.950 E-C 9 \\
1.954 E-C 9 \\
1.970 E-C 9 \\
2.002 E-09 \\
2.052 E-09 \\
2.125 E-09 \\
2.230 E-C 9 \\
2.378 E-09 \\
2.586 E-C 9 \\
2.875 E-09 \\
3.301 E-09 \\
3.920 E-09 \\
4.888 E-09 \\
6.457 E-C 9 \\
9.237 E-09 \\
1.521 E-C 8 \\
5.462 E-C 8\end{array}$ \\
\hline 43 & $3.263 E-08$ & $7.579 \mathrm{E}-08$ & $6.911 \mathrm{E}-\mathrm{C} 8$ & $5.590 \mathrm{E}-08$ \\
\hline
\end{tabular}

PANGE (METERS)

OOC. 0

$120 \mathrm{C} . \mathrm{C}$

$15 C \mathrm{C} . \mathrm{C}$

1800.0 
COSINE

\section{$-1.0 C 000 E$ CO $-9.89401 \mathrm{E}-01$ $-9.44575 E-01$ $-7.55 C 44 E-01$ $-6.17876 E-01$ $-4.58017 \mathrm{E}-01$ $-2.81605 E-01$ $-9.50125 E-02$ $9.50125 E-02$ $2.81605 E-01$ $4.58 \mathrm{C} 17 \mathrm{E}-01$ $6.17876 \mathrm{E}-01$ $7.55044 \mathrm{E}-01$ $8.65631 E-31$ $9.44575 E-01$
$9.89401 E-01$}

TOTAL

COSINE

\section{$-1.00000 \mathrm{E}$ CO} $-9.89401 E-21$ $-9.44575 E-01$ $-8.65631 E-01$ $-7.55044 E-01$ $-6.17876 E-01$ $-4.58017 E-01$ $-2.81605 \mathrm{E}-31$ $9.50125 E-02$ $9.50125 \mathrm{E}-02$ $2.81605 E-01$ 4.58017E-O1 $6.17876 E-01$ 7.55044E-OI $8.65631 E-01$ $9.89401 E-01$

TOTAL
75.0

$1.136 E-10$ $1.136 E-10$ $1.136 \mathrm{E}-10$ $1.136 \mathrm{E}-10$ $1.141 \mathrm{E}-10$ $1.153 \mathrm{E}-10$ $1.180 \mathrm{E}-10$ $1.230 E-10$ $1.316 E-10$ $1.761 E-1 C$ $1.984 E-10$ $2.107 E-1 C$ $2.779 E-1 C$ $5.002 \mathrm{E}-1 \mathrm{C}$ $1.247 E-C 9$ 3.015E-C9 $1.327 E-C 8$

$6.256 E-09$ 100.0 150.0 RANGE (METERS) 250.0 300.0 $400 . C$ $1.302 E-10$ $1.302 E-10$ $1.306 \mathrm{E}-10$ $1.314 E-10$ $1.331 E-10$ $1.366 \mathrm{E}-1 \mathrm{C}$ $1.428 \mathrm{E}-1 \mathrm{C}$ $1.530 \mathrm{E}-1 \mathrm{C}$ $2.873 E-10$ $2.034 E-10$ $3.103 E-10$ $4.3 C 1 E-10$ $8.511 E-10$ $2.710 E-C 9$ $1.305 \mathrm{E}-08$ 6.C $35 E-09$ $1.529 \mathrm{E}-10$ 1. $532 \mathrm{E}-10$ $1.541 E-10$ $1.588 E-10$ $1.640 \mathrm{E}-10$ $1.725 E-10$ $1.859 E-10$ $2.234 E-10$ $2.451 E-10$ $2.761 E-10$ $3.721 E-10$ $5.438 \mathrm{E}-10$ $8.129 E-10$ $1.915 \mathrm{E}-09$ $1.129 E-C 8$

5. $821 E-09$ $1.644 \mathrm{E}-10$ $1.652 E-10$ $1.666 \mathrm{E}-10$ $1.692 \mathrm{E}-10$ $1.733 \mathrm{E}-10$ 1. $8 \mathrm{COE}-10$ $1.904 \mathrm{E}-10$ $2.059 E-10$ $2.194 \mathrm{E}-10$ $2.716 \mathrm{E}-10$ $3.108 E-10$ 4.C $C 98 E-10$ $5.548 \mathrm{E}-10$ $8.471 \mathrm{E}-10$ $1.661 \mathrm{E}-09$ $9.047 E-09$

$5.565 E-09$ $1.676 \mathrm{E}-10$ $1.678 \mathrm{E}-1 \mathrm{C}$ $1.688 \mathrm{E}-1 \mathrm{C}$ $1.739 E-10$ $1.79 \mathrm{CE}-10$ $1.868 \mathrm{E}-10$ $1.983 \mathrm{E}-1 \mathrm{C}$ $2.151 \mathrm{E}-10$ $2.290 E-10$ $2.839 E-10$ $3.228 \mathrm{E}-10$ $4.211 E-10$ $5.561 E-10$ $8.3 C 4 E-10$ $1.489 E-09$ 7.119E-09 5. $249 E-09$

RANGE (METERS)

500.0

$60 \mathrm{C} .0$ 900.0

1200.0

1500.0 $3.91 C E-12$ 3.923E-12 3. $978 E-12$ $4.081 \mathrm{E}-12$ 4. $235 \mathrm{E}-12$ $4.449 E-12$ $4.732 E-12$ $5.100 E-12$ 6. $165 \mathrm{E}-12$ $6.165 E-12$ $6.917 E-12$ $7.871 \mathrm{E}-12$ $9.090 E-12$ $\begin{array}{llllll}5.215 \mathrm{E}-10 & 3.951 \mathrm{E}-1 \mathrm{C} & 1.452 \mathrm{E}-10 & 4.537 \mathrm{E}-11 & 1.281 \mathrm{E}-11 & 3.373 \mathrm{E}-12 \\ 7.830 \mathrm{E}-10 & 5.701 \mathrm{E}-10 & 1.938 \mathrm{E}-10 & 5.802 \mathrm{E}-11 & 1.596 \mathrm{E}-11 & 4.136 \mathrm{E}-12 \\ 2.164 \mathrm{E}-\mathrm{C} 9 & 1.365 \mathrm{E}-\mathrm{Cg} & 3.533 \mathrm{E}-10 & 9.173 \mathrm{E}-11 & 2.329 \mathrm{E}-11 & 5.754 \mathrm{E}-12\end{array}$ $\begin{array}{llllll}5.215 \mathrm{E}-10 & 3.951 \mathrm{E}-1 \mathrm{C} & 1.452 \mathrm{E}-10 & 4.537 \mathrm{E}-11 & 1.281 \mathrm{E}-11 & 3.373 \mathrm{E}-12 \\ 7.830 \mathrm{E}-10 & 5.701 \mathrm{E}-10 & 1.938 \mathrm{E}-10 & 5.802 \mathrm{E}-11 & 1.596 \mathrm{E}-11 & 4.136 \mathrm{E}-12 \\ 2.164 \mathrm{E}-\mathrm{C} & 1.365 \mathrm{E}-\mathrm{Cg} & 3.533 \mathrm{E}-10 & 9.173 \mathrm{E}-11 & 2.329 \mathrm{E}-11 & 5.754 \mathrm{E}-12\end{array}$ $\begin{array}{llllll}5.215 \mathrm{E}-10 & 3.951 \mathrm{E}-1 \mathrm{C} & 1.452 \mathrm{E}-10 & 4.537 \mathrm{E}-11 & 1.281 \mathrm{E}-11 & 3.373 \mathrm{E}-12 \\ 7.830 \mathrm{E}-10 & 5.701 \mathrm{E}-10 & 1.938 \mathrm{E}-10 & 5.802 \mathrm{E}-11 & 1.596 \mathrm{E}-11 & 4.136 \mathrm{E}-12 \\ 2.164 \mathrm{E}-\mathrm{Cg} & 1.365 \mathrm{E}-\mathrm{Cg} & 3.533 \mathrm{E}-10 & 9.173 \mathrm{E}-11 & 2.329 \mathrm{E}-11 & 5.754 \mathrm{E}-12\end{array}$ $1.651 \mathrm{E}-10 \quad 1.484 \mathrm{E}-10$ $1.653 E-1 C \quad 1.487 E-1 C$ $1.664 \mathrm{E}-10 \quad 1.500 \mathrm{E}-10$ $1.724 \mathrm{E}-10 \quad 1.566 \mathrm{E}-10$ $1.781 \mathrm{E}-10 \quad 1.626 \mathrm{E}-10$ $1.865 \mathrm{E}-10 \quad 1.712 \mathrm{E}-10$ $1.987 \mathrm{E}-10 \quad 1.831 \mathrm{E}-10$ $2.161 \mathrm{E}-10 \quad 1.996 \mathrm{E}-10$ $2.434 \mathrm{E}-10 \quad 2.221 \mathrm{E}-10$ $2.740 E-10 \quad 2.546 E-1 C$ 3. $306 E-10 \quad 2.999 E-10$ 4. C9OE-10 3.686E-10 $5.454 \mathrm{E}-10 \quad 4.758 \mathrm{E}-10$ $7.835 E-10 \quad 6.596 E-10$ $1.338 \mathrm{E}-\mathrm{C} 9 \quad 1.043 \mathrm{E}-09$ $5.593 \mathrm{E}-\mathrm{C} 9 \quad 3.462 \mathrm{E}-09$ $4.893 E-09 \quad 4.068 E-09$

1800.0 $1.060 E-12$ $1.075 E-12$ $1.103 E-12$ $1.146 \mathrm{E}-12$ $1.204 E-12$ $1.281 E-12$ $1.381 \mathrm{E}-12$ $1.508 \mathrm{E}-12$ $1.667 \mathrm{E}-12$ $1.867 E-12$ $2.119 E-12$ $2.436 \mathrm{E}-12$ $\begin{array}{llllll}5.215 \mathrm{E}-10 & 3.951 \mathrm{E}-1 \mathrm{C} & 1.452 \mathrm{E}-10 & 4.537 \mathrm{E}-11 & 1.281 \mathrm{E}-11 & 3.373 \mathrm{E}-12 \\ 7.830 \mathrm{E}-10 & 5.701 \mathrm{E}-10 & 1.938 \mathrm{E}-10 & 5.802 \mathrm{E}-11 & 1.596 \mathrm{E}-11 & 4.136 \mathrm{E}-12 \\ 2.164 \mathrm{E}-\mathrm{C} & 1.365 \mathrm{E}-\mathrm{Cg} & 3.533 \mathrm{E}-10 & 9.173 \mathrm{E}-11 & 2.329 \mathrm{E}-11 & 5.754 \mathrm{E}-12\end{array}$ $\begin{array}{llllll}5.215 \mathrm{E}-10 & 3.951 \mathrm{E}-1 \mathrm{C} & 1.452 \mathrm{E}-10 & 4.537 \mathrm{E}-11 & 1.281 \mathrm{E}-11 & 3.373 \mathrm{E}-12 \\ 7.830 \mathrm{E}-10 & 5.701 \mathrm{E}-10 & 1.938 \mathrm{E}-10 & 5.802 \mathrm{E}-11 & 1.596 \mathrm{E}-11 & 4.136 \mathrm{E}-12 \\ 2.164 \mathrm{E}-\mathrm{C} & 1.365 \mathrm{E}-\mathrm{Cg} & 3.533 \mathrm{E}-10 & 9.173 \mathrm{E}-11 & 2.329 \mathrm{E}-11 & 5.754 \mathrm{E}-12\end{array}$ $\begin{array}{llllll}5.215 \mathrm{E}-10 & 3.951 \mathrm{E}-1 \mathrm{C} & 1.452 \mathrm{E}-10 & 4.537 \mathrm{E}-11 & 1.281 \mathrm{E}-11 & 3.373 \mathrm{E}-12 \\ 7.830 \mathrm{E}-10 & 5.701 \mathrm{E}-10 & 1.938 \mathrm{E}-10 & 5.802 \mathrm{E}-11 & 1.596 \mathrm{E}-11 & 4.136 \mathrm{E}-12 \\ 2.164 \mathrm{E}-\mathrm{C} & 1.365 \mathrm{E}-\mathrm{Cg} & 3.533 \mathrm{E}-10 & 9.173 \mathrm{E}-11 & 2.329 \mathrm{E}-11 & 5.754 \mathrm{E}-12\end{array}$ $2.164 \mathrm{E}-\mathrm{C} 9$

$2.482 E-C 9$

$9.491 E-10$

$3.057 E-10$

8. $809 E-11 \quad 2.354 \mathrm{E}-11$ 
COSINE

$-1.00000 E$ CO $-9.89401 E-1) 1$ $-9.44575 E-01$ $-8.65631 \mathrm{E}-01$ $-7.55 C 44 \mathrm{E}-\mathrm{C} 1$ $-6.17876 \mathrm{E}-\mathrm{C} 1$ $-4.58017 \mathrm{E}-01$ $-2.81605 E-01$ $-9.50125 E-02$ $2.81605 E-01$ $4.58017 \mathrm{E}-01$ $6.17876 E-C 1$ $8.65631 \mathrm{E}-\mathrm{C} 1$ $9.44575 E-01$

\section{TOTAL}

COSINE

\section{-1.00000 CO} $-9.894 \mathrm{C} 1 \mathrm{E}-01$ $-9.44575 E-01$ $8.65631 E-01$ $-6.17876 \mathrm{E}-\mathrm{O} 1$ 6.17876201 $-2.81605 \mathrm{E}-21$ $-9.50125 \mathrm{E}-02$ . 5C125E-02 $2.81605 E-01$ $4.58017 \mathrm{E}-01$ $4.58017 \mathrm{E}-01$ $7.55044 \mathrm{E}-01$ $7.55044 E-01$ $9.44575 \mathrm{E}-01$ 9.89401E-01

TOTAL
75.0

1 no.c

$1.668 \mathrm{E}-10$ $1.667 \mathrm{E}-10$

$1.663 \mathrm{E}-10$

$1.659 \mathrm{E}-10$

$1.658 E-10$

$1.670 E-10$

$1.701 \mathrm{E}-10$

$1.766 \mathrm{E}-10$

$1.879 \mathrm{E}-1 \mathrm{C}$

$2.440 E-1 C$

.

2.847E-10

$3.555 E-1 C$

.

.

$1.405 E-C 8$

$7.348 \mathrm{E}-\mathrm{CO}$

$500 . C$

$1.949 E-10$

$1.973 E-10$

$2.012 \mathrm{E}-10$

2. C71E-1C

$2.158 \mathrm{E}-10$

. $444 E-10$

$2.665 \mathrm{E}-10$

$2.951 E-10$

3.358E- 10

$3.904 \mathrm{E}-1 \mathrm{C}$

$4.671 \mathrm{E}-10$

$5.801 E-10$

$5.801 E-10$

$7.591 \mathrm{E}-10$

$2.788 E-C 9$

$4.840 E-C 9$
RANGE (METERS)
$2 C 0 . C$

$25 \mathrm{C} .0$

$3 c 0.0$

$400 . \mathrm{C}$

$2.310 E-10 \quad 2.514 E-10 \quad 2.586 E-10$

$1.535 \mathrm{E}-1 \mathrm{C} \quad 2.310 \mathrm{E}-10 \quad 2.516 \mathrm{E}-10 \quad 2.589 \mathrm{E}-1 \mathrm{C}$

$\begin{array}{llll}1.932 E-10 & 2.313 E-10 & 2.524 E-10 & 2.6 n 2 E-10\end{array}$

.936E-10 2.341E-10 $2.577 \mathrm{E}-10 \quad 2.676 \mathrm{E}-1 \mathrm{C}$

$1.955 \mathrm{E}-10 \quad 2.381 \mathrm{E}-10 \quad 2.637 \mathrm{E}-1 \mathrm{C} \quad 2.752 \mathrm{E}-10$

$2.455 \mathrm{E}-10$ 2.736E-10 $2.869 \mathrm{E}-1 \mathrm{C}$

$2.223 \mathrm{E}-1 \mathrm{C}$

$2.876 \mathrm{E}-1 \mathrm{C}$

$3.768 \mathrm{E}-1 \mathrm{C}$

$4.272 E-10$

(1)

1. 11 (O)

$3.037 E-C 9$
$1.4 C 7 E-C .8$

$2.576 \mathrm{E}-10$

$2.766 \mathrm{E}-10$

$3.533 \mathrm{E}-10$

$4.026 E-10$

$5.194 \mathrm{E}-10$

$7.362 \mathrm{E}-10$

$1.654 E-C 9$

$1.246 E-08$

$2.387 \mathrm{E}-10$

3. $113 E-10$

$3.284 \mathrm{E}-10$

$4.064 \mathrm{E}-10$

$4.547 E-10$

$5.886 \mathrm{E}-10$

$7.669 \mathrm{E}-12$

$1.126 \mathrm{E}-09$

$1.021 \mathrm{E}-08$

$3.042 \mathrm{E}-1 \mathrm{C}$

$3.291 \mathrm{E}-1 \mathrm{C}$

$4.301 E-10$

$4.792 E-1 C$

$6.148 E-10$

$7.837 E-10$

$1.128 \mathrm{E}-09$

$1.93 E-09$

$7.359 E-C 9$

$7.497 E-C 9$

$7.452 E-09$

7. 25CE-09

RANGE (METERS)

600.0

900.0

$12 C \mathrm{C} . \mathrm{C}$

1500.0

$6.095 \mathrm{E}-12$ $6.115 \mathrm{E}-12$ $6.201 E-12$ $6.607 \mathrm{E}-12$ $6.947 \mathrm{E}-12$

$6.318 \mathrm{E}-11 \quad 2.091 \mathrm{E}$

$6.398 E-11$ 2.120E-11

$2.174-11$

1.606 -10

$6.787 \mathrm{E}-11$

$2.369 E-11$

$731 \mathrm{E}-10$

1. $832 \mathrm{E}-1 \mathrm{C}$

$1.968 \mathrm{E}-10$

$2.148 E-10$
$2.387 E-1 C$

$2.703 \mathrm{E}-10$

$3.129 E-1 C$

$3.71 \in E-1 C$

$4.558 \mathrm{E}-1 \mathrm{C}$

$5.850 \mathrm{E}-1 \mathrm{C}$

$8.17 C E-1 C$

$1.818 \mathrm{E}-\mathrm{C} 9$

$7.117 \mathrm{E}-11$

8.

(11

$7.94 \mathrm{E}-12$

$7.980 \mathrm{E}-12$

$9.883 \mathrm{E}-11$

$3.296 E-11$

$1.280 \mathrm{E}-10$

$1.498 E-10$

$3.7 C 8 E-11$

$8.725 E-12$

$9.668 \mathrm{E}-12$

$1.086 \mathrm{E}-11$

$1.236 \mathrm{E}-11$

$1.427 E-11$

$1.794 \mathrm{E}-10$

$4.235 E-11$

$5.808 \mathrm{E}-11$

$1.69 \mathrm{E}-11$

$2.898 E-10$

8.860E-11

$2.464 E-11$

$3.765 E-09$

$1.470 F-09$

4. $764 E-10$

$1.376 E-10$

$1.643 \mathrm{E}-12$

$1.648 \mathrm{E}-12$

$783 \mathrm{E}-12$

$998 \mathrm{E}-12$

$2.156 \mathrm{E}-12$

.357E-12

. $610 \mathrm{E}-12$

. $928 \mathrm{E}-12$

326E-12

$3.825 E-12$

$4.456 \mathrm{E}-12$

$6.423 \mathrm{E}-12$

$8.784 \mathrm{E}-12$

3. $677 E-11$
$2.562 \mathrm{E}-10 \quad 2.321 \mathrm{E}-10$

$2.566 E-1 C \quad 2.325 E-10$

$2.582 E-10 \quad 2.345 E-10$

$2.616 \mathrm{E}-10 \quad 2.385 \mathrm{E}-10$

$2.672 E-10 \quad 2.447 \mathrm{E}-10$

$2.541 \mathrm{E}-10$

2.758E-10 $2.541 E-10$

2.858E-10

. $3.111 E-10$

$4.742 E-10$ 3.450E-10

4.174E-10 $3.931 E-10$

$4.98 E-10 \quad 4.585 E-10$

.032E-10 5.546E-10

1.026E-10 $6.991 E-10$

$1.082 \mathrm{E}-\mathrm{C} 9 \mathrm{9} \quad 9.389 \mathrm{E}-10$

$1.75 \mathrm{E}-\mathrm{CS} \quad 1.422 \mathrm{E}-09$

$6.924 E-C 9 \quad 5.954 E-09$

1800.0 
COSINE

$-1.000 C O E$ OO $-9.89401 E-01$ $-9.44575 \mathrm{E}-01$ $-8.65631 \mathrm{E}-0$ $-7.55044 \mathrm{E}-\mathrm{J}$ $-6.17876 E-01$ $-2.81605 \mathrm{E}-01$ $-2.81605 E-01$ $9.50125 \mathrm{E}-02$ $2.81605 E-01$ 4. $58017 \mathrm{E}-01$ $6.17876 \mathrm{E}-01$ $7.55044 \mathrm{E}-\mathrm{Cl}$ $8.65631 E-01$ $9.44575 E-O$ $9.89401 E-01$

TOTAL

COSINE

-1.00000 E 00 $-9.89401 \mathrm{E}-01$ $-9.44575 E-01$ $-8.65631 \mathrm{E}-0$ $-7.55044 E-01$ $-6.17876 E-01$ $-4.58017 E-01$ $-2.81605 E-01$ $-9.50125 \mathrm{E}-02$ $9.50125 E-02$ $2.81605 E-3$ 4.58017E-O $7.55044 E-O$ $7.55044 E-01$ $8.65631 E-01$ $9.44575 E-01$

TOTAL
75.0

$1.460 E-13$ $.465 \mathrm{E}-13$ $482 E-13$

. $563 \mathrm{E}-13$

$1.628 \mathrm{E}-13$

1.712E-13

$1.823 \mathrm{E}-13$

$1.971 \mathrm{E}-13$

$2.206 \mathrm{E}-13$

$2.651 E-13$

$2.997 E-13$

$3.111 E-13$

$3.763 E-13$

$5.511 \mathrm{E}-13$

$8.059 E-13$

1. $151 \mathrm{E}-12$

$3.388 E-12$

500.0

$1.1835-12$

$1.189 \mathrm{E}-12$

$1.212 \mathrm{E}-12$

$1.256 E-12$

$1.320 \mathrm{E}-12$

$1.407 \mathrm{E}-12$

$1.521 \mathrm{E}-12$

$1.670 \mathrm{E}-12$

$1.862 E-12$

$2.106 E-12$

$2.412 E-12$

$2.787 E-12$

$3.779 \mathrm{E}-12$

$3.779 E-12$

$4.424 E-12$

$5.282 E-12$

$2.969 E-11$
100.0

$2.103 E-13$

$2.109 \mathrm{E}-13$

2.180E-13

246E-1

$2.332 \mathrm{E}-13$

$2.443 \mathrm{E}-13$

$2.586 \mathrm{E}-13$

2.773E-13

$.351 \mathrm{E}-13$

. $336 \mathrm{E}-13$

. $308 E-13$

$4.369 E-13$

$5.622 E-13$

$6.776 \mathrm{E}-13$

$1.743 \mathrm{E}-12$

$4.754 E-12$

600.0

. $168 \mathrm{E}-12$

$1.174 E-12$

$1.199 \mathrm{E}-12$

. $247 \mathrm{E}-12$

. $316 \mathrm{E}-12$

$1.41 C E-12$

$1.537 \mathrm{E}-12$

$1.795 E-12$

$1.926 E-12$

. $215 E-12$

.

.

उ6OE 12

.200E-12

$6.203 \mathrm{E}-12$

$7.862 \mathrm{E}-12$

150.0

RANGE (METERS)
$200 . \mathrm{C}$

$25 C . C$

300.0

400.0

\section{$3.626 E-13$}

$3.637 E-13$

$5.31 C E-13$

$6.989 \mathrm{E}-13$

$5.328 \mathrm{E}-13$

$.015 E-13$

$.763 E-13$

$3.878 \mathrm{E}-13$

$4.211 \mathrm{E}-13$

$5.528 \mathrm{E}-13$

$7.302 E-13$

$7.505 \mathrm{E}-13$

$7.565 \mathrm{E}-13$

$7.904 E-13$

$8.869 \mathrm{E}-13$

$9.534 \mathrm{E}-13$

$1.041 \mathrm{E}-12$

$1.127 \mathrm{E}-12$

$1.257 E-12$

$1.398 \mathrm{E}-12$

4.995E-13

$6.189 \mathrm{E}-13$

$902 E-13$

. $214 \mathrm{E}-12$

$1.613 \mathrm{E}-12$

$1.941 \mathrm{E}-12$

2. $604 \mathrm{E}-12$

$4.289 E-12$

$2.755 \mathrm{E}-12$

$3.578 \mathrm{E}-12$

$7.653 E-12$

$1.1 \mathrm{COE}-11$

$1.456 E-11$

RANGE (METERS)

900.0

1200.0

1500.0

$7.620 \mathrm{E}-13$

$7.672 \mathrm{E}-13$

$3.544 E-13$

$3.579 E-13$

3. $717 \mathrm{E}-13$

$8.268 \mathrm{E}-13$

$8.812 \mathrm{E}-13$

$9.553 \mathrm{E}-1$

$1.057 E-1$

$4.237 E-13$

$4.6 \mathrm{C5E}-13$

$5.112 \mathrm{E}-1$

$5.860 E-13$

$6.974 \mathrm{E}-13$

$8.6 C 9 E-13$

$1.102 \mathrm{E}-12$

$1.466 E-12$

$2.033 E-12$

$2.919 E-12$

$4.231 E-12$

$7.953 \mathrm{E}-12$

. 226E-12

. $377 E-13$

$1.401 \mathrm{E}-13$

$1.487 \mathrm{E}-13$

$1.614 E-13$

$1.744 \mathrm{E}-13$

$1.882 \mathrm{E}-13$

$2.069 \mathrm{E}-13$

$2.376 \mathrm{E}-13$

$2.876 E-13$

$3.64 C E-13$

4.

.754E-13

53 E 12

$.920 \mathrm{E}-12$

$5.505 E-12$

$1.745 E-11$

$9.092 E-12$

$\begin{array}{ll}8.527 \mathrm{E}-13 & 1.082 \mathrm{E}-12 \\ 8.561 \mathrm{E}-13 & 1.087 \mathrm{E}-12 \\ 8.692 \mathrm{E}-13 & 1.106 \mathrm{E}-12 \\ 8.940 \mathrm{E}-13 & 1.142 \mathrm{E}-12 \\ 9.291 \mathrm{E}-13 & 1.194 \mathrm{E}-12 \\ 9.750 \mathrm{E}-13 & 1.263 \mathrm{E}-12 \\ 1.033 \mathrm{E}-12 & 1.353 \mathrm{E}-12 \\ 1.107 \mathrm{E}-12 & 1.468 \mathrm{E}-12 \\ 1.198 \mathrm{E}-12 & 1.613 \mathrm{E}-12 \\ 1.308 \mathrm{E}-12 & 1.793 \mathrm{E}-12 \\ 1.444 \mathrm{E}-12 & 2.012 \mathrm{E}-12 \\ 1.602 \mathrm{E}-12 & 2.275 \mathrm{E}-12 \\ 1.798 \mathrm{E}-12 & 2.587 \mathrm{E}-12 \\ 2.048 \mathrm{E}-12 & 2.963 \mathrm{E}-12 \\ 2.422 \mathrm{E}-12 & 3.447 \mathrm{E}-12 \\ 3.120 \mathrm{E}-12 & 4.208 \mathrm{E}-12 \\ 4.927 \mathrm{E}-12 & 6.049 \mathrm{E}-12 \\ & \\ 1.818 \mathrm{E}-11 & 2.482 \mathrm{E}-11\end{array}$

1800.0 


COSINE
-1. CO00OE OO
$-9.89401 E-01$
$-9.44575 E-C 1$
$-8.65631 E-01$
$-7.55044 E-C 1$
$-6.17876 E-01$
$-4.58017 E-01$
$-2.81605 E-01$
$-9.50125 E-02$
$9.50125 E-02$
$2.81605 E-01$
$4.58017 E-01$
$6.17876 E-01$
$7.55044 E-01$
$8.65631 E-C 1$
$9.44575 E-01$
$9.89401 E-01$

TOTAL

COSINE

$-1.0000 C E C O$ $-9.89401 \mathrm{E}-01$ $-9.44575 \mathrm{E}-01$ $-8.65631 E-01$ $-6.17876 E-01$ $-4.58017 E-01$ $-2.81605 E-01$ $-9.5 \mathrm{C} 125 \mathrm{E}-\mathrm{O}$ $9.50125 \mathrm{E}-\mathrm{C} 2$ $2.816 C 5 E-C 1$ $4.58017 \mathrm{E}-01$ $6.17876 \mathrm{E}-\mathrm{C} 1$ $7.55 C 44 E-C 1$ $8.65631 E-01$ $9.44575 E-01$ $9.894 C 1 E-01$

TOTAL

$\begin{array}{ll}75.0 & 1 C 0 . C \\ 1.466 E-11 & 2.137 E-11 \\ 1.471 E-11 & 2.143 E-11 \\ 1.487 E-11 & 2.167 E-11 \\ 1.519 E-11 & 2.211 E-11 \\ 1.565 E-11 & 2.275 E-11 \\ 1.627 E-11 & 2.358 E-11 \\ 1.707 E-11 & 2.465 E-11 \\ 1.813 E-11 & 2.6 C 3 E-11 \\ 1.953 E-11 & 2.782 E-11 \\ 2.175 E-11 & 3.327 E-11 \\ 2.593 E-11 & 3.317 E-11 \\ 2.919 E-11 & 4.232 E-11 \\ 3.029 E-11 & 4.295 E-11 \\ 3.642 E-11 & 5.472 E-11 \\ 5.279 E-11 & 6.56 C E-11 \\ 7.663 E-11 & 1 . C 51 E-1 C \\ 1 . C 89 E-10 & 1.653 E-1 C \\ 3.3 C 9 E-10 & 4.689 E-1 C\end{array}$

500.6

$1.251 \mathrm{E}-1 \mathrm{C}$ $1.257 \mathrm{E}-1 \mathrm{C}$ $1.280 E-1 C$ $1.324 E-1 C$ $1.388 \mathrm{E}-1 \mathrm{C}$ $1.475 E-1 C$ $1.590 E-1 C$ $1.739 E-1 C$ $1.932 \mathrm{E}-1 \mathrm{C}$ $2.176 \mathrm{E}-1 \mathrm{C}$ $2.480 E-1 C$ $2.854 E-10$ $3.3 C 4 E-1 C$ $3.837 E-1 C$ $4.471 E-1 C$ $5.302 \mathrm{~F}-1 \mathrm{C}$ $6.961 E-1 C$

3. $C 48 E-C 9$

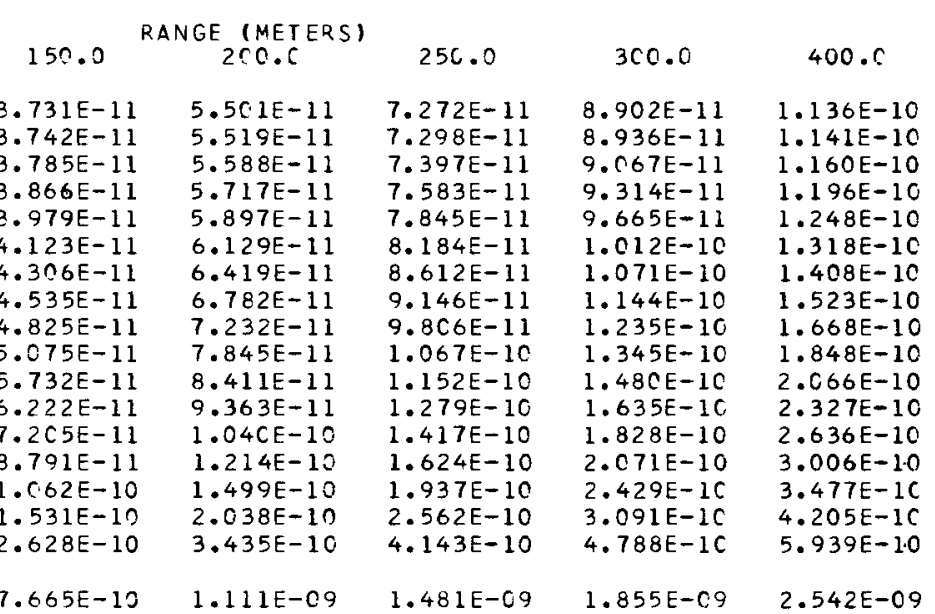

RANGE (METERS)

$$
\text { OCO.O }
$$

$12 C . C . C$

1500.0

1800.6
$8.370 E-11$ $8.423 E-11$ $8.635 \mathrm{E}-11$ 9. $(26 E-11$ $9.577 E-11$ $1.033 F-10$ $1.135 \mathrm{E}-10$ $1.278 E-10$ $1.476 \mathrm{E}-10$ $1.752 \mathrm{E}-10$ $2.136 \mathrm{E}-10$ $2.672 \mathrm{E}-10$ $3.421 E-13$ $4.443 E-17$ $5.757 E-10$ $7.269 \mathrm{E}-10$ $8.865 E-15$

2. $904 E-C 9$
$1.687 \mathrm{E}-11$ $1.711 E-11$ $1.797 \mathrm{E}-11$ $1.927 \mathrm{E}-11$ $2.061 E-11$ 2. $2 C 5 E-11$ 2. $398 \mathrm{E}-11$ 2. $712 \mathrm{E}-11$ $3.216 E-11$ $3.982 E-11$ $5.129 E-11$ $6.944 \mathrm{E}-11$ 1. CO6E-1C $1.559 \mathrm{E}-10$ $2.507 E-1 C$ $5.506 \mathrm{E}-12$ $3.314 E-C 9$
$6.476 \mathrm{E}-12$ $6.635 E-12$
$7.147 E-12$ $7.880 E-12$ $8.458 \mathrm{E}-12$ 8. $877 E-12$ $9.446 \mathrm{E}-12$ 1. $056 \mathrm{E}-11$ $1.265 \mathrm{E}-1$ $1.587 \mathrm{E}-11$ $2.059 \mathrm{E}-1$ $2.816 \mathrm{E}-11$ $4.233 \mathrm{E}-1$ $7.103 \mathrm{E}-11$ $1.277 \mathrm{E}-10$ $2.258 \mathrm{E}-10$
$3.495 \mathrm{E}-10$ $4.530 E-1 C$ 
4 PI R**2 AIR KERMA (GAMMAS)
(CM**2 ERGS/GRAM/STERADIAN/SOURCE NEUTRON)
2.350 TO 4.065 MEV NEUTRON SOURCE

$\begin{array}{ccccc}\text { RANGE (METERS) } & & & \\ & 200.0 & 25 C .0 & 300 . \mathrm{C} & 40 \mathrm{C} .0 \\ 1 & 6.863 \mathrm{E}-11 & 9.144 \mathrm{E}-11 & 1.129 \mathrm{E}-1 \mathrm{C} & 1.471 \mathrm{E}-10 \\ 1 & 6.881 \mathrm{E}-11 & 9.17 \mathrm{CE}-11 & 1.132 \mathrm{E}-10 & 1.476 \mathrm{E}-10 \\ 1 & 6.953 \mathrm{E}-11 & 9.272 \mathrm{E}-11 & 1.146 \mathrm{E}-10 & 1.496 \mathrm{E}-10 \\ 1 & 7.086 \mathrm{E}-11 & 9.464 \mathrm{E}-11 & 1.171 \mathrm{E}-10 & 1.533 \mathrm{E}-10 \\ 1 & 7.273 \mathrm{E}-11 & 9.735 \mathrm{E}-11 & 1.207 \mathrm{E}-10 & 1.586 \mathrm{E}-10 \\ 1 & 7.514 \mathrm{E}-11 & 1.009 \mathrm{E}-10 & 1.254 \mathrm{E}-1 \mathrm{C} & 1.657 \mathrm{E}-10 \\ 1 & 7.817 \mathrm{E}-11 & 1.053 \mathrm{E}-10 & 1.314 \mathrm{E}-10 & 1.749 \mathrm{E}-10 \\ 1 & 8.193 \mathrm{E}-11 & 1.108 \mathrm{E}-10 & 1.389 \mathrm{E}-10 & 1.866 \mathrm{E}-10 \\ 1 & 8.660 \mathrm{E}-11 & 1.175 \mathrm{E}-10 & 1.482 \mathrm{E}-10 & 2.012 \mathrm{E}-10 \\ 1 & 9.291 \mathrm{E}-11 & 1.264 \mathrm{E}-10 & 1.593 \mathrm{E}-10 & 2.191 \mathrm{E}-10 \\ 1 & 9.882 \mathrm{E}-11 & 1.351 \mathrm{E}-10 & 1.729 \mathrm{E}-10 & 2.408 \mathrm{E}-10 \\ 1 & 1.086 \mathrm{E}-10 & 1.48 \mathrm{CE}-10 & 1.886 \mathrm{E}-10 & 2.665 \mathrm{E}-10 \\ 1 & 1.192 \mathrm{E}-10 & 1.62 \mathrm{CE}-10 & 2.079 \mathrm{E}-10 & 2.969 \mathrm{E}-10 \\ 1 & 1.367 \mathrm{E}-10 & 1.829 \mathrm{E}-10 & 2.322 \mathrm{E}-10 & 3.332 \mathrm{E}-10 \\ 0 & 1.653 \mathrm{E}-10 & 2.143 \mathrm{E}-10 & 2.680 \mathrm{E}-10 & 3.793 \mathrm{E}-10 \\ 0 & 2.187 \mathrm{E}-10 & 2.762 \mathrm{E}-10 & 3.334 \mathrm{E}-10 & 4.508 \mathrm{E}-10 \\ 0 & 3.558 \mathrm{E}-10 & 4.314 \mathrm{E}-10 & 5.001 \mathrm{E}-10 & 6.207 \mathrm{E}-10 \\ 10 & 1.293 \mathrm{E}-\mathrm{C} 9 & 1.726 \mathrm{E}-09 & 2.163 \mathrm{E}-\mathrm{C} 9 & 2.964 \mathrm{E}-09\end{array}$

RANGE (METERS)

$900.0 \quad 1200.0$

1500.0

1800.0

$1.713 E-1 C$

$1.313 \mathrm{E}-10$

$7.497 E-11$

3. $7 C 5 E-11$

$1.707 E-11$

$1.671 \mathrm{E}-10 \quad 1.72 \mathrm{CE}-10$

$1.696 \mathrm{E}-10 \quad 1.746 \mathrm{E}-10$

$1.796 \mathrm{E}-1 \mathrm{C}$

更

$7.536 E-11$

$3.730 E-11$

$1.724 E-11$

$1.779 E-11$

$1.384 \mathrm{E}-10 \quad 7.954 \mathrm{E}-11 \quad 3.974 \mathrm{E}-11 \quad 1.862 \mathrm{E}-11$

$1.444 E-10$ 8.296E-11 4.14CE-11 $1.937 E-11$

$2.013 \mathrm{E}-1 \mathrm{C} \quad 2.097 \mathrm{E}-10 \quad 1.635 \mathrm{E}-10 \quad 9.328 \mathrm{E}-11 \quad 4.578 \mathrm{E}-11 \quad 2.096 \mathrm{E}-11$

$2.164 \mathrm{E}-10 \quad 2.268 \mathrm{E}-1 \mathrm{C} \quad 1.784 \mathrm{E}-10$ 10 $1.016 \mathrm{E}-10 \quad 4.952 \mathrm{E}-11 \mathrm{l} 2.244 \mathrm{E}-11$

$2.35 \mathrm{E}-10 \quad 2.490 \mathrm{E}-10 \quad 1.987 \mathrm{E}-10 \quad 1.136 \mathrm{E}-10 \quad 5.514 \mathrm{E}-11 \quad 2.488 \mathrm{E}-11$

$2.59 E-10$ 2.264E-10 1.30E-10 $6.332 \mathrm{E}-11$ 2.845E-11

$2.89 \mathrm{E}-10$ 3.139E-10 2.642E-10 1.546E-10 7.513E-11 $3.347 \mathrm{E}-11$

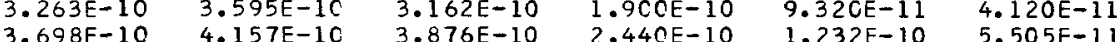

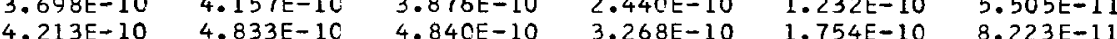

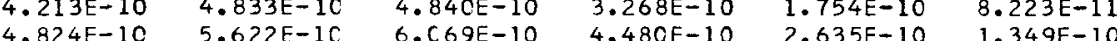

$\begin{array}{llllll}4.824 \mathrm{E}-10 & 5.622 \mathrm{E}-1 \mathrm{C} & 6.669 \mathrm{E}-10 & 4.480 \mathrm{E}-10 & 2.635 \mathrm{E}-10 & 1.349 \mathrm{E}-10 \\ 5.627 \mathrm{E}-10 & 6.558 \mathrm{E}-10 & 7.477 \mathrm{E}-10 & 6.041 \mathrm{E}-10 & 3.943 \mathrm{E}-10 & 2.249 \mathrm{E}-10\end{array}$

$\begin{array}{llllll}5.627 \mathrm{E}-10 & 6.558 \mathrm{E}-10 & 7.477 \mathrm{E}-10 & 6.241 \mathrm{E}-10 & 3.943 \mathrm{E}-10 & 2.249 \mathrm{E}-10 \\ 7.245 \mathrm{E}-1 \mathrm{C} & 8.589 \mathrm{E}-10 & 8.978 \mathrm{E}-10 & 7.618 \mathrm{E}-10 & 5.387 \mathrm{E}-10 & 3.377 \mathrm{E}-10\end{array}$

$3.56 \mathrm{CE}-09 \quad 3.888 \mathrm{E}-\mathrm{C} 9$

$3.490 E-C 9 \quad 2.242 E-09$

$1.2 C 7 E-C 9$

$5.911 E-10$ 
$\begin{aligned} & 4 \text { PI R*\#2 SILICON KERMA (GAMMAS) } \\ & \text { (CM**2 ERGS/GRAM/STERADIAN/SOURCE NEUTRON) }\end{aligned}$

COS INE

$-1.00000 \mathrm{E} 00$ $-9.89401 \mathrm{E}-01$ $-9.44575 E-C 1$ $-8.65631 E-01$ $-7.55044 E-C 1$ $-6.17876 \mathrm{E}-\mathrm{C}$ $-4.58017 \mathrm{E}-\mathrm{C} 1$ $-2.81605 E-01$ $-9.50125 \mathrm{E}-02$

$9.50125 \mathrm{E}-02$

$2.81605 E-01$

$4.58017 E-01$

$6.17876 \mathrm{E}-\mathrm{O}$

$7.55044 E-01$

$8.65631 \mathrm{~L}-01$

$9.44575 E-01$

$9.89401 E \rightarrow 01$

TOTAL

COSINE

$-1.00000 E$ OC $-9.89401 \mathrm{E}-01$ $-9.44575 E-C 1$ $-8.65631 \mathrm{E}-01$ $-7.55044 \mathrm{E}-01$ $-6.17876 E-01$ $-4.58017 \mathrm{E}-01$ $-2.81605 E-01$ $-9.50125 E-C 2$ . $50125 E-02$ $2.81605 E-01$ $4.5817 E-01$ $7.55044 \mathrm{E}-01$ . $55044 E-01$ $9.44575 \mathrm{E}-01$ $9.89401 \mathrm{E}-01$

TOTAL
75.

1. $505 \mathrm{E}-11$ 1. $50.9 E-11$ $1.526 \mathrm{E}-11$ $1.558 \mathrm{E}-11$ $1.604 \mathrm{E}-11$ $1.666 \mathrm{E}-11$ $1.747 \mathrm{E}-11$ $1.853 \mathrm{E}-11$

$1.994 \mathrm{E}-11$

$2.218 E-11$

$2.638 \mathrm{E}-11$

$2.967 \mathrm{E}-11$

3.C77E-11

$3.692 \mathrm{E}-11$

$5.338 \mathrm{E}-11$

$7.735 E-11$

$3.366 E-10$

500.0

$1.307 E-10$

$1.313 \mathrm{E}-10$

$1.337 E-10$

$1.382 E-10$

$1.448 \mathrm{E}-10$

$1.536 E-10$

$1.654 E-10$

$1.806 \mathrm{E}-10$

$2.003 E-10$

$2.252 E-1 C$

2. $945 E-10$

$2.945 E-10$

$3.949 E-10$

. $949 E-10$

5.

$7.108 E-10$

$3.149 E-C 9$
100.0

2. 2C1E-11 $2.207 E-11$ 2. $231 E-11$ . $34 C E-11$ $2.424 E-11$ $2.532 E-11$ $2.671 \mathrm{E}-11$ $2.852 \mathrm{E}-11$ 3. $400 \mathrm{E}-11$ .391E-11 $4.310 E-11$ $4.374 E-11$ $5.557 E-11$ $6.649 E-11$ $1.667 \mathrm{E}-10$ $4.782 E-1 C$

150.0 RANGE (METERS)
$200 . \mathrm{C}$

250.0

300.0

400.6

3. $860 \mathrm{E}-11$ $3.915 \mathrm{E}-11$ $3.996 \mathrm{E}-11$

$5.7 C 5 E-11$

$7.555 E-11$ $5.723 E-11$
$5.794 E-11$ $5.925 \mathrm{E}-11$ $6.108 \mathrm{E}-11$ $4.258 E-11$ $4.442 \mathrm{E}-11$ $9.294 \mathrm{E}-11$ $1.209 \mathrm{E}-10$ $.080 E-11 \quad 1.246 E-10$ $1.051 \mathrm{E}-1 \mathrm{C} \quad 1.370 \mathrm{E}-1 \mathrm{C}$ $1.110 \mathrm{E}-10 \quad 1.462 \mathrm{E}-10$ $1.185 \mathrm{E}-10$ 1.580E-10 $1.278 \mathrm{E}-10 \quad 1.728 \mathrm{E}-1 \mathrm{C}$ $1.390 \mathrm{E}-1 \mathrm{C} \quad 1.912 \mathrm{E}-1 \mathrm{C}$ $1.527 \mathrm{E}-1 \mathrm{C} \quad 2.134 \mathrm{E}-10$ $1.685 \mathrm{E}-1 \mathrm{C} \quad 2.400 \mathrm{E}-10$ $1.881 E-1 C \quad 2.715 E-10$ $2.126 \mathrm{E}-1 \mathrm{O} \quad 3.092 \mathrm{E}-10$ $2.489 \mathrm{E}-10$ 3.569E-10 $3.154 E-10 \quad 4.304 E-10$ 4.861E-1C 6.C49E-1C $1.912 E-C 9 \quad 2.625 E-09$

$\begin{array}{lllll}1.551 E-10 & 2.071 E-10 & 2.009 E-10 & 3.861 E-1 C & 6.049 E-10 \\ 2.654 E-10 & 3.475 E-10 & 4.199 E-10 & 4.861 E & \\ 7.854 E-10 & 1.142 E-09 & 1.524 E-09 & 1.912 E-C 9 & 2.625 E-09\end{array}$

\section{RANGE (METERS)}

600.0

SOO.C $1200 . \mathrm{C}$

1500.0

1800.0

$1.303 E-10$ 1. $309 \mathrm{E}-10$ $1.335 E-10$ $1.384 \mathrm{E}-10$ $1.455 \mathrm{E}-1 \mathrm{C}$ $1.552 E-1 C$ $1.682 \mathrm{E}-1 \mathrm{C}$ $1.855 \mathrm{E}-1 \mathrm{C}$ $2.081 \mathrm{E}-1 \mathrm{C}$ $2.376 \mathrm{E}-1 \mathrm{C}$ $3.235 \mathrm{E}-10$ $3.235 E-10$ $4.557 E-10$ $4.557 E-10$ $6.40 \mathrm{E}-10$ $7.409 E-10$ 8.864E-11 $8.917 E-11$ $9.134 \mathrm{E}-11$ $9.533 E-11$ $1.010 E-10$ $1 . C 86 E-10$ $1.191 E-10$ $1.336 E-10$ $1.539 E-10$ $1.820 E-10$ $2.760 E-10$ $2.760 E-10$ $4.572 E-10$ $4.572 E-10$ $7.464 E-10$ $9.091 E-10$ $3.426 E-C 9$ $4.380 E-11$ 4. $417 E-11$ $4.559 E-11$ 4.798E-11 $5.099 E-11$ $5.482 \mathrm{E}-11$ $6.008 \mathrm{E}-11$ $6.779 E-11$ $7.921 E-11$ $9.590 E-11$ $1.204 E-10$ $1.574 E-10$ 2.15OE-10 $4.383 E-10$ 4.3 7.117E-10

$1.877 E-09$

$1.849 E-11 \quad 7.265 \mathrm{E}-12$ $1.874 \mathrm{E}-11 \quad 7.429 \mathrm{E}-12$ $1.962 \mathrm{E}-11$ 7.953E-12 $2.094 E-11 \quad 8.703 E-12$ $2.231 E-11 \quad 9.290 E-12$ $2.572 \mathrm{E}-11 \quad 1.028 \mathrm{E}-11$ $1.028 E-11$ 3.404E-11 $1.352 \mathrm{E}-11$ $1.352 E-11$ $1.680 \mathrm{E}-11$ $2.927 E-11$ $2.927 E-11$ 7. $302 \mathrm{E}-11$ 7.302E $1.310 E-10$ $5.651 \mathrm{E}-10 \quad 3.588 \mathrm{E}-10$ $9.829 E-10 \quad 4.704 E-10$ 
INEUTRONS/MEV/STERADIAN/SOURCE NEUTRON

\begin{abstract}
ENERGY
GROUP (MEV)

$1.22 \mathrm{E} \quad 01--1.50 \mathrm{E} \quad 01$ $\begin{array}{lll}1.00 E & 01--1.22 E & 01 \\ 8.19 E & 00--1.00 E & 01\end{array}$

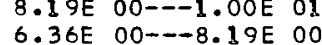
$4.97 \mathrm{E} 00--6.36 \mathrm{E}$ OO $4.07 \mathrm{E} 00--4.97 \mathrm{E} 00$ $3.01 \mathrm{E} 00--4.07 \mathrm{E}$ OO $2.46 E$ EO- $3.01 \mathrm{E}$ OO $2.35 \mathrm{E} 00--2.46 \mathrm{E}$ 00 $1.83 \mathrm{E} 00---2.35 \mathrm{E} 00$ $1.11 \mathrm{E} 00---1.83 \mathrm{E} 00$ 5.50E-0I--1.11E 00 $1.50 E-01--1.11 E$
$1.11 \mathrm{E}-01-00-5.50 \mathrm{E}-01$ $3.35 E-02--1.11 E-01$ 5.83E-04---3.35E-02 $1.01 \mathrm{E}-04--5.83 \mathrm{E}-04$ $2.90 E-05--1.01 E-04$ $3.07 E-05--2.90 E-05$ $3.06 \mathrm{E}-0 \mathrm{E}---1.07 \mathrm{E}-05$ $4.14 \mathrm{E}-07---1.12 \mathrm{E}-06$
0.0
\end{abstract}

ENERGY

GROUP (MEV)

$1.22 E$ O1---1.5OE O1 1. OOE O1-O-1.22E OI $8.19 E$ OO- $-1.00 E$ O1 6.36E $00--8.19 \mathrm{E}$ OO 4.97E $00--6.36 \mathrm{E}$ OO 4.07E $00--4.97 E$ EO 3.01E $00--4.07 E$ OO $2.46 \mathrm{E} 00--3.01 \mathrm{E} 00$ $2.35 \mathrm{E} 00--2.46 \mathrm{E}$ 00 $1.83 \mathrm{E} \quad 00--2.35 \mathrm{E} \quad 00$ $1.11 \mathrm{E} 00--1.83 \mathrm{E} 00$ 5.50E-01- $1.111 \mathrm{E}$ OO $1.11 \mathrm{E}-01--5.50 \mathrm{E}-01$
$3.35 \mathrm{E}-02--1.11 \mathrm{E}-01$ $3.35 E-02--1.11 E-01$
$5.83 E-04---3.35 E-02$ $5.83 E-04--3.35 E-02$ $1.01 E-04--5.83 E-04$
$2.90 E-05--1.01 E-04$ $2.90 E-05--1.01 E-04$
$1.07 E-05--2.90 E-05$ $1.07 E-05--2.90 E-05$
$3.06 E-06--1.07 E-05$ $3.06 E-06--1.07 E-05$ $1.12 E-06--3.06 E-06$
$4.14 E-07-\cdots-1.12 E-06$ $4.24 \mathrm{E}-07---1.12 \mathrm{E}-06$
0.0
ANGLE 1 $M U=-1$

0.0

0.0

0.0

0.0

0.0

0.0

$3.679 \mathrm{E}-04$

$2.281 \mathrm{E}-02$

$3.960 \mathrm{E}-0$

$3.288 \mathrm{E}-02$

$1.487 \mathrm{E}-01$

$1.140 E-01$
$6.283 E 00$

$2.577 \mathrm{E} O 1$

$7.249 E$ OI

$1.867 \mathrm{E} \mathrm{O2}$

4.860 E 02

$1.038 \mathrm{E} \mathrm{O3}$

ANGLE 10 $M U=0.0950$

0.0

0.0

0.0

0.0

0.0

0.0

0.0

7.809E-03

$3.941 \mathrm{E}-02$

$7.703 \mathrm{E}-02$

$4.059 \mathrm{E}-02$

$1.615 \mathrm{E}-01$

$1.199 \mathrm{E}-01$

$6.535 \mathrm{E} 00$

$2.665 \mathrm{E} \mathrm{O1}$

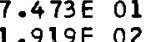

$1.919 E \quad 22$

$4.58 E$ O2

1. $.502 E \quad 03$
ANGLE $\quad 2$
$M U=-0.9894$ 0.0 0.0

0.0

0.0

0.0

0.0

0.0

0.0

3. $761 \mathrm{E}-04$ 2. $287 \mathrm{E}-02$

$3.965 \mathrm{E}-02$

$3.294 \mathrm{E}-02$

$1.488 \mathrm{E}-01$

$6.285 \mathrm{E} 00$

$2.578 \mathrm{E} 01$

$7.251 \mathrm{E}$ 01

$1.867 \mathrm{E} 02$

4. $861 \mathrm{E} 02$

1. 038603

ANGLE 11 $M U=0.2816$

0.0

0

0.0

0.0

0.0

0.0

0.0

$7.118 E-03$

$4.444 \mathrm{E}-02$

$6.175 E-02$

$4.276 \mathrm{E}-02$

$1.642 \mathrm{E}-01$

$1.210 E-01$
$6.581 E C O$

$6.581 \mathrm{E} \mathrm{CO}$

$2.681 \mathrm{E} 01$

$7.514 E$ OI

$1.928 \mathrm{E} 02$

$1.067 \mathrm{E} \mathrm{O3}$

$1.508 E 03$
ANGLE 3 ANGLE 4 U $=-0.9446$ 0.0

0.0

0.0

0.0

0.0

0.0

$4.142 E-04$

$2.316 \mathrm{E}-02$

$3.315 \mathrm{E}-02$

$1.492 \mathrm{E}-01$

6.294E 00

$2.581 \mathrm{E} \mathrm{OI}$

7.259 E 01

$1.269 E$ O2

$4.866 \mathrm{E} \quad 2$

1.039 E 03

$1.472 E \quad 03$

ANGLE 12

0.

0.0

0.0

0.0

0.0

0.0

0.0

0.0

$9.467 E-03$

$5.602 E-02$

$6.866 E-02$

$4.499 E-02$

$1.669 \mathrm{E}-01$

$1.222 E-01$

$6.626 E 00$

$2.696 \mathrm{E} \quad 1$

$7.552 \mathrm{E} 01$

$1.937 E \quad 02$

$.071 \mathrm{E} 03$

$1.513 \mathrm{E} \quad 03$

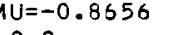
0.0

0.0

0.0

0.0

0.0

(2.0

0.0

$5.073 \mathrm{E}-04$

$2.376 \mathrm{E}-02$

$.030 \mathrm{E}-02$

$3.357 E-O 2$

$1.500 \mathrm{E}-01$

$6.312 E 00$

$2.587 \mathrm{E}$ O1

$7.275 \mathrm{E}$ OI

$1.873 \mathrm{E} \mathrm{O2}$

$4.875 \mathrm{E} \mathrm{O2}$

$1.475 \mathrm{E} 03$

ANGLE 13 $M U=0.6179$ 0.0

0.0

0.0

0.0

0.0

.0

0.0

$3.327 \mathrm{E}-02$

$7.627 \mathrm{E}-02$

$7.184 \mathrm{E}-02$

4.727E-02

$1.696 \mathrm{E}-01$

$1.232 \mathrm{E}-01$

$6.666 E$ OD

$2.710 \mathrm{E}$ OI

$7.587 \mathrm{E}$ OI

$1.945 \mathrm{E} 02$

$1.048 \mathrm{E} \mathrm{O2}$

$\begin{array}{lll}1.075 E & 03 \\ 1.518 E & 03\end{array}$ $\begin{array}{cc}\text { ANGLE } 5 & \text { AVGLE } 6 \\ M U=-0.7550 & M U=-0.6179\end{array}$ 0.0

0.0

0.0

0.0

0

0

$6.879 E-04$

$2.475 E-02$

$4.104 \mathrm{E}-02$

1.512E-01

$1.152 \mathrm{E}-01$

$6.336 \mathrm{E} 00$

$2.596 \mathrm{E} 01$

$7.297 E$ OI

$1.878 \mathrm{E} 02$

4.887E 02

$1.478 \mathrm{E} \quad 03$

ANGLE 14

0.

0.0

0.0

0.0

.0

.0

0.0

.261E-02

$1.087 \mathrm{E}-01$

$8.653 E-02$
$5.065 E-02$

$1.241 E-01$

$6.702 E 00$

2.72 2E O1

7.617E 01

.

$.078 \mathrm{E} 03$

1. $522 \mathrm{E} 03$

0.0

0.0

0.0

0.0

0.0

(2)

$1.003 E-03$

2. $621 \mathrm{E}-02$

$4.214 \mathrm{E}-02$

$.500 E-02$

1.527E-01

$6.367 E 00$

2.607E OI

$7.325 \mathrm{E}$ O1

$1.885 \mathrm{E}$ O2

4.903E 02

$\begin{array}{ll}1.046 \mathrm{E} & 03 \\ 1.482 \mathrm{E} & 03\end{array}$

AVGLE 15 $M U=0.8656$

0.0

0.0

0.0

0

0.0

0.0

$1.683 E-01$

2.050E-01

$1.191 E-01$

$5.478 \mathrm{E}-02$

$1.745 \mathrm{E}-01$

$6.732 E$ OO

$2.732 E$ OI 
(NEUTRJNS/MEV/STERADIAN/SOURCE NEUTRON)

ENERGY GROUP (MEV) $1.22 E$ O1-D-1.50E 01 1.00E $01---1.22 \mathrm{E}$ OI $8.19 E$ OO---3.00E 02 6.36E $00--8.19 E$ DO 4.97E $00--6.36$ E 00 4.07E $00--4.97 E$ OO 3.ClE $00--4.07 E$ OO $2.46 E$ OO-C. $1.83 E$ OO- $0.46 E$ DO $1.11 \mathrm{E} 00-2.33 \mathrm{E} 00$ 1.11E $00--1.83 E$ E 1.1 1 E-CI- $-5.50 \mathrm{E}-01$ $3.35 \mathrm{E}-02--1.11 \mathrm{E}-01$ $5.83 E-04---3.35 E-02$ $1.01 E-04---5.83 E-04$
$2.90 E-05---1.01 E-04$ $2.90 E-05--1.01 E-04$
$1.07 E-05---2.50 E-05$ $1.07 E-05--2.50 E-05$
$3.06 E-06--1.07 E-05$ 1.12E-06---3.06 -06 4.14E-07---1.12E-06 $4.14 \mathrm{E}-07---1.12 \mathrm{E}-06$
$0.0 \quad-0.14 \mathrm{E}-07$

\section{ENERGY} GROUP (MFV)

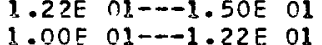
$8.19 \mathrm{G} 00--1.00 E$ OI $6.36 E$ OO-- $8.19 E$ OO $4.97 \mathrm{E}$ OO-- $6.36 \mathrm{E}$ CO 4.07E 00---4.97F 00 $3.01 \mathrm{E} 00--4.07 \mathrm{E} 00$ $2.46 \mathrm{E} \quad 00--3.01 \mathrm{E} C \mathrm{C}$ $2.35 \mathrm{~F} 00--2.46 \mathrm{E} 00$ $1.83 \mathrm{E} 00--2.35 \mathrm{E}$ तO $1.11 E$ OO-- 1.83 E 00 5.50E-01--1.11E OO 1.11E-01-- -5.50E-01 $3.35 \mathrm{E}-02--1.11 \mathrm{E}-01$ $5.83 E-04--3.35 E-02$ $2.90 E-05--1.01 E-04$ $1.07 F-05--2.90 E-05$ $3.06 \mathrm{E}-06---1.07 \mathrm{E}-05$ $1.12 \mathrm{E}-06---3.06 \mathrm{E}-06$ $4.14 \mathrm{E}-07--1.12 \mathrm{E}-06$ 0.0 0.0 0.0 0.0 0.0 0.0 0.0 $5.421 E-04$ $2.633 \mathrm{E}-\mathrm{O}$ ? $5.937 \mathrm{E}-\mathrm{C2}$ $7.940 \mathrm{E}-02$ $4.509 E-01$ $3.688 \mathrm{E}-03$ $2.108 E$ OI $8.812 E$ OI $2.508 \mathrm{E} 02$ $6.534 \mathrm{E}$ O? $1.714 E$ O3 $3.683 \mathrm{E} \mathrm{O3}$
$5.256 \mathrm{E} 03$

ANGLE 10 $M U=0.0950$ 0.0

0.0

0.0

$0.0705-03$ $7.950 E-03$
$5.935 \mathrm{~F}-02$
$7.216 \mathrm{E}-02$ $7.216 \mathrm{E}-02$
$1.031 \mathrm{E}-01$ $1.031 \mathrm{E}-01$ $4.008 \mathrm{E}-01$ $2.258 \mathrm{~F}$ OI $9.362 \mathrm{E} 01$ $2.653 \mathrm{E} \mathrm{O2}$ $6.879 E \quad 02$ $1.799 \mathrm{E} \mathrm{O3}$ $\begin{array}{ll}3.853 \mathrm{E} & 03 \\ 5.472 \mathrm{E} & 03\end{array}$
$\begin{array}{cc}A N G L E & 2 \\ M U=-0.0894\end{array}$ 0.0

0.0

0.0

0.0

0.0

0.0

0.0

5.530E- 04 $2.642 \mathrm{E}-02$ $5.949 \mathrm{E}-02$ $7.956 \mathrm{E}-\mathrm{C} 2$ $4.514 \mathrm{E}-\mathrm{Cl}$ $3.651 \mathrm{E}-0$ ? 2.ICSE OI $8.817 E 01$ $2.509 E 02$ $6.527 \mathrm{E} 02$ $1.715 \mathrm{E} 03$ $5.684 E$ C3

ANGLE 11 $U=-0.9446$ 0.0
0.0
0.0 0.0 0.0 0.0 0.0 0.0 $6.032 E-04$ $2.680 E-02$ $5.998 \mathrm{E}-02$ $8.021 \mathrm{E}-0$ ? $4.533 \mathrm{E}-01$ $3.702 \mathrm{E}-01$ $2.115 \mathrm{E} 01$ $8.837 E$ OI 2.515E O2 6.550 E 02 $1.718 E \quad 03$ $3.691 \mathrm{~F} 03$

ANGLE $1 ?$ ANGLE \&
$M U=-0.8656$ 0.0 0.0 0.0
0.0
0.0 0.0 0.0 0.0 0.0 0.0 7.230E-04 $2.758 \mathrm{E}-02$ $6.099 \mathrm{E}-02$ $8.147 \mathrm{E}-\mathrm{C2} 2$ $4.569 \mathrm{E}-01$ $3.723 \mathrm{E}-01$ 2.125E O1 $8.875 \mathrm{E}$ O1 $2.525 \mathrm{E} \mathrm{O2}$ $6.573 E$ O2 $1.724 E 03$ $3.703 E$ O $M U=0.2816 \quad M U=0.4580$ 0.0 0.0 0.0 0.0 0.0 0.0 $9.619 \mathrm{~F}-03$ $9.619 E-03$ $5.190 \mathrm{E}-02$
$1.081 \mathrm{E}-01$ $1.085 \mathrm{E}-01$ $5.216 \mathrm{E}-01$ $4.072 \mathrm{E}-01$ 2.287501 $9.465 \mathrm{E} 01$ $2.680 E$ O2 $6.943 E 02$ $1.814 \mathrm{E} 03$ $3.884 \mathrm{E} \quad 03$
0.0

0.0
0.0

0.0

0.0

0.0

0.0

$1.611 \mathrm{E}-02$ 7.600E-02 $9.811 \mathrm{E}-02$ $1.156 \mathrm{E}-0$ ? $5.345 \mathrm{E}-01$ 4.1 $35 \mathrm{E}-01$ $9.566 \mathrm{E} 01$ 2.706 E $0 ?$ $7.004 E 02$ $1.829 E \quad 03$ $3.914 \mathrm{E} O 3$
$5.549 \mathrm{E} \cap 3$
ANGLE 13 0.0 0.0
0.0 0.0

0.0

0.0

0.0

2. $082 E-02$ $8.940 E-02$ $1.388 \mathrm{E}-01$ $1.245 \mathrm{E}-01$ $5.477 \mathrm{E}-01$ $2.342 \mathrm{E} O 1$ $9.661 \mathrm{E} \mathrm{O1}$ $2.731 \mathrm{E} \mathrm{O2}$ $7.061 \mathrm{E} \mathrm{O2}$ $1.843 E 03$ $3.941 \mathrm{E}$
$5.584 \mathrm{~F}$

ANGLE 5
MU=-0.7550
0.0
0.0
0.0
0.0
0.0
0.0
0.0
0.0
0.0
$9.503 E-04$
$2.883 E-02$
$6.257 E-02$
$8.330 E-02$
$4.621 E-01$
$3.753 E-01$
$2.139 E 01$
$8.927 E 01$
$2.539 E 02$
$6.607 E 02$
$1.732 E 03$
$3.719 E 03$
$5.306 E 03$
ANGLE 14

ANGLE 14 0.0

0.0
0.0
0.0

0

0.0

.0

3. $965 \mathrm{E}-02$ $1.335 \mathrm{E}-01$ $1.452 \mathrm{E}-01$ $1.326 \mathrm{E}-01$ $5.599 \mathrm{E}-01$ 2.366E 01 $9.744 \mathrm{E}$ Ol $2.752 \mathrm{E} \quad 02$ $7.111 E 02$ 1.855E 03 $3.965 \mathrm{E} \quad 03$
AVGLE 6 $U=-0.6179$ 0.0 0.0

0.0

0.0

0.0
0.0
0.0

0.0

0.0

1. $341 \mathrm{E}-03$ 3.069E-02 $6.487 E-02$ $8.575 \mathrm{E}-02$ $4.688 \mathrm{E}-01$ 3.791E-01 $2.157 E$ OI $8.994 \mathrm{E}$ OI $2.556 E 02$ $6.649 E$ O2 $1.742 E$ O3 $3.740 E$
$5.333 E$

ANGLE 15 $M U=0.8656$ 0.0

0.0

.3

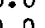

(

-1
0

0.0

$7.394 E-02$ $1.789 \mathrm{E}-01$ $2.016 E-01$ $1.437 \mathrm{E}-01$ 2.711E-C1 $2.385 E$ OI $9.812 \mathrm{E}$ OI $2.770 \mathrm{E} O 2$ $7.152 E$ O2 $1.865 \mathrm{E} \mathrm{O3}$ $3 .{ }^{\circ} 85 \mathrm{E} \quad 3$

ANGLE 7 $U=-0.4580$
0.0 0.0

0.0
0.0

0.0

0.0

$.969 E-03$ $3.333 \mathrm{E}-02$ $6.804 E-02$ $8.883 \mathrm{E}-02$ $4.770 E-01$ 
ENERGY GROUP (MEV) $1.22 \mathrm{E} 01-1.50 \mathrm{E}$ O $1.00 E$ O1-D-1.22E OI 1.1SE $00-1$ - 00 E $6.36500-1.00$ E 1 $4.97 E$ OO-- 6.36E 00 $4.07 F$ 00---4. $3.01 E$ O0-- $4.07 E 00$ 2.46 OO-- 3 . OTE 00 $2.35 E$ DO- $-2.46 E$ E 0 1.83 E $00--2.35 E 00$ $1.11 \mathrm{E} 00-\cdots-1.83 \mathrm{E} 00$ $5.50 E-01--1.11 E 00$ $1.11 E-01--5.50 E-01$ $3.35 \mathrm{E}-02---1.11 \mathrm{E}-01$ $5.83 \mathrm{E}-04--3.35 \mathrm{E}-02$ $1.01 E-04-5.83 E-04$ 2.9CE-05--1.01E-04 D6 -06- $=1.07 E-05$ $1.12 \mathrm{E}-06-\cdots 3.06 \mathrm{E}-06$ $4.14 \mathrm{E}-07--1.12 \mathrm{E}-06$
0.0 ENERGY GROUP (MEV) 1.22E 01---1.50E O1 $1.00 E$ O1---1.22E OI $8.19 \mathrm{E} \quad 00--1.00 \mathrm{E} \quad 1$ $6.36 F \quad 00--8.19 E$ OO $4.97 \mathrm{E}$ 00-- 6.36E 00

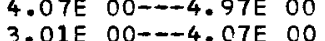
$\begin{array}{lll}3.01 E & 00---4.07 E & 00 \\ 2.46 E & 00-\cdots 3.01 E & 00\end{array}$

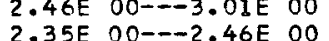

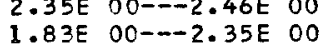
$1.83 E \quad 00-\cdots 2.35 E 00$ $1.11 E$ OO---1.83E 00 $1.11 E-01--5.50 E-01$ $3.35 \mathrm{E}-02-\cdots 1.11 \mathrm{E}-01$ $5.83 E-04--3.35 E-02$ $1.01 E-C 4---5.83 E-04$ 2.90E-05--1.01E-04 $1.07 E-05--2.90 E-05$ $3.06 E-06-1.07 E-05$ $1.12 E-06--3.06 E-06$ $4.14 \mathrm{E}-07--1.12 \mathrm{E}-06$
$0.0 \quad--4.14 \mathrm{E}-07$

ANGLE 1 $M U=-1.0000$ 0.0 0.0 0.0 0.0 0.0 0.0 0.0 0.0 $5.724 E-04$ 2.511E-02 $6.384 \mathrm{E}-02$ $1.029 \mathrm{E}-\mathrm{Cl}$ $0.499 E-C 1$
$5.499 E-01$ $3.214 \mathrm{E}$ OI $1.361 \mathrm{E} \mathrm{O2}$ $3.907 \mathrm{E} 02$ $1.027 E$ O3 2.710E 33 $8.399 E$ O3 ANGLE 10 $U=0.0950$

0.0

0.0

0.0
0.0
0.0
0.0

0.0

0.0

0.0

0.0 $4.130 \mathrm{E}-02$ $1.032 \mathrm{E}-01$
$1.350 \mathrm{E}-01$ $7.464 \mathrm{E}-01$ $6.068 \mathrm{E}-01$ $3.495 \mathrm{E}$ OI $4.192 \mathrm{E} O 2$ $1.096 \mathrm{E} 03$ $6.201 \mathrm{E} 03$ $8.843 E \quad 03$
(NEUTRONS/MEV/STERADIAN/SOURCE NEUTRON)

$\begin{array}{cc}\text { ANGLE } & 2 \\ M U=-0.9894\end{array}$ 0.0

0.0

0.0

0.0

0.0

0.0
0.0
0.0

0.0

$5.843 E-04$ $2.520 \mathrm{E}-02$ $6.399 E-02$ $1.031 \mathrm{E}-01$ $5.504 \mathrm{E}-01$ $3.216 \mathrm{E} 01$ $1.362 E 02$ $3.909 E \quad 02$ $1.027 E \quad 03$ $2.712 \mathrm{E} 03$ $8.403 \mathrm{E} 03$

LE $J=-0.9446$ 0.0

0.0

0.0

0.0

0.0

0.0

0.0

0.0

$6.382 E-04$ $2.558 \mathrm{E}-02$ 6. $461 E-02$ $1.040 E-01$ $6.539 E-01$ $3.226 \mathrm{E}$ 01 $1.366 E \quad 02$ $3.920 E$ O2 1.030 E 03 2.718E 03 $\begin{array}{lll}5.869 E & 03 \\ 8.422 E & 03\end{array}$ ANGLE 4 0.0 $U=-0.8656$
0.0 NGLE $U=-O$ MU $=-0.6179$ 0.0 0.0 0.0 0.0 0.0 0.0 0.0 $7.635 E-04$ $2.637 \mathrm{E}-02$ $6.586 \mathrm{E}-02$ 1.058E-01 $6.599 E-01$ 5.561E-DI $3.245 \mathrm{E} \mathrm{Ol}$ $1.373 E 02$ $3.939 E \quad 2$ $1.035 \mathrm{E} 03$ $2.730 \mathrm{E} 03$ $5.893 \mathrm{E} \mathrm{O3}$
$8.455 \mathrm{E} \mathrm{O3}$ ANGLE 11 ANGLE 12 ANGLE 13 $M U=0.2816$ 0.0

0.0

0.0

0.0

0.0

0.0 0.0 $1.032 E-02$ $0.167 \mathrm{E}-02$ $1.010 \mathrm{E}-01$
$1.445 \mathrm{E}-01$ $7.676 E-01$ $6.184 E-01$ 3.550E 01 $1.487 E$ O2 $4.246 \mathrm{E} \quad 02$ $1.109 \mathrm{E} 03$ $2.913 E 03$ $8.925 E \quad 3$

$M U=0.4580$

0.0
0.0

0.0

0.0

0.0

0.0

0.0

0.0

0.0

$1.408 \mathrm{E}-02$ $6.593 \mathrm{E}-02$ $1.289 E-01$ $7.593 E-01$ 6. $299 \mathrm{E}-01$ 3.290 01 . $507 E 02$ 4. 4.2 02 1.122E 03 $6.329 \mathrm{E} 03$ $9.005 E 03$

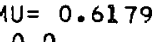

0.0

0.0

0.0

0.0

0.0

0.0

0.0

0.0 $9.381 \mathrm{E}-02$ $1.398 E-01$ $1.658 \mathrm{E}-01$ $6.4105-01$ $6.410 \mathrm{E}-01$ 526E 1.349E 02 1.133E 03 1.135 03 $9.080 E^{03}$
$0.0 \quad 0.0$

0.0

0.0

0.0

0.0

0.0

0

$9.954 E-04$

2. $762 \mathrm{E}-02$

$6.78 \mathrm{LE}-02$

$1.083 \mathrm{E}-01$

5.613E-01 3. $271 \mathrm{E} 01$ 1.383E 02 3.966E 02 $1.041 \mathrm{E} 03$ $\begin{array}{ll}2.746 E & 03 \\ 5.927 E & 03\end{array}$ $8.500 E^{03}$

0.0

0.0
0.0
0.0

0.3

0.0

0.0

1. $388 \mathrm{E}-03$ 2. $947 E-02$ $7.061 E-02$ 5.681E-01 $3.305 \mathrm{E} \mathrm{Ol}$ $1.396 E \quad 2$ 4.000E 02 $1.050 E \quad 03$ 2.767 E 03 $5.969 \mathrm{E} 03$
$8.555 \mathrm{E} 03$ ANGLE 14 ANGLE 15 $M U=0.7550 \quad M U=0.8656$ 0.0

0.0

0.0

0.0

0.0

0.0

0.0

$3.659 E-02$ $1.188 \mathrm{E}-01$ $1.778 \mathrm{E}-01$ $8.319 E-01$ $6.511 \mathrm{E}-01$ $3.702 \mathrm{E} 01$ 1.543 E 02 4. 393 E 02 $1.144 E$ E 3 $2.998 E 03$ $9.144 \mathrm{E} \quad 03$ 
(NEUTRONS/MEV/STERADIAN/SOURCE NEUTRON)

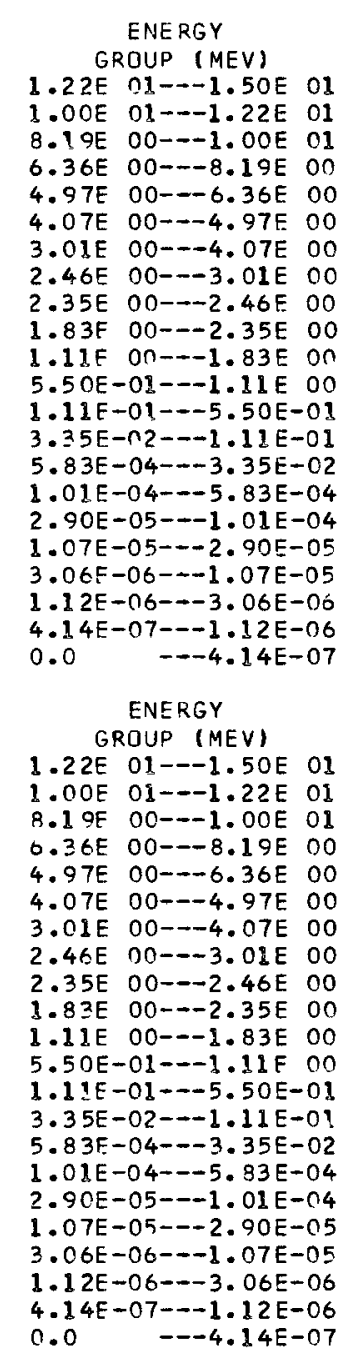

ANGLE 2 $M U=-0$
0.0

0.0

0.0

0.0

0.0

0.0

0.0

0.0

0.0

0.0

$5.111 E-04$

$1.953 \mathrm{E}-02$

$5.968 \mathrm{E}-02$

1. $244 E-01$

$8.227 \mathrm{E}-01$

$4.993 E$ OI

$2.164 E 02$

$6.314 \mathrm{E} 02$

$4.514 E$ O3

$4.514 E \quad 03$

9.856 E 03

ANGL 10

$M U=0.0950$

0.0

0.0

0.0

0.0

0.0

0.0

0.0

0.0

$5.590 E-03$

3.945E-02

$9.168 \mathrm{E}-02$

$1.685 \mathrm{E}-01$

$1.086 \mathrm{E} 00$

$9.278 \mathrm{E}-\mathrm{O} 1$

$5.552 E$ OL

2.384E 02

$6.927 \mathrm{E} 0$ ?

$1.843 \mathrm{E} 03$

4.904E 03

$1.067 \mathrm{E} 04$

$1.067 \mathrm{E} 04$
$1.536 \mathrm{E} 04$

0.0

0.0

0.0

0.0
0.0
0.0
0.0

0.0

.0

$1.961 \mathrm{E}-\mathrm{n} 2$
$5.985 \mathrm{E}-02$

$5.985 E-02$

.

.

4.

2.165E C2

.

(1)

$9.863 \mathrm{E} 03$

ANGLE 11

ก.0

0.0

0.0
0.0
0.0

0.0

0.0

0.0

0.0

$1.084 \mathrm{E}-01$

$1.122 \mathrm{E} 00$

$9.494 E-01$

$1.872 \mathrm{E} \mathrm{O3}$
$5.222 E-04$

$1.247 E-01$

$1.432 \mathrm{E} \mathrm{O4}$

ANGLE 3 $J=-0.9446$ $A N G L E \quad 4$
$M U=-0.8656$ 0.0

0.0
0.0

0.0
0.0

0.0

0.0

0.0

0.0

0.0

0.0

$5.724 E-04$

$1.993 E-0$ ?

$6.055 \mathrm{E}-02$
$1.260 \mathrm{E}-01$

$9.297 \mathrm{E}-01$

$8.272 E-01$

$5.017 E$ O1

$2.173 E$ O2

6.341 E 02

$1.696 \mathrm{E} 03$

4.532E O3

$9.893 \mathrm{E} 03$

0.0
0.0
0.0
0.0

0.0
0.0

0.0

0.0

0.0

0.0

$6.848 E-04$

2.060E-02

$6.196 \mathrm{E}-02$
$1.283 \mathrm{E}-01$

$9.397 E-01$

8.340E-01

$5.054 \mathrm{E} \quad 01$

2.188 E

$1.706 \mathrm{E} 03$

$4.558 \mathrm{E} 03$

$9.947 E 03$

ANGLE 12

ANGLE 13

ANGLE 5

$M U=-0.7$
0.0
0.0
0.0

0.0

0.0

0.0

0.0

0.0

0.0

$8.855 \mathrm{E}-04$

$2.164 \mathrm{E}-02$

$6.411 \mathrm{~F}-0$ ?
$1.318 \mathrm{E}-01$

$9.539 \mathrm{E}-01$

$8.435 E-01$

$5.105 \mathrm{E} 01$

$6.439 E$ O2

$1.720 E^{03}$

$4.595 \mathrm{E} 03$

$\begin{array}{ll}1.002 \mathrm{E} & 04 \\ 1.454 \mathrm{E} & 04\end{array}$

ANGLE 14

$M U=0.4580$

ANGLE 14

$7.807 E-03$

4.391E-0?

$7.047 E \quad 02$

$1.082 \mathrm{E} 04$

0.0

0.0

0.0

0.0
0.0

$1.212 E-02$

5.711E-02

$1.218 \mathrm{E}-01$

$1.159 \mathrm{E} 00$

$9.712 \mathrm{E}-01$

$5.776 \mathrm{E}$ OI

$7.166 \mathrm{E} 02$

$1.902 \mathrm{E} 03$

$1.097 E 04$

0.0

0.0
0.0

0.0
0.0
0.0

0.0

0

$1.833 \mathrm{E}-02$

$7.068 \mathrm{E}-02$

$2.088 \mathrm{E}-01$

$1.196 \mathrm{E} 00$

$9.923 \mathrm{E}-01$

$5.883 \mathrm{E}$ OL

$7.278 \mathrm{E}$ O2

$1.929 E 03$

5.121 E 03

1.111E 04

0.0

0.0
0.0
0.0

0.0

0.0

0

2. $955 \mathrm{E}-02$

$9.615 \mathrm{E}-02$

$1.734 \mathrm{E}-01$

$1.232 \mathrm{E} 0 \mathrm{O}$

$1.012 E$ DO

$5.980 E$ OI

$2.549 \mathrm{E} \quad 02$

$1.954 \mathrm{E} \mathrm{O3}$

$5.183 \mathrm{~F} 03$

$1.124 \mathrm{E} \quad 04$

$1.609 E \quad 04$
AVGLE 6 $M U=-0.6179$

0.0

0.0

0.0

0.0

0.0

0.0

0.0

$1.217 E-03$

$2.317 \mathrm{E}-02$

$6.717 \mathrm{E}-02$

$9.725 \mathrm{E}-01$

$8.558 \mathrm{E}-01$

(1)

DE

(7)

4.7342E 03

$1.012 \mathrm{E} 04$

$1.467 \mathrm{E} 04$

ANGLE 15

ANGLE 16

0.0

0.0

0.0

0.0

0.0

5.004E-02

$1.337 E-01$

$2.153 \mathrm{E}-01$

$2.426 E-01$

$1.263 \mathrm{E} 00$
$1.028 \mathrm{E} 00$

$6.361 E 01$

$2.579 \mathrm{E} \quad 02$

$1.974 \mathrm{E} \mathrm{O3}$

$5.233 \mathrm{E} \mathrm{O3}$

$1.134 \mathrm{E} 04$

$1.622 \mathrm{E} 04$

ANGLE 7 0.0
0.0 .4580

0.0

0.0

0.0

0.0

0.0

0.0

$1.735 E-03$

$2.531 \mathrm{E}-02$

$1.423 \mathrm{E}-01$

$9.9525-01$

8.706E-01

.

$6.600 \mathrm{E} 02$

$1.761 E$ O3

$4.698 \mathrm{E} 03$

$1.481 \mathrm{E} 04$

0.0

0.0

0.0

ANGLE 8 $M U=-0.2816 \quad M U=-0.0950$

0.0

0.0

0.0
0.0

0.0

0.0

0.0

$2.516 E-03$

2. $824 E-02$

$7.694 \mathrm{E}-02$

$1.495 E-01$

$8.879 E-01$

$5.342 E 01$

2.302E O2

$1.786 \mathrm{E} \quad 03$

$4.761 \mathrm{E} 03$

1.498 E 04

ANGLE 17

$M U=0.9446 \quad M U=0.9894$ 
(NEUTRJNS/MEV/STERADIAN/SOURCE NEUTRON)

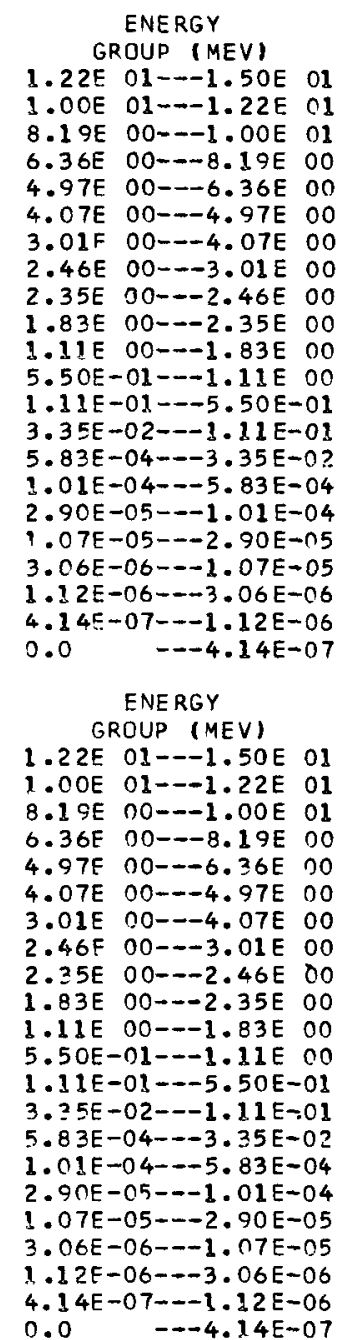

ANGLE 1 ANGLE 2 0.0 0.0 0.0 0.0 0.0 0.0 0.0 0.0

$3.899 E-04$

$3.899 \mathrm{E}-04$
$1.360 \mathrm{E}-02$

$1.360 \mathrm{E}-02$
$4.788 \mathrm{E}-02$

$1.194 \mathrm{E}-01$

$9.964 E-01$ $9.221 E-01$ $5.763 \mathrm{E} 01$ $2.545 \mathrm{E} \quad 02$ $7.532 \mathrm{E} 02$ $2.047 E \quad 03$ $5.535 E \quad 03$ $\begin{array}{ll}1.221 \mathrm{E} & 04 \\ 1.792 \mathrm{E} & 04\end{array}$

ANGLE 10 0.0

0.0

0.0

0.0

0.0

0.0

0.0

0.0

0.0
$3.930 E-03$

$2.643 E-02$

$7.844 \mathrm{E}-02$

$1.645 \mathrm{E}-01$

$1.191 E$ OO

$1.055 E$ DO

$6.509 E$ OI

$2.849 E$ O2

$8.399 E$ C2

2.270E O3

$1.343 E \quad 04$

$1.953 E 04$
0.0

0.0
0.0
0.0

0.0

0.0

0.0
0.0
0.0

0.0

. $987 E-04$

$1.365 \mathrm{E}-02$
$4.803 \mathrm{E}-02$

$1.197 \mathrm{E}-01$

$9.079 E-01$

$.232 E-01$

$.769 E$ Ol

$.548 \mathrm{E} 02$

$7.539 E$ O2

$5.54 \mathrm{CE} 03$

$1.222 E 04$

1.794E 04

ANGLE 11. $M U=0.2816$

0.0
0.0

0.0
0.0

0.0

0.0

0.0

0.0

$5.836 E-03$

$3.219 \mathrm{E}-02$

$8.900 E-02$

$1.768 \mathrm{E}-01$

$1.235 E 00$

$6.661 \mathrm{E} \mathrm{O?}$

2.920 E

$8.571 E$ E

$\because .984 \mathrm{E} 04$

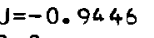

0.0

0.0

0.0

0.0

0.0
0.0
0.0

0.0
0.0

$4.382 \mathrm{E}-04$

$1.390 E-02$
$4.867 E-02$

1.210E-01

$1.004 E 00$

$9.278 E-01$

$5.796 E 01$

2.559E 02

7.570 E 02

$2.057 E 03$

$5.561 \mathrm{E} 03$

$1.227 \mathrm{E} 04$

ANGLE 12

$U=0.4580$

0.0
0.0
0.0

0.0

0.0

0.0

0.0

0.0

$8.698 \mathrm{E}-\mathrm{n3}$

$3.946 \mathrm{E}-02$

$1.038 \mathrm{E}-01$

$1.906 \mathrm{E}-01$

$1.280 E 00$

1.111E 00

$6.813 E$ OI

2.971E 12

$8.743 E$ E

2.358 E

6.3

$1.390 E$
$2.015 E$
ANGLE $4 \quad$ ANGLE 5 0.0

0.0
0.0
0.0
0.0

0.0

0.0
0.0
0.0

0.0
0.0
0.0

$5.245 \mathrm{E}-04$

$1.438 \mathrm{E}-02$
$4.993 \mathrm{E}-02$

$4.993 \mathrm{E}-02$
$1.234 \mathrm{E}-01$

$1.016 E 00$

$9.363 \mathrm{E}-01$

$2.578 \mathrm{E} \mathrm{O2}$

$7.627 \mathrm{E} 02$

$2.071 E 03$

5.599E 03

$1.235 \mathrm{E} \mathrm{O4}$

$M U=-0.7$
0.0
0.0

0.0
0.0

0.0

0.0

0.0

0

$6.753 E-04$

$1.513 \mathrm{E}-02$
$5.183 \mathrm{E}-02$

$5.183 \mathrm{E}-02$
$1.269 \mathrm{E}-01$

$1.033 \mathrm{E}$ on

$9.482 \mathrm{E}-01$

$2.606 \mathrm{E} \mathrm{O2}$

$7.707 E \quad 02$

$2.092 E 03$

$5.653 \mathrm{E} 03$

1.827E 04

ANGLE 13

0.0
0.0

0.0
0.0

0.0

0.0

0.0

0.0

0.0

$1.336 \mathrm{E}-02$

$5.056 \mathrm{E}-02$

$1.223 \mathrm{E}-01$

2.061 E 01

$1.325 \mathrm{E} 00$

$6.958 \mathrm{E} 01$

$3.029 \mathrm{E} 02$

$8.906 E$ E2

.

$1.412 \mathrm{E} 04$

$2.044 E \quad 04$

ANGLE 14

0.0

0.0

0.0

0.0
0.0
0.0
0.0

0.0

0.0

$2.116 \mathrm{E}-02$

$6.651 \mathrm{E}-02$

$1.473 \mathrm{E}-01$

$2.227 \mathrm{E}-01$

$1.367 E 00$

$1.163 E$ OO

7.091E OS

OSB

$2.436 E \quad 03$

1. $332 \mathrm{E} 04$

2.069504
ANGLE 6

\begin{tabular}{l} 
ANGLE \\
$M J=-0.6179 \quad$ ANGLE 7 \\
\hline$U=-0.4580$
\end{tabular} 0.0 0.0

0.0

0.0

0.0

$9.208 E-04$

$9.208 E-04$

$1.624 E-02$
$5.455 E-02$

$5.455 \mathrm{E}-02$

$1.355 \mathrm{E} 00$

$9.638 \mathrm{E}-01$

$6.000 E 01$

.642E 02

$.810 E$ O2

.119E O3

$1.261 \mathrm{E} \mathrm{O4}$

$1.846 \mathrm{E} 04$
ANGLE 15

$M U=0.8656$

0.0
0.0

0.3

0.0

0.0

0.5

0.0

$3.557 E-02$

$9.205 \mathrm{E}-02$

$1.801 E-01$

$1.404 E$ OO

$1.184 E$ OO

$7.202 E$ OL

$3.125 \mathrm{E}$ O2

$2.467 \mathrm{E} \quad 03$

$6.515 E \quad 03$

1. $448 E$ O 04 0.0

0.0

0.0

0.0

(

$300 E-03$

$1.778 \mathrm{E}-02$

$5.825 \mathrm{E}-02$
$1.376 \mathrm{E}-01$

$1.082 \mathrm{E} \mathrm{OO}$

$9.825 \mathrm{E}-01$

$6.105 \mathrm{E} 01$

$7.933 \mathrm{E} \mathrm{O2}$

$2.150 E \quad 03$

$5.804 \mathrm{E} \mathrm{O3}$

$\begin{array}{ll}.278 E & 04 \\ .869 E & 04\end{array}$

ANGLE 16

MU $=0.9446$
0.0

0.0

0.0

0.0

0.0

0.0

0.0

0.0

$6.976 \mathrm{E}-02$

$.379 E-01$

$2.266 E-01$

$2.558 \mathrm{E}-\mathrm{Ol}$

$1.433 \mathrm{E} 00$

$1.200 E$ OO

$7.285 E$ OI

$3.158 \mathrm{E} 02$

$3.490 \mathrm{E} 03$

2.490E 03

$6.673 E$ O3

$2.104 E 04$ $-0.2816 \quad M U=-0.0950$ 0.0

$0.0 \quad 0.0$

0.0

0.0 
(NEUTRONS/MEV/STERADIAN/SOURCE NFUTRON)

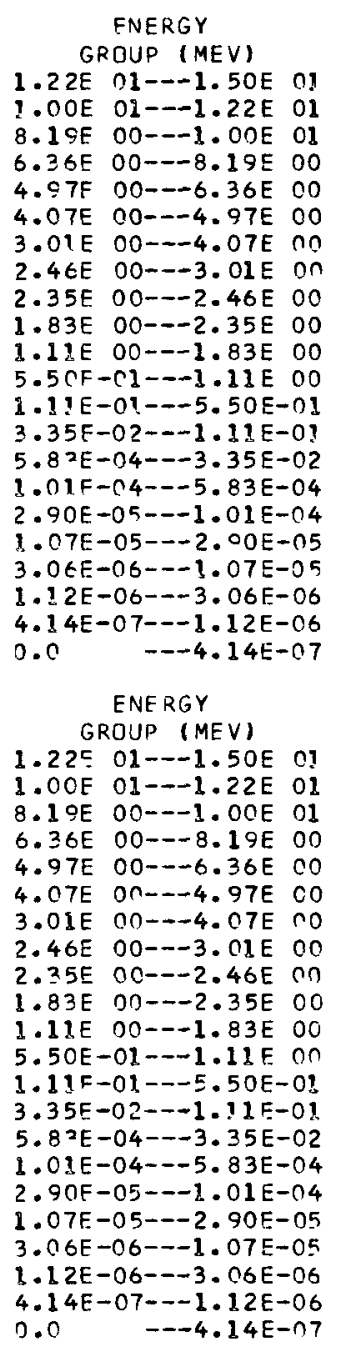

ANGLE 1
$M U=-1.0000$ 0.0

0.0

0.0

0.0

0.0

0.0

0.0

0.0

$2.732 E-04$

$8.858 \mathrm{E}-03$

1.505E-O

1.0OSE-

.

$5.751 E$ ol

$2.6011 \mathrm{E} 01$

7.5165 O2

$2.070 \mathrm{E} 03$

$5.653 \mathrm{E} \mathrm{O3}$

$5.653 \mathrm{E} 03$

$1.866 \mathrm{E}$ O4

ANGLE 10 $M U=0.0950$

$0 . n$

0.0

0.0

0.0

0.0

0.0

0.0

. 0

$2.700 E-03$

$1.762 \mathrm{E}-0$

$5.896 \mathrm{E}-0$

$1.404 \mathrm{E}-01$

$1.112 E$ OO

$1.013 E$ CO

$6.396 \mathrm{E} \mathrm{OI}$

$2.343 E \quad 02$

$8.481 \mathrm{E} 02$

$2.324 E$ OS

$6.321 E$ O3

$1.4 \mathrm{C2F} 04$
$2.056 \mathrm{E} 04$
ANGLE 2 ANGLE 3 $M U=-C .9894$ 0.0

0.0

0.0

0.0

0.0

0.0

0.0

0.0

$2.796 \mathrm{E}-\mathrm{CH}$

$8.936 \mathrm{E}-03$

.

$9.213 \mathrm{E}-0$

. $7 \in 85-01$

$5.607 \mathrm{E} 01$

$7.524 E \quad 22$

$2.072 E 03$

5.650 O3

$1.650 \mathrm{E}$ O4

1.868 E 04

ANGLE 11

$U=0.2816$

0.0

0.0

0.0

0.0

$0 . n$

0.0

0.7

0.0

$3.964 F-03$

$2.117 \mathrm{E}-02$

$0.764 \mathrm{E}-\mathrm{n} 2$
$1.512 \mathrm{E}-01$

$1.155 \mathrm{E}$ กO

$1.041 \mathrm{E} 00$

$6.559 E$ กI

$2.911 E$ त2

$8.676 \mathrm{~F} 02$

$2.375 \mathrm{E} 03$

6.45 2E 03

$1.430 E \quad 04$
$2.094 \mathrm{E} 04$
AUU $=-0.9446$
0.0

0.0
0.0

0.0

$0 . n$

0.0

0.0

0.0

$3.078 E-04$

$9.100 E-03$

$3.583 E-0$

$1.019 E-01$

$9.276 \mathrm{E}-01$

$5.635 \mathrm{E}$

$2.525 \mathrm{E}$ O2

$7.558 \mathrm{E} \quad 2$

$5.583 \mathrm{E} 03$

$1.265 \mathrm{E} \mathrm{O4}$

$1.875 \mathrm{E} \mathrm{O4}$

ANGLE 12

$M U=0.4580$

0.0

0.0
0.0
0.0

0.0

0.0

0.0

0.0

0.0

$5.918 E-03$

$2.515 \mathrm{E}-02$

7. $386 \mathrm{E}-0$ ?

$1.635 \mathrm{E}-01$

$1.200 \mathrm{E} 00$

$1.070 E 00$

$6.723 E$ E

2.97BE 02

.

2.425E O2

$.458 E \quad 04$

$2.131 \mathrm{E} O 4$ $\triangle N G L E \quad \&$
$M U=-0.8656$

0.0

0.0

0.0

0.0

0.0

0.0

0.0

$3.686 \mathrm{E}-04$

$9.426 E-03$

1.082E-02

$9.303 \mathrm{E}-01$

$8.902 E-01$

$5.683 E$ O1

$7.621 \mathrm{E} \mathrm{O2}$

$2.097 \mathrm{E} \mathrm{O3}$

$5.727 E \quad 03$

$1.274 \mathrm{E}$ O4

1.888 O4

$A N G L E \quad 13$
$M U=0.6179$

0.0

0.0

0.0

9.0

0.0

0.0

0.0

$9.039 E-03$

3. $321 \mathrm{E}-02$

$9.346 \mathrm{E}-02$

$1.245 E$ DO

.

$6.881 \mathrm{E}$ O1

$3.043 E$ O2

.

2.473E 03

.485 E 04

$2.167 E$ O4 $\triangle V G L E \quad 5$
$M U=-0.7550$ 0.0

0.0

0.0

0.1

0.0

4. $733 E-04$

$9.933 E-03$

1.072E-OI

$9.560 E-01$

$5.758 E$ I

$2.577 E$ O?

$7.709 E 02$

.121E 03

$5.788 E \quad 03$

$.288 E 04$

ANGLE 14

$M U=0.7550$

0.0

0.0

0.0

0.0

0.0

0.0

0.0

1. $427 E-02$

4.351E-02

$1.124 E-01$

$1 . ? 87 E$ Co

$1.124 \mathrm{E}$ OO

3.024E 01

3.101E O2

..222E 02

2.517 ह 03

$1.509 E 04$

2.198E 04
ANGLE 6 $M U=-0.6$ 0.0
0.0 0.0 0.0

0.0

0.0

0.0

0.0

$6.421 E-04$

$1.067 E-02$

1. $113 \mathrm{E}-01$

$9.778 \mathrm{E}-01$

$5.851 E$ OI

(16E 02

$7.823 E 02$

$2.151 E 03$

$5.867 \mathrm{E} \mathrm{O3}$

$1.929 \mathrm{E}$ O4

ANGLE 15

0.0

0.0

0.0

0.0

0.0

0.3

2. $375 \mathrm{E}-02$

5. $921 \mathrm{E}-02$

$1.371 \mathrm{E}-01$

$2.063 E-01$

$7.145 E$ OI

9.362 O2

$1.528 \mathrm{E} 04$

$2.223 E 04$

ANGLE $M U=-0.4580$ 0.0

0.0

0.0

0.0

0.0

0.0

0.0

$9.009 \mathrm{E}-04$

$1.170 \mathrm{E}-02$

$1.166 E-01$

$1.005 \mathrm{E} \mathrm{OD}$

$5.964 \mathrm{E} \mathrm{O1}$

$2.664 \mathrm{E} 02$

$7.960 E \quad 02$

$2.187 E 03$

$1.325 \mathrm{E} \mathrm{O4}$

$1.955 \mathrm{E} \mathrm{O4}$

ANGLE 16

$M U=0.9446$

0.0

0.0

0.0

0.0

0.0

0.0

0.0

$4.489 E-02$

8.591E-02

$1.701 \mathrm{E}-01$

$2.196 \mathrm{E}-01$

$7.236 E$ O1

$3.186 \mathrm{E} \mathrm{O2}$

$9.466 \mathrm{E} 02$

$1.543 \mathrm{E} 04$

$2.241 \mathrm{E} 04$ 
(NEUTRONS/MEV/STERADIAN/S QURCE NEUTRON)

ENERGY GROUP (MEV) 1.2 E $01--1.50 \mathrm{E} 01$ $1.00 E$ O1---1.22E 01 $6.36 \mathrm{E} 00--8.10 \mathrm{E}$ OD $4.97 E$ O0-- $6.36 \mathrm{E} 00$ $4.07 E 00---4.97 E 00$ $3.03 E \quad 00--4.07 E$ CO $2.46 E$ EO- -3.01E 00 $2.35 \mathrm{E} \quad 00--2.46 \mathrm{E} \quad 00$ $1.83 \mathrm{E} \quad 00--2.35 \mathrm{E}$ 00 $1.11 \mathrm{E} \quad 00--1.83 \mathrm{E}$ 00 $5.50 E-01-1.11 \mathrm{E} \quad 00$ $1.11 \mathrm{E}-01--5.50 \mathrm{E}-01$ $3.35 \mathrm{E}-02--1.11 \mathrm{E}-01$ $5.83 \mathrm{E}-\mathrm{Ca}---3.35 \mathrm{E}-02$ $1.01 E-04--5.83 E-04$ $2.90 E-05--1.01 E-C 4$ $1.07 E-05--2.90 E-05$ $3.06 \mathrm{E}-06--1.07 \mathrm{E}-05$ $1.12 \mathrm{E}-06--3.06 \mathrm{E}-06$ $4.14 \mathrm{E}-07---1.12 \mathrm{E}-06$
$0.0 \quad---4.14 \mathrm{E}-07$

$$
\text { ENERGY }
$$$$
\text { GROUP (MEV) }
$$

$1.22 \mathrm{E} 01--1.50 \mathrm{E}$ O1 $1.00 E$ OL-W-1.22E 01 $8.19 E$ OO-- $1.00 E$ Ol $6.36 \mathrm{E} \quad 00---8.19 \mathrm{E}$ 00 4.97E $00---6.36 E$ CO

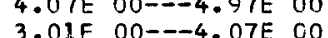
2. $26 \mathrm{E}$ OO---3.01E 00 $2.46 \mathrm{E} \quad 00--3.01 \mathrm{E} O 0$ $2.35 \mathrm{E} C 0--2.46 \mathrm{E}$ OO
$1.83 \mathrm{E} 00--2.35 \mathrm{E} 00$

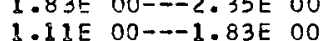
$1.11 \mathrm{E} \quad 00--1.83 \mathrm{E}$
$5.50 \mathrm{E}-01--1.11 \mathrm{E} 00$ 1.11E-01---5.50E-01 $3.35 \mathrm{E}-02-\cdots 1.11 \mathrm{E}-01$ $5.83 E-04-\cdots 3.35 E-02$ $1.01 E-04--5.83 E-04$ $2.90 E-05--1.01 E-04$ 1.07E-05- $2.90 E-05$ $1.12 \mathrm{E}-06---3.06 \mathrm{E}-06$ $4.14 \mathrm{E}-07--1.12 \mathrm{E}-06$ $4.14 E-07--1.12 E-06$
$0.0 \quad--4.14 \Xi-07$
ANGLE 1 0.0 0.0 0.0 0.0 0.0 0.0 0.0 0.0 1. $215 E-04$ $5.594 \mathrm{E}-03$ $2.444 \mathrm{E}-02$ $7.783 \mathrm{E}-02$ $7.668 E-01$
$7.462 \mathrm{E}-01$ 7.462E-01 $2.205 E 02$ $6.662 \mathrm{~F} 02$ 1.855E 03 $5.109 E 03$ $\begin{array}{ll}1.145 \mathrm{E} & 04 \\ 1.714 \mathrm{E} & 04\end{array}$

ANGLE 10 $M U=0.0950$ 0.0

0.0
0.0
0.0

0.0
0.0
0.0

0.0

0.0 0.0

$1.761 \mathrm{E}-03$ $1.114 \mathrm{E}-02$ $4.179 E-02$ $1.098 \mathrm{E}-01$ $9.355 E-01$ $5.597 \mathrm{E} 01$ $2.519 E$ O2 7.586E 02 2.1.2E 03 S.767E 03 $\begin{array}{ll}1.289 \mathrm{E} & 04 \\ 1.906 \mathrm{E} & 04\end{array}$ $M U=-0.9894$ 0.0 0.0 0.0 0.0 0.0 0.0 0.0 0.0

1. $858 \mathrm{E}-04$ $5.619 \mathrm{E}-03$ $2.452 E-02$ $7.804 \mathrm{E}-02$ $7.681 \mathrm{E}-01$ $7.473 E-01$ 4.866E 0I $2.208 E \quad 02$ $6.670 E 02$ $1.857 \mathrm{E} 03$ $5.115 \mathrm{E} 03$ $1.148 \mathrm{E} \mathrm{C4}$ $1.716 \mathrm{E}^{04}$ ANGLE 11 $\begin{aligned} M U & = \\ 0.0 & \end{aligned}$ 0.0
0.0

0.0

0.0

0.0

0.0

0.0

0.0

0.0
$2.582 E-03$ $1.340 E-02$ $4.808 E-02$ $1.185 E-01$ $9.733 E-1$ $8.95 E E-01$ 5.749E 01 $2.583 \mathrm{E} 02$ $7.774 \mathrm{E} 02$ $2.152 \mathrm{E} 03$ S.89E 1.944E 04
ANG

$J=-0.9446$

0.0

0.0

0.0

0.0

0.0

0.0

$2.049 E-04$ $5.725 \mathrm{E}-03$ $2.49 J E-C 2$ $7.894 \mathrm{E}-02$ $7.736 \mathrm{E}-01$ $7.515 \mathrm{E}-01$ 4.892 E O1 2.219E 02 $6.703 E 02$ 1.865 E 03 5.138 E 03 $1.153 E 04$ $1.723 \mathrm{E} 04$

ANGLE 12 $M U=0.4580$ $M U=$
0.0
0.0
0.0 0.0

0.0

0.0

0.0 0.0

0.0 1. $653 \mathrm{E}-02$ $5.624 E-02$ $1.284 E-01$ $1.013 E-00$ $9.218 E-01$ 5.902E 01 2.647E O2 7.961E 02 2.202E 03 1. 030 E 03 $1.346 \mathrm{E}$
$1.982 \mathrm{E}$

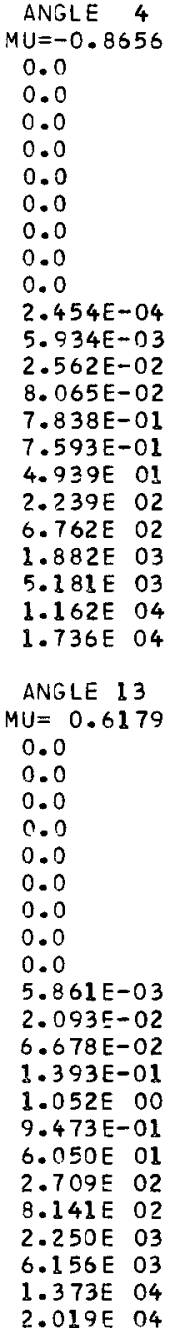

$\begin{aligned} & \text { ANGLE } 5 \\ & M U=-0.7550\end{aligned}$ 0.0 0.0 0.0 0.0 0.0 0.0 0.0 0.0

$3.145 \mathrm{E}-04$ $6.259 E-03$ .672E-02 $8.312 \mathrm{E}-02$ $7.984 \mathrm{E}-01$ $7.703 \mathrm{E}-01$ $5.006 E$ OL $2.268 E \quad 02$ $6.846 E$ O2 $1.904 \mathrm{E} 03$ 5.241 E 03 $1.175 \mathrm{E} \quad 04$ $1.754 \mathrm{E} 04$

ANGLE 14 MU $=0.7550$ 0.0
0.0 0.0 0.0 0.0 0.0 0.0 0.0 0.0
$9.214 E-03$ 9. $214 E-03$
$2.724 E-02$ $8.035 \mathrm{E}-02$ $1.508 E-01$ $1.089 \mathrm{E}$ 00 $9.707 E-01$ $6.184 E$ OI $2.764 E$ O2 $8.304 \mathrm{E} 02$ 2.292E 03 ..268E 03 $2.051 \mathrm{E} 04$
$\begin{array}{ll}A V J L E & 6 \\ M U=-0.6179\end{array}$ 0.0 0.0
0.0
0.0 0.0 0.0 0.0
0.0 0.0 0.0 4.251E-04 $6.731 E-03$ 2. $828 \mathrm{E}-02$ $8.546 \mathrm{E}-02$ 8.175E-01 $7.846 E-01$ $5.092 E 01$ $2.305 \mathrm{E} 02$ $6.955 E \quad 02$ $1.934 \mathrm{E} 03$ $5.319 E 03$ $1.192 E \quad 04$ $1.777 \mathrm{E} 04$

ANGLE 15 $M U=0.8656$ 0.0
0.0

0.0

0.0

0.0

0.0

0.0

0.0

1. $523 \mathrm{E}-02$ $3.663 \mathrm{E}-02$ $9.776 E-02$ $1.523 E-01$ $1.121 E 00$ $9.906 E-01$ 6.297E O1 2.811E 02 $8.440 E$ O2 $2.328 \mathrm{E} 03$ $6.362 E 03$ 2.077 E 04

ANGLE $\quad 7$
$M U=-0.4580$ 0.0 0.0 0.0 0.0 0.0 0.0 0.0 5. $938 E-04$ $7.387 \mathrm{E}-03$ 3.040E-02 $9.071 \mathrm{E}-02$ $8.019 E-01$ $5.196 \mathrm{E}$ O1 $2.349 E \quad 02$ $7.086 \mathrm{E} \mathrm{O2}$ $1.969 \mathrm{E} 03$ $5.413 \mathrm{E} 03$ $1.212 \mathrm{E} 04$ $1.804 \mathrm{E} 04$ 
(NEUTRONS/MEV/STERADIAN/SOURCE NEUTRON)

ENERGY

GROUP (MEV)

$1.22 E 01--1.50 E$ OI

$1.00 E$ O1--1.22E O1

8.1 SE $00--1.00 E$ OI

6.36E 00---8.19E 00

4.97E OO---6.36E OO

4.07E $00--4.97 E$ DO

3.01E $00--4.07 E$ OO

$1.83 \mathrm{E} 00--2.35 \mathrm{E} 00$

$1.11 \mathrm{E} 00--1.83 \mathrm{E} 00$

$5.50 \mathrm{E}-01--1.11 \mathrm{E}$ OO

$1.11 \mathrm{E}-01--5.50 \mathrm{E}-01$

$3.35 \mathrm{E}-02--1.11 \mathrm{E}-0$ !

$5.83 E-04--3.35 E-02$

$1.01 \mathrm{E}-04--5.83 \mathrm{E}-04$

$2.90 \mathrm{E}-05--1.01 \mathrm{E}-04$

$1.07 E-05--2.90 E-05$

$3.06 E-06--1.07 E-05$

$1.12 F-06--3.06 E-06$

$1.14 \mathrm{E}-07---1.12 \mathrm{E}-06$
$0.0 \quad--4.14 \mathrm{E}-07$

ENERGY

GROUP (MEV)

$1.22 E$ 01--1.50E 01

$1.00 E$ 01--1.22E 01

8.19E O0--1.00E O1

6.36E $00--8.19 E \quad 00$

4.97E $00--6.36 E$ OO

4.07E $00--4.97 E$ CO

$3.01 \mathrm{E}$
$2.40--4.07 E$

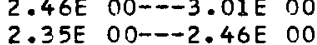

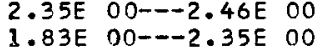

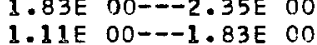

$1.11 \mathrm{E} 00--1.83 \mathrm{E}$ OO

$1.11 \mathrm{E}-01--5.50 \mathrm{E}-01$

$3.35 \mathrm{E}-02--1.11 \mathrm{E}-01$

$5.83 E-04---3.35 E-02$

$1.01 E-04--5.83 E-04$

$1.907-05--1.01 E-04$

$1.07 E-05-2.90 E-05$

3.06E-06---1.07E-05

1.12E-06-- $3.06 E-06$

$4.14 E-07--1.12 E-06$
$0.0 \quad--4.14 E-07$
ANGLE 1 ANGLE 2 0000

0.0

0

0.0

0.0

0.0

0.0
$. .434 E-05$

$1.211 \mathrm{E}-03$

.658E-03

$3.082 \mathrm{~F}-01$

$3.133 \mathrm{E}-01$

$2.116 \mathrm{E} \mathrm{OI}$

$9.838 \mathrm{E} \mathrm{O1}$

$3.028 \mathrm{E} 02$

.625E 02

.418E

$\begin{array}{ll}5.514 E & 03 \\ 8.392 E & 03\end{array}$

ANGLE 10

0.

0.0

0.0

0.0

0.0

0.0

0.0
0.0
0.0
0.0

.0

4. $168 \mathrm{E}-04$

$2.433 \mathrm{E}-03$

$1.185 \mathrm{E}-02$

.

(

$.475 \mathrm{E} \mathrm{OI}$

$1.142 E$ O2

$3.506 \mathrm{E} 02$

$9.947 \mathrm{E} \mathrm{O2}$

2.778 E 03

$9.494 \mathrm{E} 03$

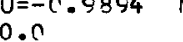

0.0

0.0

0.0

0.0

0.0

0.0

0.0

$.217 E-03$

$6.685 \mathrm{E}-0$

2.680E-02

$2.128 \mathrm{~F}-01$

2.1198 OL

. $851 E$ E

$8.636 E$ O2

$2.421 E 03$

$5.521 E 03$

8.402 F 03

ANGLE 11

0.0

0.0

0.0

0.0

0.0

0.0

0.0

6.090E-04

$2.922 \mathrm{E}-02$

$1.373 E-n 2$

$4.170 E-02$

$3.991 \mathrm{E}-\mathrm{OI}$

$3.825 E-01$

1.174E 02

.

.022E 03

.

$6.477 \mathrm{E} \mathrm{O}$

$9.717 \mathrm{E} 03$
$\begin{array}{ll}A N G L E & 3 \\ M U=-0.9446\end{array}$

0.0

0.0

0.0

0.0

0.0

0.0

0.0

$5.021 E-05$

$1.240 E-03$

$2.713 \mathrm{E}-0$

$3.112 \mathrm{E}-01$

3.157E-01

.132E OI

$.906 E$ O1

$3.049 E$ O2
$8.682 E$ O2

$2.433 E \quad 03$

$8.443 \mathrm{E} 03$

ANGLE 4 $M U=$

$U=-0.7550$

MUN $=-0.8$
0.0
0.0

ANGLE 12

$M U=0.4580$

0.0

0.0

0.0

0.0

0.0

0.0

0.0

0.0

$9.042 E-04$

$3.590 E-03$

$1.616 \mathrm{E}-02$

$4.53 E-02$

4.165E-01

.625E 01

. $225 E$ O

1.203E 02

.

$6.640 E$ O3

$.941 \mathrm{E} 03$
0.0

0.0

0.0

0.0

0.0

0.0

$6.018 \mathrm{E}-05$

$.287 E-03$
$7.015 E-03$

2.775E-02

$3.357 E-01$

3.153E-01

.154E O1

$3.079 \mathrm{E} \mathrm{O}$

$8.767 E 02$

$2.456 \mathrm{E} \quad 03$

$8.517 \mathrm{E} 03$

ANGLE 13

$M U=0.6$
0.0
0.0

0.0

0.0

0.0

0.0

0.0

0.0

$7.688 \mathrm{E}-05$

1. $359 \mathrm{E}-03$

$7.344 E-03$

$3.220 E-01$

$2.187 E$ O1

$1.015 \mathrm{E} \mathrm{O2}$

3.123E 02

8.887E 02

$\begin{array}{ll}2.489 E & 03 \\ 5.6735 & 03\end{array}$

8.620 E 03

ANGLE 14

$M U=0.7550$

0.0

0.0

0.0

0.0

0.0

0.0

0.0

0

$1.371 \mathrm{E}-03$

4.511E-03

$4.933 E-02$

.

4.068 O

.

..79

.

.

$6.795 \mathrm{E} 03$

$1.015 E 04$

0.0

$2.138 \mathrm{E}-03$

$5.784 \mathrm{E}-03$

$2.321 E-02$
$5.350 E-02$

$4.503 \mathrm{E}-01$

$4.177 \mathrm{E}-01$

$2.765 \mathrm{E} 01$

3.884E O?

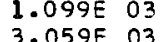

ANGLE 6
$M U=-3.6179$ 0.0

0.0
0.0
0.0
0.0

0.0

0.0

0.0

0.0

$1.032 E-04$

$1.464 \mathrm{E}-03$

7.813E-03

$3.304 \mathrm{E}-01$

$3.310 E-01$

$2.228 E$ O 1

$1.033 \mathrm{E} \mathrm{O2}$

$3.179 \mathrm{E} O 2$
$9.042 \mathrm{E} 02$

$2.531 \mathrm{E} 03$

$5.767 E$ E3
$8.750 E$ O3

ANGLE 7

0.0

0.0

0.0

0.0

0.0

0.0

0.0

0.0

$1.431 E-04$

$1.609 E-03$

$8.447 E-03$
$3.144 E-02$

$3.407 E-01$

$3.390 \mathrm{E}-01$

$2.279 E \quad 01$
$1.056 E \quad 02$

$3.246 \mathrm{E} 02$

$9.228 \mathrm{E} 02$

$\begin{array}{lll}2.582 E & 03 \\ 5.881 E & 03\end{array}$

$8.904 E \quad 3$

ANGLE 15

ANGLE 16

$U=0.8656$

$\mathrm{AN}=0$
0.0

0.0

0.0

0.0

0.0

0.0

0.0

0.0

0.0 
FNERGY GROUP (MEV) $1.22 E \quad 01--1.50 E$ OI $1.00 E$ OL---1.22E OI $8.19 E$ OO- -1. OOE O1 $6.36 \mathrm{E} \quad 00--8.19 \mathrm{E}$ OO $4.07 E$ OO$3.01 E$ O0--4. 07 E 00 $2.46 \mathrm{E} O \mathrm{OC}--3.01 \mathrm{E} 00$ $2.35 \mathrm{E} 00---2.46 \mathrm{E} 20$ $1.83 \mathrm{E} 00---2.35 \mathrm{E} 00$ $1.11 \mathrm{E}$ 00---1.83E 00 $5.5 \mathrm{CE}-01--1.11 \mathrm{E}$ OO $1.11 \mathrm{E}-01-25.50 \mathrm{E}-01$ $3.35 \mathrm{E}-02-\cdots 1.11 \mathrm{E}-01$ $5.83 E-04--3.35 E-02$ $1.01 \mathrm{E}-04-\cdots 5.83 E-04$ $2.90 E-05--1.01 E-04$ $1.07 E-05---2.00 \mathrm{E}-05$ $3.06 \mathrm{E}-06---1.07 \mathrm{E}-05$ $1.12 E-06--3.06 E-06$ $4.14 \mathrm{E}-07--1.12 \mathrm{E}-06$
0.0 ENERGY GROUP (MEV)

$1.22 E$ 01--1.50E 01 $1.00 E$ OJ---1.22E OI $8.19 E$ OO---1. OOE OL $6.36 E$ DO---8.19E OO 4.97E $00--6.36 E$ OD 4.07E $00--4.97 E$ OO 3.O1E $00--4.07 E$ OO $2.46 E$ OO- $-3.01 E$ OO $2.35 E$ OO-D. $2.46 E$ DO $1.83 \mathrm{E} 00--2.35 \mathrm{E} 00$ $1.11 E$ OO- $1.83 E$ OO 5.SOE-O1--1.11E OO $1.11 E-01-C .50 E-01$ $3.35 E-02--1.11 E-01$ $5.83 E-04-C .35 E-02$ $1.01 E-04--5.83 E-04$ $1.90=-05--1.01 E-04$ 1.06 $-06-1.07 E-05$ .06 $06-1.07 E-05$ 1.12E-06$0.0 \quad--4.14 E-07$
ANGLE 1 ANGLE 2 $M U=-1.0000$ 0.0

0.0

0.0

0.0

0.0

$9.328 E-06$ $2.341 \mathrm{E}-04$ $1.520 \mathrm{E}-03$ $7.086 E-03$ $9.049 E-02$ $9.436 \varepsilon-02$ $6.513 E 00$ $3.070 E$ OI $9.556 E$ O1 $2.758 \mathrm{E} \mathrm{O2}$ $7.813 E \quad 02$ $1.799 E 03$
$2.768 E \quad 03$ ANGLE 10 $U=0.0950$

0.0

0.0

0.0

0.0

0.0

0.0

0.0
0.0

0.0

$8.622 \mathrm{E}-05$ $4.707 E-04$ $2.771 E-03$

I.133E-OI

$1.125 \mathrm{E}-01$

$7.680 \mathrm{E} 00$

$3.594 \mathrm{E} O 1$

$1.116 E 02$

$3.211 \mathrm{E} \quad 02$

2.063E 02

$3.160 E^{03}$ 0.0

0.0

0

0.0

0.0

0.0

0.0

0.0

$2.352 \mathrm{E}-04$

$1.52 \in E-C 3$

$7.108 \mathrm{E}-03$

$9.067 E-02$

$9.451 \mathrm{E}-02$

$6.522 E 00$

$3.075 \mathrm{E} \mathrm{O1}$

9.56 SE 01

$2.762 E \quad 0 ?$

$7.823 \mathrm{E} 02$

$1.802 \mathrm{E} 03$

2.772E 03

ANGLE 11

$M U=$
0.0
0.0

0.0
0.0
0.0

0.0
0.0
0.0

0.0

0.0

0.0

0.0

1.258E-04

$5.642 E-04$

$3.223 E-03$

$1.122 E-02$

$1.163 E-01$

$1.163 E-01$

$7.925 E$ OO

$3.703 E 01$

3.304E 02

9.319E O2

2.138E 03
(NEUTRONS/MEV/STERADIAN/SOURCE NEUTRON)

$M U=-0.9894$

$9.559 E-06$

$3.240 E 03$ $=-0.9446 \quad M U=-0.8556$ 0.0

0.0

0.0

0.0

0.0

0.0

0.0

$.058 \mathrm{E}-05$

$2.399 \mathrm{E}-04$

$1.554 E-03$

$7.199 E-03$

$9.513 E-02$

$6.562 \mathrm{E} 00$

$3.093 \mathrm{E} 01$

$9.625 \mathrm{E} \mathrm{O1}$

$2.778 \mathrm{E} 02$

$7.867 \mathrm{E} 02$

$1.812 \mathrm{E} 03$

$2.786 \mathrm{E} 03$

ANGLE 12

MU $=$

0.0

0.0
0.0

0.0

0.0

0.

0.0

1. $864 E-04$

6.907E-04

$3.806 \mathrm{E}-03$

$1.222 E-02$

$1.202 E-01$

$8.172 E 00$

$3.812 E$ E

$1.183 E 02$

$3.398 \mathrm{E} 02$

2.196E 03

$3.321 \mathrm{E} 03$

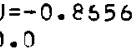

0.0

0.0

0.0

0.0

0.0

$1.268 \mathrm{E}-05$

$2.490 E-04$

$1.606 \mathrm{E}-03$

7.369E-03

$9.277 \mathrm{E}-02$

$9.625 \mathrm{E}-02$

.636E OO

$9.727 \mathrm{E}$ OI

$2.807 \mathrm{E} \mathrm{O2}$

$7.947 E \quad 02$

$3.830 \mathrm{E} \mathrm{O3}$

$2.812 E \quad 03$

ANGLE 13

$M U=0.6179$

0.0

0.0

0.0

0.0

0.0

0.

0.0

2.818E-04

$8.622 E-D 4$

$4.550 E-03$

$1.332 E-02$

$1.293 \mathrm{E}-01$

$8.412 \mathrm{E} 00$

$3.918 E$ O

$1.215 E$ O2

$3.488 E$ O

$2.824 E$ O2

$3.398 \mathrm{E} 03$
$A N G L E \quad 5$
$M U=-0.7550$

0.0

0.0

0.0

0.0

0.0

0.0

$1.618 E-05$

$2.631 E-04$

$1.685 E-03$

$7.618 \mathrm{E}-03$

$9.786 E-02$

$6.740 E$ OO

$3.173 E$ O1

$9.872 E$ OI

$2.848 E \quad 02$

$8.060 E \quad 02$

$\begin{array}{lll}1.855 E & 03 \\ 2.849 E & 03\end{array}$

ANGLE 14

0.0

0.0

0.0

0.0

0.0

0.0

0

0.0

4. $371 E-04$

$1.093 E-03$

$1.446 \mathrm{E}-02$

1.343E-01

$8.630 E 00$

4. $014 E 01$

$1.245 E 02$

$3.570 E .02$

$1.005 E$ O

$3.467 E \quad 03$

ANजLE 6 $M U=-0.6179$ ANGLE 7 ANGLE 8 ANGLE 9 $0.0 .2816 \quad M U=0$ $\begin{array}{llll}0.0 & 0.0 & 0.0 & 0.0 \\ 0.0 & 0.0 & 0.0 & 0.0\end{array}$

$\begin{array}{llll}0.0 & 0.0 & 0.0 & 0.0\end{array}$

$\begin{array}{llll}0.0 & 0.0 & 0.0 & 0.0 \\ 0.0 & 0.0 & 0.0 & 0.0\end{array}$

0.0

0.0

$2.165 E-05$ $2.835 E-04$ $1.798 \mathrm{E}-03$ $7.955 \mathrm{E}-03$ $9.730 E-02$ .955E-02 6.876E 00 $.234 \mathrm{E} 01$
$.006 \mathrm{E} 02$ $2.901 \mathrm{E} 02$ $8.206 \mathrm{E} 02$ $1.888 E \quad 03$
$2.895 E \quad 03$

0.0 0.0 0.0

$2.986 E-05$ $3.116 \mathrm{E}-04$ $1.951 \mathrm{E}-03$ $8.384 \mathrm{E}-03$ $1.005 \mathrm{E}-01$ 
(NEUTRJNS/MEV/STERADIAN/SOURCE NEUTRON)

FNE RGY GROUP (MEV) $1.22 E$ OI---1.5OE 01 $1.00 E$ O1--1.22E OI $6.36 \mathrm{E} 00---8.19 \mathrm{E}$ OD 4.97E $00--6.36 E$ OO $4.07 E$ 00--4. $.97 E$ OO $3.01 \mathrm{E} 00--4.07 E$ OO $2.46 \mathrm{E} \quad 00--3.01 \mathrm{E} 00$ $2.35 \mathrm{E} \quad 00---2.46 \mathrm{E}$ OO $1.8 \geq E$ EO--2.35E 00 $1.11 \mathrm{E} \quad 00--1.83 \mathrm{E}$ OO $5.50 E-01--1$. IIE 00 $1.11 E-01--5.50 E-01$
$3.35 \mathrm{E}-02--1.11 \mathrm{E}-01$ $3.35 \mathrm{E}-02---1.11 \mathrm{E}-01$
$5.83 \mathrm{E}-04---3.35 \mathrm{E}-02$ $5.83 E-04---3.35 E-02$
$1.01 E-04---5.83 E-04$ $2.90 E-05--1.01 E-04$ $1.07 E-05--2.90 E-05$ $3.06 \mathrm{E}-06--1.07 \mathrm{E}-05$
$1.12 \mathrm{E}-06--3.06 \mathrm{E}-06$ $1.12 \mathrm{E}-06--3.06 \mathrm{E}-06$
$4.14 \mathrm{E}-07--1.12 \mathrm{E}-\mathrm{C6}$ $4.14 E-07---1.125-C 6$
$0.0 \quad--4.14 E-07$ ENERGY

GROUP (MEV) 1.22E $01--3.50 E$ OI 1.0OE
$8.10 \mathrm{O}$ OO---1.22E 01 $6.36 \mathrm{E} 00--8.19 \mathrm{E}$ OO $4.97 \mathrm{E} O 0--6.36 \mathrm{E} \mathrm{GO}$ 4.07E 00--4. 07 E 00 $3.01 \mathrm{~F}$ ON- - -4.07E 00 2.46E OO--3. O1E NO $2.35 \mathrm{~F} O O--2.46 E$ OO $1.83 \mathrm{E}$ 00-- $2.35 \mathrm{E}$ nO $1.11 \mathrm{E} 00--1.83 \mathrm{E}$ DO 5.50E-O1-- -1.11E 0O $1.11 \mathrm{E}-0$ ! $--5.50 \mathrm{E}-\mathrm{Cl}$ $3.35 E-02--1.11 \mathrm{E}-01$ $5.83 E-04--3.35 E-02$ $1.01 E-04--5.83 E-C 4$ 2.90E-05--1.01E-04 $1.07 E-05--2.90 E-05$ $2.06 E-06--1.07 E-05$ $1.12 \mathrm{E}-06--3.06 \mathrm{E}-06$ $4.14 E-07--1.12 E-06$
$0.0 \quad--4.14 E-07$ ANGLE 1 $M U=-1.0000$ 0.0 0.0 0.0 0.0 0.0 0.0 $1.811 E-06$ $4.266 E-05$ $3.140 \mathrm{E}-04$ $1.627 \mathrm{E}-03$ $2.228 \mathrm{E}-02$ $2.362 \mathrm{E}-02$ $1.653 \mathrm{E} \mathrm{OO}$ $7.863 \mathrm{E} \mathrm{OO}$ $2.464 \mathrm{~F} \quad 01$ $7.175 E$ OI $2.046 E 02$ $4.741 \mathrm{E}$
$7.343 \mathrm{E}$ ANGLE 10 $M U=0.0950$ 0. 0.0 0.0 0.0 0.0 0.0 0.0 0 1.656 E- 05 $8.558 \mathrm{E}-05$ $5.813 E-04$ $2.391 \mathrm{E}-03$ $2.807 E-02$ $2.829 E-02$ $1.959 \mathrm{E} \mathrm{OO}$ $9.252 \mathrm{E} O 0$ $2.894 E \quad 01$ $8.399 E$ OI $2.386 E \quad 02$ $5.514 \mathrm{E}$ O2
ANGLE $\quad 2$
$M U=-0.9894$ 0.0 0.0 0.0
0.0
0.0

0.0

0.0

0.0

0.0

0.0
$1.856 \mathrm{E}-06$

4.286E-05

$3.154 \mathrm{E}-04$

$1.633 \mathrm{E}-03$

$2.233 \mathrm{E}-02$

2. $366 \mathrm{E}-02$

$1.655 \mathrm{E}$ OD 7.875E OO $2.468 \mathrm{E}$ OI $7.185 E$ Ol $2.048 \mathrm{E}$ O2 $4.747 \mathrm{E}$
$7.352 \mathrm{E}$

ANGLE 11 $M U=0.2816$ 0.0

0.0

0.0

0.0

0.0

0.0

0.0

2.413E-05 $2.413 E-05$
$1.024 E-04$
$6.780 E-04$ $2.600 E-03$ $2.928 E-02$ $2.928 \mathrm{E}-02$ $2.023 E$ กO $9.541 \mathrm{~F}$ OO $2.984 \mathrm{E} \mathrm{Ol}$ $8.652 \mathrm{E} \mathrm{OQ}$ $2.456 \mathrm{~F} \mathrm{O2}$ $5.672 \mathrm{E} \mathrm{O2}$ $8.651 E$ O2
$M U=-0.9446$ 0.0 0.0 0.0

0.0

0.0

0.0

0.0

$2.056 E-06$ $4.372 E-05$ $3.212 E-04$ $1.654 \mathrm{E}-03$ $2.251 E-02$ $2.382 E-02$ $1.666 \mathrm{E} 00$ $7.923 \mathrm{E} \mathrm{OO}$ $2.483 \mathrm{E}$ 01 T.228E OD 2.060 E O2 $4.774 E$ O2

ANGLE 12 $M U=0.4580$ 0.0 0.0 0.0 0.0 0.0 0.0 0.0 $3.572 E-05$ $1.249 E-04$ $1.249 E-04$
$3.024 E-04$ $2.837 E-03$ $3.375 \mathrm{E}-02$ $3.029 E-02$ $2.089 E 00$ $9.832 E 00$ $3.074 E$ OI $8.905 E$ OI $2.527 E$ O2 $5.832 \mathrm{E} O 2$ 8.875 E O2
ANGLE $\quad 4$
$M U=-0.8556$ 0.0 0.0

0.0

0.0

0.0

(

0.0

2. $465 E-06$

$4.539 E-05$

$3.324 E-04$

$1.594 \mathrm{E}-03$

$2.286 \mathrm{E}-02$

2.411E-02

$1.685 \mathrm{E} 00$ $8.010 E$ OO $2.510 E$ OI T.3 $2.082 E$ O2

4.3238 Q
$7.464 \mathrm{E}$ $\begin{array}{ccc}\text { ANGLE } & 5 & \text { AVGLE } \\ M U=-0.7550 & \text { MU }=-0.6179\end{array}$

ANGLE 13

.

0.0

0

0.0

0.0

5.391E-05 $1.551 E-04$
$0.506 E-04$ $3.095 \mathrm{E}-03$ $3.212 \mathrm{E}-02$ $3.128 E-02$ $2.151 \mathrm{E} 00$ 1.0 IE OI $3.160 E 01$ $9.151 \mathrm{E}$ OI $2.595 E \quad 02$ $5.985 \mathrm{E} \mathrm{O2}$ $9.089 \mathrm{E} 02$ 0.0
0.0 0.0

0.0

0.0

0.0

0.0

$3.140 E-06$

$4.796 \mathrm{E}-05$

$3.493 E-04$

$1.752 \mathrm{E}-03$
$2.335 \mathrm{E}-02$

$2.452 \mathrm{E}-02$

$1.712 E 00$ $8.135 E 00$ $2.549 E$ OI $7.416 \mathrm{E} 01$ 2.113E $4.893 \mathrm{E} 02$
$7.564 \mathrm{E} 02$

ANGLE 14

$U=-0.6$ 0.0

0.0

0.0

0.0

0.0

0.0

$4.193 E-06$

$5.167 E-05$

3. $734 \mathrm{E}-04$

$1.831 \mathrm{E}-03$

$2.400 E-02$

$2.506 \mathrm{E}-02$

$1.748 E$ E 00 $8.297 E$ OO 7. 7.558E 01 $4.984 E 02$ 4.984E O2

\section{ANजLE 15}

0.0

0.0

0.0

0

8. $327 E-05$

1. $950 \mathrm{E}-04$

$1.157 \mathrm{E}-03$

$3.340 E-02$

$3.21 \mathrm{BE}-\mathrm{n} 2$ $2.209 E 00$ .037E 01 $9.374 \mathrm{E}$ OI $2.656 \mathrm{E} \mathrm{O2}$ $6.124 \mathrm{E} 02$ $9.281 E 02$ $\begin{array}{ccc}\text { ANGLE } & 7 & \text { ANGLE } \\ M U=-0.4580 & M\end{array}$ 0.0 0.0 0.0 0.0 0.0 0 $5.769 \mathrm{E}-06$ $5.678 E-05$ $4.060 E-04$ $1.932 \mathrm{E}-03$ $2.481 E-02$ 
ENERGY

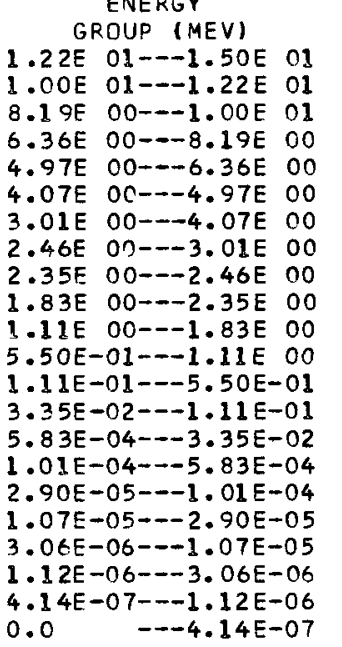

$0.0 \quad--4.14 \mathrm{E}-07$

ENERGY

GROUP (MEV)

$1.22 E$ O1---1.50E OI

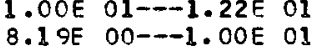
8.19E O0---1.00E O1 $6.36 \mathrm{E}$ 00-- $0.19 \mathrm{E}$ OO 4.97E $00--6.36 E$ OO

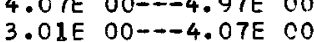
$2.46 \mathrm{E} 00--3.01 \mathrm{E} 00$ $2.35 E$ OO--2.46E 00 $1.83 \mathrm{E} 00--2.35 \mathrm{E} 00$ $1.11 E$ EO-D-1.83E OO 5.5OE-O1- - -1.11E OO $1.11 \mathrm{E}-01---5.50 \mathrm{E}-0$ .35E-02- - $1.11 \mathrm{E}-01$ 5.8 $1.01 E-04-25.83 E-04$ . $3.06 \mathrm{E}-06--1.07 \mathrm{E}-05$ 1.06 $1.12 E-06-2.06 E-06$ 1.14E-07-- $1.12 E-06$ $=-1.0000$ 0.0 0.0 0.0 0.0 0.0 0.0 0.0 $3.343 \mathrm{E}-07$ $7.502 E-06$ $6.087 E-05$ $3.411 E-04$ 4.908E-03 $5.262 E-03$ 3.717E-0 $1.779 E$ OD $5.602 \mathrm{E} 00$ $1.640 E$ OI $4.698 \mathrm{E}$ OI 1.093 E 02 $1.701 \mathrm{E} \mathrm{O2}$ ANGLE 10 $U=0.0950$ 0.0
0.0
0.0 0.0
0.0 0.0
0.0
0.0 0.0
0.0
0.0 0.0 0.0 $3.036 E-06$ $1.5 E-04$ $1.139 E-04$ 6. $08 E-03$ $6.322 E-03$ . $322 E-03$ $4.420 E-01$ 2.10OE OO 1.927E 01 5.500 E $1.276 \mathrm{E}$ O2 $1.959 \mathrm{E} 02$
ANGLE 2 $U=-0.9894$
0.0 0.0
0.0
0.0 0.0 0.0 0.0 0.0

0.0

$3.427 E-07$

7. $537 \mathrm{E}-06$

$6.114 E-05$

$3.421 \mathrm{E}-04$

$4.918 E-03$

$5.271 \mathrm{E}-03$

$1.782 \mathrm{E} 00$

$5.610 \mathrm{E} O 0$

$1.643 \mathrm{E}$ O1

$4.704 \mathrm{E}$ OI

$1.005 \mathrm{E} \mathrm{C2}$

$1.703 E$ O

ANGLE 11

ANGLE
$M U=0$
0.0
0.0

0.0

0.0
0.0
0.0
0.0

0.0

0.0

0.0

4.421E-06

$1.792 E-05$

$1.330 E-04$

$6.503 E-03$

$6.548 \mathrm{E}-03$

$4.568 \mathrm{E}-01$

2.167E

1.8106 (1)

$1.665 \mathrm{E} 01$

$1.314 \mathrm{E} 02$

$2.012 E \quad 02$
(NEUTRONS/MEV/STERADIAN/SOURCE NEUTRON)

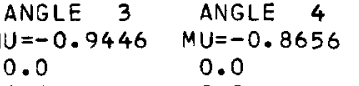

0.0

0.0
0.0
0.0

0.0

0.0

0.0

0.0

3.800E-07

$7.690 \mathrm{E}-06$

$6.230 E-05$

$3.467 E-04$
$4.961 E-03$

$5.307 E-03$

3.747E-01

$5.645 \mathrm{E} 00$

$\begin{array}{lll}5.645 E & 00 \\ 1.653 \mathrm{E} & 1\end{array}$

$4.732 E$ E 1

$1.101 \mathrm{E} 02$

$1.713 E 02$

0.0

0.0
0.0
0.0

0.0

0.0

0.0

0.0

0.0

4.554E-07

$7.983 E-06$

$6.451 E-05$

$3.552 E=04$
$5.038 \mathrm{E}-03$

$5.372 E-03$

3.791E-01

$5.708 \mathrm{E} 00$
$1.671 \mathrm{E}$

$1.671 \mathrm{E}$ O1

$4.783 \mathrm{E} 01$

$1.730 \mathrm{E} 02$

ANGLE 5
$M=-0.7550$

0.0

0.0

0.0

0.0

0.0

0.0

$5.797 E-07$

$8.434 E-06$
$5.787 E-05$

$3.787 E-05$

$3.676 E-04$
$5.149 E-03$

$5.466 \mathrm{E}-03$

3.853E-01

$1.842 \mathrm{E} \mathrm{OO}$

$5.798 \mathrm{E} 00$
$1.697 \mathrm{E} 01$

$1.697 \mathrm{E} 01$

$1.129 \mathrm{E} O 2$

$1.754 \mathrm{E} O 2$

ANGLE 6
$M U=-0.6179$

0.0

0.0

0.0
0.3
0.0

0.0

0.0

0.0

0.0

$7.730 E-07$

$9.085 E-06$
$7.265 E-05$

$3.845 \mathrm{E}-04$

$5.295 \mathrm{E}-03$

$5.589 E-03$
$3.935 E-01$

$1.879 E$ OO

$5.914 \mathrm{E}$ OO

$1.730 E$ O 1

$4.949 \mathrm{E} 01$

$1.784 \mathrm{E} 02$

ANGLE 14
$M U=0.7550$

$M U=0.4580$

ANGLE 13

0.0

0.0

0.0

0.0

0.0

0.0

0.0

$6.538 \mathrm{E}-06$

$2.181 \mathrm{E}-05$

1.577E-04

0.0

0.0

0.0

0.0

0.0

0.0

0.0

$9.852 E-0 S$

$2.696 \mathrm{E}-05$

$1.889 E-04$

0.0

0.0

0.0

0.0

0.0

0.0

0.0

$1.517 E-05$

$3.365 \mathrm{E}-05$

$2.275 E-04$

$7.405 E-03$

$6.811 E-03 \quad 7.117 E-03 \quad 7.405 E-03$

4.01E-03

7.206E-03

$4.862 E-01$

$4.994 E-01$

$2.359 E 00$

7.4

政

$2.104 \mathrm{E} \quad 1$

$2.156 \mathrm{E} 01$

$1.421 \mathrm{E} 02$

$5.832 \mathrm{E}$ O1 $5.992 \mathrm{E}$ O1

1.366 O2 $1.380 E$ O2

0.0

0.0

0.0

0.3

0.0

0.0

0.0

0.0

$2.406 \mathrm{E}-05$

$4.209 E-05$

2.731E-04

$7.644 \mathrm{E}-04$

$7.655 E-03$

$5.106 E-01$

$2.409 E$ EO

$2.500 \mathrm{E} 01$

$6.259 E$ O1

$1.449 E$ O2

$2.201 \mathrm{E} 02$

ANGLE 7
$M U=-0.4580$$\quad$ ANGLE 8 AN $=-0.2816 \quad M U=-0.0950$

$\begin{array}{lll}0.0 & 0.0 & 0.0\end{array}$

$\begin{array}{lll}0.0 & 0.0 & 0.0\end{array}$

$\begin{array}{lll}0.0 & 0.0 & 0.0\end{array}$

$\begin{array}{lll}0.0 & 0.0 & 0.0 \\ 0.0 & 0.0 & 0.0\end{array}$

$0.0 \quad 0.0$

$\begin{array}{lll}0.0 & 0.0 & 0.0\end{array}$

0.0

$\begin{array}{ll}0.0 & 0.0 \\ 1.062 E-06 & 1.488 E-06\end{array}$

$9.981 E-06 \quad 1.120 E-05$

7.911E-05 8.775E-05

$\begin{array}{ll}4.060 \mathrm{E}-04 & 4.328 \mathrm{E}-04 \\ 5.475 \mathrm{E}-03 & 5.689 \mathrm{E}-03\end{array}$

$5.737 E-03 \quad 5.912 E-03$

$4.034 E-01$

$1.924 \mathrm{E} 00$

$6.055 E 00$

$\begin{array}{lll}1.771 E & 01 \\ 5.062 E & 01\end{array}$

$1.177 \mathrm{E} 02$

$1.820 \mathrm{E} 02$

$4.149 E-01$
$1.977 \mathrm{E} 00$

$6.219 \mathrm{E} \quad 00$

$1.818 \mathrm{E}$ 01

$5.194 E 01$

$1.862 \mathrm{E} 02$

ANGLE 16
$M U=0.9446$

MU $=0$
0.0 
(GAMMAS/MEV/STERADIAN/SOURCE NEUTRON)

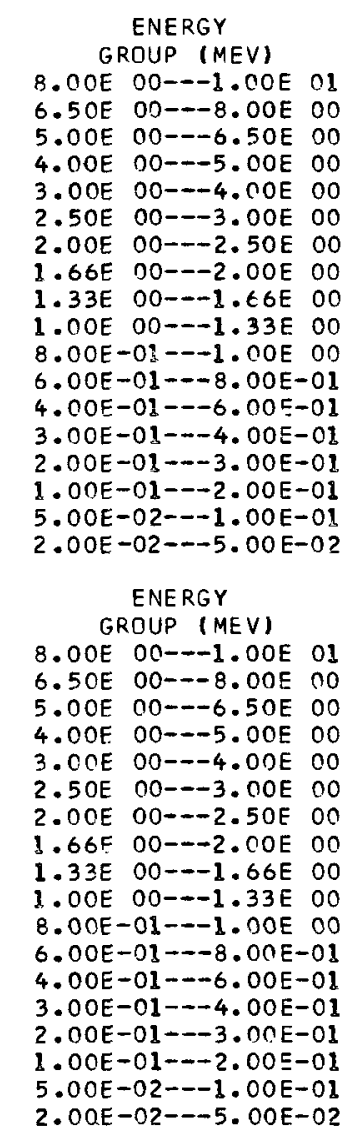

ANGLE 1
$M U=-1.0000$
$1.762 \mathrm{E}-06$
$3.645 \mathrm{E}-06$
$4.080 \mathrm{E}-05$
$1.144 \mathrm{E}-05$
$2.007 \mathrm{E}-05$
$5.651 \mathrm{E}-06$
$6.358 \mathrm{E}-06$
$7.390 \mathrm{E}-06$
$8.853 \mathrm{E}-06$
$1.151 \mathrm{E}-05$
$1.551 \mathrm{E}-05$
$1.551 \mathrm{E}-05$
$2.155 \mathrm{E}-05$
$1.501 \mathrm{E}-04$
$9.712 \mathrm{E}-05$
$1.822 \mathrm{E}-04$
$9.799 \mathrm{E}-04$
$2.014 \mathrm{E}-03$

ANGLE 2

ANGLE 3 ANGLE 4 ANGLE 5 1.765E-06 $M U=-0.9446$ $M U=-0.8656$ $M U=-0.7550$ ANजLE (1)

ANGLE 7 $3.668 E-06$ $1.792 \mathrm{E}-06$
$3.705 \mathrm{E}-06$ $3.650 E-06$ $4.086 \mathrm{E}-05$ $2.010 \mathrm{E}-05$ 2.010E-05 $5.662 E-06$ $6.371 E-06$
$7.405 E-06$ $7.405 E-06$
$8.871 E-06$ $8.871 E-06$
$1.153 E-05$ $1.153 \mathrm{E}-05$ $1.554 \mathrm{E}-05$ $2.209 E-05$ $1.592 \mathrm{E}-04$ $9.720 \mathrm{E}-05$ $1.822 \mathrm{E}-04$ $9.802 \mathrm{E}-04$ 2.014 E-03 $4.106 E-05$ $3.705 E-06$
$4.146 E-05$ 1.164E-05 $2.020 \mathrm{E}-05$ $5.707 E-06$ $6.420 \mathrm{E}-06$
$7.461 \mathrm{E}-06$ $7.461 \mathrm{E}-06$
$8.937 \mathrm{E}-06$ $8.937 E-06$
$1.161 E-05$ $1.564 \mathrm{E}-05$ $1.564 \mathrm{E}-05$ $1.564 \mathrm{E}-05$
$2.222 \mathrm{E}-05$ $1.598 E-04$ $9.748 E-05$ 1. $824 \mathrm{E}-04$ $9.814 E-04$ $2.016 E-03$ .041E-05 $5.792 \mathrm{E}-08$ $6.516 E-06$ 7.5
$9.01 \mathrm{E}-06$ $9.065 \mathrm{E}-06$ $9.065 \mathrm{E}-06$
$1.177 \mathrm{E}-05$ $1.177 E-05$ $1.585 \mathrm{E}-05$ $2.249 E-05$ $1.610 \mathrm{E}-04$ $9.803 E-05$ $1.827 E-04$ $9.837 E-04$ ANGLE 10 ANGLE 11 $\begin{array}{ccc}A N G L E ~ 12 & \text { ANGLE 13 } & \text { ANGLE } 14 \\ M U=0.4580 & M U=0.6179 & M U=0.7550\end{array}$ $2.020 E-03$ 1. $818 \mathrm{E}-06$ $3.758 \mathrm{E}-06$ 作E-05 $1.182 E-05$ $2.071 \mathrm{E}-05$
$5.918 \mathrm{E}-06$ $6.656 \mathrm{E}-06$ $6.656 E-06$
$7.730 E-06$ $7.730 E-06$
$9.252 E-06$ $9.252 \mathrm{E}-06$
$1.201 \mathrm{E}-05$ $1.615 E-05$ $1.615 \mathrm{E}-05$ 1. $827 \mathrm{E}-40$ $1.626 \mathrm{E}-04$ $9.881 E-05$ $1.832 \mathrm{E}-04$ $9.870 E-04$ $\begin{aligned} & M U=-0.6179 \\ & 1.851 E-06\end{aligned}$ $4.279 E-05$ 1.27 $2.109 E-05$ $6.082 \mathrm{E}-06$ $6.837 E-06$ $7.937 \mathrm{E}-06$ $7.937 E-06$ $9.494 \mathrm{E}-06$
$1.231 \mathrm{E}-05$ $1.653 \mathrm{E}-05$ $1.653 E-05$ 4.977E-40 $1.647 E-04$ $9.979 E-05$ $1.839 E-04$ $9.912 E-04$
$2.031 E-03$ $=-0.4580$ $1.892 \mathrm{E}-06$ $4.370 E-05$ 1. $35 \mathrm{E}-05$ . $6.280 E-06$ $7.057 E-06$ $8.188 \mathrm{E}-06$ $9.786 \mathrm{E}-06$ $1.267 \mathrm{E}-05$ $1.698 \mathrm{E}-05$ $1.698 \mathrm{E}-05$ $8.686 \mathrm{E}-40$ $8.686 E-40$
$1.672 E-04$ $1.009 \mathrm{E}-04$ $1.846 \mathrm{E}-04$ $9.960 \mathrm{E}-04$ 2.038E-03 .2816 $2.048 \mathrm{E}-06$ $4.230 E-06$ .714E-05 $1.344 E-05$ $7.041 E-06$ $7.897 \mathrm{E}-06$ $9.140 \mathrm{E}-06$ $1.089 E-05$ $1.403 \mathrm{E}-05$ $1.867 \mathrm{E}-05$ $1.867 \mathrm{E}-05$ $2.605 E-05$ $1.759 \mathrm{E}-04$ $1.049 \mathrm{E}-04$ $1.874 \mathrm{E}-04$ $1.013 \mathrm{E}-03$ 2.107F-06 4. 349E-06 4.842E-05 $1.385 E-05$ $2.400 \mathrm{E}-05$ $7.328 \mathrm{E}-06$ $8.213 E-06$ $9.497 E-06$ $1.130 E-05$ $1.453 E-05$ 1. $928 \mathrm{E}-05$ $1.928 \mathrm{E}-05$ $2.680 \mathrm{E}-05$ $1.789 E-04$ $1.062 \mathrm{E}-04$ 1. $884 \mathrm{E}-04$ $1.019 E-03$ $2.073 E-03$ ANGLE 15 $4.968 \mathrm{E}-05$ $1.425 E-05$ $2.466 \mathrm{E}-05$ $7.610 \mathrm{E}-06$ $8.524 E-06$ $9.847 \mathrm{E}-06$ $1.170 E-05$ $1.501 \mathrm{E}-05$ $1.987 \mathrm{E}-05$ $1.987 \mathrm{E}-05$ $2.751 \mathrm{E}-05$ $1.818 \mathrm{E}-04$ $1.075 \mathrm{E}-04$ $1.894 E-04$ $1.025 \mathrm{E}-03$ $2.081 E-03$ $2.217 E-06 \quad 2.264 E-06$ $4.574 E-06$ $1.462 \mathrm{E}-05$ $2.525 \mathrm{E}-05$
$7.871 \mathrm{E}-06$ $8.810 \mathrm{E}-06$ $1.017 \mathrm{E}-05$ $1.207 \mathrm{E}-05$ $1.545 \mathrm{E}-05$ $2.040 \mathrm{E}-05$ $2.040 E-05$ $2.815 \mathrm{E}-05$ $1.843 E-04$ $1.086 \mathrm{E}-04$ $1.902 \mathrm{E}-04$ $1.030 \mathrm{E}-03$ 2.088E-03 4.757E-06 $2.580 E-05$ .109E-06 $9.069 E-06$ $1.046 E-05$ $1.240 E-05$ $2.087 E-05$ 2.087E-05 $2.871 E-05$ $1.866 \mathrm{E}-04$ $1.096 \mathrm{E}-04$ $1.910 \mathrm{E}-04$ $1.034 \mathrm{E}-\mathrm{O}$ 2. $095 E-03$

ANGLE 16 $M U=0.9446$ $4.813 E-06$ $5.341 E-05$ $5.281 E-05$ $1.525 E-05$ $2.628 \mathrm{E}-05$ $8.318 E-06$ $1.071 E-05$ $1.269 E-05$ 2.128E-05 $2.128 \mathrm{E}-05$ $2.920 \mathrm{E}-05$ $1.885 \mathrm{E}-04$ $1.104 E-04$ $1.916 \mathrm{E}-04$ $1.038 E-03$ 2. $100 E-03$

ANGLE 8 $M U=-0.2816$
$1.940 E-06$ $4.008 \mathrm{E}-06$ $4.474 E-05$ $.268 \mathrm{E}-05$ $6.510 E-06$ $7.311 \mathrm{E}-06$ $8.476 \mathrm{E}-06$ $1.012 \mathrm{E}-05$ $1.309 \mathrm{E}-05$ $1.750 E-05$ $1.750 E-05$ $2.460 E-05$ $1.699 \mathrm{E}-04$ $1.022 E-04$ $1.855 E-04$ 2.046 E-03

ANGLE 17 $M U=0.9894$ $4.811 \mathrm{E}-06$ $5.340 \mathrm{E}-05$ $1.543 \mathrm{E}-05$ $2.658 \mathrm{E}-05$ $8.445 E-06$ $9.439 E-06$ $1.087 E-05$ $1.288 \mathrm{E}-05$ $1.642 \mathrm{E}-05$ $2.155 \mathrm{E}-05$ $2.155 \mathrm{E}-05$ $2.952 E-05$ $1.897 \mathrm{E}-04$ $1.110 \mathrm{E}-04$ 1.920E-04 $1.041 E-03$
$2.105 E-03$

ANGLE 9 0.0950 . $992 E-06$ $4.115 E-06$ $1.304 E-05$ 2.270E-05 $6.765 E-06$ $7.594 \mathrm{E}-06$ $8.797 \mathrm{E}-06$ $1.049 \mathrm{E}-05$ $1.049 E-05$
$1.354 E-05$ $1.354 E-05$
$1.807 E-05$ $1.807 E-05$ 2.531E-05 $.728 E-04$ $1.035 E-04$ 1. $864 \mathrm{E}-04$ 1. $007 E-03$ 2. 055E-03

SCALAR FLUX $5.274 E-05$ $5.878 \mathrm{E}-04$ $1.675 \mathrm{E}-04$ 2. $910 E-04$ $8.755 E-05$ $9.819 E-05$ $1.136 E-04$ $1.354 \mathrm{E}-04$ 1.743E-04 . $319 E-04$ 
(GAMMAS/MEV/STERADIAN/SOURCE NEUTRON)

ENERGY GROUP (MEV) 8. OOE $30--1.00 E$ OI 6.5 CE $00---8.00 E$ EO 5.OOE OO- - 6.50E DO 4.OOE OO---5.0OE OO 2.5OE $00--3.00 E$ OD

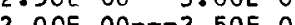
1.66E $00-12$. 00 E $1.33 \mathrm{E} 00-1-1.66 \mathrm{E} O 0$ $1.00 E$ OO- $1.33 E$ OO $8.00 E-01--1.00 E 00$ $6.00 E-01--8.00 E-01$ $4.00 E-01--6.00 E-01$ 3.00E-01-- 4 . OOE -01 $2.00 E-01-3.00 E-01$ $1.00 E-01-2.00 E-01$ 5. $00 E-02--1.00 E-01$
$2.00 E-02--5.00 E-02$

ENERGY$$
\text { GROUP (MEV) }
$$

8.00E OO---1.00E 6.5OE DO---8.COE OO 5.00 E $00---6.50 E$ CO $4.00 E$ OO- $-5.00 E$ OO 3.00 E OD- $-4.00 E$ OO $2.50 E$ OO-- -3. COE OO $2.00 E$ DO---2.50E 00 $1.66 \mathrm{E}$ OO- $-2.00 \mathrm{E}$ Oก $1.33 \mathrm{E} \quad 00---1.66 \mathrm{E} 00$ 1.00 E $00--1.33 \mathrm{E}$ OO $8.00 E-01--1.00 E$ OD $6.00 E-01--8.00 E-01$ $4.00 E-01---6.00 E-01$ $3.00 E-01--4.00 E-0$ $2.00 E-01--3.00 E-C$ $1.00 E-01--2.00 E-O 1$ $5.00 E-02---1.00 E-01$
$2.00 E-02-1-5.00 E-02$
ANGLE 1 ANGLE 2 $.718 \mathrm{E}-06$ $1.184 \mathrm{E}-05$ . 6.583E-05 $1.745 E-05$ $1.745 E-05$ 1.969E-05 $2.766 E-05$ $3.623 E-05$ $3.623 E-05$ $7.136 \mathrm{E}-05$ $2.734 E-04$ $2.734 E-04$
$3.420 E-04$ $6.740 E-04$ $1.811 \mathrm{E}-03$ $4.524 \mathrm{E}-03$ ANGLE 10 $M U=0.0950$ $7.577 \mathrm{E}-06$ $1.564 E-05$ $4.741 E-04$ $4.984 E-05$ $8.640 E-05$
$2.650 E-05$ 2.65E-05 $3.450 E-05$ $4.119 E-05$ $5.323 E-05$ $7.107 \mathrm{E}-05$ $9.951 \mathrm{E}-05$ $3.334 E-04$ $3.994 E-04$ $7.100 E-04$ $1.932 \mathrm{E}-03$ 4. $744 \mathrm{E}-03$
$1.268 \mathrm{E}-03$
ANGLE 2 ANGLE 3 $5.722 E-06$ $1.187 \mathrm{E}-05$ $3.603 E-05$ $0.527 E-05$ 1.751E-05 1. $2.307 E-05$ $2.776 \mathrm{E}-05$ $3.636 \mathrm{E}-05$ $4.954 \mathrm{E}-05$ $7.159 \mathrm{E}-05$ $2.740 E-04$ $2.740 E-04$
$3.426 E-04$ $6.743 E-04$ $1.812 \mathrm{E}-03$ $4.526 E-03$ 1.242E-03 ANGLE 11 $M U=0.2816$ $8.008 \mathrm{E}-06$
$1.652 \mathrm{E}-05$ 5.287E-05 $9.130 \mathrm{E}-05$ $2.867 E-05$ $3.215 E-05$ $3.720 E-05$ $4.431 E-05$ $5.701 E-05$ $7.569 E-05$ 1. $052 \mathrm{E}-04$ $3.447 F-04$ $4.093 \mathrm{E}-04$ $7.175 E-04$ $1.955 \mathrm{E}-03$ $4.784 \mathrm{E}-03$
$1.272 \mathrm{E}-03$ ANGLE 12 $1.935 E-04$
$\begin{array}{lll}A N G L E & 3 & \text { ANGLE } \quad 4 \\ U=-0.9446 & M U=-0.8656\end{array}$ $1.198 \mathrm{E}-05$ $1.345 E-04$ $6.71 E-05$ $1.777 E-05$ $1.777 E-05$ $2.340 E-05$ $2.816 \mathrm{E}-05$ $3.687 E-05$ $5.021 E-05$ $7.251 E-05$ $2.7 \in 0 \mathrm{E}-04$ $2.7 \in 0 E-04$
$3.447 F-04$ $6.754 E-04$ $1.817 E-03$ 4. $534 E-03$ 1. $243 \mathrm{E}-03$ $M U=0.4580$ $8.460 E-06$ $5.606 E-05$ $9.645 E-05$ $3.096 \mathrm{E}-05$ $3.467 \varepsilon-05$ $4.003 E-05$ 4.755E-05 $6.090 E-05$ $8.037 \mathrm{E}-05$ $1.107 E-04$ $3.557 E-04$ $4.189 E-04$ $7.251 \mathrm{E}-04$ $1.976 E-03$ $4.823 E-03$ $1.277 E-03$ $5.892 \mathrm{E}-06$ $1.220 E-05$ 3.805E-05 $6.712 \mathrm{E}-05$ 1.827E-05 2.062E-05 $2.893 E-05$ $3.786 E-05$ $5.152 E-05$ $7.428 \mathrm{E}-05$ $7.428 E-05$
$2.800 E-04$ $2.800 E-04$
$3.489 E-04$ $6.776 E-04$ $1.825 E-03$ $4.549 E-03$ ANGLE 13 $M U=0.6179$ $8.915 E-06$ $5.927 E-05$ $1.016 E-04$ 3. $328 \mathrm{E}-05$ $3.720 \mathrm{E}-05$ $4.286 E-05$ $5.076 E-05$ $6.471 E-05$ 1.487E-05 $1.160 \mathrm{E}-04$ . 4. $277 \varepsilon-04$ 1. 1.997E-03 1.281E-03
ANGLE 5 MU $=-0.6179$ $1.252 E-05$ $1.403 E-04$ $3.92 E-05$ $1.902 \mathrm{E}-05$ 2.145E-05 2.145E-05 $3.006 \mathrm{E}-05$ $3.931 \mathrm{E}-05$ $5.343 \mathrm{E}-05$ $7.684 \mathrm{E}-05$ $7.684 \mathrm{E}-05$
$2.858 \mathrm{E}-04$ $2.684 E-05$
$3.547 E-04$ $6.808 \mathrm{E}-04$ $1.836 \mathrm{E}-03$ $4.570 \mathrm{E}-\mathrm{C}$ $1.247 \mathrm{E}-03$ ANGLE 14 $M U=0.7550$ $9.335 \mathrm{E}-06$ $2.126 \mathrm{E}-04$ $6.225 E-05$ $3.544 \mathrm{E}-05$ $3.955 E-05$ 4. $546 E-05$ $5.369 \mathrm{E}-05$ $6.814 E-05$ 8. $888 \mathrm{E}-05$ 1. $207 \mathrm{E}-04$ $3.750 E-04$ 4. $353 E-04$ $7.388 \mathrm{E}-04$ $2.015 \mathrm{E}-03$ $1.284 E-03$ $6.513 E-06 \quad$ S. 0.2816 $1.347 E-05$ $1.507 E-04$ $4.239 E-05$ $7.425 E-05$ $2.125 E-05$ 2.394E-05 $2.786 \mathrm{E}-05$ 3.344E-05 $5.898 E-05$ $8.418 E-05$ $8.418 E-05$
$3.017 E-04$ $3.703 E-04$ $3.703 E-04$
$6.901 E-04$ $1.868 E-03$ $4.629 E-03$ $1.254 \mathrm{E}-03$ ANGLE 16 $\begin{aligned} & M U=0.9446 \\ & 9.976 E-06\end{aligned}$ $9.976 E-06$ $2.053 E-05$ $2.265 E-04$
$6.678 E-05$ $1.136 E-04$ $3.871 E-05$ 4.309E-05 $4.937 \mathrm{E}-05$ $5.806 E-05$ $7.320 E-05$ $9.468 E-05$ $1.273 E-04$ $3.876 \mathrm{E}-04$ $4.459 \mathrm{E}-04$ $7.481 E-04$ $2.040 E-03$ $4.933 E-03$ $1.289 \mathrm{E}-0$ ?

ANGLE 9 $M U=-0.0950$ $1.483 E-05$ $1.653 E-04$ $4.703 E-05$ ..183E-05 $2.755 E-05$ 2.755E-05 $3.828 E-05$ $4.964 E-05$ $6.663 E-05$ $9.397 E-05$ $3.222 E-04$ 3. $893 \mathrm{E}-04$ $7.027 E-04$ 1. $909 E-03$ 4. $704 \mathrm{E}-03$ SCALAR FLUX $1.961 \varepsilon-04$ $2.182 E-03$ $6.251 E-04$ 


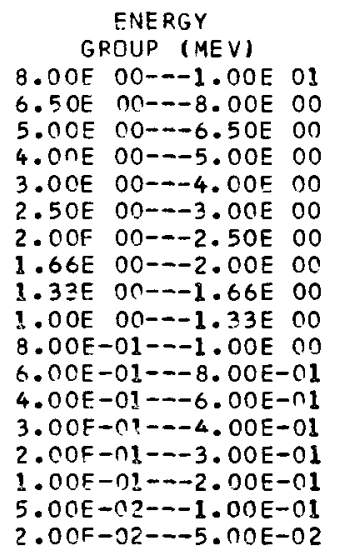

ENERG $Y$$$
\text { GROUP (MEV) }
$$

8. OOE OO---1.00E O1 6.5OE O0- - $8.00 E$ OO 5. COE OO--6.50E OO 4.00E OO---5. OOE OO 3.0 E OO--4. 0 E 0 2.00 OO- 2 . 50 OE 00 $1.66 \mathrm{~F}$ 00-0-2.00E 00 1.33 E $00--1.66$ E 00 $1.00 E$ OO-W-1.33E 00 $8.00 E-01-1.00 E 00$ $6.00 E-01--8.00 E-01$ 4. OCE-01 - $-6.00 E-01$ $3.00 E-01--4.00 E-n\}$ $2.00 E-01-2.00 E-01$ $1.00 E-01-2.00 E-01$ 2. COE $-02--5.00 E-02$

(1)

ANGLE 2 $8.678 \mathrm{E}-06$ $1.708 E-05$ $2.022 E-04$ $5.567 E-05$ $0.879 E-05$ $2.577 \mathrm{~F}-05$ $2.915 E-05$ $2.915 E-05$
$3.410 E-05$ $4.115 E-C 5$ $5.413 E-05$ $7.421 \mathrm{E}-05$ $1.083 \mathrm{~F}-04$ $4.31 .5 E-C 4$ $5.484 \mathrm{~F}-\mathrm{C} 4$ $1.118 \mathrm{E}-03$ $3.007 E-03$ $7.581 \mathrm{E}-03$ $2.097 \mathrm{E}-03$ $2.097 E-03$ ANGLE 10 ANGLE 11 $1.238 \mathrm{E}-05$
$2.555 \mathrm{E}-05$ $2.842 E-04$ $8.160 E-C .5$ $1.412 E-04$ $4.391 E-05$ $4.938 E-C 5$ 5.735E-05 6.863 E-OS $8.900 \mathrm{E}-05$ $1.193 \mathrm{E}-04$ $1.678 \mathrm{E}-04$ $5.593 E-04$ -.734E-04 103 . $2.154 E-03$

$1.333 E-05$
$2.749 F-05$ $3.050 \mathrm{E}-04$ $8.829 E-C 5$ $1.520 E-04$ $4.877 E-05$ $5.475 E-05$ $7.567 E-C 5$ 5.844E-04 $6.953 E-04$ 1. $110 \mathrm{E}-03$ 3. $117 E-03$ $8.151 E-03$
(GAMMAS/MEV/STERADIAN/SOURCE NEUTRON)

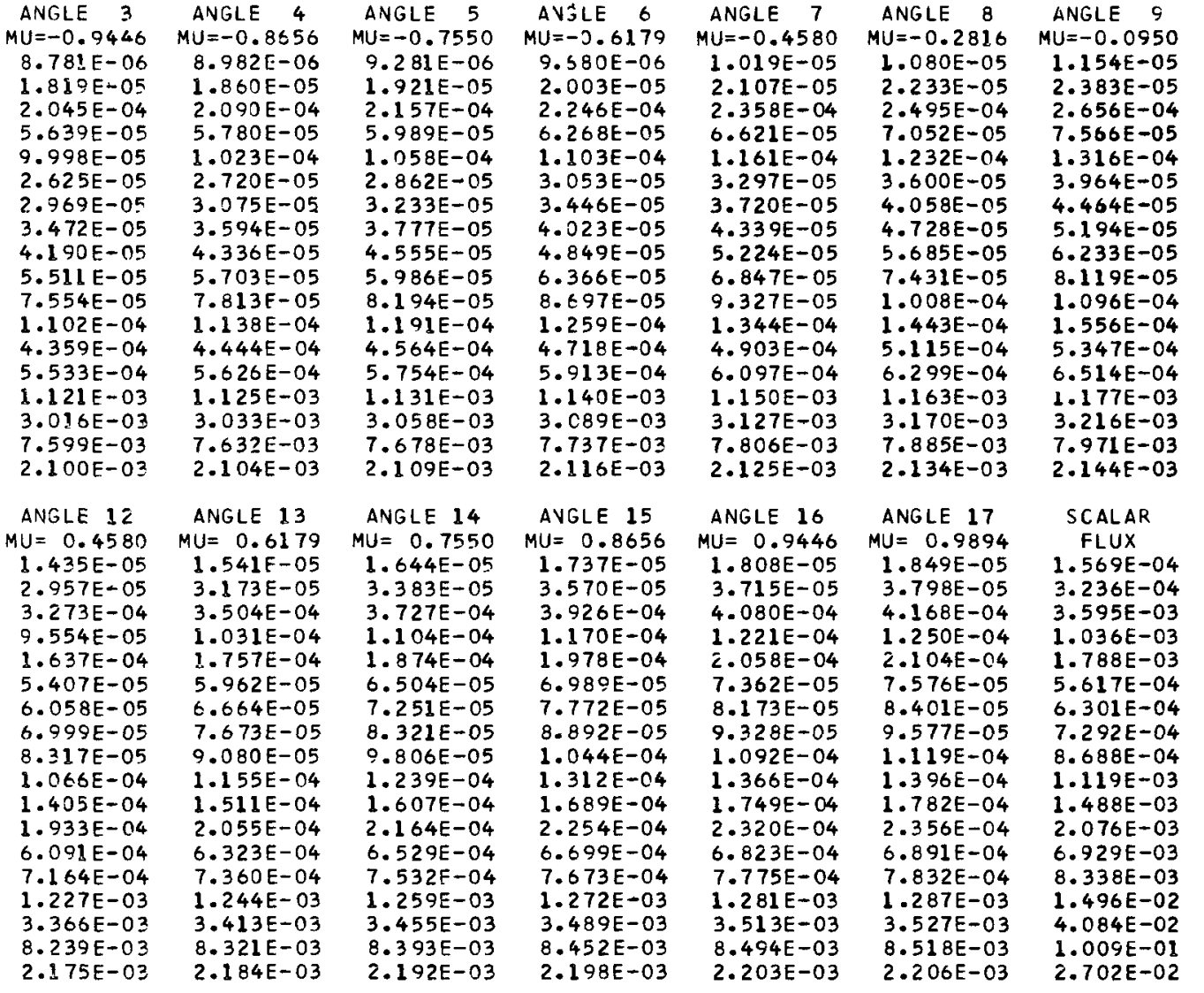
$2.175 E-03$ 
(GAMMAS/MEV/STERADIAN/SOURCE NEUTRON)

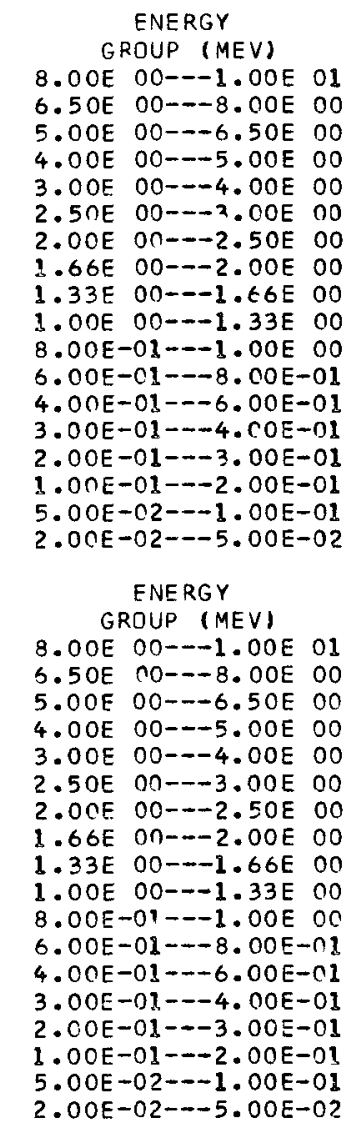

ANGLE 1 $U=-1.0000$ $1.348 E-05$
$2.795 E-05$ $3.154 \mathrm{E}-04$ $8.576 \mathrm{E}-05$ 3.805E-05 $4.328 \mathrm{E}-05$ $5.090 \mathrm{E}-05$ $6.173 E-05$ $8.168 \mathrm{E}-05$ $1.130 \mathrm{E}-04$ $1.680 \mathrm{E}-04$ $7.279 \mathrm{E}-04$ $9.556 E-04$ $2.110 \mathrm{E}-03$ $5.676 \mathrm{E}-03$ $1.462 \mathrm{E}-02$ $4.112 \mathrm{E}-03$ ANGLE 10 MU $=0.0950$ $2.202 E-05$ $4.542 E-05$ $5.043 E-04$ $1.459 \mathrm{E}-04$ $2.516 E-04$ $8.071 E-05$ $9.114 E-05$ $1.064 E-04$ $1.282 E-04$ $1.679 E-04$ $2.277 E-04$ 1.070E-03 1.070E-03 $1.305 E-03$ . 1. $597 \mathrm{E}-02$ $1.597 E-02$
ANGLE 2 $M U=-C .9894$ $1.354 \mathrm{E}-05$ $3.165 \mathrm{E}-04$ $8.614 \mathrm{E}-05$ $1.540 \mathrm{E}-04$ $3.829 \mathrm{E}-05$ $4.355 E-05$ $5.120 E-05$ $6.209 E-05$ $8.218 E-05$ $1.138 E-04$ $1.691 E-04$ $9.506 E-04$ $2.11 \mathrm{E}-03$ $5.682 E-03$ $1.682 E-03$ $1.464 E-02$
$4.113 E-03$ ANGLE 11 $M U=0.2816$ $2.454 E-05$ $5.056 \mathrm{E}-05$ $5.591 E-04$ $1.638 \varepsilon-04$ $2.804 E-04$ $9.420 E-05$ $1.062 \mathrm{E}-04$ . $481 \mathrm{E}-04$ $1.425 E-04$ . $1.140 E-03$ $1.365 E-03$ 2. $341 \mathrm{E}-03$ . $528 E-03$ 4. $303 E-03$ $\begin{array}{lclll}A N G L E & 3 & \text { ANGLE } 4 & \text { ANGLE } 5 \\ M U=-0.9446 & M U=-0.8656 & M U=-0.7550\end{array}$ $2.850 E-05$ $3.929 \mathrm{E}-05$ $4.463 E-05$ $5.245 E-05$ $6.360 E-05$ $8.425 \mathrm{E}-05$ $1.167 E-04$ 1.735E-04 $7.417 E-04$ $9.733 E-04$ $2.116 \mathrm{E}-03$ $5.706 \mathrm{E}-03$ $1.468 \mathrm{E}-02$
$4.119 \mathrm{~F}-03$ ANGLE 12 $M U=0.4580$ 2.745E-05 $5.648 \mathrm{E}-05$ $6.222 E-04$ $1.846 E-04$ $3.135 \mathrm{E}-04$ $1.101 E-04$ $1.733 E-04$ . 1. $210 \mathrm{E}-03$ $1.423 E-03$ $2.392 E-03$ 1. $6478-02$ $1.643 E-02$ $M U=-0.8656 \quad M U$ $2.936 \mathrm{E}-05$ $3.307 E-04$ $9.062 E-05$ $1.613 \mathrm{E}-04$ $4.125 E-05$ $4.680 E-05$ 5.494E-05 $8.836 E-05$ $1.226 E-04$ $7.631 E-04$ $9.998 \mathrm{E}-04$ $2.125 E-03$
$5.752 E-03$ 1. $477 \mathrm{E}-02$ $4.130 \mathrm{E}-03$ ANGLE 13 MU $=0.6179$ 3.069E-05 $6.305 E-05$ $6.917 E-04$ 2.079E-04 1. $1.650 E-04$ $3.232 E-04$ $4.36 ? E-04$ $1.479 \mathrm{E}-03$ $2.442 E-03$ $1.811 E-03$ $4.359 E-03$
ANGLE 5 MU $=-0.6179$ $3.064 E-05$ $3.447 E-04$ $1.686 \mathrm{E}-04$ $4.420 E-05$ $5.008 E-05$ $5.875 \mathrm{E}-05$ $7.124 \mathrm{E}-05$ $9.456 \mathrm{E}-05$ $1.312 \mathrm{E}-04$ $1.947 E-04$ $1.036 \mathrm{E}-03$ $2.036 \mathrm{E}-03$ $5.816 \mathrm{E}-03$ $1.490 E-02$ $4.145 \mathrm{E}-03$ ANGLE 14 $M U=0.7550$
MU $3.407 E-05$
$6.990 E-05$ $7.641 \mathrm{E}-04$ $2.323 E-04$ $3.883 E-04$ $1.871 E-04$ 2.198E-04 3. $541 E-04$ 3.541E-04 4. $697 \mathrm{E}-04$ $1.337 E-03$ $1.528 E-03$ $2.48 \mathrm{E}-03$ $1.63 \mathrm{E}-03$ $4.383 \mathrm{E}-03$

\begin{tabular}{|c|c|c|c|}
\hline $\begin{array}{l}\text { ANGLE } 6 \\
M U=-0.6179 \\
1.564 E-05 \\
3.239 E-05 \\
3.637 E-04 \\
1.010 E-04 \\
1.784 E-04 \\
4.828 E-05 \\
5.466 E-05 \\
6.408 E-05 \\
7.770 E-05 \\
1.031 E-04 \\
1.430 E-04 \\
2.116 E-04 \\
8.336 E-04 \\
1.081 E-03 \\
2.158 E-03 \\
5.399 E-03 \\
1.506 E-02 \\
4.165 E-03\end{array}$ & $\begin{array}{r}\text { ANGLE } 7 \\
M U=-0.4580 \\
1.675 E-05 \\
3.466 \mathrm{E}-05 \\
3.884 \mathrm{E}-04 \\
1.087 \mathrm{E}-04 \\
1.912 \mathrm{E}-04 \\
5.369 \mathrm{E}-05 \\
6.076 \mathrm{E}-05 \\
7.121 \mathrm{E}-05 \\
8.630 \mathrm{E}-05 \\
1.144 \mathrm{E}-04 \\
1.583 \mathrm{E}-04 \\
2.331 \mathrm{E}-04 \\
8.823 \mathrm{E}-04 \\
1.132 \mathrm{E}-03 \\
2.183 \mathrm{E}-03 \\
6.000 \mathrm{E}-03 \\
1.525 \mathrm{E}-02 \\
4.188 \mathrm{E}-03\end{array}$ & $\begin{array}{r}\text { ANGLE } 8 \\
M U=-0.2816 \\
1.816 \mathrm{E}-05 \\
3.754 \mathrm{E}-05 \\
4.195 \mathrm{E}-04 \\
1.186 \mathrm{E}-04 \\
2.073 \mathrm{E}-04 \\
6.070 \mathrm{E}-05 \\
6.868 \mathrm{E}-05 \\
8.043 \mathrm{E}-05 \\
9.738 \mathrm{E}-05 \\
1.288 \mathrm{E}-04 \\
1.775 \mathrm{E}-04 \\
2.592 \mathrm{E}-04 \\
9.389 \mathrm{E}-04 \\
1.187 \mathrm{E}-03 \\
2.214 \mathrm{E}-03 \\
6.116 \mathrm{E}-03 \\
1.547 \mathrm{E}-02 \\
4.214 \mathrm{E}-03\end{array}$ & $\begin{array}{r}\text { ANGLE } 9 \\
M U=-0.0950 \\
1.990 E-05 \\
4.110 E-05 \\
4.579 E-04 \\
1.308 E-04 \\
2.273 E-04 \\
6.961 E-05 \\
7.870 E-05 \\
9.207 E-05 \\
1.113 E-04 \\
1.466 E-04 \\
2.007 E-04 \\
2.898 E-04 \\
1.002 E-03 \\
1.246 E-03 \\
2.251 E-03 \\
6.245 E-03 \\
1.571 E-02 \\
4.243 E-03\end{array}$ \\
\hline $\begin{array}{l}\text { ANGLE 15 } \\
\text { MU= } 0.8656 \\
3.731 E-05 \\
7.646 \mathrm{E}-05 \\
8.330 \mathrm{E}-04 \\
2.557 \mathrm{E}-04 \\
4.247 \mathrm{E}-04 \\
1.647 \mathrm{E}-04 \\
1.825 \mathrm{E}-04 \\
2.077 \mathrm{E}-04 \\
2.422 \mathrm{E}-04 \\
3.006 \mathrm{E}-04 \\
3.808 \mathrm{E}-04 \\
4.978 \mathrm{E}-04 \\
1.388 \mathrm{E}-03 \\
1.570 \mathrm{E}-03 \\
2.530 \mathrm{E}-03 \\
7.040 \mathrm{E}-03 \\
1.713 \mathrm{E}-02 \\
4.402 \mathrm{E}-03\end{array}$ & $\begin{array}{r}\text { ANGLE } 16 \\
M U=0.9446 \\
4.000 E-05 \\
8.189 E-05 \\
8.900 E-04 \\
2.751 E-04 \\
4.545 E-04 \\
1.795 E-04 \\
1.981 E-04 \\
2.243 E-04 \\
2.595 E-04 \\
3.197 E-04 \\
4.010 E-04 \\
5.185 E-04 \\
1.426 E-03 \\
1.601 E-03 \\
2.561 E-03 \\
7.117 E-03 \\
1.726 E-02 \\
4.416 E-03\end{array}$ & $\begin{array}{r}\text { ANGLE } 17 \\
\text { MU=0 } 17.9894 \\
4.166 E-05 \\
8.525 E-05 \\
9.251 E-04 \\
2.871 E-04 \\
4.730 E-04 \\
1.886 E-04 \\
2.076 E-04 \\
2.343 E-04 \\
2.705 E-04 \\
3.310 E-04 \\
4.127 E-04 \\
5.304 E-04 \\
1.448 E-03 \\
1.620 E-03 \\
2.579 E-03 \\
7.162 E-03 \\
1.734 E-02 \\
4.424 E-03\end{array}$ & $\begin{array}{c}\text { SCALAR } \\
\text { FLUX } \\
2.889 E-04 \\
5.952 E-04 \\
6.584 E-03 \\
1.924 E-03 \\
3.295 E-03 \\
1.093 E-03 \\
1.227 E-03 \\
1.420 E-03 \\
1.692 E-03 \\
2.180 E-03 \\
2.898 E-03 \\
4.041 E-03 \\
1.323 E-02 \\
1.608 E-02 \\
2.886 E-02 \\
7.979 E-02 \\
1.997 E-01 \\
5.355 E-02\end{array}$ \\
\hline
\end{tabular}


(GAMMAS/MEV/STERADIAN/SOURCE NEUTRONI

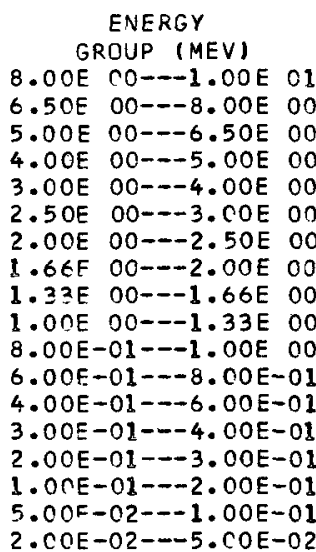

ENERGY

GROUP (MEV)

8. OCE OO- -1.0OE OI

6.5 CF 00---8.00E 00 5. COE OO- - - 5.5OE OO $4.00 E$ OO- --5. COE OD $3.00 E$ OO---4. COE 00 $2.5 O E$ OC-D-3.OOE OO 2. OOE OO---2.5OE OO $1.66 E$ OO---2.00E OO $1.33 \mathrm{E} \quad 00--1.66 \mathrm{E} 00$ $1.00 E$ OO- $-1.33 E$ OO $8.00 E-01=-1.00 E$ OO $6.00 E-01--1.00 E-01$ 4. $00 \mathrm{E}-01---6.00 \mathrm{E}-01$ $3.00 E-01--4.00 E-01$ $2.00 E-01---3.00 E-01$ $1.00 E-01---2.00 E-01$ $5.00 E-12-1.00 E-C 1$ $2.00 E-02---5.00 E-02$
ANGLE 1 $M U=-1.0000$ $1.578 \mathrm{E}-05$ $3.273 \mathrm{E}-0$ $9.957 E-05$ $1.792 \mathrm{E}-04$ $4.279 E-05$ $4.279 E-05$ $5.811 E-05$ $5.811 E-05$
$7.076 \mathrm{E}-05$ $7.076 E-05$ $1.305 E-04$ $1.305 \mathrm{E}-04$ $1.968 \mathrm{E}-04$
$9.284 \mathrm{E}-04$ $1.256 \mathrm{E}-03$ $3.016 \mathrm{E}-03$ $8.123 \mathrm{E}-\mathrm{C} 3$ $2.141 \mathrm{E}-02$ $6.106 \mathrm{E}-03$

ANGLE 2 $M U=-0.9894$ $1.586 E-C 5$ 3.28 1.71 1.001E-04 1.313E-05 $4.943 E-05$ $5.849 E-05$ $7.121 \mathrm{E}-05$ $9.453 \mathrm{E}-05$ $9.453 E-05$
$1.315 E-04$ $1.315 E-04$ $1.585 \mathrm{E}-04$
$9.329 \mathrm{E}-\mathrm{C} 4$ $1.263 \mathrm{E}-03$ $3.018 \mathrm{E}-\mathrm{C}$ $8.134 E-03$ $2.143 E-02$ $6.109 E-C^{-3}$

$\triangle N G L E \quad 3$ $M U=-0.0446$ $1.615 \mathrm{E}-05$ 3. 1. $023 E-04$ $1.837 \mathrm{E}-04$ $4.455 E-05$ $5.089 \mathrm{E}-05$ $6.010 E-05$ $7.318 \mathrm{E}-05$ $9.741 E-05$ $9.741 E-05$
$1.360 \mathrm{E}-04$ $1.360 \mathrm{E}-04$ $2.056 \mathrm{E}-04$
$9.515 \mathrm{E}-04$ 9.
$1.290 \mathrm{E}-03$ $3.025 \mathrm{E}-03$ $8.176 E-03$ $2.151 \mathrm{E}-02$ 6.1.20E-03 ANGLE 10 $U=0.0950$ $2.873 E-C 5$
$5.922 E-05$
$0.5612-04$ $6.561 E-04$ $1.913 \mathrm{E}-04$ $3.288 \mathrm{E}-04$ $1.091 \mathrm{E}-04$ $1.239 E-04$ $1.457 \mathrm{E}-04$ $1.771 \mathrm{E}-04$ $2.352 \mathrm{E}-04$ $3.241 E-04$ 4.699E-04 $1.534 E-03$ $1.902 E-03$ $3.315 E-03$ $9.402 E-C 3$ $2.391 \mathrm{E}-02$ $6.408 \mathrm{E}-03$
ANGLE 11 ANGLE 12 $M U=0.4580$ $3.838 E-05$ $7.882 E-05$ $8.638 E-04$ $2.609 E-04$ $4.301 \mathrm{E}-04$ $1.642 \mathrm{E}-04$ $1.853 \mathrm{E}-04$ $2.159 E-04$ $2.593 \mathrm{E}-04$ $3.358 \mathrm{E}-04$ $4.468 E-04$ $6.160 \mathrm{E}-04$ $1.794 \mathrm{E}-03$ $2.115 \mathrm{E}-03$ $3.497 E-03$ $9.951 \mathrm{E}-0$ $2.492 E-O$ $6.522 E-03$ $6.466 \mathrm{E}-\mathrm{O}$ $1.674 E-05$ . $471 \mathrm{E}-0$ $.066 \mathrm{E}-04$ $1.906 E-04$ 4.732E-05 $5.384 \mathrm{E}-0$ $6.343 E-05$ $1.725 \mathrm{E}-05$ $1.032 \mathrm{E}-04$ $1.448 E-04$ $2.192 \mathrm{E}-04$
$9.873 \mathrm{E}-04$ $1.341 \mathrm{E}-03$ $3.038 \mathrm{E}-03$ $3.038 \mathrm{E}-03$
$8.256 \mathrm{E}-0$ $2.168 \mathrm{E}-02$ $6.140 \mathrm{E}-03$ ANGLE 13 $M U=0.617$ $4.469 E-05$ $9.160 \mathrm{E}-05$ . $980 E-04$ 5.069E-04 5.108E-04 $2.259 E-04$ $2.611 \mathrm{E}-04$ $3.099 E-04$ $3.949 E-04$ $5.138 \mathrm{E}-04$ $6.893 E-04$ 1.919t-03 $2.217 E-03$ $3.595 \mathrm{E}-03$ $1.022 \mathrm{E}-02$ $6.575 \mathrm{E}-03$
ANGLE 5

$5 \quad$ ANGLE 6 $1.761 \mathrm{E}-05$ 3.651 E 05 4.114E-04 1. 5. $5.842 \mathrm{E}-05$ $6.872 E-05$ $8.374 \mathrm{E}-05$ $1.1235-04$ $1.580 \mathrm{E}-04$ $2.394 \mathrm{E}-04$ $2.394 \mathrm{E}-04$
$1.039 \mathrm{E}-03$ $1.410 E-03$ 3. $059 \mathrm{E}-03$ $8.370 \mathrm{E}-0$ $2.190 E-02$ $6.169 E-03$ ANGLE 14 $U=0.7550$ $1.059 E-04$ $1.148 E-03$ $3.589 E-04$ $5.907 E-04$ $2.431 E-04$ . $709 E-04$ $3.637 E-04$ 4. 546E-04 $5.784 E-04$ $7.566 E-04$ 2. $035 E-03$ 2. $312 \mathrm{E}-03$ $3.690 \mathrm{E}-03$ $1.047 \mathrm{E}-0$ $2.583 \mathrm{E}-02$ $6.622 E-03$ $1.883 \mathrm{E}-05$ $4.305-04$ 1.31 E- 04 $1.214 E-04$
$2.149 E-04$ $5.732 E-05$ $6.501 \mathrm{E}-05$ $7.547 E-05$ $9.326 \mathrm{E}-05$ $1.252 \mathrm{E}-04$ $1.252 E-04$
$1.765 E-04$ $1.765 E-04$
$2.671 E-04$ $1.671 E-04$
$1.109 E-03$ $1.494 E-03$ $3.087 E-03$ $8.517 E-03$ $2.220 E-02$
$6.205 E-03$

ANGLE 7 $2.045 \mathrm{E}-05$ 1.327E-04 2.336E 04 $6.529 E-05$ $7.410 E-05$ $8.724 E-05$ $1.065 \mathrm{E}-04$ $1.429 \mathrm{E}-04$ $2.012 E-04$ $3.033 E-04$ $1.194 \mathrm{E}-03$ $1.589 \mathrm{E}-03$ $3.126 \mathrm{E}-03$ $8.697 E-03$ $2.255 E-02$ $6.248 E-03$ ANGLE 16 $\begin{array}{ll}A V G L E & 15 \\ U=0.8656\end{array}$ $5.512 E-05$ 1. $207 E-04$ $1.301 E-03$
$4.126 E-04$ $4.126 E-04$
$6.723 E-04$ $0.723 E-04$ $2.860 E-04$
$3.151 E-04$ 3. $581 E-04$ $4.148 E-04$ $5.092 E-04$ $8.133 E-04$ $2.134 E-03$ $2.394 E-03$ 3. $774 \mathrm{E}-03$ $1.068 E-02$ $2.619 \mathrm{E}-02$ $6.660 E-03$

$M U=0.9446$ $6.563 E-05$ $1.338 \mathrm{E}-04$ $1.436 E-03$ $4.603 E-04$

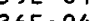
3.550E-04 $3.550 E-04$
$3.985 E-04$ $4.567 E-04$ $5.525 E-04$ $6.783 E-04$ $8.556 E-04$
ANGLE 8 ANGLE 9 $2.256 E-05$ 2.527E-05 $4.664 E-05 \quad 5.527 E-05$ $5.211 E-04 \quad 5.808 E-04$ $1.475 E-04 \quad 1.667 E-04$ 2.579E-04 $2.891 E-04$ $7.602 E-05 \quad 9.032 E-05$ $8.637 \mathrm{E}-05 \quad 1.026 \mathrm{E}-04$ $1.017 \mathrm{E}-04 \quad \mathrm{~L} 209 \mathrm{E}-04$ $1.241 E-04 \quad 1.473 E-04$ $1.665 \mathrm{E}-04 \quad 1.969 \mathrm{E}-04$ $2.334 E-04 \quad 2.743 E-04$ $3.492 E-04 \quad 4.051 E-04$ $1.296 E-03 \quad 1.410 E-03$ $1.690 \mathrm{E}-03 \quad 1.795 \mathrm{E}-03$ $3.176 \mathrm{E}-03 \quad 3.239 \mathrm{E}-03$ $8.907 E-03 \quad 9.143 E-03$ $2.296 E-02 \quad 2.342 E-02$ $6.298 E-03 \quad 6.352 E-03$ 
(GAMMAS/MEV/STERADIAN/SOURCE NEUTRONI

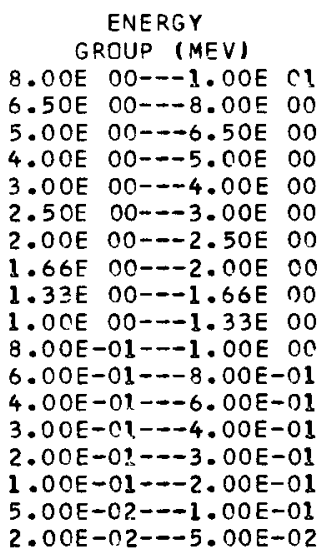

ENERGY GROUP (MEV)

8.00E OO---1. COE O1 $6.50 E$ OO- - - 8.00E 00 $5.00 F \quad 00---6.50 E \quad 00$ $4.00 E$ OC- $-5.00 E$ OO $3.00 E$ OO-- 4. $00 E$ OO $2.50 E$ OO- $0-3.00 E$ OO $2.00 E$ OO---2.50E OO $1.66 \mathrm{E} \quad 00--2.00 \mathrm{E} O 0$ $1.33 \mathrm{E} \quad 00--1.66 \mathrm{E} \quad 00$ $1.00 E$ ON- $-1.33 \mathrm{E}$ OO 8.00E-01---1.00E OO $6.00 \mathrm{E}-01--8.00 \mathrm{C}-01$ $4.00 E-01---6.00 E-01$ $3.00 E-01--4.00 E-01$ $2.00 \mathrm{E}-0 \mathrm{l}--3.00 \mathrm{E}-0$ $1.00 \mathrm{E}-01--2.00 \mathrm{E}-01$

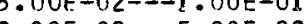
$2.00 E-02---5.00 E-02$

\begin{abstract}
ANGLE 1 $1.560 \mathrm{E}-05$
$3.234 \mathrm{E}-05$ $3.664 \mathrm{E}-04$ $9.740 \mathrm{E}-05$ $1.766 \mathrm{E}-04$ $4.083 \mathrm{E}-05$ 4.749E-05 $5.680 \mathrm{E}-05$ $0.943 E-05$ $9.182 \mathrm{E}-05$ $1.273 \mathrm{E}-04$ $1.273 E-04$ $1.944 \mathrm{E}-04$
$1.004 \mathrm{E}-03$ $1.004 E-03$ $3.664 \mathrm{E}-03$ $9.867 \mathrm{~F}-03$ $2.659 E-02$ $7.684 \mathrm{E}-03$
\end{abstract}

ANGLE 10 $M U=0.0950$ $3.106 E-05$ $7.073 E-04$ $2.079 \mathrm{E}-04$ $3.562 E-C 4$ $3.224 E-04$ $1.399 \mathrm{E}-04$ $1.658 \mathrm{E}-04$ $2.037 \mathrm{E}-04$ $2.753 E-04$ $3.871 \mathrm{E}-\mathrm{C} 4$ $5.740 E-04$ $1.856 \mathrm{E}-03$ $2.340 E-03$ $4.040 \mathrm{E}-03$ $1.169 E-02$ $3.027 E-02$
$8.131 E-03$
ANGLE 2 $M U=-0.9894$ $3.251 E-05$ $3.683 E-04$ 1.777E-04 $4.124 E-05$ $4.785 \mathrm{E}-\mathrm{C} 5$ $5.714 E-05$ $6.983 E-05$ $9.253 \mathrm{E}-\mathrm{C} 5$ $1.287 \mathrm{E}-04$ $1.966 E-04$ $1.010 \mathrm{E}-03$ $1.406 \mathrm{E}-03$ $3.666 \mathrm{E}-03$ $9.881 \mathrm{E}-03$ $2.662 \mathrm{E}-02$
$7.688 \mathrm{E}-03$ ANGLE 11 $M U=0.2816$ $3.680 \mathrm{E}-05$ $7.564 E-05$ $8.306 E-04$ $4.223 E-04$ $1.564 \mathrm{E}-04$ $1.785 \mathrm{E}-04$ $2.109 E-04$ $2.577 E-04$ $3.443 E-04$ 4. $752 \mathrm{E}-\mathrm{C} 4$ $6.831 E-04$ $2.044 E-03$ $2.489 \mathrm{E}-03$ $4.165 \mathrm{E}-03$ $1.210 \mathrm{E}-02$ $3.104 \mathrm{E}-02$ $8.218 \mathrm{E}-03$
ANGLE 3 ANGLE 4 1.601 E-05 $.321 E-05$ .759E-04 . $1.818 E-04$
$4.289 E-05$ $4.938 E-05$ $5.867 E-05$ $7.169 E-05$ $9.561 \mathrm{E}-05$ 1. $341 E-04$ $2.057 \mathrm{E}-04$ $1.034 \mathrm{E}-03$ $1.448 E-03$ $3.673 E-03$ $9.941 \mathrm{E}-03$ $2.675 \mathrm{E}-02$ $7.704 E-03$

ANGLE 12 $M U=0.4580$ $4.425 E-05$
$9.072 E-05$ $9.889 \mathrm{E}-04$ 3. $042 \mathrm{E}-04$ 5.375E-04 2.021E-04 $2.297 \mathrm{E}-04$ $2.697 E-04$ $3.265 E-04$ $4.283 E-04$ $5.757 E-04$ $7.982 E-04$ $2.232 E-03$ $2.638 \mathrm{E}-03$ $4.306 E-03$ $1.251 E-0$. $3.181 \mathrm{E}-02$ $8.304 E-03$ $3.457 E-05$ $1.898 \mathrm{E}-04$ $5.254 \mathrm{E}-05$ $6.205 E-05$ $1.020 \mathrm{E}-04$ $1.020 E-04$ $1.081 E-03$ $3.688 \mathrm{E}-03$ $1.005 \mathrm{E}-02$ $2.698 \mathrm{E}-02$ ANGLE 13 $.098 E-04$ $3.739 \mathrm{E}-04$ $6.150 E-04$ $2.611 \mathrm{E}-04$ $2.947 \mathrm{E}-04$ 4. $089 \mathrm{E}-04$ $9.120 E-04$ $2.415 E-03$ $2.786 \mathrm{E}-03$ $4.455 \mathrm{E}-03$ $1.292 \mathrm{E}-02$ $3.255 \mathrm{E}-02$ $8.385 E-03$
ANGLE 4 ANGLE 5 ANGLE 6 $\begin{array}{rrr}M U=-0.8656 & M U=-0.7550 & M U=-0.6179 \\ 1.667 E-05 & 1.765 E-05 & 1.902 E-05\end{array}$ 1. $765 \mathrm{E}-05$ 3.661E-05 $4.608 E-05$ $7.584 E-05$ $2.230 E-04$ $1.525 E-03$ $7.734 E-03$ $M U=0.6179$ 1.369E-05 $3.425 \mathrm{E}-04$ $5.237 E-04$ $6.826 E-04$
$4.128 E-04$
$1.130 E-04$

2. $014 E-04$ $2.014 E-04$
$5.080 E-05$ $5.760 E-05$ $6.785 E-05$ $6.785 E-05$
$8.302 E-05$ $8.302 E-05$ $1.125 E-04$
$1.607 E-04$ $2.486 \mathrm{E}-04$ $1.152 E-03$ $1.629 \mathrm{E}-03$ $3.710 E-03$ $1.021 E-02$ $2.731 E-02$ ANGLE 14 $M U=0.7550$ -. $509 E-05$ . $424 E-03$ $1.424 E-03$ $4.587 E-04$
$7.432 E-04$ $3.326 \mathrm{E}-04$ 3. $715 \varepsilon-04$ 4. $260 E-04$ 4. $998 \mathrm{E}-04$ $6.232 E-04$ $7.873 E-04$ 1. $017 \mathrm{E}-03$ $2.587 E-03$ $2.927 \mathrm{E}-03$ $4.600 E-03$ $1.330 E-02$ $3.323 E-02$ $8.456 E-03$ $.943 E-05$ $4.436 E-04$ $1.227 E-04$ $2.174 \mathrm{E}-04$
$5.744 \mathrm{E}-0.5$ $6.516 \mathrm{E}-05$ $6.516 E-05$ $9.421 E-05$ $9.421 E-05$ $1.835 E-04$ $2.843 E-04$ $1.247 \mathrm{E}-03$ $3.754 E-03$ $3.742 \mathrm{E}-03$ 1. $042 E-02$ $2.774 \mathrm{E}-02$
$7.829 \mathrm{E}-03$ ANGLE 15 $M U=0.8656$ 1.581E-04 $1.684 \mathrm{E}-03$ $5.532 E-04$ $8.837 E-04$ 4.111E-04 $4.534 \mathrm{E}-04$ $5.897 E-04$ $7.163 E-04$ $8.800 E-04$ $1.105 E-03$ $2.736 \mathrm{E}-03$ $3.052 \mathrm{E}-03$ $4.731 E-03$ $1.353 E-02$ $3.380 \mathrm{E}-02$ $8.516 E-03$
ANGLE 7 $A=-0.4580$ ANGLE 8 $.089 E-05$ .849E-04 $1.356 E-04$ $2.388 \mathrm{E}-04$ $6.670 E-05$ $7.591 E-05$ $8.978 E-05$ $1.500 E-04$ $2.149 E-04$ $3.327 E-04$ $1.368 E-03$ $1.067 \mathrm{E}-02$ $2.826 E-02$ $7.892 \mathrm{E}-03$ ANGLE 16 $M U=0.9446$ $.990 E-05$ $1.931 E-03$ $6.432 E-04$ $1.016 \mathrm{E}-03$ 4.841E-04 $5.276 \mathrm{E}-04$ 5. $872 E-04$ $7.912 \mathrm{E}-04$ $9.512 \mathrm{E}-04$ $1.171 E-03$
$2.853 E-03$ $3.149 \mathrm{E}-03$ $4.833 E-03$ $1.387 \mathrm{E}-02$ $3.422 E-02$ $8.559 \mathrm{E}-03$ 
(GAMMAS/MEV/STERADIAN/SOURCE NEUTRON)

ENERGY

GROUP (MEV) $9.0 O E$ ON---1.0OE OI S.SOE $00-2.00$. OO 0 $4.00 E$ OO- $-5.00 E$ OO 3.OOE OO- $4.00 E$ OO $2.50 E$ OO--3.00E 00 2.00E $00--2.50 E$ OO $1.66 \mathrm{E} 00--2.00 \mathrm{E}$ OD $1.33 E$ EO- $-1.66 E$ CO $1.00 E$ O0- - 1.33E OO 8. OOE $-C 1--1.00 E$ OO $6.00 E-01--8.00 E-01$ $4.00 F-01--6.00 E-n 1$ $3.00 E-01--4.00 E-01$ .00E-01---3.00E-01 $1.00 E-01--2.00 E-01$ $5.00 E-02--1.00 E-01$
$2.00 E-02--5.00 E-02$

$$
\text { ENERGY }
$$

$$
\text { GROUP (MEV) }
$$

8.0TE OO---1.COE OI 6.5OE $00--9.00 E$ CO $5.00 E$ OO- - - 5.5OE OO 4.0OE OO-D- . . OOE OO 3.00 E OO- - 4.00E OD $2.50 E$ OO-- 3.00E OO $2.00 E$ OO--2.5CE 00 $1.66 E$ OO--2. NOF OO $1.32 E$ OO---1. 6 GE OD 1.0 E $O 0--1.33 E$ N 8.00E-01-- $1.00 E$ OO $6.00 E-01--8.00 E-01$ $4.00 E-01--6.00 E-01$ 3.00E-01- $-4.00 E-01$ $2.00 E-C 1--3.00 E-01$ $1.00 E-01-2.00 E-O 1$ $2.00 \mathrm{E}-02---5.00 \mathrm{E}-02$ $\triangle N G L E \quad 1 \quad A N G L E ?$
$M U=-1.0000 \quad M U=-0.9894$ $M U=-1.0000$ $1.376 \mathrm{E}-05$ 2.849E-O5 $3.235 E-04$
$8.464 E-05$ $1.550 \mathrm{E}-04$ $3.479 \mathrm{E}-05$ $4.144 E-05$ $5.037 E-05$ $6.184 E-n 5$ $8.095 \mathrm{E}-05$ $1.108 E-04$ $1.708 E-04$ $9.737 E-04$ $1.386 \mathrm{E}-03$ $3.993 \mathrm{E}-03$ $1.073 \mathrm{E}-02$ $2.956 E-02$ $8.638 \mathrm{E}-03$ ANGLE 10 $M U=0.0950$ $2.954 \overline{\mathrm{E}}-0$ $6.081 E-05$ $6.708 \mathrm{E}-04$ $1.988 \mathrm{E}-04$ $3.395 \mathrm{E}-04$ $1.208 \mathrm{E}-04$ $1.390 E-04$ $1.663 \mathrm{E}-04$ $2.068 \mathrm{~F}-04$ $2.851 E-04$ $4.108 E-04$ $6.256 \mathrm{E}-04$ $2.006 \mathrm{E}-03$ $2.569 E-03$ $4.392 E-03$ $1.297 E-02$ $3.419 E-02$
$9.203 E-03$ $1.384 E-05$ (866-0 $3.253 \mathrm{E}-04$ $8.541 E-0$ $1.561 \mathrm{E}-04$ $4.173 \mathrm{E}-05$ $5.055 \mathrm{E}-05$ $6.204 E-05$ $8.152 E-05$ $1.123 E-04$ $1.733 E-04$ $9.801 \mathrm{E}-04$ $1.400 \mathrm{E}-\mathrm{C} 3$ $3.995 E-03$ $1.075 E-02$ $2.960 E-02$
$8.643 E-03$ ANGLE 11 $M U=0.2816$ $3.595 \mathrm{E}-05$ $7.380 E-05$
$8 . C 75 E-04$ $8 . C 75 E-04$ $2.459 E-04$ $4.136 \mathrm{~F}-04$ $1.608 \mathrm{E}-04$ $1.849 \mathrm{E}-04$ $2.207 E-04$ $2.732 E-C 4$ 3.723F-04 $5.246 E-04$ $7.680 E-04$ $2.237 E-03$ 2.744E-03 $4.545 E-03$ $3.517 E-C 2$ $3.517 E-C 2$
$9.315 E-C^{3}$
ANGLE 3 $M U=-0.944 t$ $1.415 E-05$ $2.536 E-05$ $3.328 E-04$
$8.841 E-05$ $8.841 E-05$
$1.605 E-04$ $1.605 E-04$
$3.699 E-05$ 4. $306 E-05$ $5.158 \mathrm{E}-05$ $6.322 E-05$ $8.418 E-05$ $1.181 E-04$ $1.836 \mathrm{E}-04$ $1.007 E-03$ $1.454 F-03$ 4. $001 \mathrm{E}-0$ $1.082 \mathrm{~F}-02$ $2.975 E-02$
$8.663 E-03$ ANGLE 4
$M U=-0.8656$
$1.480 E-05$
$3.071 \mathrm{E}-05$
$3.474 \mathrm{E}-04$
$9.379 \mathrm{E}-05$
$1.686 \mathrm{E}-04$
$4.025 \mathrm{E}-05$
$4.591 \mathrm{E}-05$
$5.428 \mathrm{E}-05$
$6.651 \mathrm{E}-05$
$9.011 \mathrm{E}-05$
$1.293 \mathrm{E}-04$
$2.028 \mathrm{E}-04$
$1.061 \mathrm{E}-03$
$1.554 \mathrm{E}-03$
$4.014 \mathrm{E}-03$
$1.096 \mathrm{E}-02$
$3.004 \mathrm{E}-0 ?$
$8.700 \mathrm{E}-03$ ANGLE 12 $M U=0.4580$ ANGLE 13 $M U=0.6179$
$5.647 \mathrm{~F}-05$ $5.647 \mathrm{~F}-05$ $1.151 E-04$
$1.236 E-03$ $1.236 \mathrm{E}-03$
$3.989 \mathrm{E}-04$ $3.989 \mathrm{E}-04$
$6.487 \mathrm{E}-04$ $2.9 \in 2 E-04$ $3.369 E-04$ 3.948E-04 4.752E-04 $6.137 E-04$ $8.028 \mathrm{E}-04$ $1.069 E-03$ $2.695 \mathrm{E}-03$ $3.105 E-03$ $4.910 E-03$ $1.45 \mathrm{E}-02$ $3.715 E-02$ ANGLE 5 1.575E-05 3.269E-05 $3.689 E-04$ $3.689 E-04$ $1.800 E-04$ $4.491 E-05$ $5.071 E-05$ $5.934 E-05$ $7.323 E-05$ $1.004 \mathrm{E}-04$ $1.460 E-04$ $2.306 \mathrm{E}-04$ $1.142 E-03$ $1.689 E-03$ $4.033 E-02$ $1.115 E-02$ $3.045 \mathrm{E}-02$
$8.752 \mathrm{E}-03$ ANGLE 14 $M U=0.7550$ $7.173 \mathrm{E}-05$ $1.457 \mathrm{E}-04$
$1.547 \mathrm{E}-03$ $5.142 \mathrm{E}-04$ $8.206 E-04$ $3.981 E-04$ $4.467 E-04$ $5.140 E-04$ 7. . $1.206 \mathrm{E}-03$ $2.908 E-03$ $3.283 E-03$ $5.098 E-03$ $1.503 E-02$ $9.625 \mathrm{E}-03$
ANILE 6
$M U=-0.6179$ $1.709 E-05$ $3.544 E-05$ $3.988 \mathrm{E}-04$ $1.104 \mathrm{E}-04$ $1.956 E-04$
$5.140 E-05$ $5.819 E-05$ $6.867 E-05$ $1.165 E-04$ $2.699 E-04$ $1.255 E-03$ $1.851 E-03$ $43 E-02$ $3.098 \mathrm{E}-02$ $8.819 E-03$ AVGLE 15

ANGLE 7 $U=-0.4580$ $1.893 \mathrm{E}-05$ $4.396 E-04$ $1.230 \mathrm{E}-04$ 政 . $400 E-04$ .042E-04 $3.250 E-04$ .028E-03 $3.163 \mathrm{E}-02$
$8.900 \mathrm{E}-03$ ANGLE 16 $M U=0.8656$ $9.003 \mathrm{~F}-05$ $1.923 E-04$
$1.916 E-03$ $6.528 \mathrm{E}-04$ $1.022 \mathrm{E}-03$ $5.175 \mathrm{E}-04$ $5.704 E=04$ $6.423 E-04$ $8.962 E-04$ $073 E-03$ $1.322 \mathrm{E}-03$ $3.098 E-03$ . 1.546E 02 $9.703 \mathrm{E}-03$
ANGLE 8 ANGLE 9 $2=-0.2816 \quad M U=$ $4.432 E-05 \quad 5.486 E-05$ $4.948 \mathrm{E}-04 \quad 5.696 \mathrm{E}-04$ $1.404 E-04 \quad 1.648 E-04$ $2.455 E-04 \quad 2.852 E-04$ $7.385 E-05 \quad 9.306 E-05$ $8.482 E-05 \quad 1.071 E-04$ $1.013 E-04 \quad 1.281 E-04$ $1.257 E-04 \quad 1.593 E-04$ $1.734 E-04$
$2.202 E-04$ $2.528 E-04 \quad 3.205 E-04$ $5.015 E-04$ $1.784 E-03$ $2.211 E-03 \quad 2.392 \mathrm{E}-03$ $1.250 \mathrm{E}-02$ $\begin{array}{ll}3.239 E-02 & 3.325 \mathrm{E}-02 \\ 8.992 \mathrm{E}-03 & 9.094 \mathrm{E}-03\end{array}$ ANGLE 17 $\begin{array}{rr}A N=0.9446 & M U=0.9894 \\ 1.089 E-04 & 1.232 E-04\end{array}$ $2.198 E-04 \quad 2.481 E-04$ $2.291 E-03 \quad 2.573 E-03$ $\begin{array}{ll}7.948 E-04 & 9.005 E-04 \\ 1.225 E-03 & 1.373 E-03\end{array}$ $6.354 E-04 \quad 7.198 E-04$ $6.882 \mathrm{E}-04 \quad 7.701 \mathrm{E}-04$ $7.593 E-04 \quad 8.378 E-04$ $9.942 E-04 \quad 1.061 E-03$ $1.171 \mathrm{E}-03 \quad 1.228 \mathrm{E}-03$ $1.409 E-03 \quad 1.460 \mathrm{E}-03$ $3.250 E-03 \quad 3.344 E-03$ $3.572 E-03 \quad 3.648 E-03$ $1.405 E-03 \quad 5.420 E-03$ $1.578 E-02$ $1.598 E-02$ $3.937 E-02$ $3.970 E-02$

SCALAR

4.701E-04 $9.611 E-04$ $1.040 E-02$ 
(GAMMAS/MEV/STERADIAN/SOURCE NEUTRONI

\begin{tabular}{|c|c|c|}
\hline & ENERGY & \\
\hline$G R C$ & JUP (MEV) & \\
\hline $8.00 E$ & $00---1.00 E \quad 0$ & 01 \\
\hline $6.50 E$ & $O O-=-8, \operatorname{COE} 0$ & 00 \\
\hline $5.00 \mathrm{E}$ & $00---6.50 E$ & 00 \\
\hline $4.00 E$ & $00---5.00 E$ & 00 \\
\hline $3.00 E$ & $00---4.00 E \quad 0$ & 00 \\
\hline $2.5 \mathrm{CE}$ & $00---3.00 E$ & \\
\hline $2.00 E$ & $00---2.50 E 0$ & 00 \\
\hline $1.66 E$ & $00---2.00 E 0$ & \\
\hline $1.3 \geq E$ & $00=--1.66 \mathrm{E}$ & \\
\hline $1.00 E$ & $0---1.33 E 0$ & 00 \\
\hline $8.00 E-C$ & $01--1.00 \mathrm{E} 0$ & \\
\hline $6.00 E-C$ & $01---8.00 E-0$ & \\
\hline $4.00 E-C$ & $-6.00 E-0$ & \\
\hline $3.00 E-C$ & $-4.00 E-0$ & \\
\hline $2.00 E-C$ & $-3.00 E-0$ & \\
\hline $1.00 E-C$ & 2.0 & \\
\hline $\begin{array}{l}5.00 E-C \\
2.00 E-C\end{array}$ & $\begin{array}{l}02---1.00 E-0 \\
02---5.00 E-0\end{array}$ & \\
\hline & & \\
\hline$G R C$ & $(M E V)$ & \\
\hline $\begin{array}{l}8.00 E \\
6.50 E\end{array}$ & $-1.00 E 0$ & 01 \\
\hline $\begin{array}{l}5.50 E \\
5.00 E\end{array}$ & $00--8.00 E$ & \\
\hline $4.00 E$ & OO- $-5.00 E \quad 0$ & \\
\hline $3.0 \mathrm{EE}$ & $00---4.00 E 0$ & \\
\hline $2.50 E$ & $00---3.00 E$ & 00 \\
\hline $2.00 E$ & $00---2.50 E \quad 0$ & \\
\hline $1.66 \mathrm{E}$ & $00---2.00 E O$ & \\
\hline $1.33 E$ & $00--1.66 E 0$ & \\
\hline $1.00 E$ & $00---1.33 \mathrm{E} 0$ & \\
\hline 8.0OE- & $01--1.00 \mathrm{E} O$ & \\
\hline $\begin{array}{l}6.00 \mathrm{E}-\mathrm{C} \\
4.00 \mathrm{E}-\mathrm{C}\end{array}$ & $01---8.00 \mathrm{E}-\mathrm{O}$ & \\
\hline $3.00 E-$ & & \\
\hline 2. ONE- & $-3.0 O E-$ & \\
\hline & & \\
\hline $\begin{array}{l}5.00 E-c \\
2.00 E-c\end{array}$ & $\begin{array}{l}02---1.00 \mathrm{E}-0 \\
02---5.00 \mathrm{E}-0\end{array}$ & \\
\hline
\end{tabular}

ANGLE 1

$\triangle N G L E 2$ $6.170 \mathrm{E}-06$ $1.263 \mathrm{E}-05$ $1.450 E-04$
$3.389 E-05$ $6.667 E-05$ $1.332 \mathrm{E}-05$ $1.951 \mathrm{E}-05$ $2.671 \mathrm{E}-05$ $3.357 E-05$ $3.965 \mathrm{E}-05$ $4.598 \mathrm{E}-05$ $7.069 E-05$ $6.086 \mathrm{E}-04$ $9.072 E-04$ $3.500 E-03$ $9.257 E-03$ $2.689 \mathrm{E}-02$ $8.055 \mathrm{E}-03$ ANGLE 10 $M U=0.0950$ $1.581 \mathrm{E}-05$
$3.249 \mathrm{E}-05$ $3.249 \mathrm{E}-05$
$3.561 \mathrm{E}-04$ $3.561 \mathrm{E}-04$
$1.084 \mathrm{E}-04$ $1.833 \mathrm{E}-04$ $7.185 \mathrm{E}-05$ $8.426 \mathrm{E}-05$ $1.036 \mathrm{E}-04$ $1.345 \mathrm{E}-04$ 2.011E-04 $3.200 E-04$ $5.396 E-04$ $1.69 \mathrm{E}-03$ $3.732 \mathrm{E}-03$ $1.163 \mathrm{E}-02$ $3.213 \mathrm{E}-02$ 8.701E-03 $6.221 \mathrm{E}-06$
$1.277 \mathrm{E}-05$ $1.463 \mathrm{E}-04$ $3.488 \mathrm{E}-05$ $6.775 E-05$ $1.381 E-05$ $1.949 E-05$ $2.618 \mathrm{E}-05$ $3.939 E-05$ $4.731 E-C 5$ $7.359 E-C 5$ $6.132 E-04$ $9.235 E-04$ $3.501 E-03$ $9.274 E-03$ $2.693 \mathrm{E}-\mathrm{C}$ $8.061 \mathrm{E}-03$ ANGLE 11 $M U=0.2816$ $2.058 \varepsilon-05$ $4.214 \mathrm{E}-05$ $4.560 E-04$ $1.448 E-04$ 2.3. $1.063 E-04$ $1.252 \mathrm{E}-04$ $1.551 E-04$ $2.024 E-04$ $3.003 E-04$ $4.609 E-04$ $7.223 E-04$ $1.945 E-03$ $3.408 E-03$ $3.88 \mathrm{E}-03$ $3.330 \mathrm{E}-02$ $8.833 \mathrm{E}-03$ $\begin{array}{ccc}\triangle N & \text { ANGLE } & 3\end{array} \quad 4$ $1.415 \mathrm{E}-05$ 3.839E-05 $7.176 \mathrm{E}-05$ $1.558 \varepsilon-05$ $1.959 \mathrm{E}-05$ $3.044 \mathrm{E}-05$ 3. $900 \mathrm{E}-05$ $5.252 E-05$ $8.449 \mathrm{E}-05$ $6.337 \mathrm{E}-04$ $9.915 \mathrm{E}-04$ $3.501 \mathrm{E}-03$ $9.349 E-03$ $2.710 E-02$ $8.083 E-03$ ANGLE 12 $M U=0.4580$
$2.799 E-05$ $2.799 E-05$
$5.696 E-05$ $6.071 E-04$ $2.013 \mathrm{E}-04$ $3.261 E-04$ $1.636 \mathrm{E}-04$ $1.945 E-04$ $2.412 E-04$ $3.112 E-04$ $4.434 E-04$ $6.375 E-04$ $9.165 E-04$ $2.185 E-03$ 2.575E-03 1.075E-03 $1.280 E-02$ $8.965 E-03$
7.336E-06 $1.403 \mathrm{E}-04$ $4.354 E-05$ $1.825 E-05$ $2.032 E-05$ $2.357 E-05$ $-05$ $4.039 E-05$
$6.142 E-05$ $1.018 \mathrm{E}-04$ $6.775 \mathrm{E}-04$ $1.116 E-03$ $3.501 E-03$ $9.487 E-03$ $2.742 E-02$ $8.124 E-03$ ANGLE 13 $M U=0.6179$ $3.975 \mathrm{E}-05$ $8.035 \mathrm{E}-05$ $8.411 E-04$ $2.922 E-04$ $4.629 E-04$ $2.594 E-04$ 3.737E-04 $6.271 E-04$ $8.353 E-04$ $1.103 \mathrm{E}-03$ $2.412 \mathrm{E}-03$ 2.762E-03 1. $3.570 \mathrm{E}-02$ $9.092 \mathrm{E}-03$ $7.336 E-06$
$1.529 E-05$ $1.724 E-04$ 4.848E-05 $8.507 E-05$ $2.110 E-05$ $2.251 E-05$ $2.545 \mathrm{E}-05$ $3.134 E-05$ $4.603 E-05$ $7.301 E-05$ 1.229E-04 $1.286 E-03$ $3.498 \mathrm{E}-03$ $9.685 \mathrm{E}-03$ $2.787 E-02$ $8.182 E-03$ ANGLE 14 $M U=0.7550$
$5.838 E-05$ $5.838 E-05$
$1.173 E-04$ 1. $203 \mathrm{E}-03$ 4. $405 E-04$ $6.761 E-04$ $4.104 E-04$ 4.722E-04 5. $557 E-04$ $6.644 E-04$ ..335E-04 $1.032 E-03$ $2.629 E-03$ $2.961 E-03$ $4.39 \mathrm{E}-03$ $3.382 \mathrm{E}-02$ $3.682 E-02$
$9.205 E-03$

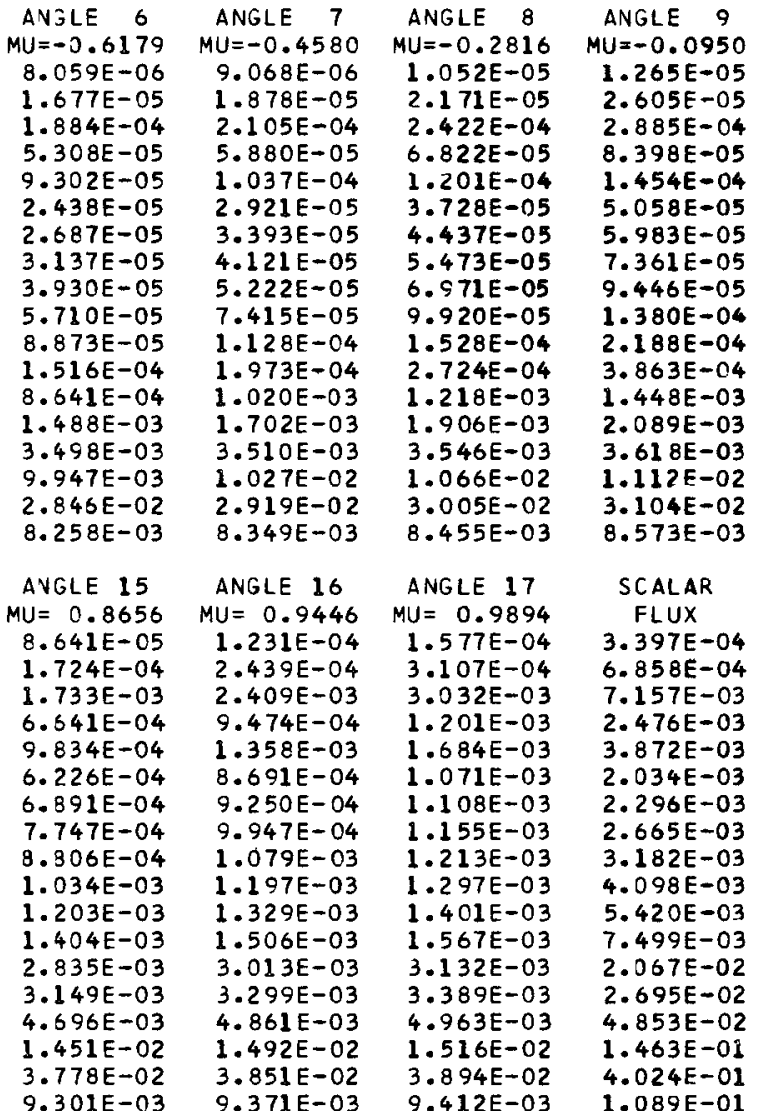


ENERGY GROUP (MEV) $8.00 E$ OO---1.00E 01 5.00 O $00---6.00 E$ E 00 $4.00 E$ OO- - $5.00 E$ OO $3.0 \cap E$ OO- $-4.00 E 00$ $2.50 E$ OO- $-3.00 E$ OO $2.00 E$ OO- $-2.50 E 00$ $1.66 E \quad 00---2.00 E$ OO $1.33 \mathrm{E} 00-\cdots-1.66 \mathrm{E} 00$ $1.00 \mathrm{E} 00---1.33 \mathrm{E} 00$ 8.00E-01---1. 00 E0 00 6.0กE-01-- - 8.00E-01 4.00E-01- - - $.00 \mathrm{E}-01$ $3.00 \mathrm{E}-01---4.00 \mathrm{E}-0 \mathrm{I}$ $2.00 E-01--3.00 E-n 1$ $1.00 \mathrm{E}-01---2.00 \mathrm{E}-01$ $5.00 E-02--1.00 E-C$ $2.00 \mathrm{E}-02---5.00 \mathrm{E}-02$ ENERGY GROUP (MEV) 8.00E OO---1.00E 01 $6.50 E$ OO---8. OOE OO $5.00 E$ OO- -6.50 E OO 4. OOE OO---5. 00 OO $3.00 E$ ON-T-T. 4.0OE ON $2.50 E$ OO- -3.00 E 00 $2.00 E$ OO---2.5กE OD $1.66 \mathrm{E}$ 00-- 2.00E 00 $1.32 E$ EO-- 1.66 E OO $1.00 E$ OO--1.33E OO $8.00 E-O 1--1.00 E$ OD $6.00 E-01--8.00 E-01$ $4.00 E-01--6.00 E-O 1$ 3. OOE-O1- -4 . OOE-OI 2.0OE O1- 3 . OOE $5.00 E-02-1.005-01$ 2.0OE-02---5.00E-02
ANG LE 1 $1.856 \mathrm{E}-06$ $3.629 \mathrm{E}-06$ $4.331 \mathrm{E}-\mathrm{C5}$ $1.690 \mathrm{E}-05$ $2.057 \mathrm{E}-06$ $7.595 \mathrm{E}-0 \mathrm{~S}$ $1.354 E-05$ $1.676 \mathrm{E}-05$ $9.860 \mathrm{E}-06$ $1.261 \mathrm{E}-05$ $2.882 \mathrm{E}-04$ $4.306 \mathrm{E}-04$ $2.231 E-03$ $5.728 \mathrm{E}-03$ $1.723 \mathrm{E}-02$
$5.231 \mathrm{E}-03$ ANG LE 10 $M U=0.095 U$ $5.599 \mathrm{E}-06$ $1.254 \mathrm{E}-04$ $3.978 \mathrm{E}-05$ $6.625 \mathrm{E}-05$ $2.833 \mathrm{E}-05$ $3.306 \mathrm{E}-05$ $4.116 E-05$ $5.628 \mathrm{E}-05$ $9.560 \mathrm{E}-05$ $1.765 \mathrm{E}-04$ $3.375 \mathrm{E}-04$ $1.409 E-03$ $2.264 E-03$ 2.092E-02 $2.092 E-02$
ANGLF 2 1. $887 \mathrm{E}-\mathrm{CS}$ 3.725E-06 $4.413 E-05$ $6.070 E-C 6$ $2.578 \mathrm{E}-0 \mathrm{O}$ $7.395 \mathrm{E}-06$ $1.261 \mathrm{E}-05$ $1.638 \mathrm{E}-05$ $1.503 E-05$ $1.057 E-05$ $2.899 \mathrm{E}-04$ $4.429 E-04$ $2.231 E-03$ $5.740 \mathrm{E}-\mathrm{C}$ $1.7265-02$ $5.235 \mathrm{E}-03$ ANGLE 11 $M U=0.2816$ $7.704 \mathrm{E}-06$
$1.578 \mathrm{E}-05$ $1.688 \mathrm{E}-04$ $5.683 E-C 5$ $5.174 E-05$ 4. $533 E-05$ $5.407 E-05$ $6.980-05$ . 4.860 04 4. (. 3 $7.724 \mathrm{E}-03$ $2.176 \mathrm{E}-02$
$5.782 \mathrm{E}-03$
(GAMMAS/MEV/STERADIAN/SOURCE NEUTRON) $M U=-0.9446$ $2.003 E-06$ $4.073 E-06$
$4.714 E-05$ $1.045 \mathrm{E}-05$ $2.135 \mathrm{E}-05$ $4.235=-06$ $6.751 E-06$ . $1.176 \mathrm{E}-05$ $1.504 E-05$
$2.510 E-05$ $2.985 E-04$ $4.952 E-04$ $2.228 E-03$ $5.791 E-03$ $1.738 \mathrm{E}-02$
$5.251 \mathrm{E}-03$ ANGLE 12 $M U=0.4580$ $1.129 E-05$
$2.286 E-05$ $2.286 \mathrm{E}-05$ $2.395 E-04$ $8.362 \mathrm{E}-05$ $1.337 \mathrm{E}-04$ $7.804 E-05$ $9.778 E-05$ (1. $2.824 E-04$ $4.32 ? E-04$ $6.402 E-04$ $1.368 E-03$ $1.585 \mathrm{E}-03$ $2.485 E-03$ $2.264 \mathrm{E}-02$ $2.264 E-02$
$5.878 F-03$ ANGLE $\quad 4$
$M U=-0.8656$ ANGLE 5
$M U=-0.7550$ 2.395E-06 $5.067 E-06$ $5.652 \mathrm{E}-05$ $1.765 E-05$ 2. $911 \mathrm{E}-05$ $7.572 E-06$ $6.446 E-06$ $5.801 E-06$ $1.333 E-05$ $2.680 E-05$ 4. $827 \mathrm{E}-05$ $3.637 E-04$ $7.282 E-04$ $2.205 E-03$ $6.017 E-03$ $5.320 E-03$

AVSLE 6
$M U=-0.6179$ $2.634 E-06$ $5.524 E-06$ $1.838 \mathrm{E}-05$ $8.191 E-06$ $9.146 E-06$ $1.188 E-05$ $1.919 \mathrm{E}-05$ $3.269 E-05$ 5. $965 E-05$ $4.380 \mathrm{E}-04$ $8.904 E-04$ $2.184 E-03$ $6.193 E-03$ $1.832 E-02$ $1.760 E-02$
$5.279 E-03$ ANGLE 13 ANGLE 130 ANGLE 14 $M U=0.6170$
$1.788 \mathrm{E}-05$ $3.576 E-05$ 3.650E-04 $1.353 \mathrm{E}-04$ $2.125 E-04$ I. $445 E-04$ . 2.361E-04 $4.122 E-04$ 4 $2.622 E-03$ DE-03 $2.352 E-02$ ANGLE 14 $M U=0.7550$ $6.060 \mathrm{E}-05$ $5.983 E-04$ $2.422 E-04$ $3.639 E-04$ $2.707 E-04$ 3. $4.884 E-04$ $6.206 E-04$ $7.581 E-04$ $1.968=-04$ $1.632 E-03$ $1.841 E-03$ $2.764 E-03$ $2.434 E-02$ $6.052 \mathrm{E}-03$
ANGLE 7 ANGLE 8 ANGLE 9 $2.957 E-06 \quad 3.466 E-06 \quad 4.290 E-06$ $6.104 E-06 \quad 7.089 E-06 \quad 8.790 E-06$ $6.845 E-05 \quad 7.943 E-05 \quad 9.738 E-05$ $1.848 \mathrm{E}-05 \quad 2.094 \mathrm{E}-05 \quad 2.791 \mathrm{E}-05$ $3.321 E-05 \quad 3.853 E-05 \quad 4.919 E-05$ $9.184 \mathrm{E}-06 \quad 1.218 \mathrm{E}-05 \quad 1.826 \mathrm{E}-05$ $1.150 E-05 \quad 1.620 E-05 \quad 2.259 E-05$ $\begin{array}{lll}1.498 E-05 & 2.146 E-05 & 2.862 E-05\end{array}$ $1.970 E-05 \quad 2.794 E-05 \quad 3.753 E-05$ $2.784 E-05 \quad 3.916 E-05 \quad 5.797 E-05$ $4.185 E-05 \quad 6.182 E-05 \quad 1.032 E-04$ $8.187 E-05 \quad 1.299 E-04 \quad 2.148 E-04$ 5.487E-04 6.946E-04 8.656E-04 $1.056 \mathrm{E}-03 \quad 1.203 \mathrm{E}-03 \quad 1.320 \mathrm{E}-03$ $2.167 E-03 \quad 2.168 E-03 \quad 2.198 E-03$ $6.411 E-03 \quad 6.675 E-03 \quad 6.983 E-03$ $1.883 E-02 \quad 1.944 E-02 \quad 2.014 E-02$ $5.437 E-03 \quad 5.512 E-03 \quad 5.596 E-03$ ANGLE 16 $M U=0.9446$ ANGLE 27 $\begin{array}{ll}9.411 E-05 & M U=0.9894 \\ 1.413 E-04\end{array}$ $1.828 \mathrm{E}-04 \quad 2.711 \mathrm{E}-04$ $1.695 \mathrm{E}-03 \quad 2.464 \mathrm{E}-03$ $7.563 E-04 \quad 1.089 E=03$ $1.015 E-03$ 1.408E-03 $7.569 E-04 \quad 1.014 E-03$ $7.936 \mathrm{E}-04 \quad 1.013 E-03$ $8.338 E-04 \quad 1.010 E-03$ $8.765 E-04$ 1.007E-03 $9.773 E-04$ 1.004E 03 $9.770 E-04$ I.020E $1.05 E-03$ 1.095E $1.07 E-03$ 1. $2.072 E-03$ 2.128E 03 O. $2.560 \mathrm{E}-02 \quad 2.592 \mathrm{E}-02$ $\begin{array}{ll}2.560 E-02 & 2.592 E-02 \\ 6.173 E-03 & 6.203 E-03\end{array}$

\section{SCALAR}

$1.807 E-04$ 3. $582 \mathrm{E}-04$ $3.542 \mathrm{E}-03$ $1.388 \mathrm{E}-03$ $2.041 E-03$
$1.337 E-03$ $1.337 E-03$ $1.514 E-03$ $1.759 E-03$ 2.100E-03 . $555 E-03$ . $886 \mathrm{E}-03$ $1.238 E-02$ $1.657 E-02$ $246 E-02$ $2.623 E-01$ 
(GAMMAS/MEV/STERADIAN/SOURCE NEUTRON)

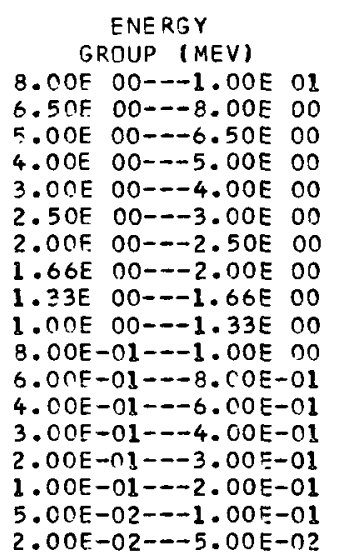

ENERGY

$$
\begin{aligned}
& \text { ENERGY } \\
& \text { GROUP (MEV) }
\end{aligned}
$$

8. OOE OO---1.0OE 01 6.50 E $00---8.00$ E 00 $5.00 E$ OO- -6.50 E 00 $4.00 E$ O0--5. 00 EO 00 $3.00 E$ OO- $-4.00 E$ OO $2.50 E$ OD- $-3.00 E$ OO $2.00 E$ OO- -2.50 E 00 1.66 E $00-2.00 E$ OO $1.32 F$ O0- $-1.66 E$ CO $1.00 \mathrm{E}$ 00--1.33E OO 3. OOE-01---1.0OE OO $6.00 E-01---8.00 E-01$ 4.00E-01---6.00E-01 $3.00 E-01--4.00 E-01$ $2.00 \mathrm{E}-01--3.00 \mathrm{E}-01$ $1.00 \mathrm{E}-01---2.00 \mathrm{E}-01$ $5.00 E-02--1.00 E-01$ 2. ONE-02---5. OOE-D2 $M U=-1.0000$ 3.962E . $3.155 E-06$ 1.676E-06 1.047E-05 $-1.326 \mathrm{E}-06$ $-5.326 E-06$ 1.25 $1.793 \mathrm{E}-04$ $1.720 E-04$ $3.024 \mathrm{E}-03$ $9.024 \mathrm{E}-03$ $2.831 E-03$

ANGLE 10 $M U=0.0950$ $1.587 \mathrm{E}-06$ $3.297 \mathrm{E}-06$ 3.553E-05 $1.241 \mathrm{E}-05$ $1.990 \mathrm{E}-05$ $.922 E-06$ $1.173 \mathrm{E}-05$ $1.713 E-05$ $3.669 E-05$
$8.380 E-05$ $3.380 E-05$ $1.828 E-04$
$5.579 E-04$
$7.504 E-04$ $7.504 E-04$ $1.174 E-03$ $3.900 E-03$ $1.132 \mathrm{E}-02$ $3.084 E-03$
ANGLE 2 ANGLE 3 $A U=-0.9894$
$4.170 E-07$ $6.983 E-07$ $9.641 \mathrm{E}-06$ $1.237 \mathrm{E}-06$ $859 E-06$ $-6.242 E-07$ $6.707 E-06$ $6.707 E-06$ 6.163E-06 $6.986 E-06$ $4.213 E-07$ $1.252 E-04$ 1. $866 \mathrm{E}-04$ $1.219 \mathrm{E}-03$ $3.031 \mathrm{E}-03$
$9.288 \mathrm{E}-03$ $2.833 E-0$

ANGLE 11 $M U=0.2816$ $2.296 \mathrm{E}-06$ $4.760 E-06$ $4.994 E-05$ $1.881 E-05$ $2.865 E-05$ $1.521 E-05$ $1.750 E-05$ $2.3=4 E-05$ $3.791 E-05$ $7.793 E-05$ $2.783 E-04$ $6.521 E-04$ $7.802 \mathrm{E}-04$ $1.224 E-03$ $4.112 \mathrm{E}-03$ $1.180 E-02$ $3.137 E-03$ 4. $340 \mathrm{E}-03$
ANGLE 3
$M U=-0.9446$ MU $=-0.9446 \quad M U=-0.8656$ $9.456 E-07$ 1.151E-C5 1.351E-06 $4.456 \mathrm{E}-06$ 2. 2. 4. $71 E-06$ 4. $3.881 E-06$ $2.402 E-06$ 1. $271 E-04$ 1.271 E-04 1.217E-03 $3.059 E-03$ $3.059 E-03$
$9.353 E-03$ $9.353 E-03$
$2.841 E-03$ ANGLE 12 $M U=0.4580$ $3.556 E-06$ $7.380 \mathrm{E}-05$ $2.706 E-05$ $4.265 E-05$ $2.346 E-05$ $3.854 E-05$ $5.651 E-05$ $8.752 E-05$ $1.521 E-04$ $3.741 E-04$ $7.280 E-04$ $8.222 \mathrm{E}-\mathrm{C} 4$ 1. $288 E-03$ 1.229E-02 $3.191 E-03$ ANGLE 4 $5.866 \mathrm{E}-07$
$1.259 \mathrm{E}-06$ $1.259 E-06$ 1. $396 E-05$ 4. $959 E-06$ $7.58 E-06$ 1.326E-06 $1.326 E-06$ $6.303 \mathrm{E}-08$ $1.643 \mathrm{E}-06$ $6.548 E-06$ $1.351 E-04$ $1.351 \mathrm{E}-04$ $2.785 E-04$
$1.209 E-03$ $3.111 \mathrm{E}-03$ $3.111 E-03$
$9.473 E-03$ $2.857 \mathrm{E}-03$ ANGLE 13 $M U=0.6179$ $6.210 \mathrm{~F}-06$ $1.219 \mathrm{E}-04$ $4.696 E-05$ $7.554 E-05$ $6.277 E-05$ $8.716 \mathrm{E}-05$ $1.231 E-04$ $1.739 E-04$ $2.573 E-04$ $3.545 E-04$ $4.554 E-04$ $7.891 E-04$ $8.849 E-04$ $1.358 E-03$ 4. $574 E-03$ $1.278 \mathrm{E}-02$ $3.242 E-03$

ANGLE 5
MU=-0.7550
$6.629 E-07$
$1.466 E-06$
$1.572 E-05$
$6.138 E-06$
$9.166 E-06$
$2.600 E-06$
$1.045 E-06$
$-1.490 E-07$
$-3.848 E-07$
$2.399 E-06$
$9.125 E-06$
$1.742 E-05$
$1.559 E-04$
$3.6411 E-04$
$1.193 E-03$
$3.184 E-03$
$9.647 E-03$
$2.880 E-03$
ANGLE 14
$M U=0.7550$
$1.246 E-05$
$2.434 E-05$
$2.309 E-04$
$1.033 E-04$
$1.563 E-04$
$1.414 E-04$
$1.807 E-04$
$2.315 E-04$
$2.935 E-04$
$3.776 E-04$
$4.541 E-04$
$5.165 E-04$
$8.465 E-04$
$9.605 E-04$
$1.431 E-03$
$4.802 E-03$
$1.325 E-02$
$3.289 E-03$

AVGLE 6 $M U=-0.6179$
$7.132 E-07$ $7.132 E-07$
$1.531 E-06$ $1.531 \mathrm{E}-06$ 5.500E-06 $8.671 E-06$ $2.238 \mathrm{E}-06$ $1.744 \mathrm{E}-06$ $1.566 \mathrm{E}-06$ 2.251E-06 $1.045 E-05$ $1.045 E-05$ $1.965 \mathrm{E}-04$ $4.672 E-04$ $4.672 \mathrm{E}-04$ $3.280 E-03$ $0.876 \mathrm{E}-03$ $2.909 E-03$

ANGLE 15 $M U=0.8656$ $2.726 \mathrm{E}-05$ $5.288 E-05$
$4.760 E-04$ $4.750 E-04$
$2.373 E-04$ 3. $273 E-04$ $2.905 E-04$ $3.322 E-04$ $3.796 E-04$ $4.304 E-04$ $4.878 E-04$ $5.288 E-04$ $5.605 E-04$ $9.111 E-04$ $1.031 \mathrm{E}-03$ $1.502 E-03$ $5.006 E-03$ $1.365 E-02$ $3.328 E-03$
ANGLE 7 $M U=-0.4580$ $7.691 \mathrm{E}-07$ 1.767E-05 4.032E-06 $7.878 \mathrm{E}-06$ 1. $914 \mathrm{E}-06$ $3.260 E-06$ $7.204 E-06$ $9.204 E-06$ 1. $301 \mathrm{E}-05$ $2.706 \mathrm{E}-05$ $2.613 E-04$ $5.698 E-04$ $1.149 E-03$ $3.398 \mathrm{E}-03$ $1.016 \mathrm{E}-02$ 2. $945 E-03$ ANGLE 16 $M U=0.9446$ $1.112 E-04$ $9.607 E-04$ $4.853 E-04$ $6.172 E-04$
$5.131 E-04$ $5.314 E-04$ $5.469 E-04$ $5.587 E-04$ $5.659 E-04$
$5.702 E-04$ $5.931 E-04$ $9.805 \mathrm{E}-04$ $1.080 \mathrm{E}-03$ $1.563 \mathrm{E}-03$ $5.166 \mathrm{E}-03$ $1.396 \mathrm{E}-02$ $3.357 E-03$

ANGLE 8 ANGLE 9 $8.2816 \quad M U=-0.0950$ $1.761 E-06 \quad 2.148 E-06$ 1.761E-06 $2.319 E-06$ 1. $2.599 E-05$ B. $1.343 E-06$ 506 $5.172 E-06$ T. $325 E-06$ $7.987 E-06$ O.200E-06 $1.053 E-05 \quad 1.191 E-05$ $1.302 E-05$ l. $1.913 E-05$ 1.933E-05 $4.048 E-05$ $5.215 E-05 \quad 1.040 E-04$ $3.494 E-04 \quad 4.526 E-04$ $6.556 E-04 \quad 7.143 E-04$ $1.137 E-03 \quad 1.144 E-03$ $3.541 E-03 \quad 3.709 E-03$ $1.050 \mathrm{E}-02 \quad 1.089 \mathrm{E}-02$ $2.987 \mathrm{E}-03 \quad 3.034 \mathrm{E}-03$

ANGLE 17 SCALAR $M U=0.9894 \quad F L U X$ $1.049 E-04 \quad 8.594 E-05$ $\begin{array}{ll}1.954 E-04 & 1.663 E-04 \\ 1.644 E-03 & 1.530 E-03\end{array}$ $7.947 E-04 \quad 6.876 E-04$ $9.554 E-04 \quad 9.515 E-04$ $7.405 E-04 \quad 7.463 E-04$ $7.155 E-04 \quad 8.473 E-04$ $6.835 E-04 \quad 9.851 E-04$ $6.476 \mathrm{E}-04$ $1.507 \mathrm{E}-03$ $5.845 E-04 \quad 1.973 E-03$ $6.145 E-04 \quad 2.693 E-03$ 6.371E-03 $1.603 \mathrm{E}-03 \quad 1.568 \mathrm{E}-02$ $5.263 E-03 \quad 4.918 E-02$ $\begin{array}{ll}1.414 E-02 & 1.420 E-01 \\ 3.374 E-03 & 3.862 E-02\end{array}$ 
(GAMMAS/MEV/STERADIAN/SOURCE NEUTRONI

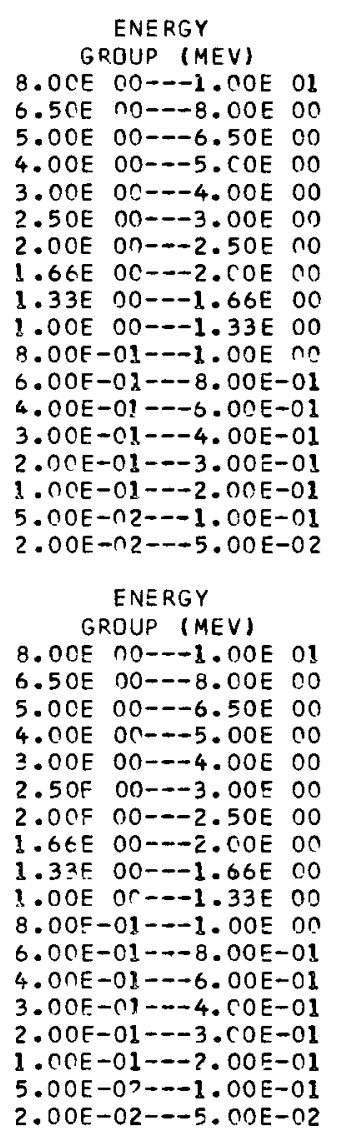
ANGLE 10 $3.050 E-08$ $-7.757 E-08$
$6.913 E-07$ $6.913 \mathrm{E}-07$ $-2.924 E-06$ $-1.938 E-06$ $-1.288 \mathrm{E}-06$ $1.514 E-06$
$4.528 E-06$ $4.528 E-06$ $6.304 E-06$ $4.123 E-06$ $-2.828 E-06$ $-6.639 E-06$ $5.426 E-05$ $7.236 E-05$ $6.175 E-04$ $1.481 E-03$ $4.578 E-03$

ANGLE 10 $M U=0.0950$ $4.025 E-07$
$8.654 E-07$ $9.080 E-06$ $4.106 \mathrm{E}-06$ $5.938 E-06$ $2.988 \mathrm{E}-06$ $2.374 \mathrm{E}-06$ $2.199 \mathrm{E}-06$ $3.324 \mathrm{E}-06$ $1.2395-05$ $3.776 \mathrm{E}-05$ 9.778E-05 $3.780 E-04$ $2.780 E-04$
$3.698 E-04$ $5.669 E-04$ 1. $0611 E-03$ $1.911 E-03$
$5.591 E-03$ 1.524E-03
ANGLE 2 $M U=-0.9894$ $4.5375-C 8$ $-1.934 E-C 8$ $-2.261 E-06$ $-1.330 \mathrm{E}-06$ $-9.736 E-07$ $1.363 \mathrm{E}-06$ $3.896 \mathrm{E}-\mathrm{O}$ 5.3 6OE-06 $3.486 \mathrm{E}-06$ 2.234E-OG 5. 6.618E-05 6.173E$4.585 E=03$ $4.585 E-C 3$
$1.400 E-03$

ANGLE II $M U=0.2816$ $6.140 \mathrm{E}-07$ $1.328 \mathrm{E}-06$ 1. $331 E-05$ $6.2930-06$ ..432E-06 $4.473 E-06$ $5.896-06$ 1.243E-05 $3.356 E-05$ $7.704 E-05$ $1.466 \mathrm{E}-04$ $3.450 E-04$ $3.250 E-04$ $3.778 E-04$
$5.902 E-04$ $2.014 \mathrm{E}-03$ $2.014 \mathrm{E}-03$
$5.824 \mathrm{E}-03$ $1.550 \mathrm{E}-03$ $M U=-0.944 \mathrm{~S}$ ANGLE 4 I CJE-08 $1.35 J E-07$ $2.173 \mathrm{E}-06$ $-1.773 E-07$ $3.524 \mathrm{E}-07$ $7.522 \mathrm{E}-07$ $1.443 \mathrm{E}-06$ $1.784 E-06$ 1.176E-06 9.065E-08 $4.618 E-13$

ANGLE 12 $M U=0.4580$ $9.633 E-07$ $1.938 E-05$ $1.95 E-05$ $1.431 \mathrm{E}-06$ $1.1395-05$ $1.5105-C 5$ 1.31 $3.933 \mathrm{E}-05$ 3. $933 \mathrm{E}-05$ $1.304 E-04$ $1.304 E-04$ $3.584 E-04$ $3.584 E-04$ $6.198 \mathrm{E}-04$ $2.125 \mathrm{E}-\mathrm{n} 3$ $6.067 E-03$ 1.576E-03 I. $481 \mathrm{E}-07$ $3.514 E-07$ 3.545E-06 $1.393 \mathrm{E}-06$ $2.435 E-06$ $6.775 \mathrm{E}-07$ $1.083 \mathrm{E}-07$ $-1.746 E-05$ $-1.166 E-06$ - $.584 E-07$ 5.525E-06 ( 6. $4.677 \mathrm{E}-03$ $4.677 E-03$

ANGLE 13 $M U=0.6179$ $1.807 E-0 S$ $3.381 E-05$ 1.381E-05 $2.224 E-05$ 2.224E-05 2.357 $5.770 E-05$ . $1.371 \mathrm{E}-04$ $1.399 \mathrm{E}-04$ $2.392 E-04$ $3.317 E-04$ 4. $245 \mathrm{E}-04$ $6.519 E-04$ $2.238 \mathrm{E}-03$ t. $310 \mathrm{E}-03$ 1. $602 \mathrm{E}-03$

ANGLE 5
$M U=-0.7550$
$1.808 E-07$
$4.307 E-07$
$4.272 E-06$
$2.725 E-06$
$2.912 E-06$
$1.125 E-06$
$-1.407 E-07$
$-1.205 E-06$
$-1.648 E-06$
$-2.255 E-07$
$3.702 E-06$
$7.278 E-06$
$6.495 E-05$
$1.736 E-04$
$5.992 E-04$
$1.561 E-03$
$4.763 E-03$
$1.423 E-03$
ANGLE 14
MU $=0.7550$
$4.331 E-06$
$8.284 E-06$
$7.554 E-05$
$3.724 E-05$
$5.936 E-05$
$6.553 E-05$
$9.015 E-05$
$1.215 E-04$
$1.586 E-04$
$2.058 E-04$
$2.431 E-04$
$2.664 E-04$
$4.035 E-04$
$4.622 E-04$
$6.846 E-04$
$2.348 E-03$
$6.538 E-03$
$1.624 E-03$

ANILE 6 $M U=-3.6179$ 3.963E-07 4.106E-06 $1.391 \mathrm{E}-06$ $2.276 \mathrm{E}-06$ 4. $007 E-07$ $1.345 \mathrm{E}-07$ $3.654 E-08$ $6.067 E-08$ $1.257 E-06$ 3. $256 E-06$ $5.752 E-06$ $8.526 \mathrm{E}-05$ $2.311 \mathrm{E}-04$ $5.831 E-04$ $1.603 E-03$ 1. $438 \mathrm{E}-03$

AVGLE 15 $M U=0.8656$ $1.192 E-05$ . $120 \mathrm{E}-04$ 1.53 E-04 $1.534 E-04$ 1.547E-04 $1.810 E-04$ 2.374E-04 $2.374 E-04$ $2.788 E-04$ $2.788 E-04$ $4.320 E-04$ $4.955 E-04$ $7.169 \mathrm{E}-04$ $2.446 E-03$ $2.446 E-03$ $1.644 \mathrm{E}-03$
ANGLE 7 $M U=-0.4580$ $3.099 E-07$ 3.721E-06 $1.130 \mathrm{E}-07$ $1.045 \mathrm{E}-06$ 3.551E-08 $8.903 E-07$ 1. $869 \mathrm{E}-06$ $3.119 E-06$ $3.399 \mathrm{E}-06$ $2.643 \mathrm{E}-06$ $7.593 \mathrm{E}-06$ $1.197 \mathrm{E}-04$ $2.874 \mathrm{E}-04$ $5.661 E-04$ $1.667 E-03$ $1.455 \mathrm{E}-03$

ANGLE 16 $M U=0.9446$ .205E-05 $4.99 E-05$ 4. $803 E-04$ $2.739 E-04$ 3.340E-04 $3.076 E-04$ 3. 3. $3.017 E-04$ 2. $932 E-04$ $2.976 \mathrm{E}-04$ $4.667 E-04$ $5.151 E-04$ $5.151 \mathrm{E}-04$
$7.459 \mathrm{E}-04$ $2.523 E-03$ $2.523 E-03$ $1.658 E-03$
ANGLE 8 ANGLE 9 $M=-0.2816 \quad M U=-0.0950$ $3.036 E-07$ $4.099 \mathrm{E}-06 \quad 5.873 \mathrm{E}-06$ $8.546 E-08 \quad 1.346 E-06$ $1.114 \mathrm{E}-06 \quad 3.081 \mathrm{E}-06$ $3.848 \mathrm{E}-07 \quad 1.499 \mathrm{E}-06$ $1.758 \mathrm{E}-06 \quad 2.197 \mathrm{E}-06$ $3.164 E-06 \quad 2.973 E-06$ $4.309 E-06 \quad 3.181 E-06$ $4.102 E-06 \quad 5.202 E-06$ $4.643 E-06 \quad 1.460 E-05$ $1.940 E-05 \quad 4.803 E-05$ $1.674 E-04 \quad 2.232 E-04$ $3.319 \mathrm{E}-04 \quad 3.582 \mathrm{E}-04$ $5.547 E-04 \quad 5.544 E-04$ 1.1 $\begin{array}{ll}1.476 E-03 & 1.3769 E-03\end{array}$

ANGLE 17 $M U=0.9894$ $6.961 E-05$
$1.256 E-04$ (2) . $025 \mathrm{E}-04$ (.)611E-04 3. $584 E-04$ $35 E-04$ $2.933 E-04$ (2) 5.218E-04 (.657E-04 $2.570 E-03$ $6.979 \mathrm{E}-\mathrm{C3}$

\section{SCALAR} FLUX $3.997 E-05$
$7.516 E-05$ $7.516 E-05$
$6.334 E-04$ $6.334 E-04$ $4.270 E-04$ $3.278 E-04$ 4.361E-04 6.071E-04 $6.038 \mathrm{E}-04$ $7.710 E-04$ 1.004E-03 $362 E-03$ $3.065 \mathrm{E}-03$ 
4 PI R*2 HENDERSON DOSE (NEUTRONS)
(CM* 2 RAD/STERADIAN/SOURCE NEUTRON)
1.108 TO 2.35 C MEV NEUTRON SOURGE
$\operatorname{COSINE}$

$-1.00000 E 00$ $-9.89401 \mathrm{E}-01$ $-9.44575 \mathrm{E}-01$ $-7.55044 E-01$ $-6.17876 E-01$ $-4.58017 \mathrm{E}-01$ $-2.81605 E-01$ $-9.50125 \mathrm{E}-02$ $9.50125 \mathrm{E}-02$ $2.81605 E-01$ 4.58017E-01 $6.17876 E-01$ $7.55 C 44 E-01$ $8.65631 \mathrm{E}-01$ $9.44575 E-01$ $9.89401 E-01$

TOTAL

COSINE

\section{$-1 . \operatorname{CCCOOE} 00$} $-9.89401 \mathrm{E}-01$ $-9.44575 E-01$ $-8.65631 \mathrm{E}-01$ $-7.55044 E-01$ $-6.17876 \mathrm{E}-\mathrm{Cl}$ $4.58 C 17 E-01$ $-2.81605 E-01$ 9.50125E-02 政 $2.81017 \mathrm{E}-01$ 7.55044E-O1 $8.65631 E-01$ $9.89401 \mathrm{E}-01$

TOTAL

$\begin{array}{lc}75.0 & 100.0 \\ 1.064 \mathrm{E}-10 & 1.299 \mathrm{E}-1 \mathrm{C} \\ 1.067 \mathrm{E}-1 \mathrm{C} & 1.302 \mathrm{E}-1 \mathrm{C} \\ 1.076 \mathrm{E}-10 & 1.314 \mathrm{E}-1 \mathrm{C} \\ 1.096 \mathrm{E}-10 & 1.340 \mathrm{E}-10 \\ 1.129 \mathrm{E}-10 & 1.381 \mathrm{E}-1 \mathrm{C} \\ 1.178 \mathrm{E}-10 & 1.443 \mathrm{E}-1 \mathrm{C} \\ 1.249 \mathrm{E}-10 & 1.53 \mathrm{CE}-1 \mathrm{C} \\ 1.348 \mathrm{E}-10 & 1.651 \mathrm{E}-1 \mathrm{C} \\ 1.483 \mathrm{E}-10 & 1.817 \mathrm{E}-1 \mathrm{C} \\ 1.953 \mathrm{E}-10 & 2.29 \mathrm{E}-1 \mathrm{C} \\ 1.880 \mathrm{E}-1 \mathrm{C} & 2.410 \mathrm{E}-1 \mathrm{C} \\ 2.225 \mathrm{E}-1 \mathrm{C} & 2.848 \mathrm{E}-1 \mathrm{C} \\ 3.052 \mathrm{E}-10 & 3.518 \mathrm{E}-1 \mathrm{C} \\ 4.467 \mathrm{E}-10 & 4.758 \mathrm{E}-1 \mathrm{C} \\ 8.216 \mathrm{E}-10 & 6.971 \mathrm{E}-10 \\ 2.054 \mathrm{E}-09 & 1.726 \mathrm{E}-\mathrm{C} 9 \\ 7.792 \mathrm{E}-\mathrm{C} 9 & 7.662 \mathrm{E}-\mathrm{Cg} \\ & \\ 4.734 \mathrm{E}-09 & 4.920 \mathrm{E}-\mathrm{C} 9\end{array}$

$\begin{array}{lcc} & \text { RANGE (METERS) } \\ 150.0 & 200.0 & 25 C .0 \\ & & \\ 1.635 E-10 & 1.812 E-10 & 1.859 E-10 \\ 1.639 E-10 & 1.817 E-10 & 1.864 \mathrm{E}-10 \\ 1.656 \mathrm{E}-10 & 1.837 \mathrm{E}-10 & 1.886 \mathrm{E}-10 \\ 1.690 \mathrm{E}-10 & 1.877 \mathrm{E}-10 & 1.929 \mathrm{E}-10 \\ 1.745 \mathrm{E}-10 & 1.940 \mathrm{E}-10 & 1.996 \mathrm{E}-10 \\ 1.825 \mathrm{E}-10 & 2.032 \mathrm{E}-10 & 2.091 \mathrm{E}-10 \\ 1.937 \mathrm{E}-10 & 2.157 \mathrm{E}-10 & 2.221 \mathrm{E}-10 \\ 2.090 \mathrm{E}-10 & 2.327 \mathrm{E}-10 & 2.396 \mathrm{E}-10 \\ 2.295 \mathrm{E}-10 & 2.553 \mathrm{E}-10 & 2.626 \mathrm{E}-10 \\ 2.649 \mathrm{E}-10 & 2.812 \mathrm{E}-10 & 2.885 \mathrm{E}-10 \\ 2.960 \mathrm{E}-10 & 3.299 \mathrm{E}-10 & 3.379 \mathrm{E}-10 \\ 3.449 \mathrm{E}-10 & 3.799 \mathrm{E}-10 & 3.866 \mathrm{E}-10 \\ 4.274 \mathrm{E}-10 & 4.656 \mathrm{E}-10 & 4.762 \mathrm{E}-10 \\ 5.528 \mathrm{E}-10 & 5.830 \mathrm{E}-10 & 5.799 \mathrm{E}-10 \\ 7.620 \mathrm{E}-10 & 7.858 \mathrm{E}-10 & 7.652 \mathrm{E}-10 \\ 1.391 \mathrm{E}-09 & 1.264 \mathrm{E}-09 & 1.145 \mathrm{E}-09 \\ 6.359 \mathrm{E}-09 & 4.989 \mathrm{E}-09 & 3.854 \mathrm{E}-09 \\ & & \\ 5.161 \mathrm{E}-09 & 5.184 \mathrm{E}-09 & 4.998 \mathrm{E}-09\end{array}$

300.0

$1.813 E-10$ $1.819 \mathrm{E}-10$ $1.841 \mathrm{E}-1 \mathrm{C}$ $1.950 E-10$ $2.044 E-10$ $2.172 \mathrm{E}-10$ $2.342 \mathrm{E}-10$ $2.566 E-10$ 2. $872 \mathrm{E}-10$ $3.241 E-1 C$ $3.783 \mathrm{E}-1 \mathrm{C}$ $4.492 E-10$ $5.542 \mathrm{E}-1 \mathrm{C}$ $7.137 E-10$ $1.024 E-C 9$ $2.976 E-C 9$

4.673E-C9 $400 . \mathrm{C}$ 1. $557 \mathrm{E}-10$ $1.561 E-10$ $1.561 E-1 C$
$1.62 C E-10$ $1.678 \mathrm{E}-10$ $1.760 E-10$ $1.87 \mathrm{CE}-10$ $2.016 E-10$ $2.206 E-10$ $.451 E-10$ $2.770 \mathrm{E}-10$ $3.774 E-10$ 作 $5.736 E-10$ $7.754 E-10$
$1.777 E-09$

3.787E-09

\begin{tabular}{|c|c|c|c|c|c|}
\hline $500 . C$ & 600.0 & $\begin{array}{l}\text { RANGE } \\
\text { SOC.0 }\end{array}$ & $\begin{array}{l}\text { ERSI } \\
12 \mathrm{CO} . \mathrm{C}\end{array}$ & 1500.0 & 1800.0 \\
\hline & $976 \mathrm{E}-11$ & $2.833 E-11$ & $7.227 \mathrm{E}-12$ & $1.626 \mathrm{E}-12$ & $3.367 \mathrm{E}-13$ \\
\hline & & $842 \mathrm{E}-11$ & & $1.631 \mathrm{E}-12$ & $3.378 \mathrm{E}-13$ \\
\hline $1.239 \mathrm{E}-10$ & $9.122 \mathrm{E}-11$ & $2.880 \mathrm{E}-11$ & $7.349 E-12$ & $1.654 \mathrm{E}-12$ & $3.425 \mathrm{E}-13$ \\
\hline & $9.35 C E-11$ & $2.953 \mathrm{E}-11$ & $7.535 \mathrm{E}-12$ & $1.695 E-12$ & $3.511 \mathrm{E}-13$ \\
\hline 3 & $9.692 \mathrm{E}-$ & $1 E-11$ & 7. & $58 E-12$ & $3.640 \mathrm{E}-13$ \\
\hline 1.38 & $7 E-1 C$ & $2 E-11$ & 8. & 1.84 & $3.817 \mathrm{E}-13$ \\
\hline 1.4 & $1 . C 81 \mathrm{E}-10$ & $3.411 \mathrm{E}-11$ & $8.697 \mathrm{E}-12$ & $1.956 \mathrm{E}-12$ & $4.047 \mathrm{E}-13$ \\
\hline 1.58 & $1.164 \mathrm{E}-10$ & $3.668 \mathrm{E}-11$ & $9.344 E$ & 2.0 & $42 E-13$ \\
\hline 1.72 & $1.270 E-10$ & $3.995 E-11$ & 1.0 & $E-12$ & $11 E-13$ \\
\hline 9$]$ & $1.407 \mathrm{E}-10$ & $4.408 \mathrm{E}-11$ & 1.1 & 2.5 & $9 E-13$ \\
\hline 2.1 & 1.58 & 4.92 & 1.24 & 2. & $5.730 \mathrm{E}-13$ \\
\hline 2.4 & 1.80 & $9 E-11$ & 1.4 & 3.1 & $6.412 E-13$ \\
\hline 28 & $2.100 E-1$ & $6.399 E-11$ & & & \\
\hline 3.46 & $2.487 \mathrm{E}-10$ & $7.433 \mathrm{E}-11$ & $1.832 \mathrm{E}-11$ & $4.027 E-12$ & $8.200 E-13$ \\
\hline 4.26 & 3.01 & $8.751 \mathrm{E}-11$ & & & $9.317 \mathrm{E}-13$ \\
\hline & 3.81 & 1. $049 \mathrm{E}-10$ & & & $1.055 \mathrm{E}-12$ \\
\hline & $6.467 \mathrm{E}-$ & $1.429 \mathrm{E}-10$ & $3 . C 46 E-1$ & $6.181 \mathrm{E}-12$ & $1.199 E-12$ \\
\hline $.859 E-0$ & $2.051 E-C 9$ & $6.182 \mathrm{E}-10$ & $1.539 \mathrm{E}-10$ & $3.409 E-11$ & $6.985 \mathrm{E}-12$ \\
\hline
\end{tabular}


COS INE

$$
\begin{aligned}
& -1 . C C C O O E \text { OC } \\
& -9.89401 \mathrm{E}-01 \\
& -9.44575 \mathrm{E}-01 \\
& -8.65631 \mathrm{E}-01 \\
& -7.55044 \mathrm{E}-01 \\
& -6.17876 \mathrm{E}-01 \\
& -4.58017 \mathrm{E}-01 \\
& -2.81605 \mathrm{E}-01 \\
& -9.50125 \mathrm{E}-02 \\
& 9.50125 \mathrm{E}-02 \\
& 2.81605 \mathrm{E}-01 \\
& 4.58017 \mathrm{E}-01 \\
& 6.17876 \mathrm{E}-01 \\
& 7.55044 \mathrm{E}-01 \\
& 8.65631 \mathrm{E}-01 \\
& 9.44575 \mathrm{E}-01 \\
& 9.89401 \mathrm{E}-01
\end{aligned}
$$

TOTAL

COSINE

\section{$-1.00000 E$ C 0} $-9.894 \mathrm{C1E}-01$

\begin{tabular}{|c|c|c|c|c|c|c|}
\hline 75.0 & $100 \cdot c$ & $15 C .0$ & $\begin{array}{l}\text { NGE }(\text { METERS ) } \\
2 C \mathrm{C} \cdot \mathrm{C}\end{array}$ & $25 c \cdot c$ & $3 c 0.0$ & $400 \cdot C$ \\
\hline$E-10$ & $2.237 E-1 C$ & $2.944 E-10$ & $3.405 E-10$ & $3.637 E-1 C$ & $3.685 E-10$ & $3.391 E-$ \\
\hline & $2.242 E-1 C$ & $2.951 \mathrm{E}-1 \mathrm{C}$ & $3.412 \mathrm{E}-10$ & $3.646 \mathrm{E}-\mathrm{IC}$ & $3.694 \mathrm{E}-1 \mathrm{~B}$ & $3.399 \mathrm{E}-10$ \\
\hline $1.809 E-10$ & $2.261 E-16$ & $2.979 E-10$ & $3.446 \mathrm{E}-10$ & $3.682 E-10$ & $3.732 E-10$ & $3.435 E-10$ \\
\hline IE-IC & $2.302 E-1 C$ & $3.035 \mathrm{E}-10$ & $3.513 E-10$ & $3.756 \mathrm{E}-10$ & $3.807 E-10$ & $3.504 E-10$ \\
\hline $2 E-1 a$ & $2.367 E-1 C$ & $3.123 E-10$ & $3.617 E-10$ & $3.867 E-10$ & $3.920 E-10$ & $3.608 \mathrm{E}-10$ \\
\hline & $2.464 E-10$ & $3.251 \mathrm{E}-10$ & $3.766 \mathrm{E}-10$ & $4.026 E-10$ & $4.080 E-10$ & $3.752 \mathrm{E}-10$ \\
\hline 2.07 & $2.60 C E-10$ & $3.429 \mathrm{E}-10$ & $3.969 E-10$ & $4.241 \mathrm{E}-1 \mathrm{C}$ & $4.295 \mathrm{E}-10$ & $3.945 \mathrm{E}-10$ \\
\hline $2.229 \mathrm{E}-10$ & $2.788 E-10$ & $3.670 \mathrm{E}-10$ & $4.242 E-10$ & $4.527 E-1 C$ & $4.579 E-10$ & $4.196 \mathrm{E}-10$ \\
\hline & $4 E-1 C$ & $3.993 E-10$ & $3 E-10$ & $4.9 C 1 E-10$ & $4.948 E-10$ & $4.518 E-10$ \\
\hline $3.188 \mathrm{E}$ & $3.752 E-16$ & $4.513 \mathrm{E}-10$ & $5.034 E-10$ & $5.34 C E-10$ & $5.441 \mathrm{E}-10$ & $4.930 E-10$ \\
\hline 3.05 & $3.968 E-1 C$ & $5.653 \mathrm{E}-10$ & $5.765 \mathrm{E}-10$ & $6.086 E-10$ & $6.046 E-10$ & $5.465 \mathrm{E}-10$ \\
\hline $1 E-10$ & $7 E-1 C$ & $3 E-10$ & $6.575 E-10$ & 6.888 & $6.902 E-10$ & $156 E-10$ \\
\hline $4.779 E-10$ & 5.65 & & & 8.165 & & $7.076 \mathrm{E}-10$ \\
\hline $6.849 E-10$ & $7.482 E-1 C$ & $8.038 \mathrm{E}-10$ & $9.697 \mathrm{E}-10$ & $9.882 E-10$ & $9.653 E-1 C$ & $8.327 \mathrm{E}-10$ \\
\hline $3 E-C 9$ & 1.C $75 E-09$ & $1.212 \mathrm{E}-$ & $1.276 E-09$ & $1.269 E-09$ & $1.210 \mathrm{E}-\mathrm{C} 9$ & $1.014 E-09$ \\
\hline & $2.578 E-C 9$ & $2.133 E-$ & $1.987 E-09$ & $1.839 E-09$ & $1.678 E-09$ & $1.320 E-09$ \\
\hline & $1.113 E-C 8$ & $9.292 \mathrm{E}-09$ & $7.352 E-09$ & $5.742 E-09$ & $4.491 \mathrm{E}-\mathrm{Cg}$ & $2.768 \mathrm{E}-\mathrm{C} 9$ \\
\hline $7.256 \mathrm{E}-\mathrm{Cg}$ & $7.698 \mathrm{E}-\mathrm{C}^{\circ}$ & $8.398 E-C 9$ & $8.750 E-n 9$ & $8.731 \mathrm{E}-09$ & $8.429 E-C 9$ & $7.256 \mathrm{E}-09$ \\
\hline
\end{tabular}
$-9.44575 E-C 1$ $-8.65631 \mathrm{E}-01$ $-6.17876 \mathrm{E}-01$ $-6.17876 E-01$ $-4.58017 \mathrm{E}-\mathrm{CI}$ $-9.50125 \mathrm{E}-02$ $0.50125 \mathrm{E}-02$ 2.81605E-01 $2.81005 E-01$ $6.17876 \mathrm{E}-01$ $7.55044 E-01$ $8.65631 \mathrm{E}-01$ $9.89401 E-01$

TOTAL

\section{RANGE (METERS)}

$500 . \mathrm{C}$

$2.822 \mathrm{E}-1 \mathrm{C}$

$2.829 \mathrm{E}-10$

$2.859 E-10$

$2.916 \mathrm{E}-10$

$3 . n 02 E-10$

$3.120 E-10$

$3.276 E-10$

$3.478 E-1 C$

3.735E-10

$4.060 E-10$

$4.474 E-1 C$

$5 . C O 2 E-1 C$

5.687E-1C

$6.594 \mathrm{E}-10$
$7.852 \mathrm{E}-10$

$1.726 \mathrm{E}-\mathrm{C} 9$

$5.782 E-C 9$
$6 C C .0$

$2.191 \mathrm{E}-1 \mathrm{C}$

$2.196 \mathrm{~F}-1 \mathrm{C}$

$2.21 \mathrm{SE}-1 \mathrm{C}$

$2.263 \mathrm{E}-10$

2.

2.

.

.

.

$3.425 \mathrm{E}-1 \mathrm{C}$

$806 E-1$

$4.291 E-10$

$5.758 E-10$

$6.987 E-1 C$

$1 . C 84 \mathrm{E}-\mathrm{C}$ )

$4.351 E-C 9$
$120 \mathrm{C} \cdot \mathrm{C}$

$7.808 \mathrm{E}-11$

$7.827 E-11$

$8.062 \mathrm{E}-11$

$8.287 \mathrm{E}-11$

$8.596 \mathrm{E}-11$

Q.503E-11

1.

1.

$1.186 \mathrm{E}-10$

$1.302 E-10$

$1.444 E-10$

列 10

$2.6695-10$

$1.468 \mathrm{E}-\mathrm{C} 9$
$2.164 E-11$

$2.169 E-11$

$2.191 \mathrm{E}-11$

$2.294 \mathrm{E}-11$

$2.377 \mathrm{E}-11$

$2.484 E-11$

$2.619 E-11$

$2.785 E-11$

$3.229 \mathrm{E}-11$

$3.521 E-11$

. 86 E 11

$4.285 \mathrm{E}-11$

$4.765 E-1$

$6.195 \mathrm{E}-11$

$3.954 E-10$
$1500 . C$

5. $158 \mathrm{E}-12$ $5.171 \mathrm{E}-12$ $5.223 E-12$ 5 (6)

$1.339 E-11$

$9.265 E-11$
1800.0

$1.114 E-12$ $1.116 \mathrm{E}-12$ $1.128 \mathrm{E}-12$ $1.149 \mathrm{E}-12$ $1.179 E-12$ $1.221 \mathrm{E}-12$
$1.274 \mathrm{E}-12$ $1.34 \mathrm{CE}-12$ $1.34 C E-12$ $1.420 E-12$ $1.631 \mathrm{E}-12$ $1.631 \mathrm{E}-12$ $1.765 E-12$ $1.92 C E-12$ $2.09 \mathrm{E}-12$ $2.499 \mathrm{E}-12$ $2.724 E-12$ $1.978 \mathrm{E}-11$ 
COSINE

$-1.0 C C C O E$ DO $-9.89401 E-01$ $-9.44575 E-01$ $-8.65631 \mathrm{E}-01$ $-7.55044 E-01$ $-6.17876 \mathrm{E}-01$ $-4.58017 E-G 1$ $-2.81605 E-01$ $-9.50125 E-02$ $9.50125 \mathrm{E}-02$ $2.81605 E-01$ $4.58017 \mathrm{E}-\mathrm{C} 1$ $7.55044 \mathrm{E}-01$ $8.65631 \mathrm{E}-01$ $9.894 \mathrm{ClE}-01$

TOTAL

COSINE

-1. OCOCOE OO $-9.89401 E-01$ $-9.44575 E-01$ $-8.65631 E-01$ $-7.55044 E-01$ $-6.17876 E-01$ $-4.58017 E-01$ $-2.81605 E-01$ $-9.50125 E-02$ $9.50125 \mathrm{E}-02$ $2.81605 \mathrm{E}-01$ 4.58017E-01 $6.17876 \mathrm{E}-01$ $7.55044 E-01$ $8.65631 E-01$ $9.44575 \mathrm{E}-01$
$9.89401 \mathrm{E}-01$

TOTAL
75.0

$1.123 E-C 8$ $1.125 E-C 8$ $1.135 \mathrm{E}-\mathrm{C} 8$ $1.189 \mathrm{E}-\mathrm{C}$ 1.240E-O8 $1.312 \mathrm{E}-\mathrm{CB}$ $1.312 \mathrm{E}-\mathrm{CB}$
$1.412 \mathrm{E}-\mathrm{OB}$ $1.550 E-C 8$ 1.550E-C8 $1.954 \mathrm{E}-\mathrm{C} 8$ $2.303 \mathrm{E}-08$ $3.126 \mathrm{E}-\mathrm{C} 8$ $4.540 E-C 8$ $8.279 \mathrm{E}-\mathrm{C} 8$ $2.054 E-C 7$ $7.746 \mathrm{E}-\mathrm{C} 7$

4. $808 E-07$

500.

$.431 \mathrm{E}-\mathrm{C} 8$ $1.435 \mathrm{E}-\mathrm{C}$ $1.453 E-C B$ $1.487 \mathrm{E}-08$ $1.538 \mathrm{E}-0$ $1.610 E-C$ $1.705 E-C B$ $1.828 \mathrm{E}-\mathrm{C}$ $1.987 \mathrm{E}-\mathrm{C} 8$ $2.191 E-C 8$ $2.452 \mathrm{E}-\mathrm{CB}$ . $789 E-C 8$ $3.229 E-C 8$ $3.819 E-C 8$ $4.643 E-C 8$ $5.947 E-C B$

$3.210 \mathrm{E}-\mathrm{CT}$
100.0

$1.385 E-C 8$ $1.388 E-C 8$ $1.40 C E-C 8$ $1.427 E-C .8$ $1.533 \mathrm{E}-\mathrm{C} 8$ $1.623 E-C 8$ $1.623 \mathrm{E}-\mathrm{C} 8$
$1.747 \mathrm{E}-\mathrm{C} 8$ $1.916 E-C 8$ $2.389 \mathrm{E}-\mathrm{C} 8$ $2.521 \mathrm{E}-\mathrm{CE}$ $2.958 \mathrm{E}-\mathrm{C} 8$ $3.643 E-08$ $4.884 E-08$ 7. $C 96 \mathrm{E}-08$ $1.735 \mathrm{E}-\mathrm{C7}$

$7.626 \mathrm{E}-07$

$5.041 E-C 7$

$60 C .0$

1. $C 68 E-C 8$ 1. $C 72 E-C 8$ l. $C 85 E-C 8$ 1.111E-08 1. $149 \mathrm{E}-0$ $1.202 E-08$ $1.273 \mathrm{E}-\mathrm{C} .8$ $1.364 E-C 8$ $1.481 \mathrm{E}-08$ $1.63 C E-C 8$ $1.818 E-C 8$ $2.059 E-C 8$ 2. $368 \varepsilon-C 8$ 2.772E-C8 $4.32 C E-C 8$ $4.133 E-C 8$ $2.335 E-C 7$

\begin{tabular}{|c|c|c|c|c|}
\hline $15 \% .0$ & $\begin{array}{l}\text { JGE (METERS) } \\
2 \mathrm{CO} 0.0\end{array}$ & 250.0 & $3 c 0.0$ & 400.0 \\
\hline $\begin{array}{l}.775 E-08 \\
.779 E-08\end{array}$ & $\begin{array}{l}1.997 E-08 \\
2.602 E-C 8\end{array}$ & $\begin{array}{l}2.077 E-08 \\
2.083 E-08\end{array}$ & $\begin{array}{l}2.051 E-C 8 \\
2.056 E-C 8\end{array}$ & $\begin{array}{l}1.797 E-08 \\
1.802 E-08\end{array}$ \\
\hline $\begin{array}{l}.779 \mathrm{E}-08 \\
.797 \mathrm{E}-08\end{array}$ & $\begin{array}{l}2.002 \mathrm{E}-\mathrm{C} 8 \\
2.024 \mathrm{E}-08\end{array}$ & $\begin{array}{l}2.083 \mathrm{E}-08 \\
2.106 \mathrm{E}-08\end{array}$ & $\begin{array}{l}2.056 E-C 8 \\
2.080 E-C 8\end{array}$ & $\begin{array}{l}1.802 \mathrm{E}-08 \\
1.824 \mathrm{E}-\mathrm{C} 8\end{array}$ \\
\hline $.833 E-C 8$ & $2.067 E-c 8$ & $2.153 \mathrm{E}-08$ & $2.127 E-C 8$ & $1.866 E-08$ \\
\hline $.891 \mathrm{E}-08$ & $2.134 E-08$ & $2.224 E-08$ & $2.198 \mathrm{E}-\mathrm{C} 8$ & $1.929 E-C 8$ \\
\hline & $2.23 \mathrm{CE}-08$ & $2.325 E-08$ & $2.299 \mathrm{E}-\mathrm{C} 8$ & $2.019 E-08$ \\
\hline $.090 E-08$ & $2.361 \mathrm{E}-08$ & $2.463 E-08$ & $2.436 E-C 8$ & $2.138 \mathrm{E}-\mathrm{C} 8$ \\
\hline$E-c 8$ & $2.538 \mathrm{E}-08$ & $2.646 \mathrm{E}-08$ & $2.616 \mathrm{E}-\mathrm{CB}$ & $2.295 \mathrm{E}-\mathrm{CE}$ \\
\hline $.459 E-08$ & $2.773 E-C 8$ & $2.888 E-08$ & $2.853 E-C 8$ & $2.498 E-08$ \\
\hline & $3.047 E-08$ & $3.164 E-08$ & $3.173 E-C 8$ & $2.760 \mathrm{E}-08$ \\
\hline $.147 E-C 8$ & $3.54 \mathrm{CE}-08$ & $3.666 E-08$ & $3.561 \mathrm{E}-08$ & $3.102 E-C 8$ \\
\hline $35 E-C 8$ & $4.061 E-08$ & $4.178 E-08$ & $4.121 E-C 8$ & $3.547 \mathrm{E}-08$ \\
\hline $.484 E-08$ & $4.929 E-08$ & $5.028 \mathrm{E}-08$ & $4.854 E-C 8$ & $4.144 E-C 8$ \\
\hline $.740 E-C 8$ & $6.127 E-C 8$ & $6.153 E-08$ & $5.928 \mathrm{E}-\mathrm{C} 8$ & $4.963 \mathrm{E}-08$ \\
\hline & $8.172 E-C 8$ & $8.026 E-08$ & $7.550 \mathrm{E}-\mathrm{C} 8$ & $6.158 E-08$ \\
\hline $.413 E-C 7$ & $1.297 \mathrm{E}-07$ & $1.185 E-07$ & $1.068 \mathrm{E}-\mathrm{C7}$ & $8.200 E-c 8$ \\
\hline $.347 E-07$ & $4.997 E-07$ & $3.878 E-07$ & $3.009 E-C 7$ & $1.818 \mathrm{E}-\mathrm{C} 7$ \\
\hline $.375 E-C 7$ & $5.478 E-07$ & $5.349 E-07$ & $5.058 E-C 7$ & $4.181 E-C 7$ \\
\hline
\end{tabular}

RANGE (METERS)
$900.0 \quad 1200$.

$15 C C .0$

$1800 \cdot C$ 
COS INE

$-1 . \operatorname{coccoe} r 0$ $-9.89401 E-01$ $-9.44575 E-01$ $-8.65631 E-01$ $-7.55044 \mathrm{E}-0$ $-6.17876 E-01$ $-4.58017 E-01$ $-2.81605 \mathrm{E}-01$ $-9.50125 E-02$ . $2.81605 E-01$ $6.17876 E-01$ $6.17876 E-01$ $8.65631 E-?$ $8.65631 E-91$ $9.89401 \mathrm{E}-01$

TOTAL

COSINE

$-1.000 C 0 \mathrm{E} C 0$
$-9.89401 \mathrm{E}-01$
$-9.44575 \mathrm{E}-01$
$-8.65631 \mathrm{E}-01$
$-7.55044 \mathrm{E}-01$
$-6.17876 \mathrm{E}-01$
$-4.58017 \mathrm{E}-01$
$-2.81605 \mathrm{E}-01$
$-9.50125 \mathrm{E}-02$
$9.50125 \mathrm{E}-02$
$2.81605 \mathrm{E}-01$
$4.58017 \mathrm{E}-01$
$6.17876 \mathrm{E}-01$
$7.55644 \mathrm{E}-01$
$8.65631 \mathrm{E}-01$
$9.44575 \mathrm{E}-01$
$9.89401 \mathrm{E}-01$

total

$\begin{array}{cc}75 . C & 1 C C . C \\ 2 . C 75 E-11 & 2.625 E-11 \\ 2.080 E-11 & 2.631 E-11 \\ 2.698 E-11 & 2.654 E-11 \\ 2.137 E-11 & 2.7 C 4 E-11 \\ 2.202 E-11 & 2.785 E-11 \\ 2.299 E-11 & 2.966 E-11 \\ 2.439 E-11 & 3 .(77 E-11 \\ 2.635 E-11 & 3.316 E-11 \\ 2.966 E-11 & 3.644 E-11 \\ 3.729 E-11 & 4.62 C E-11 \\ 3.672 E-11 & 4.780 E-11 \\ 4.355 E-11 & 5.792 E-11 \\ 6.340 E-11 & 7 . C 61 E-11 \\ 9.657 E-11 & 9.882 E-11 \\ 1.836 E-1 C & 1.484 E-1 r \\ 4.630 E-1 C & 3.874 E-1 r \\ 1.877 E-C 9 & 1.831 E-C 9 \\ & \\ 1.033 E-C 9 & 1 . C 62 E-C 9\end{array}$

$1.033 E-C 9$

$500 . c$

$3.965 \mathrm{E}-1$ $3.974 \mathrm{E}-11$ $4.97 E-11$ $4.084 E-11$ $4.170 E-11$ $4.338 \mathrm{E}-1$ $4.532 E-11$ $4.781 \mathrm{E}-11$ 5. $95 \mathrm{E}-1$ $5.493 E-1$ $5.995 \mathrm{E}-1$ $6.639 \mathrm{E}-11$ $7.479 \mathrm{E}-1$ $8.612 E-11$ 1.C $23 E-10$ 1. $292 \mathrm{E}-10$ $2.553 E-10$

$7.819 \mathrm{E}-10$

600.0
$15 C .0$ RANGE (METERS)

$25 \mathrm{C} \cdot \mathrm{C}$

300.0

$400 . C$

$3.551 E-11$

$4.22 C E-11$

$4.627 E-11$

$4.638 E-11$

4. $B C 2 E-11$

4.813E-11

4. $859 \mathrm{E}-1$

4. $950 \mathrm{E}-11$

5.087E-11

$5.281 E-11$

$5.543 E-11$

$5.887 E-11$

$6.336 \mathrm{E}-11$

. $057 \mathrm{E}-11$

1. $014 \mathrm{E}=10$

$1.014 E-10$

$1.556 \mathrm{E}-10$

$2.218 \mathrm{E}-10$

$1.102 \mathrm{E}-\mathrm{C} 9$

$1.138 E-09$

$1.144 F-C 9$

RANGE (METERS)

900.0

$12 \mathrm{CO} C \mathrm{C}$

$1500 . \mathrm{C}$

$1800 . \mathrm{C}$
$606 E-11$

$4.616 E-11$

$4.745 E-11$

$4.873 E-11$

$5.051 E-11$

$5.593 \mathrm{E}-11$

$5.985 E-11$

$7.139 \mathrm{E}-11$

9.

1. $070 E-10$

$1.307 \mathrm{E}-1 \mathrm{O}$

$1.734 E-10$

$9.626 E-10$
$1.192 \mathrm{E}-11$

$1.195 E-11$

3.199

$3.339 \mathrm{E}-11$

. 6 C $3 \mathrm{E}-11$

$3.793 \mathrm{E}-11$

$4 .(3 C E-11$

$4.327 \mathrm{E}-11$

$4.696 \mathrm{E}-11$

$5.16 \mathrm{CE}-1$

$5.753 E-11$

$6.530 \mathrm{E}-11$

$7.599 \mathrm{E}-1$

$4.263 \mathrm{E}-11$

1. $575 \mathrm{E}-1 \mathrm{C}$

$5.958 \mathrm{E}-1 \mathrm{C}$

$.416 \mathrm{E}-11$

$1.497 \mathrm{E}-11$

$1.595 \mathrm{E}-11$

$1.713 \mathrm{E}-1$

$1.856 \mathrm{E}-11$

$2.030 \mathrm{E}-11$

$2.244 \mathrm{E}-1$

2. $513 \mathrm{E}-1$

$2.877 \mathrm{E}-11$

$2.123 E-10$
$3.417 \mathrm{E}-12$

$3.455 E-12$

$8.321 E-13$

$8.339 E-13$

$8.415 E-13$

$8.758 E-13$

$3.227 \mathrm{E}-11 \quad 3.513 \mathrm{E}-12$

1.258E-11 $3.712 E-12$

$1.350 \mathrm{E}-11 \quad 3.857 \mathrm{E}-12$

$4.037 \mathrm{E}-1$

$4.037 \mathrm{E}-12$
$4.255 \mathrm{E}-12$

$4.516 \mathrm{E}-12$

$4.826 \mathrm{E}-12$

$5.621 \mathrm{E}-12$

$6.127 \mathrm{E}-12$

$6.728 \mathrm{E}-12$

$7.465 \mathrm{E}-12$
$8.838 \mathrm{E}-12$

$9.031 \mathrm{E}-13$

$0.804 E-13$

$1.032 \mathrm{E}-12$

$1.092 \mathrm{E}-12$

$1.164 \mathrm{E}-12$

$1.246 E-12$

$1.342 \mathrm{E}-12$

$1.451 \mathrm{E}-12$

$1.575 E-12$

$1.93 C E-12$

$5.91 C E-11$

$1.823 E-13$

$1.827 \mathrm{E}-13$

1.84

$2.052 \mathrm{E}-13$

列

$2.253 \mathrm{E}-13$

$2.383 \mathrm{E}-13$

$2.533 \mathrm{E}-13$

$2.7 C 6 E-13$

$2.9 C_{2} \mathrm{E}-12$

$3.121 \mathrm{E}-13$

$3.364 E-13$

$3.966 \mathrm{E}-13$

3. $073 E-12$ 
4 PI R**2 CONCRETE KERMA (NEUTRONS)
(CM**2 ERGS/GRAM/STERADIAN/SOURCE NEUTRON)

1.108 TO 2.350 MEV NEUTRON SOURCE

COS INE

$-1.00000 \mathrm{E} 00$
$-9.89401 \mathrm{E}-01$
$-9.44575 \mathrm{E}-01$
$-8.65631 \mathrm{E}-01$
$-7.55044 \mathrm{E}-01$
$-6.17876 \mathrm{E}-01$
$-4.58017 \mathrm{E}-01$
$-2.81605 \mathrm{E}-01$
$-9.50125 \mathrm{E}-02$
$9.50125 \mathrm{E}-02$
$2.81605 \mathrm{E}-01$
$4.58017 \mathrm{E}-01$
$6.17876 \mathrm{E}-01$
$7.55044 \mathrm{E}-01$
$8.65631 \mathrm{E}-01$
$9.44575 \mathrm{E}-01$
$9.89401 \mathrm{E}-01$

TOTAL

COSINE

$-1.00000 \mathrm{E} 00$ $-9.89401 E-01$ $-9.44575 E-01$ $-8.65631 E-01$ $-7.55044 E-01$ $-6.17876 \mathrm{E}-01$ $-4.58017 \mathrm{E}-01$ $-2.81605 E-01$ $9.50125 \mathrm{E}-02$ $2.81605 \mathrm{E}-01$ $4.58017 E-01$ $4.58017 E-01$ $7.55044 \mathrm{E}-01$ 7. $55044 E-01$ $8.65631 E 01$ $9.89401 E-01$

TOTAL
75.0

$1.355 E-C 9$ 1. $358 \mathrm{E}-\mathrm{Cg}$ $1.369 E-C 9$ $1.394 \mathrm{E}-\mathrm{C} 9$ $1.435 \mathrm{E}-\mathrm{C} 9$ $1.405 E-C 9$ $1.702 \mathrm{E}-\mathrm{C} 9$ $1.868 \mathrm{E}-\mathrm{C} 9$ $2.466 \mathrm{E}-\mathrm{Cg}$ $2.358 E-C 9$ $2.778 \mathrm{E}-\mathrm{O} 9$ $3.747 \mathrm{E}-\mathrm{C} 9$ $5.421 \mathrm{E}-\mathrm{C} 9$ $9.846 \mathrm{E}-\mathrm{Cg}$ $2.433 E-C 8$ $9.107 E-C 8$

$5.729 E-C 8$

$10 C .0$

$1.663 E-C 9$ $1.667 \mathrm{E}-\mathrm{C} 9$ $1.682 E-C 9$ $1.714 \mathrm{E}-\mathrm{C} 9$ $1.765 \mathrm{E}-\mathrm{C} 9$ $1.842 \mathrm{E}-09$ $1.950 \mathrm{E}-\mathrm{C} 9$ .C99E-C $2.3 \mathrm{C} 2 \mathrm{E}-09$ 2.862E-09 3. $C 37 \mathrm{E}-\mathrm{C} 9$ $3.549 \mathrm{E}-09$ $4.386 E-C 9$ $5.856 \mathrm{E}-\mathrm{CO}$ $8.482 \mathrm{E}-\mathrm{C} 9$ $2.060 E-C 8$ $8.977 E-08$

500.0

$$
60 \mathrm{C} .0
$$

$1.632 \mathrm{E}-09$ $1.637 E-C 9$ $1.658 \mathrm{E}-\mathrm{C9}$ $1.698 \mathrm{E}-09$ $1.758 \mathrm{E}-09$ $1.855 \mathrm{E}-\mathrm{CO}$ $1.955 E-C 9$ 2.1 $2 E-09$ 2.53 E-CO 2.846E-CQ 3.251E-CO $3.251 E-C 9$ $4.491 E-C 9$ $7.051 E-C O$ $1.310 \mathrm{E}-\mathrm{C} 8$

$3.735 E-C 8$

$1.209 E-09$

$1.212 \mathrm{E}-09$

$1.228 E-C 9$
l. $258 E-09$

1. $30.3 \mathrm{E}-\mathrm{C} 9$

$1.365 \mathrm{E}-09$

$1.448 \mathrm{E}-\mathrm{C} 9$

$1.556 \mathrm{E}-09$

$1.695 E-C 9$

$1.871 E-C 9$

.

$2.75 \mathrm{E}-09$

.

(3)

$4.881 E-09$

$8.01 C E-09$ $6.012 \mathrm{E}-\mathrm{C8}$

$15 \mathrm{C.0}$ RANGE (METERS) 2.113E-09 $2.119 E-09$ $2.140 E-09$ $2.184 \mathrm{E}-09$ $2.253 E-C 9$ $2.353 \mathrm{E}-09$ $2.493 \mathrm{E}-09$ $2.682 \mathrm{E}-\mathrm{C} 9$ $2.937 \mathrm{E}-09$ $3.350 \mathrm{E}-09$ 3. $777 \mathrm{E}-09$ $4.343 E-09$

$5.387 E-09$

$6.875 E-C 9$

$9.424 E-C 9$

$1.685 \mathrm{E}-\mathrm{C} 8$

$7.482 E-08$

20C.0

250.0

300.0

$400 \cdot C$

$2.36 C E-09$
$2.366 E-09$

$2.437 E-09$ $2.471 E-09$

$2.392 \mathrm{E}-09$

$2.527 \mathrm{E}-09$

$2.523 \mathrm{E}-09 \quad 2.612 \mathrm{E}-09$

$2.639 E-09 \quad 2.733 E-09$

$2.898 \mathrm{E}-09$

$3.118 \mathrm{E}-09$

$3.292 \mathrm{E}-09 \quad 3.409 \mathrm{E}-09$

$3.631 \mathrm{E}-09 \quad 3.75 \mathrm{CE}-09$

$4.2 C 9 E-09 \quad 4.338 E-09$

$4.850 E-09 \quad 4.969 E-09$

$5.885 \mathrm{E}-09 \quad 5.982 \mathrm{E}-09$

$7.336 E-09 \quad 7.347 E-09$

$9.783 \mathrm{E}-09 \quad 9.591 \mathrm{E}-0$

$1.55 C E-08 \quad 1.417 E-08$

4.582E-08

$6.408 E-08$

$6.516 \mathrm{E}-08$

$6.341 E-08$

RANGE (METERS)

900.0

1200.0

1500.0

3. $861 E-10$ $3.873 \mathrm{E}-10$ $3.924 \mathrm{E}-10$

$4.021 E-10$

$9.93 C E-11$

$9.961 \mathrm{E}-11$

2. $247 \mathrm{E}-11$

$2.254 \mathrm{E}-11$

$2.284 E-11$

$2.340 E-11$

1.034E-10

$1.071 E-10$

$2.424 E-11$

$4.365 \mathrm{E}-10$

$1.123 \mathrm{E}-10$

$2.539 E-11$

$2.689 E-11$

$4.967 \mathrm{E}-10 \quad 1.275 \mathrm{E}-10$

4. $1.383 \mathrm{E}-10$

$1.518 E-10$

(

$3.422 E-11$

$3.420 E-11$

$1.685 E-10$

4.232E-11

$4.232 E-11$

2.14

$2.446 \mathrm{E}-10$
$2.814 \mathrm{E}-10$
$3.254 \mathrm{E}-10$

$6.410 E-11$

$3.254 E-10$

$3.254 E-10$

. 0 C 6E-1

$1.819 E-09$

$2.076 E-09$

$4.628 \mathrm{E}-10$

$4.685 \mathrm{E}-12$

$4.748 \mathrm{E}-12$

$5.039 E-12$

$5.277 E-12$

(5.

$6.477 \mathrm{E}-12$

$7.086 \mathrm{E}-12$

. $731 \mathrm{E}-12$

$807 \mathrm{E}-12$

1. 1 C TE-11

$1.251 \mathrm{E}-11$
$1.4 C 8 \mathrm{E}-11$

$1.4 C 8 E-11$
$1.585 \mathrm{E}-11$

8.261E-09

9. $526 \mathrm{E}-11$
$.070 E-09$

$2.076 \mathrm{E}-09$

$2.101 \mathrm{E}-09$

$2.151 \mathrm{E}-09$

$2.226 \mathrm{E}-\mathrm{Cg}$

$2.332 \mathrm{E}-09$

$2.475 E-C 9$

$2.662 E-09$

$2.905 E-09$

$3.218 \mathrm{E}-09$

$3.629 E-09$

$4.164 E-09$

4.884E-09

$5.870 E-C 9$

7.310E-O9

$9.760 E-09$

$2.151 E-C 8$

4. $900 E-08$

$1800 . \mathrm{C}$ 
COS INE

$-1.00 O C O E$ CO $-9.89401 \mathrm{E}-01$ $-9.44575 E-0$ $-7.55044 \mathrm{E}-01$ $-6.17876 \mathrm{E}-01$ $-4.58017 E-01$ $-2.81605 \mathrm{E}-01$ $-9.50125 \mathrm{E}-02$ $9.50125 \mathrm{E}-\mathrm{C}^{2}$ $2.81605 E-01$ 4.58017E-O $6.17876 E-01$ $7.55044 \mathrm{E}-01$ $8.65631 \mathrm{E}-21$ $9.44575 \mathrm{E}-01$ $9.894 C 1 E-01$

TOTAL

COSINE

-1.00000 E 00 $-0.89401 E-01$ $-9.44575 E-0$ $-8.65631 E-01$ $-7.55044 \mathrm{E}-01$ $-6.17876 E-C$ -4.58017E-O $-2.81605 E-01$

$-9.50125 E-02$

$2.81605 \mathrm{E}-01$

4.58017E-0

$6.17876 \mathrm{E}-01$

..55044E- 01

$8.65631 \mathrm{E}-01$

$9.89401 E-O 1$
TOTAL
75.0 1. $C 66 \mathrm{E}-\mathrm{C} 9$ 1. C $76 \mathrm{E}-\mathrm{C} 9$ $1.096 \mathrm{E}-\mathrm{CO}$

$1.130 \mathrm{E}-09$

$1.181 \mathrm{E}-09$

$1.253 \mathrm{E}-\mathrm{C} 9$

$1.354 \mathrm{E}-\mathrm{C} 9$

$1.494 \mathrm{E}-\mathrm{Cg}$

$1.921 E-C 9$

$1.888 \mathrm{E}-\mathrm{Cg}$

$3.149 E-C 9$

$4.661 E-C 9$
$8.606 E-C 9$

$2.223 \mathrm{E}-\mathrm{C} 8$

8. 547E-08

4. $985 E-08$

$50 \mathrm{C} \cdot \mathrm{C}$

$.636 E-C 9$ $1.640 \mathrm{E}-\mathrm{C} 9$

$1.657 E-C 9$

$1.689 \mathrm{E}-\mathrm{C} 9$

$1.738 \mathrm{E}-\mathrm{C} 9$

$1.805 E-C 9$

$1.894 E-C 9$

2. C10E-C9

$2.158 \mathrm{E}-09$

$2.348 E-C 9$

2. $590 E-C 9$

2. $905 \mathrm{E}-\mathrm{C} 9$

$3.320 \mathrm{E}-\mathrm{C} 9$

$3.882 E-C 9$

$5.688 \mathrm{E}-09$

1. $149 E-C 8$

$10 \mathrm{C} \cdot \mathrm{C}$

150.0

RANGE (METERS)

250.0

360.0

$400 . c$

$1.21 C E-09$

$1.313 E-C 9$

$351 E-C \theta$

.393E-C9

$1.455 \mathrm{E}-\mathrm{C} 9$

$.544 \mathrm{E}-09$

$1.667 \mathrm{E}-\mathrm{C} 9$

$.836 \mathrm{E}-\mathrm{CO}$

$2.368 \mathrm{E}-09$

$8.379 E-C 8$

5.149E-C 8

600.0

$1.692 E-09$ $1.696 \mathrm{E}-09$

$.713 E-C 9$

. $147 E-09$

$1.882 \mathrm{E}-\cap 9$

$1.994 E-09$

$2.146 E-09$

$2.352 E-C 9$

$2.761 E-C 9$

$2.983 E+C 9$

$3.553 E-C 9$

$4.329 \mathrm{E}-09$

$5.692 E-C 9$

$7.816 \mathrm{E}-09$

$1.46 C E-C 8$

$6.905 E-08$

$1.937 \mathrm{E}-09$

$1.942 \mathrm{E}-09$

$2.0 C 2 E-09$

$2.065 E-09$

$2.155 \mathrm{E}-\mathrm{Cg}$

$2.280 E-C 9$

$2.448 E-C 9$

$2.672 \mathrm{E}-09$

$2.900 \mathrm{E}-09$

$3.442 E-09$

$3.898 \mathrm{E}-09$

4. $804 E-C 9$

$5.974 \mathrm{E}-\mathrm{C} 9$

8.096E-C 9

$1.314 E-C 8$

$5.384 E-C 8$

5. $374 E-08$

$5.425 E-C 8$

RANGE (METERS)

900.0

1200.0

1500.0

1800.0

C - 293E-C9

. $331 \mathrm{E}-\mathrm{C} 9$

$.368 \mathrm{E}-09$

$1.419 E-C 9$

$1.486 E-C 9$

$1.573 E-09$

$1.683 \mathrm{E}-\mathrm{C9}$

$1.822 \mathrm{E}-\mathrm{Cg}$

$1.998 \mathrm{E}-\mathrm{CS}$

2.222E-CS

$2.512 \mathrm{E}-\mathrm{CO}$

3.

$4.224 \mathrm{E}-09$

$7.045 E-C 9$

. $812 E-10$

$1.381 \mathrm{E}-10$

3. $376 E-1$

$.424 E-12$

4.823E-10 $1.384 E-10$

$4.870 E-10$

$1.397 \mathrm{E}-10$

3.383E-11

$7.44 \mathrm{CE}-12$

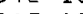

$1.421 \mathrm{E}-10$

$3.414 E-11$

$7.507 \mathrm{E}-12$

1.5C4E-10 $3.669 \mathrm{E}-11 \quad 8.058 \mathrm{E}-12$

$5.777 E-10 \quad 1.643 E-10$

$6.135 E-10$ 1.737E-10

$7.790 E-10$

(6)

.

$1.852 \mathrm{E}-10$

$1.991 E-10$

$2.158 \mathrm{E}-10$

$2.358-10$

2.58 E-10

$1.095 \mathrm{E}-09 \quad 2.884 \mathrm{E}-10$

$1.26 \mathrm{E}-09$ 3.233E-10

$8.368 \mathrm{E}-12$

$.994 E-11 \quad 8.753 E-12$

$4.214 \mathrm{E}-11 \quad 9.218 \mathrm{E}-12$

1.774E-12

4.

5.

6.109E-11 1.209E-11

$695 \mathrm{E}-11$ 1. $424 \mathrm{E}-11$

$364 \mathrm{E}-11 \quad 1.547 \mathrm{E}-11$

$1.694 \mathrm{E}-11$

$1.938 \mathrm{E}-09$

$1.943 \mathrm{E}-\mathrm{C} 9$

$1.963 E-09$
$2.003 E-09$

$2.062 E-C 9$

$2.256 \mathrm{E}-\mathrm{C} 9$

$2.591 \mathrm{E}-\mathrm{C} 9$

$2.833 \mathrm{E}-\mathrm{C} 9$

$3.156 E-C 9$

$3.573 E-09$

$4.143 E-09$
$4.932 E-09$

$6.111 \mathrm{E}-\mathrm{C} 9$

$8.183 \mathrm{E}-09$
$1.897 \bar{E}-08$

$4.281 E-C 8$

$3.400 E-C 8$

2.567E-08

$8.866 E-09$

$2.448 \mathrm{E}-09$

5. $854 \mathrm{E}-10$

$1.269 E-1 C$ 
COSINE

$-1.00000 E 00$ $-9.89401 E-01$ -8.656 $-7.55044 \mathrm{E}-01$ $-6.17876 \mathrm{E}-01$ $-4.58017 E-01$ $-2.81605 \mathrm{E}-\mathrm{Cl}$ $-9.50125 E-02$ $9.50125 \mathrm{E}-02$ $2.81605 E-01$ 4. $58017 \mathrm{E}-01$ $6.17876 \mathrm{E}-01$ 7. $55044 \mathrm{E}-01$ $8.65631 \mathrm{E}-01$ 9.89401E-01

TOTAL

COSINE

-1.00000 C CO $-9.894 \mathrm{C} 1 \mathrm{E}-01$ $-9.44575 \mathrm{E}-01$ $-8.65631 \mathrm{E}-01$ $-7.55044 \mathrm{E}-01$ $-6.17876 E-01$ $-4.58017 E-01$ $-2.81605 E-01$ $-9.5 \mathrm{C} 125 \mathrm{E}-02$ $2.81605 E-01$ 4.58017E-0 1 $6.17876 \mathrm{E}-01$ $7.55044 E-01$ $8.65631 E-01$ $9.89401 E-01$

TOTAL

\begin{tabular}{|c|c|c|c|c|c|c|}
\hline 75.0 & $100 . C$ & $150 . C$ & $\begin{array}{l}\text { JGE (MEIERS) } \\
2 C \mathrm{C} \cdot \mathrm{C}\end{array}$ & $250 . c$ & $300 \cdot 0$ & 400.0 \\
\hline $290 E-11$ & $1.145 E-10$ & $1.459 E-10$ & $1.628 E-10$ & $1.675 E-10$ & $1.635 E-1 C$ & $1.401 E-10$ \\
\hline $310 \mathrm{E}-11$ & $1.147 \mathrm{E}-1 \mathrm{C}$ & $1.463 E-10$ & $1.632 \mathrm{E}-10$ & $1.68 \mathrm{CE}-10$ & $1.640 E-10$ & $1.405 \mathrm{E}-10$ \\
\hline & $1.158 E-10$ & $1.478 E-10$ & $1.650 \mathrm{E}-10$ & $1.700 E-10$ & $1.660 \mathrm{E}-10$ & $1.423 \mathrm{E}-10$ \\
\hline & $1.180 E-10$ & $1.508 \mathrm{E}-10$ & $1.687 \mathrm{E}-10$ & $1.739 \mathrm{E}-10$ & $1.700 \mathrm{E}-10$ & $1.458 \mathrm{E}-10$ \\
\hline-11 & $1.216 \mathrm{E}-10$ & $1.557 \mathrm{E}-10$ & $1.743 \mathrm{E}-10$ & $1.799 \mathrm{E}-10$ & $1.760 E-10$ & $1.512 \mathrm{E}-10$ \\
\hline $27 E-10$ & $1.27 C E-10$ & $1.627 E-10$ & $1.824 \mathrm{E}-10$ & $1.885 \mathrm{E}-10$ & $1.845 \mathrm{E}-1 \mathrm{C}$ & $1.587 \mathrm{E}-10$ \\
\hline $87 E-1 C$ & $1.345 E-1 C$ & $1.725 \mathrm{E}-10$ & $1.936 E-10$ & $2.001 E-10$ & $1.961 \mathrm{E}-1 \mathrm{C}$ & $1.687 \mathrm{E}-10$ \\
\hline-10 & $1.448 \mathrm{E}-1 \mathrm{C}$ & $1.858 \mathrm{E}-10$ & $2.085 \mathrm{E}-10$ & $2.157 \mathrm{E}-10$ & $2.113 \mathrm{E}-10$ & $1.820 \mathrm{E}-10$ \\
\hline $285 E-10$ & $1.590 E-10$ & $2 . C 36 E-10$ & $2.283 E-10$ & $2.361 \mathrm{E}-10$ & $2.313 E-10$ & $1.991 E-10$ \\
\hline & $1.972 \mathrm{E}-1 \mathrm{C}$ & $2.330 E-10$ & $2.517 E-10$ & & & $2.212 \mathrm{E}-10$ \\
\hline $1.623 \mathrm{E}-10$ & 2.C95E-1C & $2.616 \mathrm{E}-10$ & $2.927 \mathrm{E}-10$ & $3.015 \mathrm{E}-10$ & $2.912 \mathrm{E}-10$ & $2.502 \mathrm{E}-10$ \\
\hline $1.910 \mathrm{E}-10$ & $2.458 \mathrm{E}-1 \mathrm{C}$ & $3.020 E-10$ & $3.368 \mathrm{E}-10$ & $3.451 \mathrm{E}-1 \mathrm{C}$ & $3.385 E-10$ & $2.879 E-10$ \\
\hline & 3.C $34 E-1 C$ & $3.731 \mathrm{E}-10$ & 4.C $92 E-10$ & $4.162 \mathrm{E}-1 \mathrm{C}$ & $4.003 E-10$ & $3.384 \mathrm{E}-10$ \\
\hline 3.79 & $4.072 E-10$ & $4.772 \mathrm{E}-10$ & $5.097 E-10$ & $5.108 \mathrm{E}-10$ & $4.905 \mathrm{E}-1 \mathrm{C}$ & $4.074 E-10$ \\
\hline & $5.916 E-10$ & $6.545 E-10$ & $6.799 \mathrm{E}-10$ & $6.671 \mathrm{E}-10$ & $6.265 E-10$ & $5.079 \mathrm{E}-10$ \\
\hline $1.707 E-C 9$ & $1.448 \mathrm{E}-\mathrm{C} 9$ & $1.180 \mathrm{E}-09$ & $1.082 E-C 9$ & $9.874 E-10$ & $8.885 \mathrm{E}-10$ & $6.793 \mathrm{E}-10$ \\
\hline 5035 & $6.397 E-09$ & $5.332 E-C 9$ & $4.2 C 4 E-09$ & $3.264 E-09$ & $2.533 E-C 9$ & $1.527 \mathrm{E}-09$ \\
\hline $010 E-C 9$ & $4.20 \mathrm{CE}-\mathrm{Cg}$ & $4.472 E-09$ & $4.542 E-09$ & $4.413 E-09$ & $4.148 \mathrm{E}-\mathrm{C} 9$ & $3.383 E-09$ \\
\hline
\end{tabular}

500.0

$600 . c$

RANGE
900.0

(METERS)

7.981E-11 8. $C 07 E-11$ $1.095 \mathrm{E}-10$

$1.109 \mathrm{E}-1 \mathrm{C}$

$1.138 \mathrm{E}-10$

$1.180 E-10$

$1.240 E-1 C$

$1.319 \mathrm{E}-1 \mathrm{C}$

$1.423 \mathrm{E}-10$

$1.557 \mathrm{E}-10$

$1.729 E-10$

$1.949 \mathrm{E}-10$

$2.234 \mathrm{E}-1 \mathrm{C}$

$2.607 E-10$

$3.1 C 6 \mathrm{E}-1 \mathrm{C}$

4.89BE-10

$9.269 \mathrm{E}-1 \mathrm{C}$

$8.116 \mathrm{E}-11$

$8.643 \mathrm{E}-1$

$9 . C 84 E-11$

$9.67 C E-11$

1. $C 43 E-10$

1. $141 \mathrm{E}-1 \mathrm{C}$

$1.266 E-1 C$

$1.425 \mathrm{E}-1 \mathrm{C}$

$1.628 E-1 C$

$1.891 \mathrm{E}-1 \mathrm{C}$

$2.233 E-10$

$3.382 \mathrm{E}-1 \mathrm{C}$

$5.646 E-10$

2. $560 \mathrm{E}-\mathrm{C} 9$

$1.836 E-09$
$2.465 \mathrm{E}-11$

$2.474 \mathrm{E}-11$

$2.509 \mathrm{E}-11$
$2.576 \mathrm{E}-11$

$2.677 \mathrm{E}-11$

2.816E-11

3. $C 00 E-11$

$3.540 \mathrm{E}-11$

3. $920 \mathrm{E}-1$

$4.398 E-11$

4. $995 E-11$

$6.672 E-11$

7.672E-11

$0.371 \mathrm{E}-11$

$1.265 \mathrm{E}-10$

$5.496 E-10$
$12 \mathrm{CC}$. C

$6.169 \mathrm{E}-12$

$6.191 \mathrm{E}-12$

$6.452 E-12$

$6.7 C 7 E-12$

$7.060 E-12$

$7.523 E-12$

$8.12 \mathrm{CE}-12$

$8.875 E-12$

$9.820 E-12$

$1.099 E-11$

$1.244 E-11$

$1.637 E-11$

$1.637 E-11$

$2.208 E-11$

2.208E-11

1.35 E 10
1500.0

. $366 \mathrm{E}-12$

$1.371 \mathrm{E}-12$
$1.391 \mathrm{E}-12$

$1.430 \mathrm{E}-12$

$1.486 \mathrm{E}-12$

$1.565 \mathrm{E}-12$

$1.668 \mathrm{E}-12$

$1.801 E-12$

$1.968 \mathrm{E}-12$

$2.176 \mathrm{E}-12$

2.747E-12

$2.747 E-12$

3.58CE-12

$4.1 C 8 E-12$

$4.708 E-12$

2.973E-1
$1800 . \mathrm{C}$

2.794E-13

2. $804 E-13$

$2.845 E-13$

$3.041 E-13$

$3.203 \mathrm{E}-13$

$3.686 E-13$

4. C26E-13

$4.450 E-13$

4.970E-13

$5.603 E-13$

$7.254 \mathrm{E}-13$

$8.272 E-13$

$9.383 \mathrm{E}-13$

6. $C 43 E-12$ 
COSINE

-1. CODONE OO $-9.894 \mathrm{C} 1 \mathrm{E}-0$ $-9.44575 E-01$ $-8.65631 \mathrm{E}-01$ $-7.55044 \mathrm{E}-91$ $-6.17876 E-01$ $-4.58017 \mathrm{E}-01$ $-2.81605 \mathrm{E}-01$ $-9.50125 \mathrm{E}-\mathrm{C} 2$ $9.50125 \mathrm{E}-02$ $2.81605 \mathrm{E}-01$ $4.58017 \mathrm{E}-01$ $6.17876 E-01$ $7.55044 \mathrm{E}-01$ $8.65631 \mathrm{E}-01$ $9.44575 E-01$

TOTAL

COSINE

$-1.00000 E$ OC $-9.894 C 1 E-01$ $-8.65631 \mathrm{E}-01$ $-7.55044 \mathrm{E}-01$ - $.55044 \mathrm{E}-01$ . . $0125 E-O 2$ . $4.58017 E-01$ $4.58017 E-01$ $7.55044 E-01$ $8.65631 \mathrm{E}-01$ $9.44575 \mathrm{E}-01$ $9.894 \mathrm{CLE}-01$

TOTAL

$\begin{array}{lc}75.0 & 1 C C . C \\ 1.542 E-1 C & 1.889 E-10 \\ 1.546 E-1 C & 1.894 E-1 C \\ 1.559 E-10 & 1.011 E-1 C \\ 1.587 E-1 C & 1.948 E-1 C \\ 1.633 E-1 C & 2 . C C 7 E-10 \\ 1.7 C 2 E-10 & 2.094 E-1 C \\ 1.801 F-1 C & 2.217 E-1 C \\ 1.938 E-1 C & 2.388 E-1 C \\ 2.127 E-10 & 2.620 F-1 C \\ 2.829 E-10 & 3.231 E-1 C \\ 2.691 E-1 C & 3.465 E-1 C \\ 3.165 E-1 C & 4 . C 35 E-1 C \\ 4.272 E-10 & 5 . C 11 E-1 C \\ 6.194 E-10 & 6.685 E-1 C \\ 1.124 E-09 & 9.662 E-1 C \\ 2.751 E-C 9 & 2.343 E-C 9 \\ 1.036 E-C 8 & 1 . C 21 E-C 8 \\ 6.521 E-09 & 6.841 E-C 9\end{array}$

RANGE (METERS)

$2.384 E-10$

$2.390 E-10$

$2.415 E-10$

$2.465 \mathrm{E}-10$

$2.660 E-10$

$2.820 \mathrm{E}-10$

$3.038 \mathrm{E}-10$

$3.331 E-10$

$3.785 \mathrm{E}-10$

$4.310 E-10$

$4.931 E-10$

$6.146 E-10$

$7.819 E-13$

1. C 75E-C

$1.921 \mathrm{E}-0$

$7.286 E-09$

$2.638 \mathrm{E}-10$

$2.645 \mathrm{E}-10$

$2.675 \mathrm{E}-10$

$2.826 \mathrm{E}-10$

$2.959 E-10$

$3.141 E-10$

$3.386 E-10$

3. $712 \mathrm{E}-10$

$4.113 \mathrm{E}-10$

4.757E-10

$5.511 E-10$

$6.683 E-10$

$1.114 E=09$

$1.768 \mathrm{E}-\mathrm{C}$

$6.741 E=09$

$7.388 \mathrm{E}-09$

RANGE (METERS)

500.0

$60 \mathrm{c} .0$

GOC.

$120 \mathrm{C} . \mathrm{C}$

$9.454 \mathrm{E}-12$

1. $718 E-10$

$1.723 E-10$

$1.248 \mathrm{E}-10$

$1.253 \mathrm{E}-10$

3. $858 \mathrm{E}-11$

$3.821 E-11$

$9.487 E-12$

$9.628 E-12$

1.029E- 1

$1.354 E-1 C$

$1.029 E-11$

.

1.641E-1C

$2.747 E-1 C \quad 2.7 C 1 E-10$

. $107 E-10$

$3.574 E-1 C$

$4.188 E-1 C$

$5 . C 10 E-10$

$6.159 E-1 C$

$7.965 \mathrm{E}-1 \mathrm{C}$

$1.497 \mathrm{E}-\mathrm{C} 9$

4.092E-09

. C C $1 E-10$

$2.592 \mathrm{E}-10$

3. $23 \mathrm{E}-1 \mathrm{C}$

$3.587 \mathrm{E}-1 \mathrm{C}$

$4.351 \mathrm{E}-10$

$5.482 \mathrm{E}-1 \mathrm{C}$

$9.115 E-1 C$

$4.361 \mathrm{E}-11$

$1.158 \mathrm{E}-11$

. $3 \cap E-11$

$1.252 E-11$

$5.513 \mathrm{E}-11$

$1.252 \mathrm{E}-1$

$1.522 \mathrm{E}-11$

$6.8945-11$

$1.522 E-11$

$7.863 E-11$

$1.71 \mathrm{CE}-11$
$1.944 \mathrm{~F}-11$

$1.944 \mathrm{~F}-11$
$2.232 \mathrm{E}-11$

9. $081 \mathrm{E}-11$

$1.261 E-1 C$

$2.232 E-11$

$3.011 \mathrm{E}-1$

$1.506 E-10$

$3.524 \mathrm{E}-11$

$4.335 \mathrm{E}-11$

$2.921 E-09$

$8.643 E-10$

$2.114 E-10$
$1500 . C$

256.0

$.697 E-10$

$2.7 C 5 E-10$

$2.8 C 0 E-10$

$2.898 \mathrm{E}-1 \mathrm{C}$

$3.037 E-10$

$3.227 E-10$

$3.481 E-10$

$3.816 \mathrm{E}-10$

$4.223 E-10$

$4.877 E-10$

$5.624 E-10$

$6.772 E-10$

1.

1.615E-09

.244E-09

7.150E-C9

$2.082 \mathrm{E}-12$

2.09RE 12

$2.181 \mathrm{E}-12$

$2.26 \mathrm{E}-12$

$2.553 \mathrm{E}-12$

$2.761 \mathrm{E}-12$

$3.024 \mathrm{E}-12$

$3.024 E-12$

$3.763 \mathrm{E}-12$

$4.268 \mathrm{E}-12$

$4.883 E-12$

$5.62 \mathrm{CE}-12$

列 72

$7.475 \mathrm{E}-12$
$8.760 \mathrm{E}-12$

$4.613 E-11$
$40 \mathrm{c} . \mathrm{C}$

$2.221 E-10$ $2.257 E-1 C$ $2.399 E-10$ $2.520 E-10$ $2.683 E-10$ $2.898 \mathrm{E}-10$ 3.177E-10 $4.012 E-10$ $4.632 E-10$ $5.463 E-10$ $6.603 E=10$ $1.108 E-09$ $5.437 E-C 9$

1800.0

. $242 E-13$ 4. ( 4.625E-13 .205E-13 $630 \mathrm{E}-13$ . $6.833 \mathrm{E}-13$ $7.66 \mathrm{CE}-13$ $8.672 E-13$ $9.895 E-13$ $1.134 E-12$ $1.301 \mathrm{E}-12$ $1.483 \mathrm{E}-12$

$9.339 E-12$ 
COSINE

$-1.00 C O O E O O$
$-9.89401 E-01$
$-9.44575 E-01$
$-8.65631 E-01$
$-7.55044 E-01$
$-6.17876 E-01$
$-4.58017 E-01$
$-2.81605 E-01$
$-9.50125 E-02$
$9.50125 E-02$
$2.81605 E-01$
$4.58017 E-01$
$6.17876 E-01$
$7.55044 E-01$
$8.65631 E-01$
$9.44575 E-01$
$9.89401 E-01$

TOTAL

COSINE

-1.00000 E 00 $-9.89401 \mathrm{E}-01$ $-9.44575 \mathrm{E}-01$ $-8.65631 \mathrm{E}-01$ $-7.55044 \mathrm{E}-01$ $-6.17876 E-01$ $-4.58017 E-01$ $-2.81605 E-01$ $-9.50125 E-02$ $2.81605 \mathrm{E}-01$ $2.81605 \mathrm{E}-01$ $4.58017 E-01$ $7.55044 \mathrm{E}-01$ $8.65631 \mathrm{E}-01$ $8.65631 E-01$ $9.89401 E-01$

TOTAL

$$
\text { 75. C }
$$

$1.991 E-13$ $1.994 \mathrm{E}-13$ $2.004 E-13$ $2.024 E-13$ $2.052 E-13$ 2. $089 E-13$ $2.134 E-13$ $2.185 \mathrm{E}-13$ $2.242 E-13$ $2.303 E-13$ $2.366 E-13$ $8 \mathrm{E}-13$ $2.485 E-13$ $2.536 E-13$ $2.581 E-13$ $2.611 \mathrm{E}-13$ $2.610 E-13$

$2.872 E-12$

$$
100.0
$$

3. $325 \mathrm{E}-13$ $3.33 \mathrm{CE}-13$ 3. $352 \mathrm{E}-13$ 3.394E-13 $3.537 \mathrm{E}-13$ $3.636 \mathrm{E}-13$ $3.751 E-13$ $3.880 \mathrm{E}-13$ $4.020 \mathrm{E}-13$ $4.165 \mathrm{E}-13$ $4.311 E-13$ $4.455 \mathrm{E}-13$ $4.581 E-13$ $4.684 E-13$ $4.766 E-13$ 4.791E-13 5.C. $13 E-12$

500.0

$1.883 E-12$ $1.893 E-12$ $1.933 E-12$ $2.011 \mathrm{E}-12$ $2.126 \mathrm{E}-12$ 2. $286 E-12$ $2.501 \mathrm{E}-12$ $2.787 \mathrm{E}-12$ $3.164 E-12$ $3.657 \mathrm{E}-12$ $4.297 E-12$ $6.13 \mathrm{E}-12$ $6.132 E-12$ $8.649 \mathrm{E}-12$ $8.649 E-12$ $9.879 \mathrm{E}-12$

5. $281 E-11$

\section{$1.705 \mathrm{E}-12$} $1.715 \mathrm{E}-12$ $1.755 \mathrm{E}-12$ $1.833 \mathrm{E}-12$ $1.948 \mathrm{E}-12$ $2.157 E-12$ $2.323 E-12$ $2.616 E-12$ $3.014 E-12$ 4. $275 \mathrm{E}-12$ $4.275 E-12$ . $244 E-12$ $6.520 E-12$ 1.132E-12 1.तO1E-11 $1.329 E-11$

$5.475 E-11$

150.0
ANGE $6.510 E-13$ $6.525 \mathrm{E}-13$
$6.585 \mathrm{E}-13$ $6.702 \mathrm{E}-13$ $6.875 \mathrm{E}-13$ $7.104 \mathrm{E}-13$ $7.386 \mathrm{E}-13$ $7.723 \mathrm{E}-13$ $8.112 \mathrm{E}-13$ $8.547 \mathrm{E}-1$ 9. $.14 E-13$ . $502 \mathrm{E}-13$ $9.990 E-13$

1. $C 44 E-12$

$1.083 E-12$

$112 \mathrm{E}-1$

$1.128 \mathrm{E}-12$

1.C71E-11

$2 \mathrm{C} 0.0$
(METERS)
250.0

300.0 400.0 $.907 E-13$ $1.005 \mathrm{E}-12$ $1.028 \mathrm{E}-12$ $1.061 \mathrm{E}-12$ $1.1 \mathrm{C} 5 \mathrm{E}-12$ $1.161 \mathrm{E}-12$ $1.229 \mathrm{E}-12$ $1.309 \mathrm{E}-12$ $.401 \mathrm{E}-12$ $1.5 C 4 E-12$ $1.615 \mathrm{E}-12$ $1.729 E-12$ $1.839 \mathrm{E}-12$ $1.936 \mathrm{E}-12$ $2.055 \mathrm{E}-12$

$1.772 \mathrm{E}-11$

\section{RANGE (METERS)} 900.0 $1200 . \mathrm{C}$

\section{$8.564 \mathrm{E}-13$} $8.643 E-13$ $8.949 E-13$ $9.484 \mathrm{E}-13$ $1.019 \mathrm{E}-12$ $1.111 E-12$ $1.237 \mathrm{E}-12$ $1.420 E-12$ $1.687 \mathrm{E}-12$ 2.C78E-12 $2.651 E-12$ $3.510 E-12$ $4.822 E-12$ $6.805 E-12$ $1.311 \mathrm{E}-11$ $1.622 \mathrm{E}-11$ $3.152 \mathrm{E}-13$ $3.21 \mathrm{CE}-13$ $3.415 \mathrm{E}-13$ $3.723 \mathrm{E}-13$ $4.042 E-13$ $4.376 E-13$ $4.819 E-13$ $5.543 E-13$ $6.725 E-13$ $8.553 E-13$ $1.134 \mathrm{E}-12$ $1.583 E-12$ 2. 6.74E 12 $6.123 E-12$

$9.694 E-12$

. $306 \mathrm{E}-12$ $1.310 \mathrm{E}-12$
$1.328 \mathrm{E}-12$ $1.363 \mathrm{E}-12$ $1.415 \mathrm{E}-12$ $1.485 E-12$ $1.574 \mathrm{E}-12$ $1.684 \mathrm{E}-12$ $1.818 \mathrm{E}-12$ 1. $975 \mathrm{E}-12$ 2. $157 \mathrm{E}-12$ $2.357 E-12$ 2. $571 \mathrm{E}-12$ $2.785 \mathrm{E}-12$ $2.982 \mathrm{E}-12$ $3.138 \mathrm{E}-12$ 3. $231 \mathrm{E}-12$

2. $532 E-11$

1500.0

$1.012 \mathrm{E}-13$ $1.055 \mathrm{E}-13$ $1.183 \mathrm{E}-13$ $1.371 \mathrm{E}-13$ 1. $503 \mathrm{E}-13$ $1.583 E-13$ $1.682 \mathrm{E}-13$ $1.909 E-13$ $2.364 E-13$ 3. $198 \mathrm{E}-13$ $4.190 E-13$ 5.97CE-1 $9.4 C 1 E-13$ $1.661 E-12$ $5.79 \mathrm{CE}-12$ $5.744 E-12$

$4.062 E-11 \quad 2.200 E-11 \quad 1.048 E-11 \quad 4.774 E-12$

$3.111 \mathrm{E}-14$

$3.397 \mathrm{E}-14$

$4.234 \mathrm{E}-14$

$5.197 E-14$

(5.

(5.8

6.5

6.

(1)

$.529 \mathrm{E}-13$

$2.135 E-13$

$3.423 \mathrm{E}-13$

$1.620 E-13$

$3.061 \mathrm{E}-12$

$5.061 E-12$

$4.062 E-11$

$4.774 \mathrm{E}-12$ $.566 \mathrm{E}-12$ $1.596 \mathrm{E}-12$ $1.643 E-12$ $1.714 \mathrm{E}-12$ $1.809 \mathrm{E}-12$ $1.934 \mathrm{E}-12$ $2.090 \mathrm{E}-12$ $2.283 \mathrm{E}-12$ $2.517 \mathrm{E}-12$ 2.792E-12 3. $107 \mathrm{E}-12$ $3.454 \mathrm{E}-12$ $3.813 \mathrm{E}-12$ 4. $154 \mathrm{E}-12$ $4.434 \mathrm{E}-12$ 4. $607 \mathrm{E}-12$

3. $283 E-11$

1800.0

$1.874 \mathrm{E}-12$

$.908 \mathrm{E}-12$

.976E-12

$2.215 \mathrm{E}-12$

$2.399 \mathrm{E}-12$

$2.638 \mathrm{E}-12$

$2.942 E-12$

$3.804 \mathrm{E}-12$

$4.383 E-12$

$5.061 E-12$

. $813 E-12$

$6.577 \mathrm{E}-12$

$7.685 \mathrm{E}-12$

4. 54CE-1.1 
COS INE

$-1.00000 E$ CO $-9.89401 E-01$ $-9.44575 \mathrm{E}-01$ $-8.65631 E-01$ $-7.55044 E-01$ $-6.17876 E-01$
$-4.58017 E-01$ $-4.58017 \mathrm{E}-01$ $-2.81605 \mathrm{E}-01$ $-9.50125 \mathrm{E}-02$ $9.50125 \mathrm{E}-02$ $2.81605 E-01$ $4.58017 \mathrm{E}-01$ $7.55044 \mathrm{E}-01$ $8.65631 \mathrm{E}-01$ $9.44575 E-01$ $9.89401 E-01$

TOTAL

\section{COSINE}

-1.00000 E 00 $-9.89401 \mathrm{E}-01$ $-9.44575 E-C 1$ $-8.65631 \mathrm{E}-\mathrm{O} 1$ $-7.55044 \mathrm{E}-01$ $-6.17876 E-01$ $-2.81605 E-01$ $-2.81605 \mathrm{E}-01$ $9.50125 \mathrm{E}-02$ $2.81605 \mathrm{E}-01$ $4.58017 E-01$ 4.58017E-OI $7.55044 E-01$ . $55044 E-01$ $8.65631 E-01$ $9.89401 \mathrm{E}-01$

TOTAL
75.0

100.0

$2.036 \mathrm{E}-11$ $2.038 \mathrm{E}-11$ 2. $C 49 E-11$ $2.668 \mathrm{E}-11$ $2.134 \mathrm{E}-11$ $2.178 \mathrm{E}-1$ $2.230 \mathrm{E}-1$ $2.287 \mathrm{E}-1$ $2.347 \mathrm{E}-1$ $2.410 E-11$ $2.472 E-11$ $2.529 \mathrm{E}-11$ 2. $580 \mathrm{E}-1$ $2.625 \mathrm{E}-1$ $2.654 \mathrm{E}-1$ $2.654 \mathrm{E}-1$ $2.927 E-10$

$500 . \mathrm{C}$

$1.990 \mathrm{E}-1 \mathrm{C}$ 2.00OE-1C 2.C40E-10 $2.118 \mathrm{E}-1 \mathrm{C}$ $2.234 E-1 C$ $2.395 E-1 C$ $2.611 E-1 C$ 2.897E-10 $3.274 E-10$ $3.767 E-10$ $4.406-10$ $5.221 E-10$ $7.235 E-10$ $8.742 \mathrm{E}-10$ 80

1. C82E-O

$5.415 E-C 9$
$3.403 E-1$ $3.409 E-11$ $3.431 \mathrm{E}-11$ $3.535 \mathrm{E}-1$ $3.616 E-11$ 3. $714 \mathrm{E}-11$ $3.829 E-11$ 3.958E-1 4. $098 \mathrm{E}-11$ $4.243 E-11$ $4.388 \mathrm{E}-11$ $4.532 E-1$ $4.658 E-11$ $4.761 \mathrm{E}-1$ $4.842 E-11$ E

$5.111 E-10$

600.0

$1.824 E-1 C$ $1.834 \mathrm{E}-1 \mathrm{C}$ $1.875 \mathrm{E}-10$ $1.954 E-10$ 2. $669 E-10$ $2.229 E-10$ $2.446 \mathrm{E}-10$ $2.741 E-10$ $3.139 E-10$ $3.676 E-10$ $4.398 E-10$ $6.636 \mathrm{E}-10$ 8. $8.241 \mathrm{E}-1 \mathrm{C}$ 1.C11E-C9 1. $338 \mathrm{E}-\mathrm{C}$

$5.625 \mathrm{E}-\mathrm{C} 9$
150.0 RANGE (METERS)
$200 . \mathrm{C}$

250.0

$3 c r .0$

$400 . C$ $6.681 \mathrm{E}-11$
$6.696 \mathrm{E}-11$ $.023 E-10$ $1.348 \mathrm{E}-10$
$1.353 \mathrm{E}-10$ $1.623 \mathrm{E}-10$ $6.756 \mathrm{E}-11 \quad 1.034 \mathrm{E}-10$ 1. $1.371 \mathrm{E}-1 \mathrm{C}$ $6.873 \mathrm{E}-11 \quad 1.057 \mathrm{E}-10 \quad 1.406 \mathrm{E}-10$ $7.274 E-11 \quad 1.134 E-10 \quad 1.527 E-10$ $7.557 E-11 \quad 1.19 C E-10 \quad 1.617 E-1 C$ $7.894 \mathrm{E}-11 \quad 1.258 \mathrm{E}-10 \quad 1.727 \mathrm{E}-10$ $8.282 E-11 \quad 1.338 E-10 \quad 1.861 E-10$ $8.717 E-11 \quad 1.43 C E-10 \quad 2.018 E-10$ $9.182 \mathrm{E}-11 \quad 1.533 \mathrm{E}-10 \quad 2.199 \mathrm{E}-1 \mathrm{C}$ $9.670 E-11 \quad 1.643 E-10 \quad 2.399 E-10$ $1.016 \mathrm{E}-10 \quad 1.757 \mathrm{E}-10 \quad 2.613 \mathrm{E}-10$ $1 .(61 E-10 \quad 1.867 E-10 \quad 2.826 E-10$ $1.100 \mathrm{E}-10 \quad 1.964 \mathrm{E}-10 \quad 3.022 \mathrm{E}-10$ $1.129 \mathrm{E}-10 \quad 2.039 \mathrm{E}-10 \quad 3.178 \mathrm{E}-10$ $1.144 \mathrm{E}-10 \quad 2.082 \mathrm{E}-10 \quad 3.271 \mathrm{E}-10$

$1 . C 92 E-C 9$

1. $808 \mathrm{E}-09$

2. $585 E-09$

RANGE (METERS)

$$
\begin{aligned}
& \text { RANGE } \\
& 900.0
\end{aligned}
$$

$1200 . \mathrm{C}$

$15 \mathrm{CC.} 0$

$9.669 \mathrm{E}-11$ $9.748 \mathrm{E}-11$ $1 . \operatorname{COSE}-10$ 1. C $60 \mathrm{E}-10$ $1.132 \mathrm{E}-10$ $1.225 E-10$ $1.354 \mathrm{E}-10$ $1.538 \mathrm{E}-10$ $1.8 C 7 E-10$ $2.198 E-10$ $2.770 E-10$ $4.626 E-10$ 4.93010 $6.901 E-10$ $9.709 E-10$ $1.628 \mathrm{E}-09$ $3.867 \mathrm{E}-11$ $3.924 \mathrm{E}-11$ $4.132 \mathrm{E}-11$ $4.772 E-11$ 5. $120 E-11$ $5.5805-11$ 6. $7.514 E-11$ 7. $1.213 E-10$ 1. $660 \mathrm{E}-10$ 2. $4290-10$ 政 $0.717 E-10$ $1.357 \mathrm{E}-09$ $4.201 E-C 9 \quad 2.289 E-09$ $5.035 E-12$ 5. $318 \mathrm{E}-12$ $6.152 \mathrm{E}-12$ $7.123 \mathrm{E}-12$ $7.831 \mathrm{E}-12$ $7.840 \mathrm{E}-12$ $7.908 \mathrm{E}-12$ $8.687 E-12$ $1.051 E-11$ 1. $745 \mathrm{E}-11$ 2. $347 \mathrm{E}-11$ 3.616E-11 $6.763 \mathrm{E}-11$ $1.437 \mathrm{E}-10$ $5.574 \mathrm{E}-1 \mathrm{C}$ 5. $005 E-10$ 
4 PI R**2 AIR KERMA (GAMMAS)
(CM**2 ERGS/GRAM/STERADIAN/SOURCE NEUTRON)

COSINE

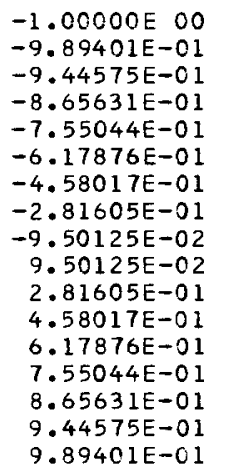

TOTAL

COSINE

\section{$-1.00000 E$ OC $-9.894 \mathrm{ClE}-01$ $-9.44575 E-01$ $-8.65631 \mathrm{E}-01$ $-7.55044 \mathrm{E}-01$ $-6.17876 \mathrm{E}-01$ $-4.58017 \mathrm{E}-01$ $-9.50125 \mathrm{E}-\mathrm{C}$ $9.50125 \mathrm{E}-02$ $2.81605 \mathrm{E}-0$ 4.58017E-0 $6.17876 \mathrm{E}-01$ $7.55044 \mathrm{E}-01$ $8.65631 E-0$ $9.44575 \mathrm{E}-01$ 9.89401E-01}

TOTAL
75.0

$2.247 \mathrm{E}-11$ $2.250 E-11$ $260 \mathrm{E}-11$ $2.278 E-11$ .341E-11 $2.383 \mathrm{E}-1$ $2.432 \mathrm{E}-11$ $2.486 \mathrm{E}-1$ $2.544 \mathrm{E}-11$ $2.603 \mathrm{E}-11$ $2.662 \mathrm{E}-11$ 2. $715 \mathrm{E}-11$ $2.763 \mathrm{E}-1$ 2.8C6E-11 $2.833 \mathrm{E}-11$ $2.833 \mathrm{E}-11$

$3.175 \mathrm{E}-1 \mathrm{C}$

500.0

$2.633 \mathrm{E}-10$ $2.643 \mathrm{E}-10$
$2.684 \mathrm{E}-1 \mathrm{C}$ $2.684 \mathrm{E}-1 \mathrm{C}$
$2.764 \mathrm{E}-10$ $2.764 E-10$
$2.882 E-10$ $2.882 E-10$
$3 . C 44 E-10$ 3.C44E-10 . $260 E-10$ $3.544 E-10$ . $914 \mathrm{E}-10$ 4. $392 \mathrm{E}-10$ $5.006 E-10$ $5.781 E-10$ $6.737 E-10$ $7.861 \mathrm{E}-10$ $9.078 \mathrm{E}-1 \mathrm{C}$ $1.100 \mathrm{E}-\mathrm{C}$

$6.136 \mathrm{E}-\mathrm{C} 9$
100.0

$3.784 E-11$ $3.789 \mathrm{E}-1$ $3.810 E-11$ $3.851 \mathrm{E}-11$ $3.987 \mathrm{E}-11$ 4. $981 \mathrm{E}-11$ 4.190E-11 4. $312 \mathrm{E}-11$ 4.312E-11 $4.582 \mathrm{E}-11$ 4.72CE-11 4. $856 \mathrm{E}-11$ 4.975E-1 5.C 71E-11 5. $148 \mathrm{E}-1$ $5.172 E-11$

$5.548 E-10$

600.0

$2.563 \mathrm{E}-1 \mathrm{C}$ $2.573 E-10$ $2.616 \mathrm{E}-10$ $2.698 \mathrm{E}-1 \mathrm{C}$ $2.982 \mathrm{E}-10$ $3.2 C 4 E-10$ $3.5 C 1 E-10$ $3.896 \mathrm{E}-1 \mathrm{C}$ $4.422 E-1 C$ $5.121 E-10$

$6.044 \mathrm{E}-10$

$7.245 \mathrm{E}-1 \mathrm{C}$

$8.747 \mathrm{E}-10$

$1.049 E-09$

$1.223 E-0$

1. $351 \mathrm{E}-\mathrm{C}$

$6.472 E-C 9$
1. 108 TO 2.350 MEV NEUTRON SOURCE

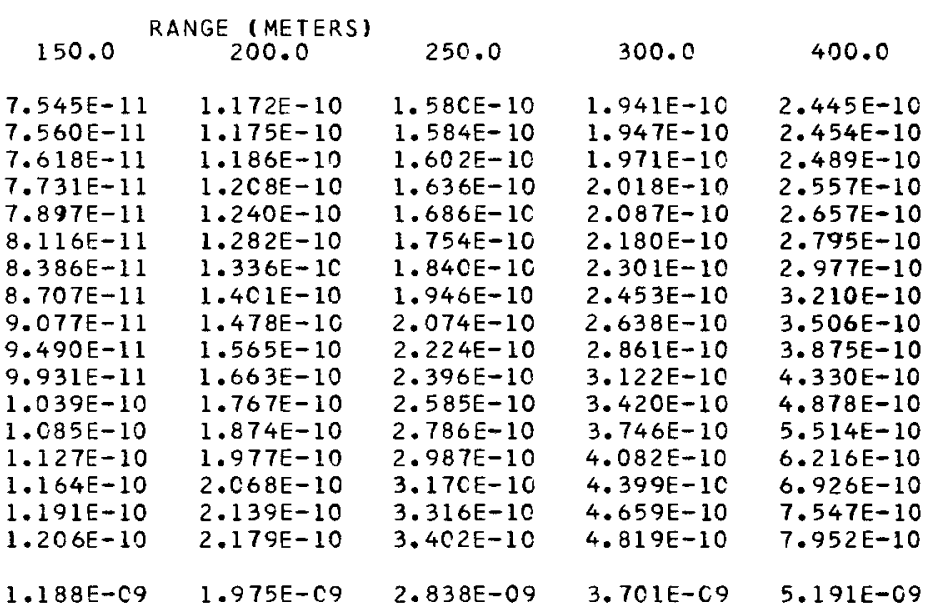

1500.0

1800.0

RANGE (METERS)
$900.0 \quad 12 \mathrm{CO} .0$

$8.521 E-11$

$.676 E-10$

$1.685 E-10$

. $583 E-11$

$8.810 E-11$

$9.567 \mathrm{E}-11$

1. $002 E-10$

$1.965 E-10$

$2.107 E-10$

$1.061 \mathrm{E}-10$

$1.149 \mathrm{E}-10$

$1.283 E-10$

$1.478 \mathrm{E}-10$

$1.765 \mathrm{E}-10$

$2.2 \mathrm{C} 8 \mathrm{E}-10$

$2.95 \mathrm{CE}-10$

$4.243 E-10$

$6.434 \mathrm{E}-10$

$9.698 \mathrm{E}-10$

$1.322 \mathrm{E}-09$

$1.311 \mathrm{E}-\mathrm{C}$

$2.883 \mathrm{E}-09$

$3.936 \mathrm{E}-11$ $3.981 \mathrm{E}-11$

$4.330 E-11$

4. $511 \mathrm{E}-1$

4. $667 E-11$

$4.860 \mathrm{E}-1$

$5.744 \mathrm{E}-11$

$6.573 \mathrm{E}-11$

$7.748 \mathrm{E}-11$

$9.566 \mathrm{E}-1$

1. $29 \mathrm{CE}-10$

$1.964 \mathrm{E}-10$

$3.321 E-10$

$5.742 E-1 C$

$8.883 E-10$

$5 . C 68 E-C 9$

$1.426 E-09$

$1.767 \mathrm{E}-1$

$1.881 \mathrm{E}-11$

$1.989 \mathrm{E}-1$

2.0B2E-1

$2.119 \mathrm{E}-1$

2.172E- 1

.298E-1

$2.530 E-1$

. 877 E- 11

$3.326 \mathrm{E}-1$

$5.254 \mathrm{E}-11$

$8.262 \mathrm{E}-11$

$1.533 \mathrm{E}-10$

$5.304 E-10$

$6.671 E-10$ 
COS INE

$-1.000 C 0 E 00$ $-9.894 C 1 E-01$ $-9.44575 \mathrm{E}-01$ $-8.65631 E-01$ $-7.55044 \mathrm{E}-01$ $-6.17876 E-01$ $-4.58017 E-01$ $-2.81605 \mathrm{E}-01$ $-9.50125 E-02$ $9.50125 E-02$ 2.81605E-01 4.58017E-त1 $7.55044 \mathrm{E}-01$ $8.65631 \mathrm{E}-01$ -1
-15 $9.89401 E-01$

TOTAL

COSINE

\section{$-1.00000 E$ OC} $-9.89401 \mathrm{E}-01$ - $.44575 E-C 1$ $-8.65631 \mathrm{E}-01$ $-6.17876 E-01$ . $-9.50125 \mathrm{E}-\mathrm{C} 2$ $.50125 \mathrm{E}-02$

.

$4.58017 \mathrm{E}-01$

$6.17876 \mathrm{E}-01$

$7.55044 \mathrm{E}-01$

$8.65631 \mathrm{E} \rightarrow 01$

$8.65631 E-01$

$9.89401 \mathrm{E}-01$

TOTAL

$\begin{array}{lc}75.0 & 100.0 \\ 2.102 \mathrm{E}-11 & 3.515 \mathrm{E}-11 \\ 2.105 \mathrm{E}-11 & 3.521 \mathrm{E}-11 \\ 2.115 \mathrm{E}-11 & 3.544 \mathrm{E}-11 \\ 2.135 \mathrm{E}-11 & 3.587 \mathrm{E}-11 \\ 2.165 \mathrm{E}-11 & 3.65 \mathrm{CE}-11 \\ 2.202 \mathrm{E}-11 & 3.733 \mathrm{E}-11 \\ 2.248 \mathrm{E}-11 & 3.834 \mathrm{E}-11 \\ 2.301 \mathrm{E}-11 & 3.952 \mathrm{E}-11 \\ 2.359 \mathrm{E}-11 & 4.083 \mathrm{E}-11 \\ 2.421 \mathrm{E}-11 & 4.227 \mathrm{E}-11 \\ 2.486 \mathrm{E}-11 & 4.37 \mathrm{E}-11 \\ 2.549 \mathrm{E}-11 & 4.525 \mathrm{E}-11 \\ 2.607 \mathrm{E}-11 & 4.672 \mathrm{E}-11 \\ 2.660 \mathrm{E}-11 & 4.802 \mathrm{E}-11 \\ 2.706 \mathrm{E}-11 & 4.907 \mathrm{E}-11 \\ 2.736 \mathrm{E}-11 & 4.990 \mathrm{E}-11 \\ 2.735 \mathrm{E}-11 & 5.016 \mathrm{E}-11 \\ 3.620 \mathrm{E}-1 \mathrm{C} & 5.272 \mathrm{E}-10\end{array}$

150.0
$6.907 E-11$
$6.923 E-11$
$.984 E-11$
$.105 E-11$
$.282 E-11$
$7.515 E-11$
$7.805 E-11$
$8.150 E-11$
$8.547 E-11$
$8.993 E-11$
$9.470 E-11$
$9.969 E-11$
$1.047 E-10$
$1.093 E-10$
$1.133 E-10$
$1.163 E-10$
$1.178 E-10$
$1.126 F-09$

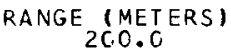

$25 \mathrm{C} . \mathrm{C}$

300.0

400.0

\section{$1.055 \mathrm{E}-10$}

1. $C 58 \mathrm{E}-10$

$1.07 C E-10$

$1.093 E-10$

$1.127 E-10$

$1.172 \mathrm{E}-10$

$1.230 \mathrm{E}-10$

$1.299 E-10$

$1.381 \mathrm{E}-10$

$1.476 \mathrm{E}-10$

$1.581 E-10$

$1.694 \mathrm{E}-10$

$1.81 C E-10$
$1.923 \mathrm{E}-10$

$1.923 \mathrm{E}-10$

2.C23E-10

2.1 CCE -10

$2.144 E-10$

$1.397 E-10$
$1.4 C 2 E-10$

$.42 \mathrm{CE}-10$

$1.456 \mathrm{E}-10$

$1.509 \mathrm{E}-1 \mathrm{C}$

$1.58 \mathrm{CE}-10$

$1.672 \mathrm{E}-10$

$1.785 E-10$

$1.922 \mathrm{E}-10$

$2.083 \mathrm{E}-10$

$2.268 \mathrm{E}-10$

$2.473 E-10$

$2.692 \mathrm{E}-1 \mathrm{C}$

$2.911 \mathrm{E}-10$

$3.112 \mathrm{E}-10$

$3.272 E-10$
$3.367 E-10$

$1.865 E-09$

$2.668 E-09$

RANGE (METERS)

$500 . c$

$600 . n$

$120 \mathrm{C} . \mathrm{C}$

$150 \mathrm{C} .0$

$180 \% .0$

$2.029 \mathrm{E}-10$ $2.073 E-10$ $2.143 E-10$ $2.389 \mathrm{E}-10$ $2.577 E-1 C$ $3.133 E-10$ $3.526 E-10$ $4.014 E-10$ $6.066 \mathrm{E}-10$ $6.847 \mathrm{E}-10$ 7. $979 \mathrm{E}-10$ $4.798 E-09$

$1.914 E-10$ $1.033 E-10$ 4. $241 E-11$ 1. $585 \mathrm{E}-11$

$5.907 E-12$ $2.089 \mathrm{E}-10 \quad 1.924 \mathrm{E}-1 \mathrm{C} \quad 1.041 \mathrm{E}-10 \quad 4.3 \mathrm{C} 1 \mathrm{E}-11$ $2.130 E-1 C$ 1.966E-1C $1.073 E-10 \quad 4.513 E-11$

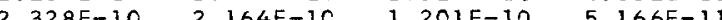
$2.492 E-102.328 E-10 \quad 1.297 E-10 \quad 5.519 E-11$ $2.79 \mathrm{E}-1 \mathrm{C} 2.35 \mathrm{CE}-1 \mathrm{C}-1.428 \mathrm{E}-10 \mathrm{O}-5.984 \mathrm{E}-11$ $3.006 \mathrm{E}-10 \quad 2.851 \mathrm{E}-1 \mathrm{C} \quad 1.616 \mathrm{E}-10 \quad 6.735 \mathrm{E}-11$

$3.896 E-10$

$4.550 E-10$

$5.385 \mathrm{E}-10$

$6.423 \mathrm{E}-10$

$7.653 \mathrm{E}-10$

$8.993 \mathrm{E}-10$

$1.025 \mathrm{E}-\mathrm{CO}$

$1.112 \mathrm{E}-\mathrm{C} 9$

$3.257 E-10$

$3.807 \mathrm{E}-10$

$4.546 \mathrm{E}-10$

$5.535 \mathrm{~F}-10$

$6.837 E-10$
$8.481 \mathrm{~F}-1 \mathrm{C}$

1. $040 E-09$

$1.233 E-C 9$

$1.375 \mathrm{E}-\mathrm{C} 9$ $1.629 \mathrm{E}-11$ $1.955 \mathrm{E}-11$ $6.197 E-12$ (.05E-12 10.768E-12 $2.184 \mathrm{E}-11 \quad 8.767 \mathrm{E}-12$ $2.292 \mathrm{E}-11 \quad 8.820 \mathrm{E}-12$ $2.53 \mathrm{IE}-1 \mathrm{l}$. $597 \mathrm{E}-12$ $1.616 E-10 \quad 6.735 E-11$ $2.5305-11 \quad 1.144 E-11$ $1.89 E-10$ 7.949E-11 $2.99 \mathrm{E}-11 \quad 1.144 \mathrm{E}-11$ $2.873 E-10 \quad 1.265 E-10 \quad 4.856 E-11 \quad 1.846 E-11$ $3.748 \mathrm{E}-10 \quad 1.720 \mathrm{E}-10 \quad 6.652 \mathrm{E}-1120.454 \mathrm{E}-11$ $5 . C 82 \mathrm{E}-10 \quad 2.5 \mathrm{C} 6 \mathrm{E}-10 \quad 10.011 \mathrm{E}-10 \quad 3.740 \mathrm{E}-11$ $7.0 .99 E-10 \quad 3.910 E-10 \quad 1.738 E-10 \quad 6.947 E-11$ $9.977 E-10 \quad 6.330 E-10 \quad 3.234 E-10 \quad 1.473 E-10$

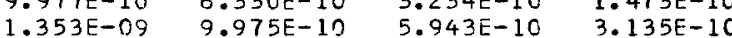
$1.672 \mathrm{E}-09 \quad 1.394 \mathrm{E}-09 \quad 9.489 \mathrm{E}-10 \quad 5.723 \mathrm{E}-10$

$5.594 E-C 9$

$5.814 E-C 9$

$4.350 E-C 9$

$2.375 E-09$

$1.137 E-C 9$

$5.201 E-10$ 
(NEUTRONS/MEV/STERADIAN/SOURCE NEUTRON)

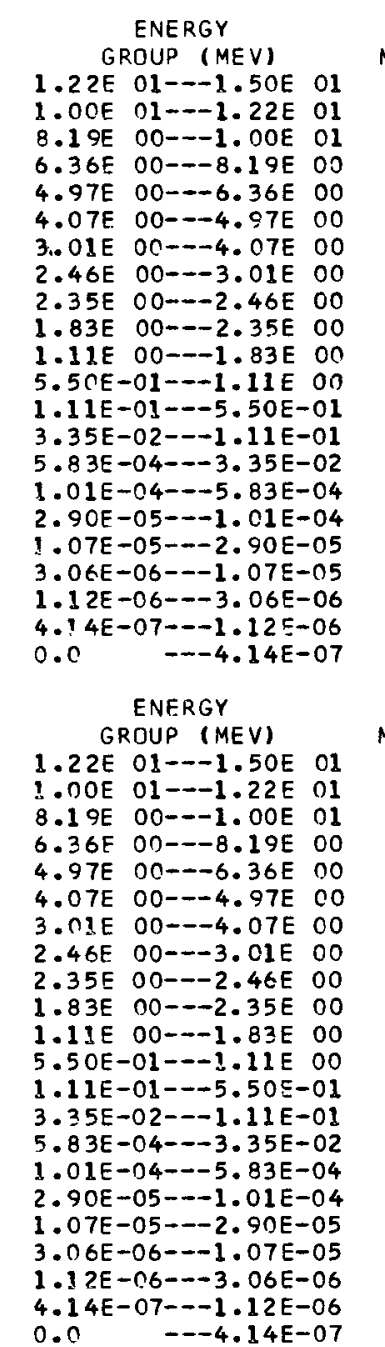

\begin{tabular}{|c|c|c|c|}
\hline ANGLE ? & $\triangle N G L E \quad 2$ & ANGLE 3 & $\triangle N G L E \quad 4$ \\
\hline$M U=-1.0000$ & $M U=-c .9894$ & $M U=-0.9446$ & $M U=-0.8656$ \\
\hline 0.0 & 0.0 & 0.0 & 0.0 \\
\hline 0.0 & 0.0 & 0.0 & 0.0 \\
\hline 0.0 & 0.0 & 0.0 & 0.0 \\
\hline 0.0 & 0.0 & 0.0 & 0.0 \\
\hline 0.0 & 0.0 & 0.0 & 0.0 \\
\hline 0.0 & 0.0 & 0.0 & 0.0 \\
\hline 0.0 & 0.0 & 0.0 & 0.0 \\
\hline 0.0 & 0.0 & 0.0 & 0.0 \\
\hline 0.0 & 0.0 & 0.0 & 0.0 \\
\hline 0.0 & 0.0 & 0.0 & 0.0 \\
\hline 0.0 & 0.0 & 0.0 & 0.0 \\
\hline $2.333 E-02$ & $2.340 E-02$ & $2.370 E-02$ & $2.433 E-02$ \\
\hline $1.048 \mathrm{E}-01$ & $1.051 \mathrm{E}-01$ & $1.064 \mathrm{E}-01$ & $1.090 E-01$ \\
\hline $5.205 E-01$ & $5.213 \mathrm{E}-01$ & $5.246 E-01$ & $5.312 E-01$ \\
\hline $3.610 E-01$ & $3.614 E-01$ & $3.629 \mathrm{E}-01$ & $3.658 \mathrm{E}-01$ \\
\hline $1.802 \mathrm{E} 01$ & $1.804 \mathrm{E} \quad 01$ & $1.809 E 01$ & $1.820 \mathrm{E} \quad 01$ \\
\hline $6.957 \mathrm{E}$ ol & $6.961 E$ o1 & $6.978 \mathrm{E} \mathrm{O1}$ & $7.010 E 01$ \\
\hline $1.874 E \quad 02$ & $1.875 \mathrm{E} \quad 02$ & $1.879 E \quad 02$ & $1.887 E \quad 02$ \\
\hline $4.624 E \quad 02$ & $4.626 \mathrm{E} 02$ & $4.635 E 02$ & $4.651 E \quad 02$ \\
\hline $1.169 E 03$ & $1.169 \mathrm{E} \quad 03$ & $1.171 \mathrm{E} 03$ & $1.175 \mathrm{E} \quad 03$ \\
\hline $2.433 \mathrm{E} \quad 03$ & $2.434 \mathrm{E} \quad 03$ & $2.438 \mathrm{E} \quad 0.3$ & $2.445 \mathrm{E} \quad 03$ \\
\hline $3.374 \mathrm{E} \quad 03$ & $3.376 E \quad 03$ & $3.380 E \quad 03$ & $3.389 E \quad 03$ \\
\hline ANGLE 10 & ANGLE 11 & ANGLE 12 & ANGLE 13 \\
\hline$M U=0.0950$ & $M U=0.2816$ & $M U=0.4580$ & $M U=0.6179$ \\
\hline 0.0 & 0.0 & 0.0 & 0.0 \\
\hline 0.0 & 0.0 & 0.0 & 0.0 \\
\hline 0.0 & 0.0 & 0.0 & 0.0 \\
\hline 0.0 & 0.0 & 0.0 & 0.0 \\
\hline 0.0 & 0.0 & 0.0 & 0.0 \\
\hline 0.0 & 0.0 & 0.0 & 0.0 \\
\hline 0.0 & 0.0 & 0.0 & 0.0 \\
\hline 0.0 & 0.0 & 0.0 & 0.0 \\
\hline 0.0 & 0.0 & 0.0 & 0.0 \\
\hline 0.0 & 0.0 & 0.0 & 0.0 \\
\hline 0.0 & 0.0 & 0.0 & 0.0 \\
\hline $4.155 E-02$ & 4.779E-02 & $6.081 E-02$ & $8.223 E-02$ \\
\hline $2.455 \mathrm{E}-01$ & $1.977 E-01$ & $2.362 E-01$ & $2.811 E-c 1$ \\
\hline $6.474 \mathrm{E}-01$ & $6.891 \mathrm{E}-01$ & $7.173 E-01$ & $7.393 \mathrm{E}-01$ \\
\hline $4.090 E-01$ & $4.203 E-01$ & $4.315 \mathrm{E}-01$ & $4.423 \mathrm{E}-01$ \\
\hline $1.964 \mathrm{E} \quad 01$ & $1.997 \mathrm{E} 01$ & $2.030 E 01$ & $2.062 \mathrm{E} \quad 01$ \\
\hline $7.439 \mathrm{E}$ ol & $7.533 \mathrm{E} \mathrm{O1}$ & $7.625 E \quad 01$ & $7.712 E$ OI \\
\hline $1.985 \mathrm{E} \quad 02$ & $2.006 E \quad 02$ & $2.026 \mathrm{E} 02$ & $2.045 \mathrm{E} \quad 02$ \\
\hline $4.856 \mathrm{E} \quad 02$ & $4.899 E \quad 02$ & $4.941 \mathrm{E} 02$ & $4.978 \mathrm{E} \quad 02$ \\
\hline $1.221 E \quad 03$ & $1.230 E \quad 03$ & $1.240 E \quad 03$ & $1.248 E \quad 03$ \\
\hline $2.532 E \quad 03$ & $2.549 E \quad 03$ & $2.566 \mathrm{E} \quad 03$ & $2.582 \mathrm{E}$ \\
\hline $3.492 E \quad 03$ & $3.513 E \quad 03$ & $\begin{array}{lll}3.533 E & 03\end{array}$ & $3.551 \mathrm{E}$ \\
\hline
\end{tabular}

$\begin{array}{ll}\begin{array}{ll}\text { ANGLE } 5 \\ M U=-0.7550\end{array} & \text { MUU } \\ 0.0 & 0 \\ 0.0 & 0.0 \\ 0.0 & 0.0 \\ 0.0 & 0.0 \\ 0.0 & 0 \\ 0.0 & 0.0 \\ 0.0 & 0 \\ 0.0 & 0.0 \\ 0.0 & 0.0 \\ 0.0 & 0 \\ 0.0 & 0.0 \\ 2.535 E-02 & 2.6 \\ 1.130 E-01 & 1 \\ 5.407 E-01 & 5.5\end{array}$

ANSLE 6
$M U=-0.6179$
0.0
0.0
0.0
0.0
0.0
0.0
0.0
0.0
0.0
0.0
0.0
$2.688 E-02$
$1.185 E-01$

ANGLE 7

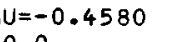

0.0

0.0

0.0

0.0

0.0

0.0

0.0

0.0

$1.130 \varepsilon-01$

(

2.910E-02

$1.259 E-01$

3.700E-01

3.754E-01

$5.696 \mathrm{E}-01$

$1.834 \mathrm{E}$ OI
$7.056 \mathrm{E}$ OI

$1.853 \mathrm{E} 01$

$1.897 \mathrm{E} 02$

$\begin{array}{ll}4.673 \mathrm{E} & 02 \\ 1.180 \mathrm{E} & 03\end{array}$

$1.180 \mathrm{E}^{0} \mathrm{O}$

$\begin{array}{ll}2.454 E & 03 \\ 3.402 E & 03\end{array}$

$7.113 \mathrm{E} 01$
$1.911 \mathrm{E} 02$

$4.701 \mathrm{E} 02$

$1.186 \mathrm{E} \quad 03$

$2.466 \mathrm{E} 03$

$3.417 E^{03}$

$1.876 \mathrm{E}$ o1

$7.182 \mathrm{E}$ 01

$1.927 \mathrm{E} \mathrm{O2}$

$\begin{array}{ll}4.735 E & 02 \\ 1.194 E & 03\end{array}$

$1.194 E \quad 03$
$2.481 E 03$

$3.433 \mathrm{E} 03$

ANGLE 8 $M U=-0.2816$

0.0

0.0
0.0

0.0

0.0

0.0

0.0

0.0

0.0

$3.224 E-02$

$1.355 E-01$

3.899E-01

$1.903 E$ O1

$7.261 \mathrm{E} 01$
$1.945 \mathrm{E} 02$

$1.945 \mathrm{E} 02$

$4.773 \mathrm{E} 02$
$1.202 \mathrm{E} 03$

$1.202 \mathrm{E} 03$

$\begin{array}{ll}2.497 E & 03 \\ 3.452 E & 03\end{array}$

ANGLE 15

ANGLE 16

ANGLE 17

ANGLE 14
$M U=0.7550$

0.0

$0.0 \quad 0.0$

0.0

0.0

0.0

0.0

0.0
0.0

0.0

0.0

0.0

$1.138 E-01$
$2.620 E-01$

0.0

0.0

0.0

0.0

0.0

0.0

0.0

0.0

0.0

$2.222 E-01$

01 3.9

0.0

$\begin{array}{ll}0.0 & 0.0 \\ 0.0 & 0.0\end{array}$

0.0

0

0.0

0.0

0.0

0.0

0.0

0.0

0.0

$7.340 E-01$ $9.086-01$ $4.360 E-01$ $8.658 \mathrm{E}-01$ $4.762 E-01$ $2.138 E$ O1 $2.092 E$ Ol 7.787 Q 1 $2.118 \mathrm{E} 01$ 2.075E 02 7. $901 \mathrm{E} 01$ $5.059 \mathrm{E} 02$ $5.011 E 02$ $1.255 \mathrm{E} \quad 03$
$2.595 \mathrm{E}$ $3.567 \mathrm{E}^{03}$

$5.039 E 02$ $1.261 E 03$ $2.506 E 03$ $1.265 \mathrm{E} \quad 03$ $2.514 E \quad 03$
$3.588 E$

0.0

0.0

0.0

0.0

0.0

0.0

0.0

0.0

$2.535 \mathrm{E} 00$

$2.175 E 00$ $9.801 E-01$ $4.815 E-01$ $7.923 E$ OI 2.090 OZ $2.090 E$ O2 1.267E 03 $2.618 E \quad 03$ $3.591 \mathrm{E} 03$
ANGLE 9 $=-0.0950$ 0.0 0.0 0.0 0.0 3.664E-02 $1.481 \mathrm{E}-01$ $6.131 \mathrm{E}-01$ $3.989 \mathrm{E}-01$ $1.932 \mathrm{E} 01$ $7.347 E$ O1

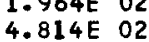
$1.211 \mathrm{E} 03$ $2.514 \mathrm{E} \quad 03$ $3.472 E 03$

SCALAR FLUX 0.0 0.0 0.0 0.0 0.0 0.0 0.0 0.0 0.0 0.0 1.372E 00 2.995E 00 $5.254 E 00$ S.141E 00 $2.459 E$ O2 $2.311 E$ O2 6.081 E 03 $1.529 \mathrm{E} 04$ $3.172 E 04$ 4.377E 04 

GROUP (MEV

$1.22 E \quad 01---1.50 E$ OI $1.00 E$ O1--1.22E 01 $8.19 E$ DO---1. OOE OI $6.36 \mathrm{E}$ OC- $-8.19 \mathrm{E}$ OO $4.97 E$ EO- $-6.36 E$ EO 4.07E $00--4.97 E$ OO $3.01 E$ EO- $-4.07 E$ EO $2.4 \mathrm{EE}$ 00--3.01E 00 $2.35 E$ OC- $-2.46 E$ OD $1.82 \mathrm{E} \quad 00--2.35 \mathrm{E} \quad 00$ $1.11 \mathrm{E} O \mathrm{C}--1.83 \mathrm{E}$ OO 5.5OE-C1- - 1.11E 00 $1--5.50 \mathrm{E}-01$ $3.35 E-02--1.11 E-01$ $1.01 \mathrm{E}-04---5.83 \mathrm{E}-04$ 1. $.07 \mathrm{E}-05--2.90 \mathrm{E}-05$ $3.06 E-06--1.07 E-05$ $1.12 E-06--3.06 E-06$ $4.14 E-07--1.12 E-06$ $0.0 \quad--4.14 E-07$ ENERGY GROUP (MEV) $1.22 E$ O1-- $1.50 E$ OI $\begin{array}{lll}1.0 O E & 01--1.22 E & 01 \\ 8.19 E & 00--1.00 E & 01\end{array}$ $8.19 E$
$6.360--1 . C O E$ 01

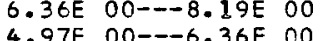
4.97E DO---6.36E 00 4.07E $00--4.97 E$ CO $2.01 E$ OO- -4. C7E CO $2.46 E$ OO-- $3.01 E$ E 00 $1.3 \mathrm{2E} 00--2.35 \mathrm{E} 00$ 1.8 E $00--2.35 E$ OO 5.50E-01- $1.11 \mathrm{E} 00$ $1.11 \mathrm{E}-01--5.50 \mathrm{E}-01$ $3.35 \mathrm{E}-02--1.11 \mathrm{E}-01$ $5.83 \mathrm{E}-04--3.35 \mathrm{E}-02$ $1.01 \mathrm{E}-04-\ldots-5.83 \mathrm{E}-04$ $2.90 \mathrm{E}-05--1.01 \mathrm{E}-04$ $1.07 E-05-2.90 E-05$ $3.06 \mathrm{E}-06--1.07 \mathrm{E}-05$ $1.12 \mathrm{E}-06-0-3.06 \mathrm{E}-06$ $4.14 E-07--1.12 E-06$ $4.14 E-07---1.12 E-06$
0.0
$-0-4.14 E-07$
ANGLE 1
$M U=-1.0000$ 0.0 0.0 0.0 0.0 0.0 0.0 0.0 0.0 $2.765 E-02$ $1.080 \mathrm{E} \mathrm{CO}$ $5.020 E$ OI $2.056 E$ O2 $5.741 \mathrm{E} 02$ $1.463 E \quad 03$ $3.774 \mathrm{E} \quad 03$ $7.987 E \quad 03$ $1.125 \mathrm{E} \quad 04$ ANGLE 10 $M U=0.0950$ 0.0

0.0

0.0

0.0

0.0
0.0

0.0

0.0 0.0 0.0 $6.284 E-02$ E-01 $1.485 \mathrm{E}$ 0O $1.054 E$ OO $2.290 \mathrm{E} 02$ $2.290 E$ O ..327E 02 $4.083 E 03$ 4.0B3E 03 $1.198 \mathrm{E} \quad 04$
ANGLE 2
$M U=-0.9894$

0.0

0.0
0.0
0.0

0.0

0.0

0.0

0.0

0.0

0.0

0.0

$2.775 E-02$

$1.478 \mathrm{E}-01$

$1.082 \mathrm{E} 00$

$8.994 \mathrm{E}-01$

$5.026 \mathrm{E} \mathrm{O1}$

$2.058 E \quad 02$

$5.746 \mathrm{E} \mathrm{O2}$

$1.464 \mathrm{E} \mathrm{O3}$

$3.777 F \quad 03$

$1.12 \in \mathrm{E} \quad 04$

ANGLE 11

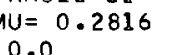

0.0

0.0
0.0

0.0

0.0

0.0

0.0

0.0

0.0

0.0

$5.586 E-02$ $3.021 E-01$ 1.371E 00 5.041E 01 ..841E 01 2.337 O2 6.443 E 02 1.620 E $4.142 E$ OS $8.701 \mathrm{E}$
1.23
(NEUTRJNS/MEV/STERADIAN/SOURCE NEUTRON)

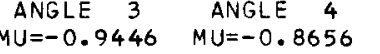$$
0.0
$$

0.0

0.0

0.0

0.0

0.0

0.0

0.0

0.0

$2.815 E-02$

$1.497 \mathrm{E}-01$

$1.090 \mathrm{E} 00$

$9.043 \mathrm{E}-01$

$5.049 E$ O1

$2.066 \mathrm{E} \mathrm{O2}$

$5.766 E$ O2
$1.469 E$ O2

$3.788 E \quad 03$

$8.014 E \quad 03$

$1.129 E 04$

ANGLE 12

$M U=0.4580$

0.0
0.0

0.0

0.0

0.0
0.0

0.0
0.0

0.0

0.0

0.0

0.0 $2.444 E-01$ $1.507 \mathrm{E} 00$ 5.980 O $5.989 \mathrm{E} 01$ 2.385E O2 1.560 E 02 $1.640 E 03$ $8.812 \mathrm{E} 03$ $1.225 \mathrm{E} 04$$$
0.0
$$

0.0

0.0

0.0

0.0

0.0

0.0

0.0

$2.900 E-02$ $1.534 E-01$

$1.105 \mathrm{E} 00$

$9.136 \mathrm{E}-01$

$2.081 \mathrm{E}$ OI

$2.081 \mathrm{E} O 2$
$5.805 \mathrm{E} \mathrm{O2}$

$1.4077 \mathrm{E} \mathrm{O3}$

$3.809 \mathrm{E} \mathrm{O3}$

$8.055 E$ O3

(

ANGLE 13

$M U=C .6179$

0.0

0.0

0.0

0.0

0.0

0.0

0.0

0.0

$9.058 E-02$

$3.716 E-01$ $1.620 E$ OD $0.136 \mathrm{E} 01$ $6.136 \mathrm{E} 01$ $6.672 \mathrm{E} 02$ $1.670 E$ O3 $1.676 \mathrm{E} 03$ $4.256 E$ OS 1. $17 E$ O

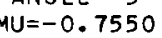

$$
0.0
$$

0.0

0.0

0.0

0.0

0.0

0.0

0.0

. $590 E-01$

$1.128 \mathrm{E} 00$
$9.269 \mathrm{E}-01$

$5.152 E$ OI

$2.102 E 02$

$1.490 E$ O3

$3.838 \mathrm{E} \quad 03$

$8.111 E$ E

$141 E$ E

ANGLE 14

$M U=0.7550$

0.0
0.0

0.0

0.0

0.0

0.0

0.0

0.0

0.0

0.0

3. $766 \mathrm{E}-01$

$1.663 E$ OO

$0.272 E 01$

$6.272 E$ O1

$2.475 E$
$6.772 E$ 02

$1.691 \mathrm{E} 03$

$1.691 E 03$

4.304E 03

$1.249 E \quad 04$

ANGLE ${ }^{6}$
$M=-0.6179$ 0.0

0.0
0.0

0.0

0.0
0.0
0.0

0.0

0.0

0.0

0.0

3.232E-02

$1.66 \in E-01$

.157E 00

..230E OI

$2.130 E$ O2

$5.928 \mathrm{E} \mathrm{O2}$

$1.505 \mathrm{E} \mathrm{O3}$

$3.875 \mathrm{E} \quad 03$

$8.184 E$ O3

SOE 04

ANGLE 15

0.0
0.0

0.0

0.0

0.0

0.0

0.0

0.0

0.0

$1.865 \mathrm{E}-01$ 
(NEUTRJNS/MEV/STERADIAN/SOURCE NEUTRON)

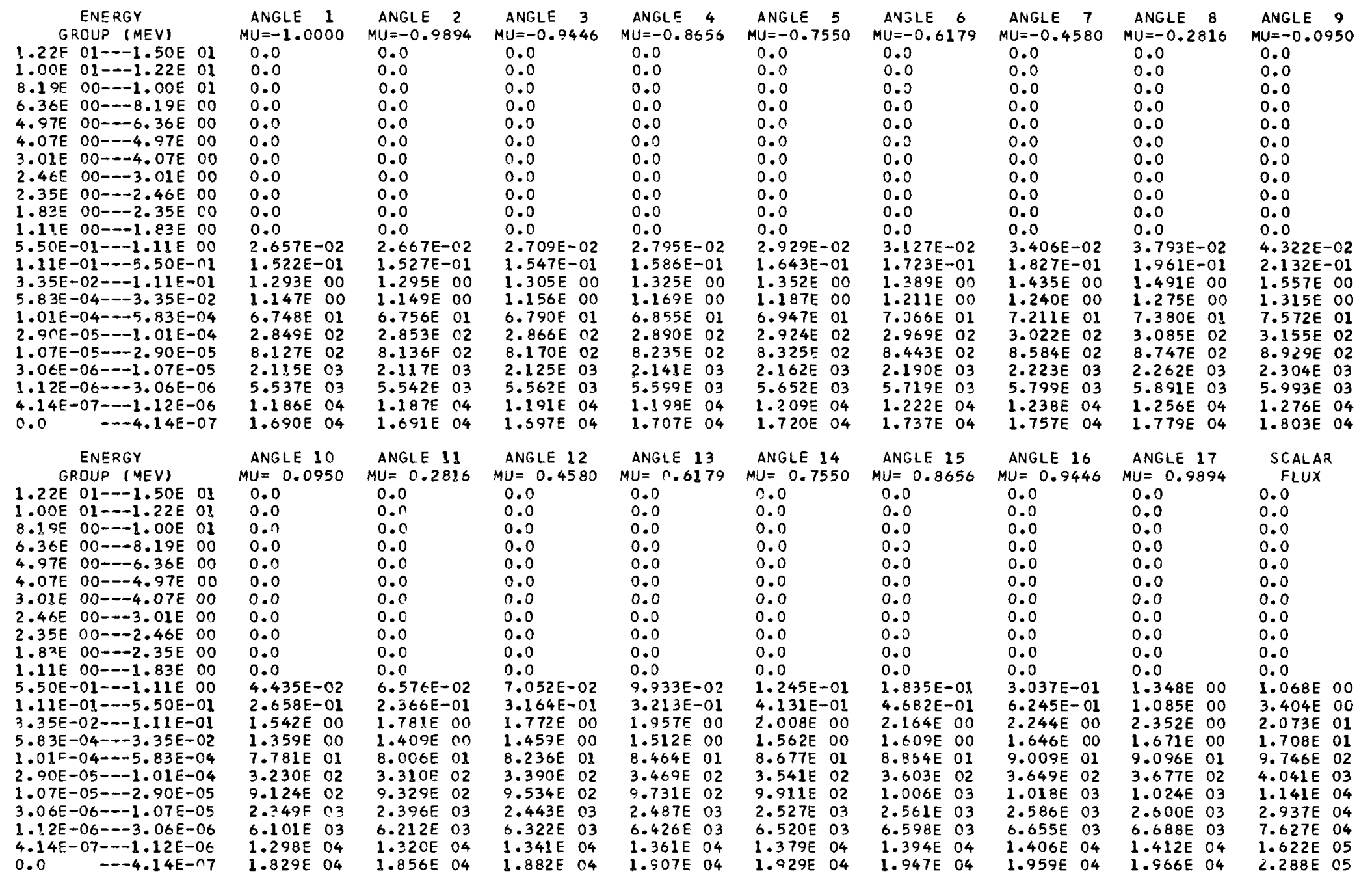


(NEUTRJNS/MEV/STTEKADIAN/SOURCE NEUTRON) $1.22 E$ O1- $-1.50 E$ OI $1.00 \mathrm{E}$ 01--1.22E 01 $6.36 \mathrm{E}$ OO---8.0OE OI $4.97 E$ OO- $-5.36 E$ OO 4.07E 00---4.97E 00 $3.01 \mathrm{E} 00---4.07 E 00$ $2.46 E$ DO-- $3.01 E$ OO $2.35 E \quad 00--2.46 E \quad 00$ $1.83 \mathrm{E} \quad 00--2.35 \mathrm{E} 00$ $1.11 \mathrm{E} 00--1.83 \mathrm{E} 00$ $5.50 E-01--1.11 \mathrm{E}$ CO $1.11 E-01---5.50 E-01$ $3.35 E-02--1.11 E-01$ $5.83 E-04--3.35 E-02$ $1.01 \mathrm{E}-04---5.83 \mathrm{E}-04$ $2.90 E-05--1.01 E-04$ 1.07E-05--2.90E-05 $3.06 E-06--1.07 E-05$
$1.12 E-06--3.06 E-06$

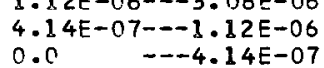
ENERGY GROUP (MEV) $1.22 E$ OL--1.5OE 01 $1.00 E$ OL--1.22E 01 8.1 9E OO---1.00E OI 6.36F NO---8.19E OO 4.97E $00-2.36 \mathrm{~F}$ OO 4.07E 00- $-4.97 E$ OO 2.46E $00-3.01 E 0$ 2.46E 0O- 3 . O1E 00 1.35E $00-2.36$ E 00 $1.11 \mathrm{E} 00--1.83 \mathrm{E} 00$ $1.11 \mathrm{E} 00-1.83 \mathrm{E} 00$ 1.11E-01- 5 . $50 \mathrm{E}-01$ 1.3 3. 1.01E-04 $-5.35 E-04$ $2.00 E-05-1.01 E-04$ 1.07E-05--2. $00 \mathrm{E}-05$ $3.06 E-06--1.07 E-05$ $1.12 \mathrm{E}-06--3.06 \mathrm{E}-06$ $4.14 E-07--1.12 E-06$ $0.0 \quad--4.14 \mathrm{E}-07$
ANGLE 2
$M U=-1.0000$ 0.0 0. 0.0 0.0 0.0 2.0 0.0 0.0 0.0 2.075E-02 $1.314 \mathrm{E}-01$ $1.363 E$ DO $1.308 E$ OO $8.241 E 01$ $3.639 \mathrm{E} 02$ $1.073 E \quad 03$ 2.895E 03 $7.779 E 03$ $1.704 \mathrm{E} \mathrm{O4}$ $2.485 E \quad 04$ ANGLE 10 $U=0 . C 950$ 0.0 0.0 0.0 0.0 0.0 0.0 0.0 0.0 $4.201 \mathrm{E}-02$ $4.201 E-02$ $1.905 E-01$ $1.572 E 00$ 1.5 $06 \mathrm{E} \mathrm{O1}$ $4.222 \mathrm{E} 02$ $1.235 \mathrm{E} 03$ $3.302 \mathrm{E} \mathrm{O3}$ $8.804 E 03$ $1.916 E \quad 04$ $2.756 \mathrm{E} \mathrm{O4}$
ANGLE 2
$M U=-C .9894$ 0.0 0.0
0.0
0.0 0.0 0.0 0.0 0.0 0.0 0.0 0.0 c. $2.083 E-02$ $1.319 E-01$ $1.366 E 00$ $1.310 \mathrm{E} \mathrm{CO}$ $8.252 E$ O1 $3.644 E$ C2 $1.074 \mathrm{E} \mathrm{O3}$ 2.899E 03 $7.787 \mathrm{E} \quad 03$ $2.487 \mathrm{E}^{\circ}$

ANGLE 11 0.0 0.0
0.0 0.0 0.0 0.0 0.0 0.0 0.0 0.0 0.0 $0.078 E-02$ $4.678 E-02$
$2.323 E-01$ $2.323 E-01$ $\begin{array}{ll}1.804 \mathrm{E} & 00 \\ 1.631 \mathrm{E} & 00\end{array}$ $1.001 \mathrm{E} 02$ $4.345 E$ O2 $1.269 E 03$ $3.386 \mathrm{E} \quad 03$ $9.013 E 03$ $1.958 \mathrm{E} 04$ 2.810E 04
$M U=-0.0,446$ 0.0 0.0 0.0 0.0
0.0
0.0 0.0 0.0 0.0 0.0 0.0 $2.119 E-02$ $1.336 \mathrm{E}-01$ $1.377 E$ OO $1.319 \mathrm{E} 00$ $8.301 \mathrm{E} \mathrm{O1}$ $3.664 E \quad 02$ $1.080 \mathrm{E} \quad 03$ $2.913 E 03$ $7.823 \mathrm{E} 03$ $1.713 E$ O4 $=0.4580 \quad M U=0.6179$ 0.0 0.0
0.0 0.0 0.0 0.0 0.0 0.0 0.0 0.0 $6.047 E-02$ $6.047 E-02$
$2.457 E-01$ $2.457 E-01$
$1.953 E 00$ $1.953 \mathrm{E}$
1.60 $1.034 \mathrm{E} \mathrm{O2}$ $4.472 E$ O2 $1.304 \mathrm{E} \mathrm{O3}$ $3.470 E 03$ $9.223 E 03$ $2.001 \mathrm{E} \mathrm{O4}$ 2. $865 E 04$ $M U=-0.8656$ 0.0 0.0 0.0 0.0
0.0 0.0 0.0 0.0 0.0 $2.191 E-02$ $1.370 E-01$ $1.399 \mathrm{E} 00$ $1.335 \mathrm{E} \mathrm{OO}$ $8.391 \mathrm{E} 01$ $3.700 \mathrm{E} \mathrm{O2}$ $1.090 \mathrm{E} 03$ $2.939 E \quad 03$ $7.889 \mathrm{E} \mathrm{O3}$ $1.727 E$ O4 2.51SE 04 ANGLE 13
$M U=0.6179$ 0.0 0.0
0.0 0.0
0.0

0.0

0.0

0.0

0.0

$7.422 E-02$ $2.917 E-01$ $2.026 \mathrm{E} 00$ $1.758 \mathrm{E} 00$ $4.596 E 02$ $1.338 \mathrm{E} 03$ $3.552 \mathrm{E} 03$ $9.424 \mathrm{E} 03$ $2.042 \mathrm{E} \mathrm{O4}$ $2.916 E 04$

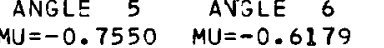$$
0.0
$$

0.0

0.0

0.0

0.0

0.0

0.0

0.0

$2.303 E-02$

$1.421 E-01$

$1.429 E 00$

$1.357 \mathrm{E} O O$

$8.518 E$ O1

$3.752 E$ O2

$1.105 E \quad 03$

$2.976 E$ O3

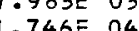

$2.541 E \quad 04$

ANGLE 14

$=0.7550$ MUNG 15 0.0 0.0

0.0

0.0
0.0

0.0
0.0
0.0

0.0

.0

0.0

$9.964 E-02$ $3.255 \mathrm{E}-02$ $2.165 \mathrm{E} 00$ $1.097 \mathrm{E} \mathrm{O2}$ 4.711E 02 $1.369 E 03$ $3.626 E$ O3 $9.606 E$ O3 $2.079 E$ O4 2.962E 04 0.0

0.0

0.0
0.0
0.0

0.0

0.0

0.0

.0

$2.466 \mathrm{E}-02$

$1.490 \mathrm{E}-0 \mathrm{l}$

$1.470 \mathrm{E} \mathrm{OO}$

$8.685 \mathrm{E} \mathrm{O1}$

.819E 02

$1.124 \mathrm{E} 03$

$8.103 \mathrm{E} \mathrm{O3}$

$8.103 E$ O

$2.574 \mathrm{E} \quad 04$

$A N G L E \quad 15$
$M=0.8656$ 0.0

0.0
0.0
0.0
0.0
0.0

0.0

0.0

0.0

0.0

MU $=-0.6179 \quad M U=-0.4580$ 

GROUP (MEV) $1.22 E$ N1---1.5OE 01 $8.19 E$ OO- $1.00 E$ OI $6.36 \mathrm{E} 00--8.19 \mathrm{E} 00$ 4.97E $00--6.36 E$ OO 4.07E $00--4.97 E 00$ $3.01 \mathrm{E} 00--4.07 \mathrm{E} 00$ $2.46500-3.01 E$ OO $2.35 \mathrm{E} \quad 00--2.46 \mathrm{E} 00$ $1.83 \mathrm{E} 00--2.35 \mathrm{E} 00$ $1.11 \mathrm{E} 00-1.83 \mathrm{E} 00$ $5.50 E-01-1.11 E$ OO $1.11 \mathrm{E}-01 \cdots 5.50 \mathrm{E}-01$ $3.35 E-02--1.11 \mathrm{E}-01$ $5.83 E-04--3.35 E-02$ $1.01 E-04--5.83 E-04$
$2.00 E-05--1.01 E-04$ 2. $90 \mathrm{E}-05--1.01 \mathrm{E}-04$ 1.07E-05- $-2.90 \mathrm{E}-05$ $3.06 \mathrm{E}-06--1.07 \mathrm{E}-05$
$1.12 \mathrm{E}-06-\cdots-3.06 \mathrm{E}-06$ $4.14 \mathrm{E}-07---1.12 \mathrm{E}-06$ $0.0 \quad-4.14 \mathrm{E}-07$ ENERGY GROUP (MEV) $1.22 \mathrm{E} \mathrm{O1---1.50E} 01$

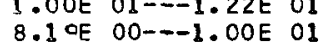

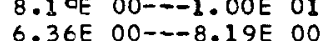
$4.36 E$ OD- $00-3.36 E$ DO $4.07 E$ OO- - 4.97E 00 $3.01 \mathrm{E} 00--4.07 \mathrm{E} 00$ $2.46 \mathrm{E} 00---3.01 \mathrm{E} 00$ $2.35 \mathrm{E} 00--2.46 \mathrm{E} \quad 00$ $1.83 \mathrm{E} \quad 00--2.35 \mathrm{E} \quad 00$ $1.11 E 00-1.83 E 00$ $5.50 E-01--1.11 E 00$ 1.1?E-01--5.50E-01 $3.35 E-02-\cdots 1.11 E-01$ $5.83 \mathrm{~F}-04--3.35 \mathrm{E}-02$ $1.01 \varepsilon-04--5.83 E-04$ 2. $90 \mathrm{CE}-05--1.01$ E-04 1. $07 \mathrm{E}-05--2.90 \mathrm{E}-05$ 3.06E-06--1.07E-05 $1.12 \mathrm{E}-06-0-3.06 \mathrm{E}-06$ $4.14 E-07--1.12 E-n S$ $0.0 \quad--4.14 \mathrm{E}-07$
ANGLE 0.0 0.0 0.0 0.0 0.0 0.0 0.0 0.0 0.0 $1.437 E-02$ $9.792 E-02$ $1.157 E 00$ $1.162 \mathrm{E} 00$ $7.640 E$ O1 $3.474 \mathrm{E} 02$ $1.048 \mathrm{E} \quad 03$ $\begin{array}{ll}2.905 E & 03 \\ 7.965 E & 03\end{array}$ $7.965 \mathrm{E} 03$ $2.642 E 04$ ANGLE 10 $M U=0.0950$ 0.0 0.0 0.0 0.0 0.0 0.0 2.795E-02 $1.524 E-01$ $1.471 \mathrm{E} 00$ $1.407 E$ OO $9.089 E$ OI $4.083 \mathrm{E} 02$ 1.225E 03 $3.369 E \quad 03$ $9.174 E$ O3 $2.981 E 04$
(NEUTRONS/MEV/STERADIAN/SOURCE NEUTRON) ANGLE 2
$M U=-0.9894$ 0.0 $M U=-0.9446$ 0.0 0.0
0.0
0.0 0.0 0.0 0.0
0.0

0.0

0.0

0.0

0.0

0.0

$1.444 \mathrm{E}-02$ $\begin{array}{ll}.444 E-02 & 1.470 E-02 \\ .824 E-02 & 9.959 E-02\end{array}$ $160 \mathrm{E} 00$ $1.164 \mathrm{E} \mathrm{OO}$ $7.651 \mathrm{E} \quad 01$ $3.479 \mathrm{E} 02$ $1.050 E \quad 03$ $\begin{array}{ll}2.909 \mathrm{E} & 03 \\ 7.975 \mathrm{E} & 03\end{array}$ $7.975 \mathrm{E} \quad 03$ $1.770 E$
$2.645 E$ $1.170 \mathrm{E} 00$ $\begin{array}{lll}1.1725 & 00 \\ 7.699 E & 01\end{array}$ 7.699E O1 $3.500 \mathrm{E} 02$

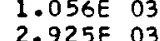
$\begin{array}{ll}2.925 E & 03 \\ 8.017 E & 03\end{array}$

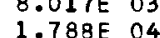
$\begin{array}{ll}1.788 E & 04 \\ 2.657 E & 04\end{array}$ ANGLE 11 ANGLE 11 ANGLE 12 $M U=0.2816$ 0.0 0.0 0.0 0.0 0.0
0.0 c.0 0.0 0.0 3. $353 \mathrm{E}-02$ $1.652 \mathrm{E}-01$ $1.571 \mathrm{E} 00$ $1.462 \mathrm{E}$ กO $9.407 E$ Ol 4.214E 02 $1.263 \mathrm{E} 03$ $3.466 E$ O3 $9.425 \mathrm{E} \mathrm{O3}$ $2.088 E 04$ $3.051 E$ O4 0.0 0.0

0.0

0.0
0.0

0.0

0.0

0.0

0.0

$4.135 E-02$ 1.873E-01 $1.646 E 00$ $1.520 \mathrm{E}$ OC $9.734 \mathrm{E} \mathrm{Ol}$ 4.348E 02 $1.301 \mathrm{E} 03$ $3.565 \mathrm{E} 03$ $9.679 \mathrm{E} 03$ $2.141 \mathrm{E} 04$ $3.120 \mathrm{E} 04$

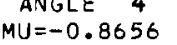
0.0 0.0
0.0
0.0 0.0

0.0

0.0

0.0

0.0

$1.522 \mathrm{E}-02$ $1.022 \mathrm{E}-01$ $1.189 \mathrm{E} 00$ $1.187 E$ OO $7.789 \mathrm{E}$ O1 $3.537 \varepsilon 02$ $1.067 E \quad 03$ $2.954 \mathrm{E} 03$ 8.094 E 03 $1.805 \mathrm{E} 04$ 2.580E 04 0.0
0.0 0.0

0.0
0.0
0.0
0.0

0.0
0.0
0.0

0.0

(

0

$5.245 E-02$ $2.095 E-01$ 1.745 E 00 1.578 E 00 $1.0 \cap 6 E$ O2 4. 480 E 02 $1.338 \mathrm{E} 03$ $3.560 E 03$ $9.924 \mathrm{E} 03$ $2.192 E 04$ 3.187 E 04

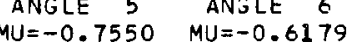

0.0 0.0 0.0 0.0 0.0 0.0 0.0 1.603E-02 $1.059 E-01$ $1.215 E 00$ $7.915 \mathrm{E} 01$ $3.591 \mathrm{E} \mathrm{O2}$ $1.082 \mathrm{E} \quad 03$ $2.996 \mathrm{E} 03$ $8.202 E$ O3 . $828 \mathrm{E} 04$ ANGLE 14
$M U=0.7550$ 0.0 0.0
0.0 0.0
0.0 0.0 0.0 0.0 0.0

0.0

6.809E-02 2.399E-01

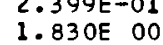
$1.633 \mathrm{E} 00$ $1.036 \mathrm{E} \mathrm{O2}$ 4.601E O2 $1.373 E 03$ $3.748 \mathrm{E} 03$ 1. $015 E 04$ $2.239 \mathrm{E} \mathrm{O4}$ $3.247 \mathrm{E} 04$

M.J
0.0
0.0
0.0
0.0
0.3
0.0
0.0
0.0
0.0
0.0
$1.720 E-02$
$1.111 E-01$
$1.251 E 00$
$1.235 E 00$
$8.081 E 01$
$3.662 E 02$
$1.103 E 03$
$3.050 E 03$
$8.344 E 03$
$1.858 E 04$
$2.751 E \quad 04$
ANGLE 15
MU= 0.8656
0.0

0.0
0.0

0.3

0.0
0.0

0.0

0.0

0.3

0.0

9. $282 E-02$ 2. $736 \mathrm{E}-01$ 1. $220 \mathrm{E} \mathrm{O0}$ $1.681 \mathrm{E} 00$ $1.362 E 02$ $1.402 E \quad 03$ $3.823 \mathrm{E} \mathrm{O3}$ $1.034 E 04$ $1.034 E 04$
$2.278 E 04$ $3.297 E \quad 04$

\begin{tabular}{|c|c|c|c|}
\hline $\begin{array}{l}\text { ANGLE } 7 \\
M U=-0.4580\end{array}$ & $\begin{array}{l}\text { ANGLE } \\
M U=-0.28\end{array}$ & $\begin{array}{c}8 \\
816\end{array}$ & $\begin{array}{l}\text { ANGLE } 9 \\
M U=-0.0950\end{array}$ \\
\hline 0.0 & 0.0 & & 0.0 \\
\hline 0.0 & 0.0 & & 0.0 \\
\hline 0.0 & 0.0 & & 0.0 \\
\hline 0.0 & 0.0 & & 0.0 \\
\hline 0.0 & 0.0 & & 0.0 \\
\hline 0.0 & 0.0 & & 0.0 \\
\hline 0.0 & 0.0 & & 0.0 \\
\hline 0.0 & 0.0 & & 0.0 \\
\hline 0.0 & 0.0 & & 0.0 \\
\hline 0.0 & 0.0 & & 0.0 \\
\hline 0.0 & 0.0 & & 0.0 \\
\hline $1.883 \mathrm{E}-02$ & $2.106 E-$ & -02 & $2.408 E-02$ \\
\hline $1.178 \mathrm{E}-01$ & $1.264 \mathrm{E}-$ & -01 & $1.371 E-01$ \\
\hline $1.295 \mathrm{E} 00$ & $1.349 E$ & 00 & $1.412 E 00$ \\
\hline $1.269 E 00$ & $1.310 E$ & 00 & $1.356 \mathrm{E} 00$ \\
\hline $8.283 E \quad 01$ & $8.521 \mathrm{E}$ & 01 & $8.792 E \quad 01$ \\
\hline $3.747 E \quad 02$ & $3.847 \mathrm{E}$ & 02 & $3.960 E \quad 02$ \\
\hline $1.128 \mathrm{E} \quad 03$ & $1.157 \mathrm{E}$ & 03 & $1.189 \mathrm{E} \quad 03$ \\
\hline $3.115 E \quad 03$ & $3.191 \mathrm{E}$ & 03 & $3.276 E \quad 03$ \\
\hline $8.514 E \quad 03$ & $8.712 \mathrm{E}$ & 03 & $8.934 E \quad 03$ \\
\hline $1.894 E \quad 04$ & $1.937 \mathrm{E}$ & 04 & $1.984 E \quad 04$ \\
\hline $2.799 E \quad 04$ & $2.854 E$ & 04 & $2.915 E \quad 04$ \\
\hline ANGLE 16 & ANGLE I & 17 & SCALAR \\
\hline$M U=0.9446$ & $M U=0.98$ & 894 & FLUX \\
\hline 0.0 & 0.0 & & 0.0 \\
\hline 0.0 & 0.0 & & 0.0 \\
\hline 0.0 & 0.0 & & 0.0 \\
\hline 0.0 & 0.0 & & 0.0 \\
\hline 0.0 & 0.0 & & 0.0 \\
\hline 0.0 & 0.0 & & 0.0 \\
\hline 0.0 & 0.0 & & 0.0 \\
\hline 0.0 & 0.0 & & 0.0 \\
\hline 0.0 & 0.0 & & 0.0 \\
\hline 0.0 & 0.0 & & 0.0 \\
\hline 0.0 & 0.0 & & 0.0 \\
\hline $1.372 E-01$ & $3.468 \mathrm{E}-$ & -01 & $5.059 E-01$ \\
\hline $3.165 E-01$ & $3.807 \mathrm{E}-$ & -01 & $2.045 E \quad 00$ \\
\hline $1.987 \mathrm{E} 00$ & $2.036 \mathrm{E}$ & 00 & $1.875 \mathrm{E} 01$ \\
\hline $1.719 \mathrm{E} \quad 00$ & $1.742 \mathrm{E}$ & 00 & $1.764 \mathrm{E} \mathrm{Ol}$ \\
\hline $1.083 E 02$ & $1.095 \mathrm{E}$ & 02 & $1.138 E \quad 03$ \\
\hline $4.785 E \quad 02$ & $4.832 E$ & 02 & $5.108 \mathrm{E} \quad 03$ \\
\hline $1.425 \mathrm{E} \quad 03$ & $1.438 \mathrm{E}$ & 03 & $1.532 \mathrm{E} \quad 04$ \\
\hline $3.879 E \quad 03$ & $3.911 \mathrm{E}$ & 03 & $4.211 E 04$ \\
\hline $1.048 \mathrm{E} \quad 04$ & $1.056 \mathrm{E}$ & 04 & $1.147 \mathrm{E} \quad 05$ \\
\hline $2.308 E \quad 04$ & $2.325 E$ & 04 & $2.542 E \quad 05$ \\
\hline & & & \\
\hline
\end{tabular}


(NEUTRJNS/MEV/STERADIAN/SOURCE NEUTRON)

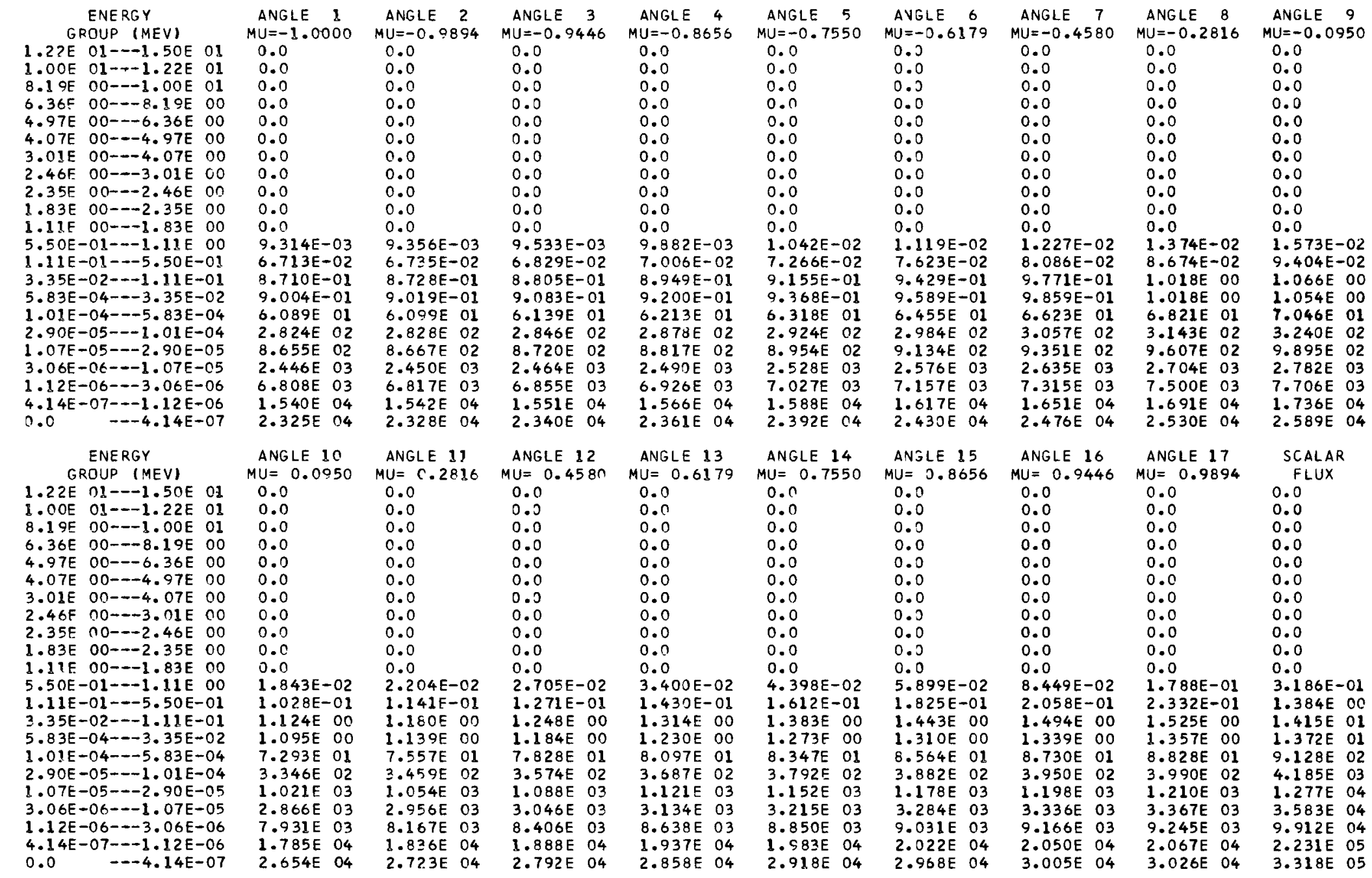




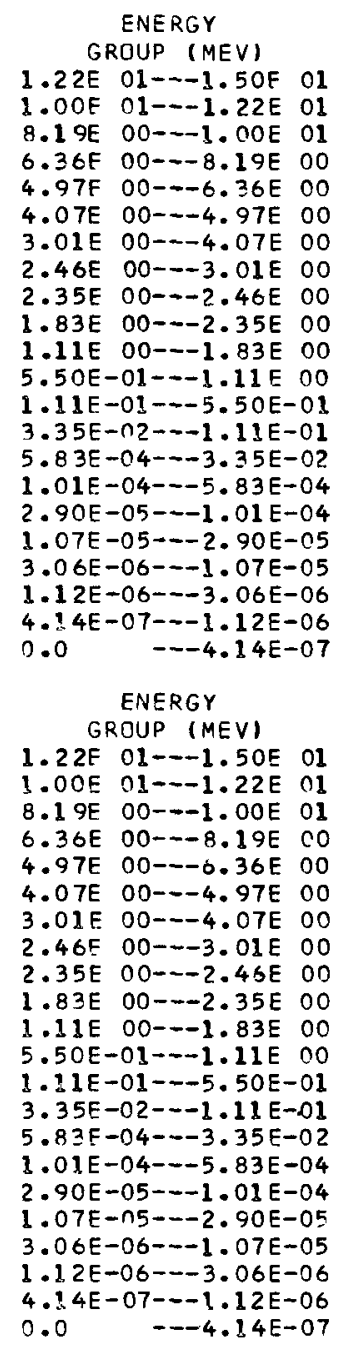

ANGLE 1 0.0 0.0

0.0

0.0

0.0

0.0

0.0

0.0

0.0

0.0

0.0

$5.781 E-03$

$4.364 \mathrm{E}-02$

$6.070 \mathrm{E}-01$

$6.403 E-01$

$4.415 E$ OI

$2.076 \mathrm{E} 02$

$6.432 E$ O2

$1.844 \mathrm{E} \quad 03$

$5.187 \mathrm{E} \quad 03$

l.186E OL

1.811E 04

ANGLE 10 $M U=0.0950$ 0.0

0.0

0.0

0.0

0.0

0.0

0.0

0.0

0.0

0.0

$1.150 \mathrm{E}-02$

$6.699 E-02$

$7.828 \mathrm{E}-01$

$5.311 E 01$

$2.472 \mathrm{E} 02$

$7.633 \mathrm{E}$ O2

$2.175 \mathrm{E} 03$

$6.089 \mathrm{E} 03$

$1.386 E^{0} 04$

$2.084 \mathrm{ECH}^{2}$
(NEUTRJNS/MEV/STERADIAN/SOURCE NEUTRONI E 2 $M U=-0.9446$ $0.0 \quad 0.0$

0.0

0.0

0.0

0.0

0.0

0.0

0.0

0.0

5. $808 \mathrm{E}-03$

$4.379 \varepsilon-02$

$6.083 E-01$

$6.414 E-01$

$4.422 E$ O1
$2.079 E$ C?

6.442E 02

$5.194 \mathrm{E} 03$

$1.188 \mathrm{E} \mathrm{O4}$

$1.813 \mathrm{E} \mathrm{O4}$

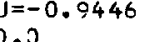

$M U=-0.8656$

ANGLE 5
$M U=-0.7550$

ANGLE 6

0.0

0.0
0.0
0.0

0.0

0.0

0.0

0.0

0.0

0.0

$5.921 E-03$

4.440E-02

$6.138 E-01$

$6.460 \mathrm{E}-01$

$4.452 \mathrm{E} 01$

$2.092 \mathrm{E} 02$

6.483E O2

$5.225 E \quad 03$

$1.194 E 04$

$1.823 E 04$

ANGLE 11

ANGLE 12

0.0

0.0
0.0
0.0

0.0
0.0

0.0
0.0

0.0

0.0

0.0

0.0

0.0

0.0
$1.377 E-02$

$7.400 E-02$

$8.124 E-01$

$5.507 \mathrm{E}$ OI

5.507E O1

$7.891 \mathrm{E} 2$

T.891E 22

$6.279 E \quad 03$

1.27 04

2.141E 04
ANGLE 13

0.0

0.0
0.0

0.0
$n .0$

0.0

0.0

0.0

0.0

0.0

0.0

1. $686 E-02$

$8.241 E-02$

$8.712 E-01$

$5.709 \mathrm{E} 01$

$5.709 E$ O1

$2.645 E$ O2

$2.338 \mathrm{E} 03$

$6.473 E$ O3

1.470 E 4

$2.199 E$ O 4

0.0

0.0

0.0

0.0

0.0

0.0

0.0

0.0

0.0

$2.113 E-02$

$.228 \mathrm{E}-02$

$8.185 \mathrm{E}-01$

$8.775 E-01$

$5.908 E$ OI

.7

$2.412 E$ O2

$6.661 \mathrm{~F} 03$

$1.511 \mathrm{E} 04$

$2.254 \mathrm{E} 04$
0.0

0.0
0.0

0.0

0.0

0.0

0.0

0.0

0.0

$6.142 \mathrm{E}-03$

$4.556 \mathrm{E}-02$

$6.239 \mathrm{E}-01$

$4.507 \mathrm{E} 01$

$2.117 E 02$

$6.557 \mathrm{E} 02$

$1.878 \mathrm{E} \mathrm{O3}$

$5.282 \mathrm{E} 03$

$1.207 E 04$

$1.841 E$ O4

0.0

0.0

0.0

0.0

0.0

0.0

0.0

0.0

6. $482 E-03$

$4.726 E-02$

$6.385 \mathrm{E}-01$

6.666E-01

$4.585 E$ O1
$2.152 E$ O2

$6.663 E \quad 02$

$1.908 E \quad 03$

$5.362 E 03$

$1.225 \mathrm{E} 04$

1. $865 \mathrm{E} 04$

ANGLE 14

$\begin{array}{cc}\text { ANGLE } 14 & \text { ANGLE } 15 \\ M U=0.7550 & M U=0.8656\end{array}$
0.0
0.0

0.0

0.0

0.0

0.0

0.0

0.0

0.0

$0.07 \mathrm{E}-02$

$1.036 E-01$

$9.645 E-01$

$6.093 E 01$

$2.811 E$ O2

$8.650 E \quad 02$

2.453E O3

$1.549 E$ O4

2.305E 04

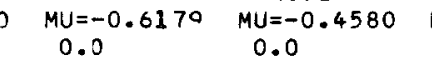

$0.0 \quad 0.0$

0.0

0.0

(1)

0.0

0.0

0.0

$.972 \mathrm{E}-03$

作

$2.197 E \quad 02$

.946E 03

$1.248 E$
$1.897 E$ 04

0.0

0.0

0.0
0.0

0.0

0.0

0.0

0.0

0.0

0.0

$3.604 E-02$
$1.162 E-01$

$9.348 \mathrm{E}-01$

$6.253 \mathrm{E} \mathrm{O1}$

2.879E 02

$8.854 E$ O2

$6.580 E \quad 03$

. $581 \mathrm{E} 04$

2.347E O4

NGLE ${ }^{7}$

0.0

0.0

$7.650 E-03$

$5.261 \mathrm{E}-02$
$6.819 \mathrm{E}-01$

$7.021 E-01$

$4.813 E$ O1

$2.253 E \quad 2$

$6.970 E \quad 02$

$1.993 E_{03}$

$5.593 E O_{3}$

$1.276 E 04$
$1.935 E 04$

ANGLE 16

0.0
0.0 ANGLE 8
$M U=-0.2816$ 0.0 .2816 MU 0.0 $\begin{array}{lll}0.0 & 0.0 & 0.0\end{array}$ $\begin{array}{lll}0.0 & 0.0 & 0.0\end{array}$ $\begin{array}{lll}0.0 & 0.0 \\ 0.0 & 0.0 & 0.0\end{array}$

0.0

0.0

0.0

0.0

0.0

8.5 74E-03

$5.643 E-02$

$7.110 E-01$

$4.253 E-01$

OE

7.167

$2.047 E \quad 03$

$5.741 E 03$

$1.309 E 04$

ANGLE 17

$M U=0.9446 \quad M U=0.9894$ 
(NEUTRONS/MEV/STERADIAN/SCURCF NEUTRON) GROUP (MFV) $1.22 E$ OL-W-1.5OE O1 1.0NE $01--1.22 \mathrm{E}$ O1 $6.36 \mathrm{~F} 00--8.19 \mathrm{E}$ OO $4.97 F \quad 00--6.36 \mathrm{E}$ OO 4.07E DO-- 4.97E 00 $3.01 \mathrm{~F}$ DO---4. $07 \mathrm{E} \mathrm{CO}$ $2.46 E$ OO-- $3.01 E 00$ $2.35 \mathrm{E} 00--2.46 \mathrm{E} 00$ $1.83 \mathrm{E} 00---2.35 \mathrm{E}$ 00 $1.11 \mathrm{E} 00--1.83 \mathrm{E}$ 00 $5.50 \mathrm{E}-01---1.11 \mathrm{E}$ 00 $1.11 \mathrm{E}-01---5.50 \mathrm{E}-01$ $3.35 \mathrm{E}-02--1.11 \mathrm{E}-01$
$5.83 \mathrm{E}-04-\cdots-3.35 \mathrm{E}-02$ 1. $01 E-04--5.83 E-04$ $2.90 F-05--1.01 E-04$ $1.07 E-05--2.90 E-05$ $3.06 \mathrm{E}-06---1.07 \mathrm{E}-05$
$1.12 \mathrm{E}-06---3.06 \mathrm{E}-06$ $1.12 \mathrm{E}-06---3.06 \mathrm{E}-06$ $4.24 \mathrm{E}-07---1.12 \mathrm{E}-06$
$0.0 \quad--4.14 \mathrm{E}-07$ ENERGY GROUP (MEV) $1.22 E$ O1---1.50E Ol $1.00 \mathrm{E}$ O1- $-1.22 \mathrm{E}$ OI $8.19 \mathrm{E}$ 00---1.00E 01 6.36F $00--8.19 E$ DO 4.97E OO--6. $36 E$ OO 4.07E OO-C. . 2.46E 00- $3.01 E$ OO 2.46E OO $3.01 E$ OO $1.83 \mathrm{E} 00--2.35 \mathrm{E}$ on 1.1 I $00--1.83 \mathrm{E}$ on 5.50E-01--1.11E 00 1.11E-01--5.50E-01 3.35E-02---1.11E-01 $5.83 E-04-\cdots 3.35 E-02$ 1.0 E-04-- $5.83 \mathrm{E}-04$ 2.90E-05--1.01E-04 1.07E-05--2.90E-05 $3.06 \mathrm{~F}-06--1.07 \mathrm{E}-05$ $1.12 \mathrm{~F}-06---3.06 \mathrm{E}-06$ $4.14 \mathrm{E}-07---1.12 \mathrm{E}-06$ $0.0 \quad--4.14 E-07$
ANGLE
$M U=-1.0000$ 0.0 0.0 0.0 0.0 0.0 0.0 0.0 0.0 0.0 $1.189 \mathrm{E}-03$ $9.947 \mathrm{E}-03$ 1.577E-01 $1.720 \mathrm{E}-01$ $1.225 \mathrm{E} 01$ $5.886 \mathrm{E} \mathrm{O1}$ $1.857 E$ O2 $5.448 \mathrm{E} 02$ $1.561 \mathrm{E} 03$ $3.634 \mathrm{E} 03$ $5.658 \mathrm{E} \quad 3$ ANGLE 10 $M U=0.0950$ 0.0 0.0 0.0 0.0 0.0 0.0 0.0 0.1 0.0 2.390E-03 $1.527 E-02$ 2. $043 \mathrm{E}-01$ $2.106 E-01$ $1.482 \mathrm{E}$ O1 $7.061 \mathrm{E} \mathrm{O1}$ $2.223 \mathrm{E} 02$ $6.493 E 02$ $1.853 \mathrm{E} \mathrm{O3}$ $4.297 E 03$ $6.588 E \quad 03$
ANGLE 2
$M U=-0.5894$ $M U$
0.0
0.0 0.0
0.0 0.0 0.0 c. 0 0.0 0.0 0.0 0.0 $1.195 \mathrm{E}-03$ $9.98 C E-03$ $1.580 E-01$ $1.723 E-01$ $1.227 \mathrm{E} O 1$ $5.896 \mathrm{~F} 01$ $1.860 \mathrm{E} \quad 02$ $5.457 \mathrm{E} \quad 2$ $1.564 E \quad 03$ $3.640 E$ O3 $5.666 \mathrm{E} \mathrm{O3}$

ANGLE 11 0.0 0.0
0.0 0.0
0.0 0.0 0.0 0.0 0.0 0.0 0.0 $2.859 \varepsilon-03$ 1. $684 \mathrm{E}-02$ $2.154 E-01$ $2.1925-01$ 1. $538 \mathrm{E} O 1$ $7.31 .5 E$ OI $2.301 \mathrm{E} O 2$ $1.915 \mathrm{E} \mathrm{O3}$ 4.437E C3 6.785 E 03

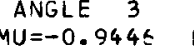

$M U=-0.8656$ 0.0 0.0 0.0
0.0
0.0 0.0 0.0 0.0 0.0 1. $219 \mathrm{E}-03$ $1.012 \mathrm{E}-02$ $1.595 \mathrm{E}-01$ $1.735 \mathrm{E}-01$ $1.235 \mathrm{E}$ OI $1.873 \mathrm{E}$ O2 $5.492 \mathrm{~F} O 2$ $1.574 \mathrm{E} O 3$ .662E 03 $.699 \mathrm{E} \mathrm{O3}$ 0.0 0.0 0.0 0.0
0.0 0.0

0.0

ANGLE 13

0.0
0.0
0.0
0.0

3.490E-02 $1.868 \mathrm{E}-02$ $2.272 \mathrm{E}-01$ $2.280 \mathrm{~F}-01$ $1.595 \mathrm{E}$
$7.574 \mathrm{E}$ ol $2.382 \mathrm{E}$ O? $6.944 \mathrm{E} \mathrm{O2}$ $1.978 \mathrm{E} \mathrm{O3}$ $4.579 \mathrm{E} 03$ $6.984 \mathrm{E} 03$ $=-0.8656$

0.0

0.0

$1.267 \mathrm{E}-03$ $1.039 E-02$ $1.622 \mathrm{E}-01$ $1.759 \mathrm{E}-01$ 1.251 E 01 $6.008 \mathrm{E}$ a $1.895 \mathrm{E}$ O2 $5.557 \mathrm{E}$ O2 $1.592 \mathrm{E} \quad 03$ $3.704 \mathrm{E} \quad 03$ $5.759 \mathrm{~F} 03$ ANGLE $13 \quad$ ANGLE 14
$M=0.6179$ $0.0 \quad 0.0$ 0.0

0.0

0.0

0.0

0.0

ANGLE 5 0.0 0.0
0.0
0.0 0.0 0.0 0.0 0.0 0.0 0.0 $1.339 \varepsilon-03$ 1. $078 \mathrm{E}-02$ $1.661 \mathrm{E}-01$ $1.792 \mathrm{E}-01$ $1.274 \mathrm{E} O 1$ $6.111 \mathrm{E}$ OI $1.928 E \quad 02$ $5.650 \mathrm{E} \mathrm{O2}$ $1.618 \mathrm{E} \quad 03$
$3.763 \mathrm{E}$ $.763 \mathrm{E} 03$ $5.843 E$ O 3 0.0
0.0 0.0 0.0 0.0 0.0 0.0 0.0 0 4. $348 \mathrm{E}-03$ $2.080 \mathrm{~F}-02$ $.392 \mathrm{E}-01$ $.368 \mathrm{E}-01$ $1.652 \mathrm{~F}$ Ol
$7.827 \mathrm{E}$ Ol 2.460 E 02 $2.460 \mathrm{E} 02$
$7.165 \mathrm{~F} 02$ 2.039 E 03 $4.718 E$ O3 $7.177 \mathrm{E} \quad 3$

$$
0.0
$$

$5.5205-03$ $2.313 \mathrm{E}-02$ $2.509 E-01$ $.449 \mathrm{E}-01$ $8.060 E$ OI $2.532 \mathrm{E} O 2$ $7.369 \mathrm{E} \mathrm{O2}$ $2.095 \mathrm{E} \mathrm{O}$ 4. $2.094 \mathrm{E}$ O3 $7.353 \mathrm{E} 03$

$\begin{array}{ll}A N G L E & 6 \\ M U=-0.6179\end{array}$ 0.3 0.0 0.0
0.0 0.0
0.0 0.0
0.0 0.0 0.0
0.0
0.0 0.0 0.0

1. $443 \mathrm{E}-03$ $1.132 \mathrm{E}-02$ $1.712 \mathrm{E}-01$ $1.836 \mathrm{E}-01$ 1.303E OL $6.247 \mathrm{E}$ OI 1.970 E 02 5.770 E O2 .6 $1 E$ E $5.951 \mathrm{E} 03$

ANजLE 15 $M=0.8656$ 0.0 0.0 0.0 0.0 0.0 0.0 0.0 0.0
0.0 0.0 0.0

$7.146 E-03$ $2.557 E-02$ 
(NEUTRONS/MEV/STERADIAN/SOURCE NEUTRON)

\section{ENERGY} GROUP (MEV) $1.22 \mathrm{E} 01--1.50 \mathrm{E}$ $1.00 E$ D1---1.22E O1 8.1 9E $00--3.00 E$ OI 4. $.97 E$ E $00--6.36 \mathrm{E}$. 00 4.07E $00---4.97 E 00$ 3.01E $00---4.07 E$ OO $2.46 E$ O0---3.01E 00 $2.35 E$ OO---2.46E 00 $1.83 \mathrm{E} \quad 00--2.35 \mathrm{E} 00$ $1.11 \mathrm{E} \quad 00---1.83 \mathrm{E} 00$ $5.50 \mathrm{E}-01--1.11 \mathrm{E} 00$ $1.11 \mathrm{E}-01---5.50 \mathrm{E}-01$ $3.35 \mathrm{E}-02---1.12 \mathrm{E}-01$ $5.83 \mathrm{E}-04---3.35 \mathrm{E}-02$ $1.01 \mathrm{E}-04---5.83 \mathrm{E}-04$ $2.90 E-05--1.01 \mathrm{E}-04$ $1.07 E-05--2.90 E-05$ 3.06E $-06--1.07 E-05$ $1.12 \mathrm{E}-06--3.06 \mathrm{E}-06$ $4.14 \mathrm{E}-07---1.12 \mathrm{E}-06$
$0.0 \quad--2.14 \mathrm{E}-07$ ENERGY GROUP (MEV) $1.22 \mathrm{E}$ 01--1.50E 01 $1.00 F$ O1--1.22E 01 8.1 QE $00--1.00 E$ O1 $6.36 \mathrm{E} \quad 00---8.19 \mathrm{E} \quad 00$ 4.97 E $00--6.36 E$ OO 4.07E $00---4.97 E$ OO $3.01 \mathrm{E}$ 00---4. $2.46 E$ EO- $3.01 E$ DO $1.83 \mathrm{E} 00---2.35 \mathrm{E} 00$ $1.83 \mathrm{E} 00--2.35 \mathrm{E}$ 00 $5.5 \mathrm{CE}-01=-1.11 \mathrm{E}$ 00 $1.1 \mathrm{EE}-01---5.50 \mathrm{E}-01$ 3.35E-02---1.11E-01 $5.83 \mathrm{E}-\mathrm{C4}-\mathrm{-}-\mathrm{3} .35 \mathrm{E}-02$ $1.01 \mathrm{E}-04--5.83 \mathrm{E}-04$ $2.90 \mathrm{E}-05-1.01 \mathrm{E}-04$ $1.07 E-05--2.90 E-05$ $3.06 \mathrm{E}-06--1.07 \mathrm{E}-05$ $1.12 E-06---3.06 E-06$ $4.14 \mathrm{E}-07=-1.12 \mathrm{E}-06$ $0.0 \quad--4.14 E-07$
ANGLE 1 0.0 0.0 0.0 0.0 0.0 0.0 0.0 0.0 0.0 $2.157 E-04$ $1.931 E-03$ $3.294 E-02$ $3.649 E-02$ $2.638 \mathrm{E} 00$ $1.281 \mathrm{E} 01$ $4.074 E$ OI $1.207 E 02$ $8.184 E$ O2 $1.285 E$ O 3 ANGLE 10
$M U=0.0950$ 0.0

0.0

0.0

0.0

0.0

0.0
0.0
0.0
0.0 0.0 0.0

4. $359 E-04$ $2.966 E-03$ $4.273 E-02$ $4.476 E-02$ $1.198 E$ E $4.890 \mathrm{E}$ OI 4.890E O1 $4.156 \mathrm{E} \mathrm{O2}$ $9.717 \mathrm{E} \mathrm{O2}$ $1.503 E 03$
ANGLE 3 $\begin{array}{ll}A N G L E & 2 \\ M U=-0.9894\end{array}$ 0.0 0.0 0.0 0.0 0.0 0.0 0.0 0.0 0.0 $2.168 E-04$ $1.937 E-03$ $3.301 E-02$ $3.656 \mathrm{E}-02$ $2.642 \mathrm{E} 00$ $1.283 E 01$ $4.080 E$ OL $1.209 \mathrm{E} 02$ $3.494 \mathrm{E} 02$ $8.156 E$ O2
$1.287 E$ O3

$U=-0.9446$ 0.0 0.0 0.0 0.0 0.0 0.0 0.0 0.0 0.0 $2.213 E-04$ $1.965 E-03$ $3.332 \mathrm{E}-02$ $3.683 E-02$ $2.661 \mathrm{E} 00$ $1.292 \mathrm{E} 01$ $4.108 E$ O1 1.21 JE 02 $3.517 E 02$ $8.249 E$
$1.295 E$ ANGLE 4 ANGLE 5

AVGLE 6 ANGLE 11 $M U=0.2816$

0.0
0.0
0.0
0.0

0.0
0.0
0.0
0.0

0.0

0.0

0.0

0.0 0.0

$5.20 S E-C 4$ $3.268 E-03$ 4.505E-0? $4.658 \mathrm{E}-02$ $3.320 E 00$ 1.596E O1 $3.404 \mathrm{E} 02$ $4.207 E 02$ 1.2904 03 $3.549 \mathrm{E} 03$
ANGLE 12 $M U=0.4580$ 0.0

0.0
0.0 0.0

0.0

0.0

0.0

0.0

0.0 0.0

0.04 $3.619 E-03$ $4.751 E-02$ $4.845 E-02$ $3.445 E$ EO $1.653 E$ O1 $1.546 E 02$ $4.441 \mathrm{E} 02$ $1.037 \mathrm{E} 03$ 1.595E 03
0.0

0.0

0.0

0.0

0.0

0.0

0.0

0.0

0.0

0.0

$2.303 E-04$

$2.017 E-03$

$3.388 \mathrm{E}-02$

3. 733 E-02

2.595E OO

$4.159 E_{01}$

$1.232 \mathrm{E} \mathrm{O2}$

$3.558 \mathrm{E} \mathrm{O2}$

$1.309 \mathrm{E} 03$

ANGLE 13

ANGLE
$M U=0$
0.0
0.0

0.0
0.0
0.0
0.0

0.0
0.0

0.0

0.0

0.0

0.0

0.0

0.0
$7.871 E-04$

4. $016 \mathrm{E}-03$

5.00E-02

..03OE-02

1.7095 OI

5.4175 o1 1.596E O2 4.581E 02

$1.641 \mathrm{E} 03$

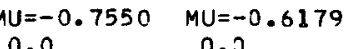

0.0

0.0

0.0

0.0

0.0

0.0

0.0

0.0

0.0

0.0

$2.434 E-04$

2. $094 \mathrm{E}-03$

$3.470 E-02$
$3.805 E-02$

$2.745 E 00$

$1.330 \mathrm{E}$ O1

$\begin{array}{lll}4.231 E & 01 \\ 1.253 E & 02\end{array}$

$1.253 \mathrm{E}$ O2

$8.481 \mathrm{E} 02$

$1.328 E$ O3

ANGLE 14 $M U=0.7550$ MUN $=0$
0.0
0.0

0.0

0.0

0.0

0.0

0.0

0.0

0.0

0.0

0.0
$9.911 E-04$

4. $446 E-03$

$5.235 \mathrm{E}-02$

$5.202 E-02$

1.7595 O1

$5.576 E$ OI

1.641E O2

1. $098 \mathrm{E} 03$

$1.682 \mathrm{E} \mathrm{O3}$

0.0

0.0
0.0
0.0

0.0
0.0
0.0

0.0

0.0

0.0

0.0

0.0

0.0

$2.625 E-04$

$2.198 E-03$

$3.579 E-02$
$3.899 E-02$

2.809E 00

1.360 E 01

$4.325 \mathrm{E} 01$

$1.280 E$ O2

.

$1.354 \mathrm{E} 03$

ANGLE 15

MU $=0.8$
0.0

0.0
0.0
0.3
0.0

0.0
0.0

0.0

0.0 
(NEUTRONS/MEV/STERADIAN/SOURCE NEUTRON)

ENERGY GNERGY $1.22 E$ O1- 1 - $1.50 E$ OI 1.OOE $01--1.22 \mathrm{E}$ OI 8.1SE $00--1.00 E$ OI 6.36E $00--8.19 E \quad 00$ 4.07E $00--4.97 E$ OO $3.01 E$ 00-- $4.07 E 00$ $2.46 E 00--3.01 E 00$ $2.35 \mathrm{E} 00--2.46 E 00$ $1.83 E$ OO- $-2.35 E \quad 00$ $1.11 E$ 00- $1.83 E$ E 00 $5.50 E-01-21.11 E 00$ $1.11 \mathrm{E}-01---5.50 \mathrm{E}-01$ $3.35 \mathrm{E}-02--1.11 \mathrm{E}-01$ $5.83 E-04--3.35 E-02$ $1.01 E-04---5.83 E-04$ $2.90 E-05--1.01 E-04$ 1.07E-05--2. $2.90 \mathrm{E}-05$ $3.06 \mathrm{E}-06--1.07 \mathrm{E}-05$ $1.12 E-06--3.06 E-06$ $4.14 E-07--1.12 E-06$
$0.0 \quad--04.14 E-07$ ENERGY $1.22 E$ O1---1.50E 01 $1.00 \mathrm{E} 01--1.22 \mathrm{E} 01$

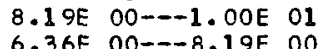
$6.36 E^{0} 00--8.19 E \quad 00$

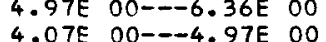
$3.01 E$ O0- $-4.07 E 00$ $2.46 \mathrm{~F} \mathrm{CO--}-3.01 \mathrm{E} 00$ $2.35 E$ OO---2.46E OO $1.83 E$ DO---2.35E 00 1.11E $00-D-1.83 E$ OO 5.5E-01-C-1.11E 0 $1.112-01--5.50 E-01$ $3.35 E-02--1.11 E-01$ $5.83 E-94--3.35 E-02$ 1.01E-042.9CE-05- $1.01 E-04$ 3.06E-06-- $1.07 E-05$ $1.12 \mathrm{E}-06-\cdots 3.06 \mathrm{E}-06$ $4.14 \mathrm{E}-07--1.12 \mathrm{~F}-06$ $4.14 E-07--1.12 F-06$
$0.0 \quad--4.14 E-07$
ANGLE 1 0.0

0.0

0.0

0.0

0.0
0.0
0.0

0.0

0.0

0.0

0.0
$3.653 E-05$

$3.433 E-04$

$6.118 \mathrm{E}-03$

$6.840 E-03$

$4.984 \mathrm{E}-01$

$7.774 E$ OO

$2.317 \mathrm{E}$ O1

$6.721 E 01$

$2.497 E 02$

ANGLE 10 $M U=0 . C 950$

0.0

0.0

0.0

0.0

0.0

0.0
0.0
0.0

0.0

0.0

0.0

0.0
$7.404 E-05$ $7.404 E-05$
$5.276 E-04$ $7.944 \mathrm{E}-03$ $8.393 E-03$ $6.049 E-01$ $2.929 E$ OO $9.342 E 00$ $2.774 E$ O1 $1.883 E 02$ $2.925 E 02$
ANGLE 2 ANGLE 3 $U=-0.9894$ 0.0

0.0

0.0

0.0

0.0

0.0

0.0

0.0

0.0

0.0

3.671E-05

$3.445 E-04$

$6.1 \geq 2 E-03$

$6.8 \leq 2 E-03$

$2.437 \mathrm{E} \mathrm{OO}$

$7.786 \mathrm{E}$ 00

$2.320 E$ O1

$6.732 \mathrm{E}$ Ol

$2.501 \mathrm{E} \mathrm{O2}$

ANGLE 11

$M U=$
$C .0$

$C .0$
0.0
0.0

0.0
0.0
0.0
0.0
0.0

0.0
0.0

0.0

0.0

0.0

0.0

0.0
$8.843 E-05$

$5.809 E-04$

$8.374 E-03$

$8.733 \mathrm{E}-03$

$6.279 E-01$

$3.035 E 00$ 2.271E O1 $8.294 E$ O1 $1.946 E$ O2 $3.015 \mathrm{E} 02$ $\begin{array}{lc}\text { ANGLE } & 3 \\ M U=-0.9446\end{array}$

0.0
0.0
0.0

0.0

0.0

0.0

0.0

0.0

0.0

0.0

$3.749 E-05$

$3.495 E-04$

$6.190 \mathrm{E}-03$

$5.029 E-01$

$2.454 \mathrm{E} 00$

$7.840 E \quad 00$

$2.336 \mathrm{E} 01$

$6.776 \mathrm{E}$ OI

2.515E 02

ANGLE 12 $M U=0.4580$

0.0

0.0

0.0

0.0

0.0

0.0

0.0

0.0

0.0

1.0 $76 E-04$

$6.425 \mathrm{E}-04$

$8.828 E-03$

$6.514 E-01$

$3.143 E$ OO

$2.970 E$ OI

$8.574 E$ OI

2.
3.1071 E 02
ANGLE 4

0.0

0.0
0.0

0.0

0.0

0.0

0.0

0.0

0.0

0.0

3. $898 E-05$

$3.588 \mathrm{E}-04$

$6.296 F-03$

$.998 \mathrm{E}-03$

$5.094 E-01$
$2.485 E ~$

$7.937 \mathrm{E} 00$

$2.364 E$ Ol

$6.857 \mathrm{E} 01$

$\begin{array}{ll}1.615 \mathrm{E} & 02 \\ 2.544 \mathrm{E} & 02\end{array}$

ANGLE 13 $M U=0.6179$

0.0
0.0

0.0

0.0

0.0

0.0

0.0

0.0

0.0

$1.330 E-04$

$7.118 \mathrm{E}-04$

9.

$6.744 E-01$

$3.249 \mathrm{E}$ OO

$1.035 \mathrm{E}$ OL

$3.065 E$ EI

$8.845 E$ OI

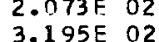

ANGLE 5
MU $=-0.7550$

0.0

0.0
0.0
0.0
0.0

0.0

0.0

0.0

0.0

0.0

$4.127 E-05$

3. $725 E-04$

6.449E-03

$5.188 \mathrm{E}-01$

$2.528 E 00$

$8.076 E$ OO
$2.405 E$ OI

$6.973 E$ O1

$1.641 \mathrm{E} \mathrm{O2}$

2.582E 02

ANGLE 14

$M U=0.7550$

0.0
0.0

0.0

0.0

0.0

0.0

0.0

0.0

0.0

0.0

1. $665 E-04$

$7.859 E-04$

$9.725 E-03$

$6.956 \mathrm{E}-01$

$3.345 \mathrm{E}$ OO

$1.065 \mathrm{E} 01$

3.155E 01

2.092E O1

$3.275 E$ O2
$\begin{array}{ll}A N G L E & 6 \\ M=-0.6179\end{array}$ 0.0

0.0

0.3

0.0

0.0

0.0

0.0
0.3

0.0

4. $454 E-05$

$3.911 E-04$

$6.651 E-03$
$7.311 E-03$

$5.310 \mathrm{E}-01$

$2.586 E 00$

$8.256 E$
$2.458 E$

.458E O1

$7.123 E$ O1
$1.676 E ~ 02$

2.632E 02

ANGLE 15

$M U=0.8656$

0.0
0.0

0.0

0.0

0.0

0.0

0.0

0.0

0.0

0.0

2.098E-04

$8.597 E-04$

$1.011 E-02$

$1.0 O 2 E-02$

$3.137 E-01$

$1.091 \mathrm{E} 01$

$3.229 \mathrm{E}$ O1

$9.303 E$ OI

$3.343 E 02$

ANGLE 7 0.0 .4580 ANGLE 8 ANGLE 9 
(NEUTRONS/MEV/STERADIAN/SOURCE NEUTRON)

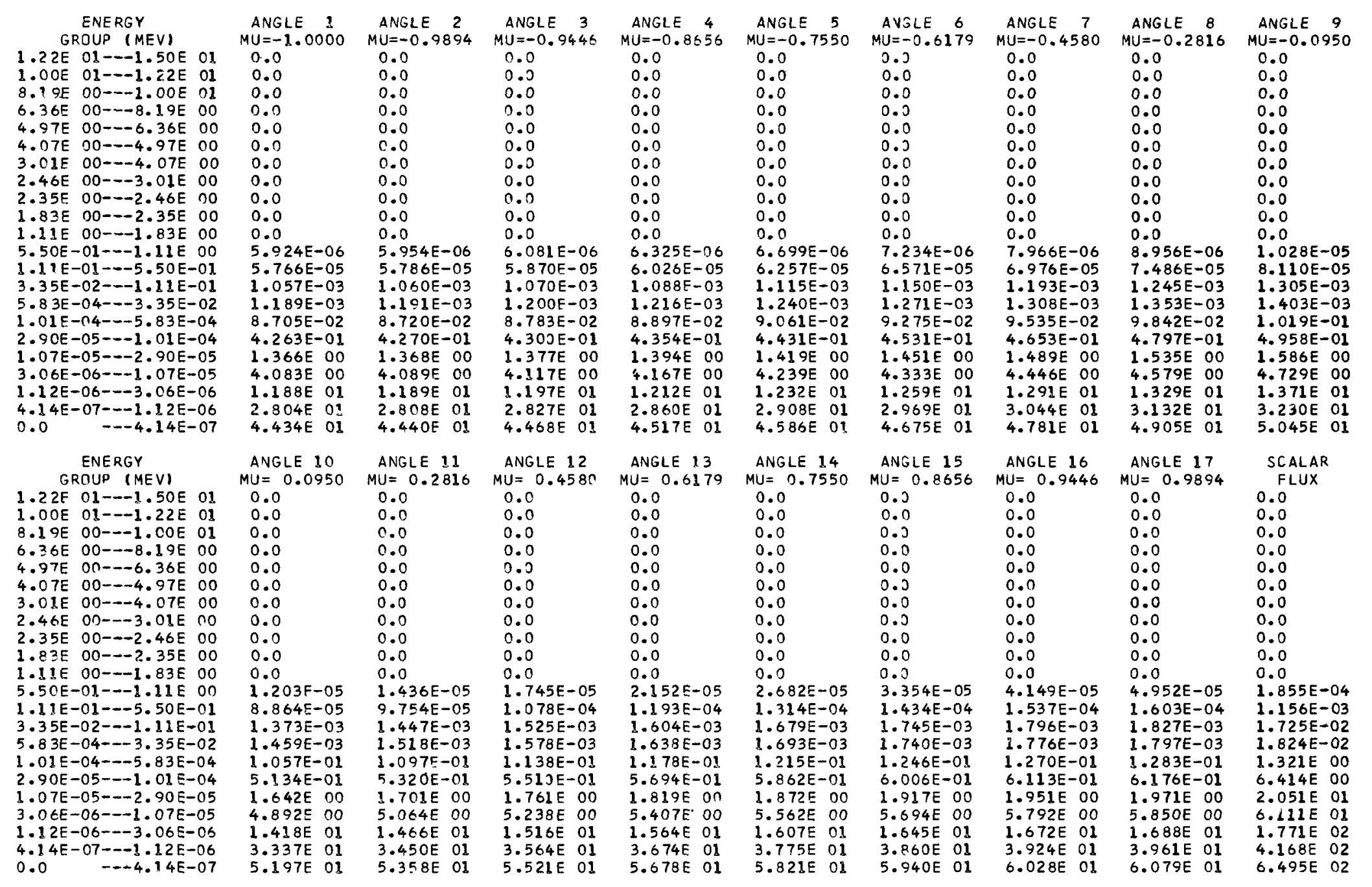


ENERGY GROUP (MEV) 8. $.00 E$ OO---1.00E OI 6.50E OO--8. 00 OO 00 4.0CE $00--6.50 E$ OO $3.00 E$ OO- $-4.00 E$ OO $2.50 \mathrm{OF}$ 00---3.00E 00 $2.00 E$ OO---2.50E OO $1.66 \mathrm{E} 00--2.00 \mathrm{E} \mathrm{OO}$ $1.33 E$ O0- $-1.66 \mathrm{E} \mathrm{O0}$ $1.00 \mathrm{E} 00---1.33 \mathrm{E} \mathrm{O0}$ 8.00E-01--1. 1.00 E 00 $6.00 E-01--8.00 E-01$ 4.0CE-O1---6. COE-CI $3.00 \mathrm{E}-01--4.00 \mathrm{E}-01$ $2.00 E-01---3.00 E-01$ $1.00 E-01--2.00 E-01$ $5.00 E-02--1.00 E-01$
$2.00 E-02--5.00 E-02$

$$
\begin{aligned}
& \text { ENERGY } \\
& \text { GROUP (MEV) }
\end{aligned}
$$

8. OOE DO---1. COE 01 $6.50 \mathrm{OE}$ OO-D-8. COE OO $5.00 E$ OO- -6.50 E 00 $4.0 O E$ OO- $-5.00 E$ OO 3.00 OOO- 2.50E DO---3.00E OO 2.0CE OO- 2.5OE ON $1.66 \mathrm{E}$ OO- $-2.00 \mathrm{E}$ OO $1.33 E$ OO1. OOE OO- - - 33 E OD S.OOF 1 3.0OF-CI-C- . COE-DI $4.00 E-01--6.00 E-O 1$ 2.0OF $01-3.00 E-01$ 2.0CF $01-3.00 E-01$ $1.00 E-02-1.00 E-01$ 2. $00 \mathrm{E}-02---5.00 \mathrm{E}-\mathrm{O} 2$
ANGLE 1 $M U=-1.0000$ $3.444 \mathrm{E}-06$ .145E-06 作 $3.916 \mathrm{E}-05$ $.426 \mathrm{E}-06$ $1.062 \mathrm{E}-05$ $1.236 \mathrm{E}-05$ $1.482 \mathrm{E}-05$ $1.929 \mathrm{E}-05$ $2.609 \mathrm{E}-05$ $3.731 E-05$ $1.444 E-04$ $1.730 \mathrm{E}-04$ $3.309 \mathrm{E}-04$ 3.592E-04 $2.053 \mathrm{E}-03$ $5.566 E-04$ ANGLE 10 $M U=0.0950$ $4.224 \mathrm{E}-06$ $8.750 E-06$ $9.836 \mathrm{E}-05$ $2.718 \mathrm{E}-05$ 4.807E-05 $1.268 \mathrm{E}-05$ $1.422 \mathrm{E}-05$ $1.645 \mathrm{E}-05$ $1.960 E-05$ 2.525E-05 $3.364 \mathrm{E}-05$ $4.702 E-05$ $1.663 \mathrm{E}-04$ 1.935E-04 . (2) $2.122 \mathrm{E}-03$
$5.648 \mathrm{E}-04$
ANGLE 2
$M U=-0.0894$ $3.451 \mathrm{E}-06$ $7.158 E-06$ $8.087 \mathrm{E}-05$ $2.192 E-05$ $3.923 E-05$ $9.452 \mathrm{E}-\mathrm{C} 6$ $1.065 \mathrm{E}-05$ $1.239 E-05$ $1.486 \mathrm{E}-05$ $1.934 E-05$ $2.615 E-05$ $3.739 E-05$ $.446 \mathrm{E}-04$ $1.732 E-04$ $3.310 E-04$ $.596 E-04$ . $054 E-03$ $5.567 E-C 4$ ANGLE 11 $M U=0.2816$
$4.303 E-06$ $4.303 E-06$ $9.057 E-06$ $1.022 E-04$ $2.833 E-05$ $5.000 E-O 5$ $1.339 E-05$ $1.500 E-05$ $1.734 E-05$ $2.063 E-05$ $2.049 E-05$ ..517E-05 1.704E-04 $1.971 E-04$ 1.971E-04 $0.476 \mathrm{E}-\mathrm{C4}$ $2.135 \mathrm{E}-03$ $5.663 E-04$
(GAMMAS/MEV/STERADIAN/SOURCE NEUTRON)

ANGLE ANGLE ANGLE 5 AVJLE 6 $7.208 \mathrm{E}-06$ $8.141 \mathrm{E}-0.05$ $2.208 \mathrm{E}-05$ $3.950 E-05$ $9.550 \mathrm{E}-\mathrm{C6}$ $1.076 E-05$ $1.252 E-05$ $1.501 \mathrm{E}-05$ $1.953 \mathrm{E}-05$ $2.640 \mathrm{E}-05$ $3.772 E-05$ $1.454 \mathrm{E}-04$ $1.740 \mathrm{E}-04$ $3.315 \mathrm{E}-04$ $8.610 \mathrm{E}-04$ $2.056 \mathrm{E}-03$ $5.570 E-04$ 3.521E-06 $7.304 \mathrm{E}-06$ 8.247E-05 $2.240 \mathrm{E}-05$ 4.004E-05 $9.743 E-05$ $1.097 \mathrm{E}-0$. $1.276 \mathrm{E}-0$ $1.530 \mathrm{E}-0$ $1.989 \mathrm{E}-05$ $2.687 \mathrm{E}-05$ $3.834 E-05$ $1.469 \mathrm{E}-04$ $1.754 \mathrm{E}-04$ $3.324 \mathrm{E}-04$ $8.638 F-04$ $2.061 \mathrm{E}-0$ $5.576 \mathrm{E}-04$

ANGLE 12 ANGLE 12 ANGLE 13 $4.563 E-06$ $9.445 \mathrm{E}-06$ $1.060 \mathrm{E}-04$ $2.948 \mathrm{E}-05$ $5.192 \mathrm{E}-05$ $1.411 \mathrm{E}-05$ $1.579 E-05$ $1.823 E-05$ $2.164 \mathrm{E}-05$ $2.772 E-05$ $5.666 E-05$ $\mathrm{U}=0.6179$ $4.722 \mathrm{~F}-06$ $9.772 \mathrm{E}-06$ $1.096 \mathrm{E}-04$ $3.057 \mathrm{E}-05$ $5.374 \mathrm{E}-05$ $1.480 E-05$ $1.654 \mathrm{E}-05$ $1.906 E-05$ $2.260 E-05$ $2.886 E-05$ $3.803 E-05$ $5.237 E-05$ $2.035 \mathrm{E}-04$ 2.00BE-04 $2.035 E-04$ 0.148E-04 $2.147 E-03$ 2.157E-03 $\begin{array}{ll}2.147 E-03 & 2.157 E-03 \\ 5.676 E-04 & 5.688 E-04\end{array}$ AN=-0.7550 MU $=-3.6179$ 3. $590 \mathrm{E}-06 \quad 3.680 \mathrm{E}-06$ 7.631E-06 $2.287 E-05 \quad 2.348 E-05$ $4.083 \mathrm{E}-05 \quad 4.186 \mathrm{E}-05$ $1.003 E-05 \quad 1.040 E-05$ $1.129 \mathrm{E}-05 \quad 1.170 \mathrm{E}-05$ $1.312 E-05 \quad 1.359 E-05$ $1.572 E-05 \quad 1.627 E-05$ $2.043 E-05 \quad 2.112 E-05$ $2.756 \mathrm{E}-05 \quad 2.846 \mathrm{E}-05$ $3.925 E-05 \quad 4.042 E-0$ $1.490 \mathrm{E}-04 \quad 1.517 \mathrm{E}-04$ $1.775 \mathrm{E}-04 \quad 1.800 \mathrm{E}-04$ $3.337 E-04 \quad 3.354 E-04$ $8.678 \mathrm{E}-04 \quad 8.728 \mathrm{E}-04$ $2.068 E-03 \quad 2.076 E-03$ $5.584 \mathrm{E}-04$ $5.594 E-04$ ANGLE 14 ANGLE 14 ANGLE 15 4. $870 \mathrm{E}-06$ $1.008 \mathrm{E}-05$ $1.129 E-04$ 3.158E-05 $5.542 \mathrm{E}-05$ $1.543 E-05$ $1.722 \mathrm{E}-05$ $1.983 E-05$ $2.346 E-05$ $2.990 E-05$ $3.926 E-05$ $1.383 E-05$ $1.810 E-04$ $3.550 E-04$ 3.55 $9.269 E-04$ $2.167 E-03$
$5.699 E-04$ $5.004 E-06$ $1.035 \mathrm{E}-05$ $1.159 E-04$ $3.249 E-05$ $5.692 E-05$ 1.783E-05 1.78 2.423E-O5 . $080 E-05$ 5.510E-05 $1.837 \mathrm{E}-04$ $2.084 \mathrm{E}-04$ $3.568 E-04$ $9.317 E-04$ $2.175 E-03$
$5.707 E-04$

ANGLE 7 $M U=-0.4580$ $3.790 E-06$ $7.858 E-06$ $2.423 E-05$ 4.312E-0 $1.086 \mathrm{E}-05$ $1.220 \mathrm{E}-05$ $1.417 E-05$ $1.695 E-05$ $2.954 E-05$ 4 $4.183 E-05$ $1.830 E-04$ $3.373 E-04$ $8.787 E-04$ $2.087 E-03$ $5.606 \mathrm{E}-04$ ANGLE 16

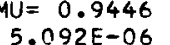
$1.053 \mathrm{E}-05$ $1.178 \mathrm{E}-04$ $3.308 \mathrm{E}-05$ $5.791 E-05$ $1.635 E-05$ $1.823 \mathrm{E}-05$ 2.095E-05 3. 4.103E-05 T.5 $1.854 E-04$ 2.098E- 4 3.5 $2.180 E-03$

ANGLE 8 ANGLE 9 $M U=-0.2816 \quad M U=-0.0950$ $3.919 E-06 \quad 4.065 E-06$ $8.123 E-06 \quad 8.423 E-06$ $9.149 \mathrm{E}-05 \quad 9.477 \mathrm{E}-05$ $2.510 E-05 \quad 2.609 E-05$ $4.460 E-05 \quad 4.626 E-05$ $1.140 \mathrm{E}-05 \quad 1.201 \mathrm{E}-05$ $1.280 E-05 \quad 1.347 E-05$ $1.485 E-05 \quad 1.561 E=05$ $1.774 \mathrm{E}-05 \quad 1.863 \mathrm{E}-05$ $2.296 E-05 \quad 2.406 E-05$ 
(GAMMAS/MEV/STERADIAN/SOURCE NEUTRON)

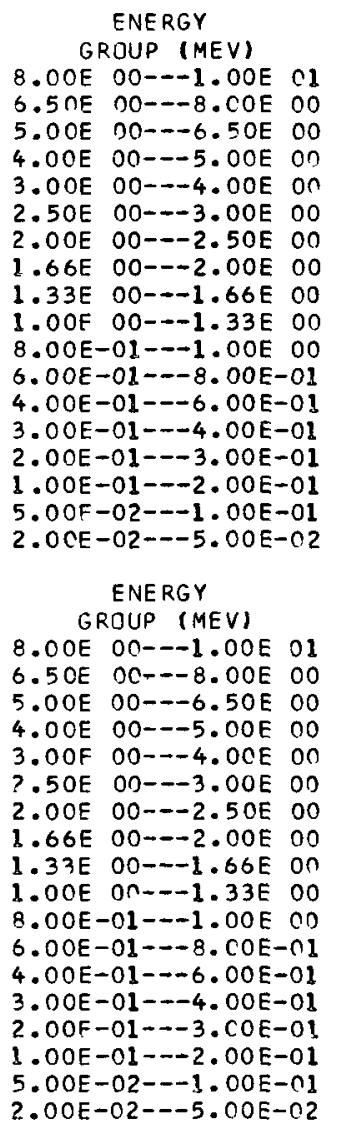

$\begin{array}{lr}\text { ANGLE } 1 & \text { ANGLE } \\ \text { MU=-1.0000 } & \text { MU=-0.9894 } \\ 1.019 E-05 & 1.022 E-05 \\ 2.115 E-05 & 2.122 E-05 \\ 2.396 E-04 & 2.404 E-04 \\ 6.415 E-05 & 6.437 E-05 \\ 1.157 E-04 & 1.161 E-04 \\ 2.631 E-05 & 2.643 E-05 \\ 2.981 E-05 & 2.995 E-05 \\ 3.490 E-05 & 3.506 E-05 \\ 4.209 E-05 & 4.228 E-05 \\ 5.524 E-05 & 5.549 E-05 \\ 7.561 E-05 & 7.596 E-05 \\ 1.103 E-04 & 1.108 E-04 \\ 4.638 E-04 & 4.650 E-04 \\ 5.735 E-04 & 5.749 E-04 \\ 1.181 E-03 & 1.181 E-03 \\ 3.076 E-03 & 3.078 E-03 \\ 7.503 E-03 & 7.508 E-03 \\ 2.070 E-03 & 2.070 E-03 \\ & \\ \text { ANGLE } 10 & A N G L E 11 \\ M U=0.0950 & M U=0.2816 \\ 1.475 E-05 & 1.591 E-05 \\ 3.053 E-05 & 3.292 E-C 5 \\ 3.427 E-04 & 3.688 E-04 \\ 9.529 E-05 & 1.033 E-04 \\ 1.680 E-04 & 1.813 E-04 \\ 4.566 E-05 & 5.082 E-05 \\ 5.130 E-05 & 5.7 C 0 E-05 \\ 5.955 E-05 & 6.600 E-05 \\ 7.122 E-05 & 7.868 E-05 \\ 9.230 E-05 & 1.014 E-04 \\ 1.238 E-04 & 1.351 E-04 \\ 1.744 E-04 & 1.882 E-04 \\ 6.105 E-04 & 6.396 E-04 \\ 7.176 E-04 & 7.430 E-04 \\ 1.270 E-03 & 1.290 E-03 \\ 3.357 E-03 & 3.412 E-03 \\ 7.995 E-03 & 8.087 E-03 \\ 2.128 E-03 & 2.139 E-03\end{array}$

ANGLE 3 ANGLE 5 AVGLE 6 6179 $2.147 E-05 \quad 2.197 E-05 \quad 2.272 E-05 \quad 2.372 E-05$ $2.432 E-04 \quad 2.487 E-04 \quad 2.570 E-04 \quad 2.6807-04$ $6.522 E-05 \quad 6.689 E-05 \quad 6.936 E-05 \quad 7.267 E=05$ $1.175 E-04 \quad 1.203 E-04 \quad 1.245 E-04 \quad 1.301 E-04$ 2.694E-05 2.794E-05 2.943E-05 3.144E-05 $3.05 J E-05$ 3.161E-05 3.326E-05 3.55OE-05 $3.570 E-05 \quad 3.696 \mathrm{E}-05 \quad 3.886 \mathrm{E}-05 \quad 4.144 \mathrm{E}-05$ 4.305E-05 4.457E-05 4.684E-05 4.991E-05

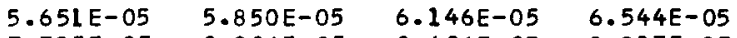

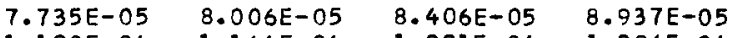
$\begin{array}{llll}1.128 E-04 & 1.166 E-04 & 1.221 E-04 & 1.294 E-04\end{array}$ $4.699 E-04 \quad 4.793 E-04 \quad 4.928 E-04 \quad 5.102 E-04$ $5.804 E-04$ 5.908E-04 6.053E-04 $6.234 E-04$ $1.184 E-03$ I.189E-03 $1.197 E-03$ $1.207 E-03$ $3.088 E-03$ 3.106E-03 $3.133 E-03$ 3.166E-03 7.525E-03 7.558E-03

$\triangle N G L E 12$

ANGLE 13 1.00503 $7.665 E-03$ $M U=0.4580$ $M U=0.6179$ ANGLE 14

AVGLE 15 1.75 $3.973 \mathrm{E}-04$ $1.852 \mathrm{E}-05$ $4.271 E-04$ $1.212 \mathrm{E}-04$ 1.212E-04 $6.253 E-05$ 5.652E-05 $6.325 \mathrm{E}-05$ $7.303 E-05$ $8.033 \mathrm{E}-05$ $9.501 \mathrm{E}-05$ $1.111 E-04$ $1.467 \mathrm{E}-04$ $2.322 F-04$ $6.687 E-04$ $7.676 E-04$ 1. $310 E-03$ $3.466 E-03$ $8.177 \mathrm{E}-03$ $2.149 E-03$

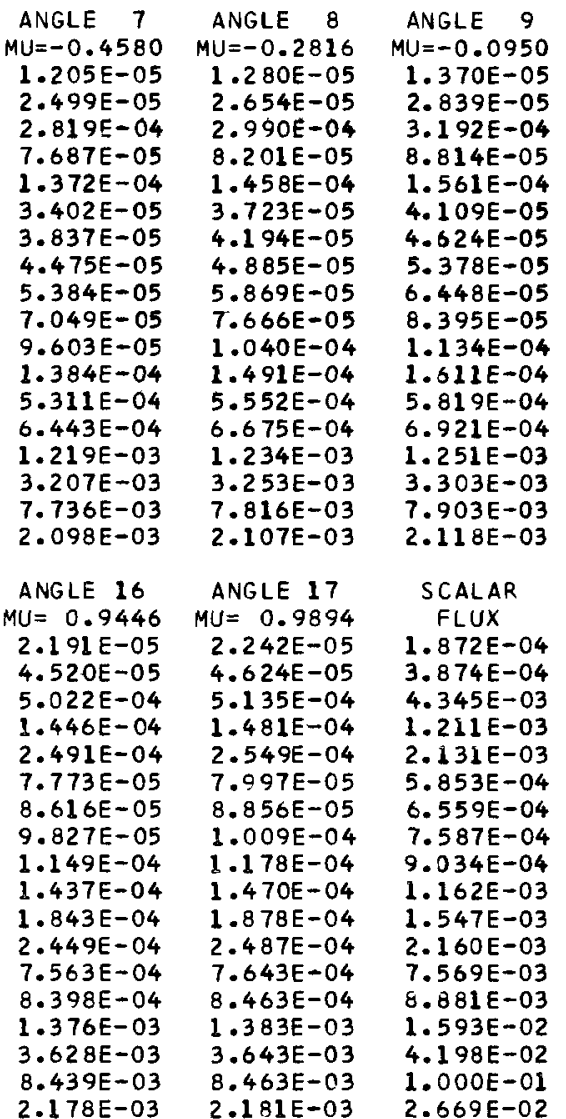


(GAMMAS/MEV/STERADIAN/SOURCE NEUTRON) GROUP (MEV) 8.0OE OO---1.00E 01 6.50E $00--8.00 E$ OO $5.00 E$ EO- -6.50 E 00 $3.00 E$ OO- $0-4.00 E$ OE $3.50 E$ OO- $3.00 E$ OO $2.00 E$ OO- $-2.50 E$ OO $1.66 \mathrm{E} 00---2.00 \mathrm{E}$ 00 $1.33 E 00--1.66 E 00$ $1.00 \mathrm{E}$ ON--1.33E OD 8.00E-01---1.00E 00 $6.00 E+01--8.00 E-01$ $4.00 E-01--6.00 E-01$ $3.005-01--4.00 E-01$ $2.00 E-01--3.00 E-01$ $1.00 \mathrm{E}-01---2.00 \mathrm{E}-\mathrm{Cl}$ $2.00 E-02--5.00 E-02$

$$
\text { ENERGY }
$$

$$
\text { GROUP (MEV) }
$$

8.0CE DO---1.00E 01 6.5OE OO- - 8.00E 00 $5.00 E$ OO- $-6.50 E$ OO $4.00 E$ DO- $-5.00 E$ DO $3.00 E$ OO- $-4.00 \mathrm{E} 00$ $2.50 E$ OO---3.0DE 00 $2.00 E$ OCN--2.5OE OO $1.66 E$ OO- $-2.00 E$ ON $1.33 \mathrm{E} 00--1.66 \mathrm{E}$ OO $1.00 E$ OO-D-1.33E 00 $8.00 E-01-2.00 E$ OO $6.00 \mathrm{E}-01--8.00 \mathrm{E}-01$ 4.0OE $01--6.00 E-01$ $2.00 \mathrm{E}-01-2-3.00 \mathrm{E}-01$ $1.00 E-01-0.2 .00 E-C 1$ $5.00 E-02-1.00 E-01$ $2.00 E-02---5.00 E-02$
ANGLE 1
$M U=-1.0000 \quad$ ANGLE 2
MU $=-0.9894$ $3.624 \mathrm{E}-05$ $4.118 E-04$ . $1.979 \mathrm{E}-04$ $4.247 E-05$ $6.977 E-05$ $9.206 \mathrm{E}-05$ $1.271 E-04$ $1.896 E-04$ $8.930 E-04$ $1.150 E-03$ $6.939 \mathrm{E}-03$ $1.748 \mathrm{E}-02$ $1.748 E-02$
$4.927 E-03$ ANGLE 10 $M U=0.0950$ $3.002 \mathrm{E}-0$ $6.210 \mathrm{E}-05$ $6.953 \mathrm{E}-04$ $1.953 \mathrm{E}-04$ $3.427 E-04$ $9.784 \mathrm{E}-05$ $1.105 \mathrm{E}-04$ $1.292 E-04$ $1.55 E-04$ . 4.017E-04 $1.387 E-03$ $1.668 \mathrm{E}-03$ . 1.927E-O3 $1.928 \mathrm{E}-02$ $1.752 \mathrm{E}-\mathrm{CD}$ $3.640 E-05$ $4.135 \mathrm{E}-04$ $1.093 E-04$
$1.987 E-04$ $4.278 E-05$ 4.896E-05 $5.782 E-05$ $7.019 E-05$ $0.266 \mathrm{E}-05$ $1.28 \mathrm{CE}-04$ $1.910 E-04$ $8.968 E-04$
$1.155 \mathrm{E}-03$ $1.15 \mathrm{E}-03$ $6.647 \mathrm{E}-03$ $1.750 \mathrm{E}-02$ $4.929 E-03$ ANGLE 11 $M U=0.2816$ $3.395 E-05$
$7.016 E-05$ $7.016 E-05$ $7.828 \mathrm{E}-04$ $2.226 E-04$ $3.876 \mathrm{E}-04$ $1.165 E-04$ $1.530 E-04$ $1.838 \mathrm{E}-04$ 2.397E-04 $3.230 E-04$ . . . $1.965 \mathrm{E}-0$ ? $5.187 \mathrm{E}-03$
ANGLE 3 $1.782 E-0.5$ $3.702 E-05$ 4.204E-04 $2.023 E-04$ $4.402 E-05$ $5.027 E-05$ $5.928 \mathrm{E}-05$ $7.196 E-05$ $9.517 E-05$ $1.318 E-04$ $1.967 \mathrm{E}-04$ $9.120 E-04$ $1.176 E-03$ $2.654 E-03$
$6.979 E-03$ $1.756 \mathrm{E}-02$ 4.937E-03 ANGLE 12 $M U=0.4580$ $3.861 E-05$
$7.968 E-05$ $8.858 E-04$ $2.551 \mathrm{E}-04$ 4.407E-04 $1.390 \mathrm{E}-04$ $1.811 \mathrm{E}-04$ $2.163 \mathrm{E}-04$ $2.788 E-04$ $3.700 E-04$ $5.105 E-04$ $2.000 \mathrm{E}-02$ $5.227 E-03$ $M U=-0.8656$ $1.841 \mathrm{E}-05$ $4.339 E-04$ $2.092 E-04$ 4.647E-05 $5.289 E-05$ $6.224 E-05$ $7.555 E-05$ $1.002 E-04$ $1.392 E-04$ $2.079 E-04$ $9.414 E-04$ $1.215 \mathrm{E}-03$ $2.667 \mathrm{E}-03$ $7.042 \mathrm{E}-03$ $1.768 E-02$
$4.952 E-03$ ANGLE 13 $M U=0.6179$ $4.395 E-05$ $9.058 E-05$ $1.003 E-03$ $2.725 \mathrm{E}-04$ $5.013 E-04$ $1.652 \mathrm{E}-04$ $1.848 \mathrm{E}-04$ 2.130 E- 04 2.522E-04 $4.185 E-04$ 5.652E-04 1.933E-03 3.156E-O3 $2.034 \mathrm{E}-02$ $5.265 \mathrm{E}-03$
$\begin{array}{ll}A N G L E & 5 \\ M U=-0.7550\end{array}$ $1.930 E-05$ $4.008 E-05$ $4.542 E-04$ $1.218 \mathrm{E}-04$ $2.196 E-04$ $5.016 E-05$ $5.692 E-05$ $6.687 E-05$ $8.117 E-05$ $1.079 E-04$ $1.503 E-04$ $2.244 E-04$ $9.843 E-04$ $1.268 \mathrm{E}-03$ $2.688 E-03$ $7.130 \mathrm{E}-03$ $1.784 E-02$
$4.972 E-03$ ANGLE 14 $M U=0.7550$ 4. $971 E-05$ $1.023 E-04$ $1.130 E-03$
$3.330 E-04$ 5.665E-04 $1.936 E-04$ $2.153 \mathrm{E}-04$ $2.464 E-04$ $3.627 E-04$ 6.15EE-04 . (6.73日E-03 $2.065 E-02$ $5.298 \mathrm{E}-03$
AVGLE 6 2.052E-05 4.260E-05 4. $820 \mathrm{E}-04$ $2.337 E-04$ $5.530 E-05$ $6.263 \mathrm{E}-05$ $7.350 E-05$ $8.922 E-05$ $1.187 \mathrm{E}-04$ $1.655 E-04$ $1.041 E-03$ $1.334 E-03$ $2.716 E-03$ $7.245 \mathrm{E}-03$ $1.806 E-02$
$4.999 E-03$ ANGLE 15 $M U=0.8656$ $5.544 E-05$ $1.140 \mathrm{E}-04$ $1.255 \mathrm{E}-03$ $3.732 E-04$ 6.307E-04 $2.217 E-04$ $2.451 E-04$ $2.783 E-04$ $3.237 E-04$ $4.006 \mathrm{E}-04$ . 8 2. $090 \mathrm{E}-02$ 5. $325 E-03$

ANGLE 7 $2.213 \mathrm{E}-05$ $4.591 E-05$ .184E-04 $1.412 \mathrm{E}-04$ $2.522 \mathrm{E}-04$ $6.217 E-05$ $7.037 E-05$ 8.254E-05 $1.002 \mathrm{E}-04$ $1.333 \mathrm{E}-04$ $1.855 E-04$ $2.752 E-04$ $1.110 \mathrm{E}-03$ $1.410 \mathrm{E}-03$ $2.752 \mathrm{E}-03$ $7.384 \mathrm{E}-03$ $1.831 E-02$
$5.030 E-03$ ANGLE 16 $M U=0.9446$ $1.240 E-04$ $1.361 E-03$ 4. $077 \mathrm{E}-04$ $6.855 E-04$ $2.455 \mathrm{E}-04$ $2.699 E-04$ 3. $046 \mathrm{E}-04$ $3.515 \mathrm{E}-04$ 4. $303 E-04$ 
(GAMMAS/MEV/STERADIAN/SOURCE NEUTRON)

ENERGY

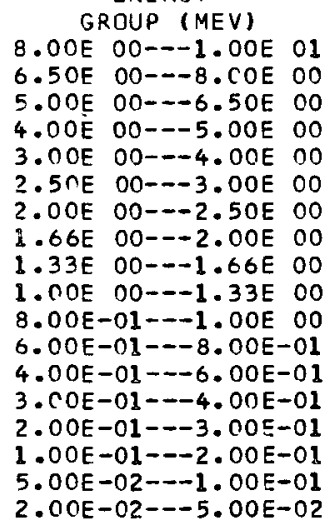
$2.00 E-02--5.00 E-02$

FNERGY

GROUP (MEV)

8.0OE OO---1.00E O1 6.50 E OO- - - 8. OOE OO $5.00 E$ OO-- -6.50E OO
$4.00 E$ OO---5.00E OO $4.00 E$ OO-- 5. OOE OO
$3.00 E$ OO-- 4.0OE 00 3.00E O0--3.00E 00 $2.00 E$ OO---2.50E 00 $1.66 E$ OO- - 2.00E OO $1.36 \mathrm{E} 00---1.66 \mathrm{E} \quad 00$ $1.00 E$ OO- - 1.33E DO $8.00 E-01--1.00 E$ OO $6.00 E-n 1--8.00 E-01$ $4.00 E-01--6.00 E-01$ 3.00E-01---4.00E-C1 $2.00 E-01-3.00 E-01$ $1.00 \mathrm{E}-01--2.00 \mathrm{E}-01$ $5.00 \mathrm{E}-02-2-1.00 \mathrm{E}-\mathrm{Cl}$ $2.00 \mathrm{E}-02---5.00 \mathrm{E}-.0$ ?
ANGLE 1 ANGLE 2 $M U=-1.0000$ $1.920 E-05$ $3.988 \mathrm{E}-05$ $4.537 E-04$ $1190 \mathrm{E}-04$ .1 $5.270 \mathrm{E}-05$ $6.265 \mathrm{E}-05$ $7.627 E-05$ $1.006 E-04$ 1.389E-04 $2.091 E-04$ $1.045 E-03$ $1.371 E-03$ $3.347 E-03$ .812E-03 $2.258 \mathrm{E}-02$ $6.428 E-03$ ANGLE 10 $M U=0.0950$ $3.545 \mathrm{E}-05$ $7.331 \mathrm{E}-05$ $8.196 \mathrm{E}-04$ $2.316 \mathrm{E}-04$ 4.0 $12 \mathrm{E}-04$ $1.388 E-04$ $1.347 E-04$ $1.581 \mathrm{E}-04$ $1.920 E-04$ $2.550 E-04$ . $520 E-04$ $1.756 E-03$ $1.756 \mathrm{E}-03$ $2.73 \mathrm{E}-03$ $1.026 \mathrm{E}-02$ $2.529 \mathrm{E}-02$ $6.757 E-03$ MU $=-0.9894$ ANGLE 3 $1.929 E-05$ 4. $007 E-05$ .106E-04 $2.185 E-04$ 4.600E-05 $5.307 E-05$ $6.304 E-05$ $7.674 \mathrm{E}-05$ $1.013 E-04$ $1.401 \mathrm{E}-04$ $2.110 E-04$ $1.050 E-03$ $1.379 E-03$ $3.349 E-03$ $8.824 \mathrm{E}-0$ $2.261 \mathrm{E}-0$ $6.431 \mathrm{E}-03$ ANGLE 11 $M U=C .2816$
$4.094 E-05$ $4.094 E-05$
$8.455 E-05$ $8.455 E-05$ $9.414 E-04$ ..69 E-04 $4.681 \mathrm{E}-04$ $1.459 E-04$ $1.650 E-04$ $1.931 E-04$ 2. . 5.1 1. $4 E-03$ 1. $3.262 E-03$ $1.058 \mathrm{E}-02$ $2.584 \mathrm{E}-02$ $4.084 \mathrm{E}-05$ $4.042 E-04$ ..229E-04 . $756 E-05$ $5.461 E-05$ $7.875 \mathrm{E}-05$ $1.043 E-04$ $1.450 E-04$ $2.187 \mathrm{E}-04$ $1.071 \mathrm{E}-03$ $1.410 \mathrm{E}-03$ $3.358 \mathrm{E}-0$ $.270 E-02$ $6.443 \mathrm{E}-03$ ANGLE 12 $M U=0.4580$ $4.774 E-05$ $9.842 E-05$ $1.091 \mathrm{E}-03$ 3.1 $5.457 \varepsilon-04$ ..802E-04 2.J31E-04 ..365E-04 $2.836 E-04$ . $678 \mathrm{E}-04$ $4.706-04$ 2.075E-03 . $400 \mathrm{E}-03$ $3.054 \mathrm{E}-03$ 1.090E-02 $1.090 E-02$

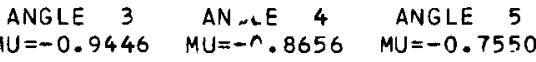
$6.883 \mathrm{E}-03$ $2.038 E-05$ $4.235 \mathrm{E}-05$ $4.809 \mathrm{E}-04$ $2.316 E-04$ $5.062 \mathrm{E}-05$ $5.777 \mathrm{E}-05$ $6.817 \mathrm{E}-05$ $8.298 E-05$ $1.105 E-04$ $1.546 \mathrm{E}-04$ $2.337 E-04$ $1.112 E-03$ $1.469 E-03$ $3.375 E-03$ 8. $960 \mathrm{~F}-03$ $6.465 E-03$ ANGLE 13 $M U=0.6179$ $5.586 \mathrm{E}-05$ $1.15 J E-04$ $1.269 \mathrm{E}-03$ $3.751 E-04$ $6.381=-04$ $2.219 E-04$ $2.488 E-04$ $2.874 E-04$ $3.411 E-04$ $5.676 \mathrm{E}-04$ 5. $676 E-04$ . $333 \mathrm{E}-03$ $.529 \mathrm{E}-03$ $4.080 \mathrm{E}-03$ $1.121 \mathrm{E}-02$ $6.941 \mathrm{E}-03$ $2.148 E-05$ $1.354 E-04$ $2.444 E-04$ $5.521 E-05$ $6.270 E-05$ $7.379 E-05$ $8.986 E-05$ $1.202 \mathrm{E}-04$ . $690 E-04$ $2.559 E-04$ $1.172 \mathrm{E}-03$ $1.550 E-03$ $3.401 \mathrm{E}-03$ $9.088 \mathrm{E}-03$ $6.496 \mathrm{E}-03$

\section{ANGLE 14}

$M U=0.7550$ $6.509 E-05$ $1.338 \mathrm{E}-04$ $1.469 E-03$ $4.405 E-04$ $7.422 E-04$ $2.695 \mathrm{E}-04$ . $432 E-04$ . 432 E- 04 . $435 E-04$ 2. 2. $049 \mathrm{E}-03$ . $151 \mathrm{E}-02$ $2.741 E-02$ $6.093 E-03$
ANGLE 6
$M U=-0.6179$ 2.299E-05 $4.775 E-05$ $1.458 E-04$ $2.620 E-04$ $6.164 E-05$ $6.986 E-05$ $8.214 E-05$ .001E-0 $1.342 E-04$ $1.891 \mathrm{E}-04$ 2. 862E-04 $1.251 E-03$ $1.649 E-03$ $3.437 E-03$ $2.343 E-02$ $6.535 \mathrm{E}-03$ ANGLE 15 $M U=0.8656$ $7.470 \mathrm{E}-05$ $1.533 E-04$ $1.677 E-03$ 5.089E-04 $8.499 E-04$ $3.188 E-04$ (3. 3.984E-04 4.615F-04 $7.091 \mathrm{E}-04$ 7.091 $9.111 E-04$ (2.755E-03 $4.312 E-03$ 1.176E-02 $2.781 E-02$ 7. $036 \mathrm{E}-03$ $M U=-0.4580 \quad M U=-0.2816 \quad M U=-0.0950$ $\begin{array}{lll}2.502 E-05 & 2.767 E-05 & 3.108 E-05 \\ 5.191 E-05 & 5.735 E-05 & 6.437 E-05\end{array}$ $5.861 E-04 \quad 6.458 E-04 \quad 7.224 E-04$ $1.597 E-04 \quad 1.778 E-04 \quad 2.013 E-04$ $2.853 E-04 \quad 3.158 E-04 \quad 3.550 E-04$ $7.040 E-05 \quad 8.220 E-05 \quad 9.796 E-05$ $7.979 E-05 \quad 9.323 E-05 \quad 1.111 E-04$ $9.384 E-05 \quad 1.097 E-04 \quad 1.307 E-04$ $\begin{array}{lll}1.144 E-04 & 1.337 E-04 & 1.592 E-04\end{array}$ $1.535 E-04 \quad 1.792 E-04 \quad 2.126 E-04$ $2.161 E-04 \quad 2.515 E-04 \quad 2.965 E-04$ $3.260 E-04 \quad 3.766 E-04 \quad 4.388 E-04$ $1.351 E-03 \quad 1.470 E-03 \quad 1.606 E-03$ $1.761 \mathrm{E}-03 \quad 1.883 \mathrm{E}-03 \quad 2.009 \mathrm{E}-03$ $3.486 E-03 \quad 3.548 E-03 \quad 3.627 E-03$ $9.456 E-03 \quad 9.694 E-03 \quad 9.964 E-03$ $0.694 E-03$
$2.426 E-02$ $6.636 E-03 \quad 6.695 E-03$ ANGLE 16 $8.337 E-05$ $1.709 \mathrm{E}-04$ $1.863 E-03$ . $9.459 E-04$ $3.624 E-04$ 4. 
(GAMMAS/MEV/STERADIAN/SOURCE NEUTRON)

ENERGY GRDUP (MEV) 8.0OE $00--1.00 E$ OI 6.5 OE OO---8. OOE OO 5.00 EO- $06.50 E$ OO 4.0OE OO--5. 00 EO 3.00 ON- 0 . 00 EO 00 $2.50 E$ OO- $-3.00 E$ OO 2. $.00 E$ OC- --2.50E OO $1.66 \mathrm{E} 00--2.00 \mathrm{E} 00$ $1.33 \mathrm{E} 00---1.66 \mathrm{E} 00$ $1.00 E$ O0--1.33E 00 8. OCE-O1---1. OOE OO 6.00E-n1---8.00E-01 4.0NE-01---6.00E-01 3. $00 \mathrm{E}-01---4.00 \mathrm{E}-01$ $2.00 E-01---3.00 E-01$ 1.00E-01---2.00E-01 $5.00 E-02--1.00 E-01$ $2.00 E-02---5.00 E-02$ ENERGY GRQUP (MEV) 8.0OE OO---1.00E OI $6.50 E$ OO- $-8.00 E$ OO 5. COE OO-C. 4.OOE OO---5. OOE OO 3.OOE $00--4.00 E$ OO 2.5OE OO--3. 00 E 00 2.0OE OO- $2.50 E$ OD $1.66 E$ OOA- $2.00 E$ OO $1.33 E$ OOC-1.66E OO $1.00 E$ ON- $1.33 E$ OO G. OOE $4.00 E-01-2.00 \mathrm{O}-01$ $3.00 E-01--4.00 E-01$ $2.00 \mathrm{E}-\mathrm{OI}-\mathrm{C}-\mathrm{O}$. $1.00 E-01---2.00 E-01$ 5.0OE-02- - $1.00 \mathrm{E}-01$ $2.00 E-02---5.00 E-02$
ANGLE 1 ANGLE 2 $M U=-1.0000 \quad M U=-0.9894$ $1.914 \mathrm{E}-05$ .972E-05 $4.528 \mathrm{E}-04$ $1.167 \mathrm{E}-04$
$2.156 \mathrm{E}-04$ $4.348 \mathrm{E}-05$ $5.169 E-05$ $6.266 E-05$ $7.674 \mathrm{E}-05$ $1.002 \mathrm{E}-04$ $1.368 \mathrm{E}-04$ $2.086 \mathrm{E}-04$ $1.186 \mathrm{E}-03$ $1.606 \mathrm{E}-03$ $4.444 \mathrm{E}-03$ $1.177 \mathrm{E}-02$ $3.121 E-02$ $9.042 \mathrm{E}-03$ ANGLE 10 $M U=0.0950$ $3.971 \mathrm{E}-05$
$8.206 \mathrm{E}-05$ $8.206 E-05$ $9.149 E-04$ $2.615 E-04$ $4.551 E-04$ $1.408 E-04$ 1. $609 E-04$ 1.910E-04 $3.198 \mathrm{E}-04$ ..2 $980-04$ $4.540 E-04$ 2.31IE-03 $2.891 \mathrm{E}-03$ $4.972 \mathrm{E}-03$ $1.415 \mathrm{E}-02$ $1.415 \mathrm{E}-02$
$3.584 \mathrm{E}-02$ $9.610 \mathrm{E}-03$
ANGLE 3 $1.925 \mathrm{E}-05$ .95E-05 $4.553 \mathrm{~F}-04$ 1.170E-04 $4.398 E-05$ 5.206E-05 $6.293 E-05$ $7.705 \mathrm{E}-05$ $1.009 E-04$ $1.384 \mathrm{E}-04$ $2.113 \mathrm{E}-04$ $1.194 E-03$ $1.620 \mathrm{E}-03$ $4.447 \mathrm{E}-03$ $1.177^{\circ} \mathrm{E}-02$ $3.12 .4 E-02$ ANGLE 11 $M U=0.2816$
$4.768 E-05$ $9.832 E-05$ $1.090 \mathrm{E}-03$ $3.179 E-04$
$5.469 E-04$ $5.469 E-14$ $1.831 E-04$ 2. 2.4 3.0B E 04 $5.082 \mathrm{E}-04$ 5. $2.572 \mathrm{E}-03$ $3.097 E-03$ $5.153 \mathrm{E}-03$ $1.469 E-02$ 3.683E-02 $3.683 \mathrm{E}-02$
$9.723 \mathrm{E}-03$ $M U=-0.9446$ 1. $968 E-05$ $4.086 E-05$ $4.653 \mathrm{E}-04$ $1.213 \mathrm{E}-04$ $4.599 \mathrm{E}-05$ $5.366 E-05$ $6.430 \mathrm{E}-05$ $7.866 \mathrm{E}-05$ $1.041 \mathrm{E}-\mathrm{C4}$ $1.449 \mathrm{E}-04$ $2.225 E-04$ $1.223 \mathrm{E}-03$ $1.676 \mathrm{E}-03$ $4.458 \mathrm{E}-03$ $1.187 \mathrm{E}-02$ $3.143 \mathrm{E}-02$ 9.067E-03 ANGLE 12 $M U=0.4580$
$5.925 E-05$ $5.825 E-05$
$1.109 E-04$ $1.199 E-04$
$1.320 E-03$ $1.320 E-03$
$3.934 E-04$ $3.934 E-04$ $6.683 E-04$ $2.414 E-04$ $2.747 E-04$ 3.23 E-04 5. . . $805 \mathrm{E}-04$ . $3.308 \mathrm{E}-03$ 5. $360 E-03$ $1.525 E-02$ $3.783 E-02$ $9.835 \mathrm{~F}-03$ ANGLE 4 $M U=-0.8656$ 2. $053 \mathrm{E}-05$ $4.265 E-05$ $4.849 E-04$ . $280 E-04$ 列 . .763E- 05 . . $574 E-04$ $1.574 E-04$ .. $382 \mathrm{E}-03$ $1.779 \mathrm{E}-03$ $4.478 \mathrm{E}-03$ 1. $201 \mathrm{E}-02$ $3.169 E-02$ $9.104 \mathrm{E}-03$ ANGLE 13 $M U=0.6179$
$7.203 E-05$ $7.203 E-05$
$1.478 E-04$ . $478 E-04$ $1.617 \mathrm{E}-03$ $4.925 E-04$ . $254 E-04$ $3.191 E-04$ 3.50SE-04 $5.200 E-04$ 5. $464 E-04$ . $464 E-04$ . $36 \mathrm{E}-03$ $3.107 E-03$ $3.523 \mathrm{E}-03$ $5.582 E-03$ $1.582 \mathrm{E}-02$ $3.881 E-02$ $9.941 E-03$ $M U=-0.7550$ $2.180 \mathrm{E}-05$ $4.531 E-05$ $5.142 E-04$ $2.484 E-04$ 5. $536 \mathrm{E}-05$ $6.272 E-05$ $7.385 \mathrm{E}-05$ $9.040 E-05$ . $227 E-04$ 1. $763 \mathrm{E}-04$ $2.742 \mathrm{E}-04$ $1.371 E-03$ $1.920 \mathrm{E}-03$ $4.509 \mathrm{E}-03$ 1. $221 \mathrm{E}-02$ $3.210 E-02$ $9.156 E-03$ ANGLE 14 $M U=0.7550$ . $827 \mathrm{E}-04$ $.984 \mathrm{E}-03$ $6.171 E-04$ . $165 \mathrm{E}=04$ $4.165 E=04$ . .653E-04 . 7.833E-04 ..83 . $3.362 \mathrm{E}-03$ . $5.804 E-03$ $1.635 E-02$ $3.970 E-02$ 1. $004 \mathrm{E}-02$
ANGLE 6 $M U=-3.6179$ 2.360E-05 .550E $1.500 E-04$ $2.693 \mathrm{E}-04$ $6.309 E-05$ $7.136 \mathrm{E}-05$ $8.403 E-05$ $1.031 \mathrm{E}-04$ $1.407 E-04$ $2.029 E-04$ $3.168 \mathrm{E}-04$ $1.493 E-03$ $2.090 E-03$ $4.554 \mathrm{E}-03$ $1.248 E-02$ $9.224 \mathrm{E}-03$

AVILE 15 $M U=0.8656$ $2.227 E-04$ $2.404 E-03$ $7.610 \mathrm{E}-04$ $1.240 E-03$ $5.270 E-04$ 5. $804 E-04$ 7.53 E -04 . ..142E-04 1.122E 03 . (2) $6.007 \mathrm{E}-03$ $1.681 \mathrm{E}-02$ 4. $346 E-02$ $1.011 \mathrm{E}-02$

ANGLE 7 ANGLE 8 ANGLE 9 $M U=-0.4580 \quad M U=-0.2816 \quad M U=-0.0950$ $\begin{array}{lll}5.404 E-05 & 6.031 E-05 & 6.989 E-05\end{array}$ $6.101 E-04 \quad 6.842 E-04 \quad 7.831 E-04$ $1.666 \mathrm{E}-04 \quad 1.891 \mathrm{E}-04 \quad 2.197 \mathrm{E}-04$ $2.975 E-04 \quad 3.354 E-04 \quad 3.865 E-04$ $7.388 E-05 \quad 8.907 E-05 \quad 1.105 E-04$ $8.390 E-05 \quad 1.016 E-04 \quad 1.263 E-04$ $9.916 E-05 \quad 1.205 E-04 \quad 1.501 E-04$ $1.220 E-04 \quad 1.485 E-04 \quad 1.851 E-04$ $\begin{array}{lll}1.666 E-04 & 2.028 E-04 & 2.525 E-04\end{array}$ $2.402 E-04 \quad 2.919 E-04 \quad 3.621 E-04$ $3.752 E-04 \quad 4.539 E-04 \quad 5.561 E-04$ $1.651 E-03 \quad 1.843 E-03 \quad 2.065 E-03$ $2.281 E-03 \quad 2.482 E-03 \quad 2.686 E-03$ $4.617 E-03 \quad 4.704 E-03 \quad 4.822 E-03$ $1.281 E-02 \quad 1.320 E-02 \quad 1.365 E-02$ $3.328 E-02 \quad 3.405 E-02 \quad 3.490 E-02$ $9.305 E-03 \quad 9.398 E-03 \quad 9.501 E-03$

ANGLE 16 ANGLE 17 SCALAR 
IGAMMAS/MEV/STERADIAN/SOURCE NEUTRONI

\begin{abstract}
ENERGY
GROUP (MEV) 8. OOE OO---1.00E 01 6.5OE $10--8.00 E$ DO S.OOE OO4.OOE OO. 2.0OE OO- 2 . 50 E 00 .66E $00-02.00 E$ OO 1.33E $00-01.66 \mathrm{E}$ OO 1.33E DO- 1.66E CO 1. 0 E $-01-2-1.00 E$ OO $6.00 E-01-28.00 E-01$ 4. OOE $-01--26.00 E-01$ $3.00 \mathrm{E}-01--4.00 \mathrm{E}-01$ $2.00 \mathrm{E}-01-23.00 \mathrm{E}-01$ $1.00 \mathrm{E}-01--2.00 \mathrm{E}-01$

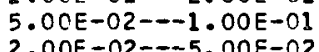

ENERGY

GROUP (MEV)

8.OCE OO-- 1. 00 E 01 6.5OE OO---8. COE OO $5.00 E$ OO- -6.50F $\mathrm{F}$ 4.0OE OO- $-5.00 E$ CO $3.00 E$ OO- $4.00 E$ CO $2.50 E$ 00- - 3.00E 00 2.0OE O0-- 2.50E OO $1.66 E$ OO- $2.00 E$ EO $1.33 \mathrm{E} 00--1.66 \mathrm{E}$ OO $1.00 \mathrm{E}$ OC $-1.33 \mathrm{E}$ OO 8.00E-01--1. $00 E$ OO $6.00 E-01=8.00 E-01$ 4.0 OE-01---6.00E-01 $3.00 \mathrm{E}-01--4.00 \mathrm{E}-0 \mathrm{I}$ 2. $00 \mathrm{E}-01--3.00 \mathrm{E}-\mathrm{C}$ $1.00 E-01-2.00 E-0$ . $00 \mathrm{E}-02-1.00 \mathrm{E}-01$ $2.00 E-02---5.00 E-02$
ANGLE 1 $M U=-1.0000$ $1.610 E-05$ . . $540 \mathrm{E}-05$ $1.705 \mathrm{E}-04$ $3.480 \mathrm{E}-05$ $4.373 E-05$ 4. $6.770 \mathrm{E}-05$ $8.608 E-05$ $1.131 E-04$ $1.736 \mathrm{E}-04$ $1.148 E-03$ $1.503 \mathrm{E}-03$ $5.020 \mathrm{E}-03$ $1.333 \mathrm{E}-02$ $3.648 \mathrm{E}-02$ $1.073 E-02$

ANGLE 2 $M U=-0.9894$ $1.620 E-05$ $3.834 E-04$ $0.657 E-05$ . 3.53 E- 05 $4.396 \mathrm{E}-05$ $5.479 E-05$ $6.755 E-05$ $8.647 E-05$ $1.149 \mathrm{E}-04$ $1.770 \mathrm{E}-04$ $1.15 \mathrm{EE}-03$ $1.611 \mathrm{E}-03$ $5.022 \mathrm{E}-\mathrm{C} 3$ $1.336 \mathrm{E}-02$ $3.653 \mathrm{E}-02$ $3.653 \mathrm{E}-02$
$1.074 \mathrm{E}-02$ $M U=-0.9446$ $1.662 E-05$ 3.447E-05 1. 1.010E$1.871 E-04$ $4.512 \mathrm{E}-05$ $5.500 \mathrm{E}-05$ $6.760 E-05$ $8.863 \mathrm{E}-05$ $1.222 \mathrm{E}-04$ $1.905 E-04$ $1.189 \mathrm{E}-03$ $1.689 \mathrm{~F}-03$ $5.032 \mathrm{E}-03$ $1.345 \mathrm{E}-02$ $3.674 E-02$ $1.076 \mathrm{E}-02$

$5.593 \mathrm{E}-03$

$1.642 \mathrm{E}-02$

$4.275 \mathrm{E}-02$

ANGLE 11 ANGLE 12 $=0.2816$ $9.378 E-05$ $1.035 E-03$ $3.071 E-04$ $5.244 E-04$ $1.881 \mathrm{E}-04$ $2.170 E-04$ $2.606 E-04$ $3.255 E-04$ $4.504 \mathrm{E}-04$ $6.468 E-04$ $9.662 E-04$ $2.908 \mathrm{E}-03$ $3.546 \mathrm{E}-03$ $5.826 E-03$ $1.714 \mathrm{E}-02$

$4.412 \mathrm{E}-\mathrm{C}$

$1.166 \mathrm{E}-02$ ANGLE 13 $=0.4580$ $M U=0.6179$ $.193 E-04$ $1.305 E-03$ $3.981 E-04$ $6.693 E-04$ $2.636 E-04$ $3.037 E-04$ $3.628 E-04$ 4. $484 \mathrm{E}-04$ $6.056 \mathrm{E}-04$ $8.372 E-04$ $1.185 E-03$ $3.254 E-03$ $3.810 E-03$ $6.099 E-03$ $1.791 \mathrm{E}-02$ $4.554 \mathrm{E}-02$ $1.182 E-02$ $1.550 E-04$ .680E-03 $.753 E-04$ $1.049 \mathrm{E}-03$ $1.405 \mathrm{E}-03$ $3.599 E-03$ $4.088 \mathrm{E}-03$ $6.399 \mathrm{E}-03$ $1.869 E-02$ $4.693 \mathrm{E}-0$

\begin{tabular}{cc} 
ANGLE 5 & \multicolumn{1}{c}{ ANGLE 6} \\
$M U=-0.7550$ & $M U=-3.6179$ \\
$1.863 E-05$ & $2.032 E-05$ \\
$3.875 E-05$ & $4.225 E-05$ \\
$4.398 E-04$ & $4.782 E-04$ \\
$1.181 E-04$ & $1.300 E-04$ \\
$2.129 E-04$ & $2.326 E-04$ \\
$4.724 E-05$ & $5.463 E-05$ \\
$5.294 E-05$ & $6.131 E-05$ \\
$6.195 E-05$ & $7.202 E-05$ \\
$7.610 E-05$ & $8.893 E-05$ \\
$1.054 E-04$ & $1.238 E-04$ \\
$1.555 E-04$ & $1.835 E-04$ \\
$2.487 E-04$ & $2.956 E-04$ \\
$1.361 E-03$ & $1.511 E-03$ \\
$2.025 E-03$ & $2.260 E-03$ \\
$5.077 E-03$ & $5.117 E-03$ \\
$1.389 E-02$ & $1.423 E-02$ \\
$3.766 E-02$ & $3.838 E-02$ \\
$1.088 E-02$ & $1.097 E-02$
\end{tabular}

ANGLE 7 $M U=-0.4580$ $2.266 E-05$ $4.704 E-05$ 1.454E-04 $1.454 E-04$ $6.504 E-05$ $7.401 E-05$ $8.790 E-05$ $1.091 E-04$ $1.516 E-04$ $1.516 E-04$ $3.629 E-04$ $3.629 E-04$
$1.710 E-03$ $2.518 E-03$ $2.518 E-03$ $5.178 \mathrm{E}-03$
$1.466 \mathrm{E}-02$ $3.925 \mathrm{E}-02$ $1.108 \mathrm{E}-02$

ANGLE ANGLE 9 $2.589 E-05 \quad 3.038 E-05$ $3.038 E-05$ $7.285 E-05$ 1. $3.483 E-04$ $1.033 \mathrm{E}-04$ $1.192 \mathrm{E}-04$ $1.431 E-04$ $1.431 E-04$ $2.832 \mathrm{E}-04 \quad 3.695 \mathrm{E}-04$ $4.598 E-04 \quad 5.933 E-04$ $2.247 \mathrm{E}-03$ $2.782 E-\cap 3 \quad 3.041 E-03$ $5.272 E-03 \quad 5.408 E=03$ $1.516 \mathrm{E}-02 \quad 1.575 \mathrm{E}-02$ $4.028 \mathrm{E}-02 \quad 4.246 \mathrm{E}-02$ $4.028 \mathrm{E}-02$
$1.121 \mathrm{E}-02 \quad 1.135 \mathrm{E}-02$ ANGLE 34 AVSLE 15 $9.992 \mathrm{E}-05$ $1.307 E-04$ $\begin{array}{ll}A N G L E & 16 \\ M & =0.9446\end{array}$ ANGLE 17 SCALAR $M U=0.9894$ $1.921 E-04$ $3.885 E-04$
$4.078 E-03$ FLUX $2.187 E-03$ $2.825 E-03$ $4.078 E-03$ $9.357 \mathrm{E}-04$ $1.185 \mathrm{E}-03$ $1.383 \mathrm{E}-03$ $1.488 E-03$ $1.854 \mathrm{E}-03$ $2.140 E-03$ $9.739 E-04$ S. $807 E-04$ $1.069 E-03$ $1.113 E-03$ $1.200 E-03$ $1.313 E-03$ $1.487 E-03$ $1.703 E-03$ $8.033 E-04$ $1.008 E-03$ $1.381 E-03$ $1.612 \mathrm{E}-03$ $.264 E-03 \quad 1.459 E-03$ $1.610 \mathrm{E}-03 \quad 1.786 \mathrm{E}-03$ $3.935 \mathrm{E}-03 \quad 4.248 \mathrm{E}-03$ $4.371 E-03 \quad 4.637 E-03$ $6.705 E-03 \quad 6.990 E-03$ $1.944 \mathrm{E}-02$ 2.010E-02 $4.821 E-02 \quad 4.932 E-02$ $4.510 E-03$ $4.852 \mathrm{E}-0$ $7.222 \mathrm{E}-03$ $2.061 \mathrm{E}-02$ $\begin{array}{lll}4.932 E-02 & 5.016 E-02 & 5.065 E-02 \\ 1.221 E-02 & 1.229 E-02 & 1.234 E-02\end{array}$ $2.003 E-03$ $4.678 E-03$ $4.985 \mathrm{E}-03$ $7.367 \mathrm{E}-03$ $2.092 \mathrm{E}-02$ $1.221 E-02 \quad 1.229 E-02 \quad 1.234 E-02$ $6.257 \mathrm{E}-04$
$1.283 \mathrm{E}-03$ $1.398 \mathrm{E}-02$ 
(GAMMAS/MEV/STERADIAN/S OURCE NEUTRON)

ENERGY 8. OOE OO---1. COE 01 $6.50 E$ OO- $-8.00 E$ OO 5.COE O0-- -6.50E OO $3.00 E$ OO$3.50 E$ OO- $-3.00 E$ OO $2.00 E$ OO- $-2.50 E$ OO $1.66 \mathrm{E} 00--2.00 E$ OO $1.33 \mathrm{E} 00--1.66 \mathrm{E}$ OO $1.00 E$ OO- $-1.33 E 00$ $8.00 \mathrm{E}-01-1.00 \mathrm{E} 00$ $6.00 E-01-8.00 E-01$ $4.00 E-01--6.00 E-01$ $3.00 E-01--4.00 E-01$ 2.00E-01-- -3.00E-01 $1.00 E-01-2.00 E-01$ $5.00 E-02--1.00 E-01$

ENERGY GROUP IMEV I

8. OOE OO-- -1.COE O? $6.50 E$ OO- $-8.00 E$ OO $5.00 E$ DO-- $6.50 E$ OO $4.00 E$ OO-D. $5.00 E$ OO 3.00E $00--4.00 E$ OO $2.50 E$ OO--3.00E OO 2.0CE OO---2.5OE DO $1.66 \mathrm{E}$ 00-D-2.00E 00 $1.33 \mathrm{E} \quad 00-1.66 \mathrm{E} \quad 00$ $1.00 E$ DO- $-1.33 E \quad 00$ 8.00E-01---1.00E OO 6.00E-01- - 8.00E-01 4.00E-01-- -6. COE-01 $3.00 E-01--4.00 E-01$ $2.00 E-01--3.00 E-01$ 1.00E-01--2.00E-01 $2.00 E-02--5.00 E-02$
ANGLE 1 ANGLE 2 $1.212 E$ $2.496 \mathrm{E}-05$ $6.825 \mathrm{E}-05$ (326E-04 .439E-05 $3.396 E-05$ $4.512 E-05$ $5.630 E-05$ $6.813 E-05$ $8.257 E-05$ $1.261 \mathrm{E}-04$ $1.007 E-03$ $1.419 E-03$ $5.110 E-03$ $1.356 \mathrm{E}-02$ $3.817 \mathrm{E}-02$ $1.136 \mathrm{E}-02$

ANGLE 10 $M U=0.0950$ $2.984 E-05$ $6.156 \mathrm{E}-05$ $6.824 E-04$ $1.995 \mathrm{E}-04$ $3.441 E-04$ $1.178 \mathrm{E}-04$ $1.368 \mathrm{E}-04$ $2.637 E-04$ $3.072 E-04$ 4.707E-04 $7.693 E-04$ $2.566 \mathrm{E}-03$ $3.360 E-03$ $5.631 \mathrm{E}-03$ $1.701 \mathrm{E}-02$ $4.542 E-02$
$1.226 E-02$ $1.221 \mathrm{E}-05$ $2.518 E-05$ $2.886 \mathrm{E}-04$ $6.965 \mathrm{E}-05$ $1.342 \mathrm{E}-04$ $3.401 E-05$ $4.458 \mathrm{E}-05$ $5.548 E-05$ $6.801 E-05$ $8.446 E-C 5$ $1.301 E-04$ $1.014 \mathrm{E}-03$ $1.441 \mathrm{E}-03$ $5.112 \mathrm{E}-03$ 1.359E-02 3.823E-02
$1.137 \mathrm{E}-02$ ANGLE 11 $M U=0.2816$ $3.817 E-05$ $7.851 E-05$ $8.625 E-04$ $.606 E-04$ $4.416 E-04$ $1.690 E-04$ $1.971 E-04$ $2.404 E-04$ $3.070 E-04$ 4. $402 E-04$ $6.564 E-04$ $1.012 \mathrm{E}-03$ $2.944 E-03$ $3.622 \mathrm{E}-03$ $5.286 E-03$ $1.784 \mathrm{E}-0$ ? $4.705 E-02$ 1.245E-02 1. 4 $4.508 E-04$ $3.325 \mathrm{E}-03$

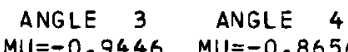
$M U=-0.9446$
$1.257 E-05$ $2.602 \mathrm{E}-05$ $2.974 E-04$ $7.472 E-05$ $1.404 \mathrm{E}-04$ $2.758 E-05$ $2.758 E-05$
$3.447 E-05$ $4.312 E-05$ $5.324 E-05$ $6.837 E-05$ $9.195 E-05$ $1.453 E-04$ $1.045 E-03$ $1.533 \mathrm{E}-03$ $5.120 \varepsilon-03$ $1.370 E-02$ $3.846 \mathrm{E}-02$ 1.140E-02

$\begin{aligned} M U & =-0.8656 \\ 1.325 E-05 & \end{aligned}$ $2.755 \mathrm{E}-05$ $3.137 \mathrm{E}-04$ $8.273 E-05$ $1.509 \mathrm{E}-04$ 3.166E-05 3.607E-05 4.255E-05 $5.216 \mathrm{E}-05$ $7.151 \mathrm{E}-05$ $1.053 \mathrm{E}-04$ $1.707 E-04$ $1.111 \mathrm{E}-03$ 1. $702 \mathrm{E}-03$ $5.133 E-03$ $1.389 E-02$ $3.890 E-02$ $1.146 \mathrm{E}-02$

ANGLE 12 ANGLE 13 $M U=0.4580$

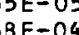
. . $.867 E-04$ 2.507E-04 ..269E-04 . $1.277 \mathrm{E}-03$ $3.901 \mathrm{E}-03$ $6.192 \mathrm{E}-03$ $1.873 \mathrm{E}-02$ $1.264 E-02$ $M U=0.6179$ $.954 E-05$ $1.523 E-03$ $4.931 E-04$ $8.052 E-04$ 3.797E-04 $4.410 \mathrm{E}-04$ $5.289 E-04$ $6.519 E-04$ $8.648 E-04$ $1.152 E-03$ $1.541 \mathrm{E}-03$ 3.702E-0 $4.208 E-03$ $6.533 \mathrm{E}-03$ $1.964 \mathrm{E}-02$ $5.042 \mathrm{E}-02$ $1.281 \mathrm{E}-02$
ANGLE 5 $M U=-0.6179$ $1.563 \mathrm{E}-05$ $3.255 \mathrm{E}-05$ 3.681E-04 1. $011 \mathrm{E}-04$ $1.798 E-04$ $4.252 E-05$ $4.698 E-05$ $5.473 E-05$ $0.800 E-05$ $9.721 E-05$ $1.486 E-04$ $2.470 E-04$ $1.378 \mathrm{E}-03$ 2. $212 E-03$ $5.175 \mathrm{E}-03$ $1.455 \mathrm{E}-02$ 4. $034 \mathrm{E}-02$ $1.165 \mathrm{E}-02$

ANGLE 15

ANGLE 7 $M U=-0.4580$ $1.756 \mathrm{E}-05$ 3.647E-0 $1.130 E-04$ $2.011 \mathrm{E}-04$ 2.011E-04 $5.825 E-05$ $6.963 E-05$ $8.728 E-05$ $1.232 E-04$ $1.856 \mathrm{E}-04$ $3.130 E-04$ $1.597 \mathrm{E}-03$ 2.513E-03 $5.221 E-C 3$ $1.502 \mathrm{E}-02$ 4.135E-02 $1.154 \mathrm{E}-02$

ANGLE 16 $9.772 E-05$ $1.985 E-04$ $2.103 E-03$ 5.725E-04 6.512E-04 $.595 E-04$ $1.032 E-04$ .134E-03 $.421 \mathrm{E}-03$ $1.784 \mathrm{E}-03$ $4.072 E-03$ $4.532 E-03$ 6.88E-03 $2.052 \mathrm{E}-02$ $5.198 \mathrm{E}-02$ $1.297 E-02$ . $778 E-04$ . $906 \mathrm{E}-03$ $9.178 \mathrm{E}-04$ ..033E-03 $1.179 E-03$ $1.403 E-03$ $1.664 E-03$ $1.991 E-03$ $4.428 E-03$ $4.843 E-O 3$ $7.220 E-03$

ANGLE 8 $M U=-0.2816$ $4.201 E-05$ $4.719 E-O 4$ $1.308 \mathrm{E}-04$ 2.320E-O4 $6.427 E-05$ 7.492E-05 $9.087 E-05$ $1.145 E-04$ $1.614 E-04$ $2.440 E=04$ $4.159 E-04$ $1.875 E-03$ $2.813 E-03$ 1.55ge-O2 $4.254 \mathrm{E}-02$ ANGLE 17 $3786564 \quad M U=0.9446 \quad M U=0.9894$
$2.374 E-04$ 
(GAMYAS/MEV/STERADIAN/SOURCE NEUTRON)

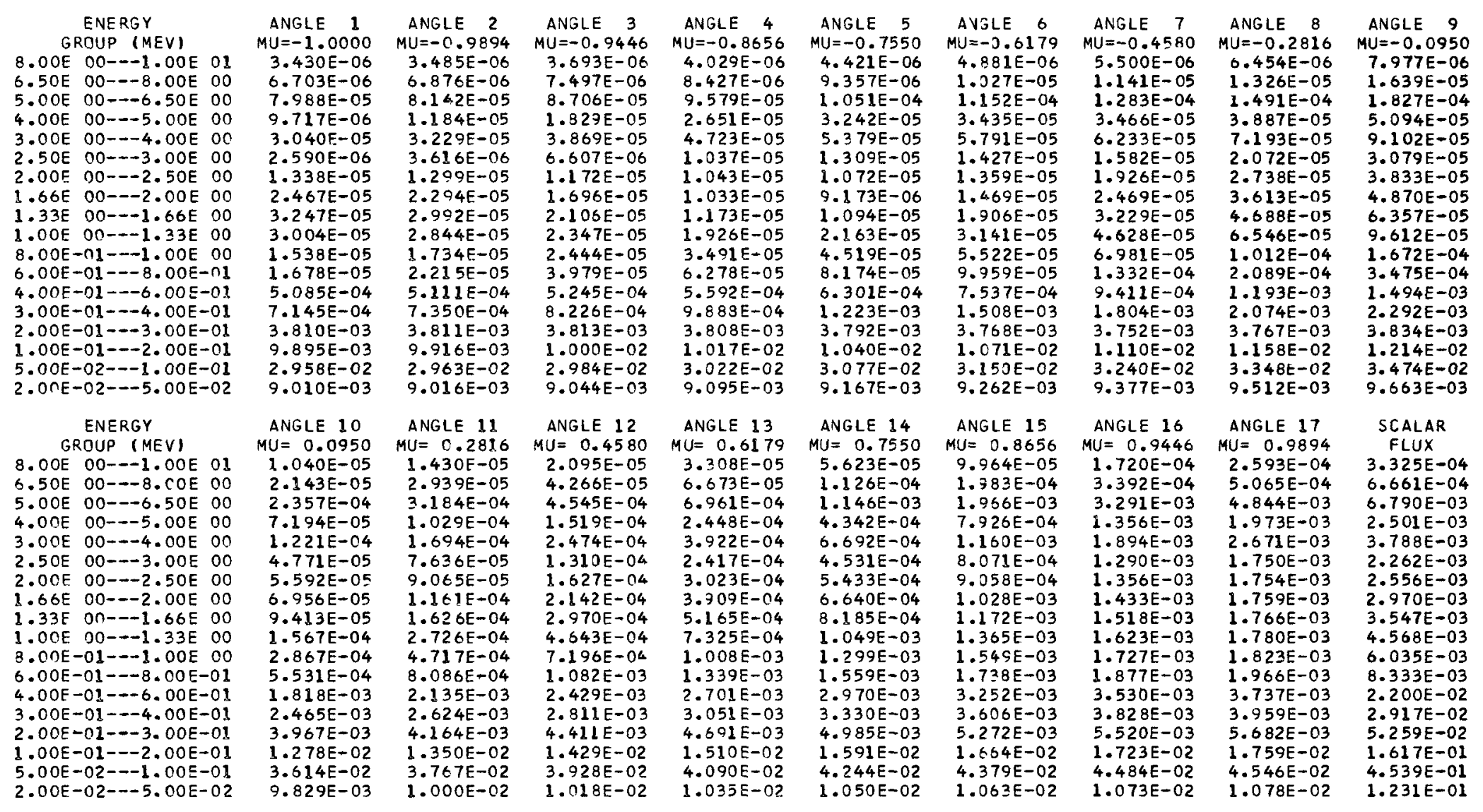


(GAMMAS/MEV/STERADIAN/S QURCE NEUTRON)

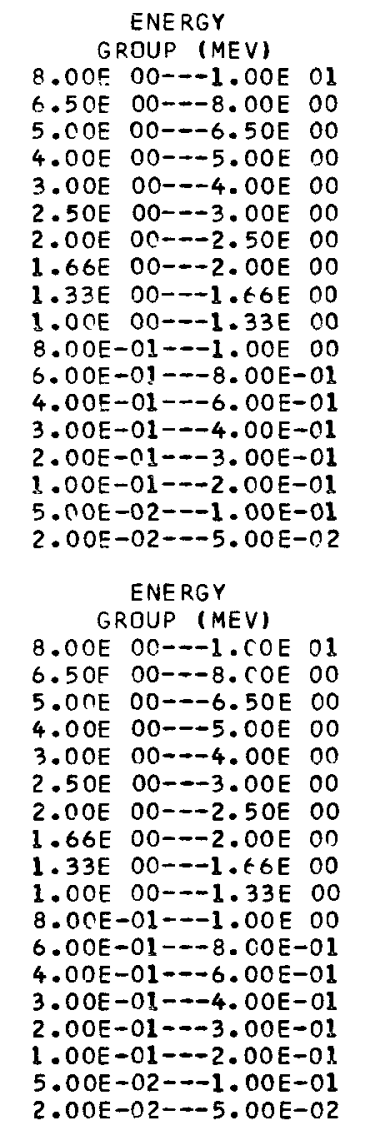

ANGLE 1 $A N=-1.0000$
$5.790 E-07$ $7.822 \mathrm{E}-07$ 醇 $6.839 E-06$ $5.784 E-06$ $1.545 \mathrm{E}-05$ $2.140 E-05$ $1.556 \mathrm{E}-05$ $-5.248 \mathrm{E}-06$ $-1.638 \mathrm{E}-05$ $2.233 \mathrm{E}-04$ $2.930 \mathrm{E}-04$ $2.203 E-03$ $5.511 \mathrm{E}-03$ $1.696 \mathrm{E}-0$ 5.207E-03 ANGLE 10 $M U=0.0950$ $2.584 \mathrm{E}-06$ $5.380 E-06$ $5.853 E-05$ $2.020 \mathrm{E}-05$ $3.274 E-05$ $1.454 E-05$ $1.537 \mathrm{E}-05$ $1.754 \mathrm{E}-05$ $2.530 \mathrm{E}-05$ $5.624 E-05$ $1.364 \mathrm{E}-04$ 1.1 $47 E-04$ $1.018 E-03$ 1.382E-03 $7.176 \mathrm{E}-03$ $2.088 \mathrm{E}-02$ $5.697 \mathrm{E}-03$

ANGLE 2
$M U=-0.9894$ $6.1 \subseteq 3 E-C 7$
$9.351 E-07$ $9.351 \mathrm{E}-07$ $4.672 E-06$ $5.976 \mathrm{E}-07$ $2.330 E-C 6$ $5.347 E-06$ $1.360 E-05$ 1.863E-05 $1.365 \mathrm{E}-05$ $-3.442 E-06$ $-1.120 E-05$ $2.231 F-04$ $3.058 \mathrm{E}-04$ $2.204 \mathrm{E}-\mathrm{C3}$ $5.523 E-03$ $1.699 E-02$ $5.211 E-03$ ANGLE 11 $M U=0.2816$ $3.786 \mathrm{E}-06$ $7.891 E-06$ 8.375E-05 $3.165 E-05$ $4.772 E-05$ $2.384 E-05$ $2.640 \mathrm{E}-05$ $3.511 E-05$ .800E-05 2.632E-04 $2.632 E-04$ $1.203 E-03$ $1.23 \mathrm{E}-03$ $1.438 E-03$ $7.592 \mathrm{E}-\mathrm{O}$ $2.181 \mathrm{E}-02$ 5.801E-03 $M=-0.9446$ $1.765 E-05 \quad 2.258 E-05$ $9.774 E-07 \quad 8.246 E-06$ $5.725 E-06 \quad 1.268 E-05$ $3.184 E-07 \quad 3.595 E-06$ 6.014E-06 $7.540 E-06$ $6.285 E-06$ $3.298 E-06$ $2.244 E-04$ $3.619 E-04$ $2.203 E-03$ $5.575 E-03$ $1.711 E-02$ 228 E 03

ANGLE 12 $M U=0.4580$ $5.935 E-06$ $1.204 E-05$ 1.256E-04 4. $466 \mathrm{E}-05$ $7.135 E-05$ $4.477 E-05$ $6.08 J E-05$ $1.055 E-05$ $2.509 E-04$ $2.599 E-C 4$ 4.395E-04 6.80E-04 $1.355 E-03$ $1.521 E-03$ (2.3 $2.278 \mathrm{E}-02$ 5.906E-03

ANGLE 5
$M U=-0.7550$
$1.080 E-06$
$2.436 E-06$
$2.604 E-05$
$1.049 E-05$
$1.581 E-05$
$4.287 E-06$
$1.153 E-06$
$-1.783 E-06$
$-2.761 E-06$
$2.237 E-06$
$1.488 E-05$
$2.933 E-05$
$2.688 E-04$
$6.322 E-04$
$2.169 E-03$
$5.808 E-03$
$1.767 E-02$
$5.301 E-03$
ANGLE 14
$M U=0.7550$
$2.169 E-05$
$4.272 E-05$
$4.160 E-04$
$1.771 E-04$
$2.751 E-04$
$2.425 E-04$
$3.150 E-04$
$4.103 E-04$
$5.282 E-04$
$6.915 E-04$
$8.429 E-04$
$9.653 E-04$
$1.599 E-03$
$1.811 E-03$
$2.691 E-03$
$8.983 E-03$
$2.470 E-02$
$6.101 E-03$

\begin{tabular}{|c|c|c|c|}
\hline $\begin{array}{l}A=-0.6179 \\
1.165 \mathrm{E}-06 \\
2.535 \mathrm{E}-06 \\
2.755 \mathrm{E}-05 \\
1.007 \mathrm{E}-05 \\
1.451 \mathrm{E}-05 \\
3.678 \mathrm{E}-06 \\
2.085 \mathrm{E}-06 \\
1.274 \mathrm{E}-06 \\
1.999 \mathrm{E}-06 \\
7.114 \mathrm{E}-06 \\
1.704 \mathrm{E}-05 \\
3.110 \mathrm{E}-05 \\
3.389 \mathrm{E}-04 \\
8.290 \mathrm{E}-04 \\
2.130 \mathrm{E}-03 \\
5.988 \mathrm{E}-03 \\
1.810 \mathrm{E}-02 \\
5.357 \mathrm{E}-03\end{array}$ & $\begin{array}{r}M U=-0.4580 \\
1.244 E-06 \\
2.516 E-06 \\
2.866 E-05 \\
5.465 E-06 \\
1.278 E-05 \\
2.533 E-06 \\
4.897 E-06 \\
8.007 E-06 \\
1.153 E-05 \\
1.498 E-05 \\
1.805 E-05 \\
3.950 E-05 \\
4.556 E-04 \\
1.029 E-03 \\
2.091 E-03 \\
6.213 E-03 \\
1.864 E-02 \\
5.426 E-03\end{array}$ & $\begin{array}{r}M U=-0.2816 \\
1.433 E-06 \\
2.806 E-06 \\
3.252 E-05 \\
5.820 E-06 \\
1.348 E-05 \\
3.542 E-06 \\
8.198 E-06 \\
1.358 E-05 \\
1.803 E-05 \\
2.086 E-05 \\
2.750 E-05 \\
7.883 E-05 \\
6.188 E-04 \\
1.197 E-03 \\
2.070 E-03 \\
6.485 E-03 \\
1.929 E-02 \\
5.507 E-03\end{array}$ & $\begin{array}{r}M U=-0.0950 \\
1.849 E-06 \\
3.721 E-06 \\
4.200 E-05 \\
1.095 E-05 \\
2.062 E-05 \\
7.994 E-06 \\
1.139 E-05 \\
1.518 E-05 \\
1.932 E-05 \\
2.919 E-05 \\
6.141 E-05 \\
1.596 E-04 \\
8.146 E-04 \\
1.312 E-03 \\
2.087 E-03 \\
6.806 E-03 \\
2.004 E-02 \\
5.598 E-03\end{array}$ \\
\hline $\begin{array}{l}\text { ANGLE } 15 \\
M U=0.8656 \\
4.935 \mathrm{E}-05 \\
9.686 \mathrm{E}-05 \\
9.031 \mathrm{E}-04 \\
4.292 \mathrm{E}-04 \\
6.070 \mathrm{E}-04 \\
5.230 \mathrm{E}-04 \\
6.036 \mathrm{E}-04 \\
6.971 \mathrm{E}-04 \\
7.985 \mathrm{E}-04 \\
9.149 \mathrm{E}-04 \\
9.977 \mathrm{E}-04 \\
1.057 \mathrm{E}-03 \\
1.741 \mathrm{E}-03 \\
1.967 \mathrm{E}-03 \\
2.845 \mathrm{E}-03 \\
9.409 \mathrm{E}-03 \\
2.553 \mathrm{E}-02 \\
6.180 \mathrm{E}-03\end{array}$ & $\begin{array}{r}A N G L E 16 \\
M U=0.9446 \\
1.113 E-04 \\
2.152 E-04 \\
1.941 E-03 \\
9.286 E-04 \\
1.213 E-03 \\
9.679 E-04 \\
1.005 E-03 \\
1.038 E-03 \\
1.065 E-03 \\
1.081 E-03 \\
1.088 E-03 \\
1.128 E-03 \\
1.901 E-03 \\
2.079 E-03 \\
2.982 E-03 \\
9.750 E-03 \\
2.617 E-02 \\
6.238 E-03\end{array}$ & $\begin{array}{r}\text { ANGLE } 17 \\
M U=0.9894 \\
2.116 \mathrm{E}-04 \\
4.009 \mathrm{E}-04 \\
3.541 \mathrm{E}-03 \\
1.604 \mathrm{E}-03 \\
1.989 \mathrm{E}-03 \\
1.455 \mathrm{E}-03 \\
1.401 \mathrm{E}-03 \\
1.335 \mathrm{E}-03 \\
1.260 \mathrm{E}-03 \\
1.170 \mathrm{E}-03 \\
1.123 \mathrm{E}-03 \\
1.178 \mathrm{E}-03 \\
2.031 \mathrm{E}-03 \\
2.135 \mathrm{E}-03 \\
3.075 \mathrm{E}-03 \\
9.959 \mathrm{E}-03 \\
2.655 \mathrm{E}-02 \\
6.272 \mathrm{E}-03\end{array}$ & $\begin{array}{c}\text { SCALAR } \\
\text { FLUX } \\
1.578 E-04 \\
3.083 E-04 \\
2.914 E-03 \\
1.261 E-03 \\
1.772 E-03 \\
1.343 E-03 \\
1.524 E-03 \\
1.772 E-03 \\
2.117 E-03 \\
2.718 E-03 \\
3.569 E-03 \\
4.888 E-03 \\
1.176 E-02 \\
1.601 E-02 \\
2.890 E-02 \\
9.081 E-02 \\
2.624 E-01 \\
7.136 E-02\end{array}$ \\
\hline
\end{tabular}


(GAMMAS/MEV/STERADIAN/SOURCE NEUTRON)

\begin{abstract}
ENERGY GROUP (MEV) 8.0OE OO---1.00E OI $6.50 E$ OO-- $8.00 E$ OO $5.00 E$ OO- $-6.50 E$ OO 4.0OE $00---5.00 E$ EO $3.00 \mathrm{E}$ OO- $-4.00 \mathrm{E}$ OO 2.50 E $00--3.00 E$ EO

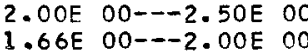
$1.33 E$ E0- $-1.66 E$ OO $1.00 E$ O0-- $-1.33 \mathrm{E}$ 00 $8.00 \mathrm{E}-01--1.00 \mathrm{E}$ OO $6.00 F-01---8.00 E-01$ $4.00 E-01--6.00 E-01$ $3.00 E-01--4.00 E-01$ $2.00 E-01--3.00 E-01$ 1. OCE $-01--2.00 E-01$ $5.00 \mathrm{E}-02--1.00 \mathrm{E}-0$ $2,00 E-02---5.00 E-n 2$ ENERGY GROUP (MEV) 8. OOE OO---1.00E 01 $6.50 E$ OO-D-8.0OE OO $5.00 E$ OO- $-6.50 \mathrm{E} 00$ 4.0OE $00-2-5.00$ E 00 3.0OE OO-C-4.0OE 00 2.5OE DO- $-3.00 E$ OO 2.0OE OO-C $2.50 E$ OO 1.66 E OO-C 2.00E $1.33 E$ OO $1.66 E$ OO B. $6.00 E-01-2.00 E-01$ $4.00 E-01-2.00 E-01$ 4. OOE $01-2.00 \mathrm{E}-01$ 2.00E-01- $-3.00 E-01$ 1.0OE-01- $3.00 E-01$ $5.00 E-02--1.00 E-01$ $2.00 E-02--5.00 E-02$
\end{abstract}

ANGLE 1 ANGLE 2 $M U=-1.0000 \quad M U=-0.9894$ $-2.431 E-08$ $-3.502 E-07$ $-9.321 E-07$ $-7.238 E-06$ $-5.563 E-06$ $-3.272 E-06$ $1.372 E-05$ 8.998E-06 $-6.897 E-06$ 1.692E-05 $1.179 \mathrm{E}-04$ $1.146 \mathrm{E}-03$ $2.760 \mathrm{E}-03$ $8.578 \mathrm{E}-03$ $2.635 \mathrm{E}-03$ ANGLE 10 $M U=0.095$ $5.675 \mathrm{E}-07$ $1.301 E-06$ $1.300 E-05$ $6.400 E-06$ $8.985 E-16$ $3.119 E-06$ 1.13 $1.464 E-06$ 1.657E-0 1.657E-05 $1.126 E-05$ $5.213 E-04$ 6.215E-04 $1.057 \mathrm{E}-03$ $3.589 E-03$ $3.589 \mathrm{E}-03$
$1.055 \mathrm{E}-02$ $2.880 E-03$ $8.400 E-09$ $-2.282 E-07$ ..758E-06 $1.165 \mathrm{E}-05$ $7.533 \mathrm{E}-06$ $-5.668 E-06$ $1.331 E-05$
$9.800 E-05$ $1.246 \mathrm{E}-\mathrm{O}$ $1.146 E-03$ $2.767 \mathrm{E}-\mathrm{O}$ $8.592 \mathrm{E}-0$ $2.636 \mathrm{E}-03$ ANGLE 11 $M U=0.2816$ $8.899 \mathrm{E}-0$ 1.97 E-06 $1.986 \mathrm{E}-05$ $1.043 E-05$ $1.326 E-05$ $6.530 E-06$ 4.9OEE-06 1.573E- 05 $5.384 \mathrm{E}-05$ 1.34 E 05 $2.707 E-04$ $6.139 E-04$ $7.082 \mathrm{E}-04$ $1.106 E-03$ $3.793 \mathrm{E}-03$ $1.101 \mathrm{E}-02$ $2.931 E-03$
ANGLE 3 $9.507 \mathrm{E}-08$ $9.476 E-08$ $2.152 E-06$ $1.154 \mathrm{E}-06$ $6.149 E-09$ $-3.725 E-07$ $1.291 E-06$ $2.887 E-06$ $3.633 E-06$ $2.119 E-06$ $-1.461 E-06$ $9.599 E-05$ $1.549 \mathrm{E}-04$ $1.145 \mathrm{E}-03$ $2.794 \mathrm{E}-03$ $8.654 E-03$ $2.645 \mathrm{E}-03$ ANGLE 12 $M U=0.4580$ $1.405 E-06$ $2.859 E-06$ $2.895 E-05$ $1.111 E-05$ $1.654 \mathrm{E}-05$ $1.159 E-05$ $1.837 E-05$ 6.384E-05 $1.324 E-04$ 1.324E- 04 2.386E- 04 $0.742 E-04$ $7.394 E-04$ $1.167 \mathrm{E}-03$ $4.013 E-03$ $4.013 \mathrm{E}-03$
$1.150 \mathrm{E}-02$ $2.984 \mathrm{E}-03$

$M U=-0.8656 \quad M U=$

$5.222 \mathrm{E}-0$

5.297E-06

$2.180 E-06$

$3.104 \mathrm{E}-06$

$1.119 E-06$

$-3.237 \mathrm{E}-0$

$1.805 E-06$

$-3.006 \mathrm{E}-06$

$2.583 \mathrm{E}-06$

$7.448 E-06$

$9.769 \mathrm{E}-05$

$2.162 \mathrm{E}-04$

$1.138 \mathrm{E}-03$

$2.843 \mathrm{E}-03$

$8.769 \mathrm{E}-0$

$2.660 \mathrm{E}-03$

ANGLE 13

$M U=0.6179$ $2.671 \mathrm{E}-06$ $5.055 E-06$ $2.069 E-05$ $1.675 E-05$ $3.216 E-05$ 3.547E-OS ..108E-0S $1.006 E-04$ 1.576E-04 2.517E-04 3.565E-04 $7.565 E-04$ 8. $13 \mathrm{E}-04$ $1.234 \mathrm{E}-03$ $4.242 E-03$ $1.199 \mathrm{E}-02$ $3.035 \mathrm{E}-03$ ANGLE $\quad 5$
$\mathrm{U}=-0.7550$ $2.698 \mathrm{E}-07$ $6.604 \mathrm{E}-07$ $6.550 E-06$ $5.243 E-06$ $5.758 \mathrm{E}-06$ $2.193 \mathrm{E}-06$ $6.702 E-07$ $-3.146 E-06$ 6. $553 E-06$
$1.379 E-05$ $1.319 E-04$ $3.080 \mathrm{E}-04$ $1.118 E-03$ 2. $912 E-03$ $8.934 E-03$ $2.682 \mathrm{E}-03$ ANGLE 14 $M U=0.7550$ $6.772 \mathrm{E}-06$ $1.298 E-05$ 1. $214 E-04$ $5.723 E-05$ $9.627 E-05$ $1.104 E-04$ 1.5 $22 E-04$ 2.202E-04 3.906E-04 $4.669 E-04$ $5.114 E-04$ $7.688 E-04$ $1.302 \mathrm{E}-0$ $4.466 \mathrm{E}-03$ $1.245 \mathrm{E}-02$ 3.081E-03
$M U=-0.6179$

ANGLE 7 $M U=-0.4580$ ANGLE 8 ANGLE 9

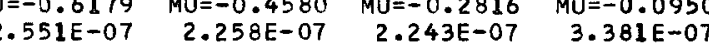
$6.151 E-07 \quad 3.713 E-07 \quad 3.041 E-07 \quad 7.071 E-07$ $6.060 E-06 \quad 4.904 E-06 \quad 4.801 E-06 \quad 7.578 E-06$ $2.427 E-06 \quad-5.604 E-07-1.034 E-06 \quad 1.252 E-06$ $3.151 E-06 \quad 6.724 E-07 \quad 9.449 E-07 \quad 3.712 E-06$ $5.608 E-07 \quad-4.452 E-07 \quad 1.002 E-07 \quad 2.103 E-06$

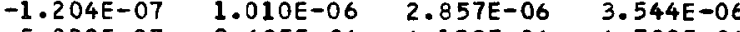

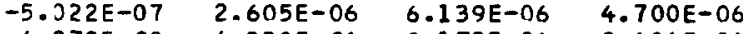
$-4.272 E-08 \quad 4.228 E-06 \quad 8.172 E-06 \quad 3.686 E-06$ $1.319 E-06 \quad 5.746 E-06 \quad 6.655 E-06 \quad 6.582 E-06$ $5.441 E-06 \quad 2.358 E-06 \quad 4.921 E-06 \quad 2.016 E-05$ $8.523 E-06$ 8.208E-06 $2.694 E-05 \quad 7.889 E-05$ $1.476 E-04 \quad 2.113 E-04 \quad 3.026 E-04 \quad 4.116 E-04$ $\begin{array}{llll}4.214 E-04 & 5.343 E-04 & 6.237 E-04 & 6.755 E-04\end{array}$ $1.088 E-03 \quad 1.055 E-03 \quad 1.032 E-03 \quad 1.031 E-03$ $3.003 E-03 \quad 3.114 E-03 \quad 3.249 E-03 \quad 3.408 E-03$ $2.710 E-03 \quad 9.424 E-03 \quad 9.749 E-03 \quad 1.013 E-02$ $2.744 E-03 \quad 2.785 E-03 \quad 2.830 E-03$

AVGLE 1 $M U=0.8656$ 2.030E-05 $3.950 \mathrm{E}-0 \mathrm{~S}$ $3.444 \mathrm{E}-04$ $1.966 \mathrm{E}-04$ $2.767 E-04$ $2.826 \mathrm{E}-04$ $3.369 E-04$ ..963E-04 5. $5.421 E-04$ $5.474 E-04$ 8.290E-04 $1.371 E-03$ $4.6711 E-03$ $1.286 \mathrm{E}-02$ 3.119E-03

ANGLE 16 $M U=0.9446$ $0.046 E-05$
$1.149 E-04$ $1.149 E-04$ $9.583 E-04$ $5.309 E-04$ . $5.865 \mathrm{E}-04$ $6.036 E-04$ $6.121 E-04$ 5. . $5.726 E-04$ . $075 E-04$ 
(GAMMAS/MEV/STERADIAN/SOURCE NEUTRON)

ENERGY 8.00E O0---1.00E O1 6.50 E $00---8.00 E$ OO 5.00E $00---6.50 E$ OO 4.00E $00--5.00 E 00$ $3.00 \mathrm{E} 00--4.00 E$ OO $2.50 E$ OO-- $3.00 E$ OO 2.00E O0---2.50E OO $1.66 \mathrm{E} 00--2.00 E 00$ $1.33 E$ OO- - $1.66 E$ EO $1.00 E$ OO---1.33E 00 $8.00 E-01--1.00 E$ OO $6.00 E-01-8.00$ E-01 $4.00 E-01--6.00 E-01$ $3.00 \mathrm{E}-01--4.00 \mathrm{E}-01$ $2.00 E-01--3.00 E-01$ 1. $00 \mathrm{E}-01--2.00 \mathrm{E}-\mathrm{Cl}$ $5.00 E-02--1.00 E-01$
$2.00 E-02=-5.00 E-02$

ENERGY

GROUP (MEV)

8.0OE O0---1.0OE OI $6.50 E$ OO- $-8.00 E$ OO 5.00 E $00---6.50 E$ OO $4.00 E$ OO- $-5.00 E$ OO $3.00 E$ OO- $-4.00 E$ OO $2.50 E$ OO-- $3.00 E$ OO $2.00 E$ OO---2.50E OO $1.66 \mathrm{E}$ O0---2.00E OO $1.33 \mathrm{E} \quad 00--1.66 \mathrm{E} \mathrm{OO}$ $1.00 \mathrm{E} \mathrm{OO--1.33E} \mathrm{OO}$ $8.00 E-01-1.00 E$ OO $6.00 E-01---8.00 E-O 1$ $4.00 E-01--6.00 E-01$ $2.00 E-01--4.00 E-01$ $2.00 E-01--3.00 E-01$ $1.00 E-01--2.00 E-O 1$ $5.00 E-02--1.00 E-01$
$2.00 E-02---5.00 E-02$ $\triangle A N G L E \quad 1$ $M U=-1.0000$
$-8.818 E-08$ $-8.818 E-08$
$-3.709 E-07$ $-2.065 \mathrm{E}-06$ $-4.688 \mathrm{E}-06$ $-4.054 \mathrm{E}-06$ $-2.092 E-06$ $1.600 E-06$ $5.638 E-06$ $8.047 \mathrm{E}-06$ $5.056 E-06$ $-4.724 E-D 6$ $-1.103 E-05$ $4.954 \mathrm{E}-05$ $5.680 E-04$ $1.327 \mathrm{E}-03$ $4.126 \mathrm{E}-03$ $1.264 E-03$

ANGLE 2 $M U=-0.9894$ $-6.756 \mathrm{E}-08$ $-2.947 E-07$ $-1.578 E-06$ $-3.817 E-06$ $-3.252 E-06$ $-1.666 \mathrm{E}-06$ $1.406 \mathrm{E}-06$ $6.760 E-06$ $4.149 E-06$ $-3.983 E-06$ $-8.878 \mathrm{E}-06$ $5.287 E-05$ $5.681 E-04$ $1.330 E-03$ $4.133 E-03$
$1.265 E-03$

ANGLE 10 $M U=0.0950$ . $340 E-07$ 3.451E-0 $2.260 E-06$ . $3.256 E-06$ $7.090 \mathrm{E}-08$ $-1.730 E-06$ $-1.730 E-06$ $4.412 E-06$ $2.794 E-05$ $8.329 \mathrm{E}-05$ $2.565 E-04$ $3.351 \mathrm{E}-04$ $5.026 \mathrm{~F}-04$ $1.718 \mathrm{E}-03$ $1.378 \mathrm{E}-03$ ANGLE 11 $M U=0.2816$ $5.987 \mathrm{E}-07$
ANGLE 3 $M U=-0.9446$ $-2.852 \mathrm{E}-09$ $-5.538 E-08$ $-3.839 E-08$ $-1.067 E-06$ $-3.510 E-07$ $6.003 \mathrm{E}-07$ $1.509 E-06$ $1.892 \mathrm{E}-06$ $8.693 \mathrm{E}-07$ $-1.436 E-06$ $-1.971 E-06$ $4.258 E-05$ $6.818 E-05$ $5.674 \mathrm{E}-04$ $1.343 E-03$ $4.163 E-03$ 1.269 E-03

ANGLE $M U=-0.8656$ $1.225 \mathrm{E}-07$ $1.146 E-06$ $9.854 E-07$ $9.854 E-07$
$1.150 E-06$ $1.150 E-06$ $-2.263 E-07$ $-1.394 E-06$ $-2.219 E-06$ $-1.581 \mathrm{E}-06$ $3.570 \mathrm{E}-06$ 4.205E-05 9. $969 \mathrm{E}-05$ $5.625 E-04$ $1.367 E-03$ 4. $217 E-03$ ANGLE 12 $M U=0.4580$ ANGLE 13 $M U=0.617$ $5.487 E-07$ $8.792 \mathrm{E}-07$ $9.360 E-06$ $7.744 E-07$
$5.429 E-06$ $5.429 E-06$ $1.099 E-05$ $2.517 E-05$ $4.760 \mathrm{E}-05$ $7.937 \mathrm{E}-05$ 1. $304 \mathrm{E}-04$ $1.846 \mathrm{E}-04$ 2. $299 \mathrm{E}-04$ $3.417 E-04$ 3.757E-04 $5.795 \mathrm{E}-04$ 2. $016 \mathrm{E}-03$ $5.716 \mathrm{E}-03$
$1.450 \mathrm{E}-03$
ANGLE 5 $M=-0.7550$ $2.864 \mathrm{E}-07$ $2.864 E-07$
$2.189 E-06$ $2.189 E-06$
$2.702 E-06$ $2.702 E-06$
$2.597 E-06$ 2.597E-06 $-6.562 \mathrm{E}-07$ $-2.236 \mathrm{E}-06$ $-3.050 E-06$ $-1.495 E-06$
$3.225 E-06$ $6.709 E-06$ 4.803E-05 $1.478 \mathrm{E}-04$ 5.504E-04 $1.400 E-03$ $4.295 E-03$
$1.286 E-03$ ANGLE 14 $M U=0.7550$ $1.824 \mathrm{E}-06$ . $3.014 E-05$
$1.505 E-05$ $3.163 \mathrm{E}-05$ 4.771E-0 $7.578 \mathrm{E}-05$ $1.115 \mathrm{E}-04$ $1.535 \mathrm{E}-04$ 2.040E-04 $2.387 E-04$ $2.516 \mathrm{E}-04$ 3.557E-04 $4.141 \mathrm{E}-04$ $6.077 \mathrm{E}-04$ $2.117 \mathrm{E}-03$ $1.471 E-03$ $6.147 E-08 \quad 9.243 E-09$ $1.409 E-07-2.321 E-0$ $1.323 \mathrm{E}-06 \quad 1.628 \mathrm{E}-\mathrm{C} 7$ $7.913 E-07-1.162 E-06$ $7.478 E-07-9.024 E-07$ $1.537 E-08 \quad-6.816 E-07$ $-3.753 E-07 \quad 2.665 E-07$ $-6.179 E-07 \quad 1.301 E-06$ $-3.838 E-07 \quad 2.246 E-06$ $2.733 E-07 \quad 2.695 E-06$ $2.073 E-06 \quad-5.547 E-07$ $1.890 \mathrm{E}-06 \quad 7.959 \mathrm{E}-07$ $6.553 E-05 \quad 9.826 E-05$ $2.073 E-04 \quad 2.657 E-04$ $1.444 E-03 \quad 1.496 E-03$ $4.398 \mathrm{E}-03 \quad 4.525 \mathrm{E}-03$

ANGLE 15 $\mathrm{A}=0.8656$
$7.600 \mathrm{E}-06$ $1.480 \mathrm{E}-05$ $1.200 E-04$ $8.412 \mathrm{E}-05$ $1.212 E-04$ $1.410 E-04$ $1.731 \mathrm{E}-04$ $2.067 E-04$ $2.380 \mathrm{E}-04$ $2.647 E-04$ $2.709 E-04$ $2.638 E-04$ 3.806E-0 $4.458 \mathrm{E}-04$ $6.367 \mathrm{E}-04$ $2.208 \mathrm{E}-03$ $6.114 E-03$
$1.489 E-03$ ANGLE 16 $\triangle N G L E \quad 8$ $6.529 E-09 \quad 4.298 E-08$ $-6.040 E-08 \quad 5.887 E-08$ $1.108 E-07 \quad 1.037 E-06$ $-1.566 E-06 \quad 3.581 E-07$ $-9.757 E-07 \quad 4.499 E-07$ $-3.549 E-07 \quad 5.496 E-07$ $1.272 E-06 \quad 1.365 E-06$ $3.243 E-06 \quad 1.017 E-06$ $4.240 E-06 \quad 2.714 E-07$ $2.356 E-06 \quad 3.647 E-07$ $-2.848 \mathrm{E}-07 \quad 6.406 \mathrm{E}-06$ $9.361 E-06 \quad 3.692 E-05$ $1.456 E-04 \quad 2.017 E-04$ $3.096 \mathrm{E}-04 \quad 3.313 \mathrm{E}-04$ $4.920 E-04$ $1.560 E-03 \quad 1.633 E-03$ $\begin{array}{ll}4.678 E-03 & 4.854 E-03 \\ 1.334 E-03 & 1.355 E-03\end{array}$ ANGLE 17 $M U=0.9894$ $9.112 E-05$ $1.615 E-04$ $1.206 E-03$ $6.332 E-04$ $6.828 \mathrm{E}-04$ $5.480 E-04$ $4.940 \mathrm{E}-04$ $4.324 E-04$ $3.688 \mathrm{E}-04$ $3.008 \mathrm{E}-04$ $2.675 \mathrm{E}-04$ $2.848 \mathrm{E}-04$ 4.512E-04 $4.601 \mathrm{E}-04$ $6.847 E-04$ 2.324E-03 $6.342 E-03$
$1.510 E-03$

SCALAR

3. $471 E-05$ $6.394 \mathrm{E}-05$ $5.000 E-04$ 2. $888 \mathrm{E}-04$ $3.623 E-04$ $3.563 E-04$ $4.056 \mathrm{E}-04$ 4. $714 \mathrm{E}-04$ 5. $609 \mathrm{E}-04$ $7.143 E-04$ $9.273 E-04$
$1.252 E-03$ $2.725 \mathrm{E}-03$ 3.792E-03 $6.846 E-03$ $2.166 \mathrm{E}-02$ 
COSINE

-1. COOCOE CO $-9.89401 E-01$ $-9.44575 E-01$ $-7.55044 E-01$ $-6.17876 E-01$ $-4.58017 E-01$ $-2.81605 E-01$ $-9.50125 \mathrm{E}-\mathrm{C}$ $9.50125 \mathrm{E}-02$ 2.81605E-01 4.58017E-0 $6.17876 E-01$ $7.55044 E-01$ $8.65631 \mathrm{E}-01$ $9.44575 \mathrm{E}-\mathrm{O}$ $9.89401 E-01$

TOTAL

$\operatorname{COSINE}$

$-1.00000 E$ OC $-9.89401 \mathrm{E}-01$ $-9.44575 E-01$ $-7.55044 E-01$ $-7.55044 E-01$ $-4.58017 E-01$ $-4.58017 E-01$ $-2.81005 E-01$ $9.50125 \mathrm{E}-02$ $90165 \mathrm{E}-01$ $4.58017 \mathrm{E}-01$ $6.17876 \mathrm{E}-01$ $6.17876 \mathrm{E}-\mathrm{Ol}$
$7.55044 \mathrm{E}-\mathrm{Cl}$ $7.55044 \mathrm{E}-\mathrm{Cl}$ $8.65631 \mathrm{E}-\mathrm{Cl}$ $9.89401 \mathrm{E}-01$

TOTAL

75.0
$8.649 E-11$
$8.674 E-11$
$8.778 E-11$
$8.987 E-11$
$9.311 E-11$
$9.774 E-11$
$1.041 E-1 C$
$1.127 E-10$
$1.241 E-10$
$1.780 E-1 C$
$1.622 E-10$
$1.959 E-1 C$
$2.420 E-10$
$2.684 E-10$
$4.567 E-1 C$
$1.273 E-C 9$
$3.883 E-C 9$
$3.133 E-C 9$

100.0

1. $C 28 \mathrm{E}-10$ 1. $C 31 E-1 C$ 1.C $43 \mathrm{E}-1 \mathrm{C}$ 1. $C 68 \mathrm{E}-10$ $1.1 C 7 E-1 C$ $1.161 \mathrm{E}-10$ $1.235 \mathrm{E}-1 \mathrm{C}$ $1.335 E-1 C$ $1.466 \mathrm{E}-10$ $1.717 E-10$ $1.996 \mathrm{E}-1 \mathrm{C}$ $2.006 \mathrm{E}-10$ $2.658 \mathrm{E}-1 \mathrm{C}$ $3.473 \mathrm{E}-1 \mathrm{C}$ $4.486 \mathrm{E}-10$ $9.341 E-10$ . $762 E-09$ $3.231 E-C 9$

500.0

600.0

$5.874 E-11$ $5.893 \mathrm{E}-11$ $5.972 E-11$ $6.123 E-11$ $6.657 \mathrm{E}-11$ $7.064 \mathrm{E}-11$ $7.588 \mathrm{E}-11$ . . 1. $14 E-10$ $1.145 E-10$ .311E-10 1. $523 \mathrm{E}-10$ . $804 \mathrm{E}-10$ $2.209 E-10$ $3.39 C E-10$

$1.283 \mathrm{E}-\mathrm{Cg}$

$8.285 E-1 C$

\begin{abstract}
150.0
RANC

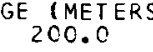

$25 \mathrm{C} .0$

300.3

$400 . C$

$1.221 \mathrm{E}-10$
$1.225 \mathrm{E}-10$
$1.240 \mathrm{E}-10$

$1.240 E-10$

$1.271 \mathrm{E}-10 \quad 1.222 \mathrm{E}-10$

$1.117 \mathrm{E}-10$

$1.291 E-10 \quad 1.242 E-10$

$1.323 E-10 \quad 1.273 E-10$

$1.371 \mathrm{E}-10$ 1. $1.319 \mathrm{E}-10$

$1.384 \mathrm{E}-10$

$.381 E-10$

$1.439 E-10$

$1.468 E-10 \quad 1.529 E-10 \quad 1.470 E-10$

$1.583 E-10 \quad 1.647 E-10 \quad 1.583 E-10$

$1.734 E-10$ 1.8C1E-10 $1.729 E-10$

$1.826 E-10$ $2.07 C E-10$ 1.996E-10

$2.345 E-10$ 2.205E-10 2.09gE-10

$2.257 E-10 \quad 2.648 E-10$ 2.522E-10

$3.173 E-10$ 3.023E-10 2.843E-10

$3.683 E-10$ 3.761E-10 $3.507 E-10$

$4.863 E-10$ 4.71OE-10 4.3CCE-10

$7.696 E-10$ 6.819E-10 $5.93 \mathrm{CE}-10$

$3.320 E-09$

$3.220 E-09$

$2.956 \mathrm{E}-09$

$1.164 \mathrm{E}-10$

$1.206 \mathrm{E}-10$

$1.265 E-10$

$1.344 E-1 C$

$1.577 \mathrm{E}-10$

$1.717 \mathrm{E}-10$

$1.978 \mathrm{E}-10$

2. $222 \mathrm{E}-10$

$2.612 \mathrm{E}-1 \mathrm{C}$

$3.084 \mathrm{E}-10$

$3.811 E-10$

$5.017 E-10$

$2.610 E-09$

$8.481 E-11$

$8.594 E-11$

$9.133 \mathrm{E}-11$

$9.580 \mathrm{E}-11$

$1.017 \mathrm{E}-10$

$1.093 \mathrm{E}-10$

$1.190 \mathrm{E}-10$

$1.319 \mathrm{E}-10$

$1.466 E-10$

$1.669 \mathrm{E}-10$

$1.919 \mathrm{E}-10$

$2.256 \mathrm{E}-10$

$2.710 \mathrm{E}-10$

$6.059 \mathrm{E}-10$

RANGE (METERS)

$900.0 \quad 1200.0$

1500.0

1800.0

$1.896 E-09$

\section{9. $001 E-12$}

$9.153 \mathrm{E}-12$

$9.384 E-12$

$9.725 \mathrm{E}-12$

1.C $19 \mathrm{E}-1$

1.C $81 \mathrm{E}-11$

$1.256 E-11$

$1.377 E-11$

$1.525 \mathrm{E}-11$

$1.525 \mathrm{E}-11$

$1.707 \mathrm{E}-11$
$1.927 \mathrm{E}-11$

$2.192 \mathrm{E}-11$

$2.192 \mathrm{E}-11$

$2.892 \mathrm{E}-11$

$3.465 E-11$

$1.375 \mathrm{E}-1 \mathrm{C}$

$1.914 E-1 C$

$1.872 \mathrm{E}-10$
$1.772 E-12$

$1.777 \mathrm{E}-12$

. $8 C 2 E-12$

.847E-12

$2.006 \mathrm{E}-12$

$2.125 \mathrm{E}-12$

$2.465 \mathrm{E}-12$

$2.465 \mathrm{E}-12$
$2.698 \mathrm{E}-12$

$2.981 E-12$

$3.323 \mathrm{E}-12$

$3.730 \mathrm{E}-12$

$4.2(9 E-12$

$4.761 \mathrm{E}-1$

$5.375 \mathrm{E}-12$

$3.177 \mathrm{E}-13$

$3.187 \mathrm{E}-13$

$3.311 E-13$

$3.431 \mathrm{E}-13$

$3.595 E-13$

$3.807 E-13$

$4.077 E-13$

$4.41 E-13$

$5.318 \mathrm{E}-13$

$5.913 E-13$

$6.615 \mathrm{E}-13$

$7.427 \mathrm{E}-13$

$8.336 \mathrm{E}-13$

$9.296 E-13$

$1.021 \mathrm{E}-12$

$3.625 \mathrm{E}-11 \quad 6.435 \mathrm{E}-12$

$5.365 E-14$

$5.382 E-14$

$5.456 \mathrm{E}-14$

$5.794 E-14$

$6.069 \mathrm{E}-14$

$6.069 E-14$

$6.879 E-14$

$7.438 \mathrm{E}-14$

$8.123 \mathrm{E}-14$

$8.949 \mathrm{E}-14$

$9.933 E-14$

$1.109 \mathrm{E}-13$

$1.240 E-13$

$1.385 \mathrm{E}-13$

$1.532 \mathrm{E}-13$ 
COSINE

$-1 . C 0000 E C C$ $-9.89401 E-0$ $-9.44575 \mathrm{E}-01$ $-7.55044 E-0$ $-6.17876 \mathrm{E}-01$ $-4.58017 E-01$ $-2.81605 E-0$ $-9.50125 \mathrm{E}-\mathrm{C}$ $9.50125 \mathrm{E}-0$ $2.81605 \mathrm{E}-0$ $4.58017 \mathrm{E}-01$ $6.17876 \mathrm{E}-0$ $7.55044 E-01$ $8.65631 \mathrm{E}-0$ $9.44575 E-C$ $9.894 C 1 E-01$

TOTAL

COSINE

$-1.00000 E$ OC $-9.89401 \mathrm{E}-\mathrm{C}$ $-9.44575 E-01$ $-8.65631 \mathrm{E}-01$ $-7.55044 \mathrm{E}-01$ $-6.17876 E-01$ -4.58017E-C 2. $9.5 \mathrm{Cl} 5 \mathrm{E}-\mathrm{O}$ $2.81605 \mathrm{E}-\mathrm{O}$ $4.58017 E-0$ 6. $7.55044 \mathrm{E}-01$ $8.65631 E-01$

$9.65631 \mathrm{E}-01$

$9.89401 E-0$

TOTAL

$$
75.0
$$

$1.709 E-10$ $1.714 \mathrm{E}-10$ $1.732 \mathrm{E}-1 \mathrm{C}$ $1.768 \mathrm{E}-1 \mathrm{C}$ $1.824 \mathrm{E}-10$ $1.903 \mathrm{E}-10$ $2.011 E-10$ . $156 \mathrm{E}-10$ . $350 \mathrm{E}-10$ .202E-1C . $982 \mathrm{E}-1 \mathrm{C}$ $3.533 \mathrm{E}-1 \mathrm{C}$ $4.286 E-1 C$ $4.755 E-1 C$ $7.845 \mathrm{E}-10$ $2.122 E-C$ $6.413 \mathrm{E}-\mathrm{C} 9$

$5.532 \mathrm{E}-\mathrm{C} 9$

$$
500.0
$$

$1.875 \mathrm{E}-10$ $1.879 E-10$ $1.898 \mathrm{E}-10$ $1.935 \mathrm{E}-1 \mathrm{C}$ $1.98 \mathrm{E}-1 \mathrm{C}$ . . $269 \mathrm{E}-10$ . $588 \mathrm{E}-1 \mathrm{C}$ $801 \mathrm{E}-10$ . $3.375 \mathrm{E}-1 \mathrm{C}$ . $4.260 \mathrm{E}-10$ $4.954 E-10$ $6.933 \mathrm{E}-10$

$3.481 E-C 9$

$1 C 0.0$
$2.139 E-1 C$
$2.144 E-1 C$
$2.166 E-1 C$
$2.211 E-1 C$
$2.279 E-1 C$
$2.375 E-1 C$
$2.5 C 4 E-1 C$
$2.676 E-1 C$
$2.902 E-1 C$
$3.363 E-1 C$
$3.775 E-1 C$
$3.978 E-1 C$
$4.901 E-1 C$
$6.231 E-1 C$
$7.911 E-1 C$
$1.592 E-C 9$
$6.243 E-C 9$
$5.94 C E-C 9$

150.0

2CC.C

$2.773 \mathrm{E}-10$

$2.809 E-10$

$2.866 \mathrm{E}-10$

$2.951 E-13$

3. $070 E-10$

$3.229 \mathrm{E}-10$

$3.437 E-10$

$3.706 \mathrm{E}-1 \mathrm{C}$

$3.941 E-10$

$4.717 E-10$

$4.824 E-10$

$6.154 \mathrm{E}-10$

$7.030 \mathrm{E}-10$

$8.975 \mathrm{E}-10$

$1.364 E-C$

$4.760 E-09$

2GC.C

$250 . C$

$300 . \mathrm{C}$

400.0

$6.561 E-C 9$

$3.103 E-10$
$.111 E-10$

$3.216 E-10$
$.266 E-10 \quad 3.28 C E-10$

$3.3 C C E-10$

$3.429 E-10$

$.601 E-10$

.823E-10

$4.1 C 6 E-10$

$4.541 E-10$

$4.870 E-10$

$5.583 \mathrm{E}-10$

$6.279 \mathrm{E}-10$

$7.492 E-10$

$9.105 \mathrm{E}-10$

$1.257 \mathrm{E}-09$
$3.544 \mathrm{E}-09$

$6.769 E-09$

$3.374 \mathrm{E}-10$

3. $5 \mathrm{C} 4 \mathrm{E}-10$

.674E-10

$4.171 \mathrm{E}-10$

$4.171 E-10$
$4.605 E-10$

$4.893 \mathrm{E}-10$

$5.583 \mathrm{E}-1 \mathrm{C}$

$6.205 \mathrm{E}-10$

$7.30 \mathrm{CE}-10$

$8.670 E-10$

. $136 \mathrm{E}-09$

RANGE IMETERS

600.0

SOO.O

$12 \mathrm{CO} \mathrm{C}$

$3.062 \mathrm{E}-10$ $3.1 C 2 E-10$

$2.533 E-10$ $3.252 E-1 C \quad 2.681 E-10$

$3.375 \mathrm{E}-10 \quad 2.779 \mathrm{E}-10$

$3.535 \mathrm{E}-10 \quad 2.908 \mathrm{E}-10$

3.070E-10

.998E-10 3.271E-10

$4.292 E-10 \quad 3.523 E-10$

$4.743 \mathrm{E}-10 \quad 3.821 \mathrm{E}-1 \mathrm{C}$

$5.214 E-10$ 4.203E-10

$5.892 \mathrm{E}-10 \quad 4.667 \mathrm{E}-10$

$6.727 E-10$ 5.206E-10

$7.955 E-10 \quad 6.057 \mathrm{E}-10$

$6.112 E-C \theta \quad 4.822 E-09$

$1.296 E-1 C$

$1.299 \mathrm{E}-1 \mathrm{C}$

$1.312 E-1 C$

3.329E-11

1500.0

1800.0 6.927E-12

$1.279 \mathrm{E}-12$

2. $206 \mathrm{E}-13$ $2.211 E-13$ $2.233 E-13$ $2.332 E-13$ $2.332 E-13$ $\begin{array}{llll}.423 \mathrm{E}-1 \mathrm{C} & 3.646 \mathrm{E}-11 & 7.561 \mathrm{E}-12 & 1.399 \mathrm{E}-12\end{array}$ $\begin{array}{llll}1.485 E-10 & 3.802 \mathrm{E}-11 & 7.879 \mathrm{E}-12 & 1.457 \mathrm{E}-12\end{array}$ $1.564 \mathrm{E}-1 \mathrm{C}-3.996 \mathrm{E}-11 \mathrm{C} .273 \mathrm{E}-12 \quad 1.529 \mathrm{E}-12 \mathrm{2} 2.63 \mathrm{EE}-13$ $1.661 \mathrm{E}-1 \mathrm{C} \quad 4.231 \mathrm{E}-11 \quad 8.746 \mathrm{E}-12 \quad 1.615 \mathrm{E}-12 \quad 2.779 \mathrm{E}-13$ $1.919 \mathrm{E}-1 \mathrm{C} \quad 4.844 \mathrm{E}-11$ $9.965 \mathrm{E}-12 \quad 120.834 \mathrm{E}-12 \quad 32.9515 \mathrm{E}-13$ $2.98 \mathrm{E}-10 \quad 4.844 \mathrm{E}-11 \quad 9.965 \mathrm{E}-12 \quad 1.834 \mathrm{E}-12 \quad 3.150 \mathrm{E}-13$ $2.088 \mathrm{E}-1 \mathrm{C} \quad 5.233 \mathrm{E}-11 \quad 1.072 \mathrm{E}-11 \quad 1.969 \mathrm{E}-12 \quad 3.377 \mathrm{E}-13$ $2.534 \mathrm{E}-1 \mathrm{C} \quad 6.202 \mathrm{E}-11 \quad 1.155 \mathrm{E}-11 \quad 2.121 \mathrm{E}-12 \quad 3.630 \mathrm{E}-13$

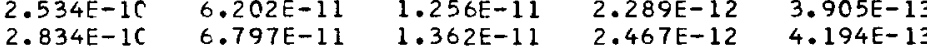
$\begin{array}{lllll}2.834 \mathrm{E}-1 \mathrm{C} & 6.797 \mathrm{E}-11 & 1.362 \mathrm{E}-11 & 2.467 \mathrm{E}-12 & 4.194 \mathrm{E}-13 \\ 3.23 \mathrm{C}-1 \mathrm{C} & 7.483 \mathrm{E}-11 & 1.475 \mathrm{E}-11 & 2.648 \mathrm{E}-12 & 4.475 \mathrm{E}-13\end{array}$ $\begin{array}{lllll}4.138 \mathrm{E}-1 \mathrm{C} & 8.468 \mathrm{E}-11 & 1.597 \mathrm{E}-11 & 2.848 \mathrm{E}-12 & 4.475 \mathrm{E}-13 \\ & & & & \end{array}$

. $468 E-11$ 1.597E-11 $2.812 E-1204.707 E-13$ 
4 PI R**2 TISSUE KERMA (NEUTRONS I
(CM**2 ERGS/GRAM/STERADIAN/SOURCE NEUTRON

COSINE

$-1.00000 E C C$
$-9.89401 E-01$
$-9.44575 E-01$
$-8.65631 E-01$
$-7.55044 E-01$
$-6.17876 E-01$
$-4.58017 E-C 1$
$-2.81605 E-01$
$-9.50125 E-02$
$9.50125 E-02$
$2.81605 E-01$
$4.58017 E-C 1$
$6.17876 E-01$
$7.55044 E-01$
$8.65631 E-C 1$
$9.44575 E-01$
$9.89401 E-01$

TOTAL

COSINE

$-1.0 C C O O E C C$ $-9.89401 \mathrm{E}-01$ $-9.44575 \mathrm{E}-\mathrm{Cl}$
$-8.65631 \mathrm{E}-01$ $-7.55044 \mathrm{E}-\mathrm{Cl}$ $-6.17876 \mathrm{E}-01$ $4.58017 \mathrm{E}-01$ $-2.81605 \mathrm{E}-01$ $-9.50125 \mathrm{E}-02$

$0.50125 \mathrm{E}-02$ $2.81605 E-01$ $4.58017 \mathrm{E}-\mathrm{C}$ $6.17876 \mathrm{E}-01$ $7.55044 \mathrm{E}-01$ $8.65631 \mathrm{E}-01$ $9.456375 \mathrm{E}-01$ $9.89401 \mathrm{E}-01$

TOTAL

$75 . C$
$1.016 E-08$
$1.019 E-C 8$
$1.031 E-08$
$1.055 E-C 8$
$1.092 E-C 8$
$1.144 E-08$
$1.216 E-08$
$1.312 E-C 8$
$1.440 E-C 8$
$2.05 C E-08$
$1.868 E-C 8$
$2.241 E-C 8$
$2.744 E-C 8$
$3.015 E-C 8$
$5.048 E-C 8$
$1.379 E-C 7$
$4.153 E-C 7$
$3.505 E-C 7$

$10 \mathrm{C} .0$

$1.223 E-08$ $1.226 \mathrm{E}-\mathrm{C} .8$ 1. $241 E-08$ 1. $270 \mathrm{E}-08$ $1.314 E-C 8$ $1.376 \mathrm{E}-08$ $1.46 C E-C 8$ $1.572 \mathrm{E}-\mathrm{C} .8$ $1.72 C E-C 8$ $1.991 \mathrm{E}-\mathrm{C} 8$ $2.312 E-C 8$ $2.414 \mathrm{E}-\mathrm{C} 8$ $3.05 C E-C 8$ $3.941 E-C 8$ $5.03 \mathrm{CE}-\mathrm{C} 8$ 1.C $21 E-C 7$ $3.659 E-C 7$

$500 . \mathrm{C}$

600.0

$7.605 \mathrm{E}-\mathrm{C} 9$ $7.628 E-c 9$ $7.725 \mathrm{E}-\mathrm{Cg}$ 8. $8.557 E-C 9$ $9.047 \mathrm{E}-\mathrm{CO}$ $1.074 \mathrm{E}-\mathrm{C}$ $1.046 E-C B$ $1.167 E-C 8$ $1.4107 E-C 8$ 1. $045 E-C B$ $2.155 E-C 8$ $2.594 E-C 8$ $3.839 \mathrm{E}-\mathrm{C} 8$

$1.590 E-C 7$

I. $(37 \mathrm{E}-\mathrm{C} 7$
150.0 RANGE (METERS)

$250 . C$

300.0

400.0

$1.482 E-08$ $\begin{array}{lll}1.486 E-C 8 & 1.57 C E-08 & 1.527 E-08\end{array}$ $\begin{array}{lll}1.5 C 4 E-C 8 & 1.589 \mathrm{E}-08 & 1.546 \mathrm{E}-08\end{array}$ $1.539 \mathrm{E}-68 \quad 1.626 \mathrm{E}-08 \quad 1.583 \mathrm{E}-08$ $1.593 \mathrm{E}-08 \quad 1.683 \mathrm{E}-08 \quad 1.638 \mathrm{E}-08$ $1.667 E-C 8 \quad 1.761 E-08 \quad 1.714 E-08$ $\begin{array}{lll}1.767 E-08 & 1.866 E-08 & 1.815 E-08\end{array}$ $1.899 \mathrm{E}-\mathrm{C} 8 \quad 2.003 \mathrm{E}-\mathrm{C} 8 \quad 1.946 \mathrm{E}-08$ $2.070 E-08 \quad 2.179 E-08 \quad 2.115 E-08$ $2.177 E-08 \quad 2.481 E-08 \quad 2.416 E-08$ $\begin{array}{lll}2.760 \mathrm{E}-08 & 2.639 \mathrm{E}-08 & 2.536 \mathrm{E}-08\end{array}$ $2.764 E-08 \quad 3.136 E-08 \quad 3.016 E-C 8$ $3.686 \mathrm{E}-\mathrm{C} 8 \quad 3.552 \mathrm{E}-08 \quad 3.375 \mathrm{E}-08$ $4.224 E-C 8 \quad 4.369 E-08 \quad 4.111 E-08$ $5.530 E-08 \quad 5.4 C 2 E-C 8 \quad 4.975 E-08$ 8.568E-C 8 T.678E-08 $6.737 E-08$

$3.840 E-0.7 \quad 3.782 E-07$

$3.516 E-07$

$1.406 \mathrm{E}-\mathrm{C} 8$ $1.410 \mathrm{E}-\mathrm{CB} \quad 1.081 \mathrm{E}-08$ $1.428 \mathrm{E}-\mathrm{C} 8 \quad 1.098 \mathrm{E}-08$ $1.462 \mathrm{E}-\mathrm{C} 8 \quad 1.125 \mathrm{E}-08$ $1.513 E-C 8 \quad 1.163 E-08$ $1.583 \mathrm{E}-08 \quad 1.217 \mathrm{E}-08$ $1.675 \mathrm{E}-\mathrm{C} 8 \quad 1.287 \mathrm{E}-08$ $1.795 E-C 8 \quad 1.378 E-08$ $1.947 E-C 8 \quad 1.492 E-08$ $2.112 \mathrm{E}-\mathrm{C} 8 \quad 1.640 \mathrm{E}-08$ $2.407 \mathrm{E}-08 \quad 1.812 \mathrm{E}-\mathrm{CB}$ $2.686 \mathrm{E}-08 \quad 2.044 \mathrm{E}-08$ $3.123 \mathrm{E}-\mathrm{C} 8 \quad 2.327 \mathrm{E}-08$ $3.647 \mathrm{E}-\mathrm{C} 8 \quad 2.703 \mathrm{E}-08$ $4.443 \mathrm{E}-\mathrm{C} 8 \quad 3.203 \mathrm{E}-08$ $5.745 E-C 8 \quad 3.972 E-08$

$3.139 E-07 \quad 2.320 E-07$

RANGE (METERS)

900.0

$120 \mathrm{C} . \mathrm{C}$

1500.0

1800.0 $\begin{array}{lllll}.062 \mathrm{E}-\mathrm{CO} & 1.202 \mathrm{E}-09 & 2.393 \mathrm{E}-1 \mathrm{C} & 4.32 \mathrm{CE}-11 & 7.330 \mathrm{E}-12\end{array}$ $\begin{array}{lllll}5.126 \mathrm{E}-\mathrm{Cg} & 1.218 \mathrm{E}-\mathrm{C} 9 & 2.424 \mathrm{E}-10 & 4.376 \mathrm{E}-11 & 7.423 \mathrm{E}-12\end{array}$ $5.429 E-C O \quad 1.247 E-C 9 E-2.481 E-10 \quad 4.478 E-11 \quad 7.597 E-12$ $5.429 E-C 9 \quad 1.289 E-C 92.565 E-10 \quad 4.63 C E-11$ $7.854 E-12$ $5.999 E-C O \quad 1.423 E-C 9$ 2. $5.911 .423 E-C 9$ 2.828E-10 5.1C2E-11 $8.650 E-12$ $6.411 E-C 9$ 1.518E-C $3.016 E-10$ $5.437 E-119.215 E-12$ $7.563 \mathrm{E} C \mathrm{CO} \quad 1.782 \mathrm{E}-\mathrm{CO} \quad 3.528 \mathrm{E}-10 \mathrm{O} 2.348 \mathrm{E}-11 \mathrm{l}$ $8.351 E-09 \quad 1.782 E-C O 2.528 E-10$ 6.348E-11 $1.074 E-11$

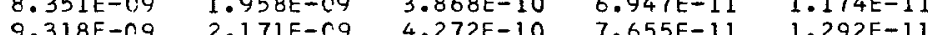

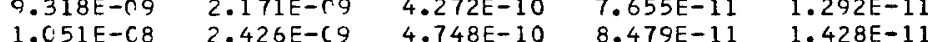

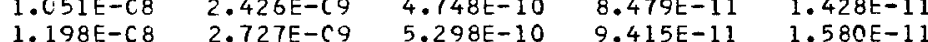
$1.198 E-C 8$ 2.727E-C9 $5.298 E-10 \quad 9.415 E-1101.580 E-11$

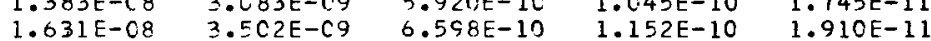
$2.200 E-C 8 \quad 4.113 E-09 \quad 7.346 E-10 \quad 1.251 E-10 \quad 2.049 E-11$

$$
\begin{array}{llll}
4.113 E-09 & 7.346 E-10 & 1.251 E-10 & 2.049 E-11 \\
2.391 E-98 & 4.686 E-09 & 8.381 E-1 C & 1.413 E-10
\end{array}
$$

$2.391 E-28 \quad 4.686 E-09$

$1.413 E-10$ 
COSINE

$-1.00000 \mathrm{E} O C$
$-9.894 \mathrm{C}-\mathrm{E}-\mathrm{C}$
$-9.44575 \mathrm{E}-01$
$-8.65631 \mathrm{E}-01$
$-7.55044 \mathrm{E}-01$
$-6.17876 \mathrm{E}-01$
$-4.58017 \mathrm{E}-01$
$-2.81605 \mathrm{E}-\mathrm{C} 1$
$-9.50125 \mathrm{E}-02$
$9.50125 \mathrm{E}-02$
$2.81605 \mathrm{E}-01$
$4.58017 \mathrm{E}-01$
$6.17876 \mathrm{E}-01$
$7.55044 \mathrm{E}-01$
$8.65631 \mathrm{E}-01$
$9.44575 \mathrm{E}-01$
$9.89401 \mathrm{E}-01$

TOTAL

COS INE

$-1.0 C O C O E$ DO $-9.89401 E-01$ $-9.44575 E-01$ $-8.65631 \mathrm{E}-01$ $-7.55044 \mathrm{E}-01$ $-6.17876 \mathrm{E}-01$ $-4.58017 E-01$ $-2.81605 \mathrm{E}-01$ $-9.50125 \mathrm{E}-02$ $9.50125 \mathrm{E}-02$ $2.81605 E-01$ 4.58017E-01 $6.17876 E-01$ $7.55044 E-01$ $8.65631 \mathrm{E}-01$ $9.44575 E-01$
$9.89401 E-01$

TOTAL

$\begin{array}{ll}75.0 & 10 C .0 \\ 2.161 \mathrm{E}-11 & 2.805 \mathrm{E}-11 \\ 2.166 \mathrm{E}-11 & 2.811 \mathrm{E}-11 \\ 2.187 \mathrm{E}-11 & 2.837 \mathrm{E}-11 \\ 2.228 \mathrm{E}-11 & 2.889 \mathrm{E}-11 \\ 2.291 \mathrm{E}-11 & 2.967 \mathrm{E}-11 \\ 2.379 \mathrm{E}-11 & 3.077 \mathrm{E}-11 \\ 2.498 \mathrm{E}-11 & 3.223 \mathrm{E}-11 \\ 2.656 \mathrm{E}-11 & 3.414 \mathrm{E}-11 \\ 2.864 \mathrm{E}-11 & 3.662 \mathrm{E}-11 \\ 3.835 \mathrm{E}-11 & 4.091 \mathrm{E}-11 \\ 3.560 \mathrm{E}-11 & 4.614 \mathrm{E}-11 \\ 4.135 \mathrm{E}-11 & 4.767 \mathrm{E}-11 \\ 4.893 \mathrm{E}-11 & 5.801 \mathrm{E}-11 \\ 5.274 \mathrm{E}-11 & 7.149 \mathrm{E}-11 \\ 8.255 \mathrm{E}-11 & 8.749 \mathrm{E}-11 \\ 2.083 \mathrm{E}-10 & 1.616 \mathrm{E}-10 \\ 6.022 \mathrm{E}-10 & 5.891 \mathrm{E}-1 \mathrm{C} \\ & \\ 6.083 \mathrm{E}-10 & 6.812 \mathrm{E}-1 \mathrm{C}\end{array}$

\begin{tabular}{|c|c|c|c|c|}
\hline 150.0 & $\begin{array}{l}\text { IGE (METERS) } \\
2 C 0.0\end{array}$ & 250.0 & 300.0 & 400.0 \\
\hline $\begin{array}{l}838 E-11 \\
847 E-11\end{array}$ & $\begin{array}{l}4.466 \mathrm{E}-11 \\
4.476 \mathrm{E}-11\end{array}$ & $\begin{array}{l}4.711 \mathrm{E}-11 \\
4.722 \mathrm{E}-11\end{array}$ & $\begin{array}{l}4.654 E-11 \\
4.664 E-11\end{array}$ & $\begin{array}{l}3.979 \mathrm{E}-11 \\
3.988 \mathrm{E}-11\end{array}$ \\
\hline $82 E-11$ & $4.517 \mathrm{E}-11$ & $4.765 E-11$ & $4.707 E-11$ & $4.024 E-11$ \\
\hline & $4.596 \mathrm{E}-11$ & $4.847 \mathrm{E}-11$ & $4.788 \mathrm{E}-11$ & $4.092 \mathrm{E}-11$ \\
\hline $54 E-11$ & $4.713 \mathrm{E}-11$ & $4.968 E-11$ & $4.906 E-11$ & $4.192 \mathrm{E}-11$ \\
\hline $96 \mathrm{E}-11$ & $4.873 E-11$ & $5.133 E-11$ & $5.066 E-11$ & $4.326 \mathrm{E}-11$ \\
\hline $83 E-11$ & $5.082 E-11$ & $5.347 \mathrm{E}-11$ & $5.273 E-11$ & $4.497 \mathrm{E}-11$ \\
\hline $23 E-11$ & $5.347 \mathrm{E}-11$ & $5.617 \mathrm{E}-11$ & $5.532 \mathrm{E}-11$ & $4.711 \mathrm{E}-11$ \\
\hline $29 \mathrm{E}-11$ & $5.681 E-11$ & $5.953 E-11$ & $5.851 E-11$ & $4.971 \mathrm{E}-11$ \\
\hline $166 \mathrm{E}-11$ & $6.19 \mathrm{CE}-11$ & $6.470 \mathrm{E}-11$ & $6.213 E-11$ & $5.286 \mathrm{E}-11$ \\
\hline-11 & $6.54 \mathrm{CE}-11$ & $6.789 \mathrm{E}-11$ & $6.741 \mathrm{E}-11$ & $5.657 \mathrm{E}-11$ \\
\hline & $7.354 E-11$ & $7.59 C E-11$ & $7.283 \mathrm{E}-11$ & $6.109 E-11$ \\
\hline $613 E-11$ & $8.068 \mathrm{E}-11$ & $8.241 \mathrm{E}-11$ & $8.038 \mathrm{E}-11$ & $6.643 \mathrm{E}-11$ \\
\hline $403 \mathrm{~F}-11$ & $9.361 E-11$ & $9.425 E-11$ & $8.928 E-11$ & $7.299 E-11$ \\
\hline & $1.095 \mathrm{E}-10$ & $1.079 E-10$ & 1. C I GE- 10 & $8.124 \mathrm{E}-11$ \\
\hline $85 E-10$ & $1.432 \mathrm{E}-10$ & $1.341 \mathrm{E}-10$ & $1.214 E-10$ & $9.303 \mathrm{E}-11$ \\
\hline & $3.486 \mathrm{E}-10$ & $2.688 E-10$ & $2.107 \mathrm{E}-1 \mathrm{C}$ & $1.322 \mathrm{E}-10$ \\
\hline $67 E-10$ & $8.754 E-10$ & $8.846 E-10$ & $8.487 E-10$ & $7.014 \mathrm{E}-10$ \\
\hline
\end{tabular}

RANGE (METERS)

500.0

$3.025 \mathrm{E}-11$

$3.032 \mathrm{E}-11$

$3.059 E-11$

$3.111 \mathrm{E}-11$

$3.186 \mathrm{E}-11$

$3.287 \mathrm{E}-11$

$3.415 \mathrm{E}-11$
$3.573 \mathrm{E}-11$

$3.765 \mathrm{E}-11$

$3.994 \mathrm{E}-11$

$4.263 \mathrm{E}-11$

$4.580 E-11$

$4.951 E-11$

$5.388 E-11$

$6.604 E-11$

$8.374 \mathrm{E}-11$

$5.230 E-10$
600.0

2.129E-11

$2.133 \mathrm{E}-11$

$2.153 E-11$

$2.241 \mathrm{E}-11$

$2.311 \mathrm{E}-11$

$2.400 E-11$

$2.510 \mathrm{E}-11$

$2.642 E-11$

$2.980 \mathrm{E}-11$

$3.192 \mathrm{E}-11$

$3.436 \mathrm{E}-11$

$3.715 \mathrm{E}-11$

$4.438 E-11$

5.258E-11

$3.635 E-10$
$1200 . \mathrm{C}$

1500.0

$5.636 \mathrm{E}-12$

$5.648 E-12$

$5.699 \mathrm{E}-12$

$5.794 \mathrm{E}-12$

$5.931 \mathrm{E}-12$

$6.114 E-12$

$6.344 \mathrm{E}-12$

$6.626 \mathrm{E}-12$

$6.960 \mathrm{E}-12$

$.352 E-12$

.802E-12

$8.311 E-12$

.880E-12

$9.503 E-12$

$1.018 \mathrm{E}-11$

$1.182 \mathrm{E}-11$

$9.436 E-11$
$1.188 \mathrm{E}-12$

$1.191 \mathrm{E}-12$

$1.221 \mathrm{E}-12$

$1.250 \mathrm{E}-12$

$1.288 \mathrm{E}-12$

$1.336 E-12$

$1.394 \mathrm{E}-12$

$1.463 \mathrm{E}-12$

$1.544 \mathrm{E}-12$

$1.635 \mathrm{E}-12$

$1.737 E-12$

$1.96 \mathrm{E}-12$

$1.968 \mathrm{E}-12$

$2.036 E-12$

2. $336 \mathrm{E}-12$

$1.971 \mathrm{E}-11$
$2.219 E-13$

$2.224 \mathrm{E}-13$

$2.244 \mathrm{E}-13$

$2.281 \mathrm{E}-13$

$2.334 E-13$

$2.4 C 5 E-13$

$2.493 \mathrm{E}-13$

$2.601 E-13$

$2.728 \mathrm{E}-13$

.

.

.

.

.

$052 E-13$

$3.661 E-12$
$.848 E-14$

. $890 E-14$

$3.954 E-14$

.046E-14

$4.168 E-14$

$4.321 E-14$

4.

$4.724 E-14$

..976E-14

.

$5.573 E-14$

.

$6.260 E-14$

$6.930 \mathrm{E}-14$

$.177 \mathrm{E}-14$

$6.324 E-13$
1800.0 


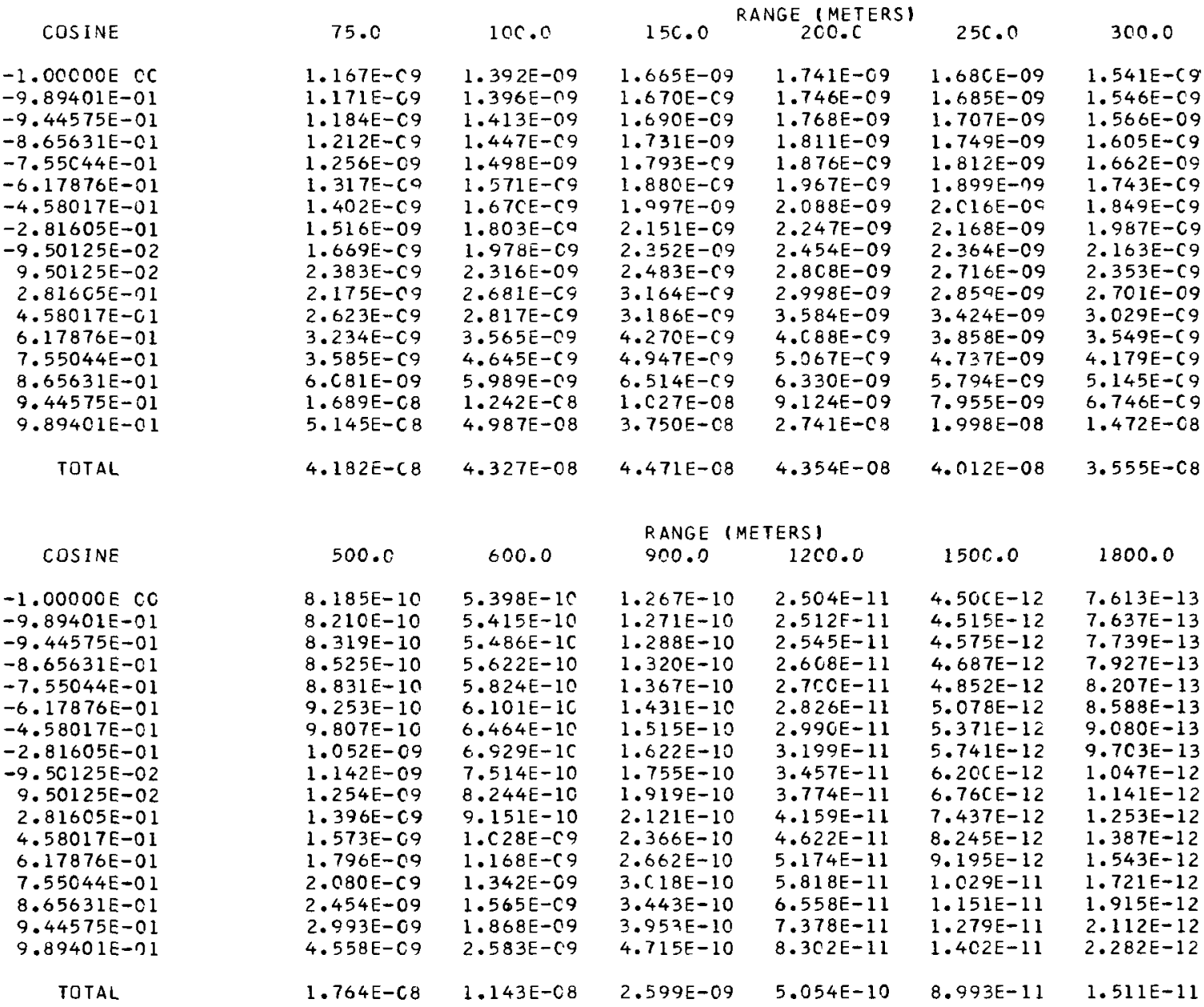


COSINE

$-1.00000 \mathrm{E} O C$
$-9.89401 \mathrm{E}-01$
$-9.44575 \mathrm{E}-01$
$-8.65631 \mathrm{E}-01$
$-7.55044 \mathrm{E}-01$
$-6.17876 \mathrm{E}-01$
$-4.58017 \mathrm{E}-01$
$-2.81605 \mathrm{E}-01$
$-9.50125 \mathrm{E}-02$
$9.50125 \mathrm{E}-02$
$2.81605 \mathrm{E}-01$
$4.58017 \mathrm{E}-01$
$6.17876 \mathrm{E}-01$
$7.55644 \mathrm{E}-01$
$8.65631 \mathrm{E}-01$
$9.44575 \mathrm{E}-01$
$9.89401 \mathrm{E}-01$

TOTAL

COSINE

-1.00000 E 00 $-9.89401 \mathrm{E}-01$ $-9.44575 E-01$ $-8.65631 E-01$ $-6.17876 \mathrm{E}-01$ $-6.17876 E-01$ $-2.81605 \mathrm{E}-01$ $-9.50125 \mathrm{E}-02$

9.50125E-02 $2.81605 \mathrm{E}-01$ $4.58017 E-01$ $6.17876 \mathrm{E}-01$

$7.55044 E-01$

$8.65631 E-01$

$8.65631 \mathrm{E}-01$

$9.89401 E-01$

TOTAL
75. C

100.6

$8.756 E-10$
$8.779 E-10$

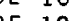
$8.872 \mathrm{E}-10$

$9.346 \mathrm{E}-1 \mathrm{C}$

$9.756 \mathrm{E}-1 \mathrm{C}$

1. $531 E-09$

$1.106 E-C 9$

$1.206 \mathrm{E}-\mathrm{C} 9$

$1.679 E-C 9$

$1.539 E-C 9$

$1.829 E-C 9$

$2.222 \mathrm{E}-09$

$2.435 E-C 9$

$4.021 E-C 9$

$1.085 E-C 8$

$3.257 \mathrm{E}-08$

2. $84 \mathrm{CE}-\mathrm{C} 8$

500.0

$1.158 \mathrm{E}-\mathrm{C} 9$ $1.161 \mathrm{E}-\mathrm{C} 9$

$1.171 E-09$

$1.191 E-09$

$1.220 \mathrm{CO}$

1.259E-C9

1. $373 \mathrm{E} C \mathrm{C}$

$1.450 \mathrm{E}-09$

$1.543 E-C 9$

$1.556 E-09$

$1.75 \mathrm{E}-09$

$1.957 \mathrm{E}-\mathrm{C} 9$

$2.158 \mathrm{E}-09$

$2.413 \mathrm{E}-09$

$2.766 \mathrm{E}-\mathrm{CO}$

$3.747 E-C 9$

$2.053 E-C 8$

600.0
RANGE 2 (METERS)

1.02
$1.10 C E-C 2$

$1.112 E-C 9$

$.134 E-C 9$

.169E-C9

. $218 E-C 9$

. $373 \mathrm{E}-09$

$.488 E-C 9$

. 7 CCE-C9

$1.951 \mathrm{E}-\mathrm{C}$ ?

$2.032 \mathrm{E}-09$

$2.525 \mathrm{E}-\mathrm{C} 9$

$3.210 \mathrm{E}-09$

4.C C $1 E-C 9$

$8.118 E-C 9$

$3.166 \mathrm{E}-\mathrm{C} 8$

3. C4CE-C 8

$8.283 E-10$ $8.301 E-1 C$ $8.515 \mathrm{E}-1 \mathrm{C}$ $8.719 E-1 C$ 8. 9.343E-1C $9.778 \mathrm{E}-1 \mathrm{C}$ $1.031 \mathrm{E}-\mathrm{C} 9$ $1.261 E-0.9$ $1.368 \mathrm{E}-\mathrm{CO}$ $1.495 \mathrm{E}-\mathrm{C} Q$ $1.651 \mathrm{E}-\mathrm{C} 9$ $1.852 \mathrm{E}-\mathrm{C} 9$ $2.303 E-C 9$

$1.439 \mathrm{E}-\mathrm{C} 8$ $2.270 E-10$ 4.855E-11 $2.27 C E-10$
$2.290 E-10$ $2.290 E-10$ $2.327 E-10$ $2.381 E-10$ $2.454 E-10$ $2.545 E-10$ $2.657 E-10$ $2.792 E-10$ $3.138 E-10$ $3.353 E-10$ $3.600 \mathrm{E}-10$ $3.879 \mathrm{E}-10$ $4.193 E-1 C$ $4.549 \mathrm{E}-10$ $5.043 \mathrm{E}-10$ $4.865 E-11$ $4.9 C 8 E-11$ 5.9BE-11 9. $15 C E-12$ $9.249 E-12 \quad 1.612 E-12$ $9.395 \mathrm{E}-12 \quad 1.638 \mathrm{E}-12$ $9.607 E-12 \quad 1.675 E-12$ $5.252 E-11$ S. $890 E-12$ 1.723E-12 $5.442 E-111.024 E-11 \quad 1.784 E-12$ $5.955 \mathrm{E}-11 \mathrm{1} 11 \mathrm{CE}-11 \quad 1.947 \mathrm{E}-12$ $6.282 E-11$ 1. $179 E-1122.950 E-12$ $6.282 E-11$ 1. $248 E-11 \quad 2.167 E-12$ $6.659-11$ 1.248E-11 2.167E-12 $\begin{array}{lll}7.089 E-11 & 1.325 E-11 & 2.298 E-12 \\ 7.571 E-11 & 1.411 E-11 & 2.442 E-12\end{array}$ $8.1 C 1 E-11 \quad 1.504 E-1122.596 E-12$ $8.672 \mathrm{E}-11 \quad 1.601 \mathrm{E}-1122.754 \mathrm{E}-12$ $9.265 \mathrm{E}-11 \quad 1.697 \mathrm{E}-11 \quad 2.964 \mathrm{E}-12$ $9.888 \mathrm{E}-11 \quad 1.782 \mathrm{E}-11 \quad 3.026 \mathrm{E}-12$

$8.065 E-10 \quad 1.508 E-10 \quad 2.616 E-11$
$25 C \cdot C$

$300 \cdot \mathrm{C}$

400.0

$15 C .0$

CUC.C $1.737 E-09$ $1.741 \mathrm{E}-09$ $1.758 E-09$ $1.791 \mathrm{E}-09$ $1.839 E-C 9$ $1.905 E-09$ $1.992 \mathrm{E}-09$ 2.103E-09 2.244E-09 $2.492 \mathrm{E}-09$ $2.592 \mathrm{E}-09$ 2. $98 \mathrm{CE}-09$ $3.268 \mathrm{E}-09$ 3.851E-09 $4.532 \mathrm{E}-09$ $5.913 E-09$ 1. $342 \mathrm{E}-08$

$3.495 E-08$

$1.722 \mathrm{E}-\mathrm{C} 9$ $1.726 \mathrm{E}-\mathrm{CS} \quad 1.499 \mathrm{E}-09$ $1.742 \mathrm{E}-\mathrm{C} 9 \mathrm{1.513 \textrm {E } - 0 9}$ $1.774 \mathrm{E}-\mathrm{C} 9 \quad 1.539 \mathrm{E}-09$ $1.820 \mathrm{E}-69 \quad 1.578 \mathrm{E}-\mathrm{C9}$ $1.883 \mathrm{E}-\mathrm{C} 9 \quad 1.630 \mathrm{E}-09$ $1.966 \mathrm{E}-\mathrm{C9} \quad 1.698 \mathrm{E}-09$ $2.071 \mathrm{E}-\mathrm{CS} \quad 1.783 \mathrm{E}-\mathrm{C} 9$ $2.203 E-C 9 \quad 1.888 E-09$ $2.344 \mathrm{E}-\mathrm{C} 9 \quad 2.022 \mathrm{E}-09$ $2.590 E-C 9 \quad 2.173 E-C 9$ $2.821 E-C 9 \quad 2.373 E-09$ $3.176 \mathrm{E}-\mathrm{C} 9 \quad 2.611 \mathrm{E}-09$ $3.596 \mathrm{E}-\mathrm{C9} \quad 2.920 \mathrm{E}-09$ $4.227 E-C 9 \quad 3.323 E-C 9$ $5.251 E-C 9 \quad 3.934 E-09$ $1.023 \mathrm{E}-\mathrm{C} 8 \quad 6.118 \mathrm{E}-09$ $3.321 E-C 8 \quad 2.736 E-08$

RANGE (METERS) SOO.O

1200.0

$150 \mathrm{C} 0$

$1800 . \mathrm{C}$ 
4 PI R**2 IONIZING SILICON KERMA (NEUTRONS)
(CM\#\#2 ERGSS/GRAM/STERADIAN/SOURCE NEUTRONI)

COS INE

$-1.00000 E$ CO $-9.89401 E-01$ $-9.44575 E-01$ $-8.65631 \mathrm{E}-01$ $-7.55044 \mathrm{E}-01$ $-6.17876 \mathrm{E}-01$ $-4.58017 \mathrm{E}-01$ $-2.816 C 5 E-01$ $9.50125 E-02$ $2.81605 E-01$ 4.58C17E-01 $6.17876 E-01$ $7.55 C 44 E-01$ $8.65631 \mathrm{E}-\mathrm{Cl}$ $9.894 \mathrm{ClE}-\mathrm{Cl}$

TOTAL

COS INE

-1. COOOOE CO $-9.894 \mathrm{C} 1 \mathrm{E}-01$ $-8.65631 \mathrm{E}-01$ $-7.55044 \mathrm{E}-\mathrm{Cl}$ $-6.17876 \mathrm{E}-\mathrm{Cl}$ $-4.58017 E-C 1$ $-2.816 C 5 E-C 1$ $-9.50125 E-C 2$ $9.50125 \mathrm{E}-02$ $2.81605 E-01$ 4.58017E-01 $6.17876 E-01$ $7.55044 E-C 1$ $8.65631 \mathrm{E}-01$ $9.44575 E-01$

TOTAL

$\begin{array}{lc}75.0 & 1 C C . C \\ 8.660 E-11 & 1 . C 12 E-1 C \\ 8.687 E-11 & 1 . C 15 E-1 C \\ 8.796 E-11 & 1 . C 28 E-10 \\ 9.017 E-11 & 1.054 E-1 C \\ 9.357 E-11 & 1 . C 94 E-1 C \\ 9.842 E-11 & 1.15 C E-1 C \\ 1.051 E-10 & 1.227 E-10 \\ 1.140 E-10 & 1.329 E-10 \\ 1.259 E-1 C & 1.465 E-1 C \\ 1.904 E-10 & 1.648 E-1 C \\ 1.67 C E-10 & 2 . C 46 E-1 C \\ 2.030 E-1 C & 2 . C 82 E-1 C \\ 2.510 E-10 & 2.7 C 9 E-10 \\ 2.694 E-10 & 3.56 C E-1 C \\ 4.568 E-10 & 4.54 C E-10 \\ 1.257 E-C 9 & 9.167 E-1 C \\ 3.750 E-C 9 & 3.62 C E-C 9 \\ 3.147 E-C 9 & 3.205 E-C 9\end{array}$

$500 . \mathrm{C}$

$5 . C 13 E-11$ $5.030 \mathrm{E}-11$ $5.104 \mathrm{E}-11$ $5.247 E-11$ $5.458 E-11$ $5.751 \mathrm{E}-1$ $6.138 \mathrm{E}-11$ $6.639 \mathrm{E}-11$ $8.073 \mathrm{E}-11$ $9.111 \mathrm{E}-1$ $1.039 \mathrm{E}-10$ $1.202 E-10$ $1.410 E-10$ $1.683 \mathrm{E}-10$ $2 . \mathrm{C} 695-1 \mathrm{C}$
$3.135 \mathrm{E}-10$

1. $154 \mathrm{E}-\mathrm{C} 9$

600.0

$3.233 E-11$

$3.244 \mathrm{E}-11$

$3.385 E-11$

$3.522 \mathrm{E}-11$

$3.711 \mathrm{E}-11$

$3.962 \mathrm{E}-11$
$4.284 \mathrm{E}-1$

$4.695 \mathrm{E}-11$

$5.212 \mathrm{E}-11$

$5.858 E-11$
$6.670 E-11$

$7.683 \mathrm{E}-1$

8. $.55 \mathrm{E}-11$

$1.273 E-1 C$

$1.759 E-10$

$7.338 \mathrm{E}-10$

$\begin{array}{lcc} & \text { RANGE (METERS) } \\ 150.0 & 200.0 & 256.0 \\ 1.169 \mathrm{E}-10 & 1.188 \mathrm{E}-10 & 1.12 C \mathrm{E}-1 \mathrm{C} \\ 1.173 \mathrm{E}-10 & 1.192 \mathrm{E}-10 & 1.123 \mathrm{E}-10 \\ 1.188 \mathrm{E}-10 & 1.2 \mathrm{C} 9 \mathrm{E}-10 & 1.139 \mathrm{E}-1 \mathrm{C} \\ 1.219 \mathrm{E}-10 & 1.241 \mathrm{E}-10 & 1.17 \mathrm{CE}-10 \\ 1.266 \mathrm{E}-10 & 1.289 \mathrm{E}-10 & 1.216 \mathrm{E}-10 \\ 1.332 \mathrm{E}-10 & 1.357 \mathrm{E}-10 & 1.280 \mathrm{E}-10 \\ 1.42 C \mathrm{CE}-10 & 1.447 \mathrm{E}-10 & 1.366 \mathrm{E}-10 \\ 1.537 \mathrm{E}-10 & 1.566 \mathrm{E}-10 & 1.478 \mathrm{E}-10 \\ 1.690 \mathrm{E}-10 & 1.722 \mathrm{E}-10 & 1.624 \mathrm{E}-10 \\ 1.685 \mathrm{E}-10 & 2.056 \mathrm{E}-10 & 1.964 \mathrm{E}-10 \\ 2.388 \mathrm{E}-10 & 2.080 \mathrm{E}-10 & 1.934 \mathrm{E}-10 \\ 2.269 \mathrm{E}-10 & 2.616 \mathrm{E}-10 & 2.462 \mathrm{E}-1 \mathrm{C} \\ 3.198 \mathrm{E}-10 & 2.92 \mathrm{CE}-10 & 2.702 \mathrm{E}-10 \\ 3.640 \mathrm{E}-10 & 3.725 \mathrm{E}-10 & 3.434 \mathrm{E}-10 \\ 4.880 \mathrm{E}-10 & 4.624 \mathrm{E}-10 & 4.167 \mathrm{E}-10 \\ 7.640 \mathrm{E}-10 & 6.729 \mathrm{E}-10 & 5.797 \mathrm{E}-10 \\ 2.687 \mathrm{E}-\mathrm{C} 9 & 1.95 \mathrm{CE}-09 & 1.412 \mathrm{E}-09 \\ 3.242 \mathrm{E}-09 & 3.109 \mathrm{E}-09 & 2.814 \mathrm{E}-\mathrm{C} 9 \\ & & \end{array}$

RANGE (METERS)

$$
900.0
$$

7.245E-12

$7.271 \mathrm{E}-12$

$7.591 \mathrm{E}-12$

$7.900 \mathrm{E}-12$

$8.329 \mathrm{E}-12$

$8.890 \mathrm{E}-12$

$9.612 E-12$

$1.166 \mathrm{E}-11$

$1.307 E-11$

$1.480 E-11$

$1.692 \mathrm{E}-11$

$2.252 E-11$

$2.613 E-11$
$3.132 E-11$

$1.605 E-10$
1200.0

$15 C C .0$

$2.458 \mathrm{E}-13$ $2.467 \mathrm{E}-13$

$2.505 E-13$

$2.576 E-13$

$2.828 \mathrm{E}-13$

$3.018 \mathrm{E}-13$

$3.263 E-13$

$3.569 \mathrm{E}-13$

$3.948 \mathrm{E}-13$

$4.412 E-13$

$4.973 E-13$
$5.641 E-13$

$6.415 E-13$

$7.281 \mathrm{E}-13$

$8.186 E-13$
$9.031 E-13$

$9.031 E-13$
300.0

400.0

$1.005 E-1 C \quad 7.387 E-11$

1. $009 \mathrm{E}-10 \quad 7.412 \mathrm{E}-11$

$1.023 E-10 \quad 7.520 E-11$

$1.051 E-10$ 7.728E-11

$1.092 E-10 \quad 8.037 E-11$

$1.15 C E-10$ 0.466E-11

$1.227 E-10$ 9.035E-11

$1.328 E-10$ 9.774E-11

$1.457 E-10$ 1.072E-10

$1.571 E-10$ 1.203E- 10

$1.873 E-10$ 1.339E-10

$2.086 E-10$ 1.545E-10

$2.499 E-10$ 1.787E-1C

$2.949 E-10$ 2.123E-10

$3.682 E-10$ 2.565E- 10

1.841E-1C $3.251 E-10$

$2.445 E-C 9 \quad 1.739 E-09$

1800.0 
COS INE

$$
\begin{aligned}
& -1.00000 E 00 \\
& -9.89401 \mathrm{E}-01 \\
& -9.44575 \mathrm{E}-01 \\
& -8.65631 \mathrm{E}-01 \\
& -7.55044 \mathrm{E}-01 \\
& -6.17876 \mathrm{E}-01 \\
& -4.58017 \mathrm{E}-01 \\
& -2.81605 \mathrm{E}-01 \\
& -9.50125 \mathrm{E}-02 \\
& 9.50125 \mathrm{E}-02 \\
& 2.81605 \mathrm{E}-01 \\
& 4.58017 \mathrm{E}-01 \\
& 6.17876 \mathrm{E}-01 \\
& 7.55044 \mathrm{E}-01 \\
& 8.65631 \mathrm{E}-01 \\
& 9.44575 \mathrm{E}-01
\end{aligned}
$$
$9.89401 E-01$

TOTAL

COSINE

$-1.00000 \mathrm{E} D 0$ $-9.89401 E-0$ $-9.44575 E-0$ $-8.65631 \mathrm{E}-0$ $-7.55044 E-0$ $-6.17876 E-0$ $-4.58017 \mathrm{E}-\mathrm{Cl}$ $-2.81605 E-0$ $-9.50125 \mathrm{E}-0$ $9.50125 \mathrm{E}-0$ $2.81605 E-0$ $6.17876 \mathrm{E}-0$ $7.55044 E-J 1$ $8.65631 \mathrm{E}-0$ $9.44575 E-0$ $9.89401 E-01$

TOTAL

\section{0} . $325 \mathrm{E}-10$ $1.329 \mathrm{E}-10$ 1. $380 \mathrm{E}-10$ $1.432 \mathrm{E}-10$ $1.507 E-10$ $1.610 \mathrm{E}-10$ $1.750 \mathrm{E}-10$ $1.936 \mathrm{E}-10$ $2.877 E-10$ 2. $566 \mathrm{E}-10$ $3.129 \mathrm{E}-10$ $3.895 \mathrm{E}-10$ 4. $276 \mathrm{E}-10$ $7.361 \mathrm{E}-10$ $2.072 \mathrm{E}-09$ $6.310 E-09$

$5.011 E-C 9$

500.0

. $511 \mathrm{E}-1$ $7.538 \mathrm{E}-11$ $7.650 E-1$ $7.867 \mathrm{E}-1$

.188E-11 $8.637 \mathrm{E}-1$ $.230 E-11$ $1.000 \mathrm{E}-10$ $1.099 \mathrm{E}-10$ . $223 E-10$ 1. $384 \mathrm{E}-1 \mathrm{C}$ $1.585 \mathrm{E}-10$ $1.845 E-10$ $2.179 E-10$ $2.626 \mathrm{E}-10$ $5.151 \mathrm{E}-10$

$1.769 E-C 9$
100.0 $1.547 \mathrm{E}-1 \mathrm{C}$ $1.567 \mathrm{E}-10$ $1.607 \mathrm{E}-10$ $1.756 \mathrm{E}-10$ $1.875 \mathrm{E}-1 \mathrm{C}$ $2.035 E-1 C$ $2.247 \mathrm{E}-1 \mathrm{C}$ $2.602 E-1 C$ $3.135 E-10$ $3.255 E-10$ $4.197 \mathrm{E}-1 \mathrm{C}$ $5.551 \mathrm{E}-1 \mathrm{C}$ $7.192 \mathrm{E}-10$ $1.505 \mathrm{E}-09$ $6.098 E-C 9$

$5 . C 83 E-C 9$

600.0

$4.835 E-11$ $4.853 E-11$ $4.926 E-11$ $.275 E-11$ $5.564 E-11$ $5.947 \mathrm{E}-1$ $6.443 E-1$ 7. $C 76 \mathrm{E}-11$ $7.878 \mathrm{E}-1$ $8.887 E-11$ $1.016 E-10$ $1.177 \mathrm{E}-10$ $1.381 E-10$ $1.646 \mathrm{E}-1 \mathrm{C}$ $2.008 \mathrm{E}-10$ $2.864 \mathrm{E}-1 \mathrm{C}$

$1.121 \mathrm{E}-09$
150.0 RANGE (METERS)
$2 \mathrm{CO} O \mathrm{C}$

$25 C . C$

300.0

$400 . c$

$1.774 \mathrm{E}-10$

$1.780 \mathrm{E}-10$

$1.851 \mathrm{E}-10$

$1.923 \mathrm{E}-10$

$2.024 \mathrm{E}-10$

$2.161 \mathrm{E}-10$

$2.343 \mathrm{E}-10$

$2.582 E-10$

$2.643 E-10$

$3.622 E-10$

$3.543 E-10$

$4.929 E-10$

$5.723 \mathrm{E}-10$

$7.672 E-10$

$1.229 \mathrm{E}-09$
$4.543 \mathrm{E}-09$

$1.798 \mathrm{E}-10$

$1.689 \mathrm{E}-10$

$1.695 \mathrm{E}-10$

$1.719 \mathrm{E}-10$

$1.766 \mathrm{E}-10$

$1.766 \mathrm{E}-10$
$1.935 \mathrm{E}-1 \mathrm{C}$

$1.935 \mathrm{E}-10$
$2.067 \mathrm{E}-10$

$2.241 \mathrm{E}-10$

$2.467 E-1 C$

$2.953 E-1 C$

2. $992 \mathrm{E}-1 \mathrm{O}$

$3.756 \mathrm{E}-10$

4. $2 \mathrm{C} 1 \mathrm{E}-1 \mathrm{C}$

$5.324 \mathrm{E}-1 \mathrm{C}$

$6.566 \mathrm{E}-10$

$9.241 E-10$

$2.378 E-09$

4. $363 \mathrm{E}-09$

1. $514 E-10$

$1.519 \mathrm{E}-10$

$1.541 \mathrm{E}-10$

$1.584 E-10$

$1.647 E-10$
$1.736 E-10$

$1.736 E-10$

$2.010 E-10$

$2.211 E-10$

$2.402 \mathrm{~F}-10$

$2.851 E-10$

$3.207 \mathrm{E}-10$

3.849E-10

$5.773 \mathrm{E}-1 \mathrm{C}$
$7.714 \mathrm{E}-10$

$7.714 \mathrm{E}-10$

$1.735 E-C 9$

$109 \mathrm{E}-10$

$1.113 \mathrm{E}-10$

$1.161 \mathrm{E}-10$

$1.208 \mathrm{E}-10$

$1.274 E-10$

$1.362 \mathrm{E}-10$

$1.476 \mathrm{E}-10$

$1.622 \mathrm{E}-10$

$2.040 E-10$

$2.363 \mathrm{E}-10$

$2.751 E-10$

$3.289 E-10$

$4.014 E-10$

$5.160 E-10$
$9.362 E-10$

5.C $91 E-09$

4.843E-09

$900.0 \quad 1200 . \mathrm{C}$
$150 C .0$

$1.080 \mathrm{E}-11$

$1.084 E-11$

$1.132 E-11$

$1.244 \mathrm{E}-11$

$1.330 \mathrm{E}-11$

$1.44 \mathrm{CE}-11$

$1.580 E-11$

$1.756 \mathrm{E}-11$

$2.246 \mathrm{E}-11$

$2.581 \mathrm{E}-1$

$2.989 E-11$

$3.485 E-11$

4. $C 88 E-11$

4. $994 \mathrm{~F}-1$

$2.070 \mathrm{E}-12$

$2.077 E-12$

$3.648 E-13$

$3.662 E-13$

3. $827 E-13$

$2.170 E-12$

$2.261 E-12$

$3.986 \mathrm{E}-13$

4.207E-13

$.550 \mathrm{E}-1$

$2.762 \mathrm{E}-12$

$3.028 \mathrm{E}-1$

$3.361 \mathrm{E}-12$

$4.868 \mathrm{E}-13$

$5.336 E-13$

$5.918 E-13$

$6.635 E-13$

$7.509 E-13$

$4.279 E-12$

$4.893 E-12$

$5.625 E-12$

I. $119 E-12$

$1.16 E-12$

$1.412 \mathrm{E}-12$

$E-12$

8. $057 E-12$
$3.782 E-C 9$

1800.0

2.674E-09

. $389 E-14$

$6.657 E-14$

政

. $08 \mathrm{E}-14$

$8.127 E-14$

$8.905 E-14$

$9.872 E-14$

$1.106 E-13$

$1.249 E-13$

$1.421 E-13$

$1.619 E-13$

$1.841 E-13$

$2.267 \mathrm{E}-13$

$1.337 E-12$ 


\begin{tabular}{|c|c|c|c|c|c|c|}
\hline COSINE & 75.0 & \multicolumn{5}{|c|}{$\begin{array}{l}\text { RANGE (METERS) } \\
200 . \mathrm{C}\end{array}$} \\
\hline $\begin{array}{l}-1.00000 E ~ O C \\
-9.89401 E-01 \\
-9.44575 E-01 \\
-8.65631 \mathrm{E}-01 \\
-7.55044 \mathrm{E}-01 \\
-6.17876 \mathrm{E}-01 \\
-4.58017 \mathrm{E}-01 \\
-2.81605 \mathrm{E}-01 \\
-9.50125 \mathrm{E}-02 \\
9.50125 \mathrm{E}-02 \\
2.81605 \mathrm{E}-01 \\
4.58017 \mathrm{E}-01 \\
6.17876 \mathrm{E}-01 \\
7.55044 \mathrm{E}-01 \\
8.65631 \mathrm{E}-01 \\
9.44575 \mathrm{E}-01 \\
9.89401 \mathrm{E}-01\end{array}$ & $\begin{array}{l}3.843 \mathrm{E}-13 \\
3.850 \mathrm{E}-13 \\
3.877 \mathrm{E}-13 \\
3.927 \mathrm{E}-13 \\
4.002 \mathrm{E}-13 \\
4.100 \mathrm{E}-13 \\
4.220 \mathrm{E}-13 \\
4.359 \mathrm{E}-13 \\
4.516 \mathrm{E}-13 \\
4.688 \mathrm{E}-13 \\
4.869 \mathrm{E}-13 \\
5.051 \mathrm{E}-13 \\
5.221 \mathrm{E}-13 \\
5.379 \mathrm{E}-13 \\
5.521 \mathrm{E}-13 \\
5.614 \mathrm{E}-13 \\
5.617 \mathrm{E}-13\end{array}$ & $\begin{array}{l}6.239 \mathrm{E}-13 \\
6.253 \mathrm{E}-13 \\
6.307 \mathrm{E}-13 \\
6.413 \mathrm{E}-13 \\
6.569 \mathrm{E}-13 \\
6.775 \mathrm{E}-13 \\
7.629 \mathrm{E}-13 \\
7.331 \mathrm{E}-13 \\
7.677 \mathrm{E}-13 \\
8.064 \mathrm{E}-13 \\
8.474 \mathrm{E}-13 \\
8.898 \mathrm{E}-13 \\
9.329 \mathrm{E}-13 \\
9.718 \mathrm{E}-13 \\
1 . \mathrm{CO} \mathrm{E}-12 \\
1 . C 31 \mathrm{E}-12 \\
1 . \mathrm{C} 40 \mathrm{E}-12\end{array}$ & $\begin{array}{l}1.148 \mathrm{E}-12 \\
1.152 \mathrm{E}-12 \\
1.165 \mathrm{E}-12 \\
1.192 \mathrm{E}-12 \\
1.232 \mathrm{E}-12 \\
1.286 \mathrm{E}-12 \\
1.353 \mathrm{E}-12 \\
1.435 \mathrm{E}-12 \\
1.533 \mathrm{E}-12 \\
1.646 \mathrm{E}-12 \\
1.772 \mathrm{E}-12 \\
1.909 \mathrm{E}-12 \\
2.051 \mathrm{E}-12 \\
2.189 \mathrm{E}-12 \\
2.314 \mathrm{E}-12 \\
2.409 \mathrm{E}-12 \\
2.463 \mathrm{E}-12\end{array}$ & $\begin{array}{l}1.633 \mathrm{E}-12 \\
1.639 \mathrm{E}-12 \\
1.663 \mathrm{E}-12 \\
1.710 \mathrm{E}-12 \\
1.78 \mathrm{CE}-12 \\
1.875 \mathrm{E}-12 \\
1.997 \mathrm{E}-12 \\
2.150 \mathrm{E}-12 \\
2.337 \mathrm{E}-12 \\
2.560 \mathrm{E}-12 \\
2.821 \mathrm{E}-12 \\
3.116 \mathrm{E}-12 \\
3.436 \mathrm{E}-12 \\
3.762 \mathrm{E}-12 \\
4.069 \mathrm{E}-12 \\
4.318 \mathrm{E}-12 \\
4.468 \mathrm{E}-12\end{array}$ & $\begin{array}{l}2.003 \mathrm{E}-12 \\
2.012 \mathrm{E}-12 \\
2.046 \mathrm{E}-12 \\
2.112 \mathrm{E}-12 \\
2.212 \mathrm{E}-12 \\
2.349 \mathrm{E}-12 \\
2.528 \mathrm{E}-12 \\
2.757 \mathrm{E}-12 \\
3.043 \mathrm{E}-12 \\
3.396 \mathrm{E}-12 \\
3.824 \mathrm{E}-12 \\
4.327 \mathrm{E}-12 \\
4.897 \mathrm{E}-12 \\
5.508 \mathrm{E}-12 \\
6.108 \mathrm{E}-12 \\
6.619 \mathrm{E}-12 \\
6.943 \mathrm{E}-12\end{array}$ & $\begin{array}{l}2.230 \mathrm{E}-12 \\
2.240 \mathrm{E}-12 \\
2.283 \mathrm{E}-12 \\
2.366 \mathrm{E}-12 \\
2.490 \mathrm{E}-12 \\
2.662 \mathrm{E}-12 \\
2.889 \mathrm{E}-12 \\
3.185 \mathrm{E}-12 \\
3.565 \mathrm{E}-12 \\
4.047 \mathrm{E}-12 \\
4.649 \mathrm{E}-12 \\
5.385 \mathrm{E}-12 \\
6.255 \mathrm{E}-12 \\
7.231 \mathrm{E}-12 \\
8.236 \mathrm{E}-12 \\
9.131 \mathrm{E}-12 \\
9.725 \mathrm{E}-12\end{array}$ \\
\hline TOTAL & $5.848 E-12$ & $1 . \cos E-11$ & $2 . C 86 E-11$ & $3.314 E-11$ & $4.519 E-11$ & $5.568 \mathrm{E}-11$ \\
\hline COSINE & 500.0 & 600.0 & $\begin{array}{l}\text { RANGE } \\
900.0\end{array}$ & $\begin{array}{l}\text { TERS I } \\
1200.0\end{array}$ & $15 C 0.0$ & 1800.0 \\
\hline $\begin{array}{l}-1.00000 \mathrm{E} 00 \\
-9.89401 \mathrm{E}-01 \\
-9.44575 \mathrm{E}-01 \\
-9.65631 \mathrm{E}-01 \\
-7.55044 \mathrm{E}-01 \\
-6.17876 \mathrm{E}-\mathrm{Cl} \\
-4.58317 \mathrm{E}-01 \\
-4.51 \\
-2.81605 \mathrm{E}-01 \\
-9.50125 \mathrm{E}-02 \\
9.50125 \mathrm{E}-02 \\
2.81605 \mathrm{E}-01 \\
4.58017 \mathrm{E}-01 \\
6.17876 \mathrm{E}-01 \\
7.55044 \mathrm{E}-01 \\
8.65631 \mathrm{E}-01 \\
9.44575 \mathrm{E}-01 \\
9.89401 \mathrm{E}-21\end{array}$ & $\begin{array}{l}1.996 \mathrm{E}-12 \\
2.009 \mathrm{E}-12 \\
2.062 \mathrm{E}-12 \\
2.163 \mathrm{E}-12 \\
2.309 \mathrm{E}-12 \\
2.510 \mathrm{E}-12 \\
2.785 \mathrm{E}-12 \\
3.163 \mathrm{E}-12 \\
3.686 \mathrm{E}-12 \\
4.410 \mathrm{E}-12 \\
5.416 \mathrm{E}-12 \\
6.813 \mathrm{E}-12 \\
8.737 \mathrm{E}-12 \\
1.133 \mathrm{E}-11 \\
1.448 \mathrm{E}-11 \\
1.788 \mathrm{E}-11 \\
2.057 \mathrm{E}-11\end{array}$ & $\begin{array}{l}1.574 \mathrm{E}-12 \\
1.586 \mathrm{E}-12 \\
1.636 \mathrm{E}-12 \\
1.728 \mathrm{E}-12 \\
1.854 \mathrm{E}-12 \\
2.624 \mathrm{E}-12 \\
2.257 \mathrm{E}-12 \\
2.585 \mathrm{E}-12 \\
3.653 \mathrm{E}-12 \\
3.724 \mathrm{E}-12 \\
4.694 \mathrm{E}-12 \\
6.110 \mathrm{E}-12 \\
8.194 \mathrm{E}-12 \\
1.120 \mathrm{E}-11 \\
1.527 \mathrm{E}-11 \\
2.006 \mathrm{E}-11 \\
2.418 \mathrm{E}-11\end{array}$ & $\begin{array}{l}5.602 \mathrm{E}-13 \\
5.711 \mathrm{E}-13 \\
6.087 \mathrm{E}-13 \\
6.657 \mathrm{E}-13 \\
7.265 \mathrm{E}-13 \\
7.898 \mathrm{E}-13 \\
8.713 \mathrm{E}-13 \\
1.002 \mathrm{E}-12 \\
1.215 \mathrm{E}-12 \\
1.546 \mathrm{E}-12 \\
2.055 \mathrm{E}-12 \\
2.880 \mathrm{E}-12 \\
4.307 \mathrm{E}-12 \\
6.864 \mathrm{E}-12 \\
1.130 \mathrm{E}-11 \\
1.810 \mathrm{E}-11 \\
2.567 \mathrm{E}-11\end{array}$ & $\begin{array}{l}1.629 \mathrm{E}-13 \\
1.722 \mathrm{E}-13 \\
1.978 \mathrm{E}-13 \\
2.354 \mathrm{E}-13 \\
2.6 \mathrm{C} 3 \mathrm{E}-13 \\
2.745 \mathrm{E}-13 \\
2.868 \mathrm{E}-13 \\
3.239 \mathrm{E}-13 \\
4.02 \mathrm{CE}-13 \\
5.290 \mathrm{E}-13 \\
7.194 \mathrm{E}-13 \\
1.029 \mathrm{E}-12 \\
1.639 \mathrm{E}-12 \\
2.967 \mathrm{E}-12 \\
5.838 \mathrm{E}-12 \\
1.138 \mathrm{E}-11 \\
1.925 \mathrm{E}-11\end{array}$ & $\begin{array}{l}4.568 \mathrm{E}-14 \\
5.212 \mathrm{E}-14 \\
7.022 \mathrm{E}-14 \\
8.898 \mathrm{E}-14 \\
1.044 \mathrm{E}-13 \\
1.009 \mathrm{E}-13 \\
9.765 \mathrm{E}-14 \\
1.089 \mathrm{E}-13 \\
1.384 \mathrm{E}-13 \\
1.963 \mathrm{E}-13 \\
2.576 \mathrm{E}-13 \\
3.551 \mathrm{E}-13 \\
5.660 \mathrm{E}-13 \\
1.126 \mathrm{E}-12 \\
2.589 \mathrm{E}-12 \\
6.021 \mathrm{E}-12 \\
1.206 \mathrm{E}-11\end{array}$ & $\begin{array}{l}1.324 \mathrm{E}-14 \\
1.697 \mathrm{E}-14 \\
2.838 \mathrm{E}-14 \\
3.722 \mathrm{E}-14 \\
4.668 \mathrm{E}-14 \\
4.119 \mathrm{E}-14 \\
3.648 \mathrm{E}-14 \\
4.138 \mathrm{E}-14 \\
5.447 \mathrm{E}-14 \\
7.684 \mathrm{E}-14 \\
1.037 \mathrm{E}-13 \\
1.316 \mathrm{E}-13 \\
1.985 \mathrm{E}-13 \\
4.175 \mathrm{E}-13 \\
1.088 \mathrm{E}-12 \\
2.922 \mathrm{E}-12 \\
6.810 \mathrm{E}-12\end{array}$ \\
\hline TOTAL & $7.251 E-11$ & $6.819 E-11$ & 4.C46E-11 & $1.950 E-11$ & $8.933 E-12$ & $4.081 E-12$ \\
\hline
\end{tabular}


COS INE

$-1.00000 E 00$ $-9.89401 \mathrm{E}-01$ $-8.65631 \mathrm{E}-01$ $-7.55044 E-01$ $-6.17876 \mathrm{E}-01$ $-4.58017 \mathrm{E}-01$ $-2.81605 \mathrm{E}-01$ $-9.50125 \mathrm{E}-02$ $9.50125 \mathrm{E}-02$ $2.81605 E-01$ 4. $58017 \mathrm{E}-01$ $6.17876 \mathrm{E}-01$ $7.55044 \mathrm{E}-01$ $8.65631 E-01$ $9.44575 \mathrm{E}-01$ $9.894 \mathrm{ClE}-01$

TOTAL

COS INE

$-1.00000 E 00$ $-9.89401 \mathrm{E}-01$ $-9.44575 E-01$ $-8.65631 E-01$ $-7.55044 \mathrm{E}-01$ $-6.17876 E-01$ - $2.81605 \mathrm{E}-01$ $-2.81605 E-01$ $-9.50125 E-02$ 2.816C5E-01 4.58017E-01 $6.17876 \mathrm{E}-01$ 7. $55044 \mathrm{E}-01$ $8.65631 E-01$ 9.894 C1E-O1

TOTAL

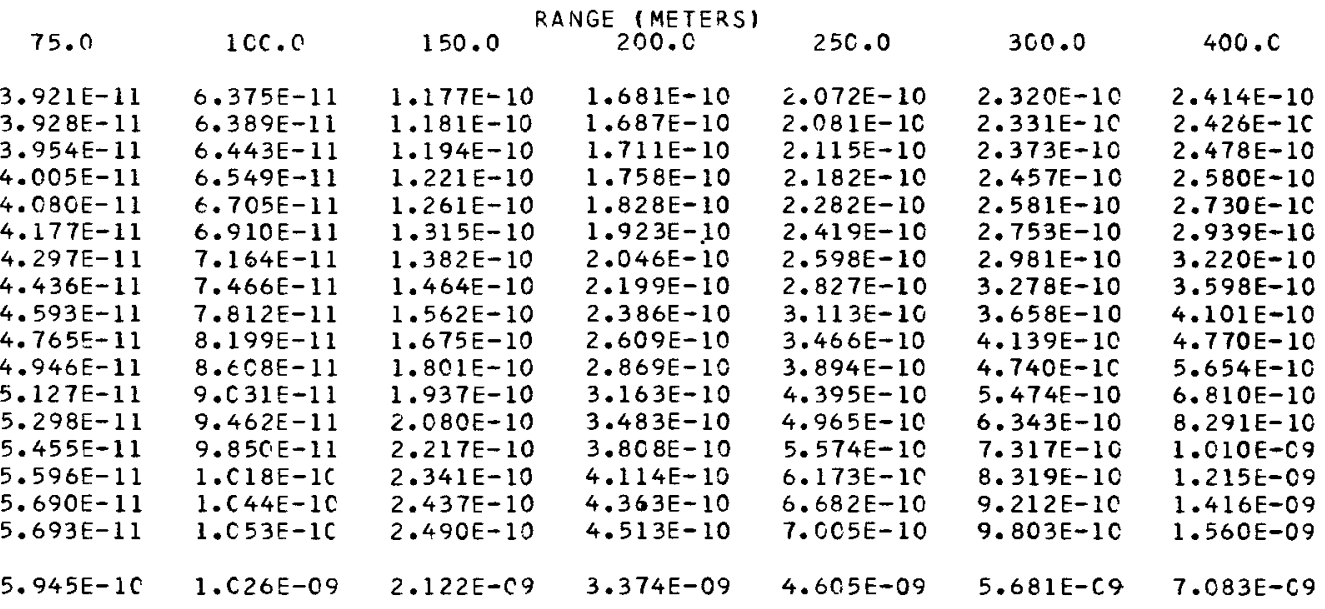

RANGE (METERS)

500.0

606.0

2.145E-1C $2.158 \mathrm{E}-10$ $2.211 \mathrm{E}-10$ $2.313 E-10$ $2.460 E-10$ $2.663 \mathrm{E}-10$ $2.940 \mathrm{E}-10$ $3.320 E-1 C$ $3.844 E-10$ $4.569 E-1 C$ $5.573 E-1 C$ $6.967 E-10$ $8.884 \mathrm{E}-10$ $1.144 \mathrm{E}-\mathrm{C}$ $1.460 \mathrm{E} C \mathrm{C}$ 2.C67E-O

$7.440 E-C$ ? $1.743 \mathrm{E}-1 \mathrm{C}$ $1.794 \mathrm{E}-10$ 1. $886 \mathrm{E}+10$ 2. $14 \mathrm{E}-1 \mathrm{C}$ $2.186 E-10$ $2.422 E-10$ 2.752E $1 \mathrm{C}$ $3.222 E-1 C$ $4.862 E-10$ $6.872 E-10$ $6.275 E-10$ $1.134 E-C$ 1.54CE 09 $2.017 E-C 9$ $2.427 E-C 9$

$7.018 \mathrm{E}-\mathrm{Cg}$
$1.731 \mathrm{E}-1 \mathrm{C}$
1200.0

$6.832 \mathrm{E}-11$

$6.941 \mathrm{E}-11$

$7.897 \mathrm{E}-11$

$8.521 \mathrm{E}-11$

$9.179 \mathrm{E}-11$

$1.002 E-10$

$1.136 E-10$

$1.351 E-10$

1.

.

$4.433 E=10$

$6.970 E-10$

$1.814 E-09$

$4.202 E-C 9$
$2.340 E-11$

$2.432 \mathrm{E}-11$

$3.067 \mathrm{E}-11$

$3.326 \mathrm{E}-11$

$3.485 E-11$

$3.629 E-11$

4.021E-11

6.0

$3.026 E-10$

$1.138 E-09$

$1.924 E-09$

$2.039 E-09$
$150 \mathrm{C} . \mathrm{C}$

$8.187 E-12$

$8.823 E-12$

.062E-11

$.252 E-11$

$1.385 \mathrm{E}-11$

$1.364 \mathrm{E}-11$

$1.486 \mathrm{E}-11$

$1.789 \mathrm{E}-11$

2.

.

.

$6.010 E-1 C$

$1.205 \mathrm{E}-09$

$9.371 E-1 C$
1800.0

$3.074 \mathrm{E}-12$

$3.442 E-12$

$4.572 E-12$

$5.462 E-12$

$6.421 E-12$

$5.929 E-12$

$5.516 \mathrm{E}-12$

$6 . C 49 E-12$

$7.391 \mathrm{E}-12$

$1.234 E-11$

$1.513 E-11$

$2.168 E-11$

1. $090 \mathrm{E}-10$

$1.090 \mathrm{E}-10$

$2.911 E-10$

$4.288 E-10$ 
4 PI R**2 AIR KERMA IGAMMAS)
(CM**2 ERGS/GRAM/ST ERADIAN/SOURCE NEUTRON)

COSINE

$-1.0 C 000 E$ OC $-9.89401 \mathrm{E}-01$ $-9.44575 E-01$ $-8.65631 E-01$ $-7.55044 \mathrm{E}-01$ $-6.17876 E-01$ $-4.58017 E-01$ $-2.81605 E-01$ $9.50125 \mathrm{C}$ 9.50125 4.58C17E-01 $6.17876 \mathrm{E}-01$ $7.55044 E-01$ $8.65631 \mathrm{E}-01$ $9.89401 E-01$

TOTAL

COSINE

$-1.00000 E 00$ $-9.89401 \mathrm{E}-01$ $-9.44575 \mathrm{E}-01$ $-8.65631 \mathrm{E}-01$ $-7.55044 E-01$ $-6.17876 E-01$ $-4.5817 E-01$ $-2.81605 \mathrm{E}-01$ $-9.50125 E-02$ $9.50125 \mathrm{E}-02$
$2.81605 \mathrm{E}-01$ $4.58017 E-01$ $6.17876 \mathrm{E}-01$ 6.17876E-01 7.55044E-01 $8.65631 E-01$ $9.89401 \mathrm{E}-01$

TOTAL

$\begin{array}{cc}75.0 & 1 C C .0 \\ 4.250 \mathrm{E}-11 & 6.977 \mathrm{E}-11 \\ 4.256 \mathrm{E}-11 & 6.991 \mathrm{E}-11 \\ 4.281 \mathrm{E}-11 & 7.042 \mathrm{E}-11 \\ 4.330 \mathrm{E}-11 & 7.143 \mathrm{E}-11 \\ 4.401 \mathrm{E}-11 & 7.292 \mathrm{E}-11 \\ 4.493 \mathrm{E}-11 & 7.487 \mathrm{E}-11 \\ 4.606 \mathrm{E}-11 & 7.728 \mathrm{E}-11 \\ 4.738 \mathrm{E}-11 & 8.014 \mathrm{E}-11 \\ 4.886 \mathrm{E}-11 & 8.341 \mathrm{E}-11 \\ 5.048 \mathrm{E}-11 & 8.705 \mathrm{E}-11 \\ 5.218 \mathrm{E}-11 & 9.091 \mathrm{E}-11 \\ 5.388 \mathrm{E}-11 & 9.488 \mathrm{E}-11 \\ 5.548 \mathrm{E}-11 & 9.891 \mathrm{E}-11 \\ 5.695 \mathrm{E}-11 & 1.026 \mathrm{E}-10 \\ 5.828 \mathrm{E}-11 & 1.056 \mathrm{E}-1 \mathrm{C} \\ 5.915 \mathrm{E}-11 & 1.080 \mathrm{E}-1 \mathrm{C} \\ 5.918 \mathrm{E}-11 & 1.089 \mathrm{E}-1 \mathrm{C} \\ 6.301 \mathrm{E}-10 & 1.089 \mathrm{E}-\mathrm{C} 9\end{array}$

150.0 $1.317 \mathrm{E}-10$ $1.321 E-10$ $1.334 \mathrm{E}-10$ $1.360 E-10$ $1.398 \mathrm{E}-10$ $1.449 \mathrm{E}-10$ $1.513 E-10$ $1.592 \mathrm{E}-10$
$1.684 \mathrm{E}-10$ $E-10$ $1.909 \mathrm{E}-10$ 2.C $38 \mathrm{E}-10$ $2.171 \mathrm{E}-10$ $2.299 E-10$ $2.415 \mathrm{E}-10$ $2.504 \mathrm{E}-10$ $2.554 E-10$

$2.265 E-09$ RANGE (METERS)
200.0 25 C. C 300.0 400.0

$\begin{array}{ll}1.929 \mathrm{E}-10 & 2.448 \mathrm{E}-10 \\ 1.935 \mathrm{E}-10 & 2.456 \mathrm{E}-10 \\ 1.959 \mathrm{E}-10 & 2.490 \mathrm{E}-10 \\ 2.004 \mathrm{E}-10 & 2.555 \mathrm{E}-10\end{array}$
$2.004 \mathrm{E}-10 \quad 2.555 \mathrm{E}-10$ $2.072 E-10 \quad 2.653 E-10$ $2.164 \mathrm{E}-10 \quad 2.786 \mathrm{E}-10$ $2.281 E-10 \quad 2.959 E-10$ $2.428 \mathrm{E}-10 \quad 3.18 \mathrm{CE}-10$ $2.606 \mathrm{E}-10 \quad 3.454 \mathrm{E}-10$ $2.817 E-10 \quad 3.790 E-10$ $3.063 E-10 \quad 4.19 E E-10$ $3.339 E-10 \quad 4.667 E-1 C$ $3.639 \mathrm{E}-10 \quad 5.201 \mathrm{E}-10$ $3.943 E-10 \quad 5.770 E-10$ $4.228 E-10 \quad 6.327 E-10$ $4.459 E-10 \quad 6.799 E-10$ $4.597 \mathrm{E}-10 \quad 7.098 \mathrm{E}-1 \mathrm{C}$

$3.624 E-09 \quad 4.987 E-09$

RANGE (METERS)

500.0

600.0

900.0

$1200 . \mathrm{C}$

1500.0

$3.191 \mathrm{E}-1$ 3. $254 \mathrm{E}-11$ $3.437 E-11$ $3.645 \mathrm{E}-11$ $3.843 \mathrm{E}-11$ $3.891 E-11$ 3.96CE-11 $4.12 C E-11$ $4.581 \mathrm{E}-11$ 5.205E-11 $5.992 \mathrm{E}-11$ $7.077 E-11$ $1.451 E-10$ $1.451 E-10$ $2.796 E-10$ $1.141 E-09$

1. $252 \mathrm{E}-09$

$1.456 \mathrm{E}-1$

$1.491 E-11$

$1.604 E-11$

$1.815 \mathrm{E}-11$

$1.803 \mathrm{E}-11$

1.808E-11

1.907E-11 2.361E-11 $2.686 \mathrm{E}-11$ $5.834 \mathrm{E}-11$ $2.869 \mathrm{E}-10$

$6.4 C C E-10$ $1.807 E-C 9$ $1.579 E-C 9$ $2.395 E-09$ $1.180 \mathrm{E}-09$

$8.478 \mathrm{E}-\mathrm{C} 9$

$8.179 E-C 9$

$5.213 E-09$

$2.648 E-09$

$5.813 E-10$
$2.830 E-10$ $2.883 E-10 \quad 3.236 E-10$ $2.965 E-10 \quad 3.339 E-10$ $3.089 \mathrm{E}-10 \quad 3.491 \mathrm{E}-10$ $3.257 E-10 \quad 3.701 E-10$ $3.480 E-10 \quad 3.983 E-10$ $3.768 \mathrm{E}-10 \quad 4.356 \mathrm{E}-10$ $4.134 E-10 \quad 4.848 E-10$ $4.594 E-10 \quad 5.495 E-10$ $5.165 E-10 \quad 6.342 E-10$ $5.858 E-1 C \quad 7.439 E-10$ $6.673 E-10 \quad 8.831 E-10$ $7.581 \mathrm{E}-10 \quad 1.052 \mathrm{E}-\mathrm{C} 9$ $8.513 E-10 \quad 1.243 E-09$ $9.339 E-1 C \quad 1.428 E-09$ $9.886 \mathrm{E}-1 \mathrm{C} \quad 1.561 \mathrm{E}-09$ $6.207 E-C 9 \quad 7.896 E-09$ 
COS INE

$-1.00000 \mathrm{E} 00$
$-9.89401 \mathrm{E}-01$
$-9.44575 \mathrm{E}-01$
$-8.65631 \mathrm{E}-01$
$-7.55044 \mathrm{E}-01$
$-6.17876 \mathrm{E}-01$
$-4.58017 \mathrm{E}-01$
$-2.81605 \mathrm{E}-01$
$-9.50125 \mathrm{E}-02$
$9.50125 \mathrm{E}-02$
$2.81605 \mathrm{E}-01$
$4.58017 \mathrm{E}-01$
$6.17876 \mathrm{E}-01$
$7.55044 \mathrm{E}-01$
$8.65631 \mathrm{E}-01$
$9.44575 \mathrm{E}-01$
$9.89401 \mathrm{E}-01$

TOTAL

COSINE

$-1.00000 E$ OO $-9.89401 \mathrm{E}-01$ $-9.44575 E-01$ $-8.65631 E-01$ $-7.55044 E-01$ $-6.17876 \mathrm{E}-01$ $-4.58017 E-01$ $-2.81605 E-01$ $9.50125 \mathrm{E}-02$ $2.81605 \mathrm{E}-01$ $2.81605 E-01$ $4.58017 E-01$ $7.55044 \mathrm{E}-01$ $8.5564 \mathrm{E}-01$ $8.65631 \mathrm{E}-01$ $9.89401 E-01$

TOTAL
$4.047 E-11$ 4. $054 \mathrm{E}-11$ $4.080 \mathrm{E}-11$ $4.133 E-11$ $4.209 \mathrm{E}-11$ $4.432 \mathrm{E}-11$ $4.575 \mathrm{E}-11$ $4.736 E-11$ 4. $911 \mathrm{E}-11$ 5.C97E-11 $5.283 E-11$ $5.458 \mathrm{E}-11$ $5.619 \mathrm{E}-1$

$5.764 E-11$ $5.860 \mathrm{E}-11$

$6.128 \mathrm{E}-10$
100.0

150.0 (METERS)
$2 C r .0$ 250.0 300.0 400.0 $.582 \mathrm{E}-11$
$.597 \mathrm{E}-11$ $1.217 \mathrm{E}-10$
$1.221 \mathrm{E}-10$ $1.235 \mathrm{E}-10$ $1.262 \mathrm{E}-10$ $6.761 \mathrm{E}-11$ $6.921 E-11$ $7.131 \mathrm{E}-11$ $7.392 \mathrm{E}-11$ $7.701 \mathrm{E}-11$ 8.C55E-11 $8.452 E-11$ $8.871 E-11$ 9.305 E 11 $9.747 E-11$ $1.015 E-1 C$ 1.C $48 E-1 C$ 1.C $75 E-10$ -

500.0 $1.358 \mathrm{E}-10$ $1.427 \mathrm{E}-10$ $1.511 E-10$ $1.611 \mathrm{E}-10$ $1.727 \mathrm{E}-10$ $1.996 E-10$ $2.142 E-10$ $2.282 \mathrm{E}-10$ $2.410 E-10$ $2.508 E-10$ 2. $563 E-10$

$2.188 \mathrm{E}-09$ $1.746 \mathrm{E}-10$ $1.771 E-10$
$1.819 E-10$ $1.891 \mathrm{E}-10$ $1.988 \mathrm{E}-10$ $2.114 E-10$ $2.271 \mathrm{E}-10$ $2.462 \mathrm{E}-10$ $2.691 E-10$ $2.958 \mathrm{E}-10$ $3.259 E-10$ $3.586 \mathrm{E}-10$ $3.920 \mathrm{E}-10$ $4.234 E-10$ $4.489 E-10$ 4. $643 E-10$ $3.479 E-09$ $2.149 \mathrm{E}-10$ $2.158 \mathrm{E}-10$ $2.192 E-10$ $2.364 \mathrm{E}-10$ $2.504 \mathrm{E}-10$ $2.687 \mathrm{E}-10$ $2.921 \mathrm{E}-10$ $3.215 E-10$ $3.577 \mathrm{E}-10$ $4.528 E-10$ $5.112 \mathrm{E}-1 \mathrm{C}$ $5.737 E-1 C$ $6.351 E-10$ $6.874 \mathrm{E}-10$ $7.205 E-10$

$4.75 C E-09$ $2.421 E-10$ $2.465 \mathrm{E}-10$ $2.550 \mathrm{E}-10$ $2.678 E-10$ $2.854 \mathrm{E}-10$ .087E-10 .391E-10 . $780 E-10$ $4.273 E-10$ $4.888 \mathrm{E}-10$ $5.641 E-10$ $6.531 \mathrm{E}-10$ $7.529 \mathrm{E}-10$ $8.557 \mathrm{E}-10$ $.473 E-10$ $1.008 \mathrm{E}-\mathrm{CO}$

$5.862 \mathrm{E}-\mathrm{C} 9$ $.520 E-1 C$
$2.533 E-10$ . $586 \mathrm{E}-10$ $2.690 \mathrm{E}-1 \mathrm{C}$ $2.844 E-10$ $3.057 \mathrm{E}-10$ $3.345 \mathrm{E}-10$ $3.731 E-10$ $4.246 E-10$ $4.931 \mathrm{E}-10$ $5.836 \mathrm{E}-10$ $7.02 C E-10$ $8.538 \mathrm{E}-10$ $1.040 \mathrm{E}-09$ $1.250 \mathrm{E}-09$ $1.455 \mathrm{E}-09$
$1.604 \mathrm{E}-09$

RANGE (METERS) 1200.0 1500.0 $1800 . \mathrm{C}$

\section{$7.484 E-11$} $7.595 \mathrm{E}-11$ $7.983 E-11$ $8.574 E-11$ $9.209 E-11$ $9.877 E-11$ $1.073 E-10$ $1.209 E-10$ $1.767 E-10$ $2.286 E-10$ $2.286 E-10$ 3.123E-10 7. $168 \mathrm{E}-10$ $1.169 \mathrm{E}-09$ $1.863 \mathrm{E}-09$ $2.639 \mathrm{E}-09$

$4.356 E-C 9$
$2.679 \mathrm{E}-11$ $2.773 E-11$ $3.036 \mathrm{E}-11$ $3.423 E-11$ $3.687 E-11$ $3.847 E-11$ $3.988 \mathrm{E}-11$ $4.382 E-11$ $6.488 E-11$ $6.488 E-11$ $0.422 E-11$ $1.155 E-10$ $3.111 \mathrm{E}-10$ 6.111E-10 $6.022 \mathrm{E}-10$ $1.168 E-09$

2.117E-09
9. $806 \mathrm{E}-12 \quad 3.825 \mathrm{E}-12$ $1.046 E-11 \quad 4.203 E-12$ $1.23 \mathrm{CE}-11 \quad 5.363 \mathrm{E}-12$ $1.425 \mathrm{E}-11$ 6.276E-12 $1.585 \mathrm{E}-11$ $7.255 \mathrm{E}-12$ $1.559 \mathrm{E}-11$ 6.741E-12 $1.534 E-11$ 6.302E-12 1.655E-11 $6.828 E-12$ $2.490 E-11 \quad 1.046 E-11$ 2.172E-11 1.046E 11 4.150E-11 $1.517 \mathrm{E}-11$ 6.253E-11 2.250E 11 .25 $2.250 E-11$ 2.663E-10 $1.116 E-10$ $0.163 \mathrm{E}-10$ 2. $2.98 \mathrm{E}-10$ $1.238 \mathrm{E}-09 \quad 6.99 \mathrm{E}-10$

$9.741 E-1 C \quad 4.459 E-1 C$

$2.493 E-09$

$7.688 \mathrm{E}-\mathrm{C} 9$

$7.258 \mathrm{E}-09$ 
(NEUTRONS/MEV/STERADIAN/SOURCE NEUTRON)

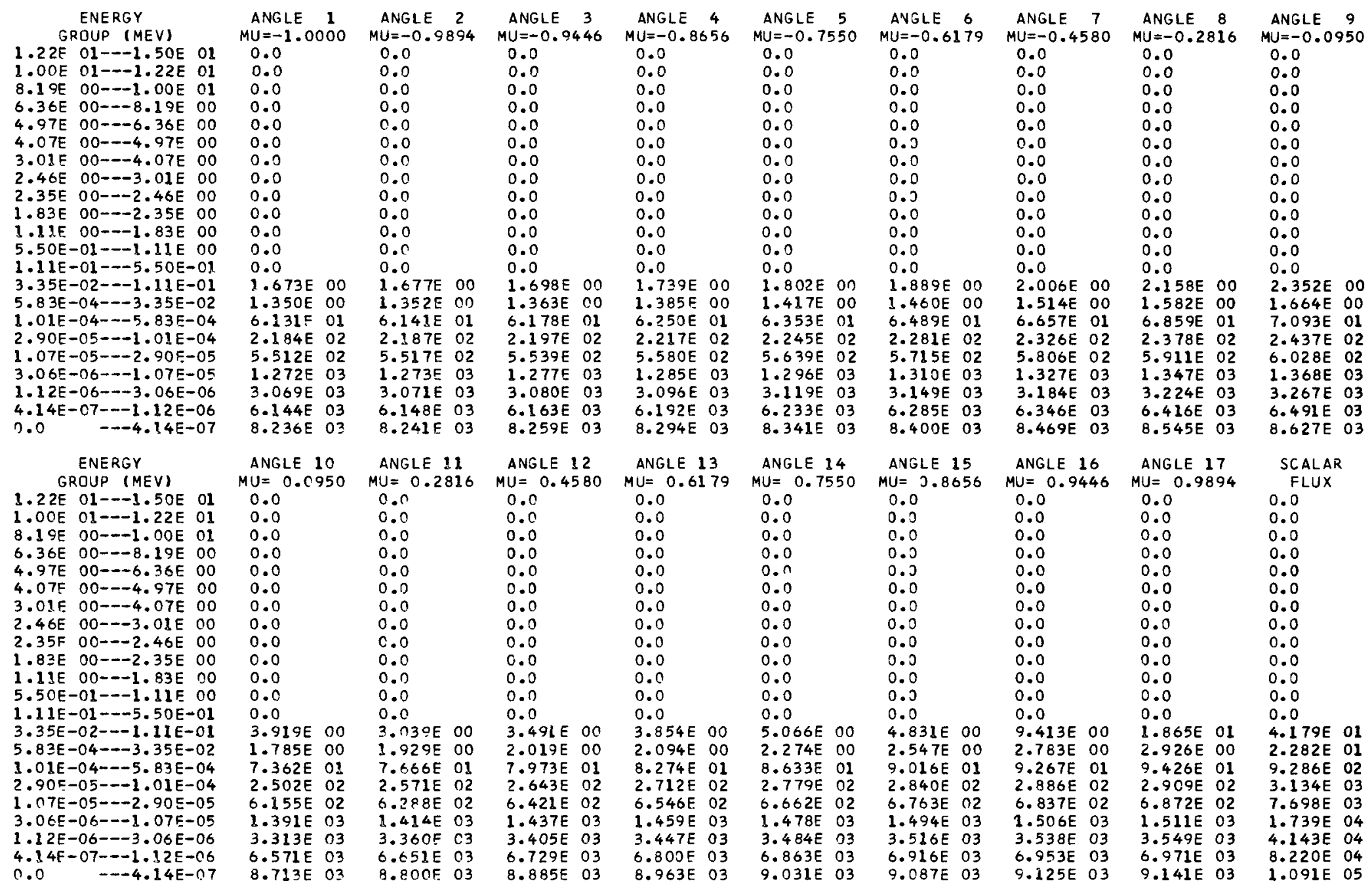


(NEUTRJNS/MEV/STERADIAN/SOURCE NEUTRON)

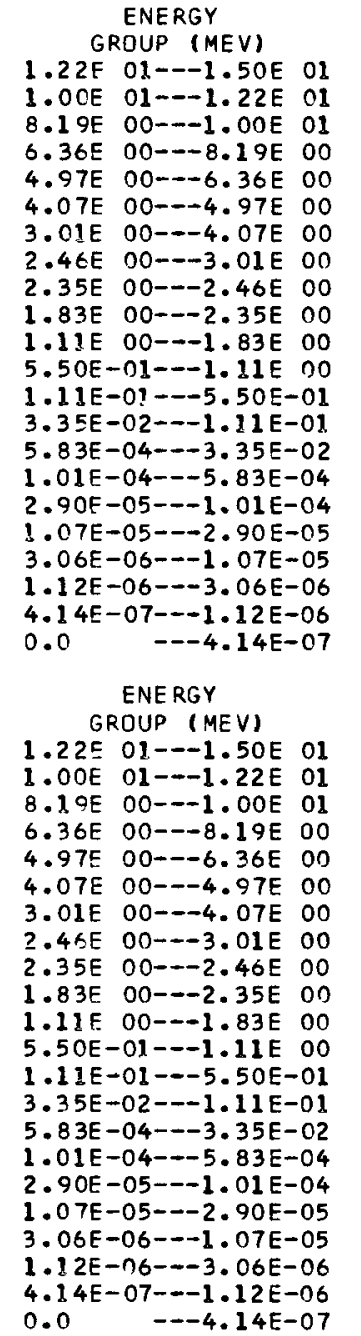

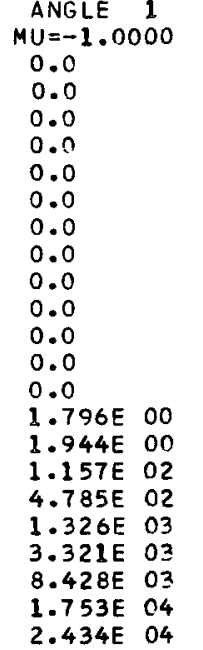

$\begin{array}{cc}\text { ANGLE } & 2 \\ M U=-0.9894 & \end{array}$ $M U=-0.98$
0.0
0.0

$$
0.0
$$

0.0

0.0
0.0

0.0
0.0

0.0

0.0

0.0

0.0

$1.802 \mathrm{E} 00$

$1.948 \mathrm{E}$
$1.159 \mathrm{E}$

$\begin{array}{lll}1.159 E & 02 \\ 4.793 E & 02\end{array}$

$1.328 \mathrm{E} 03$

$1.328 E$
$3.325 \mathrm{E} \mathrm{O3}$

$8.438 \mathrm{E} \mathrm{O3}$

$1.755 \mathrm{E} 04$

$2.436 \mathrm{E} 04$

ANGLE 10

$M U=0.0950$

0.0

0.0

0.0

0.0

0.0

0.0

0.0

0.0

0.0

0.0

0.0

0.0

$1.784 \mathrm{E}$ OO

$3.116 E$ OO

$1.461 \mathrm{E} \mathrm{O2}$

$5.805 E 02$

$1.573 \mathrm{E} \mathrm{O3}$

$9.614 \mathrm{E} \quad 03$

$1.975 \mathrm{E} 04$

$2.693 E 04$
ANGLE 11

ANGLE 12

0.0

0.0

0.0

0.0

0.0

0.0

0.0

0.0

0.0

0.0

0.0

$3.733 \mathrm{E} 00$

$.568 E 00$
$.528 E 02$

$6.036 \mathrm{E} \mathrm{O2}$

$1.627 \mathrm{E} \mathrm{O3}$

$3.960 \mathrm{E} 03$ $9.857 \mathrm{E} 03$

$2.743 E 04$

0.0

0.0

0.0

0.0

. C

0.0

0.0

0.0

0.0

0.0

0.0

$2.959 \mathrm{E} O 0$
$1.605 \mathrm{E} O 2$

$6.281 \mathrm{E} 02$

$1.683 \mathrm{E} \mathrm{O3}$

1.010E 04
MU $=-0.9446$

$M U=-0.94$
0.0
0.0

0.0

0.0

0.0

0.0

0.0

0.0

0.0

0

$1.824 \mathrm{E} 00$

$1.966 \mathrm{~F} \mathrm{O0}$

$4.825 \mathrm{E} \mathrm{O2}$

$1.336 \mathrm{E} 03$

$3.343 \mathrm{E} 03$

$.763 E 04$

$2.446 \mathrm{E} \mathrm{O4}$

$2.714 \mathrm{E} 00$

$4.073 E 03$

$2.064 \mathrm{E} 04$
ANGLE $\quad 4$
$M U=-0.8556$
0.0

0.0
0.0

0.0

0.0

0.0

0.0
0.0
0.0

0.0

0.0

0.0

0.0

$1.863 E 00$

$2.001 E 00$

$4.886 E$ O2

$1.352 E$ O3

.

$1.777 E 04$

$2.463 E_{04}$

ANGLE 13

$A U=0.6179 \quad M U=0.75$

0.0

0.0

0.0

0.0

0.0

0.0

0.0

0.0

0.0

0.0

0.0

$4.252 E 00$

$3.273 E$ OO

$1.697 E 02$

6.537E O2

$1.740 E^{03}$

$4.185 E 03$

$1.034 \mathrm{E} \mathrm{O4}$

$2.106 E$
$2.843 E$

0.0

0.0
0.0
0.0

0.0

0.0
0.0
0.0

0.0

0.0

0.0

0.0

0.0

$8.662 \mathrm{E} 03$

. 488 E

ANGLE 14

0.0

0.0

0.0
0.0
0.0
0.0

0.0

0.0

0.0

0.0

0.0

0
$\begin{array}{lll}A N G L E & 5 & \text { AN } \\ M U=-0.75 E D & M U=-0.6179\end{array}$

$1.932 \mathrm{E}$ OO

$2.052 E \quad 00$

1.21DE 02

..373E 03

$3.423 E 03$

NGLE $14 \quad$ ANGLE 15

$\begin{array}{ll}0.0 & 0.0 \\ 0.0 & 0.0\end{array}$

$4.058 E 00$

$\begin{array}{ll}3.341 E & 00 \\ 1.775 E & 02\end{array}$

$6.778 E 02$

$1.792 \mathrm{E} 03$

4. $287 \mathrm{E} \quad 03$

$1.055 \mathrm{E} 04$

$2.145 \mathrm{E} 04$

2.886E 04
$M U=-J$
0.0

0.5
0.0
0.0
0.0

0.0

0.0

0.0

0.0

0.0

0.0

0.0
$2.020 E \quad 00$

$2.121 E 00$

$5.087 E$ O2

$1.401 \mathrm{E} 03$

.

(8)

1.

0.0
0.0

0.0

0.0

0.0

0.0

0.0

0.0

0.0

0.0

0.0

$3.746 \mathrm{E} 00$

$1.862 E 02$

$6.999 E \quad 02$

.839E 03

4.375E 03

$1.074 \mathrm{E} 04$

$2.177 E$ O4
$2.921 E 04$

$A N G L E \quad 7$
$M U=-0.4580$

0.0

0.0

0.0
0.0

0.0
0.0
0.0
0.0

0.0

0.0

0.0

0.0

$2.135 E 00$

$2.208 E 00$

$1.283 \mathrm{E} 02$

$5.228 \mathrm{E} 02$

$1.435 E$ E 3

.

$1.855 \mathrm{E} \mathrm{O4}$

$2.555 \mathrm{E} 04$

ANGLE
$M U=-0.2816 \quad$ ANGLE 9
MU

$0.0 \quad 0.0$

$\begin{array}{ll}0.0 & 0.0 \\ 0.0 & 0.0\end{array}$

$0.0 \quad 0.0$

$\begin{array}{ll}0.0 & 0.0 \\ 0.0 & 0.0\end{array}$

$\begin{array}{ll}0.0 & 0.0 \\ 0.0 & 0.0\end{array}$

$0.0 \quad 0.0$

$\begin{array}{ll}0.0 & 0.0 \\ 0.0 & 0.0\end{array}$

$2.282 E 00$

2.448E OO

5.335E O2 1.390E O2

1.476E 03 S.

1.675E 03 3.522E O3

(..742E O3

$1.891 E 04$ 1.931E O4

$1.891 E$ O4 $1.931 E$ O4

ANGLE 16

ANGLE 17

SCALAR

$M U=0.9446 \quad M U=0.9894$

0.0

0.0
0.0

0.0

0.0

0.0

0.0

0.0

0.0

0.0

0.0

$6.196 \mathrm{E} 00$

$3.934 E \quad 00$

$1.931 \mathrm{E} 02$

$7.176 \mathrm{E} 02$

$1.875 \mathrm{E} 03$

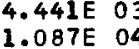

$2.201 \mathrm{E} \mathrm{O4}$

$2.946 E \quad 04$

0.0

0.0

0.0
0.0
0.0
0.0

0.0
0.0
0.0

0.0 
ENERGY GROUP (MEV)

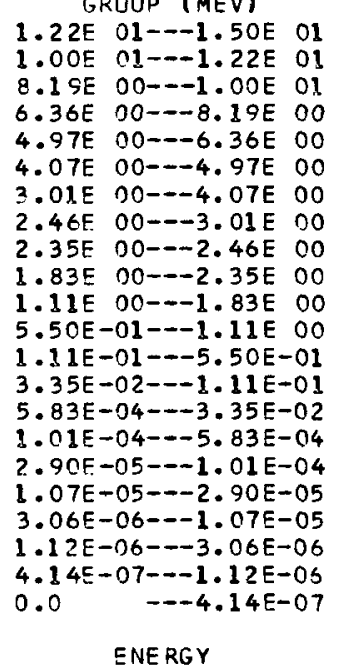
ANGLE 1 0.0 0.0 0.0 0.0 0.0
0.0
0.0 0.0 0.0 0.0 0.0 0.0 0.0 $1.576 \mathrm{E} 00$ $1.856 E 00$ $1.223 \mathrm{E} 02$ $5.4 \mathrm{COE} 02$ $1.569 \mathrm{E} 03$ $\begin{array}{ll}4.121 E & 03 \\ 1.080 E & 04\end{array}$ $2.308 E \quad 04$ $3.285 E \quad 04$ ANGLE 10 ENERGY 1.22 E O1---1.50E O1 $1.00 E$ O1- $-1.22 E$ OI $8.19 E$ O0---1.00E OL 6.36 E $00--8.19 E$ E 00 $4.97 E$ OO---6.36E NO 4.07E 00---4.97E 00 $3.01 \mathrm{E}$ OO- $-4.07 \mathrm{E} 00$ $2.46 \mathrm{E} \quad 00--3.01 \mathrm{E} 00$ $2.35 \mathrm{E} \quad 00--2.46 \mathrm{E} 00$ $1.83 \mathrm{E}$ OO---2.35E 00 $1.13 \mathrm{E} 0 \mathrm{C}-\mathrm{-1.83 \textrm {E }} 00$ $5.50 \mathrm{E}-01---1.11 \mathrm{E}$
$1.11 \mathrm{E}-01---5.50 \mathrm{E}-01$ $3.35 E-02--1.11 \mathrm{E}-01$ $5.33 E-04=-3.35 E-02$ $1.01 \mathrm{E}-04-2-5.83 \mathrm{E}-04$ $1.9 \mathrm{E}-05-1.01 \mathrm{E}-\mathrm{C}$ $1.07 E-05-2.90 E-05$ $1.12 E-06---3.06 E-06$ 4.1 4E-07--1.12E-06 $4.14 E-07---1.12 E-06$
$0.0 \quad--0.14 E-07$ 0.0

0.0
0.0
0.0

0.0

0.0

0.0
0.0
0.0

0.0

0.0

0.0

0.0

$2.140 E 00$ $2.086 E \quad 00$ $1.559 E$ O2 $6.687 \mathrm{E}$ O? $1.907 E 03$ $4.902 E$ E $1.265 E$ O 4 3.720 O4 $^{2}$
(NEUTRONS/MEV/STERADIAN/SOURCE NEUTRONI

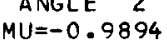

O.0. $M U=-0.9446$

$0.0 \quad 0.0$

0.0

0.0

0.0

0.0

0.0

0.0

0.0
0.0

0.0

0.0

0.0

0.0

$1.581 E 00$

1. 8 EDE 00

$1.225 E$ O2

$1.572 \mathrm{E} 03$

$4.127 E 03$

$1.082 \mathrm{E} 04$

$2.311 E 04$

0.0
0.0
0.0

0.0
0.0
0.0

0.0

0.0

0.0

0.0

0.0

0.0

0.0

0.0

0.0

$1.601 \mathrm{E} \quad 00$

$1.878 \mathrm{E} 00$

$1.235 E$ O2

$\begin{array}{ll}5.450 \mathrm{E} & 02 \\ 1.583 \mathrm{E} & 03\end{array}$

$4.153 \mathrm{E} 03$

$1.088 \mathrm{E} 04$

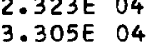

ANGLE 4

$\triangle N G L E \quad 5$
$M U=-0.7550$

$0.0 \quad 0.0$

.0

0.0

0.0

0.0

0.0

0.0

0.0

0.0

0.0

0.0

$1.637 \mathrm{E} 00$

$1.912 \mathrm{E} 00$

$5.527 E 02$

$1.603 \mathrm{E} \quad 03$

$4.201 \mathrm{E} 03$

$1.100 E \quad 04$

$3.334 \mathrm{E} O 4$

0.0

0.0

0.0

0.0

0.0

0.0

0.0
0.0

0.0

0.0

0.0

0.0

ANGLE 11

ANGLE 12

ANGLE 13

$1.695 E 00$

$1.283 E \quad 02$

5. $635 \mathrm{E} 02$

$\begin{array}{ll}1.632 E & 03 \\ 4.270 E & 03\end{array}$

$1.116 \mathrm{E} 04$

$2.378 \mathrm{E} \quad 04$
$3.374 \mathrm{E}$

AVGLE 6

ANGLE 7

ANGLE 8

MU $=-0.2816 \quad$ ANGLE 9

0.0 .0179 MU 0.0

$M U=-0.4580$
0.0

0.0

0.0

0.0

0.0
0.0
0.0

0.0

0.0

0.0

0.0

0.0

$1.771 E 00$

2. $328 \mathrm{E} 00$

1.320E 02

$\begin{array}{ll}5.779 E & 02 \\ 1.670 E & 03\end{array}$

$4.359 E$ O3
1.63

$1.137 \mathrm{E} 04$

2.420 E 04

$3.425 E \quad 04$

0.0

0.0

0.0

0.0

0.0

0.0

0.0
0.0
0.0

0.0

0.0

0.0

$1.871 E 00$

$2.113 E$ EO

$\begin{array}{ll}1.366 \mathrm{E} & 02 \\ 5.957 \mathrm{E} & 02\end{array}$

$\begin{array}{lll}5.957 E & 02 \\ 1.717 E ~ & 03\end{array}$

$4.468 E 03$

$1.163 \mathrm{E} 04$

$2.471 E \quad 04$

3.486 E 04

$\begin{array}{lll}0.0 & M U=0.4580 \\ 0.0 & 0.0 & M U=0\end{array}$

0.0

0.0

0.0

0.0

0.0

0.0

0.0

0.0

0.0

0.0

0.0

$2.506 \mathrm{E} 00$

1.649 E O2 $6.992 \mathrm{E} \mathrm{O2}$ 1.984E -3 1.074E 03 $1.305 E$ O 3.81OE O4
0.0

0.0

0.0

0.0

0.0

0.0

0.0

0.0

0.0

0.0

0.0

2.677E 00

$1.736 \mathrm{E} 02$ T.310E O2 $2.064 E$ O3 $1.250 E$ E $1.345 E$ E $3.900 E$ O4
0.0

0.0

0.0

0.0

0.0

0.0

0.0

0.0

0.0

0.0

0.0

0.0

$2.914 E 00$

$3.176 \mathrm{E} 00$

$1.835 E$
7.62 $3.144 E 03$ $5.424 E 03$ 1.384E 04 $3.986 \mathrm{E} \quad 04$

0.0

0.0

0.0

0.0

0.0

0.0

0.0
ANGLE 14

ANGLE 15

ANGLE 16

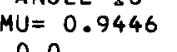

0.0

0.0

0.0

0.0

$3.864 \mathrm{E} \quad 00$

$3.188 \mathrm{E} 00$

$1.957 E 02$ $2.221 \mathrm{E} 03$ 5.5 B6E O3 $1.420 E$ O4 $4.064 E 04$

0.0

0.0
0.0
0.0
0.0

0.0
0.0
0.0
0.0

0.0

0.0

0.0

0.0

0.3

0.0

0.0

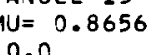


(NEUTRONS/MEV/STERADIAN/SOURCE NEUTRON) GROUP IMEV

$1.22 E$ 01---1.50E Ol $1.00 E$ OL $--1.22 \mathrm{E}$ O1 $8.19 E$ OOM--1.00E 01 $6.36 \mathrm{E} 00--8.19 \mathrm{E} 00$ $4.97 E$ O0-- $6.36 E$ E 00 $3.01 E$ O0--4. $07 E$ OO $2.46 E$ OO-- $3.01 E 00$ 2.35E $00---2.46 E$ OO $1.82 \mathrm{E} \quad 00--2.35 \mathrm{E} 00$ $1.11 \mathrm{E} 00--1.83 \mathrm{E} 00$ $5.50 E-01--1.11 E 00$ $1.11 \mathrm{E}-01--5.50 \mathrm{E}-01$ 3. 35E-02--1.11E-01 $5.83 \mathrm{~F}-04--3.35 \mathrm{E}-02$ 1.01E-04-- $5.83 \mathrm{E}-04$ 2.90E-05--1. $01 E-04$ 1.07E-05--2. $90 E-05$ $3.06 E-06--1.07 E-05$
$1.12 E-06--3.06 E-06$ $1.12 \mathrm{E}-06--3.06 \mathrm{E}-06$ $4.14 E-07---1.12 E-06$
$0.0 \quad--04.14 E-07$

\section{ENERGY GROUP (MEV)} $1.2 \mathrm{ZE} \mathrm{O1---1.50E} \mathrm{O1}$

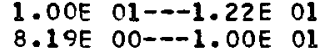

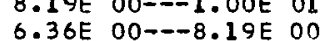
$6.36 \mathrm{E}$
$4.90---8.19 E$ $4.07 E$ OO---4.97E 00 $3.01 \mathrm{E} 00--4.07 \mathrm{E}$ OD 2.46 E $00---3.01 E$ EO $2.36 \mathrm{E} 00-2.01 \mathrm{EE} 00$ $1.83 E$ OO-- $2.35 \mathrm{E} 00$ $1.11 \mathrm{E} 00--1.83 \mathrm{E}$ 00 $5.50 \mathrm{~F}-01--1.11 \mathrm{E} 00$ $1.1 \mathrm{ME}-01--5.50 \mathrm{E}-01$ $3.35 \mathrm{E}-02--1.11 \mathrm{E}-01$ $5.83 \mathrm{E}-04--3.35 \mathrm{E}-02$ $1.01 E-04--5.83 E-04$ 2.90E-05--1.01E-04 $1.07 E-05-2.90 E-05$ $3.06 \mathrm{E}-0 \mathrm{~S}--1.07 \mathrm{E}-05$ $1.12 \mathrm{E}-06-0-3.06 \mathrm{E}-06$ $4.14 \mathrm{E}-07--1.12 \mathrm{E}-06$ $0.0 \quad--4.14 E-07$
ANGLE 1
$M U=-1.0000$ 0.0 0.0 0.0 0.0 0.0 0.0 0.0 0.0 0.0 0.0 $9.919 E-01$ $1.281 E$ OO $9.536 \mathrm{E} \mathrm{Ol}$ $4.589 E \quad 02$ $1.426 \mathrm{E} 03$ $4.038 \mathrm{E} 03$ $1.118 E 04$ $2.503 E 04$ $3.735 E \quad 04$ ANGLE 10 $M U=0.0950$ 0.0 0.0 0.0 0.0 0.0 0.0 0.0 0.0 0.0 0.0 0.0 0.0 $1.556 \mathrm{E} 00$ $1.955 \mathrm{E} 00$ $1.240 E 02$ $5.826 \mathrm{E} 02$ $1.786 E_{03}$ $4.973 \mathrm{E} \mathrm{O3}$ $1.358 \mathrm{E} \mathrm{O4}$ $3.005 E \quad 04$ $4.379 E \quad 04$
ANGLE 2
MU $=-0.9894$ 0.0

0.0

0.0
0.0
0.0

0.0

0.0

$0 . \mathrm{C}$

0.0

0.0

0.0

0.0

0.0

$0.949 \mathrm{E}-01$

$1.284 \mathrm{E} 00$

$9.557 \mathrm{E} O 1$

$4.598 \mathrm{E} 02$

$4.045 \mathrm{E} 03$

$1.045 E$ O

$2.507 E 04$

$3.740 E$ O4

ANGLE 11

$M U=0.2816$

0.0

0.0

0.0

0.0

0.0

0.0

0.0

0.0

0.0

0.0

$1.530 E$ OO $1.5307 E 00$ $1.308 \mathrm{E} \mathrm{O2}$ $6.117 E 02$ $1.870 E$ O3 $5.184 E \quad 03$ $1.411 \mathrm{E} O 4$ $3.114 E$ O4
ANGLE 3
$M U=-0.9446$ 0.0 0.0 0.0 0.0 0.0 0.0 0.0 0.0 0.0 0.0 $1.007 \mathrm{E} 00$ $1.297 E 00$ $9.644 \mathrm{E} 01$ $4.637 \mathrm{E} 02$ 1.440 E 03 $4.075 \mathrm{E} \quad 03$ $1.127 E$ O4 $\begin{array}{ll}2.524 \mathrm{E} & 04 \\ 3.762 \mathrm{E} & 04\end{array}$ ANGLE 12 $M U=0.4580$ 0.0 0.0 0.0 0.0 0.0 0.0 0.0 0.0 0.0 $1.791 \mathrm{E} 00$ 2.347E 00 $1.386 \mathrm{E}$ O2 $6.432 \mathrm{E} 02$ 1.959 E 03 $5.405 \mathrm{E} 03$ $1.466 \mathrm{E} 04$ 4.659E O4
$M U=-0.8656$ 0.0

0.0

0.0
0.0

0.0

0.0
0.0

0.0

0.0

0.0

0.0
$1.031 \mathrm{E}$

$1.321 \mathrm{E} 00$

$9.806 \mathrm{E} \mathrm{O1}$

$1.461 \mathrm{E} 03$

$4.132 \mathrm{E} \quad 3$

$1.142 \mathrm{E} 04$

3.803 E 04

ANGLE 13
$M U=0.6179$

0.0

0.0
0.0

0.0

0.0
0.0

0.0

0.0

0.0

0.0

1.897E 00 $2.025 \mathrm{E} \mathrm{OO}$

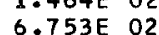
$2.048 E 03$ $5.627 \mathrm{E} 03$ 1.521E 04 $3.337 E$ O4

$4.797 E$ E
$M U=-0.7550 \quad M U=-0.6179$ 0.0

0.0

0.0

0.0

0.0

0.0

0.0

0.0

0.0

$1.066 \mathrm{E} \quad 00$

$1.356 \mathrm{E} 00$

$1.004 E$ O2

$1.492 \mathrm{E} \quad 3$

$4.212 \mathrm{E} O 3$

$1.163 \mathrm{E} 04$

3.860 E 04

ANGLE 14

ANGLE 14
$M J=0.7550 \quad M U=0.8656$

0.0

0.0

0.0

0.0
0.0
0.0
0.0

0.0

0.0

0.0

0.0

$2.169 E 00$ $2.288 E 00$ $1.545 E$ O2 2.066E 02 $5.836 \mathrm{E} 03$ $\begin{array}{ll}5.836 E & 03 \\ 1.572 E & 04\end{array}$ 3. $440 E$ E

4.924E 04

$M U=-0.6179$ MU

0.0

0.0

0.0

0.0

0.0

0.0

0.0

$1.113 E 00$

$1.403 \mathrm{E} 00$

.

..532E O3

$4.317 \mathrm{E} \quad 03$

1.190E 04

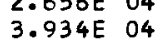

$M U=0$
0.0

0.0
0.0

0.0

0.0
0.0

0.0

0.0

0.0

0.0

$2.356 E 00$ $2.334 \mathrm{E} 00$ $1.618 \mathrm{E} 02$ $7.349 E \quad 2$ $2.212 E 03$ $6.020 E 03$ 1.S17E 04 $3.529 E \quad 04$ 5.032E O4 
(NEUTRONS/MEV/STERADIAN/SOURCE NEUTRON

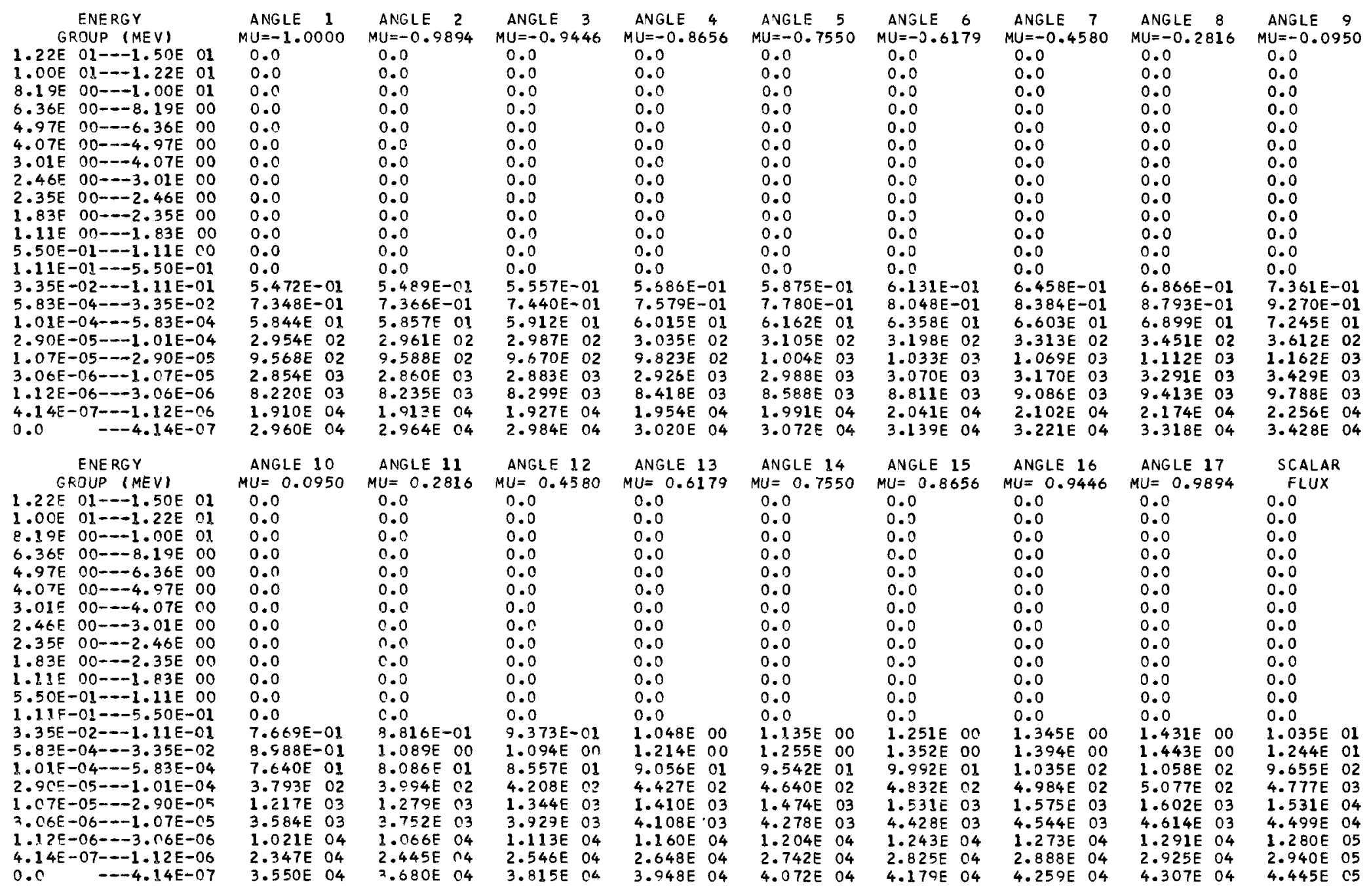


(NEUTRONS/MEV/STERADIAN/SOURCE NEUTRON)

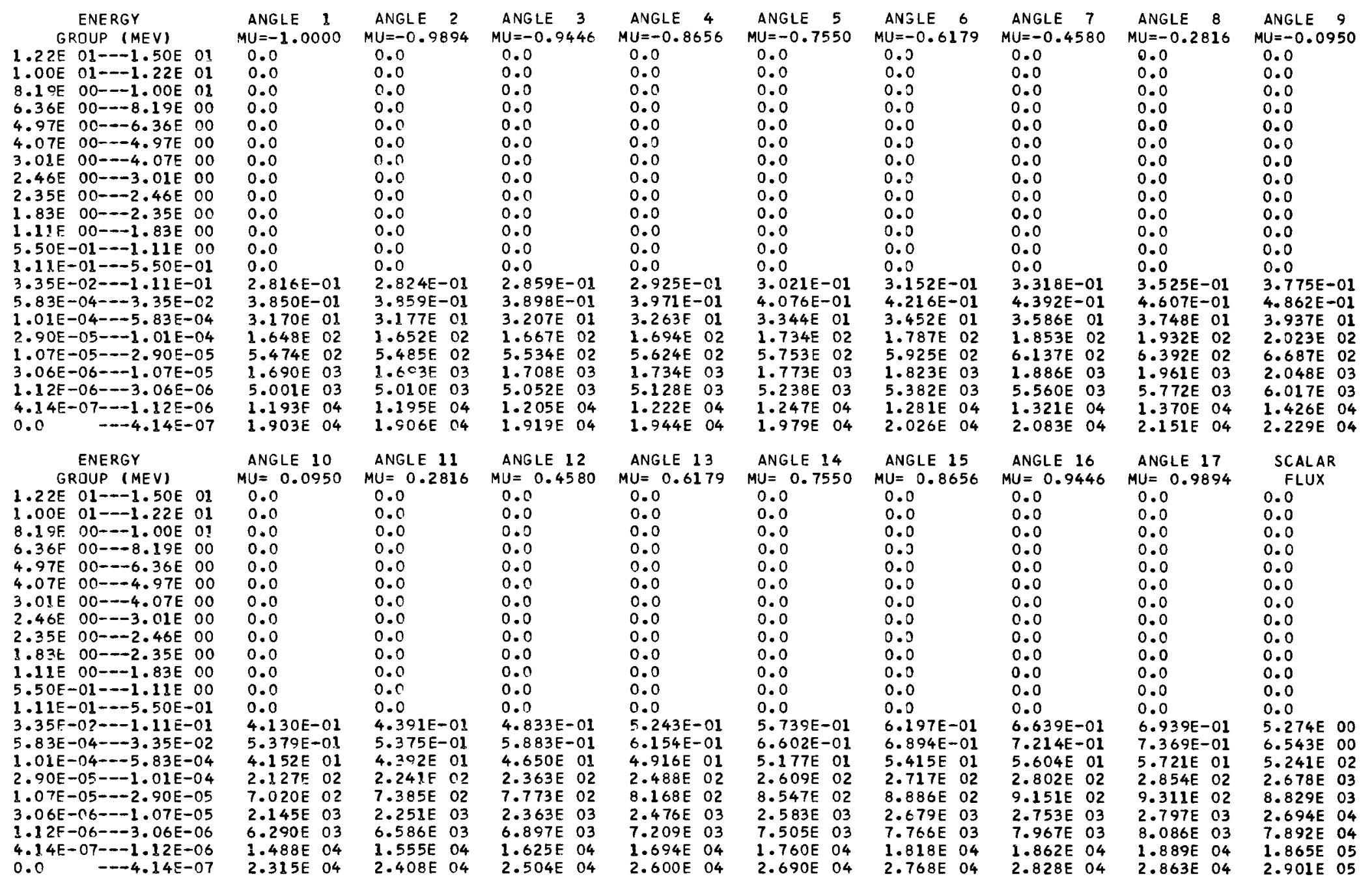


(NEUTRONS/MEV/STERADIAN/SOURCE NEUTRON)

ENERGY GROUP $1.22 E$ O1--1.50E OI $1.00 E$ O1---1.22E OL 8.19E $00-1.00 E$ OI 6.36E $00--8.19 E$ OO 4.97E $00--6.36 E$ E 00 4.07E $00--4.97 E$ OO $3.01 \mathrm{E} 00--4.07 \mathrm{E}$ Oᄋ

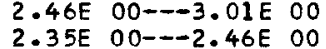

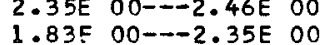
$\begin{array}{lll}1.83 E & 00--2.35 E & 00 \\ 1.11 \mathrm{E} & 00--1.83 \mathrm{E} & 00\end{array}$ $5.50 E-01--1.11 E$ OO $1.11 \mathrm{E}-01--5.50 \mathrm{E}-01$ $3.35 \mathrm{E}-02--1.11 \mathrm{E}-01$ $5.83 \mathrm{E}-04---3.35 \mathrm{E}-02$ $1.01 E-04--5.83 E-04$ 2.9CE-0.5- $1.01 E-04$ $1.07 E-05-2.90 \varepsilon-05$ $1.06 \mathrm{E}-06-2.07 \mathrm{E}-05$ $4.14 E-07--1.12 E-06$ $4.14 E-07---1.12 E-06$
$0.0 \quad--4.14 E-07$

ENERGY

GROUP (MEV) $1.22 E$ 01---1.50E Ol $1.00 E$ O1- - 1.22E O1 $8.19 \mathrm{E} \quad 00--1.00 \mathrm{E}$ 01 $4.97 E$ OO- $-6.36 \mathrm{E}$ OO $4.07 E$ 00- $4.07 E$ OO $3.01 E$ OO- $4.07 E$ E 00 $2.46 \mathrm{E} 00--3.01 \mathrm{E}$ OO $2.35 E$ E0- $2.46 E 00$ $1.83 \mathrm{E} \quad 00--2.35 \mathrm{E} 00$ $1.11 \mathrm{E} 00--1.83 \mathrm{E} 00$ 5.50E-01-- $1.11 \mathrm{E}$ OO $3.35 E-02--1.11 E-01$ $5.83 E-04-\cdots-35 E-02$ $1.01 E-04--5.83 E-04$ $2.90 E-05--1.01 E-04$ $1.07 E-05--2.90 \mathrm{E}-05$ $3.06 \mathrm{E}-06---1.07 E-05$ $1.12 \mathrm{E}-06---3.06 \mathrm{E}-06$ $4.14 E-07--1.12 E-06$ $M U=-1.0000$

0.0

0.0

0.0

0.0

0.0

0.0

0.0

0.0

0.0

$0 . \mathrm{C}$

0.0

$1.386 E-01$
$1.909 E-01$

$1.603 E 01$

$8.468 \mathrm{E}$ OI

$2.854 E$ O2

2.714E 03

$2.714 E$ E

6.5

ANGLE 10

$M U=0.0950$

0.0

0.0

0.0

0.0

0.0

0.0

0.0

0.0

0.0

0.0

0.0

1.987E-01

$2.494 \mathrm{E}-01$
$2.100 \mathrm{E} 01$

$1.095 \mathrm{E} 02$

$3.674 \mathrm{E} \quad 22$

$3.438 E \quad 03$

8.291 E 03

$1.317 E \quad 04$
$A N G L E \quad 2$
$M U=-0.0894$ 0.0

0.0

0.0

0.0

0.0

0.0

0.0

0.0

0.0

0.0
0.0

1.390E-01

$1.014 E-01$

$1.606 E$ E1

$8.486 E$ O1

$2.861 E 02$

$9.024 E 02$

$6.604 E 03$

$1.075 \mathrm{E} 04$

ANGLE 11

0.0

0.0

0.0

0.0

0.0

0.0

0.0

0.0
0.0

0.0

0.0

2.166E-01

$2.722 E-01$

$2.220 E$ OI

$1.154 E 02$

$3.867 E \quad 02$

$1.208 \mathrm{E} 03$

$3.605 \mathrm{E} 03$

$8.681 E$
$1.373 E \quad 04$
$M U=-0.9446$

0.0

0.0

0.0

0.0

0.0

0.0

0.0

0.0

1. $408 \mathrm{E}-01$

$1.933 \mathrm{E}-01$
$1.622 \mathrm{E} 01$

$8.564 \mathrm{E} \mathrm{O1}$

$2.886 E$ O2

$2.742 \mathrm{E} 03$

6.659 E 03

ANGLE 12

0.0

0.0

0.0

0.0

0.0

0.0

0.0

0.0

0.0

0.0

0.0

2.348E-01 2. $863 \mathrm{E}-01$ $2.348 E$ OI $1.217 \mathrm{E} 02$ $4.073 \mathrm{E} \mathrm{O2}$ $1.269 E \quad 03$ $3.781 \mathrm{E} \quad 03$ 9.089 E 03 $1.431 E$ O4

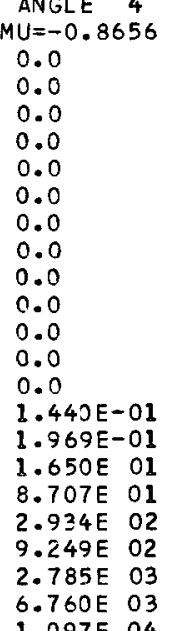

$\begin{array}{cc}\text { ANGLE } & 5 \\ M U=-0.7550\end{array}$ 0.0
0.0

0.0

0.0
0.0

0.0
0.0

0.0

0.0
0.0

0.0

0.0

0.0

1. $487 E-01$

$2.020 \mathrm{E}-01$

$1.691 E$ OI
$8.914 E$ 01

$3.003 E 02$

$9.459 E \quad 02$

$2.846 E$ E

1. $118 \mathrm{E} 04$

ANGLE 13

ANGLE 14

$M U=0.6179$

0.0

0.0

0.0

0.0

0.0

0.0
0.0

0.0

0.0

0.0

0.0

$2.558 \mathrm{E}-01$

$3.061 \mathrm{E}-01$

$2.481 \mathrm{E}$
$1.281 \mathrm{E}$

$4.281 E 02$

$1.331 E 03$

$3.959 \mathrm{E} 03$

$9.498 \mathrm{E} \quad 3$
$M U=0.7550$

0.0

0.0

0.0

0.0

0.0

0.0

0.0

0.0

0.0

0.0

2. $773 E-01$

3.224E-01

$2.610 E 01$

$1.343 E 02$

$1.391 E 03$

4.127E 03

$9.885 \mathrm{E} \mathrm{O3}$
AVSLE 6 $M U=-0.6179$ 0.0
0.0

0.3

0.0

0.0

0.0

0.0

0.0

0.0

0.0

0.0

0.0

$2.089 E-01$

$1.746 E$ ol

$9.188 \mathrm{E}$ OI

$3.093 \mathrm{E} O 2$

$2.927 \mathrm{E} 03$

$7.095 \mathrm{E} \mathrm{O3}$

ANOLE 15

0.0

0.0

0.0

0.0

0.0

0.3

0.0

0.0

0.0

0.0

2.988E-01 $3.395 \mathrm{E}-01$ $\begin{array}{ll}2.727 E & 01 \\ 1.398 E & 02\end{array}$ 4.660 E 02 $1.443 \mathrm{E} 03$ $4.275 E \quad 03$ 1. $0.22 \mathrm{E} \quad 04$ $1.590 \mathrm{E} 04$

ANGLE 7 $M U=-0.4580$ 0.0

0.0

0.0

0.0

0.0

0.0

0.0

0.0 0.0

$1.631 \mathrm{E}-01$ $2.176 \mathrm{E}-01$ $9.528 E$ O1 $3.206 E 02$ $1.0 \mathrm{CBE} 03$ $7.330 E$ O3 $1.179 E$ O

ANGLE 8 $M U=-0.2816 \quad M U=-0.0950$ 0.0 
(NEUTRONS/MEV/STERADIAN/SOURCE NEUTRONI

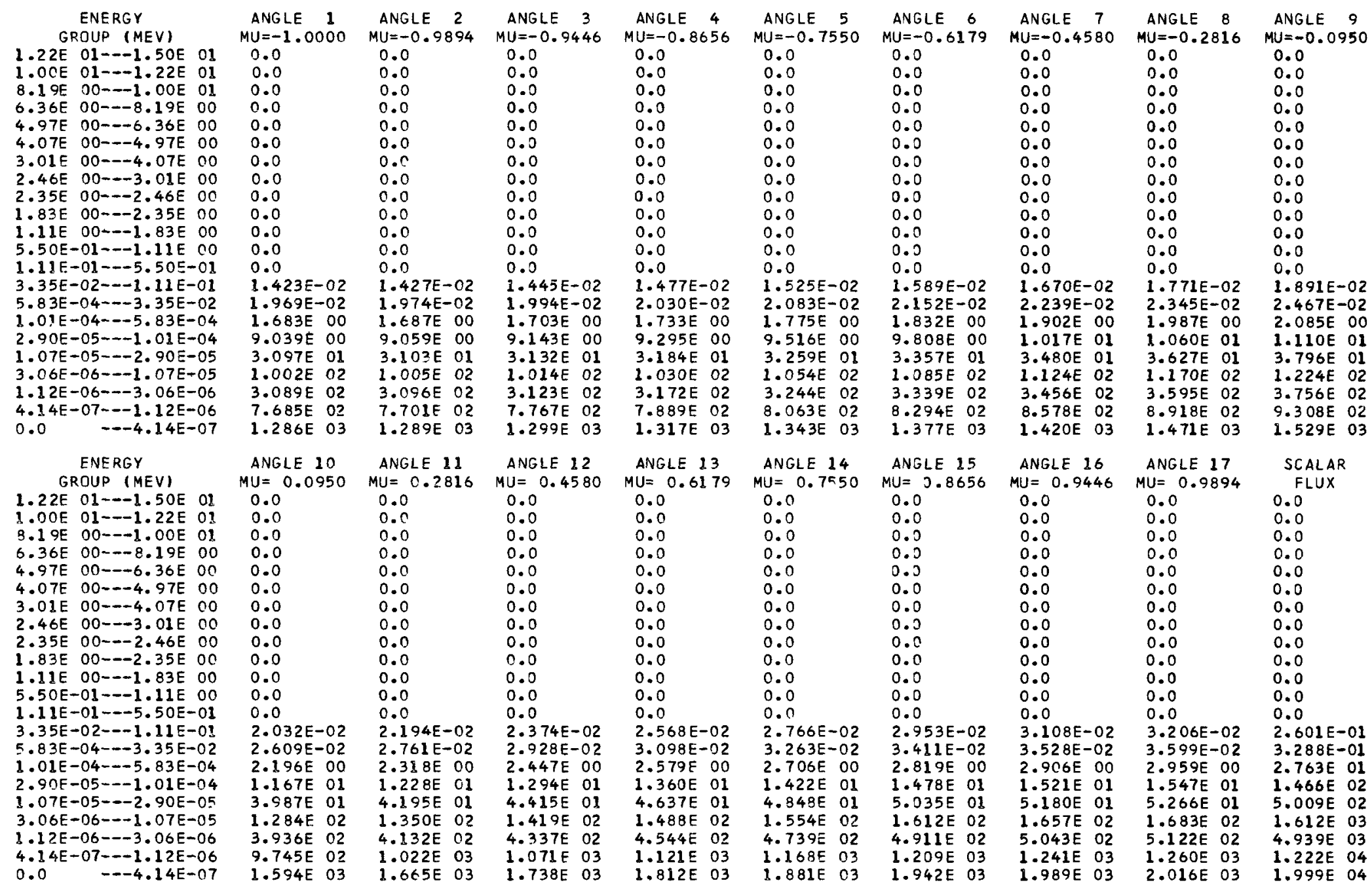




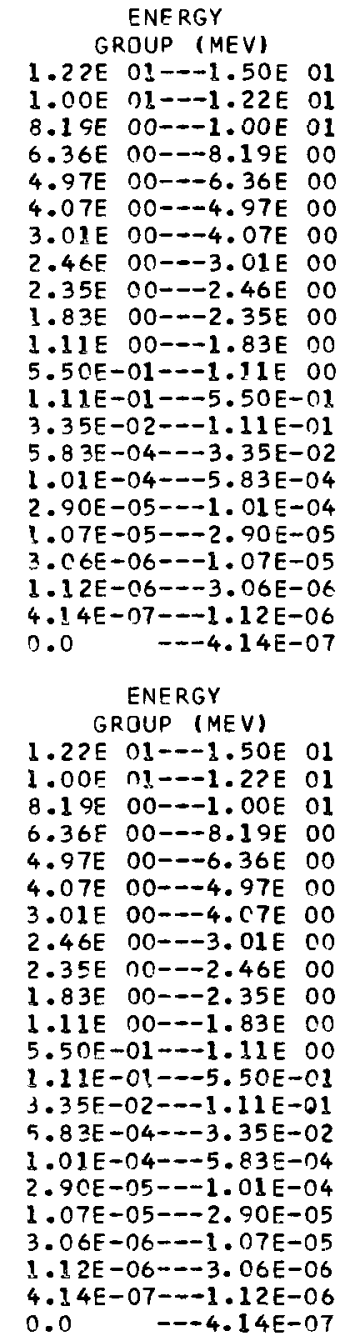
ANGLE $\quad 2$
$M U=-0.5894$ MU $=-1$ 0.0 0.0 0.0 0.0 0.0 0.0 0.0 0.0 0.0 0.0 0.0 0.0 $1.290 \mathrm{E}-03$ $1.529 \mathrm{E}-\mathrm{O}$ $8.232 E-01$ $2.828 \mathrm{~F} O 0$ $9.196 \mathrm{E} \quad 00$ $2.846 E$ OI $7.116 E$ OI $1.198 \mathrm{E}$ O2

ANGLE 10 $M U=0.0950$ 0.0 0.0 0.0 0.0 0.0 0.0 0.0 0.0 0.0 0.0 0.0 $1.835 E-03$ $2.353 \mathrm{E}-03$ $1.980-01$ $1.059 E$ OD $1.675 \mathrm{E} O$ $3.620 \mathrm{E}$ OI $9.013 E$ O $1.484 \mathrm{E} 02$ 0.0 0.0 0.0 0.0 0.0 0.0 0.0 0.0 0.0 0.0 0.0 $1.204 \mathrm{E}-03$ $1.789 \mathrm{E}-\mathrm{C3}$ $1.533 E-01$ 8.250E-C1 $2.852 \mathrm{E}$ OI $7.130 E$ OI
$1.200 E$ O2 ANGLE 11
$M U=0.2816$

$M U=0$
$0 . n$
0.0
0.0
0.0

0.0
0.0
0.0

0.0

0.0

0.0

0.0

0.0

0.0

0.0

0.0

$1.077 E-03$

$2.489 \mathrm{E}-03$

2.095E-OL

$3.815 E 00$

$1.234 E$ O1

$3.707 E$ OI

$9.446 \mathrm{E} \mathrm{OI}$

$1.550 \mathrm{E} \mathrm{O2}$
(NEUTRJNS/MEV/STERADIAN/SOURCE NEUTRON)

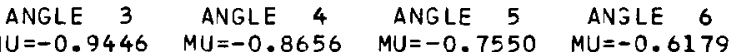
$U=-0.9446$ $9.215 \mathrm{E} 00$

0.0

0.0
0.0
0.0

0.0

0.0

0.0
0.0
0.0
0.0

0.0
0.0

0.0

0.0

0.0

1.310E-03

$1.807 E-03$

$=-0.8656$

0.0

0.0
0.0
0.0
0.0

0.0

0.0
0.0
0.0
0.0

0.0
0.0
0.0

0.0

0.0

0.0

1.547E-01

$1.339 \mathrm{E}-03$

$1.839 \mathrm{E}-03$

$1.574 \mathrm{E}-01$

$=-0.7550 \quad M U=-0.6179$

ANGLE 7

ANGLE $8 \quad$ ANGLE 9

$2.859 \mathrm{E} 00 \quad 2.906 \mathrm{E} 00$

$9.448 \mathrm{E} 00$

0.0

0.0
0.0
0.0

0.0

0.0

0.0
0.0
0.0

0.0

0.0

0.0
0.0
0.0

0.0

9.297E 00

$2.923 E$ ol

$7.304 \mathrm{E}$ ol

$1.210 E$ O2

$1.226 \mathrm{E} 02$

0.0

0.0
0.0
0.0

0.0
0.0
0.0

0.0

0.0

0.0
0.0
0.0
0.0

0.0

$1.382 E-03$

0.0

$1.612 \mathrm{E}-01 \quad 1.663 \mathrm{E}-01$

$8.925 E-01$

$2.974 E$ OO $3.064 E$ OD

..664E OO 9.950E OO

3.075E OL

1.251E 02 $1.283 \mathrm{E}$ 02

$-0.4580$

$A U=-0.2816$ $M U=-0.0950$

ANGLE 12

ANGLE 13

ANGLE 14

ANGLE 15

0.0

$M U=$
0.0
0.0

0.0

0.0
0.0
0.0

0.0
0.0

0.0

0.0

0.0

0.0

0.0

0.0
0.0

0.0
0.0
0.0

$2.134 E-03$

$2.533 E-03$

$2.209 E-01$

$4.010 \mathrm{~F} 00$

$4.010 F$ OO

$1.296 E$ O

9.898 E 01

$M U=0.7550$

MU $=$
0.0
0.0

0.0

0.0

0.0

0.0

0.0

0.0

0.0

0.0

0.0

0.0

$2.302 E-03$

$2.781 \mathrm{E}-03$

$1.324 E-01$

$4.205 E 00$

1.358E

$4.169 \mathrm{E} O$

$1.035 \mathrm{E} \mathrm{O2}$

0.0

0.0

0.0

0.0

0.0

0.0

$2.470 E-03$

$2.922 E-03$
$2.433 E-01$

$2.433 E-01$

$1.416 E$

$1.078 \mathrm{E}$ O2

C. 0

0.0
0.0

0.0
0.0
0.0

0.0
0.0

0.0
0.0
0.0

0.0

0.0
0.0

0.0
0.0
0.0

$1.513 E-03$

$2.027 E-03$

$1.726 \mathrm{E}-01$

$3.174 \mathrm{E} O 0$

$1.030 E$ O1

$3.182 \mathrm{E}$ OI

$7.940 \mathrm{E} 01$
$1.323 \mathrm{E} 02$

0.0
0.0

0.0
0.0
0.0
0.0

0.0
0.0
0.0

0.0

0.0

0.0

0.0

0.0

0.0

$1.603 \mathrm{E}-03$

$2.120 \mathrm{E}-03$

$1.802 E-01$

$3.306 E O O$

.072E 01

$3.310 E$ OI

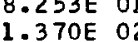

0.0

0.0

0.0

0.0

0.0

0.0

0.0

0.0

0.0

0.0

0.0

$1.710 \mathrm{E}-03$

$2.229 \mathrm{E}-03$
$1.889 \mathrm{E}-01$

$.009 E 00$

$3.459 E$ OO

$1.121 E$ OI

$3.456 \mathrm{E}$ OL

$8.612 E$ OI

ANGLE 16

ANGLE 17

MU $=0$
0.0
0.0

0.0
0.0
0.0
0.5

0.0

0.0

0.0

0.0

0.0

0.0

0.0

0

$2.627 E-03$

$3.049 E-03$

2.530E-01

$4.554 E 00$

.

$496 E 0$

$1.115 \mathrm{E} 02$
$1.804 \mathrm{E} 02$

0.0

0.0

0.0

0.0 
INEUTRONS/MEV/STTERADIAN/SOURCE NEUTRONI

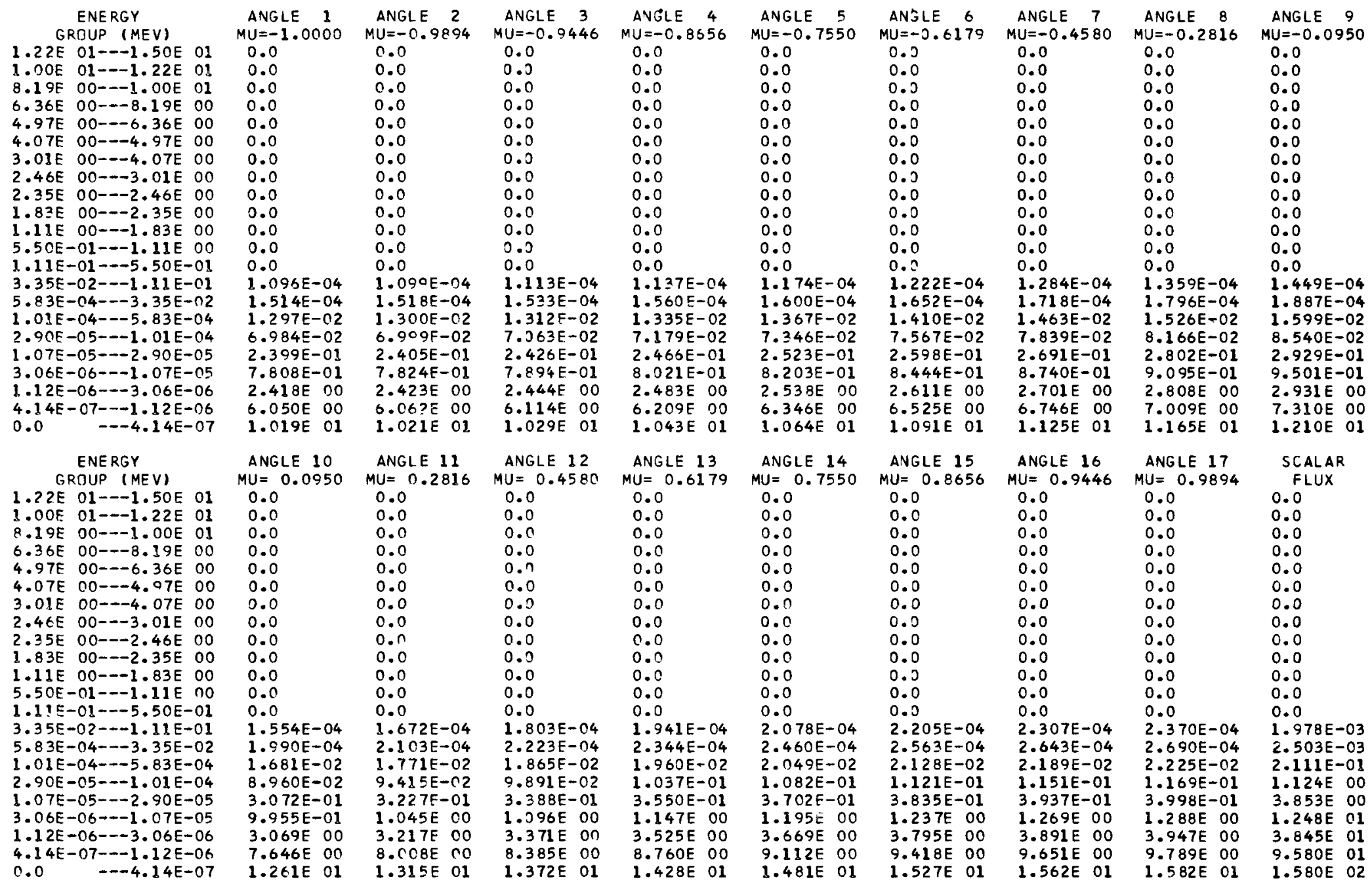


(NEUTRONS/MEV/STERADIAN/SOURCE NEUTRONI

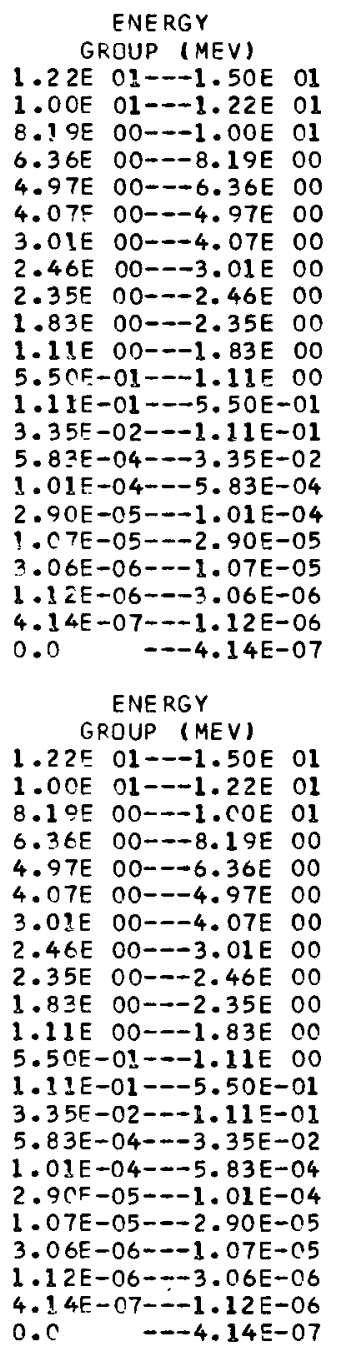

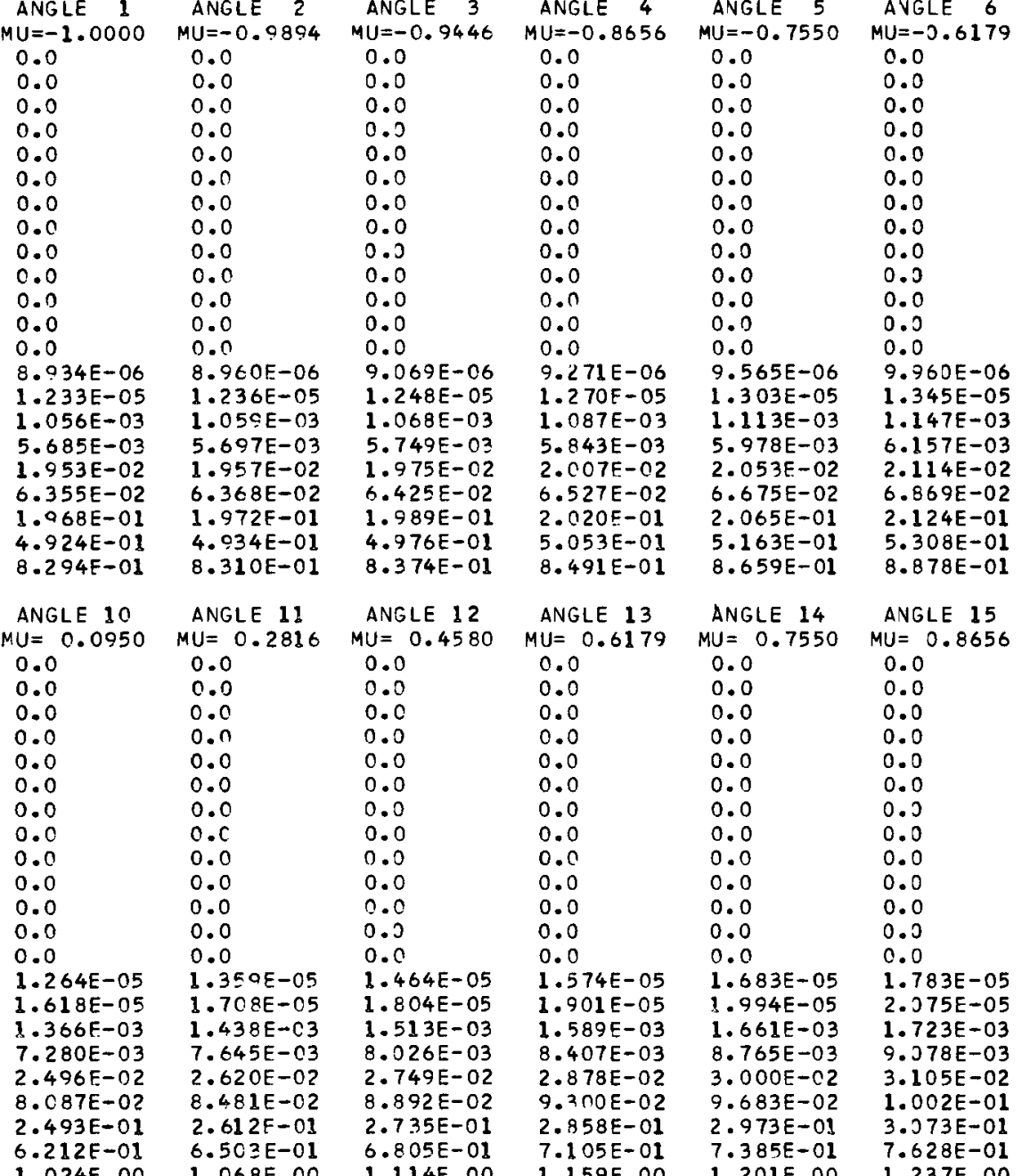

ANGLE 7
$M U=-0.4580$
0.0
0.0
0.0
0.0
0.0
0.0
0.0
0.0
0.0
0.0
0.0
0.0
0.0
$1.046 E-05$
$1.398 E-05$
$1.190 E-03$
$6.377 E-03$
$2.189 E-02$
$7.109 E-02$
$2.197 E-01$
$5.487 E-01$
$9.149 E-01$

ANGLE 8 $M U=-0.2816 \quad M U=-0.0950$ $0.0 \quad 0.0$

$0.0 \quad 0.0$

$0.0 \quad 0.0$

$\begin{array}{ll}0.0 & 0.0 \\ 0.0 & 0.0\end{array}$

$0.0 \quad 0.0$

$0.0 \quad 0.0$

$\begin{array}{ll}0.0 & 0.0 \\ 0.0 & 0.0\end{array}$

$\begin{array}{ll}0.0 & 0.0 \\ 0.0 & 0.0\end{array}$

0.0

0 .0

$1.107 \mathrm{E}-05$

$1.461 \mathrm{E}-05$

$1.241 E-03$
$6.640 E-03$

$6.640 \mathrm{E}-03$

$2.279 E-02$

$7.395 \mathrm{E}-02$

$2.284 E-01$
$5.699 E-01$

$9.149 \mathrm{E}-01$

$9.470 E-01$

0.0

0.0

$1.180 \mathrm{E}-05$

$1.535 E-05$

$1.300 \mathrm{E}-03$

$6.942 \mathrm{E}-03$

$2.381 \mathrm{E}-02$

$7.722 \mathrm{E}-02$

2. $383 \mathrm{E}-01$

$.942 E-01$

ANGLE 16

ANGLE 17

SCALAR

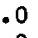

0.0

0.0

0.0 .

0.0

0.0

0.0

0.0

0.0

0.0

0.0

1. $864 \mathrm{E}-05$

$1.864 E-05$
$2.138 E-05$

1. $.771 \mathrm{E}-03$

$9.316 E-03$

$3.186 \mathrm{E}-02$

$1.027 \mathrm{E}-01$
$3.149 \mathrm{E}-01$

$7.813 \mathrm{E}-01$

$1.265 E$ OO

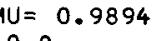

0.0

0.0
0.0

0.0
0.0

0.0

0.0

0.0

0.0

0.0

0.0

0.0

1. $913 E-05$

$1.913 E-05$
$2.175 E-05$

$1.800 \mathrm{E}-03$

$9.457 \mathrm{E}-03$

3.234E-02

$1.042 \mathrm{E}-01$

$3.194 \mathrm{E}-01$

$7.922 E-01$

FLUX

0.0

0.0

0.0

0.0

0.0

0.0

0.0

0.0
0.0

0.0

0.0

0.0

0.0

1.607 E-04

$2.033 E-04$

$1.714 \mathrm{E}-02$

$3.126 \mathrm{E}-02$

$3.128 E-01$
$1.013 E ~$

$3.123 \mathrm{E} 00$

$3.123 E$
$7.780 E$

$7.780 E$
$1.283 E$ 
(GAMMAS/MEV/STERADIAN/SOURCE NEUTRONI

ENERGY

GROUP (MEV)

$8.00 E$ OO- - 1.00E 01

6.50 E $00--8.00 E$ OD

.0OE OD- $-6.50 E$ OO 4.0OE OO- - - 5. OOE OO 2.5OE OO- - 3 . OOE OO $2.00 E$ OO- $-2.50 E$ OO $1.66 \mathrm{E} 00--2.00 \mathrm{E} 00$ $1.33 E$ OO-- 1.66E 00 . OCE 00--1.33E OD 8.00E-01-D-1.0OE 00 $6.00 E-01--8.00 E-01$ $4.00 E-01-2-6.00 E-01$ $3.00 E-01--4.00 E-01$ $2.00 E-01---3.00 E-01$ $1.00 E-01--2.00 E-01$ $5.00 E-02--1.00 E-01$

ENERGY

GROUP (MEV)

8.00E $00--1.00 E$ OL $6.50 E$ O0- - 8.00E OO $5.00 E$ OO-- -6.50E ON 4.0OE $00--5.00 E$ OO $3.00 E$ OO- $-4.00 E$. OD $2.50 E$ OO-- $3.00 E$ OO $2.00 E$ OO---2.5OE OO $1.66 E$ OO---2.00E CO .33E $00--1.66 \mathrm{E} 00$ $1.00 E$ O0-D-1.33E OO $8.00 E-01---1.00 E$ OO $6.00 E-01---8.00 E-01$ 4.00E-01---6.00E-01 $3.00 E-01--4.00 E-01$ $2.00 E-01--3.00 E-01$ $1.00 E-01--2.00 E-01$ $5.00 E-02--1.00 E-O 1$ 2. $00 \mathrm{E}-02---5.00 \mathrm{E}-02$
ANGLE 1 ANGLE 2 MU $=-1.0000$ MU $=-C .9894$ $1.432 \mathrm{E}-05 \quad 1.435 \mathrm{E}-05$ $1.432 E-05 \quad 1.435 E-05$ $4.634 E-04$ 1.638E-04 $4.249 E-05$ 4.260E-0 $1.796 \mathrm{E}-05 \quad 7.816 \mathrm{E}-05$ $1.490 \mathrm{E}-05 \quad 1.496 \mathrm{E}-05$ $1.688 \mathrm{~F}-05$
$1.973 \mathrm{E}-05$ $1.973 E-05$
$2.370 E-05$ 3.086E-05 $3.379 E-05$ $4.184 E-05 \quad 4.199 E-05$ $6.030 E-05 \quad 6.050 E-05$ $2.600 \mathrm{E}-04 \quad 2.605 \mathrm{E}-04$ $3.023 \mathrm{E}-04 \quad 3.028 \mathrm{E}-04$ $6.002 \mathrm{E}-04 \quad 6.005 \mathrm{E}-\mathrm{C}$ $1.474 \mathrm{E}-03 \quad 1.475 \mathrm{E}-03$ $3.360 \mathrm{E}-03 \quad 3.361 \mathrm{E}-03$ $9.115 E-04$

$3.361 E-03$
$9.117 \mathrm{E}-04$

ANGLE 10 $M U=0.0950$ $9.190 \mathrm{E}-06$ $1.910 \mathrm{E}-05$ $2.168 E-04$ $1.044 E-04$ $2.276 \mathrm{E}-05$ $2.554 \mathrm{E}-05$ $2.958 \mathrm{E}-05$ $3.526 \mathrm{E}-05$ $4.543 \mathrm{E}-05$ $6.066 E-05$ $8.502 E-05$ $3.208 E-04$ $3.598 E-04$ $6.388 \mathrm{E}-04$ $1.578 \mathrm{E}-03$ $3.522 \mathrm{E}-0$ $9.309 E-04$ ANGLE 11 $=0.2816$ $2.031 E-05$ 2.303E-04 $6.153 \mathrm{E}-05$ $1.110 E-04$ . $2.762 \mathrm{E}-05$ $3.194 E-C 5$ $3.798 E-05$ $4.876 E-05$ $9.014 E-05$ $3.328 E-04$ $3.701 \mathrm{E}-04$ $6.470 \mathrm{E}-04$ $1.598 \mathrm{E}-03$ $3.552 \mathrm{E}-03$ $9.344 \mathrm{E}-04$
ANGLE 3 $M U=-0.9446$ $0.963-06$ $1.653 E-04$ $4.204 E-05$ $4.304 \mathrm{E}-05$
$7.892 \mathrm{E}-05$ $7.892 \mathrm{E}-05$
$1.518 \mathrm{E}-05$ $1.518 E-05$ $2.007 E-05$ $2.007 E-05$ $2.411 E-05$
$3.139 E-05$ $3.139 E-05$ $4.256 E-05$ $2.625 E-04$ $3.049 E-04$ $6.017 E-04$ $1.479 E-03$ $3.367 \mathrm{E}-03$ $9.124 E-04$

ANGLE 4 $M U=-0.8656$ $1.476 E-05$ $1.684 E-04$ $4.390 E-05$ $8.042 \mathrm{E}-05$ $1.561 \mathrm{E}-05$ $1.561 \mathrm{E}-05$ $1.766 E-05$
$2.062 E-05$ $2.062 E-05$
$2.476 E-05$ $2.476 E-05$
$3.223 E-05$ $3.223 E-05$
$4.368 E-05$ $4.368 E-05$
$6.282 E-05$ $6.282 E-05$
$2.665 E-04$ $2.665 E-04$
$3.090 E-04$ $6.041 E-04$ $1.486 E-0$ $3.378 \mathrm{E}-0$ $9.138 \mathrm{E}-04$

ANGLE 5 ANGLE 12 ANGLE 13
$M U=0.4580 \quad M U=0.617$ $1.033 E-05 \quad 1.077 E-05$
$2.145 E-05$ $6.518 E-05$ $1.173 E-04$ $2.664 E-05$ $2.980 E-05$ $3.438 E-05$ $4.078 E-05$ $5.215 \mathrm{E}-05$ $6.890 E-05$ $9.517 E-05$ $3.445 \mathrm{E}-04$ $3.800 E-04$ $6.552 \mathrm{E}-04$ $1.617 \mathrm{E}-0$ $3.581 E-03$ $9.377 E-04$ 7.285E-06 7.541E-06 $1.516 \mathrm{E}-05$ $1.728 \mathrm{E}-04$ $4.518 E-05$ $8.263 E-05$ $1.626 E-05$ $1.837 E-05$ $2.572 E-05$ D $4.532 E-05$ $6.505 \mathrm{E}-05$ $2.721 E-04$ $3.147 E-04$ $1.496 E-03$ $9.157 E-04$ ANGLE 14

$M U=-0.6179$
$7.541 E-06$ $1.559 E-05$
$1.787 E-04$ $4.786 E-05$ $4.686 E-05$
$8.555 E-05$ $1.712 \mathrm{E}-05$ $1.712 E-05$ 2.251E-05 $2.700 E-05$ 2.700E-05 $4.747 E-05$
$6.795 E-05$ $6.795 E-05$
$2.794 E-04$ $2.794 E-04$
$3.219 E-04$ $3.219 \varepsilon-04$ $1.508 E-03$ $3.414 E-03$ ANGLE 15 $U=0.8656$ $M U=0.7550$ $1.136 E-05$
$2.357 E-05$ $2.665 E-04$ $7.206 \varepsilon-05$ $1.290 E-04$ 3.051E-05 $3.400 E-05$ $3.905 \mathrm{E}-05$ $4.607 E-05$
$5.842 E-05$ $7.635 E-05$ 1.040E-04 $3.651 E-04$ $3.968 \mathrm{E}-04$ $6.700 E-04$ 1. $650 \mathrm{E}-03$ $3.630 \varepsilon-03$ $9.432 \mathrm{E}-04$
ANGLE 7 MU $=-0.4580$ $7.859 E-06$ $1.635 \mathrm{E}-05$ $1.861 E-04$ 4.896E-05 $1.820 E-05$ $1.820 \mathrm{E}-05$ $2.051 E-05$ 2.387E-05 $2.860 E-05$ $3.714 E-05$ $7.147 E-05$ $7.147 E-05$ $2.881 E-04$
$3.302 E-04$ $3.302 E-04$
$6.174 E-04$ $6.174 E-04$
$1.523 E-03$ $3.437 E-03$ $9.209 E-04$ ANGLE 16 $M U=0.9446$ $2.546 \mathrm{E}-05$ $2.876 E-04$ $7.799 E-05$ $1.393 E-04$ $3.340 E-05$ $3.712 E-05$ $4.250 E-05$ $4.994 E-05$ $6.292 E-05$ $8.260 E-05$ $1.101 E-04$ $3.791 E-04$ 4.080E-04 $6.801 E-04$ $1.672 \mathrm{E}-03$ $3.662 E-03$ $9.468 E-04$
ANGLE 8 AU $=-0.2816 \quad$ MU $=0.0950$ $8.240 E-06 \quad 8.683 E-06$ $1.714 \mathrm{E}-05 \quad 1.805 \mathrm{E}-05$ 5.147E-05 $2.051 E-04$ $9.355 E-05 \quad 9.859 E-05$ $1.951 E-05 \quad 9.103 E-05$ $2.195 E-05 \quad 2.103 E-05$ 2.551E-05 $2.363 E-05$ 3.552E-05 $2.742 E-05$ $3.956 E-05 \quad 3.275 E-05$ $5.324 E-05 \quad 5.234 E-05$ $7.555 E-05$
$7.5578 E-05$ $2.981 E-04 \quad 3.091 E-04$ $3.395 E-04 \quad 3.495 E-04$ $0.310 E-04$
$2.558 E-03$ $1.540 \mathrm{E}-03$ 1.558E-03 $3.464 E-03 \quad 3.492 E-03$ ANGLE 17 SCALAR $M U=0.9894 \quad F L U X$ $1.226 \mathrm{E}-05$
$2.544 \mathrm{E}-05$ $2.873 E-04 \quad 2.720 E-03$ $7.799 E-05 \quad 7.243 E-04$ $1.392 \mathrm{E}-04 \quad 1.309 \mathrm{E}-03$ $3.357 E-05 \quad 2.860 E-04$ $3.731 E-05 \quad 3.206 E-04$ $4.272 E-05 \quad 3.708 E-04$ $\begin{array}{ll}5.020 E-05 & 4.411 E-04 \\ 0.321 E-05 & 5.665 E-04\end{array}$ $8.190 E-05 \quad 7.533 E-04$ $1.104 E-04 \quad 1.052 E-03$ $3.798 E-04 \quad 3.984 E-03$ $4.085 E-04 \quad 4.465 E-03$ $6.808 E-04 \quad 8.006 E-03$ $1.673 E-03 \quad 1.974 E-02$ $\begin{array}{ll}3.665 E-03 & 4.411 E-02 \\ 9.473 E-04 & 1.168 E-02\end{array}$ 
(GAMMAS/MEV/STERADIAN/SOURCE NEUTRON)

ENERGY GROUP (MEV)
$.00 E$ OO---1.00E 6.5OE 00---8.00E 00 $5.00 E$ OO- $-6.50 E$ OD $4.00 E$ OO- $-5.00 E$ OO $3.00 E$ OO- $-4.00 E$. 00 $2.50 E$ OO- $-3.00 E$ OO 2.00 E $00---2.50 E \quad 00$ $1.66 E$ E0-- 2.00E OO $1.33 E$ O0- - - 1.66E on 1.00 E $00-\cdots 1.33$ E 00 $8.00 E-01--1.00 E 00$ $6.00 E-01 \cdots-3.00 E-01$ $4.00 E-01--6.00 E-01$ $3.00 E-01---4.00 E-02$ $2.00 E-01---3.00 E-01$ $1.00 E-01--2.00 E-01$ $5.00 E-02--1.00 E-01$ $2.00 E-02---5.00 E-02$

ENERGY

GROUP (MEV)

8.00E 00---1.00E O1

6.5 OE OO- $-8.00 E$ OO $5.00 E$ DO- $-6.50 E$ OO $4.00 E$ OO- $05.00 E$ OO $3.00 E$ OO- $-4.00 E$ OO $2.50 E$ OO- $-3.00 E 00$ $2.00 E$ OO- $-2.50 E$ OO $1.66 \mathrm{E} \quad 00--2.00 \mathrm{E}$ CO $1.33 E$ OO- $-1.66 E 00$ $1.00 E$ 00- $-1.33 E 00$ 8.0OE-O1-C-1.00E OO $6.00 E-01-\rightarrow-8.00 E-01$ $4.00 E-01--6.00 E-01$ $3.00 E-C 1--4.00 E-01$ 2.0NE-01-C-3.00E-01 $1.00 E-01--2.00 E-O 2$ $2.00 \mathrm{E}-02=-1.00 E-01$
ANGLE 1 ANGLE 2 $M U=-1.0000 \quad M U=-0.9894$ $1.723 \mathrm{E}-05$ $3.587 \mathrm{E}-05$ $4.105 E-04$ ( $1.948 \mathrm{E}-04$ $3.972 E-05$ $7.451 \mathrm{E}-05$ $1.020 E-04$ $1.507 E-04$ $7.395 E-04$ $8.960 E-04$ $2.007 E-03$ 4.997E-03 $1.180 E-02$
$3.282 E-03$ ANGLE 10 MU $=0.0950$ $2.861 E-05$ $5.942 E-05$ $6.732 E-04$ $1.808 \mathrm{E}-04$ $3.255 E-04$ $7.464 E-05$ $8.404 E-05$ $9.787 E-05$ $1.176 E-04$ $1.535 \mathrm{E}-04$ $2.081 E-04$ $2.966 \mathrm{E}-04$ $1.103 E-03$ $1.268 \mathrm{E}-03$ $2.225 E-03$ $5.653 E-03$ $1.289 E-02$ .121E-04 $.056 E-04$ $1.956 E-04$ .479E-05 $3.996 \mathrm{E}-05$ $4.723 \mathrm{E}-05$ .720E-05 $.496 \mathrm{E}-05$ .027E-04 $1.518 E-04$ $7.422 E-C 4$ $8.996 \mathrm{E}-04$ $2.009 E-03$ $5.002 E-03$ $1.181 E-02$
$3.283 E-03$ ANGLE 11 $M U=0.2816$ $3.1+8 E-05$ $6.574 E-05$ $7.430 E-C 4$ $2.025 E-04$ $3.605 E-04$ $8.710 E-05$ $9.784 E-05$ $1.136 E-04$ 1. 1.31 OE-02 $3.438 \mathrm{E}-03$
ANGLE 3

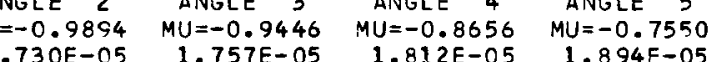
1. $312 E-05$ 1.894E-05

.186E-04 $1.075 E-04$ $1.989 E-04$ 3. $573 E-05$ $4.094 E-05$ $4.832 E-05$ $5.851 \mathrm{E}-05$ $7.680 E-05$ $1.054 E-04$ $1.558 \mathrm{E}-04$ $7.533 E-04$ $9.138 \mathrm{E}-04$ $2.015 \mathrm{E}-03$ $.024 E-03$ $1.185 \mathrm{E}-02$ $3.288 E-03$ ANGLE 12 $\begin{aligned} & M U=0.4580 \\ & 3.551 E-05\end{aligned}$ $7.363 E-05$ $8.303 E-04$ $2.271 E-04$ $4.041 \mathrm{E}-04$ $1.018 \mathrm{E}-04$ $1.140 E-04$ $1.318 \mathrm{E}-04$ $1.568 \mathrm{E}-04$ $2.014 E-04$ $2.669 E-04$ $3.684 \mathrm{E}-04$ $1.263 E-03$ $1.30 \mathrm{E}-03$ $2.340 E-03$ $1.331 \mathrm{E}-02$ $3.462 E-03$ 4.504E-04 1.168E-04 $4.758 \mathrm{E}-05 \quad 4.039 \mathrm{E}-05$ $4.291 E-05 \quad 4.593 E-05$ $5.054 \mathrm{E}-05 \quad 5.398 \mathrm{E}-05$ $6.118 \mathrm{E}-05 \quad 6.532 \mathrm{E}-05$ $8.049 E-05 \quad 8.612 E-05$ $1.108 E-04$ $1.638 E-04 \quad 1.755 E-04$ $2.026 \mathrm{E}-03$ $5.065 E-03$ $2.043 E-03$ $5.125 \mathrm{E}-03$ $1.202 E-02$
$3.309 E-03$ ANGLE 14 ANGLE 13 $M U=0.6179$ 3.9.94E-05 8.276E-05 $9.313 E-04$ $2.566 \mathrm{E}-04$ 4. $544 E-04$ $1.183 E-04$ $1.320 \mathrm{E}-04$ $1.519 \mathrm{E}-04$ $1.795 \mathrm{E}-04$ $2.282 E-04$ $2.983 E-04$ $6.063 E-03$ $3.485 E-03$ $4.406 \mathrm{E}-05$ $9.123 E-05$ $1.024 E-03$ $2.846 \mathrm{E}-04$ $5.011 \mathrm{E}-04$ $1.355 E-04$ $1.506 \mathrm{E}-04$ $1.723 E-04$ $2.022 E-04$ $2.542 E-04$ $3.279 E-04$ $1.415 E-03$ 1. $45 \mathrm{E}-03$ $6.183 E-03$ 3. $504 \mathrm{E}-03$

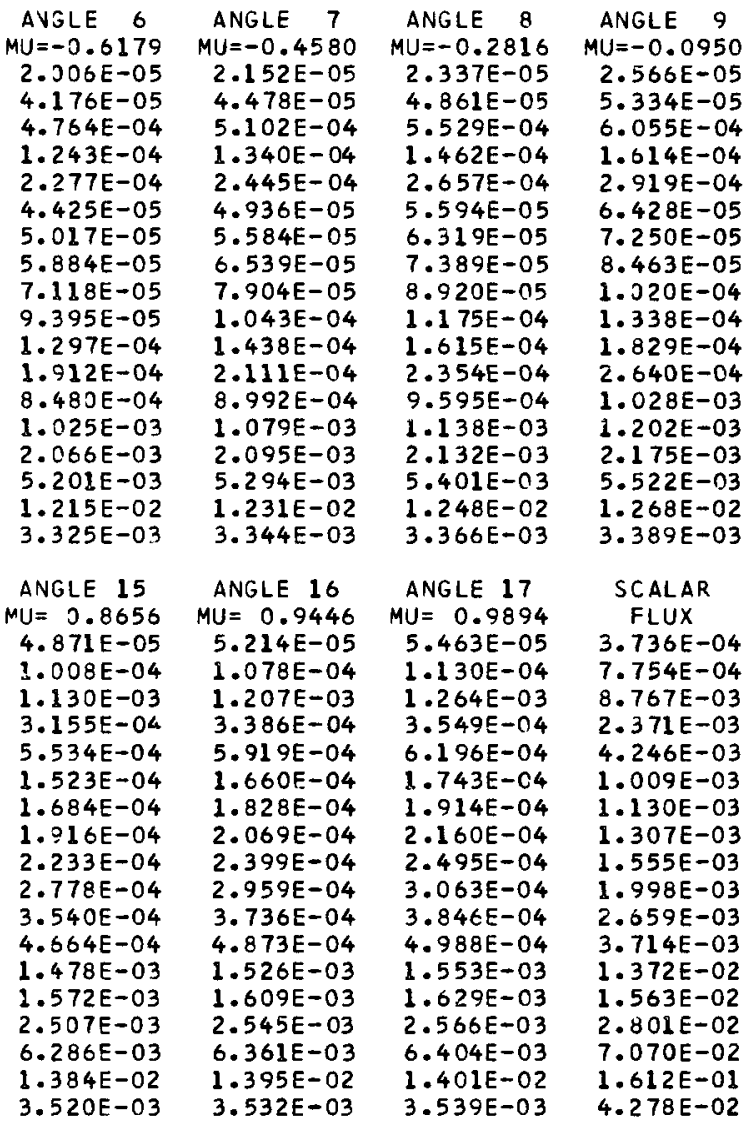


(GAMMAS/MEV/STERADIAN/SOURCE NFUTRON)

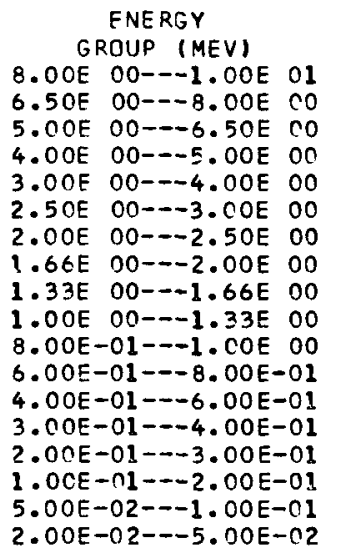

ENERGY

$$
\begin{aligned}
& \text { ENERGY } \\
& \text { GROUP (MEV) }
\end{aligned}
$$

8.00E 00-- -1.00E

6.50 E $00--8.00 E$ OO

5.00 E $00--6.50$ E 00

$4.00 E$ OO- $-5.00 E$ OO

3. COE OO- - 4. DOE OO

2.5OE OO-- $3.00 E$ OO

2.00 E $00--2.50 E \quad 00$

1. 6 GE OO- - 2.00E $0 O$

.33F $00---1.66 \mathrm{E} \mathrm{OO}$

. OOE 00--1.33E OO

8. COE-01---1.00E OO

$6.00 F-01--8.00 E-01$

4. COE-01 - - 6. 00E-O

$3.00 E-01--4$. COE-O

$2.00 E-01--3.00 E-01$

$1.00 \mathrm{E}-01--2.00 \mathrm{E}-01$

$2.0 \mathrm{CE}-02--5.00 \mathrm{E}-02$
ANGLE 1 ANGLE 2 MUV-1.0000 MU $=-0.9894$ $2.140 E-05$ 4.455E(1) . 2.414E-04 $4.796 E-05$ $5.737 E-05$ $6.981 E-05$ $9.126 \mathrm{E}-0 \mathrm{~S}$ $9.126 \mathrm{E}-05$ $1.248 \mathrm{E}-04$ $1.868 \mathrm{E}-04$ $1.008 E-03$
$1.251 E-03$ $1.251 E-03$
$3.068 E-03$ $7.068 E-03$ $1.873 \mathrm{E}-02$ $5.876 E-03$

ANGLE 10 $M U=0.0950$ $3.954 \mathrm{E}-05$ $8.209 E-C 5$ $2.514 E-04$ 4.506E-04 $1.083 E-04$ $1.224 \mathrm{E}-04$ $1.433 \mathrm{E}-04$ $1.735 \mathrm{E}-04$ $2.295 \mathrm{E}-0$ $3.160 E-04$ $4.582 E-04$ $1.691 \mathrm{E}-03$ $1.985 \mathrm{E}-03$ $3.457 \mathrm{E}-03$ $9.001 \mathrm{E}-0$ $2.093 \mathrm{E}-0$ $5.556 \mathrm{E}-03$
ANGLE 3 $M U=-0.9446$ $2.192 \mathrm{E}-05$ $4.563 E-05$ 1. $332 \mathrm{E}-04$ 1. $332 E-04$ $2.476 E-04$ $4.969 E-05$ 5.916E-05 $4.916 E-05$
$7.197 E-05$ $7.197 E-05$
$9.460 E-05$ $9.460 E-05$ $1.303 E-04$
$1.956 E-04$ $1.956 E-04$
$1.032 E-03$ $1.032 E-03$
$1.288 E-03$ $1.288 E-03$
$3.080 E-03$ $3.080 E-03$
$7.768 E-03$ $7.768 E-03$
$1.882 \mathrm{E}-02$ $5.298 E-03$ ANGLE 12 $M U=0.4580$ $5.303 E-05$ $1.099 \mathrm{E}-04$ 1. $235 E-03$ $0.423 E-04$ $6.046 E-04$ $1.630 E-04$ $1.832 E-04$ $2.545 E-04$ $3.293 E-04$ $4.392 E-04$ 6. $088 E-04$ $2.011 \mathrm{~F}-03$ 2. $247 E-03$ $3.692 \mathrm{E}-03$ $9.574 \mathrm{E}-03$ $2.183 E-02$ $5.660 \mathrm{E}-03$ $U=-0.8656$ $2.273 E-05$ $4.734 E-05$ $1.389 E-04$ $4.573 E-04$ $4.571 E-05$ $5.254 E-05$ $6.224 E-05$ .5 $50 \mathrm{E}-05$ $1.001 E-04$ $1.391 E-04$ $2.092 E-04$ $1.070 E-03$ $1.342 \mathrm{E}-03$ $3.099 \mathrm{E}-03$ $7.847 E-03$ 1.896E-02 ANGLE 13 $M U=0.6179$ $6.192 \mathrm{E}-05$ $1.281 E-04$ $1.435 \mathrm{E}-03$ $7.056 E-04$ $1.906 \mathrm{E}-04$ $2.232 E-04$ $2.574 E-04$ $3.050=-04$ $3.886 \mathrm{E}-04$ $5.080 E-04$ $6.867 \mathrm{E}-04$ $2.174 E-03$ $2.377 E-03$ $3.820 \mathrm{E}-03$ $9.861 \mathrm{E}-03$ $2.227 \mathrm{E}-02$ $5.708 \mathrm{E}-03$
ANGLE 5 AVILE 6 $M U=-0.7550$ $2.397 E-05$
$4.991 E-05$ 4.991E-05 $1.474 E-04$ $5.700-04$ $5.703 E-05$ $6.727 E-05$ $6.727 E-05$ $1.01 E-05$ $1.089 \mathrm{E}-04$ $1.522 E-04$ $2.294 E-04$ $1.127 E-03$
$1.418 E-03$ $3.126 \mathrm{E}-03$ $7.959 \mathrm{E}-03$ $1.916 \mathrm{E}-02$ $5.342 E-03$ ANGLE 14 $M U=0.7550$ $7.164 \mathrm{E}-05$ $1.653 \mathrm{E}-03$ 4.687E-04 $8.156 E-04$ $2.408 \mathrm{E}-04$ $2.675 E-04$ $3.058 \mathrm{E}-04$ $3.585 E-04$ $4.491 E-04$ $5.753 E-04$ $7.596 \mathrm{E}-04$ $2.328 E-03$ $2.499 \mathrm{E}-03$ $3.945 E-03$ $1.013 \mathrm{E}-02$ $2.266 E-02$
$5.751 E-03$ $2.568 \mathrm{E}-05$ $5.347 \mathrm{E}-05$ $6.104 E-04$ 1.590E-04 $2.917 E-04$ $6.356 E-05$ $7.477 \mathrm{E}-05$ $7.477 \mathrm{E}-05$ $1.215 \mathrm{E}-04$ $1.215 E-04$ $1.704 E-04$
$2.563 E-04$ $2.568 E-04$
$1.203 E-03$ $1.203 E-03$
$1.511 E-03$ $3.165 \mathrm{E}-03$ $8.106 E-03$ $1.942 \mathrm{E}-02$ $5.374 E-03$ ANGLE 15 $M U=0.8656$ $8.190 \mathrm{E}-05$ $1.691 E-04$ $1.884 E-03$ $9.309 E-04$ 2.831 E-04 $3.121 E-04$ $3.536 E-04$ $4.100 E-04$ $5.053 E-04$ $6.355 \mathrm{E}-04$ $8.226 \mathrm{E}-04$ $2.465 E-03$ $2.606 E-03$ $4.058 \mathrm{E}-03$ $1.035 \mathrm{E}-02$ $2.299 E-02$ $5.786 \mathrm{E}-03$
ANGLE 7 $M U=-0.4580$ $2.796 E-05$ 6. $332 E-04$ $6.632 E-04$ 1.742E-04 $6.180 E-04$ 6.410E-05 $8.536 \mathrm{E}-05$ $1.038 E-04$ $1.038 E-04$ $1.388 E-04$ $1.948 \mathrm{E}-04$
$2.925 \mathrm{E}-04$ $2.925 \mathrm{E}-04$
$1.298 \mathrm{E}-03$ $1.618 E-03$ $1.618 \mathrm{E}-03$
$3.215 \mathrm{E}-03$ $8.285 E-03$ $1.973 \mathrm{E}-02$ $5.412 \mathrm{E}-03$ ANGLE 16 $M U=0.9446$ .104E-05 $2.088 \mathrm{E}-03$ . $006 \mathrm{E}-04$ $1.033 E-03$ $.203 E-04$ $3.507 \mathrm{E}-04$ .940E-04 $4.525 E-04$ $5.502 E-04$ $6.821 E-04$ $8.703 E-04$ $2.572 E-03$ $2.689 \mathrm{E}-03$ $4.146 \mathrm{E}-03$ $1.052 \mathrm{E}-02$ $2.323 E-02$ $5.811 E-03$

ANGLE 8 ANGLE 9 $3.094 E-05 \quad M U=-0.0950$ $6.435 E-05 \quad 7.225 E-05$ $7.317 E-04$ T.195E-O4 $1.940 E-04 \quad 2.194 E-04$ $3.521 E-04 \quad 3.960 E-04$ $7.497 E-05$ Q.939E-05 $8.490 E-05 \quad 1.012 E-04$ $9.972 E-05 \quad 1.187 E-04$ $1.213 E-04 \quad 1.441 E-04$ $1.619 E-04 \quad 1.918 E-04$ $2.265 E-04 \quad 2.667 E-04$ $3.378 \mathrm{E}-04 \quad 3.932 \mathrm{E}-04$ $1.412 \mathrm{E}-03 \quad 1.544 \mathrm{E}-03$ $1.735 \mathrm{E}-03 \quad 1.858 \mathrm{E}-03$ $3.280 E-03 \quad 3.361 E-03$ $8.497 E-03 \quad 8.737 E-03$ $2.009 E-02 \quad 2.049 E-02$ ANGLE 17 SCALAR MU $=0.9894 \quad$ FLUX $2.009 E-04 \quad 1.468 E-04$ $2.230 E-03 \quad 1.277 E-02$ $6.432 E-04 \quad 3.502 E-03$ $1.103 E-03 \quad 6.221 E-03$ $3.445 \mathrm{E}-04 \quad 1.587 \mathrm{E}-03$ $3.753 E-04 \quad 1.778 E-03$ $4.193 E-04 \quad 2.055 E-03$ 4.785E-04 2.446E-03 $5.770 E-04 \quad 3.146 E-03$ $7.093 E-04 \quad 4.187 E-03$ $8.978 E-04 \quad 5.849 E-03$ $2.636 E-03 \quad 2.113 E-02$ $2.738 E-03 \quad 2.442 E-02$ 
(GAMMAS/MEV/STERADIAN/SOURCE NEUTRON)

ENERGY 8. OOE 00---1.0OE 6.50E 00---8.00E 00 5.00 E $00--6.50$ E 00 $4.00 E$ OO- $-5.00 E$ OO $3.00 E$ O0- $4.00 E$ OD

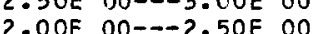
$1.66 E$ DO- - 2.00E 00 $1.33 \mathrm{E} 00---1.66 \mathrm{E} 00$ $1.0 O E$ OO- - $1.33 \mathrm{E}$ OO 8.00E-01---1.00E 00 $6.00 E-01--8.00 E-C 1$ 4.00E-01-- $6.00 \mathrm{E}-01$ $3.00 E-01--4.00 E-01$ $2.00 E-01=-3.00 \mathrm{E}-01$ 1.00E-01--2. $2.00 \mathrm{E}-01$ $5.00 \mathrm{E}-02--1.00 \mathrm{E}-01$
$2.00 \mathrm{E}-02--5.00 \mathrm{E}-02$

$$
\text { ENERGY }
$$

GROUP (MEV)

8. OOE 00---1. OOE O1 6.50 E $00--8.00 E$ DO 5.0 E ON- -6.50 E 00 4. COE $00--75.00 E$ OO $3.00 E$ OO- $-4.00 E$ OO $2.50 E$ OO- - -3. OOE OO $2.00 E$ OD---2.5OE 00 $1.66 E 00--2.00 E 00$ $1.33 E$ E $00--1.66$ E OD $1.00 E 00--1.33 \mathrm{E} 00$ $8.00 E-01--3.00 E$ DO $6.00 E-01--8.00 E-01$ $4.00 E-01=-6.00 E-01$ $3.00 \mathrm{E}-01--4.00 \mathrm{E}-01$ $2.00 E-01--3.00 E-01$ $5.00 E-02-2.00 E-01$ $2.00 \mathrm{E}-02--5.00 \mathrm{E}-02$
ANGLE 1 $2.158 E-05$ $4.483 \mathrm{E}-05$ $4.483 E-05$ $1.265 E-04$ $2.409 E-04$ $3.780 \mathrm{E}-05$ $4.737 E-05$ $5.915 E-05$ $7.270 E-05$ $9.240 \mathrm{E}-05$ $1.216 \mathrm{E}-04$ $1.849 E-04$ $1.252 \mathrm{E}-03$ $1.615 \mathrm{E}-03$ $4.830 E-03$ $1.240 E-02$ $3.180 E-02$ $9.224 E-03$ ANGLE 10 $M U=0.0950$ 4.796E-05 $9.950 \mathrm{E}-05$ $1.122 \mathrm{E}-03$ 3.082E-04 $5.488 E-04$ $1.427 E-04$ $1.632 \mathrm{E}-04$ $1.940 E-04$ $2.399 E-04$ $3.286 \mathrm{E}-04$ $4.718 E-04$ $7.185 E-04$ $2.618 E-03$ $5.51 E-03$ 5.51 8E-D3 $1.517 E-02$ $9.859 E-03$
ANGLE 2 $2.171 \mathrm{E}-05$ 4.511E-05 $5.187 \mathrm{E}-04$ $1.277 \mathrm{E}-04$ $2.427 E-04$ $3.837 E-05$ 4.767E-05 $5.923 E-05$ $7.276 E-05$ $9.295 E-05$ $1.234 E-04$ $1.881 E-04$ $1.260 E-03$ $1.631 \mathrm{E}-03$ $4.833 \mathrm{E}-03$ $1.242 \mathrm{E}-02$ $3.184 E-02$ $9.229 \mathrm{E}-03$ ANGLE 11 $M U=0.2816$ $5.866 \mathrm{E}-05$ $1.215 E-04$
$1.363 E-03$ $1.363 \mathrm{E}-03$
$3.815 \mathrm{E}-04$ $3.815 E-04$
$6.720 E-04$ $6.720 E-04$ $1.909 E-04$ $2.180 E-04$ $2.586 E-04$ $3.183 E-04$ $4.310 E-C 4$ $6.065 E-04$ $8.917 E-04$ $2.958 E-03$ $3.492 E-03$ $5.761 E-03$ $1.583 E-02$ $9.988 E-03$ ANGLE 3 $2.224 E-05$ $4.625 \mathrm{E}-05$ $5.313 E-04$ $1.324 E-04$ 2.4 4 .05E- 04 4. $902 E-05$ $5.996 \mathrm{E}-05$ $7.355 E-05$ $9.565 E-05$ $1.304 E-04$ 2.007E-04 $1.293 E-03$ $1.599 E-03$ $4.848 E-03$ $1.250 E-02$ $3.200 E-02$ $9.251 E-02$ ANGLE 12 $M U=0.4580$
$7.327 E-05$ $7.327 E-05$
$1.514 E-04$ $1.690 \mathrm{E}-03$ $4.822 E-04$ $8.396 \mathrm{E}-04$ $2.5918-04$ $2.949 E-04$ $3.478 \mathrm{E}-04$ $4.238 E-04$
$5.623 E-04$ 5.623E-04 $7.677 E-04$ $1.082 E-03$ 3. $771 \mathrm{E}-03$ $6.044 E-03$ $1.652 \mathrm{E}-02$ $3.017 E-02$ 1. $312 E-02$

\begin{tabular}{|c|c|c|}
\hline $\begin{array}{l}\text { ANGLE } 4 \\
4 U=-0.8656 \\
2.328 E-05 \\
4.848 E-05 \\
5.560 E-04 \\
1.411 E-04 \\
2.631 E-04 \\
4.470 E-05 \\
5.208 E-05 \\
6.236 E-05 \\
7.639 E-05 \\
1.019 E-04 \\
1.438 E-04 \\
2.241 E-04 \\
1.360 E-03 \\
1.825 E-03 \\
4.875 E-03 \\
1.267 E-02 \\
3.232 E-02 \\
9.292 E-03\end{array}$ & $\begin{array}{r}\text { ANGLE } 5 \\
M U=-0.7550 \\
2.487 E-05 \\
5.181 \mathrm{E}-05 \\
5.931 \mathrm{E}-04 \\
1.530 \mathrm{E}-04 \\
2.824 \mathrm{E}-04 \\
5.059 \mathrm{E}-05 \\
5.748 \mathrm{E}-05 \\
6.781 \mathrm{E}-05 \\
8.313 \mathrm{E}-05 \\
1.132 \mathrm{E}-04 \\
1.638 \mathrm{E}-04 \\
2.575 \mathrm{E}-04 \\
1.462 \mathrm{E}-03 \\
1.999 \mathrm{E}-03 \\
4.916 \mathrm{E}-03 \\
1.290 \mathrm{E}-02 \\
3.276 \mathrm{E}-02 \\
9.350 \mathrm{E}-03\end{array}$ & $\begin{array}{r}\text { AVGLE } 6 \\
M U=-0.6179 \\
2.710 E-05 \\
5.646 E-05 \\
6.450 E-04 \\
1.684 E-04 \\
3.086 E-04 \\
5.865 E-05 \\
6.615 E-05 \\
7.778 E-05 \\
9.560 E-05 \\
1.315 E-04 \\
1.920 E-04 \\
3.034 E-04 \\
1.605 E-03 \\
2.212 E-03 \\
4.973 E-03 \\
1.320 E-02 \\
3.335 E-02 \\
9.425 E-03\end{array}$ \\
\hline $\begin{array}{l}\text { ANGLE } 13 \\
\text { MU= } 0.6179 \\
9.269 \mathrm{E}-05 \\
1.912 \mathrm{E}-04 \\
2.121 \mathrm{E}-03 \\
6.171 \mathrm{E}-04 \\
1.061 \mathrm{E}-03 \\
3.525 \mathrm{E}-04 \\
3.983 \mathrm{E}-04 \\
4.646 \mathrm{E}-04 \\
5.572 \mathrm{E}-04 \\
7.192 \mathrm{E}-04 \\
9.470 \mathrm{E}-04 \\
1.277 \mathrm{E}-03 \\
3.583 \mathrm{E}-03 \\
4.062 \mathrm{E}-03 \\
6.354 \mathrm{E}-03 \\
1.723 \mathrm{E}-02 \\
4.029 \mathrm{E}-02\end{array}$ & $\begin{array}{r}A N G L E 14 \\
M U=0.7550 \\
1.176 \mathrm{E}-04 \\
2.420 \mathrm{E}-04 \\
2.670 \mathrm{E}-03 \\
7.912 \mathrm{E}-04 \\
1.343 \mathrm{E}-03 \\
4.728 \mathrm{E}-04 \\
5.278 \mathrm{E}-04 \\
6.059 \mathrm{E}-04 \\
7.121 \mathrm{E}-04 \\
8.907 \mathrm{E}-04 \\
1.130 \mathrm{E}-03 \\
1.463 \mathrm{E}-03 \\
4.047 \mathrm{E}-03 \\
4.354 \mathrm{E}-03 \\
6.370 \mathrm{E}-03 \\
1.791 \mathrm{E}-02 \\
4.133 \mathrm{E}-02\end{array}$ & $\begin{array}{r}\text { ANGLE } 15 \\
M U=0.8656 \\
1.470 \mathrm{E}-04 \\
3.020 \mathrm{E}-04 \\
3.315 \mathrm{E}-03 \\
9.975 \mathrm{E}-04 \\
1.673 \mathrm{E}-03 \\
6.130 \mathrm{E}-04 \\
6.738 \mathrm{E}-04 \\
7.591 \mathrm{E}-04 \\
8.728 \mathrm{E}-04 \\
1.058 \mathrm{E}-03 \\
1.299 \mathrm{E}-03 \\
1.527 \mathrm{E}-03 \\
4.386 \mathrm{E}-03 \\
4.627 \mathrm{E}-03 \\
6.966 \mathrm{E}-03 \\
1.851 \mathrm{E}-02 \\
4.222 \mathrm{E}-02\end{array}$ \\
\hline
\end{tabular}

$\begin{array}{lll}4.029 E-02 & 4.133 E-02 & 4.222 E-02 \\ 1.024 E-02 & 1.035 E-02 & 1.044 E-02\end{array}$
ANGLE 7 ANGLE 8 ANGLE 9 $M U=-0.4580 \quad M U=-0.2816 \quad M U=-0.0950$ $3.017 \mathrm{E}-05 \quad 3.437 \mathrm{E}-05 \quad 4.010 \mathrm{E}-05$ $6.282 E-05 \quad 7.149 E-05 \quad 8.331 E-05$ $\begin{array}{lll}7.159 E-04 & 8.123 E-04 & 9.433 E-04\end{array}$ $\begin{array}{lll}1.888 E-04 & 2.165 E-04 & 2.549 E-04 \\ 3.440 E-04 & 3.922 E-04 & 4.582 E-04\end{array}$ $6.990 \mathrm{E}-05 \quad 8.598 \mathrm{E}-05 \quad 1.092 \mathrm{E}-04$ $7.915 \mathrm{E}-05 \quad 9.794 \mathrm{E}-05 \quad 1.248 \mathrm{E}-04$ $9.343 E-05 \quad 1.162 E-04 \quad 1.484 E-04$ $1.152 E-04 \quad 1.436 E-04 \quad 1.836 E-04$ $\begin{array}{lll}1.587 E-04 & 1.976 E-04 & 2.525 E-04\end{array}$ $2.317 E-04 \quad 2.879 E-04 \quad 3.664 E-04$ $3.670 E-04 \quad 4.547 E-04 \quad 5.714 E-04$ $1.794 E-03 \quad 2.028 E-03 \quad 2.305 E-03$ $2.450 E-03 \quad 2.703 E-03 \quad 2.962 E-03$ $5.054 E-03 \quad 5.167 E-03 \quad 5.320 E-03$ $1.358 \mathrm{E}-02 \quad 1.404 \mathrm{E}-02 \quad 1.457 \mathrm{E}-02$ $3.406 E-02 \quad 3.490 E-02 \quad 3.586 E-02$ $9.515 E-03 \quad 9.619 E-03 \quad 9.735 E-03$

ANGLE 16

ANGLE 17

SCALAR

$\begin{array}{rrr}M U=0.9446 & M U=0.9894 & \text { FLUX } \\ 1.773 E-04 & 2.008 E-04 & 7.635 E-04\end{array}$

$\begin{array}{lll}1.638 \mathrm{E}-04 & 4.115 \mathrm{E}-04 & 1.577 \mathrm{E}-03\end{array}$

$3.976 \mathrm{E}-03 \quad 4.485 \mathrm{E}-03 \quad 1.760 \mathrm{E}-02$

$1.209 E-03 \quad 1.370 E-03 \quad 5.008 E-03$

$2.007 E-03 \quad 2.260 E-03 \quad 8.706 E-03$

$\begin{aligned} & 7.516 E-04 \\ & 8.518 E-04\end{aligned} 2.618 E-03$

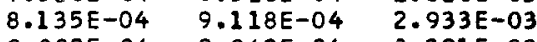
$9.002 E-04 \quad 9.963 E-04 \quad 3.391 E-03$ $1.015 E-03 \quad 1.108 E-03 \quad 4.038 E-03$ $1.198 \mathrm{E}-03 \quad 1.286 \mathrm{E}-03 \quad 5.200 \mathrm{E}-03$ $1.433 E-03 \quad 1.515 E-03 \quad 6.924 E-03$ $1.755 E-03$ 1.832E-03 $9.669 E-03$ $4.670 E-03 \quad 4.851 E-03 \quad 3.311 E-02$ $4.850 \mathrm{E}-03$ 7.9 $1.20 \mathrm{EE}-02$ 1.35E-03 $1.083 \mathrm{E}-02$ $4.280 \mathrm{E}-02$ $4.328 \mathrm{E}-02$ $\begin{array}{lll}1.051 E-02 & 1.055 E-02 & 1.235 E-01\end{array}$ 
(GAMMAS/MEV/STERADIAN/SOURCE NEUTRON]

ENERGY

$8.00 E$ OO- - - 1.0OE 01 $6.50 E$ OO- $-8.00 E$ OO $5.00 E$ OO- $-6.50 E$ OO $4.00 E$ OO- $-5.00 E$ OO $3.00 E$ OO- $-4.00 E$ OO $2.50 E$ OO---3. OOE OO $2.00 \mathrm{E} \quad 00---2.50 \mathrm{E}$ OO $1.66 \mathrm{E}$ 00---2.00E 00 $1.33 \mathrm{E} 00--1.66 \mathrm{E} 00$ $1.00 E$ OD- $-1.33 E$ OO 8.0OE-01- - 1.00E 00 6.00E-01- $--8.00 E-01$ 4.00E-01-- $6.00 E-01$ $3.00 E-01---4.00 E-01$ $2.00 E-01=-3$. COE -01 $1.00 E-01--2.00 E-01$ $5.00 E-02 \rightarrow-1.00 E-01$ $2.00 E-02--5.00 E-02$

$$
\text { ENERGY }
$$$$
\text { GROUP (MEV) }
$$

8.00E OO---1.00E OI $6.50 E$ OO- - 8.00E OO $5.00 E$ OO- - 6.5OE OO $4.00 E$ OO- $-5.00 E$ OO $3.00 E$ OO--4. $2.50 E$ OO- $-3.00 E$ OO $2.00 E$ OO- $-2.50 E$ OO 1.66 E OO-D2.00E 00 1.33 E $00--1.66 \mathrm{E}$ OO 1.00 E $00--1.33 E$ OD $8.00 E-01--1.00 E$ OO $6.00 E-01-2.00 E-01$ 4.00E-01 - 6.00E-01 3.00E-01- $-4.00 E-01$ . 00 E $01--3.00 E-01$ 1 . OOE $01--2.00 E-O 1$ $2.00 E-02--5.00 E-02$
ANGLE 1 $M U=-1.0000$ $3.256 \mathrm{E}-05$ $3.763 E-04$ $8.569 \mathrm{E}-05$ $1.713 E-04$ $2.399 E-05$ $3.626 \mathrm{E}-05$ $4.971 E-05$ $6.204 \mathrm{E}-05$ $7.269 E-05$ $8.348 \mathrm{E}-05$ $1.255 E-04$ $1.177 \mathrm{E}-03$ $1.544 \mathrm{E}-03$ $5.734 E-03$ $1.494 \mathrm{E}-02$ $4.037 E-02$ 1.197E-02

ANGLE 10 $M U=0.0950$ 4.038E-05 $8.370 E-05$ $9.406 E-04$ $2.625 \mathrm{E}-04$ $4.643 E-04$ $1.309 \mathrm{E}-04$ 1. $518 \mathrm{E}-04$ $1.841 \mathrm{E}-04$ $2.339 \mathrm{E}-04$ $3.354 E-04$ $5.105 \mathrm{E}-04$ $8.312 E-04$ $3.010 E-03$ $3.876 \mathrm{E}-03$ 6.512E-03 1.8 $4.817 \mathrm{E}-02$
$1.295 \mathrm{E}-02$
ANGLE 2 $\mathrm{MU}=-0.98994$
$1.590 \mathrm{E}-05$ $3.284 E-05$ $3.792 E-04$ $8.750 E-05$ $1.734 E-04$ $2.479 E-05$ $3.625 \mathrm{E}-05$ $4.900 \mathrm{E}-05$ $6.102 \mathrm{E}-05$ $7.248 E-05$ $8.563 E-05$ $1.301 \mathrm{E}-04$ $1.185 \mathrm{E}-03$ $1.569 \mathrm{E}-03$ $5.738 \mathrm{E}-03$ $1.497 \mathrm{E}-0$ $4.043 E-02$ $1.198 \mathrm{E}-02$

ANGLE 11 SU $=0.2816$ $5.207 E-05$
$1.077 E-04$ 1. $077 E-04$ $1.202 \mathrm{E}-03$ $3.442 E-04$ 6. $002 \mathrm{E}-04$ $1.896 \mathrm{E}-\mathrm{n} 4$ $2.202 \mathrm{E}-04$ $2.673 \mathrm{E}-04$ $3.392 E-04$ $4.819 \mathrm{E}-04$ $7.143 E-04$ $1.102 E-03$ $3.489 \mathrm{E}=03$ $6.225 E-03$ $6.854 \mathrm{E}-03$ $4.988 E-02$ 1. $316 E-02$
ANGLE 3 ANGLE 3
$M U=-0.9446 \quad M U=-0.8656$ $1.538 \mathrm{E}-05$ $3.394 \mathrm{E}-05$ 3.911E-04 $1.814 \mathrm{E}-04$ $2.766 E-05$ $3.653 \mathrm{E}-05$ 4.697E-05 $5.809 \mathrm{E}-05$ $7.261 \mathrm{E}-05$ $9.401 E-05$ $1.474 \mathrm{E}-04$ $1.220 \mathrm{E}-0$ $1.671 \mathrm{E}-03$ $5.753 E-03$ $1.509 \mathrm{E}-02$ $4.067 E-0$ 1.201E-02

ANGLE 12 $U=0.4580$ $6.954 E-05$
$1.434 E-04$ $1.434 \mathrm{E}-04$
$1.588 \mathrm{E}-03$ $4.670 E-04$ $8.025 E-04$ 2.828E-04 $3.285 E-04$ $3.971 E-04$ $4.980 E-04$ $6.873 E-04$ $9.724 E-04$ $1.405 E-03$ $3.991 \mathrm{E}-03$ $4.601 \mathrm{E}-03$ $2.267 \mathrm{E}-03$ 5.1 IIE-OD $1.336 \mathrm{E}-02$ $1.729 \mathrm{E}-05$ $4.135 \mathrm{E}-04$ $1.043 \mathrm{E}-04$ $1.953 \mathrm{E}-04$ $3.235 E-05$ $3.795 \mathrm{E}-\mathrm{O}$ 4.549E-05 $5.584 E-05$ $7.544 \mathrm{E}-0$ $1.091 \mathrm{E}-04$ $1.764 E-04$ $1.295 E-03$ $1.864 \mathrm{E}-03$
$5.779 \mathrm{E}-03$ . 4.114E-02 4.114E-02 ANGLE 13 $\mathrm{U}=0.6179$ $9.568 E-05$ $1.967 E-04$ $2.159 E-03$ $6.530 \mathrm{E}-04$ $1.104 E-03$ 4.278E-04 $4.930 E-04$ $5.871 \mathrm{E}-0$ $7.193 \mathrm{E}-04$ 1.271E-03 $4.510 E-03$ $5.017 E-03$ . 7.730E-0 $5.365 \mathrm{E}-02$ $1.356 \mathrm{E}-02$
ANGLE 5 1. $865 \mathrm{E}-05$ 3. $893 E-05$ $4.457 E-04$ 1.163E-04 $3.806 E-05$ $4.181 E-05$ 4. $803 E-05$ $5.903 E-05$ $8.436 \mathrm{E}-05$ $1.296 \mathrm{E}-04$ $2.135 E-04$ $2.130 \mathrm{E}-03$ 5. $815 E-03$ 1. 563E-02 $4.180 E-02$ $1.216 E-02$

ANGLE 14

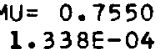
$1.338 E-04$
$2.741 E-04$ $2.983 E-03$ 9. ?.78E-04 $1.538 E-03$ $6.405 E-04$ $7.239 E-04$ $8.405 E-04$ 1. 254E-03 $1.583 E-03$ $2.013 \mathrm{E}-03$ $5.040 E-03$ $5.462 E-03$ $8.225 E-03$ 2. $316 \mathrm{E}-02$ 1. $374 \mathrm{E}-02$
ANGLE 6 2.057E-05 4.293E-05 4.902E-04 . $358 E-04$ 4.514E-05 $4.943 E-05$ $5.701 E-05$ $7.072 \mathrm{E}-05$ $1.021 \mathrm{E}-04$ $1.577 \mathrm{E}-04$ $2.626 E-04$ $1.599 E-03$ $5.465 E-03$ $1.606 \mathrm{E}-02$ $1.228 \mathrm{E}-02$ ANGLE 15 $M U=0.8656$ $1.857 E-04$
$3.794 E-04$ 4. $094 \mathrm{E}-03$ $1.304 E-03$ $2.120 E-03$ $9.207 E-04$ $1.013 E-03$ $1.140 \mathrm{E}-03$ $1.561 \mathrm{E}-03$ $1.874 \mathrm{E}-03$ $2.276 \mathrm{E}-03$ 5.562E-O3 $5.899 \mathrm{E}-03$ $8.701 E-03$ $2.415 E-02$ $1.389 \mathrm{E}-02$

ANGLE 7 ANGLE 8 ANGLE 9 $M U=-0.4580 \quad M U=-0.2816 \quad M U=-0.0950$ $\begin{array}{lll}2.324 E-05 & 2.703 E-05 & 3.247 E-05 \\ 4.844 E-05 & 5.622 E-05 & 6.742 E-05\end{array}$ $5.516 \mathrm{E}-04 \quad 6.381 \mathrm{E}-04 \quad 7.618 \mathrm{E}-04$ $1.467 \mathrm{E}-04 \quad 1.710 \mathrm{E}-04 \quad 2.076 \mathrm{E}-04$ 2.660E-04 $3.091 E-04 \quad 3.720 E-04$ $5.501 E-05 \quad 7.009 E-05 \quad 9.375 E-05$ 6.191E-05 $8.071 E-05 \quad 1.087 E-04$ $7.320 E-05 \quad 9.715 E-05 \quad 1.316 E-04$ $9.160 E-05 \quad 1.223 E-04 \quad 1.665 E-04$ $1.305 E-04 \quad 1.731 E-04 \quad 2.373 E-04$ $\begin{array}{lll}1.988 E-04 & 2.629 E-04 & 3.626 E-04\end{array}$ $3.349 E-04 \quad 4.462 E-04 \quad 6.100 E-04$ $1.852 E-03 \quad 2.177 E-03 \quad 2.568 E-03$ 3.177E-03 $3.532 E-03$ $6.064 E-03 \quad 6.250 E-03$ $1.801 E-02$ $\begin{array}{lll}4.376 E-02 & 4.504 E-02 & 4.652 E-02 \\ 1.241 E-02 & 1.257 E-02 & 1.276 E-02\end{array}$

ANGLE 16 ANGLE 17 SCALAR $\begin{array}{rrc}2.472 E-04 & 3.011 E-04 & 7.832 E-04\end{array}$ $5.037 E-04 \quad 6.122 E-04 \quad 1.611 E-03$ $5.397 \mathrm{E}-03 \quad 0.531 \mathrm{E}-03 \quad 1.772 \mathrm{E}-02$ 
(GAMMAS/MEV/STERADIAN/SOURCE NEUTRON)

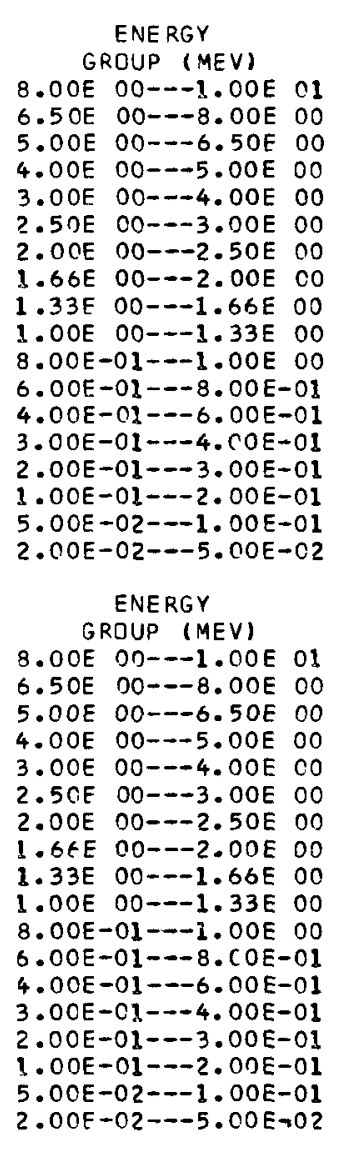

$\begin{array}{cc}\text { ANGLE 1 } & \text { ANGLE } \\ M U=-1.0000 & M U=-0.9894 \\ 9.522 E-06 & 9.619 E-06 \\ 1.931 E-05 & 1.957 E-05 \\ 2.255 E-04 & 2.281 E-04 \\ 4.197 E-05 & 4.460 E-05 \\ 9.620 E-05 & 9.872 E-05 \\ 1.005 E-05 & 1.125 E-05 \\ 2.544 E-05 & 2.504 E-05 \\ 4.107 E-05 & 3.930 E-05 \\ 5.245 E-05 & 4.996 E-05 \\ 5.327 E-05 & 5.103 E-05 \\ 4.257 E-05 & 4.512 E-05 \\ 5.772 E-05 & 6.427 E-05 \\ 9.661 E-04 & 9.721 E-04 \\ 1.258 E-03 & 1.288 E-03 \\ 5.846 E-03 & 5.850 E-03 \\ 1.535 E-02 & 1.538 E-02 \\ 4.336 E-02 & 4.343 E-02 \\ 1.308 E-02 & 1.309 E-02 \\ & \\ \text { ANGLE 10 } & 4 N 6 L E 11 \\ M U=0.0950 & M U=0.2816 \\ 2.750 E-05 & 3.712 E-05 \\ 5.696 E-05 & 7.670 E-05 \\ 6.379 E-04 & 8.514 E-04 \\ 1.806 E-04 & 2.501 E-04 \\ 3.180 E-04 & 4.317 E-04 \\ 9.674 E-05 & 1.508 E-04 \\ 1.140 E-04 & 1.781 E-04 \\ 1.412 E-04 & 2.223 E-04 \\ 1.851 E-04 & 2.938 E-04 \\ 2.817 E-04 & 4.459 E-04 \\ 4.628 E-04 & 7.080 E-04 \\ 8.190 E-04 & 1.157 E-03 \\ 2.964 E-03 & 3.499 E-03 \\ 3.955 E-03 & 4.304 E-03 \\ 6.516 E-03 & 6.892 E-03 \\ 1.984 E-02 & 2.099 E-02 \\ 5.275 E-02 & 5.496 E-02 \\ 1.426 E-02 & 1.452 E-02 \\ & \end{array}$

ANGLE 3 $M U=-0.9446$ MUVLE 4 $1.000 \mathrm{E}-05$ $2.056 E-05$
$2.382 E-04$ $2.382 E-04$
$5.299 E-05$ $5.299 E-05$
$1.076 E-04$ $1.492 \mathrm{E}-05$ 2.388E-05 $3.333 E-05$ $4.149 \mathrm{E}-05$ $4.149 \mathrm{E}-05$ $5.451 E-05$ $8.629 E-05$ 1.00OE-03 $1.410 \mathrm{E}-03$ $5.864 \mathrm{E}-03$ 1. $551 \mathrm{E}-02$ $4.372 E-02$
$1.313 E-02$ ANGLE 12 $M U=0.4580$ 1.086E-04 $1.193 \mathrm{E}-03$ $3.620 E-04$ $6.147 E-04$ $2.460 E-04$ $2.936 \mathrm{E}-04$ $3.671 E-04$ $4.793 E-04$ $6.976 \mathrm{E}-04$ $1.033 E-03$ 1. $536 E-03$ $4.047 E-03$ $4.694 \mathrm{E}-03$ 7.35 SE-03 $2.227 \mathrm{E}-02$ $5.729 \mathrm{E}-02$ $1.478 \mathrm{E}-02$

\begin{tabular}{|c|c|c|c|}
\hline $\begin{array}{l}\text { ANGLE } \\
\text { MU }=-0.8 \\
1.069 \mathrm{E} \\
2.226 \mathrm{E} \\
2.560 \mathrm{E} \\
6.469 \mathrm{E} \\
1.212 \mathrm{E} \\
1.998 \mathrm{E} \\
2.303 \mathrm{E} \\
2.640 \mathrm{E} \\
3.188 \mathrm{E} \\
4.532 \mathrm{E} \\
6.968 \mathrm{E} \\
1.183 \mathrm{E} \\
1.064 \mathrm{E} \\
1.642 \mathrm{E} \\
5.884 \mathrm{E} \\
1.576 \mathrm{E} \\
4.426 \mathrm{E} \\
1.320 \mathrm{E}\end{array}$ & $\begin{array}{l}4 \\
8656 \\
E-05 \\
E-05 \\
E-04 \\
-05 \\
E-04 \\
-05 \\
-05 \\
-05 \\
-05 \\
=05 \\
E-05 \\
-05 \\
-04 \\
-04 \\
-03 \\
E-03 \\
E-03 \\
E-02 \\
E-02 \\
E-02\end{array}$ & $\begin{array}{c}\text { ANGLE } 5 \\
M U=-0.7550 \\
1.165 \mathrm{E}-05 \\
2.443 \mathrm{E}-05 \\
2.793 \mathrm{E}-04 \\
7.564 \mathrm{E}-05 \\
1.356 \mathrm{E}-04 \\
2.496 \mathrm{E}-05 \\
2.453 \mathrm{E}-05 \\
2.530 \mathrm{E}-05 \\
3.081 \mathrm{E}-05 \\
5.001 \mathrm{E}-05 \\
8.754 \mathrm{E}-05 \\
1.522 \mathrm{E}-04 \\
1.180 \mathrm{E}-03 \\
1.966 \mathrm{E}-03 \\
5.902 \mathrm{E}-03 \\
1.622 \mathrm{E}-02 \\
4.505 \mathrm{E}-02 \\
1.330 \mathrm{E}-02\end{array}$ & $\begin{array}{r}\text { ANजLE } \quad 6 \\
M U=-0.6179 \\
1.295 E-05 \\
2.714 E-05 \\
3.092 E-04 \\
8.483 E-05 \\
1.506 E-04 \\
2.974 E-05 \\
2.992 E-05 \\
3.299 E-05 \\
4.162 E-05 \\
6.482 E-05 \\
1.092 E-04 \\
1.919 E-04 \\
1.369 E-03 \\
2.364 E-03 \\
5.926 E-03 \\
1.659 E-02 \\
4.609 E-02 \\
1.344 E-02\end{array}$ \\
\hline $\begin{array}{l}\text { ANGLE } \\
4 U=0.6 \\
7.889 \mathrm{E} \\
1.615 \mathrm{E} \\
1.753 \mathrm{E} \\
5.518 \mathrm{E} \\
9.195 \mathrm{E} \\
4.141 \mathrm{E} \\
4.913 \mathrm{E} \\
6.036 \mathrm{E} \\
7.623 \mathrm{E} \\
1.040 \mathrm{E} \\
1.419 \mathrm{E} \\
1.921 \mathrm{E} \\
4.608 \mathrm{E} \\
5.156 \mathrm{E} \\
7.898 \mathrm{E} \\
2.361 \mathrm{E} \\
5.965 \mathrm{E} \\
1.502 \mathrm{E}\end{array}$ & $\begin{array}{l}13 \\
b 179 \\
E-05 \\
E-04 \\
E-03 \\
-04 \\
E-04 \\
E-04 \\
E-04 \\
-04 \\
E-04 \\
-03 \\
-03 \\
-03 \\
-03 \\
-03 \\
-03 \\
-03 \\
-02 \\
-02 \\
-02 \\
-02\end{array}$ & $\begin{array}{r}\text { ANGLE } 14 \\
M U=0.7550 \\
1.223 E-04 \\
2.493 E-04 \\
2.672 E-03 \\
8.751 E-04 \\
1.421 E-03 \\
6.541 E-04 \\
8.006 E-04 \\
9.473 E-04 \\
1.141 E-03 \\
1.450 E-03 \\
1.826 E-03 \\
2.282 E-03 \\
5.193 E-03 \\
5.679 E-03 \\
8.477 E-03 \\
2.495 E-02 \\
5.190 E-02 \\
1.525 E-02\end{array}$ & $\begin{array}{r}\text { ANSLE } 15 \\
\text { MU=0.8656 } \\
1.905 E-04 \\
3.869 \mathrm{E}-04 \\
4.096 \mathrm{E}-03 \\
1.386 \mathrm{E}-03 \\
2.188 \mathrm{E}-03 \\
1.110 \mathrm{E}-03 \\
1.228 \mathrm{E}-03 \\
1.385 \mathrm{E}-03 \\
1.582 \mathrm{E}-03 \\
1.876 \mathrm{E}-03 \\
2.208 \mathrm{E}-03 \\
2.602 \mathrm{E}-03 \\
5.800 \mathrm{E}-03 \\
6.212 \mathrm{E}-03 \\
9.050 \mathrm{E}-03 \\
2.619 \mathrm{E}-02 \\
6.389 \mathrm{E}-02 \\
1.544 \mathrm{E}-02\end{array}$ \\
\hline
\end{tabular}
ANGLE 7 $M U=-0.4580$
$1.476 \mathrm{E}-05$ 3. $080 \mathrm{E}-05$ $3.502 E-04$ $9.434 E-05$ $1.696 E-04$
$3.577 E-05$ $3.577 \mathrm{E}-05$ $3.995 E-05$ $4.815 \mathrm{E}-05$ $6.159 E-05$ $8.961 E-05$ $1.410 E-04$ $2.539 E-04$ $1.645 E-03$
$2.796 E-03$ $2.796 E-03$
$5.972 E-03$ $1.720 \mathrm{E}-02$
$4.738 \mathrm{E}-02$ $4.738 \mathrm{E}-02$
$1.361 \mathrm{E}-02$ ANGLE 16 $M U=0.9446$ $2.839 E-04$ $0.744 E-04$
$6.021 E-03$ 2.070E-03 $3.188 E-03$ $1.619 E-03$ $1.719 \mathrm{E}-03$ $1.850 E-03$ $2.013 E-03$ $2.248 \mathrm{E}-03$ $2.513 E-03$ $2.861 E-03$ $6.375 E-03$ $6.672 \mathrm{E}-03$ $9.549 \mathrm{E}-03$ $2.719 \mathrm{E}-02$ $6.543 E-02$
$1.559 E-02$

$\begin{array}{lc}\text { ANGLE } 8 & \text { ANGLE } 9 \\ \text { MU=-0.2816 } & \text { MU=-0.0950 } \\ 1.740 E-05 & 2.138 E-05 \\ 3.617 E-05 & 4.435 E-05 \\ 4.101 E-04 & 5.003 E-04 \\ 1.094 E-04 & 1.363 E-04 \\ 1.986 E-04 & 2.451 E-04 \\ 4.625 E-05 & 0.499 E-05 \\ 5.517 E-05 & 7.767 E-05 \\ 6.874 E-05 & 9.656 E-05 \\ 8.808 E-05 & 1.246 E-04 \\ 1.262 E-04 & 1.837 E-04 \\ 1.971 E-04 & 2.974 E-04 \\ 3.654 E-04 & 5.510 E-04 \\ 2.012 E-03 & 2.460 E-03 \\ 3.218 E-03 & 3.604 E-03 \\ 6.069 E-03 & 6.243 E-03 \\ 1.794 E-02 & 1.881 E-02 \\ 4.893 E-02 & 5.073 E-02 \\ 1.380 E-02 & 1.402 E-02 \\ & \\ \text { ANGLE } 17 & S C A L A R \\ M U=0.9894 & \text { FLUX } \\ 3.776 E-04 & 6.850 E-04 \\ 7.610 E-04 & 1.400 E-03 \\ 7.927 E-03 & 1.513 E-02 \\ 2.725 E-03 & 4.788 E-03 \\ 4.131 E-03 & 7.843 E-03 \\ 2.062 E-03 & 3.366 E-03 \\ 2.124 E-03 & 3.782 E-03 \\ 2.211 E-03 & 4.382 E-03 \\ 2.322 E-03 & 5.228 E-03 \\ 2.490 E-03 & 6.742 E-03 \\ 2.700 E-03 & 8.960 E-03 \\ 3.029 E-03 & 1.248 E-02 \\ 6.791 E-03 & 3.775 E-02 \\ 6.967 E-03 & 4.791 E-02 \\ 9.878 E-03 & 8.612 E-02 \\ 2.782 E-02 & 2.517 E-01 \\ 6.635 E-02 & 6.628 E-01 \\ 1.567 E-02 & 1.787 E-01\end{array}$


(GAMMAS/MEV/STERADIAN/SOURCE NEUTRON)

ENERGY 8.00 GROUP (MEV) 6.5OE OD- - 8. COE 00 $5.00 E$ OO- - 6.50 E OO 4. OOE $00--5.00 E$ OO $3.00 E$ OO- - 4.0OE OO 2.5OE $00-1-3.00 E$ OO $2.00 E$ OO---2.50E 00 $1.66 \mathrm{E} 00---2.00 \mathrm{E}$ OO $1.33 \mathrm{E} 00--1.66 \mathrm{E}$ OO $1.00 E$ OO- $01.33 E$ OO $8.00 E-01--1.00 E$ OO 6.00E-01-- - 8. 00E-OI $4.00 E-01--6.00 E-01$ $3.00 E-01--4.00 E-0$ $2.00 E-01-\triangle-3.00 E-01$ $1.00 E-01--2.00 E-01$ $5.00 E-02--1.00 E-01$
$2.00 E-02---5.00 E-02$

\section{ENERGY}

GROUP (MEV)
.0OE OO--1.00E 01 $8.00 E$ OO-D-1.00E 01
6.50 E $00---8.00 E$ OD $6.50 E$
$5.00 E$ OD- $00-6.50 E$ OO $4.00 E$ OO- $-5.00 E$ OO 3.0OE OO- - 4. OOE OO $2.50 E$ OO- - 3. OOE OO $2.00 E$ OO--2.5OE OO $1.66 \mathrm{E}$ 00--2.00E 00 $1.33 \mathrm{E} 00--1.66 \mathrm{E}$ 0O $1.33 E$ OOT- $1.06 E$ OO $8.00 E-01--1.00 E$ OO G. OEE $01-2-8.00 E-01$ $4.00 E-01--6.00 E-01$ $3.00 E-01--4.00 E-01$ 2.0OE-01- $-3.00 E-01$ $1.00 E-01-2.00 E-01$ $5.00 E-02-1.00 E-01$ $2.00 \mathrm{E}-02--5.00 \mathrm{E}-02$
ANGLE 1 ANGLE 2 $M U=-1.0000 \quad M U=-0.9804$ $5.002 E-06$ $9.710 E-06$ $1.164 E-04$ $9.833 E-06$ $4.147 E-C 5$ $1.227 \mathrm{E}-07$ $3.607 \mathrm{E}-05$ . $4.079 \mathrm{E}-05$ $4.079 \mathrm{E}-05$
$1.218 \mathrm{E}-05$ 4.135E-0 $7.459 E-04$ $9.420 \mathrm{E}-04$ $5.452 \mathrm{E}-03$ $1.430 \mathrm{E}-02$ $4.188 \mathrm{E}-02$ $1.279 \mathrm{E}-02$ ANGLE 10 $U=0.0950$
$1.636 \mathrm{E}-05$ $1.636 E-05$
$3.387 E-05$ $3.781 E-04$ 1.781E-04 $1.089 E-04$ $271 \mathrm{E}-05$ $7.442 E-05$ $9.429 \mathrm{E}-05$ $1262 \mathrm{E}-04$ $2.087 \mathrm{E}-04$ $3.795 \mathrm{E}-04$ $7.389 E-04$ $2.680 E-03$ $3.664 \mathrm{E}-03$ $5.918 E-03$ $1.874 E-O 2$ $5.161 \mathrm{E}-02$ $1.402 \mathrm{E}-02$ .086E-06 $9.975 E-06$ $1.345 \mathrm{E}-05$ $4.456 E-0$ $1.723 \mathrm{E}-0 \mathrm{~S}$ 1.739E-05 $3.332 \mathrm{E}-05$ $4.348 E-05$ 3.831E-05 $1.531 \mathrm{E}-05$ $1.409 E-05$ $7.491 \mathrm{E}-04$ $9.713 \mathrm{E}-04$ $5.456 \mathrm{E}-03$ $1.433 \mathrm{E}-02$ $4 . ? 95 E-0$ $1.280 \mathrm{E}-02$

$\triangle$ ANGLE 11 $A U=0.2816$
$2.301 \mathrm{E}-05$ $4.754 \mathrm{E}-05$ $5.247 \mathrm{E}-04$ $1.592 \mathrm{E}-04$ $2.709 \mathrm{E}-04$ 1.04OE $1.240 E-04$ 1.5 3. $6.365 E-04$ $1.108 \mathrm{E}-03$ $3.202 \mathrm{E}-03$ $3.057 \mathrm{E}-03$ $6.275 \mathrm{E}-03$ $1.990 \mathrm{E}-0$ $5.395 \mathrm{E}-02$ $1.429 \mathrm{E}-02$
MU $=-0.9446$ $5.395 \mathrm{E}-06$ $1.091 \mathrm{E}-05$ $1.278 E-04$
$2.367 E-05$ $5.450 E-05$ $5.951 \mathrm{E}-06$ $1.499 \mathrm{E}-05$ $2.265 \mathrm{E}-0.5$ $2.782 \mathrm{E}-05$ $2.988 \mathrm{E}-05$ $2.554 \mathrm{E}-05$ 3.921 E- 05 $7.663 \mathrm{E}-04$ $1.096 \mathrm{E}-03$ $5.469 \mathrm{E}-03$ $1.446 \mathrm{E}-02$ $4.225 \mathrm{E}-\mathrm{C}$ 1.284 E- 02 ANGLE 12 $A U=0.4580$
$3.464 E-05$ $3.464 E-05$
$7.110 E-05$ $7.746 \mathrm{E}-04$ $7.746 E-04$
$2.423 E-04$ $2.423 E-04$ $4.079 E-04$ $1.839 E-04$ 2.2 $4.083 E-04$ 4.083E 04 $9.07 E-04$ $1.517 \mathrm{E}-03$ $3.717 \mathrm{E}-03$ $4.301 \mathrm{E}-03$ $6.728 E-03$ $2.119 \mathrm{E}-02$ $5.644 E-0$ $1.456 \mathrm{E}-02$
$\mathrm{U}=-0.8656$ $1.233 \mathrm{E}-05$ $1.418 \mathrm{E}-04$ 3.672E-05 $6.792 E-05$ $1.182 \mathrm{E}-05$ $1.212 \mathrm{E}-05$ $1.097 \mathrm{E}-05$ $1.223 E-05$ $4.054 \mathrm{E}-05$ $7.486 \mathrm{E}-05$ $8.125 \mathrm{E}-04$ $1.338 \mathrm{E}-03$ $5.480 E-03$ $1.470 \mathrm{E}-0$ $4.281 E-02$ 1.291E-02 ANGLE 13 MU $=0.6179$
$5.613 \mathrm{E}-05$ $1.144 \mathrm{E}-04$ 1.2.27E-03 $4.011 E-04$ 6.630 E 04 $4.456 E-04$ 5.272E-04 $5.468 E-04$ $1.018 \mathrm{E}-03$ $1.418 \mathrm{E}-03$ $1.923 E-03$ $4.231 E-03$ 4.740E-03 $7.255 \mathrm{E}-03$ $2.257 \mathrm{E}-02$ $2.257 E-02$
$5.897 E-02$ 1.483E-02

\begin{tabular}{|c|c|c|c|c|}
\hline $\begin{array}{l}=-0.7550 \\
.533 E-06 \\
.384 E-05 \\
.574 E-04 \\
.727 E-05 \\
.932 E-05 \\
.600 E-05 \\
.158 E-05 \\
.134 E-06 \\
.125 E-05 \\
.387 E-05 \\
.558 E-05 \\
.036 E-04 \\
.094 E-04 \\
.682 E-03 \\
.481 E-03 \\
.505 E-02 \\
.361 E-02 \\
.302 E-02\end{array}$ & $\begin{array}{r}\text { MU }=-0.6179 \\
7.288 E-06 \\
1.540 E-05 \\
1.746 E-04 \\
5.076 E-05 \\
8.746 E-05 \\
1.748 E-05 \\
1.522 E-05 \\
1.576 E-05 \\
2.003 E-05 \\
3.670 E-05 \\
6.917 E-05 \\
1.2611 E-04 \\
1.083 E-03 \\
2.109 E-03 \\
5.473 E-03 \\
1.552 E-02 \\
4.468 E-02 \\
1.316 E-02\end{array}$ & $\begin{array}{r}M U=-0.4580 \\
8.311 E-06 \\
1.737 E-05 \\
1.970 E-04 \\
5.208 E-05 \\
9.555 E-05 \\
1.990 E-05 \\
2.314 E-05 \\
2.832 E-05 \\
3.721 E-05 \\
5.617 E-05 \\
8.793 E-05 \\
1.691 E-04 \\
1.351 E-03 \\
2.564 E-03 \\
5.480 E-03 \\
1.612 E-02 \\
4.601 E-02 \\
1.334 E-02\end{array}$ & $\begin{array}{r}\text { ANGLE } \\
M U=-0.2816 \\
8.868 E-06 \\
2.044 E-05 \\
2.316 E-04 \\
5.964 E-05 \\
1.107 E-04 \\
2.664 E-05 \\
3.438 E-05 \\
4.508 E-05 \\
5.955 E-05 \\
8.239 E-05 \\
1.291 E-04 \\
2.682 E-04 \\
1.720 E-03 \\
2.994 E-03 \\
5.534 E-03 \\
1.684 E-02 \\
4.762 E-02 \\
1.354 E-02\end{array}$ & $\begin{array}{r}\text { ANGLE } 9 \\
M U=-0.0950 \\
1.235 E-05 \\
2.554 E-05 \\
2.878 E-04 \\
7.715 E-05 \\
1.405 E-04 \\
3.960 E-05 \\
4.958 E-05 \\
6.447 E-05 \\
8.264 E-05 \\
1.252 E-04 \\
2.172 E-04 \\
4.534 E-04 \\
2.174 E-03 \\
3.359 E-03 \\
5.673 E-03 \\
1.772 E-02 \\
4.949 E-02 \\
1.377 E-02\end{array}$ \\
\hline $\begin{array}{l}\text { ANGLE } 14 \\
J=0.7550 \\
9.675 E-05 \\
1.962 E-04 \\
2.069 E-03 \\
7.152 E-04 \\
1.144 E-03 \\
6.513 E-04 \\
7.729 E-04 \\
9.382 E-04 \\
1.154 E-03 \\
1.487 E-03 \\
1.870 E-03 \\
.294 E-03 \\
4.771 E-03 \\
5.265 E-03 \\
7.826 E-03 \\
2.396 E-02 \\
6.141 E-02 \\
1.507 E-02\end{array}$ & $\begin{array}{r}\text { ANGLE } 15 \\
M U=0.8656 \\
1.707 E-04 \\
3.446 E-04 \\
3.574 E-03 \\
1.291 E-03 \\
1.983 E-03 \\
1.158 E-03 \\
1.294 E-03 \\
1.468 E-03 \\
1.581 E-03 \\
1.979 E-03 \\
2.286 E-03 \\
2.617 E-03 \\
5.360 E-03 \\
5.813 E-03 \\
8.403 E-03 \\
2.526 E-02 \\
6.359 E-02 \\
1.528 E-02\end{array}$ & $\begin{array}{r}\text { ANGLE } 16 \\
M U=0.9446 \\
2.882 E-04 \\
5.787 E-04 \\
5.924 E-03 \\
2.170 E-03 \\
3.223 E-03 \\
1.845 E-03 \\
1.944 E-03 \\
2.068 E-03 \\
2.214 E-03 \\
2.409 E-03 \\
2.612 E-03 \\
2.883 E-03 \\
5.957 E-03 \\
6.286 E-03 \\
8.916 E-03 \\
2.633 E-02 \\
6.529 E-02 \\
1.543 E-02\end{array}$ & $\begin{array}{r}\text { ANGLE } 17 \\
\text { MU= } 0.9894 \\
4.236 E-04 \\
8.455 E-04 \\
8.583 E-03 \\
3.115 E-03 \\
4.529 E-03 \\
2.494 E-03 \\
2.520 E-03 \\
2.560 E-03 \\
2.612 E-03 \\
2.691 E-03 \\
2.808 E-03 \\
3.064 E-03 \\
6.415 E-03 \\
6.588 E-03 \\
9.264 E-03 \\
2.700 E-02 \\
6.631 E-02 \\
1.552 E-02\end{array}$ & $\begin{array}{c}\text { SCALAR } \\
\text { FLUX } \\
5.516 E-04 \\
1.119 E-03 \\
1.183 E-02 \\
3.993 E-03 \\
6.319 E-03 \\
3.202 E-03 \\
3.606 E-03 \\
4.184 E-03 \\
4.993 E-03 \\
6.440 E-03 \\
8.545 E-03 \\
1.187 E-02 \\
3.380 E-02 \\
4.396 E-02 \\
7.914 E-02 \\
2.385 E-01 \\
6.496 E-01 \\
1.757 E-01\end{array}$ \\
\hline
\end{tabular}


IGAMMAS/MEV/STERADIAN/SOURCE NEUTRON)

ENERGY GROUP (MEV) 8.00E $00---1.00 E$ O1 $6.50 E$ OO-- $8.00 E$ DO 4.0OE $00---5$. . COE 00 $3.00 E$ OO $-4.00 E$ OO $2.50 \mathrm{E} 00--3.00 \mathrm{E} \quad 00$ $2.00 E \quad 00--2.50 E \quad 00$ $1.66 E$ O0-- $2.00 E$ OO $1.33 \mathrm{E} \mathrm{O0--1.66 \textrm {E } ~} 00$ $1.00 \mathrm{E} \quad 00--1.33 \mathrm{E} \quad 00$ 8.0OE-01--1. 1.00E 00 $6.00 E-01--8.00 E-01$ 4. $00 E-01---3.00 E-01$ $3.00 E-01-4.00 E-01$ $1.00 \mathrm{E}-01--2.00 \mathrm{E}-01$ $5.00 \mathrm{E}-02--1.00 \mathrm{E}-01$ $2.00 E-02--5.00 E-02$

$$
\text { ENERGY }
$$

GROUP (MEV)

8.00E $00--1.00 E 01$ $6.50 E$ OO- $-8.00 E$ DO $5.00 E$ OO-- -6.50E 00 $4.00 E$ OO- - 5. COE 00 $3.00 E$ OOO- $4.00 E$ DO $2.50 E$ OO- $-3.00 E$ OO $2.00 E$ OO- $-2.50 E$ OO $1.66 E$ OO---2. $00 E$ OO 1.33 E $00--1.66$ E 00 $1.00 E$ OO- $-1.33 \mathrm{E}$ OO $8.00 E-01--1.00 E$ OO $6.00 E-01--2.00 E-01$ $4.00 E-01--6.00 E-01$ 3. 0 E $-01-3.00 E-01$ $1.0 E-01-3.00 E-01$ $1.00 E-01-2.00 E-01$ $2.00 \mathrm{E}-02---5.00 \mathrm{E}-02$ ANGLE 1
$M U=-1.0000 \quad$ ANGLE ${ }^{2}$
$M U=-0.9804$ $1.475 \mathrm{E}-07 \quad 2.294 \mathrm{E}-07$ $-5.451 E-C 7 \quad-2.107 E-C 7$ $2.635 \mathrm{E}-07 \quad 2.936 \mathrm{E}-06$ $-2.155 E-05-1.699 E-05$ $-1.537 E-05-1.126 E-05$ $2.905 E-05$ $2.753 E-05$ $-1.898 E-1$ $3.266 E-04$ $3.428 E-03$ $8.682 \mathrm{~F}-03$ $8.682 F-03$ $2.696 E-02$
$8.363 \mathrm{E}-03$ ANGLE 10 $M U=0.0950$ $2.276 \mathrm{E}-06$ $4.651 \mathrm{E}-06$ $5.247 F-05$ $1.923 E-C 5$ $3.025 E-05$ $1.318 \mathrm{E}-05$ $1.242 E-05$ $1.262 E-05$ $1.870 E-05$ $5.497 E-05$ $1.661 E-04$ $4.437 E-04$ 1.60 $2.215 E-03$ $3.401 \mathrm{E}-03$ $1.151 E-02$ $9.213 E-03$
ANGLE 3 $4.557 E-07 \quad 7.522 E-07$ $6.033 E-07$

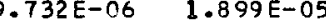
$5.406 \mathrm{E}-06$ $2.308 E-07 \quad 1.087 E-05$ $-1.529 E-06 \quad 2.350 E-06$ $3.985 E-06 \quad 8.982 E-08$ $9.162 \mathrm{E}-06 \quad-4.184 \mathrm{E}-06$ $1.170 E-05 \quad-7.064 E-06$ 7.264E-06 -4.221E-06 $-4.147 E-06 \quad 6.088 E-06$ $-6.489 E-06 \quad 1.855 E-05$ $3.200 E-04 \quad 3.253 E-04$ 4.511E-04 6.269E-04 $3.44 J \mathrm{E}-03 \quad 3.438 \mathrm{E}-0$ $8.788 E-03 \quad 8.945 E-03$ $2.721 E-02 \quad 2.759 E-02$ $8.397 \mathrm{E}-03 \quad 8.449 \mathrm{E}-0$ ANGLE 12 MU 6.096E-06 $1.251 \mathrm{E}-05$ $1.323 \mathrm{E}-04$ $4.644 E-05$ $7.396 \mathrm{E}-05$ $4.494 E-C 5$ $6.436 E-05$ $3.046 \mathrm{E}-04$ $1.824 E-04$ $3.63 ! \mathrm{E}-04$ $6.603 E-04$ $1.077 \mathrm{E}-03$ $2.206 E-03$ 2.450E- 03 $3.861 E-03$ $1.308 E-02$ $9.591 E-03$

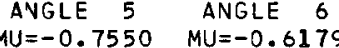
$9.591 \varepsilon-07$ $2.229 E-06$ $2.426 \mathrm{E}-05$ $1.539 E-05$ $1.637 E-05$ $6.143 E-06$ $-1.727 \mathrm{E}-06$ $-8.501 E-06$ $-1.181 E-05$ $-4.536 E-06$ $1.814 E-05$ 4.1 $00 E-05$ $3.646 E-04$ $8.954 E-04$ $3.408 E-03$ $9.171 E-03$ $2.814 E-02$ $8.522 E-03$ ANGLE 14 $M U=0.7550$ 2. $936 E-05$ $5.829 E-05$ $5.833 E-04$ $2.369 \mathrm{E}-04$ 3.851E-04 $3.424 \mathrm{E}-04$ $4.631 E-04$ $6.238 E-04$ I. $132 E-03$ $1.112 E-03$ $1.384 E-03$ $1.600 E-03$ $2.678 E-03$ $4.477 E-03$ $4.477 E-03$ $1.487 E-02$ $9.949 E-03$ $9.902 \mathrm{E}-07$ $2.287 \mathrm{E}-06$ $2.411 E-05$ $1.125 \mathrm{E}-05$ $1.562 E-05$ $2.786 E-06$ 作 $-2.007 E-06$ $-1.316 E-06$ $3.086 E-05$ $4.639 E-04$ $1.237 E-03$ $3.349 E-03$ $9.470 E-03$ $2.887 E-02$

\section{ANGLE 15} $M U=0.8656$
$7.803 E-05$ $1.552 \mathrm{E}-04$ $1.502 \mathrm{E}-03$ $6.781 E-04$ $9.907 E-04$ $8.270 E-04$ $9.685 \mathrm{E}-04$ $1.136 E-03$ $1.325 \mathrm{E}-03$ $1.53 \mathrm{E}-03$ $1.687 E-03$ $1.782 E-03$ 2.985E-03 . $368 E-03$ $1.572 \mathrm{E}-02$ $4.224 \mathrm{E}-02$ $1.010 \mathrm{E}-02$
ANGLE 7 ANGLE 8 ANGLE 9 $M U=-0.4580$ $9.875 E-07$ $.113 E-06$
$.312 E-05$ $1.292 \mathrm{E}-06$ $6.856 \mathrm{E}-06$ $1.718 \mathrm{E}-07$ $2.825 E-06$ $5.573 E-06$ $9.127 E-06$ $.258 \mathrm{E}-05$ $2.748 \mathrm{E}-05$ $6.447 E-04$ $1.591 E-03$ $3.284 E-03$ $9.845 \mathrm{E}-03$ $2.978 \mathrm{E}-02$ . ANGLE 16 $M U=0.9446$
$1.993 E-04$ $3.916 \mathrm{E}-04$ $3.707 \mathrm{E}-03$ $1.648 E-03$ $2.234 \mathrm{E}-03$ $1.664 E-03$ $1.730 E-03$ $1.792 \mathrm{E}-03$ $1.844 E-03$ $1.878 E-03$ $1.037 E-03$ $1.937 \mathrm{E}-03$ 3.634E-03 3.634E-03 I. $119 \mathrm{E}-03$ $4.349 \mathrm{E}-02$ $1.021 E-02$
$M U=-0.2816 \quad M U=-0.0950$ $1.119 E-06 \quad 1.502 E-06$ $1.997 \mathrm{E}-06 \quad 2.735 \mathrm{E}-06$ $4.513 E-07 \quad 3.363 E-05$ 7.751E-06 1.533E-05 $1.313 E-06 \quad 5.706 E-06$ 7.983E-06 $1.102 E-05$ $1.593 E-05 \quad 1.621 E-05$ $2.167 E-05 \quad 1.975 E-05$ $2.126 E-05 \quad 2.573 E-05$ $1.681 E-05 \quad 5.728 E-05$ $7.369 E-05 \quad 2.090 E-04$ $9.117 E-04 \quad 1.244 E-03$ $1.894 E-03 \quad 2.099 E-03$ $3.249 E-03 \quad 3.282 E-03$ $1.031 E-02 \quad 1.086 E-02$ $3.089 \mathrm{E}-02 \quad 3.219 \mathrm{E}-02$ ANGLE 17 $M U=0.9894$ $7.961 \mathrm{E}-04$ $7.439 E-03$ $3.086 \mathrm{E}-03$ 4.007E-03 $2.657 \mathrm{E}-03$ $2.546 \mathrm{E}-03$ $2.414 E-03$ $2.266 E-03$ $2.084 E-03$ $1.974 E-03$ $2.057 E-03$ 3.700E-O3 $3.780 E-03$ $1.687 \mathrm{E}-02$ 1. $425 \mathrm{E}-02$ $4.425 E-02$ 
(GAMMAS/MEV/STERADIAN/SOURCE NEUTRON)

ENERGY GROUP (MEV) 8.00E 00---1.00E 01 6.5OE OD---8.00E 00 4.0OE OO-- 5.00 E 00 3.00E 00--4.00E 00 $2.50 E$ OO-D- 3. OOE OO 2.0OE 00---2.50E OO $1.66 \mathrm{E} 00-\cdots 2.00 \mathrm{E} 00$ $1.33 \mathrm{E} 00--1.66 \mathrm{E} 00$ $1.00 E$ 00- $-1.33 E 00$ $8.00 E-01--1.00 E$ OO 6.00E-01- - - . C0E-01 $4.00 E-01--6.00 E-01$ $2.00 \mathrm{E}-01---3.00 \mathrm{E}-01$ $1.00 \mathrm{E}-01--2.00 \mathrm{E}-0$ $5.0 \mathrm{CE}-02--1.00 \mathrm{E}-01$ $2.00 E-02---5.00 E-02$

\section{ENERGY}

$$
\text { GROUP (MEV) }
$$

8.00E 00- - - 1. OOE OI

$6.50 E$ OO- - - 8. OOE OO $5.00 E$ OO- $-6.50 E$ OO $4.00 E$ OO- $-5.00 E$ OO 3.00 E $00---4.00 E$ OO $2.50 E$ OO- $-3.00 E$ OO 2.00E $00---2.50 E$ OO $1.66 \mathrm{E} \quad 00---2.00 \mathrm{E} O 0$ 1.33 E $00--1.66 \mathrm{E} 00$ $1.00 \mathrm{E} 00--1.33 \mathrm{E} 00$ $8.00 \mathrm{E}-01---1.00 \mathrm{E}$ OC $6.00 E-01--8.00 E-0$ $4.00 E-01--6.00 E-01$ 3.00E-01- $-4.00 E-01$ 2. 0 ONE-01- $-3.00 \mathrm{E}-01$ 1.0OF-01---2.0OE-01 $5.00 \mathrm{E}-02--1.00 \mathrm{E}-01$ $2.00 E-02---5.00 E-02$
ANGLE 1 ANGLE 2 $M U=-1.0000$ $-1.263 E-06$ $-8.739 \mathrm{E}-06$ $-1.762 \mathrm{E}-05$ $-1.541 \mathrm{E}-05$ $-8.019 E-06$ $5.422 E-06$ $2.000 \mathrm{E}-05$ $2.895 E-05$ $1.907 E-05$ $-1.622 E-05$ $1.534 E-04$ $1.848 \mathrm{E}-03$ $4.472 E-03$ $1.405 E-02$ $4.351 \mathrm{E}-03$

ANGLE 10 $2.943 E-07$ $8.327 E-07$ $7.594 \mathrm{E}-06$ $5.368 E-06$ $6.738 E-06$ $4.761 E-06$ $2.557 E-08$ $-5.256 E-06$ $-6.674 E-06$ $9.108 \mathrm{E}-06$ $7.331 \mathrm{E}-05$ $2.442 E-04$ $8.497 E-04$ $1.145 \mathrm{E}-03$ $1.708 \mathrm{E}-03$ $5.885 \mathrm{E}-03$ $1.746 \mathrm{E}=02$ $4.780 \mathrm{E}-03$ $-2.412 E-07$ $-1.018 \mathrm{E}-06$
$-6.939 \mathrm{E}-06$ $-1.441 E-05$ $-1.249 \mathrm{E}-05$ $-6.444 E-06$ $4.775 E-06$ $1.712 \mathrm{E}-05$ $2.453 E-05$ $1.580 \mathrm{~F}-05$ $-1.381 E-05$ $1.511 \mathrm{E}-04$ $1.518 \mathrm{E}-04$ $4.482 \mathrm{~F}-03$ $1.407 E-02$ $4.354 E-C 3$ ANGLE 11 $M U=0.2816$ 5.551E-07 . $1.383 E-05$ $1.206 \mathrm{E}-05$ $5.018 E-06$ $-7.096 \mathrm{E}-07$ $7.253 E-06$ $6.011 \mathrm{E}-05$ $1.942 \mathrm{E}-04$ $4.333 E-04$ $1.016 E-03$ $1.153 E-03$ $1.79 E-03$ $6.244 \mathrm{E}-03$ $4.873 E-03$
ANGLE 3 ANGLE 4 $M U=-0.9446$ $-2.712 E-07$ $-2.712 E-07$
$-1.408 E-06$ $-1.408 E-06$
$-4.565 E-06$ $-3.519 E-06$ $-3.519 E-06$
$-1.689 E-06$ $-1.689 E-06$
$1.978 E-06$ 5.470E-06 $7.061 E-06$ $3.418 E-06$ $-5.610 E-06$ $-9.003 E-06$ $1.436 E-04$ 作 $1.855 E-03$ $4.527 E-03$ $1.418 E-02$
$4.368 E-03$ ANGLE 12 $M U=0.4580$ $1.794 \mathrm{E}-06$ $1.769 \mathrm{E}-05$ $7.735 \mathrm{E}-06$ $9.636 E-06$ $5.311 E-06$ 1.157E-05 $2.972 E-05$ $7.568 \mathrm{E}-05$ $1.879 \mathrm{E}-04$ $3.749 E-04$ $6.220 E-04$ $1.133 E-03$ $1.200 \mathrm{E}-03$ $1.915 E-03$ $6.640 E-03$ $4.968 \mathrm{E}-03$ $8.910 E-08$ 2.380 E- 07 $2.495 \mathrm{E}-06$ $2.232 E-06$ $2.673 \mathrm{E}-06$ $1.416 E-06$ $-8.301 E-07$ $-4.632 E-06$ $-7.456 E-06$ $-5.832 E-06$ ( ANGLE 13 $1.690 \mathrm{E}-06$ $1.690 E-06$ $3.953 E-05$ $3.440 E-06$ $1.745 E-05$ $3.089 E-05$ $7.064 E-05$ $1.351 E-04$ $2.319 E-04$ $3.980 E-04$ $5.915 \mathrm{E}-04$ $7.726 \mathrm{E}-04$ $1.207 \mathrm{E}-03$ $1.316 \mathrm{E}-03$ $2.041 \mathrm{E}-03$ $7.060 \mathrm{E}-03$ $2.007 E-02$ $5.060 E-03$ ANGLE $M U=-0.7550$ $7.867 E-07$ $6.519 E-06$ $9.040 E-06$ $8.490 E-06$ $3.909 \mathrm{E}-06$ $-2.358 E-06$ $-7.941 E-06$ $-1.105 E-05$ $-6.235 E-06$ $9.955 \mathrm{E}-06$ $1.536 \mathrm{E}-04$ 4. $539 \mathrm{E}-04$ $1.820 \mathrm{E}-03$ 4. $726 \mathrm{E}-03$ $1.465 \mathrm{E}-02$
$4.432 \mathrm{E}-03$ ANGLE 14

AVGLE 6 $M U=-0.6179$ 1.672E-07 $3.692 \mathrm{E}-06$ $3.385 E-06$ $2.923 E-06$ 4. $409 \mathrm{E}-07$ $-2.997 E-06$ $-2.711 E-06$ $6.602 E-07$ $7.258 E-U 6$
$5.636 E-06$ $2.036 E-06$ 6.571E-04 $1.768 E-03$ $4.878 \mathrm{E}-03$ $1.502 E-02$
$4.481 E-03$ ANGLE 15 AU = 0.7550 .063E-06 1. 3 E 05 $4.900 \mathrm{E}-05$ $1.008 E-04$ $1.417 E-04$ $2.267 E-04$ $3.391 E-04$ 4.761E-04 $6.558 \mathrm{E}-04$ $7.985 E-04$ $8.723 E-04$ $1.280 \mathrm{E}-03$ $1.478 \mathrm{E}-03$ $2.171 \mathrm{E}-03$ $7.482 \mathrm{E}-03$ $2.094 \mathrm{E}-02$ $5.145 \mathrm{E}-03$ A 2.593E-05 5.134E-O5 $4.598 E-04$ 4. $034 E-04$ $4.034 E-04$ $4.402 E-04$
$5.452 E-04$ $6.620 \mathrm{E}-04$ $7.805 E-04$ $8.971 E-04$ $9.460 E-04$ $9.380 \mathrm{E}-04$ $1.398 \mathrm{E}-03$ $1.630 \mathrm{E}-03$ $2.308 \mathrm{E}-03$ T.877E-03 2.172E-02 $5.217 E-03$ $-1.571 E-06$

ANGLE 7 $M U=-0.4580$ $1.662 E-08$
$-6.670 E-08$ $7.651 E-08$ $-3.455 E-06$ $-2.919 E-06$ $-2.204 E-06$ $2.848 E-06$ $5.506 E-06$ $6.132 \mathrm{E}-0.6$ $-2.358 \mathrm{E}-06$ $-2.675 E-06$ $3.064 E-04$ .643E-04 $1.707 E-03$ $5.066 \mathrm{E}-03$ $1.549 E-02$
$4.541 E-03$ ANGLE 16

ANGLE 8 $M U=-0.2826$ $-8.197 E-09$ $-2.286 E-07$ 
(GAMMAS/MEV/STERADIAN/SOURCE NEUTRON)

\begin{abstract}
ENERGY
SRROUP (MEV)

8.00E O0--1.

$6.50 E$ OO-- $-8.00 E$ OO
5. . OE $00--6.50 E$ OO 4.0OE OO- - $5.00 E$ OO $3.00 E$ OO---4.0OE 00 2.5 OE $00--3.00 E$ OO $2.00 E$ OO---2.5OE OO $1.66 \mathrm{E} 00---2.00 \mathrm{E}$ CO $1.33 \mathrm{E} 00--1.66 \mathrm{E} 00$ $1.00 \mathrm{E}$ 00---1.33E 00 $8.00 \mathrm{E}-01--1.00 \mathrm{E}$ OO 6.00E-01---8.00E-01 4.0CE-01---6. COE-OI $3.00 \mathrm{E}-02---4.00 \mathrm{E}-01$ $2.00 \mathrm{E}-01--3.00 \mathrm{E}-01$ 1. OCE-01-- 2.0OE-01 $5.00 E-02--1.00 E-01$
$2.00 E-02---5.00 E-02$
\end{abstract}

ENERGY

GROUP (MEV)

8. OOF 00---1.00E O1 6.50 OE $00--8.00 E$ OO $5.00 E$ OO- $-6.50 E$ OO 4.0OE OO- - -5. OOE OO $3.00 E$ OO- - - . . 00 E 00 $2.50 E$ OO-- 3. $00 E$ OO 2.0OE $00--2.50 E$ OO $1.66 E$ OO---2.00E 00 $1.3 . E$ OO- 1.66 E 00 $1.00 E$ OO- $-1.33 E$ OO 8.0OE-O1-CI. 6.0OE-O1-C$4.00 E-01---6.00 E-01$ $3.00 E-01=-4.0 N E-01$ $2.00 E-01-C-3.00 E-01$ $1.00 E-01--2.00 E-O 1$ $2.00 E-02---5$. COE -02 $M U=-1.0000$ $-2.255 E-07$
$-8.528 E-07$ $-5.684 E-06$ $-1.036 E-05$ $-9.303 \mathrm{E}-06$ $-4.740 E-06$ $3.044 E-06$ $1.167 \mathrm{E}-05$ $1.702 \mathrm{E}-05$ $1.111 E-05$ $-9.913 E-06$ $-2.498 E-05$ $7.431 \mathrm{E}-05$ $6.520 E-05$ 政 $2.197 E-03$ $6.876 E-03$
$2.117 E-03$

ANGLE 2 A $-1.850 E-07$ $-7.008 E-07$ $660 \mathrm{E}-06$ C9E-06 $-7.601 E-06$ $-3.814 \mathrm{E}-06$ $2.671 \mathrm{E}-06$ $1.433 \mathrm{E}-05$ $9.420 \mathrm{E}-06$ $-8.477 \mathrm{E}-06$ $-2.035 \mathrm{E}-05$ $7.274 E-05$ $6.099 E-05$ $9.411 \mathrm{E}-04$ $2.202 E-03$ $6.888 E-03$ $2.119 E-03$ $M U=-0.9446$ $-2.286 \mathrm{E}-07$ $-1.457 E$ $-1.457 E-06$ $-2.724 E-06$ $-2.289 E-06$
$-9.734 E-07$ $1.093 \mathrm{E}-06$ $3.085 E-06$ $3.974 \mathrm{E}-06$ $1.781 \mathrm{E}-06$ $-3.529 E-06$ $-5.521 E-06$ $6.755 \mathrm{E}-05$ $9.316 \mathrm{E}-05$ $9.427 \mathrm{E}-04$ $2.225 \mathrm{E}-03$ $6.938 \mathrm{E}-03$ $2.126 \mathrm{E}-03$

ANGLE 4
$M U=-0.8656$
$2.902 E-08$
$1.072 E-07$
$8.728 E-07$
$1.480 E-06$
$1.545 E-06$
$9.684 E-07$
$-5.557 E-07$
$-2.979 E-06$
$-4.798 E-06$
$-3.798 E-06$
$1.198 E-06$
$6.105 E-06$
$6.336 E-05$
$1.446 E-04$
$9.377 E-04$
$2.265 E-03$
$7.031 E-03$
$2.138 E-03$
$M U=-0.7550$ $1.189 E-07$ $4.386 E-07$ 5. $4.761 E-06$ $4.731 E-06$ 2. $-4.914 \mathrm{E}-06$ $-6.851 E-06$ $-3.943 E-06$ $5.788 \mathrm{E}-06$ 1. $311 E-05$ $1.311 E-05$
$6.944 E-05$ $2.281 E-04$ $9.186 E-04$ 2. $321 E-03$ $7.164 \mathrm{E}-0$ $2.155 \mathrm{E}-03$

AVGLE 6 MU $=-0.6179$ ANGLE 7 ANGLE 10 ANGLE 11 ANGLE 12 ANGLE 13 $M U=0.0950$ $6.620 E-08$ $2.313 E-07$ $3.920 \mathrm{E}-06$ $M U=n .2816$ OLE 12 $M U=0.6179$ ANGLE 14 $1.749 \varepsilon-07$ $1.482 \mathrm{E}-07$ $7.726 \mathrm{E}-08$ $-2.213 E-07$ 作 $3.340 E-07$ $2.897 E-06$ $-5.629 E-07$ $-7.693 \mathrm{E}-06$ $3.360 E-06$ $2.766 E-06$ $-1.272 E-06$ 7. $7.392 E-07$ 1.527E-OS $1.298 E-04$ 4.2 $5.623 E-04$ (2.0) $.477 \mathrm{E}-03$ $2.316 E-03$ -3.886E-06 5.869-06 $2.537 \mathrm{E}-05$ $1.015 E-04$ $2.315 \mathrm{E}-04$ $5.081 E-04$ $5.550 \mathrm{E}-04$ $8.702 E-04$ $3.029 E-03$ $2.359 E-03$
$-1.713 E-06 \quad-2.213 E-06$ -1.705E-06 $2.861 E-05$ $9.909 E-06 \quad 6.552 E-05$ $3.184 E-05 \quad 1.209 E-04$ $9.723 E-05$ 2.131E-04 $1.994 E-04$ 3.136E-04 $3.276 E-04 \quad 3.967 E-04$ $5.545 E-04$ $5.758 E-04$ $5.713 \mathrm{E}-04 \quad 0.252 \mathrm{E}-04$ 9.712E-04 .255E-03 $9.660 \mathrm{E}-03$ $2.402 E-03 \quad 2.444 E-03$ $M U=0.7550$ $9.016 \mathrm{E}-07$ $1.094 E-04$ $1.757 E-04$ 2.549E-04 $3.510 E-04$ $4.160 E-104$ 4.341E-04 5.958E-04 $7.010 E-04$ $1.022 \mathrm{E}-03$ $3.577 E-03$ $2.482 E-03$ $4.866 E-08$

$1.616 E-07$

$1.471 E-06$ 1.003E-06 $-3.019 E-08$ $-1.060 \mathrm{E}-06$ $-1.809 \mathrm{E}-06$ $-1.559 E-06$ $3.071 \mathrm{E}-07$ 3.063E-06 $1.315 E-06$ $9.540 E-0.5$ 8.854E-04 2.395E-03 $7.341 \mathrm{E}-03$ $-4.372 \mathrm{E}-08 \quad-7.887 \mathrm{E}-08$ $-1.296 E-06-2.005 E-06$ $1.296 E-06$ $2.085 E-06$ $-2.674 \mathrm{E}-06-3.283 \mathrm{E}-06$ $-1.676 E-06 \quad-1.256 E-06$ $8.254 E-0822.010 E-06$ $6.074 E-06$ $3.745 E-06 \quad 8.187 E-06$ $3.848 \mathrm{E}-06 \quad 3.161 \mathrm{E}-06$ $-2.410 E-06 \quad-5.082 E-06$ $-5.371 E-06 \quad 5.073 E-06$ $1.492 \mathrm{E}-042.310 \mathrm{E}-04$ 4.429E-04 5.238E-04 $8.468 \mathrm{E}-04 \quad 8.174 \mathrm{E}-04$ $2.484 E-03 \quad 2.592 E-03$ $7.560 E-03$ 2.206 E-03

$7.825 \mathrm{E}-03$
$2.239 \mathrm{E}-03$

ANGLE 16

.865 $7.753 E-06$ $M U=0.9446$

ANGLE 17 $M U=0.9894$ .869E-04 $2.643 \mathrm{E}-03$ $1.304 \mathrm{E}-0$ $8.861 E-05$ $7.045 E-04$ $4.658 \mathrm{E}-04$ $5.761 E-04$ $5.619 E-04$ $5.805 \mathrm{E}-04$ $5.834 E-04$ $5.691 E-04$ $5.295 E-04$ $4.858 E-04$ 4. $736 \mathrm{E}-04$ $7.185 E-04$ $7.893 E-04$ $1.131 \mathrm{E}-03$ $3.882 \mathrm{E}-03$ $1.066 \mathrm{E}-02$ $2.538 \mathrm{E}-03$ $9.715 \mathrm{E}-04$ $8.322 E-04$ $6.894 E-04$ $5.372 \mathrm{E}-0$ 4.619E-04 $4.966 E-04$ $7.960 \mathrm{E}-04$ $7.846 \mathrm{E}-04$ $1.173 \mathrm{E}-03$ $3.965 \mathrm{E}-0.3$ 作 $2.514 \mathrm{E}-03$

$2.552 \mathrm{E}-03$ $-1.214 E-08$ $-5.705 E-08$ $-1.904 E-07$ 
(GAMMAS/MEV/STERADIAN/SOURCE NEUTRON)

ENERGY GROUP (MEV) 8. 00 E $00---1.00 E$ O1 $5.00 E$ OO- 00.50 . 0 CO 4. 00 E OO---5. OOE OO $3.00 E$ OO- - 4. 00 EO 00 2.50E $00---3.00 E$ OO $2.00 E$ OO- - -2.50E OO $1.66 E$ OO---2.00E 00 $1.33 E$ OO- $-1.66 E$ OO $1.00 E$ OO---1.33E OO 8.00E-OX---1.00E CO 6.00E-01---8.00E-01 4. $00 \mathrm{E}-01--6.00 \mathrm{E}-01$ $3.00 E-01---4.00 E-01$
$2.00 E-01-\cdots 3.00 E-01$ $2.00 E-01--3.00 E-01$
$1.00 E-01--2.00 E-01$ 1. $00 \mathrm{E}-01--2.00 \mathrm{E}-01$ $2.00 \mathrm{E}-02--5.00 \mathrm{E}-02$

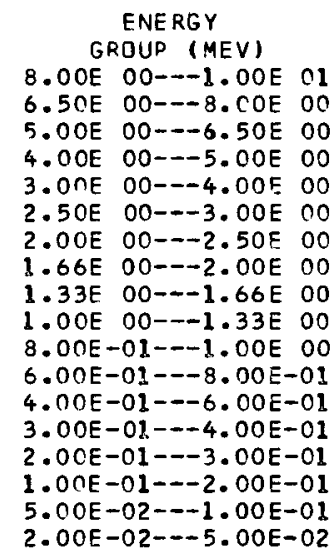

$\triangle N G L E \quad 1$ $M U=-1.0000$ $-4.896 E-07$
$-3.015 E-06$ $-5.434 E-06$ $-4.914 E-06$ $-2.467 E-06$ $6.216 \mathrm{E}-06$ $6.216 E-06$ $5.774 \mathrm{E}-06$ $-5.412 \mathrm{E}-06$ $-1.313 E-05$ $3.602 E-05$ $3.123 \mathrm{E}-05$ $4.644 \mathrm{E}-04$ $1.060 \mathrm{E}-03$ $3.299 \mathrm{E}-03$
$1.010 \mathrm{E}-03$ ANGLE 10 $M U=0.0950$ $3.513 E-08$ $1.375 E-07$ $9.765 E-07$ $1.881 E-06$ $1.978 \mathrm{E}-\mathrm{C6}$ $1.623 E-06$ $-9.080 E-07$ $-5.645 E-06$ $-6.255 E-07$ $1.779 \mathrm{E}-0 \mathrm{~S}$ $2.115 E-04$ $2.701 E-04$ $3.979 E-04$ $1.372 \mathrm{E}-03$ $4.037 \mathrm{E}-03$ $1.101 \mathrm{E}-03$
ANGLE 2 ANGLE 3 $M U=-0.9894 \quad M U=-0.9446$ $-1.094 E-67 \quad-3.608 E-08$ $-4.034 E-07$ $-2.483 E-06$ $-4.468 \mathrm{E}-06$
$-4.018 \mathrm{E}-06$ $-4.018 E-06$
$-1.983 E-06$ $-1.983 E-06$ $5.271 E-06$ $7.576 \mathrm{E}-06$ 4. $713 \mathrm{E}-06$ $-4.624 E-06$ $-1.070 E-05$ $3.515 E-05$ $3.363 \mathrm{E}-\mathrm{CL}$ $4.648 \mathrm{E}-04$ $1.063 \mathrm{E}-03$ $3.305 E-03$ ANGLE 11 $M U=0.2816$
$9.721 \mathrm{E}-08$ $9.721 \mathrm{E}-08$ $3.546 E-07$ $2.244 E-06$ $3.927 E-06$ 3.075E-06 $2.278 E-07$ $-2.825 E-06$
$-4.400 E-06$ $-1.450 E-06$ 1.191E-05 5 . $14 \mathrm{E}-05$ .181E-04 $1.181 E-04$ $2.636 \mathrm{E}-04$ $2.636 \mathrm{E}-04$ $1.446 \mathrm{E}-03$ $4.208 \mathrm{E}-03$ $1.121 E-03$
ANGLE 4 $M U=-0.8656$ $1.774 E-08$ $6.893 E-08$
$4.770 E-07$ $9.770 E-07$ 9.066E-07 $9.202 E-07$ $5.758 \mathrm{E}-07$ $-3.146 E-07$ $-1.660 E-06$ $-2.659 E-06$ $-2.065 E-06$ $7.649 \mathrm{E}-07$ $3.436 E-06$

$2.970 E-05$ $7.128 \mathrm{E}-05$ $4.616 \mathrm{E}-04$ $1.093 \mathrm{E}-03$ $3.371 \mathrm{E}-0$ $1.020 \mathrm{E}-03$

ANGLE 13 MUN= $2.743 E-08$ MU $=0.6179$ $1.165 E-08 \quad-5.150 E-07$ $1.566 E-07-3.246 E-06$ 1.029E-06 -7.073E-06 - $2.306 E-06 \quad 1.5282 E-06$ 1.001E-08 $1.191 \mathrm{E}-05$ ..01E-0B $1.191 \mathrm{E}-05$ 1.555E-05 $4.569 E-05$ 1.097E-04 $1.020 \mathrm{E}-04 \quad 1.592 \mathrm{E}-04$ 1.023E-04 $1.098 E-04$ $2.655 E-04$ 2.711E-04 $2.75 \mathrm{E}-04$ 2.711E-04 $4.375 \mathrm{E}-04 \quad 4.586 \mathrm{E}-04$ $1.526 \mathrm{E}-03 \quad 1.608 \mathrm{E}-03$ $4.387 \mathrm{E}-03 \quad 4.567 \mathrm{E}-03$ $1.38 \mathrm{E}-03$
$1.140 \mathrm{E}-03$
ANGLE 5 $M U=-0.7550$ $6.839 E-08$ $.585 \mathrm{E}-06$ $2.824 \mathrm{E}-06$ $2.530 \mathrm{E}-06$ $1.210 E-06$ $-8.212 E-07$ $-2.671 E-06$ $-3.698 E-06$ $3.163 E-06$ $6.779 E-06$ 3. $256 \mathrm{E}-05$ $1.133 E-04$ 4. $505 \mathrm{E}-04$ $1.120 \mathrm{E}-03$ $3.434 E-03$ 1. $028 \mathrm{E}-03$ ANGLE 14 $\begin{aligned} & M U=0.7550 \\ & 3.185 E-08\end{aligned}$ $3.185 E-08$
$-1.115 \mathrm{E}-07$ $-1.427 E-07$ $-3.352 E-07$ $096 \mathrm{E}-06$ $627 E-05$ 5. $366 E-05$ .306E-0 $1.31 E-04$ 1.78 $2.059 E-04$ 2.765E-04 $3.302 \mathrm{E}-04$ $4.791 E-04$ $1.689 E-03$ $4.737 E-03$ $4.737 E-03$
$1.175 E-03$
ANTLLE 6 $M U=-0.6179 \quad$ ANGLE 7 $6.497 E-07$ $3.890 E-07$
$-9.830 E-08$ $-9.830 E-08$ $-5.820 E-07$ $-9.231 E-07$ $6.908 E-08$ $1.524 \mathrm{E}-06$ $2.799 \mathrm{E}-0$ 4.571E-05 $1.670 E-04$ $4.321 E-04$ $1.154 E-03$ $3.516 \mathrm{E}-03$ $1.038 \mathrm{E}-03$ AVGLE 15 $M U=0.8656$ $2.291 E-06$ 3. $655 \mathrm{E}-05$ $3.655 E-05$ $4.240 E-05$ 1.398E-05 $1.063 E-04$ $1.712 E-04$ 1.708E-04 $2.081 E-04$ 2.313E-04 . 2. 3. $564 E-04$ $5.013 E-04$ $1.761 \mathrm{E}-03$ 4. $886 \mathrm{E}-03$ 1.190E-03 $1.221 E-06$ $2.273 E-06$
$2.448 E-06$ $2.448 E-06$
$-1.596 E-06$ $-3.519 E-06$ $7.307 E-05$ $2.198 \mathrm{E}-04$ $4.108 \mathrm{E}-04$ $1.196 \mathrm{E}-03$ $3.617 E+03$ ANGLE 16 $M U=0.9446$
$1.999 E-05$ $1.999 E-05$ $3.884 E-05$ $2.816 \mathrm{E}-04$ $2.259 E-04$ $2.749 E-04$ $2.844 E-04$ 2.928E-04 2.906 04 2.778E-04 $2.258 E-04$ $2.258 \mathrm{E}-04$ $3.299 E-04$ $3.625 \mathrm{E}-04$ $5.245 E-04$ $1.819 E-03$ $1.819 E-03$
$5.000 E-03$

5.000E-03
$1.200 E-03$ $-1.317 E-07 \quad-2.033 E-07 \quad-4.247 E-08$ $-1.643 E-06 \quad-2.274 E-06 \quad-2.048 E-07$ $-.752 E-07$ - $6.894 E-07$ 2.853E-07 $1.140 \mathrm{E}-06$ $3.405 E-06$ $4.508 E-06$ $1.414 E-06$ $-3.268 E-06$ $1.889 \mathrm{E}-0$ $1.143 \mathrm{E}-04$ $2.579 \mathrm{E}-04$ $3.945 E-04$ 1. $246 \mathrm{E}-03$ $3.739 E-03$ $1.066 \mathrm{E}-03$ ANGLE 17 $A N=0.9894$
$1.080 E-04$ $1.878 E-04$ $1.350 E-03$ $7.076 E-04$ 7.287E-04 $5.740 E-04$ $4.957 E-04$ $3.269 E-04$ . $428 E-04$ 2.068E-04 $2.303 E-04$ $3.647 E-04$ $3.551 E-04$ $1.854 \mathrm{E}-03$ $5.069 \mathrm{E}-03$ $1.206 E-03$
$2.853 E-07$

$1.186 E-06$
$6.653 E-07$ $-6.566 E-07$ $-2.011 E-06$ $8.688 E-07$ $2.468 E-05$ $1.638 E-04$ 
4 PI R*\#2 HENDERSON DOSE (NEUTRONS)
(CM *2 RAD/STERADIAN/SOURCE NEUTRON)

COS INE

\begin{abstract}
$-1.00000 \mathrm{E} 00$ $-9.89401 E-01$ $-9.44575 E-0$ $-8.65631 \mathrm{E}-0$ $-7.55044 E-01$ $-6.17876 E-O$ $-4.58017 E-01$ $-2.81605 E-01$ $-9.50125 E-02$ $9.50125 \mathrm{E}-02$ $2.81605 E-01$ 4.58017E-01 6. $17876 \mathrm{E}-01$ $7.55044 \mathrm{E}-01$ $8.65631 E-01$ $9.44575 E-01$
$9.89401 E-O 1$
\end{abstract}

TOTAL

COSINE

$-1.00000 E 00$ $-9.89401 \mathrm{E}-01$ $-8.65631 E-0$ $-7.55044 E-01$ $-6.17876 E-01$ $-4.58017 E-01$ $-2.81605 E-0$ $-9.50125 \mathrm{E}-0$

$9.50125 \mathrm{E}-02$

$2.81605 \mathrm{E}-01$

4.58017E-0

$6.17876 \mathrm{E}-01$

$7.55 C 44 E-01$

$8.65631 E-01$
$9.44575 E-01$

$9.89401 \mathrm{E}-01$

TOTAL

75.0

2.968E-11 2.977E- 11

$3.014 E-11$

3.086E-11

3. $197 \mathrm{E}-11$

$3.353 \mathrm{E}-11$

3. $829 \mathrm{E}-11$

$4.174 \mathrm{E}-11$

6. $955 \mathrm{E}-11$

5. $394 E-11$

$6.196 \mathrm{E}-11$

$6.84 C E-11$

$8.991 \mathrm{E}-11$

$8.575 \mathrm{E}-11$
$1.670 \mathrm{E}-10$

3. $310 \mathrm{E}-10$

$7.416 E-10$

100.0

$3.214 \mathrm{E}-11$ $3.224 \mathrm{E}-11$

$3.264 \mathrm{E}-11$

$3.46 C E-11$

$3.624 \mathrm{E}-11$

3. $840 E-11$

$4.117 \mathrm{E}-11$

$4.469 \mathrm{E}-11$

$4.046 E-11$

$6.213 E-11$

$7.516 E-11$

8.5

1. $53 E-10$

1. $314 E-10$

. $60 \mathrm{OE}-10$

4. $997 E-12$

$2460-12$

$1.1 C O E-11$

$1.231 E-11$

$5.631 \mathrm{E}-12$

$5.855 \mathrm{E}-12$

$9.360 E-11$
150.0

RANGE (METERS)
$20 \mathrm{C} .0$

$25 \mathrm{C} .0$

300.0

400.0

$3.188 \mathrm{E}-11$

$2.797 E-11$

2.267E-11

$.237 E-11$

2. $805 \mathrm{E}-11$

$2.274 E-11$

$1.760 E-11$
$1.766 \mathrm{E}-11$

$9.712 E-12$

$.314 \mathrm{E}-11 \quad 2.908 \mathrm{E}-11$

$3.428 \mathrm{E}-11$

$3.585 E-11$

$3.008 \mathrm{E}-1$

$2.436 \mathrm{E}-1$

$3.789 E-11$

$3.144 \mathrm{E}-11$

$2.545 \mathrm{E}-11$

$4.050 E-11$

$4.381 E-11$

. $166 \mathrm{E}-11$

$6.626 E-11$

$4.816 E-11$

$2.685 E-11$

$3.543 E-11$

$2.861 \mathrm{E}-11$

$3.798 E-11$

$2.911 E-11$
$3.754 E-11$

$3.754 E-11$
$4.183 E-11$

$4.183 \mathrm{E}-11$
$4.25 \mathrm{CE}-11$

$7.201 \mathrm{E}-11$

6.857E-11

$6.857 E-11$

5. $338 \mathrm{E}-11$

(10

$1.571 E-10$

$.728 \mathrm{E}-11$

$1.042 \mathrm{E}-10$

$6.559 \mathrm{E}-11$

$7.175 \mathrm{E}-11$

. $788 \mathrm{E}-11$

1. $830 \mathrm{E}-11$

$1.891 \mathrm{E}-11$

$1.975 E-11$

.

$2.219 E-11$

$2.386 E-11$

$2.762 E-11$

$2.716 E-11$

$3.179 E-11$

$3.367 \mathrm{E}-11$

3. $180 \mathrm{E}-11$

4. $680 \mathrm{E}-11$

5.116E-11

5. $600 \mathrm{CE}-10$

$4.418 \mathrm{E}-10$

$3.420 E-10$

(

$1.009 \mathrm{E}-11$

$1.043 E-11$

$1.088 \mathrm{E}-11$

$1.146 \mathrm{E}-11$

$1.219 \mathrm{E}-11$

$1.306 \mathrm{E}-11$

$1.361 \mathrm{E}-11$

$1.565 \mathrm{E}-11$

$1.663 E-11$

$1.860 \mathrm{E}-11$

$2.014 \mathrm{E}-11$

2.20

$2.540 E-11$

RANGE (METERS)

$900.0 \quad 1200.0$

1500.0

1800.0

$2.525 E-13$

$2.290 E-14$

$1.945 \mathrm{E}-15$

$1.586 E-16$

$\begin{array}{llll}2.533 \mathrm{E}-13 & 2.297 \mathrm{E}-14 & 1.951 \mathrm{E}-15 & 1.590 \mathrm{E}-16 \\ 2.564 \mathrm{E}-13 & 2.325 \mathrm{E}-14 & 1.975 \mathrm{E}-15 & 1.610 \mathrm{E}-16\end{array}$

$2.622 E-13 \quad 2.377 E-14 \quad 2.019 E-15$

$2.706 \mathrm{E}-13 \quad 2.453 \mathrm{E}-14$

2.965E-13 2.585E-14 $2.278 E-15 \quad 1.856 E-16$

$3.143 \mathrm{E}-13 \quad 2.844 \mathrm{E}-14 \quad 2.412 \mathrm{E}-15 \quad 15065 \mathrm{E}-16$

$3.356 \mathrm{E}-13 \quad 3.034 \mathrm{E}-14 \quad 2.572 \mathrm{E}-15 \quad 2.093 \mathrm{E}-16$

$3.607 E-13 \quad 3.256 E-14 \quad 2.757 E-15 \quad 2.243 E-16$

$3.893 \mathrm{E}-13 \quad 3.509 \mathrm{E}-14 \quad 2.968 \mathrm{E}-15 \quad 2.412 \mathrm{E}-16$

$4.213 E-13 \quad 3.788 E-14 \quad 3.199 E-15 \quad 2.598 E-16$

$4.557 E-13 \quad 4.085 E-14 \quad 3.444 E-15 \quad 2.793 E-16$

$4.909 \mathrm{E}-13 \quad 4.384 \mathrm{E}-14 \quad 3.688 \mathrm{E}-15 \quad 2.987 \mathrm{E}-16$

$5.241 E-13 \quad 4.663 E-14 \quad 3.914 E-15 \quad 3.165 E-16$

$\begin{array}{llll}5.517 \mathrm{E}-13 & 4.889 \mathrm{E}-14 & 4.095 \mathrm{E}-15 & 3.307 \mathrm{E}-16\end{array}$

$5.691 E-13 \quad 5.029 E-14 \quad 4.206 E-15 \quad 3.394 E-16$

$2.852 \mathrm{E}-15$ 
COSINE

$-1.00000 \mathrm{E} 00$ $-9.89401 E-01$ $-9.44575 \mathrm{E}-01$ $-7.55044 \mathrm{E}-01$ $-6.17876 \mathrm{E}-0$ $-4.58017 E-01$ $-2.81605 E-0$ $-9.50125 \mathrm{E}-0$ $9.50125 \mathrm{E}-02$ $2.81605 \mathrm{E}-01$ $4.58017 E-01$ $6.17876 \mathrm{E}-0$ $7.55044 \mathrm{E}-01$ $8.65631 E-0$ $9.44575 E-01$ $9.89401 E-01$

TOTAL

COSINE

$-1.00000 \mathrm{E}$ OC $-9.89401 \mathrm{E}-01$ $-9.44575 \mathrm{E}-0$ $-8.65631 \mathrm{E}-01$ $-7.55044 \mathrm{E}-01$ -6.17876E-01 $-2.81605 E-01$ $-2.81605 E-01$ $0.50125 \mathrm{E}-0$ 2.81605E-01 7.55044E-0 . $8.45575 E-01$ $9.89401 E-01$

TOTAL

\section{0}

1. $576 \mathrm{E}-10$ $1.576 E-10$
$1.580 E-10$ $1.596 \mathrm{E}-10$ $1.626 \mathrm{E}-10$ $1.672 \mathrm{E}-1 \mathrm{C}$ $1.735 E-10$ $1.818 \mathrm{E}-10$ $1.924 \mathrm{E}-1 \mathrm{C}$ $2.057 E-10$ $2.945 \mathrm{E}-10$ $2.511 E-10$ 2.789E-10 3. $\mathrm{C} 16 \mathrm{E}-10$ .725E-10 $6.212 \mathrm{E}-10$ $1.126 \mathrm{E}-09$

$3.304 E-C 9$

$50 \mathrm{c} .0$

$5.629 E-11$ $5.642 E-11$ $5.698 \mathrm{E}-11$ $5.803 E-11$ $5.954 E-11$ $6.157 E-11$ $6.410 E-11$ $6.719 E-11$ $7.085 \mathrm{E}-11$ $7.582 E-11$ 7.944E- 1 $9.038 \mathrm{E}-11$ .629E-11 1.014E- 10 1.061 E- 10 $1.061 \mathrm{E}-10$

$9.537 E-10$
100.0 $1.901 \mathrm{E}-10$ 1.905E-1C $1.960 \mathrm{E}-10$ $2.013 \mathrm{E}-10$ 2.C86E-1C $2.181 E-10$ $2.301 \mathrm{E}-10$ $2.450 \mathrm{E}-10$ $2.423 \mathrm{E}-10$ $3.069 \mathrm{E}-10$ $2.940 \mathrm{E}-10$ $3.623 \mathrm{E}-10$ $3.973 \mathrm{E}-1 \mathrm{C}$ $5.547 E-10$ $9.538 \mathrm{E}-10$

$3.629 E-0.9$

600.0

$2.881 \mathrm{E}-11$ $2.888 \mathrm{E}-11$ $2.917 \mathrm{E}-11$ $2.970 \mathrm{E}-11$ 3.C47E-11 $3.151 \mathrm{E}-11$ $3.280 \mathrm{E}-11$ $3.437 E-11$ $3.622 \mathrm{E}-11$ $3.821 \mathrm{E}-11$ $4.33 E-11$ $4.613 E-11$ $.353 E-11$

$.490 E-11$

4. 86 CE- 10
150.0 RANGE (METERS)
200.0

250.0

300.0

400.0

$2.228 \mathrm{E}-10$

$\begin{array}{ll}2.228 \mathrm{E}-10 & 2.213 \mathrm{E}-10 \\ 2.234 \mathrm{E}-10 & 2.219 \mathrm{E}-10\end{array}$ $2.255 \mathrm{E}-10$ $2.297 E-10$ $2.357 \mathrm{E}-10$ .439E- 10 $.544 \mathrm{E}-10$ $.676 E-10$ $2.838 E-10$ $.641 \mathrm{E}-10$ 列 .199E- 10 -146E-10 $4.098 \mathrm{E}-10$ $6.970 E-10$

$2.24 \mathrm{CE}-10$
$2.281 \mathrm{E}-10$

$2.341 \mathrm{E}-10$

$2.421 E-10$

$2.524 \mathrm{E}-10$

$2.650 E-10$

$2.802 E-10$

$2.794 \mathrm{E}-10$

$3.259 \mathrm{E}-10$

$3.604 E-10$

$4.179 E-10$

$4.346 \mathrm{E}-10$

$4.947 E-10$

$5.536 \mathrm{E}-10$

3.997E-09

$3.875 \mathrm{E}-0.9$

$1.983 E-10$ $1.987 E-10$ .007E- 10 $2.097 \mathrm{E}-10$ $2.168 \mathrm{E}-10$ $.259 \mathrm{E}-10$ .370E- 10 .5C1E-1C 2. . $055 E-10$ . $246 E-10$ $3.621 E-10$ $4.176 E-10$
$4.431 E-10$

$3.415 E-09$

$1.664 \mathrm{E}-10$ $1.668 \mathrm{E}-10$ $1.684 \mathrm{E}-10$ $1.760 \mathrm{E}-10$ 1.820E-10 $1.895 \mathrm{E}-10$ $1.988 \mathrm{E}-10$ $2.100 E-10$ $2.330 \mathrm{E}-10$ $2.319 \mathrm{E}-1 \mathrm{C}$ $2.586 E-10$

$2.865 E-C 9$

$1.024 \mathrm{E}-10$ $1.026 E-10$ $1.056 \mathrm{E}-10$ $1.083 E-10$ $1.120 \mathrm{E}-10$ 1. $166 \mathrm{E}-10$ $1.223 E-10$ $1.289 E-10$ $1.334 \mathrm{E}-10$ $1.472 E-10$ $1.668 \mathrm{E}-10$ $1.763 E-10$ $1.881 \mathrm{E}-10$ $1.969 E-10$ $2.043 \mathrm{E}-10$

$\begin{array}{rr}\text { RANGE } & \text { (METERS) } \\ 900.0 & 1200.0\end{array}$

$150 \mathrm{C} . \mathrm{C}$

1800.0

$1.740 E-C 9$

\section{3. $C 82 \mathrm{E}-12$} $3.089 \mathrm{E}-12$ $3.120 \mathrm{E}-12$ $3.177 \mathrm{E}-12$

$2.811 E-13$ $2.818 \mathrm{E}-13$

$2.846 \mathrm{E}-13$

$2.897 \mathrm{E}-13$

$2.972 \mathrm{E}-13$

$3.07 \mathrm{CE}-13$

$3.193 \mathrm{E}-13$

$3.342 E-13$

$3.516 E-13$

$3.713 E-13$

$3.932 \mathrm{E}-13$

$4.166 \mathrm{E}-13$

$4.641 E-13$

$4.641 E-13$

$5.017 E-13$

$5.117 E-13$

$5.161 E-11 \quad 4.682 E-12 \quad 3.96 C E-13 \quad 3.217 E-14$

$2.387 \mathrm{E}-14$

$2.392 \mathrm{E}-14$

$2.416 \mathrm{E}-14$

$2.459 \mathrm{E}-14$

.

$2.700 E-14$

.

(2.07)

(3)

.

3. $520 \mathrm{E}-14$

$3.911 \mathrm{E}-14$

$4.082 \mathrm{E}-14$

$4.217 \mathrm{E}-14$

4. $297 E-14$

$5.705 \mathrm{E}-12$

$5.161 E-11 \quad 4.682 E-12 \quad 3.96 C E-13 \quad 3.217 E-14$

$1.944 E-15$

$1.948 \mathrm{E}-15$

$1.967 \mathrm{E}-15$

$2.053 \mathrm{E}-15$

$2.121 \mathrm{E}-15$

$2.204 E-15$

$2.305 \mathrm{E}-15$

$2.423 E-15$

$2.556 E-15$

$2.857 \mathrm{E}-1$

. $169 E-15$

$3.169 E-15$

$3.410 \mathrm{E}-15$

3. $474 \mathrm{E}-15$ 
$\operatorname{COS} I N E$

$-1.00000 E 00$ $-9.89401 \mathrm{E}-\mathrm{C} 1$ $-9.44575 E-01$ $-8.65631 E-01$ $-7.55044 \mathrm{E}-01$ $-6.17876 \mathrm{E}-01$ $-4.58017 E-0$ $-9.50125 \mathrm{E}-02$ $9.50125 \mathrm{E}-02$ $2.81605 E-01$ $4.58017 E-01$ $6.17876 \mathrm{E}-01$ $7.55044 \mathrm{E}-\mathrm{O}$ $8.65631 E-0$ $9.44575 E-01$ $9.89401 E-01$

TOTAL

COS INE $-1.0000 C E$ OC $-9.89401 E-01$ $-9.44575 E-0$ $-8.65631 \mathrm{E}-0$ $-7.55044 E-01$ $-6.17876 E-01$ - $51605 E-O$ - $510125 E-02$ .50125E-O2 (50125E-02 4.58017E-O $6.17876 \mathrm{E}-0$ . $55044 E-01$ $8.65631 E-0$ $9.44575 E-01$

TOTAL

$$
75.0
$$

$5.294 E-C 9$ $5.30 .9 \mathrm{E}-\mathrm{Cg}$ $5.374 \mathrm{E}-\mathrm{Cg}$ $5.503 \mathrm{E}-\mathrm{C} 9$ $5.699 \mathrm{E}-09$ $5.973 \mathrm{E}-\mathrm{C} 9$ $6.339 \mathrm{E}-\mathrm{C} 9$ $6.815 \mathrm{E}-\mathrm{C} 9$ $7.422 E-C 9$ $1.229 \mathrm{E}-\mathrm{C} 8$ $9.572 \mathrm{E}-\mathrm{C} 9$ $1.098 \mathrm{E}-\mathrm{C} 8$ $1.211 \mathrm{E}-\mathrm{C} 8$ $1.588 \mathrm{E}-\mathrm{C} 8$ 1. $517 E-C 8$ $2.939 \mathrm{E}-\mathrm{CB}$ $5.804 \mathrm{E}-\mathrm{C} 8$

$1.313 \mathrm{E}-\mathrm{C} 7$

100.0

$5.755 E-C 9$ $5.772 \mathrm{E}-\mathrm{C} 9$ $5.843 \mathrm{E}-\mathrm{C} 9$ $5.982 \mathrm{E}-\mathrm{C} 9$ $6.191 \mathrm{E}-\mathrm{C}$ $6.481 \mathrm{E}-09$ $6.864 E-C 9$ $7.355 \mathrm{E}-\mathrm{C}$ $7.078 \mathrm{E}-\mathrm{C}$ $7.265 \mathrm{E}-\mathrm{C} 9$ $1.1 C 4 E-0$ $9.979 E-C$ $1.335 E-C$ 1. $5 \mathrm{C} 6 \mathrm{E}-\mathrm{C}$ $1.865 \mathrm{E}-\mathrm{C}$ $2.322 \mathrm{E}-\mathrm{C}$ 4.57CE-C8

$1.273 E-C 7$

500.0

600.

$9.215 \mathrm{E}-10$ $9.241 E-1 C$ $9.355 \mathrm{E}-10$ $9.568 \mathrm{E}-10$ $9.878 \mathrm{E}-1 \mathrm{C}$ $1.030 E-09$ 1. $C 83 \mathrm{E}-\mathrm{C} 9$ $1.150 \mathrm{E}-\mathrm{C} 9$ $1.230 E-C 9$ $1.345 \mathrm{E}-\mathrm{C} 9$ $1.427 E-C 9$ $1.569 E-09$ $1.857 \mathrm{E}-\mathrm{C} 9$ 2. $03 \mathrm{E}-09$ 2.CO3E. $237 \mathrm{E}-0$ $2.237 E-09$

$1.715 \mathrm{E}-08$

\begin{tabular}{|c|c|c|c|c|}
\hline 150.0 & $\begin{array}{c}\text { NGE (METERS) } \\
2 \mathrm{CO} O . \mathrm{C}\end{array}$ & 250.0 & 300.0 & $400 . C$ \\
\hline & $5.065 E-C 9$ & $4.123 E-09$ & $3.214 E-C 9$ & $1.784 \mathrm{E}-09$ \\
\hline & $5 . C 80 E-C 9$ & $4.136 E-09$ & $3.223 E-C 9$ & $1.789 \varepsilon-09$ \\
\hline $5.833 E-C 9$ & $5.143 \mathrm{E}-\mathrm{C9}$ & $4.187 \mathrm{E}-09$ & $3.263 \mathrm{E}-09$ & $1.811 \varepsilon-09$ \\
\hline $5.970 E-29$ & $5.264 E-09$ & $4.284 E-09$ & $3.339 E-C 9$ & $1.852 E-09$ \\
\hline $6.173 \mathrm{E}-\cap 9$ & $5.442 E-C 9$ & $4.428 E-09$ & $3.450 E-C 9$ & $1.913 E-09$ \\
\hline $6.452 \mathrm{E}-09$ & $5.686 \mathrm{E}-09$ & $4.623 \mathrm{E}-09$ & $3.601 \mathrm{E}-\mathrm{C} 9$ & $1.995 \mathrm{E}-09$ \\
\hline $6.816 \mathrm{E}-09$ & $6.001 E-09$ & $4.874 \mathrm{E}-\mathrm{C} 9$ & $3.795 \mathrm{E}-\mathrm{C} 9$ & $2.100 \mathrm{E}-09$ \\
\hline $280 E-09$ & $6.399 E-09$ & $5.188 \mathrm{E}-09$ & $4.039 \mathrm{E}-\mathrm{C} 9$ & $2.231 \mathrm{E}-09$ \\
\hline $869 E-09$ & $6.887 \mathrm{E}-09$ & $5.571 c-09$ & $4.339 E-C 9$ & $2.389 \mathrm{E}-09$ \\
\hline $5.791 E-09$ & $6.841 \mathrm{IE}-\mathrm{C} 9$ & $5.265 \mathrm{E}-09$ & $5.020 \mathrm{E}-\mathrm{C} 9$ & $2.485 \mathrm{E}-09$ \\
\hline $1.180 E-08$ & $8.309 E-C 9$ & $6.8 C O E-09$ & $4.926 \mathrm{E}-\mathrm{C} 9$ & $2.855 \mathrm{E}-\mathrm{C} 9$ \\
\hline $8.668 \mathrm{E}-n 9$ & $9.815 E-C 9$ & $7.532 \mathrm{E}-09$ & $5.760 \mathrm{E}-\mathrm{C} 9$ & $3.031 \mathrm{E}-09$ \\
\hline $1.346 E-C 8$ & $9.319 E-09$ & $7.687 \mathrm{E}-09$ & C.C92E-CO & $3.384 E-09$ \\
\hline & $1.227 \mathrm{E}-08$ & $9.585 \mathrm{E}-09$ & $6.955 \mathrm{E}-\mathrm{C} 9$ & $3.658 \mathrm{E}-\mathrm{C9}$ \\
\hline $1.649 E-C 8$ & $1.251 \mathrm{E}-08$ & $9.658 \mathrm{E}-09$ & $7.540 \mathrm{E}-\mathrm{Cg}$ & $4.026 \mathrm{E}-09$ \\
\hline $1.953 \mathrm{E}-08$ & $1.557 E-08$ & $1.175 E-08$ & $8.425 E-C 9$ & $4.321 E-09$ \\
\hline & $1.854 E-08$ & $1.284 \mathrm{E}-08$ & $9.190 \mathrm{E}-\mathrm{CO}$ & $4.594 E-09$ \\
\hline $1.173 E-07$ & 1. COBE-C7 & $7.985 E-08$ & $6.204 E-08$ & $3.351 E-C 8$ \\
\hline
\end{tabular}

$4.548 E-10$
$4.562 E-10$
RANGE (METERS)

$$
900.0 \quad 1200 . \mathrm{C}
$$

1500.0

$180 \mathrm{C} . \mathrm{n}$

4. $683 \mathrm{E}-11$

4.249E-12

4. $261 \mathrm{E}-12$

$4.313 \mathrm{E}-12$
$4.408 \mathrm{E}-12$

4.547E-12

$4.734 E-12$

4. $97 \mathrm{CE}-12$

5. 26CE-12

$5.606 E-12$

$6.009 \mathrm{E}-12$

$6.467 E-12$

$6.973 E-12$

7.51 CE-12

$8.049 E-12$

$8.551 \mathrm{E}-12$
$8.959 \mathrm{E}-12$

$9.211 \mathrm{E}-12$

3. $609 \mathrm{SE}-13$

$3.619 \mathrm{E}-13$
$3.663 \mathrm{E}-13$

$3.743 \mathrm{E}-13$

3.861E-13

$4.018 \mathrm{E}-13$

$4.461 E-13$

$4.751 E-13$

5.75

$5.47 C E-13$

.

$6.332 \mathrm{E}-13$

$6.772 E-13$

$7.179 E-13$

$7.5 \mathrm{C} 5 \mathrm{E}-1$

$7.705 \mathrm{E}-13$

$2.942 \mathrm{E}-14$

2. $95 \mathrm{CE}-14$

$2.985 E-14$

$3.051 \mathrm{E}-14$

$3.274 \mathrm{E}-14$

$3.435 E-14$

$3.633 E-14$

$3.867 E-14$

$4.446 E-14$

$4.782 \mathrm{E}-14$

$5.135 E-14$

$5.485 E-14$

6. $62 \mathrm{E}-14$

.

$5.260 E-13$ 
COSINE

\section{$-1.00000 \mathrm{E}$ OO $-9.44575 E-01$ $-8.65631 E-01$ $-7.55044 \mathrm{E}-01$ $-6.17876 \mathrm{E}-01$ $-4.58017 \mathrm{E}-01$ $-2.81605 E-01$ $-9.50125 E-02$ $9.50125 \mathrm{E}-02$ $2.81605 E-01$ $4.58017 \mathrm{E}-01$ $6.17876 \mathrm{E}-0$ $7.55044 E-0$ $8.65631 \mathrm{E}-0$ \\ $.44575 E-01$ \\ $9.89401 \mathrm{E}-01$}

TOTAL

COSINE

-1.0 COOOE 00 $-9.89401 \mathrm{E}-01$

$-9.44575 \mathrm{E}-01$

$-8.65631 \mathrm{E}-01$

$-7.55044 \mathrm{E}-01$

$-6.17876 E-01$

$-4.58017 \mathrm{E}-01$

$-2.81605 \mathrm{E}-01$

$-9.50125 E-02$

$9.50125 E-02$

$2.81605 E-01$

$6.57876 \mathrm{E}-01$

$7.55044 \mathrm{E}-01$

7.55044E-O

$8.65631 \mathrm{E}-01$

$9.89401 \mathrm{E}-01$

TOTAL

\begin{tabular}{|c|c|c|c|c|c|c|}
\hline 75.0 & $10 c .0$ & 150.0 & $\begin{array}{l}\text { E (METERS) } \\
2 C \mathrm{CC} . \mathrm{C}\end{array}$ & 256.0 & 300.0 & 400.0 \\
\hline $\begin{array}{l}840 \mathrm{E}-11 \\
846 \mathrm{E}-11 \\
873 \mathrm{E}-11 \\
926 \mathrm{E}-11 \\
005 \mathrm{E}-11 \\
115 \mathrm{E}-11 \\
260 \mathrm{E}-11 \\
444 \mathrm{E}-11 \\
676 \mathrm{E}-11 \\
200 \mathrm{E}-11 \\
459 \mathrm{E}-11 \\
939 \mathrm{E}-11 \\
330 \mathrm{E}-11 \\
549 \mathrm{E}-11 \\
454 \mathrm{E}-11 \\
082 \mathrm{E}-10 \\
947 \mathrm{E}-10\end{array}$ & $\begin{array}{l}3.471 \mathrm{E}-11 \\
3.479 \mathrm{E}-11 \\
3.511 \mathrm{E}-11 \\
3.575 \mathrm{E}-11 \\
3.669 \mathrm{E}-11 \\
3.797 \mathrm{E}-11 \\
3.964 \mathrm{E}-11 \\
4.173 \mathrm{E}-11 \\
4.434 \mathrm{E}-11 \\
4.395 \mathrm{E}-11 \\
5.507 \mathrm{E}-11 \\
5.291 \mathrm{E}-11 \\
6.469 \mathrm{E}-11 \\
7.073 \mathrm{E}-11 \\
8.254 \mathrm{E}-11 \\
9.780 \mathrm{E}-11 \\
1.662 \mathrm{E}-10\end{array}$ & $\begin{array}{l}4.165 \mathrm{E}-11 \\
4.174 \mathrm{E}-11 \\
4.213 \mathrm{E}-11 \\
4.288 \mathrm{E}-11 \\
4.397 \mathrm{E}-11 \\
4.544 \mathrm{E}-11 \\
4.732 \mathrm{E}-11 \\
4.967 \mathrm{E}-11 \\
5.256 \mathrm{E}-11 \\
4.932 \mathrm{E}-11 \\
6.637 \mathrm{E}-11 \\
5.913 \mathrm{E}-11 \\
7.549 \mathrm{E}-11 \\
7.478 \mathrm{E}-11 \\
8.783 \mathrm{E}-11 \\
9.825 \mathrm{E}-11 \\
1.242 \mathrm{E}-10\end{array}$ & $\begin{array}{l}4.215 \mathrm{E}-11 \\
4.224 \mathrm{E}-11 \\
4.264 \mathrm{E}-11 \\
4.339 \mathrm{E}-11 \\
4.449 \mathrm{E}-11 \\
4.596 \mathrm{E}-11 \\
4.784 \mathrm{E}-11 \\
5.014 \mathrm{E}-11 \\
5.289 \mathrm{E}-11 \\
5.292 \mathrm{E}-11 \\
6.11 \mathrm{CE}-11 \\
6.604 \mathrm{E}-11 \\
6.739 \mathrm{E}-11 \\
7.742 \mathrm{E}-11 \\
8.043 \mathrm{E}-11 \\
9.087 \mathrm{E}-11 \\
1.01 \mathrm{CE}-10\end{array}$ & $\begin{array}{l}3.833 \mathrm{E}-11 \\
3.842 \mathrm{E}-11 \\
3.878 \mathrm{E}-11 \\
3.947 \mathrm{E}-11 \\
4.047 \mathrm{E}-11 \\
4.180 \mathrm{E}-11 \\
4.348 \mathrm{E}-11 \\
4.554 \mathrm{E}-11 \\
4.797 \mathrm{E}-11 \\
4.693 \mathrm{E}-11 \\
5.578 \mathrm{E}-11 \\
5.81 \mathrm{CE}-11 \\
6.161 \mathrm{E}-11 \\
6.824 \mathrm{E}-11 \\
7.082 \mathrm{E}-11 \\
7.81 \mathrm{CE}-11 \\
8.256 \mathrm{E}-11\end{array}$ & $\begin{array}{l}3.255 \mathrm{E}-11 \\
3.262 \mathrm{E}-11 \\
3.294 \mathrm{E}-11 \\
3.352 \mathrm{E}-11 \\
3.437 \mathrm{E}-11 \\
3.551 \mathrm{E}-11 \\
3.694 \mathrm{E}-11 \\
3.869 \mathrm{E}-11 \\
4.078 \mathrm{E}-11 \\
4.494 \mathrm{E}-11 \\
4.494 \mathrm{E}-11 \\
4.975 \mathrm{E}-11 \\
5.188 \mathrm{E}-11 \\
5.651 \mathrm{E}-11 \\
5.947 \mathrm{E}-11 \\
6.357 \mathrm{E}-11 \\
6.652 \mathrm{E}-11\end{array}$ & $\begin{array}{l}2.037 \mathrm{E}-11 \\
2.042 \mathrm{E}-11 \\
2.062 \mathrm{E}-11 \\
2.099 \mathrm{E}-11 \\
2.152 \mathrm{E}-11 \\
2.223 \mathrm{E}-11 \\
2.312 \mathrm{E}-11 \\
2.421 \mathrm{E}-11 \\
2.549 \mathrm{E}-11 \\
2.642 \mathrm{E}-1.1 \\
2.896 \mathrm{E}-11 \\
3.035 \mathrm{E}-11 \\
3.267 \mathrm{E}-11 \\
3.447 \mathrm{E}-11 \\
3.664 \mathrm{E}-11 \\
3.826 \mathrm{E}-11 \\
3.960 \mathrm{E}-11\end{array}$ \\
\hline $54 \mathrm{E}-10$ & $6.521 \mathrm{E}-1 \mathrm{C}$ & $7.356 \mathrm{E}-10$ & $7.273 \mathrm{E}-10$ & $6.518 \mathrm{E}-10$ & $5.537 E-10$ & $3.428 \mathrm{E}-10$ \\
\hline
\end{tabular}

\section{RANGE (METERS)}

$\begin{array}{cc}500.0 & 600.0 \\ 1.131 \mathrm{E}-11 & 5.822 \mathrm{E}-12 \\ 1.134 \mathrm{E}-11 & 5.835 \mathrm{E}-12 \\ 1.145 \mathrm{E}-11 & 5.892 \mathrm{E}-12 \\ 1.165 \mathrm{E}-11 & 5.998 \mathrm{E}-12 \\ 1.195 \mathrm{E}-11 & 6.151 \mathrm{E}-12 \\ 1.235 \mathrm{E}-11 & 6.356 \mathrm{E}-12 \\ 1.284 \mathrm{E}-11 & 6.611 \mathrm{E}-12 \\ 1.345 \mathrm{E}-11 & 6.921 \mathrm{E}-12 \\ 1.416 \mathrm{E}-11 & 7.284 \mathrm{E}-12 \\ 1.511 \mathrm{E}-11 & 7.677 \mathrm{E}-12 \\ 1.583 \mathrm{E}-11 & 8.179 \mathrm{E}-12 \\ 1.693 \mathrm{E}-11 & 8.671 \mathrm{E}-12 \\ 1.793 \mathrm{E}-11 & 9.213 \mathrm{E}-12 \\ 1.905 \mathrm{E}-11 & 9.735 \mathrm{E}-12 \\ 2.003 \mathrm{E}-11 & 1.023 \mathrm{E}-11 \\ 2.090 \mathrm{E}-11 & 1.063 \mathrm{E}-11 \\ 2.145 \mathrm{E}-11 & 1.089 \mathrm{E}-11\end{array}$

$2.145 \mathrm{E}-11$ 11 $1.08 \mathrm{EE}-11$

$1.900 E-10 \quad 9.747 E-11$
$6.265 E-13$

$6.279 E-13$

$6.341 \mathrm{E}-13$

$6.454 \mathrm{E}-13$

$6.618 E-13$

$6.837 E-13$

$7.108 \mathrm{E}-13$

$7.438 E-13$

$7.822 E-13$

$8.261 E-13$

$8.746 E-13$

$9.269 E-13$

$9.00 \mathrm{E}-13$

$1.034 \mathrm{E}-12$

$1.081 \mathrm{E}-12$

$1.123 \mathrm{E}-12$

$1.042 E-11$
$5.720 E-14$

$5.733 E-14$

$5.789 E-14$

$5.892 \mathrm{E}-14$

$6.041 E-14$

$6.238 \mathrm{E}-14$

$6.77 \mathrm{E}-14$

6.77gE 14

7.516E-14

$7.948 E-14$

$8.410 E-14$

$8.883 E-14$

$0.34 \mathrm{E}-14$

.

$1.007 E-13$

$9.47 C E-13$
150.0 .0

$4.856 E-15$ $4.867 E-15$ 4.915E-15 $5.0 C 1 E-15$ $5.127 E-15$ $5.293 \mathrm{E}-15$ $5.499 E-15$ $5.748 E-15$ $6.037 E-15$ $6.365 E-15$ $7.1 C 8 E-15$ $7.498 E-15$ $7.874 E-15$ $8.209 E-15$ $8.47 C E-15$ $8.627 \mathrm{E}-15$

8.011 E-14
$18 \mathrm{CO} . \mathrm{C}$

$3.954 \mathrm{E}-16$ $3.963 \mathrm{E}-16$ 4. $002 E-16$ 46 $4.174 E-16$ $4.475 E-16$ $4.676 E-16$ $4.676 E-16$ $5.173 E-16$ $5.462 E-16$ $5.769 E-16$ $6.081 \mathrm{E}-16$ $6.379 E-16$ $6.851 E-16$ $6.975 E-16$

6.507E-15 
COS INE

\section{$-1.00000 E 00$ $-9.89401 E-01$ $-9.44575 \mathrm{E}-01$ $-8.65631 \mathrm{E}-01$ $-7.55044 \mathrm{E}-01$ $-6.17876 E-01$ $-4.58017 E-01$ $-2.81605 \mathrm{E}-01$ $-9.50125 \mathrm{E}-02$ $9.50125 \mathrm{E}-02$ 2.81605E-01 4. $58017 \mathrm{E}-01$ $7.55044 E-01$ $0.44575 \mathrm{E}-01$ $9.89401 E-01$}

TOTAL

COSINE

-1. CCOOOE 00 $-9.89401 E-0$ $-8.44575 E-01$ $7.55044 E-01$ $-6.17876 E=01$ $-4.58017 E-0$ - $81605 E-O$ $-0.50125 \mathrm{E}-0$ $50125 E=02$ $2.81605 \mathrm{E}-01$ $4.58017 E-O$ $6.17876 \mathrm{E}-01$ $7.55044 \mathrm{E}-01$ $8.65631 \mathrm{E}-0$ $8.65631 E-0$ $9.89401 \mathrm{E}-01$

TOTAL

75.0
$4.685 E-1 C$
$4.699 E-10$
$4.756 E-10$
$4.871 E-10$
$5.044 E-10$
$5.288 E-10$
$5.612 E-10$
$6.034 E-10$
$6.572 E-10$
$1 . C 89 E-C 9$
$8.478 E-10$
$9.727 E-10$
$1.073 E-C 9$
$1.4 C 7 E-C 9$
$1.344 E-C 9$
$2.606 E-C 9$
$5.149 E-09$

$1 C C .0$

5. C9OE-10 $5.105 E-10$ $5.168 \mathrm{E}-10$ $5.291 E-10$ $5.476 E-10$ $5.733 E-10$ $6 . C 72 E-1 C$ $6.507 E-10$ $7.059 \mathrm{E}-1 \mathrm{C}$ $6.425 E-1 C$ $9.774 E-1 C$ $8.832 E-10$ $1.182 E-C 9$ $1.334 E-C 9$ $2.058 E-C O$ 2. $553 \mathrm{E}-\mathrm{CO}$ $5.149 E-09$

$1.164 E-C 8$

$1.127 \mathrm{E}-\mathrm{C} 8$

$500 \cdot C$

$60 c .0$

$8.066 \mathrm{E}-11$ . 8. . $9.019 \mathrm{E}-1$ . l.0 $7 \mathrm{E}-1 \mathrm{C}$ C78E-10 $1.179 \mathrm{E}-10$ . $252 \mathrm{E}-1 \mathrm{C}$ 1. $377 \mathrm{E}-10$ $1.492 \mathrm{E}-10$ $1.631 \mathrm{E}-1 \mathrm{C}$ $1.760 \mathrm{E}-10$ $1.884 E-10$ $1.967 \mathrm{E}-10$ $1.504 E-C 9$

$3.976 \mathrm{E}-11$ $4.037 E-1$ .262E-1 $4.442 E-11$ $4.956 E-11$ $5.298 \mathrm{E}-1$ $5.676 \mathrm{E}-1$ $6.184 \mathrm{E}-11$ $6.696 E-11$ $7.291 \mathrm{E}-1$ $7.893 \mathrm{E}-1$ $8.5 C C E-1$ $9.016 E-11$

9.369E-11

$7.341 \mathrm{E}-1 \mathrm{C}$
150.0 RANGE (METERS I
$20 \mathrm{C} . \mathrm{C}$ 250. C 300.0 400.0 $5.073 E-10$ $5.088 \mathrm{E}-10$ $5.150 E-10$ $5.272 \mathrm{E}-10$ $5.452 \mathrm{E}-10$ $5.699 \mathrm{E}-10$ $6.021 \mathrm{E}-10$ $6.432 \mathrm{E}-10$ $6.953 E-10$ $5.106 \mathrm{E}-10$ $1.044 E-09$ $7.659 E-10$ $1.191 E-09$ $1.138 E-09$ $1.730 \mathrm{E}-\mathrm{CO}$ $1.730 E-C 9$

\section{$4.466 E-10$} $4.479 E-10$ $4.535 \mathrm{E}-10$ $4.642 \mathrm{E}-10$ $4.800 E-10$ $5.015 \mathrm{E}-10$ $5.294 E-10$ $5.646 E-10$ $6.078 \mathrm{E}-10$ $6.038 \mathrm{E}-10$ $7.335 \mathrm{E}-10$ $8.672 \mathrm{E}-10$ $1.084 E-09$ $1.0 .84 E-09$ $1.1 C 6 E-09$ $1.377 \mathrm{E}-09$

$\begin{array}{lll}3.63 C E-10 & 2.825 \mathrm{E}-10 & 1.564 \mathrm{E}-10 \\ 3.641 \mathrm{E}-10 & 2.834 \mathrm{E}-10 & 1.569 \mathrm{E}-10 \\ 3.686 \mathrm{E}-10 & 2.869 \mathrm{E}-10 & 1.588 \mathrm{E}-10 \\ 3.772 \mathrm{E}-10 & 2.936 \mathrm{E}-10 & 1.625 \mathrm{E}-10 \\ 3.899 \mathrm{E}-1 \mathrm{C} & 3.034 \mathrm{E}-10 & 1.678 \mathrm{E}-10 \\ 4.071 \mathrm{E}-1 \mathrm{C} & 3.167 \mathrm{E}-10 & 1.751 \mathrm{E}-10 \\ 4.293 \mathrm{E}-10 & 3.339 \mathrm{E}-10 & 1.843 \mathrm{E}-10 \\ 4.571 \mathrm{E}-10 & 3.554 \mathrm{E}-10 & 1.958 \mathrm{E}-10 \\ 4.909 \mathrm{E}-10 & 3.819 \mathrm{E}-10 & 2.098 \mathrm{E}-10 \\ 4.639 \mathrm{E}-10 & 4.421 \mathrm{E}-10 & 2.182 \mathrm{E}-10 \\ 5.996 \mathrm{E}-10 & 4.338 \mathrm{E}-10 & 2.509 \mathrm{E}-10 \\ 6.646 \mathrm{E}-10 & 5.075 \mathrm{E}-10 & 2.664 \mathrm{E}-10 \\ 6.78 \mathrm{CE}-10 & 5.369 \mathrm{E}-10 & 2.976 \mathrm{E}-10 \\ 8.464 \mathrm{E}-10 & 6.132 \mathrm{E}-10 & 3.218 \mathrm{E}-10 \\ 8.526 \mathrm{E}-10 & 6.651 \mathrm{E}-10 & 3.544 \mathrm{E}-10 \\ 1.638 \mathrm{E}-09 & 7.434 \mathrm{E}-10 & 3.805 \mathrm{E}-10 \\ 1.135 \mathrm{E}-09 & 8.113 \mathrm{E}-10 & 4.047 \mathrm{E}-10 \\ 7.041 \mathrm{E}-09 & 5.463 \mathrm{E}-\mathrm{C} 9 & 2.945 \mathrm{E}-\mathrm{C}-10\end{array}$

RANGE (METERS)

$900.0 \quad 12 C \mathrm{C} .0$

$150 \mathrm{C} .0$

1800.0

4. $C 88 \mathrm{E}-12$

$3.708 \mathrm{E}-13$

3.149E-14 $3.158 E-14$ $3.267 E-14$ $4.242 E-12$ 3. $847 \mathrm{E}-13$ $3.370 E-14$ $3.508 E-14$

$4.133 E-13 \quad 3.508 E-14$

$4.595 E-13 \quad 3.897 E-14$

$5.076 \mathrm{E}-12$

$5.417 E-12$

$5.817 E-12$

$6.274 \mathrm{E}-12$

3.897E-14

$4.448 E-14$

$4.783 \mathrm{E}-14$

$5.152 E-14$

5. $541 E-14$

$5.541 \mathrm{E}-14$
$5.929 \mathrm{E}-14$

$7.888 \mathrm{E}-12$

$8.415 \mathrm{E}-12$

$6.10 C \cdot E-13$

$7.048 \mathrm{E}-13$

$7.49 \mathrm{CE}-13$

$6.574 \mathrm{E}-14$

$6.751 E-14$

$8.071 E-13$

$7.439 E-1$

$6.696 \mathrm{E}-12$

5. $66 \mathrm{CE}-13$

2.567E-15

2.574E-15

$2.663 \mathrm{E}-15$

$2.746 E-15$

$2.858 \mathrm{E}-15$

3. $000 \mathrm{E}-15$

3. $174 E-15$

$3.618 E-15$

$3.888 \mathrm{E}-15$

$4.184 \mathrm{E}-1$

4. $494 E-15$

$4.802 \mathrm{E}-1$

$5.085 E-1$

$5.310 \mathrm{E}-15$

4. 599E-14 
COS INE

$-1.00600 \mathrm{E} 00$
$-9.89401 \mathrm{E}-01$
$-9.44575 \mathrm{E}-01$
$-8.65631 \mathrm{E}-01$
$-7.55044 \mathrm{E}-01$
$-6.17876 \mathrm{E}-01$
$-4.58017 \mathrm{E}-01$
$-2.81605 \mathrm{E}-01$
$-9.50125 \mathrm{E}-02$
$9.50125 \mathrm{E}-02$
$2.81605 \mathrm{E}-01$
$4.58017 \mathrm{E}-01$
$6.17876 \mathrm{E}-01$
$7.55044 \mathrm{E}-01$
$8.65631 \mathrm{E}-01$
$9.44575 \mathrm{E}-01$
$9.89401 \mathrm{E}-01$

TOTAL

COS INE

$-1.00000 E 00$ $-9.89401 E-01$ $-9.44575 \mathrm{E}-01$ $-8.65631 E-01$ $-7.55044 \mathrm{E}-01$ $-6.17876 \mathrm{E}-01$ $-4.58017 \mathrm{E}-01$ $-2.81605 \mathrm{E}-01$ $-9.50125 \mathrm{E}-02$ $9.50125 \mathrm{E}-02$ $2.81605 E-01$ $4.58017 E-01$ $6.17876 \mathrm{E}-01$ $8.65631 \mathrm{E}-01$ $9.44575 \mathrm{E}-01$

TOTAL

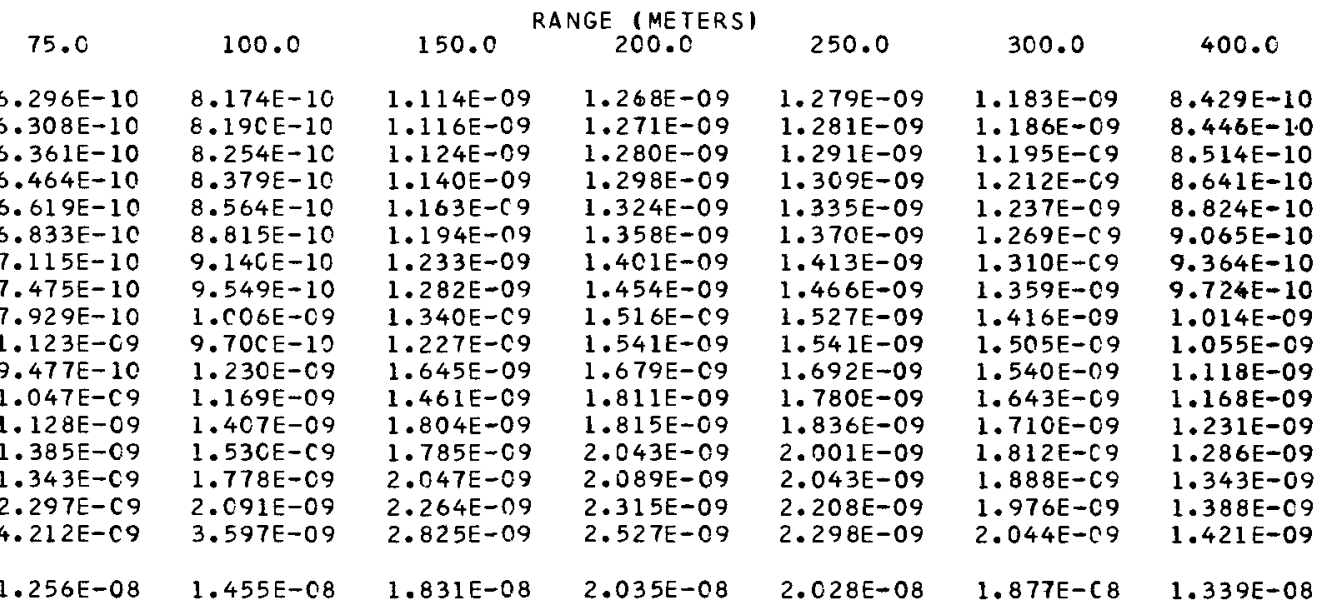

\section{RANGE (METERS)}

500.0

600.0

$5.090 E-10$

$5.101 E-10$

$5.143 E-10$

$5.222 \mathrm{E}-10$

$5.335 E-10$

$5.485 \mathrm{E}-10$

$5.671 \mathrm{E}-1 \mathrm{C}$

$5.895 E-10$

$6.155 \mathrm{E}-10$

$6.463 \mathrm{E}-10$

$6.764 \mathrm{E}-10$

$7.121 \mathrm{E}-10$

$7.43 \mathrm{E}-10$

$7.823 E-10$

$8.138 \mathrm{E}-1 \mathrm{C}$

$8.563 E-1 C$

$8.124 E-C 9$
2.764E-10

$2.77 \mathrm{CE}-10$

$2.793 \mathrm{E}-1 \mathrm{C}$
$2.837 \mathrm{E}-1 \mathrm{C}$

$2.899 \mathrm{E}-10$

$2.982 \mathrm{E}-1 \mathrm{C}$
$3.085 \mathrm{E}-10$

$3.209 \mathrm{E}-10$

$3.353 \mathrm{E}-10$

$3.512 E-10$

.

. $078 E-10$

$4.078 E-1 C$

$4.266 E=10$

$4.437 E-10$

$4.658 \mathrm{E}-1 \mathrm{O}$

$4.424 E-C 9$
1200.0

3.17OE- 1

3. $176 \mathrm{E}-11$

$3.255 \mathrm{E}-11$

3. $328 \mathrm{E}-11$

$3.425 \mathrm{E}-11$

$3.544 E-11$

$3.689 \mathrm{E}-11$

$3.855 E-11$

$4.043 E-11$

$4.249 E-11$

$4.467 E-11$

$4.688 E-11$

.

$5.090-11$

$5.327 E-11$

$5.083 E-10$
$2.928 \mathrm{E}-12$

$2.934 \mathrm{E}-12$

$2.96 \mathrm{CE}-12$

$3.006 \mathrm{E}-12$

$3.074 E-12$

$3.162 E-12$

$3.272 E-12$

$3.557 E-12$

$3.728 \mathrm{E}-12$

$4.11 E-12$

$4.499 E-12$

$4.797 E-12$

$4.875 \mathrm{E}-12$

$4.682 E-11$
1500.0

$2.489 E-13$

$2.494 E-13$

$2.55 \mathrm{EE}-13$

$2.612 E-13$

$2.687 \mathrm{E}-13$

$2.780 E-13$

$2.891 \mathrm{E}-13$

$3.018 \mathrm{E}-13$

$3.162 \mathrm{E}-13$

$3.318 E-13$

$3.482 E-13$

.

.

$4.109 \mathrm{E}-13$

3. $969 E-12$
$2.026 E-14$
$2.030 E-14$

$2.048 \mathrm{E}-14$

$2.080 \mathrm{E}-14$

$2.126 \mathrm{E}-14$

$2.261 E-14$

$2.351 \mathrm{E}-14$

$2.454 E-14$

2.569E-14

.

$2.826 E-14$

3.082E-14

3.191E-14

3. $324 E-14$

$3.223 E-13$
1800.0 


COSINE
$-1.00000 E$ OC
$-9.89401 \mathrm{E}-01$
$-9.44575 \mathrm{E}-01$
$-8.65631 \mathrm{E}-01$
$-7.55044 \mathrm{E}-01$
$-6.17876 \mathrm{E}-01$
$-4.58017 \mathrm{E}-01$
$-2.81605 \mathrm{E}-01$
$-9.50125 \mathrm{E}-02$
$9.50125 \mathrm{E}-02$
$2.81605 \mathrm{E}-01$
$4.58017 \mathrm{E}-01$
$6.17876 \mathrm{E}-01$
$7.55044 \mathrm{E}-01$
$8.65631 \mathrm{E}-01$
$9.44575 \mathrm{E}-01$
$9.89401 \mathrm{E}-01$

TOTAL

COSINE

$-1.00000 E$ OC $-9.89401 \mathrm{E}-0$

$-9.44575 E-0$

$-8.65631 E-01$

$-7.55044 E-01$

- $177876 E-01$

$-2.81605 E-01$

$-9.50125 \mathrm{E}-02$

$9.50125 E-02$

$2.81605 \mathrm{E}-01$
$4.58017 \mathrm{E}-01$

$6.17876 \mathrm{E}-01$

$7.55 \mathrm{C} 44 \mathrm{E}-0$

$8.65631 \mathrm{E}-01$

$9.44575 \mathrm{E}-01$

TOTAL

\begin{tabular}{|c|c|}
\hline 75.0 & 100.0 \\
\hline$n . r$ & C. 0 \\
\hline 0.0 & 0.5 \\
\hline 0.2 & C.C \\
\hline 0.0 & $c .0$ \\
\hline 0.0 & C.C \\
\hline $0 . c$ & r.c \\
\hline 0.0 & $c .0$ \\
\hline 0.0 & $C \cdot C$ \\
\hline 2.0 & C.C \\
\hline 0.0 & $c . c$ \\
\hline 0.0 & 0.0 \\
\hline $0 . c$ & c.c \\
\hline 0.0 & C.O \\
\hline 0.0 & $c . c$ \\
\hline 0.0 & $0 . c$ \\
\hline $0 . c$ & c.o \\
\hline 0.0 & $c . c$ \\
\hline $0 . c$ & $0 . \mathrm{C}$ \\
\hline
\end{tabular}

\begin{tabular}{|c|c|c|}
\hline 150.0 & $\begin{array}{c}\text { RANGE (ME TERS) } \\
20 \mathrm{C} .0\end{array}$ & $25 \mathrm{C} .0$ \\
\hline$c .0$ & 0.0 & C.O \\
\hline 0.6 & C.0 & C.C \\
\hline $0 . c$ & 0.0 & 0.0 \\
\hline C. $\mathrm{C}$ & $0 . C$ & 0.0 \\
\hline $0 . c$ & 0.0 & C.0 \\
\hline$C . C$ & 0.0 & 0.0 \\
\hline$C . C$ & 0.0 & C.C \\
\hline$c \cdot c$ & 0.0 & 0.0 \\
\hline 0.0 & 0.0 & 0.0 \\
\hline 0.0 & 0.0 & 0.0 \\
\hline 0.0 & 0.0 & 0.0 \\
\hline 0.0 & 0.0 & 0.0 \\
\hline $0 . \mathrm{C}$ & 0.0 & C.O \\
\hline$C \cdot C$ & 0.0 & 0.0 \\
\hline C.C & 0.0 & 0.0 \\
\hline$C . C$ & 0.0 & $c .0$ \\
\hline$c .0$ & 0.0 & 0.0 \\
\hline$C \cdot C$ & 0.0 & C.O \\
\hline
\end{tabular}

$\begin{array}{ll}300.0 & 400.0 \\ 0.0 & 0.0 \\ 0.0 & 0.0 \\ 0.0 & 0.0 \\ 0.0 & 0.0 \\ 0.0 & 0.0 \\ 0.0 & 0.0 \\ 6.0 & 0.0 \\ 0.0 & 0.0 \\ 0.0 & 0.0 \\ 0.0 & 0.0 \\ 0.0 & 0.0 \\ 0.0 & 0.0 \\ 0.0 & 0.0 \\ 0.0 & 0.0 \\ 0.0 & 0.0 \\ 0.0 & 0.0 \\ 0.0 & 0.0 \\ 0.0 & 0.0\end{array}$

530.0

orc.0

RANGE (METERS)

SOO. $12 \mathrm{CO} . \mathrm{C}$

1500.0

1800.0

$\begin{array}{ll}0 . c & c . c \\ 0.0 & c . c \\ 0.0 & 0.0 \\ 0 . c & 0 . c \\ 0.0 & 0.0 \\ 0 . c & c .0 \\ 0.0 & 1.0 \\ 0.0 & c . c \\ 0.0 & c . c \\ 0.0 & c .0 \\ 0.0 & 0.0 \\ 0.0 & 0.0 \\ 0.0 & 0.0 \\ 0.0 & 0 . c \\ 0.0 & 0.0 \\ 0.0 & c . c \\ 0.0 & 0.0 \\ 0.0 & \end{array}$

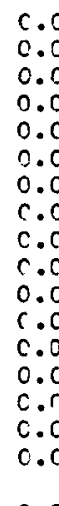

0.0
0.0
0.0
0.0
0.0
0.0
0.0
0.0
0.0
0.0
0.0
0.0
0.0
0.0
0.0
0.0
$c .0$

0.0

$0.0 \quad 0.0$

0.0
0.0

$\begin{array}{ll}0.0 & 0.0 \\ 0.0 & 0.0\end{array}$

$0.0 \quad n .0$

$\begin{array}{ll}0.0 & 0.0 \\ 0 . \mathrm{C} & \mathrm{c} .0\end{array}$

C. $0 \quad 0.0$

$0.0 \quad 0.0$

$\begin{array}{ll}0.0 & 0.0 \\ 0.0 & 0.0\end{array}$

$0.0 \quad 0.0$

$\begin{array}{ll}0.0 & c .0 \\ c .0 & 0.0\end{array}$

$0.0 \quad 0.0$

$0 . \mathrm{C} \quad 0.0$

c. 0.0

$\begin{array}{ll}0.0 & c .0 \\ 0.0 & c .0\end{array}$

$0 . r$

C. 0

0.0

c.o 
4 PI R**2 NON IONIZING SILICON KERMA (NEUTRONS)
(CM**2 ERGS/GRAM/STERADI AN/SOURCE NEUTRON)

0.00335 TO 0.111 MEV NEUTRON SOURCE

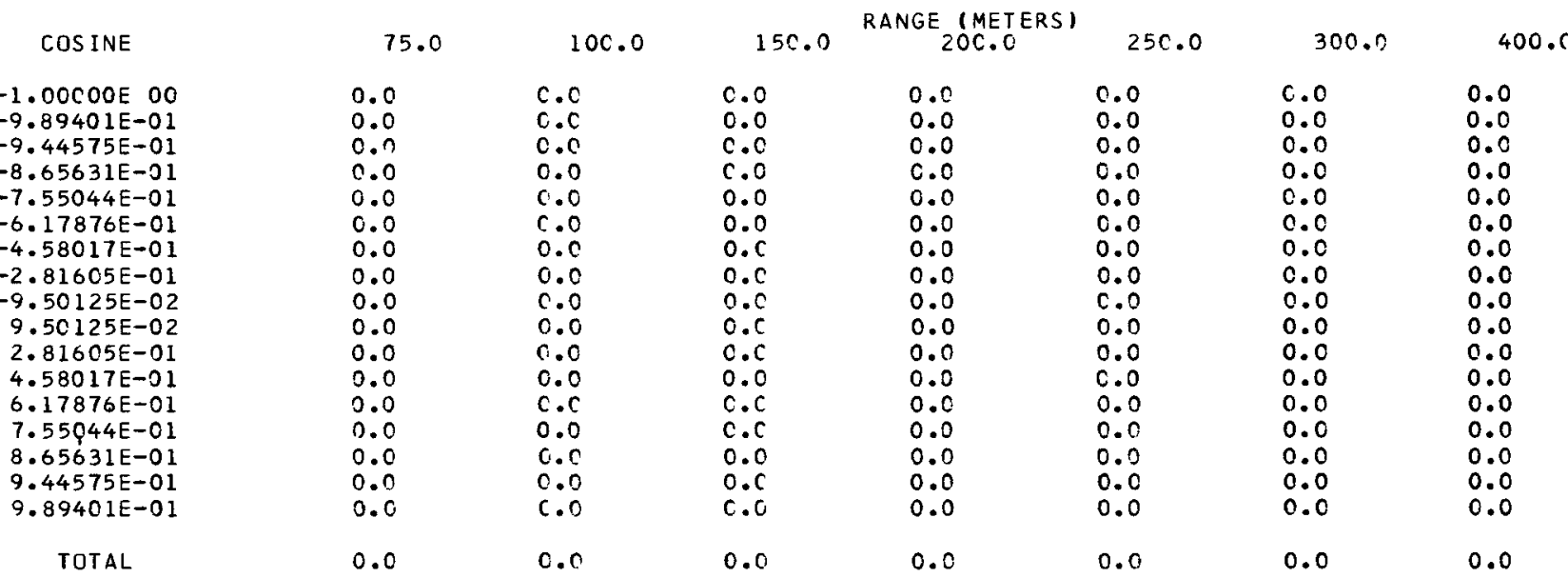

COSINE

$-1.00000 E 00$ $-9.89401 \mathrm{E}-01$ $-9.44575 E-01$ $-8.65631 \mathrm{E}-01$ $-7.55044 \mathrm{E}-01$ $-6.17876 \mathrm{E}-01$ $-4.58017 E-01$ $-2.81605 E-01$ $-9.50125 \mathrm{E}-02$ $9.50125 E-02$ $2.81605 E-01$ $4.58017 E-01$ $7.55044 E-01$ $7.55044 E-01$ $0.45575 \mathrm{E}-01$ $9.89401 E-01$

TOTAL
500.0

600.0

\section{0}

0.0

0.0

0.0

0.0

0.0

0.0

0.0

0.0
0.0
0.0

0.0

0.0

0.0

0.0

0.0

0.0

$0 . C$

0.0
0.0
0.0
0.0
0.0
$c .0$
$0 . c$
0.0
0.0
$c .0$
$c . c$
$c .0$
$c .0$
0.0
$c .0$
$c .0$
$c .0$
0.0

RANGE (METERS)

$$
\begin{array}{rr}
\text { RANGE (METERS) } \\
900.0 & 1200.0
\end{array}
$$

1500.0

1800.0

$\begin{array}{llll}c . c & 0 . c & 0 . c & 0.0 \\ c .0 & 0.0 & c .0 & c . c \\ 0 . c & 0 . c & 0.0 & 0.0 \\ 0.0 & 0.0 & 0.0 & 0.0 \\ c . c & 0.0 & 0.0 & 0.0 \\ 0.0 & 0.0 & c .0 & 0.0 \\ 0 . c & 0.0 & 0.0 & 0.0 \\ 0 . c & 0.0 & 0.0 & 0.0 \\ c .0 & 0.0 & 0.0 & 0.0 \\ c . c & 0.0 & 0.0 & 0.0 \\ c . c & 0.0 & 0.0 & 0.0 \\ c .0 & 0.0 & 0.0 & 0.0 \\ 0 . c & 0.0 & 0.0 & c .0 \\ 0 . c & 0.0 & 0.0 & c .0 \\ c . c & 0 . c & c .0 & 0.0 \\ c . c & 0.0 & 0.0 & 0.0 \\ 0.0 & 0.0 & 0.0 & c .0 \\ c . c & 0.0 & c .0 & 0.0\end{array}$


4 PI R**2 HENDER SON DOSE (GAMMAS)
(CM**2 RAD/STERADIAN/SOURCE NEUTRON)

COSINE

-1.00000 E 00

$-9.89401 \mathrm{E}-01$

-9.44575 E- 01

$-8.65631 E-01$

$-7.55044 \mathrm{E}-01$

$-6.17876 \mathrm{E}-01$

$-4.58017 E-01$

$-2.81605 E-01$

. $50125 E-02$

$2.81605 \mathrm{E}-01$

4.58017E-O1

$6.17876 \mathrm{E}-01$

7. $55044 \mathrm{E}-01$

$8.65631 \mathrm{E}-01$

$9.44575 E-01$
$9.894 C 1 E-01$

TOTAL

COS INE

-1. COCCCE 00 $-9.89401 E-01$ $-9.65631 E-01$ $-7.55044 \mathrm{E}-01$ $-6.17876 \mathrm{E}-01$ $-4.58017 E-01$ $-2.81605 \mathrm{E}-01$ $-9.50125 \mathrm{E}-02$ $9.50125 \mathrm{E}-02$ $2.81605 E-01$ 4. $58017 \mathrm{E}-\mathrm{Cl}$ 6.17876E-01 7.55044E-01 $8.65631 \mathrm{E}-01$ $9.44575 E-01$ $9.89401 E-01$

TOTAL

$\begin{array}{ll}75.2 & 100.0 \\ 7.552 \mathrm{E}-13 & 1.165 \mathrm{E}-12 \\ 7.570 \mathrm{E}-13 & 1.169 \mathrm{E}-12 \\ 7.642 \mathrm{E}-13 & 1.183 \mathrm{E}-12 \\ 7.782 \mathrm{E}-13 & 1.210 \mathrm{E}-12 \\ 7.990 \mathrm{E}-13 & 1.250 \mathrm{E}-12 \\ 8.265 \mathrm{E}-13 & 1.304 \mathrm{E}-12 \\ 8.608 \mathrm{E}-13 & 1.373 \mathrm{E}-12 \\ 9.017 \mathrm{E}-13 & 1.456 \mathrm{E}-12 \\ 9.490 \mathrm{E}-13 & 1.555 \mathrm{E}-12 \\ 1.003 \mathrm{E}-12 & 1.678 \mathrm{E}-12 \\ 1.065 \mathrm{E}-12 & 1.795 \mathrm{E}-12 \\ 1.124 \mathrm{E}-12 & 1.923 \mathrm{E}-12 \\ 1.172 \mathrm{E}-12 & 2.091 \mathrm{E}-12 \\ 1.233 \mathrm{E}-12 & 2.214 \mathrm{E}-12 \\ 1.307 \mathrm{E}-12 & 2.329 \mathrm{E}-12 \\ 1.328 \mathrm{E}-12 & 2.468 \mathrm{E}-12 \\ 1.328 \mathrm{E}-12 & 2.481 \mathrm{E}-12 \\ 1.258 \mathrm{E}-11 & 2.115 \mathrm{E}-11\end{array}$

$500 . c$

600.0

$1.320 E-12$

$1.380 E-12$

$1.483 E-12$

$1.614 E-12$

$1.780 E-12$

$2.328 \mathrm{E}-12$

2.818E-12

$3.564 \mathrm{E}-1$

$4.710 \mathrm{E}-12$

$6.517 \mathrm{E}-12$

$9.435 E-12$

$1.411 E-1$

$1.411 \mathrm{E}-1$

3. $045 \mathrm{E}-11$

3. $937 \mathrm{E}-11$

8.C. $52 E-11$
$0 . C 0335$ TO C.111 MEV NEUTRON SOURCE

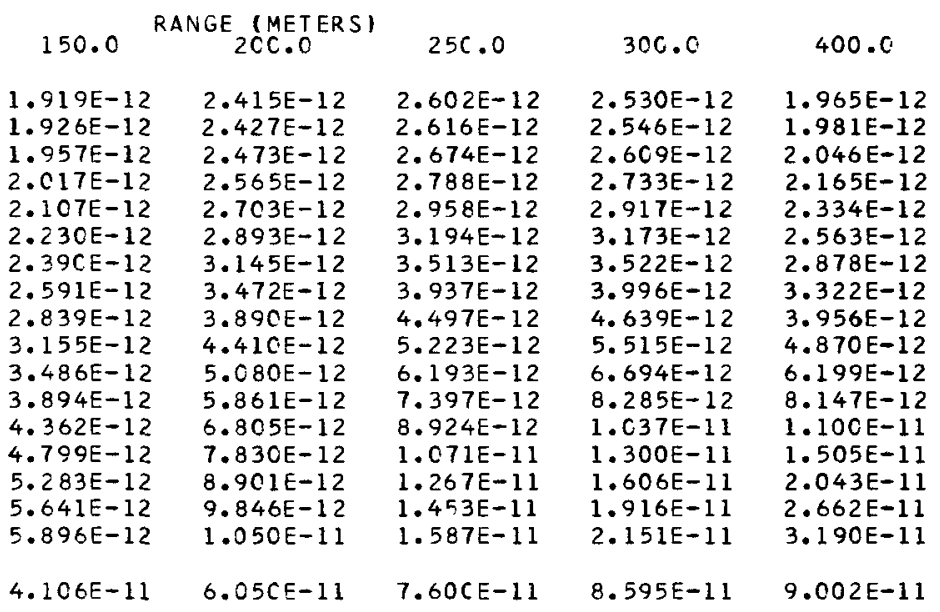

RANGE (METERS)

$000.0 \quad 120 \mathrm{C} .0$

1500.0

1800.0
$1.574 E-13$

$.572 E-13$

$1.057 E-12$

$1.29 C E-12$

$1.497 \mathrm{E}-12$

$1.830 \mathrm{E}-12$

$2.36 C E-12$

$4.615 E-12$

$4.615 E-12$

$1.157 \mathrm{E}-1$

$1.157 \mathrm{E}-1$

1. $924 \mathrm{E}-1$

$4.324 E-11$

$6.627 E-11$
$2.293 E-13 \quad 8.8 C 6 E-14$

$1.163 \mathrm{E}-13$

$1.522 E-13$

$3.394 \mathrm{E}-13 \quad 1305 \mathrm{E}-13$

$3.814 \mathrm{E}-13 \quad 1.319 \mathrm{E}-13$

$4.714 E-13$

$6.335 \mathrm{E}-13$

$8.789 E-13$

$1.282 E-12$

$2.153 E-12$

$4.3 \Gamma 4 E-12$

$9.561 \mathrm{E}-12$

$2.092 \mathrm{E}-11$

$3.869 E-11$

3.125E-11

$1.319 E-13$

$8.133 E-15$

$1.959 \mathrm{E}-15$

$6.046 \mathrm{E}-15$

$1.854 \mathrm{E}-14$

$5.657 \mathrm{E}-14 \quad 2.797 \mathrm{E}-14$

$7.612 E-14 \quad 3.779 E-14$

5. $118 E-14 \quad 3.006 E-14$

$5.118 E-14 \quad 2.320 E-14$

$2.540 \mathrm{E}-14$

$2.351 E-13 \quad 1.083 E-13 \quad 5.314 E-14$

$\begin{array}{lll}3.221 \mathrm{E}-13 & 1.488 \mathrm{E}-13 & 7.296 \mathrm{E}-14\end{array}$

$\begin{array}{lll}4.175 E-13 & 1.678 E-13 & 7.366 \mathrm{E}-14 \\ 6.4 C 4 \mathrm{E}-13 & 2.348 \mathrm{E}-13 & 9.426 \mathrm{E}-14\end{array}$

$1.384 E-12 \quad 4.97 C E-13 \quad 2.1 C 1 E-13$

$3.771 E-12 \quad 1.479 E-12 \quad 6.151 E-13$

$1.065 \mathrm{E}-11 \quad 4.898 \mathrm{E}-12 \quad 2.196 \mathrm{E}-12$

$6.723 E-12 \quad 3.165 E-12$ 
COSINE

$-1.000 C O E 00$ $-9.89401 E-01$ $-9.44575 \mathrm{E}-01$ $-8.65631 E-01$ $-7.55044 \mathrm{E}-01$ $-6.17876 E-01$ $-4.58017 \mathrm{E}-01$ $-2.81605 E-01$ $-9.50125 E-02$ $9.50125 \mathrm{E}-02$ $2.81605 \mathrm{E}-01$ $4.58017 E-01$ $6.17876 \mathrm{E}-01$ $7.55044 E-01$ $8.65631 \mathrm{E}-01$ $9.44575 E-0$

$9.89401 E-01$

TOTAL

\section{COSINE}

$-1.00000 E 00$ $-9.89401 \mathrm{E}-01$ $-9.44575 \mathrm{E}-01$ $-8.65631 E-01$ $-7.55044 E-01$ $-6.17876 E-01$ $-2.81605 E-01$ $-9.50125 E-02$ $9.50125 \mathrm{E}-02$ $.81605 \mathrm{E}-01$ 4.58017E-01 $6.17876 E-01$ $8.65631 E-01$ $0.65631 E-01$ $9.89401 \mathrm{E}-01$

TOTAL
75.0

$7.683 \mathrm{E}-11$ $7.701 \mathrm{E}-11$ $7.773 \mathrm{E}-11$ $7.913 \mathrm{E}-11$ $8.121 \mathrm{E}-11$ $8.396 \mathrm{E}-1$ $8.739 E-11$ $9.148 \mathrm{E}-1$ $9.621 E-1$ $1.016 \mathrm{E}-10$ 1. $078 \mathrm{E}-10$ $1.137 \mathrm{E}-10$ $1.185 \mathrm{E}-10$ 1. $246 \mathrm{E}-10$ $1.320 \mathrm{E}-10$ $1.341 E-10$ 1. $341 \mathrm{E}-1 \mathrm{C}$ $1.274 \mathrm{E}-09$

500.0

$1.484 \mathrm{E}-10$ 1. $501 \mathrm{E}-1 \mathrm{C}$ $1.562 \mathrm{E}-1 \mathrm{C}$ $1.665 E-10$ $1.799 E-10$ $1.968 \mathrm{E}-10$ 2.195E-10 $2.524 \mathrm{E}-10$ $3.765 \mathrm{E}-10$ $3.765 E-10$ $4.911 E-10$ $6.715 E-10$ $1.428 \mathrm{E}-\mathrm{C}$ $1.428 E-C 9$ . $3.946 \mathrm{E}-\mathrm{C}$

$8.284 E-C 9$
100.0

$1.188 \mathrm{E}-1 \mathrm{C}$ $1.191 E-1 C$ $1.232 \mathrm{E}-10$ $1.273 \mathrm{E}-10$ 1. 327E-10 $1.396 \mathrm{E}-10$ $1.479 \mathrm{E}-10$ $1.578 \mathrm{E}-10$ 1.70CE-IC $1.817 \mathrm{E}-1 \mathrm{C}$ $1.946 \mathrm{E}-10$ $2.113 \mathrm{E}-1 \mathrm{C}$ $2.236 \mathrm{E}-10$ $2.35 \mathrm{CE}-1 \mathrm{C}$ $2.5 C 3 E-1 C$ $2.143 E-09$ 600.0

$9.692 E-11$ $9.873 \mathrm{E}-11$ $1.044 \mathrm{E}-10$ $1.134 \mathrm{E}-10$ $1.236 \mathrm{E}-10$ $1.341 \mathrm{E}-1 \mathrm{C}$ $1.477 E-1 C$ $1.689 \mathrm{E}-1 \mathrm{C}$ $2 . C 26 E-10$ $2.560 \mathrm{E}-1 \mathrm{C}$ $3.406 \mathrm{E}-1 \mathrm{O}$ $4.811 E-10$ $1.287 \mathrm{E}-10$ $1.937 \mathrm{E}-0$ $1.937 E-09$ $4.331 E-09$

6.853 E- 09
RANGE (METERS)
$200 . \mathrm{C}$

$25 \mathrm{C} . \mathrm{C}$

300.0

400.0

$1.966 E-10$

$2.491 E-10$ $2.707 E-10$ $2.661 \mathrm{E}-10$ $1.974 E-10 \quad 2.503 E-10 \quad 2.721 E-10 \quad 2.67$ $\begin{array}{lllll}2.004 \mathrm{E}-10 & 2.549 \mathrm{E}-10 & 2.779 \mathrm{E}-10 & 2.741 \mathrm{E}-10 & 2.214 \mathrm{E}-10 \\ 2 . \mathrm{C} 65 \mathrm{E}-10 & 2.641 \mathrm{E}-10 & 2.893 \mathrm{E}-10 & 2.865 \mathrm{E}-10 & 2.334 \mathrm{E}-10\end{array}$ $2.155 \mathrm{E}-10 \quad 2.780 \mathrm{E}-10 \quad 3.064 \mathrm{E}-10 \quad 3.050 \mathrm{E}-10 \quad 2.505 \mathrm{E}-10$ $\begin{array}{lllll}2.278 E-10 & 2.97 \mathrm{CE}-10 & 3.301 \mathrm{E}-1 \mathrm{C} & 3.307 \mathrm{E}-1 \mathrm{C} & 2.736 \mathrm{E}-10\end{array}$ $2.437 E-10 \quad 3.222 E-10 \quad 3.621 E-10 \quad 3.657 E-10 \quad 3.054 E-10$ $2.638 \mathrm{E}-10 \quad 3.549 \mathrm{E}-10 \quad 4.046 \mathrm{E}-10 \quad 4.133 \mathrm{E}-1 \mathrm{C} \quad 3.501 \mathrm{E}-10$ $2.886 E-10 \quad 3.967 E-10 \quad 4.606 E-10 \quad 4.777 E-10 \quad 4.137 E-10$ $3.202 \mathrm{E}-10 \quad 4.487 \mathrm{E}-10 \quad 5.331 \mathrm{E}-10 \quad 5.652 \mathrm{E}-10 \quad 5.052 \mathrm{E}-10$ $3.533 E-10 \quad 5.157 E-10 \quad 6.300 E-10 \quad 6.831 E-10 \quad 6.380 E-10$ $3.941 \mathrm{E}-10 \quad 5.936 \mathrm{E}-10 \quad 7.503 \mathrm{E}-10 \quad 8.419 \mathrm{E}-10 \quad 8.324 \mathrm{E}-10$ $4.409 \mathrm{E}-10 \quad 6.879 \mathrm{E}-10 \quad 9.027 \mathrm{E}-1 \mathrm{C} \quad 1.050 \mathrm{E}-\mathrm{C} 9 \quad 1.117 \mathrm{E}-09$ $4.844 E-10 \quad 7.902 E-10 \quad 1.08 C E-09 \quad 1.312 E-09 \quad 1.521 E-09$ $\begin{array}{lllll}5.328 \mathrm{E}-10 & 8.971 \mathrm{E}-10 & 1.277 \mathrm{E}-09 & 1.617 \mathrm{E}-09 & 2.057 \mathrm{E}-09\end{array}$ $\begin{array}{lllll}5.685 E-10 & 9.915 E-10 & 1.462 E-09 & 1.926 E-C 9 & 2.675 E-09\end{array}$

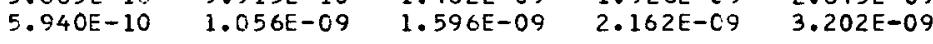
$4.165 E-09$

$6.146 E-09$

7. $732 \mathrm{E}-09$

8. 760E-C9

$9.216 E-C 9$

\section{RANGE (METERS)}

$$
9 \text { RANGE }
$$$$
1200.0
$$

1500.0

1800.0

$\begin{array}{llll}2.711 E-11 & 9.4 C 1 E-12 & 3.75 C E-12 & 1.609 E-12 \\ 2.909 E-11 & 1.075 E-11 & 4.52 C E-12 & 2.011 E-12 \\ 3.425 E-11 & 1.471 E-11 & 6.842 E-12 & 3.245 E-12 \\ 3.985 E-11 & 1.756 E-11 & 8.558 E-12 & 4.187 \mathrm{E}-12 \\ 4.491 \mathrm{E}-11 & 2.120 \mathrm{E}-11 & 1.053 \mathrm{E}-11 & 5.176 \mathrm{E}-12 \\ 4.621 \mathrm{E}-11 & 1.981 \mathrm{E}-11 & 9.360 \mathrm{E}-12 & 4.456 \mathrm{E}-12 \\ 4.619 \mathrm{E}-11 & 1.834 \mathrm{E}-11 & 8.252 \mathrm{E}-12 & 3.822 \mathrm{E}-12 \\ 5.074 \mathrm{E}-11 & 1.976 \mathrm{E}-11 & 8.804 \mathrm{E}-12 & 4.077 \mathrm{E}-12 \\ 6.007 \mathrm{E}-11 & 2.402 \mathrm{E}-11 & 1.125 \mathrm{E}-11 & 5.380 \mathrm{E}-12 \\ 7.654 \mathrm{E}-11 & 3.037 \mathrm{E}-11 & 1.415 \mathrm{E}-11 & 6.886 \mathrm{E}-12 \\ 1.013 \mathrm{E}-10 & 3.915 \mathrm{E}-11 & 1.823 \mathrm{E}-11 & 8.882 \mathrm{E}-12 \\ 1.416 \mathrm{E}-10 & 4.873 \mathrm{E}-11 & 2.019 \mathrm{E}-11 & 8.991 \mathrm{E}-12 \\ 2.279 \mathrm{E}-10 & 7.068 \mathrm{E}-11 & 2.669 \mathrm{E}-11 & 1.096 \mathrm{E}-11 \\ 4.405 \mathrm{E}-10 & 1.435 \mathrm{E}-1 \mathrm{C} & 5.213 \mathrm{E}-11 & 2.213 \mathrm{E}-11 \\ 9.616 \mathrm{E}-10 & 3.788 \mathrm{E}-10 & 1.483 \mathrm{E}-10 & 6.151 \mathrm{E}-11 \\ 2.092 \mathrm{E}-\mathrm{C} 9 & 1.062 \mathrm{E}-09 & 4.872 \mathrm{E}-10 & 2.179 \mathrm{E}-1 \mathrm{C} \\ 3.867 \mathrm{E}-\mathrm{Cg} & 2.539 \mathrm{E}-09 & 1.435 \mathrm{E}-09 & 7.489 \mathrm{E}-1 \mathrm{C} \\ 3.270 \mathrm{E}-\mathrm{Cg} & 1.511 \mathrm{E}-09 & 7.071 \mathrm{E}-1 \mathrm{C} & 3.329 \mathrm{E}-10\end{array}$


4 PI R**2 AIR KERMA (GAMMAS)
(CM\#\#2 ERGS/GRAM/STERAOI AN/ SUURCE NEUTRON)
0.00335 TO $r .111$ MEV NEUTRON SOURCE

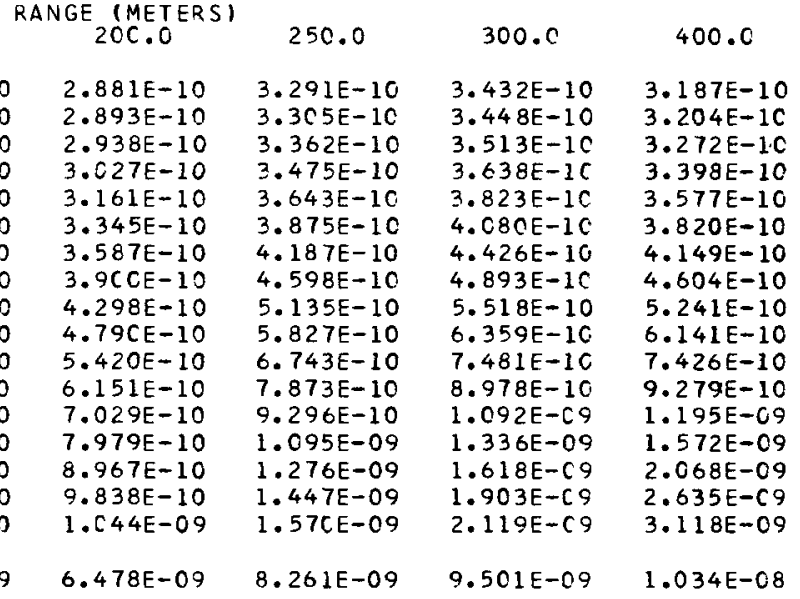

\section{0} 1200.0 1500.0

\section{$2.655 \mathrm{E}-10$} $2.673 \mathrm{~F}=10$ $2.738 \mathrm{E}-10$ $2.851 \mathrm{E}-10$ 3. C $0 E-10$ $3.190 E-10$ $3.442 E-10$ $3.798 \mathrm{E}-1 \mathrm{C}$ 4. $315 E-10$ $5.075 E-10$ $6.208 E-10$ 1.C71E-09 $1 . C 7 E-C 9$ $1.506 \mathrm{C}-\mathrm{C}$ $2.156 E-C 9$ $3.819 E-09$

$9.637 E-C 9$

$2.117 \mathrm{E}-10$ $2.136 \mathrm{E}-10$ $2.198 \mathrm{E}-1 \mathrm{C}$ $2.298 \mathrm{E}-1 \mathrm{C}$ $2.417 E-1 C$ $2.549 E-1 C$ $2.718 E-1 C$ $2.968 E-1 C$ $3.342 E-10$ $3.907 E-10$ $4.771 E-10$ 8. $528 \mathrm{E}-10$ 1. $528 \mathrm{E}-10$ 1. $976 \mathrm{E}-\mathrm{C} 9$ $1.976 E-C 9$ 3.C27E-C9 RANGE (METERS) $1.086 E-11$ $1.017 E-10 \quad 4.839 E-11$ $1.036 \mathrm{E}-10$ $1 .-89 \mathrm{E}-10$ $1.152 E-10$ $1.216 \mathrm{E}-10$ $1.251 \mathrm{E}-10$ $1.280 E-10$ $1.358 E-10$ $1.688 E-10$ $1.688 E-10$ $1.974 E-10$ 3. $282 E-10$ $3.282 E-10$ $1.314 E-10$ $1.016 E-C 9$ 2.052E-09 $4.97 \mathrm{CE}-11$ $5.363 E-11$ $5.683 E-11$ $6.105 E-11$ $6.097 E-11$ $6.11 \mathrm{E}-11$ $6.425 \mathrm{E}-11$ $7.029 \mathrm{E}-11$ $7.859 E-11$ $8.957 E-11$ 1. $260 \mathrm{E}-10$ $1.260 E-10$ $4.178 E-10$ 1. $C 44 \mathrm{E}-09$ 2. 295E-11 2. $369 E-11$ $2.596 \mathrm{E}-11$ $2.781 \mathrm{E}-11$ $3.004 E-11$ $2.954 \mathrm{E}-11$ $2.924 \mathrm{E}-11$ $3.062 \mathrm{E}-11$ $3.385 \mathrm{E}-11$ 3.765E-11 $4.274 E-11$ 4.611E-11 5.4C3E-11 $1.973 E-11$ 1.704E-10 $4.816 E-10$
$1.341 \mathrm{E}-\mathrm{C} 9$ 1. $125 \mathrm{E}-11$ 1. $244 E-11$ $1.344 \mathrm{E}-11$ $1.455 \mathrm{E}-11$ $1.416 \mathrm{E}-11$ $1.391 \mathrm{E}-11$ $1.455 E-11$ $1.620 \mathrm{E}-11$ $1.810 E-11$ 2.C54E-11 $2.136 \mathrm{E}-11$ $2.408 E-11$ 7.546E-11 $2.289 \mathrm{E}-11$ $2.166 \mathrm{E}-10$

$8.255 E-50$

$2.385 E-C 9$

$9.637 E-10$

$4.558 E-1 C$ 
COSINE

$-1.00000 E 00$ $-9.89401 E-01$ $-9.44575 E-01$ $-8.65631 E-01$ $-7.55044 \mathrm{E}-01$ $-6.17876 \mathrm{E}-01$ $-4.58017 E-01$ $-2.81605 E-01$ $-9.50125 \mathrm{E}-02$ $9.50125 \mathrm{E}-02$ $2.816 C 5 E-01$ 4.58017E-01 $6.17876 \mathrm{E}-01$ $7.55044 \mathrm{E}-01$ $8.65631 \mathrm{E}-01$ $9.44575 E-01$
$9.89401 E-01$

TOTAL

COSINE

$-1.00000 E$ OC $-9.89401 \mathrm{E}-01$ $-9.44575 E-01$ $-8.65631 \mathrm{E}-01$ $-6.17876 \mathrm{E}-01$ $-6.17876 E-01$ $-2.81605 \mathrm{E}-01$ $-9.50125 E-02$ $-9.50125 E-02$ . $0125 E-02$ $2.81605 E-01$ $4.5017 \mathrm{E}-01$ $7.55044 \mathrm{E}-01$

$8.5563 \mathrm{E}-01$

$8.65631 \mathrm{E}-01$
$9.44575 \mathrm{E}-01$

$9.89401 E-01$

TOTAL

$\begin{array}{lcc}75.0 & 100.0 & 150.0 \\ 7.924 \mathrm{E}-11 & 1.226 \mathrm{E}-1 \mathrm{C} & 2.032 \mathrm{E}-10 \\ 7.943 \mathrm{E}-11 & 1.23 C \mathrm{E}-10 & 2.040 \mathrm{E}-10 \\ 8.016 \mathrm{E}-11 & 1.244 \mathrm{E}-10 & 2.072 \mathrm{E}-10 \\ 8.160 \mathrm{E}-11 & 1.272 \mathrm{E}-1 \mathrm{C} & 2.133 \mathrm{E}-10 \\ 8.374 \mathrm{E}-11 & 1.313 \mathrm{E}-1 \mathrm{C} & 2.226 \mathrm{E}-10 \\ 8.656 \mathrm{E}-11 & 1.369 \mathrm{E}-1 \mathrm{C} & 2.352 \mathrm{E}-10 \\ 9.007 \mathrm{E}-11 & 1.439 \mathrm{E}-1 \mathrm{C} & 2.516 \mathrm{E}-10 \\ 9.426 \mathrm{E}-11 & 1.524 \mathrm{E}-10 & 2.722 \mathrm{E}-10 \\ 9.911 \mathrm{E}-11 & 1.626 \mathrm{E}-1 \mathrm{C} & 2.976 \mathrm{E}-10 \\ 1.047 \mathrm{E}-10 & 1.751 \mathrm{E}-1 \mathrm{C} & 3.299 \mathrm{E}-10 \\ 1.110 \mathrm{E}-10 & 1.871 \mathrm{E}-1 \mathrm{C} & 3.639 \mathrm{E}-10 \\ 1.170 \mathrm{E}-10 & 2.003 \mathrm{E}-1 \mathrm{C} & 4.057 \mathrm{E}-10 \\ 1.219 \mathrm{E}-1 \mathrm{C} & 2.175 \mathrm{E}-1 \mathrm{C} & 4.537 \mathrm{E}-10 \\ 1.282 \mathrm{E}-10 & 2.301 \mathrm{E}-1 \mathrm{C} & 4.984 \mathrm{E}-10 \\ 1.358 \mathrm{E}-10 & 2.418 \mathrm{E}-10 & 5.48 \mathrm{E}-10 \\ 1.380 \mathrm{E}-10 & 2.562 \mathrm{E}-10 & 5.846 \mathrm{E}-10 \\ 1.380 \mathrm{E}-10 & 2.575 \mathrm{E}-10 & 6.109 \mathrm{E}-10 \\ 1.313 \mathrm{E}-\mathrm{CO} & 2.208 \mathrm{E}-\mathrm{CO} & 4.291 \mathrm{E}-\mathrm{C} 0\end{array}$

RANGE (METERS)
200.0

250.0

$300 . \mathrm{C}$

400.0

\subsection{E-10} 2.593E-10 $2.641 E-10$ $2.735 \mathrm{E}-10$ $2.876 \mathrm{E}-10$ $3.071 \mathrm{E}-10$ 3. $33 \mathrm{CE}-10$ $3.665 \mathrm{E}-10$ $4.093 \mathrm{E}-10$ $4.626 \mathrm{E}-10$ $5.313 \mathrm{E}-10$ $6.112 E-10$ $7.079 E-10$ $8.128 E-10$ $9.225 E-10$ $1.019 E-09$ $1.086 \mathrm{E}-09$

6.334E-09 $2.812 E-10$ $2.827 \mathrm{E}-1 \mathrm{C}$ 3. $C 03 E-10$ $3.178 E-10$ $3.421 \mathrm{E}-10$ $3.748 \mathrm{E}-10$ $4.183 \mathrm{E}-10$ 4. $757 \mathrm{E}-10$ $5.501 \mathrm{E}-1 \mathrm{C}$ $6.494 E-10$ $7.726 \mathrm{E}-10$ $9.289 \mathrm{E}-10$ $1.111 \mathrm{E}-09$ $1.312 \mathrm{E}-09$ $1.563 E-09$ $1.640 E-09$

$7.972 E-09$

$2.775 E-1 C$ $2.791 \mathrm{E}-1 \mathrm{C} \quad 2.265 \mathrm{E}-1 \mathrm{C}$ $2.857 \mathrm{E}-1 \mathrm{C} \quad 2.332 \mathrm{E}-10$ $2.984 \mathrm{E}-1 \mathrm{C} \quad 2.455 \mathrm{E}-10$ 3. $173 E-10 \quad 2.629 E-10$ $3.437 \mathrm{E}-10 \quad 2.866 \mathrm{E}-10$ $3.795 \mathrm{E}-1 \mathrm{C} \quad 3.190 \mathrm{E}-1 \mathrm{C}$ $4.281 E-10 \quad 3.647 E-10$ $4.941 \mathrm{E}-10 \quad 4.297 \mathrm{E}-10$ $5.838 \mathrm{E}-10 \quad 5.233 \mathrm{E}-10$ $7.045 \mathrm{E}-1 \mathrm{C} \quad 6.592 \mathrm{E}-10$ $8.672 \mathrm{E}-1 \mathrm{C} \quad 8.583 \mathrm{E}-10$ $1.08 C E-C 9 \quad 1.149 E-09$ $1.349 \mathrm{E}-\mathrm{C} 9 \quad 1.564 \mathrm{E}-09$ $1.662 \mathrm{E}-\mathrm{C} 9 \quad 2.114 \mathrm{E}-09$ $1.980 E-C 9 \quad 2.748 E-09$ $2.221 \mathrm{E}-\mathrm{C} 9 \quad 3.289 \mathrm{E}-09$ $9.037 E-69 \quad 9.517 E-09$
(1) $2.598 \mathrm{E}-12$ $3.867 \mathrm{E}-12$ $4.834 \mathrm{E}-12$ $5.845 E-12$ $5.099 E-12$ $4.436 E-12$ 4.680E-12 $0.000 \mathrm{E}-12$ ..522E-12 $9.570 \mathrm{E}-12$ . $146 E-11$ $2.270 \mathrm{E}-11$ 6. $278 \mathrm{E}-11$ $2.231 E-10$ $7.697 \mathrm{E}-10$

$3.462 E-10$ 
(NEUTRONS/MEV/STERADIAN/SOURCE NEUTRON)

ENERGY GROUP (MEV) $1.22 E \quad 01--1.50 E$ OI $1.00 E$ 01- $-1.22 E \quad 01$ $8.19 E$ OO- -1.00E 01 4.97E $00---6.36 E$ OO 4.07E $00--4.97 E$ OO 3.01 E $00---4.07 E 00$ $2.46 \mathrm{E} 00--3.01 \mathrm{E} 00$ $2.35 \mathrm{E} 00---2.46 \mathrm{E} 00$ $1.83 \mathrm{E} 00--2.35 \mathrm{E} 00$ $1.11 E$ 00-- $1.83 E$ OO $5.50 E-01--1.11 E$ OO $1.11 E-01--5.50 E-01$ .35E-02--1.11E-0 $5.83 E-04--3.35 E-02$ $1.01 \mathrm{E}-04---5.83 \mathrm{E}-04$ $2.90 E-05--1.01 E-04$ $1.07 E-05--2.90 E-05$ $3.06 \mathrm{E}-06--1.07 \mathrm{E}-05$ $1.12 E-06-=3.06 E-06$ $4.14 \mathrm{E}-07---1.12 \mathrm{E}-06$
$0.0 \quad--4.14 \mathrm{E}-07$ ENERGY

GROUP (MEV)

$1.22 E$ O1- $-1.50 E$ O1 $\begin{aligned} & 1.00 E \\ & 8.19 E\end{aligned} 00---1.22 E$ OI $8.19 E$ OO-- $1.00 E$ OI
$6.36 E$ OO---8.19E 00 6.36E $00---8.19 E$ E 00 4.07E $00--4.97 E$ OO 3.01E OD-C. . O7E OO 2.46E $00--3.01 E$ OO 1.35E OOO- $2.46 E$ E 1.2E $00-2.35 E$ OO 1.11E 00- $1.83 E$ OO 1. $1.11 E-01--5.50 E-01$ . . $01 E-04--3.35 E-02$ 1. 1.07E-051.06E-06- $-1.07 E-05$ $1.12 \mathrm{E}-06 \mathrm{E}-3.06 \mathrm{E}-06$ $4.14 E-07=-1.12 E-06$ $0.0 \quad---4.14 \mathrm{E}-07$
ANGLE 1 ANGLE 2 $M U=-1.0000 \quad M U=-0.9894$ $-1.165 E-07-9.541 E-08$ $-6.374 E-07 \quad-5.005 E-07$ $-5.775 E-07$ $9.574 E-06$ $9.133 \mathrm{E}-05$ $3.465 E-04 \quad 3.503 E-04$ $9.267 \mathrm{E}-04$ $4.009 \mathrm{E}-03 \quad 4.002 \mathrm{E}-03$ $5.997 E-03 \quad 5.970 E-03$ $5.080 E-03 \quad 5.075 E-03$ $9.674 \mathrm{E}-03 \quad 9.698 \mathrm{E}-03$ $2.423 E-02 \quad 2.427 E-02$ $4.906 E-02 \quad 4.919 E-02$ $2.707 \mathrm{E}-01 \quad 2.711 \mathrm{E}-01$ $1.982 \mathrm{E}-01 \quad 1.984 \mathrm{E}-01$ $1.008 \mathrm{E} 01$ 1.009E 01 $3.927 \mathrm{E}$ 01 3.930E 01 $1.065 \mathrm{E} 02 \quad 1.065 \mathrm{E} 02$ $2.646 \mathrm{E} \mathrm{O2} 2.647 \mathrm{E} 02$ $6.727 \mathrm{E} \quad 02$ $1.408 E \quad 03$ $1.962 \mathrm{E} 03$

$6.729 E \quad 02$ $1.408 \mathrm{E} \quad 03$ $1.963 \mathrm{E} 03$ ANGLE 10 $M U=0.0950$
$8.085 \mathrm{E}-08$ $8.085 \mathrm{E}-08$
$1.315 \mathrm{E}-06$ $1.030 \mathrm{E}-05$ $3.721 E-05$ $2.139 \mathrm{E}-04$ $1.088 E-03$ $1.311 E-03$ 1.0 $047 \mathrm{E}-02$ $1.047 E-02$ $1.221 \mathrm{E}-02$ $2.383 E-02$ $7.74 \mathrm{E}-02$ $3.57 E-02$ $2.249 \mathrm{E}-01$ 2.249E-01 $4.194 \mathrm{E} \quad 1$ $1.125 \mathrm{E} 02$ $1.125 \mathrm{E} 02$ $2.771 \mathrm{E} 02$
$7.005 \mathrm{E} 02$ $1.460 \mathrm{E} 03$ $2.025 \mathrm{E} 03$ ANGLE 11 $M U=0.2816$
$1.296 \mathrm{E}-07$ $1.256 \mathrm{E}-07$
$1.381 \mathrm{E}-00$ $1.381 \mathrm{E}-06$ $9.123 \mathrm{E}-06$ ..160E-05 $2.835 E-04$ $7.415 E-04$ $2.376 \mathrm{E}-03$ 5.492E-03 $8.939 E-03$ $2.104 \mathrm{E}-02$ $4.560 \mathrm{E}-02$ .137E-02 (2.314E-O $2.314 \mathrm{E}-01$ 1.119E OI $4.248 E$
$1.137 \mathrm{O}^{2}$ $2.794 \mathrm{E}$ O2 $7.050 \mathrm{E} \mathrm{O2}$ $1.470 \mathrm{E} O 3$

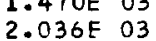

ANGLE 3 $M U=-0.9446 \quad M U=-0.8656$ $-4.165 E-08 \quad 7.575 E-09$ $6.162 E-08$ $2.948 \mathrm{E}-07 \quad 1.003 \mathrm{E}-06$ $1.069 E-05 \quad 1.178 E-0$ $8.823 \mathrm{E}-05 \quad 8.476 \mathrm{E}-05$ $3.646 E-04 \quad 3.897 E-04$ $9.351 E-04 \quad 9.507 E-04$ $3.975 E-03 \quad 3.925 E-03$ $\begin{array}{ll}5.872 E-03 & 5.710 E-03 \\ 5.063 E-03 & 5.065 E-03\end{array}$ $9.797 E-03$ $2.446 E-02$
$4.971 E-02$ $2.728 \mathrm{E}-01$ $1.992 \mathrm{E}-01$ $1.012 \mathrm{E}$ O1 $3.939 \mathrm{E} 01$ $1.067 E 02$ $2.652 \mathrm{E} 02$ $6.739 \mathrm{E} 02$ $1.410 E$ O $1.965 E \quad 03$
ANGLE 12 $M U=0.4580$
$1.255 E-07$ $1.255 E-07$
$1.900 E-06$ $1.073 \mathrm{E}-05$ $6.979 E-05$ $3.788 \mathrm{E}-04$ $.467 E-04$ $1.979 \varepsilon-03$ $0.005 E-03$ 1.510E-03 $1.161 \mathrm{E}-02$ $4.230 E-02$ $8.671 E-02$ $8.671 E-02$ $2.407 \mathrm{E}-01$ $4.301 E$ OI $4.301 E$
$1.148 \mathrm{EE} 02$ $2.817 \mathrm{E} \quad 02$ $7.105 \mathrm{E}$ O2 $7.105 E \quad 02$ $2.047 \mathrm{E} 03$
ANGLE 5 ANGLE 6 $2.167 E-08$ MU $=-0.6179$ $2.411 E-07 \quad 3.481 E-07$ $1.971 E-06 \quad 2.279 E-06$ $1.266 E-05 \quad 1.372 E-05$ $8.508 E-05 \quad 9.316 E-05$ $4.217 E-04 \quad 4.592 E-04$ $9.730 E-04 \quad 1.004 E-03$ $3.860 \mathrm{E}-03 \quad 3.794 \mathrm{E}-03$ $5.535 E-03 \quad 5.402 E-03$ $5.122 E-03 \quad 5.281 E-03$ $2.550 E-02 \quad 2.647 E-02$ $5.229 E-02 \quad 5.444 E-02$ $2.485 E-02$ $5.074 \mathrm{E}-02$ $2.760 \mathrm{E}-01$ $2.008 \mathrm{E}-01$ $1.018 \mathrm{E} 01$ $3.957 \mathrm{E}$ 01 $1.072 \mathrm{E} 02$ $2.660 \mathrm{E} 02$ $6.759 \mathrm{E} \quad 02$ $1.414 \mathrm{E} \quad 03$ $1.970 E 03$ ANGLE 13 $\mathrm{A}=0.6179$
$2.712 \mathrm{E}-07$ $2.712 E-07$
$3.320 E-06$ $3.320 E-06$
$2.585 E-05$ $9.737 E-05$ $5.136 \mathrm{E}-04$ $1.282 E-03$ . $1.436 E-02$ $1.436 \mathrm{E}-02$ $1.882 \mathrm{E}-02$ $5.882 E-02$ $1.046 \mathrm{E}-01$ $1.046 E-01$ $2.467 E-01$ $1.160 \mathrm{E} 01$ 4.352E O1 $1.159 \mathrm{E} \quad 02$ $7.150 E$ O 02 $1.487 \mathrm{E} 03$ $\begin{array}{ll}1.487 E & 03 \\ 2.057 E & 03\end{array}$ $6.786 E$ O2 $6.620 E$ O2 $1.419 \mathrm{E} 03 \quad 1.426 \mathrm{E} O 3$ $\begin{array}{ll}1.419 E & 03 \\ 1.977 E & 03\end{array}$ $1.985 \mathrm{E} 03$ ANGLE 14 ANGLE 15 $M U=0.7550$ $1.691 \mathrm{E}-00$ $1.155 \mathrm{E}-05$ $7.506 \mathrm{E}-05$ $2.987 \mathrm{E}-04$ 7. $1.863 E-03$ $1.655 \mathrm{E}-02$ 2.5 $1.833 E-02$ $4.202 E-02$ $4.436 \mathrm{E}-01$ $2.550 E-01$ $4.398 E$ O $1.168 \mathrm{E} 02$ $2.856 \mathrm{E} 02$ $7.190 E^{02}$ $1.494 E 03$ 2.065E 03
$U=0.8656$ 2. $041 E-06$ C.003E-05 $8.603 E-05$ $5.188 E-04$ $1.568 E-03$ $5.652 E-03$ $2.305 \mathrm{E}-02$ 2.305E-02 $4.450 E-02$ $6.108 \mathrm{E}-02$ $1.099 \mathrm{E}-0$ $1.571 \mathrm{E}-01$ 2.06OE-OI $2.060 \mathrm{E}-01$ $4.437 E$ OI $1.176 \mathrm{E} \mathrm{O2}$ $2.871 \mathrm{E} 02$ $7.222 \mathrm{E} O 2$
$1.500 \mathrm{E} O 3$ $2.072 E 03$ $1.037 \mathrm{E}-02 \quad 1.093 \mathrm{E}-02$

ANGLE 7 ANGLE 8 ANGLE 9 $M U=-0.4580 \quad M U=-0.2816 \quad M U=-0.0950$ 2.861E-09 $-9.781 E-09 \quad 2.200 E-09$ $2.632 \mathrm{E}-07 \quad 2.233 \mathrm{E}-07 \quad 3.956 \mathrm{E}-07$ $\begin{array}{lll}2.190 E-06 & 2.531 E-06 & 3.794 E-06 \\ 1.609 E-05 & 2.100 E-05 & 2.880 E-05\end{array}$ $1.116 E-04 \quad 1.407 E-04 \quad 1.781 E-04$ 5.029E-04 5.548E-04 6.185E-04 $1.048 E-03 \quad 1.111 E-03 \quad 1.206 E-03$ $3.757 E-03$ 3.789E-03 3.951E-03 $5.375 E-03$ 5.522E-03 5.930E-03 $\begin{array}{ll}5.107 E-03 & 6.890 E-03\end{array}$ $1.173 E-02 \quad 1.285 E-02 \quad 1.439 E-02$ $2.786 E-02$ 2.981E-02 $3.252 E-02$ $5.727 E-02$ 6.095E-02 $6.567 E-02$ $2.957 E-01$ 3.060E-01 3.185E-01 $1.098 E-01$ 2.143E-01 2.195E-01 4.045E OI 1.064E O1 1.081E OI 4.051E OI 4.095E O1 4.143E OI $1.03 E$ O2 $1.103 E$ O2 $1.114 E$ O2 2.706E O2 2.726E O2 2.748E O2 1.430 E 02 6.905E 02 6.954E O2

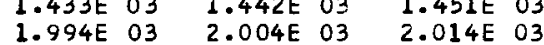
$1.379 \mathrm{E}-01$

\section{ANGLE 16}

$M U=0.9446$ $1.096 \mathrm{E}-05$
$8.412 \mathrm{E}-05$ 4. $162 \mathrm{E}-04$ $1.852 \mathrm{E}-03$ 
(NEUTRONS/MEV/STERADIAN/SOURCE NEUTRON)

ENERGY GROUP (MEV) $1.22 E$ O1- 1.50E $1.00 E$ 01---1.22E 01 $8.19 E$ OO- - 1.00E 01 $6.36 \mathrm{E} \quad 00---8.19 \mathrm{E} \quad 00$ $4.97 E$ OO- $-6.36 E$ EO 4.07E $00---4.97 E$ OO $2.46 E$ OO- $-3.01 E$ O $2.35 \mathrm{E} 00--2.46 \mathrm{E} 00$ $1.83 \mathrm{E} 00--2.35 \mathrm{E} 00$ $1.11 \mathrm{E} 00--1.83 \mathrm{E} 00$ $5.50 E-01--1.11 \mathrm{E} 00$ $1.11 \mathrm{E}-01---5.50 \mathrm{E}-01$ $3.35 \mathrm{E}-02--1.11 \mathrm{E}-01$ $5.83 E-04---3.35 E-02$ $1.01 E-04---5.83 E-04$ $2.90 \mathrm{E}-05-=-1.01 E-04$ $1.07 E-05--2.90 E-05$ $3.06 E-06---1.07 E-05$ $1.12 \mathrm{E}-06---3.06 \mathrm{E}-06$ $4.14 E-07--1.12 E-06$
0.0

ENERGY GROUP (MEV)

$1.22 E$ O1-- - 1.50E OI $1.00 E$ 01---1.22E 01 $8.19 E$ OO-- 1.00 E 6.36 E $00--8.19 E$ OO 4.97E 0O-- -6.36E OD 4.07E 00---4.97E 00 3.01E 00---4.07E OO $2.46 \mathrm{E} 00-3.01 \mathrm{E}$ OO $2.35 \mathrm{E} 00-2.46 \mathrm{E} 00$ 1.83 E $00-2.35 E$ OO $1.11 E$ OO- -1.83 E 00 5.50E $01-1.11 E$ OO $1.11 E-01--5.50 E-01$ $3.35 \mathrm{E}-02-2.11 \mathrm{E}-01$ ..83E-04-- $3.35 E-02$ $1.01 E-04--5.83 E-04$ 1.07E-05. $1.12 \mathrm{E}-06-3.06 \mathrm{E}-06$ $1.12 E-06-3.06 E-06$

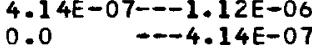

ANGLE 1 $M U=-1.0000$

ANGLE 2 政 $\begin{array}{ll}4.702 E & 03 \\ 6.652 E & 03\end{array}$ ANGLE 10 $\begin{aligned} M U & =0.0950 \\ 8.506 E-08 & \end{aligned}$ $6.118 E-07$ $9.174 \mathrm{E}-06$ $6.030 E-05$ $3.389 \mathrm{E}-04$ $5.801 \mathrm{E}-04$ $1.890 \mathrm{E}-03$ $3.108 E-03$ $5.679 E-03$ $7.878 \mathrm{E}-03$ $1.699 \mathrm{E}-02$ $1.782 E-02$ $1.482 E-01$ . . 1.304E 02 1.304E 02 . $2.375 E$ E $.021 \mathrm{E} 03$ $7.043 E \quad 03$
ANGLE 3 $M U=-0.9446$ $-3.478 E-08$
$-1.199 E-07$ $1.199 \mathrm{E}-07$
$1.477 \mathrm{E}-07$ $1.219 \mathrm{E}-05$ $1.021 E-04$ $4.298 E-04$ $1.048 \mathrm{E}-03$ $4.076 E-03$ 6.112E-03 $6.248 E-03$ $1.309 \mathrm{E}-02$ 3.595E-02 $8.586 E-02$ $6.024 E-01$ 5.070E-01 2.873E 01 $1.187 E \quad 02$ $3.336 \mathrm{E} \mathrm{O2}$ $8.558 E$ O2 $2.219 E \quad 03$ $4.717 E 03$ $6.672 \mathrm{E} 03$

ANGLE 4 $M U=-0.8656$ $8.453 \mathrm{E}-08$ $1.604 E-06$ $1.350 \mathrm{E}-05$ $9.877 E-05$ $4.586 \mathrm{E}-04$ $4.586 \mathrm{E}-04$
$1.062 \mathrm{E}-03$ 4.024E-03 4. $987 \mathrm{E}-03$
$6.3800 \mathrm{E}-03$ $6.300 E-03$ $1.341 E-02$ $3.667 \mathrm{E}-02$ $8.764 \mathrm{E}-02$ $6.100 \mathrm{E}-01$ $5.117 E-01$ $2.895 E$ OI $1.195 \mathrm{E} 02$ $3.356 E$ E2 $8.604 E$ O2 $2.230 \mathrm{E} 03$ $4.739 \mathrm{E}$
6.703

ANGLE 5 $2.771 E-08$ $3.823 E-07$ $2.439 E-06$ $1.455 E-05$
$1.008 E-04$ $1.008 E-04$
$4.951 E-04$ $4.951 E-04$
$1.082 \mathrm{E}-03$ $1.082 \mathrm{E}-03$
$3.957 \mathrm{E}-03$ $3.957 \mathrm{E}-03$
$5.877 \mathrm{E}-03$ $6.436 \mathrm{E}-03$ $6.436 E-03$
$1.393 E-02$ $3.781 \mathrm{E}-02$ $9.026 E-02$ $6.209 E-01$ $5.183 E-01$ $2.926 \mathrm{E}$ OI $1.206 E$ O2 $3.384 E$ O2 $8.669 E$ O2 4.246E O3 $6.739 \mathrm{E} 03$ $6.656 \mathrm{E} 03$ ANGLE 12 $U=0.2816$ $8.190 \mathrm{E}-08$ $1.761 E-06$
$1.005 E-05$ $6.452 \mathrm{E}-05$ $3.571 \mathrm{E}-04$ $9.839 \mathrm{E}-04$ 1. $531 E-03$ $7.114 E-03$ $1.011 \mathrm{E}-02$ $1.327 E-02$ $3.190 \mathrm{E}-02$ $6.344 \mathrm{E}-02$ $1.152 E-01$ $6.631 E-01$ $6.043 E-01$ $3.285 E$ OI $1.329 E$ OL $3.692 E \mathrm{E}$ . 3507 OS $5.082 \mathrm{E} 03$ $7.116 \mathrm{E} \quad 03$ $M U=0.4580$ $1.591 \mathrm{E}-07$ $1.056 \mathrm{E}-05$ $6.409 \mathrm{E}-05$ $6.409 E-05$
$3.574 E-04$ $1.446 \mathrm{E}-03$ $2.789 \mathrm{E}-03$ $5.481 E-03$ $1.505 \mathrm{E}-02$ $1.839 \mathrm{E}-02$ $2.490 E-02$ $7.304 \mathrm{E}-0$ $1.550 E-01$ $8.3173 E-01$ 6. .362E 01 $1.354 E$ E $9.494 \mathrm{E}$ $438 E$ $5.141 \mathrm{E} 03$ $7.187 E_{03}$ $M U=0.6179$ ANGLE 14 $M U=0.7550$ $1.573 \mathrm{E}-07$ $3.322 E-06$
$2.262 E-05$ $1.402 \mathrm{E}-04$ $7.573 \mathrm{E}-04$ $1.291 \mathrm{E}-03$ 3.377 E-0 $1.138 \mathrm{E}-02$ $1.360 \mathrm{E}-02$ $4.796 \mathrm{E}-02$ $4.796 E-02$ $1.663 \mathrm{E}-01$ $8.706 \mathrm{E}-01$ $6.366 \mathrm{E}-01$ $3.440 E$ OL $3.813 E$ O2 3.813E O2 $9.622 E$ O2 $2.467 E$ O 7.255 E O3
ANGLE 6 2. $984 E-08$ $3.917 E-07$ 1.591E-05 $1.129 \mathrm{E}-04$ $5.380 \mathrm{E}-04$ $1.113 \mathrm{E}-03$ $3.895 E-03$ $3.895 E-03$
$5.840 E-03$ $6.711 E-03$ $6.711 E-03$
$1.471 E-02$ $3.947 \mathrm{E}-02$
$9.385 \mathrm{E}-02$ $6.353 E-01$ $5.271 E-01$ $2.967 \mathrm{E} 01$ $1.220 \mathrm{E} \mathrm{O2}$ $3.421 \mathrm{E} O 2$ $8.752 E$ O2 $2.265 \mathrm{E} 03$ $6.787 \mathrm{E}_{03}$ ANGLE 15
$5.207 E-06$

$3.134 E-05$
$1.999 E-04$

$1.999 E-04$
$9.930 E-04$

$2.979 E-03$

$6.104 E-03$ $1.317 E-02$ 3.300E-02 $4.418 E-02$ $9.342 E-02$ $8.411 E-01$ $6.614 \mathrm{E}-01$ $3.514 E$ OI $1.402 E$ O2 $3.867 E$ O2 $9.736 E$ O2 $5.246 E 03$ $7.313 E \quad 03$
ANGLE 7 ANGLE 8

$8.819 \mathrm{E}-09$
$2.459 \mathrm{E}-07$ $2.459 \mathrm{E}-07$ $2.295 E-06$ $1.909 E-05$ $1.381 E=04$
$5.882 E-04$ $5.882 E-04$
$1.161 E-03$ $1.161 E-03$
$3.872 E-03$ $5.946 E-03$ $7.186 E-03$ $1.583 E-02$ $4.177 \mathrm{E}-02$ $9.852 E-02$ $6.535 \mathrm{E}-01$ $3.016 \mathrm{E}$ O1 1.237E 02 $3.464 E \quad 02$ $\begin{array}{lll}8.852 E & 02 \\ 2.289 E & 03\end{array}$ 4.285E 03 $\begin{array}{ll}4.853 E & 03 \\ 6.842 E & 03\end{array}$ ANGLE 16 $1.889 \mathrm{E}-07$
$2.704 \mathrm{E}-06$ $2.704 E-06$ $2.763 E-05$ $6.484 E-04$ $1.233 E-03$ $3.940 E-03$ $6.274 E-03$ $7.928 \mathrm{E}-03$ $1.736 E-02$ $4.491 E-02$ $1.045 E-01$ $6.757 E-01$
$5.506 E-01$ 3.073E OI 
(NEUTRONS/MEV/STERADIAN/SOURCE NEUTRON)

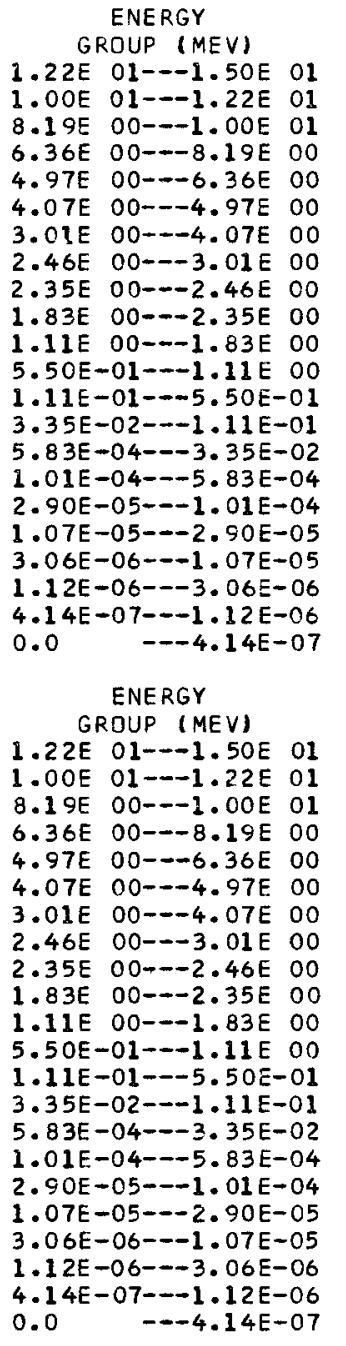

\begin{tabular}{|c|c|c|c|}
\hline ANGLE 1 & ANGLE 2 & ANGLE $\quad 3$ & ANGLE \\
\hline 0000 & $M U=-0.9894$ & $M U=-0.9446$ & $M U=-0.8656$ \\
\hline $3 E-08$ & $-7.601 E-08$ & $-2.801 E-08$ & $7.834 E-09$ \\
\hline & $-4.237 E-07$ & $-8.925 E-08$ & $1.066 \mathrm{E}-0$ \\
\hline & $-5.065 E-07$ & & $1.617 E-0$ \\
\hline $2 E-05$ & $1.083 E-05$ & $1.185 E-05$ & $1.314 E-05$ \\
\hline $043 E-04$ & $1.034 \mathrm{E}-04$ & $1.003 E-04$ & 9.76 \\
\hline $038 \mathrm{E}-04$ & $4.079 E-04$ & $4.238 \mathrm{E}-04$ & $4.515 \mathrm{E}-04$ \\
\hline $.960 E-04$ & $9.973 E-04$ & $1.003 E-03$ & $1.014 \mathrm{E}-03$ \\
\hline $3.744 E-03$ & $3.738 E-03$ & $3.714 \mathrm{E}-03$ & $3.669 E-03$ \\
\hline $694 E-03$ & $5.677 E-03$ & $5.619 \mathrm{E}-03$ & $5.536 E-03$ \\
\hline $230 E-03$ & $6.234 E-03$ & $6.261 E-03$ & $6.339 E-03$ \\
\hline $331 E-02$ & $1.335 E-02$ & $1.351 E-02$ & $1.386 E-02$ \\
\hline $.828 \mathrm{E}-02$ & $3.838 \mathrm{E}-02$ & $3.880 E-02$ & $3.966 E-02$ \\
\hline & $9.799 \mathrm{E}-02$ & $9.906 \mathrm{E}-02$ & \\
\hline $7.576 \mathrm{E}-01$ & $7.588 E-01$ & $7.639 \mathrm{E}-01$ & $7.736 E-01$ \\
\hline $.697 \mathrm{E}-01$ & $6.705 E-01$ & $6.740 \mathrm{E}-01$ & $E-01$ \\
\hline $.961 E 01$ & $3.965 E \quad 01$ & $3.983 E$ & $4.016 \mathrm{E}$ \\
\hline $1.681 \mathrm{E} \quad 02$ & $1.682 \mathrm{E} \quad 02$ & $1.689 E$ & $1.702 E \quad 02$ \\
\hline $4.815 E \quad 02$ & $4.819 E \quad 02$ & $4.838 \mathrm{E}$ & $4.872 E \quad 02$ \\
\hline $1.260 \mathrm{E} \quad 03$ & $1.261 \mathrm{E} 03$ & $1.265 E$ & $1.273 E \quad 03$ \\
\hline $3.310 E$ O3 & $3.313 \mathrm{E} \quad 03$ & $3.323 \mathrm{E}$ & $3.343 E \quad 03$ \\
\hline $7.113 \mathrm{E} \quad 03$ & $7.119 \mathrm{EE} 03$ & $7.140 E$ & $7.180 E \quad 03$ \\
\hline $1.017 E \quad 04$ & $1.018 \mathrm{E} \quad 04$ & $1.021 \mathrm{E} 04$ & $1.026 \mathrm{E} 04$ \\
\hline ANGLE 10 & ANGLE 11 & ANGLE 12 & ANGLE 13 \\
\hline & $M U=0.2816$ & $M U=0.4580$ & .6179 \\
\hline $3.897 E-08$ & $1.095 E-07$ & $1.278 E-07$ & $1.877 \mathrm{E}-$ \\
\hline $1.042 E-06$ & $1.301 \mathrm{E}-06$ & $2.044 E-06$ & $2.656 \mathrm{E}-06$ \\
\hline $850 F-06$ & $1.100 E-05$ & $1.219 E-05$ & $1.945 E-05$ \\
\hline & $6.929 E-C$ & & \\
\hline $12 E-$ & $3.793 \mathrm{E}-\mathrm{C}$ & $4 \cdot 322 E-04$ & $6.823 E-04$ \\
\hline & $8.43 \% E-Q$ & $1.302 E-03$ & \\
\hline $1.277 E-03$ & $1.975 \mathrm{E}-0$ & $2.266 \varepsilon-03$ & \\
\hline $5.258 E-03$ & $4.634 E-$ & $7.591 E-03$ & $16 E-03$ \\
\hline & 8.8 & & \\
\hline $1.247 E-02$ & $1.210 E-02$ & $1.792 \mathrm{E}-02$ & $2.106 \mathrm{E}-02$ \\
\hline $2.614 \mathrm{E}-02$ & $2.463 E-02$ & $3.499 E-02$ & $3.903 E-02$ \\
\hline & $6.530 \mathrm{E}-02$ & $8.280 E-02$ & $9.391 \mathrm{E}-02$ \\
\hline $2 E-01$ & $1.646 \mathrm{E}-01$ & $1.566 \mathrm{E}-01$ & $1.957 \mathrm{E}-01$ \\
\hline $7.915 \mathrm{E}-\mathrm{C}$ & $1.060 \mathrm{E}$ & $9.470 \mathrm{E}-01$ & \\
\hline $1.0505-01$ & $7.967 E-01$ & $8.308 \mathrm{E}-01$ & $8.506 \mathrm{E}-01$ \\
\hline $4.494 \mathrm{E} \quad 01$ & $4.609 E \quad 01$ & $4.727 E \quad 01$ & $4.844 E \quad 01$ \\
\hline $1.879 E \quad 02$ & $1.921 \mathrm{EO2}$ & & \\
\hline $5.339 E \quad 02$ & $5.446 E \quad 02$ & $5.553 \mathrm{E}$ & $5.657 \mathrm{E} \quad 02$ \\
\hline $1.383 \mathrm{E} \quad 03$ & $1.408 E 03$ & 1.43 & $1.456 E 03$ \\
\hline $3.610 E \quad 03$ & $3.668 \mathrm{E} 03$ & $3.727 E$ & $3.782 \mathrm{E}_{03}$ \\
\hline $7.712 \mathrm{E} \quad 03$ & $7.828 E \quad 03$ & $7.942 E$ & $8.050 E \quad 03$ \\
\hline $1.091 \mathrm{E} 04$ & $1.106 \mathrm{E} 04$ & $1.120 \mathrm{E}$ & $1.133 E 04$ \\
\hline
\end{tabular}

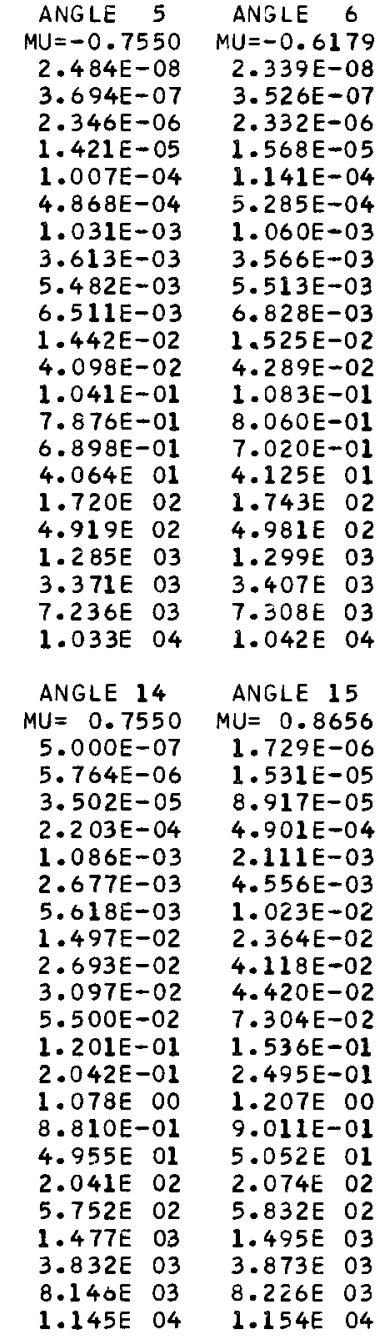
ANGLE 8

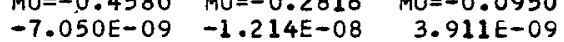
$2.028 E-07 \quad 1.565 E-07 \quad 3.908 E-07$ $2.104 E-06 \quad 2.541 E-06 \quad 4 .<32 E-06$ i. $907 \mathrm{E}-05 \quad 2.591 \mathrm{E}-05 \quad 3.647 \mathrm{E}-05$ $1.404 \mathrm{E}-04 \quad 1.793 \mathrm{E}-04 \quad 2.277 \mathrm{E}-04$ $5.776 E-04 \quad 6.369 E-04 \quad 7.112 E-04$ $1.104 \mathrm{E}-03 \quad 1.175 \mathrm{E}-03 \quad 1.287 \mathrm{E}-03$ $3.562 E-03 \quad 3.653 E-03 \quad 3.910 E-03$ $5.695 E-03 \quad 6.104 E-03 \quad 6.843 E-03$ $7.353 E-03 \quad 8.155 E-03 \quad 9.327 E-03$ $1.643 E-02 \quad 1.804 E-02 \quad 2.022 E-02$ 4.553E-02 4.909E-02 5.385E-02 $1.136 \mathrm{E}-01 \quad 1.203 \mathrm{E}-01 \quad 1.286 \mathrm{E}-01$ $8.289 E-01 \quad 8.564 E-01 \quad 8.880 E-01$ $4.200 E-01 \quad 4.348 E$ O1 $4.547 E-01$

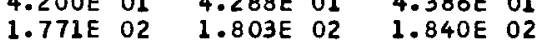
$\begin{array}{lllllll}5.055 E & 02 & 5.141 \mathrm{E} & 02 & 5.236 E & 02\end{array}$

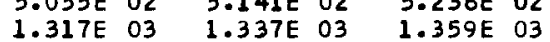
$\begin{array}{llllll}3.450 E & 03 & 3.498 E & 03 & 3.552 E & 03\end{array}$

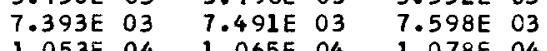
$1.053 E 04$ ANGLE 16 SCALAR $6.373 E-06 \quad 9.647 E-05$ $5.157 E-05 \quad 7.850 E-04$ $2.824 E-04 \quad 4.241 E-03$ $1.343 \mathrm{E}-03 \quad 1.763 \mathrm{E}-02$ 4.849E-03 5.388E-02 $9.861 E-03 \quad 7.763 E-02$ 2.093E-02 $1.559 E-01$ 4.963E-02 3.902E-01 $8.157 \mathrm{E}-02 \quad 6.110 \mathrm{E}-01$ $7.799 E-02 \quad 4.677 E-01$
$1.180 E-01$ $1.180 \mathrm{E}-01 \quad 4.581 \mathrm{E}-01$ $\begin{array}{ll}2.186 E-01 & 6.572 E-01 \\ 2.902 E-01 & 4.627 E-01\end{array}$ $2.902 E-01$ $1.211 \mathrm{E} 00$ $9.232 \mathrm{E}-01$ $5.129 E$ OI $\begin{array}{ll}2.098 \mathrm{E} & 02 \\ 5.892 \mathrm{E} & 02\end{array}$ $\begin{array}{ll}5.892 E & 02 \\ 1.508 E & 03\end{array}$ 1.508E 03 $\begin{array}{ll}3.903 E & 03 \\ 8.285 E & 03\end{array}$ $1.161 E 04$ 
ENERGY GROUP (MEV)

$1.22 \mathrm{E} 01---1.50 \mathrm{E} 01$

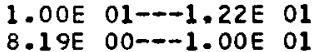
6.36E $00--8.19 E$ OO 4.97E $00--6.36 \mathrm{E} 00$ 4.07E 00--4.97E 00 3.01E 00---4.07E 00 $2.46 \mathrm{E}$ 00---3.01E 00 $2.35 E$ 00-- 2.46 E 00 $1.83 \mathrm{E} \quad 00---2.35 \mathrm{E} 00$ $1.11 \mathrm{E} 00--1.83 \mathrm{E} 00$ $5.50 E-01--1.11 \mathrm{E} 00$ $1.11 \mathrm{E}-01--5.50 \mathrm{E}-01$ $3.35 \mathrm{E}-02-0-1.11 \mathrm{E}-01$ $5.83 E-04--3.35 E-02$ $1.01 E-04--5.83 E-04$ 2.90E-05--1.01E-04 $1.07 E-05--2.90 \mathrm{E}-05$ $3.06 E-06--1.07 E-05$ $1.12 \mathrm{E}-06-0-3.06 \mathrm{E}-06$ $0.0 \quad \cdots-14 \mathrm{E}-07$

ENERGY

GROUP (MEV)

$1.22 \mathrm{E} 01--1.50 \mathrm{E} 01$ $1.00 \mathrm{E}$ 01-- $1.22 \mathrm{E}$ 01

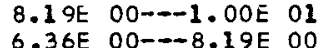

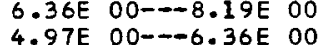

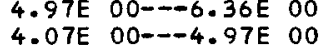

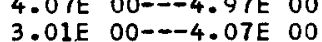
3.01E $00--4.07 E$ EO $2.46 \mathrm{E} 00--3.01 \mathrm{E} 00$

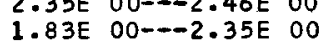

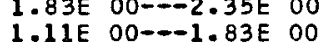
5.50E-01- $-1.11 \mathrm{E} 00$ $1.11 \mathrm{E}-01=-5.50 \mathrm{E}-01$ $3.35 \mathrm{E}-02=-1.11 \mathrm{E}-01$ $5.83 E-04=-3.35 E-02$ $1.01 \mathrm{E}-04--5.83 \mathrm{E}-04$ 2. $90 \mathrm{E}-05--1.01 \mathrm{E}-04$ 1. $07 \mathrm{E}-05--2.90 \mathrm{E}-05$ $3.06 \mathrm{E}-06---1.07 \mathrm{E}-05$ $1.12 \mathrm{E}-06--3.06 \mathrm{E}-06$ $4.14 E-07--1.12 E-06$ $0.0 \quad--4.14 E-07$
ANGLE 1 $M U=-1.0000$ $5.970 \mathrm{E}-08$ $-3.629 E-07$
$-6.496 E-07$ $6.496 \mathrm{E}-07$
$8.775 \mathrm{E}-06$ $8.775 \mathrm{E}-06$
$8.844 \mathrm{E}-05$ $8.844 \mathrm{E}-05$
$3.448 \mathrm{E}-04$ $3.448 E-04$
$8.036 E-04$ $2.762 \mathrm{E}-03$ $4.246 \mathrm{E}-03$ $5.365 \mathrm{E}-03$ $1.190 \mathrm{E}-02$ $3.659 E-02$ $1.032 \mathrm{E}-\mathrm{Cl}$ $9.075 E-01$ $8.463 E-01$ $5.271 E 01$ $2.316 \mathrm{E} 02$ $6.814 E 02$ $1.836 \mathrm{E} 03$ $4.930 E \quad 03$ $1.080 E 04$ 1.573E 04 ANGLE 10 $M U=0.0950$ $4.693 \mathrm{E}-08$ $7.005 E-07$ $6.285 E-06$ $4.594 \mathrm{E}-05$ $2.606 E-04$ $6.533 \mathrm{E}-04$ $1.256 E-03$ $3.364 E-03$ $6.720 E-03$ $9.600 \mathrm{E}-03$ $1.998 E-02$ $5.776 \mathrm{E}-02$ $1.523 \mathrm{E} 01$ $9.835 E-01$ $6.058 \mathrm{E}$ O $6.058 E$ OI $7.697 \mathrm{E} 02$ $2.058 E$ O3 2.058 03 $1.196 \mathrm{E} 04$ $1.723 E 04$
(NEUTRONS/MEV/STERADIAN/SOURCE NEUTRON)

$\triangle A N G L E 2$ 作 $\quad M U=-0.9446 \quad M U=-0.8656$ $-2.815 \mathrm{E}-07 \quad-1.655 \mathrm{E}-08 \quad 3.332 \mathrm{E}-09$ $-3.346 E-07$ $9.034 E-06$ $8.773 E-05$ $3.481 E-04$
$8.042 \mathrm{E}-04$ $3.670 \mathrm{E}-02$ $1.035 \mathrm{E}-01$ $9.090 E-01$ $8.474 E-01$ $5.277 E$ O1 6.319E 02 $6.822 \mathrm{E} \mathrm{O2}$
$1.838 \mathrm{E} \mathrm{O3}$ $4.935 \mathrm{E} 03$ $1.081 E 04$ $1.575 \mathrm{E} 04$ ANGLE 11 $M U=0.2816$ $8.067 \mathrm{E}-08$
$1.225 \mathrm{E}-06$ $8.406 \mathrm{E}-06$ $5.633 E-05$ $3.081 \mathrm{E}-04$ $8.458 E-04$ $1.442 \mathrm{E}-03$ $4.595 \mathrm{E}-03$ $9.102 E-03$ $1.250 E-02$
$2.509 E-02$ $2.509 E-02$ 1.545E-01 $1.545 E-01$ $1.023 E 00$ $1.023 E$ OD $2.226 E 01$ $7.880 E$ O2 $2.803 E$ O3 5.605E 03 $1.210 E 04$ $1.753 E 04$ ANG LE 5
$M U=-0.7550$ $M U=-0.7550$ $2.739 E-08$ $2.854 \mathrm{E}-07$
$1.856 \mathrm{E}-06$ $1.856 \mathrm{E}-06$ $8.851 E-05$ $2.898 E-07$ $9.892 \mathrm{E}-06 \quad 1.1$ $8.561 \mathrm{E}-05 \quad 8.440 \mathrm{E}-05$ $3.610 E-04$ $8.070 E-04$ -14
2 . $418 \mathrm{E}-03$ $3.716 \mathrm{E}-02$ $9.154 \mathrm{E}-01$ $8.522 \mathrm{E}-01$ $5.304 \mathrm{E} 01$ $2.330 \mathrm{E} \mathrm{O2}$ $6.852 \mathrm{E} 02$ $1.846 E 03$ 4.955E 03 $\begin{array}{ll}1.085 \mathrm{E} & 04 \\ 1.580 \mathrm{E} & 04\end{array}$ ANGLE 12 $M U=0.4580$
$1.100 E-07$ $1.100 E-07$
$1.566 E-06$ $1.566 E-06$
$1.153 E-05$ $1.153 E-05$
$7.689 E-05$ $7.689 \mathrm{E}-05$
$4.188 \mathrm{E}-04$ $1.019 \mathrm{E}-03$ $2.033 \mathrm{E}-03$ $5.717 E-03$ $1.153 \mathrm{E}-02$ $1.510 \mathrm{E}-02$ $2.875 E-02$ 1.772E-01 $1.245 E$ E 00 $1.051 \mathrm{E} 00$ $6.399 E$ O1 2.765E O2 $2.149 E \quad 03$ $5.718 \mathrm{E} 03$ $1.243 E 04$ 1.782E 04
ANGLE 6 $\begin{aligned} M U & =-0.6179 \\ 1.162 \mathrm{E}-08 & \end{aligned}$ $1.162 \mathrm{E}-08$
$2.450 \mathrm{E}-07$ $1.450 E-07$
$1.778 E-06$ $1.347 \mathrm{E}-05$ $1.347 \mathrm{E}-05$
$1.017 \mathrm{E}-04$ $1.017 E-04$
$8.470 E-04$ $8.463 E-04$ $2.671 E-03$ $4.362 \mathrm{E}-03$ $6.044 \mathrm{E}-03$ $1.372 \mathrm{E}-02$ $4.146 \mathrm{E}-02$ $1.144 \mathrm{E}-01$ $9.675 \mathrm{E}-01$ $8.896 \mathrm{E}-01$ $5.514 E$ OI $2.415 E$ O2 $7.092 \mathrm{E} 02$ $1.906 \mathrm{E} \mathrm{O3}$ $5.109 \mathrm{E} 03$ $1.623 \mathrm{E} 04$

ANGLE 15 $M U=0.8656$ $1.380 \mathrm{E}-06$ $1.316 E-05$ $7.877 E-05$ 4. $545 \mathrm{E}-04$ $1.895 \mathrm{E}-03$ $3.9 \in 1 \mathrm{E}-03$ $7.952 E-03$ $2.080 \mathrm{E}-02$ $3.872 E-02$ $3.984 E-02$ $6.260 \mathrm{E}-02$ $1.436 E-01$ $2.373 E-01$ $1.367 E$ OD 1.867E $2.867 \mathrm{E}$ O1 2.943E 02 $2.266 \mathrm{E} 03$ $6.007 E$ O3 $6.301 \mathrm{E} 04$ 1.856 E 04
ANGLE 7 $M U=-0.4580 \quad M U=-0.2816$ $M U=-0.2816$ $-8.810 \mathrm{E}-09$
$1.069 \mathrm{E}-07$ $2.039 E-06$ $2.314 E-05$ $2.314 E-05$ $5.379 \mathrm{E}-04$ $9.409 E-04$ $2.813 E-03$ $5.079 \mathrm{E}-03$ $7.325 E-03$ $1.626 \mathrm{E}-02$ $4.781 E-02$ $1.271 \mathrm{E}-01$ $1.030 \mathrm{E} 00$ $9.329 E-01$ $5.753 E$ O1 $2.511 E \quad 02$ $7.361 \mathrm{E} 02$ $1.974 \mathrm{E} 03$ $5.281 E \quad 03$ $1.153 E \quad 04$ $1.668 E \quad 04$ ANGLE 17 $\begin{aligned} & M U=0.9894 \\ & 5.401 E-05\end{aligned}$ $4.604 E-04$ $2.666 \mathrm{E}-03$ $1.129 \mathrm{E}-02$ $3.315 E-02$ $4.584 E-02$ $8.165 E-02$ $2.498 E-01$ $4.272 E-01$ $2.814 E-01$ $2.492 E-01$ $4.041 E-01$ $1.456 E-00$ $1.456 E 0$ 1.181E 00 $3.005 E 02$ . $2.306 E 03$ 6.103E 03 $6.320 E$ OS $1.878 \mathrm{E} 04$

ANGLE ? $M U=-0.0950$ $5.546 \mathrm{E}-09$ $3.586 E-06$ $3.275 E-05$ $2.035 \mathrm{E}-04$ $6.012 E-04$ $1.038 \mathrm{E}-03$ $3.075 E-03$ $5.830 \mathrm{E}-03$ 
(NEUTRONS/MEV/STERADIAN/SOURCE NEUTRON)

ENERGY GROUP (MEV) $1.22 \mathrm{E} 01--1.50 \mathrm{E}$ OI $1.00 E$ O1-- 1.22E 01 $8.19 E$ OD-- 1.00E 01 6.36E $00--8.19 \mathrm{E}$ OO $4.07 E$ OO- $-4.36 \mathrm{EE}$ OO $3.01 E$ OO- $-4.07 E$ OO $2.46 \mathrm{E} 00--3.01 \mathrm{E} 00$ $2.35 \mathrm{E} 00-02.46 \mathrm{E}$ 00 $1.83 \mathrm{E} 00--2.35 \mathrm{E} 00$ $1.11 \mathrm{E} 00-\cdots 1.83 \mathrm{E} 00$ $5.50 \mathrm{E}-01--1.11 \mathrm{E}$ OO $1.11 \mathrm{E}-01--5.50 \mathrm{E}-01$ $3.35 \mathrm{E}-02--1.11 \mathrm{E}-01$ $5.83 \mathrm{E}-04--3.35 \mathrm{E}-02$ $1.01 E-04--5.83 E-04$ $2.90 \mathrm{E}-05---1.01 \mathrm{E}-04$ $1.07 E-05--2.90 E-05$ $3.06 \mathrm{E}-06--1.07 \mathrm{E}-05$ $1.12 \mathrm{E}-06--3.06 \mathrm{E}-06$ $4.14 \mathrm{E}-07--1.12 \mathrm{E}-06$
0.0
$---4.14 \mathrm{E}-07$

$$
\text { ENERGY }
$$$$
\text { GROUP (MEVI }
$$

$1.22 \mathrm{E}$ O1---1.50E 01 $1.00 \mathrm{E}$ 01---1.22E OI $8.19 E$ OO---1.00E OI $6.36 \mathrm{E}$ OO- $-8.19 \mathrm{E}$ OO $4.97 \mathrm{E}$ OO- $-6.36 \mathrm{E}$ OO 4.07E $00--4.97 E$ OO 3.01E $00--4.07 E$ OO $2.46 \mathrm{E}$ 00-- $3.01 \mathrm{E} 00$ $2.35 E$ EO-D-2.46E 00 $1.83 \mathrm{E} 00--2.35 \mathrm{E} 00$ $1.11 E$ OO-C-1.83E 00 $5.50 \mathrm{E}-01--1.11 \mathrm{E} 00$ $1.11 \mathrm{E}-01-2.50 \mathrm{E}-01$ $3.35 \mathrm{E}-02--1.11 \mathrm{E}-01$ $5.83 E-04-C-35 E-02$ $1.01 \mathrm{E}-04--5.83 \mathrm{E}-04$ 2.90E-05- - $1.01 E-04$ 3.06E-06-1. $07 \mathrm{E}-05$ $1.12 \mathrm{E}-06-1.06 \mathrm{E}-06$ $1.12 E-06-2.06 E-06$ $4.14 E-07--1.12 E-06$
ANGLE 1 $-3.594 \mathrm{E}-08$ - $2.274 E-07$ $4.263 \mathrm{E}-07$ $6.735 \mathrm{E}-06$ $6.902 E-05$ $2.689 E-04$ $5.948 E-04$ $1.889 \mathrm{E}-03$ $2.936 \mathrm{E}-03$ $3.045 E-02$ $.223 E-02$ $8.798 \mathrm{E}-01$ $5.433 E$ OI $2.438 E 02$ $7.295 E$ O2 2.006 E 03 $5.469 E$ E3 $\begin{array}{ll}1.215 \mathrm{E} & 04 \\ 1.797 \mathrm{E} & 04\end{array}$ ANGLE 10 $M U=0.0950$ $3.329 E-08$
$5.648 E-07$ $5.648 \mathrm{E}-07$ $4.675 \mathrm{E}-06$
$3.591 \mathrm{E}-05$ $2.025 \mathrm{E}-04$ $5.373 E-04$ $8.868 \mathrm{E}-04$ $2.678 \mathrm{E}-03$ $5.540 E-03$ $8.105 E-03$ $1.670 \mathrm{E}-02$ $1.254 E-01$ $1.254 E-01$ $1.062 \mathrm{E} 00$ $9.951 E-O 1$ $3.795 \mathrm{E} 02$ 2. $326 \mathrm{E}$ O2 2.326E 02 2.275E 03 1.171E O3

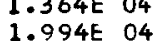

$-0.9894$ $2.930 \mathrm{E}-08$ $-1.755 E-07$ $-2.089 E-07$
$6.932 E-06$ $6.854 \mathrm{E}-05$ $0.854 \mathrm{E}-05$
$2.713 \mathrm{E}-04$ $5.950 E-04$ $2.950 E-04$ $2.937 \mathrm{E}-03$ $4.187 E-03$ $9.471 E-03$ $3.055 \mathrm{E}-02$ $9.248 \mathrm{E}-02$ $8.814 E-01$ 2.441E O2 . $5.475 \mathrm{E} 03$ $1.216 E$
$1.799 E \quad 04$ ANGLE 11 $M U=0.2816$ $6.124 \mathrm{E}-08$ $8.896 E-07$
$6.759 E-06$ $6.759 E-06$
$4.696 E-05$ $4.696 \mathrm{E}-05$ $2.547 E-04$ $6.367 E-04$ $1.116 \mathrm{E}-03$ $3.345 \mathrm{E}-03$ $7.035 \mathrm{E}-0$ $9.843 \mathrm{E}-03$ $1.929 E-02$ $5.682 E-02$ $1.422 \mathrm{E}-01$ $1.139 E$ OO 1.46 E 01 $2.469 E$ OI $2.331 \mathrm{E} 03$ $6.314 E \quad 03$ . $314 E$ E $2.034 E \quad 04$
ANGLE 3 $-9.367 E-09$ $-2.061 E-08$ $2.600 \mathrm{E}-07$
$7.593 \mathrm{E}-06$ $6.593 E-06$ $6.7 .24 \mathrm{E}-05$ $2.809 E-04$ $1.961 E-04$ $2.943 E-03$ 作 $9.603 \mathrm{E}-03$ $3.096 \mathrm{E}-02$ $9.352 \mathrm{E}-02$ $8.878 t-01$ $8.525 E-01$ $5.468 \mathrm{E} \mathrm{Ol}$ $2.453 \mathrm{E} \mathrm{O2}$ $7.339 \mathrm{E} 02$ $2.017 E \quad 03$ $5.499 \mathrm{E} \quad 03$

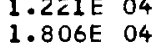

ANGLE 12 $M U=0.4580$ $7.909 \mathrm{E}-08$ $1.197 \mathrm{E}-06$
$8.894 \mathrm{E}-06$ $8.894 E-06$
$6.247 E-05$ $6.247 \mathrm{E}-05$
$3.363 \mathrm{E}-04$ $3.363 E-04$
$8.228 E-04$ $1.490 E-03$ 4.536E- 03 $9.489 \mathrm{E}-03$ $1.248 \mathrm{E}-02$ $2.321 \mathrm{E}-02$ $6.625 \mathrm{E}-02$ $1.538 E-01$ $1.173 E$ OO $6.055 \mathrm{E} O$ $6.655 E$ OI $2.945 E$ O2 $2.736 \mathrm{E}$ O3 $6.458 E$ O 3 $1.458 E$ O $1.425 E$ O
$M U=-0.8656$ $3.736 \mathrm{E}-09$ $8.375 \mathrm{E}-08$ $1.022 E-06$ $6.696 \mathrm{E}-05$ $2.978 \mathrm{E}-04$ $5.994 \mathrm{E}-04$ $1.867 \mathrm{E}-03$ $2.970 \mathrm{E}-03$ $4.334 E-03$ 9.871E-03 $3.177 \mathrm{E}-02$ SLIE-O $8.997 \mathrm{E}-01$ $8.615 E-01$ $5.522 \mathrm{E} 01$ $2.476 \mathrm{E}$ O2
$7.405 \mathrm{E} 02$ $2.035 \mathrm{E} \mathrm{O3}$ $5.545 E \quad 03$ $1.819 E$ O4

ANGLE 13 $M U=0.6179$ $1.239 \mathrm{E}-07$
$1.787 \mathrm{E}-06$ $1.330 \mathrm{E}-05$ $9.610 \mathrm{E}-05$ 4. $977 \mathrm{E}-04$ $1.153 \mathrm{E}-03$ $2.177 \mathrm{E}-03$ $6.466 \mathrm{E}-03$ $1.336 \mathrm{E}-02$ $1.621 \mathrm{E}-02$ $3.832 E-02$ 1.827E-02 $1.696 E-01$ 1.237E OO $6.838 \mathrm{E} 01$ $3.019 \mathrm{E} 02$ $8.968 \mathrm{E} 02$ $2.440 E \quad 03$ $1.454 E$ E 1.111E 04
ANGLE 5 ANGLE 6 1.7925 $M U=-0.6179$ $0.381 \mathrm{E}-09$
$.962 \mathrm{E}-08$ $1.334 E-06 \quad 1.563 E-07$ $9.309 E-06 \quad 1.063 E-06$ $7.107 E-05 \quad 8.232 E-05$ $3.195 \mathrm{E}-04 \quad 3.458 \mathrm{E}-04$ $6.069 E-04 \quad 0.458 E-04$ $1.856 E-03 \quad 1.859 E-03$ $3.041 \mathrm{E}-03 \quad 3.187 \mathrm{E}-03$ $4.511 \mathrm{E}-03 \quad 4.799 \mathrm{E}-03$ $1.092 \mathrm{E}-02$ $\begin{array}{ll}.301 E-02 & 3.476 E-02\end{array}$ $9.167 E-01$ $8.743 E-01 \quad 8.911 E-01$ $5.598 \mathrm{E}$ O1 $5.697 \mathrm{E}$ OI $2.508 E$ OL $2.549 E$ O2 $7.497 E$ O2 2.059E O3 $5.608 E 03$ $\begin{array}{ll}1.245 E & 04 \\ 1.838 E & 04\end{array}$ ANGLE 14 $M U=0.7550$ 3. $271 \mathrm{E}-07$ $2.636 E-05$ $1.786 E-04$ $8.300 E-04$
$1.794 E-03$ $1.794 \mathrm{E}-0$ $3.391 E-03$ $9.885 \mathrm{E}-03$
$1.999 \mathrm{E}-02$ $2.200 E-02$ $3.585 E-02$ $9.467 E-02$ $1.279 E 00$ $1.279 E$ E 1.122E 00 $7.007 E$ OI T. $9.160 E$ O2 6.48 6.721E 03 $1.480 E$ O

ANGLE 7 336E-09 $-5.231 E-09$ $7.397 E-08 \quad 7.670 E-08$ $1.133 \mathrm{E}-06 \quad 1.535 \mathrm{E}-06$ $1.346 \mathrm{E}-05 \quad 1.874 \mathrm{E}-05$ $1.021 \mathrm{E}-04$ 3. $772 E-04$ $6.478 E-04$ $1.899 \mathrm{E}-03$ $3.445 \mathrm{E}-03$ $5.236 E-03$ $1.178 \mathrm{E}-02$ 1.713E-02 ( .669E-01 $5.816 E$ O 
(NEUTRONS/MEV/STERADIAN/SOURCE NEUTRON)

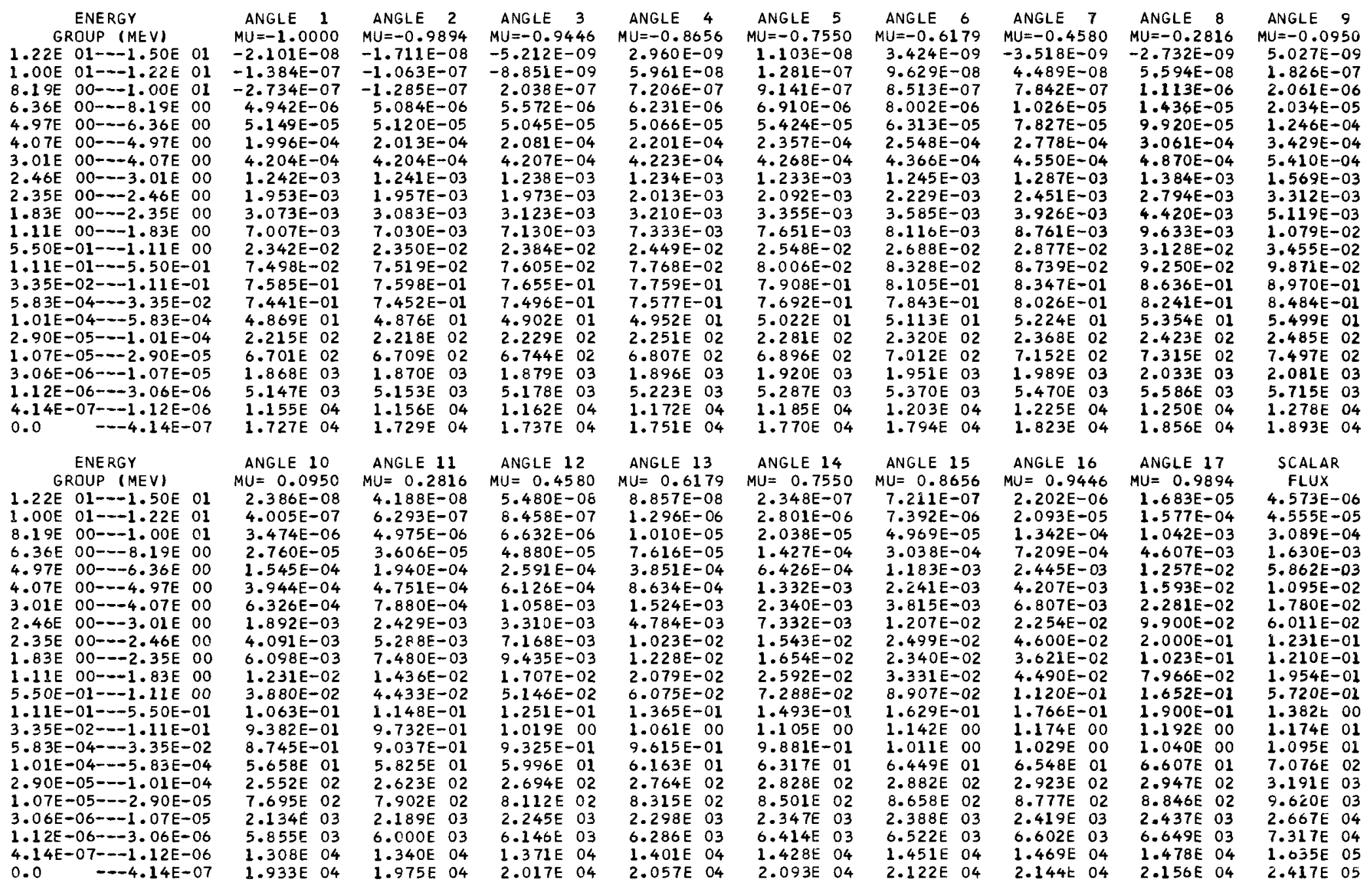


(NEUTRONS/MEV/STERADIAN/SOURCE INEUTRON)

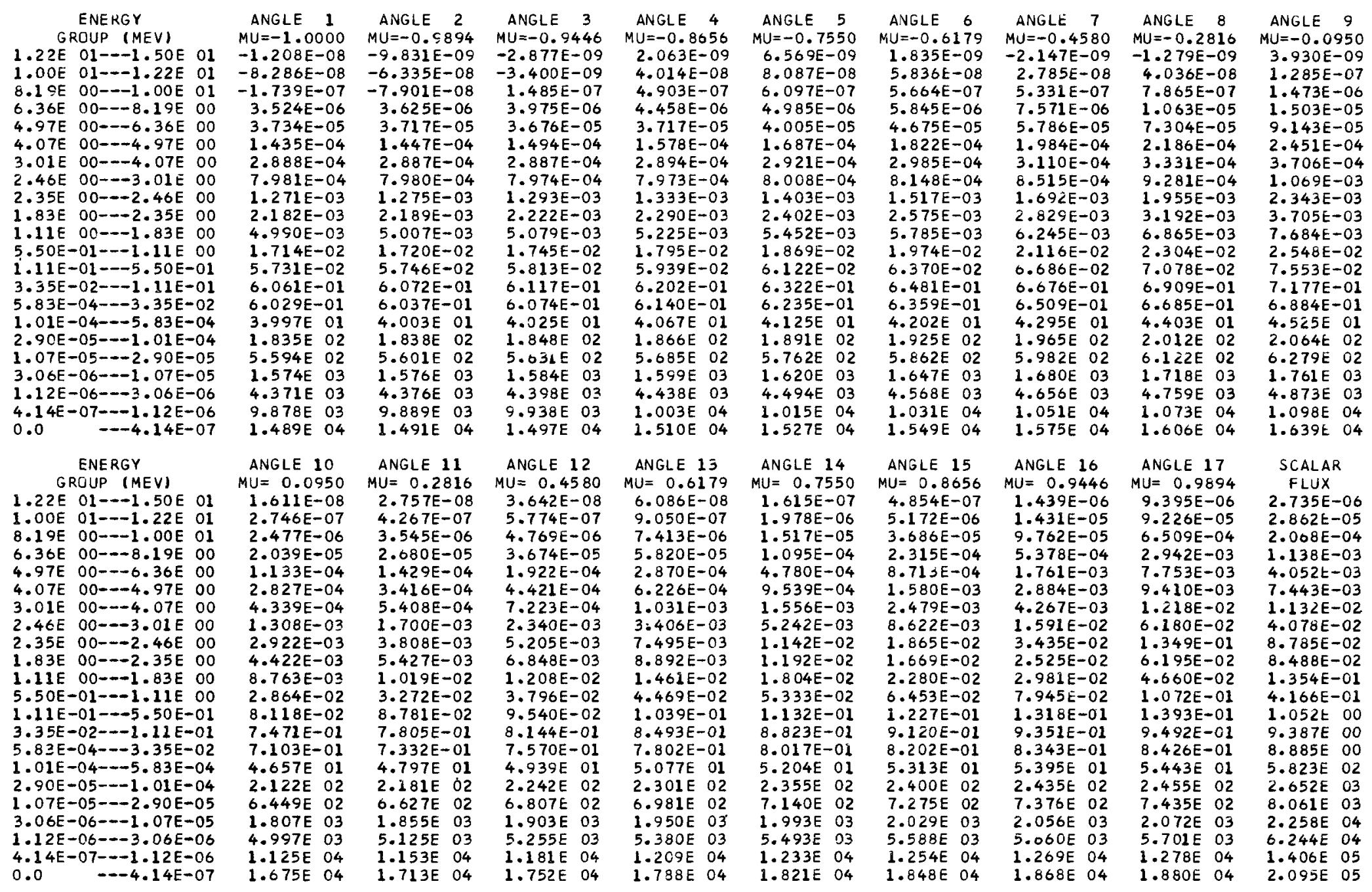


(NEUTRONS/MEV/STERADIAN/SOURCE NEUTRON)

ENERGY GROUP (MEV) $1.22 E$ O1-- $1.50 E$ E $1.00 \mathrm{E} 01---1.22 \mathrm{E} 01$ $8.19 \mathrm{E} 00-\cdots 1.00 \mathrm{E} \mathrm{O1}$ $--8.19 E$ OO 4.97E $00--6.36 \mathrm{E} \quad 00$ $4.07 \mathrm{E} 00--4.97 \mathrm{E} \quad 00$ $3.01 \mathrm{E} 00--4.07 \mathrm{E} 00$ $2.46 \mathrm{E} 00--3.01 \mathrm{E} 00$ $2.35 \mathrm{E} \quad 00--2.46 \mathrm{E} \quad 00$ $1.83 \mathrm{E} 00---2.35 \mathrm{E} 00$ $1.11 \mathrm{E} \quad 00---1.83 \mathrm{E} \quad 00$ $5.50 E-01--1.11 \mathrm{E} 00$ $1.11 \mathrm{E}-01-\cdots 5.50 \mathrm{E}-01$ $3.35 \mathrm{E}-02--1.11 \mathrm{E}-0$ $5.83 E-04--3.35 E-02$ $1.01 E-04--5.83 E-04$ $2.90 E-05--1.01 E-04$ $1.07 \mathrm{E}-05-2.90 \mathrm{E}-05$ $3.06 E-06--1.07 E-05$ $1.12 E-06--3.06 E-06$ $0.0 \quad--4.14 E-07$ ENERGY GROUP (MEV) $1.22 \mathrm{E} \mathrm{CI-D-1.50E} 01$ $1.00 \mathrm{E}$ 01-- $1.22 \mathrm{E}$ OI $8.19 \mathrm{GE}$ 00---1.00E 01 6.36 E $00--8.19 E$ OO 4.97E $00--6.36 \mathrm{E} 00$ $4.07 \mathrm{E}$ 00- $4.97 \mathrm{E} \mathrm{OO}$ $3.01 \mathrm{E} 00--4.07 \mathrm{E} 00$ 2.46E $00--3.01 E$ OO $2.35 \mathrm{E}$ 00--2.46E 00 $1.83 \mathrm{E} 00--2.35 \mathrm{E} 00$ 1.11E $00--1.83 E$ OO . 1 E $01-1.11 \mathrm{E} O 0$ 1.11E-01-D-5.50E-O1 $5.83 \mathrm{E}-04=-3.35 \mathrm{E}-02$ 1. 1. $0 E-05-1.01 E-04$ 2.9E $05-1.01 E-04$ $3.06 \mathrm{E}-06-0-1.07 \mathrm{E}-05$ 1.12E-06 $4.14 E-07-01.12 E-06$ $0.0 \quad---4.14 E-07$
ANGLE 1 $U=-1.0000$ $-2.194 E-09$
$-1.701 E-08$ $-4.433 E-08$ $1.290 E-05$ $4.783 E-05$ $1.568 E-03$ .645E-03 2.061E-O2 2.370E-OI $1.637 \mathrm{E} 01$ $6.733 E$ O2 $6.611 \mathrm{E} 03$ ANGLE 10 $4.173 \mathrm{E}-09$ $7.643 \mathrm{E}-08$ $7.920 \mathrm{E}-07$ $7.288 E-06$ $3.965 \mathrm{E}-05$ $9.318 E-05$ $1.267 E-04$ . 1. $453 \mathrm{E}-0$ . . $567 E-03$ $2.932 \mathrm{E}-01$ 1.915E 01 . 731 E 02 $7.780 E$ OR $4.971 \mathrm{E} \mathrm{O3}$ 7.499 E 03
ANGLE 2
$M U=-0.9894$ $-1.780 \mathrm{E}-09$ $-1.286 \mathrm{E}-08$ $1.930 \mathrm{E}-08$ $1.191 \mathrm{E}-06$ 1.2818E-05 $8.529 \mathrm{E}-05$ $1.991 E-04$ $3.307 E-04$ $6.907 \mathrm{E}-04$ $1.574 \mathrm{E}-03$ $5.665 E-03$ $2.067 \mathrm{E}-0$ $2.374 \mathrm{E}-0$ $2.418 \mathrm{E}-01$ $1.639 \mathrm{E} \mathrm{O}$ $7.643 \mathrm{E} \mathrm{OI}$ $2.359 \mathrm{E} \mathrm{O2}$ $6.741 \mathrm{E} 02$ $1.895 \mathrm{E} 03$ $4.335 E \quad 03$ $6.619 E$ O3 ANGLE 11 $M U=0.2816$ $6.800 \mathrm{E}-09$
$1.165 \mathrm{E}-07$ $1.165 E-07$ $1.136 \mathrm{E}-06$ $9.758 \mathrm{E}-06$ $5.049 \mathrm{E}-05$ $1.133 E-04$ $1.567 E-04$ $5.196 \mathrm{E}-04$ $1.786 E-03$ $1.786 E-03$ $3.161 E-03$ $1.093 E-02$ 3.160E-02 . $2.945 \mathrm{E}-01$ $1.973 E$ OI $2.809 E$ O2 7.096 O2 2.239E 03 $5.102 E \quad 03$ $7.681 \mathrm{E} 03$ $U=-0.9446$ $4.74 L E-10$
$2.158 E-10$ $2.158 E-10$
$4.636 E-08$ $1.309 \mathrm{E}-06$ $1.284 E-05$ 4.961E-05 $8.513 E-05$ $2.000 E-04$ $7.037 \mathrm{E}-04$ $1.597 \mathrm{E}-03$ $5.754 \mathrm{E}-0$ $2.092 \mathrm{E}-02$ $2.392 \mathrm{E}-01$ $2.433 \mathrm{E}-01$ $1.649 \mathrm{E} \mathrm{Ol}$ $7.686 \mathrm{E} 01$ $2.372 \mathrm{E} \quad 02$ . .778E 02 .. $6.652 \mathrm{E} 03$

ANGLE 12 $M U=0.4580$ $9.377 \mathrm{E}-09$
$1.622 \mathrm{E}-07$ $1.578 E-06$ 1. $387 \mathrm{E}-05$ $6.889 \mathrm{E}-05$ $1.470 \mathrm{E}-04$ $2.054 E-04$
$7.302 E-04$ $1.720 \mathrm{E}-03$ $2.251 \mathrm{E}-03$ 1. $1.265 E-02$ $3.424 E-02$ $3.040 E-01$ 2.0 2.032E $2.887 E \quad 02$ . $2.298 \mathrm{E} 03$ 5.234E 03 $7.863 \mathrm{E} \mathrm{O3}$ $M=-0.8656$ $5.174 \mathrm{E}-10$ $1.039 \mathrm{E}-08$ $1.483 \mathrm{E}-06$ $1.316 \mathrm{E}-05$ $5.216 \mathrm{E}-05$ 8.511E-05 . $603 \mathrm{E}-04$ .297E-04 $1.643 E-03$ $5.926 E-03$ $2.138 \mathrm{E}-02$ $2.460 \mathrm{E}-01$ $1.666 \mathrm{E} \mathrm{O1}$ $7.763 \mathrm{E}$ 01 $2.396 \mathrm{E}$ O2 $1.923 \mathrm{E} \mathrm{O3}$ $4.398 \mathrm{E} 03$ $6.711 \mathrm{E} 03$ ANGLE 13 $M U=0.6179$ $1.704 \mathrm{E}-08$
$2.703 \mathrm{E}-07$ $2.593 \mathrm{E}-06$ $2.273 E-05$ $1.036 \mathrm{E}-04$ $2.053 E-04$ $2.838 \mathrm{E}-04$
$1.078 \mathrm{E}-03$ 2. $534 \mathrm{E}-03$ $2.907 \mathrm{E}-03$ $4.431 E-03$ $1.481 E-02$ $3.715 E-02$ 3 $2.089 \mathrm{E} 01$ $9.630 \mathrm{E} \mathrm{O1}$ $2.963 E \quad 2$ $8.422 E \quad 02$ 5.361E 03 $8.038 E 03$
ANGLE 5 1.267E-09 $1.855 \mathrm{E}-08$ $1.662 \mathrm{E}-07$ $1.694 \mathrm{E}-06$ $1.434 \mathrm{E}-05$ $8.566 \mathrm{E}-05$ $2.057 E-04$ $3.908 \mathrm{E}-04$ $7.707 E-04$ $.714 E-03$ $6.183 E-03$ $2.204 \mathrm{E}-02$ $2.474 E-01$ $1.691 \mathrm{E} \mathrm{O1}$ $2.874 E$ OI $2.429 E$ O2 $1.949 E$ O3 $6.793 E \quad 03$ ANGLE 14 $M U=0.7550$ 4. $497 E-08$ $5.465 E-06$ 4.305E-05 1.710E-04 $3.073 E-04$ 4. $092 E-04$ 1.671E-03 $2.142 \mathrm{E}$ ol $9.858 \mathrm{E} \mathrm{O1}$ $3.032 E 02$ $8.613 \mathrm{E} \mathrm{O2}$

ANGLE 6 $2.993 E-10$ $1.256 \mathrm{E}-08$ $1.561 E-07$ $2.044 \mathrm{E}-06$ $1.678 \mathrm{E}-05$ $5.983 E-05$ $8.734 E-05$ $2.138 E-04$
$4.361 E-04$ $8.318 \mathrm{E}-04$ $1.819 E-03$ $6.545 E-03$ $2.295 \mathrm{E}-02$ $2.537 \mathrm{E}-01$ $2.549 \mathrm{E}-01$ $1.723 \mathrm{E}$ OI $2.473 \mathrm{E} 02$ 2.473E 02 1.983 E 03 $6.898 \mathrm{E} 03$ 
ENERGY GROUP (MEV) $1.22 \mathrm{E} 01--1.50 \mathrm{E}$ OI $1.00 \mathrm{E}$ O1-- $1.22 \mathrm{E}$ O1 $8.1 \mathrm{SE}$ OO---1.00E 01 $4.97 \mathrm{E} 00---6.36 \mathrm{E} 00$ $4.07 E$ OO- $-4.97 E$ O 00 $3.01 E$ O0-- $4.07 E$ OO $2.46 E \quad 00-3.01 E 00$ $2.35 \mathrm{E} \quad 00--2.46 \mathrm{E} 00$ $1.83 \mathrm{E} 00--2.35 \mathrm{E} 00$ $1.11 \mathrm{E} 00--1.83 \mathrm{E} 00$ $5.50 E-01--1.11 E$ OO 1.11E-01-- $5.50 \mathrm{E}-01$ $3.35 \mathrm{E}-02---1.11 \mathrm{E}-01$ $5.83 E-04---3.35 E-02$ $1.01 E-04---5.83 E-04$ $2.90 E-05--1.01 E-04$ $1.07 E-05--2.90 E-05$ $3.06 E-06---1.07 E-05$ $1.12 E-06--3.06 E-06$ $4.14 E-07---1.12 E-06$ $0.0 \quad-4.14 \mathrm{E}-07$ ENERGY GROUP (MEV) $1.22 E$ 01--1.50E O1 $1.00 \mathrm{E}$ 01---1.22E 01 $8.19 E$ OO- $-1.00 E$ OI 6.36 E $00 \sim-1.19 E$ OO 4.97E $00---6.36 E$ OO 4.07E $00---4.97 E$ OD 3.01E 00---4.07E 00 $2.46 \mathrm{E} 00--3.01 \mathrm{E} 00$ $2.35 \mathrm{E} 00--2.46 \mathrm{E} 00$ $1.83 E$ OO-T-2.35E 00 $1.11 \mathrm{E} 00--1.83 \mathrm{E} 00$ $5.50 E-01--1.11 E$
$1.11 E-01--5.50 E-01$ $1.11 E-01--5.50 E-01$
$3.35 E-02--1.11 E-01$ $3.35 \mathrm{E}-02--1.11 \mathrm{E}-01$
$5.83 \mathrm{E}-04-\cdots-3.35 \mathrm{E}-02$ $5.83 \mathrm{E}-04--3.35 \mathrm{E}-02$
$1.01 \mathrm{E}-04--5.83 \mathrm{E}-04$ $1.01 E-04--5.83 E-04$ $1.07 \mathrm{E}-05-2.90 \mathrm{E}-05$ $3.06 \mathrm{E}-06--1.07 \mathrm{E}-05$ $1.12 \mathrm{E}-06--3.06 \mathrm{E}-06$ $4.14 \mathrm{E}-07--1.12 \mathrm{E}-06$ $4.14 \mathrm{E}-07---1.12 \mathrm{E}-06$
$0.0 \quad---4.14 \mathrm{E}-07$
ANGLE 1 $M U=-1 . C 000$ $-3.859 E-10$ $3.508 \mathrm{E}-07$ $4.091 \mathrm{E}-06$ $1.453 E-05$ $2.353 E-05$ $4.823 E-05$ .204E-05 . $951 E-04$ $4.419 E-04$ (6) $6.180 \mathrm{E}-03$ $7.428 \mathrm{E}-02$ $7.658 E-02$ $5.249 E$ OO $2.466 \mathrm{E} 01$ $2.204 E 02$ $2.204 E$ O $0.231 E 02$ $\begin{array}{ll}1.433 E & 03 \\ 2.201 E & 03\end{array}$ ANGLE 10 $M U=0.0950$ $9.439 E-10$ $1.887 E-08$ $2.288 \mathrm{E}-07$ $2.359 E-06$ 1.254E-05 2. $800 \mathrm{E}-05$ $3.444 E-05$ $1.069 \mathrm{E}-04$ $2.647 E-04$ $4.229 E-04$ $7.647 E-04$ $2.753 \mathrm{E}-03$ Q 9.1 S.055E 02 (2.869 01 2.869 OI $2.552 \mathrm{E} \quad 02$ $7.194 \mathrm{E} 02$

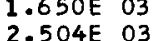

ANGLE 2 $M=-0.9894$ $-2.546 E-09$ $-5.088 E-09$ $3.608 \mathrm{E}-07$ S. $087 E-06$ $1.463 E-05$ $2.351 E-05$ $4.832 E-05$ $8.287 E-05$ $1.961 E-04$ $4.434 E-04$ $1.622 \mathrm{E}-03$ $6.198 E-03$ $7.441 E-02$ $7.669 E-02$ $5.257 \mathrm{E} 00$ $2.469 E \quad 01$ $7.664 E$ O1 $2.207 E 02$ $6.239 E 02$ $1.435 E 03$ $2.204 E 03$ ANGLE 11 $M U=0.2816$ $1.502 E-09$
$2.863 E-08$ $2.863 E-08$ $3.309 E-07$
$3.218 E-06$ $3.218 \mathrm{E}-06$
$1.608 \mathrm{E}-05$ $3.408 E-05$ $4.211 \mathrm{E}-05$ $1.447 E-04$ $3.551 E-04$
$5.198 E-04$ $5.198 \mathrm{E}-04$ $8.801 E-04$ $3.141 E-03$ 9.597E-02 . $350 E-02$ 6.337E 02 $2.952 \mathrm{E} 01$ $9.148 E$ OI $2.624 \mathrm{E} 02$ 1.392E 02 $2.566 \mathrm{E} \quad 03$
(NEUTRONS/MEV/STERADIAN/SOURCE NEUTRON)

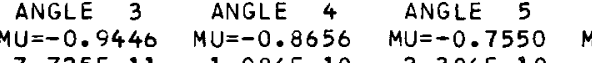
$0.399 E-11$
$.239 E-08$ $1.239 \mathrm{E}-08$
$3.978 \mathrm{E}-07$ $4.102 \varepsilon-06$ $4.102 \varepsilon-06$
$1.504 E-05$ $2.345 \mathrm{E}-05$ $4.872 \mathrm{E}-05$ $8.636 \mathrm{E}-05$ $2.003 \mathrm{E}-04$ $4.499 E-04$ $1.648 \mathrm{E}-03$ $6.272 \mathrm{E}-03$ $7.500 \mathrm{E}-02$ $7.717 E-02$ $5.288 \mathrm{E} 00$ $2.483 \mathrm{E}$ ol $7.708 \mathrm{E} 01$ $2.219 E 02$ 6.273 E 02 $\begin{array}{lll}1.443 E & 03 \\ 2.215 E & 03\end{array}$ ANGLE 12 $M U=0.4580$ $4.123 E-08$
$4.152 E-07$ 4. $752 \mathrm{E}-07$ 4.713E-06 $2.211 E-05$ 4.408 E- 05 $5.409 E-05$ $2.055 \mathrm{E}-04$ $5.020 E-04$ $6.542 E-04$ $1.027 E-03$ $3.627 E-03$ $1.025 \mathrm{E}-02$ $1.001 E-01$ $9.650 E-02$ 6.527E OO $3.036 E$ OI $9.405 E$ OI $2.696 \mathrm{E} 02$ 1.530E 02 $2.628 \mathrm{E} 03$
$2.533 E-09$

$3.565 E-08$
$4.546 E-07$

$4.540 E-06$
$1.576 E-05$

1. $576 \mathrm{E}-05$

.342E-05 4.556E-05 .295E-05 $2.085 E-04$ $4.627 \varepsilon-04$ $1.098 E-03$ $6.411 E-03$
$7.606 E-02$ $7.804 \mathrm{E}-02$ $5.345 \mathrm{E} 00$ $2.509 E \quad 01$ $7.786 \mathrm{E} 01$ $2.241 E 02$ $6.334 \mathrm{E} \mathrm{O2}$ $1.456 \mathrm{E} \mathrm{O3}$ $2.235 E 03$ $2.306 \mathrm{E}-10$ $3.905 E-09$ $4.250 E-08$ $1.674 E-05$ $2.354 \bar{c}-05$ $5.112 E-05$ $1.028 \mathrm{E}-04$ $2.212 E-04$ $4.828 \mathrm{E}-04$ $1.773 E-03$ $6.613 \mathrm{E}-03$ $7.758 \mathrm{E}-02$ $7.929 E-02$ $5.425 \mathrm{E} 00$ $2.545 E 01$ $7.898 E$ OI $2.273 E$ O2 $0.422 \mathrm{E} \mathrm{O2}$ $1.476 E \quad 03$
$2.263 E \quad 03$ $A N G L E 14$
$M U=0.7550$ $M U=0.6179$ 7.213E-08 $8.171 E-07$ 7.898E-06 $3.329 E-05$ $6.085 \mathrm{E}-05$ $7.252 E-05$ $3.055 \mathrm{E}-04$ $7.536 E-04$ $8.406 E-04$ $1.214 E-03$ $4.228 \mathrm{E}-03$ $1.104 \mathrm{E}-01$ $1.042 E-01$ 6.710E 00 6.710E 0 $9.652 \mathrm{E} \mathrm{O1}$ $2.766 E$ E 2 7.780 O2 $\begin{array}{lll}1.782 E & 03 \\ 2.687 E \quad 03\end{array}$

$1.028 \mathrm{E}-07$

. $751 \mathrm{E}-06$

. $496 E-05$ 5.428E-05 1. $746 \mathrm{E}-04$ . $205 \mathrm{E}-0$ 1. $101 \mathrm{E}-03$ $1.444 \mathrm{E}-03$ 1. $445 E-03$ $1.196 E-02$ $6.077 \mathrm{E}$ 9.877E 01 7. $1.820 \mathrm{E} 03$

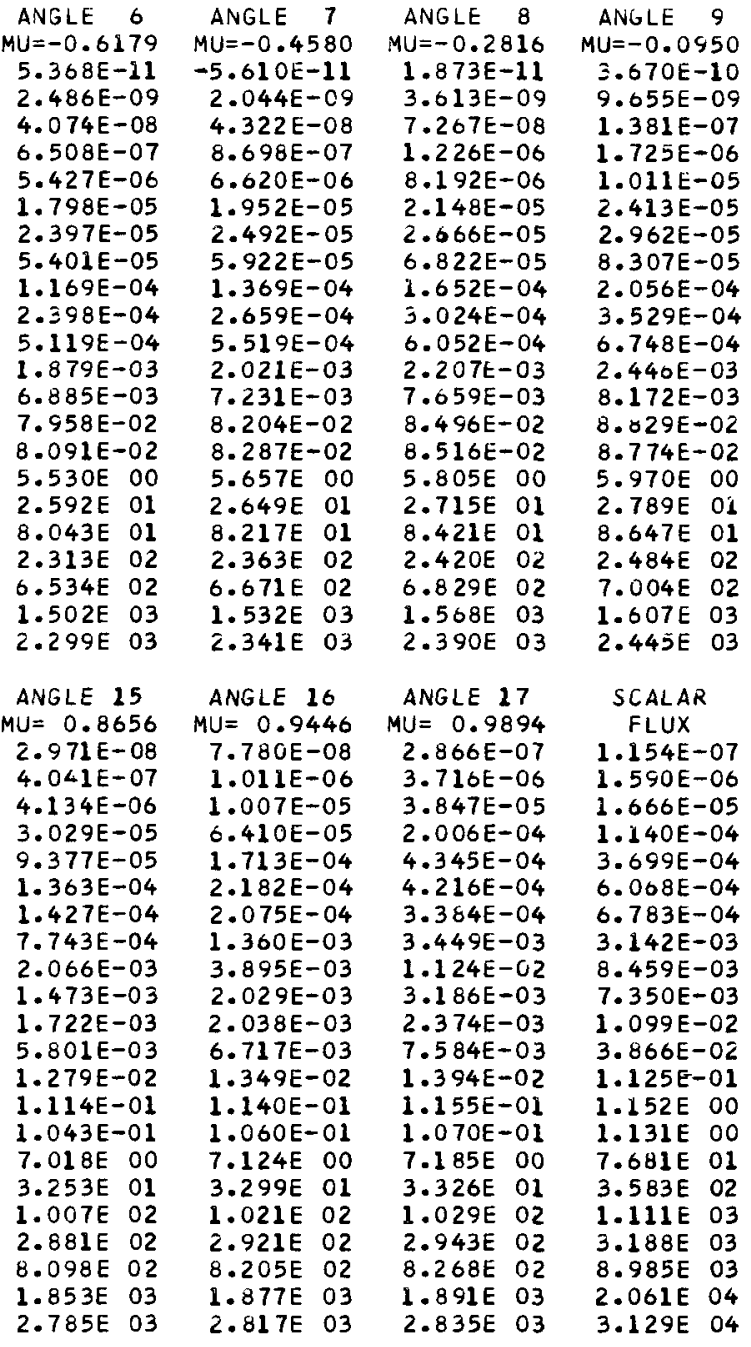
2.785 E 03 
(NEUTRONS/MEV/STERADIAN/SOURCE NEUTRON)

ENERGY

GROUP (MEV) $1.22 \mathrm{E}$ 01-- $1.50 \mathrm{E}$ 01 $1.00 \mathrm{E}$ 01-- -1.22E 01 .1SE $00--1.00 E$ OI $4.97 E$ O0- $-6.36 E$ OD $4.07 E$ O0- $-4.97 E$ O $3.01 E$ O0- $-4.07 E$ OD $46 \mathrm{E} 00--3.01 \mathrm{E} 00$ $2.35 \mathrm{E} \quad 00--2.46 \mathrm{E}$ 00 $1.83 \mathrm{E} \quad 00--2.35 \mathrm{E} 00$ $1.11 \mathrm{E} 00--1.83 \mathrm{E} 00$ $5.50 \mathrm{E}-01--1.11 \mathrm{E}$ OO $1.11 \mathrm{E}-01---5.50 \mathrm{E}-01$ $3.35 E-02-\cdots-1.11 E-01$ $5.83 E-04---3.35 E-02$ $1.01 E-04--5.83 E-04$ $2.90 E-05--1.01 E-04$ $1.07 E-05--2.90 E-05$ $3.06 \mathrm{E}-06---1.07 \mathrm{E}-05$ $1.12 \mathrm{E}-06---3.06 \mathrm{E}-06$ $4.14 E-07--1.12 E-06$
0.0

$$
\text { ENERGY }
$$

GROUP (MEV)

$1.22 E$ O1- $1.50 E$ O1 $1.00 E$ 01---1.22E OI 8.1 OE OO--1.00E OI $6.36 \mathrm{E}$ 00---8.19E 00 4.97E $00--6.36 \mathrm{E} \mathrm{OO}$ 4.07E 00---4.97E 00 $3.01 \mathrm{E} 00--4.07 \mathrm{E} \mathrm{CO}$ $2.46 \mathrm{E}$ 00- $-3.01 \mathrm{E}$ OO $2.35 \mathrm{E}$ 00---2.46E OO $1.83 E$ OO- $-2.35 E$ OO $1.11 \mathrm{E} 00--1.83 \mathrm{E} 00$ $5.50 \mathrm{E}-01-1.11 \mathrm{E}$ OO $1.11 E-01--5.50 E-01$ $3.35 E-02--1.11 E-01$ $5.83 E-04--3.35 E-02$ $1.01 E-04--5.83 E-04$ $2.90 E-05--1.01 E-04$ $1.07 E-05-D-2.90 E \rightarrow 05$ $3.06 E-06-1.07 E-05$ $1.12 E-06--3.06 E-06$ $4.14 E-07--1.12 E-06$
0.0
$---4.14 E-07$
ANG LE 1 $M U=-1.0000$ $-6.735 E-10$ $-2.957 \mathrm{E}-09$ $.015 \mathrm{E}-0$ $1.236 \mathrm{E}-06$ $6.292 \mathrm{E}-06$ $1.175 \mathrm{E}-05$ $2.042 \mathrm{E}-05$ $5.235 \mathrm{E}-0$ $1.178 E-04$ 4.306E-04 . 1.483E 00 $6.993 E O O$ . $178 \mathrm{E}$ O $6.294 \mathrm{E}$
$1.785 \mathrm{E}$ $4.117 E$ O2 $6.343 E \quad 02$ ANGLE 10 $M U=0.0$ $4.374 \mathrm{E}-09$ $6.276 \mathrm{E}-0$ $7.231 \mathrm{E}-0$ $3.756 \mathrm{E}-06$ $8.002 E-06$ $9.109 \mathrm{E}-06$ $2.820 \mathrm{E}-05$ $7.104 E-05$ $1.155 \mathrm{E}-04$ $2.025 E-04$ $7.345 E-04$ $2.391 \mathrm{E}-03$ $2.568 \mathrm{E}-02$ $2.543 E-02$ $1.738 \mathrm{E} 00$ $8.140 E$ OO 2.531E O1 $7.295 E$ OI $2.063 E$ O2 $7.223 \mathrm{E} 02$
ANGLE 2 MUNGE $5.009 E-10$ $1.395 \mathrm{E}-0$ $1.044 \mathrm{E}-07$ $1.236 \mathrm{E}-06$ $4.226 \mathrm{E}-06$ $6.287 E-06$ $1.178 \mathrm{E}-05$ $2.067 E-05$ $5.263 \mathrm{E}-05$ $4.323 E-04$ $1.689 \mathrm{E}-03$ $2.078 \mathrm{E}-02$ $2.154 \mathrm{E}-02$ $7.003 E 00$ $2.181 \mathrm{E} 01$ $6.303 E$ OI $1.787 \mathrm{E} 02$ $4.122 \mathrm{E} 02$
$6.351 \mathrm{E} 02$ ANGLE 11 $M=0.2816$
$3.135 \mathrm{E}-10$ $6.681 E-09$ $9.178 E-08$ $1.004 E-06$ $4.833 \mathrm{E}-06$ $9.727 E-06$ $1.102 \mathrm{E}-05$ $3.846 E-05$ $9.603 \mathrm{E}-05$ $1.419 \mathrm{E}-04$ $2.323 \mathrm{E}-04$ $8.369 E-04$ $2.578 \mathrm{E}-03$ $2.678 \mathrm{E}-02$ $2.026 \mathrm{E}-02$ $1.791 E$ OO $8.376 E$ OO 2.604E O1 7.500 OI $2.119 \mathrm{E}$ O2

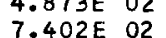

$-1.255 E-11$

$9.362 \mathrm{E}-12$

$1.155 \mathrm{E}-07$

$1.155 \mathrm{E}-07$

$4.336 \mathrm{E}-06$

$4.336 E-06$
$6.270 E-06$

$1.192 \mathrm{E}-05$

$2.170 E-05$

$5.386 \mathrm{E}-05$ $1.199 \mathrm{E}-04$ $4.394 E-04$ $1.710 \mathrm{E}-03$ $2.094 \mathrm{E}-02$ $2.168 \mathrm{E}-02$ $.494 E$ OO $.043 E$ EO .193E OI $6.338 \mathrm{E}$ O1 $4.145 \mathrm{E} \quad 02$

ANGLE 12 $4.754 \mathrm{E}-10$ $9.925 \mathrm{E}-0$ $1.361 \mathrm{E}-07$ $1.506 \mathrm{E}-06$ $6.668 \mathrm{E}-06$ $1.251 \mathrm{E}-05$ $1.390 \mathrm{E}-05$
$5.487 \mathrm{E}-05$ $1.371 \mathrm{E}-04$ $1.783 \mathrm{E}-04$ $2.699 \mathrm{E}-04$ $2.787 E-03$ $2.793 \mathrm{E}-02$ $2.710 E-02$ $1.844 \mathrm{E} 00$ $8.614 \mathrm{E} 00$ $2.677 \mathrm{E}$ O1 $7.706 E$ OI $2.176 \mathrm{E} 02$ $5.001 E$ O2
$7.582 \mathrm{E} \quad 02$ $\triangle A N G E \quad 4$ $M U=-0.8656$ $5.853 \mathrm{E}-10$ $8.844 E-09$ $1.332 \mathrm{E}-07$ $1.294 \mathrm{E}-06$ $4.532 \mathrm{E}-06$ $6.259 \mathrm{E}-06$ $1.220 \mathrm{E}-05$ $2.361 \mathrm{E}-05$ $5.624 E-05$ $1.233 E-04$ $1.748 E-03$ $2.124 \mathrm{E}-02$ 2.192E-02 $1.510 E 00$ $2.216 \mathrm{E} 01$ $6.402 E \quad 01$ $1.815 \mathrm{E} 02$ $\begin{array}{ll}4.185 \mathrm{E} & 02 \\ 6.442 \mathrm{E} & 02\end{array}$ ANGLE 13 $U=0.6179$ $9.547 \mathrm{E}-10$ $2.424 \mathrm{E}-07$ $2.563 E-06$ $1.001 \mathrm{E}-0$ $1.706 E-05$ $1.818 \mathrm{E}-05$ $8.172 \mathrm{E}-05$ 2.090E-04 $2.280 E-04$ $3.165 E-04$ $3.011 E-03$ $2.906 \mathrm{E}-02$ $2.790 E-02$ 1.895 E $2.747 \mathrm{E} 01$ $7.904 \mathrm{E}$ O1 2.231E O2 $5.124 \varepsilon$ O2
$7.754 \mathrm{E}$ O2
ANGLE $5 \quad$ ANGLE 6 4.118E-11 $7.852 E-10$ $1.054 \mathrm{E}-08$ $1.571 \mathrm{E}-07$ $1.422 E-06$ $4.802 \mathrm{E}-06$ $6.289 \mathrm{E}-06$ $1.269 E-05$ $2.639 E-05$
$5.987 E-05$ $1.286 E-04$ $4.731 E-04$ $1.803 \mathrm{E}-03$ $2.166 \mathrm{E}-02$ $1.533 \mathrm{E} 00$ $7.219 E \quad 00$ $2.247 E$ OI $6.492 E \quad 01$ 1.840 E 02 $4.242 E \quad 02$
$6.524 \mathrm{E} 02$

ANGLE 14 $M U=0.7550$ $4.082 E-08$ $5.238 \mathrm{E}-07$ $4.843 E-06$ $1.613 \mathrm{E}-05$ $2.444 E-05$ 2.436E-05 $1.268 E-04$ . $3.729 \mathrm{E}-04$ $1.305 \mathrm{E}-03$ $3.239 \mathrm{E}-03$ $3.012 \mathrm{E}-02$ $2.864 E-02$ 1.942E 00 . $2.280 E$ O2 $5.235 E$ O2 $1.029 \mathrm{E}-11$ $5.360 \mathrm{E}-10$ $1.964 \mathrm{E}-07$ $1.656 \mathrm{E}-06$ $5.149 E-06$ $0.401 E-06$ $1.357 E-05$ $3.033 E-05$ 6.510E-05 $5.016 \mathrm{E}-04$ $1.877 \mathrm{E}-03$ $2.222 E-02$ $2.273 E-02$ $1.563 \mathrm{E} 00$ $7.353 \mathrm{E} \mathrm{O0}$ $2.289 E \quad 01$ $6.609 E 01$ $1.873 E 02$ $4.315 E 02$
$6.628 E 02$ ANGLE 15 $M U=0.8656$ $9.907 \mathrm{E}-08$ $1.219 E-06$ $9.630 \mathrm{E}-06$ $2.726 \mathrm{E}-05$ $3.628 \mathrm{E}-05$ $3.313 \mathrm{E}-05$ 2.054E-04 
(NEUTRONS/MEV/STEKADIAIN/SOURCE NEUTRON)

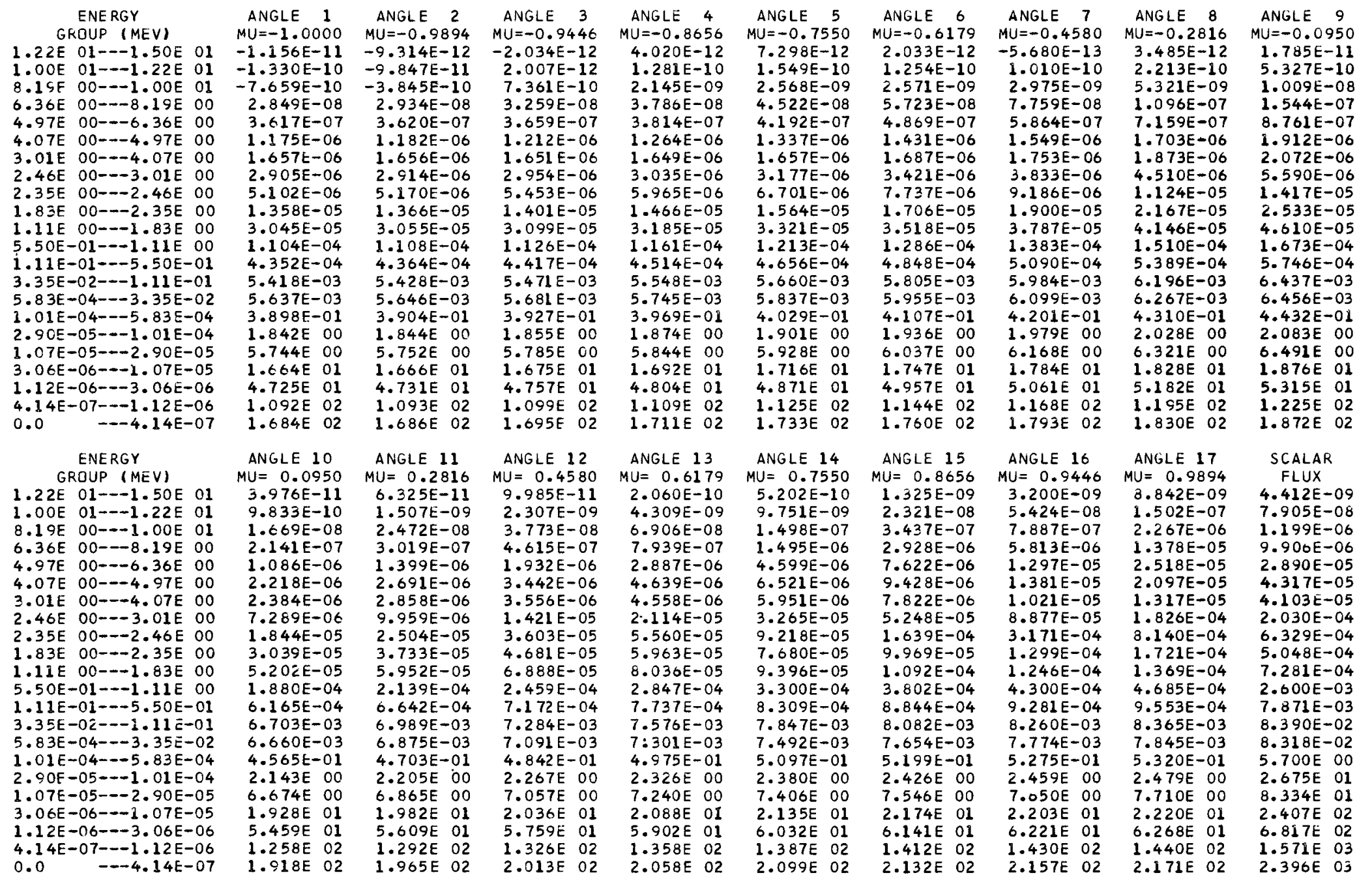


(GAMMAS/MEV/STERADIAN/SOURCE NEUTRON)

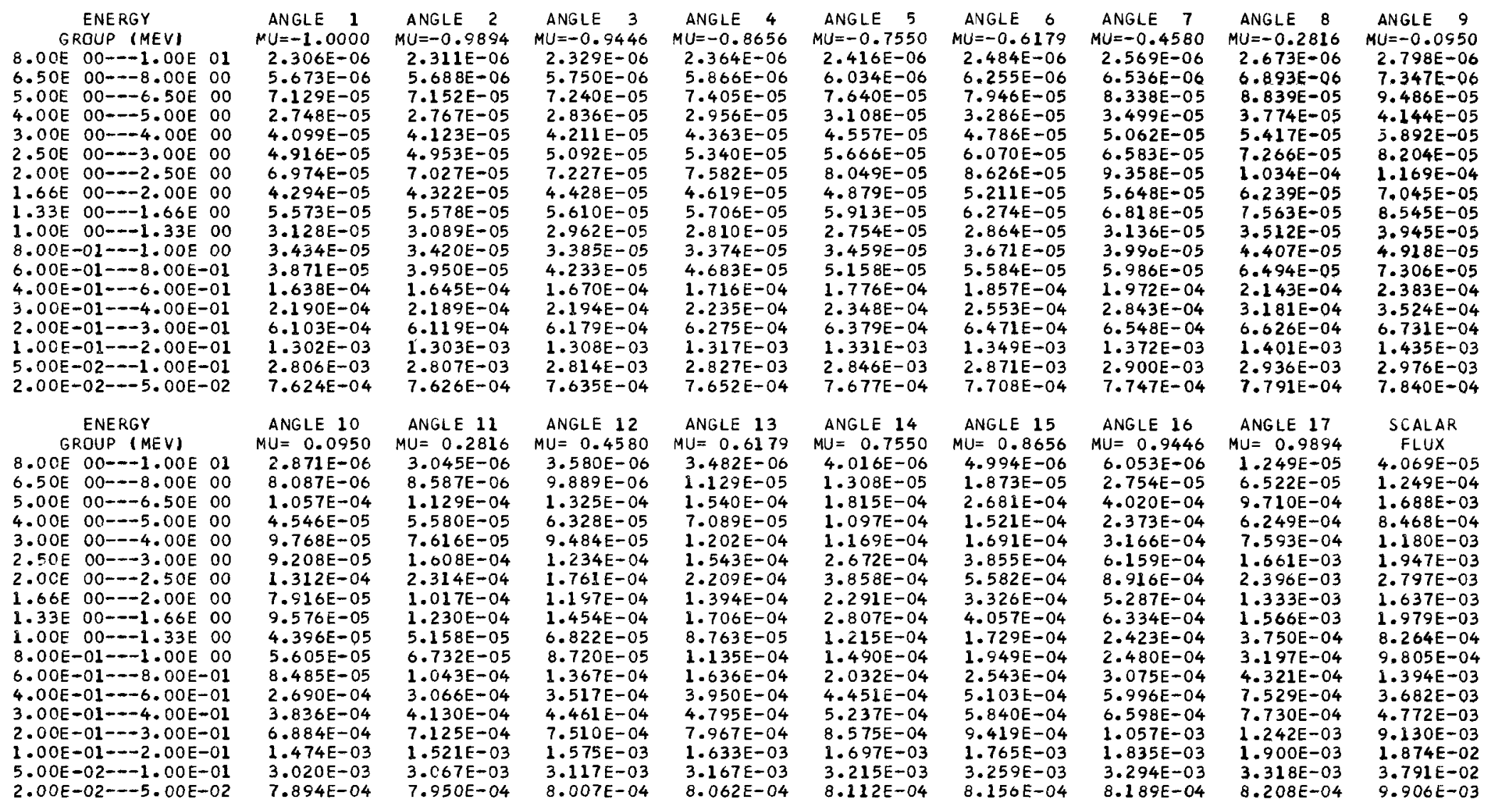


(GAMMAS/MEV/STERADIAN/SOURCE NEUTRON)

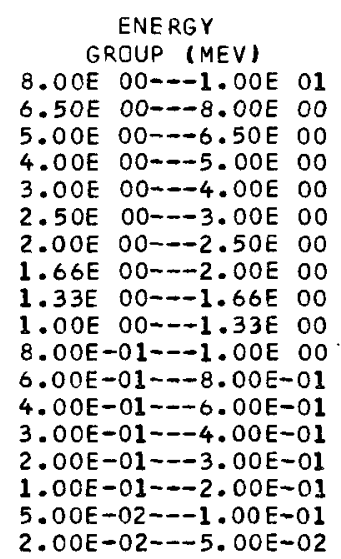

ANGLE 1 ANGLE 2

ANGLE 3

ENERGY GROUP (MEV)

$8.00 E$ OO- -1.00E 01 5.00 E $00--6.50 E$ OO 4.0OE $00--5.00 E 00$ 3.00 E $00--4.00 E 00$ $2.50 E$ OO---3.00E OO 2.0OE 0O- -2.50E 00 $1.66 \mathrm{E}$ 0O--D2.00E 00 1.33 E $00--1.66$ E 00 $1.00 E$ 00-- $1.33 E$ OO 8.00 E-01- $1.00 \mathrm{E} 00$ $6.00 E-01--8.00 E-01$ $4.00 E-01--6.00 E-01$ $3.00 E-01--4.00 E-01$ $2.00 E-01--3.00 E-01$ $1.00 E-01-2.00 E-01$ $2.00 \mathrm{E}-02-2.00 \mathrm{E}-02$

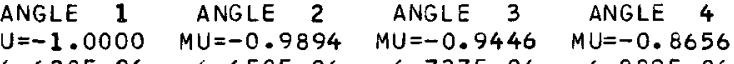
$1.470 \mathrm{E}-05 \quad 1.6576 \mathrm{E}-05$

$3.885 \mathrm{E}-03$ $9.258 E-03$
$2.599 E-03$ $2.600 \mathrm{E}-03$ ANGLE 10 $M U=0.0950$ $8.228 E-06$
$2.429 E-05$ $3.019 E-04$ $7.848 \mathrm{E}-05$ $1.321 E-04$ $1.583 E-04$ $2.202 E-04$ $9.068 \mathrm{E}-05$ $1.089 E-04$ $9.585 \mathrm{E}-05$ $1.382 E-04$ $1.947 \mathrm{E}-04$ $7.129 \mathrm{E}-04$ 1.026 E-03 $1.781 \mathrm{E}-03$ $4.532 E-03$ $1.030 E-02$
$2.733 E-03$
ANGLE 11 U $=0.2816$ $1.119 \mathrm{E}-05$
$2.136 \mathrm{E}-05$ $2.522 E-04$ $1.264 E-04$ $1.757 E-04$ $1.393 E-04$ $1.915 \mathrm{E}-04$ $1.926 E-04$ $2.324 E-04$ $1.385 \mathrm{E}-04$ $1.793 \mathrm{E}-04$ $2.892 \mathrm{E}-04$ $8.324 \mathrm{E}-04$ $2.117 E-03$ $1.872 E-03$ $4.705 E-03$ $1.054 \mathrm{E}-02$
$2.761 \mathrm{E}-03$ $6.737 \mathrm{E}-06$ $1.495 E-05$ 1.786E-04 $0.424 E-05$ $6.424 E-05$ $9.562 E-05$ $6.714 E-05$ $8.550 \mathrm{E}-05$ $6.418 E-05$ $7.801 \mathrm{E}-05$ $1.020 \mathrm{E}-04$ 4.297E-04 $5.690 E-04$ $1.601 \mathrm{E}-03$ $3.907 E-03$ $9.259 E-03$ 2.605E-03

ANGLE 12 $M U=0.4580$ $2.834 \mathrm{E}-05$ $3.543 E-04$ $1.002 E-04$ $1.833 E-04$ $2.168 E-04$ $3.023 \mathrm{E}-04$ $1.455 E-04$ $1.766 E-04$ $1.588 \mathrm{E}-04$ $2.234 \mathrm{E}-04$ 3.354E-04 $9.307 E-04$ $2.182 E-03$ $1.951 E-03$ 4.895E-03 $2.791 \mathrm{E}-03$ 6.889E-06 $1.533 E-05$ $1.03 E-04$ 9. $7.367 \mathrm{E}-05$ $1.017 E-04$ $1.017 E-04$ $7.058 E-05$ $8.644 E-05$ $7.842 \mathrm{E}-05$ $1.134 \mathrm{E}-04$ $4.426 E-04$ $5.845 \mathrm{E}-04$ $1.626 \mathrm{E}-03$ $3.943 \mathrm{E}-03$ $9.362 \mathrm{E}-03$ $2.613 E-03$

ANGLE 5 $7.114 \mathrm{E}-06$ $2.588 E-05$ $0.432 E-05$ $1.032 \mathrm{E}-04$ $7.901 \mathrm{E}-05$ $1.089 E-04$ $7.499 E-05$ $8.949 E-05$ $6.061 \mathrm{E}-05$ $8.167 E-05$ $1.251 E-04$ $4.599 \mathrm{E}-04$ $6.209 E-04$ $1.653 E-03$ $3.995 \mathrm{E}-03$ $9.452 \mathrm{E}-03$ $2.625 E-03$ ANGLE 13

ANGLE 6 $7.413 E-06$ $1.659 \mathrm{E}-05$ 1. $888=04$ 1.084E-04 $8.505 \mathrm{E}-05$ 1.171E-04 $8.044 \mathrm{E}-05$ $9.558 \mathrm{E}-05$ $6.483 \mathrm{E}-05$ $8.841 E-05$ $1.355 \mathrm{E}-04$ $1.355 E-04$
$4.832 E-04$ $6.825 E-04$ $1.676 \mathrm{E}-03$ $4.063 E-03$ $9.569 \mathrm{E}-03$ $2.640 E-03$ $U=0.6179$
$1.221 \mathrm{E}-05$ $2.766 \bar{\varepsilon}-05$ $3.379 E-04$ $1.682 \mathrm{E}-04$
$2.160 \mathrm{E}-04$ $2.388 E-04$ $3.335 E-04$ $2.837 E-04$ $3.485 \mathrm{E}-04$ $2.210 \mathrm{E}-04$ $2.996 \mathrm{E}-04$ 4. $\hat{3} 32 \mathrm{E}-04$ $1.052 \mathrm{E}-03$ $1.279 E-03$ $2.073 E-03$ $5.121 \mathrm{E}-03$ $1.106 \mathrm{E}-02$
$2.820 \mathrm{E}-03$
ANGLE 14

$M U=0.7550$

ANGLE 15 $3.530 E-05 \quad 4.006 E-05$ $4.512 \mathrm{E}-04 \quad 5.198 \mathrm{E}-04$ $1.693 E-04$ $2.716 E-04$ 5.029E-04 $3.025 \mathrm{E}-04$ 3.758E-04 $3.141 \mathrm{E}-04$ $4.021 E-04$ $5.400 E-04$ $1.181 E-03$ $1.402 E-03$ $2.233 \mathrm{E}-03$ $5.360 \mathrm{E}-03$ $2.847 E-03$
ANGLE 7 $7.791 \mathrm{E}-06 \quad 8.255 \mathrm{E}-06$ $1.749 E-05 \quad 1.861 E-05$ $7.251 E-05 \quad 7.236 E-04$ $1.148 E-04 \quad 1.229 E-04$ $9.237 E-05 \quad 1.022 E-04$ $1.271 E-04 \quad 1.407 E-04$ $1.051 E-04 \quad 1.180 E-04$ $7.314 E-05 \quad 8.373 E-05$ $1.314 E-05$
$9.808 E-05$ $1.808 E-05$
$1.455 E-04$ $\begin{array}{ll}1.455 E-04 & 1.590 E-04 \\ 5.169 E-04 & 5.663 E-04\end{array}$ $7.655 E-04 \quad 8.595 E-04$ $1.690 \mathrm{E}-03 \quad 1.718 \mathrm{E}-03$ $4.150 E-03 \quad 4.256 E-03$ $9.713 E-03$ 2.659E-03 ANGLE 16 $M U=0.9446$ $5.846 \mathrm{E}-05$ $7.991 E-04$ 4. $210 \mathrm{E}-04$ $5.699 E-04$ $9.332 \mathrm{E}-04$ $1.329 E-03$ $9.050 \mathrm{E}-04$ $1.073 E-03$ $6.428 \mathrm{E}-04$ $6.805 E-04$ $8.062 \mathrm{E}-04$ $1.570 \mathrm{E}-03$ $1.761 E-03$ $2.696 \mathrm{E}-03$ $5.887 \mathrm{E}-03$ $1.176 \mathrm{E}-02$ $2.890 E-03$ $2.681 E-03$

ANGLE 9 AU 20.0950 . $2.408 E-04$ $8.561 E+05$ $1.336 E-04$ $1.599 E-04$ $1.107 E-04$ $1.343 E-04$ $9.522 E-05$ $1.239 E-04$ $1.814 E-04$ $9.518 \mathrm{E}-04$ $1.748 \mathrm{E}-03$ ( $1.008 \mathrm{E}-02$ SCALAR $M U=0.5894 \quad$ FLUX $\begin{array}{ll}2.793 E-05 & 1.250 E-04 \\ 1.222 E-04 & 3.142 E-04\end{array}$ $1.750 E-03 \quad 3.930 E-03$ $1.073 \mathrm{E}-03 \quad 1.603 \mathrm{E}-03$ $1.283 \mathrm{E}-03 \quad 2.340 \mathrm{E}-03$ $2.610 \mathrm{E}-03 \quad 2.798 \mathrm{E}-03$ $3.684 E-03 \quad 3.919 E-03$ $2.198 E-03 \quad 2.592 E-03$ $2.504 E-03 \quad 3.118 E-03$ $2.040 E-03$ $8.671 E-04 \quad 2.547 E-03$ $1.018 E-03 \quad 3.526 E-03$ $1.925 E-03 \quad 9.719 E-03$ 3.010E-03 $1.275 E-02$ 2.365E-02 $6.123 E-03$ $5.773 E-02$ $\begin{array}{ll}1.901 E-03 & 3.429 E-02\end{array}$ 
(GAMMAS/MEV/STERADIAN/SOURCE NEUTRON) ENERGY
GROUP (MEV) 8.00E $00---1.00 E$ OI 6.50 OO $00--8.00$ E 00 $5.50 E$ DO-- $8.00 E$ E $4.00 E$ OO- 5 . 00 E $3.00 E$ OO- $-4.00 E$ E $2.50 \mathrm{E}$ O0- $-3.00 E$ OO $2.00 E$ OO-D-2.50E 00 $1.66 \mathrm{E} 00--2.00 \mathrm{E}$ OO $1.33 \mathrm{E} 00--1.66 \mathrm{E}$ OD $1.00 E$ OO- $-1.33 E$ E 00 $8.00 E-01--1.00 E 00$ $6.00 \mathrm{E}-01--8.00 \mathrm{E}-01$ $4.00 \mathrm{E}-01--6.00 \mathrm{E}-01$ 3. $00 \mathrm{OE}-01=-4.00 \mathrm{E}-0$ $2.00 E-01--3.00 E-01$ $1.00 E-01--2.00 E-01$ $5.00 \mathrm{E}-02---1.00 \mathrm{E}-01$ $2.00 E-02--5.00 E-02$

$$
\text { ENERGY }
$$$$
\text { GROUP (MEV }
$$

8.0OE OO---1.00E OI 6.5 OE $00---8.00 E$ OO 5.00E $00---6.50 E$ OO ..00E $00--5.00 E$ OO $3.00 E$ OO- $-4.00 E$ OD $2.50 E$ OO- $-3.00 E$ OO $2.00 E$ OO-W2.50E 00 $1.66 E$ DO- $-2.00 E$ OO $1.33 \mathrm{E} 00--1.66 \mathrm{E} \mathrm{OO}$ $1.00 \mathrm{E}$ 00---1.33E 00 $8.00 E-01--1.00 E 00$ $6.00 \mathrm{E}-01--8.00 \mathrm{E}-0$ 4.00E-01---6.00E-01 $3.00 E-01-4.00 E-01$ $2.00 E-01--3.00 E-01$ $1.00 \mathrm{E}-01--2.00 \mathrm{E}-01$ $2.00 \mathrm{E}-02---5.00 \mathrm{E}-02$
ANGLE 1 $9.511 \mathrm{E}-06$ . $2.424 \mathrm{E}-04$ $2.424 \mathrm{E}-04$
$7.270 \mathrm{E}-05$ $1.228 \mathrm{E}-04$ $1.228 E-04$ $9.671 \mathrm{E}-05$ $7.401 \mathrm{E}-05$ $9.858 \mathrm{E}-05$ $8.799 \mathrm{E}-05$ $1.040 \mathrm{E}-04$ $1.258 \mathrm{E}-04$ $5.931 E-04$ $8.016 \mathrm{E}-04$ $2.257 \mathrm{E}-03$ $5.776 \mathrm{E}-03$ $1.434 \mathrm{E}-02$ 4.084E-03 ANGLE 10 $M U=0.0950$

$1.524 E-05$
$3.100 E-05$ $3.100 E-05$ $1.386 \mathrm{E}-04$ $1.386 E-04$ $1.410 \mathrm{E}-04$ $1.894 E-0$ $1.747 \mathrm{E}-04$ $2.113 \mathrm{E}-04$ $1.606 \mathrm{E}-0$ $2.091 E-04$ $3.263 \mathrm{E}-04$ $1.059 \mathrm{E}-0$ $1.498 \mathrm{E}-03$ $2.573 \mathrm{E}-03$ $6.825 \mathrm{E}-0$ $1.619 E-02$
$4.320 E-03$
ANGLE 2 $M U=-0.9894$
$9.545 E-06$ $2.068 E-05$ $2.434 \mathrm{E}-04$ $7.337 \mathrm{E}-0 \mathrm{~S}$ $7.337 \mathrm{E}-05$
$1.237 \mathrm{E}-04$ $1.237 E-04$ $9.792 \mathrm{E}-0 \mathrm{~S}$ $7.467 E-05$ $9.843 E-05$ $8.681 E-05$ $1.037 \mathrm{E}-04$ $1.286 \mathrm{E}-04$ $5.960 E-04$ $8.028 \mathrm{E}-04$ $2.263 E-03$ $5.784 \mathrm{E}-03$ $1.436 E-02$ $4.085 \mathrm{E}-03$

\section{ANGLE 11} $M U=0.2816$ $1.552 E-05$
$3.698 E-05$ $4.423 E-04$ $1.405 \mathrm{E}-04$ $2.263 E-04$ $1.943 \mathrm{E}-04$ $2.644 \mathrm{E}-04$ $1.674 E-04$ $2.009 \mathrm{E}-04$ $1.777 \mathrm{E}-04$ $2.508 E-04$ $3.828 E-04$ $1.198 \mathrm{E}-03$ $1.594 \mathrm{E}-03$ $2.657 \mathrm{E}-03$ $7.099 \mathrm{E}-03$ $1.661 E-02$
$4.370 E-03$
ANGLE 3 $M U=-0.9446$ $9.679 E-06$ $2.473 \mathrm{E}-04$ $7.585 E-05$ $7.585 \mathrm{E}-05$ $1.271 E-04$
$7.520 E-05$ $1.024 E-04$ $7.713 E-05$ $9.823 E-05$ $8.311 E-05$ $034 E-04$
0 6.072E-04 $.814 E-03$ $4.093 \mathrm{E}-03$ ANGLE 12 $A=0.4580$
$1.830 E-05$ $1.830 E-05$ 4. $597 E-04$ $1.788 E-04$ $2.725 \mathrm{E}-04$ $2.159 E-04$ $2.928 E-04$ $2.434 E-04$ $2.056 \mathrm{E}-04$ 2. $395 \mathrm{E}-04$ $3.297 E-04$ $5.083 \mathrm{E}-04$ $1.364 \mathrm{E}-03$ $1.710 E-03$ $2.801 E-03$ $7.409 \mathrm{E}-03$ $1.707 E-02$ $4.423 E-03$
ANGLE 4 $M U=-0.8656$
$9.941 E-06$ . $2.548 \mathrm{E}-04$ $7.998 \mathrm{E}-05$ $1.328 \mathrm{E}-04$ $8.073 E-05$ $1.096 \mathrm{E}-04$ $8.138 \mathrm{E}-05$ $9.915 E-05$ $7.941 \mathrm{E}-05$ $1.049 \mathrm{E}-04$ $1.547 E-04$ $6.271 E-04$ $8.379 E-04$ $2.318 \mathrm{E}-03$ $5.872 E-03$ $4.108 E-03$ ANGLE 13 $M U=0.6179$
$1.923 \mathrm{E}-05$ $4.607 \mathrm{E}-05$ 5. 562 E- 04 $1.966 \mathrm{E}-04$ $3.029 E-04$ 2.967E-04 4. $071 \mathrm{E}-04$ 2. 800 E-04 $3.461 E-04$ $3.121 E-04$ 4. $331 E-04$ $6.161 \mathrm{E}-04$ $1.518 E-03$ $1.832 \mathrm{E}-03$ $2.956 \mathrm{E}-03$ $7.752 E-03$ $4.474 \mathrm{E}-03$
ANG LE 5 $1.033 \mathrm{E}-05$ $2.250 E-05$ $2.654 E-04$ $8.490 E-05$ 1. $398 \mathrm{E}-04$ $8.715 E-05$ $1.180 E-04$ $8.680 E-05$ $1.029 E-04$ $8.006 \mathrm{E}-05$ $1.105 \mathrm{E}-04$ $1.713 E-04$ $6.542 \mathrm{E}-04$ $8.950 E-04$ $2.354 \mathrm{E}-03$ 5. $956 \mathrm{E}-03$ $1.468 E-02$ $4.129 E-03$ ANGLE 14 $M U=0.7550$ $5.089 \mathrm{E}-05$ $6.171 E-04$ 2.549E-04 3. $756 \mathrm{E}-04$ 3. $863 \mathrm{E}-04$ $5.342 E-04$ $4.193 \mathrm{E}-04$ $5.221 E-04$ $4.465 \mathrm{E}-04$ $5.793 E-04$ $7.733 \mathrm{E}-04$ $1.696 \mathrm{E}-03$ 2.004E-03 $3.168 E-03$ $8.124 E-03$ 1.79g 02
ANGLE 6 MU $=-0.6179$ . $2.792 \mathrm{E}-04$ $9.029 E-05$ $1.479 \mathrm{E}-04$ $9.417 \mathrm{E}-05$ $1.272 \mathrm{E}-04$ 9.355E-05 $108 \mathrm{E}-04$ $8.719 E-05$ $1.213 E-04$ $6.910 E-04$ $2.387 E-03$ $6.068 \mathrm{E}-03$ $4.156 E-03$ ANGLE 15 $M U=0.8656$
$2.415 \mathrm{E}-05$ $6.200 E-05$ $7.758 \mathrm{E}-04$ $3.304 E-04$ 4. $771 E-04$ $6.077 E-04$ $8.508 E-04$ $6.146 \mathrm{E}-04$ $7.539 E-04$ $6.213 \mathrm{E}-04$ 7.504E-04 $9.191 \mathrm{E}-04$ 1.897E-03 2.210E-03 $3.412 E-03$ $8.514 E-03$ $4.564 E-03$

ANGLE 7 $M U=-0.4580$ . $517 \mathrm{E}-05$ $2.968 \mathrm{E}-04$ $.655 \mathrm{E}-05$ $1.575 \mathrm{E}-04$ $.026 \mathrm{E}-04$ $1.026 E-04$
$1.384 E-04$ $1.025 \mathrm{E}-04$ $1.232 E-04$ $1.000 E-04$ $1.362 E-04$ $2.018 E-04$ $7.438 \mathrm{E}-04$ $2.417 E-03$ 
(GAMMAS/MEV/STERADIAN/SOURCE NEUTRON)

\begin{abstract}
ENERGY GROUP (MEV) 8.00E $00---1.00 E$ O1 $6.50 E$ OO- - 8.00E OD $5.00 E$ OO- $-6.50 E$ OO 4.0OE OO- $-5.00 E$ OO . NOE OO-C-4.0OE OO .5OE OO- -3.00E OO 1. 1.66E $00-2.00 E$ OO 1. . . OOE $01--8.00 E-01$ $4.00 E-01--6.00 E-01$ $3.00 E-01---4.00 E-01$ .0OE $-01-\cdots 3.00 E-01$ $1.00 \mathrm{E}-01--2.00 \mathrm{E}-01$ $5.00 E-02--1.00 E-01$ $2.00 E-02--5.00 E-02$
\end{abstract}

ENERGY$$
\text { GROUP (MEV) }
$$

.0OE DO--D. 1.00E OI $6.50 E$ OO-- - 8. OOE OO $5.00 E$ OO-- $6.50 E$ OO 4.0OE 0O- $-5.00 E$ OO 3.00 E $00--4.00 E$ OO .5OE OO- $-3.00 E$ EO . .0OE 00---2.50E OO $1.66 \mathrm{E} 00--2.00 \mathrm{E} 00$ $.33 E$ E0- $-1.66 E 00$ $1.00 \mathrm{E} 00--1.33 \mathrm{E} 00$ 8.00E-01--1.0OE OO $6.00 E-01--8.00 E-01$ $4.00 E-01--6.00 E-01$ $3.00 E-01=-4.00 E-01$ $2.00 \mathrm{E}-01--3.00 \mathrm{E}-01$ $1.00 E-01--2.00 E-01$ $2.0 C E-02--5.00 E-02$
ANGLE 1 ANGLE 2 $M U=-1.0000 \quad M U=-0.9894$ - $350 E-05$ $2.866 \mathrm{E}-05$ 3.3 9. 7. 0.534E-0.5 (179E-05 $1.096 E-04$ 1.12E-04 $1.342 E-04$ $1 . E S 3 E-04$ 8.670E- 04 1. $191 E-03$
$3.461 E-03$ $3.467 \mathrm{E}-03$ $9.217 \mathrm{E}-03$ $2.414 E-02$ $7.005 E-03$

ANGLE 10 $M U=0.0950$ $2.347 E-05$ . $.858 E-04$

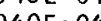
. (2) 1.872E-04 $2.268 \mathrm{E}-04$ $2.304 \mathrm{E}-04$ $3.186 \mathrm{E}-04$ $4.880 \mathrm{E}-04$ $1.663 \mathrm{E}-03$ 2.312E-03 $3.937 \mathrm{E}-03$ $1.110 E-02$ $2.7 \varepsilon 1 E-02$ $7.471 E-03$

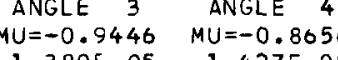
. 9.866E-05 . $866 \mathrm{E}-05$ $7.754 E-05$ ..654E- 05 1.053E-05 1.087E-04 $1.055 \mathrm{E}-04$ $1.055 E-04$
$1.350 E-04$ $1.350 E-04$
$1.883 E-04$ $1.883 E-04$ 1.217E-03 3.504E-03 $9.285 \mathrm{E}-03$ $2.429 E-02$ $7.024 E-03$ ANGLE 12 $M U=0.4580$ 3.080E-05 $6.671 E-05$ $7.655 E-04$ $2.554 E-04$ $4.105 E-04$ $2.667 E-04$ $3.488 \mathrm{E}-04$ $2.904 E-04$ $3.535 E-04$ $3.690 \mathrm{E}-04$ $5.246 E-04$ $7.922 E-04$ $2.149 E-03$ $2.642 E-03$ $4.299 E-03$ $1.212 \varepsilon-02$ 2.956E-02 $7.672 \mathrm{t}-03$ $1.049 E-04$ L.096E-04 $1.021 \mathrm{E}-04$ $394 t-04$ $2.119 E-04$ 9.259E-04 . $273 \mathrm{E}-03$ . $549 E-03$ 9.391E-03 $2.451 E-02$ 7.053E-03

ANGLE 13 $3.578 E-05$ $7.722 E-05$ $3.093 E-04$ $3.390 E-04$ $4.791 E-04$ $5.041 \mathrm{E}-04$ 6. $971 E-04$ . 2. 35 E 03 2. $7.771 E-03$

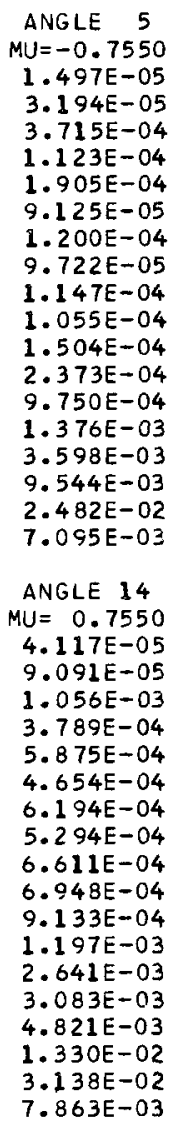

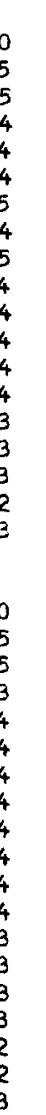
$.950 E-04$

\begin{tabular}{|c|c|c|}
\hline $\begin{array}{r}\text { ANGLE } 7 \\
\text { MU=-0.4580 } \\
1.720 E-05 \\
3.670 E-05 \\
4.255 E-04 \\
1.304 E-04 \\
2.192 E-04 \\
1.094 E-04 \\
1.431 E-04 \\
1.183 E-04 \\
1.434 E-04 \\
1.399 E-04 \\
1.944 E-04 \\
2.906 E-04 \\
1.139 E-03 \\
1.724 E-03 \\
3.690 E-03 \\
1.000 E-02 \\
2.574 E-02 \\
7.214 E-03\end{array}$ & $\begin{array}{r}\text { ANGLE } \\
M U=-0.2816 \\
1.885 E-05 \\
4.020 E-05 \\
4.652 E-04 \\
1.434 E-04 \\
2.401 E-04 \\
1.234 E-04 \\
1.614 E-04 \\
1.357 E-04 \\
1.069 E-04 \\
1.664 E-04 \\
2.256 E-04 \\
3.308 E-04 \\
1.273 E-03 \\
1.934 E-03 \\
3.745 E-03 \\
1.031 E-02 \\
2.634 E-02 \\
7.290 E-03\end{array}$ & $\begin{array}{r}\text { ANGLE } 9 \\
M U=-0.0950 \\
2.094 \mathrm{E}-05 \\
4.470 \mathrm{E}-05 \\
5.164 \mathrm{E}-04 \\
1.615 \mathrm{E}-04 \\
2.684 \mathrm{E}-04 \\
1.440 \mathrm{E}-04 \\
1.883 \mathrm{E}-04 \\
1.598 \mathrm{E}-04 \\
1.960 \mathrm{E}-04 \\
1.959 \mathrm{E}-04 \\
2.047 \mathrm{E}-04 \\
3.945 \mathrm{E}-04 \\
1.450 \mathrm{E}-03 \\
2.135 \mathrm{E}-03 \\
3.824 \mathrm{E}-03 \\
1.068 \mathrm{E}-02 \\
2.703 \mathrm{E}-02 \\
7.376 \mathrm{E}-03\end{array}$ \\
\hline $\begin{array}{l}\text { ANGLE } 16 \\
M U=0.9446 \\
5.499 E-05 \\
1.356 E-04 \\
1.653 E-03 \\
7.368 E-04 \\
1.049 E-03 \\
1.221 E-03 \\
1.666 E-03 \\
1.336 E-03 \\
1.558 E-03 \\
1.317 E-03 \\
1.441 E-03 \\
1.651 E-03 \\
3.279 E-03 \\
3.682 E-03 \\
5.507 E-03 \\
1.449 E-02 \\
3.288 E-02 \\
8.003 E-03\end{array}$ & $\begin{array}{r}\text { ANGLE } 17 \\
\text { MU= } 0.9894 \\
7.216 E-05 \\
2.286 E-04 \\
2.990 E-03 \\
1.614 E-03 \\
2.038 E-03 \\
3.151 E-03 \\
4.244 E-03 \\
2.887 E-03 \\
3.139 E-03 \\
1.870 E-03 \\
1.767 E-03 \\
1.954 E-03 \\
3.794 E-03 \\
4.021 E-03 \\
5.937 E-03 \\
1.495 E-02 \\
3.333 E-02 \\
8.039 E-03\end{array}$ & $\begin{array}{c}\text { SCALAR } \\
\text { FLUX } \\
3.275 E-04 \\
7.272 E-04 \\
8.517 E-03 \\
3.004 E-03 \\
4.698 E-03 \\
3.533 E-03 \\
4.697 E-03 \\
3.826 E-03 \\
4.586 E-03 \\
4.309 E-03 \\
5.547 E-03 \\
7.661 E-03 \\
2.104 E-02 \\
2.818 E-02 \\
5.135 E-02 \\
1.409 E-01 \\
3.496 E-01 \\
9.309 E-02\end{array}$ \\
\hline
\end{tabular}


(GAMMAS/MEV/STERADIAN/SOURCE NEUTRONI

ENERGY 8. OOE OO6.50 OO $00--8.00 E$ OO $5.00 E$ OO- $-6.50 E$ OO 4.0OE $00--5.00 E$ OO . 00 OO- $0 .-4.00 E$ OO $.50 E$ OO---3.00E 00 $2.00 E$ OO- - 2.50E 00 $1.66 \mathrm{E} 00--2.00 \mathrm{E} 00$ $1.33 \mathrm{E} 00--1.66 \mathrm{E} 00$ $1.00 \mathrm{E} 00--1.33 \mathrm{E} 00$ 8.00E-01 - - 1. OOE 00 $6.00 \mathrm{E}-01--8.00 \mathrm{E}-01$ $4.00 E-01--6.00 E-01$ $3.00 E-01--4.00 E-01$ $2.00 \mathrm{E}-01--3.00 \mathrm{E}-01$ $1.00 \mathrm{E}-01 \cdots-2.00 \mathrm{E}-01$ 1.00E-02- $-1.00 E-01$ $2.00 \mathrm{E}-02---5.00 \mathrm{E}-02$

$$
\text { ENERGY }
$$

$$
\text { GRDUP (MEV) }
$$

8. OOE OO---1.00E 01 $6.50 E$ OO- - 8. OOE 00 5.00 E $00--6.50 E$ OO 4.0OE OO---5.0OE OO .0OE OO---4.00E 00 $2.50 E$ OO---3. OOE OO $2.00 E$ OO--2.50E 00 $1.66 E$ OO- $-2.00 E$ OO $1.33 \mathrm{E} 00--1.66 \mathrm{E}$ OO $1.00 E$ O0--1.33E 00 $8.00 E-01--1.00 E$ OO $6.00 E-01--8.00 E-01$ $4.00 E-01--6.00 E-01$ 2.00E-01-C-3.00E 01 . $1.00 E-01-C 2.00 E-01$ $2.00 \mathrm{E}-02---5.00 \mathrm{E}-02$
ANGLE 1 ANGLE 2 $M U=-1.0000$ $1.462 \mathrm{E}-05$ $3.076 \mathrm{E}-05$ $3.076 E-05$
$3.558 E-04$ $9.661 \mathrm{E}-05$ $1.725 \mathrm{E}-04$ $6.284 E-05$ $8.431 E-05$ $7.862 E-05$ $1.058 \mathrm{E}-04$ $1.144 \mathrm{E}-04$ $1.383 \mathrm{E}-04$ $1.787 \mathrm{E}-04$ $1.004 E-03$ $1.405 \mathrm{E}-03$ $4.322 \mathrm{E}-03$ $1.165 \mathrm{E}-02$ $3.160 E-02$ $9.286 \mathrm{E}-03$ ANGLE 10 $M U=0.0950$ $2.823 E-05$ $5.952 \mathrm{E}-05$ $6.781 E-04$ $2.120 E-04$ $3.528 \mathrm{E}-04$ $1.752 E-04$ $2.233 \mathrm{E}-04$ $2.075 E-04$ $2.536 \mathrm{E}-04$ $2.812 E-04$ 6. $35 \mathrm{E}-04$ 2. $2.102 E-03$ $4.892 \mathrm{E}-03$ $1.425 \mathrm{E}-02$ $1.425 E-02$ $9.958 E-03$
ANGLE 2
$M U=-0.9894$ $1.469 \mathrm{E}-05$ $3.093 \mathrm{E}-05$ $3.578 E-04$
$9.787 E-05$ $1.742 E-04$ $6.414 E-05$ 8.573E-05 $7.927 E-05$ $1.053 \mathrm{E}-04$ $1.129 E-04$ $1.386 \mathrm{E}-04$ $1.836 E-04$ $1.010 \mathrm{E}-03$ $1.413 \mathrm{E}-03$ $4.330 \mathrm{E}-03$ $1.167 \mathrm{E}-02$ $3.164 E-02$ $9.291 E-03$ ANGLE 11 $M U=0.2816$ $3.317 \mathrm{E}-05$
$7.005 \mathrm{E}-05$ $7.005 \mathrm{E}-05$ $7.954 E-04$
$2.546 E-04$ $2.546 E-04$
$4.183 E-04$ $2.215 E-04$ $2.813 E-04$ $2.611 E-04$ $3.168 \mathrm{E}-04$
$3.576 \mathrm{E}-04$ $3.576 E-04$ $5.143 E-04$ $8.063 E-04$ $2.413 E-03$ S.09E-03 1. $490 \mathrm{E}-02$ $1.49 E-02$ $3.817 E-02$
$1.010 E-02$
ANGLE 3 $M U=-0.9446$ $1.503 E-05$ $3.162 E-05$ $1.025 \mathrm{E}-04$ $1.805 \mathrm{E}-04$ $6.890 \mathrm{E}-05$ $9.096 E-05$ $8.184 \mathrm{E}-05$ $1.041 E-04$ $1.086 E-04$ $1.408 \mathrm{E}-04$ 2.016E-04 $1.036 \mathrm{E}-03$ $1.452 \mathrm{E}-03$ 4.361E-03 $1.175 \mathrm{E}-02$ $3.181 E-02$ $9.314 E-03$ ANGLE 12 $M U=0.4580$ $3.961 E-05$
$8.366 E-05$ $8.366 E-05$ $9.465 E-04$
$3.105 E-04$ 5.032E-04 $2.837 E-04$ $3.597 \mathrm{E}-04$ 3.378E-04 $4.139 E-04$ 4.777E-04 $6.838 \mathrm{E}-04$ 1.730 $2.72 \exists E-03$ $5.353 \mathrm{E}-03$ $1.562 E-02$ 1.562E-02 $1.025 \mathrm{E}-02$ ANGLE
$M U=-0.8656$ 1.559E-05 3.294E-05 $3.811 E-04$ $1.101 E-04$ $1.912 \mathrm{E}-04$ $7.636 E-05$ $9.927 E-05$ $8.661 E-05$ $1.045 \mathrm{E}-04$ $1.059 \mathrm{E}-04$ $1.479 E-04$ $2.302 E-04$ $1.083 \mathrm{E}-03$ $1.540 E-03$ .409E-03 $1.189 \mathrm{E}-02$ $3.213 E-02$ $9.356 E-03$ ANGLE 13 $M U=0.6179$ $4.778 \mathrm{E}-05$ $1.012 \mathrm{E}-04$
$1.142 \mathrm{E}-03$ $1.142 \mathrm{E}-03$ $3.842 \mathrm{E}-04$
$6.138 \mathrm{E}-04$ $6.138 E-04$
$3.741 E-04$ $3.741 E-04$ $4.514 E-04$ 6.613E-04 $6.570 E-04$ ..102E-04 . $3.563 \mathrm{E}-03$ $5.650 \mathrm{E}-03$ 1.638 E-02 1.638E-02 1. $039 E-02$
ANGLE 5 $M U=-0.7550$
$1.647 \mathrm{E}-05$ $3.485 E-05$ $4.028 E-04$ $1.189 \mathrm{E}-04$ $2.044 \mathrm{E}-04$ $8.441 E-05$ $1.085 E-04$ $9.348 E-05$ $1.101 \mathrm{E}-04$ $1.115 E-04$ $1.628 \mathrm{E}-04$ $2.615 E-04$ $1.152 \mathrm{E}-03$ $1.687 \mathrm{E}-03$ $4.461 E-03$ $1.210 \mathrm{E}-02$ $3.258 E-02$ DE-O3 ALE 14 $A=0.7550$
$5.787 \mathrm{E}-05$ $1.234 E-04$ $1.393 \mathrm{E}-03$ $4.871 \mathrm{E}-04$ $7.643 \mathrm{E}-04$ $5.162 E-04$ $6.626 \mathrm{E}-04$ $6.329 E-04$ $7.885 \mathrm{E}-04$ $9.052 E-04$ $1.185 E-03$ 1.537E-03 $3.342 E-03$ . $1.716 E-02$ 1.716E-02 $4.207 \mathrm{E}-02$
$1.052 \mathrm{E}-02$
AVGLE 6 $M U=-0.6179$ $1.770 E-05$
$3.743 E-05$ $4.318 E-04$ $1.286 E-04$ $2.198 E-04$ $9.283 E-05$ $.188 E-04$ $1.034 E-04$ $1.231 \mathrm{E}-04$ $1.285 E-04$ $1.870 E-04$ $2.945 E-04$ $1.896 E-03$ $4.512 E-03$ $9.493 E-03$

ANGLE 15 $1.514 \mathrm{E}-04$ $1.721 E-03$ $6.425 E-04$ $9.813 E-04$ $7.669 E-04$ $9.975 E-04$ $9.366 E-04$ $1.139 E-03$ $1.225 E-03$ $1.487 \mathrm{E}-03$ $1.792 E-03$ . $4.188 E-03$ $1.792 \mathrm{E}-02$ $4.323 \mathrm{E}-02$ $1.063 \mathrm{E}-02$

ANGLE 7 $M U=-0.4580$ $1.934 E-05$
$4.087 E-05$ $4.702 E-04$ $1.403 E-04$ .557E-04 $1.387 E-03$ . 
(GAMMAS/MEV/STERADIAN/SOURCE NEUTRONI

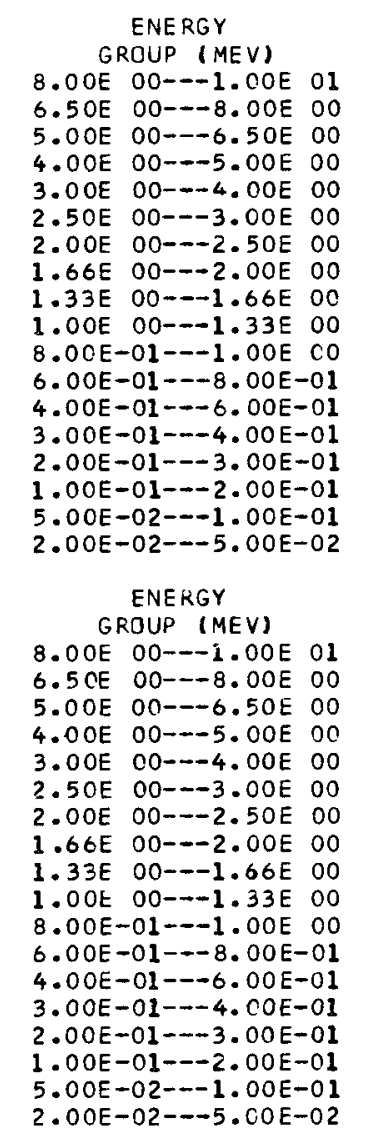

ANGLE 1 ANGLE 2 $M U=-1.0000 \quad M U=-0.5894$ $2.840 \mathrm{E}-05$ $3.279 E-04$ $8.618 E-05$ $1.568 E-04$ $5.098 \mathrm{E}-05$ $6.949 E-05$ $6.944 \mathrm{E}-05$ $9.399 \mathrm{E}-05$ $1.047 E-04$ $1.245 \mathrm{E}-04$ $1.625 \mathrm{E}-04$ $1.015 \mathrm{E}-03$ $1.445 E-03$ $4.760 E-03$ $1.288 \mathrm{E}-02$ $3.587 E-02$ 1.064E-02 $1.365 \mathrm{E}-05$ $2.858 \mathrm{E}-05$ $3.300 E-04$ $8.760 E-05$ $1.586 \mathrm{E}-04$ $5.230 E-05$ $7.073 E-05$ $6.982 \mathrm{E}-05$ $9.319 \mathrm{E}-05$ $1.032 \mathrm{E}-04$ $1.251 \mathrm{E}-04$ $1.678 E-04$
$1.022 \mathrm{E}-03$ $1.457 \mathrm{E}-03$ $4.767 \mathrm{E}-03$ $1.290 \mathrm{E}-02$ $3.592 \mathrm{E}-02$
$1.065 \mathrm{E}-02$ ANGLE 10 $M U=0.0950$ $2.836 E-05$ $5.942 E-05$ $6.718 \mathrm{E}-04$ $2.084 E-04$ $3.485 \mathrm{E}-04$ $1.634 E-04$ $2.052 E-04$ $2.022 E-04$ $2.488 E-04$ $2.965 E-04$ $4.304 E-04$ $6.533 E-04$ 2.320E-03 3.182E-03 $1.555 \mathrm{E}-02$ $1.535 E-02$ $1.146 \mathrm{E}-02$

$4.390 E-02$
$1.163 E-02$
ANGLE 3 $1.397 E-05$ $2.931 \mathrm{E}-05$ $3.384 \mathrm{E}-04$ $9.276 \mathrm{E}-05$ $5.707 E-05$ $7.532 \mathrm{E}-05$ 7.149E-05 $9.103 E-05$ $9.880 \mathrm{E}-05$ $1.284 E-04$
$1.873 E-04$ $1.873 E-04$
$1.050 E-03$ $1.511 \mathrm{E}-03$ 4.796E-03 $1.3 \mathrm{GOE}-02$ $3.612 \mathrm{E}-02$
$1.067 \mathrm{E}-02$ ANGLE 12 $M U=0.4580$ $4 . \angle 32 \mathrm{E}-05$
$8.851 \mathrm{E}-05$ $8.851 E-05$
$9.888 E-04$ $3.238 \mathrm{E}-04$ $5.256 E-04$ $2.822 E-04$ 3.534E-04 3. $534 E-04$ $5.414 \mathrm{E}-04$ $5.414 \mathrm{E}-04$ 1. $178 \mathrm{E}-03$ $1.178 \mathrm{E}-03$ $3.03 E-03$ $5.807 E-03$ $5.807 E-03$
$1.756 E-02$ $1.756 E-02$ 1. $181 \mathrm{E}-02$ $\begin{array}{ccc}\text { ANGLE } & 3 & \text { ANGLE } \quad 4 \\ M U=-0.9446 & M U=-0.8656\end{array}$ $1.458 \mathrm{E}-05$
$3.069 \mathrm{E}-05$ $3.541 \mathrm{E}-04$ $1.010 E-04$ $1.767 E-04$ $6.439 \varepsilon-05$ $8.258 E-05$ $7.502 E-05$
$9.028 E-05$ $9.028 \mathrm{E}-05$ $9.630 E-05$ 1. $372 E-04$ $1.104 \mathrm{E}-03$ $1.626 E-03$ $4.840 \mathrm{E}-03$ $1.317 \mathrm{E}-02$ $3.651 \mathrm{E}-02$ $1.072 \mathrm{E}-02$ ANGLE 13 $A U=0.6179$ $5.322 E-05$
$1.113 E-04$ $1.113 E-04$
$1.235 E-03$ $4.157 E-04$ $6.645 \varepsilon-04$ $3.849 E-04$ $4.796 E-04$ $4.89 E-04$ $0.121 E-04$ $7.5915-04$ 1.051E 03 $1.462 \mathrm{E}-03$ $3.937 \mathrm{E}-03$ G. 1.844E-02 $4.709 E-02$ $4.709 E-02$
$1.198 E-02$ ANGLE 5
$M U=-0.7550$ 1. $550 \mathrm{E}-05$ $3.266 \mathrm{E}-05$ 3. $761 \mathrm{E}-04$ $1.101 E-04$ $7.907 E-05$ 7.197E-05 $8.108 \mathrm{E}-05$ $9.537 \mathrm{E}-05$
$1.028 \mathrm{E}-04$ $1.028 \mathrm{E}-04$
$1.540 \mathrm{E}-04$ $2.518 E-04$ $1.186 E-03$ $1.809 \mathrm{E}-03$ $4.887 \mathrm{E}-0$ 1. $342 \mathrm{E}-02$ $3.706 E-02$ $1.080 E-02$ ANGLE 14 $M U=0.7550$
$6.763 E-05$ $0.763 E-05$
$1.418 E-04$ 1. $568 \mathrm{E}-03$ $5.479 E-04$ $8.595 E-04$ $5.466 E-04$ $6.835 E-04$ $8.005 E-04$ . $1.049 E-03$ $1.748 \mathrm{E}-03$ 3. $717 \mathrm{E}-03$ 4.264E-03 $6.552 E-03$ 1. 3 हE 02 $4.864 E-02$
$1.213 E-02$
ANGLE 6 $M U=-0.6179$ $1.677 E-05$ $3.531 \mathrm{E}-05$ $4.057 E-04$ $1.197 \mathrm{E}-04$ $2.055 \mathrm{E}-04$
$7.969 \mathrm{E}-05$ $1.001 E-04$ $9.092 \mathrm{E}-05$ $1.088 \mathrm{E}-04$ $1.214 \mathrm{E}-0$ $1.802 \mathrm{E}-04$ $2.885 \mathrm{E}-04$ $1.305 \mathrm{E}-03$ 2. $059 \mathrm{E}-03$ $4.932 \mathrm{E}-03$ $1.375 \mathrm{E}-02$ $3.780 \mathrm{E}-02$ $1.089 \mathrm{E}-02$

ANGLE 15 $M U=0.8656$ $8.573 \mathrm{E}-05$
$1.817 \mathrm{E}-04$ $2.011 E-03$ $7.459 \mathrm{E}-04$ $1.140 E-03$ $8.187 E-04$ $1.030 E-03$ $1.034 \mathrm{E}-03$ $1.247 E-03$ $1.405 \mathrm{E}-03$ $2.018 \mathrm{E}-03$ $4.066 \mathrm{E}-03$ $4.611 E-03$ $6.918 \mathrm{E}-03$ $5.002 \mathrm{E}-02$ $1.227 \mathrm{E}-02$
ANGLE 7 $M U=-0.4580$ $3.889 E-05$ $4.453 E-04$ $1.312 E-04$ $8.939 E-05$ 8. $939 E-05$ $1.060 E-04$ $1.309 E-04$ $1.508 \mathrm{E}-04$ 2.162E-04 $1.473 E-03$ $2.352 E-03$ 4.984E-03 $1.417 \mathrm{E}-02$ $3.870 \mathrm{E}-02$ 1.

ANGLE 16 $M U=0.9446$ $1.068 E-04$
$2.344 E-04$ Z. $631 E-03$ $1.075 E-03$ $1.571 E-03$ $1.342 \mathrm{E}-03$ $1.701 E-03$ $1.601 E-03$ $1.824 E-03$ $1.826 \mathrm{E}-03$ $2.008 E-03$ $2.267 E-03$ $4.450 E-03$ $7.272 E-03$ 2.089E-02 $5.112 E-02$ 5.
$1.236 E-02$

ANGLE 9 $M U=-0.0950$ 2.403E-05 $5.038 E-05$
$5.722 E-04$ $1.726 E-04$ $2.926 \mathrm{E}-04$ $1.282 E-04$ $1.620 \mathrm{E}-04$ $1.590 E-04$ $1.983 E-04$ $2.340 E-04$ 3.316E-04 $5.248 E-04$
$1.987 E-03$ 2. $934 \mathrm{E}-03$ 5.1 $74 E-03$ $1.527 E-02$ $4.101 \mathrm{E}-02$ SCALAR FLUX 4. $537 E-04$ $9.672 E-04$ $1.090 \mathrm{E}-02$ . $3.616 E-03$ $4.544 E-03$ $4.348 E-03$ $5.205 \mathrm{E}-03$ $7.026 E-03$ $1.054 \mathrm{E}-02$ $2.943 E-02$ $3.840 E-02$ $6.953 E-02$
$2.018 E-01$ $5.325 \mathrm{E}-01$ 1. $436 E-01$ 
(GAMMAS/MEV/STERADIAN/SOURCE NEUTRON)

ENERGY GROUP (MEV) 8.0OE OO---1.00E 01 $6.50 E$ OO- - - 8. DOE 00 4.0OE $00--6.50 E$ OO $3.00 E$ OO- $-4.00 E$ OO $3.50 E$ OO $\rightarrow-3.00 E$ OO $2.00 E$ OO- $-2.50 E$ OO 1.66 E $00-2-2.00 E$ OO $1.33 E$ OD- $-1.66 E$ OO $1.00 E$ OO- $-1.33 E$ OO $6.00 E-01--1.00 E$ OD $6.00 \mathrm{E}-01---8.00 \mathrm{E}-01$ 4.00E-01-- $-6.00 E-01$ 3.00E-01---4.00E-01 $2.00 \mathrm{E}-01--3.00 \mathrm{E}-0$ $1.00 E-01--2.00 E-01$ $5.00 E-02=-1.00 E-01$ 2. $00 \mathrm{E}-02---5.00 E-02$

$$
\text { ENERGY }
$$$$
\text { GROUP (MEV }
$$

8.00E $00--1.00 E$ O1 $6.50 E$ OO- $-8.00 E$ OO $5.00 E$ O0- $-6.50 E$ OO 4.00E OO-- -5.00E OD $3.00 E$ OOC--4.0OE OO $2.50 E$ OO- $-3.00 E$ OO $2.00 E$ OO---2.50E OO $1.66 E$ O0-- 2. 00 E OO $1.33 E$ CO---1.66E 00 $1.00 \mathrm{E} 00---1.33 \mathrm{E} 00$ 8.00E-01---1.00E 00 $6.00 E-01--8.00 E-01$ 4.00E-01- -6. $60 \mathrm{E}-01$ $3.00 E-01--4.00 \mathrm{E}-01$ 2.00E-01- -3.00E-01 $1.00 E-01--2.00 E-01$ $5.00 E-02-1.00 E-01$
ANGLE 1 ANGLE 2 $1.142 E-05$
$2.377 E-05$ 2.377E-05 $6.947 E-05$ $6.947 E-05$ $3.860 E-05$ $3.860 E-05$
$5.456 E-05$ $5.456 E-05$
$5.823 E-05$ $5.823 E-05$
$7.935 E-05$ $8.910 E-05$ 1.356E-03 . $304 E-02$ $3.708 \mathrm{E}-02$
$1.108 \mathrm{E}-02$ ANGLE 10 $2.546 E-05$ 5 $1.854 E-04$ $3.102 E-04$ $1.422 E-04$ $1.770 \mathrm{E}-04$ $1.821 \mathrm{E}-04$ $2.262 \mathrm{E}-04$ $2.842 E-04$ $4.258 \mathrm{E}-04$ $7.064 E-04$ $2.343 E-03$ $3.216 E-03$ $5.360 E-03$ $1.632 E-02$ $1.422 E-02$
$1.197 E-02$ $2.395 \mathrm{E}-05$ $1.371 E-03$
ANGLE $2 \quad$ ANGLE 3 $1.149 \mathrm{E}-05$ $2.764 E-04$
$7.098 E-05$ 1. $310 E-04$ $1.310 E-04$ $5.988 E-05$ $5.825 E-05$ 7.821E-05 $8.751 E-05$ 1.029E-04 $1.384 E-04$ $9.458 \mathrm{E}-04$ $1.307 E-02$ $3.713 \mathrm{E}-02$ $1.109 E-02$

ANGLE 11 $M U=0.2816$ $3.150 E-05$
$6.561 E-05$ $7.322 E-04$ $2.349 E-04$ $3.864 E-04$ $1.911 \mathrm{E}-04$ $2.363 E-04$ $2.454 \mathrm{E}-04$ $3.048 \mathrm{E}-04$ $3.918 \mathrm{E}-04$ 5. $913 E-04$ $9.515 E-04$ $2.718 \mathrm{E}-03$ $3.447 E-03$ $5.601 E-03$ $1.714 \mathrm{E}-02$ 4.587E-02 $1.216 \mathrm{E}-02$ ANGLE 12 $1.179 \mathrm{E}-05$ $2.465 \mathrm{E}-05$ $2.844 E-04$
$7.644 E-05$ $7.644 E-05$
$1.379 E-04$ $1.379 E-04$
$4.446 E-05$ $4.446 E-05$ $5.918 E-05$
$5.868 E-05$ $7.486 E-05$ $8.291 E-05$ $1.070 E-04$ $1.584 E-04$ $9.737 E-04$ (4) .316E-02 $3.736 E-02$ $112 E-02$ ANGLE 4 $M U=-0.8656$ $2.595 E-05$ 2. $8.482 \mathrm{E}-05$ $1.489 E-04$ $5.127 E-05$ $6.496 E-05$ $6.048 \mathrm{E}-05$ $6.048 E-05$
$7.253 E-05$ 8.012E-05 $1.166 \mathrm{E}-04$ $1.892 \mathrm{E}-04$ $1.029 E-03$ $1.571 \mathrm{E}-03$ 4.892E-03 $1.335 E-02$ $3.778 E-02$
$1.117 E-02$ ANGLE 13 $4.013 \mathrm{E}-05$ $8.334 E-05$ $9.220 E-04$ $3.043 E-04$ $4.926 \mathrm{E}-04$ $2.626 \mathrm{E}-04$ $3.241 E-04$ $3.431 E-04$ 4. $305 E-04$ $5.608 \mathrm{E}-04$ $8.220 \mathrm{E}-04$ $1.236 \mathrm{E}-03$ $3.087 \mathrm{E}-03$ $3.695 E-03$ $5.898 E-03$ $1.802 E-02$ $4.761 E-02$
$1.235 E-02$ $5.244 E-05$ $1.087 E-04$ $1.192 \mathrm{E}-03$ $4.052 E-04$ $6.454 E-04$ $3.728 E-04$ $4.599 \varepsilon-04$ 4.952E-04 $6.242 E-04$ $8.046 \mathrm{E}-04$ $1.114 E-03$ $1.537 \mathrm{E}-03$ $3.440 \mathrm{E}-03$ $3.986 E-03$ $6.235 E-03$ $1.896 E-02$ $4.936 \mathrm{E}-02$ ANGLE 5 ANGLE 6

MU $=-0.6179$ $1.319 \mathrm{E}-05 \quad 1.435 \mathrm{E}-05$ 2.776E-05 3.017E-05 $9.342 \mathrm{E}-05 \quad 1.018 \mathrm{E}-04$ $9.342 E-05$ $1.613 \mathrm{E}-04$
$5.794 \mathrm{E}-05$ $7.157 \mathrm{E}-05$ $6.515 E-05$ $7.646 E-05$ $8.639 \mathrm{E}-05$ $1.333 E-04$ $2.221 E-04$ $1.115 E-03$ $1.778 \mathrm{E}-03$ 4. $927 \mathrm{E}-03$ 1. $362 E-02$ $3.839 \mathrm{E}-02$
$1.125 \mathrm{E}-02$ ANGLE 14

ANGLE 7 $M U=-0.4580$ $1.594 \mathrm{E}-05$
$3.342 \mathrm{E}-05$ $3.815 \mathrm{E}-04$ $1.117 E-04$ $1.923 E-04$ 7.262E-05 $9.086 E-05$ $8.875 E-05$ $1.107 E-04$ $1.332 \mathrm{E}-04$ $1.933 E-04$ $3.076 E-04$ $1.429 E-03$ $2.364 \mathrm{E}-03$ $5.003 E-03$ $1.442 E-02$ 4. $017 E-02$ $1.148 E-02$

ANGLE 16 $6.991 \mathrm{E}-05$ $1.450 \mathrm{E}-04$ $5.582 E-04$ $8.708 E-04$ 5. $500 \mathrm{E}-04$ $6.770 E-04$ $7.288 E-04$ $9.050 E-04$ $1.121 \mathrm{E}-03$ $1.446 \mathrm{E}-03$ $1.830 \mathrm{E}-03$ 3. $782 \mathrm{E}-03$ $4.320 E-03$ $6.594 E-03$ $1.988 \mathrm{E}-02$ $5.104 \mathrm{E}-02$
ANGLE 8 $M U=-0.2816$ $3.792 E-05$ 4.311E-0 $1.266 E-04$ $2.176 E-04$ $8.610 E-05$ $1.086 E-04$ $1.099 \mathrm{E}-04$ $1.392 E-04$ $1.700 E-04$ $2.424 E-04$ $3.879 E-04$ $1.678 E-03$ $2.679 E-03$ $5.070 \mathrm{E}-03$ $4.135 E-02$ ANGLE 17 $M U=0.9894$ $3.831 \mathrm{E}-04$ $4.363 E-03$ $2.046 \mathrm{E}-03$ $2.684 E-03$ 2.769E-03 $3.352 \mathrm{E}-03$ $2.752 E-03$ $2.837 \mathrm{E}-03$ $2.378 \mathrm{E}-03$ 2.343E-03 $2.534 E-03$ 4. $864 E-03$ $5.188 \mathrm{E}-03$ $7.544 E-03$ $2.194 E-02$ $5.442 E-02$

ANGLE 9 $M U=-0.0950$ 
(GAMMAS/MEV/STERAUIAN/SOURCE NEUTRON)

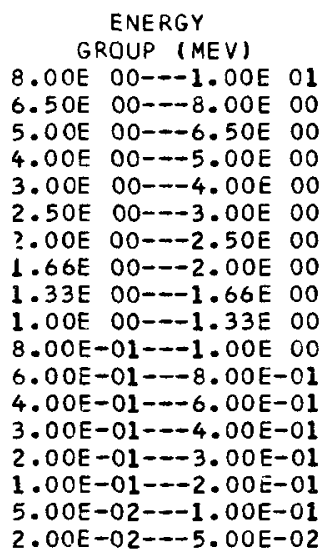

ENERGY

GROUP (MEV)

$8.00 E$ O0---1.00E 01

$6.50 E$ OO-- $-8.00 E$ OO
$5.00 E$ OD- $-6.50 E$ OO 4.00E $00--5.00$ E 00 3.00 E $00---4.00 E$ EO $2.50 E$ OO- $-3.00 E \quad 00$ $2.00 E$ O0---2.50E 00 1.66 E $00---2.00 E$ EO $1.33 E$ 00---1.66E 00 $1.00 E$ OO- -1.33 E 00 $8.00 E-01--1.00 E 00$ $6.00 E-01=-2.00 E-01$ 4.00E-01---6.00E-01 $3.00 E-01--4.00 E-01$ $2.00 E-01--3$. . COE-O $1.00 E-01--2.00 E-01$ $5.00 E-02---1.00 E-01$
$2.00 E-02-\cdots 5.00 E-02$
ANGLE 1 ANGLE 2 $4.806 E-006$
.9000 $4.806 E-06$
$9.759 E-06$ 1.140E-04 $1.140 E-04$
$2.278 E-05$ $1.152 \mathrm{E}-05$ $1.152 \mathrm{E}-05$ $2.247 E-05$
$3.042 E-05$ $4.256 E-05$ $4.458 E-05$ $3.812 \mathrm{E}-05$ $3.812 E-05$
$4.417 E-05$ $4.417 E-05$
$5.541 E-04$ $8.172 E-04$ $3.726 E-03$ $9.871 \mathrm{E}-03$ $2.932 E-02$ $8,892 E-03$

ANGLE 10 $1.248 \mathrm{E}-05$ $2.589 E-05$ $2.879 \mathrm{E}-04$ $9.140 E-05$ $1.520 E-04$ $8.697 \mathrm{E}-05$ $9.577 \mathrm{E}-05$ $1.227 E-04$ $1.754 E-04$ $2.960 E-04$ $5.463 \mathrm{E}-04$ $1.769 \mathrm{E}-03$ $2.436 \mathrm{E}-03$ $3.959 E-03$ $1.261 E-02$ $3.558 \mathrm{E}-02$ $9.673 \mathrm{E}-03$ $4.855 \mathrm{E}-06$ $9.901 \mathrm{E}-06$ $1.154 E-04$
$2.440 E-05$ $2.440 E-05$ $1.259 \mathrm{E}-05$ $2.272 \mathrm{E}-05$ $2.949 \mathrm{E}-05$ $4.056 E-05$ $4.056 E-05$ $3.931 E-05$ $4.918 E-05$ $4.918 E-05$
$5.578 E-04$ $8.332 E-04$ $3.729 E-03$ $9.891 E-03$ $2.937 E-02$ 8.899E-03

ANGLE 11 $M U=0.2816$ $3.380 E-05$ $3.713 E-04$ $1.238 \mathrm{E}-04$ $2.006 E-04$ $1.031 E-04$ $1.259 E-04$ $1.421 \mathrm{E}-04$ $1.860 E-04$ $2.765 E-04$ $4.600 \mathrm{E}-04$ $7.828 E-04$ $2.074 \mathrm{E}-03$ $2.592 \mathrm{E}=03$ $4.143 E-03$ $1.329 \mathrm{E}-02$ $3.704 \mathrm{E}-02$ $9.838 \mathrm{E}-03$
ANGLE 3 ANGLE 4

1

ANGLE 5 $5.046 \mathrm{E}-06$ $1.043 \mathrm{E}-05$ $1.208 \mathrm{E}-04$ 2.997E-05 $5.683 \mathrm{E}-05$ $1.626 E-05$
$2.370 E-05$ $2.370 E-05$
$2.650 E-05$ $3.410 E-05$ $3.726 \mathrm{E}-05$ $3.726 E-05$
$4.414 E-05$ $4.414 E-05$
$6.708 E-05$ $6.708 E-05$
$5.745 E-04$ $9.031 E-04$ $3.739 t-03$ $9.975 \mathrm{E}-03$ $2.956 \mathrm{E}-02$ 8. $925 E-03$ ANGLE 12 $M U=0.4580$ $2.243 E-05$
$4.609 E-05$ 4. $987 E-04$ $1.715 E-04$ $2.738 E-04$ $1.562 E-04$ $1.941 \mathrm{E}-04$ $2.287 E-04$ $3.044 E-04$ $4.462 E-04$ $6.874 E-04$ $1.044 \mathrm{E}-03$ $2.357 \mathrm{E}-03$ $2.770 E-03$ $4.373 E-03$ $1.403 E-02$ $3.857 \mathrm{E}-02$
$1.000 \mathrm{E}-02$ $5.379 \mathrm{E}-06$ $1.129 E-05$ 3. $745 \mathrm{E}-05$ $6.529 E-05$ $2.110 \varepsilon-05$ 2. $535 \mathrm{E}-05$ $2.535 E-05$ $2.354 E-05$
$2.748 E-05$ $2.748 E-05$
$3.268 E-05$ $3.268 E-05$
$5.270 E-05$
$9.0164 E-05$ $5.270 E-05$
$9.164 E-05$ $9.164 E-05$
$6.116 E-04$ $6.116 E-04$
$1.041 E-03$ 3. $749 \mathrm{E}-03$ $1.013 \mathrm{E}-02$ $2.993 E-02$ $8.974 \mathrm{E}-03$ ANGLE 13 $M U=0.6179$ $3.251 \mathrm{E}-05$
$6.630 \mathrm{E}-05$ $7.041 E-04$ 2. $517 E-04$ $3.958 \mathrm{E}-04$ 2.525E-04 $3.162 E-04$ $3.797 E-04$ 4. $984 E-04$ $6.918 \mathrm{E}-04$ $9.644 E-04$ $1.301 E-03$ $2.615 E-03$ $2.095 \mathrm{E}-03$ $4.532 E-03$ $1.480 \mathrm{E}-02$ 1.017E-02
5.814E-06 MU $=-0.6179$ $1.228 \mathrm{E}-05 \quad 1.342 \mathrm{E}-05$ $1.403 E-04 \quad 1.528 \mathrm{E}-04$ $7.3248 E-05 \quad 4.613 E-05$ $2.468 E-05 \quad 2.694 E-05$ $2.769 \mathrm{E}-05 \quad 3.143 \mathrm{E}-05$ $2.413 E-05 \quad 2.979 E-05$ $2.756 E-05 \quad 3.635 E-05$ $3.571 \mathrm{E}-05 \quad 4.781 \mathrm{E}-05$ $6.391 E-05 \quad 7.805 E-05$ $1.131 E-04 \quad 1.340 E-04$ $6.789 E-04 \quad 7.904 E-04$ $1.245 E-03 \quad 1.504 E-03$ $3.750 E-03 \quad 3.743 E-03$ $1.035 \mathrm{E}-02 \quad 1.065 \mathrm{E}-02$ $3.046 \mathrm{E}-02 \quad 3.115 \mathrm{E}-02$ $9.043 E-03 \quad 9.133 E-03$

ANGLE 14 $M U=0.7550$ $1.008 \mathrm{E}-04$ $1.049 \mathrm{E}-03$ $3.998 E-04$ $6.106 \mathrm{E}-04$ 4. $288 E-04$ $5.264 E-04$ $6.190 E-04$ $7.765 E-04$ $9.970 E-04$ 1.259E-03 $2.863 \mathrm{E}-03$ $3.257 E-03$ $4.903 E-03$ $1.556 E-02$ $4.157 \mathrm{E}-02$ $1.031 \mathrm{E}-02$ ANGLE 15 $M U=0.8656$ $7.862 \mathrm{E}-05$
$1.594 \mathrm{E}-04$ $1.627 \mathrm{E}-03$ $6.678 \mathrm{E}-04$ $9.771 E-04$ $7.330 E-04$ $8.059 \mathrm{E}-04$ $9.662 E-04$ $1.134 \mathrm{E}-03$ $1.326 \mathrm{E}-03$ $1.528 \mathrm{E}-03$ $1.726 \mathrm{E}-03$ $3.120 \mathrm{E}-03$ 3.517E-03 $5.169 \mathrm{E}-03$ $1.625 \mathrm{E}-02$ $4.286 \mathrm{E}-02$ $1.044 \mathrm{E}-02$ ANGLE 6

ANGLE 7 ANGLE 8 $7.147 E-06 \quad 8 . \angle 73 E-06$
$1.491 E-05$ $1.491 E-05$ $1.694 t-04$ 4.879E-05 $8.462 E-05$ $2.986 E-05$ $3.767 E-05$ $5.169 E-05$ $6.662 E-05$ $9.782 \mathrm{E}-05$ $1.677 \mathrm{E}-04$ $9.576 \mathrm{E}-04$ $1.783 E-03$ $3.741 \mathrm{E}-03$ $1.102 E-02$ $3.202 E-G 2$ $9 . \angle 43 E-03$
ANGLE 16 $U=0.9446$
$1.231 E-04$ $2.515 E-04$ $2.54 \angle E-03$ $1.113 t-03$ $1.548 \mathrm{E}-\mathrm{C3}$ $1.220 E-03$ $1.387 \mathrm{E}-03$ $1.437 \mathrm{E}-0 \mathrm{O}$ $1.559 E-03$ $1.638 \mathrm{E}-03$ $1.737 E-03$ $3.365 \mathrm{E}-03$ $3.726 \mathrm{E}-03$ $5.402 E-03$ $1.681 E-02$ $4.387 E-02$
$1.053 E-02$

MU $=-0.0950$ $1.942 E-04 \quad 2.318 E-04$ $5.515 E-05 \quad 6.882 E-05$ $9.668 E-05 \quad 1.183 E-04$ $3.657 E-05 \quad 40$ S66E-05 $4.774 E-05 \quad 0.320 E-05$ $5.350 E-05 \quad 7.034 E-05$ $6.965 E-05 \quad 9.028 E-05$ $8.963 E-05 \quad 1.209 E-04$ $1.310 E-04 \quad 1.913 E-04$ $2.365 E-04 \quad 3.004 E-04$ $1.185 E-03 \quad 1.463 E-03$ $2.045 E-03 \quad 2.263 E-03$ $3.763 E-03 \quad 3.832 E-03$ $1.147 E-02$ 1.200E-02 $3.305 E-02 \quad 3.424 E-02$ 
(GAMMAS/MEV/STERADIAN/SOURCE NEUTRON)

ENERGY GROUP (MEV) $8.00 E$ OO- $-1.00 E$ OI 5. $4.00 E$ OO- $00-5.00 E$ OO 00 $3.00 E$ OO- $-4.00 E$ OO $2.50 E$ OO- $-3.00 E$ OO $2.00 E$ OO- - 2.50E 00 .66E $00--2$. . COE 00 $1.33 \mathrm{E} 00--1.66 \mathrm{E} 00$ $1.00 E$ OO- $-1.33 E$ OO 8. OOE-01--1.0OE OO $6.00 \mathrm{E}-01--8.00 \mathrm{E}-01$ $4.00 E-01--6.00 E-01$ $3.00 E-01--4.00 E-01$ $2.00 E-01--3.00 E-01$ $1.00 E-01--2.00 E-01$ $5.00 E-02--1.00 E-01$ 2. $00 \mathrm{E}-02---5.00 \mathrm{E}-02$

$$
\text { ENERGY }
$$

$$
\text { GROUP (MEV) }
$$

$8.00 E$ OO-D-1.00E OI 6.5OE OO---8.00E OO $5.00 E$ OO- $-6.50 E$ OD $4.00 E$ OO- $-5.00 E$ OO $3.00 E$ OO- - 4.COE OO 2.5OE OO--3.00E OO $2.00 E$ OO---2.50E OO 1.66 E OO---2.00E OO $1.33 \mathrm{E} 00--1.66 \mathrm{E}$ OO $1.00 E$ OO- $1.33 \mathrm{E} O 0$ $8.00 E-01--1.00 E$ OO $6.00 E-01--8.00 E-01$ 4.00E-01- $6.00 E-01$ 3.00E-O1- - 4. DOE-OI $2.00 E-01--3.00 E-01$ $1.00 E-01--2.00 E-01$ $2.00 E-02--1.00 E-01$ $\triangle N G L E \quad 1$ $1.476 \mathrm{E}-06$
1.0000 $2.795 \mathrm{E}-06$ $3.437 \mathrm{E}-05$ 3.437E-05 $1.091 \mathrm{E}-05$ $8.500 \mathrm{E}-07$ $8.644 E-06$ $1.602 \mathrm{E}-05$ $2.295 \mathrm{E}-05$ $2.065 \mathrm{E}-05$ $7.374 E-06$ $1.650 E-06$ $2.656 E-04$ $3.864 \mathrm{E}-04$ $2.251 E-03$ $5.776 \mathrm{E}-03$ $1.758 \mathrm{E}-02$ ANGLE 10 $U U=0.0950$

$4.484 E-06$ $9.305 \mathrm{E}-06$ $1.027 \mathrm{E}-04$ $3.439 \mathrm{E}-05$ $5.610 \mathrm{E}-05$ $2.764 E-05$ $3.283 \mathrm{E}-05$ $3.633 \mathrm{E}-05$ $4.795 \mathrm{E}-05$ $7.962 \mathrm{E}-05$ 1.587E-04 $3.299 \mathrm{E}-04$ $1.044 \mathrm{E}-03$ 1.432E-0 $2.270 E-03$ $2.150 \mathrm{E}-02$ $2.150 E-02$
$5.856 E-03$
ANGLE 2 $M U=-0.9894$ $2.902 \mathrm{E}-06$ $3.531 \mathrm{E}-05$ 3.257E-O $1.231 \mathrm{E}-05$ $1.721 E-06$ $8.559 \mathrm{E}-06$ $1.482 \mathrm{E}-0$ $2.087 E-05$ $1.901 E-05$ $8.507 E-06$ 5.949E-06 $2.666 \mathrm{E}-04$ $3.978 E-04$ $2.252 E-03$ $5.789 \mathrm{E}-0$ $1.761 E-02$
$5.373 E-03$

\section{ANGLE 11} $M U=0.2816$ $0.135 E-06$ $1.271 E-05$
$1.377 E-04$ $1.377 \mathrm{E}-04$
$4.936 \mathrm{E}-05$ $4.936 E-05$
$7.739 E-05$ $7.739 \mathrm{E}-05$ $4.219 \mathrm{E}-05$ $5.034 E-05$ $5.930 E-05$ $8.399 E-05$ $1.473 \mathrm{E}-04$ $2.761 \mathrm{E}-04$ $4.980 \mathrm{E}-04$ $1.230 \mathrm{E}-03$ $1.503 \mathrm{E}-03$ 2.36E-O . $241 \mathrm{E}-02$ $5.959 E-03$
ANGLE 3 $U=-0.9446$ $3.283 \mathrm{E}-06$ 3.871E-O 7.830E-0 7.830E-06 $4.398 \mathrm{E}-06$ $8.276 E-06$ $1.073 \mathrm{E}-05$ $1.374 \mathrm{E}-05$ $1.375 \mathrm{E}-0$ $1.272 \mathrm{E}-0$ $1.953 \mathrm{E}-05$ 2.725E-04 $4.478 \mathrm{E}-04$ $2.255 E-03$ $5.841 \mathrm{E}-03$ $1.773 \mathrm{E}-02$
$5.390 \mathrm{E}-03$ ANGLE 12 $U=0.4580$ $8.907 E-06$ $1.819 \mathrm{E}-05$ $6.966 \mathrm{E}-05$ $1.095 \mathrm{E}-04$ $6.866 \mathrm{E}-05$ 8.840E-05 $1.143 E-04$ $1.653 \mathrm{E}-04$ $2.704 \mathrm{E}-04$ $4.397 E-04$ 6.75SE-04 $1.388 E-03$ $1.594 E-03$ 2.506E-03 . $306 \mathrm{E}-03$ $6.063 E-03$
ANGLE 4 $1.800 \mathrm{E}-06$ $3.814 E-06$ $4.357 \mathrm{E}-05$ $1.356 \mathrm{E}-05$ $1.356 E-05$ $2.286 E-05$
$7.577 \mathrm{E}-06$ $8.007 E-06$ $6.195 E-06$ $6.257 E-06$ $8.871 E-06$ $1.899 \mathrm{E}-05$ $3.633 E-05$ $2.893 E-04$ $5.465 \mathrm{E}-04$ $2.252 \mathrm{E}-03$ $5.937 \mathrm{E}-03$ $1.796 \mathrm{E}-02$
$5.420 \mathrm{E}-03$ ANGLE 13 $M=0.6179$ $1.406 \mathrm{E}-05$
$2.826 \mathrm{E}-05$ $2.826 \mathrm{E}-05$ $1.092 \mathrm{E}-04$ $1.720 \mathrm{E}-04$ $1.270 E-04$
$1.687 E-04$ $1.687 E-04$
$2.222 E-04$ $3.082 \mathrm{E}-04$ $4.516 E-04$ $6.341 \mathrm{E}-04$ $8.376 E-04$ $1.521 \mathrm{E}-03$ $1.723 E-03$ $2.652 E-03$ $8.770 E-03$ $6.163 E-03$
ANGLE 5 MU $=-0.617$ 1.979E-06 2.163E-06 $4.260 E-06$ $4.797 E-05$ $2.638 E-05$ $2.638 E-05$
$9.300 E-06$ $8.286 E-06$ $5.174 \mathrm{E}-06$ $5.238 \mathrm{E}-06$ $9.774 E-06$ $2.499 E-05$ 4. $722 \mathrm{E}-05$ $3.270 E-04$ $6.918 \mathrm{E}-04$ $2.237 E-03$ $6.075 E-03$ $1.829 E-02$
$5.463 E-03$ ANGLE 14 $M U=0.7550$ $2.448 E-05$
$4.884 E-05$ $4.885 \mathrm{E}-04$ $2.022 \mathrm{E}-04$ $3.070 E-04$ $2.527 \mathrm{E}-04$ $3.215 \mathrm{E}-04$ 4.016E-04 $6.708 E-04$ (6.7 (2.702E 04 $1.648 \mathrm{E}-03$ $1.878 \mathrm{E}-03$ $2.804 \mathrm{E}-03$ $9.225 \mathrm{E}-03$

\begin{tabular}{|c|c|c|c|}
\hline $\begin{array}{l}A V=-0.6179 \\
2.163 E-06 \\
4.594 E-06 \\
5.179 E-05 \\
1.674 E-05 \\
2.718 E-05 \\
9.240 E-06 \\
9.700 E-06 \\
8.627 E-06 \\
1.099 E-05 \\
1.668 E-05 \\
3.010 E-05 \\
5.330 E-05 \\
3.969 E-04 \\
8.728 E-04 \\
2.212 E-03 \\
6.256 E-03 \\
1.873 E-02 \\
5.519 E-03\end{array}$ & $\begin{array}{r}\text { ANG }=-0.4580 \\
2.400 E-06 \\
4.972 E-06 \\
5.649 E-05 \\
1.526 E-05 \\
2.742 E-05 \\
9.157 E-06 \\
1.268 E-05 \\
1.510 E-05 \\
2.040 E-05 \\
2.649 E-05 \\
3.650 E-05 \\
6.704 E-05 \\
5.081 E-04 \\
1.061 E-03 \\
2.187 E-03 \\
6.482 E-03 \\
1.927 E-02 \\
5.588 E-03\end{array}$ & $\begin{array}{r}\text { ANG }=-0.2816 \\
2.7 .88 E-06 \\
5.696 \mathrm{E}-06 \\
6.478 \mathrm{E}-05 \\
1.052 \mathrm{E}-05 \\
3.093 \mathrm{E}-05 \\
1.158 \mathrm{E}-05 \\
1.729 \mathrm{E}-05 \\
2.179 \mathrm{E}-05 \\
2.895 \mathrm{E}-05 \\
3.627 \mathrm{E}-05 \\
5.153 \mathrm{E}-05 \\
1.097 \mathrm{E}-04 \\
6.618 \mathrm{E}-04 \\
1.227 \mathrm{E}-03 \\
2.179 \mathrm{E}-03 \\
6.756 \mathrm{E}-03 \\
1.991 \mathrm{E}-02 \\
5.668 \mathrm{E}-03\end{array}$ & $\begin{array}{r}\text { MUE }=-0.0950 \\
3.440 E-06 \\
7.065 E-06 \\
7.943 E-05 \\
2.289 E-05 \\
4.045 E-05 \\
1.776 E-05 \\
2.356 E-05 \\
2.755 E-05 \\
3.551 E-05 \\
4.949 E-05 \\
8.745 E-05 \\
1.960 E-04 \\
8.474 E-04 \\
1.349 E-03 \\
2.204 E-03 \\
7.077 E-03 \\
2.066 E-02 \\
5.758 E-03\end{array}$ \\
\hline $\begin{array}{l}\text { ANGLE } 15 \\
1 U=0.8656 \\
4.601 E-05 \\
9.153 E-05 \\
8.839 E-04 \\
4.076 E-04 \\
5.787 E-04 \\
4.918 E-04 \\
5.788 E-04 \\
6.624 E-04 \\
7.700 E-04 \\
8.912 E-04 \\
9.919 E-04 \\
1.073 E-03 \\
1.787 E-03 \\
2.027 E-03 \\
2.953 E-03 \\
9.638 E-03 \\
2.604 E-02 \\
6.330 E-03\end{array}$ & $\begin{array}{r}\text { ANGLE } 16 \\
M U=0.9446 \\
8.753 E-05 \\
1.736 E-04 \\
1.635 E-03 \\
7.944 E-04 \\
1.051 E-03 \\
8.812 E-04 \\
9.612 E-04 \\
9.977 E-04 \\
1.048 E-03 \\
1.075 E-03 \\
1.098 E-03 \\
1.152 E-03 \\
1.938 E-03 \\
2.136 E-03 \\
3.083 E-03 \\
9.967 E-03 \\
2.666 E-02 \\
6.387 E-03\end{array}$ & $\begin{array}{r}\text { ANGLE } 17 \\
M U=0.9894 \\
1.515 \mathrm{E}-04 \\
3.108 \mathrm{E}-04 \\
2.935 \mathrm{E}-03 \\
1.439 \mathrm{E}-03 \\
1.747 \mathrm{E}-03 \\
1.473 \mathrm{E}-03 \\
1.511 \mathrm{E}-03 \\
1.390 \mathrm{E}-03 \\
1.326 \mathrm{E}-03 \\
1.204 \mathrm{E}-03 \\
1.150 \mathrm{E}-03 \\
1.206 \mathrm{E}-03 \\
2.062 \mathrm{E}-03 \\
2.192 \mathrm{E}-03 \\
3.171 \mathrm{E}-03 \\
1.017 \mathrm{E}-02 \\
2.703 \mathrm{E}-02 \\
6.421 \mathrm{E}-03\end{array}$ & $\begin{array}{c}\text { SCALAR } \\
\text { FLUX } \\
1.572 E-04 \\
3.174 E-04 \\
3.174 E-03 \\
1.336 E-03 \\
1.910 E-03 \\
1.428 E-03 \\
1.667 E-03 \\
1.876 E-03 \\
2.242 E-03 \\
2.826 E-03 \\
3.705 E-03 \\
5.076 E-03 \\
1.229 E-02 \\
1.6711 E-02 \\
3.018 E-02 \\
9.410 E-02 \\
2.699 E-01 \\
7.335 E-02\end{array}$ \\
\hline
\end{tabular}
$6.254 \mathrm{E}-03$ 
(GAMMAS/MEV/STERADIAN/SOURCE NEUTRON)

ENERGY GROUP (MEV) 8.0OE OO---1. COE OI $5.00 E$ OO---6.50E OD $4.00 E$ OO- $-5.00 E$ OO 3.00 OE OO---4.0OE 00 $2.50 \mathrm{E} \quad 00--3.00 \mathrm{E} \quad 00$ $2.00 E$ OO-- $2.50 E$ OO $1.66 \mathrm{E} \quad 00---2.00 \mathrm{E} 00$ .33E $00--1.66$ E 00 $1.00 E$ O0 $0-1.33 E$ EO $8.00 \mathrm{E}-01--1.00 \mathrm{E} 00$ $6.00 \mathrm{E}-01---8.00$ $4.00 E-01--6.00 E-01$ $3.00 E-01--4.00 E-01$ $.00 E-01--3.00 E-01$ $1.00 \mathrm{E}-01-\cdots 2.00 \mathrm{E}-01$ $5.00 \mathrm{E}-02-1.00 \mathrm{E}-0 \mathrm{I}$ $2.00 E-02--5.00 E-02$

\section{ENERGY}

GROUP (MEV)

8.00E O0--1. 1.00E 01 $6.50 \mathrm{OE}$ 00-- $-8.00 \mathrm{E}$ OO 5.0 E $00--6.50 E$ EO 4.00 E $00--5.00 E$ OO $3.0 O F$ OO-C. $2.50 E$ EO- $-3.00 E$ EO 2.OOE $00-2.50 E$ OO $1.66 \mathrm{E}$ OO- $2.00 \mathrm{E}$ OO 1.33 E $00--1.66 E$ OO 1.0 CE OD-D-1.33E OO $8.00 E-01--1$. COE 00 $6.00 E-01-8.00 E-01$ $4.00 E-01--0.00 E-01$ .0OE O12.0OE-O1-O3. OOE-OI $1.00 E-01-2.00 E-O I$ $2.00 E-02--5.00 E-02$
ANGLE 1 $\begin{aligned} M U & =-1.0000 \\ 3.434 E-07 & \end{aligned}$ $4.937 \mathrm{E}-07$ 7.745E-06 $-3.328 \mathrm{E}-06$ $-4.347 E-07$ $-1.561 E-06$ . $561 E-06$ (1) $1.287 E-05$ $9.963 E-06$
$-1.687 E-06$ $-8.341 E-06$ $1.191 \mathrm{E}-04$ $1.660 \mathrm{E}-04$ $1.205 \mathrm{E}-03$ $2.988 \mathrm{E}-03$ $.206 E-03$ $2.817 E-03$ ANGLE 10 $M U=0.0950$ $1.384 E-06$
$2.907 E-06$ $2.907 E-06$ $3.174 E-05$ $1.202 \mathrm{E}-05$ $1.856 E-05$ $9.788 E-06$ $1.047 E-05$ $1.057 E-05$ $1.429 E-05$ $3.055 E-05$ $7.620 E-05$ $1.776 E-04$ 7.5 $156 E-03$ $3.862 \mathrm{E}-03$ 1.127E-0 $3.073 E-03$
ANGLE 3 $M U=-0.9446$ $4.385 \mathrm{E}-07$ $8.369 \mathrm{E}-07$ $1.043 E-05$ $8.762 E-07$ $3.724 E-06$ $6.909 \mathrm{E}-07$ $2.857 E-06$ $3.909 E-06$ 4. $999 \mathrm{E}-06$ $4.244 E-06$ $2.164 E-06$ $3.233 E-06$ $1.198 \mathrm{E}-04$ $2.021 E-04$ $1.205 \mathrm{E}-03$ $3.023 \mathrm{E}-03$ $9.287 \mathrm{E}-03$ $2.828 \mathrm{E}-03$ $M U=-0.8656$ $5.364 E-07$
$1.167 E-06$ $1.309 \mathrm{E}-05$ $5.142 \mathrm{E}-06$ .885E-06 $2.153 \mathrm{E}-06$ $5.855 \mathrm{E}-07$ $-3.398 E-08$ $6.589 E-06$ $6.589 E-06$
$1.451 E-05$ $1.451 E-05$ 1.257E-04 $2.607 \mathrm{E}-04$ 1. $200 \mathrm{E}-03$ $3.074 E-03$ $9.407 E-03$
$2.844 E-03$ ANGLE 12 $2.963 E-06$ $6.031 E-06$ $6.299 \mathrm{E}-05$ 2.376E-O5 $3.649 E-05$ $2.489 E-05$ $3.408 E-05$ $4.927 E-05$ $7.932 E-05$ $1.444 E-04$ $2.462 E-04$ $3.803 E-04$ T.218E-04 ..084E-04 $1.271 E-03$ $A=0.617$ $5.003 E-06$ $9.856 \mathrm{E}-06$ $1.000 E-04$ $3.751 E-05$ $6.124 E-05$ $5.354 E-05$ $7.854 E-05$ $1.140 \mathrm{E}-04$ $1.678 E-04$ 3.612E-04 $3.612 E-04$ $7.785 \mathrm{E}-04$ $7.785 E-04$ $1.342 E-03$ $1.342 E-03$ $4.542 E-03$ $1.276 E-02$
$3.234 E-03$
$\begin{array}{rl}A N G L E & 5 \\ M U & =-0.7550\end{array}$ $-1.729 E-07$ $M U=0.7550$ $1.175 E-02$
$3.127 E-03$ $6.087 E-07$ $1.372 \mathrm{E}-06$ $1.485 E-05$ $6.470 E-06$ $9.343 \mathrm{E}-06$ $3.427 E-06$ $1.852 E-06$ 1.904E-07 $1.687 E-06$ $9.435 E-06$ $1.851 E-05$ $1.437 E-04$ $3.476 \mathrm{E}-04$ $1.185 \mathrm{E}-03$ $3.147 \mathrm{E}-03$ $9.581 \mathrm{E}-03$
$2.867 \mathrm{E}-03$ ANGLE 14 $9.909 \mathrm{E}-06$ $1.941 E-05$ $1.871 \mathrm{E}-04$ $8.379 E-05$ $1.302 E-04$ $1.271 E-04$ $1.711 \mathrm{E}-04$ $2.256 E-04$ 2. $959 E-04$ $4.707 E-04$ $4.707 E-04$ . $15 E-04$ . $15 E-04$ . $531 E-04$ $1.414 E-03$ 1.324E-O

ANGLE 6
$M U=-0.6179$ $6.485 \mathrm{E}-07$ $1.411 E-06$ 1. $552 \mathrm{E}-05$ $5.574 \mathrm{E}-06$ $8.392 \mathrm{E}-06$ $2.807 E-06$ $2.376 E-06$ $1.672 \mathrm{E}-06$ $2.281 E-06$ 4. $972 E-06$ $1.065 \mathrm{E}-0$ $1.888 \mathrm{E}-05$ $1.816 \mathrm{E}-0$ 4.552E-04 $1.161 \mathrm{E}-03$ $3.242 E-03$ $9.811 E-03$ $2.896 \mathrm{E}-03$ ANGLE 15 $M U=0.8656$ $2.232 E-05$ 4. $379 \mathrm{E}-05$ $3.993 E-04$ $2.098 E-04$ $2.935 E-04$ $2.814 \mathrm{E}-04$ $3.346 E-04$ $3.889 E-04$ $4.498 E-04$ 5.127E-04 5.526E-04 $8.959 E-04$ . $027 \mathrm{E}-03$ $1.027 E-03$ $1.48 E-03$ $1.365 \mathrm{E}-02$ $3.281 \mathrm{E}-03 \quad 3.321 \mathrm{E}-03$
ANGLE 7 ANGLE 8 ANGLE 9 $M U=-0.4580 \quad M U=-0.2816 \quad M U=-0.0550$ $\begin{array}{lll}6.870 E-07 & 7.861 E-07 & 1.006 E-06\end{array}$ $1.391 \mathrm{E}-06 \quad 1.536 \mathrm{E}-06 \quad 2.028 \mathrm{E}-06$ $1.600 E-05 \quad 1.805 E-05 \quad 2.313 E-05$ $3.387 \mathrm{E}-06 \quad 2.835 \mathrm{E}-06 \quad 6.183 \mathrm{E}-06$ 7.042E-06 7.625E-06 $1.161 E-05$ $\begin{array}{lll}2.048 E-06 & 2.779 E-06 & 5.622 E-06\end{array}$ $5.913 E-06$
$8.792 E-06$ $8.174 E-06 \quad 1.183 E-05 \quad 1.197 E-05$ $9.983 E-06 \quad 1.312 E-05 \quad 1.679 E-05$ $1.121 E-05 \quad 1.642 E-05 \quad 3.483 E-05$ $2.268 E-05 \quad 4.473 E-05 \quad 9.596 E-05$ $2.452 E-04 \quad 3.342 E-04 \quad 4.407 E-04$ $\begin{array}{lll}5.640 E-04 & 6.542 E-04 & 7.130 E-04\end{array}$ $1.136 E-03 \quad 1.121 E-03 \quad 1.126 E-03$ $3.361 \mathrm{E}-03 \quad 3.503 \mathrm{E}-03 \quad 3.670 \mathrm{E}-03$ $1.010 \mathrm{E}-02 \quad 1.044 \mathrm{E}-02 \quad 1.083 \mathrm{E}-02$ 2.932E-03 2.975E-03 3.022E-03 ANGLE 16 ANGLE 17 SCALAR $M U=0.9446 \quad M U=0.9894 \quad F L U X$ $5.198 \mathrm{E}-05 \quad 1.078 \mathrm{E}-04 \quad 7.606 \mathrm{E}-05$ $1.008 E-04 \quad 2.110 E-04 \quad 1.499 E-04$ $8.829 E-04 \quad 1.829 E-03 \quad 1.397 E-03$ $4.804 E-04 \quad 9.462 E-04 \quad 6.634 E-04$ $6.085 E-04 \quad 1.087 E-03 \quad 9.010 E-04$ $5.446 E-04 \quad 9.136 E-04 \quad 7.614 E-04$ $5.791 E-04 \quad 8.911 E-04 \quad 8.810 E-04$ $6.067 E-04 \quad 7.426 E-04 \quad 1.002 E=03$ 4 $1.524 E-03$ $2.710 \mathrm{E}-03$ $2.710 E-03$ $6.234 E-03$ I. $550 E-02$ $1.397 E-02 \quad 1.249 E-03$ 4.875E-02 $\begin{array}{lll}3.351 E-03 & 3.468 E-03 & 3.849 E-02\end{array}$ 
(GAMMAS/MEV/STERADIAN/SOURCE NEUTRON)

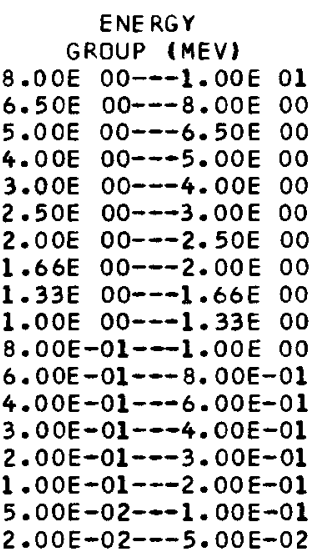

ENERGY

GROUP (MEV)

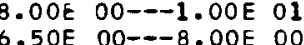
$5.00 E$ OO- $-6.50 E$ OO 4.0OE $00---5.00 E \quad 00$ $3.00 E$ OO- $-4.00 E$ OO $2.50 E$ OO- - 3.00E OO $2.00 E$ OO- $-2.50 E$ OO $1.33 \mathrm{E} 00---1.66 \mathrm{E} 00$ $1.00 \mathrm{E} 00--1.33 \mathrm{E} \mathrm{OO}$ 8.00E-O1---1.00E 00 $6.00 E-01--8 . C O E-01$ 4.00E-01---6.00E-01 $3.00 E-01--4.00 E-01$ $2.00 E-01--3.00 E-01$ $1.00 E-01--2.00 E-01$ $5.00 E-02--1.00 E-01$
$2.00 E-02---5.00 E-02$
ANGLE 1 $M U=-1.0000$ $3.808 \mathrm{E}-0 \mathrm{~B}$ $-6.739 E-0$ $-3.143 E-06$ $-2.173 E-06$ $-1.446 \mathrm{E}-06$ $-1.446 \mathrm{E}-06$
$1.638 \mathrm{E}-06$ $1.638 \mathrm{E}-06$ 7.190E-06 $4.990 E-06$ $-2.764 \mathrm{E}-06$ $-7.503 E-06$ $5.298 \mathrm{E}-05$ $6.946 \mathrm{E}-05$ $6.055 E-04$ $1.455 \mathrm{E}-03$ 4.503E-0 $1.377 \mathrm{E}-03$

ANGLE 2 $M U=-0.9894$ $-8.411 E-09$ $1.162 \mathrm{E}-06$ $-2.425 E-06$ $-1.496 E-06$ $-1.073 E-06$ $1.512 \mathrm{E}-0 \mathrm{G}$ $4.302 E-06$ $4.231 E-06$ $-2.165 E-06$ $-5.696 E-06$ $5.265 \mathrm{E}-05$ $7.294 E-05$ $6.056 \mathrm{E}-04$ $1.458 \mathrm{E}-03$ 4.511E-O3 $1.378 \mathrm{E}-03$

ANGLE 10 $\begin{aligned} M U & =0.0950 \\ 4.009 E-07 & \end{aligned}$ $8.721 E-07$ $9.277 E-06$ $4.409 \mathrm{E}-0 \mathrm{6}$ $6.281 E-06$ $3.520 E-06$ $2.924 E-06$ $2.018 E-06$ $2.725 E-06$ $1.058 E-05$ $3.515 E-O 5$ ..029E-05 $2.735 E-04$ $3.641 E-04$ $5.563 E-04$ $1.878 \mathrm{E}-03$ $5.505 E-03$
$1.501 E-03$ ANGLE 11 $A .2816$
$-962 E-07$ 1. $310 \mathrm{OE}-06$ $1.333 E-05$ $6.728 \mathrm{E}-06$ $8.795 E-06$ $4.847 \mathrm{E}-06$ $4.345 \mathrm{E}-06$ $4.961 E-06$ $1.045 E-05$ $3.081 E-05$ $7.407 E-05$ $1.452 E-04$ $3.200 E-04$ $3.703 E-04$ $5.797 \mathrm{E}-04$ $1.980 E-03$ $5.736 \mathrm{E}-03$ $1.527 E-03$
ANGLE 3 MU $=-0.9446$ $9.848 \mathrm{E}-0$ $2.366 \mathrm{E}-06$ 2.366E-06 $-1.616 E-07$
$3.398 E-07$ $2.160 \mathrm{E}-08$ $2.160 \mathrm{E}-08$
$1.052 \mathrm{E}-06$ $1.052 \mathrm{E}-06$
$1.706 \mathrm{E}-06$ $2.168 \mathrm{E}-06$ $1.469 \mathrm{E}-06$ $-8.544 E-08$ $1.048 \mathrm{E}-07$ 5.2COE-O $8.863 E-05$ $6.049 E-04$ $1.472 \mathrm{E}-03$ $4.543 E-03$ 1. $382 \mathrm{E}-03$

ANGLE $M U=-0.8656$ 1.553E- 07 $3.816 E-06$ $1.559 E-06$ $2.743 \mathrm{E}-06$ $1.043 E-06$ $3.865 \mathrm{E}-07$ $-5.494 E-07$ $-5.494 E-07$
$-1.133 E-06$ $-1.133 E-06$ $2.033 E-06$ $5.154 E-06$ $5.353 E-05$ $1.201 E-04$ $6.006 \mathrm{E}-04$ $1.498 \mathrm{E}-03$ $4.602 E-03$ $1.390 \mathrm{E}-03$

ANGLE 5 $M U=-0.7550$ $1.881 E-07$
$4.554 E-07$ $4.582 E-06$ $3.021 \mathrm{E}-06$ 3. $300 E-06$ 1.344E-06 $1.585 E-07$ $-1.140 E-06$ $-1.140 E-06$ $-3.298 \mathrm{E}-07$ $-3.298 E-07$
$4.070 E-06$ $8.195 E-06$ $6.162 \mathrm{E}-05$ $6.162 E-05$
$1.673 E-04$ $5.901 \mathrm{E}-04$ $1.534 \mathrm{E}-03$ $4.686 \mathrm{E}-03$ $1.401 \mathrm{E}-03$

ANGLE 6 $M U=-0.6179$
$1.861 \mathrm{E}-07$ $4.164 E-07$ 4.421E-06 $1.563 E-06$ 2.533E-06 6.859E-07 $4.055 \mathrm{E}-07$ $5.645 \mathrm{E}-08$ $5.645 \mathrm{E}-08$
$1.624 \mathrm{E}-07$ $1.624 E-07$
$1.406 E-06$ $1.406 E-06$
$3.680 E-06$ $3.680 E-06$
$5.969 E-06$ $5.969 E-06$
$8.076 \mathrm{E}-05$ $8.076 E-05$
$2.254 E-04$ $5.740 \mathrm{E}-04$ $1.580 E-03$ $4.798 E-03$ $1.415 \mathrm{E}-03$ ANGLE 12 ANGLE 13 ANGLE 14
$M U=0.7550$ 0.4580
$8.926 \mathrm{E}-07$ $M U=0.6179$ ANGLE 15 $1.817 \mathrm{E}-06$ $1.858 \mathrm{E}-05$ $7.267 \mathrm{E}-06$ $1.072 E-05$ $1.562 E-06 \quad 3.545 E-06$ $.963 E-06$ ..984E-05 $6.773 E-06$ $6.321 E-05$ $3.025 \mathrm{E}-05$ $5.040 E-05$ $5.885 E-05$ $2.026 E-05$ $3.428 \mathrm{E}-05$ $5.514 E-05$ $74 \mathrm{E}-04$ $1.571 E-04$ $2.068 E-04$ $2.452 E-04$ $2.665 E-04$ $3.939 \mathrm{E}-04$ $4.554 \mathrm{E}-04$ $6.722 E-04$ $2.310 \mathrm{E}-03$ $6.445 \mathrm{E}-0$ $M U=0.8656$ $9.725 E-06$ $1.624 \mathrm{E}-04$ $9.845 E-05$ $1.380 E=04$ $1.470 E-04$ $1.773 E-04$ $2.084 E-04$
$2.400 E-04$ $2.692 E-04$ $2.821 E-04$ $2.840 \mathrm{E}-04$ $4.219 E-04$ $4.888 \mathrm{E}-04$ $7.040 \mathrm{E}-04$ $2.407 E-03$ $6.090 \mathrm{E}-04$ $2.089 E-03$ $5.977 E-0$ $1.578 \mathrm{E}-03$
ANGLE 7 $A U=-0.4580$
$1.717 \mathrm{E}-07$ $3.271 E-07$ $3.947 E-06$ $9.35 \mathrm{EE}-07$ $1.263 E-07$ $1.142 E-06$ $2.320 E-06$ $3.626 E-06$ $3.946 \mathrm{E}-06$ $2.835 E-06$ $6.670 E-06$ $1.142 E-04$ $2.829 E-04$ $1.433 \mathrm{E}-03$

ANGLE 16 $M U=0.9446$ $2.785 E-05$
$5.311 E-05$ 4.312E-04 $2.631 E-04$ $3.223 E-04$ $3.038 E-04$ $3.174 E-04$ $3.211 E-04$ $3.196 \mathrm{E}-04$ $3.078 E-04$ $2.954 E-04$ $2.977 \mathrm{E}-04$ $4.583 E-04$ $5.068 \mathrm{E}-04$ $7.332 E-04$ $2.484 E-03$ $6.795 E-03$ $1.634 \mathrm{E}-03$

ANGLE 8 $M U=-0.2816$ $3.155 \mathrm{E}-07$ $4.251 E-06$ $4.251 E-06$ $1.311 E=06$ $4.987 E-07$ $2.120 E-06$ $2.120 E-06$
$3.667 E-06$ $3.667 E-06$
$5.079 E-06$ $4.579 E-06$ $4.143 E-06$ $1.718 \mathrm{E}-05$ $1.616 \mathrm{E}-04$ $3.281 \mathrm{E}-0$ $1.706 E-03$ $5.102 \mathrm{E}-03$ 
COSINE
$-1.00000 \mathrm{E} 00$
$-9.89401 \mathrm{E}-01$
$-9.44575 \mathrm{E}-01$
$-8.65631 \mathrm{E}-01$
$-7.55044 \mathrm{E}-01$
$-6.17876 \mathrm{E}-01$
$-4.58017 \mathrm{E}-01$
$-2.81605 \mathrm{E}-01$
$-9.50125 \mathrm{E}-02$
$9.50125 \mathrm{E}-02$
$2.81605 \mathrm{E}-01$
$4.58017 \mathrm{E}-01$
$6.17876 \mathrm{E}-01$
$7.55044 \mathrm{E}-01$
$8.65631 \mathrm{E}-01$
$9.44575 \mathrm{E}-01$
$9.89401 \mathrm{E}-01$

$9.89401 \mathrm{E}-01$

TOTAL

COSINE

-1. CONOCOE 00 $-9.89401 E-01$ $-9.44575 E-01$ $-8.65631 \mathrm{E}-01$ $-6.17876 E-01$ $-4.58017 \mathrm{E}-01$ $-2.81605 E-01$ $-9.50125 \mathrm{E}-02$ $9.50125 \mathrm{E}-02$ $2.81605 \mathrm{E}-01$ $4.58017 \mathrm{E}-01$ $6.17876 \mathrm{E}-01$ $7.55044 \mathrm{E}-01$
$8.65631 \mathrm{E}-01$ $8.65631 \mathrm{E}-01$
$9.44575 \mathrm{E}-01$ $9.89401 \mathrm{E}-01$

TOTAL

75.0
$9.761 \mathrm{E}-11$
$9.777 \mathrm{E}-11$
$9.843 \mathrm{E}-11$
$9.985 \mathrm{E}-11$
$1.022 \mathrm{E}-10$
$1.060 \mathrm{E}-10$
$1.115 \mathrm{E}-10$
$1.194 \mathrm{E}-10$
$1.305 \mathrm{E}-10$
$1.760 \mathrm{E}-10$
$1.790 \mathrm{E}-10$
$1.922 \mathrm{E}-10$
$2.445 \mathrm{E}-1 \mathrm{C}$
$3.518 \mathrm{E}-10$
$5.214 \mathrm{E}-10$
$1.132 \mathrm{E}-09$
$9.810 \mathrm{E}-09$
$4.258 \mathrm{E}-09$

100.0

1. $166 \mathrm{E}-10$ $1.168 \mathrm{E}-10$ $1.177 E-10$ $1.195 E-10$ $1.226 E-10$ $1.339 \mathrm{E}-10$ $1.33 \mathrm{E}-10$ $1.434 E-10$ $1.897 E-10$ $1.997 \mathrm{E}-10$ $2.271 \mathrm{E}-10$ 3.268E-10 3. $577 \mathrm{E}-10$ $5.869 E-10$ $1.089 \mathrm{E}-09$ 8.511E-09 4. 359E-O9

500.0

$1.003 E-1 C$ $1.018 \mathrm{E}-10$ $1.041 \mathrm{E}-1 \mathrm{O}$ $1.07 \in E-1 C$ $1.125 \mathrm{E}-10$ $1.193 E-10$ $1.282 \mathrm{E}-1 \mathrm{C}$ $1.399 \mathrm{E}-10$ 1. $552 \mathrm{E}-10$ $1.750 \mathrm{E}-10$ $2.015 \mathrm{E}-10$ $2.375 \mathrm{E}-10$ $2.886 \mathrm{E}-10$ $3.656 \mathrm{E}-10$ $4.999 \mathrm{E}-10$ $1.150 \mathrm{E}-\mathrm{CO}$

$2.409 E-C 9$

600.0

150.0
$1.425 \mathrm{E}-10$
$1.428 \mathrm{E}-10$
$1.440 \mathrm{E}-10$
$1.465 \mathrm{E}-10$
$1.506 \mathrm{E}-10$
$1.566 \mathrm{E}-10$
$1.652 \mathrm{E}-10$
$1.771 \mathrm{E}-10$
$1.935 \mathrm{E}-10$
$2.074 \mathrm{E}-10$
$2.502 \mathrm{E}-10$
$2.839 \mathrm{E}-10$
$3.757 \mathrm{E}-10$
$4.257 \mathrm{E}-10$
$6.772 \mathrm{E}-10$
$1.05 \mathrm{CE}-09$
$6.515 \mathrm{E}-09$
$4.492 \mathrm{E}-09$

RANGE (METERS) 200.0 250.0

$1.544 \mathrm{E}-10$ $1.548 \mathrm{E}-10$ $1.562 \mathrm{E}-10$
$1.592 \mathrm{E}-10$ $1.592 E-10$ $1.639 E-10$ $1.707 E-10$ $1.935 \mathrm{E}-10$ $2.113 \mathrm{E}-10$ $2.386 E-10$ $2.386-10$ $3.174 \mathrm{E}-10$ $3.777 \mathrm{E}-10$ $4.863 \mathrm{E}-10$ $6.603 \mathrm{E}-10$ $1.046 \mathrm{E}-09$ 5.017E-09

4.457E-09

1. $559 \mathrm{E}-10$ $1.563 \mathrm{E}-10$ $1.579 \mathrm{E}-10$ $1.610 E-10$ $1.659 \mathrm{E}-10$ $1.731 E-10$ $1.83 \mathrm{E}-10$ 1.965-10 $2.417 \mathrm{E}-10$ $2.417 E-10$ $3.198 \mathrm{E}-10$ $3.778 \mathrm{E}-10$ $4.814 \mathrm{E}-10$ $4.814 E-10$ $0.765 \mathrm{E}-10$ $3.879 \mathrm{E}-09$

$4.246 \mathrm{E}-09$

300.0 400.0

1. 504E-10 $1.508 \mathrm{E}-10$ $1.524 \mathrm{E}-10 \quad 1.295 \mathrm{E}-10$ $1.555 E-10 \quad 1.324 \mathrm{E}-10$ $1.604 E-10$ 1.367E-10 $1.675 E-10$ 1.429E-10 $1.97 E-10$ 1.5 $1.5 E-10$ $1.07 \mathrm{E}-10$ 1. $1.627 \mathrm{E}-10$ $2.314 E-10$ 1. $1067 E-10$ $2.614 \mathrm{E}-10$ 10 $3.023 E-10 \quad 2.231 E-10$ $3.650 E-10 \quad 3.058 E-10$ $4.537 E-10 \quad 3.753 E-10$ 5.994E-10 4.839E-10 $8.837 \mathrm{E}-10 \quad 4.839 \mathrm{E}-10$ $3.022 \mathrm{E}-09 \quad 1.829 \mathrm{E}-10$ $3.933 E-09 \quad 3.170 E-09$

RANGE (METERS)

1200.0

1500.0

1800.0

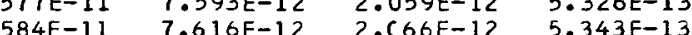

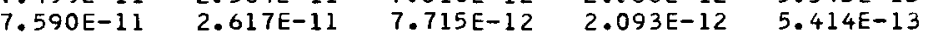
$7.765 \mathrm{E}-11 \quad 2.680 \mathrm{E}-11 \quad 7.903 \mathrm{E}-12 \quad 2.144 \mathrm{E}-12 \quad 5.547 \mathrm{E}-1 \mathrm{~s}$ $8.031 E-11 \quad 2.774 E-11 \quad 8.184 E-12 \quad 2.221 E-12 \quad 5.747 E-13$ $8.407 \mathrm{E}-11 \quad 2.907 \mathrm{E}-11 \quad 8.57 \mathrm{SE}-12 \quad 2.328 \mathrm{E}-12 \quad 6.026 \mathrm{E}-13$ $8.914 \mathrm{E}-11 \quad 3.083 \mathrm{E}-11 \quad 9.102 \mathrm{E}-12 \quad 2.471 \mathrm{E}-12 \quad 6.394 \mathrm{E}-13$ $9.584 E-11 \quad 3.315 E-11 \quad 9.783 E-12 \quad 2.655 E-12 \quad 6.871 E-13$ $\begin{array}{lllll}1.045 \mathrm{E}-10 & 3.612 \mathrm{E}-11 & 1.065 \mathrm{E}-11 & 2.890 \mathrm{E}-12 & 7.477 \mathrm{E}-13\end{array}$ $1.158 \mathrm{E}-10 \quad 3.992 \mathrm{E}-11 \quad 1.176 \mathrm{E}-11 \quad 3.187 \mathrm{E}-12 \quad 8.242 \mathrm{E}-13$ $\begin{array}{lllll}1.304 E-10 & 4.478 E-11 & 1.316 E-11 & 3.563 \mathrm{E}-12 & 9.205 \mathrm{E}-13\end{array}$ $\begin{array}{lllll}1.496 \mathrm{E}-10 & 5.107 \mathrm{E}-11 & 1.495 \mathrm{E}-11 & 4.040 \mathrm{E}-12 & 1.042 \mathrm{E}-12 \\ 1.755 \mathrm{E}-10 & 5.931 \mathrm{E}-11 & 1.727 \mathrm{E}-11 & 4.651 \mathrm{E}-12 & 1.198 \mathrm{E}-12\end{array}$ $\begin{array}{lllll}2.115 \mathrm{E}-10 & 7.037 \mathrm{E}-11 & 2.032 \mathrm{E}-11 & 5.445 \mathrm{E}-12 & 1.399 \mathrm{E}-12\end{array}$ $2.645 \mathrm{E}-10 \quad 8.584 \mathrm{E}-11 \quad 2.445 \mathrm{E}-11 \quad 6.504 \mathrm{E}-12 \quad 1.663 \mathrm{E}-12$ $3.531 \mathrm{E}-10 \quad 1.096 \mathrm{E}-10 \quad 3.048 \mathrm{E}-11 \quad 7.999 \mathrm{E}-12 \quad 2.030 \mathrm{E}-12$ 7.20.0E-10 $1.800 \mathrm{E}-10 \quad 4.499 \mathrm{E}-11 \quad 1.112 \mathrm{E}-11 \quad 2.722 \mathrm{E}-12$
$1.756 E-09$
$5.824 \mathrm{E}-10$
$1.684 E-10$
4. $522 \mathrm{E}-11$
$1.163 \mathrm{E}-11$ 
COSINE $-1.0 C 000 E$ OO $-9.89401 E-01$ $-9.44575 E-01$ $-8.65631 \mathrm{E}-01$ $-6.17876 \mathrm{E}-01$ $-4.58017 \mathrm{E}-01$ $-2.81605 E-01$ $-9.50125 \mathrm{E}-02$ $9.50125 \mathrm{E}-02$ $2.81605 \mathrm{E}-01$ $4.58017 \mathrm{E}-01$ $6.17876 \mathrm{E}-01$ $7.55044 \mathrm{E}-01$ $8.65631 \mathrm{E}-\mathrm{C}$ $9.44575 \mathrm{E}-01$

TOTAL

COSINE

$-1.00000 E 00$ $-9.89401 E-01$ $-9.44575 E-01$ $-8.65631 \mathrm{E}-01$ $-7.55044 \mathrm{E}-01$ $-6.17876 \mathrm{E}-01$ . $-2.81605 E-01$ $-9.50125 \mathrm{E}-02$ $2.81605 \mathrm{E}-01$ 4.58017E-01 $6.17876 \mathrm{E}-01$ $8.65631 \mathrm{E}-01$ $8.65631 \mathrm{E}-01$ $9.44575 \mathrm{E}-01$

TOTAL

75.0
$1.667 \mathrm{E}-1 \mathrm{C}$
$1.670 \mathrm{E}-10$
$1.681 \mathrm{E}-10$
$1.705 \mathrm{E}-10$
$1.744 \mathrm{E}-10$
$1.804 \mathrm{E}-10$
$1.892 \mathrm{E}-10$
$2.017 \mathrm{E}-10$
$2.191 \mathrm{E}-10$
$2.867 \mathrm{E}-10$
$2.939 \mathrm{E}-10$
$3.132 \mathrm{E}-10$
$3.919 \mathrm{E}-10$
$5.539 \mathrm{E}-10$
$7.977 \mathrm{E}-10$
$1.662 \mathrm{E}-09$
$1.395 \mathrm{E}-08$
$6.516 \mathrm{E}-09$

$2.059 \mathrm{E}-10$ $2.063 \mathrm{E}-10$ $2.078 \mathrm{E}-10$ $2.109 E-10$ $2.160 \mathrm{E}-10$ $2.236 E-10$ $2.345 \mathrm{E}-10$ $2.497 \mathrm{E}-10$ $2.707 \mathrm{E}-10$ 3. $268 \mathrm{E}-10$ $3.356 \mathrm{E}-10$ $3.772 \mathrm{E}-10$ $5.337 \mathrm{E}-10$ $5.712 \mathrm{E}-10$ $9.156 \mathrm{E}-10$ $1.630 \mathrm{E}-09$ $1.210 \mathrm{E}-\mathrm{C} 8$

$6.852 \mathrm{E}-09$

$5 \mathrm{co.0}$

600.0

$2.327 E-10$ $2.333 \mathrm{E}-10$ $2.356 \mathrm{E}-10$ $2.400 \mathrm{E}-10$ $2.466 \mathrm{E}-10$ $2.559 \mathrm{E}-10$ $2.683 E-10$ $2.843 E-10$ $3.049 \mathrm{E}-10$ $3.312 E-10$ 3.641E-10 $4.074 E-10$ $4.644 E-10$ $5.430 E-10$ $6.581 E-10$ $8.527 \mathrm{E}-10$ $1.755 E-09$

$4.817 \mathrm{E}+09$

\begin{tabular}{ccccc}
\multicolumn{5}{c}{ RANGE (METERS) } \\
150.0 & 206.0 & 250.0 & 300.0 & 400.0 \\
$2.661 \mathrm{E}-10$ & $3.022 \mathrm{E}-10$ & $3.173 \mathrm{E}-10$ & $3.166 \mathrm{E}-10$ & $2.841 \mathrm{E}-10$ \\
$2.666 \mathrm{E}-10$ & $3.028 \mathrm{E}-10$ & $3.180 \mathrm{E}-10$ & $3.173 \mathrm{E}-10$ & $2.848 \mathrm{E}-10$ \\
$2.688 \mathrm{E}-10$ & $3.054 \mathrm{E}-10$ & $3.209 \mathrm{E}-10$ & $3.203 \mathrm{E}-10$ & $2.875 \mathrm{E}-10$ \\
$2.732 \mathrm{E}-10$ & $3.106 \mathrm{E}-10$ & $3.266 \mathrm{E}-10$ & $3.261 \mathrm{E}-10$ & $2.929 \mathrm{E}-10$ \\
$2.801 \mathrm{E}-10$ & $3.188 \mathrm{E}-10$ & $3.353 \mathrm{E}-10$ & $3.349 \mathrm{E}-10$ & $3.009 \mathrm{E}-10$ \\
$2.903 \mathrm{E}-10$ & $3.305 \mathrm{E}-10$ & $3.478 \mathrm{E}-10$ & $3.475 \mathrm{E}-10$ & $3.123 \mathrm{E}-10$ \\
$3.045 \mathrm{E}-10$ & $3.468 \mathrm{E}-10$ & $3.649 \mathrm{E}-10$ & $3.646 \mathrm{E}-10$ & $3.275 \mathrm{E}-10$ \\
$3.241 \mathrm{E}-10$ & $3.688 \mathrm{E}-10$ & $3.878 \mathrm{E}-10$ & $3.872 \mathrm{E}-10$ & $3.474 \mathrm{E}-10$ \\
$3.504 \mathrm{E}-10$ & $3.979 \mathrm{E}-10$ & $4.178 \mathrm{E}-10$ & $4.167 \mathrm{E}-10$ & $3.731 \mathrm{E}-10$ \\
$3.785 \mathrm{E}-10$ & $4.380 \mathrm{E}-10$ & $4.575 \mathrm{E}-10$ & $4.572 \mathrm{E}-10$ & $4.049 \mathrm{E}-10$ \\
$4.363 \mathrm{E}-10$ & $4.855 \mathrm{E}-10$ & $5.070 \mathrm{E}-10$ & $5.046 \mathrm{E}-10$ & $4.492 \mathrm{E}-10$ \\
$4.924 \mathrm{E}-10$ & $5.634 \mathrm{E}-10$ & $5.835 \mathrm{E}-10$ & $5.727 \mathrm{E}-10$ & $5.041 \mathrm{E}-10$ \\
$6.343 \mathrm{E}-10$ & $6.594 \mathrm{E}-10$ & $6.770 \mathrm{E}-10$ & $6.656 \mathrm{E}-10$ & $5.802 \mathrm{E}-10$ \\
$6.996 \mathrm{E}-10$ & $8.196 \mathrm{E}-10$ & $8.300 \mathrm{E}-10$ & $8.012 \mathrm{E}-10$ & $6.861 \mathrm{E}-10$ \\
$1.082 \mathrm{E}-09$ & $1.078 \mathrm{E}-09$ & $1.066 \mathrm{E}-09$ & $1.016 \mathrm{E}-09$ & $8.478 \mathrm{E}-10$ \\
$1.609 \mathrm{E}-09$ & $1.633 \mathrm{E}-09$ & $1.552 \mathrm{E}-09$ & $1.428 \mathrm{E}-09$ & $1.136 \mathrm{E}-09$ \\
$9.292 \mathrm{E}-09$ & $7.195 \mathrm{E}-09$ & $5.606 \mathrm{E}-09$ & $4.408 \mathrm{E}-09$ & $2.762 \mathrm{E}-09$ \\
$7.399 \mathrm{E}-09$ & $7.632 \mathrm{E}-09$ & $7.528 \mathrm{E}-09$ & $7.194 \mathrm{E}-09$ & $6.094 \mathrm{E}-09$
\end{tabular}

\section{RANGE (METERS)}

900.0

1200.0

1500.0

1800.0

$\begin{array}{lllll}1.793 \mathrm{E}-10 & 6.592 \mathrm{E}-11 & 2.010 \mathrm{E}-11 & 5.544 \mathrm{E}-12 & 1.443 \mathrm{E}-12 \\ 1.798 \mathrm{E}-10 & 6.608 \mathrm{E}-11 & 2.015 \mathrm{E}-11 & 5.557 \mathrm{E}-12 & 1.447 \mathrm{E}-12 \\ 1.816 \mathrm{E}-10 & 6.675 \mathrm{E}-11 & 2.035 \mathrm{E}-11 & 5.614 \mathrm{E}-12 & 1.462 \mathrm{E}-12 \\ 1.850 \mathrm{E}-10 & 6.802 \mathrm{E}-11 & 2.074 \mathrm{E}-11 & 5.721 \mathrm{E}-12 & 1.490 \mathrm{E}-12 \\ 1.901 \mathrm{E}-10 & 6.991 \mathrm{E}-11 & 2.131 \mathrm{E}-11 & 5.879 \mathrm{E}-12 & 1.531 \mathrm{E}-12 \\ 1.973 \mathrm{E}-10 & 7.251 \mathrm{E}-11 & 2.210 \mathrm{E}-11 & 6.096 \mathrm{E}-12 & 1.587 \mathrm{E}-12 \\ 2.067 \mathrm{E}-10 & 7.592 \mathrm{E}-11 & 2.313 \mathrm{E}-11 & 6.376 \mathrm{E}-12 & 1.660 \mathrm{E}-12 \\ 2.189 \mathrm{E}-10 & 8.027 \mathrm{E}-11 & 2.443 \mathrm{E}-11 & 6.732 \mathrm{E}-12 & 1.752 \mathrm{E}-12 \\ 2.344 \mathrm{E}-10 & 8.573 \mathrm{E}-11 & 2.605 \mathrm{E}-11 & 7.174 \mathrm{E}-12 & 1.866 \mathrm{E}-12 \\ 2.540 \mathrm{E}-10 & 9.253 \mathrm{E}-11 & 2.806 \mathrm{E}-11 & 7.717 \mathrm{E}-12 & 2.006 \mathrm{E}-12 \\ 2.788 \mathrm{E}-10 & 1.010 \mathrm{E}-10 & 3.053 \mathrm{E}-11 & 8.382 \mathrm{E}-12 & 2.177 \mathrm{E}-12 \\ 3.104 \mathrm{E}-10 & 1.115 \mathrm{E}-10 & 3.358 \mathrm{E}-11 & 9.198 \mathrm{E}-12 & 2.386 \mathrm{E}-12 \\ 3.518 \mathrm{E}-10 & 1.250 \mathrm{E}-10 & 3.739 \mathrm{E}-11 & 1.021 \mathrm{E}-11 & 2.643 \mathrm{E}-12 \\ 4.075 \mathrm{E}-10 & 1.423 \mathrm{E}-10 & 4.222 \mathrm{E}-11 & 1.147 \mathrm{E}-11 & 2.962 \mathrm{E}-12 \\ 4.870 \mathrm{E}-10 & 1.658 \mathrm{E}-10 & 4.852 \mathrm{E}-11 & 1.309 \mathrm{E}-11 & 3.366 \mathrm{E}-12 \\ 6.156 \mathrm{E}-10 & 2.003 \mathrm{E}-10 & 5.733 \mathrm{E}-11 & 1.528 \mathrm{E}-11 & 3.903 \mathrm{E}-12 \\ 1.124 \mathrm{E}-09 & 2.980 \mathrm{E}-10 & 7.753 \mathrm{E}-11 & 1.964 \mathrm{E}-11 & 4.872 \mathrm{E}-12 \\ 3.622 \mathrm{E}-09 & 1.278 \mathrm{E}-09 & 3.819 \mathrm{E}-10 & 1.042 \mathrm{E}-10 & 2.699 \mathrm{E}-11\end{array}$


COSINE

-1. COOCOE OO $-9.44575 \mathrm{E}-01$ $-8.65631 E-01$ $-6.17876 \mathrm{E}-01$ $-4.58017 \mathrm{E}-01$ $-2.81605 E-01$ $-9.50125 \mathrm{E}-02$ $9.5 \mathrm{C125E-02}$ $4.58017 \mathrm{E}-01$ $6.17876 \mathrm{E}-01$ $7.55044 \mathrm{E}-01$ $8.65631 E-01$ $9.44575 \mathrm{E}-01$ $9.89401 E-01$

TOTAL

$$
75.0
$$

100.0

$$
\text { COSINE }
$$

$-1.00000 \mathrm{E} 00$ $-9.89401 \mathrm{E}-01$ $-9.44575 E-01$ $-8.65631 \mathrm{E}-01$ $-7.55044 E-01$ $-6.17876 \mathrm{E}-01$ $-4.58017 \mathrm{E}-01$ $-2.81605 E-01$ $-9.50125 \mathrm{E}-02$ $9.5 C 125 \mathrm{E}-02$ 2.81605E-01 4.58017E-O1 $7.55044 E-01$ 8.6563 IE-OI $8.65631 E-01$ $9.44575 E-01$

TOTAL
$1.056 \mathrm{E}-08$ $1.058 \mathrm{E}-08$ $1.065 \mathrm{E}-08$ $1.081 E-08$ $1.081 \mathrm{E}-08$ $1.146 \mathrm{E}-08$ $1.205 \mathrm{E}-08$ $1.288 \mathrm{E}-08$ $1.405 \mathrm{E}-08$ $1.871 \mathrm{E}-08$ $1.913 \mathrm{E}-08$ $2.047 \mathrm{E}-08$ $2.593 \mathrm{E}-08$ $3.707 \mathrm{E}-08$ $5.427 E-08$ $1.161 \mathrm{E}-07$ $9.963 \mathrm{E}-07$

$4.434 E-07$

500.0

1. $274 E-C 8$ $1.276 \mathrm{E}-08$ $1.285 \mathrm{E}-\mathrm{C} 8$ 1. $338 \mathrm{E}-08$ 1. $388 \mathrm{E}-08$ $1.460 E-08$ $1.561 \mathrm{E}-08$ 1. $701 \mathrm{E}-08$ $2.071 E-08$ $2.140 \mathrm{E}-\mathrm{C} 8$ $3.474 E-08$ $3.778 E-08$ $6.152 \mathrm{E}-08$
$1.124 \mathrm{E}-07$ $8.641 E-07$

4.577E-07

600.0

$1.170 E-08$ $1.173 \mathrm{E}-08$ $1.187 \mathrm{E}-08$ $1.212 \mathrm{E}-08$ $1.251 \mathrm{E}-08$ $1.307 \mathrm{E}-08$ $1.381 \mathrm{E}-08$ $1.479 E-08$ $1.606 \mathrm{E}-08$ $1.771 \mathrm{E}-08$ $1.982 \mathrm{E}-08$ $2.263 E-08$ $2.640 E-08$ $3.171 E-08$ . $5.333 E-08$

$2.691 E-07$
$8.791 E-C 9$ $8.815 \mathrm{E}-09$ $8.918 \mathrm{E}-\mathrm{C} 9$ $9.115 \mathrm{E}-09$ $9.413 E-C 9$ $9.832 \mathrm{E}-09$ $1.039 \mathrm{E}-08$ $1.113 E-08$ $1.208 \mathrm{E}-08$ $1.329 \mathrm{E}-08$ $1.486 \mathrm{E}-\mathrm{C} 8$ $1.690 E-08$ $2.337 E-08$ $2.883 \mathrm{E}-08$ $2.883 E-08$ $3.788 E-08$

\begin{tabular}{|c|c|c|c|c|}
\hline 150.0 & $\begin{array}{l}\text { E IMETERS } \\
200.0\end{array}$ & 250.0 & 300.0 & 400.0 \\
\hline $\begin{array}{l}579 \mathrm{E}-08 \\
582 \mathrm{E}-08 \\
596 \mathrm{E}-08 \\
623 \mathrm{E}-08 \\
668 \mathrm{E}-08 \\
733 \mathrm{E}-08 \\
825 \mathrm{E}-08 \\
953 \mathrm{E}-08 \\
127 \mathrm{E}-08 \\
319 \mathrm{E}-08 \\
697 \mathrm{E}-08 \\
.086 \mathrm{E}-08 \\
028 \mathrm{E}-08 \\
532 \mathrm{E}-08 \\
133 \mathrm{E}-08 \\
092 \mathrm{E}-07 \\
621 \mathrm{E}-07\end{array}$ & $\begin{array}{l}1.731 \mathrm{E}-08 \\
1.735 \mathrm{E}-08 \\
1.751 \mathrm{E}-08 \\
1.784 \mathrm{E}-08 \\
1.835 \mathrm{E}-08 \\
1.909 \mathrm{E}-08 \\
2.013 \mathrm{E}-08 \\
2.155 \mathrm{E}-08 \\
2.345 \mathrm{E}-08 \\
2.605 \mathrm{E}-08 \\
2.926 \mathrm{E}-08 \\
3.444 \mathrm{E}-08 \\
4.101 \mathrm{E}-08 \\
5.198 \mathrm{E}-08 \\
7.000 \mathrm{E}-08 \\
1.091 \mathrm{E}-07 \\
5.108 \mathrm{E}-07\end{array}$ & $\begin{array}{l}1.764 \mathrm{E}-08 \\
1.768 \mathrm{E}-08 \\
1.786 \mathrm{E}-08 \\
1.821 \mathrm{E}-08 \\
1.875 \mathrm{E}-08 \\
1.953 \mathrm{E}-08 \\
2.061 \mathrm{E}-08 \\
2.206 \mathrm{E}-08 \\
2.399 \mathrm{E}-08 \\
2.655 \mathrm{E}-08 \\
2.987 \mathrm{E}-08 \\
3.491 \mathrm{E}-08 \\
4.128 \mathrm{E}-08 \\
5.172 \mathrm{E}-08 \\
6.815 \mathrm{E}-08 \\
1.023 \mathrm{E}-07 \\
3.958 \mathrm{E}-07\end{array}$ & $\begin{array}{l}1.715 \mathrm{E}-08 \\
1.720 \mathrm{E}-08 \\
1.737 \mathrm{E}-08 \\
1.772 \mathrm{E}-08 \\
1.826 \mathrm{E}-08 \\
1.904 \mathrm{E}-08 \\
2.010 \mathrm{E}-08 \\
2.153 \mathrm{E}-08 \\
2.341 \mathrm{E}-08 \\
2.604 \mathrm{E}-08 \\
2.912 \mathrm{E}-08 \\
3.364 \mathrm{E}-08 \\
3.988 \mathrm{E}-08 \\
4.915 \mathrm{E}-08 \\
6.403 \mathrm{E}-08 \\
9.304 \mathrm{E}-08 \\
3.091 \mathrm{E}-07\end{array}$ & $\begin{array}{l}1.476 \mathrm{E}-08 \\
1.480 \mathrm{E}-08 \\
1.496 \mathrm{E}-08 \\
1.527 \mathrm{E}-08 \\
1.576 \mathrm{E}-08 \\
1.644 \mathrm{E}-08 \\
1.737 \mathrm{E}-08 \\
1.860 \mathrm{E}-08 \\
2.021 \mathrm{E}-08 \\
2.223 \mathrm{E}-08 \\
2.509 \mathrm{E}-08 \\
2.869 \mathrm{E}-08 \\
3.377 \mathrm{E}-08 \\
4.096 \mathrm{E}-08 \\
5.213 \mathrm{E}-08 \\
7.239 \mathrm{E}-08 \\
1.907 \mathrm{E}-07\end{array}$ \\
\hline $781 \mathrm{E}-0$ & $4.789 E-07$ & $4.603 \mathrm{E}-07$ & $4.300 E-07$ & $3.507 E-07$ \\
\hline
\end{tabular}

1.977 E-07
RANGE (METERS)

$$
900.0 \quad 1200.0
$$

1500.0

1800.0

$\begin{array}{llll}3.075 \mathrm{E}-09 & 9.133 \mathrm{E}-10 & 2.486 \mathrm{E}-10 & 6.437 \mathrm{E}-11 \\ 3.084 \mathrm{E}-09 & 9.159 \mathrm{E}-10 & 2.493 \mathrm{E}-10 & 6.456 \mathrm{E}-11 \\ 3.121 \mathrm{E}-09 & 9.272 \mathrm{E}-10 & 2.524 \mathrm{E}-10 & 6.537 \mathrm{E}-11 \\ 3.192 \mathrm{E}-09 & 9.486 \mathrm{E}-10 & 2.583 \mathrm{E}-10 & 6.689 \mathrm{E}-11 \\ 3.299 \mathrm{E}-09 & 9.806 \mathrm{E}-10 & 2.670 \mathrm{E}-10 & 6.916 \mathrm{E}-11 \\ 3.448 \mathrm{E}-09 & 1.025 \mathrm{E}-09 & 2.792 \mathrm{E}-10 & 7.231 \mathrm{E}-11 \\ 3.645 \mathrm{E}-09 & 1.084 \mathrm{E}-09 & 2.951 \mathrm{E}-10 & 7.645 \mathrm{E}-11 \\ 3.901 \mathrm{E}-09 & 1.159 \mathrm{E}-09 & 3.157 \mathrm{E}-10 & 8.177 \mathrm{E}-11 \\ 4.228 \mathrm{E}-09 & 1.256 \mathrm{E}-09 & 3.417 \mathrm{E}-10 & 8.847 \mathrm{E}-11 \\ 4.642 \mathrm{E}-09 & 1.377 \mathrm{E}-09 & 3.743 \mathrm{E}-10 & 9.686 \mathrm{E}-11 \\ 5.168 \mathrm{E}-09 & 1.529 \mathrm{E}-09 & 4.150 \mathrm{E}-10 & 1.073 \mathrm{E}-10 \\ 5.839 \mathrm{E}-09 & 1.721 \mathrm{E}-09 & 4.662 \mathrm{E}-10 & 1.204 \mathrm{E}-10 \\ 6.711 \mathrm{E}-09 & 1.967 \mathrm{E}-09 & 5.312 \mathrm{E}-10 & 1.369 \mathrm{E}-10 \\ 7.867 \mathrm{E}-09 & 2.286 \mathrm{E}-09 & 6.145 \mathrm{E}-10 & 1.580 \mathrm{E}-10 \\ 9.466 \mathrm{E}-09 & 2.715 \mathrm{E}-09 & 7.244 \mathrm{E}-10 & 1.855 \mathrm{E}-10 \\ 1.189 \mathrm{E}-08 & 3.332 \mathrm{E}-09 & 8.779 \mathrm{E}-10 & 2.231 \mathrm{E}-10 \\ 1.903 \mathrm{E}-08 & 4.806 \mathrm{E}-09 & 1.196 \mathrm{E}-09 & 2.935 \mathrm{E}-10 \\ 6.655 \mathrm{E}-08 & 1.94 \mathrm{CE}-08 & 5.228 \mathrm{E}-09 & 1.346 \mathrm{E}-09\end{array}$




COSINE
$-1.00000 E 00$
$-9.89401 \mathrm{E}-01$
$-9.44575 \mathrm{E}-01$
$-8.65631 \mathrm{E}-01$
$-7.55044 \mathrm{E}-01$
$-6.17876 \mathrm{E}-01$
$-4.58017 \mathrm{E}-01$
$-2.81605 \mathrm{E}-01$
$-9.50125 \mathrm{E}-02$
$9.50125 \mathrm{E}-02$
$2.81605 \mathrm{E}-01$
$4.58017 \mathrm{E}-01$
$6.17876 \mathrm{E}-01$
$7.55044 \mathrm{E}-01$
$8.65631 \mathrm{E}-01$
$9.44575 \mathrm{E}-01$
$9.89401 \mathrm{E}-01$

TOT AL

COSINE

-1.00000 E 00 $-9.89401 \mathrm{E}-01$ $-9.44575 E-01$ $-8.65631 E-01$ $-7.55044 E-01$ $-6.17876 E-01$ $-4.58017 E-01$ $-2.81605 E-01$ $-9.50125 E-02$ 9.50125E-02 $2.81605 \mathrm{E}-01$ $4.58017 \mathrm{E}-01$ $6.17876 \mathrm{E}-01$ $7.55044 E-01$ $8.65631 \mathrm{E}-01$ $9.44575 E-01$
$9.89401 E-01$

TOTAL

75.0
$2.524 \mathrm{E}-11$
$2.528 \mathrm{E}-11$
$2.542 \mathrm{E}-11$
$2.573 \mathrm{E}-11$
$2.625 \mathrm{E}-11$
$2.707 \mathrm{E}-11$
$2.833 \mathrm{E}-11$
$3.016 \mathrm{E}-11$
$3.279 \mathrm{E}-11$
$4.456 \mathrm{E}-11$
$4.448 \mathrm{E}-11$
$4.809 \mathrm{E}-11$
$6.188 \mathrm{E}-11$
$8.930 \mathrm{E}-11$
$1.405 \mathrm{E}-10$
$3.415 \mathrm{E}-10$
$3.269 \mathrm{E}-09$
$1.238 \mathrm{E}-09$

100.0

3. $.887 \mathrm{E}-11$ .

3. $11 E-11$

$3.218 \mathrm{E}-11$

$3.321 \mathrm{E}-11$

.

$4.011 \mathrm{E}-11$

$4.739 \mathrm{E}-11$

5.064E-

$5.869 E-11$

$8.006 \mathrm{E}-11$

$9.396 \mathrm{E}-11$

$1.545 \mathrm{E}-10$

$3.158 \mathrm{E}-10$

$2.864 \mathrm{E}-09$

$1.250 \mathrm{E}-09$

500.0

600.0

. $559 \mathrm{E}-1$ $3.567 E-11$

$3.599 \mathrm{E}-11$

.660E-11

$3.752 \mathrm{E}-11$

$3.883 \mathrm{E}-11$

$4.058 \mathrm{E}-1$

4.28 SE-11

4.587E-1

$4.976 \mathrm{E}-1$

$5.471 \mathrm{E}-11$

$6.147 \mathrm{E}-1$

$7.093 \mathrm{E}-11$

$8.508 \mathrm{E}-11$

$1.080 \mathrm{E}-10$

$1.516 \mathrm{E}-10$

$4 \mathrm{E}-10$

$7.683 E-10$
$2.765 E-11$

$2.796 E-11$

.844E-11

$2.916 \mathrm{E}-11$

3.017E-11

$3.152 \mathrm{E}-11$

$3.554 \mathrm{E}-11$

3. $842 E-11$

$4.216 \mathrm{E}-11$

4. $710 \mathrm{E}-11$

$5.397 \mathrm{E}-11$

$6.402 \mathrm{E}-11$

$7.995 \mathrm{E}-11$

$2.543 \mathrm{EL}^{\mathrm{L}} 10$

$5.768 \mathrm{E}-10$
150.0

RANGE (METERS)

$3.960 E-11$

$4.540 \mathrm{E}-11$

$4.143 E-11$ 4.717E-11

$4.281 \mathrm{E}-11 \quad 4.877 \mathrm{E}-11$

$4.480 \mathrm{E}-11 \quad 5.104 \mathrm{E}-11$

$4.761 E-11$

$5.149 \mathrm{E}-11$

$5.560 E-11$

$6.479 \mathrm{E}-11$

$7.401 \mathrm{E}-11$

$9.458 \mathrm{E}-11$

$1.778 \mathrm{E}-10$

$3.008 \mathrm{E}-10$

$2.214 \mathrm{E}-09$

$5.104 \mathrm{E}-11$

$5.419 E-11$
$5.845 E-11$

$6.445 \mathrm{E}-11$

$6.445 \mathrm{E}-11$

$7.160 \mathrm{E}-11$
$8.400 \mathrm{E}-11$

$1.000 \mathrm{E}-10$

$1.000 \mathrm{E}-10$
$1.289 \mathrm{E}-10$

$1.289 \mathrm{E}-10$

$2.978 \mathrm{E}-10$

$1.720 \mathrm{E}-09$

1. 282E-09

$1.282 E-09$

RANGE (METERS)

900.0

1200.0
$1.034 \mathrm{E}-11$

$1.046 \mathrm{E}-11$

$1.064 \mathrm{E}-11$

$1.091 \mathrm{E}-11$

$1.128 \mathrm{E}-11$

$1.178 \mathrm{E}-11$

$1.322 \mathrm{E}-11$

$1.423 \mathrm{E}-11$

$1.551 \mathrm{E}-11$

$1.717 \mathrm{E}-11$

$1.941 \mathrm{E}-11$

$2.258 \mathrm{E}-11$

$2.736 E-11$

$3.549 \mathrm{E}-11$
$6.431 \mathrm{E}-11$

$3.184 E-12$

$3.191 \mathrm{E}-12$

$3.277 \mathrm{E}-12$

$3.361 E-12$

$3.476 \mathrm{E}-12$

$3.626 \mathrm{E}-12$

$3.819 \mathrm{E}-12$

$4.060 E-12$

$4.360 \mathrm{E}-12$

$4.738 \mathrm{E}-12$

$5.223 \mathrm{E}-12$

$5.867 \mathrm{E}-12$

$6.761 \mathrm{E}-12$

$8.076 \mathrm{E}-12$

$1.020 \mathrm{E}-11$

$2.040 E-10$

$6.131 E-11$
1800.0

250.0

300.0

$40 \mathrm{C} .0$

$4.739 \mathrm{E}-11$

$4.786 \mathrm{E}-11$

4.862E-11

$5.150 E-11$

5

$6.153 \mathrm{E}-11$

$7.486 \mathrm{E}-1$

$8.697 \mathrm{E}-11$

$1.026 \mathrm{E}-10$

$1.300 \mathrm{E}-10$
$1.762 \mathrm{E}-10$

$2.802 \mathrm{E}-10$

$8.838 E-13$

8.858E-13

$9.098 \mathrm{E}-13$

$9.330 \mathrm{E}-13$

$9.650 \mathrm{E}-13$

$1.007 E-12$

$1.126 \mathrm{E}-12$

$1.208 \mathrm{E}-12$

$1.311 \mathrm{E}-12$

$1.442 \mathrm{E}-12$

$1.616 \mathrm{E}-12$

$1.854 \mathrm{E}-12$

$2.199 \mathrm{E}-12$

$2.742 \mathrm{E}-1$

$1.683 E-11$

4. 306E-11

$4.352 \mathrm{E}-11$

$4.352 E-11$

$4.536 \mathrm{E}-11$

$4.694 \mathrm{E}-1$

$4.908 \mathrm{E}-11$

$5.193 \mathrm{E}-1$

6.701E-11

$8.824 E-11$

$2.027 \mathrm{E}-10$

$9.775 E-10$

$2.315 \mathrm{E}-13$

$2.337 E-13$

$.378 E-13$

.439E-13

$2.523 \mathrm{E}-13$

$2.631 \mathrm{E}-13$

$2.770 E-13$

$2.942 E-13$

$3.156 \mathrm{E}-13$

$3.423 \mathrm{E}-13$

3. $765 \mathrm{E}-13$

$4.831 \mathrm{E}-13$

$7.076 \mathrm{E}-13$

1.001E-12

4. $381 \mathrm{E}-12$ 
4 PI R* 2 CONCRETE KERMA (NEUTRONS)

(CM**2 ERGS/GRAM/STERADIAN/SOURCE NEUTRON)
FISSION SOURCE

300.0

400.0

$\begin{array}{ll}1.979 E-09 & 1.691 \mathrm{E}-09 \\ 1.984 \mathrm{E}-09 & 1.695 \mathrm{E}-09 \\ 2.005 \mathrm{E}-09 & 1.714 \mathrm{E}-09 \\ 2.046 \mathrm{E}-09 & 1.751 \mathrm{E}-09 \\ 2.110 \mathrm{E}-09 & 1.808 \mathrm{E}-09 \\ 2.202 \mathrm{E}-09 & 1.888 \mathrm{E}-09 \\ 2.327 \mathrm{E}-09 & 1.998 \mathrm{E}-09 \\ 2.497 \mathrm{E}-09 & 2.144 \mathrm{E}-09 \\ 2.721 \mathrm{E}-09 & 2.335 \mathrm{E}-09 \\ 3.028 \mathrm{E}-09 & 2.578 \mathrm{E}-09 \\ 3.410 \mathrm{E}-09 & 2.918 \mathrm{E}-09 \\ 3.950 \mathrm{E}-09 & 3.354 \mathrm{E}-09 \\ 4.714 \mathrm{E}-09 & 3.968 \mathrm{E}-09 \\ 5.843 \mathrm{E}-09 & 4.847 \mathrm{E}-09 \\ 7.682 \mathrm{E}-09 & 6.221 \mathrm{E}-09 \\ 1.128 \mathrm{E}-08 & 8.732 \mathrm{E}-09 \\ 3.835 \mathrm{E}-08 & 2.35 \mathrm{C}-08 \\ 5.091 \mathrm{E}-08 & 4.121 \mathrm{E}-08\end{array}$

1800.0

$-1 \cdot C 0000 E 00$ $-9.89401 \mathrm{E}-01$ $-9.44575 \mathrm{E}-01$ $-8.65631 \mathrm{E}-01$ $-7.55044 \mathrm{E}-0$ $-6.17876 E-0$ $-4.58017 E-01$ $-2.81605 \mathrm{E}-\mathrm{O} 1$ $-9.50125 E-02$ $9.5 C 125 \mathrm{E}-02$ $2.81605 \mathrm{E}-01$ $4.58017 \mathrm{E}-01$ $6.17876 \mathrm{E}-01$ $7.55044 E-01$ $8.65631 \mathrm{E}-01$ $9.44575 E-01$

TOTAL

$\begin{array}{ll}1.254 \mathrm{E}-09 & 1.504 \mathrm{E}-09 \\ 1.256 \mathrm{E}-09 & 1.567 \mathrm{E}-09 \\ 1.265 \mathrm{E}-09 & 1.519 \mathrm{E}-\mathrm{C9} \\ 1.283 \mathrm{E}-09 & 1.543 \mathrm{E}-09 \\ 1.315 \mathrm{E}-09 & 1.583 \mathrm{E}-09 \\ 1.363 \mathrm{E}-09 & 1.642 \mathrm{E}-09 \\ 1.433 \mathrm{E}-09 & 1.729 \mathrm{E}-09 \\ 1.534 \mathrm{E}-09 & 1.850 \mathrm{E}-09 \\ 1.675 \mathrm{E}-09 & 2.019 \mathrm{E}-09 \\ 2.229 \mathrm{E}-09 & 2.471 \mathrm{E}-09 \\ 2.299 \mathrm{E}-09 & 2.551 \mathrm{E}-\mathrm{Cg} \\ 2.455 \mathrm{E}-09 & 2.966 \mathrm{E}-09 \\ 3.114 \mathrm{E}-09 & 4.177 \mathrm{E}-\mathrm{C} 9 \\ 4.483 \mathrm{E}-09 & 4.557 \mathrm{E}-\mathrm{Cg} \\ 6.611 \mathrm{E}-09 & 7.470 \mathrm{E}-09 \\ 1.439 \mathrm{E}-08 & 1.384 \mathrm{E}-08 \\ 1.250 \mathrm{E}-07 & 1.084 \mathrm{E}-\mathrm{C} 7 \\ 5.432 \mathrm{E}-08 & 5.574 \mathrm{E}-08\end{array}$

500.0

$1.333 \mathrm{E}-09$ $1.337 \mathrm{E}-\mathrm{CS}$ $1.352 \mathrm{E}-0 \mathrm{~S}$ $1.382 \mathrm{E}-09$ $1.428 \mathrm{E}-\mathrm{C} 9$ $1.493 \mathrm{E}-09$ $1.580 \mathrm{E}-\mathrm{C} 9$ $1.696 \mathrm{E}-09$ $1.846 \mathrm{E}-\mathrm{C} 9$ $2.043 \mathrm{E}-\mathrm{C} 9$ $2.295 \mathrm{E}-09$ $2.633 \mathrm{E}-09$ $3.090 \mathrm{E}-09$ . 737 E-09 $4.710 \mathrm{E}-09$ $6.403 E-09$

. $143 E-08$

600.0

RANGE
900.0

1200.0

$9.971 \mathrm{E}-10$ $9.999 E-10$ $1.012 \mathrm{E}-\mathrm{C} 9$ $1.035 \mathrm{E}-09$ $1.070 \mathrm{E}-09$ $1.119 \mathrm{E}-09$ $1.184 E-09$ 1. $271 \mathrm{E}-09$ $1.383 \mathrm{E}-09$ $1.528 \mathrm{E}-\mathrm{C} 9$ $1.715 \mathrm{E}-09$ $1.960 \mathrm{E}-09$ $2.289 E-09$ $2.745 E-C 9$ $3.414 \mathrm{E}-0$ $4.529 \mathrm{E}-\mathrm{C} 9$

$2.258 \mathrm{E}-08$

$1.851 \mathrm{E}-09$ $1.855 \mathrm{E}-09$ $1.904 \mathrm{E}-09$ $1.957 \mathrm{E}-09$ $2.035 \mathrm{E}-09$ $2.145 \mathrm{E}-09$ 2.5C6E-09 $2.716 \mathrm{E}-09$ $3.664 E-0$ $1.336 E-0$ $8.286 E-08$
$.017 \mathrm{E}-09$ $2.021 \mathrm{E}-09$ $2.079 \mathrm{E}-09$

$2.140 \mathrm{E}-09$

$2.228 \mathrm{E}-09$

$2.352 \mathrm{E}-09$

$2.521 \mathrm{E}-09$

$2.749 \mathrm{E}-09$

$3.076 \mathrm{E}-09$
$3.438 \mathrm{E}-09$

$4.082 \mathrm{E}-09$

4.870E-O $6.375 \mathrm{E}-08$

$5.735 E-08$

1500.0

$.184 E-12$ $\begin{array}{ll}.458 E-10 & 1.022 E-10 \\ 3.468 E-10 & 1.025 E-10\end{array}$ $3.511 \mathrm{E}-10 \quad 1.038 \mathrm{E}-10$ $3.593 \mathrm{E}-10 \quad 1.063 \mathrm{E}-10$ $3.717 \mathrm{E}-10 \quad 1.100 \mathrm{E}-10$ $3.890 \mathrm{E}-10 \quad 1.151 \mathrm{E}-10$ $4.120 \mathrm{E}-10$ 1.220E-10 $4.421 E-10 \quad 1.308 E-10$ $4.806 \mathrm{E}-10 \quad 1.421 \mathrm{E}-10$ $5.296 \mathrm{E}-10 \quad 1.564 \mathrm{E}-10$ $5.920 \mathrm{E}-10 \quad 1.744 \mathrm{E}-10$ $6.723 \mathrm{E}-10 \quad 1.974 \mathrm{E}-10$ $7.772 \mathrm{E}-10 \quad 2.269 \mathrm{E}-10$ $9.172 E-10 \quad 2.654 E-10$ $1.112 E-09$ 3.175E-10 $1.410 E-09 \quad 3.928 E-10$ 2.784E-11 $7.206 \mathrm{E}-1$ $2.820 \mathrm{E}-11 \quad 7.299 \mathrm{E}-12$ $2.888 \mathrm{E}-11 \quad 7.474 \mathrm{E}-12$ $2.989 E-11 \quad 7.736 E-12$ 8.101E-12 $3.315 E-11 \quad 8.582 E-12$ $3.860 \mathrm{E}-11$ $9.990 \mathrm{E}-12$ $4.244 \mathrm{E}-11 \quad 1.098 \mathrm{E}-11$ $4.727 \mathrm{E}-11 \quad 1.222 \mathrm{E}-1$ 5.337E-11 1.378E-11 $7.116 \mathrm{E}-11$ 1.576E-11 7.121E-II $1.830 E-11$ $1.053 E-11$ 2.162E-11 $1.416 \mathrm{E}-10 \quad 3.466 \mathrm{E}-11$

$7.664 \mathrm{E}-09 \quad 2.223 \mathrm{E}-\mathrm{C} 9 \quad 5.976 \mathrm{E}-10 \quad 1.537 \mathrm{E}-10$ 
COSINE

$-1.000 C 0 E 00$ $-9.89401 \mathrm{E}-01$ $-8.65631 \mathrm{E}-01$ $-8.65631 E-01$ $-6.55076 E=01$ $-4.58017 E-01$ $-2.81605 E-01$ $-2.80125 \mathrm{E}-02$ $9.50125 \mathrm{E}-02$ $2.81605 E-01$ $4.58017 \mathrm{E}-01$ $4.58017 \mathrm{E}-01$
$6.17876 \mathrm{E}-01$ $7.55044 \mathrm{E}-01$ $8.65631 E-01$ $9.44575 E-01$ $9.89401 E-01$

TOTAL

COSINE

$-1.00000 E 00$ $-9.89401 \mathrm{E}-\mathrm{O}$ -9.44575 E- 01 $-8.65631 \mathrm{E}-01$ $-7.55044 E-01$ $-6.17876 E-01$ $-4.58017 \mathrm{E}-01$ $-2.81605 E-01$ -9.50125E-02 $9.5 \mathrm{C} 125 \mathrm{E}-02$ $2.81605 E-01$ 4.58017E-O1 $6.17876 \mathrm{E}-01$ 7.55044E-Ol $8.65631 \mathrm{E}-01$ $9.44575 \mathrm{E}-01$
$9.89401 \mathrm{E}-01$

TOTAL

$$
75.0
$$
$1.138 \mathrm{E}-09$ $1.14 E E-09$ $1.162 \mathrm{E}-09$ $1.188 E-09$ $1.229 E-09$ $1.370 E-C 9$ $1.379 E-C 9$ $2.053 \mathrm{E}-09$ $2.094 \mathrm{E}-09$ $2.238 \mathrm{E}-09$ $2.891 \mathrm{E}-09$ $4.224 E-C 9$ $6.686 \mathrm{E}-09$ $1.643 E-08$ $1.540 \mathrm{E}-07$ $5.811 \mathrm{E}-08$

500.0

$1.471 \mathrm{E}-09$ $1.475 E-09$
$1.489 E-09$ $1.489 \mathrm{E}-09$ $1.515 \mathrm{E}-09$ $1.555 \mathrm{E}-09$ $1.613 \mathrm{E}-09$ $1.690 \mathrm{E}-\mathrm{C} 9$ $1.792 \mathrm{E}-09$ $1.92 \in E-09$ 2.101 E-09 $2.329 \mathrm{E}-09$ $2.642 \mathrm{E}-09$ $3.083 \mathrm{E}-09$ $3.741 \mathrm{E}-09$ $4.798 \mathrm{E}-09$ $6.775 \mathrm{E}-09$ $.764 \mathrm{E}-0$ $3.295 E-08$
100.0 $1.367 \mathrm{E}-09$ $1.369 E-09$
$1.379 E-09$ 399E-0 $1.432 E-O 9$ $482 \mathrm{E}-09$ 1.556E-09 $1.662 \mathrm{E}-0$ $1.810 \mathrm{E}-09$ $2.166 \mathrm{E}-0$ $2.324 \mathrm{E}-09$ $2.679 \mathrm{E}-09$ $3.783 \mathrm{E}-09$ 7. $326 \mathrm{E}-09$ $1.506 \mathrm{E}-08$ 1. $342 \mathrm{E}-07$

5. $809 \mathrm{E}-08$

600.0 $1.142 \mathrm{E}-09$
$1.145 \mathrm{E}-09$ 1. $155 \mathrm{E}-\mathrm{C} 9$ $1.176 \mathrm{E}-09$ $1.207 \mathrm{E}-09$ $1.251 E-09$ 1. 310 E-O9 $1.388 \mathrm{E}-\mathrm{C}$ $1.489 \mathrm{E}-09$ $1.619 \mathrm{E}-09$ $1.789 \mathrm{E}-09$ $2.016 \mathrm{E}-09$ $2.334 E-C 9$ 2.798E-O $3.524 E-O$ $4.830 E-09$ $1.096 \mathrm{E}-0$

$2.460 E-08$
150.0 RANGE (METERS)
200.0

250.0

300.0

400.0

1. $710 E-09$ $1.713 E-09 \quad 1.910 E-09$ $1.930 \mathrm{E}-09$ $1.992 \mathrm{E}-09$ $1.996 \mathrm{E}-09$ $2.048 \mathrm{E}-09$ $2.101 \mathrm{E}-09$ $2.179 \mathrm{E}-09$ $2.287 \mathrm{E}-09$ $2.436 E-09$ $2.637 \mathrm{E}-09$ $2.946 \mathrm{E}-09$ $3.235 \mathrm{E}-09$ $3.849 \mathrm{E}-09$ $4.563 \mathrm{E}-09$ 5. $900 E-09$ $8.081 E-09$ $1.300 \mathrm{E}-08$ $6.080 \mathrm{E}-08$

$5.492 E-08$

$1.983 E-09$ 2.040 E-09 1.837E-09 2.171E-09 1.856E-09 $2.171 E-09 \quad 1.956 E-09$ $2.425 E-09 \quad 2.179 E-09$ 2.620 E- 09 2.179E-09 $2.882 \mathrm{E}-09 \quad 2.564 \mathrm{E}-09$ $3.239 \mathrm{E}-09 \quad 2.866 \mathrm{E}-09$ $3.727 \mathrm{E}-09 \quad 3.272 \mathrm{E}-09$ 4.474E-09 $3.862 \mathrm{E}-09$ $5.622 \mathrm{E}-09 \quad 4.760 \mathrm{E}-09$ $\begin{array}{ll}7.622 E-09 & 4.760 E-09\end{array}$ $1.178 \mathrm{E}-08 \quad 9.173 \mathrm{E}-09$ $4.716 \mathrm{E}-08 \quad 2.865 \mathrm{E}-08$ $5.127 E-08 \quad 4.229 E-08$

RANGE (METERS)

1500.0

1800.0

$9.589 \mathrm{E}-12$

$3.671 \mathrm{E}-11$

$\begin{array}{llll}4.321 \mathrm{E}-10 & 1.333 \mathrm{E}-10 & 3.707 \mathrm{E}-11 & 9.704 \mathrm{E}-12 \\ 4.399 \mathrm{E}-10 & 1.357 \mathrm{E}-10 & 3.773 \mathrm{E}-11 & 9.877 \mathrm{E}-12\end{array}$

$4.515 \mathrm{E}-10 \quad 1.392 \mathrm{E}-10 \quad 3.87 .1 \mathrm{E}-11 \quad 1.014 \mathrm{E}-11$

$4.676 \mathrm{E}-10 \quad 1.442 \mathrm{E}-10 \quad 4.008 \mathrm{E}-11 \quad 1.049 \mathrm{E}-11$

$4.890 \mathrm{E}-10 \quad 1.506 \mathrm{E}-10 \quad 4.186 \mathrm{E}-11 \quad 1.096 \mathrm{E}-11$

$5.167 E-10$ 1.590E-10 4.415E-11 $1.156 E-11$

$5.520 \mathrm{E}-10 \quad 1.695 \mathrm{E}-10 \quad 4.703 \mathrm{E}-11 \quad 1.230 \mathrm{E}-11$

$5.970 E-10 \quad 1.828 \mathrm{E}-10 \quad 5.065 \mathrm{E}-11 \quad 1.324 \mathrm{E}-11$

$6.546 \mathrm{E}-10$ 1.996E-10 $5.521 \mathrm{E}-11 \quad 1.442 \mathrm{E}-11$

7.299E-10 2.214E-10 6.105E-11 $1.593 \mathrm{E}-11$

$9.317 E-10$ 2.502E-10 $6.874 E-11$ 1.791E-11

$1.744 E-10$ 2.898E-10 $7.913 E-112.056 \mathrm{E}-11$

$1.1836 \mathrm{E}-09 \quad 4.355 \mathrm{E}-10 \quad 1.378 \mathrm{E}-1102.426 \mathrm{E}-11$

$\begin{array}{llll}1.736 E-09 & 4.355 E-10 & 1.159 \mathrm{E}-10 & 2.969 \mathrm{E}-11 \\ 2.700 \mathrm{E}-09 & 6.716 \mathrm{E}-10 & 1.659 \mathrm{E}-10 & 4.061 \mathrm{E}-11\end{array}$

$4.061 \mathrm{E}-11$

$8.621 E-09$

$2.581 E-09$

7.072E-10

$1.839 \mathrm{E}-10$ 
4 PI R*\#2 IONIZING SILICON KERMA (NEUTRCNS) (CM* 2 ERGS/GRAM/STERAOIAN/SOURCE NEUTRON)
FISSION SOURCE

1800.0

\begin{tabular}{|c|c|c|c|c|c|c|}
\hline 75.0 & 100.0 & 150.0 & $\begin{array}{c}\text { IGE (METER } \\
200.0\end{array}$ & 250.0 & 300.0 & 400.0 \\
\hline $\begin{array}{l}9.562 \mathrm{E}-11 \\
9.589 \mathrm{E}-11 \\
9.681 \mathrm{E}-11 \\
9.847 \mathrm{E}-11 \\
1.011 \mathrm{E}-10 \\
1.051 \mathrm{E}-10 \\
1.109 \mathrm{E}-1 \mathrm{C} \\
1.195 \mathrm{E}-10 \\
1.317 \mathrm{E}-10 \\
1.771 \mathrm{E}-10 \\
1.833 \mathrm{E}-10 \\
2.0 \mathrm{CCE}-10 \\
2.586 \mathrm{E}-10 \\
3.944 \mathrm{E}-10 \\
6.085 \mathrm{E}-10 \\
1.520 \mathrm{E}-09 \\
1.583 \mathrm{E}-08\end{array}$ & $\begin{array}{l}1.133 \mathrm{E}-10 \\
1.136 \mathrm{E}-10 \\
1.147 \mathrm{E}-10 \\
1.169 \mathrm{E}-10 \\
1.202 \mathrm{E}-10 \\
1.25 \mathrm{CE}-10 \\
1.320 \mathrm{E}-10 \\
1.423 \mathrm{E}-10 \\
1.567 \mathrm{E}-10 \\
1.951 \mathrm{E}-10 \\
2.028 \mathrm{E}-10 \\
2.336 \mathrm{E}-10 \\
3.342 \mathrm{E}-10 \\
3.911 \mathrm{E}-10 \\
6.705 \mathrm{E}-10 \\
1.403 \mathrm{E}-09 \\
1.386 \mathrm{E}-08\end{array}$ & $\begin{array}{l}1.367 \mathrm{E}-10 \\
1.371 \mathrm{E}-10 \\
1.385 \mathrm{E}-10 \\
1.413 \mathrm{E}-10 \\
1.456 \mathrm{E}-10 \\
1.517 \mathrm{E}-10 \\
1.606 \mathrm{E}-10 \\
1.732 \mathrm{E}-10 \\
1.907 \mathrm{E}-10 \\
2.113 \mathrm{E}-10 \\
2.479 \mathrm{E}-10 \\
2.875 \mathrm{E}-10 \\
3.830 \mathrm{E}-10 \\
4.565 \mathrm{E}-10 \\
7.499 \mathrm{E}-10 \\
1.319 \mathrm{E}-09 \\
1.074 \mathrm{E}-08\end{array}$ & $\begin{array}{l}1.467 \mathrm{E}-10 \\
1.471 \mathrm{E}-10 \\
1.487 \mathrm{E}-10 \\
1.519 \mathrm{E}-10 \\
1.567 \mathrm{E}-10 \\
1.636 \mathrm{E}-10 \\
1.734 \mathrm{E}-10 \\
1.873 \mathrm{E}-10 \\
2.062 \mathrm{E}-10 \\
2.323 \mathrm{E}-10 \\
2.645 \mathrm{E}-10 \\
3.153 \mathrm{E}-10 \\
3.861 \mathrm{E}-10 \\
5.095 \mathrm{E}-10 \\
7.363 \mathrm{E}-10 \\
1.283 \mathrm{E}-09 \\
8.367 \mathrm{E}-09\end{array}$ & $\begin{array}{l}1.468 \mathrm{E}-10 \\
1.473 \mathrm{E}-10 \\
1.490 \mathrm{E}-10 \\
1.523 \mathrm{E}-10 \\
1.573 \mathrm{E}-10 \\
1.645 \mathrm{E}-10 \\
1.745 \mathrm{E}-10 \\
1.886 \mathrm{E}-10 \\
2.076 \mathrm{E}-10 \\
2.334 \mathrm{E}-10 \\
2.658 \mathrm{E}-10 \\
3.155 \mathrm{E}-10 \\
3.838 \mathrm{E}-10 \\
5.017 \mathrm{E}-10 \\
7.107 \mathrm{E}-10 \\
1.191 \mathrm{E}-09 \\
6.517 \mathrm{E}-09\end{array}$ & $\begin{array}{l}1.406 \mathrm{E}-10 \\
1.411 \mathrm{E}-10 \\
1.428 \mathrm{E}-10 \\
1.461 \mathrm{E}-10 \\
1.510 \mathrm{E}-10 \\
1.580 \mathrm{E}-10 \\
1.679 \mathrm{E}-10 \\
1.815 \mathrm{E}-10 \\
1.998 \mathrm{E}-10 \\
2.247 \mathrm{E}-10 \\
2.561 \mathrm{E}-10 \\
3.008 \mathrm{E}-10 \\
3.668 \mathrm{E}-10 \\
4.730 \mathrm{E}-10 \\
6.629 \mathrm{E}-10 \\
1.077 \mathrm{E}-09 \\
5.101 \mathrm{E}-09\end{array}$ & $\begin{array}{l}1.181 \mathrm{E}-10 \\
1.185 \mathrm{E}-10 \\
1.200 \mathrm{E}-10 \\
1.229 \mathrm{E}-10 \\
1.272 \mathrm{E}-10 \\
1.333 \mathrm{E}-10 \\
1.418 \mathrm{E}-10 \\
1.535 \mathrm{E}-10 \\
1.690 \mathrm{E}-10 \\
1.889 \mathrm{E}-10 \\
2.161 \mathrm{E}-10 \\
2.523 \mathrm{E}-10 \\
3.055 \mathrm{E}-10 \\
3.890 \mathrm{E}-10 \\
5.340 \mathrm{E}-10 \\
8.329 \mathrm{E}-10 \\
3.141 \mathrm{E}-09\end{array}$ \\
\hline $.547 \mathrm{E}-09$ & $5.483 E-09$ & $5.371 \mathrm{E}-09$ & $5.142 \mathrm{E}-09$ & $4.776 E-09$ & $4.343 E-09$ & $3.407 E-09$ \\
\hline
\end{tabular}

-1.00000 E 00

$-9.89401 \mathrm{E}-01$

$-9.44575 E-01$

$-8.65631 E-01$

$-7.55044 \mathrm{E}-01$

$-6.17876 E-01$

$-4.58017 \mathrm{E}-01$

$-2.81605 E-01$

$9.50125 E-02$

$9.50125 E-02$

$2.81605 E-01$

$4.17876 E-01$

$6.17876 \mathrm{E}-01$

$8.65631 \mathrm{E}-01$

$8.65631 \mathrm{E}-01$

$9.89401 \mathrm{E}-01$

TOTAL

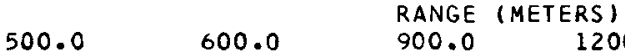

$9.194 E-11$

6. $8 C 7 E-11$

$2.314 E-11$

1200.0

1500.0

$6.774 E-12$

$1.833 E-12$

$4.746 E-13$

$9.346 \mathrm{E}-11$

$6.921 \mathrm{E}-11$

$2.355 \mathrm{E}-1$

$6.798 \mathrm{E}-12$

$1.840 \mathrm{E}-12$

$4.762 E-13$

$7.097 \mathrm{E}-11$

2.417E-1

$7.081 \mathrm{E}-12$

$9.923 E-11$

$7.358 \mathrm{E}-1$

$2.509 \mathrm{E}-11$

$7.357 \mathrm{E}-12$

$1.041 \mathrm{E}-10$

$8.234 \mathrm{E}-11$

$2.816 \mathrm{E}-11$

$7.746 \mathrm{E}-12$

$1.918 \mathrm{E}-12$

$1.993 \mathrm{E}-1$

$4.966 \mathrm{E}-13$
$5.165 \mathrm{E}-13$

$1.200 \mathrm{E}-10$

$8.917 E-11$

$3.051 E-11$

$8.272 \mathrm{E}-12$

$2.244 \mathrm{E}-12$

$5.445 E-13$

$1.321 \mathrm{E}-10$

$9.814 \mathrm{E}-11$

$3.357 \mathrm{E}-11$

$8.969 \mathrm{E}-12$

$2.435 E-12$

$5.822 \mathrm{E}-13$

$1.479 E-10$

$1.098 \mathrm{E}-10$

.750E-11

$9.869 \mathrm{E}-12$

$2.680 \mathrm{E}-12$

$2.993 \mathrm{E}-12$

$3.396 \mathrm{E}-12$

$1.250 \mathrm{E}-11$

$1.962 \mathrm{E}-10$

$.452 E-10$

$4.931 E-11$

$1.446 \mathrm{E}-1$

$1.741 E-10$

.

$1.722 \mathrm{E}-11$

. $932 \mathrm{E}-12$

$4.685 \mathrm{E}-12$

$5.80 \mathrm{CE}-12$

$2.774 \mathrm{E}-11$

$2.983 \mathrm{E}-10$

$2.921 E-10$

$7.548 \mathrm{E}-12$

$1.837 \mathrm{E}-11$

$7.420 \mathrm{E}-11$

$6.960 \mathrm{E}-1$

$7.777 \mathrm{E}-13$

$8.831 \mathrm{E}-13$

$1.224 \mathrm{E}-12$

$1.520 \mathrm{E}-1 \mathrm{~L}$

$1.982 \mathrm{E}-12$

$4.543 \mathrm{E}-12$

$2.544 E-09$

1. $833 E-09$

$5.970 E-10$

$1.721 \mathrm{E}-10$

$4.643 E-11$

$1.206 \mathrm{E}-11$ 
COSINE

-1.0 COOOE 00 $-9.89401 \mathrm{E}-01$ $-9.44575 E-01$ $-7.55044 E-01$ $-6.17876 \mathrm{E}-01$ $-4.58017 \mathrm{E}-01$ $-2.81605 \mathrm{E}-01$ $-9.50125 \mathrm{E}-02$ $9.50125 \mathrm{E}-02$ $2.81605 \mathrm{E}-01$ $4.58017 \mathrm{E}-01$ $6.17876 \mathrm{E}-01$ $7.55044 \mathrm{E}-01$ $8.65631 \mathrm{E}-01$
$9.44575 \mathrm{E}-01$ $9.89401 E-01$

TOTAL

C CSINE

$-1.00000 E 00$ $-9.89401 \mathrm{E}-01$ $-9.44575 E-01$ $-8.65631 E-01$
$-7.55044 E-01$ $-7.55044 E-01$
$-6.17876 E-01$ $-4.58017 E-01$ $-2.81605 E-01$ $-9.50125 E-02$ $2.81605 E-01$ 4.58017E-01 $6.17876 \mathrm{E}-01$ 7.55044E-O1 $8.65631 E-01$ $9.89401 \mathrm{E}-01$

TOTAL
75.0 $1.409 \mathrm{E}-10$ $1.419 \mathrm{E}-10$ $1.475 E-10$ $1.530 \mathrm{E}-10$ $1.610 E-10$ $1.725 \mathrm{E}-10$ $1.886 \mathrm{E}-1 \mathrm{C}$ $2.517 E-10$ $2.583 \mathrm{E}-10$ $2.781 E-10$ $3.523 \mathrm{E}-10$ $5.089 \mathrm{E}-10$ $7.424 \mathrm{E}-10$ $1.345 E-08$

$5.989 E-09$

500.0

$1.382 \mathrm{E}-10$ $1.386 \mathrm{E}-10$ $1.403 \mathrm{E}-10$ $1.436 \mathrm{E}-10$ $1.487 \mathrm{E}-10$ $1.559 \mathrm{E}-10$ $1.657 E-10$ $1.787 E-10$ $1.957 E-10$ $2.178 \mathrm{E}-10$ $2.466 E-10$ $2.849 E-10$ $3.366 E-10$ 4.095E-10 5.182E-10 $1.593 \mathrm{E}-09$

3.381E-C9
100.0

$1.676 \mathrm{E}-10$ $1.679 \mathrm{E}-10$ $1.692 \mathrm{E}-10$
$1.719 \mathrm{E}-10$ $1.763 E-10$ $1.763 E-10$ $1.929 E-10$ $2.068 \mathrm{E}-10$ $2.260 \mathrm{E}-10$ $2.760 \mathrm{E}-10$ $2.868 \mathrm{E}-10$ $3.277 \mathrm{E}-10$ 4. $706 \mathrm{E}-10$ $5.133 E-10$ $8.392 \mathrm{E}-10$ $1.528 \mathrm{E}-\mathrm{C} 9$ $1.164 \mathrm{E}-08$ $6.154 E-09$

600.0

$1.023 E-10$ $1.026 \mathrm{E}-10$ $1.039 E-10$ $1.064 E-10$ $1.103 \mathrm{E}-10$ $1.157 \mathrm{E}-10$ $1.231 \mathrm{E}-10$ $1.328 \mathrm{E}-10$ $1.454 \mathrm{E}-10$ $1.617 E-10$ $1.829 E-10$ $2.107 E-10$ $2.480 E-10$ $2.994 E-10$ $3.743 E-10$ $4.978 E-10$
$9.997 E-10$

$2.456 \mathrm{E}-09$

$\begin{array}{lcc} & \text { RANGE (METERS) } \\ 150.0 & 200.0 & 250.0 \\ .034 \mathrm{E}-10 & 2.191 \mathrm{E}-10 & 2.199 \mathrm{E}-10 \\ .038 \mathrm{E}-10 & 2.196 \mathrm{E}-10 & 2.204 \mathrm{E}-10 \\ .056 \mathrm{E}-10 & 2.218 \mathrm{E}-10 & 2.227 \mathrm{E}-10 \\ .093 \mathrm{E}-10 & 2.261 \mathrm{E}-10 & 2.273 \mathrm{E}-10 \\ .153 \mathrm{E}-10 & 2.329 \mathrm{E}-10 & 2.345 \mathrm{E}-10 \\ .241 \mathrm{E}-10 & 2.429 \mathrm{E}-10 & 2.449 \mathrm{E}-10 \\ .367 \mathrm{E}-10 & 2.570 \mathrm{E}-10 & 2.594 \mathrm{E}-10 \\ .541 \mathrm{E}-10 & 2.762 \mathrm{E}-10 & 2.791 \mathrm{E}-10 \\ .778 \mathrm{E}-10 & 3.021 \mathrm{E}-10 & 3.054 \mathrm{E}-10 \\ .986 \mathrm{E}-10 & 3.420 \mathrm{E}-10 & 3.454 \mathrm{E}-10 \\ .584 \mathrm{E}-10 & 3.792 \mathrm{E}-10 & 3.825 \mathrm{E}-10 \\ .107 \mathrm{E}-10 & 4.553 \mathrm{E}-10 & 4.575 \mathrm{E}-10 \\ .396 \mathrm{E}-10 & 5.423 \mathrm{E}-10 & 5.411 \mathrm{E}-10 \\ .096 \mathrm{E}-10 & 6.958 \mathrm{E}-10 & 6.878 \mathrm{E}-10 \\ .658 \mathrm{E}-10 & 9.411 \mathrm{E}-10 & 9.107 \mathrm{E}-10 \\ .482 \mathrm{E}-09 & 1.476 \mathrm{E}-09 & 1.378 \mathrm{E}-09 \\ .914 \mathrm{E}-09 & 6.873 \mathrm{E}-09 & 5.322 \mathrm{E}-09 \\ & & \\ .359 \mathrm{E}-09 & 6.313 \mathrm{E}-09 & 6.009 \mathrm{E}-09\end{array}$

2.109E-10 $2.115 \mathrm{E}-10$ $2.138 E-10$ $2.184 \mathrm{E}-10$ $2.256 E-10$ $2.358 \mathrm{E}-10$ $2.500 E-10$ $2.692 \mathrm{E}-10$ $2.947 E-10$ $3.282 \mathrm{E}-10$ $3.740 \mathrm{E}-10$ $4.339 E-10$ $6.475 E-10$ $8.525 E-10$ $1.247 E-09$ $4.153 E-09$

$5.558 \mathrm{E}-09$
400.0

$1.774 \mathrm{E}-10$

$1.779 \mathrm{E}-10$

$1.800 E-10$

$1.842 \mathrm{E}-10$

$1.905 \mathrm{E}-10$
$1.995 \mathrm{E}-10$

$2.118 E-10$

$2.282 \mathrm{E}-10$

.499E- 10

780E-10

$.159 E-10$

.658E-10

$5.341 \mathrm{E}-10$

$6.870 \mathrm{E}-10$

$9.636 E-10$
$2.556 E-09$

$4.465 \mathrm{E}-09$

$$
\begin{array}{rr}
\text { RANGE } & \text { (METERS) } \\
900.0 & 1200.0
\end{array}
$$

3. $470 E-11$

$3.481 \mathrm{E}-11$

$3.528 \mathrm{E}-1$

3. $618 \mathrm{E}-11$

3. $754 \mathrm{E}-11$

$4.199 \mathrm{E}-11$

$4.533 E-11$

4. $964 E-11$

5.514E-11

$7.219 E-11$

0.

9.

9.

$1.541 \mathrm{E}-10$

$.514 \mathrm{E}-10$

$8.075 E-10$

\section{$1.013 E-1$}

$1.016 \mathrm{E}-11$

$1.030 \mathrm{E}-11$

$1.057 \mathrm{E}-11$

$1.098 \mathrm{E}-11$

$1.229 \mathrm{E}-11$

$1.327 \mathrm{E}-11$

$1.453 \mathrm{E}-11$

$1.816 \mathrm{E}-11$

$2.075 \mathrm{E}-11$

$2.409 \mathrm{E}-11$

$2.846 E-11$

$4.281 \mathrm{E}-11$

OOE- 1

$2.322 E-10$
1500.0

$2.733 \mathrm{E}-12$

$2.742 \mathrm{E}-12$

$2.854 \mathrm{E}-12$

$2.964 \mathrm{E}-12$

$3.321 \mathrm{E}-12$

$3.587 \mathrm{E}-12$

. $925 E-12$

$4.897 E-12$

$5.586 \mathrm{E}-12$

$6.466 \mathrm{E}-12$

.110E-

$1.559 \mathrm{E}-11$

$6.210 E-11$
1800.0

$7.051 \mathrm{E}-13$

$7.075 \mathrm{E}-13$

$7.365 \mathrm{E}-13$

$7.651 E-13$

$8.576 E-13$

$9.261 \mathrm{E}-13$

$1.013 \mathrm{E}-12$

$1.124 \mathrm{E}-12$

$1.438 \mathrm{E}-12$

$1.662 \mathrm{E}-12$

.

$2.843 \mathrm{E}-12$

$1.593 E-11$ 
4 PI $R *$ * 2 HENDERSON DOSE (GAMMAS)

( CM $\$ 2$ RAD/STERADIAN/SOURCE NEUTRON )

COSINE

-1. OCCCOE OO $-9.89401 \mathrm{E}-01$ $-9.44575 E-01$ $-8.65631 E-01$ $-7.55044 E-01$ $-6.17876 E-01$ $-4.58 C 17 E-01$ $-2.816 C 5 E-01$ $9.50125 \mathrm{E}-02$ 9 $4.58 \mathrm{Cl} 7 \mathrm{E}-01$ $4.58 C 17 E-01$ $7.55044 \mathrm{E}-01$ $8.65631 \mathrm{E}-01$ $8.6531 E-01$ $9.89401 E-01$

TOTAL

COSINE

$-1 . \operatorname{COCCSE} 00$ $-9.89401 E-01$ $-9.44575 E-01$ $-8.65631 E-01$ $-7.55044 \mathrm{E}-01$ $-6.17876 E-01$ $-4.58017 \mathrm{E}-31$ $-2.81605 E-01$ $-9.50125 E-02$ $9.50125 E-02$ $2.81605 \mathrm{E}-01$ $4.58017 \mathrm{E}-01$ $6.17876 \mathrm{E}-01$ $7.55044 E-01$ $8.65631 E-21$ $9.44575 E-01$ 9.89401E-01

TOTAL

$\begin{array}{cc}75.0 & 100.0 \\ 4.368 \mathrm{E}-13 & 6.107 \mathrm{E}-13 \\ 4.386 \mathrm{E}-13 & 6.134 \mathrm{E}-13 \\ 4.455 \mathrm{E}-13 & 6.234 \mathrm{E}-13 \\ 4.583 \mathrm{E}-13 & 6.417 \mathrm{E}-13 \\ 4.760 \mathrm{E}-13 & 6.667 \mathrm{E}-13 \\ 4.987 \mathrm{E}-13 & 6.984 \mathrm{E}-13 \\ 5.277 \mathrm{E}-13 & 7.383 \mathrm{E}-13 \\ 5.654 \mathrm{E}-13 & 7.896 \mathrm{E}-13 \\ 6.152 \mathrm{E}-13 & 8.567 \mathrm{E}-13 \\ 7.228 \mathrm{E}-13 & 1.012 \mathrm{E}-12 \\ 8.315 \mathrm{E}-13 & 1.032 \mathrm{E}-12 \\ 9.005 \mathrm{E}-13 & 1.261 \mathrm{E}-12 \\ 1.063 \mathrm{E}-12 & 1.452 \mathrm{E}-12 \\ 1.393 \mathrm{E}-12 & 1.836 \mathrm{E}-12 \\ 1.988 \mathrm{E}-12 & 2.483 \mathrm{E}-12 \\ 3.085 \mathrm{E}-12 & 4.190 \mathrm{E}-12 \\ 7.552 \mathrm{E}-12 & 9.514 \mathrm{E}-12 \\ 1.185 \mathrm{E}-11 & 1.586 \mathrm{E}-11\end{array}$

150.0
$9.765 \mathrm{E}-13$
$9.811 \mathrm{E}-13$
$9.986 \mathrm{E}-13$
$1.030 \mathrm{E}-12$
$1.074 \mathrm{E}-12$
$1.128 \mathrm{E}-12$
$1.196 \mathrm{E}-12$
$1.282 \mathrm{E}-12$
$1.394 \mathrm{E}-12$
$1.601 \mathrm{E}-12$
$1.660 \mathrm{E}-12$
$2.013 \mathrm{E}-12$
$2.244 \mathrm{E}-12$
$2.839 \mathrm{E}-12$
$3.696 \mathrm{E}-12$
$5.716 \mathrm{E}-12$
$1.303 \mathrm{E}-11$
$2.443 \mathrm{E}-11$

RANGE (METERS)
200.0

250.0

300.0

400.0

$1.317 \mathrm{E}-12$

$.323 E-12 \quad 1.591 E-12$

$1.323 \mathrm{E}-12 \mathrm{1} \quad 1.600 \mathrm{E}-12$

$1.349 E-12 \quad 1.634 E-12$

$1.46 C \mathrm{CE}-12 \quad 1.779 \mathrm{E}-12$

$1.886 E-12$

$1.772 E-12$

$1.940 E-12$

$2.199 \mathrm{E}-12$

$2.689 E-12$

$2.740 \mathrm{E}-12$

$3.245 E-12$

$3.857 E-12$

$4.994 \mathrm{E}-12$

$1.583 E-11$

$3.314 \mathrm{E}-11$

$3.123 \mathrm{E}-12$

$3.531 \mathrm{E}-12$

4.

$6.319 \mathrm{E}-12$

6.3

$1.813 \mathrm{E}-11$

4. $138 E-11$

$1.783 \mathrm{E}-12$

$1.793 \mathrm{E}-12$

$1.834 \mathrm{E}-12$
$1.908 \mathrm{E}-12$

$2.009 \mathrm{E}-12$

$2.139 \mathrm{E}-12$

$2.306 E-12$

$2.526 \mathrm{E}-12$

$3.193 E-12$

$4.657 E-12$

$4.269 E-12$

$6.083 \mathrm{E}-12$

$7.08 \mathrm{E}-12$

$1.050 \mathrm{E}-1$

$2.005 \mathrm{E}-1$

$4.856 \mathrm{E}-11$

$1.918 \mathrm{E}-12$

$1.931 \mathrm{E}-12$

$1.980 E-12$

$2.069 \mathrm{E}-12$

$2.191 E-12$

.

$2.838 \mathrm{E}-12$

$3.214 E-12$

$4.713 E-12$

$5.224 \mathrm{E}-12$

$7.841 E-12$

$9.841 E-12$

$1.331 \mathrm{E}-1$

$2.301 E-11$

$5.822 E-11$
500.0

600.0

$1.801 E-12$

$1.814 \mathrm{E}-12$

$1.866 E-12$

$2.084 E-12$

$2.248 E-12$

$2.464 E-12$

$2.760 E-12$

$3.169 \mathrm{E}-12$

$4.483 \mathrm{E}-12$

$5.503 \mathrm{E}-12$

$6.893 \mathrm{E}-12$

$8.799 E-12$

$1.144 E-11$

$1.538 \mathrm{E}-1$

$2.489 E-11$

$6 \cdot 142 \mathrm{E}-11$
1200.0

$1.549 \mathrm{E}-12$

$1.612 \mathrm{E}-12$

$1.700 \mathrm{E}-12$

$1.966 \mathrm{E}-12$

$2.165 \mathrm{E}-12$

$2.443 E-12$

$3.388 \mathrm{E}-12$

$4.154 \mathrm{E}-12$

$5.226 \mathrm{E}-12$

$6.747 E-12$

$8.923 E-12$

$1.202 \mathrm{E}-1$

$2.572 \mathrm{E}-11$

$5.937 E-11$
$7.306 E-13$

$.367 E-13$

$.032 E-13$

$9.789 E-13$

$1.079 E-12$
$1.230 E-12$

$1.464 E-1$

$1.811 \mathrm{E}-12$

2. $322 \mathrm{E}-12$

$3.092 \mathrm{E}-12$

$4.320 \mathrm{E}-12$

$6.347 \mathrm{E}-12$

$9.661 \mathrm{E}-12$

$1.478 \mathrm{E}-11$

$3.949 E-11$
$2.741 E-13$
$2.815 E-13$

$3.060 \mathrm{E}-13$

$3.409 \mathrm{E}-13$

$3.984 E-13$

$4.308 \mathrm{E}-13$

$4.900 \mathrm{E}-13$

$7.940 \mathrm{E}-13$

$9.968 \mathrm{E}-13$

$1.372 \mathrm{E}-12$

$2.029 \mathrm{E}-12$

$3.276 \mathrm{E}-12$

$5.648 \mathrm{E}-12$

$9.848 \mathrm{E}-1$

$1.662 \mathrm{E}-1$

$2.080 E-11$
1500.0

$9.321 E-14$

$9.824 \mathrm{E}-14$

$1.126 \mathrm{E}-13$
$1.340 \mathrm{E}-13$

$1.474 \mathrm{E}-13$

$1.534 \mathrm{E}-13$

$1.597 \mathrm{E}-13$

$1.791 E-13$

$2.918 \mathrm{E}-13$

$3.897 \mathrm{E}-13$

$5.806 \mathrm{E}-12$

$9.950 E-12$
1800.0

$3.053 \mathrm{E}-14$

$3.360 E-14$

$4.262 E-14$
$5.311 E-14$

$6.023 E-14$

$5.945 E-14$

$5.864 E-14$
$6.596 E-14$

8.361 E-14

$1.134 \mathrm{E}-1$

$1.516 \mathrm{E}-13$

5. $909 \mathrm{E}-13$

$5.970 E-12$

$4.587 E-12$ 
COSINE

$-1.00000 E 00$ $-9.89401 \mathrm{E}-01$ . $-7.55044 \mathrm{E}-01$ $6.17876 \mathrm{E}-01$ $-4.58017 \mathrm{E}-01$ $-2.81605 \mathrm{E}-01$ $9.50125 \mathrm{E}-02$ $2.81605 \mathrm{E}-01$ $2.81605 E-01$
$4.58017 \mathrm{E}-01$ $6.17876 E-01$ $6.17876 E-01$ $7.55044 E-01$
$8.65631 E-01$ $8.65631 E-01$ $9.89401 \mathrm{E}-01$

TOTAL

COSINE

$-1.00000 E 00$ $-9.89401 \mathrm{E}-01$ $-9.44575 E-01$ $-8.65631 \mathrm{E}-01$ $-7.55044 E-01$ $-6.17876 E-01$ $-4.58017 \mathrm{E}-01$ $-2.81605 E-01$ $-9.50125 E-02$ $9.50125 \mathrm{E}-02$ 2.81605E-01 $4.58017 \mathrm{E}-01$ $6.17876 \mathrm{E}-01$ $7.55044 E-01$ $8.65631 E-01$ $9.44575 \mathrm{E}-01$
$9.89401 \mathrm{E}-01$

TOTAL

$\begin{array}{cc}75.0 & 100.0 \\ 4.429 \mathrm{E}-11 & 6.240 \mathrm{E}-11 \\ 4.447 \mathrm{E}-11 & 6.266 \mathrm{E}-11 \\ 4.514 \mathrm{E}-11 & 6.364 \mathrm{E}-11 \\ 4.639 \mathrm{E}-11 & 6.544 \mathrm{E}-11 \\ 4.813 \mathrm{E}-11 & 6.791 \mathrm{E}-11 \\ 5.036 \mathrm{E}-11 & 7.103 \mathrm{E}-11 \\ 5.320 \mathrm{E}-11 & 7.496 \mathrm{E}-11 \\ 5.690 \mathrm{E}-11 & 8.002 \mathrm{E}-11 \\ 6.178 \mathrm{E}-11 & 8.661 \mathrm{E}-11 \\ 7.212 \mathrm{E}-11 & 1.019 \mathrm{E}-10 \\ 8.264 \mathrm{E}-11 & 1.036 \mathrm{E}-10 \\ 8.966 \mathrm{E}-11 & 1.263 \mathrm{E}-10 \\ 1.054 \mathrm{E}-10 & 1.445 \mathrm{E}-10 \\ 1.374 \mathrm{E}-10 & 1.825 \mathrm{E}-10 \\ 1.956 \mathrm{E}-10 & 2.449 \mathrm{E}-10 \\ 3.021 \mathrm{E}-10 & 4.026 \mathrm{E}-10 \\ 7.368 \mathrm{E}-10 & 9.294 \mathrm{E}-10 \\ 1.177 \mathrm{E}-09 & 1.585 \mathrm{E}-09\end{array}$

500.0

$1.945 \mathrm{E}-10$ $1.958 \mathrm{E}-10$ $2.010 \mathrm{E}-10$ $2.103 \mathrm{E}-10$ $2.230 \mathrm{E}-10$ $2.395 \mathrm{E}-10$ $2.613 E-10$ $2.911 E-10$ $3.321 E-10$ 3.880E-10 $4.634 E-10$ $5.651 \mathrm{E}-10$ $7.032 \mathrm{E}-10$ $8.923 \mathrm{E}-10$ $1.154 \mathrm{E}-09$ $1.541 \mathrm{E}-0$ $2.473 E-09$

$6.313 E-09$

600.0

$6.119 E-09$
$150.0 \quad$ RANGE (METERS)
200.0

$1.008 \mathrm{E}-10$ $1.030 \mathrm{E}-10 \quad 1.401 \mathrm{E}-10$ $10 \quad 1.448 \mathrm{E}-10$ $1.104 \mathrm{E}-10 \quad 1.511 \mathrm{E}-10$ $1.158 \mathrm{E}-10 \quad 1.592 \mathrm{E}-10$ $1.225 \mathrm{E}-10 \quad 1.693 \mathrm{E}-10$ $1.823 \mathrm{E}-10$ $1.630 \mathrm{E}-10 \quad 2.170 \mathrm{E}-10$ $1.681 \mathrm{E}-10 \quad 2.500 \mathrm{E}-10$ $2.034 \mathrm{E}-10 \quad 2.780 \mathrm{E}-10$ $2.255 \mathrm{E}-10 \quad 3.280 \mathrm{E}-10$ $2.840 \mathrm{E}-10 \quad 3.877 \mathrm{E}-10$ $3.670 \mathrm{E}-10 \quad 4.989 \mathrm{E}-10$ $5.642 \mathrm{E}-10 \quad 7.262 \mathrm{E}-10$ $1.277 \mathrm{E}-09 \quad 1.555 \mathrm{E}-09$

2.462E-09 3.359E-09

RANGE (METERS)

900.0

1200.0

1500.0

1800.0

$\begin{array}{lllll}1.712 \mathrm{E}-10 & 8.613 \mathrm{E}-11 & 3.472 \mathrm{E}-11 & 1.318 \mathrm{E}-11 & 4.949 \mathrm{E}-12 \\ 1.72 & & & & \end{array}$

$1.762 \mathrm{E}-10 \quad 8.986 \mathrm{E}-11 \quad 3.791 \mathrm{E}-11 \quad 1.511 \mathrm{E}-11 \quad 6.148 \mathrm{E}-12$

$\begin{array}{lllll}1.851 E-10 & 9.581 \mathrm{E}-11 & 4.142 \mathrm{E}-11 & 1.725 \mathrm{E}-11 & 7.201 \mathrm{E}-12\end{array}$

$\begin{array}{lllll}1.969 \mathrm{E}-10 & 1.026 \mathrm{E}-10 & 4.467 \mathrm{E}-11 & 1.865 \mathrm{E}-11 & 7.936 \mathrm{E}-12\end{array}$

$1.104 \mathrm{E}-10$ 4.743E-11 $1.934 \mathrm{E}-11 \quad 7.911 \mathrm{E}-12$

$2.321 \mathrm{E}-10 \quad 1.207 \mathrm{E}-10 \quad 5.087 \mathrm{E}-11 \quad 2.009 \mathrm{E}-11 \quad 7.889 \mathrm{E}-12$

$2.602 \mathrm{E}-10 \quad 1.361 \mathrm{E}-10 \quad 5.697 \mathrm{E}-11 \quad 2.213 \mathrm{E}-11 \quad 8.666 \mathrm{E}-12$

$2.997 \mathrm{E}-10 \quad 1.596 \mathrm{E}-10 \quad 6.750 \mathrm{E}-11 \quad 2.651 \mathrm{E}-11 \quad 1.046 \mathrm{E}-11$

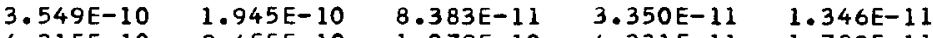

$4.315 \mathrm{E}-10 \quad 2.455 \mathrm{E}-10 \quad 1.079 \mathrm{E}-10 \quad 4.331 \mathrm{E}-11 \quad 1.729 \mathrm{E}-11$

$6.382 \mathrm{E}-10 \quad 3.222 \mathrm{E}-10 \quad 1.452 \mathrm{E}-10 \quad 5.822 \mathrm{E}-11 \quad 2.261 \mathrm{E}-11$

6.
$.055 \mathrm{E}-10$

$9.055 \mathrm{E}-10 \quad 6.452 \mathrm{E}-10 \quad 3.337 \mathrm{E}-10 \quad 10476 \mathrm{E}-10 \quad 6.053 \mathrm{E}-11$

$1.653 \mathrm{E}-0.737 \mathrm{E}-10$ 5.685E-10 $2.820 \mathrm{E}-10$ 10 $1.286 \mathrm{E}-10$

$\begin{array}{lllll}1.653 E-09 & 1.481 E-09 & 9.852 E-10 & 5.565 E-10 & 2.861 E-10 \\ 2.561 E-09 & 2.302 \mathrm{E}-09 & 1.659 \mathrm{E}-09 & 1.040 \mathrm{E}-09 & 5.958 \mathrm{E}-10\end{array}$

$4.098 E-09$

$2.170 E-09$

$1.042 E-09$

$4.811 \mathrm{E}-10$ 
4 PI R*\#2 AIR KERMA (GAMMAS)

(CM* 2 ERGS/GRAM/STERADIAN/SOURCE NEUTRON)
FISSION SOURCE

$1500.0 \quad 1800.0$
$-1 . r 0000 E$ OC $-9.89401 E-01$ $-9.44575 \mathrm{E}-01$ $-8.65631 \mathrm{E}-01$ $-7.55044 E-01$ $-4.58017 \mathrm{E}-01$ $-2.81605 \mathrm{E}-01$ $-9.50125 E-02$ $9.50125 \mathrm{E}-02$ $.81605 \mathrm{E}-\mathrm{C}$ $4.58017 E-01$ $7.17576 \mathrm{E}-01$ 8.65631 $8.6537 \mathrm{E}-01$ $9.89401 \mathrm{E}-\mathrm{O} 1$

TOTAL
75.0 $5.103 \mathrm{E}-11$ $5.169 \mathrm{E}-11$ $5.462 \mathrm{E}-11$ $5.682 \mathrm{E}-11$ $5.964 E-11$ $.328 \mathrm{E}-11$ $7.8 \mathrm{C} 1 \mathrm{E}-11$ . $843 \mathrm{E}-11$ .5C1E- 1

. $402 \mathrm{E}-10$ $.957 \mathrm{E}-10$ $2.962 \mathrm{E}-1 \mathrm{C}$ $7.042 \mathrm{E}-10$

$1.236 \mathrm{E}-09$

500.0

$2.862 \mathrm{E}-10$ $2.875 \mathrm{E}-10$ $2.930 \mathrm{E}-1 \mathrm{C}$ 3.02 SE-1C $3.163 \mathrm{E}-10$ $3.339 E-10$ $3.570 E-10$ $3.881 E-10$ $4.301 E-10$ 5.864E-10 $6.607 \mathrm{E}-1 \mathrm{C}$ $7.607 E-1 C$ .745E- 10 $1.745 \mathrm{E}-10$ 1.58EE- 0 1. $454 \mathrm{E}-\mathrm{C} 9$

7.447 E-09
$1 \mathrm{cos} 0$

7. $365 \mathrm{E}-11$ 7. $39 \mathrm{CE}-11$ $7.487 \mathrm{E}-11$ $7.666 E-11$ . $8.617 \mathrm{E}-1$ $.122 E-1$ $1.124 \mathrm{E}-10$ . $149 E-10$ . $363 E-10$ $1.546 E-10$ 2.504E-10 $3.990 E-10$
$8.933 E-10$

1. $703 \mathrm{E}-09$

600.0

$2.660 \mathrm{E}-10$ $2.674 \mathrm{E}-10$ $2.727 \mathrm{E}-10$ $2.823 \mathrm{E}-10$ $2.951 \mathrm{E}-10$ $3.116 E-10$ $3.334 E-10$ $3.632 E-10$ $4.042 E-10$ $4.603 E-10$ $5.366 E-10$ $7.869 \mathrm{E}-10$ . $869 E-10$ 1.281 E-C $1.693 \mathrm{E}-\mathrm{C}$ $1.693 E-C 9$
$2.536 E-09$

7.319E-09

\begin{tabular}{ccccc}
\multicolumn{5}{c}{ RANGE (METERS) } \\
150.0 & 200.0 & 250.0 & 300.0 & 400.0 \\
$1.233 \mathrm{E}-10$ & $1.718 \mathrm{E}-10$ & $2.141 \mathrm{E}-10$ & $2.472 \mathrm{E}-10$ & $2.837 \mathrm{E}-10$ \\
$1.237 \mathrm{E}-10$ & $1.725 \mathrm{E}-10$ & $2.150 \mathrm{E}-10$ & $2.483 \mathrm{E}-10$ & $2.850 \mathrm{E}-10$ \\
$1.254 \mathrm{E}-10$ & $1.751 \mathrm{E}-10$ & $2.184 \mathrm{E}-10$ & $2.524 \mathrm{E}-10$ & $2.901 \mathrm{E}-10$ \\
$1.286 \mathrm{E}-10$ & $1.798 \mathrm{E}-10$ & $2.246 \mathrm{E}-10$ & $2.600 \mathrm{E}-10$ & $2.994 \mathrm{E}-10$ \\
$1.329 \mathrm{E}-10$ & $1.862 \mathrm{E}-10$ & $2.332 \mathrm{E}-10$ & $2.7 \mathrm{C} 4 \mathrm{E}-10$ & $3.123 \mathrm{E}-10$ \\
$1.384 \mathrm{E}-10$ & $1.944 \mathrm{E}-10$ & $2.441 \mathrm{E}-10$ & $2.838 \mathrm{E}-10$ & $3.290 \mathrm{E}-10$ \\
$1.452 \mathrm{E}-10$ & $2.048 \mathrm{E}-10$ & $2.581 \mathrm{E}-10$ & $3.011 \mathrm{E}-10$ & $3.508 \mathrm{E}-10$ \\
$1.539 \mathrm{E}-10$ & $2.180 \mathrm{E}-10$ & $2.760 \mathrm{E}-10$ & $3.235 \mathrm{E}-10$ & $3.797 \mathrm{E}-10$ \\
$1.650 \mathrm{E}-10$ & $2.348 \mathrm{E}-10$ & $2.991 \mathrm{E}-10$ & $3.526 \mathrm{E}-10$ & $4.179 \mathrm{E}-10$ \\
$1.850 \mathrm{E}-10$ & $2.532 \mathrm{E}-10$ & $3.254 \mathrm{E}-10$ & $3.904 \mathrm{E}-10$ & $4.679 \mathrm{E}-10$ \\
$1.916 \mathrm{E}-10$ & $2.854 \mathrm{E}-10$ & $3.678 \mathrm{E}-10$ & $4.363 \mathrm{E}-10$ & $5.328 \mathrm{E}-10$ \\
$2.252 \mathrm{E}-10$ & $3.138 \mathrm{E}-10$ & $4.082 \mathrm{E}-10$ & $4.961 \mathrm{E}-10$ & $6.161 \mathrm{E}-10$ \\
$2.479 \mathrm{E}-10$ & $3.623 \mathrm{E}-10$ & $4.715 \mathrm{E}-10$ & $5.702 \mathrm{E}-10$ & $7.240 \mathrm{E}-10$ \\
$3.038 \mathrm{E}-10$ & $4.209 \mathrm{E}-10$ & $5.471 \mathrm{E}-10$ & $6.703 \mathrm{E}-10$ & $8.662 \mathrm{E}-10$ \\
$3.839 \mathrm{E}-10$ & $5.275 \mathrm{E}-10$ & $6.733 \mathrm{E}-10$ & $8.148 \mathrm{E}-10$ & $1.062 \mathrm{E}-09$ \\
$5.700 \mathrm{E}-10$ & $7.433 \mathrm{E}-10$ & $9.143 \mathrm{E}-10$ & $1.081 \mathrm{E}-09$ & $1.375 \mathrm{E}-09$ \\
$1.239 \mathrm{E}-\mathrm{Cg}$ & $1.521 \mathrm{E}-09$ & $1.756 \mathrm{E}-09$ & $1.955 \mathrm{E}-09$ & $2.262 \mathrm{E}-09$ \\
$2.723 \mathrm{E}-09$ & $3.779 \mathrm{E}-09$ & $4.790 \mathrm{E}-09$ & $5.683 \mathrm{E}-09$ & $6.941 \mathrm{E}-09$
\end{tabular}

RANGE (METERS)

10

3.837E-11

$1.737 \mathrm{E}-11$

$1.644 E-10$

$1.684 \mathrm{E}-10$

$1.750 \mathrm{E}-10$

$8.309 E-11$

$8.573 \mathrm{E}-1$

$8.967 \mathrm{E}-11$

$9.369 \mathrm{E}-11$

$3.887 E-11$

$1.767 \mathrm{E}-11$

$1.859 \mathrm{E}-11$

$1.974 \mathrm{E}-11$

$2.068 \mathrm{E}-11$

. $924 \mathrm{E}-10$

1.025E-10

4.

$2.102 \mathrm{E}-11$

.

1.

$2.145 \mathrm{E}-11$

2.272E-11

$2.479 \mathrm{E}-10$

$.224 \mathrm{E}-10$

$2.500 E-11$

$3.366 E-10$

$1.403 \mathrm{E}-10$

$2.847 \mathrm{E}-11$

$4.129 \mathrm{E}-10$

3.282E-11

$4.870 \mathrm{E}-11$

$7.98 \mathrm{E}-11$

$1.394 \mathrm{E}-10$

$\begin{array}{llll}1.028 \mathrm{E}-09 & 6.036 \mathrm{E}-10 & 3.022 \mathrm{E}-10 & 1.394 \mathrm{E}-10 \\ 1.497 \mathrm{E}-09 & 9.875 \mathrm{E}-10 & 5.545 \mathrm{E}-10 & 2.840 \mathrm{E}-10\end{array}$

$2.253 E-09 \quad 1.604 E-09$

$9.953 E-10$

$6.463 \mathrm{E}-10$ 


COSINE
$-1.00000 E 00$
$-9.89401 E-01$
$-9.44575 E-01$
$-8.65631 E-01$
$-7.55044 E-01$
$-6.17876 E-01$
$-4.58017 E-01$
$-2.81605 E-01$
$-9.50125 E-02$
$9.50125 E-02$
$2.81605 E-01$
$4.58017 E-01$
$6.17876 E-01$
$7.55044 E-01$
$8.65631 E-01$
$9.44575 E-01$
$9.89401 E-01$

TOTAL

COSINE

$-1.00000 E 00$ $-9.89401 E-01$ $-9.44575 \mathrm{E}-01$ $-8.65631 E-01$ $-7.55044 E-01$ $-6.17876 E-01$ $-4.58017 \mathrm{E}-01$ $-2.81605 E-01$ $-9.50125 E-02$ $9.50125 \mathrm{E}-02$ $2.81605 \mathrm{E}-01$ $4.58017 \mathrm{E}-01$ $6.17876 E-01$ $7.55044 \mathrm{E}-01$ 8.65631 E-01 $9.89401 \mathrm{E}-01$ TOTAL

$\begin{array}{cc}75.0 & 100.0 \\ 4.555 E-11 & 6.434 \mathrm{E}-11 \\ 4.573 \mathrm{E}-11 & 6.461 \mathrm{E}-11 \\ 4.642 \mathrm{E}-11 & 6.561 \mathrm{E}-11 \\ 4.769 \mathrm{E}-11 & 6.744 \mathrm{E}-11 \\ 4.945 \mathrm{E}-11 & 6.995 \mathrm{E}-11 \\ 5.172 \mathrm{E}-11 & 7.313 \mathrm{E}-11 \\ 5.462 \mathrm{E}-11 & 7.713 \mathrm{E}-11 \\ 5.837 \mathrm{E}-11 & 8.227 \mathrm{E}-11 \\ 6.333 \mathrm{E}-11 & 8.898 \mathrm{E}-11 \\ 7.386 \mathrm{E}-11 & 1.046 \mathrm{E}-10 \\ 8.446 \mathrm{E}-11 & 1.062 \mathrm{E}-10 \\ 9.168 \mathrm{E}-11 & 1.293 \mathrm{E}-10 \\ 1.077 \mathrm{E}-10 & 1.477 \mathrm{E}-10 \\ 1.4 \mathrm{C} 1 \mathrm{E}-10 & 1.865 \mathrm{E}-10 \\ 1.993 \mathrm{E}-10 & 2.497 \mathrm{E}-10 \\ 3.076 \mathrm{E}-10 & 4.1 \mathrm{E}-10 \\ 7.498 \mathrm{E}-10 & 9.462 \mathrm{E}-10 \\ 1.203 \mathrm{E}-09 & 1.623 \mathrm{E}-09 \\ & \end{array}$

$50 \mathrm{C} .0$

$2.048 \mathrm{E}-10$ $2.061 \mathrm{E}-10$ $2.114 \mathrm{E}-10$ $2.210 \mathrm{E}-10$ $2.339 \mathrm{E}-10$ $2.507 \mathrm{E}-10$ $2.730 \mathrm{E}-10$ $3.034 \mathrm{E}-10$ $3.453 E-10$ $4.023 \mathrm{E}-10$ $4.793 \mathrm{E}-10$ $5.832 \mathrm{E}-10$ $7.243 E-10$ $9.175 \mathrm{E}-10$ $1.184 \mathrm{E}-09$ $1.580 \mathrm{E}-09$ 2.531E-09 $6.524 E-09$

600.0

$$
150.0
$$

RA

1. $043 \mathrm{E}-10$ 1. $048 \mathrm{E}-10$ $1.065 \mathrm{E}-10$
$1.098 \mathrm{E}-10$ 1. $141 \mathrm{E}-10$ 1.196E-10 $1.265 \mathrm{E}-10$ 1. $352 \mathrm{E}-10$ $1.465 E-10$ $1.678 \mathrm{E}-10$ $1.728 \mathrm{E}-10$ 2. $089 \mathrm{E}-10$ $2.312 \mathrm{E}-10$ $2.908 E-10$ $3.748 \mathrm{E}-10$ 1. $300 \mathrm{E}-09$

$2.527 \mathrm{E}-09$

200.0
(METERS)

250.0

300.0

400.0 $1.421 \mathrm{E}-10 \quad 1.732 \mathrm{E}-10$ $1.454 \mathrm{E}-10$ $1.501 \mathrm{E}-10$ $1.566 \mathrm{E}-10$ $1.648 E-10$ $1.752 E-10$ $1.884 E-10$ $2.237 \mathrm{E}-10$ $2.575 \mathrm{E}-10$ $2.859 E-10$

$3.368 \mathrm{E}-10$

$3.975 E-10$

$5.105 \mathrm{E}-10$

$7.415 \mathrm{E}-10$

$1.585 \mathrm{E}-09$ $1.741 E-10$ $1.775 \mathrm{E}-10$
$1.838 \mathrm{E}-10$ $1.923 \mathrm{E}-10$ $2.033 \mathrm{E}-10$ $2.172 \mathrm{E}-10$ $2.352 \mathrm{E}-10$ $2.585 \mathrm{E}-10$ $2.848 \mathrm{E}-10$ $3.701 E-10$ $4.365 E-10$ $5.35 \mathrm{E}-10$ $0.490 E-10$ $9.069 \mathrm{E}-10$

$1.822 \mathrm{E}-0$

$3.455 E-09$

$4.336 E-09$

1. $957 \mathrm{E}-10$ $1.967 \mathrm{E}-10$ $2.008 \mathrm{E}-10$ $2.084 \mathrm{E}-10$ $2.187 \mathrm{E}-10$ $2.492 \mathrm{E}-10$ $2.492 E-10$ $2.717 E-10$ 3.397E-10 $3.867 E-10$ $4.86 \mathrm{E}-10$ $4.489 E-10$ 5. $7.848 \mathrm{E}-10$ $1.071 E-09$ $2.021 \mathrm{E}-09$

$5.108 \mathrm{E}-09$

$2.140 \mathrm{E}-10$ $2.153 \mathrm{E}-10$ $2.203 \mathrm{E}-10$ $2.295 E-10$ $2.420 E-10$ $2.798 E-10$ $3.084 E-10$ $3.084 E-10$ $3.979 E-10$ $3.979 E-10$ 4.647E-10 $6.515 E-10$ $6.653 \mathrm{E}-10$ $8.164 E-10$ $1.026 \mathrm{E}-09$
$1.364 \mathrm{E}-09$ $2.331 E-09$

$6.159 E-09$

\section{RANGE (METERS)}

900.0

1200.0

1500.0

1800.0 $1.812 \mathrm{E}-10$ 9.299E-11 $3.921 \mathrm{E}-11 \quad 1.552 \mathrm{E}-11 \quad 6.119 \mathrm{E}-12$ $1.863 \mathrm{E}-10$ 9.681E-11 4.173E-11 $1.700 \mathrm{E}-11 \quad 7.039 \mathrm{E}-12$ $\begin{array}{lllll}1.954 \mathrm{E}-10 & 1.029 \mathrm{E}-10 & 4.532 \mathrm{E}-11 & 1.919 \mathrm{E}-11 & 8.118 \mathrm{E}-12\end{array}$ $2.228 \mathrm{E}-10 \quad 1.177 \mathrm{E}-10 \quad 5.141 \mathrm{E}-11 \quad 2.130 \mathrm{E}-11 \quad 8.829 \mathrm{E}-12$ $\begin{array}{lllll}2.434 E-10 & 1.282 \mathrm{E}-10 & 5.488 \mathrm{E}-11 & 2.203 \mathrm{E}-11 & 8.790 \mathrm{E}-12\end{array}$ $2.720 \mathrm{E}-10 \quad 1.439 \mathrm{E}-10 \quad 6.106 \mathrm{E}-11 \quad 2.408 \mathrm{E}-11 \quad 9.565 \mathrm{E}-12$ $3.124 \mathrm{E}-10 \quad 1.678 \mathrm{E}-10 \quad 7.175 \mathrm{E}-11 \quad 2.852 \mathrm{E}-11 \quad 1.138 \mathrm{E}-11$ $3.687 \mathrm{E}-10$ 2.033E-10 $8.835 \mathrm{E}-11 \quad 3.561 \mathrm{E}-11 \quad 1.442 \mathrm{E}-11$ 2.468E-10 2.553E-10 $1.128 \mathrm{E}-10 \quad 4.555 \mathrm{E}-11 \quad 1.828 \mathrm{E}-11$ $5.559 \mathrm{E}-10 \quad 3.336 \mathrm{E}-10$ 1. $1.508 \mathrm{E}-10$ 6.066E-11 $2.365 \mathrm{E}-11$ $7.104 \mathrm{E}-10$ 4.582E-10 $2.172 \mathrm{E}-10$ $8.928 \mathrm{E}-11 \quad 3.456 \mathrm{E}-11$ $9312 \mathrm{E}-10$ 6.636E-10 3.432E-10 1.517E-10 $6.219 \mathrm{E}-11$ $1.245 E-09 \quad 9.997 \mathrm{E}-10 \quad 5.834 \mathrm{E}-10 \quad 2.892 \mathrm{E}-10 \quad 100317 \mathrm{E}-10$ $2.622 \mathrm{E}-09$ 1.519E-09 $1.010 \mathrm{E}-09$ 5.706E-10 $2.933 \mathrm{E}-10$ $2.360 \mathrm{E}-09$ 1.701E-09 $1.067 \mathrm{E}-09$ 6.116E-10 $6.329 E-09 \quad 4.247 E-09 \quad 2.252 E-09 \quad 1.082 E-09 \quad 5.000 E-10$ 
(NEUTRGNS/MEV/STERADIAN/SOURCE NEUTRON)

ENERGY

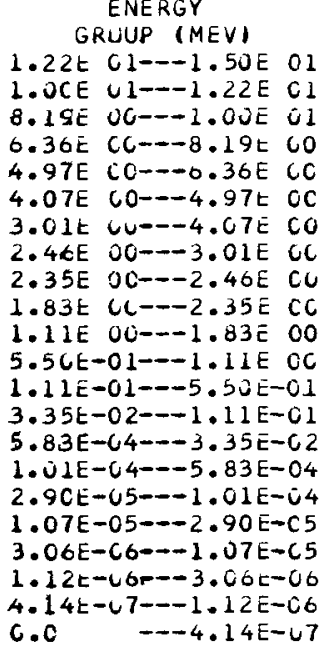

\section{ENERGY}

GROUP (MEV)
1.225 C $1=-1.50 \mathrm{E}$ C

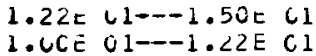
$1 . U C E$ O $1--1.22 E ~ C 1$
$8.19 E$ CO---1.00E CI $8.19 E$ CO-- $-1.00 E$ C
$6.36 \mathrm{E}$ CU $--8.19 E$ OO $6.36 \mathrm{E} \mathrm{CU}--8.19 \mathrm{E}$
$4.97 \mathrm{E} \mathrm{CO--3.36 \textrm {E }} \mathrm{CO}$ 4.07E CO-- $0.97 \mathrm{E}$ CO $3.01 E 00--4.077 E$ EO 2.4 EE 00--3.01E CO $2.35 \mathrm{E} O C--2.46 \mathrm{E}$ CO 1.83E COC- $2.35 E$ OO 1 .11E UO- $-1.83 E$ CO 1.5CE-01-CD $1.11 E$ OC 1.1E-01-C-5.5OE-O . 1.01E 1.01E $04-0.015$ 1.07E-65$3.06 E-66=-1.07 E-05$ 1.12E-C $6---1.06 \mathrm{E}-\mathrm{C}$ $4.14 E-07-\cdots-1.06 E-C 6$ $0.0 \quad--4.14 E-07$
ANGLE 1 ANGLE 2 $M U=-1.0 \mathrm{COC}$ $-5.280 t-05$ $5.220 E-C 5$ $1.9639 \mathrm{E}-04$ $2.228 \mathrm{E}-04$ $3.703 E-04$ $0.501 E-04$ $1.458 E-03$ $2.087 E-03$ $1.583 \mathrm{E}-03$ $2.838 \mathrm{E}-03$ $8.336 \mathrm{E}-03$ $2.495 E-02$ $7.123 \mathrm{E}-01$ $8.835 E-01$ $5.082 E$ OL $1.909 E 02$ $4.806 E$ OL $1.084 \mathrm{E} 03$ 2.559E O3 $5.029 E \quad 03$ $0.641 E$ C3

ANGLE 10

$M U=0.0950^{\circ}$ $3.064 E-05$ $3.054 \mathrm{E}-04$ $3.562 E-04$ $3.753 \mathrm{E}-04$ $6.071 E-04$ . $925 c-04$ $1.302 E-03$ . $35 \mathrm{EE}-\mathrm{C}$ $3.436 E-C 3$ $3.457 E-03$ $6.844 \mathrm{E}-03$ $1.793 E-02$ 1.088E 00 $1.201 \mathrm{E} C \mathrm{O}$ $9.906 E 01$ $2.370 \mathrm{E} 02$ $5.671 E$ C $1.230 \mathrm{E} O 3$ $2.837 \mathrm{E} \mathrm{O3}$ $5.484 E$ CD
$7.119 E$ C
$U=-6.9894$ $-4.324 t-C 5$ $1.88 C E-\hat{~}$ $1.882 E-: 4$ $2.217 \mathrm{E}-04$ $3.8 C 7 E-C 4$ $6.543 E-C$ $1.457 E-C 3$ 2. CBCE-03 $1.582 \mathrm{E}-03$ $2.846 E-03$ $8.353 \mathrm{E}-\mathrm{O}$ $2.502 E-02$ $7.142 \mathrm{E}-\mathrm{Cl}$ $8.856 t-01$ $5.092 E$ LI $1.913 E \quad 02$ $4.813 \mathrm{E} \mathrm{O2}$ $1 . C 85 E 03$ 2.562E O3 $5 . C 33 E \mathrm{CB}$ $6.645 \mathrm{E} 03$ ANGLE 11 $A N=C .2816$
$5.871 \mathrm{E}-05$ $5.871 E-05$
$2.828 \mathrm{E}-04$ $2.828 E-04$
$2.422 \mathrm{E}-\mathrm{O} 4$ $2.422 \mathrm{E}-04$
$2.081 \mathrm{E}-04$ $2.681 E-04$
$4.432 E-04$ $4.432 E-04$
$8.597 E-04$ $9.225 \mathrm{E}-\mathrm{C} 4$ $2.144 E-03$ 3.CCSE-O3 . $1<6 E-03$ $1.563 E-02$ $4.395 E-O 2$ l. $324 \mathrm{E} C \mathrm{C}$ 1.32 $7 . C B C E$ OI $2.576 t 0$ $5.877 \mathrm{E} \mathrm{O2}$ $1.262 \mathrm{E} \mathrm{O} 3$ $2.895 \mathrm{E} \mathrm{C} 3$ $5.574 \mathrm{E} C 3$
$7.21 \mathrm{CE}$
ANGLE $\quad 3$
$M U=-C .9440$

3
ANOLE 4
440
$M U=-6.8656$ 1. $087 E-05$ 5. $58 \mathrm{z} \mathrm{t}-05$ $1.795 E-04$
$1.59 L E-04$ $1.59 L E-04$
$2.184 E-C 4$ $2.184 E-C 4$
$3.90 C E-O 4$ $6.51 \angle \mathrm{E}-\mathrm{C}$ $1.453 E-C 3$ $2.05 b E-03$ 1. $582 \mathrm{E}-03$ $2.877 E-C$ $8.423 E-03$ $2.531 E-02$ $7.219 E-G 1$ $8.942 \mathrm{E}-01$ $5.134 E 01$ $1.925 t_{02} 02$ $4.839 E 02$ $2.571 E 03$ $5.049 \mathrm{E}$ U3 $0.663 \mathrm{E} \mathrm{C3}$

ANGLE 12 $A N=0.458 U$
$.088 \mathrm{E}-05$ $3.688 \mathrm{E}-05$ $3.454 \mathrm{E}-04$ $3.069 E-04$ 3. $339 E-04$ $5.553 E-04$ $9.034 E-04$ $1.173 E-03$ 2.8UOE-CB $3.132 \mathrm{E}-03$ . $379 E-03$ 1.4975 1. 30 E-O . $2.099 E$ OU 7.377 E OI 2.505E 02 6.119E 02 $1.290 \mathrm{E} \mathrm{O3}$ $2.953 E$ O3 $5.663 E$ C 3
$7.30 C$ E 03 $3.432 t-C 6$ $1.657 E-C$ $1.535 \mathrm{E}-64$ $\angle .157 \mathrm{E}-04$ $4.071 E-C$ $6.541 E-0$ $1.447 \mathrm{E}-03$ <.415t-03 1.589E-03 $2.943 t-c 3$ $0.57 \mathrm{CE}-03$ $2.509 E-C 2$ $7.372 \mathrm{E}-\mathrm{Cl}$ $9.112 \mathrm{E}-01$ $5.215 \mathrm{E} \mathrm{Cl}$ $1.950 \mathrm{E} \mathrm{OZ}$ $1.699 E \quad 03$ 2.589 C3 $5.479 E$ O $6.697 \mathrm{E} \mathrm{C3}$

ANULE 13 $A U=C .6179$
$1.225 E-04$ $1.224 E-04$
$4.225 E-C 4$ $4.488 \mathrm{E}-\tilde{U}^{4}$ $4.791 \mathrm{E}-0$ $8.026 E-04$ 1. $109 \mathrm{E}-03$ $1.752 \mathrm{~L}-03$ 3.6 $4.048 E-O 3$ 4. 3 C6E-O . 5.985E . $16 E-C 2$ $1.524 E$ $7.846 E$ 2.680 E C2 $6.337 E$ C 2 3. $0 C A E C$ $5.747 E$ OJ $7.384 E$ O 作 $9.0 \angle C E-06$ $6.359 E-05$ $1.518 E-04$ $1.499 \mathrm{E}-\mathrm{U}$ $2.19 \mathrm{CE}-\mathrm{C} 4$ $4.3 C 5 E-04$ $6.0 C 8 E-C 4$ $1.440 E-U 3$ $1.972 \mathrm{E}-03$ $1.61<E-03$ 3. $052 \mathrm{E}-\mathrm{C}$ $8.811 E-03$ $2.677 E-C L$ $7.605 \mathrm{E}-01$ $9.367 \mathrm{E}-\mathrm{Cl}$ $5.334 \mathrm{E} \mathrm{Ol}$ $4.964 E \mathrm{C} 2$ 1.112E O3 $2.614 \mathrm{E} \mathrm{U3}$ $5.1 \angle 1 E$ C 3 $6.743 E$ U3 ANGLE 14 $A U=0.7550$
$7.662 E-C 4$ 5.662E-C4
5.995E-C4 $8.2215-64$ $8.755 E-C 4$ $1.463 \mathrm{t}-\mathrm{U3}$ $1.746 \mathrm{E}-\mathrm{C} 3$ 2.551E-03 0.73 EE-O 6.C97e-0 $1.216 t-c 2$ 3. $C 44 E-02$ $6.742 E-C$ $1.623 E 00$ 9.141E 3. CO\&E C 6.57UE 02 $1.36 C E 03$ $3 . C 59 E ~ C 3$ $5.8 \angle 2 E<3$ 7.458 E $O 3$ 1. $333 t-C 5$ $1.43 \mathrm{CE}-\mathrm{C}$ $1.43 C E-C 4$
$1.516 \mathrm{E}-\mathrm{C} 4$ $2.328 \mathrm{E}-\mathrm{L} 4$ $4.600 E-04$ $6.757 E-C 4$ $1.438 E-C 3$ $1.944 E-C 3$ . $665 E-C$ 3. $216 E-C$ $.172 \mathrm{E}-\mathrm{O}$ . $600 E-C 2$ $9.718 \mathrm{E}-\mathrm{Cl}$ .034E $2.034 E$ C2
$5.061 E$ C2 $1.128 \mathrm{E} C \mathrm{C}$ $2.647 E$ OS $5.176 E ~ C 3$
$6.802 E ~ C 3$ ANGLE 15 $M U=0.8656$ $9.248 \mathrm{E}-04$ $1.181 E-C S$ $1.159 \mathrm{E}-03$ $1.238 \mathrm{E}=\mathrm{O} 3$ 2.047E-C 3 $2.993 \mathrm{~L}-\mathrm{C}$ . $550 E-03$ $6.400 E-03$ $9.243 E-C 3$ .. 777 ES $1.757 E-02$ 4.115e-C2

ANGLE $6 \quad$ ANGLE 7 
ENERGY GROUP (MEVI $1.22 E$ OI-- 1. 5OE GI $1.0 C E$ O1-D- $1.22 E$ C 8.19E 00---1.00E G 6.36E $00--8.19 E \mathrm{CO}$ 4.97E UO- 6.36 E CO $3.01100--4.07 E 00$ 2.465 $60-0-3.01 E$ OO $2.35 \mathrm{E} 00--2.46 \mathrm{E} O 0$ I.83E $00--2.35 E$ OO $1.11 \mathrm{E} 00-1.83 \mathrm{E} 00$ 5.5CE-01--1.11E CO $1.11 E-U 1--5.50 E-0$ $3.35 \mathrm{E}-02--1.11 \mathrm{E}-0$ $5.83 E-04--3.35 E-02$ $1.01 \mathrm{E}-04---5.83 \mathrm{E}-\mathrm{C}_{4}$ $2.90 E-05=-1.01 E-i 4$ $1.07 E-05--2.90 E-05$ $3.06 \mathrm{E}-06=-1.07 E-05$ $1.1<E-06=-3.06 E-C 6$ $4.14 E-07---1.12 \mathrm{E}-\mathrm{C}$ $0.6 \quad--4.14 E-0$

$$
\text { ENERGY }
$$

$$
\text { GRUUP (MEV) }
$$

$1.22 \mathrm{E} \mathrm{O1-D-1.50E} 0$ 1. COE U L-1.22E O 8.19E OC-D-1.00E O $6.36 \mathrm{E}$ 00-- $8.19 \mathrm{E}$ OC 4.97E OO-D. 0.36 E OO 4.07E $00-4.97 E$ OO $3.01 E$ OC-- $4.07 E$ CO 2.46E OO--3. OIE OO $2.35 E$ OO- $2.46 E$ EO 00 $1.83 \mathrm{E}$ OC-D-2.35E 00 1.11E 00-- $-1.83 \mathrm{E} 00$ 5.5OE-O1$1.11 E-O 1--5.50 E-O 1$ . . $83 E-04-3.35 E-C 2$ 1.01 C $04-5.83 E-C 4$ 2.96E $05-1.01 E-04$ $1.07 E-05=-2.90 E-05$ 1.06E $06-1.07 E-C 5$ $1.12 E-06-2.06 E-C 6$ $4.14 \mathrm{E}-\mathrm{C} 7---1.12 \mathrm{E}-\mathrm{C} 6$
0.0 $M U=-1.0000 \quad M U=-C \cdot 989$ $4.961 E=C 5 \quad-4.058 E-05$ $4.772 E-C 5 \quad 4.894 E-05$ $1.955 E-04 \quad 1.942 E-C 4$ $2.883 E-04 \quad 2.870 E-04$ $5.092 \mathrm{E}-04 \quad 5.123 \mathrm{E}-\mathrm{O} 4$ $8.270 \mathrm{E}-04 \quad 8.27 \mathrm{CE}-04$ $1.783 E-03 \quad 1.783 E-C$ $2.570 E-03$ $2.212 \mathrm{E}-03$ 4.019E-03 $1.217 E-02$ $3.899 \mathrm{E}-02$ $9.253 \bar{E}-01$ $1.118 E$ OO $7.603 E$ OI $3.362 E$ O $9.647 E \quad 02$ $2.472 E$ OS $1.325 E \quad 04$ $1.845 E 04$ ANGLE 10 $M U=C .0950$ $3.854 \mathrm{E}-\mathrm{C}$ $2.695 \mathrm{E}-\mathrm{C} 4$ $1.797 E-0$ $2.366 E \rightarrow 04$ $4.222 \mathrm{E}-0$ $6.938 E-04$ $8.414 E-04$ $1.674 \mathrm{E}-\mathrm{C} 3$ $2.581 E-03$ 2.856E-O 5. $334 \mathrm{E}-0 \mathrm{~S}$ $7.40 E-O 2$ $7.406 E-02$ $2.017 E$ OO $1.302 E$ CO .5 58 E 1. 1.174E 03 7. 1.521E 04 $1.521 \mathrm{E} \mathrm{O4}$
$2.071 \mathrm{E} \mathrm{G4}$ $475 \mathrm{E} \mathrm{O}$ E 1.847E C4 ANGLE 11 $3.622 \mathrm{E}-04$ 3.326E-O4 $3.967 \mathrm{E}-\mathrm{C} 4$ $6.796 E-04$ $1.295 \mathrm{E}-03$ $1.325 \mathrm{E}-03$ $3.159 \mathrm{E}-03$ $4.7 C 2 E-03$ $5.071 E-03$ $9.581 E-03$ 2.55OE-C2 . 1.321E OO 1.341E OU 4.519E 02 $1.238 E$ U3 $7.632 E$ OS $1.563 E$ $2.118 E$ i 4
(NEUTRONS/MEV/STERADIAN/SOURCE NEUTRDN) ANGLE 4 ANGLE $\quad 5$
$M U=-0.7550$ ANGLE 6 $1.352 E-C S$ ANGLE 7 $M U=-0.4580$ I. $576 \mathrm{E}-05$ 5.296E-05 $1.821 \mathrm{E}-04$ $2.834 \mathrm{E}-04$ $5.245 \mathrm{E}-04$ $8.276 \mathrm{E}-04$ $1.779 \mathrm{E}-0.3$ $2.544 \mathrm{E}-03$ $2.222 \mathrm{E}-03$ 4. $C 79 E-03$ $1.233 \mathrm{E}-02$ $3.956 \mathrm{E}-02$ $9.377 \bar{E}-01$ $1.131 \mathrm{E} 00$ $7.683 E$ O1 $3.394 E$ O $9.73 L E ~ O 2$ 2. $490 \mathrm{E} 03$ $6.380 E$ O AN $=-0.8656$ $5.834 E-C 5$ $1.687 \mathrm{E}-\mathrm{C} 4$ $1.687 E-C 4$ $1.835 E-C 4$ $5.469 E-O 4$ $0.305 E-04$ $1.775 \mathrm{E}-\mathrm{C} 3$ $2.513 E-0$ $2.247 E-03$ $4.180 \mathrm{E}-\mathrm{C}$ $1.259 \mathrm{E}-\mathrm{C} 2$ 4.C45E-C $9.570 E-01$ $1.152 E C O$ $7.8 C 7 E$ UI $3.442 E$ OL $9.857 \mathrm{E} \mathrm{O2}$ $2.519 E$ C3 $1.333 E$
$1.855 E$

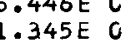
$1.8 .70 \mathrm{E} 04$ $1.256 E-05$ $6.329 E-05$ $1.558 E-04$ $1.804 E-04$
$2.884 E-04$ $5.776 E-C 4$ $8.383 E-04$ $1.772 E-03$ $2.3 C 0 E-03$ $4.342 E-03$ $1.300 E-02$ $4.178 E-02$ $9.855 \mathrm{E}-01$ $1.181 E 00$ $3.513 E 02$ $3.513 E$ O 2 2.560 C 3 $6.539 E 03$ $1.890 E \quad 04$ $7.01 C E-C 5$ $8.480 E-05$ $1.849 E-C 4$ $6.165 E-C_{4}$ $8.544 E-04$ $778 E-C 3$ $2.397 E-C 3$ $4.582 E-03$ $1.359 E-02$ 4. $360 E-02$ $1.024 E$ OC $8.224 E$ OC $3.606 \mathrm{E} \mathrm{CL}$ $1.028 \mathrm{t} 03$ $2.613 \mathrm{E} 03$ $6.661 \mathrm{E} 03$ $1.386 \mathrm{E} \mathrm{C4}$
$1.917 \mathrm{E} \mathrm{C} 4$ ANGLE 12 ANGLE 13 ANGLE 14 $M U=0.2816$
$3.711 \mathrm{E}-05$
$M U=0.4580$
$7.211 \mathrm{E}-05$ $2.739 E-04$ 4. $769 \mathrm{E}-04$ $5.364 E-04$ $9.120 E-04$ $9.888 E-04$ $1.877 E-03$ 2.515E-O $3.959 E-03$ $7.724 E-03$ $7.724 \mathrm{E}-03$ $7.810 E-02$ 7.81CE-OO2 $1.486 E$ OO $1.097 E$ E 4.072 O $1.289 \mathrm{E} \mathrm{O3}$ $3.165 E \quad$ $7.871 \mathrm{E} 03$

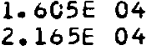

$M=0.6179$ $5.747 E-04$ $3.812 \mathrm{E}-04$ $4.868 E-04$ $8.691 \mathrm{E}-04$ $\angle .012 \mathrm{E}-03$ $1.730 E-O S$ $4.622 \mathrm{E}-03$ $7.725 \mathrm{E}-\mathrm{C} 3$ $1.420 E-C$ $3.572 \mathrm{E}-02$ 7.83 E-C2 $1.742 E$ CO $1.79 \mathrm{E}$ OL $5.494 \mathrm{E}$ C 2 .347E C $811 \mathrm{E}$ $1.647 E$ G $2.210 E 04$
$M U=0.8656$

$M U=0.8656$
$G .212 E-04$

$0.758 E-04$

. $01 E-04$

$1.762 E-03$

2. C1 1E-03

$3.496 \mathrm{E}-03$

$7.492 \mathrm{E}-03$ $7.772 E-03$ $1.334 \mathrm{E}-02$ $3.441 E-C 2$ $1.009 E-01$ 2.09CE OO $2.022 E 0$ $4.612 \mathrm{E}$ O2 $1.410 \mathrm{E} \mathrm{O3}$ $1.335 \mathrm{E} G 3$ $1.685 \mathrm{E} 04$ 2. 252E 04

$1.444 \mathrm{E}-03$

$1.198 \mathrm{E}-\mathrm{C} 3$ $1.403 E-03$ 2.364E-03 $4.161 E=C 3$ $4.666 \mathrm{E}$ प3 $1.426 \mathrm{E}=02$ $1.402 E=02$ 5.770E $C 2$ $1.128 \mathrm{E}-\mathrm{Cl}$ $\angle .257 \mathrm{E} \mathrm{CO}$ 2.400 E OC $1.390 \mathrm{E} \mathrm{C2}$ 
(NEUTRONS/MEV/STERADIAN/SOURCE NEUTRUN)

$1.22 E$ CI-- $1.50 E$ OL $1.0 \mathrm{VE}$ G $1---1.22 \mathrm{E}$ OI 6.365 OO---8.19E CO 4.97E $00--6.36 \mathrm{E} \mathrm{GO}$ 4.07E OC- $-4.97 \mathrm{E} \mathrm{CO}$ 3.01 E UO-- $-4.07 E$ CO $2.46 E$ UO- -3.01 E OU $2.35 \mathrm{E} \quad 00--2.46 \mathrm{E}$ CO $1.83 E$ EO--O-2.35E CC $1.11 E$ OO- $1.83 E$ OO 3.5CE-01-- -1.11E 0O $1.11 E-61---5.50 E-01$ $3.35 E-02--1.11 E-C 1$ $5.83 E-C 4 \sim-3.35 E-02$ $1.01 E-04---5.83 E-C 4$ 2.9 CE- $65--1.01 E-04$ $1.07 \mathrm{~L}-05--2.90 \mathrm{E}-\mathrm{C}-\mathrm{S}$ $3.06 E-46--1.07 E-05$ $1.12 \mathrm{E}-06--3.06 \mathrm{E}-\mathrm{CO}$ $4.14 E-U 7--1.12 E-06$ U.C $\quad--4.14 E-07$

$$
\text { ENERGY }
$$

$$
\text { GROUP (MEV) }
$$

$1.22 \mathrm{E} \mathrm{O1-D-1.5CE} 01$ $1.00 E$ Q1-D-1.22E O1 8.1 TE OC-D-1.00E OI $6.36 \mathrm{E} \mathrm{OO---8.19E} \mathrm{CO}$ 4.97 E $00-6.36 E$ OO $4.07 E$ CO-- 4.97E CO 3.CIE OO- $-4.07 E$ CO $2.4 E E$ OO--3.01E CO $2.35 \mathrm{E} 00--2.46 \mathrm{E} 00$ 1.83 E OO-D2.35E CO $1.11 E$ UO-D-1.83E 60 5.5OE-OI-O1.11E-CI-C-5. SUE-C S. $35 E-02-1$ - $11 E-C 1$ . $33 E-04-3.35 E-02$ .01E-04-C . 9CE- $05=-1.012-C 4$ 1.07 $-05-=-2.90 E-C 5$ 12E-06-O-3.06E-C6 4.14E-C 7-- - $1.12 E-C 6$ $4.14 E-C 7=-1.12 E-C 6$
ANGLE 1 $U=-1.0000$ $4.114 E-05$ $.928 E-04$ $2.973 E-04$ $5.356 \mathrm{E}-04$ $.783 E-0$. .580E-03 .360E-03 $4.299 \mathrm{E}-03$ 1. $317 E-02$ $4.303 E-02$ 9.111E-U1 $1.086 E$ UO 7.717E O1 $3.577 E$ OZ $1.074 E \quad 03$ $2.902 \mathrm{E} \mathrm{O3}$ $7.734 E \quad 03$ $1.671 \mathrm{E} \mathrm{O4}$ $2.401 E 04$ ANGLE 10 $M U=0.0950$ $1.766 \mathrm{E}-05$ $1.849 E-04$ $3.064 E-04$ $3.763 \mathrm{E}-04$ $6.593 \mathrm{E}-\mathrm{C} 4$ $1.071 E-03$ 1.3UGE-03 2.576t-03 $3.937 E-03$ 4.360 E-03 $8.169 E-C 3$ $2.301 \mathrm{E}-\mathrm{CL} 2$ . $313 E-02$ $1.370 E$ DO 1. $116 \mathrm{E} 02$ $1.116 E \mathrm{O}$ 1. $338 \mathrm{E} 03$ 1. g. $207 E$ OS 9.207E 03 1.960 04
ANGLE $\angle$ $-3.444 E-05$ $4.242 E-05$ $1.725 \mathrm{E}-04$ $.915 E-04$ . $962 E-04$ $5.388 \mathrm{E}-04$ . $1.78 \angle \mathrm{E}-\mathrm{U}$ $2.576 \mathrm{E}-03$ $2.363 E-C 3$ $4.312 \mathrm{E}-03$ $1.321 t=02$ $4.315 E-C 2$ $9.133 \mathrm{E}-\mathrm{Cl}$ $1.688 \mathrm{E}$ GO $7.733 \mathrm{E} O 1$ $3.584 \mathrm{E}$ O2 1. C76E C3 $2.5 C 7 E$ C3 7.746t 03 $1.673 \mathrm{E} C 4$ $2.4 C 4 E \quad O$ ANGLE 11 $M U=C .2816$ $4.964 \mathrm{E}-05$ $2.978 E-04$ $2.837 E-04$ .590-C4 $6.372 E-C 4$ $1.011 \mathrm{E}-\mathrm{C}$ $1.223 E-03$ $2.435 E-03$ $3.512 E-03$ 7.799E-0 7.79ge-03 $2.216 E-02$ . $324 \mathrm{E} \mathrm{OC}$ $1.324 E$ OC $1.475 E$ OO 9.786 E O 1.865E C2 1.385E O3 $9.536 \mathrm{E} 03$ C23E 04 $2.826 \mathrm{t} \mathrm{C4}$ $-1.269 E-05$ $4.660 \mathrm{E}-05$ .653E-04 $1.872 E-04$ 2.93CE-04 $.514 E-04$ $.461 L-04$ .781E-03 2.561E-03 $4.37 \mathrm{E}-\mathrm{C}$ 1. $336 \mathrm{E}-02$ 1. $36 \mathrm{E}-02$ $9 . \angle 29 \mathrm{E}-01$ $9 . \angle 29 E-01$
$1.098 E 00$ $7.798 \mathrm{E} 01$ $3.611 \mathrm{E} 02$ $1.084 \mathrm{E} 03$ $2.926 E 03$ 7.794 E 03 .683E O4 $2.416 \mathrm{E} 04$

ANGLE 12

$M U=0.4580$ $3.793 \mathrm{E}-05$ $3.366 E-04$ 4. $342 \mathrm{E}-04$ . $9.134 E-C 4$ $1.444 E-03$ 1.786E-U $3.534 \mathrm{E}-03$ 6. $C 45 E-03$ 6.C $45 E-03$ 1.CBIE-02 2.953E-02 . $1.635 \mathrm{E} O C$ 164E 02 4.905 E 02 4.450 E 03 $3.803 E$ O3 9.8795 O3 $2 . C 88 E$ J4 $2.9 C 2 E \quad 04$ $A N G L E$
$M U=-C .8050$ $3.55 \mathrm{CE}-\mathrm{Ct}$ $5.228 \mathrm{E}-\mathrm{C5}$ $1.54 U E-C 4$ $1.817 \mathrm{E}-04$ $.745 E-04$ $2.543 E-03$ 2.411 E-CS $1.478 \mathrm{E}-\mathrm{CS}$ $1.366 \mathrm{E}-\mathrm{C2}$ $4.464 E-C 2$ $9.413 E-C 1$ 1117 OO $7.922 L 01$ $3.064 \mathrm{E} \mathrm{C2}$ $1.099 \mathrm{E}$ OS 2.963 E O3 $1.701 E$ C $1.701 E$ C4
$\angle .439$ C 4 ANGLE 13 $M U=0.6179$ $8.508 E-C 5$ $4.846 \mathrm{E}-04$ $4.913 E-C 4$ $6.094 E-C 4$ $.688 \mathrm{E}-\mathrm{C}$ $.133 E-C$ $6.667 E-C 3$ $6.667 E-C 3$ $1.205 E-C$ $3.236 \mathrm{E}-\mathrm{C2} 2$ $9.331 E-O L$ $1.678 \mathrm{E}$ OC $1.800 E$ CL $.269 \mathrm{E} \mathrm{C2}$ $1.521 \mathrm{E} \mathrm{C3}$ $3.954 E$ C $1.022 \mathrm{E}$ $2.976 \mathrm{E} \mathrm{O4}$
ANGLE 5 $1.126 E-05$ $.723 E-C 5$ $.435 E-O 4$ . 568 E-04 2.538E-CO 2.479E 03 . $655 E-C 3$ $1.413 \mathrm{~L}-02$ $9.682 E-01$ 1.144E CO 8.1C1E O $3.740 E$ O 1.120E U3 $8.011 E 03$ $1.726 \mathrm{E} \mathrm{O4}$ $\begin{array}{lll}1.726 E & 04 \\ 2.47 C E & 04\end{array}$ ANGLE 14 $M U=0.7550$ $2.266 E-64$
$7.301 E-04$ $7.301 E-04$ $7.872 E-C 4$ $9.561 E-04$ $1.661 E-03$ $2.571 E-03$ 6. $160 \mathrm{E}-\mathrm{C} 3$ $6.160 E-C_{3}$ (. 849E-G3 $668 \mathrm{E}-\mathrm{C} 2$ $4.296 E-C 2$ 9.721 E 02 $1.784 \mathrm{E}$ CO .832 UO $5.507 t$ Q 2 1.593E 03 1. U55E U4 $3.212 E$ C 4 $.06 C E-05$ $6.368 \mathrm{E}-05$ $.856 E-04$ $.257 E-C_{4}$ 列 
(NEUTRONS/MEVASTERADIAN/SGURCE NEUTRON)

\begin{tabular}{|c|c|c|c|c|c|c|c|c|c|}
\hline 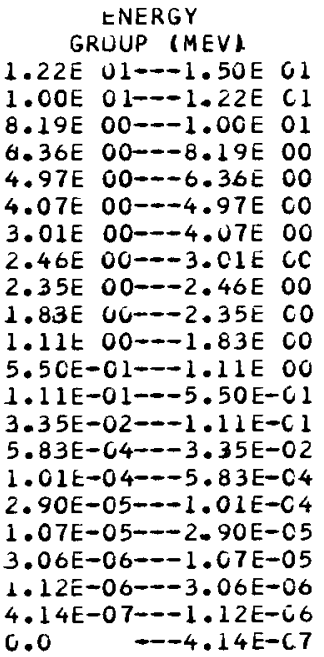 & $\begin{array}{l}\text { ANGLE } 1 \\
M U=-1.0000 \\
-2.765 \mathrm{E}-05 \\
2.782 \mathrm{E}-05 \\
1.279 \mathrm{E}-04 \\
1.631 \mathrm{E}-04 \\
2.723 \mathrm{E}-04 \\
5.052 \mathrm{E}-04 \\
7.631 \mathrm{E}-04 \\
1.545 \mathrm{E}-03 \\
2.253 \mathrm{E}-03 \\
2.268 \mathrm{E}-03 \\
4.122 \mathrm{E}-03 \\
1.280 \mathrm{E}-02 \\
4.279 \mathrm{E}-02 \\
7.523 \mathrm{E}-01 \\
8.665 \mathrm{E}-01 \\
6.339 \mathrm{E} 01 \\
3.066 \mathrm{E} \quad 02 \\
9.618 \mathrm{E} 02 \\
2.763 \mathrm{E} 03 \\
7.734 \mathrm{E} 03 \\
1.750 \mathrm{E} 04 \\
2.635 \mathrm{E} 04\end{array}$ & $\begin{array}{l}\text { ANGLE } 2 \\
1 \mathrm{U}=-7 C .9894 \\
2.208 \mathrm{E}-05 \\
2.898 \mathrm{E}-05 \\
1.267 \mathrm{E}-04 \\
1.622 \mathrm{E}-04 \\
2.714 \mathrm{E}-04 \\
5.08 \mathrm{E}-04 \\
7.631 \mathrm{E}-04 \\
1.546 \mathrm{E}-03 \\
2.252 \mathrm{E}-03 \\
2.272 \mathrm{E}-03 \\
4.135 \mathrm{E}-03 \\
1.284 \mathrm{E}-02 \\
4.291 \mathrm{E}-02 \\
7.541 \mathrm{E}-01 \\
8.682 \mathrm{E}-01 \\
6.351 \mathrm{E} 01 \\
3.072 \mathrm{E} 02 \\
9.635 \mathrm{E} 02 \\
2.768 \mathrm{E} 03 \\
7.747 \mathrm{E} 03 \\
1.752 \mathrm{E} 04 \\
2.639 \mathrm{E} \mathrm{O4}\end{array}$ & $\begin{array}{l}\text { ANGLE } 3 \\
M U=-C .9446 \\
-7.502 E-06 \\
3.287 \mathrm{E}-05 \\
1.223 \mathrm{E}-04 \\
1.589 \mathrm{E}-04 \\
2.694 \mathrm{E}-04 \\
5.195 \mathrm{E}-04 \\
7.634 \mathrm{E}-04 \\
1.546 \mathrm{E}-03 \\
2.25 \mathrm{E}-03 \\
2.292 \mathrm{E}-03 \\
4.190 \mathrm{E}-03 \\
1.306 \mathrm{E}-02 \\
4.341 \mathrm{E}-02 \\
7.615 \mathrm{E}-01 \\
8.756 \mathrm{E}-01 \\
6.402 \mathrm{E} 01 \\
3 . \mathrm{C} 95 \mathrm{E} 02 \\
9.708 \mathrm{E} 02 \\
2.788 \mathrm{E} 03 \\
7.800 \mathrm{E} \mathrm{C3} \\
1.764 \mathrm{E} 04 \\
2.654 \mathrm{E} 04\end{array}$ & 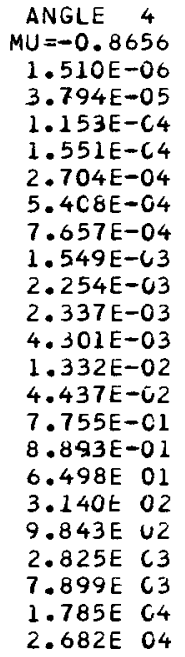 & $\begin{array}{l}\text { ANGLE } 5 \\
M U=-C .7550 \\
1.241 E-C 5 \\
4.213 E-05 \\
1.094 E-04 \\
1.548 E-C 4 \\
2.812 E-04 \\
5.702 E-04 \\
7.730 E-04 \\
1.558 E-03 \\
2.278 E-03 \\
2.417 E-03 \\
4.475 E-03 \\
1.381 E-C 2 \\
4.578 E-C 2 \\
7.958 E-01 \\
9.091 E-01 \\
6.636 E-11 \\
3.203 E 02 \\
1.064 E 03 \\
2.878 E 03 \\
8.039 E 03 \\
1.815 \mathrm{E} 04 \\
2.722 E 04\end{array}$ & $\begin{array}{l}\text { ANGLE } 6 \\
1 U=-0.6179 \\
5.267 E-06 \\
4.717 E-05 \\
1.087 E-04 \\
1.623 E-C 4 \\
3.068 E-C 4 \\
6.081 E-C 4 \\
7.887 E-04 \\
1.580 E-03 \\
2.339 E-03 \\
2.545 E-03 \\
4.728 E-03 \\
1.451 E-02 \\
4.770 E-02 \\
8.232 E-01 \\
9.354 E-01 \\
6.820 E \quad 01 \\
3.268 E \quad 02 \\
1.029 E \quad C 3 \\
2.947 E \quad C 3 \\
8.224 E \quad C 3 \\
1.855 E \quad 04 \\
2.774 E \quad C 4\end{array}$ & $\begin{array}{l}\text { ANGLE } 7 \\
\text { AU }=-0.4580 \\
-3.183 \mathrm{E}-06 \\
5.797 \mathrm{E}-05 \\
1.181 \mathrm{E}-04 \\
1.808 \mathrm{E}-04 \\
3.494 \mathrm{E}-04 \\
6.561 \mathrm{E}-04 \\
8.173 \mathrm{E}-04 \\
1.627 \mathrm{E}-03 \\
2.458 \mathrm{E}-03\end{array}$ & 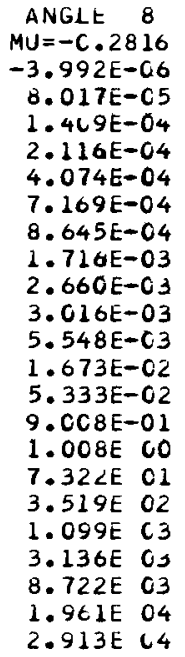 & $\begin{array}{l}\text { AWGLE } 9 \\
1 U=-C . C 950 \\
2.513 E-06 \\
1.161 E-04 \\
1.771 E-C 4 \\
2.536 E-04 \\
4.781 E-04 \\
7.955 E-04 \\
9.393 E-C 4 \\
1.871 E-C 3 \\
2.981 E-03 \\
3.464 E-C 3 \\
6.172 E-C 3 \\
1.841 E-C 2 \\
5.724 E-02 \\
9.521 E-01 \\
1 . C 55 E \quad C L \\
7.638 E 01 \\
3.667 E \quad 02 \\
1.143 E \quad 03 \\
3.254 E \quad 03 \\
9.031 E \quad 03 \\
2 . C 27 E \quad C 4 \\
2.998 E \quad 4\end{array}$ \\
\hline 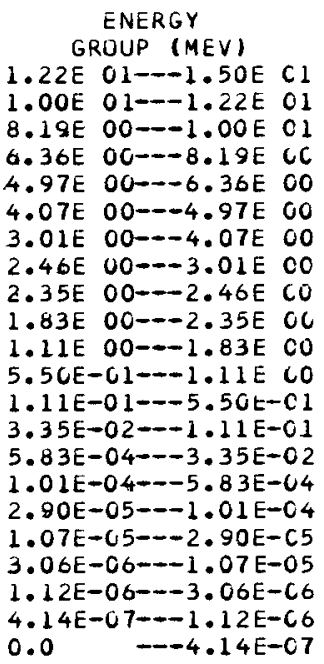 & $\begin{array}{l}1.691 \mathrm{E}-C 4 \\
2.128 \mathrm{E}-04 \\
2.947 \mathrm{E}-04 \\
5.452 \mathrm{E}-\mathrm{C} 4 \\
8.721 \mathrm{E}-04 \\
1.016 \mathrm{E}-03 \\
2.055 \mathrm{E}-03 \\
3.383 \mathrm{E}-03 \\
3.834 \mathrm{E}-03 \\
6.752 \mathrm{E}-03 \\
2.001 \mathrm{E}-02 \\
6.564 \mathrm{E}-02 \\
9.638 \mathrm{E}-01 \\
1.024 \mathrm{E} 00 \\
7.277 \mathrm{E} 01 \\
4.022 \mathrm{E} 02 \\
1.187 \mathrm{E} 03 \\
3.386 \mathrm{E} 03 \\
9.376 \mathrm{E} 03 \\
2.099 \mathrm{E} 04 \\
3.092 \mathrm{E} 04\end{array}$ & $\begin{array}{l}3.655 \mathrm{E}-05 \\
2.105 \mathrm{E}-04 \\
2.874 \mathrm{E}-04 \\
3.773 \mathrm{E}-04 \\
6.846 \mathrm{E}-04 \\
1.079 \mathrm{E}-03 \\
1.279 \mathrm{E}-03 \\
2.581 \mathrm{E}-03 \\
4.280 \mathrm{E}-03 \\
4.767 \mathrm{E}-03 \\
8.30 \angle \mathrm{E}-03 \\
2.4 \mathrm{CE}-02 \\
6.523 \mathrm{E}-02 \\
1.115 \mathrm{E} 00 \\
1.22 \mathrm{CE} 00 \\
8.833 \mathrm{EE} 01 \\
3.92 \mathrm{CE} 02 \\
1.251 \mathrm{E} 03 \\
3.531 \mathrm{E} 03 \\
9.75 \mathrm{EE} 03 \\
2.178 \mathrm{EE} 04 \\
3.192 \mathrm{E} 04\end{array}$ & 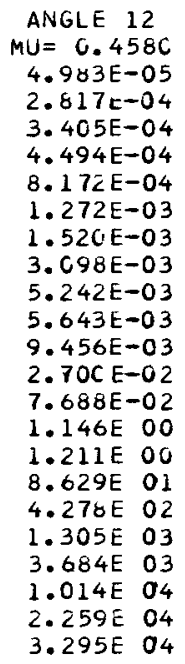 & 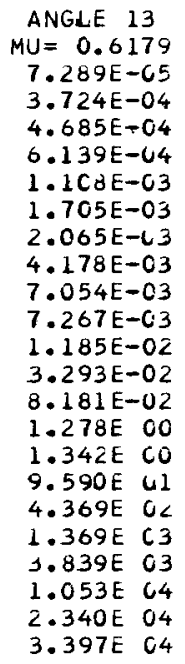 & 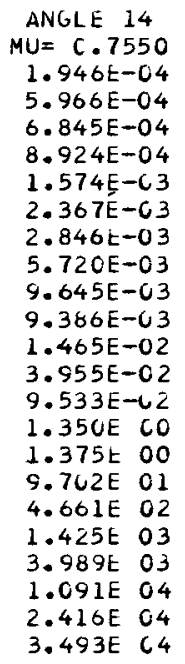 & 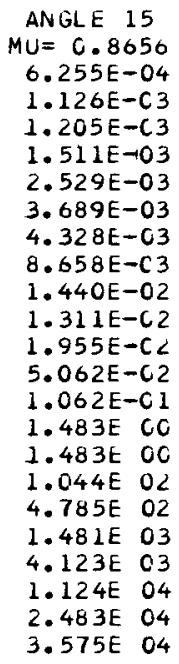 & $\begin{array}{l}2.1 \\
2.5 \\
2.5 \\
2.5 \\
4.6 \\
6.5 \\
7.2 \\
1.4 \\
2.4 \\
2.0 \\
2.7 \\
6.8 \\
1.2 \\
1.5 \\
1.5 \\
1.0 \\
4.5 \\
1.5 \\
4.2 \\
1.2 \\
2.5 \\
3.6\end{array}$ & 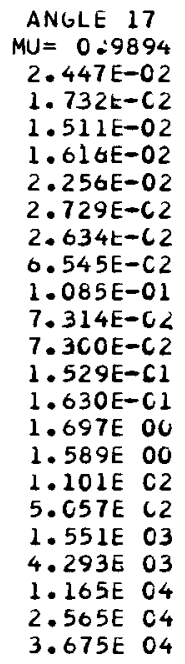 & 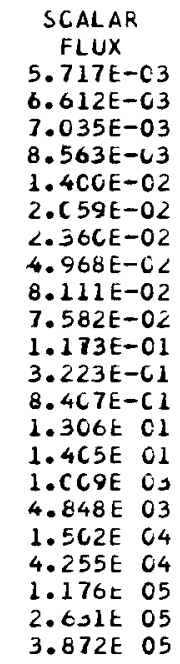 \\
\hline
\end{tabular}



GROUP (MEV) $1 . \angle 2 E$ O1-- - 1.5CE O1 1. VOE O1-D-1.22E CI $8.19 E$ CO- $-1.00 E$ OI $6.36 E$ OO---8.19E CL $4.97 E$ UC-- - 6.36E OO $3.01 \mathrm{E} 00---4.07 \mathrm{E} 00$ $2.46 \mathrm{E}$ UO--- $3.01 \mathrm{E} 00$ $2.35 E$ OU $--2.46 E$ OC $1.83 \mathrm{E} \mathrm{CO}---2.35 \mathrm{E} \mathrm{CO}$ $1.11 E$ JO-O- $1.83 E 00$ $5.50 E-01-=-1.11 E$ OO $1.11 c-01--5.5 U E-C$ $3.35 E-02-m-1.11 E-0$ $5.83 \mathrm{E}-04---3.35 \mathrm{E}-02$ $1.01 E-04--5.83 E-C 4$ 2.9CE-05---1. CIE-C $1.07 E-05---2.90 E-05$ $3.06 E-06---1.07 E-C$ $1.12 E-06---3.06 E-C O$ $4.14 \mathrm{E}-07---1.12 \mathrm{E}-\mathrm{C}$ $0.0 \quad--4.14 \mathrm{E}-07$ ENERGY GROUP IMEVI $1.22 E$ CI-- $1.50 E$ CI $1.0 O E$ C1- $1.22 E$ CI $8.19 \mathrm{E} \mathrm{CO}---1.00 \mathrm{E} \mathrm{OI}$ $6.36 E^{0}$ OO- $-8.19 E$ OO $4.97 E C 0-06.36 E$ CO $4.07 E$ OO- $-4.97 E$ CC $3.01 \mathrm{E} 00--4.07 \mathrm{E}$ OC $2.46 E$ OO---3.01E OC $2.35 \mathrm{E} \quad 00--2.46 \mathrm{E}$ CO $1.83 \mathrm{E}$ CO---2.35E CO $1.11 \mathrm{E} \mathrm{OO-C-1.83E} \mathrm{CC}$ . $50 \mathrm{E}-01--1.11 \mathrm{E}$ OO $1.11 \mathrm{E}-01--5.50 \mathrm{E}-01$ $3.35 E-02--1.11 E-C 1$ $3.83 E-04--3.35 E-02$ $1.01 E-04=-5.83 E-C 4$ 1. $07 E-05-1.01 E-04$ $1.07 E-05=-2.90 E-05$ 1.0OE C6$4.14 E-C 7-9-1.12 E-06$ $4.14 E-C 7--1.12 E-06$

ANGLE 1 $A U=-1.00 C \mathrm{C}$ $1.028 E-05$ 8.7.24E-05 $1.256 \mathrm{E}-04$ $2.248 \mathrm{E}-\mathrm{C} 4$ $4.263 E-04$ $6.222 E-04$ $1.217 \mathrm{E}-03$ $1.786 \mathrm{E}-03$ $1.935 \mathrm{E}-03$ $1.092 E-62$ $5.683 E-01$ $6.347 \mathrm{E}-01$ $4.622 E$ O.1 $2.250 E \mathrm{CZ}$ $7.151 E$ O2 2.1C4E O3 6. $024 E 03$ $1.395 E \quad 04$ $2.158 E \quad 04$ $\triangle N G L E$ IC $M U=0.0950$ $1.5 C 9 E-05$ $1.149 E-04$ $1.732 \vec{E}-0.4$ $2.520 E-04$ $4.799 E-04$ $7.654 E-04$ O.774E-04 $1.276 E-03$ $3.008 E-03$ $5.461 E-03$ $5.951 E-03$ $1.7 .81 E-02$ 7. $232 \mathrm{E}-02$ (.619 $6.037 E 01$ $2.697 E$ OD $0.828 \mathrm{E} 02$ 2.573E 03 $1.684 E C^{\circ}$ $2.553 E \quad 04$
(NEUTRONS/MEV/STERAOIAN/SOURCE NEUTKON)

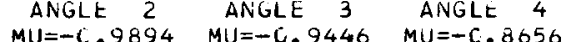
$1.863 E-C 5 \quad 2.172 E-05$ $8.661 E-05 \quad 8.419 E-C 5$ $04 \quad 1.227 E-04$ $2.242 E-04$ $1.787 \mathrm{E}-03$ $1.94 C E-O 3$ $3.509 E-03$ $1.055 E-02$ $3.7 C 2 E-U 2$ $5.695 \mathrm{E}-\mathrm{Cl}$ $6.35 \mathrm{GE}-\mathrm{C} 1$ $4.63 \mathrm{CE} \mathrm{Cl}$ $7.163 \mathrm{E} 02$ $2.1 C 8 E 03$ $6 . C 34 E 03$ $1.398 \mathrm{E} 04$ $2.162 \mathrm{EO}_{4}$ ANGLE 11 $M U=0.2816$
$2.775 E-45$ $2.775 \mathrm{E}-\mathrm{L} 5$
$1.537 \mathrm{E}-\mathrm{C} 4$ $2.154 \mathrm{E}-04$ $3.045 E-C 4$ $5.711 E-04$ $8.928 E-04$ $1.627 E-03$ 2.12CE-O3 $3.660 E-03$ $4.094 E-03$ 6.8O3E-C3 $2 . C 14 E-C 2$ $3.81 C E-C 2$ . $5.884 E 01$ $2.933 \mathrm{E} \mathrm{C2}$ $9.172 t$ C 2 $2.681 \mathrm{E} \mathrm{C} 3$ 7.611 E 03 $1.749 \mathrm{E} \mathrm{O4}$ 2.640 E 04 $J=-0.7550$ $M U=-0.0179$
$2.892 E-C 6$ $.093 E-C O$
$.570 E-C 5$ . $.2 C 4 E-04$ $2.252 E-04$ $4.556 E-C_{4}$ $6.243 E-C_{4}$ $1.225 \mathrm{E}-0 \mathrm{3}$ 1.8 IUE-G 3 2. $\quad 0 C 7 E-63$ $3.653 E-03$ $1.235 E-02$ $3.827 \mathrm{E}-\mathrm{C} 2$ $5.847 E-C I$ $6.5 \mathrm{C} 2 \mathrm{E}-\mathrm{C} 1$ $4.731 E$ UI 2.3C1E 02 7.313E O2 2.151E O3 $6.153 \mathrm{E} \mathrm{C3}$ $1.424 E$
$2.199 E$ ANGLE 13 $M U=0.0179$ $5.616 t-65$ $2.733 E-04$ $3.675 E-C 4$ $5.153 E-04$ $9.491 \mathrm{E}-\mathrm{C} 4$ $1.438 \mathrm{E}-\mathrm{C} 3$ $1.074 E-03$ $3.532 E-03$ $6.278 E-C 3$ $0.2 .78 E-C 3$ $9.669 E-03$ $2.763 E-02$ $8.086 \mathrm{E}-\mathrm{O}$ $9.93 \mathrm{E}-\mathrm{Cl}$ $6.534 E$ OI $3.182 \mathrm{O} O \mathrm{O}$ $1.0 C O E C 3$ 2. $10 \mathrm{E}$ O 8.229 C C $_{3}$ $1.884 E O 4$ $2.821 E \quad C 4$ $.844 t-05$ $7.751 E-65$ $1.213 \mathrm{E}-04$ $2.357 E-04$ ( $6.303 t-04$ $.849 E-03$ ..084E-03 $.802 E-03$ . $183 E-G 2$ $.947 E-U 2$ . 0 OCE 01 $4.826 \mathrm{E} \mathrm{OI}$ $2.346 E$ C2 $7.452 \mathrm{E} \mathrm{U2}$ $2.191 E 03$ $6.264 E$ C 3 $1.449 E$ O4 $\angle .233$ E $G 4$ ANGLE 14 $M U=C .7550$ $1.483 \mathrm{E}-\mathrm{C} 4$
$4.412 \mathrm{E}-04$ $5.621 E-C 4$ $7.739 E-C .4$ 1.375E-03 .C2OE-O3 .. $4.932 E-63$ .670E-C3 . 1.19E $3.338 E-02$ . . . 384 C2 L.C4lE C 3 1.CUIE 03 $8.523 E$ OS $1.948 E 04$ 2. 906 E 4 $.287 E-04$ E. $2.203 E-03$ $4.018 E-03$ 1.244E-C2 t. $181 \mathrm{E}-01$ $6.814 E-01$ 4.951E C1 $7.636 E_{02}$ $2.243 E \quad 03$ 6.410 E US $1.482 \mathrm{E} \mathrm{O4}$ $2.278 \mathrm{E} 04$ ANGLE 15 $M U=0.8650$ $4.602 E \neg C 4$
$8.558 E-C 4$ 9. $922 E-C 4$ $1.305 E-C 3$ $2.174 E-C 3$ $3.065 E-63$ $3.393 E-C 3$ 1. $266 \mathrm{E}-02$ $1.110 \mathrm{E}-02$ $4.128 \mathrm{E}-\mathrm{C2}$ 4.128E-02 $1.02 \mathrm{CE} 00$ $1.002 \mathrm{E} \mathrm{OC}$ $7.123 E \mathrm{CD}$ $3.423 \mathrm{E} 02$ $3.117 E$ O3 $8.782 E 03$ $2.004 E 04$ 2. $979 E \quad C_{4}$

ANGLE 7 ANGLE 8 ANGLE 9 2.418E- $-40 \quad-2.370 E-C 6 \quad M U=-U . \angle 75 C$ $4.000 E-05 \quad 5.589 E-C 5 \quad 8.178 E-05$ $8.713 E-05 \quad 1.660 E-C 4 \quad 1.351 E-04$ $1.453 E-04 \quad 1.718 E-6.4 \quad 2.675 E-04$ $2.958 E-04 \quad 3.456 E-C 4 \quad 4 . C 6 C E-04$ $5.52 \angle E-C 4 \quad 6.037 E-64 \quad 6.709 E-C 4$ $6.678 E-04 \quad 7.082 E-C 4 \quad 7.723 E-C 4$ $1.311 E-C 3 \quad 1.397 E-C 3 \quad 1.541 E-03$ $2.047 E-03 \quad 2.246 E-C 3 \quad 2.549 E-03$ $2.379 E-C 3 \quad 2.629 E-C 3 \quad 2.975 E-C 3$ $4.314 E-C 3$ 4.71IE-C 3 5.233E-C 3 $1.328 E-02 \quad 1.439 E-C L \quad 1.584 E-C 2$ $4.320 E-C 2 \quad 4.583 E-C 2 \quad 4.9 C 5 E-C 2$ $6.422 \mathrm{E}-\mathrm{Cl} 1$
$7.034 \mathrm{E}-01$ $5.105 \mathrm{E} C \mathrm{C}$ 5.2.2L CI $5.511 \mathrm{E} \mathrm{Cl}$ $2.476 \mathrm{E} \mathrm{O2} \mathrm{2.563E} \mathrm{02} \mathrm{2.662E} \mathrm{O2}$ $7.863 \mathrm{E} \mathrm{O}_{2} \quad 8.134 \mathrm{E}$ C2 $8.447 \mathrm{E} \mathrm{O2}$

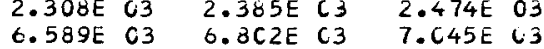
$1.522 E$ O4 $1.569 E$ C4 $1.624 E$ O 
(NEUTRUNS/MEV/SI ERADIAIN/SOURCE NEUTRON)

ENERGY GROUP (MEV) $1.22 E$ O1-- 1.50E OI $1.00 \mathrm{E} 01--1.22 \mathrm{E} \mathrm{O1}$ 8.19E OO---1.00E CI $6.36 E$ OC- $-8.19 E$ LO 4.97E OC-- 6.36 E OC $4.07 E$ OO- $-4.97 \mathrm{E}$ CO $3.01 E$ OO- $4.07 E \mathrm{CO}$ $2.46 \mathrm{E} 00---3.01 \mathrm{E}$ CO $2.35 \mathrm{C} 00--2.46 \mathrm{E} \mathrm{CO}$ $1.83 \mathrm{E} 00--2.35 \mathrm{E} \mathrm{CO}$ $1.11 \mathrm{E} 00--1.83 \mathrm{E}$ GO $5.5 C E-C 1--1.11 E 00$ $1.11 E-01---5.50 E-C 1$ $2.35 E-02-\cdots-1.11 E-C$ $5.83 E-64--3.35 E-C 2$ 1. $01 \mathrm{E}-04=--5.83 \mathrm{E}-\mathrm{O} 4$ $2.90 E-35--1.01 E-C 4$ $1.07 E-05--2.90 \bar{E}-05$ $3.06 \mathrm{E}-06--1.07 \mathrm{E}-65$ $1.12 \mathrm{E}-06---3.66 \mathrm{E}-06$ $4.14 \mathrm{E}-07--1.12 \mathrm{E}-\mathrm{C}$ $0.0 \quad---4.14 \mathrm{E}-07$

ENERGY GROUP (MEV) $1.22 E$ OI-D-1.50E CI 1.OCE OL--1.22E CI 8.1 19E OU-D1.00E C1 $6.36 E$ OC- $-8.19 E$ OC 4.97E OC- $-6.36 E$ OO 4.07E OC- - 4.97E OO 3. CIE OO-- 4. U7E CO $2.46 E$ O0- $3.01 \mathrm{E} \mathrm{OO}$ $2.35 \mathrm{E} 00--2.46 \mathrm{E}$ CO $1.83 E$ O0-- $2.35 E$ CO 1.11E 00--1.83E CO $5.50 E-01=-1.11 E$ OC $1.11 E-01=-5.50 E-01$
$3.35 E-02=-1.11 E-01$ $5.33 E-04---3.35 E-C 2$ $5.83 E-04---3.35 E-62$
$1.015-04 m--5.83 E-04$ $2.90 \mathrm{E}-05-2.01 \mathrm{E}-04$ $.07 E-05=-2.90 E-C 5$ 1.12E $06=-3.06 E-C 6$ $1.12 E-06-2.06 E-C 6$ $4.14 E-07=-1.12 E-06$
ANGLE 1 $M U=-1.006 \mathrm{O}$ $-9.52 C E-06$ $1.093 E-05$ . $180 E-05$ $1.752 \mathrm{E}-04$ $4.796 E-04$ $9.796 \mathrm{E}-04$ $1.344 \mathrm{E}-0.3$ $1.545 \mathrm{E}-03$ $2.775 E-03$ $8.663 \mathrm{E}-03$ $2.949 E-02$ $4.133 E-01$ $4.501 E-0$ $3.232 E$ OI $1.566 \mathrm{t} \mathrm{O2}$ $4.976 \mathrm{E} 02$ $1.471 E$ O3 4.240E O3 $9.909 E \quad O 3$ 1.550 OU ANGLE 10 $M U=C . C 950$ $1.081 E-05$ $7.781 E-0.5$ $1.252 E-04$ $1.942 E-C 4$ $3.812 \mathrm{E}-04$ $6.054 E-04$ $6.783 \mathrm{E}-04$ $1.387 E-03$ $2.420 \mathrm{E}-03$ $2.806 E-03$ $4.674 E-03$ $1.413 E-02$ $4.211 \mathrm{E}-02$ $5.307 \mathrm{E}-01$ $5.461 E-01$ $3.874 E$ E $6.041 E$ O2 $6.041 E$ E $5.119 E$ OS ..119E U $\begin{array}{ll}1.191 E & 04 \\ 1.831 E & 04\end{array}$
ANGLE 2 $-7.753 E-C 6 \quad M U 2.36 \angle E-06$ $1.160 E-05$ $5.694 \mathrm{E}-05$ $9.136 E-C 5$ $1.749 E-04$ $3.398 E-04$ $4.796 E=04$ 9.102E-04 $1.346 E-03$ $59 E-O 3$ $2.784 E-C 3$ .692E-03 $2.957 \mathrm{E}-\mathrm{C}$ $4.142 E-01$ $4.509 \mathrm{E}-01$
$3.237 \mathrm{E} 01$ $1.569 E 02$ 4.984E O2 $1.474 \mathrm{E} \mathrm{O3}$ 4.247E O3 $9.924 \mathrm{E} \mathrm{O}$ $1.552 \mathrm{t} 04$ ANGLE 11 $M U=0.2816$ $1.898 E-05$ 1. $635 \mathrm{E}-04$ $1.582 \mathrm{E}-04$ $2.376 E-04$ $4.581 \mathrm{E}-04$ $7.132 \mathrm{E}-\mathrm{C}$ 8.C25E-04 $1.681 E-03$ $2.986 \mathrm{E}-03$ $3.339 \mathrm{E}-03$ 5.373E-03 $1.608 \mathrm{E}-\mathrm{O}$ $4.558 \mathrm{E}-\mathrm{C} 2$ $5.651 E-01$ $5.828 E-01$ $4.158 E$ E $1.975 E$ O2 6.299E O2 $1.854 E$ O3 . $1.236 \mathrm{E} \mathrm{O}$
$1.894 \mathrm{E} \mathrm{C}$

$9.990 E \mathrm{C} 3$
$1.561 \mathrm{E} \mathrm{O4}$

ANGLE 12

$1.347 \mathrm{E}-\mathrm{Q} 4$

2.017E-04

3. $C$ C $8 E-C 4$

$5.741 E-04$

$8.796 E-04$

$9.944 \mathrm{E}-04$

2. 13 CE-0 3

$3.832 \mathrm{E}-03$

$4.071 E-03$

$6.287 E-03$

$1.857 \mathrm{E}-\mathrm{C2}$

$5.959 \mathrm{E}-01$

$6 . C 32 E-01$

4.282E C1

$6.552 \mathrm{E} \mathrm{O2}$

1.928E O3

1.283E 04
$A N G L E \quad 4$
$M U=-0.8656$

$1.341 E-C$

$1.341 E-C 6$

5.397E-C5

$8.871 E-05$

$1.767 \mathrm{E}-\mathrm{C} 4$

$3.608 t-04$

$4.812 E-04$

$9.197 E-C$

$1.377 \mathrm{E}-03$

$1.610 E-03$

2. 9 COE-03

$9.049 E-03$

$3.057 E-C 2$

$4.246 E-C$

$3.3 C 4 E C 1$

$1.600 \mathrm{E} \mathrm{C2}$

$5.084 \mathrm{E} \mathrm{C2}$

$1.503 E \quad 03$

4.329E O3

$1.011 E$ C4
$1.579 E$ O4

$M U=0.4580$
$2.483 E-05$

ANGLE 13

$U=0.6179$

$4.013 E-05$

$1.885 \mathrm{E}-04$

$2.763 \mathrm{E}-\mathrm{C} 4$

$4.112 E-04$

$7.695 \mathrm{E}-04$

$1.150 E-03$

1.294E-03

.

(2.031

(2.509E-OS

.

$5.462 E-02$

$6.325 \mathrm{E}-01$

6.337E-OI

2.494E

$6.815 E 0$

2.003E C3

1.

$1.958 E$ C4
NGLE MU $=-C$ C 6 . $998 E-C 6 \quad 1.552 E-C 6$ 1.896E-05 $5.278 E-0.5$ $9.014 E-05$ 1.859E-04 .799E-04 政 ( (. $419 E=03$ (2) 作 ..151E-02 $4.344 E-01$ (1) (1)

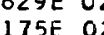
$1.530 \mathrm{E} \mathrm{O3}^{2}$ $1.029 E 04$ $1.6 C 3 E 04$ ANGLE 14 $U=0.7550$
$1.064 E-04$ $3.120 E-04$ $4.301 E-04$ $6.249 E-C 4$ $1.115 \mathrm{E}-\mathrm{C}$ $1.604 \mathrm{E}-03$ $1.762 E-C 3$ $7.250-193$ $6.571 E-03$ (1) 610E-02 6.0670E-O 4.634E 01 $7.059 E \quad 02$ 5. 1. $373 E$ O4

$1.373 E O 4$
$2.082 E$ C 4

ANGLE 15

$M U=0.8656$
$3.268 E-04$

$6.143 E-C$

$7.713 E-C$

$1.060 \mathrm{E}-03$

$1.755 \mathrm{E}-\mathrm{C} 3$
$2.400 \mathrm{E} \rightarrow \mathrm{O}$

$2.521 E-03$

$5.866 \mathrm{E}-03$

$1.072 \mathrm{E}-\mathrm{C2}$

$8.822 E-03$

$1.147 \mathrm{E}-\mathrm{C} 2$

$3.171 \mathrm{E}-\mathrm{C} 2$

$6.604 \mathrm{E}-02$

$6.822 \mathrm{E}-\mathrm{Cl}$

ANGLE 7 $-1.238 E-06 \quad 2.278 E-06$ $3.720 E-C 5 \quad 5.486 E-05$ $7.557 E-05 \quad 9.742 E-05$ $1.315 \mathrm{E}-04 \quad 1.597 \mathrm{E}-\mathrm{C} 4$ $2.748 E-C 4 \quad 3.231 E-04$ $4.779 E-C 4 \quad 5.317 E-04$ $5.478 E-04 \quad 5.989 E-C 4$ $1.675 E-03 \quad 1.197 E-0.3$ $1.778 \mathrm{E}-\mathrm{CS} \quad \mathrm{LC} 38 \mathrm{E}-03$ $2.132 E-C 3 \quad 2.417 E-03$ $1.147 \mathrm{E}-02 \quad 1.264 \mathrm{E}-02$ $3.651 E-C 2 \quad 3.9 C 2 E-C$ 5. $C 67 \mathrm{E}-01$ 5.124E-01 $3.665 E$ CI $3.801 E$ OI $1.771 E$ C2 $1.835 E$ O2 $5.825 \mathrm{E} \mathrm{O2}$ $1.659 \mathrm{O} \mathrm{O}_{3}$ 4. $935 E$ O3

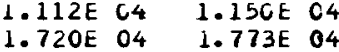

SCALAR 
(NEUTRUNS/MEV/STERALIAN/SOUKCE NEUTRUN)

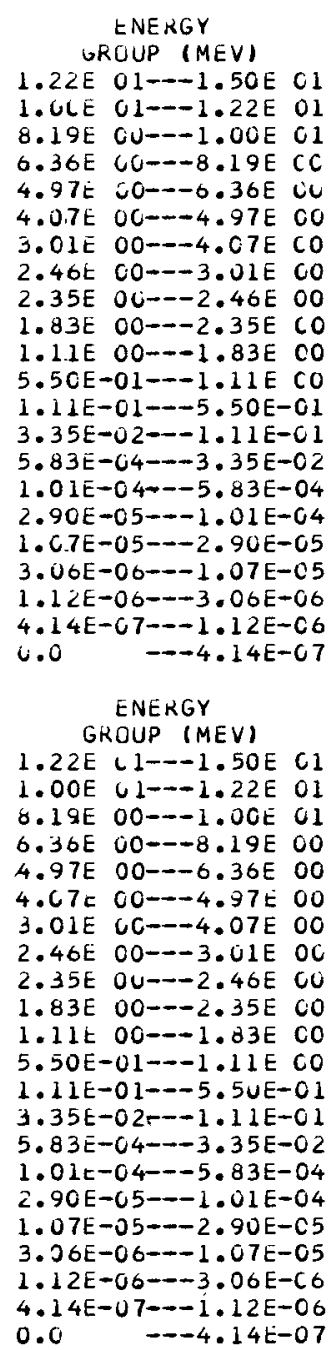

ANGLE 1 $M U=-1.0000$ $-5.476 \mathrm{E}-06$ $3.666 E-C 5$ $3.666 E-C 5$ $6.495 \mathrm{E}-05$ $1.316 \mathrm{E}-04$ $2.575 E-04$ $3.567 E-04$ $6.58=E-04$ $9.793 \mathrm{E}-\mathrm{C4}$ $1.181 E-03$ 2.11OE-03 $6.569 E-03$ $2.244 \mathrm{E}-02$ 2.949E-O 3.151E-C1 $2.232 E$ OI $1.073 E 02$ . A.95 02 $2.878 E$ O3 $.1 .054 E$ O 4

ANGLE 10 $M U=0.0950$ $7.299 E-26$ $.081 E-C 5$ $8.767 E-05$
$1.445 E-04$ $2.916 \mathrm{E}-04$ $4.619 E-04$ 5. $078 E-04$ $1.046 E-03$ $1.866 \mathrm{E}-03$ $2.178 \mathrm{E}-03$ $3.540 E-03$ I. $075 \mathrm{E}-\mathrm{G} 2$ $3.182 \mathrm{E}-0.2$ $3.758 \mathrm{E}-01$ $3.852 E-C 1$ $2.717 E 01$ $1.284 \mathrm{E} 02$ 4.080E 02 $1.199 \mathrm{C}^{03}$ $3.443 E \quad 03$ $8.029 E 03$ $1.239 \mathrm{E}^{4}$ $M U=-C .9894$ $-4.455 E-C 6$ . C $82 \mathrm{E}-06$ $.655 E-05$ $0.468 E-05$ $1.315 \mathrm{E}-\mathrm{C} 4$ $.589 E-04$ $3.566 E-04$ $6.591 E-04$ $9.815 E-C 4$ $1.185 E-03$ 2.117E-03 $6.591 E-03$ 2.250E-OL $2.555-C 1$ $3.157 E-C 1$ $2.230 E$ E $1.675 E$ OL . COE C2 1.COIE C3 $6.740 \mathrm{E} O$ $6.740 E$ OS

\section{ANGLE 11} $M U=C .2816$ 1. $\angle 49 E-C 5$ $1.114 E-04$ $1.114 E-04$
$1.774 E-04$ $3.515 \mathrm{E}-04$ $5.454 E-C 4$ $5.454 E-C 4$ 6. $205 E-04$ $2.316 E-03$ 2.592E-C 4.C55E-C3 $1.22 \mathrm{CE}-02$ 3.445E-02 3. $248 E-C$ $3.981 \mathrm{E}-\mathrm{O} 1$ $2.795 E$ it 1 $1.340 \mathrm{E} \mathrm{CZ}$ 4.225E O2 $1.244 \mathrm{E} \mathrm{O3}$ $3.568 E \quad 03$ $8.315 E$ $1.279 \mathrm{E} \mathrm{G}^{4}$
3 $-1.3 C 4 E-06$ $8.675 E-C 6$ 3. 0 C9E-05 6. $381 \mathrm{E}-05$ $1.314 \mathrm{E}-04$ $2.644 E-04$ $3.567 \mathrm{E}-04$ $6.62 L E-04$ $9.913 \mathrm{E}-04$ . $147 \mathrm{E}-0$ . 6. C.276E-02 2.98E E-01 $3.179 E-01$ . $3.421 E$ O . 00 E $1.001 E$ OS $6.784 E 03$ $1.062 \mathrm{E} \mathrm{O}$

ANGLE 12 $M U=C .4580$ 1.65CE-OS 1. $435 \mathrm{E}-0$ $2.272 \mathrm{E}-04$ $4.43 C E-04$ $6.742 \mathrm{E}-04$ $6.742 E-04$ $7.425 E-04$ $1.027 E-03$ 3. $158 E-03$ 4. $723 \mathrm{E}-\mathrm{C} 3$ $1.4 \mathrm{C} 6 \mathrm{E}-\mathrm{C} 2$ $3.749 E-02$ $4.163 E-01$ $4.155 \mathrm{E}-\mathrm{C}$ $2.915 E 01$ $1.387 \mathrm{E} \mathrm{OL}$ $4.390 \mathrm{E} \mathrm{O2}$ 1.29UE OS $3.697 E_{03}$ $1.321 \mathrm{E} 04$
ANGLE 4 $M U=-0.8656$ $9.347 E-C 7$
$1.067 E-65$ $1.067 E-C 5$
$3.535 E-C 5$ $1.0675 E-C 5$
$6.325 E-05$ $6.325 E-05$
$1.336 E-C 4$ $1.336 \mathrm{E}-\mathrm{C} 4$ $2.746 \mathrm{E}-04$ $3.578 \mathrm{E}-04$ $6.683 E-64$ $1.013 \mathrm{E}-03$ $1.235 \mathrm{E}-03$ . $6.868 E-03$ $2.326 E-02$ .026E-01 $3.219 E-01$ .279E 01 $1.463 \mathrm{E} C 2$ . 2.036 C3 $.863 \mathrm{E} \mathrm{O3}$ 1. $0.73 \mathrm{E} 04$

ANGLE 13 $M U=0.6179$ $1.262 E-04$ $1.995 E-C 4$ $3.147 E-04$ $5.969 E-04$ $8.812 E-04$ $9.591 E-C 4$ $2.169 E-03$ $4 . C_{34}-C_{3}$ $3.942 \mathrm{E}-\mathrm{C} 3$ $5.597 \mathrm{E}-03$ $1.643 E-02$ 4. $093 \mathrm{E}-\mathrm{C} 2$ $4.381 \mathrm{E}-\mathrm{Cl}$ 4.313E-01 $3.018 \mathrm{E} 01$ $1.44 \mathrm{CE} \mathrm{C2}$ $4.547 \mathrm{E} \mathrm{O2}$ i. $336 E$ C3 $3.826 \mathrm{E} 03$ $8.966 E \mathrm{C}$ $1.362 E \quad 44$ $2.977 E-06$ $1.215 E-05$ $3.5 \mathrm{C} 3 \mathrm{E}-\mathrm{C} 5$ $6.474 \mathrm{E}-05$ $1.411 \mathrm{E}-\mathrm{C} 4$ 2.890E-O4 $3.614 E-C 4$ $6.8 C 0 E-04$ 1.C5IE-C3 $1.289 \mathrm{E}-03$ $7.142 E-03$ $.397 \mathrm{E}-\mathrm{C} 2$ 3.C92E-O . $278 E-O 1$ . $319 E$ E 1.114E 02 $2.985 \mathrm{E} 03$ 6.976E O3 1. CACE 04

ANGLE 14 $M U=C .7550$ $7.317 E-C 5$ $.134 \mathrm{E}-\mathrm{O} 4$ $3.167 \mathrm{E}-\mathrm{U} 4$ $4.841 E-04$ $1.222 E-03$ $1.290 \mathrm{E}-\mathrm{C} 3$ $3.633 \mathrm{E}-03$ $5.693 E-03$ $5.054 E-03$ $6.749 E-03$ $1.946 E-02$ 4. $470 \mathrm{E}-\mathrm{C} 2$ $4.6 \mathrm{C} 2 \mathrm{E}-\mathrm{Cl}$ 4. $475 \mathrm{E}-01$ $3.127 \mathrm{E} \mathrm{Cl}$ $1.486 \mathrm{E} \mathrm{C2}$ $1.379 \mathrm{E} 03$ $3.947 \mathrm{E} \mathrm{C} 3$ $9.181 \mathrm{E} 03$ $1.4 \mathrm{CCE} \quad 04$ $8.315 \mathrm{E}-\mathrm{CC} 7$ $1.308 \mathrm{E}-\mathrm{C} .5$ $3.651 E=05$ $7.006 E-05$ $1.561 \mathrm{E}-04$ $3.080 E-04$ $3.694 E-C 4$ $7.010 E-C 4$ $1.112 E-C 3$ 1. $37 \mathrm{E}-03$ $2.425 E-03$ 7.52 E-03 $2.494 E-02$ $3.181 E-01$ . $355 E-O 1$ 2.372E CI $1.130 E$ O 2 1. C60E 03 1.060E 03 $7.124 \mathrm{E} C 3$
$1.111 \mathrm{E}$ C4

\section{ANGLE 15} $M U=0.8656$ $2.200 \mathrm{E}-04$ $5.757 E-04$ $8.256 E-04$ $8.256 E-04$
$1.357 E-03$ $1.357 E-03$ $1.867 \mathrm{E}-\mathrm{C} 3$ $1.867 E-C 3$
$4.485 E-C 3$ $8.512 E-03$ $6.707 E-03$ $8.295 E-03$ $2.338 \mathrm{E}-\mathrm{G} 2$ 4.868E-02 4. $864 E-01$ $4.610 E-01$ $3.215 E$ OI $1.5 \angle 8 E \quad O 2$ $4.828 E$ C2 $1.416 E_{03}$ $4.052 \mathrm{E} \mathrm{O} 3$ $9.420 E$ C $1.434 E$ C4 $A U=-0.4580$ $-9.728 E-07$ 
(NEUTKONSAMEV/STERADIAN/SOURCE NEUTRON)

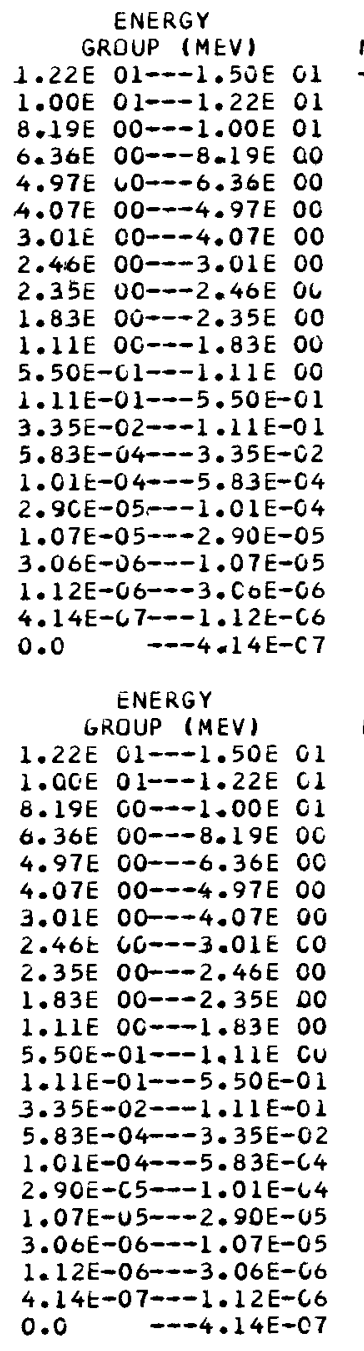

ANGLE 1 AU $.368 \mathrm{E}-06$ $.862 \mathrm{E}-06$ .05 . $08 E-05$ 889E-05 $.294 E-04$ $1.294 E-04$ $2.245 E-04$
$3.395 E-04$ $.395 E-04$
$.0537 E-04$ $8.011 \mathrm{E}-04$ $2.459 E-03$ $8.421 E-03$ $1.009 \mathrm{E}-01$ $1.046 \mathrm{E}-01$ $7.221 E 00$ $3.413 E 01$ $1.065 E 02$ $3.091 E \mathrm{C} 2$ 8.8COE 02 $2.038 E_{03}$
$3.159 E_{03}$

ANGLE 10 $M U=C .095 C$ $1.891 E-C 6$ . $273 E-05$ . . $128 \mathrm{E}-04$ $1.778 \mathrm{E}-04$ $1.862 E-04$ 3. $9 \mathrm{COE}-\mathrm{C} 4$ $7.276 \mathrm{E}-04$ $8.610 E-04$ $1.331 E-03$ $4.027 E-03$ $1.181 \mathrm{E}-02$ $1.254 \mathrm{E}-01$ 1. $243 \mathrm{E}-01$ $8.51 \mathrm{BE} 00$ $4.004 E O 1$ $1.249 E \mathrm{CZ}$ $3.618 E$ E2 1.027 E O3 $3.635 E \quad 03$
ANGLE 2 -8.066 E-07 $1.503 E-06$ $8.897 \mathrm{E}-06$ $.051 E-65$ . $906 \mathrm{E}-05$ $9.938 \mathrm{E}-05$ $.294 E-C 4$ $3.409 E-64$ $3.409 E-C 4$
$4.554 E-04$ $8.038 \mathrm{E}-04$ $2.467 \mathrm{E}-03$ $8.444 E-C 3$ 1. $011 E-01$ $.047 E-01$ $7.232 \mathrm{E} \mathrm{OO}$ $3.417 E 01$ $1.067 \mathrm{E} \mathrm{O2}$ $3.696 \mathrm{E} \mathrm{O2}$ $8.812 \mathrm{E} \mathrm{C2}$ 2.C41E 03 $3.163 E 03$

ANGLE 11 $M U=C .2816$ $1.717 E-05$ . $.487 \mathrm{E}-\mathrm{C} 5$ $1.372 E=04$ $2.11 \mathrm{CE}-\mathrm{C} 4$ $2.197 E-G 4$ $4.825 E-04$ $9.165 E-C 4$ 1. $C 25 E-03$ $1.514 \mathrm{E}-\mathrm{C}$ $4.553 \mathrm{E}-\mathrm{C} 3$ $1.272 \mathrm{E}-02$ $1.310 E-01$ $1.286 E-01$ $8.798 E$ OU $4.129 E$ OI $1.208 \mathrm{E} \mathrm{C2}$ $3.729 E \mathrm{C} 2$ $1.059 E$ C3 $3.736 \mathrm{E} 03$
ANGLE 3 $1.963 E-06$ .CO4E-OS . $037 E-05$ $4.924 E-05$ 1.C1 $4 E-04$ $1.295 E-04$ $2.265 E-04$ $3.467 \mathrm{E}-\mathrm{O}$ $4.629 E-04$ $8.152 E-04$ $2.504 E-C 3$ $8.541 E-03$ $1.019 \mathrm{E}-01$ $1.054 \mathrm{E}-01$ $7.276 \mathrm{E} \mathrm{OO}$ $3.438 \mathrm{E} \mathrm{OI}$ $1.073 E 02$ $3.114 \mathrm{E} \mathrm{O2}$ $8.863 \mathrm{E} \mathrm{O2}$ 2.C53E 03 $3.18 \mathrm{CE} \quad 03$

ANGLE 12 $M U=0.4580$
$4.249 E-66$ . 249E-06 作 .518E-05 $1.752 \mathrm{E}-04$ $2.616 E-04$ $2.693 E-04$ $6.224 E-04$ $1.202 \mathrm{E}-03$ $1.247 E-03$ $1.745 \mathrm{E}-03$ $3.211 E-03$ $1.375 E-C 2$ $1.369 E-01$ $1.33 C E-01$ $9.681 \mathrm{E} 00$ 4.257E DI $1.328 E$ O $3.843 \mathrm{E} \mathrm{O2}$ 1.CSOE OZ $3.838 E \quad 03$
ANGLE 4 $2.344 E-07$ $.526 E-C 6$ $9.134 \mathrm{t}-06$ $2.045 \mathrm{E}-\mathrm{CS}$ $.042 E-05$ $1.052 \mathrm{E}-04$ $1.299 \mathrm{E}-\mathrm{C} 4$ $3.585 E-04$ $4.777 E-C 4$ $8.375 E-C 4$ $2.574 \mathrm{E}-03$ $8.722 \mathrm{E}-03$ $1.033 E-01$ $1.066 \mathrm{E}-01$ $7.356 \mathrm{E} \mathrm{OO}$ $3.475 E$ OI $1.084 \mathrm{E} 02$ $3.147 \mathrm{E} \mathrm{O2}$ $8.955 \mathrm{E} \mathrm{C2}$ $2.074 \mathrm{E} \mathrm{O}$ 3. $211 \mathrm{t} 03$

ANGLE 13 $A U=0.6179$
$7.724 E-C 6$ $6.586 E-C 5$ $1.221 E-04$ $2.388 E-04$ $3.406 E-04$ $3.414 E-04$ $8.366 E-04$ $1.647 E-03$ $1.548 E-03$ $2.035 E-03$ $0.025 E-C 3$ $1.487 \mathrm{E}-02$ $1.429 \mathrm{E}-01$ $1.373 E-0$ $9.359 \mathrm{E} \mathrm{OU}$
$4.382 \mathrm{E} \mathrm{OL}$ 4.382E CI $1.366 E$ CL $1.121 \mathrm{E} 03$ 2.589 E 03 $3.937 \mathrm{E} \mathrm{O3}$
ANGLE 5 5.740E-C 7 $2.915 \mathrm{E}-\mathrm{C6}$ y.348E-06 $2.134 E-C 5$ $5.369 \mathrm{E}-05$ $1.106 E-04$ $1.313 E-C 4$
$2.358 E-04$ $3.775 \mathrm{E}-\mathrm{C} 4$ 5. CO9E-0 4 $8.716 \mathrm{E}-\mathrm{C} 4$ $2.680 E-03$ $8.985 t-03$ $1.054 \mathrm{E}-01$ 1. $084 \mathrm{E}-01$ $7.471 \mathrm{E} \mathrm{OO}$ $3.527 \mathrm{E} \mathrm{O1}$ $1.101 E$ C2 $3.193 \mathrm{E} 02$ . C87E 02 $2.104 \mathrm{E} \mathrm{O3}$
$3.254 \mathrm{E} \mathrm{C3}$ ANGLE 14 $M U=C .7550$ $2.038 E-05$ 1.1 COE -04 $1.937 E-04$ $3.478 E-04$ $4.653 E-04$ $4.451 \mathrm{E}-04$ $1.172 \mathrm{E}-0 \mathrm{~S}$ $2.363 E-03$ $1.958 \mathrm{t}-03$ $2.396 E-C 3$ $7.016 \mathrm{E}-03$ $1.605 E-02$ $1.486 E-O 1$ $1.413 E-C 1$ $9.616 E 00$ 4.497 E OI $1.4 C 2 E$ C2 $4.055 E$ O2 $1.150 E$ CS $4.0 \angle O E O 3$

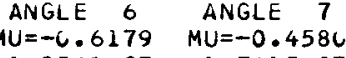
$1.356 \mathrm{E}-07$ $-1.741 E-07$ 1.003E-05 $\quad 1.177 \mathrm{E}-\mathrm{G}$ $2.775 \mathrm{E}-05$ $5.978 E-05 \quad 6.888 E-05$ $1.178 E-04 \quad 1.271 E-04$ $1.343 \mathrm{E}-04 \quad 1.398 \mathrm{E}-04$ $2.457 E-04 \quad 2.621 E-04$ $4.064 E-04 \quad 4.490 E-04$ $5.350 E=04 \quad 5.829 E-04$ $9.205 E-04 \quad 9.867 E-04$ $2.827 t-03 \quad 3.023 E-03$ $9.340 E-03 \quad 9.791 E-03$ $1.115 \mathrm{E}-\mathrm{Cl}$ $1.134 E-01$ $7.803 E 00$ $3.678 E$ OI $1.148 E 02$ $3.328 E$ O2 9.257 C2 $9.464 E$ O2 $3.309 \mathrm{E} 03 \quad 3.375 \mathrm{E} \mathrm{O}$ ANGLE 15 $M U=0.8656$ $5.797 \mathrm{E}-\mathrm{C5}$ $1.247 E-04$
$2.070 E \rightarrow 04$ 3. $346 \mathrm{E}-\mathrm{C}$ $5.373 E-C 4$ $6.64 \mathrm{VE} \neg \mathrm{C} 4$ $5.933 \mathrm{E}-\mathrm{C} 4$ $1.718 \mathrm{E}-03$ $3.577 E-C 3$ $2.532 \mathrm{E}-03$ $2.835 E-C 3$ $8.199 \mathrm{E}-03$ $1.721 \mathrm{E} \rightarrow \mathrm{C} 2$ $1.537 E-01$ 1.447E 01 4.595E 01 $1.433 \mathrm{E} \mathrm{O} 2$ $4.142 E$ C2 $1.174 E$ C $4.104 \mathrm{E} \mathrm{O} 3$

ANGLE 8 $M U=-C \cdot 2816$ $5.2711-09$ 1. $5 C 9 E-05$ 8.C71E-C . $491 E-04$ $.881 E-04$ 2.106E-C4 $6.491 E-0$ .280E-C3 $1.035 \mathrm{E}-\mathrm{O}$ $.156 \mathrm{E}-\mathrm{C}$ .160E-C .016E 00 
ENERGY GROUP (MEVI. $1.22 E$ O1--1.50E C1 $8.19 \mathrm{E} \mathrm{OC-D-1.00E} \mathrm{O1}$ $6.30 E$ OO- $-8.19 E$ CO $4.9 .7 \mathrm{E} 00--6.36 \mathrm{E} 00$ $4.07 E 00-04.97 \mathrm{E} \mathrm{CO}$ 3. C1E $60---4.07 E$ OU $2.46 E$ OO---3.U1E CO $2.35 \mathrm{E} 00--2.46 \mathrm{E} 00$ 1.83 E $00-2.35 \mathrm{E} 00$ $1.21 E$ DO- $-1.83 E \mathrm{E} \mathrm{CO}$ $5.5 C E-01--1.11 E$ OO $1.11 E-C 1---5.50 E-C$ $3.35 E-02--1.11 E-C$ $5.83 \mathrm{t}-04--3.35 \mathrm{E}-02$ $1.01 E-U 4=-5.83 E-04$ $2.9 C E-05-1.01 E-C$ $1.07 E-05=-2.90 E-05$ $3.06 E-06--1.07 E-C 5$ $1.12 E-06=--3.06 E-06$ $4.14 \mathrm{E}-07---1.12 \mathrm{E}-66$
$0.0 \quad--4.14 \mathrm{E}-07$

$$
\text { ENERGY }
$$

GROUP (MEV)

$1.22 E$ O1-- 1.5CE 01 $1.0 \mathrm{CE} \mathrm{O1--1.22E} \mathrm{O1}$ $8.19 \mathrm{E}$ 00-- $1.00 \mathrm{E}^{\circ}$
$6.36 \mathrm{E} 00--8.19 \mathrm{E}$ OU $6.36 \mathrm{E}$ OO-- $-8.19 \mathrm{E}$ OU
$4.97 \mathrm{E}$ OO---6.36E 00 $4.97 E$ OC-D-6.36E OO 4.07E WO--4.97E OO 3.46E OO---9.UIE 00 2.35 E $00--2.46$ E 00 $.83 E$ NO-C-2.35E UO 1.11 E 00-- 1.83 E 60 .SUE-O11.11E-01-- 5 . $50 E-O$ . . $83 \mathrm{E}-04--3.35 E-02$ $1.01 E-04-2-5.83 E-04$ 2.90 $05-1.01 E-04$ $1.07 E-05-2.00 E-05$ 1. $4.14 E-07=-1.12 E-06$ $4.14 E-07-0-1.12 E-06$
$C .0 \quad-04.14 E-C 7$
ANGLE 1 ANGLE 2 $M U=-1.000 \mathrm{C}$ $1.749 E-07$ $1.997 \mathrm{E}-\mathrm{C} 6$ $5.943 \mathrm{E}-06$ $1.640 \mathrm{E}-05$ $3.367 \mathrm{E}-\mathrm{C} 5$ $4.231 E-05$ 7.C43E-C 5 $1.078 \mathrm{t}-04$ $1.532 \mathrm{E}-04$ $2.696 \mathrm{E}-04$ $8.152 \mathrm{E}-04$ $2.775 E-C 3$ $3.231 E-62$ $3.316 E-02$ $2.271 E 00$ $1.067 \mathrm{E} \mathrm{OI}$ $3.314 E$ C. $9.554 \mathrm{E} \mathrm{O1}$ $2.7 C 5 E \quad 02$ $6.233 \mathrm{E} \mathrm{O}$ $9.596 E$ O2 ANGLE 10 $M U=0.0950$ $4.277 E-0$ $2.928 E-06$ $7.212 \mathrm{E}-06$ 1. $662 \mathrm{E}-05$ $3.860 E-05$ $6.058 E-05$ $6.112 \mathrm{E}-65$ $1.294 E-04$ $2.479 E-04$ 2.960E-U4 $4.449 E-04$ $1.332 E-03$ $3.863 E-03$ . . 65 E 02 $1.238 \mathrm{E} O 1$ $1.238 E$ OI $1.105 \mathrm{E} \mathrm{O2}$ $3.122 E$ $7.178 \mathrm{E} \mathrm{O}$ $1.092 \mathrm{E}$ OS

ANGLE 11 $U=C .2816$ 6.807E-0 . . $114 E-C 5$ $4.728 E-C 5$ $7.728 E-C 5$ 7.204E 05 1.614E 0 .527E-C . 4 CE-O 4 1.5C1E -03 (14 $.139 E-C 2$ .C28E-C 2.731E CO $3.952 E C$ $1.136 \mathrm{E} \mathrm{U2}$ $3.208 \mathrm{E} 02$ $7.372 E$ OL
(NEUTKLNSAMEV/STERADIAN/SOURCE NEUTRON) $=-0.9894 \quad M U=-C .9446 \quad M U=-0.0656$

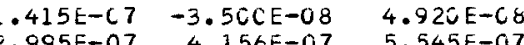
$2 . C 2 C E-06$ 2.1CGE-06 $2.206 E-C 6$ $5.936 E-06 \quad 5.930 E-06 \quad 6.024 t-16$ $1.641 \mathrm{E}-\mathrm{C} 5 \quad 1.651 \mathrm{E}-05 \quad 1.699 \mathrm{E}-05$ $3.383 E-05 \quad 3.451 E-05 \quad 3.576 E-05$ $7.058 E-05 \quad 7.122 E-05 \quad 7.254 E-05$ $1.084 E-04 \quad 1.107 E-04 \quad 1.153 E-64$ $1.539 E-04 \quad 1.507 E-04 \quad 1.621 E-04$ $2.7 C 5 E-C 4 \quad 2.743 E-04 \quad 2.818 E-04$ $8.101 E-C 4 \quad 8.3 U 5 E-04 \quad 8.54 C E-C 4$ $2.782 \mathrm{E}-03 \quad \angle .814 \mathrm{E}-03 \quad 2.873 \mathrm{E}-\mathrm{G}$ $3.237 E-02 \quad 3.261 E-02$ $3.321 t-02 \quad 3.341 E-02 \quad 3.378 E-C$ 2. $\angle 87 E$ OO $2.311 E$ OC 1.C74E OI 1.C8DE CI $3.316 E$ OI 3.336E O1 3.370E OL 9.366 E CI 9.619E OI $9.714 E$ O $2.723 E$ O2 $2.750 E$ OL

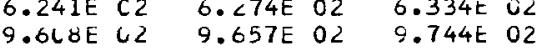
S.850E-07 $5.485 E-06$ $1.289 \mathrm{t}-05$ $2.844 E-05$ $0.091 E-05$ $8.934 E-05$ $8.742 E-05$ $4.177 E-04$ $4.177 E-04$ $4.283 E-04$ S.773E-04 $4.468 E-C 3$ $4.468 E-O 3$ $4.31<E-02$ 2.812E 00 2. $312 E$ O 4. CG3E OI 4. $168 \mathrm{E} 02$ $3.295 \mathrm{C} 2$ $7.568 \mathrm{C} 2$ $1119 E \mathrm{C} 3 \mathrm{Z}$. $147 \mathrm{E}$ O3
ANGLE 5 ANGLE 6 1. $C 45 E-67$ $6.461 E-07$ $2.309 E-C 6$ $6.385 E-06$ $1.819 E-05$ $3.756 \mathrm{E}-\mathrm{C} 5$ $4.295 E-U 5$ $7.480 E-65$ $1.225 E-C 4$ 1. $7 \mathrm{C} 4 \mathrm{E}-\mathrm{C} 4$ $2.933 \mathrm{E}-04$ $8.892 \mathrm{E}-\mathrm{C} 4$ $2.958 \mathrm{E}-\mathrm{C} 3$ $3.37 \mathrm{CE}-\mathrm{C} 2$ $3.430 \mathrm{E}-\mathrm{C} 2$ $2.345 E 00$ $1.1 C C E$ U 1 $3.418 E$ CI 9.8500 UI 2.788 E C $6.42 \mathrm{CE} \mathrm{C2}$ 9.867 t C2 ANGLE 14 $M U=0.7550$ $4.949 \mathrm{E}-16$ $1.575 E-65$ $3.401 E-05$ $6.814 \mathrm{E}-65$ $1.219 E-04$ $1.561 \mathrm{E}-\mathrm{C} 4$ $1.393 E-04$ $3.962 E-C 4$ $8.422 E-C 4$ $7.635-04$ $7.741 E-U 4$ 5.16OE-0 $4.645 E-U 2$ $4.045 E-U 2$ 2. $901 E$ OO 2.961E OO 4.266E O $4.266 \mathrm{E}$ 3.4535 O2 $7.926 \mathrm{E} C 2$ $1.197 \mathrm{E} \mathrm{C} 3$ $7.256 \mathrm{E}-67$ $2.524 E-06$ $7.195 E-06$ .033E-05 3.998E- 65 ..853E-O $.825 E-04$ .074 EAC $3.454 E-C$ $3.498 \mathrm{E}-\mathrm{CL}$ .39CE OO 1.120E OI .479E CI 1. OC2E 02 $6.530 E$ O2 ANGLE 15 $3.274 t-05$ 1.187E-04 $2.172 \mathrm{E}-\mathrm{C4}$ 1. $794 E-C 4$ . $762 E-U 4$ $1.290 E-C 3$ $8.421 E-C 4$ . ¿.596E-CI $4.786 \mathrm{E}-\mathrm{O}$ $4.786 E-02$ $3.0 \angle 1 E 00$ $.403 E$ ol $4.348 E$ O1
$1.248 E$ C2 $3.517 t 02$ $8.07 \mathrm{CE} C$
ANGLE $6 \quad$ ANGLE 7 $2.433 \mathrm{E} \rightarrow \mathrm{CB}-2.542 \mathrm{E}-\mathrm{C} 8$ $1.002 \mathrm{C} C 3$ $M U=0.8656$
$1.347 \mathrm{E}-05$ $6.569 E-05$ $1.863 E-04$ $1.217 \mathrm{E} \mathrm{C3}$

\section{AIVGLE 16} $3.526 E-05$ $7.191 \mathrm{E}-05$ $1.354 \mathrm{E}-04$ $2.180 \mathrm{E}-\mathrm{C} 4$ $2.966 \mathrm{E}-\mathrm{C} 4$ $3.10 C E-C 4$ $2.316 E-C 4$ . $798 \mathrm{E}-04$ 2.117E-0 $1.084 \mathrm{ECC} 3$ . $5.77<E-O S$

ANULE 8 ANGLE 9 $8.4915-C Q \quad M U=-C . C 954$ $1.316 E-C 6 \quad 2.014 E-06$ $3.958 E-C O \quad 5.381 E-66$ $1.064 E-C 5$ 1. $330 E-115$ $2.75 C E-C 5 \quad 3 . \angle 42 E-C 5$ $4.723 E-C 5 \quad 5.278 E-05$ $4.893 E-C 5 \quad 5.379 E-U 5$ $9.378 E-C 5$ 1. $C 8 C E-04$ 1. $7 C 7 t-C 4 \quad 2 . C 23 E-C 4$ $2.225 E-04 \quad 2.536 E-C 4$ $3.0 C 7 E-C 4 \quad 3.578 E-C 4$ $1.088 E-C 3$ 1. $196 E-03$ $3.398 \mathrm{~L}-\mathrm{C} 3 \quad 3.612 \mathrm{E}-03$ $3.818 \mathrm{E}-02$ $3.677 E-C 2 \quad 3.786 E-02$ $2.5 C 6 E$ OC $\angle .576 E$ OC $1.173 E$ C1 1.204E O $3.738 \mathrm{E} \mathrm{O1}$ $1 . C 76 t \mathrm{C2}$ $2.904 E L_{2}$

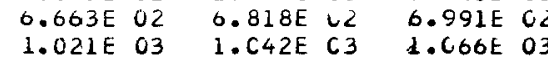


(NEUTRONS/MEV/STEKADIAN/SOURCE NEUTRON)

\begin{abstract}
ENERGY
GROUP (MEV)

$1.22 \mathrm{E} 61-1.50 \mathrm{E} 01$ $1.00 E$ O1-D-1.22E Cl $6.36 E$ OO-D- $8.19 E 00$ $4.9 .7 \mathrm{E}$ OO---6.36E CO $3.01 E$ 00-- $4.07 E 00$ $2.46 E$ 00-- $3.01 E$ OO $2.35 \mathrm{E} \mathrm{OOO-} 2.46 \mathrm{E} \mathrm{CO}$ $1.83 E$ COD-- $2.35 E$ OO
$1.11 E$ OOO--1.83E CO $5.5 C E-01--1.11 E$ OC 1. $11 E-01--5.50 E-C 1$ $3.35 E-C 2--1.11 E-01$ $5.83 \mathrm{E}-04--3.35 \mathrm{E}-02$ $1.01 E-04--5.83 E-04$ $1.07 E-C 5 \cdots-2.90 E-C 5$ $3.06 E-06-\cdots-1.07 E-05$ $1.12 E-00--3.06 E-06$ $4.14 E-07--1.12 E-C 6$ 0.0
\end{abstract}

$\triangle N G L E \quad 1$ $-3.03 .6 E-08$ $4.934 E-08$ $1.636 \mathrm{E}-\mathrm{C} 6$ $5.155 E-06$ $.071 \mathrm{E}-\mathrm{C5}$ $2.116 E-05$ $3.269 E-05$ $4.822 E-05$ $8.486 E-05$ $2.536 \mathrm{E}-04$ $8.572 \mathrm{E}-\mathrm{C}$ $9.860 \mathrm{E}-0.3$ $1.009 \mathrm{E}-02$ $6.889 E-01$ $3.230 \mathrm{E} \mathrm{OO}$ $1.002 E O 1$ $2.882 E$ O $8.147 E$ CI $2.878 E \quad 02$

ENERGY GRUUP (MEV)
$1.22 E$ O1-D- $1.50 E$ $1.00 E$ OI-- $1.22 E$ O 8.1 QE O0---1.00E 01 6.36 E $00--8.19 E$ OO 4.97E 00---6.36E 00 4.07E 00---4.97E 00 3.01 E OO- $4.07 E$ OO $2.46 \mathrm{E}$ OO---3.01E CO $2.35 \mathrm{E} \mathrm{OOW-2.46E} \mathrm{OC}$ $1.83 \mathrm{E}$ OC---2.35E CO $1.11 E$ OO-D-1.83E OC $5.50 E-01--1.11 E$ OO $1.11 E-01-C-50 E-O$ $3.35 E-02-C-1.11 E-C 1$ $1.83 E-04--3.35 E-C 2$ $1.01 E-C 4-5.83 E-04$ $2.90 E-05-1.01 E-C 4$ 1.07E-05 1.12E-06 $4.14 E-07--1.12 E-06$ $4.14 \mathrm{E}-07---1.12 \mathrm{E}-06$
0.0
ANGLE 10 $M U=0.0950$ $8.982 E-08$ Q.433E-07 $1.862 E-C 6$ $5.021 E-C 6$ $1.234 \mathrm{E}-05$ $1.927 \mathrm{E}-05$ $1.891 \mathrm{E}-05$ $4.031 \mathrm{E}-05$ $7.845 E-C 5$ $9.434 E-05$ $1.395 \mathrm{E}-04$ $4.133 E-04$ 1.187E-03 $1.206 E-02$ $1.181 \mathrm{E}-02$ . $3.730 \mathrm{E}$ OO $1.156 E$ OI .317E 9.351E 01 $3.259 \mathrm{E} \mathrm{O2}$
ANGLE $M U=-C .9894$
$-2.451 E-C 8$ $5.727 t-08$ $4.404 \mathrm{E}-07$ $1.636 \mathrm{E}-\mathrm{CO}$ $5.159 \mathrm{E}-06$ $1.159 \mathrm{E}-06$
$1.076 \mathrm{E}-\mathrm{CS}$ 1.3 C6E-05 2.3 CEE-05 $3.288 \mathrm{E}-05$ $4.844 E-05$ $8.514 E-C 5$ $2.545 \mathrm{E}-\mathrm{C} 4$ $8.594 E-04$ $9.883 \mathrm{E}-03$ $1.010 E-C 2$ $3.234 E 00$ $1.0 C 3 E$ OL 2.886E OI $8.157 E$ OI $1.876 E 0$ $2.882 \mathrm{E} \mathrm{O2}$

ANGLE $-5.685 \mathrm{E}-\mathrm{C} 9$ $8.451 E-08$
$4.710 E-07$ $1.645 \mathrm{E}-06$ 5. $205 \mathrm{E}-06$ 5. $205 E-06$
$1 . C 97 E-05$ $1.3 C 7 E-05$ $2.143 E-05$ $3.368 E-05$ $4.936 E-05$ $8.636 E-05$ 2.584E-04 $8.691 \mathrm{E}-04$ $9.956 \mathrm{E}-03$ $1.016 E-02$ $6.937 E-01$ $3.251 \mathrm{E} \mathrm{OO}$ 1. COBE 0 2.901E 01 $.886 \mathrm{E} \mathrm{G}$ $2.896 \mathrm{E} \mathrm{O} 2$

$A N G L E \quad 4$
$M=-0.8656$ $9.633 \mathrm{E}-\mathrm{C} 9$ $1.165 \mathrm{E}-07$ $1.165 E-07$
$5.097 E-07$ $1.689 \mathrm{E}-06$ $5.374 \mathrm{E}-\mathrm{CO}$ $1.136 \mathrm{E}-\mathrm{C} 5$ 1. $312 \mathrm{E}-05$ $2.188 \mathrm{E}-\mathrm{C} 5$ 3. $524 E-G 5$ $5.115 \mathrm{E}-05$ $8.871 \mathrm{E}-05$ $2.658 \mathrm{E}-\mathrm{C} 4$ $8.871 \mathrm{E}-\mathrm{C} 4$ $1.009 \mathrm{E}-\mathrm{C} 2$ $1.027 \mathrm{E}-02$ $7.008 t-01$ $3.283 \mathrm{E} 00$ 1.018 E C $2.929 E$ O $8.277 E$ E $1.903 E$ O2 ANGLE 13 ANGLE 11
$M U=C .2816$ $1.42 C E-C 7$ $2.495 E-06$ $2.495 E-06$ $0.484 \mathrm{E}-06$ $2.294 \mathrm{E}-05$ $2.210 E-05$ $5.054 E-05$ $1.004 E-C 4$ $1.124 \mathrm{E}-04$ $1.576 \mathrm{E}-\mathrm{C} 4$ $4.647 E-04$ $1.272 \mathrm{E}-03$ $1.255 E-02$ $1.217 E-02$ $8.233 \mathrm{E}-\mathrm{Cl}$ 1.187t 01 1.187E 3.4C5E OI $2.2 \mathrm{ClE}$ O2 $3.337 E_{02}$ ANGLE 12 $M U=0.4580$ $2.155 \mathrm{E}-07$ $1.253 \overline{\bar{E}}-06$ 3. $48 \mathrm{CE}-0 \circ$ $8.914 \mathrm{E}-06$ $1.969 \mathrm{E}-05$ $2.843 E-05$ 2. $68 \mathrm{BCE}-05$ $6.586 E-05$ 1. $341 E-04$ 1. $363 E-04$ 1. $798 \mathrm{E}-04$ $5.273 E-04$ $1.366 E-03$ $1.354 \mathrm{E}-02$ 1.254E-02 $3.464 E-01$ $257 \mathrm{E} O$ $3.414 \mathrm{E} \mathrm{O} 2$ $A N=0.6179$
$4.326 \mathrm{E}-C 7$ $2.018 E-00$ $5.467 E-06$ $1.347 \mathrm{E}-05$ $2.716 E-C 5$ $3.668 \mathrm{E}-05$ $3.315 E-C 5$ $8.904 t-C 5$ $1.876 \mathrm{E}-\mathrm{C}$ $1.679 \mathrm{E}-04$ $2.066 E-04$ $6.019 E-C 4$ $1.466 E-03$ $1.354 E-C 2$ $1.289 E-C 2$ $8.687 E-C 1$ 4.034E OC 1.24 E C $1.008 \mathrm{E} \mathrm{O2}$ 1.008E 02
ANGLE

5
ANGLE $1.866 E-C 8$ $5.368 E-C 7$ $5.464 E-07$
$1.815 E-06$ $5.772 \mathrm{E}-06$ $1.193 E-05$ 1. $327 E-05$ $2.264 E-C 5$ 3.763E-05 $5.389 E-05$ $9.231 E-C 5$ 2.767E-04 $9.132 E=04$ $1.028 E-0$ 7. IC $8 E-01$ $3.329 E 00$ $1.032 \mathrm{E} \mathrm{CI}$ $1.928 \mathrm{E} O 2$
$2.957 \mathrm{E} \mathrm{O2}$ $M U=-0.4580$ $1.532 E-07$
$6.058 E-07$ $2.077 E-06$ $2.077 E-06$
$0.467 E-06$ $6.467 E-06$
$1.269 E-05$ $1.359 E-05$ $2.389 E-05$ $4.115 \mathrm{E}-05$ 5.785E-O $9.744 \mathrm{E}-05$ $2.920 E-04$ $9.483 E-O$ $1.063 \mathrm{E}-02$ $3.387 E$ O $1.050 E$ O $3.019 \mathrm{E} \mathrm{O}$ .528E OI $3.002 \mathrm{E} \mathrm{C2}$ ANGLE 14 3.488 E 2

$=0.7550$ ANGLE 15

$1.109 \mathrm{E}-06$
$3.845 \mathrm{E}-06$ $9.865 E-06$ $2.228 \mathrm{E}-05$ $3.957 E-C 5$ $4.892 E-05$ $4.157 E-C 5$ $1.245 E-C 4$ $2.758 \mathrm{E}-04$ $2.089 E-04$ $2.376 E-04$ 6.879E-04 1. $400 \mathrm{E}-\mathrm{C}$ $1.400 E-C 2$ $4.123 E C 0$ $1.276 E$ O1 
(NEUTRGNS/MEV/STERADIAN/SOURCE NEUTRON)

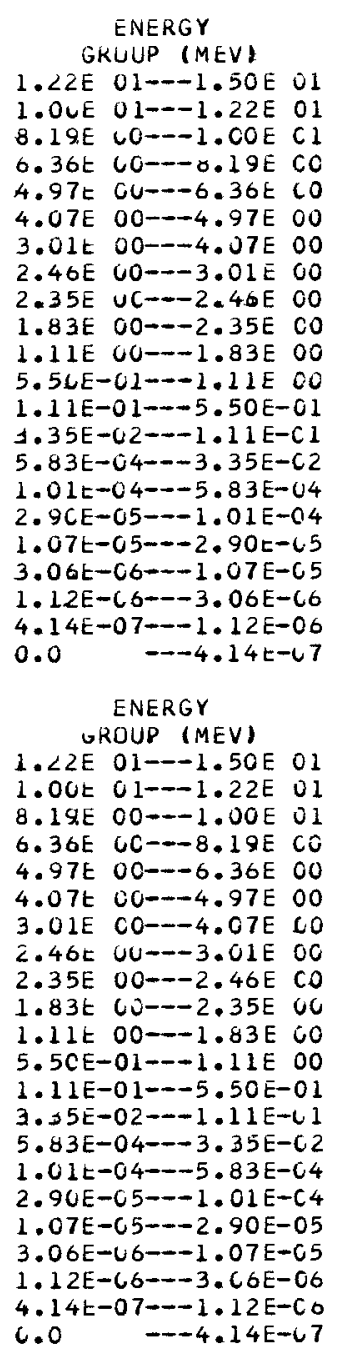

\begin{tabular}{|c|c|c|c|}
\hline $\begin{array}{l}\text { ANGLE } 1 \\
U=-1.000 C \\
5.24 U E-09 \\
8.820 E-09 \\
9.06 C E-08 \\
4.374 E-07 \\
1.557 E-06 \\
3.261 E-06 \\
3.886 E-06 \\
6.186 E-06 \\
9.619 E-06 \\
1.452 E-05 \\
2.559 E-05 \\
7.592 E-C 5 \\
2.548 E-04 \\
2.914 E-C 3 \\
2.974 E-03 \\
2.028 E-C 1 \\
9.541 E-01 \\
2.945 E \quad 00 \\
8.407 E \quad 00 \\
2.392 E \quad 11 \\
5.498 E \quad 01 \\
8.436 E \quad 11\end{array}$ & $\begin{array}{l}\text { ANGLE } 2 \\
M U=-C, 9894 \\
-4.221 E-09 \\
1 . C 6 C E-C 8 \\
9.341 E-U 8 \\
4.381 E-07 \\
1.559 E-06 \\
3.276 E-06 \\
3.887 E-C 6 \\
6.202 E-06 \\
9.679 E-06 \\
1.459 E-05 \\
2.567 E-05 \\
7.62 C E-05 \\
2.555 E-04 \\
1.919 E-05 \\
2.978 E-03 \\
2 . C 31 E-01 \\
9.513 E-01 \\
2.949 E \quad 0 C \\
8.477 E \text { OC } \\
2.395 E O 1 \\
5.544 E \quad 01 \\
8.446 E 01\end{array}$ & $\begin{array}{r}\text { ANGLE } 3 \\
M U=-C .9446 \\
-9.216 E-1 C \\
1.671 E-08 \\
1 . C 32 E-07 \\
4.429 E-07 \\
1.575 E-06 \\
3.339 E-06 \\
3.89 C E-06 \\
6.272 E-06 \\
9.933 E-06 \\
1.488 E-05 \\
2.604 E-05 \\
7.736 E-05 \\
2.584 E-04 \\
2.941 E-03 \\
2.995 E-03 \\
2.642 E-01 \\
9.564 E-01 \\
2.964 E \quad 00 \\
8.522 E \quad 00 \\
2.407 E \quad 01 \\
5.532 E \quad 01 \\
8.487 E \quad 01\end{array}$ & $\begin{array}{l}\text { ANGLE } 4 \\
M U=-G .8656 \\
1.8<2 E-09 \\
2.380 E-C 8 \\
1.153 E-U 7 \\
4.593 E-07 \\
1.631 E-C 6 \\
3.456 E-06 \\
3.9 C 7 E-06 \\
6.415 E-C 6 \\
1.042 E-C 5 \\
1.543 E-05 \\
2.675 E-05 \\
7.956 E-05 \\
2.637 E-04 \\
2.979 E-03 \\
3.027 E-03 \\
2.063 E-01 \\
9.656 E-C 1 \\
2.993 E \quad C 0 \\
8.6 C 2 E \quad U C \\
2.429 E \quad 11 \\
5.582 E \quad 01 \\
8.561 E \text { U1 }\end{array}$ \\
\hline $\begin{array}{l}\text { ANGLE } 10 \\
U=0.0950 \\
1.802 \mathrm{E}-C 8 \\
1.374 \mathrm{E}-07 \\
4.664 \mathrm{E}-07 \\
1.461 \mathrm{E}-06 \\
3.772 \mathrm{E}-06 \\
5.867 \mathrm{E}-06 \\
5.64 \mathrm{E}-06 \\
1.207 \mathrm{E}-05 \\
2.372 \mathrm{E}-05 \\
2.867 \mathrm{E}-05 \\
4.195 \mathrm{E}-05 \\
1.234 \mathrm{E}-04 \\
3.513 \mathrm{E}-04 \\
3.55 \mathrm{CE}-03 \\
3.472 \mathrm{E}-03 \\
2.349 \mathrm{E}-01 \\
1.094 \mathrm{E} \quad 00 \\
3.387 \mathrm{E} \text { CO } \\
9.713 \mathrm{E} 00 \\
2.737 \mathrm{E} \quad 01 \\
6.276 \mathrm{E} \quad 01 \\
9.525 \mathrm{E} 01\end{array}$ & $\begin{array}{r}\text { ANGLE } 11 \\
M U=C .2816 \\
2.866 E-08 \\
1.928 E-07 \\
6.360 E-07 \\
1.915 E-06 \\
4.661 E-06 \\
6.986 E-06 \\
6.66 C E-06 \\
1.510 E-C 5 \\
3.049 E-C 5 \\
3.416 E-05 \\
4.732 E-C 5 \\
1.385 E-C 4 \\
3.760 E-04 \\
3.609 E-03 \\
3.575 E-03 \\
2.414 E-01 \\
1.123 E 00 \\
3.476 E 00 \\
9.965 E \quad 00 \\
2.806 E 01 \\
6.433 E 01 \\
9.745 E O 1\end{array}$ & $\begin{array}{r}\text { ANGLE } 12 \\
M U=0.4580 \\
4.525 \mathrm{E}-08 \\
2.783 \mathrm{E}-\mathrm{CC} 7 \\
9.1 \mathrm{COE}-\mathrm{C} 7 \\
2.685 \mathrm{E}-06 \\
6.07 \angle \mathrm{E}-06 \\
8.648 \mathrm{E}-06 \\
7.937 \mathrm{E}-06 \\
1.982 \mathrm{E}-05 \\
4.095 \mathrm{E}-05 \\
4.14 \mathrm{C}-05 \\
5.385 \mathrm{E}-05 \\
1.568 \mathrm{E}-04 \\
4.031 \mathrm{E}-04 \\
3.831 \mathrm{E}-03 \\
3.678 \mathrm{E}-03 \\
2.48 \mathrm{E}-01 \\
1.15 \angle \mathrm{E} 00 \\
3.566 \mathrm{E} 00 \\
1.222 \mathrm{E} 01 \\
2.876 \mathrm{E} 01 \\
6.59 \mathrm{E} 01 \\
9.965 \mathrm{E} 01\end{array}$ & $\begin{array}{l}\text { ANGLE } 13 \\
M U=C .6179 \\
9.333 E-C 8 \\
4.029 E-C 7 \\
1.479 E-06 \\
4.142 E-C 0 \\
8.402 E-C 6 \\
1.111 E-05 \\
9.740 E-06 \\
2.68 C E-C 5 \\
5.774 E-05 \\
5.084 E-C 5 \\
0.161 E-05 \\
1.783 E-C 4 \\
4.316 E-C 4 \\
3.972 E-C 3 \\
3.778 E-03 \\
2.543 E-01 \\
1.18 U E O C \\
3.652 E \quad C \\
1.046 E 01 \\
2.942 E \quad 1 \\
6.739 E \quad 1 \\
1.017 E 02\end{array}$ \\
\hline
\end{tabular}

ANGLE 5 $M U=-0.755 \mathrm{C}$ $3.3 C 7 E-C 9$ $2.812 E-08$
$1.259 E-07$ $5.001 E-07$ $1.756 \mathrm{E}-\mathrm{C} 6$ $3.626 \mathrm{E}-\mathrm{C} 6$ $3.953 E-C 6$ $6.656 \mathrm{E}-\mathrm{C} 6$ $1.116 \mathrm{E}-\mathrm{C} 5$ $1.629 E-05$ $2.783 E-05$ $8.285 \mathrm{E}-\mathrm{C} 5$ $2.714 E-64$ $3.035 E-03$ $3.072 E-03$ L.O92E-OI 9.787E-01 $3 . C 33 E$ OO $8.715 E$ CO $2.461 E$ CI $8.663 E \mathrm{E} O 1$

ANGLE 14 $M U=0.7550$ $2.357 E-C 7$ $9.024 t-C 7$ $6.946 E-06$ $1.222 E-05$ $1.469 E-C 5$ 1. $207 t-05$ $3.737 E-C 5$ 8. $559 E-C 5$ $0.292 E-05$ 7. $C 47 E-05$ $2.027 E-04$ 4. 600 E-O 4 4. $1 \mathrm{C} 2 \mathrm{E}-63$ $3.869 \varepsilon-03$ $2.600 E-C 1$ $1.265 E 00$ $3.729 E$ UO $1.068 E_{01}$ $3.602 \mathrm{E} \mathrm{Cl}$ $6.873 E$ OL 1. C36E C2

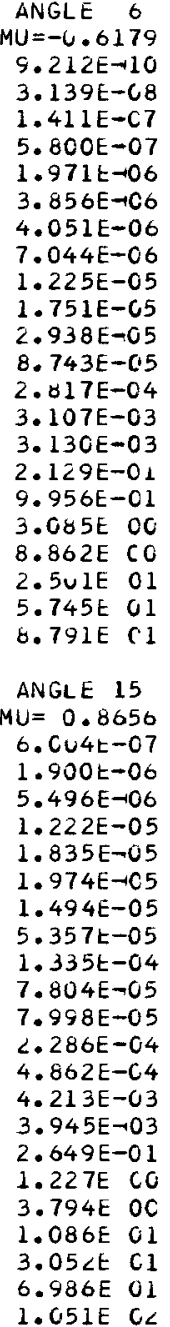

ANGLe 7 $-2.573 \mathrm{E}-1 \mathrm{C}$ $3.918 E-08$
$1.736 \mathrm{E}-07$ $7.113 \mathrm{E}-67$ $2.278 E-06$ $4.157 \mathrm{E}-\mathrm{G} 6$ $4.228 \mathrm{E}-06$
$7.649 \mathrm{E}-06$ $1.380 \mathrm{E}-65$ $1.9195-05$ $3.145 E-U 5$ 9.346E-C5 $2.947 E-04$ $3.19 a E-03$ $3.20 \mathrm{CE}-\mathrm{C} 3$ $\angle .175 \mathrm{E}-\mathrm{Cl}$ $1.016 E$ CO $3.147 E$ OC $9.038 \mathrm{E}$ CO $2.550 E$ OI $5.856 E$ CI

ANGLE 16 $M U=0.9446$ $1.45 \mathrm{CE}-46$ .047E-06 1.125E-05 $2.792 E-05$ $2.647 \mathrm{E}-05$ 1.815E-05 $7.874 E-65$ $2.200 E-04$ $0.611 E-05$ (8.906E-C5 $2.533 E-C 4$ $5.074 E-C 4$ $4.298 E-03$ $4.002 \mathrm{E}-03$ $2.684 \mathrm{E}-01$ $1.242 E$ OC $3.842 E$ OO $1.099 E$ UI 3.089 E C1 $7.069 \mathrm{E} \mathrm{Cl}$ $1.062 \mathrm{E}$ C2
ANGLE 8 ANGLE 9 1. $=-2810 \quad M U=-$ L.C 950 1.579E-C9 8.09LE-09 $2.372 E-C 7 \quad 3.361 E-C 7$ $8.955 E-C 7$ 1.14bE-U6 $2.671 E-C 6 \quad 3.252 E-06$ 4.557E-06 5.C99E-06 $4.520 E-16 \quad 4.971 E-06$ $8.579 E-C 6$ 9.981E-06 $1.601 \mathrm{E}-05 \quad 1.916 \mathrm{E}-45$ $2.148 E-C 5$ 2.455E-05 $3.416 E-C 5 \quad 3.762 E-05$ $1.013 E-C 4 \quad 1.111 E-04$ $3.166 \mathrm{E}-\mathrm{C} 4 \quad 3.295 \mathrm{E}-\mathrm{C} 4$ $3.3 C 1 E-C 3$ 3.419E-O3 $3.282 E-03 \quad 3.373 E-03$ $2.227 E-C 1 \quad 2 . \angle 86 E-C 1$ 1.03 E OO 1. COGE OO $3.220 E$ OO 3.3CCE CO $9.243 E$ OO $9.469 E$ OU $2.6 C 7 E$ C1 $2.669 E$ OI $9.120 E$ O1 $9.314 E 01$

ANGLL 17 SCALAR $1.999 \mathrm{E}-06$ $2.572 E-C 5$ 0. $460 E-06$ 2.572E-CS 1.SC8E-C5 $4.399 E-C 5 \quad 7.348 E-05$ $3.5 C E E-C 5 \quad 1.74 E-C 5$ 3. $120 E-C 5$ 1.CC4E-C 4 $1.236 E-04 \quad 2.400 E-C 5$ $4.182 E-04 \quad 5.403 E-04$ $\begin{array}{ll}1.162 E-04 & 5.531 E-04 \\ 1.164 E-U 4 & 4.395 E-C 4\end{array}$ $9.581 E-05 \quad 5.709 E-04$ $2.716 E-C 4 \quad 1.667 E-03$ $5.204 E-04 \quad 4.463 E-03$ $\begin{array}{ll}4.347 E-03 & 4.463 E-03 \\ 4 & 4.44 E-U 2\end{array}$ $4.036 E-C 3 \quad 4.335 E-02$ $2.765 \mathrm{E}-01 \quad 2.933 \mathrm{E}$ UC 1.251E OC $1.366 \mathrm{E} \mathrm{Cl}$ $3.870 E$ GO $4.229 E$ OI $1.1 C 7 E$ O 1 1.213E C2 3.1IUE C1 3.417E O2 $7.117 E$ GL $7.838 \mathrm{E}$ C2 $1.069 \mathrm{E} \mathrm{C2}$ 
(GAMMASAMEV/STERADIAN/SGURCE NEUTRDN)

\begin{abstract}
ENERGY
GROUP ME
OCRDUP (MEVI

8.OCE OO-D-1.00E 01

6.5CE OO- - 5 . COE OO

5. OOE OO---6.50E CO

3. OOE OO---4.0OE CO

2.56 E OO- $-3.00 E$ CC

2. OOE OO---2.5OE CO

$1.66 \mathrm{E}$ OO-- $2.00 \mathrm{E}$ CO

$1.33 \mathrm{E} \mathrm{OO-D-1.66E} 00$

1. OCE OC--1.33E CO

6.OUE-O1---1.COE OO

4. 0 CE-O1-- $6.00 \mathrm{E}-01$

$3.00 E-01--4.00 E-C 1$

$2.00 \mathrm{E}-01--3.00 \mathrm{E}-01$

$1.0 \mathrm{CE}-\mathrm{C1} 1-2.00 \mathrm{E}-\mathrm{C} 1$

$5.00 E-C 2--1.00 E-01$
$2.00 E-02=-5.00 E-C 2$
\end{abstract}

ENERGY

GROUP (MEV)

8.0CE CO-D-1.00E CI 6.5OE OO---3.COE OO 5.0CE OO- -6.50 E CO 4.OCE OC-D-5. DUE OU
3.UCE OO---4. OOE CO 2.5OE OO-D. 3. OUE OO 2.COE OO- $-2.50 \mathrm{E}$ OO 1.66 E CO-D-2.00E OO $1.33 \mathrm{E}$ 00-- $1.66 \mathrm{E} 00$ 1.00 E $00-1.33 E$ OO $8.00 \mathrm{E}-\mathrm{C} 1=-1 . \mathrm{COE}$ OO $6.00 E-C 1--8.00 E-01$ 4.0CE-U1--6.00E-CI . OOE $01-2.00 E-C 1$ . COE-O1- $3.00 E-C 1$ 1.0CE-01-C 2.00E-C1 2. $00 E-C 2=-1.00 E-C 1$
$2.0 G E-02=-5.00 E-C 2$
ANGLE I $2.025 E-05$ $3.385 E-04$ $1.809 E-04$ $1.713 E-04$ $3.477 E-0.4$ $2.7 C 1 E-C 4$ $3.487 \mathrm{E}-04$ $4.716 E-04$ $2.88 C E-04$ $1.820 \mathrm{E}-04$ $4.468 \mathrm{E}-04$ $9.581 E-04$ $1.23 C E-03$ $4.173 E-03$ $8.614 E-03$ $1.691 \mathrm{E}-02$ $4.6 C 7 t-03$

ANGLE 10 $M U=0 . C 950$

$3.666 \mathrm{E}-05$ $1.641 E-04$ $5.864 \mathrm{E}-04$ $3.548 E-04$ 3. $189 \mathrm{E}-04$ $6.782 E-04$ $5.166 E-04$ $6.501 E=04$ $8.634 E-04$ $5.062 E-U 4$ 3. $556 \mathrm{E}-04$ $1.048 \mathrm{E}-\mathrm{C3}$ $1.908 \mathrm{E}-03$ $2.748 E-03$ . $1.013 E-02$ $4.850 E-C \mathrm{I}$

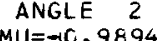
$2 . C 35 E-05$ 8.699E-O $3.4 \mathrm{C} 4 \mathrm{E}-04$ 1. $828 \mathrm{E}-04$ $1.828 E-04$ $3.5 \mathrm{C} 4 \mathrm{E}-04$ $2.721 E-04$ $3.5 \mathrm{C} 2 \mathrm{E}-\mathrm{C} 4$ 4.729E-04 $2.865 E-04$ $1.807 t-04$ 4. $542 E-04$ $9.633 \mathrm{E}-04$ $1 . \angle 31 E-03$ $4.19 C E-03$ $8.623 E-03$ $1.693 \mathrm{E}-\mathrm{C} 2$ $4.609 \mathrm{E}-\mathrm{C} 3$

ANGLE 11 $M U=C .2816$ $.734 E-G 4$ $1.734 \mathrm{E}-\mathrm{C} 4$
$7.336 \mathrm{E}-\mathrm{C} 4$ . $617 E-04$ 4.CC1E-04 $1.200 \mathrm{E}-\mathrm{C} 3$ $6.677 \mathrm{E}-\mathrm{C}$ $1.158 \mathrm{E}-\mathrm{C} 3$ $1.553 t-0 \mathrm{~J}$ $6.632 \mathrm{E}-04$ $4.496 \mathrm{E}-\mathrm{C} 4$ $1.411 E-C 3$ $2.268 \mathrm{E}-0$ $3 . C 65 E-03$ $5.414 E-03$ $1.058 E-C 2$ 4. $962 E-03$
ANGLE 3 $M U=-0.9446$ $2.077 E-05$ $3.477 \mathrm{E}-04$ $1.895 E-04$ $1.791 E-04$ $3.607 E-04$ $2.798 \mathrm{E}-04$ $3.561 E-04$ $4.784 \mathrm{E}-04$ $2.823 E-04$ 1. $77 \mathrm{CE}-04$ 4.812E-04 $9.832 \mathrm{E}-04$ $1.243 E-03$ $4.25 C E-03$ $8.658 E-03$ $1.699 \mathrm{E}-02$ 4.617E-03 $M U=-0.8650$ .297E-05 .611E-C $2.012 \mathrm{E}-\mathrm{C}$ $1.894 E-04$ $3.791 E-04$ $2.934 E-04$ $3.682 E-04$ $4.911 \mathrm{E}-\mathrm{C}$ $2.797 E-C 4$ $1.749 \mathrm{E}-\mathrm{C}_{4}$ $5.259 E-04$ $1.020 \mathrm{E}-\mathrm{C} 3$ $1.287 \mathrm{E}-03$ $4.351 E-03$ $8.729 E-C 3$ $4.632 \mathrm{E}-\mathrm{C} 3$ ANGLE 12 $M U=0.4580$ ANGLE 13 5. 543E-05 3.763E-04 8.460 E-C4 5. $400 E-04$ $5.414 E-04$ .147E-03 . 6.606E-04 6.177E-04 2.735E-O3 . 5. $.973 \mathrm{E}-02$

$4.956 E-03$ $U=C .6179$ $6.352 E-C 5$ $9.588 E-04$ $6.193 E-04$ $6.948 \mathrm{E}-04$ $1.430 \mathrm{E}-\mathrm{C} 3$ $1.231 \mathrm{E}-03$ $1.136 \mathrm{E}-0$ $1.518 \mathrm{E}-03$ $1.310 \mathrm{E}-\mathrm{C} 3$ $8.408 E-O$ $2.664 E-C 3$ $3.211 \mathrm{E}-03$ . $795 \mathrm{E}-03$ $6.277 E-C 3$ $1.168 \mathrm{E}-02$ $5.008 \mathrm{E}-\mathrm{OS}_{2}$
ANGLE 5 .847E-0 $2.156 E-04$ $2.021 E-04$ $4.036 E-04$ $3.115 E-C 4$ $3.869 E-04$ $5.135 \mathrm{E}-04$ $2.855 E-C 4$ $1.803 E-04$ . $775 E-04$ $1.071 \mathrm{E}-03$ .390E-03 . $468 E-03$ $8.838 E-C 3$ $.726 E-02$
$4.654 E-03$

$2.425 E-0$ $4.037 E-04$ $2.323 \mathrm{E} \neg 04$ $2.164 \mathrm{E}-0$ $4.343 \mathrm{E}-04$ $3.341 \mathrm{E}-\mathrm{C} 4$ $4.140 \mathrm{E}-\mathrm{C}$ $5.487 E=04$ $3.045 E-04$ $1.963 \mathrm{E}-04$ $6.315 \mathrm{E}-\mathrm{C}$ $1.144 \mathrm{E}-\mathrm{C} 3$ $1.569 \mathrm{E} \rightarrow 03$ 4. $587 \mathrm{E}-\mathrm{C} 3$ $8.990 \mathrm{E}-\mathrm{C} 3$ $1.748 E-02$ $4.682 E-03$

ANGLE 7 $M U=-0.4580$ $1.153 \mathrm{E}-\mathrm{O}$ $4.343 E-04$ $2.525 E-C 4$ $2.334 \mathrm{E}-04$ $4.738 \mathrm{E}-04$ $3.632 \mathrm{E}-04$ 4. $520 E-04$ 5.997E-04 . 221E-0 4 . $250 E-03$ .702E-C3 $9.189 E-03$ 1.774E-02 ANGLE 14 ANGLE 15

1550 MU $=0.8656$ ANGLE 16

$1.526 \mathrm{E}-\mathrm{C}$

$7.208 E \neg 04$

2.097E-03

$1.482 E-U 3$

$1.017 \mathrm{E}-03$

1.591E-03 2.962E-03

$1.214 E-03 \quad 1.876 E-03$

$1.991 E-03 \quad 2.920 E-103$ 1.68CE-O3 $3.923 E-03$ 1.408E-O3 $2.176 \mathrm{E}-03$ $1.157 E-C 3$ 1.589E-O3 $3.865 E-03$ $4.615 E-103$ $7.900 E-03$ $1.306 E-02$ $5.057 \mathrm{E}-03 \quad 5.100 \mathrm{E} \rightarrow 03$
0 $1.284 E-U 3$ $1.234 E-62 \quad 1.306 E-02$

ANGLE 8 $M U=-C .2816$ $2.204 \mathrm{E}-\mathrm{C} 5$ $4.746 E=04$ . $788 \mathrm{E}-\mathrm{C} 4$ . $554 E-C 4$ . $268 E-04$ $5.04 C E=04$ . $7.726 \mathrm{E}-04$ $1.805 E-C 2$
$4.756 E-C 3$ ANGLE 17 $M U=0.9446 \quad M U=6.9894$ $2.468 E-04 \quad 6.9 C 7 E-C 4$ 
(GAMMAS/MEVASTERADIAN/SOURCE NEUTRON)

\begin{abstract}
ENERGY GROUP (MEV). 8. OCE CO--1.00E OI . 50 E $00--8.00$ E CO 4 OCE OO- 6.500 CO $3.0 \mathrm{CE}$ 00-- 4 .OOE CO 2.0 5 CE OL2.00 UO-- $2.50 E$ OO $1.66 E$ OO-OC $2.00 E$ OC 1.33E OC-D-1.06E OL I. OCE OC-D- 1.33E CO 8.OCE-O1-D-1.0OE OC $6.0 G E-C 1--8.00 E-C$ $4.00 E-01--6.00 E-01$ 3. ULE-C1-O- 4 . COE-01 $2.0 C E-U 1--3.00 E-C 1$ $1.00 t-G 1--2.00 E-01$ 5. OCE-U2-- $1.00 E-C I$
$2.0 C E-C 2-D-5.0 O E-C 2$
\end{abstract}

$$
\text { ENERGY }
$$$$
\text { GROUP (MEV) }
$$

8. OCE OO---1.0OE G $0.50 E$ CO-D. COE OO 5. OCE UO-- 6. 50 OE OO 4. OCE $00--5.00 E 0 O$ 3. COE OO- - 4. LOE OO 2.50 E UO---3. OUE OO 2.0UE OO---2.50E OO $1.66 \mathrm{C}$ CO---2.0UE 00 $1.33 E 00--1.06 E \mathrm{CO}$ $1.0 C E$ CO---1.33E CO
$8.00 E-01--1.00 E$ OO
$0.0 C E-O 1-O-8.00 E-01$ $8.00 E-01-1 .-1.00 E$
$0.0 C E-01=-8.00 E-01$ 6. OCE E-O1--8.00E-O1
4.00E-U1---6.00E-01 $4.00 E-U 1---6.00 E-01$
$3.00 E-C 1---4.00 E-01$ 3.00E-C1---4.00E-01
$2.00 E-01-O-3.0 C E-01$ 1.00E-01-D-2.00E-0 5.0CE-C2-D-1.0OE-C 2. OOE-O2--5. OUE-C2

ANGLE 1 $M U=-1.0000$ $2.819 \mathrm{E}-05$ $.047 E-C 4$ $5.397 \mathrm{E}-04$ $2.306 E-04$ $2.624 \mathrm{E}-04$ $.909 \mathrm{E}-\mathrm{C}$ $3.434 E-04$ $5.8 U 1 E-04$ $4.0 U 15 E-04$ $3.046 \mathrm{E}-04$ $5.402 \mathrm{E}-04$ $1.803 E-03$ $2.396 \mathrm{E}-03$ $8.990 E-03$ $2.212 E-02$ $5.068 \mathrm{E}-02$ $1.450 E-02$

ANGLE 10 $M U=0.0950$
$3.745 E-C 5$ S41E-C $2.641 E-C 4$
$7.968 E-04$ . $4.277 E-04$ 1. $.052 E-03$ $4.882 E-04$ $4.882 E-04$ 1. $369 \mathrm{E}-\mathrm{C} 3$ $5.274 E-O 4$ $6.639 \mathrm{E}-\mathrm{U}$ $1.134 \mathrm{E}-03$ $3.999 \mathrm{E}-\mathrm{C}$ $6.227 E-0$ $1.107 E-02$ 2.727E-O2 1.55 E-CL $\triangle N G L E 2$ $M U=-C .9894$ $1.056 \mathrm{E}-04$ $5.438 E-04$ $2.347 E-04$ $2.664 \mathrm{E}-\mathrm{C}$ $3.958 \mathrm{E}-04$ $3.470 \mathrm{E}-04$ $4.285 \mathrm{E}-04$ $5.798 E-04$ 3. $964 E-04$ $3.018 \mathrm{E}-04$ $5.562 E-C 4$ $1.815 \mathrm{E}-03$ $2.4 C 1 E-C S$ $9.029 \mathrm{E}-03$ $2.215 \mathrm{E}-\mathrm{C} 2$ $5.074 E-02$ $1.451 E-U 2$

ANGLE 11 $\begin{aligned} M U & =656 \mathrm{E}-05\end{aligned}$ $2.242 \mathrm{E}-\mathrm{C}$ $1.458 E-03$ $8.229 E-C$ $7.943 E-04$ $8.912 E-C$ $1.157 E-03$ 9.C12E-04 $1.174 E-03$ $1.215 \mathrm{E}-03$ $9.966 E-04$ $2.7 C 6 E-03$ $5.113 E-03$ $7.127 E-03$ $1.2 C O E-O 2$ $2.881 E-C$ $1.584 E-02$
ANuLE 3 $2.911 \mathrm{E}-05$ 1.090 E-04 $5.591 E-04$ 2. BICE-O $4.136 \mathrm{E}-04$ $3.605 E-04$ 4. $348 \mathrm{E}-04$ $5.811 \mathrm{E}-04$ 3. $806 \mathrm{E}-04$ 2. $947 E-04$ $6.136 t-04$ $1.859 \mathrm{E}-03$ $2.439 E-03$ $9.172 \mathrm{E}-03$ $2.228 \mathrm{E}-02$ $5.099 E-02$ $1.454 E-02$

ANGLE 12 ANGLE 13 $M U=0.4580$ $.12 L E-05$
$3.785 E-04$ 1. $185 \mathrm{E}-03$ $5.807 E-04$ $6.657 E-04$ $1.516 \mathrm{E}-03$ $8.718 \mathrm{E}-0$ $1.495 \mathrm{E}-03$
$1.971 \mathrm{E}-03$ $1.073 \mathrm{E}-03$
$1.338 \mathrm{E}-03$ $1.338 \mathrm{E}-03$ $2.416 E-03$ 3. $993 E-03$ $7.796 E-03$ 1. 3. $054 \mathrm{E}-02$ 6. $326 E-02$
$1.6 C 9 E-O D$$$
\text { ANGLE }
$$
$=-C .8650$ . $152 t-04$ $5.152 E-C 4$ $2.732 E-04$ $3.045 E-04$ $4.434 \mathrm{E}-\mathrm{C} 4$ 3. $833 \mathrm{E}-\mathrm{CC}_{4}$ $4.485 E-C 4$ $5.894 E-G 4$ $3.047 E-C_{4}$ 2. $924 E-C 4$ $7.035=-04$ $1.94 \mathrm{CE}-03$ $2.571 E-C$ $9.407 E-C 3$ $2.253 t-C 2$ $5.146 E-O 2$ $1.461 E-C 2$

ANGLE $M U=-C .7550$ $1.233 E-04$ $6.225 \mathrm{E}-04$ $6.225 \mathrm{E}-04$ $3.305 \mathrm{E}-0$ $4.789 \mathrm{E}-\mathrm{C} 4$ $4.112 E-C$ $4.717 E-04$ $6.131 \mathrm{E}-\mathrm{C} 4$ $3.678 E-C 4$ 3. $C 83 E-C 4$ $7.965 t-04$ $2.053 \mathrm{E}-\mathrm{O}$ $2.864 t-C$ $9.670 \mathrm{E}-\mathrm{a}$ $2.291 \mathrm{E}-02$ $5.216 E-02$
$1.47 C E-C 2$ $M U=-0.6179$ $1.333 E-0$ $6.668 \mathrm{E}-0$ $3.251 \mathrm{E}-04$ 3. $567 E-04$ $5.190 E-04$ 4. $440 E-04$ 5. $440 E=04$ $6.591 E-04$ $4.005 E-04$ .477E-04 作

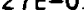
. $343 E-02$ $1.483 \mathrm{E}-02$ ANGLE 14

ANGLE 7 $M U=-0.4580$ $3.796 E-05$ $1.46 \mathrm{CE}-\mathrm{U} 4$ $3.225 E-C 4$ $3.859 E-04$ $5.682 E-04$ $4.857 E-04$ $5.610 E-C 4$ $7.311 E-04$ $4.615 E-C 4$ $4.072 E-04$ $2.471 E-03$ $1.498 E-02$

ANGLE 15 $274 \mathrm{E}-04$ 2.069E-C $1.211 E-0$ $1.104 t-0 \mathrm{~J}$ $1.558 E-03$ $1.676 E-C$ $1.709 E-C 3$ $2.273 E-C$ . प. 4. . $2 U 5 E-O S$ 1. $1.394 \varepsilon-02$ 3.260 E-O2 $1.635 \mathrm{t}-\mathrm{C2} 2$
$M U=0.7550$ $6.453 E-04$ $2.120 \mathrm{E}-\mathrm{C}$ 1. $207 \mathrm{E}-\mathrm{C}$ $1.273 \mathrm{E}-\mathrm{U}$ $2.802 \mathrm{E}-\mathrm{C}$ $1.998 \mathrm{E}-\mathrm{C}$ $2.685 E-U 3$ 2.714E-O 2.838E-O . $644 E-O 3$ 1. . $6.795 E-G 2$ $1.66 \mathrm{Ct}-02$ ANGLE 16 $.334 E-04$ .056E-U3 . $542 E-03$

ANGLE 8 $M U=-C .2810$ 7. $959 E-C 4$ 3.911E-O 淿 $5.436 \mathrm{E}-\mathrm{C}_{4}$ $6.351 E-C$ $8.308 E-C 4$ $4.815 E-C 4$ $4.786 \mathrm{E}-\mathrm{C}$ $1.043 \mathrm{E}-\mathrm{C}$ 
(GAMMAS/MEV/STERADIAN/SOURCE NEUTRON)

ENERGY GROUP IMEVI 6.00E COW-1.00E O1 $6.50 \mathrm{E} O 0-8.00 \mathrm{E}$ OO 5. 00 EO-- $6.50 E$ OO 4.0OE OO--5.00E CO 3. OCE OC- - 4. OOE OO 2.5OE OO-- -3.00E CO $2.00 E$ OO---2.50E OO 1.66E 00--D2. COE CO $1.33 \mathrm{E} 00--1.66 \mathrm{E}$ OO $1.00 \mathrm{E} 00=1.33 \mathrm{E}$ CO 8.00E-01---1.0CE CO $6.00 E-C 1---8.00 E-01$ 4.00E-01-- - $0.00 E-01$ $2.00 \mathrm{E} \rightarrow 01--3.00 \mathrm{E}-01$ 1. OOE-O1--2. UOE-01 $5.00 E-02--1.00 E-01$ 2.0OE $-02--5.00 E-C 2$

\section{ENERGY \\ GROUP (MEV)}

8.00E 00--1.00E O1 $6.50 E$ CO-- -8. OCE OO
$5.00 E$ OO-O-6.5CE OO

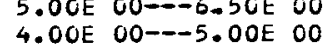
$4.00 E$ OO- $-5.00 E$
$3.00 E$ OO $2.50 E$ CO- $3.00 E$ OO 2.00 CO-- 2.5OE CO $2.00 E$ CO---2.5OE CO
$1.66 E$ OO-D-2.0CE OO $1.66 E$ OO-D-2. OCE OO $1.0 C E$ OO- $1.33 E 00$

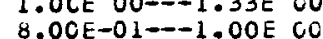
$6.00 E-01--8.00 E-01$ $4.00 E-01=0.00 E-01$ $3.00 E-01=-4.00 E-63$ $2.00 E-01-\cdots 3.00 E-C 1$ 1.0CE-O1- $-2.00 \mathrm{E}-01$ $2.00 E-02--5.00 E-02$
ANGLE 1 $M U=-1.00 C 0$ $2.971 \mathrm{E}-05$ (.) . . $736 E-04$ . $290 E-04$ $4.274 \mathrm{E}-04$ $3.365 \mathrm{E}-04$ $5.296 \mathrm{E}-04$ $2.150 E-03$ $2.9 \mathrm{C} 9 \mathrm{E}-03$ $1.152 \mathrm{E}-02$ $2.996 \mathrm{E}-02$ $7.336 \mathrm{E}-02$ $2.144 \mathrm{E}-02$ ANGLE 10 $M U=0.0950$ $7.127 E-05$ $2.084 E-04$ $1.325 \mathrm{E}-03$ $0.632 E-04$ $7.168 E-04$ $7.886 E-04$ . $221 E-04$ $8.187 E-04$ 1.0 . 2.197E-03 $5.395 E-03$ 5.317E-03 $1.447 E-02$ $3.769 E-02$ $8.711 \mathrm{E}-02$ 2. $324 E-02$
ANGLE 2 2.994E-05 $1.047 \mathrm{E}-04$ $6.014 E-04$ $2.371 E-04$ $2.893 E-04$ $3.786 E-04$ $3.518 E-04$ $4.301 E-04$ $5.847 E-C_{4}$ $4.198 E-04$ $3.334 E-04$ 5. 5C 3E- 04 $2.165 \mathrm{E}-03$ $2.917 E-03$ $.157 \mathrm{E}-02$ $3.001 \mathrm{E}-02$ $.346 \mathrm{E}-02$ $2.145 \mathrm{E}-\mathrm{C} 2$ ANGLE 11 $M U=0.2816$ $6.675 \mathrm{E}-05$ $3.021 E-04$ $1.327 E-03$ . $19 C E-04$ $1.247 E-04$ $1.174 E+03$ $1.200 \mathrm{E}-\mathrm{O}$ 1. $200 E-03$ $1.563 E-03$ . $03 E-04$ 1.1S6E 03 . 9.144E-O $1.518 \mathrm{E}-02$ $3.992 E-02$ $9.051 E-C 2$ $2.365 E-C 2$ $M U=-0.9446$ $3.082 E-05$ 1. $089 \mathrm{E}-04$ $6.212 \mathrm{E}-04$ $2.565 \mathrm{E}-04$ 3. $C 90 E-04$ $4.002 E-04$ $3.679 E-04$ 4. $352 E-04$ 5. $809 \mathrm{E}-04$ 3. $962 \mathrm{E}-04$ $3.254 \mathrm{E}-04$ $6.245 \mathrm{E}-04$ $2.223 E-03$ $2.978 \mathrm{E}-03$ $1.176 \mathrm{E}-02$ $3 . \mathrm{C} 21 \mathrm{E}-02$

ANGLE 12 $M U=0.4580$ $1.014 \mathrm{E}-04$ $3.402 E-04$ $1.849 E-03$ . 1.015E-0 1. $313 E-03$ $1.33 \mathrm{E}-03$ $1.799 \mathrm{E}-03$ $1.79 E-03$ 1. $798 \mathrm{E}-03$ 1.79E-03 7. $1.023 \mathrm{E}-0$ $1.651 \mathrm{E}-02$ $4.255 \mathrm{E}-02$ $9.422 \mathrm{E}-02$

$2.408 E-02$
ANGLE 4 $M U=-0.8656$ $.242 E-C 5$ .16UE-04 . . .351E-0 4 . $942 E-04$ $4.473 E-04$ . $830 E-04$ $3.715 E-C 4$ $3.248 E-04$ $7.3 .79 E-04$ $2.327 E-C 3$ $3.175 E-C 3$ $1.205 \mathrm{E}-02$ $3.059 \mathrm{E}-\mathrm{O} 2$ $.465 E-C 2$ $2.162 E-02$ ANGLE 13 $M U=0.6179$
$1.148 E-C 4$ $1.148 E-C 4$ $4.916 E-04$ 2.110E-C 1.0TIE-OS 1.168E-03 1. 1.555E-CB 2.04OE 03 2.706E-C 2. $509 E-C 3$ 4.564E-O3 $9.307 E-03$ $1.139 E-G 2$ $1.792 E-C 2$ $4.554 \mathrm{E}-\mathrm{C} 2$ $9.814 \mathrm{E}-\mathrm{C}$ $2.451 E-C 2$
ANGLE 5 $M U=-0.755 C$ $1.250 E-04$ $7.003 E-04$ $3.182 E-04$ $4.725 E-04$ $4.250 E-04$ . $3.482 E-04$ $2.476 E-03$ $3.599 \mathrm{E}-03$ $1.238 \mathrm{E}-\mathrm{C2} 2$ $3.116 \mathrm{E}-02$ 2.177E-C2

ANGLE 14 $A N=0.7550$
$1.723 E-64$ $6.627 E-C_{4}$ $2.956 E-03$ $1.630 E-03$ $1.671 E-03$ $2.626 E-C 3$ . $2.858 E-03$ 3.79 $3.805 E-C 3$ $6.480 E-03$ $1.485 E-03$ $1.3 C \mathrm{E}-02$ $1.996 E-02$ $4.890 E-02$ 1.C21t-0

$2.492 \mathrm{E}-\mathrm{O} 2$
ANGLE 6 $M U=-0.6179$ $3.740 E-05$ $1.357 E-04$ 7. $541 E-04$ $3.471 E-04$ $4.038 E-04$ $5.150 E-C 4$ $4.600 E-04$ $6.526 E-04$ 4.133Eन $4.013 E-04$ $9.451 E=04$ $2.697 \mathrm{E} \neg 03$ $4.290 E-C 3$ $1.270 \mathrm{E}-0$ $3.194 \mathrm{E} \sim 02$ $2.197 E-02$

ANGLE 15 $M U=0.8656$ $2.472 E-04$ $1.103 E-C 3$ $4.041 E-03$ $2.396 E-03$ 2. $379 E-03$ 4. $4.73 \cup E=103$ . $215 E-03$ 6. 5.293E-O $8.338 \mathrm{E}-10$ 1. $336 \mathrm{E}-02$ $1.504 E-0$ $5.255 \mathrm{E}-\mathrm{C} 2$ $1.059 \mathrm{E}-0$ $2.528 \mathrm{E}-\mathrm{C}$

ANGLE 7 ANGLE 8 ANGLE 9 $M U=-C .4580$ $4.105 \mathrm{E}-65$ 5.641E-04 .662E-04 $4.788 \mathrm{E}-04$ $1.041 E-03$ 
(GAMMAS/MEK/STEKAUIAN/SOURCE NEUTRON)

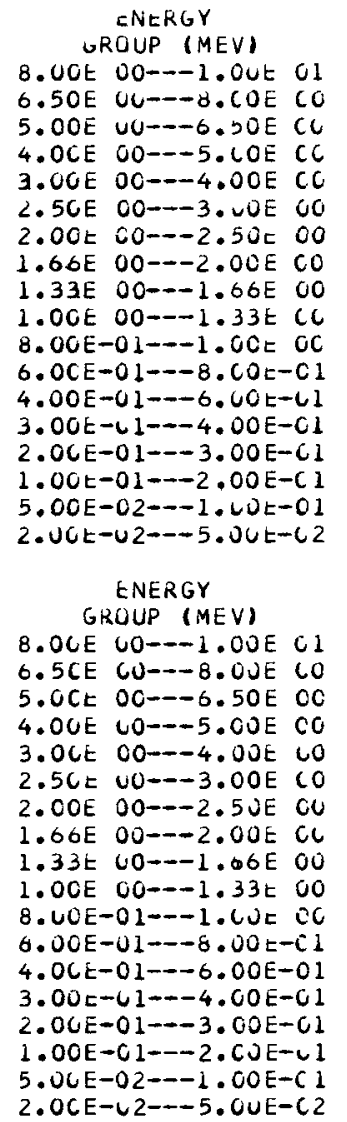
$M=-1 . \angle C O O$ $2.045 E-05$
$8.515 E-05$ $5.594 t-04$ $1.880 \mathrm{E}-04$ $2.547 E-04$ $2.933 E-64$ $3.037 E-04$ $3.782 \mathrm{E}-04$ $5.257 E-04$ $4.085 \mathrm{E}-04$ $3.191 \mathrm{E}-04$ $4.188 \mathrm{E}-0$ $2.386 \mathrm{E}-0 \mathrm{~S}$ $3.299 \mathrm{E}-0 \mathrm{~S}$ $1.461 \mathrm{E}-\mathrm{J} 2$ $4.016 \mathrm{E}-0$ $1.076 E-01$ ANGLE 10 $M U=0.1950$ $5.950 E-05$ C.139E-04 $5.401 E-04$ G. $721 E=04$ $0.721 E-04$ . $7.327 t-04$ $1.122 E-03$ 1. $122 \mathrm{E}-03$ 1.049E-03 $1.049 E-03$ $6.765 E-03$ $1.001 \mathrm{E}-\mathrm{C}$ $1.797 E-02$ $5.180 E-O 2$ $1.310 \mathrm{E}-0$ 3.539t-a
ANGLE 2 $M U=-C .9894$ $2.672 E-C 5$
$8.647 E-C 5$ $5.606 E-04$ $1.955 c-04$ $2.623 E-04$ 3.CC5E-O4 3. C85E- 04 $2.778 E-C 4$ $5.205 E-04$ 3. $971 E-04$ $3.162 E-C 4$ $4.458 E-04$ $2.403 t-03$ $3.318 \mathrm{E}-03$ $1.467 E-C 2$ $4.024 E-C 2$ $3.234 E-C 2$

ANGLE 11 $M U=C .2816$ $7.804 E-C 5$ $2.59 \mathrm{E}-\mathrm{C} 4$ $7.519 E-04$ $7.219 E-\mathrm{CH}_{4}$ $9.853 E-C 4$ $9.8 C 3 E-04$ 1.65 1. $1.2<1 \mathrm{E}-03$ $1.543 \mathrm{t}-03$ $3.147 E-03$ $1.176 E-C 2$ $1.919 E-02$ $5.515 t-02$ $1.369 \mathrm{E}-\mathrm{Cl}$ $3.609 E-C<$
ANGLE 3 $M U=-0.9440$ $9.132 E-05$ 5.9C5E-0 4 $2.217 E-04$ $2.89 C E-G 4$ $3.263 E-04$ 3. $26 C E-04$ $3.777 E-04$ 5. $648 E-04$ $3.609 E-04$ 3. $100 \mathrm{E}-\mathrm{C} 4$ $5.423 t-04$ $2.474 \mathrm{E}-03$ $3.430 E-03$ $1.489 E-02$ $4.054 \mathrm{E}-02$ 3. $244 \mathrm{E}-\mathrm{C} 2$ ANGLE 12 $M U=0.458 \mathrm{C}$ $3.093 E-05$ 1. $941 E-03$ $8.918 E-04$ l. $1.318 \mathrm{E}-03$ $1.318 E-03$ $1.231 E-03$ $1.446 \mathrm{E}-03$ $1.736 E-C 3$ $2.337 E-03$ 2. 3 1. C11E-C2 $1.297 \mathrm{E}-02$ 2. CTCE-O2 5. $50<\mathrm{E}-02$ $1.433 \mathrm{E}-01$ $3.681 E-U 2$
ANGLE 4 $M U=-0.8656$ $2.952 E-05$
$9.927 E-05$ $6.319 E-04$ $2.6065-64$ $3.292 \mathrm{E}-04$ $3.655 E-04$ $3.533 E-64$ $3.824 E-C 4$ 4.911E-C 4 $3.220 E-04$ $3.150 E-C 4$ $0.837 E-04$ $2.602 \mathrm{E}-\mathrm{CH}$ $3.750 E-63$ $1.524 E-C 2$ $4.113 \mathrm{E}-\mathrm{C2}$ $3.262 \mathrm{E}-0$ $M U=0.6179$ $1.332 \mathrm{E}-\mathrm{C} 4$ $4.647 t-64$ $1.22 U E-C 3$ $1.774 E-C 3$ $1.774 \mathrm{E}-\mathrm{C3}$ $1.753 E-03$ 2.7CE-CB 2.7C1E-OB $3.553 \mathrm{E}-\mathrm{OS}$ $5.9 \angle 4 E-03$ $1.20 C E-C 2$ $1.454 \mathrm{E}-0 \mathrm{C}$ $0.341 t-0$ $1.5 \mathrm{C}<\mathrm{E}-\mathrm{Cl}$ $3.754 E-C 2$
ANGLE 5 $U=-C .7550$ 1. $182 E-05$ $6.821 E-04$ $6.821 E-04$ $3.679 E-04$ $4.044 E-U 4$ $3.831 E-04$ $3.992 E-04$ $5.018 E-04$ $3.194 E-04$ $3.493 E-04$ $8.118 E-U 4$ $2.793 E-03$ $4.393 E-03$ $1.561 E-C 2$ $4.200 E-C 2$ $1.117 E-01$ $M U=C .7550$ $1.856 t-04$ $0.86 \mathrm{CE}-\mathrm{C} 4$ $1.717 \mathrm{E}-\mathrm{C} 3$ $1.717 E-C 3$ $1.97 \mathrm{E}-03$ $2.586 \mathrm{E}-\mathrm{C3}$ $3.152 \mathrm{E}-03$ $4.183 E-C 3$ $4.222 \mathrm{E}-03$ $5.222 E-03$ $7.697 t-C 3$ 1.44 t $-\mathrm{C} 2$ $2.501 E-62$ $6.0<4 E-C 2$ $6.0<4 E-C 2$
$1.570 E-01$ $3.023 r-u 2$ . $1.183 \mathrm{E}-\mathrm{C} 4$ $7.401 E-C 4$ $3.247 E-04$ $4.004 E=04$ $4.400 E-04$ $4.351 E-C 4$ 5.5CIE-O4 $3.695 E-C 4$ $4.171 E-04$ 9.09 $3.094 E-03$ . 1. l. $142 E$ E $3.323 E-02$ ANGLE 15 $M U=6.8656$ $2.888 \mathrm{E}-04$
$1.115 \mathrm{E}-03$ 1. $115 E-03$ $2.706 E-03$ 2.706E-03 $4.037 E-03$ $4.43 E-03$ $5.155 E-C 3$ $6.690 E-103$ $6.523 E-C 3$ OCGE-C2 $1.673 t-02$ $1.906 \mathrm{E}-02$ 2.787E-G2 $7.334 E-02$ $1.630 E-C 1$ , $.884 E-G 2$
ANGLE 7 $M U=-U .4580$ $3.835 E-C 5$ $8.126 \mathrm{E}-04$ $3.504 t-C 4$ 4. $337 E-04$ $4.807 E-04$ $4.582 E-04$ $6.369 E-04$ $4.639 E-04$ $5.109 E-04$ 1. $106 \mathrm{t}-03$ $3.582 E=03$ $1.687 \mathrm{E}=02$ $1.627 E-02$ $1.174 E-01$ $3.365 \mathrm{E}-\mathrm{UL} 2$

ANGLE 16 $M U=0.9446$ $5.299 E-04$ $2.245 E-C 3$ $8.420 E-03$ $5.207 E-03$ $8.699 \mathrm{E}-03$ $7.65 E-03$ . 1.170E-02 $1.014 \mathrm{E}-\mathrm{C} 2$ $9.651 \mathrm{E}-\mathrm{CS}$ $1.042 \mathrm{E}-\mathrm{C} 2$ $2.200 \mathrm{E}-\mathrm{C} 2$ $3.131 \mathrm{E}-02$ $7.840 \mathrm{E}-\mathrm{LS}$ $1.69<E-01$ $3.9315-C<$ $4=-C .2510$ 1. $C 57 E-03$ $4.886 E-C 4 \quad 4.547 E-C 4$ $5.4<4 E-C 4 \quad 6.413 E-04$ $5 . \angle \angle 9 E-C 4 \quad 6.212 E-C 4$ 7. $5.8 C 5 E-C 4 \quad 3 . C C 6 E-C 4$ $6.267 E-C 4 \quad 7.867 E-C 4$ $1.18 C E-C 3 \quad 1.533 E-01$ $4.338 E-C 3 \quad 5.410 E-C 3$ $1.084 E-C 3$. $425 E-03$ $1.664 E-C_{2} \quad 1.718 E-02$ $1.6312 \mathrm{CL}$ $3.416 E-C<\quad 3.474 E-02$

ANGLE 17 $M U=C .9894$ $1.597 t-C 3$
$7.223 E-C 3$ $2.1 \mathrm{CSE}-\mathrm{C} \angle$ $1.455 E-6 \angle$ $2.442 \mathrm{E}=02$ $1.06 \mathrm{E}=02$ 2. 
(GAMMASAMEV/STERADIAN/SOURCE NEUTRON)

ENERGY GROUP (MEVI 8.00E OO-- 1.00E O1 6.5 UE OO-D-8.00E OO 5.00E DOT$4.00 E$ OO- $-5.00 E$ OO 2.50E $00--3.00 E C O$ $2.00 E$ OOM- $\angle .50 E$ CO $1.66 \mathrm{E} 00---2.00 \mathrm{E} C O$ $1.33 \mathrm{E} \mathrm{VO}=-1.66 \mathrm{E} \mathrm{OO}$ 1.00 E $00---1.33 E \mathrm{CO}$ $8.00 \mathrm{E}-01-2-1.00 \mathrm{E} C$ $6.0 C E-01--8.00 E-C$ $4.00 \mathrm{E}-01---6.00 \mathrm{E}-0$ $3.00 E-01=-4.00 E-01$ $2.00 E-C 1---3.00 E-0$ $1.00 E-01=-2.00 E-C$ $5.00 E-02=-1.00 E-01$ $2.00 E-C 2--5.00 E-02$ ENERGY GROUP (MEV) 8. OCE OO- 1. 00 E 01 6.5OE COD-8.00E CO 5.00 E $00--6.50 E$ OO $4.00 E$ OO-D. $500 E$ OC $3.00 E$ OON- 4.00E OO $2.5 C E$ OOD- $3.00 E$ OC $2.00 E$ EO-D2.50E CO $1.66 E$ OC-C-2.00E OC $1.33 E$ EO- $1.06 E$ OC 1.00 E O0-D- $1.33 E$ CO $8.0 C E-01--1.00 E$ OO 6.00E-U1--0.0UE-U1 4.0CE-O1C- $6.00 E-O 1$ $3.00 E-01-4.00 E-O 1$ $1.00 E-01=-2.00 E-01$ $1.00 E-01=-2.00 E-01$ 1.00E-02- $-1.00 E-01$
ANGLE 1 ANGLE 2 $M U=-1 . C 0 O C \quad M U=-6.9894$ $1.992 \mathrm{E}-05$ $0.076 E-65$ $1.247 E-04$ ..847E-04 $2.389 E-C 4$ $4.352 E-04$ $3.504 E-04$ . $2.736 E-04$ $2.238 \mathrm{E}-03$ 1.124E-03 $1.546 E-C$ $3.759 E-02$ ANGLE 10 $M U=0 . C 950$ $5.050 \mathrm{E}-05$ $1.654 \mathrm{E}-0$ $1.074 E-03$ $4.648 \mathrm{E}-04$ $5.896 \mathrm{E}-04$ 6. $318 E-04$ $6.458 E-G$ $7.014 E-04$ $8.992 E-04$ I. $042 E=03$ $1.042 E-03$ $2.151 \varepsilon-03$ $1.124 E=02$ $1.124 E-0.2$ $5.679 E-02$ . $679 E-02$ $4.137 \mathrm{E}-\mathrm{C} 2$ $\triangle A N G L E \quad 3$ 2.11SE-05 $6.712 E-05$ 4. $656 E-C 4$ $1.637 E-04$ 2. $24 C E-04$ $2.411 E-04$ 2. $588 E-04$ $2.981 E-04$ $3.997 t-04$ 2.923E-04 $2.490 E-04$ 4. $109 t-C 4$ 2. $320 E-03$ 3. $3 C 3 E-03$ 1. $574 \mathrm{E}-02$ 4. $384 E-C 2$ 1. $241 E-01$ $3.773 \hat{E}-02$ ANGLE 12 $M U=U .458 \mathrm{C}$ 8.601E-05 $2.850 E-04$ $1.707 \mathrm{E}-03$ $7.976 \mathrm{E}-04$ $9.895 E-04$ $1.093 E-03$ $1.116 E-03$ 1. $262 E-G 3$ $1.666 E-03$ $1.765 E-03$ $2.565 E-03$ $4.611 E-03$ $1.075 E-02$ $2.153 E-02$ $2.153 E-02$ .. $4950-02$ $4.312 E-02$

ANGLE
$M U=-C .8656$
$2.280 E-C 5$
$7.485 E-05$
$5.065 E-C 4$
$2.064 E-04$
$2.076 E-04$
$2.798 E-04$
$2.822 E-04$
$2.933 E-C 4$
$3.710 E-C 4$
$2.442 E-C 4$
$2.600 E-04$
$5.612 E-C 4$
$2.445 E-03$
$3.710 E-03$
$1.608 E-02$
$4.453 E-02$
$1.257 E-01$
$3.796 E-02$
ANGLE 13
$M U=0.0179$
$1.2 C 0 E-04$
$4.013 E-04$
$2.397 E-03$
$1.095 E-03$
$1.351 E-03$
$1.555 E-03$
$1.6 C 9 E-03$
$1.905 E-03$
$2.559 E-03$
$2.864 E-C 3$
$3.964 E-03$
$6.262 E-C 3$
$1.263 E-02$
$1.515 E-02$
$2.353 E-C 2$
$6.991 E-02$
$1.760 E-01$
$4.402 E-C 2$

4

列

5 $=-0.7550$ $.488 E-05$ .289E-05 5 $.418 E-04$ .053E-C4 . $240 E-C 4$ 3.C1 8E-04 3. $710 \mathrm{E}-\mathrm{C} 4$ $2.381 E-C 4$ $2.985 \mathrm{E}-04$ $6.853 E-C 4$ $2.644 E-C 3$ $4.485 \mathrm{E}-03$ $.641 E-C 2$ $4.553 E-02$ $1.281 E-01$ $.828 E-C 2$ ANGLE 14 $M U=0.7550$ $1.784 E-C 4$
$6.094 E-04$ $0.094 \mathrm{E}-04$
$3.436 \mathrm{E}-\mathrm{Cl}$ $3.436 E-13$ $1.642 E-03$ $1.998 E-U 3$ $2.423 E-03$ $.537 E-03$ .065E-0 . 4.55 . - $156 \mathrm{E}-03$ $1.470 \mathrm{E}-02$ $1.724 \mathrm{E}-02$ $2.589 \mathrm{E}-\mathrm{C2}$ $.524 E-02$ $4.485 \mathrm{E}-02$

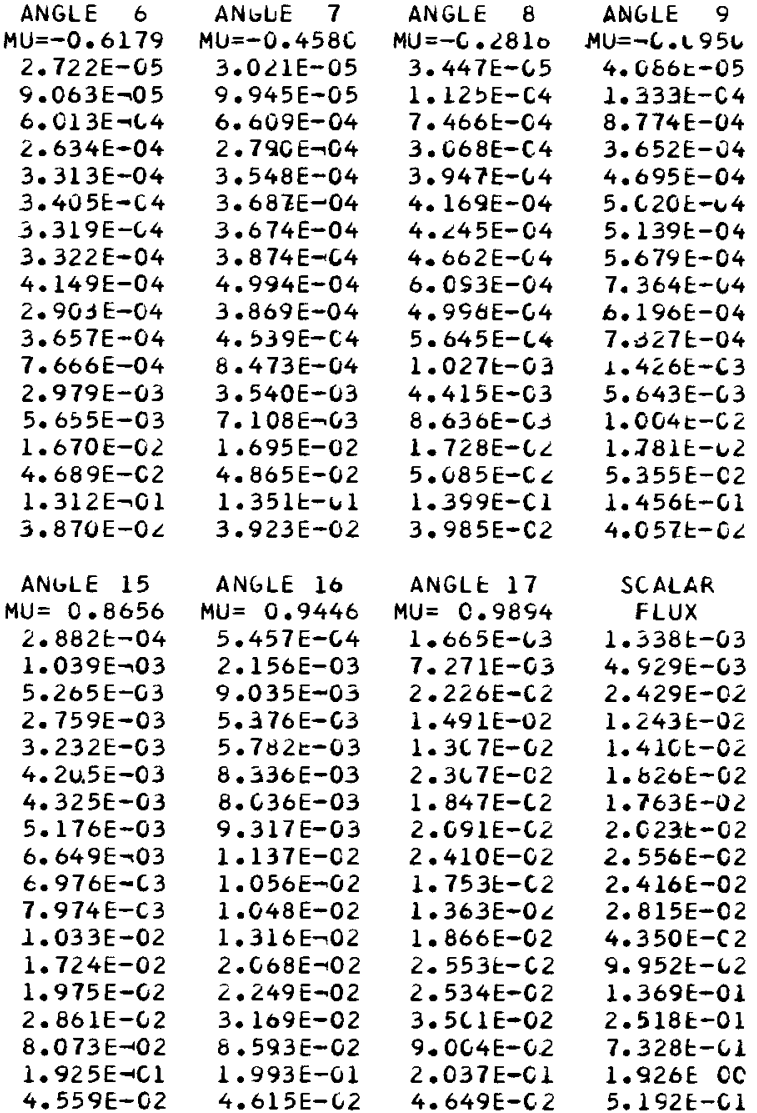


(GAMMAS/MEV/STERADIAN/SOUKCE NEUTRUN) ENERGY 8.00E OC-D-1.00E O1 $6.5 \mathrm{CE}$ CO-- -8.COE CO 5. OCE OO- - 6.5OE CO 4. $\mathrm{COE} 00--5.00 E \mathrm{CO}$ 3. COE VO-- -4.0OE CO 2.50 E $00--3.00$ E CO $2.00 E$ OO-D-2.5OE CO $1.66 E$ OO- 2 . COE CO $1.33 E$ OC-D-1.6OE 20 $1.00 E$ UOSO-1.33E CC $1.30 E$ OO 6.0CE-01-- -8.00E-C1 4. OCE-C1---6.0OE-C2 $3.00 E-61---4.00 E-C 1$
$2.00 E-C 1=-3.00 E-01$ $1.00 E-01---2$. . OOE -01 $5.00 E-02-\cdots 1.00 t-01$ 2. OLE-C2--D. OUE-C 2 ENERGY GROUP IMEVI $0.00 E$ UC- $-1.00 E$ CI $6.5 C E$ CO-- - 8. OCE CO 5.0CE OO-O-6.5CE LC 4. UCE OC---5. JOE CO 3.OCE OC--4. OUE OO 2.5CE OC- $3.00 E$ UO $2.00 E$ UC-D-2.5UE CO 1.66 E OO-D-2.COE CC $1.33 E$ 00-- $1.66 E$ OC 1.00 EO . COE-UI6. OOE O1 $4.00 E-61-6.00 E-61$ 2.0OE-OI- $3.00 E-01$ 2.0CE-O1- 3 . OCE $5.00 E-U 2=1.00 E-01$ 5.
$2.00 E-02--5.00 E-C 2$.
ANGLE 1 $1.373 \mathrm{E}-\mathrm{C} 5$ $3.975 E-05$ $3.044 E-04$ $2.418 E-04$ $3.497 E-04$ $2.871 t-04$ $1.802 E-04$ $1.455 E-C 4$ $1.932 E T O 3$ $2.694 \mathrm{E}-03$ $.484 \mathrm{~L}-0.2$ 1. $179 E-02$ $3.797 E-6.2$ ANGLE 10 $M U=0.0950$ $3.752 \mathrm{E}-05$ $1.209 E-04$ $8.160 E-04$ $3.531 E-04$ $4.556 E-04$ $4.784 E-04$ $5.043 E-04$ $0.428 E-04$ $0.938 E-04$ $9.460 E-04$ 1. $984 \mathrm{E}-03$ 1.984E-03 $1.069 E-02$ $1.045 E-02$ $5.513 \mathrm{E}-02$ 1.535t-01 $4.191 E-02$
$A N G L E{ }^{3}$
$M U=-0.9446$ $1.399 E-05$ 4.110E-C5 $3.11<E-04$ $7.815 E-05$ $1.256 E=04$ $1.423 t-C 4$ $1.819 \mathrm{E}-04$ $2.378 E-C 4$ $3.388 E-04$ $2.7<2 E-C 4$ $1.799 E-C 4$ $1.771 \mathrm{E}-\mathrm{C} 4$ $1.945 \mathrm{E}-03$ $2.727 E-03$ $1.489 E-02$ $4.187 E-02$ $1.233 E-C 1$ 3. 8 COE-G 2 ANGLE 11 $M U=C .2816$
$4.913 E-O D$ $4.913 E-C D$
$1.593 E-C 4$ $1.553 E-C 4$
$1 . C 53 E-C 3$ $4.730 t-04$ 6. CC1E-O4 $6.316 E-04$ 6. . $143 E-04$ $9.704 E-04$ . $704 E-04$ 1.5 . . $1.863 E-G$ $5.889 E-O 2$ $1.612 E-01$ 4. $28 \mathrm{CE}-02$ $1.491 E-05$ . $591 E=05$ . $156 E-04$ $.103 E-04$ . $685 \mathrm{E}-04$ . $685 E-04$ $.25 C E-04$ $3.031 E-04$ 2.23 YE-04 1. $825 E-04$ 2. $869 E-04$ $1.998 E-03$ C. $901 E-03$ 1. 5U9E-02 $4.224 E-02$ $1.242 E-01$ 3. $813 E-02$ ANGLE 12 $M U=0.4580$
$6.724 E-05$ $2.175 E-04$ $1.4 \mathrm{CTE}-03$ C. $354 E-04$ $8.021 E-04$ $8.6 C 0 E-04$ $9.138 E-04$ 1.038E-03 1. $1.630 E-03$ 2. 1. C2OE-O2 $1.272 E-02$ 1.272E-02 $0.32 C E-02$ $1.696 \mathrm{E}-01$ 4. $373 E-02$
$\begin{array}{ll}A N G L E & 4 \\ M U=-0.8656 & \end{array}$ $1.639 E-C 5$
$5.3 C 3 E-C 5$ $5.3 C 3 E-C 5$ $3.730 \mathrm{E}-\mathrm{O} 4$ $1.535 E-64$ $2.013 E-C 4$ . $045 E-04$ $2.120 E-04$ $2.115 t-04$ $2.628 \mathrm{E}-\mathrm{C} 4$ 1.711E-04 $1.985 E-C 4$ $4.345 E-C 4$ $2.103 E-03$ $3.346 E-C 3$ $1.538 \mathrm{E}-\mathrm{C} 2$ $4.293 t-C L$ $3.836 E-C 2$ ANGLE 13 $M U=0.6179$ $9.756 \mathrm{E}-\mathrm{C} 5$ $3.147 E-04$ $1.98 C E-03$ $8.969 E-C 4$ $1.136 \mathrm{E}-03$ $1.264 E-03$ $1.383 E-03$ $2.267 E-03$ $2.267 E-03$ $2.771 E-03$ G. $6.103 E-03$ $1.196 \mathrm{C}$ $2.198 \mathrm{E}-02$ $0.798 \mathrm{E}-\mathrm{C2}$ $1.704 E-C 1$ $4.464 E-02$
ANGLE 5 ANGLE 6 $M U=-0.7550 \quad M U=-6.6179$ $.8 C 2 E-C 5 \quad 1.973 E-05$ $.968 E-05 \quad 6.511 E-05$ 4.111E-04 4.473E-04 $1.856 \mathrm{E}-04 \quad 1.998 \mathrm{E}-04$ $1.598 E-04$
$2.523 E$ 2.501E-04 $2.501 E-04$
$2.481 E-04$ .126E-U4 2.373E-04 $2.546 E-04 \quad 2.935 E-04$ 1.627E-C4 $2.124 E-04$ 2.366E-04 2.947E-04 . $0.433 E-04$ 6.023E-04 C.288E-C3 $2.020 E-C 3$ 1. $564 \mathrm{E}-\mathrm{C} 2 \mathrm{C} \quad \mathrm{1.583 \textrm {E } - 0 2}$ $4.304 E-G 2$ $4.530 \mathrm{E}-02$ $4.394 E-02$ 4.530E-O2 $3.869 \mathrm{E}-\mathrm{C} 2 \quad 3.913 \mathrm{E}-\mathrm{C} 2$ ANGLE 14 ANGLE 15 $M U=0.7550 \quad M U=0.8650$ $1.528 E-C 4 \quad 2.626 E-04$ 1.995E-C4 $8.981 E-04$ . $.986 E-C 3 \quad 4.881 E-03$ $1.415 E-C 3 \quad 2.541 E-C 3$ . $1.778 E-03$ (.29)

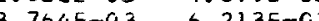
. (5.814E-G3 7. 1.84 1.612E-U2 $1.59 \mathrm{E} E \mathrm{C}$ $1.40 E-U 2$ 1.837E 22 $7.3+0 E-C 2 \quad 7.827 E-02$ $1.313 E-C 1 \quad 1.827 E-02$ $4.551 E-02 \quad 4.655 E-62$
ANGLE 7 ANGLE 8 ANGLE 9 $M U=-0.458 C \quad M U=-C .2810 \quad M U=C D C S 5 C$ $7.671 E-05 \quad 7.962 E-C 5 \quad 9.539 E-C 5$ 4.891E-04 5.525E-C4 $6.559 E-04$ $2.050 E-C 4 \quad 2.215 E-C 4 \quad 2.684 E-04$ $2.639 E-04 \quad 2.9 C 9 E-04 \quad 3.524 E-04$ $2.667 E-04 \quad 3.013 E-C 4 \quad 3.697 E-04$ $2.756 \mathrm{E}-\mathrm{C}_{4} \quad 3.226 \mathrm{E}-\mathrm{C}_{4} \quad 3.968 \mathrm{E}-04$ $2.866 E-C 4 \quad 3.545 E-C 4 \quad 4.364 E-C 4$ $3.718 E-C 4 \quad 4.672 E-C 4 \quad 5.667 E-C 4$ $3.024 E-04 \quad 3.598 E-C .4 \quad 4.966 t-04$ $3.659 E-04 \quad 4.575 E-04 \quad 6.246 E-C_{4}$ $6.589 E-04 \quad 8.267 E-C 4 \quad 1.237 E-03$ $3.195 E-C 3 \quad 4.095 E-C 3 \quad 5.344 E-03$ $6.839 E-03$ d.326E-C3 $9.631 \mathrm{E}-03$ $1.60 C E-C 2 \quad 1.624 E-C 2 \quad 1.669 E-C 2$ 4.7O5E-C2 4.924E-C2 5.192E-C2 $1.357 E-01 \quad 1.407 E-01 \quad 1.467 E-C 1$ $3.968 \mathrm{E}-\mathrm{C} 2 \quad 4.033 \mathrm{E}-\mathrm{C2} \quad 4.1 \mathrm{CBE}-02$

ANGLE 16 $=0.9446$ ANGLE 17 $5.225 \mathrm{t}-04 \quad 1.63 \mathrm{UE}-03$ $1.955 \mathrm{E}-03$ $8.835 E-03$ $5.104 E-03$ 7. $7.638 \mathrm{E}-03$ $7.694 E-C 3$ $1.753 E-03$ 1.C44E-02 (.009E-C2 $1.018 E-02$ $1.200 \mathrm{C} 2$ 1.80 $2.00 E-C$ $8.300 \mathrm{E}-\mathrm{C2}$ $4.024 E-C 1$ $4.683 E-C 2$

SCALAR FLUX $1.161 \mathrm{E}-\mathrm{CSS}_{3}$ $4.163 E-03$ $2.107 E-C 2$ $1 . C 86 \mathrm{E}-\mathrm{CL}$ $1.255 E-C 2$ $1.549 E-02$ $1.559 E-02$ 1.773e-v2 $2 . \angle 19 t-C 2$ $2.7 C B E-C 2$ $4 . \mathrm{C} 28 \mathrm{t}-\mathrm{C} 2$ . $285 \mathrm{C}$ $1.287 t-01$ $1.287 E-01$ 7. 7. $943 E$ CC $5.258 \mathrm{E}-\mathrm{Cl}_{1}$ 
GGAMMAS/MEVISTERADIAN/SOURCE NEUTRUNI

ENERGY

0.5 CE UC- $-8.60 E$ CO

$5.0 C E$ OO- $6.50 E$ OC

4. OCE OO- -5.0OE 00

3. COE OOC- $4.00 E$ LO

$2.5 \mathrm{CE} \quad 00 \rightarrow-3.00 \mathrm{~B}$ CO

$2.00 \mathrm{E} 00--2.50 \mathrm{E} 00$

$1.66500-\cdots 2.00 E$ OO

$1.33 \mathrm{E} \mathrm{00-D-1.66E} \mathrm{00}$

$1.00 E \quad 00 \rightarrow-1.33 E \mathrm{CO}$

$8.00 E-C 1--1.00 E$ CO

6.00E-01- $-8.00 E-0$

$4.00 E-01-6.00 E-C$

3.00E-01-- - 4.0UE-O

$2.00 E-01--3.00 E-0$

$1.00 \mathrm{E}-01-2.00 \mathrm{E}-01$

$5.00 E-02--1.00 E-01$

2.00E-02---5.00E-02

ENERGY

GRDUP (MEV)

8.00E OO-D-1.00E 01

$6.50 E$ OOM- $8.00 E$ OC

$5.00 E$ OO---6.50E $\mathrm{CO}$

4.00 E UO- $-5.00 E$ CO

3. OOE OO- - 4.0OE CO

2.5CE OO-W3. CCE OO

2.0UE OO-D-2.5OE CO

$1.66 E$ OO-D2.00E CO

$1.33 E$ EO- $1.66 E \mathrm{EOO}$

$1.00 E$ OO- $1.33 E$ OO

8.00E-01--1.00E CO

6.0OE-O1- $-8.00 E-O 1$

4.0CE-O1-D-6.00E-O

3. OE $01-2.00 E-U 1$

2.00E-O1=-3.00E-O

$1.00 E-01-2$. COE-C

$2.00 \mathrm{E}-\mathrm{C} 2---5.0 \mathrm{C} \mathrm{E}-0$
ANGLE 1

ANGLE 2

$9.235 \mathrm{E}-06$

$2.562 \mathrm{E}-05$

2. C $88 \mathrm{E}-0$

3.757E-C5

7.316E-05

8.9C8E-05

$1.325 \mathrm{E}-0$

1. $837 \mathrm{E}-04$

$2.662 E-04$

$2.157 E-04$

$1.193 E-04$
$8.262 E-05$

$1.606 \mathrm{E}-\mathrm{C} 3$

$2.241 \mathrm{E}-03$

$1.597 E-03$

2.207E-03

$1.342 E-02$

3.754E-O2

3. $52 \mathrm{CE}-02$

ANGLE 10

$2.659 E-05$

$8.464 \mathrm{E}-05$

$5.891 E-C 4$

$2.571 \mathrm{E}-04$

$3.356 \mathrm{E}-04$

$3.486 E-04$

$3.777 \mathrm{E}-04$
$3.998 \mathrm{E}-04$

$5.086 \mathrm{E}-04$

$4.985 E-04$

$7.868 \mathrm{E}-\mathrm{C} 4$

$1.760 E-03$

..199E-03

$1.538 \mathrm{E}-02$

$1.538 E-02$

$4.973 E-02$

$3.889 E-02$ $3.523 \mathrm{E}-02$ ANGLE 11 $M U=C .2816$

$3.973 E-G 2$
ANGLE
3 $\quad$ ANGLE 4 ANGLE 5 3.C13E-05 $2.315 E-04$ $6.970 E-05$ $1.050 E-04$ $1.413 E-04$ $1.663 \mathrm{E}-04$ $2.250 E-04$ $1.665 \mathrm{E}-04$ $1.261 E-04$ $1.891 E-04$ $1.643 \mathrm{E}-03$ $2.419 E-03$ $1.362 E-02$

$3.762 E-02 \quad 3.796 E-02$

$1.145 E-0$ 3.535E-02 ANGLE 12 $M U=0.458$ 4. $946 \mathrm{E}-05$ $1.570 \mathrm{OE}-04$ $1.049 \mathrm{E}-03$ 4. $794 E-04$ $6.092 \mathrm{E}-04$ $6.458 E-04$ $7.101 E-04$ $8.13 \mathrm{CE}-04$ $1.117 E-03$ $1.433 E-0$ 2.35CE-03 . 9.189E-C3 $1.118 E-02$ $5.700 E-02$ $5.700 E-02$

1. $.059 \mathrm{E}-02$

ANGLE 13
$1.135 E-C 5$
$3.650 E-C 5$

$2.648 \mathrm{E}-04$

$1.108 \mathrm{E}-04$

$1.467 E-04$
$1.459 E-04$

$1.536 E-04$

$1.464 \mathrm{E}-\mathrm{C} 4$

$1.778 E-04$
$1.131 E-04$

$1.458 \mathrm{E}-04$

$3.263 E-C 4$

$1.724 \mathrm{E}-03$

$1.864 E-C 3$
$1.385 E-C 2$

$3.860 E-C 2$

$1.161 \mathrm{E}$

$3.556 E-C$

$M U=C .6179$

$7.398 E-C 5$

$1.514 E-C 3$

$6.871 E-04$

$8.852 E-04$

$9.824 \mathrm{E}-04$

$1.129 E-03$

1.

1.937E

.

5.

. $060 E-02$

(

926E-02

$A N=-0.7550$
$1.263 E-05$

$1.263 E-05$
$4.186 E-05$

.953E-04

$1.394 \mathrm{E}-04$

$1.754 E-04$
$1.685 E-04$

$1.651 E-04$

$1.421 E-C 4$

64 $6 E-04$

$1.812 E-04$

$1.2 \mathrm{C} 1 \mathrm{E}-04$
$1.883 \mathrm{E}-03$

$3.667 E-C 3$

$1.403 \mathrm{E}-\mathrm{C} 2$

$3.952 E-G 2$

$3.588 \mathrm{E}-02$

ANGLE 14

$M U=0.7550$

$1.217 E-04$
$3.861 E-C 4$

$2.393 E-03$

$1.141 \mathrm{E}-03$

$1.473 \mathrm{E}-03$

$1.7 C 6 \mathrm{E}-03$

$1.985 \mathrm{E}-\mathrm{C} 3$

.

.

5.441E-C

100

$6.579 E-02$

$1.738 \mathrm{E}-01$

$1.655 \mathrm{E}-\mathrm{C} 1$

$4.225 E-62$ $-6.6179$ ANGLE 7 $M U=-0.4580$
$1.516 E-C 5$ $4.840 \mathrm{E}-\mathrm{C} 5$ $3.461 \mathrm{E}-04$ $1.445 \mathrm{E} \rightarrow 04$
$1.8720 \mathrm{E}-04$ $1.820 \mathrm{E}-04$ $1.858 E-04$ $1.989 E-04$
$2.057 E-04$ $2.698 \mathrm{E}-04$ $2.290 E-04$ $2.788 E-04$ $4.863 E-04$ $2.739 E-03$ $6.195 \mathrm{E}-\mathrm{C} 3$ $1.421 E-62$ $4.238 E-02$ $1.253 E-01$ $3.681 E-U 2$ BE-02 ANGLE 16 $M U=0.8656$ $7.374 \mathrm{E}-04$ $4.181 E \rightarrow C 3$ $2.208 E-C 3$ $2.755 \mathrm{E}-03$ $3.276 E-03$ $3.683 \mathrm{E}-\mathrm{C} 3$ 4. $404 \mathrm{E}-03$ 6. (3) 296E-02 1. $294 E-02$ $M U=0.9446$ $4.747 \mathrm{E}-04$
$1.700 \mathrm{E}-03$ $8.087 E-03$ $4.765 \mathrm{E}-\mathrm{C} 3$ $5.473 \mathrm{E}-\mathrm{CB}$ $6.781 \mathrm{E}-03$ 7.039E-U3 7. $891 E-03$ $9.215 E-03$ $9.100 E-03$ $9.214 E-03$ $1.039 E-02$ $1.624 E-02$ $3.495 \mathrm{E}-0 \mathrm{Z}$ $2.495 E-02$ 1. $428 E-02$ $1.877 E-C 1$

ANGLE 8 ANGLE 9 $M U=-C .2816 \quad M U=-C .095 \mathrm{C}$ $1.7 .27 E-C 5$ 2.C85E-05 $5.397 E-C 5 \quad 6.532 t-05$ $3.8 \times 2 E-C 4 \quad 4.659 E-04$ $1.506 E-C 4 \quad 1.866 E-04$ $\begin{array}{ll}2.028 E-04 & 2.5 C 9 E-C 4 \\ 2.092 E-C 4 & 2.626 E-04\end{array}$ $2.361 E-04 \quad 2.946 E-G 4$ $2.624 E-C 4 \quad 3.239 E-04$ $3.493 \mathrm{E}-\mathrm{C} 4 \quad 4.2 \mathrm{C6E}-04$ $3.074 E-C 4 \quad 3.779 E-C_{4}$ $3.474 E-04 \quad 4.845 E-04$ $6.342 E-C 4 \quad 1 . C 31 E-03$ $3.6 C U E-C 3 \quad 4.780 E-C 3$ $8.675 E-03$ $1.472 E-02$ $4.438 \mathrm{E}-02 \quad 4.682 \mathrm{E}-02$ $\begin{array}{ll}1.301 E-C 1 & 1.357 E-C 1 \\ 3.742 E-C 2 & 3.612 E-C 2\end{array}$ ANGLE 17 $A U=0.9894$
$1.531 \mathrm{E}-03$ $0.236 \mathrm{E}-03$ $2.058 \mathrm{E}-\mathrm{C} 2$ $1.326 \mathrm{E}-\mathrm{C2}$ $1.234 E-C 2$ $1.784 \mathrm{E}-\mathrm{C} 2$ $1.524 E-02$ $1.605 E-02$ $1.723 E-02$ $1.345 \mathrm{E}-02$ $1.29 C E-C 2$ $1.912 \mathrm{E}-\mathrm{C} 2$ $1.933 \mathrm{E}-\mathrm{C} 2$ $2.672 \mathrm{E}-\mathrm{C2} 2$ 1.917E-O1 1.917E-0 
(GAMAISSMEV/STERADIAIV/SUURCE NEUTRON) GROUP (MEV) 8. OCE GO---1.00E OI 6.5 CE NO $--8.00 E$ CO 5. OCE OO-T-6.5OE CO $4.00 E$ OU $---5.00 E$ CO 3.OCE OC---4. VOE CO $2.5 \mathrm{CE} \mathrm{OO--3.00E} \mathrm{CO}$ $2.0 \mathrm{CE} \mathrm{CO}--2.50 \mathrm{E}$ WO $1.66 E$ OC-D-2. COE UO 1.33E COS-D1.66E OU

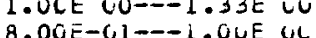
$6.00 E-01---8$. COE-O1 3. OCE-O1- - 4.00E-CL 2.0CE-GI-- -3. $00 E-01$ 1.0CE-O1-C2.0UE-C 1 $5.00 E-62--1.00 E-01$
$2.00 E-02--5.00 E-U 2$

$$
\text { ENERGY }
$$

GKUUP (MEV)

8.00E 0O---1. UUE CI 6.5CE OO-D-D. JOE VO 5.OUE OUD- 6.5OE CO 4.0 UE VO-D-5. CUE OU $3.00 E$ UO-C- 4 . JUE $6 O$ $2.5 \mathrm{CE}$ OO-- $3.00 \mathrm{E}$ OC $2.0 \mathrm{CE}$ CO-O $\angle .5 \mathrm{CE} C O$ $1.66 E$ OU-D-2. OUE CO $1.33 E$ OO-D-1.06E CU 1.0CE OO-D-1. J3E OO $8.00 E-01-1.00 E$ OO $0.00 E-01---8.00 E-61$ $4.00 E-01-2.00 E-01$ 3.0OE-CI-C2.0CE CI $3.00 E-1$ 1 . OOE-O1 2 . OOE OI 2.0UE-02-D. 00 . U -42 $M U=-1.000 \mathrm{C}$ $2.025 E-06$ $2.991 E-06$ $4.723 E-C 5$ $-2.274 E-C b$ $7.479 E-06$ $9.2 C 4 E-C 5$ $1.412 E-04$ $1.515 \mathrm{E}-05$ $-0.621 E-0 S$ $8.066 E-C 4$ $1.057 E-C 3$
$8.182 E-03$ $8.182 E-03$ $2.195 \mathrm{E}-02$ $6.863 \mathrm{E}-02$
$2.14 C E-02$ ANGLE 10 $M U=0.1950$ $8.602 \mathrm{E}-\mathrm{C} 6$ $2.654 \mathrm{E}-\mathrm{CS}$ $2.011 E-04$ $9.282 E-C 5$ $1.243 E-04$ $1.237 E-04$ $1.379 E-C 4$ $1.343 E-04$ $1.646 \mathrm{E}-04$ $1.885 E-C 4$ $4.092 E-C 4$ $1.006 \mathrm{E}-03$ $5.556 E-U S$ 8.5 $2.909 E-V^{2}$ $2.909 E-U 2$ $0.616 E-02$
ANGLE 2
$M U=-0.9894$ $2.198 E-06$ 4. $002 E-06$ 7.312E-05 $4.849 t-05$ $8.588 \mathrm{E}-05$
$1.292 \mathrm{E}-04$ $1.292 E-04$ $1.854 E-C 5$ $-3.916 E-05$ $8.067 E-04$ $1.084 E-03$ $8.204 E-C 3$ $2.2 C O E-C 2$ $0.87 G E=02$ $2.142 \mathrm{E}-0<$ ANGLE 11 $M U=C .2816$ $1.197 E-C 5$
$3.785 E-C 5$ $3.785 E-C 5$
$2.741 E-04$ $2.741 E-04$
$1.384 E-C 4$ $1.384 E-C 4$
$1.737 t-04$ $1.737 t-04$ $1.692 E-C 4$ $1.812 E-04$ $1.8 C 7 E-04$ $2.466 E-C 4$ $3.812 t-04$ . $391 E-C 4$ $4.63 C E-03$ $5.835 E-03$ $5.855 E-03$ .187E-CS . $101 \mathrm{E} C 2$ $2.412 \mathrm{E}-\mathrm{C} 2$ ANGLE 3
$M U=-0.9446 \quad$ ANGLE $\quad 4$
MU $=-C .8656$ $1.142 \mathrm{E}-05$ $4.397 E-65$ $2.352 E-05 \quad 5.541 E-C 5$ . COC E-05 5.U3CE-C5 $0.014 E-05 \quad 5.187 E-C 5$ $6.491 E-05 \quad 3.941 E-05$ .777E-U5 $4.077 E-C 5$ $5.779 E-05 \quad 1.957 E-05$ $.142 E-05$ $3.332 E-05 \quad 1.384 E-C 4$ $8.111 E-04 \quad 8.361 E-04$ $1.222 E-03 \quad 1.551 E-03$ $8 .<78 E-03$ 8.300t-03 $2.221 E-02$ 2.260E-C2 $6.928 E-02 \quad 7.026 E-C 2$ $2.149 E-02 \quad 2.162 E-02$ ANGLE 12 $M=C .458 \mathrm{C}$ ANGLE 13 $1.713 E-05 \quad 2.076 \mathrm{E}-65$ $5 . \angle 95 E-C 5$ 3. $757 E-04$ $1.789 \mathrm{E}-04$ $2.277 E-04$ $2.342 \mathrm{E}-04$ $2.777 E-04$ 3. $357 E-04$ $5.12 \angle E-04$ $1.535 E-03$ $\angle .664 \mathrm{E}-03$ $3.386 \mathrm{E}-03$ $0.230 E-C 3$ $9.859 \mathrm{E}-\mathrm{C} 3$ $3.315 E-02$ $2.463 \mathrm{E}-\mathrm{C} C$
ANuLE 5
$M U=-0.7550$ $4.2 \mathrm{COE}-\mathrm{C6}$ $1.417 \mathrm{E}-\mathrm{C} 5$ $.04 E-C 4$ $7.289 E-C 5$ $6.086 E-45$ $5.327 E-05$ $2.959 E-05$ $2.7 C 8 E-C 5$ $2.1 C 4 E-C 5$ $7.701 E-05$ 1.812E-04 $9.195 \mathrm{E}-04$ $2.123 \mathrm{E}-0 \mathrm{C}$ $8.384 t-C 3$ $2.316 \mathrm{E}-\mathrm{C} 2$ $7.168 \mathrm{E}-\mathrm{G} 2$ 2.181E-02 ANGLE 14 $M U=0.755 C$
$4.934 E-C 5$ $4.934 E-C 5$
$1.468 E-04$ $1.468 E-04$
$9.684 E-C 4$ $9.684 E-04$
$4.785 E-C 4$ $4.785 E-04$
$0.656 t-04$ $0.656 \mathrm{E}-04$ $8.254 E-04$ 1. C92E-U3 $1.456 E-03$ $2 . C 29 E-C 3$ $2.732 E-03$ $4.285 E-43$ $4.285 E-C 3$ $6.651 E-U 3$ 1.746E-OD $1.146 E-02$ $3.786 E-02$ $2.559 E-02$ $\triangle N$ NOLE 6 $4.481 \mathrm{E}-06$ $1.472 \mathrm{E}-05$ $1.095 E-04$ $5.507 E-05$ $6.750 E-05$ $5.929 E-C 5$ $5.677 E-05$ 4. 3 JE -05 5. $3 C 6 E-05$ $4.693 E-05$ $9.454 \mathrm{E}-05$ $1.746 E-C 4$ $1.118 E-03$ $2.916 E-C 3$ $0.336 E-03$ 2. $39 C E \neg 02$ 7. $358 E-02$ $\angle .206 E-C 2$ ANGLE 15 $M U=0.8656$ $1.1 C O E-04$
$3.398 E-04$ $2.033 E-03$ $1.185 \mathrm{E}-03$ $1.557 t-C 3$ $1.871 E-C 3$ $\angle .270 E-C 3$ 3.43 $3.438 E-C 3$ $4.566 t-03$ $7.448 E-C 3$ $8.668 \mathrm{E}-\mathrm{C} 3$ $1.233 E-02$ 4.C1SE-02 $2.598 E-02$
AINGLE 7 ANGLE $\triangle$ ANGLE $S$ $M U=-0.458 C \quad M U=-C .2016 \quad M U=-C . C>5 C$ $4.645 \mathrm{E}-\mathrm{C6} \quad 5.1<8 \mathrm{z}-\mathrm{C6} \quad 0.366 \mathrm{t}-\mathrm{C6}$ $1.109 E-04 \quad 1.451 E-C 5 \quad 1.849 E-05$ 4.087E-05 3.5د7E-C5 $5.301 E-05$ $5.773 E-C 5 \quad 5.5 C 5 E-C 5 \quad 7.518 E-05$ $5.401 E-05 \quad 5.972 E-C 5 \quad 8.445 E-05$ $6.011 E-C 5 \quad 8.373 E-C 5 \quad 1.083 E-C 4$ $7.305 E-05 \quad 1.0 \angle 4 E-C 4 \quad 1 . \angle 07 t-C 4$ $1.009 t-04 \quad 1.406 E-C 4 \quad 1.543 E-04$ $9.331 E-05 \quad 1.271 E-C 4 \quad 1.394 E-04$ $1.032 \mathrm{E}-\mathrm{C} 4 \quad 1.213 \mathrm{E}-\mathrm{C} 4 \quad 1.575 \mathrm{E}-04$ $1.623 E-04 \quad 2.4 E 4 E-04 \quad 5.342 E-04$ $1.495 E-C 3 \quad 2.086 E-C_{3} 2.870 E-C 3$ $3.801 E-C 3 \quad 4.6 C 5 E-63 \quad 5.192 E-C 3$ $8.254 \mathrm{E}-03 \quad 8.226 \mathrm{E}-\mathrm{C3} \quad 8.346 \mathrm{E}-\mathrm{U} 3$ $2.485 E-02 \quad 2.6 C 2 E-C 2 \quad 2.743 E-C 2$ $\begin{array}{lll}7.596 E-C 2 & 7.886 E-C 2 & 8.226 E-C 2 \\ 2.237 E-G 2 & 2.274 E-C 2 & 2.316 E-C 2\end{array}$ $M U=0.9446 \quad M U=0.9894$ $\angle .910 E-C 4$ 1.C95E-C $9.588 \mathrm{E}-04 \quad 4.069 \mathrm{E}-\mathrm{L} 3$ $4.827 E-03 \quad 1.4 \angle O E-02$ $3.084 E-C S \quad 8.999 E-C 3$ $3.657 t-43 \quad 8.652 E-13$ $4.188 E-03$ 1.CO4E-CL $4.925 \mathrm{t}-03 \quad 8.992 \mathrm{E}-03$ $5.448 E-03 \quad 8.846 E-03$ $5.448 E-03 \quad 7.15 C E-C 3$ $5.410 E-03$ O. $399 \mathrm{C}-\mathrm{C}_{3}$ $5.645 E-03$ 6. $361 E-C 3$ $8.302 E=C 3$ O.63CE 1. $317 E-0<$ L $1.317 E-02$ l. $383 E-C 2$ $4.208 E-C 2$ 4.33bE-C $2.628 E-0<\quad \angle .645 E-C \angle$

\section{SCALAR}

5. $C 21 \mathrm{LLC}-\mathrm{C}_{4}$

$1.683 E-63$

$8.488 E-0.5$

4.791t-03

5. $646 \mathrm{E}-\mathrm{C} 3$

$6.5 C 2 E-C 3$

T.107E-OS $9.924 E-C S$ $1.151 E-L^{2}$
$1.474 E-04$ $1.474 E-0<$ $2.059 \mathrm{t}-0<2$ $6.45 \mathrm{CE}-\mathrm{C2}$ $1.174 E-01$ 3.718E-C1 2. $902 \mathrm{E}-\mathrm{C} 1$ 
IGAMMAS/MEVISTERADIAN/SOURCE NEUTRON I

ENERGY GROUP IMEV 8.0CE 00---1.00E 01 $6.50 \mathrm{E}$ UO-- -8.00E 00 5.00 E CO- - 6.50 E CO $4.00 E$ OO- $-5.00 E$ OO 3. OCE $00--4.00 E$ CO 2.5CE OO-D-3. COE $1.66 \mathrm{E}$ 00---2.00E CL $1.33 \mathrm{E} 00--1.06 \mathrm{E} 00$ 1.0 CE $00--1.33 \mathrm{E} 00$ $8.00 E-01---1.00 E$ OO $6.00 \mathrm{E}-01--8.00 \mathrm{E}-01$ $4.00 E-C 1---6.00 E-O 1$ 3.0UE-01-- -4.00E-01 $2.00 E-01---3.00 E-01$ 1. OCE-01---2.00E-O $5.00 E-02-\cdots-1.00 E-01$ $2.00 t-02---5.00 E-0$

\section{ENERGY}

GKOUP (MEV)

8. OOE OO-- - 1.00E C1 $6.50 \mathrm{E}$ OC---8.COE CO 5. OCE OO- - 6.50E OC 4.00E 00---5.00E 00 3.0 CE $00--4.00 E 0 O$ 2. SOE OO-D-3.00E CQ $2.0 \mathrm{CE}$ OO---2.50E 00 $1.66 E$ OO-D-2.00E 00 $1.33 E$ OO-D-1.06E 00 $1.00 E$ OO- $-1.33 \mathrm{E}$ OO $6.00 E-01-1.00 E$ OC $6.0 C E-01-C-8.00 E-C 1$ $4.00 E-01--6.00 E-01$ 3.OOE OIC 4 .0OE-OI 1. OOE $01-2.00 E-C$ $1.00 E-O 1-2.00 E-C 1$ 5. CQE-02---1.00E-01
ANGLE 1 $M U=-1.000$ $1.611 E-07$ 4. $484 \mathrm{E}-06$ $4.428 \mathrm{E}-05$
$-1.825 \mathrm{E}-05$ $1.839 E-05$
$4.661 E-05$ $4.661 \mathrm{E}-05$ $7.226 \mathrm{E}-05$
$5.615 \mathrm{E}-05$ (6. $-6.547 E-05$ $3.842 \mathrm{E}-04$ $4.710 E-04$ $4.345 E-03$. $1.111 \mathrm{E}-\mathrm{C} 2$ $3.495 E-C$ $1.086 \mathrm{E}-02$ ANGLE 10 $M U=C .0950$ $2.719 E-06$ $8.240 E-C 0$ $6.676 \mathrm{E}-05$ $3.526 \mathrm{E}-05$ $4.676 E-05$ $4.348 E-05$ $4.532 E-05$ $3.649 E-05$ $3.967 E-05$ $6.040 E-05$ 1.774E 04 $5.774 E-04$ $1.98 \mathrm{E}-03$ $2.816 E-03$ $1.463 E-02$ $1.463 E-Q 2$ $4.361 E-C 2$
$1.194 E-02$
$M U=-0.9894$ 2.861E-C7 $-7.611 E-07$ $7.622 E-06$ $-1.827 E-05$ $-1.249 E-05$ $-3.571 E-06$ $4.192 E-05$ 4.192E 05 $4.867 E-05$ $-7.998 E-06$ $3.819 E-C 4$ $4.879 E-04$ $4.354 \mathrm{E}-03$ $1.114 E-02$ $3.5 C \angle E-C 2$ 1.C87E-C2 ANGLE 11 $M U=C .2816$
$3.991 E-C 6$ $3.991 E-C 6$
$1.269 E-05$ $9.504 E-05$ $5.602 E-C 5$ $0.752 E-05$ $5.786 \mathrm{E}-\mathrm{C} 5$ $5.545 E-05$ $4.716 \mathrm{E}-\mathrm{C} 5$ $7.247 E-05$ $1.704 E-04$ 1.571E-04 $1.012 E-03$ $2.414 E-03$ $4.537 E-03$ 1.53 $4.571 E-02$ $4.571 \mathrm{E}-02$
$1.218 \mathrm{E}-02$ ANGLE 3
$M U=-0.9446 \quad M U=-0.8656$ $6.687 E-C 7$ . $.727 \mathrm{E}-05$ $2.394 E-05$ .000E-C5 $.992 E-05$ $.715 E-06$ $.449 E-07$ $3.760 \mathrm{E}-04$ $5.725 E-04$ $4.381 E-03$ $1.124 E-C 2$ $3.528 E-C 2$ 1.090 E-02 ANGLE 12 $M U=C .4580$ $5.638 E-06$
$1.692 E-05$ $1.692 \mathrm{E}-05$
$1.266 \mathrm{E}-\mathrm{C} 4$ $1.266 E-C 4$ $6.272 \mathrm{E}-05$ $7.715 E-05$ $7.434 \mathrm{E}-05$ $9.376 E-05$ 1. $264 E-04$ $2.243 E-04$ $4.460 E-04$ $0.636 E-04$ $1.467 E-03$ $2.741 E-03$ $4.834 E-03$ $1.654 \mathrm{E}-02$ $4.795 \mathrm{E}-\mathrm{C} 2$ 1. $242 \mathrm{E}-02$ ANGLE $\quad 4$
$\mathrm{HU}=-0.8656$ $1.152 t-06$
$3.876 E-06$ $3.876 E-06$ $3.002 E-05$ . 375E-05 .817E-05 $.567 E-05$ $5.378 E-00$ $2.539 E-.06$ $-1.322 E-06$ $5.622 \mathrm{E}-05$ $3.790 E-C 4$ $.689 E-04$ $4.400 E-C_{3}$ $1.144 E-C 2$ $3.577 E-02$ $1.097 \mathrm{E}-02$ ANGLE 13 $M U=0.6179$ $8.707 E-C 6$ $2.389 E+C 5$ $1.812 \mathrm{E}-\mathrm{C} 4$ $7.214 E-65$ $1.075 \mathrm{E}-04$ $1.394 E-04$ $2.208 E-04$ $3.435 E-04$ $5.600 E-04$ 1. $383 \mathrm{E}-03$ 1.877E-C3 $1.877 E-C 3$ 3.343E-C3 $3.343 E-C 3$ 1. 761 E $0<$ $5.025 \mathrm{E}-\mathrm{C} 2$ $1.266 E-C 2$
ANGLE 5 $=-0.7550$ $.460 E-06$ . $2.184 E-05$ . 1.371E-0 $-3.5 C .0 E-06$ $3.759 E-05$ $7.512 \mathrm{E}-05$ $4.185 E-C 4$ $1.102 E-03$ $4.374 E-C 3$ $1.173 E-02$ $.648 E-C$ $1.106 E-02$

ANGLE 14 $M U=0.7550$ $1.738 \mathrm{E}-05$ $4.8 C O E-05$ .379E-04 $1.674 E-04$ $2.633 E-04$ $3.673 \mathrm{E}-04$ $5.441 E-04$ . $100 E-03$ 1. (202E-O3 . $203 E-03$ $5.500 E-03$ . $1.868 E-02$ $1.288 E-C 2$
ANGLE 6 $M U=-0.6179$ $1.464 \mathrm{E}-\mathrm{C} 6$ $3.673 E-C 5$ ..105t-O5 $2.392 \mathrm{E}-05$ $1.817 E-C 5$ 1. $529 E-C 5$ $8.563 E-06$ $1.052 \mathrm{E}-05$ $1.348 E-05$ $3.789 E-05$ $6.504 E-05$ . $299 \mathrm{E}-\mathrm{C}$ 1.550t-C ..210E-C2 $3.743 \mathrm{E}-\mathrm{O}$ $1.119 \mathrm{E}-02$

ANGLE 15

$M U=0.8656$ 1. $375 \mathrm{E}=0 \mathrm{E}$ $1.375 e-04$ 5. $561 E=04$ $7.561 E-04$ . $9.691 E-04$ 1. $517 E-03$ 1.517E-03 $.852 \mathrm{E}-\mathrm{O}$ $1.969 E-02$ $5.448 E-02$

ANGLE 7 $1.314 E-06$ $3.514 E-66$ $.242 E-05$ $1.032 \mathrm{E}-05$ $1.244 \mathrm{E}-05$ 2.C1OE-C 5 $2.605 E-05$ $3.948 \mathrm{E}-05$ . $939 E-05$ $4.282 E=05$ $4.174 E R 05$ 7.479E-04 $4.030 E-03$ $1.257 E-02$ 
(GAMMASJMEV/STERADIAN/SOURCE NEUTRON)

\begin{abstract}
ENERGY
B OORUP IMEVI.

8.00E CO-D-1. CUE C1

6.5CE CO---8. 80 E OO
5.0 CE OO-- 6.50 OC OC

4. UCE CO--5. COE CL

4.

3.

2.0UE OOR $2.50 E$ CO

1.60E $00-2.002 .00 \mathrm{CO}$

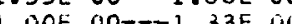

8.0C $-01--1.30$ E 0

8. OCE-O1-- - $0.00 \mathrm{E}-\mathrm{C}$

$4.0 C E=01-2.00 E-C$

4.OCE

2.0CE-01-O-3.0OE- 1

1.OCE-C1--2. 20 E 01

$5.00 \mathrm{E}-02--1.00 \mathrm{E}-01$

, $2.00 E-C 2--5.30 E-C 2$
\end{abstract}

GROUP (MEV)

8. OCE U0--1. OOE 01 6.5 GE UO-- 8 . CUE CO
$5.00 E$ OU-- $6.50 E$ OC 4. UCE OU-D-5.00E CO $3.00 E$ CO-- $4.00 E$ OO $2.5 C E$ CO $\rightarrow-3.00 E ~ C C$ 2.00E $00--2.50 \mathrm{E} \mathrm{CO}$ $.33 \mathrm{E} \mathrm{OC-D-1.66E} 00$ . OOE 0O-D-1.33E OO $8.0 C E-01--1.00 E$ CO $6.00 E-01--8.00 E-C$ $4.00 E-C 1=-6.00 E-0$ 3.0 CE-01-- $4.00 E-O$ $2.00 \mathrm{E}-61---3.00 \mathrm{E}-01$ 1.0 CE-U1-- $2.00 E-01$ $5.00 E-02=-1.00 E-01$ $2.00 \mathrm{E}-62--5.00 \mathrm{E}-\mathrm{C} 2$
ANGLE 1 ANGLE 2 ANGLE $1.0000 \quad M U=-i .9894$ $1.974 E-07-1.141 E-C 7$ $1.698 E-06$ - $1.265 E-06$ $1.62 \mathrm{CE}-05-1 .<82 \mathrm{E}-05$ $3.672 E-C 5$ 2.69lE-0

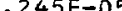
$.799 \mathrm{E}-04$ $2.069 E-C 4$
$2.171 E-03$ $5.328 E-03$ .672E-02 $5.165 \mathrm{E}-03$ ANGLE 10 $M U=0.0950$ $8.709 E-07$ $.834 E-C 6$
$1.234 E-C 5$ $1.478 \mathrm{E}-05$ $1.891 \mathrm{E}-\mathrm{C5}$ $1.623 \mathrm{E}-05$ $1.387 \mathrm{E}-05$ $5.097 \mathrm{E}-06$ $1.581 E-06$ $1.676 \mathrm{E}-05$ $9.547 t-05$ $3.027 E-04$ 9. $962 E-C_{4}$ $1.350 E-03$ $2.038 \mathrm{E}-03$ $6.965 \mathrm{E}-03$ $5.661 E-03$ . $341 E-03$

ANGLE 11 $M U=C .2816$ 4.6C $3 E-0.6$ $3.349 \mathrm{E}-05$ $2.414 E-05$ $\angle .678 \mathrm{E}-05$ $1.952 \mathrm{E}-05$ $1.393 E-C 5$ $0.396 E-C 6$ $1.875 t-05$ $7.661 \mathrm{E}-05$ $2.369 E-04$ $5.258 \mathrm{E}-\mathrm{C}$ $1.184 E-03$ $1.363 E-03$ $2.139 E-C 3$ $7.373 E-03$ $5.768 E-03$
ANGLE 3 $M U=-0.9446$
$9.509 E-08$ 3.071E- 08 3.07E-08 3. $-3.4945-07$ $-3.454 L-07$ $0.453 E-U 6$ $6.153 E-66$
$9.187 E-06$ I. $226 E-G 5$ $1.226 E-C 5$
$7.362 E-06$ $-1.958 E-06$ $-3.628 E-06$ $-3.628 E-06$
$1.717 E-04$ $1.717 E-04$
$2.63 J E-64$ $2.183 E-03$ $5.394 E-03$ $1.688 \mathrm{E}-\hat{\mathrm{U} 2}$ $5.186 E-03$

ANGLE 4 $-1.140 E-06$ $5.169 E-03$

ANGLE 12 $2.266 \mathrm{E}-03$
$7.818 \mathrm{E}-03$ $2.265 \mathrm{E}-0$ $5.875 E-03$ $5.980 \mathrm{E}$ $4 U=-0.8650$ $4.086 E-\dot{0} 7$ $1.217 E-06$ $5.067 E-05$ $7.196 E-C 6$ $6.196 \mathrm{E}-06$ $4.232 E-C 6$

ANGLE 5 $M U=-C .7550$ $5.314 E-C$ $1.950 E-06$ $1.277 E-05$ $7.37 E-C 5$ $2.878 E-C 6$ $2.057 E-06$ $-7.677 E-06$ $-4.356 E-C 6$ l. $573 E-C 5$ $3.711 E-65$ $1.878 E-04$ $5.402 E-C 4$ $2.155 E-03$ $5.625 E-C$ $5.625 E-C 3$ $\begin{array}{ll}1.711 E-C 2 & 1.744 E-C 2 \\ 5.216 E-03 & 5.260 E-63\end{array}$ ANGLE 14 $M U=U .7550$ $5.677 E-C 6$ $1.392 E-C 5$ 1. $087 E-C 4$ 5. C9OE-CS
$9.9 C 2 E-05$ $1.016 E-C 4$ $2.642 E-64$ $3.985 E-C 4$ $5.755 E-C 4$ $7.948 E-64$ $9.695 E-04$ 1. $C 56 E-C 3$ $1.474 E-63$ $1.736 t-U$ $2.534 E-C$. $8.746 \mathrm{E}-\mathrm{O}$ $2.464 E-12$
$6.075 E-C 3$ $M U=-0.6179$ $4.786 E-07$ $1.678 E \rightarrow 06$ $6.187 E-06$ . 6. 2.853E-G7 $3.946 E-06$ $1.468 \mathrm{E}-05$ $1.9411-05$ $2.463 \mathrm{E}-04$ 2.463E-04 $2.099 E \rightarrow G 3$ 5.803E-G . $797 \mathrm{E}-02$ $5.317 E-C 3$

ANGLE 15 $M U=0.8656$ $.842 \mathrm{E}-\mathrm{C} 5$ $3.271 \mathrm{E}-\mathrm{C} 4$ $2.5 C 3 E=C 4$ $3.666 \mathrm{E}-04$ $4.837 E-04$ $0.253 E-C 4$ $.828 E-C 4$ ..095E-O3 . $154 \mathrm{E}-03$ 1.143E-03 $1.597 t-03$ $1.893 \mathrm{E}-\mathrm{C}$ .675E-O3 $9.170 E-C 3$ . $550 E-02$
ANGLE 7 $M U=-U .458 C$ $3.289 E-C 7$
$4.467 E-C 7$ $8.353 \mathrm{E}-\mathrm{CG}$ -1. CO1E-06 6.743E-OT ..21CE-U6 ..02 1.712E-05 1. $6.652 E-00$ $7.545 \mathrm{E}-06$ $3.625 E-04$ $3.625 E-04$ $2.033 E-03$ $6.022 \mathrm{E}-03$ $1.842 \mathrm{E}-\mathrm{C} 2$ $5.386 E-03$

ANGLE 16 $M U=0.9446$ $7.452 E-65$ $.137 \mathrm{E}-03$ . $945 \mathrm{E}-04$ $1.077 \mathrm{E}-03$ $1.198 \mathrm{E}-03$ $1 . \angle 97 E-03$ $1.349 E-C 3$ . $314 \mathrm{E}-\mathrm{C}$ .220E-03 $1.782 \mathrm{~L}-\mathrm{U}$ .811E-03 $9.511 E-C 3$ $2.617 E-C$ $6.216 \mathrm{E}-03$

ANGLE 8 ANGLE 9 $2.617 E-07$ MU $=-C . C 950$ 2. $117 E-07$ U. $421 E-07$ $7.092 E-06$ I. $196 \mathrm{E}-05$ 7. $5.06 C E-C 7$. D. $365 E-C 6$ $1.362 E-05$ 1. $362 E-05$ 1.4ICE-O5 $1.885 E-C 5 \quad 1.115 E-05$ $0.452 E-C O \quad 2.517 E-U 5$ . $328 E-05 \quad 1.296 E-04$ $5.40 \mathrm{CE}-C_{4} \quad 7.615 t-04$ $.212 t-C_{3}+3.315 t-63$ $1.985 E-C^{2} \quad 1.983 E-C^{3}$ $6.6 C 2 E-C$. $1.9 C 8 E-62 \quad 1.984 E-02$ $5.46 \& E-C 3 \quad 5.56 C t-C 3$

ANGLE 17 SCALAK $M U=0.9894 \quad$ FLUX 
(GAMMAS/MEV/STERAGIAN/SOURCE NEUTRONI

ENERGY (MEV) 8.00E OO---1.00E O1 $5.00 E$ OO- $0.0 .50 E$ CO $4.00 E$ OO- $-5.00 E$ CO $3.00 E$ OO- $-4.0 O E$ CO .5OE $00---3.00 E$ OO 1.00E $00---2.50 E$ CU $1.66 \mathrm{E} 00--2.00 \mathrm{E} \mathrm{OO}$ $.33 \mathrm{E}$ UOC- $-1.66 \mathrm{E} \mathrm{OC}$ $1.00 \mathrm{E}$ CO-- $1.33 \mathrm{E}$ UO .00E-01- $-1.00 E$ CO $6.00 E-01-\cdots-8.00 E-01$ $4.00 E-01-06.00 E-01$ $3.00 E-01---4.00 E-C 1$ $2.00 t-C 1--3.00 t-C 1$ $1.00 E-01--2.00 E-01$ $2.00 \mathrm{E}-02--5.00 \mathrm{E}-\mathrm{C2}$

\section{ENERGY}

GROUP IMEVK

8.00E 00--1.00E C1 6.5OE OO---8.00E OO 5.OCE CO--6.50E OO 4.0OE OO---5.0UE LC 3.00E CO-D-4.00E OO 2.5OE OO-Z-3.00E CO 2.00E UO-CO $2.50 E$ OC $1.06 E$ OO-D-2.00E $\angle O$ 1.33E UO- 1.06 E OO $1.00 E$ OO $1.33 E$ OO 8. OOE O1- 1 .OOE OO 4.ULE-C1-C-6.0OE-C 4.ULE CI . 1.00E $01-2.00 E-C$ $1.00-02-1.00 E-1$ 2.00E-02 $1.00 E-01$ $M U=-1.0000$ $-1.143 E-06$ $-3.741 E-06$ $3.616 E-06$ $1.224 E-O 5$ 1.839E-05 $1.271 E-C 5$ $-8.391 E-06$ 2.359E-05 $8.420 E-05$ $9.140 E-05$ $1.053 E-03$ 2.502E-03 $2.402 E-C 3$ ANGLE $1 C$ $M U=.0 .095 \mathrm{C}$ $3.086 E-07$
$1.060 E-06$ $1.060 E-06$ $7.890 E-06$ $5.695 E-06$ $8.143 E-06$ $6.402 E-06$ $-2.164 E-06$ $-3.829 E-C 6$ $.014 E-06$ 1. $523 \mathrm{E}-04$ $4.787 E-04$ 4.787E-04 . $487 \mathrm{E}-04$ $3.248 t-03$ 9. $2.623 \mathrm{E}-\mathrm{C3}$
ANGLE 3

ANGLE 2 $-1.429 E-07$ -9.05 SEE-07 -2.705E-06 -7.419E-06 $-6.405 E-06$ 3.074E 06 $3.346 \mathrm{E}-\mathrm{CO}$ 1.055E-C5 $1.565 E-05$ $1.058=05$ $-7.017 \mathrm{E}-06$ $-1.883 \mathrm{E}-\mathrm{O}$ $8.286 E-05$ $.647 \mathrm{E}-05$ $1.055 E-03$ $2.508 \mathrm{E}-03$ $.83 L E-C 3$ $M U=C .2816$ $M U=C .2816$ $.873 \mathrm{E}-\mathrm{C} 6$ $1.232 E-C 5$ $1.106 \mathrm{E}-05$ $1.111 E-C 5$ $2.032 E-C 6$ $2.032 E-C 6$ $4.255 \mathrm{E}-\mathrm{C} 6$ 3. $539 E-C 5$ $1.194 E-04$ $2.630 E-G 4$ $5.646 E-04$ $6.30 \mathrm{JE}-04$ $9.924 \mathrm{E}-04$ $3.429 E-03$ $2.670 \mathrm{E}-03$
$M U=-C .9446$ $3.913 \mathrm{E}-10$ $-1.557 E-07$ $-1.783 E-06$ $-1.056 \mathrm{E}-06$ $-6.437 E-08$ 2. $342 \mathrm{E}-06$ 3.984E-06 $5.21 C E-06$ 2. $807 \mathrm{E}-06$ $-2.205 E-06$ $-3.406 E-06$ $7.858 \mathrm{E}-05$ 1. $211 E-04$ 1. $057 \mathrm{E}-03$ $2.533 E-0$ .888E-0 $M U=0.458 \mathrm{C}$ MU $=0.458 C$
$0 . C 91 \mathrm{E}-\mathrm{C} 7$ $1.804 \mathrm{E}-06$ $1.34 C E-C 5$ 6.611E-06 $0.725 E-06$ . 1.847E-06 $4.635 E-05$ $4.635 E-05$ 2. $275 E-04$ 3. $81 E-04$ 6.172E-04 $6.528 \mathrm{E}-\mathrm{C}$ 1. $046 E-03$ $3.624 \mathrm{E}-03$ $3.624 \mathrm{E}-03$
$1.646 \mathrm{E}-0$ $2.717 \mathrm{E}-03$
ANGLE 4 $M U=-0.8656$ $1.303 \mathrm{E}-07$ 4.131E-07 $2.505 E-06$ $3.006 E-06$ $2.132 t-06$ $7.887 E-07$ $-3.646 E-C 6$ . $2.709 E-C 6$ $9.311 E-06$ $7.608 \mathrm{E}-05$ $1.759 \mathrm{E}-04$ $1.052 \mathrm{E}-\mathrm{C} 3$ $2.578 \mathrm{E}-0$ $\triangle N G L E$ is $M U=C .6179$
$7.005 E-07$ $1.15 C E-06$ $1.388 E-05$ $-1.029 E-06$ . $3.458 E-05$ 7.968E-CS $4.537 E-04$ .435E-O4 $7.131 E-04$ $1.100 E-C 3$ . $1.090 \mathrm{E}-\mathrm{C}$ $2.763 E-.03$
ANGLE 5 $M U=-0.7550$ $9.341 \mathrm{E}-\mathrm{O}$ 4. $921 E-06$ $6.086 E-C 6$ $6.132 \mathrm{E}-06$ $3.503 E-06$ $7.796 \mathrm{E}-\mathrm{C} 8$ $-3.621 E-06$ $-5.513 E-06$ $3.044 E-06$ $7.462 E-06$ $1.670 E-05$ $8.469 E-C 5$ $2.651 E-U 4$ $1.033 \mathrm{E}-03$ $2.641 E-C 3$ $8.142 E-C$ $2.444 E-03$

ANGLE 14 $M U=0.7550$ $1.683 E-C 6$ $3.272 E-06$ 1. $360 \mathrm{E}-05$ $1.360 E-05$ 3.641E-05 $1.275 E-04$ $.953 E-C 4$ $4.681 E-0$ $4.911 E-C$ $6.681 E-04$ $7.932 t-04$ $4.0<3 E-C$ $4.0<3 E-C .3$
$1.132 E-C 2$

$2.804 E-C$
ANGLE 6 $M U=-C .6179$

ANGLE 7 $M U=-0.458 C$ ANGLE $\triangle$ ANGLE 9

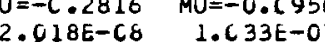
$4.738 E-07-6.016 E-08-2.079 E-07 \quad 1.509 E-C 7$ $4.097 E-06 \quad 1.144 E-06 \quad 1.149 E-66 \quad 3.657 E-06$ $2.202 E-106 \quad-1.829 E-06 \quad-2.7 C 7 E-06 \quad 1.379 E-C 6$ $2.235 E-06 \quad-1.303 E-06 \quad-1.495 E-06 \quad 1.679 E-06$ $1.025 E-06 \quad-5.619 E-07 \quad 8.088 E-C 8 \quad 2.894 E-06$ $4.190 E-07 \quad 1.849 E-06 \quad 4.017 E-06 \quad 5 . C 95 E-C 6$

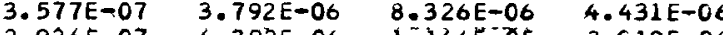
$2.926 E-07$ 6.392E-06 $1.144 E-905$ 3.019E-06 $1.275 E-06 \quad 7.505 E=06 \quad 7.160 E-06 \quad 0.613 E-07$ $\begin{aligned} & 5.863 E-06 \\ & 5.015 E-07\end{aligned}-8.273 E-07 \quad 9.303 E-C 6$ $5.402 E-06 \quad-1.130 E-08$ 1.331E-C5 $6.285 E-05$ $1.144 E-C 4 \quad 1.736 E-04 \quad 2.625 E-C 4 \quad 3.7 C 8 E-04$ 3.902E-04 4.959E-04 $5.841 E-C 4 \quad 6.27 G E-04$ $9.607 E=04$ $9.318 E-04$ 9.264E-C4 2.723E-124E-03 $2.945 E-03 \quad 3.086 E-03$ $\begin{array}{llll}8.341 E-C 3 & 8.587 E-03 & 8.883 E-C 3 & 9.224 E-03 \\ 2.470 E-03 & 2.501 E-03 & 2.538 E-03 & 2.579 E-C 3\end{array}$ $2.579 E-C 3$

ANGLE 15 $\mathrm{U}=0.8656$ $7.173 \mathrm{E}-\mathrm{C6}$ $2.031 \mathrm{E}-\mathrm{C} 5$ $1.252 E-C 4$ 1.116E-04 $1.733 E-04$ $2.377 E-04$ . $4.060 \mathrm{E}=4$ $5.395-C 4$ $7.179 E-04$ 8.571E -04 $1.211 E-03$ 4. $202 \mathrm{E} \rightarrow \mathrm{O}$ $4.202 \mathrm{E}-03$
$1.169 \mathrm{E}-02$ $2.839 \mathrm{E}-0$

ANGLE 16 $M U=0.9446$ $3.533 E-C 5$ $1.053 E-04$ $5.119 \mathrm{E}-04$ $4.494 E-C 4$ $5.424 E-04$ $6.000 E-04$ $6.415 E-04$ $6.473 E-04$ $6.473 E-04$ $5.541 \mathrm{E}-04$ 
COSINE

$-1 . C C O O C E$ OC
$-9.89401 E-01$
$-9.44575 E-01$
$-8.65631 E-01$
$-7.55044 E-C 1$
$-6.17876 E-01$
$-4.58017 E-01$
$-2.81605 E-C 1$
$-9.50125 E-02$
$9.50125 E-02$
$2.81605 E-01$
$4.58017 E-01$
$6.17876 E-01$
$7.55 C 44 E-01$
$8.05631 E-01$
$9.44575 E-C 1$
$9.89401 E-01$

$9.89401 \mathrm{E}-01$

TOTAL

COSINE

-1. COOOOE OO $-9.89401 \bar{c}-01$ $-9.44575 \mathrm{E}-01$ $-7.65044 E-01$ $-6.17876 E-01$ $-4.58017 E-01$ $-2.81655 \mathrm{E}-01$ $-9.50125 E-02$ $9.50125 \mathrm{E}-\mathrm{C} 2$ $2.81605 \mathrm{E}-01$ 4.58017E-O1 $6.17876 \mathrm{E}-01$ $7.55044 E-01$ $8.65631 E-01$ $9.44575 \mathrm{E}-01$ $9.89401 E-01$

TOTAL
75.0

100.0

$6.343 E-11$

$6.368 \mathrm{E}-11$

$6.452 E-11$

$6.585 E-11$

$6.762 \mathrm{E}-11$

$7.034 \mathrm{E}-11$

$7.412 \mathrm{E}-11$

$7.985 \mathrm{E}-11$

$8.786 \mathrm{E}-11$

$9.972 E-11$

$1.173 E-1 C$

$1.37 \mathrm{E}-1 \mathrm{C}$

$1.691 E-1 C$

(3.165E-10

$3.162 E-1 C$

$6.036 E-1 C$

$2.857 \mathrm{E}-10$

$5.824 E-C 9$

$2.465 E-C 9$

$2.481 E-C S$

500.0

600.0

$4.694 \mathrm{E}-11$

4. $709 \mathrm{E}-11$

$4.874 \mathrm{E}-11$

$5.038 \mathrm{E}-11$

$5.265 \mathrm{E}-11$

$5.587 E-11$

$6.031 E-11$

$6.619 E-11$

$7.380 E-11$

$8.366 \mathrm{E}-11$

$1.148 E-1 C$

$1.425 E-10$

$1.886 \mathrm{E}-1 \mathrm{C}$

$2.780 \mathrm{E}-1 \mathrm{C}$

$7.475 \mathrm{E}-1 \mathrm{C}$

$1.207 E-C 9$

$1.39 C E-1 C$

$2.008 E-1 C$
$4.769 E-1 C$
150.0 RANGE (METERS)
290.0

250.0

300.0

400.0

$7.473 E-11$
$7.501 E-11$

$7.501 \mathrm{E}-11$

$7.757 \mathrm{E}-11$

$7.977 \mathrm{E}-11$

$8.3 \mathrm{C5E}-1$

$8.761 \mathrm{E}-11$

$9.439 \mathrm{E}-11$

1.C 3 EE-10

$1.239 E-10$

$1.373 E-10$

$1.467 E-10$

$2.355 E-10$

3.

$5.521 E-10$

$3.912 \mathrm{E}-\mathrm{C} 9$

$7.844 E-11$

$7.711 \mathrm{E}-11$
$7.737 \mathrm{E}-11$

7. $285 \mathrm{E}-11$

$7.309 \mathrm{E}-11$

$7.402 \mathrm{E}-11$
$7.558 \mathrm{E}-11$

$7.972 \mathrm{E}-11$

7. $836 \mathrm{E}-11$

$7.997 \mathrm{E}-11$

.

.

$8.733 E-11$

$9.946 E-11$

$1.093 \mathrm{~F}-10$

$1.263 \mathrm{E}-10$

$1.362 \mathrm{E}-10$

$1.64 \mathrm{EE}-10$

$1.937 E-10$

2.4

$3.439 E-10$

$5.734 E-10$

$2.490 E-C 9$

$2.393 E-09$

.253E-11

$9.096 E-11$

$9.808 \mathrm{E}-11$

$1.077 \mathrm{E}-10$

$1.248 E-10$

$1.336 E-10$

$1.62 C E-10$

$1.885 \mathrm{E}-10$

$2.428 E-10$

$5.309 E-10$

$2.392 E-09$

$8.135 \mathrm{E}-11$

$8.613 \mathrm{E}-11$

$9.290 \mathrm{E}-11$

(3OE

.

$1.304 E-10$

$1.495 \mathrm{E}-10$

.

$4.795 E-10$

$1.886 E-09$

$2.025 E-C C$

$6.032 E-11$

$6.051 E-11$

$6.261 \mathrm{E}-11$

$6.468 \mathrm{E}-11$

$6.752 \mathrm{E}-11$

$7.159 \mathrm{E}-11$

$7.725 \mathrm{E}-11$

$9.483 \mathrm{E}-11$

$1.073 E-10$

$1.243 E-10$

$1.479 E-10$

$1.847 E-10$

$2.471 E-10$

$1.181 \mathrm{E}-09$

RANGE (METERS)

$2.229 E-09$

$1.599 E-09$

$3.514 \mathrm{E}-11 \quad 1.29 \mathrm{CE}-11 \quad 4.239 \mathrm{E}-12 \quad 1.311 \mathrm{E}-12 \quad 3.904 \mathrm{E}-13$

$3.524 E-11 \quad 1.294 E-11 \quad 4.251 E-12 \quad 1.315 E-12 \quad 3.915 E-13$

$3.650 \mathrm{E}-11 \quad 1.341 \mathrm{E}-11 \quad 4.4 \mathrm{CBE}-12 \quad 12364 \mathrm{E}-12 \quad 4.062 \mathrm{E}-13$

$3.774 \mathrm{E}-11 \quad 1.388 \mathrm{E}-11 \quad 4.564 \mathrm{E}-12 \quad 12412 \mathrm{E}-12 \quad 4.209 \mathrm{E}-13$

$3.947 \mathrm{E}-11 \quad 1.454 \mathrm{E}-11 \quad 4.786 \mathrm{E}-12 \quad 1.482 \mathrm{E}-12 \quad 4.417 \mathrm{E}-13$

$4.191 \mathrm{E}-11 \quad 1.546 \mathrm{E}-11 \quad 5.089 \mathrm{E}-12 \quad 12576 \mathrm{E}-12 \quad 4.698 \mathrm{E}-13$

$4.525 \mathrm{E}-11 \quad 1.669 \mathrm{E}-11 \quad 5.494 \mathrm{E}-12 \quad 1.701 \mathrm{E}-12 \quad 5.070 \mathrm{E}-13$

$4.965 \mathrm{E}-11 \quad 1.829 \mathrm{E}-11 \quad 6.018 \mathrm{E}-12 \quad 1.862 \mathrm{E}-12 \quad 5.548 \mathrm{E}-13$

$5.534 \mathrm{E}-11 \quad 2.035 \mathrm{E}-11 \quad 6.688 \mathrm{E}-12 \quad 2.068 \mathrm{E}-12 \quad 6.157 \mathrm{E}-13$

$\begin{array}{lllll}6.264 \mathrm{E}-11 & 2.3 \mathrm{COE}-11 & 7.548 \mathrm{E}-12 & 2.332 \mathrm{E}-12 & 6.940 \mathrm{E}-13\end{array}$

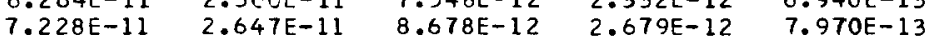

$8.573 E-11 \quad 3.128 \mathrm{E}-11 \quad 1.023 \mathrm{E}-11 \quad 3.154 \mathrm{E}-12 \quad 9.372 \mathrm{E}-13$

$\begin{array}{lllll}1 . C 60 E-10 & 3.834 E-11 & 1.247 E-11 & 3.830 E-12 & 1.135 E-12\end{array}$

$4.934 E-11$

$1.247 E-11$

4. $83 \mathrm{CE}-12$

$1.422 \mathrm{E}-12$

$6.837 E-11$
$1.275 E-10$

3. $479 E-11$

$6.381 E-12$
$9.549 E-12$

$2.620 \mathrm{E}-12$

$3.134 E-10$

$1.010 E-10$

$3.086 E-11$

$9.118 \mathrm{E}-12$ 
COS INE

$-1.00000 \mathrm{E} 00$
$-9.89401 \mathrm{E}-01$
$-9.44575 \mathrm{E}-01$
$-8.65631 \mathrm{E}-01$
$-7.55044 \mathrm{E}-01$
$-6.17876 \mathrm{E}-01$
$-4.58017 \mathrm{E}-01$
$-2.81605 \mathrm{E}-01$
$-9.50125 \mathrm{E}-02$
$9.50125 \mathrm{E}-02$
$2.81605 \mathrm{E}-01$
$4.58017 \mathrm{E}-01$
$6.17876 \mathrm{E}-01$
$7.55044 \mathrm{E}-01$
$8.65631 \mathrm{E}-01$
$9.44575 \mathrm{E}-01$
$9.894 \mathrm{C} 1 \mathrm{E}-01$

TOTAL

COSINE

$-1.00000 E 00$ $-9.89401 E-01$ $-9.44575 E-01$ $-8.65631 E-01$ $-7.55044 E-01$ $-6.17876 \mathrm{E}-01$ $-4.58017 \mathrm{E}-01$ $-2.81605 \mathrm{E}-01$ $-9.50125 E-C 2$ $9.50125 E-02$ $2.81605 \mathrm{E}-01$ $4.58017 E-01$ 6.17876E-01 . $55044 E-01$ $8.65631 \mathrm{E}-01$ $9.89401 \mathrm{E}-01$

TOTAL

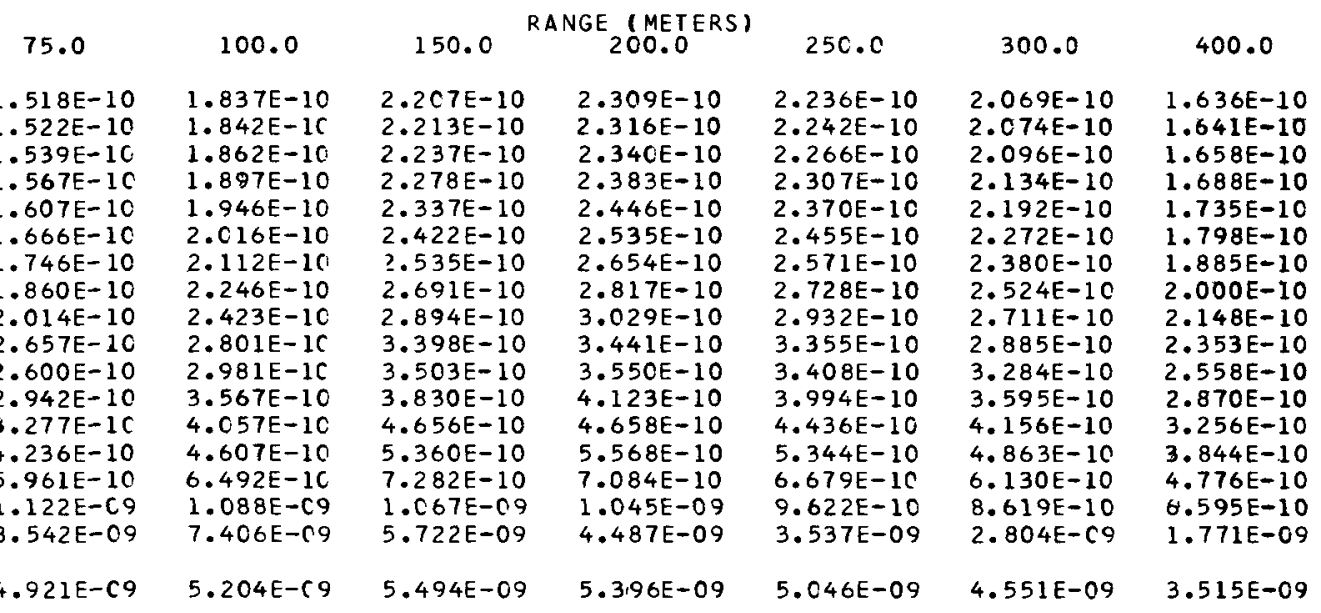

500.0

$1.223 \mathrm{E}-10$ $1.226 \mathrm{E}-10$ $1.239 \mathrm{E}-10$ $1.262 E-10$ $1.297 \mathrm{E}-10$ $1.345 \mathrm{E}-10$ $1.410 \mathrm{E}-10$ $1.497 \mathrm{E}-10$ $1.607 E-10$ $1.742 E-10$ $1.923 E-10$ $2.141 E-10$ $2.440 E-10$ $2.868 \mathrm{E}-10$ $3.553 E-10$ $4.828 \mathrm{E}-10$

$2.589 E-C 9$ 600.0

$8.877 E-11$ 900.0 1200.0 1500.0 1800.0 $3.108 E-11 \quad 1.005 E-11 \quad 3.087 E-12 \quad 9.157 E-13$ $3.116 E-11 \quad 1.007 E-11 \quad 3.094 E-12 \quad 9.180 E-13$ $3.148 \mathrm{E}-11 \quad 1.018 \mathrm{E}-11 \quad 3.127 \mathrm{E}-12 \quad 9.276 \mathrm{E}-13$ $3.208 \mathrm{E}-11$ 1.038E-11 3.187E-12 $9.456 \mathrm{E}-13$ $3.299 E-11 \quad 1.067 E-11 \quad 3.278 E-12 \quad 9.728 E-13$ $3.424 \mathrm{E}-11 \quad 1.108 \mathrm{E}-11 \quad 3.405 \mathrm{E}-12 \quad 1.011 \mathrm{E}-12$ $3.593 E-11$ 1.163E-11 3.574E-12 $1.061 E-12$ $3.815 \mathrm{E}-11 \quad 1.234 \mathrm{E}-11 \quad 3.793 \mathrm{E}-12 \quad 1.125 \mathrm{E}-12$ 4.C95E-11 1.325E-11 4.068E-12 1.207E-12 $4.447 E-11$ 1.437E-11 4.412E-12 $1.308 E-12$ $4.887 E-11 \quad 1.578 E-11 \quad 4.841 E-12 \quad 1.435 E-12$ $5.447 E-11$ 1.758E-11 $5.390 E-12$ 1.597E-12 $6.196 \mathrm{E}-11$ 1.997E-11 6.118E-12 $1.811 \mathrm{E}-12$ $7.256 \mathrm{E}-112.331 \mathrm{E}-11 \mathrm{~T} 2.123 \mathrm{E}-12 \mathrm{2} 2.104 \mathrm{E}-12$ $8.861 E-11 \quad 2.824 E-11 \quad 8.573 E-12 \quad 2.520 E-12$ $1.155 E-10$ 3.605E-11 $1.078 E-11 \quad 3.132 E-12$

$6.393 E-10 \quad 2.042 E-10 \quad 6.222 E-11 \quad 1.835 E-11$ 
COS INE

$$
\begin{aligned}
& -1.06000 \mathrm{E} 00 \\
& -9.894 \mathrm{C} 1 \mathrm{E}-01 \\
& -9.44575 \mathrm{E}-01 \\
& -8.55631 \mathrm{E}-01 \\
& -7.55044 \mathrm{E}-01 \\
& -6.17876 \mathrm{E}-01 \\
& -4.58017 \mathrm{E}-01 \\
& -2.81605 \mathrm{E}-01 \\
& -9.50125 \mathrm{E}-02 \\
& 9.50125 \mathrm{E}-\mathrm{C} 2 \\
& 2.81605 \mathrm{E}-01 \\
& 4.58017 \mathrm{E}-01 \\
& 6.17876 \mathrm{E}-01 \\
& 7.55044 \mathrm{E}-01 \\
& 8.65631 \mathrm{E}-01 \\
& 9.44575 \mathrm{E}-01 \\
& 9.89401 \mathrm{E}-01
\end{aligned}
$$

TOTAL

COSINE

$-1.00 C 00 E$ OO $-9.89401 \mathrm{E}-01$

$-9.44575 E-01$

$-8.65631 E-01$

$-7.55044 E-01$

$-6.17876 \mathrm{E}-01$

$-2.816 C 5 E-01$

$-9.50125 \mathrm{E}-02$

$9.50125 \mathrm{E}-\mathrm{C} 2$

$2.51605 \mathrm{E}-01$

. $58017 E-01$

$6.17876 \mathrm{E}-01$

$7.55044 \mathrm{E}-01$

$0.65631 E-01$

$9.44575 E-01$

$9.89401 E-01$

TOTAL

$$
75.0
$$

100.0

$7.798 E-09$ $7.829 E-09$

$7.935 E-C 9$

$8.1 C 4 E-09$

$8.329 E-C 9$

$9.128 E-09$

$9.815 E-C$

$1.076 \mathrm{E}-\mathrm{C} 8$

$1.254 E-C 8$

$1.412 \mathrm{E}-08$

$1.711 \mathrm{E}-\mathrm{C} 8$

$1.985 \mathrm{E}-\mathrm{C} 8$

$2.413 E-C 8$

$3.64 C E-08$

$6.76 C E-C 8$

$5.50 C E-07$

$2.866 E-07$

$2.830 E-C 7$

$$
500.0
$$

600.0

$5.531 \mathrm{E}-\mathrm{CO}$ $5.548 \mathrm{E}-\mathrm{C} 9$ $5.616 E-C 9$ $5.926 E-09$ . $926 E-09$ . $182 E-09$ 7. . 7.683E-C . . 1. $.584 \mathrm{E}-08$ $.072 \mathrm{E}-\mathrm{C} B$ $3.015 \mathrm{E}-08$ $8.032 \mathrm{E}-\mathrm{C} 8$

$1.364 E-07$
150.0 RANGE (METERS)
200.0

250.0

300.0

$400 . \mathrm{C}$

$9.133 E-09$ $9.326 E-09$ 9.676E-09 $9.445 E-09$ $9.759 E-\cap 9 \quad 1.017 E-08 \quad 9.946 E-09$ $\begin{array}{lll}1.016 E-08 & 1.059 E-08 & 1.035 E-08\end{array}$ $1.070 \mathrm{E}-08 \quad 1.118 \mathrm{E}-08 \quad 1.093 \mathrm{E}-08$ $1.150 \mathrm{E}-08 \quad 1.201 \mathrm{E}-\mathrm{C8} \quad 1.175 \mathrm{E}-08$ $1.259 E-08 \quad 1.314 E-C 8 \quad 1.285 E-08$ $1.582 \mathrm{E}-\mathrm{C} 8 \mathrm{~B} \quad 1.512 \mathrm{E}-08 \quad 1.483 \mathrm{E}-08$ $\begin{array}{lll}1.618 E-C 8 & 1.621 E-08 & 1.575 E-08\end{array}$ $1.754 E-C 8 \quad 1.932 E-08 \quad 1.887 E-08$ $2.275 E-C 8 \quad 2.267 E-08 \quad 2.183 E-08$ $2.757 E-C 8 \quad 2.858 E-08 \quad 2.761 E-08$ $\begin{array}{lll}3.999 E-08 & 3.889 E-08 & 3.695 E-08\end{array}$ $\begin{array}{lll}4.228 E-07 & 3.295 E-07 & 2.579 E-07\end{array}$

$2.882 \mathrm{E}-07$

$2.756 E-07$

$2.558 E-07$

$8.727 E-09$ $7.156 E-C 9$ $7.156 E-09$
$8.756 E-C 9$ $9.054 \mathrm{E}-09 \quad 7.425 \mathrm{E}-09$ $9.342 \mathrm{E}-09 \quad 7.664 \mathrm{E}-09$ $9.727 E-C 9 \quad 7.989 E-09$ $1.028 \mathrm{E}-\mathrm{C} 8 \quad 8.448 \mathrm{E}-\mathrm{C} 9$ $1.105 E-C 8 \quad 9.083 E-09$ $1.207 \mathrm{E}-08 \quad 9.924 \mathrm{E}-09$ $1.326 E-C 8 \quad 1.105 E-08$ $1.525 \mathrm{E}-\mathrm{C} 8 \quad 1.239 \mathrm{E}-\mathrm{C} 8$ $1.732 \mathrm{E}-08 \quad 1.424 \mathrm{E}-08$ $2.067 \mathrm{E}-\mathrm{C} 8 \quad 1.677 \mathrm{E}-08$ $2.548 \mathrm{E}-08 \quad 2.068 \mathrm{E}-08$ $3.421 E-C 8 \quad 2.729 E-08$ $5.254 \mathrm{E}-\mathrm{C} 8 \quad 4.068 \mathrm{E}-08$ 2.032E-07 1.271E-07

RANGE (METERS)

QOOC.O 1200.0

$150 \mathrm{C} \cdot \mathrm{C}$

1800.0 4.133E-09 $1.507 E-09 \quad 4.938 E-10 \quad 1.525 E-10 \quad 4.538 E-11$ $4.184 \mathrm{E}-\mathrm{C} 9 \mathrm{1}-526 \mathrm{E}-09 \quad 4.999 \mathrm{E}-10 \quad 1.544 \mathrm{E}-10 \quad 4.594 \mathrm{E}-11$ $4.276 E-C 9$ 1.560E $4.416 E-C 9$ 1.612E-C9 $5.286 E-10$ 1.633E-10 $4.861 E-11$

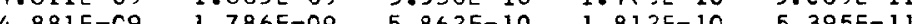
$5.25 \mathrm{CE}-\mathrm{CO} \quad 1.920 \mathrm{E}-09$ 6.301E-10 $1.812 \mathrm{E}-10 \mathrm{O}-5.395 \mathrm{E}-11$ 2.094E-09 6.367E-10 2.121E $1000.311 E-11$ $6.731 E-C$ 2. $2.317 E-09$ 7.586E-10 $2.121 E-10$ 6.311E-11 $7.130 E-C O \quad 2.317 E-C 9$ (.5BE-10 $2.341 E-10$ 6.962E-11 2.170E-C $2.968 \mathrm{E}-09$ $9.696 \mathrm{E}-10 \quad 2.987 \mathrm{E}-10 \mathrm{O}-8.875 \mathrm{E}-11$ $8.170 E-C 9.968 E-09$ 9.696E-10 $2.987 E-10 \quad 8.875 E-11$ $1.173 E-08 \quad 4.214 E-09 \quad 1.367 E-09 \quad 4.190 E-10 \quad 1.034 E-10$ $\begin{array}{lllll}1.173 E-08 & 4.214 E-09 & 1.367 E-09 & 4.190 E-10 & 1.240 E-10 \\ 1.521 E-C 8 & 5.370 E-C 9 & 1.7211 E-09 & 5.232 E-10 & 1.538 E-10\end{array}$ $\begin{array}{lllll}1.521 \mathrm{E}-\mathrm{CB} & 5.370 \mathrm{E}-\mathrm{C} 9 & 1.721 \mathrm{E}-09 & 5.232 \mathrm{E}-10 & 1.538 \mathrm{E}-10 \\ 2.173 \mathrm{E}-\mathrm{C8} & 7.365 \mathrm{E}-\mathrm{Cg} & 2.298 \mathrm{E}-09 & 6.852 \mathrm{E}-10 & 1.986 \mathrm{E}-10\end{array}$ $5.119 E-C 8 \quad 1.364 E-C 8 \quad 3.716 E-09 \quad 1.018 E-09 \quad 2.981 E-10$ $9.969 E-C 8 \quad 3.511 E-08 \quad 1.129 E-08 \quad 3.445 E-09 \quad 1.017 E-C 9$ 
COSINE

$-1.00000 \mathrm{E} 00$ $-9.89401 E-01$ $-9.44575 \mathrm{E}-01$ $-8.65631 E-01$ $-7.55044 \mathrm{E}-01$ $-6.17876 E-01$ $-4.58017 E-01$ $-2.81605 E-01$ $-9.50125 E-02$ $9.50125 E-02$ $2.81605 \mathrm{E}-01$ 4.58017E-01 $6.17876 \mathrm{E}-01$ 7.55044E-01 $8.65631 E-01$ $9.44575 E-01$ $9.89401 E-01$

TOTAL

COSINE

-1.00000 E 00 $-9.89401 \mathrm{E}-01$ $-9.44575 E-01$ $-8.65631 E-01$ $-7.55044 E-01$ $-6.17876 E-01$ $-4.58017 E-01$ $-2.81605 E-01$ $-9.50125 \mathrm{E}-02$ . $2.81605 E-01$ $6.17876 \mathrm{E}-01$ $7.17576 \mathrm{E}-01$ $8.65631 \mathrm{E}-01$ $8.65631 \mathrm{E}-01$ $9.89401 \mathrm{E}-01$

TOTAL

$\begin{array}{cc}75.0 & 100.0 \\ 2.834 E-11 & 3.419 E-11 \\ 2.850 E-11 & 3.437 E-11 \\ 2.897 E-11 & 3.491 E-11 \\ 2.958 E-11 & 3.564 E-11 \\ 3.024 E-11 & 3.646 E-11 \\ 3.127 E-11 & 3.769 E-11 \\ 3.263 E-11 & 3.933 E-11 \\ 3.490 E-11 & 4.199 E-11 \\ 3.816 E-11 & 4.575 E-11 \\ 5.282 E-11 & 5.213 E-11 \\ 5.014 E-11 & 5.777 E-11 \\ 5.731 E-11 & 7.044 E-11 \\ 6.638 E-11 & 7.925 E-11 \\ 9.572 E-11 & 9.967 E-11 \\ 1.364 E-10 & 1.496 E-10 \\ 3.281 E-10 & 3.008 E-10 \\ 3.323 E-09 & 2.921 E-09 \\ & \\ 1.303 E-09 & 1.310 E-09\end{array}$

150.0

RANGE (METERS)
200.0

250.0

300.0

400.0

4.099E-11

4.277E-11

4.126E-11

$4.142 E-11$

$4.272 E-11$

$4.391 E-11$

$4.177 E-11 \quad 4.354 E-1$

$4.362 E-11 \quad 4.547 E-11$

$4.510 E-11 \quad 4.703 E-11$

4.708E-11

$4.918 E-11$

$5.244 E-11$

$4.534 \mathrm{E}-11$

4.747E-11

$5.064 E-11$

$5.694 E-11$
$6.594 E-11$

6.496E-11

$5.450 E-11$

$6.792 \mathrm{E}-1$

$6.5 C 1 \mathrm{E}-11$

$6.842 \mathrm{E}-11$

$8.076 \mathrm{E}-11$

7.81CE-11

$9.198 \mathrm{E}-11$

$1.611 E-10$

$2.815 E-10$

$8.778 \mathrm{E}-11$

$1.118 E-10$

$1.529 \mathrm{E}-10$

$1.418 \mathrm{E}-09$
1.09

$2.907 E-10$

$1.800 E-09$

1.139E-09

$3.798 \mathrm{E}-11$

$2.979 \mathrm{E}-11$

$3.060 E-11 \quad 3.015 E-11$

$3.931 \mathrm{E}-11 \quad 3.071 \mathrm{E}-11$

4.039E-11 3.156E-11

$4.173 \mathrm{E}-11 \quad 3.265 \mathrm{E}-11$

$4.373 \mathrm{E}-11 \quad 3.426 \mathrm{E}-11$

$4.665 \mathrm{E}-11 \quad 3.659 \mathrm{E}-11$

$5.060 \mathrm{E}-11 \quad 3.972 \mathrm{E}-11$

$6.294 E-11 \quad 4.408 E-11$

$6.968 \mathrm{E}-11 \quad 5.535 \mathrm{E}-11$

$8.261 \mathrm{E}-11 \quad 6.458 \mathrm{E}-11$

$1.017 E-10 \quad 8.066 \mathrm{E}-11$

$1.413 E-10 \quad 1.110 E-10$

$2.334 E-10 \quad 1.800 E-10$

$1.120 E-09 \quad 6.988 E-10$

$1.307 E-09$

$1.244 E-09$

$1.011 E-09$

$7.632 E-10$

\section{0}

1. $572 E-13$

1.576E-13

$1.592 E-13$

$1.672 \mathrm{E}-13$

$1.741 \mathrm{E}-13$

$1.836 E-13$

(2)

$2.337 E-13$

.

$2.091 E-13$

$3.558 \mathrm{E}-13$

$4.450 E-13$

$5.907 \mathrm{E}-13$

8. $340 \mathrm{E}-13$

3. $638 \mathrm{E}-12$ 
COSINE

$-1.0000 C E$ CO $-9.89401 \mathrm{E}-01$ $-9.44575 E-01$ $-8.65631 E-01$ $-7.55 C 44 E-01$ $-6.17876 E-01$ $-4.58017 \mathrm{E}-01$ $-2.81605 E-01$ $-9.50125 \mathrm{E}-02$ $9.50125 \mathrm{E}-02$ 2.81605E-01 $4.58017 \mathrm{E}-01$ $6.17876 \mathrm{E}-01$ $7.55044 \mathrm{E}-01$ $8.65631 \mathrm{E}-01$ $9.89401 E-01$

TOTAL

COSINE

$-1.00000 E$ OC $-9.89401 E-C 1$ $-9.44575 \mathrm{E}-01$ $-8.65631 E-01$ $-7.55044 \mathrm{E}-01$ $-4.58017 \mathrm{E}-01$ $2.81605 E-01$ $-2.81605 E-01$ $0.50125 \mathrm{E}-02$ . 4. $58 \mathrm{C} 17 \mathrm{E}-01$ $6.17876 \mathrm{E}-01$ $7.55044 \mathrm{E}-\mathrm{O} 1$ $7.55044 E-01$ $8.65631 E-C 1$ $9.89401 E-01$

TOTAL
75.0

100.0

$7.351 E-10$
$7.404 E-10$

$7.561 \mathrm{E}-10$

$7.766 \mathrm{E}-1 \mathrm{C}$

$7.981 \mathrm{E}-10$

$8.305 \mathrm{E}-10$

$8.719 E-1 C$

$9.408 \mathrm{E}-10$

$1.041 \mathrm{E}-09$

$1.485 E-09$

$1.445 \mathrm{E}-\mathrm{C9}$

$1.611 \mathrm{E}-09$

$1.974 \mathrm{E}-09$

$2.929 E-09$

$4.230 E-C 9$

1.050 E- 07

$3.931 E-08$

500.0

$6.307 \mathrm{E}-10$

$6.332 \mathrm{E}-10$

$6.425 E-10$

$6.577 E-10$

7.

7. $521 \mathrm{E}-10$

8. $122 E-10$

.

$9.978 \mathrm{E}-1 \mathrm{C}$

$1.133 E-C 9$

$1.133 E-C 9$

$1.556 \mathrm{E}-\mathrm{CQ}$

$1.951 E-C O$

$2.651 E-09$

$4.097 E-C 9$

$1.263 E-C 8$

$1.696 \mathrm{E}-08$
$8.655 E-1 C$ $8.713 \mathrm{E}-1 \mathrm{C}$

$8.89 \mathrm{CE}-1 \mathrm{C}$

0

$9.77 \mathrm{CE}-10$

$1.026 \mathrm{E}-09$

$1.106 \mathrm{E}-\mathrm{C} 9$

$1.222 \mathrm{E}-\mathrm{C} 9$

$1.4 C 5 \mathrm{E}-\mathrm{C} 9$

$1.639 \mathrm{E}-\mathrm{C} 9$

$1.958 \mathrm{E} C \mathrm{C}$

$2.353 E-C 9$

.

$4.63 C E-09$

$9.134 \mathrm{E}-\mathrm{C} 8$

$3.875 \mathrm{E}-08$

600.0

$4.712 E-1 C$ $4.729 E-1 C$ $97 E-1 C$ $4.911 E-16$ $5.305 E-1 C$ $5.626 \mathrm{E}-10$ $6.076 E-10$ $6.675 \mathrm{E}-1 \mathrm{C}$ $7.455 \mathrm{E}-1 \mathrm{C}$ $8.444 E-10$ $9.738 \mathrm{E}-10$ $1.157 \mathrm{E}-\mathrm{CO}$ $1.445 \mathrm{E}-\mathrm{C} 9$ $1.944 \mathrm{E}-\mathrm{C} 9$ $2.938 E-C 9$
$7.9 C O E-C 9$

$1.232 \mathrm{E}-\mathrm{C} 8$
150.0 RANGE (METERS)
$2 \mathrm{COO} 0.0$

250.0

300.0

400.0

$1.018 \mathrm{E}-09$
$.024 \mathrm{E}-\mathrm{C} 9$

$1.065 \mathrm{E}-09$

$1.045 E-09$
$1.05 C E-09$

$9.850 E-10$

$8.127 E-10$

$.043 E-C 9 \quad 1.09$

1.090-OQ $1.068 E-09$

- $C 1 E-C 9$

.147E-O

.206E-09

$1.433 E-09$

$1.754 \mathrm{E}-09$

1.9 C 1E-C9

$2.054 \mathrm{E}-09$

$2.689 E-09$

$3.334 E-C 9$

$5.694 E-09$

7.C $10 E-08$

.1151E-09

$.092 E-09$
$.130 E-09$

$1.175 \mathrm{E}-09$

1.19G-C9

.264E-09

$1.363 E-09$

$1.502 E-09$

$1.889 \mathrm{E}-09$

$2.273 E-09$

$2.686 \mathrm{E}-09$

$3.483 E-09$

$4.945 E-09$

$5.443 E-08$

$1.339 E-09$

$1.339 \mathrm{E}-09$
$1.475 \mathrm{E}-09$

$1.713 \mathrm{E}-09$

$1.843 \mathrm{E}-09$

$2.226 \mathrm{E}-09$

$2.601 E-09$

3. $373 \mathrm{E}-09$

$4.711 E-09$

$4.234 \mathrm{E}-08$

$3.556 \mathrm{E}-08$

$3.263 E-08$

8.160E-10

$1.006 E-C 9 \quad 8.286 E-10$

8.480E-10

$1.065 E-C 9 \quad 8.772 E-10$

$9.140 E-10$
$1.1710-C 9$

$1.171 \mathrm{E}-09 \quad 9.675 \mathrm{E}-10$

$1.391 E-09 \quad 1.149 E-09$

$1.545 \mathrm{E}-09 \quad 1.289 \mathrm{E}-09$

$1.787 \mathrm{E}-09 \quad 1.460 \mathrm{E}-09$

$2.049 E-09 \quad 1.691 E-09$

$2.472 E-C 9 \quad 2.015 E-09$

$2.542 E-C 9$

$7.371 E-09$ 3.494E-09

$3.310 \mathrm{E}-\mathrm{CB} \quad 2.035 \mathrm{E}-08$

$2.931 E-C 8 \quad 2.274 E-08$

RANGE (METERS)

$1.725 \mathrm{E}-10$

$12 C 0.0$

$15 C C . C$

1800.0

5.745E-11 $1.753 E-11 \quad 5.216 E-12$

$\begin{array}{llll}1.796 \mathrm{E}-10 & 5.887 \mathrm{E}-11 & 1.818 \mathrm{E}-11 & 5.410 \mathrm{E}-12\end{array}$

$1.858 \mathrm{E}-10$ 6.093E-11 $1.882 \mathrm{E}-11 \quad 5.602 \mathrm{E}-12$

$1.944 E-10 \quad 6.382 E-11 \quad 1.973 E-11 \quad 5.873 E-12$

$\begin{array}{llll}2.065 E-10 & 6.780 E-11 & 2.096 E-11 & 6.241 E-12\end{array}$

$2.444 E-10$ 8.013E-11 $2.267 E^{2}-112.728 E-12$

$2.722 \mathrm{E}-10 \quad 8.9 \mathrm{C}$ E-11 $2.745 \mathrm{E}-1128.156 \mathrm{E}-12$

$3.074 E-10$ 1.004E-10 $3.092 E-11 \quad 9.180 E-12$

$3.537 \mathrm{E}-10$ 1. $154 \mathrm{E}-10$ 3.549E-11 $1.053 \mathrm{E}-11$

$4.188 \mathrm{E}-10 \quad 1.363 \mathrm{E}-10 \quad 4.184 \mathrm{E}-11 \quad 1.240 \mathrm{E}-11$

$5.179 E-10 \quad 1.674 E-10 \quad 5.113 E-11 \quad 1.509 E-11$

$6.812 E-10 \quad 2.166 E-10 \quad 6.54 C E-11 \quad 1.913 E-11$

$\begin{array}{llll}9.792 \mathrm{E}-10 & 3.008 \mathrm{E}-10 & 8.853 \mathrm{E}-11 & 2.540 \mathrm{E}-11 \\ 2.000 \mathrm{l}-09 & 5.229 \mathrm{E}-10 & 1.39 \mathrm{CE}-10 & 3.725 \mathrm{E}-11\end{array}$

$4.283 E-C 9 \quad 1.366 E-09 \quad 4.144 E-1 C \quad 1.218 E-1 C$ 


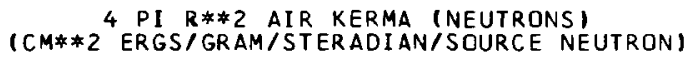

THERMONUCLEAR SOURCE

$\begin{array}{cc}75.0 & 100.0 \\ 9.124 E-10 & 1.139 E-C 9 \\ 9.204 E-10 & 1.147 E-09 \\ 9.424 E-1 C & 1.172 E-C 9 \\ 9.679 E-10 & 1.202 E-C 9 \\ 9.897 E-10 & 1.23 C E-C 9 \\ 1.023 E-C 9 & 1.270 E-C 9 \\ 1.062 E-C 9 & 1.319 E-09 \\ 1.137 E-09 & 1.407 E-C 9 \\ 1.249 E-C 9 & 1.538 E-C 9 \\ 1.788 E-09 & 1.691 E-C 9 \\ 1.678 E-09 & 2.012 E-C 9 \\ 1.876 E-09 & 2.356 E-09 \\ 2.312 E-C 9 & 2.799 E-C 9 \\ 3.604 E-09 & 3.732 E-C 9 \\ 5.121 E-09 & 5.767 E-C 9 \\ 1.397 E-C 8 & 1.255 E-C 8 \\ 1.548 E-67 & 1.357 E-07 \\ 5.274 E-C 8 & 5.226 E-C 8\end{array}$

150.0 RANGE (METERS)
$200 . \mathrm{C}$

250.0

300.0

400.0

$\begin{array}{lll}1.466 E-09 & 1.623 E-09 & 1.636 E-09 \\ 1.475 E-09 & 1.631 E-09 & 1.644 E-09\end{array}$

$1.475 E-09 \quad 1.631 E-09 \quad 1.644 E-09$

$1.537 \mathrm{E}-09 \quad 1.692 \mathrm{E}-09 \quad 1.069 \mathrm{E}-09$

$1.573 \mathrm{E}-\mathrm{C} 9 \quad 1.734 \mathrm{E}-09 \quad 1.749 \mathrm{E}-09$

$1.623 E-09 \quad 1.791 E-09 \quad 1.803 E-09$

$1.687 \mathrm{E}-09 \quad 1.866 \mathrm{E}-09 \quad 1.882 \mathrm{E}-09$

$1.797 E-09 \quad 1.986 E-09 \quad 2 . C C 3 E-09$

$1.954 E-09 \quad 2.155 E-09 \quad 2.171 E-09$

$2.204 \mathrm{E}-09 \quad 2.461 \mathrm{E}-\mathrm{C9} \quad 2.482 \mathrm{E}-09$

$2.534 E-09 \quad 2.6 C 3 E-09 \quad 2.605 E-09$

$2.706 E-09 \quad 3.089 E-C 9 \quad 3.095 E-09$

$3.364 E-09 \quad 3.543 E-09 \quad 3.521 E-09$

4.265E-09 4.587E-09 4.536E-09

$6.515 E-09 \quad 6.533 E-09 \quad 6.348 E-0$

$1.222 E-08 \quad 1.196 E-08 \quad 1.113 E-08$

$1.052 E-07 \quad 8.234 E-08 \quad 6.439 E-08$

$5.223 E-08$

$5.056 E-08$

$4.712 E-08$

$1.554 \mathrm{E}-\mathrm{C} 9$

$1.560 E-C 9$

$1.583 \mathrm{E}-09$

$1.613 \mathrm{E}-\mathrm{C} 9$

$1.659 \mathrm{E}-\mathrm{C} 9$

$1.711 \mathrm{E}-\mathrm{CO}$

$1.788 \mathrm{E}-09$

$1.904 \mathrm{E}-09$

$2.063 E-09$

$2.251 \mathrm{E}-09$

$2.555 E-09$

. $864 E-09$

.390E-C9

$4.229 \mathrm{E}-09$

$5.957 E-C 9$

$1.007 E-08$

$5.050 \mathrm{E}-08$

$4.257 E-C 8$

$.255 \mathrm{E}-09$

$1.277 \mathrm{E}-09$

$1.302 E-C 9$

$1.339 \mathrm{E}-\mathrm{OQ}$

$1.383 \mathrm{E}-09$

$1.448 E-09$

$1.544 E-09$

$1.674 \mathrm{E}-09$

$1.848 \mathrm{E}-09$

$2.056 \mathrm{E}-09$

2.337E-09

$2.741 \mathrm{E}-09$

3.434E-09

4.767E-09

$7.805 E-C 9$

3. $109 \mathrm{E}-08$

RANGE (METERS)

500.0

600.0

900.0

1200.0

1500.0

1800.0

$3.266 E-08$

$9.364 \mathrm{E}-10 \quad 6.733 \mathrm{E}-1 \mathrm{O}$

$9.397 E-10$

$9.519 E-10$

$9.707 E-10$

$9.983 \mathrm{E}-10$

$1.033 E-09$

$1.083 \mathrm{E}-\mathrm{C} 9$

$1.157 E-09$

$1.255 \mathrm{E}-\mathrm{C} 9$

$1.383 \mathrm{E}-\mathrm{Cg}$

$1.546 E-09$

1.757E-09

2.591E-09

$2.591 E-09$

5.573E-CO

$1.923 E-C 8$

$6.755 \mathrm{E}-10$

$6.84 \mathrm{CE}-10$

$6.975 E-1 C$

$7.175 \mathrm{E}-10$

$7.432 E-1 C$

$7.807 E-10$

$8.342 E-10$

$9.058 E-10$

1.117E-CO

$1.273 E-C 9$

$1.502 \mathrm{E}-00$

$1.884 E-09$

$2.591 E-09$

$2.299 \mathrm{E}-10 \quad 7.360 \mathrm{E}-11$

$2.306 \mathrm{E}-10 \quad 7.380 \mathrm{E}-11$

$2.258 \mathrm{E}-11$
$2.283 \mathrm{E}-11$

$6.669 E-12$

$6.686 \mathrm{E}-12$

$7.462 \mathrm{E}-11$ 2.283E-11 $6.758 \mathrm{E}-12$

$2.379 \mathrm{E}-10 \quad 7.611 \mathrm{E}-11 \quad 2.328 \mathrm{E}-11 \quad 6.893 \mathrm{E}-12$

$2.448 \mathrm{E}-10 \quad 7.834 \mathrm{E}-11 \quad 2.397 \mathrm{E}-11 \quad 7.098 \mathrm{E}-12$

2.542E-10 $8.146 \mathrm{E}-11$ 2.495E-11 $7.391 \mathrm{E}-12$

$2.676 \mathrm{E}-10 \quad 8.585 \mathrm{E}-11 \quad 2.63 \mathrm{CE}-11 \quad 7.793 \mathrm{E}-12$

9.184E-11 2.813E-11 $8.332 \mathrm{E}-12$

. $.111 E-10$ $9.92 E-11$ 3.052E-11 $9.034 E-12$

$3.431 E-10$ 1.09E-10 3.360E-11 $9.939 E-12$

$3.841 E-10$ 1.230E-10 3.760E-11 $1.112 E-11$

$5.393 E-10$ 1.408E-10 4.3C7E-11 $1.274 E-11$

$6.53 E-10$ 2.071EE 10 5.

$6.531 E-10$ 2.088E-10 6.357E-11 $1.872 E-11$

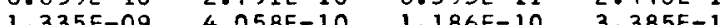

$\begin{array}{llll}1.335 \mathrm{E}-09 & 4.058 \mathrm{E}-10 & 1.186 \mathrm{E}-10 & 3.385 \mathrm{E}-11 \\ 2.956 \mathrm{E}-09 & 7.567 \mathrm{E}-10 & 1.977 \mathrm{E}-1 \mathrm{C} & 5.227 \mathrm{E}-11\end{array}$

$1.195 E-08$

$2.376 \mathrm{E}-\mathrm{C} 8$

$5.560 E-C 9$

$1.737 \mathrm{E}-09$

5. $225 E-10$

1. $528 \mathrm{E}-1 \mathrm{C}$

$5.5605-09$

(n)

\begin{abstract}
T28 -10
\end{abstract}


COSINE

$-1.00000 E \quad 00$
$-9.89401 \mathrm{E}-\mathrm{Cl}$
$-9.44575 \mathrm{E}-01$
$-8.65631 \mathrm{E}-01$
$-7.55044 \mathrm{E}-01$
$-6.17876 \mathrm{E}-01$
$-4.58017 \mathrm{E}-01$
$-2.81605 \mathrm{E}-01$
$-9.50125 \mathrm{E}-02$
$9.50125 \mathrm{E}-02$
$2.81605 \mathrm{E}-01$
$4.58017 \mathrm{E}-01$
$6.17876 \mathrm{E}-01$
$7.55644 \mathrm{E}-01$
$8.65631 \mathrm{E}-01$
$9.44575 \mathrm{E}-01$
$9.89401 \mathrm{E}-01$

TOTAL

COSINE

$-1.00000 E 00$
$-9.89401 E-01$
$-9.44575 E-01$
$-8.65631 E-01$
$-7.55044 E-01$
$-6.17876 E-01$
$-4.58017 E-01$
$-2.81605 E-01$
$-9.50125 E-02$
$9.50125 E-02$
$2.81605 E-01$
$4.58017 E-01$
$6.17876 E-01$
$7.55044 E-01$
$8.65631 E-01$
$9.44575 E-01$
$9.89401 E-01$

$9.89401 E-01$

TOTAL

\begin{tabular}{|c|c|c|c|c|c|c|}
\hline 75.0 & $100 \cdot \mathrm{C}$ & 150.0 & $\begin{array}{c}\text { NGE } \\
2(\mathrm{ME} T \mathrm{O} \text { (MRS) }\end{array}$ & 250.0 & $30 c .0$ & 400.0 \\
\hline $562 E-11$ & $9.633 E-11$ & 1. $C 8 \mathrm{CE}-1 \mathrm{C}$ & $1.102 E-10$ & $1.062 \mathrm{E}-10$ & $9.889 E-11$ & $7.997 \mathrm{E}-11$ \\
\hline $.772 E-11$ & $9.851 \mathrm{E}-11$ & $1.101 \mathrm{E}-10$ & $1.120 \mathrm{E}-10$ & $1.678 \mathrm{E}-1 \mathrm{C}$ & $1.002 \mathrm{E}-10$ & $8.085 \mathrm{E}-11$ \\
\hline $.282 \mathrm{E}-11$ & $1.040 E-10$ & $1.157 \mathrm{E}-10$ & $1.172 \mathrm{E}-10$ & $1.124 \mathrm{E}-10$ & $1.042 \mathrm{E}-1 \mathrm{C}$ & $8.364 \mathrm{E}-11$ \\
\hline $.710 E-1$ & $1.088 \mathrm{E}-10$ & $1.206 \mathrm{E}-10$ & $1.214 \mathrm{E}-10$ & $1.156 \mathrm{E}-10$ & $1.073 E-10$ & $8.630 E-11$ \\
\hline $.829 \mathrm{E}-11$ & $1.104 \mathrm{E}-10$ & $1.230 \mathrm{E}-10$ & $1.247 \mathrm{E}-1 \mathrm{C}$ & $1.209 \mathrm{E}-10$ & $1.121 \mathrm{E}-1 \mathrm{C}$ & $9.012 \mathrm{E}-11$ \\
\hline $.012 \mathrm{E}-10$ & $1.138 \mathrm{E}-1 \mathrm{C}$ & $1.271 \mathrm{E}-10$ & $1.291 \mathrm{E}-10$ & $1.240 E-10$ & $1.154 \mathrm{E}-10$ & $9.339 \mathrm{E}-11$ \\
\hline $.044 E-10$ & $1.177 \mathrm{E}-1 \mathrm{C}$ & $1.326 \mathrm{E}-10$ & $1.363 \mathrm{E}-10$ & $1.32 \mathrm{rE}-1 \mathrm{C}$ & $1.235 \mathrm{E}-10$ & $1.006 \mathrm{E}-10$ \\
\hline .169 & $1.321 E-1 C$ & $1.497 E-10$ & $1.543 E-10$ & $1.499 \mathrm{E}-10$ & $1.404 \mathrm{E}-10$ & $1.146 \mathrm{E}-10$ \\
\hline $.385 \mathrm{E}-10$ & $1.569 \mathrm{E}-1 \mathrm{C}$ & $1.780 \mathrm{E}-10$ & $1.835 \mathrm{E}-10$ & $1.782 \mathrm{E}-10$ & $1.667 \mathrm{E}-10$ & $1.357 \mathrm{E}-10$ \\
\hline $.472 \mathrm{E}-1 \mathrm{C}$ & $1.732 \mathrm{E}-1 \mathrm{C}$ & $2.069 E-10$ & $2.305 \mathrm{E}-10$ & $2.236 \mathrm{E}-1 \mathrm{C}$ & $2.019 E-10$ & $1.642 \mathrm{E}-10$ \\
\hline . $13 C E-10$ & $2.425 E-10$ & $2.855 \mathrm{E}-1 \mathrm{C}$ & $2.673 E-10$ & $2.579 \mathrm{E}-10$ & $2.471 \mathrm{E}-10$ & $1.987 \mathrm{E}-10$ \\
\hline $.534 E-10$ & 3.C15E-1C & $3.224 \mathrm{E}-10$ & $3.400 \mathrm{E}-10$ & $3.283 \mathrm{E}-10$ & $2.990 \mathrm{E}-10$ & $2.422 \mathrm{E}-10$ \\
\hline & $3.71 C E-1 C$ & $4.030 E-10$ & $4.2 C 7 E-10$ & $4.080 E-10$ & $3.868 \mathrm{E}-1 \mathrm{C}$ & $3.129 \mathrm{E}-10$ \\
\hline $.722 \mathrm{E}-10$ & $6.231 \mathrm{E}-10$ & $6.275 E-1 C$ & $6.335 \mathrm{E}-10$ & $6.097 E-10$ & $5.635 E-10$ & $4.581 E-10$ \\
\hline $.798 \mathrm{E}-10$ & $1 . C 73 E-09$ & $1.128 \mathrm{E}-99$ & $1.1 \mathrm{C} 1 \mathrm{E}-09$ & $1.056 \mathrm{E}-09$ & $9.826 \mathrm{E}-10$ & $7.893 E-10$ \\
\hline & $2.899 \mathrm{E}-09$ & $2.714 \mathrm{E}-09$ & $2.566 E-09$ & $2.349 E-09$ & $2.114 E-C 9$ & $1.637 \mathrm{E}-09$ \\
\hline $.384 E-08$ & $3.863 E-08$ & $3.013 E-C 8$ & $2.366 E-08$ & $1.852 E-08$ & $1.451 \mathrm{E}-\mathrm{CB}$ & $8.919 E-09$ \\
\hline $.173 E-C B$ & 1.C79E-C8 & $9.54 \mathrm{CE}-09$ & $8.43 \mathrm{CE}-09$ & $7.348 \mathrm{E}-09$ & $6.346 \mathrm{E}-\mathrm{C} 9$ & $4.624 \mathrm{E}-\mathrm{C} 9$ \\
\hline
\end{tabular}

RANGE (METERS)

$1200 . \mathrm{C}$

1500.0

1800.0

$6.102 \mathrm{E}-11$

$600 \cdot C$

900.0

$5.0 C 6 E-12$

$1.577 E-11$

$5.035 E-12$

$5.146 E-12$

$5.320 \mathrm{E}-12$

$5.566 \mathrm{E}-12$

$5.912 \mathrm{E}-12$

6.467E-12

$7.315 E-12$

$8.498 E-12$

$1.003 E-11$

$1.199 E-11$

$1.479 E-11$

1.959E-11

$2.879 E-11$

$\begin{array}{lllll}8.651 \mathrm{E}-10 & 2.832 \mathrm{E}-10 & 8.483 \mathrm{E}-11 & 2.431 \mathrm{E}-11 & 6.795 \mathrm{E}-12 \\ 3.404 \mathrm{E}-\mathrm{Cg} & 8.235 \mathrm{E}-13 & 2.041 \mathrm{E}-10 & 5.148 \mathrm{E}-11 & 1.314 \mathrm{E}-11\end{array}$

$\begin{array}{lllll}8.651 E-10 & 2.832 E-10 & 8.483 E-11 & 2.431 E-11 & 6.795 E-12 \\ 3.404 E-C 9 & 8.235 E-10 & 2.041 E-10 & 5.148 E-11 & 1.314 E-11\end{array}$

$1.509 E-12$

$1.517 \mathrm{E}-12$

$1.548 \mathrm{E}-12$
$1.6 \mathrm{COE}-12$

$1.676 \mathrm{E}-12$

$1.785 E-12$

$1.054 E-12$

$2.2 C 4 E-12$

$2.547 \mathrm{E}-12$

$2.992 \mathrm{E}-12$

$3.573 \mathrm{E}-12$

$4.427 E-12$

$5.889 E-12$

8.635E-12

$\begin{array}{lllll}8.651 \mathrm{E}-10 & 2.832 \mathrm{E}-10 & 8.483 \mathrm{E}-11 & 2.431 \mathrm{E}-11 & 6.795 \mathrm{E}-12 \\ 3.404 \mathrm{E}-\mathrm{Cg} & 8.235 \mathrm{E}-10 & 2.041 \mathrm{E}-10 & 5.148 \mathrm{E}-11 & 1.314 \mathrm{E}-11\end{array}$

$\begin{array}{lllll}8.651 E-10 & 2.832 E-10 & 8.483 E-11 & 2.431 E-11 & 6.795 E-12 \\ 3.404 E-C 9 & 8.235 E-10 & 2.041 E-10 & 5.148 E-11 & 1.314 E-11\end{array}$

$4.405 \mathrm{E}-13$

$4.427 E-13$

$4.515 E-13$

$4.668 \mathrm{E}-13$

4. $891 \mathrm{E}-13$

$5.218 \mathrm{E}-13$

5.711E-13

$7.426 E-13$

.

(

1.034E-12

(2)

2.507E-12

(2)

$\begin{array}{lllll}8.651 E-10 & 2.832 E-10 & 8.483 E-11 & 2.431 E-11 & 6.795 E-12 \\ 3.404 E-C 9 & 8.235 E-10 & 2.041 E-10 & 5.148 E-11 & 1.314 E-11\end{array}$

$3.289 E-C 9$

$2.299 E-09$

$6.373 E-11$

$1.808 E-11$ 
COSINE

$-1.00000 \mathrm{E} 00$
$-9.89401 \mathrm{E}-01$
$-9.44575 \mathrm{E}-01$
$-8.65631 \mathrm{E}-01$
$-7.55044 \mathrm{E}-01$
$-6.17876 \mathrm{E}-01$
$-4.58017 \mathrm{E}-01$
$-2.81605 \mathrm{E}-01$
$-9.50125 \mathrm{E}-02$
$9.50125 \mathrm{E}-02$
$2.81605 \mathrm{E}-01$
$4.58017 \mathrm{E}-01$
$6.17876 \mathrm{E}-01$
$7.55044 \mathrm{E}-01$
$8.55631 \mathrm{E}-01$
$9.44575 \mathrm{E}-01$
$9.89401 \mathrm{E}-01$

TOTAL

COSINE

$-1.00000 E$ CO $-9.89401 \mathrm{E}-01$ $-9.44575 \mathrm{E}-01$ $-8.65631 \mathrm{E}-01$ $-7.55044 E-01$ $-6.17876 E-01$ $-2.81605 \mathrm{E}-01$ $-2.81605 E-01$ $9.50125 E-02$ $9.50125 E-02$ 2.81605E-OI 4. $58017 E-01$ $6.17876 E-01$ $8.65631 E-01$ $8.65631 \mathrm{E}-01$ $9.894 \mathrm{C} 1 \mathrm{E}-01$

TOTAL

\begin{tabular}{|c|c|c|c|c|c|c|}
\hline 75.0 & 100.0 & 150.0 & $\begin{array}{l}\text { IGE (METERS) } \\
200 . \mathrm{C}\end{array}$ & $25 C .0$ & $300 . \mathrm{C}$ & $40 C .0$ \\
\hline $229 \mathrm{E}-11$ & $\begin{array}{l}7.388 E-11 \\
7.419 E-11\end{array}$ & $\begin{array}{l}8.906 E-11 \\
8.941 E-11\end{array}$ & $9.547 E-11$ & $9.556 \mathrm{E}-11$ & $9.165 \mathrm{E}-11$ & $7.758 \mathrm{E}-11$ \\
\hline $\begin{array}{l}257 \mathrm{E}-11 \\
343 \mathrm{E}-11\end{array}$ & $\begin{array}{l}7.419 E-11 \\
7.520 E-11\end{array}$ & $\begin{array}{l}8.941 E-11 \\
9.051 E-11\end{array}$ & $\begin{array}{l}9.582 E-11 \\
9.709 E-11\end{array}$ & $\begin{array}{l}59 C E-11 \\
716 E-11\end{array}$ & $\begin{array}{l}9.197 E-11 \\
9.318 E-11\end{array}$ & $\begin{array}{l}7.783 \mathrm{E}-11 \\
7.886 \mathrm{E}-11\end{array}$ \\
\hline & $7.675 \mathrm{E}-11$ & $9.253 \mathrm{E}-11$ & & $9.922 \mathrm{E}-11$ & $9.521 \mathrm{E}-11$ & $8.065 E-11$ \\
\hline$=-11$ & $7.877 \mathrm{E}-11$ & $9.515 \mathrm{E}-11$ & $1.022 \mathrm{E}-10$ & $1.025 E-10$ & $9.841 E-11$ & $8.343 \mathrm{E}-11$ \\
\hline & $8.191 \mathrm{E}-11$ & & & & $1.028 E-10$ & $8.726 E-11$ \\
\hline 1 & $8.632 E-11$ & $1.047 \mathrm{E}-10$ & $1.128 \mathrm{E}-10$ & $133 E-10$ & $1.091 \mathrm{E}-10$ & $9.275 E-11$ \\
\hline 836 & $9.320 E-11$ & $1.131 \mathrm{E}-10$ & $1.219 \mathrm{E}-10$ & $1.226 \mathrm{E}-10$ & $1.180 \mathrm{E}-10$ & $1.004 E-10$ \\
\hline & & $9 E-10$ & & & $302 \mathrm{E}-1$ & $1.107 E-10$ \\
\hline$=-10$ & $1.134 \mathrm{E}-10$ & $1.372 \mathrm{E}-10$ & $527 E-10$ & $1.530 E-10$ & $1.467 E-10$ & $1.239 E-10$ \\
\hline .181 & $1.389 E-10$ & $1.691 \mathrm{E}-10$ & $1.721 \mathrm{E}-10$ & $1.726 \mathrm{E}-10$ & $1.671 \mathrm{E}-10$ & $1.417 \mathrm{E}-10$ \\
\hline & & $C E-10$ & $2.059 \mathrm{E}-10$ & $2.056 \mathrm{E}-1$ & $1.951 \mathrm{E}-1 \mathrm{C}$ & $1.646 E-10$ \\
\hline & $2.093 E-10$ & $2.432 \mathrm{E}-10$ & $459 E-10$ & $2.446 E-10$ & $2.351 \mathrm{E}-10$ & $1.975 \mathrm{E}-10$ \\
\hline & $2.608 \mathrm{E}-10$ & $2.932 \mathrm{E}-10$ & $3.206 \mathrm{E}-10$ & $3.158 \mathrm{E}-10$ & $2.976 E-10$ & $2.476 E-10$ \\
\hline & $3.950 E-10$ & $4.523 \mathrm{E}-10$ & $4.478 \mathrm{E}-10$ & $4.343 E-10$ & $4.079 E-1 C$ & $3.330 E-10$ \\
\hline 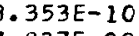 & $7.822 E-10$ & $7.705 E-10$ & $7.579 E-10$ & $7.071 E-10$ & $6.429 E-10$ & $5.047 E-10$ \\
\hline & $6.831 E-C 9$ & $5.272 E-09$ & $4.118 E-09$ & $3.226 \mathrm{E}-09$ & $2.543 E-C 9$ & $1.594 E-09$ \\
\hline $11 E-0$ & $3.115 E-C 9$ & $3.144 E-C 9$ & $3.061 E-C 9$ & $2.879 E-09$ & $2.646 \mathrm{E}-\mathrm{Cg}$ & $2.118 \mathrm{E}-0 \mathrm{~g}$ \\
\hline
\end{tabular}

RANGE (METERS)

500.0

600.0

900.0

1200.0

1500.0

$1.759 E-12$ $1.764 \mathrm{E}-12$ $1.788 E-12$ $1.833 \mathrm{E}-12$ $1.902 \mathrm{E}-12$ $2.000 \mathrm{E}-12$ $2.130 \mathrm{E}-12$ $2.309 E-12$ $2.536 \mathrm{E}-12$ $2.826 E-12$ 3.197E-12 $4.345 E-12$ 4.345E-12 $6.652 E-12$ $6.672 E-12$ $8.73 C E-12$$$
\text { 4. } 221 E-11
$$

$.259 E-13$

$5.330 \mathrm{E}-13$

.

.

5. $364 E-13$

6.36 E 13

6.

$.421 E-13$

$9.521 E-13$

$.291 E-12$

$1.565 E-12$

$2.546 \mathrm{E}-12$

2.546E-12

$1.248 \mathrm{E}-11$ 
COSINE

$-1.0 C C O O E$ CO $-9.89401 E-01$ $-9.44575 \mathrm{E}-01$ $-8.65631 E-01$ $-6.17876 \mathrm{E}-01$ -4.58017E-01 $-2.81605 E-C 1$ $-50125 \mathrm{E}-02$ $2.81605 \mathrm{E}-01$ 4.58017E-01 $0.17876 \mathrm{E}-01$ 7. $55044 \mathrm{E}-01$ $8.65631 \mathrm{E}-01$ $9.44575 E-01$ $9.89401 \mathrm{E}-\mathrm{C} 1$

total

COSINE

$-1.0 C C O O E O O$ $-9.894 \mathrm{C} 1 \mathrm{E}-01$ $-7.55044 E-01$ $-6.17876 E-01$ $-4.58017 E-01$ $-2.816 C 5 E-01$ $-9.50125 E-02$ $9.50125 E-02$ $2.81605 E-01$ 4. $58017 \mathrm{E}-01$ $6.17876 \mathrm{E}-01$ $7.55044 \mathrm{E}-01$ $8.65631 E-01$ $9.44575 E-01$

TOTAL

$$
75.0
$$

$2.563 E-12$ $2.563 E-12$ $2.579 E-12$
$2.639 E-12$ $2.639 \mathrm{E}-12$ $2.750 \mathrm{E}-12$ $2.902 \mathrm{E}-12$ $3.098 \mathrm{E}-12$ $3.350 E-12$ $3.684 E-12$ $4.136 \mathrm{E}-12$ $4.629 E-12$ $6.077 E-12$ $7.433 E-12$ ..451E-12 $1.160 E-11$ $1.736 E-11$ $7.339 \mathrm{E}-11$

$9.258 E-11$

500.0 $2.544 \mathrm{E}-12$
$2.598 \mathrm{E}-12$ $2.792 \mathrm{E}-12$ $3.083 \mathrm{E}-12$ $3.373 E-12$ $3.645 \mathrm{E}-12$ $3.960 \mathrm{E}-12$ 4. 444E- 12 $5.232 \mathrm{E}-12$ $6.438 \mathrm{E}-12$ $8.192 \mathrm{E}-12$ $1.079 E-11$ $1.495 \mathrm{E}-11$ $2.229 E-11$ $3.612 \mathrm{E}-11$ $6.523 E-11$ $1.609 \mathrm{E}-10$ 160.0 3. $C 78 E-12$ $3.100 \mathrm{E}-12$ $3.180 \mathrm{E}-12$ $3.326 \mathrm{E}-12$ $3.52 C \mathrm{E}-12$ $3.765 \mathrm{E}-12$ $4.076 E-12$ $5.488 E-12$ $5.603 E-12$ 7. $584 \mathrm{E}-12$ $7.695 \mathrm{E}-12$ $1.142 \mathrm{E}-1$ $1.412 \mathrm{E}-1$ 2. $85 E-11$ $9.176 E-11$

1.133E-1C

$60 \mathrm{C} .0$

1.879E-12 $1.931 \mathrm{E}-12$ $2.112 \mathrm{E}-12$ $2.613 \mathrm{E}-12$ $2.811 \mathrm{E}-12$ 3. $.26 \mathrm{E}-1$ 3. $380 \mathrm{E}-12$ $4 . \mathrm{COIE-12}$ 4. $977 \mathrm{E}-12$ $6.397 \mathrm{E}-12$ $8.509 E-12$ $1.201 \mathrm{E}-1$ $1.852 E-11$ $3.145 \mathrm{E}-11$ $5.925 E-11$ $1.475 E-1 C$

$1.596 \mathrm{E}-10$

\begin{tabular}{|c|c|c|c|c|}
\hline 150.0 & $\begin{array}{l}\text { NGE (METERS) } \\
2 C O . C\end{array}$ & $250 . C$ & 300.0 & 400.0 \\
\hline & $4.171 E-12$ & 4. $2 C 2 E-12$ & $4.015 \mathrm{E}-12$ & $3.317 E-12$ \\
\hline & $4.211 \mathrm{E}-12$ & $4.249 E-12$ & $4.066 \mathrm{E}-12$ & $3.372 \mathrm{E}-12$ \\
\hline & $4.363 E-12$ & $4.423 E-12$ & $4.254 E-12$ & $3.570 E-12$ \\
\hline $178 \mathrm{E}-12$ & $4.623 \mathrm{E}-12$ & $4.716 \mathrm{E}-12$ & $4.565 E-12$ & $3.882 \mathrm{E}-12$ \\
\hline $449 E-12$ & $4.947 E-12$ & $5.071 \mathrm{E}-12$ & $4.928 E-12$ & $4.221 E-12$ \\
\hline 4. $777 \mathrm{E}-12$ & $5.331 E-12$ & $5.479 E-12$ & $5.334 \mathrm{E}-12$ & $4.572 \mathrm{E}-12$ \\
\hline $5.188 \mathrm{E}-12$ & $5.810 E-12$ & 5. $987 E-12$ & $5.835 E-12$ & $4.995 E-12$ \\
\hline $737 \mathrm{E}-12$ & $6.458 E-12$ & $0.684 E-12$ & $6.537 \mathrm{E}-12$ & $5.612 \mathrm{E}-12$ \\
\hline $6.491 E-12$ & $7.364 E-12$ & $7.681 \mathrm{E}-12$ & $7.563 \mathrm{E}-12$ & $6.562 \mathrm{E}-12$ \\
\hline $6.498 \mathrm{E}-12$ & $9.138 E-12$ & $9.633 E-12$ & $8.929 E-12$ & $7.979 \mathrm{E}-12$ \\
\hline & $9.875 E-12$ & & & $9.990 E-12$ \\
\hline $9.750 E-12$ & $1.319 \mathrm{E}-11$ & $1.423 \mathrm{E}-11$ & $1.395 \mathrm{E}-11$ & $1.294 \mathrm{E}-11$ \\
\hline $1.497 \mathrm{E}-11$ & $1.592 \mathrm{E}-11$ & $1.741 \mathrm{E}-11$ & $1.849 \mathrm{E}-11$ & $1.748 \mathrm{E}-11$ \\
\hline $1.774 E-11$ & $2.23 \mathrm{CE}-11$ & $2.46 \mathrm{CE}-11$ & $2.542 \mathrm{E}-11$ & $2.510 E-11$ \\
\hline $2.84 C E-11$ & $3.220 \mathrm{E}-11$ & 3. $565 \mathrm{E}-11$ & $3.815 \mathrm{E}-11$ & $3.889 \mathrm{E}-11$ \\
\hline & $5.628 E-11$ & $6.202 \mathrm{E}-11$ & $6.554 E-11$ & $6.797 E-11$ \\
\hline & $1.426 \mathrm{E}-10$ & $1.563 E-10$ & $1.645 E-10$ & $1.681 E-10$ \\
\hline $1.464 E-10$ & $1.707 \mathrm{E}-10$ & $1.841 E-10$ & $1.888 E-10$ & $1.808 E-10$ \\
\hline
\end{tabular}

$1.344 E-1 C$
RANGE (METERS I

$900.0 \quad 1200.0$

1500.0

1800.0

$6.859 \mathrm{E}-13 \quad 2.332 \mathrm{E}-13 \quad 7.618 \mathrm{E}-14 \quad 2.429 \mathrm{E}-14$

$\begin{array}{llll}7.287 \mathrm{E}-13 & 2.605 \mathrm{E}-13 & 9.177 \mathrm{E}-14 & 3.239 \mathrm{E}-14\end{array}$

$\begin{array}{llll}8.471 E-13 & 3.357 E-13 & 1.353 E-13 & 5.625 E-14 \\ 1.024 E-12 & 4.298 E-13 & 1.816 E-13 & 7.880 E-14\end{array}$

$1.148 \mathrm{E}-12 \quad 4.947 \mathrm{E}-13 \quad 2.166 \mathrm{E}-13 \quad 9.602 \mathrm{E}-14$

$\begin{array}{llll}1.200 E-12 & 4.996 \mathrm{E}-13 & 2.035 \mathrm{E}-13 & 8.625 \mathrm{E}-14\end{array}$

$\begin{array}{llll}1.241 E-12 & 4.779 E-13 & 1.885 E-13 & 8.378 E-14 \\ 1.357 E-12 & 5.279 E-13 & 2.052 E-13 & 8.317 E-14\end{array}$

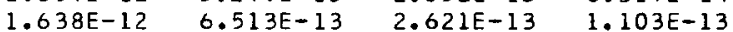

$\begin{array}{llll}2.107 E-12 & 8.723 E-13 & 3.670 E-13 & 1.579 E-13\end{array}$

$2.757 \mathrm{E}-12 \quad 1.156 \mathrm{E}-12 \quad 4.897 \mathrm{E}-13 \quad 2.130 \mathrm{E}-13$

$\begin{array}{llll}3.679 E-12 & 1.511 E-12 & 6.183 E-13 & 2.557 E-13\end{array}$

$5.293 \mathrm{E}-12 \quad 2.151 \mathrm{E}-12 \quad 8.562 \mathrm{E}-13 \quad 3.395 \mathrm{E}-13$

$8.760 \mathrm{E}-12 \quad 3.726 \mathrm{E}-12 \quad 1.542 \mathrm{E}-12 \quad 6.378 \mathrm{E}-13$

$\begin{array}{llll}1.682 \mathrm{E}-11 & 7.872 \mathrm{E}-12 & 3.54 \mathrm{CE}-12 & 1.578 \mathrm{E}-12\end{array}$

$3.632 \mathrm{E}-11 \quad 1.9(4 \mathrm{E}-11 \quad 9.332 \mathrm{E}-12 \quad 4.442 \mathrm{E}-12$

$9.732 E-11 \quad 5.6 C 0 E-11 \quad 2.99 \mathrm{CE}-11 \quad 1.527 \mathrm{E}-11$

$7.020 E-11$

$3.380 E-11$

$1.582 E-11 \quad 7.345 E-12$ 
COSINE

$-1.00000 \mathrm{E} 00$ $-9.89401 E-01$ $-9.44575 \mathrm{E}-01$ $-9.44575 E-01$ $-8.65631 E-01$ $-6.17876 \mathrm{E}-01$ $-4.58017 \mathrm{E}-01$ $-2.81605 E-01$ $-9.50125 \mathrm{E}-02$ $9.50125 \mathrm{E}-02$ $2.81605 \mathrm{E}-01$ 4.58017E-01 $6.17876 \mathrm{E}-01$ $8.65631 \mathrm{E}-01$ $9.44575 E-01$ $9.89401 E-01$

TOTAL

COSINE

$-1.00000 \mathrm{E}$ OC $-9.89401 E-01$ $-9.44575 \mathrm{E}-01$ $-8.65631 E-01$ $-7.55044 \mathrm{E}-01$ $-6.17876 \mathrm{E}-01$ -4.58017 E- 01 $-2.81605 \mathrm{E}-01$ $-9.50125 E-02$ $9.50125 E-02$ $2.81605 E-01$ $4.58017 E-01$ $6.17876 E-01$ $7.55044 \mathrm{E}-01$ $8.65631 \mathrm{E}-01$ $9.89401 E-01$

TOTAL
75.

$2.612 \mathrm{E}-10$ $2.612 E-10$
$2.627 \mathrm{E}-10$ $2.687 \mathrm{E}-10$ $2.797 \mathrm{E}-10$ $2.948 \mathrm{E}-10$ $3.142 \mathrm{E}-10$ $3.392 \mathrm{E}-10$ $3.723 \mathrm{E}-10$ $4.171 \mathrm{E}-10$ $4.659 \mathrm{E}-10$ 6. C67E- 10 $7.443 \mathrm{E}-10$ $8.413 \mathrm{E}-10$ $1.157 \mathrm{E}-\mathrm{C}$ $1.726 \mathrm{E}-\mathrm{C}$ $2.855 \mathrm{E}-\mathrm{O}$

$7.272 \mathrm{E}-09$

$9.255 \mathrm{E}-0.9$

500.0

$3.045 \mathrm{E}-10$ $3.099 \mathrm{E}-10$ $3.293 \mathrm{E}-10$ $3.586 E-10$ $3.882 \mathrm{E}-1 \mathrm{O}$ $4.164 E-10$ $4.493 \mathrm{E}-10$

$4.992 \mathrm{E}-10$ $5.793 E-10$ $7.010 \mathrm{E}-10$ $8.769 E-10$ 1.136E-C9 $1.551 E-C 9$ 2. $277 \mathrm{E}-\mathrm{C} 9$ $0.528 E-C 9$ .

$1.659 E-08$
100.0 $3.19 C E-10$ $3.27 \mathrm{CE}-10$ $3.607 \mathrm{E}-10$ $3.850 \mathrm{E}-10$ $4.16 C E-10$ $4.569 E-10$ $5.123 \mathrm{E}-10$ $5.671 E-10$ $7.64 \mathrm{CE}-1 \mathrm{C}$ $7.742 \mathrm{E}-10$ $1.146 \mathrm{E}-0$ $1.405 \mathrm{E}-\mathrm{C}$ $2.075 \mathrm{E}-09$ $3.633 \mathrm{E}-\mathrm{C}$ $9.096 \mathrm{E}-\mathrm{C} 9$

$1.137 E-08$

600.0

$2.343 \mathrm{E}-1 \mathrm{C}$ $2.395 E-1 C$ $2.577 \mathrm{E}-10$ $2.840 \mathrm{E}-10$ $3.085 E-10$ $3.294 \mathrm{E}-10$ $3.522 \mathrm{E}-10$ $3.891 E-10$ $4.526 \mathrm{E}-10$ $5.512 E-10$ $6.939 E-10$ $9.051 E-10$ $1.898 \mathrm{E} 09$ 1.37609 $3.176 E-09$ $1.473 E-08$

$1.403 E-C .8$

\begin{tabular}{|c|c|c|c|c|}
\hline 150.0 & $\begin{array}{l}\text { IGE (METERS) } \\
200.0\end{array}$ & 250.0 & 300.0 & 400.0 \\
\hline & $4.452 E-10$ & $4.566 \mathrm{E}-10$ & $4.443 E-10$ & $3.814 \mathrm{E}-10$ \\
\hline & $4.492 E-10$ & $4.613 \mathrm{E}-10$ & $4.494 \mathrm{E}-10$ & $3.868 \mathrm{E}-10$ \\
\hline $154 \mathrm{E}-10$ & $4.643 E-10$ & $4.786 \mathrm{E}-10$ & $4.681 \mathrm{E}-10$ & $4.066 \mathrm{E}-10$ \\
\hline & $4.902 E-10$ & $5.079 E-1 C$ & $4.992 \mathrm{E}-10$ & $4.380 E-10$ \\
\hline$E-10$ & $5.228 \mathrm{E}-10$ & $5.436 \mathrm{E}-10$ & $5.359 \mathrm{E}-10$ & $4.724 \mathrm{E}-10$ \\
\hline$O E-10$ & $5.613 E-10$ & $5.848 \mathrm{E}-10$ & $5.771 E-10$ & $5.084 E-10$ \\
\hline $2 E-10$ & $6.095 \mathrm{E}-10$ & $6.361 \mathrm{E}-10$ & $6.281 \mathrm{E}-10$ & $5.519 E-10$ \\
\hline $20 E-10$ & $6.745 E-10$ & $7.064 E-10$ & $6.991 \mathrm{E}=10$ & $6.150 E-10$ \\
\hline $671 \mathrm{E}-10$ & $7.652 \mathrm{E}-10$ & $8.065 \mathrm{E}-10$ & $8.023 \mathrm{E}-10$ & $7.110 E-1.0$ \\
\hline $680 E-10$ & $9.419 \mathrm{E}-10$ & $1.001 \mathrm{E}-09$ & $9.393 \mathrm{E}-10$ & $8.536 E-10$ \\
\hline $.020 \mathrm{E}-09$ & $1.016 E-09$ & $1.092 \mathrm{E}-09$ & $1.166 \mathrm{E}-09$ & $1.055 \mathrm{E}-09$ \\
\hline $.904 E-10$ & $1.344 E-09$ & $1.459 \mathrm{E}-09$ & $1.441 \mathrm{E}-09$ & $1.350 \mathrm{E}-09$ \\
\hline & $1.616 E-09$ & $1.775 E-09$ & $1.891 \mathrm{E}-\mathrm{Cg}$ & $1.801 E-09$ \\
\hline $780 E-09$ & $2.247 \mathrm{E}-09$ & $2.487 \mathrm{E}-09$ & $2.577 \mathrm{E}-\mathrm{C} 9$ & $2.556 \mathrm{E}-09$ \\
\hline $.838 \mathrm{E}-09$ & $3.225 \mathrm{E}-09$ & $3.579 \mathrm{E}-09$ & $3.836 \mathrm{E}-09$ & $3.920 E-09$ \\
\hline $.693 \mathrm{E}-09$ & $5.606 \mathrm{E}-09$ & $6.188 \mathrm{E}-09$ & $6.546 \mathrm{E}-\mathrm{C} 9$ & $6.799 \mathrm{E}-09$ \\
\hline & $1.418 E-08$ & $1.555 E-08$ & $1.638 \mathrm{E}-\mathrm{C} 8$ & $1.676 \mathrm{E}-08$ \\
\hline $80 E-08$ & $1.735 E-08$ & $1.882 E-08$ & $1.938 E-08$ & $1.869 E-08$ \\
\hline
\end{tabular}

RANGE (METERS)

$900.0 \quad 1200 . \mathrm{C}$

1500.0

1800.0

$\begin{array}{llll}9.704 \mathrm{E}-11 & 3.794 \mathrm{E}-11 & 1.466 \mathrm{E}-11 & 5.734 \mathrm{E}-12 \\ 1.013 \mathrm{E}-10 & 4.063 \mathrm{E}-11 & 1.620 \mathrm{E}-11 & 6.535 \mathrm{E}-12 \\ 1.131 \mathrm{E}-10 & 4.813 \mathrm{E}-11 & 2.052 \mathrm{E}-11 & 8.898 \mathrm{E}-12 \\ 1.309 \mathrm{E}-10 & 5.756 \mathrm{E}-11 & 2.518 \mathrm{E}-11 & 1.117 \mathrm{E}-11 \\ 1.437 \mathrm{E}-10 & 6.425 \mathrm{E}-11 & 2.873 \mathrm{E}-11 & 1.291 \mathrm{E}-11 \\ 1.496 \mathrm{E}-10 & 6.514 \mathrm{E}-11 & 2.766 \mathrm{E}-11 & 1.205 \mathrm{E}-11 \\ 1.546 \mathrm{E}-10 & 6.353 \mathrm{E}-11 & 2.640 \mathrm{E}-11 & 1.091 \mathrm{E}-11 \\ 1.672 \mathrm{E}-10 & 6.894 \mathrm{E}-11 & 2.826 \mathrm{E}-11 & 1.193 \mathrm{E}-11 \\ 1.961 \mathrm{E}-10 & 8.166 \mathrm{E}-11 & 3.411 \mathrm{E}-11 & 1.471 \mathrm{E}-11 \\ 2.437 \mathrm{E}-10 & 1.040 \mathrm{E}-10 & 4.469 \mathrm{E}-11 & 1.950 \mathrm{E}-11 \\ 3.092 \mathrm{E}-10 & 1.326 \mathrm{E}-10 & 5.704 \mathrm{E}-11 & 2.504 \mathrm{E}-11 \\ 4.015 \mathrm{E}-10 & 1.682 \mathrm{E}-10 & 6.994 \mathrm{E}-11 & 2.934 \mathrm{E}-11 \\ 5.616 \mathrm{E}-10 & 2.314 \mathrm{E}-10 & 9.336 \mathrm{E}-11 & 3.753 \mathrm{E}-11 \\ 9.035 \mathrm{E}-10 & 3.860 \mathrm{E}-10 & 1.603 \mathrm{E}-10 & 6.649 \mathrm{E}-11 \\ 1.699 \mathrm{E}-09 & 7.935 \mathrm{E}-10 & 3.560 \mathrm{E}-10 & 1.583 \mathrm{E}-10 \\ 3.630 \mathrm{E}-09 & 1.898 \mathrm{E}-09 & 9.286 \mathrm{E}-10 & 4.412 \mathrm{E}-10 \\ 9.728 \mathrm{E}-09 & 5.602 \mathrm{E}-09 & 2.993 \mathrm{E}-09 & 1.530 \mathrm{E}-09 \\ & & & \\ 7.384 \mathrm{E}-09 & 3.563 \mathrm{E}-09 & 1.668 \mathrm{E}-09 & 7.743 \mathrm{E}-1 \mathrm{C}\end{array}$




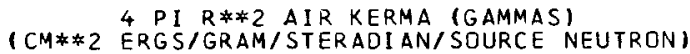

THERMONUCLEAR SOURCE

COSINE
$1.0 C 000 E$ OC
$9.89401 E-01$
$9.44575 E-01$
$8.65631 E-01$
$7.55044 E-01$
$6.17876 E-01$
$4.58017 E-01$
$2.81605 E-01$
$9.50125 E-C 2$
$9.50125 E-02$
$2.81605 E-01$
$4.58017 E-C 1$
$6.17876 E-01$
$7.55044 E-01$
$8.65631 E-01$
$9.44575 E-01$
$9.89401 E-01$

TOTAL

COS INE

-1. COOCOE CO

$-9.89401 E-01$

$-9.44575 E-01$

$-8.65631 E-01$

$-6.17876 \mathrm{E}-01$

$-4.58017 \mathrm{E}-01$

$-2.81605 \mathrm{E}-01$

$-9.50125 \mathrm{E}-02$

$9.50125 \mathrm{E}-02$

2.81605E-01

$4.58017 E-01$

$8.65631 \mathrm{E}-01$

$9.44575 \mathrm{E}-01$

$401 \mathrm{E}-01$

TOTAL

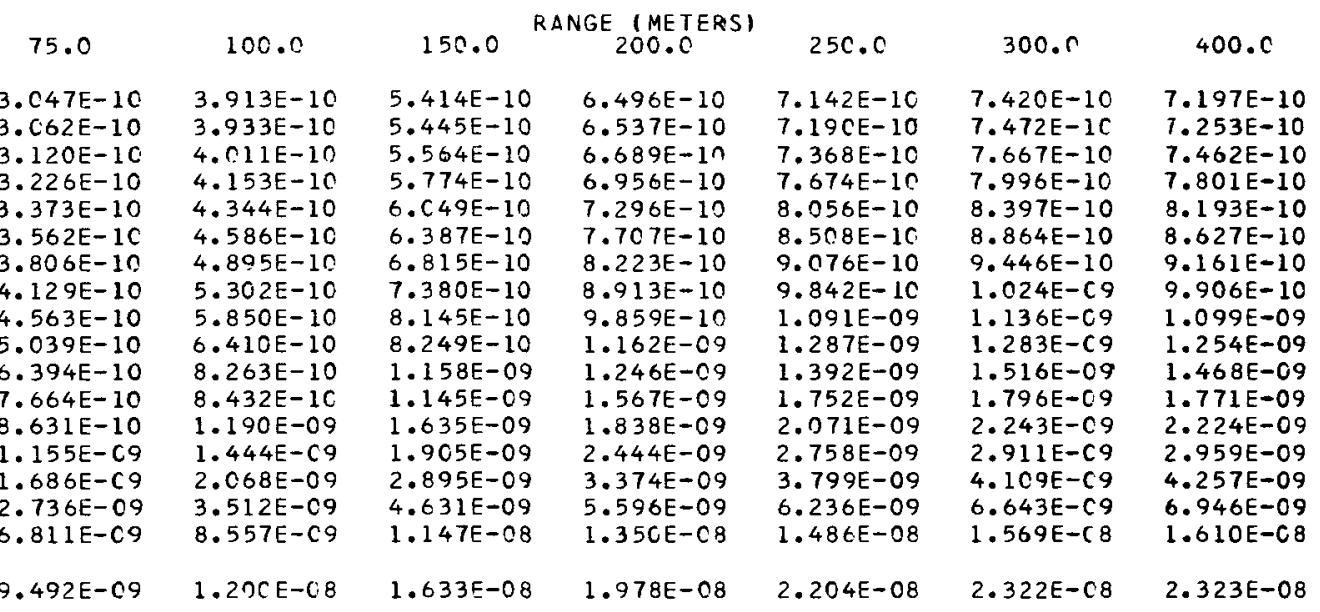

RANGE (METERS)

$500.0 \quad 600.0 \quad 900.0 \quad 120 \mathrm{C} . \mathrm{C}$

1500.0

1800.0

$2.840 E-10 \quad 1.337 E-10$

$2.883 \mathrm{E}-10$

1. $364 \mathrm{E}-10$

$1.440 E-10$
$1.541 E-10$

$1.541 E-10$
$1.624 E-10$

$1.624 E-10$
$1.662 E-10$

$1.662 \mathrm{E}-10$
$1.685 \mathrm{E}-10$

$1.685 \mathrm{E}-10$
$1.780 \mathrm{E}-10$

$1.780 E-10$
$1.951 E-10$

$1.951 E-10$
$2.219 E-10$ $2.255 \mathrm{E}-10$ $2.554 \mathrm{E}-10$
$2.966 \mathrm{E}-10$ $2.966 E-10$
$3.646 E-10$ $5.178 E-10$ (9.178E-10 $1.925 E-09$ $5.28 \mathrm{PE}-09$

$6.082 E-11$

$6.232 E-11$

$6.663 \mathrm{E}-11$

$7.588 \mathrm{E}-11$

$7.627 \mathrm{E}-11$

$7.689 \mathrm{E}-11$

$8.074 E-11$

(10 $1.158 \mathrm{E}-1 \mathrm{C}$ $1.315 \mathrm{E}-10$ $1.576 \mathrm{E}-10$ $2.244 E-10$ $4.099 E-10$

2. $809 E-09$

$6.682 \mathrm{E}-\mathrm{C}$

3.507E $C 9$

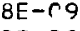

$3.697 E-09$

$4.887 . E-09$
2. $746 \mathrm{E}-11$

$2.823 \mathrm{E}-11$

$3.294 \mathrm{E}-11$

$3.502 \mathrm{E}-11$

$3.487 \mathrm{E}-11$

$3.464 \varepsilon-11$

$3.655 \mathrm{E}-11$

$4.020 E-11$

$4.58 E-11$

.

6.775E-11

.775E-11

.

$4.448 E-10$

$4.448 E-10$

$1.067 E-09$ 
4 PI R*\#2 SILICON KERMA (GAMMAS)
(CM\#*2 ERGS/GRAM/STERADIAN/SOURCE NEUTRON)

COSINE

$$
\begin{array}{r}
-1.00000 E \quad 00 \\
-9.89401 E-01 \\
-9.44575 E-01 \\
-8.65631 E-01 \\
-7.55044 E-01 \\
-6.17876 E-01 \\
-4.58017 E-01 \\
-2.81605 E-01 \\
-9.50125 E-02 \\
9.50125 E-02 \\
2.81605 E-01 \\
4.58017 E-01 \\
6.17876 E-01 \\
7.55 C 44 E-01 \\
8.65631 E-01 \\
9.44575 E-01 \\
9.89401 E-01
\end{array}
$$

TOTAL

COSINE

$-1.00000 E 00$ $-9.89401 E-01$ $-9.44575 E-01$ $-8.65631 \mathrm{E}-01$ $-7.55044 \mathrm{E}-01$ $-6.17876 E-01$ $-4.58017 E-01$ $-2.81605 E-01$ $-9.50125 E-02$ $9.50125 \mathrm{E}-02$ $2.81605 E-01$ $4.58017 E-01$ $6.17876 E-01$ $8.65631 E-01$ $8.65631 E-01$ $9.89401 \mathrm{E}-01$

TOTAL

$\begin{array}{cc}75.0 & 10 C .0 \\ 2.689 \mathrm{E}-10 & 3.273 \mathrm{E}-10 \\ 2.705 \mathrm{E}-10 & 3.295 \mathrm{E}-10 \\ 2.766 \mathrm{E}-10 & 3.376 \mathrm{E}-10 \\ 2.878 \mathrm{E}-10 & 3.524 \mathrm{E}-10 \\ 3.032 \mathrm{E}-10 & 3.721 \mathrm{E}-1 \mathrm{C} \\ 3.230 \mathrm{E}-10 & 3.969 \mathrm{E}-1 \mathrm{C} \\ 3.485 \mathrm{E}-10 & 4.285 \mathrm{E}-1 \mathrm{C} \\ 3.824 \mathrm{E}-10 & 4.702 \mathrm{E}-10 \\ 4.280 \mathrm{E}-10 & 5.267 \mathrm{E}-10 \\ 4.778 \mathrm{E}-10 & 5.826 \mathrm{E}-10 \\ 6.208 \mathrm{E}-10 & 7.838 \mathrm{E}-1 \mathrm{C} \\ 7.630 \mathrm{E}-10 & 7.936 \mathrm{E}-10 \\ 8.605 \mathrm{E}-10 & 1.175 \mathrm{E}-09 \\ 1.184 \mathrm{E}-09 & 1.437 \mathrm{E}-09 \\ 1.766 \mathrm{E}-09 & 2.123 \mathrm{E}-09 \\ 2.920 \mathrm{E}-09 & 3.718 \mathrm{E}-\mathrm{C} 9 \\ 7.443 \mathrm{E}-\mathrm{CO} & 9.310 \mathrm{E}-09 \\ 9.482 \mathrm{E}-\mathrm{C} 9 & 1.166 \mathrm{E}-\mathrm{C} 8\end{array}$

150.0
$4.162 E-10$
$4.194 E-10$
$4.316 E-10$
$4.529 E-10$
$4.804 E-10$
$5.139 E-10$
$5.559 E-10$
$6.118 E-10$
$6.884 E-10$
$6.888 E-10$
$1.049 E-09$
$1.018 E-C 9$
$1.548 E-09$
$1.822 E-09$
$2.906 E-09$
$4.800 E-09$
$1.240 E-08$
$1.520 E-08$

RANG IOC.C
$2 O C T E R$ $4.700 E-10$ $5.119 E-10$ $5.451 E-10$ $5.845 \mathrm{E}-10$ $6.336 E-10$ $6.998 \mathrm{E}-10$ $7.922 \mathrm{E}-10$ $9.726 \mathrm{E}-10$ $1.047 E-09$ $1.383 E-09$ $1.659 E-C 9$ $2.304 E-09$ $3.303 E-09$ $5.737 \mathrm{E}-09$ $1.452 \mathrm{E}-08$

$1.786 E-08$

RANGE (METERS)

500.0

600.0

900.0

1200.0

3. $322 \mathrm{E}-10$ $3.377 E-10$ $3.575 \mathrm{E}-1 \mathrm{C}$ $3.875 \mathrm{E}-10$ $4.178 E-10$ $4.464 E-10$ 4.797E-10 $5.302 E-10$ $6.117 \mathrm{E}-10$ $7.354 \mathrm{E}-10$ . $143 \mathrm{E}-10$ $1.178 E-C 9$ $1.600 E-C 9$ $2.341 E-09$ $0.738=C 9$ $1.647 \mathrm{E}-08$ $2.590 \mathrm{E}-10$ $2.644 \mathrm{E}-1 \mathrm{C}$ $2.83 C E-1 C$ $3.099 E-10$ $3.349 \mathrm{E}-1 \mathrm{C}$ $3.561 E-10$ $3.791 E-10$ $4.164 E-10$ 4.808 E-1C . $9.259 E-10$ $9.405 E-10$ $1.951 E-09$ $1.951 E-09$ $6.2570-09$ $1.512 \mathrm{E}-\mathrm{C} 8$

$1.111 \mathrm{E}-10$ 1.155E-10 $1.276 E-10$ $1.458 \mathrm{E}-10$ $1.589 \mathrm{E}-10$ $1.648 E-10$ $1.697 E-10$ $1.823 E-10$ 2.115E-10 $2.598 \mathrm{E}-10$ $3.261 E-10$ $5.816 E-10$ 5. $816 E-10$ 1. $741 E=09$ $3.719 \mathrm{E}-09$ $9.990 E-09$

1. $720 E-08$

\section{$4.478 \mathrm{E}-11$} 4. $754 E-11$ $5.525 \mathrm{E}-11$ $6.491 \mathrm{E}-11$ $7.173 \mathrm{E}-11$ $7.25 \mathrm{E}-11$ $7.080 E-11$ $7.617 E-11$ $8.902 \mathrm{E}-11$ $1.405 E-10$ $1.405 E-10$ $1.763 E-10$ $3.969 E-10$ $3.969 E-10$ $8.126 \mathrm{E}-10$ $5.756 \mathrm{E}-09$

3. 7 C8E-09
250.0

300.0

400.0 $4.811 E-10$ .858E-10 $5.335 \mathrm{E}-10$ .699E-10 $6.119 E-10$ $6.642 E-10$ $7.358 \mathrm{E}-10$ $8.378 \mathrm{E}-10$ 1.036E-09 $.128 \mathrm{E}-09$ $.503 E-C 9$ $1.824 \mathrm{E}-09$ $2.552 E-09$ $3.666 \mathrm{E}-09$ $6.334 \mathrm{E}-09$
$1.593 \mathrm{E}-08$

1. 939 E-08 $4.713 \mathrm{E}-10$ $4.957 E-10 \quad 4.361 E-10$ $5.275 E-10 \quad 4.682 E-10$ $5.649 E-10 \quad 5.033 E-10$ . C6.9E-10 $5.399 E-10$  .310E-10 6.482E-10 $8.362 E-10 \quad 7.459 E-10$ $.757 E-10 \quad 8.910 E-10$ $1.096 \mathrm{E}-\mathrm{C} 9$ $1.856 \mathrm{E}-09$ 2.626E-09 $3.931 \mathrm{E}-09 \quad 4.019 \mathrm{E}-09$ $6.703 E-C 9 \quad 6.964 E-09$ $1.679 E-C 8 \quad 1.719 E-08$ $2.000 E-C 8 \quad 1.934 E-08$

$1500.0 \quad 1800.0$

$1.780 \mathrm{E}-11 \quad 7.158 \mathrm{E}-12$ $1.939 \mathrm{E}-11 \quad 7.98 \mathrm{CE}-12$ $2.382 E-11 \quad 1.041 \mathrm{E}-11$ $2.861 \mathrm{E}-11$ 1.274E-11 $3.223 E-11 \quad 1.452 E-11$ 3.110E-11 $1.362 E-11$ $2.974 E-11$ 1.242E-11 $3.156 E-11$ 1.343E-11 $4.819 E-11$ 2. $6.066 E-11$ 2. $7.355 E-11$ (1) $9.698 \mathrm{E}-11 \mathrm{O}$ $1.647 \mathrm{E}-1 \mathrm{C} \quad 6.828 \mathrm{E}-11$ $9.509 E-10 \quad 4.516 E-10$ $3.077 \mathrm{E}-09 \quad 1.574 \mathrm{E}-\mathrm{C}$

$1.737 E-C 9 \quad 8.058 E-10$ 
(NEUTRONS/MEV/STERADIAN/SOURCE NEUTRON)

ENERGY 1.22 E C1---1.50E $C 1$ $1.00 E$ O1-- $1.22 \mathrm{E}$ O $8.29 E$ 0O-- - 1. $00 E$ E $6.36 \mathrm{E} \mathrm{OC}--8.19 \mathrm{E} \mathrm{CO}$ $4.97 \mathrm{E} 00--76.36 \mathrm{ECO}$ $4.07 \mathrm{E} 00--4.97 \mathrm{E} \mathrm{CO}$ $3.01 \mathrm{E} \mathrm{OO--4.07E} \mathrm{CO}$ $2.46 \mathrm{E} 00---3.01 \mathrm{E} \mathrm{CO}$ $2.35 \mathrm{E} 00---2.46 \mathrm{E} \mathrm{OO}$ $1.83 \mathrm{E} 00---2.35 \mathrm{E} \mathrm{CC}$ $1.11 \mathrm{E} 00---1.83 \mathrm{E} \mathrm{CO}$ $5.5 C E-01--1.11 E$ CC $1.11 E-01--5.50 E-C$ $3.35 E-02-C-1.11 E-C$ $5.83 E-04--3.35 E-C 2$ $1.01 E-04---5.83 E-C 4$ $2.90 E-05--1.01 E-04$ $1.07 E-05--2.90 E-C$ $3.06 \mathrm{E}-06--1.07 \mathrm{E}-\mathrm{C}$ $1.12 \mathrm{E}-\mathrm{C6} 6---3.06 \mathrm{E}-\mathrm{C} 6$ $4.14 E-07--1.12 E-C 6$ $0.0 \quad-4.14 E-07$ ENERGY

$$
\text { GROUP (MEV) }
$$

1.22 E O1-- $1.50 E$ C $1.00 E$ OL--1.22E C $8.19 E$ CO--1.0OE C $6.36 E$ OO-- $8.19 E$ OC

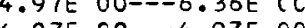
$4.07 E$ CO- $4.97 E$ CO $3.01 E$ OO-C-4.07E CO $2.46 E$ OD- $3.01 E$ CC 2.35 E OC--2.46E OO 1.83E CO- $2.35 E$ CO $1.11 \mathrm{E} C 0--1.83 \mathrm{E}$ CO 1.11E-O1$1.11 E-01$ 5.3 1004 2. $01 E-05-1.01 E-C 4$ 1. $07 E-05-2.01 E-C 4$ $1.07 E$ CSTCO $1.12 \mathrm{E}-06--3.06 \mathrm{E}-\mathrm{C} 6$ $4.12 E-07-1.12 E-C 6$ $4.14 E-07---1.12 E-C 6$
$0.0 \quad---4.14 E-C 7$
ANGLE 1 ANGLE 2 $M U=-1.0000$ $2.24 \mathrm{CE}-08$ $3.734 E-08$ $1.809 \mathrm{E}-07$ $7.309 \mathrm{E}-0$ $1.589 E-06$ $1.870 \mathrm{E}-06$ $2.820 E-96$ $4.129 \mathrm{E}-06$ $6.346 \mathrm{E}-06$ $1.169 \mathrm{E}-05$ $3.312 E-05$ $1.028 \mathrm{E}-0$ $1.064 \mathrm{E}-0$ $1.066 \mathrm{E}-03$ 7. $189 \mathrm{E}-02$ $3.342 E-0$ $1.030 E 00$ $2.942 \mathrm{E} \mathrm{OO}$ $8.266 \mathrm{E} \mathrm{OO}$ $1.891 E O$ $2.884 E$ OI ANGLE 10 $M U=0.0950$ 3. $\mathrm{CO9E}-08$ $6.7495-08$ $1.847 \mathrm{E}-07$ $7.14 C E-07$ $1.786 \mathrm{E}-06$ $2.963 \mathrm{E}-06$ $2.732 \mathrm{E}-06$ . $503 E-06$ $1.032 F-05$ 1. $254 E-05$ 1. $270 \mathrm{E}-05$ . $393 \mathrm{E}-04$ $1.393 E-04$ 1.229E-03 $1.229 \mathrm{E}-03$
$8.227 \mathrm{E}-02$ . $227 E-02$ 1.171E 00 1.171E 00 . $350 \mathrm{E} 00$ $2.134 E 0$ $3.223 \mathrm{E} \mathrm{OI}$
$M U=-C \cdot 9894$
$-6.319 E-10$ $2.258 \mathrm{E}-08$ $3.829 E-08$ $1.821 E-07$ $7.336 \mathrm{E}-07$ $1.596 \mathrm{E}-06$ $1.871 \mathrm{E}-06$ $2.827 \mathrm{E}-06$ $4.156 E-06$ $6.376 \mathrm{E}-06$ $1.173 \mathrm{E}-05$ 3.323E-05 $1.031 E-04$ 1. C66E-03 $1 . C 67 E-03$ $7.197 \mathrm{E}-\mathrm{C2}$ $3.346 \mathrm{E}-01$ $1 . C 31 E$ DO 2.945E 00 $8.275 E$ OO $2.887 E$ O ANGLE 11 $M U=0.2816$
$4.57 C E-08$ $4.57 \mathrm{CE}-0$ $8.827 \mathrm{E}-08$ $2.561 \mathrm{E}-07$ $9.581 \mathrm{E}-07$ $2.225 E-06$ $3.595 \mathrm{E}-06$ $3.168 E-06$ . $882 E-C 6$ $1.320 E-05$ 1.486-05 $2.126 E-05$ . $1.483 E-04$ $1.323 E-C 3$ $8.435 \mathrm{E}-02$ . $1.195 E$ OO $3.414 \mathrm{E}$ on . $414 \mathrm{E}$ OO $2.182 \mathrm{E} \mathrm{O}$ 3.290 E OI $\begin{array}{ccc}\text { ANGLE } & 3 & \text { ANGLE } \quad 4 \\ M U=-0.9446 & M U=-0.8656\end{array}$ $7.138 \mathrm{E}-10$ $2.317 \mathrm{E}-08$ 4. $171 E-08$ $7.471 \mathrm{E}-07$ 4.274E-06 $1.19 C E-05$ . 373 E-O .042E-04 $1 . C 73 E-03$ $7.235 \mathrm{E}-02$ 3. $363 \mathrm{E}-01$ $1.036 E 00$ 2.959E OD $8.315 E 00$ $1.902 \mathrm{E} \mathrm{O}$ $2.900 E^{01}$

\section{ANGLE 12} $M U=0.4580$
$7.048 \mathrm{E}-08$ $1.221 E-07$ $3.751 \mathrm{E}-0$ 1. $372 \mathrm{E}-06$ $2.917 E-06$ 4. $536=-96$ $3.763 E-16$ $8.906 E-06$ $1.761 \mathrm{E}-05$ $1.795 E-O 5$ $2.403 F-05$ $6.582 E-05$ $1.581 E-04$ 1. $1.296 E-03$ $8.643 E-02$ 1.227E 00 $3.492 \mathrm{E} 00$ $9.778 \mathrm{E} 00$ $2.23 \mathrm{CE}$ OI 3.356E OI
$A N G L E \quad{ }^{4}$
$M U=-C .8656$ $2.399 E-C$
$2.394 E-O$ 4. $623 \mathrm{E}-08$ 2.010 E-07 $7.803 \mathrm{E}-\mathrm{C} 7$ $1.681 \mathrm{E}-\mathrm{C} 6$ $1.892 \mathrm{E}-\mathrm{C} 6$ $2.926 \mathrm{E}-06$ $4.499 E-C 6$ $6.754 \mathrm{E}-\mathrm{C} 6$ $1.222 \mathrm{E}-05$ $3.466 \mathrm{E}-05$ $1.062 \mathrm{E}-04$ $1.086 \mathrm{E}-03$ $1.084 \mathrm{E}-03$ $7.301 \mathrm{E}-\mathrm{C} 2$ 3.393E-01 $1.046 \mathrm{E} \mathrm{OO}$ $2.985 \mathrm{E} \mathrm{OO}$ $8.385 \mathrm{E} \mathrm{OC}$ 1.917 E C $2.924 \mathrm{E} \mathrm{Ol}$ ANGLE 13 $\mathrm{AU}=\mathrm{C} .6179$
$1.219 \mathrm{E}-07$ $1.219 \mathrm{E}-07$
$1.906 \mathrm{E}-07$ $1.906 \mathrm{E}-07$ 6.141E-C7 $2.130 \mathrm{E}-06$ $4.038 E-06$ $4.928 E-06$ 4.544E-06 $1.188 E-05$ $2.460 E-C 5$ 2.186E-O5 $2.724 E-05$ . $396 \mathrm{E}-05$ $1.682 E-04$ $1.327 \mathrm{E}-03$ $8.327 E-03$ $4.074 E-01$ $4.074 E-01$ 1.25 E DO . . 975 E $3.419 E$ OI

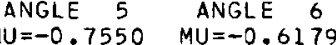
3. $143 \mathrm{E}-09$ $2.486 \mathrm{E}-0$ 5. $C 8 \mathrm{CE}-08$ $2.262 E-07$ $8.431 \mathrm{E}-07$ $1.763 \mathrm{E}-06$ $1.922 E-06$ 3. $038 \mathrm{E}-06$ $4.842 E-06$ $7.132 \mathrm{E}-06$ $1.271 \mathrm{E}-05$ $3.605 \mathrm{E}-0$ 1. $C 92 \mathrm{E}-04$ $1.105 E-03$ $1.099 \mathrm{E}-03$ $7.397 \mathrm{E}-02$ $3.435 \mathrm{E}-01$ 1.C59E 00 $3 . C 21 E 00$ $8.485 E$ OO $1.940 \mathrm{E} \mathrm{O} 1$ $2.956 \mathrm{E} \mathrm{CI}$ ANGLE 14 $\mathrm{AU}=\mathrm{C} .755 \mathrm{C}$
$2.373 \mathrm{E}-07$ 3.364E-07 $1.117 E-C 6$ $3.519 E-06$ 5.829E-06 . 1.623E-C . $523 \mathrm{E}-\mathrm{C}$ . 8.283E-05 1. $456 E-04$ $1.356 \mathrm{E}-03$ $9.018 E-C 2$ 4. $151 E-01$ $3.631 E$ OO 1. Cl6E O $2.315 \mathrm{E} \mathrm{Cl}$ $3.474 \mathrm{E}$ OI $3.719 E-09$ $2.682 E-08$
$5.759 E-08$ $2.694 \mathrm{E}-07$ $9.427 \mathrm{E}-07$ $1.876 \mathrm{E}-06$ $1.976 \mathrm{E}-06$ $3.219 E-06$ $5.338 \mathrm{E}-\mathrm{C} 6$ $7.672 \mathrm{E}-06$

ANGLE 7 
(NEUTRONS/MEV/STERADIAN/SOURCE NEUTRON)

ENERGY GROUP (MEV) $1.22 \mathrm{E} 01--1.50 \mathrm{E} \mathrm{CI}$ 1.00 E $01--1.22 \mathrm{E} \mathrm{C1}$ 8.19E OO---1.0OE CI 6.36E CO--8.19E OD 4.97E $00--6.36 E$ CO $4.07 E$ EO-- $4.97 E$ EO $3.01 E$ OO- $-4.07 E$ EO $2.46 \mathrm{E} 00--3.01 \mathrm{E} \mathrm{CC}$ 2.35E $00-3-2.46 E$ CO $1.83 E$ OC- $-2.35 E$ CC $1.11 E$ OO-C. $1.83 \mathrm{E}$ CC $5.50 E-01--1.11 E$ CO
$1.11 E-C 1---5.50 \mathrm{E}-\mathrm{Cl}$ $3.35 \mathrm{E}-02-0-1.11 \mathrm{E}-01$ $5.83 \mathrm{E}-04--3.35 \mathrm{E}-02$ $1.01 \mathrm{E}-04-05.83 \mathrm{E}-\mathrm{C} 4$ 2.90E-05--1.01E-C4 $1.07 E-05-2.90 E-C 5$ $3.06 E-06--1.07 \mathrm{E}-05$ $1.12 \mathrm{E}-06--3.06 \mathrm{E}-06$ $4.14 \mathrm{E}-07---1.12 \mathrm{E}-06$
$0.0 \quad-0-4.14 \mathrm{E}-07$ ENERGY

GROUP (MEV) $1.22 \mathrm{E} \mathrm{O1--1.50E} \mathrm{O1}$ 1.OCE O1--1.22E 01 6.36 E OO---8.19E OO $4.97 \mathrm{E} 00-6.36 \mathrm{E}$ CO $4.07 E$ OO-- $4.36 \mathrm{E}$ CO $3.01 \mathrm{E} 00--4.07 \mathrm{E} C O$ $2.46 \mathrm{E} \mathrm{OO--3.01E} \mathrm{CO}$ $2.35 \mathrm{E} \mathrm{OO--2.46E} \mathrm{OO}$ $1.83 \mathrm{E}$ OD- $-2.35 \mathrm{E} 00$ $1.11 \mathrm{E} 00--1.83 \mathrm{E} 00$ $5.50 E-01+-1.11$ E 00 $1.11 E-01--5.50 E-C 1$ 3.35E-C 2---1.11E-O 1 $5.83 E-04--3.35 E-C 2$ $1.01 E-04-\cdots 5.83 E-04$ 2.90E-05-- 1.01 E-0 4 $1.07 \mathrm{E}-05--2.90 \mathrm{E}-\mathrm{C} 5$ $3.06 \mathrm{E}-06=-1.07 \mathrm{E}-05$ $1.12 E-06---3.06 E-C 6$ $4.14 E-07---1.12 E-C 6$ $0.0 \quad--4.14 E-07$ $-925 E-13 \quad-1.855 E-13$ $2.6 C 9 E-11$ $7.979 E-10$ $4.979 E-10$ 作 I.700E-D9 $1.116 E-08$ $2.447 \mathrm{E}-08$ $3.933 \mathrm{E}-08$ $7.456 \mathrm{E}-08$ $2.181 \mathrm{E}-07$ $7.030 \mathrm{E}-07$ $7.467 \mathrm{E}-06$ $7.532 \mathrm{E}-06$ $5.109 E-04$ $2.384 E-03$ $7.370 \mathrm{E}-03$ $2.112 \mathrm{E}-02$ $5.950 E-C 2$ $1.364 E-01$
$2.087 E-01$ ANGLE 10 $M U=0.0950$ $3.776 E-11$ 1. 4. $1.098 \mathrm{E}-08$ $E-08$ $629 E-08$ 3. $328 \mathrm{E}-08$ $7.965 \mathrm{E}-08$ 1. $3.493 E-07$ 9. 55 E-07 $8.982 E-06$ $8.700 \mathrm{E}-06$ $5.855 E-C 4$ 2.717E-03 8.390E-03 $2.398 \mathrm{E}-02$ $6.741 \mathrm{E}-02$ $1.542 \mathrm{E}-\mathrm{O} 1$ 2. 335 E-O 1
ANGLE 3 $7.033 \mathrm{E}-11$ 8.C67E-10 $4.440 \mathrm{E}-0$ ? $9.742 E-09$ $1.117 E-08$ $1.653 \mathrm{E}-08$ $2.465 E-08$ 3.953E-08 $7.481 \mathrm{E}-\mathrm{C8}$ $2.189 \mathrm{E}-07$ $7.048 \mathrm{E}-\mathrm{C} 7$ $7.48 C E-06$ $7.542 \mathrm{E}-06$ $5.115 E-04$ $2.387 \mathrm{E}-03$ $7.378 \mathrm{E}-03$ $2.114 \mathrm{E}-02$ $5.957 \mathrm{E}-02$ 2. $C 89 E-01$ ANGLE 11 $M U=C .2816$
$5.799 \mathrm{E}-11$ $1.575 \mathrm{E}-10$ $9.27 \mathrm{CE}-10$ $5.566 \mathrm{E}-09$ $1.376 \mathrm{E}-08$ $2.211 E-08$ $1.885 \mathrm{E}-08$ $4.184 \mathrm{E}-08$ $8.210 \mathrm{E}-08$ $9.482 E-08$ $1.358 \mathrm{E}-07$ $3.893 E-07$ 1 . C17E-06 $8.936 E-06$ 6. $104 E-04$ $2.783 \mathrm{E}-03$ $8.592 \mathrm{E}-\mathrm{C} 3$ $2.455 \mathrm{E}-02$ $2.455 E-02$
$6.897 \mathrm{E}-02$ $1.577 \mathrm{E}-\mathrm{O}$ 2. $384 E-01$ $M U=-C .9446$ $1.175 \mathrm{E}-12$ .875E-1 $.621 \mathrm{E}-1$ $8.47 C E-10$ $4.531 E-09$ $9.921 \mathrm{E}-\mathrm{C} 9$ $1.12 \mathrm{CE}-08$ $1.674 \mathrm{E}-08$ $2.541 \mathrm{E}-\mathrm{O}$ 4.04C E-08 7. $590 \mathrm{E}-08$ $2.222 \mathrm{E}-07$ 7. 7. $532 \mathrm{E}-06$ 7.584E-06 $5.142 E-04$ . . . (2) 2.099E-01

ANGLE 12

$M U=0.4580$ $9.203 \mathrm{E}-11$ . $446 \mathrm{E}-09$ $1.446 \mathrm{E}-09$ 1.815E-O $1.815 E-08$ 227E-C8 2.227E-C8 1.110E-0 1.110E-07 1. $533 \mathrm{E}-07$ $4.365 E-07$ . $084 E-C 6$ $9.631 E-06$ . $172 \mathrm{E}-06$ $6.153 \mathrm{E}-04$ $2.849 \mathrm{E}-\mathrm{Cl}^{3}$ $2.849 E-C 3$
$8.794 E-03$ 2. $512 \mathrm{E}-02$ $2.512 \mathrm{E}-02$
$7 . \mathrm{C} 52 \mathrm{E}-02$ $1.612 \mathrm{E}-01$ ANGLE $\quad 4$
$M U=-0.8656$ $2.847 \mathrm{E}-1$ $3.123 \mathrm{E}-11$ $1.058 \mathrm{E}-10$ $9.330 \mathrm{E}-1 \mathrm{C}$ $4.746 E-C$ $1.025 E-08$ $1.129 E-08$ $1.717 E-08$ . 683 E-08 ..2C4E-CB $7.798 E-08$ $7.625 \mathrm{E}-06$ $7.659 \mathrm{E}-\mathrm{C} 6$ $5.190 \mathrm{E}-04$ $2.420 \mathrm{E}-03$ $7.481 \mathrm{E}-03$ $2.143 E-02$ $6.037 \mathrm{E}-02$ $.384 E-01$ $M U=0.6179$ $1.619 \mathrm{E}-10$ . 2.530E-O $2.522 \mathrm{E}-\mathrm{CB}$ $3.633 \mathrm{E}-\mathrm{O}$ $.667 E-08$ $.267 \mathrm{E}-08$ . $1.734 E-07$ 1.734E-07 1.153E-C6 $9.948 E-06$ $9.396 \mathrm{E}-0 \mathrm{C}$ $6.294 E-04$ $2.911 \mathrm{E}-03$ $2.911 E-03$
$8.984 E-03$ $2.565 \mathrm{E}-0$ $2.565 E-02$ $1.645 \mathrm{E}-01$
ANGLE 5
$M U=-0.7550$ $3.339 \mathrm{E}-11$ $1.227 \mathrm{E}-10$ $1.087 E-09$ $5.143 \mathrm{E}-09$ 1.C75E-08 $1.147 \mathrm{E}-0$ $1.788 \mathrm{E}-08$ $2.899 E-08$ $4.453 E-C 8$ $8.115 \mathrm{E}-08$ $2.378 E-07$ $7.471 E-07$ $7.760 E-06$ $7.765 \mathrm{E}-\mathrm{C} 6$ $5.258 \mathrm{E}-04$ $2.451 \mathrm{E}-03$ $7.575 \mathrm{E}-03$ $2.170 \mathrm{E}-02$ $6.110 E-02$ $1.400 \mathrm{E}-0$ ANGLE 14 $3.116 \mathrm{E}-10$ $7.136 \mathrm{E}-10$ $4.847 \mathrm{E}-09$ . $247 E-08$ ..630E-C8 . $198 \mathrm{E}-08$ . $1.693 \mathrm{E}-07$ $1.952 E-C 7$ $5.478 E-07$ . $220 \mathrm{E}-06$ $1.024 \mathrm{E}-0 \mathrm{~S}$ $6.421 \mathrm{E}-04$ $2.967 E-03$ $9.155 \mathrm{E}-03$ $2.613 E-C 2$ $2.613 E-C 2$
$7.330 E-C 2$ $7.330 E-C 2$
$1.675 E-01$ $2.519 E-01$ $4=-68179$ $3.664 \mathrm{E}-1$ $1.451 \mathrm{E}-10$ $1.339 E-09$ $5.761 E-09$ $1.144 \mathrm{E}-08$ 1. $180 \mathrm{E}-08$ $1.903 \mathrm{E}-08$ $3.210 \mathrm{E}-08$ 4. $807 \mathrm{E}-0$ $8.562 \mathrm{E}-08$ 2. $506 \mathrm{E}-07$ $7.744 \mathrm{E}-0$ $7.934 \mathrm{E}-06$ 7. $902 \mathrm{E}-06$ $5.346 E-04$ $2.490 E-C 3$ 
(NEUTRONS/MEV/STERADIAN/SOURCE NEUTRON)

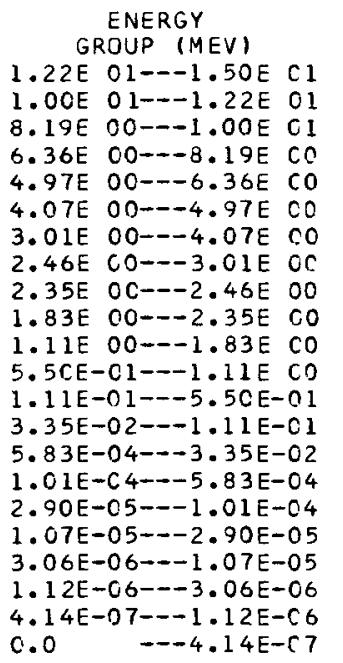

ENERGY

GROUP (MEV)

$1.22 \mathrm{E} 01--1.50 \mathrm{E} O 1$ $1.00 E$ OI- $-1.22 E$ O 8.19E CO-- 1. DOE CI $6.36 E$ OO- $-8.19 E$ OO 4.97E OO---6.36E 00 4.07E 00---4.97E 00 $3.01 E$ OO-- 4. OTE CO $2.46 E$ CO- $-3.01 E 00$ $2.35 \mathrm{E}$ 00-- $2.46 \mathrm{E} \mathrm{OO}$ $1.83 E$ OO- $2.35 E$ CO $1.11 E$ CO- $-1.83 \mathrm{E} \mathrm{OO}$ $5.5 \mathrm{CE}-01---1.11 \mathrm{E}$ CO $1.11 E-01--5.50 E-0$ $3.35 E-02-\cdots-1.11 E-6$. $5.83 \mathrm{E}-04--3.35 \mathrm{E}-\mathrm{C} 2$ $1.01 E-04--5.83 E-C 4$ $2.9 C E-C 5--1.01 E-04$ $1.07 E-05-C 2.90 E-O 5$ 1.06E-06-D-1.07E-CS $1.12 E-06-3.06 E-C 6$ $4.14 E-07--1.12 E-06$
$0.0 \quad--4.14 E-C ?$ $\triangle N G L E \quad I$ $M U=-1.0000$ (2) $.092 \mathrm{E}-1$ $2.7 C 6 E-12$ $4.290 \mathrm{E}-1$ $5 . C 15 \mathrm{E}-11$ $6.939 E-11$ $1.001 \mathrm{E}-10$ $1.729 \mathrm{E}-1 \mathrm{C}$ $1.729 E-1 C$ $9.846 E-10$ $3.296 \mathrm{E}-09$ 3.591 당 $3.657 \mathrm{E}-08$ $2.477 \mathrm{E}-06$ I. $156 \mathrm{E}-05$ $3.568 \mathrm{E}-05$ $1.021 E-C$ $2.877 E-04$ $6.595 E-04$ 1.C11E-C3

ANGLE 2 $M U=-0.9894$
$-2.363 E-17$ . 5C CE- 1 $9.620 E-14$ $2.755 \mathrm{E}-1$ $1.949 \mathrm{E}-1$ 4.317E-11 5. $226 \mathrm{E}-1$ $6.972 E-11$ $1.742 \mathrm{E}-10$ $3.42 \mathrm{CE}-10$ $3.42 C E-10$ 3. $3 C 8 \mathrm{E}-09$ $3.6 C G E-C 8$ $3.664 E-C 8$ $2.481 E-O 6$ $1.157 E-05$ $3.573 \mathrm{E}-05$ 1. C $23 E-C 4$ $2.881 \mathrm{E}-04$ $6.6 \mathrm{C} 5 \mathrm{E}-\mathrm{C}$ $1.013 \mathrm{E}-\mathrm{C} 3$ $1.239 \mathrm{E}-15$ I. $502 \mathrm{E}-13$ $1.502 E-13$ 2. $C$ CE-1 4. $432 \mathrm{E}-11$ 5.C77E-11 $7.12 \mathrm{CE}-1$ $1.798 \mathrm{E}-10$ $3.495 \mathrm{E}-10$ $3.495 E-10$
$1 . C 12 E-09$ 3. $361 \mathrm{E}-09$ 3.635E-C 8 $3.691 \mathrm{E}-\mathrm{C} 8$ $2.499 \mathrm{E}-\mathrm{C}$ $1.165 E-05$ $3.598 \mathrm{E}-05$ 1. C $3 C E-C^{-} 4$ $2.901 E-04$ $6.649 E-04$ 1. C19E-O3

ANGLE 4
$M U=-0.8656$
$2.784 E-15$
$3.385 E-14$
$2.160 E-13$
$3.430 E-12$
$2.143 E-11$
$4.651 E-11$
$5.183 E-11$
$7.416 E-11$
$1.134 E-10$
$1.905 E-10$
$3.637 E-10$
$1.055 E-09$
$3.458 E-09$
$3.698 E-08$
$3.741 E-08$
$2.531 E-06$
$1.180 E-05$
$3.642 E-C 5$
$1.042 E-04$
$2.935 E-04$
$6.726 E-04$
$1.030 E-03$
$\triangle A N G L E 5$ 3. $884 E-15$ $3.798 \mathrm{E}-14$ $2.728 E-13$ $4.238 \mathrm{E}-12$ $2.386 \mathrm{E}-1$ 4.987E-1 $5.364 \mathrm{E}-1$ $7.910 E-11$ $2.069 \mathrm{E}-10$ $3.855 \mathrm{E}-10$ $3.855 E-10$ 1.118E-0 3. $788 \mathrm{BE}-08$ $3.811 \mathrm{E}-08$ $2.577 E-06$ 1.200E-05 $3.705 \mathrm{E}-05$ 1. $C 60 \mathrm{E}-04$ $2.984 E-04$ $6.836 \mathrm{E}-04$ 1. $C 46 E-03$

ANGLE 6
$M U=-0.6179$
$5.272 E-15$
$4.358 E-14$
$3.491 E-13$
$5.580 E-12$
$2.767 E-11$
$5.465 E-11$
$5.647 E-11$
$8.688 E-11$
$1.447 E-1 C$
$2.303 E-1 C$
$4.157 E-10$
$1.205 E-09$
$3.781 E-09$
$3.902 E-08$
$3.901 E-08$
$2.634 E-C 6$
$1.226 E-05$
$3.784 E-05$
$1.082 E-04$
$3.045 E-04$
$6.975 E-04$
$1.065 E-03$

ANGLE 7 4580 MU ANGL 8 $8.261 \mathrm{E}-15$
$5.591 \mathrm{E}-14$ $5.140 \mathrm{E}-13$ $7.662 \mathrm{E}-1$ $3.297 E-1$ $6.215 \mathrm{E}-1$ $6.060 \mathrm{E}-11$ $9.864 \mathrm{E}-1$ $1.718 \mathrm{E}-10$ $2.620 E-10$ $4.550 \mathrm{E}-1 \mathrm{C}$ $1.317 E-09$ $4.005 \mathrm{E}-09$ $4.040 E-08$ $4.007 E-08$ $2.703 E-06$ $1.257 E-05$ $3.878 \mathrm{E}-05$ $1.109 E-04$ 3. $119 \mathrm{E}-04$ $7.140 E-04$ $1.088 \mathrm{E}-03$

ANGLE 16 LE 10 ANGLE 11 ANGLE 12 $4.202 \mathrm{E}-14$ $1.776 \mathrm{E}-13$ $2.141 E-12$ 2. $(24 E-1)$ $5.843 \mathrm{E}-11$ $9.578 \mathrm{E}-1$ $8.399 \mathrm{E}-1$ $1.72 C E-10$ 3. $315 \mathrm{E}-10$ 4. $205 \mathrm{E}-10$ $6.3 \mathrm{C} 5 \mathrm{E}-10$ $1.814 E=09$ 4. $906 E-09$ $4.561 E-O$ $4.402 E-08$ 2.956E-06 $1.370 \mathrm{E}-05$ 1. $206 E-04$ $1.206 E-04$ $3.388 \mathrm{E}-04$ $7.747 \mathrm{E}-04$
$1.172 \mathrm{E}-03$ $M U=0.2816$ ANGLE 13 ANGLE 14 ANGLE 15 . $588 \mathrm{E}-1$ $3.261 \mathrm{E}-12$ $2.899 E-11$ $1.173 \mathrm{E}-10$ 9.77CE-1 $2.187 E-10$ $4.343 \mathrm{E}-10$ $5 . C 51 \mathrm{E}-10$ $7.127 \mathrm{E}-10$ 2.C43E-09 $5.272 E-C 9$ $4.757 E-08$ $4.548 E-08$ 3.C48E-06 $1.411 \mathrm{E}-05$ 1. $242 E-0$ $1.242 E-04$ $3.486 \mathrm{E}-04$ $7.968 E-04$
$1.263 E-03$ $M U=0.755 C$ $3.540 E-13$ $1.307 E-12$ $1.878 \mathrm{E}-11$ $1.236 \mathrm{E}-10$ $2.549 \mathrm{E}-10$ $1.655 \mathrm{E}-10$ $5.225 \mathrm{E}-10$ $1.299 \mathrm{E}-09$ $9.083 \mathrm{E}-10$ 1.C3 5E-09 $2.920 \mathrm{E}-\mathrm{C}$ $6.433 E-09$ $5.312 E-08$ $4.947 E-C 8$ 3. $301 E-C 6$ $1.523 E-C 5$ 4.694E-C5 $1.338 E-04$ $3.751 E-04$

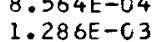
ANGLE 17

ANGLE 8 ANGLE 9 $1.495 E-14 \quad 2.604 E-14$ $8.1 C 2 E-14 \quad 1.213 E-13$ $8.489 E-13 \quad 1.386 E-12$ $1.066 \mathrm{E}-11 \quad 1.467 \mathrm{E}-11$ $3.974 E-11 \quad 4.791 E-11$ $6.973 E-11 \quad 8.078 E-11$ $6.628 \mathrm{E}-11 \quad 7.382 \mathrm{E}-11$ $.157 \mathrm{E}-10 \quad 1.393 \mathrm{E}-10$ $2.100 E-10$
$3.032 E-10$
$5.514 E-10$ $5.035 \mathrm{E}-10 \quad 3.549 \mathrm{E}-10$ $1.455 \mathrm{E}-09$
$1.619 \mathrm{E}-09$ $4.269 \mathrm{E}-09 \quad 4.569 \mathrm{E}-\mathrm{CQ}$ $4.198 E-08 \quad 4.371 E-08$ $4.129 E-08 \quad 4.257 E-C 8$ $2.781 E-06 \quad 2.863 \mathrm{E}-06$ $1.292 \mathrm{E}-05 \quad 1.328 \mathrm{E}-05$ $3.985 \mathrm{E}-05 \quad 4 . \mathrm{C} 97 \mathrm{E}-05$ $1.139 E-04 \quad 1.170 E-04$ $3.202 \mathrm{E}-\mathrm{C} 4 \quad 3.288 \mathrm{E}-04$ $\begin{array}{ll}7.328 \mathrm{E}-04 & 7.522 \mathrm{E}-04 \\ 1.114 \mathrm{E}-03 & 1.141 \mathrm{E}-03\end{array}$ SCALAR ANGLE 17 FLUX $2.003 \mathrm{E}-12 \quad 1.991 \mathrm{E}-12$ . 240E-12 7.517E-12 $1.166 \mathrm{E}-10$ 1.C $88 \mathrm{E}-10$ $4.953 E-10 \quad 6.629 E-10$ $5.004 E-10 \quad 1.152 E-09$ $2.415 E-10 \quad 1.622 E-C 9$ $1.188 \mathrm{E}-09 \quad 3.163 \mathrm{E}-\mathrm{C} 9$ $5.086 \mathrm{E}-09 \quad 7.819 \mathrm{E}-09$ $1.4 \mathrm{ClE}-09 \quad 6.173 \mathrm{E}-09$ $1.316 \mathrm{E}-09 \quad 8.337 \mathrm{E}-09$ $3.668 \mathrm{E}-\mathrm{C9} \quad 2.382 \mathrm{E}-08$ $7.188 \mathrm{E}-\mathrm{C9} \quad 6.157 \mathrm{E}-\mathrm{C} 8$ $5.626 \mathrm{E}-08 \quad 5.674 \mathrm{E}-07$ $5.164 E-08 \quad 5.477 E-07$ $1.583 E-05 \quad 1.7 C 4 E-C 4$ 4.879E-05 1.7C4E-C4 
(GAMMAS/MEV/STERADIAN/SOURCE NEUTRON)

\begin{abstract}
ENERGY
GROUP (MEV) 8.00E 00---1.00E 01 $6.50 E$ OO- $-8.00 E$ CO 5. 00 E $00---6.50 E O D$ 4.0OE CO-- $-5.00 E$ CC 3.00E OO---4.00E OO $2.50 \mathrm{E} \mathrm{OO---3.00E} \mathrm{CO}$ $2.00 E$ CO-- 2.5OE 00 $1.66 \mathrm{E} 00--2.00 \mathrm{E}$ OO $1.33 E$ EO-- $1.66 E$ EO $1.00 E$ OO-- $-1.33 E$ OO $8.00 \mathrm{E}-01--1.00 \mathrm{E}$ CO $6.00 E-01=-8.00 E-C 1$ $4.00 E-01--6.00 E-C 1$ 3.0OE-OI-- - 4.00E-O $2.00 \mathrm{E}-01--3.00 \mathrm{E}-\mathrm{Cl}$ $1.00 E-01-2-2.00 E-0$ $5.00 E-02--1.00 E-01$
$2.00 E-C 2---5.00 E-02$
\end{abstract}

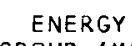

8.00E OO-- 1.00E 01 6.5OE CO-- - . DOE OO 5. DOE OO---6.5OE OO 4.DOE DO- $5.00 E$ CC . $.00 E$ OO-OC. 2. 2.OCE OO- 2 . $O$ OE CO .33E $00--1.66 E$ 1.0OE $00--1.33 E \mathrm{ECO}$ $8.00 E-C 1=-1.00 E$ COO $6.00 E-01--8.00 E-C 1$ $4.00 E-01-=-6.00 E-01$ 3.0CE-01-- $4.00 E-O 1$ .OCE-C1--3.0OE-O 1.OCE-01-- $2.00 E-C$ $5.00 \mathrm{E}-02--1.00 \mathrm{E}-\mathrm{C}$ 2.0OE-02---5. $00 \mathrm{E}-\mathrm{C} 2$
ANGLE 1 $M U=-1.0000$ $-2.940 \mathrm{E}-06$ $-7.892 E-06$ $-1.296 \mathrm{E}-05$ $-1.146 \mathrm{E}-05$ $5.044 E-06$ $5.347 E-06$ $2.395 E-05$ $1.457 E-05$ - $1.449 E-05$ .280E-05 $1.021 E-04$ . $1.261 \mathrm{E}-03$ $2.866 \mathrm{E}-03$ $2.704 \mathrm{E}-03$

ANGLE 10

$M U=0.0950$ $2.723 \mathrm{E}-07$ $1.335 E-06$ $6.117 E-06$ $6.817 E-06$ (526E-O6 $5.926 \mathrm{E}-06$ $1.031 E-06$ $-1.405 E-05$ $2.843 E-07$ $5.671 E-05$ $1.927 E-04$ $5.719 E-04$ $7.173 \mathrm{E}-04$ $1.072 \mathrm{E}-03$ $3.677 \mathrm{E}-03$ $1.078 \mathrm{E}-02$ $2.938 \mathrm{E}-03$
ANGLE 2 $-4.365 E-07 \quad-1.185 E-07$ $-2.41 C E-06$-7.111E-0 $-6.356 E-06 \quad-1.421 E-06$ $-1.059 E-05-2.984 E-06$ $-9.271 E-06-2.282 E-06$ $-3.949 E-C 6 \quad-5.255 E-07$ $4.716 \mathrm{E}-06$ $1.435 \mathrm{E}-05$ $2.006 E-05$ $1.187 E-05$ $1.226 \mathrm{E}-05$ 2.651E-05 1. $C 44 E-04$ $1.24 E-04$ $1.261 E-03$ . 2.706E-O

ANGLE 11 $M U=0.2816$
$6 . C 45 E-07$ $6 . C 45 E-07$ l.C $72 E-05$ $1.228 \mathrm{E}-05$ 1.C41E-O 2.C $97 E-06$ $-5.671 \mathrm{E}-06$ $-9.836 \mathrm{E}-06$ $-2.488 E-G 7$ $4 . C 86 E-05$ 1. $52 \mathrm{CE}-04$ $3.27 C E-04$ $6.59 C E-04$ $7.017 E-04$ $1.116 \mathrm{E}-03$ $3.864 \mathrm{E}-03$ $1.121 \mathrm{E}-02$ $2.986 \mathrm{E}-03$
$\begin{array}{ll}A N G L E & 4 \\ M U & =-0.8656\end{array}$ $1.253 \mathrm{E}-\mathrm{O}$ $6.227 E-C 7$ $2.515 E-C 6$ $3.031 \mathrm{E}-06$ $3.187 \mathrm{E}-06$ $1.977 \mathrm{E}-06$ $-4.861 E-07$ 2.151E-06 4. $59 \mathrm{CE}-06$ $5.667 \mathrm{E}-06$ $2.325 \mathrm{E}-06$ $-6.059 E-06$ $9.201 \mathrm{E}-05$ $1.375 \mathrm{E}-04$ $1.26 \mathrm{CE}-03$ 2.901E-0 $8.956 \mathrm{E}-03$

ANGLE 12 $M U=0.4580$
$4.168 \mathrm{E}-07$ $8.418 E-07$ $6.047 \mathrm{E}-06$ $-1.404 E-06$ $-4.284 E-06$ $1.097 \mathrm{E}-06$ $1.097 E-06$ $5.055 E-05$ $1.464 E-04$ $2.84 \mathrm{E}-04$ $4.428 \mathrm{E}-04$ $7.013 E-04$ $7.013 E-04$ $1.168 \mathrm{E}-03$ 4. C64E-0 4. $C 64 E-03$
$1.166 E-02$ 3.C $34 \mathrm{E}-03$
ANGLE 5 $-0.7550$ 3.297E-07 1. $555 \mathrm{E}-06$ 7.396E-06 $6.751 E-06$ 2.959 E-06 $-1.834 E-06$ $-6.641 E-06$ $-8.987 E-06$ $-4.467 E-C 6$ $9.599 \mathrm{E}-06$ $1.795 E-05$ $9.6 C 1 E-05$ $3.215 \mathrm{E}-04$ $1.215 \mathrm{E}-0$ $3.023 E-03$ $9.230 \mathrm{E}-03$ $2.750 E-0$

ANGLE 14 $M U=0.7550$
$-1.022 \mathrm{E}-\mathrm{C} 7$ $-2.316 \mathrm{E}-06$ $1.452 \mathrm{E}-06$ 3. $681 \mathrm{E}-07$ $2.539 \mathrm{E}-05$ $7.765 E-05$ 1. $545 E-04$ $2.522 E-04$ $4.63 E=04$ $5.455 E-04$ $5.420 E-04$ . $8.735 E-04$ 1.265E-03 4. $464 \mathrm{E}-03$ $1.253 E-02$ 3.123E-03
ANGLE 6 $M U=-0.6179$ $1.142 \mathrm{E}-07$
$4.863 \mathrm{E}-07$ $4.863 E-07$ $1.576 \mathrm{E}-06$ $1.096 \mathrm{E}-06$ $-4.545 E-08$ $-9.075 E-07$ $-1.571 E-06$ 6.507E-07 $1.203 E-06$ $5.260 E-06$ $2.305 E-06$ $4.341 E-04$ 1. $1621 E-04$ 醇 $3.114 E-03$ $9.444 \mathrm{E}=03$

ANGLE 15 $M U=0.8656$ $4.826 \mathrm{E}-\mathrm{C6}$ $1.894-05$ 7.969E-05 $1.959 E-04$ 1.959E-04 政 $5.587 E-04$ $6.091 \mathrm{E}-04$ $5.543 E-04$ $7.735 \mathrm{E}-04$ $9.320 E-04$ $1.317 E-03$ $4.639 E-03$ $1.289 E-C 2$ $3.158 E-03$
ANGLE 7 ANGLE 8 ANGLE 9 $M U=-0.4580 \quad M U=-0.2816$ $-1.259 E-07 \quad-2.016 E-07$

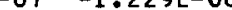
$-1.690 E-06 \quad-2.535 E-06$ -06 -5.1728 $-3.740 E-06$-3.885E-06 $1.858 E-06 \quad-6.396 E-07$ $1.272 \mathrm{E}-06 \quad 4.506 \mathrm{E}-06$ 7.736E-06 $1.045 E-05$ $7.948 \mathrm{E}-06 \quad 1.328 \mathrm{E}-05$ $8.212 E-06$ 4.142E-C6 $-3.873 E-06 \quad-7.041 E-06$ 6.315E-06 $1.122 E-05$ 2.100E-04 3.210E-04 $1.107 \mathrm{E}-03 \quad 1.064 \mathrm{E}-03$ $3.224 E-03 \quad 3.355 E-03$ $9.707 \mathrm{E}-03 \quad 1.03 \mathrm{E}-02$ $2.810 \mathrm{E}-03 \quad 2.848 \mathrm{E}-03$ $2.848 \mathrm{E}-03$ ANGLE 16 $M U=0.9446$ $4.153 E-05$
$1.665 E-04$ $4.478 E-04$ $5.498 E-04$ $7.525 E-04$ $7.737 E-04$ $7.645 \mathrm{E}-04$ $7.246 \mathrm{E}-04$ $6.443 \mathrm{E}-04$ $5.759 E-04$ $8.578 E-04$ $9.387 E-04$ $1.372 \mathrm{E}-03$ $4.776 \mathrm{E}-03$ $3.184 \mathrm{E}-03$ ANGLE 17
$M U=0.9894$ $4.6245-04$ $1.557 E-03$ $2.558 \mathrm{E}-03$ $2.064 E-03$ $1.714 E-03$ 1.525 $8.468 E-04$ $6.154 E-C 4$ $0.042 E-04$ $9.042 E-04$ $9.152 E-04$ $1.414 E-03$ $4.858 \mathrm{E}-03$ 1. $333 \mathrm{E}-02$

3. 199E-03 
(GAMMAS/MEV/STERADIAN/SOURCE NEUTRON)

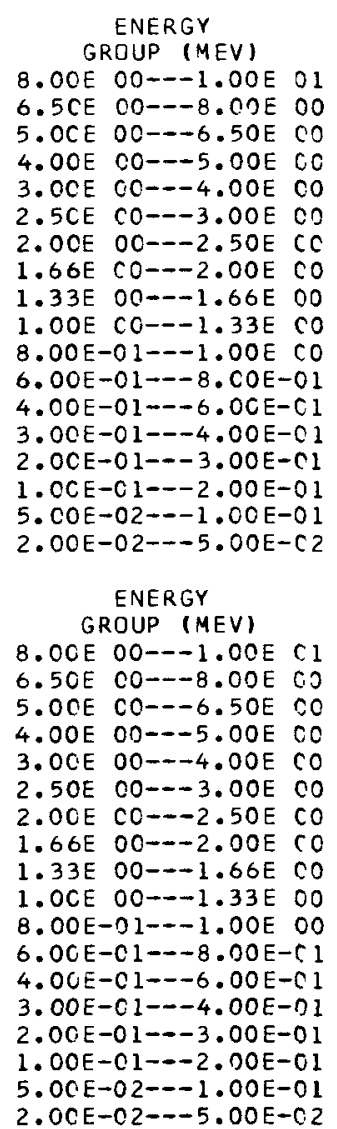
$=-1.0000$

$-1.993 E-07$ $-5.012 E-07$ $-7.232 E-07$ $-6.059 \mathrm{E}-07$ $-2.060 \mathrm{E}-07$ $3.763 \mathrm{E}-07$ $9.683 \mathrm{E}-07$ $1.241 \mathrm{E}-06$ $6.1 C 9 E-07$ $-9.214 E-07$ $-1.685 E-06$ $5.469 \mathrm{E}-06$ $4.964 \mathrm{E}-06$ $6.486 E-05$ $1.408 \mathrm{E}-04$ $1.295 \mathrm{E}-04$

ANGLE 10 $M U=C .0950$ $2.033 \mathrm{E}-08$ 2.857E-07 $4.217 E-07$ 4. $217 E-07$ 2.192E-07 $2.192 E-07$
$-2.822 E-07$ $-2.822 E-07$
$-7.915 E-07$ $-9.970 E-07$ $1.115 E-07$ 3. $452 E-06$ $2.908 E-05$ $3.460 E-05$ $5.199 E-05$ I. $777 \mathrm{E}-04$ 5. $14 \mathrm{CE}-04$ $1.397 E-04$
ANGLE 2 $M U=-0.9894$ $-3.414 E-08$ $-1.643 E-C 7$ $-4.1165-07$ $-5.932 E-07$ $-4.932 \mathrm{E}-07$ $-1.614 E-07$ $3.221 \mathrm{E}-07$
$8.1 C 7 \mathrm{E}-07$ $1.027 \mathrm{E}-06$ 4. $867 \mathrm{E}-07$ $-7.769 \mathrm{E}-07$ $-1.364 E-C 6$ $5.321 \mathrm{E}-06$ $5.399 E-C 6$ $6.481 E-05$ $1.411 E-04$ $4.311 E-C 4$

ANGLE 11 $M U=C .2816$ 4. $323 \mathrm{E}-08$ $5.167 E-07$ $6.446 E-07$ $4.021 E-07$ $-6.058 \mathrm{E}-07$ $-6.058 E-07$ $-5.8 C 2 E-C 8$ $2.475 E-06$ $8.616 \mathrm{E}-06$ $1.72 \mathrm{CE}-05$ $3.341 E-05$ $5.376 E-05$ 1. $857 \mathrm{E}-04$ 5. $323 \mathrm{E}-04$ $1.417 \mathrm{E}-\mathrm{C} 4$ $M U=-0.9446 \quad$ ANGLE 4 $-1.642 E-08 \quad 9.111 E-09$ $-4.982 \mathrm{E}-\mathrm{C} 8 \quad 4.491 \mathrm{E}-\mathrm{C} 8$ $-1.192 \mathrm{E}-\mathrm{C} 7 \quad 1.238 \mathrm{E}-\mathrm{C} 7$ $-1.697 E-07 \quad 1.8 C 9 E-07$ $-1.283 E-07 \quad 1.676 E-07$ $-2.236 E-08 \quad 7.648 E-C 8$ $\begin{array}{ll}1.144 E-07 & -9.285 E-08 \\ 2.325 E-07 & -2.905 E-C 7\end{array}$ $2.325 E-07$ $2.574 E-07-2.905 E-C 7$ $6.328 E-C 8$ $-2.775 \mathrm{E}-07$ $-3.178 \mathrm{E}-07$ $7.841 E-06$ $6.444 \mathrm{E}-05$ $1.425 \mathrm{E}-04$ $4.339 E-04$ $1.30 C E-04$ $-4.049 \mathrm{E}-07$ $-2.472 E-07$
$2.195 E-07$ $2.195 \mathrm{E}-07$
$5.600 \mathrm{E}-07$ $4.503 E-06$ $1.140 E-05$ $6.333 E-05$ $1.449 E-04$ 1. $306 \mathrm{E}-04$ ANGLE 12 $M U=0.4580$
$-2.624 E-09$ $-2.624 E-09$
$-4.003 E-08$ ANGLE 13 $M U=0.6179$
$-9.264 E-08$ $-4.713 E-07$ $-1.585 E-07-1.143 E-06$ $-6.833 E-07-1.107 E-06$ $-5.561 E-07 \quad 2.397 E-07$ $3.832 E-08 \quad 2.326 E-C 6$ $5.962 E-06$ 3.457E-06 $1.051 E-C 5$ $1.521 E-05 \quad 2.161 E-05$ 1.521E-05 $2.161 E-05$ $3.367 E-05 \quad 3.344 E-C 5$ $3.416 E-05$ 3.713E-05 $5.574 E-05 \quad 5.749 E-05$ $1.942 E-04 \quad 2.026 E-04$ $1.942 E-04 \quad 2.026 E-04$ $1.437 \mathrm{E}-04 \quad 1.457 \mathrm{E}-04$
ANGLE 5 $\begin{aligned} M U & =-6.7550 \\ 2.268 E-C 8 & \end{aligned}$ $2.268 \mathrm{E}-\mathrm{C} 8$ $2.770 \mathrm{E}-07$ 3. $854 \mathrm{E}-07$ 3.118E-07 $7.871 \mathrm{E}-\mathrm{C} 8$ $-1.786 E-07$ $-4.192 E-07$
$-5.059 E-07$ $-1.957 \mathrm{E}-07$ $5.324 \mathrm{E}-07$ $7.469 \mathrm{E}-\mathrm{C} 7$ $5.098 E-06$ $1.737 E-05$ $6.112 E-05$ $1.483 E-04$ $4.462 E-04$ $M U=C .7550$ $-1.033 \mathrm{E}-07$ $-4.911 \mathrm{E}-\mathrm{C} 7$ $-3.577 E-07$ $1.221 \mathrm{E}-0 \mathrm{~S}$ 4. $9 . C 13 E-06$ $1.935 E-05$ $2.426 E-C 5$ $2.426 E-C 5$ $2.455 E-05$ 2.45 E - 05 $4.073 E-C 5$ $5.915 \mathrm{E}-05$ $2.105 \mathrm{E}-04$ $2.105 E-04$ 1. $474 \mathrm{E}-04$ $M U=-0.6179$
$4.439 \mathrm{E}-09$ $1.867 \mathrm{E}-08$ $1.867 E-08$ $4.431 \mathrm{E}-\mathrm{C} 8$ $3.905 E-08$ $2.370 \mathrm{E}-\mathrm{CS}$ $-8.398 E-08$ $-9.081 E-08$ $2.02 C E-C 8$ $8.713 E-C 8$ $1.664 E-07$ $1.532 \mathrm{E}-08$ $7.298 E-06$ $2.737 E-05$ $1.525 \mathrm{E}-04$ 4. $557 \varepsilon-04$

ANGIE 15

$U=0.8656$ $1.736 \mathrm{E}-07$ $3.196 \mathrm{E}-06$ $6.192 \mathrm{E}-06$ $1.148 \mathrm{E}-05$ $1.640 \mathrm{E}-05$ $2.080 E-05$ $2.732 E-05$ $2.828 E-05$ $2.672 \mathrm{E}-05$ 2.672E-05 3. $549 \mathrm{E}-05$ $4.278 E-05$ 6.278E-05 $2.174 E-04$ $2.174 E-C 4$
$6.008 E-r 4$ $6.008 E-C 4$
$1.489 E-C 4$ \begin{tabular}{cc} 
ANGLE & 7 \\
$M U=-0.458 C$ & ANGLE 8 \\
\hline$U$ & $8-C .2816$
\end{tabular} $-1.457 E-08-1.983 \mathrm{E}-\mathrm{C} 8$ $-7.224 E-08 \quad-9.4968$ $-1.830 \mathrm{E}-07 \quad-2.280 \mathrm{E}-\mathrm{C} 7$ -2.800E-07 -3.192E-07 $-2.500 E-07-2.322 E-C 7$ 1.C79E-07 - $2.188 \mathrm{E}-\mathrm{C8}$ $8.615 E-08$ 2. $253 E-07$ 3.044E-07 S. 0 CEE-07 $4.508 E-07$ C. $173 E-07$ 3.499E-07 3.762E-08 -3.589E-07 $-5.088 E-C 7$ 1. $147 \mathrm{E}-07$ 7.438E-C7 $1.147 E-05$ 1.724E-05 5. $1.576 \mathrm{E}-04 \quad 1.635 \mathrm{E}-\mathrm{C4}$ $1.576 E-04$ 1.635E-C4 l. $342 E=04 \quad 4.812 E-04$ $8 E-C_{4}$ ANGLE 16 ANGLE 17 2.325E-06 MU $9.325 E-06 \quad 9.3267 E-05$ $2.294 E-05 \quad 1.305 E-04$ $3.664 E-05 \quad 1.033 E-04$ $3.664 E-05$ O.370E-05 $3.789 E-05 \quad 5.663 E-05$ $3.543 E-05$ $40413 E-05$ $3.185 E-05$ 3. $313 E-05$ $2.724 E-05$ 2.363E-05 $2.451 E-05 \quad 2.154 E-05$ $2.509 \mathrm{E}-05 \quad 2.641 \mathrm{E}-05$ $4.240 E-05$ 4. $089 E-05$ $6.327 \mathrm{E}-05 \quad 6.498 \mathrm{E}-05$ $2.228 E-04 \quad 2.26 C E-04$ $0.117 E-04 \quad 6.180 E-04$ $1.499 \mathrm{E}-04 \quad 1.5 \mathrm{C} 5 \mathrm{E}-04$
ANGLE 9 $-1.344 E-C 9$ $1.275 \mathrm{E}-09$ $1.772 E-08$ $5.265 E-08$ $4.414 E-C 8$ $1.222 E-07$ $1.254 E-C 7$ $-3.434 E-08$ $-2.947 E-07$ 3.775E-C7 $4.291 E=07$ $4.55 \mathrm{E}-06$ $3.377-05$ $5.125 \mathrm{E}-05$ 1.702E 04 4. $968 \mathrm{E}-04$ $1.377 E-04$

SCAL AR FLUX $6.567 \mathrm{E}-\mathrm{C} 6$ $3.921 E-05$ $3.2 C 2 E-05$ $3.544 E-05$ $3.544 E-05$ 4.C1OE-O5 4.556E-C5 5.25 E-OS $6.198 E-05$
$7.792 E-C 5$ $9.997 \mathrm{E}-05$ $9.997 E-05$
$1.334 E-C 4$ $2.334 E-C 4$ $3.943 E-04$ $7.109 \mathrm{E}-04$ $2.226 \mathrm{E}-03$ $2.226 \mathrm{E}-\mathrm{Cl}$
$6.430 \mathrm{E}-03$ $1.748 \mathrm{E}-03$ 
(GAMMAS/MEV/STERADIAN/SOURCE NEUTRON)

ENERGY 8. DOE OO- (MEV) 6.5 CE OO-- 8.00 E 00 5.0OE OD---6.50E OO 3.00E $00---4.00 E$ OO 2.5CE $00--3.00 \mathrm{E} 00$ 2.0 CE $00---2.50 E 00$ $1.66 E \quad 00--2.00 E 00$ $1.33 \mathrm{E} 00---1.66 \mathrm{E} 00$ $1.00 \mathrm{E} \mathrm{O0--1.33E} 00$ $8.00 \mathrm{E}-01--1.00 \mathrm{E} 00$ 6.0OE-01---8.00E-CI $4.00 E-01--6.00 E-01$ 3. $00 \mathrm{E}-01---4.00 \mathrm{E}-01$ $2.00 E-01 \rightarrow-3.00 E-C 1$ $1.00 \mathrm{E}-01=-2.00 \mathrm{E}-\mathrm{O}$ $5.00 E-02=-1.00 E-01$ $2.00 \mathrm{E}-02-\cdots-5.00 \mathrm{E}-\mathrm{C} 2$

$$
\text { ENERGY }
$$

$$
\text { GROUP (MEV) }
$$

8.00E 00--1.00E 01

$6.50 E$ OO- -8.00 E 00

$5.00 E$ CO---6.50E 00

$4.00 E$ OO- -5.00 E 00

$3.00 E$ CO---4.00E 00

2.50 E CO-- $3.00 E$ CO

2. COE OO---2.50E OO
$1.66 E$ OO---2.0OE 00

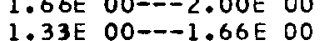

$1.33 \mathrm{E} 00---1.66$ E 00
$1.00 \mathrm{E} 00--1.33 \mathrm{E} 00$

$8.00 \mathrm{E}-01---1.00 \mathrm{E}$ OO

$6.00 \mathrm{E}-01-\cdots 8.00 \mathrm{E}-01$

4.00E-01-- $6.00 \mathrm{E}-01$

3.00E-01-- $4.00 \mathrm{E}-\mathrm{C} I$

$2.00 E-01--3.00 E-01$

1 . OOE O1 $2.00 E-01$

$2.00 \mathrm{E}-02--05.00 \mathrm{E}-02$
ANGLE 1 $U=-1.0000$ $-1.839 \mathrm{E}-09$ $-1.898 \mathrm{E}-08$ 2.612E-08 $2.182 E-08$ 4.269E-08 $2.187 \mathrm{E}-08$ $2.052 \mathrm{E}-07$ $3.052 \mathrm{E}-06$ $1.834 \mathrm{E}-05$ $1.834 E-05$
$5.810 E-06$ ANGLE 10 $M U=0.0950$ $1.713 \mathrm{E}-09$ $7.656 \mathrm{E}-09$ $1.8 C 9 E-08$ 3. $C 97 E-08$ $2.600 \mathrm{E}-08$ $6.862 E-09$ $-4.723 E-08$ (2) (2) (2) (2.656E-06 .75CE-06 $2.414 \mathrm{E}-05$ $6.481 E-06$
ANGLE 3

ANGLE 2 $-1.521 E-09$ $-6.756 E-09$ $-1.564 E-C 8$ $-2.145 E-08$ $-1.7 C 2 E-08$ $1.525 E-08$ $3.404 E-08$ $4.126 \mathrm{E}-08$ $1.7 C 7 E-08$ $-3.585 E-08$ $-5.802 E-08$
$2.575 E-07$ $2.575 \mathrm{E}-07$ $2.299 \mathrm{E}-\mathrm{C} 7$
$3.053 \mathrm{E}-06$ $1.84 C E-C 5$ $5.816 \mathrm{E}-06$ $M U=-0.9446$ $-4.418 \mathrm{E}-10$ $-1.940 E-09$ $-4.376 E-09$ $-5.771 E-09$ $-4.087 E-09$ $-4.625 E-11$ $4.909 \mathrm{E}-\mathrm{C} 9$
$9.052 \mathrm{E}-\mathrm{C} 9$ $9.414 \mathrm{E}-09$ $1.346 E-09$ $-1.196 \mathrm{E}-08$ $-1.232 E-08$
$2.354 E-07$
$3.415 E-07$ $3.415 E-07$ $3.415 E-07$ $6.343 \mathrm{E}-06$ $1.863 E-05$ 5.844E-06 $\begin{array}{ll}A N G L E & 4 \\ M U=-0.8656\end{array}$ $5.789 E-10$ $2.599 E-09$ $6.163 \mathrm{E}-09$ $8.705 E-09$ $7.430 E-09$ $2.488 \mathrm{E}-09$ $-5.387 \mathrm{E}-09$
$-1.376 \mathrm{E}-08$ $-1.376 E-08$ $-9.740 \mathrm{E}-09$ $1.299 \mathrm{E}-08$ $2.785 E-08$ $5.604 E-07$ $3.030 \mathrm{E}-\mathrm{CS}$ $6.537 E-06$ $1.904 E-C 5$ $5.892 \mathrm{E}-06$ ANGLE 11 ANGLE 12 $M U=C .2816$ $3.216 \mathrm{E}-09$ $1.343 E-08$
$2.840 E-08$ $3.204 E-08$ $1.175 \mathrm{E}-08$
$-1.544 \mathrm{E}-08$ $-1.544 E-08$ $-3.677 E-08$ $-3.900 E-08$ 1. $500 \mathrm{E}-09$ $1.609 \mathrm{E}-07$ $4.784 E-07$ I. 60 EE-06 $1.686 E-06$ $1.710 E-06$ $9.191 E-06$ $2.518 \mathrm{E}-05$ $6.6 \mathrm{C1E}-06$
$-8.722 \mathrm{E}-10 \quad-8.320 \mathrm{E}-09$

$-5.418 E-09$

1. $777 \mathrm{E}-08$

$-3.482 E-08$

$-3.138 \mathrm{E}-08$

$8.122 \mathrm{E}-10$

$6.682 E-08$

$1.989 \mathrm{E}-07$

$8.105 \mathrm{E}-07$

$1.141 E-06$

$1.725 E-06$

2.844E-06

$9.633 \mathrm{E}-06$

$2.621 \mathrm{E}-05$

$6.715 E-06$
$A N G L E \quad 5$
$M U=-C .7550$ $9.525 \mathrm{E}-1 \mathrm{C}$ $4.195 E-09$ $9.613 E-09$ $1.289 E-08$ $9.740 E-09$ $1.318 \mathrm{E}-09$ $-9.244 E-09$ $-1.884 \mathrm{E}-\mathrm{C} 8$ $-2.135 \mathrm{E}-\mathrm{C} 8$
$-6.335 \mathrm{E}-09$ . $335 E-C 9$ 2. $518 \mathrm{E}-08$ $3.213 E-C 8$ $8.822 \mathrm{E}-07$ $2.962 E-06$ $6.805 E-06$ $1.962 E-C 5$ $5.959 E-06$

ANGLE 14

$M U=0.7550$ -1. CC5E-08 $-5.756 E-08$ $-1.593 E-08$ $8.710 E-08$ $2.821 \mathrm{E}-07$ $5.155 E-07$ $7.732 \mathrm{E}-07$ $1.023 \mathrm{E}-06$ $1.239 \mathrm{E}-06$ 1.291E-06 $1.210 E-06$ $1.704 E-06$ $2.060 E-06$ 1. $45 \mathrm{E}-05$ $1.045 E-05$ $6.915 E-06$ $M U=-0.6179$ $1.526 \mathrm{E}-10$
$6.051 \mathrm{E}-10$ $1.112 E-09$ $7.871 E-10$ $-7.740 \mathrm{E}-10$ $-2.608 \mathrm{E}-09$ $-3.448 \mathrm{E}-09$
$-2.428 \mathrm{E}-09$ . $6.391 E-09$ $6.380 E-09$ $-1.700 \mathrm{E}-\mathrm{C} 9$ . $932 E-C 7$ $1.259 E-06$

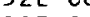
.135E-06 ANGLE 15 $M U=0.8656$ $1.095 \mathrm{E}-\mathrm{C} 8$ $5.744 \mathrm{E}-\mathrm{C} 8$ $1.908 \mathrm{E}-07$ 3. $807 \mathrm{E}-07$ $.702 \mathrm{E}-\mathrm{C} 7$ $9.116 E-07$ $1.109 E-06$ $1.269 E-06$ $1.372 E-06$ $1.390 \mathrm{E}-06$ $1.300 E-06$ $1.189 E-06$ $1.794 \mathrm{E}-06$ $2.148 \mathrm{E}-06$ 1.090E-06 $1.079 E-05$ $2.880 E-05$

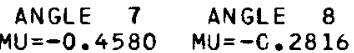
$-9.681 E-10 \quad-1.273 E-09$ $-4.305 E-09 \quad-5.467 \mathrm{E}-09$ $-1.004 \mathrm{E}-08$ - $1.200 \mathrm{E}-08$ $-1.182 \mathrm{E}-08 \quad-9.848 \mathrm{E}-0$ $-3.846 \mathrm{E}-09 \quad 5.3 \mathrm{C} 6 \mathrm{E}-10$ $1.857 \mathrm{E}-08 \quad 2.893 \mathrm{E}-08$ . $529 E-08$ $1.518 E-08$ $-2.136 E-08$ .265E-07 $1.596 E-06$ $.728 E-06$ $7.506 E-06$ 6.118E-05 ANGLE 16 $M U=0.9446$ $1.543 \mathrm{E}-07$ $5.784 \mathrm{E}-07$ $1.296 \mathrm{E}-06$ $1.751 E-06$ $1.962 \mathrm{E}-06$ $1.978 E-06$ $1.883 \mathrm{E}-06$ $1.718 \mathrm{E}-06$ $1.519 \mathrm{E}-06$ $1.290 \mathrm{E}-06$ $1.174 \mathrm{E}-06$ $1.226 E-06$ $1.959 \mathrm{E}-06$ $2.119 E-06$ .191E-06 $1.105 E-05$

$7.048 \mathrm{E}-06$

ANGLE 9 $M U=-0.0950$ $5.243 E-10$ $2.053 E-C 9$ $1.173 E-09$ 5.C12E-09 $8.870 E-09$ $-6.173 E-09$ $-1.772 E-08$ $-2.032 E-C 8$ $3.295 E-C 8$ 


CUSINE
$-1.00000 E 00$
$-9.89401 E-01$
$-9.44575 E-01$
$-8.65631 E-01$
$-7.55044 E-C 1$
$-6.17876 E-01$
$-4.58017 E-01$
$-2.816 C 5 E-01$
$-9.50125 E-02$
$9.50125 E-02$
$2.816 C 5 E-01$
$4.58017 E-01$
$6.17876 E-01$
$7.55044 E-01$
$8.65631 E-01$
$9.44575 E-01$
$9.89401 E-01$

TOTAL

COSINE

-1.00000 UO $-9.89401 E-C I$ $-9.44575 E-01$
$-8.65631 E-01$ $-7.55044 E-01$ $-6.17876 \mathrm{E}-01$ $-4.58017 E-0.1$ $-2.816 C 5 E-01$ $-9.50125 E-02$ $9.50125 E-02$ $2.81605 E-01$ $4.58017 E-01$ $6.17876 E-01$ $7.55044 \bar{E}-01$ $8.65631 E-C 1$ $9.44575 E-C 1$
$9.39401 E-01$

TOTAL

\begin{tabular}{|c|c|c|c|c|c|c|}
\hline 1800.0 & 2100.0 & 2400.0 & $\begin{array}{l}\text { VGE (METERS) } \\
27 \mathrm{CO} \cdot \mathrm{C}\end{array}$ & $300 \mathrm{C} .0$ & $3360 . c$ & $3600 . C$ \\
\hline $\begin{array}{l}1.777 \mathrm{E}-12 \\
1.782 \mathrm{E}-12 \\
1.806 \mathrm{E}-12 \\
1.850 \mathrm{E}-12 \\
1.917 \mathrm{E}-12 \\
2.012 \mathrm{E}-12 \\
2.142 \mathrm{E}-12 \\
2.315 \mathrm{E}-12 \\
2.539 \mathrm{E}-12 \\
2.827 \mathrm{E}-12 \\
3.200 \mathrm{E}-12 \\
3.696 \mathrm{E}-12 \\
4.383 \mathrm{E}-12 \\
5.364 \mathrm{E}-12 \\
6.803 \mathrm{E}-12 \\
8.937 \mathrm{E}-12 \\
1.231 \mathrm{E}-11\end{array}$ & $\begin{array}{l}5.522 \mathrm{E}-13 \\
5.539 \mathrm{E}-13 \\
5.611 \mathrm{E}-13 \\
5.749 \mathrm{E}-13 \\
5.958 \mathrm{E}-13 \\
6.255 \mathrm{E}-13 \\
6.657 \mathrm{E}-13 \\
7.189 \mathrm{E}-13 \\
7.875 \mathrm{E}-13 \\
8.755 \mathrm{E}-13 \\
9.894 \mathrm{E}-13 \\
1.140 \mathrm{E}-12 \\
1.347 \mathrm{E}-12 \\
1.638 \mathrm{E}-12 \\
2.055 \mathrm{E}-12 \\
2.65 \mathrm{CE}-12 \\
3.505 \mathrm{E}-12\end{array}$ & $\begin{array}{l}1.655 \mathrm{E}-13 \\
1.660 \mathrm{E}-13 \\
1.682 \mathrm{E}-13 \\
1.724 \mathrm{E}-13 \\
1.786 \mathrm{E}-13 \\
1.876 \mathrm{E}-13 \\
1.996 \mathrm{E}-13 \\
2.154 \mathrm{E}-13 \\
2.357 \mathrm{E}-13 \\
2.618 \mathrm{E}-13 \\
2.955 \mathrm{E}-13 \\
3.400 \mathrm{E}-13 \\
4.006 \mathrm{E}-13 \\
4.847 \mathrm{E}-13 \\
0.030 \mathrm{E}-13 \\
7.669 \mathrm{E}-13 \\
9.860 \mathrm{E}-13\end{array}$ & $\begin{array}{l}4.833 \mathrm{E}-14 \\
4.847 \mathrm{E}-14 \\
4.911 \mathrm{E}-14 \\
5.032 \mathrm{E}-14 \\
5.216 \mathrm{E}-14 \\
5.477 \mathrm{E}-14 \\
5.826 \mathrm{E}-14 \\
6.286 \mathrm{E}-14 \\
6.875 \mathrm{E}-14 \\
7.630 \mathrm{E}-14 \\
8.604 \mathrm{E}-14 \\
9.888 \mathrm{E}-14 \\
1.162 \mathrm{E}-13 \\
1.4 \mathrm{C} 1 \mathrm{E}-13 \\
1.732 \mathrm{E}-13 \\
2.180 \mathrm{E}-13 \\
2.745 \mathrm{E}-13\end{array}$ & $\begin{array}{l}1.38 Z E-14 \\
1.387 E-14 \\
1.405 E-14 \\
1.44 C E-14 \\
1.492 E-14 \\
1.567 E-14 \\
1.667 E-14 \\
1.797 E-14 \\
1.965 E-14 \\
2.18 C E-14 \\
2.456 E-14 \\
2.82 C E-14 \\
3.310 E-14 \\
3.978 E-14 \\
4.893 E-14 \\
6.107 E-14 \\
7.572 E-14\end{array}$ & $\begin{array}{l}3.891 \mathrm{E}-15 \\
3.903 \mathrm{E}-15 \\
3.955 \mathrm{E}-15 \\
4.053 \mathrm{E}-15 \\
4.201 \mathrm{E}-15 \\
4.411 \mathrm{E}-15 \\
4.691 \mathrm{E}-15 \\
5.059 \mathrm{E}-15 \\
5.529 \mathrm{E}-15 \\
6.130 \mathrm{E}-15 \\
6.905 \mathrm{E}-15 \\
7.921 \mathrm{E}-15 \\
9.284 \mathrm{E}-15 \\
1.113 \mathrm{E}-14 \\
1.364 \mathrm{E}-14 \\
1.691 \mathrm{E}-14 \\
2.071 \mathrm{E}-14\end{array}$ & $\begin{array}{l}1.681 \mathrm{E}-15 \\
1.085 \mathrm{E}-15 \\
1.099 \mathrm{E}-15 \\
1.126 \mathrm{E}-15 \\
1.168 \mathrm{E}-15 \\
1.226 \mathrm{E}-15 \\
1.304 \mathrm{E}-15 \\
1.406 \mathrm{E}-15 \\
1.536 \mathrm{E}-15 \\
1.702 \mathrm{E}-15 \\
1.917 \mathrm{E}-15 \\
2.198 \mathrm{E}-15 \\
2.573 \mathrm{E}-15 \\
3.079 \mathrm{E}-15 \\
3.76 \mathrm{EE}-15 \\
4.636 \mathrm{E}-15 \\
5.624 \mathrm{E}-15\end{array}$ \\
\hline $4.241 t-11$ & $1.298 t-11$ & $3.850 \mathrm{E}-12$ & $1.115 E-12$ & $3.17 し E-13$ & $8.882 E-14$ & $2.459 t-14$ \\
\hline
\end{tabular}

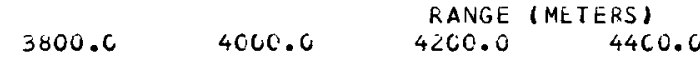

4.578E-16 1.93CE-16 $4.592 E-16 \quad 1.936 E-16$ $4.653 \mathrm{E}-16 \quad 1.962 \mathrm{E}-16$ 4.768E-16 2.C1CE-16 $4.944 E-16 \quad 2 . C 84 E-16$ $5.190 \mathrm{E}-16 \quad 2.188 \mathrm{E}-16$ $5.52 U E-16 \quad 2.327 E-16$ $.950 E-16$ $2.509 E-16$ $6.501 E-16 \quad 2.740 E-16$ $7.204 E-16$ 3.C37E-16 $8.110 E-16 \quad 3.418 E-16$ $9.295 E-16$ 3.916E-16 $1 . C 88 E-15$ 4.58CE-16 $1.300 E-15 \quad 5.470 E-16$ $1.585 E-15 \quad 6.657 E-16$ $1.948 E-15 \quad 8.159 E-16$ 2.350E-15 G.793E-16 $8.1 C 5 E-17$ $\begin{array}{ll}8.130 E-17 & 3.395 E-17 \\ 8.239 E-17 & 3.441 E-17\end{array}$ $3.528 \mathrm{E}-17$ $8.755 E-17 \quad 3.659 E-17$ $9.192 \mathrm{E}-17 \quad 3.843 \mathrm{E}-17$ $9.775 E-17$ 4.688E-17 $1.054 E-16 \quad 4.407 E-17$ $1.151 E-16$ 4.815E-17 $1.275 E-10 \quad 5.335 E-17$ $1.435 E-16 \quad 6.0 C 4 E-12$ $\begin{array}{ll}1.644 E-16 & 6.878 E-17 \\ 1.922 E-10 & 8.038 E-17\end{array}$ $\begin{array}{ll}1.922 E-10 & 8.038 E-17 \\ 2.294 E-16 & 9.580 E-17\end{array}$ $2.787 E-16 \quad 1.163 t-16$ $3.407 E-16 \quad 1.419 \mathrm{E}-16$ $4.672 \mathrm{E}-16 \quad 1.689 \mathrm{E}-16$

$1.039 E-14$ 4. $373 E-15$ $1.834 E-15 \quad 7.664 E-16 \quad 3.177 E-16 \quad 1.260 E-16$

$4600.0 \quad 4800.0$

$1.385 t-17 \quad 4.949 t-18$ 1.39CE-17 4.971E-18 $1.41 C E-17 \quad 5.068 E-18$ $1.447 \mathrm{E}-17 \quad 5.252 \mathrm{E}-18$ $1.5 C 4 E-17 \quad 5.53 C E-18$ $1.584 \mathrm{E}-17 \quad 5.918 \mathrm{E}-18$ $1.688 E-17 \quad 6.427 E-18$ $1.824 \mathrm{E}-17 \quad 7.067 \mathrm{E}-18$ $1.955 E-17 \quad 7.843 t-18$ $2.214 E-17 \quad 8.798 E-18$ $2.494 E-17 \quad 9.998 E-18$ $2.859 E-17$ 1.154E-17 3. $343 E-17 \quad 1.350 E-17$ $3.987 E-17 \quad 1.626 t-17$ $4.835 E-17 \quad 1.978 E-17$ $5.868 E-17 \quad 2.412 E-17$ $6.986 t-17 \quad 2.856 t-17$ 


\begin{tabular}{|c|c|c|c|c|c|c|c|}
\hline COSINE & 1800.0 & 2100.0 & 2400.0 & $\begin{array}{l}\text { GE (METERS) } \\
2760 . C\end{array}$ & $30 c c \cdot c$ & 3300.0 & 3600.0 \\
\hline $\begin{array}{l}1.00000 \mathrm{E} 00 \\
9.89401 \mathrm{E}-01 \\
9.44575 \mathrm{E}-01 \\
8.65631 \mathrm{E}-01 \\
7.55044 \mathrm{E}-01 \\
6.17876 \mathrm{E}-01 \\
4.58017 \mathrm{E}-01 \\
2.81605 \mathrm{E}-01 \\
9.50125 \mathrm{E}-02 \\
9.50125 \mathrm{E}-02 \\
2.81605 \mathrm{E}-01 \\
4.58017 \mathrm{E}-01 \\
6.17876 \mathrm{E}-01 \\
7.55044 \mathrm{E}-01 \\
8.65631 \mathrm{E}-01 \\
9.44575 \mathrm{E}-01 \\
9.89401 \mathrm{E}-01\end{array}$ & $\begin{array}{l}3.790 \mathrm{E}-12 \\
3.800 \mathrm{E}-12 \\
3.841 \mathrm{E}-12 \\
3.918 \mathrm{E}-12 \\
4.034 \mathrm{E}-12 \\
4.197 \mathrm{E}-12 \\
4.414 \mathrm{E}-12 \\
4.698 \mathrm{E}-12 \\
5.059 \mathrm{E}-12 \\
5.514 \mathrm{E}-12 \\
6.092 \mathrm{E}-12 \\
6.843 \mathrm{E}-12 \\
7.857 \mathrm{E}-12 \\
9.276 \mathrm{E}-12 \\
1.132 \mathrm{E}-11 \\
1.431 \mathrm{E}-11 \\
1.899 \mathrm{E}-11\end{array}$ & $\begin{array}{l}1.202 \mathrm{E}-12 \\
1.205 \mathrm{E}-12 \\
1.218 \mathrm{E}-12 \\
1.242 \mathrm{E}-12 \\
1.279 \mathrm{E}-12 \\
1.33 \mathrm{CE}-12 \\
1.398 \mathrm{E}-12 \\
1.486 \mathrm{E}-12 \\
1.598 \mathrm{E}-12 \\
1.735 \mathrm{E}-12 \\
1.916 \mathrm{E}-12 \\
2.146 \mathrm{E}-12 \\
2.454 \mathrm{E}-12 \\
2.877 \mathrm{E}-12 \\
3.472 \mathrm{E}-12 \\
4.311 \mathrm{E}-12 \\
5.505 \mathrm{E}-12\end{array}$ & $\begin{array}{l}3.655 E-13 \\
3.064 E-13 \\
3.704 E-13 \\
3.778 E-13 \\
3.889 E-13 \\
4.044 E-13 \\
4.249 E-13 \\
4.514 E-13 \\
4.848 E-13 \\
5.267 E-13 \\
5.797 E-13 \\
6.479 E-13 \\
7.384 E-13 \\
8.613 E-13 \\
1.031 E-12 \\
1.263 E-12 \\
1.571 E-12\end{array}$ & $\begin{array}{l}1.079 \mathrm{E}-13 \\
1.081 \mathrm{E}-13 \\
1.093 \mathrm{E}-13 \\
1.115 \mathrm{E}-13 \\
1.147 \mathrm{E}-13 \\
1.193 \mathrm{E}-13 \\
1.253 \mathrm{E}-13 \\
1.33 \mathrm{EE}-13 \\
1.428 \mathrm{E}-13 \\
1.550 \mathrm{E}-13 \\
1.704 \mathrm{E}-13 \\
1.9 \mathrm{C} 1 \mathrm{E}-13 \\
2.162 \mathrm{E}-13 \\
2.512 \mathrm{E}-13 \\
2.988 \mathrm{E}-13 \\
3.624 \mathrm{E}-13 \\
4.421 \mathrm{E}-13\end{array}$ & $\begin{array}{l}3.11 C E-14 \\
3.118 \mathrm{E}-14 \\
3.151 \mathrm{E}-14 \\
3.214 \mathrm{E}-14 \\
3.308 \mathrm{E}-14 \\
3.439 \mathrm{E}-14 \\
3.611 \mathrm{E}-14 \\
3.832 \mathrm{E}-14 \\
4.111 \mathrm{E}-14 \\
4.45 \mathrm{EE}-14 \\
4.897 \mathrm{E}-14 \\
5.45 \mathrm{EE}-14 \\
6.195 \mathrm{E}-14 \\
7.177 \mathrm{E}-14 \\
8.497 \mathrm{E}-14 \\
1.023 \mathrm{E}-13 \\
1.23 \mathrm{EE}-13\end{array}$ & $\begin{array}{l}8.8 C 5 \mathrm{E}-15 \\
8.82 .7 \mathrm{E}-15 \\
8.922 \mathrm{E}-15 \\
9.099 \mathrm{E}-15 \\
9.366 \mathrm{E}-15 \\
9.735 \mathrm{E}-15 \\
1.022 \mathrm{E}-14 \\
1.084 \mathrm{E}-14 \\
1.163 \mathrm{E}-14 \\
1.261 \mathrm{E}-14 \\
1.383 \mathrm{E}-14 \\
1.541 \mathrm{E}-14 \\
1.746 \mathrm{E}-14 \\
2.018 \mathrm{E}-14 \\
2.381 \mathrm{E}-14 \\
2.847 \mathrm{E}-14 \\
3.386 \mathrm{E}-14\end{array}$ & $\begin{array}{l}2.458 \mathrm{E}-15 \\
2.464 \mathrm{E}-15 \\
2.490 \mathrm{E}-15 \\
2.546 \mathrm{E}-15 \\
2.614 \mathrm{E}-15 \\
2.717 \mathrm{E}-15 \\
2.852 \mathrm{E}-15 \\
3.025 \mathrm{E}-15 \\
3.243 \mathrm{E}-15 \\
3.514 \mathrm{E}-15 \\
3.855 \mathrm{E}-15 \\
4.290 \mathrm{E}-15 \\
4.857 \mathrm{E}-15 \\
5.604 \mathrm{E}-15 \\
6.590 \mathrm{E}-15 \\
7.841 \mathrm{E}-15 \\
9.241 \mathrm{E}-15\end{array}$ \\
\hline TUTAL & $7.878 \mathrm{E}-11$ & $2.458 E-11$ & $7.392 E-12$ & $2.163 E-12$ & $6.198 \mathrm{E}-13$ & $1.74 .7 t-13$ & $4.858 E-14$ \\
\hline
\end{tabular}

COSINE

$-1.00000 \mathrm{ECC}$ $-9.89401 \mathrm{E}-\mathrm{O}$ $-9.44575 t-01$ $-8.65631 E-01$ $-7.55044 E-01$ $-6.17876 \mathrm{E}-\mathrm{O} 1$ $-4.58017 \mathrm{E}-\mathrm{O}$ $-2.81605 E-01$ $9.50125 \mathrm{E}-\mathrm{C} 2$ $90125 E-02$ $2.81605 E-01$ $4.58017 E-C 1$ $7.55044 E-01$ 8:65631E-01 $9.44575 \mathrm{E}-01$

TOTAL

$\begin{array}{ll}3800.0 & 4000 . C \\ 1.043 E-15 & 4.4 C 5 E-16 \\ 1.046 E-15 & 4.416 E-16 \\ 1.057 E-15 & 4.464 E-16 \\ 1.078 E-15 & 4.553 E-16 \\ 1.109 E-15 & 4.686 E-16 \\ 1.153 E-15 & 4.870 E-16 \\ 1.210 E-15 & 5.111 E-16 \\ 1.283 E-15 & 5.42 C E-16 \\ 1.375 E-15 & 5.8 C 7 E-16 \\ 1.490 E-15 & 6.292 E-16 \\ 1.634 E-15 & 6.899 E-16 \\ 1.818 E-15 & 7.672 E-16 \\ 2.057 E-15 & 8.676 E-16 \\ 2.3716-15 & 9.991 E-16 \\ 2.783 E-15 & 1.171 E-15 \\ 3.303 E-15 & 1.386 E-15 \\ 3.872 E-15 & 1.618 E-15 \\ 2.057 E-14 & 8.675 E-15\end{array}$
KANGE (METERSI
$4200.0 \quad 44 \mathrm{CO} .0$

\section{$1.853 E-16$}

$1.858 \mathrm{E}-16$

$1.878 E-16$

$1.915 \mathrm{E}-16$

$1.971 \mathrm{E}-16$

2.049E-16

$2.150 E-16$

$2.28 C E-16$

$2.443 E-16$

$2.646 \mathrm{E}-16$

$2.901 E-16$

$3.225 E-16$

$3.646 E-16$

$4.1950-16$

$4.911 E-10$

$6.742 E-16$

$3.045 E-15$

\section{$7.751 \mathrm{E}-17$}

$7.771 \mathrm{E}-17$

$7.855 \mathrm{E}-17$

$8.013 E-17$

$8.249 \mathrm{E}-17$

$9.001 \mathrm{E}-17$

$9.546 \mathrm{E}-17$

$1.023 E-16$

$1.108 \mathrm{E}-16$

$1.215 \mathrm{E}-16$

$1.350 E-16$

$1.526 \mathrm{E}-16$

$2.052 \mathrm{E}-16$

$2.418 \mathrm{E}-16$

1. $525 \mathrm{E}-15$
4600.0

3. $18 C E-17$

$3.189 E-17$

$3.226 E-17$

$3.294 E-17$

$3.39 E E-17$

$3.536 E-17$

3.95CE-17

4.237E-17

$4.595 E-17$

5.043E-17

$5.61 \mathrm{CE}-17$

$7.296 \mathrm{E}-17$

$8.528 E-17$

l. $16 C \mathrm{CE}-10$

$6.318 E-16$
4800.0

$147 E-17$

1.151E-17

1.169E-17

$1.202 t-17$

$1.252 t-17$

1.320E-17

$1.408 \mathrm{E}-17$

$1.647 \mathrm{E}-17$

1. $805 \mathrm{E}-17$

$1.998 \mathrm{E}-17$

$2.239 E-17$

2. $447 E-17$

.947E- 17

4.C81E-17

$4.715 E-17$

$2.478 E-16$ 


CUSINE
$-1.00000 E 00$
$-9.89401 E-01$
$9.44575 E-01$
$-8.65631 E-01$
$-7.55644 E-01$
$-6.17876 E-01$
$-4.580175-01$
$-2.01605 E-01$
$-9.50125 E-02$
$9.50125 E-02$
$2.81605 E-01$
$4.58017 E-01$
$6.17876 E-01$
$7.55044 E-01$
$8.05031 E-01$
$9.44575 E-01$
$9.89401 E-01$

TOTAL

COSINE

-1. COUUUE CL $-9.094015-C 1$ $-9.4457 \mathrm{DE}-01$ $-8.05031 L-01$
$-7.55 C 44 E-U 1$ $-6.17876 t-01$ $-4.50017 E-01$ $-2.01605 E-01$ $9.50125 E-02$ $9.50125 \mathrm{E}-\mathrm{C2} 2$
$2.81605 \mathrm{E}-01$ $4.500175-v+$ $4.580175-01$ $7.55444 E-01$ $0.65631 t-01$ $0.65631 t-01$ $9.894015-01$

tOTAL

\begin{tabular}{|c|c|c|c|c|c|c|}
\hline 1860.2 & $21 c 0 . c$ & 2400.0 & $\begin{array}{l}\text { IGE (METEXS) } \\
270 \mathrm{C} \cdot \mathrm{C}\end{array}$ & 3000.0 & 3300.0 & 3600.0 \\
\hline $\begin{array}{l}2.012 \mathrm{E}-10 \\
2.018 \mathrm{E}-10 \\
2.044 \mathrm{E}-10 \\
2.092 \mathrm{E}-10 \\
2.164 \mathrm{E}-10 \\
2.267 \mathrm{E}-10 \\
2.406 \mathrm{E}-1 \mathrm{C} \\
2.590 \mathrm{E}-10 \\
2.028 \mathrm{E}-10 \\
3.132 \mathrm{E}-10 \\
3.524 \mathrm{E}-10 \\
4.042 \mathrm{E}-10 \\
4.757 \mathrm{E}-10 \\
5.780 \mathrm{E}-10 \\
7.283 \mathrm{E}-10 \\
9.524 \mathrm{E}-10 \\
1.311 \mathrm{E}-\mathrm{C}\end{array}$ & $\begin{array}{l}6.285 \mathrm{E}-11 \\
6.363 \mathrm{E}-11 \\
6.382 \mathrm{E}-11 \\
6.532 \mathrm{E}-11 \\
6.759 \mathrm{E}-11 \\
7.079 \mathrm{E}-11 \\
7.511 \mathrm{E}-11 \\
8.079 \mathrm{E}-11 \\
8.809 \mathrm{E}-11 \\
9.74 \mathrm{CE}-11 \\
1.094 \mathrm{E}-10 \\
1.252 \mathrm{E}-1 \mathrm{C} \\
1.467 \mathrm{E}-1 \mathrm{C} \\
1.770 \mathrm{E}-10 \\
2.204 \mathrm{E}-10 \\
2.825 \mathrm{E}-10 \\
3.726 \mathrm{E}-10\end{array}$ & $\begin{array}{l}1.891 \mathrm{E}-11 \\
1.897 \mathrm{E}-11 \\
1.920 \mathrm{E}-11 \\
1.966 \mathrm{E}-11 \\
2.034 \mathrm{E}-11 \\
2.130 \mathrm{E}-11 \\
2.259 \mathrm{E}-11 \\
2.429 \mathrm{E}-11 \\
2.646 \mathrm{E}-11 \\
2.922 \mathrm{E}-11 \\
3.276 \mathrm{E}-11 \\
3.742 \mathrm{E}-11 \\
4.3736-11 \\
5.247 \mathrm{E}-11 \\
6.476 \mathrm{E}-11 \\
8.184 \mathrm{E}-11 \\
1.648 \mathrm{E}-10\end{array}$ & $\begin{array}{l}5.536 E-12 \\
5.552 E-12 \\
5.622 E-12 \\
5.754 E-112 \\
5.954 E-12 \\
6.236 E-12 \\
6.013 E-12 \\
7.1 C 5 E-12 \\
7.734 E-12 \\
8.534 E-12 \\
9.560 E-112 \\
1.09 \mathrm{CE}-11 \\
1.271 E-11 \\
1.519 E-11 \\
1.862 E-11 \\
2.328 E-11 \\
2.918 E-11\end{array}$ & $\begin{array}{l}1.587 \mathrm{E}-12 \\
1.591 \mathrm{E}-12 \\
1.611 \mathrm{E}-12 \\
1.649 \mathrm{E}-12 \\
1.767 \mathrm{E}-12 \\
1.787 \mathrm{E}-12 \\
1.895 \mathrm{E}-12 \\
2.036 \mathrm{E}-12 \\
2.215 \mathrm{E}-12 \\
2.442 \mathrm{E}-12 \\
2.734 \mathrm{E}-12 \\
3.114 \mathrm{E}-12 \\
3.624 \mathrm{E}-12 \\
4.316 \mathrm{E}-12 \\
5.266 \mathrm{E}-12 \\
6.526 \mathrm{E}-12 \\
8.051 \mathrm{E}-12\end{array}$ & $\begin{array}{l}4.473 \mathrm{E}-13 \\
4.486 \mathrm{E}-13 \\
4.543 \mathrm{E}-13 \\
4.650 \mathrm{E}-13 \\
4.812 \mathrm{E}-13 \\
5.039 \mathrm{E}-13 \\
5.342 \mathrm{C}-13 \\
5.737 \mathrm{E}-13 \\
6.239 \mathrm{E}-13 \\
6.877 \mathrm{E}-13 \\
7.693 \mathrm{E}-13 \\
8.757 \mathrm{E}-13 \\
1.018 \mathrm{E}-12 \\
1.209 \mathrm{E}-12 \\
1.409 \mathrm{E}-12 \\
1.808 \mathrm{E}-12 \\
2.203 \mathrm{E}-12\end{array}$ & $\begin{array}{l}1.244 E-13 \\
1.248 E-13 \\
1.264 E-13 \\
1.294 E-13 \\
1.339 E-13 \\
1.402 E-13 \\
1.486 E-13 \\
1.596 E-13 \\
1.735 E-13 \\
1.911 E-13 \\
2.137 E-13 \\
2.432 E-13 \\
2.823 E-13 \\
3.348 E-13 \\
4.053 E-13 \\
4.96 C E-13 \\
5.984 E-13\end{array}$ \\
\hline $.656 t-c 9$ & $1.428 \bar{E}-69$ & $4.248 t-10$ & $1.233 E-10$ & $3.511 z-11$ & $9.848 E-12$ & $2.729 \mathrm{E}-12$ \\
\hline
\end{tabular}

KANGE (METERS)

$3800.6 \quad 4006.6$

5. $271 t-14$ $5.287 E-14$ $5.480 E-14$ $5.67<E-14$ $5.939 E-14$ $6.295 t-14$ $0.750[-14$ 8. $C 93 E-14$ $1.029 t-1$ $1.194 \mathrm{E}-13$ $1.414 t-13$ $1.7698-13$ $2.485 t-13$ $2.501 E-13$ $2.23 C t-14$
$2.258 t-14$ $2.312 \mathrm{E}-14$ $2.393 E-14$ $2.505 E-14$ $2.655 E-14$ $3.098 E-14$ $3.413 E-14$ $3.814 \mathrm{E}-14$ $4.397 t-14$ 5.028E-14 $5.951 \mathrm{E}-14$ $7.175 \mathrm{E}-14$ $8.733 E-14$ 1. $C 43 c-13$

$1.154 E-12$

$4.858 E-13$
$44 \mathrm{CO} 0$

4600.0

$9.342 t-15$

$15 \quad 3.903 E-15$

9.480E-15 $3.965 E-15$

$9.713 t-15$

1.C53E-14

1.116E-14

$1.198 \mathrm{E}-14$

$1.434 E-14$

$1.3034 t-14$
$1.062 t-14$

$1.0 C 2 t-14$
$1.021 t-14$

C.111t-14 $2.496 E-14$ 3. $607 t-14$ $3.648 \mathrm{E}-14$ $4.336 \bar{c}-14$

$2.038 E-13$
$1.625 \mathrm{E}-15$

$1.815 E-15$

$4.667 E-15$

$5.011 E-15$

$3.446 t-12$

$6.764 t-15$

7.02 UE-15

$1.043 E-14$

$1.255 E-14$

$1.52 C E-14$
$1.799 E-14$

$8.519 E-14$
$5.724 E-16$

$5.749 E-16$ $5.749 E-16$ $6.056 E-16$ $6.360 \mathrm{E}-16$ $7.329 E-16$ $8.017 E-16$ $8.017 E-16$
$8.847 E-16$

$9.864 t-16$

$1.113 \mathrm{E}-15$

$1.275 t-15$

$1.487 t-15$

$1.487 E-15$
$1.766 E-15$

$2.130 \mathrm{E}-15$ $\begin{array}{ll}\text { t. } 305 \mathrm{E}-15 & 2.578 \mathrm{E}-15 \\ 7.44 \mathrm{CE}-15 & 3.04 \mathrm{CE}-15\end{array}$

$3.531=-14 \quad 1.396 t-14$ 


\begin{abstract}
COSINE
$-1.00060 E 00$ $-9.894 C 1 E-01$ $-9.44575 E-01$
$-8.65631 E-01$ $-8.65631 E-01$ $-6.17876 \mathrm{E}-01$ $-4.58017 E-01$ $-2.81605 E-01$ $-9.50125 E-02$ $9.50125 E-02$ $2.81605 \mathrm{E}-01$ $4.58017 \mathrm{E}-\mathrm{C} 1$ $6.17876 \mathrm{E}-01$ $7.55044 \mathrm{E}-01$ $8.65631 \mathrm{E}-01$ $9.89401 E=01$
\end{abstract}

TIOTAL

COSINE

-1.00000 E 00 $-9.89401 E-01$ $-9.44575 E-01$ $-8.65631 E-C 1$ $-7.55044 E-01$
$-6.17876 \mathrm{E}-01$ $-6.17876 \mathrm{E}-01$ $-4.58017 E-O 1$ $-2.816 C 5 \mathrm{E}-01$ $-9.50125 E-02$ 9.50125E-C2 $2.81605 \mathrm{E}-01$ $4.58017 E-01$ $6.17876 \mathrm{E}-\mathrm{C} 1$ $7.55044 E-C 1$ $8.65631 E-01$ $9.44575 E-01$

TOTAL

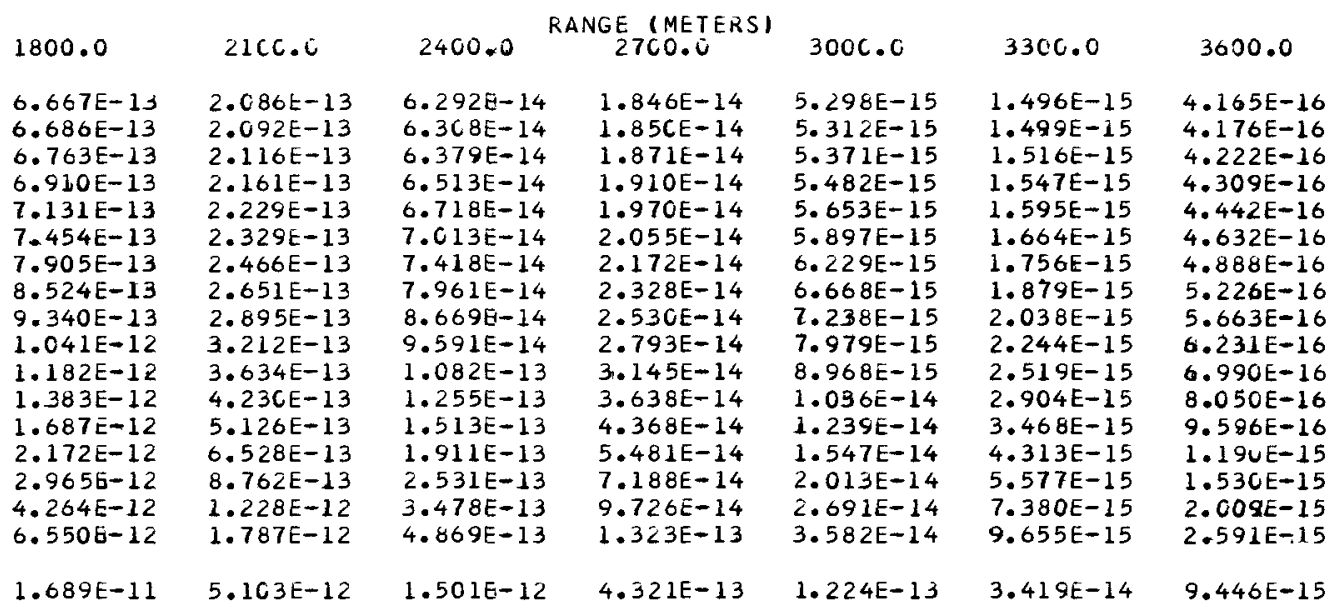

\section{RANGE (METERS)}

3800.0

4000.0

1. $765 \mathrm{E}-16$

$1.770 \mathrm{E}-16$

$1.789 E-16$

$1.826 E-16$

$1.882 \mathrm{E}-16$

$1.963 \mathrm{E}-16$

2.071E-16

$2.213 E-16$

$2.637 E-16$

$2.957 E-16$

$3.403 E-16$

$5.05 \mathrm{E}-16$

$5.015 E-16$

$6.433 E-16$

$1.075 E-15$

$3.986 E-15$

\section{$7.448 \mathrm{E}-17$}

$7.467 E-17$

$7.55 \mathrm{CE}-17$
$7.7 \mathrm{C} 5 \mathrm{E}-17$

$7.943 E-17$

$8.281 E-17$

$8.736 E-17$

1. C11E-16

$1.111 E-16$

$1.246 E-16$

$1.433 E-16$

1.

(2)

3.5CFE 16

$4.456 E-16$

$1.676 E-15$$$
4400.0
$$

3.13UE-17

3. $173 \mathrm{E}-17$

$3.238 \mathrm{E}-17$

$3.338 E-17$

$3.480 E-17$

$3.922 \mathrm{E}-17$

$4.246 E-17$

$4.667 E-17$

$6 . C 15 E-17$

$7.152 \mathrm{E}-17$

$8.826 E-17$

$1.127 \mathrm{E}-16$

1. $843 E-16$

7. $223 E-16$
$460 \mathrm{C} .0$

1. $3 \mathrm{C} 8 \mathrm{E}-17$

$1.312 \mathrm{E}-17$

$+.354 \mathrm{E}-17$

$1.396 \mathrm{E}-17$

$1.456 \mathrm{E}-17$

$1.536 \mathrm{E}-17$

$1.641 \mathrm{E}-17$

1.975E-17

1.

2.515E-17

$3.605 E-17$

$4.694 \mathrm{E}-17$

$6.063 E-17$

$2.932 \mathrm{E}-16$

\section{5. $364 E-18$} $5.378 \mathrm{E}-18$ $5.442 E-18$ $5.562 E-18$ $5.744 t-18$ $6.00 C E-18$ $6.342 E-18$ $6.786 E-18$ $7.355 E-18$ $8.093 E-18$ $9.077 \mathrm{E}-18$ $1.045 E-17$ $1.242 E-17$ $1.531 \mathrm{E}-17$ 2. 5 C9E-17 $3.134 \mathrm{E}-17$

1. $214 \mathrm{E}-16$
4800.0

$1.935 E-18$

$1.942 E-18$

$1.972 E-18$

$2.029 E-18$

$2.117 E-18$

$2.241 E-18$

$2.405 \mathrm{E}-18$

$2.615 E-18$

$2.870 E-18$

$3.192 \mathrm{E}-18$

$3.612 E-18$

$4.189 E-18$

$5.015 E-18$

$0.215 \mathrm{E}-18$

$7.938 \mathrm{E}-18$

$1.024 \mathrm{E}-17$

4. $796 E-17$ 


COSINE
$-1.0 C C O O E U C$
$-9.89401 E-01$
$-9.44575 E-01$
$-8.65631 E-U 1$
$-7.55044 E-01$
$-6.17876 E-01$
$-4.58017 E-U 1$
$-2.816 C 5 E-U 1$
$-9.50125 E-02$
$9.5 C 125 E-02$
$2.81005 E-U 1$
$4.58017 E-01$
$6.17676 E-C 1$
$7.55044 E-01$
$8.65631 E-01$
$9.44575 E-C 1$
$9.894 C 1 E-C 1$

TUTAL

CUSINEE

-1. coucot on $-9.89401 E-01$ $-8.65631 \mathrm{c}-01$ $-6.17876 E-01$ $-4.58017 E-61$ $-2.81605 \mathrm{E}-01$ $-9.50125 E-02$ $9.50125 E-U 2$ $2.81605 t-01$ $4.58017 E-01$ $0.178760-01$ $8.05631 c-01$ $9.44575 \mathrm{E}-0 \mathrm{I}$ $9.894 U 1 E-C 1$

TOTAL

\begin{tabular}{|c|c|c|c|c|c|c|}
\hline $8 \mathrm{cos} .0$ & $21 C 0.0$ & 2400.0 & $\begin{array}{l}\text { OE } \quad \text { METER } \\
\angle 7 C \mathrm{C} . \mathrm{C}\end{array}$ & 3000.0 & $330 \mathrm{c} . \mathrm{C}$ & 3600.6 \\
\hline $\begin{array}{l}2.357 \mathrm{E}-11 \\
2.364 \mathrm{E}-11 \\
2.396 \mathrm{E}-11 \\
2.454 \mathrm{E}-11 \\
2.542 \mathrm{E}-11 \\
2.667 \mathrm{E}-11 \\
2.838 \mathrm{E}-11 \\
3.068 \mathrm{E}-11 \\
3.368 \mathrm{E}-11 \\
3.754 \mathrm{E}-11 \\
4.254 \mathrm{E}-11 \\
4.923 \mathrm{E}-11 \\
5.869 \mathrm{E}-11 \\
7.273 \mathrm{E}-11 \\
9.429 \mathrm{E}-11 \\
1.280 \mathrm{E}-10 \\
1.058 \mathrm{E}-10\end{array}$ & $\begin{array}{l}7.326 \mathrm{E}-12 \\
7.349 \mathrm{E}-12 \\
7.445 \mathrm{E}-12 \\
7.626 \mathrm{E}-12 \\
7.899 \mathrm{E}-12 \\
8.287 \mathrm{E}-12 \\
8.814 \mathrm{E}-12 \\
9.517 \mathrm{E}-12 \\
1.443 \mathrm{E}-11 \\
1.159 \mathrm{E}-11 \\
1.310 \mathrm{E}-11 \\
1.512 \mathrm{E}-11 \\
1.793 \mathrm{E}-11 \\
2.26 \mathrm{E}-11 \\
2.005 \mathrm{E}-11 \\
3.707 \mathrm{E}-11 \\
5.088 \mathrm{E}-11\end{array}$ & $\begin{array}{l}2.197 \mathrm{E}-12 \\
2.204 \mathrm{E}-12 \\
2.232 \mathrm{E}-12 \\
2.287 \mathrm{E}-12 \\
2.369 \mathrm{E}-12 \\
2.485 \mathrm{E}-12 \\
2.642 \mathrm{E}-12 \\
2.850 \mathrm{E}-12 \\
3.118 \mathrm{E}-12 \\
3.461 \mathrm{E}-12 \\
2.965 \mathrm{E}-12 \\
4.494 \mathrm{E}-12 \\
5.368 \mathrm{E}-12 \\
6.466 \mathrm{E}-12 \\
8.146 \mathrm{E}-12 \\
1.056 \mathrm{E}-11 \\
1.394 \mathrm{E}-11\end{array}$ & $\begin{array}{l}6.415 \mathrm{E}-13 \\
0.435 \mathrm{E}-13 \\
6.519 \mathrm{E}-13 \\
6.677 \mathrm{E}-13 \\
6.917 \mathrm{E}-13 \\
7.256 \mathrm{E}-13 \\
7.712 \mathrm{E}-13 \\
8.314 \mathrm{E}-13 \\
9.687 \mathrm{E}-13 \\
1.468 \mathrm{E}-12 \\
1.135 \mathrm{E}-12 \\
1.304 \mathrm{E}-12 \\
1.536 \mathrm{E}-12 \\
1.061 \mathrm{E}-12 \\
2.323 \mathrm{E}-12 \\
2.966 \mathrm{E}-12 \\
3.811 \mathrm{E}-12\end{array}$ & $\begin{array}{l}1.835 \mathrm{E}-13 \\
1.841 \mathrm{E}-13 \\
1.865 \mathrm{E}-13 \\
1.916 \mathrm{E}-13 \\
1.979 \mathrm{E}-13 \\
2.076 \mathrm{E}-13 \\
2.26 \mathrm{EE-13} \\
2.377 \mathrm{E}-13 \\
2.596 \mathrm{E}-13 \\
2.877 \mathrm{E}-13 \\
3.236 \mathrm{E}-13 \\
3.715 \mathrm{E}-13 \\
4.364 \mathrm{E}-13 \\
5.265 \mathrm{E}-13 \\
6.527 \mathrm{E}-13 \\
8.242 \mathrm{E}-13 \\
1.038 \mathrm{E}-12\end{array}$ & $\begin{array}{l}5.168 E-14 \\
5.183 E-14 \\
5.251 E-14 \\
5.379 E-14 \\
5.572 E-14 \\
5.845 E-14 \\
6.210 E-14 \\
6.689 E-14 \\
7.302 E-14 \\
8.086 E-14 \\
9.094 E-14 \\
1.042 E-13 \\
1.222 E-13 \\
1.470 E-13 \\
1.812 E-13 \\
2.268 E-13 \\
2.81 \angle E-13\end{array}$ & $\begin{array}{l}1.436 \mathrm{E}-14 \\
1.441 \mathrm{E}-14 \\
1.46 \mathrm{E}-14 \\
1.495 \mathrm{E}-14 \\
1.549 \mathrm{E}-14 \\
1.625 \mathrm{E}-14 \\
1.726 \mathrm{E}-14 \\
1.359 \mathrm{E}-14 \\
2.028 \mathrm{E}-14 \\
2.245 \mathrm{E}-14 \\
2.523 \mathrm{E}-14 \\
2.890 \mathrm{E}-14 \\
3.384 \mathrm{E}-14 \\
4.059 \mathrm{E}-14 \\
4.983 \mathrm{E}-14 \\
6.195 \mathrm{E}-14 \\
7.585 \mathrm{E}-14\end{array}$ \\
\hline $746 E-10$ & $1.743 E-1 C$ & $5.140 E-11$ & $1.483 t-11$ & $4.2 \mathrm{C} \in E-12$ & $1.176 E-12$ & $3.253 E-13$ \\
\hline
\end{tabular}

\section{KANGE (METERS)}

$420 \hat{0} .0$ METERST

4600.0

4800.0

$\begin{array}{ll}\text { 6.CB1E-15 } & 2.564 E-15 \\ 6.10 U E-15 & 2.572 E-15\end{array}$

$2.6 C 5 E-15$
$2.669 E-15$

$2.669 E-15$
$2.765 E-15$

$2.900 E-15$

3. $081 \mathrm{E}-15$

$3.317 \mathrm{E}-15$

$3.018 E-15$

$4.003 E-15$

$5.147 E-15$

6. C $18 E-15$

$7.2 \cup C \bar{E}-15$

$8.799 E-15$

$1.005 E-14$

1.C $77 \mathrm{E}-15$

4. $497 E-16$

$1.841 E-16$

$1.847 E-16$
$1.873 E-16$

$1.873 E-16$
$1.922 t-16$

$1.996 E-16$

$1.121 E-15 \quad 4.084 E-16$

$1.218 \mathrm{E}-15 \quad 5.093 \mathrm{E}-16$

1.220 15 5. $512 E-16 \quad 2.235 E-16$

$1.393 E-15 \quad 5.827 E-16 \quad 2.411 E-16$

$1.519 E-15 \quad 6.356 E-16 \quad 2.634 t-16$

$1.681 \mathrm{E}-15 \quad 7.031 \mathrm{E}-16$

$2.160 E-15 \quad 9.635 E-16 \quad 3.755 E-16$

$2.525 E-15 \quad 1.055 E-15 \quad 4.388 \mathrm{t}-16$

$3.017 E-15 \quad 1.260 E-15 \quad 5.239 E-16$

$3.680 E-15$

$1.882 \mathrm{E}-15$

$5.239 E-16$
$6.375 E-16$

7. $801 E-16$
$9.3 C 4 E-16$

4. $191 \mathrm{E}-15$

$5.777 E-14$

$2.422 E-14$

$1.012 E+14$

$6.583 E-17$

$0.612 \mathrm{E}-17$

$0.739 E-17$
$6.977 E-17$
$7.340 E-17$

$7.340 \mathrm{E}-17$

$7.843 \mathrm{E}-17$

$8.504 E-17$

$9.334 E-17$

$1.034 E-16$

1. $314 \mathrm{E}-16$

$1.515 t-16$

.780E-16

$2.135 \mathrm{E}-16$

3. $191 \mathrm{E}-16$

3.80 UE- 16

$1.373 E-13$

$1.661 E-15$ 


\begin{tabular}{|c|c|c|c|c|c|c|c|}
\hline CUSINE & 1800.0 & 2100.0 & 2400.0 & $\begin{array}{l}\text { OE (METERS) } \\
2700.0\end{array}$ & $300 \mathrm{c} .0$ & $3300 . \mathrm{C}$ & 3600.0 \\
\hline $\begin{array}{l}1.00 L 0 C r \quad 00 \\
9.89401 \mathrm{E}-01 \\
9.44575 \mathrm{E}-01 \\
8.65631 \mathrm{E}-01 \\
7.55044 \mathrm{E}-01 \\
6.17876 \mathrm{E}-01 \\
4.58017 \mathrm{E}-01 \\
2.81605 \mathrm{E}-\mathrm{Cl} \\
9.50125 \mathrm{E}-\mathrm{C} 2 \\
9.50125 \mathrm{E}-02 \\
2.81605 \mathrm{E}-01 \\
4.58017 \mathrm{E}-\mathrm{C} 1 \\
6.17876 \mathrm{E}-01 \\
7.55044 \mathrm{E}-\mathrm{Cl} \\
8.65631 \mathrm{E}-01 \\
9.44575 \mathrm{E}-01 \\
9.89401 \mathrm{E}-01\end{array}$ & $\begin{array}{l}2.87 \mathrm{UE}-11 \\
2.878 \mathrm{E}-11 \\
2.912 \mathrm{E}-11 \\
2.976 \mathrm{E}-11 \\
3.072 \mathrm{E}-11 \\
3.210 \mathrm{E}-11 \\
3.403 \mathrm{E}-11 \\
3.669 \mathrm{E}-11 \\
4.022 \mathrm{E}-11 \\
4.486 \mathrm{E}-11 \\
5.102 \mathrm{E}-11 \\
5.962 \mathrm{E}-11 \\
7.246 \mathrm{E}-11 \\
9.260 \mathrm{E}-11 \\
1.250 \mathrm{E}-1 \mathrm{C} \\
1.776 \mathrm{E}-10 \\
2.706 \mathrm{E}-10\end{array}$ & $\begin{array}{l}8.967 \mathrm{E}-12 \\
8.992 \mathrm{E}-12 \\
9.095 \mathrm{E}-12 \\
9.29 \mathrm{CE}-12 \\
9.585 \mathrm{E}-12 \\
1.001 \mathrm{E}-11 \\
1.060 \mathrm{E}-11 \\
1.14 \mathrm{CE}-11 \\
1.245 \mathrm{E}-11 \\
1.382 \mathrm{E}-11 \\
1.565 \mathrm{E}-11 \\
1.820 \mathrm{E}-11 \\
2.196 \mathrm{E}-11 \\
2.772 \mathrm{E}-11 \\
3.67 \mathrm{CE}-11 \\
5.061 \mathrm{E}-11 \\
7.255 \mathrm{E}-11\end{array}$ & $\begin{array}{l}2.701 \mathrm{E}-12 \\
2.708 \mathrm{E}-12 \\
2.739 \mathrm{E}-12 \\
2.797 \mathrm{E}-12 \\
2.885 \mathrm{E}-12 \\
3.011 \mathrm{E}-12 \\
3.185 \mathrm{E}-12 \\
3.418 \mathrm{E}-12 \\
3.724 \mathrm{E}-12 \\
4.124 \mathrm{E}-12 \\
4.655 \mathrm{E}-12 \\
5.394 \mathrm{E}-12 \\
6.47 \mathrm{EE}-12 \\
8.689 \mathrm{E}-12 \\
1.655 \mathrm{E}-11 \\
1.423 \mathrm{E}-11 \\
1.955 \mathrm{E}-11\end{array}$ & $\begin{array}{l}7.917 \mathrm{E}-13 \\
7.938 \mathrm{E}-13 \\
8.026 \mathrm{E}-13 \\
8.195 \mathrm{E}-13 \\
8.451 \mathrm{E}-13 \\
8.818 \mathrm{E}-13 \\
9.319 \mathrm{E}-13 \\
9.989 \mathrm{E}-13 \\
1.086 \mathrm{E}-12 \\
1.200 \mathrm{E}-12 \\
1.352 \mathrm{E}-12 \\
1.562 \mathrm{E}-12 \\
1.866 \mathrm{E}-12 \\
2.316 \mathrm{E}-12 \\
2.988 \mathrm{E}-12 \\
3.961 \mathrm{E}-12 \\
5.274 \mathrm{E}-12\end{array}$ & $\begin{array}{l}2.272 \mathrm{E}-13 \\
2.277 \mathrm{E}-13 \\
2.303 \mathrm{E}-13 \\
2.351 \mathrm{E}-13 \\
2.424 \mathrm{E}-13 \\
2.529 \mathrm{E}-13 \\
2.671 \mathrm{E}-13 \\
2.86 \mathrm{CE}-13 \\
3.106 \mathrm{E}-13 \\
3.427 \mathrm{E}-13 \\
3.854 \mathrm{E}-13 \\
4.443 \mathrm{E}-13 \\
5.289 \mathrm{E}-13 \\
6.529 \mathrm{E}-13 \\
8.351 \mathrm{E}-13 \\
1.093 \mathrm{E}-12 \\
1.421 \mathrm{E}-12\end{array}$ & $\begin{array}{l}6.410 E-14 \\
6.426 E-14 \\
6.497 E-14 \\
6.632 E-14 \\
6.838 E-14 \\
7.131 E-14 \\
7.529 E-14 \\
8.056 E-14 \\
8.741 E-14 \\
9.634 E-14 \\
1.082 E-13 \\
1.246 E-13 \\
1.479 E-13 \\
1.818 E-13 \\
2.310 E-13 \\
2.990 E-13 \\
3.821 E-13\end{array}$ & $\begin{array}{l}1.784 \mathrm{E}-14 \\
1.789 \mathrm{E}-14 \\
1.809 \mathrm{E}-14 \\
1.846 \mathrm{E}-14 \\
1.903 \mathrm{E}-14 \\
1.985 \mathrm{E}-14 \\
2.095 \mathrm{E}-14 \\
2.240 \mathrm{E}-14 \\
2.429 \mathrm{E}-14 \\
2.675 \mathrm{E}-14 \\
3.002 \mathrm{E}-14 \\
3.451 \mathrm{E}-14 \\
4.094 \mathrm{E}-14 \\
5.011 \mathrm{E}-14 \\
6.3131 \mathrm{E}-14 \\
8.125 \mathrm{E}-14 \\
1.024 \mathrm{E}-13\end{array}$ \\
\hline TOTAL & $7.208 \mathrm{E}-10$ & $2.170 \mathrm{E}-10$ & $6.365 E-11$ & $1.83 \mathrm{CE}-11$ & $5.176 E-12$ & $1.446 E-1<$ & $3.993 E-13$ \\
\hline
\end{tabular}

COSINE

$-1.00000 \bar{E}$ NO $-9.89401 E-01$ $-9.44575 E-01$ $-8.65631 E-01$ $-7.55 C 44 E-01$ $-6.17876 \mathrm{E}-\mathrm{Cl} 1$ $-2.81605 c-01$ $-9.50125 \mathrm{E}-\mathrm{C} 2$ . Q.50125E-02 $4.58017 E-01$ $7.55044 E-01$ $8.65631 E-01$ $9.44575 E-01$

TOTAL

$\begin{array}{ll}3800 . C & 4060.0 \\ 7.561 E-15 & 3.190 E-15 \\ 7.581 E-15 & 3.199 E-15 \\ 7.664 E-15 & 3.234 E-15 \\ 7.822 E-15 & 3.300 E-15 \\ 8.065 E-15 & 3.403 E-15 \\ 8.409 E-15 & 3.547 E-15 \\ 8.873 E-15 & 3.743 E-15 \\ 9.487 E-15 & 4.001 E-15 \\ 1.028 E-14 & 4.335 E-15 \\ 1.132 E-14 & 4.771 E-15 \\ 1.270 E-14 & 5.349 E-15 \\ 1.459 E-14 & 6.143 E-15 \\ 1.727 E-14 & 7.266 E-15 \\ 2.112 E-14 & 8.871 E-15 \\ 2.660 E-14 & 1.114 E-14 \\ 3.398 E-14 & 1.417 E-14 \\ 4.247 E-14 & 1.759 E-14 \\ 1.685 E-13 & 7.084 E-14\end{array}$

RANGE (METERS) 4200.0 440C.O

$1.341 \mathrm{E}-15$ $1.344 E-15$ $1.359 E-15$ $1.387 E-15$ $1.430 \mathrm{E}-15$ $1.491 E-15$ $1.573 \mathrm{E}-15$ $1.681 \mathrm{E}-15$ $1.821 \mathrm{E}-15$ $2.003 E-15$ $2.246 E-15$ $2.578 \mathrm{E}-15$ $3.047 E-15$ $3.715 E-15$ $4.656 E-15$ $5.898 E-15$

$2.968 t-14$
$5.603 \mathrm{E}-16$ $5.617 \mathrm{E}-16$ $5.68 C E-16$ $5.798 \mathrm{t}-16$ 5. 6.2350 $7.617 \mathrm{E}=16$ (2) 1.078E 15 $3.005 E-15$

I. 239 E- 14
4600.0

$2.296 E-16$ $2.302 E-16$ $2.330 E-16$ $2.381 E-16$ $2.460 E-16$ $2.57 \mathrm{CE}-16$ $2.717 \mathrm{E}-16$ $2.909 E-16$ $3.155 E-16$ $3.475 E-16$ $3.898 E-16$ $4.477 E-16$ $5.290 E-16$ $6.440 E-16$ $1.043 E-15$ $1.013 E-15$

5. $132 \mathrm{E}-15$
4800.0

$8.260 E-17$ 8. $291 \mathrm{E}-17$ $8.423 E-17$ $8.673 E-17$ $9.055 E-17$ $9.593 E-17$ $1.031 E-16$ $1.121 \mathrm{E}-16$ $1.232 E-16$ $1.372 E-16$ $1.553 E-16$ 1. $137 E-16$ $2.137 E-16$ $3.278 E-16$ $4.134 E-16$ $4.134 E-16$
$5.039 E-16$

$2.027 E-15$ 


COSINE
$-1 . C 00 C O E \cup C$
$-9.89401 E-C 1$
$-9.44575 E-01$
$-8.65631 E-C 1$
$-7.55044 E-C 1$
$-6.17876 E-C 1$
$-4.58017 E-U 1$
$-2.81005 E-01$
$-9.56125 E-02$
$9.50125 E-02$
$2.81605 E-01$
$4.58 C 17 E-C 1$
$0.17076 E-01$
$7.55044 E-01$
$8.65631 E-01$
$9.44575 E-01$
$9.89401 E-01$

TUTAL

CUSINE

-1.00000 E OC $-9.89401 E-01$ $-8.65631 \mathrm{E}-\mathrm{O}$ $-8.65631 E-01$ $-6.17876 E-31$ $-6.17876 E-J 1$ $-2.816 C 5 \mathrm{t}-01$ $-9.50125 E-U 2$ $9.50125 \mathrm{E}-02$ . $4.58017 E-01$ $6.178765-01$ $7.550445-01$ $8.056315-w 1$ $9.44575 r-C 1$ $9.894 C 1 E-U 1$

TOTAL

1800.0
$2.292 E-12$
$2.304 E-12$
$2.35 C E-12$
$2.432 E-12$
$2.551 E-12$
$2.734 E-12$
$3.012 E-12$
$3.426 E-12$
$3.596 E-12$
$4.748 E-12$
$5.759 E-12$
$7.279 E-12$
$9.871 E-12$
$1.460 E-11$
$2.332 E-11$
$3.913 E-11$
$7.026 E-11$
$1.612 E-10$
10

$3800 . C$

$4.951 E-16$
$4.973 E-16$
$5.065 E-16$
$5.237 E-16$
$5.5016-16$
$5.89 C E-16$
$6.440 E-16$
$7.196 E-16$
$8.203 E-16$
$9.549 E-16$
$1.244 E-15$
$1.431 E-15$
$1.901 E-15$
$2.689 E-15$
$3.987 E-15$
$5.979 E-15$
$8.586 E-15$
$1.812 E-14$

$\angle 100.6$

2400.0

$.74 C t-13$ $6.77 \angle \vec{E}-13$

$6.944 t-13$

$7.145 E-13$

$7.50 C E-13$

$8 . C 38 E-13$

$8.841 \mathrm{E}-13$

1. $0(1 E-12$

$1.154 \mathrm{E}-12$

$1.369 t-12$

$1.655 t-12$

$2.087 \mathrm{E}-12$

$2.818 \mathrm{E}-12$

$4.127 E-12$

$6.477 \mathrm{E}-12$

$.05 E-11$

.787t-1

L.831E-11

$4 \operatorname{ccc} .6$

2. $C 77 E-16$

$2.086 E-16$

$\angle .124 E-16$

$2.196 E-16$

2.3C7E 16

$2.470 E-16$

. $014 \mathrm{E}-16$

$3.014 \mathrm{E}-16$

. $433 E-16$

$4.7825-16$

$5.782 E-16$

. $940 E-16$

. $12 C E-15$

$1.654 E-15$

. $4665-15$

3. $5 \iota 5 E-15$

$7.54 C E-15$
9438-13

$5.54 C E-14$
$5.645 E-14$

$5.839 \mathrm{E}-14$

$6.13 \mathrm{JE}-14$

$6.572 t-14$

$7.207 E-14$

$8.103 \mathrm{E}-14$

$9.307 E-14$

$1.090 E-13$

$1.311 t-13$

$1.047 E-13$

2.200E-13

$3.182 \mathrm{E}-13$

$4.863 E-13$

$7.636 \mathrm{E}-13$
$1.189 \mathrm{E}-12$

$4.593 t-12$

$36 \cup C \cdot c$

3360.6

$3600 . \mathrm{C}$

$1.545 t-14$

1. $552 \mathrm{E}-14$

1. $581 \mathrm{E}-14$

$1.63 \in E-14$

$1.710 \mathrm{E}-14$

.841E-14

.016E-14

$2.262 L-14$

$2.591 E-14$

$3.028 E-14$

$3.635 E-14$

4. . $6 C E-14$

. $725 \mathrm{E}-14$

8.725e-14

.

$2.042 E-13$

$2.161 E-12$

5. $91 C E-13$

4. $284 E-15$

$4.303 E-15$

$4.53 E-15-1.245 E-15$

4.762E-15 $1.308 E-15$

..400E-15

5.582E-15 1.532E-15

$6.251 E-15 \quad 1.713 E-15$

$7.144 E-15$ 1.954E-15

$8.334 E-15 \quad 2.276 E-15$

$9.94 E-15 \quad 2.728 E-15$

$1.252 E-14$ 3.415E-15

$1.669 E-14 \quad 4.542 E-15$

3. $368 E-14 \quad 6.441 E-15$

5.445E-14 9.591E-15

8.C6IE-14 $1.448 E-14$

$1.607 \bar{E}-13 \quad 4.344 E-14$

KANGE (METERS)

$$
4200.3
$$

4006.0

4800.0 $8.719 E-17 \quad 3.626 E-17 \quad 1.478 E-17 \quad 5.250 E-18$ $9.181 \mathrm{E}-17 \mathrm{3} \quad 3.82 \mathrm{CE}-17 \quad 1.561 \mathrm{E}-17 \quad 50.38 \mathrm{E}-18$ $9.646 E-17 \quad 4.615 E-17 \quad 1.645 E-17 \quad 0.025 E-18$ $1.033 \mathrm{E}-16 \mathrm{C} \quad 4.3 \mathrm{CCE}-17 \quad 1.706 \mathrm{E}-17$ $0.525 \mathrm{E}-18$ 1.36E-17 $1.966 \mathrm{E}-17 \quad 0.039 \mathrm{E}-18$ $1.250 \mathrm{E}-16 \quad 5.243 \mathrm{E}-17$ 2.164E-17 $0.4417 \mathrm{E}-18$ $1.453 E-10 \quad 5.966 E-17 \quad 2.465 E-17 \quad 9.777 E-18$ $1.067 E-16 \quad 6.936 t-17 \quad 2.869 E-17 \quad 1.156 E-17$

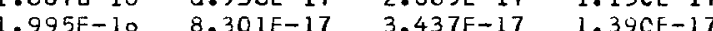
$2.485 E-16 \quad 1.038 E-16 \quad 4.3 \mathrm{C} 1 \mathrm{E}-17 \quad 1.750 \mathrm{E}-17$ $5.699 \mathrm{E}-17$ 2.331t-17 $1.933 \mathrm{E}-16 \quad 8 . \cup 6 \mathrm{CL}-17 \quad 3.280 \mathrm{E}-17$ $2.035 t-16 \quad 1.17 C E-16 \quad 4.798 E-17$ $1.010=-15 \quad 4.180 E-16 \quad 1.717 E-16 \quad 7.623 E-17$ $\begin{array}{llll}1.431 E-15 & 5.837 E-16 & 2.38 C E-16 & 9.676 E-17\end{array}$ $3.132 t-15 \quad 1.248 E-1 D \quad 5.352 E-16 \quad 2.150 t-16$ 


CUSINE
$-1.60000 E$ OO
$-9.89401 \mathrm{E}-01$
$-9.44575 \mathrm{E}-01$
$-8.65631 \mathrm{E}-01$
$-7.55044 \mathrm{E}-01$
$-6.17876 \mathrm{E}-01$
$-4.58017 \mathrm{E}-01$
$-2.81605 \mathrm{E}-01$
$-9.50125 \mathrm{E}-\mathrm{C} 2$
$9.50125 \mathrm{E}-02$
$2.81605 \mathrm{E}-01$
$4.58017 \mathrm{E}-01$
$0.17876 \mathrm{E}-01$
$7.55044 \mathrm{E}-01$
$8.65631 \mathrm{E}-01$
$9.44575 \mathrm{E}-01$
$9.89401 \mathrm{E}-01$

TOTAL

COSINE

\section{-1. COOOCE OC $-9.89401 E-01$ $-9.44575 E-01$
$-8.65631 E-01$ $-7.55044 E-01$ $-6.17876 \mathrm{E}-01$ $-4.58017 \mathrm{E}-01$ $-2.81605 \mathrm{E}-\mathrm{Cl}$ $-9.50125 E-02$ 9. $50125 E-02$ $2.81605 \mathrm{E}-\mathrm{Cl}$ $4.58017 t-01$ $6.17876 \mathrm{E}-01$ $7.55044 \bar{E}-01$ $8.65631 \bar{c}-01$ $9.44575 E-01$
$9.894 C 1 E-01$}

TOTAL

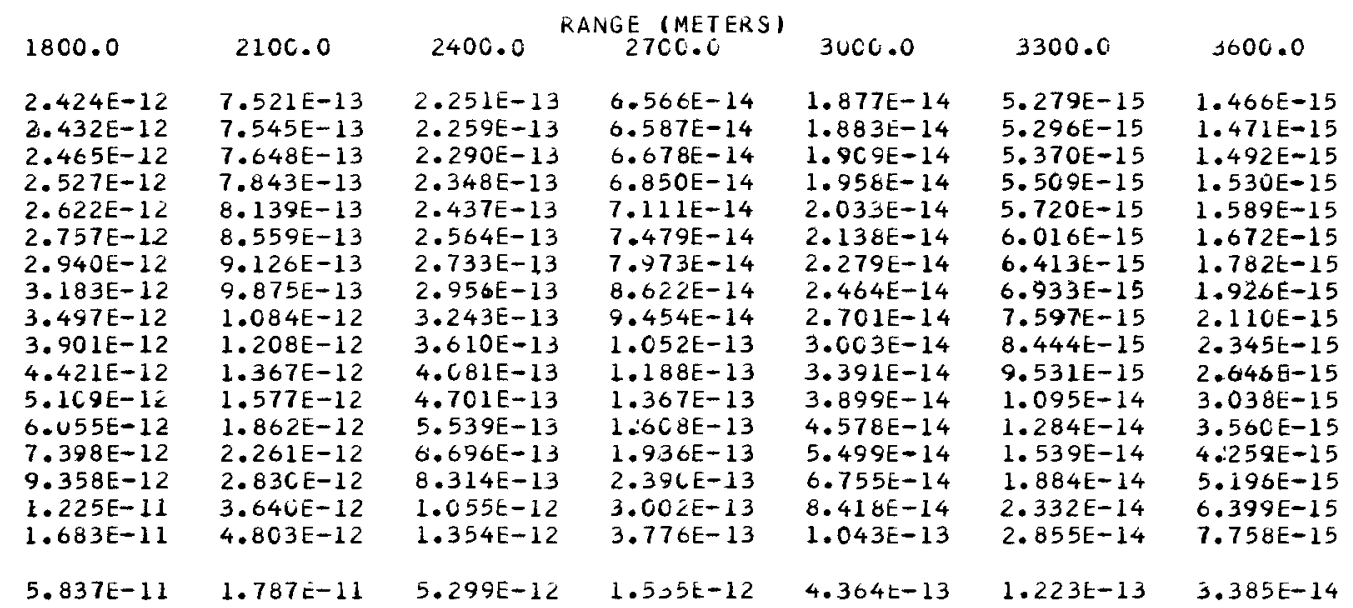

RANGE (METERS)

\begin{tabular}{|c|c|c|c|c|c|}
\hline 3800.0 & 4000.0 & 4200.0 & $44 \mathrm{CO} . \mathrm{C}$ & 4606.0 & 4800.0 \\
\hline $\begin{array}{l}206 E-16 \\
226 E-16\end{array}$ & $\begin{array}{l}2.616 E-16 \\
2.625 E-16\end{array}$ & & $\begin{array}{l}4.58 \mathrm{EE}-17 \\
4.6 \mathrm{C} 1 \mathrm{E}-17\end{array}$ & $\begin{array}{l}1.876 \mathrm{E}-17 \\
1.882 \mathrm{E}-17\end{array}$ & $\begin{array}{l}6.682 \mathrm{E}-1 \\
6.714 \mathrm{E}-1\end{array}$ \\
\hline 313 & $.661 E-16$ & & & & $6.853 E-18$ \\
\hline 6 & $2.730 \mathrm{E}$ & $1.147 t-16$ & $4.789 E-17$ & 1.964 & $7.113 E-18$ \\
\hline-16 & $=-16$ & $1.1916-16$ & $4.976 \mathrm{E}-17$ & & $7.508 E-18$ \\
\hline 0 & & $E-16$ & 5.2 & & \\
\hline 54 & & -16 & & & -18 \\
\hline 15 & -16 & -16 & 6.0 & 2.49 & $=-18$ \\
\hline-16 & $E-10$ & $1.581 \mathrm{E}$ & 6.6 & 2. & $1.078 t-17$ \\
\hline & & & & & $E-17$ \\
\hline-15 & 4.71 & $E=10$ & 8.2 & $3.442 t-17$ & $1.382 t-17$ \\
\hline 5 & 5.41 & 2.2 & 9.508 & & $1.598 E-17$ \\
\hline 50 & & & & & $1.881 \mathrm{E}-17$ \\
\hline-15 & $7.567 \mathrm{E}-16$ & -10 & $1.320 \mathrm{E}-16$ & $5.517 \mathrm{E}-17$ & $2.253 E-17$ \\
\hline & & 3.8 & 1.609 & & $2.738 E-17$ \\
\hline & $1.127 E-$ & 4.70 & $1.961 \mathrm{E}-1$ & $8.139 E-17$ & $3.336 E-17$ \\
\hline & $1.352 \mathrm{E}-$ & $=-16+2$ & & & $3.954 E-17$ \\
\hline - & $.019 E-15$ & $: 3<4 E-13$ & $1.055 \mathrm{E}-15$ & $4.373 E-16$ & $1.736 E-16$ \\
\hline
\end{tabular}




\section{PI R\#*2 HENOEKSEN DUSE (GAMMAS)
(CMM**2 RAL/STERADIAN/SOURCE NEUTKUNI}

COSINE

-1. COOLOE CC $-9.894 C 1 \mathrm{E}-01$ $-9.44575 E-01$ $-8.65631 E-01$ $55044 \mathrm{E}-U 1$ $-6.17876 \mathrm{E}-\mathrm{Ul}$ $-4.58 \cup 17 \mathrm{E}-\mathrm{O} I$ $-9.50125 t-42$ $9.50125 \mathrm{t}-\mathrm{C}^{2}$ $2.81645 \mathrm{E}-\mathrm{O} 1$ $2.816 U 5 E-01$
$4.58417 E-C 1$ $4.58617 E-01$ $7.55044 \mathrm{E}-\mathrm{Cl}$ $8.65631 E-01$ $9.44575 \mathrm{E}-01$ $9.89401 \mathrm{t}-01$

TOTAL

COSINE

$-1.0000 C E$ Cl $-9.89401 \mathrm{c}-01$ $94575 c-01$ $0.650315-01$ $-6.17876 E-G 1$ $-4.58017 E-C 1$ $4.58017 E-C 1$ $-9.501255-02$ $9.50125 E-02$ $2.81605 \mathrm{E}-\mathrm{G} 1$ $4.580175-01$ 4. $17876 \mathrm{E}-01$ $7.55 \cup 44 E-01$ $8.05031 E-01$ $8.05031 E-01$ $9.894 \mathrm{CIE}-01$

TOTAL

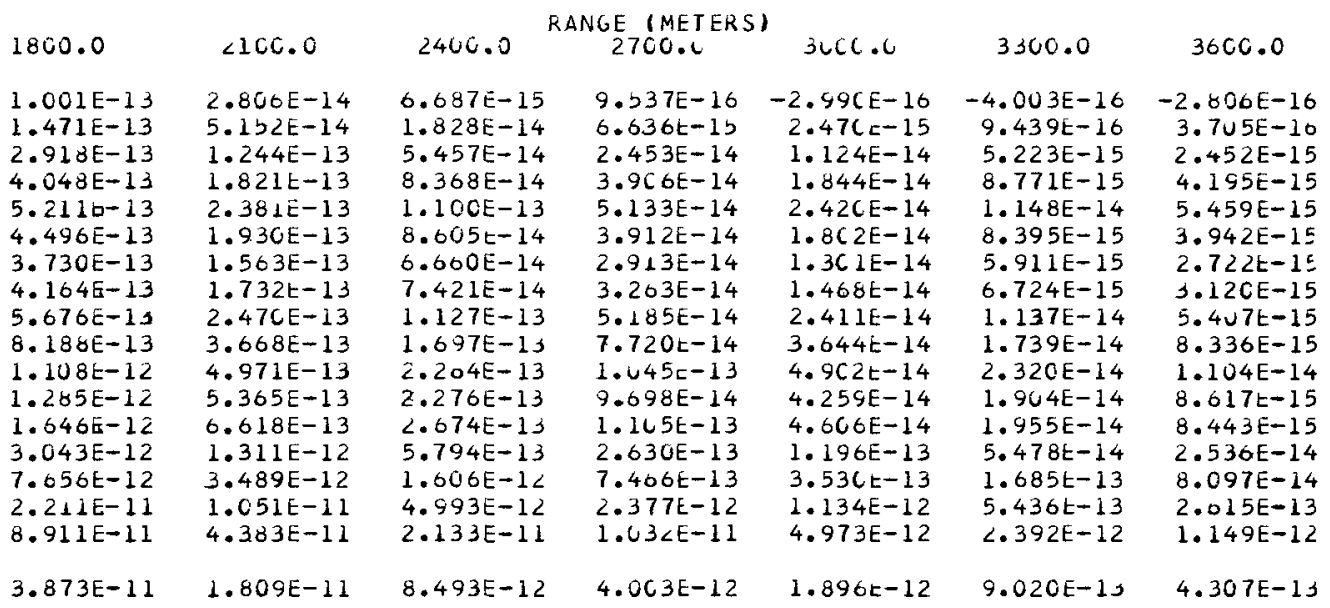

RANGE (METERS)

3800.0

4000.0

4200.0

4600.0

4860.0

$2.029 \mathrm{E}-16$

$9 .(73 E-16$

$-8.059 t-17$

$6.989 \mathrm{E}-17$
$5.555 \mathrm{E}-16$

4. $996 t-17$

$3.426 E-16$

$5.968 \mathrm{E}-16$

$7.513 \mathrm{t}-16$

2. C $26 E-15$

$2.404 E-15$

$1.635 E-15$

$1.871 E-15$

$3.313 t-15$

5.11OE-15

$6.7368-15$

$5.118 \mathrm{E}-15$

$4.876 E-15$

$1.527 \mathrm{E}-14$

$4.981 E-14$

$1.609 E-1$

7. C49E-1

$1.461 t-15$

$9.839 \mathrm{E}-16$

$1.132 E-15$

$3.142 E-15$

$4.105 \mathrm{E}-15$

$3.062 t-15$

$2.84 C E-15$

$9.231 \mathrm{E}-15$

3.C71E-14

$9.914 E-14$

$4.325 \mathrm{E}-1$

$8.830 E-10$

$5.937 E-10$

$6.868 E-16$

$1.247 E-15$

1.934E-15

$2.514 E-15$

$1.031 \mathrm{E}-15$

$1.003 E-15$

$5.066 E-12$

$1.696 \mathrm{E}-14$

$6.117 E-14$

.t5be-13

$3.591 E-16$

$4.178 E-16$

$7.005 E-10$

$1.192 E-15$

$1.542 E-15$

$1.096 E-15$

.

$1.173 t-14$

$3.779 E-14$

1.63 CE- 13

$0.092 E-14$
$-6.348 t-18$

$4.473 E-17$
$2.121 E-16$

$3.624 t-16$

4. 4 C 9t-16

$3.273 t-16$

$2.177 E-16$

$2.543 E-16$

..617t-16

$7.320 E-16$

$9.458 \mathrm{E}-16$

.

$5.78 \mathrm{EE} \rightarrow 16$

$2.087 \mathrm{E}-15$

$2.337 t-14$

1.CC $2 t-13$

$3.744 E-14$
$1.659 E-17$

4.023E-17

$1.192 E-16$
$1.960 \mathrm{E}-16$

$2.288 \mathrm{E}-16$

$1.830 E-16$

1.296E-16

$2.796 \mathrm{E}-16$

$4.546 \mathrm{E}-16$

$5.720 \mathrm{E}-16$

3. 9 C $3 E-16$

$1.274 \mathrm{E}-15$

$4.498 E-15$

$0.16 \mathrm{Ct}-14$

$2.636 \mathrm{E}-13$

$1.616 E-13 \quad 9.916 E-14$

2. $295 E-14$ 


\begin{tabular}{|c|c|c|c|c|c|c|c|}
\hline COSINE & $18 C 0.0$ & 2100.0 & $\angle 400.0$ & $\begin{array}{l}\text { IGE (METERS) } \\
27 \cup C \cdot G\end{array}$ & 3000.0 & 3300.0 & $3600 \cdot c$ \\
\hline $\begin{array}{l}-1.000 C 0 E \quad 00 \\
9.89401 \mathrm{E}-\mathrm{Cl} 1 \\
-9.44575 \mathrm{E}-01 \\
-8.65631 \mathrm{E}-01 \\
.7 .55044 \mathrm{E}-01 \\
-6.17876 \mathrm{E}-01 \\
-4.580177 \mathrm{E}-01 \\
-2.81605 \mathrm{E}-01 \\
9.50125 \mathrm{E}-\mathrm{C2} \\
9.50125 \mathrm{E}-02 \\
2.81605 \mathrm{E}-01 \\
4.58017 \mathrm{E}-\mathrm{Cl} \\
6.17876 \mathrm{E}-01 \\
7.55044 \mathrm{E}-01 \\
8.65631 \mathrm{E}-01 \\
9.44575 \mathrm{E}-01 \\
9.89401 \mathrm{E}-01\end{array}$ & $\begin{array}{l}2.758 E-11 \\
3.224 E-11 \\
4.666 E-11 \\
5.804 E-11 \\
6.986 E-11 \\
6.328 E-11 \\
5.619 E-11 \\
6.097 E-11 \\
7.647 E-11 \\
1.018 E-10 \\
1.330 E-10 \\
1.490 E-10 \\
1.840 E-10 \\
3.293 E-10 \\
7.695 E-10 \\
2.199 E-09 \\
8.975 E-C 9\end{array}$ & $\begin{array}{l}1.089 \mathrm{E}-11 \\
1.322 \mathrm{E}-11 \\
2.048 \mathrm{E}-11 \\
2.629 \mathrm{E}-11 \\
3.197 \mathrm{E}-11 \\
2.772 \mathrm{E}-11 \\
2.43 \mathrm{CE}-11 \\
2.618 \mathrm{E}-11 \\
3.372 \mathrm{E}-11 \\
4.577 \mathrm{E}-11 \\
5.891 \mathrm{E}-11 \\
6.303 \mathrm{E}-11 \\
7.467 \mathrm{E}-11 \\
1.375 \mathrm{E}-1 \mathrm{C} \\
3.496 \mathrm{E}-10 \\
1 .(43 \mathrm{E}-09 \\
4.421 \mathrm{E}-69\end{array}$ & $\begin{array}{l}4.409 E-12 \\
5.562 \mathrm{E}-12 \\
9.178 \mathrm{E}-12 \\
1.211 \mathrm{E}-11 \\
1.478 \mathrm{E}-11 \\
1.250 \mathrm{E}-11 \\
1.066 \mathrm{E}-11 \\
1.150 \mathrm{E}-11 \\
1.542 \mathrm{E}-11 \\
2.114 \mathrm{E}-11 \\
2.686 \mathrm{E}-11 \\
2.706 \mathrm{E}-11 \\
3.069 \mathrm{E}-11 \\
6.074 \mathrm{E}-11 \\
1.6 \mathrm{C} 5 \mathrm{E}-10 \\
4.952 \mathrm{E}-10 \\
2.155 \mathrm{E}-\mathrm{Cg}\end{array}$ & $\begin{array}{l}1.635 \mathrm{E}-12 \\
2.46 \mathrm{CE}-12 \\
4.186 \mathrm{E}-12 \\
5.647 \mathrm{E}-12 \\
6.895 \mathrm{E}-12 \\
5.725 \mathrm{E}-12 \\
4.773 \mathrm{E}-12 \\
5.155 \mathrm{E}-12 \\
7.107 \mathrm{E}-12 \\
9.648 \mathrm{E}-12 \\
1.241 \mathrm{E}-11 \\
1.168 \mathrm{E}-11 \\
1.286 \mathrm{E}-11 \\
2.753 \mathrm{E}-11 \\
7.446 \mathrm{E}-11 \\
2.356 \mathrm{E}-10 \\
1.644 \mathrm{E}-09\end{array}$ & $\begin{array}{l}7.832 \mathrm{E}-13 \\
1.059 \mathrm{E}-12 \\
1.935 \mathrm{E}-12 \\
2.659 \mathrm{E}-12 \\
3.246 \mathrm{E}-12 \\
2.651 \mathrm{E}-12 \\
2.170 \mathrm{E}-12 \\
2.35 \mathrm{CE}-12 \\
3.307 \mathrm{E}-12 \\
4.543 \mathrm{E}-12 \\
5.816 \mathrm{E}-12 \\
5.183 \mathrm{E}-12 \\
5.44 \mathrm{CE}-12 \\
1.25 \mathrm{CE}-11 \\
3.515 \mathrm{E}-11 \\
1.124 \mathrm{E}-10 \\
5.039 \mathrm{E}-10\end{array}$ & $\begin{array}{l}3.419 \mathrm{E}-13 \\
4.759 \mathrm{E}-13 \\
9.038 \mathrm{E}-13 \\
1.261 \mathrm{E}-12 \\
1.536 \mathrm{E}-12 \\
1.239 \mathrm{E}-12 \\
9.994 \mathrm{E}-13 \\
1.086 \mathrm{E}-12 \\
1.557 \mathrm{E}-12 \\
2.160 \mathrm{E}-12 \\
2.749 \mathrm{E}-12 \\
2.337 \mathrm{E}-12 \\
2.340 \mathrm{E}-12 \\
5.719 \mathrm{E}-12 \\
1.676 \mathrm{E}-11 \\
5.385 \mathrm{E}-11 \\
2.427 \mathrm{E}-10\end{array}$ & $\begin{array}{l}1.520 \mathrm{E}-13 \\
2.17 \mathrm{CE}-13 \\
4.254 \mathrm{E}-13 \\
6.011 \mathrm{E}-13 \\
7.300 \mathrm{E}-13 \\
5.829 \mathrm{E}-13 \\
4.648 \mathrm{E}-13 \\
5.069 \mathrm{E}-13 \\
7.386 \mathrm{E}-13 \\
1.032 \mathrm{E}-12 \\
1.307 \mathrm{E}-12 \\
1.065 \mathrm{E}-11 \\
1.023 \mathrm{E}-12 \\
2.644 \mathrm{E}-12 \\
8.044 \mathrm{E}-12 \\
2.591 \mathrm{E}-11 \\
1.168 \mathrm{E}-10\end{array}$ \\
\hline TUTAL & $4 . C 97 E-09$ & $1.912 E-09$ & $8.965 E-10$ & $4.222 E-10$ & $1.999 E-1 C$ & $9.509 E-11$ & $4.540 E-11$ \\
\hline
\end{tabular}

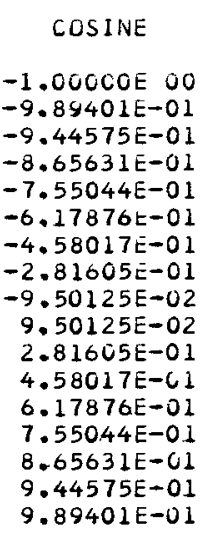

TOTAL

$\begin{array}{lc}3800 . \mathrm{C} & 400 \mathrm{C} .0 \\ 8.950 E-14 & 5.330 E-14 \\ 1.2966-13 & 7.795 \mathrm{E}-14 \\ 2.584 \mathrm{E}-13 & 1.574 \mathrm{E}-13 \\ 3.676 E-13 & 2.252 \mathrm{E}-13 \\ 4.435 \mathrm{E}-13 & 2.716 \mathrm{E}-13 \\ 3.550 \mathrm{E}-13 & 2.159 \mathrm{E}-13 \\ 2.804 \mathrm{E}-13 & 1.695 \mathrm{E}-13 \\ 3.054 \mathrm{E}-13 & 1.851 \mathrm{E}-13 \\ 4.314 \mathrm{E}-13 & 2.761 \mathrm{E}-13 \\ 6.316 \mathrm{E}-13 & 3.875 \mathrm{E}-13 \\ 7.9706-13 & 4.858 \mathrm{E}-13 \\ 6.352 \mathrm{E}-13 & 3.811 \mathrm{E}-13 \\ 5.951 \mathrm{E}-13 & 3.487 \mathrm{E}-13 \\ 1.590 \mathrm{E}-12 & 9.600 \mathrm{E}-13 \\ 4.946 \mathrm{E}-12 & 3.648 \mathrm{E}-12 \\ 1.595 \mathrm{E}-11 & 9.829 \mathrm{E}-12 \\ 7.170 \mathrm{E}-11 & 4.404 \mathrm{E}-11 \\ 2.778 \mathrm{E}-11 & 1.703 \mathrm{E}-11\end{array}$

\section{RANGE (METERS)}

$$
\begin{array}{r}
\text { RAINGE } \\
4200.0
\end{array}
$$$$
4400.0
$$

$3.24 C E-14$ $4.747 E-14$
$9.620 E-14$ $9.620 E-14$
$1.381 E-13$ $1.381 E-13$
$1.656 E-13$ $1.656 \mathrm{E}-13$
$1.313 \mathrm{E}-13$ 1. $.27 E-13$ $1.027 E-13$
$1.124 E-13$ $1.691 \mathrm{E}-13$ $2.381 \mathrm{E}-13$ $2.974 E-13$ $2.286 \mathrm{E}-13$ 2.052E-13

$5.822 \mathrm{E}-1$

$1.881 E-12$

$2.706 \mathrm{E}-11$

2. $059 E-14$ $2.963 E-14$ $5.9 C 2 E-14$ $8.472 E-14$ $1.006 E-13$ $7.987 E-14$ $0.229 E-14$ $6.842 E-14$ $1.464 \mathrm{E}-13$ $1.823 E-13$ $1.373 \mathrm{E}-13$ $1.214 \mathrm{E}-1$ 3. $542 E-13$

$3.750 \mathrm{E}-12$

$1.750 \mathrm{E}-12$
$1.663 \mathrm{E}-1$

$2.778 E-11$

1. $445 E-11$

$6.421 E-12$
$460 \mathrm{C} .0$

$1.41 G E-14$ $1.923 E-14$ $3.606 \mathrm{E}-14$
$5.128 \mathrm{E}-14$ $5.944 E-14$ 4. $835 E-14$ $3.768 E-14$ $4.152 E-14$ $8.973 E-14$ 1. $116 E-13$ (1) 2. $2.32 C E-12$ (3)

$3.945 E-12$
9. $251 E-15$ $1.164 \mathrm{E}-14$ $1.963 E-14$ $3.101 \mathrm{E}-14$ $2.672 E-14$ 2. 5. $477=-14$ 列 . $153 E-14$ $4.454 \mathrm{E}-13$ $1.436 \mathrm{E}-12$ $1.436 \mathrm{E}-12$

$2.408 \mathrm{E}-12$ 
4 PI R*\#2 AIR KEKMA (GAMMAS)
ICM* Z ERGSIGRAM/STERADIAN/SUURCE NEUTKUNI

$18 C 0.0$
$1.438 E-10$
$1.483 E-10$
$1.623 E-10$
$1.744 E-10$
$1.877 E-1 C$
$1.852 E-10$
$1.831 E-10$
$1.927 E-10$
$2.128 E-10$
$2.429 E-10$
$2.776 E-1 C$
$3.035 E-10$
$3.472 E-10$
$4.853 E-1 C$
$9.137 E-10$
$2.232 E-C 9$
$8.332 E-C 9$
$5.601 E-C 9$

2100.0 $6.467 E-11$ $6.467 E-11$
$6.688 \mathrm{E}-11$ $6.688 E-11$ $7.994 E-11$ $8.628 E-11$ $8.397 \mathrm{E}-11$ $8.281 E-11$ $8.688 E-11$ $9.639 E-11$ $1.1 \cup 4 E-10$ $1.20 \mathrm{CE}-1 \mathrm{C}$ $1.338 \mathrm{E}-1 \mathrm{C}$ $1.500 E-10$ $2.138 \mathrm{E}-1 \mathrm{C}$ $4.155 \mathrm{E}-1 \mathrm{C}$ $1.055 \mathrm{E}-\mathrm{C}$ $4.089 E-69$ $89401 E-01$

TOTAL

COSINE

\section{-1.60600 OC} $-9.89401 E-01$ $-9.44575 E-U 1$ $-8.65631 \mathrm{E}-01$ $-7.55444 E-C$ $-0.17876 E-01$ $-4.58017 E-G$ $-2.816 C 5 E-C$

$-9.50125 \mathrm{E}-02$

$9.50125 E-C 2$

$2.81605 E-C 1$ $4.58017 E-U 1$ $0.17876 t=01$ $7.55044 E-01$ $8.65631 E-U 1$ $9.44575 \mathrm{E}-01$
$9.89401 \mathrm{E}-01$

TOTAL

$2.622 t-c 9$

3800.6

$8.366 E-13$ $8.740 t-13$ $9.954 E-13$

1. $104 E-12$

$1.188 E-12$

$1.128 E-12$

$1.085 \tau-12$

$1.137 \mathrm{E}-12$

1. 3 UOE-12

$1.500 E-12$

$1.691 \mathrm{E}-12$

$1.596 \mathrm{E}-12$

$1.030 E-12$

$2.626 E-12$

$5.795 \mathrm{E}-12$

$1.588 E-11$

$3.706=-11$ $\angle 400.0$ RANGE (METERS)
$2700 . C$

12.2WU TU 15.CCC MEV NEUTKON SUURCE

$2.94 C E-11 \quad 1.352 E-11 \quad 6.271 c-12$

.C49E-11 1.405E-11

$3.697 \mathrm{E}-11$

$1.724 \mathrm{E}-11$

$1.862 \mathrm{E}-11$

$1.788 \mathrm{E}-11$

$3.857 \mathrm{E}-11$

$1.742 E-11$

$6.53 \mathrm{CE}-12$

$8.092 \mathrm{E}-12$

$8.739 E-12$

$8.351 E-12$

$8.103 \mathrm{E}-12$

$3.962 E-11 \quad 1.826 E-11 \quad 8.491 E-12$

$4.436 E-11$ 2.056E-11 9.601E-12

$5.093 c-11 \quad 2.349 E-11 \quad 1.1 C 1 E-11$

$5.772 E-11$ 2.671E-11 $1.248 E-11$

$5.975 \mathrm{E}-11$ 2.69CE-11 $1.23 \mathrm{CE}-1$

$6.551 E-11$ 2.9C9E-11 $1.3 C 6 E-11$

$4.289 E-11$ 2.C16E-11

$1.948 E-10$ 8. $848 E-11$

$\begin{array}{lll}4.990 E-10 & 2.367 E-10 & 1.126 E-10 \\ 1.987 E-C 9 & 9.6 C 2 E-1 U & 4.624 E-10\end{array}$

$4.6 \angle 4 \bar{E}-1 C$

$5.724 t-16$

$2.697 k-10$

$3306 \cdot 6$

3604.0

$2.932 \mathrm{E}-12 \quad 1.379 \mathrm{E}-12$

$.057 E-12 \quad 1.44 C E-12$

$.462 \mathrm{E}-121.636 \mathrm{E}-12$

$.820 \mathrm{E}-12 \quad 1.811 \mathrm{E}-12$

$4.123 \mathrm{E}-12 \quad 1.953 \mathrm{E}-12$

$3.795 E-12 \quad 1.737 E-12$

$3.976 \mathrm{E}-12 \quad 1.874 \mathrm{E}-12$

$4.516 E-12 \quad 2.136 E-12$

$5.193 \mathrm{E}-12 \quad 2.462 \mathrm{E}-12$

. $875 E-12$ 2.779E-12

$2.046 \mathrm{E}-12$

9.932E-12 $2.724 E-12$

$9.325 \mathrm{E}-12 \quad 4.34 \mathrm{gE}-12$

$5.385 \mathrm{E}-1129.453 \mathrm{E}-12$

$2.223 E-1 \mathrm{C} \quad 1.068 \mathrm{E}-10$

$1.277 E-1 C \quad 6.07 \perp E-11$

\begin{tabular}{|c|c|c|c|c|}
\hline $\begin{array}{l}\text { 2. } 89 \mathrm{E}-13 \\
5.319 \mathrm{E}-13 \\
6.666 \mathrm{E}-13 \\
6.738 \mathrm{E}-13 \\
7.243 \mathrm{E}-13 \\
6.868 \mathrm{E}-13 \\
6.599 \mathrm{E}-13 \\
6.917 \mathrm{E}-13 \\
7.929 \mathrm{E}-13 \\
5.155 \mathrm{E}-13 \\
1.029 \mathrm{E}-12 \\
9.656 \mathrm{E}-13 \\
9.796 \mathrm{E}-13 \\
1.591 \mathrm{E}-12 \\
3.506 \mathrm{E}-12 \\
9.773 \mathrm{E}-12 \\
4.019 \mathrm{E}-11\end{array}$ & $\begin{array}{l}3.105 \mathrm{E}-13 \\
3.245 \mathrm{E}-13 \\
3.703 \mathrm{E}-13 \\
4.118 \mathrm{E}-13 \\
4.421 \mathrm{E}-13 \\
4.187 \mathrm{E}-13 \\
4.019 \mathrm{E}-13 \\
4.213 \mathrm{E}-13 \\
4.841 \mathrm{E}-13 \\
5.597 \mathrm{E}-13 \\
6.282 \mathrm{E}-13 \\
5.830 \mathrm{E}-13 \\
5.904 \mathrm{E}-13 \\
9.672 \mathrm{E}-13 \\
2.191 \mathrm{E}-12 \\
6.023 \mathrm{E}-12 \\
2.467 \mathrm{E}-11\end{array}$ & $\begin{array}{l}1.902 \mathrm{E}-13 \\
1.986 \mathrm{E}-13 \\
2.263 \mathrm{E}-13 \\
2.517 \mathrm{E}-13 \\
2.694 \mathrm{E}-13 \\
2.553 \mathrm{E}-13 \\
2.450 \mathrm{E}-13 \\
2.571 \mathrm{E}-13 \\
2.959 \mathrm{E}-13 \\
3.425 \mathrm{E}-13 \\
3.839 \mathrm{E}-13 \\
3.540 \mathrm{E}-13 \\
3.567 \mathrm{E}-13 \\
5.892 \mathrm{E}-13 \\
1.35 \mathrm{E}-12 \\
3.716 \mathrm{E}-12 \\
1.515 \mathrm{E}-11\end{array}$ & 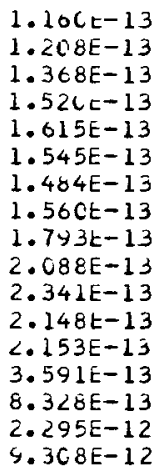 & $\begin{array}{l}6.274 \mathrm{E}-14 \\
6.506 \mathrm{E}-14 \\
7.299 \mathrm{E}-14 \\
8.143 \mathrm{E}-14 \\
8.658 \mathrm{E}-14 \\
8.524 \mathrm{E}-14 \\
8.360 \mathrm{E}-14 \\
8.917 \mathrm{E}-14 \\
1.040 \mathrm{E}-13 \\
1.234 \mathrm{E}-13 \\
1.381 \mathrm{E}-13 \\
1.262 \mathrm{E}-13 \\
1.263 \mathrm{E}-13 \\
2.157 \mathrm{E}-13 \\
5.108 \mathrm{E}-13 \\
1.416 \mathrm{E}-12 \\
5.720 \mathrm{E}-12\end{array}$ \\
\hline $265 \mathrm{E}-11$ & $1.387 E-11$ & $8.498 t-12$ & $5.200 E-12$ & $3.114 \mathrm{E}-12$ \\
\hline
\end{tabular}




\begin{tabular}{|c|c|c|c|c|c|c|c|}
\hline $\operatorname{Cos} I N E$ & 1800.0 & 2100.0 & 2400.0 & $\begin{array}{l}\text { GE (ME TERS) } \\
2700.0\end{array}$ & 3000.0 & 3300.0 & 3600.0 \\
\hline $\begin{array}{l}1.00000 \mathrm{E} 00 \\
9.89401 \mathrm{E}-01 \\
9.44575 \mathrm{E}-01 \\
8.65631 \mathrm{E}-01 \\
7.55044 \mathrm{E}-01 \\
6.17870 \mathrm{E}-01 \\
4.58017 \mathrm{E}-01 \\
2.81605 \mathrm{E}-01 \\
9.50125 \mathrm{E}-02 \\
9.50125 \mathrm{E}-02 \\
2.81605 \mathrm{E}-01 \\
4.58017 \mathrm{E}-01 \\
6.17876 \mathrm{E}-01 \\
7.55044 \mathrm{E}-01 \\
8.65631 \mathrm{E}-01 \\
9.44575 \mathrm{E}-01 \\
9.89401 \mathrm{E}-\mathrm{Cl}\end{array}$ & $\begin{array}{l}3.510 \mathrm{E}-11 \\
3.990 \mathrm{E}-11 \\
5.474 \mathrm{E}-11 \\
6.645 \mathrm{E}-11 \\
7.854 \mathrm{E}-11 \\
7.168 \mathrm{E}-11 \\
6.423 \mathrm{E}-11 \\
6.891 \mathrm{E}-1 \mathrm{1} \\
8.461 \mathrm{E}-11 \\
1.103 \mathrm{E}-10 \\
1.398 \mathrm{E}-10 \\
1.573 \mathrm{E}-10 \\
1.916 \mathrm{E}-10 \\
3.279 \mathrm{E}-10 \\
7.864 \mathrm{E}-10 \\
2.252 \mathrm{E}-09 \\
9.251 \mathrm{E}-\mathrm{C} 9\end{array}$ & $\begin{array}{l}1.425 \mathrm{E}-11 \\
1.665 \mathrm{E}-11 \\
2.412 \mathrm{E}-11 \\
3.011 \mathrm{E}-11 \\
3.592 \mathrm{E}-11 \\
3.15 \mathrm{CE}-11 \\
2.791 \mathrm{E}-11 \\
2.973 \mathrm{E}-11 \\
3.737 \mathrm{E}-11 \\
4.960 \mathrm{E}-11 \\
6.286 \mathrm{E}-11 \\
6.668 \mathrm{E}-11 \\
7.803 \mathrm{E}-11 \\
1.411 \mathrm{E}-1 \mathrm{C} \\
3.571 \mathrm{E}-10 \\
1.068 \mathrm{E}-09 \\
4.560 \mathrm{E}-\mathrm{C} 9\end{array}$ & $\begin{array}{l}5.922 \mathrm{E}-12 \\
7.109 \mathrm{E}-12 \\
1.084 \mathrm{E}-11 \\
1.385 \mathrm{E}-11 \\
1.659 \mathrm{E}-11 \\
1.422 \mathrm{E}-11 \\
1.230 \mathrm{E}-11 \\
1.310 \mathrm{E}-11 \\
1.709 \mathrm{E}-11 \\
2.296 \mathrm{E}-11 \\
2.867 \mathrm{E}-11 \\
2.867 \mathrm{E}-11 \\
3.260 \mathrm{E}-11 \\
6.225 \mathrm{E}-11 \\
1.638 \mathrm{E}-10 \\
5.071 \mathrm{E}-10 \\
2.224 \mathrm{E}-09\end{array}$ & $\begin{array}{l}2.525 \mathrm{E}-12 \\
3.107 \mathrm{E}-12 \\
4.948 \mathrm{E}-12 \\
0.454 \mathrm{E}-12 \\
7.732 \mathrm{E}-12 \\
6.518 \mathrm{E}-12 \\
5.52 \mathrm{CE}-12 \\
5.888 \mathrm{E}-12 \\
7.674 \mathrm{E}-12 \\
1.045 \mathrm{E}-11 \\
1.324 \mathrm{E}-11 \\
1.240 \mathrm{E}-11 \\
1.342 \mathrm{E}-11 \\
2.818 \mathrm{E}-11 \\
7.596 \mathrm{E}-11 \\
2.413 \mathrm{E}-10 \\
1.078 \mathrm{E}-09\end{array}$ & $\begin{array}{l}1.101 \mathrm{E}-12 \\
1.385 \mathrm{E}-12 \\
2.289 \mathrm{E}-12 \\
3.035 \mathrm{E}-12 \\
3.636 \mathrm{E}-12 \\
3.019 \mathrm{E}-12 \\
2.515 \mathrm{E}-12 \\
2.689 \mathrm{E}-12 \\
3.663 \mathrm{E}-12 \\
4.919 \mathrm{E}-12 \\
6.265 \mathrm{E}-12 \\
5.508 \mathrm{E}-12 \\
5.678 \mathrm{E}-12 \\
1.279 \mathrm{E}-11 \\
3.585 \mathrm{E}-11 \\
1.151 \mathrm{E}-10 \\
5.206 \mathrm{E}-10\end{array}$ & $\begin{array}{l}4.891 \mathrm{E}-13 \\
6.274 \mathrm{E}-13 \\
1.069 \mathrm{E}-12 \\
1.437 \mathrm{E}-12 \\
1.720 \mathrm{E}-12 \\
1.411 \mathrm{E}-12 \\
1.160 \mathrm{E}-12 \\
1.244 \mathrm{E}-12 \\
1.723 \mathrm{E}-12 \\
2.337 \mathrm{E}-12 \\
2.932 \mathrm{E}-12 \\
2.485 \mathrm{E}-12 \\
2.444 \mathrm{E}-12 \\
5.845 \mathrm{E}-12 \\
1.709 \mathrm{E}-11 \\
5.516 \mathrm{E}-11 \\
2.509 \mathrm{E}-1 \mathrm{C}\end{array}$ & $\begin{array}{l}2.208 E-13 \\
2.879 \mathrm{E}-13 \\
5.030 \mathrm{E}-13 \\
6.84 .1 \mathrm{E}-13 \\
8.163 \mathrm{E}-13 \\
6.636 \mathrm{E}-13 \\
5.39 \mathrm{gE}-13 \\
5.806 \mathrm{E}-13 \\
8.171 \mathrm{E}-13 \\
1.116 \mathrm{E}-12 \\
1.393 \mathrm{E}-12 \\
1.134 \mathrm{E}-12 \\
1.069 \mathrm{E}-12 \\
2.700 \mathrm{E}-12 \\
8.202 \mathrm{E}-12 \\
2.655 \mathrm{E}-11 \\
1.208 \mathrm{E}-10\end{array}$ \\
\hline TOTAL & $4.270 E-09$ & $1.991 \mathrm{E}-09$ & $9.338 E-10$ & $4.397 E-10$ & $2 . C 82 E-10$ & $9.902 E-11$ & $4.727 E-11$ \\
\hline
\end{tabular}

COSINE

-1.0000UE 00 $-9.89401 E-01$ $-9.44575 E-C 1$

$-8.65631 E-01$ $-7.55044 \mathrm{E}-01$ $-6.17876 E-01$ $-4.58017 \mathrm{E}-01$

$-2.81605 E-0$

$-9.50125 \mathrm{E}-0$

$9.50125 E-C$

$2.81605 E-01$

$4.58017 E-01$

$6.17876 \mathrm{E}-0$

$7.55044 \mathrm{E}-01$

$8.65631 \mathrm{E}-\mathrm{Cl}$

$9.89401 E-01$

TOTAL
3800.0

4000.0

$1.310 E-13$ $1.724 E-13$

$3.054 \mathrm{E}-13$

$4.959 E-13$

$4.039 \mathrm{E}-13$

$3.258 E-13$

3. $500 \mathrm{E}-13$

$4.990 \mathrm{E}-13$

$6.825 \mathrm{E}-13$

$8.495 \mathrm{E}-13$

$6.764 E-13$

$6.214 E-13$

$1.622 \mathrm{E}-12$

$5.044 E-12$

$1.634 E-11$
$7,419 E-11$

$2.893 E-11$
$7.844 E-14$ $1.039 \mathrm{E}-13$ $1.859 \mathrm{E}-13$ $2.559 \mathrm{E}-13$ $3.028 \mathrm{E}-13$ $2.456 E-13$ $1.97 \mathrm{CE}-13$ $2.121 E-13$ 3. $.5 C E-13$ $4.186 E-13$ $5.178 \mathrm{E}-13$

$4.058 \mathrm{E}-13$

3. $640 E-13$

$9.793 \mathrm{E}-13$

$3.108 \mathrm{E}-1$

$.007 \mathrm{E}-11$

$4.558 \mathrm{E}-11$

$1.773 E-11$

\section{RANGE IMETERS}

4200.0

$4.766 E-14$ $4.322 E-14$
$1.136 E-13$

$1.569 \mathrm{E}-13$

$1.850 \mathrm{E}-13$

$1.494 \mathrm{E}-13$
$1.194 \mathrm{E}-13$

$1.289 E-13$

1. $868 \mathrm{E}-13$

$2.571 E-13$

$3.169 \mathrm{E}-1 \mathrm{~s}$

$2.435 \mathrm{E}-13$

$2.142 \mathrm{E}-13$

(.

1.919E-12

$0.2198-12$

$1.688 \mathrm{E}-11$
$4400 . C$

$2.989 E-14$ $3.923 E-14$

$6.96 \mathrm{CE}-14$

$9.612 E-14$
$1.124 E-13$

$1.124 \mathrm{E}-13$
$9.086 \mathrm{E}-14$

$9.086 \mathrm{E}-14$
$7.244 \mathrm{E}-14$

$7.839 \mathrm{E}-14$

$1.145 E-13$

$1.58 \mathrm{CE}-13$

$1.463 E-13$

$1.267 \mathrm{E}-13$

$3.610 \mathrm{E}-13$

$1.186 \mathrm{E}-12$

$3.844 \mathrm{E}-12$

$1.723 \mathrm{E}-1$

$6.688 \mathrm{E}-12$
4600.0

$1.978 \mathrm{E}-14$

$2.507 E-14$

4. $247 E-14$

$5.818 E-14$

$0.653 E-14$

$5.498 E-14$
$4.379 t-14$

4. $752 E-14$

$6.904 E-14$

$9.675 E-14$

1. $188 \mathrm{E}-13$

$8.774 E-14$

$7.485 E-14$

$7.345 E-13$

$2.379 E-12$

$1.060 E-11$

4. $108 \mathrm{E}-12$

4800.0

$1.252 E-14$

$1.498 E-14$

$2.324 E-14$
$3.133 E-14$

$3.492 E-14$

$3.039 E-14$

$2.757 E-14$

$4.069 E-14$

$5.870 \mathrm{E}-14$

$5.142 E-14$

$4.290 E-14$

$4.540 E-13$

$1.473 \mathrm{E}-12$

6. $524 E-12$

2. $504 \mathrm{E}-12$ 
(NEUTRONS/MEV/STERADIAN/SOURCE NEUTRONI

ENERGY GROUP (MEV) $1.22 \mathrm{E} 01---1.50 \mathrm{E}$ O1 $1.00 E$ O1---1.22E 01 $8.19 \mathrm{E}$ 00---1.00E 01
$6.36 \mathrm{E} 00--8.19 \mathrm{E} 00$ 6.36E O0---8.19E 00 $4.97 \mathrm{E}$ OO---6.36E 00
$4.07 \mathrm{E} 00---4.97 E 00$ $4.07 \mathrm{E}$ 00---4.97E 00
$3.01 \mathrm{E} 00---4.07 \mathrm{E} 00$ $3.01 \mathrm{E}$ O0---4.07E 00 $2.46 \mathrm{E} O 0---3.01 \mathrm{E}$
$2.35 \mathrm{E} \mathrm{OO---2.46E} 00$ $2.35 \mathrm{E} 00---2.46 \mathrm{E} 00$
$1.83 \mathrm{E} 00---2.35 \mathrm{E} 00$ $1.11 \mathrm{E} \mathrm{CO--1.83E} 00$ $5.5 \mathrm{CE}-01--1.11 \mathrm{E}$ 00 $1.11 E-01--5.50 \mathrm{E}-01$ $3.35 \mathrm{E}-02--1.11 \mathrm{E}-01$ $5.83 \mathrm{E}-04--3.35 \mathrm{E}-02$ $1.01 \mathrm{E}-\mathrm{C} 4--5.83 \mathrm{E}-04$ $2.90 E-05--1.01 \mathrm{E}-04$ $1.07 E-C 5---2.90 E-05$ 3.06E-06--1.07E-05 $3.06 \mathrm{E}-06---1.07 \mathrm{E}-05$
$1.12 \mathrm{E}-06--3.06 \mathrm{E}-06$ $4.14 \mathrm{E}-07--1.12 \mathrm{E}-06$ $0.0 \quad--4.14 \mathrm{E}-07$ ENERGY GROUP (MEV) $1.22 E$ O 1---1. 5CE 01 $1.00 E \quad 01--1.22 \mathrm{E} \quad 01$ $6.36 \mathrm{E}$ CO-- $8.19 \mathrm{E}$ 00 $4.97 \mathrm{E}$ NC---6.36E DO 4.07E $00--4.97 E$ CO $3.01 \mathrm{E} 00--4.07 \mathrm{E} 00$ $2.46 E$ OD- $-3.01 E$ OO 2.35 E OC---2.46E 00 $1.83 E$ EO--2.35E 00 $1.11 E$ OC- $-1.83 E$ OO $5.50 E-01--1.11 E 00$ $1.11 \mathrm{E}-01---5.50 \mathrm{E}-01$ $3.35 \mathrm{E}-02--1.11 \mathrm{E}-01$ $5.83 E-04--3.35 E-02$ $1.01 \mathrm{E}-\mathrm{C4}---5.83 \mathrm{E}-04$ 2.9CE-C5---1. C1 E-04 1.C TE-05---2. 90E-05 $3.06 E-06-0-1.07 E-05$ $1.12 E-06--3.06 E-06$ $4.14 \mathrm{E}-07--1.12 \mathrm{E}-06$
0.0 $-1.0000$ $-3.394 E-13$ $5.016 \mathrm{E}-11$ $.119 \mathrm{E}-09$ $.906 \mathrm{E}-08$ $8.697 E-08$ $1.125 \mathrm{E}-07$ $1.839 \mathrm{E}-07$ $3.229 E-07$ 8.653E-07 $1.929 E-06$ . . $335 \mathrm{E}-04$ . . $406 E-02$ 2.406E-02 $3.553 \mathrm{E}-01$ . $2.929 E 00$ 6.773E 00 $1.046 \mathrm{E} \mathrm{O1}$

ANGLE 10 $M U=0.0950$ $1.491 E-12$ $4.618 \mathrm{E}-11$ $1.751 \mathrm{E}-08$ $8.487 E-08$ $1.617 E-07$ $1.615 \mathrm{E}-07$ $4.720 E-07$ $1.177 \mathrm{E}-06$ $1.975 \mathrm{E}-06$ $3.265 E-06$ $1.155 \mathrm{E}-05$ $3.764 \mathrm{E}-05$ $4.114 E-04$ $2.811 \mathrm{E}-\mathrm{C2}$ $1.321 \mathrm{E}-01$ $4.120 \mathrm{E}-01$ $1.192 \mathrm{E} 00$ $3.379 E$ DO $7.794 \mathrm{E}$ OD $1.190 E 01$ AN $=-0.9894 \quad$ ANGLE 3 $.722 E-13 \quad M U=-C .9446 \quad M U=-0.8656$ $.804 E-11$ $2.186 \mathrm{E}-09$ $2.912 \mathrm{E}-08$
$8.745 \mathrm{E}-08$ $8.745 \mathrm{E}-08$ $1.124 \mathrm{E}-07$ $1.846 \mathrm{E}-07$ $3.275 E-07$ $8.708 \mathrm{E}-07$ $1.935 \mathrm{E}-06$ $2.677 E-05$ ..677E-05 . $471 E-04$ . . $3.558 \mathrm{E}-01$ $1.032 \mathrm{E} 00$ $2.933 \mathrm{E} 00$ $6.782 E 00$ $6.782 \mathrm{E} 00$
$1.047 \mathrm{E} 01$

ANGLE 11 $M U=0.2816$ $2.410 \mathrm{E}-12$ $7.204 \mathrm{E}-11$
$1.697 \mathrm{E}-09$ $2.533 \mathrm{E}-08$ $2.533 E-08$ $1.953 E-07$ $1.913 \mathrm{E}-07$ $6.417 \mathrm{E}-07$ $1.603 \mathrm{E}-06$ $2.423 \mathrm{E}-06$ 3. $720 \mathrm{E}-06$ $1.311 \mathrm{E}-05$ $4.049 \mathrm{E}-05$ $4.286 \mathrm{E}-04$ $4.223 \mathrm{E}-04$ $2.895 \mathrm{E}-02$ $1.359 \mathrm{E}-01$ $4.236 \mathrm{E}-01$ $1.225 \mathrm{E} 00$ $3.470 E$ OD $.000 E 0$ $1.219 E 01$ $2.269 E-13$ $6.327 E-12$
$1.483 E-10$ $1.483 \mathrm{E}-10$ $3.518 \mathrm{E}-09$ $3.396 \mathrm{E}-08$ $9.811 \mathrm{E}-08$ $1.129 \mathrm{E}-07$ $2.035 \mathrm{E}-07$ 4. $260 \mathrm{E}-07$ 1.COTE-06 $2.100 E-06$ T. $490 E-06$ 2.855E-05 $3.482 E-04$ 2. $486 \mathrm{E}-02$ $2.486 \mathrm{E}-02$ $3.666 \mathrm{E}-01$ $1.062 \mathrm{E} 00$ $3.019 E 00$ $6.977 E 00$ $1.076 E 01$

ANGLE 14 ANGLE 12 $U=0.4580$ $4.041 E-12$ $1.158 \mathrm{E}-10$
$2.715 \mathrm{E}-09$ $3.981 \mathrm{E}-08$ $3.981 \mathrm{E}-08$
$1.505 \mathrm{E}-07$ $2.470 E-07$ $2.337 E-07$ $9.096 \mathrm{E}-0$ $2.330 E-06$ $3.029 E-06$ $4.282 \mathrm{E}-06$ $1.501 \mathrm{E}-05$ 4.362E-05 $4.462 \mathrm{E}-04$ 4. $354 \mathrm{E}-04$ $2.979 \mathrm{E}-\mathrm{C2}$ $1.396 \mathrm{E}-\mathrm{Cl}$ 4. $352 \mathrm{E}-01$ $1.257 E 00$ $3.561 \mathrm{E} \mathrm{DO}$ $1.248 E 01$

ANGLE 13 $M U=0.6179$ $8.590 E-12$ $2.244 \mathrm{E}-10$
$5.157 \mathrm{E}-09$ $6.942 \mathrm{E}-08$ $2.225 \mathrm{E}-07$
$3.266 \mathrm{E}-07$ $2.917 \mathrm{E}-07$ $1.340 \mathrm{E}-06$ $3.663 \mathrm{E}-06$ $3.832 E-06$ $4.959 \mathrm{E}-06$ $1.728 \mathrm{E}-05$ $4.694 \mathrm{E}-05$ $4.636 \mathrm{E}-04$ $4.479 \mathrm{E}-04$ $3.059 \mathrm{E}-02$ $1.432 \mathrm{E}-01$ $4.463 \mathrm{E}-01$ $1.289 \mathrm{E} \mathrm{OO}$ $3.648 \mathrm{E} 00$ $.403 E 00$ $1.275 \mathrm{E} \mathrm{Ol}$ $2.114 E-11$ $1.120 E-08$ $1.298 \mathrm{E}-07$ $3.470 E-07$ $4.459 E-07$ $3.679 E-07$ $6.226 \mathrm{E}-06$ 4.874E-06 $5.740 \mathrm{E}-06$ $1.987 \mathrm{E}-05$ $5.025 \mathrm{E}-05$ 4.797E-04 . $593 \mathrm{E}-04$ . $132 \mathrm{E}-02$ 4. $.563 \mathrm{E}-01$ .317E 00 $3.727 E 00$ .581E 00 1. 300E 01
$M U=-0.6179$ 8. $656 \mathrm{E}-14$ $5.364 E-12$
$1.545 E-10$ $1.545 \mathrm{E}-10$ $4.535 E-09$ $3.920 \mathrm{E}-08$ $1.048 \mathrm{E}-07$ $.151 \mathrm{E}-07$ $2.205 E-07$ $4.912 E-07$ $1.102 E-06$ 2.223E-06 $7.939 E-06$ . . 2.533E-02 . 3.732E-OI $1.081 E 00$ $3.072 E 00$ 7.097E 00 $1.093 \mathrm{E} 01$

ANGLE 15 2.114E-11 $\quad 5.148 \mathrm{E}-11$
ANGLE $M U=-0.4580$ $.990 E-12$ $1.912 E-10$ $6.208 E-09$ $4.676 \mathrm{E}-08$ $1.132 \mathrm{E}-07$ $1.197 E-07$ 2.483E-07 (. 8.535E-06 104E 00 3.135E 00 $7.241 E 00$ $1.113 E 01$

ANGLE 16

ANGLE 8 $M U=-0.2816$ $1.114 E-11$ $3.551 \mathrm{E}-10$ $8.797 \mathrm{E}-09$ $5.651 \mathrm{E}-08$ $1.243 \mathrm{E}-07$ $1.279 E-07$ 7. 
(NEUTRONS/MEV/STERADIAN/SOURCE NEUTRON)

ENERGY
GROUP (MEV)

$1.22 E$ 01-- - 1.50E O1

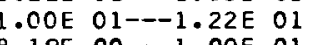

$6.36 \mathrm{E} 00-2-8.19 \mathrm{E}$ OO

4.

4.97E $00---6.36 E$ EO 00

$3.01 E$ O0- - 4.07E 00

$2.46 E$ O0---3.01E 00

$2.35 \mathrm{E} \mathrm{OO--2.46E} 00$

$1.83 \mathrm{E} 00--2.35 \mathrm{E}$ CO

5.50E-01--1.11E 00

$1.11 \mathrm{E}-01---5.50 \mathrm{E}-01$

$3.35 \mathrm{E}-02-\cdots-1.11 \mathrm{E}-01$

$5.83 \mathrm{E}-04--3.35 \mathrm{E}-02$

$2.01 E-04--5.83 E-04$

$1.07 E-05---2.90 E-05$

$3.06 E-06=-1.07 E-05$

$1.12 \mathrm{E}-06---3.06 \mathrm{E}-06$

$4.14 \mathrm{E}-07---1.12 \mathrm{E}-06$

$0.0 \quad---4.14 \mathrm{E}-07$

ENERGY

GROUP (MEV)

$1.22 \mathrm{E} 01---1.50 \mathrm{E}$ ol

$1.00 \mathrm{E}$
$81---1.22 \mathrm{E} 01$

$8.19 \mathrm{E} 00--1.00 \mathrm{E}$ OI
$6.36 \mathrm{E} 00--8.19 \mathrm{E}$ OO

$4.97 E$ OO- - $6.36 E$ OO

4.07E $00---4.97 \mathrm{E}$ OO

3.01E $00---4.07 E$ OO $2.46 \mathrm{E} 00--3.01 \mathrm{E} 00$ $2.35 \mathrm{E} 00--2.46 \mathrm{E} 00$ 1.83E $00--2.35 E$ OO $1.11 \mathrm{E}$ O0-- $1.83 \mathrm{E}$ กO .5OE-O1- $1.11 E$ OO 1 . 1 E-OI-C . $35 E-02-1.11 E-01$ $5.83 \mathrm{E}-04--3.35 \mathrm{E}-02$ $1.01 E-04--5.83 E-04$ 2.90 $-05--1.01 E-04$ $3.06 E-06--1.07 E-05$ $1.12 E-06-3.06 E-06$ $1.12 E-06-3.06 E-06$ $4.14 E-07--1.12 E-06$
$0.0 \quad--4.14 E-07$
ANGLE 1 ANGLE 2 $2.965 \mathrm{E}-16$ MU $-1.952 \mathrm{E}-13-1.226 \mathrm{E}-1$ $.030 E-11 \quad 1.066 E-11$ $1.618 \mathrm{E}-10 \quad 1.623 \mathrm{E}-10$ $4.258 E-10 \quad 4.279 E-10$ $5.046 E-10$ 7.886E-10 $7.915 \mathrm{E}-10$ $1.338 \mathrm{E}-09 \quad 1.355 \mathrm{E}-09$ $3.236 \mathrm{E}-09$ 7.108E-09 $2.386 E-08$ $9.004 E-08$ $1.104 \mathrm{E}-06$
$1.145 \mathrm{E}-06$ $7.907 \mathrm{E}-05$ $3.734 \mathrm{E}-04$ $1.164 E-03$ $3.372 E-03$ . $578 \mathrm{E}-03$ $2.213 \mathrm{E}-02$
$3.413 \mathrm{E}-02$ ANGLE 10 $M=0.0950$ $1.783 \mathrm{E}-15$ $8.827 \mathrm{E}-14$ $4.458 \mathrm{E}-12$ $9.954 \mathrm{E}-1$ $4.503 \mathrm{E}-10$ $7.793 \mathrm{E}-10$ $7.311 \mathrm{E}-10$ $1.914 \mathrm{E}-09$ $4.480 E-0$ $7.513 \mathrm{E}-0$ $1.187 E-08$ 4.COOE -08 $1.258 \mathrm{E}-07$ $1.353 E-06$ $1.342 E-06$ $4.314 E-04$ $4.314 E-04$ $3.882 E-03$ 1. $100 \mathrm{E}-02$ $1.100 E-02$ $2.535 \mathrm{E}-02$
ANGLE 3

$\begin{array}{ccc}A N G L E & 3 & \text { ANGLE } 4 \\ M U=-0.9446 & M U=-0.8656\end{array}$ $3.737 E-17$ $6.170 E-16$ $1.065 \mathrm{E}-13$ $1.214 \mathrm{E}-11$ $4.367 \mathrm{E}-10$ $5.045 E-10$ $5.045 E-10$
$8.041 E-10$ $8.041 E-10$ $1.425 \mathrm{E}-09$
$3.357 \mathrm{E}-09$ $3.357 \mathrm{E}-09$
$7.228 \mathrm{E}-09$ $2.435 E-08$ $9.135 \mathrm{E}-08$ $1.114 \mathrm{E}-06$ $1.153 \mathrm{E}-06$ 7. $963 \mathrm{E}-05$ 3. $759 \mathrm{E}-04$ 1. $172 \mathrm{E}-03$ $9.39 \mathrm{E}-03$ $2.227 \mathrm{E}-02$ 3. $435 \mathrm{E}-02$ ANGLE 12 $M U=0.4580$ $5.337 E-15$ $2.430 E-13$ $1.197 E-11$ $2.421 E-10$ $7.844 \mathrm{E}-10$ 1. $167 \mathrm{E}-09$ $1.029 \mathrm{E}-09$ $3.519 E-C 9$ 8. $878 \mathrm{E}-09$ 1. $142 E-08$ $1.538 \mathrm{E}-08$ $5.139 E-08$ $1.448 E-07$ $1.462 E-06$ $1.423 E-06$ $9.716 E-05$ 4.54 E- 04 $1.416 E-03$ 4.08BE-03 $1.157 E-02$ $4.048 \mathrm{E}-02$ $1.409 E-16$ $8.124 \mathrm{E}-15$ $3.777 \mathrm{E}-13$ $1.733 \mathrm{E}-10$ $4.527 \mathrm{E}-10$ $5.057 E-10$ $5.057 \mathrm{E}-10$
$8.297 \mathrm{E}-10$ $8.297 \mathrm{E}-10$
$1.544 \mathrm{E}-09$ $1.544 \mathrm{E}-09$
$3.539 \mathrm{E}-09$ $3.539 \mathrm{E}-09$
$7.420 \mathrm{E}-09$ $7.420 E-09$
$2.509 E-08$ $9.330 \mathrm{E}-08$ $1.129 \mathrm{E}-06$ $1.166 \mathrm{E}-06$ $8.045 E-05$ $3.796 \mathrm{E}-04$ $1.184 E-03$ $3.427 \mathrm{E}-03$ $9.732 \mathrm{E}-03$ $2.248 \mathrm{E}-02$
$3.465 \mathrm{E}-02$ ANGLE 13 $M U=0.6179$ $1.159 \mathrm{E}-14$ $2.356 \mathrm{E}-11$ $4.275 \mathrm{E}-10$ 1. $135 \mathrm{E}-09$ $1.507 E-09$ $1.255 \mathrm{E}-09$ $5.042 E-09$ $1.418 \mathrm{E}-08$ $1.430 \mathrm{E}-08$ $1.765 E-08$ $5.867 E-08$ $1.552 E-07$ $1.516 E-06$ $1.462 E-06$ $9.966 E-05$ 1.451E-03 $1.451 E-03$ $4.18 E-03$ $1.184 \mathrm{E}-02$ 4.134E-02
ANGLE 5 9.982E-15 $038 \mathrm{E}-15$ $4.788 E-13 \quad 5.336 E-13$ $1.841 E-11$ $1.899 \mathrm{E}-10$ $5.107 E-10$ $5.1 C 7 E-10$
$8.731 E-10$ $8.731 E-10$
$1.710 E-09$ $1.710 E-09$
$3.8 C 9 E-09$ $3.8 C 9 E-09$
$7.724 \mathrm{E}-09$ $2.724 \mathrm{E}-09$ $9.616 \mathrm{E}-08$ $1.151 E-06$ $1.183 E-06$ $8.162 \mathrm{E}-05$ $3.849 E-04$ $1.200 E-03$ $3.474 E-03$ $9.862 \mathrm{E}-03$ $2.277 \mathrm{E}-02$ $3.508 E-02$ ANGLE 14 $M U=0.7550$ $2.752 \mathrm{E}-14$ $5.079 \mathrm{E}-11$ $7.906 \mathrm{E}-10$ $1.714 \mathrm{E}-09$ $1.987 \mathrm{E}-09$ $1.535 E-09$ $7.435 \mathrm{E}-09$ $2.465 E-08$ $1.788 \mathrm{E}-08$ $2.020 E-08$ $0.677 \mathrm{E}-08$ $1.566 \mathrm{E}-06$ $1.498 \mathrm{E}-06$ $1.019 E-04$ 4.762E-04 $1.482 E-03$ $1.208 E-02$ $1.208 E-02$ 4. $210 \mathrm{E}-02$
ANGLE 7 $U=-0.4580$ $8.815 \mathrm{E}-17$ $9.965 \mathrm{E}-15$
$7.280 \mathrm{E}-13$ $3.380 \mathrm{E}-11$ $2.558 E-10$ $5.467 \mathrm{E}-10$ $5.467 E-10$ $5.449 \mathrm{E}-10$
$1.056 \mathrm{E}-09$ $2.277 \mathrm{E}-09$ $4.683 \mathrm{E}-09$ $8.769 \mathrm{E}-09$ $2.979 E-08$ $1.048 E-07$ $1.214 \mathrm{E}-06$ $1.234 E-06$ $8.494 E-05$ 4.000E-04 1.247E-03 $3.607 E-03$ $1.023 E-02$ $2.361 \mathrm{E}-02$
$3.625 \mathrm{E}-02$ ANGLE 16 $M U=0.9446$
$1.348 \mathrm{E}-13$ $5.014 \mathrm{E}-12$ $2.267 \mathrm{E}-10$ $2.624 E-09$ $3.953 \mathrm{E}-09$ $3.381 \mathrm{E}-09$
$2.177 \mathrm{E}-09$ $1.710 \mathrm{E}-08$ $9.173 \mathrm{E}-08$ $2.669 \mathrm{E}-08$ $2.523 \mathrm{E}-08$ $8.273 E-08$ $1.821 \mathrm{E}-07$ $1.641 \mathrm{E}-06$ 1.5 $1.052 E-04$ l.5 $1.527 E-03$ 4. $401 E-03$ $1.243 E-02$ 4. $317 E-02$

ANGLE 8 $M U=-0.2816$ 3.068E-16 $2.254 \mathrm{E}-14$ $1.407 E-12$
$4.831 E-11$ $3.052 \mathrm{E}-10$ $6.001 E-10$ $5.830 E-10$ $1.232 \mathrm{E}-09$ $1.232 \mathrm{E}-09$ 5.358E-09 $9.565 \mathrm{E}-09$ 3.244E-08 1. $107 \mathrm{E}-07$ $1.255 \mathrm{E}-06$ $1.267 \mathrm{E}-06$ 8.705E-05 $4.095 E-04$ $1.276 \mathrm{E}-03$ 
(NEUTRONS/MEV/STERADIAN/SOURCE NEUTRON)

ENERGY

1.22E O1- - 1.50E $1.00 \mathrm{E}$ 01-- $1.22 \mathrm{E}$ O1 $8.19 \mathrm{E}$ OO---1.00E 01 6.36 E $00--8.19 E$ CO $4.97 \mathrm{E}$ 00--6.36E CO 4.07E $00--4.97 E$ EO $3.01 E$ 00-- 4.07E 00 $2.46 \mathrm{E} 00--3.01 \mathrm{E} 00$

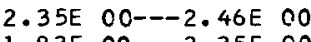

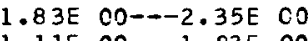
$1.11 \mathrm{E} 00--1.83 \mathrm{E}$ 00 $5.50 \mathrm{E}-01--1.11 \mathrm{E}$ DO $1.11 \mathrm{E}-01--5.50 \mathrm{E}-01$ $3.35 \mathrm{E}-02--1.11 \mathrm{E}-01$ $5.83 \mathrm{E}-04--3.35 \mathrm{E}-02$ $1.01 E-04--5.83 E-04$ $2.90 E-05--1$. ClE-04 $1.07 E-05--2.90 E-05$ $3.06 \mathrm{E}-06---1.07 \mathrm{E}-05$ $1.12 \mathrm{E}-06---3.06 \mathrm{E}-06$ $4.14 \mathrm{E}-07--1.12 \mathrm{E}-06$
$0.0 \quad--4.14 \mathrm{E}-07$

$$
\text { ENERGY }
$$

GROUP (MEV)

$1.22 \mathrm{E} 01---1.50 \mathrm{E} 01$ $1.00 E$ O1--1.22E 0 $8.19 \mathrm{E}$ 00---1.00E OI 6.36E $00---8.19 E$ OO $4.97 \mathrm{E} 00---6.36 \mathrm{E} 00$ $4.07 \mathrm{E} 00--4.97 \mathrm{E} \mathrm{OO}$ $3.01 \mathrm{E} 00---4.07 \mathrm{E} 00$ $2.46 \mathrm{E} 00--3.01 \mathrm{E}$ OO

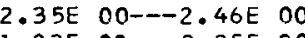
$1.83 E$ OO- $-2.35 \mathrm{E} \mathrm{CO}$ $1.11 E$ OC--1.83E ON $5.50 \mathrm{E}-\mathrm{C} 1--1.11 \mathrm{E}$ OO . $3.35 E-02--1.11 E-01$ 5.8 उE-C4-C $3.35 E-C 2$ $1.01 E-04--5.83 E-04$ $2.90 \mathrm{E}-0.5--1.01 \mathrm{E}-04$ $1.07 E-05-2.90 E-C 5$ $12 E-06--3.06 E-06$ $1.12 E-96--3.06 E-06$ $4.14 \mathrm{E}-07---1.12 \mathrm{E}-06$
$0.0 \quad--4.14 \mathrm{E}-07$
ANGLE 1 ANGLE 2 $-2.413 E-19$ $-1.139 \mathrm{E}-17$ $-6.326 \mathrm{E}-16$ $3.635 \mathrm{E}-14$ $6.715 E-13$ $.054 \mathrm{E}-12$ $2.917 \mathrm{E}-12$ $467 t-12$ 1. C $15 \mathrm{E}-11$ $2.220 E-11$ $7.009 \mathrm{E}-11$ $2.621 \mathrm{E}-10$ $3.196 \mathrm{E}-\mathrm{CO}$ $3.320 \mathrm{E}-09$ $2.280 \mathrm{E}-0$ $1.073 E-06$ $3.333 E-06$ $9.618 \mathrm{E}-06$ $2.725 \mathrm{E}-05$ $9.692 \mathrm{E}-05$ ANGLE 10 $M U=0.0950$ $1.906 \mathrm{E}-18$ $1.530 \mathrm{E}-16$ $1.643 \mathrm{E}-14$ $5.022 \mathrm{E}-13$ $2.168 \mathrm{E}-12$ $3.519 E-12$ $3.198 \mathrm{E}-12$ $7.696 \mathrm{E}-12$ $1.704 E-11$ $2.740 E-11$ $4.134 \mathrm{E}-11$ $1.321 E-10$ $3.944 E-10$ $4.093 \mathrm{E}-09$ $4.024 E-09$ $2.738 E-07$ $1.279 E-C 6$ $3.972 E-06$ $3.229 E-05$ $7.423 E-05$ $1.129 E-04$ $-8.547 E-18$ $4.289 E-16$ $6.750 E-13$ $1.665 \mathrm{E}-12$ $1.920 \mathrm{E}-12$ $2.806 E-12$ $4.528 \mathrm{E}-12$ 1. C25E-11 $2.230 E-11$ $7.047 \mathrm{E}-11$ $2.631 \mathrm{E}-1 \mathrm{~J}$ $3.203 \mathrm{E}-09$ $3.32 \mathrm{EE}-\mathrm{C}$ $2.284 \mathrm{E}-07$ $1 . C 74 E-06$ $3.339 E-06$ $9.634 \mathrm{E}-06$ $2.730 \mathrm{E}-05$ $9.707 E-05$ ANGLE 11 $3.253 \mathrm{E}-18$ $2.523 E-16$ $2.663 \mathrm{E}-14$ $7.697 \mathrm{E}-1$ $2.767 E-12$ $4.234 \mathrm{E}-12$ $3.757 E-12$ $1.011 \mathrm{E}-11$ $2.313 E-11$ $3.357 E-11$ $4.7 C 2 E-11$ $1.500 \mathrm{E}-1 \mathrm{C}$ $4.255 E-10$ $4.277 E-09$ $4.163 E-C 9$ $2.827 E-O$ $1.320 E-06$
$4 . C 96 E-06$ $1.178 \mathrm{E}-05$ $3.327 E-05$ $7.645 \mathrm{E}-05$ $7.645 E-05$
$1.16 C E-04$

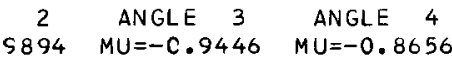
$-1.916 \mathrm{E}-19$ $U=0.2816$

$M U=-C .9446$

$-2.618 \mathrm{E}-20$

$6.618 \mathrm{E}-19$

$2.444 \mathrm{E}-16$

$4.474 E-14$

$6.934 \mathrm{E}-13$

$1.934 \mathrm{E}-12$

$2.872 \mathrm{E}-12$

4.778E-12

$1.066 \mathrm{E}-1$

$2.275 \mathrm{E}-1$

$7.211 \mathrm{E}-11$

2. $674 \mathrm{E}-10$

$3.352 \mathrm{E}-09$

2. 300E-O

$1.082 \mathrm{E}-06$

$3.363 E-06$

$9.7 C 2 E-06$

$2.748 \mathrm{E}-05$

(7)E-O5

ANGLE 12

$M=0.4580$

$6.025 E-18$
$4.458 E-16$

$4.458 E-16$

$1.273 E-12$

$3.735 \mathrm{E}-12$

$5.267 \mathrm{E}-12$

4.501E-12

$3.374 \mathrm{E}-11$

4.162E-11

$5.379 \mathrm{E}-11$

1.711E-10

$4.587 E-10$

$4.464 E-09$

4. $301 E-09$

2.916E-07

$1.359 E-06$

$4.219 \mathrm{E}-06$
$1.213 \mathrm{E}-05$

$3.423 E-05$

$7.862 E-05$

$7.862 \mathrm{E}-05$
$1.191 \mathrm{E}-04$

\section{$U=-0.8656$ \\ $1.299 E-19$ \\ $1.029 \mathrm{E}-15$ \\ $5.668 \mathrm{E}-14$ \\ $7.400 E-13$
$1.796 E-12$ \\ $1.966 \mathrm{E}-12$ \\ $3.0 C 3 E-12$ \\ $5.220 \mathrm{E}-12$
$1.143 \mathrm{E}-11$ \\ $2.363 E-11$ \\ $7.519 \mathrm{E}-11$ \\ $2.751 E-10$
$3.293 E-09$ \\ $3.398 \mathrm{E}-\mathrm{CO}$ \\ $2.331 \mathrm{E}-07$ \\ $1.096 \mathrm{E}-06$
$3.405 \mathrm{E}-06$ \\ $9.822 \mathrm{E}-06$ \\ $2.782 \mathrm{E}-05$}

$9.88 C E-05$

ANGLE 13

$U=0.6179$

$1.315 \mathrm{E}-17$
$9.092 \mathrm{E}-16$

$9.092 \mathrm{E}-16$

$2.362 \mathrm{E}-12$

$5.326 \mathrm{E}-12$

$6.737 \mathrm{E}-12$

$5.454 \mathrm{E}-12$

$1.925 \mathrm{E}-11$

$5.362 \mathrm{E}-11$

$6.162 \mathrm{E}-11$

$1.953 \mathrm{E}-10$

$4.644 E-09$

$4.644 E-09$

$3.400-07$

1.307E-06

.

$4.335 E-06$

1.246E-05

$8.067 \mathrm{E}-0$

$1.220 \mathrm{E}-04$
ANGLE 5 $1.406 \mathrm{E}-17 \quad 1.344 \mathrm{E}-17$ $1.381 E-15 \quad 1.660 E-15$ $7.524 \mathrm{E}-14 \quad 1.055 \mathrm{E}-13$ $8.308 \mathrm{E}-13 \quad 9.782 \mathrm{E}-13$ $2.025 \mathrm{E}-12 \quad 2.123 \mathrm{E}-12$ $3.224 \mathrm{E}-12 \quad 3.575 \mathrm{E}-12$ $5.866 \mathrm{E}-12 \quad 6.814 \mathrm{E}-12$ $1.259 E-11 \quad 1.421 E-11$ $.982 \mathrm{E}-11 \quad 8.621 \mathrm{E}-1$ $2.865 \mathrm{E}-10 \quad 3.013 \mathrm{E}-10$ $3.375 E-09 \quad 3.480 E-09$ $3.464 E-09 \quad 3.548 E-0$ $2.374 E-07 \quad 2.429 E-07$ 1.140E-06 . $365 E-06 \quad 342 E-06$ $9.99 \mathrm{E}-06 \quad 1.021 E-05$ 2.830E-05 $2.890 E-05$ $\begin{array}{ll}1.503 E-04 & 1.023 E-04\end{array}$

\section{ANGLE 14}

$\begin{aligned} & M U=C .7550 \\ & 3 . C 56 E-17\end{aligned}$

$2.000 E-15$

1. $590 \mathrm{E}-1$

$4.153 \mathrm{E}-12$

$7.863 \mathrm{E}-12$

$8.739 E-12$

$2.757 \mathrm{E}-11$

$9.292 \mathrm{E}-11$

$6.409 \mathrm{E}-11$

$7.021 \mathrm{E}-11$

$2.216 E-10$

5.262E-10 $4.550=09$ 4. 75 E 0 1. $430 E-06$ .. $430 E-06$ $4.438 E-06$ 1.594E-05 $8.249 E-05$ 1. $245 \mathrm{E}-04$

ANGLE 7 $M U=-0.2816$ $3.701 E-19$ $3.949 \mathrm{E}-17$ $5.098 E-15$ $2.315 \mathrm{E}-13$ $1.450 \mathrm{E}-12$ $2.641 \mathrm{E}-12$ $4.4918 \mathrm{E}-12$ $1.027 \mathrm{E}-1$ $1.917 \mathrm{E}-11$ $3.273 \mathrm{E}-11$ $1.048 \mathrm{E}-10$ $3.413 E-10$ $3.754 \mathrm{E}-09$ $3.763 \mathrm{E}-0$ $2.569 E-07$ $1.203 E-06$ 
(GAMMAS/MEV/STERADIAN/SOURCE NEUTRON)

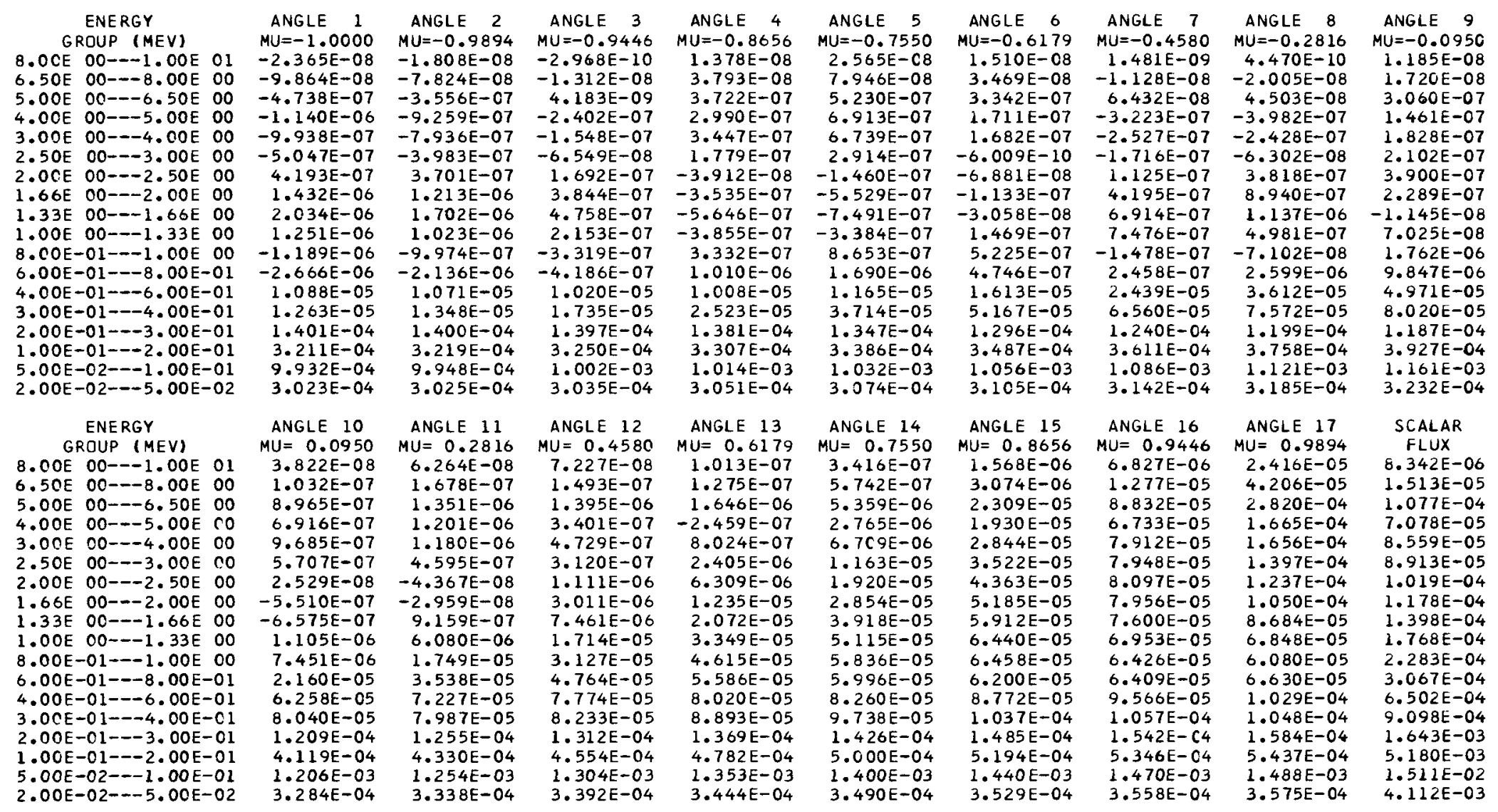


(GAMMAS/MEV/STERADIAN/SOURCE NEUTRON)

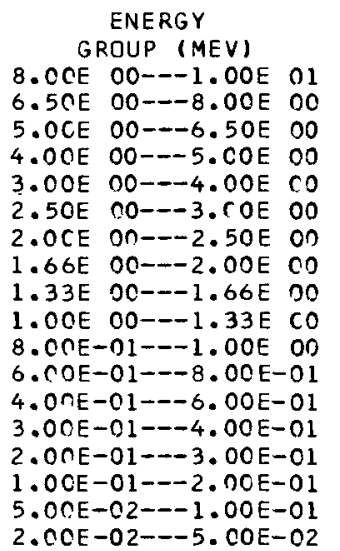

ENERGY

GROUP (MEV)

8. OCE OC---1. ODE 01 $6.50 E$ OD- - 8. OOE 00 5. COE OO- -6.50E CO 4. OCE OO- 3 - 2 . COE OO OO2.5CE OC-D-3. COE OO $2.00 E$ O0---2.50E 00 $1.66 \mathrm{E} 00--2.00 \mathrm{E}$ OO $1.33 E$ DO- $-1.66 \mathrm{E} \mathrm{CO}$ $1.0 \mathrm{CE} \mathrm{O0-D-1.33E} \mathrm{OO}$ $8.00 E-01--1.00 E 00$ $6.0 n E-01---8$. COE-O 4.00E-01-- $6.00 \mathrm{E}-01$ $3.0 \mathrm{CE}-01--4.00 \mathrm{E}-0$ 2,0 CE-01-- 3.00E-O $1.00 \mathrm{E}-01-\cdots 2.00 \mathrm{E}-0$ $5.00 E-02-1.00 E-C$ $2.0 C E-02--5.00 E-02$ $M U=-1.00 \cap 0$ $-2.748 \mathrm{E}-09$
$-9.497 \mathrm{E}-09$ $-3.954 E-0$ $-3.954 E-08$ $-6.230 E-08$ - $230 \mathrm{E}-08$ 2.68E-08 $8.740 \mathrm{E}-0$ $8.740 \mathrm{E}-08$ $6.267 \mathrm{E}-08$ 6.267E-08 $-1.583 E-07$ $5.142 \mathrm{E}-07$ $5.285 \mathrm{E}-07$ $6.774 \mathrm{E}-06$ $6.774 \mathrm{E}-06$
$1.483 \mathrm{E}-05$ $1.483 \mathrm{E}-05$
$4.549 \mathrm{E}-0.05$ $4.549 \mathrm{E}-05$
$1.372 \mathrm{E}-05$

ANGLE 10 $M U=0.0950$ $1.408 \mathrm{E}-09$
$4.938 \mathrm{E}-09$ $2.360 E-08$ $4.080 \mathrm{E}-08$ $4.943 E-08$ $2.503 \mathrm{E}-08$ $-2.225 \mathrm{E}-08$ $-7.034 \mathrm{E}-08$
$-8.816 \mathrm{E}-08$ . $16 E-08$ $3.674 \mathrm{E}-\mathrm{C}$ $1.107 \mathrm{E}-0$ $3.000 E-06$ $3.671 E-06$ $5.499 \mathrm{E}-06$ $1.879 \mathrm{E}-\mathrm{C5}$ $5.453 \mathrm{E}-0$ $1.482 \mathrm{E}-05$
ANGLE 2 $M U=-0.9894 \quad M U=-0.0446$ AN $-7.812 \mathrm{E}-09-2.326 \mathrm{E}-09$ $-3.242 E-08 \quad-9.195 E-09$ $-1.648 \mathrm{E}-08$ $-1.333 E-08$ (2.130E-08 $7.319 E-08$ $9.704 E-08$ $5.012 \mathrm{E}-08$ $-6.865 \mathrm{E}-08$ $-1.281 \mathrm{E}-07$ $5.015 \mathrm{E}-07$ $5.728 \mathrm{E}-07$
$6.770 \mathrm{E}-06$ $6.770 \mathrm{E}-06$
$1.486 \mathrm{E}-05$ $1.486 \mathrm{E}-05$
$4.556 \mathrm{E}-05$ $1.556 E-05$
$1.373 E-05$ ANGLE 11 $M U=0.2816$ $2.862 \mathrm{E}-09$ $9.376 \mathrm{E}-\mathrm{C} 9$ $4.162 \mathrm{E}-08$ $6.265 \mathrm{E}-08$ $4.264 \mathrm{E}-08$ $9.485 E-09$ $4.945 E-08$ $5.992 \mathrm{E}-0$
$3.541 \mathrm{E}-0$ $3.541 \mathrm{E}-09$ $2.840 \mathrm{E}-07$ $8.985 E-07$ $3.385 \mathrm{E}-06$ 3.570E-OS $5.684 \mathrm{E}-0$ $1.966 \mathrm{E}-05$ $5.652 E-05$ $1.505 \mathrm{E}-05$ $2.104 \mathrm{E}-08$ $7.477 \mathrm{E}-09$ $-2.479 E-08$ $-2.975 E-08$ $4.610 E-07$ $4.610 E-07$
$7.758 E-07$ $6.735 E-06$ $6.735 E-06$
$1.5 C 1 E-05$ $1.5 \mathrm{C} 1 \mathrm{E}-05$
$4.586 \mathrm{E}-05$ 4. $586 E-05$
$1.377 E-05$

ANGLE 12 $M U=0.4580$ $-1.293 E-10$ $-2.192 E-09$
$-9.905 E-09$ $-4.201 E-08$ $-6.111 E-08$ $-4.562 E-08$ 3.581E-09 $1.134 \mathrm{E}-07$ 3. $502 E-07$ $8.846 E-07$ $1.590 E-06$ 3.537E-06 $3.652 E-06$ $5.899 \mathrm{E}-06$ $2.058 \mathrm{E}-0$ $5.856 \mathrm{E}-05$ 1. $527 E-05$
ANGLE 4 $M U=-0.8656$ $6.242 \mathrm{E}-10$
$2.184 \mathrm{E}-09$ $1.013 E-08$ $1.756 \mathrm{E}-08$ $1.713 \mathrm{E}-08$ $8.954 \mathrm{E}-09$ $-6.861 E-09$

$2.624 \mathrm{E}-08$ $-3.829 E-08$ 2.477E-08 1.926E-08 $4.374 \mathrm{E}-07$ $1.185 \mathrm{E}-06$ $6.627 \mathrm{E}-06$ $0.627 \mathrm{E}-06$
$1.526 \mathrm{E}-05$ $1.526 \mathrm{E}-05$
$4.640 \mathrm{E}-05$ $1.384 \mathrm{E}-05$ ANGLE 13 $M U=0.6179$ $2.280 E-06$ $2.613 \mathrm{E}-06$
$3.543 \mathrm{E}-06$ $3.951 \mathrm{E}-06$ $6.097 \mathrm{E}-06$ $2.149 \mathrm{E}-0$ $6.056 E-05$ 1. $548 \mathrm{E}-05$ ANGLE 5

ANGLE 6 $.524 E-09$ . 2. $227 \mathrm{E}-08$ $3.241 E-08$ 1. $241 E \mathrm{E}-08$ 1. $408 \mathrm{E}-08$ $-3.804 E-08$ $-4.793 E-08$ $-2.051 E-08$ 4.747E-08 $5.061 \mathrm{E}-07$ $1.797 \mathrm{E}-06$ $6.410 \mathrm{E}-06$ $1.4102 E-06$
$1.562 E-05$ 4.719E-O $1.394 \mathrm{E}-05$

$\begin{array}{ll}A N G L E & 6\end{array} \quad$ ANGLE 77 $8.879 E-10$ $3.759 \mathrm{E}-09$ $3.884 E-09$ $6.429 \mathrm{E}-10$ $-7.567 \mathrm{E}-09$ $-8.440 E-09$ $-2.699 \mathrm{E}-09$ $7.524 \mathrm{E}-\mathrm{Cg}$ $1.559 E-08$ $7.340 \mathrm{E}-07$ $2.525 E-06$ $2.525 E-06$
$6.099 E-06$ $6.099 E-06$
$1.607 E-05$ $4.822 \mathrm{E}-05$ $1.407 \mathrm{E}-05$ ANGLE 14 ANGLE 15 $-1.9 C 7 E-08 \quad-9.152 E-09 \quad 8.368 E-08$ $-7.754 E-08 \quad-1.961 E-08 \quad 4.621 E-07$ $-1.475 E-07-1.291 E-09 \quad 7.411 E-07$ 1.652E-07 $1.281 E-06$ $3.908 \mathrm{E}-08 \quad 5.049 \mathrm{E}-07 \quad 1.775 \mathrm{E}-06$ $2.697 E-07 \quad 9.534 E-07 \quad 2.219 E-06$ $1.478 E-06$ $2.022 E-06$ $2.899 E-06$
$3.027 \mathrm{E}-06$ $2.901 E-06$ $2.678 \varepsilon-06$ $3.574 E-06$ $4.319 E-06$ 6. $287 E-06$ $2.236 \mathrm{E}-05$ 1.567E-05

$2.682 \mathrm{E}-06$ $4.545 \mathrm{E}-06$ $6.501 \mathrm{E}-06$ $2.312 \mathrm{E}-05$ $6.400 E-05$ I. $582 E-05$ ANGLE 7 ANGLE 8 $2.540 \mathrm{E}-08-2.410 \mathrm{E}-08$ $-5.284 E-09$ $0.093 \mathrm{E}-09 \quad 2.247 \mathrm{E}-0$ $2.742 E-08 \quad 5.136 E-08$ $4.234 \mathrm{E}-08$ $3.472 \mathrm{E}-08$ $-3.041 \mathrm{E}-08$ $-2.638 E-08$ $1.160 \mathrm{E}-06$ $3.193 \mathrm{E}-06$
$5.767 \mathrm{E}-06$ $5.767 \mathrm{E}-06$
$1.662 \mathrm{E}-0.05$ $4.948 E-05$ $4.948 E-05$
$1.423 \mathrm{E}-05$

ANGLE 16 $M U=0.9446$ $3.391 \mathrm{E}-07$ $3.210 \mathrm{E}-06$ $3.520 E-06$ $4.010 \mathrm{E}-06$ $4.137 E-06$ $4.062 E-06$ $3.820 E-06$ $3.470 E-06$ $3.006 E-06$ $2.714 \mathrm{E}-06$ $2.750 E-06$ $4.128 \mathrm{E}-06$ $4.537 E-06$ $6.729 E-06$ $2.370 E-05$ 1.594E-05 $U=0.9894$ $2.113 E-06$ $3.203 E-06$ $1.523 E-05$ $9.930 E-06$ $7.212 E-0$ $5.996 \mathrm{E}-06$ $4.789 \mathrm{E}-\mathrm{C} 6$ $3.690 \mathrm{E}-06$ $2.715 E-06$ $2.435 \mathrm{E}-06$ $2.862 \mathrm{E}-06$ $4.458 \mathrm{E}-06$ $4.413 \mathrm{E}-06$

ANGLE 9 $M U=-0.0950$ . 2.132E 5.004E-09 $3.797 \mathrm{E}-09$ $1.087 \mathrm{E}-0$ $1.196 \mathrm{E}-08$ $2.839 E-0$ $-2.652 E-08$ CEE $6.726 E-09$ $9.659 E-08 \quad 4.841 E-08$ $2.415 E-06$ $3.632 \mathrm{E}-06 \quad 3.760 \mathrm{E}-06$ $5.516 \mathrm{E}-06 \quad 5.424 \mathrm{E}-06$ $1.725 \mathrm{E}-05 \quad 1.798 \mathrm{E}-05$ $5.097 \mathrm{E}-05 \quad 5.266 \mathrm{E}-05$ ANGLE 17 SCALAR 
(GAMMAS/MEV/STERADIAN/SOURCE NEUTRON)

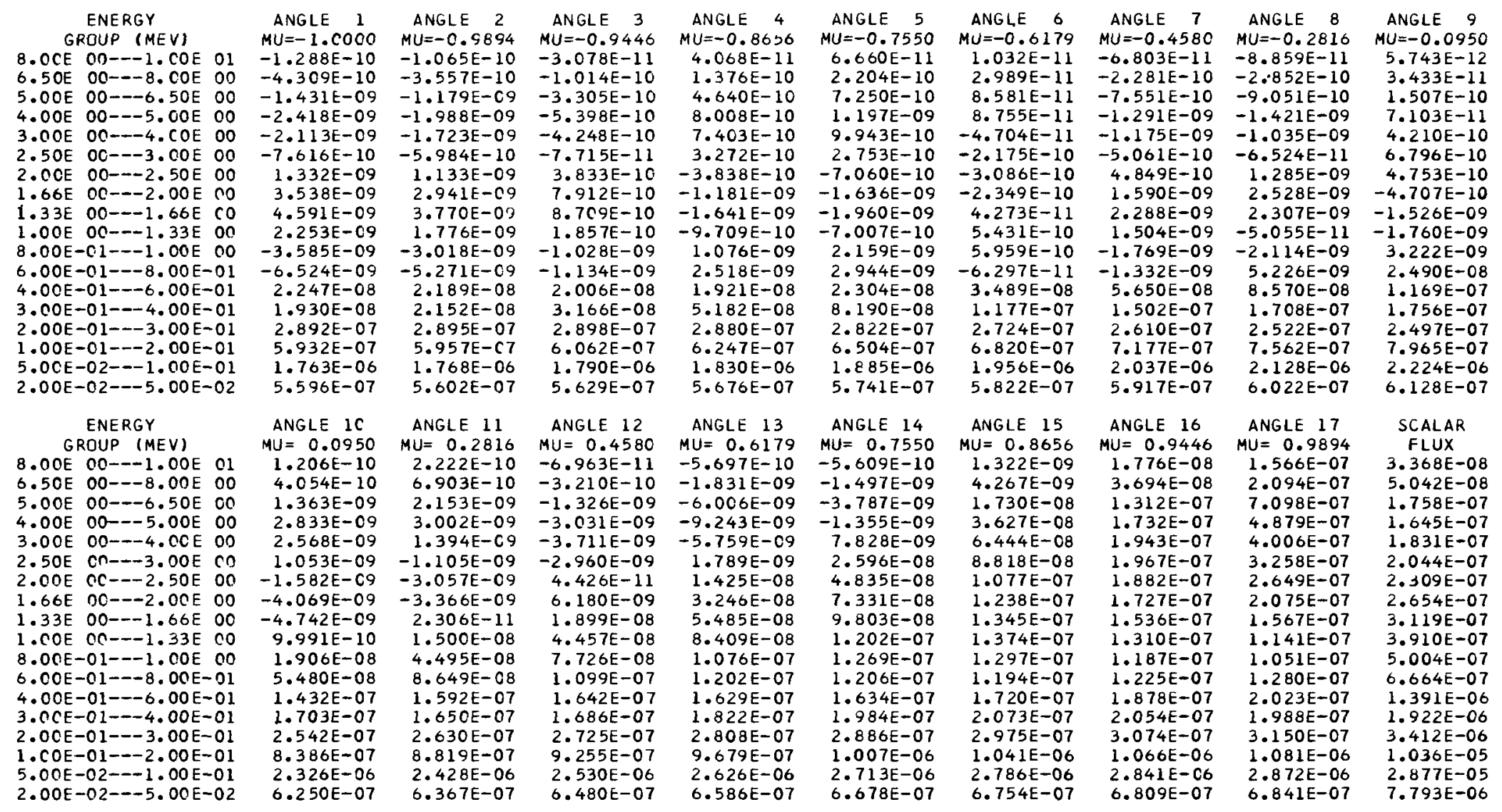




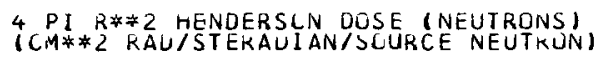

CUSINE
$-1.00 C 00 E O 0$
$-9.88401 E-01$
$-9.44575 E-01$
$-8.65631 E-C 1$
$-7.55044 E-01$
$-6.17876 E-C 1$
$-4.58617 E-01$
$-2.81605 E-U 1$
$-9.50125 E-02$
$9.50125 E-02$
$2.81605 E-01$
$4.58017 E-01$
$6.17876 E-U 1$
$7.55044 E-U 1$
$8.65631 E-01$
$9.44575 E-01$
$9.89401 E-01$

TUTAL

CUSINE

- 1. Gocuir ou $-9.894 \mathrm{C} 1 \mathrm{E}-\mathrm{J} 1$ $-9.44575 E-C 1$ $-8.65631 c-01$ $-7.55044 E-01$ $-0.17870 \mathrm{E}-\mathrm{Cl}$ $-4.58017 \mathrm{c}-01$ $-2.81605 E-01$ $9.5012 \mathrm{BE}-\mathrm{C} 2$ $9.50125 \mathrm{E}-\mathrm{C}_{2}$ $2.81005 E-01$ $4.58017 E-01$ $0.17876 E-01$ $8.65631 E-01$ $9.44575 E-01$
$9.894 \mathrm{Clt}-01$

TOTAL

$38 C 0.0$
$4.497 \mathrm{E}-17$
$4.511 \mathrm{E}-17$
$4.572 \mathrm{E}-17$
$4.696 \mathrm{E}-17$
$4.858 \mathrm{E}-17$
$5 .(96 \mathrm{E}-17$
$5.410 \mathrm{E}-17$
$5.817 \mathrm{E}-17$
$6.331 \mathrm{E}-17$
$6.979 \mathrm{E}-17$
$7.794 \mathrm{E}-17$
$8.827 \mathrm{E}-17$
$1.415 \mathrm{E}-16$
$1.184 \mathrm{E}-16$
$1.405 \mathrm{E}-16$
$1.695 \mathrm{E}-16$
$2.100 \mathrm{E}-16$

$2.100 E-16$

$40 C 0 . C$

1. 75CE-17 $1.755 \mathrm{E}-17$ $1.779 \mathrm{E}-17$ $1.823 \mathrm{E}-17$ $1.890 E-17$ $1.983 \mathrm{E}-17$ $2.1 C 5 E-17$ $2.264 E-17$ $2.404 E-17$ 3. $C 35 \mathrm{E}-17$ $3.438 \mathrm{E}-17$ $3.954 \mathrm{E}-17$
$4.617 \mathrm{E}-17$ $4.617 \mathrm{E}-17$ $5.612 \mathrm{E}-17$ $0.612 \mathrm{E}-17$
$8.162 \mathrm{E}-17$

$9.797 \mathrm{E}-16$

$3.816 \mathrm{E}-16$

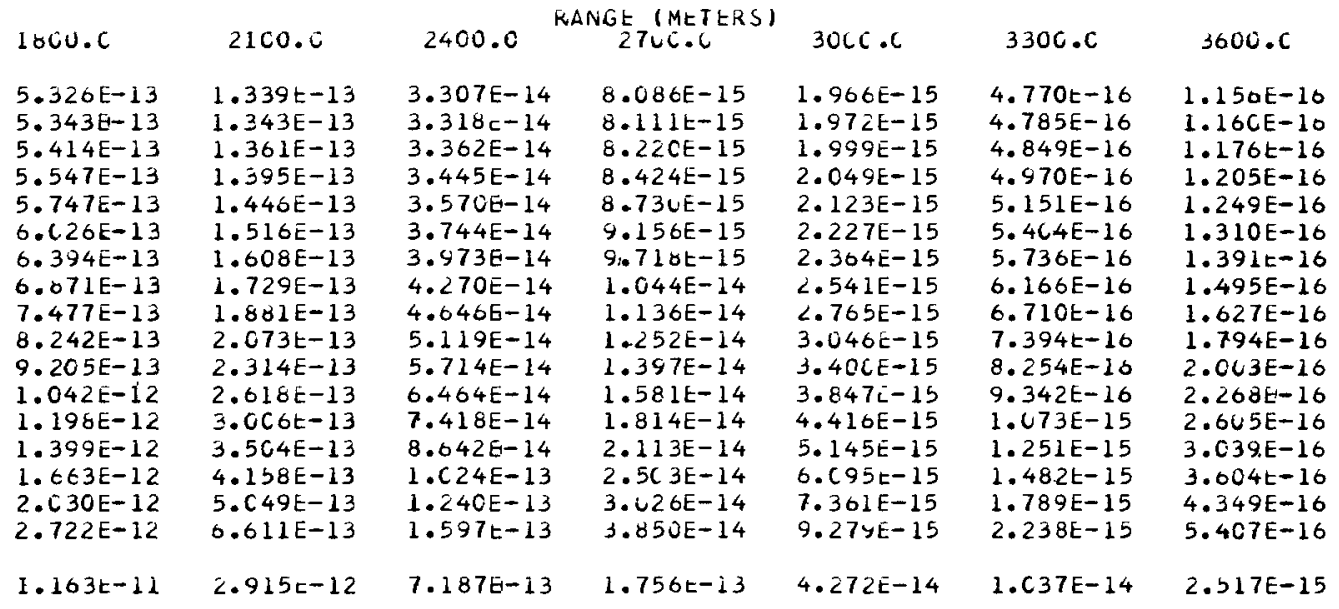

RANGE (METEKS)

FISSION SOUKCE

$\begin{array}{llll}6.812 E-18 & 2.650 E-18 & 1.017 E-18 & 3.469 E-19 \\ 6.833 E-18 & 2.658 E-18 & 1.021 E-18 & 3.484 E-19 \\ 6.925 E-18 & 2.694 E-18 & 1.035 E-18 & 3.551 E-19 \\ 7.698 E-18 & 2.762 E-18 & 1.062 E-18 & 3.674 E-19 \\ 7.359 E-18 & 2.864 E-18 & 1.104 E-18 & 3.861 E-19 \\ 7.721 E-18 & 3.065 E-18 & 1.16 C E-18 & 4.119 E-19 \\ 8.199 E-18 & 3.192 E-18 & 1.235 E-18 & 4.453 E-19 \\ 8.016 E-18 & 5.434 E-18 & 1.336 E-18 & 4.871 E-19 \\ 9.598 E-18 & 3.739 E-18 & 1.454 E-18 & 5.377 E-19 \\ 1 .(58 E-17 & 4.124 E-18 & 1.6 C 2 E-18 & 5.996 E-19 \\ 1.182 E-17 & 4.0 C 9 E-18 & 1.792 E-18 & 6.763 E-19 \\ 1.34 U E-17 & 5.226 E-18 & 2.034 E-18 & 7.729 E-19 \\ 1.542 E-17 & 6.616 E-18 & 2.344 E-18 & 8.960 E-19 \\ 1.002 \mathrm{C}-17 & 7.036 E-18 & 2.745 E-18 & 1.054 E-18 \\ 2.140 E-17 & 8.303 E-18 & 3.266 E-18 & 1.060 E-18 \\ 2.582 E-17 & 1.049 E-17 & 3.942 E-18 & 1.525 E-18 \\ 3.176 E-17 & 1.237 E-17 & 4.822 E-18 & 1.865 E-18 \\ 1.487 E-10 & 5.798 E-17 & 2.253 E-17 & 8.427 E-18\end{array}$




\begin{tabular}{|c|c|c|c|c|c|c|c|}
\hline COSINE & $1800 . \mathrm{C}$ & 2100.0 & 2400.0 & $\begin{array}{l}\text { NuE (METERS) } \\
27 \mathrm{CO} 0.0\end{array}$ & 36.00 .0 & 3300.0 & 3000.0 \\
\hline $\begin{array}{l}1 . C 0000 \mathrm{E} O 0 \\
9.89401 \mathrm{E}-01 \\
9.44575 \mathrm{E}-01 \\
8.65631 \mathrm{E}-01 \\
7.55044 \mathrm{E}-01 \\
6.17876 \mathrm{E}-01 \\
4.58017 \mathrm{E}-01 \\
2.81605 \mathrm{E}-01 \\
9.50125 \mathrm{E}-02 \\
9.50125 \mathrm{E}-02 \\
2.81605 \mathrm{E}-01 \\
4.58017 \mathrm{E}-01 \\
6.17876 \mathrm{E}-01 \\
7.55044 \mathrm{E}-01 \\
8.65631 \mathrm{E}-01 \\
9.44575 \mathrm{E}-01 \\
9.89401 \mathrm{E}-01\end{array}$ & $\begin{array}{l}1.443 \mathrm{E}-12 \\
1.447 \mathrm{E}-12 \\
1.462 \mathrm{E}-12 \\
1.490 \mathrm{E}-12 \\
1.531 \mathrm{E}-12 \\
1.587 \mathrm{E}-12 \\
1.66 \mathrm{E}-12 \\
1.752 \mathrm{E}-12 \\
1.866 \mathrm{E}-12 \\
2.006 \mathrm{E}-12 \\
2.177 \mathrm{E}-12 \\
2.386 \mathrm{E}-12 \\
2.643 \mathrm{E}-12 \\
2.962 \mathrm{E}-12 \\
3.366 \mathrm{E}-12 \\
3.903 \mathrm{E}-12 \\
4.872 \mathrm{E}-12\end{array}$ & $\begin{array}{l}3.631 \mathrm{E}-13 \\
3.64 C \mathrm{E}-13 \\
3.677 \mathrm{E}-13 \\
3.747 \mathrm{E}-13 \\
3.851 \mathrm{E}-13 \\
3.992 \mathrm{E}-13 \\
4.175 \mathrm{E}-13 \\
4.407 \mathrm{E}-13 \\
4.692 \mathrm{E}-13 \\
5.043 \mathrm{E}-13 \\
5.470 \mathrm{E}-13 \\
5.991 \mathrm{E}-13 \\
6.630 \mathrm{E}-13 \\
7.42 \mathrm{CE}-13 \\
8.417 \mathrm{E}-13 \\
9.723 \mathrm{E}-13 \\
1.192 \mathrm{E}-12\end{array}$ & $\begin{array}{l}8.935 E-14 \\
8.957 \mathrm{E}-14 \\
9.650 E-14 \\
9.222 \mathrm{E}-14 \\
9.477 \mathrm{E}-14 \\
9.825 \mathrm{E}-14 \\
1.028 \mathrm{E}-13 \\
1.085 \mathrm{E}-13 \\
1.155 \mathrm{E}-13 \\
1.241 \mathrm{E}-13 \\
1.346 \mathrm{E}-13 \\
1.474 \mathrm{E}-13 \\
1.631 \mathrm{E}-13 \\
1.824 \mathrm{E}-13 \\
2.067 \mathrm{E}-13 \\
2.384 \mathrm{E}-13 \\
2.886 \mathrm{E}-13\end{array}$ & $\begin{array}{l}2.173 E-14 \\
2.178 E-14 \\
2.200 E-14 \\
2.242 E-14 \\
2.304 E-14 \\
2.389 E-14 \\
2.499 E-14 \\
2.638 E-14 \\
2.8 C 9 E-14 \\
3.018 E-14 \\
3.273 E-14 \\
3.585 E-14 \\
3.967 E-14 \\
4.438 E-14 \\
5.030 E-14 \\
5.794 E-14 \\
6.955 E-14\end{array}$ & $\begin{array}{l}5.248 E-15 \\
5.260 E-15 \\
5.315 E-15 \\
5.416 E-15 \\
5.567 E-15 \\
5.772 E-15 \\
6.038 E-15 \\
6.374 E-15 \\
6.788 E-15 \\
7.295 E-15 \\
7.913 E-15 \\
8.667 E-15 \\
9.594 E-15 \\
1.074 E-14 \\
1.218 E-14 \\
1.402 E-14 \\
1.673 E-14\end{array}$ & $\begin{array}{l}1.264 \mathrm{E}-15 \\
1.267 \mathrm{E}-15 \\
1.280 \mathrm{E}-15 \\
1.304 \mathrm{E}-15 \\
1.341 \mathrm{E}-15 \\
1.390 \mathrm{E}-15 \\
1.454 \mathrm{E}-15 \\
1.535 \mathrm{E}-15 \\
1.635 \mathrm{E}-15 \\
1.758 \mathrm{E}-15 \\
1.907 \mathrm{E}-15 \\
2.090 \mathrm{E}-15 \\
2.315 \mathrm{E}-15 \\
2.593 \mathrm{E}-15 \\
2.942 \mathrm{E}-15 \\
3.389 \mathrm{E}-15 \\
4.023 \mathrm{E}-15\end{array}$ & $\begin{array}{l}3.04 \mathrm{CE}-16 \\
3.04 \mathrm{GE}-16 \\
3.08 \mathrm{CE}-16 \\
3.138 \mathrm{E}-16 \\
3.226 \mathrm{E}-16 \\
3.340 \mathrm{E}-16 \\
3.500 \mathrm{E}-16 \\
3.690 \mathrm{E}-16 \\
3.938 \mathrm{E}-16 \\
4.234 \mathrm{E}-16 \\
4.595 \mathrm{E}-16 \\
5.03 .7 \mathrm{E}-16 \\
5.582 \mathrm{E}-16 \\
6.258 \mathrm{E}-16 \\
7.108 \mathrm{E}-16 \\
8.192 \mathrm{E}-16 \\
9.686 \mathrm{E}-16\end{array}$ \\
\hline TOTAL & $2.699 E-11$ & $6.770 E-12$ & $1.664 E-12$ & $4.047 E-13$ & $9.783 E-14$ & $2.359 E-14$ & $5.684 E-15$ \\
\hline
\end{tabular}

COSINE

$-1.00000 E 00$ $-9.89401 E-01$ $-9.44575 E-01$ $-8.65631 \mathrm{E}-01$ $-7.55044 E-01$ $-6.17876 E-O 1$ $-2.81605 E-01$ $9.50125 \mathrm{E}-\mathrm{G} 2$ $2.81605 \mathrm{E}-\mathrm{Cl}$ 4.58017E-G1 7.55044E-CI 8.6563iE-U1 $9.89401 E-C 1$

TOIAL

$\begin{array}{lc}3800.0 & 400 C . C \\ 1.176 E-16 & 4.555 E-17 \\ 1.179 E-16 & 4.566 E-17 \\ 1.192 E-16 & 4.613 E-17 \\ 1.214 E-16 & 4.762 E-17 \\ 1.248 E-16 & 4.834 E-17 \\ 1.295 E-16 & 5.013 E-17 \\ 1.355 E-16 & 5.246 E-17 \\ 1.431 E-16 & 5.541 E-17 \\ 1.524 E-16 & 5.904 E-17 \\ 1.639 E-16 & 6.351 E-17 \\ 1.780 E-16 & 6.896 E-17 \\ 1.952 E-16 & 7.565 E-17 \\ 2.164 E-16 & 8.392 E-17 \\ 2.427 E-16 & 9.421 E-17 \\ 2.759 E-16 & 1 . C 72 E-16 \\ 3.181 E-16 & 1.236 E-16 \\ 3.752 E-16 & 1.455 E-16 \\ 2.202 E-15 & 8.535 E-16\end{array}$

\section{RANGE (METERS)} 4200.0 $44 \mathrm{C0} 0$

\section{$1.764 \mathrm{E}-17$}

$1.769 \mathrm{E}-17$

$1.787 \mathrm{E}-17$
$1.822 \mathrm{E}-17$

$1.822 \mathrm{E}-17$
$1.873 \mathrm{E}-17$

$1.873 E-17$
$1.942 E-17$

$1.942 E-17$
$2.033 E-17$

$2.147 E-17$

$2.289 E-17$

$2.462 \mathrm{E}-17$
$2.674 \mathrm{E}-17$

$2.935 \mathrm{E}-17$

$3.258 \mathrm{E}-17$

$3.659 \mathrm{E}-17$

$4.807 \mathrm{E}-17$

$5.648 E-17$

3. $311 E-16$

\section{$6.831 E-18$}

$6.848 \mathrm{E}-18$

$6.92 \mathrm{CE}-18$

7.054E-18

$7.253 E-18$

7.525E-18

$7.877 E-18$

.

$9.548 E-18$

$1.037 \mathrm{E}-17$

$1.139 E-17$

$1.265 \mathrm{E}-17$

$1.422 E-17$

$1.871 \mathrm{E}-17$

$2.195 \mathrm{E}-17$

1. $285 E-16$
4600.0

2. $614 \mathrm{E}-18$ $2.621 E-18$ $2.65 \mathrm{CE}-18$ $2.704 \mathrm{E}-18$ $2.783 t-18$ 2. . $206 E-18$ (3) . 4.010E-18 政 $7.276 \mathrm{E}-18$ $8.522 E-18$

$4.964 E-17$

$.952 E-19$

$8.983 E-19$

$9.113 E-29$

$9.355 \mathrm{E}-19$

$9.714 \mathrm{E}-19$

$1.020 E-18$

$1.082 \mathrm{E}-18$

$1.159 E-18$

$1.249 E-10$
$1.358 E-18$

$1.358 E-18$

$1.488 \mathrm{E}-18$

$1.648 \mathrm{E}-18$

$1.844 E-18$

$2.088 E-18$

$2.395 E-18$

3. $261 \mathrm{E}-18$

1. $827 \mathrm{E}-17$ 
$\operatorname{COS} I N E$

$-1.60000 E \cup U$
$-9.89401 E-01$
$-9.44575 E-U 1$
$-8.05631 E-01$
$-7.55044 E-01$
$-6.17870 E-01$
$-4.58017 E-01$
$-2.81605 E-01$
$-9.50125 E-02$
$9.50125 E-C 2$
$2.81005 E-01$
$4.58017 E-01$
$6.17870 E-01$
$7.55044 E-01$
$8.05051 E-01$
$9.44575 E-01$
$9.89401 E-01$

TOTAL

CUSINL

-1.00000 U UC $-9.89401 E-U 1$ $-9.44575 \mathrm{t}-\mathrm{C}$ $-8.65631 \mathrm{E}-01$ $-7.55044 \mathrm{E}-\mathrm{C}$. $-4.58017 \mathrm{E}-01$ $-2.81605 t-01$

$9.50125 c-02$

9.50125E-U2

$2.81605 E-01$

$4.58017 E-01$

$6.17876 \mathrm{E}-01$

$7.55044 E-01$

$8.05631 E-01$

$0.89401=0 \mathrm{C}$

TOTAL
1800.0
$6.437 t-11$
$6.456 E-11$
$6.537 \mathrm{E}-11$
$6.689 \mathrm{E}-11$
$6.916 \mathrm{E}-11$
$7.231 \mathrm{E}-11$
$7.645 \mathrm{E}-11$
$8.177 \mathrm{E}-11$
$8.847 \mathrm{E}-11$
$9.686 \mathrm{E}-11$
$1.073 \mathrm{E}-10$
$1.204 \mathrm{E}-10$
$1.369 \mathrm{E}-10$
$1.580 \mathrm{E}-10$
$1.855 \mathrm{E}-10$
$2.231 \mathrm{E}-10$
$2.935 \mathrm{E}-10$

$\angle 100.0$

$1.618 \mathrm{E}-11$ $1.623 t-11$ $1.682 \mathrm{t}-11$ $1.7395-11$ $1.818 E-11$ $1.922 \mathrm{E}-11$ $2.056 E-11$ $2.224 \mathrm{E}-11$ $2.434 \mathrm{t}-11$ 6.090E-11 3. $C 23 E-11$ $3.434 E-11$ $3.957 E-11$ $4.636 E-11$ $5.552 E-11$ $7.142 E-11$

$1.346 E-C 9$

$3.374 t-10$

3800.0

$400 C .0$

$5.37 \in E-15$ $5.392 E-15$

$5.460 E-15$

$5.588 E-15$

$5.781 \mathrm{E}-15$

$6.047 \mathrm{E}-15$

$6.396 E-15$

$6.844 E-15$

$7.406 E-15$

$8.1 C 9 E-15$

$8.986 E-15$

1. $\operatorname{COSE}-14$

$1.148 E-14$

$1.325 E-14$

$1.554 E-14$

2. $264 E-14$

$1.123 E-13$

\begin{tabular}{|c|c|c|c|c|}
\hline$\angle 4 \cup 0.0$ & $\begin{array}{l}\text { RANGE (METERS) } \\
27 C(. .\end{array}$ & 3000.0 & 3360.0 & 3600.6 \\
\hline$E-1 L$ & $9.74 C E-13$ & $2.364 E-13$ & $5.722 t-14$ & $1.384 E-14$ \\
\hline & $9.760 \mathrm{E}-13$ & $2.371 E-13$ & $5.739 t-14$ & $1.388 \mathrm{E}-14$ \\
\hline $4.153 \mathrm{E}-12$ & $9.892 E-13$ & $2.401 \mathrm{E}-1 \mathrm{~s}$ & $5.81<t-14$ & $1.406 E-14$ \\
\hline $4.148 \mathrm{E}-12$ & $1.012 \mathrm{E}-12$ & $2.457 t-13$ & $5.948 t-14$ & $1.437 E-14$ \\
\hline 4. $\angle 89 E-12$ & $1.047 E-12$ & $2.541 \mathrm{E}-13$ & $6.153 E-14$ & $1.489 E-14$ \\
\hline$+85 E-12$ & $1.095 E-12$ & $2.658 t-13$ & $6.435 E-14$ & $1.557 \mathrm{E}-14$ \\
\hline & $1.158 E-12$ & $2.816 E-13$ & $6.805 k-14$ & $1.647 t-14$ \\
\hline$E-12$ & $1.238 E-12$ & 3. $C 06 E-13$ & $7.280 E-14$ & $1.76<E-14$ \\
\hline & $1.340 t-12$ & $3.252 E-13$ & $7.877 t-14$ & $1.90 C E-14$ \\
\hline $0.604 \mathrm{E}-12$ & $1.466 t-12$ & $3.500 t-13$ & $8.622 t-14$ & L.C $87 \mathrm{E}-14$ \\
\hline $0.648 \mathrm{E}-12$ & $1.023 E-12$ & $3.942 E-13$ & $9.549 E-14$ & $2.312 \mathrm{E}-14$ \\
\hline $7.453 t-12$ & $1.820 E-12$ & $4.42 \mathrm{CE}-13$ & $1.671 t-13$ & $2.594 E-14$ \\
\hline $8.465 c-1<$ & $2.067 \mathrm{E}-12$ & $5 . C 21 E-13$ & $1.217 \mathrm{E}-13$ & $2.951 t-14$ \\
\hline $9.748 E-12$ & $2.380 E-12$ & $5.785 \mathrm{E}-13$ & $1.403 t-13$ & $3.464 E-14$ \\
\hline $1.141 \mathrm{E}-11$ & $2.785 E-12$ & $6.770 E-13$ & $1.04 \mathrm{JE}-1 \mathrm{~S}$ & $3.99 \iota k-14$ \\
\hline $1.363 E-11$ & $3.32<E-12$ & $8.671 E-13$ & $1.959 E-12$ & $4.755 E-14$ \\
\hline & $4.162 E-12$ & $1.003 c-12$ & $2.417 t-13$ & $5.834 E-14$ \\
\hline $0.310 t-11$ & $2.028 E-11$ & $4.923 E-12$ & $1.193 E-12$ & $2.889 \mathrm{~g}-13$ \\
\hline
\end{tabular}

RANGE (METERS)

$4200.0 \quad 4460.6$

4600.0

4860.6 $2.095 E-15$ 8.144E-10 $3.163 E-10 \quad 1.214 E-16 \quad 4.147 t-17$ $2.122 E-15 \quad 8 . \angle 47 E-16 \quad 3 . \angle 04 E-16 \quad 1.23 C E-16 \quad 4.221 E-17$ $2.171 E-15 \quad 8.442 E-16 \quad 3.28 C E-16 \quad 1.261 E-16 \quad 4.360 E-17$ $2.240 E-15 \quad 8.733 E-16 \quad 3.394 E-16 \quad 1.3 C E E-16 \quad 4.568 E-17$ $2.35 C E-15$ 9.130E-16 $3.551 E-10 \quad 1.369 E-16 \quad 4.853 E-17$ $2.485 E-15 \quad 9.665 E-16 \quad 3.758 E-16 \quad 1.451 E-16 \quad 5.221 E-17$ $2.66 C E-15$ 1.C34E-15 4.C23E-16 $1.556 \mathrm{E}-16 \quad 5.078 \mathrm{E}-17$

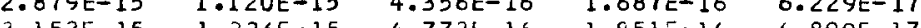
$3.152 E-15 \quad 1.226 E-15 \quad 4.772 E-16 \quad 1.851 \mathrm{E}-16 \quad 0.899 \mathrm{E}-17$ 3.92E 15 1.36OE-15 $5.293 E-16$ $2.055 E-16 \quad 7.721 E-17$ $4.923 E-15$ 1.527E-15 $2.947 t-10$ 2. $2.311 E-16 \quad 8.746 E-17$ $5.159 E-15$ 2. $011 E-15 \quad 7.842 E-16 \quad 3.655 E-16 \quad 1.004 E-16$ 6.054E-15 2.011E-15 $7.042 E-16$ 3.C5EE-16 $1.169 E-16$ $6.034 E-15$ 2.361E-15 $9.217 E-16 \quad 3.595 E-16 \quad 1.382 E-16$ $8.797 E-15 \quad 3.422 t-15 \quad 1.332 t-15 \quad 5.188 t-16 \quad$ L.Cu2t-16

$\angle . C U 2 t-16$

$9.570 E-10$ 


COSINE
$-1.00000 E C U$
$-9.89401 E-01$
$-9.44575 E-01$
$-8.65631 E-C 1$
$-7.55044 E-U 1$
$-0.17876 E-01$
$-4.58017 E-01$
$-2.81605 E-C 1$
$-9.50125 E-02$
$9.50125 E-C 2$
$2.81605 E-01$
$4.58017 E-01$
$6.17876 E-01$
$7.55044 E-01$
$8.65631 E-01$
$9.44575 E-C 1$
$9.89401 E-01$

TUTAL

COSINE

$-1.00000 E$ UO $-9.89401 E-C 1$ $-9.44575 E-01$ $-8.05631 \mathrm{E}-01$ $-7.55044 E-61$ $-6.17876 E-O$ $-2.816 C 5 \mathrm{C}-\mathrm{Cl}$ $-2.816 C 5 E-C 1$ $9.50125 \mathrm{E}-\mathrm{C} 2$ 9.50125E-C2 $4.580175-C 1$ $0.17876 \mathrm{E}-\mathrm{O}$

$0.17876 E-01$ $8.55044 E-01$

$8.44575 \mathrm{E}-0$ $9.89401 E-01$

IOTAL

\begin{tabular}{|c|c|c|c|c|c|c|}
\hline 1800.0 & 2100.0 & 2400.0 & $\begin{array}{l}\text { OE (METERS) } \\
2700.0\end{array}$ & 3000.0 & 3300.0 & $36 u c .0$ \\
\hline $\begin{array}{l}2.310 \mathrm{E}-13 \\
2.315 \mathrm{E}-13 \\
2.337 \mathrm{E}-13 \\
2.378 \mathrm{E}-13 \\
2.439 \mathrm{E}-13 \\
2.523 \mathrm{E}-13 \\
2.631 \mathrm{E}-13 \\
2.770 \mathrm{E}-13 \\
2.942 \mathrm{E}-13 \\
3.156 \mathrm{E}-13 \\
3.423 \mathrm{E}-13 \\
3.765 \mathrm{E}-13 \\
4.215 \mathrm{E}-13 \\
4.631 \mathrm{E}-13 \\
5.716 \mathrm{E}-13 \\
7.076 \mathrm{E}-13 \\
1.001 \mathrm{E}-12\end{array}$ & $\begin{array}{l}5.824 E-14 \\
5.837 \mathrm{E}-14 \\
5.893 \mathrm{E}-14 \\
5.997 \mathrm{E}-14 \\
6.151 \mathrm{E}-14 \\
6.363 \mathrm{E}-14 \\
6.639 \mathrm{E}-14 \\
6.989 \mathrm{E}-14 \\
7.425 \mathrm{E}-14 \\
7.966 \mathrm{E}-14 \\
8.643 \mathrm{E}-14 \\
9.508 \mathrm{E}-14 \\
1.065 \mathrm{E}-13 \\
1.221 \mathrm{E}-13 \\
1.445 \mathrm{E}-13 \\
1.783 \mathrm{E}-13 \\
2.45 \mathrm{CE}-13\end{array}$ & $\begin{array}{l}1.436 E-14 \\
1.439 E-14 \\
1.453 E-14 \\
1.478 E-14 \\
1.517 E-14 \\
1.569 E-14 \\
1.038 E-14 \\
1.725 E-14 \\
1.833 E-14 \\
1.907 E-14 \\
2.136 E-14 \\
2.352 E-14 \\
2.636 E-14 \\
3.631 E-14 \\
3.591 E-14 \\
4.427 E-14 \\
5.960 E-14\end{array}$ & $\begin{array}{l}3.495 \mathrm{E}-15 \\
3.5 \mathrm{C} 3 \mathrm{E}-15 \\
3.537 \mathrm{E}-15 \\
3.600 \mathrm{E}-15 \\
3.694 \mathrm{E}-15 \\
3.823 \mathrm{~L}-15 \\
3.991 \mathrm{E}-15 \\
4.2 \mathrm{C}-15 \\
4.470 \mathrm{E}-15 \\
4.3 \mathrm{C} 1 \mathrm{E}-15 \\
5.217 \mathrm{E}-15 \\
5.752 \mathrm{E}-15 \\
6.465 \mathrm{E}-15 \\
7.444 \mathrm{E}-15 \\
8.841 \mathrm{E}-15 \\
1.091 \mathrm{E}-14 \\
1.447 \mathrm{E}-14\end{array}$ & $\begin{array}{l}8.453 E-16 \\
8.472 E-16 \\
8.555 E-16 \\
8.7 C 8 E-16 \\
8.937 E-16 \\
9.251 E-16 \\
9.661 E-16 \\
1.018 E-15 \\
1.083 E-15 \\
1.164 E-15 \\
1.267 E-15 \\
1.399 E-15 \\
1.575 E-15 \\
1.819 E-15 \\
2.167 E-15 \\
2.676 E-15 \\
3.511 E-15\end{array}$ & $\begin{array}{l}2.038 \mathrm{E}-16 \\
2.043 \mathrm{E}-16 \\
2.063 \mathrm{E}-16 \\
2.100 \mathrm{E}-16 \\
2.156 \mathrm{E}-16 \\
2.232 \mathrm{E}-16 \\
2.332 \mathrm{E}-16 \\
2.459 \mathrm{E}-16 \\
2.618 \mathrm{E}-16 \\
2.816 \mathrm{E}-16 \\
3.067 \mathrm{E}-16 \\
3.393 \mathrm{E}-16 \\
3.831 \mathrm{E}-16 \\
4.437 \mathrm{E}-16 \\
5.302 \mathrm{E}-16 \\
6.558 \mathrm{E}-16 \\
8.528 \mathrm{E}-16\end{array}$ & $\begin{array}{l}4.91 \mathrm{dE}-17 \\
4.922 \mathrm{E}-17 \\
4.971 \mathrm{E}-17 \\
5.061 \mathrm{E}-17 \\
5.196 \mathrm{E}-17 \\
5.382 \mathrm{E}-17 \\
5.625 \mathrm{E}-17 \\
5.935 \mathrm{E}-17 \\
6.322 \mathrm{E}-17 \\
6.807 \mathrm{E}-17 \\
7.423 \mathrm{E}-17 \\
8.227 \mathrm{E}-17 \\
9.313 \mathrm{E}-17 \\
1.082 \mathrm{E}-16 \\
1.298 \mathrm{E}-16 \\
1.608 \mathrm{E}-16 \\
2.076 \mathrm{E}-16\end{array}$ \\
\hline $4.381 E-12$ & 1. $1 C 5 E-12$ & $2.73 G E-13$ & $6.075 E-14$ & $1.623 E-14$ & $3.935 E-15$ & $9.543 E-16$ \\
\hline
\end{tabular}

RANGE (METEKS)

$1.902 E-17 \quad 7.374 E-18$ $\begin{array}{ll}1.902 \mathrm{E}-17 & 7.374 \mathrm{E}-18 \\ 1.907 \mathrm{E}-17 & 7.391 \mathrm{E}-18\end{array}$ $1.907 \mathrm{E}-17$
$1.926 \mathrm{E}-17$
1.9617 $\begin{array}{ll}1.961 \mathrm{E}-17 & 7.601 \mathrm{E}-18 \\ 2.013 \mathrm{E}-17 & 7.806 \mathrm{E}-18\end{array}$ $2.086 \mathrm{E}-17 \quad 8.089 \mathrm{E}-18$ $2.080 E-17$. $0.085 E-18$ $2.101 \mathrm{E}-17 \mathrm{C}$ 2.302E-17 $2.453 E-17$ 2. 83 E 17 $1.246 \mathrm{E}-17$ $4.63 \mathrm{E}-17$ 1.416E-17 $5.080 \mathrm{E}-17 \mathrm{I}$ $6.302 E-17 \quad 2.472 E-17$ 8.101E-17 $3.165 \mathrm{E}-17$

$3.713 E-16 \quad 1.446 E-16$

\section{RANGE
$42 C 0.0$ 4400.0}

$2.800 \mathrm{E}-10$ $2.867 \mathrm{E}-18$ $2.095 \mathrm{E}-18$ $2.949 \mathrm{E}-18$ $3.129 E-18$ $3.139 E-18$ $3.283 E-18$ $3.46 \mathrm{dE}-18$ 3.700E-18 3. $4.356 E-10$ 5.85 E 18 $6.466 E-18$ $7.800 \mathrm{E}-18$ $9.7056-18$ $1.238 E-17$

$5.033 E-17$
$1.1 C 9 E-18$ $1.111 \mathrm{E}-18$ $1.123 \mathrm{E}-18$ $1.14 J E-18$ $1.175 \mathrm{E}-18$ $1.218 \mathrm{E}-18$ $1.275 E-18$ $1.347 t-18$ $1.438 E-18$ $1.553 \mathrm{E}-18$ $1.69 \mathrm{E}-18$ $1.159 E-18$ $2.159 E-18$ . C63E-18 $3.813 E-18$ $4.849 E-18$

$2.196 \mathrm{E}-17$
$460 \mathrm{C} . \mathrm{C}$

4. $250 E-19$ 4. $26 C E-19$ 4. $305 E-19$ $4.315 E-19$ $4.515 E-19$ $4.688 E-19$ 5.202E-19 $5.562 E-19$ 5.561E-19 $6.594 \mathrm{E}-19$

$7.362 \mathrm{E}-19$

$8.416 E-19$

$9.897 E-19$

$1.201 E-18$

$1.499 \mathrm{E}-18$

$8.527 E-18$
$4800 . \mathrm{C}$

$1.459 E-19$ $1.464 \mathrm{E}-19$ $1.484 \mathrm{E}-19$ 1. $522 \mathrm{E}-19$ 1. $657 \mathrm{E}-19$ $1.657 E-19$ 1.884E- 19 . 2. $222 \mathrm{E}-19$ 2.458E-19 . $3.189 \mathrm{E}-19$ $3.781 E-19$ $4.623 E-19$ $5.797 \mathrm{E}-19$ $7.356 E-19$ $3.164 E-18$ 


CUSINE
$-1.000 C O E O C$
$-9.89401 E-C 1$
$-9.44575 E-01$
$-8.65031 E-01$
$-7.55044 E-01$
$-6.17876 E-C 1$
$-4.58017 E-U 1$
$-2.816 C 5 E-01$
$-9.50125 E-C 2$
$9.50125 E-02$
$2.816 C 5 E-01$
$4.58017 E-01$
$0.17876 E-01$
$7.55444 E-01$
$8.65631 E-01$
$9.44575 E-01$
$9.89401 E-01$

TUTAL

COSINE

$-1.00000 E 00$ $-9.89401 E-01$ $-9.44575 E-G 1$ -8.65631 E-01 $-7.55044 E-01$ $-6.17876 E-01$ $-4.58017 E-01$ $-2.81605 E-01$ $9.5 C 125 E-02$ 9.50125E-02 $2.81605 E-01$
$4.58017 E-01$ $4.58017 \bar{E}-01$ $0.17876 E-01$
$7.55044 E-01$ 7.55044E-01 $8.65631 E-01$
$9.44575 E-01$ $9.894 C 1 E-01$

TOTAL

1800.0
$7.184 \mathrm{E}-12$
$7.206 \mathrm{E}-12$
$7.299 \mathrm{E}-12$
$7.474 \mathrm{E}-12$
$7.736 \mathrm{E}-12$
$8.1 \mathrm{C} 1 \mathrm{E}-12$
$8.582 \mathrm{E}-12$
$9.264 \mathrm{E}-12$
$9.990 \mathrm{E}-12$
$1.098 \mathrm{E}-11$
$1.222 \mathrm{E}-11$
$1.378 \mathrm{E}-11$
$1.576 \mathrm{E}-11$
$1.829 \mathrm{E}-11$
$2.162 \mathrm{E}-11$
$2.618 \mathrm{E}-11$
$3.466 \mathrm{E}-11$
$1.537 \mathrm{E}-10$

2100.0

1. 8 C6E -12 $1.812 E-12$ $1.835 E-12$ $1.879 \mathrm{E}-12$ $1.946 t-12$ 2. $\mathrm{C} 37 \mathrm{E}-12$ $2.158 \mathrm{E}-12$ $2.315 \mathrm{E}-1<$ 2.512E-12 $2.76 \mathrm{Ct}-12$ 3.C7CE-12 $3.460 E-12$ $3.953 \mathrm{E}-12$ $4.583 E-12$ $5.4 \mathrm{C5E}-12$ $6.513 \mathrm{E}-12$
$8.419 \mathrm{E}-12$ $3.853 E-11$

\section{0}

4000.0

6. $C 41 E-16$ 6. C 6UE- 16 $6.139 E-16$ $6.288 E-16$ $6.512 E-16$ . 7. 35 E 16 7.75 E-16 $8.424 E-16$ $9.259 E-16$ 1. $163 \mathrm{E}-15$ 1. $331 \mathrm{E}-15$ 1. $547 E-15$ 1.826E-15 $1.826 E-15$ $2.189 \mathrm{E}-15$
$2.679 \mathrm{E}-15$ 2.349E-16 $2.357 E-16$ $2.388 E-16$ $2.445 E-16$ 2.53 EE-16 $2.653 E-16$ $2.813 E-16$ $3.277 \mathrm{E}-16$ $3.277 E-16$ 3.603E-16 $4.011 E-16$ $5.527 E-16$ $0.18 E-16$ $7.125 \mathrm{E}-16$ $8.542 E-10$ 1. $442 E-15$

$1.292 \mathrm{E}-14 \quad 5 . \mathrm{C} 3 \mathrm{CE}-15$
RANGE (METERS)

$3000 . C$

3300.6

3600.0

$4.458 \mathrm{E}-13$ $4.530 E-13 \quad 1.1 C 7 E-13$ 2.647E-14 $6.416 E-15$ $1.174 E-13 \quad 2.852 E-14$ $1.2 \angle 9 L-13$ $1.302 E-13 \quad 3.166 E-14$ $5.715 E-13 \quad 1.397 E-13 \quad 3.396 E-14$ $1.516 \mathrm{E}-13$ 3.685E-14 $6.813 t-13 \quad 1.665 E-13 \quad 4.049 E-14$ $7.577 \mathrm{E}-13$ 1.852E-13 4.5C $3 \mathrm{E}-14$ $8.537 E-13$ 2.087E-13 5.075E-14 $9.751 \mathrm{E}-13 \quad 2.384 \mathrm{E}-13 \quad 5.299 \mathrm{E}-14$ $1.130 E-12$ 2.762E-13 6.723E-14 $1.331 E-12$ 3.253E-13 7.92CE-14 $1.600 E-12 \quad 3.9 C 3 E-13 \quad 9.496 E-14$ $2.034 \mathrm{E}-12 \quad 4.963 \mathrm{E}-13 \quad 1.182 \mathrm{E}-13$ y. $498 E-12$

C. 32OE-12

$5.64 c t-13$

RANGE (METERS)

$4 \angle 50.0 \quad 440 \mathrm{C} .0$

$400 \mathrm{C} .0$ 3. $254 E-17$ G. $142 E-17$ $9.169 \mathrm{E}-17$ $9.290 E-17$ $9.516 \mathrm{E}-17$ $9.855 E-17$ $1.0335-16$ $1.095 E-16$ $1.175 E-10$ $1.276 E-16$ $1.403 E-16$ $1.562 E-16$ $1.764 E-16$ $2.353 E-16$ $2.782 E-16$ $3.336 \mathrm{E}-16$ $4.259 E-10$ 3. $365 \mathrm{E}-17$ 3. 7 CEE -17 $3.734 \mathrm{E}-17$ $3.834 \mathrm{E}-17$ $4.261 \mathrm{E}-17$ $4.261 E-17$ $4.574 E-17$ 4. $968 E-17$ $6.087 \mathrm{E}-17$ $6.0 B 7 E-17$ $7.887 E-17$ 9.887E-17 $1.087 \mathrm{E}-16$ 1. $304 E-16$ $1.583 E-16$

1.960E-15 7.641E-16
$1.554 E-15$
$1.559 E-15$ 1. $579 \mathrm{E}-15$ $1.618 \mathrm{E}-15$ $1.675 E-15$ $1.755 E-15$ $1.86 U E-15$ $1.996 \mathrm{E}-15$ $2.166 E-15$ $2.381 \mathrm{E}-15$ $2.649 \mathrm{gE}-15$ $2.988 \mathrm{E}-15$ $3.418 \mathrm{E}-15$ $3.970 E-15$ $4.684 t-15$ $5.614 \mathrm{E}-15$ $6.895 \mathrm{E}-15$ $3.320 \mathrm{E}-14$ 


COSINE
$-1.00 C 00 E 00$
$-9.894 C 1 E-01$
$-9.44575 E-01$
$-8.65631 E-01$
$-7.55044 E-01$
$-6.17876 E-01$
$-4.58017 E-01$
$-2.816 C 5 E-C 1$
$-9.50125 E-C 2$
$9.50125 E-02$
$2.81605 E-01$
$4.58017 E-01$
$6.17876 E-01$
$7.55044 E-01$
$8.65631 E-01$
$9.44575 E-01$
$9.89401 E-01$

TOTAL

COSINE

$-1.00000 E-00$
$-9.894 C 1 \mathrm{E}-01$
$-9.44575 \mathrm{E}-01$
$-8.65631 \mathrm{E}-01$
$-7.55044 \mathrm{E}-01$
$-6.17876 \mathrm{E}-01$
$-4.58017 \mathrm{E}-\mathrm{Cl}$
$-2.81605 \mathrm{E}-01$
$-9.56125 \mathrm{E}-02$
$9.50125 \mathrm{E}-0.2$
$2.81605 \mathrm{E}-01$
$4.58017 \mathrm{E}-01$
$6.17876 \mathrm{E}-01$
$7.55044 \mathrm{E}-01$
$8.65631 \mathrm{E}-01$
$9.44575 \mathrm{E}-01$
$9.894 \mathrm{C}-01$

TOTAL

\begin{tabular}{|c|c|c|c|c|c|c|}
\hline 800.0 & 2100.0 & 2400.0 & $\begin{array}{l}\text { GE }(M E T E R S) \\
2700.0\end{array}$ & $300 \cdot 0.0$ & 3300.0 & 3600.0 \\
\hline $\begin{array}{l}9.589 \mathrm{E}-12 \\
9.611 \mathrm{E}-12 \\
9.704 \mathrm{E}-12 \\
9.877 \mathrm{E}-12 \\
1.014 \mathrm{E}-11 \\
1.049 \mathrm{E}-11 \\
1.096 \mathrm{E}-11 \\
1.156 \mathrm{E}-11 \\
1.230 \mathrm{E}-11 \\
1.324 \mathrm{E}-11 \\
1.442 \mathrm{E}-11 \\
1.593 \mathrm{E}-11 \\
1.791 \mathrm{E}-11 \\
2.056 \mathrm{E}-11 \\
2.426 \mathrm{E}-11 \\
2.969 \mathrm{E}-11 \\
4.061 \mathrm{E}-11\end{array}$ & $\begin{array}{l}2.421 \mathrm{E}-12 \\
2.427 \mathrm{E}-12 \\
2.45 \mathrm{CE}-12 \\
2.494 \mathrm{E}-12 \\
2.56 \mathrm{E}-12 \\
2.650 \mathrm{E}-12 \\
2.768 \mathrm{E}-12 \\
2.919 \mathrm{E}-12 \\
3.109 \mathrm{E}-12 \\
3.346 \mathrm{E}-12 \\
3.644 \mathrm{E}-12 \\
4.025 \mathrm{E}-12 \\
4.523 \mathrm{E}-12 \\
5.189 \mathrm{E}-12 \\
6.110 \mathrm{E}-12 \\
7.439 \mathrm{E}-12 \\
9.875 \mathrm{E}-12\end{array}$ & $\begin{array}{l}5.977 \mathrm{E}-13 \\
5.990 \mathrm{E}-13 \\
6.049 \mathrm{E}-13 \\
6.158 \mathrm{E}-13 \\
6.320 \mathrm{E}-13 \\
6.544 \mathrm{E}-13 \\
6.837 \mathrm{E}-13 \\
7.212 \mathrm{E}-13 \\
7.682 \mathrm{E}-13 \\
8.271 \mathrm{E}-13 \\
9.012 \mathrm{E}-13 \\
9.960 \mathrm{E}-13 \\
1.120 \mathrm{E}-12 \\
1.286 \mathrm{E}-12 \\
1.514 \mathrm{E}-12 \\
1.838 \mathrm{E}-12 \\
2.390 \mathrm{E}-12\end{array}$ & $\begin{array}{l}1.457 \mathrm{E}-13 \\
1.460 \mathrm{E}-13 \\
1.475 \mathrm{E}-13 \\
1.501 \mathrm{E}-13 \\
1.541 \mathrm{E}-13 \\
1.596 \mathrm{E}-13 \\
1.668 \mathrm{E}-13 \\
1.760 \mathrm{E}-13 \\
1.876 \mathrm{E}-13 \\
2.021 \mathrm{E}-13 \\
2.2 \mathrm{C} 3 \mathrm{E}-13 \\
2.437 \mathrm{E}-13 \\
2.744 \mathrm{E}-13 \\
3.154 \mathrm{E}-13 \\
3.717 \mathrm{E}-13 \\
4.548 \mathrm{E}-13 \\
5.774 \mathrm{E}-13\end{array}$ & $\begin{array}{l}3.528 \mathrm{E}-14 \\
3.536 \mathrm{E}-14 \\
3.571 \mathrm{E}-14 \\
3.636 \mathrm{E}-14 \\
3.733 \mathrm{E}-14 \\
3.867 \mathrm{E}-14 \\
4.043 \mathrm{E}-14 \\
4.268 \mathrm{E}-14 \\
4.551 \mathrm{E}-14 \\
4.905 \mathrm{E}-14 \\
5.353 \mathrm{E}-14 \\
5.929 \mathrm{E}-14 \\
6.686 \mathrm{E}-14 \\
7.700 \mathrm{E}-14 \\
9.089 \mathrm{E}-14 \\
1.102 \mathrm{E}-13 \\
1.396 \mathrm{E}-13\end{array}$ & $\begin{array}{l}8.516 \mathrm{E}-15 \\
8.536 \mathrm{E}-15 \\
8.621 \mathrm{E}-15 \\
8.778 \mathrm{E}-15 \\
9.015 \mathrm{E}-15 \\
9.342 \mathrm{E}-15 \\
9.770 \mathrm{E}-15 \\
1.032 \mathrm{E}-14 \\
1.101 \mathrm{E}-14 \\
1.188 \mathrm{E}-14 \\
1.297 \mathrm{E}-14 \\
1.439 \mathrm{E}-14 \\
1.626 \mathrm{E}-14 \\
1.876 \mathrm{E}-14 \\
2.219 \mathrm{E}-14 \\
2.692 \mathrm{E}-14 \\
3.379 \mathrm{E}-14\end{array}$ & $\begin{array}{l}2.055 \mathrm{E}-15 \\
2.059 \mathrm{E}-15 \\
2.08 \mathrm{E}-15 \\
2.118 \mathrm{E}-15 \\
2.176 \mathrm{E}-15 \\
2.255 \mathrm{E}-15 \\
2.359 \mathrm{E}-15 \\
2.493 \mathrm{E}-15 \\
2.662 \mathrm{E}-15 \\
2.874 \mathrm{E}-15 \\
3.143 \mathrm{E}-15 \\
3.49 .1 \mathrm{E}-15 \\
3.952 \mathrm{E}-15 \\
4.573 \mathrm{E}-15 \\
5.421 \mathrm{E}-15 \\
6.579 \mathrm{E}-15 \\
8.199 . \mathrm{E}-15\end{array}$ \\
\hline $.839 E-10$ & $4.635 E-11$ & $1.145 E-11$ & $2.8 C O E-12$ & $6.807 E-13$ & $1.651 E-13$ & $4.004 E-14$ \\
\hline
\end{tabular}

RANGE (METERS)

$3800.6 \quad 4000.0$

$4200.0 \quad 4400.0$

$7.965 E-10 \quad 3.09 \mathrm{CE}-16$

$7.983 E-16$

$3.128 \mathrm{E}-16$

$8.212 \mathrm{E}-16$

$8.436 E-16$

$8.746 \mathrm{E}-16$

$9.153 E-16$

9.67 EE- 16

$1.033 \mathrm{E}-15$

$1.116 \mathrm{E}-15$

$1.222 \mathrm{E}-15$

$1.359 \mathrm{E}-15$

1. $540 \mathrm{E}-15$

$1.785 \mathrm{E}-15$

$2.120 E-15$

$2.574 \mathrm{t}-15$
$3.195 \mathrm{E}-15$

$3.186 \mathrm{E}-16$

$3.394 E-16$

$3.553 \mathrm{E}-16$

$3.757 E-16$

4. C15E-16

$4.340 E-16$

$4.754 E-16$

$5.292 E-16$

$6.008 \mathrm{E}-16$

$6.975 \mathrm{E}-16$

$8.295 E-16$

$1.008 \mathrm{E}-15$

$1.246 E-15$

$1.558 E-14$

$6.066 E-15$
$1.199 \mathrm{E}-16$

$1.202 E-16$

$1.214 \mathrm{E}-16$
$1.237 \mathrm{E}-16$

$1.271 \mathrm{E}-16$

$1.318 \mathrm{E}-16$

$1.46 C E-16$

$1.561 \mathrm{E}-16$

$1.688 \mathrm{E}-10$

$1.851 \mathrm{E}-16$

$2.063 \mathrm{E}-16$

$2.345 \mathrm{E}-16$

$2.728 \mathrm{E}-16$

$3.249 E-16$

$3.951 \mathrm{t}-10$

$4.869 E-16$
$2.364 E-15$
$4.653 E-17$

$4.664 t-17$

$4.711 E-17$

$4 \cdot 80 \mathrm{CE}-17$

$4.934 \mathrm{E}-17$
$5.119 \mathrm{E}-17$

$5.362 \mathrm{E}-17$

$5.676 \mathrm{E}-17$

$6.071 \mathrm{E}-17$

$6.571 \mathrm{E}-17$

$7.211 \mathrm{E}-17$

$8.046 \mathrm{E}-17$

$9.162 \mathrm{E}-17$

$1.067 \mathrm{E}-16$

$1.274 E-16$

$1.55 \mathrm{CE}-16$

$1.904 \mathrm{E}-16$

9. $217 E-16$
$46 C^{\circ C} \cdot C$

1. $784 \mathrm{E}-17$

$1.788 \mathrm{E}-17$

$1.808 \mathrm{E}-17$

$1.844 E-17$
$1.897 \mathrm{E}-17$

$1.972 \mathrm{E}-17$

$2.069 E-17$

$2.193 \mathrm{E}-17$

$2.547 \mathrm{E}-17$

2.799E-17

3. $13 \mathrm{CE}-17$

3. $572 \mathrm{E}-17$

$4.171 \mathrm{E}-17$

4.98GE-17

$6.077 \mathrm{E}-17$

$7.449 \mathrm{E}-17$

$3.579 E-16 \quad 1.328 E-16$

$6.114 E-18$

$6.135 E-18$

$6.384 E-18$

$6.628 E-18$

$0.966 E-18$

$7.404 E-18$
$7.951 E-18$

8.6C9E-18

$9.426 E-18$

$1.045 \mathrm{E}-17$

1. $354 \mathrm{E}-17$

1. $593 \mathrm{E}-17$

1. $919 E-17$

$2.881 E-17$

$1.328 E-16$ 


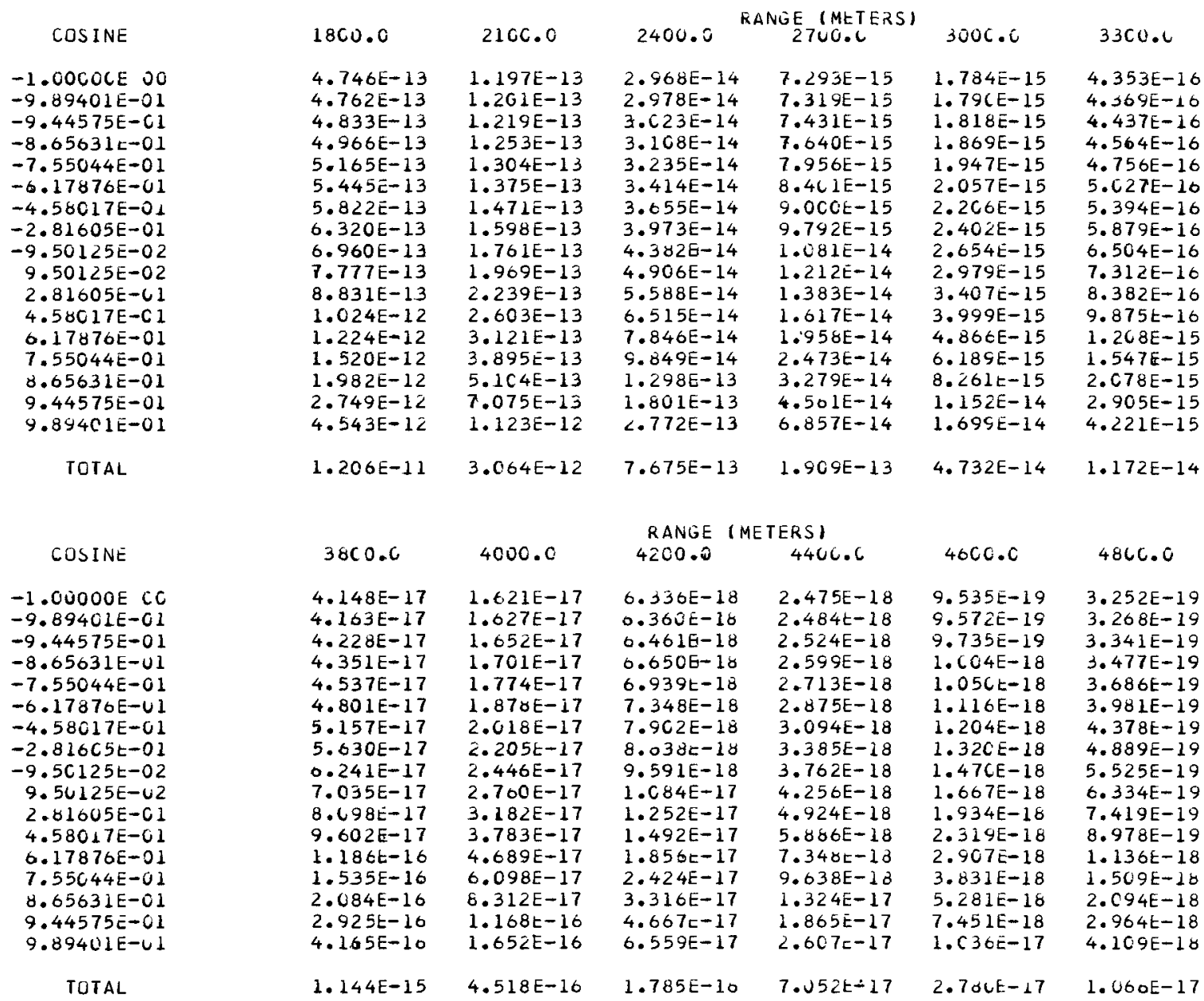




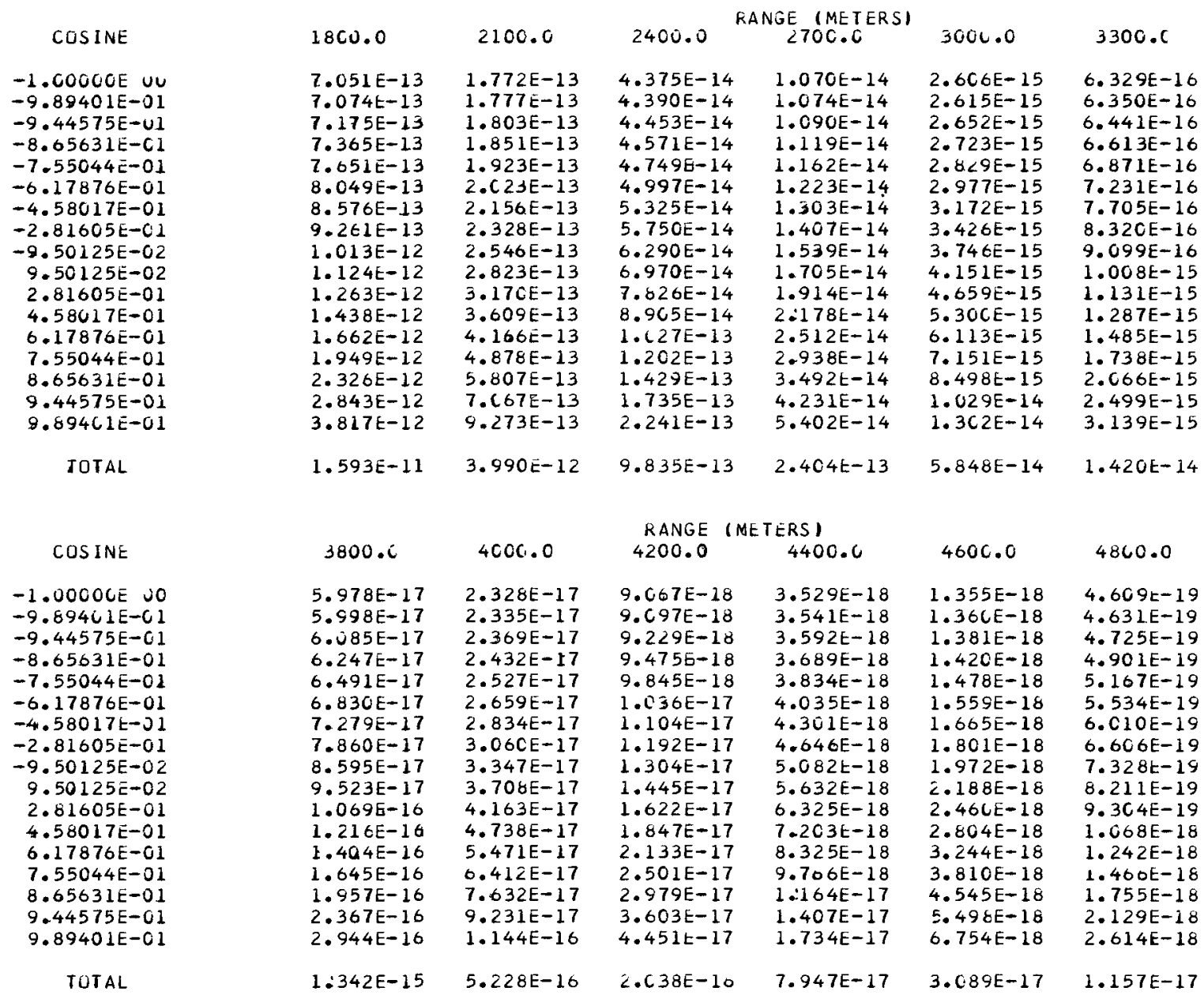




\begin{abstract}
CUSINE
$-1.00000 E$ OQ $-9.89401 \mathrm{E}-01$ $-9.44575 E-01$ $-7.55044 E-01$ $-6.17876 E-C 1$ $-4.58 \cup 17 E-01$ $-2.81605 t-41$ $-9.50125 E-C /$ $9.5 J 125 E-C 2$ 2.81605E-01 $4.58017 t-C 1$ $6.17876 \bar{c}-01$ $7.55 C 44 E-01$ 8.65631 E-01 $9.44575 E-01$ $9.894 C I E-C L$
\end{abstract}

TOTAL

COSINE

-1. CUCLOE CC $-9.89401 \mathrm{E}-01$ $-9.44575 E-01$ $-8.65631 c-C 1$ $-7.55044 E-C 1$ $-6.17876 E-U 1$ $-4.58017 \bar{c}-\mathrm{C} 1$ $-2.81005 E-61$ $9.50125 \mathrm{E}-02$ . . 2. $01605 E-01$ $4.58017 E-01$ $0.17876 E-01$ $8.55631 E-01$ $0.44575 E-C 1$ 9. 894 C IE-O 1

TOTAL

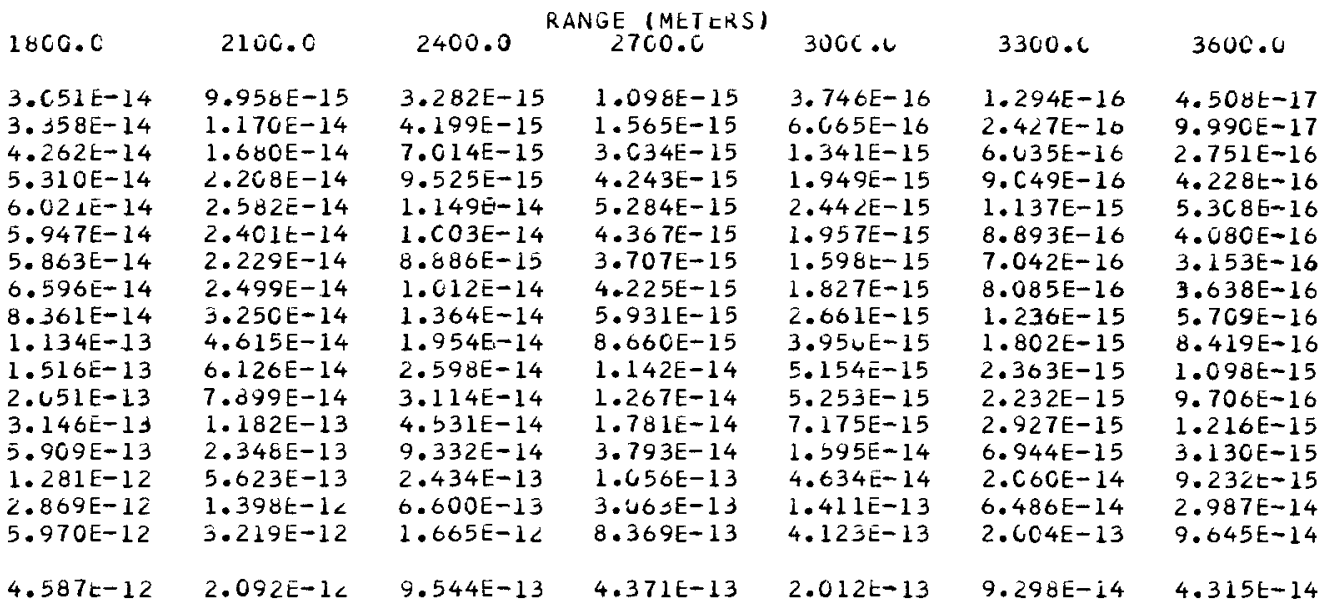

RANGE (METERS)

\begin{tabular}{|c|c|c|c|c|c|}
\hline 3800.0 & 4000.0 & $\begin{array}{l}\text { RANGE IN } \\
42 C 0.0\end{array}$ & $\begin{array}{l}\text { ERSI } \\
4400 . C\end{array}$ & $460 \mathrm{C} .0$ & 4800.0 \\
\hline & & $12 \mathrm{IE}$ & $4.004 \mathrm{E}-$ & $3.585 \mathrm{r}$ & $3.367 E-18$ \\
\hline & & -17 & & & \\
\hline & $.784 E-17$ & $.868 E-17$ & $3.539 t-17$ & $2.137 t-17$ & $1.176 \mathrm{E}-17$ \\
\hline-16 & $1.541 E-16$ & $9.3 \angle C E-17$ & $5.634 E-17$ & $3.366 E-17$ & $1.796 \mathrm{E}-17$ \\
\hline$\therefore 189 \mathrm{E}-16$ & $1.914 \mathrm{E}-16$ & $1.153 \mathrm{E}-16$ & $6.508 \mathrm{E}-17$ & $4.008 t-17$ & $2.074 t-17$ \\
\hline 16 & 1.46 & 8.767 & 5.24 & $3 E-17$ & $1.724 \mathrm{E}-17$ \\
\hline 1.8 & $E-16$ & 6.51 & 3.86 & 2. & $2 E-17$ \\
\hline 2.1 & -16 & $7.552 \mathrm{E}$ & 4.50 & & $1.580 \mathrm{E}-17$ \\
\hline 3.43 & $2.006 E-16$ & $1.245 E-16$ & 7.51 & $4.528 E-17$ & $2.629 t-17$ \\
\hline 5.0 & $3.076 E-16$ & $1.362 E-16$ & 1.12 & 6.8 & $E-17$ \\
\hline 6.607 & & 2.38 & 1.4 & & $1 E-17$ \\
\hline 5.633 & 3.29 & 1.946 & 1.1 & 6.7 & $3.964 E-17$ \\
\hline-16 & 3.886 & 2.221 & $1: 278$ & & $3 E-17$ \\
\hline & & $6.411 E-16$ & $3.804 E-16$ & $2.26 \mathrm{JE}-16$ & $1.346 \mathrm{E}-10$ \\
\hline $3 E-15$ & $3.266 E-15$ & $1.958 \bar{c}-15$ & $1.176 E-15$ & $7.111 E-16$ & $4.298 t-16$ \\
\hline$=-14$ & 1.06 & $6.384 \theta-15$ & $3.834 \mathrm{E}-$ & & $1.391 \mathrm{E}-15$ \\
\hline & & $2.199 E-14$ & $1.340 E-14$ & $8.162 E-15$ & $4.970 t-15$ \\
\hline & & & & & \\
\hline
\end{tabular}




\begin{tabular}{|c|c|c|c|c|c|c|}
\hline COSINE & $18 \mathrm{Co.0}$ & \multicolumn{5}{|c|}{$\begin{array}{l}\text { RANGE (METERS) } \\
27 C C .0\end{array}$} \\
\hline $\begin{array}{l}-1 . C O C O C E ~ O C \\
-9.89401 E-O 1 \\
-9.44575 E-01 \\
-8.65631 E-01 \\
-7.55444 E-01 \\
-6.17876 E-01 \\
-4.58017 E-01 \\
-2.81605 E-01 \\
-9.50125 E-02 \\
9.50125 E-02 \\
2.81605 E-01 \\
4.58017 E-01 \\
6.17876 E-01 \\
7.55044 E-01 \\
8.65631 E-C 1 \\
9.44575 E-C 1 \\
9.89401 E-01\end{array}$ & $\begin{array}{l}4.948 \mathrm{E}-12 \\
5.251 \mathrm{E}-12 \\
6.149 \mathrm{E}-12 \\
7.200 \mathrm{E}-12 \\
7.935 \mathrm{E}-12 \\
7.913 \mathrm{E}-12 \\
7.889 \mathrm{E}-12 \\
8.666 \mathrm{E}-12 \\
1.046 \mathrm{E}-11 \\
1.346 \mathrm{E}-11 \\
1.729 \mathrm{E}-11 \\
2.261 \mathrm{E}-11 \\
3.339 \mathrm{E}-11 \\
6.053 \mathrm{E}-11 \\
1.286 \mathrm{E}-10 \\
2.861 \mathrm{E}-10 \\
5.958 \mathrm{E}-10\end{array}$ & $\begin{array}{l}1.897 \mathrm{E}-12 \\
2.069 \mathrm{E}-12 \\
2.573 \mathrm{E}-12 \\
3.106 \mathrm{E}-12 \\
3.486 \mathrm{E}-12 \\
3.334 \mathrm{E}-12 \\
3.190 \mathrm{E}-12 \\
3.48 \mathrm{E}-12 \\
4.247 \mathrm{E}-12 \\
5.616 \mathrm{E}-12 \\
7.131 \mathrm{E}-12 \\
8.897 \mathrm{E}-12 \\
1.274 \mathrm{E}-11 \\
2.414 \mathrm{E}-11 \\
5.633 \mathrm{E}-11 \\
1.392 \mathrm{E}-10 \\
3.214 \mathrm{E}-10\end{array}$ & $\begin{array}{l}7.495 \mathrm{E}-13 \\
8.40 \mathrm{EE}-13 \\
1.119 \mathrm{E}-12 \\
1.371 \mathrm{E}-12 \\
1.570 \mathrm{E}-12 \\
1.438 \mathrm{E}-12 \\
1.337 \mathrm{E}-12 \\
1.470 \mathrm{E}-12 \\
1.827 \mathrm{E}-12 \\
2.419 \mathrm{E}-12 \\
3.065 \mathrm{E}-12 \\
3.582 \mathrm{E}-12 \\
4.960 \mathrm{E}-12 \\
9.631 \mathrm{E}-12 \\
2.434 \mathrm{E}-11 \\
6.564 \mathrm{E}-11 \\
1.664 \mathrm{E}-10\end{array}$ & $\begin{array}{l}3.053 \mathrm{E}-13 \\
3.513 \mathrm{E}-13 \\
4.968 \mathrm{E}-13 \\
6.178 \mathrm{E}-13 \\
7.235 \mathrm{E}-13 \\
6.382 \mathrm{E}-13 \\
5.783 \mathrm{E}-13 \\
6.340 \mathrm{E}-13 \\
8.071 \mathrm{E}-13 \\
1.480 \mathrm{E}-12 \\
1.358 \mathrm{E}-12 \\
1.484 \mathrm{E}-12 \\
1.980 \mathrm{E}-12 \\
3.927 \mathrm{E}-12 \\
1.054 \mathrm{E}-11 \\
3.043 \mathrm{E}-11 \\
8.373 \mathrm{E}-11\end{array}$ & $\begin{array}{l}1.279 \mathrm{E}-13 \\
1.508 \mathrm{E}-13 \\
2.235 \mathrm{E}-1 \mathrm{E} \\
2.843 \mathrm{E}-13 \\
3.344 \mathrm{E}-13 \\
2.888 \mathrm{E}-13 \\
2.557 \mathrm{E}-13 \\
2.804 \mathrm{E}-13 \\
3.648 \mathrm{E}-13 \\
4.936 \mathrm{E}-13 \\
6.148 \mathrm{E}-13 \\
6.26 \mathrm{CE}-13 \\
8.091 \mathrm{E}-13 \\
1.655 \mathrm{E}-12 \\
4.618 \mathrm{E}-12 \\
1.400 \mathrm{E}-11 \\
4.130 \mathrm{E}-11\end{array}$ & $\begin{array}{l}5.473 \mathrm{E}-14 \\
6.594 \mathrm{E}-14 \\
1.017 \mathrm{E}-13 \\
1.318 \mathrm{E}-13 \\
1.554 \mathrm{E}-13 \\
1.320 \mathrm{E}-13 \\
1.147 \mathrm{E}-13 \\
1.259 \mathrm{E}-13 \\
1.691 \mathrm{E}-13 \\
2.255 \mathrm{E}-13 \\
2.822 \mathrm{E}-13 \\
2.697 \mathrm{E}-13 \\
3.350 \mathrm{E}-13 \\
7.215 \mathrm{E}-13 \\
2.050 \mathrm{E}-12 \\
6.433 \mathrm{E}-12 \\
2.01 \mathrm{E}-11\end{array}$ \\
\hline TUTAL & $4.811 E-10$ & $2.197 \mathrm{E}-1 \mathrm{C}$ & $1.003 E-10$ & $4.594 \mathrm{E}-11$ & $2.114 \mathrm{E}-11$ & $9.773 E-12$ \\
\hline COSINE & $3800 . \mathrm{C}$ & 4000.0 & $\begin{array}{l}\text { RANGE (I } \\
4200.0\end{array}$ & $\begin{array}{l}\text { TERSI } \\
440 \mathrm{C.O}\end{array}$ & 4600.0 & 4800.0 \\
\hline $\begin{array}{l}-1.00000 \mathrm{E} 00 \\
-9.89401 \mathrm{E}-01 \\
-9.44575 \mathrm{E}-\mathrm{Cl} \\
-8.65631 \mathrm{E}-01 \\
-7.55644 \mathrm{E}-01 \\
-6.17876 \mathrm{E}-01 \\
-4.58017 \mathrm{E}-\mathrm{G} 1 \\
-2.81605 \mathrm{E}-01 \\
-9.50125 \mathrm{E}-02 \\
9.50125 \mathrm{E}-02 \\
2.81605 \mathrm{E}-01 \\
4.58017 \mathrm{E}-01 \\
6.17870 \mathrm{E}-01 \\
7.55044 \mathrm{E}-01 \\
8.65631 \mathrm{E}-01 \\
9.44575 \mathrm{E}-01 \\
9.89401 \mathrm{E}-01\end{array}$ & $\begin{array}{l}1.381 \mathrm{E}-14 \\
1.714 \mathrm{E}-14 \\
2.783 \mathrm{E}-14 \\
3.697 \mathrm{E}-14 \\
4.348 \mathrm{E}-14 \\
3.633 \mathrm{E}-14 \\
3.085 \mathrm{E}-14 \\
3.386 \mathrm{E}-14 \\
4.689 \mathrm{E}-14 \\
6.337 \mathrm{E}-14 \\
7.876 \mathrm{E}-14 \\
6.922 \mathrm{E}-14 \\
8.005 \mathrm{E}-14 \\
1.912 \mathrm{E}-13 \\
5.436 \mathrm{E}-13 \\
1.767 \mathrm{E}-12 \\
5.937 \mathrm{E}-12\end{array}$ & $\begin{array}{l}8.061 \mathrm{E}-15 \\
1.010 \mathrm{E}-14 \\
1.665 \mathrm{E}-14 \\
2.228 \mathrm{E}-14 \\
2.61 \mathrm{E}-14 \\
2.18 \mathrm{CE}-14 \\
1.833 \mathrm{E}-14 \\
2.015 \mathrm{E}-14 \\
2.817 \mathrm{E}-14 \\
3.825 \mathrm{E}-14 \\
4.74 \mathrm{E}-14 \\
4.065 \mathrm{E}-14 \\
4.571 \mathrm{E}-14 \\
1.127 \mathrm{E}-13 \\
3.241 \mathrm{E}-13 \\
1.056 \mathrm{E}-12 \\
3.630 \mathrm{E}-12\end{array}$ & $\begin{array}{l}4.761 E-15 \\
5.995 \mathrm{E}-15 \\
9.984 \mathrm{E}-15 \\
1.344 \mathrm{E}-14 \\
1.571 \mathrm{E}-14 \\
1.305 \mathrm{E}-14 \\
1.091 \mathrm{E}-14 \\
1.201 \mathrm{E}-14 \\
1.695 \mathrm{E}-14 \\
2.316 \mathrm{E}-14 \\
2.841 \mathrm{E}-14 \\
2.407 \mathrm{E}-14 \\
2.633 \mathrm{E}-14 \\
6.656 \mathrm{E}-14 \\
1.942 \mathrm{E}-13 \\
6.327 \mathrm{E}-13 \\
2.217 \mathrm{E}-12\end{array}$ & $\begin{array}{l}2.883 \mathrm{E}-15 \\
3.617 \mathrm{E}-15 \\
6.005 \mathrm{E}-15 \\
8.165 \mathrm{E}-15 \\
9.412 \mathrm{E}-15 \\
7.811 \mathrm{E}-15 \\
6.503 \mathrm{E}-15 \\
7.174 \mathrm{E}-15 \\
1.621 \mathrm{E}-14 \\
1.397 \mathrm{E}-14 \\
1.711 \mathrm{E}-14 \\
1.426 \mathrm{E}-14 \\
1.523 \mathrm{E}-14 \\
3.947 \mathrm{E}-14 \\
1.168 \mathrm{E}-13 \\
3.799 \mathrm{E}-13 \\
1.352 \mathrm{E}-12\end{array}$ & $\begin{array}{l}1.819 \mathrm{E}-15 \\
2.233 \mathrm{E}-15 \\
3.591 \mathrm{E}-15 \\
4.827 \mathrm{E}-15 \\
5.492 \mathrm{E}-15 \\
4.656 \mathrm{E}-15 \\
3.862 \mathrm{E}-15 \\
4.273 \mathrm{E}-15 \\
6.131 \mathrm{E}-15 \\
8.431 \mathrm{E}-15 \\
1.030 \mathrm{E}-14 \\
8.429 \mathrm{E}-15 \\
8.834 \mathrm{E}-15 \\
2.346 \mathrm{E}-14 \\
7.049 \mathrm{E}-14 \\
2.287 \mathrm{E}-13 \\
8.245 \mathrm{E}-13\end{array}$ & $\begin{array}{l}1.078 \mathrm{E}-15 \\
1.27 \mathrm{CE}-15 \\
1.914 \mathrm{E}-15 \\
2.553 \mathrm{E}-15 \\
2.850 \mathrm{E}-15 \\
2.533 \mathrm{E}-15 \\
2.1711 \mathrm{E}-15 \\
2.443 \mathrm{E}-15 \\
3.512 \mathrm{E}-15 \\
5.046 \mathrm{E}-15 \\
6.070 \mathrm{E}-15 \\
4.889 \mathrm{E}-15 \\
5.038 \mathrm{E}-15 \\
1.389 \mathrm{E}-14 \\
4.255 \mathrm{E}-14 \\
1.379 \mathrm{E}-13 \\
5.426 \mathrm{E}-13\end{array}$ \\
\hline TUTAL & $2.725 E-12$ & $1.639 E-12$ & $9.874 \mathrm{E}-13$ & $5.956 E-13$ & $3.594 E-13$ & $2.153 E-13$ \\
\hline
\end{tabular}




COSINE
$-1.00000 E$ OC
$-9.89401 E-U 1$
$-9.44575 E-01$
$-8.05631 E-01$
$-7.55044 E-01$
$-6.17876 E-01$
$-4.58017 E-01$
$-2.81605 E-01$
$-9.50125 E-02$
$9.50125 E-62$
$2.81605 E-01$
$4.58017 E-01$
$6.17076 E-L 1$
$7.55044 E-01$
$8.65631 E-01$
$9.44575 E-01$
$9.89402 E-01$

TUTAL

CUSINE

-1. OULCOCE No $-9.894 C 1 E-01$ $-9.44575 \mathrm{E}-01$ $-8.65031 \mathrm{E}-61$ $-7.55044 t-C$ $-6.17876 \mathrm{E}-0$ $-4.58017 E-01$ $-2.81605 E-01$ $-9.50125 E-02$ $9.50125 t-c 2$ $2.81605 \mathrm{E}-\mathrm{U}$ $4.58017 \mathrm{E}-\mathrm{C}$

$6.17876 \mathrm{E}-0$

$7.55044 \mathrm{E}-\mathrm{Cl}$

$8.65631 \mathrm{E}-01$

$9.44575 \mathrm{E}-0$

$9.89401 \mathrm{E}-G 1$

TOTAL

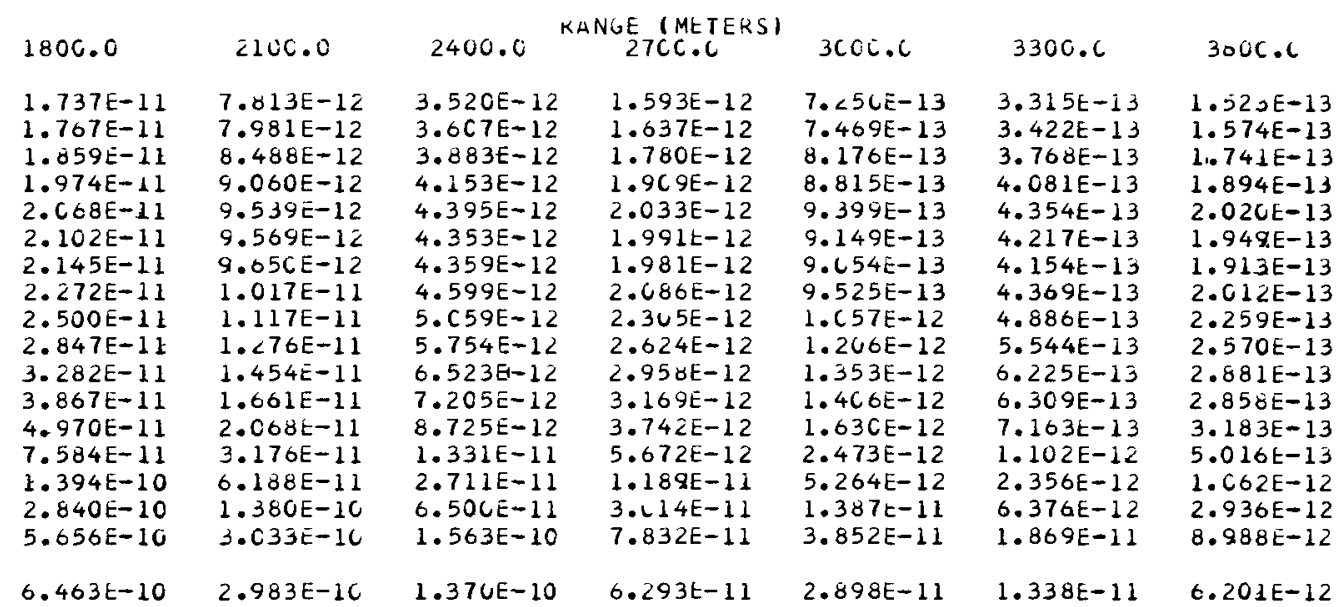

KANGE (METERS

$4060.0 \quad 4200.0 \quad 440 \mathrm{C} . \mathrm{C} \quad 460 \mathrm{C} . \mathrm{C} \quad 4800.0$

$1.166 E-14 \quad 6.175 E-15$

\section{$\begin{array}{ll}3.251 E-14 & 1.953 E-14 \\ 3.368 E-14 & \end{array}$}

$3.368 \varepsilon-14$

$2.251 E-14$

$4.101 E-14 \quad 2.464 E-14$

$4.304 E-14 \quad 2.617 E-14$

$\begin{array}{ll}4.198 E-14 & 2.517 E-14 \\ 4.096 E-14 & 2.454 E-14\end{array}$

$4.305 E-14 \quad 2.58 \mathrm{CE}-14$

$4.872 E-14 \quad 2.926 E-14$

$5.569 E-14 \quad 3.35 C E-14$

$6.207 \mathrm{E}-14 \quad 3.728 \mathrm{E}-14$

$6.604 E-14 \quad 3.584 E-14$

$t .469 E-14 \quad 3.828 E-14$

$1 . C 47 E-13 \quad 6.239 E-14$

$2.254 E-13 \quad 1.355 t-13$

$6 . \angle 72 E-13 \quad 3.765 E-13$

$2 . C 47 E-12 \quad 1.247 E-12$

6.366E-15

$1.463 E-14 \quad 7.730 E-15$

$1.546 E-14 \quad 8.189 E-15$

$8.142 E-15$

$1.539 E-14 \quad 8.649 E-15$

1.751E-14 9.931E-15

$1.167 t-14$

$2.235 E-14$ 1.296E-14

$2.134 E-14 \quad 1.234 E-14$

2.266E-14 1.307E-14

2.186E-14

8.16CE-14 $4.892 E-14$

$\begin{array}{ll}2.265 E-13 & 1.362 E-13 \\ 7.595 E-13 & 4.021 E-13\end{array}$ 
COSINE

$-1.00000 E$ UU
$-9.894 C 1 E-01$
$-9.44575 E-C 1$
$-8.65631 E-01$
$-7.55044 E-01$
$-6.17876 E-01$
$-4.58017 E-C 1$
$-2.816 C 5 E-C 1$
$-9.50125 E-02$
$9.50125 E-02$
$2.81605 E-01$
$4.58017 E-01$
$6.17876 E-01$
$7.5544 E-01$
$8.65031 E-U 1$
$9.44575 E-C 1$
$9.89401 E-01$

TUTAL

COS INE

$-1 . \operatorname{COCOCE} 00$ $-9.89401 E-U 1$ $-9.44575 E-01$ $-8.65631 E-01$ $-6.17876 E-O$ $-6.17876 E-01$ $-2.81605 E-i$ $-9.5 C 125 E-C$ $9.5 \cup 1<5 E-02$ $2.81605 E-U 1$ 0.170765-C $7.55644 t-01$ . $9.44575 E-01$ $9.84401=0\}$

TOTAL

1860.0
$5.807 E-12$
$6.117 \mathrm{E}-12$
$7.039 \mathrm{E}-12$
$8.117 \mathrm{E}-12$
$8.863 \mathrm{E}-12$
$8.831 \mathrm{E}-12$
$8.796 \mathrm{E}-12$
$9.565 \mathrm{E}-12$
$1.138 \mathrm{E}-11$
$1.442 \mathrm{E}-11$
$1.828 \mathrm{E}-11$
$2.365 \mathrm{E}-11$
$3.456 \mathrm{E}-11$
$6.219 \mathrm{E}-11$
$1.317 \mathrm{E}-10$
$2.933 \mathrm{E}-10$
$6.116 \mathrm{E}-10$
$5.600 \mathrm{E}-10$

$210 \mathrm{C} . \mathrm{C}$

$2.29 C E-12$ $2.466 \mathrm{E}-12$ $2.984 E-12$ $3.531 t-12$ 3.917E-12 $3.757 \mathrm{E}-12$ $3.601 E-12$ $3.887 E-12$ $4.662 E-12$ $6.049 \mathrm{E}-1 / 2$ $7.575 E-12$ $9.340 E-12$ 1. $3 \angle 1 E-11$ $2.478 E-11$ $5.707 E-11$ $1.427 E-10$ $3.301 E-1 C$ $2 . \angle 84 E-1 C$

$3800 . \mathrm{C}$

4000.0

$1.837 \mathrm{E}-14$ $2.180 E-14$ $3.281 E-14$ $4.220 E-14$ $4.886 E-14$ $4.144 E-14$ 3.509E-14 $5.188 E-14$ . $188 \mathrm{E}-14$ .

$7.405 E-14$

8.3

$8.344 \mathrm{E}-14$
$1.955 \mathrm{E}-13$

$5.545 \mathrm{E}-13$

1.810E-12

$6.115 \mathrm{E}-12$

$2.834 E-12$
2400.0 RANGE 2700.0 (MERS)

$3 c c c . c$

3300.0

$360 u .0$

$9.278 E-13$

$3.861 E-13$

$1.646 \mathrm{E}-13$ $1.882 \mathrm{E}-13$

$7.149 E-14$ $3.15 \cup E-14$ . $307 E-12 \quad 5.335 E-13$ $.56 t t-12$ 列 $3.254 \mathrm{E}-13$ 3. $766 \mathrm{E}-13$ $1.631 E-12$ 作 13 $7.264 \mathrm{E}-13$ $6.632 \mathrm{E}-13$ $7.179 E-13$ $8.931 \mathrm{E}-13$ 1. $170 \mathrm{E}-12$ $1.449 E-12$ $1.568 \mathrm{E}-12$ $2.057 E-12$ $4.025 \mathrm{E}-12$ $1.078 \mathrm{E}-11$ $3.118 E-1 I$ $8.607 E-11$ $2.944 E-13$ $3.186 \mathrm{E}-13$ 4. $C 42 E-13$ $5.346 \mathrm{E}-13$ $6.563 \mathrm{E}-13$ $6.629 t-13$ $8.416 \mathrm{E}-13$ $1.695 \mathrm{E}-12$
$4.717 \mathrm{E}-12$ $1.435 \mathrm{E}-11$ $4.248 \mathrm{E}-11$ $\begin{array}{ll}1.198 \mathrm{E}-13 & 5.497 \mathrm{E}-14 \\ 1.508 \mathrm{E}-13 & 7.016 \mathrm{E}-14\end{array}$ $1.748 \mathrm{E}-13 \quad 8.13 \mathrm{gE}-14$ $1.506 \mathrm{E}-1 \mathrm{~s} \quad 6.928 \mathrm{E}-14$ $1.324 \mathrm{E}-13 \quad 6.014 \mathrm{E}-14$ $1.434 \mathrm{E}-13$ 6.519E-14 $1.872 \mathrm{E}-13 \quad 8.645 \mathrm{E}-14$ $2.444 E-13 \quad 1.138 E-13$ $3.013 E-13 \quad 1.398 E-13$ $2.862 E-13 \quad 1.26 \mathrm{CE}-13$ $3.487 \mathrm{E}-13 \quad 1.47 \mathrm{CE}-13$ $1.386 \mathrm{E}-13 \quad 3.327 \mathrm{E}-1$ $2.092 \mathrm{E}-12 \quad 9.362 \mathrm{E}-13$ $3.033 \mathrm{E}-12$ $2.068 \mathrm{E}-11 \quad 9.976 \mathrm{E}-12$ $1.043 E-10 \quad 4.777 E-11 \quad 2.198 E-11 \quad 1.016 E-11 \quad 4.717 E-12$

KANGE (METERS)

$$
4200.0 \quad 4400 . \mathrm{C}
$$

4606.0

$4800 . \mathrm{C}$
$6.382 E-15$

$1.176 E-14$ 7.0CSE-15

$1.532 E-14$ $14.069 E-15$

$1.057 E-14$

$1.488 \mathrm{E}-14 \quad 8.9 \mathrm{C} 5 \mathrm{E}-15$

$1 . \angle 64 E-14$

$1.373 \mathrm{E}-14$ 14

$1.498 E-14$ 1. $510 E-14$

$2.498 E-14$ 1. $510 E-14$

2.56IE-14 $1.517 E-14$

$2.745 \mathrm{E}-14$ 1.

$6.803 E-14 \quad 4.032 E-14$

$1.981 \mathrm{E}-13 \quad 13.032 \mathrm{E}-14$

$0.482 E-13$ 3.893E-1

$2.285 \mathrm{E}-12 \quad 1.395 \mathrm{E}-12$

$1.627 E-12 \quad 0.195 E-13$
$2.399 E-15$ $4.225 E-15 \quad 2.265 E-15$

5.497E-15 $2.921 E-15$

$6.176 E-15$ 3.223E-15

$2.803 E-15$

$2.703 E-15$

$3.857 E-15$

$5.417 \mathrm{E}-15$

$6.444 E-15$

$5.172 E-15$

$9.204 E-15 \quad 5.216 E-15$

$2.395 E-14 \quad 1.414 E-14$

$7.188 \mathrm{E}-14 \quad 4.335 \mathrm{E}-14$

$1.413 E-13$

$8.507 E-13 \quad 5.188 E-1$

$3.738 E-13 \quad 2.236 E-13$ 


$$
\begin{gathered}
\text { ORNL-4464 } \\
\text { UC-34 - Physics }
\end{gathered}
$$

INTERNAL DISTRIBUTION

1. Biology Library

2-4. Central Research Library

5-6. ORNL - Y-12 Technical Library Document Reference Section

7-56. Laboratory Records Department

57. Laboratory Records, ORNL R.C.

58. L. S. Abbott

59. V. R. Cain

60. G. T. Chapman

61. H. C. Claiborne

62-66. C. E. Clifford

67-71. M. L. Gritzner

72. F. F. Haywood

73. R. F. Hibbs

74. H. H. Hubbell

75. T. D. Jones

76. W. H. Jordan
77. H. G. MacPherson

78-79. F. C. Maienschein

80. J. R. McWherter

81. S. K. Penny

82. J. W. Poston

83. H. W. Schmitt

84. M. J. Skinner

85-89. E. A. Straker

90. D. A. Sundberg

91. D. K. Trubey

92. J. W. Webster

93. A. M. Weinberg

94. H. A. Wright

95. E. R. Cohen (consultant)

96. B. C. Diven (consultant)

97. H. Feshbach (consultant)

98. H. Goldstein (consultant)

EXTERNAL DISTRIBUTION

99-287. Given DASA Shielding and Initial Radiation Distribution (updated 9-12-69)

288. A. B. Chilton, Room 207, Nuclear Engineering Laboratory, University of Illinois, Urbana, Illinois

289. R. L. French, Radiation Research Associates, Inc., 3550 Hulen St., Fort Worth, Texas

290. L. V. Spencer, National Bureau of Standards, Washington, D.C.

291. Laboratory and University Division, AEC, ORO

292-524. Given distribution as shown in TID-4500 under Physics category (25 copies - CFSTI) 